\title{
Development of a Hydrogasification Process for Co-Production of Substitute Natural Gas (SNG) and Electric Power from Western Coals
}

\author{
FINAL SCIENTIFIC/TECHNICAL REPORT \\ REPORTING PERIOD START DATE: APRIL 1, 2006 \\ REPORTING PERIOD END DATE: APRIL 30, 2011
}

PRINCIPAL AUTHOR: XIAOLEI SUN AND NANCY TURLEY RINK REPORT ISSUE DATE: JANUARY 2013

THE PROJECT WAS FUNDED BY DOE NETL COOPERATIVE AGREEMENT NO: DE-FC26-06NT42759

BY

ARIZONA PUBLIC SERVICE COMPANY 400 NORTH 5TH STREET

PHOENIX, ARIZONA, 85004

WORLEYPARSONS

2676 MORGANTOWN ROAD

READING, PA 19607 


\section{DISC LAIMER}

This report was prepared as an account of work sponsored by an agency of the United States Government. Neither the United States Government nor any agency thereof, nor any of their employees, makes any warranty, express or implied, or assumes any legal liability or responsibility for the accuracy, completeness, or usefulness of any information, apparatus, product, or process disclosed, or represents that its use would not infringe privately owned rights. Reference herein to any specific commercial product, process, or service by trade name, trademark, manufacturer, or otherwise does not necessarily constitute or imply its endorsement, recommendation, or favoring by the United States Government or any agency thereof. The views and opinions of authors expressed herein do not necessarily state or reflect those of the United States Government or any agency thereof. 


\section{ACKNOWEDG MENTS}

This project was sponsored by the National Energy Technology Laboratory (NETL) of the U.S. Department of Energy (DOE), under Cooperative Agreement No. DE-FC226-06NT42759. This financial assistance from DOE/NETL is gratefully acknowledged. NETL's Office of Research and Development (ORD) is acknowledged for their contribution of Computational Fluid Dynamic Modeling to aid in design of the bench-scale hydrogasifier, and the chemical and physical analyses of selected algae species. In addition, some cost-share for the project was by Worley Parsons. 


\section{ACRONYMS AND ABBREVIATIONS}

\begin{tabular}{|c|c|}
\hline Acronym & Description \\
\hline${ }^{\circ} \mathrm{C}$ & Degrees Celsius \\
\hline${ }^{\circ} \mathrm{F}$ & Degrees Fahrenheit \\
\hline$>$ & Greater than \\
\hline$\leq$ & Less than or equal to \\
\hline$\geq$ & Greater than or equal to \\
\hline$\%$ & Percent \\
\hline$\sim$ & Approximate \\
\hline$\xi$ & Convection coefficient \\
\hline$\xi_{0}$ & Convection factor $\xi_{0}=1$ if Ro $<0$ else $\xi_{0}=0$ \\
\hline$\xi_{0}$ & Convection factor $1-\xi_{0}$ \\
\hline$\epsilon_{g}$ & Volume fraction of the fluid phase (void fraction) \\
\hline$\epsilon_{\mathrm{m}}$ & Void fraction - where $m=g$ or $s$ for the gas or solid phase \\
\hline$\epsilon_{\mathrm{s}}$ & Volume fraction in the solid phase (void fraction) \\
\hline$\mu \mathrm{E} / \mathrm{m}^{2} / \mathrm{s}$ & Micro Einstein per square meter per second \\
\hline$\mu \mathrm{g}$ & Microgram \\
\hline$\mu \mathrm{g} / \mathrm{L}$ & Microgram/liter \\
\hline$\mu \mathrm{l}$ & Microliter \\
\hline$\mu \mathrm{m}$ & Micrometer (also known as micron) \\
\hline$\mu \mathrm{ohms}$ & Micro-ohms \\
\hline $1000 x$ & One Thousand Times \\
\hline${ }^{13} \mathrm{C}$ NMR & Carbon 13 - Nuclear Magnetic Resonance \\
\hline 1-D & One-dimensional \\
\hline $1 \mathrm{X}$ & One Time \\
\hline $2 \mathrm{x}$ & Two Times \\
\hline $3-D$ & Three-dimensional \\
\hline $4 \mathrm{C}$ & Four Corners Power Plant, Farmington, New Mexico \\
\hline $6 \mathrm{M}$ & 6 meter \\
\hline $15 X$ & 15 times \\
\hline $32 \mathrm{X}$ & 32 times \\
\hline AC & Activated Carbon \\
\hline AED & Atomic-emission detector \\
\hline AFM & Atomic Force Microscopy \\
\hline $\mathrm{Ag}$ & Silver \\
\hline AHP & Advanced Hydrogasification Process \\
\hline Al & Aluminum \\
\hline a.m. & Ante Meridiem \\
\hline $\mathrm{amu}$ & Atomic Mass Unit \\
\hline AOV & Air Operated Valve \\
\hline
\end{tabular}




\begin{tabular}{|c|c|}
\hline Acronym & Des crip tion \\
\hline APS & Arizona Public Service Company \\
\hline $\mathrm{ARCH}$ & Advanced Rapid Carbon Hydrogasification \\
\hline As & Arsenic \\
\hline $\mathrm{As}(\mathrm{OH})_{3}$ & Arsenic Hydroxide \\
\hline $\mathrm{AsCl}_{3}$ & Arsenic Trichloride \\
\hline ASME & American Society of Mechanical Engineers \\
\hline ASTM & American Society of Testing and Materials \\
\hline ASU & Arizona State University \\
\hline B & Boron \\
\hline $\mathrm{Ba}$ & Barium \\
\hline BASF & Badische Anilin- und Soda-Fabrik \\
\hline $\mathrm{Be}$ & Beryllium \\
\hline BET & Brunauer-Emmett Teller \\
\hline BHT & Butylated Hydroxytoluene \\
\hline BOM & Bill of Material \\
\hline BPVC & Boiler and Pressure Vessel Code \\
\hline $\mathrm{Br}$ & Bromine \\
\hline BSRx & Bench Scale Reactor \\
\hline Btu & British Thermal Units \\
\hline Btu/lb & British Thermal Units per Pound \\
\hline BTX & Benzene, Toluene, and Xylene \\
\hline C & Carbon \\
\hline C1 & Cultivator 1 \\
\hline $\mathrm{C}_{1}-\mathrm{C}_{6}$ & Alkane Hydrocarbon series \\
\hline $\mathrm{C} 2$ & Cultivator 2 \\
\hline $\mathrm{C}_{2} \mathrm{H}_{4}$ & Ethylene \\
\hline $\mathrm{C}_{2} \mathrm{H}_{6}$ & Ethane \\
\hline C3 & Cultivator 3 \\
\hline $\mathrm{C}_{6} \mathrm{H}_{6}$ & Benzene \\
\hline $\mathrm{C}_{7} \mathrm{H}_{8}$ & Toluene \\
\hline $\mathrm{C}_{8} \mathrm{H}_{10}$ & Ethyl Benzene \\
\hline $\mathrm{C}_{10} \mathrm{H}_{8}$ & Naphthalene \\
\hline $\mathrm{Ca}$ & Calcium \\
\hline CA & Compressed Air \\
\hline CAER & Center for Applied Energy Research, University of Kentucky \\
\hline $\mathrm{cc} / \mathrm{hr}$ & Cubic Centimeter per Hour \\
\hline $\mathrm{Cd}$ & Cadmium \\
\hline $\mathrm{Ce}$ & Cerium \\
\hline CF & Coal Feeder \\
\hline CFD & Computational Fluid Dynamics (Model) \\
\hline $\mathrm{CH}_{4}$ & Methane \\
\hline
\end{tabular}




\begin{tabular}{|c|c|}
\hline Acronym & Description \\
\hline $\mathrm{Cl}$ & Chloride \\
\hline $\mathrm{Cm}$ & Centimeter \\
\hline Co & Cobalt \\
\hline $\mathrm{CO}$ & Carbon Monoxide \\
\hline $\mathrm{CO}_{2}$ & Carbon Dioxide \\
\hline $\mathrm{CoCl}_{2} \cdot 6 \mathrm{H}_{2} \mathrm{O}$ & Cobalt (II) chloride \\
\hline COD & Chemical Oxygen Demand \\
\hline CON & Concentrator \\
\hline $\cos$ & Carbonyl Sulfide \\
\hline $\mathrm{Cr}$ & Chrome \\
\hline CSTR & Continuous Stir Tank Reactor \\
\hline $\mathrm{Cu}$ & Copper \\
\hline CVIP & Cryogenic Vessels, Instrumentation, and Piping, Inc. \\
\hline DL & Detection Limit \\
\hline $\mathrm{D}_{\mathrm{mn}}$ & Diffusion coefficient for the nth species in the $\mathrm{mth}$ ( $\mathrm{g}$ or $\mathrm{s}$ ) phase $\mathrm{kg} / \mathrm{m} \cdot \mathrm{s}$ \\
\hline DO & Dissolved Oxygen \\
\hline DOE & U.S. Department of Energy \\
\hline EDS & Energy Dispersive Spectrometry \\
\hline e.g. & Example \\
\hline EIA & Energy Information Agency \\
\hline $\mathrm{F}$ & Fluorine \\
\hline F-76 & Fuel Oil \\
\hline$f^{*}$ & $\begin{array}{l}\text { Maximum fractional amount of a component that can be gasified from the coal } \\
\text { particle }\end{array}$ \\
\hline$f^{*}{ }^{*}$ & $\begin{array}{l}\text { Maximum fractional amount of carbon matter of coal that would only react in the } \\
\text { presence of hydrogen }\end{array}$ \\
\hline FAME & Fatty Acid Methyl Ester \\
\hline FCV & Flow Control Valves \\
\hline $\mathrm{Fe}$ & Iron \\
\hline$F_{g s}$ & Coefficent for the interphase force between the fluid phase and gas phase \\
\hline FFA & Free Fatty Acids \\
\hline FID & Flame Ionization Detector \\
\hline $\mathrm{FIO}$ & Furnished and Installed by Others \\
\hline$f_{j}$ & Mass of Components/Mass of Coal \\
\hline FM & Florescence Maximal/Flow Meter \\
\hline $\mathrm{ft}^{3} / \mathrm{h}$ & Cubic Feet per Hour \\
\hline g & Gram \\
\hline $\overrightarrow{\mathrm{g}}$ & Acceleration due to gravity \\
\hline$g / L$ & Grams per Liter \\
\hline g/L/day & Grams per Liter per Day \\
\hline
\end{tabular}




\begin{tabular}{|l|l|}
\multicolumn{1}{|c|}{ Acronym } & \multicolumn{2}{c}{ Des crip tion } \\
\hline $\mathrm{g} / \mathrm{m}^{2} / \mathrm{d}$ & Grams per Square Meters per Day \\
\hline $\mathrm{g} / \mathrm{mL}$ & Grams per Milliliter \\
\hline $\mathrm{g} / \mathrm{mol}$ & Grams per Mole \\
\hline $\mathrm{gal}$ & Gallons \\
\hline $\mathrm{GC}$ & Gas Chromatography \\
\hline $\mathrm{GE}$ & General Electric \\
\hline $\mathrm{GF} 3$ & Algae Facility (name) \\
\hline $\mathrm{Ghg}$ & Greenhouse Gas \\
\hline $\mathrm{gpm}$ & Gallons per Minute \\
\hline $\mathrm{GTI}$ & Gas Technology Institute \\
\hline $\mathrm{H}$ & Hydrogen \\
\hline $\mathrm{H}_{2}$ & Coproduced Hydrogen \\
\hline $\mathrm{H}_{2} \mathrm{O}$ & Water \\
\hline $\mathrm{H}_{2} \mathrm{~S}$ & Hydrogen Sulfide \\
\hline $\mathrm{H}_{2} \mathrm{Se}$ & Hydrogen Selenide \\
\hline $\mathrm{H}_{2} \mathrm{SeO}$ & Selenious Acid \\
\hline $\mathrm{H}_{2} \mathrm{SO}$ & Sulfuric Acid \\
\hline $\mathrm{H}_{3} \mathrm{AsO} \mathrm{O}_{3}$ & Arsenite \\
\hline $\mathrm{HDPE}$ & High-Density Polyethylene (Pipe) \\
\hline $\mathrm{H}_{\mathrm{g}}$ & Mercury \\
\hline $\mathrm{HgCl}$ & Mercury (II) Chloride \\
\hline $\mathrm{HgS}$ & Mercury Sulfide \\
\hline $\mathrm{HHV}$ & High Heating Value \\
\hline $\mathrm{HMI}$ & Human Machine Interface \\
\hline $\mathrm{hp}$ & Horsepower \\
\hline $\mathrm{HPTGA}$ & High Pressure Thermogravimetric Analysis \\
\hline $\mathrm{HSeO}$ & Monobasic Selenite Oxyanions \\
\hline $\mathrm{Hz}$ & Hertz \\
\hline $\mathrm{IC}$ & Ion Chromatography \\
\hline $\mathrm{ICP}-\mathrm{MS}_{3}$ & Inductively Coupled Plasma - Mass Spectrometer \\
\hline $\mathrm{ICP}-\mathrm{OES}$ & Inductively Coupled Plasma - Optical Emission Spectrometer \\
\hline $\mathrm{ID}$ & Inside Diameter \\
\hline $\mathrm{IES}$ & Integrated Energy System with CO \\
\hline in-lb & Inch-Pound Project \\
\hline $\mathrm{JP}-5$ & Jet Propellant 5 \\
\hline $\mathrm{JP} 8$ & Military Aviation Fuel \\
\hline $\mathrm{K}$ & Potassium \\
\hline $\mathrm{kg}$ & Kilogram \\
\hline $\mathrm{kg} / \mathrm{hr}$ & Kilogram per Hour \\
\hline $\mathrm{kg} / \mathrm{kg}-\mathrm{coal}$ & Kilograms per Kilogram of Coal \\
\hline $\mathrm{kg} / \mathrm{mol}$ & Kilogram per Mole \\
\hline & \\
\hline
\end{tabular}




\begin{tabular}{|c|c|}
\hline Acronym & Des crip tion \\
\hline $\mathrm{KOH}$ & Potassium Hydroxide \\
\hline kw & Kilowatt \\
\hline L or I & Liter \\
\hline $\mathrm{lb}$ & Pound \\
\hline $\mathrm{lb} / \mathrm{ft}^{3}$ & Pounds per Cubic Feet \\
\hline $\mathrm{lb} / \mathrm{hr}$ & Pounds per Hour \\
\hline LCD & Liquid Crystal Display \\
\hline $\mathrm{LCO}_{2}$ & Liquid Carbon Dioxide \\
\hline LEED & Low-Energy Electron Diffraction \\
\hline LEIS & Low-Energy Ion Scattering Spectroscopy \\
\hline LHV & Low Heating Value \\
\hline $\mathrm{Li}$ & Lithium \\
\hline LSNE & Lyophilization Services of New England \\
\hline $\mathrm{M}$ or $\mathrm{m}$ & Meter \\
\hline $\mathrm{m}^{2}$ & Square Meters \\
\hline $\mathrm{mA}$ & Millieamp \\
\hline MATLAB & MATrix LABoratory \\
\hline MAWP & Maximum Allowable Working Pressure \\
\hline MD & Magnetic Drive \\
\hline $\mathrm{Me}$ & Methyl \\
\hline MFC & Mass Flow Controller \\
\hline $\mathrm{Mg}$ & Magnesium \\
\hline $\mathrm{mg} / \mathrm{kg}$ & Milligram/Kilogram \\
\hline $\mathrm{mg} / \mathrm{l}$ & Milligram per Liter \\
\hline $\mathrm{mg} / \mathrm{m}^{3}$ & Magnesium $/ \mathrm{m}^{3}$ \\
\hline $\min$ & Minute \\
\hline MIT & Massachusetts Institute of Technology, Cambridge, Massachusetts \\
\hline $\mathrm{mL}$ & Milliliter \\
\hline $\mathrm{mL} / \mathrm{s}$ & Milliliter per second \\
\hline $\mathrm{mm}$ & Millimeter \\
\hline MMBtu & Million British Thermal Unit \\
\hline $\mathrm{mmol}$ & Milli Mole \\
\hline MMSCFD & Million Standard Cubic Feet per Day \\
\hline $\mathrm{Mn}$ & Manganese \\
\hline Mo & Molybdenum \\
\hline MS & Mass Spectrometer/Spectroscopy \\
\hline $\begin{array}{l}\text { (MSS-SP- } \\
61)\end{array}$ & Manufacturers Standardization Society, Standard Practice 61 \\
\hline MW & Megawatt/Molecular Weight \\
\hline MWe & Megawatt Electric \\
\hline $\mathrm{n}$ & Index of the nth chemical species \\
\hline
\end{tabular}




\begin{tabular}{|c|c|}
\hline Acronym & Des crip tion \\
\hline $\mathrm{N}$ & Nitrogen \\
\hline $\mathrm{N}_{2}$ & Nitrogen \\
\hline $\mathrm{Na}$ & Sodium \\
\hline $\mathrm{NaC}_{\mathrm{i}}$ & Halite \\
\hline $\mathrm{NaCl}$ & Sodium Chloride \\
\hline $\mathrm{NaOH}$ & Sodium Hydroxide \\
\hline nd & Not Detected \\
\hline NETL & National Energy Technology Laboratory \\
\hline $\mathrm{N}_{\mathrm{g}}$ & Total number of fluid - phase chemical species \\
\hline NG & Natural Gas \\
\hline $\mathrm{ng} / \mathrm{L}$ & Nanogram per Liter \\
\hline NGCC & Natural Gas Combined Cycle \\
\hline $\mathrm{Ni}$ & Nickel \\
\hline $\mathrm{Ni} / \mathrm{Mg} / \mathrm{Al}$ & Nickel/Magnesium/Aluminum \\
\hline NJFL & New Jersey Field Laboratory \\
\hline $\mathrm{NO}_{\mathrm{x}}$ & Nitrogen Oxides \\
\hline NO & Nitric Oxide \\
\hline $\mathrm{NO}_{2}$ & Nitrogen Dioxide \\
\hline $\mathrm{NO}_{3}$ & Nitrogen Trioxide \\
\hline $\mathrm{N}_{\mathrm{s}}$ & Total number of solid-phase chemical species \\
\hline $\mathrm{O}_{2}$ & Oxygen \\
\hline O\&M & Operations and Maintenance \\
\hline OD & Outside Diameter \\
\hline ORD & Office of Research and Development, NETL \\
\hline $\mathrm{P}$ & Phosphorous \\
\hline P\&ID & Piping and Instrumentation Diagram \\
\hline PAHS & Polycyclic Aromatic Hydrocarbons \\
\hline PAR & Photosynthetically Active Radiation \\
\hline $\mathrm{Pb}$ & Lead \\
\hline $\mathrm{PbCl}_{2}$ & Lead Dichloride \\
\hline PBR & Photo BioReactor \\
\hline PBS & Pressure Balancing System \\
\hline PCS & Pressure Control System \\
\hline PCV & Pressure Control Valve \\
\hline $\mathrm{Pd}$ & Palladium \\
\hline $\mathrm{P}_{\mathrm{g}}$ & Pressure in the fluid phase; $\mathrm{Pa}$ \\
\hline $\mathrm{P}_{\mathrm{gs}}$ & Microscopic (material) density of the solid phase: $\mathrm{kg} / \mathrm{m}^{3}$ \\
\hline $\mathrm{pH}$ & Parts of Hydrogen \\
\hline PLC & Programmable Logic Control \\
\hline$P_{\mathrm{m}}$ & Microscopic density of the $m_{\mathrm{th}}$ phase $\left(\mathrm{kg} / \mathrm{m}^{3}\right)$ \\
\hline p.m. & Post Meridiem \\
\hline
\end{tabular}




\begin{tabular}{|c|c|}
\hline Acronym & Description \\
\hline $\mathrm{PO}_{4}$ & Phosphate \\
\hline POX & Partial Oxidation \\
\hline $\mathrm{pph}$ & Pounds per Hour \\
\hline ppm & Parts per Million \\
\hline ppmv & Parts per Million by Volume \\
\hline ppmw & Parts per Million by Weight \\
\hline psi & Pounds per Square Inch \\
\hline psia & Pounds per Square Inch Absolute \\
\hline psid & Pounds per Square Inch Differential \\
\hline psig & Pounds per Square Inch Gage \\
\hline $\mathrm{Pt}$ & Platinum \\
\hline PT & Pressure Transducer \\
\hline $\mathrm{Pt} / \mathrm{c}$ & Platinum on Carbon \\
\hline PVC & Polyvinyl Chloride \\
\hline QA/QC & Quality Assurance and Quality Control \\
\hline $\mathrm{R}$ & Reactor \\
\hline$R \& D$ & Research and Development \\
\hline RC & Rapid Carbon \\
\hline 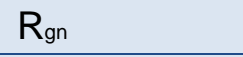 & Rate of production of the $n_{\mathrm{th}}$ chemical species in the fluid phase $/ \mathrm{kg} / \mathrm{m}^{3} \cdot \mathrm{s}$ \\
\hline RKG & Runge-Kutta-Gill \\
\hline $\mathrm{RL}$ & Reading Limit \\
\hline $\mathrm{R}_{\mathrm{mn}}$ & Rate of production of the $n_{\mathrm{th}}$ chemical species in the $\left(\mathrm{g}\right.$ or s) phase $\mathrm{Kg} / \mathrm{m}^{2} . \mathrm{s}$ \\
\hline RO & Reverse Osmosis \\
\hline $\mathrm{rpm}$ & Revolutions per Minute \\
\hline $\mathrm{Rsn}_{\mathrm{sn}}(-1)$ & Rate of production of the $n_{\mathrm{th}}$ chemical species in the solid phase $/ \mathrm{kg} / \mathrm{m}^{3} \cdot \mathrm{s}$ \\
\hline RT & Residence Time \\
\hline $\mathrm{s}$ & Seconds \\
\hline $\mathrm{S}$ & Sulfur \\
\hline $\mathrm{Sb}$ & Antimony \\
\hline scf/hr or scfh & Standard Cubic Feet per Hour \\
\hline SCFM & Standard Cubic Feet per Minute \\
\hline SE & Standard Error \\
\hline Se & Selenium \\
\hline $\mathrm{SeCl}_{2}$ & Selenium Chloride \\
\hline SEM & Scanning Electron Microscopy \\
\hline $\mathrm{SeO}_{3}{ }^{2-}$ & Monobasic Selenite Oxyanion \\
\hline SG & Sample Gas \\
\hline $\mathrm{Si}$ & Silicon \\
\hline SIMDIST & Simulated Distillation \\
\hline Sn & Tin \\
\hline SNG & Substitute Natural Gas \\
\hline
\end{tabular}




\begin{tabular}{|c|c|}
\hline Acronym & Des crip tion \\
\hline $\mathrm{SO}_{x}$ & Sulfer Oxides \\
\hline $\mathrm{SO}_{2}$ & Sulfer Dioxide \\
\hline $\mathrm{SO}_{3}$ & Sulfer Trioxide \\
\hline $\mathrm{SO}_{4}$ & Sulfate \\
\hline $\mathrm{Sr}$ & Stronium \\
\hline SS & Stainless Steel \\
\hline$\overline{\mathrm{S}}_{\mathrm{sm}}$ & Solids phase - $\mathrm{m}$ stress tensor: $\mathrm{Pa}$ \\
\hline STM & Scanning Tunneling Microscopy \\
\hline SV & Solenoid Valve \\
\hline $\mathrm{T}$ & Temperature \\
\hline TCD & Thermal Conductivity Detector \\
\hline$T_{g}$ & Gas Temperatures \\
\hline $\begin{array}{l}\overline{\bar{\tau}} \\
\tau_{g}\end{array}$ & Fluid phase deviatoric stress tensor: $\mathrm{Pa}$ \\
\hline TGA & Thermogravimetric Analysis \\
\hline Th & Thorium \\
\hline $\mathrm{Ti}$ & Titanium \\
\hline TIG & Tungsten Inert Gas \\
\hline TI & Thallium \\
\hline$T_{s}$ & Solid Temperature \\
\hline TSS & Total Suspended Solids \\
\hline TRIS & (Hydroxymethl) Aminomethane $\left(\mathrm{HOCH}_{2}\right)_{3} \mathrm{CNH}_{2}$ \\
\hline $\mathrm{U}$ & Uranium \\
\hline USU & Utah State University \\
\hline UV & Ultraviolet \\
\hline $\mathrm{V}$ & Vanadium \\
\hline $\mathrm{v} / \mathrm{v}$ & Volume to Volume \\
\hline VFD & Variable Frequency Drive \\
\hline vol. & Volume \\
\hline $\overrightarrow{\mathrm{V}}_{\mathrm{g}}$ & fluid-phase velocity vector; $\mathrm{m} / \mathrm{s}$ \\
\hline $\overrightarrow{v_{s}}$ & solid phase velocity vector; $\mathrm{m} / \mathrm{s}$ \\
\hline Vs. & versus \\
\hline VTF & Vertical Thin Film \\
\hline $\mathrm{vvm}$ & Vessel Volume per Minute \\
\hline W & Watts \\
\hline W.C. & Water Column \\
\hline wt\% & Weight Percent \\
\hline$X_{m n}$ & Mass fraction of the nth chemical species in the fluid phase \\
\hline XPS & X-Ray Photoelectron Spectroscopy \\
\hline
\end{tabular}


Development of a Hydrogasification Process for

Coproduction of SNG and Electrical Power from Westem Coals

Final Report

\begin{tabular}{|l|l|}
\multicolumn{1}{|c|}{ Acronym } & \\
\hline $\mathrm{XRD}$ & X-Ray Diffraction Analysis \\
\hline $\mathrm{X}_{\mathrm{smn}}$ & Mass fraction of the nth chemical species in the mth solids phase \\
\hline $\mathrm{Zn}$ & Zinc \\
\hline $\mathrm{ZnO}$ & Zinc Oxide \\
\hline $\mathrm{ZnCl}_{2}$ & Zinc Chloride \\
\hline
\end{tabular}




\section{ABSTRACT}

This report presents the results of the research and development conducted on an Advanced Hydrogasification Process (AHP) conceived and developed by Arizona Public Service Company (APS) under U.S. Department of Energy (DOE) contract: DE-FC26-06NT42759 for Substitute Natural Gas (SNG) production from western coal. A double-wall (i.e., a hydrogasification contained within a pressure shell) down-flow hydrogasification reactor was designed, engineered, constructed, commissioned and operated by APS, Phoenix, AZ. The reactor is ASME-certified under Section VIII with a rating of 1150 pounds per square inch gage (psig) maximum allowable working pressure at 1950 degrees Fahrenheit $\left({ }^{\circ} \mathrm{F}\right)$. The reaction zone had a 1.75 inch inner diameter and 13 feet length. The initial testing of a sub-bituminous coal demonstrated $\sim 50 \%$ carbon conversion and $\sim 10 \%$ methane yield in the product gas under $1625^{\circ} \mathrm{F}, 1000$ psig pressure, with a 11 seconds (s) residence time, and 0.4 hydrogen-to-coal mass ratio. Liquid by-products mainly contained Benzene, Toluene, Xylene (BTX) and tar. Char collected from the bottom of the reactor had 9000-British thermal units per pound (Btu/lb) heating value. A three-dimensional (3D) computational fluid dynamic model simulation of the hydrodynamics around the reactor head was utilized to design the nozzles for injecting the hydrogen into the gasifier to optimize gas-solid mixing to achieve improved carbon conversion.

The report also presents the evaluation of using algae for carbon dioxide $\left(\mathrm{CO}_{2}\right)$ management and biofuel production. Nannochloropsis, Selenastrum and Scenedesmus were determined to be the best algae strains for the project purpose and were studied in an outdoor system which included a 6-meter $(6 \mathrm{M})$ radius cultivator with a total surface area of 113 square meters $\left(\mathrm{m}^{2}\right)$ and a total culture volume between 10,000 to 15,000 liters (L); a $\mathrm{CO}_{2}$ on-demand feeding system; an on-line data collection system for temperature, $\mathrm{pH}$, Photosynthetically Activate Radiation (PAR) and dissolved oxygen (DO); and a 2 gallons per minute (gpm) algae culture dewatering system. Among the three algae strains, Scenedesmus showed the most tolerance to temperature and irradiance conditions in Phoenix and the best self-settling characteristics. Experimental findings and operational strategies determined through these tests guided the operation of the algae cultivation system for the scale-up study. Effect of power plant flue gas, especially heavy metals, on algae growth and biomass adsorption were evaluated as well. 


\section{TABLE OF CONIENTS}

DISCLAIMER

ii

ACKNOWEDGMENTS iif

ACRONYMS AND ABBREVIATIONS .. iv

ABSTRACT xiii

EXECUTIVE SUMMARY . xxiv

1 AdVANCED HYDROGASIFCATION - BENCH+SCALE TESTING ...................... 1-1

1.1 INTRODUC TION ............................................................................. 1-1

1.2 BENCH SCALE HYDROG ASIFCATION PROCESS DESCRIPIION ............................ 1-5

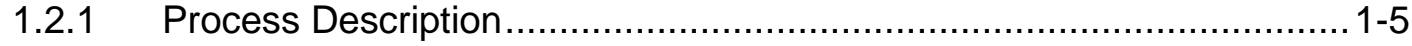

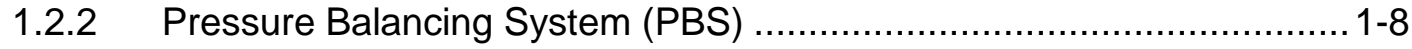

1.2.3 System Automation................................................................. 1-11

1.3 Computational Fuid DYNamics Modeung on HYDrogen INJ eCtor

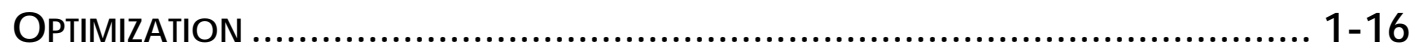

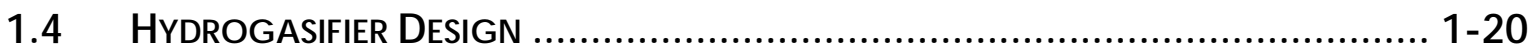

1.4.1 Hydrogasifier Design Criteria …….......................................... 1-20

1.4.2 Inner Reactor Tube Design ..................................................... 1-24

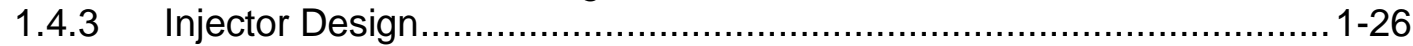

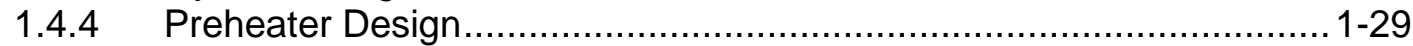

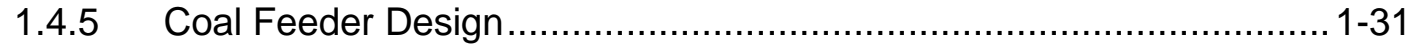

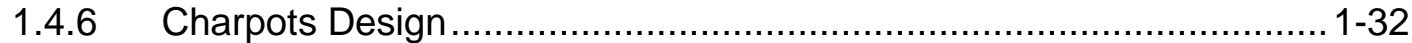

1.4.7 Liquid Collectors Design ......................................................... 1-35

1.4.8 Thermal Expansion Issues....................................................... 1-36

1.4.9 Design Flexibility ............................................................... 1-40

1.4.10 Design drawing Pressure and Temperature Limits............................ 1-43

1.5 HYDROGASIFER TEST PLAN ................................................................... 1-43

1.6 GAS, LQUID, AND SOUD ANALYSIS...................................................... 1-45

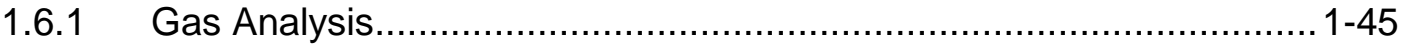

1.6.2 Liquid Condensate Analysis...................................................... 1-46

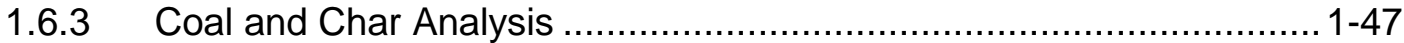

1.7 COALFEEDER CALBRATION ................................................................... 1-47

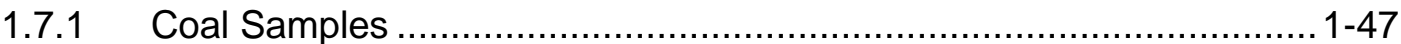

1.7.2 Coal Feeder Calibration Setup.................................................... 1-50

1.7.3 Coal Feeder Calibration with Original Auger .................................. 1-52

1.7.4 Coal Feeder Calibration with New Auger ......................................1-55

1.8 KINEIICS ONE-DIMENSIONAL SIMULATION .................................................. 1-58

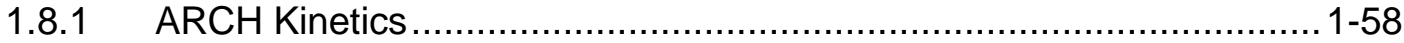

1.8.2 Results and Discussion ........................................................ 1-65

1.9 EXPERIMENTALDATA ANALYSIS............................................................ 1-70

1.9.1 System Commissioning ........................................................ 1-70

1.9.2 Preliminary Hydrogasification Tests .......................................... 1-72

1.9.3 Hydrogasification Testing on November 17, 2009 (Test 1 "After

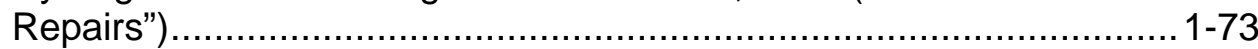


1.9.4 Hydrogasification Testing on December 1, 2009 (Test 2 - After "Repairs") .........................................................................1-75

1.9.5 Hydrogasification Testing on December 9, 2009 (Test 3 - After "Repairs") .......................................................................... $1-78$

1.9.6 Significant Dates ..................................................................

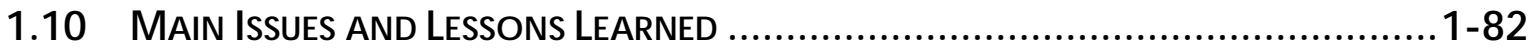

1.10 .1 Manufacturer Change .............................................................

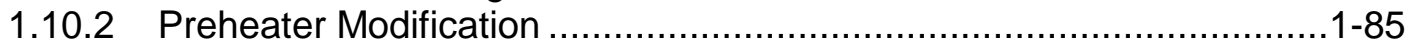

1.10.3 Coal Feeder P\&ID Modification ...............................................

1.10.4 Reactor Heater Modification ......................................................

1.10 .5 Other Process Improvements ...............................................1-100

1.11 CONCLUSIONSON HYDROGASIRCATION BENCH TESTING ..........................1-104

2 AlgAe TESTING .................................................................... 2-1

2.1 INTRODUCTION AND BACKGROUND..................................................2-1

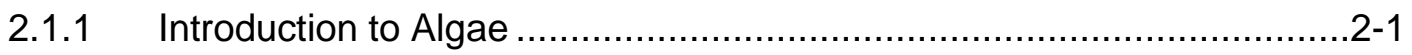

2.1.2 APS/GreenFuel Partnership ................................................ $2-4$

2.1.3 Incorporation of Algae Work into SNG Project.............................. $2-7$

2.2 INIIALALGAE ProdUCTION................................................................2-9

2.3 AlgAE LABORATORY AT3RD AVENUE RED FACIUTY............................ 2-15

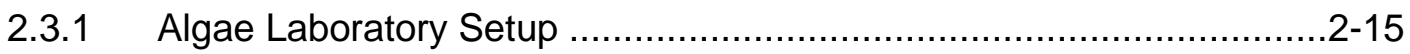

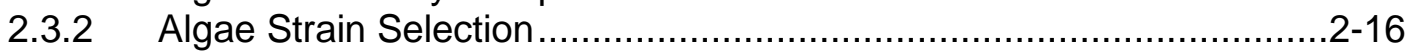

2.3.3 Algae Growth Medium .........................................................2-20

2.3.4 Algae Nursery and Bottle Culture Maintenance .............................2-24

2.3.5 Inoculum Production ...........................................................2-26

2.3.6 Summary of Algae Study at 3rd Avenue Lab Facility........................2-27

2.4 SCALABLE CARBON CAPTURE WTH AlgAE ...........................................2-29

2.4.1 3rd Avenue Outdoor Test Site Description ...................................2-29

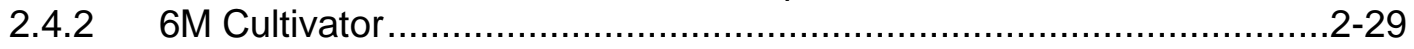

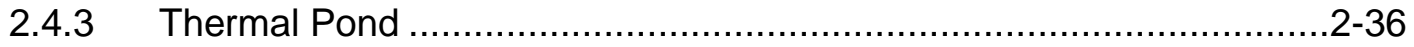

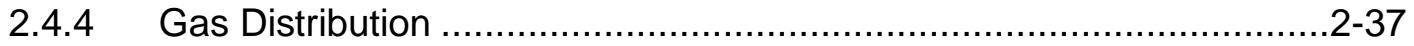

2.4.5 Harvest/Dewatering ............................................................ 29

2.4.6 3rd Avenue Test System Results and Discussion ............................2-45

2.4.7 Summary of 6M Radius Algae Cultivator Test Results ......................2-63

2.5 Algae Oil Extraction and Processing for Carbon Re-Uiluzation...........2-67

2.5.1 Outsourced Oil Extraction and Biofuel Production from Algae Biomass ....................................................................... 2-67

2.5.2 In-House Lipid Analysis and Oil Extraction Development ..................2-79

2.5.3 Summary of Oil Extraction and Biofuel Production ...........................2-82

2.6 EfeCts Of Fue Gas On Algal GroWth and Lid Production ................2-84

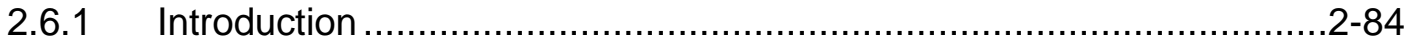

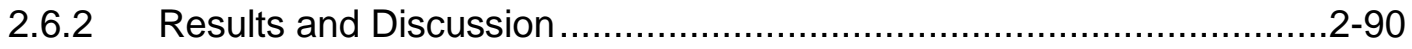

2.6.3 Summary of Heavy Metal Study ................................................. $2-103$

2.6.4 References for Chapter 2.6 .................................................. $2-103$ 


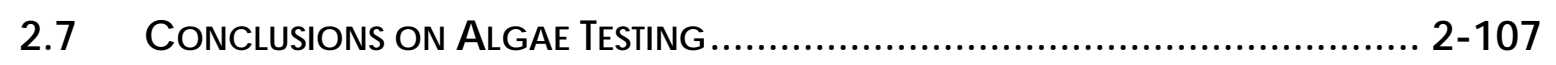

3 Preuminary Engineering Package and Systems Analysis for HYDROGASIFCATION/ SUBSITIUIE NATURAL GAS COMMERCIAL SCALE FACIUTY COMMERCIAL DESIGN ................................................... 3-1

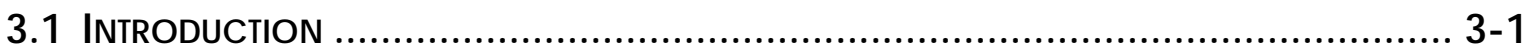




\section{LSTOF TABLES}

Table 1-1 - Temperature and Pressure Parameters for Alarm Strategy...........................1-15

Table 1-2 $-\mathrm{H}_{2}$ Injection Nozzle Optimization - Experimental Simulations ..........................1-18

Table 1-3 - Design Criteria for Reactor Components................................................

Table 1-4 - Hydrogasification Experimental Plan..................................................... 1-44

Table 1-5 - Calibration Gas Components and Concentrations......................................46

Table 1-6 - Comparison of the Two Coal Samples .....................................................

Table 1-7 - Coal Feeder Calibration at High Pressure with New Auger ............................1-56

Table 1-8 - Model Component Carbon Conversions Compared with Experimental Data......1-65

Table 1-9 - Model Component Carbon Conversions ................................................. 1-69

Table 1-10 - Proximate and Ultimate Analysis of Coal and Char .................................... 1-72

Table 2-1 - Plant Material Oil Yields ........................................................................ 2

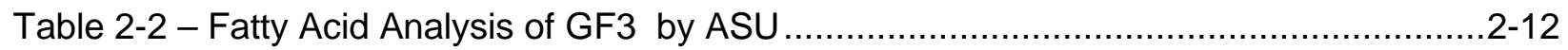

Table 2-3 - Fatty Acid Analysis of GF3 by POS ........................................................ $2-14$

Table 2-4 - Lipid Content Study for Algae Strain Selection ............................................2-20

Table 2-5 -Recipe for the Creation of the Artificial Seawater Medium Used for Marine Algae Strains ......................................................................................2-22

Table 2-6 - Medium Recipe for the Creation of the Freshwater Medium Used for Freshwater Algae Strains ...............................................................2-23

Table 2-7 - Summary of Nannochloropsis Productivity and Harvest Data from 6M Radius Cultivator ................................................................................ 2-52

Table 2-8 - Summary of $\mathrm{CO}_{2}$ Capture Data from 3rd Avenue Cultivator June 17 through June 23, 2009. Values are Derived from Averages of Measurements Taken Every Minute....

Table 2-9 - Summary of Selenastrum Productivity and Harvest Data from 6M Radius Cultivator....

Table 2-10 - Summary of Scenedesmus Productivity and Harvest Data from 6M radius Cultivator.

Table 2-11 - Summary of Mechanical Operation, Algae Cultivation, and Harvests Conducted in the 6M Radius Cultivation System at 3rd Avenue R\&D Test Site.

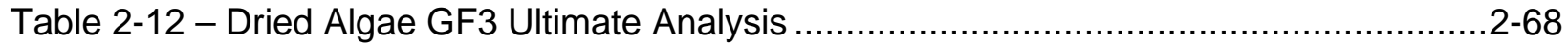

Table 2-13 - Ultimate Analysis of Western Coal ........................................................... $2-68$

Table 2-14 - Dried Algae GF3 Elemental Analysis and Comparison with Corn Stalk

(Provided by NETL) ............................................................................. $2-69$

Table 2-15 - Fresh City Water and Centrifuge Effluent Elemental Analysis (NETL) and

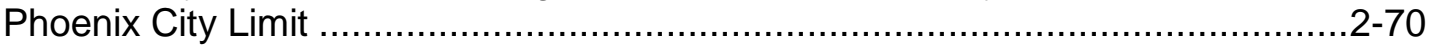

Table 2-16 - ConocoPhillips Triglyceride Specifications ............................................ 2-74

Table 2-17 - NETL/ORD, CAER, and APS Project Catalyst Testing and Product

Analysis - Summary by Facility ............................................................. $2-75$

Table 2-18 - Noble Catalyst Performance Comparison with Stearic Acid .........................2-77

Table 2-19 - Noble Catalyst Performance Comparison with Tristearin and Algae Oil .............2-77

Table 2-20 - CAER In-house Ni Catalyst Performance Comparison with Tristearin ...............2-78

Table 2-21 - Results Obtained from 5 wt\% Pd/AC Catalyst with Different Feedstocks ..........2-78

Table 2-22 - Results Obtained from the SAT002 Catalyst with Different Feedstocks ............2-79

Table 2-23 - Fatty Acid Methyl Esters in the Biomass ................................................. 2-81

Table 2-24 - Maximum Metal Concentration Likely to be Present in the Algae Growth

Medium 
Table 2-25 - Comparison of APS and USU Water Analyses on Redhawk Cooling Pond and Cholla Lake Water Sample

Table 2-26 - Anion Analysis of Water Samples........................................................ 2-94

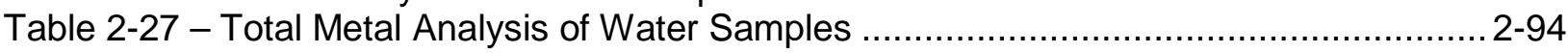

Table 2-28 - Analysis of Other Parameters on Water Samples ........................................ 2-95 


\section{LSTOF FGURES}

Figure 1-1 - AHP Conceptual Process Description...................................................

Figure 1-2 - Bench-Scale Hydrogasification Reactor Schematic ....................................1-6

Figure 1-3 - LabVIEW Interface Process Overview .................................................1-12

Figure 1-4 - LabVIEW Interface Temperatures - Overall.........................................1

Figure 1-5 - LabVIEW Interface Temperature Profile along Reactor Internal Tube

(Reactor Bottom Left and Reactor Top Right Sides of Graph, Respectively) .......1-14

Figure 1-6 - Hydrogen Injection Nozzle Orientation ................................................... 18

Figure 1-7 - A Case of Good Hydrogen and Coal Mixing .............................................1-19

Figure 1-8 - A Case of Bad Hydrogen and Coal Mixing ............................................. 1-20

Figure 1-9 - Simplified Diagram of the Bench-Scale Hydrogasification Reactor ..................1-23

Figure 1-10 - Reactor Inner Tube at Manufacturer ................................................... 1-26

Figure 1-11 - Reactor Center Hub Assembly........................................................... 1-27

Figure 1-12 - Injector and Injector Wands Assembly within the Top-Hat Section.................1-29

Figure 1-13 - Reactor Preheater at Manufacturer.................................................... $1-31$

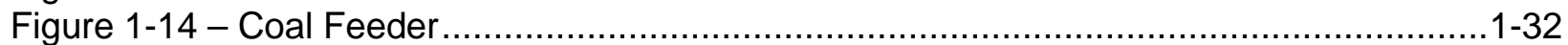

Figure 1-15 -Two-Stage Charpots with Actuated Full Port Ball Valve on Testing Site ...........1-35

Figure 1-16 - Two-Stage Condensate Collection Cylinders with Actuated Full Port Ball

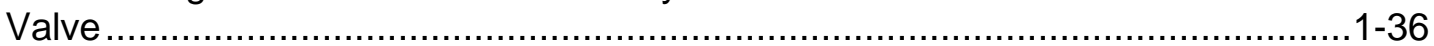

Figure 1-17 - Reactor Inner Tube Bellows..........................................................

Figure 1-18 - Flat to Groove Flange Connection Between Top Hat to Reactor Top..............1-42

Figure 1-19 - Comparison of Coal Particle Size Distributions (wt\% Retained on Screen) ......1-48

Figure 1-20 - Comparison of Coal Sieve Analysis (Cumulative wt\% Retained) ....................1-49

Figure 1-21 - Coal Feeding Testing Arrangement under Ambient Conditions......................1-50

Figure 1-22 - Higher-Pressure Test Setup ...................................................... 1-51

Figure 1-23 - Coal Feeder Calibration Using Original Auger Design ................................1-52

Figure 1-24 -Left Photo shows Auger and Right Photo Shows Auger End Cap and

Magnetic Drive Assembly .......................................................................

Figure 1-25 - Original Auger Design (Left Hand) .................................................. 1-53

Figure 1-26 - Proposed Modification of Existing Auger............................................. $1-54$

Figure 1-27 - Comparison of New and Old Augers (Front: New Auger; Back: Old Auger) .....1-54

Figure 1-28 - Coal Feeder Calibration Using New Auger..............................................1-55

Figure 1-29 - Comparison of Theoretical and Experimental Coal Delivery Rate ...................1-56

Figure 1-30 - Comparison of Coal Feed Rate under Ambient Conditions and High

Pressure with Carrier Gas Conditions ..................................................... $1-57$

Figure 1-31 - Step Size Effect on Model Gas Temperature ......................................... 1-63

Figure 1-32 - Statistical Design for Evaluating the Model ........................................... 1-66

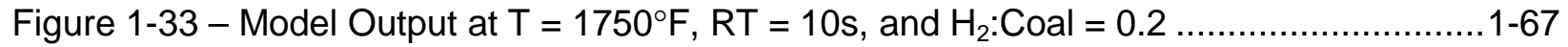

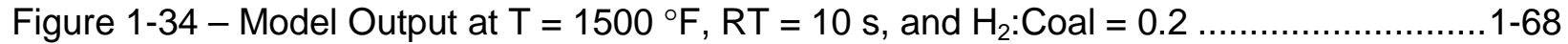

Figure 1-35 - Surface Response of Conversion to Methane .........................................1-69

Figure 1-36 - GC Output from November 17, 2009, Hydrogasification

(Test 1 After "Repairs")..........................................................................1-74

Figure 1-37 - Methane Mass Spectrometer Signal from the November 17, 2009,

Hydrogasification (Test 1 After "Repairs")....................................................1-75

Figure 1-38 - Reactor Temperature Profile from the December 1, 2009,

Hydrogasification (Test 2 After "Repairs") ............................................... 1-76

Figure 1-39 - Temperature versus Time Profile from the December 1, 2009,

Hydrogasification (Test 2 After "Repairs"). 
Figure 1-40 - Methane Concentration in the Product Stream from December 1, 2009, Hydrogasification Test 2 After "Repairs".

Figure 1-41 - Temperature-versus-Time Profile from the December 9, 2009,

Hydrogasification (Test 3 After "Repairs")

Figure 1-42 - Methane Concentration in the Product Stream from the December 9,

2009, Hydrogasification (Test Three After "Repairs") ................................... 1-80

Figure 1-43 - Hydrogasifier on June 29, 2009 ...................................................... 1-81

Figure 1-44 - Large Insulation Box of the Original Zone 5 Preheater Orientation ................. 1-86

Figure 1-45 - Reorientation of Hydrogen Crossover Line ............................................. 1-86

Figure 1-46 - Plan View Orientation of Hydrogen Crossover Lines

with New Watlow Heaters ................................................................... 1-87

Figure 1-47 - Left: Burned Heater during Preheater Zone 5 Re-commissioning. Right:

Burned Inconel 625 1/4-Inch Tubing due to an Electrical Short.......................... 1-88

Figure 1-48 - P\&ID of the Coal Feeder .............................................................. 1-89

Figure 1-49 - Ambient Pressure Coal Feeder Test with the Coal Feeder Vent Line Fully

Opened ....................................................................................... 1-90

Figure 1-50 - Ambient Pressure Coal Feeder Test with Fully Opened Coal Feeder Top ....... 1-90

Figure 1-51 - Modified P\&ID around Coal Feeder for Better Pressure Equilibrium ............... 1-91

Figure 1-52 - Temperature Profile around Preheater No. 1 (The One Closest to

Preheater Outlet) .......................................................................... 1-92

Figure 1-53 - Temperature Profile around Reactor Heater No. 1 (The One Closest to

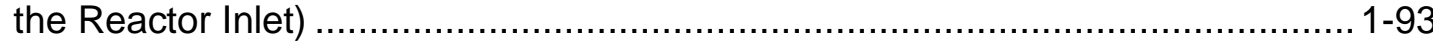

Figure 1-55 - Hot Spot Locations (In Inches) .................................................... 1-95

Figure 1-56 - Hydrogasifier Internal Tube after De-Telescoping (Left Photo) and Burned

Power Wire_Fatal Failure of Top Four Zone Heaters (Right Photo).................. 1-96

Figure 1-57 - Left: Inner Reactor Tube and Original Spider Plate; Right: Used Watlow

Heater (Black Deposition was from the Burned Insulation)............................... 1-97

Figure 1-58 - Reactor Heat Loss Estimation Under the Original Set Up......................... 1-98

Figure 1-59 - Left Photo shows the Modified Spider Plate and Newly Installed

Thermocouples; Right Photo shows New Heaters Wrapped Around the Inner Reactor Tube....

Figure 1-60 - Photo Left shows Increased Insulation Thickness; and photo right shows

Stainless Steel Heat Shield to Limit Nitrogen Natural Convection ...................... 1-99

Figure 1-61 - Left photo shows the Final Layer with all Power Wires Sheathed by

Ceramic; Right Photos show the Repaired Reactor Inner Tube Before Re-

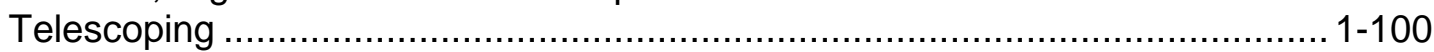

Figure 1-62 - Swagelok Valve between the Upper and Lower Charpots .......................... 1-101

Figure 1-63 - Left Photo Shows Picture of the Old Gas-Liquid Separator and the Right

Photo Shows the New Separator ....................................................... 1-103

Figure 1-64 - Mass Spectrometer $\mathrm{H}_{2} \mathrm{~S}$ Output................................................... 1-104

Figure 2-1 - Ashqelon Israel, 2000--MW Coal-Fired Power Plant......................................2-3

Figure 2-2 - Natural Beta Technologies Ltd, Elat Israel, Open Raceway Algae Farm ............ 2-3

Figure 2-3 - Aquatechnologies Algae Farm in Ketura, Israel - High-Value Products .............. 2-4

Figure 2-4 - Left photo: BPS Algae Farm in Klotze, Germany. Right photo: 10,000-M²

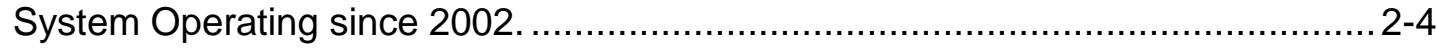

Figure 2-5 - GreenFuel Early Demonstration at Massachusetts Institute of Technology,

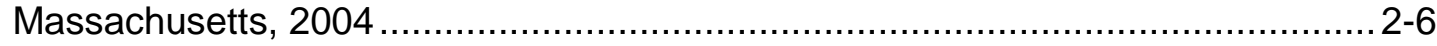

Figure 2-6 - GreenFuel Gen3 System at APS's Redhawk Power Plant in Arizona 2005-

2006 
Figure 2-7 - GreenFuel Gen5 System at APS's Redhawk Power Plant in Arizona, June 2007

Figure 2-8 - GreenFuel Algae Adaptation Study at APS's Four Corners Power Plant,

Farmington, New Mexico, December 2007

Figure 2-9 - Bag Farm at 3rd Avenue R\&D Facility ..................................................

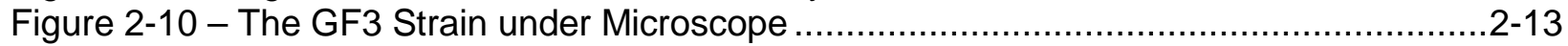

Figure 2-11 - APS 3rd Avenue Algae Lab, Phoenix, Arizona.......................................2-15

Figure 2-12 - Algae Nursery and 2 L Bottle Culture .....................................................2-26

Figure 2-13 - Thin Film, Flat Panel Culture System ................................................2-27

Figure 2-14 - Schematic of the $3^{\text {rd }}$ Avenue Outdoor Site in Phoenix, Arizona, Used for

Testing of the 6M Algae Cultivation System ..............................................2-29

Figure 2-15 - Photo of the Cultivator Floating in the Thermal Pond ................................ $2-30$

Figure 2-16 - 6M Cultivator Floating in a Thermal Pond .............................................. 2-30

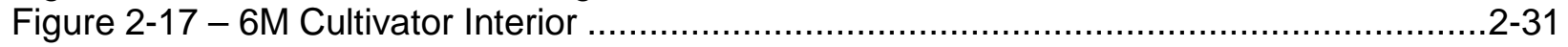

Figure 2-18 - Filtered Air Was Injected into the Canopy ............................................2-33

Figure 2-19 - Air Was Exchanged and Removed, Moderating Headspace Oxygen

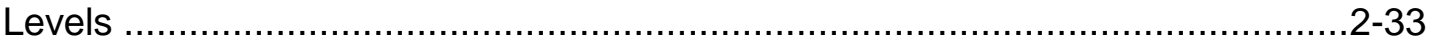

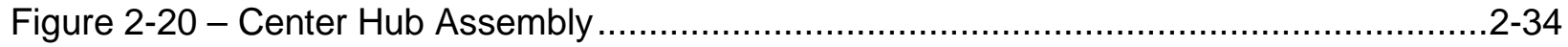

Figure 2-21 - Jets Propelled the Wands through the Culture, Creating Hydro Mixing ............2-35

Figure 2-22 - Wands Showing Discharge of Liquid Volume through Exhaust Holes ..............2-35

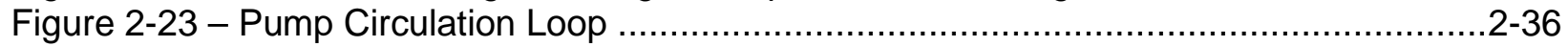

Figure 2-24 - 3rd Avenue Thermal Pond under Construction .......................................2-36

Figure 2-25 - 3rd Avenue Gas and Liquid Distribution Loop Diagram with Inline Sensors .....2-38

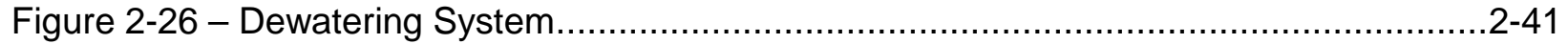

Figure 2-27 - Culture from the Cultivator Entering the Dewatering System (through

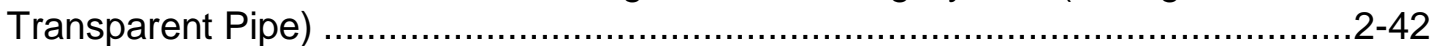

Figure 2-28 - Resulting Separated, Clear Water after Dewatering of Algae........................2-42

Figure 2-29 - Concentrated Algae in Large Tank after Dewatering (Approximately 10

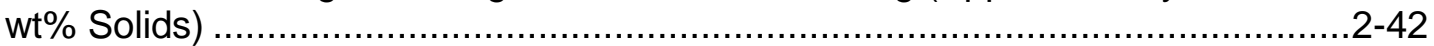

Figure 2-30 - Culture Dewatering Lavin (12-413V) Centrifuge ..........................................2-43

Figure 2-31 - Algae Paste out of Lavin Centrifuge ...................................................2-44

Figure 2-32 - Nannochloropsis Culture Density as Measured by FM (no unit) within the

Cultivation System ............................................................................. $2-46$

Figure 2-33 - Nannochloropsis Culture Density as Measured by Ash-Free Dry Weight

(grams of dry biomass per liter of culture) ............................................. $2-46$

Figure 2-34 - Nannochloropsis Culture Temperature within the Cultivation System ..............2-48

Figure 2-35 - Nannochloropsis Culture pH within the Cultivation System ...........................2-49

Figure 2-36 - Nannochloropsis Culture Volume, Estimated by Salinity within the

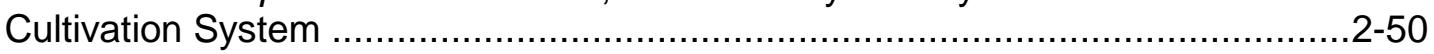

Figure 2-37 - Nannochloropsis Growth Curve by Ash-Free Dry Weight............................2-51

Figure 2-38 - Logged Culture PH for the 24-hour Period on 4/27/2009 during the

Nannochloropsis Test in the Cultivator....

Figure 2-39 - Logged Culture Condition Data for the 24-Hour Period on 4/27/2009

during the Nannochloropsis Test in the Cultivator. B: Culture Temperature;

C: PAR; D, Dissolved Oxygen (DO) Concentration in the Liquid Culture. ............2-54

Figure 2-40 - Selenastrum Culture Density as Measured by Ash-Free Dry Weight

(Grams of Dry Biomass per Liter of Culture)...............................................2-56

Figure 2-41 - Automatically Logged Culture Condition Data for the 24-hour Period on

5/25/2009 during the Selenastrum Test in the Cultivator. A: Culture pH. B:

Culture Temperature. C: PAR. D: DO Concentration in the Liquid Culture. .......2-58 
Figure 2-42 - Scenedesmus Culture Density as Measured by Dry Weight (Grams of Dry Biomass per Liter of Culture) .................................................................... 2-60

Figure 2-43 - Settled Scenedesmus Culture after 5 Hours in a Conical-Bottom Vessel ........ 2-62

Figure 2-44 - Gas Chromatographs of the Fatty Acid Methyl Esters in Scenedesmus

Analyzed with Different GC Columns ......................................................... 2-80

Figure 2-45 - SOx and NOx Reactions in the Aqueous Phase ....................................... 2-85 


\section{LSTOF APPENDIXES}

Appendix A: Feasibility of SNG Formation through Coal Hydrogasification

Appendix B: Bench Scale Reactor Design from APS

Appendix C: Bench Scale Hydrogasifier Reactor Final Design from Gaspar Inc.

Appendix D: Bench Scale Hydrogasifier Preheater Final Design from Gaspar Inc.

Appendix E: Bench Scale Hydrogasifier Coal Feeder Final Design from Gaspar Inc.

Appendix F: Bench Scale Hydrogasifier Upper CharPot Assembly Final Design from Gaspar Inc.

Appendix G: Bench Scale Hydrogasifier Lower CharPot Assembly Final Design from Gaspar

Inc.

Appendix H: Bench Scale Hydrogasification Testing Process PI\&D

Appendix I: Bench Scale Hydrogasification Testing Process Bill of Material

Appendix J: Bench Scale Hydrogasification Testing Commissioning Procedure

Appendix K: Bench Scale Hydrogasification Testing Startup Procedure

Appendix L: Bench Scale Hydrogasification Testing Shutdown Procedure

Appendix M: Process Design, Plant Design, Systems Analysis and Cost Engineering 


\section{EXECUTIVE SUMMARY}

The objective of the project was to develop and demonstrate a coal hydrogasification-based process for producing Substitute Natural Gas (SNG) from coal and evaluate an algae process. for the management of carbon dioxide, a greenhouse gas that is normally emitted to the atmosphere during power generation and other industrial processes that utilize fossil fuels, by utilizing it for biofuel production. APS conducted an extensive research effort to achieve these goals under a Cooperative Agreement with the United States Department of Energy, National Energy Technology Laboratory (NETL).

The coal hydrogasification-based process was chosen over partial oxidation (POX) gasification because it produces significantly more fuel gas and SNG product from the same amount of feedstock. On a relative basis, the hydrogasification production of SNG is about $20 \%$ more than for the POX system, and water usage/consumption is dramatically reduced.

For the coal-to-SNG portion of the project an advanced double-wall down-flow hydrogasification reactor was engineered, constructed, commissioned and operated by APS in Phoenix, Arizona. The reactor - ASME-certified under Section VIII with a maximum allowable working pressure of 1150 psig at $1950^{\circ} \mathrm{F}$ and operated by a LabVIEW automation system - had the following distinguishing characteristics:

- The hydrogasification reactor could feed pulverized coal at rates up to $15 \mathrm{lb} /$ hour.

- The hydrogen injection temperature could be varied up to $1350^{\circ} \mathrm{F}$.

- The reactor assembly had two stages of charpots and liquid pots for transient and steady state sample collection.

- The hydrogasification reaction zone had a 1.75-inch internal diameter (ID) and was 13 feet long.

- On-line gas chromatography and mass spectroscopy (GC/MS) were installed for product gas analysis.

During its early testing phase, the reactor experienced several major modifications to improve reactor heating, hydrogen preheating and a steady coal feeding rate. The successful tests demonstrated close to $50 \%$ carbon conversion and $\sim 10 \%$ methane yield in the product gas under $1625^{\circ} \mathrm{F}, 1000$ psig pressure, 11 seconds (s) residence time, and a 0.4 hydrogen-to-coal 
mass ratio. GC/MS analysis on the organic phase indicated the liquid by-products mainly contained benzene, toluene, xylene (BTX) and tar. Char collected from the bottom of the reactor had a 9000-Btu/lb heating value.

Extensive computational fluid dynamics modeling of the hydrogen nozzle design was conducted to develop a design that would provide optimal gas and solid phase mixing to result in improved carbon conversion. The hydrodynamics (solid equatorial mixing, back mixing and temperature profile) around the reactor head were studied, resulting in 0.18-inch hydrogen nozzles ID, 45-degree shooting angle, and 30-degree swirling angle as optimum configurations.

A one-dimensional (1-D) model of the coal hydrogasification reactions was further developed based on Advanced Rapid Carbon Hydrogasification ( $\mathrm{ARCH}$ ) kinetics originally proposed in the early 1990s. The proposed reaction pathways for methane formation were first through coal devolatilization and second through further reaction of rapid carbon (RC) with hydrogen and hydro cracking of BTX and tar. Result comparisons indicated the model did a reasonable job of predicting the carbon conversion to methane $\left(\mathrm{CH}_{4}\right)$, BTX, and total conversion without additional tuning. However, the model did not accurately predict the distribution of conversions to carbon monoxide (CO) and oil.

For the $\mathrm{CO}_{2}$ utilization portion of the program, a six meter (6M) radius algae cultivation system was built at APS's $3^{\text {rd }}$ Avenue algae research and development (R\&D) facility. This outdoor system included a $6 \mathrm{M}$ radius cultivator with a total surface area of $113 \mathrm{~m}^{2}$ and a total culture volume between $10,000 \mathrm{~L}$ to $15,000 \mathrm{~L}$; a $\mathrm{CO}_{2}$ on-demand feeding system; an on-line data collection and process control system that captured data for temperature, $\mathrm{pH}, \mathrm{PAR}$ and dissolved oxygen (DO) and automatically adjusted parameters to optimize algae growth; and a -2 gallons per minute (gpm) algae culture dewatering system. The 6M cultivation system demonstrated approximately 170 days of total operation since the onset of testing. Approximately $77,000 \mathrm{~L}$ of culture was harvested. Three algae species were examined and cultured throughout the duration of experimentation - Nannochloropsis, Selenastrum and Scenedesmus.

The Nannochloropsis harvested at the site gave an oil content of 9.21 weight percent (wt\%) of biomass, where $37 \mathrm{wt} \%$ of the total lipids consisted of omega 3, 6 and 9. Selenastrum produced oil content of $17 \mathrm{wt} \%$ total fat and $8 \mathrm{wt} \%$ nonpolar lipids. A total neutral lipid content of $80 \mathrm{wt} \%$ of the total fatty acids was obtained from an acetone-dried Scenedesmus biomass, which generally contains 9-17 wt\% total lipids when grown in non-stressed conditions. 
Depending on the algae growth rate, a $\mathrm{CO}_{2}$ capture rate of up to $90 \mathrm{wt} \%$ was observed utilizing the $6 \mathrm{M}$ cultivator design. Among the three algae strains, Scenedesmus showed the most tolerance to temperature and irradiance conditions in Phoenix and the best self-settling characteristics. Experimental findings and operational strategies determined through these tests guided the operation of the algae cultivation system at APS's Redhawk test facility for a scale-up study.

In-house biomass and oil analysis protocols and experimental facilities were established. These significantly assisted in monitoring algae cultivation, studying stressing effects on algae oil content and even facilitating strain selection. It has been generally recognized that oil extraction using "green" algae is very challenging. Non-flowing green gum was obtained from all-oil extraction exercises using green algae, which was probably caused by the interaction of chlorophyll and phosphor lipids. Crude oil pretreatment will be required for any oil upgrading. It is necessary to manipulate the algae biomass cultivating condition to increase the fat level and reduce chlorophyll in the biomass, thereby easing the oil extraction process.

Utah State University (USU) in Logan, Utah, investigated and developed new procedures and methods to examine the effects of heavy metals and other chemical species present in flue gas. Reported results indicated that lead $(\mathrm{Pb})$ did not adversely affect the growth of algae. Continued metal element analysis of algae culture water, algae biomass ion exchanged rinse water, algae biomass and crude algae oil would determine the deposition of metals. Metal deposition would occur in water, via physical adsorption on the algae biomass cell wall, or inside the algae cell.

Research work on the project commenced during the later part of 2009 with a particular focus on bench-scale hydrogasifier testing, 1D kinetics model simulation for coal hydrogasification, extensive algae stressing, oil extraction, and lipid analysis. The Coal-to-SNG study and carbon dioxide management via algae cultivation and conversion efforts, including the addition of efforts towards the demonstration of a one-third-acre algae farm at APS's Redhawk testing facility were continued under DOE award DE-FE0001099, "Integrated Energy System (IES) with Beneficial $\mathrm{CO}_{2}$ Use." Both projects were terminated by APS on March 31, 2010. This technical final closeout report should be reviewed in conjunction with the IES final technical closeout report for a comprehensive view of the research.

Additional work was performed by WorleyParsons Group Inc., Reading PA which advances the conceptual design for a 3000 ton-per-day commercial-scale AHP plant. The WorleyParsons 
Systems Study is a continuation of work that was initially discontinued by APS in March 2010, and at the request of DOE resumed in July 2010. This work, titled, "Preliminary Engineering Package and Systems Analysis for Hydrogasification/Substitute Natural Gas Commercial Scale Facility Conceptual Design" is presented in Chapter 3 of this report. 
Development of a Hydrogasification Process for

Coproduction of SNG and Eectrical Power from Westem Coals

Final Report

THIS PAGE INTENTIONALLY LEFT BLANK 


\section{AdVANCED HYDROGASTHCATION - BENCH+SCALE TESTING}

\subsection{INTRODUCTION}

The Advanced Hydrogasification Process (AHP) is a concept being developed to use America's abundant western coal supply to address concerns of diminishing domestic oil and natural gas (NG) resources as energy providers and reduce greenhouse gas emissions with renewable energy. APS is the prime recipient for this cooperative project with the DOE to conduct research and development on several features of the AHP. As shown in Figure 1-1, the APS AHP process is an integrated five-step systems process: (1) hydrogen production using incremental renewable energy to minimize $\mathrm{CO}_{2}$ emissions, (2) SNG production by hydrogasification of coal, (3) oxy-combustion of coal/char to produce electricity, (4) carbon recycling of $\mathrm{CO}_{2}$ emissions through biological processes, and (5) biofuels production by reuse of $\mathrm{CO}_{2}$. The combination of these steps creates an integrated process that delivers energy and fuel in a long-term sustainable process. Ultimately, the Algae Farm will need to be developed.

The AHP uses coal as a source for carbon and hydrogen produced from renewable resources in a process (hydrogasification) that produces SNG with very little greenhouse gas $\mathrm{CO}_{2}$ emissions. Hydrogen can be produced from many sources. In the AHP conceptualized facility, large-scale electrolysis produces hydrogen and oxygen at a commercial efficiency of $75 \%$ high heating value (HHV). Through incremental use of renewable energy (such as wind) this hydrogen can be produced without $\mathrm{CO}_{2}$ emissions. Grid energy (from base load nuclear or coal) can also be incrementally used to firm the hydrogen supply and increase load factor during non-peak periods. In this manner, off-peak and seasonal renewable energy can be stored indefinitely in existing infrastructure and used in a wide array of applications including electric production.

Hydrogen can be produced using renewable energy and converted to NG (a desirable fuel for power generation or industrial use) via coal hydrogasification, which is the key R\&D element under this Cooperative Agreement. This NG can be stored and distributed using existing NG infrastructure. This combination of technologies provides a strategy toward sustainability.

The need for the AHP is further supported by two inevitabilities: (1) without a viable $\mathrm{CO}_{2}$ strategy, the future use of coal to produce electricity faces regulatory and legal challenges; and (2) electric utility renewable energy portfolio requirements are likely to increase over time, making a dispatchable renewable fuel valuable. Breakthroughs such as hydrogasification are required to make large-scale dispatchable electric power generation from renewable energy a 
reality.

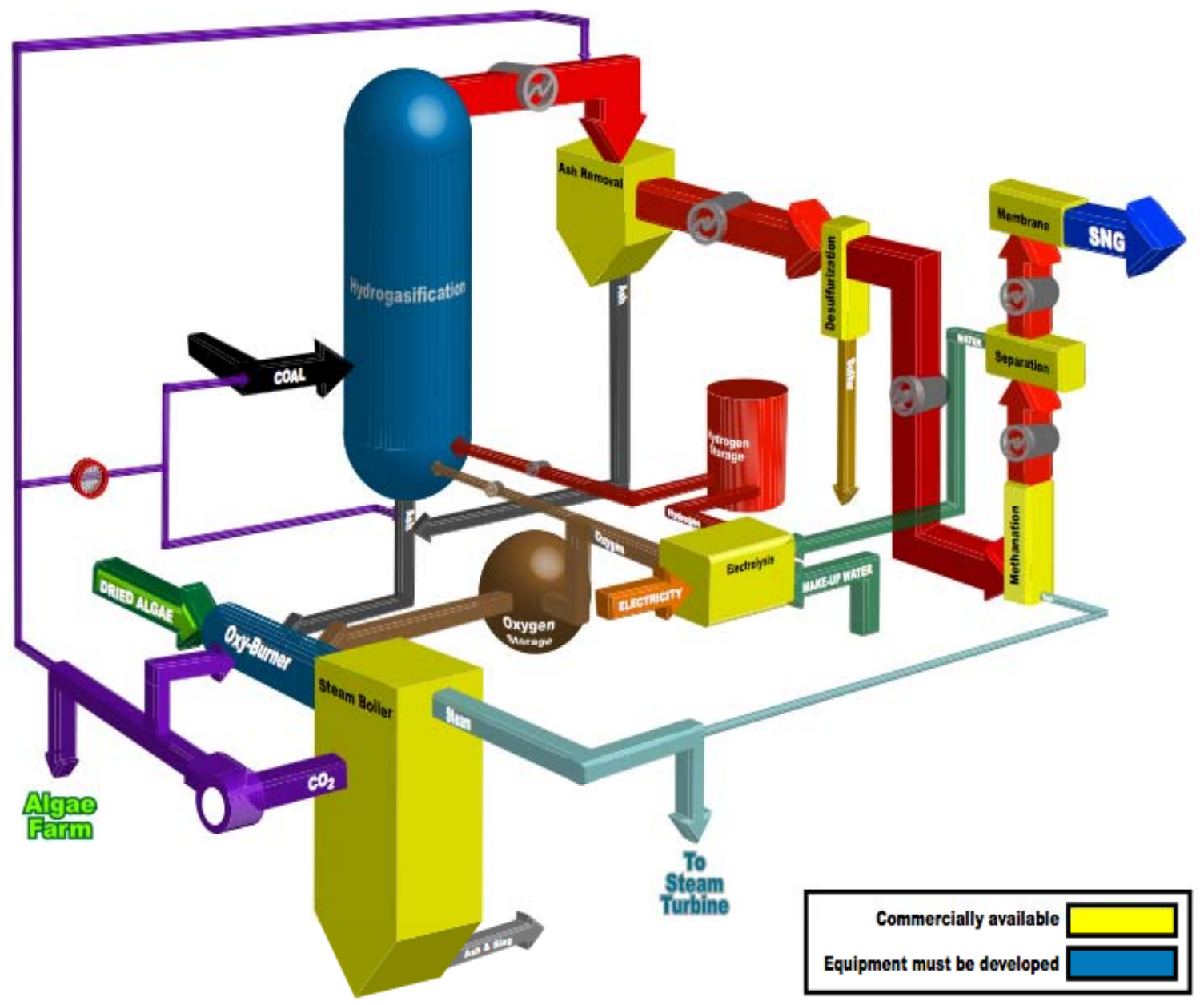

Figure 1-1 - AHP Conceptual Process Description

Gasification-based technology is the only environment-friendly technology that provides the flexibility to coproduce hydrogen $\left(\mathrm{H}_{2}\right)$, SNG, premium hydrocarbon liquids including transportation fuels, and electric power in desired combinations from coal and other carbonaceous feedstock. Rising costs and limited supply of crude oil and NG provide a strong incentive for the development of coal gasification-based co-production processes. This project evaluates the co-production of SNG and electricity from coal. SNG is a hydrogen carrier, is 
easily stored, is a fuel source for existing natural gas combined cycle (NGCC) power production and is easily transported in existing nation-wide NG pipeline networks. The efficient production of SNG from coal offers supply and price stability coupled with transportability to an electric power generation infrastructure that has grown highly dependent on NG.

Hydrogasification research was previously studied by the DOE during the 1970s. The Gas Research Institute, now the Gas Technology Institute (GTI), aggressively researched hydrogasification as a means of providing a more efficient process to produce SNG than the traditional partial oxidation process followed by methanation. DOE research stopped in 1982 when the price of NG fell. Osaka Gas \& Electric together with British Gas resumed research in 1992; the Japanese government joined the effort, and that program was renamed $A R C H$ (Advanced Rapid Coal Hydrogasification). The ARCH research program terminated when the price of NG fell again and the Energy Information Agency (EIA) of the DOE forecasted a favorable economic future and adequate future reserves for NG. A detailed literature review on hydrogasification is presented in Appendix $A$.

The AHP Project was to be conducted in three phases. In Phase I, high pressure thermogravimetric analysis (HPTGA) tests were run, a chemical process model was created and preliminary engineering and economic studies were performed. Effort was also expended in reviewing the prior research efforts of DOE in hydrogasification research from the 1960s through 2002 (Japanese and British Gas ARCH Project).

The HPTGA testing evaluated the gasification reactivity of a devolatilized western coal sample in the $1500-1700^{\circ} \mathrm{F}$ temperature range, at 500 psi pressure using a 50 vol\% $\mathrm{H}_{2} \mathrm{O}-50 \% \mathrm{~N}_{2}$ gas mixture. One additional test was also carried out at $1700^{\circ} \mathrm{F}$, but at the higher pressure of 700 psig. HPTGA test results indicated the devolatilized coal sample is highly reactive under these regular gasification conditions. Consistent with expectations, reactivity improved with increasing temperature. Reactivity was reduced at the increased pressure. It took 90 minutes to achieve $\sim 100 \%$ base carbon conversion at $1500^{\circ} \mathrm{F}$ (and 500 psig). Similar high conversions were achieved during 30 minutes at $1600^{\circ} \mathrm{F}$ and during only 15 minutes at $1700^{\circ} \mathrm{F}$. At $1700^{\circ} \mathrm{F}$, reactivity at 700 psi was lower than that at 500 psig, requiring about 25 minutes to achieve complete conversion compared to 15 minutes at the lower pressure.

In phase II, the hydrogasification kinetics were being created by using a bench-scale kinetics reactor (BSRx). Test results were intended to be used to update both the process model and 
the preliminary engineering and economics study. Also in phase II, a task was added to evaluate using the carbon dioxide $\left(\mathrm{CO}_{2}\right)$ from power plant stack gas to cultivate algae, which consumes the carbon as a method to manage carbon dioxide emission. The end goal was to then develop a scalable algae farm and a biofuel sample (military aviation fuel - JP8) was to be produced by using algae grown in the bioreactor/farm. The algae farm was to be operated for a period of one year while integrated with an APS power plant. Phase III of the project would encompass the engineering, construction, and operation of an engineering-scale hydrogasification reactor and necessary appurtenances.

This project was selected from among applications received in response to a DOE-NETL funding opportunity announcement entitled, "Co-Production Advanced Technology/Process Concepts," which was co-sponsored by the Hydrogen and Clean Fuels Program. The Program supports R\&D activities related to hydrogen from coal pathways. The primary program elements were: (1) central hydrogen production pathway, (2) alternate production pathway, and (3) hydrogen utilization. The APS AHP project supported the alternate production technology pathway in which clean syngas from coal is converted to high-hydrogen-content liquid hydrocarbon carriers, alcohols, or, as in this specific project - SNG.

The goals of this three-phase project were to develop an AHP process with the following features:

- Process efficiency greater than $50 \%$

- Production of SNG at a cost less than the market price for NG

- Capture and sequestration (through conversion) of the equivalent of $90 \%$ of $\mathrm{CO}_{2}$ emissions

- Reduction in water use by least $50 \%$ below the usage of a comparable partial-oxidation based gasification or syngas methanation process

- Ability to accept hydrogen as a supplemental source of energy

- Use of low-British thermal units (Btu) western coal

- Coproduction of electricity

- Integration of an algae process for $\mathrm{CO}_{2}$ recycle

- Use of oil from algae $\mathrm{CO}_{2}$ recovery to create high-value transportation fuels 
The research of the project was continued under DOE award "Integrated Energy System with the Beneficial $\mathrm{CO}_{2}$ Use" (DE-FE0001099) which was initiated September, 2009. Both projects were terminated by APS on March 31, 2010. Please refer to the DE-FE0001099 Technical Final Closeout Report for a more comprehensive project report.

\subsection{BenchSCALE HYDROgasifCATION PROCESS Description}

The process description is summarized in this subsection. The piping and instrumentation diagram (P\&ID) is contained in Appendix $\mathrm{H}$. Appendix I contains the bill of material (BOM). Detailed operations procedures are contained in Appendix J, Appendix $\mathrm{K}$ and Appendix L.

\subsubsection{PROCESS DESCRIPIION}

A schematic of the bench-scale hydrogasification reactor may be seen in Figure 1-2. Gas (hydrogen/nitrogen) first entered pre-heater 4 and flowed through the subsequent preheaters before entering the reactor and mixing with the coal feed at the top. In the figure the pre-heater zone 5 was a group of small heaters to cover the heat loss from hydrogen crossover lines connecting preheater and reactor. Included in the coal feeding system were the hopper, magnetic drive, and an auger. A small amount of hydrogen, which was separated from main hydrogen stream, was supplied to the discharge of coal feeder as a carrier gas. Once the hydrogen-enriched syngas produced in the hydrogasifier and the unconverted coal or char left the reactor, the solid products were collected in either the upper or lower charpot. The syngas then passed by the cooler, which condensed water and heavy hydrocarbons out of the product stream. The liquid products were subsequently collected in either the lower or upper liquid collectors. Finally, the gas passed through a ZnO sorbent bed to capture the hydrogen sulfide for odor control and then was vented. With that, there were two gas sampling lines, one upstream and one downstream of the $\mathrm{ZnO}$ sorbent bed. 


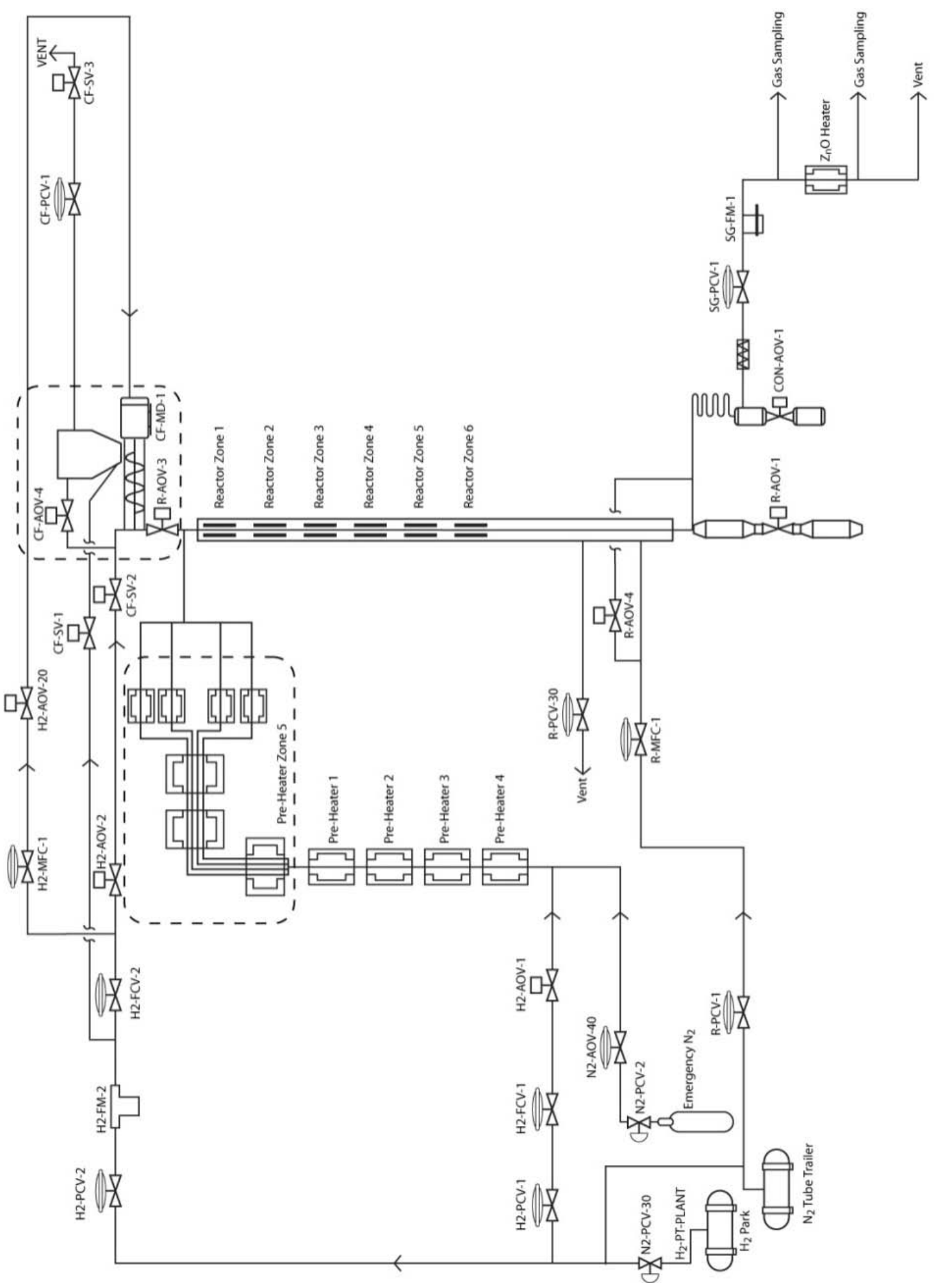

Figure 1-2 - Bench-Scale Hydrogasification Reactor Schematic 
To start up the reactor, the back-pressure control valve at the system exit (Tag number SGPCV-1) was closed. The reactor and annular space were pressurized with nitrogen. The nitrogen supply valve (Tag number R-PCV-1) was used to pressurize the annular space and additional nitrogen was fed to the bottom of the preheater through a system pressure control valve normally designated for hydrogen (Tag number H2-PCV-1) and was used to control the reactor pressurization. A small slipstream of nitrogen bypassed the pre-heater through pressure the control valve (Tag number H2-PCV-2), and was used to control the coal feeder pressurization. The pressurization was controlled to ensure that there was no pressure differential greater than 25 psig between the pressure shell and the reactor. With the shell side pressure higher, reaction gas is prevented from leaking from the reactor to the shell zone that contains electrical heaters.

Once the reactor was at the desired operating pressure the system back-pressure control valve (SG-PCV-1) at the exit of the reactor was opened and the pressure equalization between the pressure shell and the hydrogasification reactor was initiated by automating a vent valve at the bottom of the pressure shell (Tag number R-PCV-30). The gas being fed to the reactor was switched from nitrogen to hydrogen. At this point the preheaters and reactor heaters were turned on. Once the reactor reached the desired operating temperature, the coal-feed isolation valve (Tag number R-AOV-3) at the top of the reactor was opened and coal feeding commenced. At this point, the introduction of ambient temperature coal caused a heat sink in the system. As soon as the system recovered from the heat sink and a constant temperature profile was attained, the reactor was considered to be at steady state. After the reactor reached steady-state, the lower char pot and the lower condensate pot were isolated (by closing valves R-AOV-1 and CON-AOV-1) so the steady-state products would be captured in the upper charpot and upper condenser pot and thereby separated from the transient products in the lower pots.

Upon completion of coal feeding, the reactor was ready to shut down. The first step was to turn off all of the heaters. Next, the gas going into the reactor was switched from hydrogen to nitrogen. The reactor would slowly be depressurized when reactor and preheater temperature dropped below $1300^{\circ} \mathrm{F}$. Once the reactor was depressurized, pressure would be built back up to 200 psig and depressurized again. This purging process was done twice to ensure that there was no residual hydrogen left in the reactor. 
The coal and char products were collected from the charpots at the end of the test when everything cooled down. The blind flange at the bottom of the lower charpot was unbolted and removed. The coal and char in the lower charpot dropped into a sample bucket, which was then sealed and labeled for later analysis. Once the lower charpot was cleaned of residual coal and char then the upper charpot was discharged for collection of samples for analysis. The operator opened charpot isolation valve R-AOV-1, which allowed the coal and char in the upper charpot to drop through the valve and through the lower charpot. The coal and char were collected in a sample bucket and sealed for later analysis.

The charpots were typically cleaned to the extent possible. The gasket on the bottom of the lower charpot was removed and replaced with a new gasket, and the blind flange was re-installed and tightened up to the design bolt torque for the flange. This joint was pressureand leak-tested before initiating a new test. A gas sample was analyzed through an online gas chromatography (GC) and mass spectroscopy, A SRI Instruments Model 8610C Multiple Gas GC \#2, which was equipped with a gas sampling valve with dual sampling loops one which contained a Molecular Sieve column and the other a Hayesep-D column and a Standard Research Systems QMS 200 mass spectrometer were utilized.

\subsubsection{PRESSURE BALANCING SYSTEM (PBS)}

As was mentioned previously, the bench-scale hydrogasification reactor was designed to operate at extremely high temperature $\left(1950^{\circ} \mathrm{F}\right)$ and pressure $(1150 \mathrm{psig})$. This combination of high pressure and temperature rendered most metals unusable in the application because the tensile strength of metals was dramatically reduced at this temperature. The reactor design coped with this inherent limitation of metals by using a "balanced pressure" reactor configuration wherein the inner reactor tube was contained centrally inside a larger outer shell (10-inch schedule 80 pipe). The annular space between the inner tube and outer shell was pressurized with dry ambient temperature gaseous nitrogen which flowed through the annular space providing some cooling at the lower part of the reactor. Additionally, the nitrogen removed moisture that could corrode heaters and wiring as well as any oxygen from the annular space, thereby reducing the likelihood of a combustion incident in the annulus.

The balanced pressure design was intended to maintain the annular space pressure slightly higher than the inner tube reaction pressure. The pressure in the annulus was always higher than the inner tube so that if a leak occurred, inert nitrogen gas would flow into the inner tube quenching the reaction. This arrangement also prevented hydrogen or coal dust from diffusing 
into the annular space, a situation which could compromise the performance of the heaters, insulation, wiring, and temperature sensors. The annulus could be pressurized to a maximum pressure of 1200 psig. At this condition, the inner tube could operate at $1150 \mathrm{psig}$ and $1950^{\circ} \mathrm{F}$.

As described earlier, the mechanical design of the inner tube was analyzed using ASME BPVC calculations and buckling equations from Roark's Formulas for Stress and Strain. The result of the ASME analysis indicated that the inner tube could operate at $1950^{\circ} \mathrm{F}$ with a maximum 50-psid pressure differential (external pressure above internal pressure) with a safety factor consistent with ASME BPVC requirements. At lower temperatures, the allowable differential pressure was much higher. Additionally, by operating at less than 50 psid, the safety factor was increased because the inner tube was subject to lower stress. If an incident occurred where the reactor pressure differential exceeded 50 psid then it was more likely that the inner tube or the lower bellows would fail. In the case where the annular pressure exceeded the inner tube pressure, the inner tube or the lower bellows would collapse inward and nitrogen gas would flow into the reaction area, quenching the reaction and equalizing the pressure.

An alternative scenario that could happen is where the inner tube pressure exceeded the annular pressure. This would cause either the inner tube or lower bellows to rupture outward, allowing hydrogen and coal dust to flow into the annulus. Either scenario is catastrophic to the reactor and would require extensive repair. The rupture scenario that would allow coal into the annulus would likely result in more damage due to coal infiltration into the insulation, heaters, and wiring. Either failure mode points out the importance of the PBS operating properly. As such, safety systems were implemented to automatically shut-down the system in the event pre-determined pressure differential limits were exceeded.

To prevent a catastrophic failure of the reactor due to a high pressure differential, the PBS was designed to control the pressure in the inner tube and annulus. A design target to maintain the pressure difference at less than 10 psid was set. The PBS used a system of pressure transmitters (R-PT-30, R-PT-2, R-PT-3, and R-DPT-1) to sense the pressure in the inner tube, annulus and nitrogen purge system. These transmitters had an accuracy of $0.1 \%$ and provided a 4-20 mA signal to the pressure control system (PCS). The plant operator used the PCS to set the operating pressure in the inner tube and annulus.

In addition to maintaining the pressure in the inner tube and annulus, the PBS also controlled a nitrogen purge through the annulus. This nitrogen purge flow could be set by the operator and was controlled by the PCS. Nominally, the purge flowed at about 600 cubic square feet per 
hour (scf/hr), and a mass flow controller (MFC) (R-MFC-1) and PCV (R-PCV-1) controlled the nitrogen pressure and the flow rate of nitrogen into the annular space. The pressure in the annulus was controlled by a back-pressure regulator (R-PCV-30), which vented nitrogen to the atmosphere to control the flow of purge nitrogen out of the annulus. In tandem, the nitrogen supply through R-MFC-1 (nitrogen inlet) and R-PCV-30 (nitrogen exit)controlled the pressure in the annulus while simultaneously allowing the operator to change the nitrogen purge flow rate as deemed necessary to provide cooling on the lower reactor bellows. A secondary control loop was also designed into the PBS. This loop included a pressure equalization valve and a differential pressure transmitter. The equalization valve was installed in a pipeline that connected the annulus and the inner tube. When the valve was opened, this line allowed the pressure in the annulus and the inner tube to remain the same. This valve was normally open when the reactor was off. During normal operation, if the differential pressure exceeded the alarm setpoint (usually set at about 25 psid), the equalization valve would open, thereby equalizing the pressure between the inner tube and the annulus and protecting the inner tube and lower bellows from damage due to an excessive differential pressure.

Because the hydrogasification process was very dynamic, the PBS needed to react very rapidly to changes of the inner tube conditions. Further complicating the control response was the fact that the annular volume was about 8 times the volume of the inner tube which had an inside diameter of 1.75 inches. As a consequence, a small increase in the pressure in the inner tube would require a large nitrogen volume increase in the annular space to maintain the proper differential pressure. This necessitated a very tight control on the pressure differential between the inner tube and annulus with a target of less than $1 \%$ of the operating pressure $(<10$ psid at 1200 psig). To meet these demanding control requirements, Tescom control valves were chosen and configured with the Tescom ER-3000 electronic controller. Tescom was contacted to review the control strategy and control target. There was a high confidence that the Tescom valve with ER-3000 could control any gas stream with the accuracy and repeatability that was required. However, there was less confidence that the ER-3000 could control multiple, interdependent pressures and flows while maintaining the specified accuracy, control speed, and repeatability.

Based on the Tescom commitment and technical support, it was decided to proceed and build the PBS as designed using the ER-3000 electronic control. The PBS was fabricated and installed per the design and installation was completed in March 2009. Testing and commissioning proceeded through April and May 2009. During this testing, the PBS 
performance was characterized and tuned. The test results indicated that the PBS met or exceeded all of the original design requirements and could control the pressure differential to as low as 2 psid at 1000 psig (0.2\% of operating pressure). This level of control was a significant technical accomplishment for the Tescom ER-3000 controller and significantly exceeded expectations.

\subsubsection{SYSTIEM AUTOMATION}

A LabVIEW- based control system Figure 1-3, Figure 1-4, and Figure 1-5 show the three main control display panels: LabVIEW Process Overview, All Temperature Alarming Interface, and Temperature Profile along the Reactor Tube. These figures are presented to display the interface and are not for depicting actual operating conditions. The field service control engineer from Tescom participated in site system validation, including double-checking system logic and calibration. 


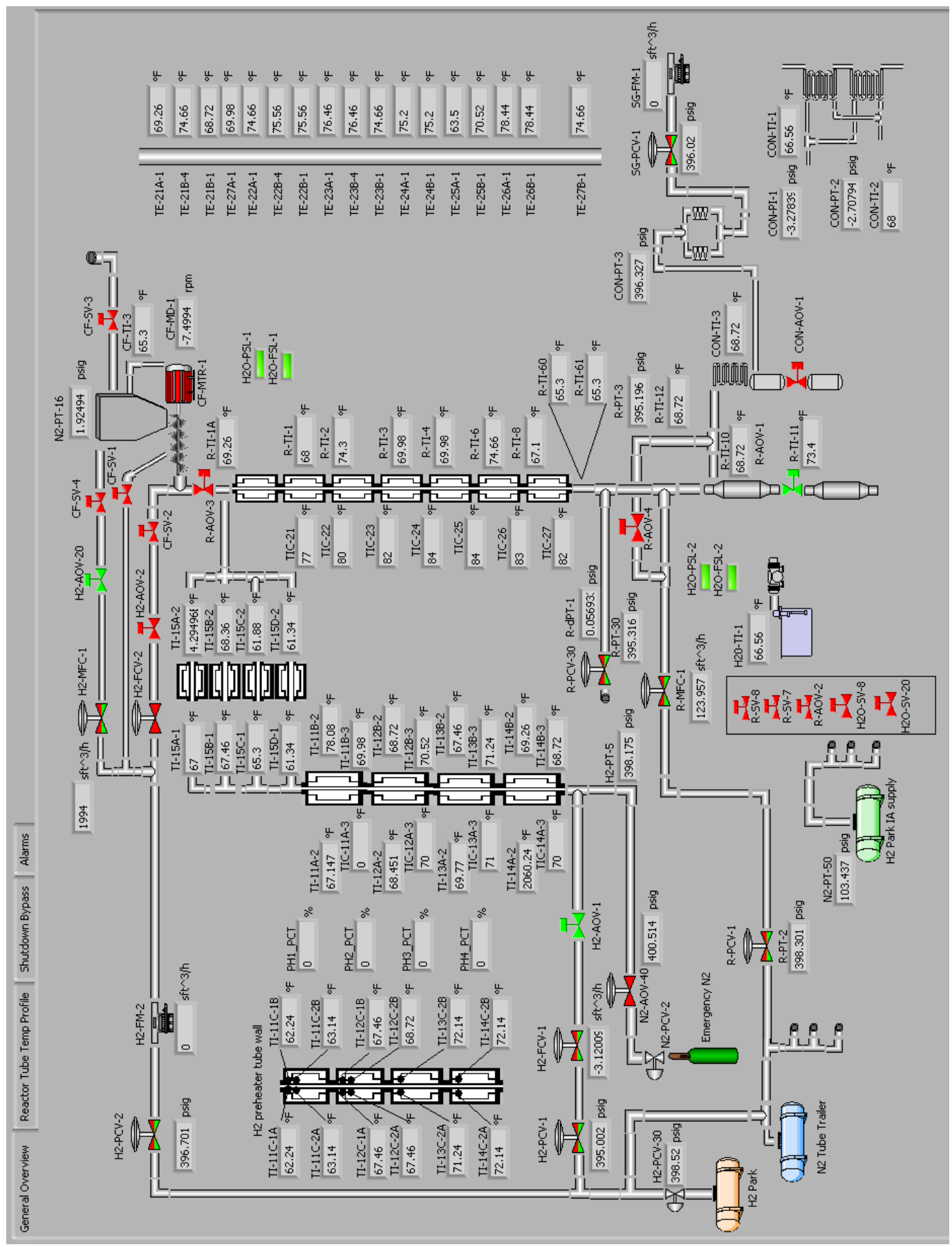

Figure 1-3 - LabVIEW Interface Process Overview 


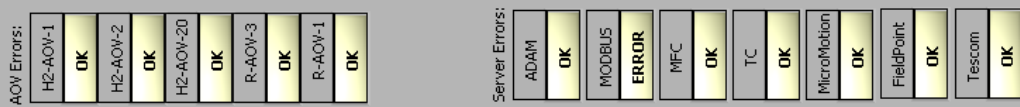<smiles>[Al+2]</smiles>

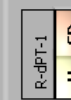

\begin{tabular}{|l|l|}
\hline$\frac{1}{\vdots}$ & जิ \\
\hline$\vdots$ & I \\
\hline
\end{tabular}

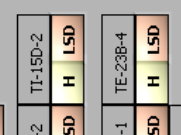

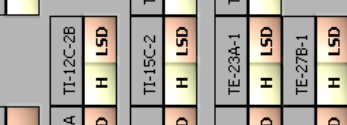

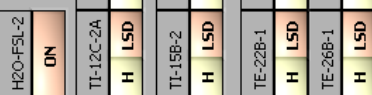

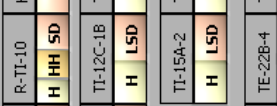

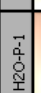

章

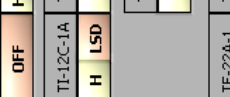

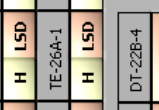

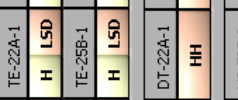

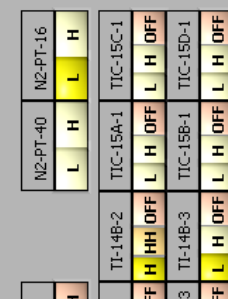

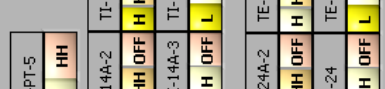

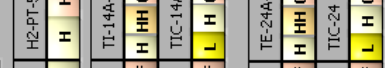

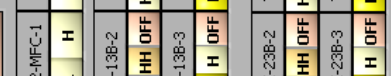

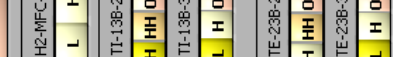

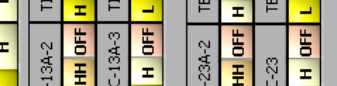

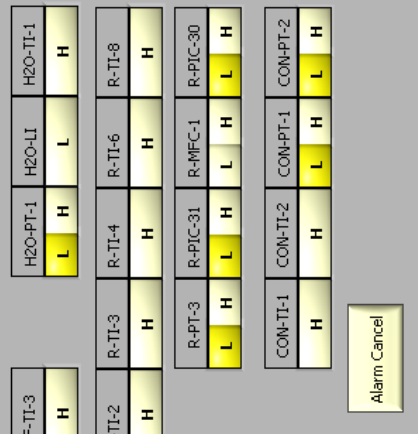

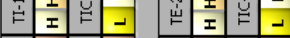

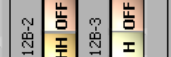

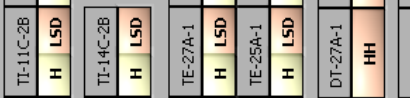

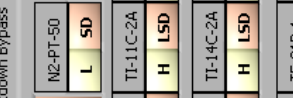

疍

管产

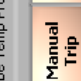

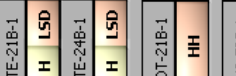

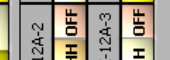

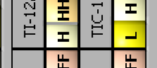

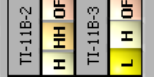

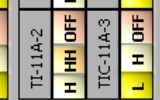

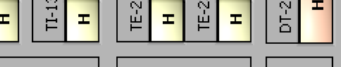
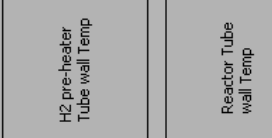

岛

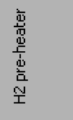

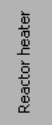

Figure 1-4 - LabVIEW Interface Temperatures - Overall 


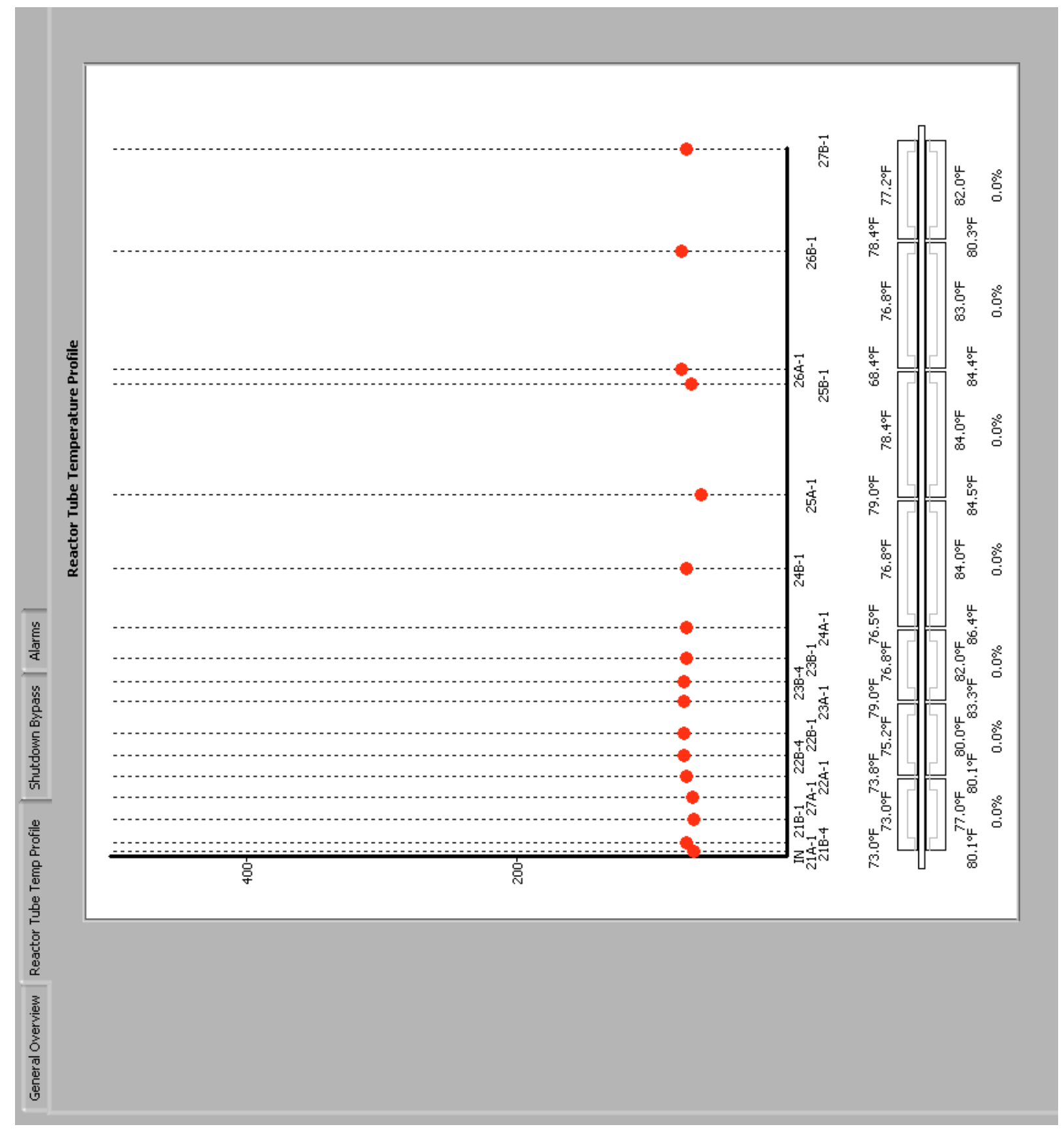

Figure 1-5 - LabVIEW Interface Temperature Profile along Reactor Internal Tube (Reactor Bottom Left and Reactor Top Right Sides of Graph, Respectively) 
Table 1-1 below, summarizes major temperature and operation parameters for which a multi-tiered alarm and automated shutdown system was implemented to ensure design and operating limits were not exceeded for personnel safety and equipment integrity.

Table 1-1 - Temperature and Pressure Parameters for Alarm Strategy

\begin{tabular}{|c|c|c|c|c|}
\hline Ves s el/Pipe & $\begin{array}{l}\text { Design } \\
\text { T(deg F) }\end{array}$ & $\begin{array}{l}\text { Des ign } \\
\text { P(psig) }\end{array}$ & $\begin{array}{l}\text { Oper. T } \\
(\text { deg F) }\end{array}$ & $\begin{array}{c}\text { Oper. P } \\
\text { (psig) }\end{array}$ \\
\hline $\mathrm{H}_{2}$-PT-Plant & 150 & 2000 & 100 & 1800 \\
\hline $\mathrm{H}_{2}$ Feed & 150 & 1150 & 100 & $1000 \sim 1100$ \\
\hline Carrier Gas & 150 & 1150 & 100 & $1000 \sim 1100$ \\
\hline $\begin{array}{c}\text { Emergency } \mathrm{N}_{2} \\
\text { Purge }\end{array}$ & 150 & 1200 & 100 & $1000 \sim 1100$ \\
\hline Instrument $\mathrm{N}_{2}$ & 150 & 154 & 100 & 107 \\
\hline $\begin{array}{c}\mathrm{H}_{2} \text { Preheater Tube } \\
\text { HTR-1 Section }\end{array}$ & 1650 & 1200 & 1470 & 1000 \\
\hline $\begin{array}{c}\mathrm{H}_{2} \text { Preheater Tube } \\
\text { HTR-2 Section }\end{array}$ & 1650 & 1200 & 1470 & 1000 \\
\hline $\begin{array}{c}\mathrm{H}_{2} \text { Preheater Tube } \\
\text { HTR-3 Section }\end{array}$ & 1650 & 1200 & 1470 & 1000 \\
\hline $\begin{array}{c}\mathrm{H}_{2} \text { Preheater Tube } \\
\text { HTR-4 Section }\end{array}$ & 1650 & 1200 & 1470 & 1000 \\
\hline $\begin{array}{l}\text { Reactor Head } \\
\text { Nozzle }\end{array}$ & 1650 & 1200 & 1470 & 1000 \\
\hline Coal Feed Hopper & 150 & 1150 & 100 & 1010 \\
\hline $\begin{array}{l}\text { Reactor Tube } \\
\text { Outside }\end{array}$ & 1000 & 1150 & $<750$ & 1000 \\
\hline Reactor Head & 1000 & 1200 & $<750$ & 1000 \\
\hline Reactor Tube & 1950 & 50 & $1450-1750$ & 20 \\
\hline Reactor Wall & $\begin{array}{c}700 \\
\text { (Gaspar) }\end{array}$ & 1200 & Under 400 & 1050 \\
\hline Reactor (annulus) & 700 & 1200 & Under 400 & 1050 \\
\hline $\begin{array}{l}\text { Pressure Balanc- } \\
\text { ing (bellows) }\end{array}$ & 1000 & 50 & 700 & 15 \\
\hline Char Pot & 1200 & 1150 & Under 1000 & 1000 \\
\hline Char Pot Outlet & 1200 & 1150 & Under 1000 & 1000 \\
\hline
\end{tabular}




\subsection{Computational fuid Dynamics Modejng on Hydrogen INJ ECTOR OPIIMIZATION}

To aid in the design and operation of the bench-scale reactor injector, a non-isothermal, reacting, transient, three-dimensional (3-D) Computational Fluid Dynamics (CFD) model of the injector zone was developed using ANSYS/FLUENT commercial CFD software. The simulation work was carried out by the Computational Science Division of the Office of Research and Development at NETL. The model was based on an Eulerian-Eulerian approach which treats both the gas and solids phases as continuous and fully interpenetrating phases. This results in mass, momentum, species, and energy balance equations for both the gas and solids phases. Under isothermal conditions the balance equations are shown below:

Gas-Phase Continuity

$$
\frac{\partial}{\partial t}\left(\varepsilon_{g} \rho_{g}\right)+\nabla \circ\left(\varepsilon_{g} \rho_{g} \vec{v}_{g}\right)=\sum_{n=1}^{N_{g}} R_{g n}
$$

Solids-Phase Continuity

$$
\frac{\partial}{\partial t}\left(\varepsilon_{s} \rho_{s}\right)+\nabla \circ\left(\varepsilon_{s} \rho_{s} \vec{v}_{s}\right)=\sum_{n=1}^{N_{s}} R_{s n}
$$

Gas-Phase Momentum

$\frac{\partial}{\partial t}\left(\varepsilon_{g} \rho_{g} \vec{v}_{g}\right)+\nabla \circ\left(\varepsilon_{g} \rho_{g} \vec{v}_{g} \vec{v}_{g}\right)=-\varepsilon_{g} \nabla P_{g}+\nabla \circ \overline{\overline{\tau_{g}}}+F_{g s}\left(\vec{v}_{s}-\vec{v}_{g}\right)+\varepsilon_{g} \rho_{g} \vec{g}-R_{0}\left(\xi_{0} \vec{v}_{g}+\bar{\xi}_{0} \vec{v}_{s}\right)$

Solids-Phase Momentum

$\frac{\partial}{\partial t}\left(\varepsilon_{s} \rho_{s} \vec{v}_{s}\right)+\nabla \circ\left(\varepsilon_{s} \rho_{s} \vec{v}_{s} \vec{v}_{s}\right)=-\varepsilon_{s} \nabla P_{g}+\nabla \circ \overline{\overline{S_{m}}}-F_{g s}\left(\vec{v}_{s}-\vec{v}_{g}\right)+\varepsilon_{s} \rho_{s} \vec{g}-R_{0}\left(\xi_{0} \vec{v}_{g}+\bar{\xi}_{0} \vec{v}_{s}\right)$

Species Balance

$\frac{\partial}{\partial t}\left(\varepsilon_{m} \rho_{m} X_{m n}\right)+\nabla \circ\left(\varepsilon_{m} \rho_{m} X_{m n} \vec{v}_{m}\right)=\nabla \circ D_{m n} \nabla X_{s m n}+R_{m n}$

where $m=g$ or $s$ for the gas or solids phase and $\bar{\xi}_{0}=1-\xi_{0}$ and $\xi_{0}=1$ if $R_{0}<0$; else $\xi_{0}=0$. The eight dependent hydrodynamic variables in the 3-D simulation - void fraction $\varepsilon_{g}$ (the solid void fraction $\varepsilon_{s}=1-\varepsilon_{g}$ ), pressure $P_{g}$, and six velocity components - are found by numerically solving the coupled non-linear partial differential equations. Constitutive relations needed to close the system, and the gas/solids energy balance equations can be found in 
Syamlal, et al. ${ }^{1}$ and Syamlal. ${ }^{2}$ A discussion on the solution procedure and further numerical references can be found in Guenther and Syamlal. ${ }^{3}$

To maximize methane yields requires rapid heating of the coal in a hydrogen-rich environment; hence proper mixing and residence times are critical in the process. This work was initially focused on conducting non-reacting CFD simulations at temperature and pressure to investigate the hydrodynamic conditions in the vicinity of the hydrogen and coal inlets. Hydrogen-to-coal mass ratio, injector diameter, injector shooting angle, and injector swirling angle were among the parameters investigated. The hydrogen nozzle ID reflects the linear velocity of the hot hydrogen stream injected into the hydrogasification reactor. The shooting angle reflects the downward angle of the attached injector with respect to the vertical flow of coal in the reactor. The swirl angle reflects the angle of hydrogen flow to produce a vortex in the reactor. Predictions from the model allowed engineers to determine the optimal placement of the hydrogen jets into the hydrogasifier.

Several simulations were performed to determine the optimal placement of the hydrogen jets. Optimal placement would maximize coal and hydrogen mixing at the inlet of the reactor. The previously mentioned factors were varied as follows: the injector diameter was varied between 0.064 and 0.18 inches, the shooting angle $\theta$ (Figure 1-6) was varied between 45 and 75 degrees, and the swirling angle $\phi$ (Figure 1-6) was varied between 30 and 60 degrees. The hydrogen-to-coal ratio factor was more complicated as it was a function of the coal feed rate, hydrogen feed rate, and hydrogen carrier gas rate. Based on a literature search, the optimal hydrogen-to-coal ratio was found to be between $0.2-0.4$. With the reasonable coal feed rate range between 5 and 15 pounds per hour ( $\mathrm{lb} / \mathrm{hr}$ ), the hydrogen feed rate was defined between $0.5-5.85 \mathrm{lb} / \mathrm{hr}$. The hydrogen carrier rate (mass basis) was evaluated as $1 \%-10 \%$ of total coal feed rate (mass/mass), which lead to $0.05 \sim 1.5 \mathrm{lb} / \mathrm{hr}$. Finally these three rates were bounded by the following conditions:

\footnotetext{
${ }^{1}$ M. Syamlal, W. Rogers, and T. O'Brien, MFIX Documentation: Theory Guide (DOE/METC-94/1004, 1993).

${ }^{2}$ M. Syamlal, MFIX Documentation Numerical Technique (EG\&G Tech. Report: DOE/MC 31346-5824, 1998).

${ }^{3}$ C. Guenther, T.O. Brien, and M. Syamlal, A Numerical Model of Silane Pyrolysis in a Gas-Solids Fluidized Bed, in International Conference on Multiphase Flow, (New Orleans, 2001).
} 
- $\mathrm{H}_{2}$ carrier gas rate $+\mathrm{H}_{2}$ feed rate -0.4 coal feed rate $\leq 0$

- $\mathrm{H}_{2}$ carrier gas rate $+\mathrm{H}_{2}$ feed rate -0.2 coal feed rate $\geq 0$

- $\mathrm{H}_{2}$ carrier gas rate -0.1 coal feed rate $\leq 0$

- $\mathrm{H}_{2}$ carrier gas rate -0.01 coal feed rate $\geq 0$
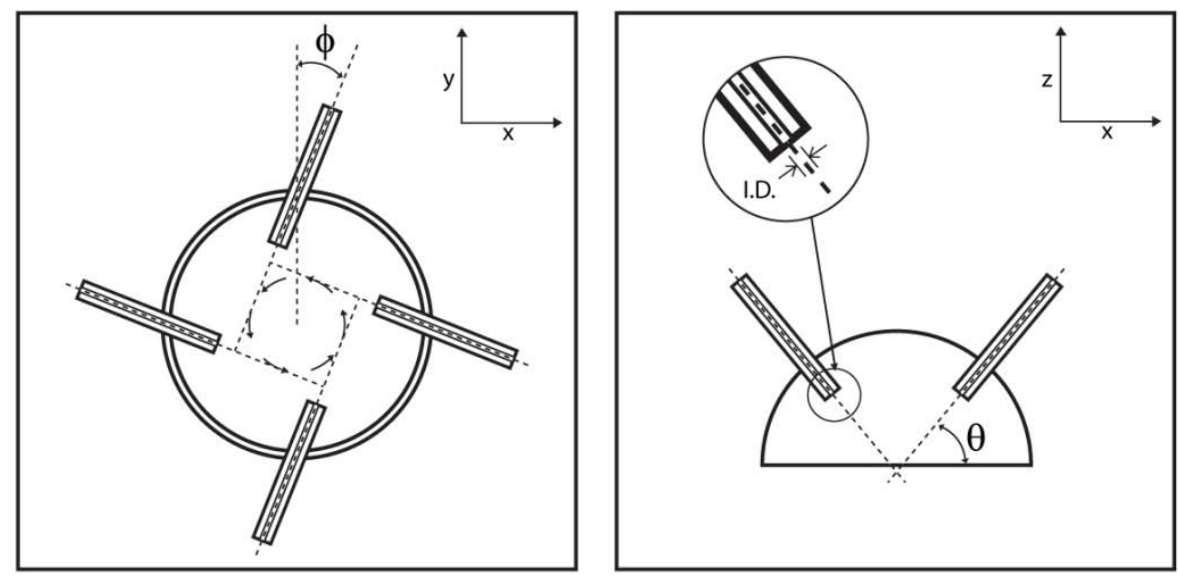

Figure 1-6 - Hydrogen Injection Nozzle Orientation

These four conditions ensured that the hydrogen-to-coal ratios ranged between 0.2 and 0.4 and that the hydrogen carrier gas-to-coal ratio ranged between 0.01 and 0.1 . The simulated conditions may be seen in Table 1-2.

Table 1-2 - $\mathrm{H}_{2}$ Injection Nozzle Optimization - Experimental Simulations

\begin{tabular}{|c|c|c|c|c|c|c|}
$\begin{array}{c}\text { Simulation } \\
\text { No. }\end{array}$ & $\begin{array}{c}\text { Injector } \\
\text { I.D. } \\
\text { (nches) }\end{array}$ & $\begin{array}{c}\text { Shooting } \\
\text { Angle } \\
\text { (degree) }\end{array}$ & $\begin{array}{c}\text { Coal Feed } \\
\text { Rate } \\
(\mathrm{lb} / \mathrm{hr})\end{array}$ & $\begin{array}{c}\mathrm{H}_{2} \text { Feed } \\
\text { Rate } \\
(\mathrm{lb} / \mathrm{hr} \text { ) }\end{array}$ & $\begin{array}{c}\mathrm{H}_{2} \text { Carrier } \\
\text { Gas Rate } \\
(\mathrm{lb} / \mathrm{hr} \text { ) }\end{array}$ & $\begin{array}{c}\text { Swirling } \\
\text { Angle } \\
\text { (degree) }\end{array}$ \\
\hline 1 & 0.06 & 75 & 15.00 & 5.85 & 0.15 & 60 \\
\hline 2 & 0.18 & 45 & 6.40 & 1.92 & 0.64 & 60 \\
\hline 3 & 0.06 & 75 & 15.00 & 4.50 & 1.50 & 30 \\
\hline 4 & 0.06 & 45 & 15.00 & 2.85 & 0.15 & 30 \\
\hline 5 & 0.06 & 45 & 15.00 & 1.50 & 1.50 & 60 \\
\hline 6 & 0.18 & 75 & 15.00 & 4.50 & 1.50 & 60 \\
\hline 7 & 0.12 & 60 & 10.00 & 3.18 & 0.78 & 45 \\
\hline 8 & 0.18 & 45 & 5.00 & 0.62 & 0.38 & 30 \\
\hline 9 & 0.18 & 75 & 5.00 & 0.91 & 0.09 & 60 \\
\hline 10 & 0.18 & 45 & 15.00 & 2.85 & 0.15 & 60 \\
\hline 11 & 0.18 & 45 & 15.00 & 5.14 & 0.86 & 30 \\
\hline 12 & 0.18 & 75 & 13.61 & 5.31 & 0.14 & 30 \\
\hline 13 & 0.06 & 45 & 5.00 & 1.95 & 0.05 & 60 \\
\hline 14 & 0.06 & 45 & 6.48 & 2.00 & 0.59 & 30 \\
\hline 15 & 0.06 & 75 & 6.37 & 0.64 & 0.64 & 60 \\
\hline 16 & 0.06 & 75 & 5.00 & 0.94 & 0.06 & 30 \\
\hline 17 & 0.18 & 75 & 15.00 & 1.50 & 1.50 & 30 \\
\hline
\end{tabular}


The results from the model were analyzed to optimize coal/hydrogen mixing at the inlet of the reactor. To evaluate the hydrodynamics around the reactor head, solid equatorial mixing along sectional areas, solid flux back mixing and sectional temperature profiles were studied. The simulations and detailed analysis were completed. The results showed that to achieve good coal and hydrogen mixing and to minimize the solid back flow (to prevent coal from clogging at reactor neck), a large injector inner diameter, a small shooting angle, and a minimal amount of swirling were recommended. The simulated experiments showed that the overall hydrogen-tocoal ratio and the hydrogen carrier gas-to-coal ratio should be minimized.

Figure 1-7 and Figure 1-8 show a good mixing case and a bad mixing case respectively. In these illustrations, the contours of the computer projections represent the average volume fraction of the solid phase. The left side of the figure is the top of the reactor and on the right the hydrogen injectors may be seen. Figure 1-7 is a case where optimal parameters were used. At the contour cross-section furthest down the reactor (the cross section furthest to the right in Figure 1-7), good mixing may be seen. The volume fraction at this point is relatively uniform. In contrast to this, Figure 1-8 shows two distinct areas in the bottom cross-sectional contour. This is representative of bad mixing, because there is a section of low volume fraction in the core and a section of high volume fraction near the wall.

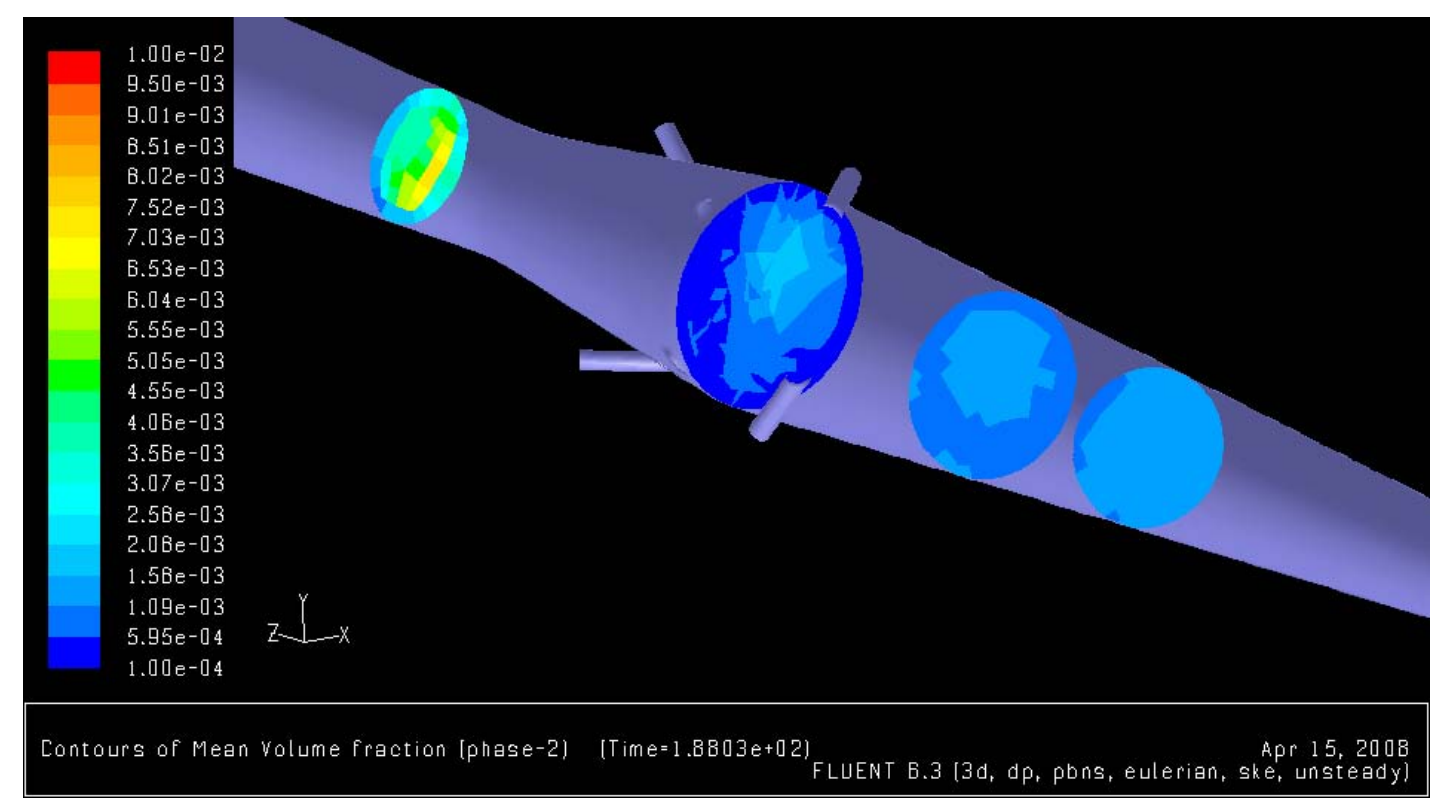

\begin{tabular}{|c|c|c|c|c|c|}
\hline Injector I.D. (in) & $\begin{array}{c}\text { Shooting Angle } \\
\left({ }^{\circ}\right)\end{array}$ & $\begin{array}{c}\text { Coal Feed Rate } \\
(\mathrm{lb} / \mathrm{hr})\end{array}$ & $\begin{array}{c}\text { H2 Feed Rate } \\
(\mathrm{lb} / \mathrm{hr})\end{array}$ & $\begin{array}{c}\text { H2 Carrier Gas } \\
\text { Rate (lb/hr) }\end{array}$ & Swirling $\left(^{\circ}\right)$ \\
\hline 0.18 & 45 & 5 & 0.62 & 0.38 & 30 \\
\hline
\end{tabular}

Figure 1-7 - A Case of Good Hydrogen and Coal Mixing 


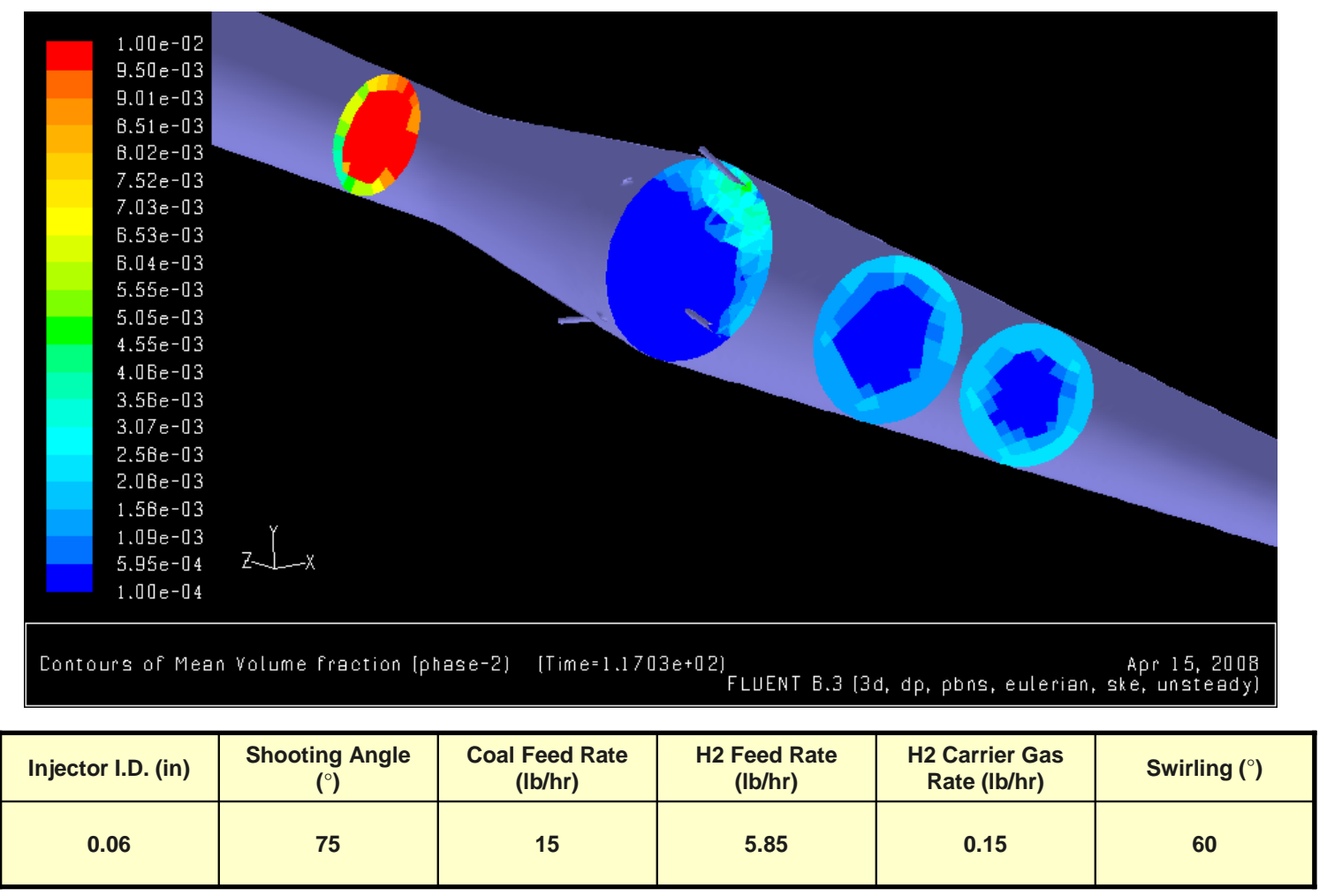

Figure 1-8 - A Case of Bad Hydrogen and Coal Mixing

Within the interested hydrogen and coal feed rate range, a smaller hydrogen nozzle ID gives higher hydrogen linear velocity, which creates unfavorable solid/gas equatorial mixing. A higher shooting angle (hydrogen injection more downward) also creates an unfavorable solid/gas mixing. The effects of swirling are more complicated. By creating a low-pressure center, a certain degree of swirling helps coal flow downward and prevents solid back flow that could cause clogging. However, too much swirling also tends to give a centrifuge effect in the reactor tube that impairs the solid/gas mixing. The reactor injector geometry was finalized as (1) 45-degree shooting angle, (2) 30-degree swirling angle, and (3) 0.18-inch injector (ID).

\subsection{HYDROGASIFER DESIGN}

\subsubsection{HYDROgasifer DESIgn CRTIERIA}

At the beginning of the project, a set of design criteria was developed for the hydrogasification reactor. The reactor was to be designed for an area zoned light industrial in Phoenix, AZ. The reactor's environmental design conditions were to include (1) a minimum 70 mile-per-hour wind threshold for the frame, (2) an earthquake zone 1 (which is very stable), (3) no snow, and (4) a lowest mean temperature of $54.2^{\circ} \mathrm{F}$. Material interface to the reactor were to include coal as 
well as (1) benzene, toluene, and xylene (BTX), (2) methane, (3) hydrogen, (4) steam, (5) heavy hydrocarbons, and (6) nitrogen.

\subsubsection{Design and Operating Conditions}

Whereas thermodynamic equilibrium calculations predict higher methane formation at higher pressures and moderate temperatures, the hydrogasification reactor was to be designed for the following operating conditions:

- $\quad$ An operating pressure of 1000 pounds per square inch (psi)

- An operating temperature of $1800^{\circ} \mathrm{F}$

- An operating pressure for coal feeding of 1000 psi

- $\quad$ An operating pressure for char removal of 1000 psi

- An operating pressure for hydrogen feeding of $1010 \mathrm{psi}$

- An operating temperature for hydrogen feeding higher than $1200^{\circ} \mathrm{F}$

Because of heat-losses associated with the small size of bench scale reactor systems, the design needed to accommodate a means to add heat to the reactor vessel walls. The desired pressure and temperature specifications added complexities for the materials of construction selection to meet ASME pressure vessel code. A refractory-lined vessel was deemed undesirable for this reactor scale. The final design, show in detail in Appendix $B$ of this report, resulted in a nominal 10.75-inch-outsidediameter SA106-B carbon steel reactor vessel contained with a 1.75-inch-insidediameter Inconel 617 pressure containment vessel. Electrical heaters were installed within the annular region between the two vessels to assist with maintaining desired process temperature. The tube and shell design required the use of bellows to accommodate the variances in thermal expansion between the two vessels.

There were many design considerations for the fatigue life of the hydrogasification reactor. These fatigue-life design considerations included:

- A testing operation time of two hours with a minimum of 24 hours between tests.

- A total of 1000 hours of testing time

- A maximum reactor temperature of $1950^{\circ} \mathrm{F}$ 


\subsubsection{Operational Design Considerations}

Operationally the reactor was designed to be purged with nitrogen before startup and at shutdown. For safety considerations, the annular space was designed to hold a volume of nitrogen that would be 30 times the volume of hydrogen in the inner reactor tube. If hydrogen leaked from the inner tube to the annulus, the differential pressure drop between the inner tube and the annulus would be detected and the reactor would be shut down. At this point the concentration of hydrogen in the annulus would be less than $4 \%$, which is not flammable. The annulus was designed to be constantly purged with nitrogen at approximately 8 Standard Cubic Feet per Minute (SCFM), further diluting the hydrogen. The worst-case scenario would be if an operator started the reactor with air at 0 psig in the annular space, heated the reactor, and turned on the hydrogen flow to a pressure that ruptures the bellows (48 pounds per square inch differential (psid)). If all of these were to happen, which would require the operator to override multiple interlocks and violate operating procedures, then at a hydrogen pressure as low as a few psi, a flammable $4 \% \mathrm{H}_{2}$ /air mixture would be attained.

\subsubsection{Administrative Control}

All operations were designed to be conducted in accordance with written operating procedures. Both a control operator and a test supervisor were present any time the reactor was above ambient temperature and pressure. Clear guidance on temperature limits was contained in the operating procedures.

An emergency shutdown procedure was also available, providing both the conditions for implementing an emergency shutdown and the sequence. The temperature and pressure limits were an integral part of the procedure.

\subsubsection{Process Controls}

The reactor processes were controlled using a distributed digital control system. Critical reactor components were monitored using thermocouples attached both inside and outside the reactor pressure boundary. Using the maximum code compliance temperatures, an emergency shutdown setpoint was developed. The emergency shutdown setpoint was reduced in consideration of both instrument error and transient response error. All alarm setpoints were set appropriately below the trip point to allow operator response to impending trip conditions.

A simplified flow diagram of the bench-scale hydrogasification reactor is presented in Figure 1-9. 


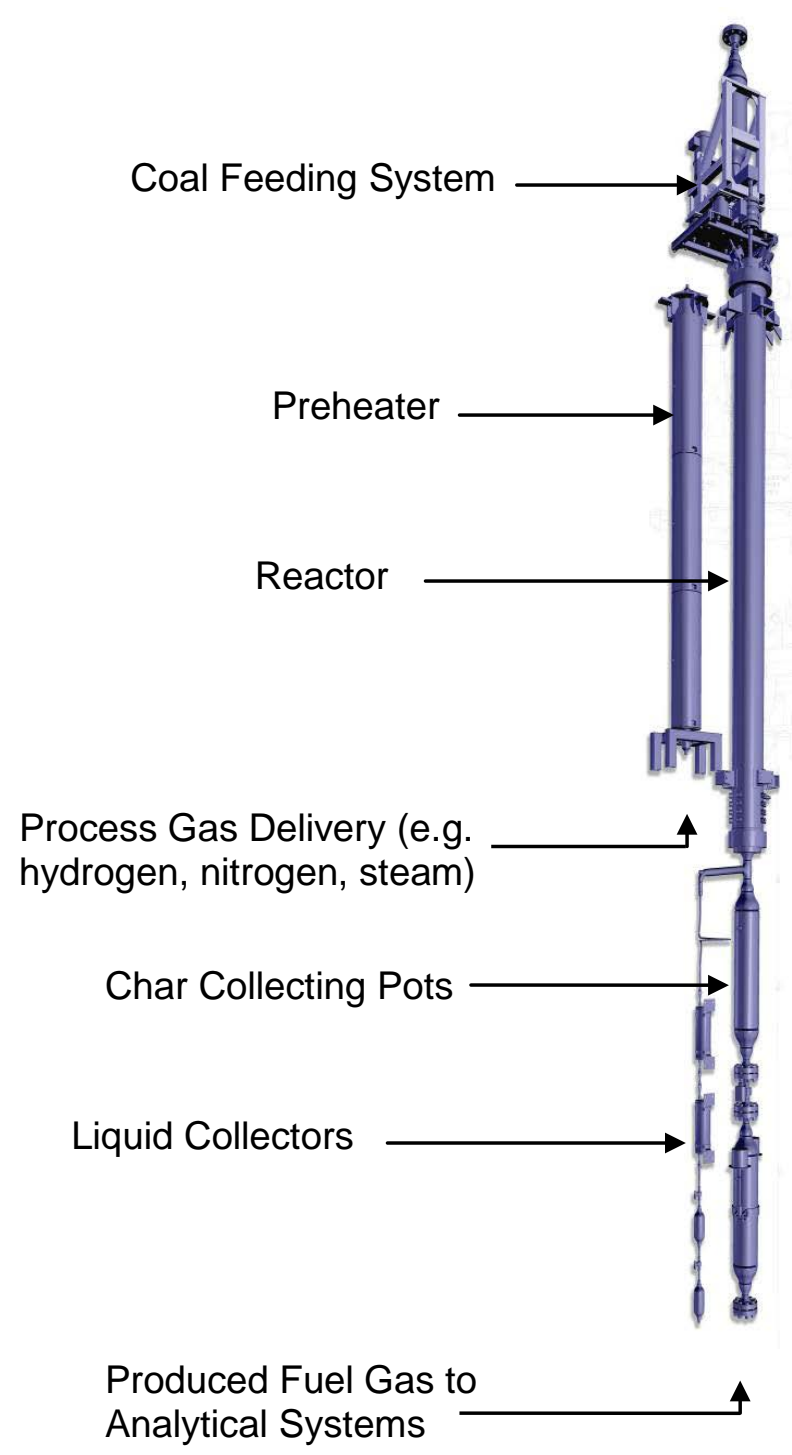

Figure 1-9 - Simplified Diagram of the Bench-Scale Hydrogasification Reactor

The hydrogasifier APS developed was a dual-wall down-flow reactor, with heating elements located in the annulus space. The coal was fed in the pulverized solid phase from the top center of the reactor. Hydrogen was preheated and injected to the reactor top through four nozzles set at a 45-degree angle. The reactor had a total length about 16 feet with a heated zone of 13 feet. The pressure of the reactor's inner tube was controlled from 5 to 15 psi lower 
than the annulus space, where nitrogen gas was used for balancing pressure. Two stages of charpots and liquid collectors were used to differentiate the transient and steady state samples. The key areas of the reactor design included (1) a preheater, (2) a reactor, (3) a coal-feeding system, (4) char collecting pots, and (5) liquid-collectors. Designing these key elements required consideration of many factors, including selection of construction materials, thermal expansion, and maintenance, etc. Following are descriptions of how and why certain design considerations were made for each of these components and how they were connected to form the bench-scale hydrogasification reactor.

\subsubsection{INNER REACTOR TUBE DESIGN}

The inner tube of the hydrogasification reactor was a 16-foot long, 2-inch outside diameter (OD) by 0.125 -inch thick Inconel 617 alloy tube that could be operated at extreme conditions. The tube was heated from the outside to $1600^{\circ} \mathrm{F}$ by ceramic electric heaters. To obtain good heat transfer from the heaters to the inner tube, the wall thickness of the tube had to be as thin as possible yet thick enough to contain the reaction pressure of 1000 to $1150 \mathrm{psig}$. Inside the tube, pulverized coal reacted with hydrogen gas. Once the hydrogasification reactions began, the exothermic reactions could boost the temperature in the tube to as high as $1950^{\circ} \mathrm{F}$. This maximum temperature point varied throughout the inner tube depending upon the coal feed rate and hydrogen-to-coal ratio.

The operating conditions on the inner tube also created significant thermal expansion of the tube both radially and longitudinally. Longitudinally, at maximum temperature the tube could expand by as much as 2.5 inches in length. To address this, the inner tube was "fixed" to the reactor at the top and the bottom of the tube was allowed to "float" as the tube was being heated and cooled. The inner tube was then sealed at the bottom using expansion bellows. The bellows exerted a thrust on the inner tube that caused more stress in the Inconel 617 tube and created a potential for inner tube buckling, especially as the tube was heated up and the tensile strength dropped. To minimize the likelihood of buckling, (1) the inner tube was supported radially by a series of "spider" plates that kept the inner tube centered in the shell; and (2) a design and specifications were developed for the dimensions and tolerances of the inner tube to minimize the eccentricity and maintain the straightness of the tube.

During the design phase the inner tube was analyzed using American Society of Mechanical Engineers (ASME), Boiler and Pressure Vessel Code (BPVC) calculations. The mechanical properties (tensile strength, Young's modulus, and thermal expansion coefficient) for Inconel 
617 were obtained from data sheets provided by Special Metals. Initially, the maximum temperature in the tube was expected to be $1600^{\circ} \mathrm{F}$. Several materials were acceptable for this temperature including Inconel 625, which has an ASME BPVC maximum operating temperature of $1600^{\circ} \mathrm{F}$. However, further research determined that the exothermic reaction could hit $1950^{\circ} \mathrm{F}$ and at this temperature Inconel 625 was not an acceptable material as its tensile strength dropped off rapidly over $1600^{\circ} \mathrm{F}$, which was the maximum allowable temperature per ASME BPVC. Several other high temperature materials were evaluated including alloy $800 \mathrm{H}$, Waspaloy and Inconel 617. Most of the exotic high-temperature materials that are used in jet engines were not readily available, especially in the size and shape required for the inner tube. Further, in assessing the high-temperature properties of these materials - including tensile strength, corrosion resistance, and rupture stress life at $1950^{\circ} \mathrm{F}$ - the Inconel 617 best met the anticipated operating conditions. Inconel 617 also had excellent resistance to both oxidation and reduction atmospheres.

Inconel 617 was not available in a 2-inch OD tube or in any pipe or tubular shape approximately this size. It was, however, available in sheets of an appropriate thickness to meet the operating pressure for the inner tube (0.125-inch thick). High Temp Metals, Los Angeles, CA had a 4-foot-wide sheet of 0.125 -inch-thick Inconel 617 , and this sheet could be cut into approximately 6 -inch-wide, 48 -inch-long strips. These strips were rolled to form a 48 -inch-long by 2 -inch OD tube.

Suppliers that could make the Inconel 617 sheet into this 2-inch OD tubular form and then weld the tube seam without using filler metal were sought. Several custom tube fabricators were identified but only one, Valley Metals, Poway, CA, could form the tube, seam-weld the 617 Inconel, and then draw the tubing to obtain a consistently round tube. Valley Metals also offered a "double draw" process for the tubing in which the tubing was drawn over a precision mandrel twice to obtain a tight tolerance on dimensions. A tube was produced that was even more precise than the design tolerances. The 48-inch-long pieces of 2-inch OD tube were shipped to Cryogenic Vessels, Instrumentation and Piping, Inc. (CVIP), Emmaus, PA, which was selected to make the circumferential welds to join the pieces of tube into one 16-foot-long tube. CVIP was an ASME BPVC code shop, had current procedures and qualified welders for welding 617 Inconel, and had orbital welding capabilities. The tube was welded together and each weld was x-rayed and examined by an independent ASME inspector. The finished 16-foot tube was shipped to Gaspar, Canton, $\mathrm{OH}$, where the bench-scale hydrogasification reactor was fabricated. The formal ASME calculations were completed by Gaspar as well. The result of the 
ASME analysis indicated that the inner tube could operate at $1950^{\circ} \mathrm{F}$ with a maximum 50 psi pressure differential (external pressure above internal pressure) and a safety factor consistent with ASME BPVC requirements.

The Inconel 617 tubing was visually inspected several times during the test phase. The inside of the tube showed little or no significant degradation, cracking, erosion or corrosion. Moreover, there was very little coal or tar accumulation on the inner wall. The deposits present on the inner wall were dry and powdery and were easily removed with a wire brush. Figure 1-10 shows the reactor inner tube at Gaspar.

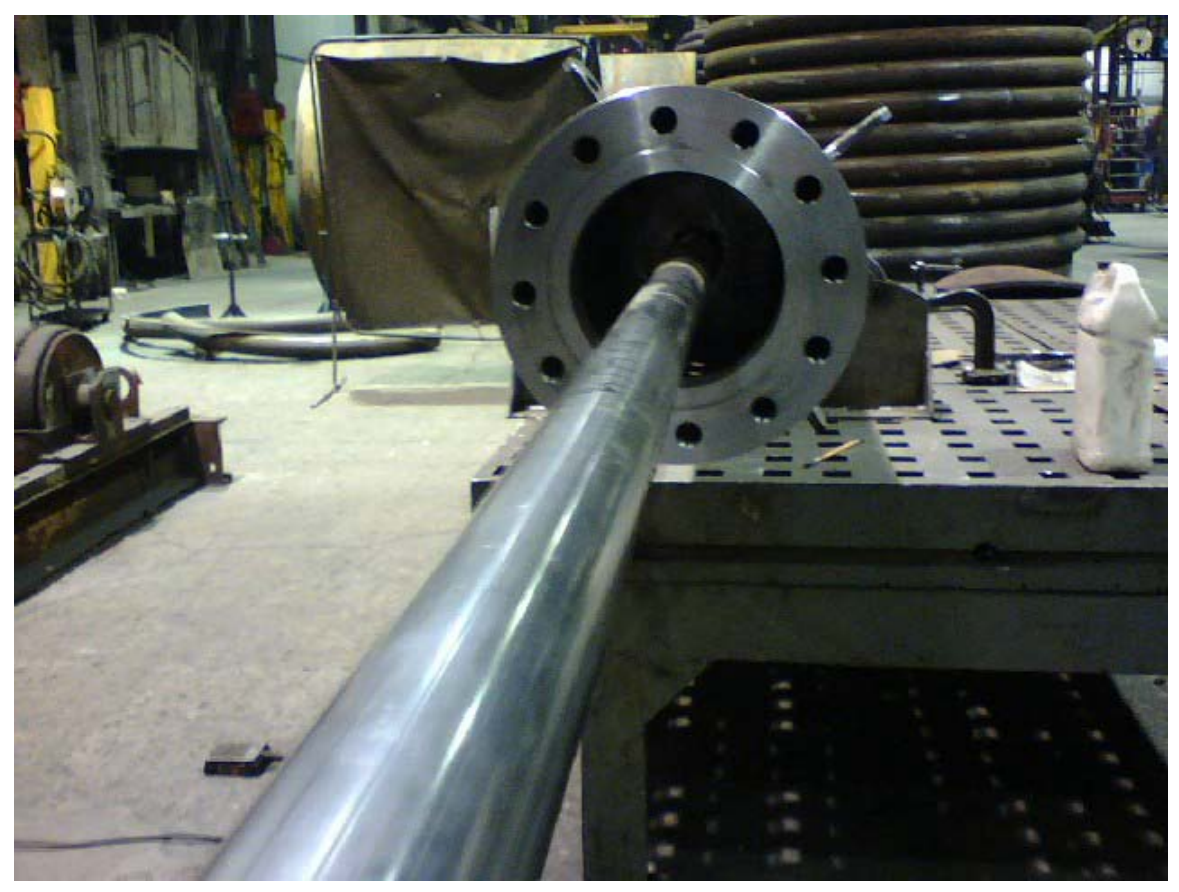

Figure 1-10 - Reactor Inner Tube at Manufacturer

Please refer to Appendix B and Appendix C for detailed reactor design drawings from APS and Gaspar.

\subsubsection{INJ ECTOR DESIGN}

The hydrogen injector hub assembly was located at the top of the bench-scale reactor and was the delivery system for the hot hydrogen gas to the reactor. Hydrogen was generated and stored on-site at the APS facility using an existing electrohydroloysis unit. The assembly design was aided by Computational Fluid-Dynamic (CFD) modeling to select injector diameters and injection angles to optimize the mixing of the coal that was introduced from the top center of the assembly via a screw feeder. The assembly was welded to the top of the Inconel 617 inner 
tube, and the inner tube was welded to the 1-inch schedule 80 stainless steel (SS) pipe that penetrates through the top center of the "top hat" assembly. The injector assembly included the hub assembly and the hydrogen injector wands. The hub assembly is shown in Figure 1-11. Bellows were manufactured by American BOA, Cumming, Georgia. The other hub assembly parts were machined by Dimension Design, Brodheadsville, Pennsylvania, and were machined from Inconel 625 forged bar. The machined parts were assembled and Tungsten Inert Gas (TIG) welded using by Gaspar, Canton, Ohio.
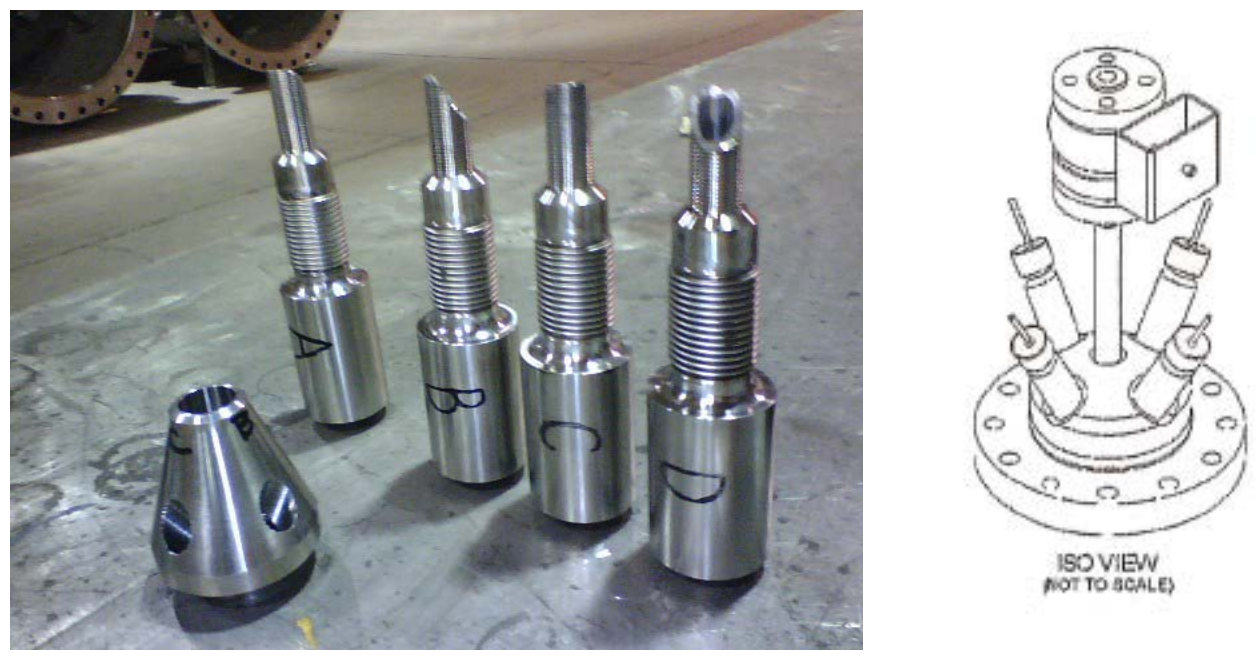

Figure 1-11 - Reactor Center Hub Assembly

The hub was machined so that its top end exactly matched the inside diameter (ID) and outside diameter (OD) of 1 inch schedule 80 pipe. It was leveled to a 37.5-degree angle for weld preparation. The bottom end of the hub was machined to the dimensions of the 2-inch Inconel 617 inner tube (2-inch OD and 1.75-inch ID). The weld at this end was a square butt weld intended for an autogenous (no filler metal) weld between the hub and the Inconel 617 inner tube.

There were four machined ports (3/4-16UNF-2B straight threads) in the hub. They were located 90 degrees apart and were angled at 45 degrees from the vertical. This 45 -degree angle was the angle selected for the best mixing of the coal powder falling down through the hub and the hot hydrogen injected into the hub. The hub was also machined on the inside to provide a smooth flow transition from the 1-inch pipe ID to the 2-inch inner tube ID. A straight thread was chosen as the thread design rather than a taper thread to allow for further adjustment if needed at final assembly. The injector nozzle ends were trial fitted into the hub. The parts were marked to indicate the extension of the part into the hub and also the angle and curvature at the end 
relative to the hub ID. Gaspar then machined the end to conform as closely as possible to the hub ID.

The center portion of the injector sub-assembly was the BOA bellows. It was designed to allow both expansion and contraction (0.10 inch) and changes in the hub and top hat dimensions as they were heated up and cooled down. The bellows was also designed to allow up to 0.060-inch lateral movement, which was the key to the injector hub design; the hub expanded downward as it was heated up and this caused the bellows to flex downward by 0.030 inch to 0.040 inch. This sub-assembly was inspected for weld integrity.

Gaspar proceeded to weld the 1-inch schedule $80316 \mathrm{H}$ SS pipe to the top of the Hub. This weld was positioned into the top hat and centered within the cap. The hub position was re-checked by Gaspar quality assurance and quality control (QA/QC) and upon approval was welded into the top hat cap. The four injector sub-assemblies were inserted through the ports on the top hat cap and threaded into the hub until they extended fully through the hub wall and flushed to the hub ID. The threads provided both a way to make precise adjustments to the position and extension into the hub and a first level of mechanical attachment and seal between the hub and the injector assembly. The four injector sub-assemblies were seal-welded at the point inside the hub where the injector matched up to the hub ID.

A second major sub-assembly within the top hat injector was the injector wand (Figure 1-12). The main wand was a 0.25 -inch OD Inconel 625 tube. This tube was slipped into the socket of the nozzle and a fillet seal weld was made between the two parts. Washers were used at the ends of the wand. The 0.25 -inch Inconel tube was wrapped with a composite wrap of 316 SS foil (0.002-inch thick) and Thermal Ceramics Superwool 1/8-inch-thick ceramic fiber paper. The alternating layers of ceramic fiber insulation and SS foil provided a radiation shield to minimize radiant heat transfer from the injector wand to the top hat cap and the ceramic fiber insulation decreases thermal conductivity between the wand and the cap. The combination was also flexible and allowed the wand to flex as the hub moved during startup and shutdown. 


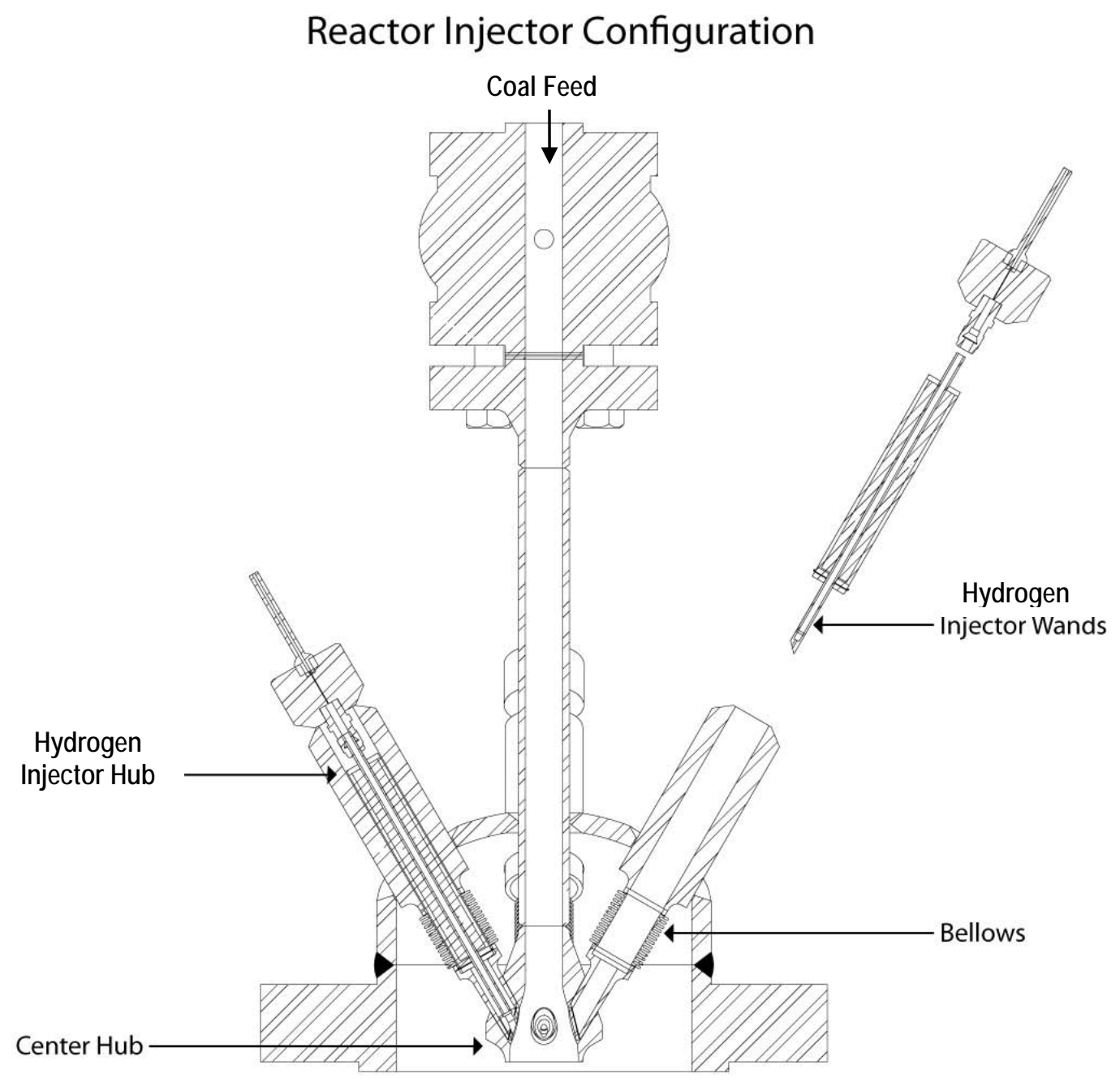

Figure 1-12 - Injector and Injector Wands Assembly within the Top-Hat Section

Please refer to Appendix $B$ for detailed reactor injector and center hub design drawings from APS.

\subsubsection{PReHeAter Design}

The preheater - a device that heated the hydrogen up to about $1600^{\circ} \mathrm{F}$ before it was injected into the reactor head - was an ASME pressure vessel and could be operated up to $1150 \mathrm{psig}$. It was a single thick-wall pressure vessel, which was designed, fabricated, and stamped to meet the ASME BPVC. The OD was 3 inches, the bore was 1 inch, and the length was 15 feet. Finally, the vessel was heated with a series of ceramic fiber clam-shell heaters mounted on the outside of the vessel.

To comply with the ASME BPVC, the preheater needed to be designed using a BPVC material. 
Moreover, the material needed to be commercially available in the size and shape required. Inconel 625 meets the BPVC for service up to $1600^{\circ} \mathrm{F}$ and is also available in 3-inch-diameter solid round bar. To fabricate the preheater and meet the BPVC, the solid round bar was heat-treated and solution-annealed by Solar Atmospheres, Hermitage, and Pennsylvania. The solution-annealed bars were then sent to Betar, Hillsborough, New Jersey, and were bored out to a 1-inch ID. These machined bars were then sent to Gaspar for final welding into the preheater vessel configuration.

The preheater design went through multiple startup-shutdown iterations to assess the heat transfer from the external heaters to the hydrogen gas. The analysis of the heat transfer through the 1-inch Inconel 625 wall indicated that there was adequate thermal conductivity through the wall to heat the hydrogen to the target temperature. A more detailed analysis on heat transfer between the inner wall and the hydrogen was also completed. The analysis indicated that the heat transfer through the boundary layer (film heat transfer coefficient) was too low to achieve the target temperature of $1600^{\circ} \mathrm{F}$. Based on this analysis, several different heat transfer models were applied and different conclusions were obtained. As the preheater was a key element of the hydrogasification testing process, two ways to enhance the heat transfer in the preheater were tried: (1) the preheater was lengthened by $25 \%$ from 12 feet to 15 feet; and (2) devices to enhance the heat transfer were investigated. APS identified several devices that were designed to enhance the gas-phase heat transfer by creating turbulence in the gas flow. This turbulence and higher wall velocity as the preheater was lengthened would result in higher film heat transfer coefficients and thus a higher heat transfer rate.

Several turbulence-inducing devices were located and included a "twisted ribbon" design from Fuel Efficiency, Davis Industrial Park Clyde, NY and a wire matrix "turbulator" provided by Cal Gavin, Warwickshire, England. The performance of both designs was analyzed and the internal heat transfer rates were re-calculated for both devices. The results indicated that the Cal Gavin turbulator provided the highest degree of enhancement of the heat transfer rate. Based on calculations, the Cal Gavin device was expected to increase the heat transfer by a factor of three. Therefore, two 1-inch-diameter hiTran wire matrix turbulators from Cal Gavin were acquired. The turbulator was fabricated with SS wire woven into a tubular shape. This design created a high degree of turbulence at the vessel wall without generating a large pressure drop through the bore. The Cal Gavin turbulator was easily inserted into the preheater. Testing results indicated that the preheater was able to achieve an outlet hydrogen temperature of $1550^{\circ} \mathrm{F}$ (Figure 1-13) 

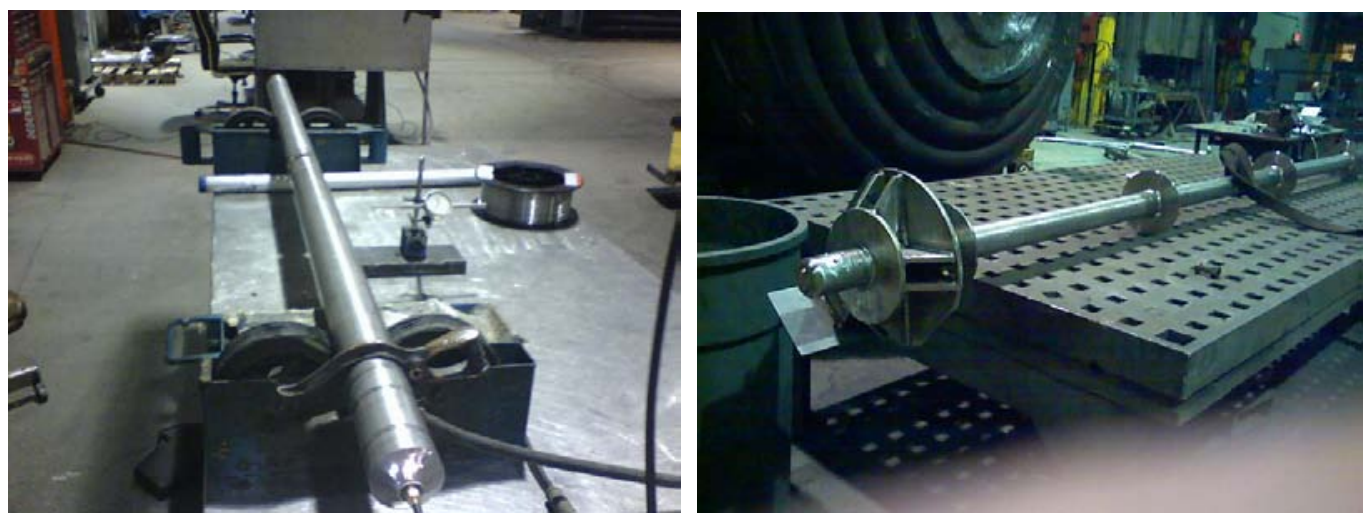

Figure 1-13 - Reactor Preheater at Manufacturer

Please refer to Appendix $B$ and Appendix $D$ for detailed hydrogen preheater design drawings from APS and Gaspar.

\subsubsection{COAL FEeDER DESIG N}

The coal feeder design was based on a screw feeder concept. Two hundred mesh New Mexico sub-bituminous pulverized coal was loaded into a hopper and delivered to the top of the reactor using an auger. The feeding system was designed to ensure that there would be no back pressure across the feeder and the feeder could provide a consistent feed rate. There were three major components of the magnetic drive and auger assembly: (1) an auger, which was designed and machined by Metalfab Inc., Vernon, New Jersey; (2) a magnetic drive, which was provided by PDC Inc., Warminster, Pennsylvania; and (3) an end cap which was machined by Dimension Design, Brodheadsville, Pennsylvania. The coal hopper was fabricated using 8-inch schedule $80316 \mathrm{H}$ SS pipe and pipe fittings. This material was selected because it provided the best combination of pressure and temperature, allowing the coal feeder to operate at up to $200^{\circ} \mathrm{F}$ and 1200 psig. The coal hopper was designed and fabricated to meet ASME BPVC Section VIII by Gaspar, as was the final assembly of the coal feeder system (Figure 1-14). 


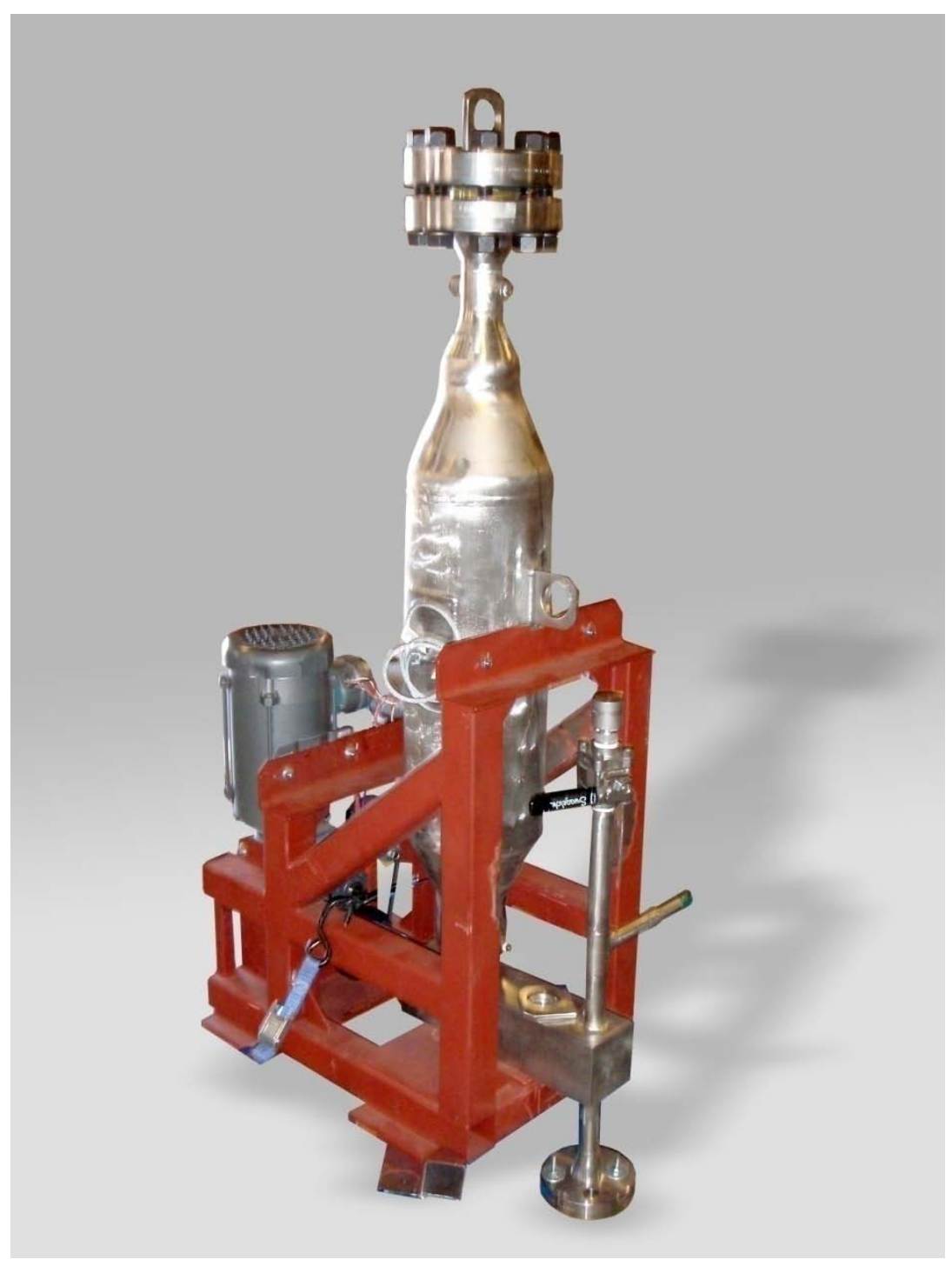

Figure 1-14 - Coal Feeder

Please refer to Appendix B and Appendix E for detailed coal feeder design drawings from APS and Gaspar.

\subsubsection{CHARPOTS DESIGN}

Char is the partially reacted coal powder. During hydrogasification, some of the carbon in the coal reacts with the hydrogen to produce methane. Also, most of the moisture and liquid hydrocarbons in the coal are volatized and removed from the coal particles. As a result the char particles tend to be fairly dry, powdery and free flowing.

There were two charpots in the reactor assembly used to capture the coal at the end of the reaction process, the upper and the lower charpot which were configured in a typical 
lock-hopper arrangement. They were attached to the bottom of the reactor assembly. The attachment was through a custom bolted flange, which bolted onto the bottom of the reactor and the top of the upper charpot. Both charpots were fabricated using 8-inch schedule $80316 \mathrm{H} \mathrm{SS}$ pipe and pipe fittings. This material was selected in order to provide the best combination of pressure and temperature, allowing the charpots to operate at up to $1000^{\circ} \mathrm{F}$ and $1150 \mathrm{psig}$. The charpots were designed and fabricated to meet ASME BPVC Section VIII. They were fabricated by Gaspar and were individually code stamped. There was an air-actuated full-port ball valve (tag number R-AOV-1) located between the upper and lower charpots. This valve allowed the operator to open and/or close the valve remotely. The valve was utilized to isolate the upper charpot from the lower charpot, accommodating collection of to collect coal samples from transient operation in the lower char pot and from steady-state operation in the upper charpot.

The charpot's design was very similar to that of the coal feed hopper. Both charpots were sized to contain the full amount of feed coal in the event that there was no conversion of the coal. Though this condition was very unlikely, the charpots were sized to safely contain the coal in a worst-case scenario.

\subsubsection{Lower Chanpot}

During startup, the reactor operates in a non-steady state condition. The coal feed rate, operating temperatures and hydrogen feed rate are continuously being adjusted to bring the reaction into a steady state. The online gas analyzers provide near real-time analysis of the product gas composition, which data is used, in part, to determine when the reactor reaches steady state. During the initial transient startup phase, the ball valve (tag number R-AOV-1) between the upper and lower charpot is open. This allows the hydrogen, coal, char, and reaction products to drop into the lower charpot. These products include a well-mixed composition of methane, partially reacted coal, coal, moisture, and heavier hydrocarbons including those that are liquid at ambient temperature. The reactor and lower charpot are designed to collect these mixed species for analysis later on. The reactor products continue to drop into the lower charpot and are collected until the reactor reaches steady state. Once steady state is reached, the operator closes the ball valve (R-AOV-1) to seal the partially reacted coal, char, and product gases into the lower charpot at about 1100 psig.

\subsubsection{Upper Charpot}

The upper charpot was configured slightly differently from the lower charpot. The upper charpot was also manufactured with 8-inch schedule $80316 \mathrm{H}$ SS pipe and fittings, but the top of the 
upper charpot was arranged with a tee fitting. The reactor's inner tube (2-inch-diameter Inconel $617)$ passed vertically downward through the run of the tee and extended about 18 inches into the upper charpot. There was a small, about 3/8-inch annular gap between the OD of the 2-inch inner tube and the ID of the tee that the inner tube passed through. This gap was sufficient to allow the product gases to exit the charpot assembly with minimal pressure drop. The gap also allowed the inner tube to expand downward into the upper charpot as it is heated up without impinging on the upper charpot tee. The reactor bottom assembly and flanges were precision machined to maintain concentricity between the inner tube and the center of the upper charpot tee.

The product gases and char drop from the hydrogasifier reactor through the inner tube into the upper charpot. As they exit the inner tube, the products go into the upper charpot, which has a much larger diameter and therefore has a much larger flow area. The upper charpot ID is about 7.6 inches versus the inner tube ID of 1.75 inch. The difference in diameters causes the product gas velocity to slow down by more than 18 times. Moreover, the product gas is forced into a reverse direction so that it will exit the upper charpot at the top tee. This combination of direction reversal and dramatically lower gas velocity allows most of the char particles to separate out of the gas flow stream and drop to the bottom of the upper charpot. The product gases exit from the upper charpot through the branch of the tee.

The product gases are at $1150 \mathrm{psig}$ and can be as hot as $1000^{\circ} \mathrm{F}$. They exit from the upper charpot through the 2-inch branch of the tee, which is sloped downward so that any heavy hydrocarbons that could begin to condense will drain to the lower part of the reactor. The 2-inch outlet reduces down to $1 / 2$-inch OD tube 316 SS. The $1 / 2$-inch OD tube has a short section of a cooling loop (8 inches in diameter and 24 inches long) that provides some initial cooling of the gas and also allows for thermal expansion and movement of the charpots as the reactor temperature increases. The $1 / 2$-inch OD tube connects into the top of the cooler and condensers.

Figure 1-15 provides a picture of the two-stage charpots from the testing site. Please refer to Appendix B, Appendix F, and Appendix $G$ for detailed charpots design drawings from APS and Gaspar. 


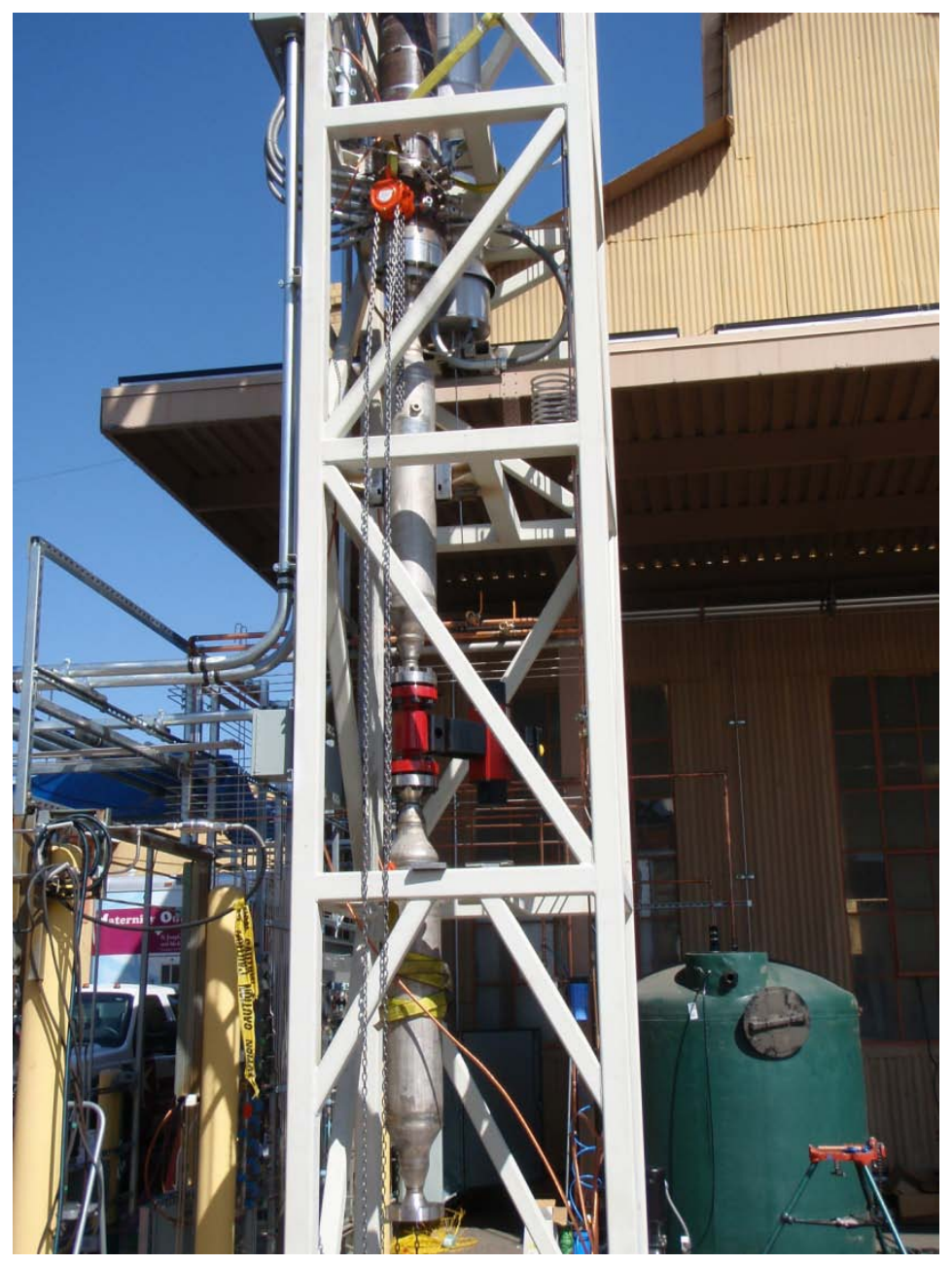

Figure 1-15 -Two-Stage Charpots with Actuated Full Port Ball Valve on Testing Site

\subsubsection{LQUID COUECTORS DESIGN}

The product gases were cooled to near ambient temperature in two identical coolers that were arranged in series. These coolers were manufactured by Sentry Equipment, Oconomowoc, Wisconsin, and they were ASME pressure vessels constructed of 316 SS. Both were Sentry Model WSW8222. The outer shell was rated to $150 \mathrm{psig}$ at $300^{\circ} \mathrm{F}$. The inner helical coiled tube was $1 / 2$-inch 316 SS tubing rated to 1200 psig at $650^{\circ} \mathrm{F}$. The product gas passed through the ID of the $1 / 2$-inch tubing. Cooling water (city water at ambient conditions) flowed around the outside of the $1 / 2$-inch tubing helical coil. The product gas and water flowed in a counter-flow arrangement to maximize the heat transfer. Generally, the first cooler cooled the product gases while the second cooler generally condensed the moisture and heavy hydrocarbons. Cooling water flows through each cooler/condenser. This water is constantly circulated back to the main water tank. The condensates that were liquefied in the coolers were separated by the 
condensate collector assembly (cylinder numbers CON-CYL-1 and -2). These condensate collectors (Figure 1-16) were arranged like the upper charpot and lower charpot with an actuated ball valve between them. During the startup non-steady-state period, the valve was open and the condensate dropped into the lower cylinder (CON-CYL-2). When the operator switched to the steady-state condition, the ball valve (tag number CON-AOV-1) closed and the condensate was collected in the upper cylinder (CON-CYL-1).

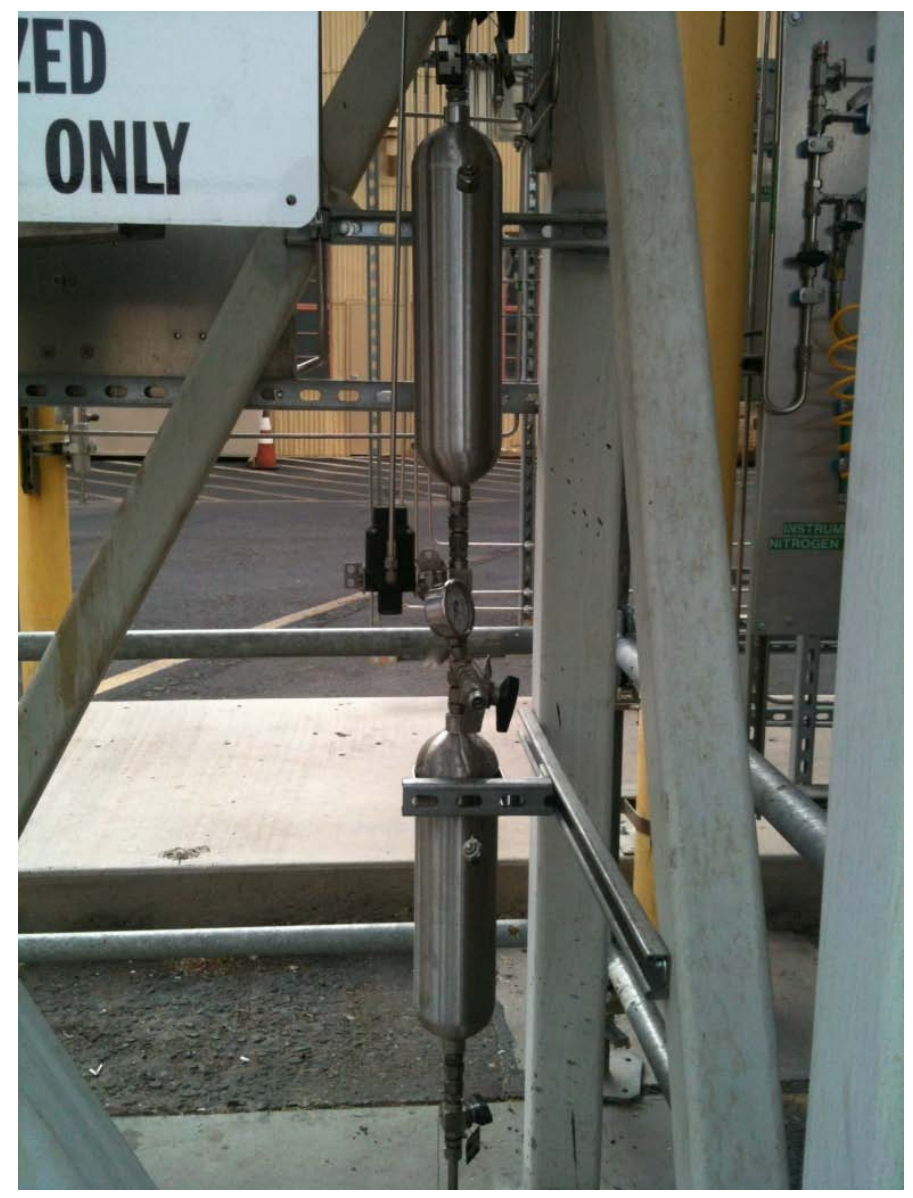

\section{Figure 1-16 - Two-Stage Condensate Collection Cylinders with Actuated Full Port Ball Valve}

Please refer to Appendix B for detailed liquid collector assembly design drawings from APS.

\subsubsection{THERMAL EXPANSION ISSUES}

The hydrogasification reactor was operated over a very wide range of temperatures. At start-up the gasifier components were at ambient temperature, 40 to $140^{\circ} \mathrm{F}$ in Phoenix. At the maximum operating conditions the inner tube could reach to $1950^{\circ} \mathrm{F}$, and the reactor shell could reach $700^{\circ} \mathrm{F}$. The preheater was designed to operate at $1600^{\circ} \mathrm{F}$. As was mentioned previously, the 
reactor was set up vertically and the inner tube, in which the reaction occurred, was 16 feet long. As this tube heats up to $1950^{\circ} \mathrm{F}$, it grows in length by about 2.5 inches. To allow for this expansion the inner tube was fixed or anchored at the top of the reactor and the tube was allowed to expand downward. A high-temperature expansion bellows was attached to the lower end of the inner tube to allow for thermal expansion. The bottom end of the bellows was attached to the reactor shell. The bellows created the pressure boundary between the inner tube and the reactor annulus.

The expansion bellows was designed per ASME BPVC Section VIII Division 1 and Section II Division D utilizing a high-temperature Alloy $800 \mathrm{H}$. It was rated to operate at $1000^{\circ} \mathrm{F}$ with an external pressure of 50 psid or with an internal pressure of 35 psid. Based on the worst-case operating conditions the bellows had a fatigue life of 166 full cycles.

The bellows (as shown in Figure 1-17) was designed to expand and contract through the full 2.5 inches that the inner tube expanded. To accommodate this amount of extension the bellows was pre-tensioned (stretched) by 1.25 inches from its normal or neutral position while at ambient temperature. It was then installed in the reactor at the pre-tensioned condition. The pre-tension imparted about 300 pounds of pull on the bellows, which was transferred to the inner tube. At ambient temperature the inner tube was also pre-tensioned by a 300-pound load; this did not change the length of the inner tube, but it did help to center the inner tube at the lower end of the reactor.

As the reactor temperature increased, the inner tube expanded downward and compressed the bellows. From ambient temperature to about $1000^{\circ} \mathrm{F}$ on the inner tube, the tube expansion relieved the pre-tension on the bellows. At about $1000^{\circ} \mathrm{F}$ the inner tube had expanded theoretically by approximately 1.25 inches, and at this temperature the inner tube would compress the bellows back to its neutral position where there was no tension or compression on the bellows. As the tube continued to expand downward, the bellows would further compress and transition into a compression state. Theoretically, at the maximum operating temperature the bellows would be compressed by about 1.25 inches from the neutral position. At this point the bellows would exert about 300 pounds of upward thrust (push) on the inner tube. This thrust force is important as an excess of thrust could cause column buckling of the inner tube and lead to a catastrophic failure.

The buckling condition was analyzed as part of the ASME BPVC evaluation. A worst-case scenario was used in which the inner tube was at its maximum temperature, $1950^{\circ} \mathrm{F}$, and the 
entire 16-foot length of the tube was unsupported. The upward thrust force of 300 pounds was well below the force required to cause buckling of the tube. The inner tube and bellows design was tested in service and met acceptance criteria for systems checkout.

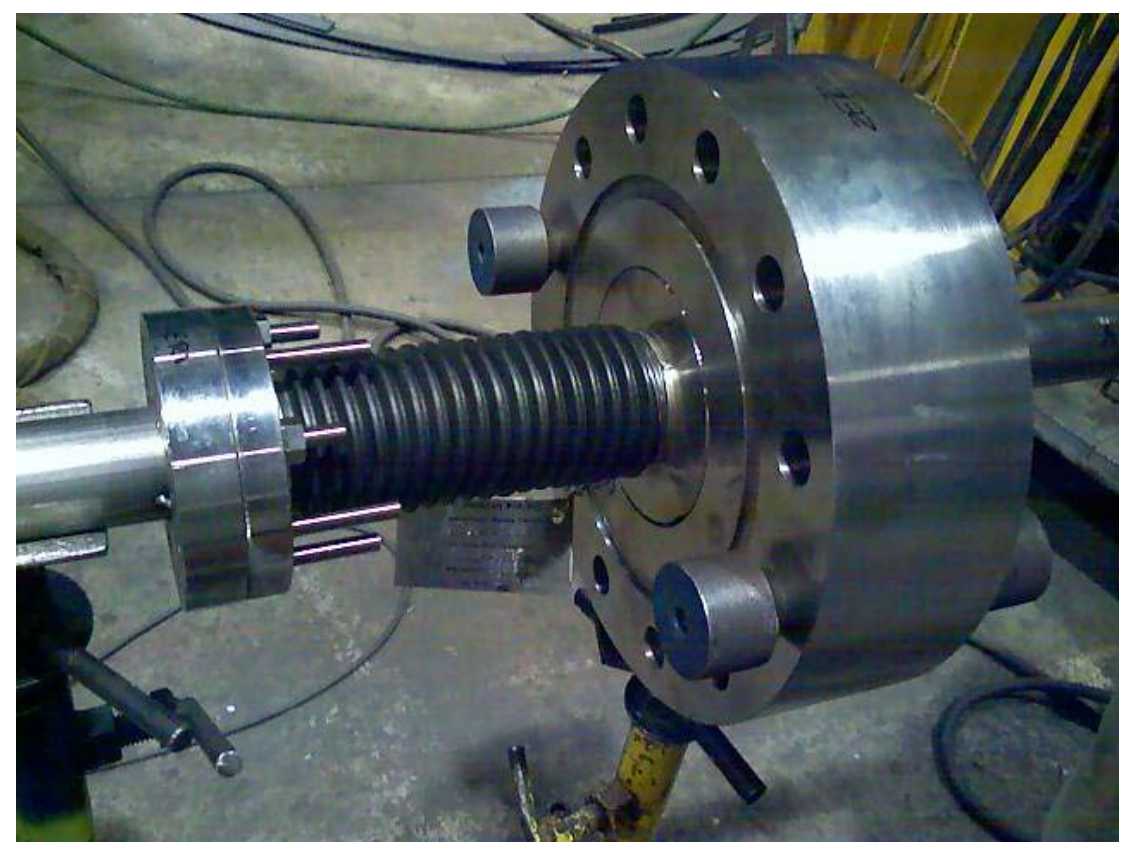

Figure 1-17 - Reactor Inner Tube Bellows

While the inner tube experienced the greatest amount of thermal expansion, the reactor external shell (10-inch schedule 80 pipe) was observed to expand longitudinally by up to 1.2 inches when it heated to its maximum operating temperature of $700^{\circ} \mathrm{F}$. As with the inner tube, the shell was also anchored at the top of the shell where it was bolted to the reactor frame. The shell was allowed to expand downward, and this longitudinal expansion of the shell was relatively straightforward. The lower reactor shell was attached to a set of rigid guide plates on the reactor frame that restrained the shell from moving laterally while allowing the shell to expand vertically without restraint. The lateral support was required to constrain the reactor shell from moving due to wind loads or seismic events.

The preheater (Inconel 625) operated at $1600^{\circ} \mathrm{F}$ under normal conditions and was 15 feet long. As with the shell, the preheater was also anchored to the reactor frame at the top of the pre-heater. As it heated up, it was observed to expand downward by nearly 2 inches. The preheater also had guide plates on the lower portion to constrain the vessel from lateral movement.

A second dimension of thermal expansion was radial expansion. For the preheater and reactor 
shell, this was a minor design consideration, but for the inner tube it was an important factor. At the time of start-up, the reactor was at ambient temperature. There were support guide plates or "spider" plates attached to the inner tube to keep it centered within the outer shell. There was a small air gap between the spider plates and the inside of the shell. As the reactor was heated, the inner tube and spider plates temperature increased faster than the outer shell. As a result the spider plates expanded radially toward the shell while the shell ID remained relatively constant. The result of this was the clearance between the spider edges and the shell ID diminished. Because of this, the spider plate shape and dimensions were engineered to provide both support and guidance for the inner tube, while preventing impingement of the spider on the shell ID.

The last area of concern for thermal expansion was the area between the top of the reactor and the coal feeder. This distance was about 30 inches, and this segment consisted of several flanges, a ball valve, and 1-inch 316 SS pipe. It was not clear during the design phase how hot this segment might become, so the thermal expansion analysis was based on a very conservative temperature of $1000^{\circ} \mathrm{F}$ throughout the entire segment. The maximum expected expansion by calculation was 0.19 inch. The reactor top was anchored to the frame and the coal feeder sat above the reactor. With this arrangement the 1-inch pipe segment expanded upward as does the coal feeder. This arrangement minimized the stress and buckling forces on the pipe. To allow the feeder to "float" relative to the top of the reactor, it was mounted on four-spring loaded pipe supports. These spring cans were pre-loaded (compressed) by the weight of the feeder and coal hopper and adjusted so that there was a neutral force on the flange sets in the segment. At ambient temperature the feeder was leveled and the springs were in mild compression. As the pipe segment heated and expanded upward it pushed the feeder system upward, thereby relieving some of the initial spring compression.

During the test phase it was observed that the connection between the feeder and reactor remained relatively cool, and the expansion was much less than the calculated 0.19 inch. The spring-loaded feeder support did work as expected, as it allowed the feeder to move as the reactor heated, preventing the feeder from tilting and keeping bending moments off the flange sets. 


\subsubsection{DESIgn FIEXIBIUTY}

During the design phase of developing the bench-scale hydrogasification reactor, the advantages and disadvantages of designing the reactor for increased design flexibility were evaluated. By implementing more flexibility into the design, certain design configurations could be reconfigured to allow for varying the range of testing conditions or perhaps use of reactants other than hydrogen and coal. Adding the extra flexibility also facilitates ease of repair, modification or upgrading of certain components as necessary.

The greater flexibility in the reactor design requires using bolted and flanged configurations where possible. At the maximum operating conditions (1200 psig, 1,600 $\mathrm{F}$ ), bolted and flanged assemblies are operated at the extreme edge of the allowable operating conditions. This type of assembly was also more prone to leaking than an all-welded construction. The alternative to using bolted and flanged assemblies is to weld all joints and points of connections. Welded joints typically are stronger and have essentially zero leakage. However, if a repair or upgrade on the reactor is required, removing the weld, doing the repair, and then re-welding the joint will be more costly than the same repair with a bolted assembly. Moreover, if the welding is done on an ASME pressure vessel, the repair must be analyzed and approved by an ASME code engineer and additionally the repair could only be completed by a contractor with an ASME "R" stamp. The vessel will also have to be re-tested per the ASME code.

Key areas of the reactor design were assessed to identify the components that were bolted and/or flanged and those that were all welded. These areas included:

- Top hat body

- Hydrogen injector guides (4)

- Hydrogen injection wands (4)

- Connection of top hat to reactor shell

- Attachment of lower bellows to inner tube

- Charpot connection to lower reactor shell

- Hydrogen inlet piping

- Product outlet piping

- Preheater 
It was deemed that several of these areas did not require a high degree of flexibility in the design and that the lower likelihood of a gas leak from a welded design was preferred. Consequently, the top hat body, hydrogen injector guides, preheater, hydrogen inlet piping, and the product outlet piping were all-welded configurations. The remaining key areas - hydrogen injection wands, connection of top hat to reactor shell, attachment of lower bellows to inner tube, charpot connection to lower reactor shell - were designed as bolted or flanged assemblies. High-temperature gaskets such as Flexitallic spiral-wound Inconel gaskets were used to seal the assemblies. They are detailed as follows.

\subsubsection{Injector Wands}

As described in the reactor injector section, the injector wands were designed with a tip nozzle welded onto the $1 / 4$-inch OD Inconel tubing. This tip nozzle could easily be removed and replaced with a nozzle having a different orifice diameter or with a varying-angle nozzle that ranges from 0 to 50 degree relative to the injector longitudinal axis. This flexibility could be used to vary the hot hydrogen injection angle and velocity into the top of the inner tube where the hot hydrogen and coal were first mixed (Figure 1-12).

\subsubsection{Top Hat to ReactorTop}

The top hat to reactor top connection point was designed as a custom bolted flange. The gasket seal design was a flat-to-groove configuration (Figure 1-18) with the groove on the reactor top flange and the flat on the top hat flange. This arrangement was chosen as it provided a superior seal and the gasket could be set into the groove for easy flange make-up. The gasket was an Inconel spiral-wound gasket with Thermiculite ${ }^{\circledR}$ filler and was rated to $2300^{\circ} \mathrm{F}$ and to over 1500 psig. The gasket was completely restrained within the groove when it was compressed per ASME and Flexitallic standards were bubble tight. This flanged arrangement allowed for the removal of the entire inner tube assembly (tube, heaters, thermocouples, and wiring) as one piece to inspect or repair the assembly. 

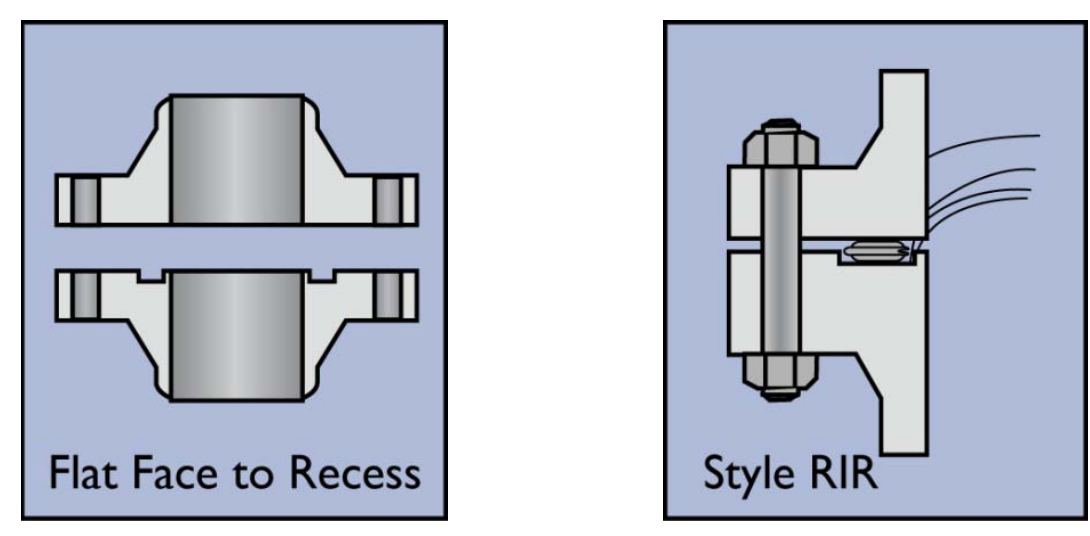

\section{Figure 1-18 - Flat to Groove Flange Connection Between Top Hat to Reactor Top}

\subsubsection{Lower Bellows to Inner Tube}

The connection of the lower bellows and inner tube consisted of a custom flange plate - welded to the inner tube at the lower end. The lower bellows (flanged at both ends) bolted onto this plate and was sealed with a Flexitallic Thermiculite gasket. This seal was exposed to as much as 1200 psig, but the pressure difference across the gasket was low, ranging from 2 to 50 psid. The inner tube ran longitudinally through the center of the lower bellows. The bottom of the bellows was bolted to a removable adapter flange on the bottom of the reactor shell. Again, a Flexitallic Thermiculite gasket was used to seal this flange assembly. With this arrangement, the lower bellows were accessible from the bottom of the reactor and could be replaced in the event of a bellows failure without de-telescoping the entire inner tube assembly. This flange design appeared to work with a minimum amount of leakage through the gasket. The leakage could be reduced to zero by welding the bellows assembly to the inner tube and to the adapter flange. However, the latter weld would have been an extremely difficult task due to the location, and a welded design would require fully de-telescoping the inner tube assembly to replace the bellows.

\subsubsection{Charpot Connection to Lower Reactor Shell}

The upper charpot connected with lower reactor shell with flanges. This made the charpot assembly relatively independent and easy to disassemble from the reactor main body in case of any unexpected clogging or reactor repair. The connection between the two charpots and the full-port actuated ball valve were also designed with flanges to facilitate any required repairs. The lower charpot was configured with flanged ends. The design anticipated that these charpots would collect char and possibly tar and would require periodic cleaning out. A flanged arrangement with replaceable gaskets was the most cost-effective way to provide easy disas- 
sembly for cleaning. Please refer to Appendix B detailed gasket design drawings from APS.

\subsubsection{DESIGN DRAMIng PRESSURE AND TEMPERATURE LIMIS}

Table 1-3 summarizes the design pressures and temperatures as well as the maximum allowable working pressures (MAWP) at the Design Temperature delineated in the table below for the various hydrogasification reactor components.

Table 1-3 - Design Criteria for Reactor Components

\begin{tabular}{|l|c|c|c|}
\multicolumn{1}{|c|}{ Component } & $\begin{array}{c}\text { Design Temperature } \\
\left({ }^{\circ} \mathrm{F}\right)\end{array}$ & $\begin{array}{c}\text { Design Pressure } \\
(\mathrm{ps} \text { ig })\end{array}$ & $\begin{array}{c}\text { MAWP } \\
\text { (psig @ design } \\
\text { temp) }\end{array}$ \\
\hline $\begin{array}{l}\text { Coal Feeder and } \\
\text { Hopper }\end{array}$ & 200 & 1200 & 1700 \\
\hline Reactor Top Hat & 1000 & 1200 & 1596 \\
\hline Reactor Top Flange & 700 & 1200 & 1255 \\
\hline $\begin{array}{l}\text { Reactor Bottom } \\
\text { Flange }\end{array}$ & 700 & 1200 & 1255 \\
\hline Pressure Shell Body & 700 & 1200 & 1382 \\
\hline $\begin{array}{l}\text { Reactor Adapter } \\
\text { Flange }\end{array}$ & 1000 & 1200 & 3319 \\
\hline $\begin{array}{l}\text { Inner Tube "Reactor" }- \\
\text { 617 Inconel }\end{array}$ & 1950 & 50 psid & 50 psid \\
\hline Reactor Bellows & 1200 & 35 psid & 35 psid \\
\hline Injector Bellows & 1600 & 48 psid & 48 psid \\
\hline Preheater & 1600 & 1150 & 1261 \\
\hline Upper Charpot & 1000 & 1150 & 1169 \\
\hline Lower Charpot & 1000 & 1150 & 1502 \\
\hline
\end{tabular}

\subsection{HYDROgASIFER TEST PLAN}

The experimental test plan designed to evaluate the effects of temperature, coal residence time and hydrogen-to-coal ( $\mathrm{lb} / \mathrm{lb})$ ratio on the quantity and quality of the product streams is presented in Table 1-4. The design of experiments utilized a three-factor central composite design. It was developed to maximize the amount of analysis that could be done in a budget-limited number of runs. Additionally, the design was blocked (i.e. some randomness in experiments was sacrificed) in the event anything unforeseen should happen, such as a critical reactor failure, so meaningful analysis could still be completed. Runs that contributed to linear analysis of the response surfaces were grouped into the first block, and runs that would affect the curvature of the response in the second. All of the runs were randomized in the respective blocks to minimize any systematic error that might arise. 
The experimental factors were chosen because previous experience as presented in literature indicated that these factors, along with being repeatable, would have a significant impact on the results. It is well known that high pressure and moderate temperatures lead to higher methane production in the produced syngas. There, the pressure was held constant at 1000 psig. The three factors were reactor temperature, hydrogen-to-coal ratio (lb/lb), and the reactor length (i.e. coal residence time). It is intuitive why the first two factors were chosen, but perhaps why reactor length was chosen is not. The reactor had six heated zones. By controlling the heater power output, the reaction zone length could be controlled and used as a coded variable for residence time. The reactor length was defined as the length from the top of the reactor (this is where the coal and hydrogen were fed) to a point where the reactor temperature was insufficient to maintain the hydrogasification reactions $\left(1300^{\circ} \mathrm{F}\right)$. There were three levels of reactor length that were attempted to be precisely controlled for every run.

Table 1-4 - Hydrogasification Experimental Plan

\begin{tabular}{|c|c|c|c|c|}
\hline Run No. & $\begin{array}{c}\mathrm{H}_{2}: \text { Coal Ratio } \\
(\mathrm{Ib} / \mathrm{lb})\end{array}$ & $\begin{array}{c}\text { Reactor } \\
\text { Temperature } \\
\left({ }^{\circ} \mathrm{F}\right)\end{array}$ & $\begin{array}{c}\text { Reactor } \\
\text { Leng th } \\
\text { (inches) }\end{array}$ & Block \\
\hline 1 & 0.5 & 1500 & 105 & 1 \\
2 & 0.4 & 1625 & 77 & 1 \\
\hline 3 & 0.5 & 1750 & 105 & 1 \\
4 & 0.3 & 1750 & 105 & 1 \\
\hline 5 & 0.4 & 1625 & 77 & 1 \\
6 & 0.5 & 1750 & 49 & 1 \\
\hline 7 & 0.3 & 1750 & 49 & 1 \\
8 & 0.3 & 1500 & 105 & 1 \\
\hline 9 & 0.3 & 1500 & 49 & 1 \\
10 & 0.5 & 1500 & 49 & 1 \\
\hline 11 & 0.4 & 1500 & 77 & 2 \\
\hline 12 & 0.4 & 1625 & 77 & 2 \\
\hline 13 & 0.4 & 1625 & 105 & 2 \\
\hline 14 & 0.4 & 1625 & 49 & 2 \\
\hline 15 & 0.5 & 1625 & 77 & 2 \\
\hline 16 & 0.3 & 1625 & 77 & 2 \\
\hline 17 & 0.4 & 1750 & 77 & 2 \\
18 & 0.4 & 1625 & 77 & 2 \\
\hline
\end{tabular}

Temperature and hydrogen-coal ratio are factors that are more identifiable with hydrogasification experimentation. The temperature setpoint was considered to be reached after there was a relatively flat temperature profile across the reactor length. Hydrogen-to-coal 
ratio had a single target coal feed rate $(8 \mathrm{lb} / \mathrm{hr})$ and the hydrogen-coal ratio was therefore set by varying the hydrogen flow rate. The coal feed rate was set to a single value to ensure:

- Approximately 2 hours of testing time with the designed coal hopper volume;

- The hot hydrogen could provide enough heat to raise the gas-solid mixing temperature to initiate the hydrogasification reactions;

- The error introduced by coal feeding system was minimized. It was determined that the precise hydrogen feed rate was much easier to achieve than coal feed rate.

\subsection{GAS, LQUID, AND SOUD ANALYSIS}

\subsubsection{GASANALYSIS}

A SRI 8610C Gas chromatography (GC) was used to determine product gas composition. It had a thermal conductivity detector (TCD) and a flame ionization detector (FID). It was also equipped with a methanizer for low-level $\mathrm{CO}$ and $\mathrm{CO}_{2}$ detection. Ultra-high-purity helium was the carrier gas, and research grade hydrogen and ultra-zero air was used for the FID flame. The GC had dual columns: Molecular Sieve $13 \mathrm{X}$ column for the separation of $\mathrm{H}_{2}, \mathrm{O}_{2}, \mathrm{~N}_{2}, \mathrm{CH}_{4}$, $\mathrm{CO}$ and $\mathrm{CO}_{2}$ and Hayesep-D column for all compounds in the $\mathrm{C}_{1}-\mathrm{C}_{6}$ range.

There were two temperature programs used during testing: one with a temperature ramp and one without. The reason why this two-temperature program method was chosen is that a higher temperature aids in the detection of larger molecules (i.e., $\mathrm{C}_{2} \mathrm{H}_{4}$ and $\mathrm{C}_{2} \mathrm{H}_{6}$ ). Therefore to make data collection more efficient, the high-temperature ramp program was not initiated until it was determined that the hydrogasification process was at a steady state. The GC was started up as recommended by the manufacturer.

For calibration of the GC, two gas standards were purchased, and the GC was calibrated on every testing date. The components of the calibration gas, with molar concentrations, are given in Table 1-5. 
Table 1-5 - Calibration Gas Components and Concentrations

\begin{tabular}{|l|c|c|}
\multicolumn{1}{|c|}{ Component } & Calibration Gas 1, \% & Calibration Gas 2, \% \\
\hline Acetylene & 1.01 & 0.00 \\
\hline Carbon Dioxide & 3.04 & 2.01 \\
\hline Carbon Monoxide & 5.08 & 1.99 \\
\hline Ethane & 0.997 & 0.00 \\
\hline Ethylene & 1.03 & 0.00 \\
\hline Methane & 22.5 & 10.0 \\
\hline Nitrogen & 9.89 & 5.04 \\
\hline Hydrogen & 56.5 & 81.0 \\
\hline
\end{tabular}

The concentration of a component in the product gas stream was determined by the area under the intensity curve from the GC. For hydrogen, the concentration was taken from the TCD, which measures the difference between the thermal conductivity of a component and a reference gas (helium in this instance). When measuring the concentration of hydrogen, there is more potential for error because: (1) the thermal conductivity of hydrogen is higher than that of helium, so the intensity curve must be inverted; and (2) the slope of the concentration versus peak area regression model is steep. For these reasons, along with the two calibration gases listed above (Table 1-5), ultra-high-purity hydrogen was used as a calibration gas.

Another piece of analytical equipment used to analyze the product gas stream was a mass spectrometer (MS). The MS can measure component generation in real time, as opposed to the GC, which required periodic injections. The MS was particularly important because it could measure sulfurous components in the product gas stream. A QMS 200 model from Stanford Research Systems was used. This device had the capability to measure components with mass up to 200 atomic mass units (amu). For all tests, the MS was run in pressure-versus-time mode with a new scan triggered every two seconds. A channel electron multiplier was used for sulfurous components analysis to provide higher resolution during the scan.

The two sulfurous components that were being screened were hydrogen sulfide $\left(\mathrm{H}_{2} \mathrm{~S}\right)$ and carbonyl sulfide (COS). To calibrate the instrument for sulfur, a gas mixture of 514 parts per million (ppm) COS, 4,970 ppm $\mathrm{H}_{2} \mathrm{~S}$, and the balance $\mathrm{H}_{2}$ was used.

\subsubsection{LQUID CONDENSATE ANALYSIS}

To analyze the liquid samples, the oil and BTX samples were first put into a separatory funnel to 
separate the oil layer from the water layer. The upper and lower layer oil samples were then dissolved into hexane and transferred into GC vials. The samples were analyzed on an HP 7890A GC equipped with an HP7673B injector, an HP-INNOWAX column, and a 5975C MS. Approximately $0.2 \mu \mathrm{L}$ of the sample was injected into the split injection mode with a split ratio of $75 / 1$. The oven temperature was programmed from 50 to $200^{\circ} \mathrm{C}\left(122\right.$ to $392^{\circ} \mathrm{F}$ ) with $25^{\circ} \mathrm{C} / \mathrm{min}$ $\left(77^{\circ} \mathrm{F} / \mathrm{min}\right)$, then to $250^{\circ} \mathrm{C}\left(482^{\circ} \mathrm{F}\right)$ with $5^{\circ} \mathrm{C} / \mathrm{min}\left(41^{\circ} \mathrm{F} / \mathrm{min}\right)$, and the temperature was held at $250^{\circ} \mathrm{C}\left(482^{\circ} \mathrm{F}\right)$ for $8 \mathrm{~min}$. The components in the oil samples were identified and quantitatively determined by MS.

\subsubsection{COALAND CHAR ANALYSIS}

The final aspect of the analysis of the hydrogasification tests was that of the coal and char. All samples of coal and the upper pot char from each test were sent to SGS Mineral Services at Denver, Colorado, for proximate and ultimate analysis. The coal particle size, heating value analyses, and metal element analyses were also obtained for the designated samples. The details relating to the gas, liquid, and solid analysis can be found in Section 3.2 of the IES Final Technical Closeout Report.

\subsection{COAL FeEder CALBRATION}

After the design, manufacture and assembly the coal feeding system (as depicted in Appendix E), coal feeder testing and calibration were performed prior to the hydrogasification test. The main scope of coal feeder testing and calibration included the following: (1) evaluation of the coal sample preparation method, (2) observation of the stability and uniformity of the coal feed, (3) calibration of the coal feeder at ambient conditions (14.11 psia in Phoenix, Arizona), and (4) calibration of the coal feeder at 1000 psig.

\subsubsection{COALSAMPLES}

The Navajo Mine subbituminous coal was used for coal feeder calibration and the hydrogasification test. Two coal samples were evaluated. Sample No. 1 was obtained through a local grinding company, Alex \& Alex, at Tucson, Arizona, by grinding Navajo coal directly from the coal mine. Sample No. 2 was the coal sample acquired directly from the APS Four Corners Power Plant pulverizer.

Figure 1-19 and Figure 1-20 show the comparison of these two coal samples. Alex \& Alex No. 1 sample was required to have a size of $100 \%$ pass through 200 mesh. Navajo No. 2 had about 
50\% pass through 200 mesh. Finer coal particles give less bulk density. As shown in Table 1-6, the Alex \& Alex No. 1 coal had a bulk density of $0.47 \mathrm{gram} / \mathrm{milliliter}(\mathrm{g} / \mathrm{mL})(29.34$ pound/cubic-feet $\left(\mathrm{lb} / \mathrm{ft}^{3}\right)$ ); lower than Navajo No. 2 of $\left.0.59 \mathrm{~g} / \mathrm{mL} 36.83 \mathrm{lb} / \mathrm{ft}^{3}\right)$.

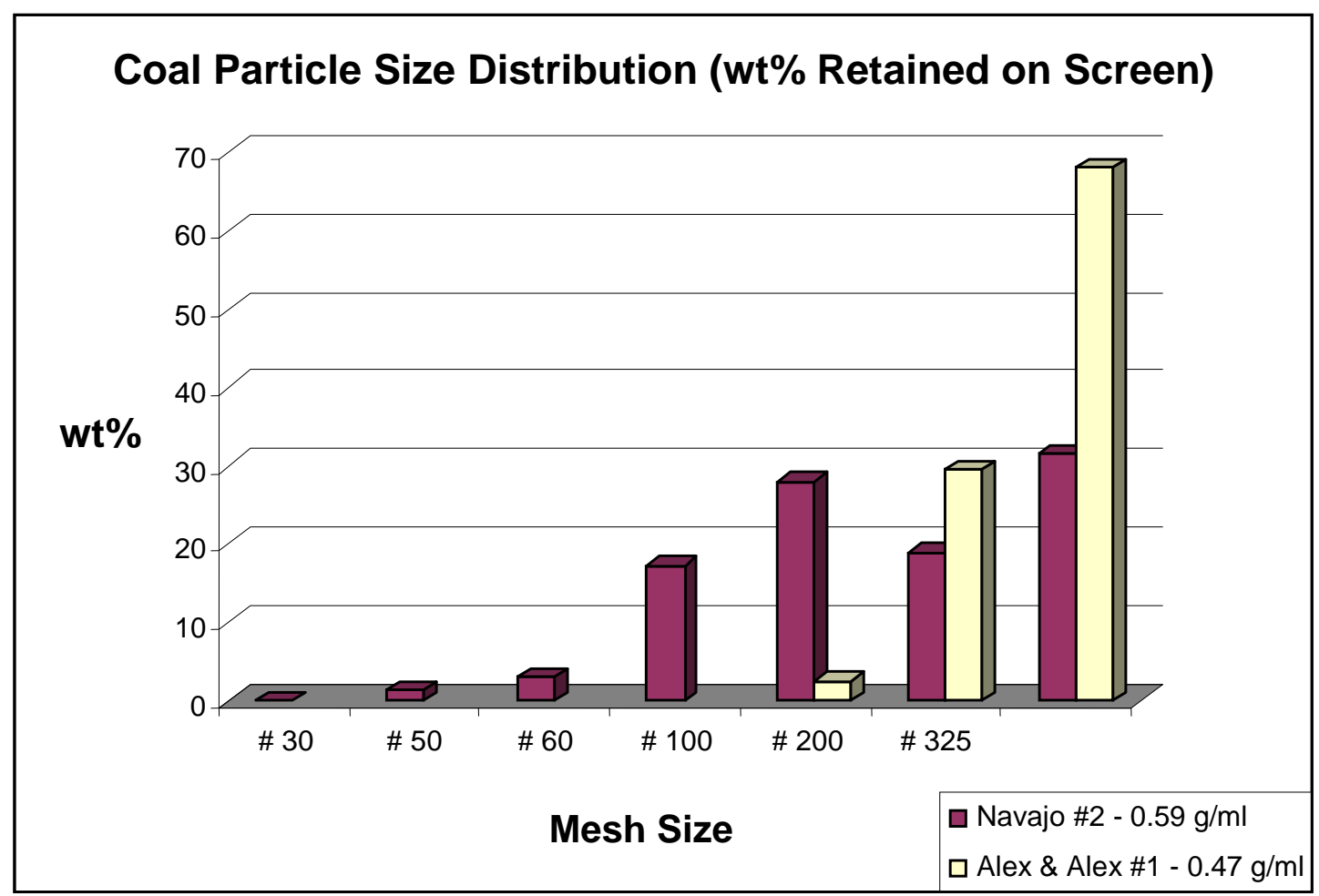

Figure 1-19 - Comparison of Coal Particle Size Distributions (wt\% Retained on Screen) 


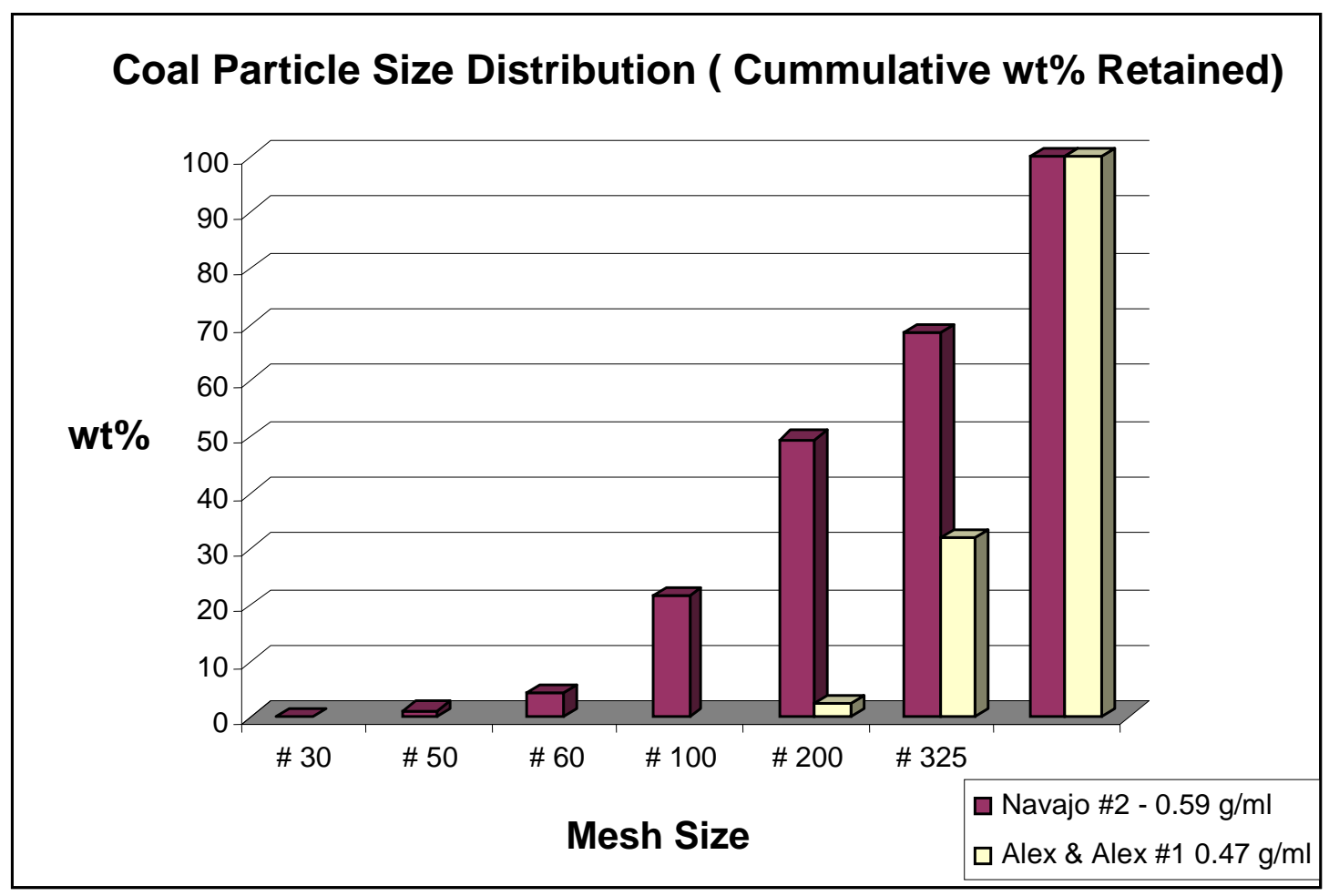

Figure 1-20 - Comparison of Coal Sieve Analysis (Cumulative wt\% Retained)

To increase coal fluidity and minimize agglomeration during hydrogasification testing, fumed silica was mixed with the coal. This addition significantly improved coal fluidity; however, it also significantly reduced the coal density. An addition of 10 weight $\%$ fumed silica caused about a $50 \%$ reduction of the bulk density. Table 1-6 compares the bulk density of pure coal with that of coal mixed with silica for the two coal samples.

Table 1-6 - Comparis on of the Two Coal Samples

\begin{tabular}{|l|c|c|}
\hline \multicolumn{1}{|c|}{ Coal Samples } & Alex \& Alex No. 1 & Nava jo No. 2 \\
\hline Bulk Density w/o Silica & $0.47 \mathrm{~g} / \mathrm{mL}$ & $0.59 \mathrm{~g} / \mathrm{mL}$ \\
\hline Silica Content (by weight) & $10 \%$ & $5 \%$ \\
\hline Bulk Density w/ Silica & $0.23 \mathrm{~g} / \mathrm{mL}$ & $0.39 \mathrm{~g} / \mathrm{mL}$ \\
\hline
\end{tabular}

Bulk density is the key factor to determine which coal samples should be used for the hydrogasification testing. The bulk density of the coal samples should be able to meet three criteria:

- Allow the coal feeder to feed variable amounts of coal feedstock accurately up to $15 \mathrm{lb} / \mathrm{hr}$; 
- Allow the coal hopper to hold at least 1.5 hours of feedstock supply;

- Produce a small coal particle and narrow particle size distribution. High bulk density correlates to bigger particle sizes, and it is difficult to achieve high carbon conversions with large particle sizes due to the inherent diffusion/mass transfer issues encountered with the large particle size.

With the current coal hopper, a bulk density of coal-silica feedstock of approximately $0.45 \mathrm{~g} / \mathrm{mL}$ $\left(28.1 \mathrm{lb} / \mathrm{ft}^{3}\right)$ was required to satisfy criterion No. 2. With a $0.23-\mathrm{g} / \mathrm{mL}\left(14.36 \mathrm{lb} / \mathrm{ft}^{3}\right)$ bulk density, Alex \& Alex No. 1 coal-silica mixture was too fine for the project. Channeling was often observed during the coal feeder test using this coal feedstock sample, which was caused by high moisture content of the sample introduced during the grinding process. To minimize the extra exercise of the coal sample preparation, Navajo No. 2 obtained directly from the mine was used for all the project hydrogasification testing.

\subsubsection{CoAl Feeder Caubration Setup}

Coal feeder tests were carried out under ambient conditions and high-pressure conditions. The ambient conditions test setup is shown in Figure 1-21.
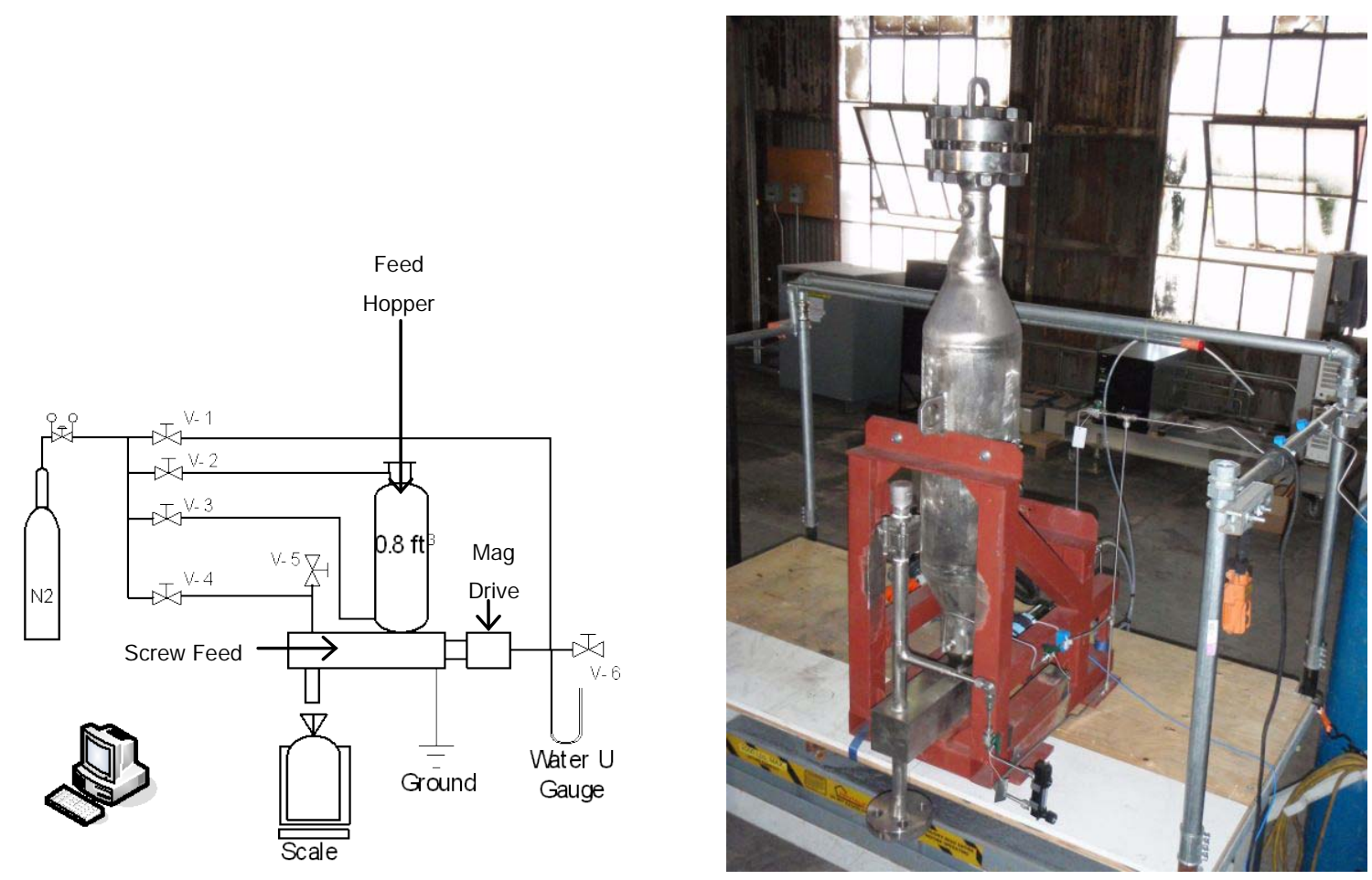

Figure 1-21 - Coal Feeding Testing Arrangement under Ambient Conditions 
During the ambient test, the magnetic drive was fed with nitrogen through valve $\mathrm{V}-1$ to create the pressure balance of magnetic drive for sealing purpose. The carrier gas was fed through V-4. The high-pressure test setup is shown in Figure 1-22. The test was performed under 1000 psig of pressure. Several pressure points - coal hopper, magnetic drive housing, auger housing, and charpot - had to have the pressure equalized. Any unnecessary pressure drop between the coal feeder and charpot could cause coal to flow in an uncontrollable manner. During the hydrogasification testing, $\mathrm{V}-1, \mathrm{~V}-2$, and $\mathrm{V}-3$ lines had hydrogen flow, and they were automatically controlled.

During high-pressure testing, the feed hopper and charpot were pressurized by flowing gas through line $\mathrm{V}-4$ to 1000 psig. The equilibrium line (V-2 line) was kept closed during the pressurizing so that gas would enter the coal feeder from the bottom and was kept open during testing to ensure the same pressure between the coal feeder and charpot. The influence of the small pressure difference between coal feeder and charpot (which was being utilized as a collection vessel for the unreacted feed material) on the coal feed rate was observed but was hard to quantify. For high-pressure testing at 1000psig, $17 \mathrm{lbs}$ of coal feed stock was loaded.
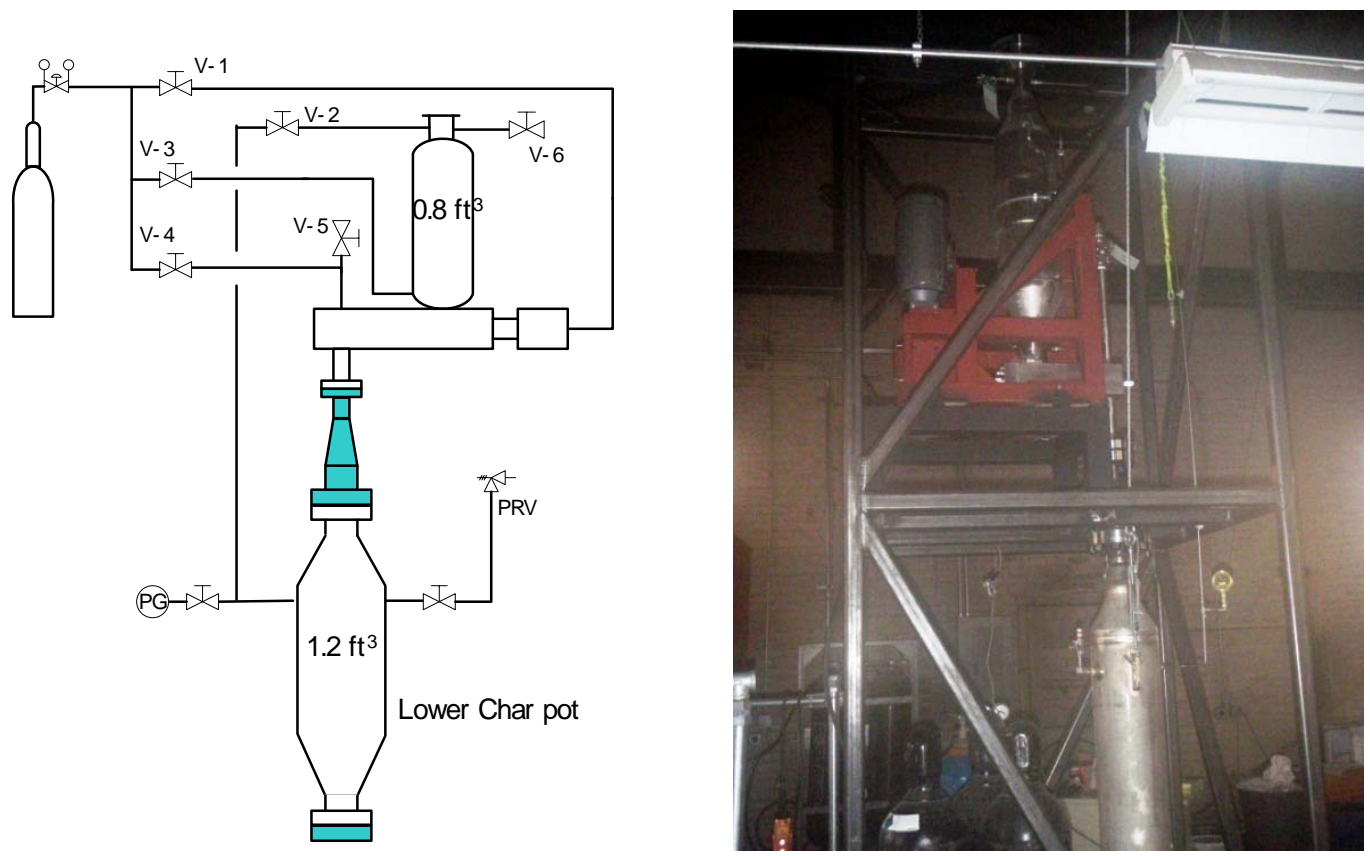

Figure 1-22 - Higher-Pressure Test Setup 


\subsubsection{CoAl Feeder Caubration WTH OriginalAuger}

Figure 1-23 shows coal feeder calibration results and reproducibility using the Navajo No. 2 coal sample mixed with $5 \mathrm{wt} \%$ silica. At the time of the calibration, this mixed coal sample had a bulk density of $0.39 \mathrm{~g} / \mathrm{mL}$. The results in Figure $1-23$ show that at 100 revolutions per minute (rpm), the recommended high end of Magdrive rotation speed, only $2.5 \mathrm{lb} / \mathrm{hr}$ of the coal feed rate was achieved under ambient conditions and less than $4 \mathrm{lb} / \mathrm{hr}$ was achieved under high-pressure conditions. This result indicated that the screw auger of the coal feeder as originally designed was significantly under designed. The need to add fumed silica to obtain flow resulted in a much-reduced bulk density for the coal-silica feed, thereby reducing flow rate.

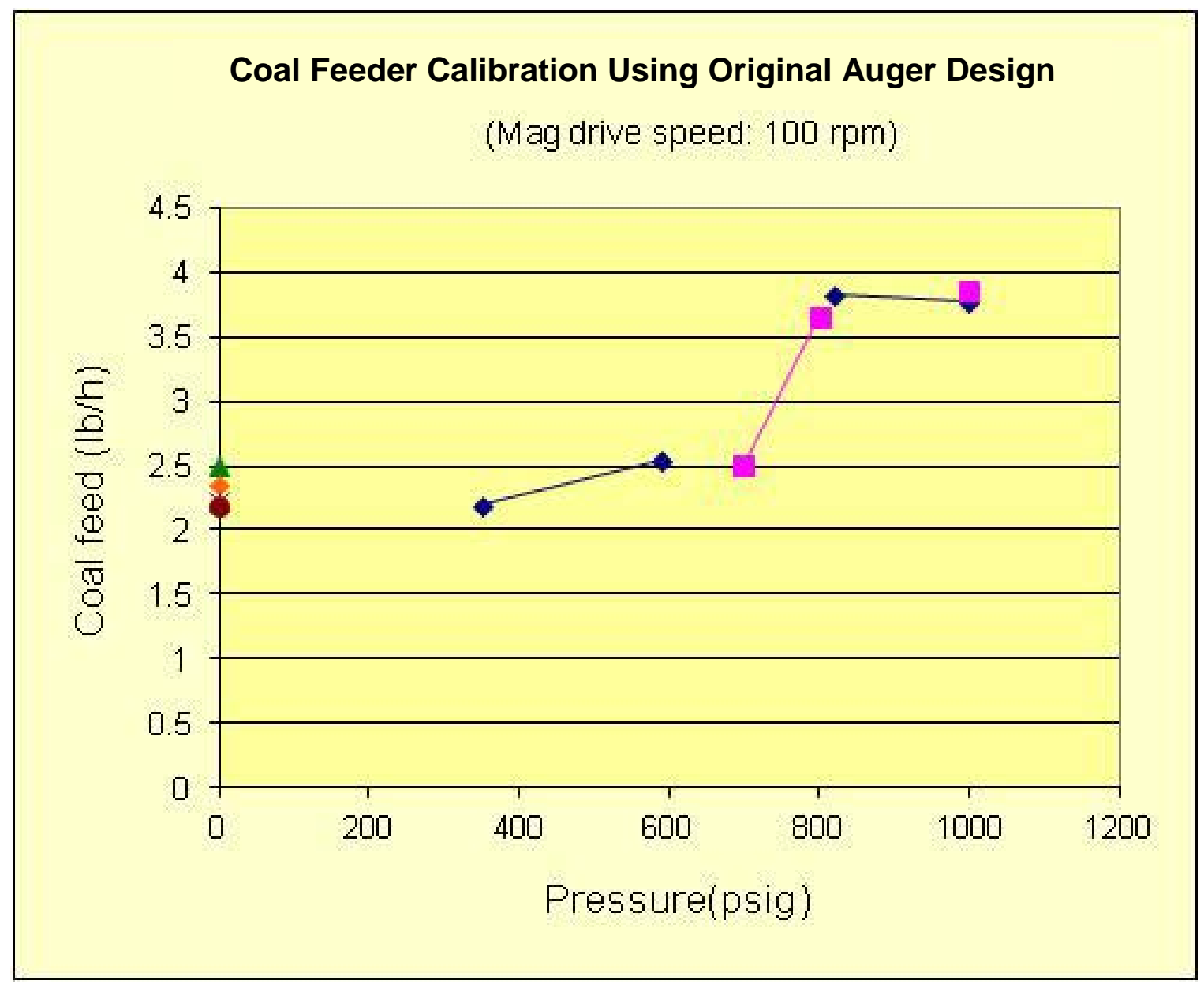

Figure 1-23 - Coal Feeder Calibration Using Original Auger Design

\subsubsection{Coal Feeder Auger Re-Design}

Figure 1-24 shows the original auger on the left, and the auger end-cap and magnetic drive assembly on the right. 

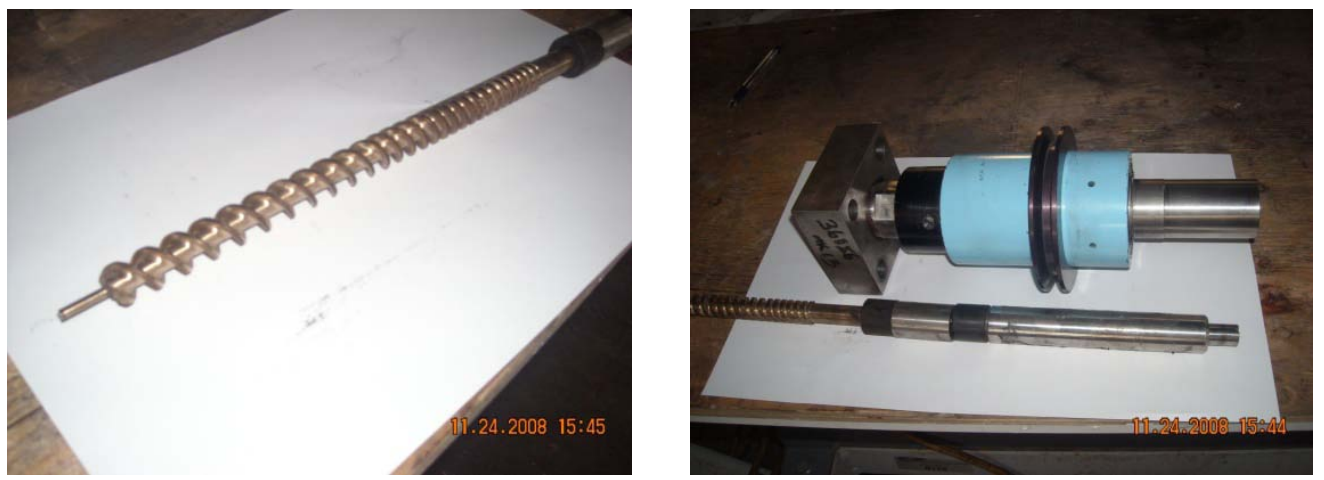

\section{Figure 1-24 -Left Photo shows Auger and Right Photo Shows Auger End Cap and Magnetic Drive Assembly}

As shown in Figure 1-25, the auger had a unique design. It had a double flight with a denser pitch on the receiving side and a center shaft tapered from 0.5-inch OD to 0.25-inch OD from the receiving port to the delivery port. This configuration was designed to ensure stable and reproducible coal feeding. Unfortunately, with this special design, the auger was not able to deliver pure coal. When using pure coal, rat holing was observed in the coal hopper. Since the addition of silica reduced the bulk density of the solid sample, the auger could not provide the designed $15 \mathrm{lb} / \mathrm{hr}$ feed rate. The unique design of the existing auger (varied pitch distance and tapered center shaft) did not seem to be necessary, since the addition of silica gave the coal much greater fluidity.

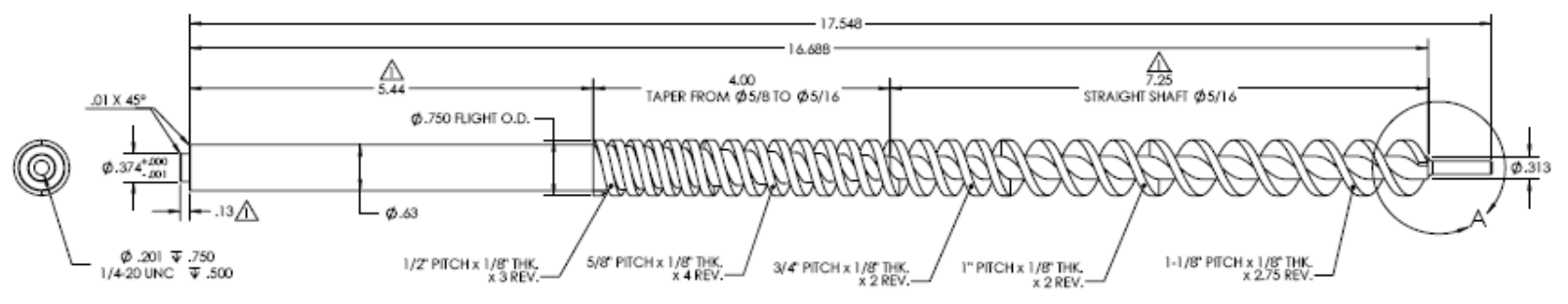

Figure 1-25 - Original Auger Design (Left Hand)

There were two strategies to improve the existing auger. The quick-fix was to modify the existing auger by machining off one flight, which would provide more volume in the receiving port, as shown in Figure 1-26. With this approach, a higher feed rate could be achieved. The other solution was to machine a new auger, which meant a completely new design using the new bulk density which was ultimately pursued. 


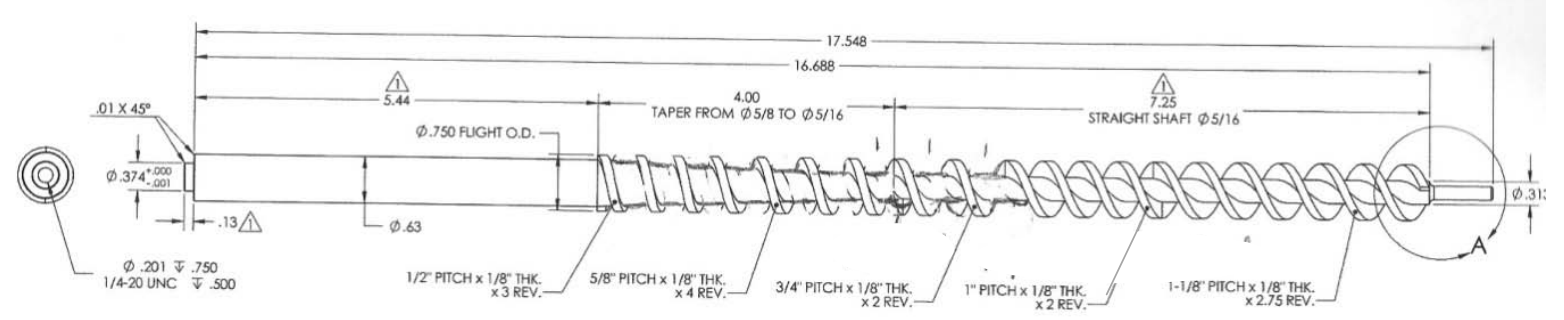

Figure 1-26 - Proposed Modification of Existing Auger

An additional issue came from the flight orientation of the auger. It had a left-hand thread which resulted in the direction of the rotation to deliver coal is counterclockwise when viewed from the discharge end of the auger. This is the same direction to unscrew the auger when driven by the magnetic drive. Inadvertent unscrewing of the auger would occur if more than 70 inch-pounds (in-lb) of torque were applied to the auger accidently. If the auger unscrewed, it would begin to get longer (as it unscrews from the magnetic drive), which would eventually lead to binding in the bore. Two approaches could be pursued to fix this problem: (1) change the auger to a righthand thread so that the coal is delivered to the reactor tube when rotating clock-wise or (2) use a mid-strength thread-locking compound to ensure that the auger would not come loose at less than $70 \mathrm{in}-\mathrm{lb}$ of torque.

With all these concerns, a new auger was designed for the coal feeder. The new auger was machined by Dimension Design with a larger pitch volume to increase the coal feed rate.

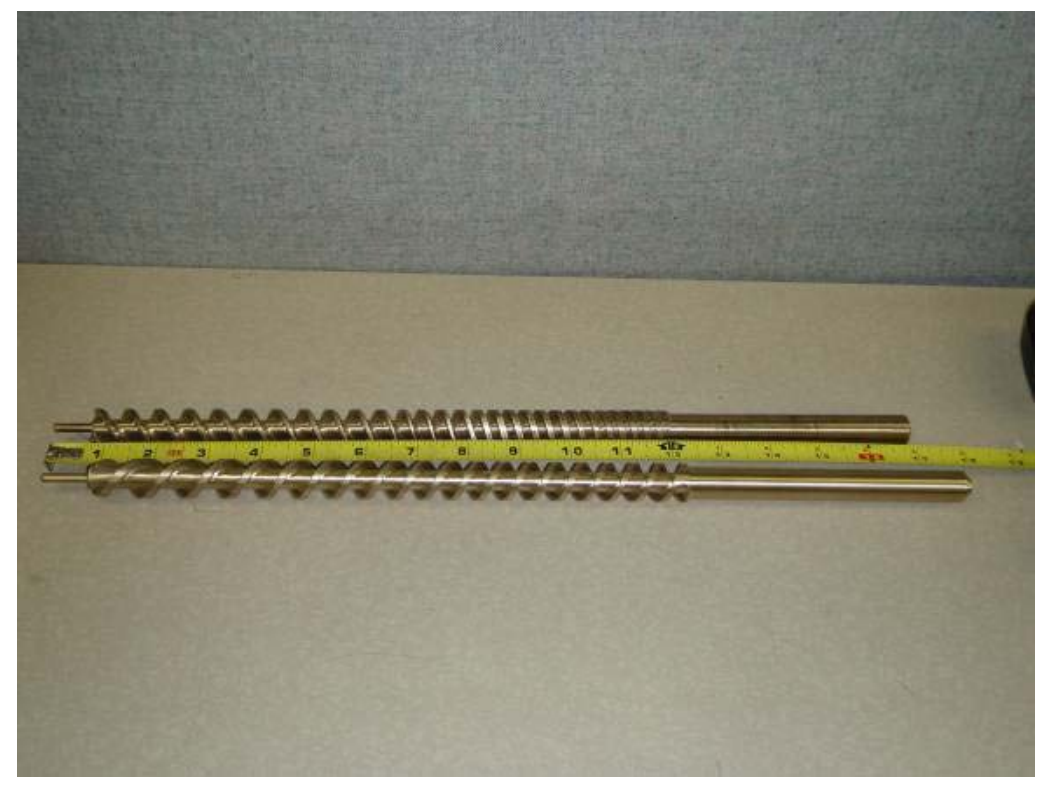

Figure 1-27 - Comparison of New and Old Augers (Front: New Auger; Back: Old Auger) 
As shown in Figure 1-27, the new auger had a double flight design and the center shaft was not tapered. Figure 1-27 shows the new and old augers. At the receiving zone, the shaft had a 0.75-inch OD and 0.375-inch ID. The flight width was 0.125 inch and the pitch distance was 1 inch. Compared with the previous auger, the new auger provided a much higher feed rate and eliminated the other issues.

\subsubsection{CoAl Feeder Caubration WTH New Auger}

Figure 1-28 presents coal feeder calibration under ambient and high-pressure conditions using the new auger. In Figure 1-28, three dark blue points delineate test results from high-pressure testing, which are also shown in Table 1-7. As can be seen from the figure, the points correlate well to the points that were measured at ambient pressure. As expected, the performance of the new auger was not influenced by the actual testing pressure. The coal feed rate was considerably lower than the theoretically calculated value based on the auger pitch volume (3.5 $\mathrm{lb} / \mathrm{hr}$ versus $10.5 \mathrm{lb} / \mathrm{hr}$ at $50 \mathrm{rpm}$, as shown in Figure 1-29), probably because each flight was not likely to be filled over each rotation or the coal sample was not fully delivered over each rotation. The void/dead volume reduced the actual available volume for delivery.

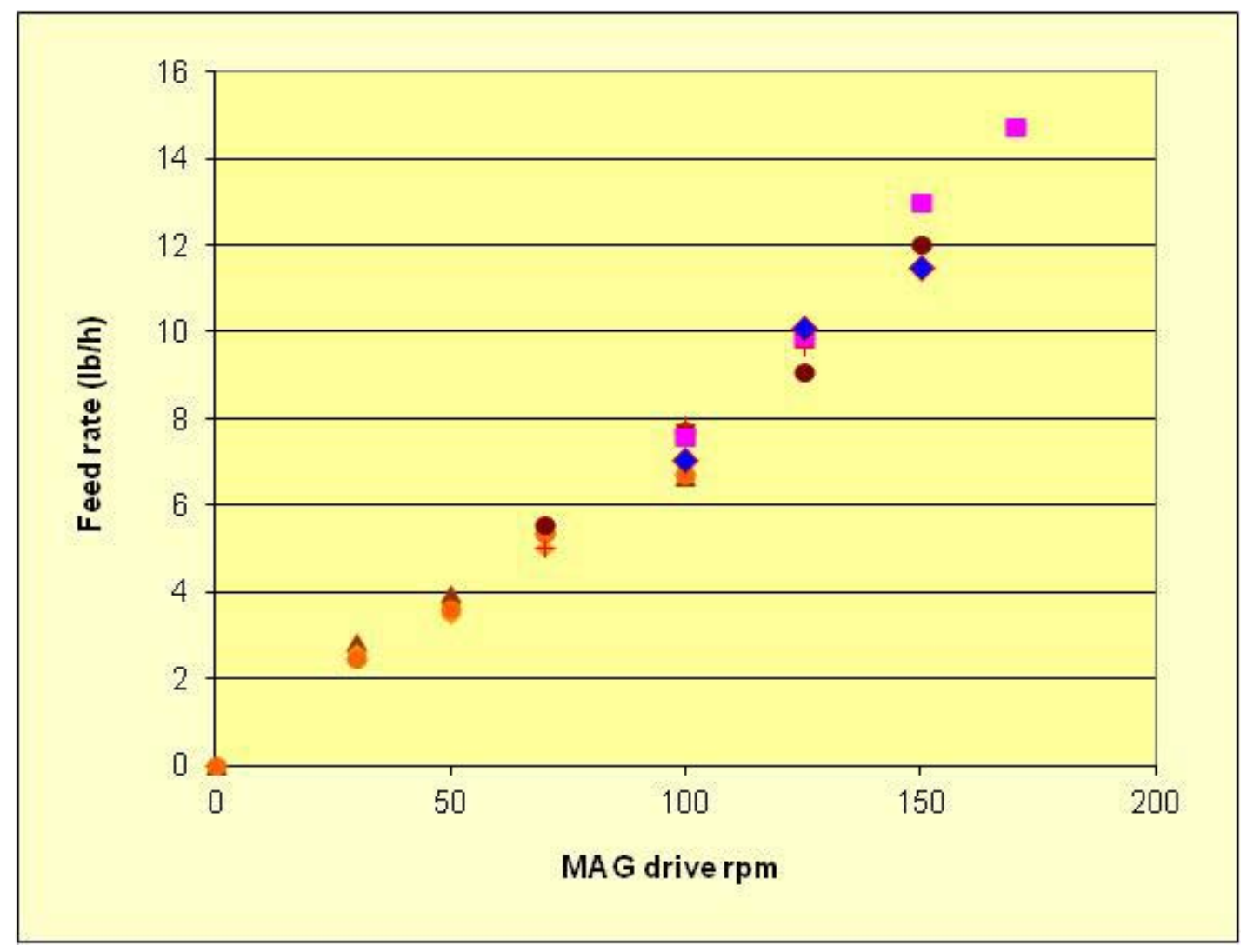

Figure 1-28 - Coal Feeder Calibration Using New Auger 
Table 1-7 - Coal Feeder Calibration at High Pressure with New Auger

\begin{tabular}{|l|c|c|c|}
\hline \multicolumn{1}{|c|}{ Item and Rating } & \multicolumn{3}{|c|}{ Meas urement } \\
\hline Mag drive rpm & 100 & 125 & 150 \\
\hline Operation $(\mathrm{min})$ & 60 & 30 & 30 \\
\hline Feed Coal $(\mathrm{lb})$ & 7.02 & 5.05 & 5.75 \\
\hline Feed Rate $(\mathrm{lb} / \mathrm{h})$ & 7.02 & 10.1 & 11.5 \\
\hline
\end{tabular}

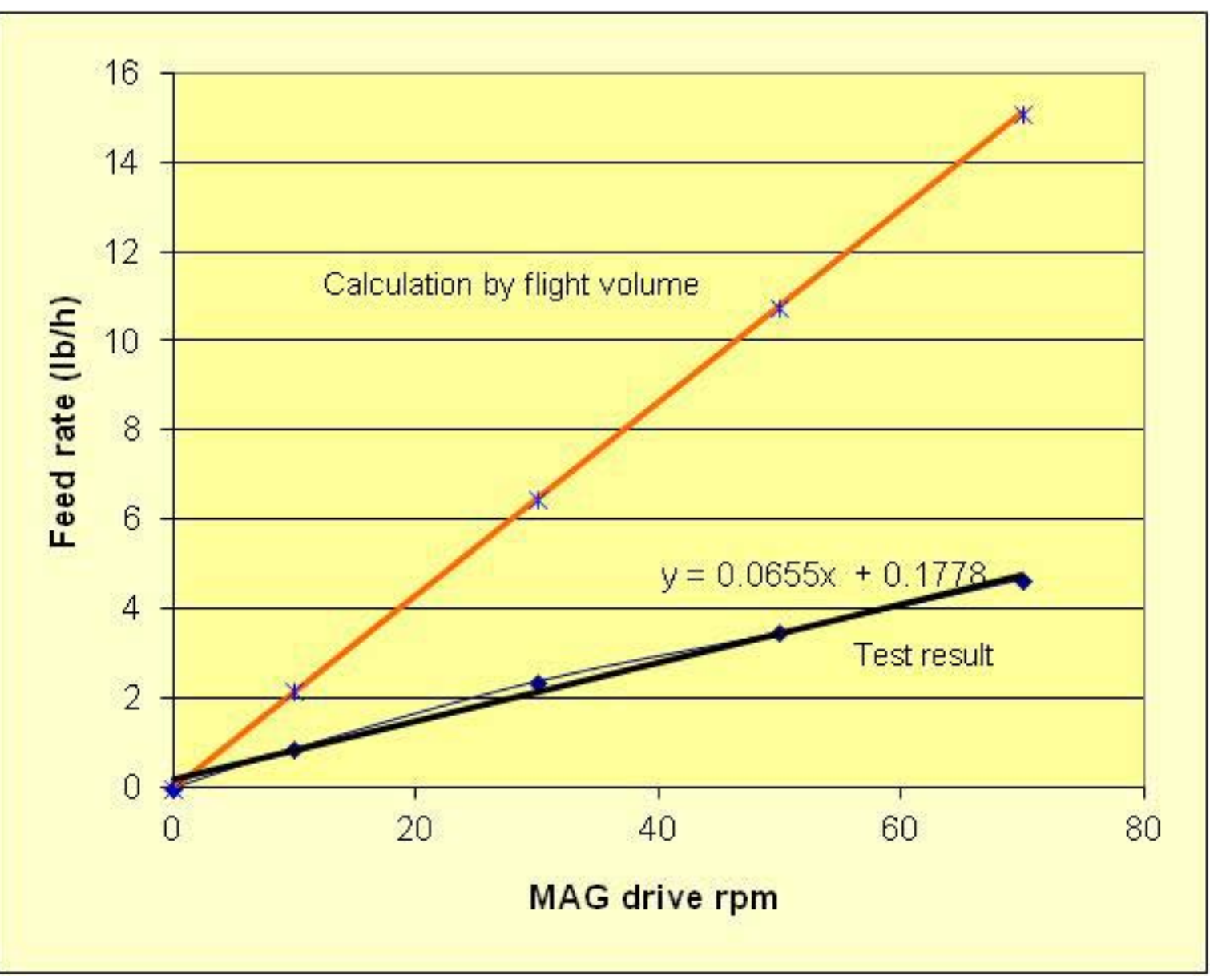

Figure 1-29 - Comparison of Theoretical and Experimental Coal Delivery Rate

Further coal feeder calibration was performed after the coal feeder was assembled with the reactor, allowing for coal feeder testing under operating conditions with carrier gas. As shown in Figure 1-30, the carrier gas flow significantly improved coal feed rate (7 lb/hr versus $3.5 \mathrm{lb} / \mathrm{hr}$ at $50 \mathrm{rpm}$ ). It was observed that the transient small pressure differential between coal hopper and reactor would significantly affect coal feed rate. As stated in the Section 1.10.3, the pressure equilibrium line between coal hopper and reactor had to change from $1 / 4$ inch size to $1 / 2$ inch size, 
filters had to be removed to achieve true dynamic equilibrium between coal hopper and reactor. The reproducibility of the coal feeding was monitored throughout the hydrogasification testing, which was further supported under DOE project DE-FE0001099, "Integrated Energy System with the Beneficial $\mathrm{CO}_{2}$ Use." Please refer to its Technical Final Closeout Report for additional information on this feeder performance.

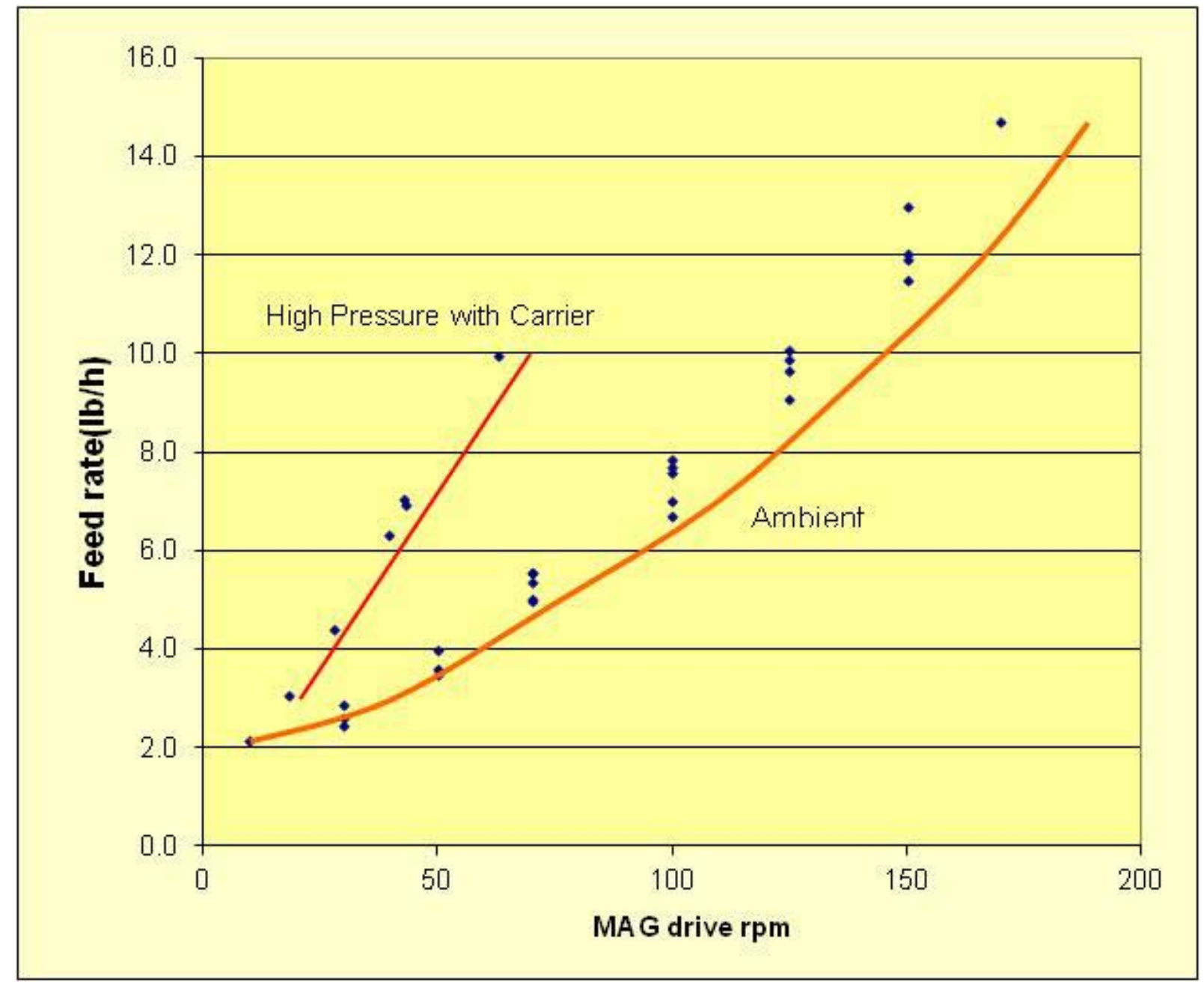

Figure 1-30 - Comparison of Coal Feed Rate under Ambient Conditions and High Pressure with Carrier Gas Conditions 


\subsection{KINEIICS ONE-DIMENSIONAL SIMULATION}

There are a finite number of experimental runs that may be carried out in the hydrogasification reactor. With this in mind, it was deemed important to develop and/or use a model that could evaluate test conditions that are not part of a limited experimental plan. A one-dimensional (1-D) model of the coal hydrogasification reactions, developed by Miura in the early 1990s as part of the Advanced Rapid Coal Hydrogasification (ARCH) project ${ }^{4}$, was used as a basis in this project.

The implementation of this model was extended over several phases. First, it was necessary to understand the proposed reaction schemes and the models for the various reactions. Next, the model was coded with MATrix LABoratory (MATLAB) and the results were compared with experimental data collected during the APS BSRx testing program. Finally, the model was evaluated with a statistical design to ensure that the predicted trends agreed with what was observed in the literature. After the completion of these steps, a form of the ARCH model was used in evaluating results from the BSRx experimental plan. Details of the 1-D modeling effort, which was based on prior hydrogasification work for the sake of efficiency, is presented in the subsections below:

\subsubsection{ARCHKINETICS}

\subsubsection{Coal Components Reactions}

The reaction scheme for the coal gasification process was assumed to be extremely elementary. The authors predicted that the initial reactions that occurred produced a series of six components that gasify from the coal particle: (1) $\mathrm{CO}$, (2) $\mathrm{CO}_{2}$, (3) $\mathrm{H}_{2} \mathrm{O}$, (4) $\mathrm{CH}_{4}$, (5) Benzene, Toluene, and Xylene, (BTX) $\mathrm{C}_{6} \mathrm{H}_{6}, \mathrm{C}_{7} \mathrm{H}_{8}$, and $\mathrm{C}_{8} \mathrm{H}_{10}$, respectively, and (6) oil $\left(\mathrm{C}_{10} \mathrm{H}_{8}\right)$. All of the components were measured with regard to fraction of the component in the coal particle $\left(f_{j}=\right.$ mass of component $j /$ mass of coal) with $f^{*}$ representing the maximum fractional amount of a component that can be gasified from the coal particle. The rate of release of these components was modeled as follows:

\footnotetext{
${ }^{4}$ Y. Asaoka, T. Azuma, H. Gray, F. Noguchi, and H. Maruyama, Development of Coal Hydrogasification Technology 1. Hydrogasification and Simulation in the ARCH Gasifier, in 16th Annual International Pittsburgh Coal Conference, 1999.

${ }^{5}$ Y. Asaoka, T. Azuma, H. Gray, F. Noguchi, and H. Maruyama, Development of Coal Hydrogasification Technology 1. Hydrogasification and Fluid Dynamics Behavior in the ARCH Gasifier, in 15th Annual International Pittsburgh Coal Conference, 1998.
} 


$$
u_{s} \frac{d\left(f_{j}\right)_{p}}{d z}=k_{j}\left\{f_{j}^{*}-\left(f_{j}\right)_{p}\right\}
$$

In Equation (1), the subscript $p$ represents gas products from the coal particle.

\subsection{Rapid Carbon}

The seventh component in the ARCH simulation is referred to as "rapid carbon (RC)". Rapid carbon is an abstract component to understand. The previously referenced studies in Japan combined the solid product from the gasification of coal in an inert atmosphere with hydrogen. Depending on the temperature and partial pressure of hydrogen in the system, $\mathrm{CH}_{4}, \mathrm{H}_{2} \mathrm{O}$, and BTX would begin forming at different rates. In order to model this phenomenon, RC was the name given to the carbon matter of coal that would only react in the presence of hydrogen. Although $\left(f_{R C}\right)_{p}$ was modeled by Equation (1), it was actually a more complex function, because $\mathrm{f}^{{ }_{\mathrm{RC}}}$ was not modeled as a constant value. Rapid carbon became activated as a function of the partial pressure of the eighth component, $\mathrm{H}_{2}$, and temperature of the particle:

$$
\mathrm{f}_{\mathrm{RC}}^{*}=5.004 \mathrm{p}_{\mathrm{H} 2}^{0.337} \exp \left(-5245 / \mathrm{T}_{\mathrm{s}}\right)
$$

The RC function was difficult to model because once RC was activated, it could not become inactivated; f* could not decrease, so a condition had to be set that indicated if the new value was less than the old value, then the old value was used. Essentially this eliminated certain methods that could be used to evaluate the differential equations, such as a stiff method. Finally, there was a maximum value for $\mathrm{f}_{\mathrm{RC}}$ which was taken to be 0.15 .

\subsection{RC Reactions}

The reaction of $\mathrm{RC}$ with $\mathrm{H}_{2}$ proceeded in two parallel pathways:

$$
\begin{aligned}
& \mathrm{CH}_{m} \mathrm{O}_{\mathrm{n}}+(2+\mathrm{n}-\mathrm{m} / 2) \mathrm{H}_{2} \rightarrow \mathrm{CH}_{4}+\mathrm{nH}_{2} \mathrm{O} \\
& \mathrm{CH}_{\mathrm{m}} \mathrm{O}_{\mathrm{n}}+(0.5+\mathrm{n}-\mathrm{m} / 2) \mathrm{H}_{2} \rightarrow(1 / 6) \mathrm{C}_{6} \mathrm{H}_{6}+\mathrm{nH}_{2} \mathrm{O}
\end{aligned}
$$

In the ARCH model, selectivity of the reaction pathways is calculated with a value alpha, with alpha being a function of the partial pressure of $\mathrm{H}_{2}$ :

$$
\alpha=1-\frac{9.26}{\left(\mathrm{p}_{\mathrm{H} 2}+8.33\right)}
$$


Another consideration with the RC reactions was that they take place on/in the coal particle, so the reaction rate parameter was calculated with the solid temperature. Additionally, the only time RC becomes gasified was after a reaction with $\mathrm{H}_{2}$. Therefore, when doing a mass balance on the coal particle it was important to understand that the activated RC from Equation (2) is still part of the particle.

\subsection{Molec ular Formula of RC}

An approach using the ultimate analysis of the coal was used to calculate the coefficients of RC. First it was assumed that the coal consisted of only carbon $(C)$, hydrogen $(H)$, and oxygen $(O)$. Secondly, RC was assumed to have the same molecular formula as char. With that, the molecular formula of char was calculated assuming all of the $f^{\star}$ values of the first six components ( $\mathrm{CO}, \mathrm{CO}_{2}, \mathrm{H}_{2} \mathrm{O}, \mathrm{CH}_{4}, \mathrm{BTX}$, oil) completely gasified out of the coal.

To calculate the coefficients of RC, the coefficients and molecular weight (MW) of coal $\left(\mathrm{CH}_{\text {Mcoal }} \mathrm{O}_{\text {Ncoal }}\right)$ were calculated first:

$$
\begin{aligned}
& \mathrm{M}_{\text {coal }}=\left(\mathrm{WFracH} / \mathrm{MW}_{\mathrm{H}}\right) /\left(\mathrm{WFracC} / \mathrm{MW}_{\mathrm{C}}\right) \\
& \mathrm{N}_{\text {coal }}=\left(\mathrm{WFracO} / \mathrm{MW}_{\mathrm{O}}\right) /\left(\mathrm{WFracC} / \mathrm{MW}_{\mathrm{C}}\right) \\
& \mathrm{MW}_{\text {coal }}=\mathrm{MW}_{\mathrm{C}}+\mathrm{M}_{\text {coal }} * \mathrm{MW}_{\mathrm{H}}+\mathrm{N}_{\text {coal }} * \mathrm{MW}_{\mathrm{O}}
\end{aligned}
$$

Again, the weight fractions (WFrac(component)) are from the ultimate analysis of the coal.

Using the coefficients and $\mathrm{MW}$ of coal, mole balances on $\mathrm{C}, \mathrm{H}$, and $\mathrm{O}$ may be done assuming all of the $\mathrm{f}^{\star}$ components gasified to calculate the coefficients and MW of char, and hence RC:

$$
\begin{aligned}
& A_{\text {Char }}=1-\left(\frac{f_{\mathrm{CO}}^{*}}{M W_{\mathrm{CO}}}+\frac{\mathrm{f}_{\mathrm{CO}_{2}}^{*}}{\mathrm{MW}_{\mathrm{CO}_{2}}}+\frac{\mathrm{f}_{\mathrm{CH}_{4}}^{*}}{\mathrm{MW}_{\mathrm{CH}_{4}}}+6 \frac{\mathrm{f}_{\mathrm{BTX}}^{*}}{\mathrm{MW}_{\mathrm{BTX}}}+10 \frac{\mathrm{f}_{\text {Oil }}^{*}}{\mathrm{MW}_{\text {Oil }}}\right) * \mathrm{MW}_{\text {Coal }} \\
& \mathrm{M}_{\text {Char }}=\left(\mathrm{M}_{\text {Coal }}-\left(2 \frac{\mathrm{f}_{\mathrm{H}_{2} \mathrm{O}}^{*}}{\mathrm{MW}_{\mathrm{H}_{2} \mathrm{O}}}+4 \frac{\mathrm{f}_{\mathrm{CH}_{4}}^{*}}{\mathrm{MW}_{\mathrm{CH}_{4}}}+6 \frac{\mathrm{f}_{\mathrm{BTX}}^{*}}{\mathrm{MW}_{\mathrm{BTX}}}+8 \frac{\mathrm{f}_{\text {Oil }}^{*}}{\mathrm{MW}_{\text {Oil }}}\right) * \mathrm{MW}_{\text {Coal }}\right) / \mathrm{A}_{\text {Char }} \\
& \mathrm{N}_{\text {Char }}=\left(\mathrm{N}_{\text {Coal }}-\left(\frac{\mathrm{f}_{\mathrm{CO}}^{*}}{\mathrm{MW}_{\mathrm{CO}}}+2 \frac{\mathrm{f}_{\mathrm{CO}_{2}}^{*}}{\mathrm{MW}_{\mathrm{CO}_{2}}}+\frac{\mathrm{f}_{\mathrm{H}_{2} \mathrm{O}}}{\mathrm{MW}_{\mathrm{H}_{2} \mathrm{O}}}\right) * \mathrm{MW}_{\text {Coal }}\right) / \mathrm{A}_{\mathrm{Char}}
\end{aligned}
$$




$$
\mathrm{MW}_{\text {Char }}=\mathrm{MW}_{\mathrm{C}}+\mathrm{M}_{\text {Char }} * \mathrm{MW}_{\mathrm{H}}+\mathrm{N}_{\text {Char }} * \mathrm{MW}_{\mathrm{O}}
$$

$A_{\text {char }}$ was calculated to normalize the coefficients for a coefficient of 1 for carbon.

\subsection{Oil Reaction}

Like RC, the gas phase hydrocracking reaction of oil with $\mathrm{H}_{2}$ happened in two parallel reactions:

$\mathrm{C}_{10} \mathrm{H}_{8}+\mathrm{H}_{2} \rightarrow(5 / 3) \mathrm{C}_{6} \mathrm{H}_{6}$

$\mathrm{C}_{10} \mathrm{H}_{8}+16 \mathrm{H}_{2} \rightarrow 10 \mathrm{CH}_{4}$

The selectivity of the reactions to $\mathrm{C}_{6} \mathrm{H}_{6}$ was determined with a parameter $\beta$. The $\beta$ parameter was a function of the partial pressure of $\mathrm{H}_{2}$ :

$$
\beta=1-\frac{4.885}{\mathrm{p}_{\mathrm{H}_{2}}^{0.64}}
$$

\subsection{BIX Reaction}

The reaction of BTX, assumed to be pure benzene for simplification, was a single reaction, with $\mathrm{CH}_{4}$ as the only product:

$\mathrm{C}_{6} \mathrm{H}_{6}+9 \mathrm{H}_{2} \rightarrow 6 \mathrm{CH}_{4}$

\subsection{Water - Gas Shift}

The water-gas shift is an equilibrium reaction involving water and $\mathrm{CO}$ reacting to produce hydrogen and $\mathrm{CO}_{2}$.

$\mathrm{H}_{2} \mathrm{O}+\mathrm{CO} \rightarrow \mathrm{H}_{2}+\mathrm{CO}_{2}$

In the original $\mathrm{ARCH}$ kinetics the equilibrium constant was used to effectively supply the rate of reaction:

$$
\mathrm{Keq}=\frac{\left[\mathrm{CO}_{2}\right]\left[\mathrm{H}_{2}\right]}{[\mathrm{CO}]\left[\mathrm{H}_{2} \mathrm{O}\right]}
$$

An empirical function for the equilibrium constant of the water-gas shift was used:

$\mathrm{Keq}=0.265^{\star} \exp (3958 / \mathrm{T})$ 
To calculate the rate of change in the original ARCH kinetics model, Equation (19) was set equal to Equation (20). Because the reaction was equimolar, the amount of moles that the reaction changed was calculated using the quadratic formula as follows:

$0.265 * \exp (3958 / \mathrm{T})=\frac{\left(\left[\mathrm{CO}_{2}\right]+\mathrm{x}\right)\left(\left[\mathrm{H}_{2}\right]+\mathrm{x}\right)}{([\mathrm{CO}]-\mathrm{x})\left(\left[\mathrm{H}_{2} \mathrm{O}\right]-\mathrm{x}\right)}$

Equation (21) was expanded on $\mathrm{x}$, set equal to zero, and solved for $\mathrm{x}$ :

$x=\frac{-B+\sqrt{B^{2}-4 A C}}{2 A}$

Where

$$
\begin{aligned}
& A=1-\mathrm{Keq} \\
& B=\mathrm{Keq} *\left([\mathrm{CO}]+\left[\mathrm{H}_{2} \mathrm{O}\right]\right)+\left[\mathrm{CO}_{2}\right]+\left[\mathrm{H}_{2}\right] \\
& C=-\mathrm{Keq} *[\mathrm{CO}] *\left[\mathrm{H}_{2} \mathrm{O}\right]+\left[\mathrm{CO}_{2}\right]+\left[\mathrm{H}_{2}\right]
\end{aligned}
$$

This was done after every step of the solver method with the values of the components subsequently either increased or decreased by the value $x$. Additionally, the rate of the water-gas shift was calculated by dividing $\mathrm{x}$ by the model step size:

$\mathrm{R}_{\text {shift }}=\frac{\mathrm{x}}{\mathrm{dz}}$

This rate was then used in the next integrating step.

The major assumption with this calculation was that the water-gas shift was instantaneously going to equilibrium; hence, an equation for thermodynamic equilibrium was used. The only problem with this assumption was how it applied to the energy balance if the value of $x$ ever became too large. An instantaneous assumption could cause $\mathrm{R}_{\text {shift }}$ to go to infinity. This in turn would cause the temperature of the gas to either go to infinity or negative infinity, depending on the simulation step size and the direction of the reaction. Figure 1-31 shows the model completed with the original step size, along with smaller step sizes of $5 \times 10^{-6}$ and $5 \times 10^{-7}$. As may be seen in this figure, the large step size allows the simulation to get past instability at a $z$ distance longer than 40 inches. Similarly, it gives impractical solutions, as the total amount of predicted carbon to react was larger than the total amount of carbon that was allowed to react. 
(The highest carbon conversion from this simulation could be calculated from $f^{\star}$ values and was equal to approximately $54.75 \%$.) Note that in Figure 1-31 as the step size became smaller the gas temperature went to zero more quickly.

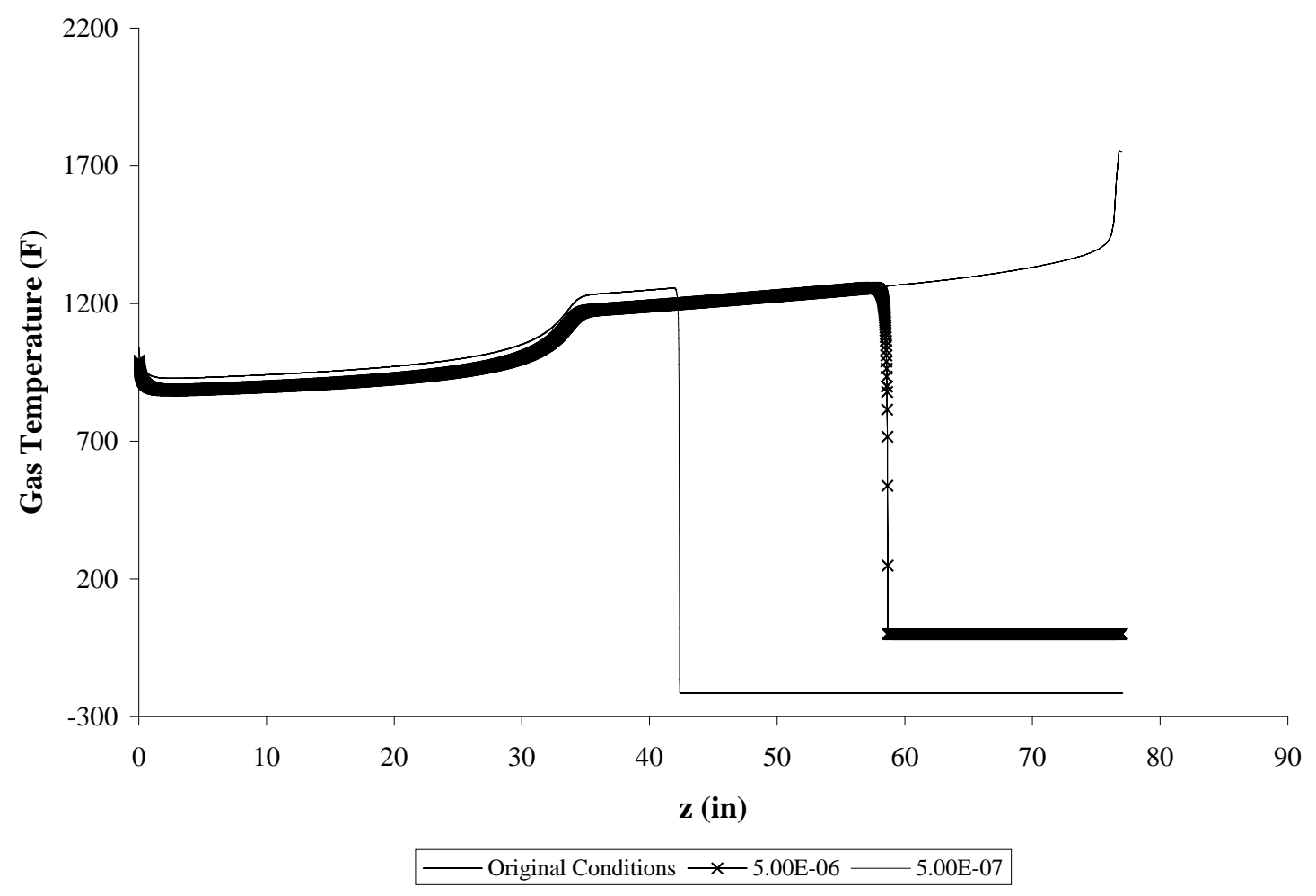

Figure 1-31 - Step Size Effect on Model Gas Temperature

To overcome problems that were encountered by the instantaneous equilibrium assumption, $R_{\text {shift }}$ was recast in terms of kinetic rates. Rate laws that were used along with the equilibrium constant; from Bustamante, et. al. ${ }^{5}$

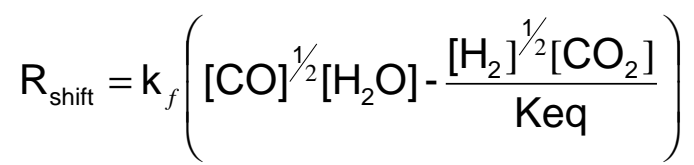

\subsection{Energy Balance}

The energy balance is a two-phase balance between the solid coal and gas phase. The solid and gas phase balances are as follows:

${ }^{5}$ F. Bustamante, R.M. Enick et al., "High-temperature kinetics of homogeneous reverse water-gas shift reaction," AIChE Journal 50(5): 1028-1041 (2004). 


$$
\begin{aligned}
& {\left[\left(\mathrm{f}_{\text {Coal }}\right)_{p}+\left(\mathrm{f}_{\mathrm{Char}}\right)_{p}\right] c_{p s} \frac{d T_{s}}{d z}=h_{c} a_{p} S\left(T_{g}-T_{s}\right) / G_{s 0}+\Sigma\left\{-\Delta h_{j}\left(T_{s}\right) r_{p, j}\right\} / u_{s}+} \\
& \frac{\left\{\left(-r_{R C 1, m}\right)\left(-\Delta \mathrm{H}_{74}\right)+\left(-r_{R C 2, m}\right)\left(-\Delta \mathrm{H}_{75}\right)\right\}}{u_{s} M_{R C}} \\
& \Sigma\left(f_{j} C_{p j}\right) \frac{d T_{g}}{d z}=h_{c} a_{p} S\left(T_{s}-T_{g}\right) / G_{s 0}+\Sigma \bar{C}_{p j}\left(T_{s}-T_{g}\right) \frac{d\left(f_{j}\right)_{p}}{d z}+ \\
& \frac{S}{G_{s 0}}\left[r_{\text {shirt }}\left(-\Delta \mathrm{H}_{\text {shirt }}\right)+r_{1}\left(-\Delta \mathrm{H}_{1}\right)+r_{2}\left(-\Delta \mathrm{H}_{2}\right)+r_{3}\left(-H_{3}\right)\right]
\end{aligned}
$$

The first term on the right side of both equations represents convectional heat transfer. For the solid heat transfer [Equation (28)], the second term represents the energy consumed to generate the variety of gasified products from the coal particle. The final term of this equation represents the energy from the reaction of activated $\mathrm{RC}$ and $\mathrm{H}_{2}$. For the gas-phase energy balance [Equation (29)], the second term represents the product gas from the coal-particle heat transfer with the bulk gas and the final term represents energy to and from the gas-phase reactions.

In order to save computational expense, when the differences between the solid and gas temperatures became small, the convection term was dropped along with the energy balance between the hydrogasification product from coal and the bulk gas. At this point, both heat balances are combined into a single energy balance.

\subsection{Solver Method}

The Runge-Kutta-Gill (RKG) predictor/corrector method was used to solve the model differential equations. The equations for the RKG method follow:

$y_{n+1}=y_{n}+1 / 6\left(k_{1}+k_{4}\right)+1 / 3\left(b k_{2}+d k_{3}\right)$

where

$$
\begin{aligned}
& \mathrm{k}_{1}=h \mathbf{f}\left(\mathrm{z}_{\mathrm{n}}, \mathrm{y}_{\mathrm{n}}\right) \\
& \mathrm{k}_{2}=h \mathrm{f}\left(\mathrm{z}_{\mathrm{n}}+h / 2, \mathrm{y}_{\mathrm{n}}+1 / 2 \mathrm{k}_{1}\right) \\
& \mathrm{k}_{3}=h \mathrm{f}\left(\mathrm{z}_{\mathrm{n}}+h / 2, \mathrm{y}_{\mathrm{n}}+a \mathrm{k}_{1}+b \mathrm{k}_{2}\right) \\
& \mathrm{k}_{4}=h \mathrm{f}\left(\mathrm{z}_{\mathrm{n}+\mathrm{h}}, \mathrm{y}_{\mathrm{n}}=c \mathrm{k}_{2}+d \mathrm{k}_{3}\right)
\end{aligned}
$$


with

$$
\begin{aligned}
& a=\frac{\sqrt{2}-1}{2} \\
& b=\frac{2-\sqrt{2}}{2} \\
& c=-\frac{\sqrt{2}}{2} \\
& d=1+\frac{\sqrt{2}}{2}
\end{aligned}
$$

The equations are solved sequentially with step size $h$. As was concluded previously, the choice of step size is important for the stability of the solution. If the chosen step size is too large, then the solution becomes unstable. Additionally, if the chosen step size is too small, the computational expense becomes higher and it takes longer for a solution. For this simulation, the initial step size was $h=5 \times 10^{-7}$ and switched to $h=5 \times 10^{-6}$ at a $z$ value of 0.01 .

\subsubsection{RESULTS AND DISCUSSION}

To verify that the model was giving reasonable estimates and would be suitable, results from it were compared with experimental data from a 1992 Osaka Gas report. Test setpoints were temperature at $1650^{\circ} \mathrm{F}$, residence time at $10.7 \mathrm{~s}$, and hydrogen-coal ratio at 0.23 . The model output results are compared with the Oaska experimental data in Table 1-8.

Table 1-8 - Model Component Carbon Conversions

Compared with Experimental Data

\begin{tabular}{|l|c|c|c|c|c|c|}
\hline \multicolumn{1}{|c|}{ Items Compared } & \multicolumn{5}{c|}{ Chemicals } & Total \\
& $\mathrm{CH}_{4}$ & $\mathrm{CO}$ & $\mathrm{CO}_{2}$ & $\mathrm{BTX}$ & $\mathrm{Oil}$ & \\
\hline Model & 28.6 & 3.2 & 1.5 & 11.5 & 10 & 54.8 \\
\hline Experimental & 30.5 & 7.1 & 0.6 & 10.6 & 2.1 & 51.6 \\
\hline
\end{tabular}

Comparing results shows that the model does a reasonable job of predicting the carbon conversion to $\mathrm{CH}_{4}, \mathrm{BTX}$, and total conversion. However, the model is off with the distribution of conversions to $\mathrm{CO}$ and oil. This error could be attributed to an excess amount of oxygen in the experimental system. The combined carbon conversion to $\mathrm{CO}$ and $\mathrm{CO}_{2}$ in the model is limited 
at approximately $4.7 \%$ as $f_{\mathrm{CO}}^{*}$ and $f_{\mathrm{CO} 2}^{*}$ determine this value. As may be seen from Table 1-8, the experimental carbon conversion from the Oaska testing to $\mathrm{CO}$ and $\mathrm{CO}_{2}$ is in excess of 7.1\%. This suggests that if there was excess oxygen in the reactor, then this could react with oil to reduce the carbon conversion of oil and increase that of $\mathrm{CO}$. As this experimental data was taken from an external source, there is no way to confirm this assumption. In spite of this, the model was considered to be reasonable and was used for further analysis.

\subsubsection{Statistical Evaluation of the Model}

In order to evaluate the model over the range of variables for the APS test plan, the model was exercised on a $2 \times 3$ full factorial design with a center point. Three factors were varied, which were: (1) temperature, (2) residence time, and (3) hydrogen-coal ratio. The design with respective factor levels is shown in Figure 1-32.

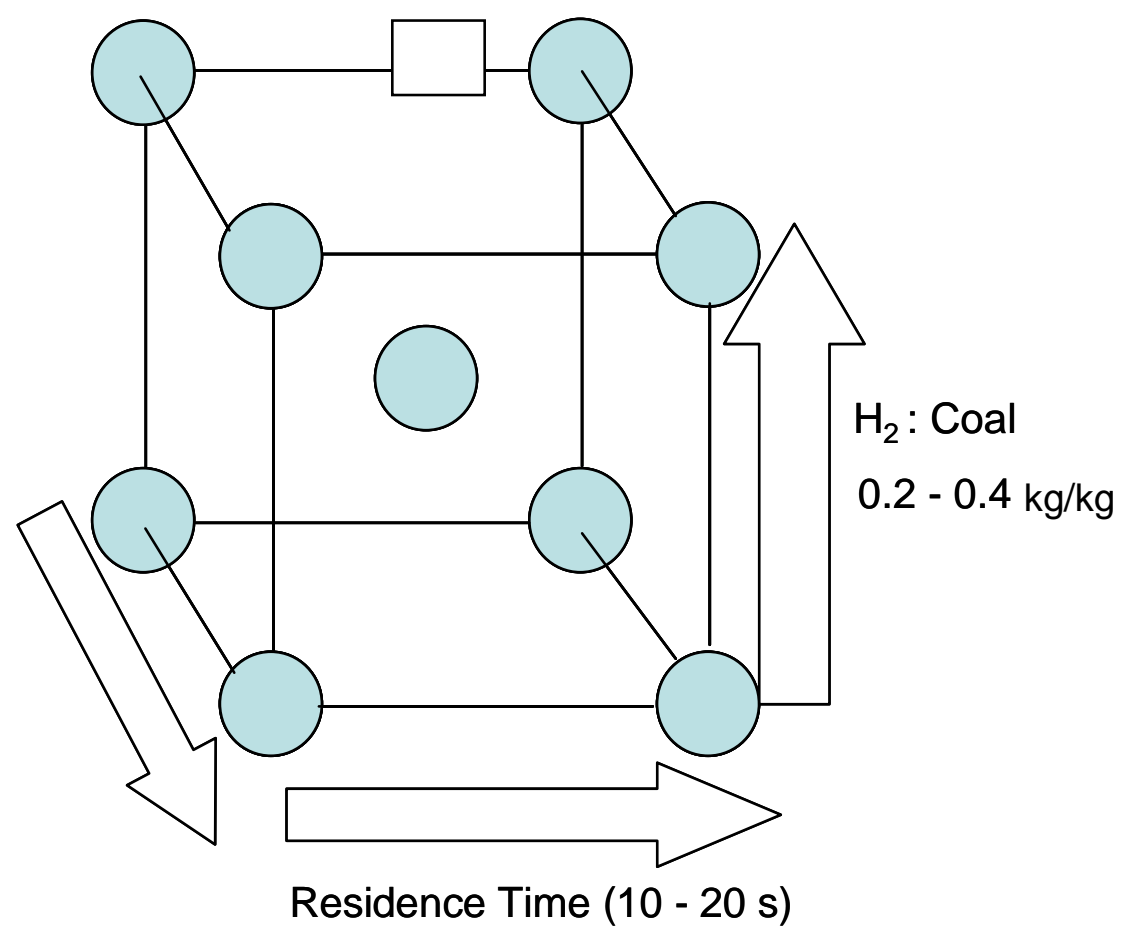

Figure 1-32 - Statistical Design for Evaluating the Model

Figure 1-33 and Figure 1-34 are representative output plots from the model at an assumed operating pressure of 1000 psig. The conditions modeled were a 10 s residence time, a 0.2 hydrogen-coal ratio, and two temperatures: $1750^{\circ} \mathrm{F}$ (Figure 1-33) and $1500^{\circ} \mathrm{F}$ (Figure 1-34). Both plots show the concentration of product gas kilograms of product gas per kilogram of coal (kg/kg-coal) as well as gas temperature versus time. The gas composition plots (Figures 1-36 
and 1-37) do not include any added carrier (i.e. hydrogen) and/or diluents gases. The focus was on predicting the incremental products that would be produced as a result of Pyrolysis and Hydropyrolysis reactions. For simplification, the results also did not consider any sulfur-related reactions (e.g. $\mathrm{H}_{2}+\mathrm{S}=\mathrm{H}_{2} \mathrm{~S}$ ). Temperature was found to have a strong effect on the results; thus, two temperature plots are presented.

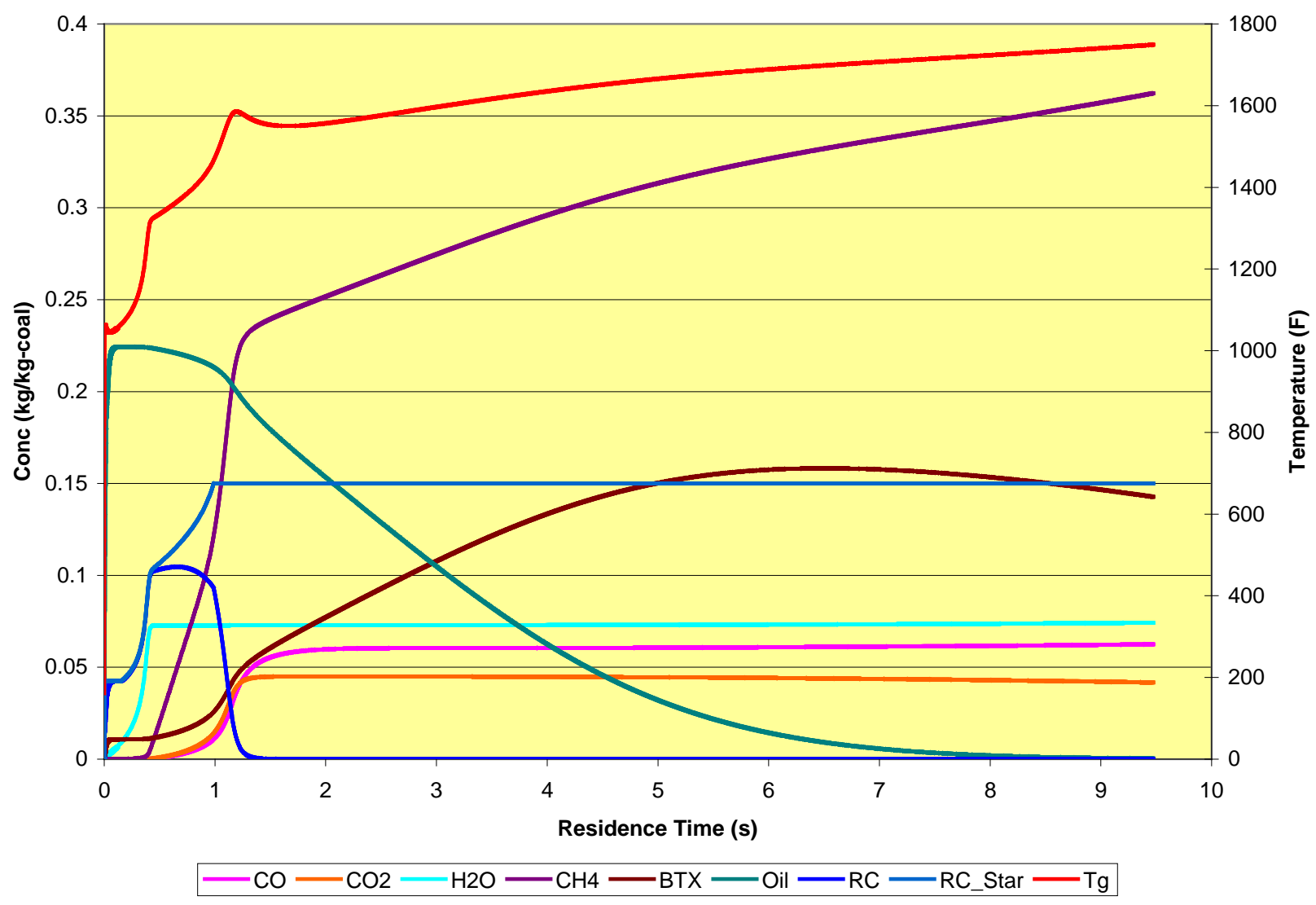

Figure 1-33 - Model Output at $\mathrm{T}=1750^{\circ} \mathrm{F}, \mathrm{RT}=10 \mathrm{~s}$, and $\mathrm{H}_{2}:$ Coal $=0.2$

The strong effect from temperature is evident with regard to RC activation and hydrocarbon cracking. First, RC is formed and consumed quickly at $1750^{\circ} \mathrm{F}$, which in turns leads to fast formation of methane. Contrast to this is Figure 1-34 at $1500^{\circ} \mathrm{F}$; which illustrates hampered RC activation and consumption. Methane formation in this instance is delayed and the maximum yield is smaller than that observed in Figure 1-33. The other major difference between the two simulated cases is the rate of hydrocarbon cracking. Hydrocarbon cracking is considered to be oil breaking down into BTX and methane, and BTX breaking down to methane. It is clear when comparing the two figures that oil is rapidly gasified out of the coal particle in the high-temperature case and subsequently consumed, whereas in the low-temperature case it is 
not. Additionally, in the high-temperature case the model predicts that the amount of oil generated in the system reaches an apex, whereas in the low-temperature case it does not.

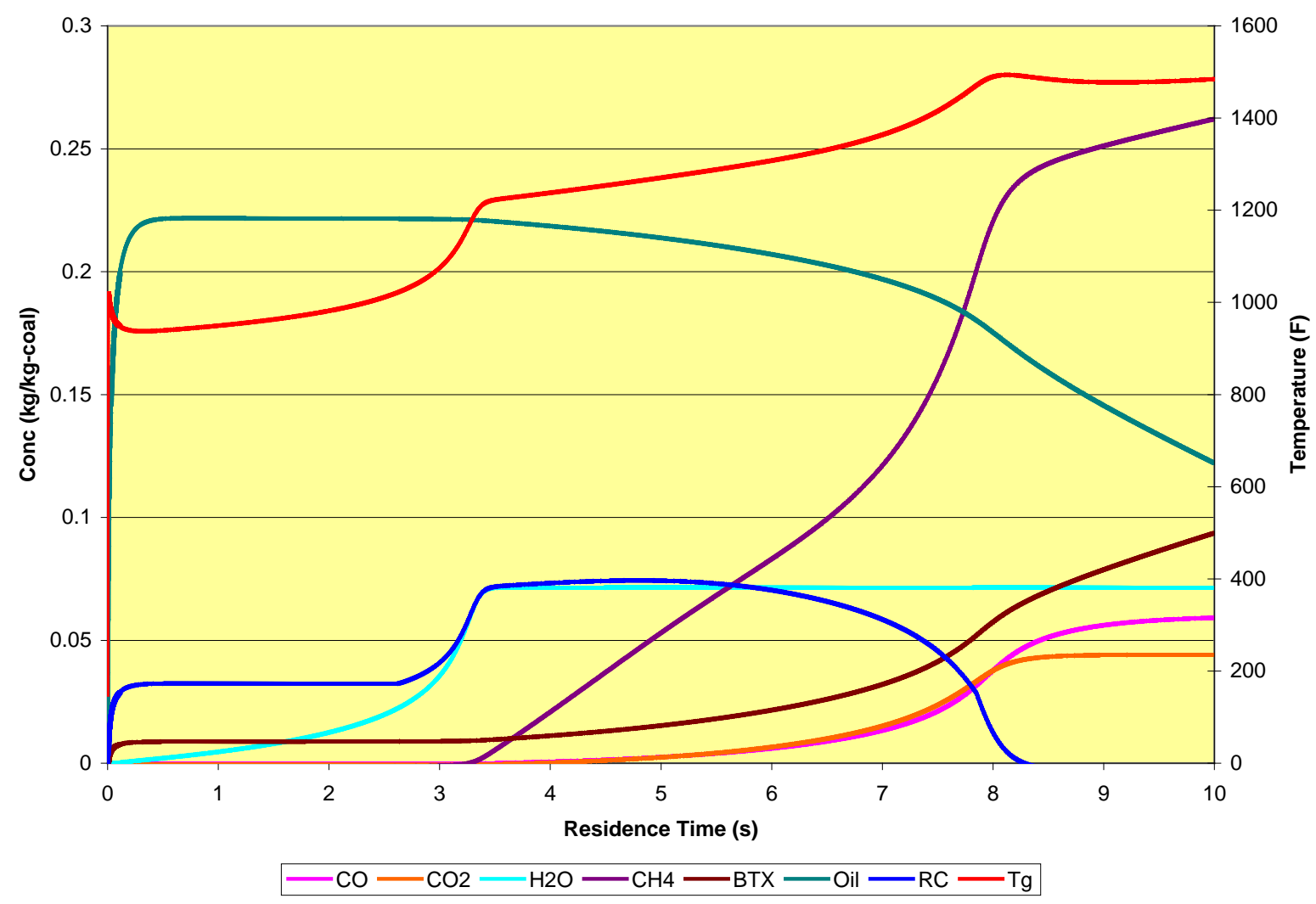

Figure 1-34 - Model Output at $\mathrm{T}=1500^{\circ} \mathrm{F}, \mathrm{RT}=10 \mathrm{~s}$, and $\mathrm{H}_{2}:$ Coal $=0.2$

The output from Figure 1-33 and Figure 1-34 agree with what is expected experimentally; that a higher temperature will generate a product gas stream that is richer in methane. Further quantifying this, the component carbon conversions are shown in Table 1-9. The major difference between the two cases is the increase of carbon conversion to methane and the decrease of carbon conversion to oil by increasing the temperature. This trend is also observed by increasing residence time and hydrogen-coal ratio. An interesting observation is that all of the modeled cases predicted approximately the same overall carbon conversion. . 
Table 1-9 - Model Component Carbon Conversions

After 10 Seconds Residence Time

\begin{tabular}{|c|c|c|}
\hline Component & $1750{ }^{\circ} \mathrm{F}$ & $1500{ }^{\circ} \mathrm{F}$ \\
\hline $\mathrm{CO}$ & $3.32 \%$ & $3.20 \%$ \\
\hline $\mathrm{CO}_{2}$ & $1.41 \%$ & $1.52 \%$ \\
\hline $\mathrm{CH}_{4}$ & $33.7 \%$ & $25.3 \%$ \\
\hline $\mathrm{BTX}$ & $16.3 \%$ & $12.3 \%$ \\
\hline Oil & $0.04 \%$ & $12.4 \%$ \\
\hline Total & $54.8 \%$ & $54.7 \%$ \\
\hline
\end{tabular}

The total carbon conversion did not significantly change between modeled cases and carbon conversion to methane was used as the response in the statistical analysis. Initial results show that all of the factors were significant, but none of the higher-order terms were significant. A relevant example of a response surface is shown below in Figure 1-35. In this instance, the hydrogen-coal ratio was held constant at 0.3 .

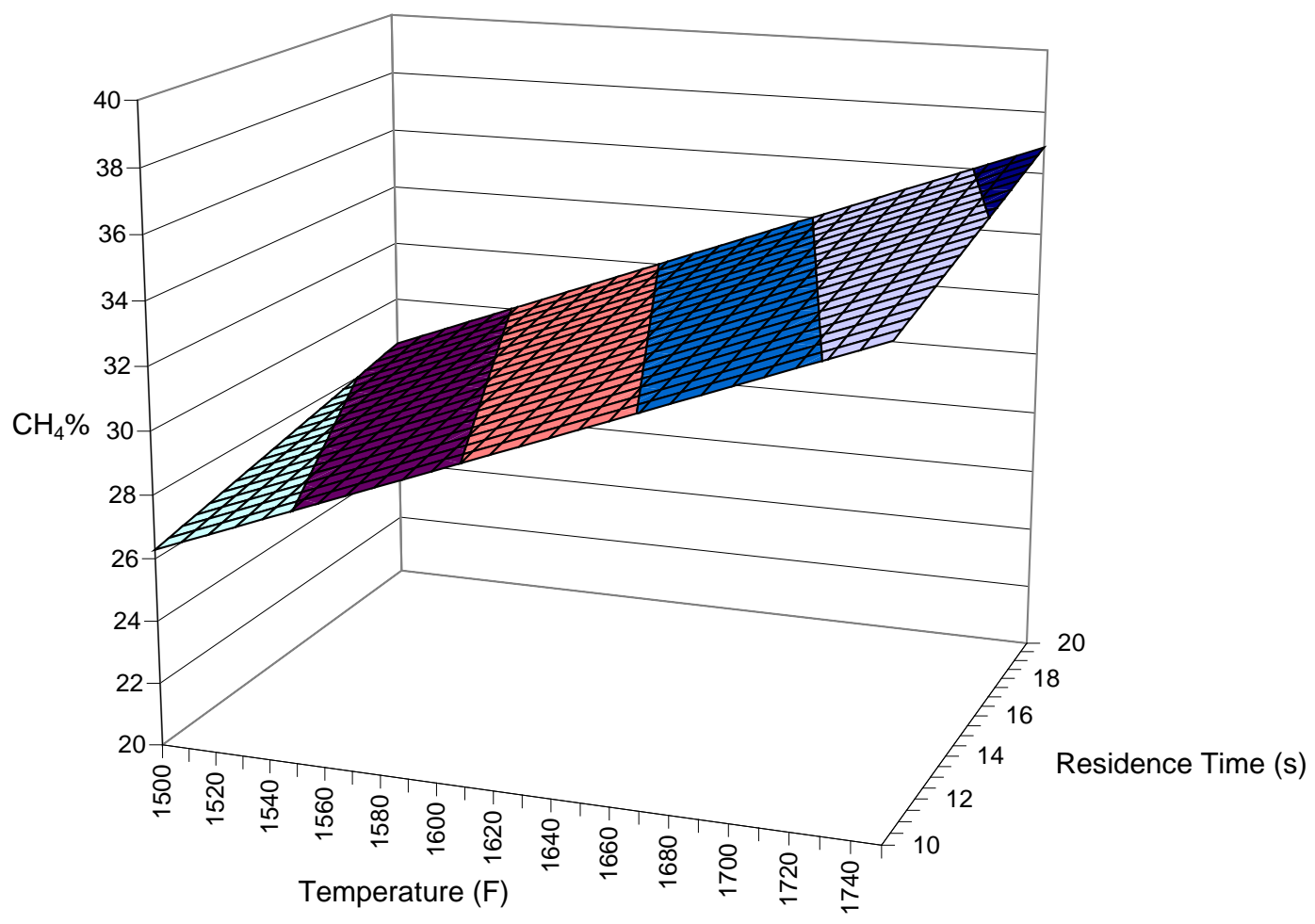

Figure 1-35 - Surface Response of Conversion to Methane

Based on reaction conditions the response surface predicts a carbon conversion to methane of approximately $37 \%$, with the highest conversion occurring at the highest temperature and 
longest residence time that were studied. Increasing the hydrogen-coal ratio also increases the conversion, but not as significantly as the other two factors. These were the trends that were expected, so the model was adequate in that regard and was thus considered to be viable tool to use in further analysis.

Further model analysis on the actual experimental data was supported by DOE project, DE-FE0001099, "IES with Beneficial $\mathrm{CO}_{2}$ Use." Please refer to its Technical Final Closeout Report for additional research on using this model to simulate real testing conditions.

\subsection{EXPERIMENTAL DATA ANALYSSS}

\subsubsection{SYSTEM COMMISSIONING}

The hydrogasifier was shipped to APS in several components. Reactor body, preheater, and top hat were shipped inside the reactor frame from Gaspar. Charpots, coal feeder assembly, condensers, liquid collectors and the reactor heater control panel were shipped separately. The full assemble of the bench-scale reactor hydrogasification system was completed at APS in March 2009. The process P\&ID, plumbing and electrical wiring were completed at the APS jobsite. The assembled bench-scale hydrogasification system is shown in Figure 1-43.

At the completion of assembly of the bench-scale reactor, prerequisite, pre-commissioning, and commissioning test procedures were executed. The prerequisite testing was performed to verify the process construction based on the P\&ID. This exercise cleared inconsistencies between the paper \& field construction, which included missing tags, inaccurate tags, and item specifications. The pre-commissioning procedure covered the functional testing of the P\&ID. This process was officially started on March 28, 2009, which was the first day that system had nitrogen flow. During pre-commissioning, all solenoid valves (SVs), PCVs, and flow control valves (FCVs) were tested. Due to a separate coal feeder calibration, part of the pre-commissioning was performed with an interim closed-reactor system - using blind-flanged upper and lower Copeland isolation valves.

Bench-scale reactor commissioning was officially started on May $6^{\text {th }}, 2009$ and included the following activities:

- $\quad$ Final adjustment of all FCVs and PCVs.

- Validation of system control during pressurization to ensure that pressure of the inner tube and annulus space increased at the same rate. 
- Validation of bench-scale reactor system pressure integrity at 1000 psig.

- Validation of the bench-scale reactor control system by diligent calibration of three main flow meters: (1) H2-FM-1 - hydrogen inlet flow, (2) H2-FM-2 - hydrogen carrier gas flow, and (3) R-MFC - N2 balancing system flow.

- Validation of the function of N2-RO-1 - restriction of nitrogen emergency purge to ensure there would not be a large pressure effect on system balancing due to the purge.

- Validation of three emergency shutdown sequences on the balancing system to identify the smallest system disturbance.

- Validation of functions for emergency local shutdown, full shutdown, and manual button shutdown.

- Validation of the following items: the use of nitrogen tube trailer (for system purge and pressure buildup); the use of 12-pack nitrogen for instrument nitrogen supply; use of 12-pack nitrogen for emergency purge; and the use of hydrogen.

- Validation of the performance of the heaters and calibration for auto-ramping under nitrogen and hydrogen.

- Establishment of temperature profiles along preheater and reactor.

- Adjustment of an existing misting system and addition of new gas purging line to control the hot spot based upon operational observations on the external reactor shell.

- Operational incorporation of the existing APS Hydrogen Park as the hydrogen supply.

- Calibration of GC and MS with standard gas.

- Testing and validation of bench-scale reactor auto-ramping shutdown and manual shutdown.

- $\quad$ Time recording of all major exercises.

The commissioning protocol was completed in approximately 5 weeks. The following significant conclusions were reached:

- At 1000 psig the system leakage rate was about $0.5 \mathrm{psig} / \mathrm{min}$ on average.

- Preheaters achieved hydrogen exit temperature of $1550^{\circ} \mathrm{F}$.

- A large heat loss was observed in hydrogen injection tubing in the crossover section, which led to low hydrogen-injection temperatures in the bench-scale reactor.

- Hot spots were observed on the external reactor shell. The single-change thermal paint 
changes colors at $464^{\circ} \mathrm{F},\left(240^{\circ} \mathrm{C}\right)$. Additionally, the observed annulus space temperature was higher than anticipated. The outer wall hot spots were speculated to be due to natural nitrogen convection in the annulus space or due to the metal-to-metal conduction through the reactor heater spider support.

- Reactor heaters, especially heaters 1 through 3, had only enough power to maintain the temperature, but not enough additional capacity to increase the hydrogen from the injectors. It was concluded that natural convection of the nitrogen in the annulus was conveying heat to the outer shell and consuming more energy than was calculated for the heater design. This situation prevented the bench scale reactor (BSRx) from achieving the target operating temperature.

- Copeland isolation valves had severe leakage.

\subsubsection{PREUMINARY HYDROGASIFCATION TESTS}

The very first hydrogasification test was completed on June 9, 2009. The coal that was utilized was a Navajo Mine sub-bituminous coal, ground to a nominal particle diameter of 100 micrometers. The Ultimate and Proximate analyses for the fresh coal, as introduced to the coal feeder, are show in Table 1-10 below:

Table 1-10 - Proximate and Ultimate Analys is of Coal and Char

\begin{tabular}{|l|c|}
\hline \multicolumn{1}{|c|}{ Attribute } & Coal \\
\hline \% Moisture, Total & 8.00 \\
\hline \% Ash & 27.90 \\
\hline \% Volatile Matter & 30.17 \\
\hline \% Fixed Carbon & 33.93 \\
\hline \multicolumn{1}{|c|}{ Total } & 100.00 \\
\hline Gross Calorific Value (Btu/lb) & 8,812 \\
\hline \% Sulfur & 0.70 \\
\hline \% Carbon & 49.29 \\
\hline \% Hydrogen & 3.74 \\
\hline \% Nitrogen & 1.07 \\
\hline \% Oxygen (Calculated) & 9.30 \\
\hline
\end{tabular}

Due to the large heat loss through the hydrogen crossover line (which connects the hydrogen preheater to the hydrogasifier), the highest hydrogen injection temperature only reached $650^{\circ} \mathrm{F}$. These temperatures were too low for rapid coal heat up and for hydrogasification to take place. Only 18\% carbon conversion was obtained. As detailed in Section 1.10.2, preheater zone 5 was redesigned and modified to increase hydrogen injection temperature, and it was able to 
reach $1350^{\circ} \mathrm{F}$ after preheater zone 5 modification. The second preliminary hydrogasification test revealed and confirmed the problem of unsteady coal feeding, which is detailed in Section 1.10.3. This was improved by increasing the pressure equilibrium line size between coal feeder and reactor and removing filters in between. In addition, both tests observed large heat loss from reactor inner tube to annulus space and therefore to reactor outer shell. This finally led to the reactor heater burning down during the third hydrogasification trial on August 6, 2009, while attempting to achieve high-reaction temperature. This caused about a three-month shutdown. As detailed in Section 1.10.4, the reactor was de-telescoped and electrical heaters in the annulus space were all redesigned and replaced.

\subsubsection{HydrogasifCATION TESTINg ON Novgmber 17, 2009 (TEST 1 "After REPAIRS")}

The fourth hydrogasification test was executed on Tuesday, November 17, 2009 to test the effectiveness of the new reactor configuration. The hydrogen temperature at the inlet of the reactor was approximately $1350^{\circ} \mathrm{F}$ and the average reactor temperature was $1600^{\circ} \mathrm{F}$. The reactor pressure was held at 1000 psig. A high temperature of $1750^{\circ} \mathrm{F}$ was observed in the reactor, which indicated that the hydrogasification reactions were exothermic. Approximately 17 pounds of coal were fed and 10 pounds of char recovered after 1 hour and 40 minutes of testing.

Initial results from a GC and $\mathrm{MS}$ indicated that $\mathrm{CH}_{4}$ was produced during the testing. Figure 1-36 shows a printout from the GC. Four components were detected by the GC: Hydrogen $\left(\mathrm{H}_{2}\right)$, $\mathrm{CH}_{4}, \mathrm{CO}$, and $\mathrm{N}_{2}$. The first three components were directly associated with hydrogasification as a reactant and products; the fourth component, $\mathrm{N}_{2}$, had a significantly high peak, indicating it was not $\mathrm{N}_{2}$ from coal, but rather is likely the result of a leak across the bellows that separates the reactor vessel from the $\mathrm{N}_{2}$ purged pressure shell. 


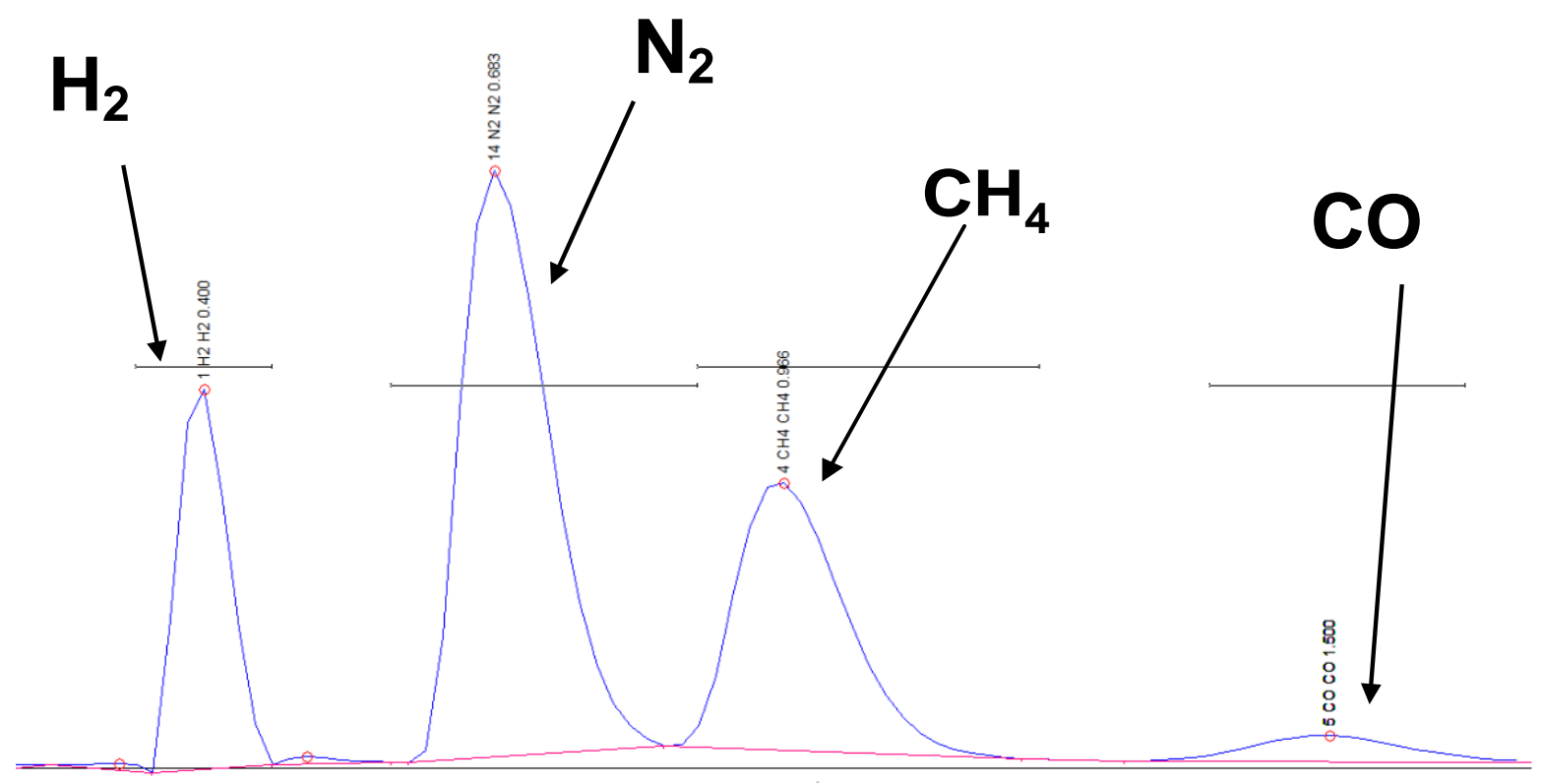

Figure 1-36 - GC Output from November 17, 2009, Hydrogasification (Test 1 After "Repairs")

The MS results also indicated that methane was being produced. As may be seen in Figure 1-37, at the onset of the reaction the methane signal from the MS increased. 


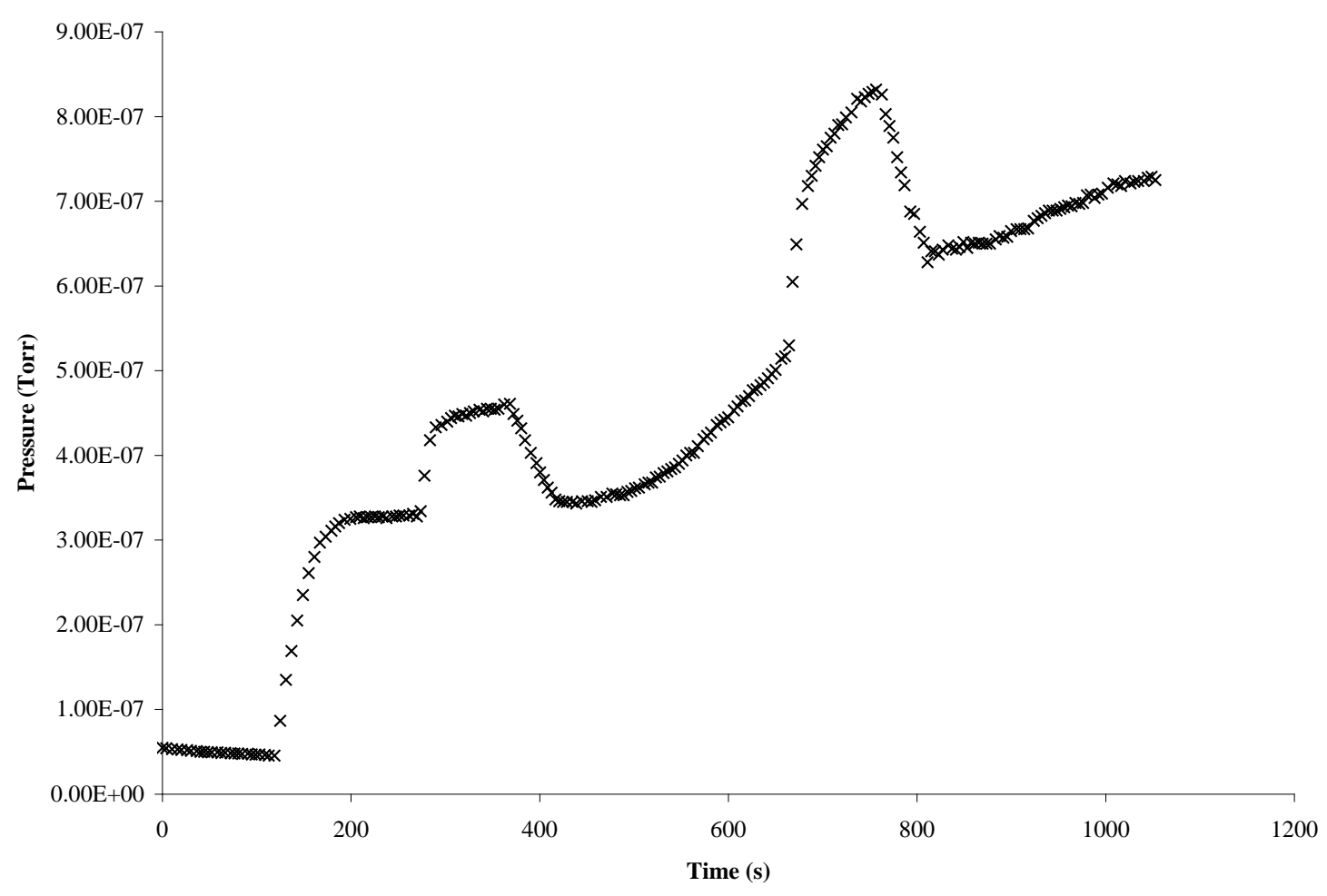

Figure 1-37 - Methane Mass Spectrometer Signal from the November 17, 2009, Hydrogasification (Test 1 After "Repairs")

Initial results indicated that this was the most successful test. An overall carbon conversion of $49 \%$ was estimated from ultimate analysis of the coal and char. Data from the GC suggested that the amount of methane produced was higher than previous tests. Additionally, the methane signal from the MS was stronger than previously observed.

\subsubsection{HYDROgasifCATION TESTING ON DeCember 1, 2009 (TEST 2 - AfieR “RE- PAIRS")}

After the first successful hydrogasification test was completed with the new reactor configuration, aspects of the experimental plan started to be executed. On December 1, 2009 a second hydrogasification run was completed. The targeted reactor conditions were a pressure of 1000 psig, a temperature of $1500^{\circ} \mathrm{F}$, hydrogen-to-coal ratio of 0.3 , and an approximate residence time of 12.8s. A temperature profile from the run (Figure 1-38) shows the temperature profile along the length of the reactor. 


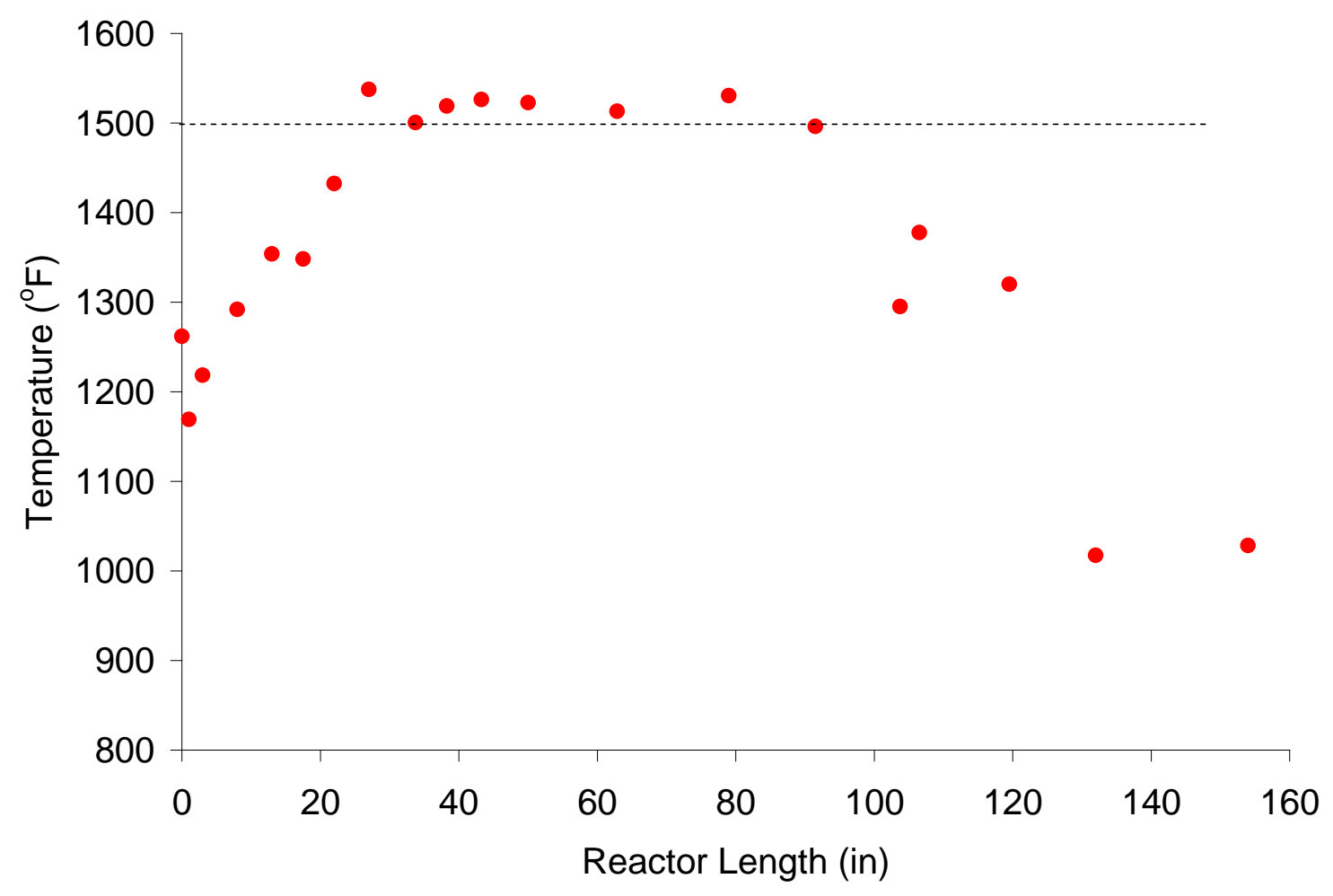

\section{Figure 1-38 - Reactor Temperature Profile from the December 1, 2009, Hydrogasification (Test 2 After "Repairs")}

The first point in Figure 1-38 is the hydrogen injection temperature, which was approximately $1250^{\circ} \mathrm{F}$. This temperature was high enough to initiate the hydrogasification reactions. Additionally, the middle of the reactor achieved a relatively constant temperature of $1500^{\circ} \mathrm{F}$, which was the target setpoint.

This was a very successful run as steady state was achieved. This observation was based on a constant temperature profile and a constant $\mathrm{CH}_{4}$ concentration measurement from the product stream. Figure 1-39 shows temperature-versus-time profiles from three points in the reactor. The three thermocouples were located at the approximate top (8 inches down from the point of coal introduction), middle (63 inches), and bottom (132 inches) of the reactor. Once coal feeding began, the reactor temperature started to drop. It has been concluded that this was due to the non-heated coal being introduced into the reactor. As the hydrogasification reactions followed, the reactor temperature increased, due to the exothermic hydrogasification reactions. At approximately 45 minutes into the experimental run, steady state was achieved and the reactor temperature in the center of the reactor was at the $1500^{\circ} \mathrm{F}$ setpoint. Finally, when the coal feed was stopped, the temperature in the top of the reactor increased and in the 
middle/bottom of the reactor the temperature decreased. At this point, as was expected, fresh coal was no longer providing a temperature sink at the top of the reactor and energy was no longer being generated from the hydrogasification reactions.

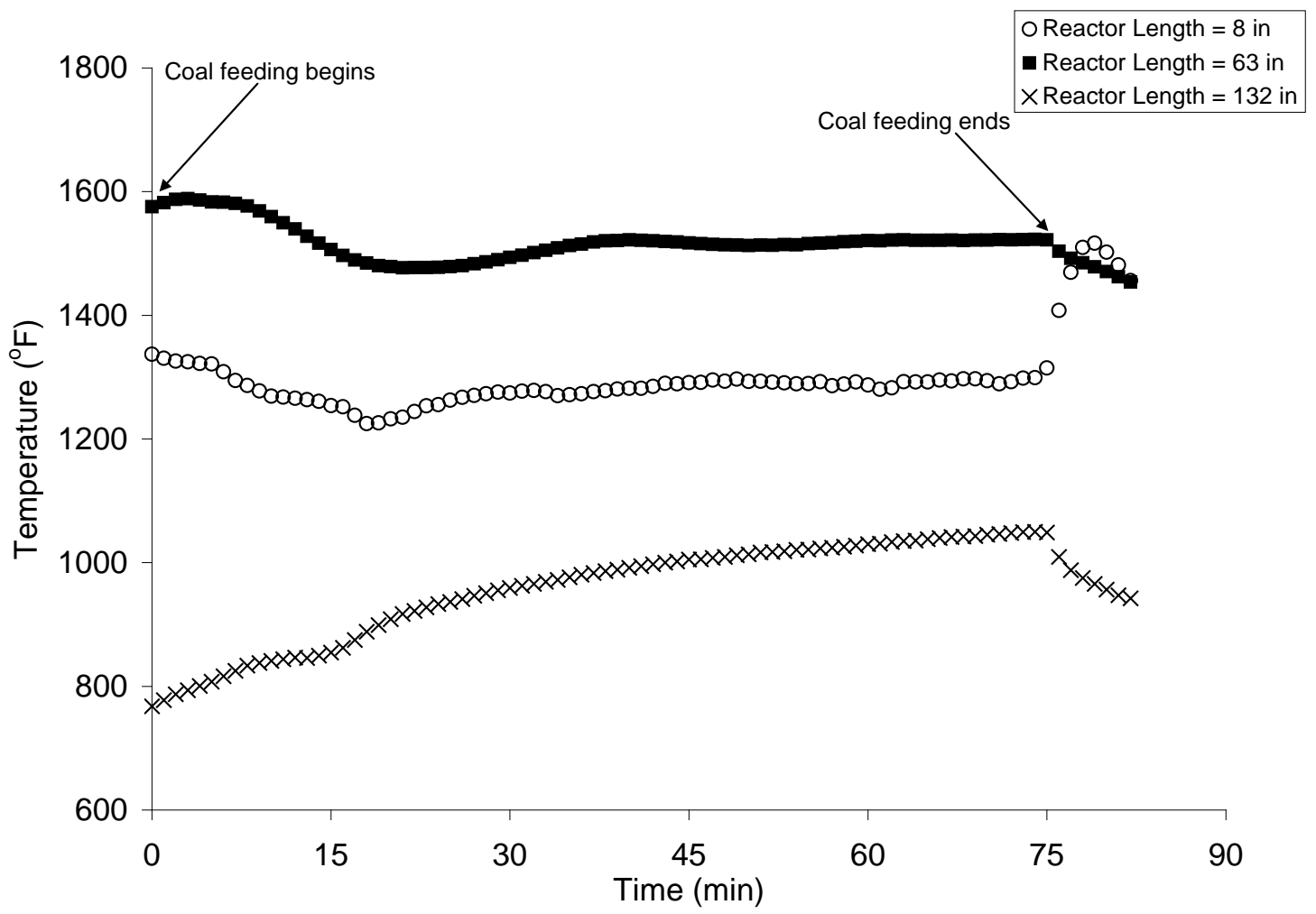

Figure 1-39 - Temperature versus Time Profile from the December 1, 2009, Hydrogasification (Test 2 After "Repairs")

Figure 1-40 shows the concentration of methane in the gas stream versus time obtained from the $\mathrm{GC}$ analysis. When coal was introduced into the reactor, the concentration of $\mathrm{CH}_{4}$ began to increase. At approximately 75 minutes into the experimental run, the methane concentration of the product stream became approximately constant. At the same time the temperature became steady, showing good agreement between the two sets of data. $\mathrm{CH}_{4}$ concentration was measured at the peak (highest point), which was about 10.5\%, and at steady state the concentration was $10 \%$. 


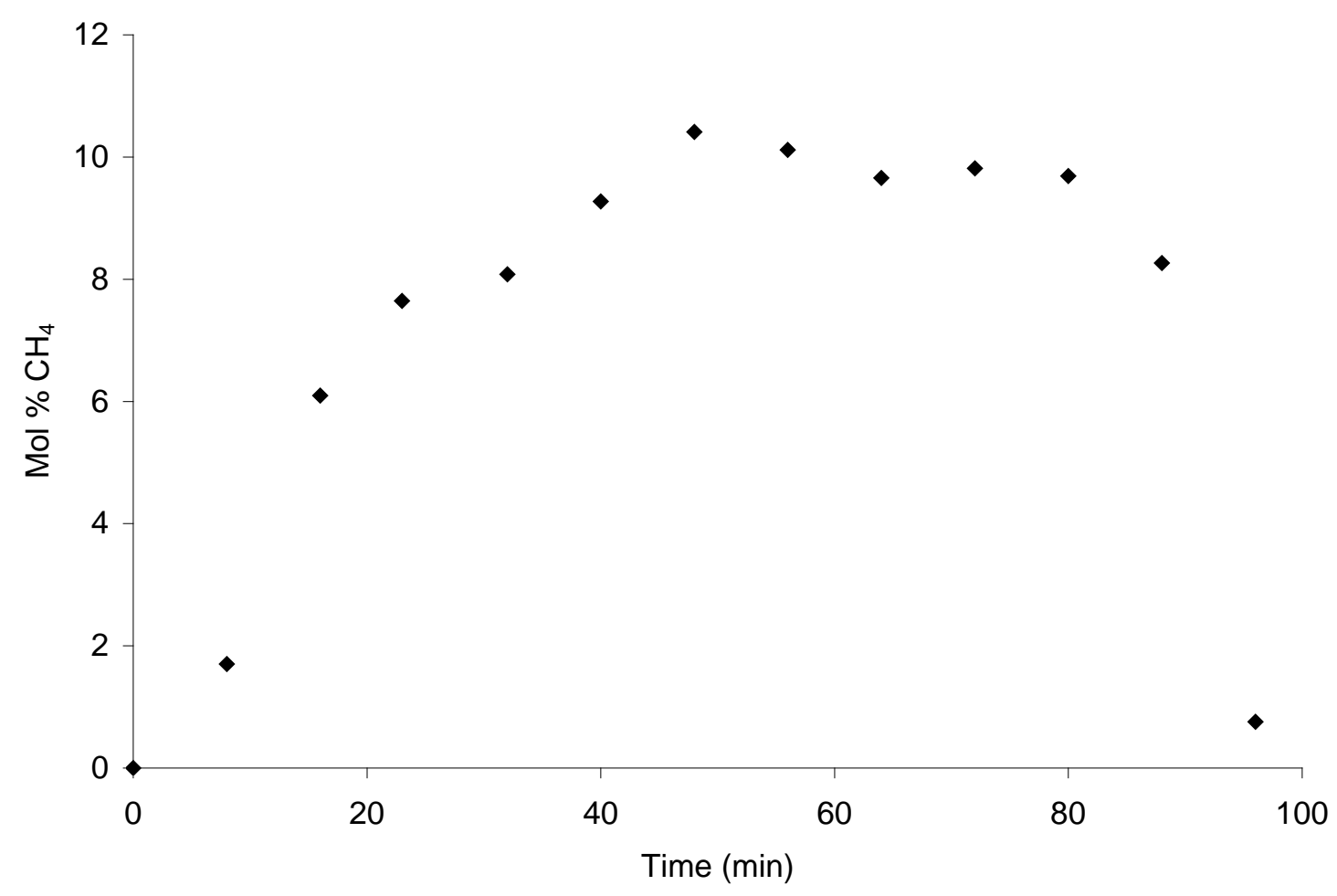

Figure 1-40 - Methane Concentration in the Product Stream from December 1, 2009, Hydrogasification Test 2 After "Repairs"

\subsubsection{Hydrogasifcation TEsting on DeCember 9, 2009 (TEST 3 - Afier "REPAIRS")}

Another hydrogasification test was completed on December 9, 2009. The reaction conditions were a reactor pressure of 1000 psig, a reactor temperature of $1625^{\circ} \mathrm{F}$, a hydrogen-to-coal ratio of 0.4 , and an approximate residence time of 11s. A temperature profile from this hydrogasification test is shown in Figure 1-41 at the same three locations in the reactor mentioned above. At the onset of coal feeding, the temperature in the reactor again dropped, as it did with the previous run, before it rose due to the hydrogasification reactions. After about 90 minutes, the temperature profile was approximately at steady state. However, in this run the coal feed rate was slightly fluctuating, which made it difficult to maintain a steady temperature. In spite of this, the temperature in the reactor remained relatively constant. When coal feeding was discontinued, the temperature in the reactor rapidly rose and the temperatures at the middle and bottom of the reactor fell. Again this was due to the elimination of the heat sink from non-heated coal at the top of the reactor and loss of the exotherm from the hydrogasification reactions, respectively. 


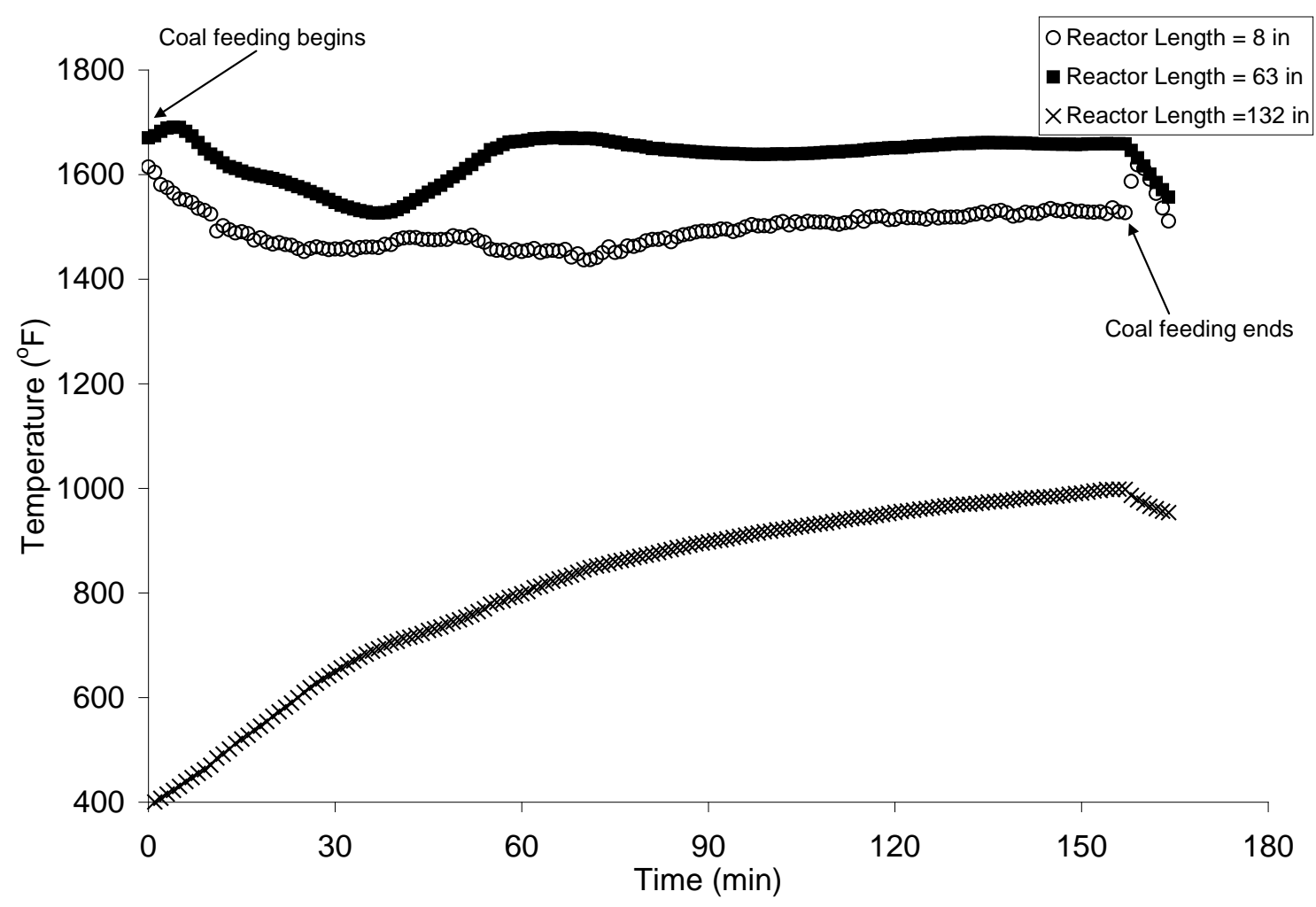

Figure 1-41 - Temperature-versus-Time Profile from the December 9, 2009, Hydrogasification (Test 3 After "Repairs")

The results of the $\mathrm{CH}_{4}$ gas concentration from the $\mathrm{GC}$ is shown in Figure 1-42. After approximately 90 minutes, a relatively constant $\mathrm{CH}_{4}$ concentration was reached, which again correlated well with observations from the temperature profiles. What is evident when looking at this experimental data compared with data from test 2 was the strong temperature effect on $\mathrm{CH}_{4}$ concentration. During the initial decline in reactor temperature, the $\mathrm{CH}_{4}$ concentration diminished as well and then increased with the temperature rise to the desired setpoint. As was mentioned previously, the reactor temperature was slightly fluctuating during steady state, and this effect was more pronounced in the $\mathrm{CH}_{4}$ concentration curve. The rising and ebbing of the temperature caused the same trend with $\mathrm{CH}_{4}$ concentration.

The concentration of methane in the gas stream peaked at $8.5 \%$ and averaged about $7.2 \%$ during steady state. A higher temperature and higher hydrogen-to-coal ratio suggested that the $\mathrm{CH}_{4}$ concentration in Test 3 should have been higher than that from Test 2. However, in this instance an experimental run with a higher hydrogen-to-coal ratio will have more hydrogen in the product stream, thus diluting the generated $\mathrm{CH}_{4}$ concentration. What is more meaningful to look at in this instance was the carbon conversion of coal to $\mathrm{CH}_{4}$. At the time of this report, a comprehensive carbon balance was needed for all analytical analyses, but it was not completed. 


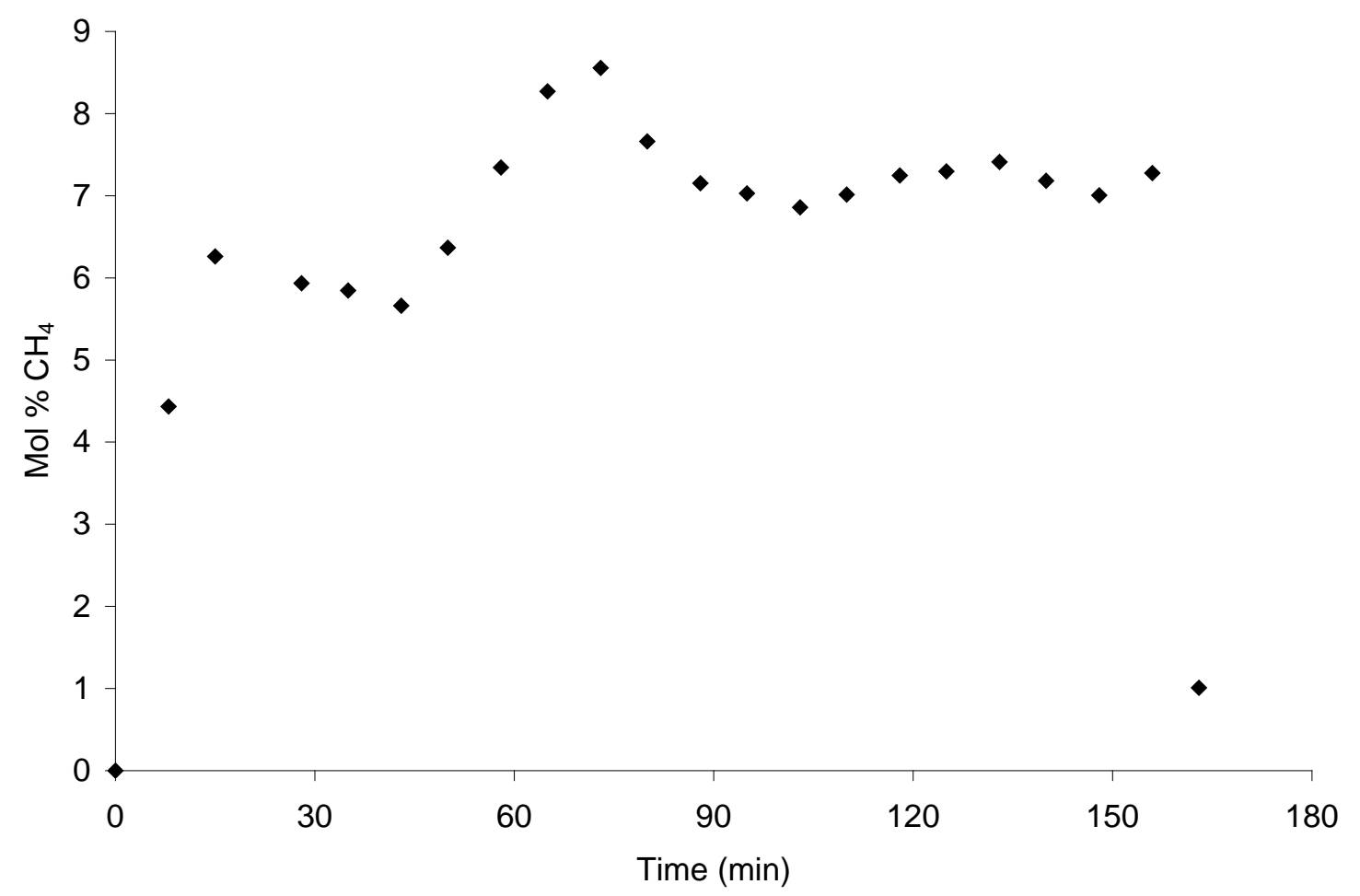

Figure 1-42 - Methane Concentration in the Product Stream from the December 9, 2009, Hydrogasification (Test Three After "Repairs")

Further tests were continued under DOE Cooperative Agreement: DE-FE0001099, "Integrated Energy System with Beneficial $\mathrm{CO}_{2}$ Use", and the results of tests with an improved gas analysis system are reported there. Please refer to its Technical Final Closeout Report for more information. 


\subsubsection{SIGNIFCANTDATES}

The list below delineates significant dates of major project accomplishments.

January 6, 2009

March 28, 2009

April 3, 2009

April 17, 2009

May 6, 2009

May 29, 2009

June 9, 2009

July 23, 2009

August 6, 2009

August 12, 2009

October 1, 2009

November 13, 2009

November 17, 2009

December 1, 2009

December 9, 2009
Reactor delivered to APS

Nitrogen flowed through the system for the first time

Heaters were turned on for the first time

Bottom charpot and coal feeder were installed

Officially began commissioning

Hydrogen flowed through the system for the first time

First hydrogasification test; preheater zone 5 was modified

Second hydrogasification test; coal feeder P\&ID was modified.

Third hydrogasification test: heater failure; began heater repair

Reactor inner tube de-telescoping

Reactor inner tube re-telescoping

Re-commissioning began

First Hydrogasification "hot" test with reconfigured reactor

Second Hydrogasification test reached steady state

Third hydrogasification test was successfully completed

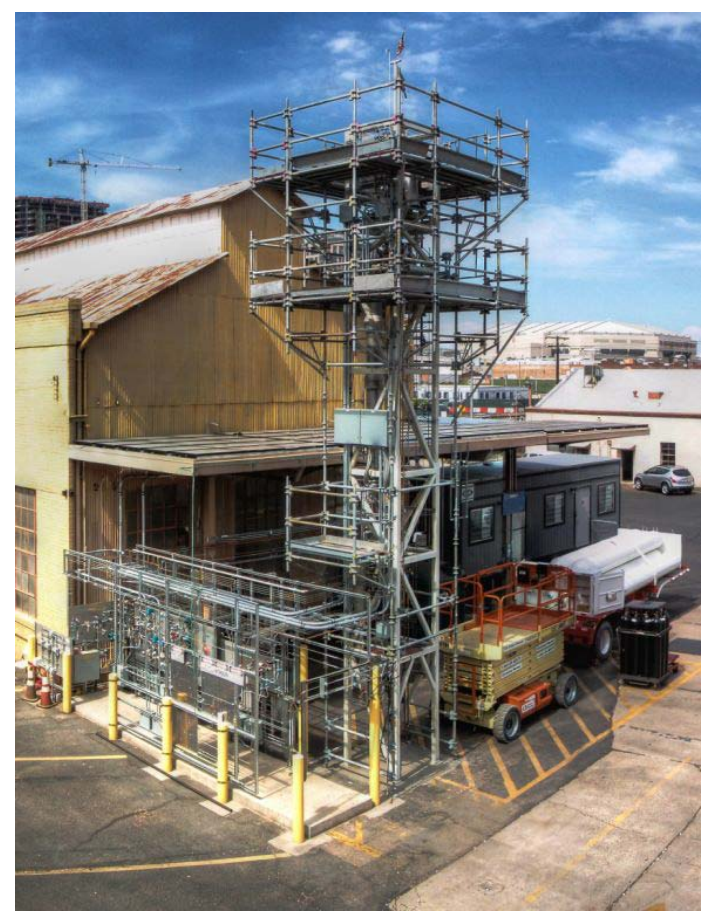

Figure 1-43 - Hydrogasifier on June 29, 2009 


\subsection{MAIN ISSUES AND LESSONS LEARNED}

The following section describes the major issues encountered in this research project during the commissioning and initial testing phases. Due to the novelty of the hydrogasifier design, resolution of these unexpected issues was not trivial. The reactor, hydrogen preheating, coal feeding and process control systems had to be modified during the process to ensure the systems could perform at design operating conditions of 1200 psig @ $1950^{\circ} \mathrm{F}$ and deliver qualified test data.

\subsubsection{MANUFACTURER C HANGE}

During the design and construction phase of the hydrogasification reactor, it was necessary to find a qualified fabricator for the reactor as the design would be complex. Also the use of exotic materials and an ASME-stamped pressure vessel were required. This was not trivial as it was difficult to find a fabricator with the experience and necessary space to build an ASME-stamped pressure vessel of this magnitude. Design and operating criteria were established for the bench-scale hydrogasification reactor as follows:

- Design the reactor and associated subsystems to meet ASME Boiler and Pressure Vessel Code (BPVC) Section VIII Div. 1, and ASME Process Piping Code B31.3.

- Design the reactor and vessels to meet a maximum allowable working pressure of 1200 psig at $1950^{\circ} \mathrm{F}$.

- Utilize construction materials that are compatible with hot, high-pressure hydrogen gas.

With the design and construction criteria established, a search for a qualified fabricator of the reactor was begun in accordance with the following:

- Fabricator must be an ASME pressure vessel shop with a "U"-stamp qualification.

- Fabricator must have experience fabricating hydrogen systems, including both hydrogen vessels and hydrogen piping systems.

- Fabricator must have experience welding high-temperature materials such as Inconel 625 and 617 and must have existing weld procedures and welders qualified for these weld procedures. 
- Fabricator must have experience in fabricating vessels and systems designed for high-temperature and high-pressure service.

Several potential fabricators were identified who met these criteria and who also had direct experience fabricating hydrogen systems for one or more of the major hydrogen gas suppliers: e.g., Air Products, PRAXAIR or Linde.

The fabricators identified initially included: ACME Cryogenics Inc., Pressure Dynamic Consultants (PDC Inc.) and CVIP Inc. Of the three fabricators, CVIP was the only one that had adequate floor space and fabrication labor to meet the project schedule. Additionally, CVIP had an extensive number of approved/tested Inconel and nickel weld procedures and qualified welders. Finally, CVIP had recent and significant experience building hydrogen vessels and systems. For all of these reasons, CVIP was initially chosen as the fabricator of the hydrogasification reactor.

A major issue with CVIP is that they are primarily a fabricator and do not staff an ASME code engineer. To ensure that a vessel would be designed and built to code it was then necessary to subcontract the code engineering to a company that specializes in pressure vessel design and analysis. The subcontractor was PMC Engineering, which specializes in the design of high- and low-temperature pressure vessels to ASME Section III and Section VIII Division 1 and 2 criteria. Relevant experience that PMC Engineering had was designing waste gas processing at temperatures above $1200^{\circ} \mathrm{F}$, cryogenic industrial gas production at temperatures below $-320^{\circ} \mathrm{F}$, and high- and low-temperature piping systems that satisfy ASME Section III, ASME B31.1 and B31.3 design criteria. PMC used a variety of pressure vessel software packages including Finite Element Analysis programs for modeling and engineering vessels. The principal vessel-code engineer from PMC also had extensive experience with high-temperature materials including Inconel used for the main reactor tube.

Preliminary fabrication drawings for the hydrogasification reactor were provided to PMC along with the design specifications indicating the desired maximum allowable working conditions: pressure, temperature and gas composition. PMC was contracted to analyze the design using appropriate ASME BPVC analysis and to determine if the design met the code at the design conditions. The initial analysis indicated that certain areas of the design could not meet both the pressure and temperature design specifications simultaneously. PMC did not provide any code calculations but instead provided a short written report. 
PMC was asked to identify the elements required in redesign to meet the operating conditions and to indicate what changes were required to meet the specifications (i.e., increase reactor shell thickness, flange thickness, etc.). The design drawings for the hydrogasification reactor were to be revised based on this feedback and then re-submitted to PMC for further code review and analysis; however, PMC did not provide sufficient feedback to effectively modify the design to meet the code. As opposed to providing the design calculations to improve the reactor, PMC provided a series of proposals to develop a new design with a time frame of 9-12 months to complete, far beyond the project schedule.

As discussions proceeded with PMC, another fabricator was identified, Gaspar of Akron, Ohio. Gaspar Inc. had recently completed fabricating a series of 16 large (up to 60-inch diameter), high-pressure (600 psig) and high-temperature $\left(600^{\circ} \mathrm{F}\right)$, flanged hydrogen reactors. These reactors were inspected and the quality of workmanship was determined to be very high. Additionally, Gaspar worked extensively with a major oil company and a major chemical company and had provided a wide array of pressure vessels and heat exchangers to these clients. In addition, in many instances the wetted surfaces of these items were fabricated from Inconel or similar materials. As a result, Gaspar had a wide range of Inconel weld procedures available. Finally, Gaspar had adequate floor space for the hydrogasification reactor as well as in-house machining capabilities to manufacture the custom high-pressure flanges. In short, Gaspar met or exceeded all of the fabricator selection criteria described above, and had an in-house, vessel engineering group.

Hydrogasification reactor design drawings and owner specifications were submitted to Gaspar for a budgetary quote and a preliminary assessment of the ASME BPVC compliance. Based upon the response from Gaspar and the on-going difficulties with CVIP/PMC, fabrication of the hydrogasification reactor was given to Gaspar.

The engineering group at Gaspar ran standard ASME BPVC calculations and determined what elements of the hydrogasification reactor design met BPVC code as designed and identified what elements required modification. In addition, the changes that were required to meet the operating conditions were identified. In some cases, Gaspar determined that the operating specifications had to be modified (hydrogasification reactor de-rated) in order for the design to comply with the BPVC. Based upon analysis and feedback from Gaspar, the hydrogasification reactor design drawings and owner specifications were modified, and subsequently the design package was re-submitted to Gaspar. After a series of iterations the design and operating 
conditions were certified to meet ASME BPVC with a Maximum Allowable Working Pressure (MAWP) rating of 1200 psig at $1950^{\circ} \mathrm{F}$.

Upon successful re-engineering of the hydrogasification reactor, Gaspar was tasked with fabricating it.

\subsubsection{PreHeAter MOdifCATION}

During initial testing of the hydrogasification reactor on June 9, 2009, a large heat sink was observed at the hydrogen crossover area in the zone 5 preheater. Figure 1-44 shows an original picture of preheater zone 5 . In the large insulation box, the four lines entering the reactor were subject to large heat losses. With a $1500^{\circ} \mathrm{F}$ delivered hydrogen temperature from the hydrogen preheater, an approximately $850^{\circ} \mathrm{F}$ temperature drop was detected when the gas stream reached the reactor head injector. To overcome this excessive heat loss, the preheater zone 5 was modified. In the original design, four gas lines were arranged separately to connect the preheater to the reactor head. Four heaters (Watlow VC400J12A, 2 inch by 12 inch, 1/2 inch ID, 350 Watts) were installed, with one on each gas line, for $1.7 \mathrm{~kW}$ of total power. The heat loss through the preheater zone 5 was estimated to be $3.0 \mathrm{~kW}$. Therefore, in the modified design eight heaters were installed on preheater zone 5 for a total of $3.6 \mathrm{~kW}$ of power; they were as follows:

- 3 full-cylindrical units of Watlow VC400J12A, 2 inch by 12 inch, $1 / 2$ inch ID, 350W

- 2 full-cylindrical units of Watlow VC400J06A, 2 inch by 6 inch, $1 / 2$ inch ID, 175W

- 4 half-cylindrical units of Watlow VS110A12S, 6 inch by 8 inch, 2 inch ID, 275 W

- 2 half-cylindrical units of Watlow VS110A12S, 6 inch by 14 inch, 2 inch ID, 550 W 


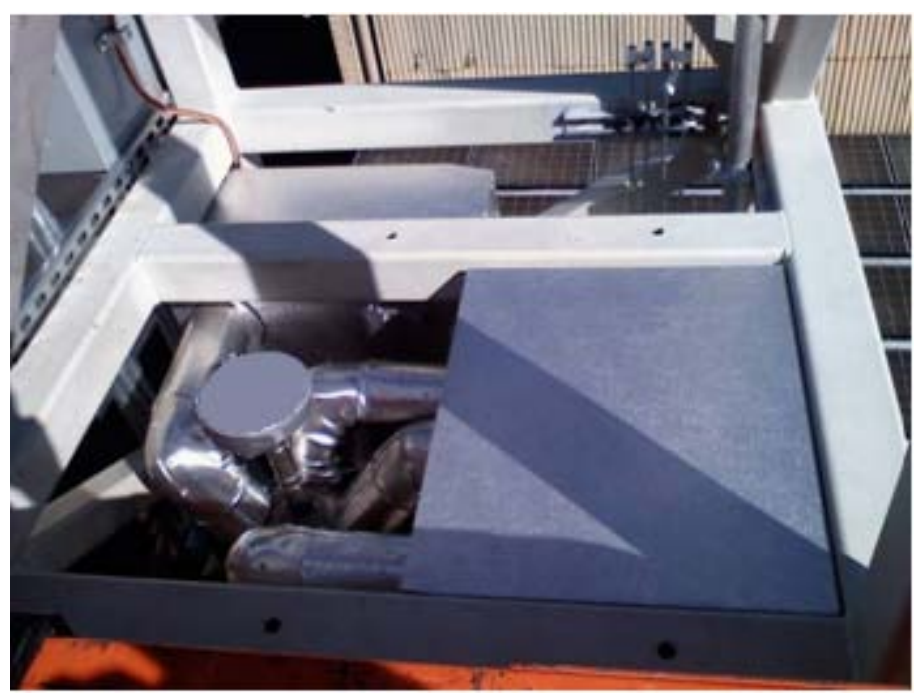

Figure 1-44 - Large Insulation Box of the Original Zone 5 Preheater Orientation

The orientations of the four gas lines were changed as well: they were bundled together in the proximity of the reactor head for better thermal management. Additionally, seven more thermal couples were installed for a total of 15 thermocouples in the preheater zone 5. (It was observed that thermocouples would break during the testing due to unknown reasons.) The modified orientation of the gas crossover lines significantly reduced the total surface area of preheater zone 5 , hence reducing the heat loss potential in this area. The new orientation of preheater zone 5 is shown in Figure 1-45. Figure 1-46 shows the orientation of hydrogen crossover with the new heaters.

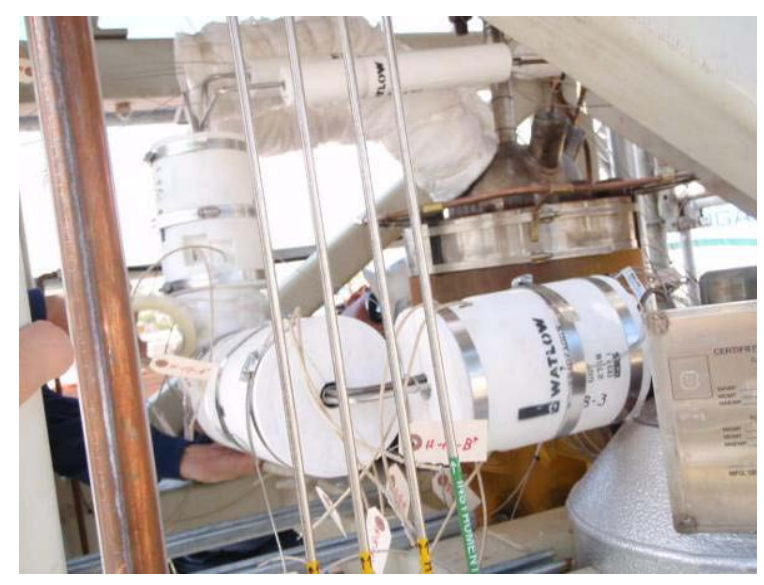

Figure 1-45 - Reorientation of Hydrogen Crossover Line 


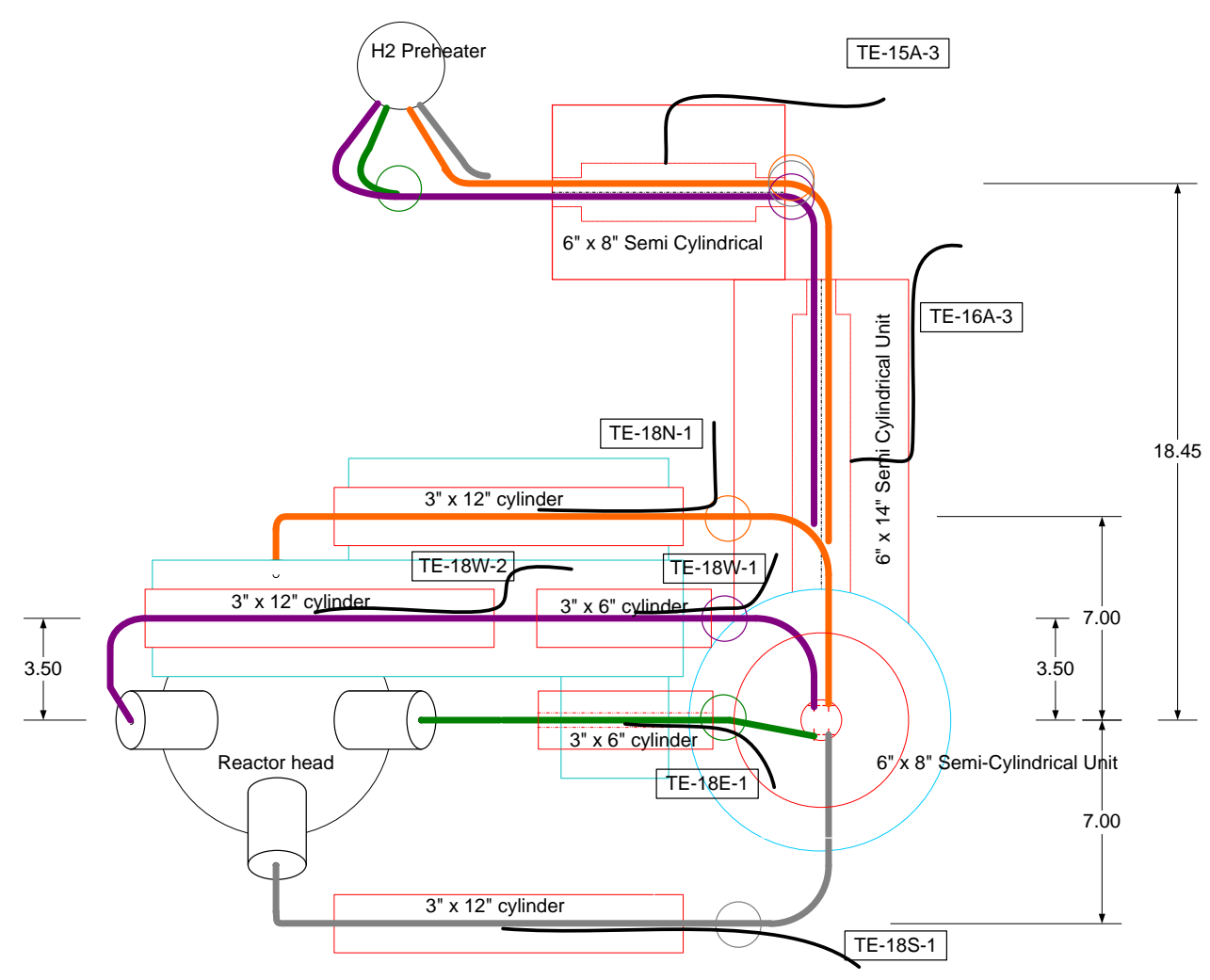

Figure 1-46 - Plan View Orientation of Hydrogen Crossover Lines with New Watlow Heaters

Re-commissioning of the preheater zone 5 was completed shortly after the modification. Unfortunately, an electrical short occurred, burning out a heater and gas line as seen in Figure 1-47 (left and right photos, respectively). An investigation of the incident found that a heating element likely made contact with the Inconel tubing to cause the short. The burned full cylindrical heater had an ID of $1 / 2$ inch and the Inconel tubing had an OD of $1 / 4$ inch. Due to this incident, all 5 full- cylindrical heaters were replaced with 1-inch diameter cylindrical heaters. A plan view diagram of the new preheater assembly is shown in Figure 1-46. Additionally, a ceramic sheet was used to cover the Inconel tubing and properly space the tubing in the center of the heaters. The burned heater incident delayed the project for several days; however, with the new set up, the hydrogen injection temperature was measured as high as $1350^{\circ} \mathrm{F}$, which was sufficient to initiate the hydrogasification reactions. 


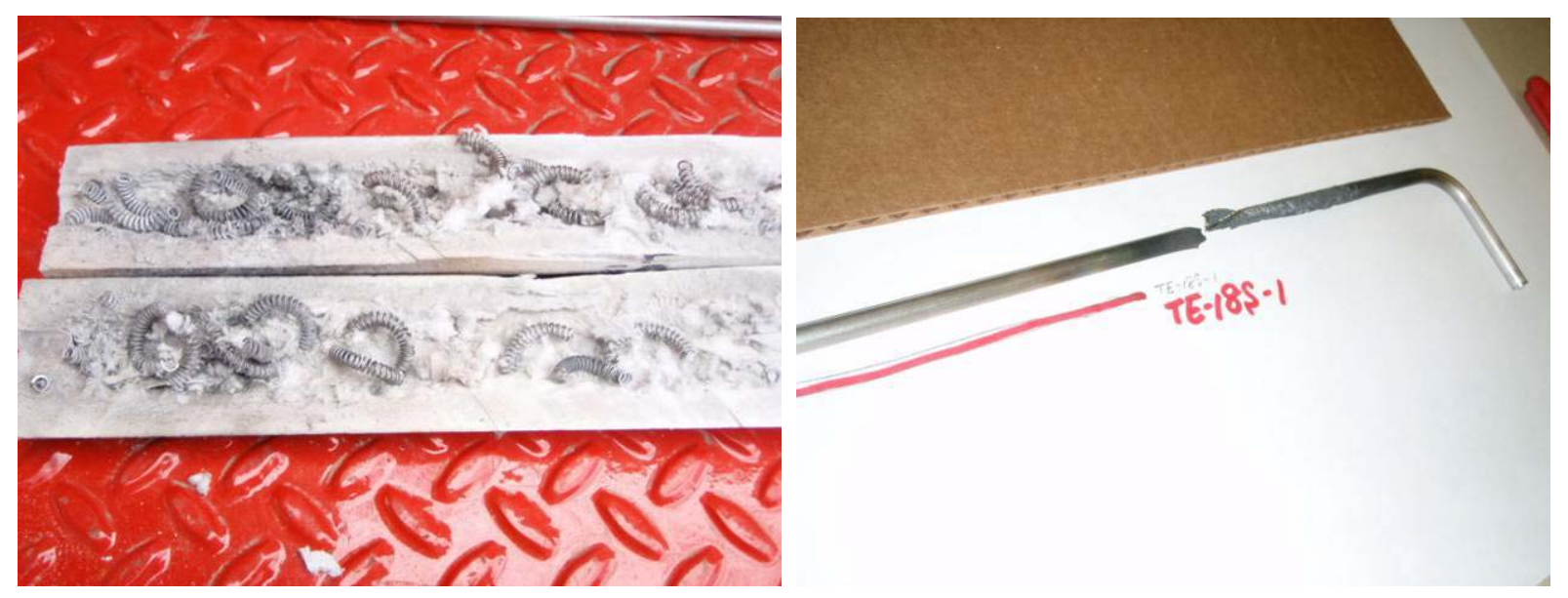

Figure 1-47 - Left: Burned Heater during Preheater Zone 5 Re-commissioning. Right: Burned Inconel 625 1/4-Inch Tubing due to an Electrical Short

\subsubsection{COALFEEDER P\&ID MODIFCATION}

During the initial hydrogasification tests, a higher than expected coal feeding rate was observed. The intended coal feed rate was $8.00 \mathrm{lb} / \mathrm{hr}$; however, during the first hydrogasification attempt, the actual feed rate was approximately $11.5 \mathrm{lb} / \mathrm{hr}$. The deviation between the set feed rate and experimental feed rate was not at first considered a significant issue. On the subsequent hydrogasification test the difference between the $8 \mathrm{lb} / \mathrm{hr}$ coal feed rate setpoint was extreme, with a $50 \mathrm{lb} / \mathrm{hr}$ feed rate. Considering the magnitude of this error, testing was halted to troubleshoot the issue.

Figure 1-48 shows the P\&ID of the coal feeding system during the initial hydrogasification tests. While feeding coal, valves CF-SV-2 and CF-SV-4 were full open. The pressure (P2 in Figure 1-48) in the coal feeder top chamber was expected to be approximately equal to the pressure (P1 in Figure 1-48) at the screw delivery port to mitigate any extra force in the coal feeding system. The higher than expected flow rate indicated that P2 was likely higher than P1. In order to confirm this hypothesis, tests were conducted under ambient conditions. 


\section{Observation at high pressure gasification test}

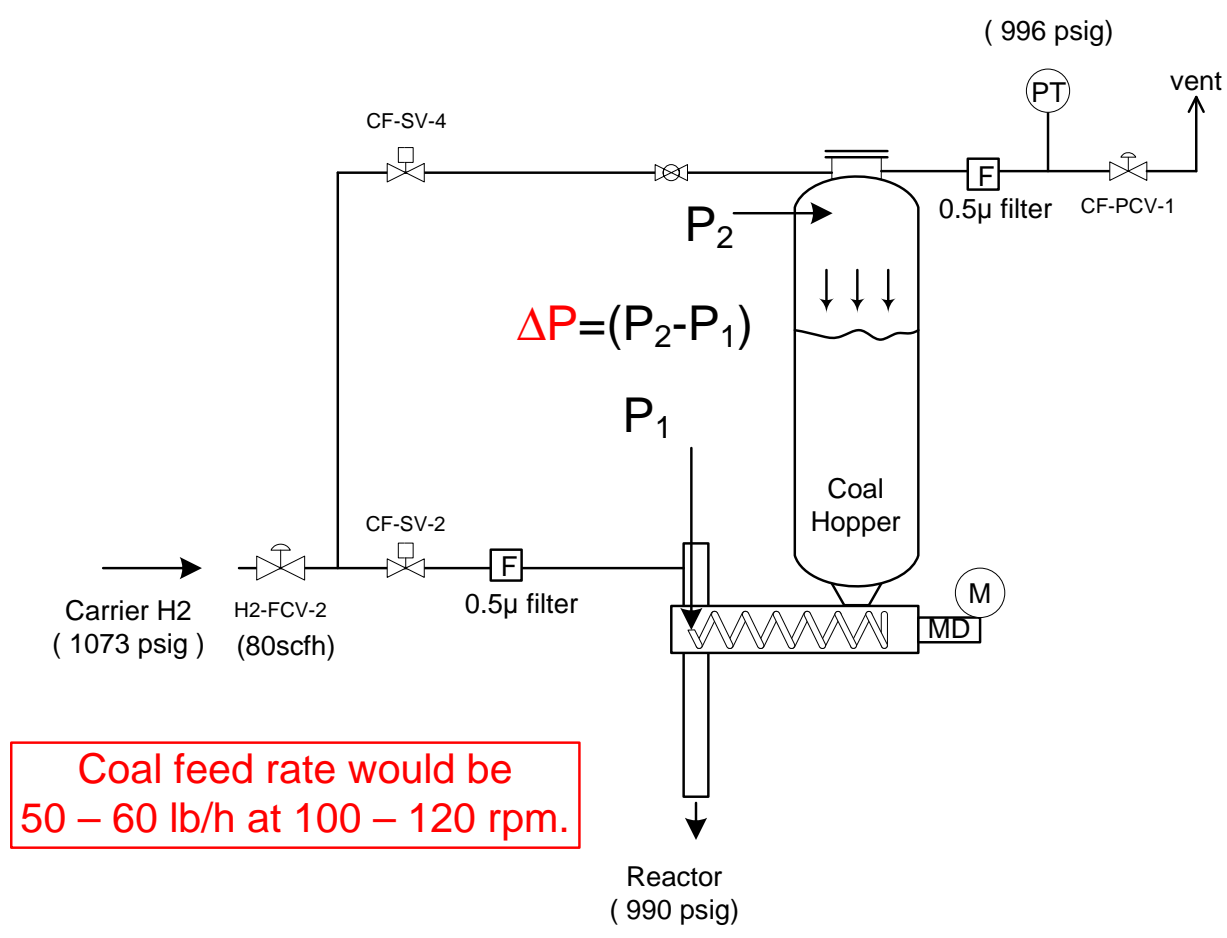

Figure 1-48 - P\&ID of the Coal Feeder

Figure 1-49 and Figure 1-50 show the feeder orientations in the two coal feeder tests. In the first test (the configuration is shown in Figure 1-49), the coal hopper top flange was closed and valve CF-PCV-1 was fully open along with the bottom flange of the reactor charpot. This led to ambient pressure at the coal feeder pressure transmitter and at the screw discharge port. By fully opening valve CF-SV-4, the system was expected to be under ambient pressure and should feed at the calibrated rates. However, testing indicated an approximately 55\% higher coal feed rate than was observed during commissioning (12 lb/hr versus $7.7 \mathrm{lb} / \mathrm{hr}$ ). This result indicates that there was likely still a pressure difference causing the higher than expected coal flow rate. In the second test (the configuration is shown in Figure 1-50), the top flange of the coal feeder was open. This made the pressure (P2) in the coal feeder definitely equal to ambient pressure, with approximately the same coal feed rate measured during commissioning. 
Ambient pressure coal feeder test - 1

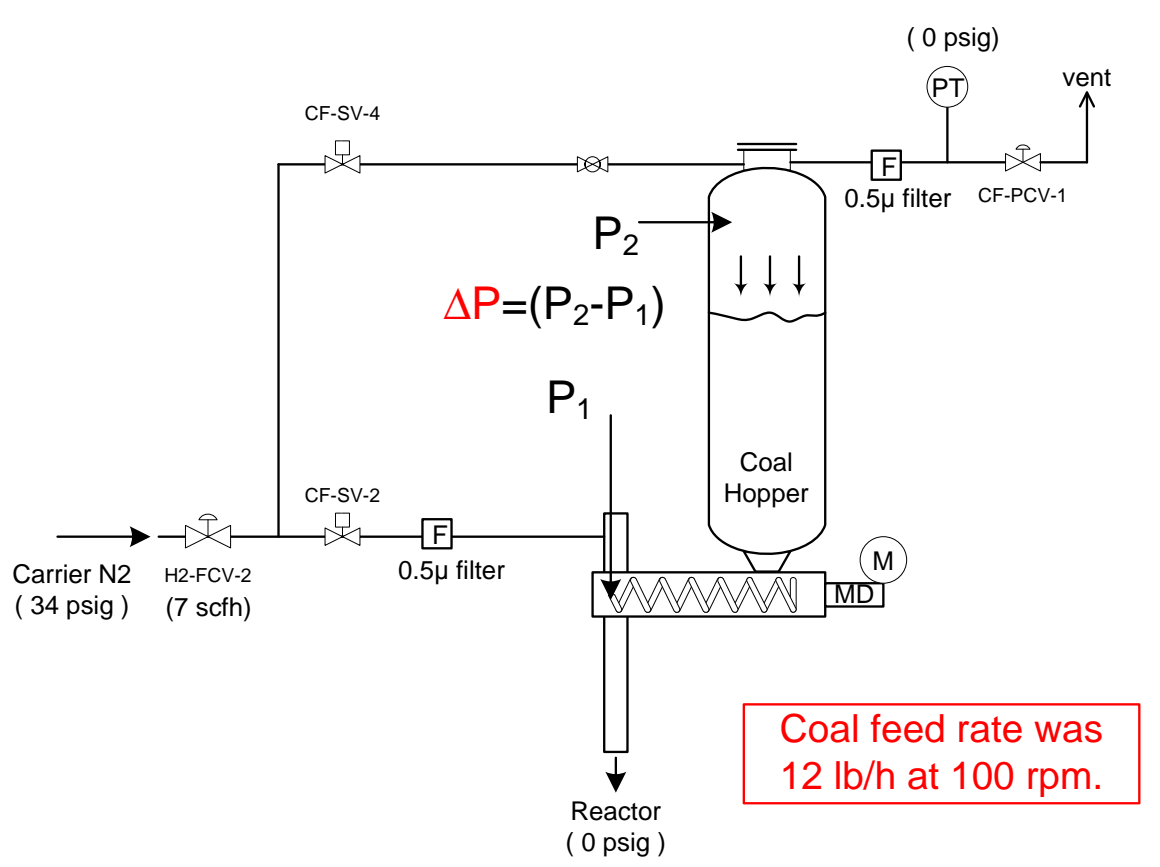

Figure 1-49 - Ambient Pressure Coal Feeder Test with the Coal Feeder Vent Line Fully Opened

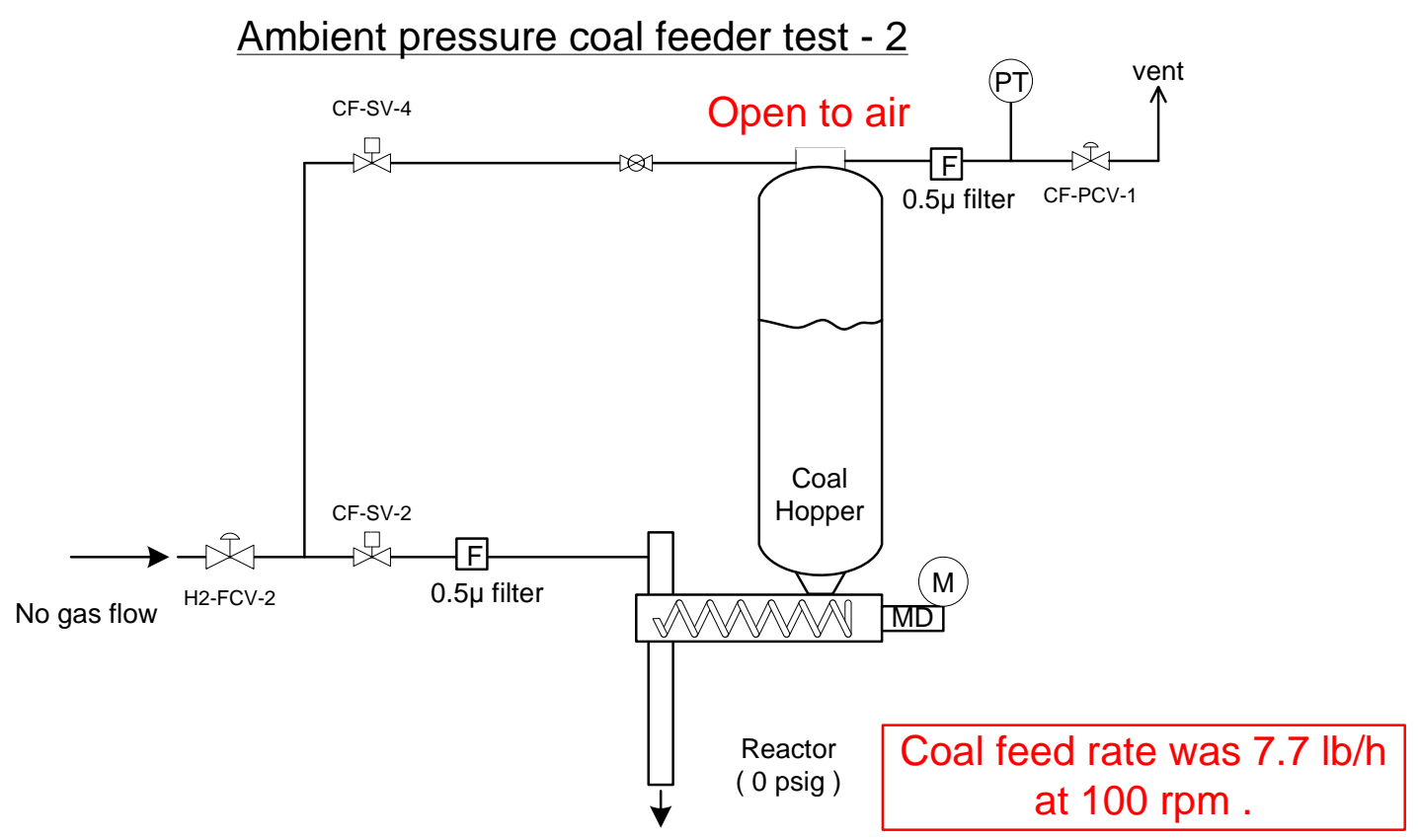

Figure 1-50 - Ambient Pressure Coal Feeder Test with Fully Opened Coal Feeder Top

After completing the two tests, it was evident that there was a large pressure drop across valve CF-SV-4 and the filter. Additionally, the equalizing line was not able to balance the pressure of 
the coal hopper and screw discharge port. To overcome this problem, the P\&ID of the coal feeder was changed to the configuration detailed in Figure 1-51. The changes included the following: (1) replacing valve CF-SV-4 with a full-bore AOV valve; (2) relocating the filter upstream of the equalizing line; and (3) replacing the existing $1 / 4$-inch line with $1 / 2$-inch tubing. Along with these changes, the pressure transducer was relocated for a better measurement of the coal hopper pressure. With these modifications, coal feeding was re-tested at 1000 psig. Finally, a reproducible coal feed rate was attained at high pressure and compared favorably with the calibration.

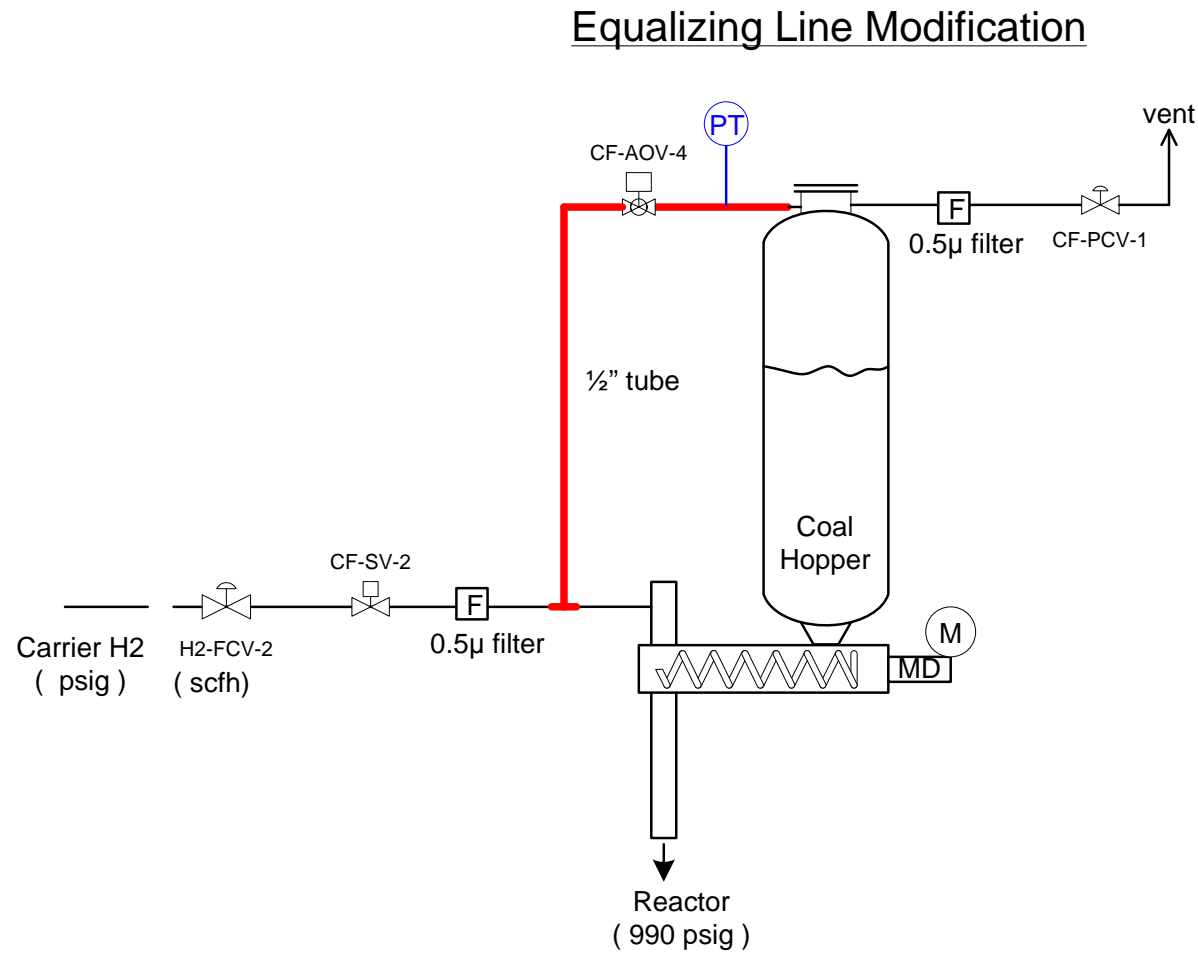

Figure 1-51 - Modified P\&ID around Coal Feeder for Better Pressure Equilibrium

\subsubsection{REACTOR HEATER MOdIFCATION}

On June 9, 2009, the testing also revealed that there was not enough energy transmitted from the heaters to the reactor wall to raise the wall to the desired temperature setpoint. The heaters that were originally installed on the reactor should have been powerful enough to increase the reactor wall to a temperature higher than the highest experimental setpoint. A phenomenon that may explain this deficiency can be observed by comparing temperature profiles from a preheater heater (Figure 1-52) and a reactor heater (Figure 1-53). The preheater was a single tube design and the temperature profile around a heater from it (shown in Figure 1-52) was not 
out of the ordinary. In the center of the heater a temperature measurement of approximately $1600^{\circ} \mathrm{F}$ was taken and $850^{\circ} \mathrm{F}$ at the external shell. Both of the temperatures are reasonable, as the temperature setpoint was $1600^{\circ} \mathrm{F}$ and insulation should limit energy transfer from the heating element to the external shell; hence the temperature at the external shell is significantly lower. In contrast, the heater around the reactor had an unusual temperature profile. In this instance, the center of the heater had a temperature of approximately $1240^{\circ} \mathrm{F}$ and the external shell temperature approached $1110^{\circ} \mathrm{F}$ (as shown in Figure 1-53). Again, because of a significant amount of insulation between these two points, energy transfer should have been limited.

The phenomenon observed in the reactor heaters only occurred when the reactor was pressurized in both inside the inner tube and annular space. During the commissioning of the reactor, the heaters were tested at atmospheric pressure, and temperature profiles similar to Figure 1-52 were observed. It was hypothesized that stronger natural convection exists in the reactor annular space when the reactor is pressurized.
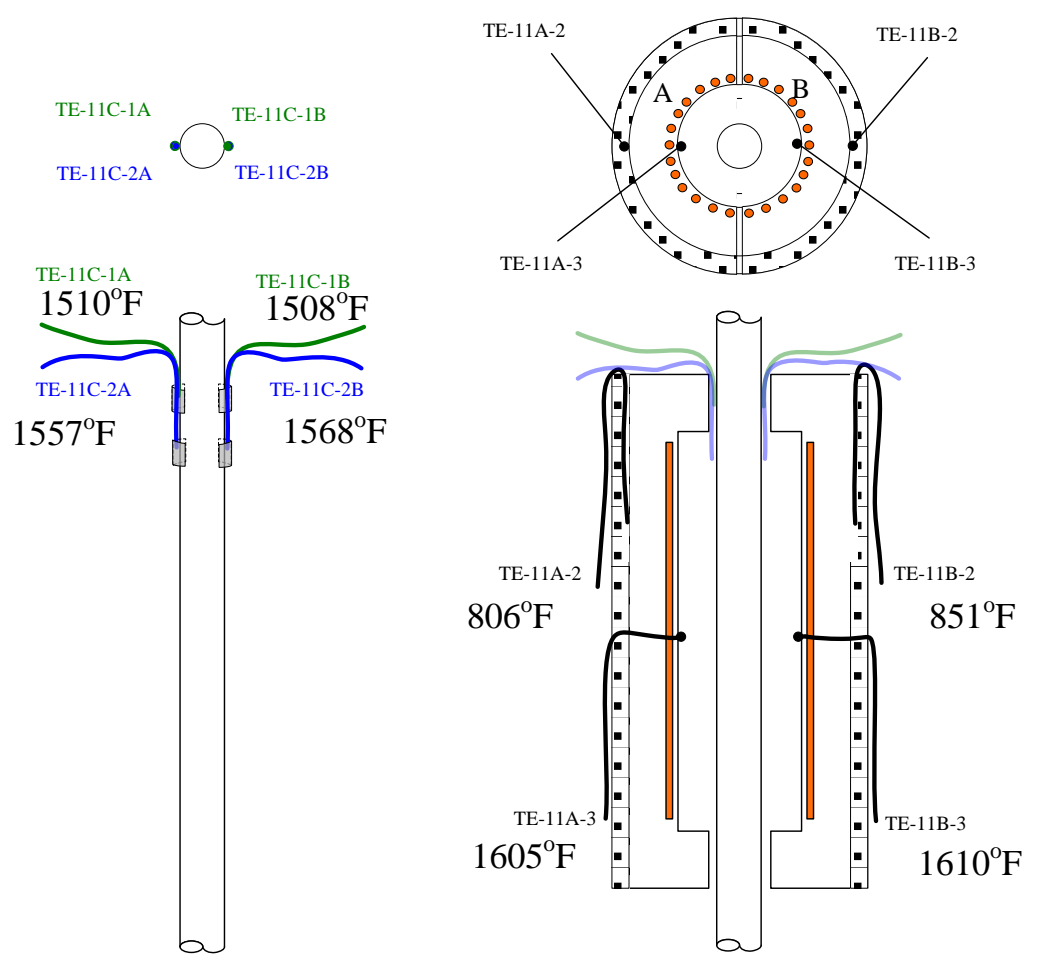

$\underline{\mathrm{H}_{2}}$ Preheater PH-HTR-1

Figure 1-52 - Temperature Profile around Preheater No. 1 (The One Closest to Preheater Outlet) 


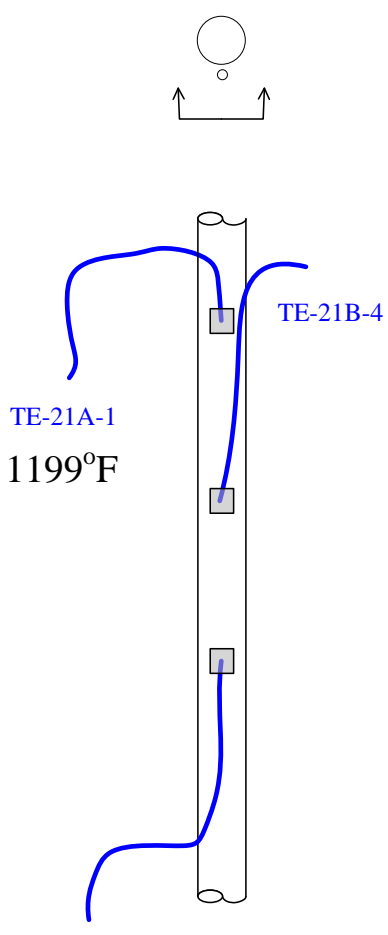

TE-21B-1

$1243^{\circ} \mathrm{F}$
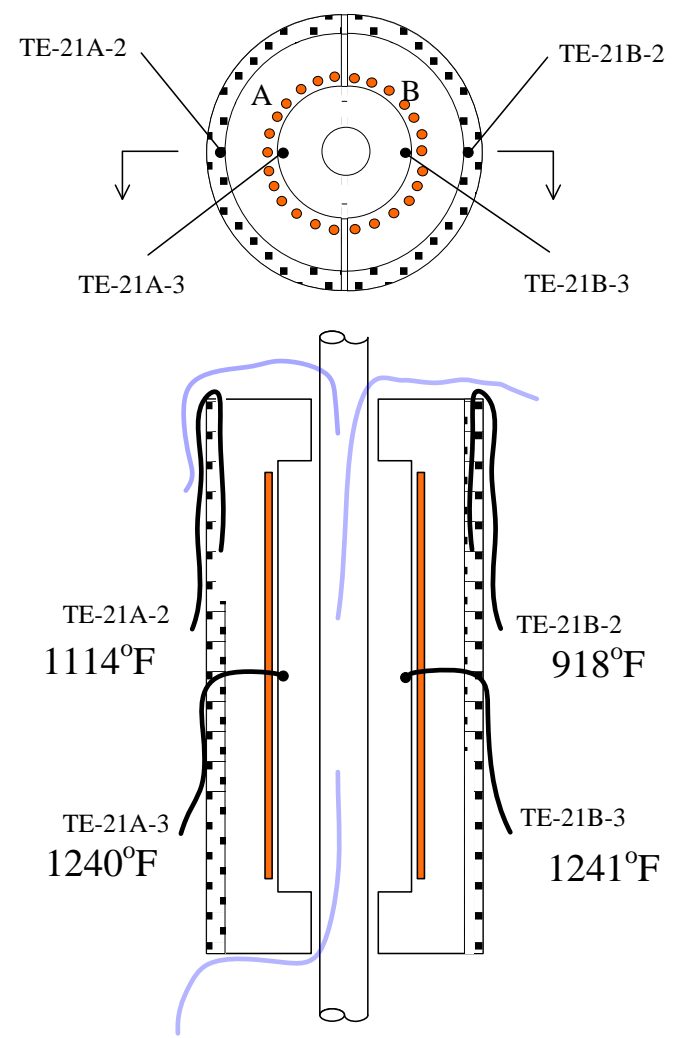

Figure 1-53 - Temperature Profile around Reactor Heater No. 1 (The One Closest to the Reactor Inlet)

The inner wall of the double-wall reactor design was physically separated into seven zones by spider plates. Spider plates, depicted at the bottom of Figure 1-54, were intended to provide support to the heaters and insulation, as well as to help center the inner tube. The plates were square and the diagonal distance of a plate was very close to the inner diameter of the external wall. Additionally, the spider plates were affixed to the outer wall of the inner tube (i.e., the reactor vessel), which resulted in the spider plates, and the supported insulation and heaters, moving downward during thermal expansion and creating a gap between heater zones. This design, therefore, forced the insulation to be separated into seven zones. As shown in Figure 1-54, gaps between the insulation and spider plate were formed due to thermal expansion differences between the metal tube and ceramic heater. This gap likely increased the natural convection flow that occurred in the annular space, which led to additional heat loss from the inner tube, raised the annular space temperature, and provided a pathway to the pressure vessel shell for the heated inert gas that provided the pressure balance between the inner tube and the pressure shell. Another problem associated with the spider plate was not enough 
insulation was wrapped over it due to its size. In fact, the hot spot location profile shown in Figure 1-55 (indicated by the yellow thermal profiles) confirmed this speculation, as all hot spots were located at zone-to-zone interfaces.

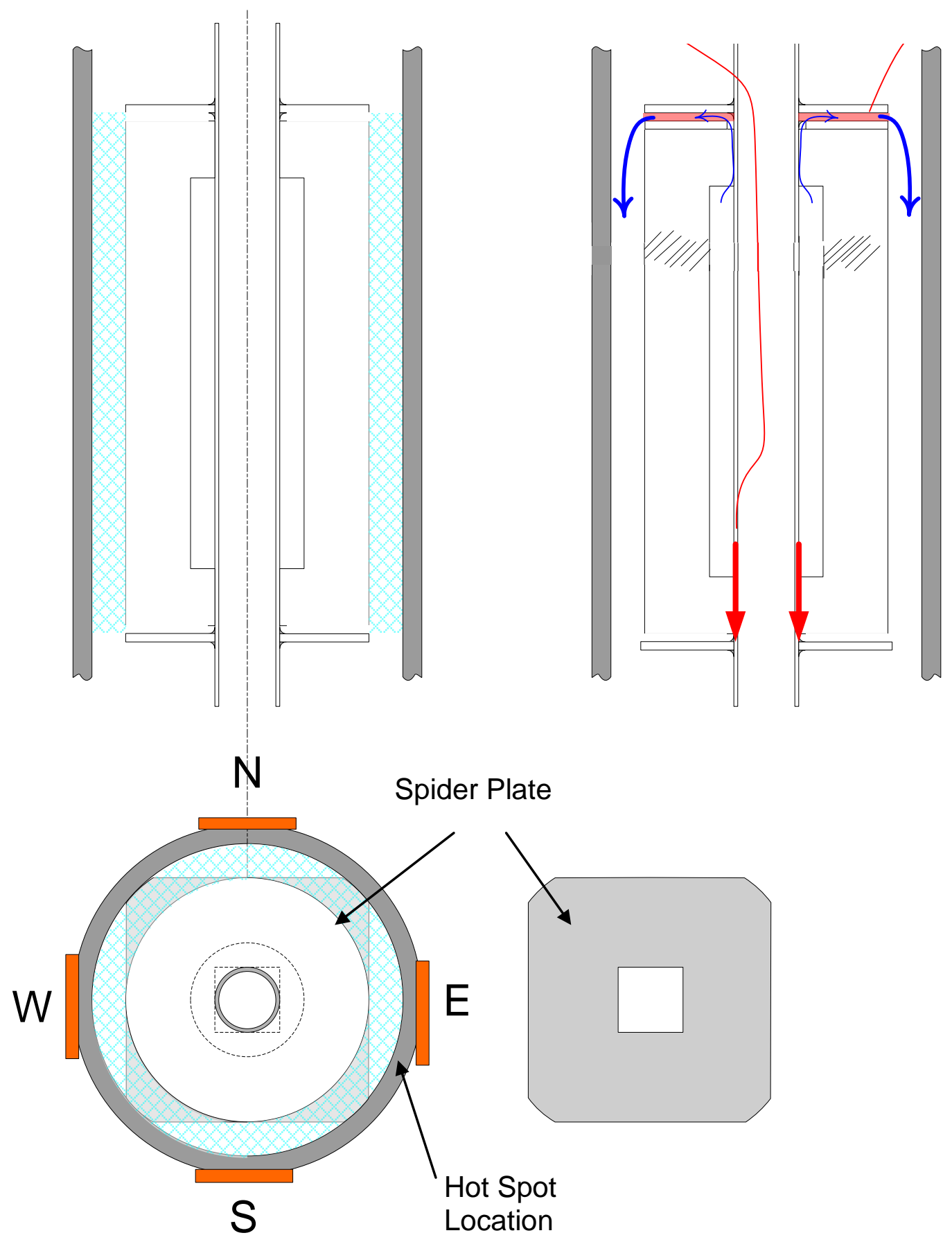

Figure 1-54 - Cause of Excess Temperature at External Shell 


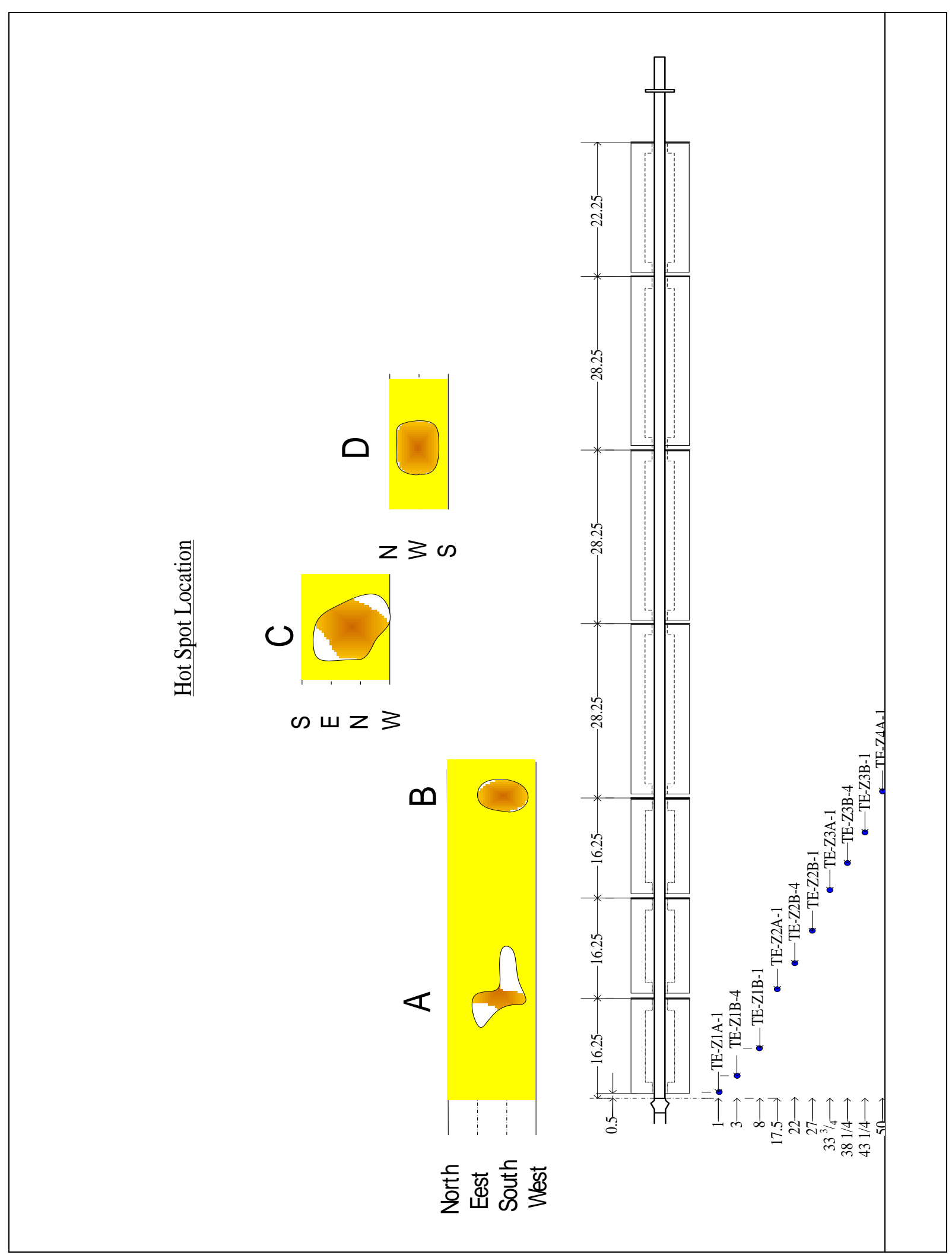

Figure 1-55 - Hot Spot Locations (In Inches) 
The issues with the reactor heaters were addressed after a critical failure occurred. When inner tube wall reached $1350^{\circ} \mathrm{F}$ with the maximum heat load of the reactor heaters, the power lines of top four heaters were burned. This incident forced a full system shut down and de-telescoping of the inner tube out of the pressure vessel for troubleshooting and repairing the reactor.

De-telescoping commenced shortly after the heater failure and went smoothly. As shown in Figure 1-56 (left photo), the inner Inconel tube with insulation came out of the external shell intact. In Figure 1-56 (right photo), the fatal cause of the heater failure can be seen. A high temperature in the annular space along with physical abrasion from Inconel wiring (the Inconel wiring was used to tighten insulation in place) caused a metal-to-metal contact of the power wires and therefore shorted the heaters. The electrical short was partially caused by the higher-than-expected temperature in the annular space, which further illustrated that the current design was not sufficient.
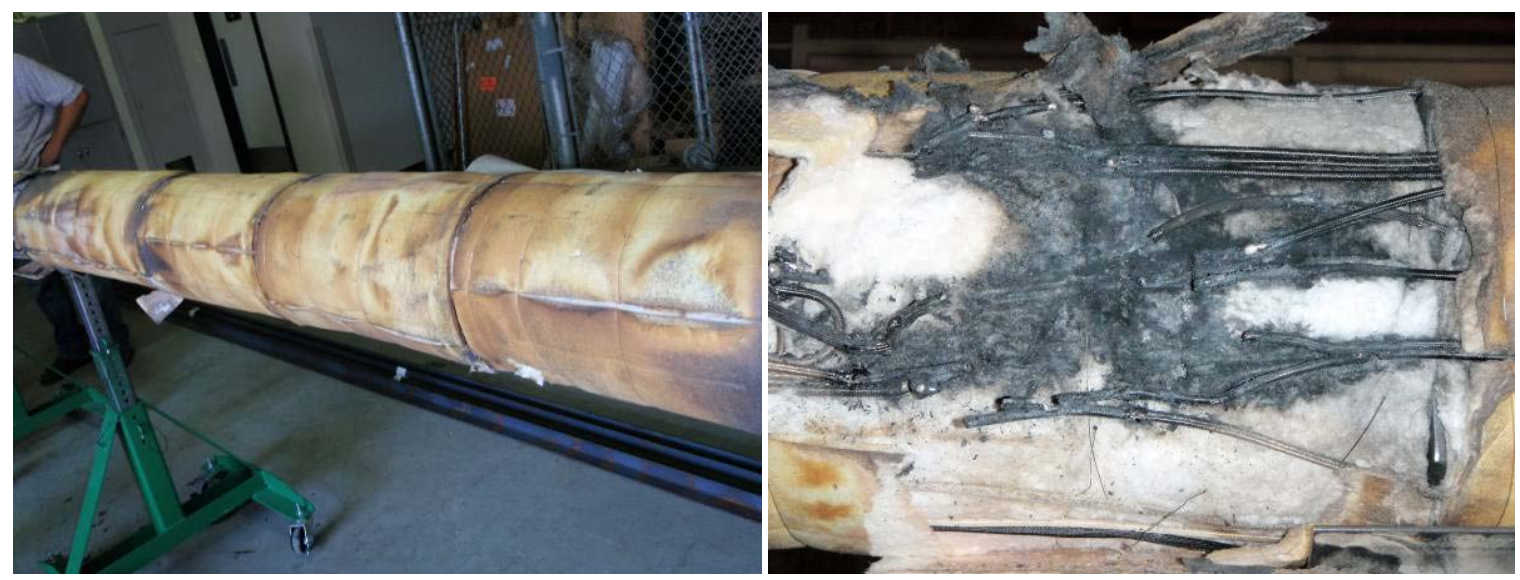

Figure 1-56 - Hydrogasifier Internal Tube after De-Telescoping (Left Photo) and Burned Power Wire-Fatal Failure of Top Four Zone Heaters (Right Photo) 
After removing the insulation and heaters, Figure 1-57 (left photo), the reactor inner Inconel tube was carefully inspected and was found to be in good condition. There was a black appearance on the heater inner surface as shown in Figure 1-57 (right photo), which was from burned organic binder from an insulation material as opposed to the heater.
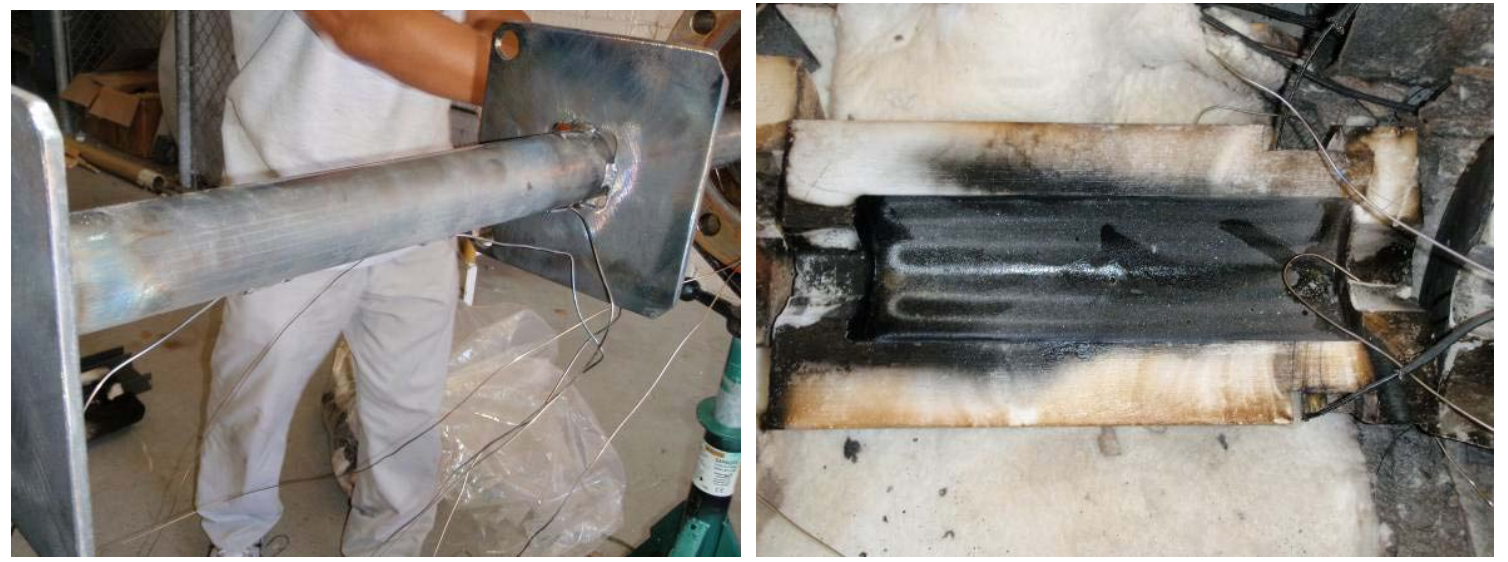

Figure 1-57 - Left: Inner Reactor Tube and Original Spider Plate; Right: Used Watlow Heater (Black Deposition was from the Burned Insulation)

The reactor repair mainly focused on better reactor insulation and more heat input. Heat loss from the reactor was estimated under open-air conditions (no misting). During the first set of experiments, a system was used to spray a fine mist of water on the reactor shell to assist with shell temperature management. The average temperature of the external reactor wall was approximately $300^{\circ} \mathrm{F}$, which, based on calculations presented in Figure 1-58, meant less than $15 \mathrm{~kW}$ of heat loss. The SNG hydrogasifier was designed as an adiabatic reactor; thus energy supplied by the heaters was supposed to make up for heat losses at the designed operating temperature $\left(1500-1750^{\circ} \mathrm{F}\right)$ as opposed to supplying additional heat. The maximum heat load from the reactor heaters was $16.2 \mathrm{~kW}$, which meant at the average reactor shell temperature there was just sufficient output to overcome this heat sink. When factoring in additional heat losses from operating the misting system, the required heat load from the reactor heaters would be too large. In fact, when utilizing $100 \%$ of the heat load, the reactor inner wall was only able to reach $1350^{\circ} \mathrm{F}$. Because of the significant heat losses, a new design approach, along with a different heater type, was used. 


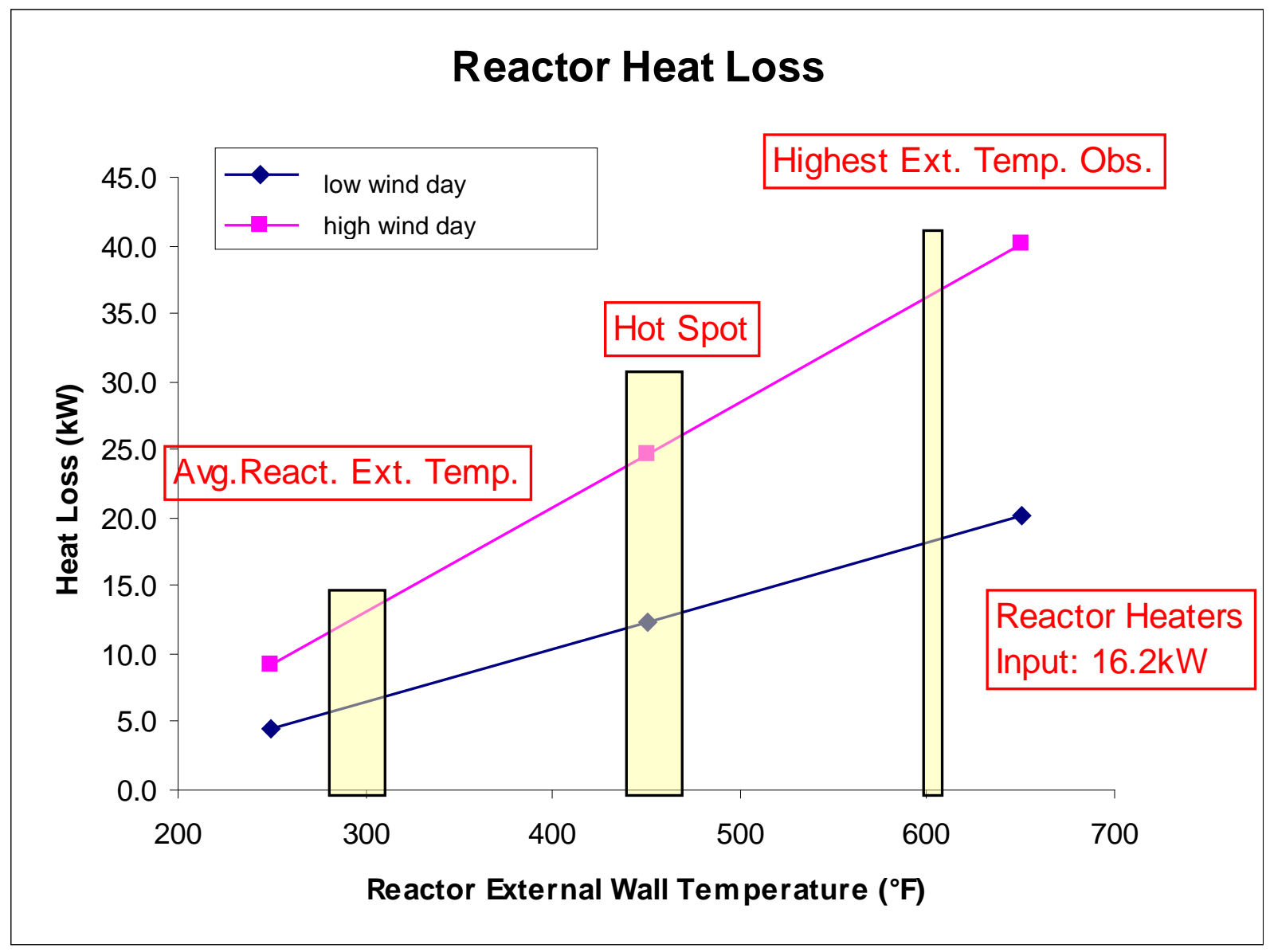

Figure 1-58 - Reactor Heat Loss Estimation Under the Original Set Up

Consequently, three signification modifications were made to the reactor design: (1) altering the spider plates, (2) replacing the clam shell heaters with mat heaters, and (3) adding more insulation. As was previously discussed, the thermal expansion of the reactor tube and heaters was different, which caused hot spots at the spider plate interfaces. To prevent this from occurring again, more insulation was needed at these points and to accomplish this, the spider plates were cut back as shown in Figure 1-59 (left photo).

To address the lack of heating load as well as the heater transfer issue, nine new heaters were purchased from Zircar Ceramic, Inc., as shown in Figure 1-59 (right photo). The heaters were ceramic-bead weaved mats, which were physically wrapped around the inner tube. This would help with heat transfer in two regards: (1) heat would be transferred via conduction; and (2) natural convection currents would be limited. The heaters have a high power density: at 8 inches x 14.75 inches they provide $3.6 \mathrm{~kW}$ of energy and require only an 80-V power supply. This heat density is four times that of the previous heaters. The ceramic beads have a 
thickness of 0.41 inch (10 centimeters $(\mathrm{cm})$ ), which left ample space to increase the amount of insulation (3.75 inches of insulation as opposed to 1 inch), thereby accomplishing the third design criterion.

Figure 1-60 (left photo) shows the insulation thickness of the modified design. Finally, reactor heating zones were reduced from seven to six. The top three zones were 15 inches long and the bottom three zones are all 28 inches long. Two heaters were installed in each bottom zone and a total of $24.2 \mathrm{~kW}$ of power was installed.
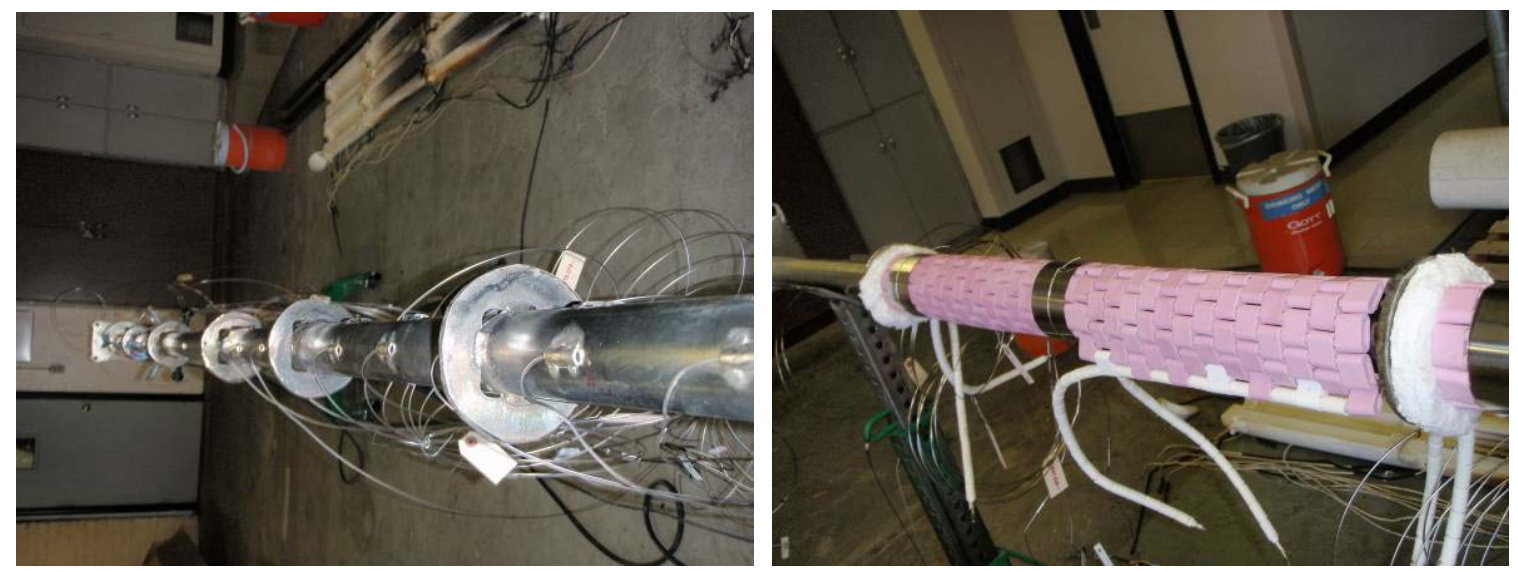

Figure 1-59 - Left Photo shows the Modified Spider Plate and

Newly Installed Thermocouples;

Right Photo shows New Heaters Wrapped Around the Inner Reactor Tube
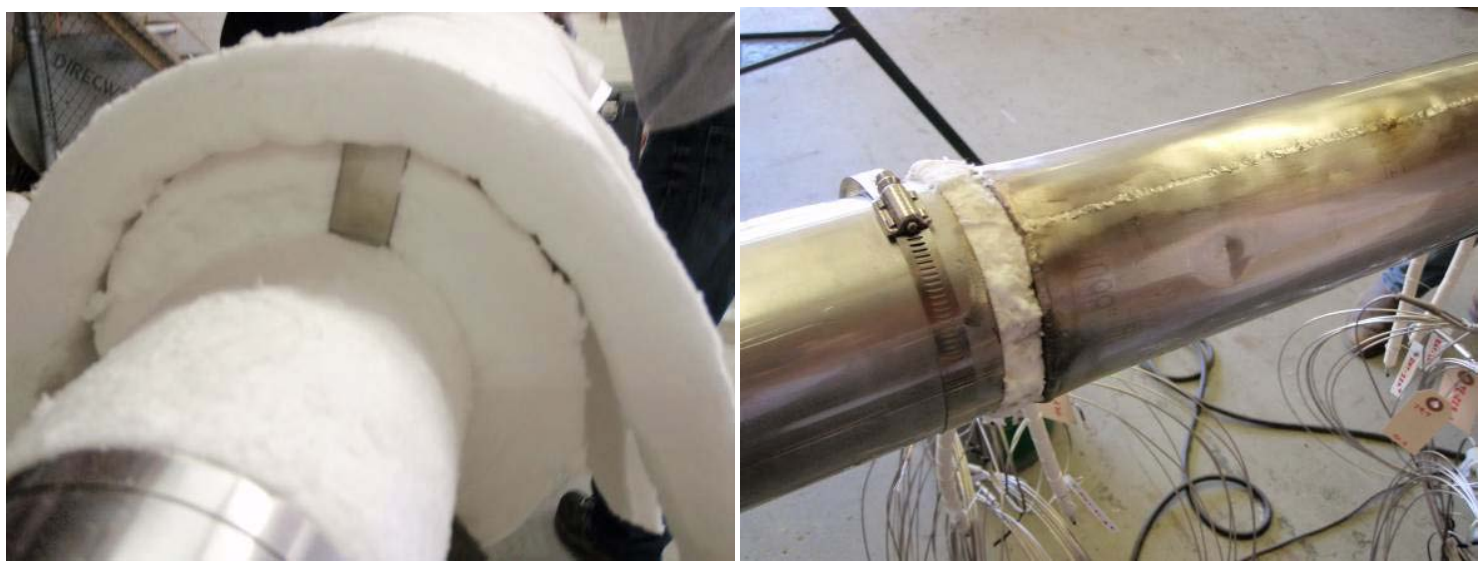

Figure 1-60 - Photo Left shows Increased Insulation Thickness; and photo right shows Stainless Steel Heat Shield to Limit Nitrogen Natural Convection

There were a few other minor modifications made to solve prior issues. First, a stainless-steel heat shield was installed. 
Figure 1-60 (right photo) shows the additional barrier to natural convection flows. Next, power wires were sheathed with ceramic to prevent short-outs, Figure 1-61 (left photo). Additionally, the power wire connections were joined by Cad welding as opposed to connectors. Finally, a very thin stainless-steel foil, Figure 1-61 (right photo), was wrapped around the reactor to reduce friction when re-telescoping the reactor into the external shell.

The new heaters were rated for a maximum current of $45 \mathrm{~A}$, which is higher than the $7.5 \mathrm{~A}$ current of the former heaters. Because of the increase in current potential, power wires, contactors, breakers, and fuses were upgraded. All of the modifications were successful and the hydrogasification reactor was able to achieve the designed temperature.
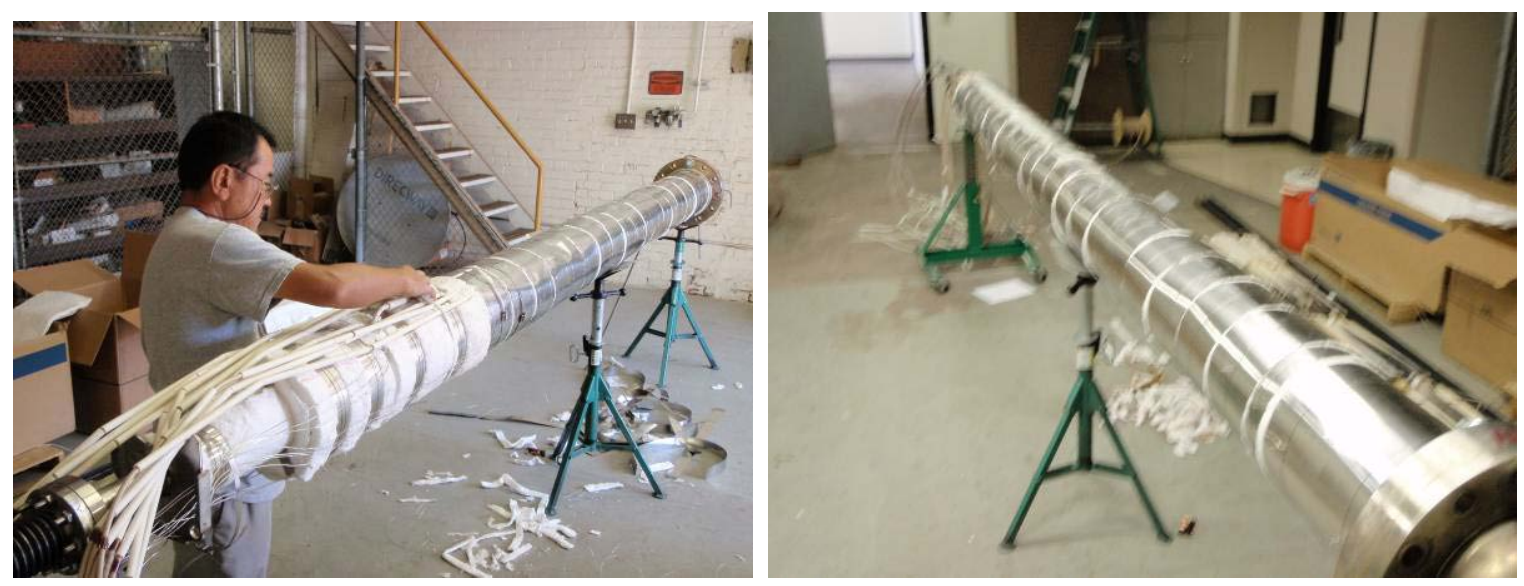

Figure 1-61 - Left photo shows the Final Layer with all Power Wires Sheathed by Ceramic; Right Photos show the Repaired Reactor Inner Tube Before Re-Telescoping

\subsubsection{OTHER PROCESS IMPROVEMENIS}

\subsubsection{Coal Feed/Charpot Isolation Valves}

After the start of hydrogasification reactor design, the coal feed and charpot isolation valves were identified as key elements in the design. The design specifications for the valves were the same as for the reactor:

- Maximum allowable working pressure of 1200 psig;

- Maximum operating temperature of $1950^{\circ} \mathrm{F}$;

- Construction materials and valve design compatible with coal and gaseous hydrogen.

In addition, the valve specification required an actuated valve, flanged ends, a low outboard-leak rate, a low seat-leak rate, and a straight-through valve bore to minimize catch 
points for the coal. The expectation was to identify a valve that would be bubble tight. The specification included the flow rate for coal and for hydrogen and a coal size of 200 mesh.

The valves were to meet or exceed Manufacturers Standardization Society, Standard Practice 61 (MSS-SP-61) that establishes requirements and acceptance criteria for shell and seat closure pressure testing of valves. This specification is key as MSS-SP-61 defines the allowable leak rate for a ball valve as $<10 \mathrm{cc} / \mathrm{hr}$ per inch of nominal pipe diameter. All shutoff or isolation valves specified to MS-SP-61 must pass this leak test at a fluid (liquid or gas) pressure no less than 1.1 times the $1000^{\circ} \mathrm{F}\left(380^{\circ} \mathrm{C}\right)$ rating rounded to the next 5 psig.

Copeland valves were ordered and installed into the hydrogasification reactor between the charpots and at the top of the reactor. They leaked noticeably during the testing. As a result, they were soon replaced because they obviously were defective and did not meet the MSS-SP-61 specifications. Ultimately, Swagelok valves, Figure 1-62, were ordered and installed to replace the Copeland valves.

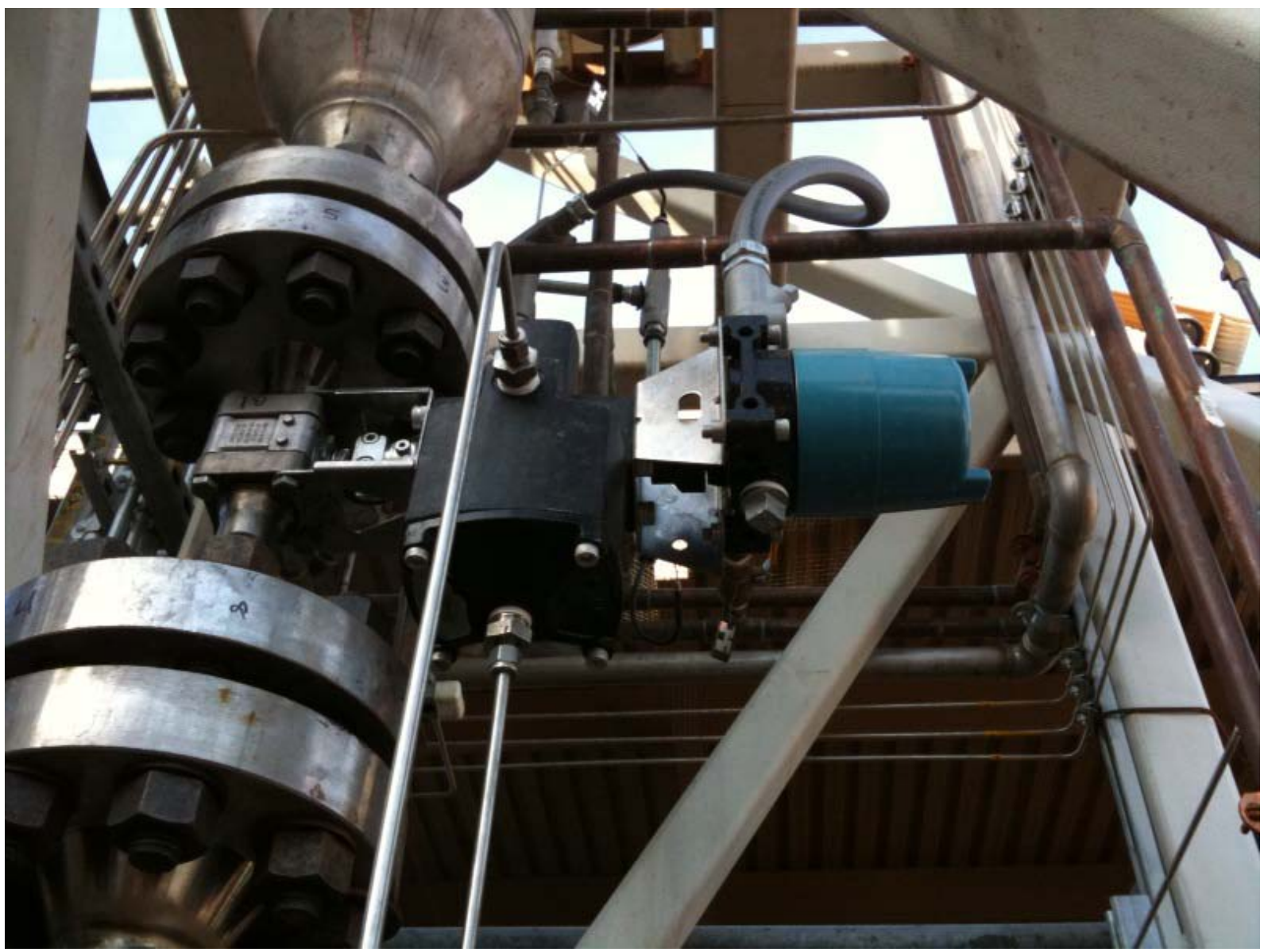

Figure 1-62 - Swagelok Valve between the Upper and Lower Charpots 
The valves were Swagelok Thermal Service 316 SS ball valves and actuators. Once installed and operated there was virtually no leakage across the valve seats even at 1200 psig.

\subsubsection{Addition of ZnO Desulfunization Bed}

A zinc oxide $(\mathrm{ZnO})$ desulfurization bed was installed for the product stream due to a strong sulfur smell during initial testing. Sud Chemie's desulfurization catalyst, G-73E (3/16-inch pellet), was purchased from Stem Chemicals. The catalyst, ZnO with Calcium Aluminate, was operated at $400^{\circ} \mathrm{F}$ to remove sulfur components in the product gas before it was vented to atmosphere. The unit was installed downstream of product gas analyzers and depressurization.

\subsubsection{Condenser Modific ations}

During initial hydrogasification tests there was a condenser pump failure. This failure occurred during the November 17, 2009 hydrogasification run and as a result some water, BTX, and oil condensed in the sample line. Some of the liquid also went into the analytical equipment causing the mass spectrometer to fail. To prevent this from occurring in the future, changes were implemented into the process, including visually checking to see whether the pump was operating. Additionally, the condenser was modified to achieve a more efficient gas-liquid separation. This was accomplished by increasing the sectional surface area of the gas-liquid separator therefore to further reduce the gas velocity in the separator and leave longer time for liquid precipitation. Shown in Figure 1-63 below are the former and latter separator configurations. 

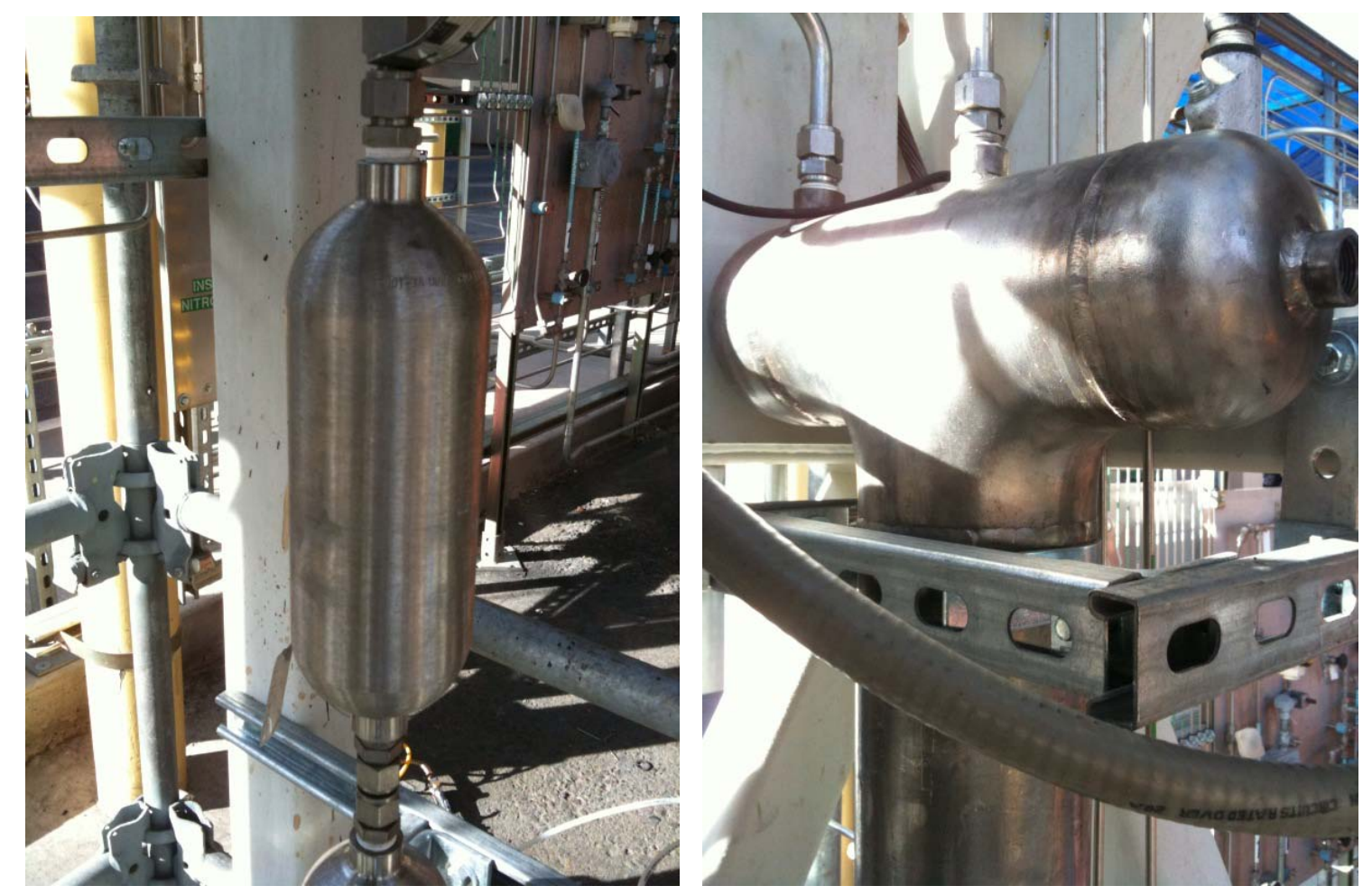

\section{Figure 1-63 - Left Photo Shows Picture of the Old Gas-Liquid Separator and the Right Photo Shows the New Separator}

\subsubsection{Addition of Sec ond Sampling Line}

While the reactor gaskets were being replaced, some other process modifications and upgrades were made. The most notable modification was the installation of a second gas sampling line downstream of the $\mathrm{ZnO}$ sorbent bed. In order to detect $\mathrm{CO}_{2}$, the gas chromatograph (GC) was equipped with a methanizer, which converted non-combustible $\mathrm{CO}_{2}$ into combustible $\mathrm{CH}_{4}$ on-line, so it was detectable by a Flame Ionization Detector (FID). In addition, the methanizer used a nickel-based catalyst for this conversion, which was susceptible to sulfur poisoning. The second gas sampling line was to deliver sulfur-free product gas to the GC. It also provided an opportunity for the mass spectrometer to monitor the sulfur removal with the ZnO sorbent bed. Figure 1-64 shows the mass spec $\mathrm{H}_{2} \mathrm{~S}$ signal for sampling from upstream, downstream and then again upstream of the ZnO sorbent bed. As seen in Figure 1-64, when the mass spec was sampling upstream of the sorbent bed, the $\mathrm{H}_{2} \mathrm{~S}$ concentration was clearly above zero parts per million. In contrast, when the mass spec was sampling downstream of the sorbent bed, the $\mathrm{H}_{2} \mathrm{~S}$ concentration becomes undetectable. This is clear evidence that the ZnO was an effective desulfurization sorbent. 


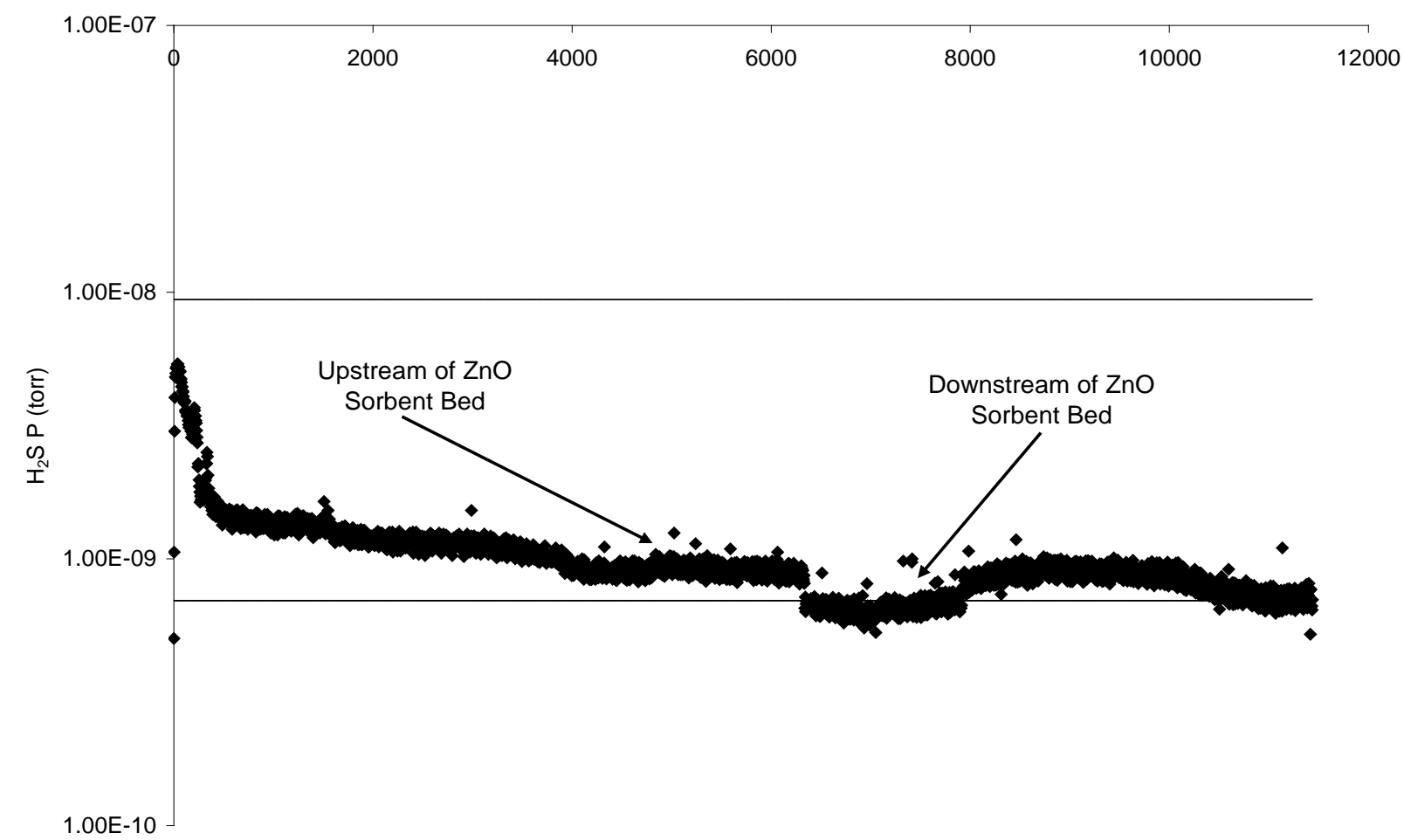

Time (s)

- H2S Experimental —-4970ppm H2S Standard $-0 \mathrm{H} 2 \mathrm{~S}$

Figure 1-64 - Mass Spectrometer $\mathrm{H}_{2} \mathrm{~S}$ Output

\subsection{CONCLUSIONS ON HYDROGASIFCATION BENCH TESTING}

An advanced high temperature, high-pressure hydrogasification reactor was engineered and constructed during this project. It was ASME certified under Section VIII with a rating of 1150 psig at $1950^{\circ} \mathrm{F}$. The hydrogasification reactor could feed 200 mesh (74 micron) pulverized coal at rates up to $15 \mathrm{lb} /$ hour. The hydrogen injection temperature could be varied up to $1350^{\circ} \mathrm{F}$. The hydrogen preheater was made of Inconel 625 and the reactor was made of Inconel 617. The hydrogasification reactor assembly included a coal feeding system, ash collection pots, and a 42 feet tall vertical design. The hydrogasification reaction zone was 1.75 -inch internal diameter (ID) by 16 feet long.

The NETL Computational Fluid Dynamic (CFD) modeling of the reactor was extremely helpful in reactor design and configuration. Extensive CFD modeling of the hydrogen nozzle design was completed and seventeen cases were designed. The hydrodynamics (solid equatorial mixing, back mixing and temperature profile) around the reactor head were studied, resulting in a design recommendation of 0.18-inch hydrogen nozzle ID, 45-degree shooting angle, and 30-degree swirling angle as optimum configurations. 
A 1-D model of the coal hydrogasification reactions was further developed based on $A R C H$ kinetics originally proposed by Miura in the early 1990s. Pathways for methane's formation proposed by the reaction scheme were through initial reactions: 1) coal decomposition and secondary reactions; 2) reaction of Rapid Carbon and 3) hydrocracking of Benzene/Toluene/Xylene (BTX) and tars and oils. Comparing results showed that the model did a reasonable job of predicting the carbon conversion to $\mathrm{CH}_{4}, \mathrm{BTX}$, and total carbon conversion. However, the model was off with the distribution of conversions to $\mathrm{CO}$ and oil.

The hydrogasification reactor was installed at an APS facility. The system was remotely operated in a control room using LabVIEW automation system. The hydrogasification reactor control room used GC/MS to determine product gas compositions.

The first hydrogasification test was completed on June 9, 2009. Experimental conditions included hydrogen injection temperature of $650^{\circ} \mathrm{F}$, reaction temperature of $1250^{\circ} \mathrm{F}$, pressure of 1000 psig, 10s residence time, 0.4 hydrogen-to-coal ratio, and $11.5 \mathrm{lb} / \mathrm{hr}$ coal flow rate. Due to the low reaction temperature, only $18 \%$ carbon conversion was achieved. GC/MS analysis on the organic phase indicated it mainly contained benzene, toluene, xylene, and tar. Char collected from the bottom of the reactor had a 9000 Btu/lb heating value.

While trying to achieve the desired hydrogasification conditions, the reactor experienced unsteady coal feed rate, low hydrogen injection temperatures and an incident where reactor heaters failed. Modifications and repairs were conducted, which included modifying preheater zone 5 , modifying the coal feeding system and reconfiguring all reactor heating zones.

Once the hydrogasification reactor was repaired and put back into operation three more hydrogasification runs were executed. These test runs were the first to reach steady state and produced $\sim 50 \%$ carbon conversions and $\sim 10 \%$ methane yield in the production gas. The hydrogen injection temperature reached $1350^{\circ} \mathrm{F}$ and reactor temperature reached $1750^{\circ} \mathrm{F}$. At this point, all of the analytical techniques were reevaluated and recalibrated. Continued hydrogasification testing became part of a follow-on project, DE-FE0001099 "Integrated Energy System with Beneficial Carbon Dioxide $\left(\mathrm{CO}_{2}\right)$ Use (IES). Thirteen successful tests were conducted from January to March 2010, under the follow-on Cooperative Agreement and the results of those hydrogasification tests are reported in the Final Topical Report for that project. The IES project was officially terminated by APS on March 31, 2010. 
Development of a Hydrogasification Process for

Coproduction of SNG and Eectrical Power from Westem Coals

Final Report

THIS PAGE INTENTIONALLY LEFT BLANK 


\section{Algae testing}

\section{$2.1 \quad$ INTRODUCTION AND BACKGROUND}

\subsubsection{INTRODUCTION TO ALGAE}

Algae have existed for 3.5 billion years, having adapted and evolved in many of earth's ecosystems. They have adapted to live on land in the snows of mountains, in cryptobiotic crusts found in deserts and grassland soils, or embedded within the surfaces of rocks in deserts. There are thousands of species of algae. They are made up of carbohydrates, lipids, and proteins, where carbohydrates can be used to make ethanol; the lipids can be used to produce diesel and aviation fuels; and proteins can be used for food additives. They produce oxygen while consuming $\mathrm{CO}_{2}$ and are the original source of most of the fossils fuels we use today.

Although there exists a market for some algal species in food additives, nutrients, cosmetics, and fish food, using algae farms to capture and recycle $\mathrm{CO}_{2}$ as a means of addressing climate change and developing a new energy source, is a new industry, creating new jobs. The concept of using algae to create biofuels has been explored in the United States since the 1970s. The idea has gained momentum over the last several years due to the escalating costs of fossil fuels and concerns about energy independence and climate change.

Algae can be cultivated on arid land using low-quality water and can be grown without compromising farmlands and forests. They do not require potable or even agricultural quality water to grow. Microalgae are single-cell plant, and do not grow but divide. In good growing conditions algae can divide every few hours. Because of this doubling capacity, algae can be harvested daily and offers a biological option for carbon recycling from $\mathrm{CO}_{2}$ emission sources. Certain species of algae can have high lipid content. These lipids can be removed and processed into liquid transportation fuels (e.g., biodiesel, ethanol, and military fuel), constituting a beneficial reuse of $\mathrm{CO}_{2}$ and offsetting an equivalent amount of imported petroleum that typically fuels the nation's transportation needs. Algae have yield of oil per acre that is approximately two orders of magnitude higher than those of traditional plant materials used to produce biofuels. Table 2-1 provides a summary of potential oil yield from algae versus yield from other plant materials. 
Table 2-1 - Plant Material Oil Yields

\begin{tabular}{|c|c|}
\hline & $\begin{array}{c}\text { Oil Yield } \\
\text { Gallon/Acre/Year }\end{array}$ \\
\hline Plant Material & $1600-6500$ \\
\hline Corn & 13 \\
\hline Soy & 47 \\
\hline Safflower & 83 \\
\hline Jojoba & 192 \\
\hline Coconut & 290 \\
\hline Palm & 640 \\
\hline
\end{tabular}

There are several operating microalgae farms in the world. The harvesting of seaweed (algae) from the ocean is a commercial process, but this operation as well as other commercial operations like shrimp farms are excluded from this discussion.

The largest of the microalgae farms are "open" systems. The term open means that the water culture is open to the air. These are low-cost systems and could be raceways, open ponds, or circular agitated ponds. Open pond systems are very good in wastewater applications. Ponds are used in fisheries applications, generally grow algae as needed for their primary business, and generally yield low growth (5 grams per square meter per day $\left(\mathrm{g} / \mathrm{m}^{2} / \mathrm{d}\right)$ ) of many species.

Raceways are also open systems and are subject to many predation problems, evaporation losses, and low $\mathrm{CO}_{2}$ capture efficiency. Raceways are commercial systems used to grow a single species. Raceways are considered to be the lowest-cost farm systems, producing algae for about $\$ 5,000 /$ ton (dry) at growth rates up to about $20 \mathrm{~g} / \mathrm{m}^{2} / \mathrm{d}$. The largest raceway system is about 50 acres. In Israel, one Seambiotic farm operates a small raceway system being fed with 
flue gas from a coal-fired power plant (see Figure 2-1 and Figure 2-2). This farm is supplied with water from the plant condenser cooling water, which uses once-through Mediterranean Sea water. Seambiotic also operates a 20-acre open raceway farm in the southern Negev desert, which uses water from the Red Sea.

$\mathrm{CO}_{2}$ is trucked to this farm from Egypt. This algae crop is used for human consumption (Figure 2-2).

There are closed algal systems that use numerous bioreactors in parallel or series combinations. These systems have several advantages, among which are higher productivity, higher purity, less water loss, and lower predator control issues. Tubular bioreactors are a type of closed-system bioreactor and are

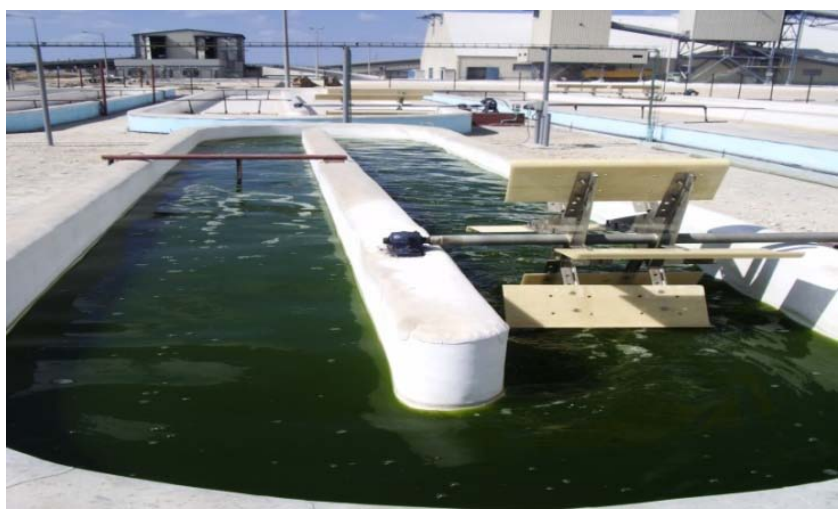

Figure 2-1 - Ashqelon Israel, 2000--MW Coal-Fired Power Plant

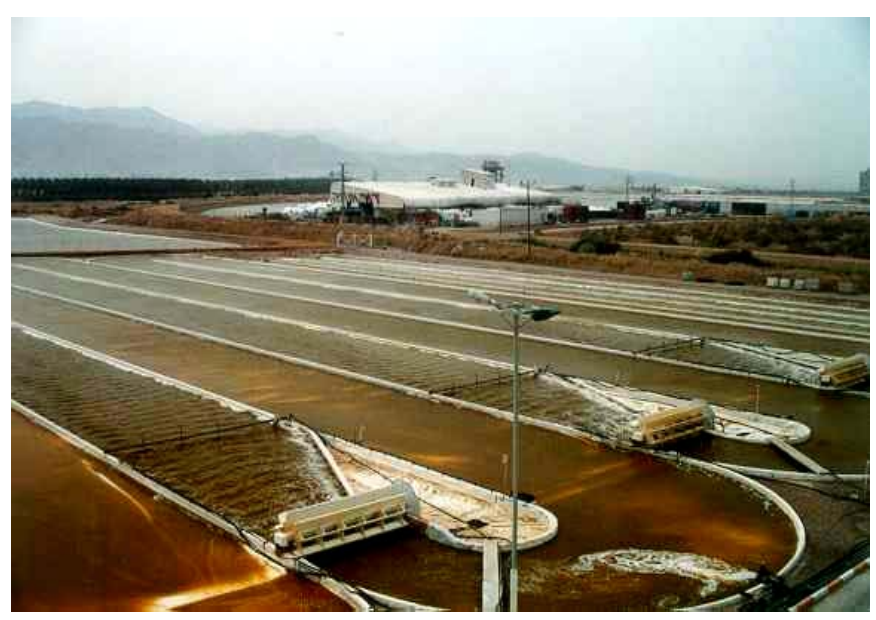

Figure 2-2 - Natural Beta Technologies Ltd, Elat Israel, Open Raceway Algae Farm typically small-diameter glass tubes (see Figure 2-3). The bioreactor systems are generally small and easily maintained, growing a small amount of algae as a high-value crop. These systems are highly effective, capturing up to $98 \mathrm{wt} \%$ of the $\mathrm{CO}_{2}$ injected to the culture. To date, these systems have high capital and operations and maintenance (O\&M) costs. The $\mathrm{CO}_{2}$ is purchased commercially and injected into the culture. Water is not recycled. These systems are very effective in growing high-value, quality algae and are good for pharmaceuticals, human food products, chemicals, etc. The growth rates of algae in these systems are higher than open systems. Production costs are about $\$ 10,000 /$ ton (dry). The largest tubular bioreactor system is in IGV Institute, Germany, at about 3 acres (See Figure 2-4). All of these types of commercial algae farms typically purchase $\mathrm{CO}_{2}$ at a cost of about $\$ 500 /$ ton. 


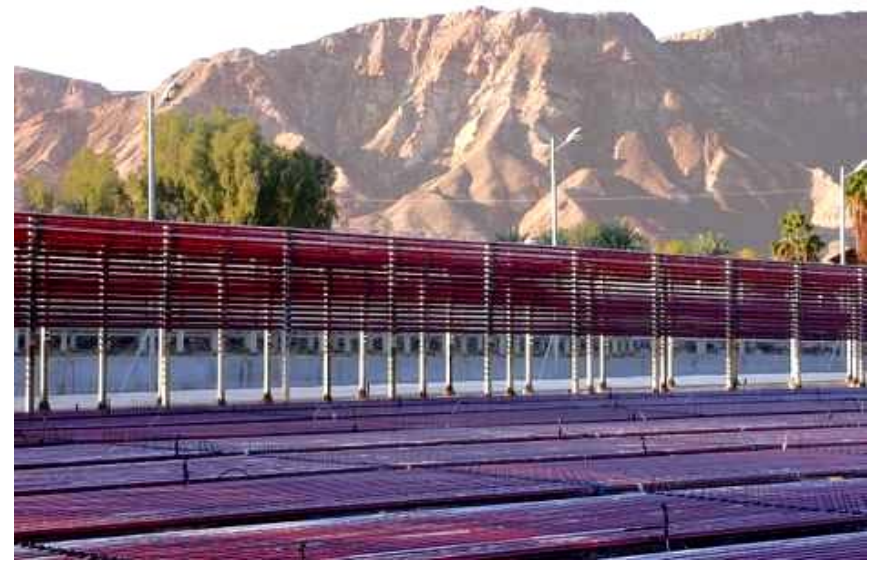

Figure 2-3 - Aquatechnologies Algae Farm in Ketura, Israel - High-Value Products

There are also a number of experimental algae systems that have been developed at universities and privately. Many of these systems are impressive in their production rates. These researchers are providing additional insight into photosynthesis efficiency. (The research applications of the University of Arizona are a good example of very effective research being performed.) As effective as these systems have been, they do not address the following key challenges: successful application of flue gas, rational economics, and proven levels of $\mathrm{CO}_{2}$ capture from flue gas, which are among the major goals of this project.
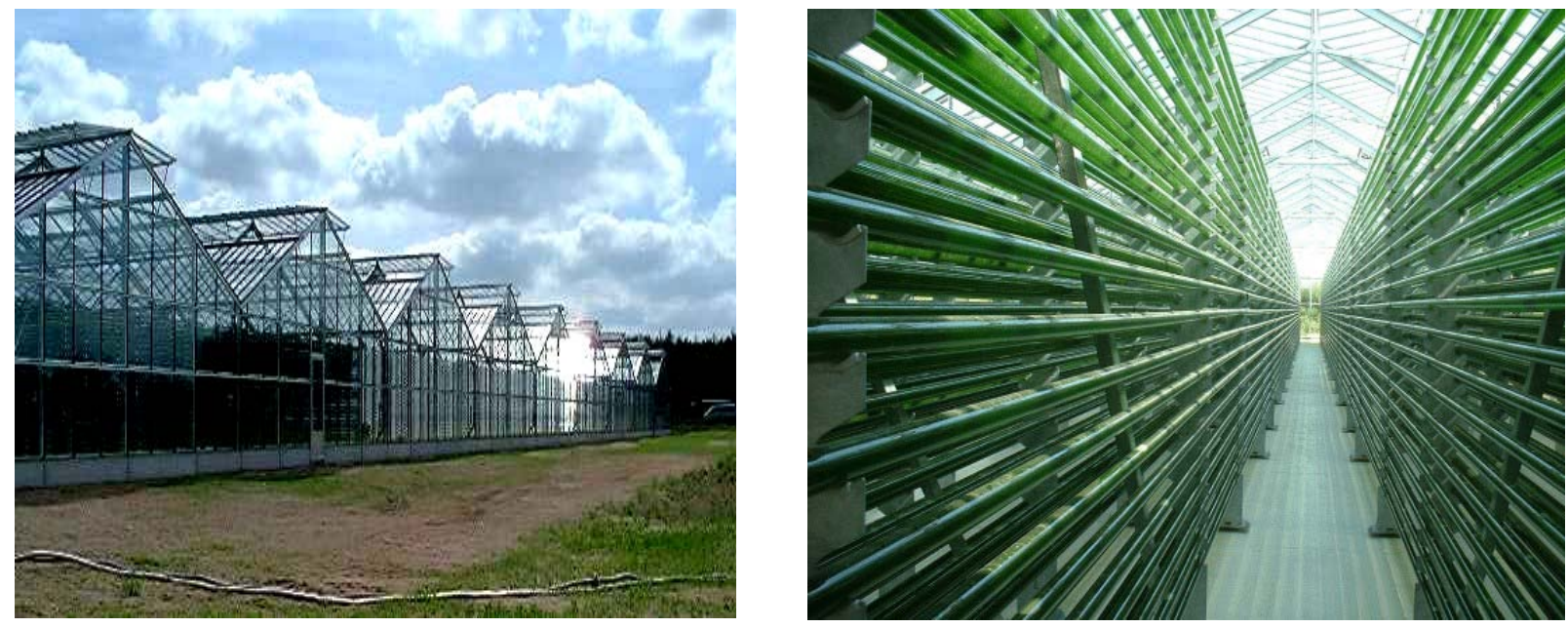

Figure 2-4 - Left photo: BPS Algae Farm in Klotze, Germany. Right photo: 10,000-M² System Operating since 2002.

\subsubsection{APS/ G REeNFuEL PARINERSHIP}

APS began its evaluation of algae as a $\mathrm{CO}_{2}$ recycling option in early 2005, when the Massachusetts Institute of Technology (MIT) initiated its algae R\&D project and began injecting flue gas from their campus utility boiler into a series of algae-filled tubes (Figure 2-5). Shortly thereafter APS contracted with the newly formed GreenFuel Technologies (formed by the MIT researchers working on the project) to perform a demonstration of $\mathrm{CO}_{2}$ recycling from algae in 
Arizona at the Redhawk Power Plant. A tubular system (GEN3, see Figure 2-6), similar to that developed in the MIT's R\&D project, was built and operation was initiated at Redhawk in August 2005. GEN3 was operated continuously with flue gas until November 2006. GEN3 was an expensive system, energy intensive and maintenance intensive, and it could not be scaled for utility use; but it grew algae at a high rate $\left(57 \mathrm{~g} / \mathrm{m}^{2} / \mathrm{d}\right)$. GreenFuel subsequently constructed a new prototype system (GEN5 or vertical thin film (VTF)) in an attempt to address some of the limitations of the GEN3 system and placed it into operation in April 2007 (Figure 2-7). GEN5 was a $1000-\mathrm{m}^{2}$ system and initially grew algae as designed, but failed by June 2007 due to challenges in maintaining the correct culture conditions for the algae. The GEN5 system was then reduced to $100 \mathrm{~m}^{2}$ and operated for 3 weeks. After the 3-week operational period at the reduced size, GreenFuel ended its GEN5 system operation in August 2007. It was estimated that several million dollars was spent in developing and demonstrating the GEN5 system. GreenFuel claimed very high growth rates with the GEN5 system, but experienced severe problems in continuous operation. Meanwhile during the contractual period between APS and GreenFuel, GreenFuel engaged Inventure Chemicals, Gig Harbor, Washington, to develop a propriety process to produce biodiesel from algal lipids and ethanol from the remaining biomass. Inventure produced products that met the American Society of Testing and Materials (ASTM) requirements for biodiesel and ethanol. Further algae testing occurred when APS hired GreenFuel to perform "adaptation studies" of several candidate algae at its 2000 MW Four Corners (4C) Power Plant in Farmington, New Mexico (Figure 2-8). After 3 months of studies using the water and flue gas at 4C, GreenFuel provided an Adaptation Report in December 2007 showing that several algal species had flourished in the $4 \mathrm{C}$ environment. In addition to their studies at Redhawk and 4C, GreenFuel assisted with the planning and provided training for the "bag farm" installed at APS's 3rd Avenue R\&D facilities in Phoenix, Arizona, in 2008. GreenFuel also performed initial strain selection for the project and provided inoculum for the first inoculation of the bag farm (see Section 2.2 of this report). 

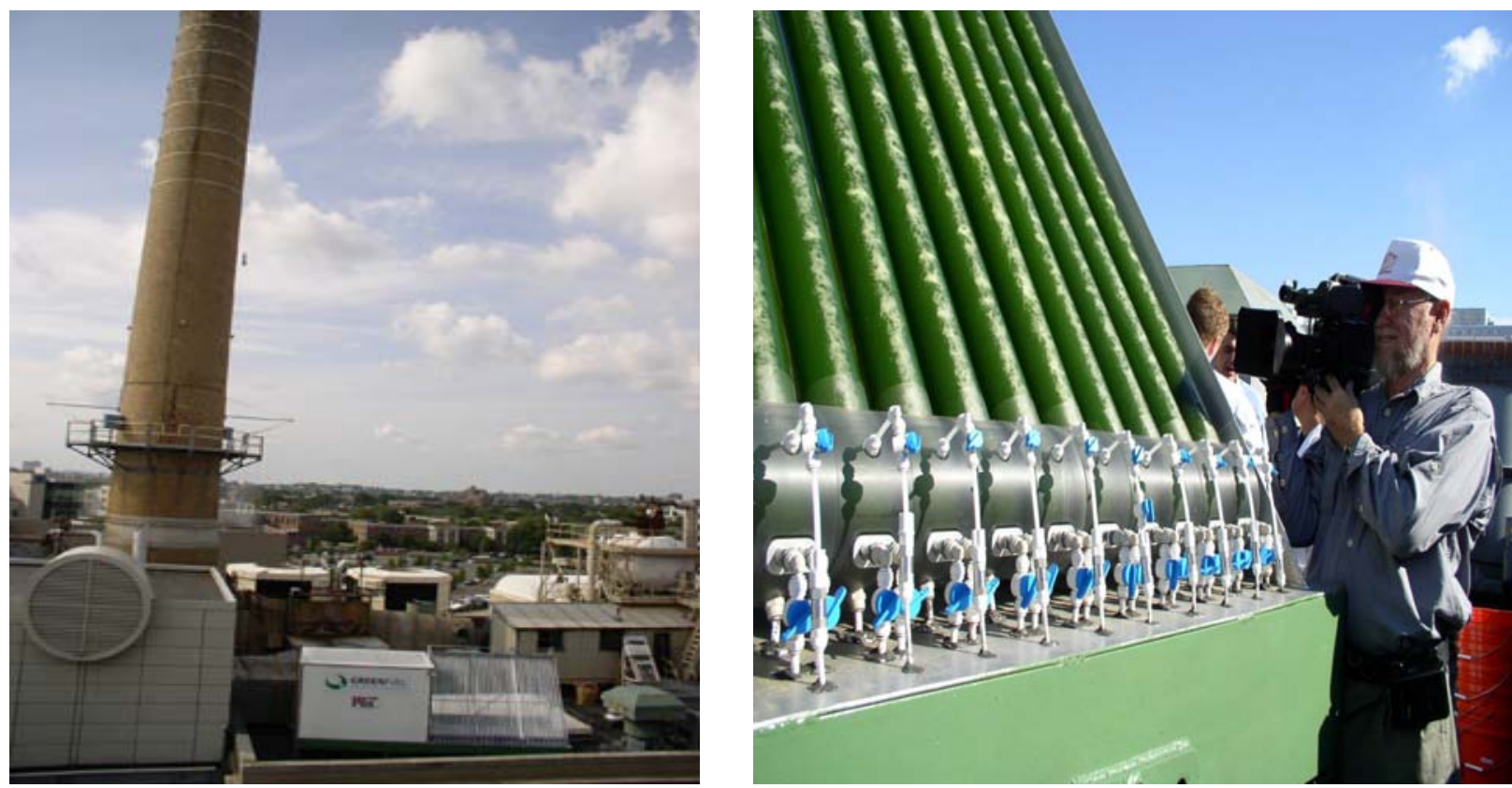

Figure 2-5 - GreenFuel Early Demonstration at Massachusetts Institute of Technology, Massachusetts, 2004

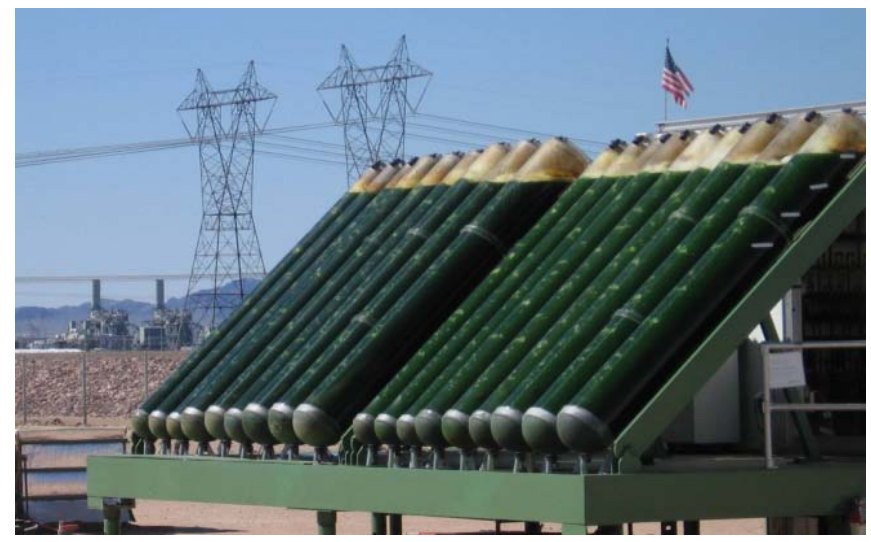

Figure 2-6 - GreenFuel Gen3 System at APS's Redhawk Power Plant in Arizona 2005-2006

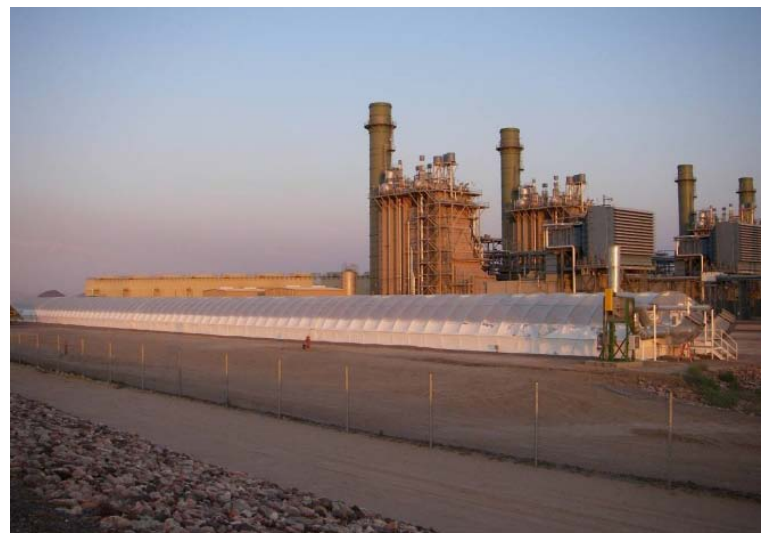

Figure 2-7 - GreenFuel Gen5 System at APS's Redhawk Power Plant in Arizona, June 2007 


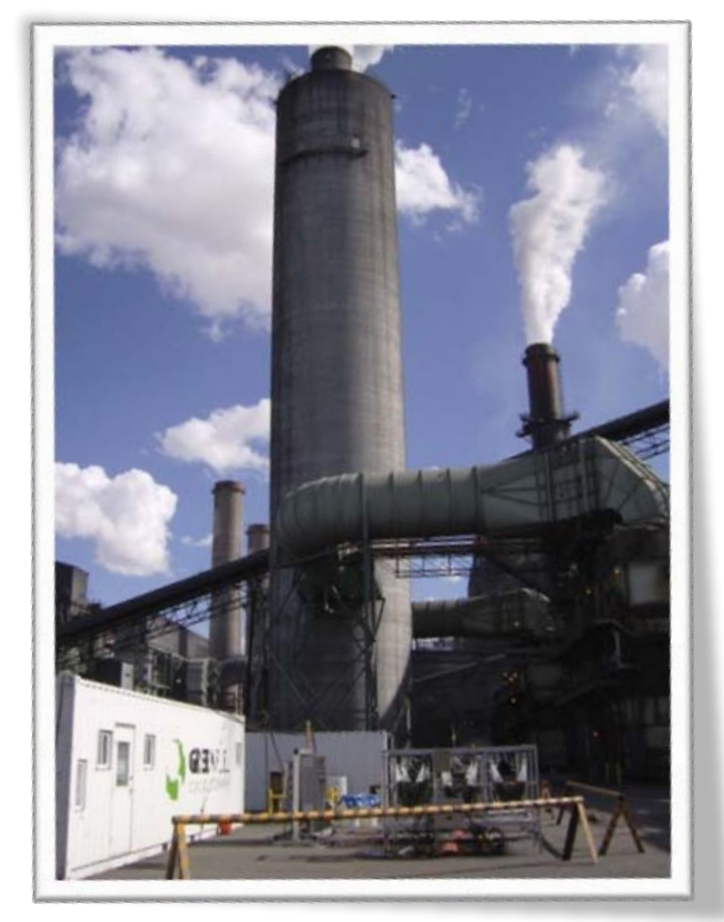

Figure 2-8 - GreenFuel Algae Adaptation Study at APS's Four Corners Power Plant, Farmington, New Mexico, December 2007

\subsubsection{INCORPORATION OF ALgAE WORK INTO SNG PROJ ECT}

Concerned about greenhouse gas (GHG) emissions from its fossil plants and potential liability if the U.S. adopts strict GHG controls to address climate change, in 2008, APS began to work with DOE's NETL to explore expanded possibilities for $\mathrm{CO}_{2}$ capture through algae, with the goal of determining whether progress on carbon capture from fossil power plant flue gas was feasible. APS was in a favorable position to work with algae experts on how to integrate an algae system into an operating power plant with the intent of capturing $\mathrm{CO}_{2}$ emissions. APS operates $4000 \mathrm{MW}$ of natural gas (NG) generation, and owns and/or operates an additional $5000 \mathrm{MW}$ of coal generation. The potential synergies of algae and power plant systems had not been previously considered. To fit into a power plant environment, the algae bioreactor system must be robust, scalable, water sensitive, and economic to be a viable alternative for $\mathrm{CO}_{2}$ capture.

APS proposed to demonstrate scalable carbon capture from a power plant slipstream with algae by investigating a scalable prototype algae farm system consisting of multiple prototype units 
(up to 120,000 liters (L) of culture on one unit). The project proposed to integrate with an operating power plant and demonstrate continuous operation capturing $\mathrm{CO}_{2}$, growing algae, harvesting algae, and managing overall operations to demonstrate control, production rates, and economics. The evaluation of the prototype system would also provide a baseline for economies of scale for increasing size and transferability to larger fossil-fuel combustion operations.

The algal biomass produced was to be collected and analyzed for oils, carbon and other significant chemical content. Limited amounts of algal oil were to be extracted to provide an algal crude oil source for processing into useful military fuel. Samples of algae paste, dried algae, algal crude oil, and algal paste would be delivered to NETL and outside labs for independent analyses and studies. These laboratories include (1) University of Kentucky Center for Applied Energy Research (CAER), Lexington, Kentucky; (2) Arizona State University (ASU) School of Life Sciences, Gilbert, Arizona; (3) POS Pilot Plant Corporation, Saskatoon, Saskatchewan, Canada; (4) DynaSep LLC, Newark, Delaware; (5) New Jersey Feed Laboratory, Ewing, New Jersey; (6) ConocoPhillips, Bartlesville, Oklahoma and (7) the National Energy Technology Laboratory (NETL).

APS also proposed to develop and implement a plan for laboratory development of high-value liquid fuel products from algae oil, development of algal oil extraction techniques and development of a process to produce liquid carbon carrier(s) certified to meet military standards (such as Jet Propellant 5 (JP-5) and/or Fuel Oil (F-76)). A sample of military fuel was to be delivered to NETL for independent analysis of liquid quality. Heavy metal absorption by algae was proposed to be re-evaluated also in a slipstream of coal flue gas.

This portion of the project aimed to address the emission of $\mathrm{CO}_{2}$ from industrial processes, including the hydrogasification process and emission from the combustion of SNG produced by hydrogasification. The integration of algae into the APS advanced hydrogasification process (AHP) for carbon recycling consumes the $\mathrm{CO}_{2}$ emissions from the AHP and combustion of SNG produced by hydrogasification. It also produces renewable biofuels and other high-value byproducts through growth of algae. Although it adds an additional expense with the accompanying land and energy use, the potential high-value by-products from algae addition would help offset some of the operational costs versus other $\mathrm{CO}_{2}$ capture methods.

Two algal R\&D facilities were built for the project. The 3rd Avenue APS R\&D facility was located in downtown Phoenix. Facilities included a laboratory, algal nursery, 70-foot-diameter 
thermal pond, a bag farm (100 bags, each with $80 \mathrm{~L}$ capacity) with support infrastructure, and a bioreactor fabrication area. The second facility was a $\mathrm{CO}_{2}$-capture demonstration project at the APS 1000 MW Redhawk Power Plant, located 55 miles west of Phoenix. This site had a 2000- $\mathrm{m}^{2}$ algae farming footprint. It incorporated water treatment, inoculation, flue gas $\mathrm{CO}_{2}$ concentration and delivery, dewatering, algae grinding, and an algae paste storage system.

\subsection{INITIALALgAE PRODUCTION}

The algae portion of the SNG project began primarily in the first quarter of 2008. At that time, efforts focused on producing algae biomass to supply the work on lipid extraction and biofuel production performed by offsite laboratories. As the project progressed, the algae nursery, strain selection and analytical work were directed to the site. Additionally, a prototype six meter radius (6M) bioreactor was designed, built, and tested at the 3rd Avenue R\&D Facility in Phoenix, Arizona (see Figure 2-15).

During the period of January 1 through March 31, 2008, a large $8000 \mathrm{~L}$ outdoor bag farm was built over a 4-week construction period at the 3rd Avenue R\&D facility. The initial function of the bag farm was to generate large quantities of algal biomass for processing to algae oil, and this product was then transported for further processing at offsite laboratories. As the project progressed, the bag farm became increasingly utilized for inoculum development.

The bag farm is depicted in Figure 2-9 and was built with a capacity of 100 bags. The farm consisted of several freestanding pallet frames assembled together and located outdoors in an east-to-west orientation. Each frame was intended to support four $80 \mathrm{~L}$ bags made of 8-millimeter thick clear polyethylene suspended from each side of the frame. 


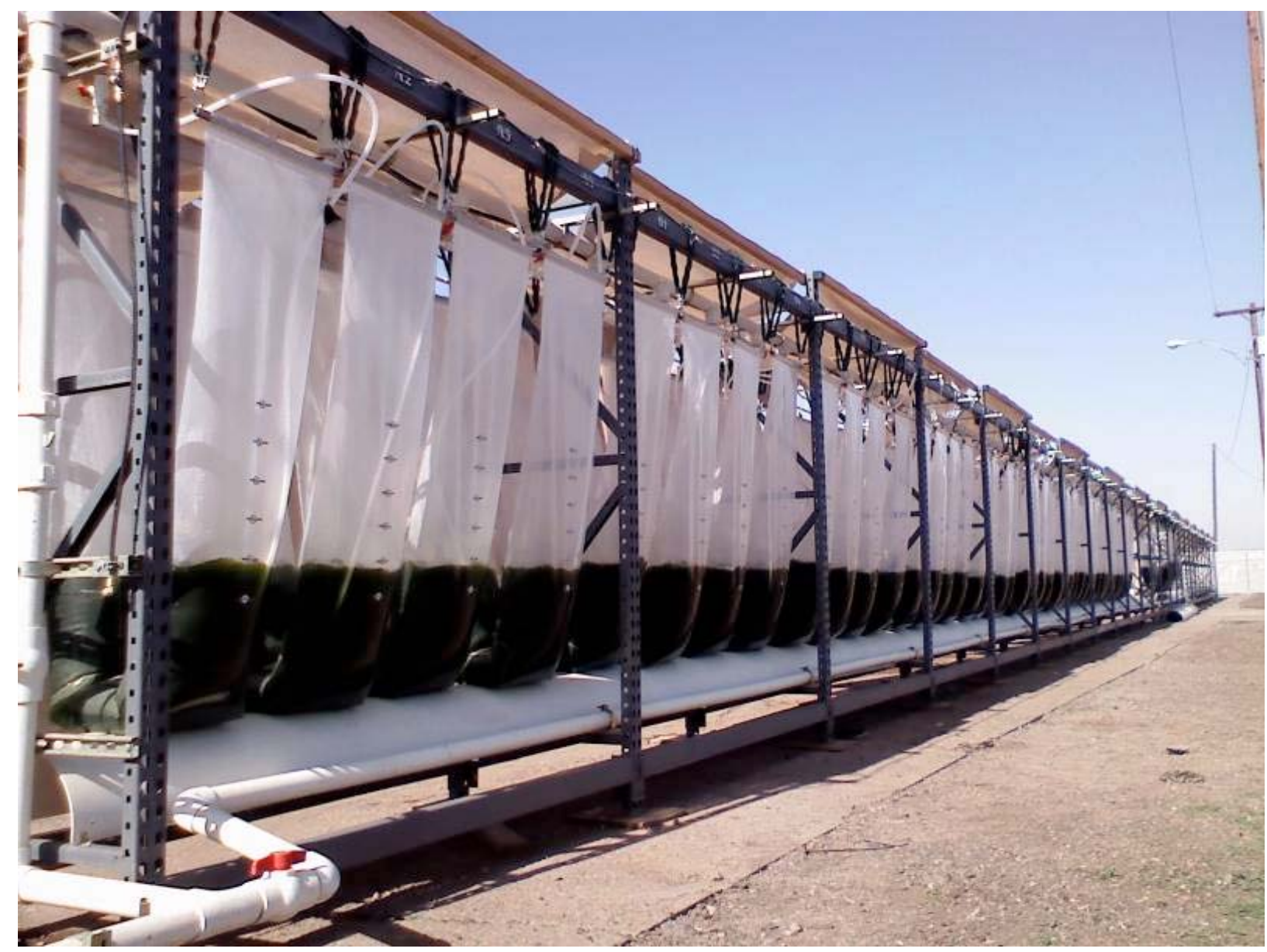

Figure 2-9 - Bag Farm at 3rd Avenue R\&D Facility

A liquid $\mathrm{CO}_{2}$ tank, blowers, polyvinyl chloride (PVC) piping and valves comprising the gas delivery system provided a $\mathrm{CO}_{2} /$ air mix and turbulence to the cultures. A water misting system was in place for the purpose of evaporative cooling, effective in the arid Arizona climate.

The farm was first commissioned on April 2, 2008. GreenFuel Technologies Corporation (GreenFuel) provided onsite training to the farm's staff over a two-week period and provided the inoculation culture which was given the identifier name GF3. Initial inoculation of the bag farm occurred April 6, 2008, and it was continuously operated with the GF3 algae strain culture until August 23, 2008. The algae strain was not identified beyond the GF3 designation due to the proprietary nature of the information. The seed culture at a density of approximately $6 \mathrm{~g} / \mathrm{L}$ was shipped from GreenFuel and was used to inoculate bags equaling $4000 \mathrm{~L}$ total culture volume (half the capacity of the system). The operational procedure included a ten-day growth period after an individual bag inoculation or longer to ensure a harvested culture with a density of at least $1 \mathrm{~g} / \mathrm{L}$. 
When maintaining cultures in continuous operation, a harvest entailed removal of $50 \mathrm{wt} \%$ of any individual bag culture followed by replenishing the removed volume with an equal volume of growth medium. Otherwise, all of the cultures were harvested and combined for appropriate use. If algal paste was desired, the cultures were concentrated and dewatered by centrifugation. The resulting paste was then frozen and stored or shipped to partner sites as necessary. Daily sampling was performed on every tenth bag to ensure the growth and health of the cultures within normal ranges.

The daily samples evaluated the following parameters: $\mathrm{pH}$, temperature, fluorescence, and microscope observations. The daily sampling technique was standardized as much as possible including collection times and analysis periods. Generally, GF3 required 10 days to grow from inoculation at $0.2 \mathrm{~g} / \mathrm{L}$ to $1.0 \mathrm{~g} / \mathrm{L}$, and 7 days from $0.5 \mathrm{~g} / \mathrm{L}$ to $1.0 \mathrm{~g} / \mathrm{L}$. Harvesting of GF3 began April 20, 2008 and included harvests of about $320 \mathrm{~L}$ of algal culture from 4 bags each day (Monday through Friday). Centrifugation of this harvest generally yielded $4 \mathrm{~L}$ of algal paste. In total, about 300 kilograms $(\mathrm{kg})$ of algae paste and 500 grams $(\mathrm{g})$ of dried algae resulted from these bag-farm harvests, which were sent to the outside labs for lipid extraction and the generation of JP-8 (jet fuel). Since lipids are the portion of the algae that is used in the production of liquid fuel, it was a goal of the project to cultivate algae in a manner that maximized lipid content. 
GF3 algae biomass and algae oil were analyzed by ASU and POS in the fiscal fourth quarter (July-September) of 2008. Based on the ASU report, the total lipid content of GF3 was found to be 20.3 wt\% based on fatty acid analysis, in which neutral lipid content in dry weight was 4.2 wt\%; and polar lipid content in dry weight was $15.4 \mathrm{wt} \%$. Table 2-2 provides the details of the analyses conducted on the cultivated algae. To obtain neutral lipid and polar lipid contents data, freeze-dried algae samples were extracted with methanol at $40^{\circ} \mathrm{C}\left(140^{\circ} \mathrm{F}\right)$ for 40 minutes. The mixture was centrifuged, the supernatant removed, and the solid phase was extracted with a mixture of hexane and ether (1:1, volume to volume (v/v). Diethyl ester, hexane, and Table 2-2 - Fatty Acid Analysis of GF3 by ASU

water were added to the combined supernatants, so as to form a ratio of 1:1:1:1 $(v / v / v / v)$. The mixture was shaken and then extracted twice with a mixture of diethyl ether and hexane $(1: 1, v / v)$. The organic phases were combined, evaporated to dryness and weighed for neutral lipid content. The methanol and water phases were combined, also evaporated to dryness and weighed for polar lipid content.

Fatty acid analyses were conducted by gas chromatography (GC) after direct transmethylation of fatty acids with sulphuric acid in methanol. The fatty acid methyl esters (FAMEs) obtained were extracted with hexane containing 0.8 wt $\%$ butylated hydroxytoluene (BHT) and analyzed by HP6890 GC (Hewlett-Packard) equipped with HP7673 injector, a flame-ionization detector, and an HP-INNOWAX ${ }^{\mathrm{TM}}$ capillary column (HP 1909N-133, 30 meter $(\mathrm{m})$ by 0.25 millimeter $(\mathrm{mm})$ by 0.25 micrometer $(\mu \mathrm{m}))$. A $2-\mu l$ sample was injected using the split-less injection mode. The inlet and detector

\begin{tabular}{|c|c|}
\hline Fatty acids & $\begin{array}{c}\text { Percentage (wt\%) of } \\
\text { Fatty Acids in Total } \\
\text { Fatty Acids }\end{array}$ \\
\hline C6:0 & 0 \\
\hline C8:0 & 0.04 \\
\hline C10:0 & 0.02 \\
\hline C11:0 & 0.02 \\
\hline C12:0 & 1.75 \\
\hline C13:0 & 1.40 \\
\hline C14:0 & 0.01 \\
\hline C14:1 & 0.04 \\
\hline C15:0 & 0.8 \\
\hline C15:1 & 0.03 \\
\hline C16:0 & 26.40 \\
\hline C16:1 & 2.60 \\
\hline C17:1 & 8.40 \\
\hline C18:0 & 21.10 \\
\hline n9t C18:1 & 0.02 \\
\hline n9c C18:1 & 0.60 \\
\hline C18:2 & 30.30 \\
\hline C20:0 & 3.25 \\
\hline n6c C18:3 & 2.80 \\
\hline n3 C18:3 & 0 \\
\hline C20:1 & 0.03 \\
\hline C21:0 & 0.02 \\
\hline C20:2 & 0.07 \\
\hline $\mathrm{C} 22: 0$ & 0 \\
\hline n6C20:3 & 0 \\
\hline n3C20:3 & 0.06 \\
\hline n9 C22:1 & 0.03 \\
\hline C23:0 & 0 \\
\hline C20:4 & 0 \\
\hline $\mathrm{C} 20: 5$ & 0 \\
\hline C24:0 & 0.03 \\
\hline C24:1 & 0.02 \\
\hline C22:6 & 0.03 \\
\hline
\end{tabular}


temperatures were kept at $250^{\circ} \mathrm{C}$ and $270^{\circ} \mathrm{C}\left(482^{\circ} \mathrm{F}\right.$ to $\left.518^{\circ} \mathrm{F}\right)$, respectively; the oven temperature was programmed from $170^{\circ} \mathrm{C}$ to $220^{\circ} \mathrm{C}\left(338^{\circ} \mathrm{F}\right.$ to $\left.428^{\circ} \mathrm{F}\right)$ increasing at $1^{\circ} \mathrm{C} / \mathrm{min}\left(34^{\circ} \mathrm{F}\right)$. High-purity nitrogen gas was used as the carrier gas. FAMEs were identified by comparing their retention times with those of the authentic standards (supplied by Sigma) and were quantified by comparing their peak areas with that of the internal standard (C17:0). Table 2-2 gives the fatty acid composition. It shows that the major species have carbon chain lengths of 16 and 18 , and about $34 \mathrm{wt} \%$ lipids contain a saturated carbon chain.

POS processed relatively large quantities of samples. After freeze-drying the paste, they normally ground the algae into fine powder using a bead grinder. This grinding process was expected to mechanically break the algae cell wall so that neutral lipids could be easily accessed and extracted by hexane. Due to the banana shape of the GF3 strain (Figure 2-10), the breakage of cell walls through this grinding process was not very effective. Extending the grinding time was required. At the end, POS obtained about 3 wt $\%$ neutral lipids based on dry basis by using hexane as solvent. This generally agreed with the ASU results. The chain length of major species was determined to be 18. In addition, POS results indicated that the GF3 strain had enriched unsaturated fatty acids of $\sim 58$ wt\%. Omega 3, 6, and 9 are about 54 wt\% total fatty acids, as shown in Table 2-3.

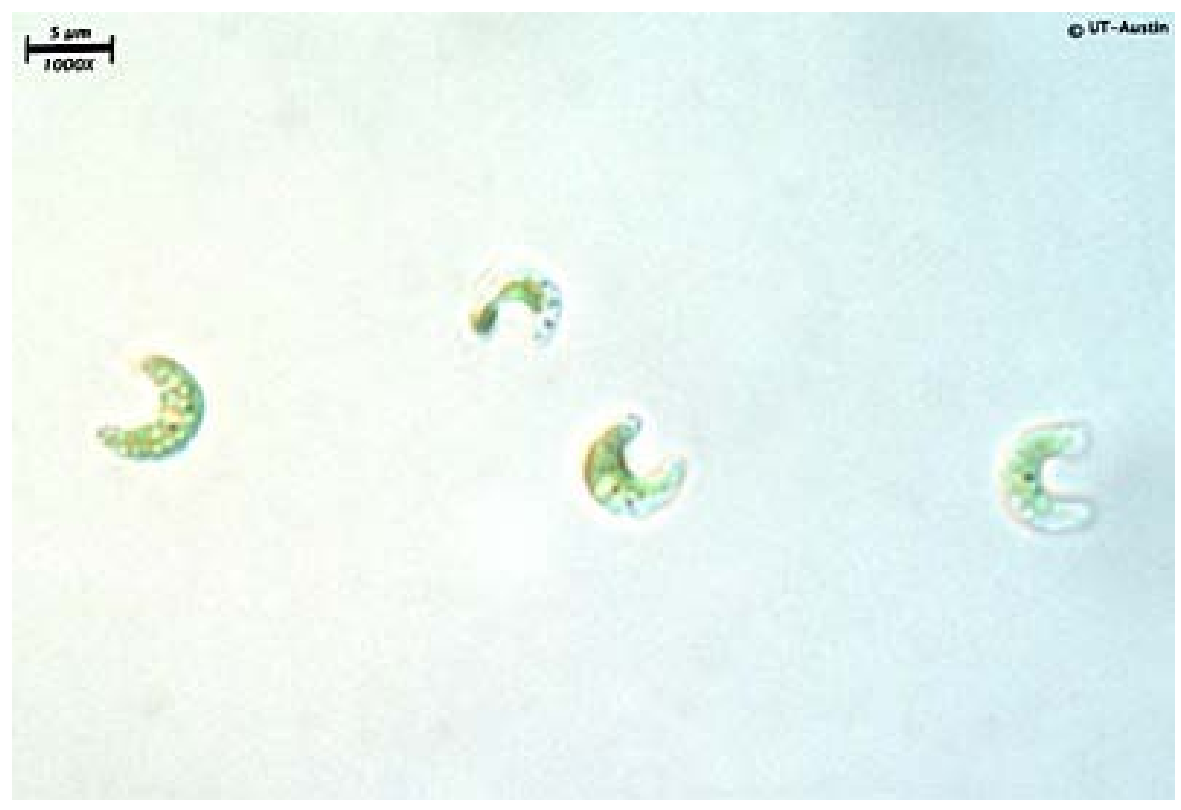

Figure 2-10 - The GF3 Strain under Microscope 
Table 2-3 - Fatty Acid Analysis of GF3 by POS

\begin{tabular}{|c|c|}
\hline Analyte & Res ult Units \\
\hline \multicolumn{2}{|l|}{ Elemental Analysis } \\
\hline Phosphorus & $22.0 \mathrm{ppm}$ \\
\hline Fatty Acid Profile & $\mathrm{wt} \%$ \\
\hline C6 Caproic & 0.03 wt $\%$ \\
\hline C14 Myristic & $1.40 \mathrm{wt} \%$ \\
\hline C14:1 Tetradecenoic & 0.07 wt $\%$ \\
\hline C15 Pentadecanoic & 0.05 wt $\%$ \\
\hline C16 Palmitic & 3.73 wt $\%$ \\
\hline C16:1 Hexadecenoic & 2.89 wt $\%$ \\
\hline C17:1n7 Heptadecenoic & 0.08 wt $\%$ \\
\hline C18 Stearic & 0.36 wt $\%$ \\
\hline C18:1n9 Oleic & $7.88 w t \%$ \\
\hline C18:10ctadecenoic & 0.40 wt $\%$ \\
\hline C18:2 Linoleic & 13.70 wt $\%$ \\
\hline C18:3n6 gamma-Linolenic & $0.73 \mathrm{wt} \%$ \\
\hline C18:3n3 alpha-Linolenic & 23.10 wt $\%$ \\
\hline C18:4 Octadecatetraenoic & $8.27 \mathrm{wt} \%$ \\
\hline C20 Arachidic & 0.05 wt $\%$ \\
\hline C20: 1 Eicosenoic & $0.15 w t \%$ \\
\hline C20:2n6 Eicosadienoic & 0.05 wt $\%$ \\
\hline C20:3n3 Eicosatrienoic & 0.09 wt $\%$ \\
\hline C20:4n3 Eicosatetraenoic & 0.03 wt $\%$ \\
\hline C20:5n3 Eicosapentaenoic & 0.27 wt $\%$ \\
\hline C22 Behenic & 0.24 wt $\%$ \\
\hline C22:1n9 Erucic & 0.03 wt $\%$ \\
\hline $\begin{array}{l}\text { C22:5n3 Docosapentaenoic } \\
7,10,13,16,19\end{array}$ & 0.09 wt $\%$ \\
\hline C24 Lignoceric & 0.41 wt $\%$ \\
\hline C24: 1 n9 Nervonic & 0.03 wt $\%$ \\
\hline Others & 35.88 wt $\%$ \\
\hline Total Saturates & 6.27 wt $\%$ \\
\hline Total Monounsaturates & $11.53 \mathrm{wt} \%$ \\
\hline Total Polyunsaturates & 46.33 wt $\%$ \\
\hline Total Omeqa 3 & 31.85 wt $\%$ \\
\hline Total Omega 6 & 14.48 wt $\%$ \\
\hline Total Omega 9 & 8.09 wt\% \\
\hline
\end{tabular}

After the evaluation of the GF3 strain, work began to assemble an in-house algae lab and to expand the 3rd Avenue R\&D Facility to facilitate algae strain selection. The GF3 strain is the only algae species cultivated in the bag farm at the 3rd Avenue site; all subsequent growth phases in the bag farm following GF3 were to support inoculum development. 


\subsection{Algae LabORATORY AT3RD AVEnUe R\&D FACIUTY}

\subsubsection{Algae Laboratory Setup}

The algae laboratory at 3rd Avenue, Phoenix was assembled from January 1 to March 31, 2008. The initial lab setup had two main purposes: (1) to monitor culture growth and health and (2) to concentrate and store biomass for shipment. To support and ensure culture growth and health, the lab was equipped with a light microscope, pH meters, temperature gun, fluorescence meter, balances, and other basic lab consumables. To concentrate, dewater, and dry the algae, two Lavin centrifuges and a tray dryer were purchased. The lab was erected with laboratory benches, a fume hood, a walk-in fume hood, a Parr high-pressure autoclave, and Agilent GC/MS for lipid analysis and fuel production work. As the project progressed, more equipment was added to keep cultures sterile, to improve density measurements, and to expand analytical work to oil extraction. This included adding an oven, a furnace, a bench-top centrifuge, a freeze-drier, an autoclave, a shaker table, a Soxhlet extraction apparatus and lab furniture. Additionally, an algae nursery and a thin-film bag culture system were also installed in the lab. Figure 2-11 shows a portion of the APS algae laboratory.

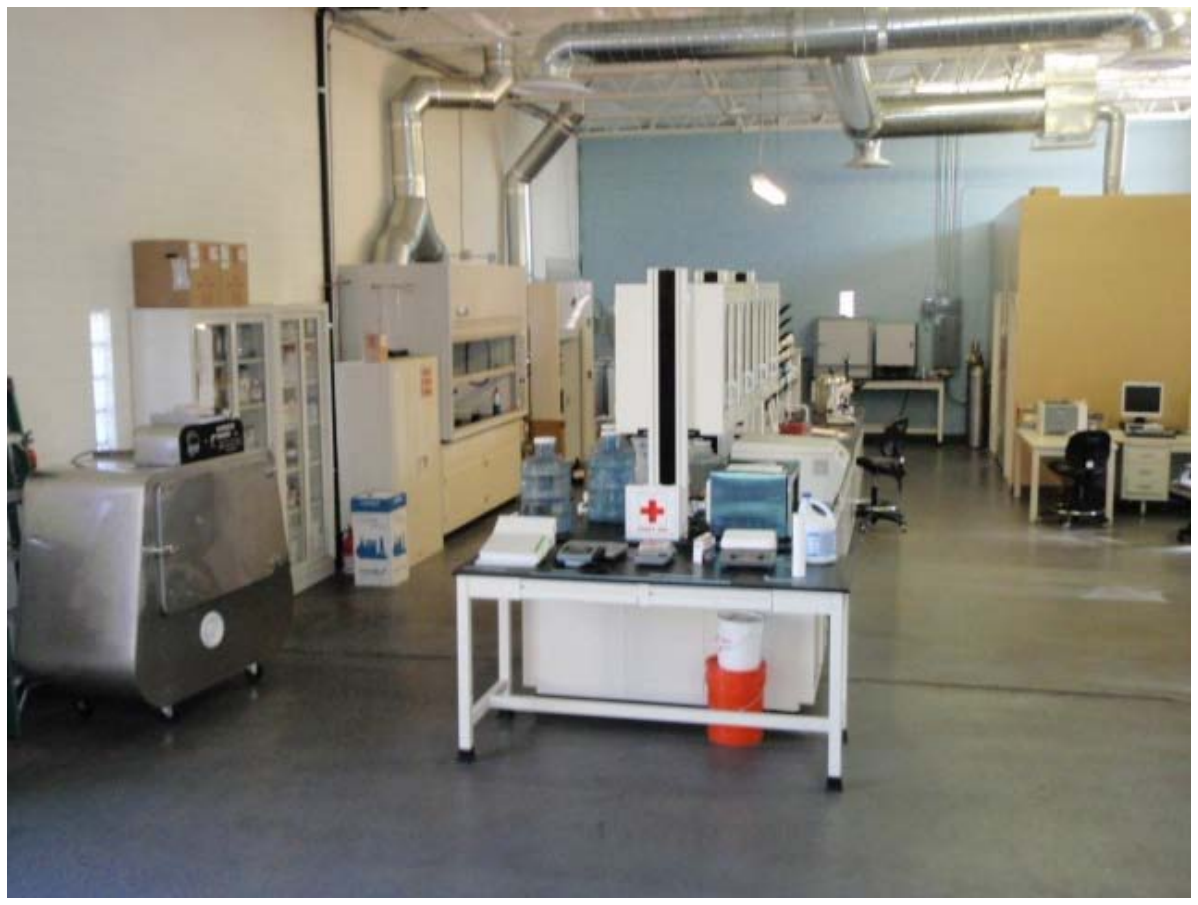

Figure 2-11 - APS 3rd Avenue Algae Lab, Phoenix, Arizona 


\subsubsection{Algae Strain Seiection}

Algae strain selection is a critical factor in the success of the project. Each strain has a constrained set of conditions in which it will grow optimally, and specific lipids and cell products that it can produce. Algal strains grown outside the ranges of its ideal conditions may grow slowly, abnormally or even die. Additionally, growing a species that does not have the high lipid content desired by the project was not viable. Therefore, choosing an algae strain that grows ideally under project conditions, such as site temperatures and water source, and that makes the desired products was an important aspect of project planning.

The specific characteristics screened in this project focused on finding algae species that can have a high growth rate, high carbon content, and high lipid content in the dried algae biomass. The lipid profile of the strain should be compatible with fuel production, mainly short to midchain fatty acids. The oil should be easily extractable. A secondary yet important screening criterion is the ability to settle naturally for ease of decanting (to minimize energy needed and associated cost for separating the algae from the water. The traditional method of utilizing a centrifuge to separate the algae from the water is an energy intensive step that on a commercial scale would not be economically viable. A culture that settles naturally by gravity to the bottom of a culture vessel when agitation is removed represents a significant opportunity for energy and cost savings in dewatering the biomass. Other desirable parameters were tolerance to solar irradiance, culture salinity, and pump-shear sensitivity. The potential candidates were intended to be robust enough to survive and overcome inadvertent system anomalies, not accumulate heavy metals that could be present in the flue gas from a coal-fired power plant, and be able to resist desert spores and local bacteria.

Initially, species selection and screening for the project was performed by GreenFuel. They selected and provided the initial strain grown on the 3rd Avenue site, GF3, in April 2008. Based on the initial algae production study, the GF3 strain was reported to have a wide temperature range tolerability of $17^{\circ} \mathrm{C}$ to $40^{\circ} \mathrm{C}\left(32^{\circ} \mathrm{F}\right.$ to $\left.104^{\circ} \mathrm{F}\right)$; be able to tolerate the Arizona heat and system fluctuations; have a fast growth rate; and produce a short carbon chain, which was ideal for military aviation fuel processing. However, initial attempts at oil extraction by outside labs yielded lower than expected neutral lipid content (less than 5 wt\% neutral lipids). Thus, future in-house species selection and screening was initiated for the project to identify novel candidates. 
The species candidates considered in this selection process took into account the history of past research from commercial, academic, and governmental projects, as well as the team's collective experience and advice from algal experts in Israel, Germany, Texas, and Arizona. The species considered were Chlorella vulgaris, Nannochloris sp., Nannochloropsis oculata, Selenastrum sp., Scenedesmus obliquus, Scenedesmus acutus, and Scenedesmus dimorphus as they were purported to have many of the desired characteristics required by the project. All of these cultures were grown onsite and evaluated for their potential.

Three species emerged as leading candidates: Nannochloropsis oculata, Selenastrum, and Scenedesmus obliquus. Chlorella was eliminated due to a lack of settling and a tendency to accumulate carbohydrates rather than lipids under stress conditions. Nannochloris was removed from consideration because it lacked the ability to settle and did not accumulate lipids under stress conditions. All three leading candidates were evaluated at the APS 3rd Avenue R\&D facility using the $6 \mathrm{M}$ cultivation and harvesting system after indoor lab evaluation.

\subsubsection{Algae Strain Evaluation - Nannochloropsis}

Nannochloropsis, a marine species, emerged as a candidate because in previous studies this strain was shown to grow successfully on coal flue gas without showing negative impacts from the heavy metals and because it accumulates significant amounts of oil. Since the species tolerates lower temperatures generally $18^{\circ} \mathrm{C}$ to $20^{\circ} \mathrm{C}\left(64.4^{\circ} \mathrm{F}\right.$ to $\left.68^{\circ} \mathrm{F}\right)$, it was proposed as a good candidate for growth in the cooler winter months.

In Table 2-4, samples 1 and 2 were Nannochloropsis using indoor cultivation. Nannochloropsis was found to reach total fat levels of $\sim 23 \mathrm{wt} \%$ biomass (sample 1), with neutral lipid content of $\sim 7.5 \mathrm{wt} \%$. When stressed (sample 2), Nannochloropsis could accumulate up to $34 \mathrm{wt} \%$ total fat and $18 \mathrm{wt} \%$ of the biomass as neutral lipids.

Nannochloropsis was selected for the first growth trial in the $6 \mathrm{M}$ cultivation system in Phoenix due to these promising laboratory results. Two growth periods were performed in the $6 \mathrm{M}$ cultivator with Nannochloropsis from February 12 to March 15, 2009 and from April 2 to May 18, 2009. (This data is detailed later in this report.) It was determined that the culture could capture close to $90 \mathrm{wt} \%$ of $\mathrm{CO}_{2}$ introduced in this outdoor cultivation system. The average growth rate of the strain reached $7 \mathrm{~g} / \mathrm{m}^{2} / \mathrm{d}$. When culture temperatures were over the safe maximum $\left(20^{\circ} \mathrm{C}\right.$ $\left.\left[60^{\circ} \mathrm{F}\right]\right)$, it affected the health and growth of the culture. On May 17, before the adverse effects of elevated summer temperatures, the culture was fully harvested to protect the quality of the 
harvested biomass. At this time, Nannochloropsis trials were discontinued and the species was replaced with a higher-temperature tolerant species.

Efforts by external laboratories to extract algal oil from the Nannochloropsis species were unsatisfactory and similar to the results of attempting oil extraction from GF3 (see Section 2.5.1.2). The resulting product proved unsuitable for fuel production. Nannochloropsis test data indicated that it was not likely a good candidate for the project due to temperature limitations and the oil extraction issue.

\subsubsection{Algae Strain Evaluation - Selenastrum}

In laboratory experiments, Selenastrum $s p$ was found to accumulate a significant amount of oil under stress. When growing exponentially, Selenastrum had approximate $18 \mathrm{wt} \%$ total fat and 14 wt\% neutral lipids (sample 3 in Table 2-4). When under nutrient stress conditions, Selenastrum was found to accumulate up to $35 \mathrm{wt} \%$ total fat, in which $30 \mathrm{wt} \%$ was neutral lipids (samples 4 and 5 in Table 2-4).

The $6 \mathrm{M}$ outdoor test of Selenastrum was started on May 22, 2009. Selenastrum was found to meet productivity expectations in an initial growth period with an average productivity of 22 $\mathrm{g} / \mathrm{m}^{2} / \mathrm{d}$. However, subsequent increasing culture temperatures due to high ambient temperatures in Phoenix corresponded to a decrease in productivity down to $6-7 \mathrm{~g} / \mathrm{m}^{2} / \mathrm{d}$.

The fact that the growth rate of the culture decreased over time indicated that a more robust species of algae may be necessary for the high temperature and irradiance conditions found in Phoenix. Additionally, Selenastrum did not exhibit a characteristic to settle, thus the dewatering energy input for this species would be high. As a result of these observations, Selenastrum did not remain a high-priority candidate and the testing focus shifted to the remaining candidate with settling characteristics - Scenedesmus.

\subsubsection{Algae Strain Evaluation - Scenedesmus}

The 6M cultivator was inoculated with Scenedesmus on August 17, 2009. Within this system, Scenedesmus demonstrated a good growth rate, peaking at $20 \mathrm{~g} / \mathrm{m}^{2} / \mathrm{d}$ and great tolerance to varying conditions. The culture was temperature and sunlight tolerant, withstanding the daytime irradiance and temperature highs of August and the night temperature lows in November. The culture demonstrated resistance to the other algal contaminants and survived in the presence of algae grazers. Scenedesmus demonstrated self-settling characteristics. An increase in density from $1.5 \mathrm{~g} / \mathrm{L}$ to $48 \mathrm{~g} / \mathrm{L}$ by self-settling was demonstrated in a period of 5 hours. However, based 
on indoor lab studies, its total fat and neutral lipid levels were lower than Selenastrum when stressed, ranging between 8 wt $\%$ to 15 wt $\%$ total fat and 7 wt $\%$ to 16 wt $\%$ neutral lipids (samples 6 through 10 shown in Table 2-4). However, both total fat and lipid levels were equal to those of Selenastrum when neither species were stressed. The analysis nevertheless indicated that a larger proportion of the total fat was made up of neutral lipids in Scenedesmus.

These studies also indicated that among the three species tested in the $6 \mathrm{M}$ cultivator in Phoenix, Scenedesmus had the characteristics that make it the optimal choice for cultivation at APS's Redhawk testing facility, especially if better algae stressing technology could be developed to significantly increase its lipid content and reduce the chlorophyll content at the same time. A high amount of lipids is essential as a high amount will yield a large quantity of fuel. The studies stressed algae by depraving it of either nitrates or phosphates which are nutrients essential for algae growth. Results showed by removing nitrates from the system that the lipid content of the biomass from this species could be increased from approximately $10 \%$ to $35 \%$. An additional advantage of stressing the system by removing nitrates is that is also causes the amount of chlorophyll to decrease. Have the algae devoid of chlorophyll makes oil extraction easier.

Study on Scenedesmus was continued under a follow-on Cooperative Agreement, DOE award DE-FE0001099, "Integrated Energy System (IES) with Beneficial $\mathrm{CO}_{2}$ Use." Please refer to its Final Technical Closeout Report for further detailed study on this species.

A complete list of the algae lipid analyses conducted for Nannochloropsis, Selenastrum and Scenedesmus along with a brief description of the imposed experimental and culture conditions can be found in Table 2-4. 
Table 2-4 - Lipid Content Study for Algae Strain Selection

\begin{tabular}{|c|c|c|c|c|c|c|c|c|}
\hline $\begin{array}{c}\text { Sample } \\
\#\end{array}$ & Species & Vessel & Location & \begin{tabular}{|l} 
Location \\
Purpose of \\
Sample
\end{tabular} & $\begin{array}{l}\text { Experimental } \\
\text { Condition Notes }\end{array}$ & Test Ordered & $\begin{array}{l}\text { Total Fat } \\
\text { (wt } \% \text { of } \\
\text { biomass) }\end{array}$ & $\begin{array}{c}\text { Neutral } \\
\text { Lipid (wt\% } \\
\text { of biomass) }\end{array}$ \\
\hline 1 & Nannochloropsis & $\begin{array}{l}\text { Thin } \\
\text { Film }\end{array}$ & Indoor & $\begin{array}{l}\text { Winter } \\
\text { Species } \\
\text { Optimization }\end{array}$ & $\begin{array}{l}\text { Dewatered } \\
\text { cultivator culture } \\
\text { placed directly in } \\
\text { thin film reactor for } \\
2 d\end{array}$ & $\begin{array}{l}\text { Fat (Acid Hydrolysis) } \\
\text { Fatty Acid Profile (and } \\
\text { extraction) }\end{array}$ & 22.85 & 7.45 \\
\hline 2 & Nannochloropsis & $\begin{array}{l}\text { Thin } \\
\text { Film }\end{array}$ & Indoor & \begin{tabular}{|l} 
Winter \\
Species \\
Optimization
\end{tabular} & $\begin{array}{l}\text { Grown until } \\
\text { nutrient depletion } \\
\text { and stationary } \\
\text { phase reached }\end{array}$ & $\begin{array}{l}\text { Fat (Acid Hydrolysis) } \\
\text { Fatty Acid Profile (and } \\
\text { extraction) }\end{array}$ & 34.81 & 18.50 \\
\hline 3 & Selenastrum & Bottle & Indoor & $\begin{array}{l}\text { Redhawk } \\
\text { Species } \\
\text { Selection }\end{array}$ & $\begin{array}{l}\text { Grown without } \\
\text { limitation, re- } \\
\text { suspended in } \\
\text { nutrient deplete } \\
\text { medium }\end{array}$ & $\begin{array}{l}\text { Fat (Acid Hydrolysis) } \\
\text { Fatty Acid Profile (and } \\
\text { extraction) } \\
\text { Amino Acid profile } \\
\text { (Methods } 1 \text { and 2) } \\
\end{array}$ & 18.09 & 13.88 \\
\hline 4 & Selenastrum & $\begin{array}{l}\text { Thin } \\
\text { Film }\end{array}$ & Indoor & $\begin{array}{l}\text { Redhawk } \\
\text { Species } \\
\text { Selection }\end{array}$ & $\begin{array}{l}\text { Grown until } \\
\text { nutrient depletion } \\
\text { and stationary } \\
\text { phase reached }\end{array}$ & $\begin{array}{l}\text { Proximate Fat (Acid } \\
\text { Hydrolysis) } \\
\text { Fatty Acid Profile (and } \\
\text { extraction) }\end{array}$ & 35.04 & 30.58 \\
\hline 5 & Selenastrum & $\begin{array}{l}\text { Thin } \\
\text { Film }\end{array}$ & Indoor & $\begin{array}{l}\text { Redhawk } \\
\text { Species } \\
\text { Optimization }\end{array}$ & $\begin{array}{l}\text { Grown until } \\
\text { nutrient depletion } \\
\text { and stationary } \\
\text { phase reached }\end{array}$ & $\begin{array}{l}\text { Proximate Fat (Acid } \\
\text { Hydrolysis) } \\
\text { Fatty Acid Profile (and } \\
\text { extraction) } \\
\end{array}$ & 37.47 & 33.90 \\
\hline 6 & Scenedesmus & Bottle & Indoor & \begin{tabular}{|l} 
Redhawk \\
Species \\
Selection
\end{tabular} & $\begin{array}{l}\text { Nonstressed } \\
\text { control bottle }\end{array}$ & $\begin{array}{l}\text { Fat (Acid Hydrolysis) } \\
\text { Fatty Acid Profile (and } \\
\text { extraction) } \\
\end{array}$ & 8.56 & 8.40 \\
\hline 7 & Scenedesmus & Bag & Outdoor & $\begin{array}{l}\text { Redhawk } \\
\text { Species } \\
\text { Selection }\end{array}$ & Old outdoor bag & $\begin{array}{l}\text { Fat (Acid Hydrolysis) } \\
\text { Fatty Acid Profile (and } \\
\text { extraction) }\end{array}$ & 12.8 & 9.17 \\
\hline 8 & Scenedesmus & Bottle & Indoor & $\begin{array}{l}\text { Redhawk } \\
\text { Species } \\
\text { Selection }\end{array}$ & $\begin{array}{l}\text { Grown without } \\
\text { limitation, re- } \\
\text { suspended in } \\
\text { nutrient deplete } \\
\text { medium for 4d }\end{array}$ & $\begin{array}{l}\text { Fat (Acid Hydrolysis) } \\
\text { Fatty Acid Profile (and } \\
\text { extraction) }\end{array}$ & 9.47 & 9.07 \\
\hline 9 & Scenedesmus & $\begin{array}{l}\text { Thin } \\
\text { Film }\end{array}$ & Indoor & $\begin{array}{l}\text { Redhawk } \\
\text { Species } \\
\text { Selection }\end{array}$ & $\begin{array}{l}\text { Grown until } \\
\text { nutrient depletion } \\
\text { and stationary } \\
\text { phase reached }\end{array}$ & $\begin{array}{l}\text { Proximate Fat (Acid } \\
\text { Hydrolysis) } \\
\text { Fatty Acid Profile (and } \\
\text { extraction) }\end{array}$ & 15.86 & 12.89 \\
\hline 10 & Scenedesmus & $\begin{array}{l}\text { Thin } \\
\text { Film }\end{array}$ & Indoor & \begin{tabular}{|l|} 
Redhawk \\
Species \\
Optimization
\end{tabular} & $\begin{array}{l}\text { Grown until } \\
\text { nutrient depletion } \\
\text { and stationary } \\
\text { phase reached }\end{array}$ & $\begin{array}{l}\text { Proximate Fat (Acid } \\
\text { Hydrolysis) } \\
\text { Fatty Acid Profile (and } \\
\text { extraction) } \\
\text { Amino Acid profile } \\
\text { (Methods } 1 \text { and 2) }\end{array}$ & 19.98 & 16.80 \\
\hline
\end{tabular}

\subsubsection{Algae GroWTh Medium}

An important aspect of an algae culture is the algae growth medium, which provides nutrients required for the algae. For this project, an artificial seawater medium was used for the marine strain of microalgae investigated, Nannochloropsis, and a freshwater medium was used for the other strains of algae investigated - Selenastrum, and Scenedesmus. 
To produce the artificial seawater medium, a series of stock solutions was created containing the appropriate levels of concentrated chemical nutrients. These stock solutions were then mixed together in the appropriate quantities with water to make the growth medium. The artificial seawater medium also required the addition of an artificial sea salt since a source of filtered seawater was not available. These artificial sea salt mixes are available from many pet stores and online aquarium supply companies. Bulk sources of $\mathrm{NaCl}$ can also be used, but the level of other constituents in these sources must be well known and examined to ensure that no harmful compounds are present in the salt which may reduce growth of the algae. The production of the artificial seawater medium began with the production of the first two nutrient stocks (see Table 2-5). For each of these nutrient stocks, the micronutrient stock and the iron solution were created separately in solutions with one part nutrient to 1000 parts water. 


\section{Table 2-5 -Recipe for the Creation of the Artificial Seawater Medium Used for Marine Algae Strains}

\section{Artificial Seawater Medium Preparation}

Micronutrients

Fill a clean $1 \mathrm{~L}$ beaker about halfway with deionized or distilled water, weigh the listed components. Mix components and bring to final volume of $1 \mathrm{~L}$ with deionized or distilled water. Transfer the solution to a labeled clean $1 \mathrm{~L}$ media bottle and autoclave.

\section{To Make 1 L of One Thous and}

Times (1000X) Stock,

Chemical Component

Add Grams (g)

Final Preparation

\begin{tabular}{l|r}
$\mathrm{ZnSO}_{4} * 7 \mathrm{H}_{2} \mathrm{O}$ & 0.0220 \\
$\mathrm{MnC1}^{*} 4 \mathrm{H}_{2} \mathrm{O}$ & 0.1800 \\
$\mathrm{Na}_{2} \mathrm{MoO}_{4}{ }^{*} 2 \mathrm{H}_{2} \mathrm{O}$ & 0.0063 \\
$\mathrm{CuSO}_{4}{ }^{*} 5 \mathrm{H}_{2} \mathrm{O}$ & 0.0098 \\
$\mathrm{CoC}_{12}{ }^{*} 6 \mathrm{H}_{2} \mathrm{O}$ & 0.0100
\end{tabular}

Iron Solution

Fill a clean $1 \mathrm{~L}$ beaker about halfway with deionized or distilled water, weigh the listed components. Mix components and bring to final volume of $1 \mathrm{~L}$ with deionized or distilled water. Transfer the solution to a labeled clean $1 \mathrm{~L}$ media bottle and autoclave.

\begin{tabular}{|c|c|c|}
\hline Chemical Component & $\begin{array}{c}\text { To Make } 1 \text { L of } 1000 X \text { Stock } \\
\text { Add Grams (g) }\end{array}$ & Final Preparation \\
\hline$\frac{\mathrm{FeC}_{13}{ }^{*} 6 \mathrm{H}_{2} \mathrm{O}}{\mathrm{Na}_{2} \mathrm{EDTA}^{*} 2 \mathrm{H}_{2} \mathrm{O}}$ & $\begin{array}{l}3.1500 \\
4.3600\end{array}$ & $\begin{array}{c}\text { Use } 1 \mathrm{~mL} \text { of this stock } \\
\text { per } 1 \mathrm{~L} \text { of media }\end{array}$ \\
\hline \multicolumn{3}{|l|}{ Macronutrient Portion } \\
\hline \multicolumn{3}{|c|}{ To the final medium vessel and volume desired, add the following components and mix well. } \\
\hline Chemical Component & Per L of Medium Needed, Add & Final Vessel \\
\hline $\mathrm{NaNO}_{3}$ & $0.075 \mathrm{~g}$ & \multirow{5}{*}{$\begin{array}{l}\text { Combine all ingredients in } \\
\text { the appropriately sized } \\
\text { vessel and volume of } \\
\text { medium required }\end{array}$} \\
\hline $\mathrm{NaH}_{2} \mathrm{PO}_{4}{ }^{*} \mathrm{H}_{2} \mathrm{O}$ & $0.005 \mathrm{~g}$ & \\
\hline Micronutrient stock & $1 \mathrm{~mL}$ & \\
\hline Iron Solution & $1 \mathrm{~mL}$ & \\
\hline Artificial sea salt mix & $36 \mathrm{~g}$ & \\
\hline
\end{tabular}

Once $1 \mathrm{~mL}$ of each of the two micronutrient liquid stocks was added per liter of macronutrient medium required, the appropriate amounts of the designated nitrogen and phosphorus sources were added, followed by the artificial sea salt mix as outlined in Table 2-5. Once all components were mixed together, the final medium was autoclaved. Alternatively, depending on the sterility of the culture desired, in some cases, each stock was autoclaved, but the final medium was not. Once the medium had cooled and been well mixed, inoculum was added to the medium in the cultivation device.

The above method for the creation of medium works well for small quantities in the laboratory and for large batches (up to $20 \mathrm{~L}$ ) of the liquid stock of the iron solution shown in Table 2-5, but 
for all other medium components, the appropriate amounts for the total desired culture volume were simply weighed and added directly to a volume of water in the large cultivation devices, along with the liquid iron solution.

Freshwater medium was created by initially making the separate liquid stocks outlined in Table 2-6. As with the marine medium, these stock solutions were then added with the macronutrients in the appropriate ratios outlined below to create the final one time (1X) freshwater culture medium.

\section{Table 2-6 - Medium Recipe for the Creation of the Freshwater Medium Used for Freshwater Algae Strains}

\begin{tabular}{|c|c|c|}
\hline \multicolumn{3}{|c|}{ Freshwater Medium Preparation } \\
\hline \multicolumn{3}{|l|}{ Micronutrients } \\
\hline \multicolumn{3}{|c|}{$\begin{array}{l}\text { Fill a clean } 1 \mathrm{~L} \text { beaker about halfway with deionized or distilled water, weigh the listed compo- } \\
\text { nents. Mix components and bring to final volume of } 1 \mathrm{~L} \text { with deionized or distilled water. } \\
\text { Transfer the solution to a labeled clean } 1 \mathrm{~L} \text { media bottle and autoclave. }\end{array}$} \\
\hline Chemical Component & $\begin{array}{c}\text { To make } 1 \text { L of } 1000 X \text { Stock, } \\
\text { Add Grams (g) }\end{array}$ & Final Preparation \\
\hline $\mathrm{ZnSO}_{4} * 7 \mathrm{H}_{2} \mathrm{O}$ & 0.2200 & \multirow{10}{*}{$\begin{array}{l}\text { Use } 1 \mathrm{~mL} \text { of this stock } \\
\text { per } 1 \mathrm{~L} \text { of media }\end{array}$} \\
\hline $\mathrm{MnC}_{12} * 4 \mathrm{H}_{2} \mathrm{O}$ & 1.8100 & \\
\hline $\mathrm{Na}_{2} \mathrm{MoO}_{4} \star 2 \mathrm{H}_{2} \mathrm{O}$ & 0.3900 & \\
\hline $\mathrm{CuSO}_{4} * 5 \mathrm{H}_{2} \mathrm{O}$ & 0.0790 & \\
\hline $\mathrm{Co}\left(\mathrm{NO}_{3}\right) 2 * 6 \mathrm{H}_{2} \mathrm{O}$ & 0.0490 & \\
\hline $\mathrm{MgSO}_{4} * 7 \mathrm{H}_{2} \mathrm{O}$ & 7.5000 & \\
\hline Citric acid $^{\star} \mathrm{H}_{2} \mathrm{O}$ & 6.0000 & \\
\hline $\mathrm{CaC}_{12} * 2 \mathrm{H}_{2} \mathrm{O}$ & 36.0000 & \\
\hline $\mathrm{Na}_{2} \mathrm{CO}_{3}$ & 20.0000 & \\
\hline $\mathrm{H}_{3} \mathrm{BO}_{3}$ & 2.6000 & \\
\hline \multicolumn{3}{|l|}{ Iron Solution } \\
\hline \multicolumn{3}{|c|}{$\begin{array}{l}\text { Fill a clean } 1 \mathrm{~L} \text { beaker about halfway with deionized or distilled water, weigh the listed compo- } \\
\text { nents. Mix components and bring to final volume of } 1 \mathrm{~L} \text { with deionized or distilled water. } \\
\text { Transfer the solution to a labeled clean } 1 \mathrm{~L} \text { media bottle and autoclave. }\end{array}$} \\
\hline Chemical Component & $\begin{array}{c}\text { To make } 1 \text { L of } 1000 X \text { Stock } \\
\text { Add Grams (g) }\end{array}$ & Final Preparation \\
\hline $\mathrm{NH}_{4}$ ferric citrate & 6.0000 & \multirow{2}{*}{$\begin{array}{l}\text { Use } 1 \mathrm{~mL} \text { of this stock } \\
\text { per } 1 \mathrm{~L} \text { of media }\end{array}$} \\
\hline $\mathrm{Na}_{2} \mathrm{EDTA}^{\star} 2 \mathrm{H}_{2} \mathrm{O}$ & 1.0000 & \\
\hline \multicolumn{3}{|l|}{ Macronutrient portion } \\
\hline \multicolumn{3}{|c|}{ To the final medium vessel and volume desired, add the following components and mix well. } \\
\hline Chemical Component & Per L of Medium Needed Add & Final Vess el \\
\hline $\mathrm{NaNO}_{3}$ & $1.5 \mathrm{~g}$ & \multirow{4}{*}{$\begin{array}{l}\text { Combine all } \\
\text { ingredients in the } \\
\text { appropriately sized } \\
\text { vessel and volume of } \\
\text { medium required }\end{array}$} \\
\hline $\mathrm{K}_{2} \mathrm{HPO}_{4}$ & $0.04 \mathrm{~g}$ & \\
\hline Micronutrient stock & $1 \mathrm{~mL}$ & \\
\hline Iron solution & $1 \mathrm{~mL}$ & \\
\hline
\end{tabular}


Once the fresh water stock solutions were made, they were added to the appropriate volume of water as outlined in Table 2-6, combined with the nitrogen and phosphorous sources, mixed well, and then autoclaved if the individual stocks had not previously been autoclaved. After cooling, if autoclaved, the medium was then combined with inoculum in the cultivation vessel. As with the preparation of the seawater medium, this technique worked well for small amounts of medium in the laboratory and in inoculum production, but a different technique had to be applied for the large-scale outdoor cultures, although the recipe was the same. For large-scale cultures, the iron solution in Table 2-6 was still prepared in a large volume of water, but all other medium components were individually weighed given the final volume of medium required, and the chemical components were added directly to the water in the cultivation vessel.

In this manner, both marine and freshwater strains of algae were cultured. As a result, the cultures could be investigated during the screening process and subsequent inoculum production and cultivation testing for this project.

\subsubsection{Algae Nursery AND Botmle Culture Maintienance}

To aid in strain selection and to keep clean seed stocks of cultures for scale-up, an algae nursery was added to the 3rd Avenue Lab facility between July 1 and September 31, 2008. The purpose of the nursery was to provide a clean, cool, and stable environment to keep and maintain algal monocultures. The nursery provided an environment unlikely to introduce contamination to the cultures while maintaining conditions optimal for robust, dense growth of most algal strains. This environment consisted of a partially enclosed, temperature-controlled room equipped with shelving and tables to support cultures and lighting fixtures. The lighting fixtures were double banks of fluorescent lighting with output of approximately 200 micro Einstein per meter squared per second $\left(\mu \mathrm{E} / \mathrm{m}^{2} / \mathrm{s}\right)$ providing illumination to the cultures on a continuous basis. A supply of $3-5 \mathrm{wt} \% \mathrm{CO}_{2} /$ air mix was bubbled from $\mathrm{CO}_{2}$ cylinders and an air pump into the bottom of the bottle cultures to provide mixing of air and $\mathrm{CO}_{2}$. A shaker table was also added to shake cultures not bubbled with the $\mathrm{CO}_{2} /$ air mix.

The nursery housed slant or plate cultures, flask cultures, and bottle cultures. All culture media and culture vessels were prepared by using steam sterilization with an autoclave. All culture transfers were performed aseptically in a fume hood. Slant and plate cultures provided a solid substrate on which to grow cultures that required very little maintenance and lasted for several months. They were prepared with $10 \mathrm{wt} \%$ agar media. Slants and plate cultures were inoculated with a sterile inoculation loop from another solid substrate culture or from a clean, 
dilute algal monoculture. It took several weeks for solid substrate cultures to grow to sufficient cell numbers to be ready for culture scale-up. Since these cultures rarely needed to be accessed, they stayed clean for long periods of time and provided a constant source of clean seed culture. The culture scale-up from agar slants or plates employed flask cultures.

Flask cultures were 50-150 mL liquid cultures in $250 \mathrm{~mL}$ Erlenmeyer flasks capped with foam plugs or other porous filter material to prevent contamination but allow air exchange. Flasks were prepared with media containing $0.5 \mathrm{~g} / \mathrm{L}$ sodium bicarbonate to provide a carbon source. Sterile inoculation loops were used to transfer cells of a slant or plate culture to flasks. Flask cultures were maintained on a continuous basis by replenishing with fresh media as necessary. Without $\mathrm{CO}_{2}$, they grew slower than bottle cultures, but they were in liquid media, which grow faster than agar or plate cultures. Flasks were mixed by placing them on an orbital shaker, providing constant gentle swirling. Once a flask culture reached sufficient density, it was used to inoculate a bottle culture as part of culture scale-up.

Bottle cultures were $2 \mathrm{~L}$ glass media bottles with rubber stoppers affixed with glass and rubber or plastic tubing to provide an inlet of $\mathrm{CO}_{2}$ /air and an outlet for waste and excess gases. Figure 2-12 shows a $2 \mathrm{~L}$ bottle culture. Both the inlet and the outlet were equipped with inline air filters to prevent the introduction of contamination into the bottle cultures and the escape of cells from the culture that could potentially contaminate surrounding cultures. Bottle culture media could be prepared with or without sodium bicarbonate; the bicarbonate as a carbon source was not necessary as these cultures were provided with $\mathrm{CO}_{2}$ and did impart some buffering. Bottle cultures represented the actively growing culture collection and provided dense culture for the next scale-up inoculation. Bottle cultures were acclimated and maintained in active growth phase and were refreshed with new media every few weeks to keep cultures healthy. 

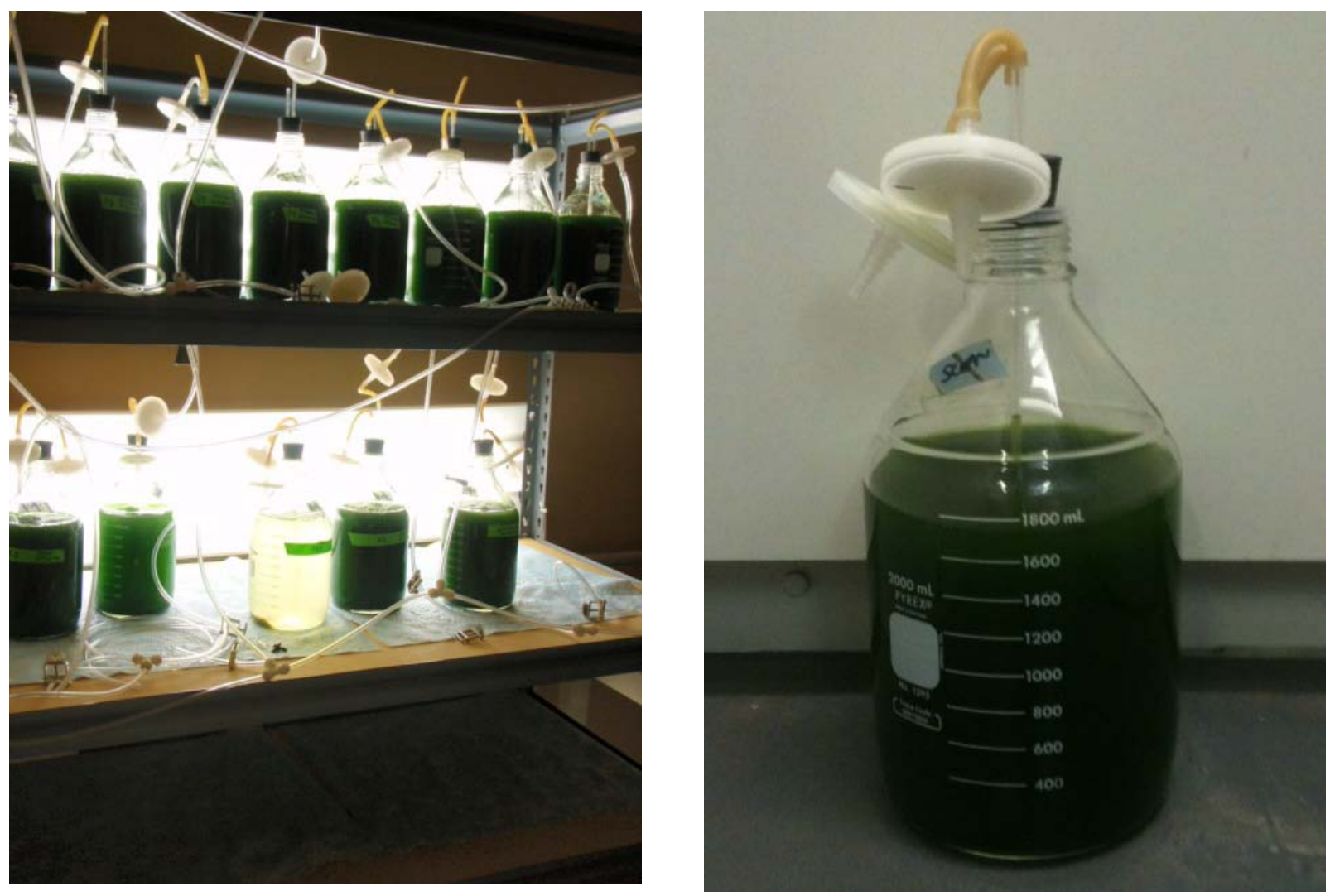

Figure 2-12 - Algae Nursery and 2 L Bottle Culture

\subsubsection{INOCULUM PRODUCTION}

The process of growing a culture from an agar slant up to a $2 \mathrm{~L}$ bottle culture required a series of steps. A culture inoculated at a too low cell density may become nonviable, fail to grow, or grow at greatly reduced rates. Therefore, culture scale-up had to be performed in stages until the desired culture volume was met. After a culture was grown in the $2 \mathrm{~L}$ bottle culture phase, it was ready for the bag culture stage. The $80 \mathrm{~L}$ outdoor hanging bag was initially used for large-quantity inoculum production; however, the outdoor bag farm showed several disadvantages: (1) being an outdoor system, contamination could produce a lower-quality inoculum for the larger growth systems; (2) a large light path providing relatively low-density culture produced at low growth rates (the light path was represented by the depth of the culture with respect to the light source); and (3) possible high-ambient temperatures not adequately controlled by the misting system could affect culture health. To aid in inoculum development, an indoor bag system of thin film, flat panel 10-15 L bag cultures was introduced in the lab in December 2008. The thin film culture system is shown in Figure 2-13. Like the outdoor bag farm, this system used polyethylene bags to contain the culture and a $\mathrm{CO}_{2} /$ air mix to provide $\mathrm{CO}_{2}$ and mixing, but the indoor system used artificial fluorescent lighting providing $200 \mu \mathrm{E} / \mathrm{m}^{2} / \mathrm{s}$ 
from each side of the culture with a short light path of $3-5 \mathrm{~cm}$. The reduced light path, 24-hour light, and double-sided illumination allowed for production of dense inoculum at high-growth rates, and being in an indoor controlled system produced a cleaner, higher quality inoculum. This system provided a high-density, clean culture to inoculate the outdoor bag farm for further scale-up or directly into the large-scale cultivation system as culture volumes and densities dictated.

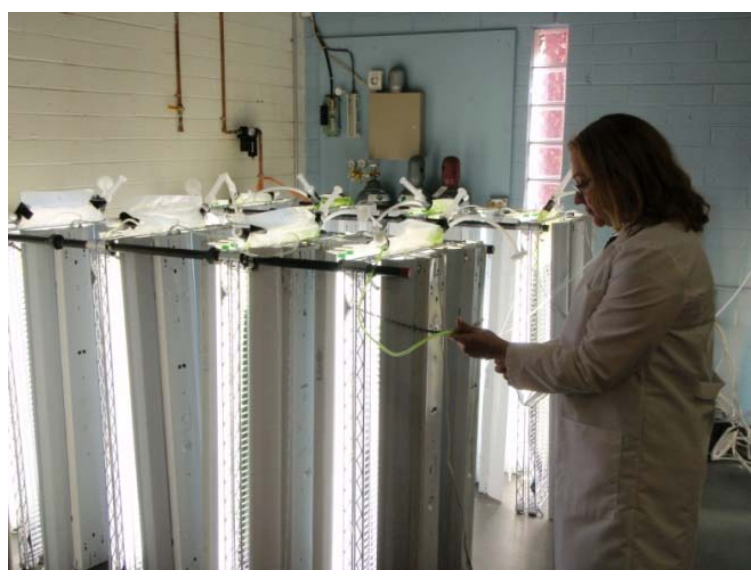

Figure 2-13 - Thin Film, Flat Panel Culture System

\subsubsection{SUMmaRY OF AlgAE STUDY AT3RD AVENUE LAB FACIUTY}

During this project, a large $8000 \mathrm{~L}$ outdoor bag farm and $\mathrm{a} \sim 1000-\mathrm{m}^{2}$ algae lab were erected at the APS $3^{\text {rd }}$ Avenue R\&D and Lab facilities. The lab was equipped to monitor culture growth and process harvested algae culture for storage and shipment. The bottle culture area of the laboratory was started and maintained to provide clean sources of inoculum in the event of contamination of any experimental cultures. The nursery housed the cultures of current interest for the ability to scale-up clean, healthy cultures as needed. Clean healthy seed stock cultures are the foundation of clean healthy large-scale cultures. Without clean seed stocks, culture contamination as well as other possible culture variances would become a barrier to successful culturing and specimen repeatability. Throughout the many scale-up processes of various cultures, inoculum was never the limiting factor for this project.

An extensive and ongoing algae selection process was conducted. In addition to the GF3 strain, Chlorella vulgaris, Nannochloris sp., Nannochloropsis oculata, Selenastrum sp., Scenedesmus obliquus, Scenedesmus acutus, and Scenedesmus dimorphus were tested, as they were purported to have many of the desired characteristics required by the project. Three species emerged as leading candidates based on the in-house lab-scale study: Nannochloropsis oculata, Selenastrum, and Scenedesmus obliquus. Lipid analysis revealed that the GF3 strain only contained about $5 \mathrm{wt} \%$ neutral lipids, which makes it difficult to extract oil. Nannochloropsis was found to reach total fat levels of $\sim 23$ wt $\%$ biomass, with neutral lipid content of $\sim 7.5 \mathrm{wt} \%$ when growing naturally. It could accumulate up to $34 \mathrm{wt} \%$ total fat and 18 $w t \%$ biomass as neutral lipids when stressed. Selenastrum had approximately $18 \mathrm{wt} \%$ total fat 
and 14 wt\% neutral lipids when growing exponentially. When under nutrient-stress conditions, Selenastrum was found to accumulate up to $35 \mathrm{wt} \%$ total fat, of which $30 \mathrm{wt} \%$ were neutral lipids. Neutral lipids are the portions that are utilized in the conversion to fuel oil. The achieved total fat and neutral lipid levels of Scenedesmus were lower than Selenastrum when stressed during this stage of the algae strain selection study, ranging between $10 \mathrm{wt} \%$ to $20 \mathrm{wt} \%$ total fats and 9 wt $\%$ to 17 wt\% neutral lipids. Scenedesmus was further found to exhibit rapid growth rates, thrive under the high temperatures found in Arizona, settle naturally, and grow for long periods of time without contamination. As a result of these characteristics, necessary for both the local growth conditions as well as the project objectives, Scenedesmus was chosen as the strain of focus. 


\subsection{SCALABIE CARBON CAPTURE MTH ALGAE}

\subsubsection{Rd AVenUe OUIDOOR TEST STIE DESCRIPIION}

Both inoculum scale-up and the first $6 \mathrm{M}$ initial algae cultivation systems were conducted at the $3^{\text {rd }}$ Avenue R\&D facility in Phoenix (Figure 2-14). The cultivator is represented by the object shown floating in the temperature-controlled pond in the center of the rendering. The hanging bag inoculum system, which is described in this report, is depicted along the left side of the rendering. The structure on the left side of the diagram and south of the bag farm was the gas distribution system. The dewatering system is depicted to the right side of the cultivator under a canopy. The primary building in the foreground houses offices, a workshop, and basic field laboratory apparatus for onsite use.

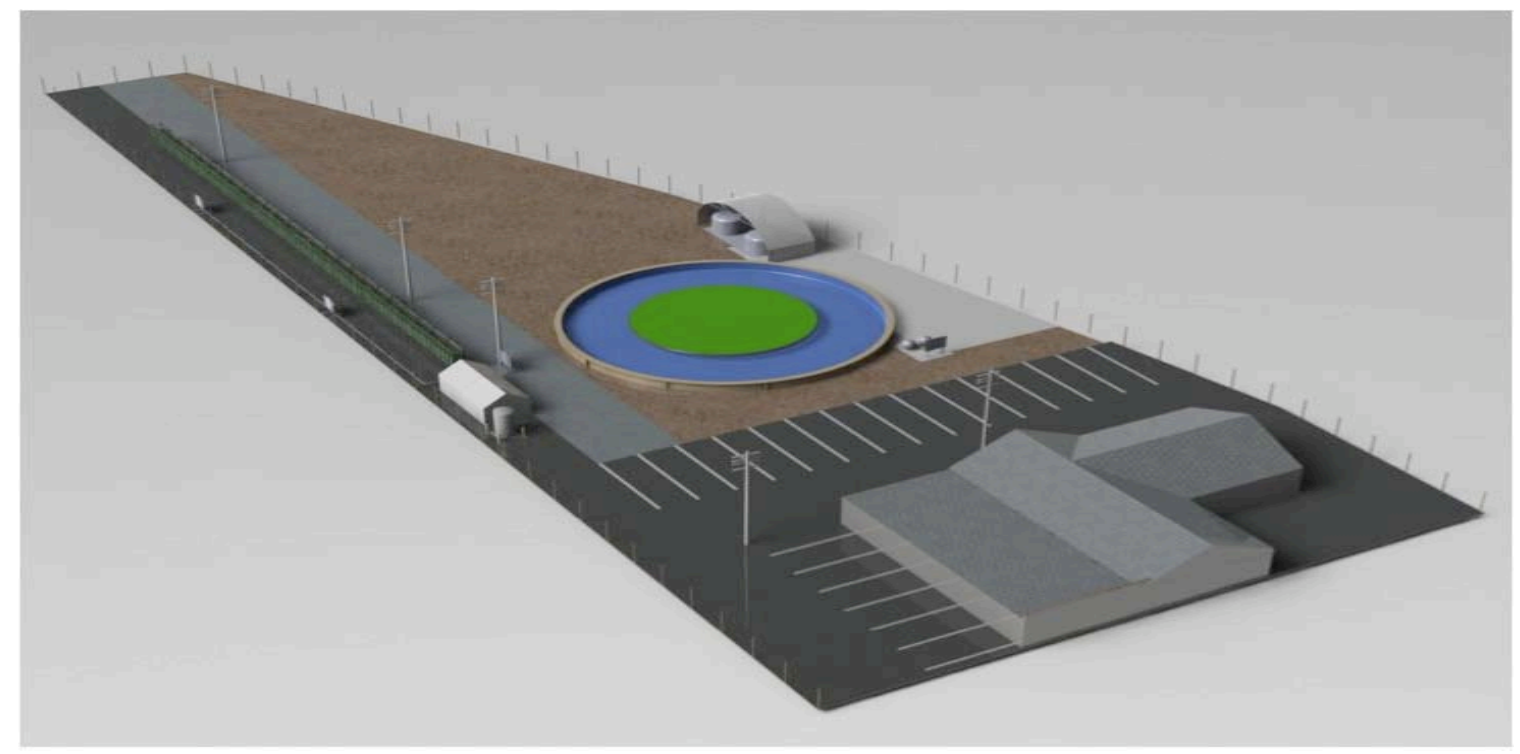

Figure 2-14 - Schematic of the $3^{\text {rd }}$ Avenue Outdoor Site in Phoenix, Arizona, Used for Testing of the $6 \mathrm{M}$ Algae Cultivation System

\subsubsection{M CULTIVATOR}

\subsubsection{6M Radius Test Cultivator Design}

The $6 \mathrm{M}$ cultivator was designed by Element Cleantech, Newark, Delaware to deliver robust and efficient algae production as an outdoor system. The cultivator provides conditions similar to a raceway pond, but it has more efficient delivery of $\mathrm{CO}_{2}$ throughout the culture growth area. Being a closed system, it also reduces the contamination and evaporation exhibited by raceway ponds. This fully operational cultivator just before inoculation is shown in Figure 2-15. 


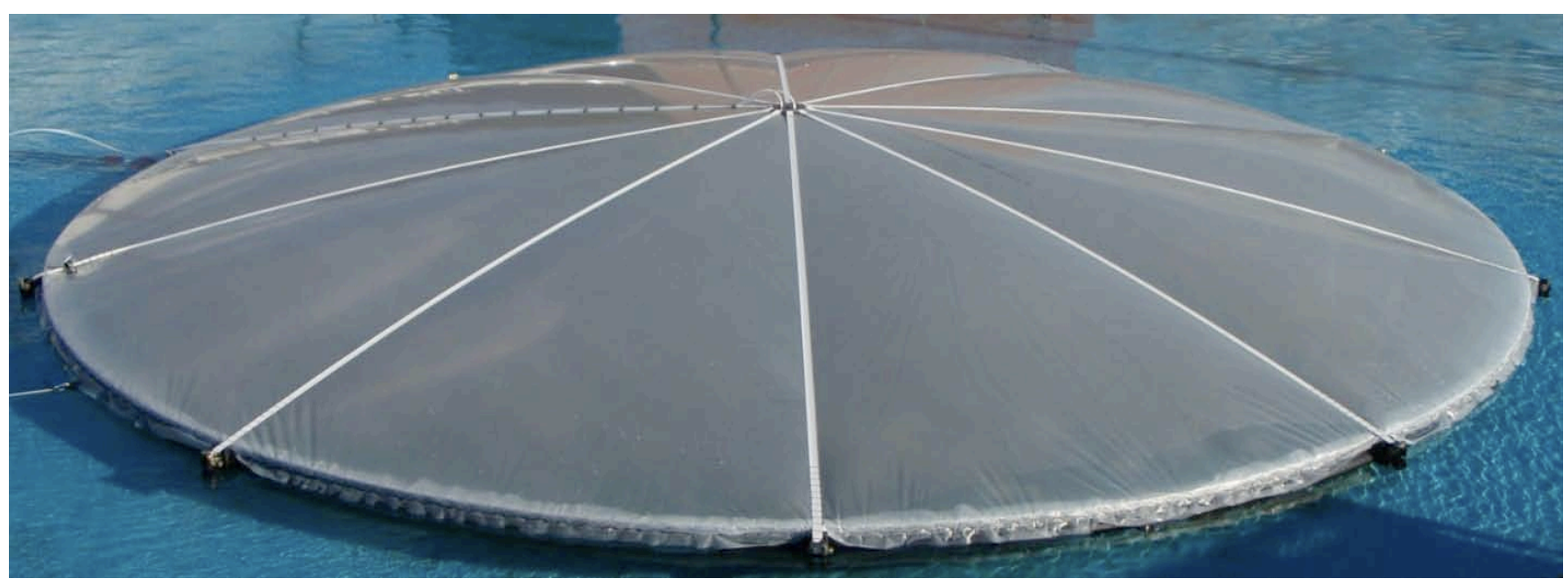

Figure 2-15 - Photo of the Cultivator Floating in the Thermal Pond

The $6 \mathrm{M}$ cultivator has a diameter of $12 \mathrm{~m}$ and a total surface area of $113 \mathrm{~m}^{2}$, with an active culture footprint of $95 \mathrm{~m}^{2}$. The cultivator is typically run at a total volume of between $10,000 \mathrm{~L}$ and $15,000 \mathrm{~L}$. The mixing technique of this design is a series of four wands revolving around a center hub in a clockwise motion. A centrifugal pump drives the wands. Liquid volume from the lower section of the center hub, which is an intake from the main cavity of the cultivator, is forced into a 2-inch PVC line that runs to the centrifugal pump. The liquid is discharged from the pump and is returned back to the cultivator by a 2-inch PVC line to a divided section of the center hub that supplies the four radially oriented wands. Each wand has a series of holes that exhaust the incoming liquid from the center hub back out into the main cavity of the cultivator. This discharge of liquid from the series of holes moves the wands and creates the revolution of all four wands, which in turn causes the mixing dynamic of the culture in the cultivator cavity. The pump runs continuously.

See Figure 2-16 and Figure 2-17 for a schematic overview of the floating 6M cultivator.

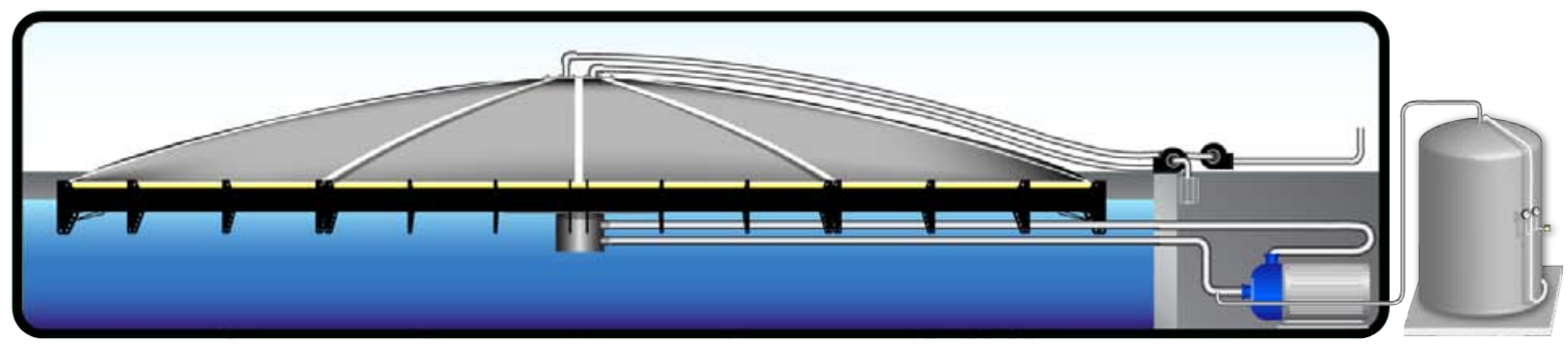

Figure 2-16 - 6M Cultivator Floating in a Thermal Pond 


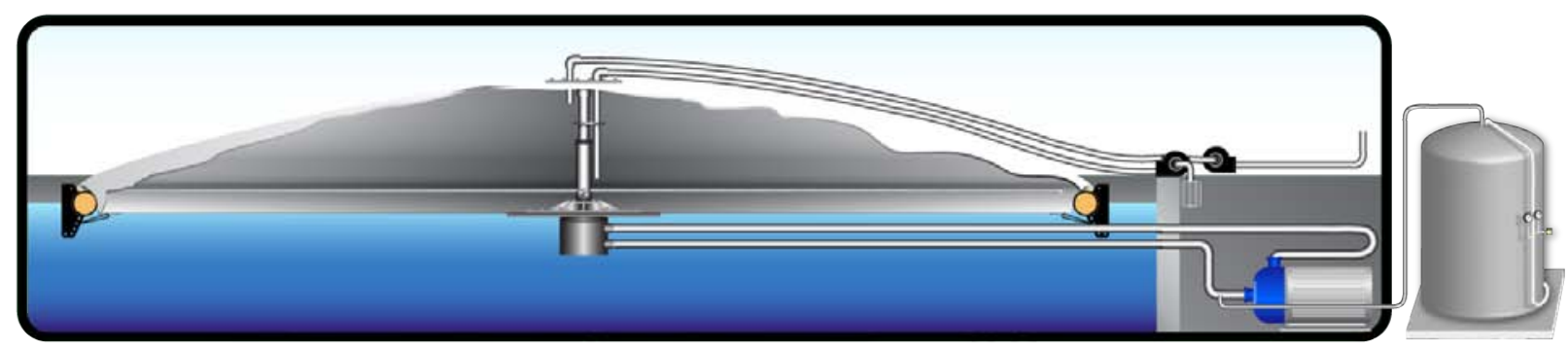

Figure 2-17 - 6M Cultivator Interior

The cultivator top cover is inflated using a regenerative air blower. Air is directed by a 2-inch gas pressure line from the blower through a regulating flow meter. Air is then metered at an approximate flow rate of $8-20$ scfh into a 2-inch PVC gas line to an air injection opening near the center hub, which finally discharges gas into the head space of the cultivator. Air exits by the canopy air exhaust line. Proper inflation of the dome is maintained by the flow meter.

In addition to the air entering the head space of the cultivator, $\mathrm{CO}_{2}$ is injected into the cultivator as well. The $\mathrm{CO}_{2}$ is distributed to the gas distribution panel of the cultivator where it is regulated by flow meters and then discharged into the gas distribution manifold and mixes with air before entering the cultivator.

For the cultivation system at $3^{\text {rd }}$ Ave, there was no filtration system for incoming water for the cultivator. City water was the source for both the cultivator and the thermal pond. Water to be pumped to the cultivator was collected in the dewatering vessel and/or feed tanks. Volumes entering or exiting a cultivator were tracked by flow totalizers.

The cultivator had associated sensors collecting and displaying data remotely by a LabVIEW network. Algae culture variables were continuously monitored using this automated data acquisition and control system. The data collected during this period included (1) liquid culture temperature, (2) $\mathrm{pH}$ in and $\mathrm{pH}$ out, (3) conductivity, (4) Photosynthetically Active Radiation (PAR) in the location of the cultivator, (5) timing of the $\mathrm{CO}_{2}$ injection into the liquid phase, and (6) percentage of $\mathrm{CO}_{2}$ in the gas-phase feed line into the cultivator and exhaust. All data were logged in one minute intervals during experimentation. The $\mathrm{pH}$ sensors communicated to a solenoid valve (SV) that controlled $\mathrm{CO}_{2}$ flow into the cultivator. The high and low $\mathrm{pH}$ setpoints ( $\mathrm{pH}$ range from 6-8) informed the communicating mechanism to either open or close the $\mathrm{CO}_{2}$-controlling $\mathrm{SV}$, and this ensured that excess $\mathrm{CO}_{2}$ was not provided to the culture, increasing the efficiency of the $\mathrm{CO}_{2}$ capture of the system. The setup and design of the data 
acquisition system was tested to determine the important variables to measure and the frequency to monitor the variables.

\subsubsection{6M Radius Test Cultivator Construction Components}

\subsection{Ring}

The circumference ring was made from 10-foot sections of 8-inch high-density polyethylene (HDPE) pipe, which was heated and shaped around a support jig to the proper arc. These 10-foot sections were then welded together forming the enclosed ring. Fittings for attaching the top and bottom liners, as well as for the tensioning system, were welded in place on the ring. The ring was floated in the thermal pond before attaching any other components. The ring was 12 feet in diameter.

\subsection{Bottom Liner}

The bottom liner consisted of a heavy-duty agricultural liner that was commercially cut to size and shaped based on ring dimensions. The liner was fastened between a bottom plate and the center hub with a nut and bolt configuration and silicone. Once the bottom liner was affixed to the center hub, heavy-duty straps were secured to the bottom plate and the top plates of the center hub. These straps run from the center hub assembly to the circumference ring and are used in tensioning the top and bottom liners.

\subsection{Top Liner}

The top liner consisted of 6- to 10-millimeter thick transparent plastic material. The top liner was affixed between the center hub assembly and the top plate with a nut and bolt configuration. The top liner was secured and tightened by a $1 \frac{1}{2} 2$-inch circumference pipe that was welded to the 8-inch floating circumference ring and had a 1/3 section of pipe removed laterally. This lateral cut allowed a $1 \frac{1}{2}$-inch PVC pipe to be inserted inside the cut pipe with the top liner material that had been unrolled around the entire float ring to be sandwiched in between. The top liner was now taut between the center hub and the circumference ring in a downward pitch allowing precipitation to run off the top liner and into the thermal pond. The top liner was then finally tightened to an appropriate degree using the straps to allow for proper inflation of the dome and to insure the center hub was upright. See Figure 2-18 and Figure 2-19 for a depiction of the air circulation with the top liner in place. 


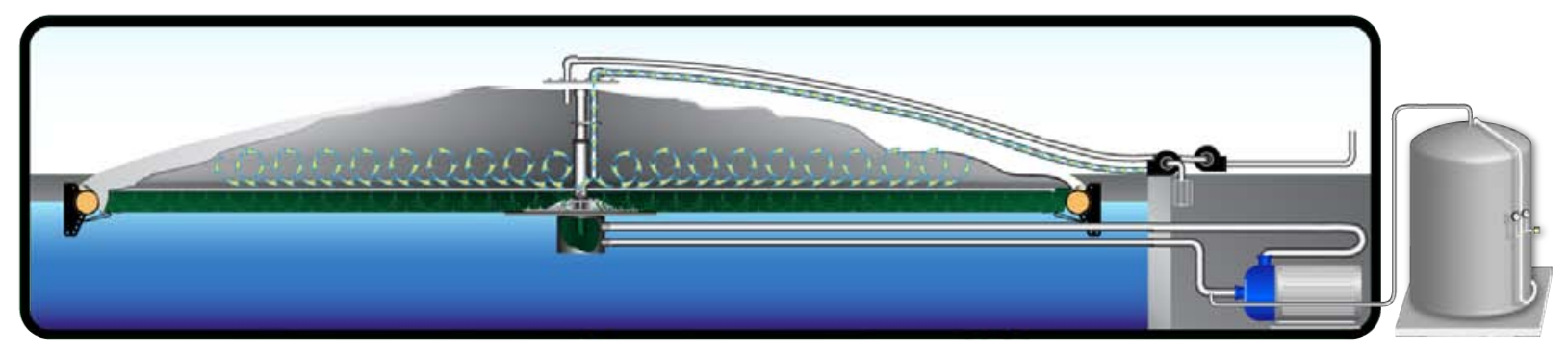

Figure 2-18 - Filtered Air Was Injected into the Canopy

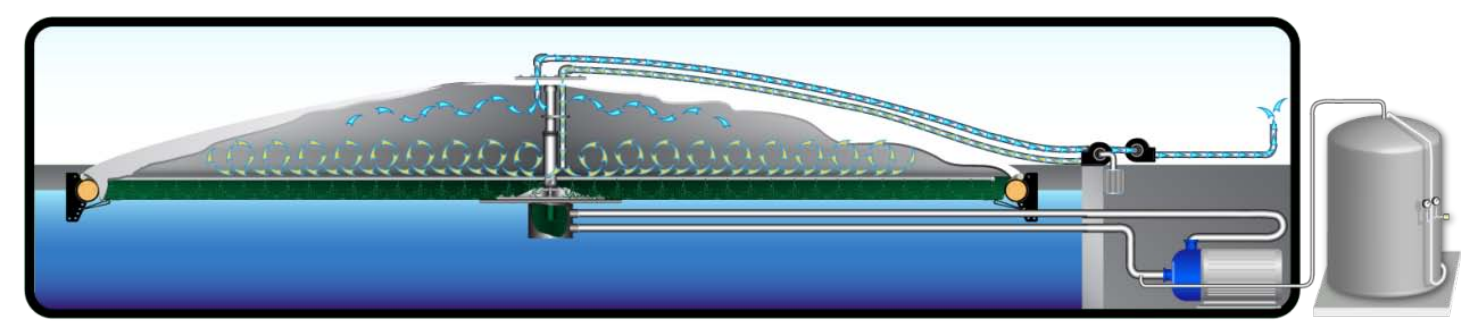

Figure 2-19 - Air Was Exchanged and Removed, Moderating Headspace Oxygen Levels

\subsection{Center Hulb}

The center hub included a cylindrical vertical central PVC pipe and flat stock assembly that functioned as follows: (1) a stem from which the wands spin; (2) intake and discharge ports for liquid and gas volumes; and (3) a central stabilizing column that allows for adjustment in depth profile based on inflation and tensioning parameters. The center hub was secured by a lateral tensioning system (see Figure 2-20). 


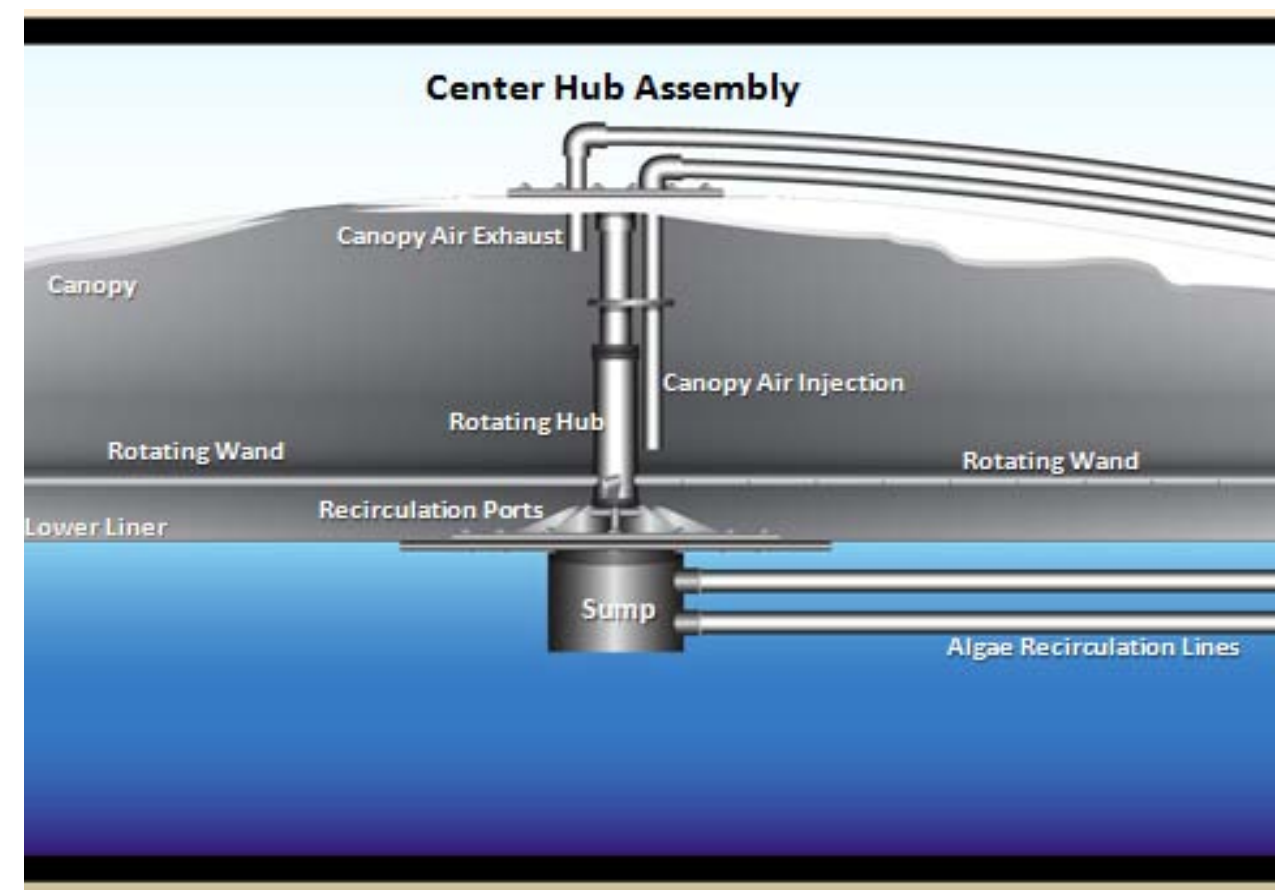

Figure 2-20 - Center Hub Assembly

\subsection{Sec uning and Tensioning System}

The tensioning system consisted of bottom and top straps affixed to the bottom and top plates respectively and running to the circumference ring. Straps could be tightened or loosened by the ratchet mechanism welded to the circumference ring in order to raise or lower the depth profile (center hub assembly) in conjunction with the inflation rate adjustment of the cultivator. The greater the amount of gas in the cultivator, the higher the center hub would rise, which created a lower depth of liquid volume across the bottom liner. The typical depth within the cultivator was $15 \mathrm{~cm}$.

\subsection{Wands with Foats}

The wands were schedule 80 PVC pipes with a 90-degree elbow on the end closest to the ring. Wands were inserted into the center hub by a compression fitting with a locking pin. Plastic encased Styrofoam floats were attached to the ends of each wand to help prevent chaffing of the liner (see Figure 2-21 and Figure 2-22). 


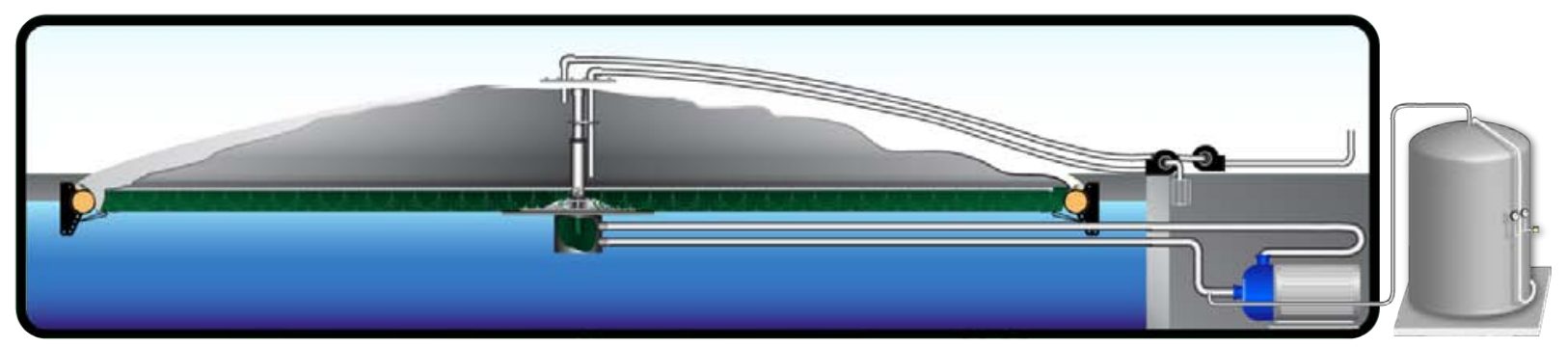

Figure 2-21 - Jets Propelled the Wands through the Culture, Creating Hydro Mixing

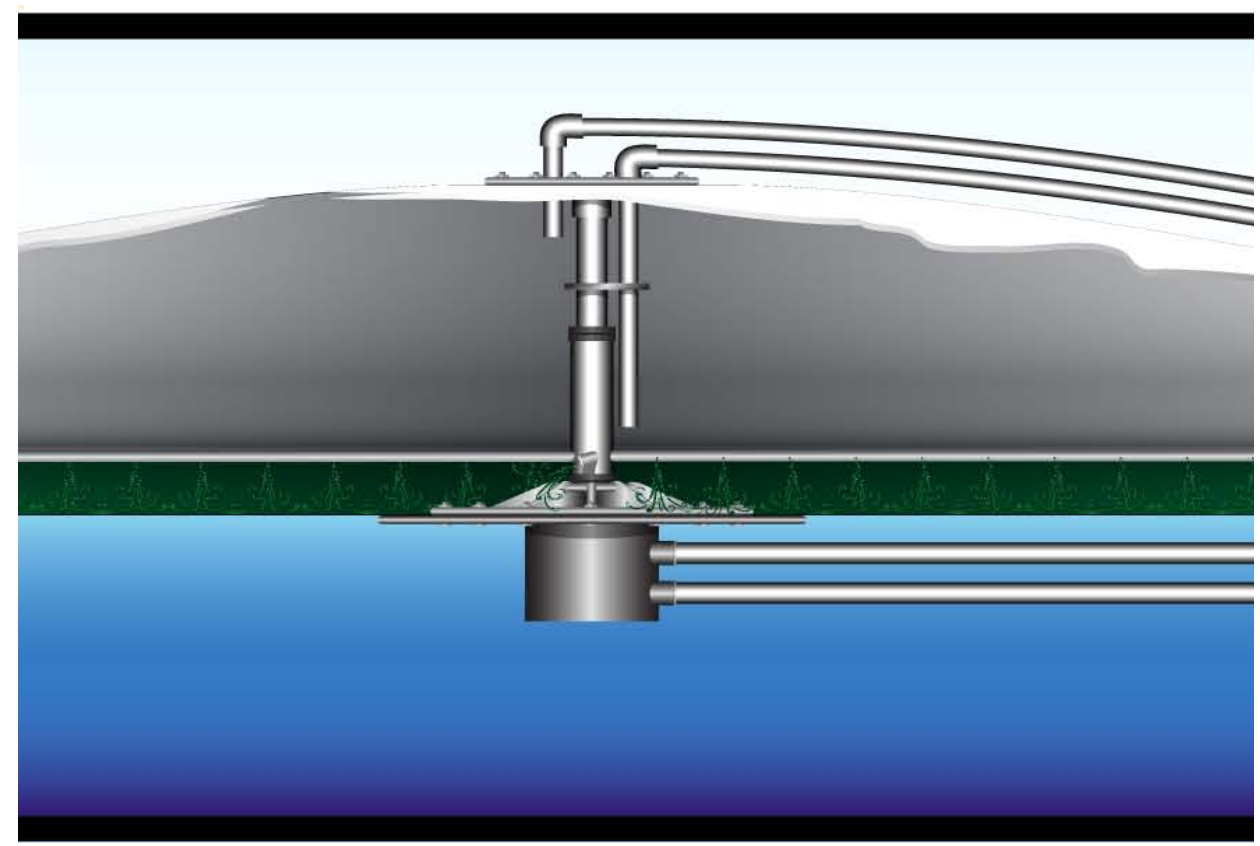

Figure 2-22 - Wands Showing Discharge of Liquid Volume through Exhaust Holes

\subsection{Gas Distribution Pipes}

A 2-inch schedule 80 PVC pipe ran from the adjacent gas distribution panel and delivered the selected gas (air/ $\mathrm{CO}_{2}$ mixture) at a rate ranging from $8-20$ scfh to the canopy air injection port adjacent to the center hub. Air was then discharged at a metered rate from the canopy air exhaust.

\subsection{Liquid Distribution loop}

A 2-inch schedule 80 PVC pipe ran from the 7.5 horse power (hp) centrifugal pump underneath the cultivator (submerged) into the center hub where liquid volume was distributed to the four 
wands. Liquid volume was returned from the center sump through a 2-inch schedule 80 return line back to the pump (see Figure 2-23).

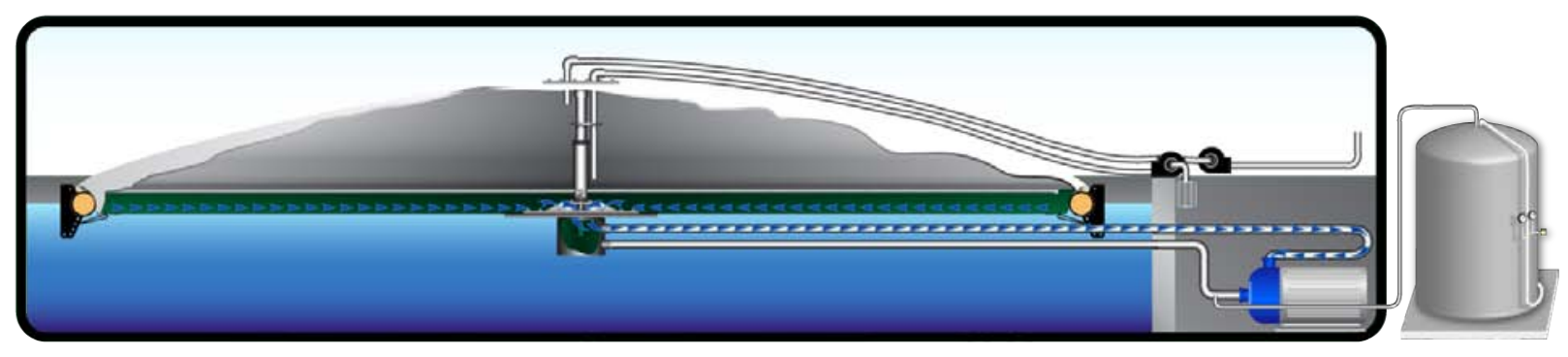

Figure 2-23 - Pump Circulation Loop

Before the inoculation of the algae, the cultivator was commissioned using water to ensure that culture growth parameters could be achieved without the threat of mechanical breakdown jeopardizing the health and productivity of the culture.

\subsubsection{THERMAL POND}

The cultivators were floating and anchored within a thermal pond. The pond was 70 feet in diameter and contained approximately 50,000 gallons (gal) of water (see Figure 2-24). The primary purpose of the thermal pond was thermal regulation of the liquid volume within the cultivators. Given the extreme Arizona desert heat, temperatures can reach over $110^{\circ} \mathrm{F}$ in the spring and summer months. The thermal pond helped to offset these extreme temperatures by using the large volumes of water to create temperature buffering. In addition, there was a chiller circulating water to keep the thermal pond at the desired temperature, and as a result the culture temperature in the cultivator stayed within

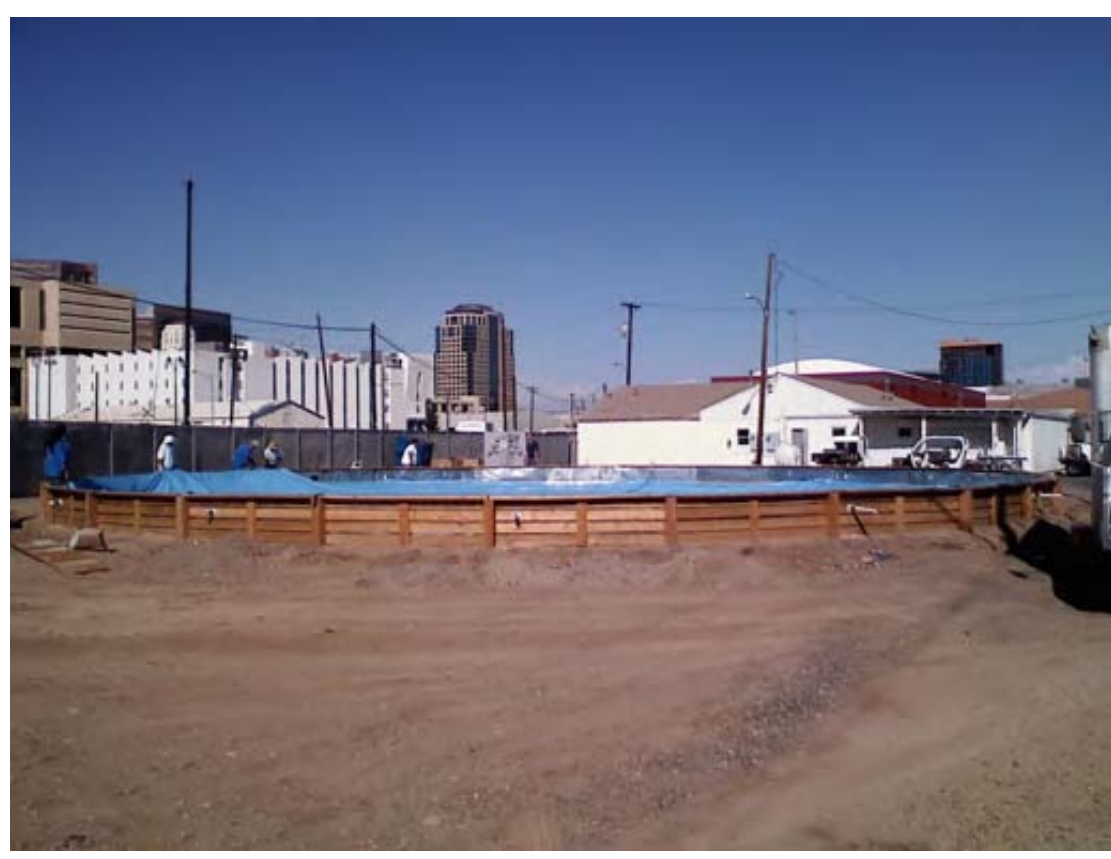

Figure 2-24 - 3rd Avenue Thermal Pond under Construction 
operational ranges. Thermal pond water never came in contact with the culture inside the cultivator. Adjacent to the thermal pond was a recirculating sand filter system. These filters kept the thermal pond clarified. The pond was also kept disinfected through hypochlorite doses and conventional pool floats with chlorine tablets.

\subsubsection{GAS DISTIRIBUIION}

At the site, a regenerative air blower supplied air to the $6 \mathrm{M}$ cultivator. $\mathrm{A} \mathrm{CO}_{2}$ storage tank supplied $\mathrm{CO}_{2}$ to the cultivator gas distribution system. $\mathrm{CO}_{2}$ and air were combined in a $1 / 2$-inch SS gas header as part of a gas distribution loop servicing the cultivator. The header ran through a control panel and then into the cultivator itself. There were sensors on the upstream and downstream sides of the cultivator that recorded $\mathrm{CO}_{2}$ concentration values.

However with regard to $\mathrm{CO}_{2}$ flow into the cultivator, a SV received signals from a $\mathrm{pH}$ meter located on the inflow pipeline to the cultivator dictating whether the solenoid was open or closed and as a result how much $\mathrm{CO}_{2}$ the cultivator was receiving. The $\mathrm{CO}_{2}$ flow was therefore dictated by $\mathrm{pH}$ setpoints. If there were a change in $\mathrm{pH}$, the solenoid would open or close accordingly (high $\mathrm{pH}$ - solenoid open, low pH - solenoid closed). Gas flowed into the distribution configuration on the center hub of the cultivator and was then dispersed through the wands and into the main cavity of the cultivator and hence the culture. There was an exhaust manifold that returned excess gas by an exhaust blower (see Figure 2-25). 


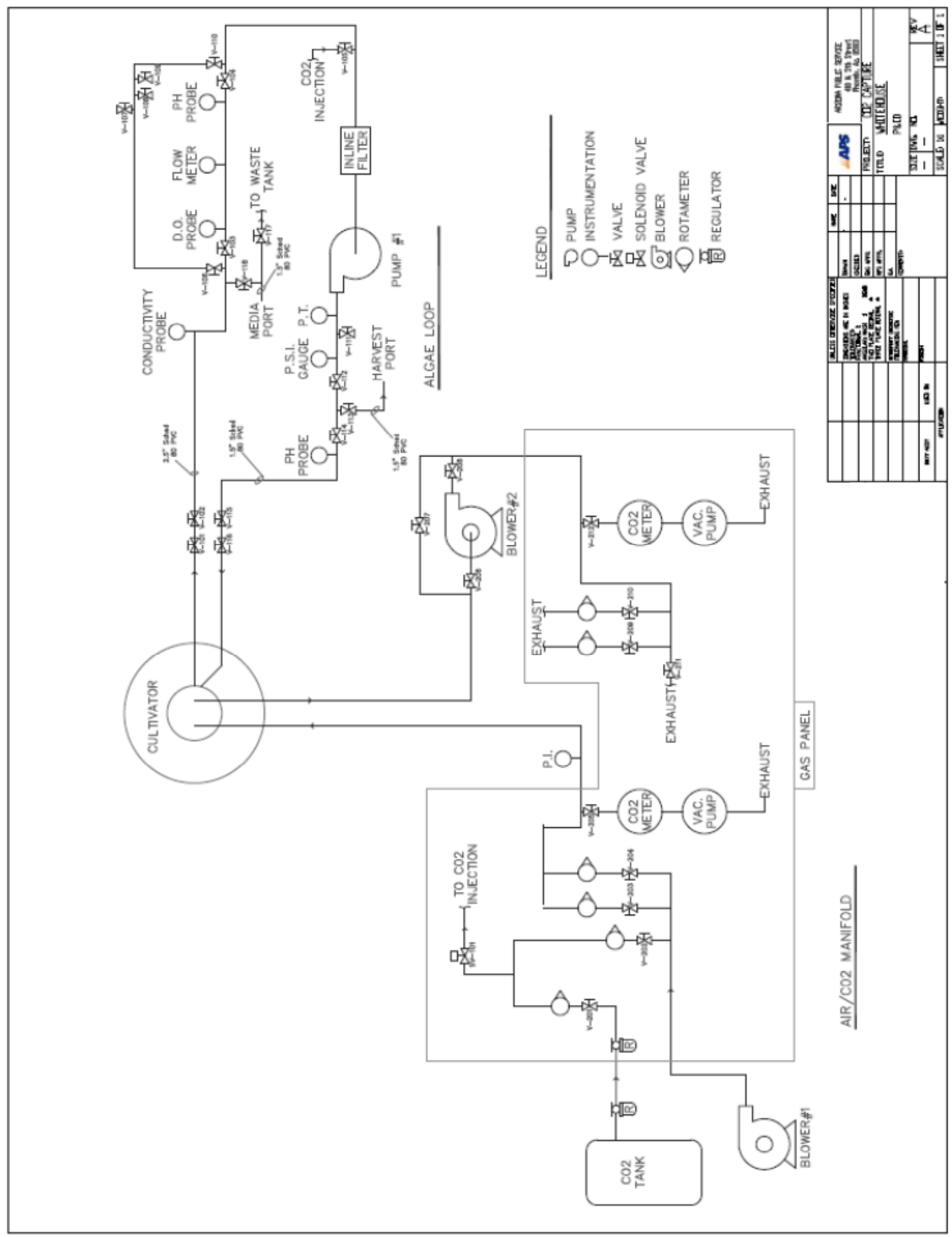

Figure 2-25 - 3rd Avenue Gas and Liquid Distribution Loop Diagram with Inline Sensors 
In addition to regulating $\mathrm{pH}$ within the optimal range of 6 to 8 within the culture, the gas distribution system also maintained dome inflation within the cultivator to a positive pressure. Regardless of whether $\mathrm{CO}_{2}$ was flowing in the line, air was always flowing at a constant measured flow into the cultivator as dictated by a flow meter. There was no automated method for maintaining a specific dome inflation profile. As a result, the dome profile was maintained through visual checks to ensure that the top liner was not underinflated, which could cause the liner to make contact with the spinning wands and could rip the liner material. Likewise, over-inflation had the potential to make the entire cultivator rise to a degree that might cause introduction of air into the liquid circulation loop, causing the pump to cavitate. Proper inflation of the dome generally was not an issue as the cultivator was monitored to the degree that aberrations in inflation could be corrected manually. Once the proper settings were reached, the cultivator profile was relatively stable with just minor adjustments being required.

\subsubsection{HARVEST/ DeWATERING}

When the cultivator culture was ready for harvest as described later in this section, the culture was pumped from the cultivator by the liquid volume circulation loop and directed to the adjacent dewatering feed tank. There was a flow totalizer monitoring the desired volume, typically $4500 \mathrm{~L}$, of culture transferred. Depending on the volume of culture removed, the primary cultivator circulation pump could be deactivated to avoid pump cavitations. Before or in conjunction with beginning the dewatering process, the cultivator was refilled with the desired makeup volume of media and the primary circulation pump again reactivated if needed and stabilized. At this point, the dewatering system could be activated, and the culture was directed from the feed tank to the dewatering unit.

The primary dewatering apparatus at 3rd Avenue was based on cross-flow filtration technology, designed and manufactured by DynaSep, Newark, Delaware. The design goal of the dewatering unit was to produce algae paste of 8-12 wt\%. By using a variable frequency drive (VFD) gear pump, the system was able to draw algae culture (green water) from a feed tank and maintain a set pressure in the filter loops. This pump discharged to the first stage filter loop, the largest loop in the system. This loop had a centrifugal pump that circulated the dilute algae repeatedly through the cross-flow filters at velocities that discouraged the formation of an impermeable cake of solid algae biomass on the filter's inner surfaces. In this loop, the equipment removed approximately $90 \mathrm{wt} \%$ of the water as permeate (clear water), which was then discharged to a collection tank (permeate tank). A slipstream of the algae concentrate 
moved from the first filter loop to the second. The second filter loop was smaller and used a second gear pump due to the increased viscosity of the material in this loop. Permeate could be routed directly to the tank or it could be run through the shell side of the second filter loop to provide cooling. The velocities were similar to those in the first loop to limit the algae solid from caking on the filter's interior surface. The second filter loop's discharge was the system's product, which was an algae paste concentrate greater than 8 wt\%. Figure 2-26 shows the entire system.

The first harvest of algae culture from the cultivator occurred on March 2, 2008, and this marked the first testing of the dewatering system. Figure 2-27 shows the harvested culture coming from the cultivator to the harvest system by a transparent pipe. The dewatering system successfully reduced the water content of the harvested culture as can be seen in Figure 2-28, which is a picture of the resulting water separated during the harvesting process. Figure 2-29 shows the resulting concentrated algae biomass at approximately $10 \mathrm{wt} \%$ solids. This particular dewatering system could process algae solution at a rate of $\sim 2 \mathrm{gpm}$. 


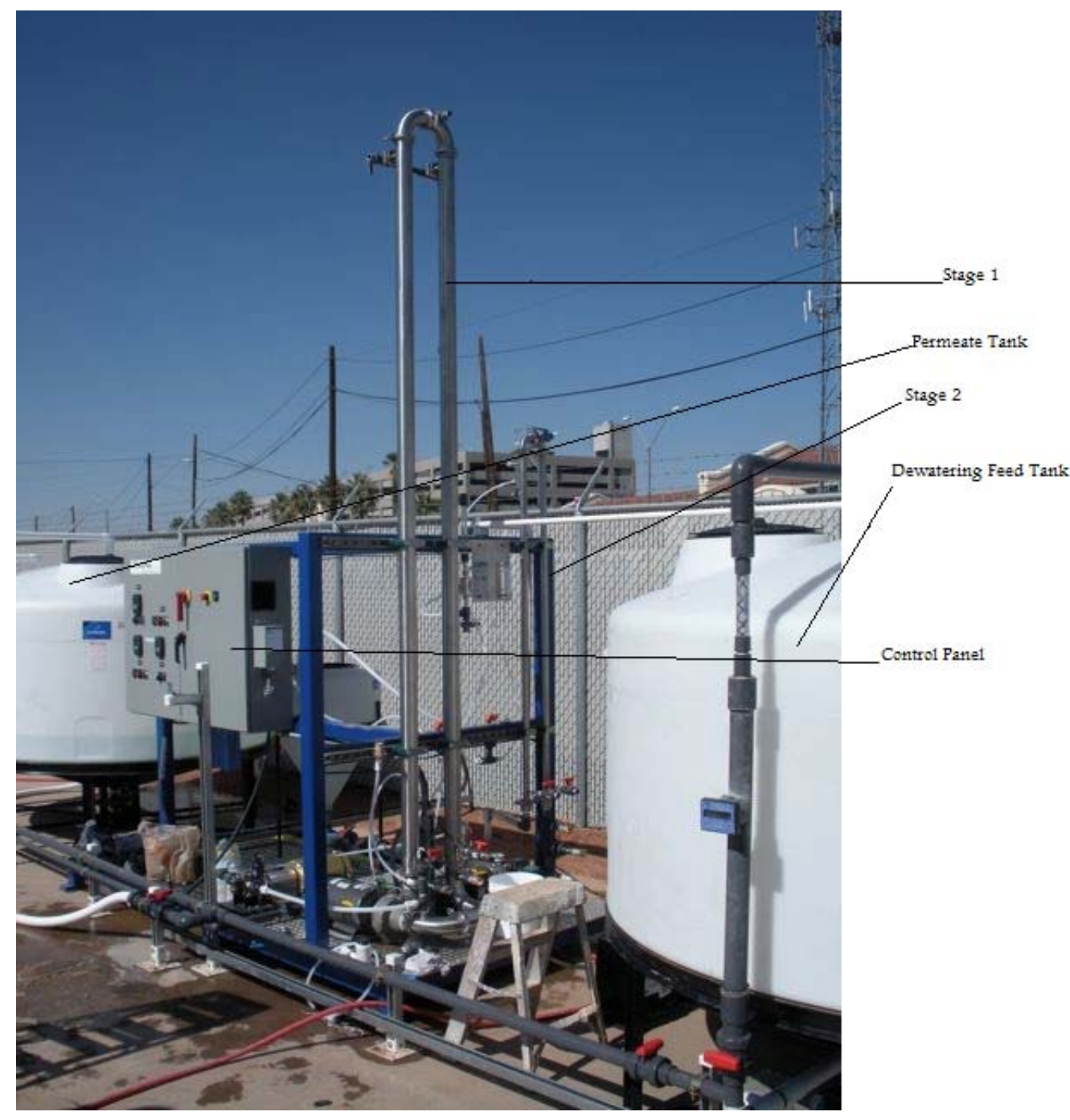

Figure 2-26 - Dewatering System 


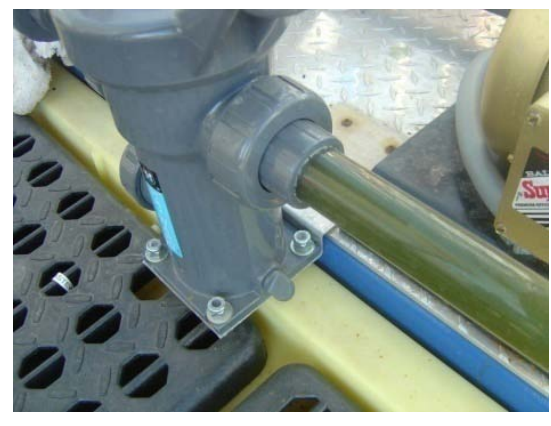

Figure 2-27 - Culture from the Cultivator Entering the Dewatering System (through Transparent Pipe)

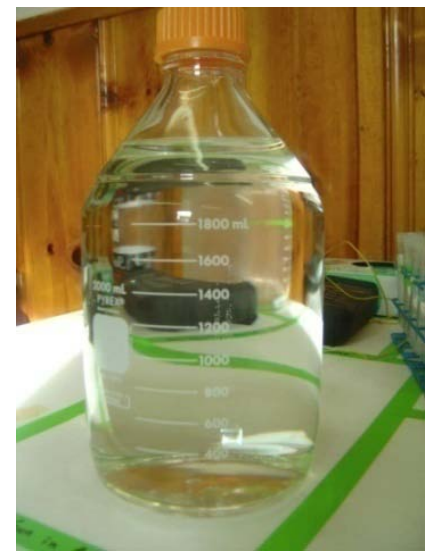

Figure 2-28 - Resulting Separated, Clear Water after Dewatering of Algae

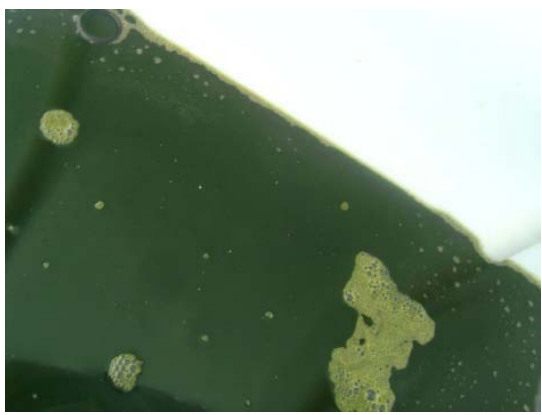

Figure 2-29 - Concentrated Algae in Large Tank after Dewatering (Approximately 10 wt $\%$ Solids)

The DynaSep dewatering system was used for large volumes of culture in order to create concentrated culture on the order of 8-12 wt\% solids as indicated. In some cases, product from this dewatering unit went on to be further concentrated using the Lavin centrifuges (see Figure 2-30). These centrifuges were effective but were not an option for dewatering large volumes of dilute culture given the time-consuming energy intensive nature of the process. The Lavin centrifuges had to be operated twice to minimize the material loss, which led to its typical processing rate of $\sim 0.02 \mathrm{gpm}$. The centrifuges were only used after product had been concentrated in the DynaSep dewatering unit. Concentrated culture from the dewatering unit was dispensed into an initial holding tank and metered into the centrifuge at a rate that allowed for concentration to occur in the bowl and created a clear discharge or permeate upon exit. In some cases, product was able to be concentrated up to as much as $30 \mathrm{wt} \%$ solids (see Figure 2-31). 


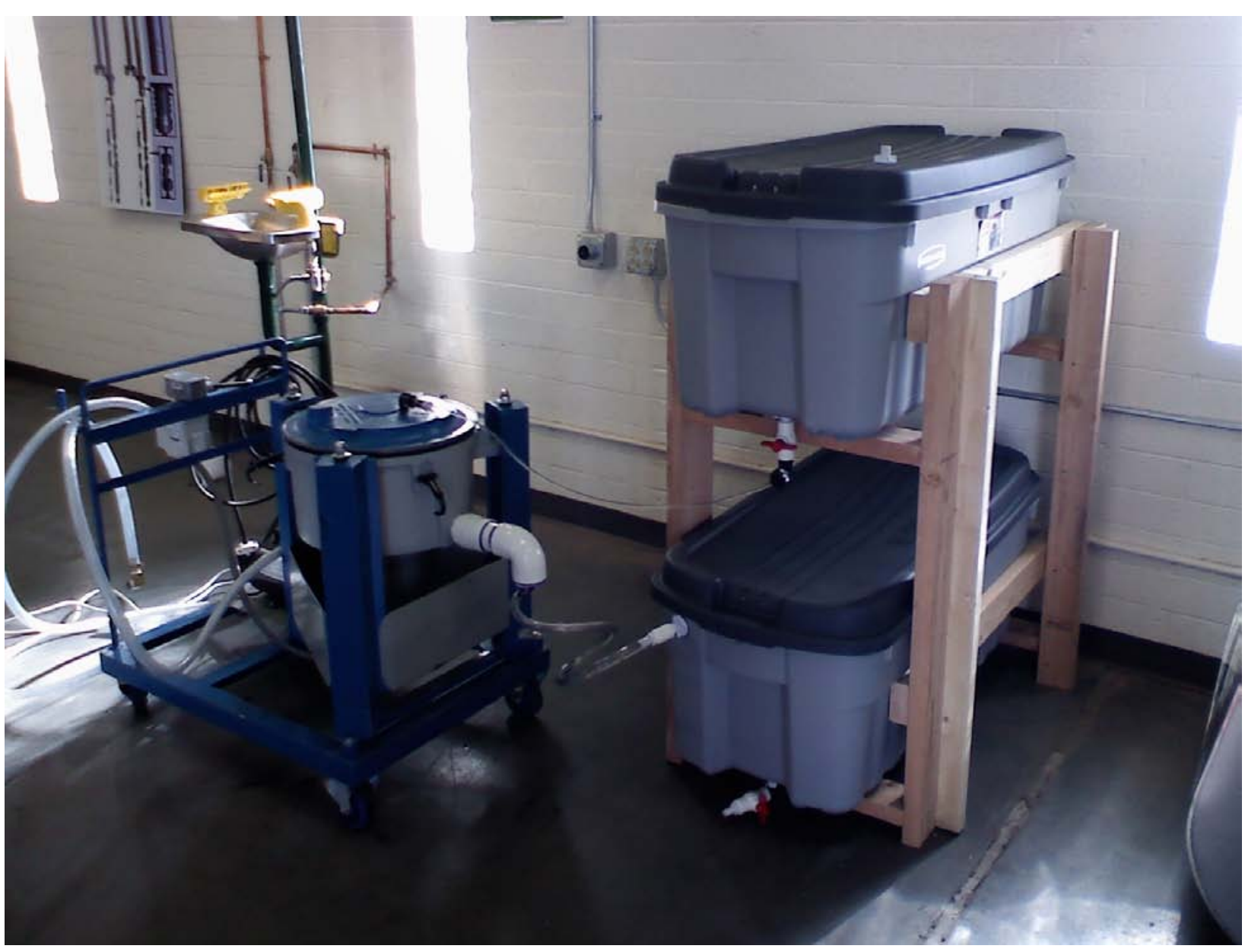

Figure 2-30 - Culture Dewatering Lavin (12-413V) Centrifuge 


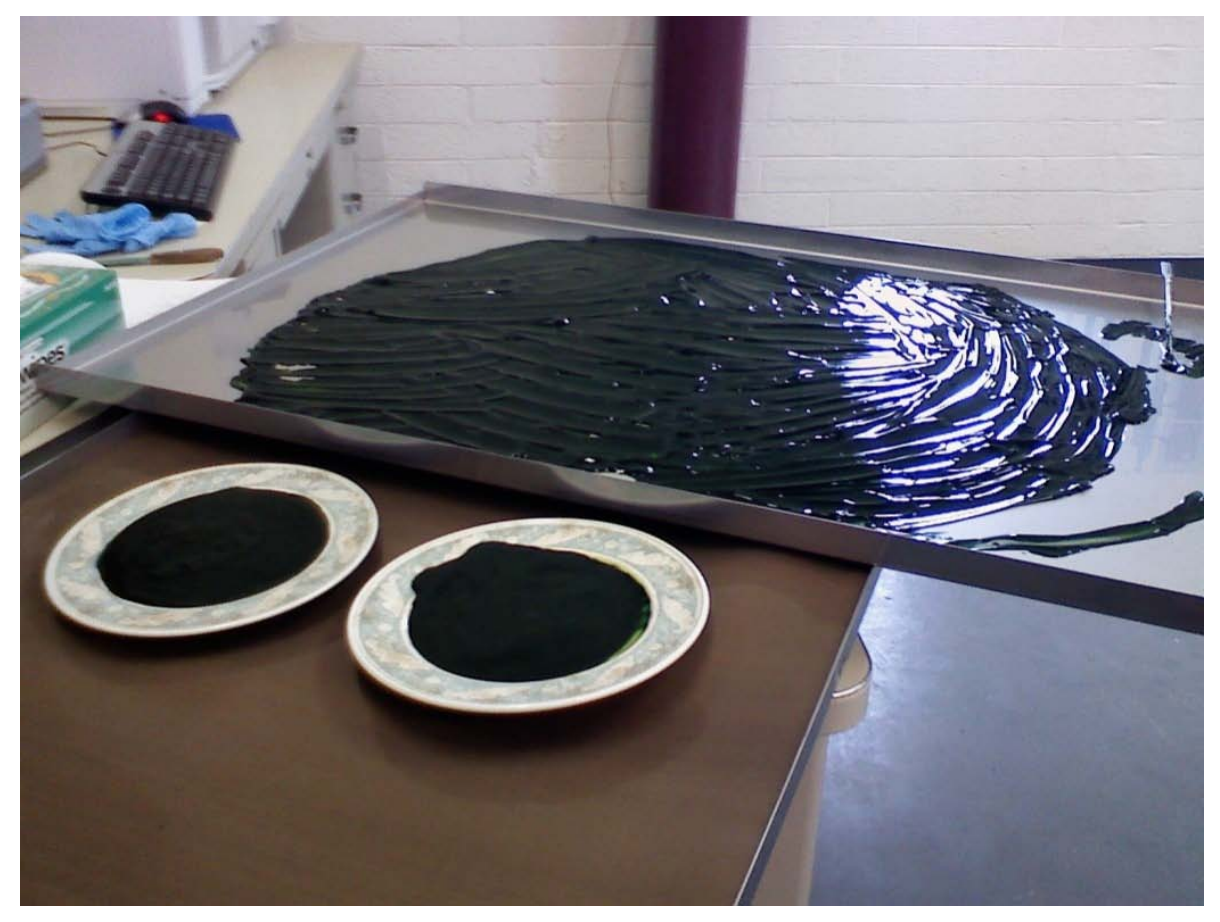

Figure 2-31 - Algae Paste out of Lavin Centrifuge 


\subsubsection{RD AVenUe TEST SYSTEM RESULTS AND DISCUSSION}

\subsubsection{6M Radius Cultivator Algae Test Run 1 - Nannoc hloropsis}

\subsection{First Batch Test of Nannoc hloropsis}

\section{Study of Growth Rate}

The first inoculation of Nannochloropsis was conducted after sunset on February 11, 2009. The initial running volume of culture within the cultivator was brought to $12,000 \mathrm{~L}$ with an estimated average culture depth of $15 \mathrm{~cm}$. Since the initial culture density was considered low (florescence maximal [FM] reading of 700) the culture was allowed to acclimate to sunlight by using a $60 \mathrm{wt} \%$ shade cloth for 8 days in order to prevent bleaching of the culture from the high irradiance provided by this system. After this 8-day acclimation period, when culture FM reading reached approximately 800 , the shade cloth was removed and the culture was exposed to full sunlight beginning on the afternoon of February 20, 2009.

Throughout this test, samples were taken hourly to track a number of physical and chemical conditions in the cultivation system. However, actual biomass density measurements in the form of ash-free dry weight were not taken until February 22, 2009, due to a lack of the necessary analytical equipment and resources at that time. The relative photosynthetic pigment density of the culture was estimated using the FM readings taken by a fluorometer, which can also be used to monitor algae growth. The FM is a unitless indication of the condition of chlorophyll as measured by a burst of white light reflected back from dark conditions where the photosynthetic process absorbs red wavelength. Waltz's Mini-PAM fluorometer was used to measure its value. The FM density curve over the period of time following inoculation of the cultivator can be seen in Figure 2-32. A decrease in density can be seen after the first harvest on March 2, 2009, followed by the subsequent increased density from growth. 


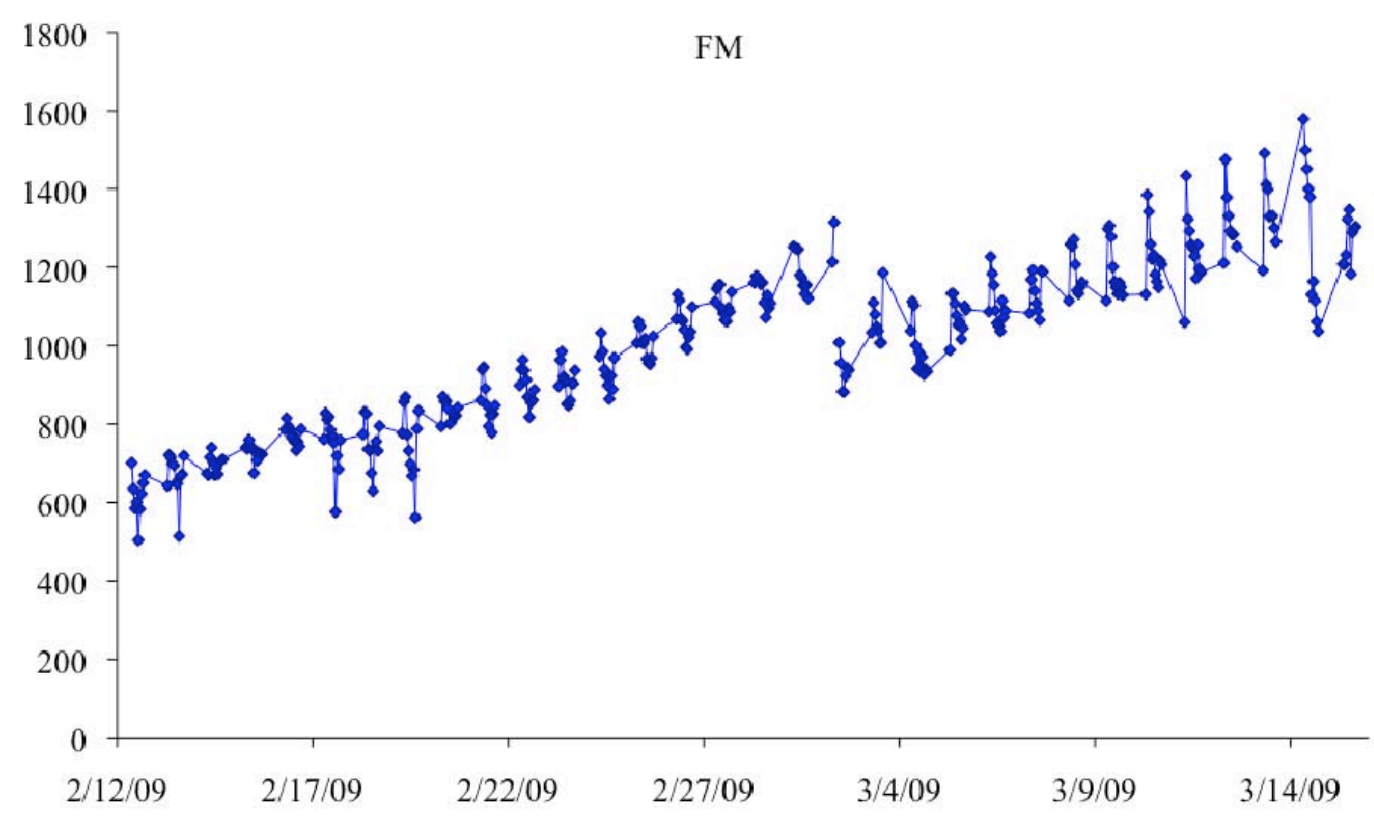

Figure 2-32 - Nannochloropsis Culture Density as Measured by FM (no unit) within the Cultivation System

Figure 2-33 shows the exact measurement of the biomass density in the form of ash-free dry weight per liter of culture that was collected until March 22, 2009.

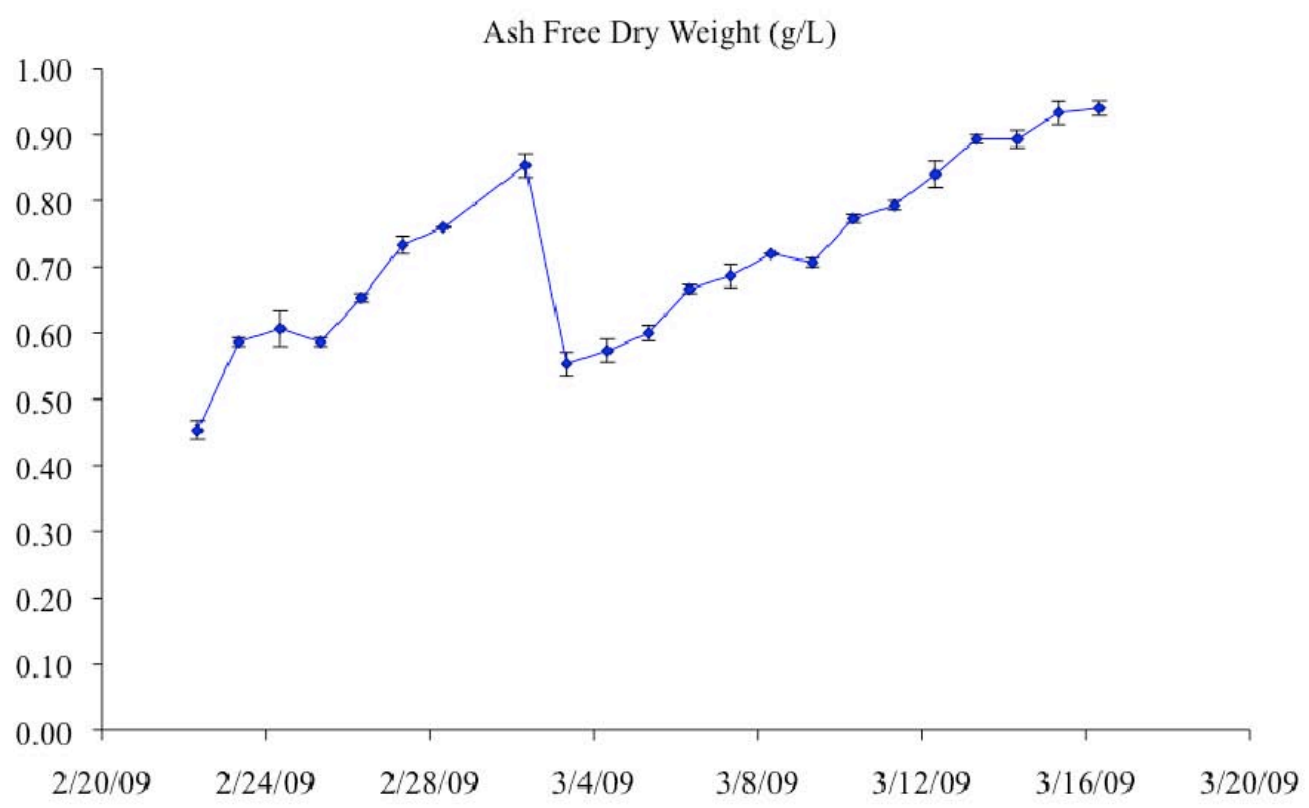

Figure 2-33 - Nannochloropsis Culture Density as Measured by Ash-Free Dry Weight (grams of dry biomass per liter of culture) 
For an initial growth curve, Nannochloropsis demonstrated $\sim 14 \mathrm{~g} / \mathrm{m}^{2} / \mathrm{d}$ growth rate. Not only was the culture able to acclimate to full sunlight under these conditions, but the cultivator also consistently provided the conditions necessary to maintain growth and health of the culture for 32 days. However, the resulting productivity for this test was a modest, $\sim 4 \mathrm{~g} / \mathrm{m}^{2} / \mathrm{d}$.

A number of factors could be responsible for this lower than expected productivity. One possibility was inconsistent depth profiles within this cultivation system. Such inconsistencies resulted from some of the culture residing in deeper areas shifting the optimal culture density for high productivity. Careful observation of the algae cultivation system revealed that indeed a majority of the culture material was at depths around two times (2X) the designed depth of 15 $\mathrm{cm}$. Interestingly, given the volumetric productivity shown, the fact the culture could still result in this level of productivity suggests that if the entire culture was run at $30 \mathrm{~cm}$ deep and thus 24,000 L volume instead of 12,000 , the target aerial productivity could still be reached. Furthermore, the highest daily growth rate of $\sim 14 \mathrm{~g} / \mathrm{m}^{2} / \mathrm{d}$ was achieved when culture density was the lowest at $0.01 \mathrm{~g} / \mathrm{L}$. Although this was a single data point, it may support the likelihood that the culture would have exhibited higher productivity at a lower density for the given light path provided by the cultivation system.

\section{Study of Culture Temperature and pH}

Culture temperature was measured throughout the testing as shown in Figure 2-34. The daily increase in temperature due to solar radiation can be seen as peaks in the temperature within each 24-hour period. Overall, temperature was maintained well within the range of safe growing temperatures for this specific species of algae of $10-20^{\circ} \mathrm{C}\left(50-68^{\circ} \mathrm{F}\right)$. 


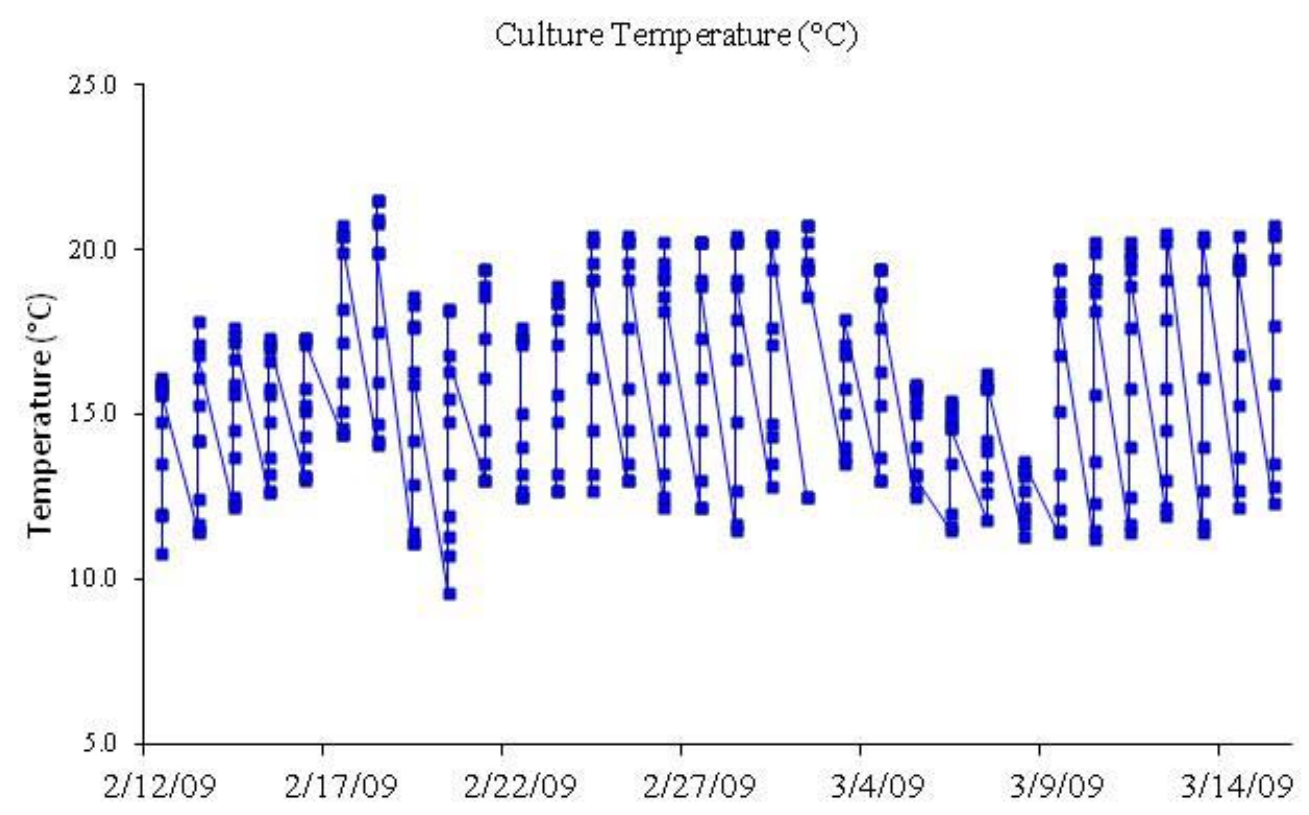

Figure 2-34 - Nannochloropsis Culture Temperature within the Cultivation System

As described previously, the $\mathrm{pH}$ of the culture was maintained by manipulating the flow of $\mathrm{CO}_{2}$ into the cultivation system. In algal culture, the consumption of $\mathrm{CO}_{2}$ as a carbon source reduces the content of $\mathrm{CO}_{2}$ in solution; therefore, as a culture grows, $\mathrm{pH}$ rises if the solution is not further supplemented with $\mathrm{CO}_{2}$. Maintaining a range of $\mathrm{pH}$ is important to culture health and growth. As can be seen in Figure 2-35, the $\mathrm{pH}$ was maintained well within the optimal range of 6 to 8 for the growth of this species of algae simply by manipulating the modest flow of $\mathrm{CO}_{2}$ into the system, ranging from 8 to 20 standard cubic feet per hour (scf/hr). Slight changes to the $\mathrm{CO}_{2}$ flow resulted in marked changes to the $\mathrm{pH}$ indicating an efficient $\mathrm{CO}_{2}$ delivery mechanism in the cultivation system.

This initial test run sought to sustain a robust and actively growing algae culture and provided initial data to guide setpoints for an on-demand $\mathrm{CO}_{2}$ feeding system that was under development. An on-demand $\mathrm{CO}_{2}$ feeding system based on $\mathrm{pH}$ value would maintain the optimal $\mathrm{pH}$ range for algae growth and significantly reduce the labor requirement. 
Culture PH

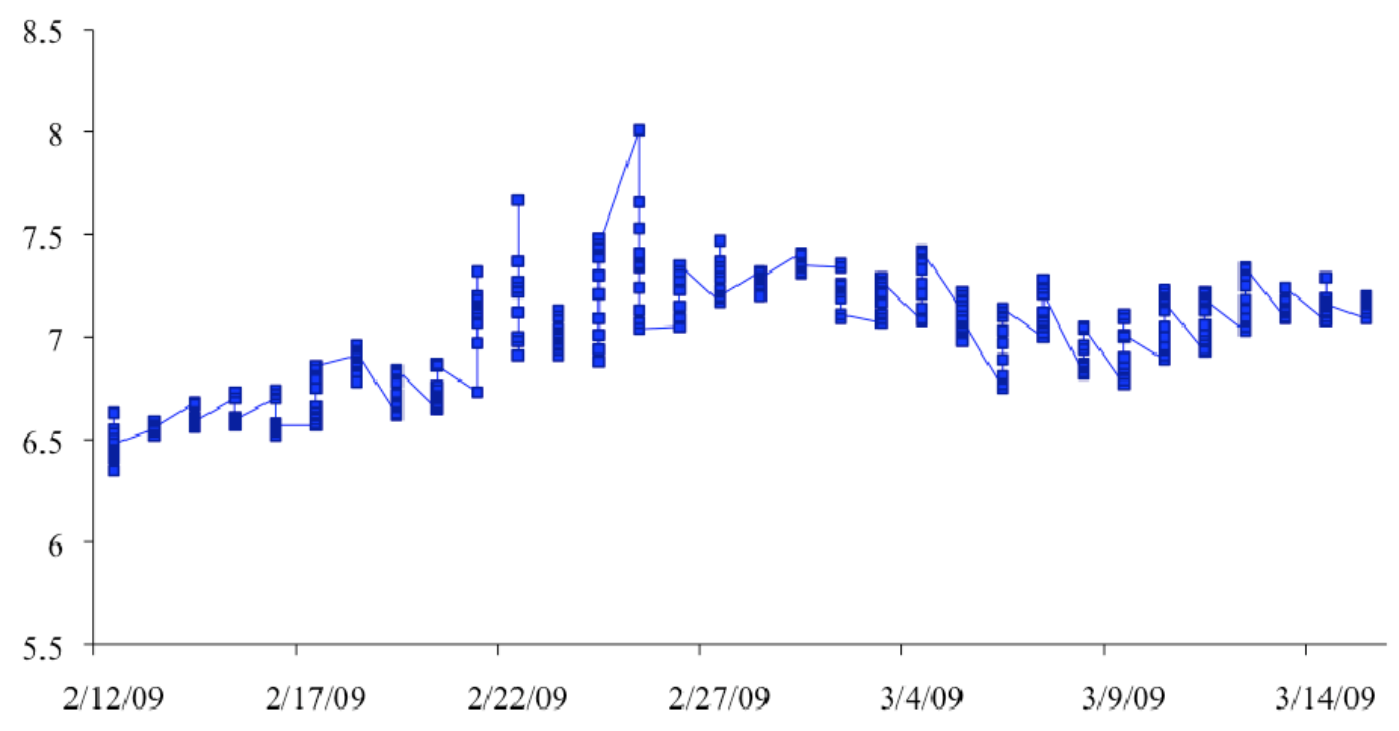

Figure 2-35 - Nannochloropsis Culture pH within the Cultivation System

\section{Study of Estimated Loss of C ulture Volume}

The culture volume was estimated by using the starting volume of $12,000 \mathrm{~L}$, the starting salinity of the culture and culture salinity measured throughout the experiment. This estimated culture volume is shown in Figure 2-36. In this figure, a slight loss of culture volume to evaporation over time is shown; however, no makeup water was ever added to the cultivator except after the harvest. This is far different than open-system algae cultivators where significant volumes of water are lost each day. Another trend that can be seen in this figure is the decrease in culture volume each day as condensation occurred on the reactor cover and as humidity increased in the gas space within the cultivator. Interestingly, much of this water was not lost from the system and returned to the culture overnight when temperatures decreased. 


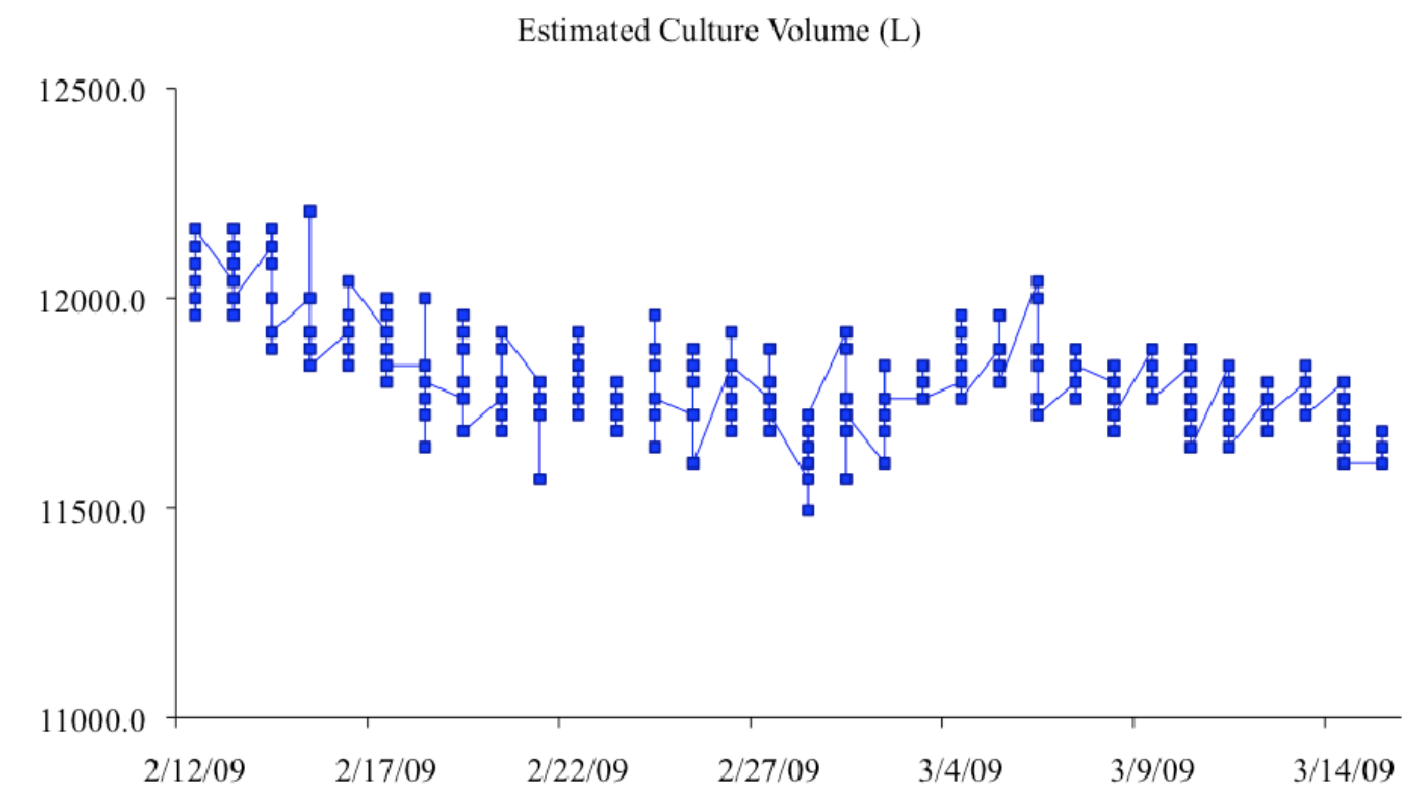

\section{Figure 2-36 - Nannochloropsis Culture Volume, Estimated by Salinity within the Cultivation System}

\section{Study of Harvesting}

The first harvest of this algae culture from the cultivator occurred on March 2, 2008. The effects of the first harvest can be seen in Figure 2-32 and Figure 2-33, where a drop in culture density is shown, resulting from removing $4500 \mathrm{~L}$ of culture for harvesting the biomass and replacing it with $4500 \mathrm{~L}$ of fresh media to begin the second growth period. This event marked two important milestones for the project; the first non-experimental operation of the dewatering system used in this project and the first reduction in density of the culture. Both of these milestones occurred successfully. The biomass density of the culture at the time of harvest was $0.85 \mathrm{~g} / \mathrm{L}$, resulting in a harvest of approximately $3.8 \mathrm{~kg}$ of Nannochloropsis biomass (by ash-free dry weight).

This first batch from the $6 \mathrm{M}$ cultivation system showed a successful 32 days of continuous operation and good algae biomass production. A number of improvements were then made to the system to ensure that the expected algae productivity, $\mathrm{CO}_{2}$ capture and lipid production were achieved in subsequent runs. These changes included a change to the cultivator installation process resulting in a more consistent and shallow culture depth throughout the cultivator to achieve the expected productivity across the high-culture densities used in this phase of the study; a change to the installation process that ensured the material used to separate the culture from the float pond was not jeopardized during the installation process; and the installation of a series of automated data acquisition systems and on-demand $\mathrm{CO}_{2}$ controls 
to automatically collect data and control $\mathrm{pH}$ inside the cultivator incrementally improving growth conditions and productivity in the cultivation systems. Once these changes were made, the system was re-inoculated with Nannochloropsis for the second batch testing.

\subsection{Sec ond Batch Test of Nannochloropsis}

\section{Study of Growth Rate}

The second operational period with the newly tensioned cultivator liner continued from inoculation on April 2, 2009 until May 18, 2009. The significant duration of this growth period without mechanical failures or required servicing marked a major step in demonstrating the robustness of this system. The culture density of the biomass in the system over the 46-day test, including the three operational harvests and subsequent growth periods, can be seen in Figure 2-37.

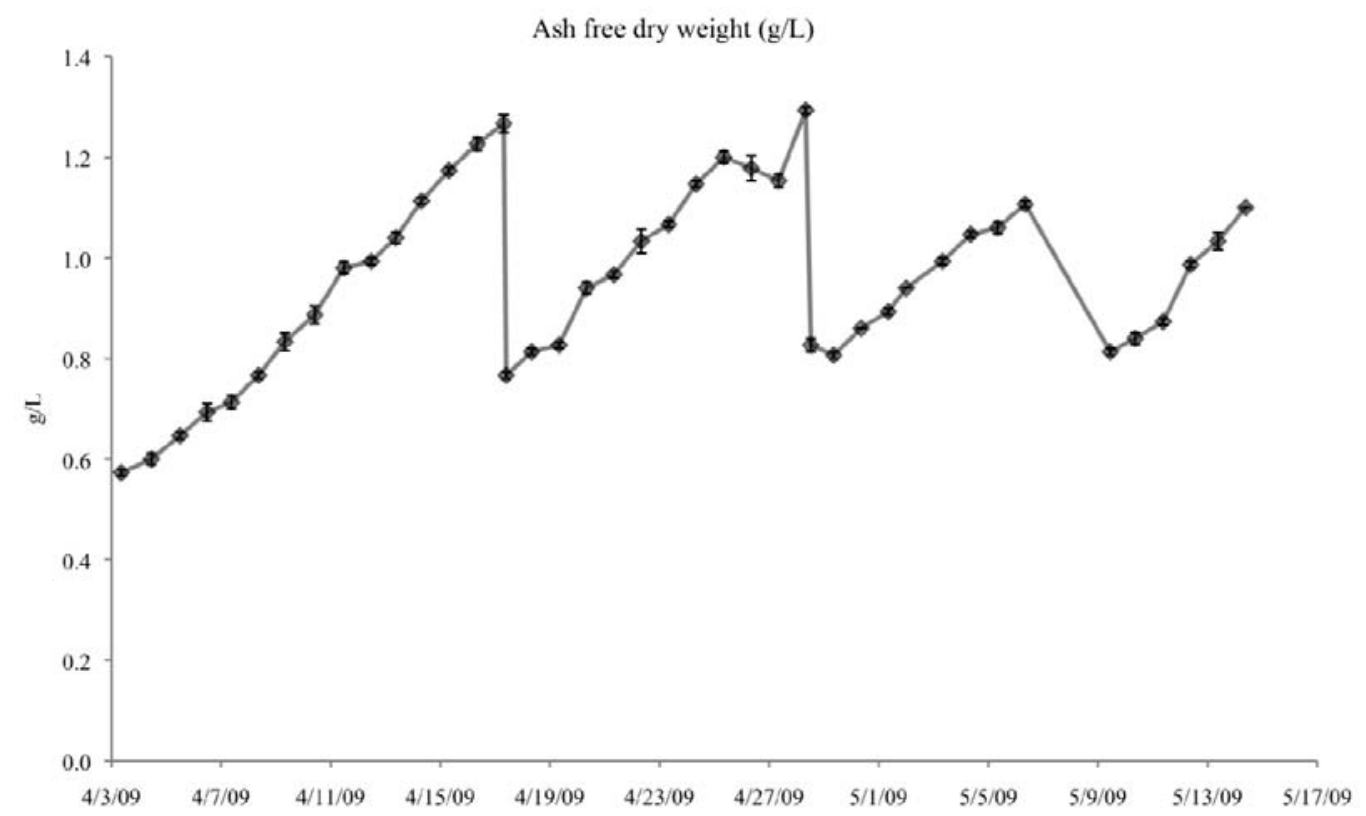

Figure 2-37 - Nannochloropsis Growth Curve by Ash-Free Dry Weight

Over the entire period of the test, the mean aerial biomass productivity of the culture was approximately $6 \mathrm{~g} / \mathrm{m}^{2} / \mathrm{d}$, which was an increase from the first test period. Numerous peaks in productivity were observed, ranging from 12 to $17 \mathrm{~g} / \mathrm{m}^{2} / \mathrm{d}$. Individual mean productivity for each growth period between harvests is summarized in Table 2-7. 
Table 2-7 - Summary of Nannochloropsis Productivity and Harvest Data from 6M Radius Cultivator

\begin{tabular}{|c|c|r|r|r|r|r|c|c|} 
& & \multicolumn{4}{|c|}{ Aerial productivity (g/m2/d) } & \multicolumn{4}{c|}{ Harvest } \\
\cline { 3 - 9 } Growth Period & Date & Mean & SE & Max & Min & Volume (L) & Density (g/L) & Biomass (g) \\
\hline 1 & $4 / 02 / 09-4 / 17 / 2009$ & 6.27 & 0.70 & 11.10 & 1.68 & 4,500 & 1.27 & 5,715 \\
\hline 2 & $4 / 17 / 09-4 / 28 / 2009$ & 6.09 & 1.96 & 17.81 & -3.14 & 4,500 & 1.29 & 5,805 \\
\hline 3 & $4 / 28 / 09-5 / 06 / 2009$ & 4.52 & 1.31 & 9.03 & -3.03 & 4500 & 1.11 & 4,995 \\
\hline 4 & $5 / 09 / 09-5 / 17 / 2009$ & 7.27 & 1.93 & 14.27 & 3.72 & 12,000 & $>1.10$ & $>13,200$ \\
\hline
\end{tabular}

Table 2-7 also summarizes the volumes of the three operational harvests and the final harvest along with the resulting biomass. After the third harvest, the permeate media that had been separated from the biomass during the harvest was recycled back to the reactor to observe its effect on growth. Although many environmental variables were involved over different growth periods, it was clear that recycling the dewatered permeate did not have a negative impact on the mean growth rate of Growth Period 4 as compared to Growth Period 3. Over the course of the entire test, approximately $30 \mathrm{~kg}(66 \mathrm{lb})$ of algae biomass was harvested. Significant amounts of data on daily environmental and physical conditions were collected throughout this test.

\section{Study of Oil Content}

The oil extraction on Nannochloropsis, performed by POS, using petroleum ether as the extraction solvent, indicated the species contains neutral lipids of $\sim 9.21 \mathrm{wt} \%$ of the total biomass. Their lipids analysis results also showed that the total unsaturated fatty acid (Monounsaturates and Polyunsaturates) of the species was $-45 \mathrm{wt} \%$ of fatty acids. The algae contained high value components of omega 3,6 , and 9 . They contributed to -37 wt $\%$ of the total oil; i.e., $\sim 2.7$ wt $\%$ of the total biomass. Nevertheless, the extracted algae oil was reported to be a non-flowing product and could not be used for further biofuel production without significant pretreatment.

\section{Study of $\mathrm{CO}_{2}$ Capture}

Figure 2-38 shows the Nannochloropsis culture's pH profile during a 24-hour period on April 27, 2009. The fluctuations in $\mathrm{pH}$ throughout the day can be seen based on the $\mathrm{pH}$ setpoints regulating the $\mathrm{CO}_{2}$ injection into the culture. During this time, the $\mathrm{pH}$ setpoints were between 7 and 7.25. As the $\mathrm{pH}$ of the culture reached $7.25, \mathrm{CO}_{2}$ was to be injected into the system until the $\mathrm{pH}$ level was lowered to 7 , at which time $\mathrm{CO}_{2}$ injection was disengaged. By monitoring $\mathrm{CO}_{2}$ input and output to the cultivator, the $\mathrm{CO}_{2}$ capture rate for the system was determined between June 17 and June 24, 2009. These results are shown in Table 2-8. The mean of the 24-hour 
averages of $\mathrm{CO}_{2}$ capture was $63.2 \mathrm{wt} \%$. The maximum 24-hour average was $69.3 \mathrm{wt} \%$, and the minimum daily average was $46.1 \mathrm{wt} \%$. However, these rates included the nighttime period in addition to the $\mathrm{CO}_{2}$ capture during active growth. The $\mathrm{CO}_{2}$ capture data for the daytime period between 11 a.m. and 4 p.m. achieved an average capture rate as high as 85.3 wt\%.

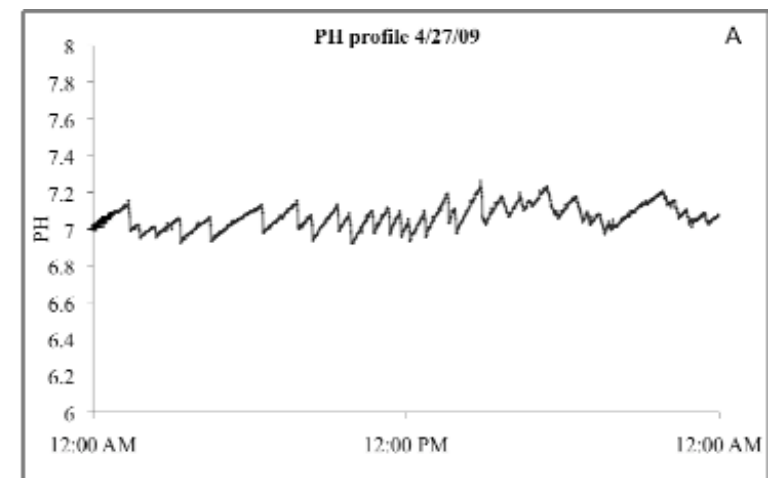

Figure 2-38 - Logged Culture PH for the 24-hour Period on 4/27/2009 during the Nannochloropsis Test in the Cultivator

The greater than $80 w t \%$ capture rates during daytime hours demonstrated the efficient introduction of $\mathrm{CO}_{2}$ into the system and its distribution throughout the growth area of the cultivator, making it available for biological uptake with a much higher rate than in open algae culturing systems currently used at similar scale. At nighttime, algae stop photosynthesis and start to breathe $\mathrm{O}_{2}$ and exhale $\mathrm{CO}_{2}$. This is why at night, as shown in Table 2-8, the cultivator gives a net $\mathrm{CO}_{2}$ emission rate.

Table 2-8 - Summary of $\mathrm{CO}_{2}$ Capture Data from 3rd Avenue Cultivator June 17 through June 23, 2009. Values are Derived from Averages of Measurements Taken Every Minute

\begin{tabular}{|c|c|c|c|}
\hline $\begin{array}{c}\text { Dates } \\
\text { June } 2009 \text { (Day) }\end{array}$ & $\begin{array}{c}\mathrm{CO}_{2} \text { Capture Daily } \\
(\text { wt\%) }\end{array}$ & $\begin{array}{c}\mathrm{CO}_{2} \text { Capture Daily } \\
11 \text { a.m. to 4 p.m. } \\
(\text { wt\%) }\end{array}$ & $\begin{array}{c}\mathrm{CO}_{2} \text { Night Emis sion } \\
\text { (scf/hr) }\end{array}$ \\
\hline 17 & 61.9 & 86.0 & 1.56 \\
\hline 18 & 67.8 & 86.2 & 0.24 \\
\hline 19 & 67.4 & 87.3 & 0.06 \\
\hline 20 & 57.7 & 81.4 & 1.30 \\
\hline 21 & 69.3 & 86.6 & 0.16 \\
\hline 22 & 67.4 & 87.2 & 1.30 \\
\hline 23 & 67.7 & 86.4 & 0.06 \\
\hline
\end{tabular}

\section{Study of Culture Temperature, pH and Dissolved Oxygen}

The variation in temperature over a 24-hour period can be seen in the temperature data presented in Figure 2-39. The temperature was lowered to around $12^{\circ} \mathrm{C}\left(54^{\circ} \mathrm{F}\right)$ at night and 
increased throughout the day to peaks around $18^{\circ} \mathrm{C}\left(64^{\circ} \mathrm{F}\right)$. Temperature profiles like this are ideal for the coldwater-species Nannochloropsis growth. The low temperature at night reduced the growth rate of any possible contaminant heterotrophic organisms, and the temperature under sunlight provided the most favorable conditions to the Nannochloropsis so that it outgrew the contaminants.
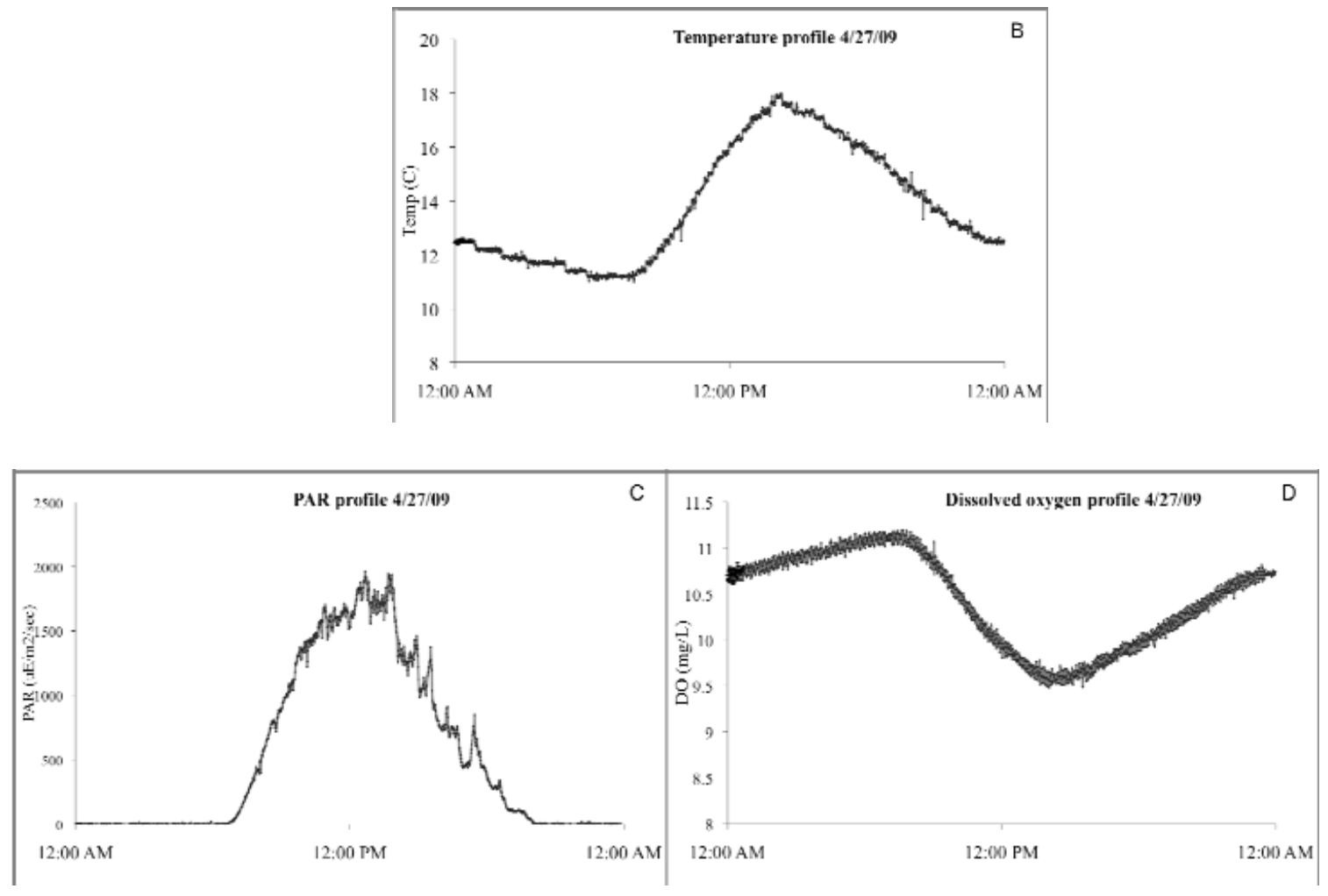

Figure 2-39 - Logged Culture Condition Data for the 24-Hour Period on 4/27/2009 during the Nannochloropsis Test in the Cultivator. B: Culture Temperature; C: PAR; D, Dissolved Oxygen (DO) Concentration in the Liquid Culture.

The driving force behind trends in many of the data sets, irradiance of the sun, can be seen in the PAR data in Figure 2-39. This 24-hour period in particular appeared to have generally high levels of PAR with some passing clouds, as shown by the small peaks and valleys in PAR. Throughout the day, PAR data similar to that shown can be correlated to growth rate data and the actual amount of PAR to determine if suboptimal growth rates are a result of less irradiance for a given period. For a given microalgae species, operating experience coupled with PAR data may be used as a control factor for culture mixing or recirculation so that mixing or recirculation will only occur when sufficient sunlight for photosynthesis is available. 
The final important variable for a closed algae cultivation system is the concentration of DO in the culture. As oxygen is produced during photosynthesis, it can accumulate within a closed system and inhibit efficient photosynthesis when DO reaches $\sim 20 \mathrm{mg} / \mathrm{L} \mathrm{O} 2$ concentration in the water. As shown in Figure 2-39, the DO of the algae culture in the system ranged from 9.5 to approximately $11 \mathrm{mg} / \mathrm{L}$. Although DO levels would be expected to accumulate in a truly closed system to levels that could suppress growth, the data suggested that this system was exhausting enough during active photosynthesis that the DO in the system was not increasing during the day. In fact, as a result of the higher demand for $\mathrm{CO}_{2}$ injection during the day, when coupled with the exhaustion of the cultivator, the DO levels actually decreased during the period of increased intensity PAR, and thus photosynthesis and growth rate were the highest. This is a promising sign for this cultivator as accumulation of DO is a major challenge in many other closed systems.

Over the course of this second test of Nannochloropsis, the ambient temperature in Phoenix increased significantly, and although temperature control was imposed on the cultivator, periodic increases in temperature above the safe maximum $\left(20^{\circ} \mathrm{C}\left[68^{\circ} \mathrm{F}\right]\right)$ for this species occurred. As a result Nannochloropsis culture was completely harvested on May 17, 2009, and replaced with Selenastrum, a species that could tolerate a higher temperature.

\subsubsection{6M Radius Cultivator Algae Test Run 2 - Selenastrum}

Based on previous algae strain selection studies, Selenastrum emerged as one of the three leading candidates due to its rapid growth rate, tolerance to high temperature, and ability to accumulate significant amount of lipids when stressed. Therefore, after the outdoor study of Nannochloropsis on May 17, 2009, the cultivation system was cleaned and re-inoculated with Selenastrum on May 22, 2009.

\subsection{Study on Growth Rate}

The culture was inoculated at a low density $(\sim 0.1 \mathrm{~g} / \mathrm{L})$. It was initially shaded to prevent photo-inhibition and bleaching, and after three days, the shade cloth was removed, resulting in significantly higher algal biomass productivity for the 6 days following than any prior instances. The initial growth period of the cultivator was conducted at a reduced culture volume of $8000 \mathrm{~L}$, rather than $12,000 \mathrm{~L}$, to use less inoculum. As a result, far less of the reactor footprint consisted of culture for this initial growth period (the culture tended to collect closer to the center of the circular reactor). Therefore, volumetric productivity and the size of the reduced culture area were used to estimate aerial productivity rather than relating the productivity of the reduced 
volume of the entire footprint of the system. The growth curve beginning with inoculation on May 22, 2009, and continuing through completion of the test run on June 25, 2009 is shown in Figure 2-40.

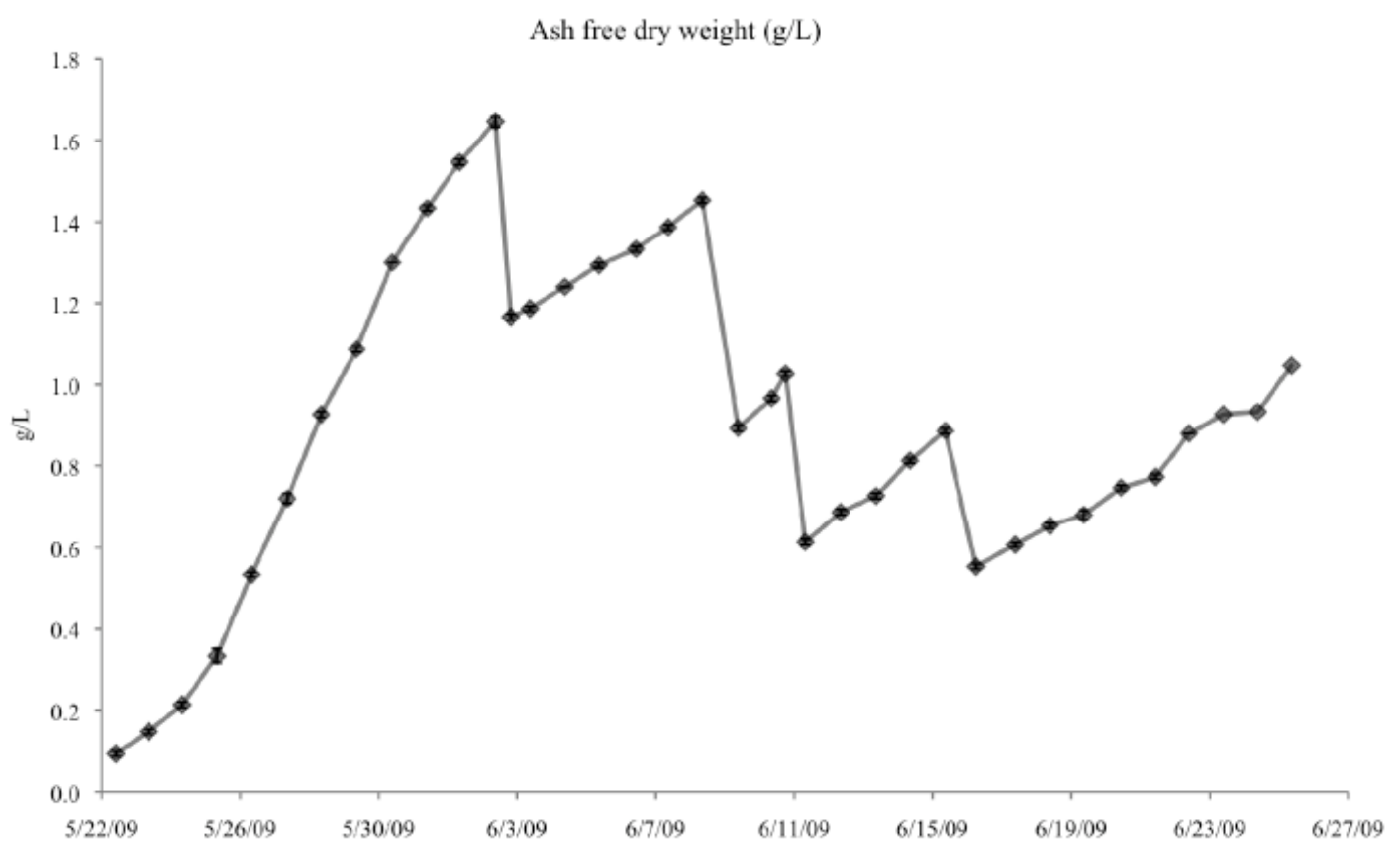

Figure 2-40 - Selenastrum Culture Density as Measured by Ash-Free Dry Weight (Grams of Dry Biomass per Liter of Culture)

A rapid growth rate from low density to a relatively high density indicated the ability of this species to use high irradiances, and the mixing and $\mathrm{CO}_{2}$ delivery conditions within the cultivator were ideal. While the Nannochloropsis daily volumetric growth rate (grams of dry biomass increase per liter per day) averaged approximately $0.05 \mathrm{~g} / \mathrm{L} /$ day during the second test period, Selenastrum in its initial growth period averaged $0.17 \mathrm{~g} / \mathrm{L} / \mathrm{day}$, which was three times more than that of Nannochloropsis. This volumetric growth rate would translate into an average aerial productivity of over $20 \mathrm{~g} / \mathrm{m}^{2} / \mathrm{d}$ when the cultivator has $12,000 \mathrm{~L}$ of culture. Given the reduced footprint of the culture at a lower density, the aerial productivity during the 6-day period from May 25 through May 30, 2009, resulted in an average aerial productivity of $22.4 \mathrm{~g} / \mathrm{m}^{2} / \mathrm{d}$, with peaks of $25.86 \mathrm{~g} / \mathrm{m}^{2} / \mathrm{d}$ and $26.38 \mathrm{~g} / \mathrm{m}^{2} / \mathrm{d}$. These results were significant because in the initial attempt at growing one of the fast-growing freshwater species, the project goal of averaging 25 $\mathrm{g} / \mathrm{m}^{2} / \mathrm{d}$ could be met when density, mixing, light, and $\mathrm{CO}_{2}$ were all managed properly. After this initial rapid growth period, the growth rates of the following periods began to decrease as shown in Table 2-9. 
Table 2-9 - Summary of Selenastrum Productivity and Harvest Data from 6M Radius Cultivator

\begin{tabular}{|c|c|r|r|r|r|r|c|c|} 
& & \multicolumn{6}{|c|}{ Aerial productivity (g/m2/d) } & \multicolumn{4}{c|}{ Harvest } \\
\cline { 3 - 9 } Growth Period & Date & Mean & \multicolumn{1}{|c|}{ SE } & Max & Min & Volume (L) & Density (g/L) & Biomass (g) \\
\hline 1 & $5 / 24 / 2009-6 / 02 / 2009$ & 19.54 & 1.93 & 26.38 & 9.82 & - & - & - \\
\hline 2 & $6 / 02 / 2009-6 / 08 / 2009$ & 6.43 & 0.63 & 8.55 & 4.55 & 4500 & 1.45 & 6525 \\
\hline 3 & $6 / 09 / 2009-6 / 10 / 2009$ & 14.27 & 4.71 & 18.98 & 9.56 & 4500 & 1.03 & 4635 \\
\hline 4 & $6 / 11 / 2009-6 / 15 / 2009$ & 7.21 & 1.10 & 9.41 & 4.17 & 4500 & 0.89 & 4005 \\
\hline 5 & $6 / 16 / 2009-6 / 22 / 2009$ & 6.13 & 1.66 & 14.01 & 2.88 & 4000 & 0.75 & 3000 \\
\hline
\end{tabular}

The decrease in growth rate during subsequent growth periods relative to the initial period may be attributed to a number of factors. Although the Selenastrum culture was demonstrated to withstand temperatures over $30^{\circ} \mathrm{C}\left(86^{\circ} \mathrm{F}\right)$, there were occasions when the chiller maintaining the temperature in the thermal pond failed, exposing the culture to a temperature approaching $40^{\circ} \mathrm{C}$ $\left(104^{\circ} \mathrm{F}\right)$. Examinations of Selenastrum cultures in the bag system indicated a sensitivity of this species to extreme ambient temperatures. Another factor reducing the growth rate was a number of cloudy days that occurred during the final three growth periods, which can significantly affect growth rates. Finally, in the last week of operation, it was observed that a seal in one of the cultivator components began to leak, reducing mixing in the cultivator. As a result, the growth period of the cultivator was ended on June 25, 2009.

\subsection{Study of Culture pH, Temperature, PAR, and DO}

Figure 2-41 shows culture $\mathrm{pH}$, culture temeprature, PAR, and the DO profile during the 24-hour period of May 25, 2009.

The environmental data for the 24-hour period specified above illustrate the culture conditions during the initial growth curve of Selenastrum when a rapid growth rate was observed. The $\mathrm{pH}$ curve from May 25, 2009 shows an interesting pattern. The pH increase due to the rapid growth rate of Selenastrum was so high that the flow of $\mathrm{CO}_{2}$ to the system was not enough to lower the $\mathrm{pH}$ to the low setpoint during the daytime. Although this pattern could be dangerous for the algae culture if the $\mathrm{pH}$ continued to increase indefinitely, the $\mathrm{CO}_{2}$ flow was enough to maintain the $\mathrm{pH}$ to less than 7.4 until the irradiance decreased near the end of the day and $\mathrm{CO}_{2}$ demand declined.

The temperature decreased at night as a result of the thermal pond; however, as irradiance increased, so did the temperature of the culture. This particular temperature curve came at a time when the temperature of the system was slowly being increased, and by the end of this run 
maximum temperatures during the day were reaching and exceeding $30^{\circ} \mathrm{C}\left(86^{\circ} \mathrm{F}\right)$ on some days despite the use of the chiller.

The PAR curve shows a completely sunny day without a decrease in PAR due to clouds. This was likely a major factor leading to the high productivity observed that day. Additionally, the DO profile exhibited a similar pattern to what was observed throughout the growth periods within the cultivator. DO concentrations (range $8.5-10 \mathrm{mg} / \mathrm{L}$ ) did not reach the toxic limit ( $20 \mathrm{mg} / \mathrm{L}$ ) and were expected to be within a safe range for the algae culture.

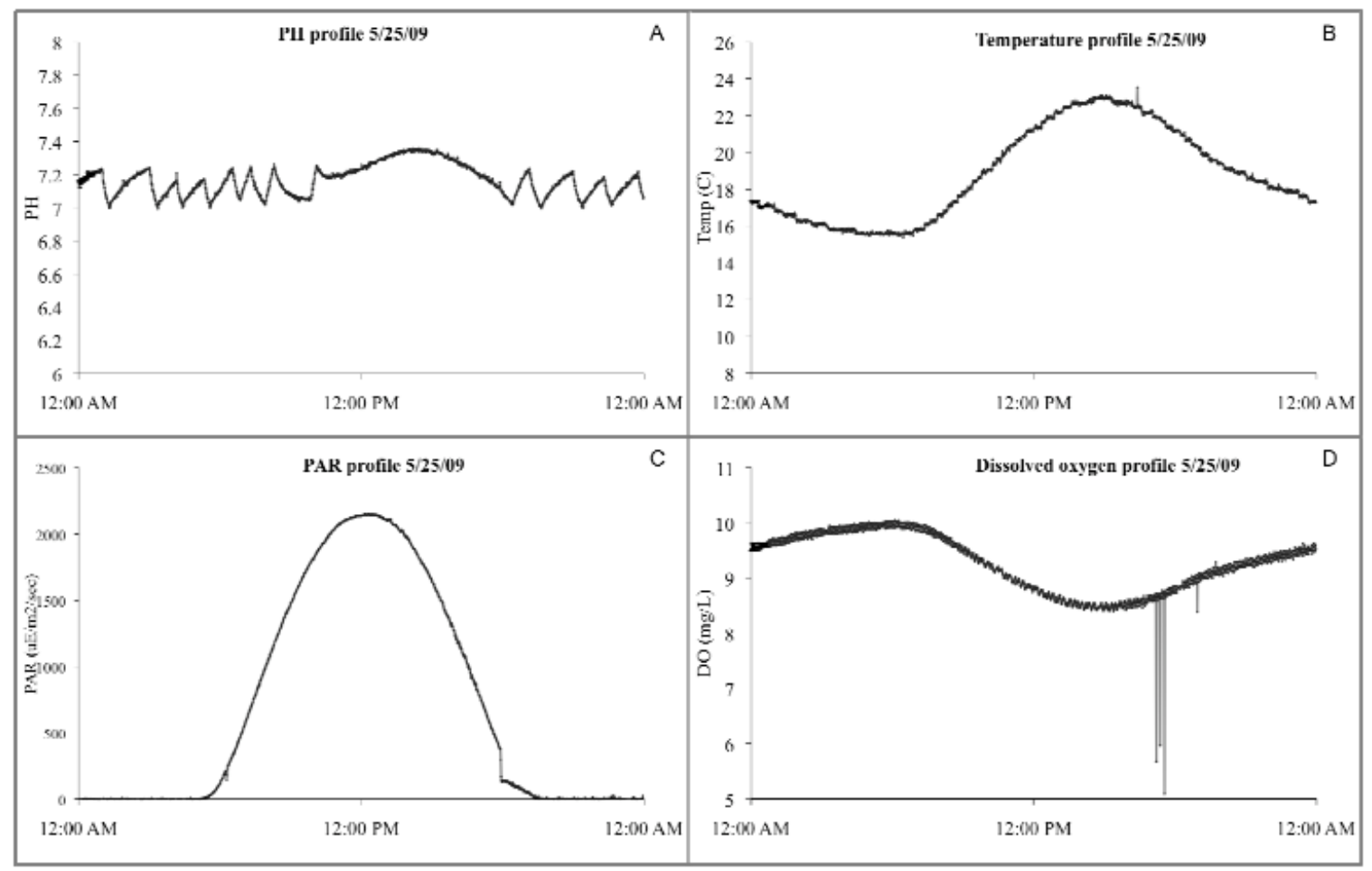

Figure 2-41 - Automatically Logged Culture Condition Data for the 24-hour Period on 5/25/2009 during the Selenastrum Test in the Cultivator. A: Culture pH. B: Culture Temperature. C: PAR. D: DO Concentration in the Liquid Culture.

The daily average carbon capture rates for Selenastrum were found to be $84 \mathrm{wt} \%, 89 \mathrm{wt} \%$, and $70 w t \%$ on separate days. The high carbon capture rates exhibited by this cultivation system demonstrated efficient delivery of $\mathrm{CO}_{2}$ to the growth area, controlled input of the amount of $\mathrm{CO}_{2}$ demanded by the algae culture, and the closed nature of the cultivating system. As mentioned before, even though the system was closed enough to exhibit high-carbon capture rates, the accumulation of DO was not found to occur. 
The lipid analysis for the first harvested Selenastrum showed Selenastrum had oil content of 17 $w t \%$ total fat and $8 \mathrm{wt} \%$ neutral lipids. Selenastrum was found to meet productivity expectations in an initial growth period: however, the fact that the growth rate of the culture decreased over time indicated that a more robust species of algae may be necessary for high temperature and irradiance conditions that would be found at the Redhawk test facility. Additionally, the importance of the species to naturally settle became clear during this time. Selenastrum did not exhibit a characteristic to settle and thus the dewatering energy input for this species would be too high. As a result of these observations, although Selenastrum remained a high-priority candidate, there was a continued effort to test a species with a high growth rate, robustness to high irradiance and temperature, and the ability to settle - Scenedesmus.

\subsubsection{6M Radius Cultivator Algae Test Run 3 - Scenedesmus}

The third algae candidate examined was Scenedesmus. Scenedesmus was found in laboratory experiments to have a similar growth rate to Selenastrum. The total fat levels and neutral lipid levels of Scenedesmus were lower than Selenastrum; however, its oil content has potential to be increased when the algae was stressed. In addition, Scenedesmus settled completely to the bottom of storage vessels in a matter of hours. As a result, the next test run of the cultivator was inoculated with Scenedesmus to examine its growth rate in the cultivation system, as well as its naturally settling behavior in a large scale.

The study of Scenedesmus was partially covered by this project and partially covered by the follow-on project DE-FE0001099, "Integrated Energy System (IES) with Beneficial $\mathrm{CO}_{2}$ Use". The following section presents only the results of the study of this species performed under this project.

The cultivator was inoculated on August 17, 2009, with at culture density of $0.1 \mathrm{~g} / \mathrm{L}$ with $8000 \mathrm{~L}$ volume. The cultivator was covered with a shade cloth for two days and was removed on August 19, 2009. This, as well as all subsequent growth periods, can be seen in Figure 2-42. 


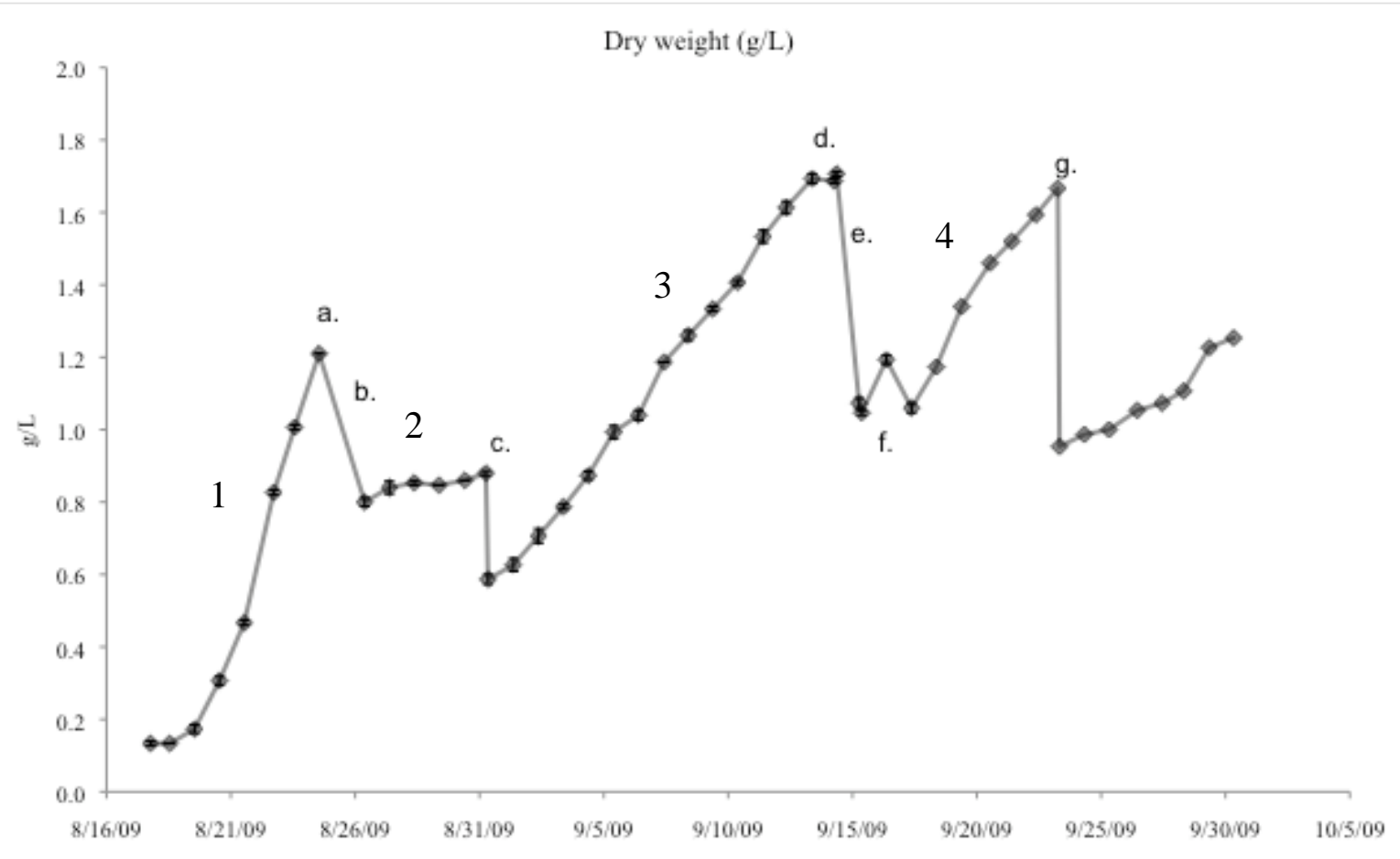

\section{Figure 2-42 - Scenedesmus Culture Density as Measured by Dry Weight (Grams of Dry Biomass per Liter of Culture)}

During growth period 1 with decreased volume, the growth rate of the Scenedesmus culture was very high, averaging approximately $20.12 \mathrm{~g} / \mathrm{m}^{2} / \mathrm{d}$ as shown in Figure 2-42. Addition of media occurred on August 24, 2009 (label b in Figure 2-42), after which the cultivator was running at a full $12,000 \mathrm{~L}$ culture volume. However, the productivity of the culture decreased markedly in the initial growth curve after this media addition (growth period $2-2.11 \mathrm{~g} / \mathrm{m}^{2} / \mathrm{d}$ ), as the culture was being stressed by limiting nitrate in the media. After a five-day period of observed slow growth, a $4000 \mathrm{~L}$ harvest was conducted, and nitrate was added to the media (label c in Figure 2-42).

The objective of growth period 3 was to determine the maximum density that Scenedesmus could reach given the conditions within the cultivator. It was critical to know the maximum culture density of a given species before productivity ceases as a result of light limitation for efficient operational management of cultures. By knowing the maximum culture density, harvests can maintain the culture below that density at which productivity of the system stops. In this case, the culture grew consistently, although sub-optimally at a rate of $10.95 \mathrm{~g} / \mathrm{m}^{2} / \mathrm{d}$, to a 
density of $1.6 \mathrm{~g} / \mathrm{L}$. The cultivator was harvested (label $\mathrm{d}$ in Figure 2-42) at a maximum density of this culture, for the given conditions, and a $12,000 \mathrm{~L}$ operational volume was reached.

As indicated by the slopes of the growth curve lines shown in Figure 2-42, the productivity of this culture, at reduced volume $(8,000 \mathrm{~L})$ in growth period 1 was higher than the running volume of $12,000 \mathrm{~L}$ during growth period 3 . Therefore, at the end of growth period 3 , a large harvest of two 4,000 $\mathrm{L}$ batches was conducted (label e in Figure 2-42). Initially to examine the effect of total culture volume and thus light path, after the large harvest, 4,000 L of fresh medium was added. Afterwards, an additional 2,000 L was added (label $f$ in Figure 2-42) for an operational culture volume of $10,000 \mathrm{~L}$, which is between the high productivity $8,000 \mathrm{~L}$ culture (growth period 1) and the lower productivity $12,000 \mathrm{~L}$ culture (growth period 3). The resulting growth period 4 showed no significant increase in productivity of the culture over growth period 3 by decreasing the volume $\left(10.95\right.$ to $\left.10.70 \mathrm{~g} / \mathrm{m}^{2} / \mathrm{d}\right)$. (It should be noted that the management of the culture during growth period 4 was for operational strategies and setpoints, before operating the pilot-scale Redhawk cultivation system, rather than to optimize productivity.) Despite the lower than hypothesized growth rate in period 4, average productivity during testing with Scenedesmus was higher and sustained over a much longer period of time than in any previous run of the cultivator.

The culture density did not seem to have significant influence on growth rate. For growth periods 1,3 , and 4 , the growth rate over the period did not slow down due to the increase of culture density (see Table 2-10). Overall, 45 days continuous operation occurred (see Figure 2-42) resulting in a total volume of approximately $16,500 \mathrm{~L}$ (yielding $23.9 \mathrm{~kg}$ biomass on a dry weight basis) harvested from the Scenedesmus culture.

\section{Table 2-10 - Summary of Scenedesmus Productivity and Harvest Data from 6M radius} Cultivator

\begin{tabular}{|c|c|r|r|r|r|r|r|r|}
\multirow{2}{*}{ Growth Period } & \multirow{2}{*}{ Date } & \multicolumn{4}{|c|}{ Aerial productivity (g/m²/day) } & \multicolumn{3}{c|}{ Harvest } \\
\cline { 3 - 9 } & Mean & SE & Max & Min & Volume (L) & Density (g/L) & Biomass (g) \\
\hline 1 & $8 / 21 / 2009-8 / 24 / 2009$ & 20.12 & 2.61 & 25.54 & 17.49 & - & - & - \\
\hline 2 & $8 / 26 / 2009-8 / 31 / 2009$ & 2.11 & 0.97 & 5.11 & -0.85 & 4000 & 0.83 & 3320 \\
\hline 3 & $9 / 01 / 2009-9 / 14 / 2009$ & 10.70 & 1.24 & 18.03 & -0.95 & 8000 & 1.71 & 13680 \\
\hline 4 & $9 / 16 / 2009-9 / 23 / 2009$ & 10.95 & 1.51 & 17.32 & 7.25 & 4500 & 1.54 & 6930 \\
\hline
\end{tabular}

A series of observational laboratory experiments conducted during the period of July 1, $2009-$ September 30, 2009 examined the feasibility of naturally settling Scenedesmus as a partial dewatering strategy. Previous species examined were found not to settle, even when un-agitated. Results showed this species to rapidly settle when left unmixed for a period of 


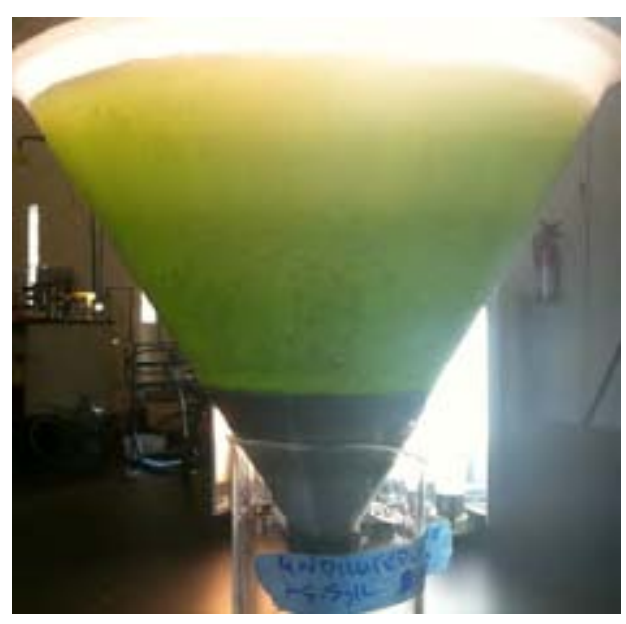

Figure 2-43 - Settled Scenedesmus Culture after 5 Hours in a Conical-Bottom Vessel time. There was significant variability in the settling rate and final settled culture density. This variability was likely due to the growth phase, culture conditions, temperature, $\mathrm{pH}$, and other unknown factors; however, the key result was that some portion of the population in a given culture consistently settled to the bottom of the vessel in a matter of hours when left un-agitated.

Representative of this series of settling experiments, a $1.5 \mathrm{~L}$ of culture, at a density of $1.5 \mathrm{~g} / \mathrm{L}$ (similar to the harvest density from an outdoor cultivation system), was placed in a covered $60^{\circ}$-angled bottom funnel to simulate settling dynamics within a $60^{\circ}$ cone-bottom settling tank. The culture was left un-agitated for 5 hours to simulate a reasonable amount of settling time that could be accommodated after a harvest. The resulting culture can be seen in Figure 2-43.

Although the culture on top is not completely clear, most of the biomass settled out after 5 hours. The settled biomass was found to be $47.7 \mathrm{~g} / \mathrm{L}$ and the non-settled portion was $0.04 \mathrm{~g} / \mathrm{L}$. This 5-hour settling period, with no energy expenditure for dewatering, resulted in a nearly $32 \mathrm{X}$ increase in the concentration of the biomass. As with any biological system, the culture dynamics affecting this settling vary, and thus, settling rates and final densities are likely to vary as well. In spite of this, any method to increase de-watering efficiency is beneficial. Over time, as culture settling is practiced on large-scale cultures, the dynamics and variability of this process will be better understood and utilized.

In conclusion, Scenedesmus was chosen as the species for the project based on observational and experimental results. Characteristics of this species that led to this choice were temperature tolerance, rapid growth rate, resistance to contamination, and the ability to settle when harvested. The continued research focused more on how to increase the species' lipid content and reduce its chlorophyll content by suppressing its cultivation conditions. This research is further supported under the follow-on DOE Cooperative Agreement, 
DE-FE0001099, "Integrated Energy System (IES) with Beneficial $\mathrm{CO}_{2}$ Use." Please refer to its final technical closeout report for the results.

\subsubsection{SUMmARY OF 6M RADIUS AlgAE CULTIVATOR TEST RESULTS}

In summary, the $6 \mathrm{M}$ radius algae cultivation system was built at APS $3^{\text {rd }}$ Avenue algae R\&D facility site. This outdoor system included a $6 \mathrm{M}$ radius cultivator with a total surface area of 113 $\mathrm{m}^{2}$ and a total culture volume capacity between $10,000 \mathrm{~L}$ to $15,000 \mathrm{~L}$; $\mathrm{C} \mathrm{CO}_{2}$ on-demand feeding system; an on-line data collection system for temperature, $\mathrm{pH}, \mathrm{PAR}$ and DO; and a $\sim 2 \mathrm{gpm}$ algae culture dewatering system. The 6M cultivation system demonstrated approximately 170 days of total operation since the onset of testing. Approximately $77,000 \mathrm{~L}$ of culture were harvested. Three algae species were examined and cultured throughout the duration of experimentation - Nannochloropsis, Selenastrum and Scenedesmus. A summary of operational periods, biomass productivities, and volumes and biomass harvested throughout testing on these species is presented in Table 2-11.

The Nannochloropsis harvested at the site gave an oil content of $9.21 \mathrm{wt} \%$ of biomass, where $37 \mathrm{wt} \%$ consisted of omega 3, 6 and 9. Selenastrum obtained an oil content of $17 \mathrm{wt} \%$ total fat and $8 w t \%$ neutral lipids. A total neutral lipid content of $80 w t \%$ of the total fatty acids was obtained from an acetone-dried Scenedesmus biomass, which generally contains 20 wt $\%$ total lipids when grown in stressed conditions. A maximum $\mathrm{CO}_{2}$ capture rate of 90 wt $\%$ was achieved with this $6 \mathrm{M}$ cultivator design.

Among these three algae, Scenedesmus showed the most tolerance of temperature and irradiance conditions in Phoenix and the best self-settling characteristics. Experimental findings and operational strategies determined through these tests guided the operation of the algae cultivation system at APS's Redhawk test facility for a scale-up study. This knowledge ensured active, consistent growth of the culture, and thus $\mathrm{CO}_{2}$ capture, while aiding in the dewatering, oil extraction, and fuel production processes. Continued extensive algae stressing to biologically increase algae oil content and reduce the chlorophyll content at the $3^{\text {rd }}$ Avenue and Redhawk test facilities was studied under DOE Cooperative Agreement: DE-FE0001099, "Integrated Energy System with Beneficial $\mathrm{CO}_{2}$ Use." Please refer to its final scientific/technical report for further results. 
Development of a Hydrogasification Process for

Coproduction of SNG and Eectrical Power from Westem Coals

Final Report

THIS PAGE INTENTIONALLY LEFT BLANK 
Table 2-11 - Summary of Mechanical Operation, Algae Cultivation, and Harvests Conducted in the 6M Radius Cultivation System at 3rd Avenue R\&D Test Site

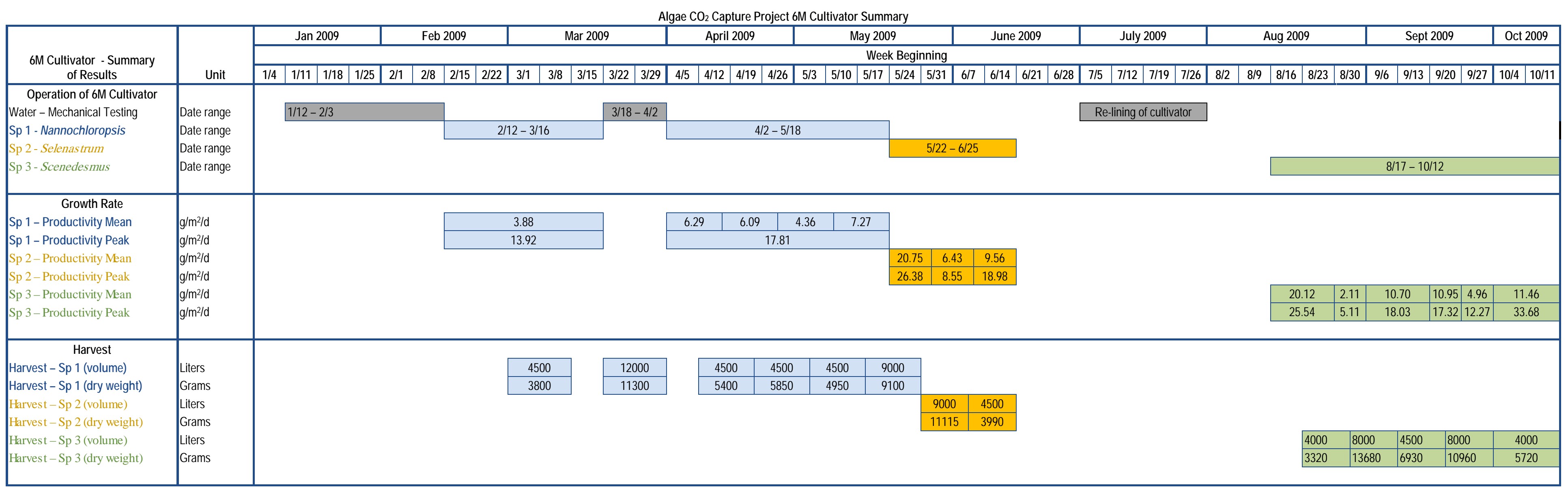


Development of a Hydrogasification Process for

Coproduction of SNG and Eectrical Power from Westem Coals

Final Report

THIS PAGE INTENTIONALLY LEFT BLANK. 


\subsection{Algae Oll Extraction and Processing for Carbon Re- UTIUZATION}

\subsubsection{Outsourced Oll Extraction and Biofuel Production rom Algae Bio- MASS}

Algal lipid and oil extraction work was initiated in the third quarter of fiscal year 2008 (March June 2008) by NETL/ Office of Research and Development (ORD), CAER at the University of Kentucky, ASU, POS, DynaSep and ConocoPhillips. APS sent about $300 \mathrm{~kg}$ of algae paste and $500 \mathrm{~g}$ of dried algae in total for algae lipid analyses and oil extraction exercises.

\subsubsection{Algae and Water Analysis}

Ultimate analysis provided by CAER is shown in Table 2-12. The GF3 algae strain was compared to local Western coal to assess difference in heating value. When comparing algae to BHP Navajo mine Western coal (Table 2-13), the analyzed GF3 strain samples have less carbon (46 wt\% versus $62 \mathrm{wt} \%$ ) and more oxygen (32 wt \% versus $10 \mathrm{wt} \%$ ). The ester group in lipids, triglyceride, explains the high oxygen content in algae. Because the GF3 strain was a freshwater species, dried algae ash content was far less than coal (8wt\% versus 21 wt\%). The marine species would have a much higher ash content if an additional washing step was not used to remove any salt remaining in the algal biomass. The heating value of the dried algae was also determined by NETL. On a dry basis, HHV was 8,600 Btu/lb and low heating value (LHV) was $7,939 \mathrm{Btu} / \mathrm{lb}$. As a comparison, Navajo mine coal has a heating value of 10,710 Btu/lb on a dry basis.

Elemental analysis of dried algae was performed by NETL and is presented in Table 2-14. Compared with corn stalk, the GF3 strain contained more Phosphorous (P), Sulfur (S), Magnesium (Mg), and Sodium ( $\mathrm{Na}$ ). However, these algae element contents can be tailored by nutrients added to the culture.

Extensive water analysis was performed by NETL/ORD (Table 2-15) on the effluent from the Lavin centrifuge to determine if the centrifuge effluent met the city of Phoenix's criteria as nonhazardous material, and could be disposed of utilizing the city wastewater system. The bag farm used Phoenix city water as its water source. As seen in Table 2-15, except for sulfur, all other components are well below the Phoenix city limit for potable water. This sulfur was introduced by sulfate in the nutrient medium and can potentially be reduced without hurting algae growth rate. 
Table 2-12 - Dried Algae GF3 Ultimate Analysis

\begin{tabular}{|l|c|c|c|c|c|c|c|}
\hline \multirow{2}{*}{ Sample } & \multicolumn{7}{|c|}{ Percentage of (wt\%) } \\
\cline { 2 - 7 } & $\mathrm{H}$ & $\mathrm{C}$ & $\mathrm{N}$ & $\mathrm{S}$ & $\mathrm{O}$ & As h & Mois ture \\
\hline Dry Algae & 7.26 & 46.14 & 5.34 & 0.66 & 32.50 & 8.10 & 8.46 \\
\hline Source: CAER, University of Kentucky, Lexington, $\mathrm{KY}$ \\
\hline
\end{tabular}

Table 2-13 - Ultimate Analysis of Western Coal

\begin{tabular}{|c|c|c|c|c|c|c|c|}
\hline \multirow{2}{*}{ Sample } & \multicolumn{7}{|c|}{ Percentage of (wt\%) } \\
\cline { 2 - 7 } & $\mathrm{H}$ & $\mathrm{C}$ & $\mathrm{N}$ & $\mathrm{S}$ & $\mathrm{O}$ & As h & Mois ture \\
\hline $\begin{array}{c}\text { Western } \\
\text { Coal }\end{array}$ & 4.61 & 61.92 & 1.28 & 0.69 & 10.18 & 21.32 & 7.27 \\
\hline
\end{tabular}


Table 2-14 - Dried Algae GF3 Elemental Analysis and Comparison with Corn Stalk (Provided by NETL)

\begin{tabular}{|c|c|c|c|}
\hline Chemical Name & Unit of Measure & Dried Algae & Corn Stalk \\
\hline $\mathrm{Ag}$ & $\mu \mathrm{g} / \mathrm{g}$ & $<\mathrm{DL}$ & - \\
\hline Al & $\mu \mathrm{g} / \mathrm{g}$ & 57.9 & - \\
\hline As & $\mu \mathrm{g} / \mathrm{g}$ & $<\mathrm{DL}$ & - \\
\hline $\mathrm{Ba}$ & $\mu \mathrm{g} / \mathrm{g}$ & 6.2 & - \\
\hline $\mathrm{Be}$ & $\mu \mathrm{g} / \mathrm{g}$ & $<\mathrm{DL}$ & - \\
\hline $\mathrm{Ca}$ & $\mu \mathrm{g} / \mathrm{g}$ & 3401 & 2,160 \\
\hline Cd & $\mu \mathrm{g} / \mathrm{g}$ & $<\mathrm{DL}$ & - \\
\hline $\mathrm{Ce}$ & $\mu \mathrm{g} / \mathrm{g}$ & $<\mathrm{DL}$ & - \\
\hline Co & $\mu \mathrm{g} / \mathrm{g}$ & $<\mathrm{DL}$ & - \\
\hline $\mathrm{Cr}$ & $\mu \mathrm{g} / \mathrm{g}$ & 945 & - \\
\hline $\mathrm{Cu}$ & $\mu \mathrm{g} / \mathrm{g}$ & 60.4 & - \\
\hline $\mathrm{Fe}$ & $\mu \mathrm{g} / \mathrm{g}$ & 4,100 & 139 \\
\hline K & $\mu \mathrm{g} / \mathrm{g}$ & 7,188 & 17,350 \\
\hline Mg & $\mu \mathrm{g} / \mathrm{g}$ & 2,788 & 1,600 \\
\hline Mn & $\mu \mathrm{g} / \mathrm{g}$ & 105 & 15.0 \\
\hline Mo & $\mu \mathrm{g} / \mathrm{g}$ & 6.2 & - \\
\hline $\mathrm{Na}$ & $\mu \mathrm{g} / \mathrm{g}$ & 8940 & - \\
\hline $\mathrm{Ni}$ & $\mu \mathrm{g} / \mathrm{g}$ & 452 & - \\
\hline $\mathbf{P}$ & $\mu \mathrm{g} / \mathrm{g}$ & 8,298 & - \\
\hline $\mathbf{P b}$ & $\mu \mathrm{g} / \mathrm{g}$ & $<\mathrm{DL}$ & - \\
\hline $\mathbf{S}$ & $\mu \mathrm{g} / \mathrm{g}$ & 4,915 & - \\
\hline Sb & $\mu \mathrm{g} / \mathrm{g}$ & 13.6 & - \\
\hline $\mathrm{Se}$ & $\mu \mathrm{g} / \mathrm{g}$ & $<\mathrm{DL}$ & - \\
\hline Si & $\mu \mathrm{g} / \mathrm{g}$ & 255 & - \\
\hline Sn & $\mu \mathrm{g} / \mathrm{g}$ & $<\mathrm{DL}$ & - \\
\hline Sr & $\mu \mathrm{g} / \mathrm{g}$ & 26.9 & 12.0 \\
\hline Ti & $\mu \mathrm{g} / \mathrm{g}$ & 4.2 & - \\
\hline TI & $\mu \mathrm{g} / \mathrm{g}$ & $<\mathrm{DL}$ & - \\
\hline V & $\mu \mathrm{g} / \mathrm{g}$ & $<\mathrm{DL}$ & - \\
\hline $\mathrm{Zn}$ & $\mu \mathrm{g} / \mathrm{g}$ & 71.4 & 32.0 \\
\hline \multicolumn{4}{|c|}{$\begin{array}{l}\text { DL = Detection Limit } \\
\mu \mathrm{g}=\text { Microgram } \\
\text { Source: NETL/ORD, }\end{array}$} \\
\hline
\end{tabular}


Table 2-15 - Fresh City Water and Centrifuge Effluent Elemental Analysis (NETL) and Phoenix City Limit

\begin{tabular}{|c|c|c|c|c|}
\hline \multicolumn{5}{|c|}{ ICP-OES } \\
\hline $\begin{array}{l}\text { Chemical } \\
\text { Name }\end{array}$ & $\begin{array}{l}\text { Unit of } \\
\text { Meas ure }\end{array}$ & City Water & $\begin{array}{c}\text { Centrifuge Efflu- } \\
\text { ent }\end{array}$ & City Limit \\
\hline $\mathrm{Ag}$ & $\mu \mathrm{g} / \mathrm{L}$ & $<\mathrm{DL}$ & $<\mathrm{DL}$ & 500 \\
\hline $\mathrm{Al}$ & $\mu \mathrm{g} / \mathrm{L}$ & 22.3 & $<\mathrm{DL}$ & - \\
\hline As & $\mu \mathrm{g} / \mathrm{L}$ & $<\mathrm{RL}$ & $<\mathrm{DL}$ & 100 \\
\hline B & $\mu \mathrm{g} / \mathrm{L}$ & 53.4 & 120 & 5,600 \\
\hline $\mathrm{Ba}$ & $\mu \mathrm{g} / \mathrm{L}$ & 46.7 & 50.4 & - \\
\hline $\mathrm{Be}$ & $\mu \mathrm{g} / \mathrm{L}$ & $<\mathrm{RL}$ & $<\mathrm{DL}$ & - \\
\hline $\mathrm{Ca}$ & $\mu \mathrm{g} / \mathrm{L}$ & 37,370 & 43,060 & - \\
\hline $\mathrm{Cd}$ & $\mu \mathrm{g} / \mathrm{L}$ & $<\mathrm{DL}$ & $<\mathrm{DL}$ & 47 \\
\hline $\mathrm{Ce}$ & $\mu \mathrm{g} / \mathrm{L}$ & $<\mathrm{DL}$ & $<\mathrm{DL}$ & - \\
\hline Co & $\mathrm{ug} / \mathrm{L}$ & $<\mathrm{DL}$ & $<\mathrm{DL}$ & - \\
\hline $\mathrm{Cr}$ & $\mu \mathrm{g} / \mathrm{L}$ & $<\mathrm{DL}$ & $<\mathrm{DL}$ & 1,400 \\
\hline $\mathrm{Cu}$ & $\mu \mathrm{g} / \mathrm{L}$ & 4.4 & 10.7 & 1,700 \\
\hline $\mathrm{Fe}$ & $\mu \mathrm{g} / \mathrm{L}$ & 4.1 & 20.8 & - \\
\hline $\mathrm{K}$ & $\mu \mathrm{g} / \mathrm{L}$ & 3,407 & 68,380 & - \\
\hline $\mathrm{Li}$ & $\mu \mathrm{g} / \mathrm{L}$ & $<\mathrm{DL}$ & 21.4 & - \\
\hline $\mathrm{Mg}$ & $\mu \mathrm{g} / \mathrm{L}$ & 14,330 & 12,570 & - \\
\hline $\mathrm{Mn}$ & $\mu \mathrm{g} / \mathrm{L}$ & $<\mathrm{DL}$ & 2.7 & - \\
\hline Mo & $\mu \mathrm{g} / \mathrm{L}$ & $<\mathrm{DL}$ & 8.4 & - \\
\hline $\mathrm{Na}$ & $\mu \mathrm{g} / \mathrm{L}$ & 81.9 & 838 & - \\
\hline $\mathrm{Ni}$ & $\mathrm{ug} / \mathrm{L}$ & $<\mathrm{DL}$ & $<\mathrm{DL}$ & 5,000 \\
\hline $\mathrm{P}$ & $\mu \mathrm{g} / \mathrm{L}$ & $<\mathrm{DL}$ & 9,340 & - \\
\hline $\mathrm{Pb}$ & $\mu \mathrm{g} / \mathrm{L}$ & $<\mathrm{DL}$ & $<\mathrm{DL}$ & 500 \\
\hline $\mathrm{S}$ & $\mu \mathrm{g} / \mathrm{L}$ & 28,430 & 29,910 & 10,000 \\
\hline $\mathrm{Sb}$ & $\mu \mathrm{g} / \mathrm{L}$ & $<\mathrm{DL}$ & $<\mathrm{DL}$ & - \\
\hline $\mathrm{Se}$ & $\mu \mathrm{g} / \mathrm{L}$ & $<\mathrm{DL}$ & $<\mathrm{DL}$ & 100 \\
\hline $\mathrm{Si}$ & $\mu \mathrm{g} / \mathrm{L}$ & 5,793 & 8,110 & - \\
\hline Sn & $\mu \mathrm{g} / \mathrm{L}$ & $<\mathrm{DL}$ & $<\mathrm{DL}$ & - \\
\hline $\mathrm{Sr}$ & $\mu \mathrm{g} / \mathrm{L}$ & 394 & 444 & - \\
\hline $\mathrm{Ti}$ & $\mu \mathrm{g} / \mathrm{L}$ & $<\mathrm{DL}$ & $<\mathrm{DL}$ & - \\
\hline $\mathrm{TI}$ & $\mu \mathrm{g} / \mathrm{L}$ & $<\mathrm{DL}$ & $<\mathrm{DL}$ & - \\
\hline V & $\mu \mathrm{g} / \mathrm{L}$ & $<\mathrm{DL}$ & $<\mathrm{DL}$ & - \\
\hline $\mathrm{Zn}$ & $\mu \mathrm{g} / \mathrm{L}$ & $<\mathrm{DL}$ & 7.5 & 5,400 \\
\hline \multicolumn{5}{|c|}{ Inductivity Coupled Plas ma - Mass Spectrometer (ICP-MS) } \\
\hline $\begin{array}{l}\text { Chemical } \\
\text { Name }\end{array}$ & $\begin{array}{l}\text { Unit of } \\
\text { Meas ure }\end{array}$ & City Water & $\begin{array}{l}\text { Centrifuge Efflu- } \\
\text { ent }\end{array}$ & City Limit \\
\hline $\mathrm{Ag}$ & $\mu \mathrm{g} / \mathrm{L}$ & 1.50 & 1.04 & - \\
\hline $\mathrm{Cd}$ & $\mu \mathrm{g} / \mathrm{L}$ & $<\mathrm{DL}$ & $<\mathrm{DL}$ & - \\
\hline $\mathrm{Sb}$ & $\mu \mathrm{g} / \mathrm{L}$ & 2.42 & 3.69 & - \\
\hline $\mathrm{TI}$ & $\mu \mathrm{g} / \mathrm{L}$ & $<\mathrm{DL}$ & 0.322 & - \\
\hline $\mathrm{Pb}$ & $\mu \mathrm{g} / \mathrm{L}$ & 1.99 & 15.8 & - \\
\hline Th & $\mu \mathrm{g} / \mathrm{L}$ & $<\mathrm{DL}$ & $<\mathrm{DL}$ & - \\
\hline$U$ & $\mu \mathrm{g} / \mathrm{L}$ & 2.36 & 16.6 & - \\
\hline \multicolumn{5}{|c|}{$\mathrm{DL}=$ Detection Limit } \\
\hline Source: NET & & & & \\
\hline
\end{tabular}




\subsubsection{Algae Oil Extraction}

Various lipid extraction methods were explored by the laboratories, including NETL/ORD, University of Kentucky CAER, and DynaSep, from March 2008 to June 2009 on the GF3 strain and Nannochloropsis.

\subsection{Oil Extraction on the GF3 Strain}

Three sets of Soxhlet extractions on GF3 were first tried by NETL as follows: one 3-hour reflux using $200 \mathrm{~mL}$ hexane plus $75 \mathrm{~mL}$ isopropanol; one 8-hour reflux using the same reagent combo; and one 8-hour reflux using a larger portion of isopropanol (160 mL hexane plus $115 \mathrm{~mL}$ isopropanol). All procedures used a $5 \mathrm{~g}$ algae specimen as the starting mass and a total volume of reagent of $275 \mathrm{~mL}$. Based on the amount of solids collected after extraction and rotary evaporation, about $9 \mathrm{wt} \%, 6 \mathrm{wt} \%$, and $5 \mathrm{wt} \%$ oil were extracted, respectively, from the biomass. The mass balances were 102 wt $\%, 103$ wt $\%$, and 102 wt $\%$, respectively.

University of Kentucky CAER also performed separate Soxhlet and reflux extraction on the GF3 strain. The details of these extractions are as follows:

- $\quad 3.17 \mathrm{~g}$ of dried algae was extracted with $400 \mathrm{~mL}$ of hexane in a Soxhlet apparatus equipped with a cellulose sample holder (thimble). The duration of the extraction was 16 hours, and the mass of oil recovered was $0.56 \mathrm{~g}$ (17.7 wt\%). A sample of this oil was sent to NETL for GC/MS analysis. The calculated mass balance was $105.4 \mathrm{wt} \%$, suggesting that either the oil or the remaining algae contained residual solvent.

- $11.10 \mathrm{~g}$ of algae paste was extracted as above using $200 \mathrm{~mL}$ of hexane mixed with 200 $\mathrm{mL}$ of methanol. After the extraction was finished, the two solvent layers were separated, and $0.54 \mathrm{~g}$ of oil (yield of $4.9 \mathrm{wt} \%$ ) was recovered from the hexane layer. In addition, a waxy substance was isolated. (This was obtained from the methanol used to wash the hexane layer before isolation of the oil.)

- $29.47 \mathrm{~g}$ of algae paste was extracted with $400 \mathrm{~mL}$ of hexane mixed with $10 \mathrm{~mL}$ of methanol in a Soxhlet apparatus equipped with a sintered glass sample holder. After 16 hours, the extraction was stopped and the resulting solvent layers were separated.

- $20.23 \mathrm{~g}$ of algae paste was mixed with $200 \mathrm{~mL}$ of methanol. The mixture was refluxed and liquid samples removed at 5 minutes, 15 minutes, 30 minutes, 1 hour, and each subsequent hour for 6 hours. 
Further, the University of Kentucky CAER conducted a preliminary pyrolysis study using GF3 algae paste. The pyrolysis was performed at $350^{\circ} \mathrm{C}\left(662^{\circ} \mathrm{F}\right)$ for about 30 minutes. Approximately $30 \mathrm{wt} \%$ bio-oil was obtained.

POS was recommended due to their previous "hands-on" experience with algae oil extraction. They also have the capability of both lab- and pilot-plant-scale oil extraction techniques. The contract scope with POS was to extract crude oil from dried algae biomass by bead milling in hexane (or other suitable solvent). In this work scope, the project was planning to provide POS $\sim 10 \mathrm{~kg}$ algae paste. Following the extraction, the crude oil was going to be analyzed for phosphorous content, peroxide value, free fatty acid value, and color to help identify the necessary oil pretreatments for the oil upgrading process.

However, the preliminary algae oil extraction performed by POS did not show promising results. With two solvents, pure hexane and mixture of hexane and ethanol, about $5.6 \mathrm{wt} \%$ and $20 \mathrm{wt} \%$ oil were extracted from the GF3 strain, respectively. Crude oil from algae contains neutral lipid (triglyceride) and polar lipids (phospholipids, glycolipids, etc.). A solvent with stronger polarity tends to extract more polar lipids as well as other substances such as sterol, chlorophyll and carotenoids, etc. This may explain the higher percentage of oil extracted when more polar solvent (mixture of hexane and ethanol) was used. Overall, the results for oil extraction of the GF3 strain were unsatisfactory. Its oil content was low and the oil was difficult to extract.

\subsection{Oil extraction of Nannoc hloropsis}

In the third quarter (April through June) of fiscal year 2009, $240 \mathrm{~L}$ of algae paste with a solids concentration of $18 \mathrm{wt} \%$ was purchased from Reed Mariculture and was sent to Lyophilization Services of New England (LSNE) for drying. About $45 \mathrm{~kg}$ of dried Nannochloropsis was obtained from LSNE. DynaSep performed the oil extraction using these dried algae. At DynaSep, the dried algae were slurried with approximately $6.6 \mathrm{lb}(3 \mathrm{~kg})$ of solvents (the solvent mixture contained $30 \mathrm{wt} \%$ hexane and $70 \mathrm{wt} \%$ methanol) and were loaded into an extraction vessel in batches of $2.76 \mathrm{lb}(1.25 \mathrm{~kg})$. The purpose of the solvent was to hydrate the algae charge and begin the extraction process. For approximately 5 hours, clean solvent was introduced at a rate of $3.3 \mathrm{lb} / \mathrm{hr}$ (1.5 kilograms per hour $(\mathrm{kg} / \mathrm{hr})$ ), resulting in $10.5 \mathrm{~kg}$ of solvent per batch. The resulting ratio of solvent to dry algae extracted was approximately 8.4 .

The solvent was removed from the extracted material in a two-step process using a rotary evaporator and vacuum oven. In the first step, a hot water bath at $140^{\circ} \mathrm{F}\left(60^{\circ} \mathrm{C}\right)$ was used to supply heat for evaporation with the bulk of the solvent evaporated using the rotary evaporator 
operated at 7.4 psi (15 in $\mathrm{Hg}$ ). The remaining solvent was evaporated in a vacuum oven operating at $12.8 \mathrm{psi}(26 \mathrm{in} \mathrm{Hg})$ and $140^{\circ} \mathrm{F}\left(60^{\circ} \mathrm{C}\right)$ for $8.2 \mathrm{lb}(3.7 \mathrm{~kg})$ of total extracted material. A total of $33 \mathrm{lb}(15 \mathrm{~kg})$ of dried algae was processed by DynaSep, which resulted in $8.2 \mathrm{lb}$ (3.7 $\mathrm{kg}$ ) of extract. In general the results were below expectation with the low quality and low flowability of the extracted oil product. The resulting oil sample was sent to ConocoPhillips for oil upgrading.

\subsection{Attemptat Oil Upgrading by Conoc oPhillips on Nannoc hloropsis Extract}

ConocoPhillips conducted fatty acid, triglyceride, and elemental analysis on the Nannochloropsis oil sample obtained by DynaSep. Around $25 \mathrm{wt} \%$ fatty acid was identified, which was close to the 27 wt\% measured by POS. ConocoPhillips claimed 25 wt $\%$ fatty acid was too low for oil upgrading and additional steps to clean the oil would make the oil yield even lower.

The main elements of the algae oil extract were as follows:

\begin{tabular}{c||cccccccc} 
Element & $\mathrm{C}$ & $\mathrm{H}$ & $\mathrm{N}$ & $\mathrm{Na}$ & $\mathrm{K}$ & $\mathrm{P}$ & $\mathrm{Mg}$ & Others \\
\hline \hline $\mathrm{Wt} \%$ & $49.06 \%$ & $7.51 \%$ & $2.66 \%$ & $9.93 \%$ & $1.68 \%$ & $0.232 \%$ & $0.0975 \%$ & $28.8 \%$
\end{tabular}

The salt content in this sample was too high and needed to be lowered to make the extracted oil fit the triglyceride specifications (Table 2-16) in ConocoPhillips' existing biofuel process. 
Table 2-16 - ConocoPhillips Triglyceride Specifications

\begin{tabular}{|l|l|}
\multicolumn{1}{|c|}{ Property } & \multicolumn{1}{c|}{$\begin{array}{c}\text { Level Max } \\
\text { Percent (wt\%) or ppm }\end{array}$} \\
\hline Moisture content & $<0.2 \mathrm{wt} \%$ \\
\hline Unsaponified materials & $<1 \mathrm{wt} \%$ \\
\hline Insoluble impurity & $<0.05 \mathrm{wt} \%$ \\
\hline Ash content & $<0.03 \mathrm{wt} \%$ \\
\hline Free fatty acids & $<4 \mathrm{wt} \%$ \\
\hline Phosphorous & $<5 \mathrm{ppm}$ \\
\hline All other metals are listed & \\
below: & \\
Sodium & \\
Calcium & \\
Potassium & \\
Magnesium & \\
Lithium & \\
Barium & \\
Strontium & \\
\hline Note: The total ppm concentration of the metals listed above \\
must be $<10$ ppm
\end{tabular}

\subsubsection{Biofuel Production}

\subsection{Algae Oil to J P-8}

Once the algae have been harvested, oil needs to be extracted from the biomass. The algae oil could then be refined into renewable jet fuel using conventional hydro-processing technology applied in petroleum refineries around the world today after the crude oil pretreatment. The process first needs to remove oxygen from the feedstock oil through decarboxylation and hydro-deoxygenation processes using bimetallic or novel metal heterogeneous catalysts. The product was then further refined through isomerisation, a process by which one molecule is cracked open and re-arranged to form another molecule shape, to meet the specifications needed for jet fuel. NETL/ORD and University of Kentucky's CAER started catalyst screening for the decarboxylation process during the third quarter of fiscal year 2008. This was the key step for crude algae oil upgrading.

\subsection{Catalyst Supply}

The catalyst supplies were obtained primarily from CAER (their in-house catalysts) and BASF. The catalysts included noble metal catalysts and Ni-based catalysts. The following delineates the catalysts that were obtained: 
- CAER in-house catalysts:

o Noble metal catalysts: 1 wt \% Pt/Al $\mathrm{O}_{3}, 1 \mathrm{wt} \% \mathrm{Pt} / \mathrm{C}, 5 \mathrm{wt} \% \mathrm{Pd} / \mathrm{C}$

o Ni catalysts: Ni (0.8) Al (0.2); Ni (0.4) Mg (0.27) Al (0.33); Ni (0.67) Al (0.33), Ni (0.54) Mg (0.13) Al (0.33); Ni (0.13) Mg (0.54) Al (0.33); Ni (0.4) Mg (0.27) Al (0.33); 10 wt\% Ni/Activated Carbon (AC)

- BASF Vendor catalysts:

o E-473P: Reduced and stabilized nickel on silica/alumina powder

o E-474TR: Reduced and stabilized nickel on silica/alumina tablet (for fixed-bed testing)

o Ni-5536P: Reduced and stabilized nickel on silica/alumina powder

o Ni-3298E: Reduced and stabilized nickel on extruded silica/alumina (for fixed-bed testing)

o X-256: 2 wt\% Pd/C containing approximately 50 wt\% moisture content.

o $2 \mathrm{wt} \% \mathrm{Pt} / \mathrm{C}$

o $5 \mathrm{wt} \% \mathrm{Pt} / \mathrm{C}$

o $5 \mathrm{wt} \% \mathrm{Pd} / \mathrm{C}$

\subsection{Testing and Analytical Facilities}

The obtained catalysts were tested and analyzed by NETL/ORD, CAER and APS project personnel as summarized in Table 2-17.

Table 2-17 - NETLIORD, CAER, and APS Project Catalyst Testing and Product Analysis - Summary by Facility

\begin{tabular}{|c|c|c|}
\hline & Testing Facility & Analytic al Facility \\
\hline NETL/ORD & $\begin{array}{l}\text { Fixed-bed, continuous stirred tank } \\
\text { reactor (CSTR), and agitated reactors: } \\
\text { ( } \mathrm{T} \text { up to } 1000^{\circ} \mathrm{C}\left(1832^{\circ} \mathrm{F}\right) \text { ), } \mathrm{P} \text { up to } \\
2000 \text { psi, liquid and gas feeds) } \\
\text { Online gas analysis } \\
\text { Automated/unattended operation. }\end{array}$ & $\begin{array}{l}\text { Materials characterization XRD with hot- } \\
\text { stage, SEM with EDS, TGA, BET, XPS, } \\
\text { LEIS, LEED, Auger, STM, AFM, gradient } \\
\text { film evaporator } \\
\text { Materials analysis: } \\
\text { GC systems with MS, AED, FIO, TCD, } \\
\text { ICP-OES }\end{array}$ \\
\hline CAER & $\begin{array}{l}300 \mathrm{~mL} \text { autoclave, } 100 \mathrm{~mL} \text { autoclave } \\
\text { 4*25 mL autoclave array }\end{array}$ & $\begin{array}{l}\text { GC (gases), SIMDIST, elemental analy- } \\
\text { sis ( } \mathrm{C} \& \mathrm{H}, 0 \text { by difference), 13C NMR }\end{array}$ \\
\hline APS Project & $\begin{array}{l}2 \mathrm{~L} \text { autoclave } \\
2 \mathrm{~L} \text { distillation equipment }\end{array}$ & GC/MS, GC \\
\hline
\end{tabular}




\subsection{Catalyst Testing Results}

The catalyst screening test results presented below are from CAER. The $100 \mathrm{~mL}$ algae oil with clear yellow color and high flowability was obtained from GreenFuel by APS in 2008. Both University of Kentucky and NETL received about $30 \mathrm{~mL}$ of the algae oil. The SIMDIST analysis on the algae oil from CAER indicated that the algae oil contained $30 \mathrm{wt} \%$ hexane solvent in the algae oil and the carbon numbers in the algae oil were from C5 to C15. Due to the limited algae oil supply, most of the decarboxylation experiments performed at CAER used model molecules tristearin (octadecanoic acid, 1, 2, 3-propanetriyl ester) and triolein. Before CAER procured a $100 \mathrm{~mL}$ autoclave reactor, the decarboxylation test was performed in their $300 \mathrm{~mL}$ autoclave setup. Generally, $0.5 \mathrm{~g}$ catalyst, $25 \mathrm{~g}$ substance (model molecule or algae oil), and $22 \mathrm{~g}$ Dodecane were used for each test. Dodecane was added in the reactor simply for diluting purposes. After flushing with $\mathrm{N}_{2}$, the reaction was started under 100 psig pressure. All tests were performed at $350^{\circ} \mathrm{C}\left(662^{\circ} \mathrm{F}\right)$ for 4 hours. The tests performed to-date included the upgrading of fatty acids, FAMEs, tristearin, and algae oil using noble metal and Ni catalysts (as listed above). The product analyses were conducted using GC (for gases), SIMDIST, elemental analysis ( $\mathrm{C} \& \mathrm{H}, \mathrm{O}$ by difference), and ${ }^{13} \mathrm{C}$ NMR. The main observations from CAER catalyst testing were as follows:

- Gas byproduct phase normally contains: $\mathrm{CO}, \mathrm{CO}_{2}, \mathrm{H}_{2}, \mathrm{CH}_{4}, \mathrm{C} 2$, and $\mathrm{C} 3$ hydrocarbons.

- $\quad$ Liquid product phase normally contains saturated hydrocarbons (C5 above).

- In triglyceride conversion, $1 \mathrm{wt} \% \mathrm{Pt} / \mathrm{C}$ and $5 \mathrm{wt} \% \mathrm{Pd} / \mathrm{C}$ show similar activity and product distributions. These catalysts are more active than the metal oxide catalysts reported in the literature (e.g., $\mathrm{Al}_{2} \mathrm{O}_{3}, \mathrm{ZnO}$ ).

- Tristearin and algae oil afford similar product distributions, although algae oil seems to be less reactive. This was unexpected and required further investigation. Main products are C5-C15 hydrocarbons. GC/MS data indicated that the hydrocarbons are saturated.

- $\mathrm{Ni} / \mathrm{Mg} / \mathrm{Al}$ catalysts show promise for deoxygenation of tristearin. Activity is better than Pd or Pt catalysts screened to-date for best Ni catalyst, selectivity to C8-C17 is similar to $\mathrm{Pd}$ and Pt catalysts.

- Fatty acids and FAMEs can also be converted to hydrocarbons over Pt/C and Pd/C catalysts. 
- The activation procedure employed is important: in situ reduction affords more active catalysts (active site is $\mathrm{NiO}$ ).

- The activity for $\mathrm{C}-\mathrm{C}$ bond scission appeared to depend on the Mg content.

Table 2-18, Table 2-19, and Table 2-20 provide the result summaries from CAER catalyst screening. Both model molecules and algae oil acquired from GreenFuel contained trace amounts of $\mathrm{S}$ and $\mathrm{P}$, which could be a concern with respect to the catalyst deactivation; however, catalyst deactivation was not evaluated.

Table 2-18 - Noble Catalyst Performance Comparison with Stearic Acid and Methyl (Me) Stearate

\begin{tabular}{|c|c|c|c|c|c|}
\hline Catalyst & $\mathrm{Pt} / \mathrm{AI}_{2} \mathrm{O}_{3}$ & $\mathrm{Pt} / \mathrm{C}$ & $\mathrm{Pd} / \mathrm{C}$ & $\mathrm{Pt} / \mathrm{C}$ & $\mathrm{Pd} / \mathrm{C}$ \\
\hline Reactant & Stearic Acid & Stearic Acid & Stearic Acid & Me Stearate & Me Stearate \\
\hline $\mathrm{CO}+\mathrm{CO}_{2}(\mathrm{mmol})$ & 51.3 & 61.7 & 50.4 & 30.8 & 27.3 \\
\hline Conversion (wt\%) & 59.0 & 70.9 & 57.9 & 36.2 & 32.1 \\
\hline \multicolumn{6}{|l|}{ Product Yields } \\
\hline Solids $(\mathrm{g})^{\star}$ & 44.1 & 38.6 & 41.5 & 44.3 & 40.3 \\
\hline Liquids (g) & 0 & 0 & 0 & 0 & 0 \\
\hline Gases $(\mathrm{g})^{\star \star}$ & 2.2 & 2.3 & 2.0 & 1.3 & 1.1 \\
\hline $\begin{array}{l}\text { Mass Balance } \\
(\mathrm{wt} \%)\end{array}$ & 97.5 & 86.2 & 91.6 & 96.2 & 87.3 \\
\hline \multicolumn{6}{|c|}{$\begin{array}{l}\text { *Solids exclude initial catalyst weight } \\
{ }^{*} \text { Gase exclude initial } \mathrm{N}_{2} \text { charge } \\
\text { Source: CAER, University of Kentucky, Lexington, KY }\end{array}$} \\
\hline
\end{tabular}

Table 2-19 - Noble Catalyst Performance Comparison with Tristearin and Algae Oil

\begin{tabular}{|c|c|c|c|c|c|}
\hline Catalyst & $\mathrm{Pt} / \mathrm{AI}_{2} \mathrm{O}_{3}$ & $\mathrm{Pt} / \mathrm{C}$ & $\mathrm{Pd} / \mathrm{C}$ & $\mathrm{Pt} / \mathrm{C}$ & $\mathrm{Pd} / \mathrm{C}$ \\
\hline Reactant & Tristearin & Tristearin & Tristearin & Algae Oil & Algae Oil \\
\hline $\mathrm{CO}_{2}+\mathrm{CO}(\mathrm{mmol})$ & 77.6 & 94.8 & 101.2 & 56.2 & 48.5 \\
\hline $\begin{array}{l}\% \text { Conversion by } \\
\text { Mass (wt } \%)\end{array}$ & 61.6 & 75.2 & 80.3 & 44.6 & 38.5 \\
\hline \multicolumn{6}{|l|}{ Product Yields } \\
\hline Solids $(\mathrm{g})^{\star}$ & 40.2 & 25.8 & 22.4 & 0 & 3.1 \\
\hline Liquids (g) & 0 & 9.7 & 14.8 & 39.8 & 20.9 \\
\hline Gases $(g)^{* *}$ & 3.2 & 3.7 & 3.8 & 2.9 & 3.4 \\
\hline Mass Balance (wt\%) & 90.8 & 88.4 & 90.9 & 95.0 & 81.0 \\
\hline \multicolumn{6}{|c|}{$\begin{array}{l}\text { *Solids exclude initial catalyst weight } \\
\star * \text { Gases exclude initial } \mathrm{N}_{2} \text { charge } \\
\text { Source: CAER, University of Kentucky, Lexington, KY }\end{array}$} \\
\hline
\end{tabular}


Table 2-20 - CAER In-house Ni Catalyst Performance Comparison with Tristearin

\begin{tabular}{|c|c|c|c|c|c|c|}
\hline Catalyst & NiO.BAIO.2 & $\begin{array}{l}\text { NiOAM- } \\
\text { g0.3AIO. } 3\end{array}$ & NiO.7AIO.3 & $\begin{array}{c}\text { Ni0.5MgO.1Al } \\
0.3\end{array}$ & $\begin{array}{c}\text { NiOAM- } \\
\text { g0.3AIO.3 }\end{array}$ & $\begin{array}{c}\text { NiO.1 } \\
\text { Mg0.5AIO.3 }\end{array}$ \\
\hline Reduction & Ex situ & Ex situ & In situ & In situ & In situ & In situ \\
\hline $\begin{array}{l}\text { Deoxygenation* } \\
\text { (wt\%) }\end{array}$ & 34.9 & 55.5 & 70.5 & 78.3 & 94.0 & 91.7 \\
\hline \multicolumn{7}{|c|}{ Gas sampling (mmol) } \\
\hline $\mathrm{H}^{2}$ & 6.4 & 3.8 & 7.0 & 6.4 & 8.3 & 11.9 \\
\hline $\mathrm{CH}^{4}$ & 49.9 & 5.1 & 33.0 & 48.1 & 11.4 & 26.2 \\
\hline$\Sigma \mathrm{C}^{2}$ & 13.4 & 4.3 & 6.0 & 7.5 & 6.5 & 21.3 \\
\hline$\Sigma C^{3}$ & 10.5 & 3.7 & 4.2 & 5.6 & 5.4 & 17.2 \\
\hline$\Sigma \mathrm{C}^{4}$ & 4.9 & 1.8 & 2.3 & 3.4 & 2.7 & 7.2 \\
\hline \multicolumn{7}{|c|}{ Products recovered } \\
\hline Gas (g) & 3.9 & 2.9 & 4.7 & 5.8 & 5.2 & 6.6 \\
\hline Oil (g) & 24.9 & 33.3 & 33.8 & 31.9 & 32.1 & 26.2 \\
\hline Solid (g) & 1.4 & 1.3 & 0.6 & 1.0 & 0.8 & 0.9 \\
\hline Total (g) & 31.5 & 37.6 & 37.9 & 37.0 & 38.0 & 33.5 \\
\hline $\begin{array}{l}\text { Mass Balance } \\
\text { (wt\%) }\end{array}$ & 73.9 & 84.4 & 88.9 & 89.0 & 90.0 & 83.5 \\
\hline
\end{tabular}

After the catalyst screening, more tests were carried out to study the behavior of different oil sources with the same catalysts. Table 2-21 and Table 2-22 give the results of tests using tristearin, triolein, and salad oil. The test results show that with higher unsaturation in the carbon chain (salad oil > triolein > tristearin), $5 \mathrm{wt} \% \mathrm{Pd} / \mathrm{AC}$ gave about same selectivity of a C8-C17 component; however, the catalyst tagged SAT002 showed significantly higher C8-C17 component selectivity using salad oil than using tristearin.

Table 2-21 - Results Obtained from 5 wt\% Pd/AC Catalyst with Different Feedstocks

\begin{tabular}{|c|c|c|c|c|c|c|c|c|c|}
\hline \multirow[b]{2}{*}{ Feedstock } & \multirow[b]{2}{*}{$\begin{array}{l}\text { Run } \\
\text { No. }\end{array}$} & \multirow[b]{2}{*}{$\begin{array}{c}\text { Pre- } \\
\text { treatment }\end{array}$} & \multicolumn{3}{|c|}{ Product } & \multicolumn{3}{|c|}{ Selectivity to Liquid Products ( wt\%) } & \multirow{2}{*}{$\begin{array}{c}\text { Liquid wt\% } \\
\text { x C8-C17 } \\
\text { wt } \%\end{array}$} \\
\hline & & & $\begin{array}{l}\text { Solid } \\
\text { ( wt\%) }\end{array}$ & $\begin{array}{c}\text { Gas } \\
(w t \%)\end{array}$ & $\begin{array}{l}\text { Liquid } \\
\text { ( wt\%) }\end{array}$ & $\leq \mathrm{C} 7$ & C8-C17 & $\geq \mathrm{C} 18$ & \\
\hline Tristearin & 48 & A & 1.3 & 10.9 & 87.7 & 2.1 & 70.3 & 27.6 & 61.7 \\
\hline Triolein & 49 & A & 6.7 & 12.5 & 80.8 & 2.6 & 68.9 & 28.5 & 55.7 \\
\hline Salad Oil & 47 & A & 5.2 & 18.6 & 76.2 & 2.8 & 67.2 & 30.0 & 51.2 \\
\hline
\end{tabular}


Table 2-22 - Results Obtained from the SAT002 Catalyst with Different Feedstocks

\begin{tabular}{|c|c|c|c|c|c|c|c|c|c|}
\hline \multirow[b]{2}{*}{ Feedstock } & \multirow[b]{2}{*}{ Run \# } & \multirow[b]{2}{*}{$\begin{array}{c}\text { Pre- } \\
\text { treatment }\end{array}$} & \multicolumn{3}{|c|}{ Product } & \multicolumn{3}{|c|}{ Selectivity to Liquid Products ( wt\%) } & \multirow{2}{*}{$\begin{array}{c}\text { Liquid wt\% } \\
\text { x C8-C17 } \\
\text { wt } \%\end{array}$} \\
\hline & & & $\begin{array}{l}\text { Solid } \\
(w t \%)\end{array}$ & $\begin{array}{c}\text { Gas } \\
\text { (wt\%) }\end{array}$ & $\begin{array}{l}\text { Liquid } \\
\text { (wt\%) }\end{array}$ & $\leq \mathrm{C} 7$ & C8-C17 & $\geq \mathrm{C} 18$ & \\
\hline Tristearin & 46 & C & 0.0 & 6.1 & 93.9 & 0.4 & 38.7 & 32.4 & 36.3 \\
\hline Salad Oil & 52 & C & 5.1 & 22.3 & 72.6 & 2.0 & 62.2 & 35.8 & 45.2 \\
\hline Salad Oil & 43 & D & 3.0 & 9.2 & 87.8 & 4.8 & 72.0 & 23.2 & 63.2 \\
\hline
\end{tabular}

Pretreatment Method C: The catalyst was calcined ex situ in air and reduced in situ at $350^{\circ} \mathrm{C}\left(662^{\circ} \mathrm{F}\right)$ under 10 wt $\% \mathrm{H}_{2} / \mathrm{He}$.

Pretreatment Method D: The catalyst was calcined ex situ in air.

Source: CAER, University of Kentucky, Lexington, KY

\subsubsection{IN-House LPID ANALYSIS AND OIL EXIRACTION DEVELOPMENT}

The project also constructed a functional laboratory and a production "farm" to produce algae oil for the lab work in the third quarter (March through June) of fiscal year 2008. The laboratory includes essential lab equipment for algae lipid analysis and oil extraction.

Starting in September 2009, in-house oil extraction and lipid analysis on algae, mainly Scenedesmus sp., were performed in the APS laboratory. The fatty acid analysis was conducted by converting fatty acids to their methyl ester through an acid catalytic approach, and FAME was quantitatively determined using GC/MS with an internal standard. Oil extraction from algae biomass was also attempted, and the extraction results from dried algae obtained from different drying methods were compared.

\subsubsection{In-House Algae Lipid Analysis}

Fatty acid analysis was performed by using an acid catalytic approach to convert all lipids and free fatty acids (FFAs) to FAMEs. Conversion of lipids and FFA into FAMEs is a common approach as it can reduce the adsorption of solute on the GC column and improve hydrocarbon separation. An internal standard was injected together with the sample to reduce analytical errors and obtain fatty acid results in weight.

Esterification techniques have acid and base catalysis approaches based on the reagents used. The reagents most commonly associated with a base catalyst are sodium hydroxide $(\mathrm{NaOH})$ or potassium hydroxide $(\mathrm{KOH})$ in methanol. The advantage of base catalysis esterification is that it can be carried out at room temperature with a short reaction time. However, a base catalysis approach will not convert FFAs into FAMEs, which limits its applicability to high FFAs containing oil. Thus, acid catalytic esterification was applied in this instance, with sulfuric acid $\left(\mathrm{H}_{2} \mathrm{SO}_{4}\right)$ in methanol chosen as the reagent. The method developed is as follows: 
$100 \mathrm{mg}$ of freeze-dried algae biomass was weighed and added with $50 \mu \mathrm{l}(40 \mathrm{mg} / \mathrm{ml})$ methyl nonadecanoate (C19:0) internal standard in hexane, and $1 \mathrm{~mL}(10 \mathrm{wt} \%(\mathrm{v} / \mathrm{v})$ methanolic $\mathrm{H}_{2} \mathrm{SO}_{4}$. The mixture was heated at $60^{\circ} \mathrm{C}$ for 1 hour and the FAMEs were extracted with hexane containing $0.2 \mathrm{wt} \%$ Butylated Hydroxytoluene (BHT) and analyzed on HP-7890A GC equipped with HP 7673B injector and 5975C MS. Two capillary columns (HP-PONA, $50 \mathrm{~m}$ by $0.2 \mathrm{~mm}$ by $0.5 \mu \mathrm{m}$ and HP-INNOWAX, $30 \mathrm{~m}$ by $0.32 \mathrm{~mm}$ by $0.25 \mu \mathrm{m}$ ) were used and the spectrums from two different GC columns were compared. The results are shown in Figure 2-44.
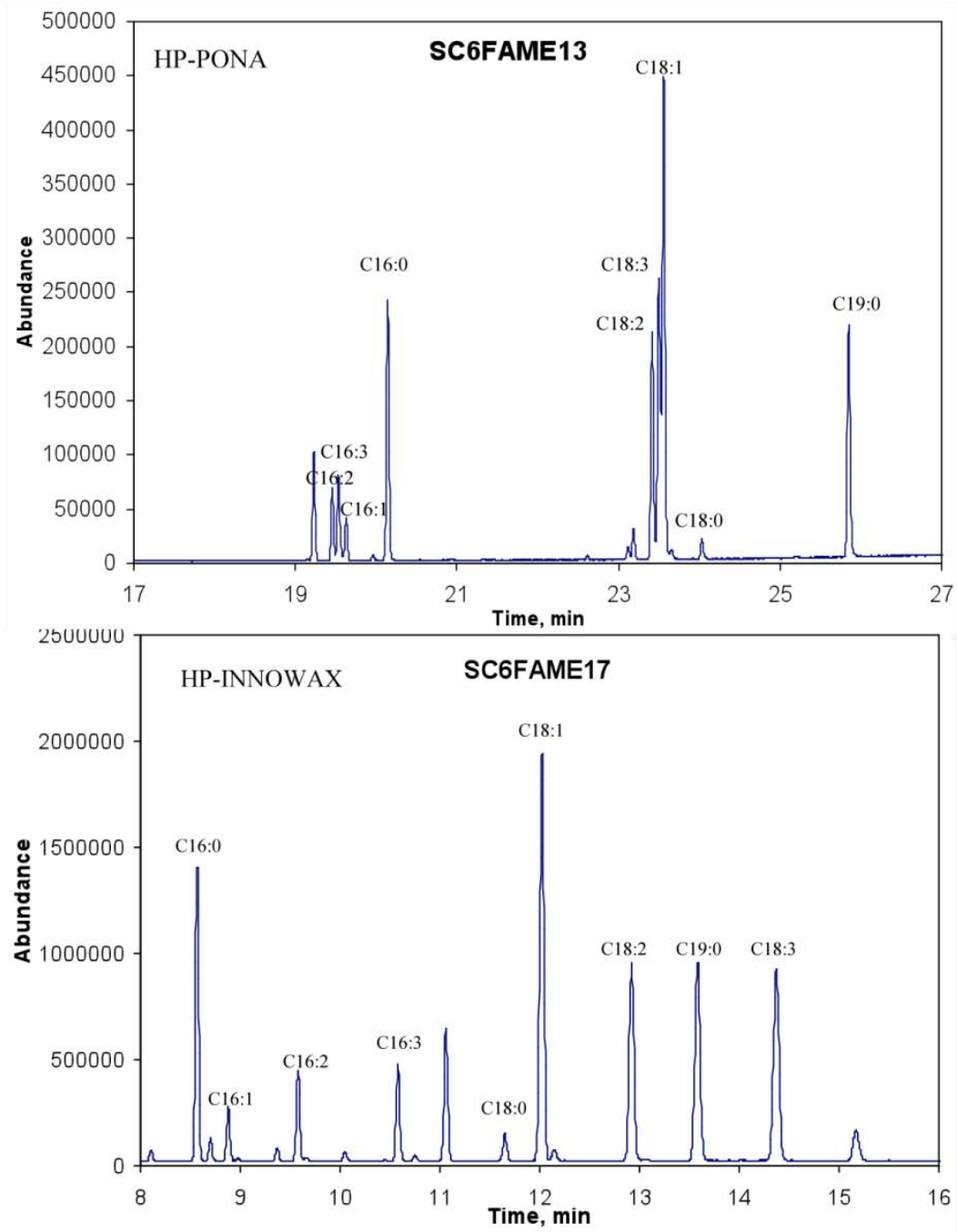

Figure 2-44 - Gas Chromatographs of the Fatty Acid Methyl Esters in Scenedesmus Analyzed with Different GC Columns 
The HP-INNOWAX gave a better separation than that from HP-PONA due to higher polarity of HP-INNOWAX. The HP-INNOWAX had shorter analysis time compared to that of HP-PONA because of the availability of thinner films on the column. Therefore, all the GC-MS analyses were performed subsequently using HP-INNOWAX.

FAMEs were identified by MS and quantified by comparing peak areas with an internal standard (C19:0), with results shown in Table 2-23. There were eight FAME derivatives in the Scenedesmus biomass resulting in total fatty acid content of $12 \mathrm{wt} \%$. This analysis compared favorably with an 11 wt\% fatty acid content analyzed by an external source. From the analysis, the most abundant fatty acid was oleic acid methyl ester with 31 wt\% of the total fatty acids. Additionally, the total content of four 18-carbon acid methyl esters; (1) oleic acid methyl ester, (2) octadecanoic acid methyl ester, (3) octadecadienoic acid methyl ester, and (4) oxtadecatrienoic acid methyl ester, was $68 \mathrm{wt} \%$ of the total fatty acids. This result was indicative of high-quality biodiesel.

Table 2-23 - Fatty Acid Methyl Esters in the Biomass

\begin{tabular}{|c|c|c|l|c|}
\hline No & $\begin{array}{c}\text { Molecular } \\
\text { formula }\end{array}$ & $\begin{array}{c}\text { Relative } \\
\text { molecular mas s }\end{array}$ & \multicolumn{1}{|c|}{ Fatty acid methyl es ter } & $\begin{array}{c}\text { Relative } \\
\text { content } \\
\text { (wt\%) }\end{array}$ \\
\hline 1 & $\mathrm{C}_{17} \mathrm{H}_{34} \mathrm{O}_{2}$ & 270 & Hexadecanoic acid methyl ester & 17.11 \\
\hline 2 & $\mathrm{C}_{17} \mathrm{H}_{32} \mathrm{O}_{2}$ & 268 & 9-Hexadecanoic acid methyl ester & 3.21 \\
\hline 3 & $\mathrm{C}_{17} \mathrm{H}_{30} \mathrm{O}_{2}$ & 266 & 7,10 -Hexadecadienoic acid methyl ester & 5.33 \\
\hline 4 & $\mathrm{C}_{17} \mathrm{H}_{28} \mathrm{O}_{2}$ & 264 & 7,10, 13-Hexadecatrienoic methyl ester & 5.95 \\
\hline 5 & $\mathrm{C}_{19} \mathrm{H}_{38} \mathrm{O}_{2}$ & 298 & Octadecanoic acid methyl ester & 1.90 \\
\hline 6 & $\mathrm{C}_{19} \mathrm{H}_{36} \mathrm{O}_{2}$ & 296 & 7-Octadecenoic acid methyl ester & 30.79 \\
\hline 7 & $\mathrm{C}_{19} \mathrm{H}_{34} \mathrm{O}_{2}$ & 294 & $9,12-$ Octadecadienoic acid methyl ester & 16.08 \\
\hline 8 & $\mathrm{C}_{19} \mathrm{H}_{32} \mathrm{O}_{2}$ & 292 & $9,12,15$-Octadecatrienoic acid methyl ester & 19.60 \\
\hline
\end{tabular}

\subsubsection{In-House Algae Oil Extraction}

The first step in extracting oil from algae is to dry the algae biopaste, which is often considered to be the most energy intensive part of this process. Currently, freeze-drying is the most widely used method to dry the wet paste ( 20 wt $\%$ solid and $~-80$ wt\% liquid by weight); however, freeze-drying is time-consuming and energy intensive. This technique is not ideal, as it does not break the algae cell wall, thus producing a limited amount of neutral lipids. A recently investigated alternative is chemical drying with acetone. This method was found to be efficient at drying algae paste, while effectively cracking the cell wall as well. Recent results yielded 20 wt\% dry algae from Scenedesmus using acetone, which was then ready for oil extraction. 
The next step of the oil extraction process was to extract the lipids from the dry algae biomass. To do this, an acetone-dried algae sample was extracted with methanol at $40^{\circ} \mathrm{C}\left(104^{\circ} \mathrm{F}\right)$ for 40 minutes. The mixture was centrifuged at $3500 \mathrm{rpm}$ for 5 minutes, after which the supernatant was removed and the solids were re-extracted with a mixture of hexane and diethyl ether (1:1 volume ratio). This mixture was centrifuged and the supernatant from it was combined with other supernatants. The supernatant mixture was then combined with an equal volume ratio of diethyl ester, hexane and water, which subsequently was vortexed, sonicated, and centrifuged. The upper phase was collected, and the bottom phases were combined, where the upper phase was a neutral lipid phase and the bottom phase was a polar lipid phase. Finally, the lipid solutions were evaporated to remove the solvents and weighed. A total neutral lipid content of $80 \mathrm{wt} \%$ of the total fatty acids was obtained from an acetone-dried sample, which was higher than that observed from a freeze-dried sample ( $27 \mathrm{wt} \%)$.

Extensive algae stressing, oil extraction, and lipid analysis were studied under DOE Cooperative Agreement: DE-FE0001099, "Integrated Energy System with Beneficial $\mathrm{CO}_{2}$ Use." Please refer to its final technical closeout report for further results.

\subsubsection{SUMMARY OF OIL EXTRACTION AND BIOFUEL PRODUCTION}

Crude oil from algae includes neutral lipids and polar lipids. Depending on the polarity of the solvents used for oil extraction, different amounts of lipids would be extracted. About $120 \mathrm{~kg}$ of algae paste and $500 \mathrm{~g}$ of dried algae (utilizing the GF3 and Nannochloropsis strains) were sent to outside labs (CAER at the University of Kentucky, NETL/ORD, DynaSep and POS) for various algae oil extraction exercises. It has been generally recognized that oil extraction using "green" algae is very challenging. Non-flowing green gum was obtained from all these exercises, which was probably caused by the interaction of chlorophyll and phosphor lipids. For one gallon Nannochloropsis extract sent to ConocoPhillips, it was found the fatty acid content was only $\sim 25 \mathrm{wt} \%$ and extract quality was too low for oil upgrading. Crude oil pretreatment will be required for any oil upgrading.

Initial catalyst screening tests for the decarboxylation process on CAER in-house catalysts and BASF vender catalysts were carried mostly by CAER using model molecules, due to the limited supply of algae oil. The model molecule, Tristearin, showed similar product distributions as that of algae oil, which were mainly C5-C15 saturated hydrocarbons. Ni/Mg/Al catalysts showed the promise for deoxygenation of Tristerarin. Their performance was better than that of $\mathrm{Pd}$ or $\mathrm{Pt}$ catalysts. 
In-house biomass and oil analysis protocol and facilities were established. They significantly assisted in monitoring algae cultivation, studying stressing effects on algae oil content and even facilitating strain selection. Development of the fatty acid analysis method was an important step in increasing the analytical capability of the laboratory to deliver consistent, reliable, and repeatable lipid content results.

The low neutral lipid contents from algae biomass that had been tested made it necessary to manipulate the algae biomass cultivating condition to increase the fat level and reduce chlorophyll in biomass, thereby easing the oil extraction process. Extensive algae stressing, oil extraction, and lipid analysis were continued under DOE Cooperative Agreement: DE-FE0001099, "Integrated Energy System with Beneficial $\mathrm{CO}_{2}$ Use." Please refer to its final technical closeout report for further results. 


\subsection{Emects Of Rue Gas On Algal Growth And Lipid Production}

\subsubsection{INTRODUCTION}

Work was planned and performed during the period between June 1, 2009 and March 31, 2010 by Biological \& Irrigation Engineering, Utah State University, Logan, Utah, to determine the effects of flue gas on algal growth and lipid production. Although flue gases are a readily available source of $\mathrm{CO}_{2}$ for growth of photoautotrophs, they also contain several other chemical species such as sulfur oxides (commonly referred to as $\mathrm{SO}_{x}$ and comprised primarily of $\mathrm{SO}_{2}$ and $\mathrm{SO}_{3}$ ), nitrogen oxides (commonly referred to as $\mathrm{NO}_{\mathrm{X}}$ and comprised primarily of $\mathrm{NO}$ and $\mathrm{NO}_{2}$ ) (Reddy 2002), heavy metal species including $\mathrm{Ag}, \mathrm{As}, \mathrm{Cd}, \mathrm{Co}, \mathrm{Cr}, \mathrm{Cu}, \mathrm{Hg}, \mathrm{Ni}, \mathrm{Pb}$, Se, and $\mathrm{Zn}$ (Jakob et al. 1995; Wang et al. 2006), and carbon compounds including polycyclic aromatic hydrocarbons (PAHs) (Mastral and Callen 2000). All these components, in addition to $\mathrm{CO}_{2}$ can influence algae growth by directly interacting with the microorganisms or indirectly by influencing the chemistry of the growth medium. In order to design stable and highly productive microalgal systems integrated with flue gases, the influence of these interactions on growth and resulting biofuel quality must be understood and quantified.

\subsubsection{SOx and NOx in Fue Gas}

Although environmental regulations on $\mathrm{SO}_{x}$ and $\mathrm{NO}_{x}$ emissions from power plants exist, their concentration limits in flue gases released into the atmosphere vary based on location and plant due to local regulations as well as trading of emissions allowed under the Clean Air Act of 1990 (USEPA 2007). However, most large power plants employ $\mathrm{SO}_{\mathrm{x}}$ and $\mathrm{NO}_{\mathrm{x}}$ emission control systems which have overall efficiencies greater than 90\% (Committee on Mine Placement of Coal Combustion Wastes 2006). Under these circumstances, total $\mathrm{SO}_{\mathrm{x}}$ and $\mathrm{NO}_{\mathrm{x}}$ concentrations are likely to be less than 200 and 60 parts per million by volume (ppmv), respectively, predominantly comprising of $\mathrm{SO}_{2}$ and $\mathrm{NO}$.

In water, oxides of sulfur and nitrogen dissociate to form sulfite, nitrate and nitrite (Babich and Stotzky 1980) and the primary chemical reactions leading to the formation of these species is shown in Figure 2-45. In unbuffered systems, these dissolved-gas reactions can decrease the $\mathrm{pH}$ of the growth medium and can cause a decrease in cell growth (Matsumoto et al. 1995; Reddy 2002). In addition, the formed sulfite and nitrite ions may inhibit algal growth at high concentrations (Reddy 2002; Yanagi et al. 1995). Reddy (Reddy 2002), for example, showed that inhibition of growth of Chlorella sp. occurred above sulfite and nitrite concentrations of 


\begin{tabular}{|l|} 
Aqueous $\mathrm{SO}_{X}$ dissociation reactions: \\
$\mathrm{SO}_{2}(\mathrm{~g})+\mathrm{H}_{2} \mathrm{O}(\mathrm{l}) \rightarrow \mathrm{SO}_{2} \cdot \mathrm{H}_{2} \mathrm{O}(\mathrm{aq})$ \\
$\mathrm{SO}_{2} \cdot \mathrm{H}_{2} \mathrm{O}(\mathrm{aq}) \rightarrow \mathrm{H}^{+}+\mathbf{H S O}_{3}{ }^{-}$ \\
$\mathrm{HSO}_{3}{ }^{-}(\mathrm{aq}) \rightarrow \mathrm{H}^{+}+\mathrm{SO}_{3}{ }^{2-}$ \\
\\
Aqueous $\mathrm{NO}_{X}$ dissociation reactions: \\
$2 \mathrm{NO}_{2}(\mathrm{~g})+\mathrm{H}_{2} \mathrm{O}(\mathrm{l}) \rightarrow 2 \mathrm{H}^{+}+\mathrm{NO}_{3}{ }^{-}+\mathbf{N O}_{2}^{-}$ \\
$\mathrm{NO}_{(\mathrm{g})}+\mathrm{NO}_{2}(\mathrm{~g})+\mathrm{H}_{2} \mathrm{O}(\mathrm{l}) \rightarrow 2 \mathrm{H}^{+}+2 \mathrm{NO}_{2}^{-}$ \\
$3 \mathrm{NO}_{2}(\mathrm{~g})+\mathrm{H}_{2} \mathrm{O}(\mathrm{l}) \rightarrow 2 \mathrm{H}^{+}+2 \mathrm{NO}_{3}{ }^{-}+\mathrm{NO}(\mathrm{g})$ \\
\hline
\end{tabular}

Figure 2-45 - SOx and NOx Reactions in the Aqueous Phase
$100 \mathrm{mg} / \mathrm{L}$. However, other studies with Phaeodactylum tricornutum and Nannochloropsis salina show no detrimental impact when $\mathrm{NO}_{\mathrm{X}^{-}}$ containing flue gas was used for growth (Matsumoto et al. 1995), either due to insufficient dissolved nitrite levels or due to greater nitrite tolerance by the organisms tested. In fact, at low concentrations, it is also possible that

nitrate, nitrite or sulfite derived from dissolved $\mathrm{SO}_{x}$ and $\mathrm{NO}_{x}$ could serve as sources of $\mathrm{N}$ and $\mathrm{S}$ for microalgal growth.

\subsubsection{Volatile Heavy Metals in Fue Gas}

Heavy metals in flue gases come from two sources - ash particles or volatilized metal species (Pavageau et al. 2002). Since most metal species are non-volatile at ambient temperatures, they are likely to condense and precipitate out, at least partially, onto ash particles when stack gases (typically at $93^{\circ} \mathrm{C}\left(200^{\circ} \mathrm{F}\right)$ ) are cooled before injection into algal growth reactors (Benemann 1993; Benemann 1997; Douskova et al. 2009). However, some species of metals such as such $\mathrm{Hg}$, As and Se can exist in a vapor phase even at low temperatures.

At ambient temperatures, mercury can exist both in elemental and oxidized forms, especially as a metal chloride. At the exit of the high temperature zone in the coal combustion reactor, $\mathrm{Hg}$ exists mostly in elemental form $(\mathrm{Hg}(0))$ but is partially oxidized to $\mathrm{HgCl}_{2}$ (Galbreath et al. 2000; Hall et al. 1991; Senior et al. 2000) or HgS (Yan et al. 2000) at lower temperatures in the stack. The $\mathrm{Hg}(\mathrm{II})$ formed under these cooler conditions is relatively stable and does not easily thermally decompose back into its elemental form (Galbreath et al. 2000). However, the extent of $\mathrm{Hg}$ oxidation depends on the chlorine content of the coal (Zhao et al. 2006) and the combustion system used (e.g., oxidizing or reducing conditions) (Yan et al. 2000). Laboratory studies suggest that $\mathrm{Hg}(0)$ may only be between $10-20 \%$ of the total $\mathrm{Hg}$ at the point of release from the stack (Kellie et al. 2004) and even lower when flue gases are further cooled down to ambient temperatures (Yan et al. 2000).

For algae systems, there is significant relevance of $\mathrm{Hg}$ speciation - the concentration of $\mathrm{Hg}$ (II) (such as from $\mathrm{HgCl}_{2}$ ) in water is a result of chemical equilibrium interactions of the ionic $\mathrm{Hg}$ 
species with the aqueous medium (Clever et al. 1985), whereas the concentration of dissolved elemental mercury is driven by vapor-liquid equilibrium and governed by Henry's Law (Andersson et al. 2008; Sanemasa 1975). As a result, while concentrations of $\mathrm{Hg}(\mathrm{II})$ in water can increase until its solubility limits are reached (approximately $75 \mathrm{~g} / \mathrm{L}$ for $\mathrm{HgCl}_{2}$ (Clever et al. 1985), the amount of $\mathrm{Hg}(0)$ dissolved in water depends on its concentration in the vapor phase. At $25^{\circ} \mathrm{C}\left(77^{\circ} \mathrm{F}\right)$, the dimensionless Henry's law constant for elemental mercury is 0.32 (Andersson et al. 2008) and assuming a gas-phase $\mathrm{Hg}(0)$ concentration of $0.016 \mathrm{mg} / \mathrm{m}^{3}$ (Kellie et al. 2004), the maximum expected $\mathrm{Hg}(0)$ concentration in the aqueous medium, assuming equilibrium between vapor and liquid phases, would only be $50 \mathrm{ng} / \mathrm{L}$.

The other major metal species likely to be present in vapor phase in cooled flue gases are As and Se. Like Hg, the specific As and Se species formed depend on the chlorine content of the parent coal as well as on the combustion processes used. Under reducing combustion conditions or when the chlorine content of coal is low, metallic As, a water-insoluble species, is likely to be dominant in cooled flue gases (Yan et al. 2000). When oxidizing combustion conditions exist or when the chlorine content of the coal is high, $\mathrm{AsCl}_{3}$ is most likely to form (Yan et al. 2000). $\mathrm{AsCl}_{3}$ is unstable in water and decomposes to $\mathrm{As}(\mathrm{OH})_{3}$ (Wiberg 2001) that stabilizes in water as arsenite $\left(\mathrm{H}_{3} \mathrm{AsO}_{3}\right)$ at near neutral $\mathrm{pH}$ (Bohn 1976) and is very soluble ( 18.5 g/L) (Pokrovski et al. 1996).

In the case of $\mathrm{Se}$, it can exist as $\mathrm{SeCl}_{2}$ or $\mathrm{H}_{2} \mathrm{Se}$ at ambient temperatures after exiting the stack, with $\mathrm{H}_{2} \mathrm{Se}$ being the dominant species only when total chlorine content of the coal is low $(<0.16$ parts per million by weight (ppmw)). If the chlorine content is $>16$ ppmw, $\mathrm{SeCl}_{2}$ is the major species formed (Yan et al. 2000). $\mathrm{H}_{2} \mathrm{Se}$, a gaseous species at ambient temperature, is partially soluble in water but is unstable in solution and decomposes rapidly in the presence of oxygen to form elemental selenium - an insoluble metal (Yost 2007). $\mathrm{SeCl}_{2}$ is also unstable and is decomposed by water to form selenious acid $\left(\mathrm{H}_{2} \mathrm{SeO}_{3}\right)$ (Booth and Morfit 1862) that stabilizes as the monobasic and dibasic selenite oxyanions $\left(\mathrm{HSeO}_{3}{ }^{-}\right.$and $\left.\mathrm{SeO}_{3}{ }^{2-}\right)$ in water at near-neutral $\mathrm{pH}$ (Zhu et al. 2004). Being reactive with water, the volatile As and Se species could react with moisture present in flue gas. Condensation of water vapor on particulates in flue gases could then result in deposition of formed As and Se oxyanions on fly ash.

\subsubsection{Other Heavy Metals in Fue Gas}

Other metal species exiting with flue gases are most likely associated with sub-micron-size ash particles that are not captured with electrostatic precipitators or fabric filters (Swaine 2000). In 
fly ash, metals are part of a solid matrix consisting of mostly fused silicates and oxides (Thompson and Argent 1999). However, some metals such as $\mathrm{Cd}, \mathrm{Cu}, \mathrm{Pb}$, and $\mathrm{Zn}$ as well as As and Se are present as ionic species (Pavageau et al. 2002) and can be leached out of solution under appropriate conditions (Wang et al. 2007a). Typically, the cationic metal species such as $\mathrm{Cu}(\mathrm{II}), \mathrm{Cd}(\mathrm{II})$ and $\mathrm{Zn}(\mathrm{II})$ are more labile under acidic conditions and remain in the solid phase under neutral or alkaline conditions. The trend is opposite for other metals such as Se and As that can exist as oxyanions such as selenite and arsenite and become soluble under alkaline pH conditions (Wang et al. 2008; Wang et al. 2007b). The presence of ammonia also influences the release of metal ions into solution - ammonia forms complexes with cationic metal ions and can solubilize metals under neutral or alkaline conditions (Wang et al. 2006). These influences on metal solubilization from fly ash are especially significant for algal systems integrated with flue gases if ammonium salts are used as a nitrogen source for culture growth since algae grow under neutral to alkaline conditions.

Although fly ash capture efficiencies (using electrostatic precipitators or filters) are generally greater than 99\% (Strand et al. 2002), the sub-micron-sized particles most likely to escape usually have higher metal content than the average fly ash particles (Jakob et al. 1995), presumably due to re-deposition of vaporized metals (Pavageau et al. 2004). However, comprehensive metal content data on exclusively un-captured fly ash is lacking. Given the lack of accurate metal speciation data under the specific conditions likely in integrated algae-flue gas systems, only estimates of potential metal concentrations can be made based on reported information. One approach to derive these estimates is to assume that in the extreme case, metals contained in $1 \%$ of the fly ash generated during coal combustion would be injected into algal reactors and would progressively accumulate in the growth media when the water is recycled. Under these conditions, the actual amount of fly ash that is likely to eventually accumulate in the algal bioreactors can be calculated using a mass balance approach with the following additional assumptions:

- Volume of flue gas generated per $\mathrm{kg}$ of coal burned $=5000 \mathrm{~L}$

- $\quad$ Total ash content of coal $=10 \%$

- $\quad$ Fly ash content of total ash $=90 \%$

- Fraction of fly ash present in flue gas entering into algal reactor $=1 \%$ 
- Gas flow rate into algae reactor $=110 \mathrm{~mL} / \mathrm{s}$ (corresponds to a sparge rate of $1.5 \mathrm{vvm}$ (vessel volume per minute)

- Total duration of one algae harvesting cycle = 10 days

Using the above information and assumptions, the amount of fly ash present in the algal reactors at the end of one harvesting cycle comes out to be $\sim 0.2 \mathrm{~g}$. Using highest reported values of metal content in fly ash (Table 2-24, column 1) (Committee on Mine Placement of Coal Combustion Wastes 2006), metal concentrations in the growth medium can be calculated by assuming that all the metals leach out - this enables calculation of maximum concentrations of metals that the algae are likely to be exposed to. These calculations for different water recycle conditions are shown in Table 2-24.

Table 2-24 - Maximum Metal Concentration Likely to be Present in the Algae Growth Medium

\begin{tabular}{|c|c|c|c|c|c|}
\hline \multirow{2}{*}{ Component } & \multirow{2}{*}{$\begin{array}{c}\text { Mass } \\
\text { fraction } \\
(\mathrm{mg} / \mathrm{kg})\end{array}$} & \multicolumn{4}{|c|}{$\begin{array}{l}\text { Es timated conc. In liquid if completely leached } \\
\text { (mg/L) }\end{array}$} \\
\hline & & $\begin{array}{l}\text { Assuming } 1 \\
\text { recycle }\end{array}$ & $\begin{array}{l}\text { Assuming } 5 \\
\text { recycles* }\end{array}$ & $\begin{array}{l}\text { As suming } \\
10 \text { recycles } *\end{array}$ & $\begin{array}{l}\text { As suming } \\
20 \text { recycles } *\end{array}$ \\
\hline Arsenic & 391.0 & 0.08 & 0.39 & 0.78 & 1.56 \\
\hline Cadmium & 76.0 & 0.02 & 0.08 & 0.15 & 0.30 \\
\hline Chromium & 651 & 0.13 & 0.65 & 1.30 & 2.60 \\
\hline Cobalt & 79.0 & 0.02 & 0.08 & 0.16 & 0.32 \\
\hline Copper & 655.0 & 0.13 & 0.66 & 1.31 & 2.62 \\
\hline Lead & 273.0 & 0.05 & 0.27 & 0.55 & 1.09 \\
\hline Nickel & 1270.0 & 0.25 & 1.27 & 2.54 & 5.08 \\
\hline Mercury & 49.5 & 0.01 & 0.05 & 0.10 & 0.20 \\
\hline Selenium & 49.5 & 0.01 & 0.05 & 0.10 & 0.20 \\
\hline Zinc & 2200.0 & 0.44 & 2.20 & 4.40 & 8.80 \\
\hline
\end{tabular}

${ }^{*}$ Recycle refers to the number of times water is reused after algae is harvested

\subsubsection{Influence of Heavy Metals on Algae}

Overall, heavy metals can influence algae and algae-derived products in the following ways:

- Toxicity due to metals may impede the growth of algae and/or their lipid production abilities.

- If metals are taken up by algae partition into the lipid fraction, the quality of the fuel may be compromised and may not meet regulatory requirements. 
- Partition of metals into the protein or other nutritional components of algal biomass (e.g., omega-3 lipids or carotenoids) may limit viable generation of these valuable co-products.

- Accumulation and magnification of metal content in algae may also pose problems with disposal of residual post-processing material.

- Reuse of water may become limited if metal concentrations increase due to recycling.

In general, algae have a high capacity to uptake metals from solution (Mehta and Gaur 2005) and can very likely be influenced, to varying degrees, by metals present in flue gases. Metal interactions with algae involve two fundamental processes - (1) sorption of metals onto algal surfaces and (2) intracellular uptake of metals (Bates et al. 1982) (see Figure 2-46).

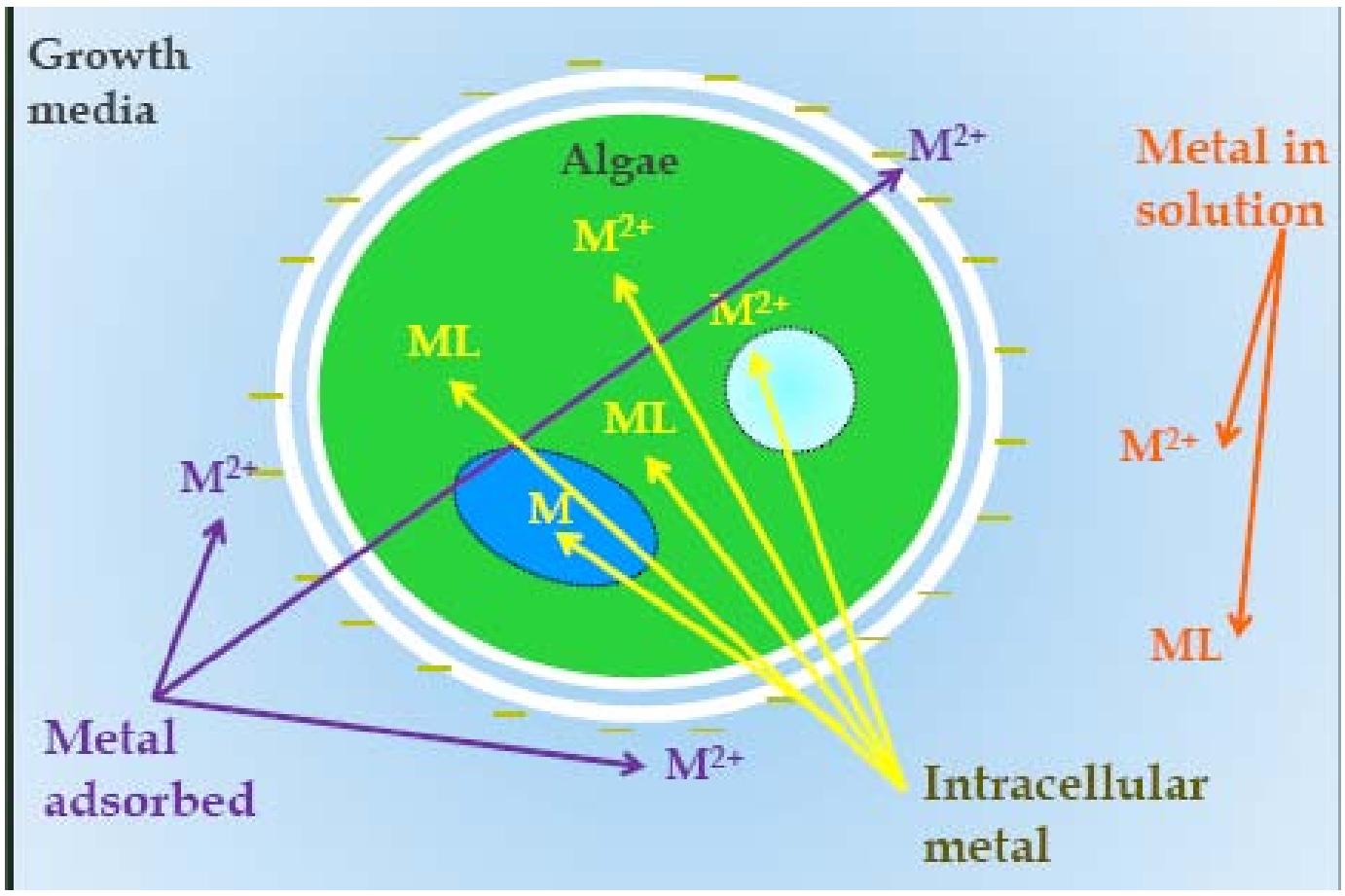

Figure 2-46 - Metal Ads orption

The sorption processes are usually reversible and involve only weak interactions with algal cell surfaces, but after cellular uptake, the associations between algae and metal species remain relatively strong and permanent (Mehta and Gaur 2005). In general, metal adsorption accounts for $>80 \%$ of the total metal associated with algal biomass during initial exposure to metals (Mehta et al. 2002), but over time adsorbed metals accumulate into the sub-cellular material (Knauer et al. 1997). 
Algae can cope with limited exposure to low concentrations of metals through various detoxification mechanisms such as induction of antioxidants, including carotenoids (Pinto et al. 2003). However, at high concentrations or under prolonged exposure, permanent cellular damage occurs, resulting in growth inhibition and cell death (Baptista and Vasconcelos 2006; Pinto et al. 2003). Although metal partitioning within algal cells is not very well understood, one study showed that in Ankistrodesmus falcatus, Sn was about $85 \%$ in the cellular polysaccharide fraction, $15 \%$ in the protein fraction, and $0.2 \%$ in lipid and low molecular weight fractions (Wong et al. 1984). Other studies have shown metal accumulation inside the vacuoles of algal cells (Gadd 1988). In addition, the presence of multiple metals affects the metal uptake and distribution characteristics. For instance, Okamura and Aoyama (Okamura and Aoyama 1994) have shown that when present in a mixture, $\mathrm{Cd}$ and $\mathrm{Cr}(\mathrm{VI})$ influence each other's concentration and distribution among membrane, cell wall, and soluble and miscellaneous fractions of Chlorella ellipsoidea. All these factors are important to consider when designing systems that that utilize flue gases for algal growth.

In order to design stable and highly productive microalgal systems integrated with flue gases, the influence of these interactions on growth, lipid synthesis and resulting biofuel quality must be understood and quantified. Proposed goals were to quantitatively establish baseline interactions between flue gas species and algae. Besides metals, interactions of $\mathrm{SO}_{\mathrm{X}}$ and $\mathrm{NO}_{\mathrm{X}}$ with algae were also to be evaluated. Simultaneous effects of multiple species were not studied, and instead the focus was on providing an accurate description of biological interactions with individual flue gas species and quantifying these effects as they relate to overall growth and lipid accumulation. The major part of the effort focused on laboratory studies under controlled and well-defined conditions; however, a small secondary effort to study algae growth with actual flue gas from a power plant was also proposed.

\subsubsection{RESULTS AND DISC USSION}

\subsubsection{Experimental Setup}

In the period of August 1 through October 31, 2010, the Utah State University (USU) research group developed an experimental set-up for growing algae on simulated flue gases from a coal-fired combustion-based power system and techniques for analysis of samples from experiments. The experimental set-up is shown in Figure 2-47, and consisted of $5 \mathrm{~L}$ vessels equipped with paddles and a sparger. A stir plate placed under the reactors rotated the stirrer 
to provide mixing. Each reactor had two double-row, 2-foot-long T12 ballasts that held the light fixtures for the reactors. Each ballast had one GE- and one Phillips-plant/aquarium fluorescent light. These were programmed to go on daily at 6:30 a.m. and shut off at 7:00 p.m. Each reactor had an inlet line for the flue gas, a sample line for doing the required daily measurements of growth, and a vent line. The $\mathrm{CO}_{2}$ in the flue gas performed two functions - (1) it served as the inorganic carbon source for algal growth and (2) it buffered the system to maintain $\mathrm{pH}$. Therefore, control of gas flow rates was critical to maintain algal growth.

Another option would be to use an external biological buffer (such as phosphate or TRIS) to keep the $\mathrm{pH}$ constant. However, use of such external buffers can lead to complexation of heavy metals, especially cationic metals like $\mathrm{Cu}^{2+}, \mathrm{Pb}^{2+}, \mathrm{Zn}^{2+}$, etc., that would alter the microbe-metal interactions and provide data that would be less relevant to envisioned large-scale commercial systems. To-date, manual control of gases maintained $\mathrm{pH}$ in the range 6.5-7.5. Future experiments would be designed to utilize an automated gas control system to maintain $\mathrm{pH}$ at $7 \pm 0.1$ and would compare reactor performance as a function $\mathrm{pH}$ controlled automatically versus manually. This test will clearly identify the importance of accurate $\mathrm{pH}$ control and the need for automated $\mathrm{pH}$ control systems in future experiments.

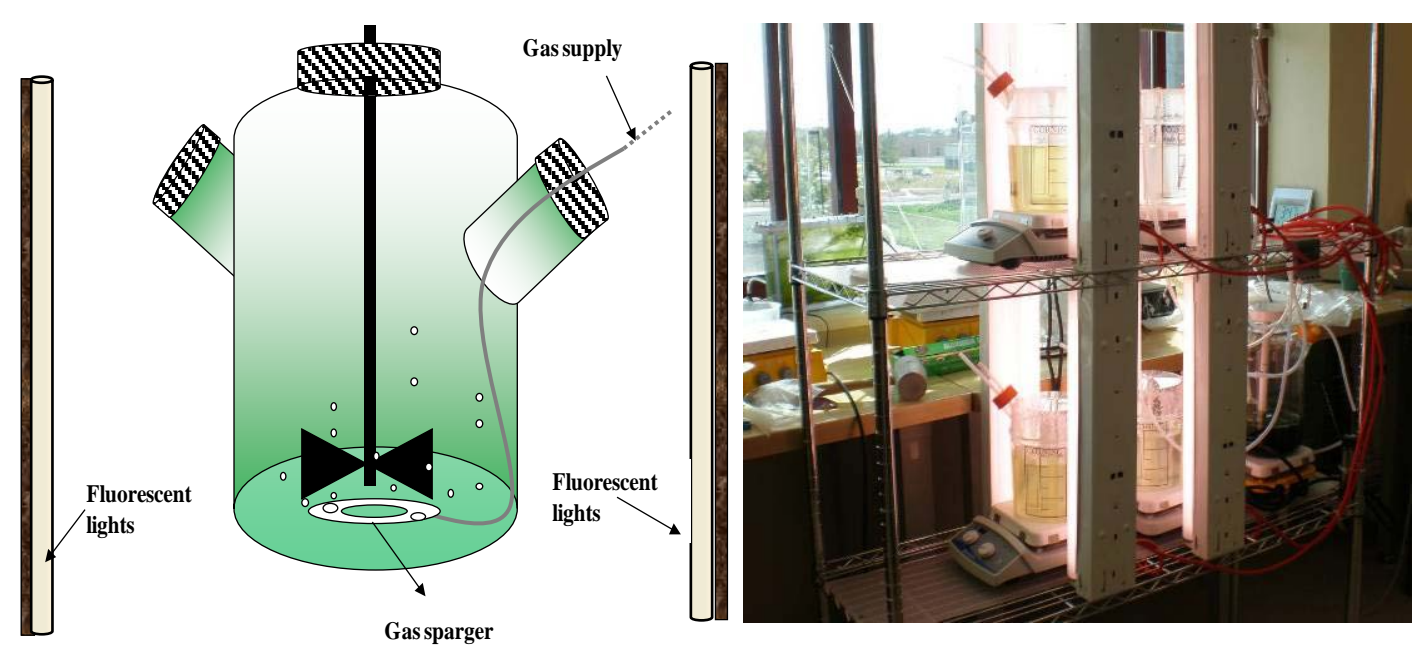

Figure 2-47 - Schematic of Experimental Reactors (Left) and Photograph of USU Experimental Set-Up (Right) 
The analytical methods that were validated and tested and included assays of total suspended solids (TSS), total phosphorus, ortho-phosphate, chemical oxygen demand (COD), anions (chloride, bromide, nitrate, nitrite, phosphate and sulfate) using ion chromatography (IC) and analysis of heavy metals (including procedures for sample digestion) using ICP-MS. Analysis of three water samples obtained from the APS Cholla and Redhawk sites have been completed using the methods developed at USU.

\subsubsection{Water Sample Analysis}

Three water samples were received from APS for analysis of metals, anions and other constituents relevant to algal growth. The samples were taken from the Redhawk cooling pond, Redhawk filter back flush (both located at a natural gas fired power plant) and Cholla Lake (located at a pulverized coal-fired power plant). Analysis of these samples enabled development of methods and calibrations useful for similar tests to be performed in samples obtained from future algae experiments.

Table 2-25 shows a comparison of USU results with measurements obtained by APS on the Redhawk pond (located at a natural gas fired power plant) and the Cholla Lake (located at a pulverized coalOfired power plant). As can be seen, most of the USU values correspond closely with data obtained independently by APS on the Cholla Lake samples. However, some difference is seen in the analysis results of the Redhawk cooling pond water. This is likely due to the difference in methods used by APS and USU. USU analysis reflects a total metal analysis obtained after thorough acid digestion of water samples and includes both soluble and insoluble metals. The APS tests on Redhawk samples were most likely performed after acidifying the samples but without any digestion. Thus the APS method might not have analyzed all the insoluble metals. It must also be noted that the samples sent to USU and the samples analyzed by APS were collected on different days although they came from the same general location.

Table 2-26, Table 2-27 and Table 2-28 below respectively show analysis results for anions, metals and other parameters in the water samples taken from the Redhawk cooling pond, Redhawk filter back flush and Cholla Lake conducted by USU. 
Table 2-25 - Comparis on of APS and USU Water Analyses on Redhawk Cooling Pond and Cholla Lake Water Sample

\begin{tabular}{|c|c|c|c|c|}
\hline \multirow[b]{2}{*}{ Parameter analyzed } & \multicolumn{2}{|c|}{ Cholla Lake } & \multicolumn{2}{|c|}{ Redhawk Cooling Pond } \\
\hline & $\begin{array}{c}\text { APS }^{1}(\mu \mathrm{g} / \mathrm{L} \\
\text { unless } \\
\text { otherwise } \\
\text { indicated) }\end{array}$ & $\begin{array}{l}\text { USU ( } \mu g / L \\
\text { unless } \\
\text { otherwise } \\
\text { indicated) }\end{array}$ & $\operatorname{APS}^{2}(\mu \mathrm{g} / \mathrm{L})$ & USU $(\mu \mathrm{g} / \mathrm{L})$ \\
\hline \multicolumn{5}{|l|}{ Metals } \\
\hline $\mathrm{Al}$ & $<100$ & 576.2 & 10.4 & 417 \\
\hline As & & 1.26 & $<\mathrm{DL}$ & 0.30 \\
\hline $\mathrm{Ba}$ & 130 & 167 & 9.23 & 9.7 \\
\hline $\mathrm{Be}$ & & 0.25 & $<\mathrm{DL}$ & 0.25 \\
\hline Co & & 0.45 & $<\mathrm{DL}$ & 3.52 \\
\hline $\mathrm{Cr}$ & & 2.6 & 6.81 & 5.9 \\
\hline $\mathrm{Cu}$ & $<50$ & 18.1 & 16.8 & 89.8 \\
\hline $\mathrm{Fe}$ & 110 & 103 & 10.1 & 260 \\
\hline $\mathrm{Mn}$ & 20 & 27.0 & $<\mathrm{DL}$ & 44.0 \\
\hline $\mathrm{Ni}$ & & 2.1 & 2.64 & 5.8 \\
\hline $\mathrm{Pb}$ & & 10.7 & $<\mathrm{DL}$ & 3.7 \\
\hline $\mathrm{Sb}$ & & 0.12 & 2.64 & 0.63 \\
\hline $\mathrm{Se}$ & & $<0.14$ & $<\mathrm{DL}$ & $<0.14$ \\
\hline $\mathrm{Ti}$ & & $<0.15$ & $<\mathrm{DL}$ & $<0.15$ \\
\hline $\mathrm{V}$ & & 6.3 & 1.67 & 4.4 \\
\hline $\mathrm{Zn}$ & & 22.2 & $<\mathrm{DL}$ & 9.96 \\
\hline \multicolumn{5}{|l|}{ Anions and others } \\
\hline $\mathrm{pH}$ & 8.2 & 7.76 & & \\
\hline $\begin{array}{c}\text { Specific conductance } \\
\text { at } 25^{\circ} \mathrm{C}, \mu \text { ohms }\end{array}$ & 3020 & 2540 & & \\
\hline $\begin{array}{c}\text { Sulfur, total, as } \mathrm{SO}_{4} \\
\text { ppm }\end{array}$ & 318 & 279.1 & & \\
\hline Chloride as $\mathrm{Cl}, \mathrm{ppm}$ & 832 & 566.6 & & \\
\hline Nitrate, as $\mathrm{NO}_{3}, \mathrm{ppm}$ & $<1$ & nd & & \\
\hline $\begin{array}{c}\text { Phosphate, total, as } \\
\mathrm{PO}_{4}, \mathrm{ppm}\end{array}$ & $<0.4$ & nd & & \\
\hline Fluoride, as F, ppm & 0.6 & 0.635 & & \\
\hline \multicolumn{5}{|c|}{$\begin{array}{l}{ }^{1} \text { Data taken from file "Cholla Lake Water Analysis rs.pdf sent to USU } \\
{ }^{2} \text { Data taken from file "ICP_APS Water sample_to Sally_032509_Red Hawk.xIs" } \\
{ }^{*} 15 x \text { dilution was necessary to bring concentration within calibration range } \\
\text { nd = not detected } \\
\text { DL = detection limit }\end{array}$} \\
\hline
\end{tabular}


Table 2-26 - Anion Analys is of Water Samples

\begin{tabular}{|c|c|c|c|c|c|c|c|}
\hline Element & $\begin{array}{l}\text { Conc } \\
\text { Units }\end{array}$ & $\begin{array}{c}\text { Redhawk } \\
\text { pond }\end{array}$ & $\begin{array}{l}\text { Standard } \\
\text { deviation }\end{array}$ & $\begin{array}{l}\text { Redhawk } \\
\text { filter back } \\
\text { flush }\end{array}$ & $\begin{array}{l}\text { Standard } \\
\text { deviation }\end{array}$ & $\begin{array}{l}\text { Cholla } \\
\text { Lake }\end{array}$ & $\begin{array}{l}\text { Standard } \\
\text { deviation }\end{array}$ \\
\hline $\mathrm{F}^{-}$ & $\mathrm{mg} / \mathrm{L}$ & 0.911 & 0.442 & 1.277 & 0.241 & 0.635 & 0.013 \\
\hline $\mathrm{Cl}^{-}$ & $\mathrm{mg} / \mathrm{L}$ & 386.002 & 8.326 & 373.162 & 12.807 & 566.625 & 2.772 \\
\hline $\mathrm{NO}_{2}^{-}$as $\mathrm{N}$ & $\mathrm{mg} / \mathrm{L}$ & nd & & nd & & nd & \\
\hline $\mathrm{Br}^{-}$ & $\mathrm{mg} / \mathrm{L}$ & 1.307 & 0.113 & 1.196 & 0.003 & nd & \\
\hline $\mathrm{NO}_{3}{ }^{-}$as $\mathrm{N}$ & $\mathrm{mg} / \mathrm{L}$ & 2.696 & 0.004 & 3.543 & 0.027 & nd & \\
\hline $\mathrm{PO}_{4}{ }^{-}$as $\mathrm{P}$ & $\mathrm{mg} / \mathrm{L}$ & nd & & nd & & nd & \\
\hline $\mathrm{SO}_{4}^{-}$ & $\mathrm{mg} / \mathrm{L}$ & 201.253 & 13.198 & 189.507 & 8.041 & 279.099 & 5.992 \\
\hline
\end{tabular}

Table 2-27 - Total Metal Analys is of Water Samples

\begin{tabular}{|c|c|c|c|c|c|}
\hline \multirow{2}{*}{ Element } & \multicolumn{1}{c}{$\begin{array}{c}\text { Conc. } \\
\text { units }\end{array}$} & $\begin{array}{c}\text { Redhawk } \\
\text { pond }\end{array}$ & $\begin{array}{c}\text { Redhawk } \\
\text { filter Back } \\
\text { flush }\end{array}$ & Cholla Lake & $\begin{array}{c}\text { Method } \\
\text { detection } \\
\text { limit }(\mu \mathrm{g} / \mathrm{L})\end{array}$ \\
\hline $9 \mathrm{Be}$ & $\mu \mathrm{g} / \mathrm{L}$ & 0.25 & 1.01 & 0.25 & 0.13 \\
\hline $27 \mathrm{Al}$ & $\mu \mathrm{g} / \mathrm{L}$ & 417 & 31030 & 576.2 & 6.37 \\
\hline $51 \mathrm{~V}$ & $\mu \mathrm{g} / \mathrm{L}$ & 4.4 & 18.2 & 6.3 & 0.11 \\
\hline $52 \mathrm{Cr}$ & $\mu \mathrm{g} / \mathrm{L}$ & 5.9 & 18.9 & 2.6 & 0.10 \\
\hline $55 \mathrm{Mn}$ & $\mu \mathrm{g} / \mathrm{L}$ & 44.0 & 269 & 27.0 & 0.28 \\
\hline $56 \mathrm{Fe}$ & $\mu \mathrm{g} / \mathrm{L}$ & 260 & 5731 & 103 & 3.08 \\
\hline $59 \mathrm{Co}$ & $\mu \mathrm{g} / \mathrm{L}$ & 3.52 & 2.62 & 0.45 & 0.11 \\
\hline $60 \mathrm{Ni}$ & $\mu \mathrm{g} / \mathrm{L}$ & 5.8 & 20.0 & 2.1 & 0.25 \\
\hline $63 \mathrm{Cu}$ & $\mu \mathrm{g} / \mathrm{L}$ & 89.8 & 32.2 & 18.1 & 0.35 \\
\hline $66 \mathrm{Zn}$ & $\mu \mathrm{g} / \mathrm{L}$ & 9.96 & 30.3 & 22.2 & 2.90 \\
\hline $75 \mathrm{As}$ & $\mu \mathrm{g} / \mathrm{L}$ & 0.30 & 2.56 & 1.26 & 0.04 \\
\hline $78 \mathrm{Se}$ & $\mu \mathrm{g} / \mathrm{L}$ & $<0.14$ & $<0.14$ & $<0.14$ & 0.14 \\
\hline $111 \mathrm{Cd}$ & $\mu \mathrm{g} / \mathrm{L}$ & $<0.18$ & $<0.18$ & $<0.18$ & 0.18 \\
\hline $121 \mathrm{Sb}$ & $\mu \mathrm{g} / \mathrm{L}$ & 0.63 & 0.73 & 0.12 & 0.17 \\
\hline $137 \mathrm{Ba}$ & $\mu \mathrm{g} / \mathrm{L}$ & 9.7 & 226 & 167 & 0.27 \\
\hline $205 \mathrm{Ti}$ & $\mu \mathrm{g} / \mathrm{L}$ & $<0.15$ & $<0.15$ & $<0.15$ & 0.15 \\
\hline $208 \mathrm{~Pb}$ & $\mu \mathrm{g} / \mathrm{L}$ & 3.7 & 13.8 & 10.7 & 0.14 \\
\hline
\end{tabular}


Table 2-28 - Analys is of Other Parameters on Water Samples

\begin{tabular}{|c|c|c|c|c|}
\hline Tes t & Units & $\begin{array}{l}\text { Redhawk } \\
\text { pond }\end{array}$ & $\begin{array}{l}\text { Redhawk } \\
\text { Filter Back } \\
\text { flush }\end{array}$ & Cholla Lake \\
\hline $\mathrm{pH}$ & & 6.66 & 6.17 & 7.76 \\
\hline Electrical conductivity & $\mu \mathrm{S} / \mathrm{cm}$ & 1877.00 & 1880.00 & 2540.00 \\
\hline Total Phosphorous & $\mathrm{mg} / \mathrm{L}$ & 0.51 & 1.70 & 0.43 \\
\hline Chemical oxygen demand & & 89.33 & 223.00 & 85.00 \\
\hline
\end{tabular}

\subsubsection{Growth Studies with Neochloris}

Growth studies were performed in the stirred reactors described above with manual pH control. The $N$. oleoabundans strain was grown in a modified Bristol medium. At the time of the study, the specific strain to be utilized for the future pilot-scale tests (i.e. Scenedesmus) at APS had not yet been decided, so a readily available strain was utilized to develop methodologies and observe trends. The composition of this medium was: $\mathrm{NaNO}_{3}(3 \mathrm{mM}), \mathrm{K}_{2} \mathrm{HPO}_{4}(1.4 \mathrm{mM})$, $\mathrm{MgSO}_{4} .7 \mathrm{H}_{2} \mathrm{O}(0.3 \mathrm{mM})$, urea $(1 \mathrm{mM}), \mathrm{CaCl}_{2} .2 \mathrm{H}_{2} \mathrm{O}(0.17 \mathrm{mM}), \mathrm{NaCl}(0.43 \mathrm{mM})$, ferric ammonium citrate (15 mg/L). Medium pH was adjusted to 7.5. This was a minimal mineral medium and contains only defined chemical components without growth factors like vitamins or amino acids. Thus, metabolic processes were easy to observe and quantify when cultures were grown in this medium. Results from growth studies are shown in Figure 2-48. Changes in optical density and Total Suspended Solids (TSS) were monitored over time to generate a growth curve. Final biomass concentrations obtained in these tests were $0.8 \mathrm{~g} / \mathrm{L}$, which is consistent with other tests in our lab of this organism. 


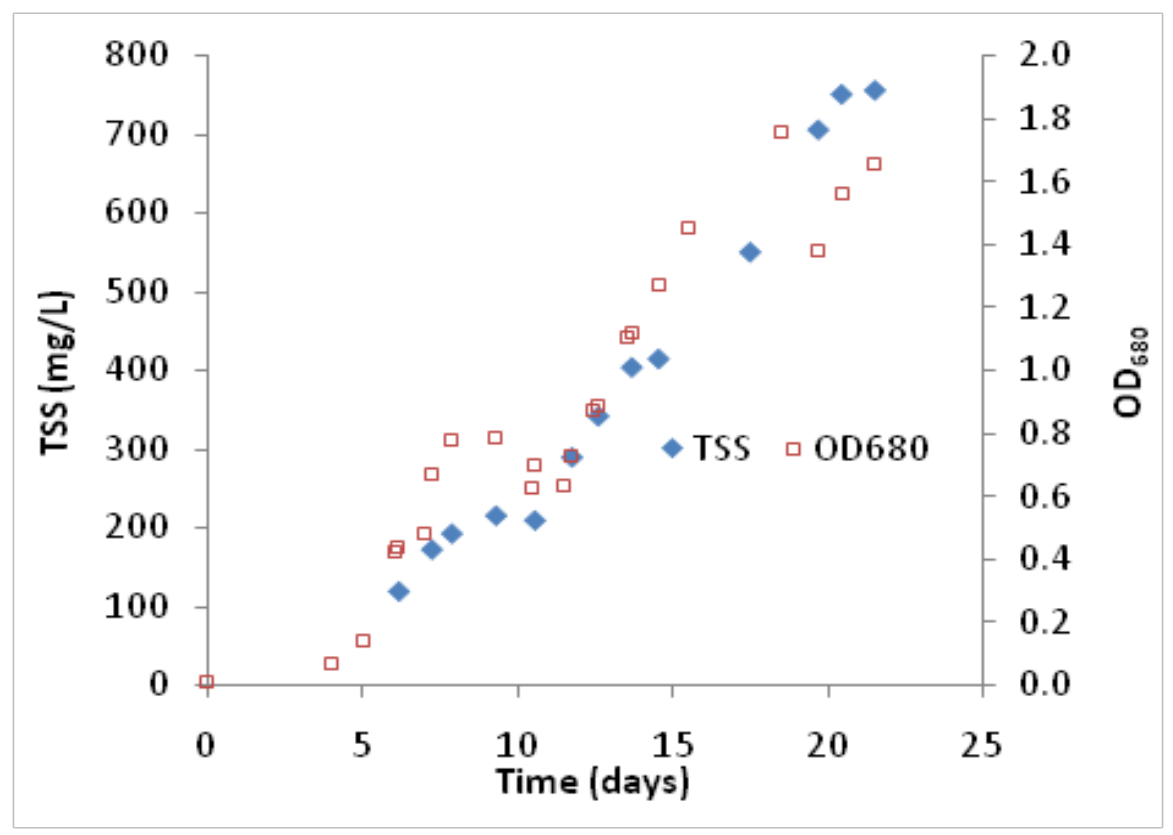

Figure 2-48 - Growth curve for Neochloris Oleoabundans

Figure 2-49 - OD-TSS Correlation for Neochloris Oleoabundans

A correlation curve between optical density (OD, measured at $680 \mathrm{~nm}$ ) and TSS values was generated to facilitate future conversion of measured OD data with actual biomass concentrations. The data plot is shown in Figure 2-49 and shows good linear correlation $\left(R^{2}=\right.$ 0.92), suggesting that simple OD measurements can be used to obtain confident estimates of culture density. Additionally, in this time period initial culturing of Scenedesmus obliquus, the 
strain of focus at APS, was completed in preparation for more comprehensive tests with simulated flue gases to be described below.

\subsubsection{Metal Uptake Study with Scenedesmus}

In the period of January 1 through March 31, 2010 cellular uptake experiments were performed to account for the distribution of heavy metals after prolonged exposure of the algae. Scenedesmus obliquus cultures were grown in the presence of metals. During growth, active metabolic transport occurs, as well as changes in cell size and number. Solution chemistry of the culture medium also occurs due to depletion of nutrients. All these factors can affect metal uptake. To test cellular uptake under growth conditions and determine effects on growth and lipid production, a culture of Scenedesmus obliquus was grown in $3 \mathrm{~L}$ reactors, with 12 hour light cycles. Three metals, $\mathrm{Zn}, \mathrm{Pb}$ and $\mathrm{Co}$, were tested in duplicate. A control with algae and no metal addition was used to monitor baseline growth. Cell free controls for each metal were also tested for abiotic interaction of metals with growth media. Figure 2-50 shows the experimental set up indicating the metals tested. Figure 2-51 shows a schematic of the procedure for testing cellular uptake. Metals were introduced using salts: $\mathrm{ZnCl}_{2}, \mathrm{PbCl}_{2}$ and $\mathrm{CoCl}_{2} \cdot 6 \mathrm{H}_{2} \mathrm{O}$. Algal cultures were to be grown in reactors and exposed to the metals for approximately 20 days until a stationary phase of growth was reached. Periodic samples were taken for analysis of metals in solution, metals adsorbed onto cell surfaces and intracellular metal. 


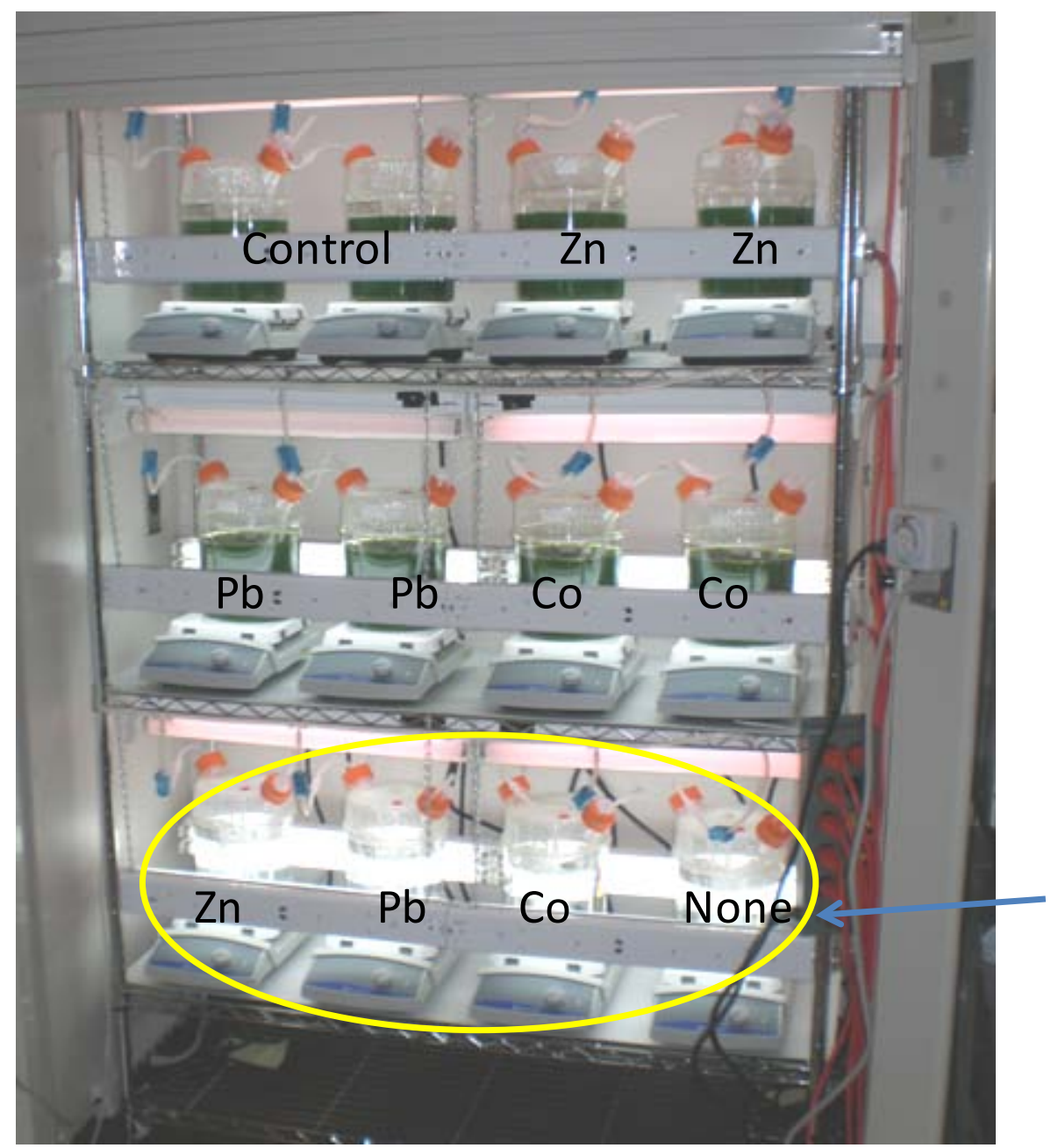

Algae-free media + metal

Figure 2-50 - Experimental Set-Up for Testing of Zn, Pb, and Co 


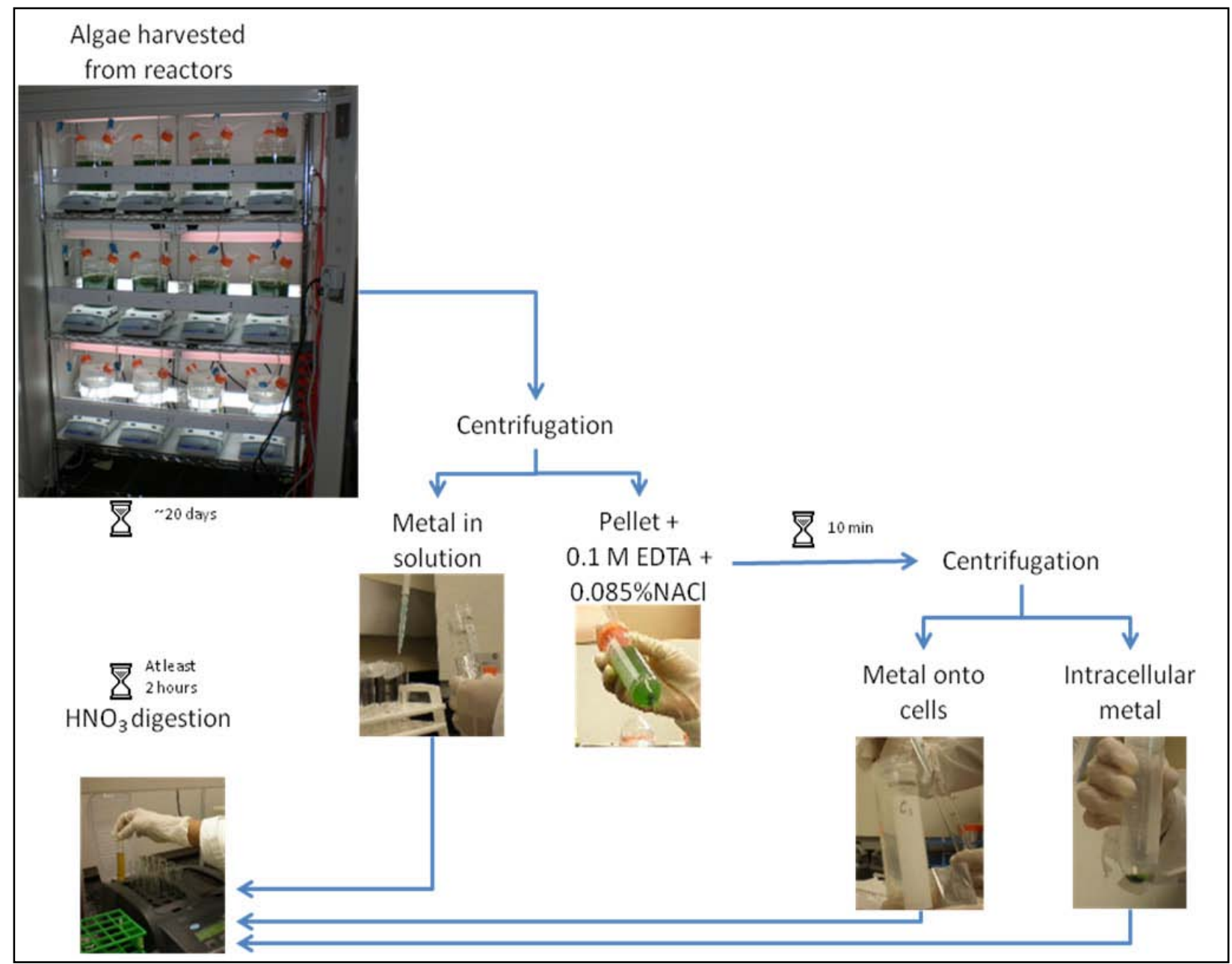

Figure 2-51 - Experimental Procedure for Analys is of Cellular Uptake of Metals

The culture grew for 18 days until it reached steady state. Figure 2-52 shows the trend of the growth curve. The control and the reactors treated with metals showed no statistically significant difference in growth. This effect may be explained due to complexation of the heavy metals with other compounds in the media, and possibly precipitation, reducing bioavailabity of ion metals $\left(\mathrm{Zn}^{2+}, \mathrm{Pb}^{2+}, \mathrm{Co}^{2+}\right)$ that cause the cellular damage. More detailed observation of the media-compound speciation was being performed at the time of this report. Secondary efforts to study algae growth with actual flue gas from a power plant were also planned.

The $\mathrm{pH}$ was controlled by manual adjustment of the $\mathrm{CO}_{2}$ input in the reactors. Figure 2-53 shows the variation of the $\mathrm{pH}$ along the period of growth. Average $\mathrm{pH}$ for reactors with biomass was $6.95 \pm 0.05$ (desired $\mathrm{pH}$ was 7 ), and all the reactors with biomass followed similar trends. The abiotic reactors did not have $\mathrm{CO}_{2}$ sparging and the $\mathrm{pH}$ stayed close to initially adjusted values. 


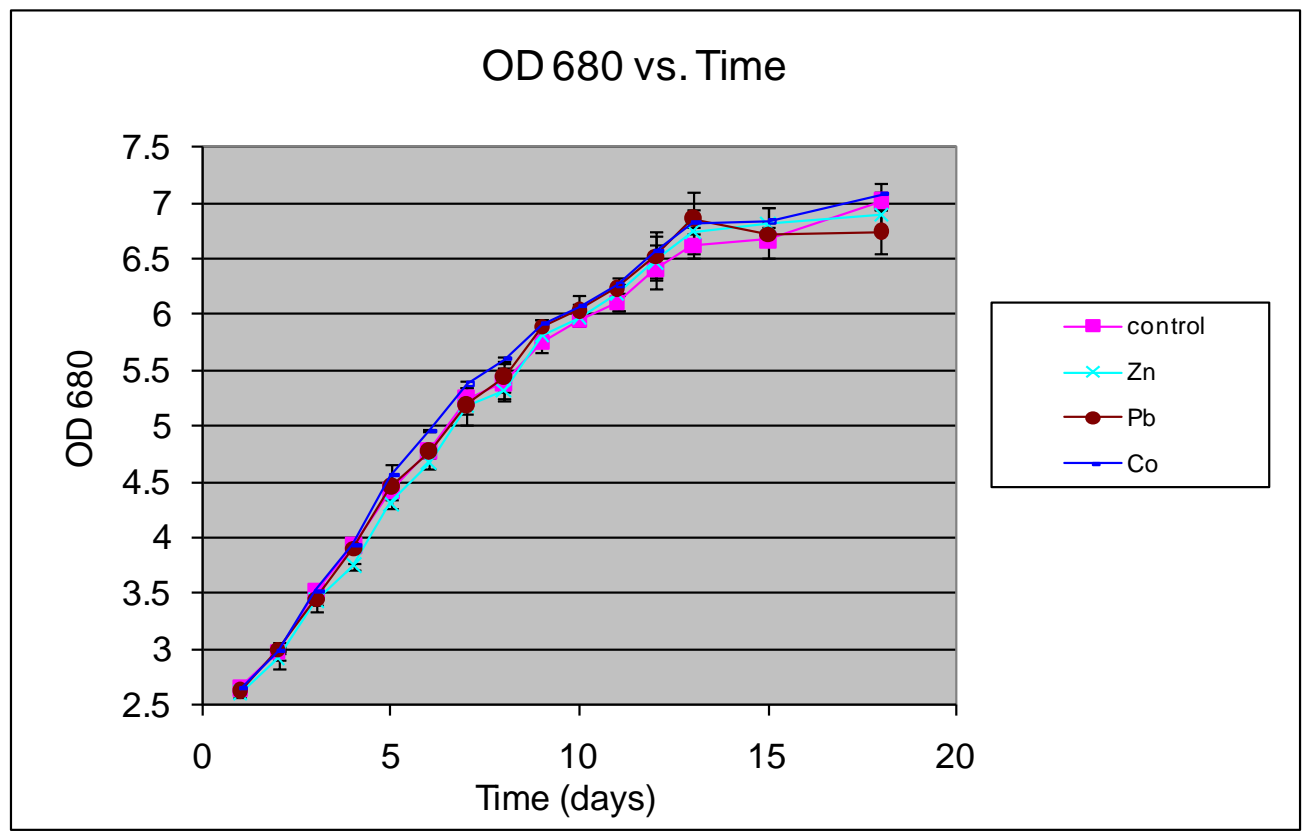

Figure 2-52 - Growth Curve of Duplicate Reactors (Error Bars as Standard Deviation)

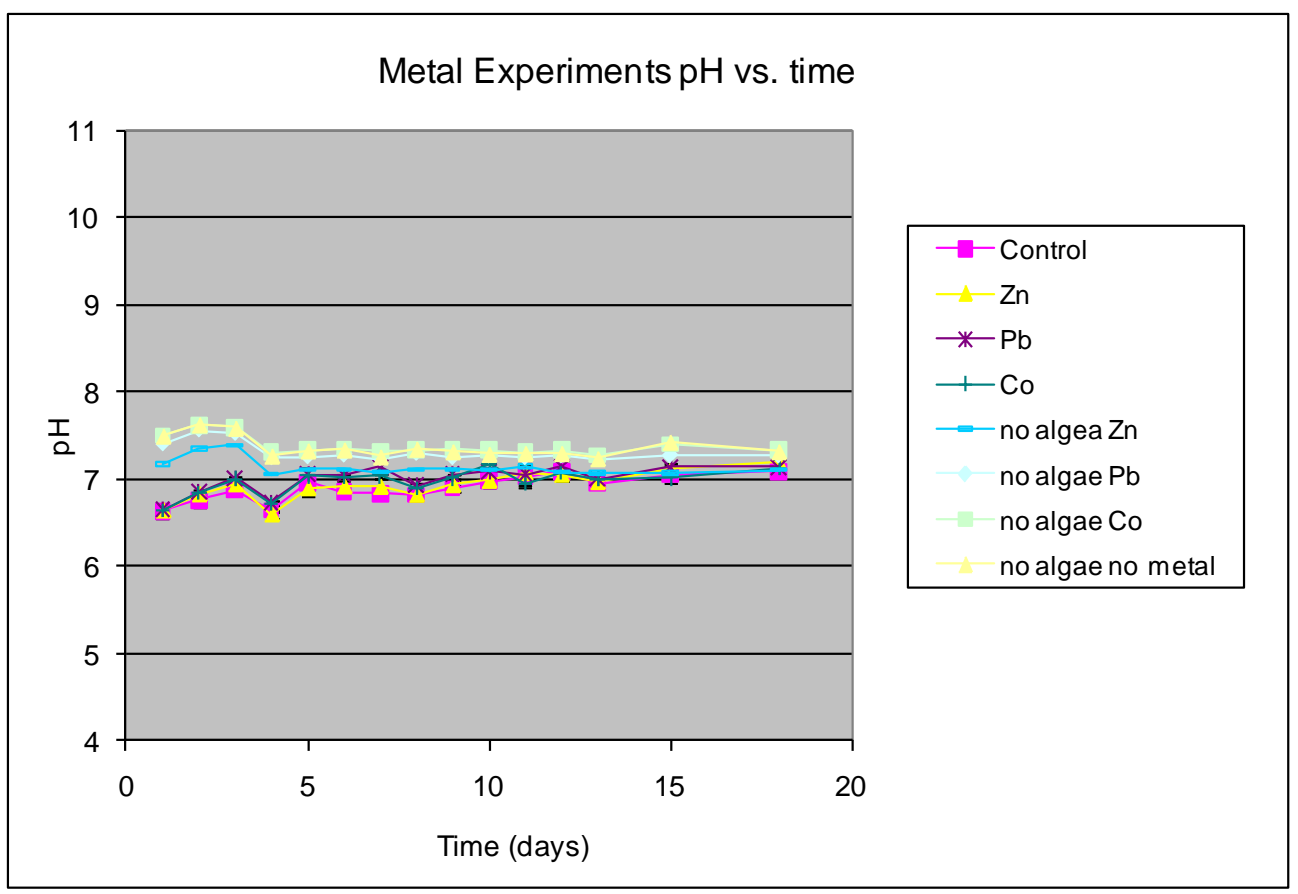

Figure 2-53 - pH Variation along the Growth Time Frame

Measurement of TSS was also done to ensure the validity of the calibration equation and increase its applicable range. Figure 2-54 shows combined data from previous and current reactors. It is observed that the new calibration equation range is $0-2500 \mathrm{mg} / \mathrm{L}$ of dry biomass. 
Lipid analysis and metal analysis for samples taken from the reactors were in progress at time of reporting. Figure 2-55 shows a chromatogram obtained from a gas chromatograph lipid analysis. Preliminary calculations have shown that approximately $8 \%$ lipids (on dry biomass basis) are present in the culture.

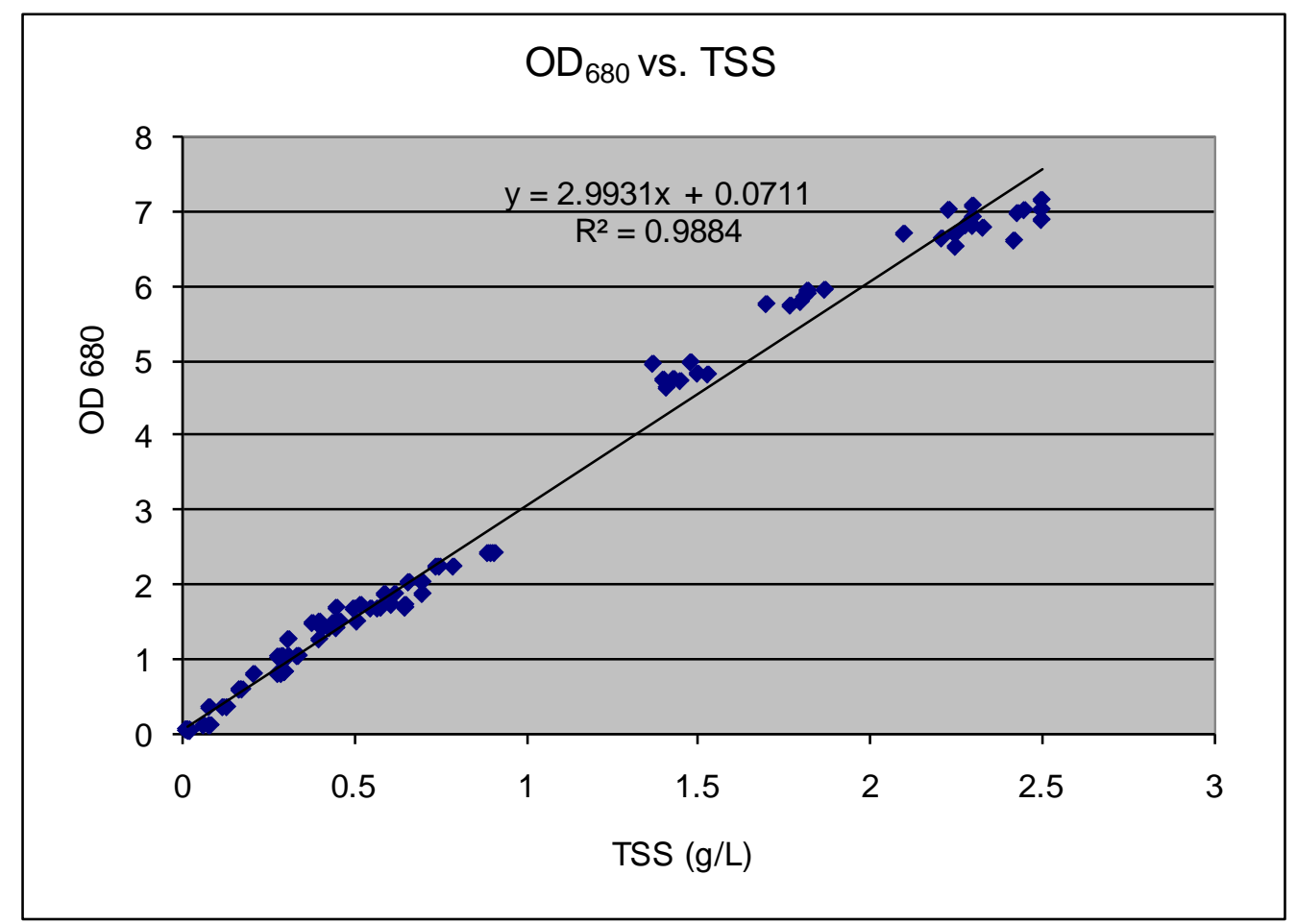

Figure 2-54 - Total Suspended Solids (TS S) with Optical Density (OD) at $680 \mathrm{~nm}$ 


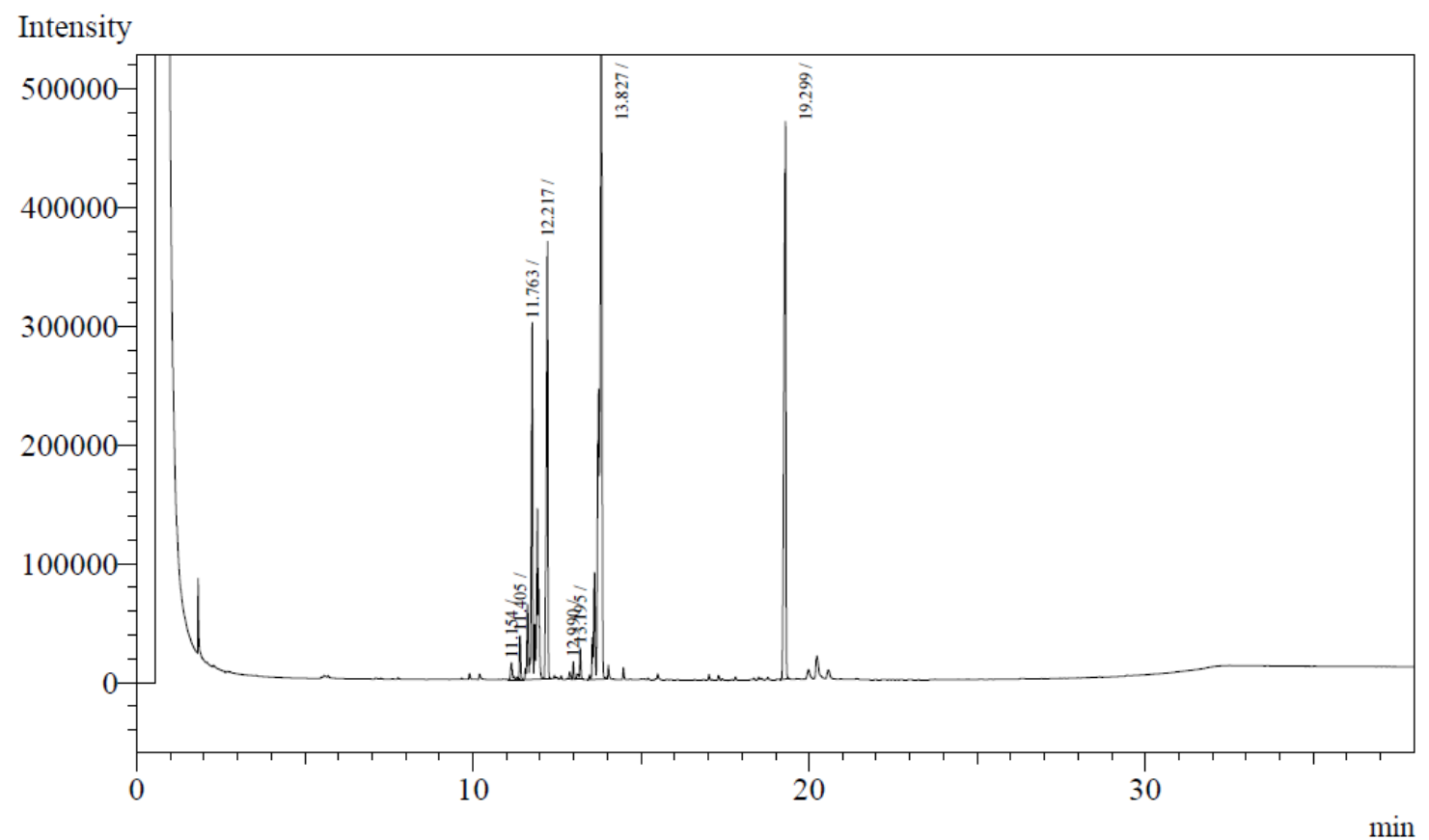

Figure 2-55 - Example of Chromatogram from Lipid Analys is of Algae Culture in Reactors At the time of project cessation (i.e., APS opted not to enter into an additional Phase of study for the project), planned work by USU was incomplete. Cellular adsorption studies were planned similar to the cellular uptake experiments but for shorter trial periods ( 1 week as opposed to 20 days), and tests to determine metal effects on lipid accumulation were underway at the time of last reporting. The aim of the adsorption experiments was to determine the sorption capacity of Scenedesmus obliquus and also determine the suitability of using sorption predictive models. The adsorption experiments were to be done under non-growth conditions to eliminate confounding effects with cell growth. Adsorption experiments for algae are usually done under light limitation to avoid confounding with cellular uptake (usually more active during photosynthesis), and this approach would be used in order to compare data with published literature. Additionally, the future studies would include analyses with other prevalent heavy metals in flue gas (e.g., $\mathrm{Hg}, \mathrm{As}, \mathrm{Ni}$ ).

However in commercial algae systems, nutrient limitation is more likely to occur due to the natural depletion of nutrients or due to agronomic management to induce lipid production. For this reason the adsorption phenomenon under nutrient limitation will also be analyzed. Additionally, adsorption equilibrium models such the Langmuir and the Freundlich models to predict metal sorption would also be analyzed. The lipid tests were planned to determine if during the active transport of the metals into the cell, algae growth/adaptation/inhibition might be 
occurring at the same time. Many cellular components can be affected by metal uptake. Therefore, the objective of these experiments was to know the final effect of a longer exposure of the algae to metals. For this purpose, growth and lipid production were to be monitored, under growth conditions and under nutrient starvation. Lipid samples from the growth samples were being analyzed at the time of this report. In addition to these tests more metals present in flue gas other than the ones tested in the studies reported here ( $\mathrm{Co}, \mathrm{Zn}$, and $\mathrm{Pb})$ would be tested for cellular uptake, adsorption, and effects on lipid accumulation.

\subsubsection{SUMMARY OF HEAVY METALSTUDY}

Utah State University investigated and developed new procedures and methods to examine the effects of heavy metals present in flue gas on algae growth. USU also successfully tested and identified the components in Redhawk cooling pond water, Redhawk filter backflush and Cholla lake water sources. While testing was still in initial stages at the time of this report, the initial results indicated that $\mathrm{Co}, \mathrm{Zn}$, and $\mathrm{Pb}$ did not adversely affect the growth of Scenedesmus when compared with control cultures.

This work was officially terminated by APS due to the closeout of the project on March 31, 2010. Continued metal element analysis of algae culture water, algae biomass ion exchanged rinse water, algae biomass and crude algae oil would determine the deposition of metals. Metal deposition would occur in water, via physical adsorption on the algae biomass cell wall, or inside the algae cell.

\subsubsection{REFRENCES FOR CHAPIER 2.6}

- Andersson ME, Gardfeldt K, Wangberg I, Stromberg D. 2008. Determination of Henry's law constant for elemental mercury. Chemosphere 73(4):587-592.

- Babich H, Stotzky G. 1980. Environmental factors that influence the toxicity of heavy metals and gaseous pollutants to microorganisms. Crc Critical Reviews in Microbiology 8(2):99-145.

- Baptista MS, Vasconcelos MT. 2006. Cyanobacteria metal interactions: Requirements, toxicity, and ecological implications. Critical Reviews in Microbiology 32(3):127-137.

- Bates SS, Tessier A, Campbell PGC, Buffle J. 1982. Zinc adsorption and transport by Chlamydomonas variabilis and Scenedesmus subspicatus (chlorophyceae) grown in semi-continuous culture. Journal of Phycology 18(4):521-529.

- Benemann JR. Utilization of carbon dioxide from fossil-fuel burning power plants with biological systems. 1993. Pergamon-Elsevier Science Ltd. p 999-1004. 
- $\quad$ Benemann JR. 1997. CO2 mitigation with microalgae systems. Energy Conversion and Management 38:S475-S479.

- Booth JC, Morfit C. 1862. Selenium. The encyclopedia of chemistry, practical and theoretical: embracing its application to the arts, metallurgy, mineralogy, geology, medicine, and pharmacy: H.C. Baird. p 978.

- $\quad$ Clever HL, Johnson SA, Derrick ME. 1985. The solubility of mercury and some sparingly soluble mercury salts in water and aqueous-electrolyte solutions. Journal of Physical and Chemical Reference Data 14(3):631-681.

- Committee on Mine Placement of Coal Combustion Wastes. Managing Coal Combustion Residues in Mines. 2006. National-Research-Council, editor. Washington, DC: The National Academies Press.

- Douskova I, Doucha J, Livansky K, Machat J, Novak P, Umysova D, Zachleder V, Vitova M. 2009. Simultaneous flue gas bioremediation and reduction of microalgal biomass production costs. Applied Microbiology and Biotechnology 82(1):179-185.

- Dukes JS. 2003. Burning buried sunshine: Human consumption of ancient solar energy. Climate Change 61: 31-44

- Gadd GM. 1988. Accumulation of metals by microorganisms and algae. In: Rehm HJ, editor. Biotechnology. Weinheim: VCH. p 401-434.

- Galbreath KC, Zygarlicke CJ, Olson ES, Pavlish JH, Toman DL. 2000. Evaluating mercury transformation mechanisms in a laboratory-scale combustion system. Science of the Total Environment 261(1-3):149-155.

- Hall B, Schager P, Lindqvist O. 1991. Chemical reactions of mercury in combustion flue gases. Water Air and Soil Pollution 56:3-14.

- Jakob A, Stucki S, Kuhn P. 1995. Evaporation of heavy metals during the heat treatment of municipal solid-waste incinerator fly ash. Environmental Science \& Technology 29(9):2429-2436.

- Kellie S, Duan YF, Cao Y, Chu P, Mehta A, Carty R, Liu KL, Pan WP, Riley JT. 2004. Mercury emissions from a $100 \mathrm{MW}$ wall-fired boiler as measured by semicontinuous mercury monitor and Ontario Hydro Method. Fuel Processing Technology 85(6-7):487499.

- Knauer K, Behra R, Sigg L. 1997. Adsorption and uptake of copper by the green alga Scenedesmus subspicatus (Chlorophyta). Journal of Phycology 33(4):596-601.

- Mastral AM, Callen MS. 2000. A review of polycyclic aromatic hydrocarbon (PAH) emissions from energy generation. Environmental Science \& Technology 34(15):30513057.

- Matsumoto H, Shioji N, Hamasaki A, Ikuta Y, Fukuda Y, Sato M, Endo N, Tsukamoto T. 1995. Carbon-dioxide fixation by microalgae photosynthesis using actual flue-gas discharged from a boiler. Applied Biochemistry and Biotechnology 51-2:681-692.

- Mehta SK, Gaur JP. 2005. Use of algae for removing heavy metal ions from wastewater: Progress and prospects. Critical Reviews in Biotechnology 25(3):113-152. 
- Mehta SK, Singh A, Gaur JP. 2002. Kinetics of adsorption and uptake of Cu2+ by Chlorella vulgaris: Influence of $\mathrm{pH}$, temperature, culture age, and cations. Journal of Environmental Science and Health Part A - Toxic/Hazardous Substances \& Environmental Engineering 37(3):399-414.

- Okamura H, Aoyama I. 1994. Interactive toxic effect and distribution of heavy metals in phytoplankton. Environmental Toxicology and Water Quality 9(1):7-15.

- Pavageau MP, Pecheyran C, Krupp EM, Morin A, Donard OFX. 2002. Volatile metal species in coal combustion flue gas. Environmental Science \& Technology 36(7):15611573.

- Pinto E, Sigaud-Kutner TCS, Leitao MAS, Okamoto OK, Morse D, Colepicolo P. 2003. Heavy metal-induced oxidative stress in algae. Journal of Phycology 39(6):1008-1018.

- Reddy MH. 2002. Application of algal culture technology for carbon dioxide and flue gas emission control [MS Thesis]. Tempe: Arizona State University. 96 p.

- Sanemasa I. 1975. The solubility of elemental mercury vapor in water. Bulletin of the Chemical Society of Japan 48(6):1795-1798.

- Senior CL, Sarofim AF, Zeng TF, Helble JJ, Mamani-Paco R. 2000. Gas-phase transformations of mercury in coal-fired power plants. Fuel Processing Technology 63 (2-3):197-213.

- Strand M, Pagels J, Szpila A, Gudmundsson A, Swietlicki E, Bohgard M, Sanati M. 2002. Fly ash penetration through electrostatic precipitator and flue gas condenser in a 6 MW biomass fired boiler. Energy \& Fuels 16(6):1499-1506.

- Swaine DJ. 2000. Why trace elements are important. Fuel Processing Technology 65:21-33.

- Thompson D, Argent BB. 1999. Coal ash composition as a function of feedstock composition. Fuel 78(5):539-548.

- USEPA. 2007. The Plain English Guide to the Clean Air Act. Research Triangle Park: USEPA. Report nr Office of Air Quality Planning and Standards EPA-456/K-07-001 http://www.epa.gov/air/caa/peg/peg.pdf.

- Wang JM, Ban H, Teng XJ, Wang H, Ladwig K. 2006. Impacts of pH and ammonia on the leaching of $\mathrm{Cu}(\mathrm{II})$ and $\mathrm{Cd}(\mathrm{II})$ from coal fly ash. Chemosphere 64(11):1892-1898.

- Wang JM, Wang T, Burken JG, Chusuel CC, Ban H, Ladwig K, Huang CP. 2008. Adsorption of arsenic (V) onto fly ash: A speciation-based approach. Chemosphere 72(3):381-388.

- Wang T, Wang HM, Ban H, Ladwig K. 2007a. Quantifying the availability and the stability of trace cationic elements in fly ash. Waste Management 27(10):1345-1355.

- Wang T, Wang J, Burken JG, Ban H, Ladwig K. 2007b. The leaching characteristics of selenium from coal fly ashes. Journal of Environmental Quality 36(6):1784-1792.

- Wiberg NH, Holleman AF, Wiberg E. 2001. Holleman-Wiberg's Inorganic Chemistry. 
- Wong PTS, Maguire RJ, Chau YK, Kramar O. 1984. Uptake and accumulation of inorganic tin by a fresh-water alga, Ankistrodesmus falcatus. Canadian Journal of Fisheries and Aquatic Sciences 41(11):1570-1574.

- Yan R, Gauthier D, Flamant G. 2000. Possible interactions between As, Se, and Hg during coal combustion. Combustion and Flame 120(1-2):49-60.

- Yanagi M, Watanabe Y, Saiki H. 1995. CO2 fixation by Chlorella sp HA-1 and its utilization. Energy Conversion and Management 36(6-9):713-716.

- Yost DM. 2007. Oxygen, Sulfur, Selenium, Tellurium and Their Compounds with Hydrogen. In: Yost DM, editor. Systematic Inorganic Chemistry: Read Books. p 440.

- Zhao YX, Mann MD, Pavlish JH, Mibeck BAF, Dunham GE, Olson ES. 2006. Application of gold catalyst for mercury oxidation by chlorine. Environmental Science \& Technology 40(5):1603-1608.

- Zhu J, Zuo W, Liang X, Li S, Zheng B. 2004. Occurrence of native selenium in Yutangba and its environmental implications. Applied Geochemistry 19(3):461-467. 


\subsection{Conclusions on Algae TESTING}

In this project APS built a large $8,000 \mathrm{~L}$ outdoor bag farm and a $\sim 1000-\mathrm{m}^{2}$ algae lab at APS $3^{\text {rd }}$ Avenue R\&D facility. The lab was equipped to monitor culture growth and process harvested algae culture for storage and shipment. In-house biomass and oil analysis protocol and facilities were established. They significantly assisted in monitoring algae cultivation, studying stressing effects on algae oil content and even facilitating strain selection.

APS demonstrated an outdoor algae cultivation and harvesting system, which included a $6 \mathrm{M}$ radius cultivator which had a total surface area of $113 \mathrm{~m}^{2}$ and a total culture volume between 10,000 L to 15,000 L; a $\mathrm{CO}_{2}$ on-demand feeding system; an on-line temperature, pH, PAR and DO collection system; and a $\sim 2$ gpm algae culture dewatering system. The $6 \mathrm{M}$ cultivation system demonstrated approximately 170 days of total operation since the onset of testing. Approximately $77,000 \mathrm{~L}$ of culture was harvested. The highest $\mathrm{CO}_{2}$ capture rate of obtained was $90 \mathrm{wt} \%$ with this $6 \mathrm{M}$ cultivator design.

Based on the in-house lab-scale algae strain selection study, Nannochloropsis oculata, Selanastrum, and Scenedesmus obliquus were determined to be the leading candidates. Among them, Scenedesmus obliquus was found to exhibit rapid growth rates, thrive under the high temperatures found in Arizona, settle naturally, and grow for long periods of time without contamination. The achieved total fat and neutral lipid levels of Scenedesmus ranged between $10 \mathrm{wt} \%$ to $20 \mathrm{wt} \%$ total fat and $9 \mathrm{wt} \%$ to $17 \mathrm{wt} \%$ neutral lipids when stressed during the test period. Continued extensive algae stressing study on Scenedesmus to biologically increase algae oil content and reduce the chlorophyll content at the $3^{\text {rd }}$ Avenue and Redhawk test facilities was performed under DOE Cooperative Agreement: DE-FE0001099, "Integrated Energy System with Beneficial $\mathrm{CO}_{2}$ Use." Please refer to its final technical closeout report for further results.

It is generally recognized that oil extraction using "green" algae is very challenging. Non-flowing green gum was obtained from all oil extraction exercises; probably caused by the interaction of chlorophyll and phosphor lipids. The one gallon Nannochloropsis extract sent to ConocoPhillips had fatty acid content only $\sim 25$ wt $\%$ and extract quality was too low for oil upgrading. Crude oil pretreatment will be required for any oil upgrading. 
Initial catalyst screening tests for the decarboxylation process were executed using model molecule Tristearin. Ni/Mg/Al catalysts showed better performance than that of $\mathrm{Pd}$ or $\mathrm{Pt}$ catalysts.

The study on effect of heavy metals on algae growth rate indicated that $\mathrm{Co}, \mathrm{Zn}$, and $\mathrm{Pb}$ did not adversely affect the growth of Scenedesmus when compared with control cultures. Continued metal element analysis of algae culture water, algae biomass ion exchanged rinse water, algae biomass and crude algae oil would determine the deposition of metals. Metal deposition would occur in water, via physical adsorption on the algae biomass cell wall, or inside the algae cell. 


\section{Preuminary Eng ineering Package and Systems Analysis FOR HYDROGASIFCATION/SUBSITUUTE NATURAL GAS COM- MERCIALSCALE FACIUTYY COMMERCIAL DESIGN}

\subsection{INTRODUCTION}

The scope of work for this project included a task to perform a systems analysis and cost engineering for a commercial scale coal-to-SNG plant based on the APS concept. The tehno-economic analysis was conducted to provide a forecast for facility equipment needs, construction costs, start-up costs and operating costs for the novel yet unproven hydrogasification / substitute natural gas (SNG) process. The plant would co-produce 119.7 MMSCFD of SNG, net electric power of 201 MWe and revenue- generating elemental sulfur product of 20.6 tons per day.

WorleyParsons was contracted to prepare this analysis for a plant based on 1000 short tons per day of dry coal feed, to evaluate the feasibility of a commercial-scale co-production facility that would co-produce SNG and electricity. The package includes a preliminary engineering and cost estimates for a commercial-scale facility. To the extent possible WorleyParsons utilized data that was collected by APS during their bench-scale hydrogasification test campaign. Whereas the experimental project was terminated by APS before the bench-scale data collection could be completed and a pilot-scale facility could be constructed under the planned follow on Cooperative Agreement DE-FE0001099 "Integrated Energy System with Beneficial Carbon Dioxide Use," only limited information was available to feed the Techno-Economic model. It is acknowledged that this is a preliminary study. The design and cost data are suited for planning and budget estimate purposes only, and are not of sufficient depth of detail to justify major capital investment.

A key observation from the study is that the source and price of hydrogen utilized for the gasification process will be a key to producing SNG at a cost that is competitive with natural gas. The APS process assumes that hydrogen is produced via electrolysis, an energy intensive process, to split water to form hydrogen and oxygen. Fifty eight percent of the costs of hydrogen production are predicted to come from the electricity costs to operate the electrolysis unit. If electricity is purchased from the grid, the resulting first year production costs for SNG from hydrogasification would be approximately $\$ 30.15 / \mathrm{MMBtu}$ (with approximately $85 \%$ of the cost of SNG coming from the hydrogen costs). This is significantly above the current price of 
natural gas of about $\$ 4$ to $\$ 5 / M M B t u$. The coal-to-SNG and electricity o-production process would become economically viable only with significant credits for utilizing renewable energy (i.e., windmills) to generate the electricity for the process, greatly decreasing the amount of hydrogen required by the process, and/or finding a lower cost method for producing hydrogen.

The Systems Analysis prepared by WorleyParson is presented in Appendix M. 


\section{APPENDIX A}

\section{Feasibility of SNG Formation through Coal Hydrogasification}


Development of a Hydrogasification Process for

Coproduction of SNG and Electrical Power from Western Coals

Final Report

THIS PAGE INTENTIONALLY LEFT BLANK 
Feasibility of SNG Formation through Coal Hydrogasification

\author{
Literature Review
}

By

Xiaolei (Sally) Sun

Arizona Public Service Company 


\section{TABLE OF CONTENTS}

1. Feasibility of SNG Formation through Coal Hydrogasification .....................5

2. Previous Work on Coal Hydrogasification (Hydropyrolysis) ..........................5

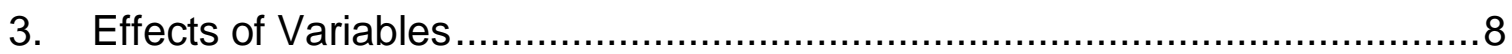

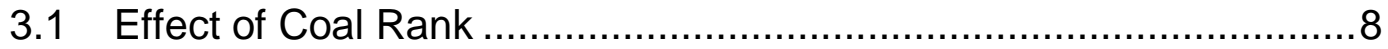

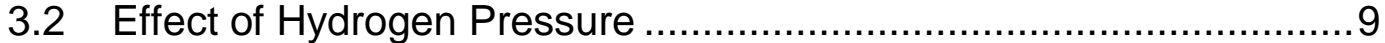

3.3 Effect of Residence Time ..................................................... 13

3.4 Effect of Temperature............................................................... 14

3.5 Effect of Coal Particle Size ........................................................17

3.6 Effect of Heating Rate ...................................................... 18

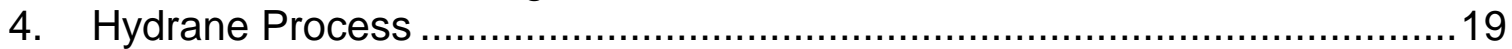

4.1 Hydrane Process..................................................................19

4.2 Advantages and Disadvantages of the Hydrane Process ..............22

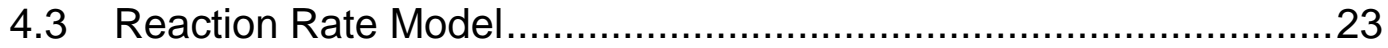

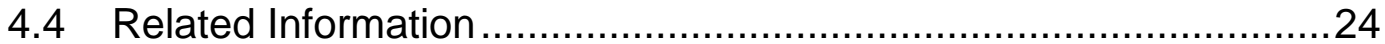

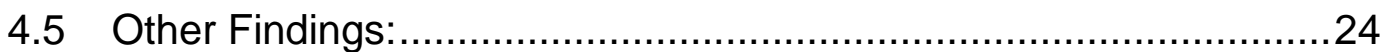

4.6 Lab/Engineer Scale Reactor Design .........................................26

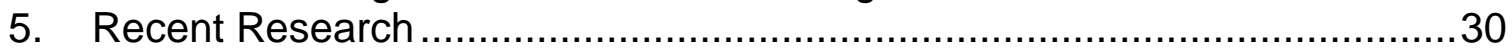

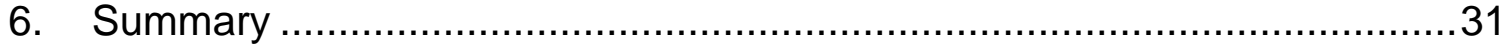

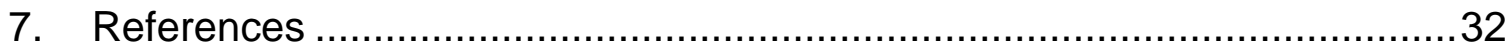

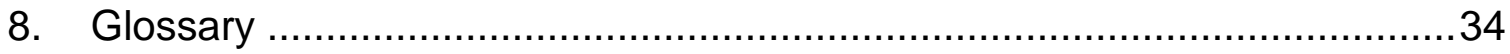




\section{List of Figures}

Figure 1 - Comparison of methane yields from hydropyrolysis and pyrolysis of Montana lignite to different peak temperatures. (Suuberg 1980)

Figure 2 - TOTAL CARBON CONVERSION AND LIQUID YIELD FROM FLASH HYDROGENATION OF DIFFERENT COALS. (CHEN 1978).

Figure 3 - Product yields at maximum conversion to BTX. Fraction converted vs. pressure. Temperature 750 to $800^{\circ} \mathrm{C}\left(1,382\right.$ to $\left.1,472^{\circ} \mathrm{F}\right)$. (Fallon 1980)......9

Figure 4 - Maximum gaseous hydrocarbon yields vs. system pressure. Temperature 875 to $900^{\circ} \mathrm{C}\left(1,607\right.$ to $\left.1,652^{\circ} \mathrm{F}\right)$; coal residence time 2.4 to 7.0 $s$ (Fallon 1980)

Figure 5 - Yield as a function of $\mathrm{H}_{2}$ pressure for Dennison medium-volatile bituminous coal: Peak temperature $825 \pm 50^{\circ} \mathrm{C}\left(1,517 \pm 122^{\circ} \mathrm{F}\right)$; Rapid heating rate $\sim 600 \mathrm{~K} / \mathrm{s}\left(\sim 1,080^{\circ} \mathrm{F} / \mathrm{s}\right) ; \bullet \mathrm{CH}_{4} ;{ }^{\circ} \mathrm{C}_{2} \mathrm{H}_{6} ;-\mathrm{C}_{6} \mathrm{H}_{6}$; ㅁ Naphthalene. (Stangeby 1981)

Figure 6 - Flash hydropyrolysis of lignite. Pressure 2500 psig; reactor temperature $825^{\circ} \mathrm{C}\left(1,517^{\circ} \mathrm{F}\right)$; preheated temperature $30^{\circ} \mathrm{C}\left(86^{\circ} \mathrm{F}\right)$. (Fallon 1980)

Figure 7 - Yield for Minto high-volatile bituminous coal: $10 \mathrm{MPa}(1,450 \mathrm{psi}) \mathrm{H}_{2}$; fast heating rate $600 \mathrm{~K} / \mathrm{s}\left(1,080^{\circ} \mathrm{Fls}\right) ; \bullet$ methane; ${ }^{\circ}$ naphthalene; - ethane; 口 benzene. (Stangeby 1981)

Figure 8 - Flash hydropyrolysis of lignite: Liquid yield vs. temperature. Pressure 2000 psig. (Fallon 1980)

Figure 9 - Flash hydropyrolysis of lignite: Maximum gaseous hydrocarbon yield $\left(\mathrm{CH}_{4}+\mathrm{C}_{2} \mathrm{H}_{6}\right)$ vs. temperature. Pressure 500 to 2500 psi; residence time 2.4 $s$ to $7.0 \mathrm{~s}$ (Fallon 1980)

Figure 10 - Formation rate of $\mathrm{CH}_{4}$ under $1.1 \mathrm{MPa}(160 \mathrm{psi}) \mathrm{H}_{2}$ : Coal, Zollverein (no. 4); heating rate, $3 \mathrm{~K} / \mathrm{min}\left(5.4^{\circ} \mathrm{F} / \mathrm{s}\right)$. ---: $\mathrm{CH}_{4}$ formation during pyrolysis in $\mathrm{N}_{2}$ (Arendt 1981)

Figure 11 - Effect of particle diameter on total yields from Pyrolysis and Hydropyrolysis of bituminous coal: •, hydropyrolysis, 69 atm (1,014 psi) H2;

$\circ$, Pyrolysis, 1 atm (14.7psi)He. (Suuberg 1980).

Figure 12 - Yield of light hydrocarbons as a function of peak temperature: Devco high-volatile bituminous coal: $1 \mathrm{~atm}(14.7 \mathrm{psi})$ helium; 20s; medium heating rate, $\sim 6000 \mathrm{~K} / \mathrm{s}\left(10,832^{\circ} \mathrm{F} / \mathrm{s}\right) ; \bullet \mathrm{CH}_{4} ;{ }^{\circ}, \mathrm{C}_{2} \mathrm{H}_{6} ;-\mathrm{C}_{2} \mathrm{H}_{4}$ (Stangeby 1981) ......19

Figure 13 - Yield of light hydrocarbons as a function of peak temperature: Devco high-volatile bituminous coal: $1 \mathrm{~atm}(14.7 \mathrm{psi})$ helium; 20s; medium heating rate, $\sim 250 \mathrm{~K} / \mathrm{s}\left(482^{\circ} \mathrm{F} / \mathrm{s}\right) ; \bullet \mathrm{CH}_{4} ;{ }^{\circ} \mathrm{C}_{2} \mathrm{H}_{6} ;-\mathrm{C}_{2} \mathrm{H}_{4}$ (Stangeby 1981) .............19

Figure 14 - Conceptual two-stage reactor. (Feldmann 1972)........................26

Figure 15 - Conceptual integrated hydrogasification unit. (Feldmann 1973) ......28

Figure 16 - Commercial Hydrane Reactor: Capacity Million scf Pipeline Gas. (Feldmann 1973) 


\section{List of Tables}

Table 1 - Flash Pyrolysis Data for North Dakota Lignite (Pyrolysis Runs at $750^{\circ} \mathrm{C}$ $\left(1382^{\circ} \mathrm{F}\right)$ ). (Sundaram 1982)....

Table 2 - Comparison of Yields from Pyrolysis and Hydropyrolysis of Montana lignite. (Suuberg 1980)

Table 3 - Comparison of Yields from Pyrolysis and Hydropyrolysis of Pittsburgh Seam Bituminous Coal. (Suuberg 1980)

Table 4 - Summary of FDR Operation Range ..........................................21

Table 5 - Operating Data for FDP Hydrogasification of Raw Coal (Feldmann 1975)

Table 6 - Typical Analyses of Coals Used in Hydrane Study. (Feldmann 1973) 24

Table 7 - Dimensional Design of FDP, Hoppers, and Fluid-Bed Reactor in Figure 14. (Feldmann 1972) 


\section{Feasibility of SNG Formation through Coal Hydrogasification}

Coal hydrogasification and coal hydropyrolysis were extensively investigated in the 1970s. In Talwalkar's U.S. Department of Energy (DOE) report (Talwalkar 1983) on coal pyrolysis, two sections included a summary of the 1970's work performed on coal pyrolysis in a hydrogen environment. The previous research efforts were primarily focused on discovering the effects of coal rank, hydrogen partial pressure, reaction temperature, heating rate, and coal particle size on coal hydrogasification. Total carbon conversion, yield of methane and other light hydrocarbon gases, light aromatic oils as well as tar were studied. Various apparatus, such as electric grids, entrained flow and fluidized beds were used in this effort. Kinetic models were described in some studies, as well as the utilization of catalysts. Another good review comes from the review of the Hydrane process. By developing the Hydrane process, Feldmann et al. spent a significant effort attempting commercialization of coal hydrogasification (Feldmann 1971; Feldmann 1972; Feldmann 1973; Feldmann 1975). Their twostage reactor design solved the raw coal agglomeration problem at elevated temperatures.

The main purpose of this review is to reexamine previous work, summarize the major discoveries from these previous studies including the highlights of the Hydrane process and therefore present the feasibility of Substitute Natural Gas (SNG) formation through coal hydrogasification process in support of future research. Due to scope limitations, catalyst involved studies are not covered in this review.

\section{Previous Work on Coal Hydrogasification (Hydropyrolysis)}

Rapid devolatilization and hydrogasification of bituminous coal were studied by Massachusetts Institute of Technology, Cambridge, Massachusetts) MIT) in the 1970s (Anthony 1976). Coal weight loss (volatile yield) was measured as a function of residence time (0-20 seconds (s)), heating rate (65-10,000 Degrees Celsius per second $\left({ }^{\circ} \mathrm{C} / \mathrm{s}\right)\left(149-18,032\right.$ Degrees Fahrenheit per second $\left.\left({ }^{\circ} \mathrm{F} / \mathrm{s}\right)\right)$, final temperature $\left(400-1100^{\circ} \mathrm{C}\left(752-2012^{\circ} \mathrm{F}\right)\right)$, total pressure $(0.0001-7 \mathrm{MPa}$ (0.015 - 1015 pounds per square inch (psi)), hydrogen partial pressure (0-7 mega Pascal (MPa) (0-1015 psi)), and particle size (70-1000 micrometer $(\mu \mathrm{m}))$. In their paper, the authors pointed out that hydrogen can interrupt the charforming sequence, thereby increasing volatile yield. They also found that volatile yield increased significantly with increasing temperature and decreasing particle 
size. Within the studied heating rate range, volatile yield increased only slightly as heating rate increased (Anthony 1976).

Early work by Dent (Dent 1944) showed that a substantial portion of the carbon in the raw coal can be converted to methane more rapidly than can the carbon in the char. Numerous experiments indicated the existence of a short-lived period of high reactivity, which was first believed simply to reflect hydrogenation of the coal's volatile matter (Birch 1960; Anthony 1976). Similarly, in Schroeder's patent (Schroeder 1962), the inventor pointed out that non-catalytic hydrogasification of raw coal can involve yields significantly exceeding the proximate volatile matter.

Fallon research group (Fallon 1980) studied the flash hydropyrolysis of lignite and sub-bituminous coals to both liquids and gaseous hydrocarbon products. Ground to 100 mesh, lignite and sub-bituminous coal were hydrogenated with preheated hydrogen in a highly instrumented 1-inch-inside-diameter by eight-foot entrained down-flow tubular reactor system designed to be operated at up to $900^{\circ} \mathrm{C}\left(1652^{\circ} \mathrm{F}\right)$ and $4000 \mathrm{psi}$. The heat-up rate of the coal particles was calculated to be in the order of 30,000 to $50,000^{\circ} \mathrm{C} / \mathrm{s}\left(54,032\right.$ to $\left.90,032^{\circ} \mathrm{F} / \mathrm{s}\right)$. Methane yield in excess of 80 percent (\%) was observed at 2500 psi and 875 to $900^{\circ} \mathrm{C}\left(1,607\right.$ to $\left.1,652^{\circ} \mathrm{F}\right)$. Coal particle residence times were in the order of 4 to $12 \mathrm{~s}$ and the effect of residence time was studied by the use of product sample taps located along the length of the reactor. Hydrogen to coal feed ratios as low as approximately 0.2 pound per pound $(\mathrm{lb} / \mathrm{lb})$ were studied and methane concentrations as high as $34 \%$ in the process stream were obtained.

Suuberg (Suuberg 1980) reported product composition after the rapid hydropyrolysis of a Montana lignite and a Pittsburgh Seam bituminous coal. Experimental conditions included temperature up to $1080^{\circ} \mathrm{C}\left(1,976^{\circ} \mathrm{F}\right)$, and holding times at peak or final temperature of 0-20 s. Most runs were performed under pure hydrogen at a pressure of $\underline{69}$ atm $(1,014 \mathrm{psi})$ with an average particle diameter of $74 \mu \mathrm{m}$. The nominal heating rate in all runs was $1000^{\circ} \mathrm{C} / \mathrm{s}$ $\left(1,832^{\circ} \mathrm{F} / \mathrm{s}\right)$. The experiment was carried out using an electric grid. Under the conditions studied, methane was the principle reaction product and the yields of light aromatic liquids were small. Comparing the methane yield from the lignite for $1 \mathrm{~atm}$ (14.7 psi) He pyrolysis and $69 \mathrm{~atm}(1,014 \mathrm{psi}) \mathrm{H}_{2}$ hydropyrolysis, Figure 1 clearly shows that the hydrogen has a substantial effect at temperatures as low as $600^{\circ} \mathrm{C}\left(1,112^{\circ} \mathrm{F}\right)$. The comparison of methane yield for $\left.69 \mathrm{~atm}(1,014 \mathrm{psi}) \mathrm{H}_{2}\right)$ hydrolysis and 69 atm $(1,014 \mathrm{psi}) \mathrm{He}$ pyrolysis, in the same figure, indicates that the increased yield of methane is far greater than could be simply attributed by the auto-hydrogenation process, which is a well-known effect whereby the yield of methane could be increased merely by increasing external inert gas pressure. 


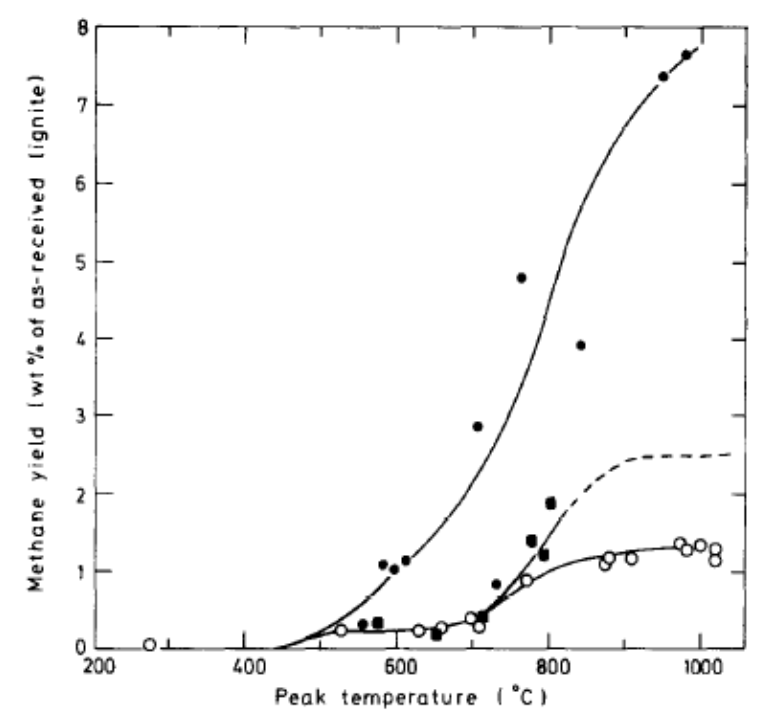

Figure 3 Comparison of methane yields from hydropyrolysis and pyrolysis of Montana lignite to different peak temperatures. [Average particle diameter, $74 \mu \mathrm{m}$; heating rate, $1000^{\circ} \mathrm{Cs}^{-1}$. Asymptote for 69 atm helium ( ---$)$ established by runs with holding times

of $2-10 \mathrm{~s}$ at the peak temperature.] $\bullet, 69 \mathrm{~atm} \mathrm{H}_{2} ; 0,1 \mathrm{~atm} \mathrm{He}$;
$\square, 69 \mathrm{~atm} \mathrm{He}$

Figure 1 - Comparison of methane yields from hydropyrolysis and pyrolysis of Montana lignite to different peak temperatures. (Suuberg 1980)

Finn et al. (Finn 1980) used a two-step hydropyrolysis process for producing Benzene, Toluene, Xylene (BTX) aromatics and light hydrocarbon gases by treating coal under $\mathrm{H}_{2}$-pressure at a lower temperature to produce highly aromatic compounds which were cracked in a separate reaction zone at more severe conditions in order to yield benzene, toluene and xylene.

Arendt et al. carried comparative investigations on coal pyrolysis using two different apparatuses (Arendt 1981), which mainly differed in the heating rates achieved (3 Kelvin per minute (K/min) (37.4 degrees Fahrenheit per minute ( $\left.{ }^{\circ} \mathrm{F} / \mathrm{min}\right)$, and $100-1000$ Kelvin per second $\left.(\mathrm{K} / \mathrm{s})\left(212^{\circ} \mathrm{F}-1,832^{\circ} \mathrm{F}\right)\right)$. The rapid heating of a small sample of finely-ground coal ( 10 milligram (mg)) was possible by using the wire net technique, where the coal particles were distributed as a layer between the folded halves of a stainless-steel screen and the wire net was heated by an electric current under a stagnant gas atmosphere at room temperature. Samples of $\sim 1$ gram (g) were investigated using a thermobalance, where the coal was fed into a perforated stainless steel basket which was connected to the thermobalance by a platinum wire and the gas and the sample were heated by a covered heating conductor that was directly attached to the outer surface of the pressurized reactor wall. During their study, the authors found that under $\mathrm{H}_{2}$, pyrolysis was influenced strongly at an elevated pressure. Additional amounts of highly aromatic products were released by hydrogenation of the coal itself, particularly between 500 to $700^{\circ} \mathrm{C}$ (932 to 
$1,292^{\circ} \mathrm{F}$ ). Due to the short residence time and $\mathrm{H}_{2}$ diffusion issues, it is also determined that the reaction was less at higher heating rates.

\section{Effects of Variables}

In the following section, the effects of coal rank, hydrogen pressure, reaction temperature, heating rate, residence time as well as coal particle size on coal hydrogasification, especially on methane (light hydrocarbon) yield and total carbon conversion, will be reviewed.

\subsection{Effect of Coal Rank}

Chen et al. (Chen 1978) investigated the flash hydrogenation yield of different coals. They concluded (as shown in Figure 2) that the product distribution from the flash hydrogenation of different coals was strongly dependent on the chemical and physical nature of the coal, as measured approximately by the rank. The total carbon conversion and yields of light paraffins decreased, while the total liquid yield increased with increasing rank. The yield of light-liquid BTX fuels was maximized for intermediate ranks, decaying for the higher and lower rank coals.

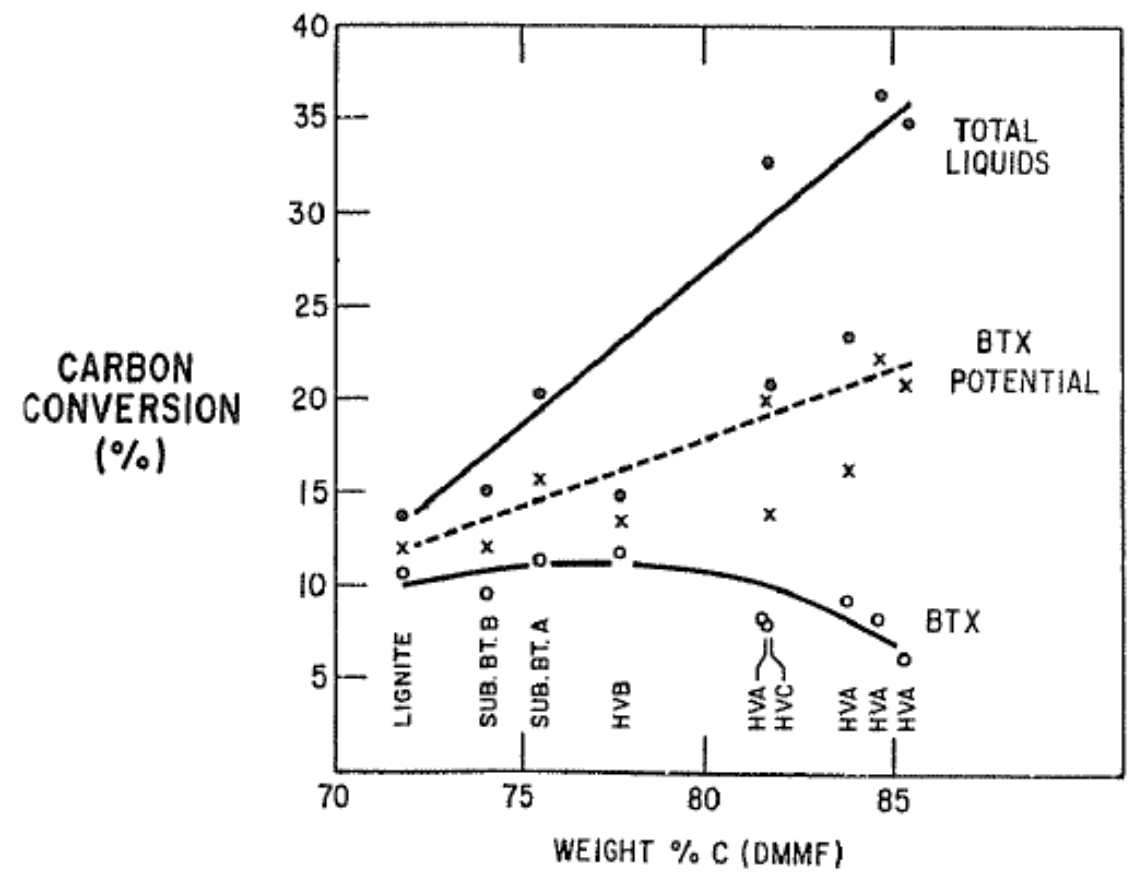

Figure 2 - Total Carbon Conversion and Liquid Yield from Flash Hydrogenation of Different Coals. (Chen 1978) 
The study of Fallon et al. on New Mexico sub-bituminous and lignite found .a constant incremental yield of BTX and gaseous hydrocarbons for sub-bituminous coal. This resulted in an overall $10 \%$ increase in yield of hydrocarbon products for sub-bituminous coal compared to the lignite coal (Figure 3).

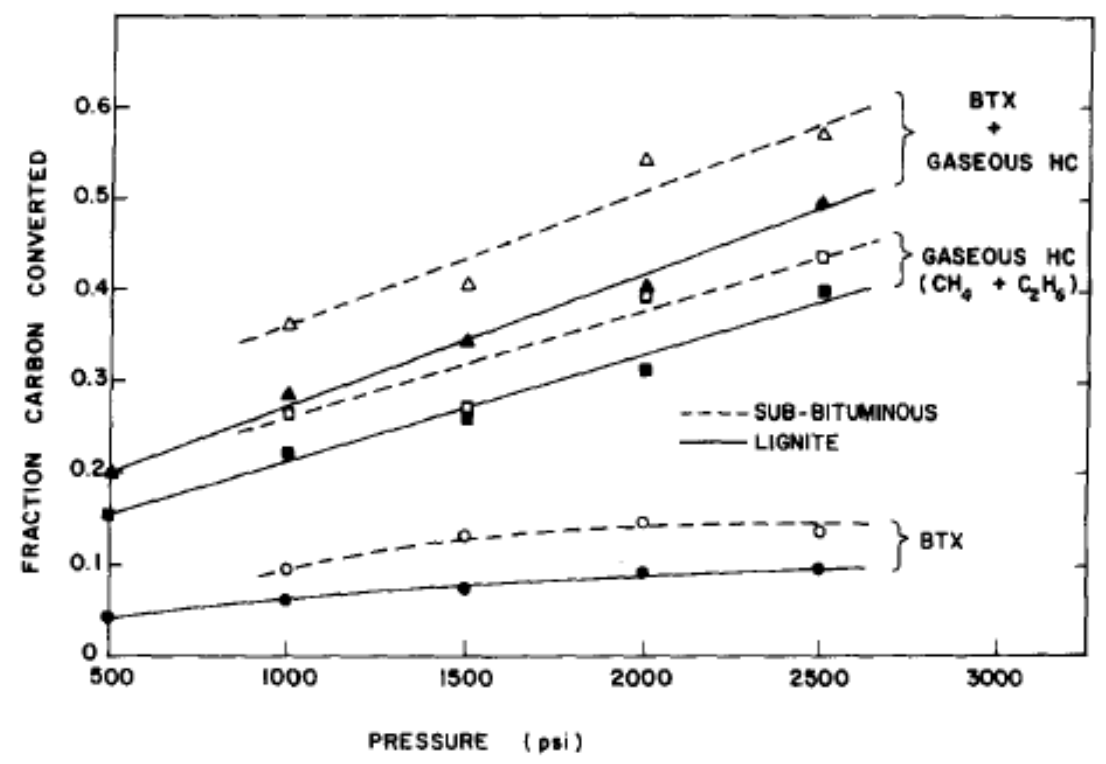

Figure 3 - Product yields at maximum conversion to BTX. Fraction converted vs. pressure. Temperature 750 to $800^{\circ} \mathrm{C}\left(1,382\right.$ to $\left.1,472^{\circ} \mathrm{F}\right)$. (Fallon 1980).

\subsection{Effect of Hydrogen Pressure}

The effect of hydrogen pressure on pyrolysis in an entrained flow reactor was demonstrated by Sundaram et al. (Sundaram 1982). Their data showed significant increases in methane, ethane, and benzene from hydropyrolysis at $750^{\circ} \mathrm{C}$, as shown in Table 1 . Total hydrocarbon yield increased from $40.4 \%$ to $53.7 \%\left(1,382^{\circ} \mathrm{F}\right)$ as testing pressure increased from 1500 psi to $2500 \mathrm{psi}$.

However, within this tested pressure range, the effect of increasing pressure on methane yield was not significant (from $24 \%$ to $26.9 \%$ ), although the relatively low methane formation could be due to a low testing temperature. 
Table 1 - Flash Pyrolysis Data for North Dakota Lignite (Pyrolysis Runs at $750^{\circ} \mathrm{C}\left(1,382^{\circ} \mathrm{F}\right)$ ) (Sundaram 1982)

\begin{tabular}{|c|c|c|c|c|c|c|}
\hline & 1500 & psi & 2000 & psi & 2500 & psi \\
\hline & $\underline{\mathrm{He}}$ & $\underline{\mathrm{H}_{2}}$ & $\underline{\mathrm{He}}$ & $\underline{\mathrm{H}_{2}}$ & He & $\underline{\mathrm{H}_{2}}$ \\
\hline $\begin{array}{l}\text { Run No. } \\
\text { Coal Feed Rate }(1 \mathrm{~b} / \mathrm{h}) \\
\text { Gas Feed Rate }(1 \mathrm{~b} / \mathrm{h}) \\
\text { Residence Time }(\mathrm{s}) \\
\text { Carbon Converted, } \%\end{array}$ & $\begin{array}{r}69 \\
1.23 \\
1.60 \\
12.3\end{array}$ & $\begin{array}{r}59 \\
1.46 \\
0.88 \\
7.4\end{array}$ & $\begin{array}{l}70 \\
1.26 \\
1.62 \\
14.3\end{array}$ & $\begin{array}{r}58 \\
1.52 \\
0.80 \\
8.7\end{array}$ & $\begin{array}{r}71 \\
1.31 \\
1.72 \\
15.2\end{array}$ & $\begin{array}{r}61 \\
1.32 \\
0.82 \\
9.6\end{array}$ \\
\hline Co & * & 7.01 & * & 8.12 & * & 6.6 \\
\hline $\mathrm{CO}_{2}$ & 7.76 & 0.44 & 8.18 & 0.32 & 7.74 & 0.26 \\
\hline $\mathrm{CH}_{4}$ & 4.28 & 24.0 & 5.45 & 27.5 & 4.99 & 26.9 \\
\hline $\mathrm{C}_{2} \mathrm{H}_{6}$ & 0.69 & 6.8 & 0.64 & 5.5 & 0.66 & 8.3 \\
\hline $\mathrm{C}_{6} \mathrm{H}_{6}$ & 0.97 & 7.3 & 1.11 & 7.6 & - & 9.0 \\
\hline $\mathrm{C}_{7} \mathrm{H}_{8}$ & 0.12 & - & 0.13 & - & 0.14 & - \\
\hline$>\mathrm{C}_{9}$ & 0.58 & 2.3 & 2.27 & 10.4 & 2.07 & 9.5 \\
\hline Total & 14.40 & 47.85 & 17.78 & 59.44 & 16.50 & 60.56 \\
\hline Total Hydrocat & 6.64 & 40.4 & 9.6 & 51.0 & 8.76 & 53.7 \\
\hline
\end{tabular}

*CO could not be measured as it has approximately the same retention time In the gas chromatograph as the heliur.

Arendt et al. (Arendt 1981) observed that with increasing pressure, tar was increasingly cracked resulting in large yields of char and light hydrocarbon gases. $\mathrm{H}_{2}$ influenced pyrolysis significantly at increased pressures. Additional amounts of highly aromatic products were released by hydrogenation of the coal itself, particularly between 500 and $700^{\circ} \mathrm{C}\left(932\right.$ and $\left.1,292^{\circ} \mathrm{F}\right)$, and the yield of light products, $\mathrm{CH}_{2}$ and $\mathrm{C}_{2} \mathrm{H}_{6}$, increased significantly. The author also pointed out that when coal was gasified with steam, high-volatile feed coal had to be degasified before being applied to the reactor. If this pretreatment was performed at high $\mathrm{H}_{2}$ pressure, a significant quantity of important gaseous and liquid products can be isolated as released compounds even if the coal was heated rapidly in an entrained phase reactor, where the residence time of the feed material and the products was short, allowing only rapid steps of hydrogenation and gasification.

The study of New Mexico sub-bituminous coal and lignite coal (Fallon 1980) indicated that greater yields of gaseous hydrocarbon product were obtained at lower pressure for sub-bituminous, as shown in Figure 4. At 1000 psi, the lignite yields approximately $35 \%$ and the sub-bituminous $55 \%$ of the gaseous products. The temperature and residence times necessary to produce the maximum gaseous products, however, were approximately the same. 


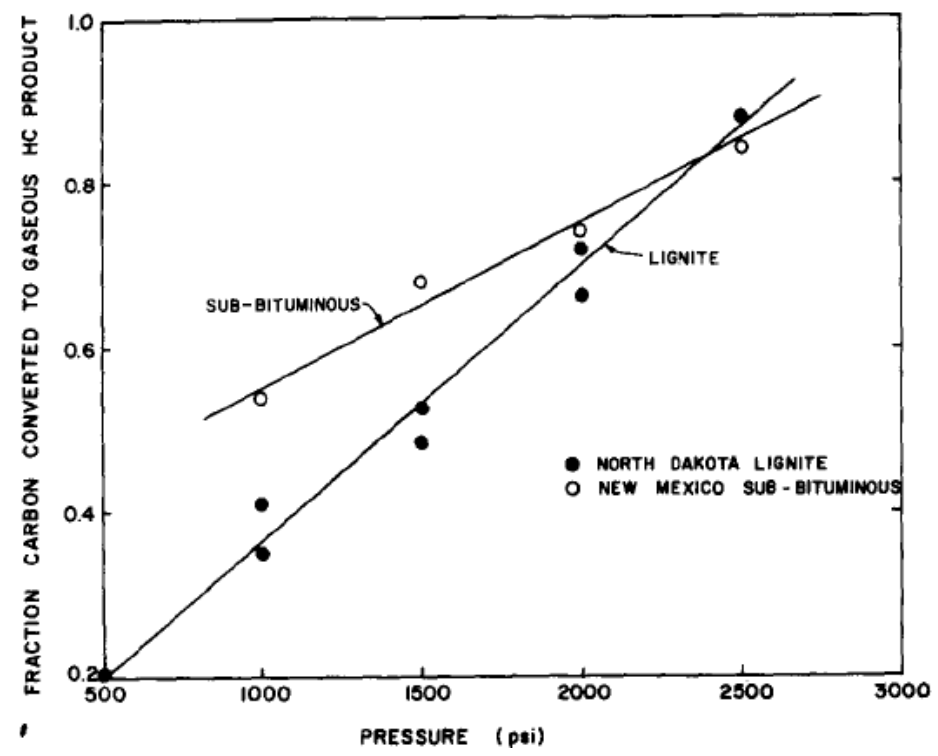

Figure 4 - Maximum gaseous hydrocarbon yields vs. system pressure. Temperature 875 to $900^{\circ} \mathrm{C}\left(1,607\right.$ to $\left.1,652^{\circ} \mathrm{F}\right)$; coal residence time 2.4 to $7.0 \mathrm{~s}$ (Fallon 1980).

The research done by Stangeby et al. (Stangeby 1981) also showed that a hydrogen pressure increase from 1-10 MPa (145-1,450 psi) caused an approximate doubling of gas and liquid yields of the coal. 


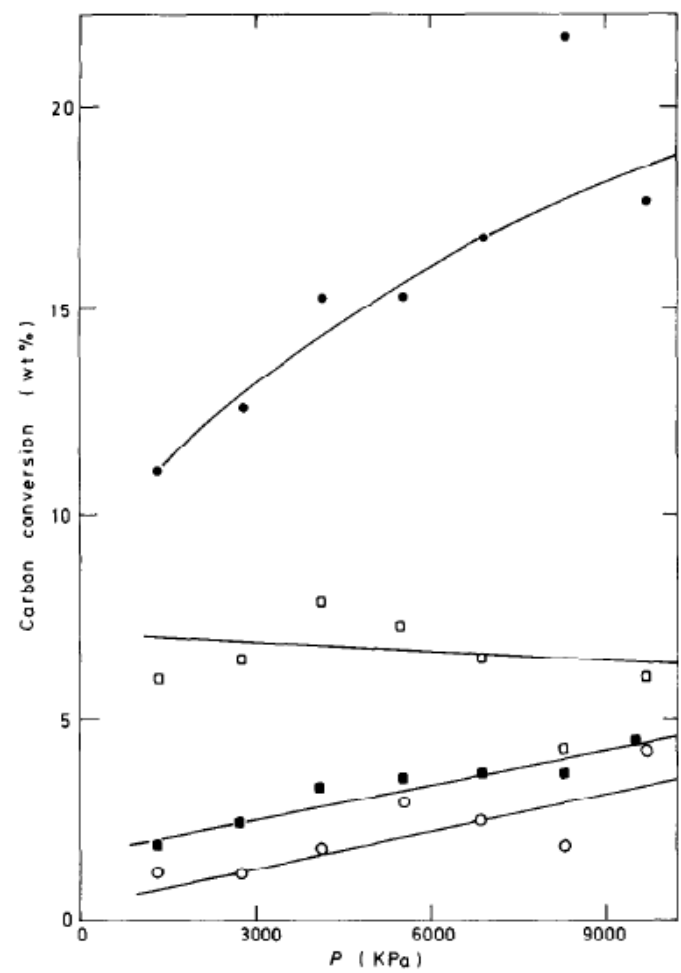

Figure 5 - Yield as a function of $\mathrm{H}_{2}$ pressure for Dennison medium-volatile bituminous coal: Peak temperature $825 \pm 50^{\circ} \mathrm{C}\left(1,517 \pm 122^{\circ} \mathrm{F}\right)$; Rapid heating rate $\sim 600 \mathrm{~K} / \mathrm{s}$ $\left(\sim 1,080^{\circ} \mathrm{F} / \mathrm{s}\right) ; \bullet \mathrm{CH}_{4} ;{ }^{\circ} \mathrm{C}_{2} \mathrm{H}_{6} ; \sim \mathrm{C}_{6} \mathrm{H}_{6}$; 口 Naphthalene. (Stangeby 1981)

Suuberg (Suuberg 1980) has compared the data from the atmospheric-pressure pyrolysis and high-pressure hydropyrolysis of the lignite and the Pittsburgh Seam bituminous. Tables 2 and 3 show the isolated effect of hydrogen on the product composition. The main increase in yield from hydropyrolysis is due to light hydrocarbon gases. 
Table 2 - Comparison of yields from pyrolysis and hydropyrolysis of Montana lignite (Suuberg 1980)

Table 2 Comparison of yields from pyrolysis and hydropyrolysis of Montana lignite. Heating rate, $1000^{\circ} \mathrm{C} \mathrm{s}^{-1}$; average particle diameter, $74 \mu \mathrm{m}$; holding time and temperature: pyrolysis under $1 \mathrm{~atm}$ $\mathrm{He}, 3-10 \mathrm{~s}$ at $\approx 900-1000^{\circ} \mathrm{C}$; pyrolysis under $69 \mathrm{~atm} \mathrm{He}, \approx 10 \mathrm{~s}$ at $875-1070^{\circ} \mathrm{C}$; hydropyrolysis under $69 \mathrm{~atm} \mathrm{H}_{2}, \approx 10 \mathrm{~s}$ at $850-1000^{\circ} \mathrm{C}$

\begin{tabular}{lccc}
\hline & \multicolumn{3}{c}{ Yield (wt \% of Lignite as-received) } \\
\cline { 2 - 4 } Product & 1 atm He & 69 atm He & 69 atm $\mathrm{H}_{2}$ \\
\hline $\mathrm{CO}$ & 9.4 & 9.0 & 7.1 \\
$\mathrm{CO}_{2}$ & 9.5 & 10.6 & 8.5 \\
$\mathrm{H}_{2} \mathrm{O}$ & 16.5 & 12.9 & 16.0 \\
$\mathrm{CH}_{4}$ & 1.3 & 2.5 & 9.5 \\
$\mathrm{C}_{2} \mathrm{H}_{4}$ & 0.6 & 0.6 & 0.2 \\
$\mathrm{C}_{2} \mathrm{H}_{6}$ & 0.2 & 0.2 & 1.4 \\
Other hydrocarbons & 0.8 & 1.7 & 4.1 \\
Tar & 5.4 & $\approx 3$ & $\approx 8$ \\
Char & 56.0 & 59.8 & 48.5 \\
\hline
\end{tabular}

Table 3 - Comparison of yields from pyrolysis and hydropyrolysis of Pittsburgh Seam bituminous coal (Suuberg 1980)

Table 3 Comparison of yields from pyrolysis and hydropyrolysis of Pittsburgh Seam bituminous coal. Heating rate, $1000^{\circ} \mathrm{Cs}^{-1}$; average particle diameter, $74 \mu \mathrm{m}$; holding time and temperature: pyrolysis under $1 \mathrm{~atm} \mathrm{He}, 2-10 \mathrm{~s}$ at $850-1000^{\circ} \mathrm{C}$; pyrolysis under $69 \mathrm{~atm} \mathrm{He}$, $2-10 \mathrm{~s}$ at $850-1070^{\circ} \mathrm{C}$; hydropyrolysis under $69 \mathrm{~atm} \mathrm{H}_{2}, 14-20 \mathrm{~s}$ at $870-930^{\circ} \mathrm{C}$

\begin{tabular}{lccc}
\hline & \multicolumn{3}{c}{ Yield (wt \% of coal as-received) } \\
\cline { 2 - 4 } Product & $1 \mathrm{~atm} \mathrm{He}$ & $69 \mathrm{~atm} \mathrm{He}$ & $69 \mathrm{~atm} \mathrm{\textrm {H } _ { 2 }}$ \\
\hline $\mathrm{CO}$ & 2.4 & 2.5 & N.M. \\
$\mathrm{CO}_{2}$ & 1.2 & 1.7 & 1.3 \\
$\mathrm{H}_{2} \mathrm{O}$ & 6.8 & 9.5 & $\mathrm{~N} . \mathrm{M}$. \\
$\mathrm{CH}_{4}$ & 2.5 & 3.2 & 23.2 \\
$\mathrm{C}_{2} \mathrm{H}_{4}$ & 0.8 & 0.5 & 0.4 \\
$\mathrm{C}_{2} \mathrm{H}_{6}$ & 0.5 & 0.9 & 2.3 \\
$\mathrm{C}_{3} \mathrm{H}_{6}+\mathrm{C}_{3} \mathrm{H}_{8}$ & 1.3 & 0.7 & 0.7 \\
Other hydrocarbon gases & 1.3 & 1.6 & 2.0 \\
Light HC liquids & 2.4 & 2.0 & 5.3 \\
Tar & 23 & 12 & 12 \\
Char & 53.0 & 62.4 & 40.2 \\
\hline
\end{tabular}

${ }^{a}$ N,M, - Not measured

\subsection{Effect of Residence Time}

Suuberg et al. pointed out in their paper (Suuberg 1980) that there was general agreement in the literature that the high yields of light aromatic oils frequently obtained during hydropyrolysis were a result of a general sequence of reactions: 


$$
\text { coal } \stackrel{\text { pyrolysis }}{\longrightarrow} \text { tarvapour } \stackrel{\text { hydrocracking }}{\longrightarrow} \text { lightoils . }
$$

When the vapor product residence times were almost zero, there was little opportunity for the hydro-cracking reactions to occur, which would lead to low yields of light aromatics and high yields of heavy tars.

Figure 6 (Fallon 1980) also shows that longer residence time favors light hydrocarbons especially methane formation. The formation of ethane and light aromatics monotonically decreases with longer residence time. In the same study, Fallon et al. also found that the residence time directly affected the extent of decomposition. At shorter residence times, the reaction couldn't reach completion; and at longer residence times, decomposition to the methane reduced total yields.

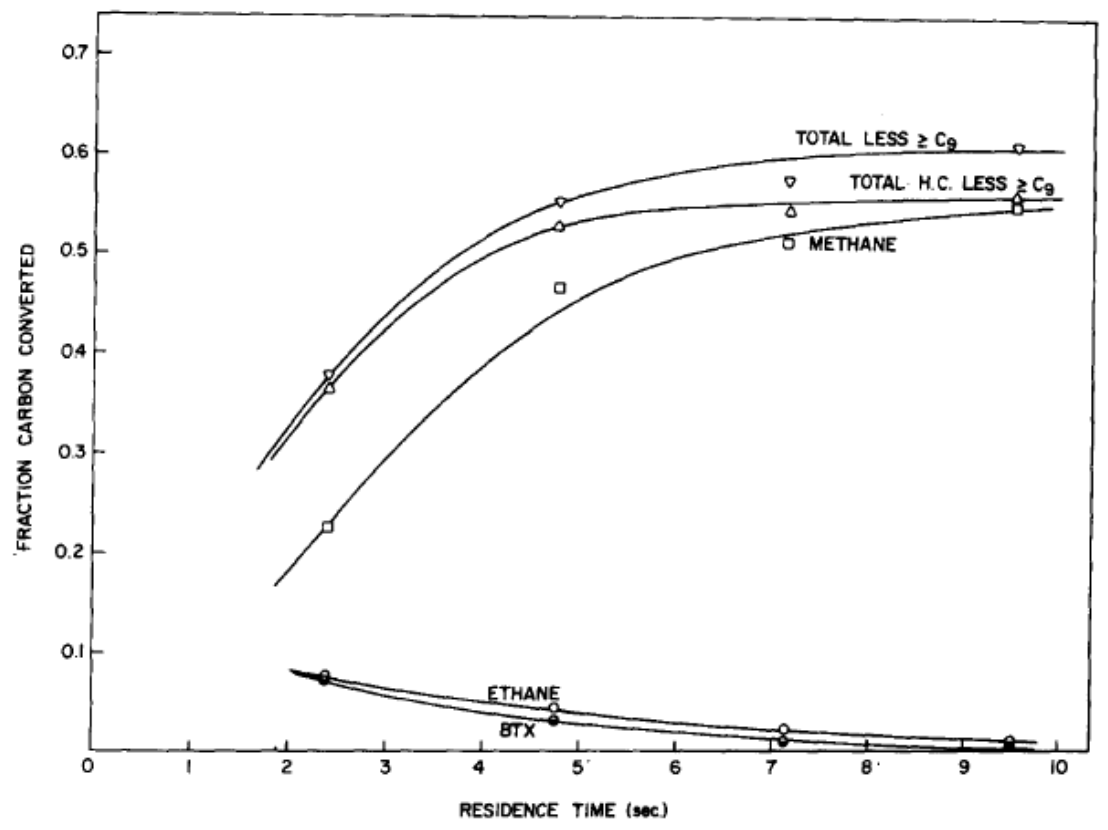

Figure 6 - Flash hydropyrolysis of lignite. Pressure 2500 psig; reactor temperature $825^{\circ} \mathrm{C}$ $\left(1,517^{\circ} \mathrm{F}\right)$; preheated temperature $30^{\circ} \mathrm{C}\left(86^{\circ} \mathrm{F}\right)$. (Fallon 1980)

\subsection{Effect of Temperature}

The study evaluating temperature effect on $\mathrm{CH}_{4}$ formation, by Arendt et al. (Arendt 1981), showed during pyrolysis in $\mathrm{H}_{2}$ atmosphere at high pressure (100 atm) $(1,470 \mathrm{psi}))$, the methane $\left(\mathrm{CH}_{4}\right)$ formation occurred in four distinguishable steps (Figure 7). $\mathrm{CH}_{4}$ formation was measured with a thermobalance reactor. The first peak was caused by decomposition reactions that occurred when only nitrogen $\left(\mathrm{N}_{2}\right)$ was present. Above $500^{\circ} \mathrm{C}\left(932^{\circ} \mathrm{F}\right)$, initial reactions with $\mathrm{H}_{2}$ were indicated. $\mathrm{H}_{2}$ reacted with the volatiles which evolved from the decomposing coal. The third peak appeared as the result of the rapid hydrogasification of very reactive carbon atoms in the char. At an even higher temperature, the unreactive 
char residue was gasified with $\mathrm{H}_{2}$ in the so-called slow hydrogasification reaction. This fourth step did not contribute to the yield of $\mathrm{CH}_{4}$ in the electric grid apparatus because the pyrolysis was completed after only a few seconds (Arendt 1981).

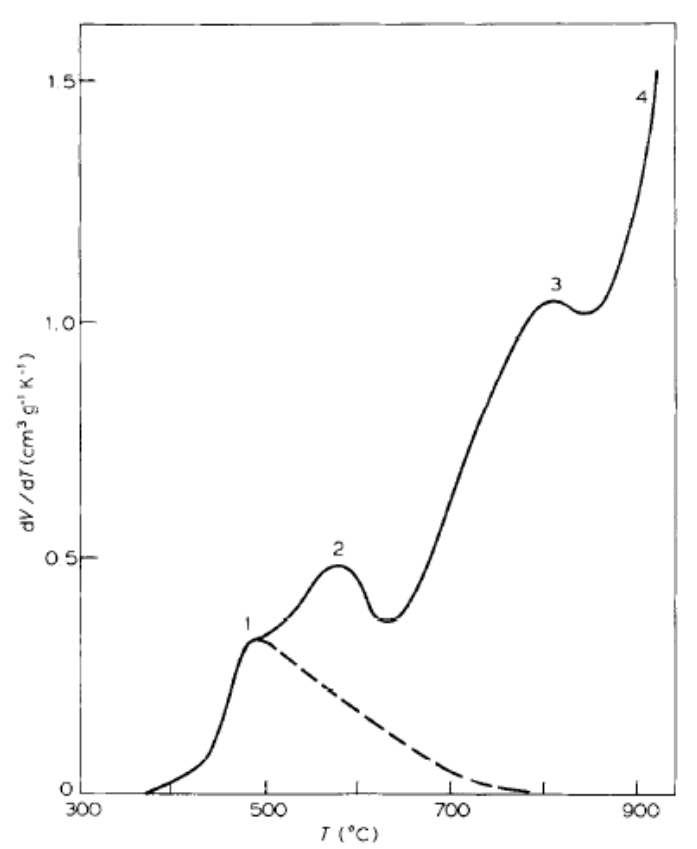

Figure 7 - Yield for Minto high-volatile bituminous coal: $10 \mathrm{MPa}(1,450 \mathrm{psi}) \mathrm{H}_{2}$; fast heating rate $600 \mathrm{~K} / \mathrm{s}\left(1,080^{\circ} \mathrm{Fls}\right) ; \bullet$ methane; $^{\circ}$ naphthalene; - ethane; $\square$ benzene. (Stangeby 1981)

Fallon's research (Fallon 1980) showed that at a hydrogen pressure of 2000 psig, the liquids of $>\mathrm{C} 9$ were seen to maximize at approximately $9 \%$ yield at a temperature of $750^{\circ} \mathrm{C}\left(1,382^{\circ} \mathrm{F}\right)$, while a BTX maximum of approximately $10 \%$ occurred at $800^{\circ} \mathrm{C}\left(1,472^{\circ} \mathrm{F}\right)$, as shown in Figure 8 . The research found that at temperatures of $850^{\circ} \mathrm{C}\left(1,562^{\circ} \mathrm{F}\right)$ and greater, the liquid hydrocarbons were seen to decompose almost entirely to produce gaseous hydrocarbons, principally methane and ethane. This, plus the additional gaseous products formed directly from the coal, resulted in maximum gaseous yields $\left(\mathrm{CH}_{4}+\mathrm{C}_{2} \mathrm{H}_{6}\right)$ shown in Figure 9. Figure 9 also shows that the formation of these products appears to be a direct function of the hydrogen pressure, increasing from about $20 \%$ at 500 psig to $>80 \%$ at $2500 \mathrm{psi}$. Since higher temperatures accelerates the decomposition, the competing reactions of formation and decomposition at 2000 to $2500 \mathrm{psi}$ results in the maximum yield occurring at $875^{\circ} \mathrm{C}\left(1,607^{\circ} \mathrm{F}\right)$. A total conversion of $88 \%$ to $\mathrm{CH}_{4}$ and $\mathrm{C}_{2} \mathrm{H}_{6}$ at $875^{\circ} \mathrm{C}\left(1,607^{\circ} \mathrm{F}\right)$ and 2500 psi was observed. 


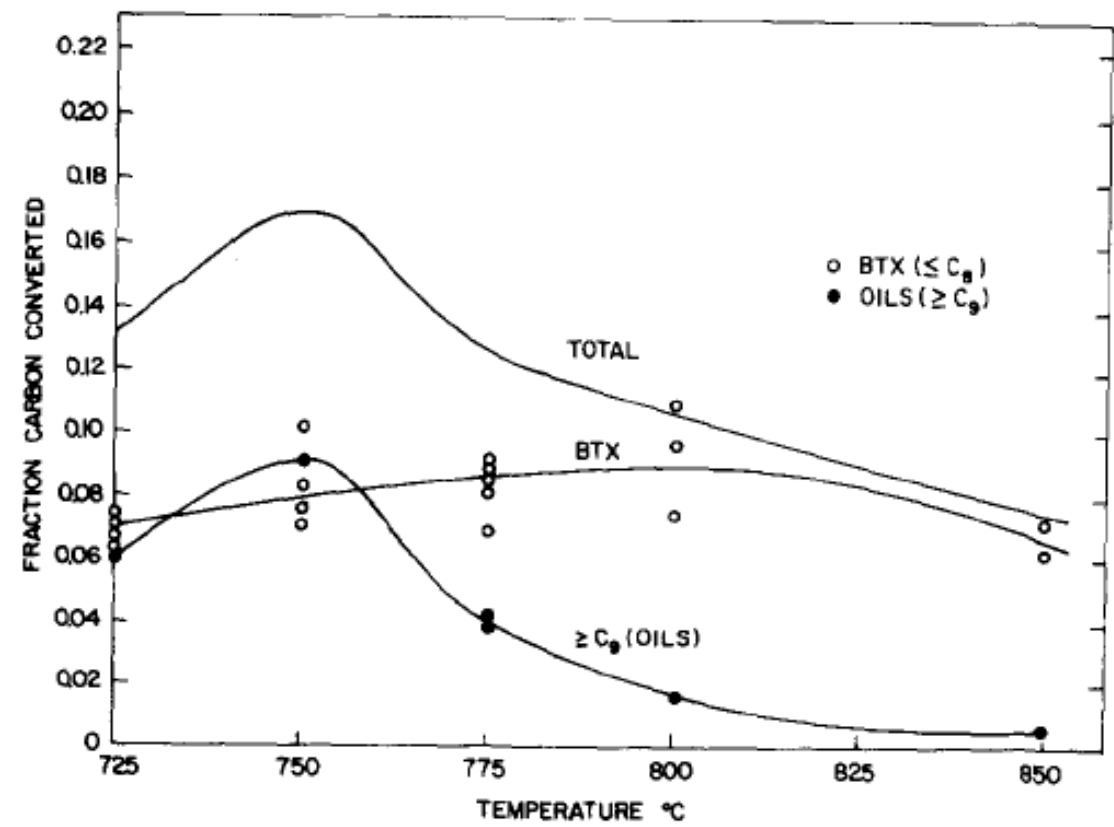

Figure 8 - Flash hydropyrolysis of lignite: Liquid yield vs. temperature. Pressure 2000 psig. (Fallon 1980)

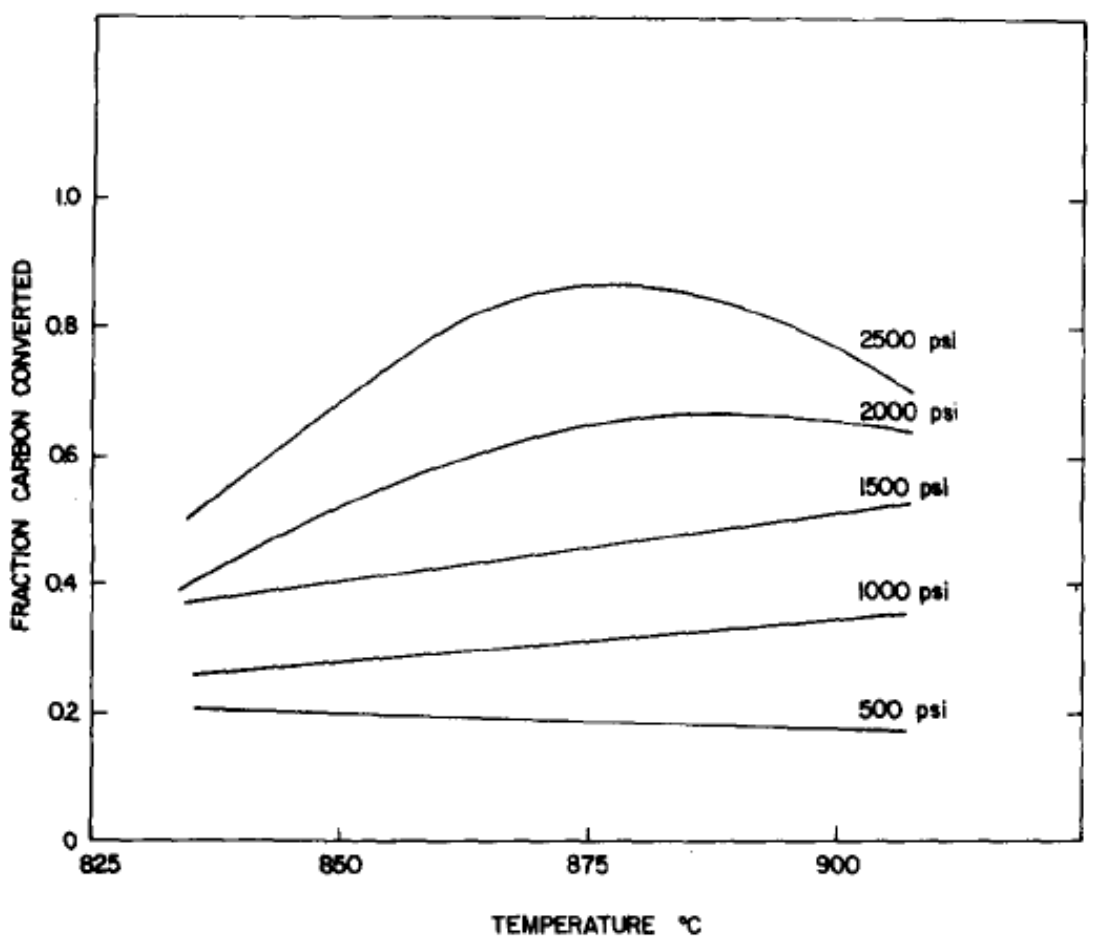

Figure 9 - Flash hydropyrolysis of lignite: Maximum gaseous hydrocarbon yield $\left(\mathrm{CH}_{4}+\right.$ $\mathrm{C}_{2} \mathrm{H}_{6}$ ) vs. temperature. Pressure 500 to $2500 \mathrm{psi}$; residence time $2.4 \mathrm{~s}$ to $7.0 \mathrm{~s}$ (Fallon 1980)

Similar results were reported by Stangeby et al. (Stangeby 1981). As shown in Figure 10 with $100 \mathrm{~atm}(1,470 \mathrm{psi}) \mathrm{H}_{2}$, hydropyrolysis of high volatile Canadian 
coal gave an optimum yield of light oils at temperatures around $800^{\circ} \mathrm{C}\left(1,482^{\circ} \mathrm{F}\right)$ while the methane production increased monotonically with peak temperature.

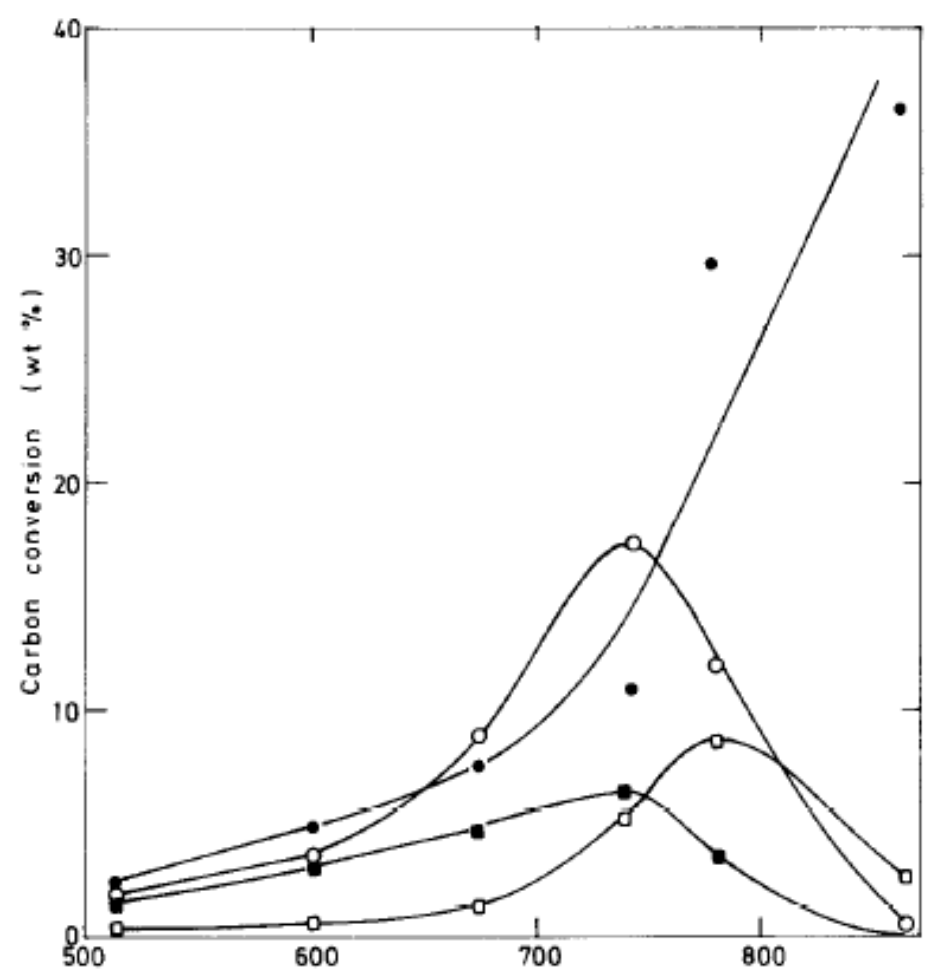

Figure 10 - Formation rate of $\mathrm{CH}_{4}$ under $1.1 \mathrm{MPa}(160 \mathrm{psi}) \mathrm{H}_{2}$ : Coal, Zollverein (no. 4); heating rate, $3 \mathrm{~K} / \mathrm{min}\left(5.4^{\circ} \mathrm{F} / \mathrm{s}\right)$. ---: $\mathrm{CH}_{4}$ formation during pyrolysis in $\mathrm{N}_{2}$ (Arendt 1981).

\subsection{Effect of Coal Particle Size}

Studies of the effect of particle diameter on the hydropyrolysis of the bituminous coal were reported (Suuberg 1980). The results for total weight loss under 69 atm $(1,014 \mathrm{psi})$ of hydrogen were compared with pyrolysis data obtained under 1 atm of helium as shown in Figure 11, where, it shows the total weight loss in hydrogen decreases dramatically with increasing particle diameter. Similarly, extrapolation to smaller particle diameters suggests opportunity for significant improvements in total conversion. The yield breakdown of the several important products: $\mathrm{CH}_{4}, \mathrm{C}_{2} \mathrm{H}_{6}$, other $\mathrm{HC}$ gases, light $\mathrm{HC}$ liquids, $\mathrm{CO}_{2}$, and tar were also compared under these two conditions. Apart from an apparent decline in the yields of ethane and other hydrocarbon gases with increasing particle diameter, which may reflect an increased contribution of secondary cracking reactions, no clear trends were observed. 


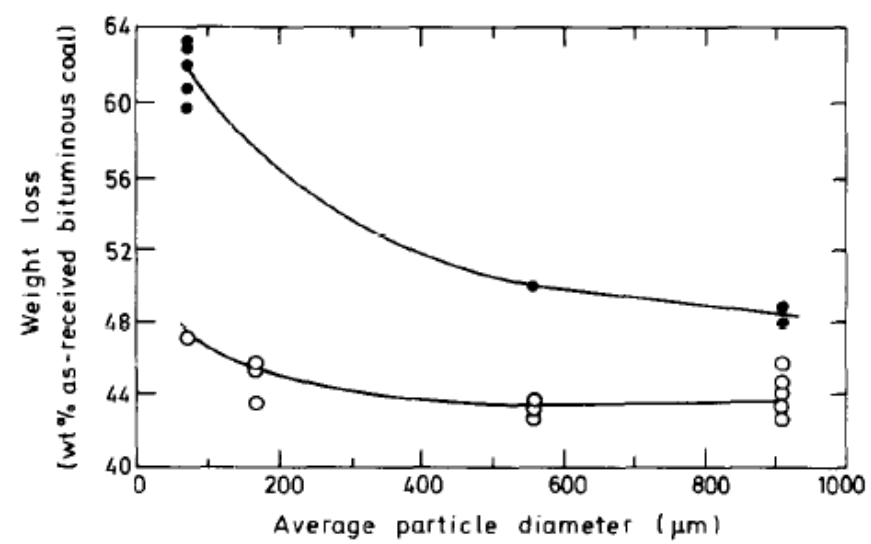

Figure 11 - Effect of particle diameter on total yields from Pyrolysis and Hydropyrolysis of bituminous coal: •, hydropyrolysis, 69 atm (1,014 psi) H2; ${ }^{\circ}$, Pyrolysis, $1 \mathrm{~atm}(14.7 \mathrm{psi}) \mathrm{He}$. (Suuberg 1980)

\subsection{Effect of Heating Rate}

Heating rate was found to have little effect on total weight loss of the coal, but a dramatic effect on the actual composition of products (Figures 12 and 13). High heating rates substantially increased the yield of light hydrocarbons. When the coal temperature was brought up "immediately" to high temperature, the $\mathrm{CH}_{4}$ and $\mathrm{C}_{2} \mathrm{H}_{4}$ formation was obviously favored thermodynamically (Stangeby 1981). 


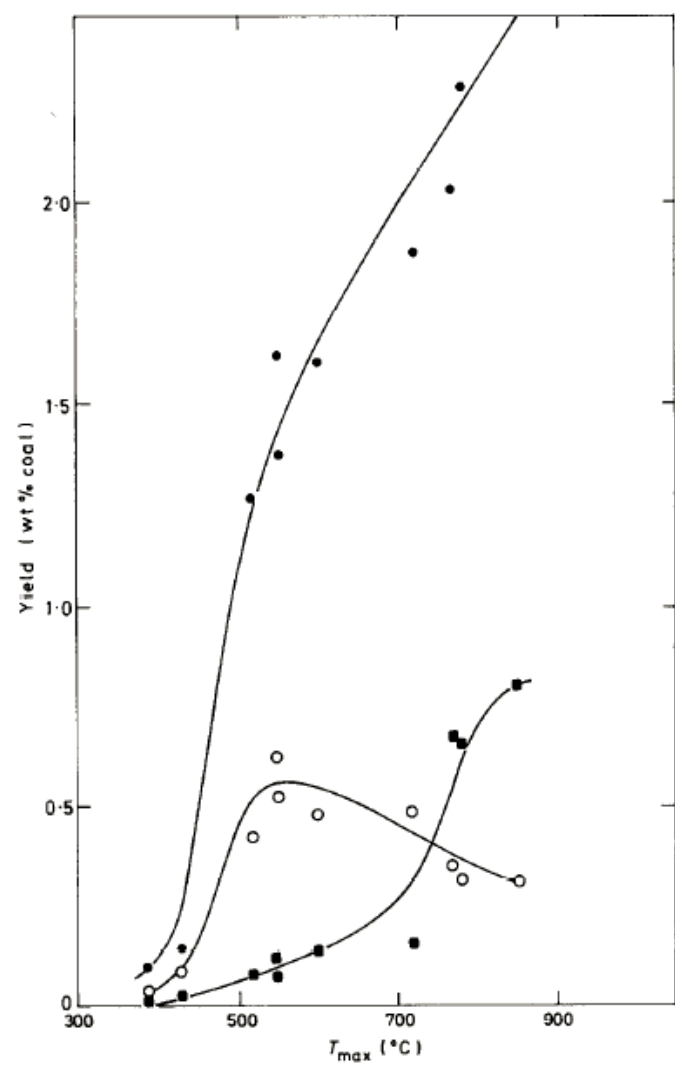

Figure 12 - Yield of light hydrocarbons as a function of peak temperature: Devco highvolatile bituminous coal: 1 atm (14.7psi) helium; 20s; medium heating rate, $\sim 6000$ $\mathrm{K} / \mathrm{s}\left(10,832^{\circ} \mathrm{F} / \mathrm{s}\right) ; \bullet \mathrm{CH}_{4} ;{ }^{\circ}, \mathrm{C}_{2} \mathrm{H}_{6} ; \varpi \mathrm{C}_{2} \mathrm{H}_{4}$ (Stangeby 1981)

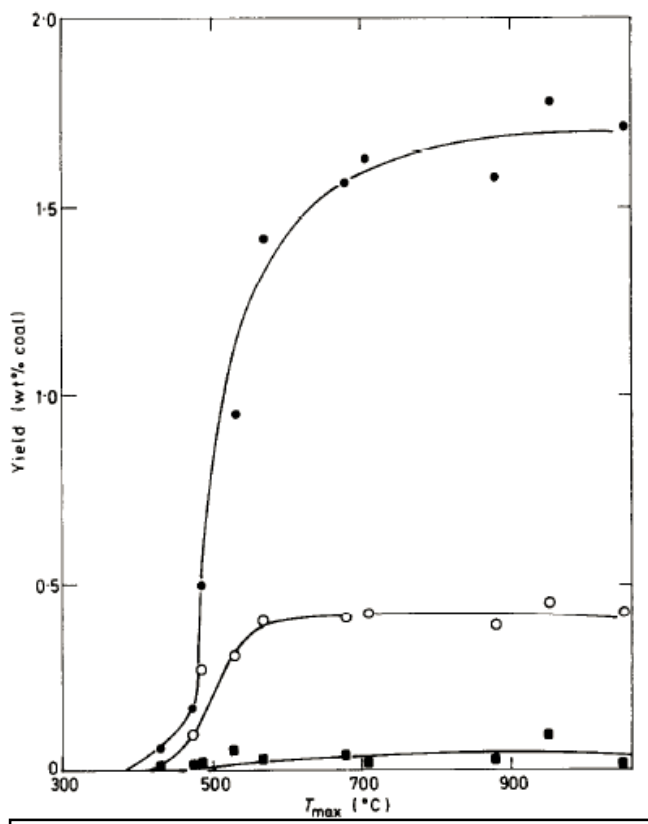

Figure 13 - Yield of light hydrocarbons as a function of peak temperature: Devco highvolatile bituminous coal: 1 atm (14.7 psi) helium; 20s; medium heating rate, $\sim 250 \mathrm{~K} / \mathrm{s}$ (482 ${ }^{\circ} \mathrm{F} / \mathrm{s}$ ); $\bullet \mathrm{CH}_{4} ;{ }^{\circ} \mathrm{C}_{2} \mathrm{H}_{6} ; \square \mathrm{C}_{2} \mathrm{H}_{4}$ (Stangeby 1981)

It is again important to note in Figure 12 that the fast heating curve for methane and ethylene rise sharply with a peak temperature at $900^{\circ} \mathrm{C}\left(1652^{\circ} \mathrm{F}\right)$. The slower heating curves, however, appear to have leveled by $700^{\circ} \mathrm{C}\left(1292^{\circ} \mathrm{F}\right)$ on increasing the temperature.

\section{Hydrane Process}

\subsection{Hydrane Process}

During the 1970s, Feldmann et al. (Feldmann 1971; Feldmann 1972; Feldmann 1973; Feldmann 1975) carried an extensive study on "Hydrane Process" - a two stage reactor design for coal hydrogasification, at the Pittsburgh Energy Research Center. In their study, they reviewed the economic advantages of methane production through coal hydrogasification; designed a free-fall dilute- 
phase reactor and thereby solved raw coal agglomeration problem at elevated temperature; studied the effect of interesting operating conditions on total carbon conversion and product distribution; investigated the reaction kinetics; examined the fluidized properties of coal char, and proposed an engineering-scale reactor design. In the following section, the highlights of their research will be reviewed and summarized.

In the Hydrane process study, the authors stated that the economic advantages arise in this process due to Hydrane minimizing both the coal and oxygen required to produce a unit of methane. The economically important quantities are reduced because:

- Elimination of pretreatment itself allows approximately $10-15 \%$ of the hydrogen-rich portion of the coal ordinarily "lost" during pretreatment to be converted to methane;

- Less hydrogen is required to produce methane if the hydrogen reacts with the carbon in the coal rather than with carbon in carbon monoxide as occurs during methanation;

- The heat generated during hydrogasification is utilizable for carrying out the endothermic carbon-steam reaction, thus reducing oxygen requirements while the heat released by methanation is not utilizable because of the relatively low temperature $\left(900^{\circ} \mathrm{F}\right.$ maximum) that the methanation reactor must operate at to protect the catalyst.

As a matter of fact, these advantages of producing methane by this approach have been documented in even earlier research: Channabasappa and Linden (Channabasappa 1956) concluded that hydrogenating coal to methane with the hydrogen produced by steam-oxygen gasification of char was more thermally efficient than steam-oxygen gasification of coal to synthesis gas followed by the water-gas shift and methanation reactions to produce methane. Henry, Louks (Henry 1970), Wen and coworkers (Wen; Wen 1972) pointed out, by a comparison of specific processes, the economic virtues of using raw coal and producing methane in the gasifier rather than by methanation.

In this Hydrane process, the first stage free-fall dilute-phase (FDP) reactor is the key to handle caking coals without pretreatment. A lab scale FDP reactor was designed to have a 3 inch inside diameter (ID) (heated tube contained in a 10 inch pressure vessel) and 5 foot length. The coal falls freely through the reactor tube concurrent with the reacting gas that is also injected at the top of the reactor. Because of rapid heating and a dilute solids phase, agglomeration is avoided; particles are plastic and sticky for only a short time, during which particle-particle collisions are few. The FDP reactor was designed to have two important functions. It must convert the coal to a non-agglomerating char for the subsequent fluid bed, and it must convert enough carbon to methane so the FDP 
product gas is, after the acid gas removal and light methanation, an acceptable pipeline gas.

Table 4 summarizes the typical FDP reactor operating range for the hydrane process and Table 5 lists the example operating data for FDP hydrogasification. As shown in Table 5, run 153 is an example of pure $\mathrm{H}_{2}$ input test, while others are mix $\left(\mathrm{H}_{2}, \mathrm{CH}_{4}\right.$ and $\left.\mathrm{N}_{2}\right)$ gas input tests. Total carbon conversion of all cases falls into the $32-36 \%$ range. Methane formation processes at $66-76 \%$ in the product gas stream for the mix gas input and $47 \%$ for the pure $\mathrm{H}_{2}$ input test. Since there was a range of $42-49 \%$ methane input in mix gas cases, the pure $\mathrm{H}_{2}$ input test actually generated $>30 \%$ more methane than the mix gas input tests.

Table 4 - Summary of FDP reactor operation range

\begin{tabular}{l|l}
\hline \hline Parameters & \\
\hline Temperature & $900{ }^{\circ} \mathrm{C}\left(1,652^{\circ} \mathrm{F}\right)$ \\
\hline Pressure & $1000-2000$ psig \\
\hline Conversion & $-32-33 \%$ (mix gas input) $\sim 38 \%$ (pure hydrogen input) \\
\hline Product methane & $>60 \%$ (mix gas input) \\
\hline Coal particle size & $100 \times 200$ mesh \\
\hline Coal source & Pittsburgh Seam coal \\
\hline
\end{tabular}


Table 5 - Operating data for FDP hydrogasification of raw coal (Feldmann 1975)

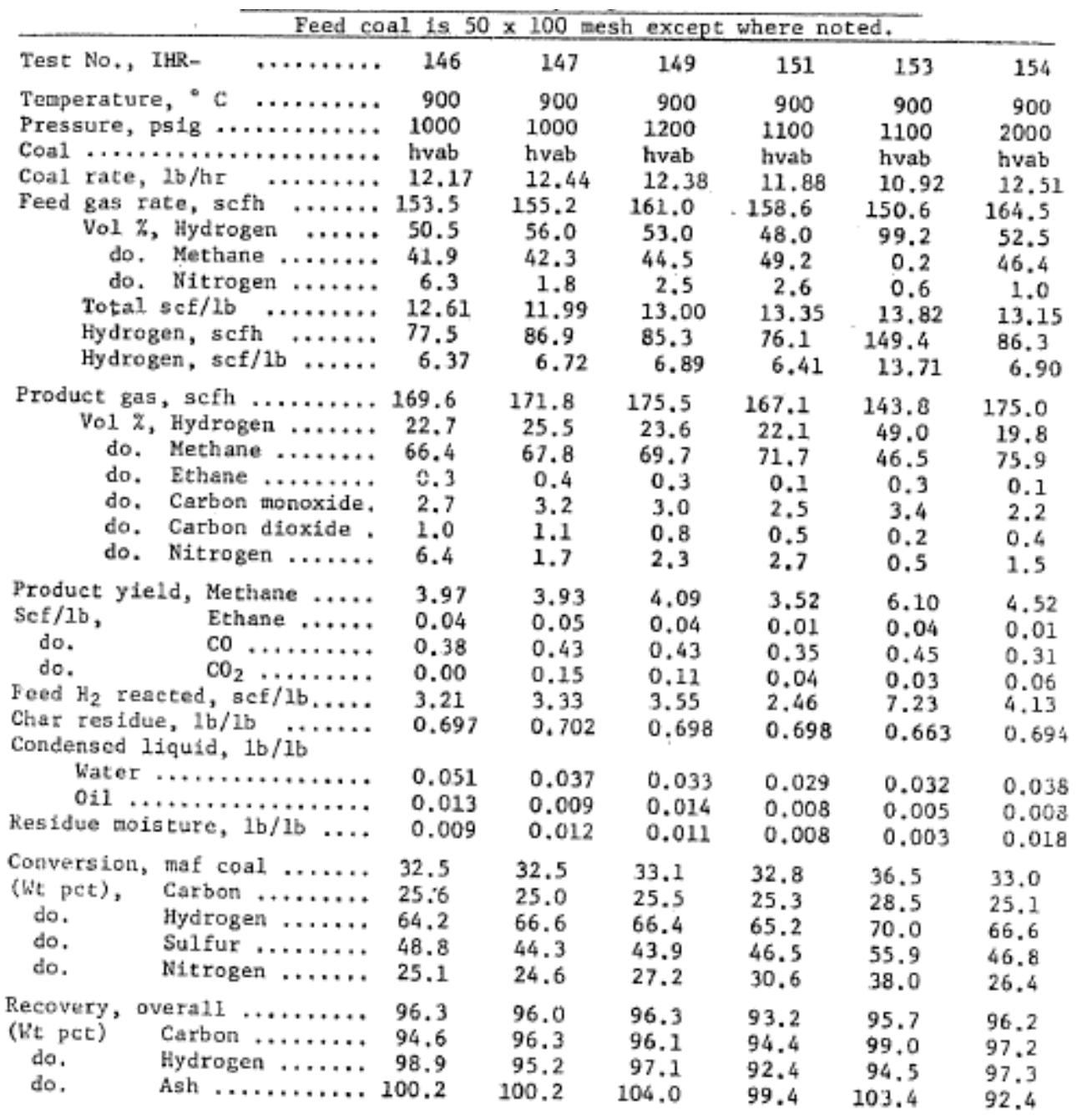

\subsection{Advantages and Disadvantages of the Hydrane Process}

The following summarize the advantages and disadvantages of adopting the Hydrane Process:

- Advantages include:

- Relatively extensive research on lab-scale testing and modeling and the availability of suggestions on commercial size design;

- Coal aggregation problem was solved by applying a Free Dropping Reactor as a first stage reactor; 
- Demonstration that a two-stage reactor can achieve total 50$55 \% \mathrm{C}$ conversion and $>60 \%$ methane, with $900^{\circ} \mathrm{C}$ $\left(1,652^{\circ} \mathrm{F}\right), 1000 \mathrm{psig}$ operating condition. Retention time is in seconds range;

- Disadvantages include:

- High temperature, high pressure, hydrogen enrich environmental operation;

- Two stage operation with 50-55\% total C conversion. Further conversion of char still is needed;

- No experience on scale up; the heating up rate would be considerably slowed down with the larger inside-diameter reactor since the impact of heat radiation from the wall will be considerably decreased with the increased reactor size. Countercurrent operation may help on this issue. However, need to be careful on small gas off take lines and removing gas from reactor where particle concentrations are high.

\subsection{Reaction Rate Model}

In order to utilize the data generated by the FDP reactor to scale-up the Hydrane process, a rate equation was developed that allowed a reasonable correlation of all FDP experiments. The rate of methane generation is given by the following equation (Feldmann 1973).

$$
\frac{d c_{A}}{d t}=c_{0} \frac{d X}{d t}=k p_{H_{2}}\left(c_{0}(1-X)-c_{i}\right)
$$

Where:

$\mathrm{C}_{\mathrm{A}}$ : concentration of active carbon in the coal or char at any time;

$\mathrm{C}_{0}$ : concentration of carbon in the coal feed;

$\mathrm{X}$ : the fraction of carbon converted to methane;

$\mathrm{k}$ : rate constant for methane formation $\mathrm{atm}^{-1} \mathrm{hr}^{-1}$;

$\mathrm{P}_{\mathrm{H} 2}$ : partial pressure of hydrogen atm;

$\mathrm{c}_{\mathrm{i}}$ : fraction of unreactive carbon which could, for example, be formed by the cross linking of solid carbon species into a very stable polymeric structure. $\mathrm{c}_{\mathrm{i}}$ was assumed to be 0 during the calculation, which means that almost all of the carbon was capable of being converted to methane at sufficiently high hydrogen partial pressure and/or char residence time. 
Integration form of the model:

$$
\int_{E}^{X} \frac{d X}{(1-X)-c_{i} / c_{0}}=k p_{H_{2}} \frac{L}{U_{T}}
$$

$\mathrm{L}$ : length of the FDP in feet;

$\mathrm{E}$ : fraction of carbon that appears to be "instantaneously" gasified;

$\mathrm{U}_{\mathrm{T}}$ : average terminal velocity of the char in $\mathrm{ft} / \mathrm{hr}$;

An agreement between the reaction rate model and the experimental data at a reactor wall temperature of $750^{\circ} \mathrm{C}\left(1,382^{\circ} \mathrm{F}\right)$ was shown. The simulation results indicated that carbon conversion to methane was proportional with pressure increase and the hydrogasification of raw coal in the FDP reactor may be limited by hydrogen diffusion, at least at the higher temperature used in this study.

\subsection{Related Information}

Table 6 - Typical analyses of coals used in Hydrane study (Feldmann 1973)

\begin{tabular}{|c|c|c|c|}
\hline & $\begin{array}{l}\text { Pittsburgh Seam } \\
\text { hvab coal }\end{array}$ & $\begin{array}{l}\text { Illinois \#6 hvcb } \\
\text { coal }\end{array}$ & N. Dakota Lignite \\
\hline \multicolumn{4}{|c|}{ Proximate Analysis } \\
\hline Moisture & 1.2 & 1.4 & 7.8 \\
\hline Volatile matter & 36.4 & 36.8 & 39.7 \\
\hline Fixed carbon & 56.7 & 55.9 & 46.9 \\
\hline Ash & 5.7 & 5.9 & 5.6 \\
\hline \multicolumn{4}{|c|}{ Ultimate Analysis (Day basis) } \\
\hline $\mathrm{C}$ & 79.09 & 75.45 & 64.64 \\
\hline $\mathrm{H}$ & 5.22 & 5.12 & 4.48 \\
\hline $\mathrm{N}$ & 1.60 & 1.72 & 0.76 \\
\hline $\mathrm{D}$ & 1.10 & 1.32 & 0.76 \\
\hline O (by difference) & 7.22 & 10.41 & 23.29 \\
\hline Ash & 5.77 & 5.98 & 6.07 \\
\hline Total & 100 & 100 & 100 \\
\hline
\end{tabular}

\subsection{Other Findings:}

- Hydrogen consumption is low because hydrogasification is of the relatively hydrogen-rich portion of the coal which is one of the great advantages of the direct hydrogasification of raw coal. 
- The higher the coal heat-up rate, the lower the hydrogen consumption to produce a unit of methane because of the improved utilization of the coal's hydrogen.

- Hydrogen consumption per unit of methane formed at the carbon conversion levels required for balanced operation is about 1.38

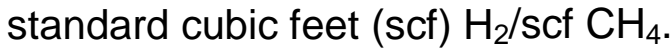

- Carbon conversion to $\mathrm{CH}_{4}$ higher than needed for balanced operation (45\%) could easily be achieved.

- Operating problems caused by the caking nature of coals were minimal.

- The type of liquids formed during FDP hydrogasification are highly aromatic with the average molecule having about 4 condensed rings. About 0.07 pounds $(\mathrm{lb})$ of these tars per $\mathrm{lb}$ of coal fed. Since these tars are free of sulfur they could be most advantageously used as a fuel supplement for the integrated Hydrane Plant's steam boilers.

- Illinois coal requires substantially less hydrogen to produce a unit of methane than does Pittsburgh Seam coal.

- Desulfurization of Illinois high-volatile C bituminous (hvcb) coal ranged from $72-90 \%$ in the two-stage reactor. (Lignite retains sulfur, probably due to the formation of sodium sulfides in the ash. Most tested coal passed Environmental Protection Agency (EPA) emission standards for solid fuel.) 


\subsection{Lab/Engineer Scale Reactor Design}

A lab-scale reactor was designed and built for the hydrane study.

Engineering-scale designs were proposed as shown in Figures 14-16. Table 7 gives the dimensional design of Figure 14.

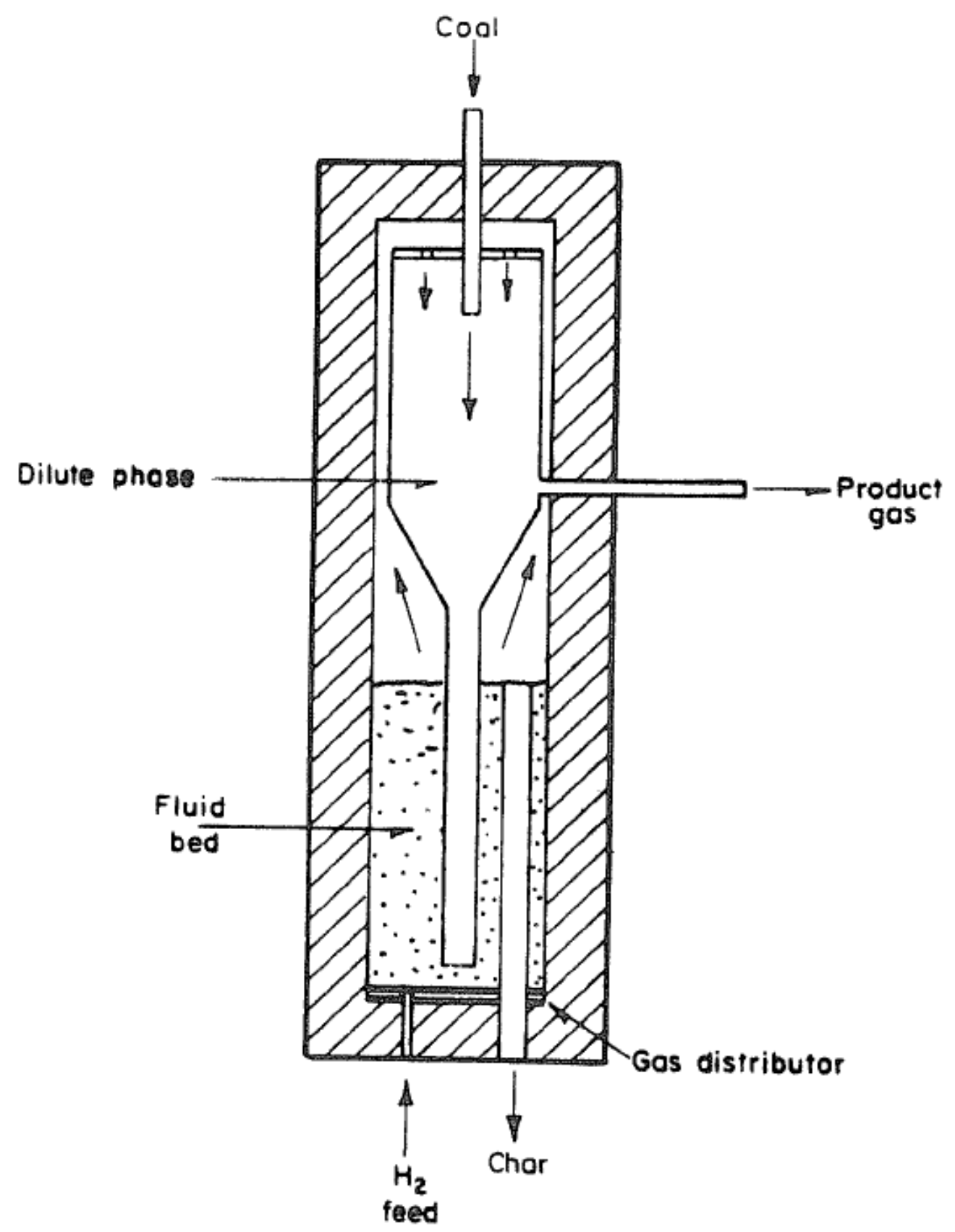

Figure 14 - Conceptual two-stage reactor. (Feldmann 1972) 
Table 7 - Dimensional design of FDP, hoppers, and fluid-bed reactor in Figure 14 (Feldmann 1972)

\begin{tabular}{|l|l|}
\hline FDP & 18 \\
\hline Number & $10 \mathrm{ft}$ \\
\hline Height & $14.3 \mathrm{ft}$ \\
\hline Inner shell ID & $3 / 8 \mathrm{in}$ \\
\hline Inner Wall thickness & $15.8 \mathrm{ft}$ \\
\hline Outer shell ID & $7.3 \mathrm{in}$ \\
\hline Outer Wall thickness & $4.5 \mathrm{in}$ \\
\hline Refractory thickness & 36 \\
\hline Hoppers & $14.2 \mathrm{ft}$ \\
\hline Number & $4.0 \mathrm{in}$ \\
\hline Height & 2 \\
\hline Wall thickness & $62 \mathrm{ft}$ \\
\hline Fluid Bed Reactor & $14.6 \mathrm{ft}$ \\
\hline Number & $6.6 \mathrm{in}$ \\
\hline Height & $4.5 \mathrm{in}$ \\
\hline ID &
\end{tabular}




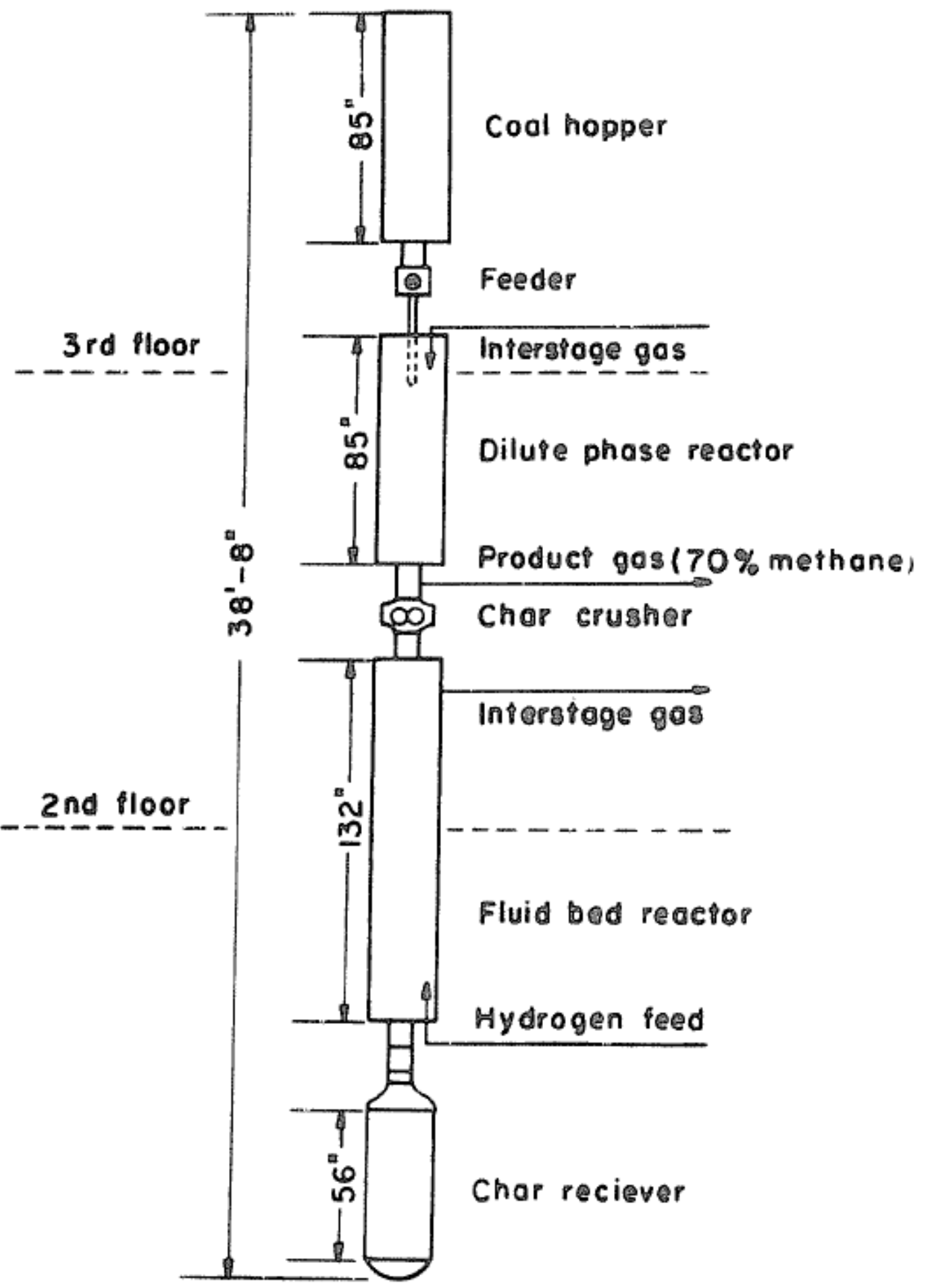

Figure 15 - Conceptual integrated hydrogasification unit. (Feldmann 1973) 


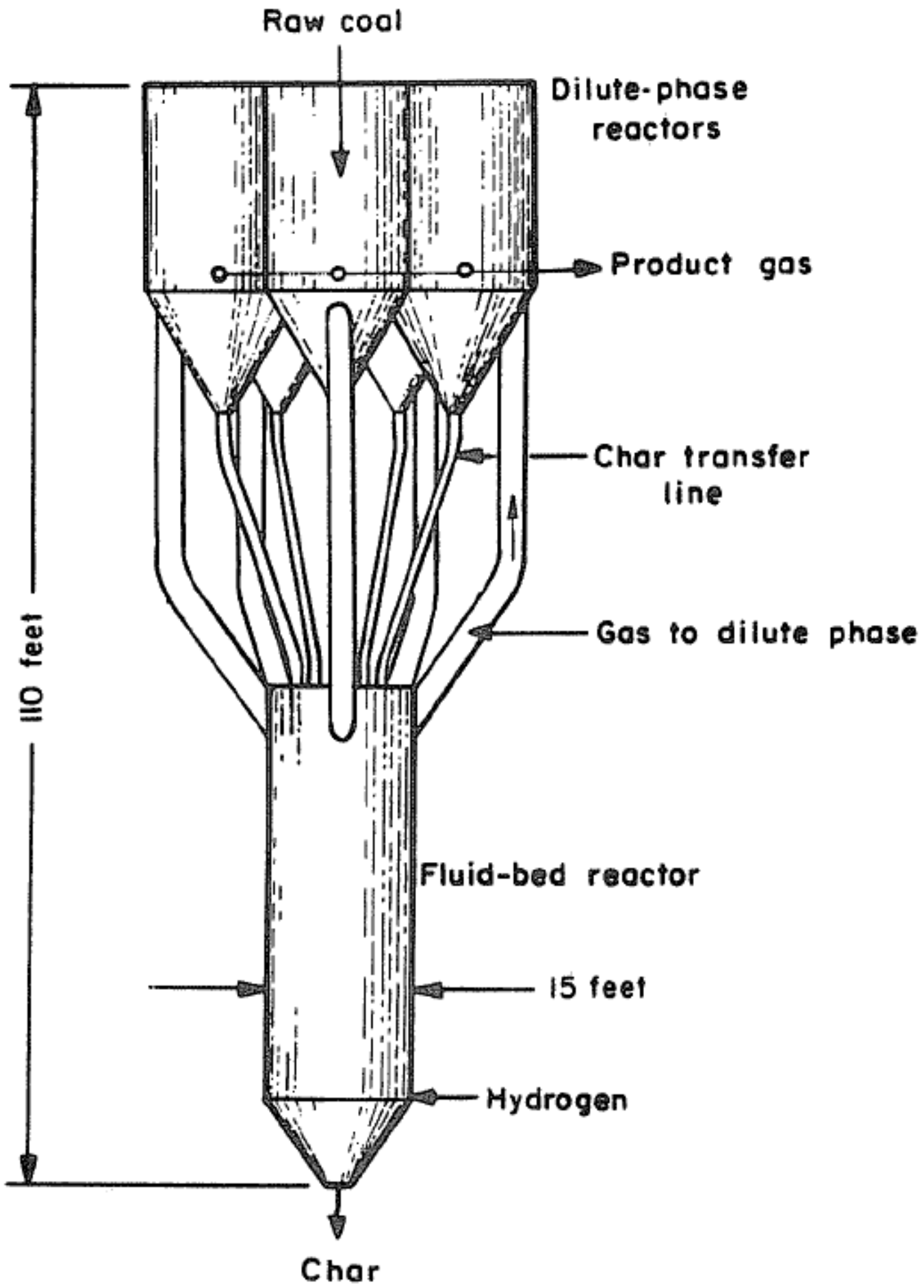

Figure 16 - Commercial Hydrane Reactor: Capacity Million scf Pipeline Gas. (Feldmann 1973) 


\section{Recent Research}

In spite of all the research carried out on coal hydrogasification to make Substitute Natural Gas (SNG) during the 1960s to early 1970's, the price dive of natural gas during the 1980's brought an end to this research within the U.S. before it was demonstrated successfully in the industry. Recently, Japan showed the interest in the idea and some studies have been published in late the 1990s to early 2000 s.

Coal hydrogasification for producing SNG is a promising technology in Japan (Noguchi 2000) to secure a stable support of natural gas by diversifying its sources. Under agreement with New Energy and Technology Development Organization (NEDO), the Japan Gas Association has completed a 5-year program in which three elemental gasifier technologies and two peripheral technologies were developed. In gasification tests, a wide range of carbon conversion was verified under various conditions influencing two-stage reaction; a new reaction model was established to estimate gasifier performance. A new injector, which incorporates a tip burner to generate high temperature hydrogen, was developed through a hot model test. The optimal internal configuration was developed to generate adequate recycling of product gas and form the two-stage reaction zone through a cold model test. Dense phase coal conveying was verified using hydrogen under high pressure between $3-7 \mathrm{MPa}$ (435-1,015 psi), and various factors for stable discharge and cooling of char, which is residue from the hydrogasification, was confirmed using a model particle. Engineering studies for a scale plant have shown the coal hydrogasification process to be technically viable with high energy conversion in addition to being economically feasible in the future.

Kaiho et al. (Kaiho 2002) investigated hydrogasification of coal using a batch type reactor in the late 1990s. They found that a feeding ratio of hydrogen to coal was an important parameter to the design of the plant for the hydrogasification process, because it determined the direct volume and diameter of the equipment in the process unit, it relates to plant cost, running cost and its thermal efficiency. There were some papers showing that the ratio effect on the conversion of coal to methane and BTX, however, there also were some objections against this observation. In their research, they have built an apparatus that can gasify coal in a reactor having a definite volume, and studied the details of this $\mathrm{H}_{2} / \mathrm{coal}$ effect and pointed out this effect was the result of competition between two kinds of reactions that occurred around volatile matter, decomposing into gas and condensating into liquid droplets. 


\section{Summary}

It has been generally agreed that high yield methane can be produced through this coal hydrogasification process with an acceptable total carbon conversion. The key operating variables affect the total carbon conversions and methane yield in this process are hydrogen partial pressure, reaction temperature and coal particle size. High reaction temperature may cause the cracking of other HC's compounds and increases methane formation. Mass transfer limitations may strongly affect the product spectra observed during hydropyrolysis, which lead to the observed effect of coal particle size. With all the advantages demonstrated on this coal hydrogasification process both theoretically and in the laboratory, challenges still remain to industrially accomplishing this high pressure, high temperature, $\mathrm{H}_{2}$-enriched operation on the raw coal. 


\section{References}

Anthony, D. B., Howard, J.B., Hottel, H.C. and Meisser, H. P. (1976). "Rapid Devolatilization and Hydrogasification of Bituminous Coal." Fuel 55: 121.

Arendt, P., van Heek, K-H (1981). "Comparative investigations of coal pyrolysis under inert gas and $\mathrm{H} 2$ at low and high heating rates and pressure up to 10MPa." Fuel 60: 779.

Birch, T. J., Hall, K.R., and Urie, R.W. (1960). J. Inst. Fuel 33: 422.

Channabasappa, K. C. a. L., H.R. (1956). Ind. Eng. Chem. 48: 900.

Chen, W., Graff, R.A., and LaCava, A. (1978). Comparative Study of U.S. Coals in Flash Hydrogenation. ACS, NY.

Dent, F. J. (1944). Gas Journal 244: 502.

Fallon, P. T., Bhatt, B., Steinberg, M. (1980). "The flash hydropyrolysis of lignite and sub-bituminous coals to both liquid and gaseous hydrocarbon products." Fuel Processing Technology 3: 155.

Feldmann, H. F., and Yavorsky, P.M. (1973). The Hydrane Process. 5th AGA/OCR Synthetic Pipeline Gas Symposium, Chicago, Illinois.

Feldmann, H. F., Kiang, K-D, and Yavorsky, P.M. (1971). Fluidization Properties of Coal Char. Symposium on Gasification of Coal, Washington, D.C.

Feldmann, H. F., Mima, J.A., and Yavorsky, P.M. (1975). Pressurized Hydrogasification of Raw Coal in Dilute Phase Reactor. DOI, Pittsburgh Energy Research Center.

Feldmann, H. F., Wen, C.Y., Simons, W.H., and Yavorsky, P.M. (1972). Supplemental Pipeline Gas from Coal by the Hydrane Process. 71st AIChE National Meeting, Dallas, Texas.

Finn, M. J., Fynes, G., Ladner, W.R. and Newman, J.O.H. (1980). "Light aromatics from the hydropyrolysis of coal." Fuel 59: 397. 
Henry, J. P. J. a. L., B.M. (1970). An Economic Comparison of Processes for Producing Pipeline Gas From Coal. ACS. Chicago, IL, Preprints of Fuel Div.

Kaiho, M., et al. (2002). "Investigation on hydrogasification of coal using batch type reactor. (2). Influence of $\mathrm{H} 2 /$ coal ratio on material balance of hydrogasification." Fuel and Energy Abstracts 43(1): 34.

Noguchi, F. (2000). Coal hydrogasification process for the production of SNG and chemicals. Annu. Int. Pittsburgh Coal Conf.

Schroeder, W. C. (1962). U.S. 3,030,297.

Stangeby, P. C. a. S., P.L. (1981). "Rapid Pyrolysis an hydropyrolysis of Canadian coals." Fuel 60(131).

Sundaram, M. S., Steinberg, M., and Fallon, P.T. (1982). Flash Hydropyrolysis of Coal for Conversion to Liquid and Gaseous Fuels. DOE/METC/82-48, Brookhaven Nat'l. Lab.

Suuberg, E. M., Peters W.A., Howard J.B. (1980). "Product compositions in rapid Hydropyrolysis of coal." Fuel 59: 405.

Talwalkar, A. T. (1983). A tropical report on coal pyrolysis: DOE/MC/19316-1408.

Wen, C. Y., Li, C.T., Tscheng, S.H., and O'Brien, W.S. (1972).

Comparison of Alternate Coal Gasification Processes for Pipeline Gas Production. 65th AIChE National Meeting. New York, NY.

Wen, C. Y. a. C.-W. Interim Rept, No. 1. OCR. Optimization of Coal Gasification Processes, Research and Development. Washington D.C., Department of Interior. 


\section{Glossary}

Aromatics: relating to, or containing one or more six-carbon_rings characteristic of the benzene series and related organic groups

BTX: Benzene, Toluene, Xylene

Bituminous coal: a relatively hard coal containing a tar-like substance called bitumen. It is of better quality than lignite coal but of poorer quality than anthracite coal;

Char: substances obtained by partially burning coal

Coal tar: a brown or black liquid of high viscosity from destructive distillation of coal

Conversion: during the chemical reaction, the conversion of molecule $A$ to molecule B

Devolatilization: process that by heating coal removes its volatiles

Entrain Flow: where fine coal particle is suspended in the gas phase, so that the gas will carry the coal to flow

FDP: Free-fall dilute-phase

Fluidized Bed: where the fluid velocity is sufficient to suspend the particles, but not large enough to carry them out of the vessel

Gasification: process that converts coal into carbon monoxide and hydrogen

Hydrogasification: process that converts coal into methane with input of hydrogen

Hydropyrolysis: chemical decomposition of coal by heating in the presence of hydrogen

Lignite: the lowest rank of coal used almost exclusively as fuel for steam-electric power generation often referred to as brown coal

Natural Gas: gaseous fossil fuel consisting primarily of methane commonly referred to as gas 
Pyrolysis: chemical decomposition of coal by heating in the absence of oxygen or any other reagents, except possibly steam

Residence Time: the average time the coal particle spends within the reactor

SNG: Substituted Natural Gas

Yield: the amount of product obtained in a chemical reaction, also known as chemical yield and reaction yield 
Development of a Hydrogasification Process for

Coproduction of SNG and Electrical Power from Western Coals

Final Report

THIS PAGE INTENTIONALLY LEFT BLANK 


\section{APPENDIX B}

\section{Bench Scale Reactor Design from APS}


Development of a Hydrogasification Process for

Coproduction of SNG and Electrical Power from Western Coals

Final Report

THIS PAGE INTENTIONALLY LEFT BLANK 
GENERAL NOTES:

1 SEE SHEET 2 FOR ORTHAGONAL VIEWS

2 FOR MATERIAL DESCRIPTIONS OF SUB-ASSEMBLIES PART NUMBER COLUMN ON BILL OF MATERIAL

93

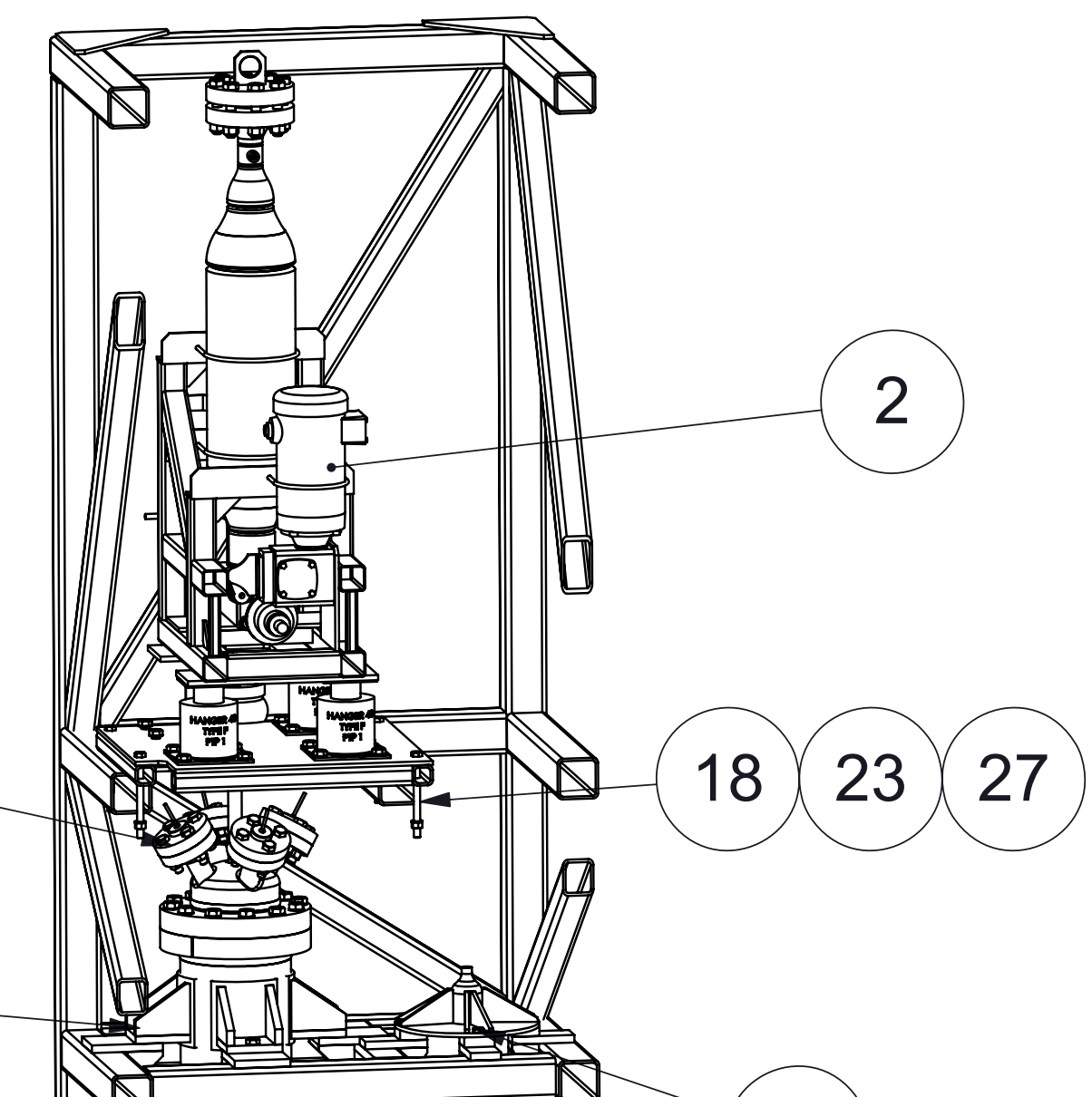

262217

262

1520

84

1410

1213

(21) 16
18

192428

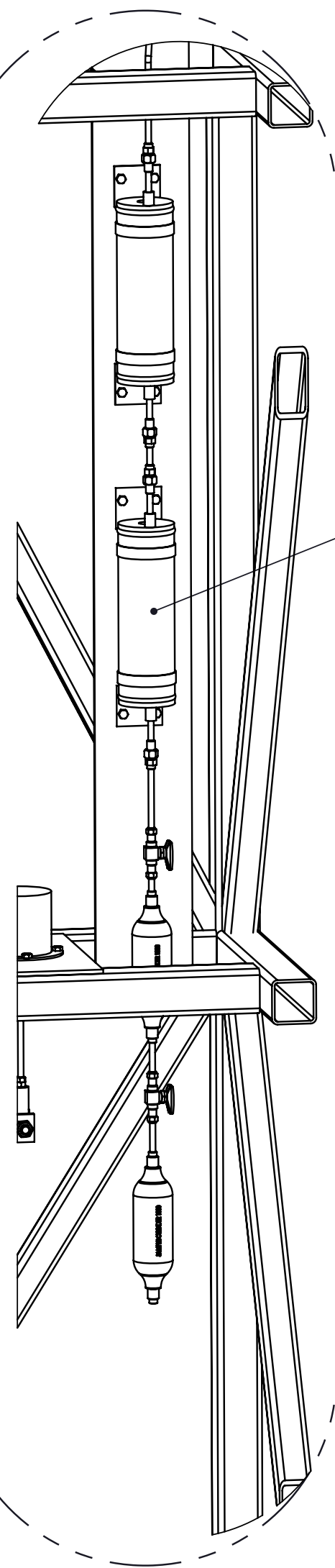

6

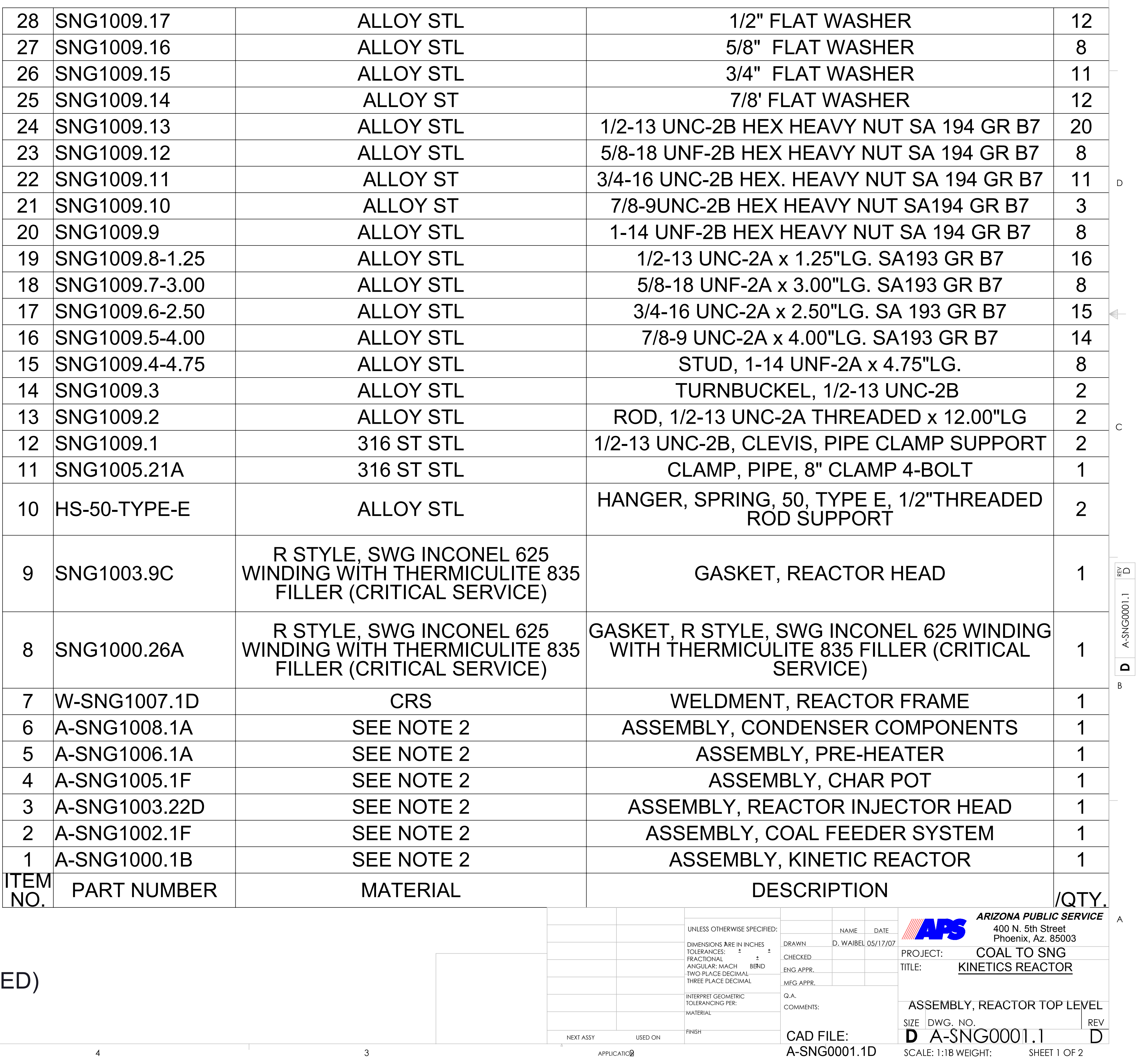
NOTE: FRAME CUT (SECTIONED)
FOR CLARITY

TEV REVISIONS

\section{DESCRIPTION}

DATE REV.BY APPROVED

ALL $B$ UPDATEDFRAME

DW

$\begin{array}{lllll}\text { ALL } & C & \text { UPDATED BOM MADE COND. } & 02 / 05 / 07 & \text { DW }\end{array}$

\begin{tabular}{l|l|l|l|l} 
ALL & F & FAME HT. 37'-5" WAS 35'-8.5" & 03/11/08 & DW \\
\hline
\end{tabular}

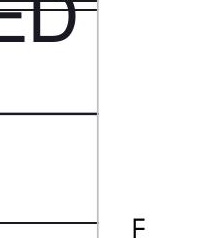




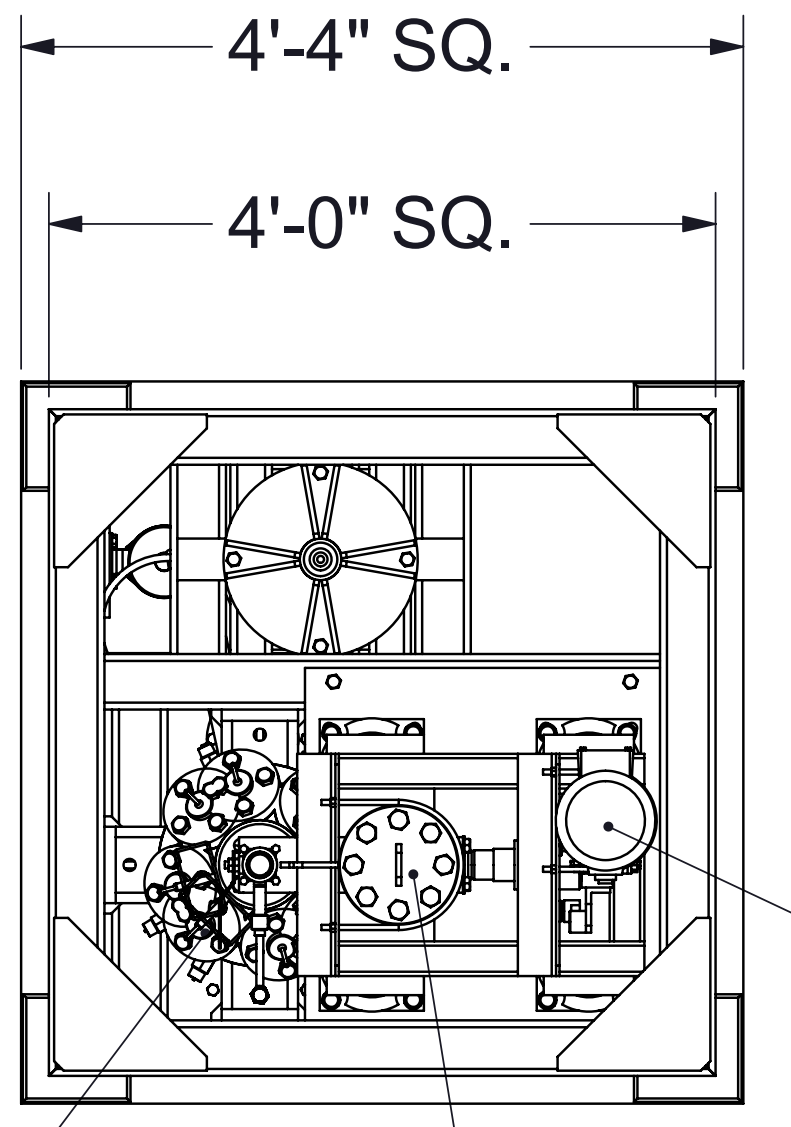

3 COAL INLET
2

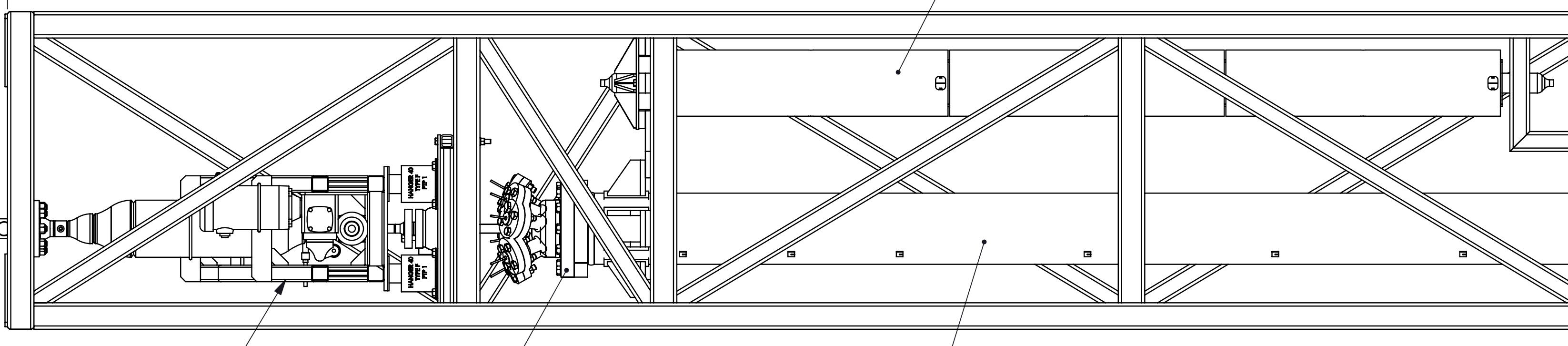

2

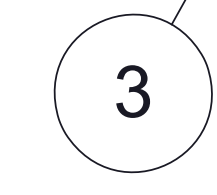

is
(1)

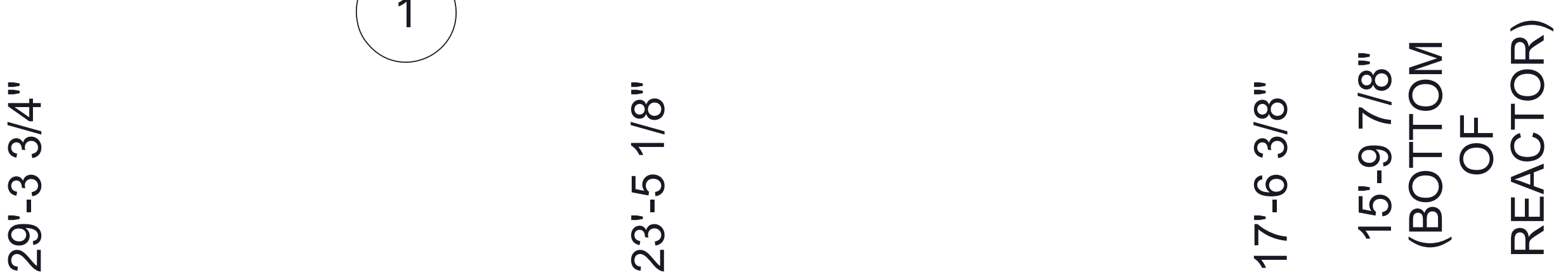

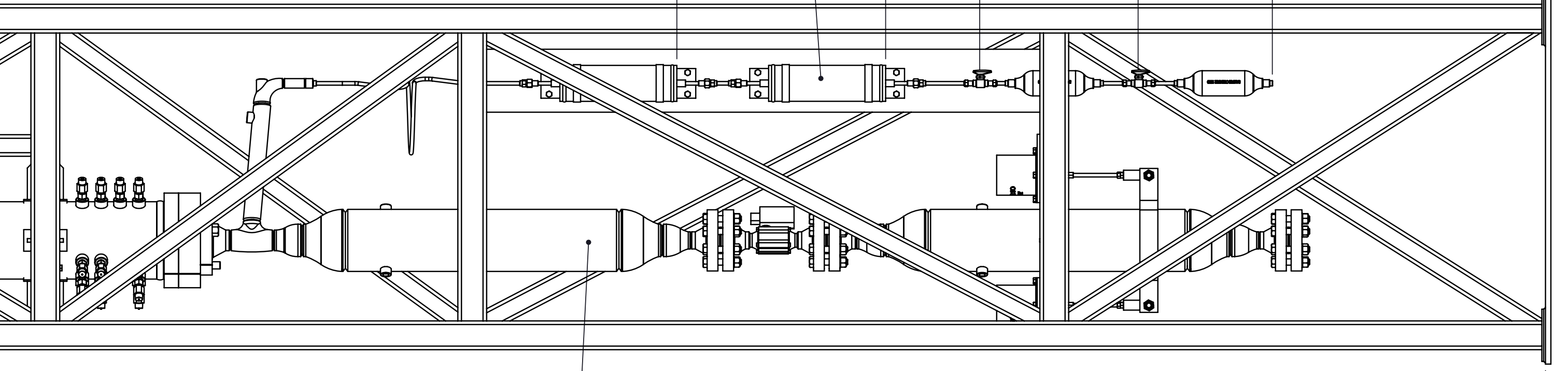

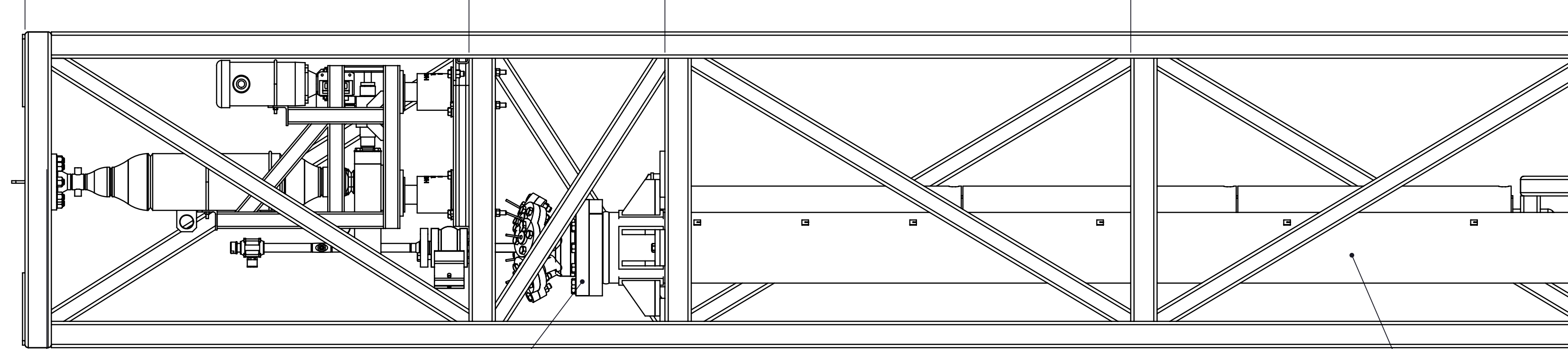

3
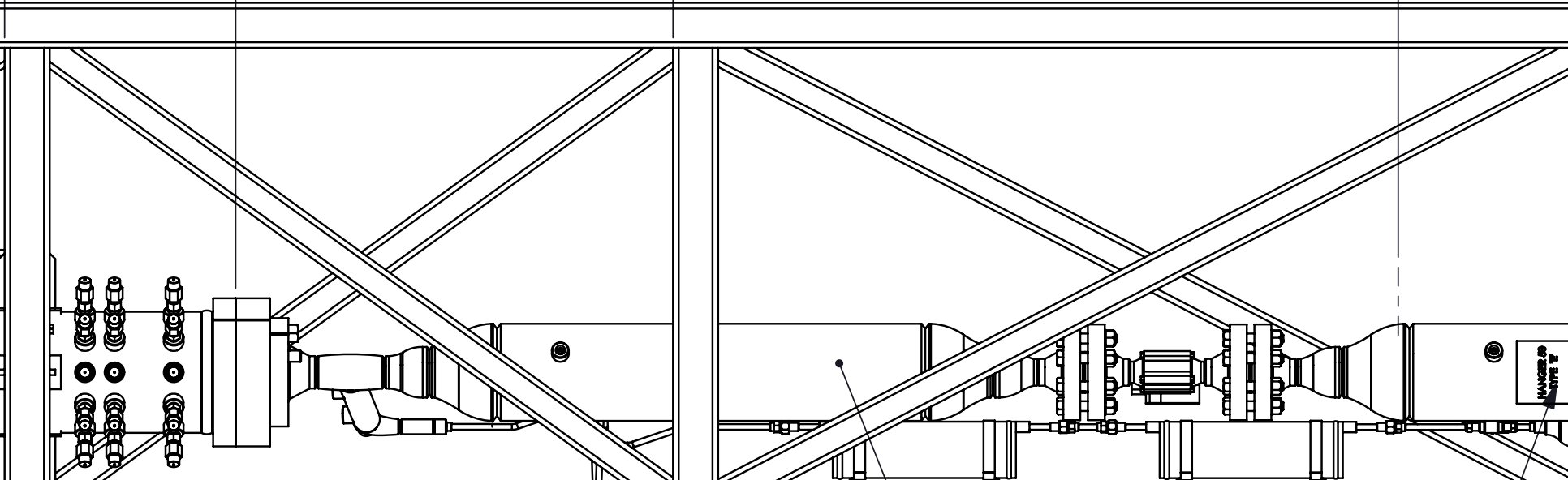

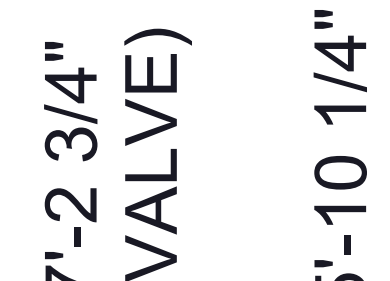

- 37'-1 5/8" TO TOP OF COAL HOPPER

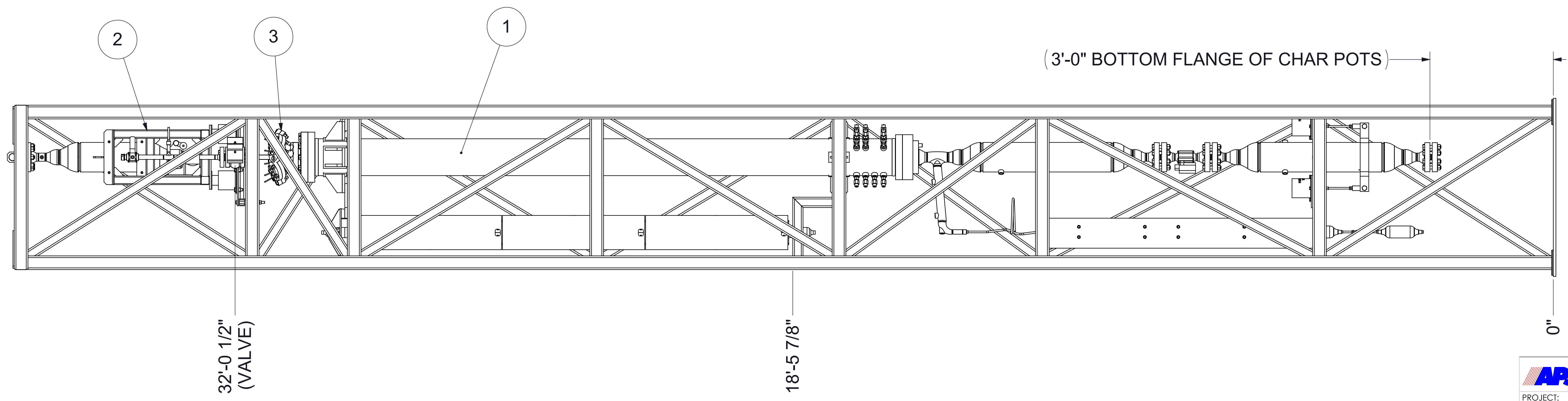


DEE SHEET 2 FOR ORTHAGONAL VIEWS

2 FOR MATERIAL DESCRIPTIONS OF SUB-ASSEMBLIES PART NUMBER COLUMN ON BILL OF MATERIA

93

262216

1

2621

1519

84

30

1312

$21 \quad 16$

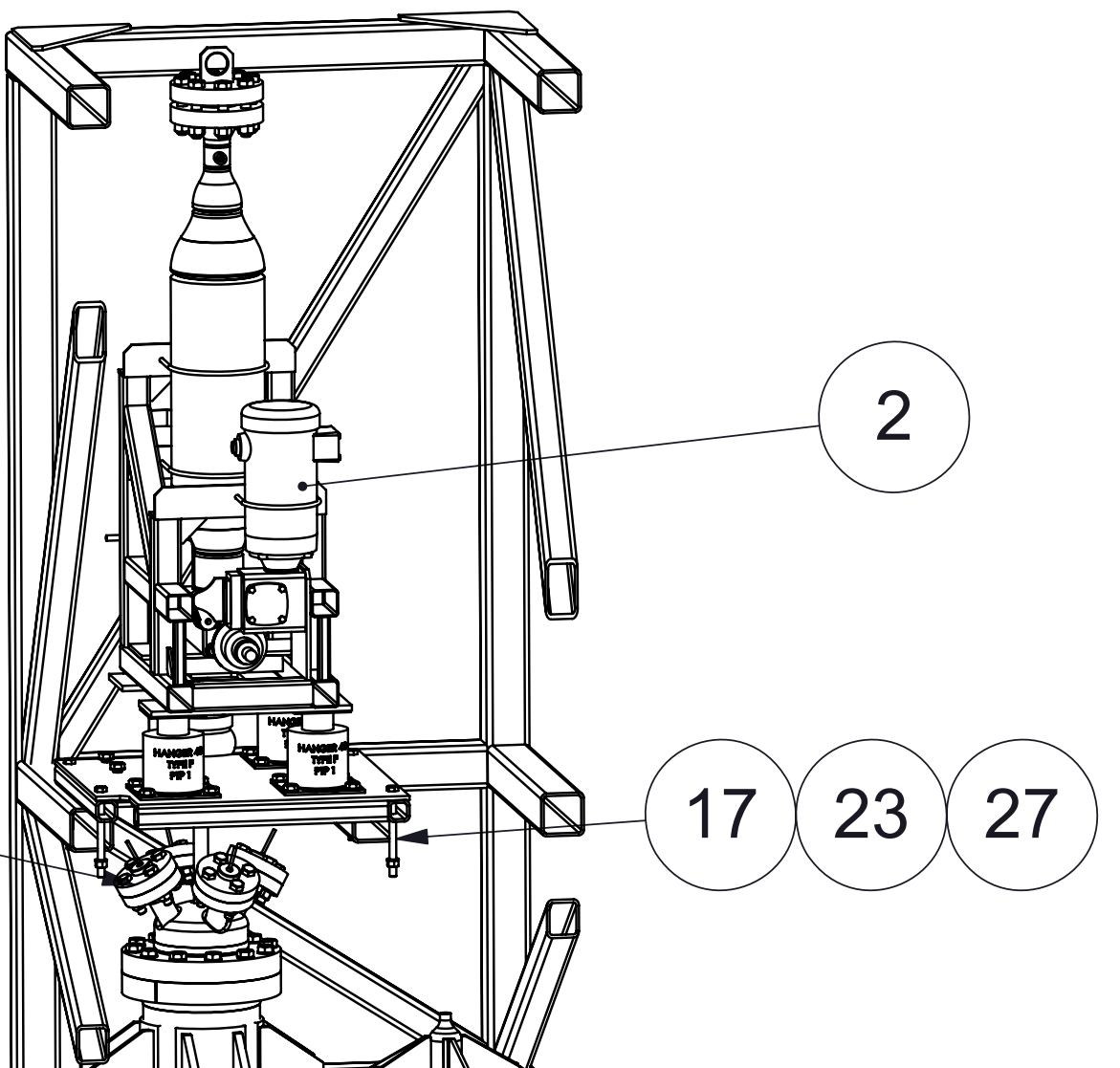

17

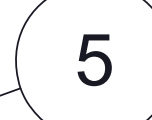

2428

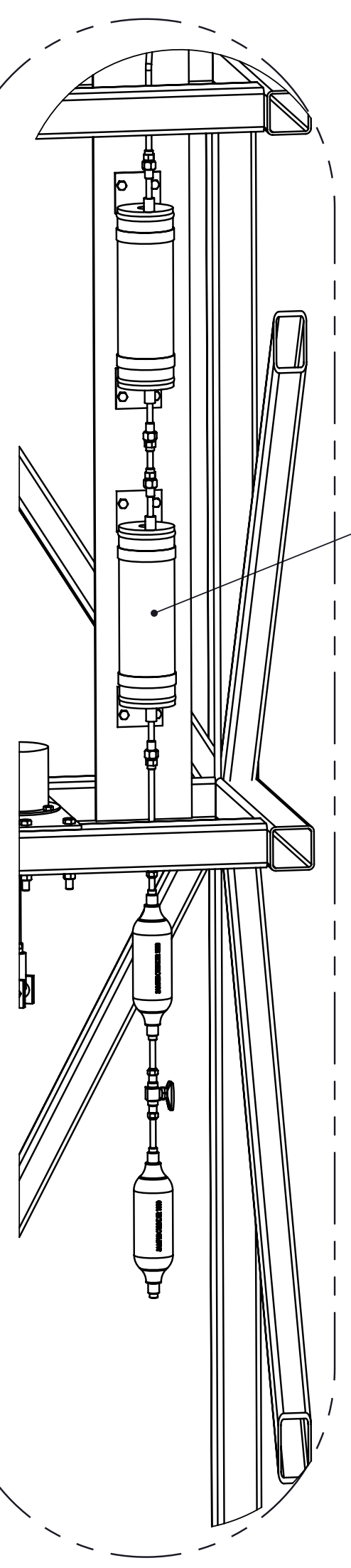

6

\begin{tabular}{|c|c|c|c|}
\hline 30 & HS-50-TYPE-E & ALLOY STL & $H$ \\
\hline 29 & SNG1007.9A & CRS & \\
\hline 28 & SNG1007.8A & CRS & \\
\hline 27 & SNG1009.17 & ALLOY STL & \\
\hline 26 & SNG1009.16 & ALLOY STL & \\
\hline 25 & SNG1009.15 & ALLOY STL & \\
\hline 24 & SNG1009.14 & ALLOY ST & \\
\hline 23 & SNG1009.13 & ALLOY STL & 1 \\
\hline 22 & SNG1009.12 & ALLOY STL & 5 \\
\hline 21 & SNG1009.11 & ALLOY ST & 31 \\
\hline 20 & SNG1009.10 & ALLOY ST & \\
\hline 19 & SNG1009.9 & ALLOY STL & \\
\hline 18 & SNG1009.8-1.25 & ALLOY STL & \\
\hline 17 & SNG1009.7-3.00 & ALLOY ST & \\
\hline 16 & SNG1009.6-2.50 & ALLOY STL & \\
\hline 15 & SNG1009.5-4.00 & ALLOY STL & \\
\hline 14 & SNG1009.4-4.75 & ALLOY STL & \\
\hline 13 & SNG1009.3 & ALLOY STL & \\
\hline 12 & SNG1009.2 & ALLOY STL & \\
\hline 11 & SNG1009.1 & 316 ST STL & $1 /$ \\
\hline 10 & SNG1005.21A & 316 ST STL & \\
\hline 9 & SNG1003.9C & $\begin{array}{l}\text { R STYLE, SWG INCONEL 625 } \\
\text { WINDING WITH THERMICULIE } 835 \\
\text { FILLER (CRITICAL SERVICE) }\end{array}$ & \\
\hline 8 & SNG1000.26A & $\begin{array}{l}\text { R STYLE, SWG INCONEL 625 } \\
\text { WINDING WITH THERMICULIE } 835 \\
\text { FILLER (CRITICAL SERVICE) }\end{array}$ & \\
\hline 7 & W-SNG1007.1F & CRS & \\
\hline 6 & A-SNG1008.1A & SEE NOTE 2 & \\
\hline 5 & A-SNG1006.1B & & \\
\hline 4 & A-SNG1005.1G & SEE BOM & \\
\hline 3 & A-SNG1003.22D & SEE NOTE 2 & \\
\hline 2 & A-SNG1002.1F & SEE NOTE 2 & \\
\hline & A-SNG1000.1D & & \\
\hline & PART NUMBER & MATERIAL & \\
\hline
\end{tabular}

ANGER, SPRING, 50, TYPE E, 1/2"THREADED PLATE, SPRING HANGER SUPPORT, TWO PLATE, SPRING HANGER SUPPORT

$$
\begin{aligned}
& \text { 1/2" FLAT WASHER } \\
& \text { 5/8" FLAT WASHER } \\
& \text { 3/4" FLAT WASHER } \\
& \text { 7/8' FLAT WASHER }
\end{aligned}
$$

1/2-13 UNC-2B HEX HEAVY NUT SA 194 GR B7

5/8-18 UNF-2B HEX HEAVY NUT SA 194 GR B7

14-16 UNC-2B HEX. HEAVY NUT SA 194 GR B7

7/8-9UNC-2B HEX HEAVY NUT SA194 GR B7

14 UNF-2B HEX HEAVY NUT SA 194 GR B7

$1 / 2-13$ UNC-2A x 1.25"LG. SA193 GR B7

5/8-18 UNF-2A × 8.00"LG. SA193 GR B7

/4-16 UNC-2A x 250"LG. SA 193 GR B7

7/8-9 UNC-2A x 4.00"LG. SA193 GR B7 STUD, 1-14 UNF-2A x 4.75"LG.

TURNBUCKEL, 1/2-13 UNC-2B

ROD, 1/2-13 UNC-2A THREADED x 12.00"LG 1/2-13 UNC-2B, CLEVIS, PIPE CLAMP SUPPORT

CLAMP, PIPE, 8" CLAMP 4-BOLT

GASKET, REACTOR HEAD

GASKET, R STYLE, SWG INCONEL 625 WINDING

WELDMENT, REACTOR FRAME ASSEMBLY, CONDENSER COMPONENTS ASSEMBLY, PRE-HEATER
ASSEMBLY, CHAR POT

ASSEMBLY, REACTOR INJECTOR HEAD ASSEMBLY, COAL FEEDER SYSTEM ASSEMBLY, KINETIC REACTOR DESCRIPTION

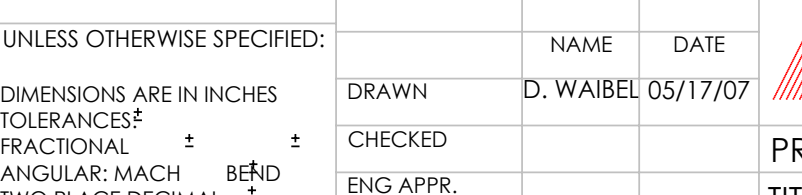

COAL TO SNG
CNETICS REACTOR

ASSEMBLY REACTOR TOP LEVE

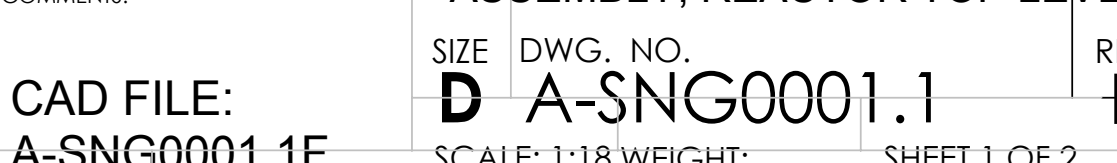




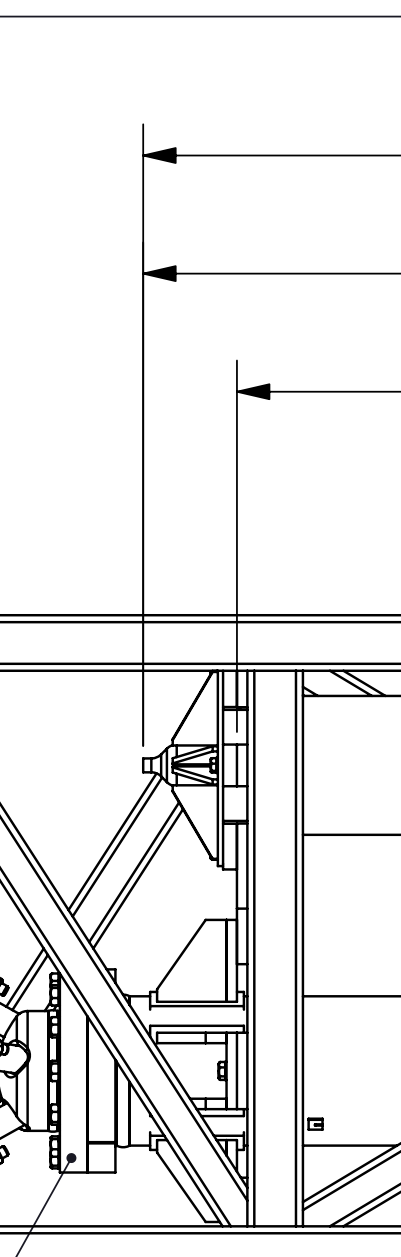

$37^{\prime}-5 "$

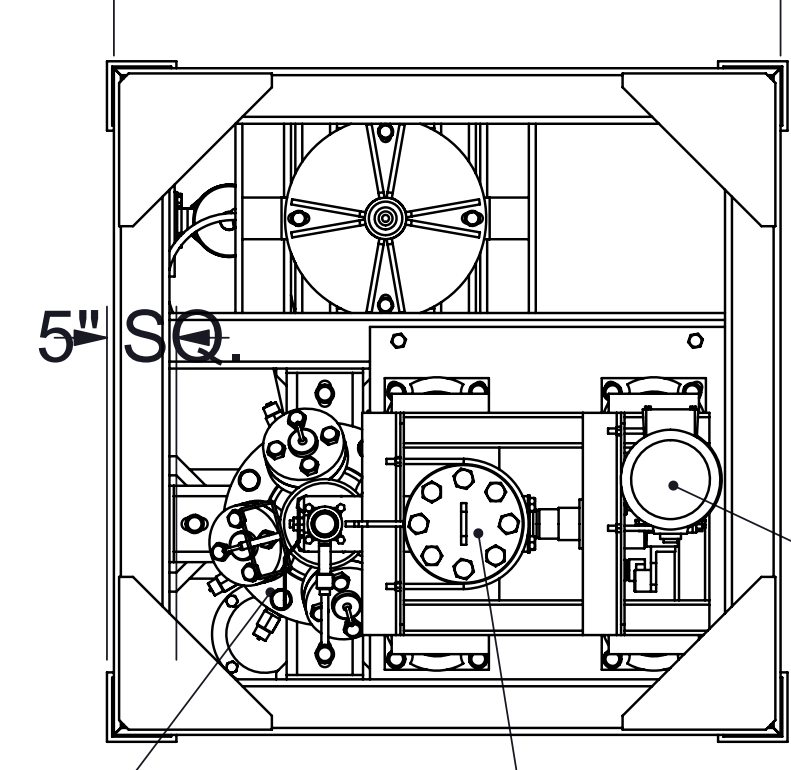

3

COAL INLET

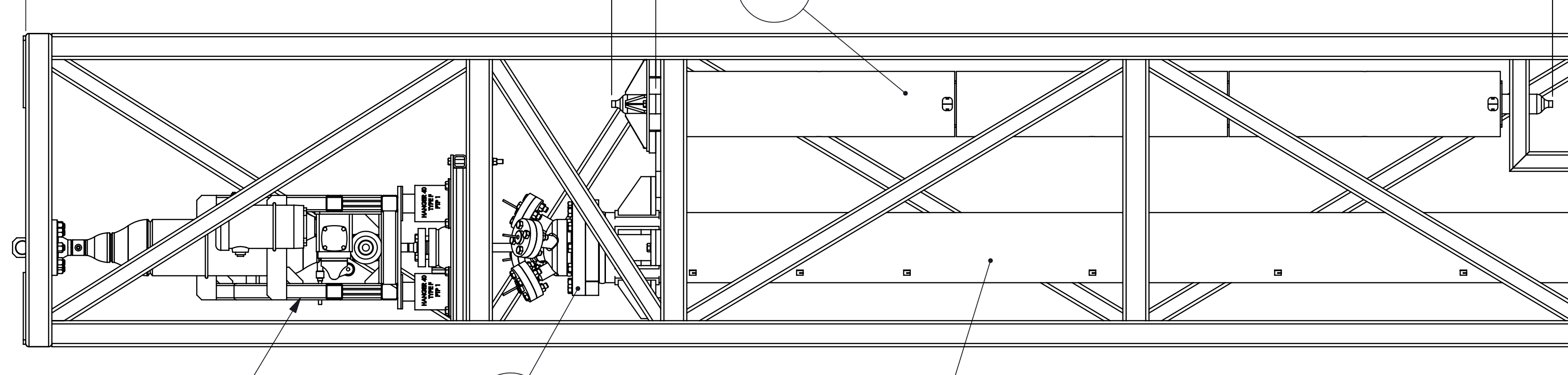

2

1

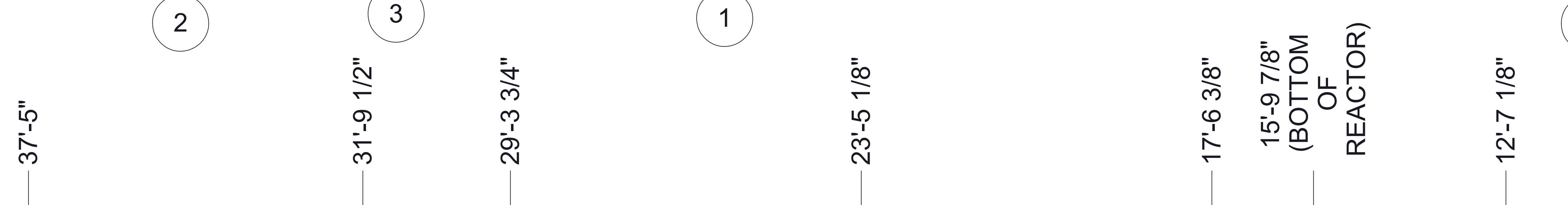

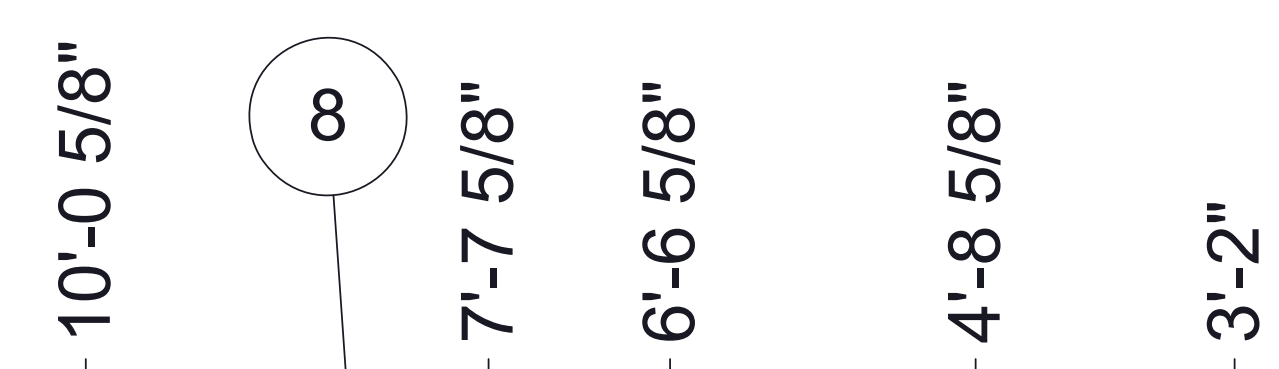

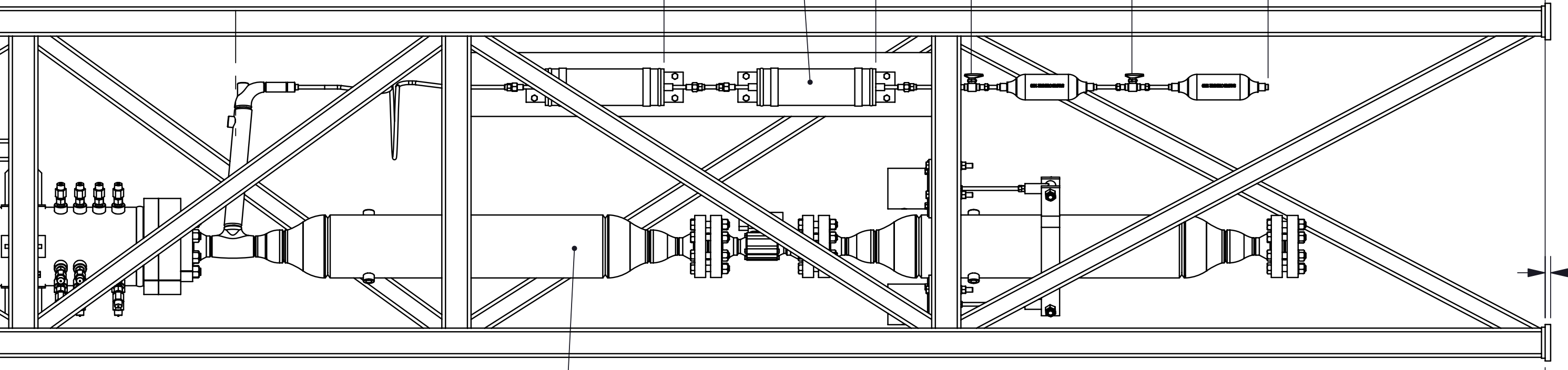

$-3 / 4^{\prime \prime}$ THK.

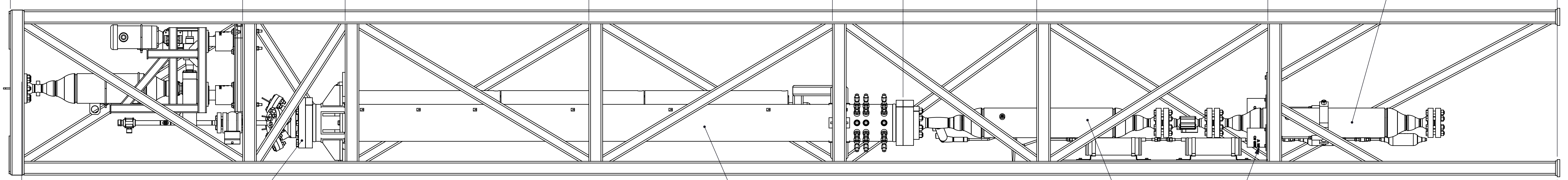

3

1

4

10

37'-1 5/8" TO TOP OF COAL HOPPER

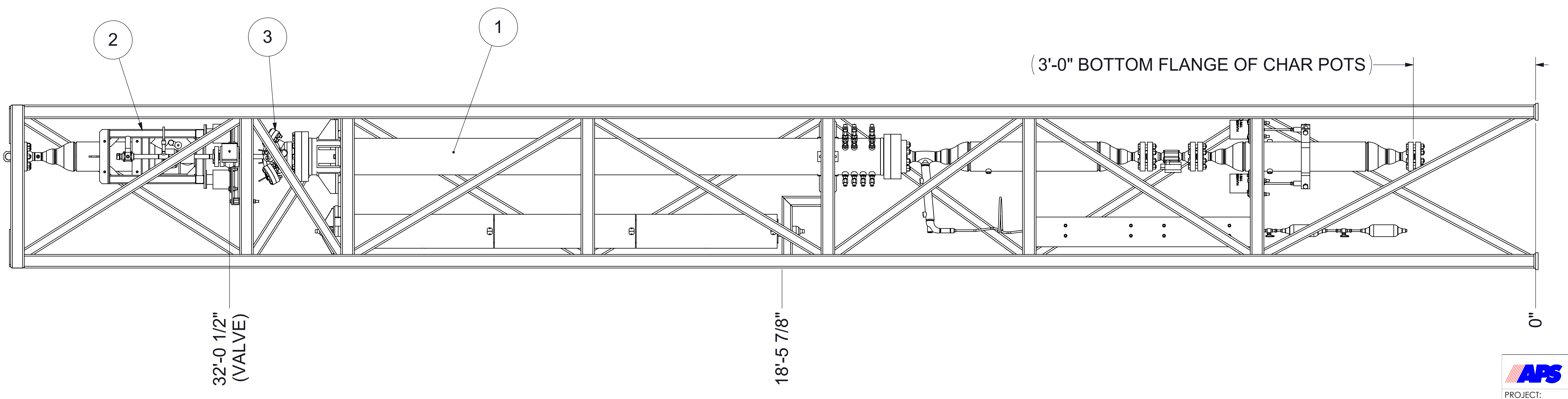




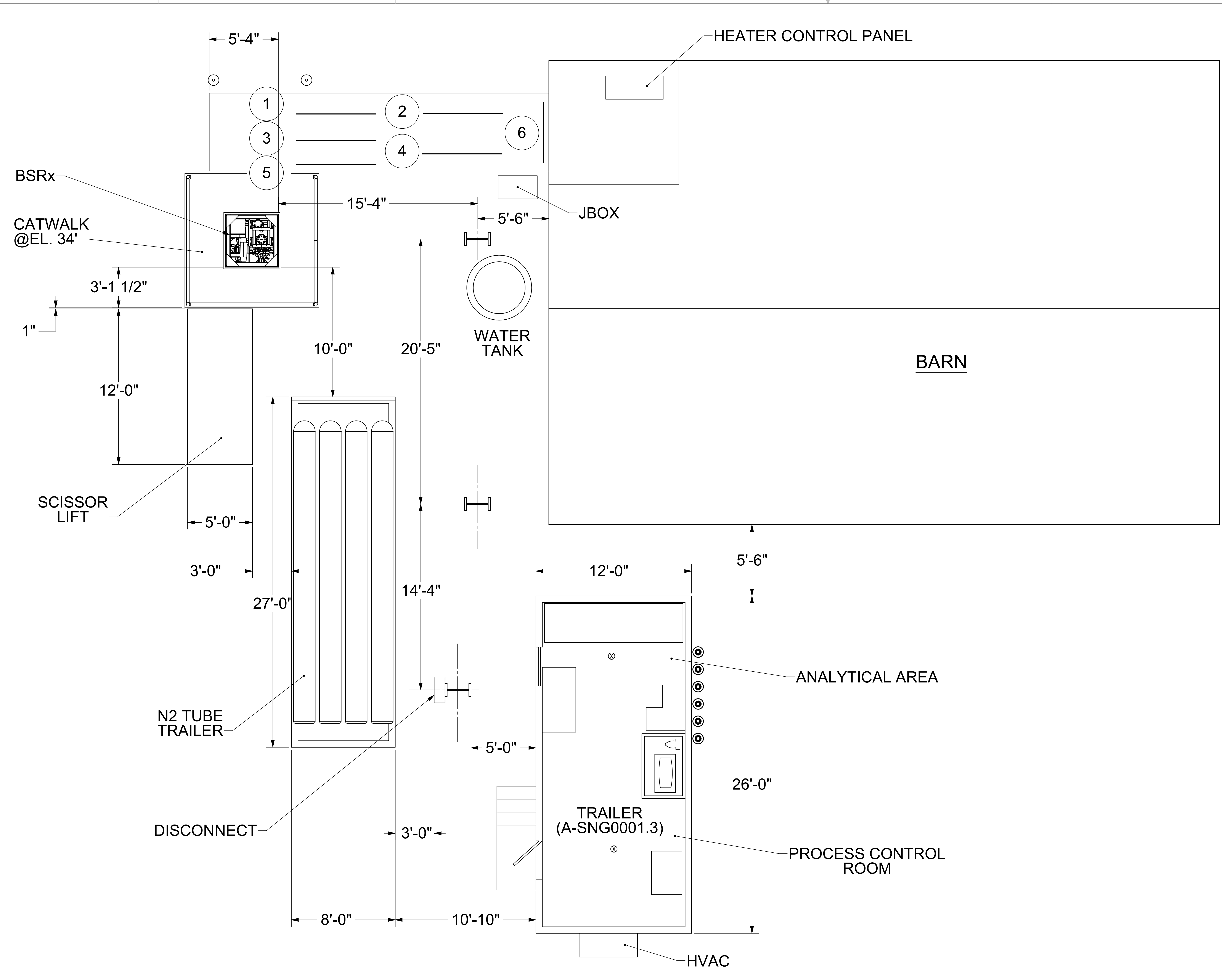

\section{LEGEND}

(1) HIGH PRESSURE N2

(2) N2 PRESSURE BALANCING

(3) EMERGENCY N2 PURGE

(4) HYDROGEN CONTROL

5 INSTRUMENT N2

6 SNG PRODUCT 


\section{GENERAL NOTES:}

1 SEE SHEET 2 FOR ORTHO VIEWS, SECTIONS, AND DETAILS.

2 FOR MATERIAL DESCRIPTIONS OF SUB-ASSEMBLIES SEE SUB-ASSEMBLY NUMBER AS LISTED IN

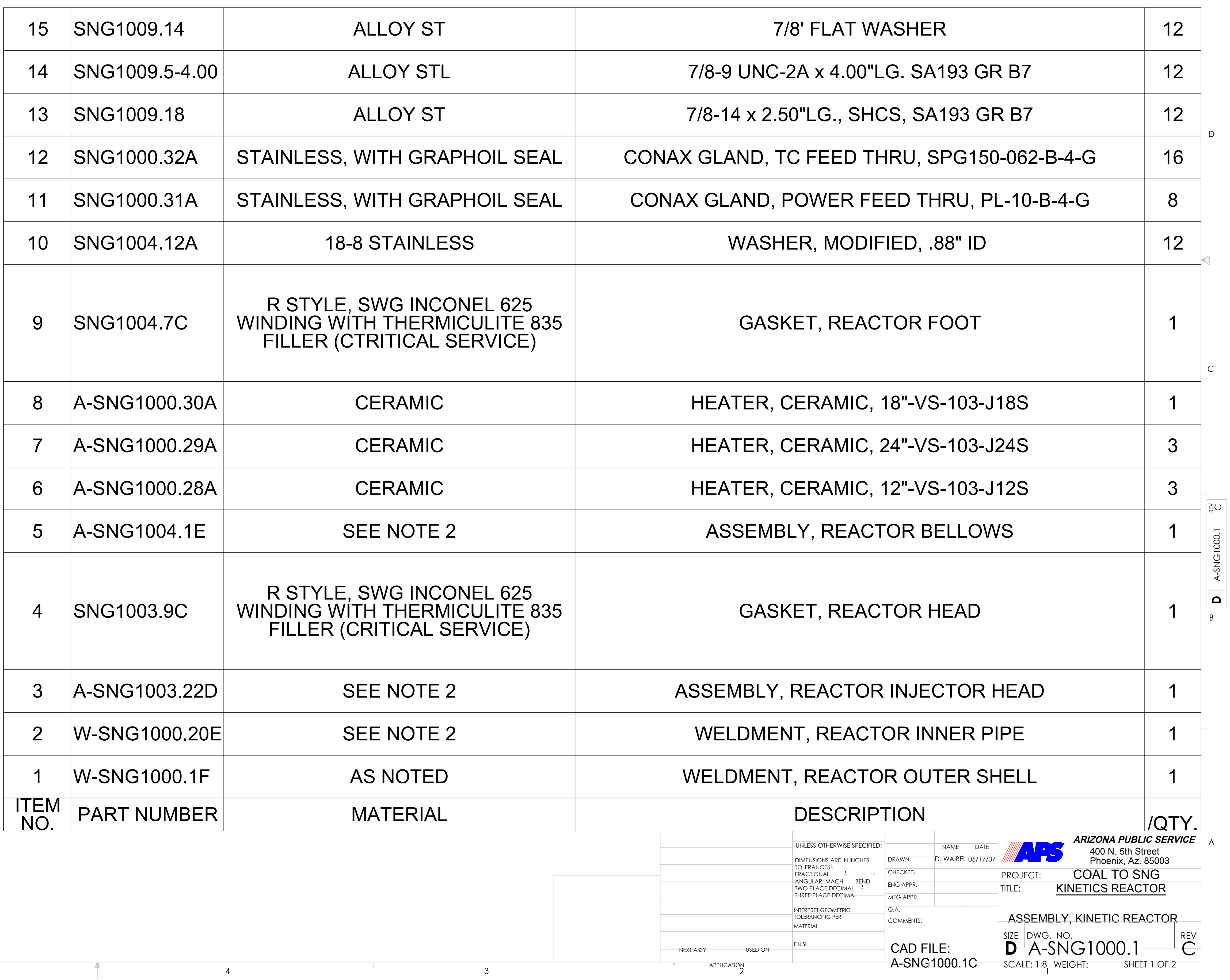


$4 \mathrm{X} 7 / 8$ " WIDE x 1.0" LONG SLOTS

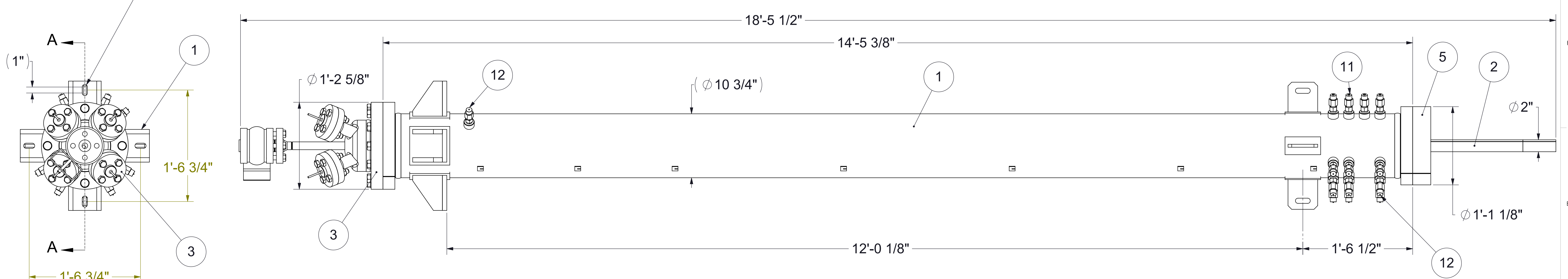

- 1'-6 3/4"
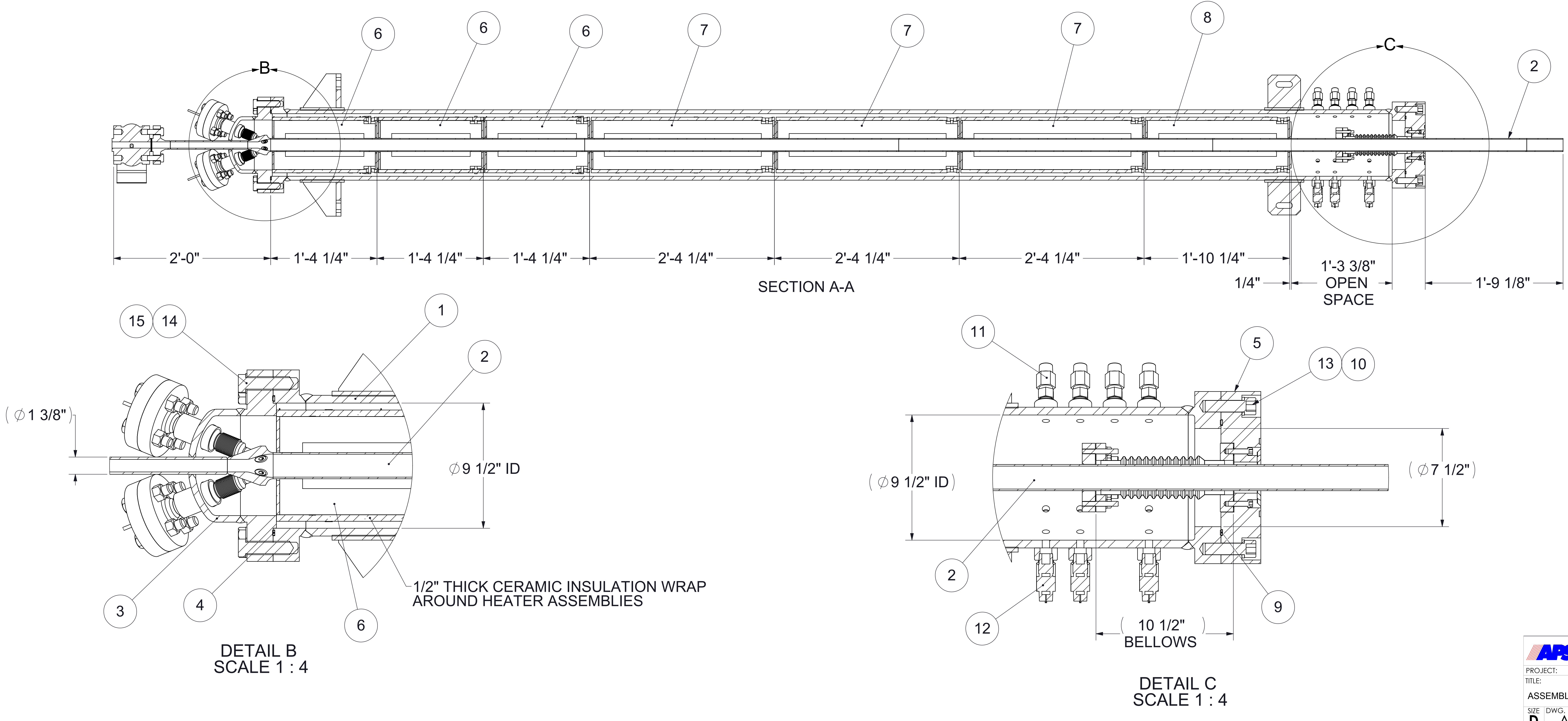


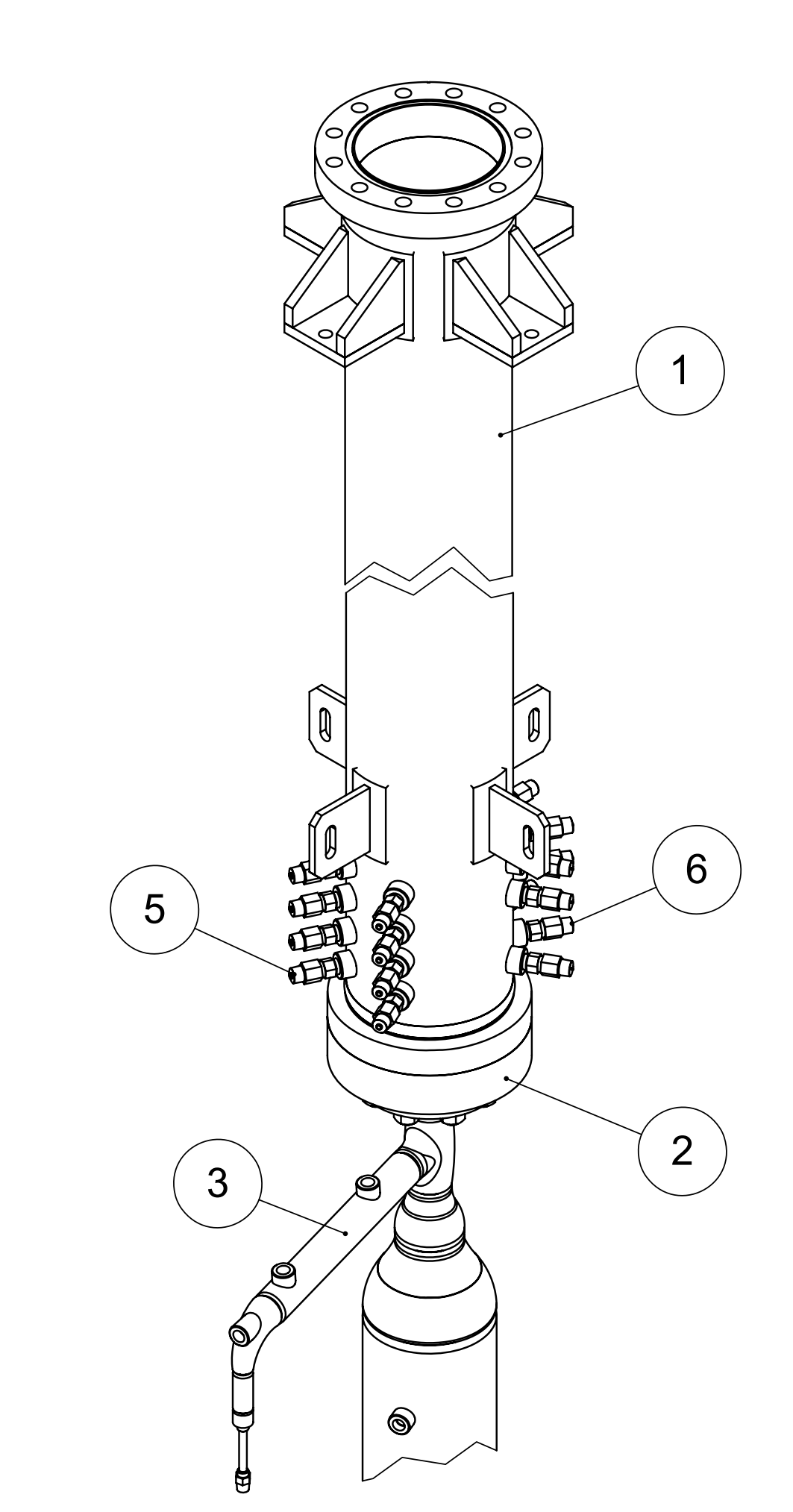

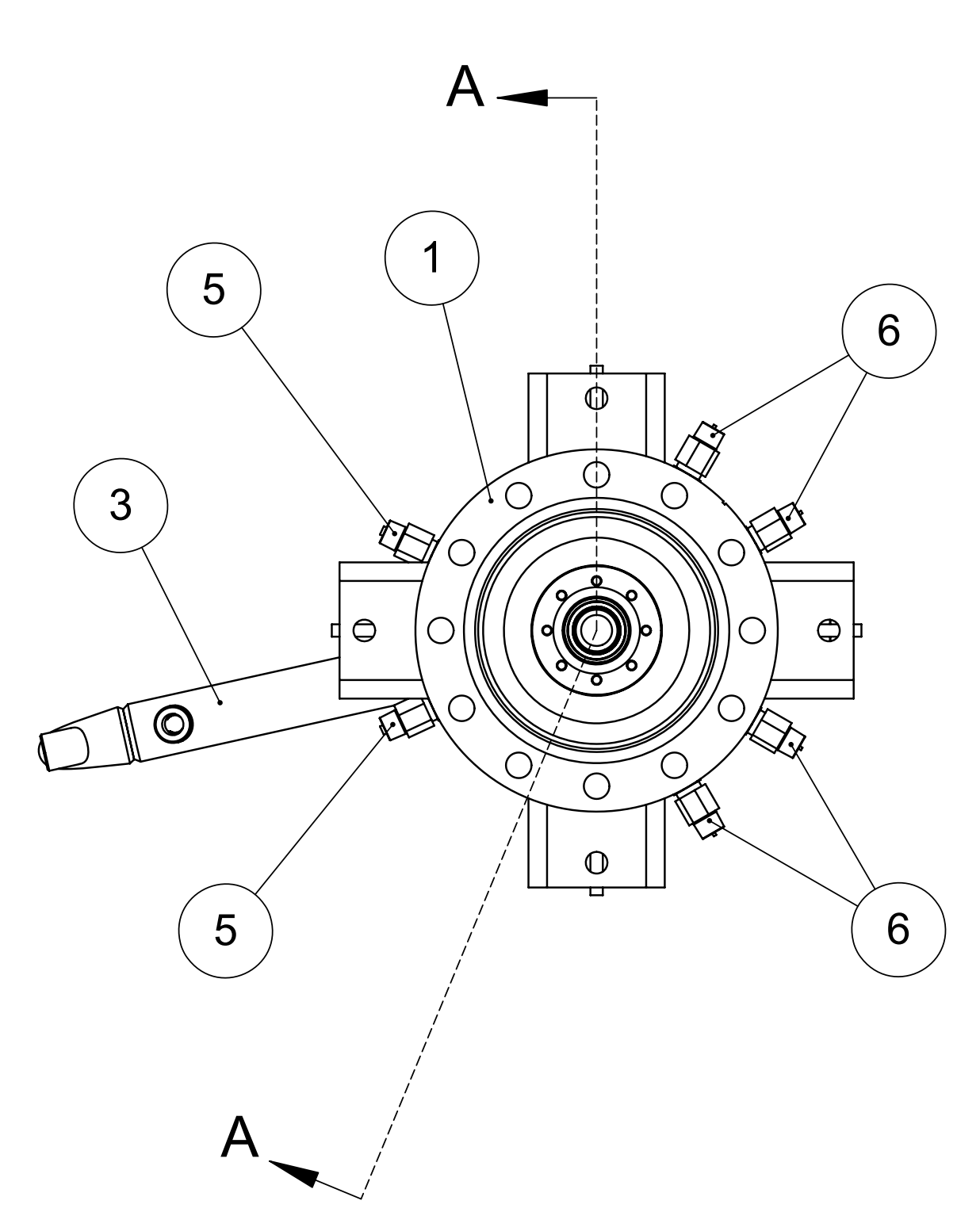

SEE SHEET 2

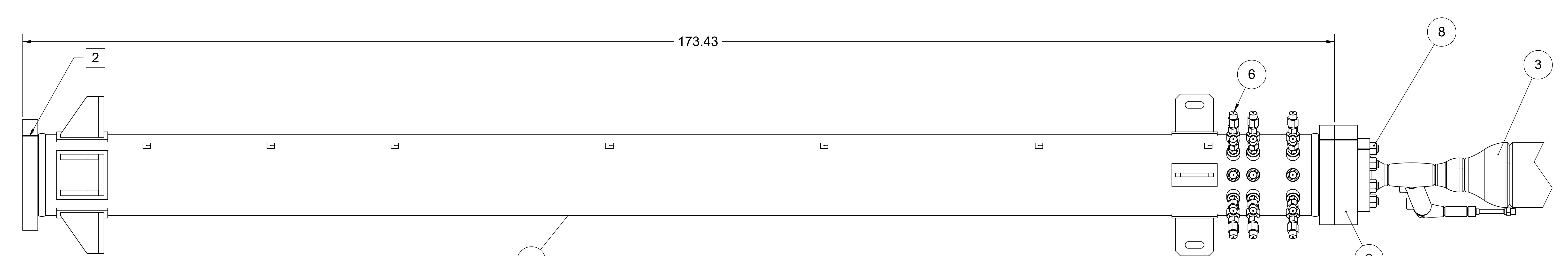

11

\begin{tabular}{|c|c|c|c|}
\multicolumn{4}{|c}{ REVISIONS } \\
\hline ZONE & REV. & DESCRIPTION & D \\
\hline & A & INITIAL RELEASE & 01 \\
\hline
\end{tabular}

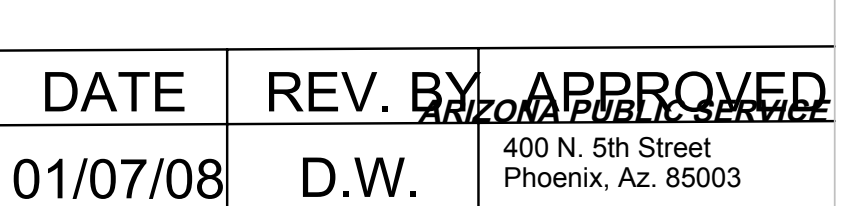

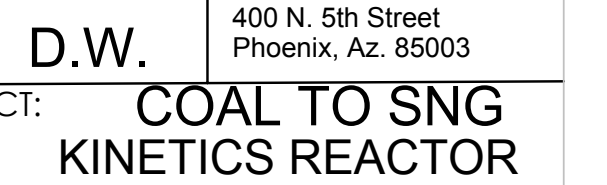




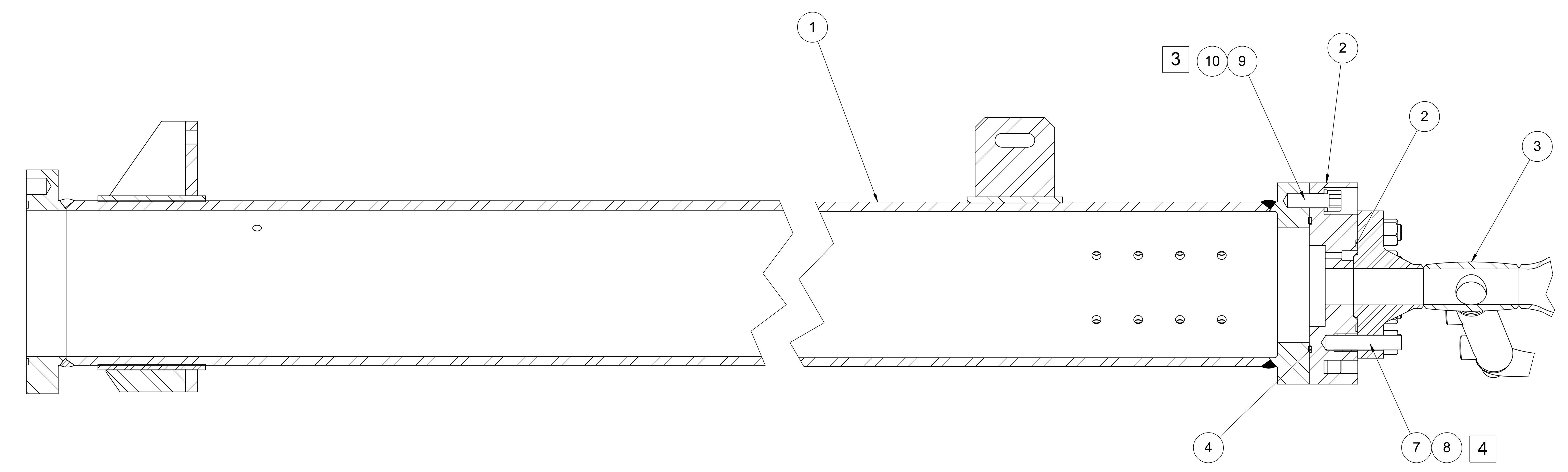

SECTION AA-A
SCALE $1: 4$ 


\section{GENERAL NOTES:}

DIMENSION AND TOLERANCING PER ANSI Y14.5-1994.

2 BREAK ALL EDGES .5 [.20] MAX. ALL INSIDE RADII 2.4 [.09] MAX. UNLESS NOTED OTHERWISE.

\begin{tabular}{|c|c|c|}
\hline \multicolumn{2}{|c|}{} & \multicolumn{2}{c}{ REVISIONS } \\
\hline ZONE & REV. & DESCRPTION \\
\hline ALL & A & INITIAL RELEASE \\
\hline ALL & B & UPDATED MATERIAL CALL OUT \\
\hline ALL & C & LENGTH CHANGE, 5.90 WAS 5.40 \\
\hline
\end{tabular}
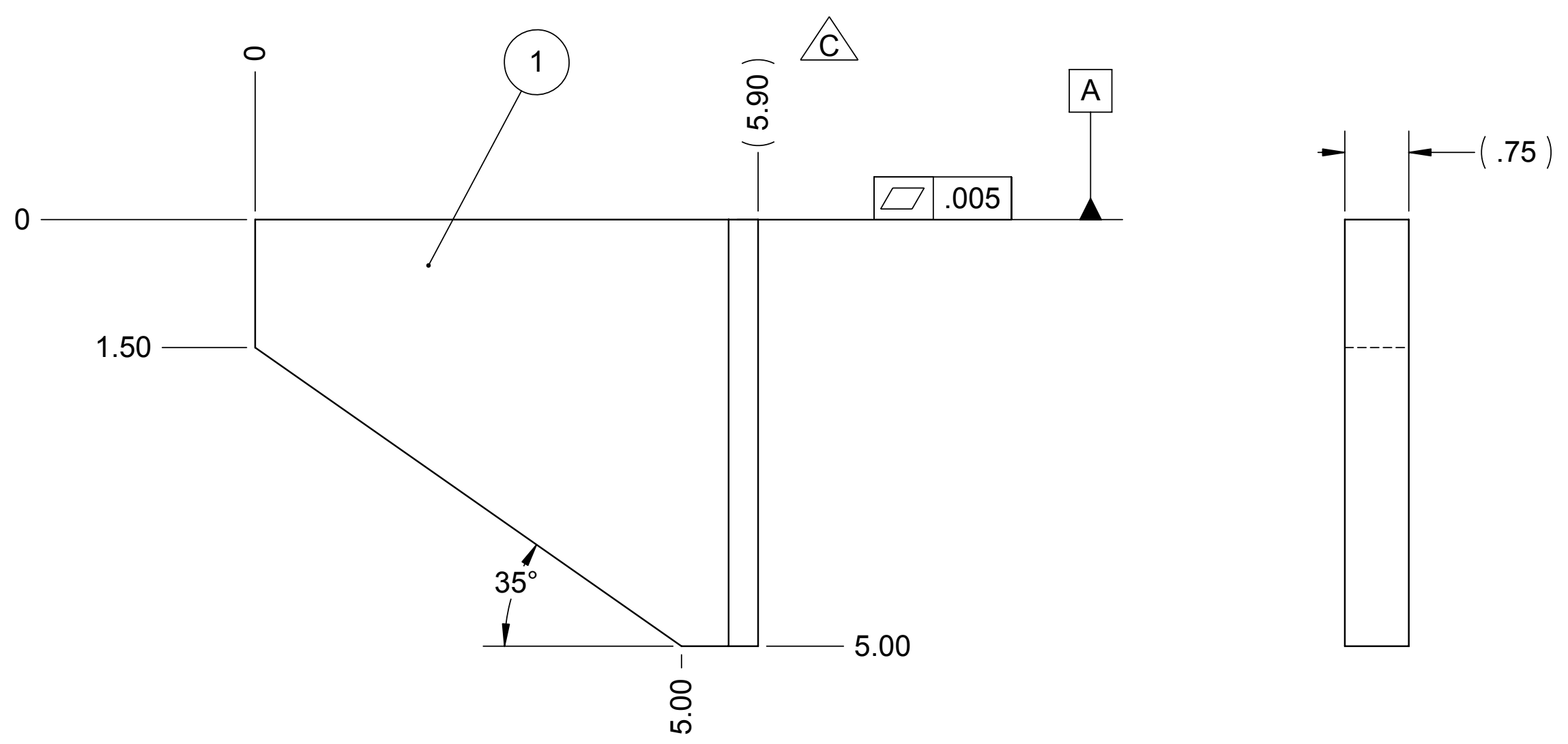

01/02/08 D.W.

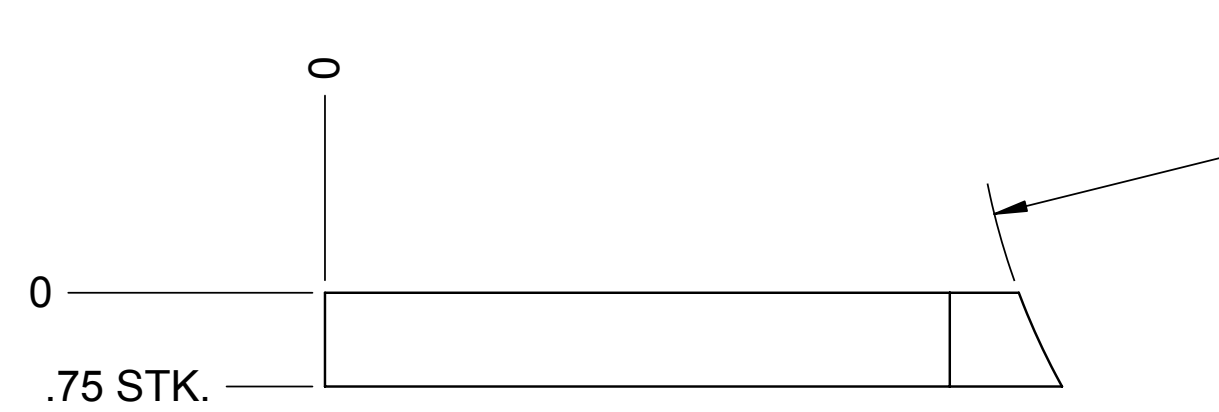

R5.69

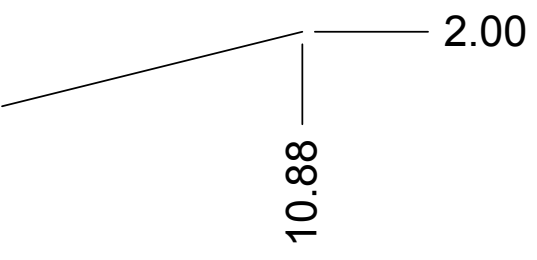

2.00

\begin{tabular}{|l|l|}
\hline 1 & SNG1000.10C \\
\hline
\end{tabular}

SA-515 GR 70 PER ASTM / SA-20 ITEM NO.

PART NUMBER
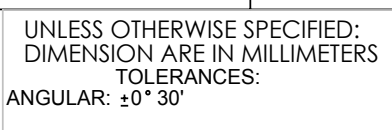

IED:

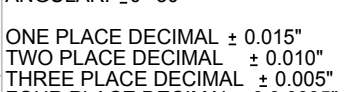

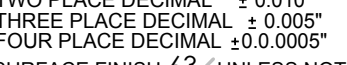

INTERPEET GEOMERRIC TOLERANCING

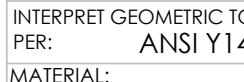

MATERAL: SEE BOM

SIMLARTO

GUSSET, LEFT, REACTOR, TOP SUPPORT

\section{DESCRIPTION}

\begin{tabular}{|l|c|}
\hline DATE & NAME \\
\hline $6 / 13 / 07$ & D. WAlBEL
\end{tabular}

IIIIII ARIZONA PUBLIC SERVICE 400 N. 5th Street PROJECT: COAL TO SNG COAL TO SNG
KINECTICS RFACTOR GUSSET, LEFT, REACTOR

\begin{tabular}{l|l}
\hline Q.A. & GUSSET, LEFT, \\
\hline SI 0 MM 25 & TOP SUPPORT
\end{tabular}

\begin{tabular}{l|l} 
SIZE & DWG. NO. \\
$\mathbf{B}$ & SNG 1000.10
\end{tabular}

B SNG1000.10 
REVISIONS

DIMENSION AND TOLERANCING PER ANSI Y14.5-1994.

2 BREAK ALL EDGES .5 [.20] MAX. ALL INSIDE RADII 2.4 [.09] MAX. UNLESS NOTED OTHERWISE.

\begin{tabular}{|c|c|c|c|c|}
\hline \multicolumn{5}{|c|}{ REVISIONS } \\
\hline ZONE & REV. & DESCRPTION & DATE & REV. BY APPROVED \\
\hline ALL & A & INITAL RELEASE & $06 / 14 / 07$ & D.W. \\
\hline ALL & B & MATERLA CALL OUT UPDATED & $01 / 02 / 08$ & D.W. \\
\hline
\end{tabular}

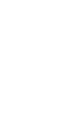
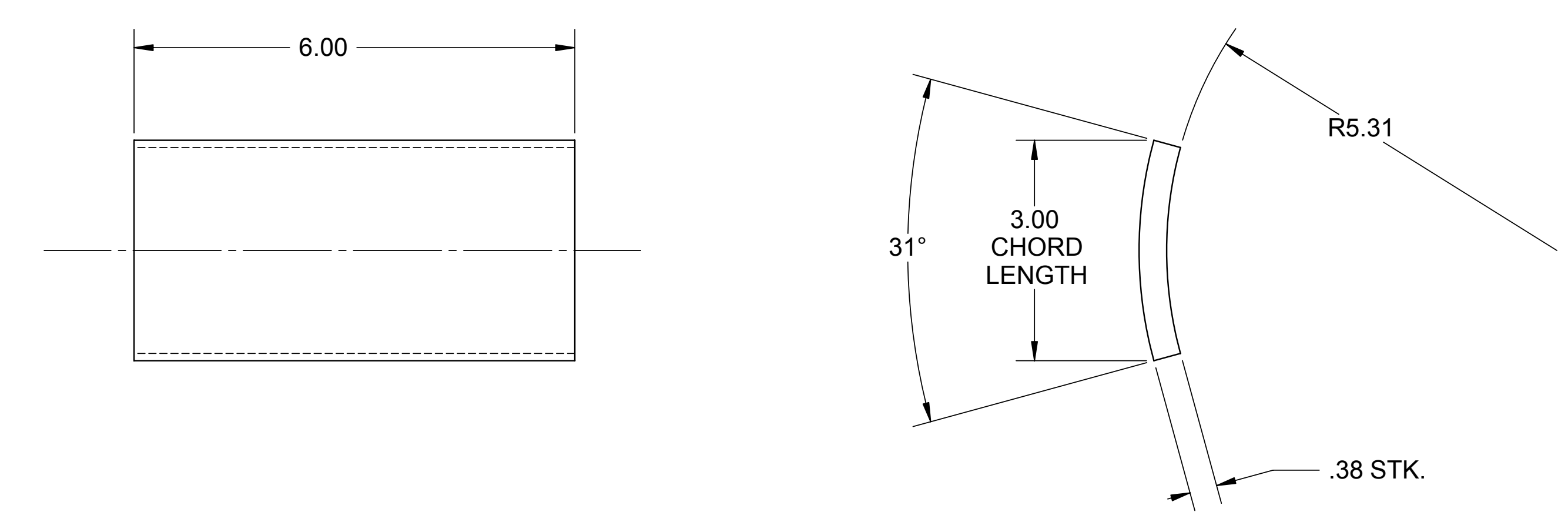

\begin{tabular}{|c|c|c|c|c|c|c|c|c|}
\hline 1 & SNG1000.11B & \multicolumn{2}{|c|}{$\begin{array}{c}\text { SA-515 GR } 70 \text { PER } \\
\text { ASTM / SA-20 CARBON } \\
\text { STEEL PLAATE } 3 / 8 " \\
\text { THICK }\end{array}$} & \multicolumn{4}{|c|}{$\begin{array}{l}\text { PLATE, REACTOR SHELL, } \\
\text { REINFORCEMENT PAD }\end{array}$} & 1 \\
\hline ITEM NO. & PART NUMBER & \multicolumn{2}{|c|}{ MATERIAL } & \multicolumn{4}{|c|}{ DESCRIPTION } & QTY. \\
\hline & & \multirow{6}{*}{ 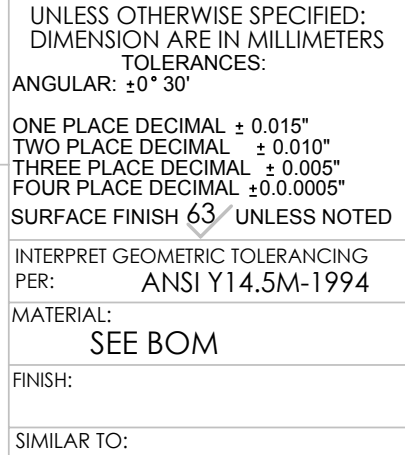 } & $\begin{array}{ll}\text { DRAWN } & 0 \\
\text { CHEKKED } & \end{array}$ & $\begin{array}{l}\text { DATE } \\
\text { Do/14/07 }\end{array}$ & $\begin{array}{l}\text { NAME } \\
\text { D. WABBEL }\end{array}$ & \multicolumn{3}{|c|}{$\begin{array}{l}\text { ARIZONA PUBLIC SERVICE } \\
400 \mathrm{~N} .5 \text { th Street } \\
\text { Phoenin, Az. } 85003\end{array}$} \\
\hline & & & ENG APP. & & & $\begin{array}{l}\text { PROJECT } \\
\text { TTILE: }\end{array}$ & \multirow{2}{*}{$\begin{array}{r}\text { COAL TO } \\
\text { KINFTICS }\end{array}$} & \\
\hline & & & $Q_{\text {Q. }}$ & & & \multirow{2}{*}{\multicolumn{3}{|c|}{$\begin{array}{l}\text { PLATE, REACTOR SHELL, } \\
\text { REINFORCING PAD }\end{array}$}} \\
\hline & & & \multirow{2}{*}{\multicolumn{2}{|c|}{$\underset{\text { METRIC }}{\mathrm{SI}}=\mathrm{MM}_{1} 25$}} & & & & \\
\hline & & & & & 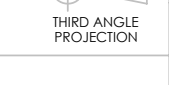 & \multirow{2}{*}{\multicolumn{2}{|c|}{$\begin{array}{l}\text { SIIE } \\
\text { B DWG. NO. } \\
\text { SCALE: } 1: 2 \text { WEIGHT: }\end{array}$}} & REV \\
\hline & & & CAD FILE & & NG1000.11B & & & SHEET 1 OF 1 \\
\hline
\end{tabular}




\section{GENERAL NOTES:}

DIMENSION AND TOLERANCING PER ANSI Y14.5-1994.

2 BREAK ALL EDGES .5 [.20] MAX. ALL INSIDE RADII 2.4 [.09] MAX. UNLESS NOTED OTHERWISE.

\begin{tabular}{|c|c|c|c|c|}
\hline \multicolumn{5}{|c|}{ REVISIONS } \\
\hline ZONE & REV. & DESCRPTION & DATE & REV. BY APPROVED \\
\hline ALL & A & INITIAL RELEASE & $06 / 14 / 07$ & D.W. \\
\hline ALL & B & MATERIAL CALL OUT UPDATED & $01 / 02 / 08$ & D.W. \\
\hline
\end{tabular}
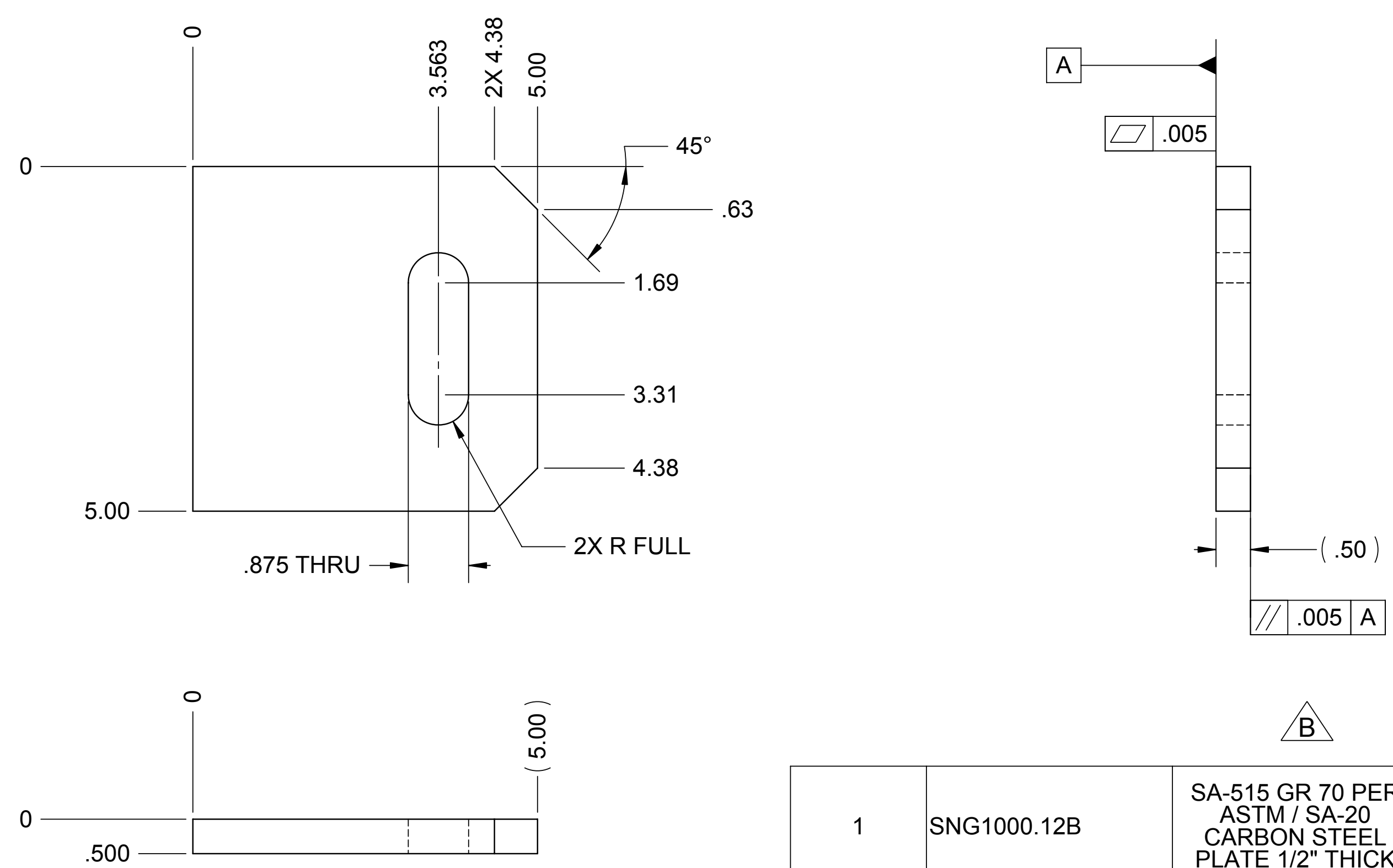

B

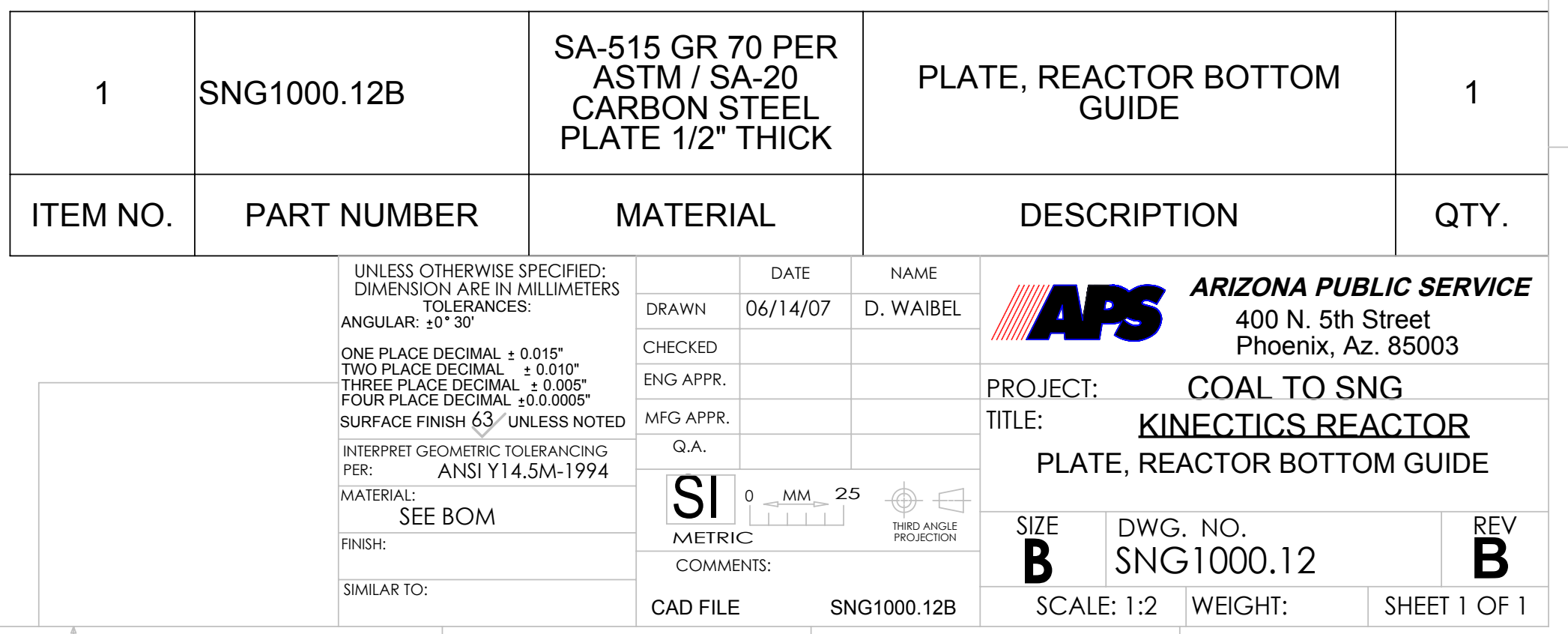


2 BREAK ALL EDGES .5 [.20] MAX. ALL INSIDE RADII
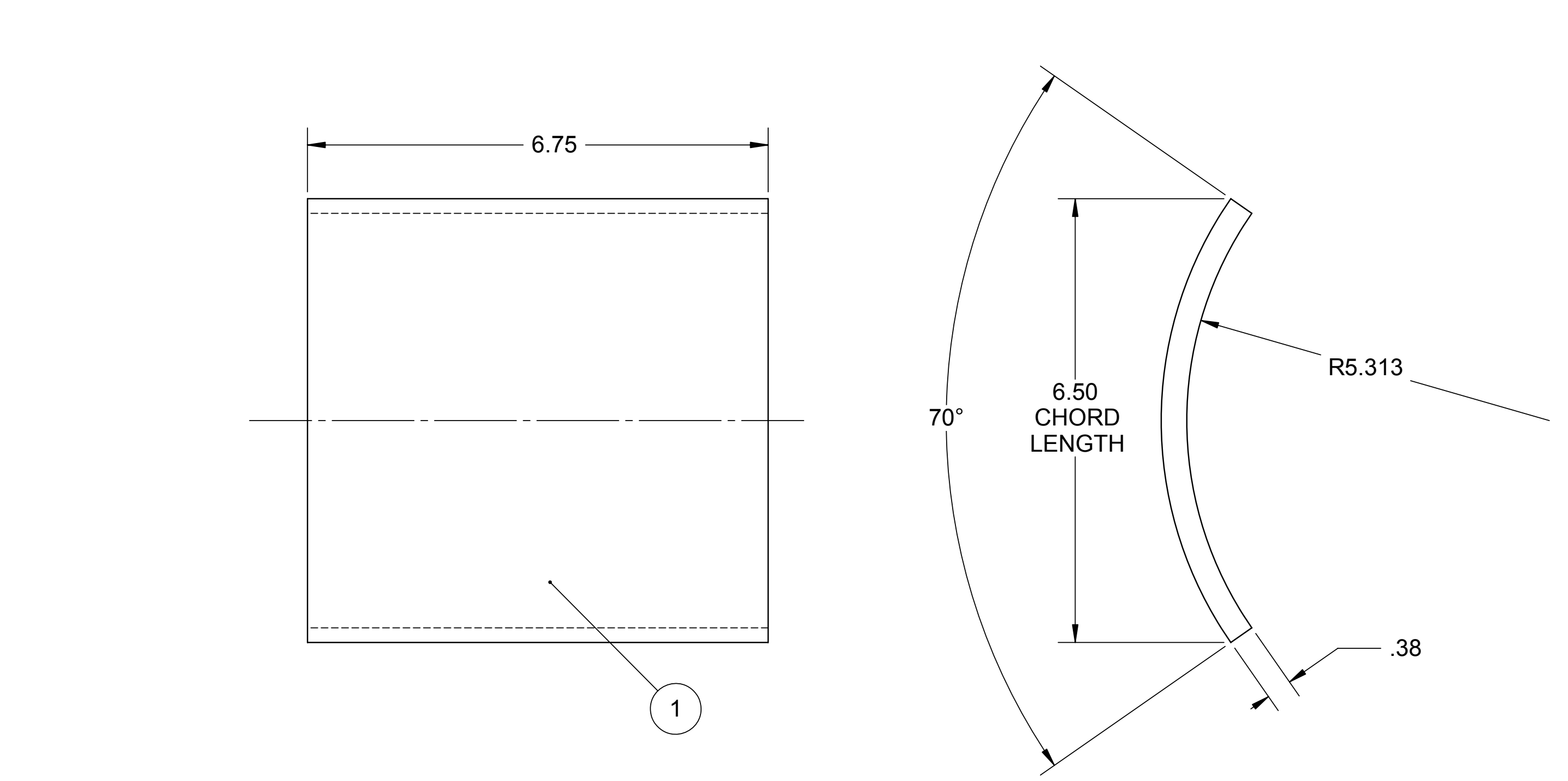

\begin{tabular}{|c|c|c|c|c|c|}
\hline 1 & SNG1000.13B & \multicolumn{3}{|c|}{$\begin{array}{c}\text { SA-515 GR } 70 \text { PER } \\
\text { ASTM / SA-20 } \\
\text { CARBON STEEL } \\
\text { PLATE, 3/8" THICK }\end{array}$} & \\
\hline ITEM NO. & PART NUMBER & \multicolumn{3}{|c|}{ MATERIAL } & \\
\hline & \multirow{5}{*}{\multicolumn{2}{|c|}{ 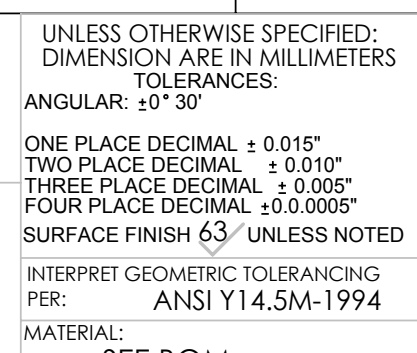 }} & DRAWN & $\begin{array}{c}\text { DATE } \\
06 / 14 / 07\end{array}$ & $\begin{array}{c}\text { NAME } \\
\text { D. WAIBEL }\end{array}$ \\
\hline & & & $\begin{array}{l}\text { CHECKED } \\
\text { ENG APPR }\end{array}$ & & \\
\hline & & & MFG APPR & & \\
\hline & & & Q.A. & & \\
\hline & $\begin{array}{l}\text { MATERIAL: } \\
\text { SEE BO }\end{array}$ & & SI & $0=M M$ & क) \\
\hline & & FEINS: & & & \\
\hline & & & & & \\
\hline
\end{tabular}

\section{ARIZONA PUBLIC SERVICE 400 N. 5th Street
Phoenix, Az. 85003} PROJECT: COAL TO SNG IITE: KINETICS REACTOR PLATE, REACTOR SHELL,

RE

\begin{tabular}{l|l} 
SIZE & DWG. NO. \\
B & SNG 1000.13
\end{tabular} 

REFERENCE; WATLOW'S THERMOCOUPLE, WELD PAD, MILLED SLOT,
ORDER CODE 5 .

BREAK ALL EDGES .5 [.20] MAX. ALL INSIDE RADII 2.4 [.09] MAX. UNLESS NOTED OTHERWISE.
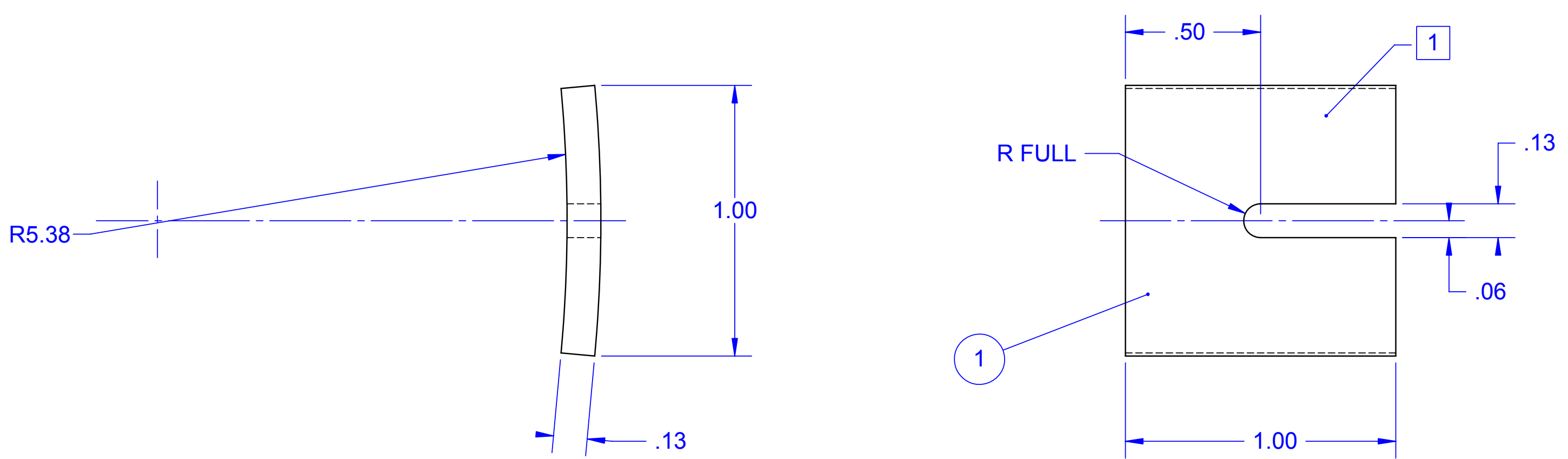

\begin{tabular}{|c|c|c|c|c|c|c|}
\hline 1 & \multicolumn{3}{|c|}{ SNG1000.14A } & PLATE & REACTOR SHELL TC PAD & 1 \\
\hline $\mathrm{MNO}$. & \multicolumn{3}{|c|}{ PART NUMBER } & & DESCRIPTION & QTY. \\
\hline \multicolumn{2}{|c|}{$\begin{array}{l}\text { UNLESS OTHERWISE SPECIFIED } \\
\text { DIMENSION ARE IN MLILIMETERS } \\
\text { TOLERANCES: }\end{array}$} & DRAWN & $\begin{array}{l}\text { DATE } \\
06 / 30 / 07\end{array}$ & $\begin{array}{l}\text { NAME } \\
\text { D. WAIBEL }\end{array}$ & & \\
\hline
\end{tabular}

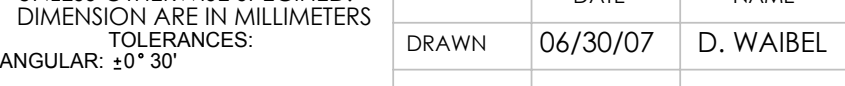

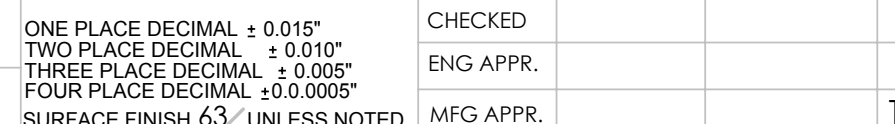

ARIZONA PUBLIC SERVICE $400 \mathrm{~N}$. 5th Street
Phoenix, Az. 85003

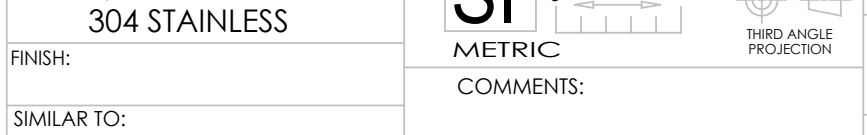




\section{GENERAL NOTES:}

DIMENSION AND TOLERANCING PER ANSI Y14.5-1994.

2 BREAK ALL EDGES .5 [.20] MAX. ALL INSIDE RADII

2.4 [.09] MAX. UNLESS NOTED OTHERWISE.

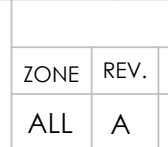

\section{REVISIONS}

DESCRPPTION

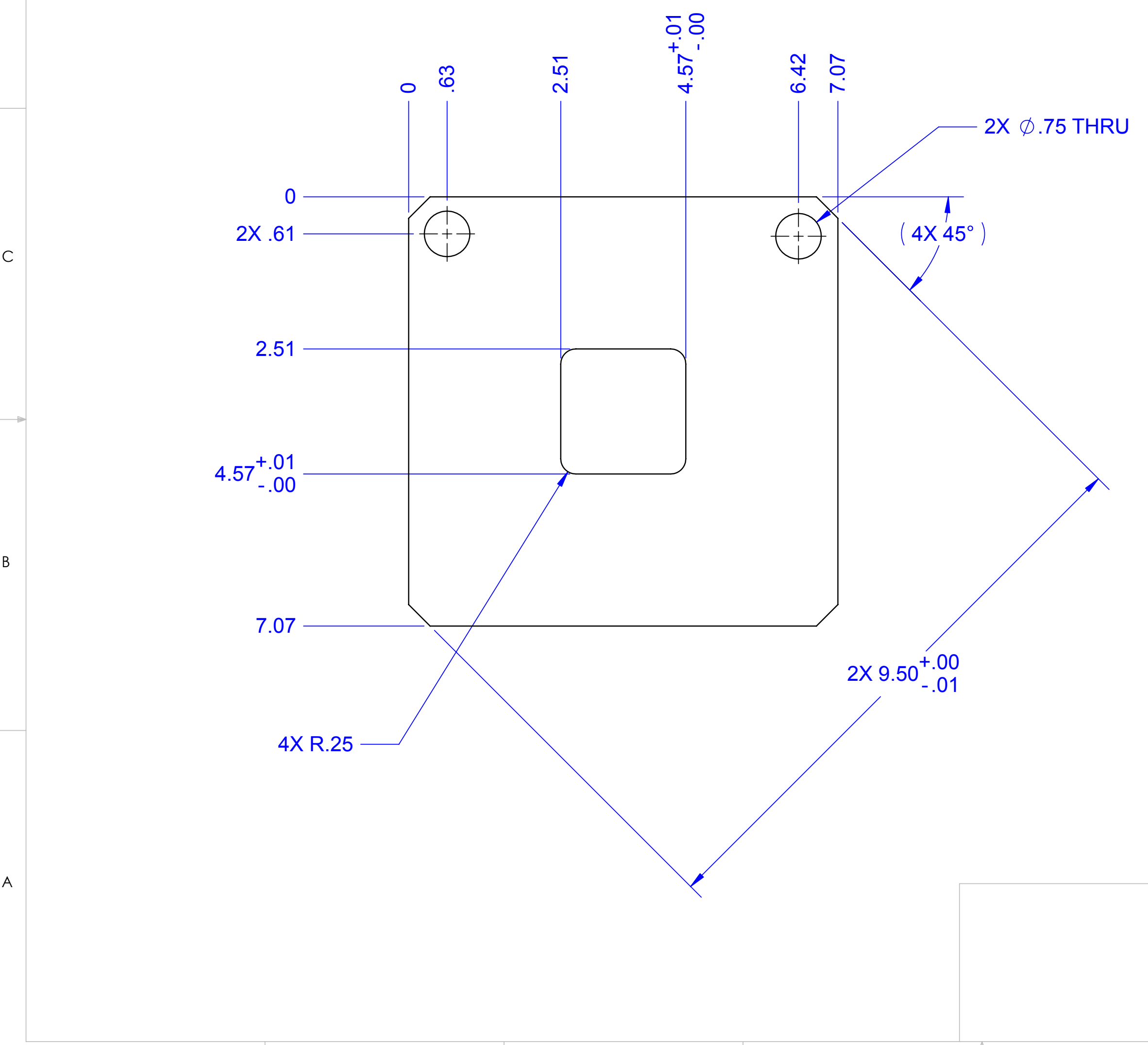

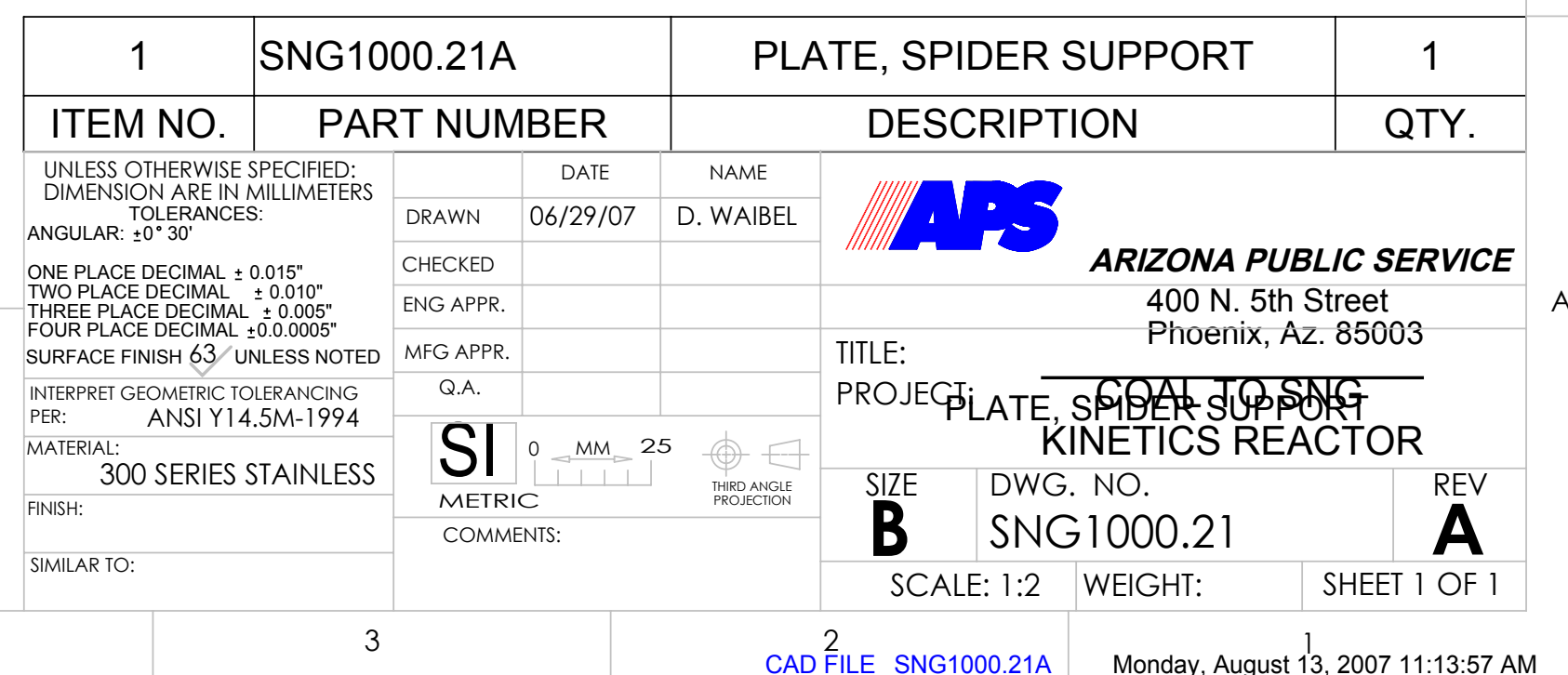




\section{GENERAL NOTES:}

1 DIMENSION AND TOLERANCING PER ANSI Y14.5-1994.

2 BREAK ALL EDGES .5 [.20] MAX. ALL INSIDE RADII

2.4 [.09] MAX. UNLESS NOTED OTHERWISE.

D $\square$ SURFACE FINISH AS INDICATED, SHOULD ALSO BE TO A

QUALITY OF A GASKET SEALING FINISH BY BEST MECHANICAL MEANS

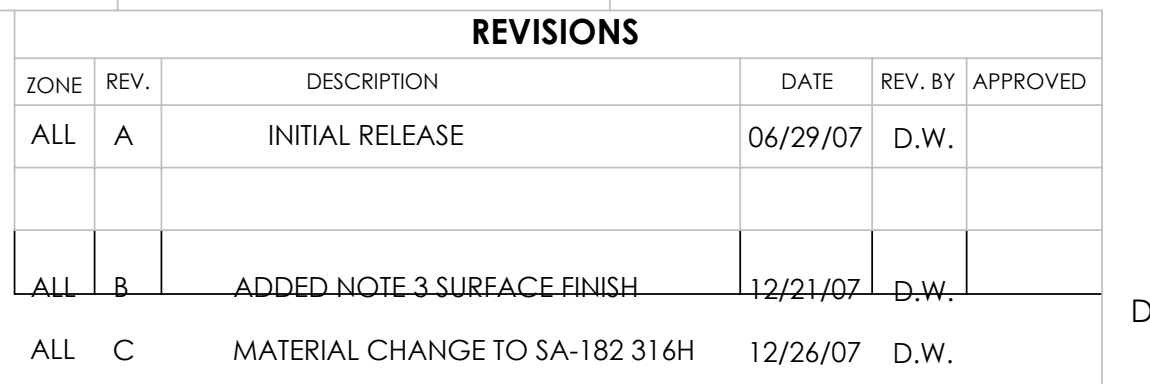

3

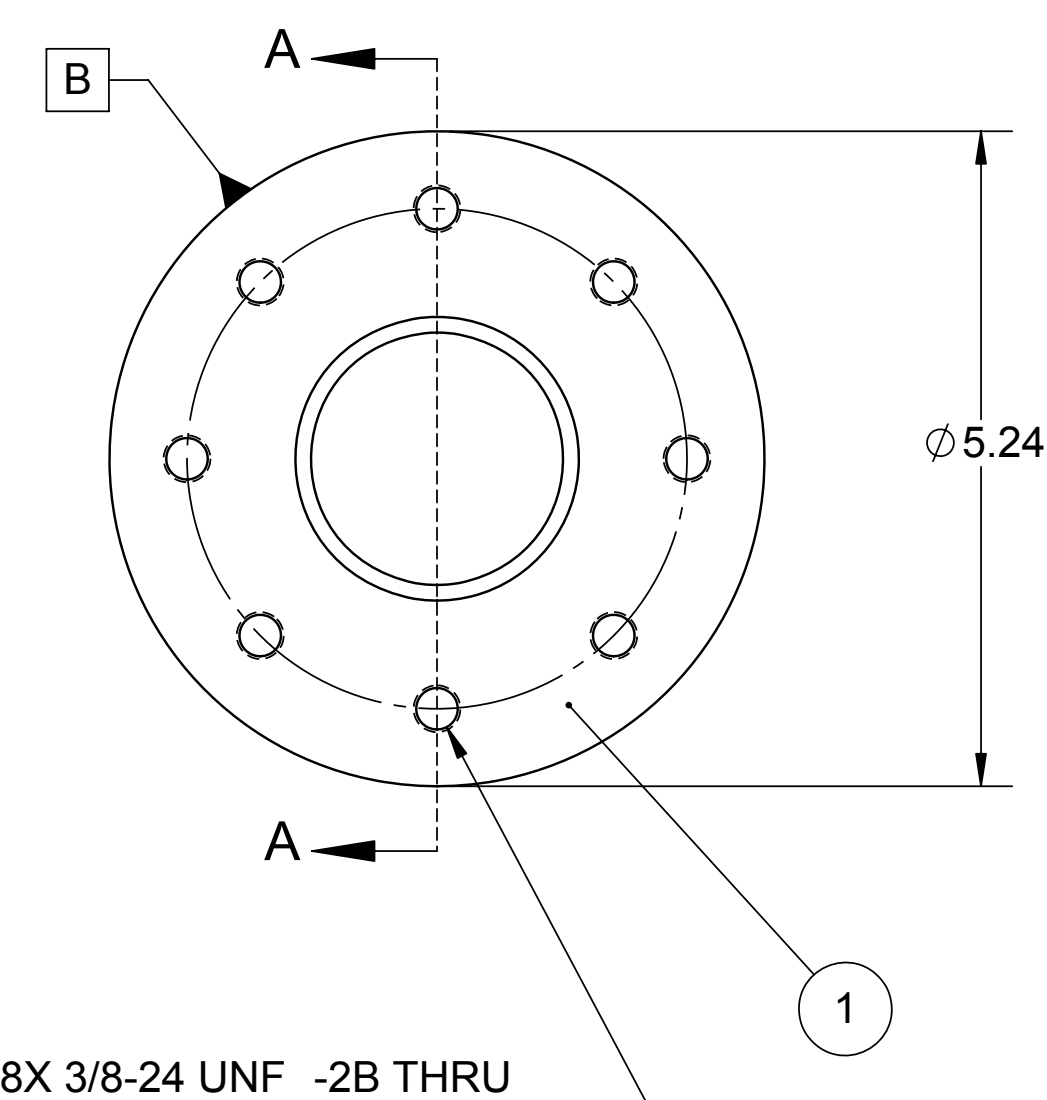

PACED ON $\varnothing 4.000$

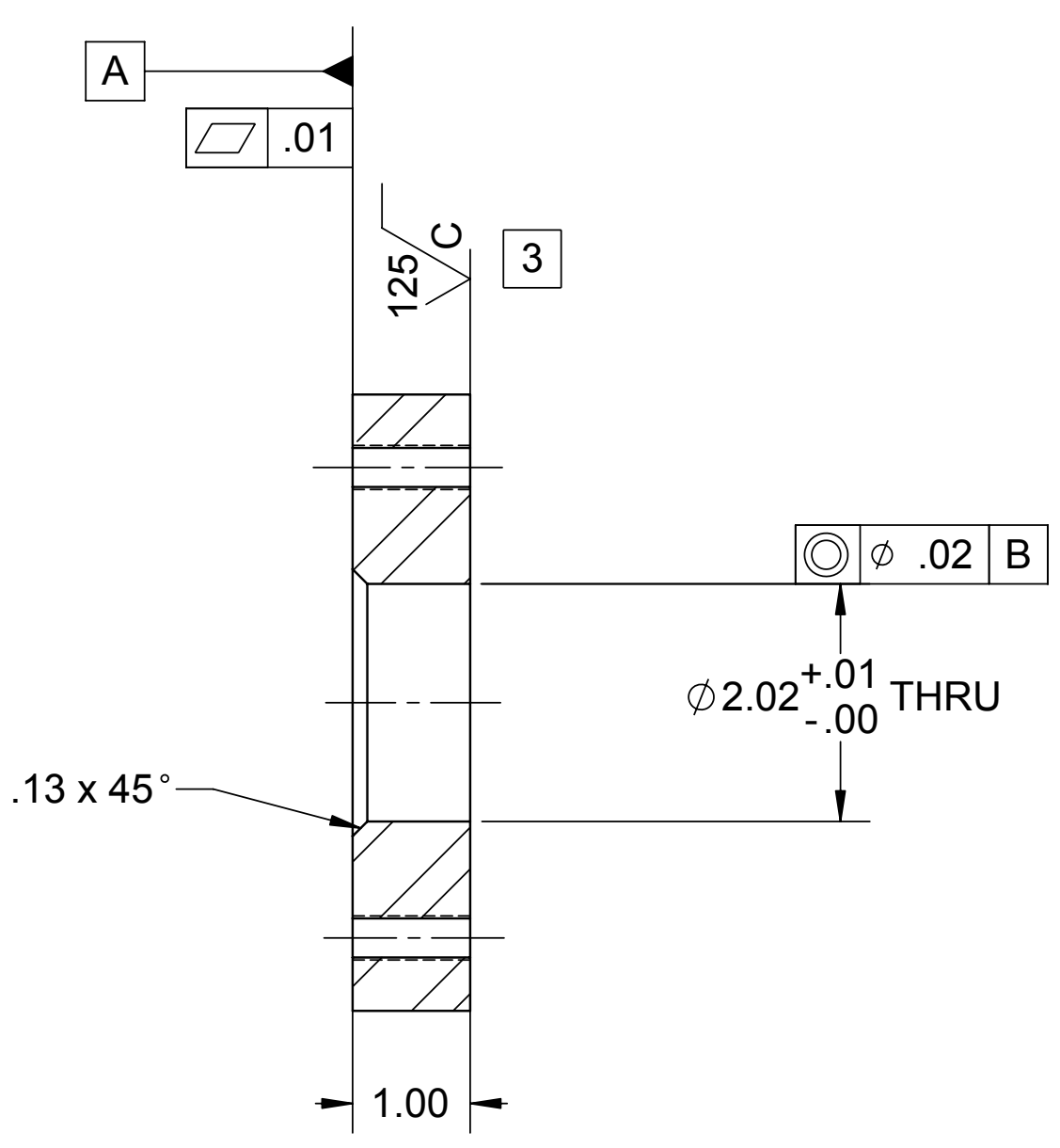

SECTION A-A

c)

\begin{tabular}{|c|c|c|c|c|c|c|c|}
\hline 1 & SNG1000.22C & \multicolumn{4}{|c|}{ SA-182, GRADE 316H SS } & FLANGE, INNER TUBE & 1 \\
\hline $\begin{array}{l}\text { TTEM } \\
\text { NO. }\end{array}$ & $\begin{array}{c}\text { PART } \\
\text { NUMBER }\end{array}$ & \multicolumn{4}{|c|}{ MATERIAL } & DESCRIPTION & QTY \\
\hline & \multirow{2}{*}{ 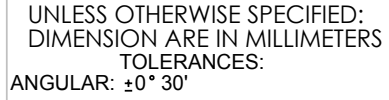 } & & DATE & NAME & \\
\hline & & DRAWN & $06 / 29 / 07$ & D. WAIBEL & & & \\
\hline & \multirow{3}{*}{ 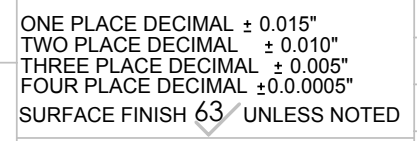 } & CHECKED & & & & & \\
\hline & & ENG APPR. & & & \multirow{2}{*}{\multicolumn{3}{|c|}{$\begin{array}{l}400 \text { N. 5th Street } \\
\text { Phoenix, Az. } 85003\end{array}$}} \\
\hline & & MFG APPR. & & & & & \\
\hline & 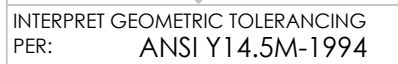 & Q.A. & & & \multirow{2}{*}{\multicolumn{3}{|c|}{$\begin{array}{l}\text { PROJECT: COALTOSNG } \\
\text { TITLE: FLANGTEININER REACTOR }\end{array}$}} \\
\hline & $\begin{array}{l}\text { MATERIAL: } \\
\text { SFF BOM }\end{array}$ & \multirow{3}{*}{\multicolumn{2}{|c|}{$\begin{array}{l}\text { SI } \\
\text { METRIC } \\
\text { COMMENS: }\end{array}$}} & \multirow{3}{*}{ 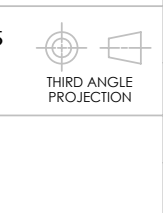 } & & & \\
\hline & $\begin{array}{l}\text { SEE BOM } \\
\text { FNISH: }\end{array}$ & & & & \multirow[t]{2}{*}{$\mathbf{S I I}^{\mathrm{B}}$} & $\begin{array}{l}\text { DWG. NO. } \\
\text { SNG1000.22 }\end{array}$ & REV \\
\hline & SIMILAR TO: & & & & & \multicolumn{2}{|l|}{ ALE: $1: 2$ WEIGHT: } \\
\hline
\end{tabular}


1 DIMENSION AND TOLERANCING PER ANSI Y14.5-1994.

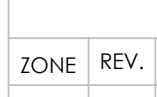

\section{REVISIONS}

$\mathrm{D}$

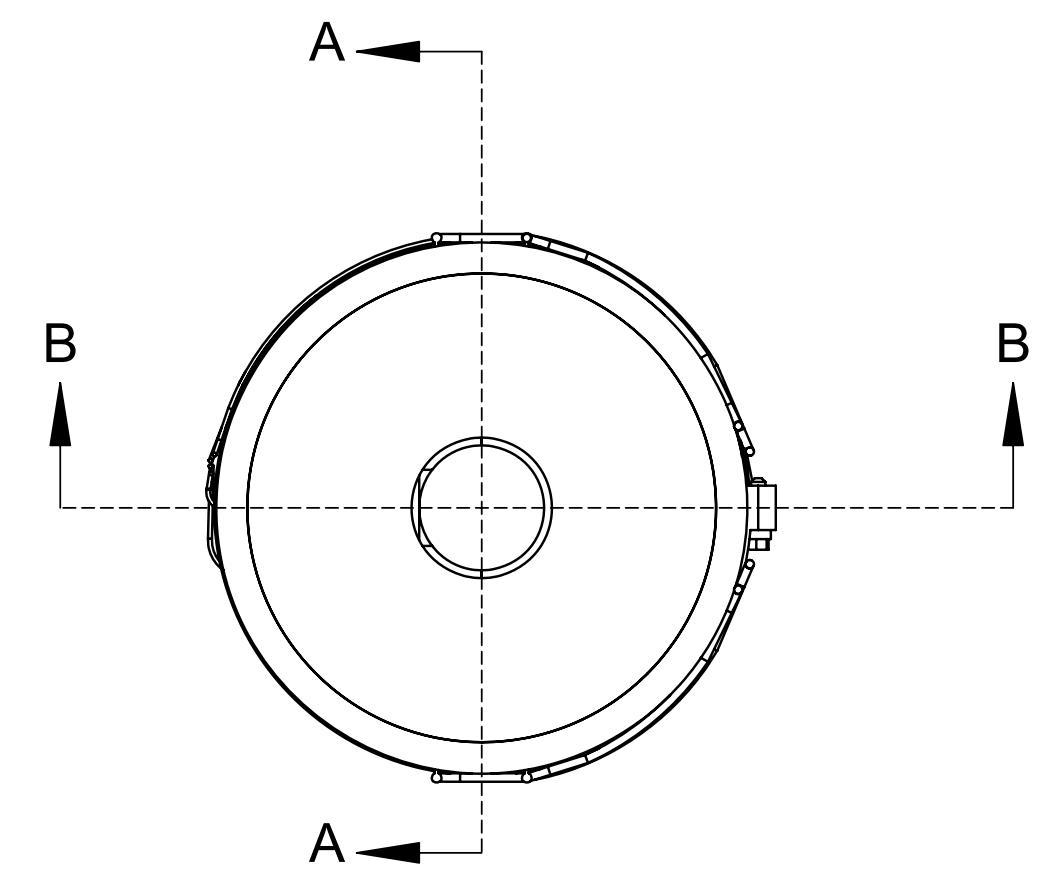

B

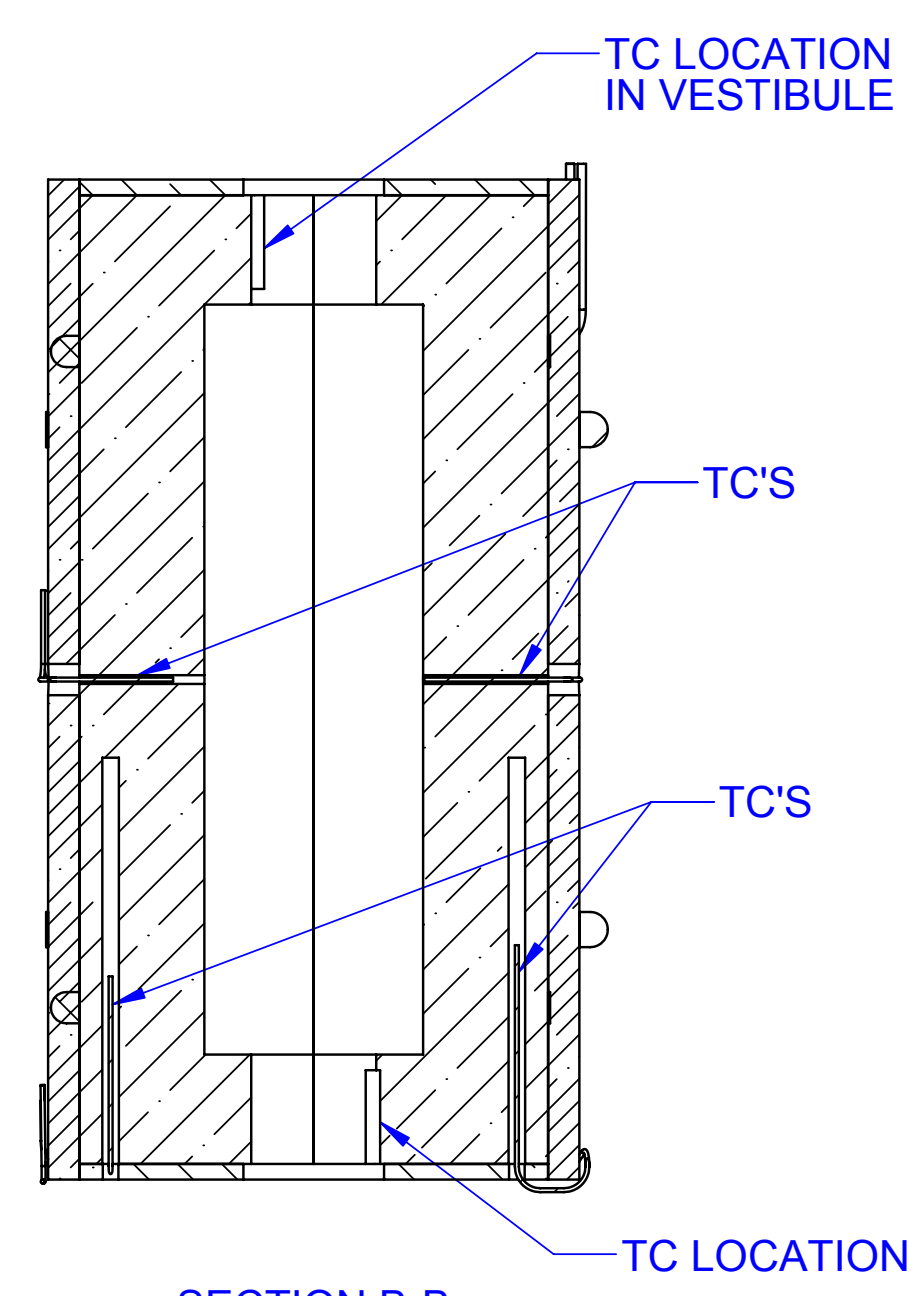

SECTION B-B

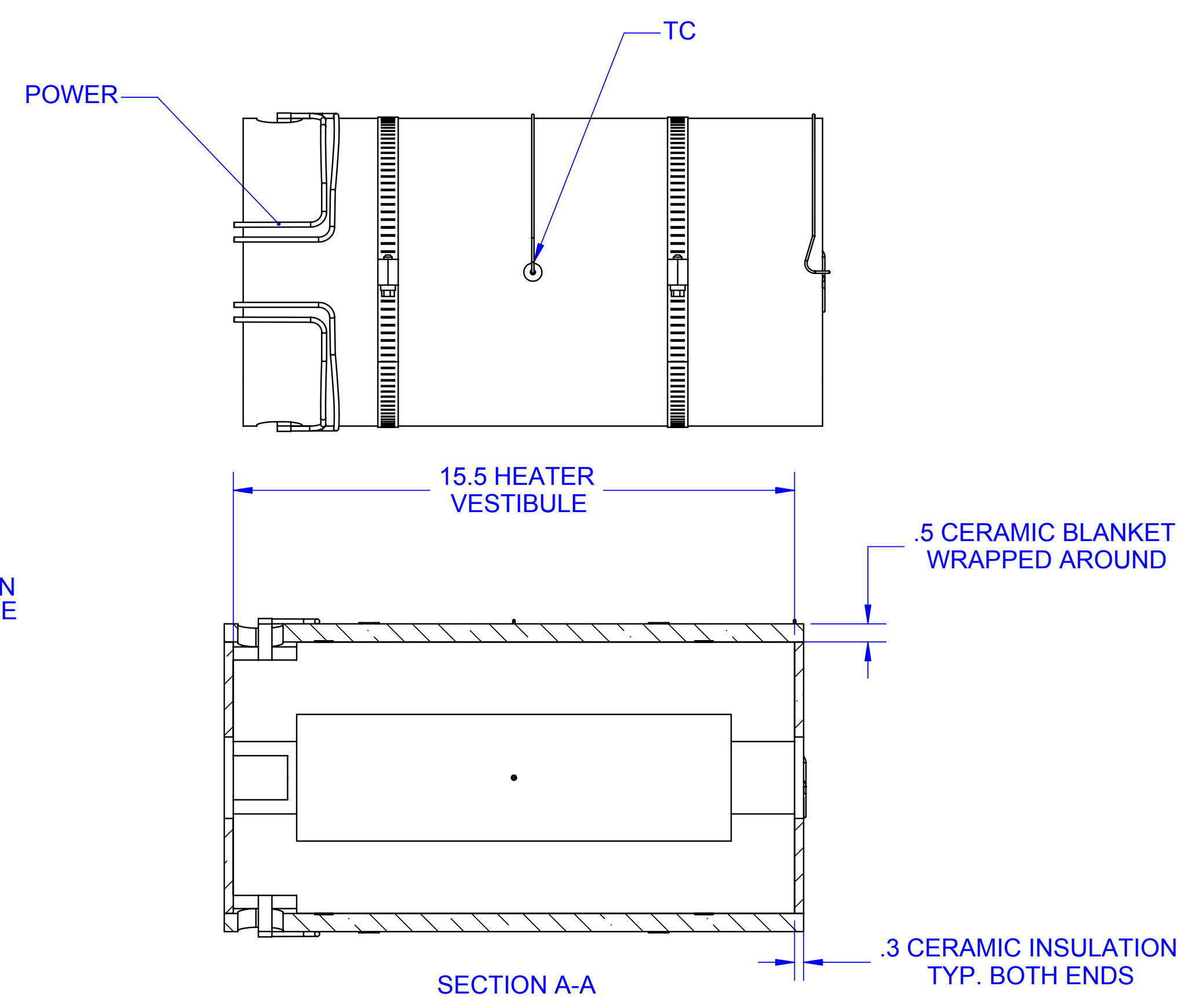

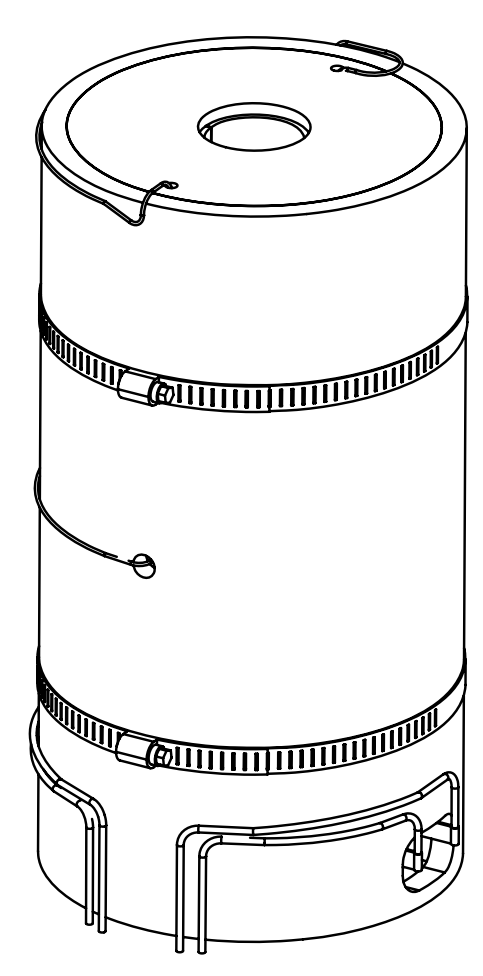

VS103J12

12" HEATER SHOWN

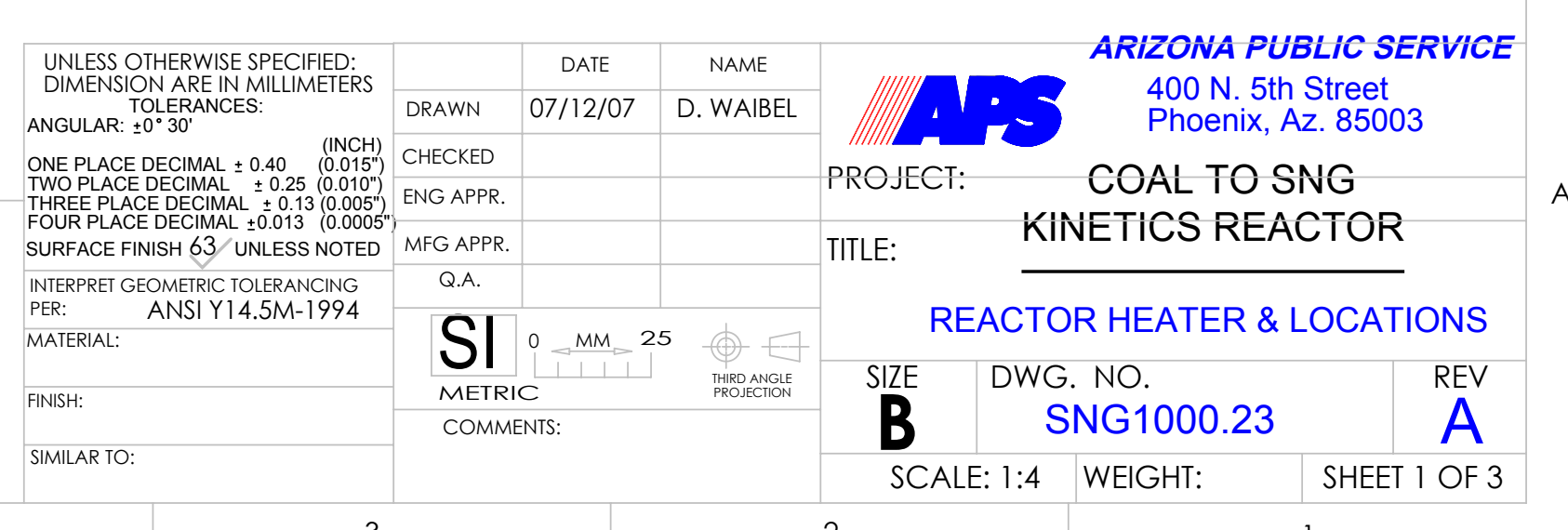




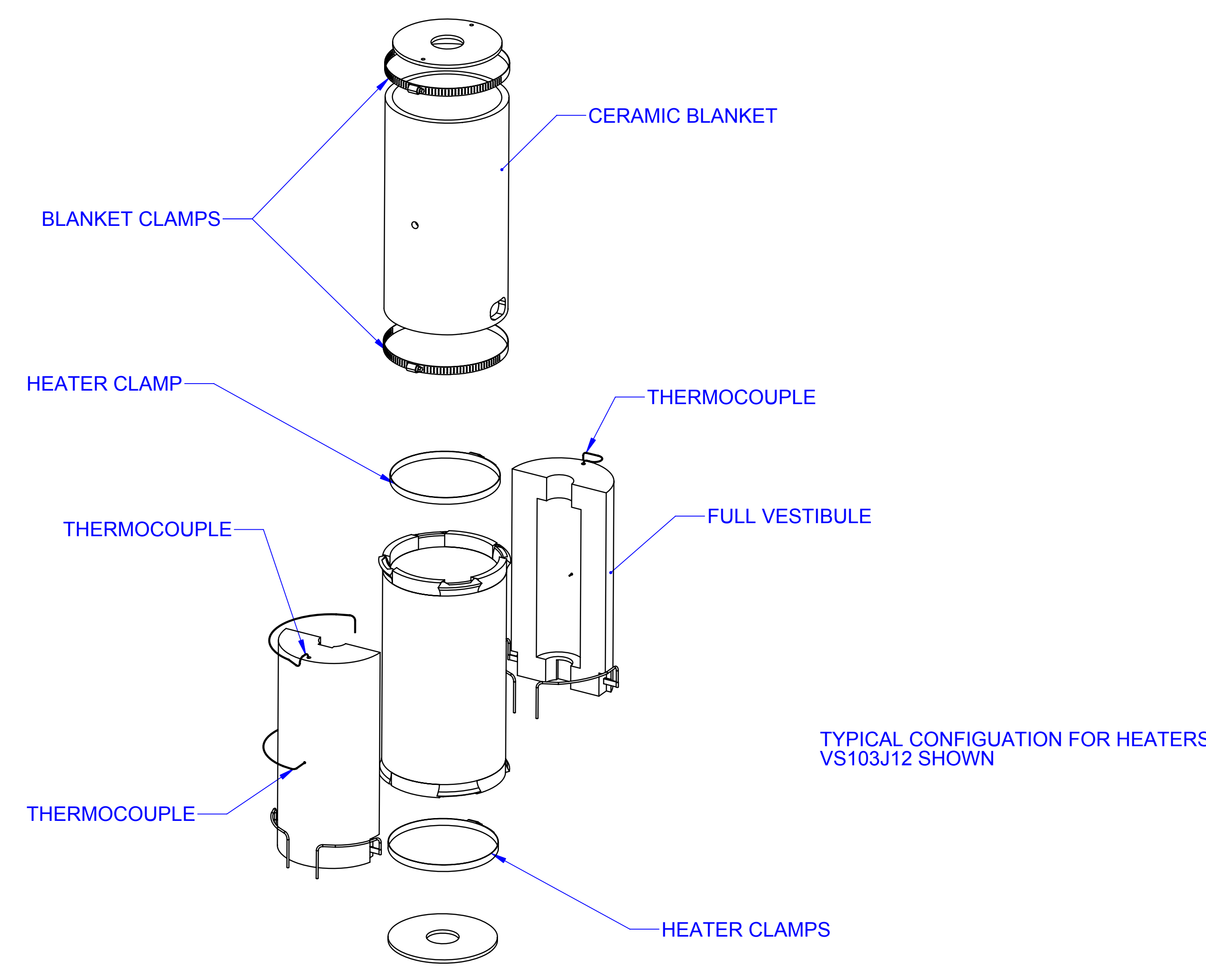
IIIIIT ARIZONA PUBLIC SERVICE $400 \mathrm{~N}$. 5th Street
Phoenix, AZ. 85003
PROJECT: COAL TO SNG
TITL: KINETICS REACTOR REACTOR HEATER \& LOCATION
\begin{tabular}{l|l|l} 
SIIZE & DWG. NO. & REV \\
\hline B & SNG1000.23 & A \\
\hline
\end{tabular} SCALE: 1:4 WEIGHT: SHEET 2 OF 3



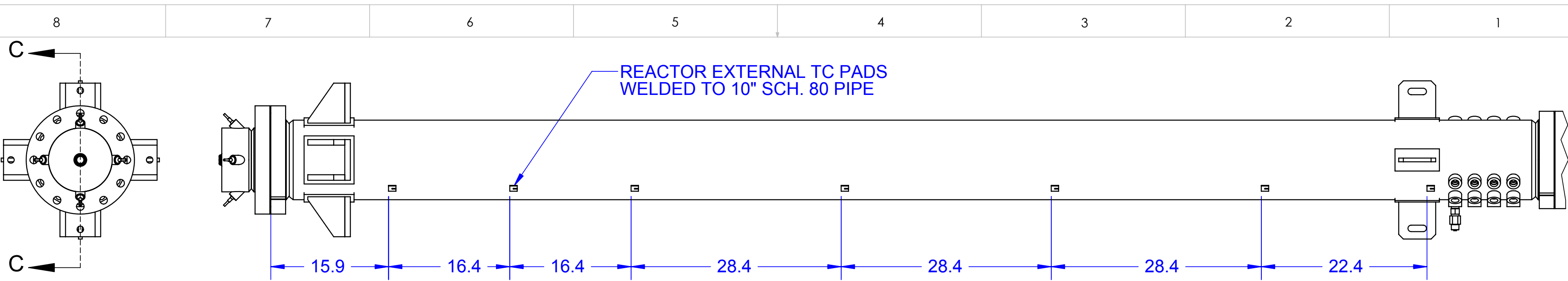

NAL TC PADS

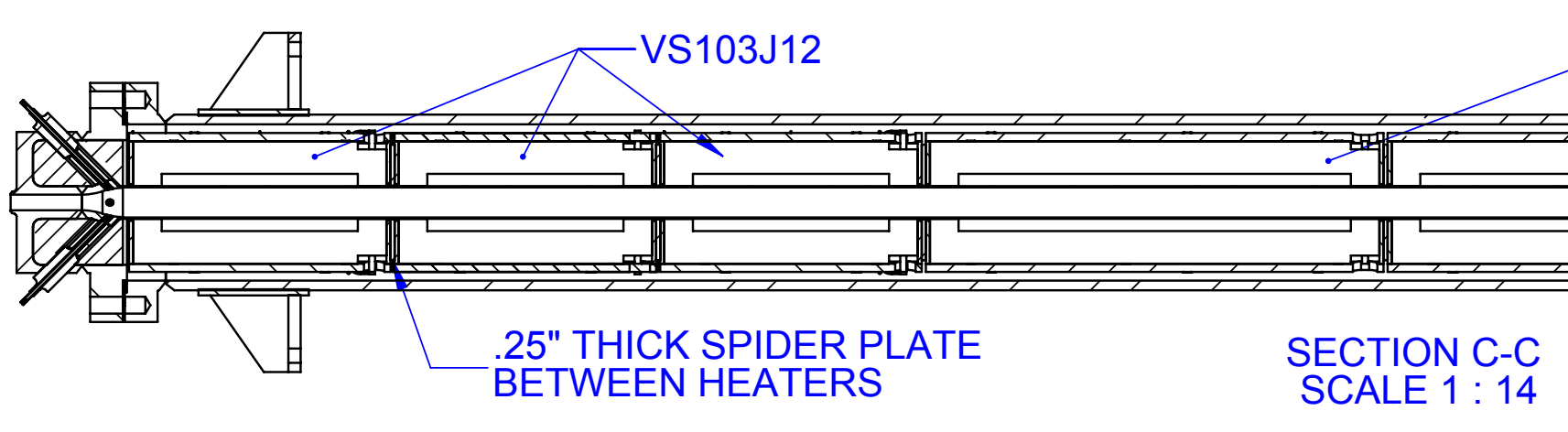

VS103J24-

VS103J18 (n) III (1) :

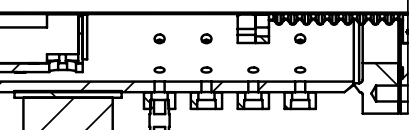
$\begin{array}{ll}\text { 25" THETWEEN HEATERS } & \text { SECTION C-C } \\ \text { SCALE } 1: 14\end{array}$

$\mathrm{D}-$

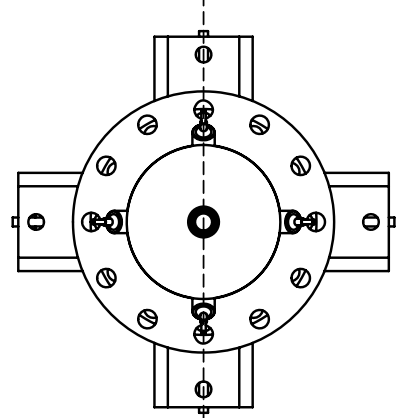

$\mathrm{D}-$

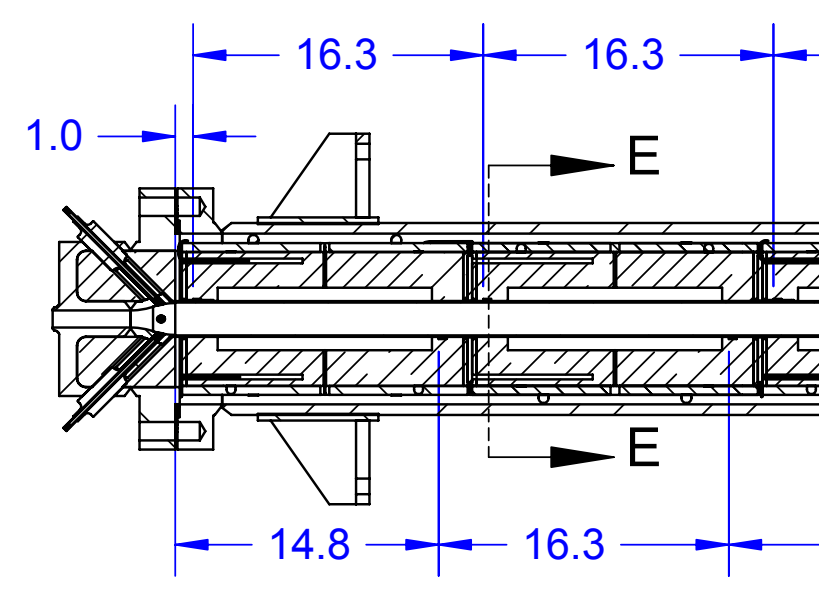

$-14.8 \longrightarrow 16.3 \longrightarrow 16.3$ $16.3 \rightarrow$ ON 2" TUBE
28.3 28.2
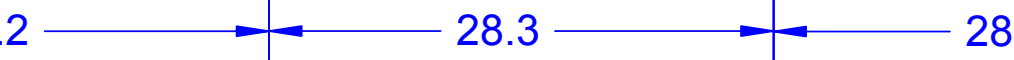

SEALED FEED THRUS

WIRING AND TC'S-

SPIDER PLATE-

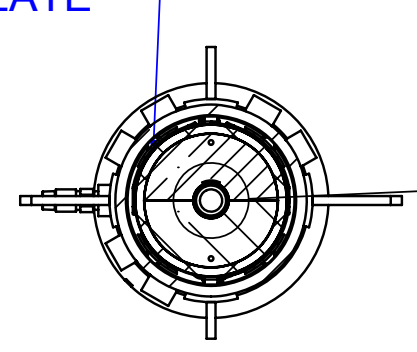

SECTION E-E SCALE $1: 14$
TC PAD TO BE WELDED
$180^{\circ}$ APART ON 2" TUBE

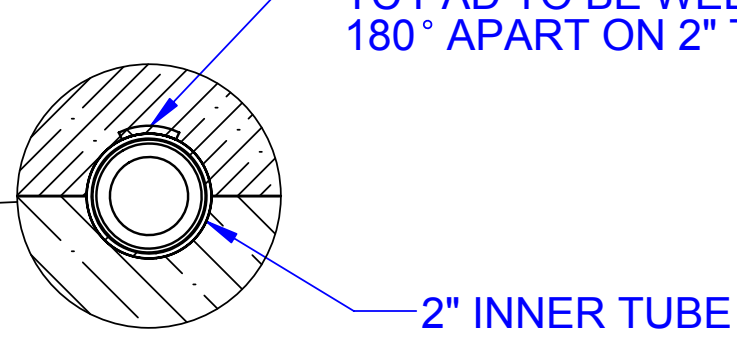

DETAIL F
ARIZONA PUBLIC SERVICE 400 N. 5th Street PROJECT: COAL TO SNG TITLE: KINETICS REACTOR REACTOR HEATER \& LOCATIONS

\begin{tabular}{l|l|l} 
SIIE & DWG. NO. & REV \\
$\mathbf{B}$ & SNG1000.23 & A \\
\hline
\end{tabular} SCALE: 1:64 WEIGHT: SHEET 3 OF 3 
2 BREAK ALL EDGES .5 [.20] MAX. ALL INSIDE RADII 2.4 [.09] MAX. UNLESS NOTED OTHERWISE.

\begin{tabular}{|c|c|c|c|c|}
\hline \multicolumn{5}{|c|}{ REVISIONS } \\
\hline ZONE & REV. & DESCRPPTION & DATE & REV. BY APPROV \\
\hline ALL & A & INITIAL RELEASE & $08 / 23 / 07$ & D.W. \\
\hline & & & & \\
\hline
\end{tabular}

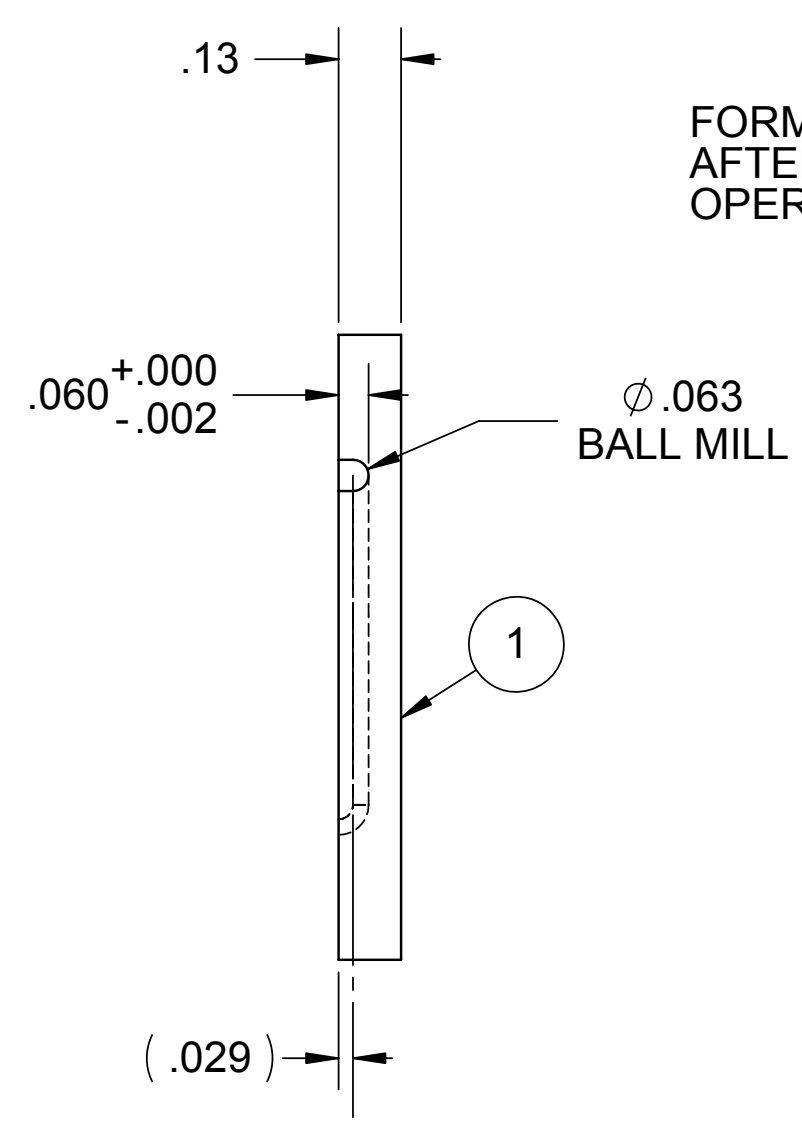

FORM COMPONENT

佶

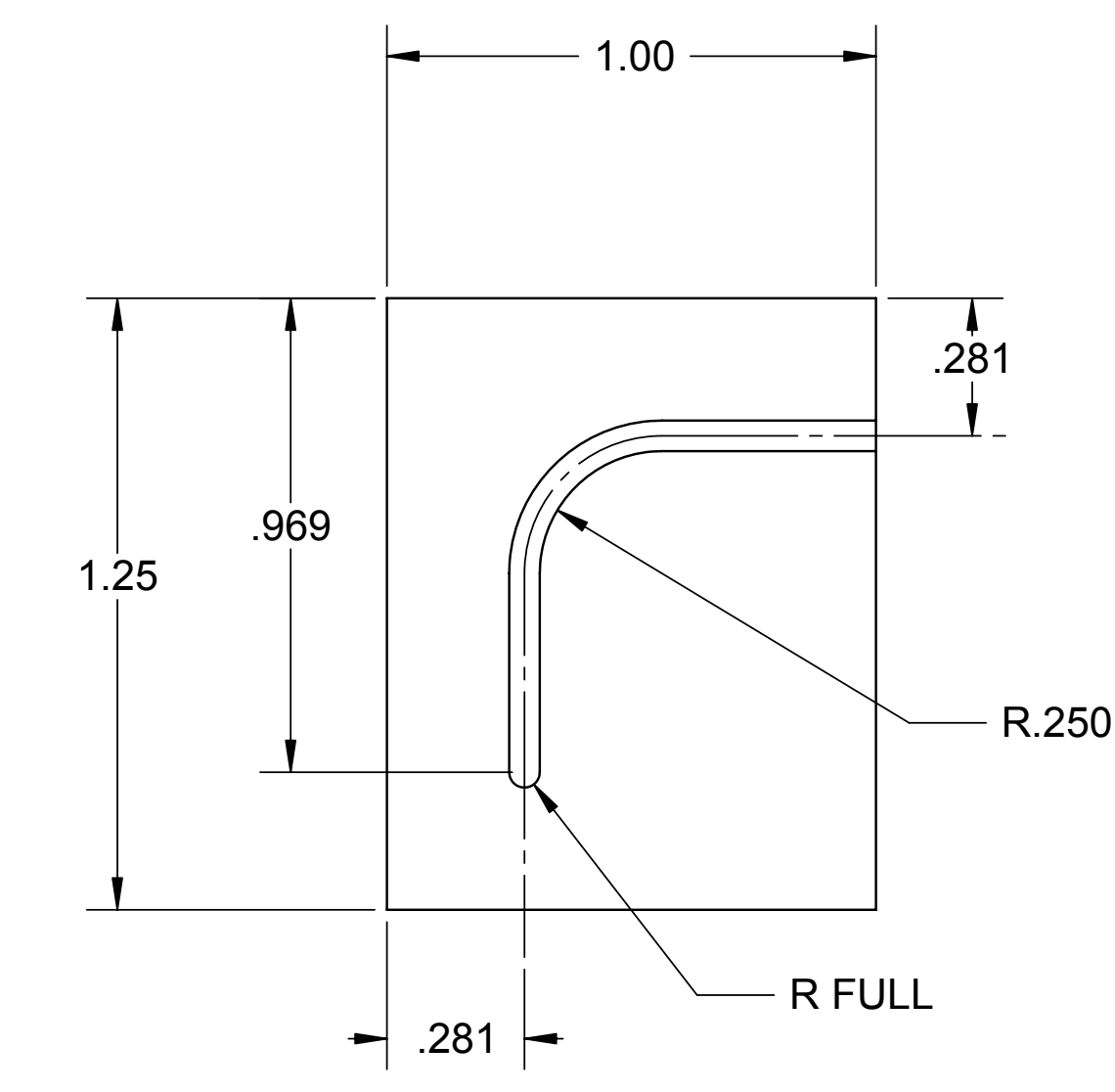

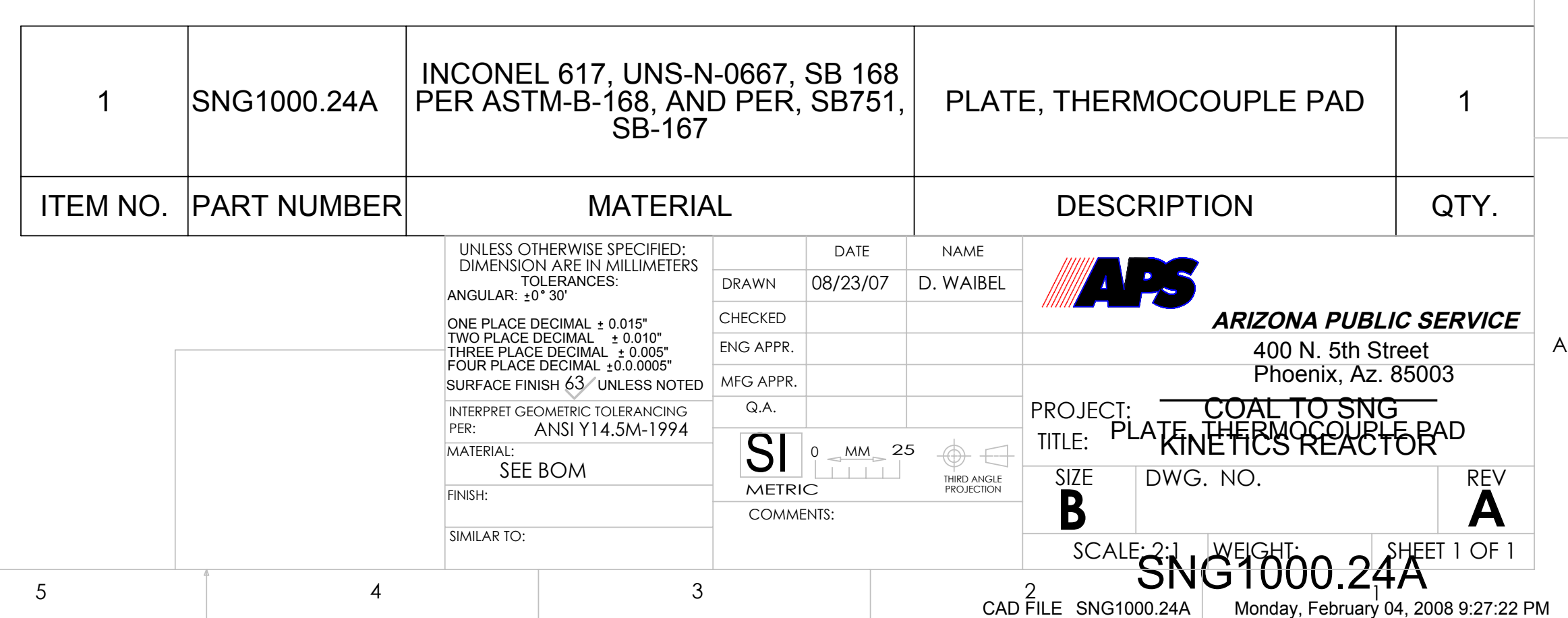




\section{GENERAL NOTES:}

1 DIMENSION AND TOLERANCING PER ANSI Y14.5-1994.

2 GASKET TO BE STYLE 'R', (CRITICAL SERVICE SERIES) SEE BOM BELOW
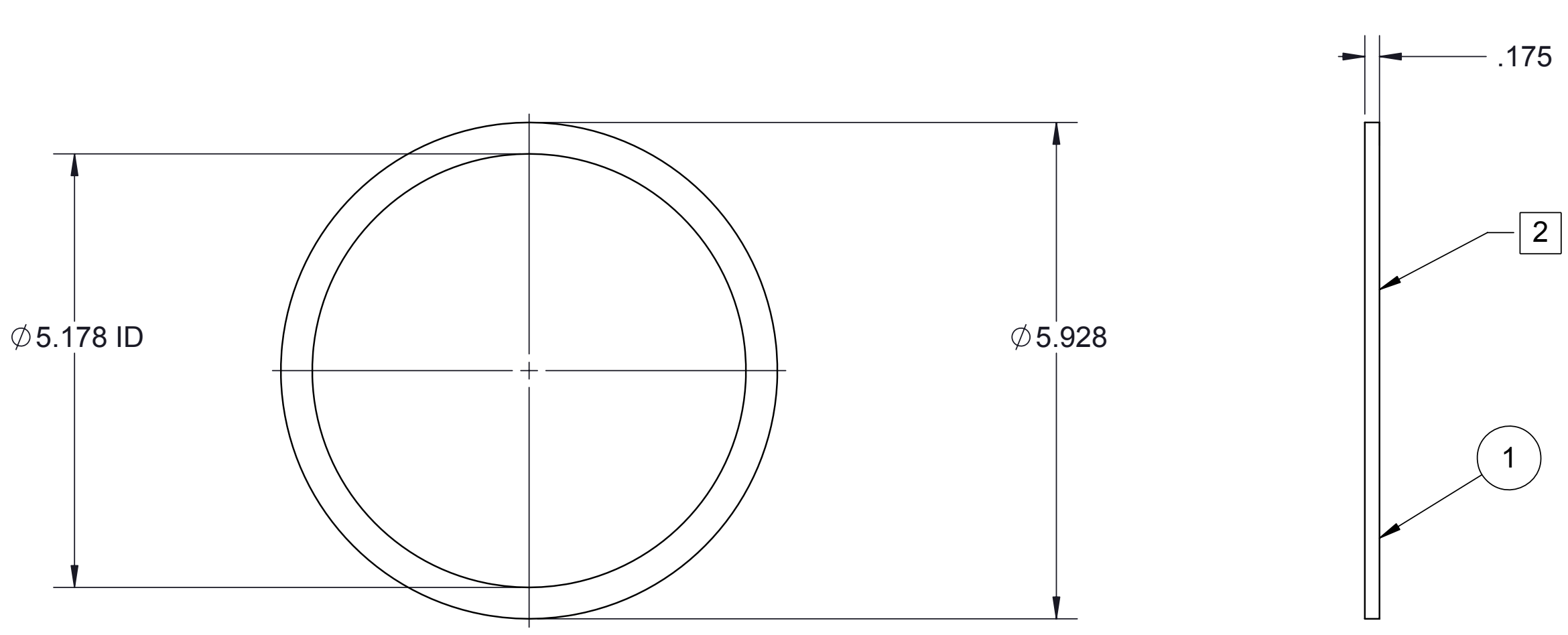

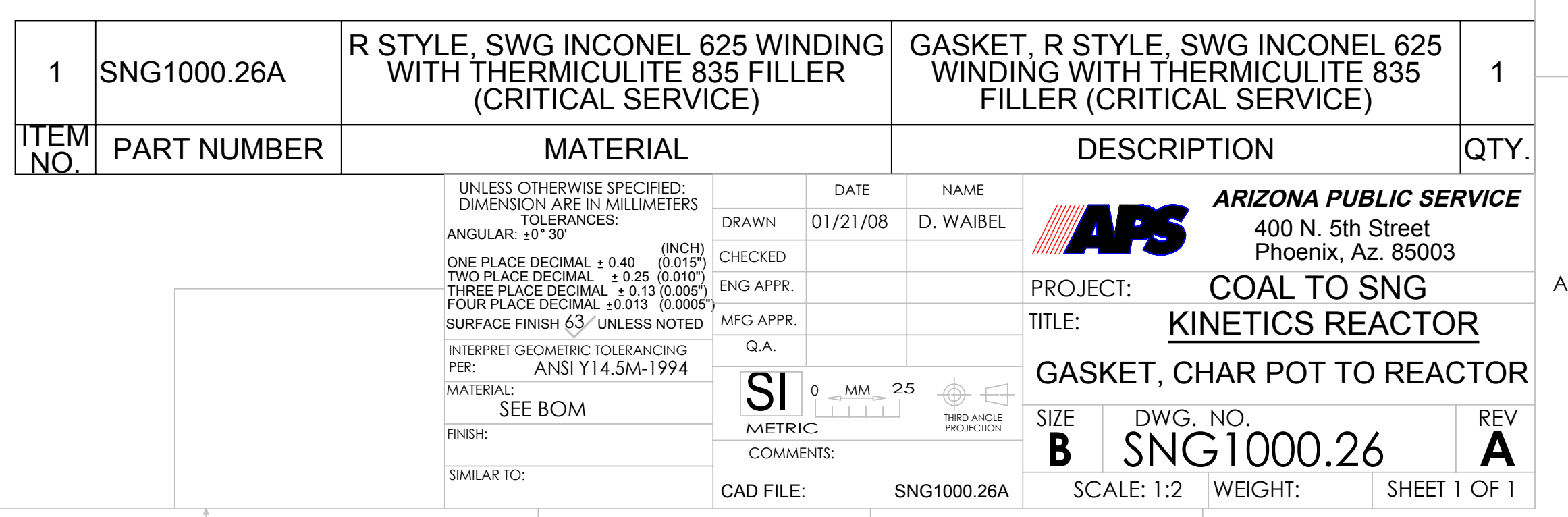


1 DIMENSION AND TOLERANCING PER ANSI Y14.5-1994.

$2 \varnothing 2 "$ TUBE HOLD ROUNDNESS.

3 TUBE DIMENSIONS AND TOLERANCES PER ASTM A269, AND SB-751, SB-167.

4 PROVIDE MILL TEST REPORT(MTR)

5 MAINTAIN STRAIGHTNESS T0 $\leq .030 " / 3 F t$. END TO END.

6 WALL THICKNESS .125" NOMINAL FROM .125" THICK SHEET (.120/.130)

C

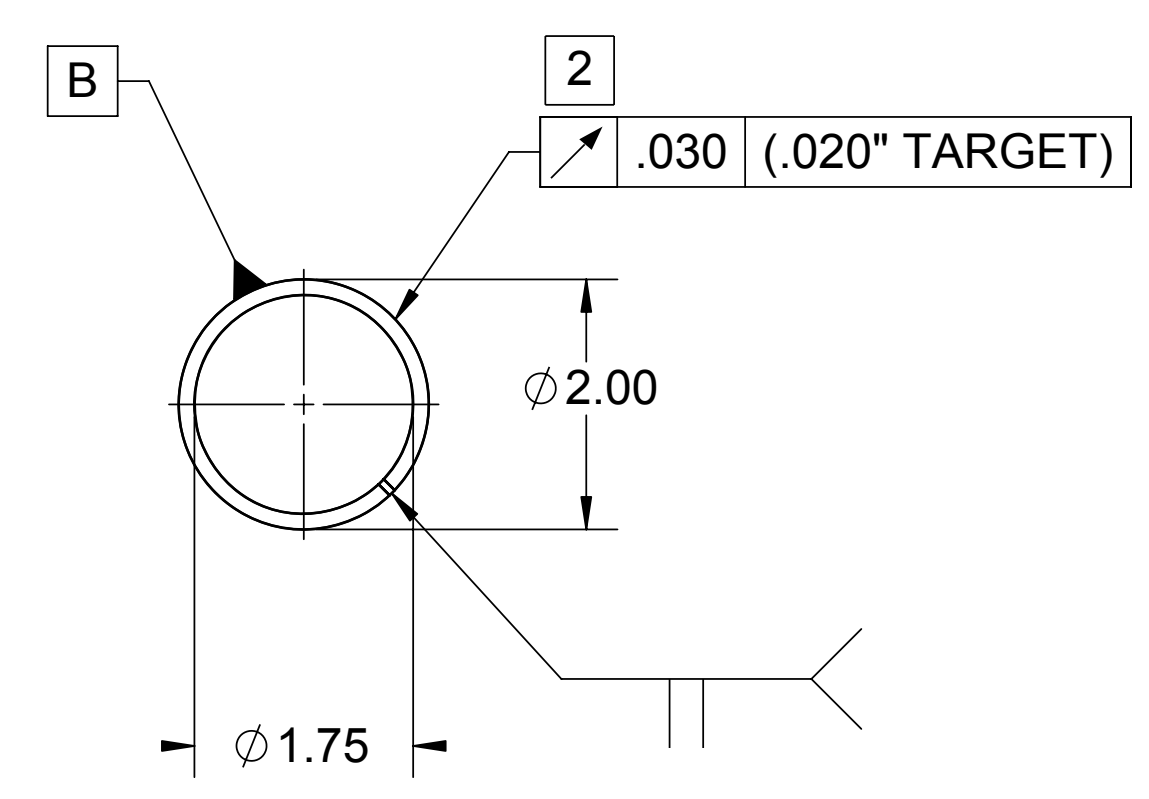

A

48.0 RANDOM LENGTH

(DIM. NOT TO SCALE)

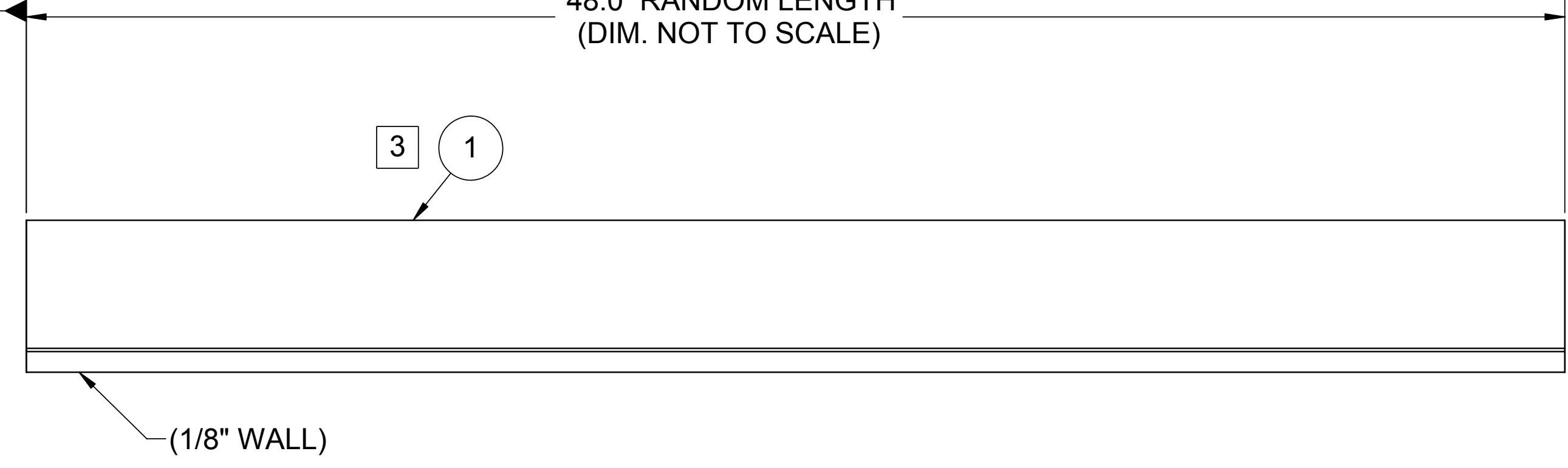

\begin{tabular}{|c|c|c|c|c|c|}
\hline 1 & SNG1000.4-1 & \multicolumn{3}{|c|}{$\begin{array}{c}\text { INCONEL 617, UNS-N-06617, } \\
\text { SB168 PER ASTM-B-168, } \\
(.125 " \text { THK. SHEET) }\end{array}$} & \\
\hline $\begin{array}{c}\text { TTEM } \\
\text { NO. }\end{array}$ & PART NUMBER & \multicolumn{3}{|c|}{ MATERIAL } & \\
\hline & & \multirow{6}{*}{ 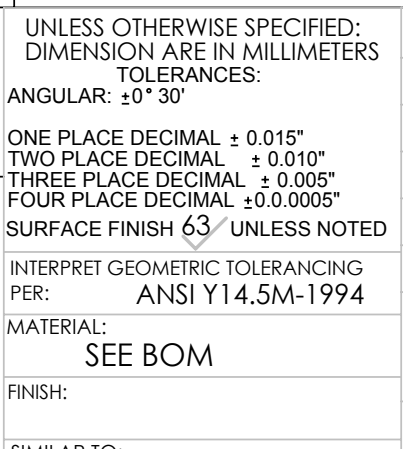 } & & \begin{tabular}{|c|c|} 
DATE \\
$1 / 20108$
\end{tabular} & NAME \\
\hline & & & $\begin{array}{l}\text { DRAWN } \\
\text { CHECKED }\end{array}$ & 01/30/08 & D. WAIBEL \\
\hline & & & ENG APPR. & & \\
\hline & & & $\begin{array}{l}\text { MFG APPR. } \\
\text { Q.A. }\end{array}$ & & \\
\hline & & & & & \\
\hline & & & $\underset{\text { METR }}{\text { COMM }}$ & & 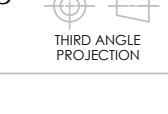 \\
\hline & & SIMLLAR TO: & & & \\
\hline
\end{tabular}



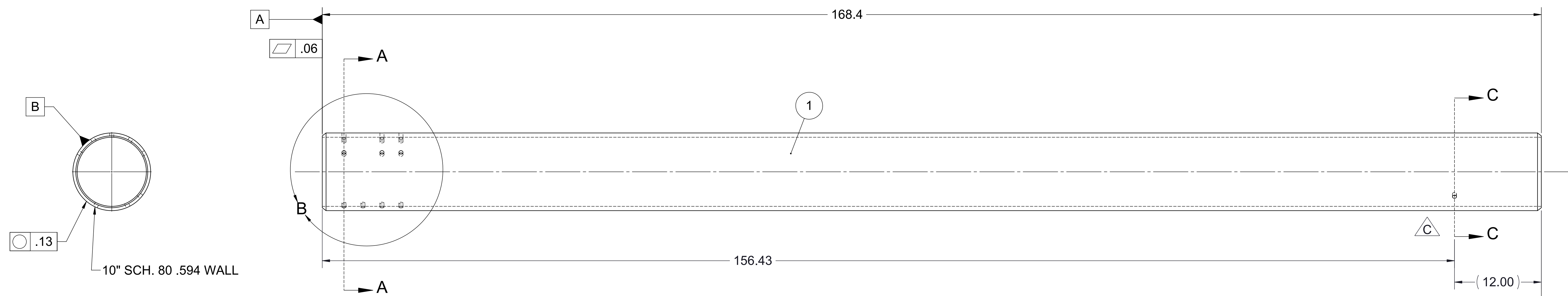

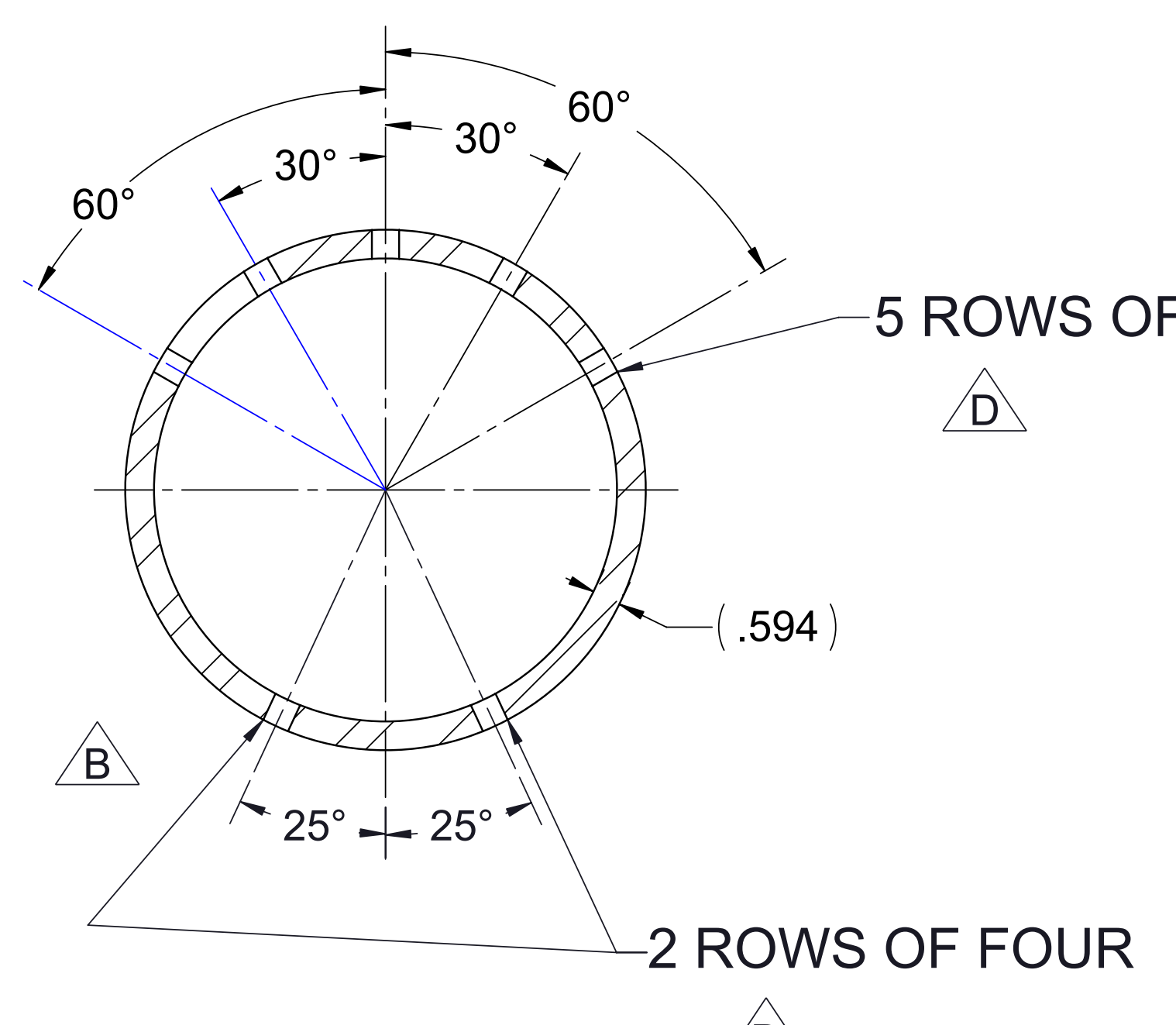

$\Delta$

SECTIONA-A
SCALE $1: 4$
TYP BOTH ENDS-

$.53 \longrightarrow$

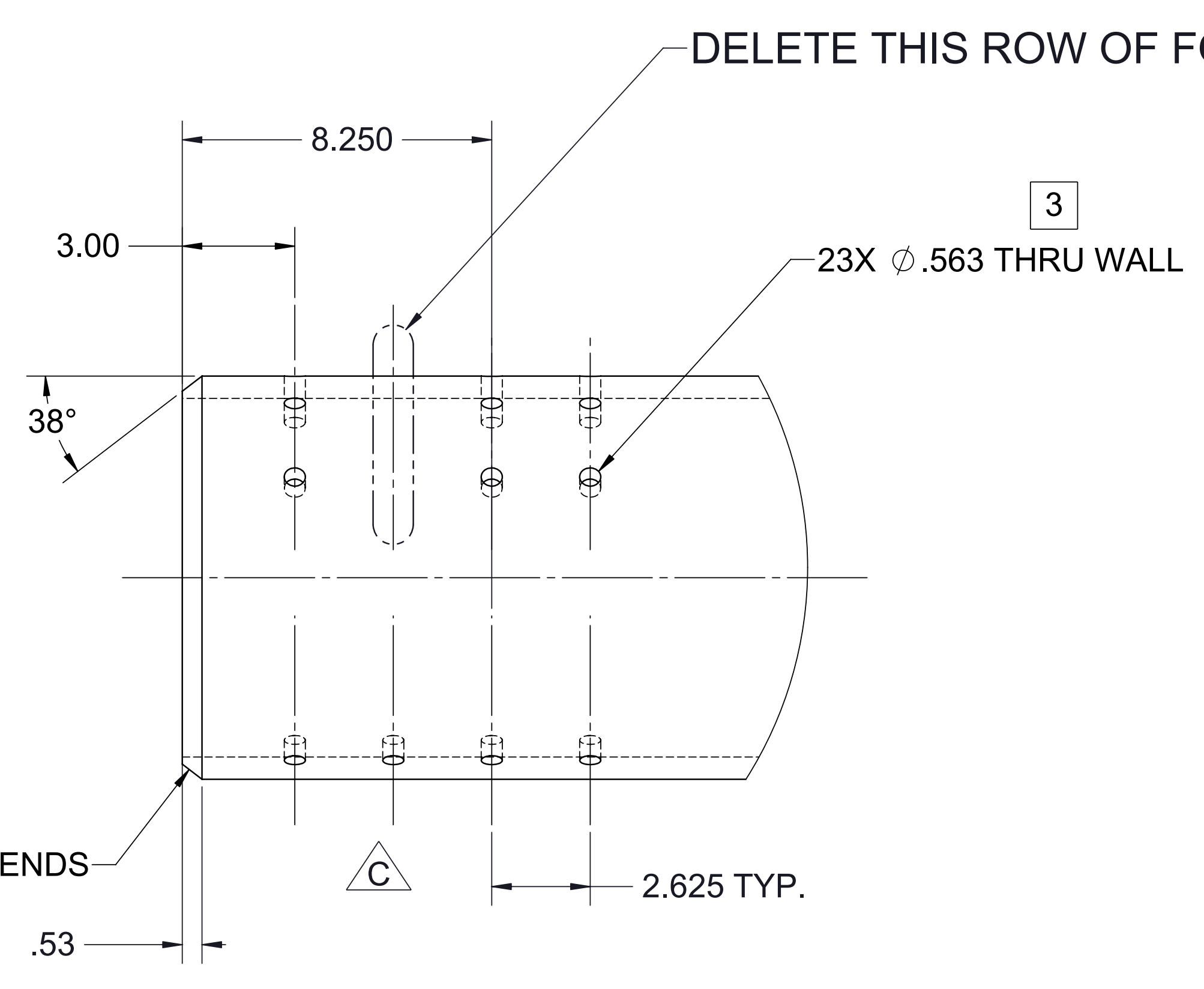

DETALL B
SCALE $1: 4$

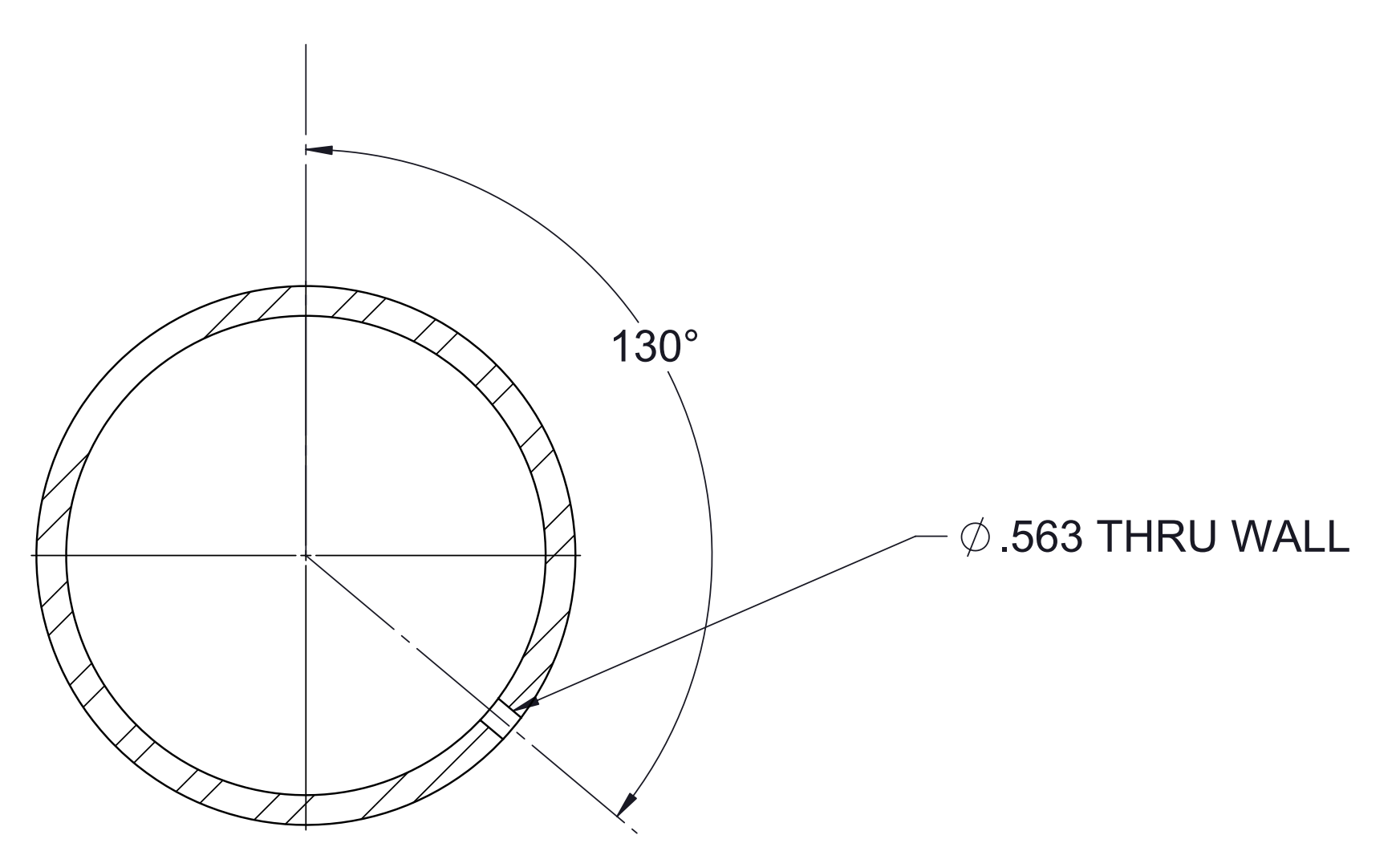

C. SECTION C-C 


\section{GENERAL NOTES:}

1 DIMENSION AND TOLERANCING PER ANSI Y14.5-1994.

2 PROVIDE MILL TEST REPORT(MTR)

3 MAINTAIN STRAIGHTNESS TO <.020" END TO END.

4 ALL WELDS TO MEET PRESSURE VESSEL CODE, ASME SECTION 8, DIVISION 1, UNLESS NOTED OTHERWISE.

5 INSPECTION OF WELDS TO BE 100\% X-RAY, UNLESS SPECIFIED OTHERWISE.

6 END PREP FOR TUBE BUTT WELD, SQUARE END PREP.

$197.5+/-.13$

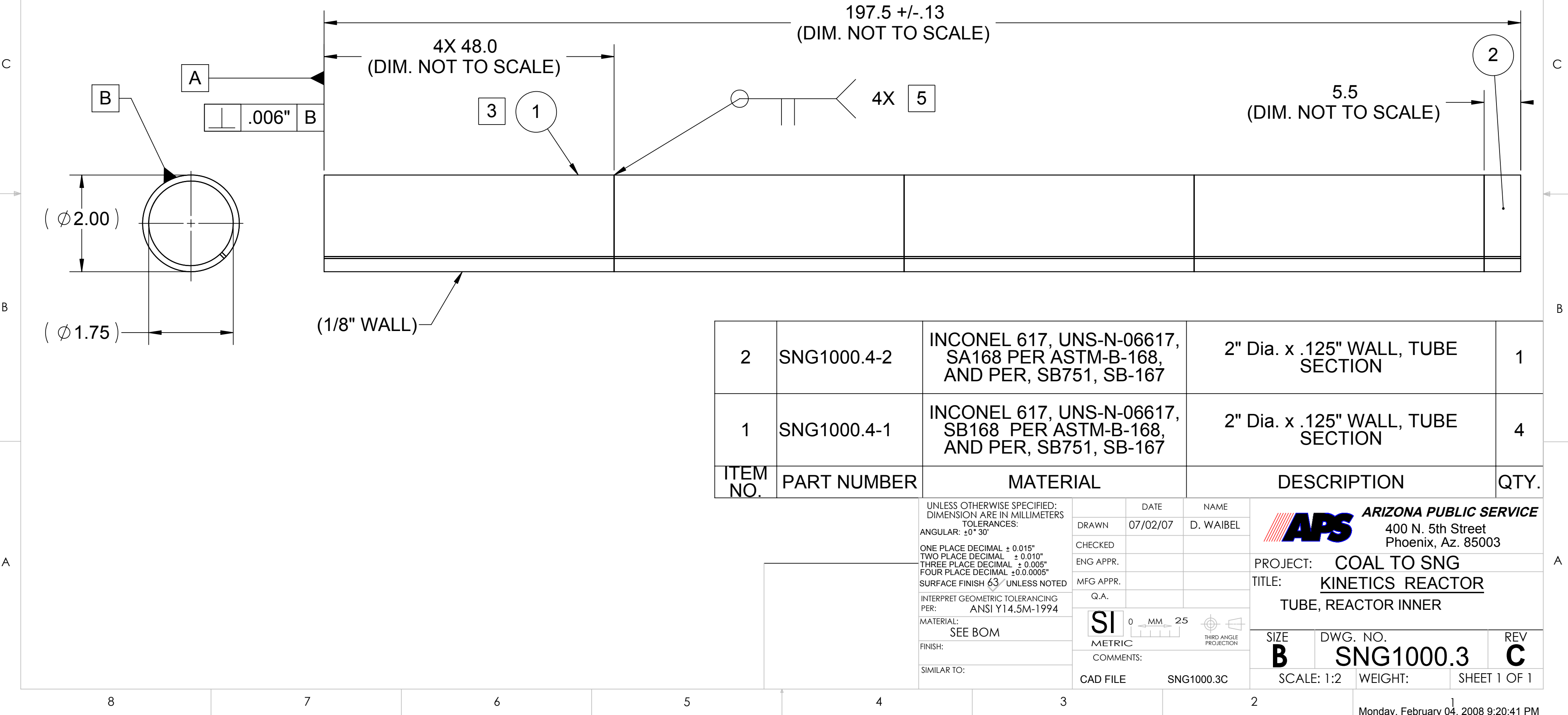




\section{GENERAL NOTES:}

DIMENSION AND TOLERANCING PER ANSI Y14.5-1994.

$2 \varnothing 2 "$ TUBE HOLD ROUNDNESS.

3 TUBE DIMENSIONS AND TOLERANCES PER ASTM A269, AND SB-751, SB-167.

4 PROVIDE MILL TEST REPORT(MTR)

5 MAINTAIN STRAIGHTNESS T0 <.030" END TO END.

6 MAKE FOUR AT 48" LONG, AND ONE AT 5.5" LONG, SEE BOM BELOW

$B$

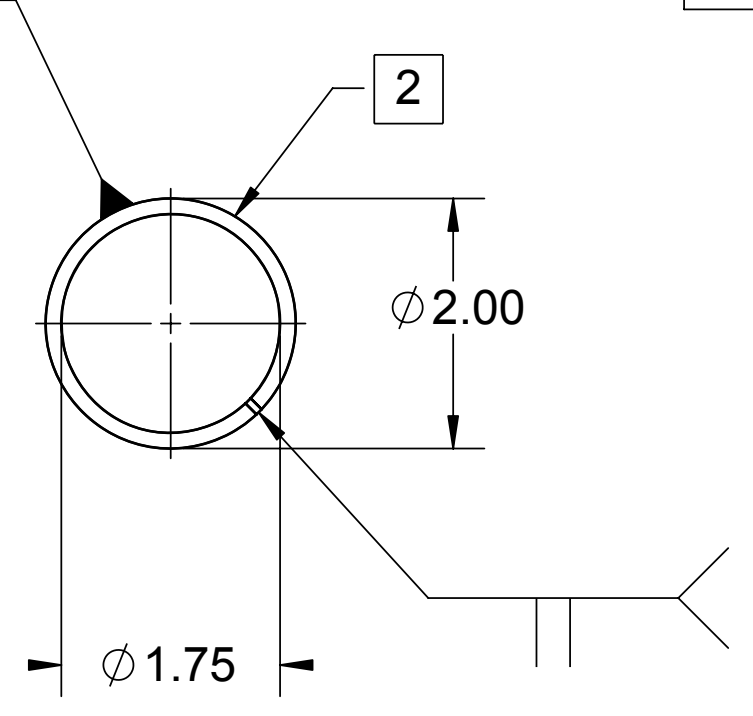

B
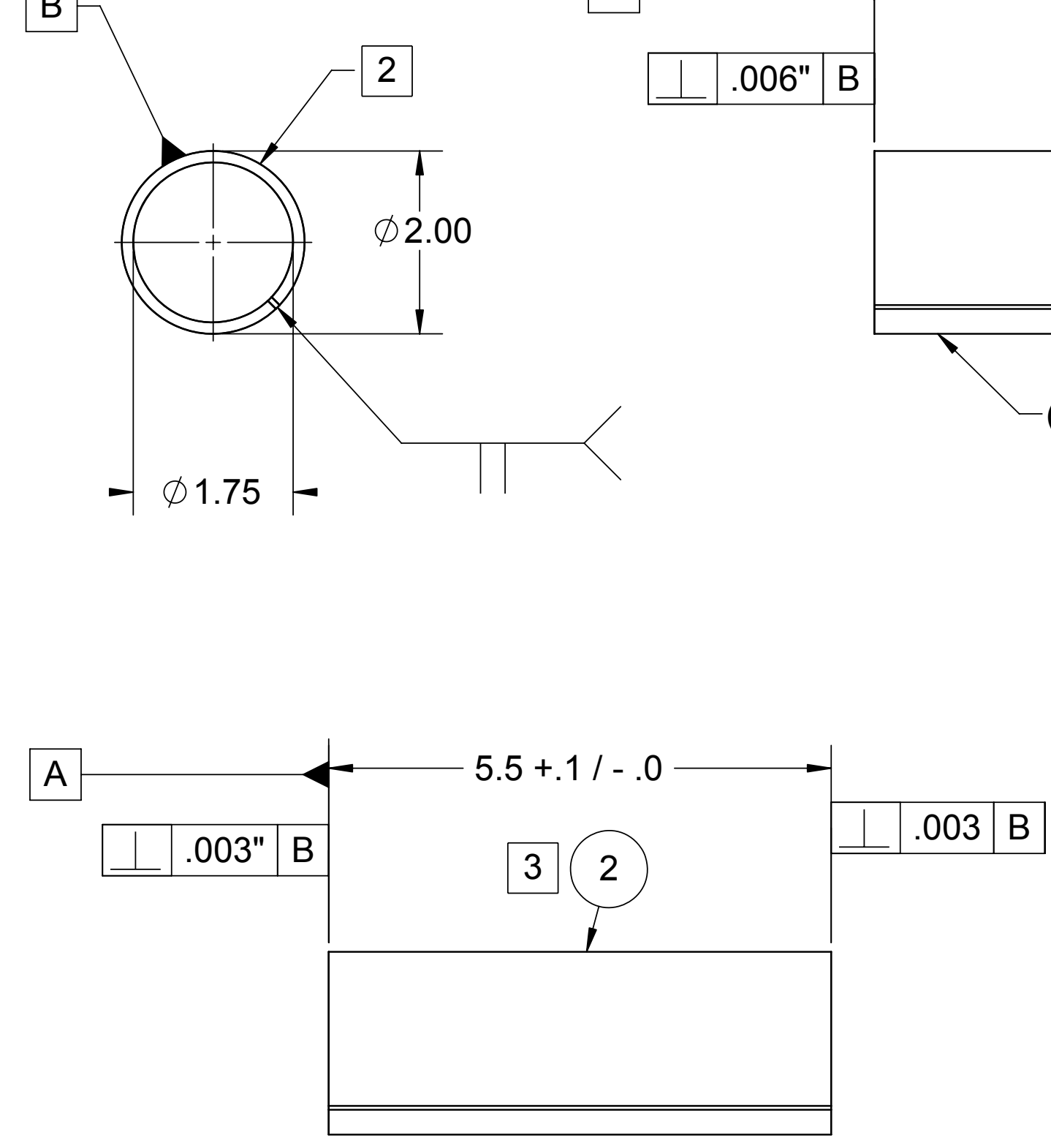

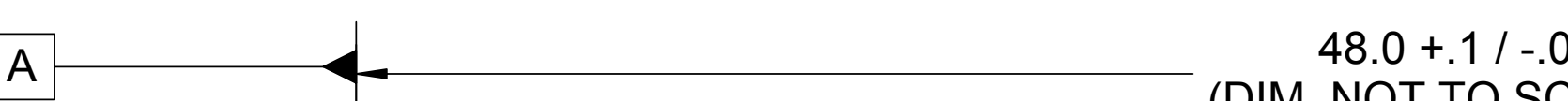

(DIM. NOT TO SCALE)

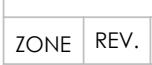

DESCRPTION INITIAL RELEASE
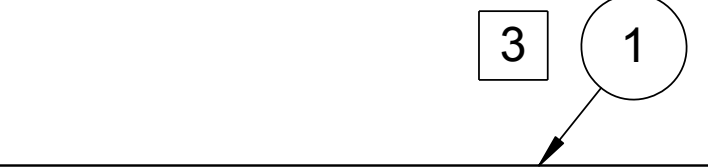

(1/8" WALL)

\begin{tabular}{|c|c|c|c|c|c|c|c|}
\hline 2 & SNG1000.4-2 & \multicolumn{3}{|c|}{$\begin{array}{l}\text { INCONEL 617, UNS-N-06617, } \\
\text { SB168 PER ASTM-B-168, } \\
\text { AND PER, SB751, SB-167 }\end{array}$} & \multicolumn{2}{|c|}{$\begin{array}{c}\text { 2" Dia. } x .125 " \text { WALL, TUBE } \\
\text { SECTION }\end{array}$} & 1 \\
\hline 1 & SNG1000.4-1 & \multicolumn{3}{|c|}{$\begin{array}{l}\text { INCONEL 617, UNS-N-06617, } \\
\text { SB168 PER ASTM-B-168, } \\
\text { AND PER, SB751, SB-167 }\end{array}$} & \multicolumn{2}{|c|}{$\begin{array}{c}\text { 2" Dia. } x .125 " \text { WALL, TUBE } \\
\text { SECTION }\end{array}$} & 4 \\
\hline \multirow{10}{*}{$\begin{array}{l}\text { TTEM } \\
\text { NO. }\end{array}$} & PART NUMBER & \multicolumn{3}{|c|}{ MATERIAL } & \multicolumn{2}{|r|}{ DESCRIPTION } & QTY. \\
\hline & & \multirow{6}{*}{ 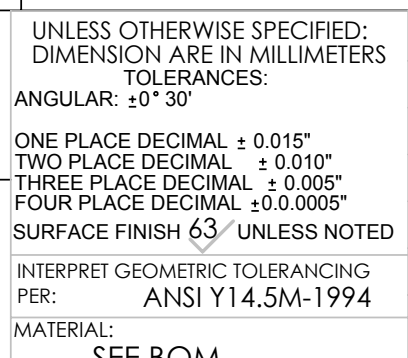 } & & DATE & NAME & \multirow{2}{*}{\multicolumn{2}{|c|}{$\begin{array}{l}\text { ARIZONA PUBLIC SERVICE } \\
400 \mathrm{~N} \text {. 5th Street } \\
\text { Phoenix A7 } 85003\end{array}$}} \\
\hline & & & DRAWN & $01 / 30 / 08$ & D. WAIBEL & & \\
\hline & & & $\begin{array}{l}\text { CHECKED } \\
\text { ENG APPR. }\end{array}$ & & & \multicolumn{2}{|l|}{ PROJECT: COAL TO SNG } \\
\hline & & & \multirow{2}{*}{$\begin{array}{l}\text { MFG APPR. } \\
\text { Q.A. }\end{array}$} & & & \multicolumn{2}{|l|}{ TIILE: $\quad$ KINETICS REACTOR } \\
\hline & & & & & & \multirow{2}{*}{\multicolumn{2}{|c|}{$\begin{array}{l}\text { 2" Dia. x 125" WALL, TUBE SECTION } \\
\text { CUT TO LENGTH }\end{array}$}} \\
\hline & & & \multirow{3}{*}{\multicolumn{2}{|c|}{$\underset{\substack{\text { METRIC } \\
\text { COMMENTS: }}}{ }$}} & \multirow{3}{*}{ 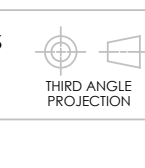 } & & \\
\hline & & FNISH: & & & & \multirow{2}{*}{ SIZE } & EV \\
\hline & & SIMIAPTO. & & & & & A \\
\hline & & & \multicolumn{2}{|l|}{ CAD FILE } & G1000.4A & SCALE: 1:2 WEIGHT: & SHEET 1 OF 1 \\
\hline
\end{tabular}




\section{GENERAL NOTES:}

DIMENSION AND TOLERANCING PER ANSI Y14.5-1994.

2 BREAK ALL EDGES .5 [.20] MAX. ALL INSIDE RADII

2.4 [.09] MAX. UNLESS NOTED OTHERWISE.

\begin{tabular}{|c|c|c|c|c|}
\hline \multicolumn{5}{|c|}{ REVISIONS } \\
\hline ZONE & REV. & DESCRIPTION & DATE & REV. BY APPROV \\
\hline ALL & A & INITIAL RELEASE & $06 / 13 / 07$ & D.W. \\
\hline ALL & B & UPDATED MATERIAL CALL OUT & $01 / 02 / 08$ & D.W. \\
\hline ALL & C & $\begin{array}{c}\text { CHANGED .88 THRU HOLE TO } \\
88 \times 1.00 \text { SLOT }\end{array}$ & $04 / 01 / 08$ & D.W. \\
\hline
\end{tabular}

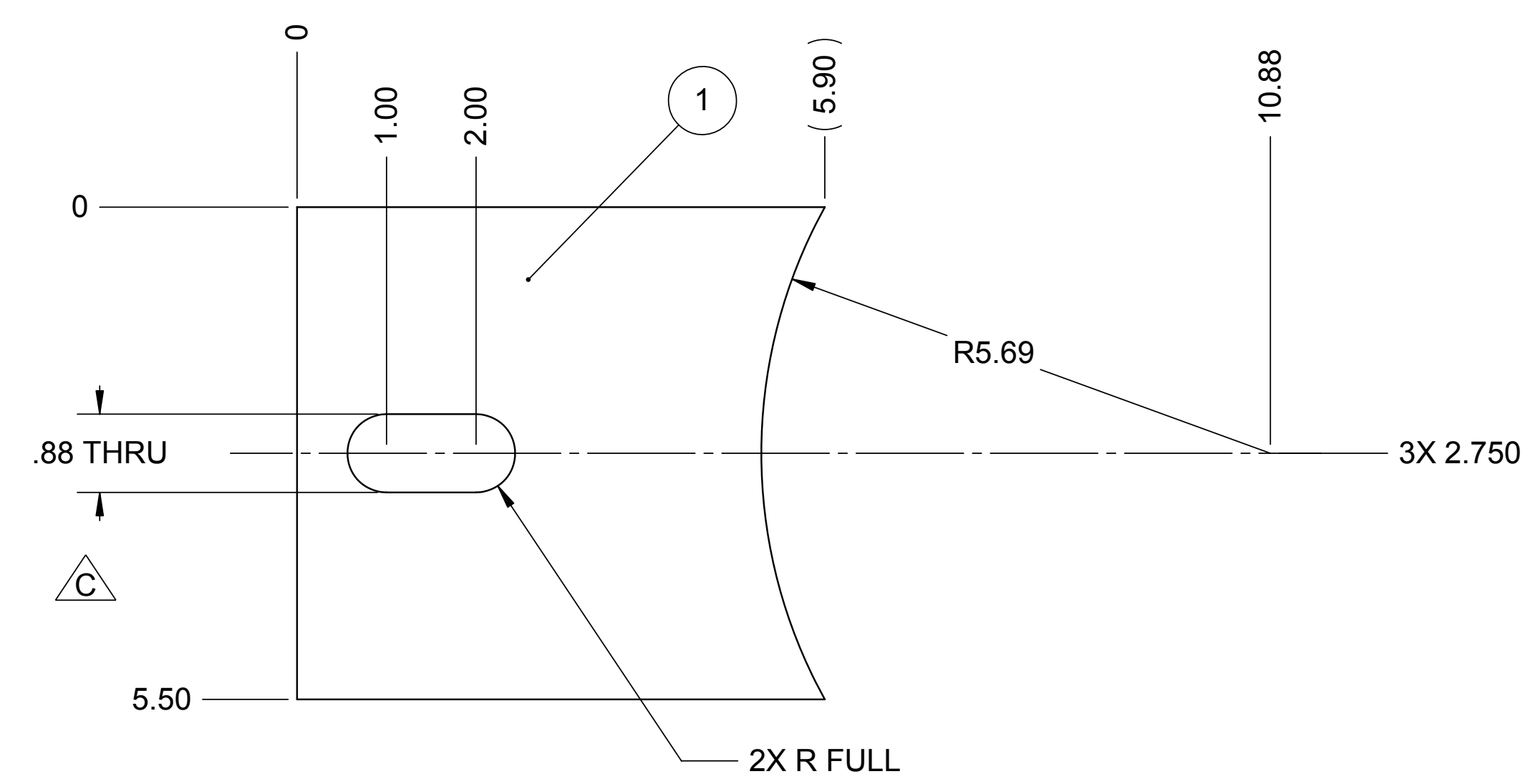

B

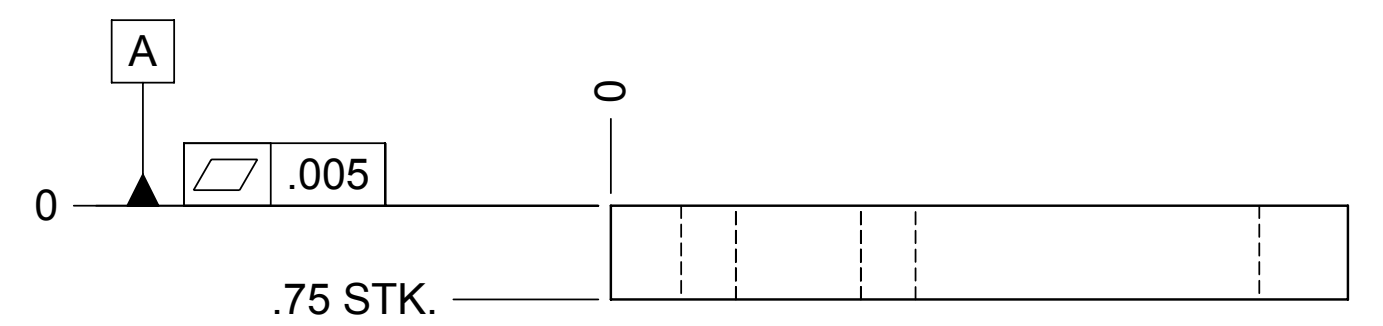

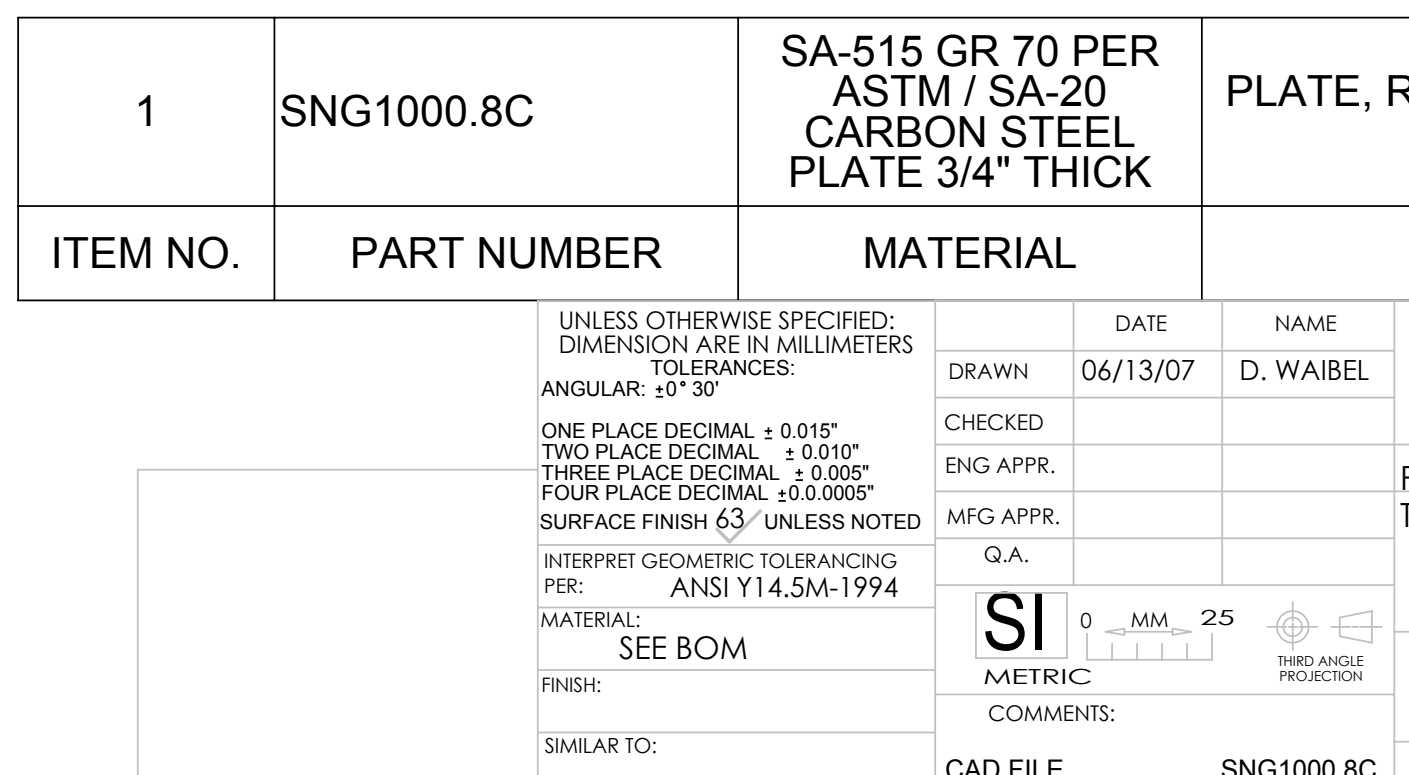
TOP SUP OUTER SHELL
TOP SUPORT

DESCRIPTION

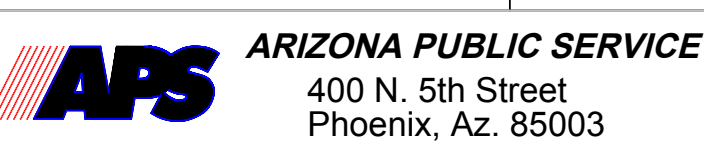
PROJECT: $\quad$ COAL TO SNG KINETICS REACTOR
PLATE, REACTOR OUTER SHELL,
TOP SUPPORT

B SNG1000.8 


\section{GENERAL NOTES:}

DIMENSION AND TOLERANCING PER ANSI Y14.5-1994.

2 BREAK ALL EDGES .5 [.20] MAX. ALL INSIDE RADII 2.4 [.09] MAX. UNLESS NOTED OTHERWISE.

D

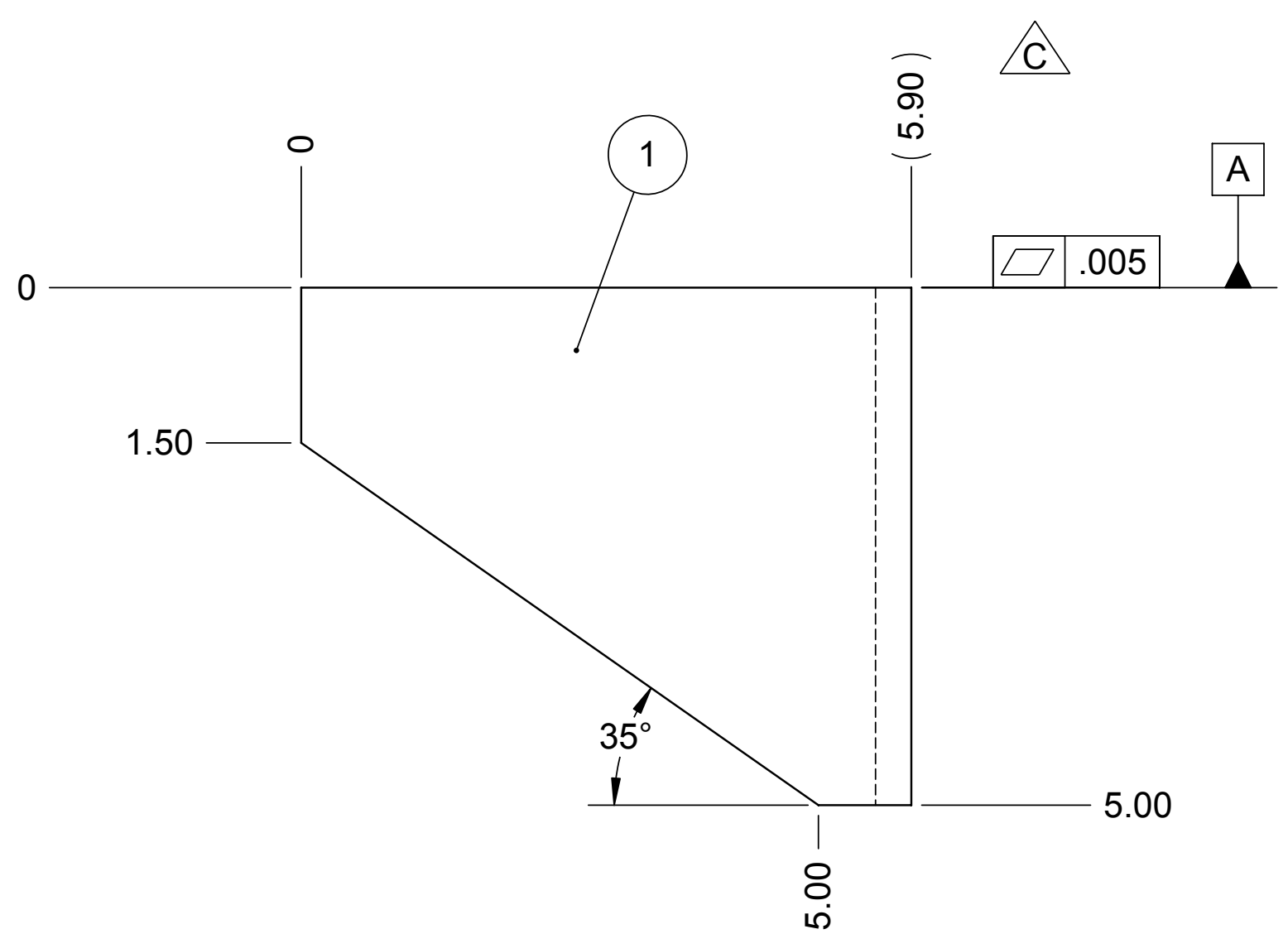

2

REVISIONS

\begin{tabular}{|l|l|l|l|l|}
\hline \multicolumn{5}{|c|}{ REVISIONS } \\
\hline ZONE & REV. & DESCRPTION & DATE & REV. BY APPROVED \\
\hline ALL & A & INITIAL RELEASE & $06 / 13 / 07$ & D.W. \\
\hline ALL & B & UPDATED MATERIAL CALL OUT & $01 / 02 / 08$ & D.W. \\
\hline ALL & C & LENGTH CHANGE, 5.90 WAS 5.40 & $04 / 01 / 08$ & D.W. \\
\hline
\end{tabular}

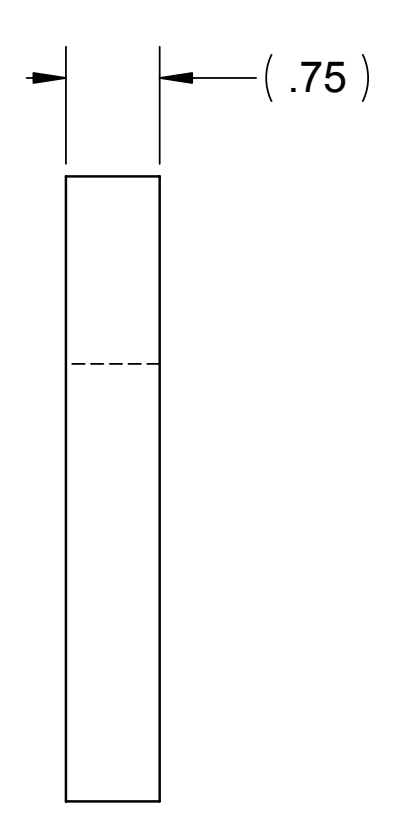

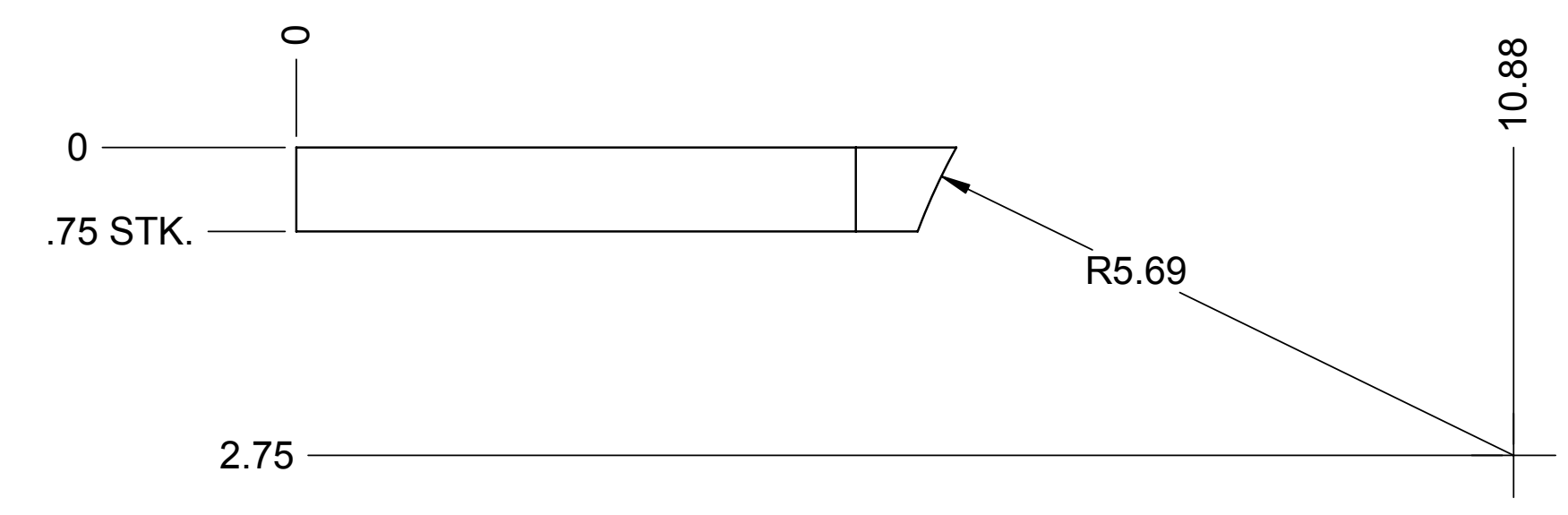

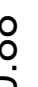

B

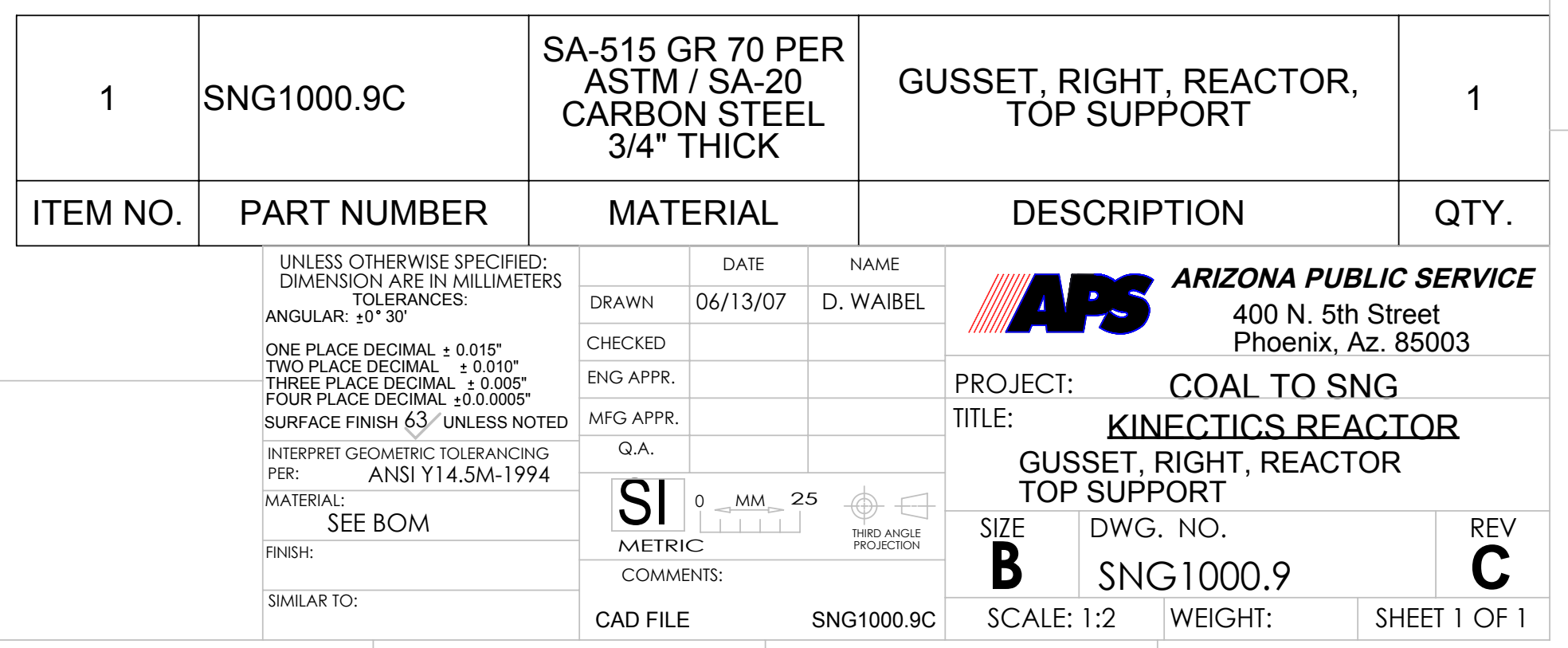



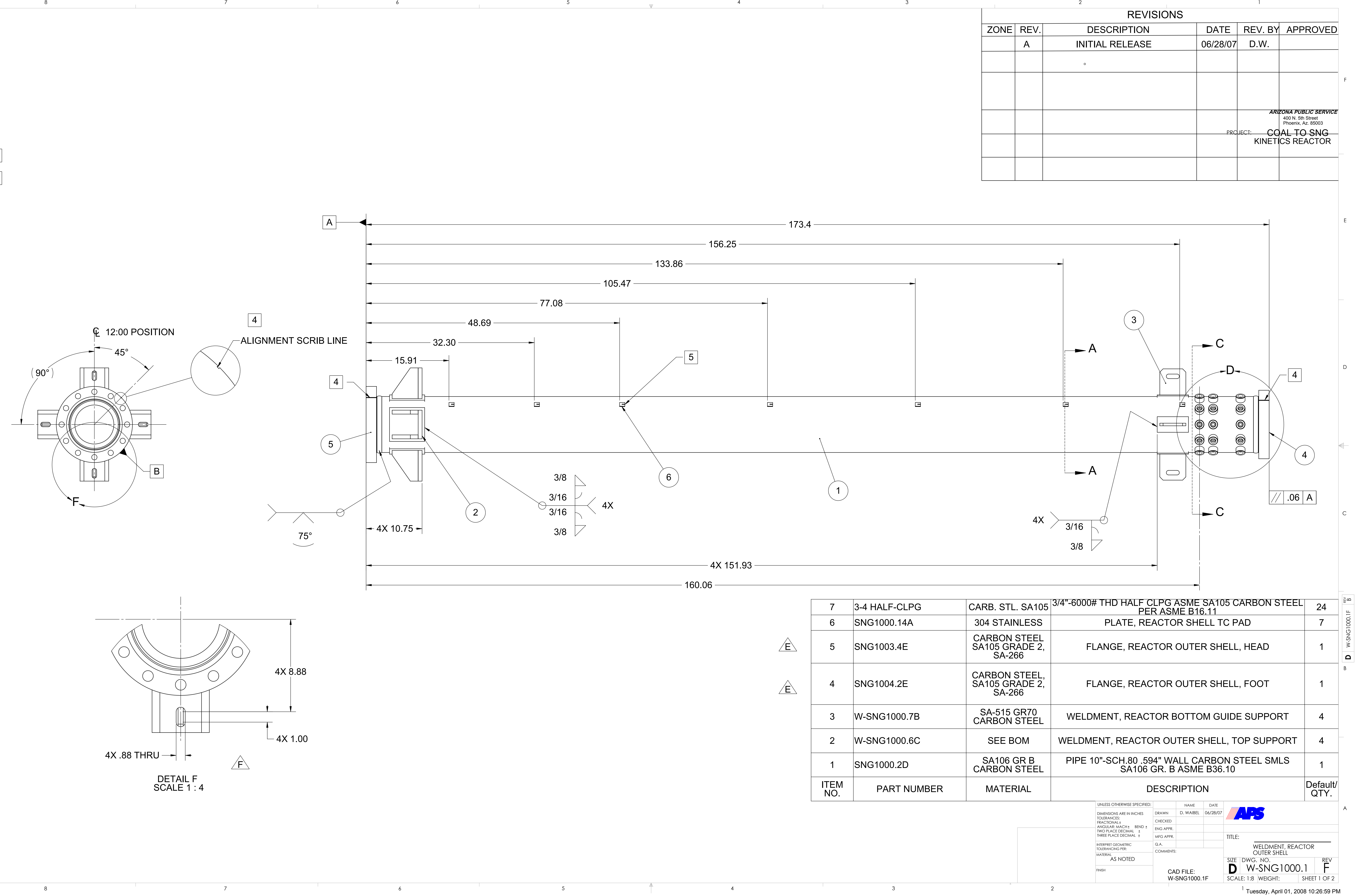


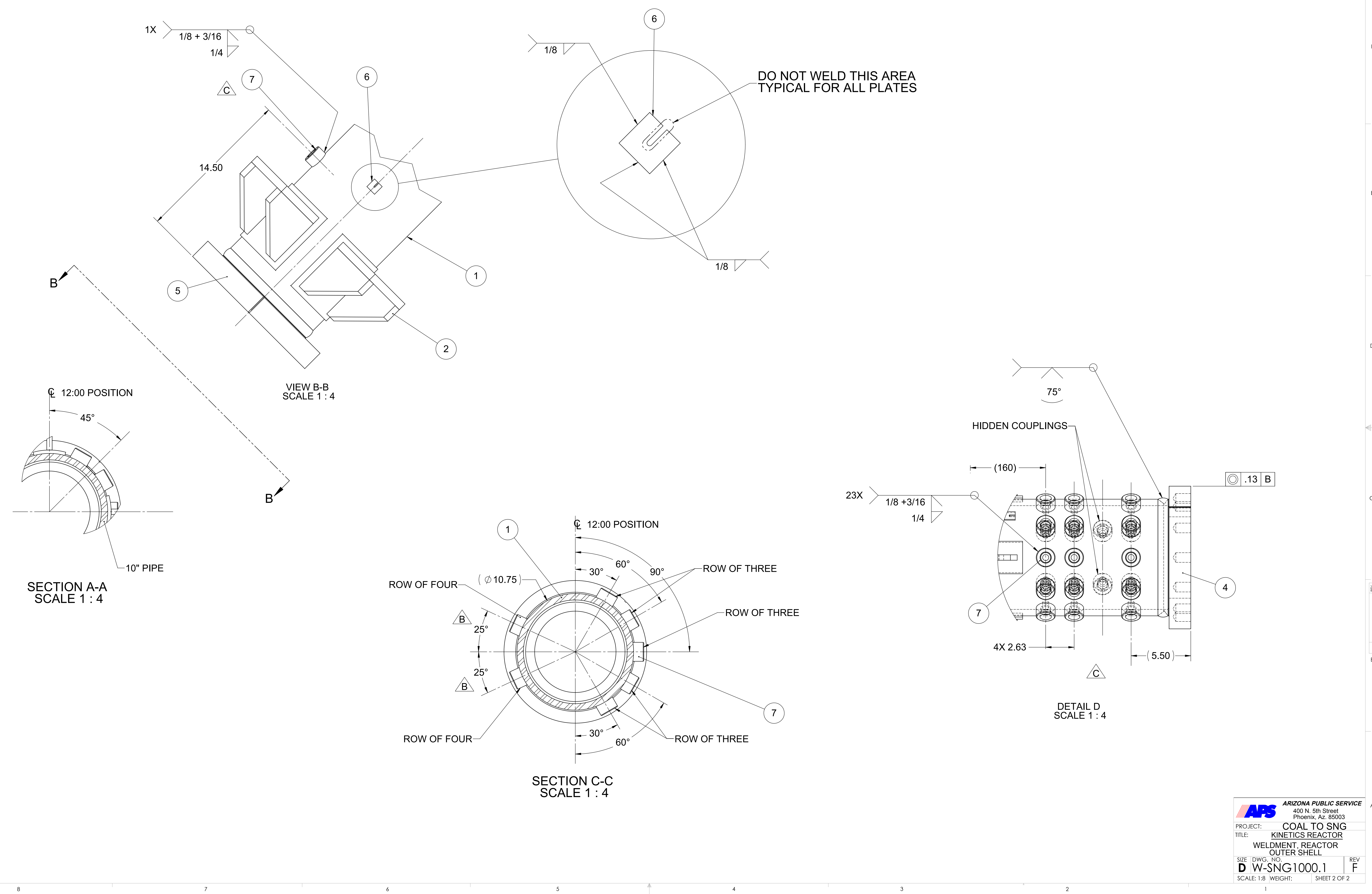



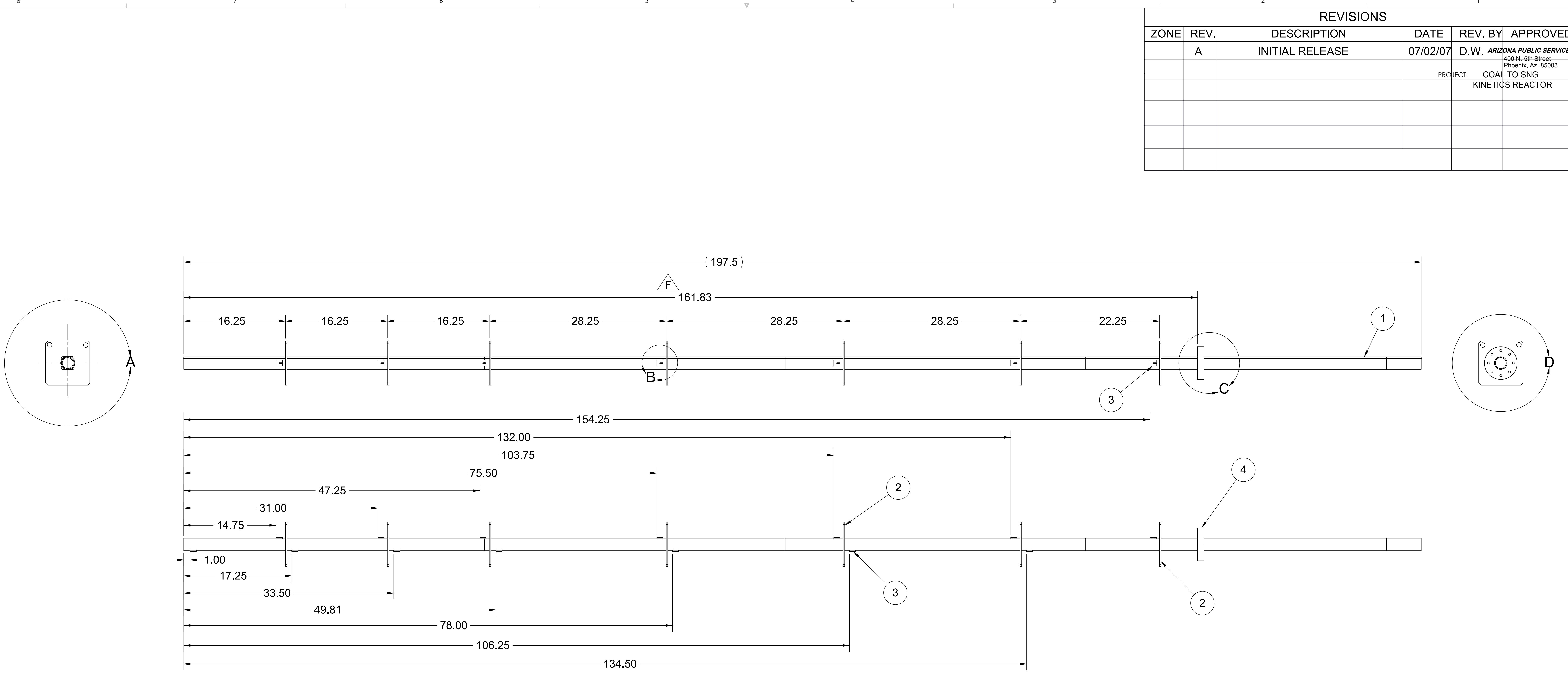

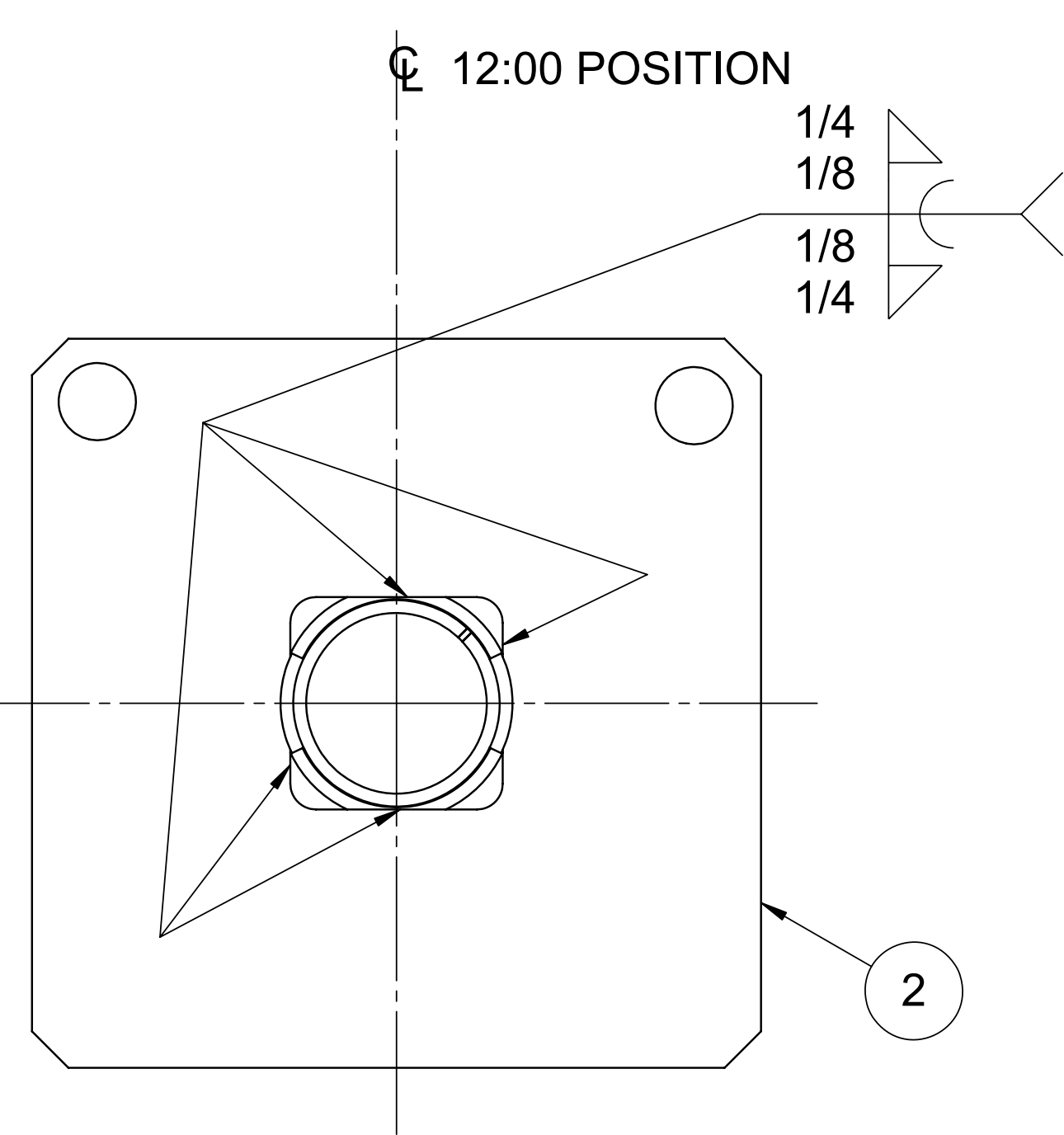

DETALLA
SCALE $1: 2$

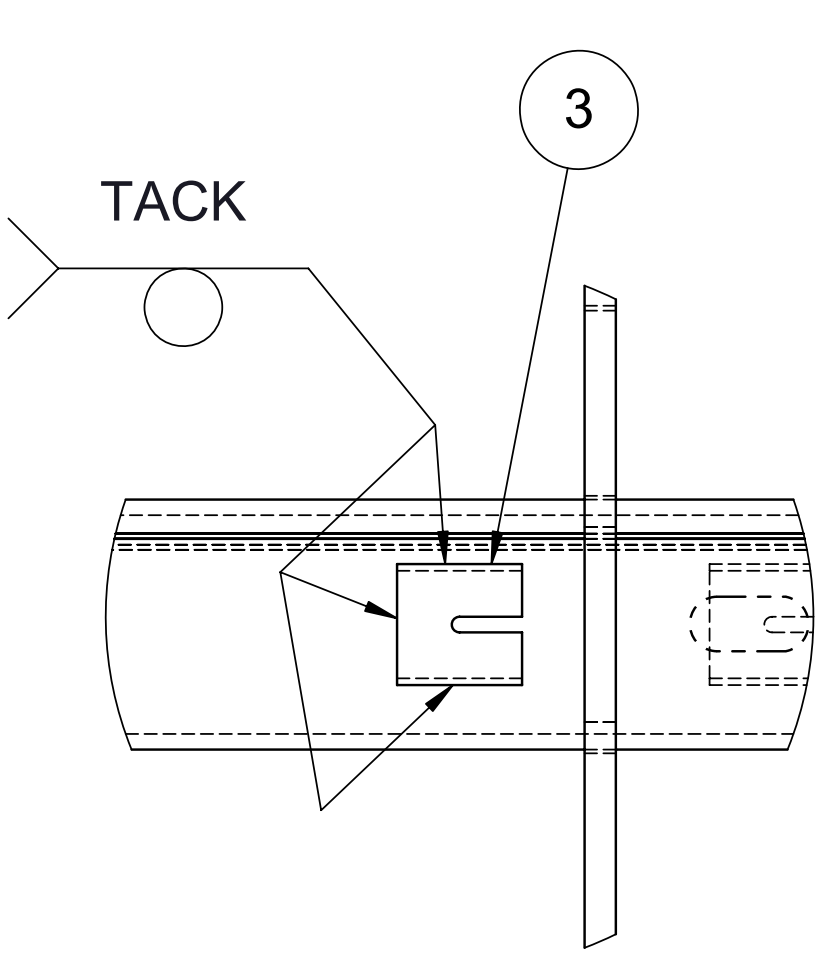

DEFALLB
SCALE $1: 2$

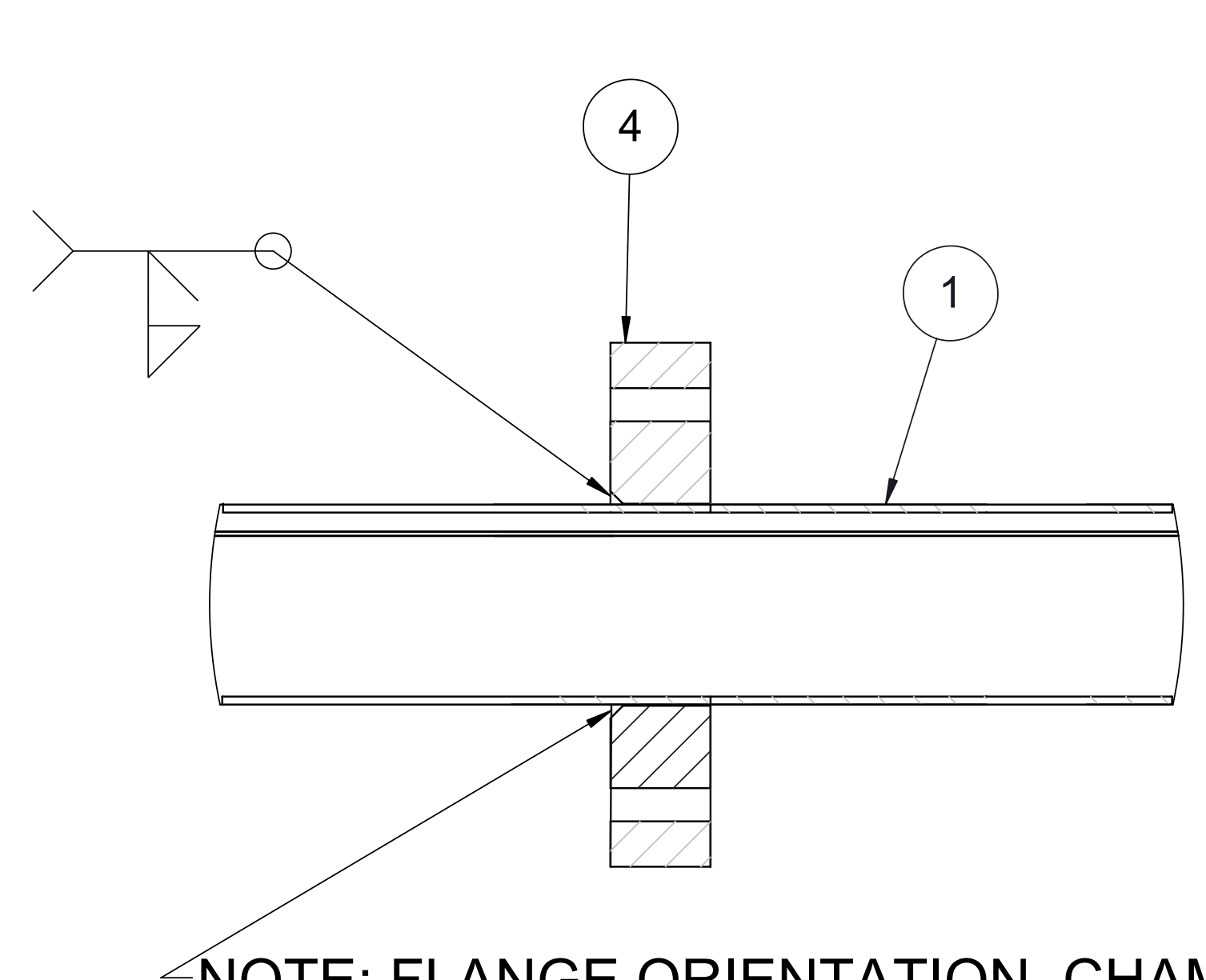

NOTE: FLANGE ORIENTATION, CHAMFER ON ID OF FLANGE TO FACE
TOWARDS PLATES

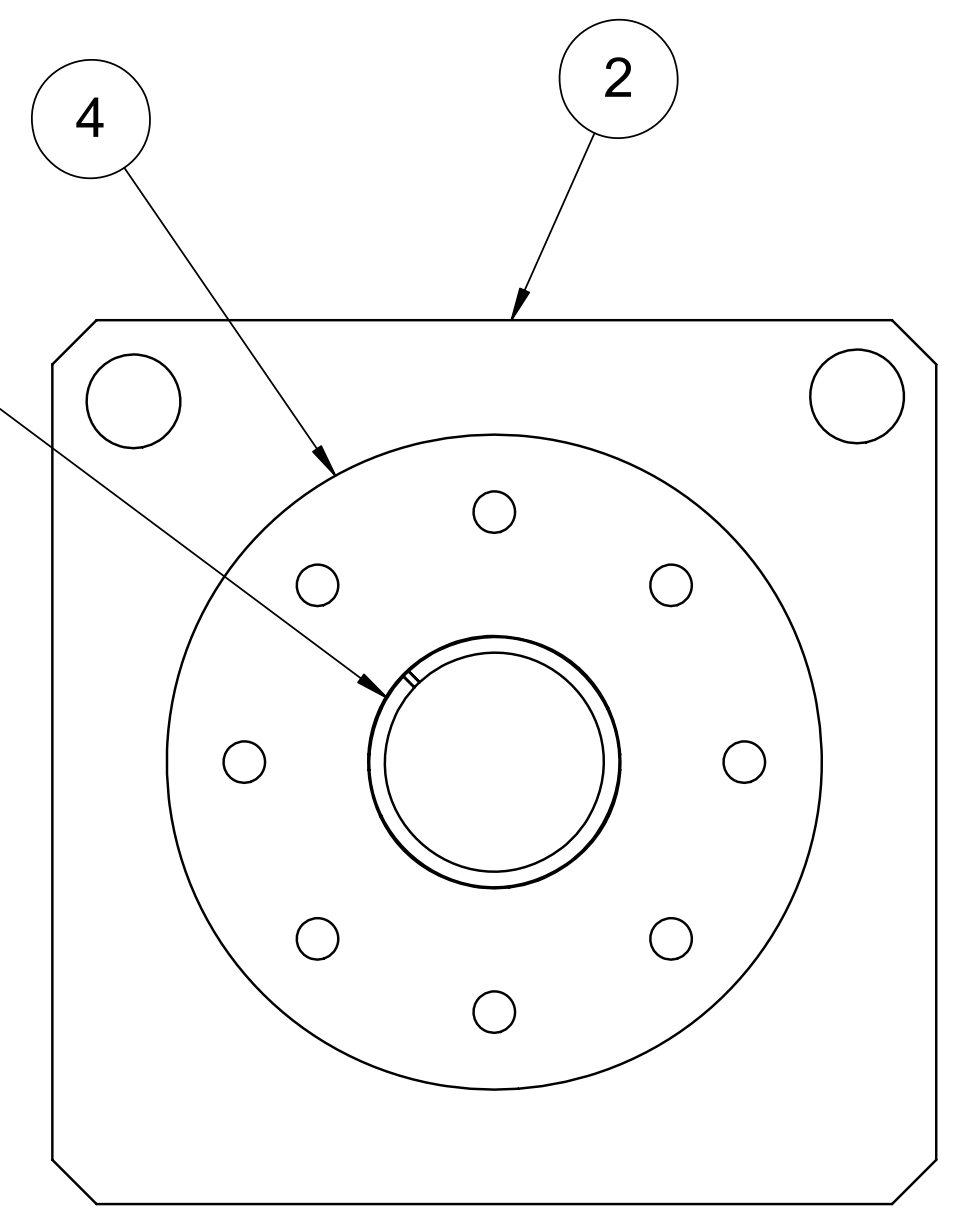

DETALLD
SCALE $1: 22$

\begin{tabular}{|c|c|c|c|c|}
\hline 4 & SNG1000.22C & SA-182, GRADE 316H SS & FLANGE, INNER TUBE & 1 \\
\hline 3 & SNG1000.24A & 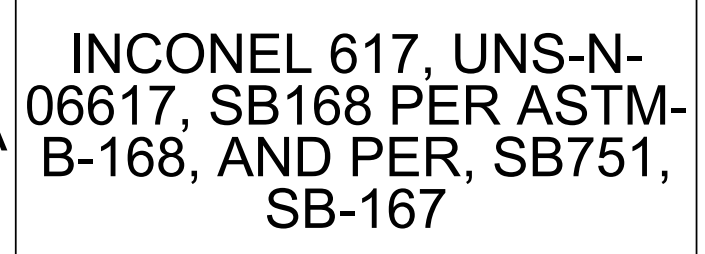 & PLATE, REACTOR, INNER TC PAD & 14 \\
\hline 2 & SNG1000.21A & SA 182 GR. 316 H S.S. & PLATE, SPIDER SUPPORT & \\
\hline 1 & SNG1000.3C & $\begin{array}{l}\text { INCONEL 617, UNS-N- } \\
\text { O6617., SB168 PER } \\
\text { ASTM-B-1688 AND PER } \\
\text { SB751, SB-167 }\end{array}$ & TUBE, REACTOR INNER & \\
\hline $\begin{array}{l}\text { TTEM } \\
\text { NO. }\end{array}$ & $\begin{array}{c}\text { PART } \\
\text { NUMBER }\end{array}$ & MATERIAL & DESCRIPTION & QTY \\
\hline & & 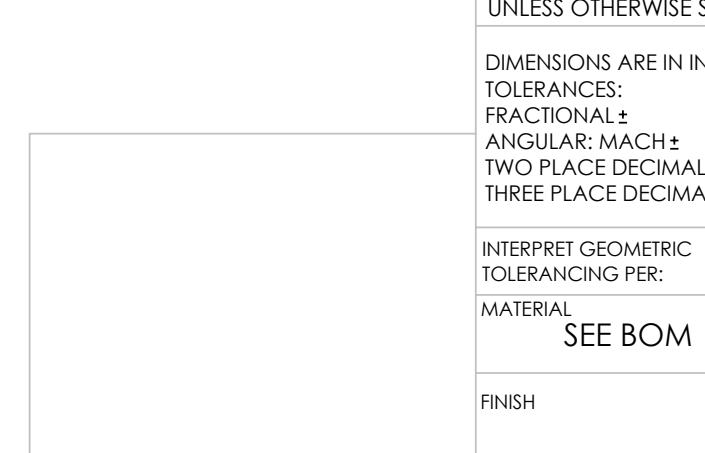 & 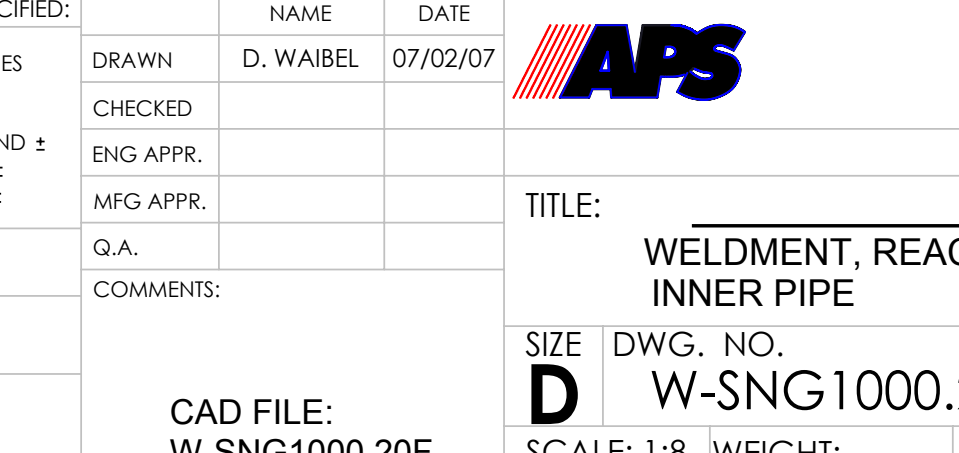 & , \\
\hline
\end{tabular}




\section{GENERAL NOTES:}

1 SHEET 1, ISO VIEW (S), BILL OF MATERIALS, NOTES. SHEET 2, DETAIL VIEWS.

2 STANDARD WELDING PRACTICE PER, ANSI / AWS D1.3 FOR ALL WELDS, U.N.O.

WELDS TO MEET ASME BPVC SECTION 8, DIVISION 1.

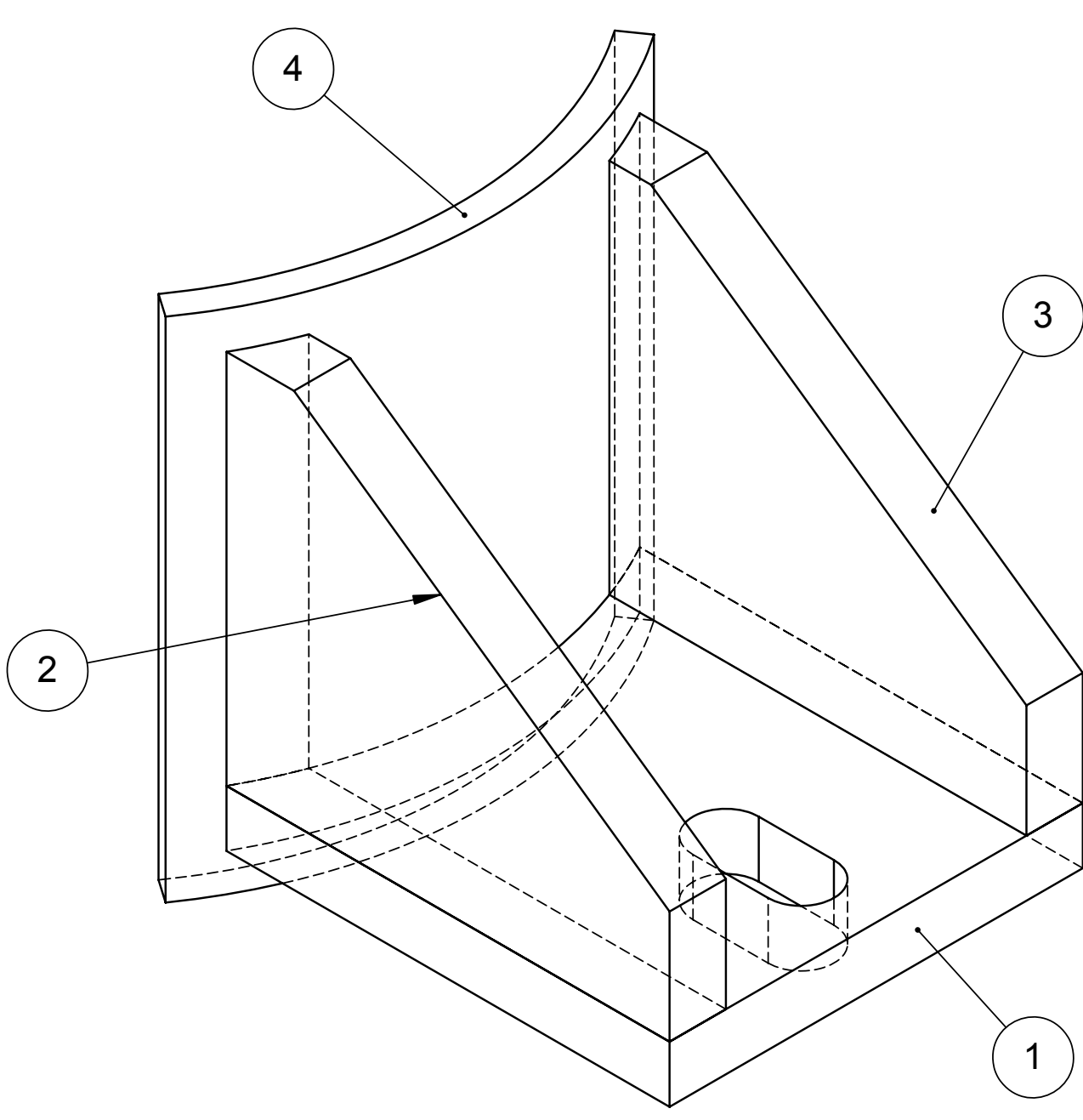

ISO VIEW DO NOT SCALE

3
B

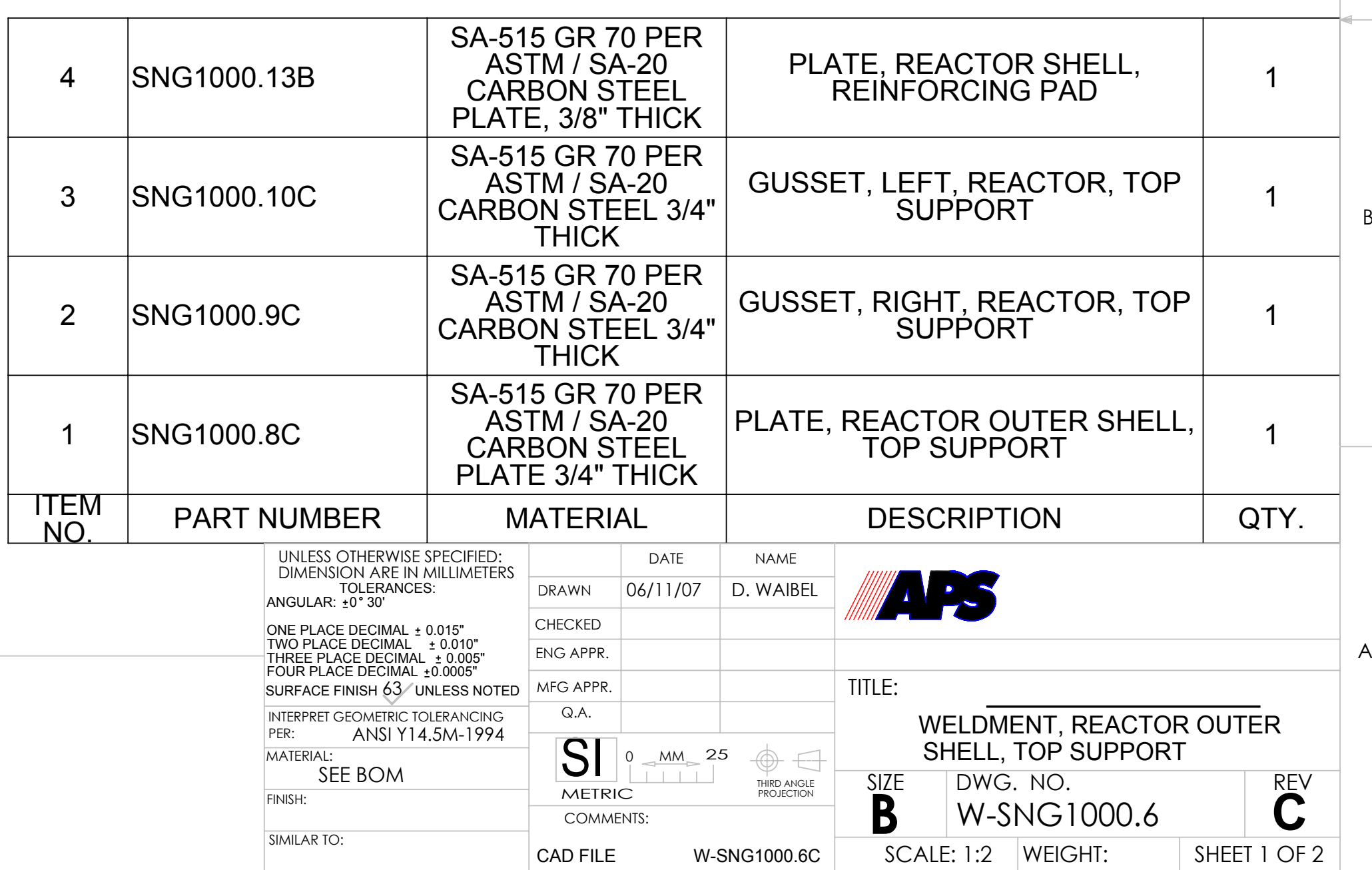



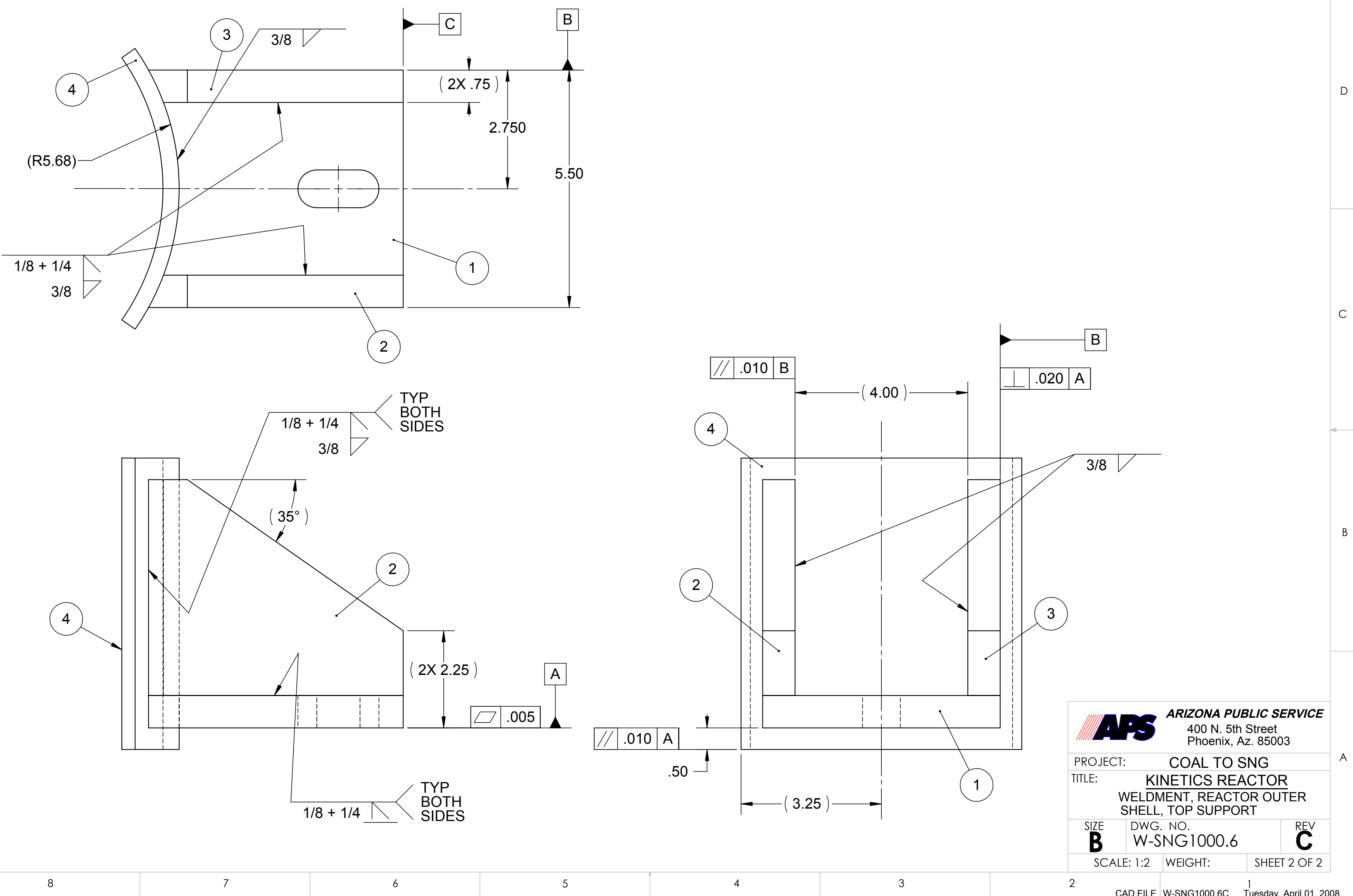
1 STANDARD WELDING PRACTICE PER, ANSI / AWS D1.3 FOR ALL WELDS, U.N.O.

2 QUADRANT ON ITEM 1, AS INDICATED, IS DATUM 'A', WHICH IS USED TO HOLD POSITIONING BETWEEN COMPONENTS, ITEM 1, AND ITEM 2.

3 WELDS TO MEET ASME BPVC SECTION 8, DIVISION 1.

5.50

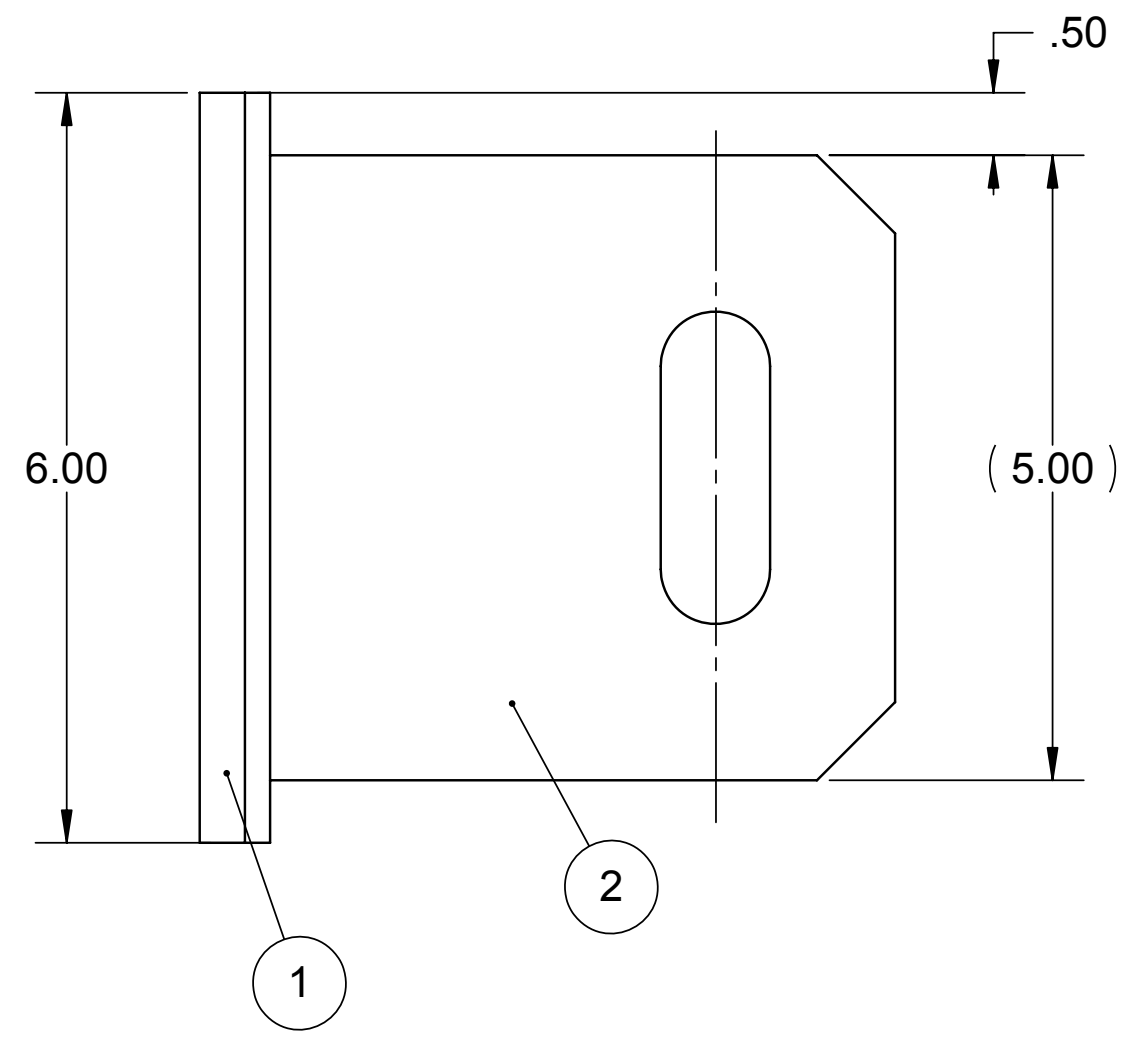

B

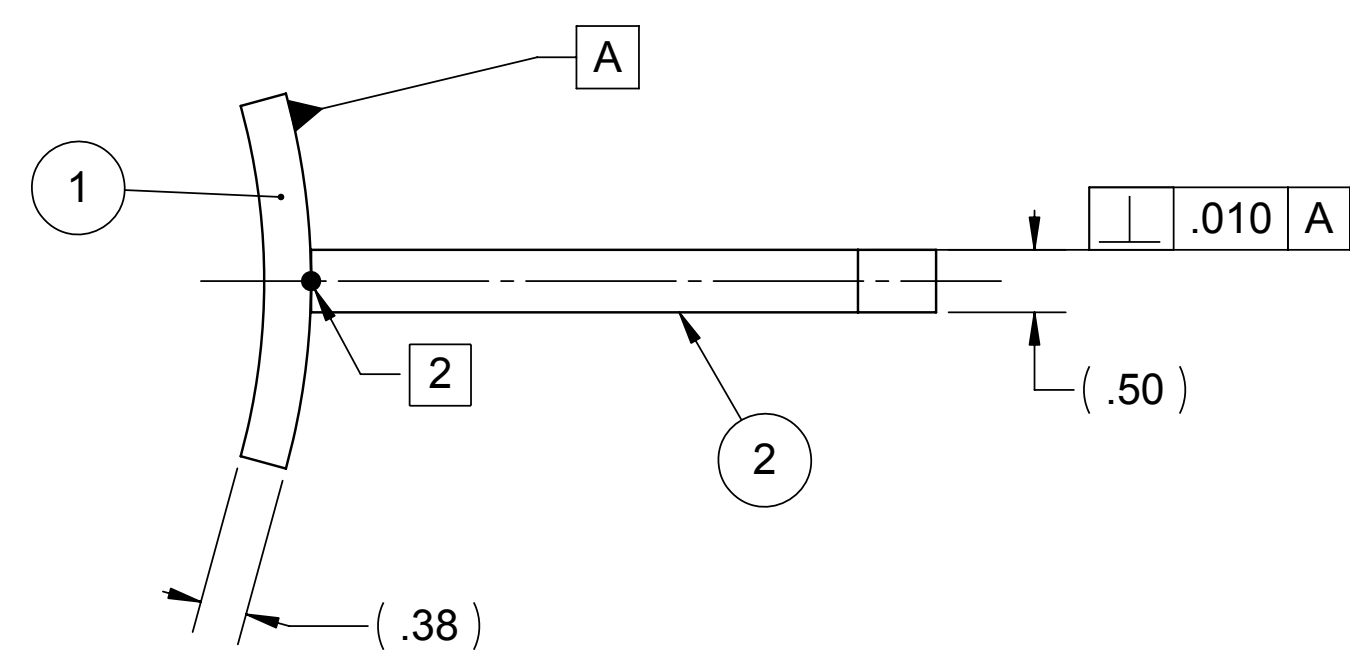

\section{REVISIONS}

\begin{tabular}{|c|c|c|c|c|}
\hline ZONE & REV. & DESCRPTION & & \\
\hline ALL & A & INITIAL RELEASE & DATE & REV. B \\
\hline & & $06 / 11 / 07$ & DW \\
\hline
\end{tabular}

\begin{tabular}{l|l|l|l|l|l|l|l} 
ALL & B ADDED NOTE 3 \& MAT'L CALLOUT UPDATED 01/02/08 & DW
\end{tabular}

B

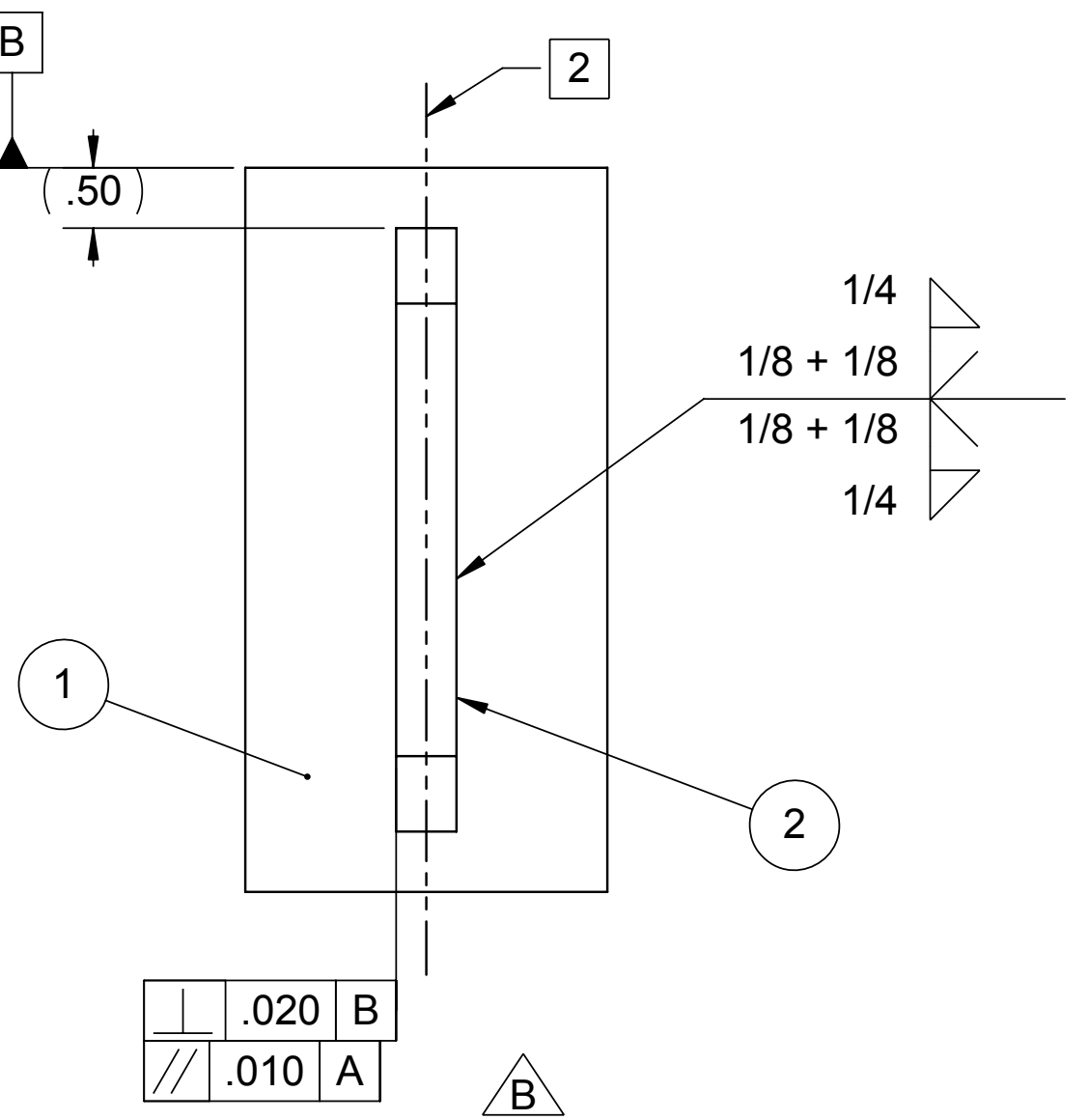

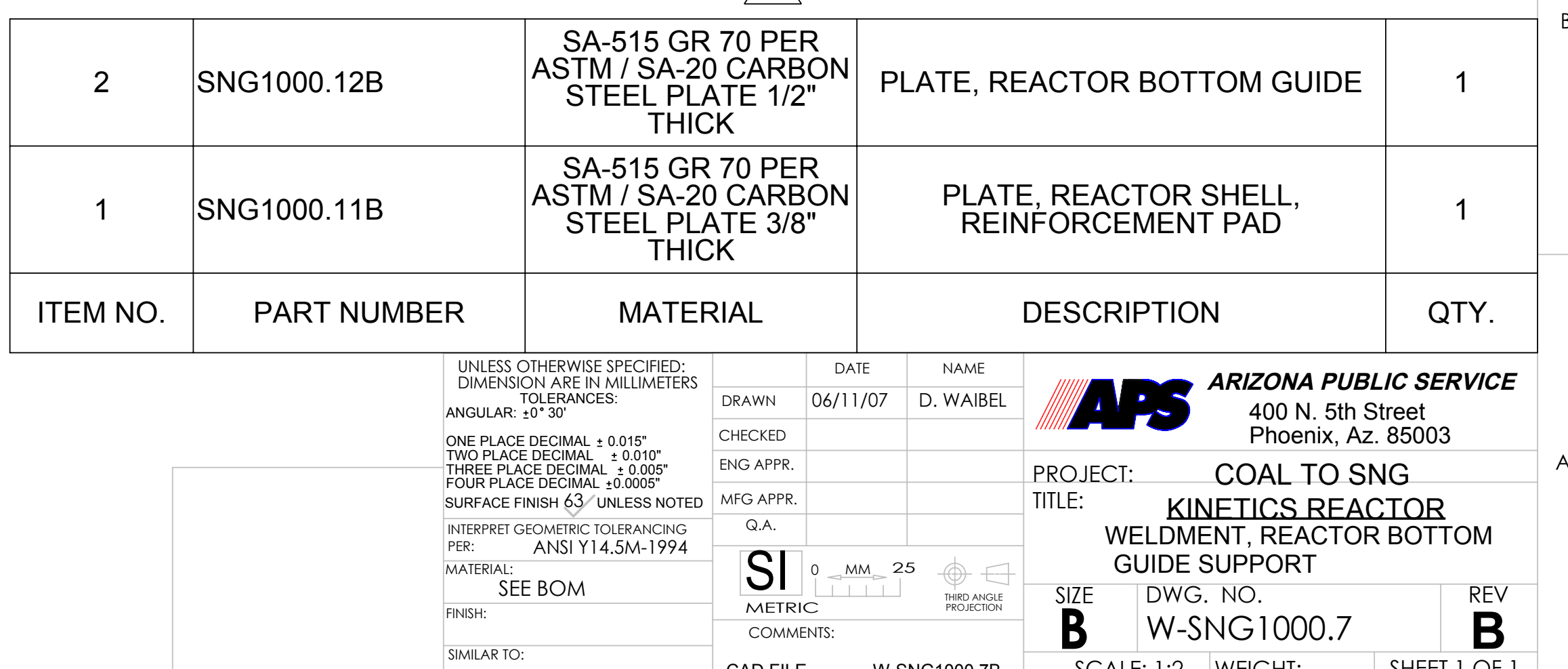




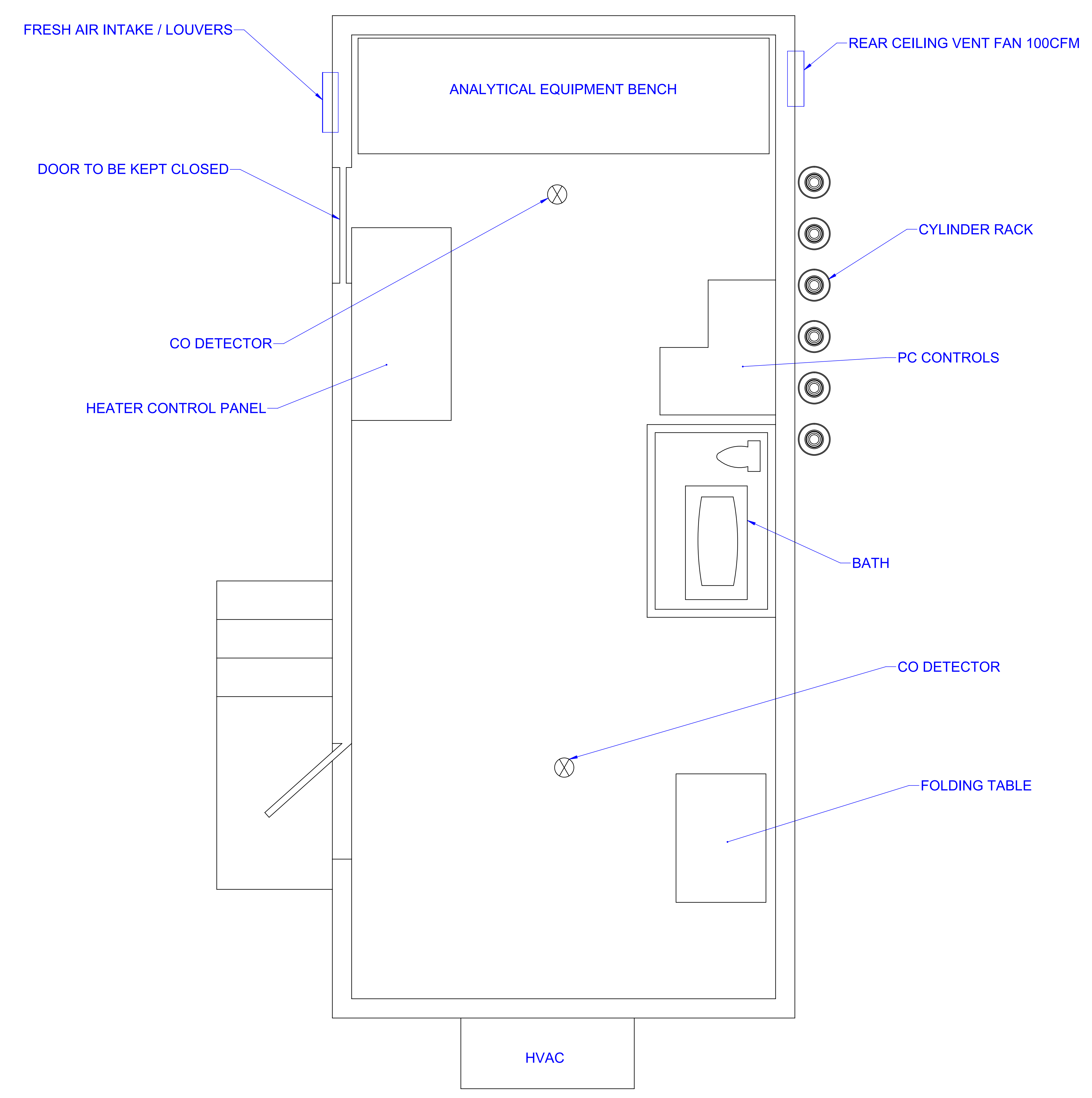

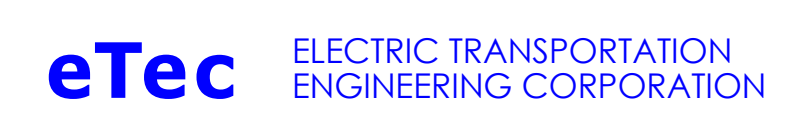

\begin{tabular}{|c|c|c|c|c|}
\hline 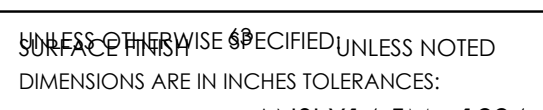 & 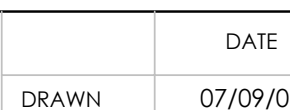 & Nowe & - & \\
\hline 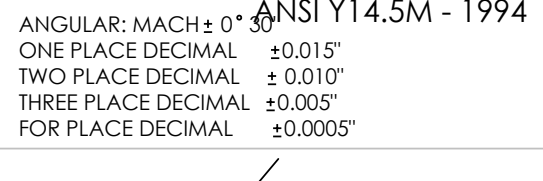 & 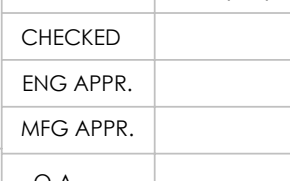 & & LAYOUT, TRAILER & \\
\hline 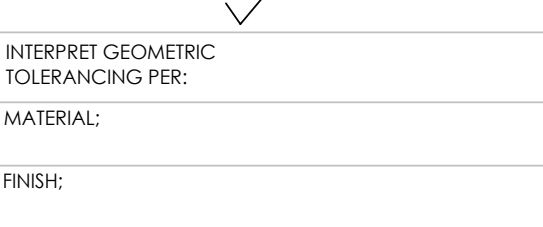 & CAD FllE; & & 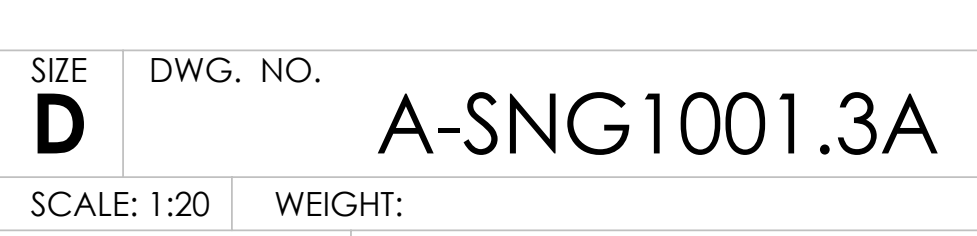 & $\begin{array}{l}\text { REV } \\
\text { OF } 1\end{array}$ \\
\hline
\end{tabular}




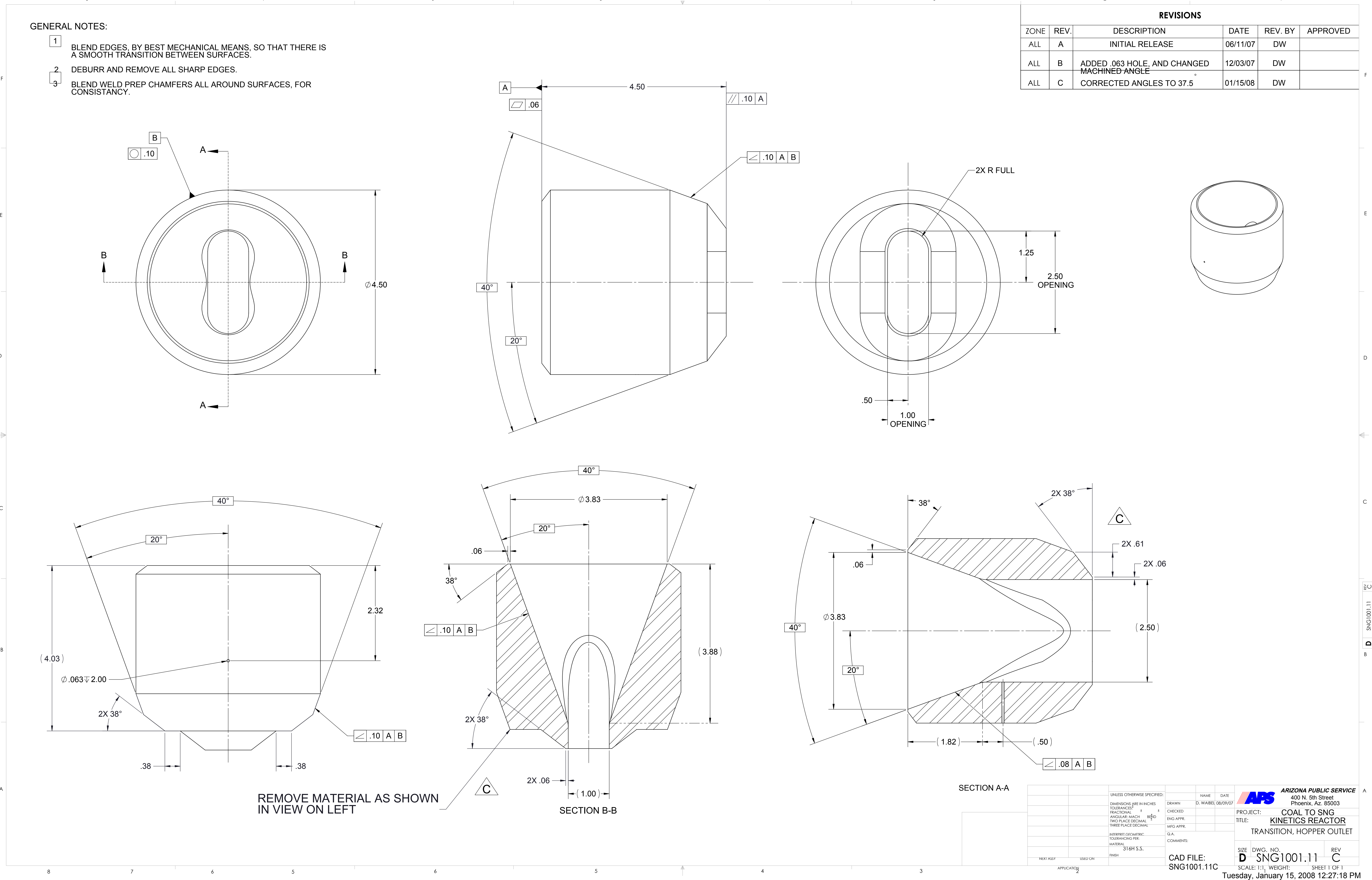




\begin{tabular}{|c|c|c|c|c|c|}
\hline \multicolumn{6}{|c|}{ REVISIONS } \\
\hline ZONE & REV. & DESCRPPTION & DATE & & APPROVED \\
\hline ALL & A & INTIALL RLLEASE & $\mid 03 / 04 / 188$ & $\mathrm{DW}$ & \\
\hline & & & & & \\
\hline
\end{tabular}

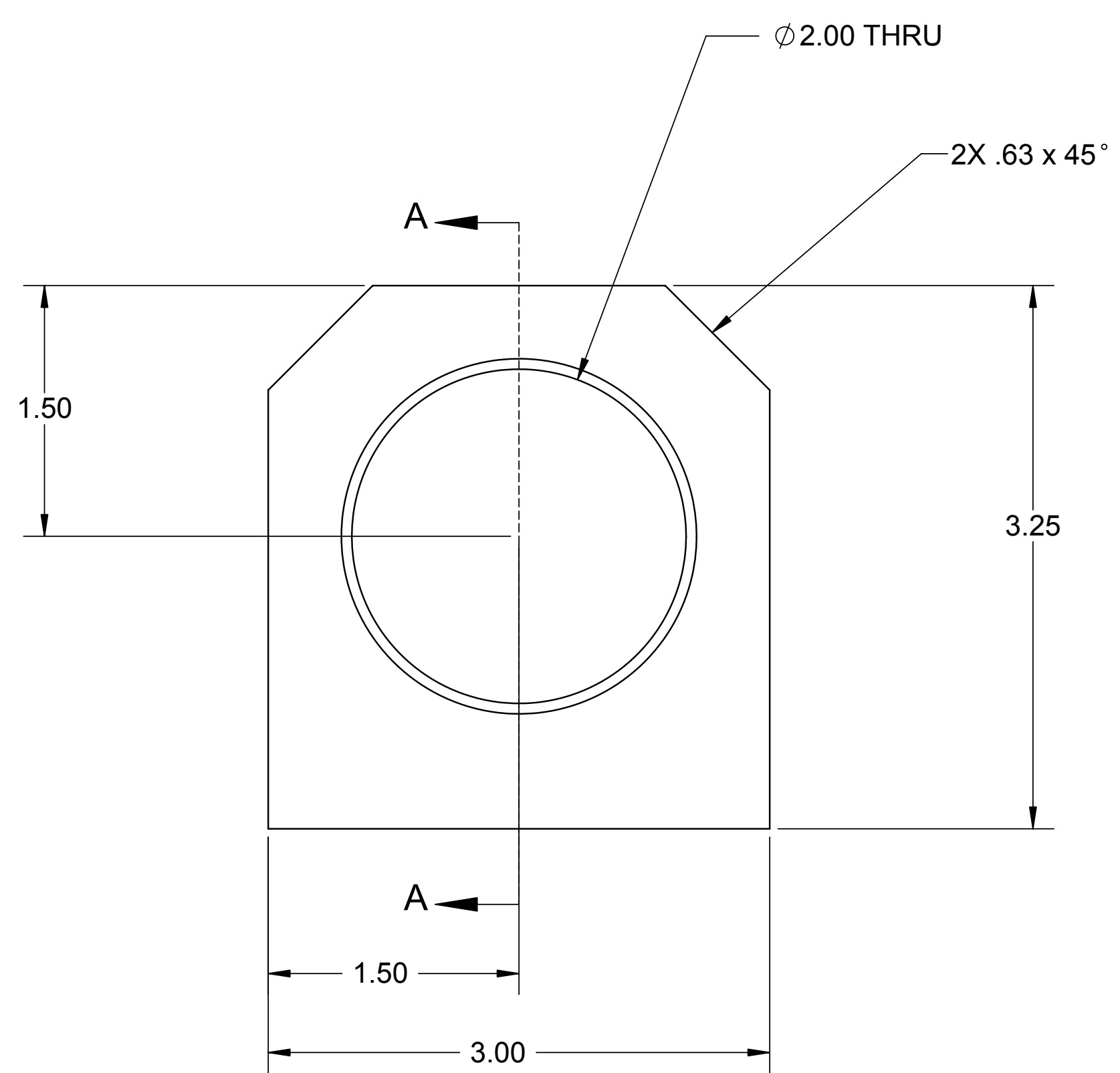

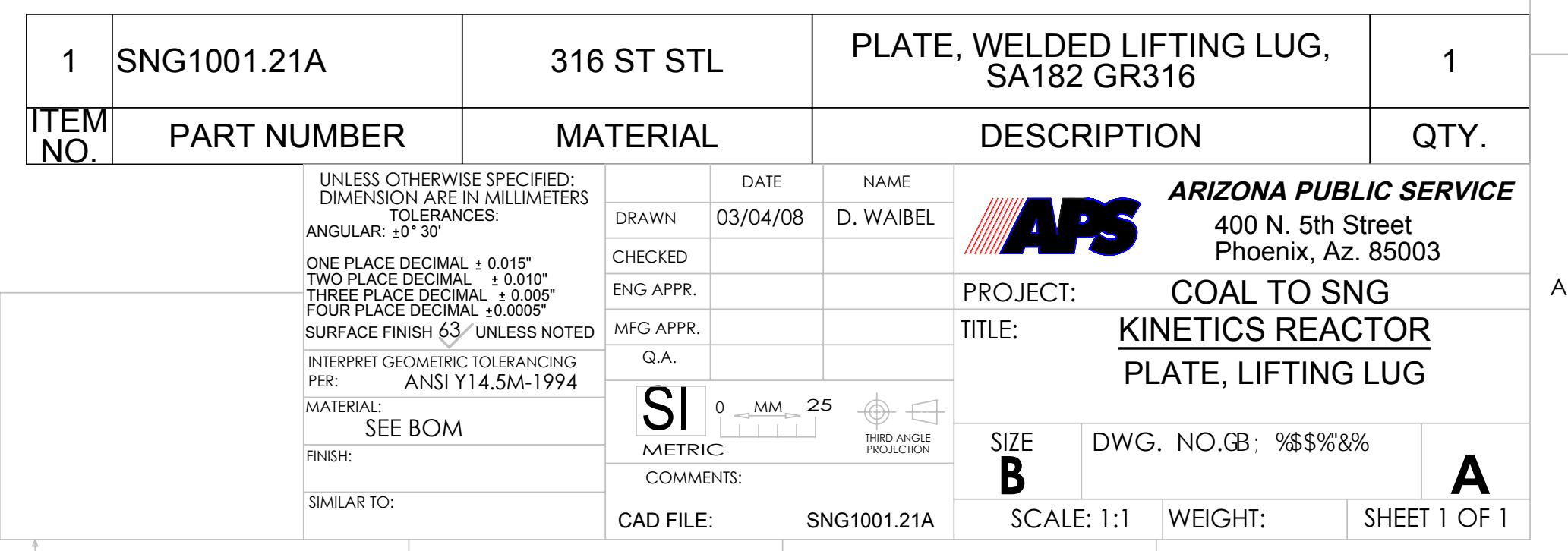


FITTINGS, AND PIPE, TO BE IN ACCORDANCE WITH ANSI B16.9. FLANGES IN

ACCORDANCE WITH ANSI B16.5. GASKETS IN ACCORDANCE WITH, ASME B16.20. PER ASME B16.25.
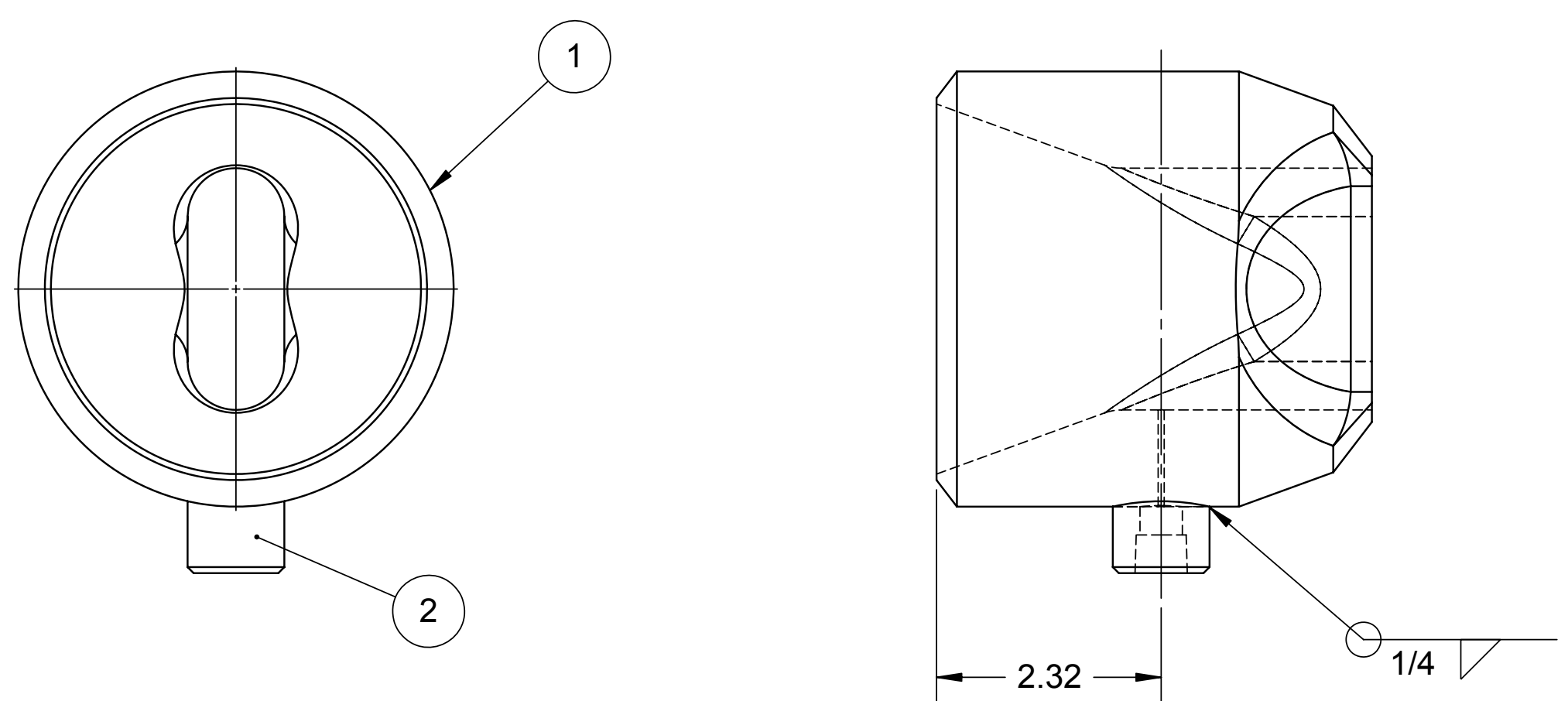

\begin{tabular}{|c|c|c|c|c|c|c|c|c|}
\hline 2 & \multicolumn{2}{|c|}{ SNG1001.20B } & \multicolumn{2}{|c|}{ SA182 QRF 316} & \multicolumn{3}{|c|}{$\begin{array}{c}\text { THREADOLET, 1/4"- } \\
\text { 3000LB, THD, HALF CLPG. }\end{array}$} & 1 \\
\hline 1 & \multicolumn{2}{|c|}{ SNG1001.11B } & \multicolumn{2}{|c|}{316 H S.S. } & \multicolumn{3}{|c|}{$\begin{array}{l}\text { TRANSITION, HOPPER } \\
\text { OUTLET }\end{array}$} & 1 \\
\hline $\begin{array}{l}\text { TTEM } \\
\text { NO. }\end{array}$ & \multicolumn{2}{|c|}{ PART NUMBER } & \multicolumn{2}{|c|}{ MATERIAL } & \multirow{2}{*}{\multicolumn{4}{|c|}{\begin{tabular}{c|c} 
DESCRIPTION & QTY. \\
ARIZONA PUBLIC SERVICE \\
400 N. 5th Street \\
Phoenix, Az. 85003
\end{tabular}}} \\
\hline \multirow{4}{*}{\multicolumn{2}{|c|}{ 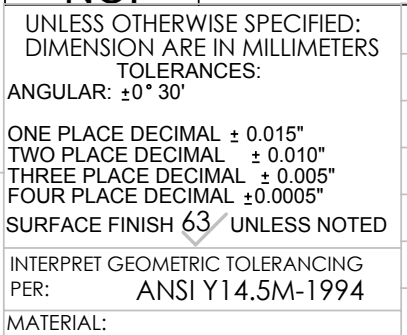 }} & $\begin{array}{l}\text { DRAWN } \\
\text { CHECKED }\end{array}$ & \begin{tabular}{|c|} 
DATE \\
$12 / 03 / 07$ \\
\end{tabular} & \begin{tabular}{|c|} 
NAME \\
D. WAIBEL \\
\end{tabular} & & & & \\
\hline & & $\begin{array}{l}\text { CHECKED } \\
\text { ENG APPR. }\end{array}$ & & & \multirow{3}{*}{\multicolumn{4}{|c|}{$\begin{array}{lc}\text { PROJECT: } & \text { COAL TO SNG } \\
\text { TITLE: } & \text { KINETICS REACTOR } \\
\text { WELDMENT, COAL FEED TRANSITION }\end{array}$}} \\
\hline & & MFG APPR. & & & & & & \\
\hline & & \multirow{2}{*}{\multicolumn{2}{|c|}{$\underset{\text { METRIC }}{\mathrm{SI}}{ }_{\text {MM }} 25$}} & & & & & \\
\hline AS NC & ED IN BOM & & & \multirow[t]{2}{*}{ 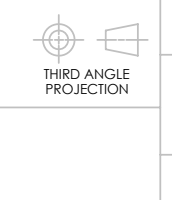 } & \multirow{2}{*}{\multicolumn{2}{|c|}{\begin{tabular}{l|l} 
SIZE & DWG. NO. \\
B & W-SNG 1001.18
\end{tabular}}} & & \multirow{2}{*}{$\underset{\text { EET I OF }}{\mathbf{R E V}_{1}}$} \\
\hline SIMLLAR TO: & & & & & & SHEE & \\
\hline
\end{tabular}


1 ALL WELDS TO MEET PRESSURE VESSEL CODE, ASME SECTION 8, DIVISION 1 ,

c

\begin{tabular}{|c|c|c|c|c|c|}
\hline \multicolumn{6}{|c|}{ REVISIONS } \\
\hline ZONE & REV. & DESCRIPTION & DATE & REV. BY & APPROVED \\
\hline ALL & A & INITIAL RELEASE & $08 / 09 / 07$ & D.W. & \\
\hline Al & $B$ & DIM 4625 WAS 3444 & 10/09/07 & DW & \\
\hline ALL & c & $\begin{array}{l}\text { ADDED MATERIAL COLUMN TO BOM, ADDED } \\
\text { FITING SPEC, AND X-RAY ISPECTION TO } \\
\text { NOTES. DELETED CAL OUT ON GROOVE WELDS }\end{array}$ & 11/27/07 & D.W. & \\
\hline ALL & D & ADDED NOTE 5; MATERIAL SPEC. AND ADDED & 12/06/07 & D.W. & \\
\hline ALL & E & ADDED LIFTING LUG & $03 / 04 / 08$ & D.W. & \\
\hline
\end{tabular}

I

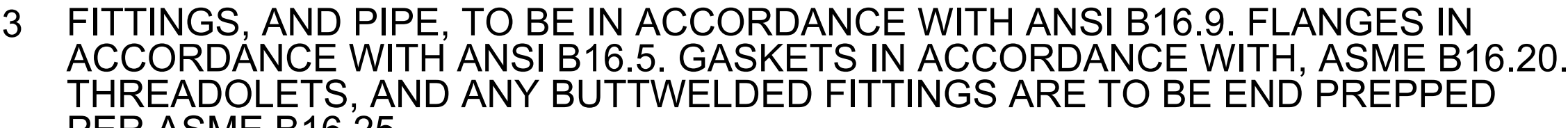
PER ASME B16.25.

4 ITEM 4, (PIPE, 2"-SCH.80), TO HAVE TWO ${ }^{\varnothing} .375$ THRU HOLES, $180^{\circ}$ APART, AS SHOWN.

5 MATERIAL: 316L S.S., CARBON CONTENT MUST BE EQUAL TO, OR ABOVE .04\%

46.25
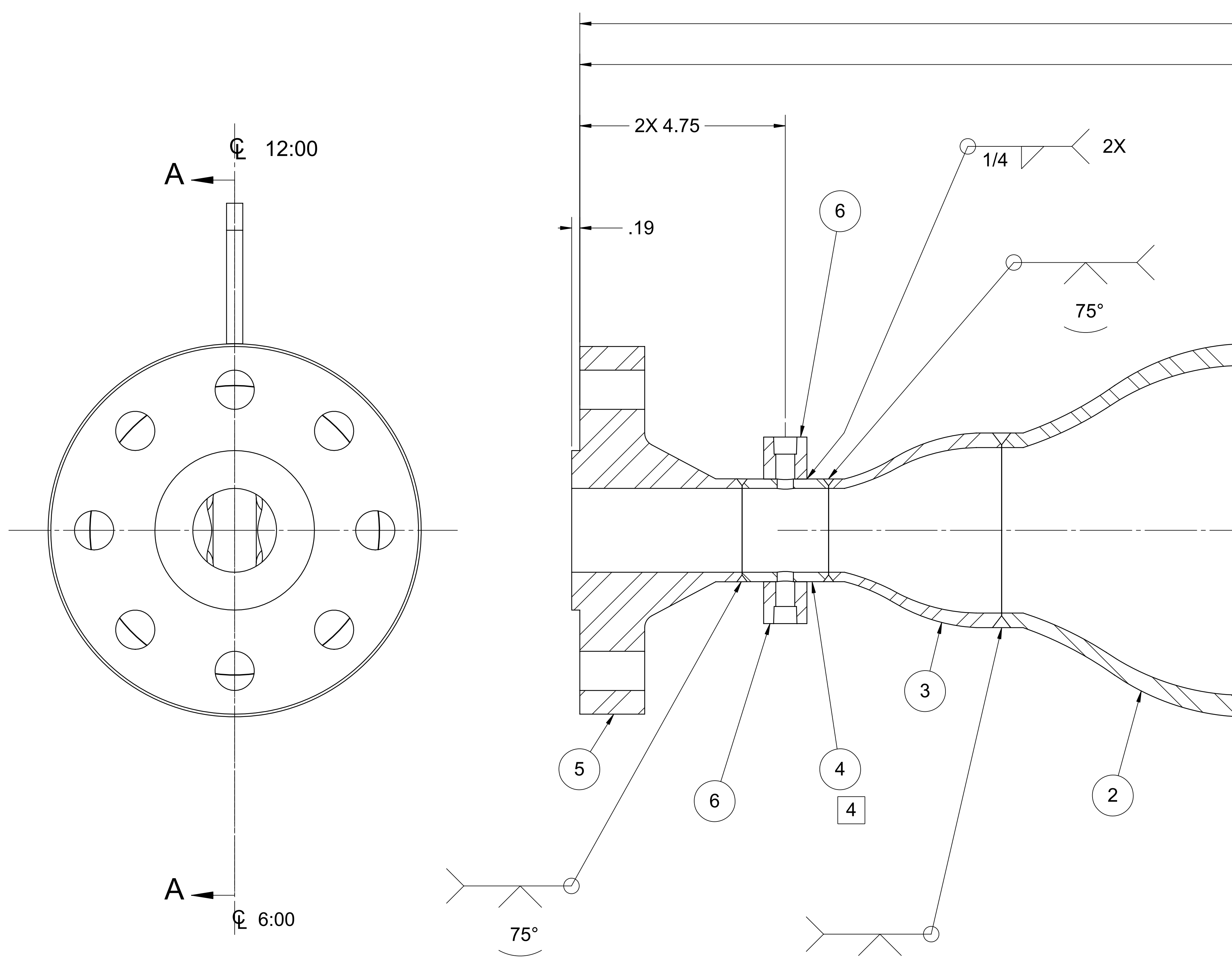

$75^{\circ}$

$75^{\circ}$

8 E

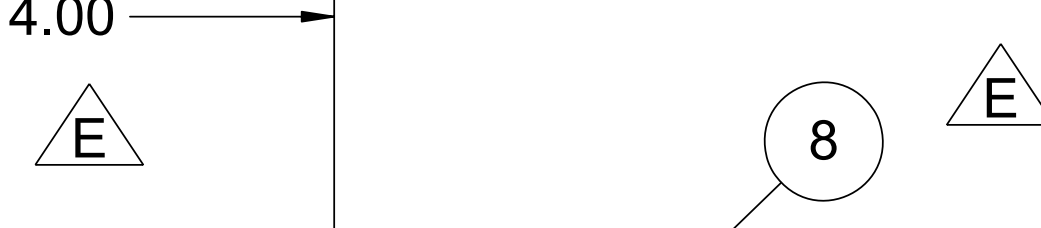

企

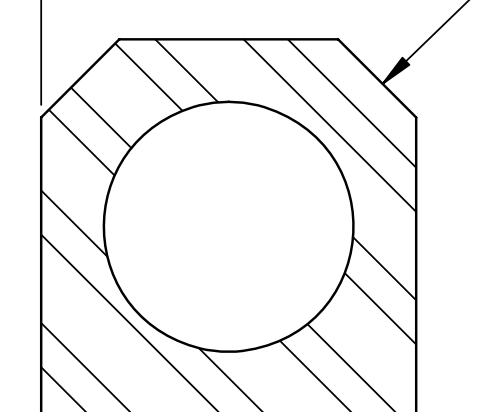

(a)

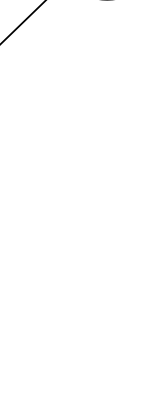

c

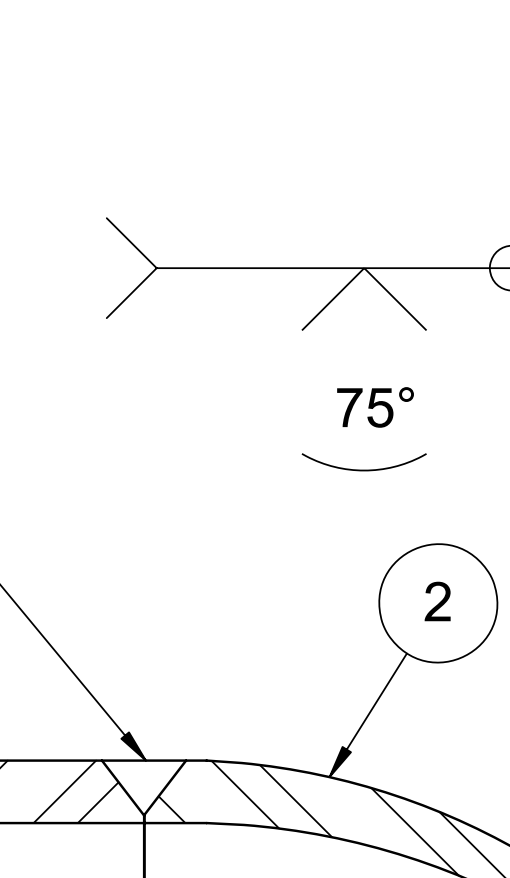

\rangle$_{1 / 4}$

2

$75^{\circ}$

C D

\begin{tabular}{|c|c|c|c|}
\hline 8 & SNG1001.21A & 316 ST STL & PLATE, LIFTING LUG \\
\hline 7 & $\begin{array}{l}\text { W- } \\
\text { SNG1001.18A }\end{array}$ & 316 s.S. 5 & AL FEED TRANSITION \\
\hline 6 & SNG1001.9A & 316 s.S. 5 & $\begin{array}{l}\text { 1/4"-6000\# FGD THD HALF CPLG SA182 GR } \\
\text { F316 ASME B16.11 }\end{array}$ \\
\hline 5 & SNG1001.7A & 316 S.S. 5 & $\begin{array}{l}\text { 2"-WNF FGD ST STL 1500\# RF BORE FOR } \\
\text { SCH.80 125 TO 250 AAR FACE FINISH } \\
\text { SA182 GRF316 ASME B16.5 }\end{array}$ \\
\hline 4 & SNG10C & 316 s.S. 5 & $\begin{array}{l}\text { PIPE 2"-SCH. } 80.218 " \text { WALL ST STL SMLS } \\
\text { SA312 TP 316L ASME B 36.19 }\end{array}$ \\
\hline 3 & SNG1001.5A & 316 s.s. 5 & $\begin{array}{l}\text { 4"x2" CONC RED. WR } \\
\text { SA403 WP-316L }\end{array}$ \\
\hline 2 & SNG100 & 316 s.S. 5 & $\begin{array}{l}\text { 8"x4" CONC RED. WRT ST STL SCH.80 BW } \\
\text { SA403 WP-316L ASME B16.9 }\end{array}$ \\
\hline 1 & SNG1 & 316 S.S. 5 & $\begin{array}{l}\text { PIPE 8"-SCH. } 80.500 " \text { WALL ST STL SMLS } \\
\text { SA312 TP 316L ASME B } 36.19\end{array}$ \\
\hline & NUMBEER & MATERIAL & NESCRIDTH \\
\hline
\end{tabular}

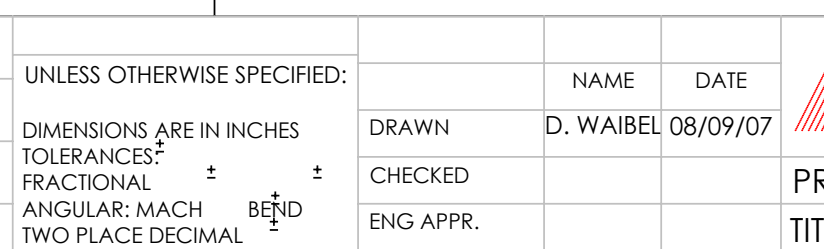

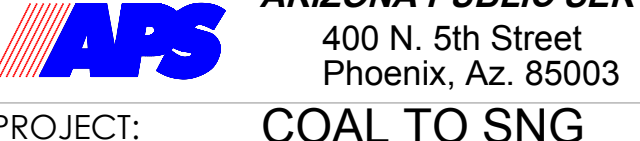

COAL TO SNG
KINETICS REACTO
WELDMENT, COAL HOPPER

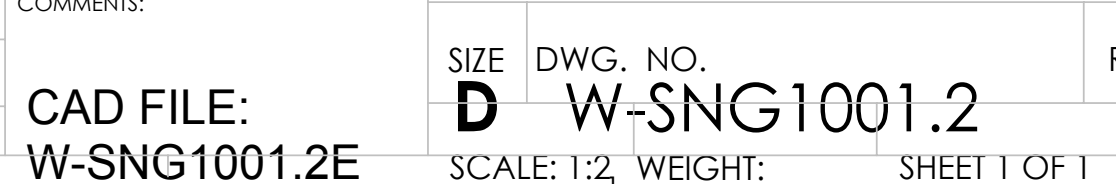




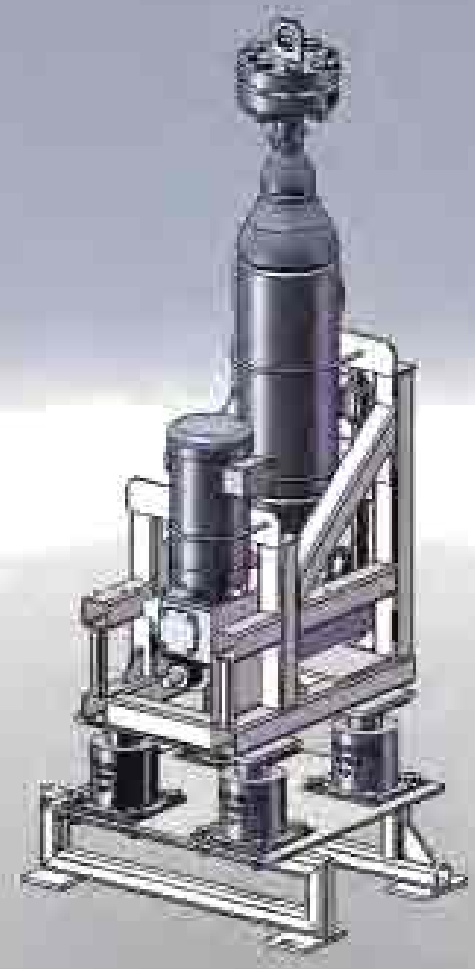




\section{GENERAL NOTES:}

FITTINGS, AND PIPE, TO BE IN ACCORDANCE WITH ANSI B16.9. FLANGES IN THREADOLETS, AND ANY BUTTWELDED FITTINGS ARE TO BE END PREPPED

PER ASME B16.25.

2 ALL WELDS TO MEET PRESSURE VESSEL CODE, PER ASME SECTION 8, DIVISION 1

\begin{tabular}{|c|c|c|c|c|c|}
\hline \multicolumn{6}{|c|}{ REVISIONS } \\
\hline ZONE & REV. & DESCRIPTION & DATE & REV. BY & APPROVED \\
\hline ALL & A & INITIAL DESIGN & 11/09/07 & D.W. & \\
\hline$A L L$ & B & DESIGN CHANGE & 11/1/9107 & D.W. & \\
\hline ALL & $c$ & DESIGN CHANGE & 11/25/07 & D.W. & \\
\hline ALL & $D$ & DESIGN CHANGE & 11130107 & D.W. & \\
\hline ALL & E & DESIGN CHANGE & $12106 / 107$ & D.W. & \\
\hline ALL & $\mathrm{F}$ & DESIGN CHANGE & $12 / 11 / 07$ & D.W. & \\
\hline ALL & G & DELETED END PIN IN SCREW & 01110108 & D.W. & \\
\hline ALL & $\mathrm{H}$ & ADDED LIFTING LUG TO & $03 / 04 / 08$ & D.W. & \\
\hline
\end{tabular}

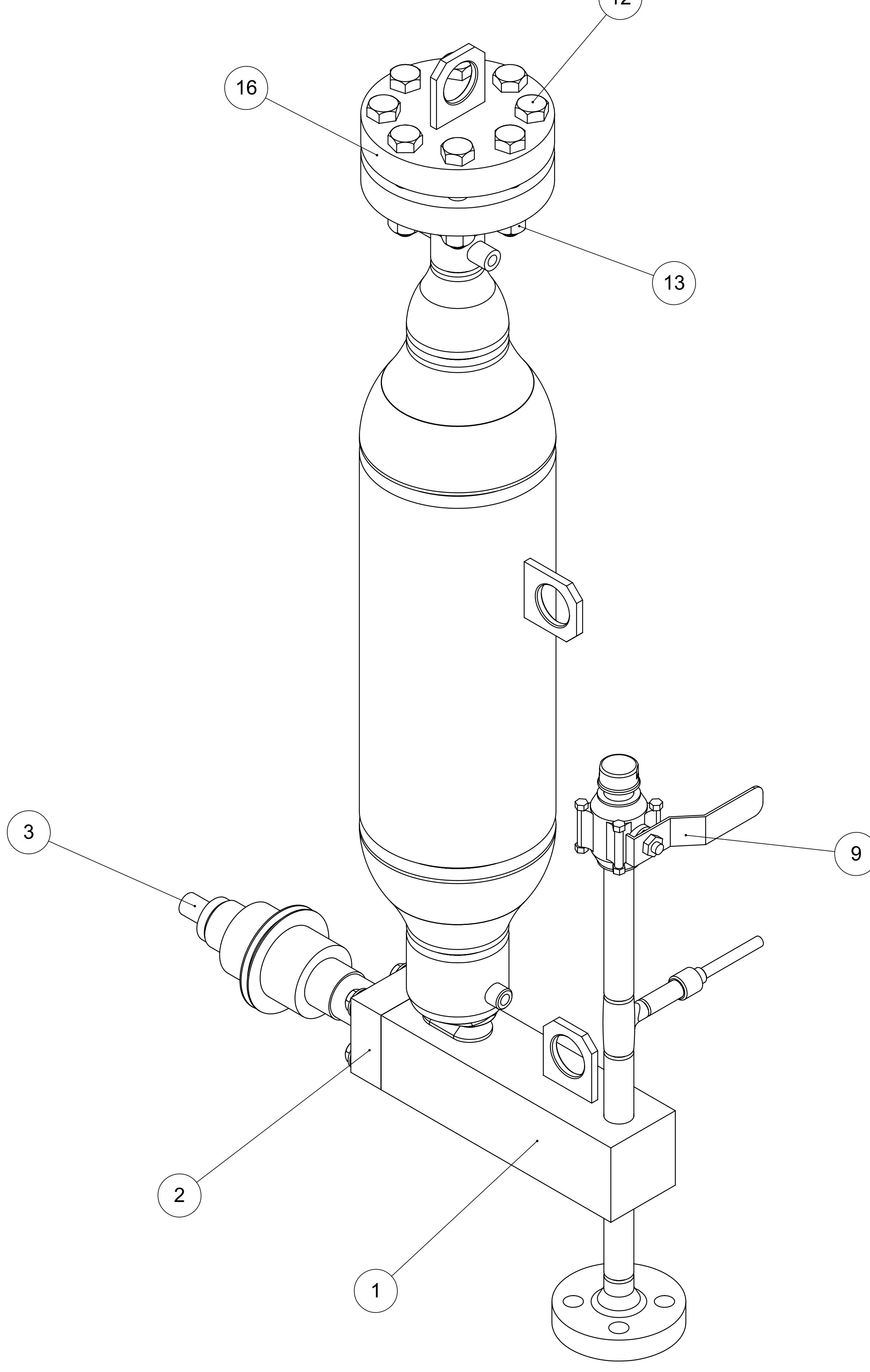

ALL $\quad H \quad$ ADDED LIFTING LUG TO

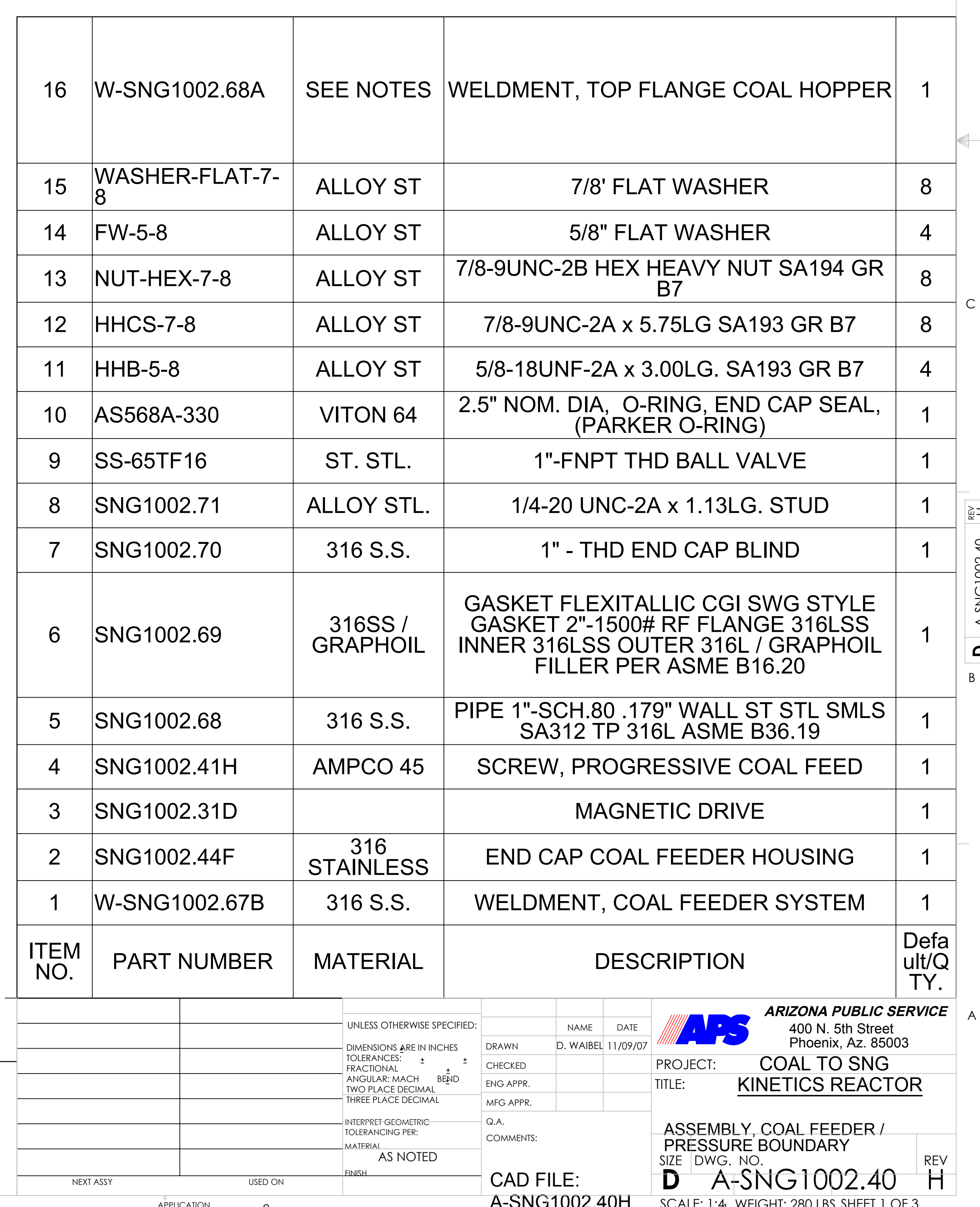




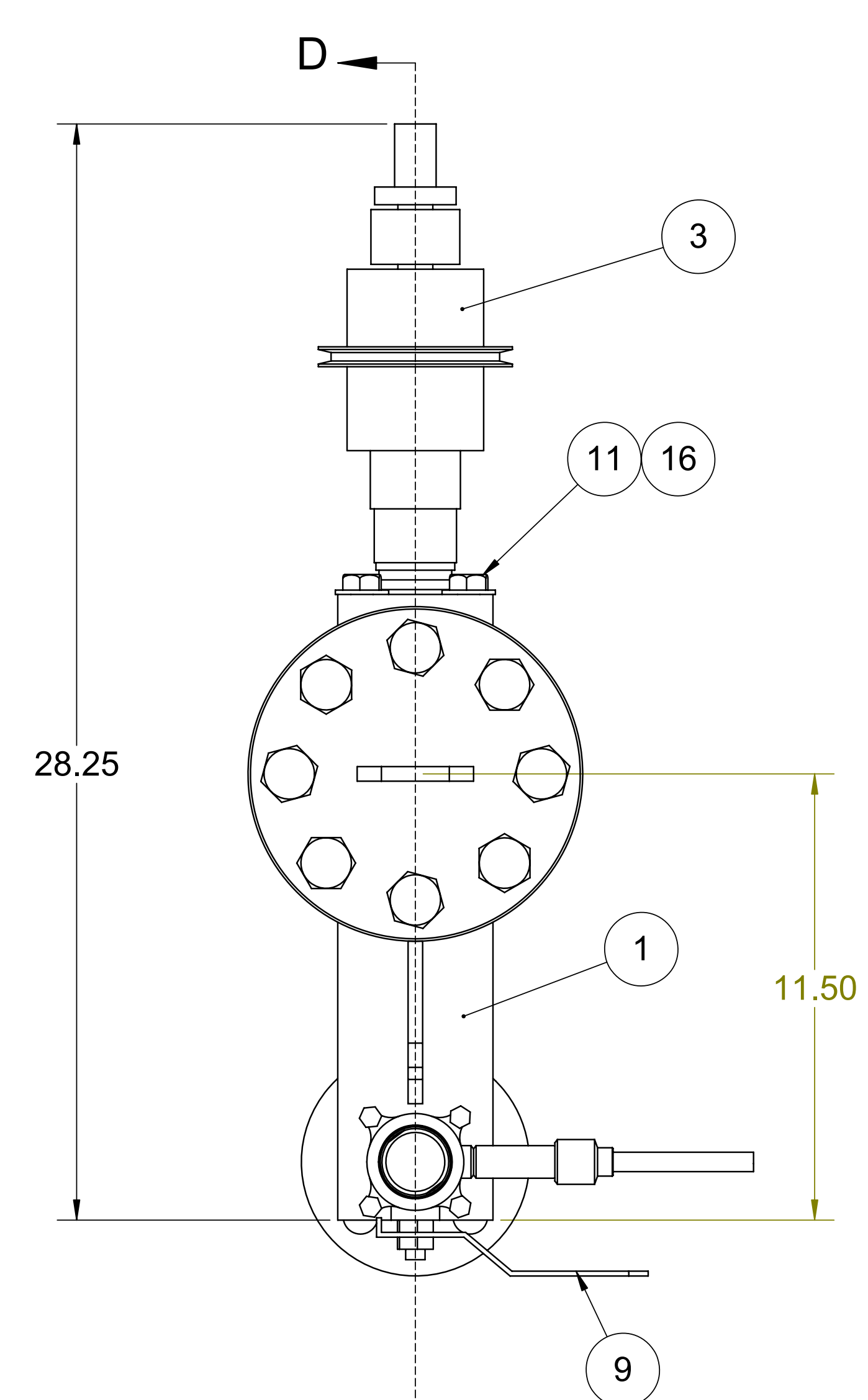

D -
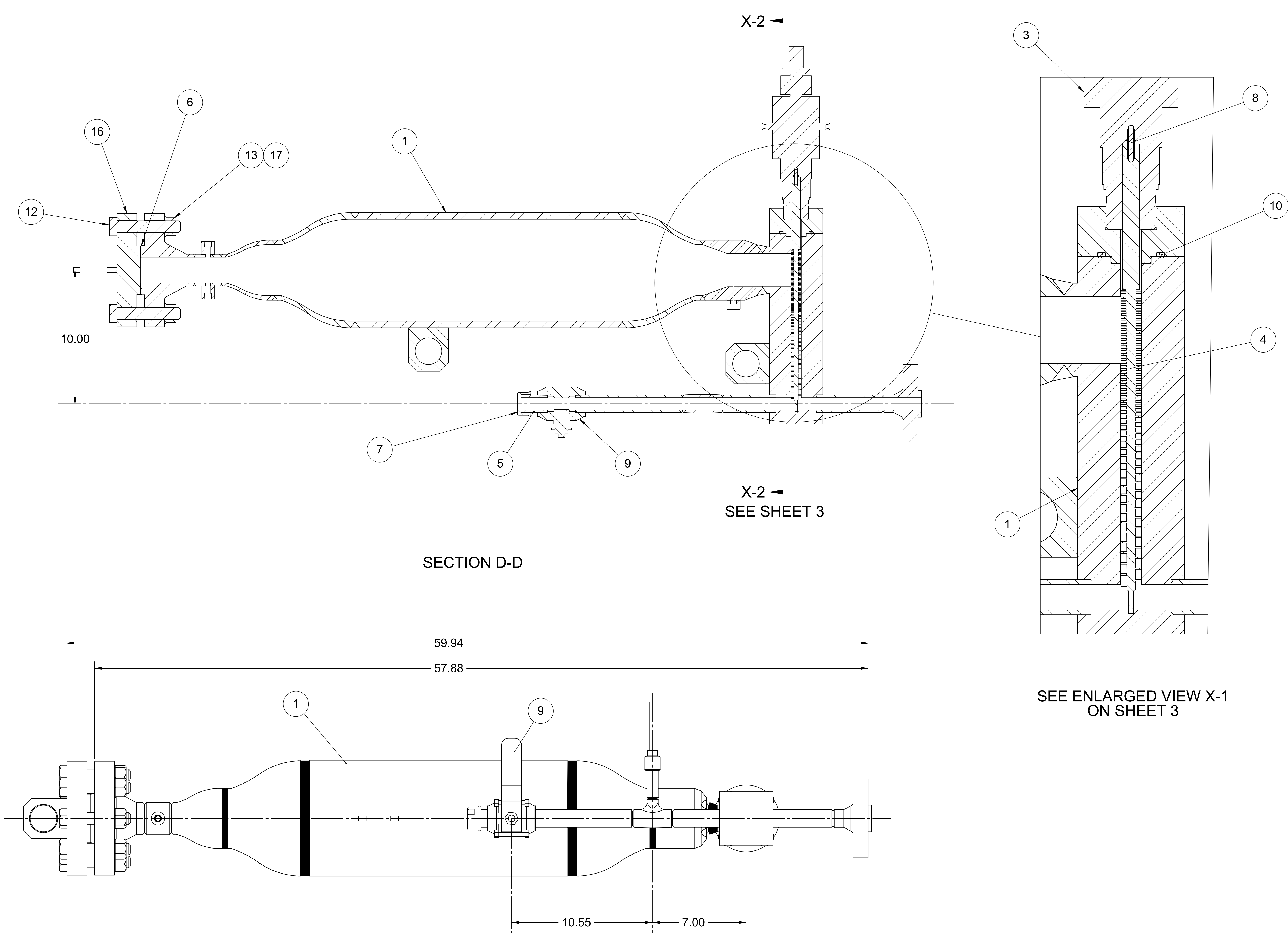

SEE ENLARGED VIEW X-1 ON SHEET 3 

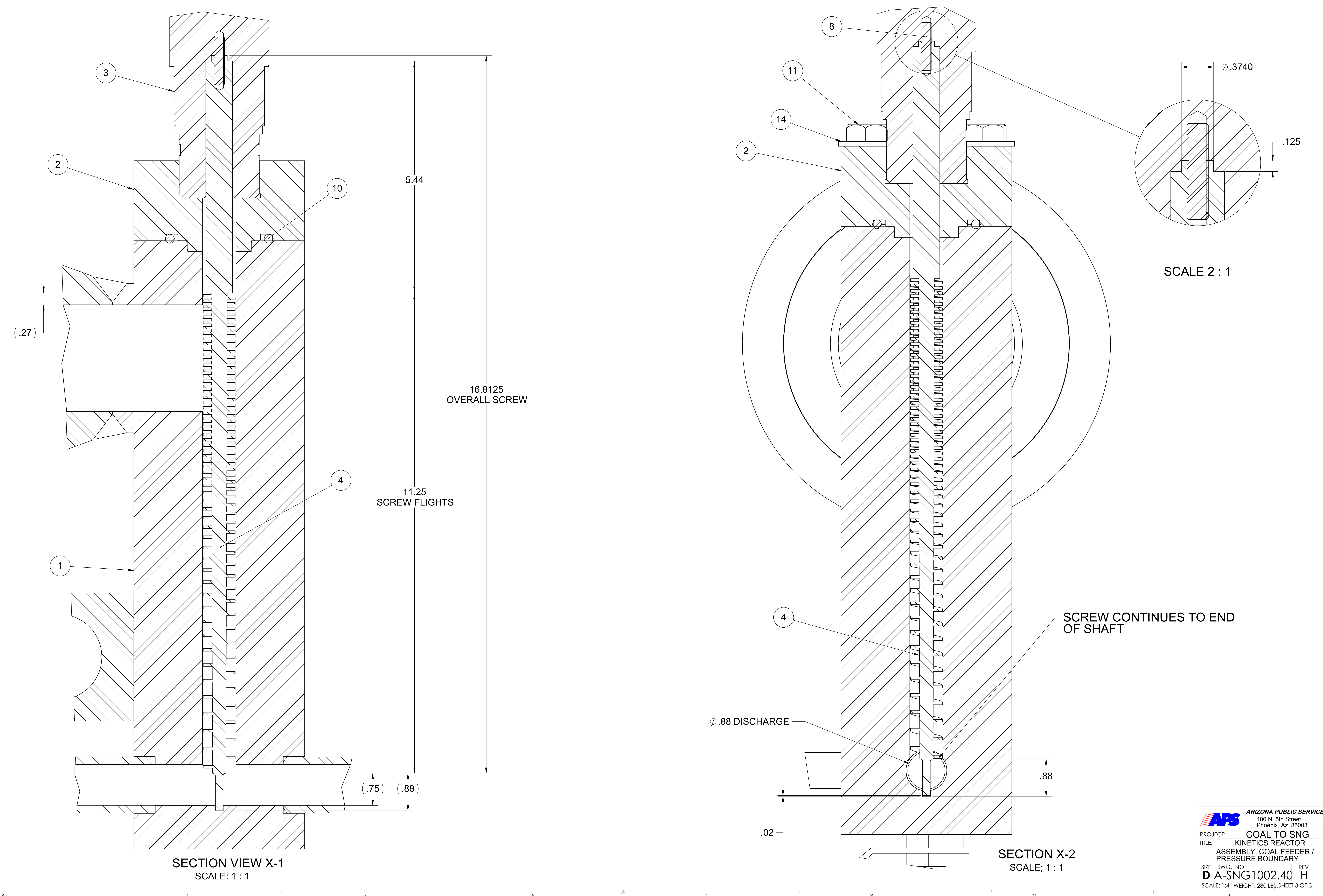


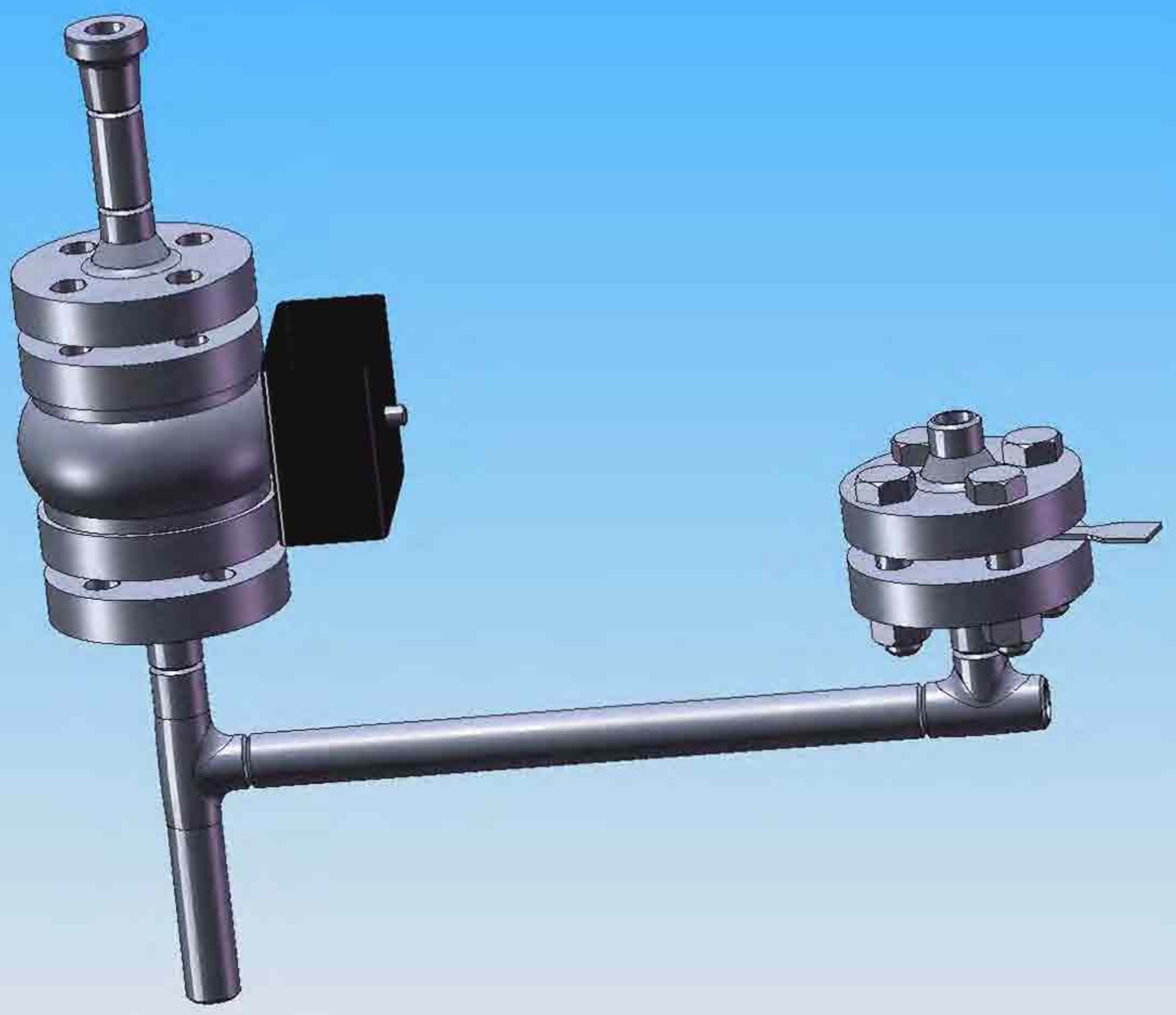



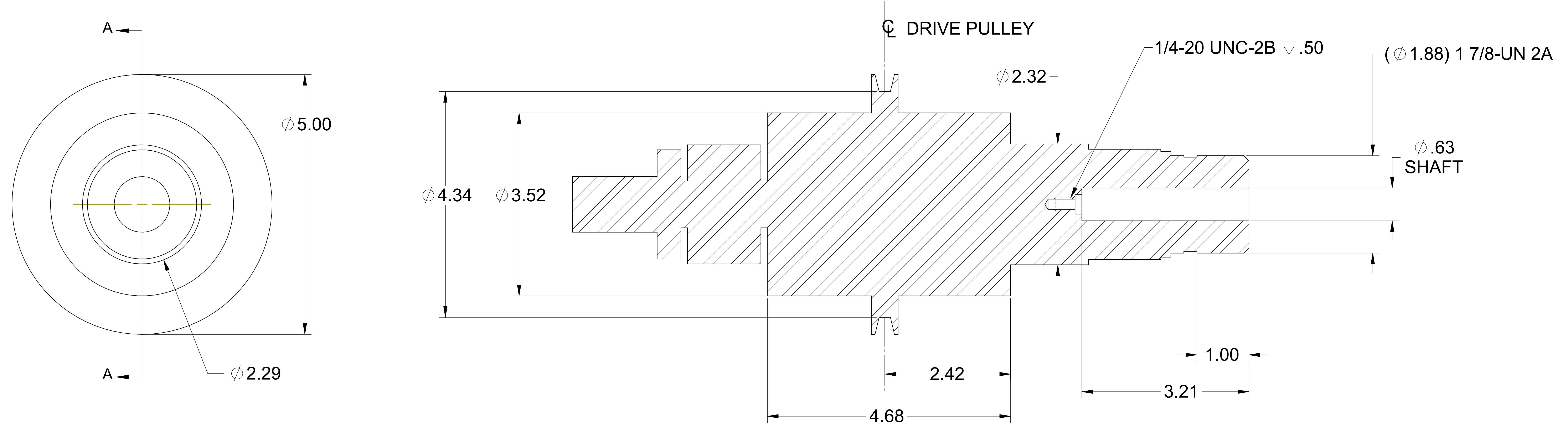

SECTION A-A

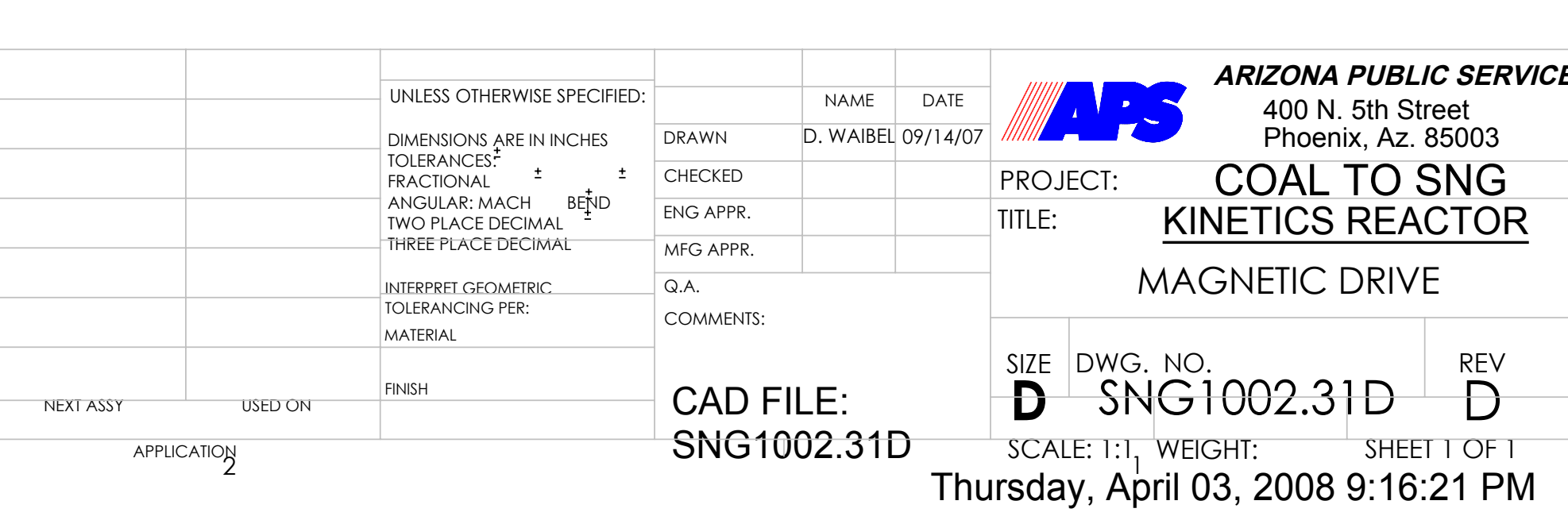




\section{GENERAL NOTES:}

1 DEBURR AND REMOVE ALL SHARP EDGES 2 USING BEST MECHANICAL METHODS, FLIGHT OF SCREW TO END

\begin{tabular}{|c|c|c|c|c|c|}
\hline & & REVISIONS & & & \\
\hline ZONE & REV. & DESGRIPTION & DATE & REV.BY & APPROVED \\
\hline ALL & $\mathrm{k}$ & 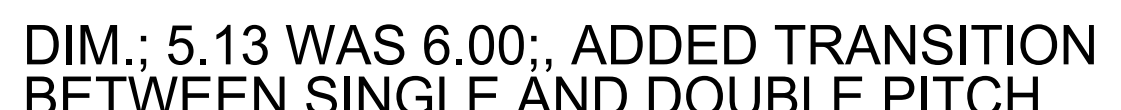 & 12/10/08 & DW & \\
\hline ALL & $\mathrm{L}$ & $\begin{array}{l}\text { CHANGE PITCH AND TO LEFT HAND } \\
\text { FLIGHTS ON AUGER }\end{array}$ & 02/26/09 & DW & \\
\hline ALL & M & $\begin{array}{l}\text { CHANGE PITCH AT DISHCHARGE TO } 1.75 \\
\text { FHIIGTS ON AUGER }\end{array}$ & 03/02/09 & DW & \\
\hline ALL & $\mathrm{N}$ & 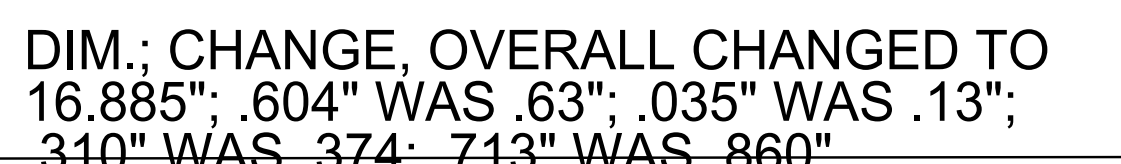 & 04/02/08 & DW & \\
\hline
\end{tabular}

\begin{tabular}{|c|c|c|c|c|c|}
\hline \multicolumn{6}{|c|}{ REVISIONS } \\
\hline ZONE & REV. & DESCRIPTION & DATE & REV. BY & APPROVED \\
\hline ALL & A & INITIAL DRAWING & $07 / 11 / 07$ & DW & \\
\hline ALL & B & INTERNAL CHANGE; 14.88 WAS 15.88 & $08 / 08 / 07$ & $D W$ & \\
\hline ALL & $c$ & INTERNAL CHANGE; 13.13 WAS 14.88 & $08 / 09 / 07$ & DW & \\
\hline ALL & D & $\begin{array}{l}\text { LENGTH } 17.88 \text { WAS } 13.13 \text {, SHAFT } \\
\text { DIA CHANGED TO .63, SCREW DIA. }\end{array}$ & 11/07/07 & DW & \\
\hline ALL & E & 17.00 WAS $17.875 ;$ ADDED STEP DIA. & 11/09/07 & DW & \\
\hline ALL & $\mathrm{F}$ & ADDED WTE 2; ADDED \#8-32 TAPPED; & 11/28/07 & DW & \\
\hline ALL & G & $\begin{array}{l}\text { ADDED NOTE 3; DIM. } 5.44 \text { WAS } 6.13 \text {, } \\
\text { DIM 16.6 WAS } 1700.00\end{array}$ & 12/11/107 & DW & \\
\hline ALL & $\mathrm{H}$ & 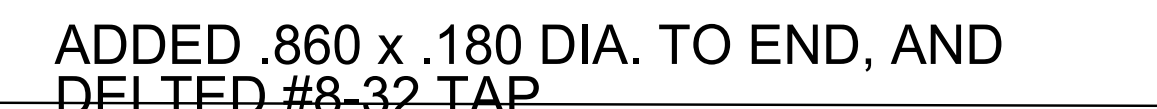 & $01 / 17 / 08$ & DW & \\
\hline ALL & J & DIM. 6.00" WAS 4.00"; CHANGED PITCH & 12/08/08 & DW & \\
\hline
\end{tabular}

114.20 UNC -28 S. 50
(RISHT HAND THREAD)

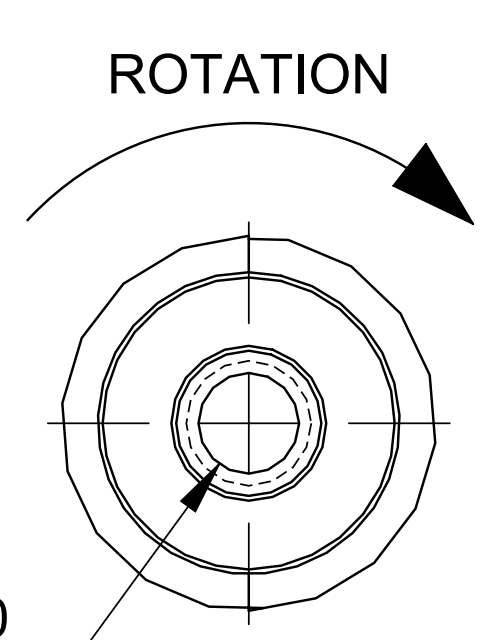

DETALA

(1)

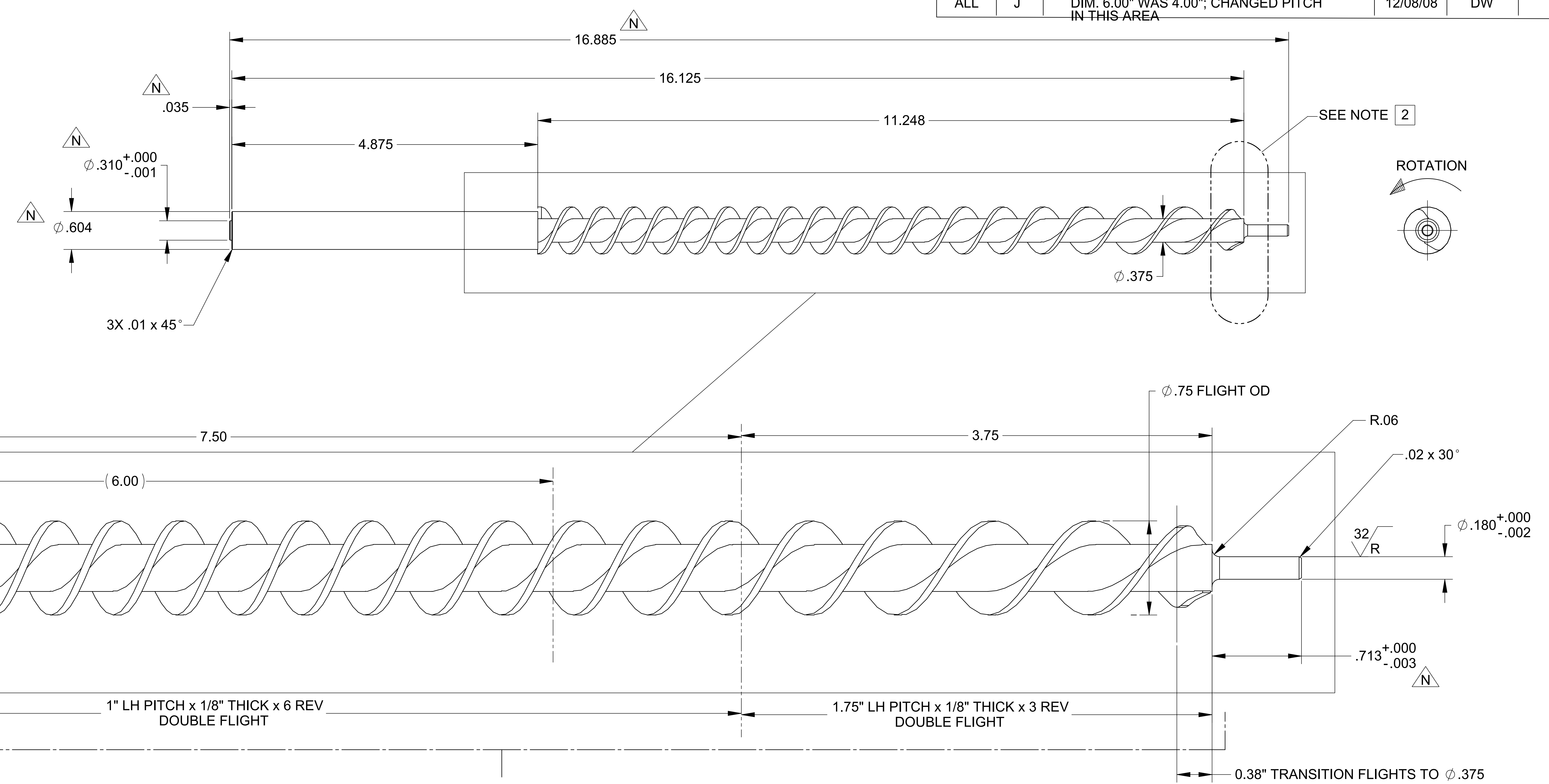

SEE NOTE 2

DETAIL B
SCALE $2: 1$ 
1 DEBURR AND REMOVE ALL SHARP EDGES.

2. FABRICATOR TO INSTALL HELLACAL INSERTS, $5 / 8-18$ THREAD,
18-8 STANINLESS STEEL, MCMASTER-CARR; 91732A238
OR EQUIVELENT.

\begin{tabular}{|c|c|c|c|c|c|}
\hline \multicolumn{6}{|c|}{ REVIS } \\
\hline ZONE & REV & $\begin{array}{l}\text { DESCRRPTION } \\
\text { DNTIAR REEASEE }\end{array}$ & 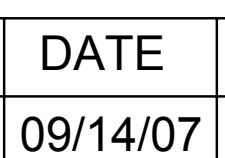 & $\frac{\mathrm{REV} \cdot \mathrm{BY}}{\mathrm{DW}}$ & APPROVE \\
\hline ALL & B & 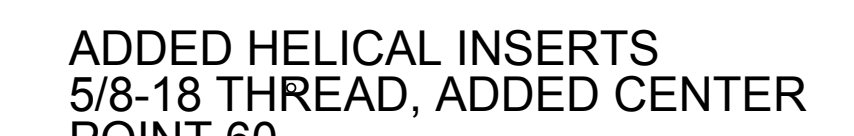 & 11128107 & DW & \\
\hline ALL & c & 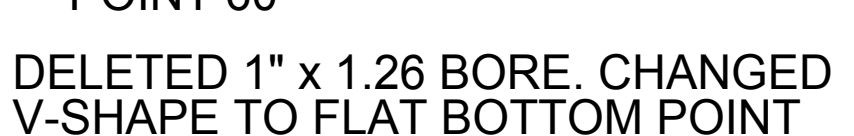 & 12103/07 & ow & \\
\hline
\end{tabular}
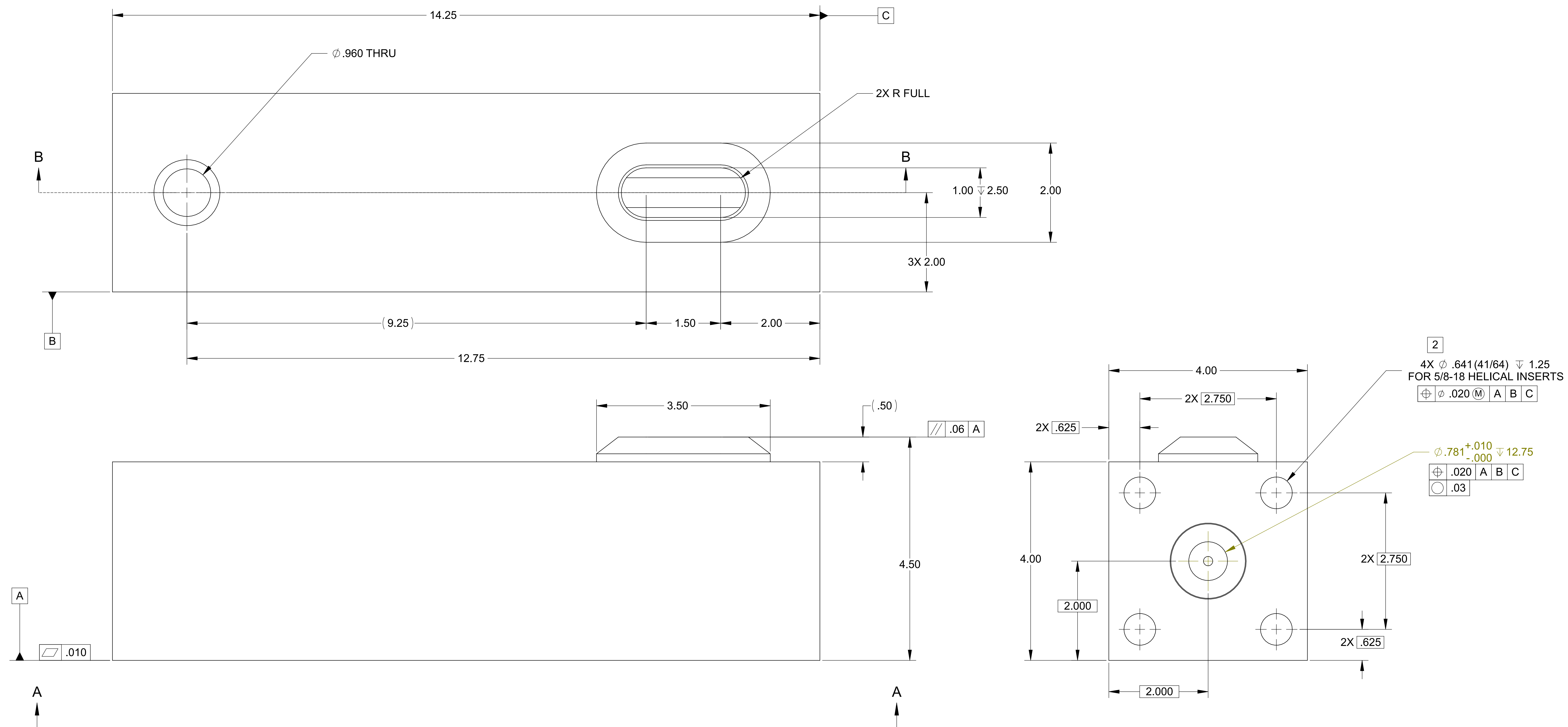

SEE SHEET 2

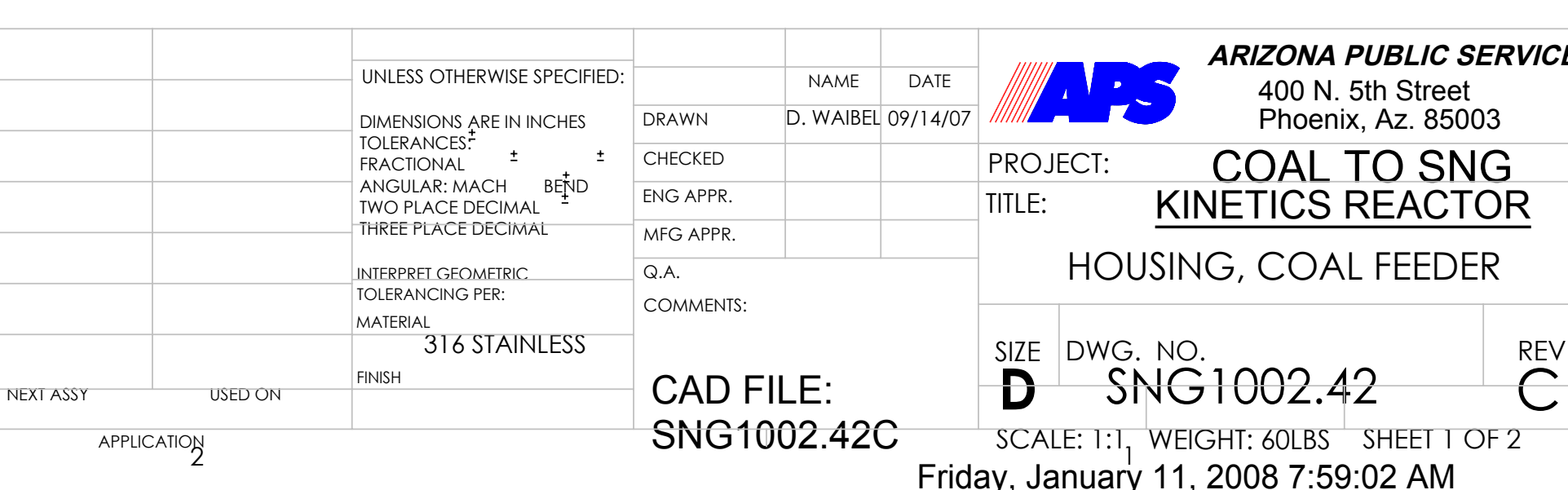




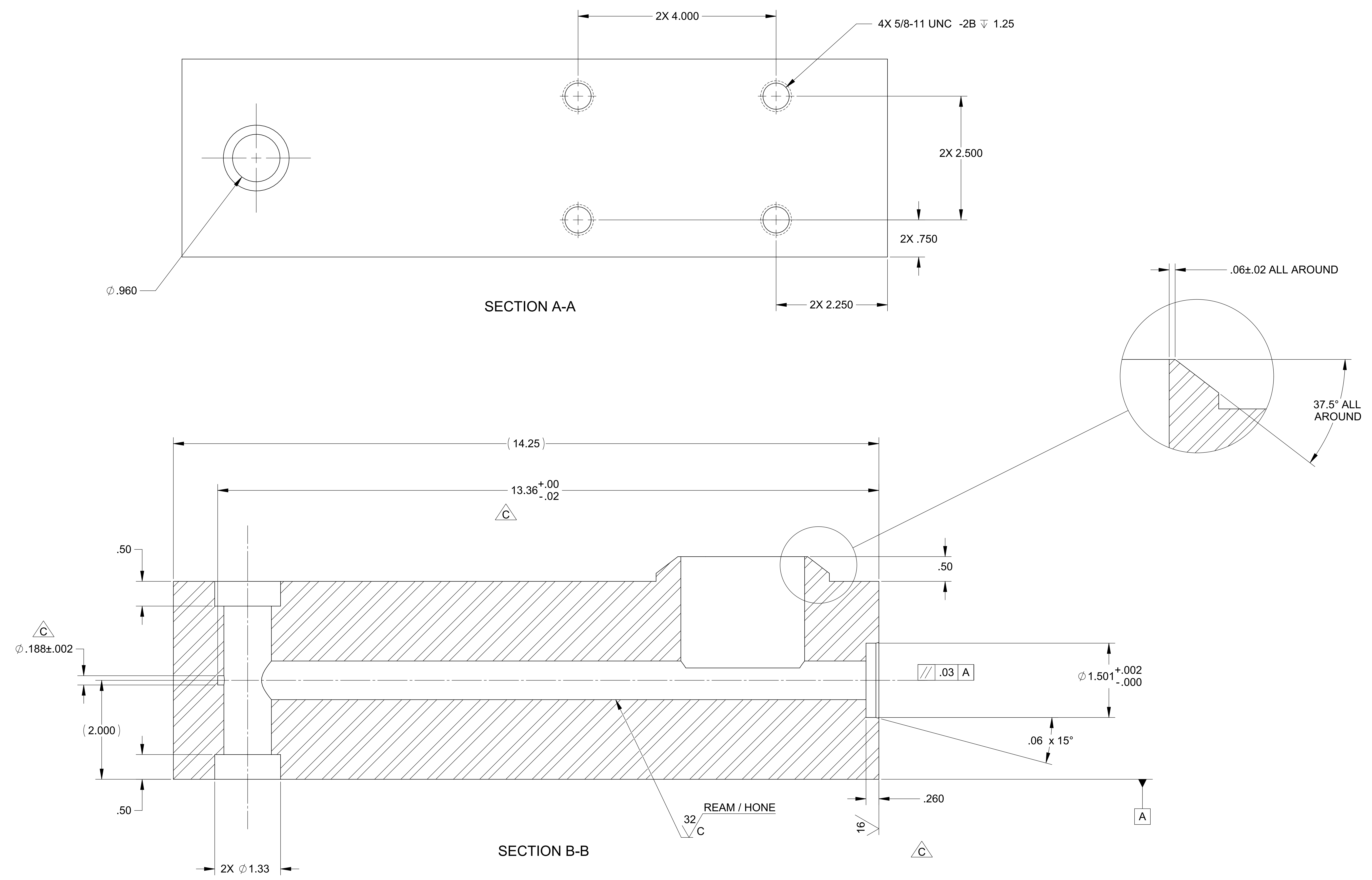



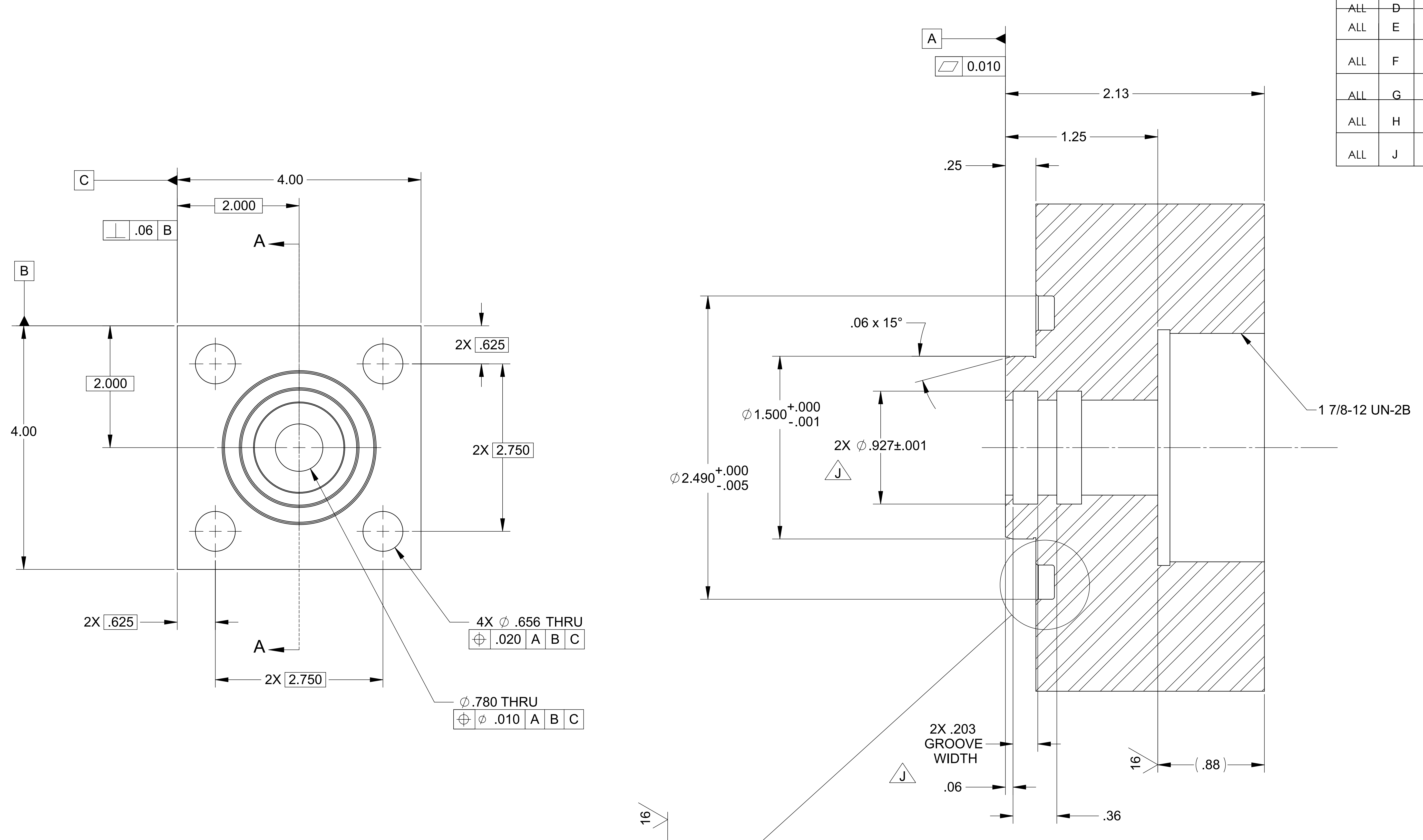

\begin{tabular}{|c|c|c|c|c|c|}
\hline \multicolumn{6}{|c|}{ REVISIONS } \\
\hline ZONE & REV. & DESCRIPTION & DATE & REV.BY & APPROVED \\
\hline ALL & A & INITIAL DESIGN CONCEPT & $07 / 18107$ & $\mathrm{DW}$ & \\
\hline ALt- & $B$ & DESIGNREVIEW & 080108107 & DW & \\
\hline ALL & $c$ & DESIGN RELEASE & 09144107 & DW & \\
\hline Alt & $D$ & DESIGNRELEASE & 101121207 & DW & \\
\hline ALL & $\mathrm{E}$ & $\begin{array}{l}\text { ADDED PILOT, 1.50; DELETED } \\
\text { SEALBORE, }\end{array}$ & 11128107 & DW & \\
\hline ALL & $\mathrm{F}$ & $\begin{array}{l}\text { CHANGED WIDTH TO } 2.125^{\prime \prime} \\
\text { CORRECTIDD O-RING GROOVE }\end{array}$ & 12/103/07 & DW & \\
\hline AlL & $G$ & ADDED GROOVE FOR SHAFT SEAL & $11 / 27108$ & DW & \\
\hline ALL & $\mathrm{H}$ & 1.125" $\times .312^{\prime \prime}$ BORE WAS & $12104 / 08$ & DW & \\
\hline ALL & J & CHANGED $1.125 "$ DIA. BORE TO & 22177/08 & DW & \\
\hline
\end{tabular}

SECTION A-A
SCALE 2:1

$.281-.005$ TO вOTTOM OF GROOVE

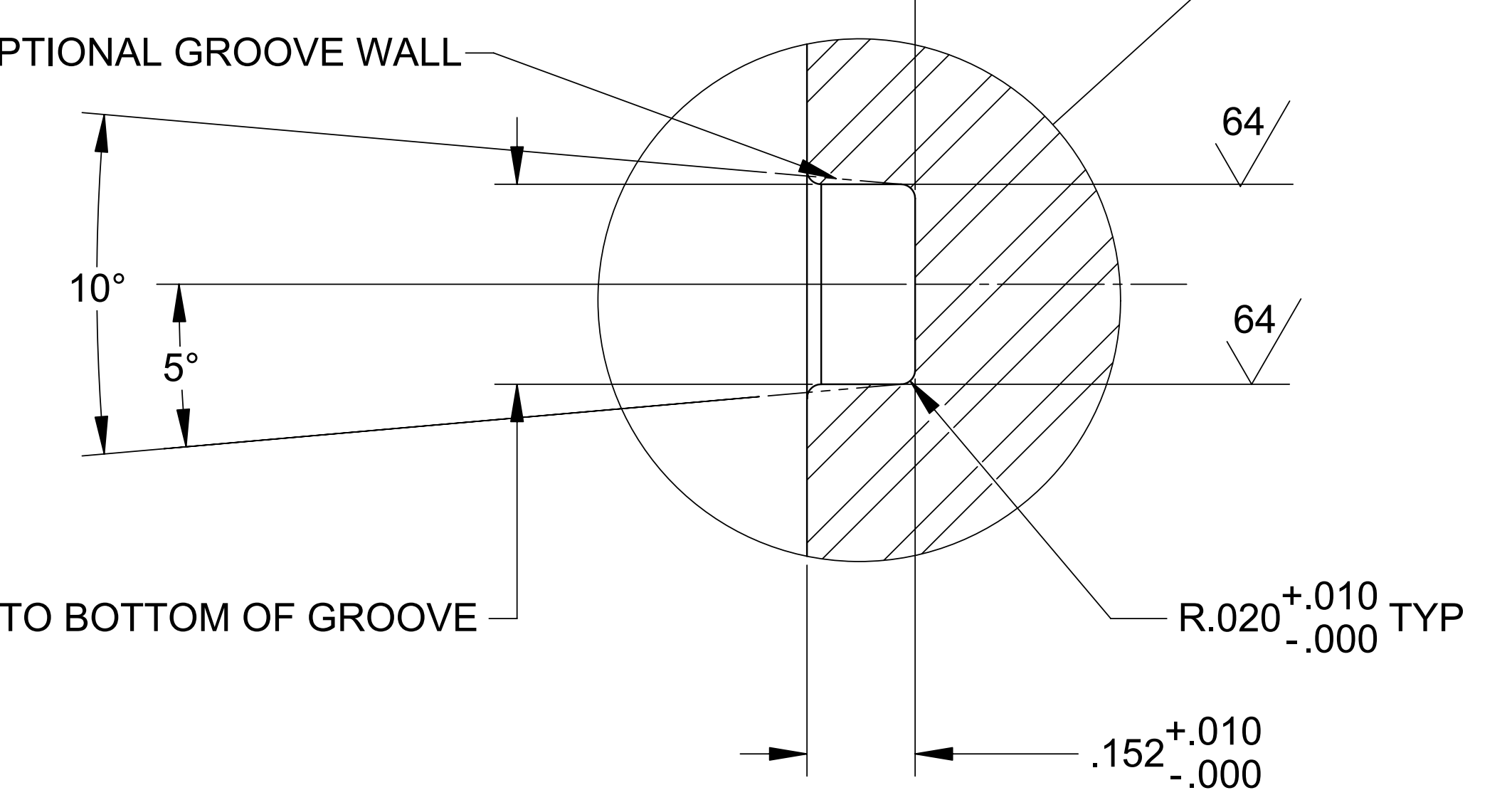


REVISIONS

1 DIMENSION AND TOLERANCING PER ANSI Y14.5-1994.
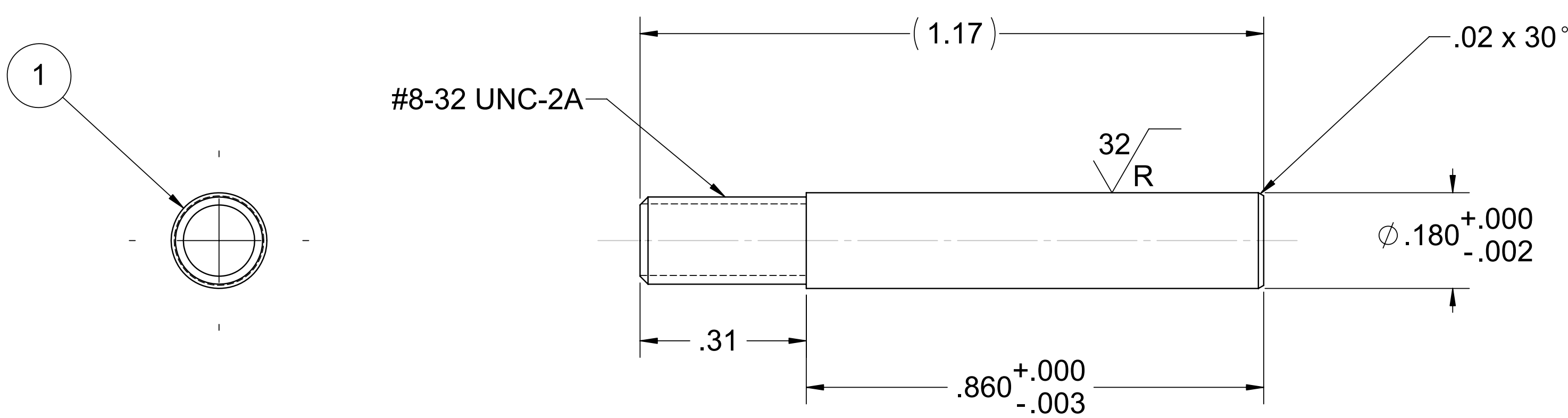

\begin{tabular}{|c|c|c|c|c|}
\hline 1 & \multicolumn{3}{|c|}{ SNG1002.72A } & \\
\hline $\begin{array}{l}\text { ITEM } \\
\text { NO. }\end{array}$ & \multicolumn{3}{|c|}{ PART NUMBER } & \\
\hline \multirow{2}{*}{\multicolumn{2}{|c|}{ 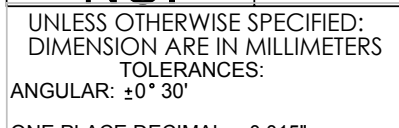 }} & & $\begin{array}{l}\text { DATE } \\
12 / 11 / 07\end{array}$ & $\begin{array}{l}\text { NAME } \\
\text { D. WABBEL }\end{array}$ \\
\hline & & CHECKED & & \\
\hline \multirow{2}{*}{\multicolumn{2}{|c|}{ 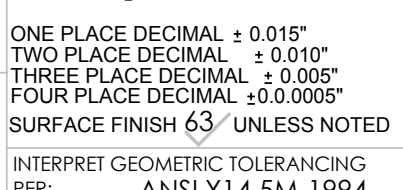 }} & ENG APPR. & & \\
\hline & MFG APPR. & & \\
\hline & & Q.A. & & \\
\hline \multicolumn{2}{|c|}{$\begin{array}{l}\text { MATERIAL: } \\
\text { FMPSH: AMPCO } 45\end{array}$} & SI & {$[-M M-25$} & 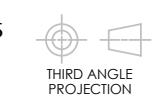 \\
\hline & & & & \\
\hline
\end{tabular}

\begin{tabular}{c|c}
\hline ND PIN, SCREW & 1 \\
SUPPORT & QTY. \\
\hline DESCRIPTION &
\end{tabular}

I.:S

TITLE: 400 N. 5th Street
Phoenix, Az. 85003

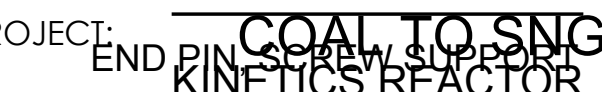

SIZE DWG. NO. 

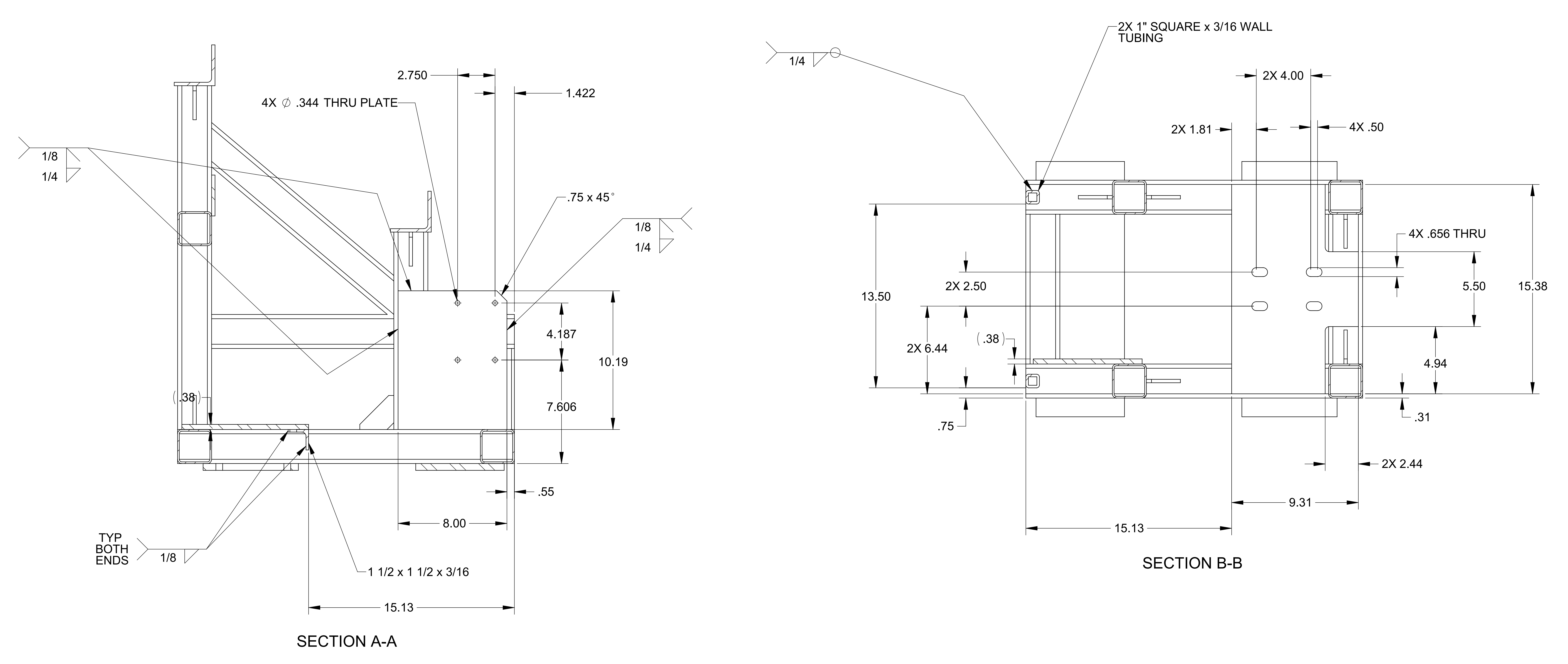

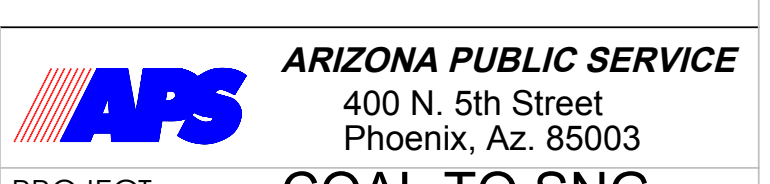




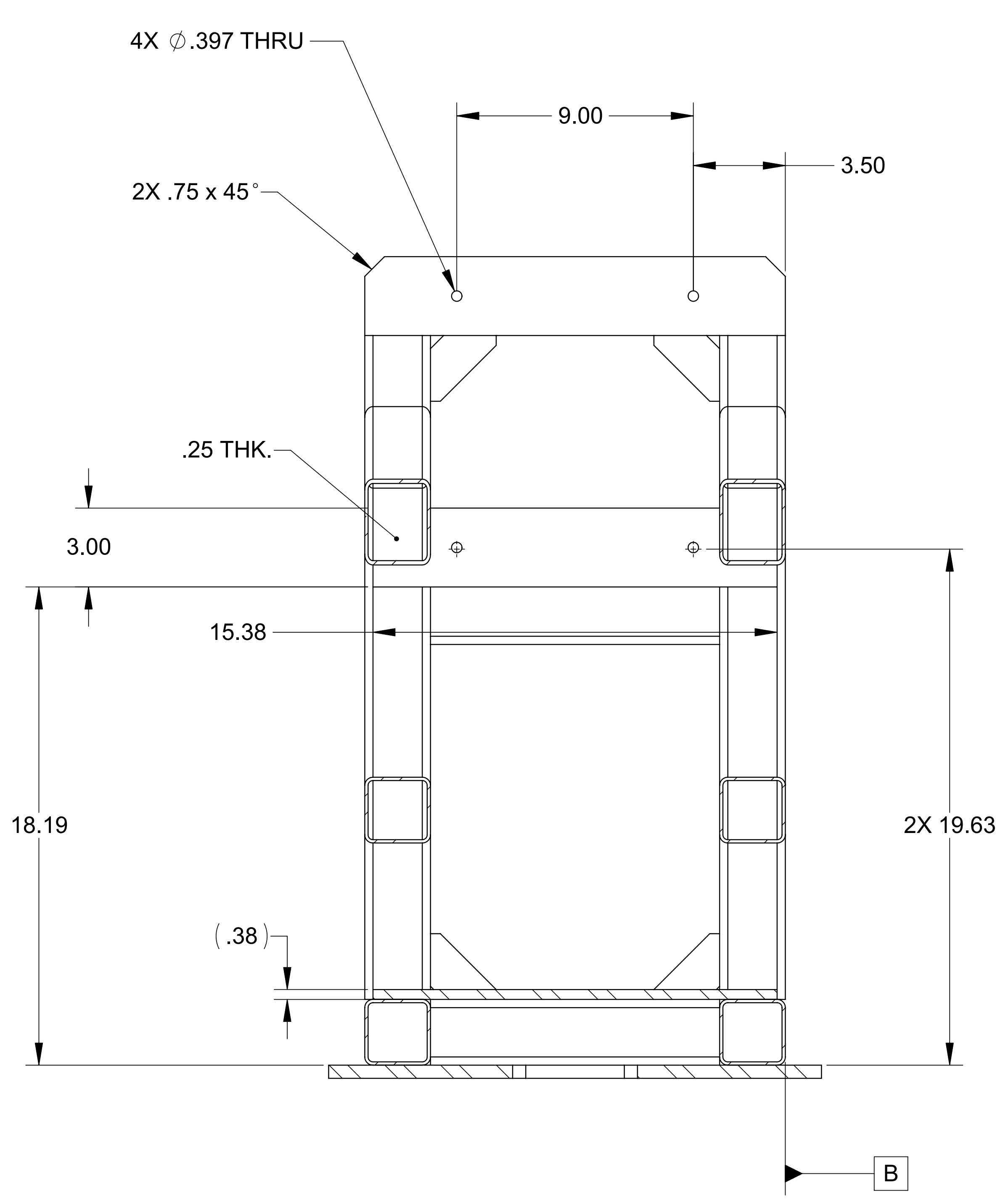

SECTION C-C

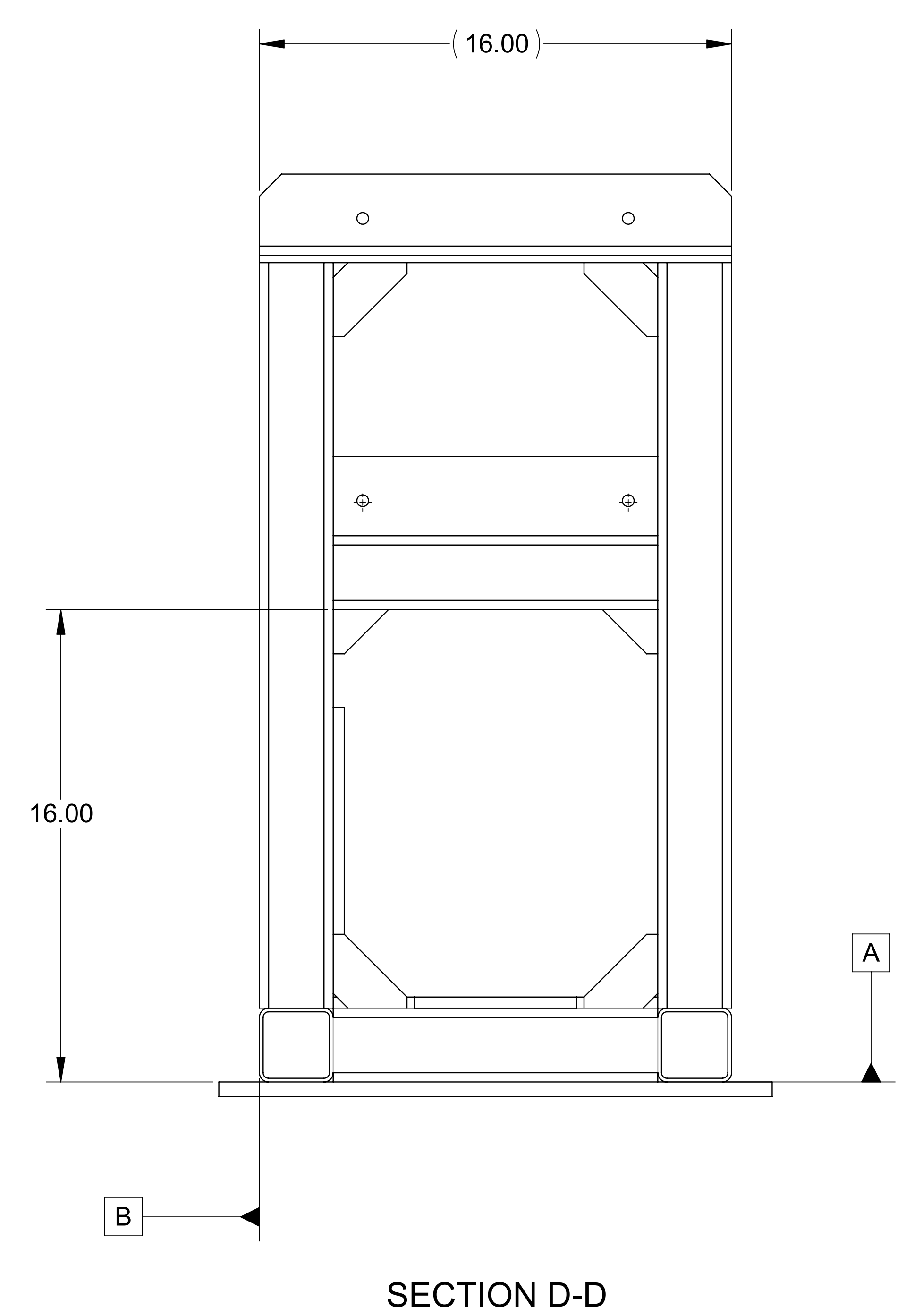


FRAME CONSTRUCTED USING 2" SQUARE TUBING WITH 1/4" THICK WALL, UNLESS NOTED OTHERWISE

DEBURR AND REMOVE ALL SHARP EDGES, PRIOR TO PRIMER COATING.

FINISH: PRIMER USING SHERWIN-WILLIAMS, KEM 400 PRIMER RED OXIDE, PRODUCT NUMBER: E61R00402 OR
EQUIVALENT. PERPARE SURFACE PER MANUFACTURES DATA SHEET.

SEE SHEET; 2 FOR EXPLODED VIEW, SHEET; 3 FOR WELDING DETAILS, SHEET; 4 FOR COMPONENTS AND CUT
LENGTH DETALLS.
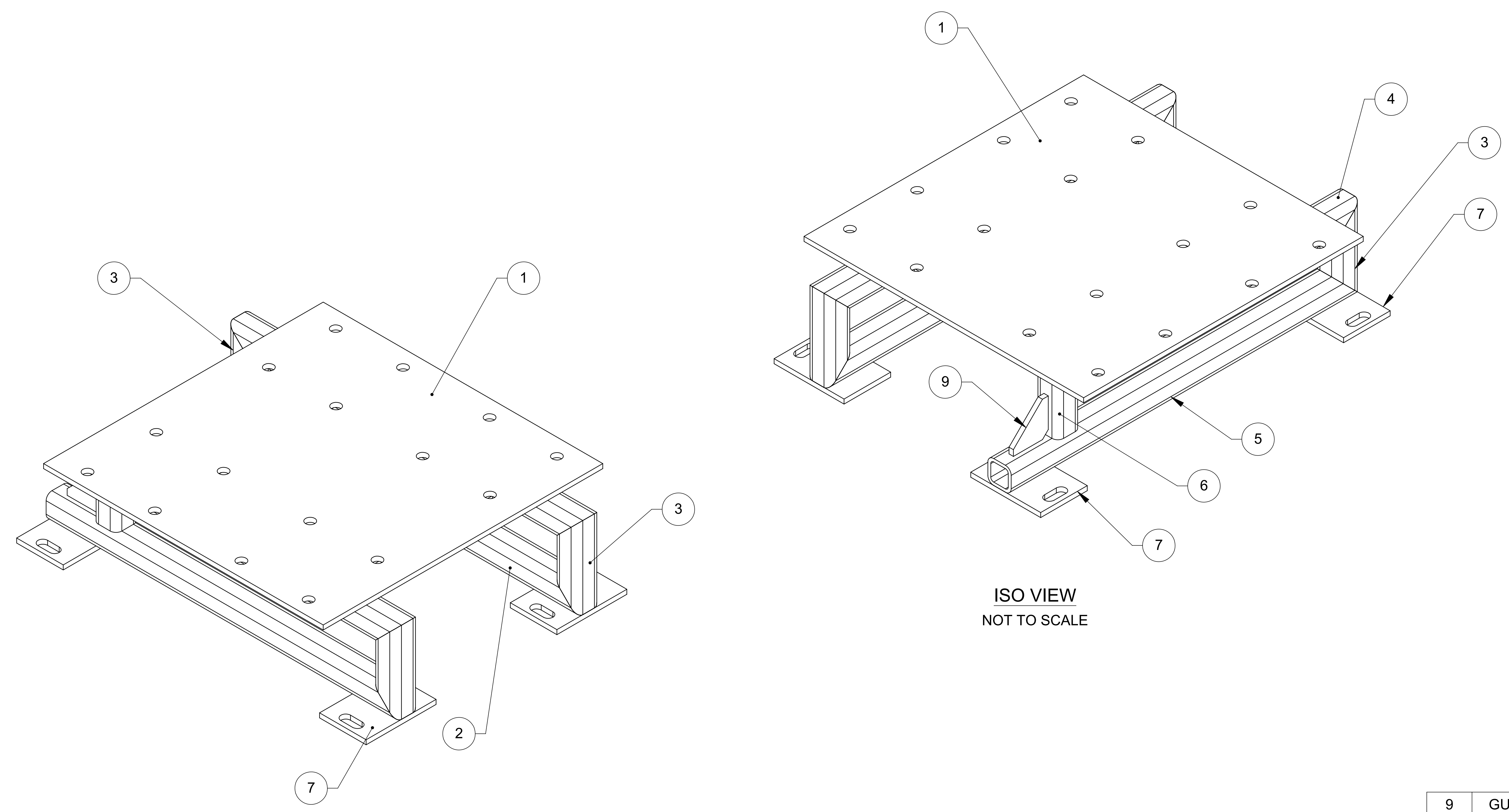

ISO VIEW

NOT TO SCALE

ISO VIEW

ISO VO SCA

\begin{tabular}{|c|c|c|c|}
\hline 9 & GUSSET-1002.49B & PLATE, GUSSET, PLAIN STEEL (CRS) & $r$ \\
\hline 8 & TUBE-F-1002.49B & & \\
\hline & PAD-1002.49B & & \\
\hline 6 & \multirow{2}{*}{$\begin{array}{l}\text { TUBE-D-1002.49B } \\
\text { TUBE-E-1002.49B }\end{array}$} & $\begin{array}{l}\text { PAD, 6" } \times 4^{\prime \prime} \times 3 / 8 \text { PLAN STEEL (CRS) } \\
\text { SQ. TUBE, 2X2X14" WALL, PLAIN }\end{array}$ & 1 \\
\hline 5 & & 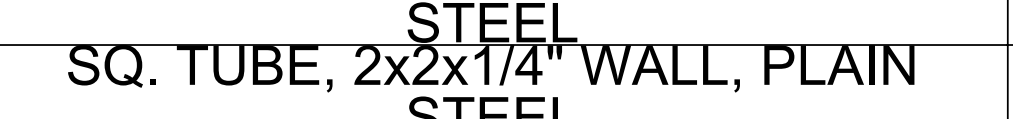 & 1 \\
\hline 4 & TUBE-C-1002.49B & & 1 \\
\hline 3 & TUBE-B-1002.49B & 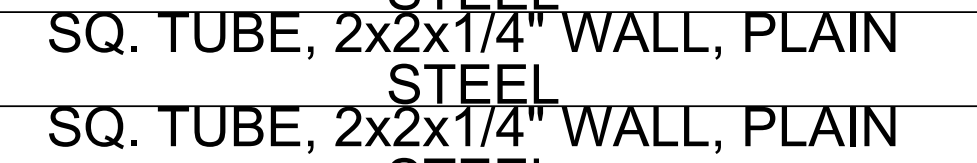 & 3 \\
\hline$?$ & TUBE-A-1002.49B & 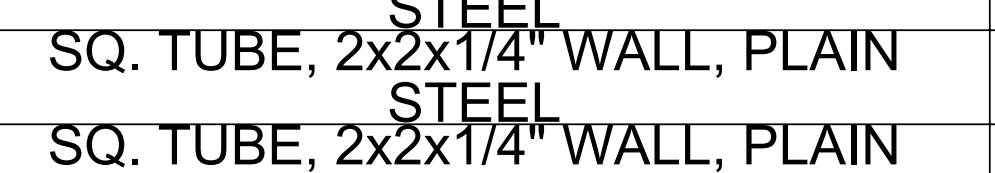 & 2 \\
\hline & PLATE-1002.49B & 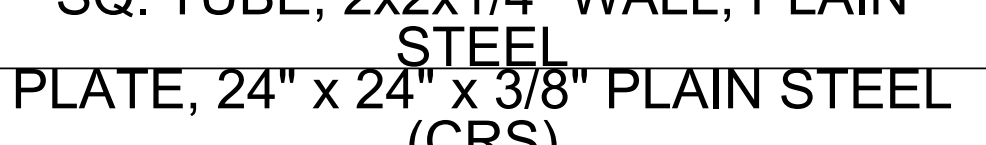 & 1 \\
\hline No & PART NUMBER & DESCRIPTION & QTY \\
\hline & & \multirow{4}{*}{\multicolumn{2}{|c|}{ 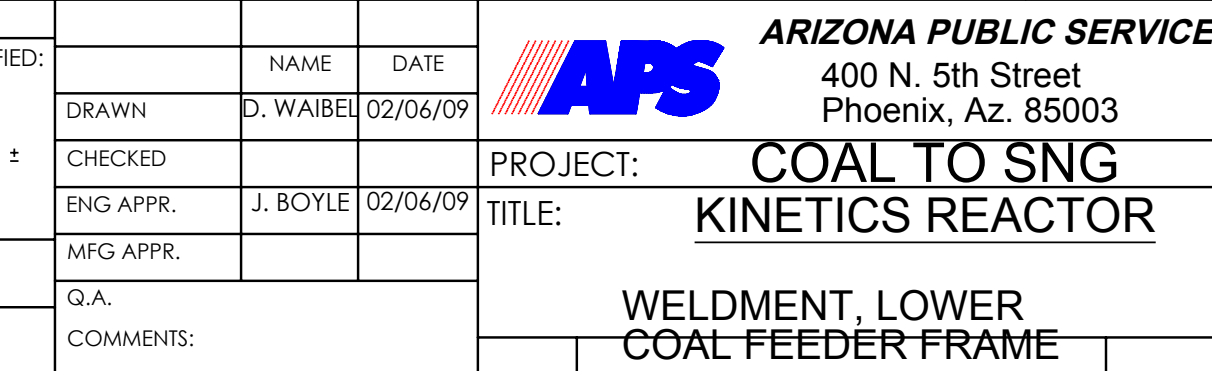 }} \\
\hline & 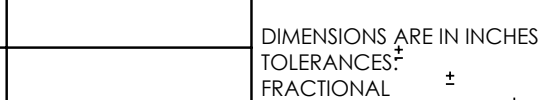 & & \\
\hline & 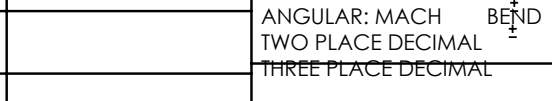 & & \\
\hline & 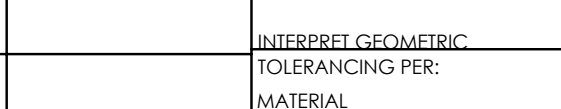 & & \\
\hline & 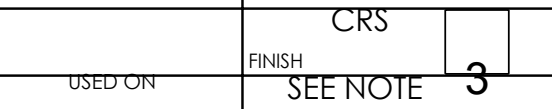 & SIE DWG. NO WNG10 & BEV \\
\hline
\end{tabular}




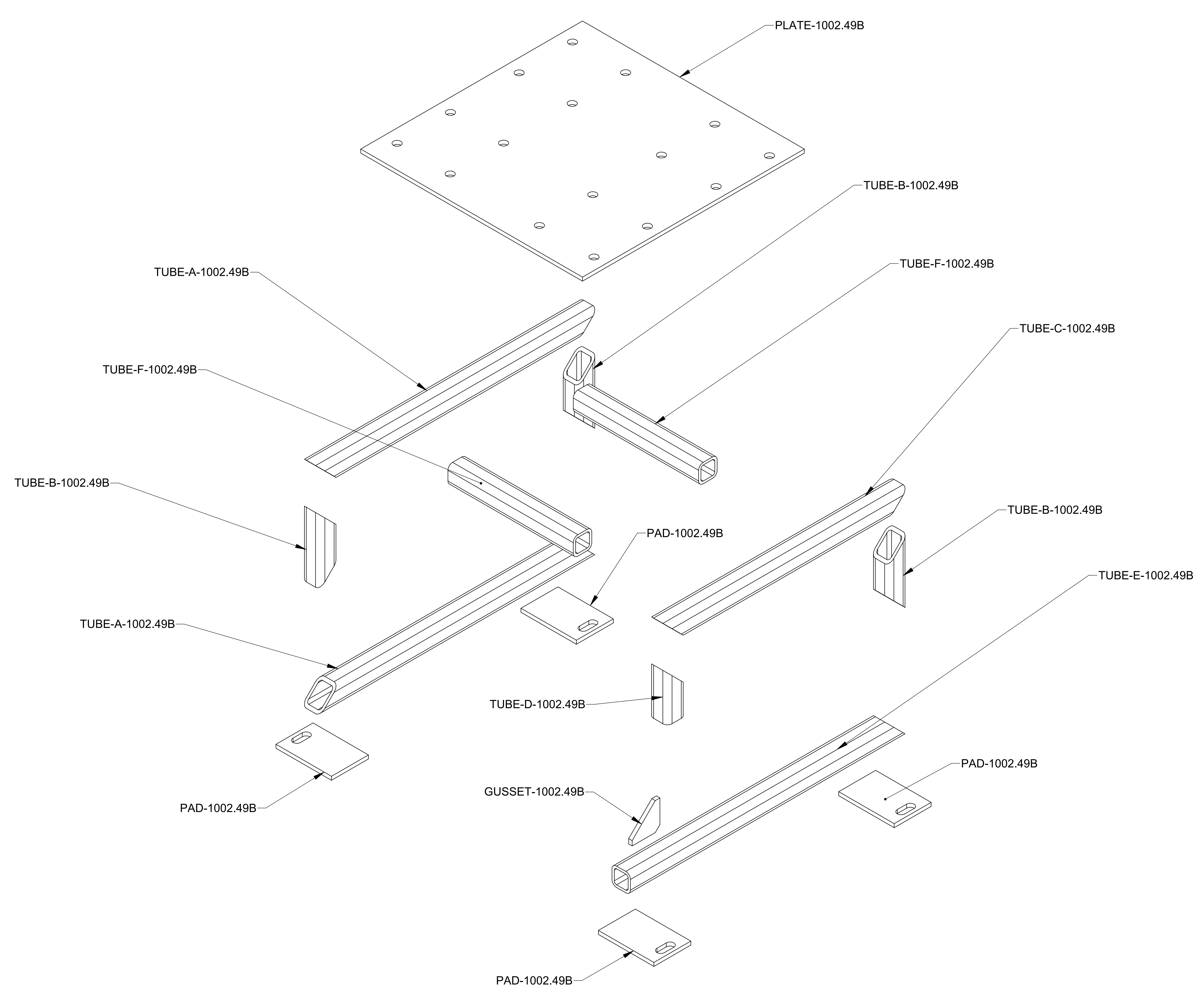

EXPLODED VIEW

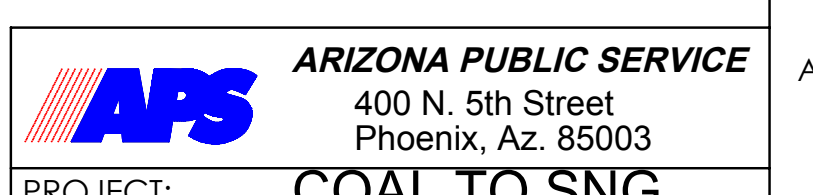

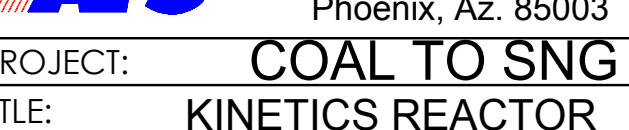



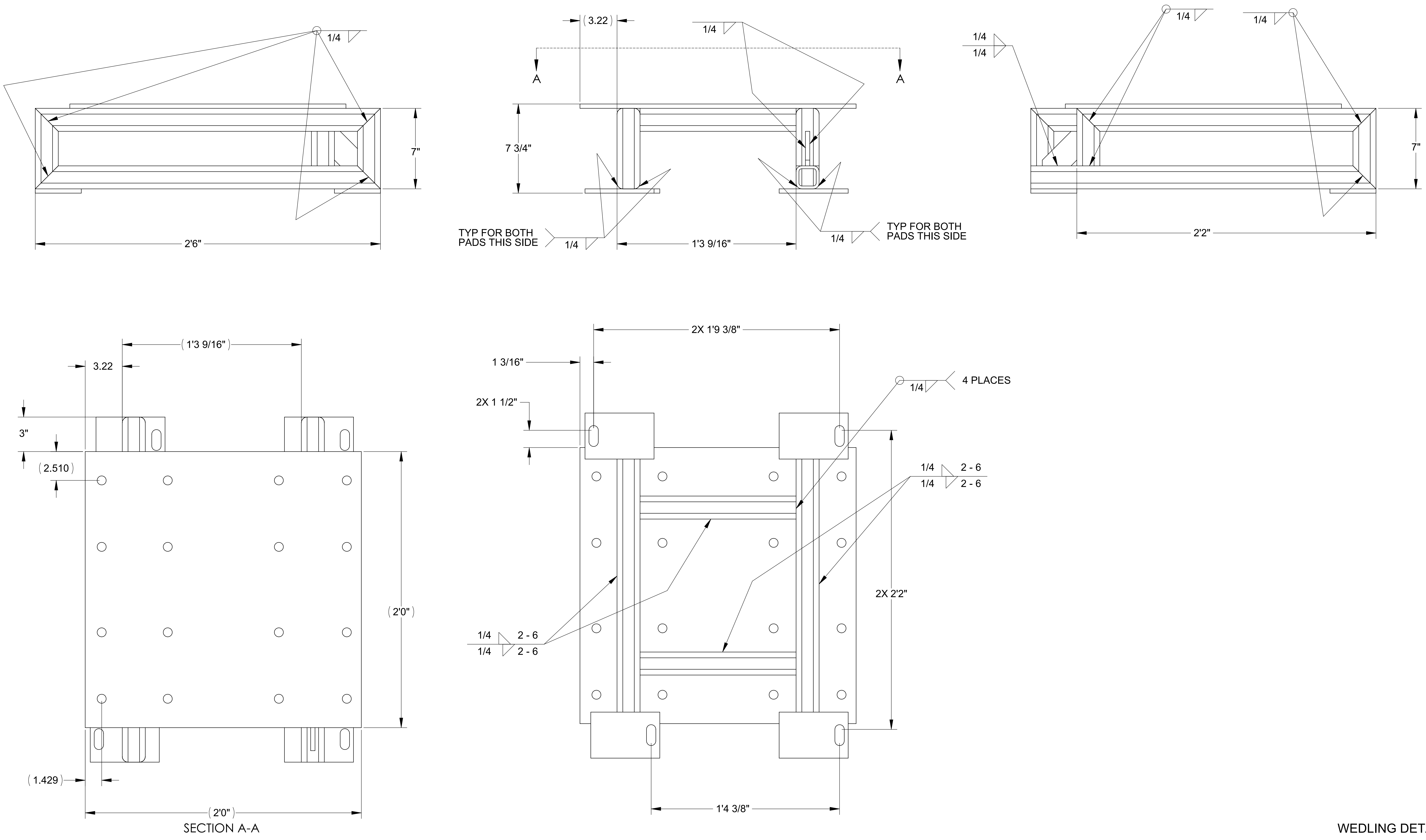


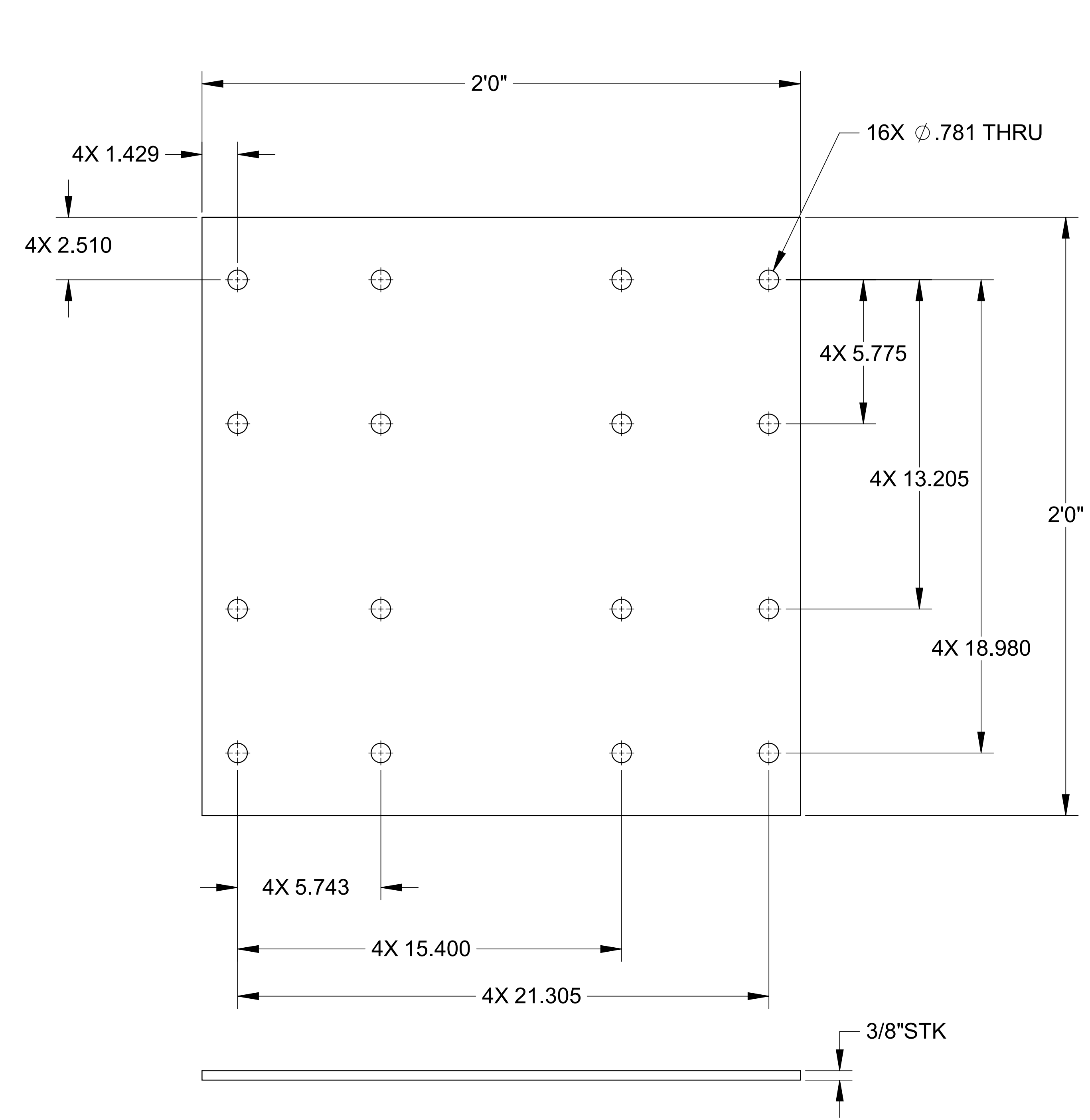

(1) PLATE

SCALE: $1: 4$

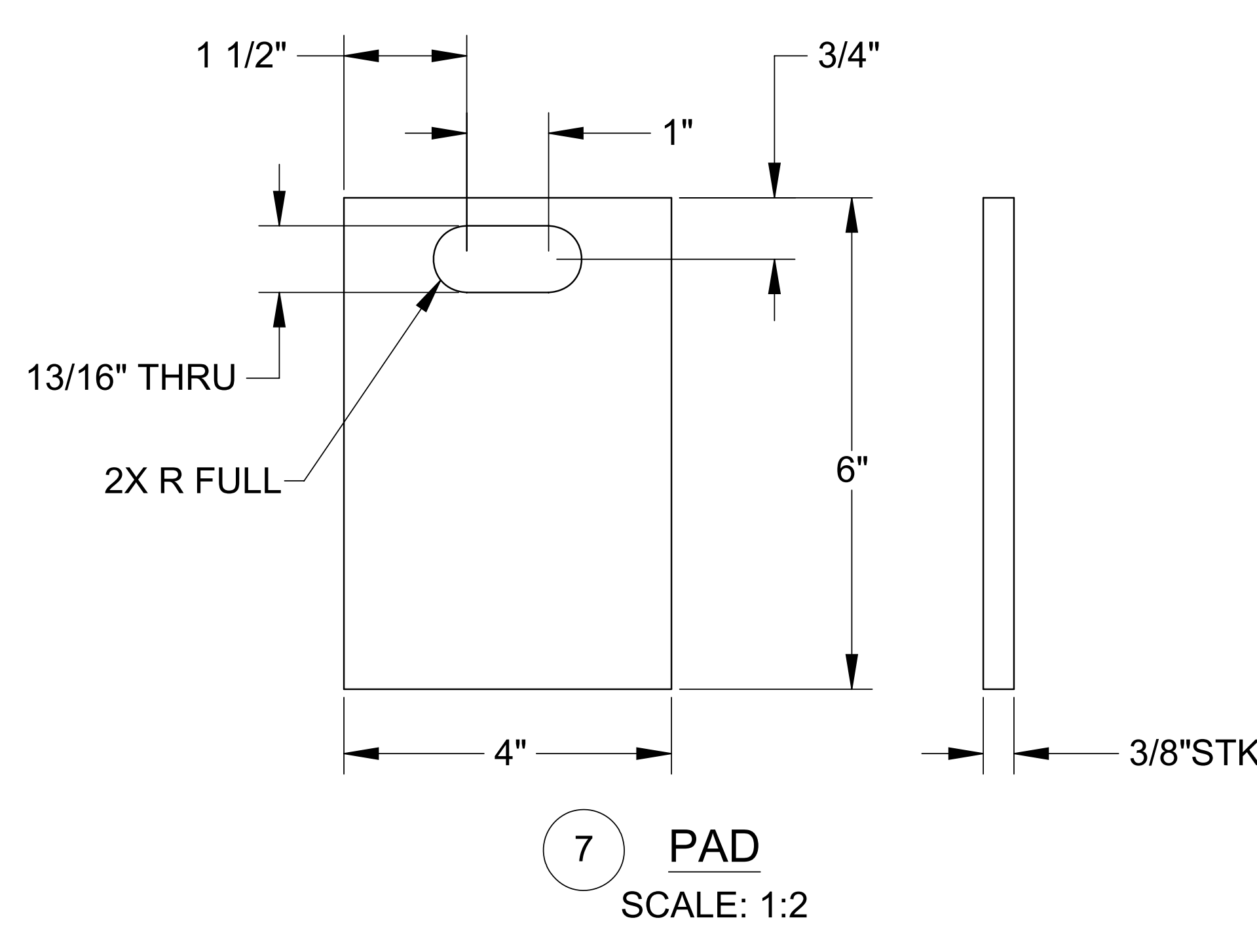

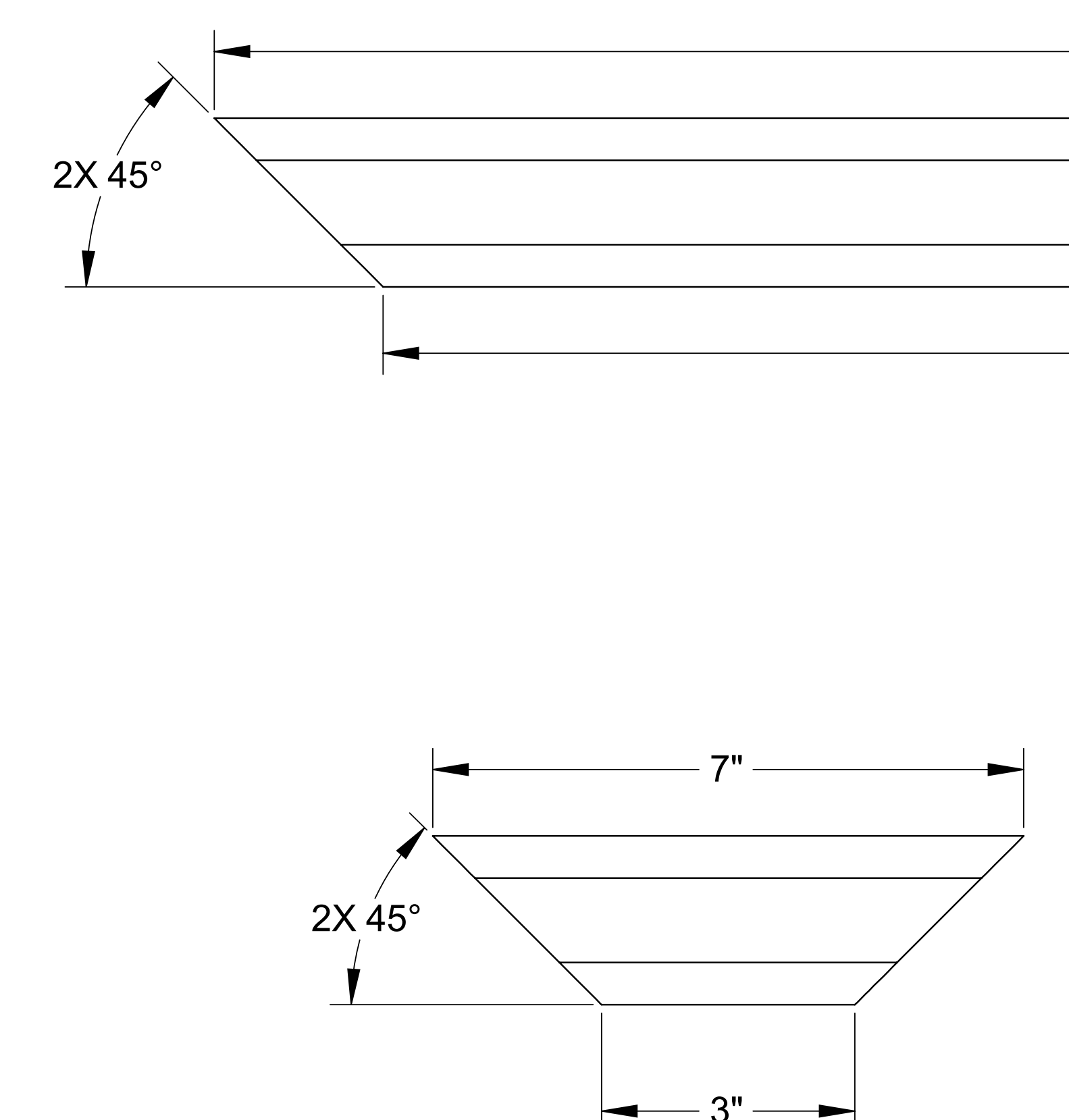

(3) TUBE B
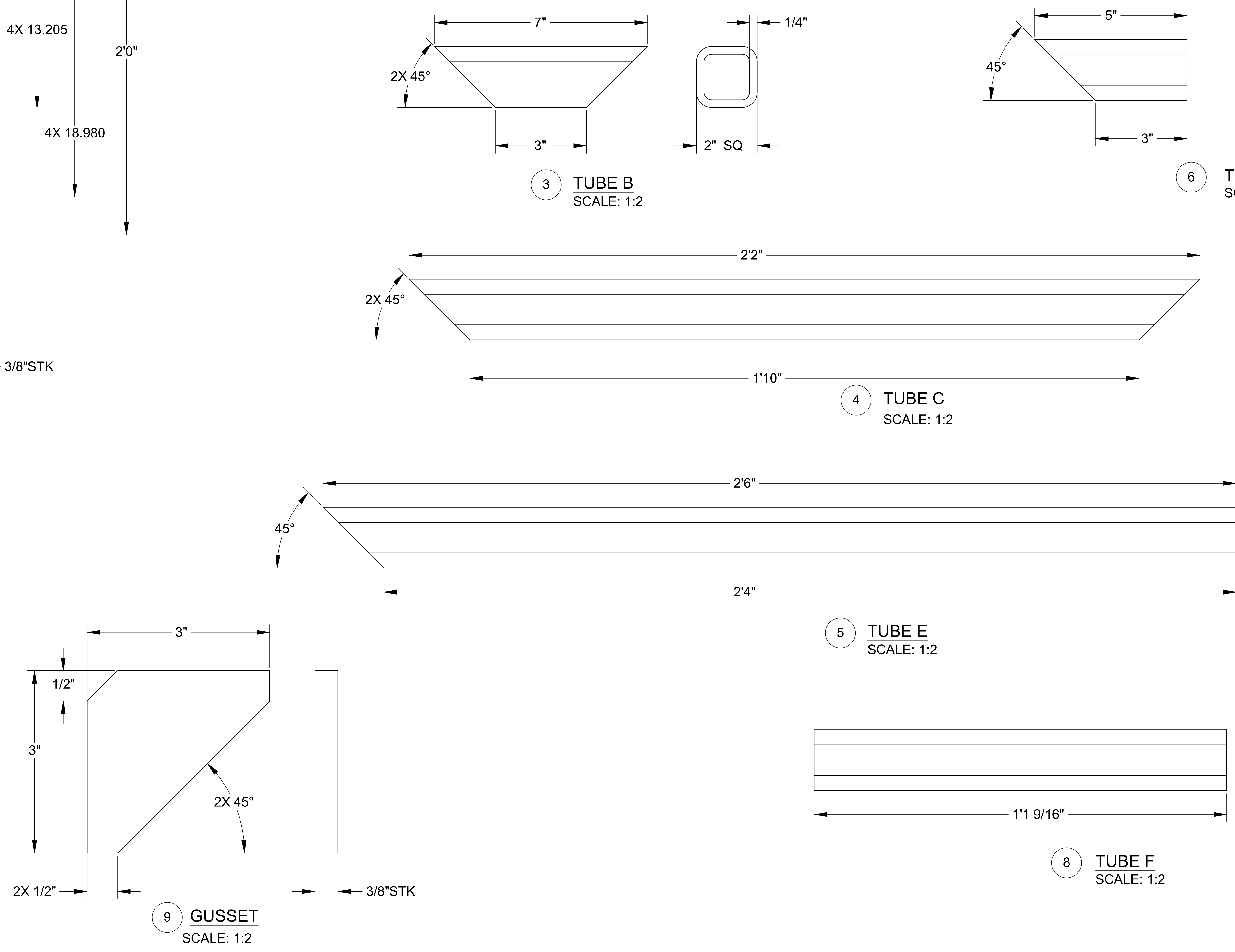

(5) TUBEE

(2) TUBE A
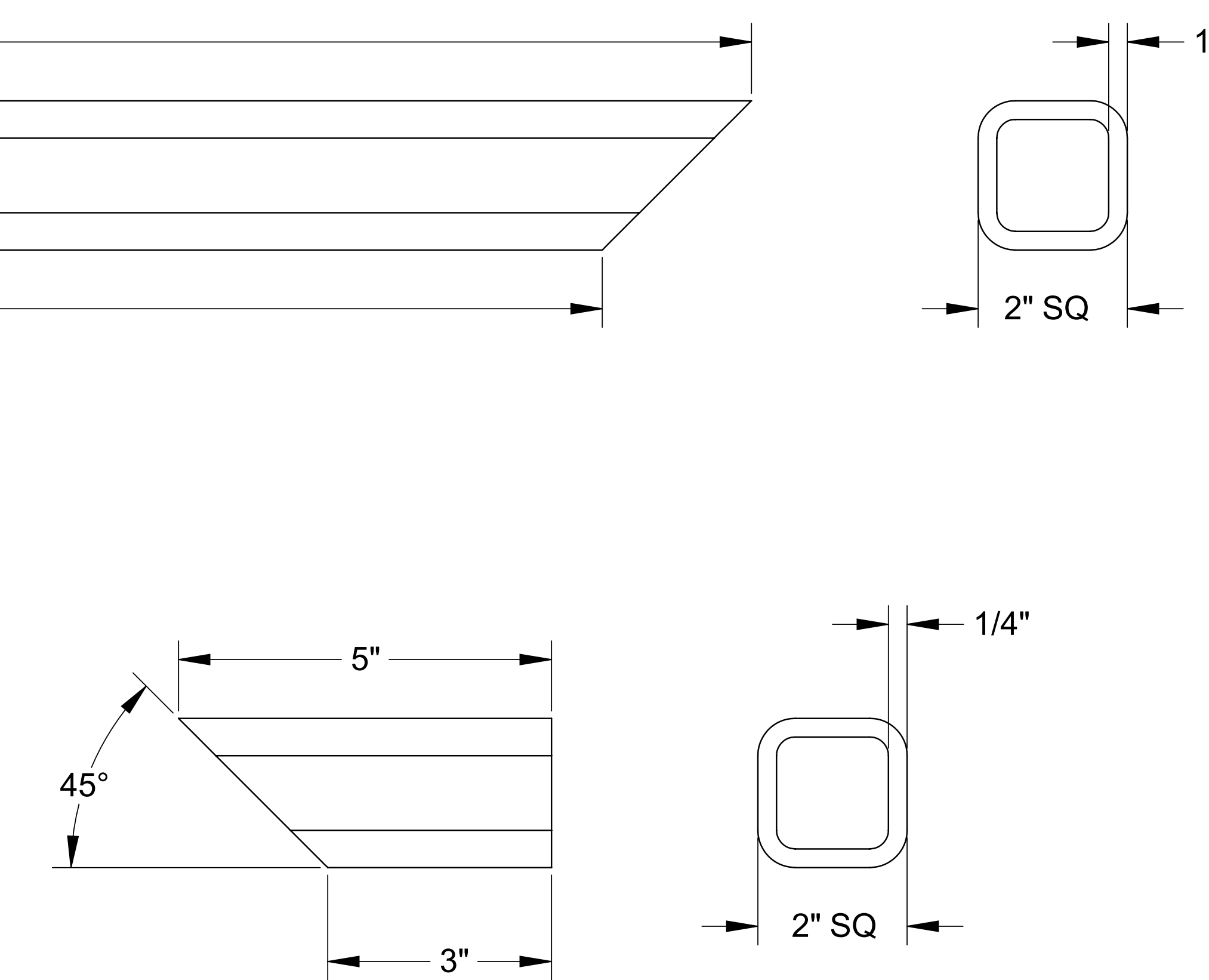

(6) TUBE D
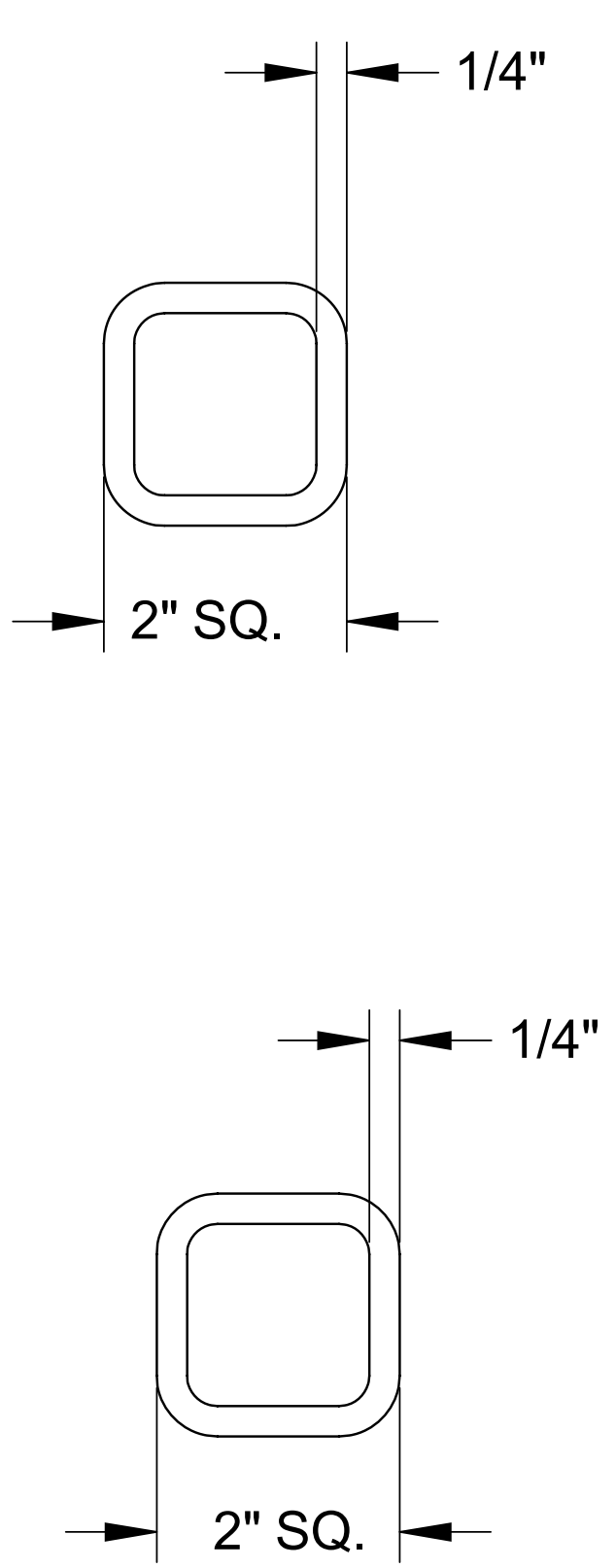

$-2^{n} \mathrm{SQ}$.

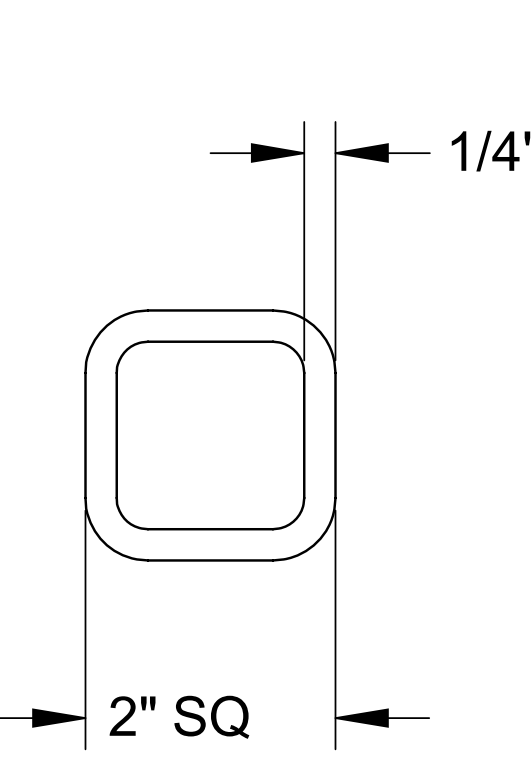

(8) $\frac{\text { TUBE } F}{\text { SCALE: } 1: 2}$ 


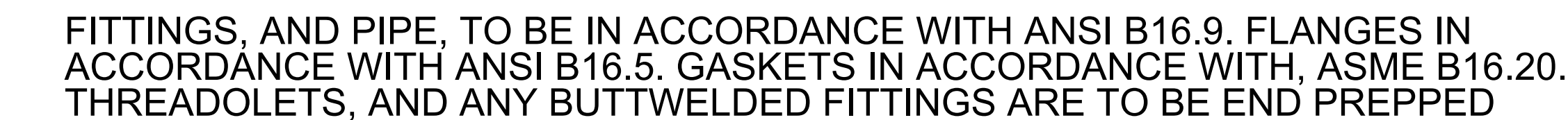
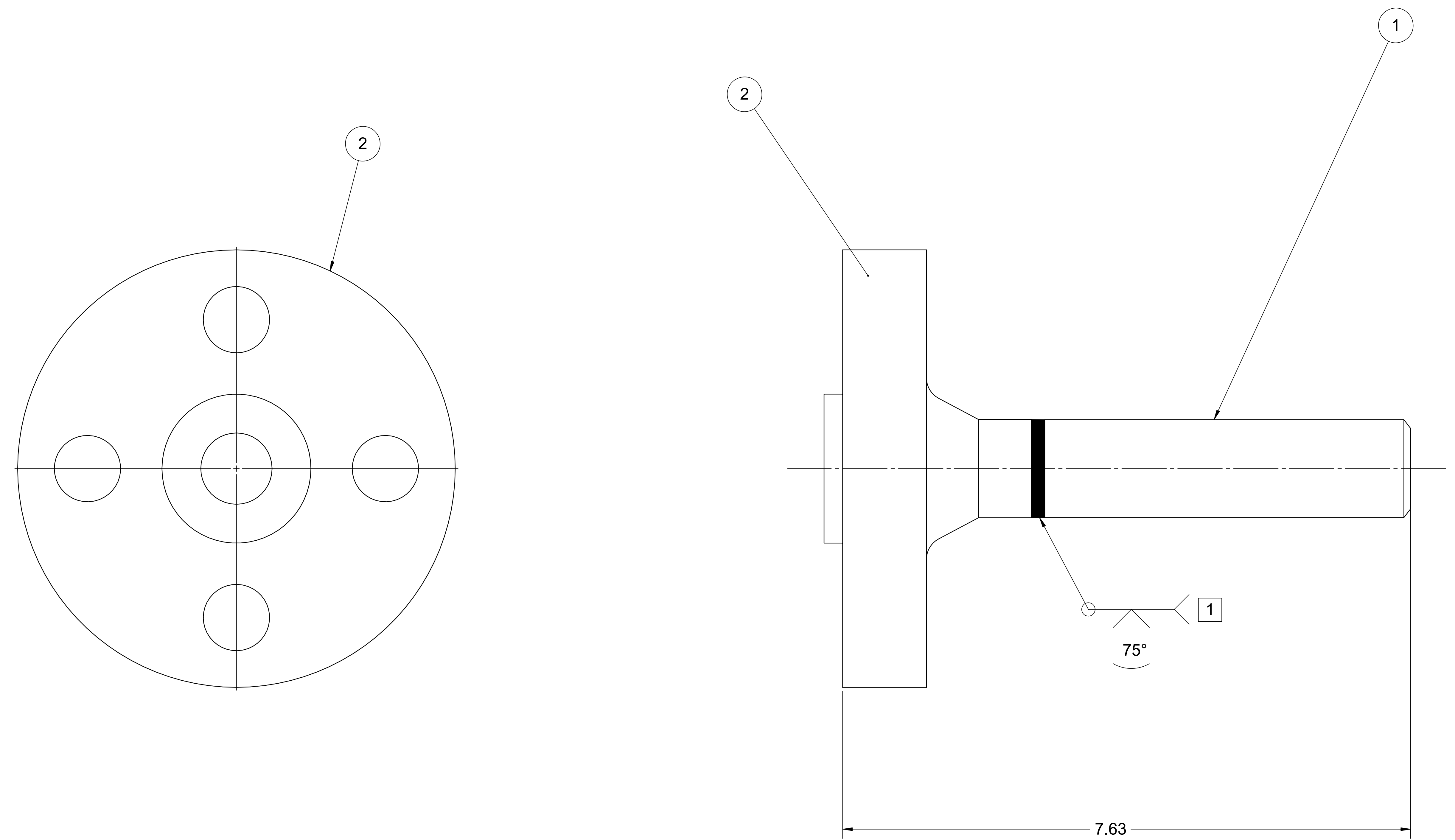

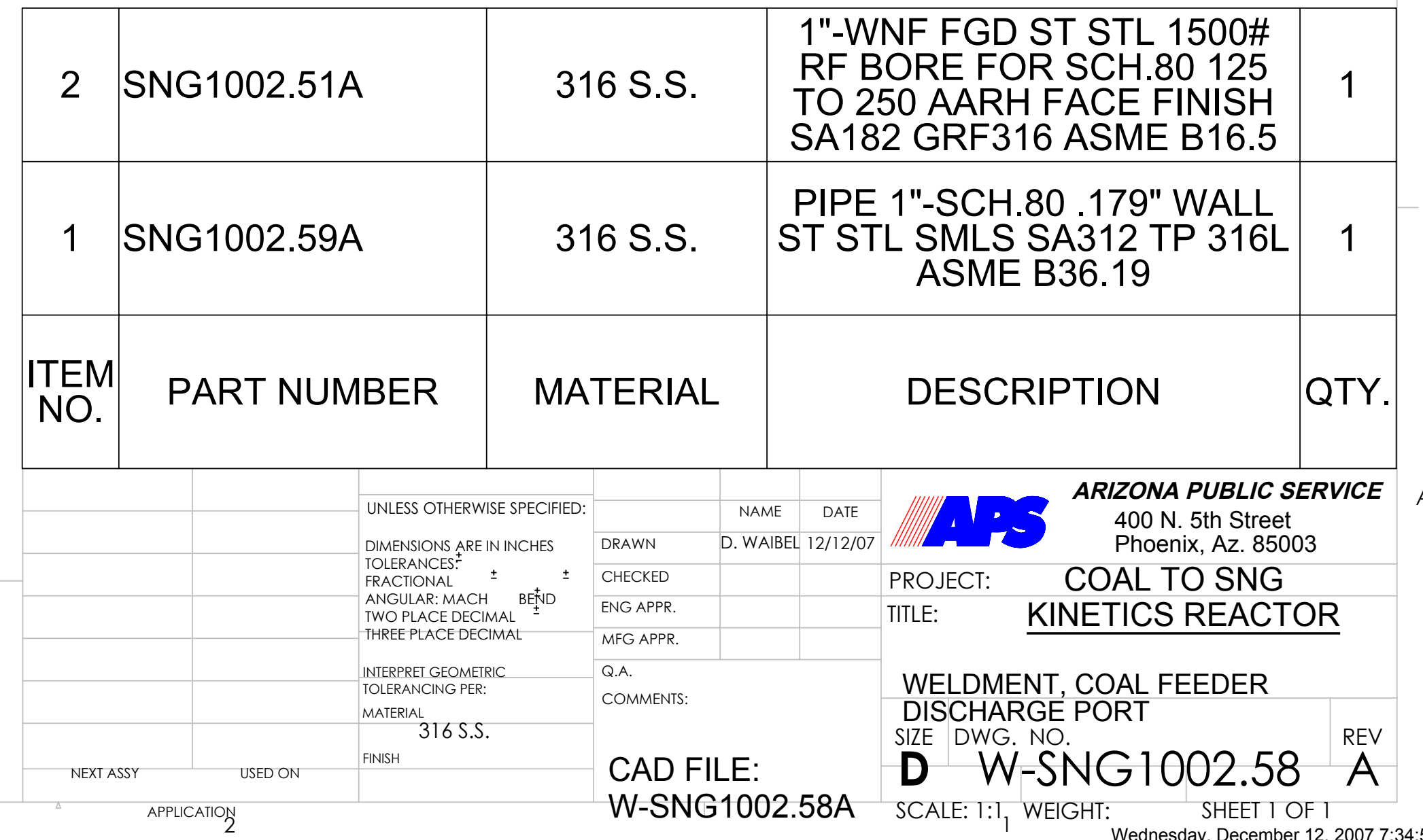



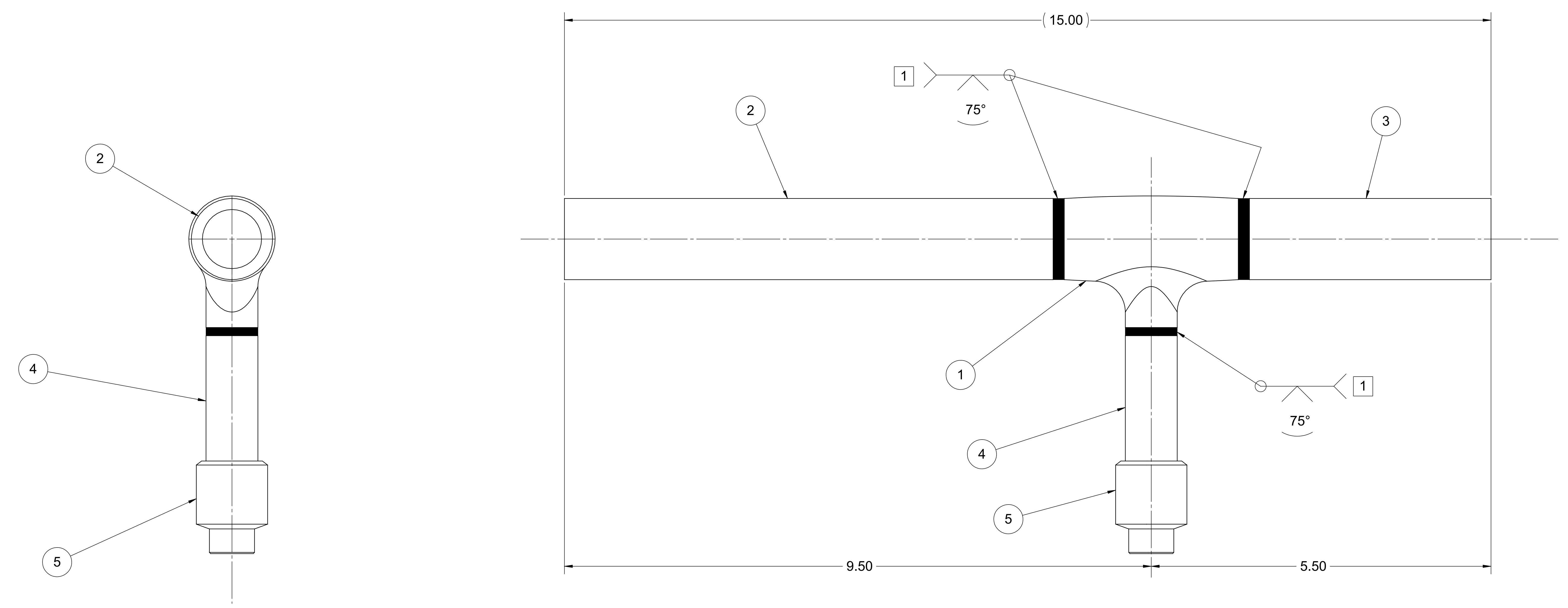

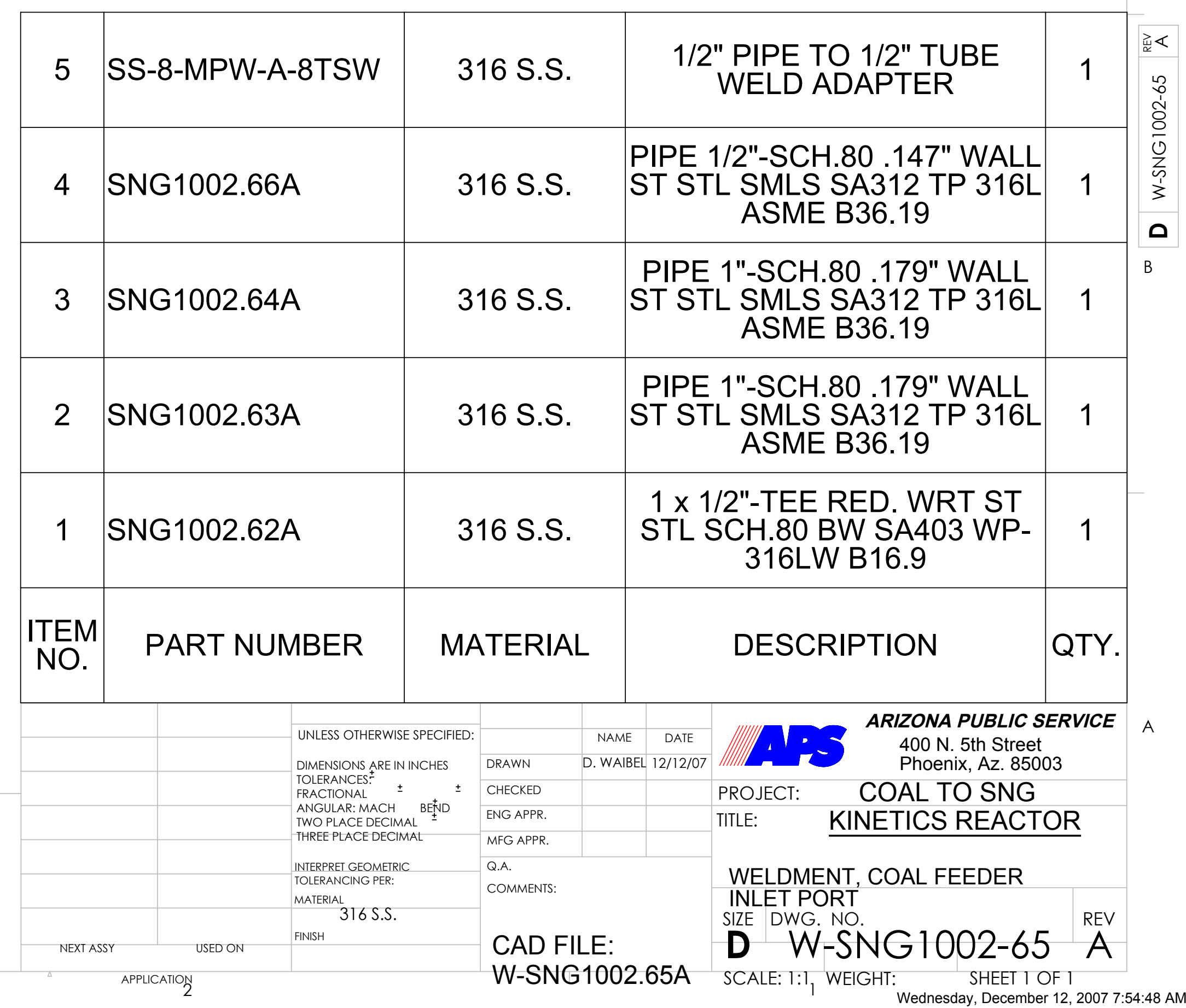


ALL WELDS TO MEET PRESSURE VESSEL CODE, ASME SECTION 8, DIVISION 1 ,
UNLSSS NOTED OTHERWISE.

2 INSPECTION OF WELDS TO BE 100\% X-RAY, UNLESS SPECIFIED OTHERWISE.

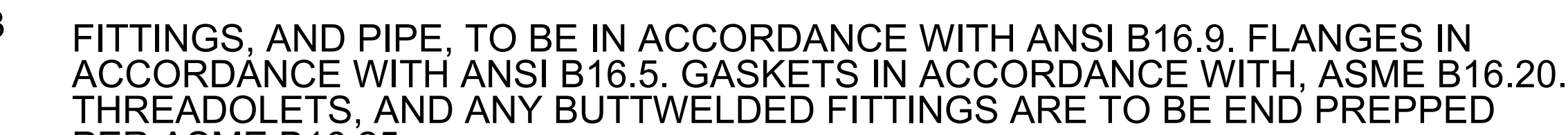

4 PER ASME B16.25.

\begin{tabular}{|c|c|c|c|c|c|}
\multicolumn{5}{|c|}{ REVISIONS } \\
\hline ZONE & REV. & DESCRIPTION & DATE & REV. BY & APPROVED \\
\hline ALL & A & INITIAL RELEASE & $12 / 06 / 07$ & D.W. & \\
\hline ALL & B & ADDED LIFTING LUG & $03 / 04 / 08$ & D.W. & \\
\hline
\end{tabular}
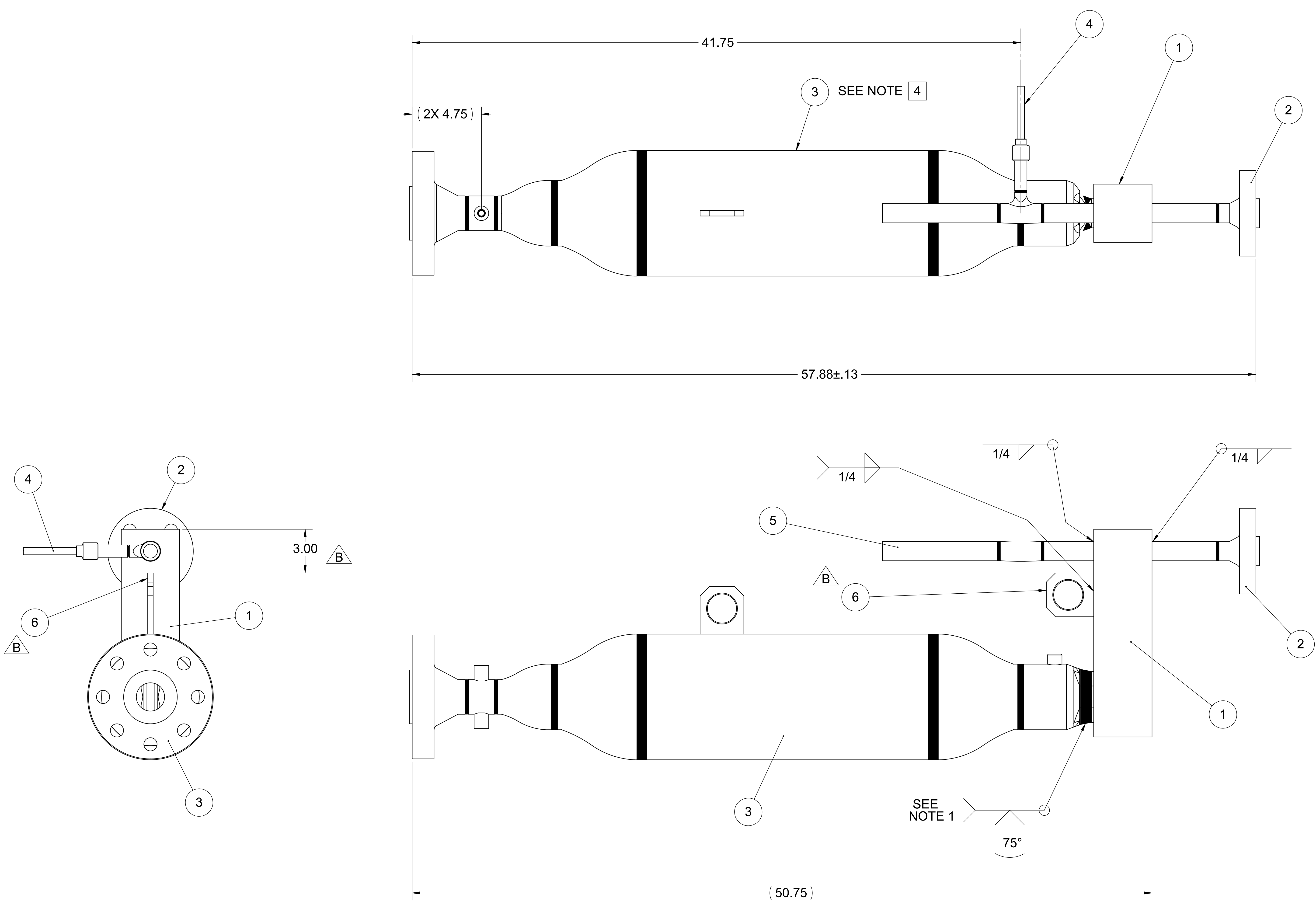

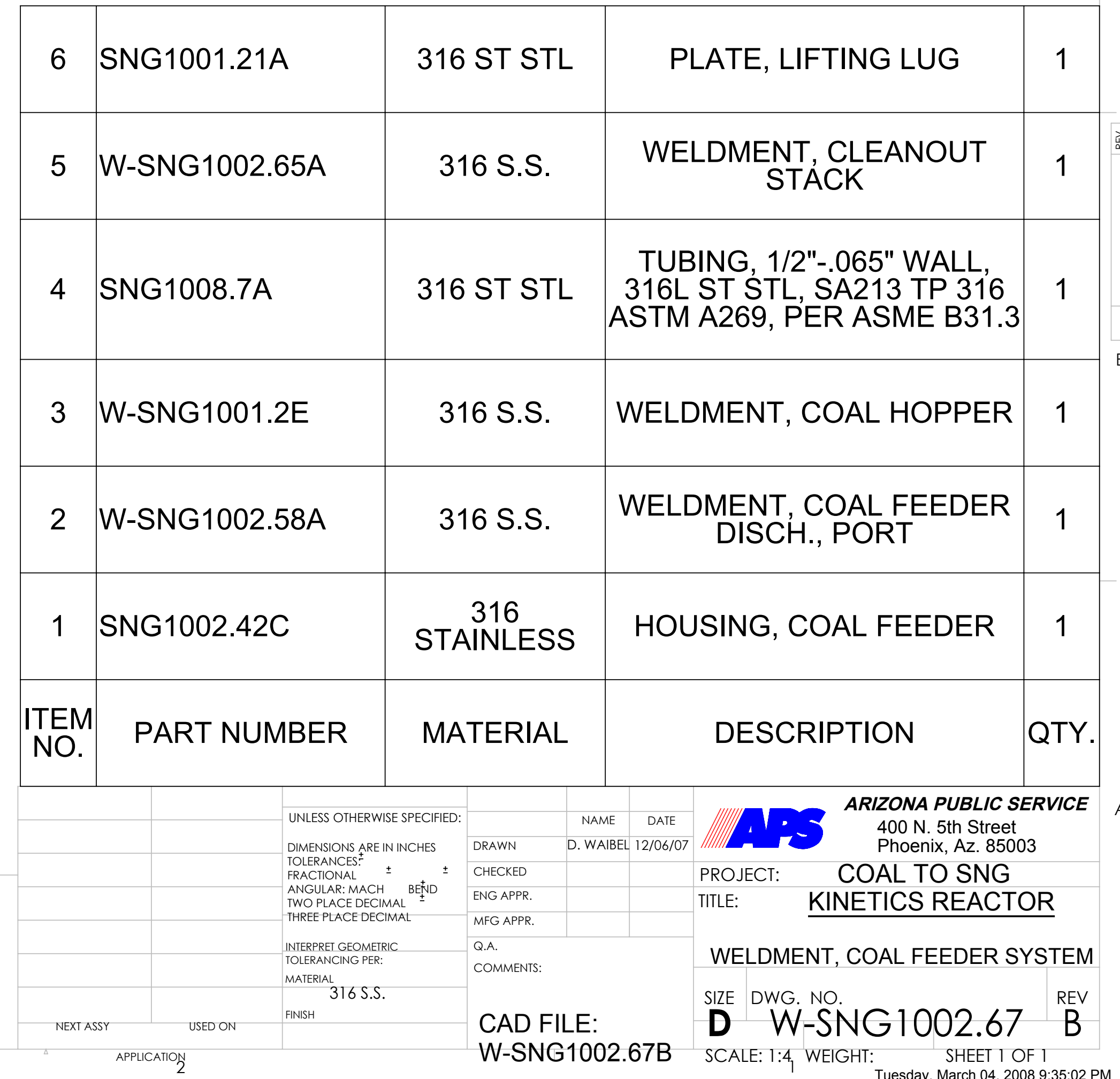




\section{GENERAL NOTES:}

MATERIAL: 316 ST STL, CARBON CONTENT MUST BE EQUAL TO, OR ABOVE .04\%

\begin{tabular}{l|l|l|l|l|l|}
\multicolumn{5}{|c}{ REVISIONS } \\
\hline ZONE & REV. & DESCRIPTION & DATE & REV. BY APPROVED \\
\hline
\end{tabular}
$03 / 17108$ DW
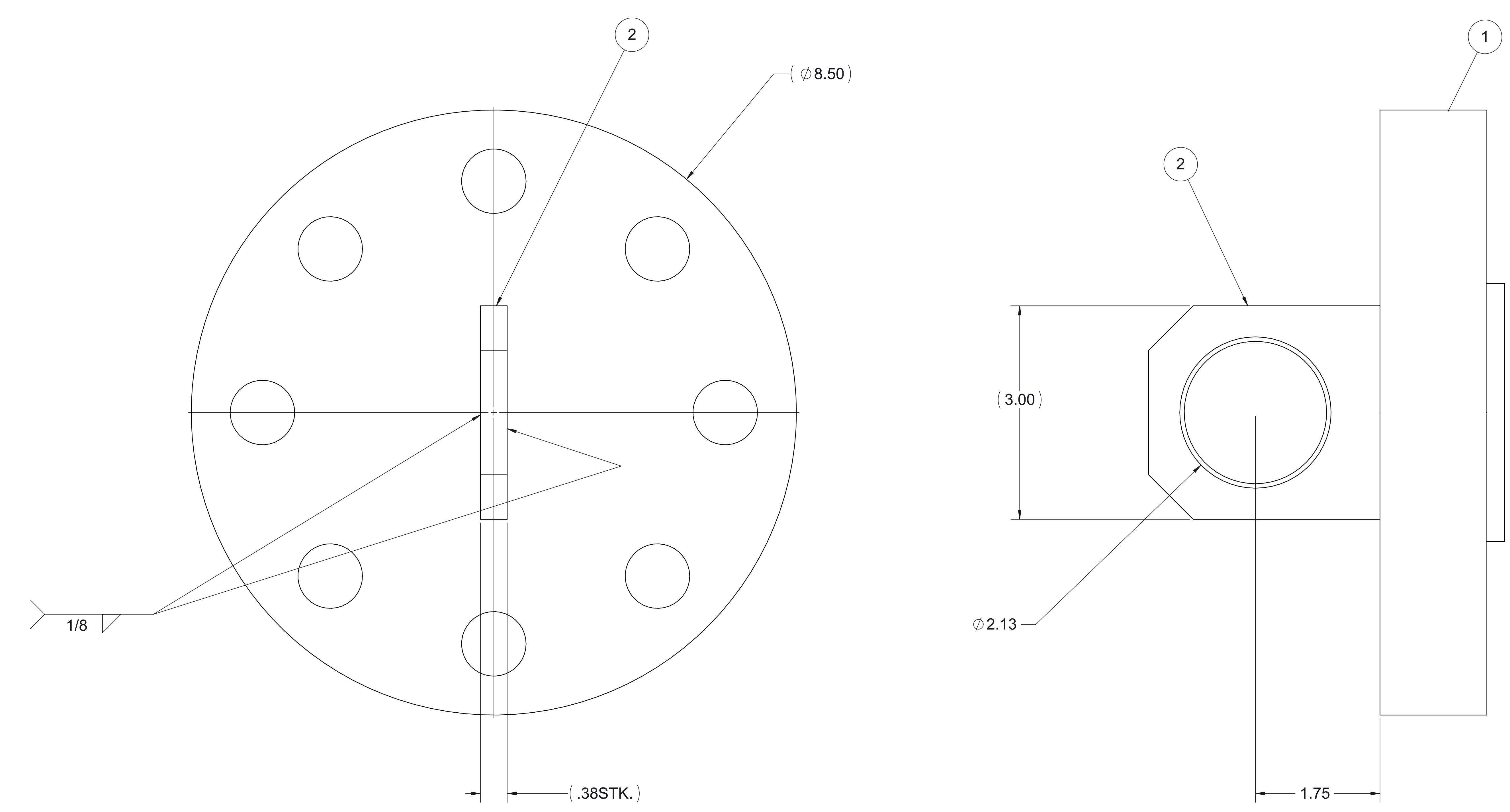

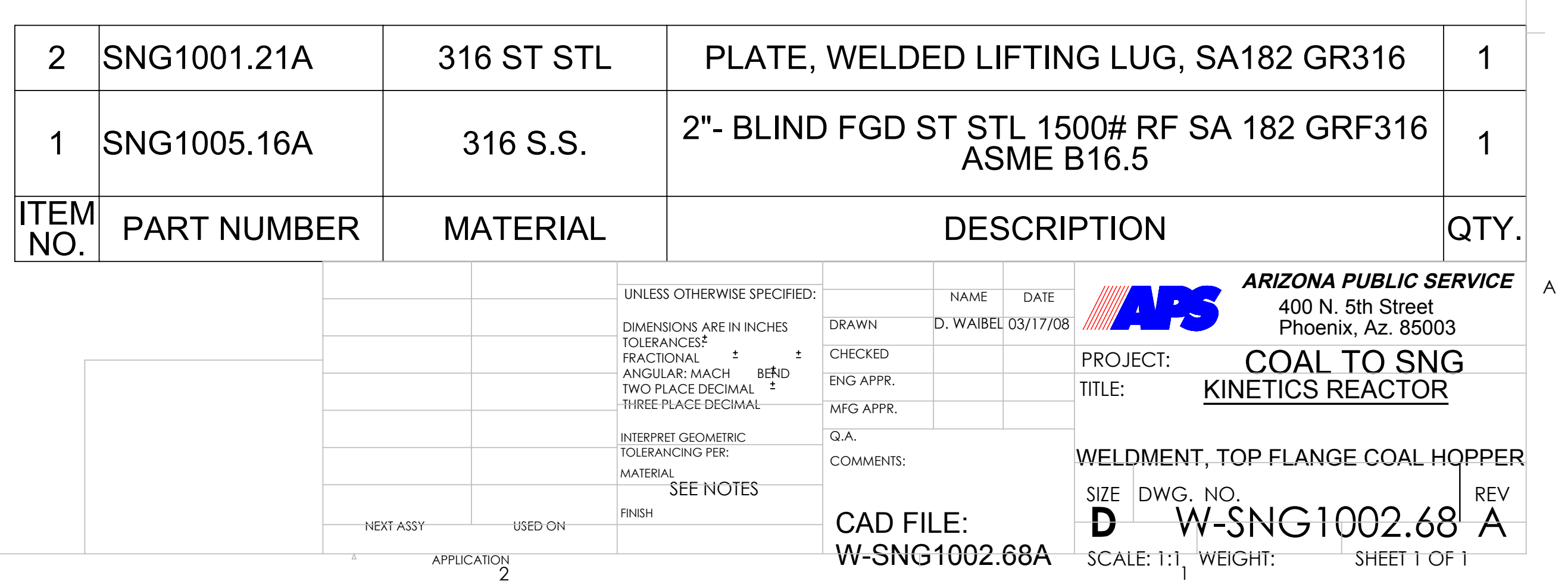


FRAME CONSTRUCTED USING 2" SQUARE TUBING WITH 1/4" THICK WALL, UNLESS NOTED OTHERWISE,

2 DEBURR AND REMOVE ALL SHARP EDGES, PRIOR TO PRIMER COATING.

FINISH; PRIMER USING SHERWIN-WILLIAMS, KEM 400 PRIMER RED OXIDE, PRODUCT NUMBER: E61R00402 OR
EQUIVALENT. PERPARE SURFACE PER MANUFACTURES DATA SHEET.

REVISIONS
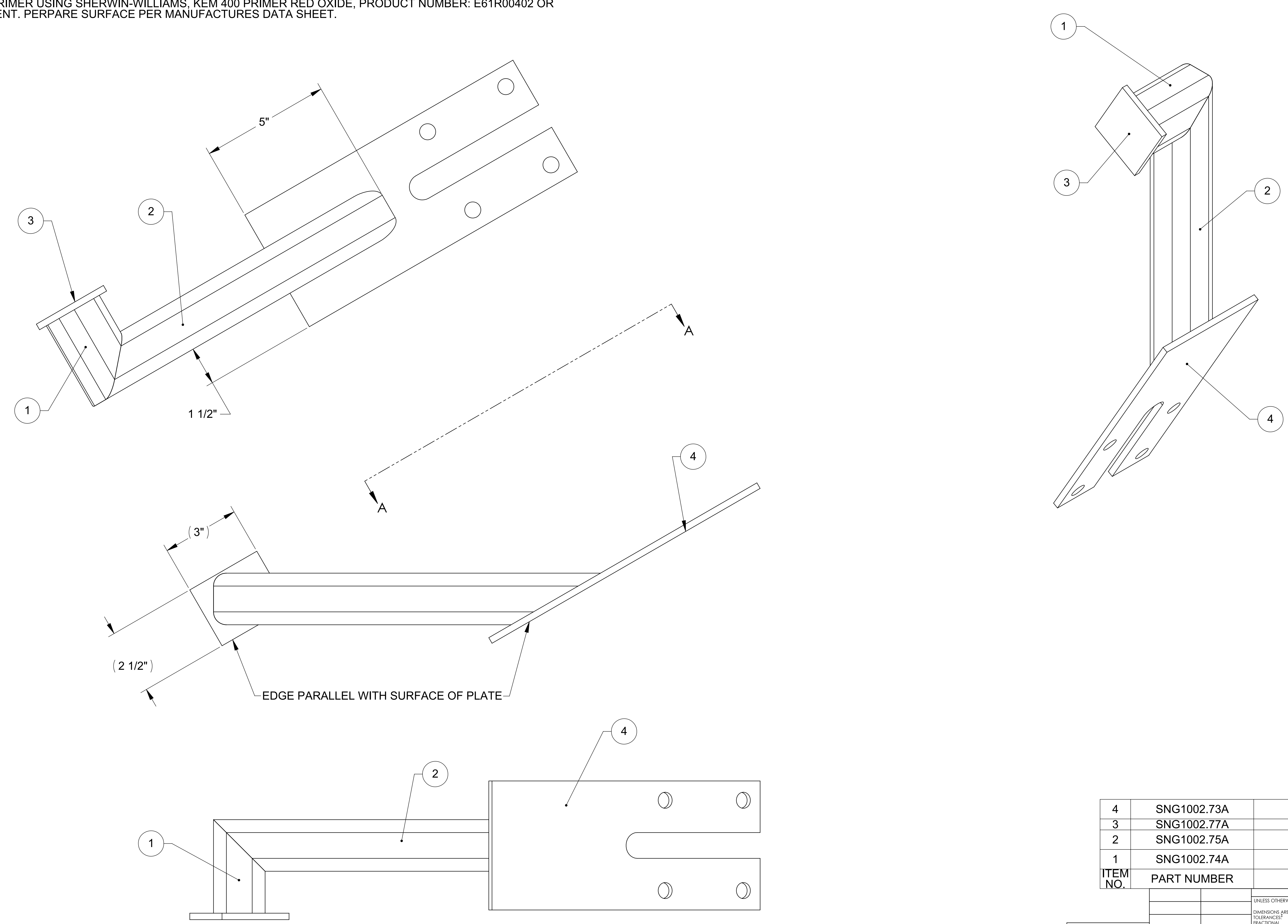

\begin{tabular}{|c|c|c|c|c|c|c|}
\hline 4 & SNG1002.73A & \multicolumn{4}{|c|}{ PLATE. ACTUATOR SUPPORT } & 1 \\
\hline 3 & SNG1002.77A & \multicolumn{4}{|c|}{ PLATE, WELDED FOOT } & \\
\hline 2 & SNG1002.75A & \multicolumn{4}{|c|}{ TUBE, SQUARE, 2x2x.25" WALL, LONG } & 1 \\
\hline & \multirow{2}{*}{$\begin{array}{l}\text { SNG1002.74A } \\
\text { PART NUMBER }\end{array}$} & TUBE, & SQAUF & $R E, 2 \times 2 \times .2$ & "WALL, SHORT & 1 \\
\hline \multirow[t]{5}{*}{$\begin{array}{l}\text { ITTM } \\
\text { NO. }\end{array}$} & & & QTY \\
\hline & & sispecened & \multicolumn{2}{|c|}{ DESCRIPTION } & 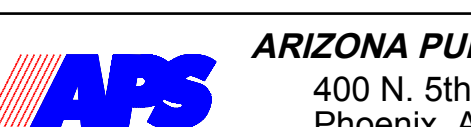 & \\
\hline & & 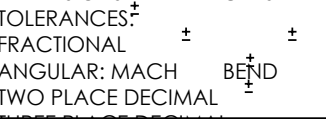 & 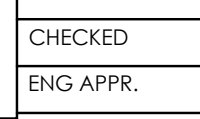 & 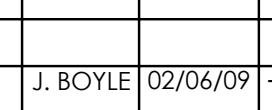 & $\begin{array}{l}\text { COAL TO } \\
\text { KIETIIS RE }\end{array}$ & OR \\
\hline & & 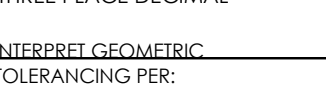 & 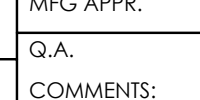 & & WELDMENT, & \\
\hline & & 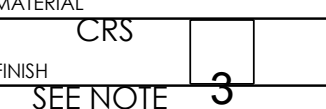 & CAD FII & & IIE DWG NO NG 1002 & REV \\
\hline
\end{tabular}




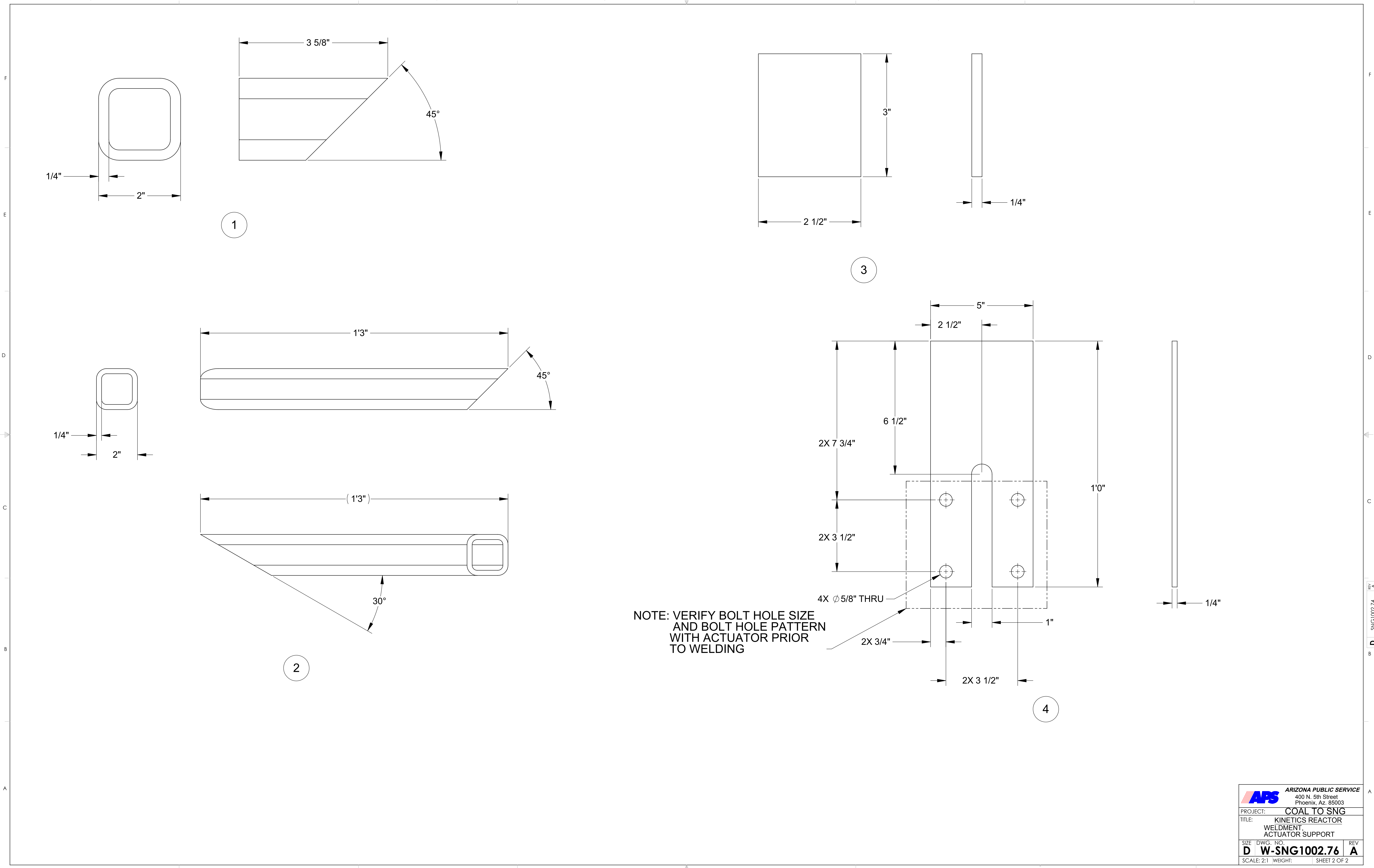


1 FOR PROCEDURE ON WRAPPING CERAMIC FIBER, AND STAINLESS FOIL,
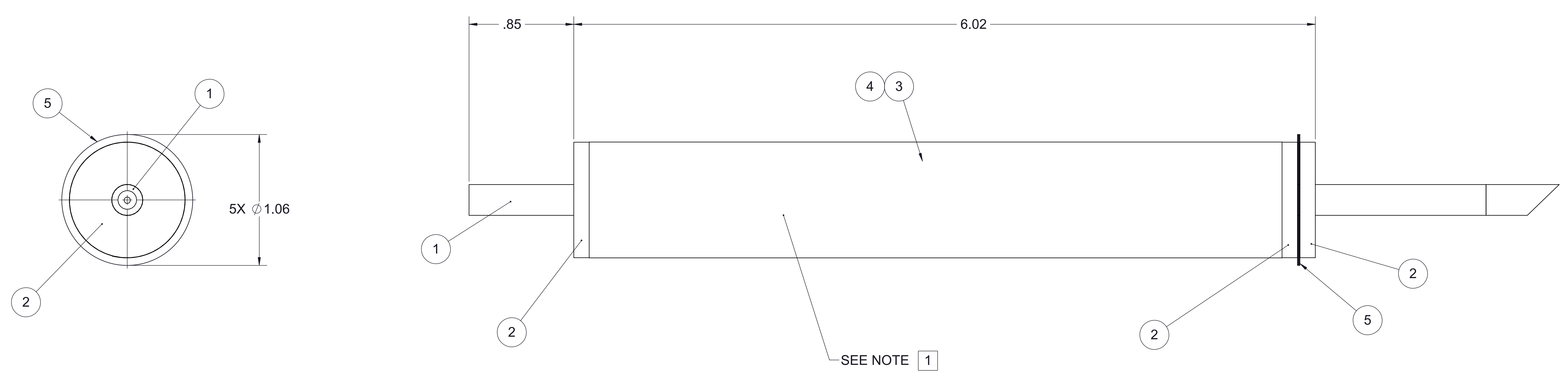

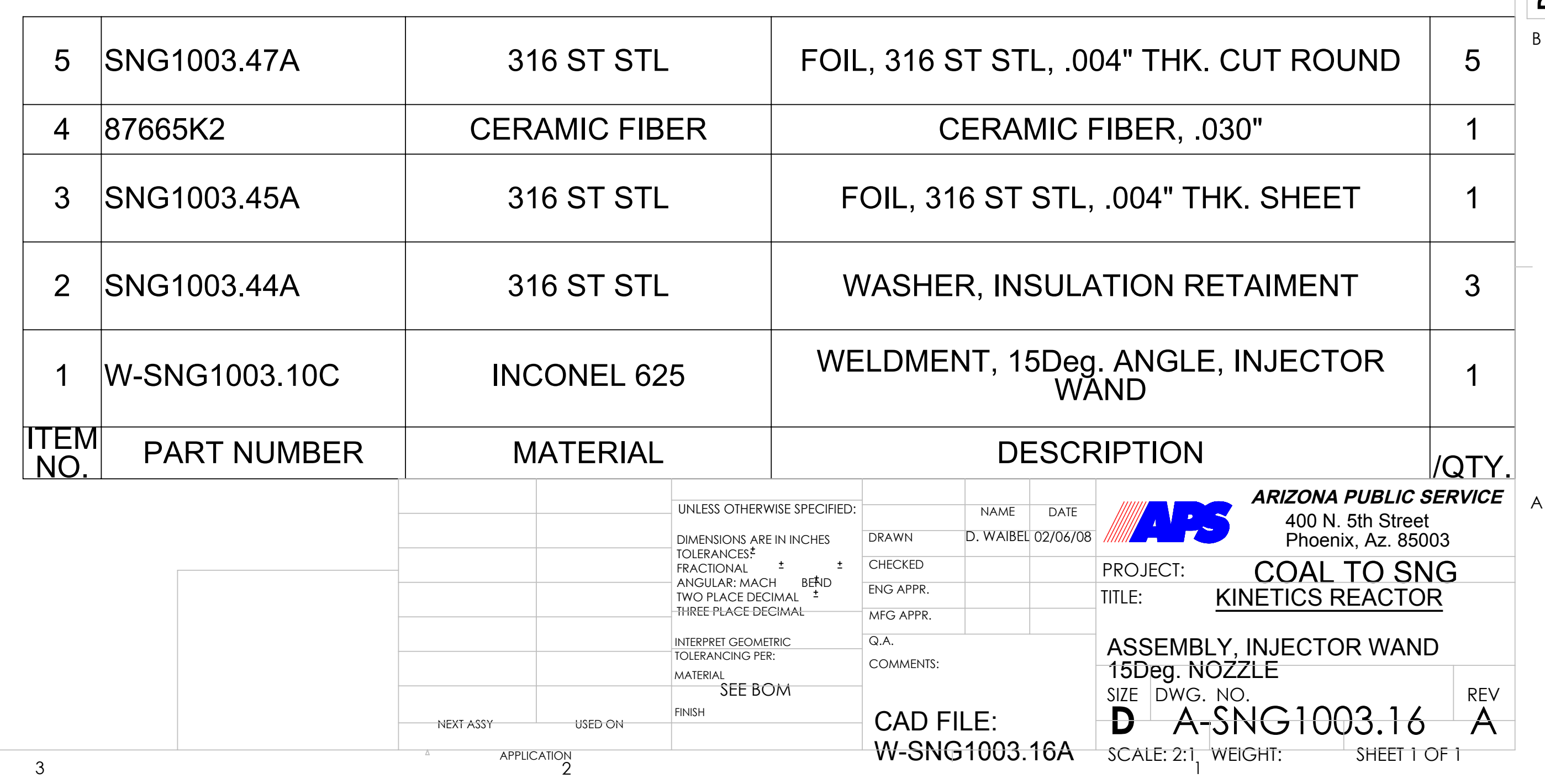


1 FOR PROCEDURE ON WRAPPING CERAMIC FIBER, AND STAINLESS FOIL,
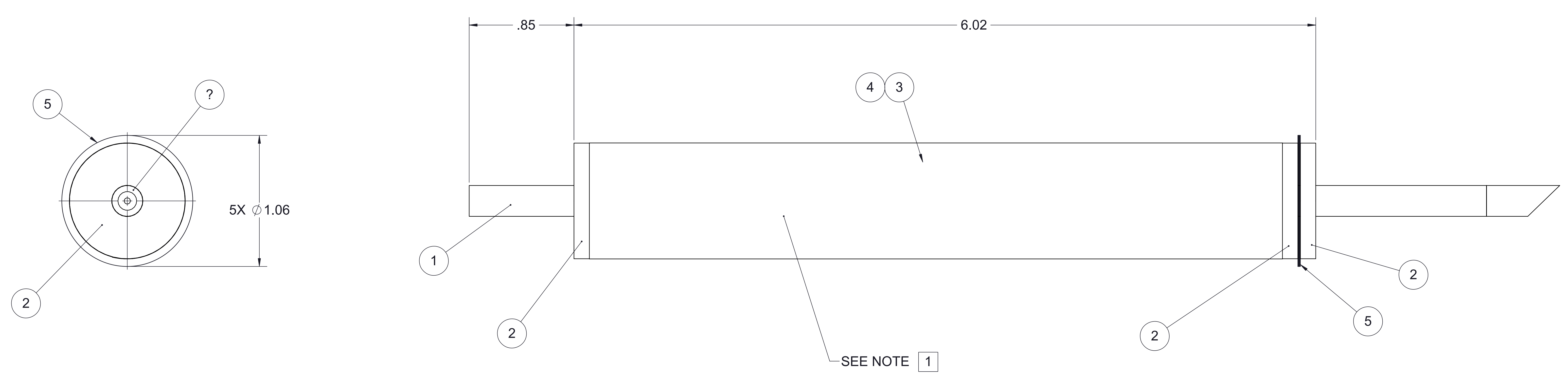

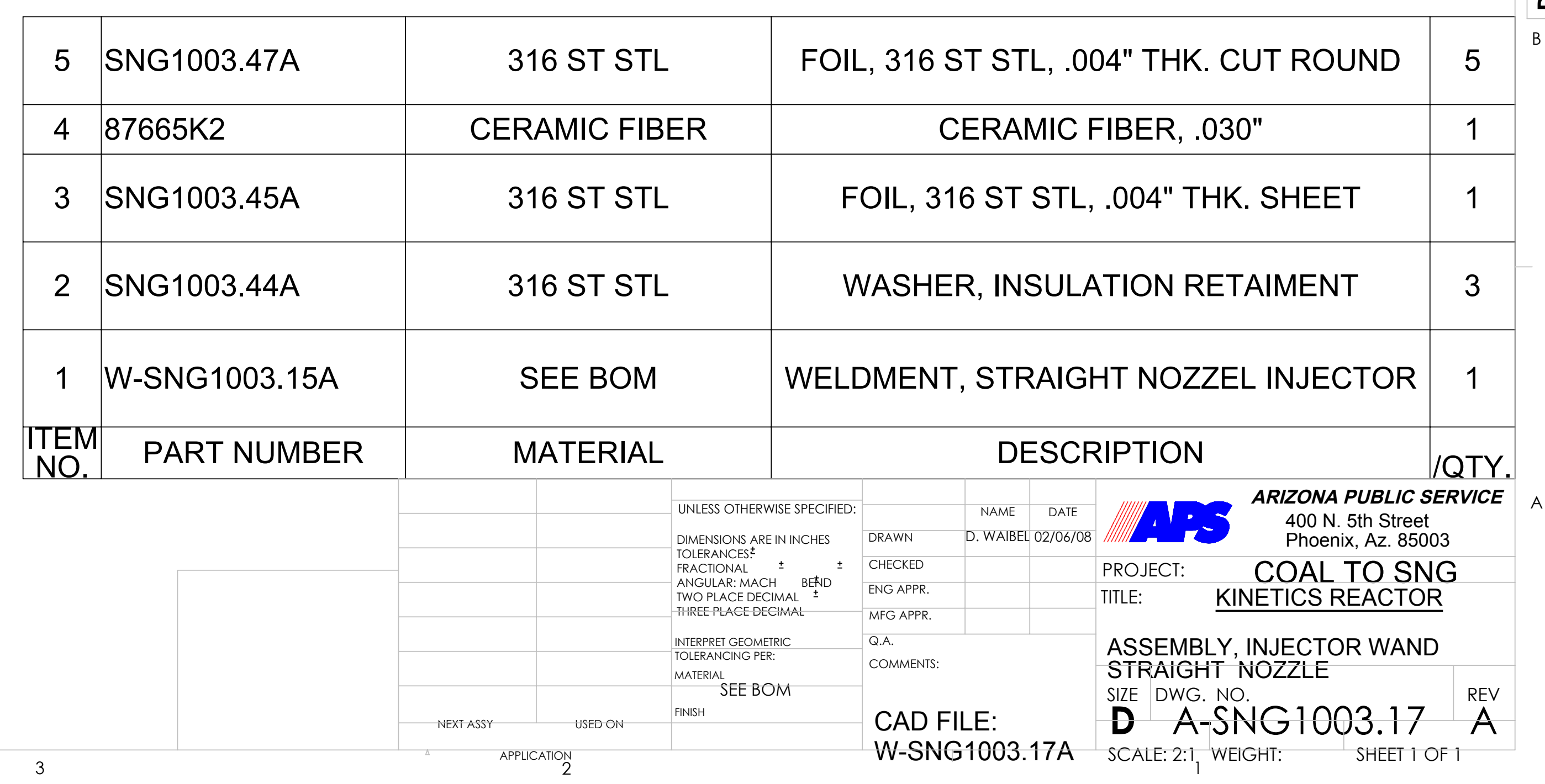




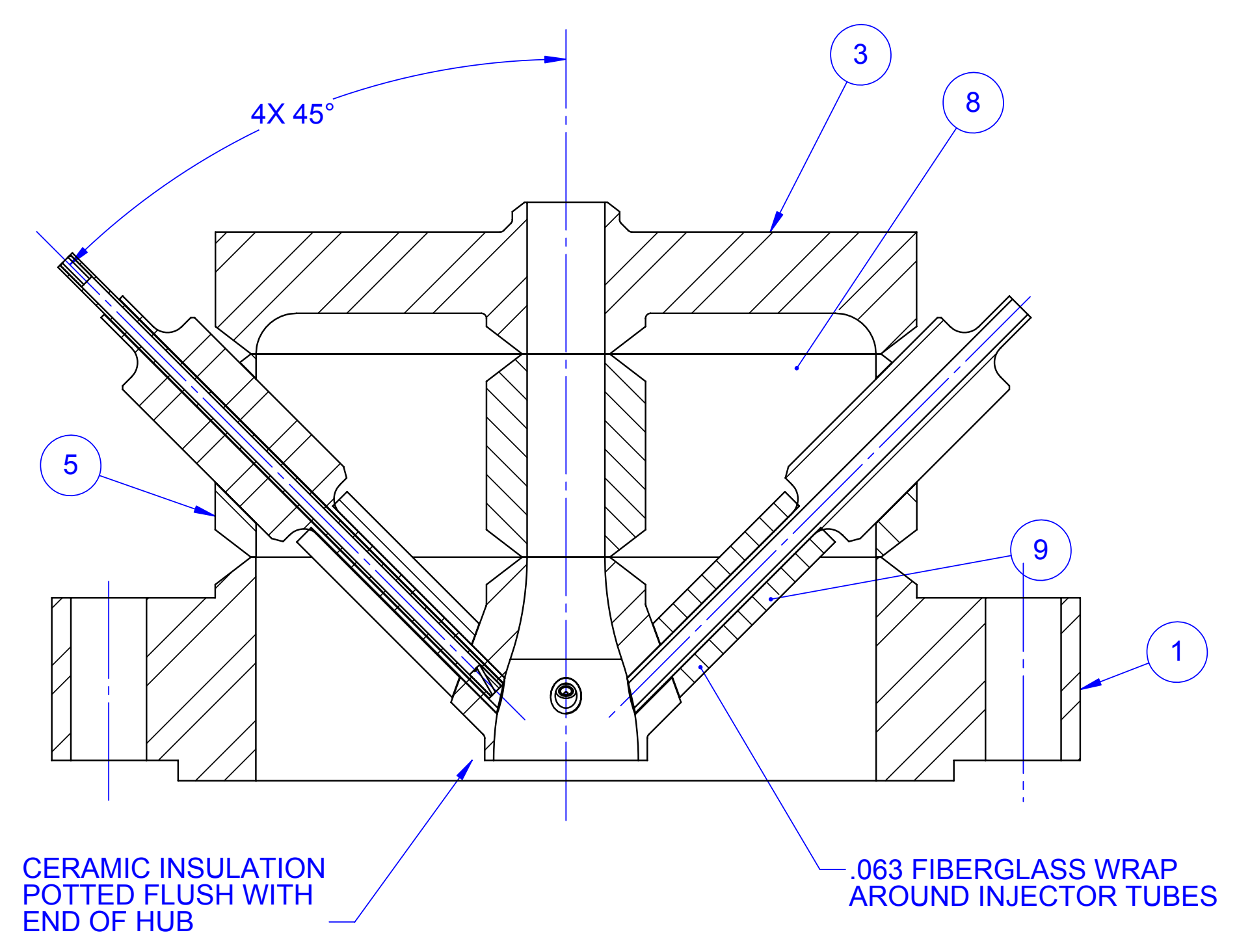

SECTION A-A

\begin{tabular}{|c|l|c|c|}
\hline 9 & FIBER-WRAP-1 & & 4 \\
\hline 8 & $8498 K 35$ & ALUMINA, CERAMIC COMPOUND & 1 \\
\hline 7 & SNG1003.6A-1 & TUBE, 3/8" INJECTOR & 4 \\
\hline 6 & SNG1003.7B & PIPE, REACTOR HEAD SPACER & 1 \\
\hline 5 & SNG1003.8B & PIPE, REACTOR HEAD INJECTOR & 1 \\
\hline 4 & SNG1NG & 1 \\
\hline 3 & SNG1003.5A & HUB, REACTOR INNER HEAD & 1 \\
\hline 2 & W-SNG1003.10A & $\begin{array}{c}\text { FLANGE, WELD NECK REACTOR } \\
\text { HEAD }\end{array}$ & 1 \\
\hline 1 & SNG1003.3A & $\begin{array}{c}\text { FLANGE, REACTOR WEATIOR INJECTOR } \\
\text { HEAD }\end{array}$ & 1 \\
\hline ITEM NO. & PART NUMBER & DESCRIPTION & QTY. \\
\hline
\end{tabular}

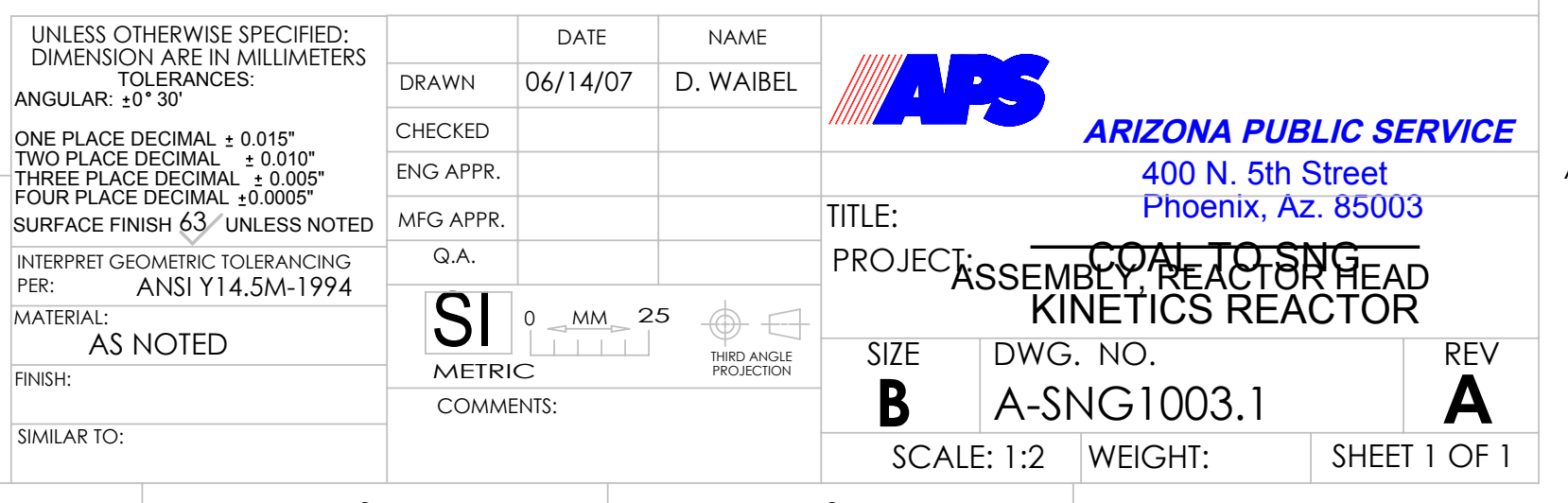




\begin{tabular}{|c|c|c|c|c|}
\hline \multicolumn{5}{|c|}{ REVISIONS } \\
\hline ZONE & REV. & DESCRIPTION & DATE & REV. B B \\
\hline & A & INITIAL DESIGN & $06 / 27 / 07$ & 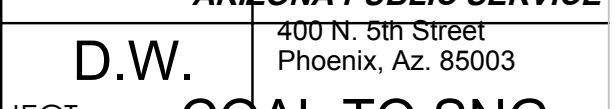 \\
\hline & & & & KINETCS REACTOR \\
\hline & & & & \\
\hline & & & & \\
\hline
\end{tabular}

A

(1) A SEET 2 (20) (ㅇ) (a)

o 100

080

5

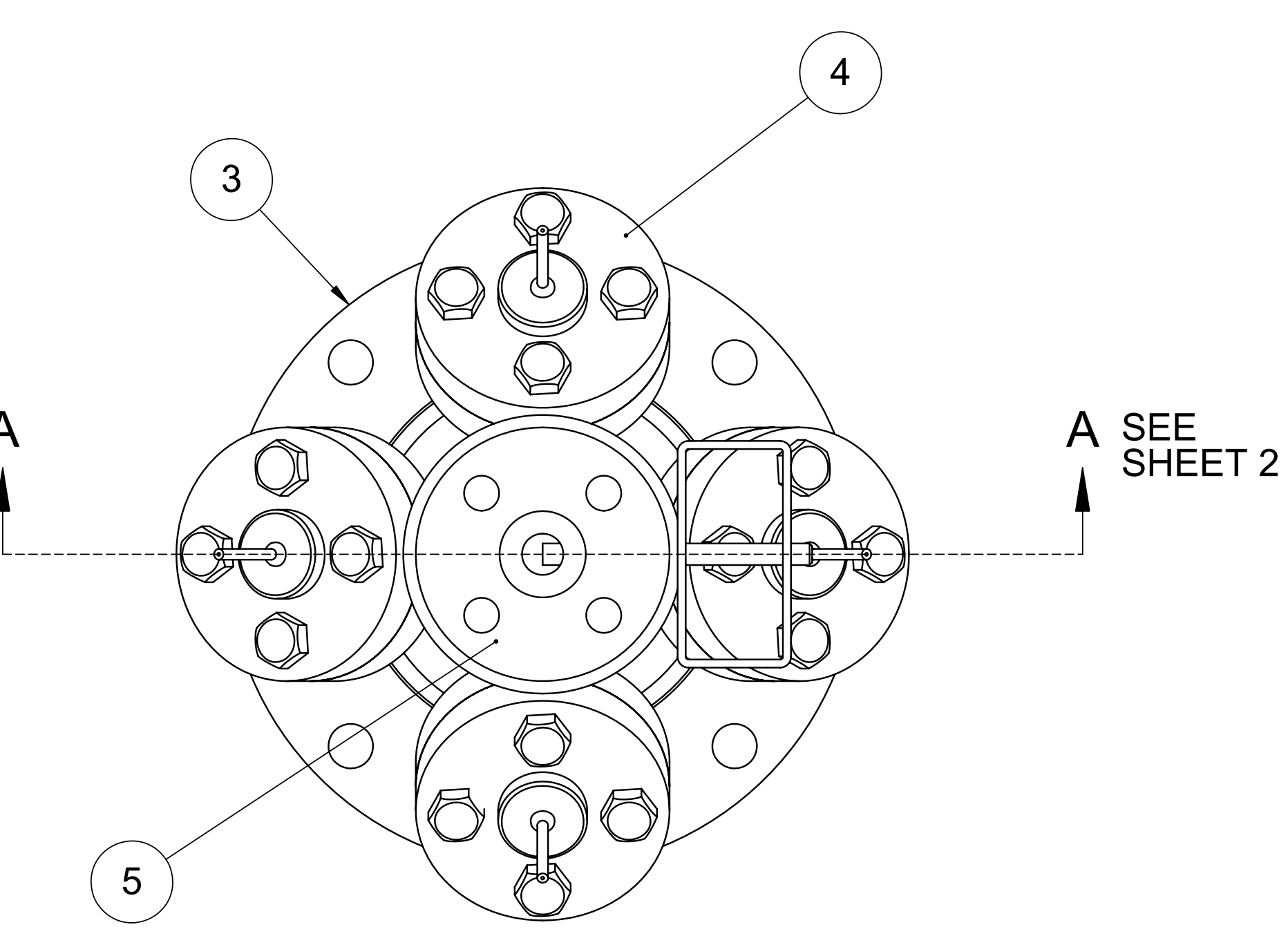

$1 0 \longdiv { 1 0 }$

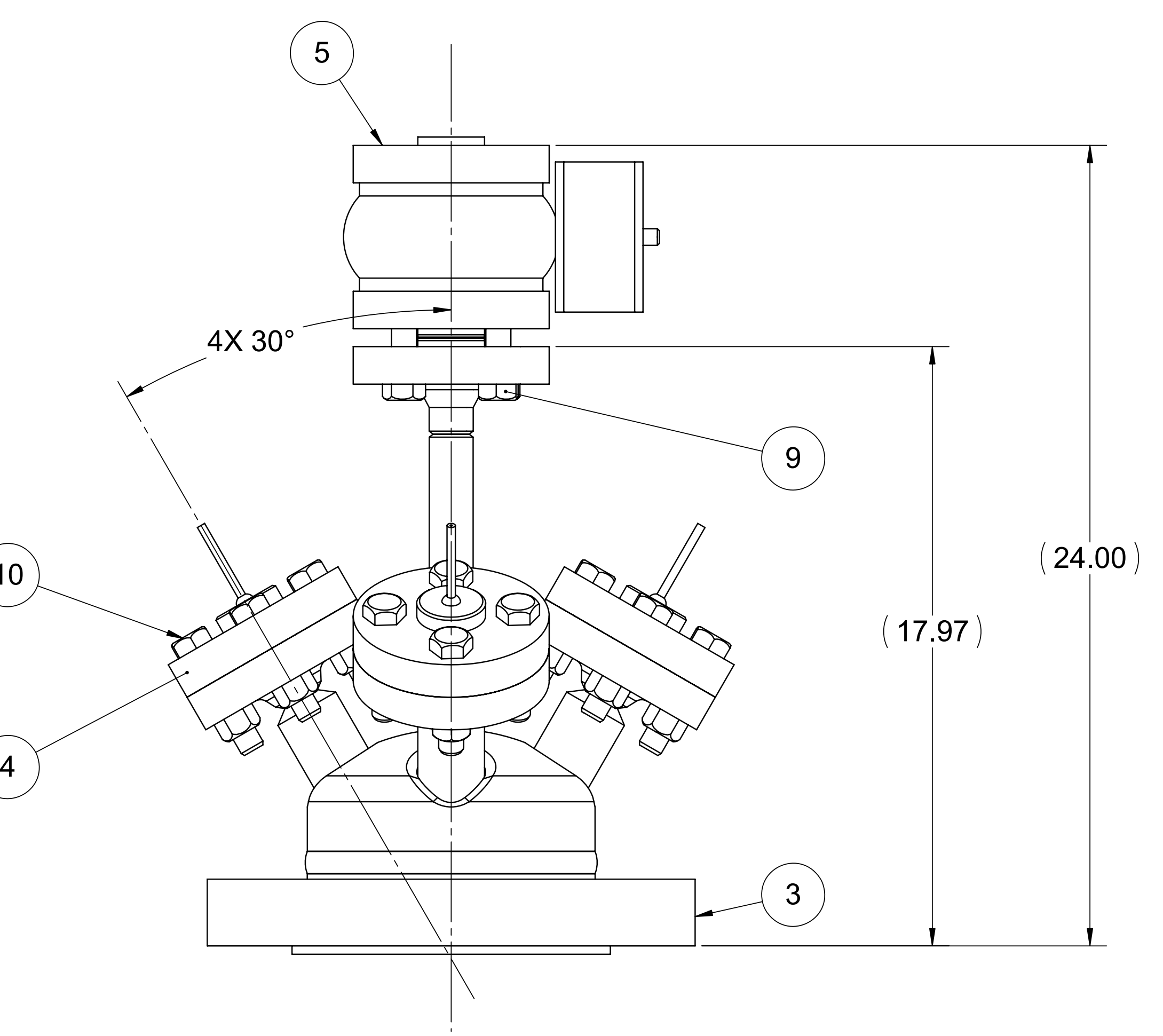

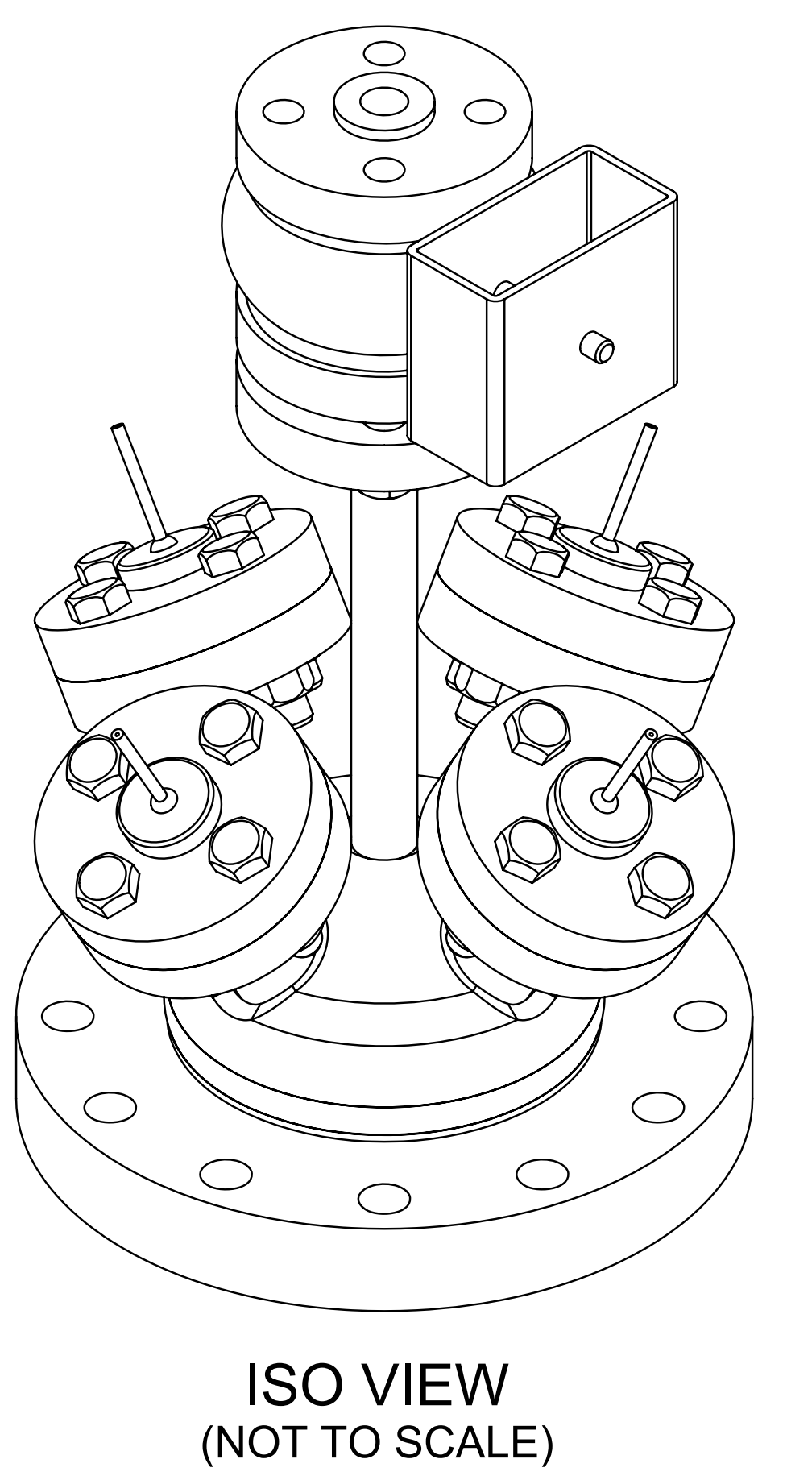

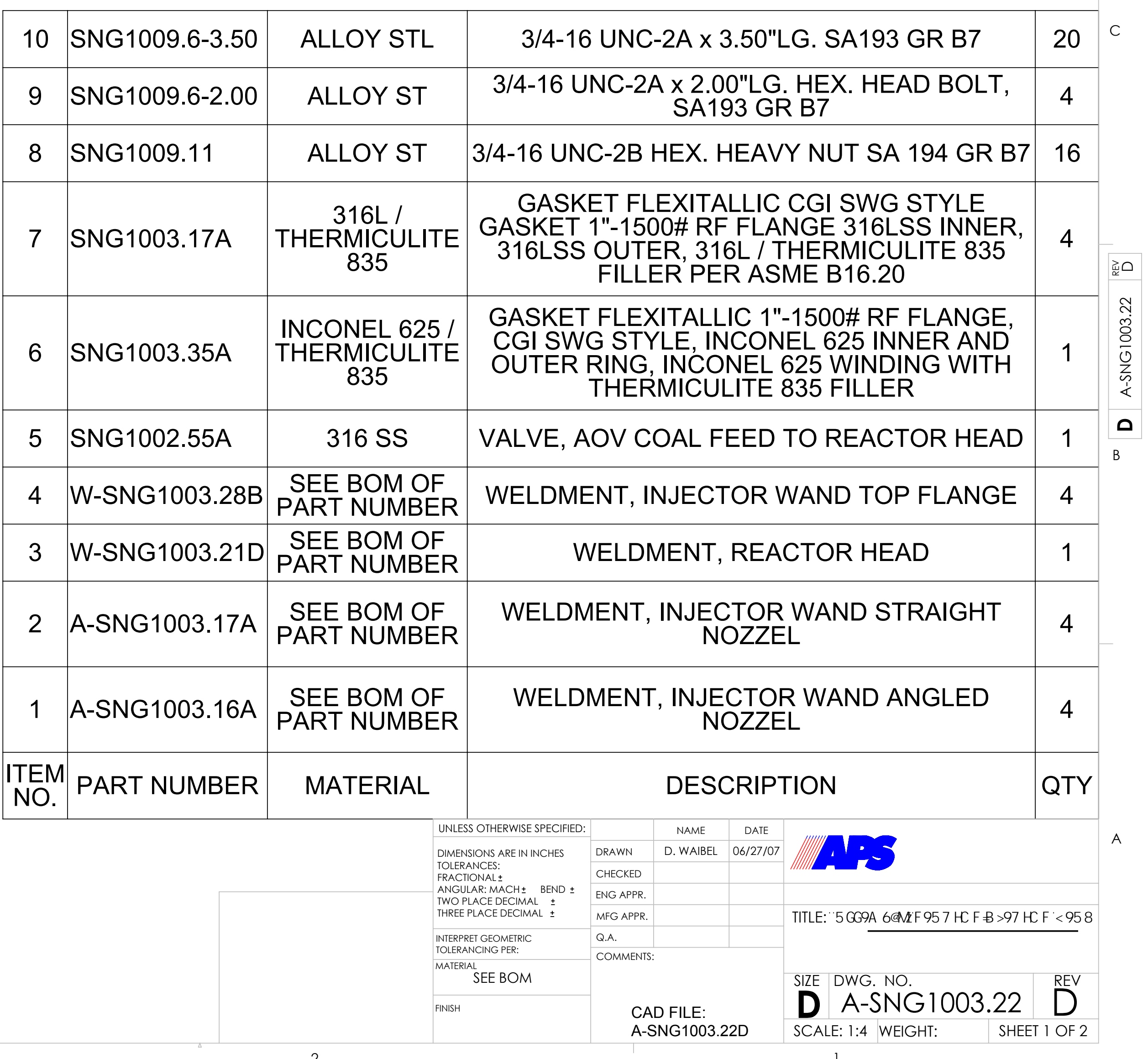




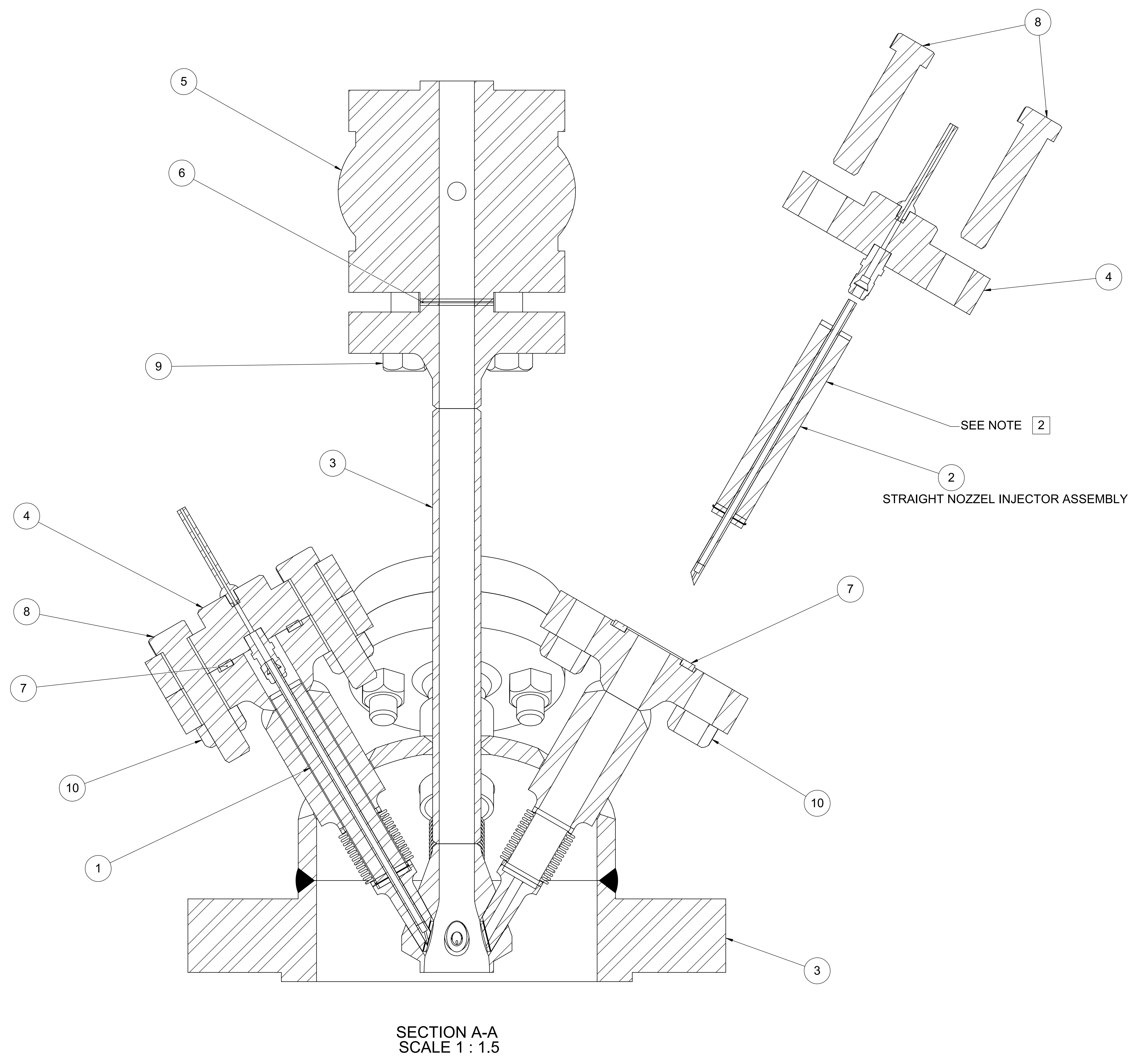



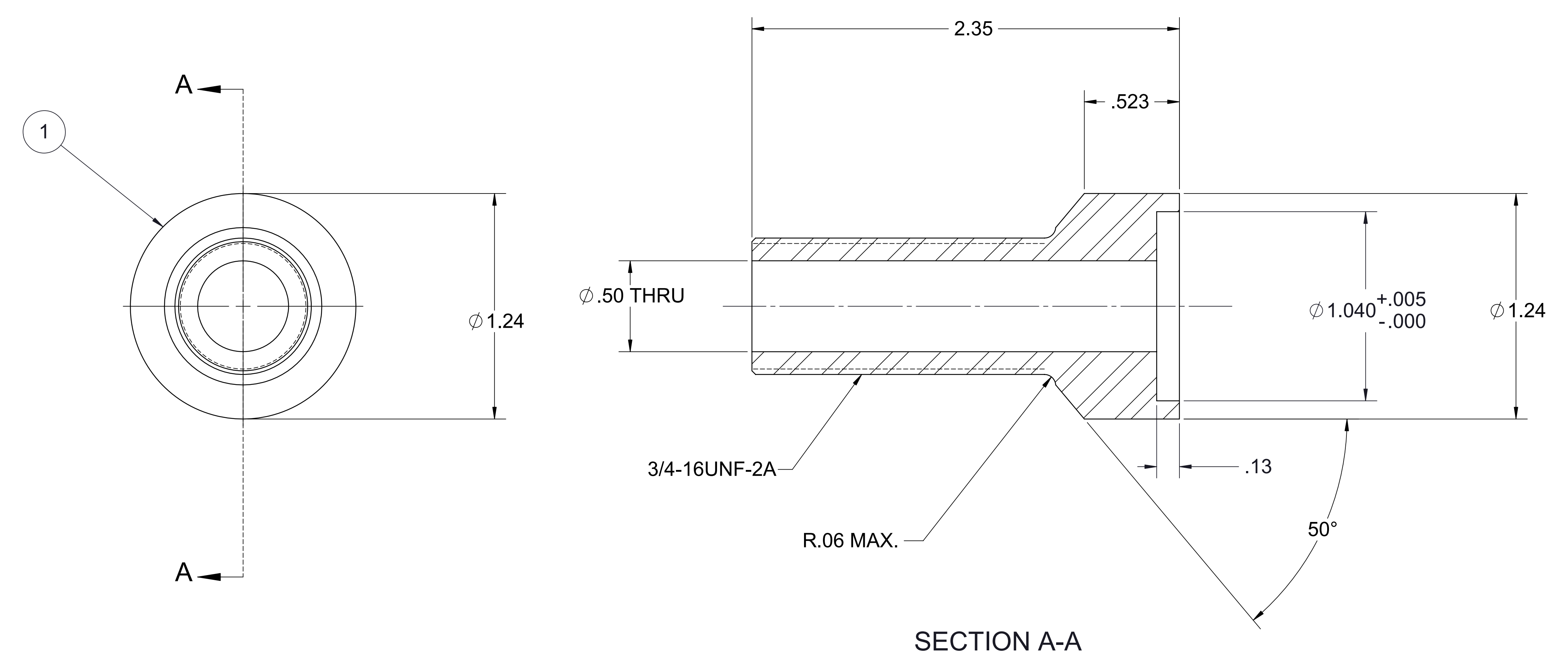

SECTION A-A

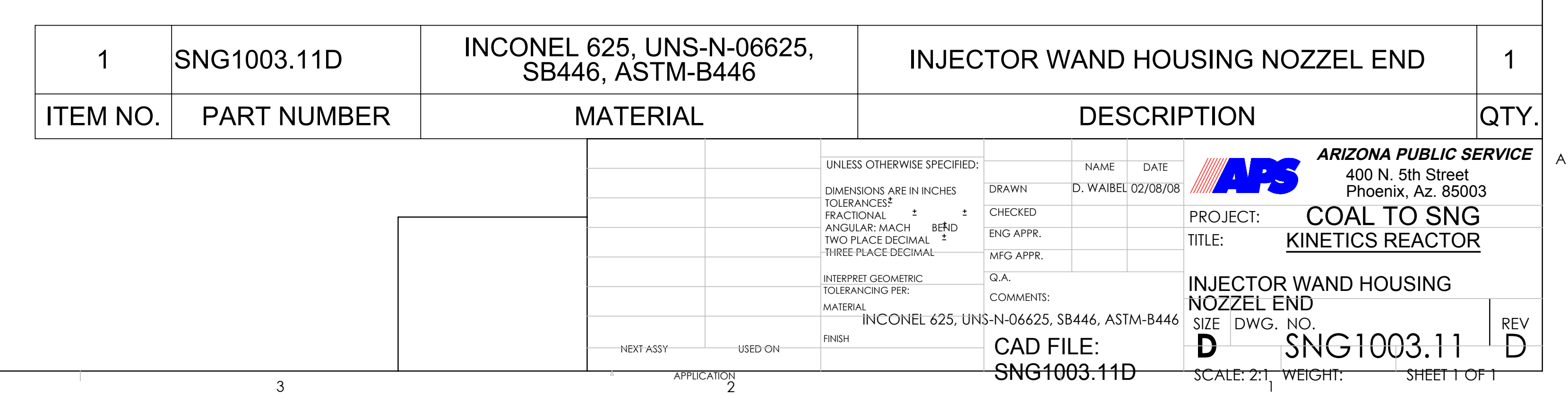




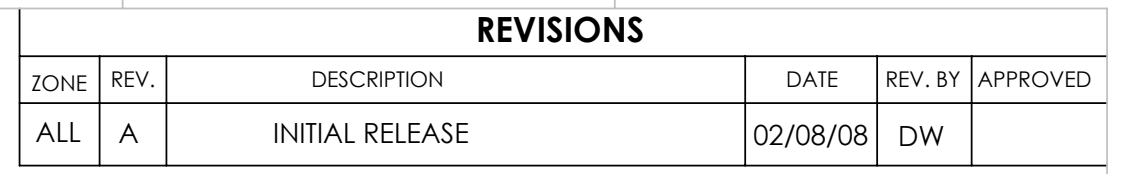
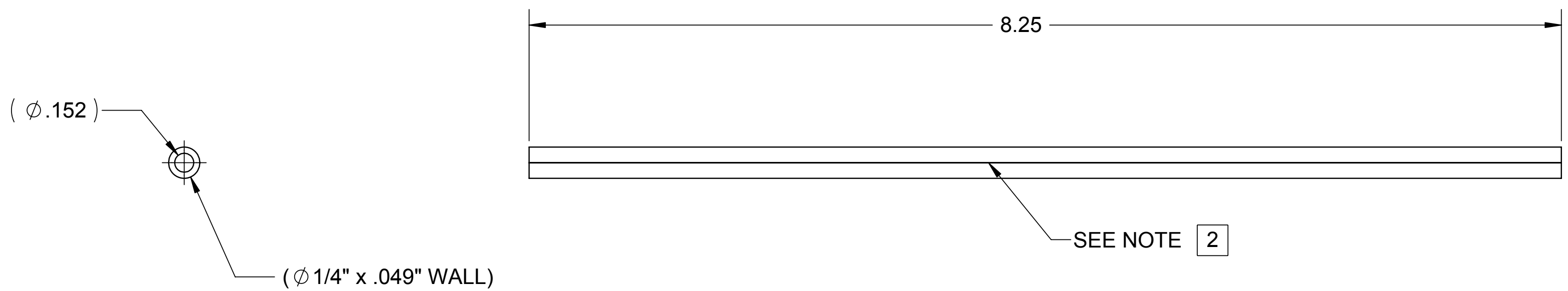

\begin{tabular}{|c|c|c|c|c|}
\hline 1 & SNG1003.12A & INCONEL 625 & $\begin{array}{c}\text { TUBE, 1/4" X.049" WALL, } \\
\text { INJECTOR WAND }\end{array}$ & 1 \\
\hline ITEM NO. & PART NUMBER & MATERIAL & DESCRIPTION & QTY. \\
\hline
\end{tabular}

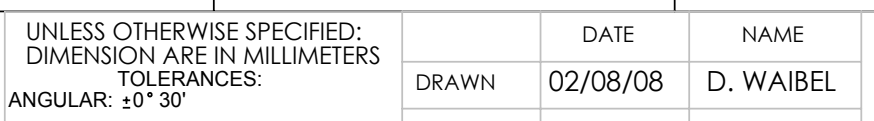

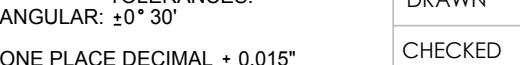

ARIZONA PUBLIC SERVICE

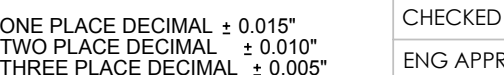

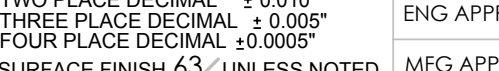
SURFACE FNST BS INERPRET GEOMERTIC TOLERANCING
PRR: MATERIAL:
SEE BOM

SI FINSH: METRIC CAD FILE: 400 N. 5th Street
Phoenix, Az. 85003 COAL TO SNG PROJECT: COAL TO SNG TITLE: KINETICS REACTOR TUBE, 1/4" x .049"WALL, INJECTOR WAND B 

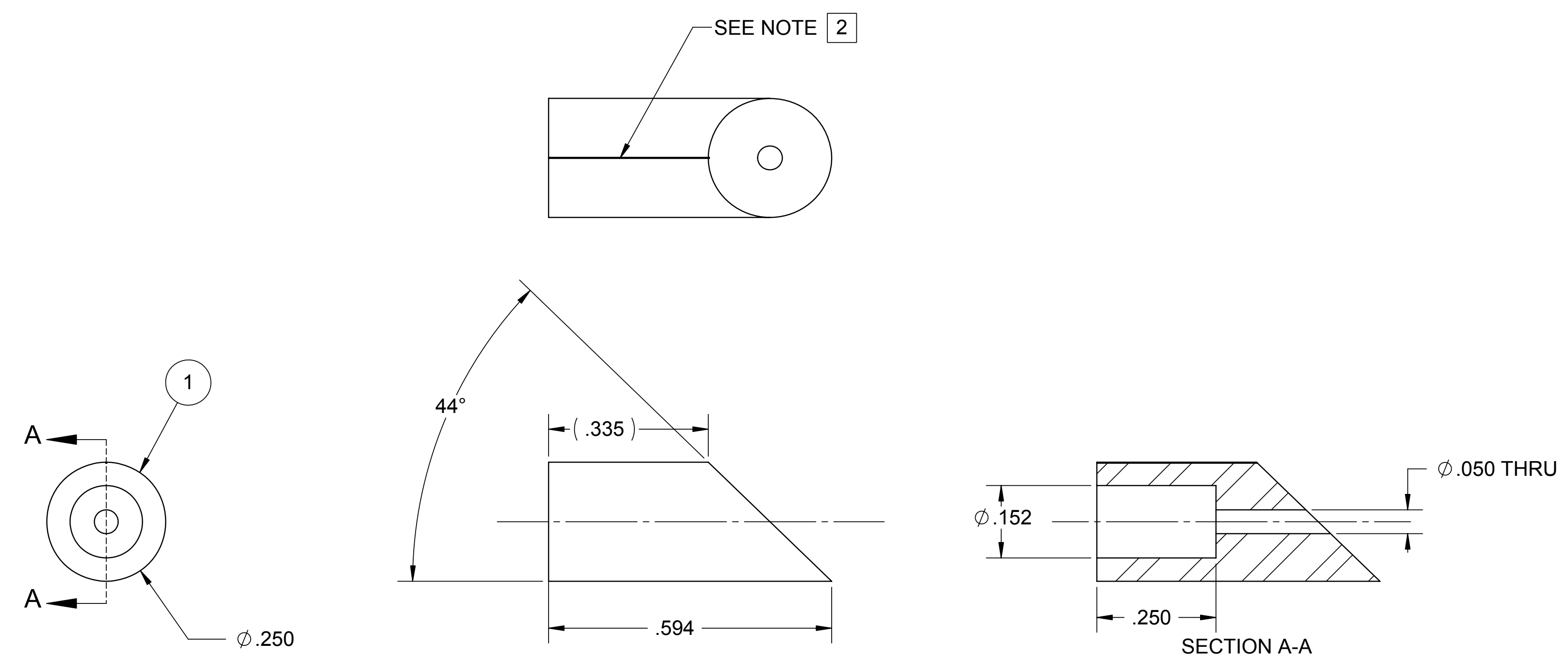

\begin{tabular}{|c|c|c|c|c|}
\hline 1 & SNG1003.13A & INCONEL 625 & NOZZEL, STRAIGHT THRU, & 1 \\
\hline ITJEM NO. & PART NUMBER & MATERIAL & DESCRIPTION & QTY. \\
\hline
\end{tabular}

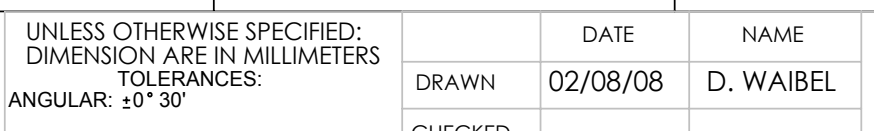

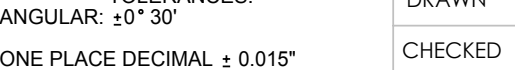

ARIZONA PUBLIC SERVICE

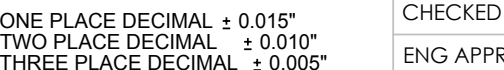
FOUR PACACE DECLMAL $\pm 0.0005^{\prime \prime}$ SURFACE FNISH 63 UN

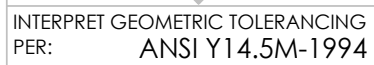
MATERIAL:
SEE BOM

Q.A. FINSH:
SIMLLAR TO: COMMENTS CAD FILE: 400 N. 5th Street COAL TO SNG PROJECT: COALTO SNG TITLE: KINETICS REACTOR INJECTOR NOZZEL, STRAIGHT 

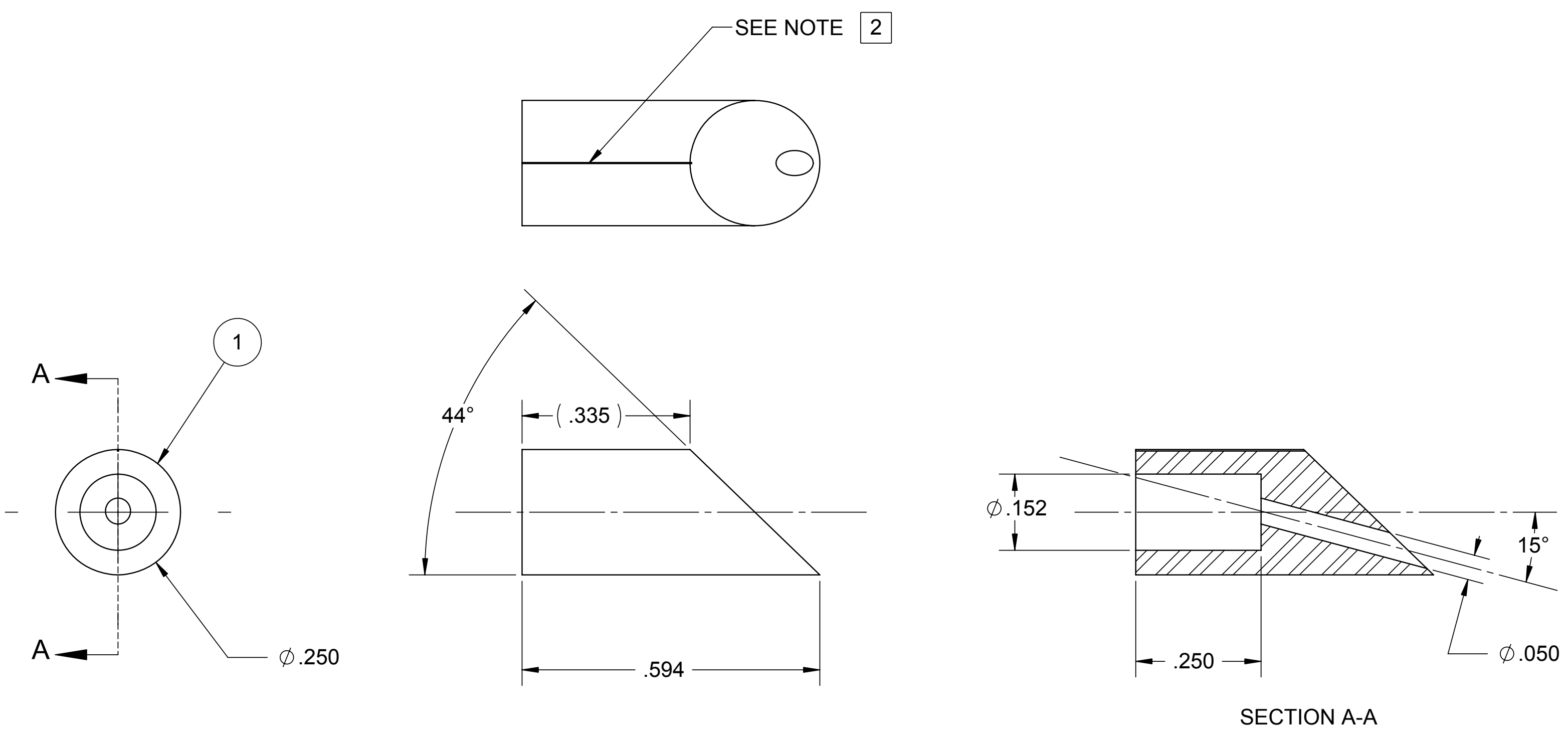

\begin{tabular}{|c|c|c|c|c|c|}
\hline 1 & SNG1003.14A & INCC & NEL $\epsilon$ & & NOZZ \\
\hline ITEM NO. & PART NUMBER & MA & TERIA & & \\
\hline & $\begin{array}{l}\text { UNESES OTh } \\
\text { DIMENSIOR }\end{array}$ & $\begin{array}{l}\text { CIFED: } \\
\text { IMEIRS }\end{array}$ & & DATE & NAME \\
\hline & $\begin{array}{l}\text { ANGULAR: TO } \pm 0 \\
\text { To }\end{array}$ & & DRAWN & 02/08/08 & D. WAIBEL \\
\hline & $\begin{array}{l}\text { ONE PAACED } \\
\text { TWOPACEED }\end{array}$ & & CHECKED & & \\
\hline & $\begin{array}{l}\text { THRE PLAAE } \\
\text { FOUR PLACE }\end{array}$ & & ENG APPR. & & \\
\hline & SURFACE FINI & SS NOTED & MFG APPR & & \\
\hline & $\begin{array}{l}\text { NIERPREE GEO } \\
\text { PERR }\end{array}$ & $\begin{array}{l}\text { ACLNG } \\
-1994\end{array}$ & Q.A. & & \\
\hline & $\begin{array}{l}\text { MATERRAL: } \\
\text { SEE }\end{array}$ & & Sl & 0 & $5 \oint_{\text {THROA ANGEE }}$ \\
\hline & FINSH: & & COMN & & \\
\hline & SIMLILAR TO: & & CAD FIL & & G1003.14A \\
\hline
\end{tabular}

$$
\begin{aligned}
& \text { DESCRIPTION } \\
& \text { DEL, 15Deg. INJEC }
\end{aligned}
$$

\begin{tabular}{l|l} 
QTY. \\
\hline
\end{tabular}

IIIIIIIST ARIZONA PUBLIC SERVICE

PROJECT: COAL TO SNG TITE: KINETICS REACTOR INJECTOR NOZZEL, 15Deg.

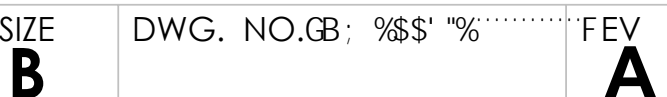

SCALE: 4:1 WEIGHT: SHEET I OF I 

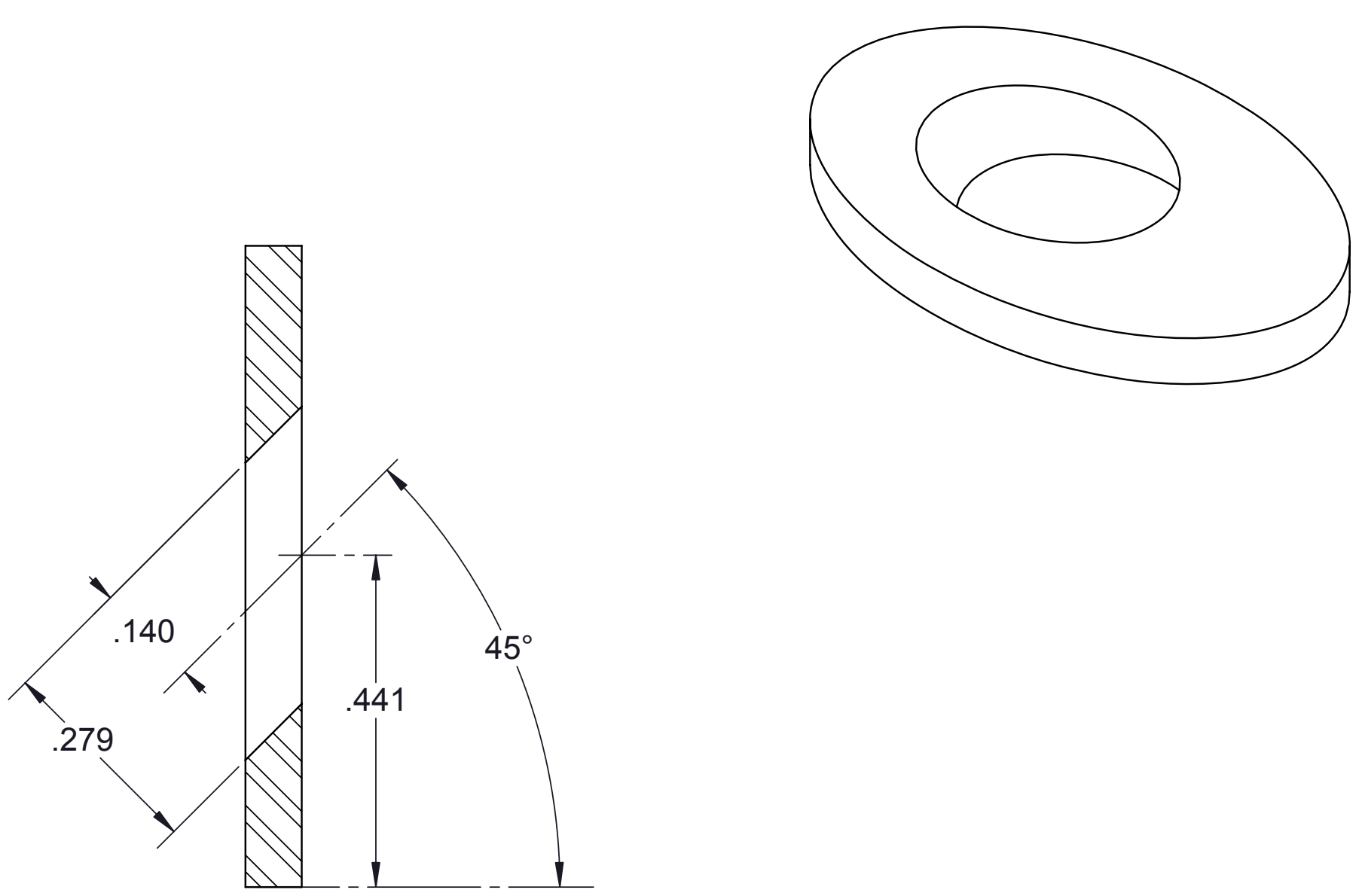

SECTION A-A

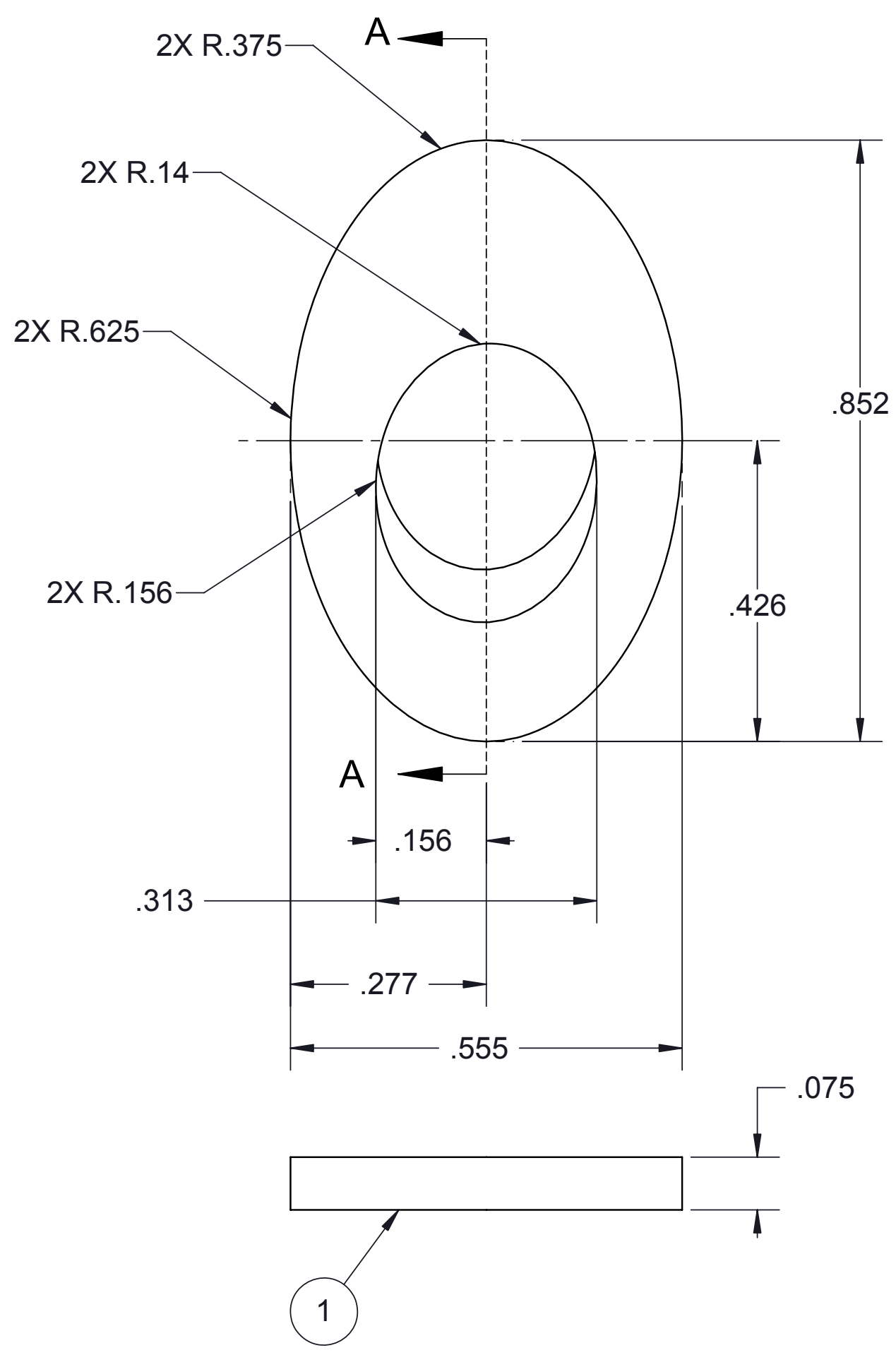

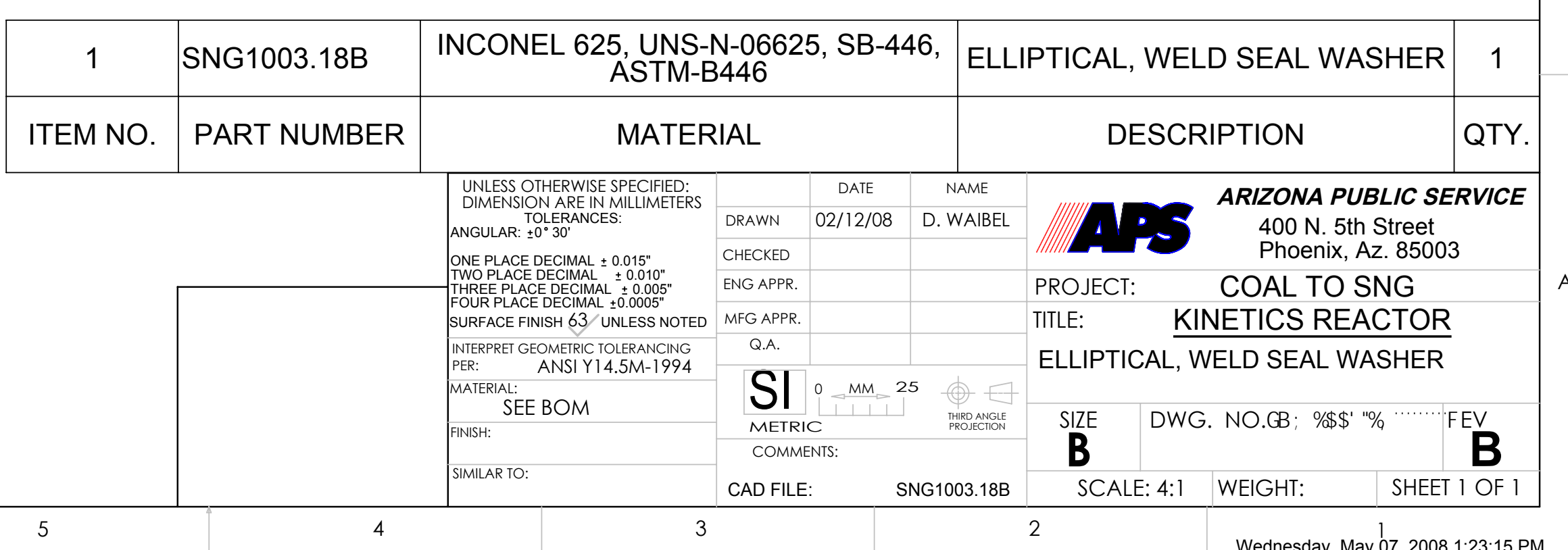




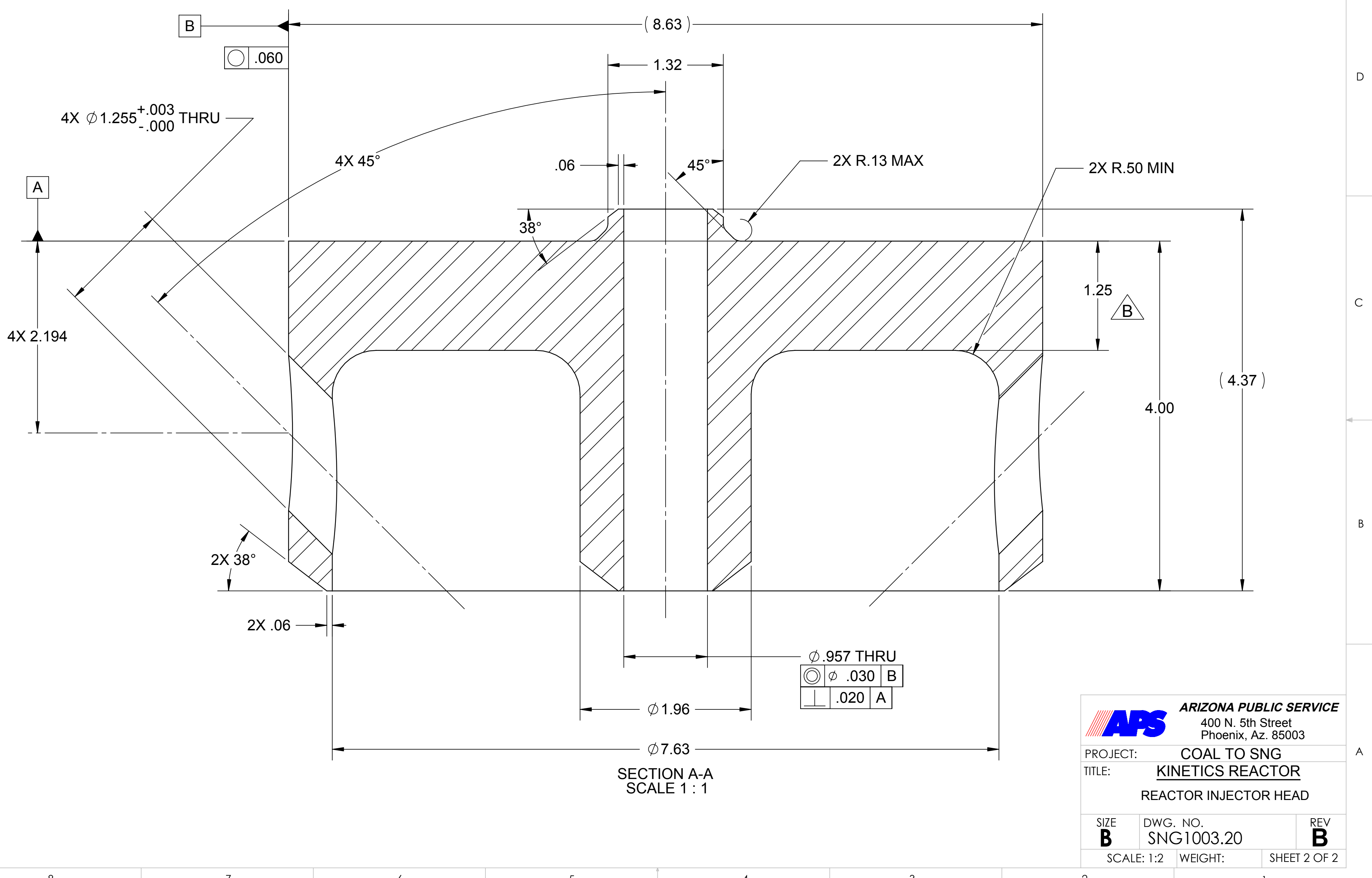



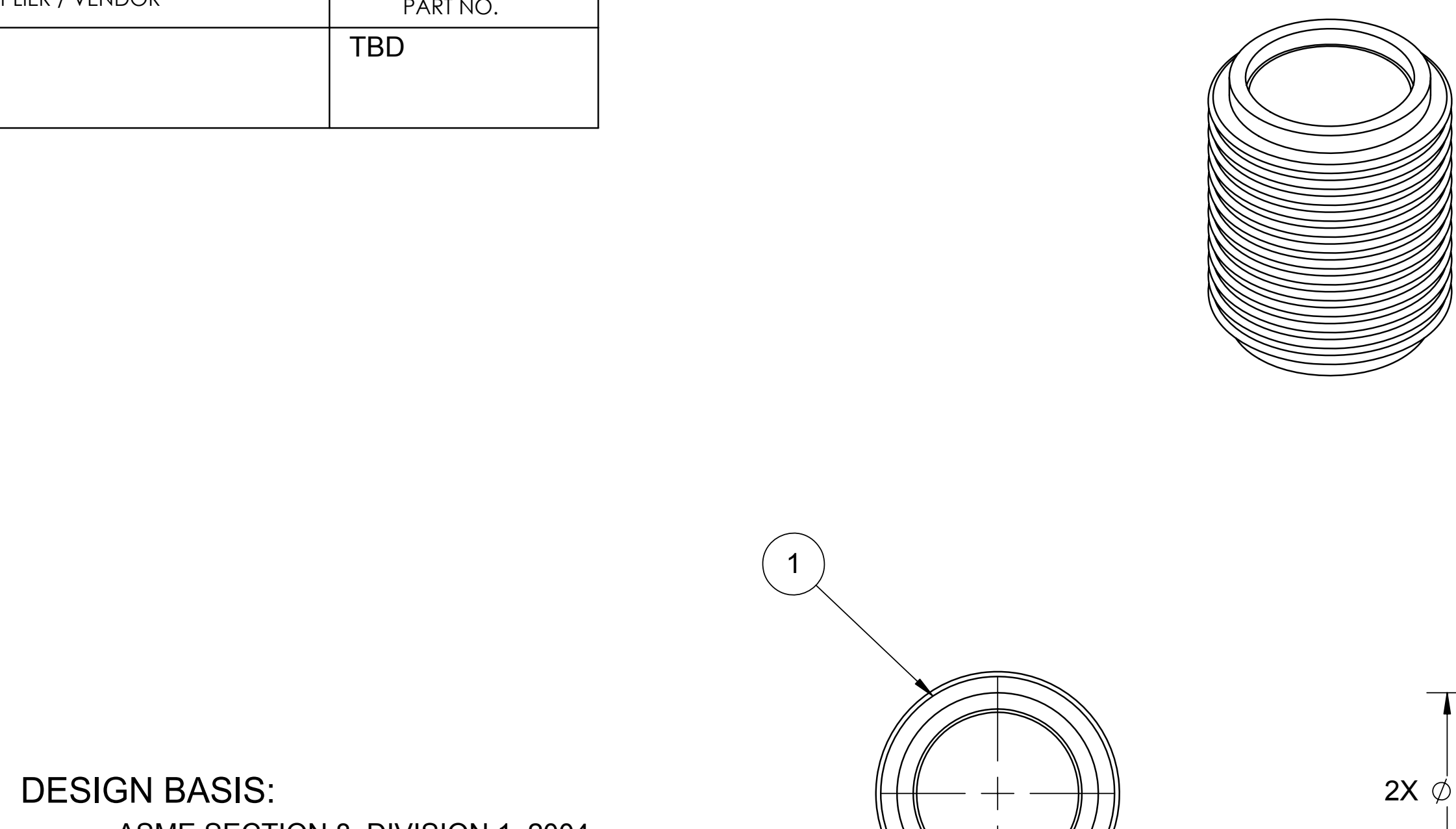

ASME SECTION 8, DIVISION 1, 2004

в GENERAL NOTES:

1 BELLOWS; BELLOWS MATERIAL,

2

3
COLLAR MATERIAL,

DESIGN TEMP (F)

AXIAL COMPRESSION (IN)

AXIAL EXTENSION (IN)

OVERALL (") IS AT ROOM TEMPERATURE

WITH NO COMPRESSION OR TENSIONS.

INSTALL BELLOWS

DESIGN BASIS: ASME SECTION 8, DIVISION 1, 2004 STRESS BASIS: ASME SECTION 2, DIVISION D, 2005

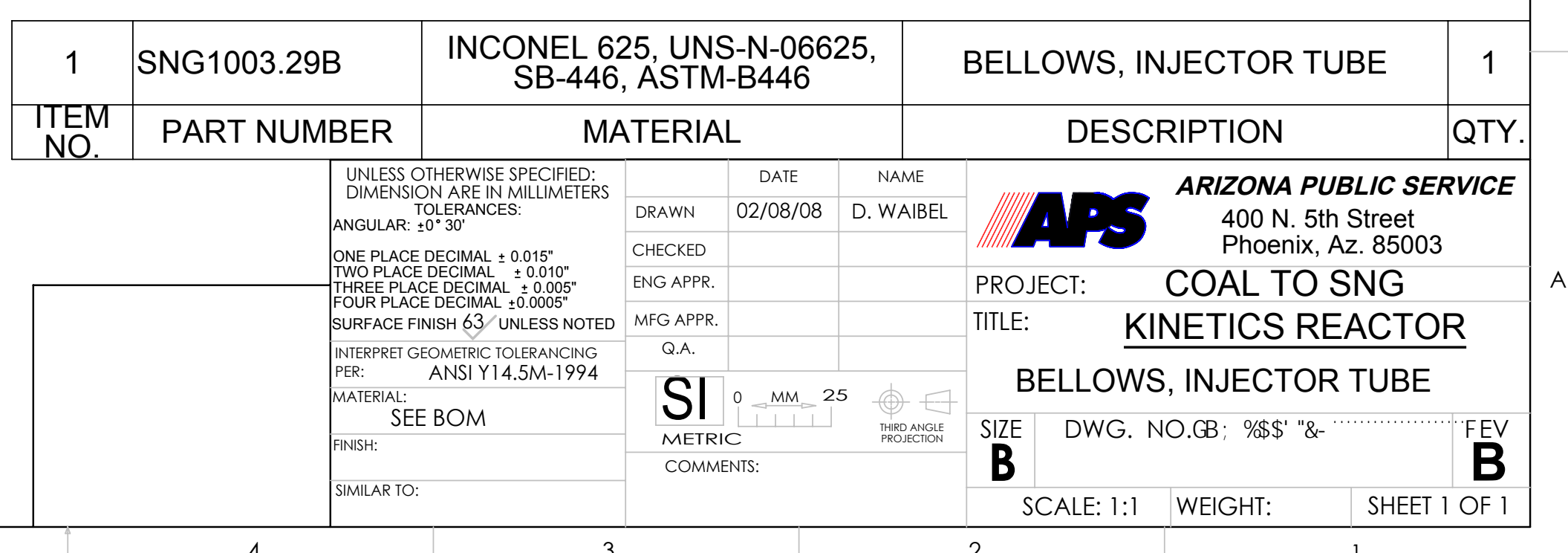




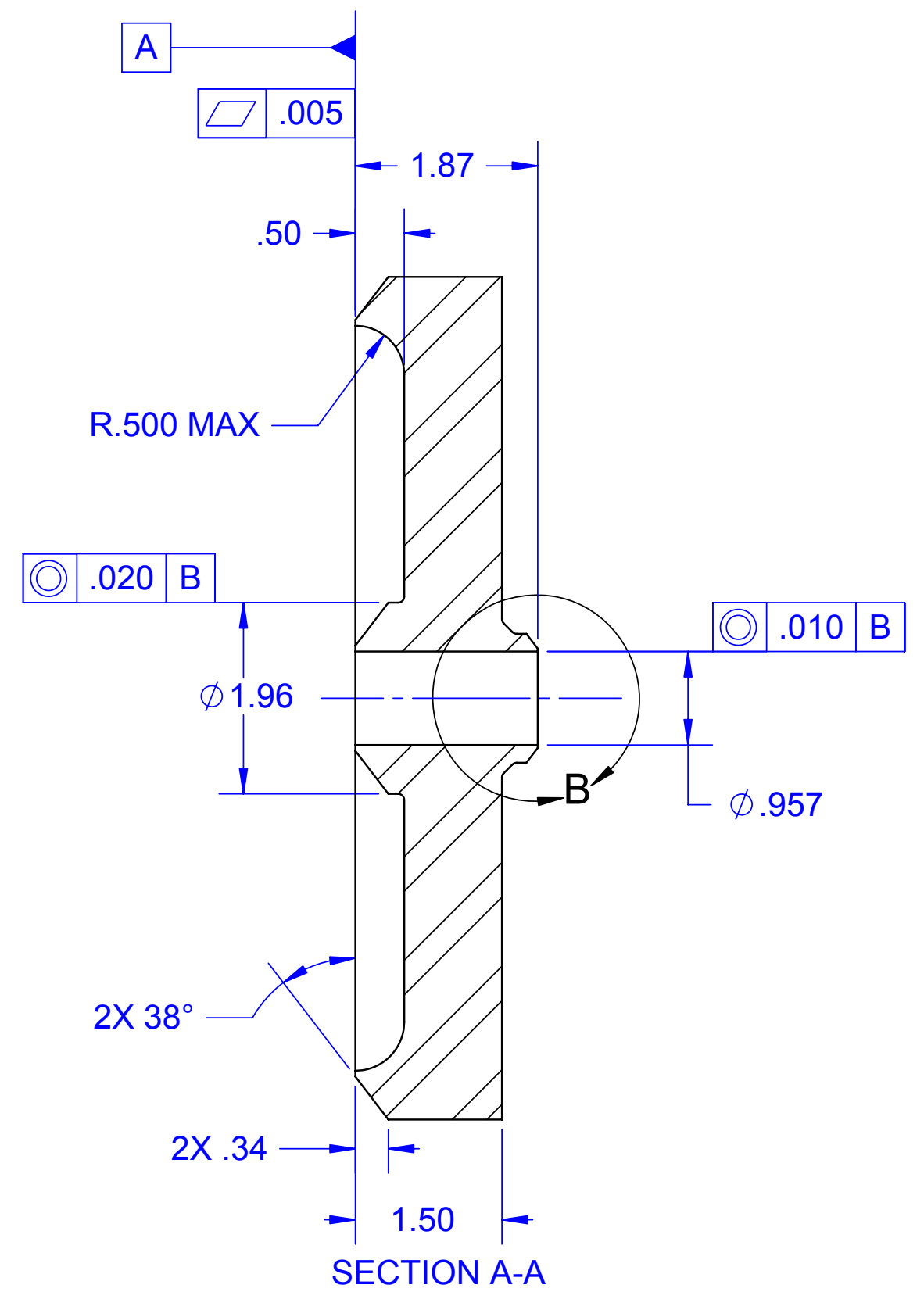

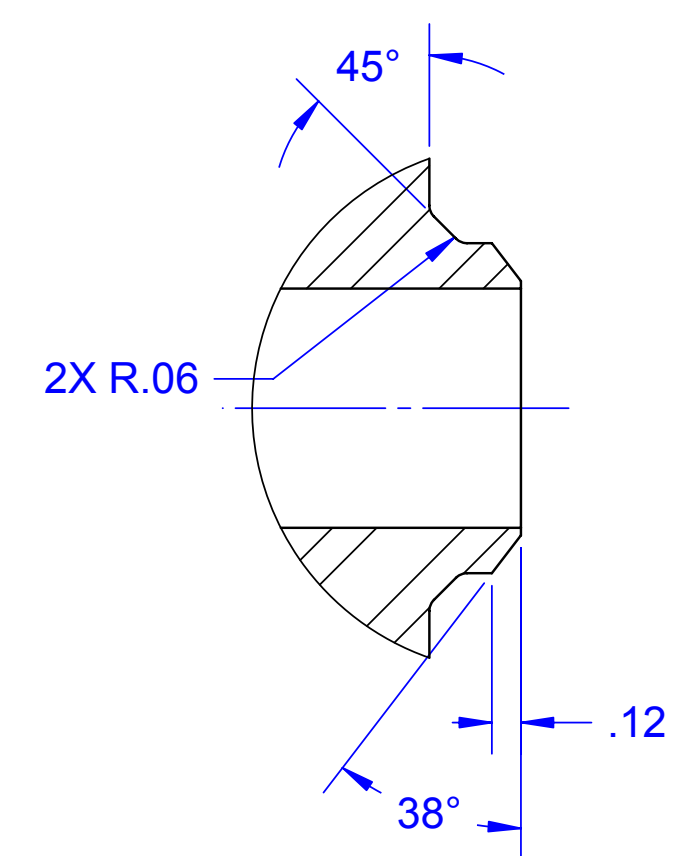

DETAIL B

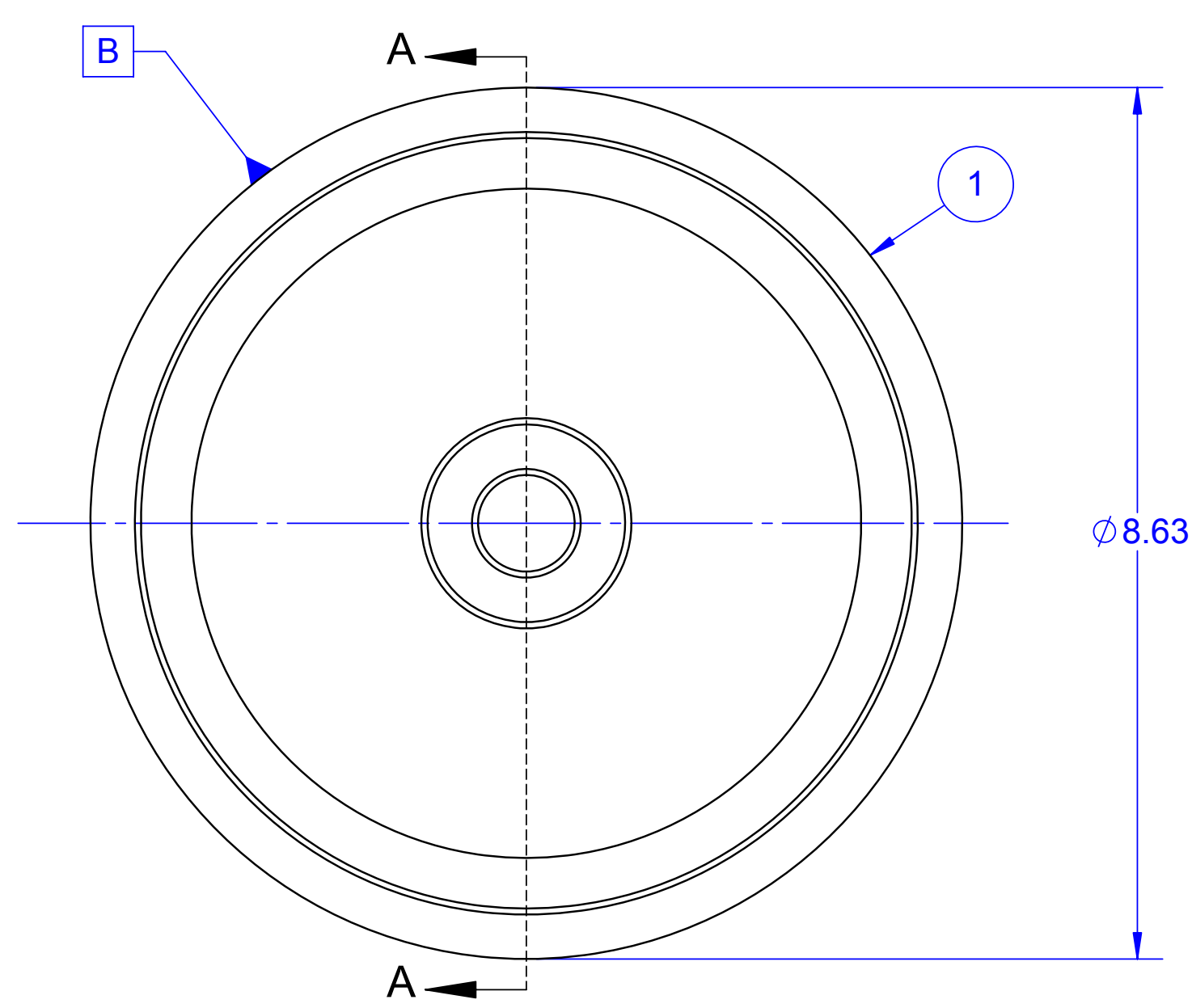

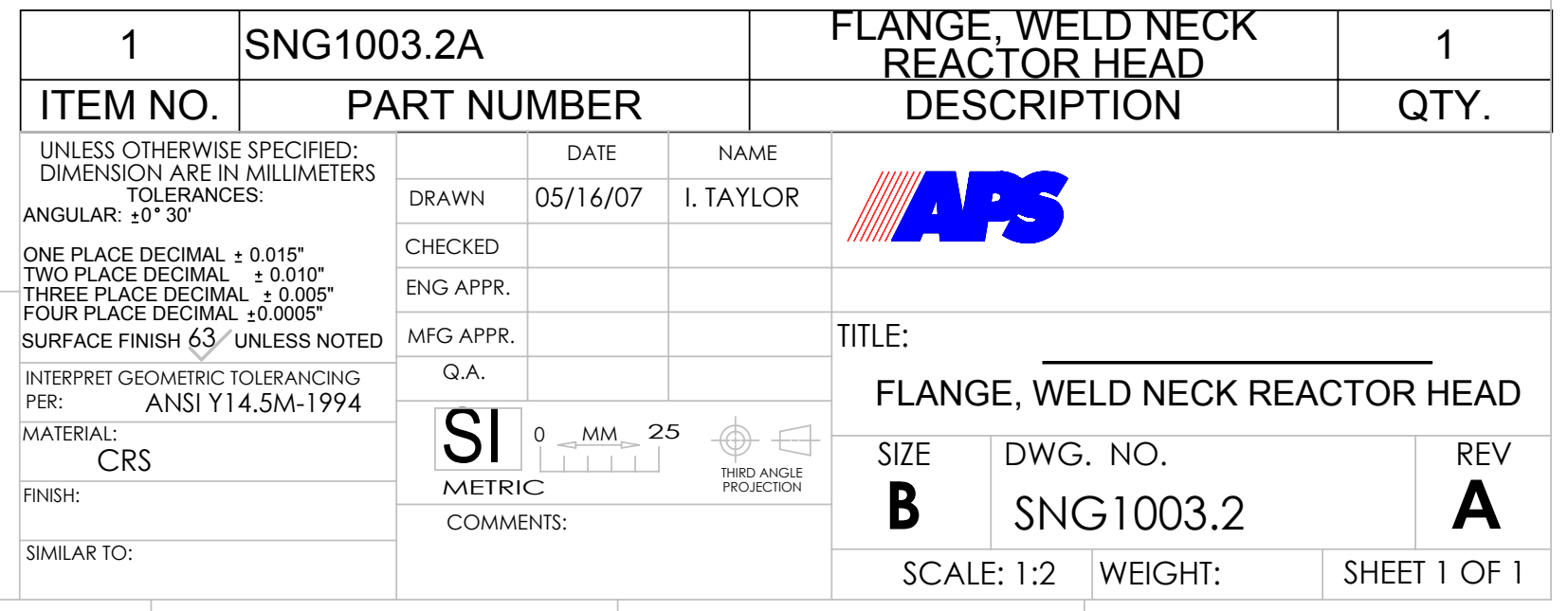




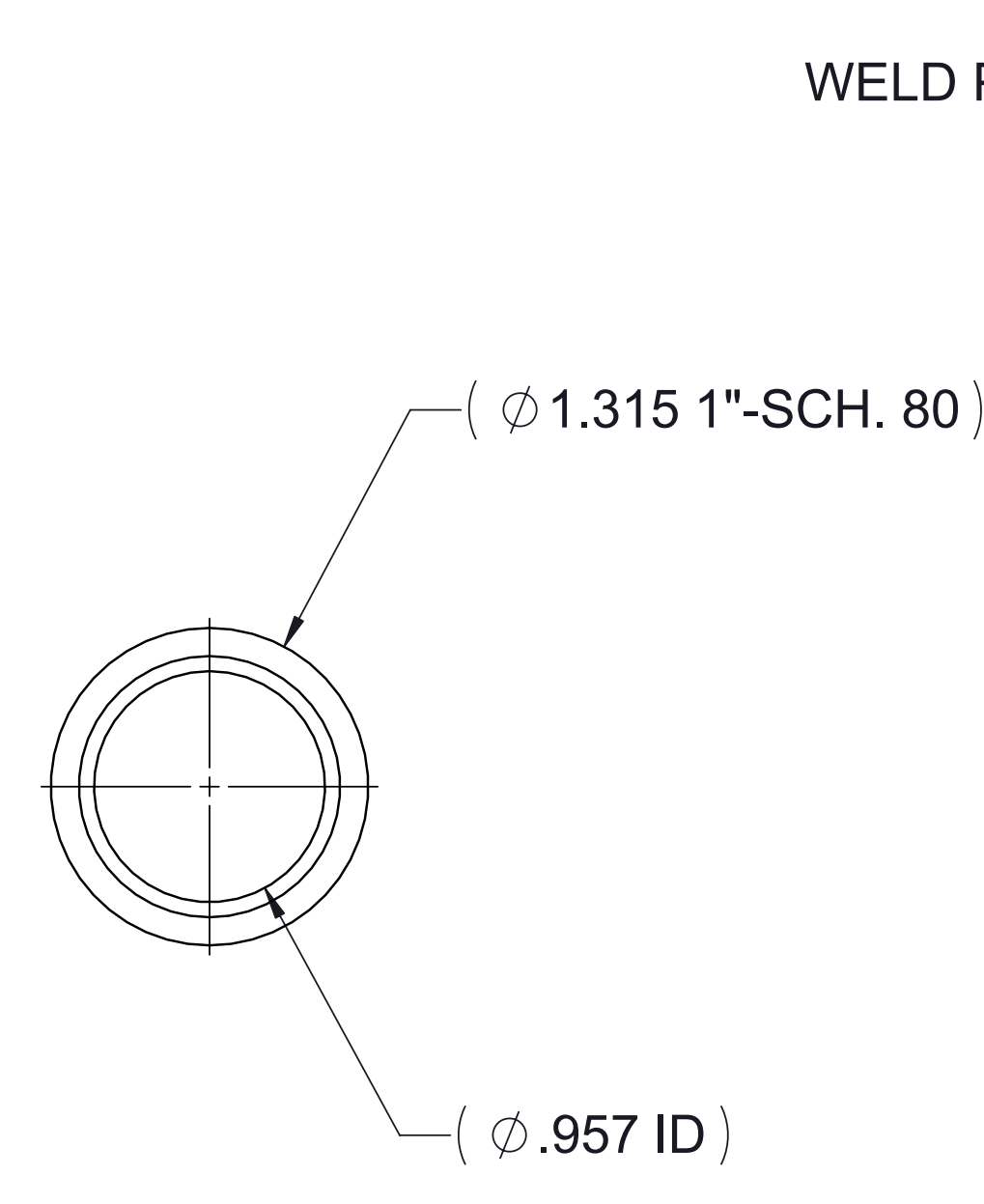

WELD PREP BOTH ENDS

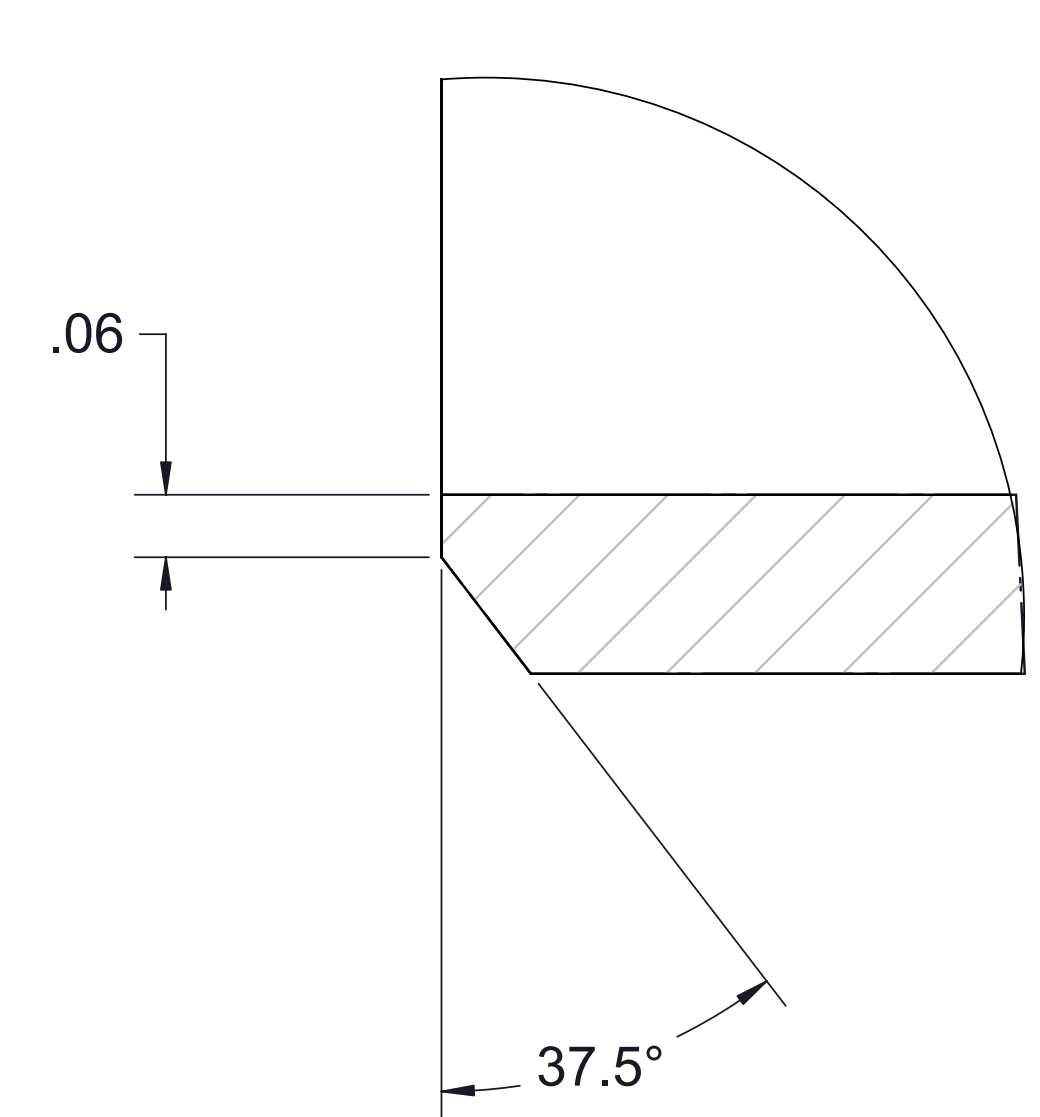

DETALAA

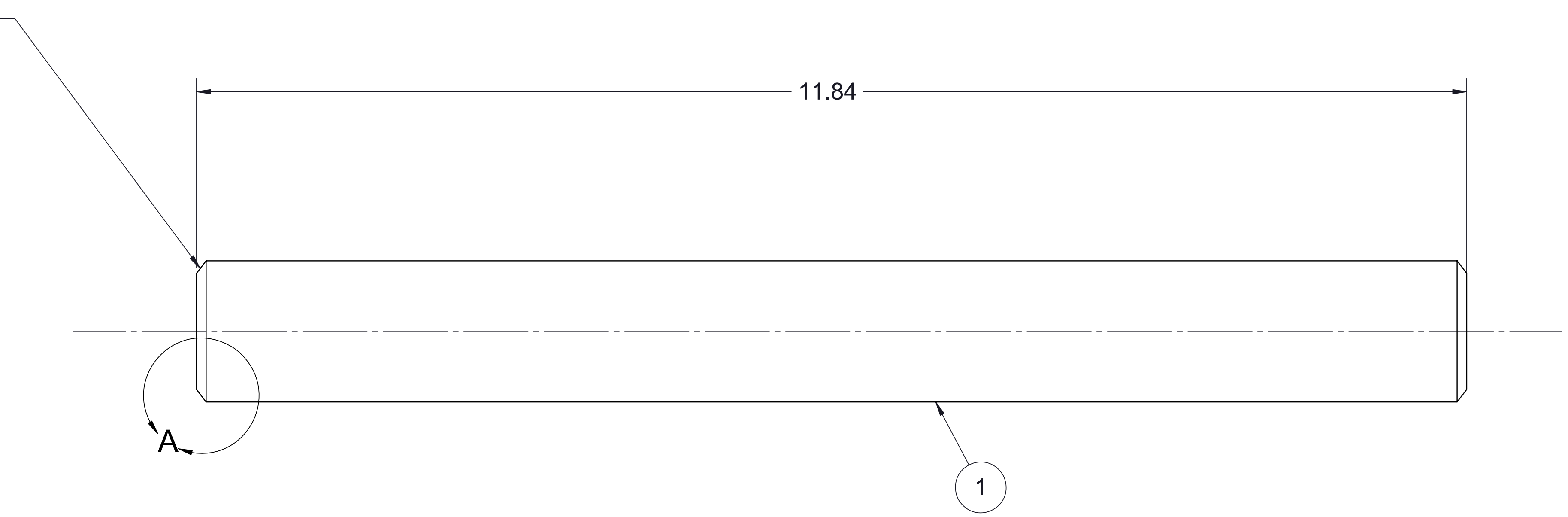



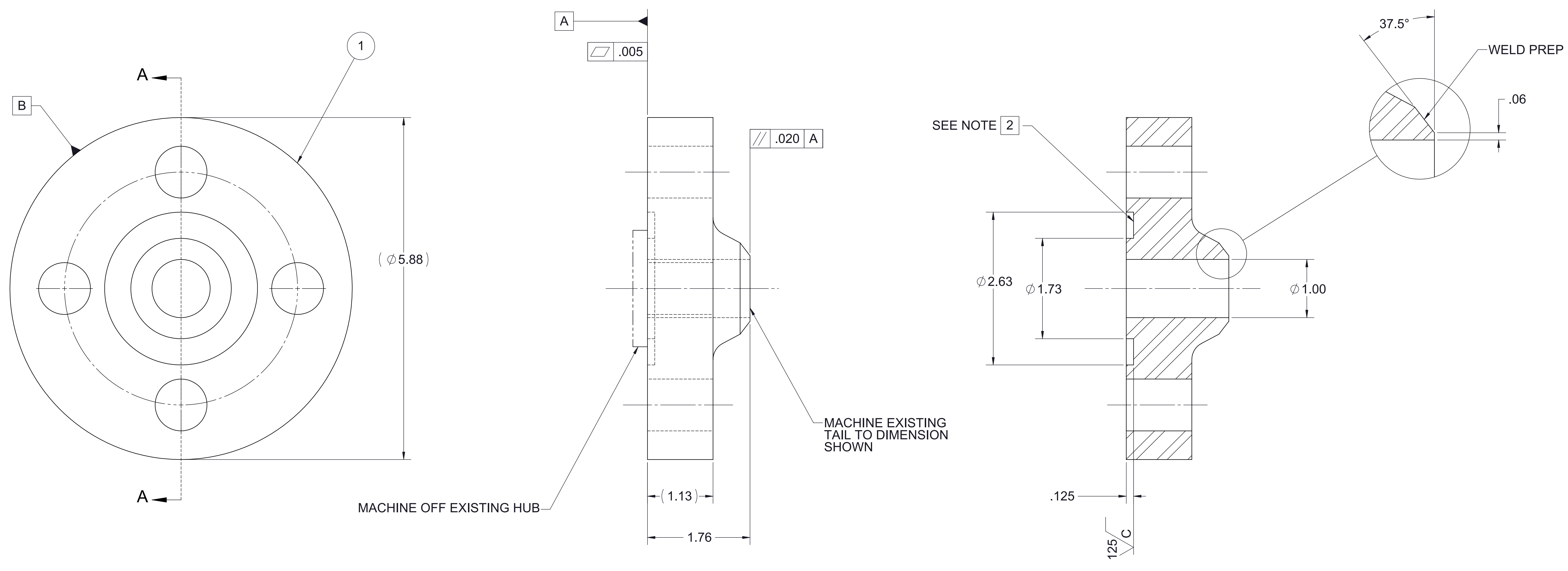

SECTION A-A

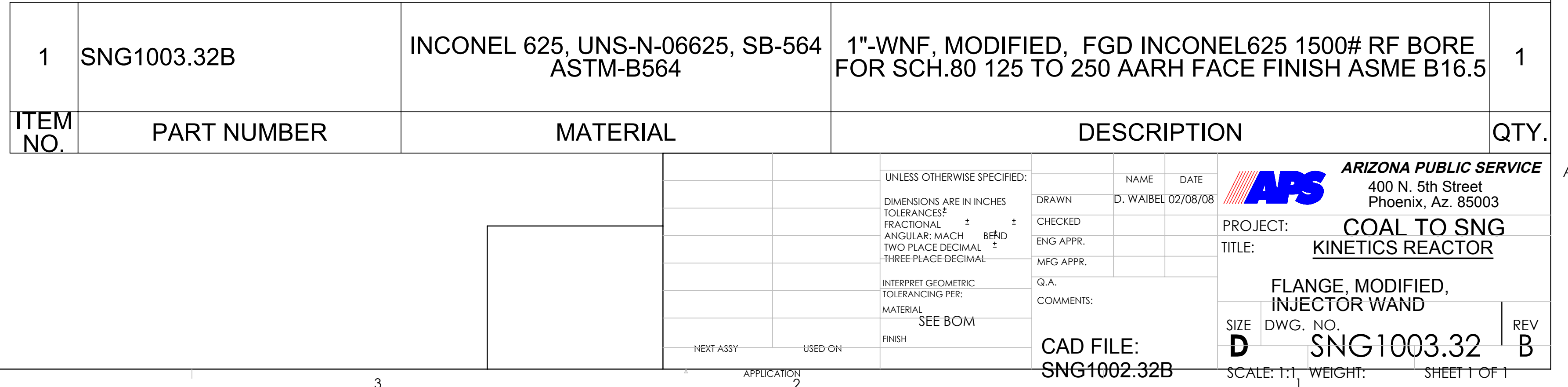



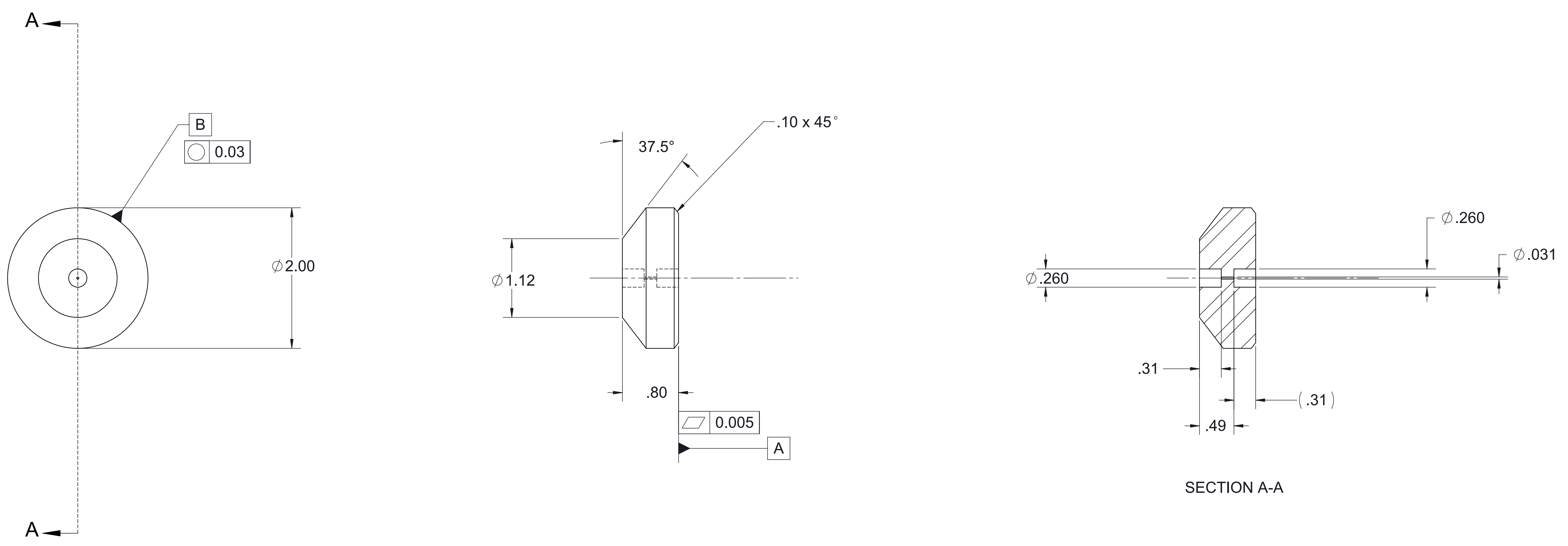

SECTION A-A

$A-$

\begin{tabular}{|c|c|c|c|c|c|c|c|c|}
\hline \multicolumn{2}{|c|}{\begin{tabular}{l|l}
1 & SNG1003.33B
\end{tabular}} & \multicolumn{2}{|c|}{$\begin{array}{l}\text { INCONEL 625, UNS-N-O6625, } \\
\text { SB-446, ASTM-B446 }\end{array}$} & \multicolumn{4}{|c|}{ 1"-SLIP-ON, 1500\#, INCONEL 625, MODIFIED } & 1 \\
\hline \multirow{5}{*}{$\begin{array}{l}\text { TTEM } \\
\text { NO. }\end{array}$} & \multirow{5}{*}{ PART NUMBER } & \multicolumn{2}{|l|}{ MATERIAL } & \multicolumn{4}{|c|}{ DESCRIPTION } & QTY. \\
\hline & & & \multicolumn{2}{|c|}{ 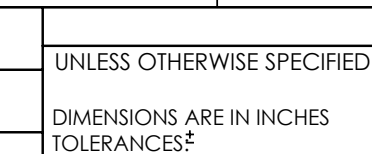 } & & 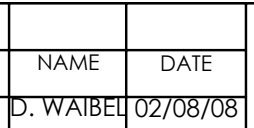 & \multicolumn{2}{|c|}{ IIT) } \\
\hline & & & 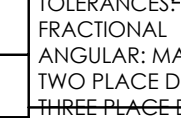 & & 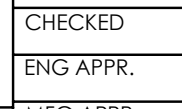 & 4 & 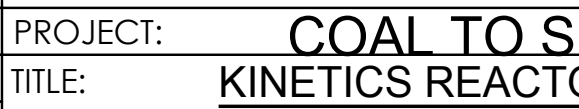 & \\
\hline & & & 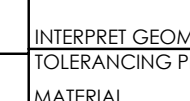 & ec & 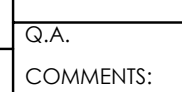 & & FLANGE, TOP INJECTION & EAD \\
\hline & & & & & & $=$ & 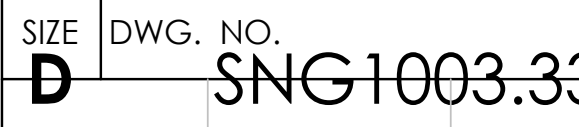 & $\mathrm{BEV}_{\mathrm{B}}^{\mathrm{REV}}$ \\
\hline
\end{tabular}


1 REMOVE AND DEBURR ALL SHARP EDGES.

\begin{tabular}{|c|c|c|c|c|c|}
\hline \multicolumn{4}{|c|}{ REVISIONS } \\
\hline ZONE & REV. & DESCRPTION & DATE & REV.BY APPRO \\
\hline ALL & A & INITIAL RELEASE & $06 / 11 / 07$ & DW \\
\hline ALL & B & DIM; 14.63 WAS 12.65, B.C. 12.570 & $07 / 23 / 07$ & DW \\
\hline
\end{tabular}
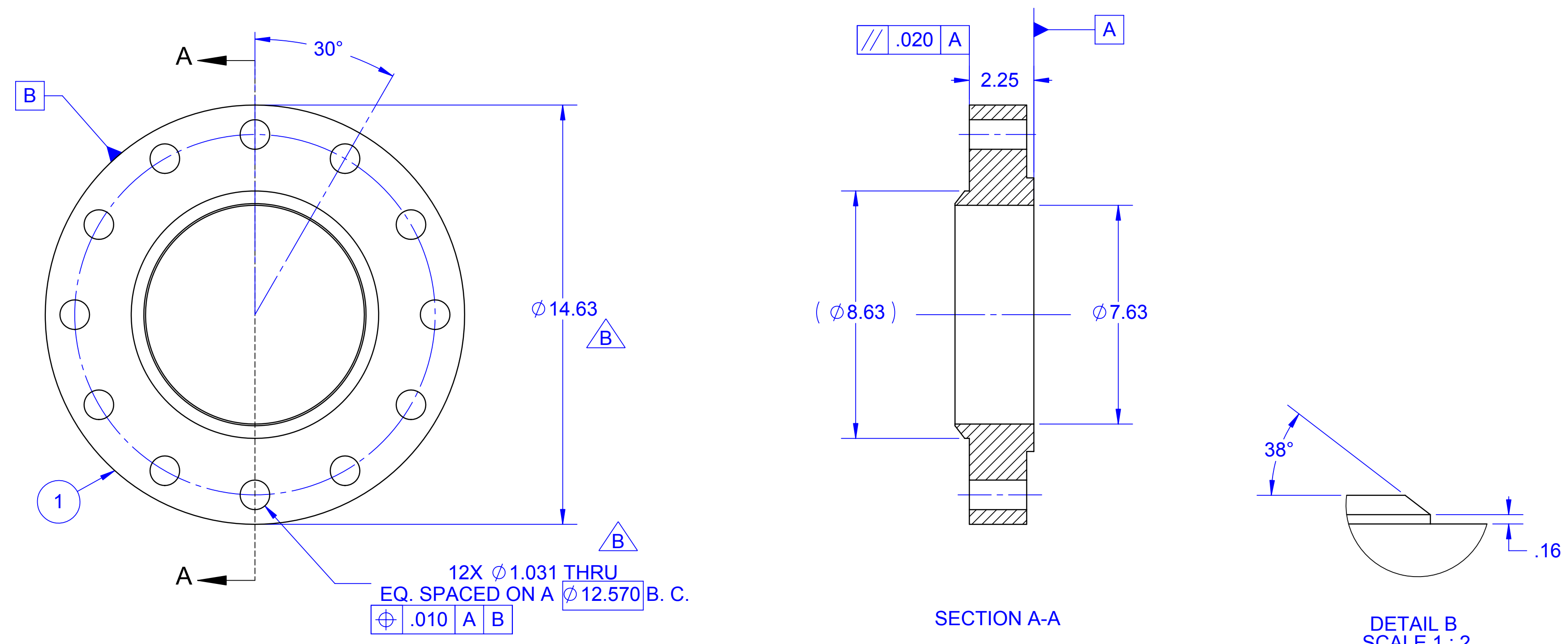

SECTION A-A

$$
\text { DETAIL B }
$$

SCALE $1: 2$

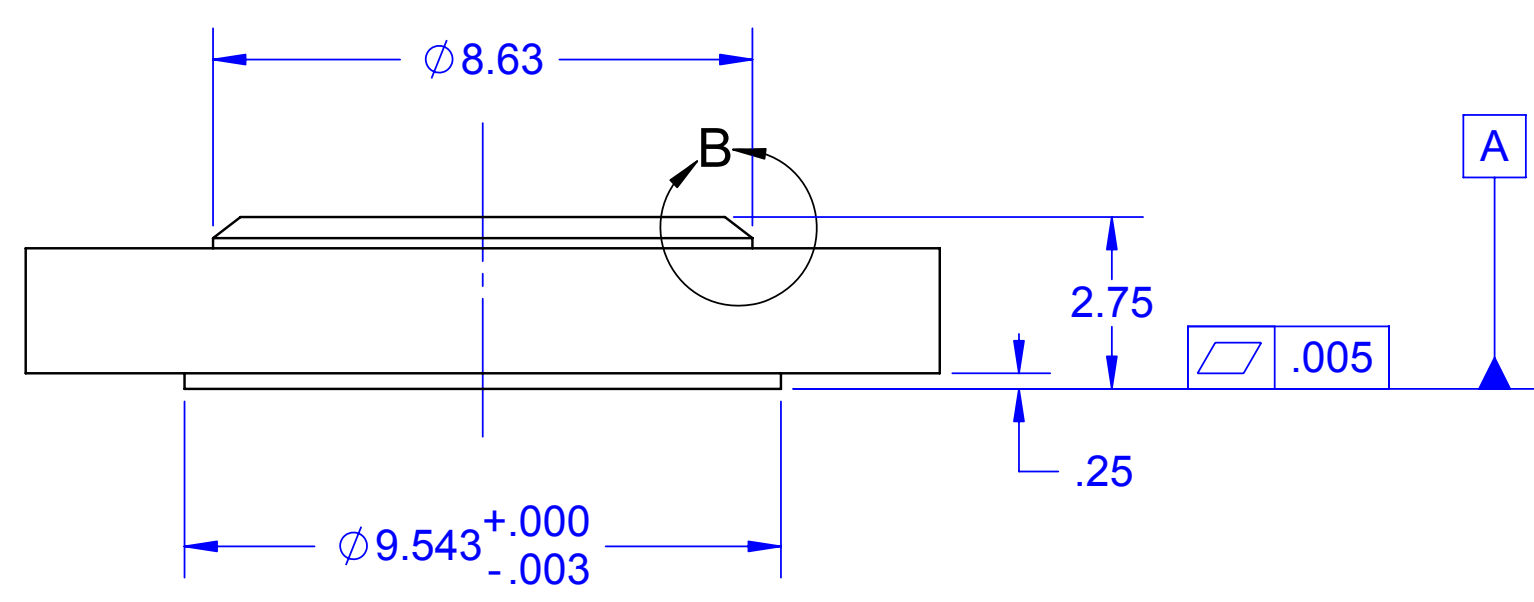

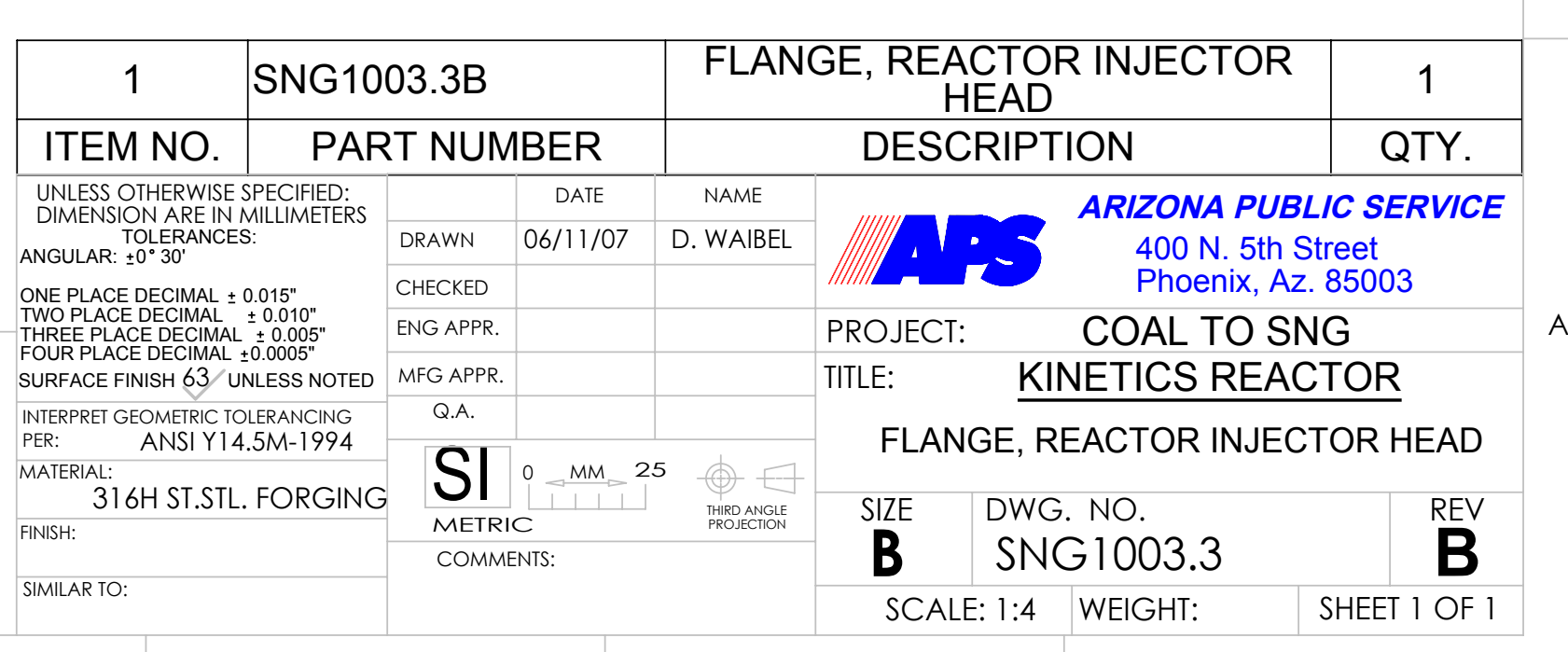


1 USE 2" DIAMETER, STEEL BALL, (TOOLING BALL, WITH NO SHOULDER) PLACE IN CENTER
HOLE AS SHOWN, TO LOCATE AND INSPECT FEATURE TO DIMENSION SHOWN.

2 MATERIAL: 316 ST STL, CARBON CONTENT MUST BE EQUAL TO, OR ABOVE. $04 \%$

SEE NOTES 2 2"-TOOLING BALL-

1
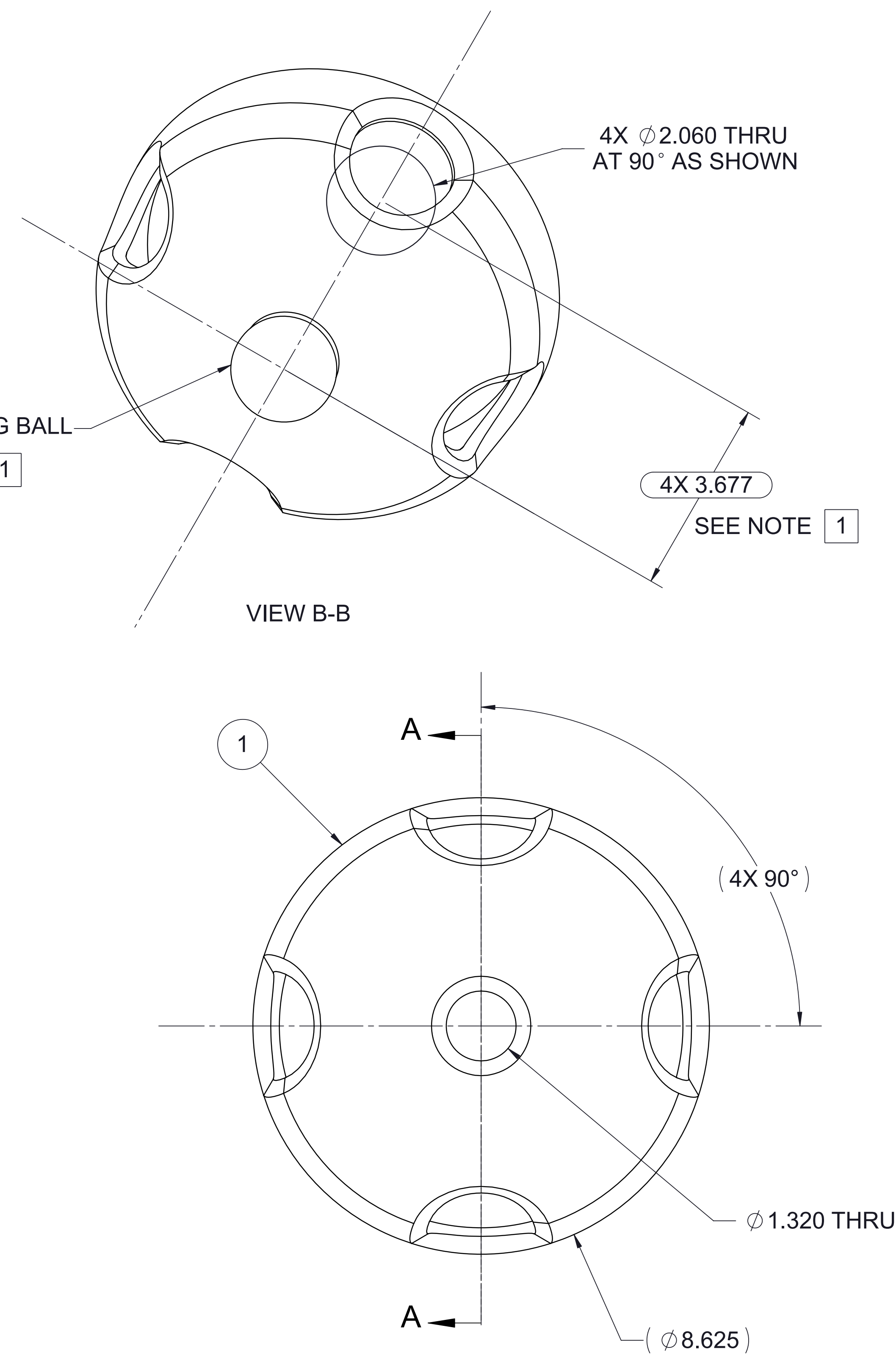

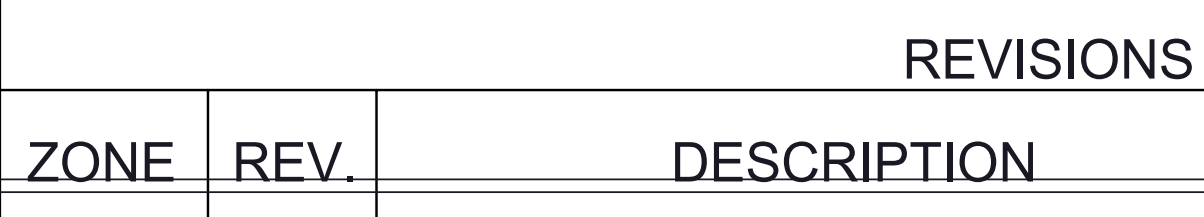

\begin{tabular}{c|c|c|}
\hline ZONE & REV & DESCRIPTION \\
\hline ALL & A & INITIAL RELEASE \\
\hline
\end{tabular}

ALL $\quad$ B CHANGED 316 TO $316 \mathrm{H}$, W/SPECS

DATE REV. BY APPROVED

$02 / 106 / 08$ DW

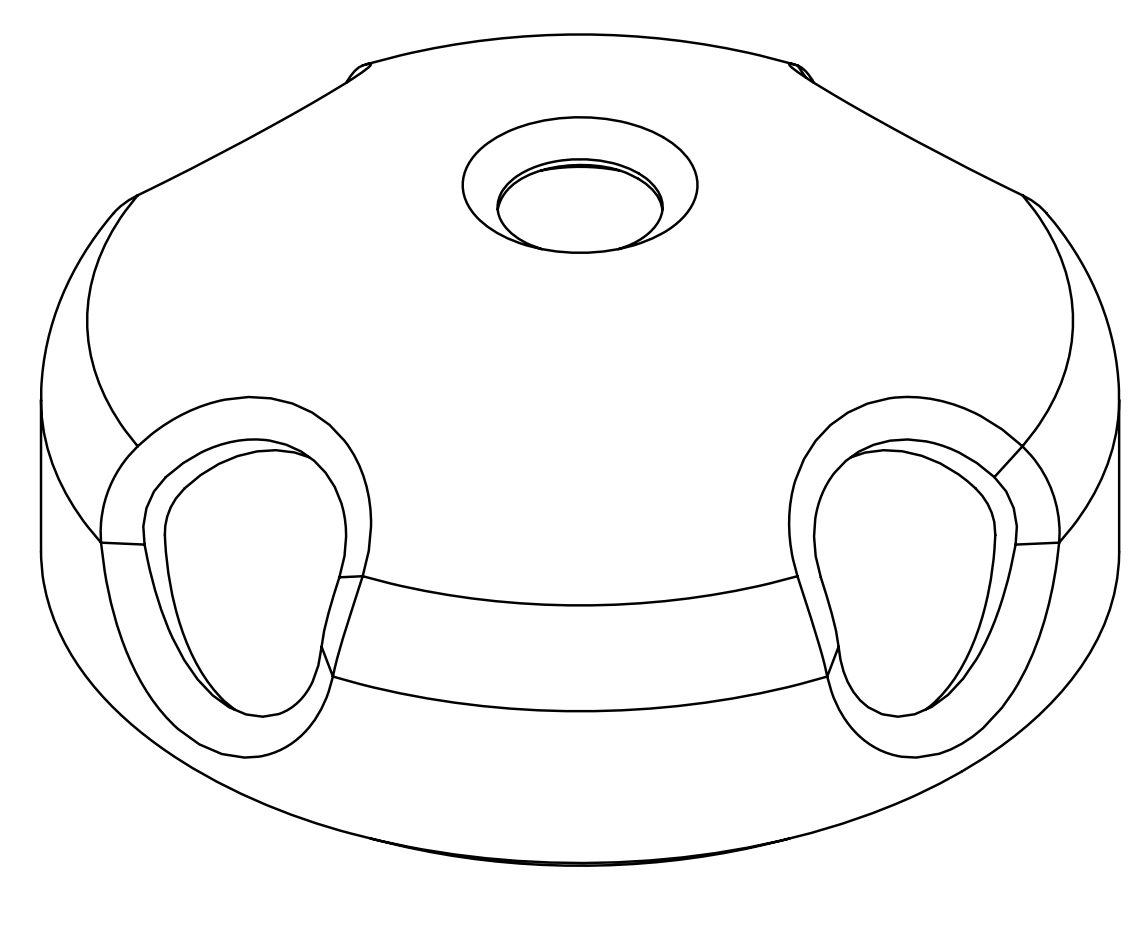

\begin{tabular}{|l|l|l|l|l|}
\hline ALL & $C$ & ANGLE CHANGED; FROM $30^{\circ}$ TO $45^{\circ}$ & $08 / 14108$ & DW
\end{tabular}

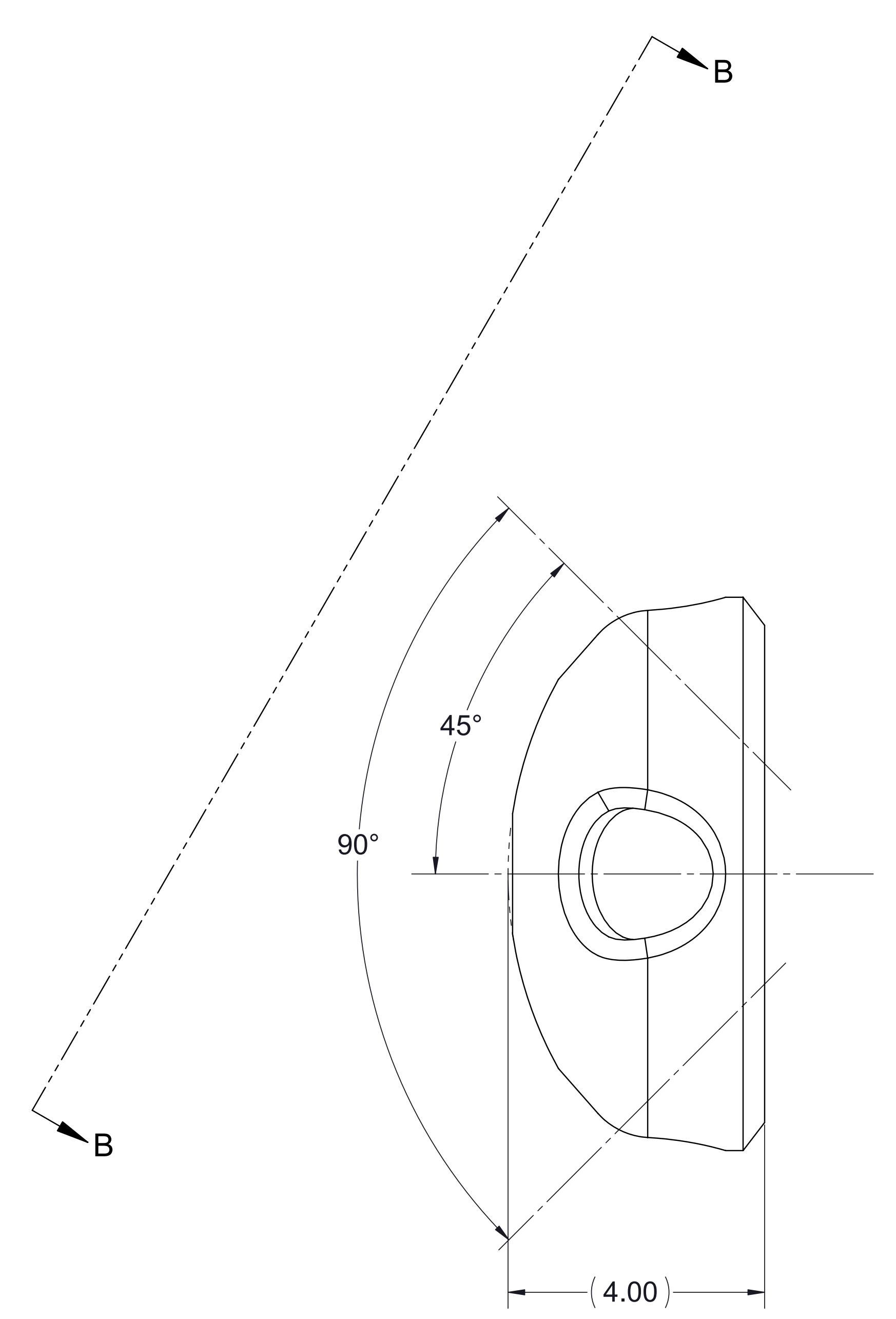

$4 \times .31 \times 37.5^{\circ}$ SECTIONAAA

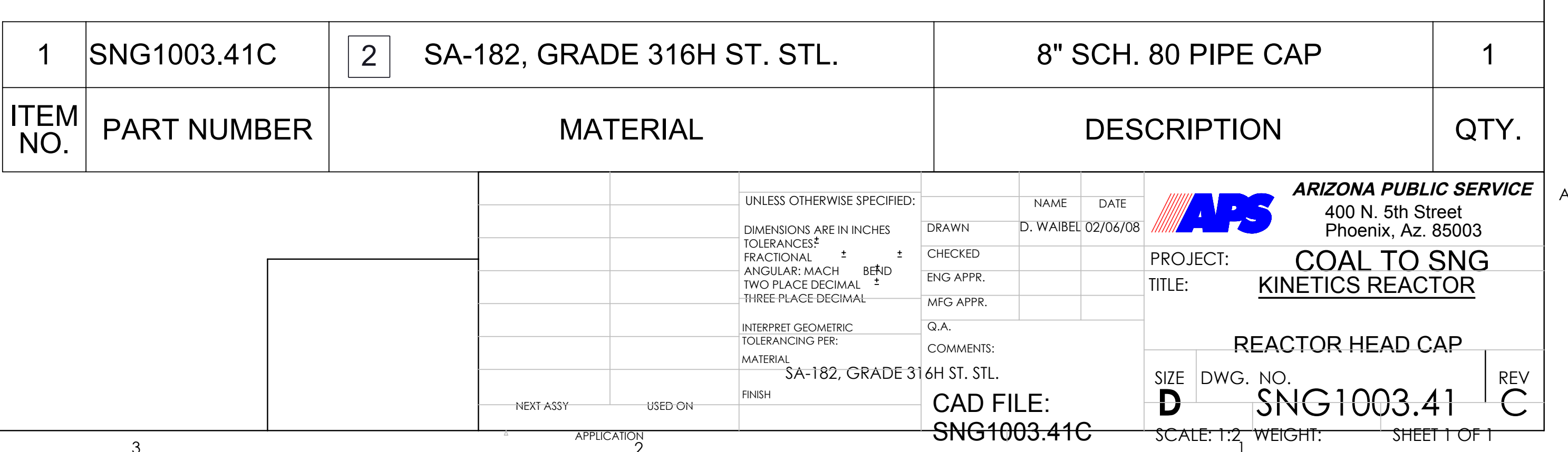




\begin{tabular}{|c|c|c|c|c|c|}
\hline \multicolumn{5}{|c|}{ REVISIONS } \\
\hline ZONE & REV. & DESCRPTION & DATE & REV. BY & APRROVED \\
\hline ALL & A & INITIAL RELEASE & $02 / 12 / 08$ & DW & \\
\hline
\end{tabular}
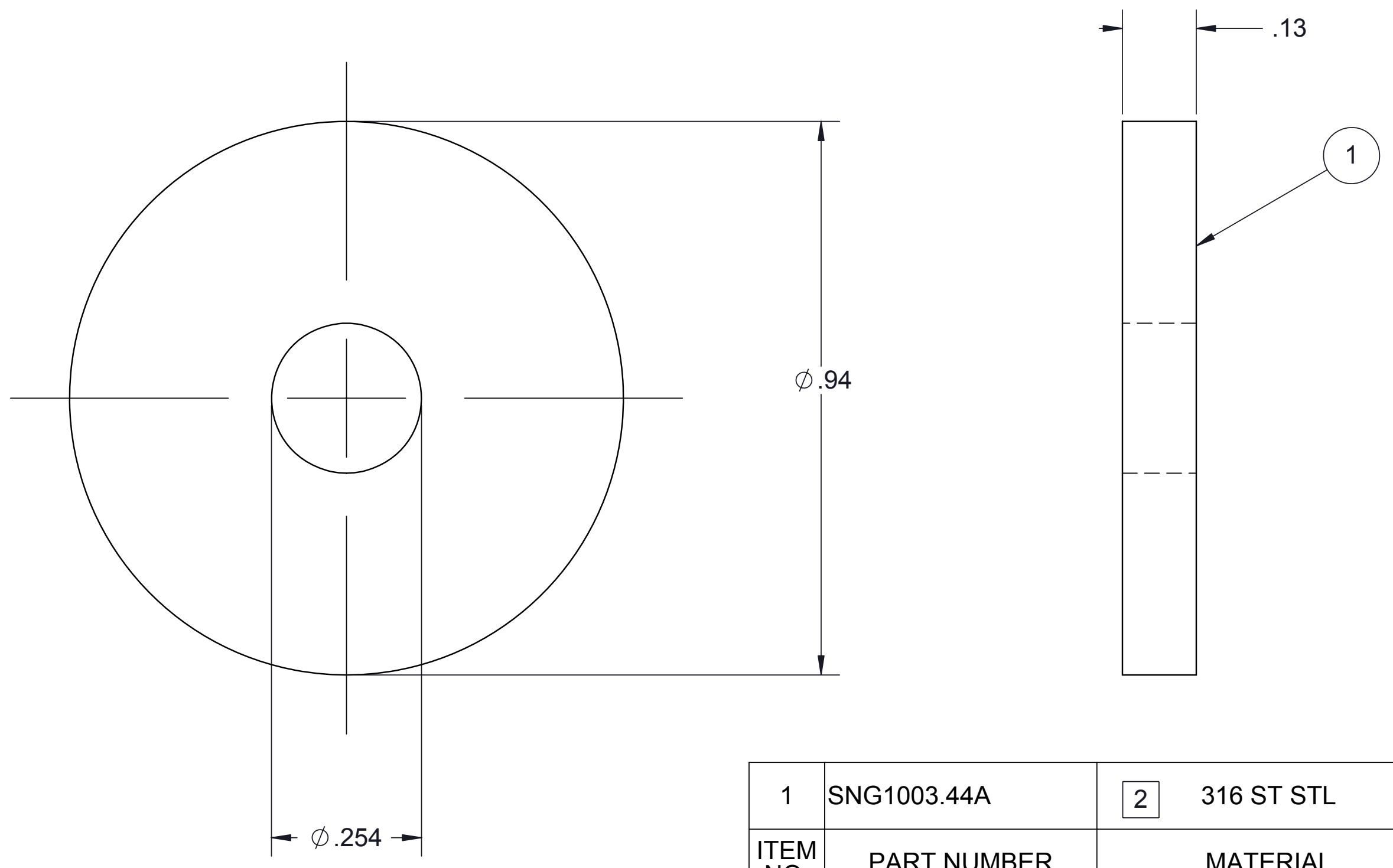

\begin{tabular}{|c|c|cc|c|c|}
\hline 1 & SNG1003.44A & 2 & 316 ST STL & $\begin{array}{c}\text { WASHER, INSULATION } \\
\text { RETAINMENT }\end{array}$ & 1 \\
\hline $\begin{array}{c}\text { ITEM } \\
\text { NO. }\end{array}$ & PART NUMBER & MATERIAL & DESCRIPTION & QTY. \\
\hline
\end{tabular}

ARIZONA PUBLIC SERVICE

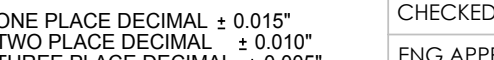

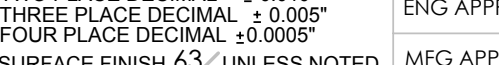

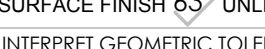

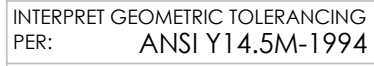
MAERALL: SEE BOM

\section{SI} FINSH:$$
\mathrm{SITRC}_{\text {MER }}
$$
CAD FILE: $400 \mathrm{~N}$. 5th Street
Phoenix, Az 85003 PROJECT: COAL TO SNG TITE: KINETICS REACTOR WASHER, INSULATION RETAINMENT

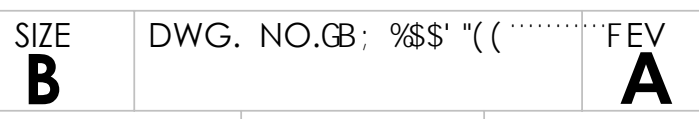
SNG1003.44A

SCALE: 4:1 WEIGHT: 


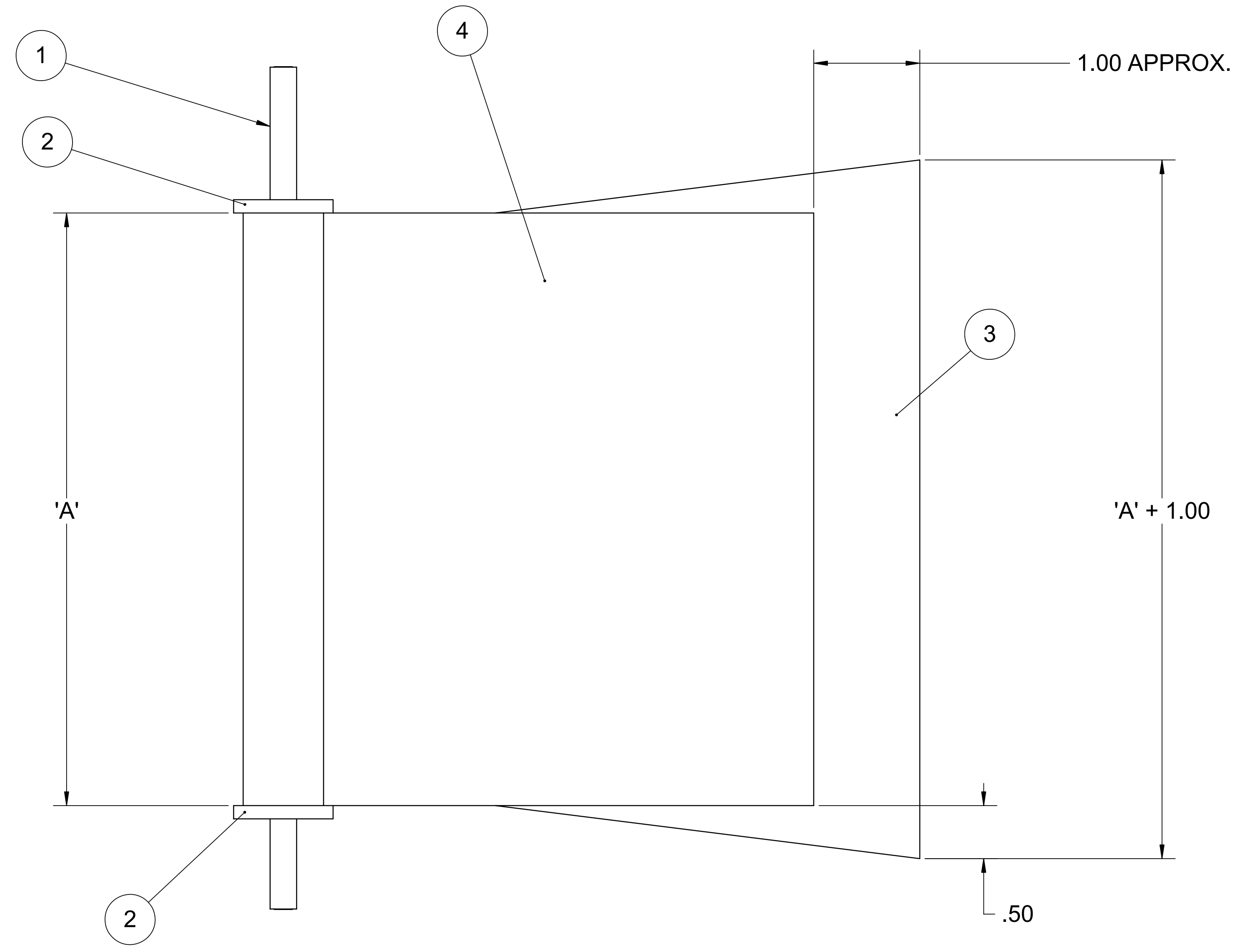

WASHER REMOVED

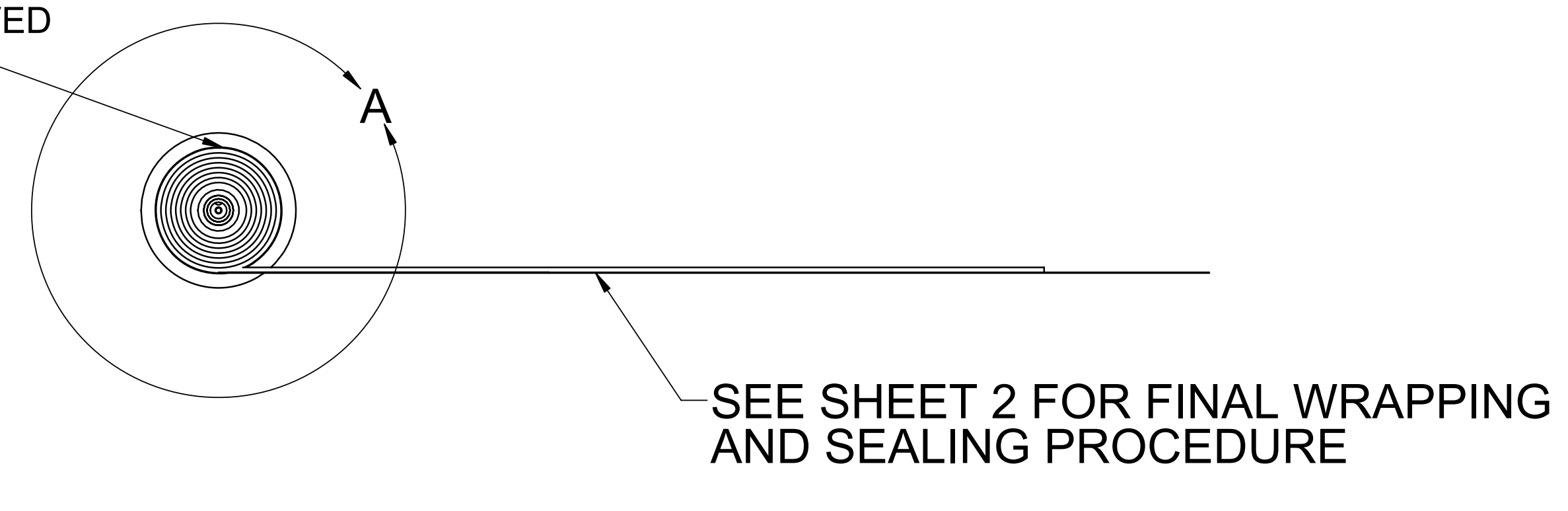

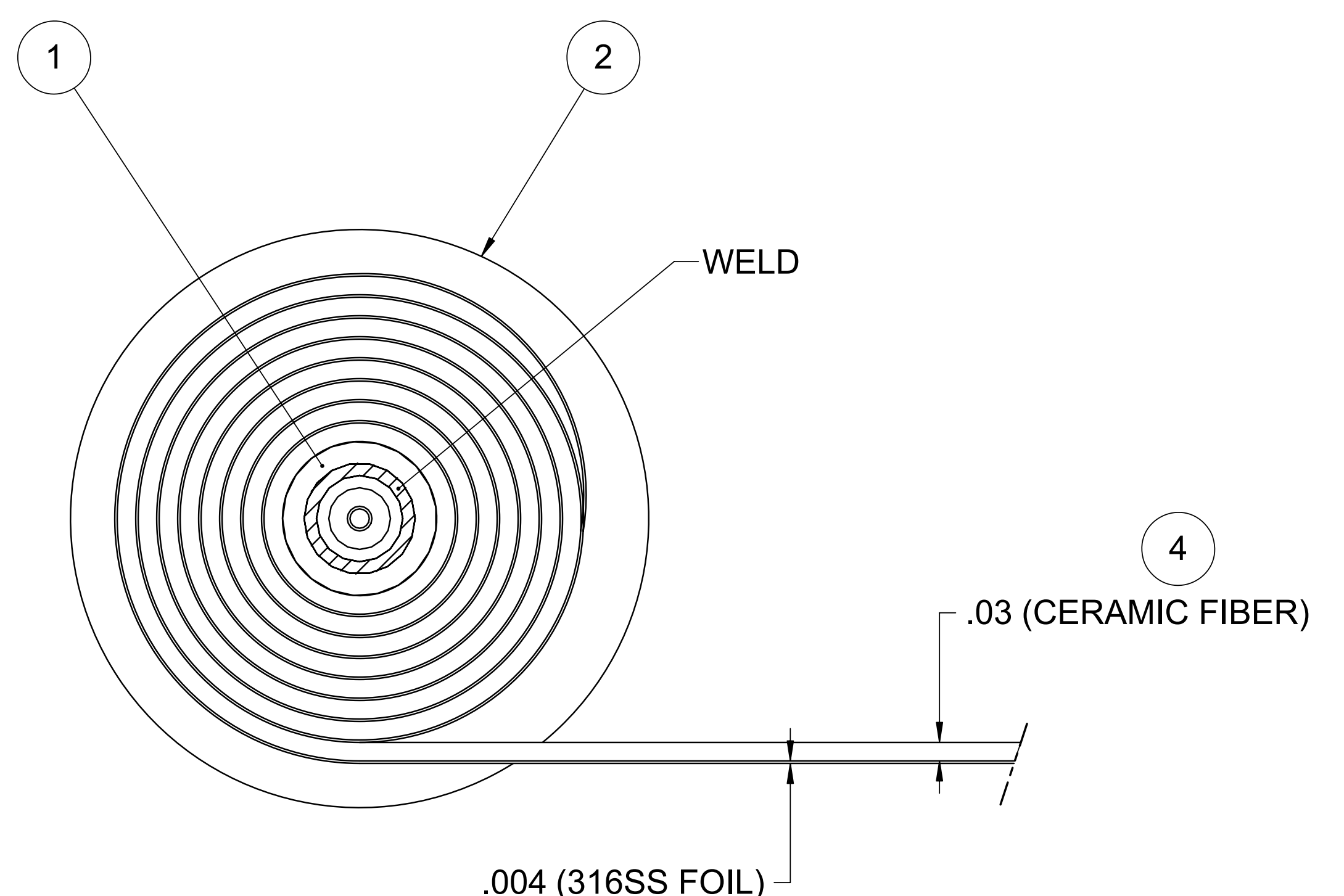

.004 (316SS FOIL)

(3)

DETALAA
SCALE $4: 1$

\begin{tabular}{|c|c|c|c|c|}
\hline 4 & 87665K2 & CERAMIC FIBER & CERAMIC FIBER, $.030 "$ & . \\
\hline 3 & 316SS-FOIL1 & 316 s.s. & $\begin{array}{l}\text { FOIL, STAINLESS, INSULATING, } \\
.002 "-.004 " \text { "THK. }\end{array}$ & 1 \\
\hline 2 & SNG1003.44A & 316 s.s. & $\begin{array}{l}\text { WASHER, INSULATION } \\
\text { RETAIMENT }\end{array}$ & 2 \\
\hline 1 & W-SNG1003.10 & 316 ST. STL. & $\begin{array}{l}\text { WELDMENT, REACTOR HEAD, } \\
\text { INJECTOR WAND }\end{array}$ & 1 \\
\hline \multirow[t]{3}{*}{$\begin{array}{l}\text { EM } \\
\text { O. }\end{array}$} & PART NUMBER & MATERIAL & DESCRIPTION & QTY \\
\hline & & 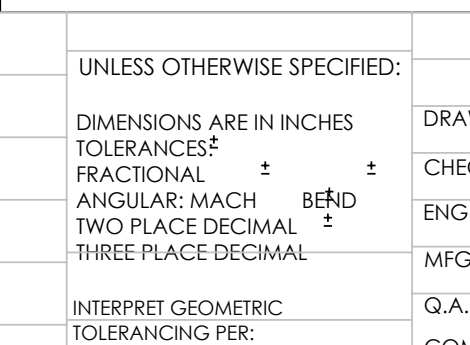 & 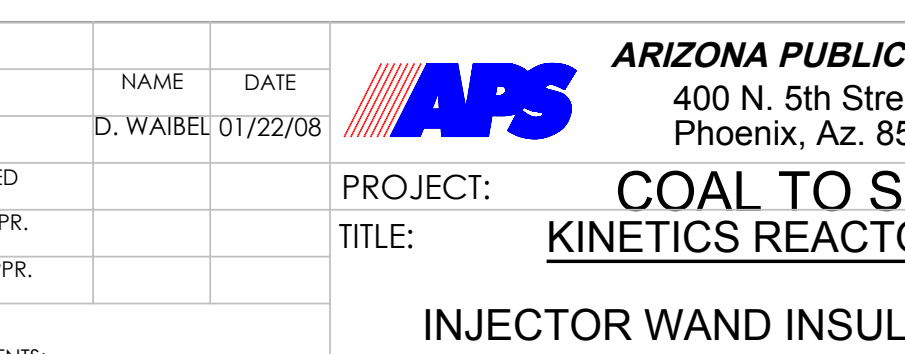 & \\
\hline & 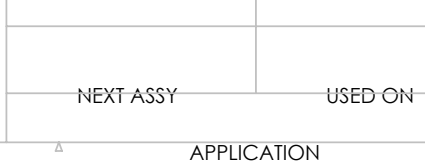 & & 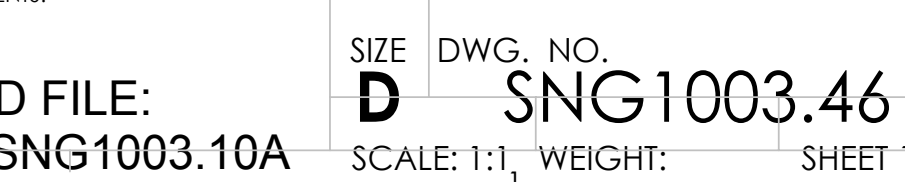 & $\underset{O F 2}{\operatorname{REV}}$ \\
\hline
\end{tabular}




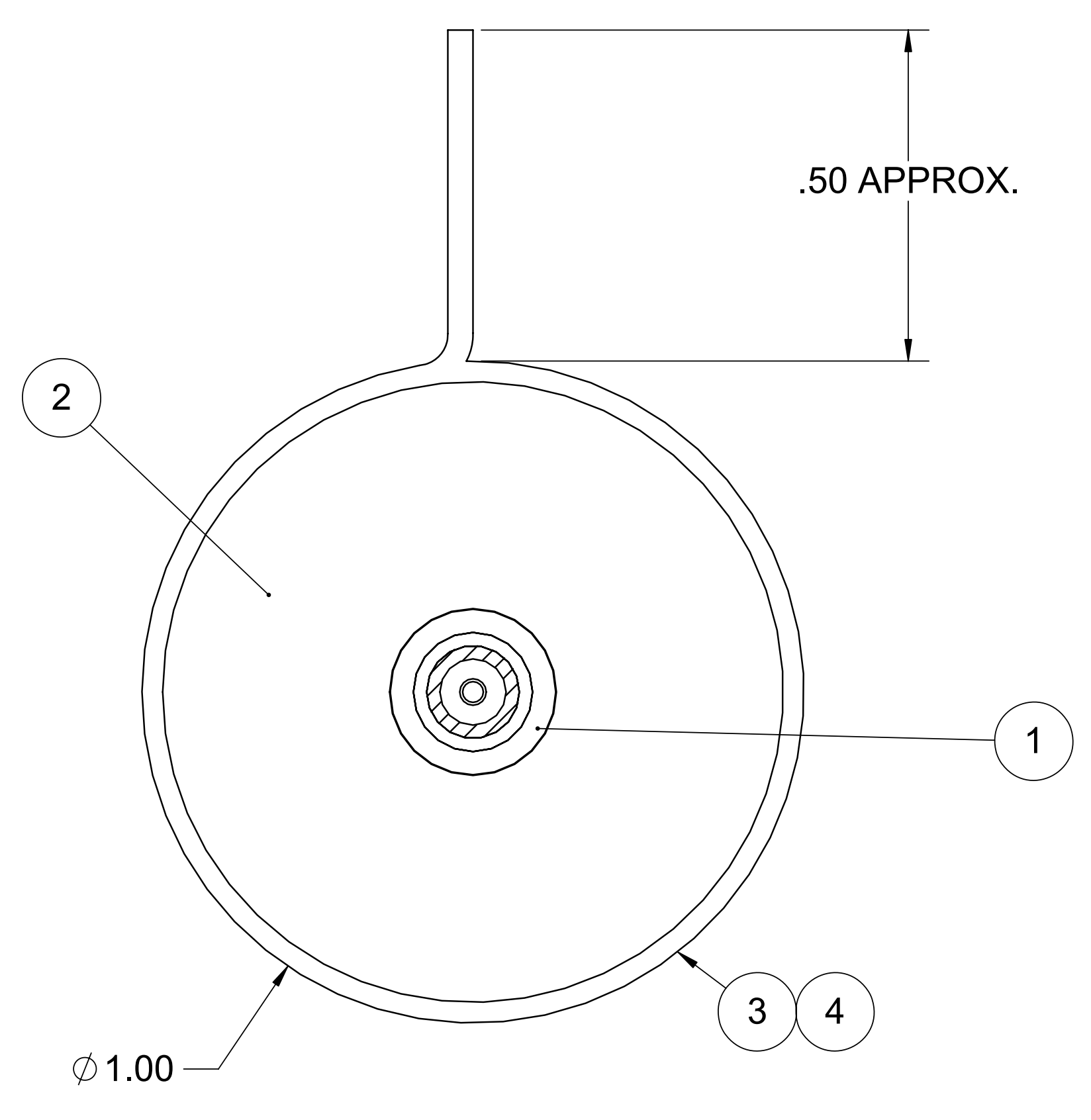

SEAM SEALING STEP 1

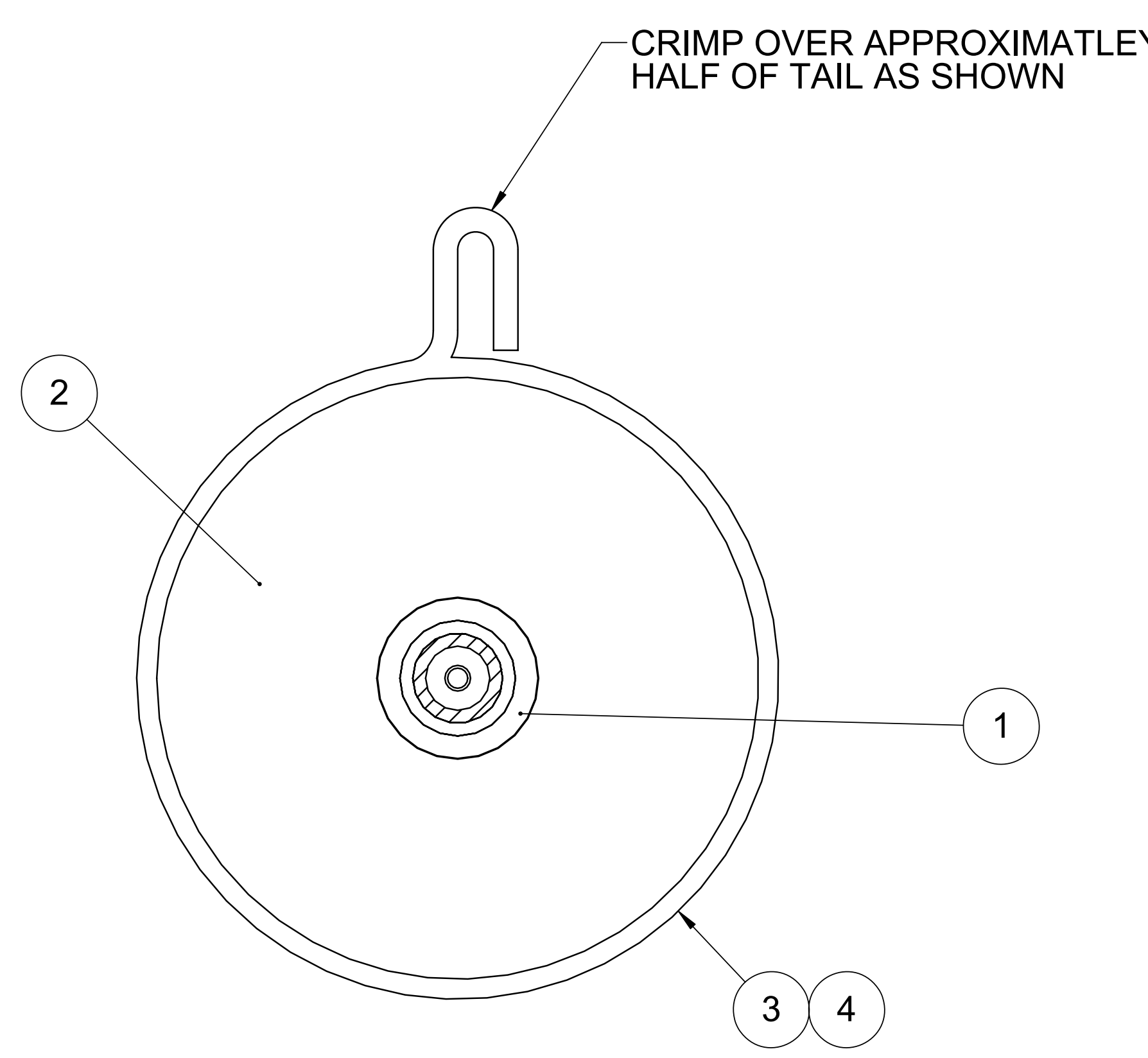

SEAM SEALING STEP 2

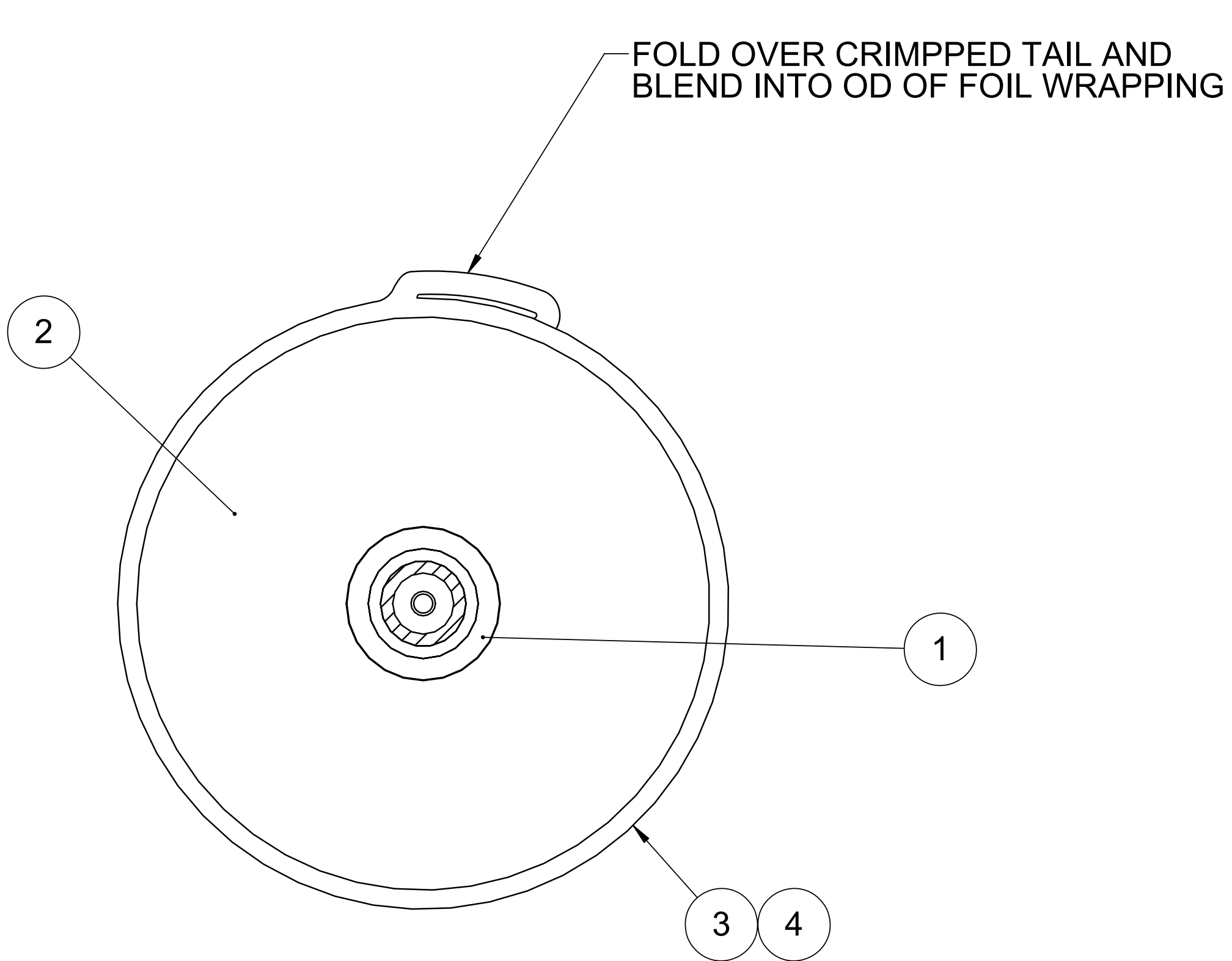

SEAM SEALING STEP 3

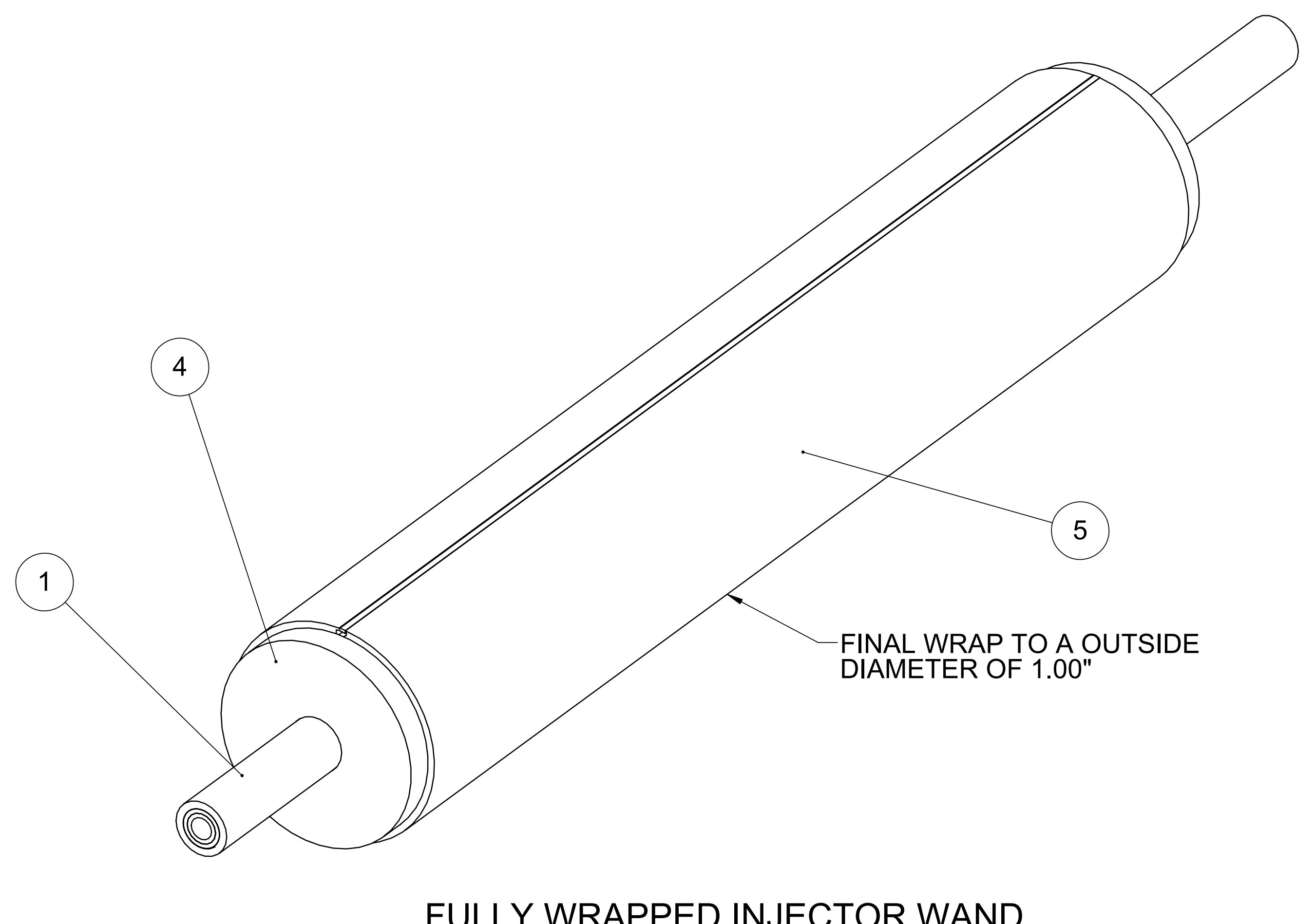




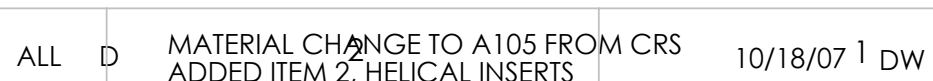
GENERAL 24 NOTES:

A SURFACE FINISH AS INDICATED, SHOULD ALSO BE TO A QUALITY
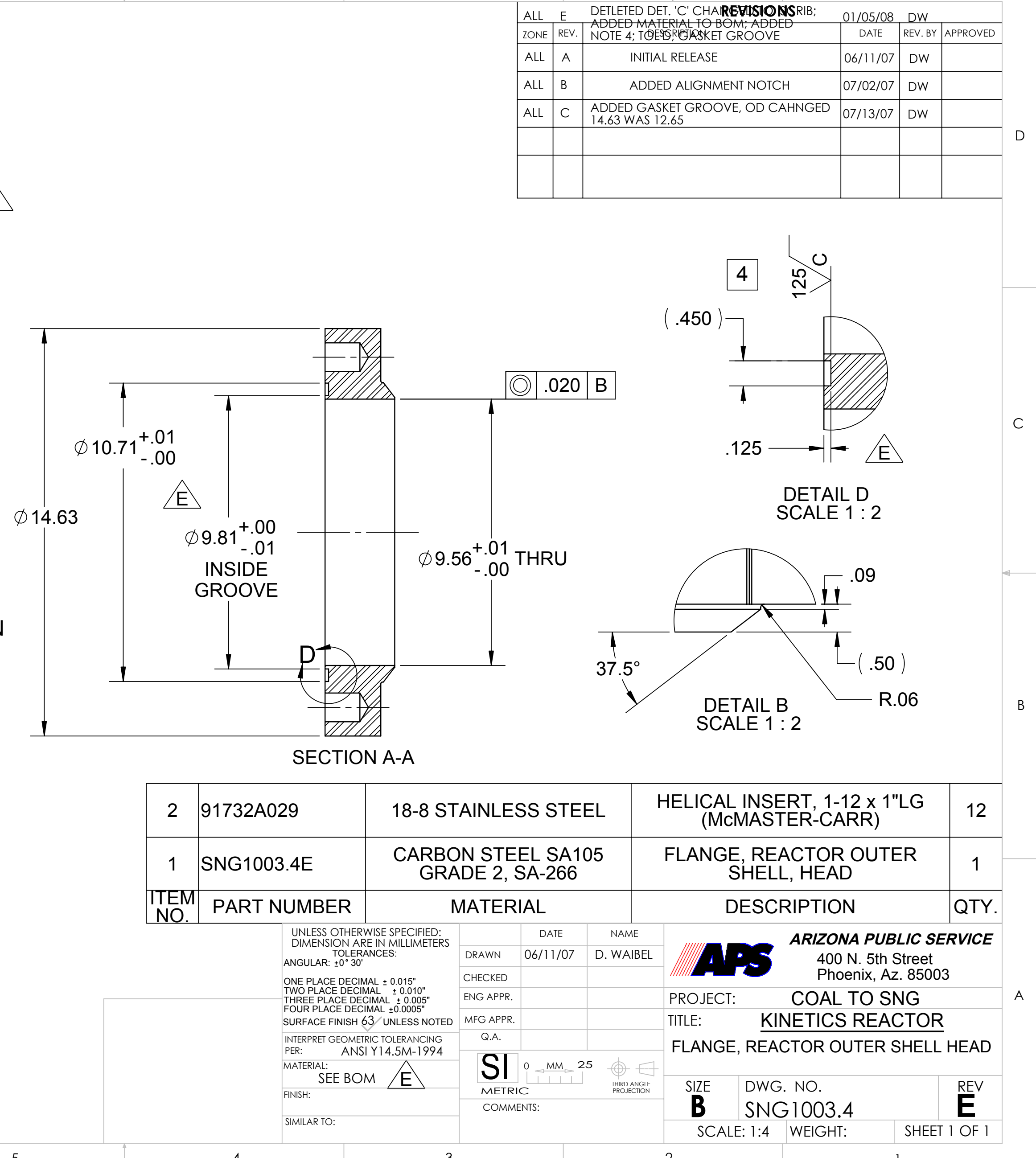

(2) E SCRIB LINE IN LOCATIONAS SHOWN FOR ASSEMBLY ALIGNMENT

2 c)

$12 X(1-1 / 32 ") \varnothing 1.031 \rrbracket 1.25$ EQ. SPACED ON $\varnothing 12.570$ B.C.

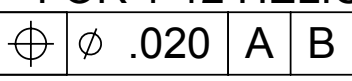

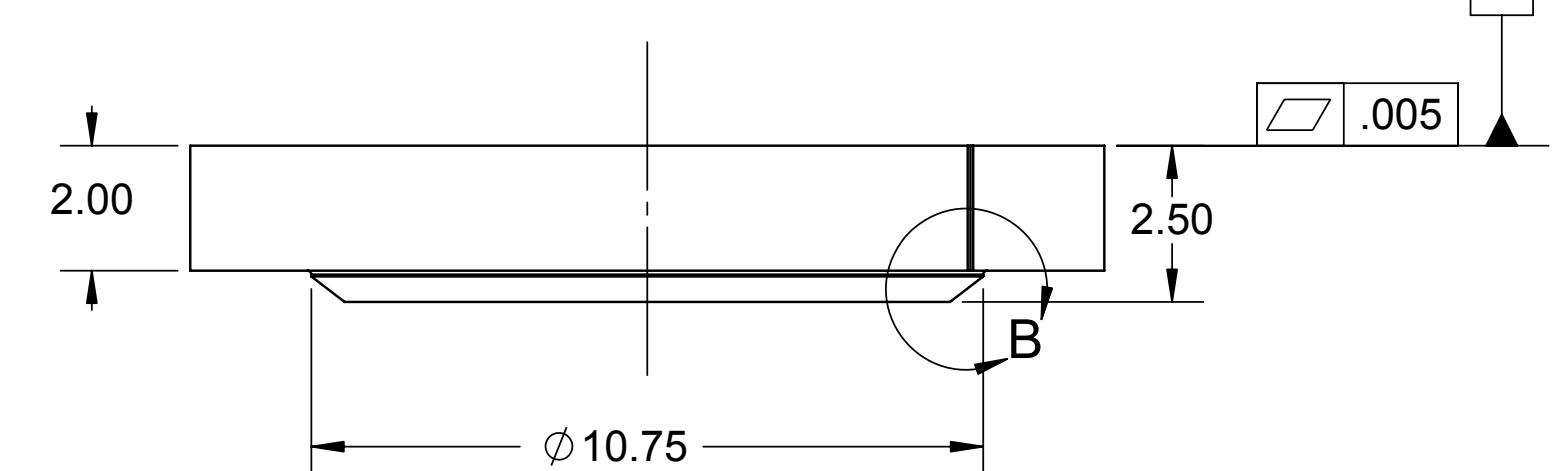

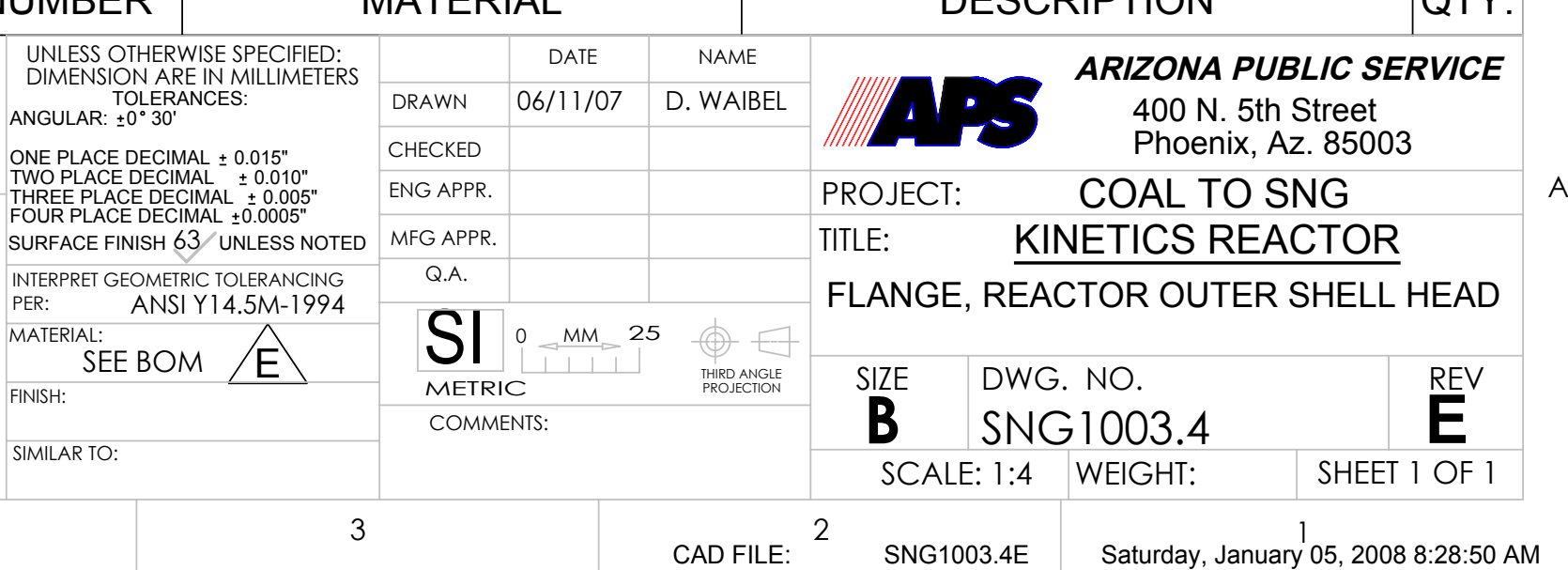




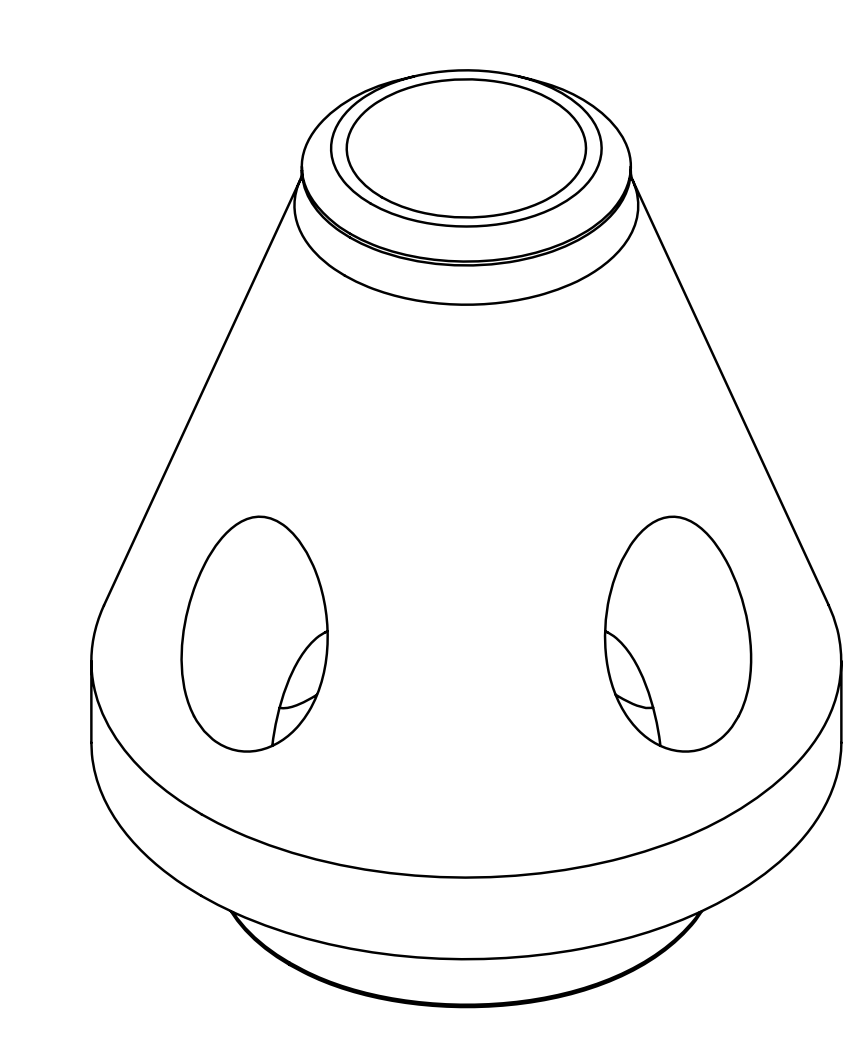

\begin{tabular}{|c|c|c|}
\hline ZONE & REV & DESCRIPTION \\
\hline ALL & A & INITIAL RELEASE \\
\hline ALL & B & DESIONS \\
\hline
\end{tabular}

ALL B DESIGN CHANGE

\begin{tabular}{ll|l} 
ALL & $C$ & DIM. 1.876 WAS 2.001 \\
\hline
\end{tabular}

\begin{tabular}{|l|l|l|}
\hline ALL & D & DESIGN CHANGE \\
\hline
\end{tabular}

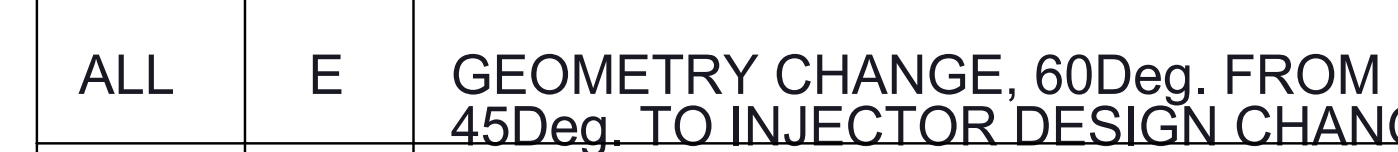

\begin{tabular}{l|l|l} 
ALL & F & MATERIAL INCONEL625 WAS 316 \\
\hline
\end{tabular}

\begin{tabular}{l|l|l} 
ALL & $G$ & ANGLE; $45^{\circ}$ WAS 30 \\
\hline
\end{tabular}

ALL $\quad H \quad$ ANGLE; $30^{\circ}$ WAS $45^{\circ}$

06/25/07 DW

ALL 3/4-16UNF WAS 38N NDT GEOMETRY

$08 / 15 / 07 \quad$ DW

$11 / 14 / 07 \quad$ DW

\begin{tabular}{|l|l|}
\hline $02 / 07 / 08$ & DW \\
\hline
\end{tabular}
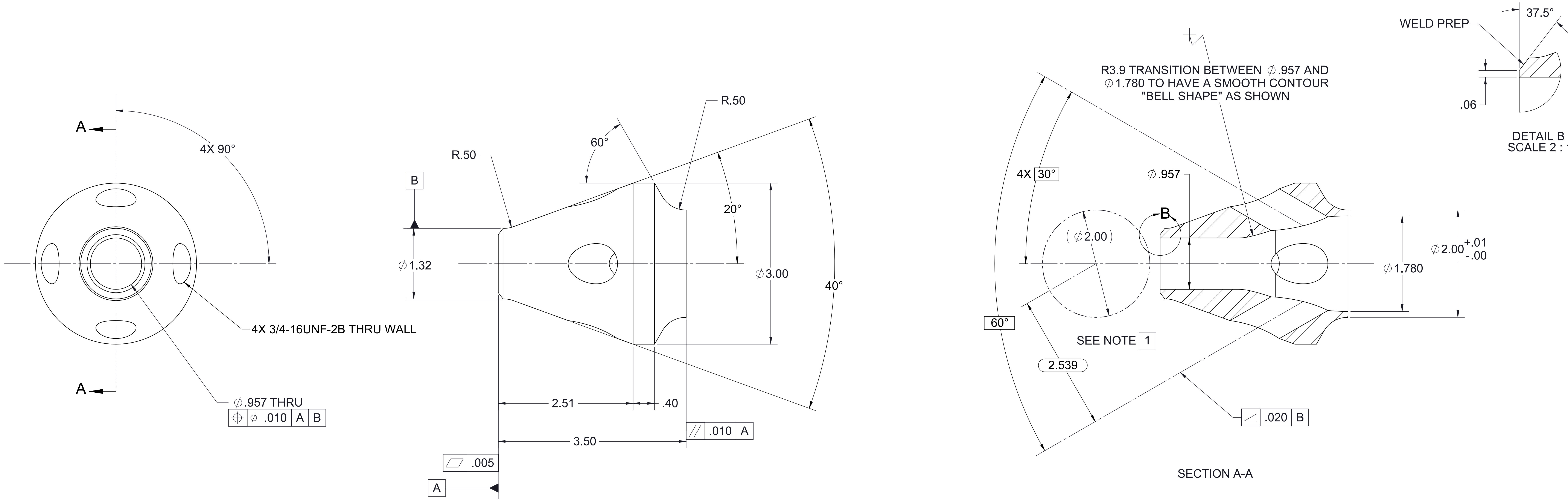

SECTION A-A

\begin{tabular}{|c|c|c|c|c|c|}
\hline & SNG1003.5K & \multicolumn{2}{|c|}{$\begin{array}{l}\text { INCONEL 625, UNS-NO6625, SB-446 } \\
\text { ASTM-B446 }\end{array}$} & HUB, REACTOR INNER HEAD & 1 \\
\hline $\begin{array}{l}\text { ITEM } \\
\text { NO. }\end{array}$ & PART NUMBER & \multicolumn{2}{|c|}{ MATERIAL } & DESCRIPTION & QTY. \\
\hline & & & \multirow{4}{*}{ 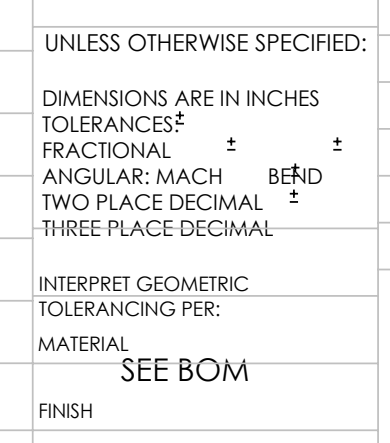 } & \multicolumn{2}{|c|}{ 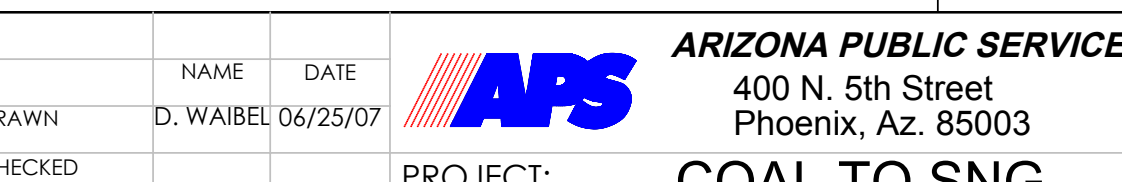 } \\
\hline & & & & \multicolumn{2}{|c|}{$\begin{array}{ll}\text { ProJECT: } & \text { COAL TO SNG } \\
\text { TILE: } & \text { KINETTCS REACTOR } \\
\end{array}$} \\
\hline & & & & \multirow{2}{*}{\multicolumn{2}{|c|}{ 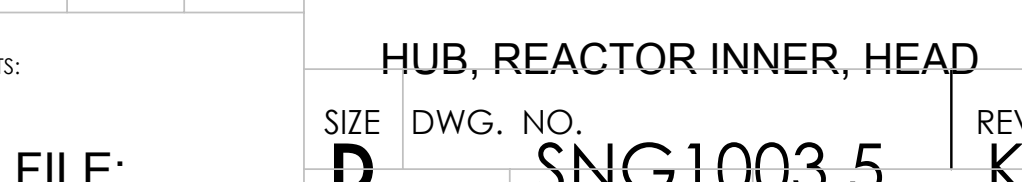 }} \\
\hline & & werestst & & & \\
\hline
\end{tabular}




\begin{tabular}{|c|c|l|l|l|l|}
\multicolumn{7}{|c|}{ REVISIONS } \\
\hline ZONE & REV & \multicolumn{1}{|c|}{ DESCRIPTION } & DATE & REV BY & APPROVED \\
\hline ALL & A & INITIAL RELEASE & $06 / 25 / 07$ & DW & \\
\hline ALL & B & DESIGN CHANGE & $07 / 21 / 07$ & DW & \\
\hline ALL & C & DESIGN CHANGE & $08 / 15 / 07$ & DW & \\
\hline ALL & D & DESIGN CHANGE & $11 / 14 / 07$ & DW & \\
\hline ALL & E & GEOMETRY CHANGE & $02 / 07 / 08$ & DW & \\
\hline ALL & F & INCONEL 625 WAS 316 ST STL & $05 / 07 / 08$ & DW & \\
\hline ALL & G & DIM. 3.38 WAS 3.56; DIM. ,25 WAS .10 & $11 / 10 / 08$ & DW & \\
\hline
\end{tabular}
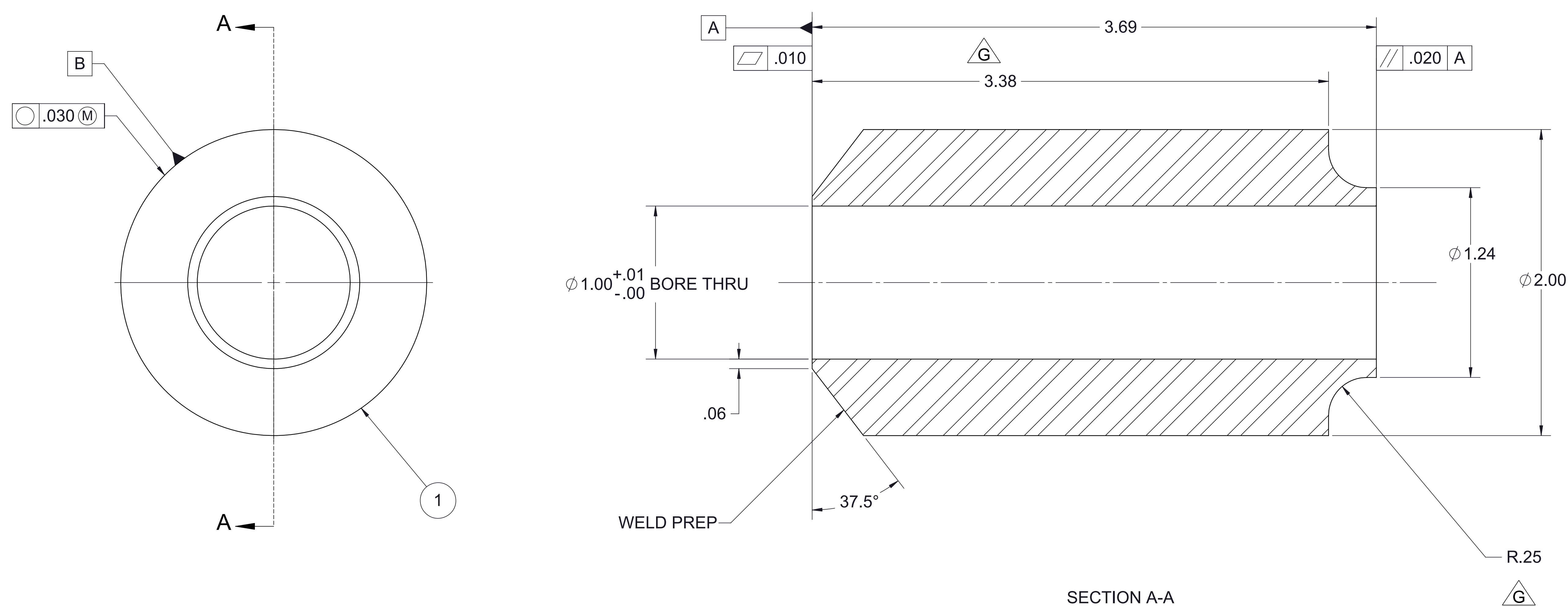

SECTION A-A

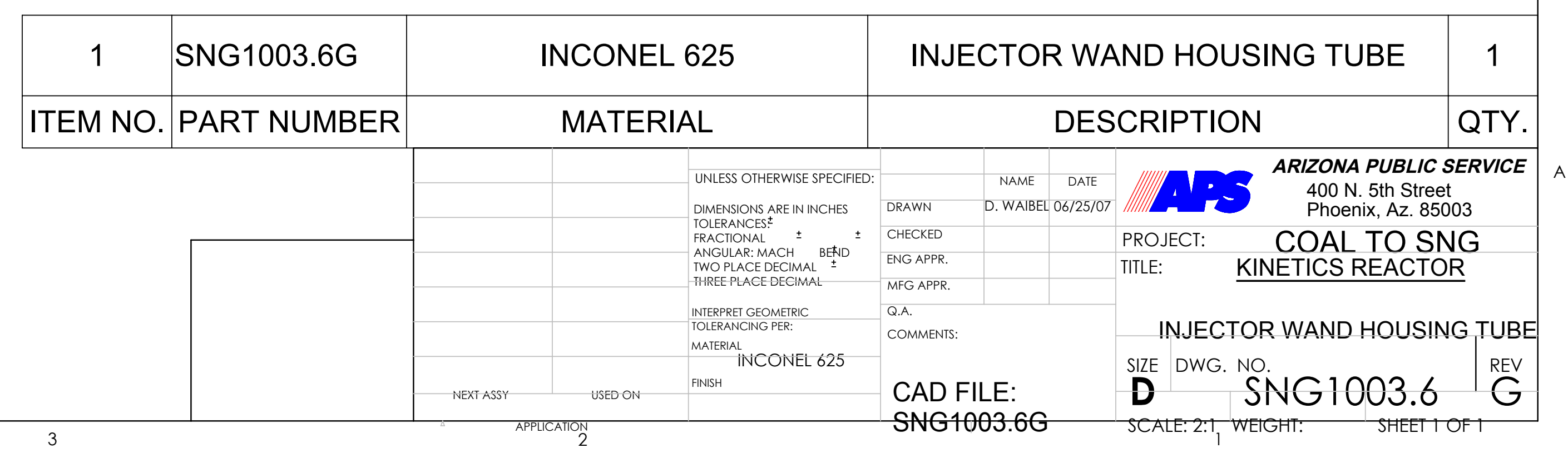




\section{GENERAL NOTES:}

DIMENSION AND TOLERANCING PER ANSI Y14.5-1994.

2 BREAK ALL EDGES .5 [.20] MAX. ALL INSIDE RADII

2.4 [.09] MAX. UNLESS NOTED OTHERWISE.
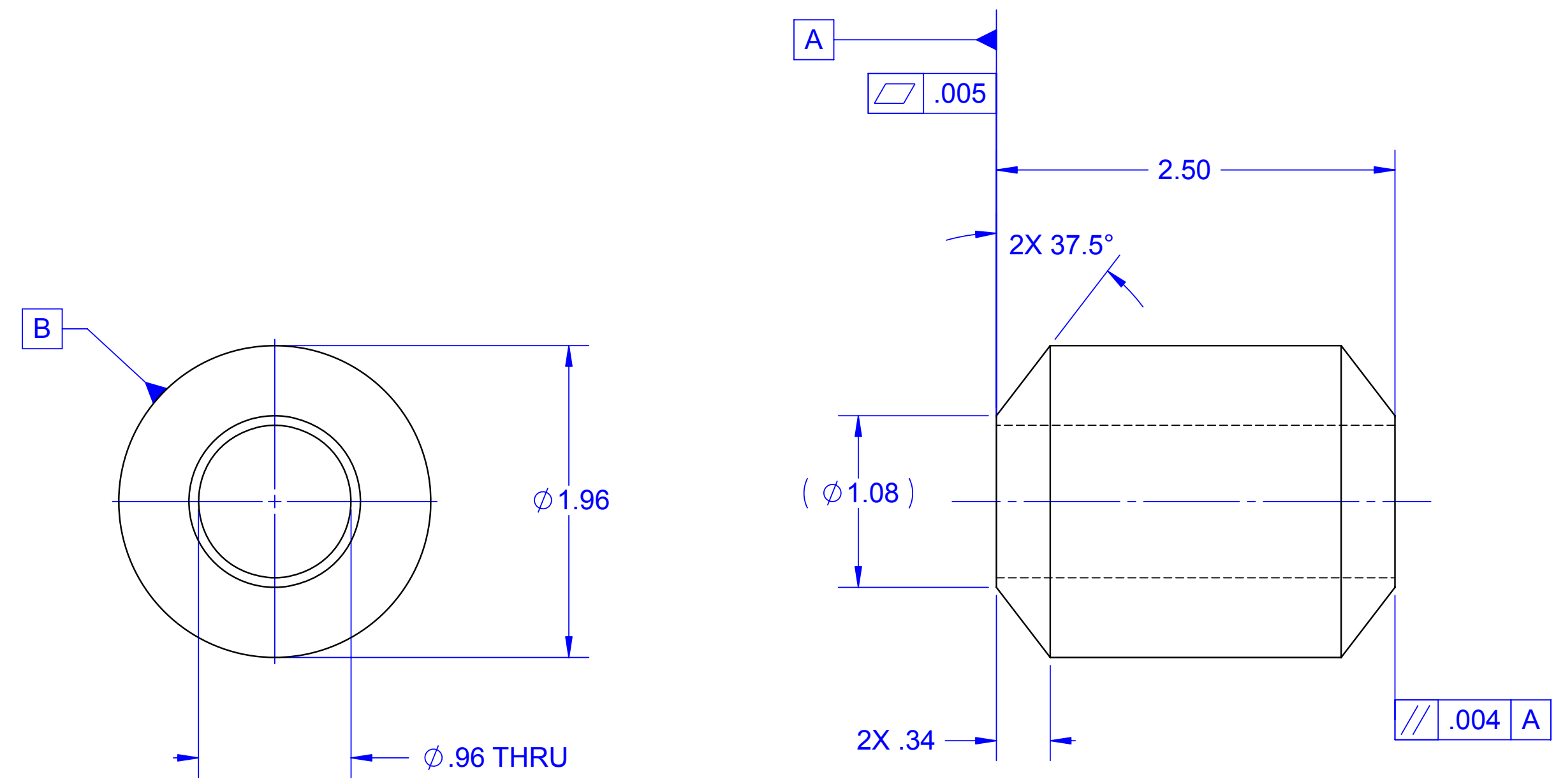

\begin{tabular}{|c|c|c|c|}
\hline 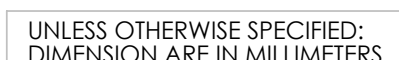 & & DATE & Name \\
\hline 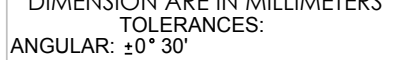 & DRAWN & 06/12/07 & D. WAIBEL \\
\hline 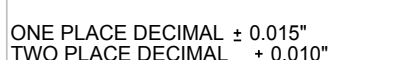 & CHECKED & & \\
\hline 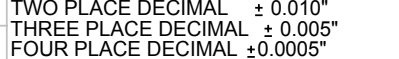 & ENG APPR. & & \\
\hline 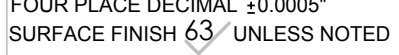 & MFG APPR & & \\
\hline $\begin{array}{l}\text { INTERPRET GEOMERTC TOLERANCING } \\
\text { PERP: } \\
\text { ANSIYY Y4.5M-1994 }\end{array}$ & Q.A. & & \\
\hline $\begin{array}{l}\text { ERRAL: } \\
\text { 316H ST. STL. }\end{array}$ & $\underset{\substack{\text { METR } \\
\text { COMN }}}{\text { CI }}$ & 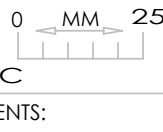 & 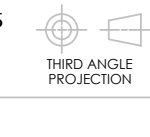 \\
\hline
\end{tabular}

ARIIIIIIINA PUBLIC SERVICE PROJECT: COAL TO SNG IITLE: KINETICS REACTOR PIPE, REACTOR HEAD SPACER SCALE: 1:1 WEIGHT: AEV 


\section{GENERAL NOTES:}

DIMENSION AND TOLERANCING PER ANSI Y14.5-1994.

2 BREAK ALL EDGES .5 [.20] MAX. ALL INSIDE RADII 2.4 [.09] MAX. UNLESS NOTED OTHERWISE.

D

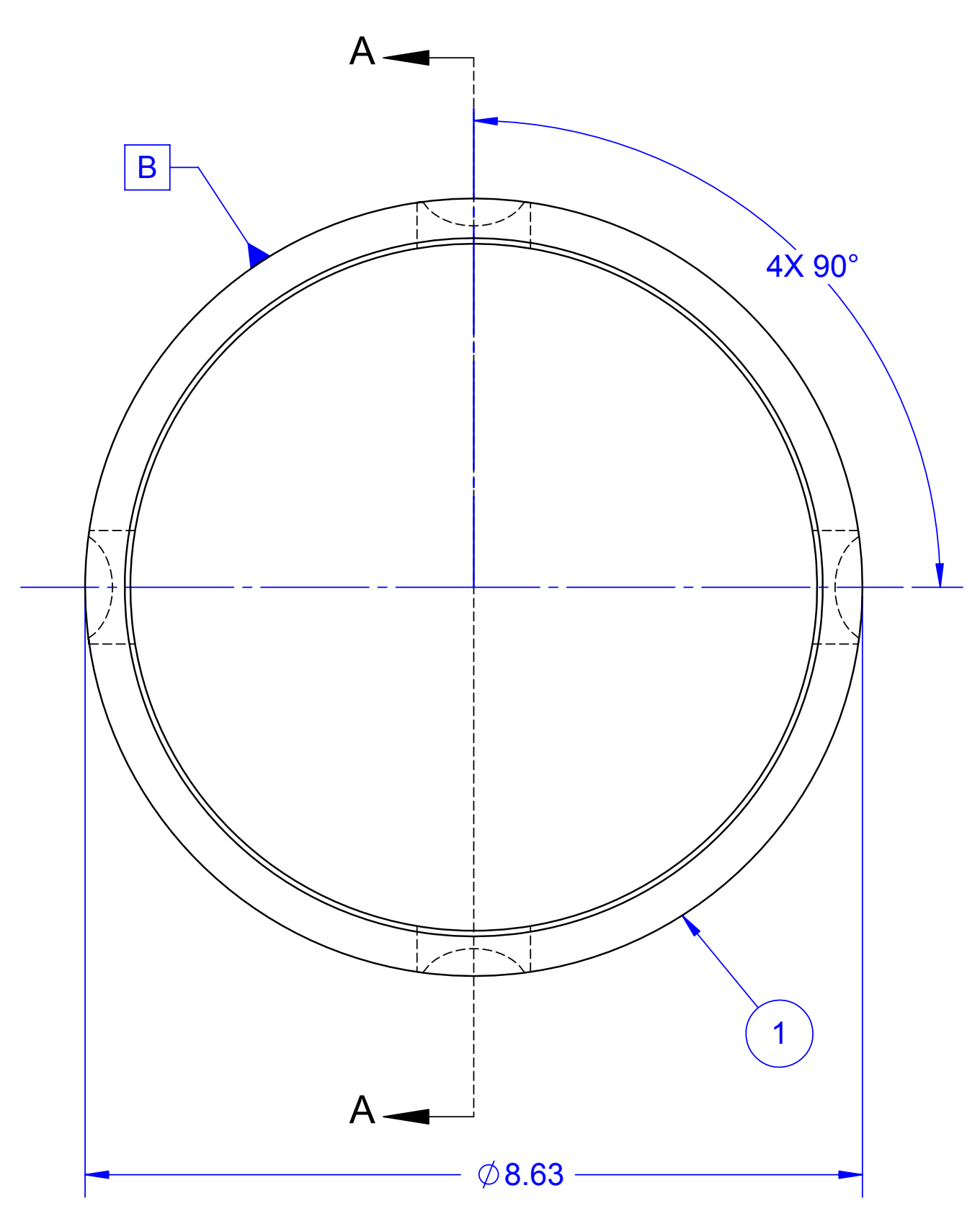

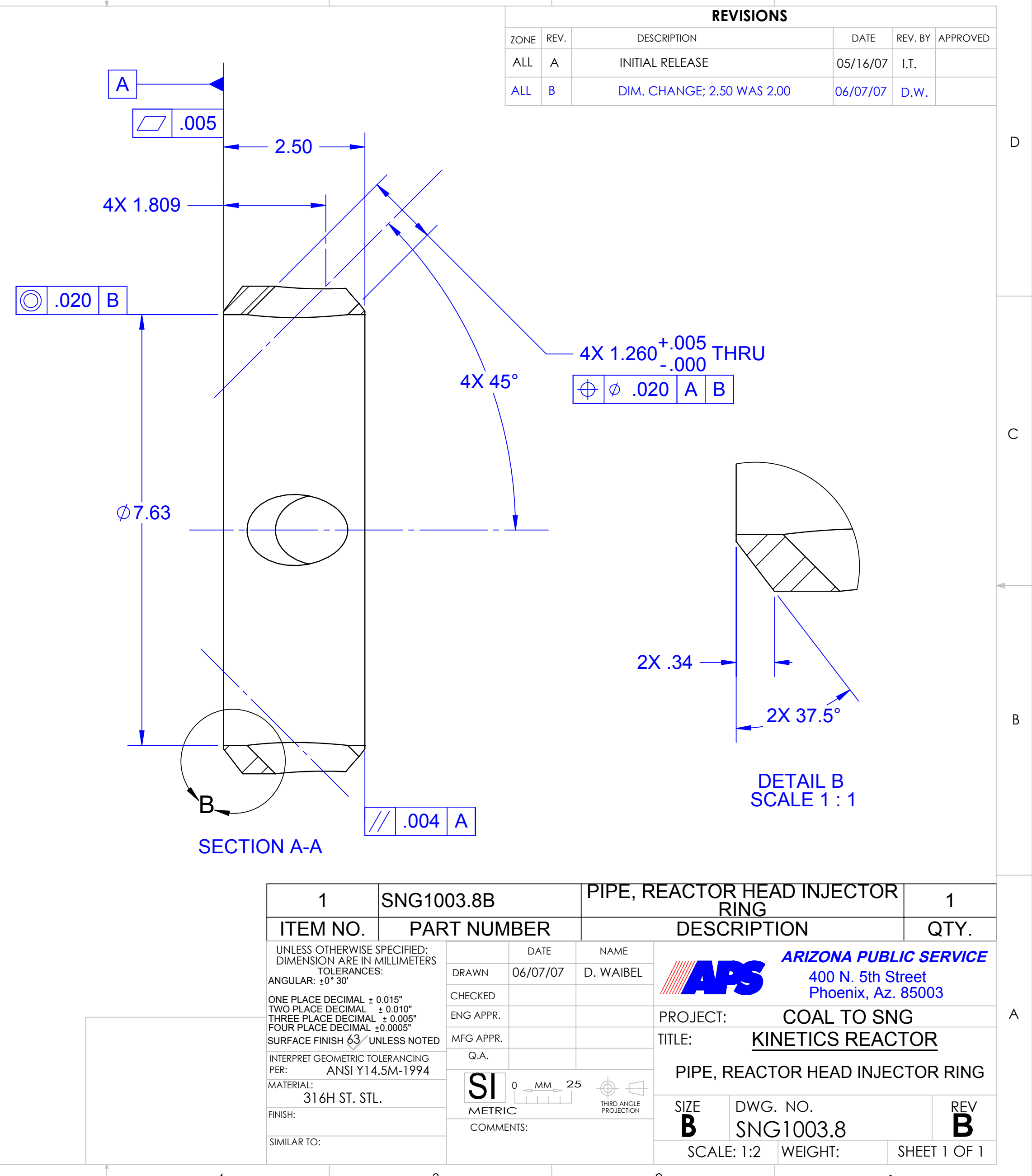


1 DIMENSION AND TOLERANCING PER ANSI Y14.5-1994.

2 GASKET TO BE STYLE 'R', (CRITICAL SERVICE SERIES) SEE BOM BELOW.
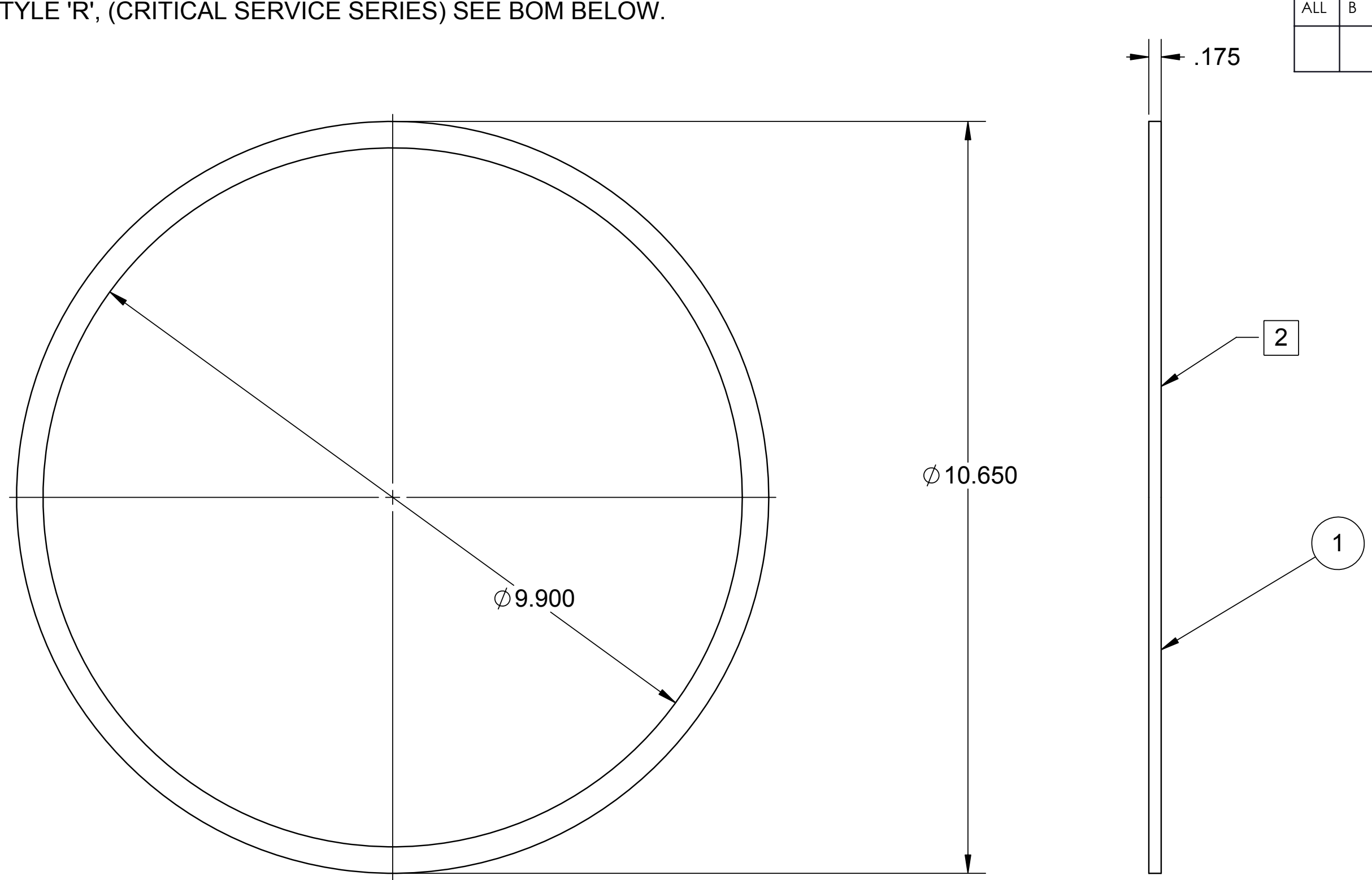

\begin{tabular}{|c|c|c|c|c|c|}
\hline 1 & SNG1003.9C & \multicolumn{4}{|c|}{$\begin{array}{l}\text { R STYLE, SWG INCONEL } 625 \\
\text { WINDING WITH THERMICULITE } 835 \\
\text { FILLER (CRITICAL SERVICE) }\end{array}$} \\
\hline $\begin{array}{l}\text { TTEM } \\
\text { NO. }\end{array}$ & PART NUMBER & \multicolumn{4}{|c|}{ MATERIAL } \\
\hline & & \multirow{7}{*}{ 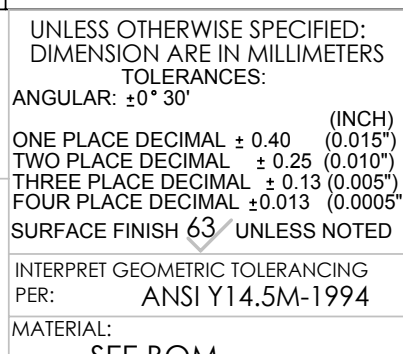 } & & DATE & NAME \\
\hline & & & DRAWN & 1. TAYLOR & 05/16/07 \\
\hline & & & CHECKED & & \\
\hline & & & ENG APPR. & & \\
\hline & & & MFG APPR & & \\
\hline & & & Q.A. & & \\
\hline & & & Sil & $c=\frac{M M}{2} 25$ & $\underbrace{}_{\substack{\text { thito } \\
\text { pron }}}$ \\
\hline & & & COMN & & \\
\hline
\end{tabular}

GASKET, REACTOR HEAD

DESCRIPTION

ARIZONA PUBLIC SERVICE IIIII $\quad \begin{aligned} & 400 \text { N. 5th Street } \\ & \text { Phoenix, Az. } 85003\end{aligned}$

PROJECT: $\quad$ COAL TO SNG IITLE: KINETICS REACTOR GASKET, REACTOR HEAD 


\begin{tabular}{|c|c|c|c|c|c|}
\hline \multicolumn{6}{|c|}{ REVISIONS } \\
\hline ZONE & REV. & DESCRIPTION & DATE & REV. B & APPROVED \\
\hline ALL & A & INITIAL RELEASE & 06/12/07 & D.W. & \\
\hline & & & & & \\
\hline & & & & & \\
\hline
\end{tabular}


${ }_{8}$ INSPECTION OF WELDS TO BE $100 \%$ X-RAY, UNLESS SPECIFIED OTHERWISE. BUTTWELDED FITTINGS ARE TO BE END PREPPED PER ASME B16.25.

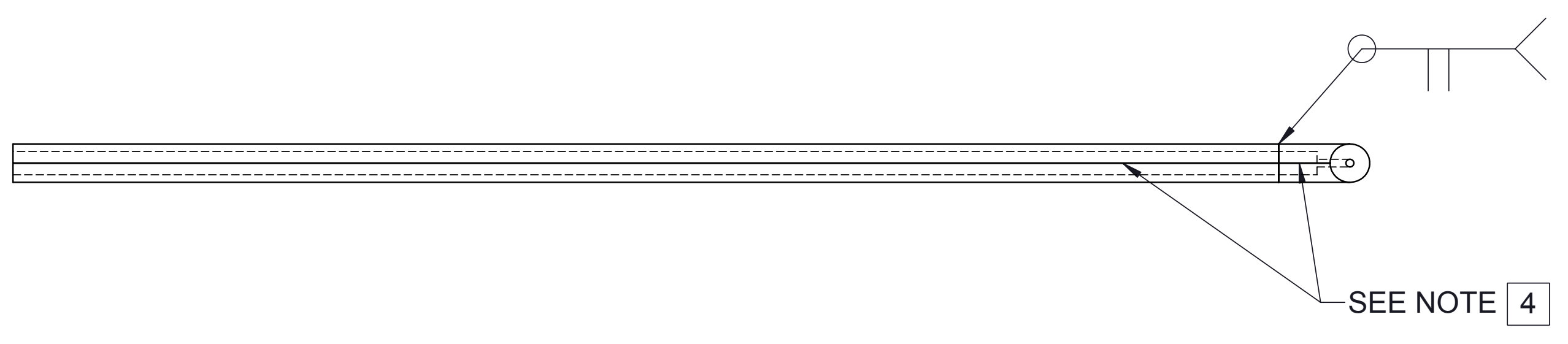

$(8.25)$

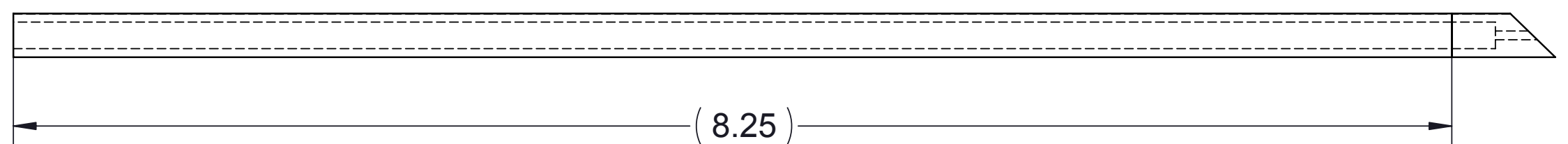

\begin{tabular}{|c|l|c|c|c|}
\hline 2 & SNG1003.13A & INCONEL 625 & NOZZEL, STRAIGHT THRU, & 1 \\
\hline 1 & SNG1003.12A & INCONEL 625 & $\begin{array}{c}\text { TUBE, 1/4" X.049" WALL, } \\
\text { INJECTOR WAND }\end{array}$ & 1 \\
\hline $\begin{array}{c}\text { ITEM } \\
\text { NO. }\end{array}$ & PART NUMBER & MATERIAL & DESCRIPTION & QTY. \\
\hline
\end{tabular}
COAL TO SNG IITLE: KINETICS REACTOR WELDMENT, STRAIGHT NOZZEL INJECTOR 
1 CONCENTRICITY TO BE MAINTAINED, AS SHOWN BETWEEN ITEMS; 1, 3, AND 5 AFTER WELDING.

2 CONCENTRICITY / COAXIALITY TO BE MAINTAINED, AS SHOWN BETWEEN ITEMS;

3 WELDS TO MEET (PV) PRESSURE VESSELS CODE, PER ANSI B16.9

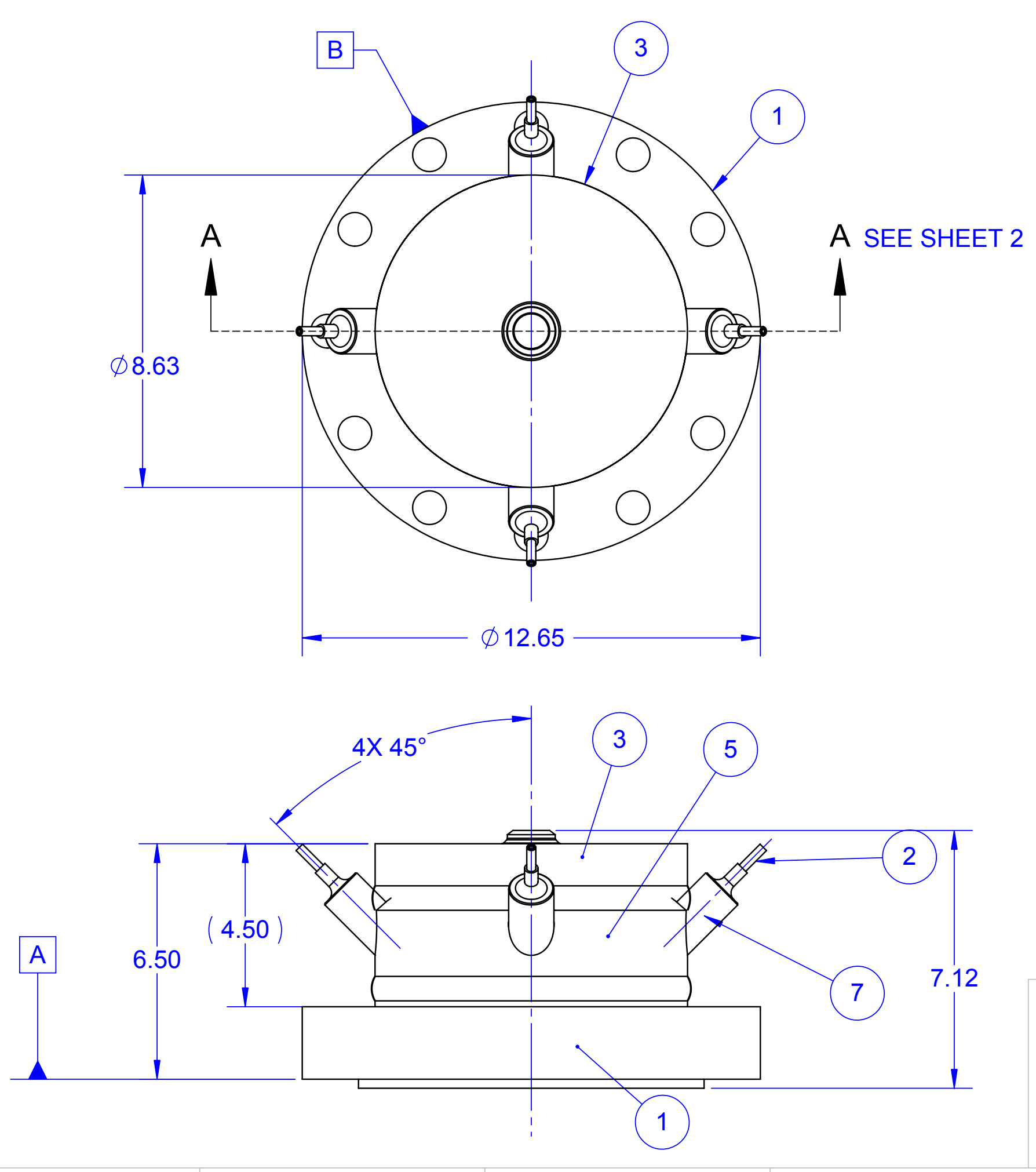

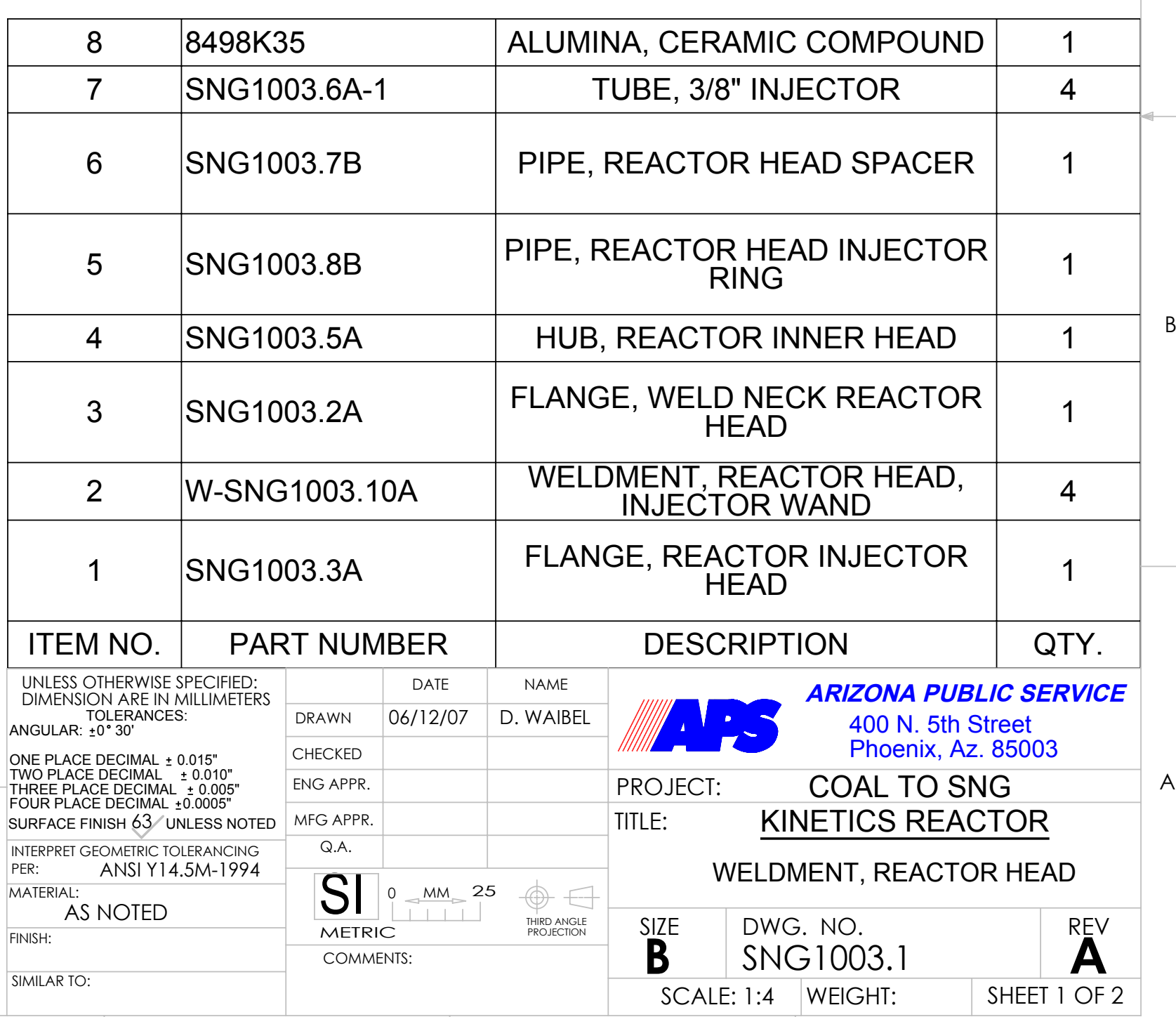



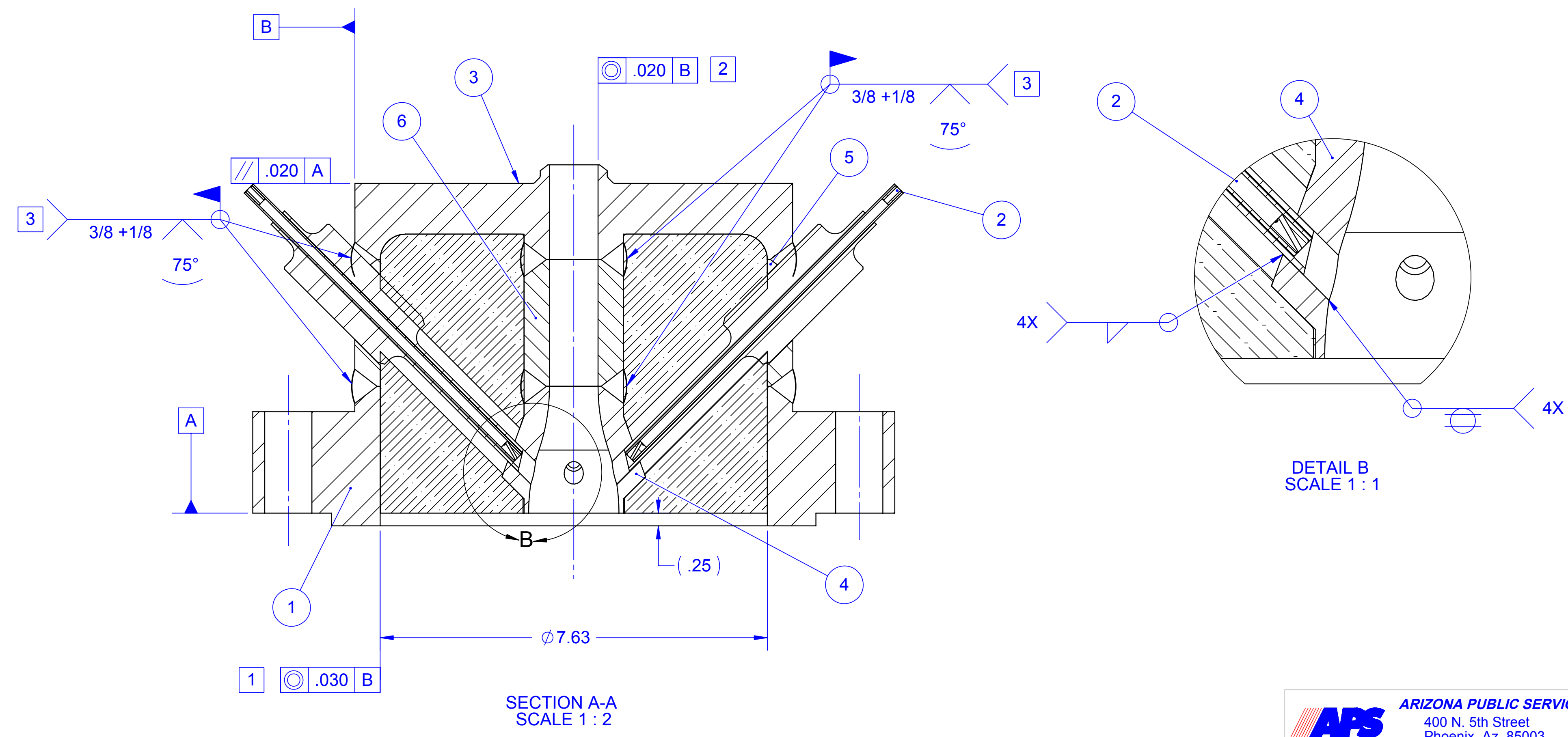

DETAIL B
SCALE $1: 1$

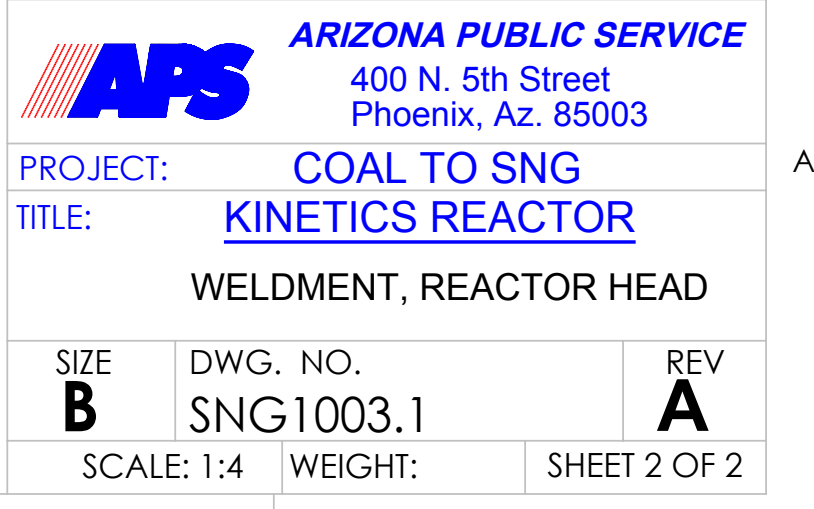




\begin{tabular}{|c|c|c|c|c|c|}
\hline & & REVI & & & 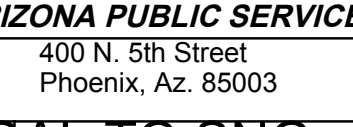 \\
\hline ZONE & REV. & DESCRIPTION & DATER & 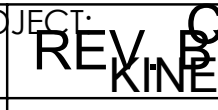 & 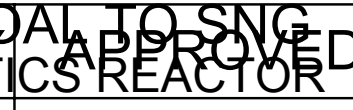 \\
\hline & A & INITIAL DESIGN & $07 / 04 / 07$ & D.W. & \\
\hline & & & & & \\
\hline & & & & & \\
\hline & & & & & \\
\hline & & & & & \\
\hline & & $\circ$ & & & \\
\hline & & 。 & & & \\
\hline
\end{tabular}

A
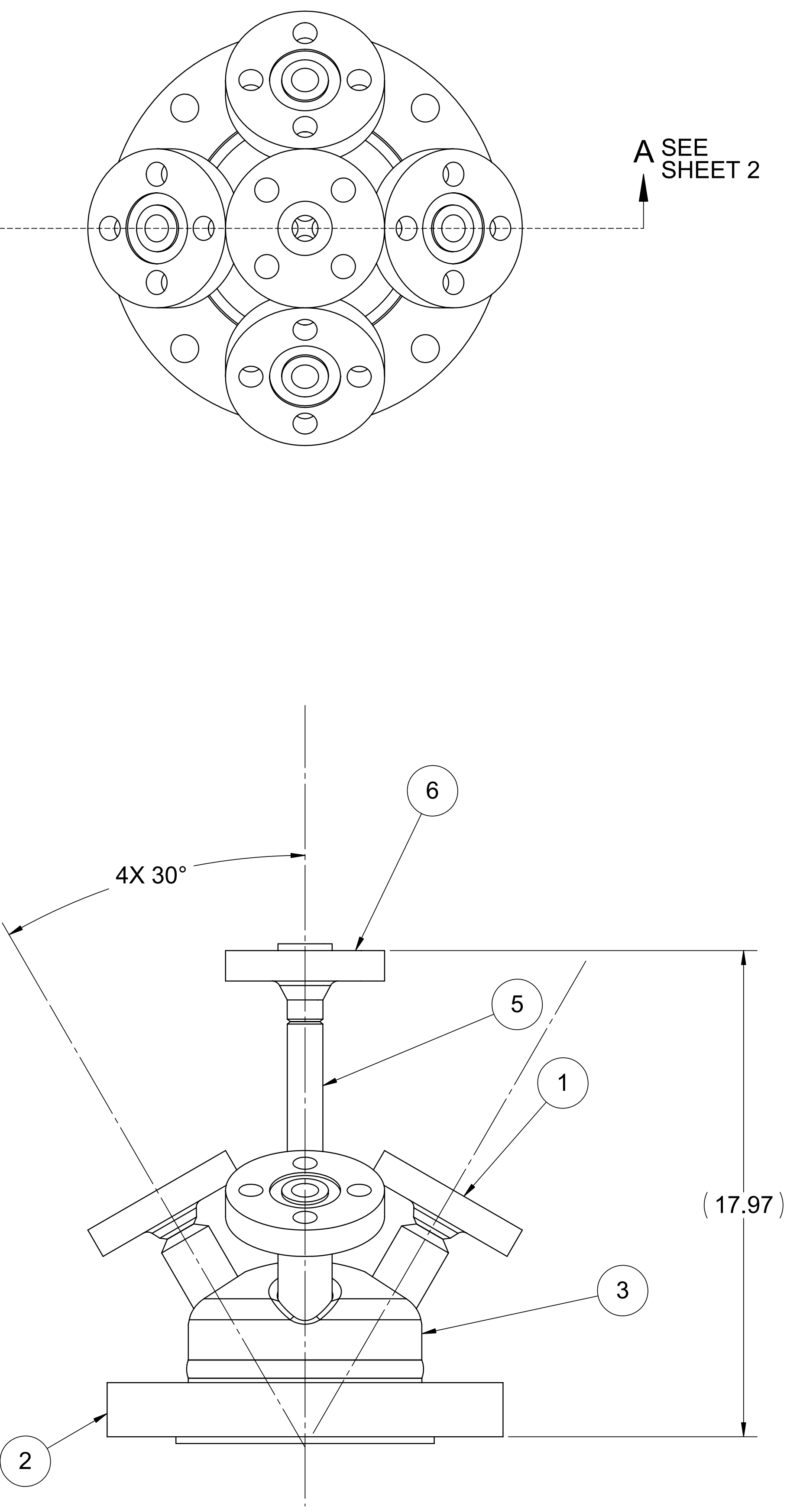

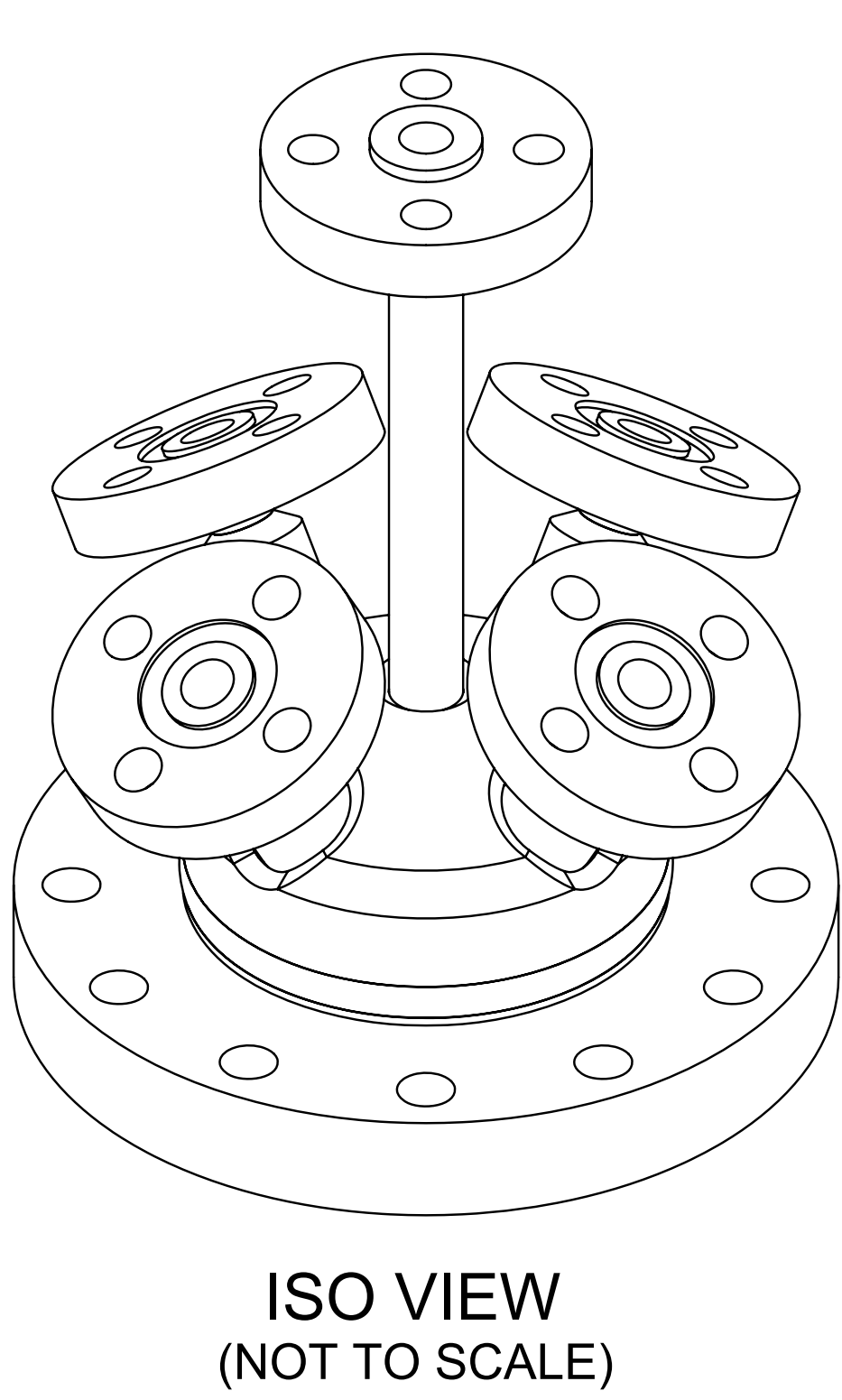

\begin{tabular}{|c|c|c|c|c|c|}
\hline 6 & SNG1002.51B & \multicolumn{2}{|c|}{ INCONEL 625, UNS-N-06625, SB-564, ASTM-B564 } & $\begin{array}{l}\text { 1"-WNF FGD INCONEL625 1500\# RF } \\
\text { BORE FOR SCH.80 } 125 \text { TO } 250 \text { AARH }\end{array}$ & \\
\hline 5 & SNG1003.31B & \multicolumn{2}{|c|}{ INCONEL 625, UNS-N-06625, SB-444, ASTM-B444 } & $\begin{array}{l}\text { PIPE 1"-SCH.80. } 19 \mathrm{WALL} \\
\text { INCONEL625 SMLS ASME B36.19 }\end{array}$ & 1 \\
\hline 4 & SNG1003.5K & \multicolumn{2}{|r|}{ INCONEL 625} & HUB, REACTOR INNER HEAD & 1 \\
\hline 3 & SNG1003.41B & 4 & SA-182, GRADE 316H ST. STL. & 8" SCH. 80 PIPE CAP & 1 \\
\hline 2 & SNG1003.3B & 4 & 316H ST.STL. FORGING & FLANGE, REACTOR INJECTOR HEAD & 1 \\
\hline 1 & W-SNG1003.27C & & SEE BOM & $\begin{array}{l}\text { WELDMENT, INJECTOR WAND } \\
\text { HOUSING }\end{array}$ & 4 \\
\hline EM NO. & PART NUMBER & & MATERIAL & DESCRIPTION & QTY \\
\hline
\end{tabular}

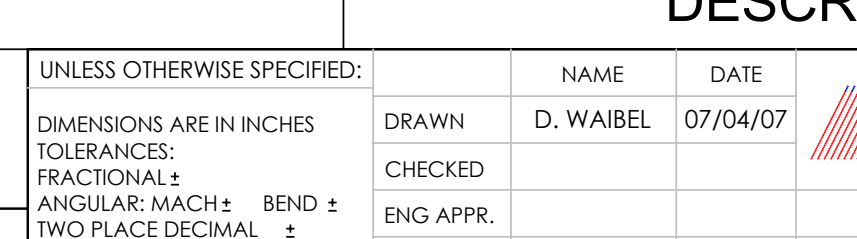

IIIE:

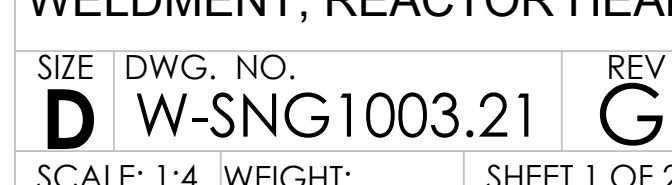




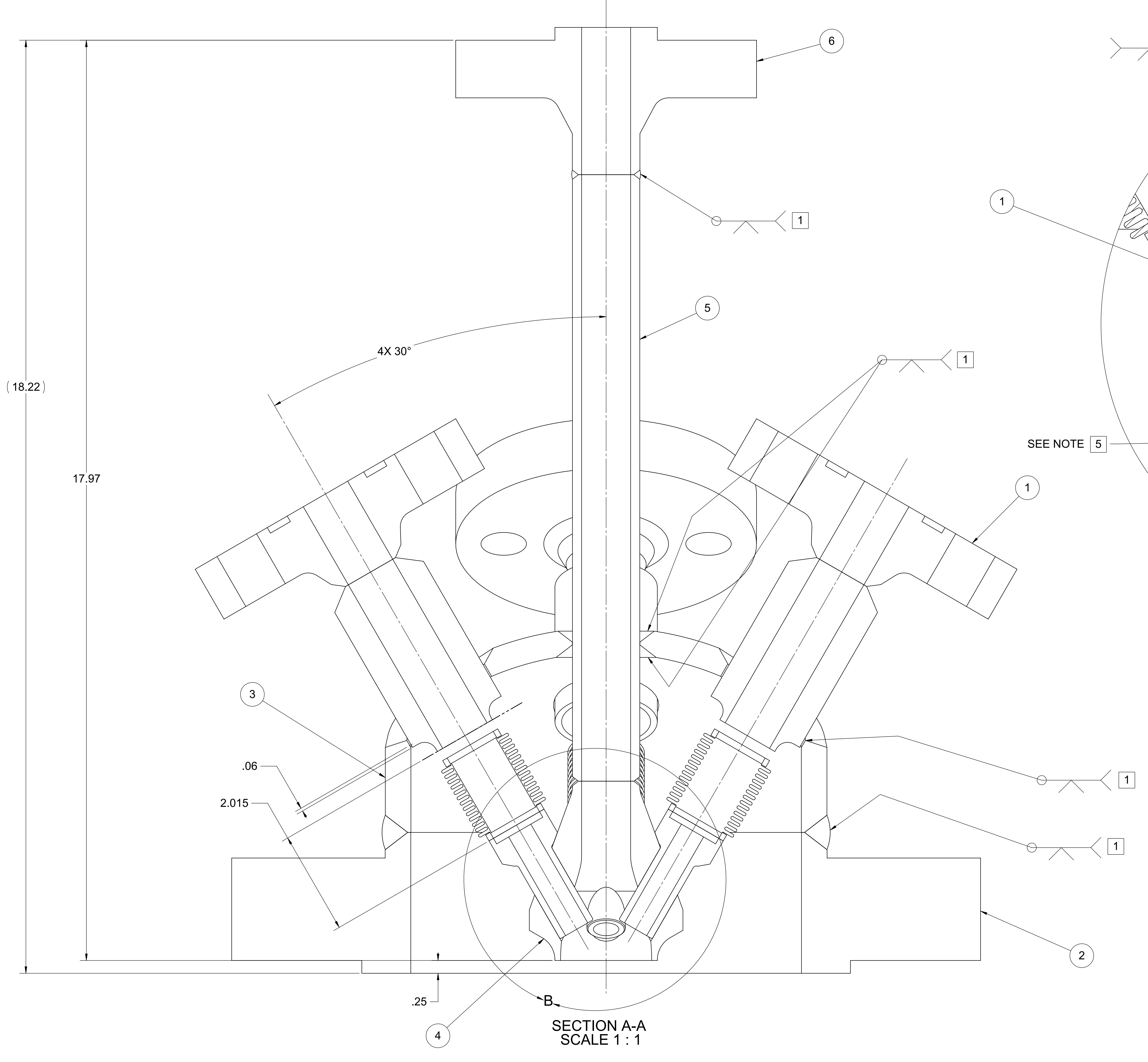

(5)

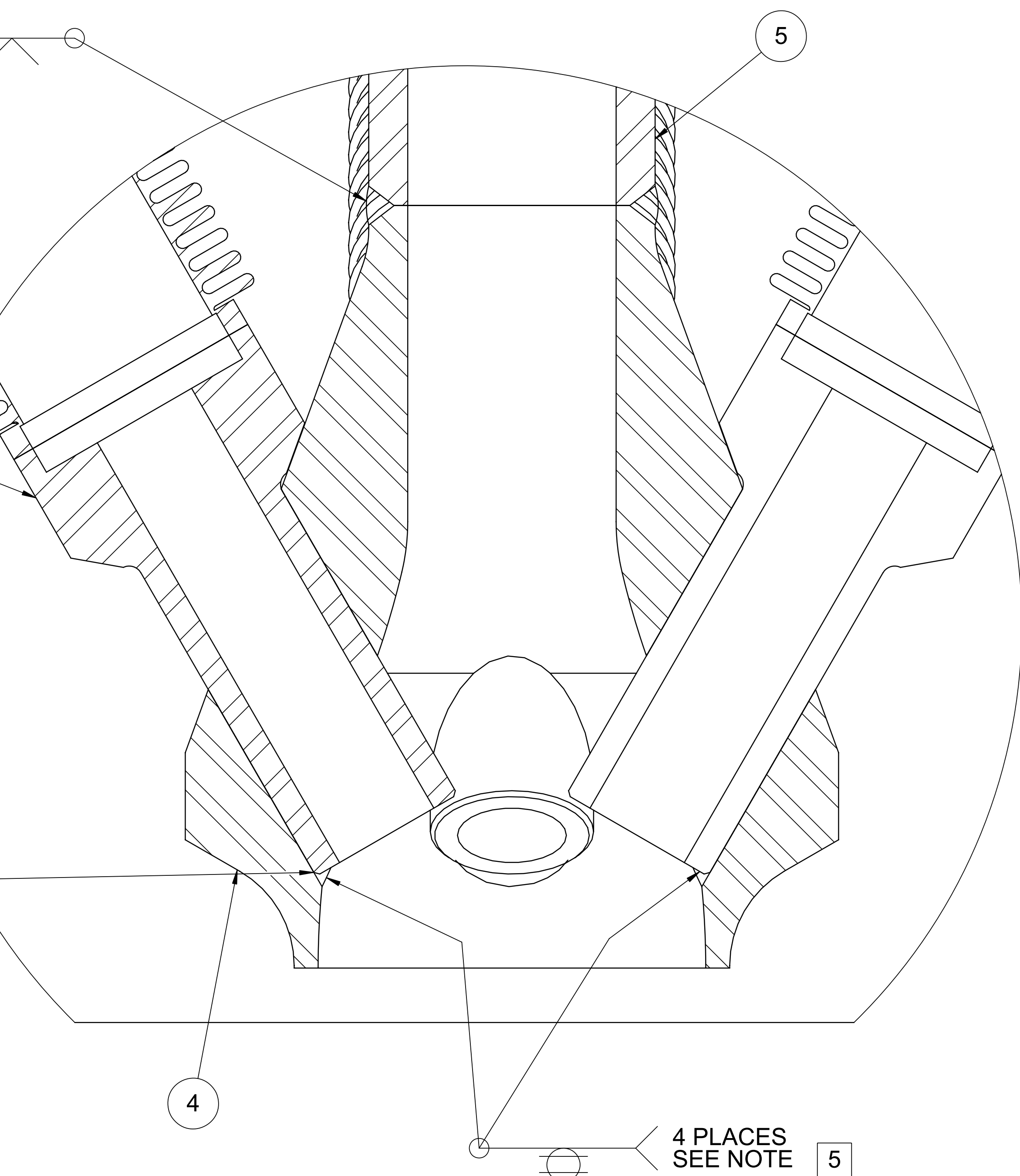

DETALL B
SCALE $2: 1$ 


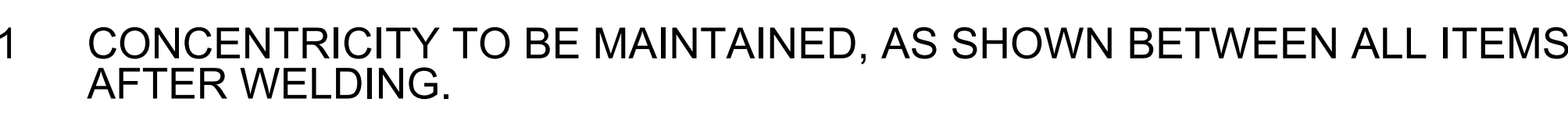

2 ALL WELDS TO MEET PRESSURE VESSEL CODE, ASME SECTION 8, DIVISION 1 ,
UNLESS NOTED OTHERWISE.

3 INSPECTION OF WELDS TO BE 100\% X-RAY, UNLESS SPECIFIED OTHERWISE,

4 FITTINGS, AND PIPE, TO BE IN ACCORDANCE WITH ANSI B16.9. FLANGES IN

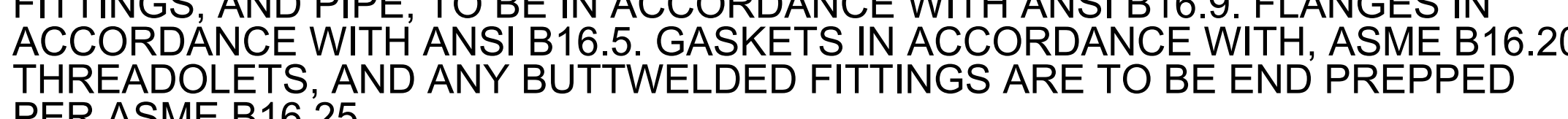

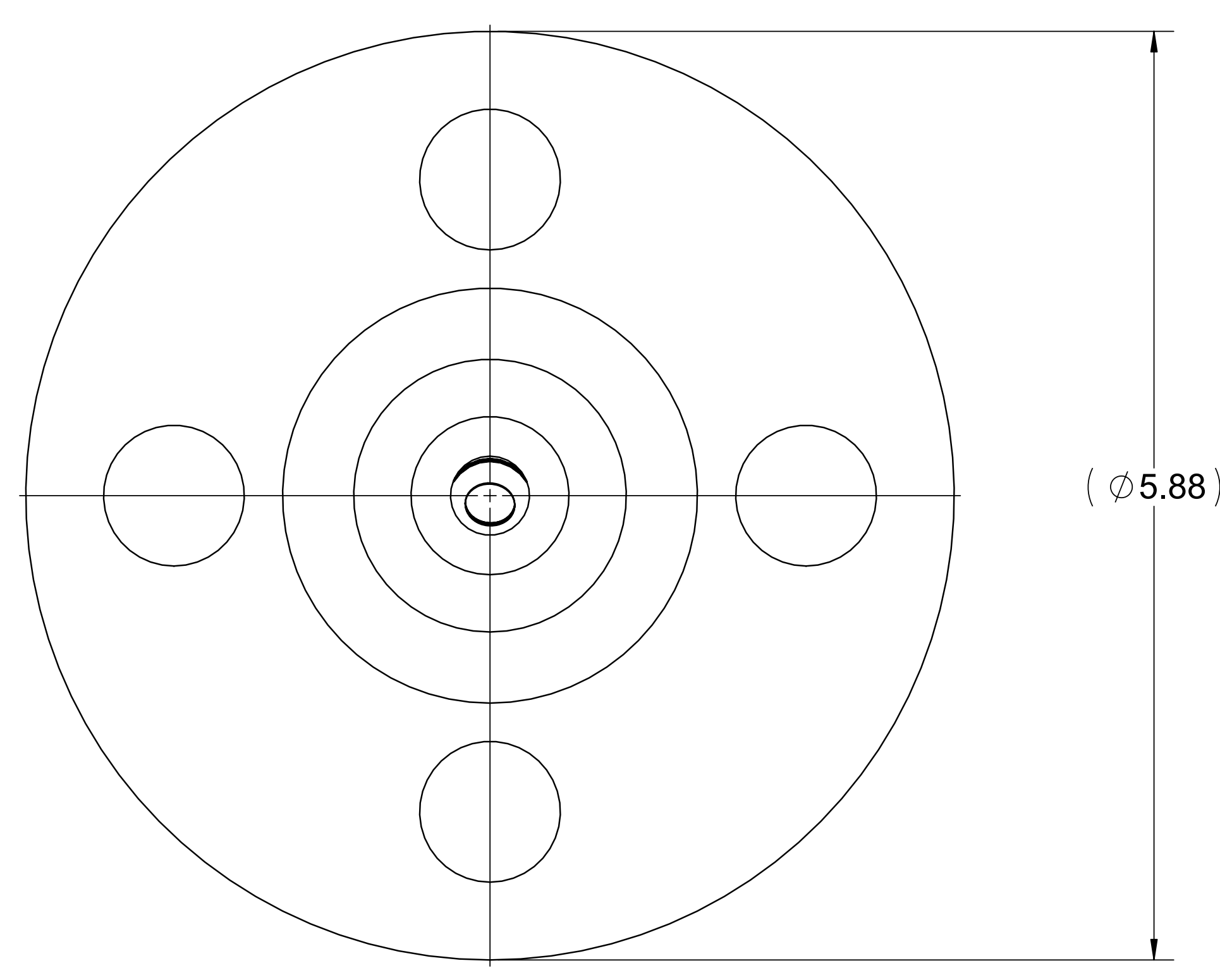

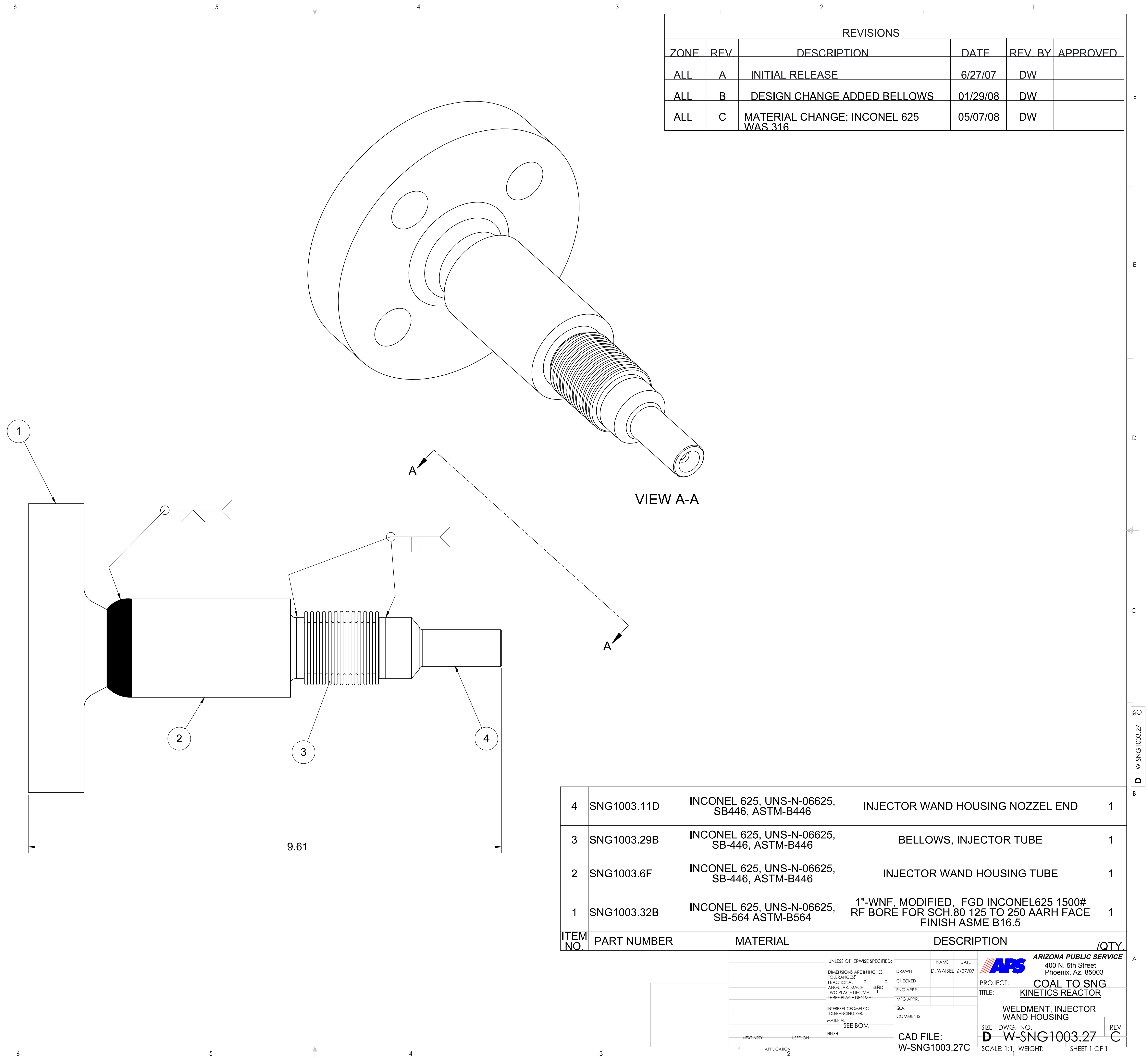


GENERAL NOTES

CONCENTRICITY TO BE MAINTAINED, AS SHOWN BETWEEN ALL ITEMS
AFTER WELDING.

2 ALL WELDS TO MEET PRESSURE VESSEL CODE, ASME SECTION 8, DIVISION 1 ,

3 INSPECTION OF WELDS TO BE 100\% X-RAY, UNLESS SPECIFIED OTHERWISE.

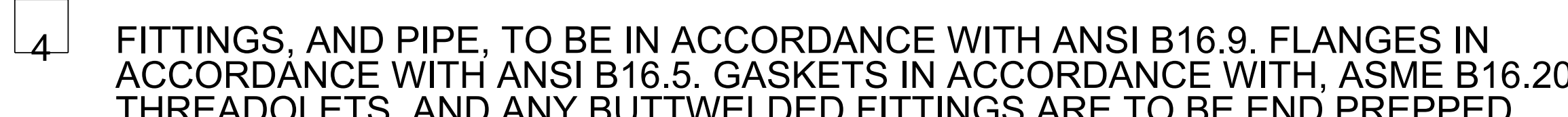
THRADOLEES, AND ANY BUTTWELDED FITTINGS ARE TO BE END PREPPED
PER ASME B16.25.

5. MATERIAL; 316 STAINLESS STEEL CARBON CONTENT MUST BE EQUAL TO, OR
ABOVE. $04 \%$

\section{ZONE REV. DESCRIPTION DATE REV. BY APPROVED}

ALL A INITIAL RELEASE

0/17/07 DW

ALL B DESIGN CHANGE, ADDED FITTING
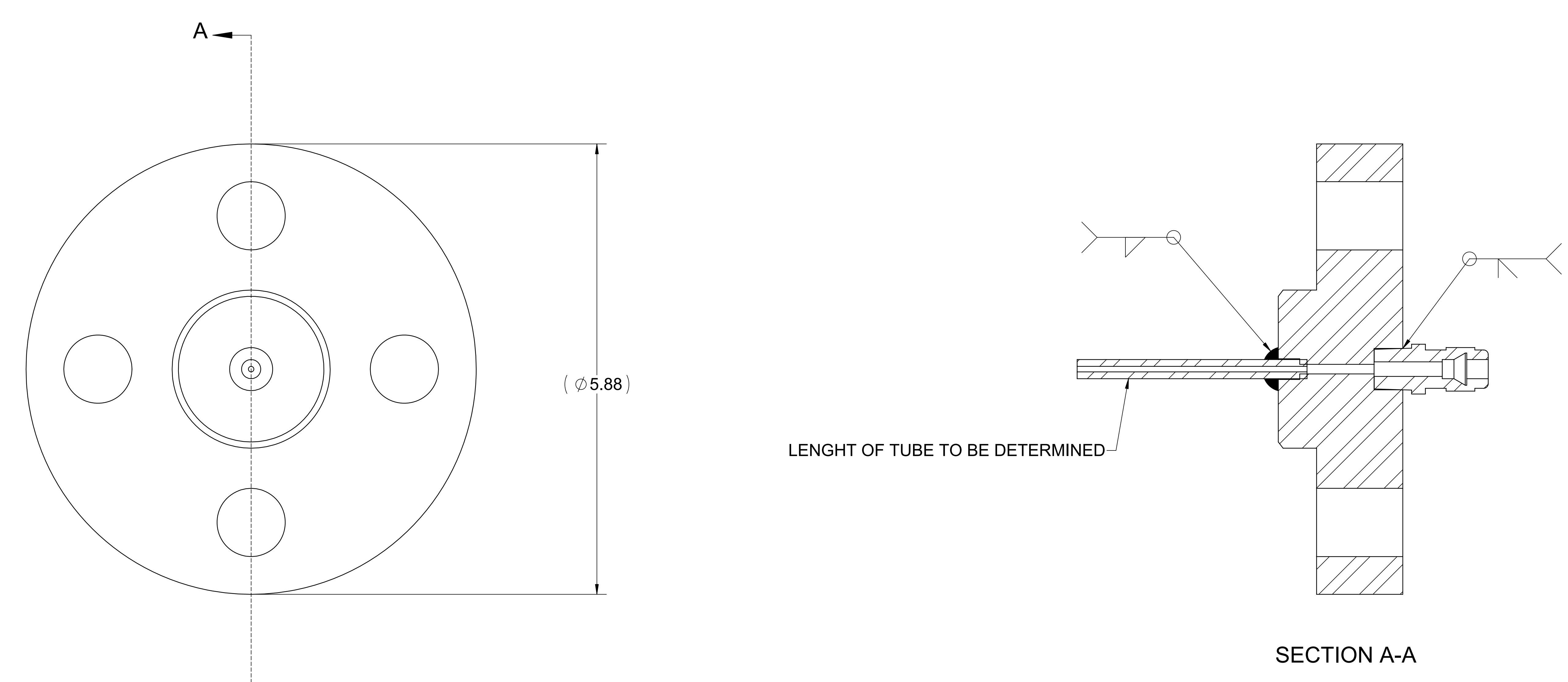

A-

SECTION A-A

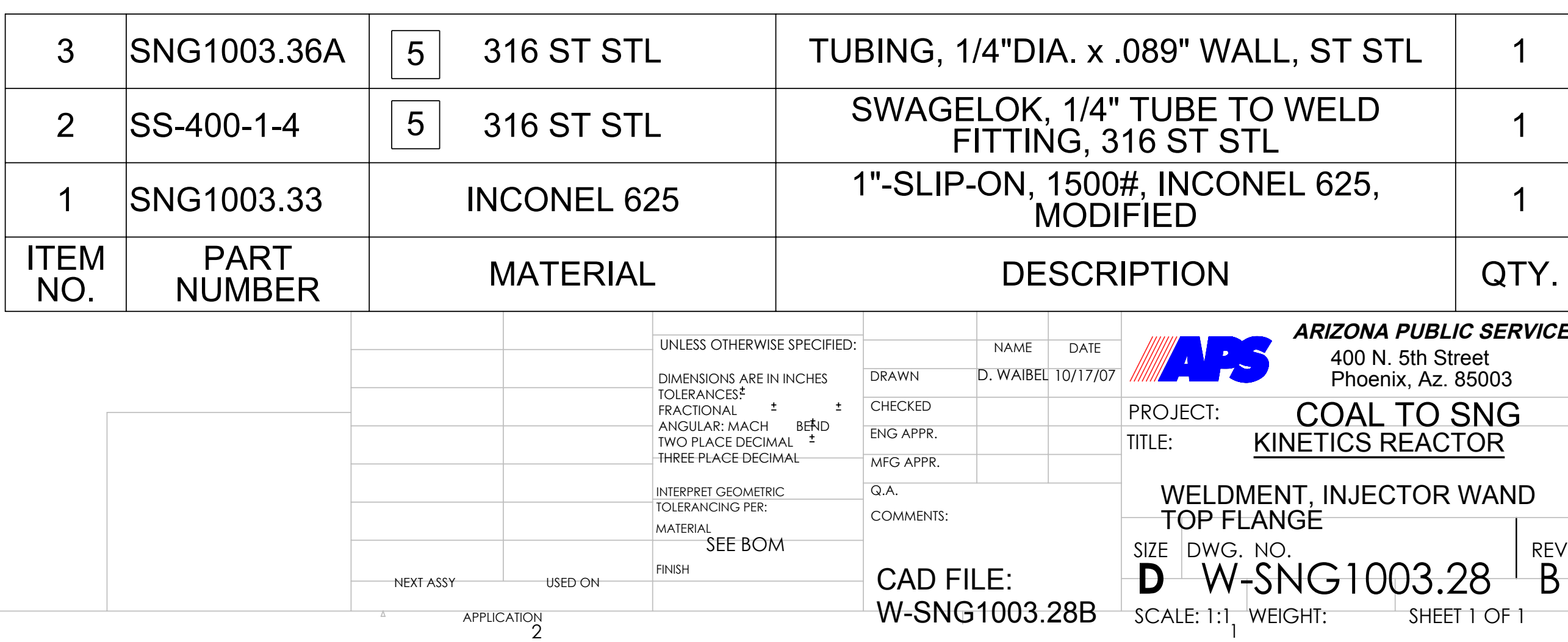


GENERAL NOTES:

CONCENTRICITY / COAXIALITY TO BE MAINTAINED, AS SHOWN BETWEEN ITEMS
1 AND 2 AFTER WELDING.

WELDS TO MEET (PV) PRESSURE VESSELS CODE, PER ANSI B16.9
ALL WELDSTOBEFRE OF ANY POROCITIES AND SLAG, DO NOT

3 REFERENCE DRAWING W-SNG1003.21, FOR DIMENSIONS AND WELDING.

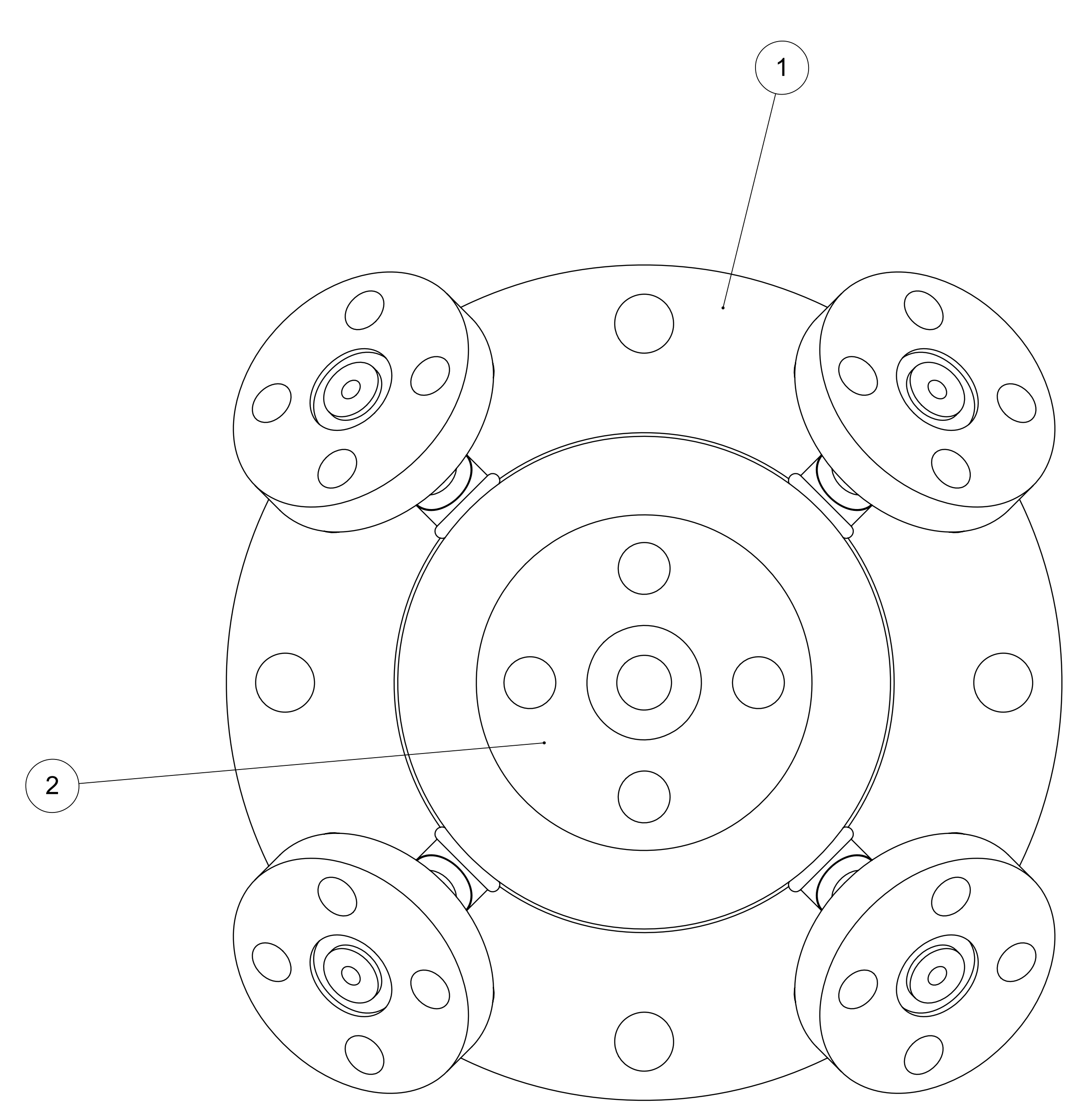

\section{ZOME REV REVISIONS}

ZONE REV. DESCRIPTION DATE REV. BY APPROVED

ALL A INITIAL RELEASE 11/20/07 DW

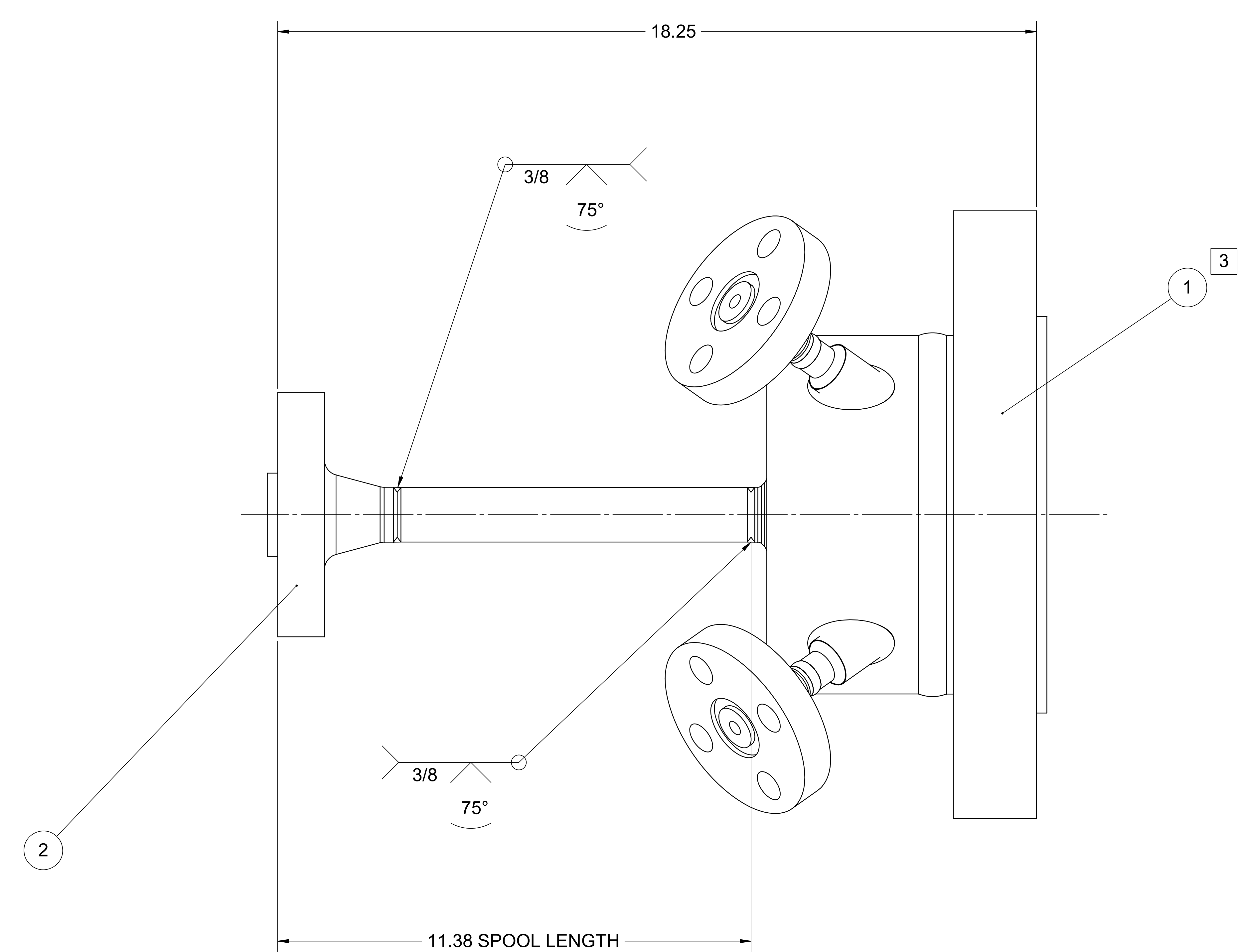

\begin{tabular}{|c|c|c|c|}
\hline 2 & W-SNG1001.15A & WELDMENT, COAL FEED PIPE & 1 \\
\hline 1 & W-SNG1003.21B & WELMENT, REACTOR HEAD & 1 \\
\hline ITEM NO. & PART NUMBER & DESCRIPTION & QTY. \\
\hline
\end{tabular}

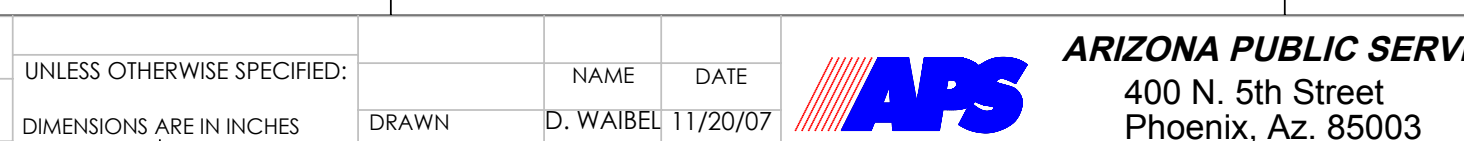

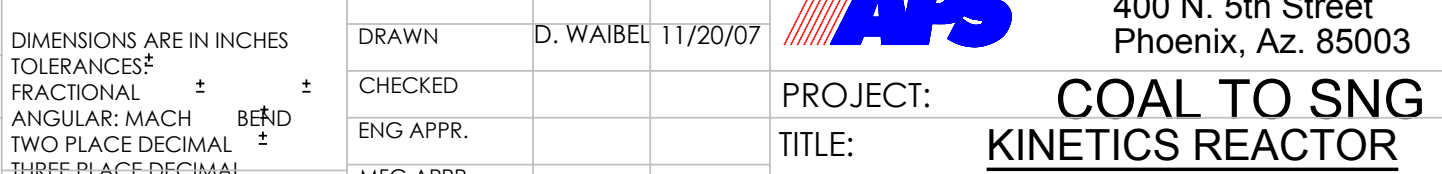

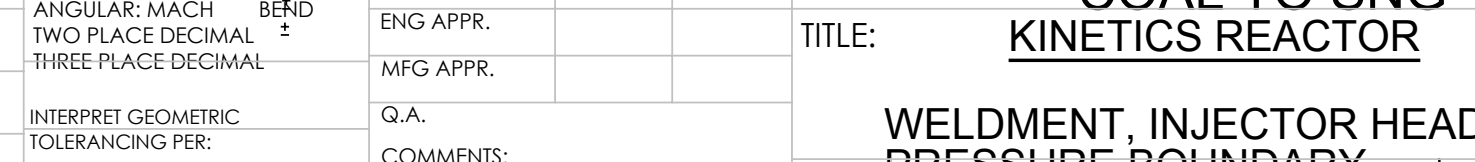

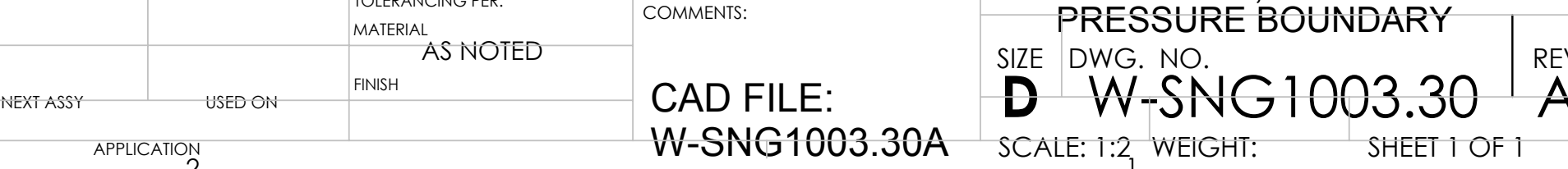


FITTINGS, AND HARDWARE, TO BE IN ACCORDANCE WITH ANSI B16.9. FLANGES IN

TREADOLTS, AND ANY BUTTWELDED FITTINGS ARE TO BE END PREPPED

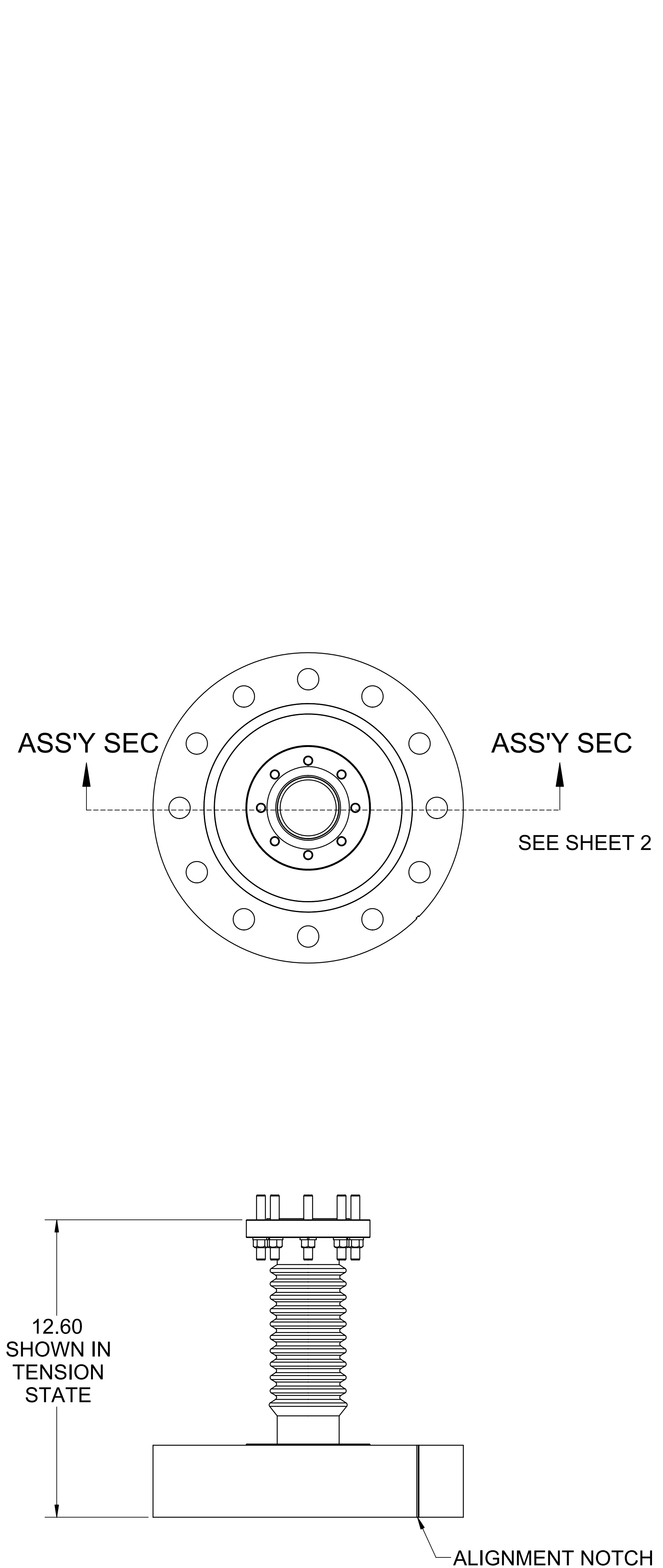

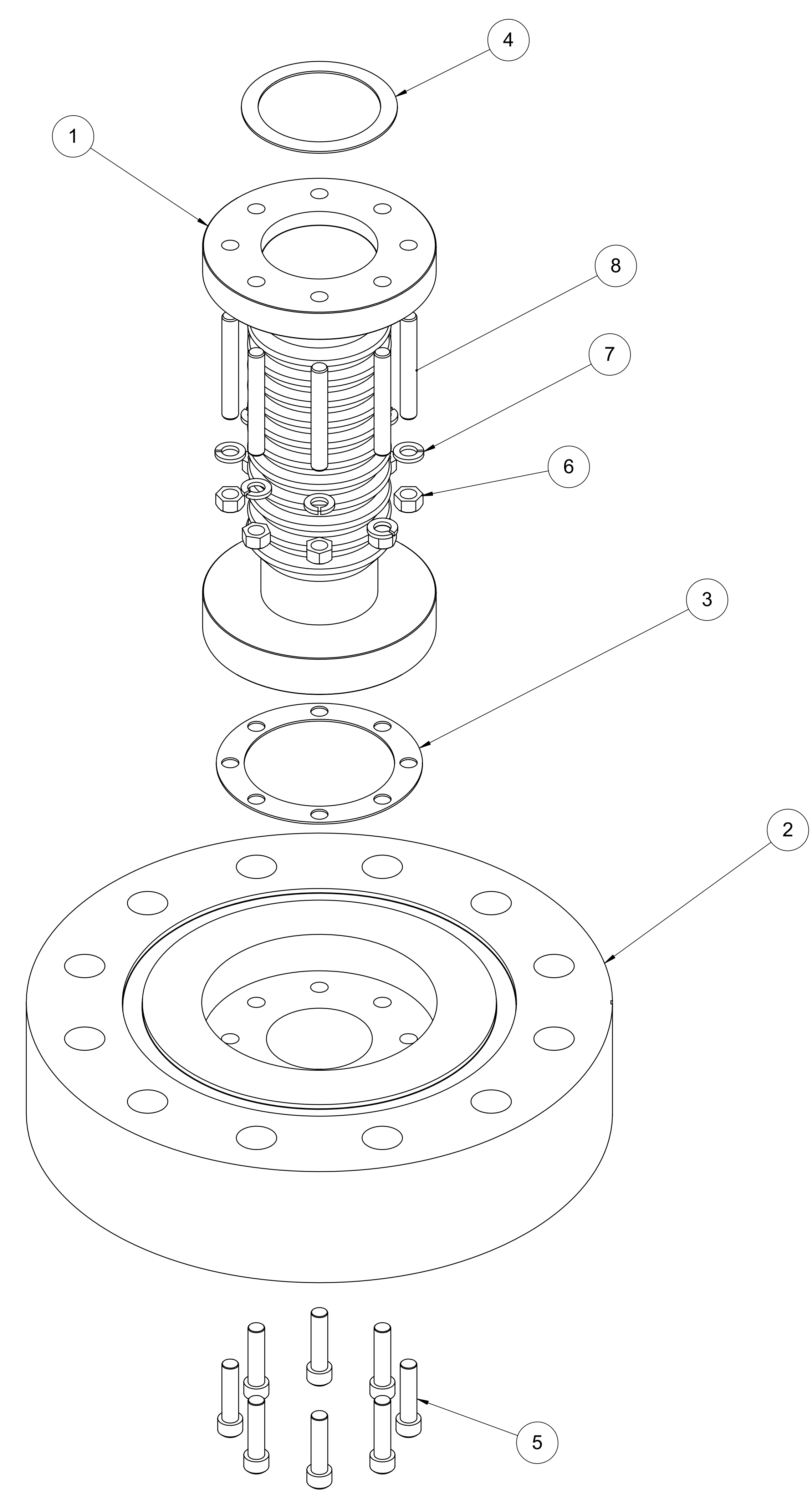

\begin{tabular}{|c|c|c|c|c|c|}
\hline \multicolumn{6}{|c|}{ REVISIONS } \\
\hline ZONE & REV. & DESCRIPTION & DATE & REV.BY & APPROV \\
\hline ALL & A & INITIAL DESIGN & $06 / 25 / 07$ & DW & \\
\hline ALL & B & $\begin{array}{l}\text { ADDEDD HARDWARE, CHANGED } \\
\text { MATERAL SEC ONBEWS }\end{array}$ & 12/20/07 & DW & \\
\hline ALL & c & $\begin{array}{l}\text { DELETED; ASME SEC III DIV. D } \\
\text { IN MATERIAL CALL OUT }\end{array}$ & 12/26//07 & DW & \\
\hline ALL & D & CHANGE TO ITEM 2; ADAPTER FLG. & 12/30/07 & DW & \\
\hline ALL & E & $\begin{array}{l}\text { DIM. CHANGE TO ADAPTER FLG } \\
\text { SEE DWG SNG1004.3 UPDATED }\end{array}$ & 01/06/08 & DW & \\
\hline
\end{tabular}

c

\begin{tabular}{|c|c|c|c|c|}
\hline 8 & BEST SOURCE & $\begin{array}{l}\text { INCONEL } 625 \text { B6 } \\
\text { GRADE } 660 \text { OR } 651\end{array}$ & 3/8-24 × 2.75LG THD STUD & \\
\hline 7 & BEST SOURCE & $\begin{array}{l}\text { INCONEL } 625 \text { B6 } \\
\text { GRADE } 660 \text { OR } 651\end{array}$ & 3/8" LOCK WASHER & \\
\hline 6 & BEST SOURCE & $\begin{array}{l}\text { INCONEL } 625 \text { B6 } \\
\text { GRADE } 660 \text { OR } 651\end{array}$ & 3/8-24 UNF, HEX NUT & \\
\hline 5 & BEST SOURCE & $\begin{array}{l}\text { INCONEL } 625 \mathrm{~B} 6 \\
\text { GRADE } 660 \text { OR } 651\end{array}$ & $\begin{array}{l}\text { 3/8-24 UNF-2A x 1.50LG. INCONEL } 625 \text { B6 } \\
\text { GRADE } 660 \text { OR } 651\end{array}$ & \\
\hline 4 & SNG1004.11 & THERMICULITE 815 & GASKET, TOP BELLOWS FLANGE & \\
\hline 3 & SNG1004.10 & THERMICULITE 815 & GASKET, BOTTOM BELLOWS FLANGE & \\
\hline 2 & SNG1004.3 & $\begin{array}{l}\text { SA } 182 \text { GR.316H } \\
\text { S.S. }\end{array}$ & $\begin{array}{c}\text { FLANGE, ADAPTER, REACTOR TO CHAR } \\
\text { POT }\end{array}$ & \\
\hline 1 & W-SNG1004.9 & $\begin{array}{l}\text { SB409-ALLOY 800H, } \\
\text { ASMESBS490 UNS } \\
\text { No88810 }\end{array}$ & WELDMENT, REATOR BELLOWS & \\
\hline ITEM & PART NUMBER & MATERIAL & DESCRIPTION & \\
\hline
\end{tabular}

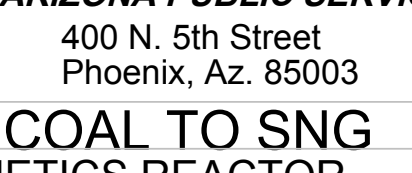

COAL TO SNG
LETICS REACTOR

SSEMBLY, REACTOR BELLOWS

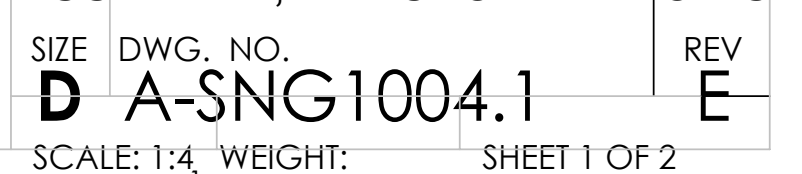



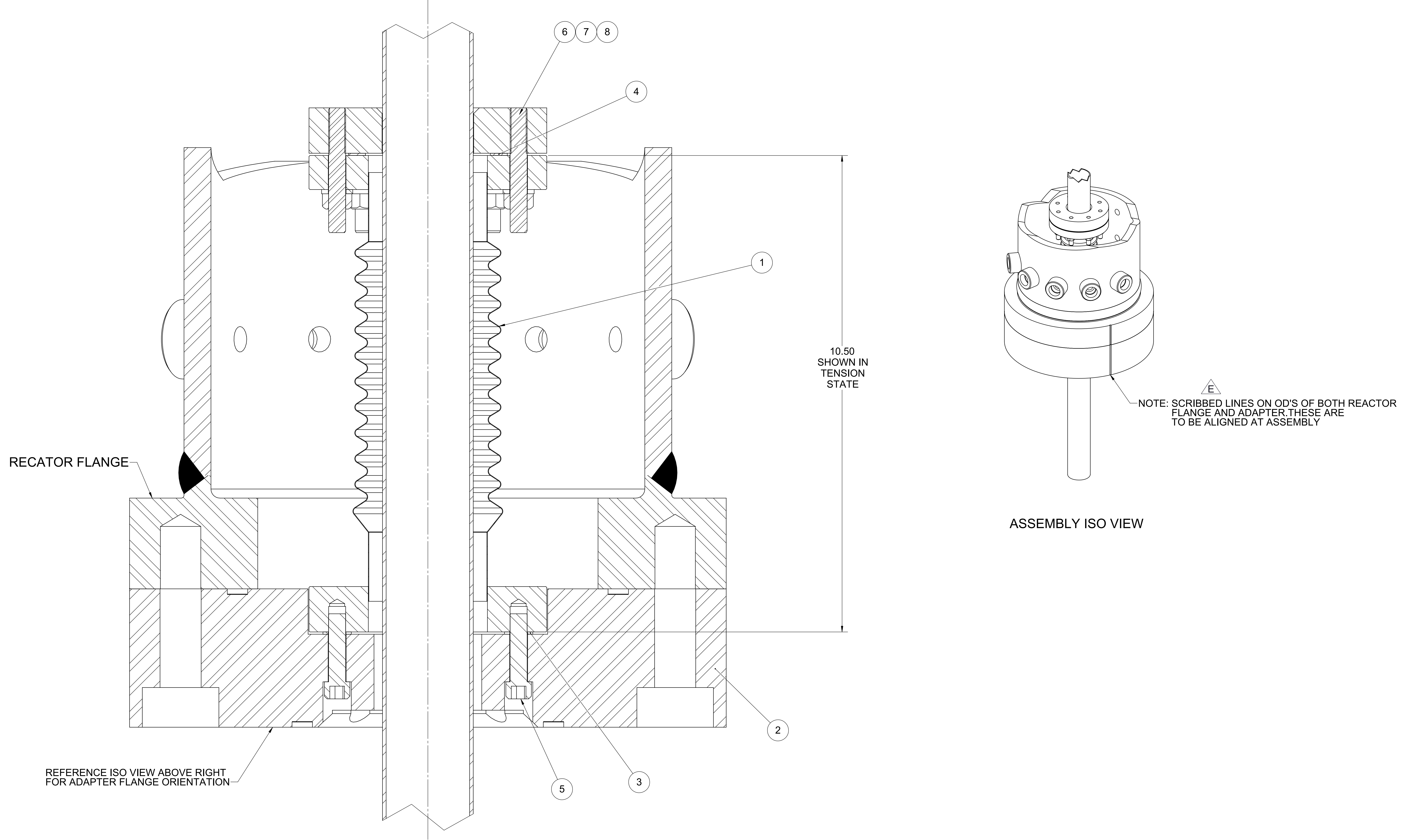

ASSEMBLY ISO VIEW 
DIMENSION AND TOLERANCING PER ANSI Y14 5-1994.

2 GASKET TO BE SOFT CUT, CRITICAL SERVICE SERIES, FLEXITALLIC THERMICULITE 815 WITH .004" 316SS TANGED REINFORCEMENT.

\begin{tabular}{|l|c|c|c|c|}
\hline \multicolumn{4}{c}{ REVISIONS } \\
\hline ZONE & REV. & DESCRPTION & & \\
\hline ALL & A & INITIAL RELEASE & REV. BY APPROV \\
\hline & & $12 / 24 / 07$ & DW \\
\hline
\end{tabular}

D

$\varnothing .391 \mathrm{THRU}$ EQ. SPACED ON $\varnothing 4.00$ B.C.
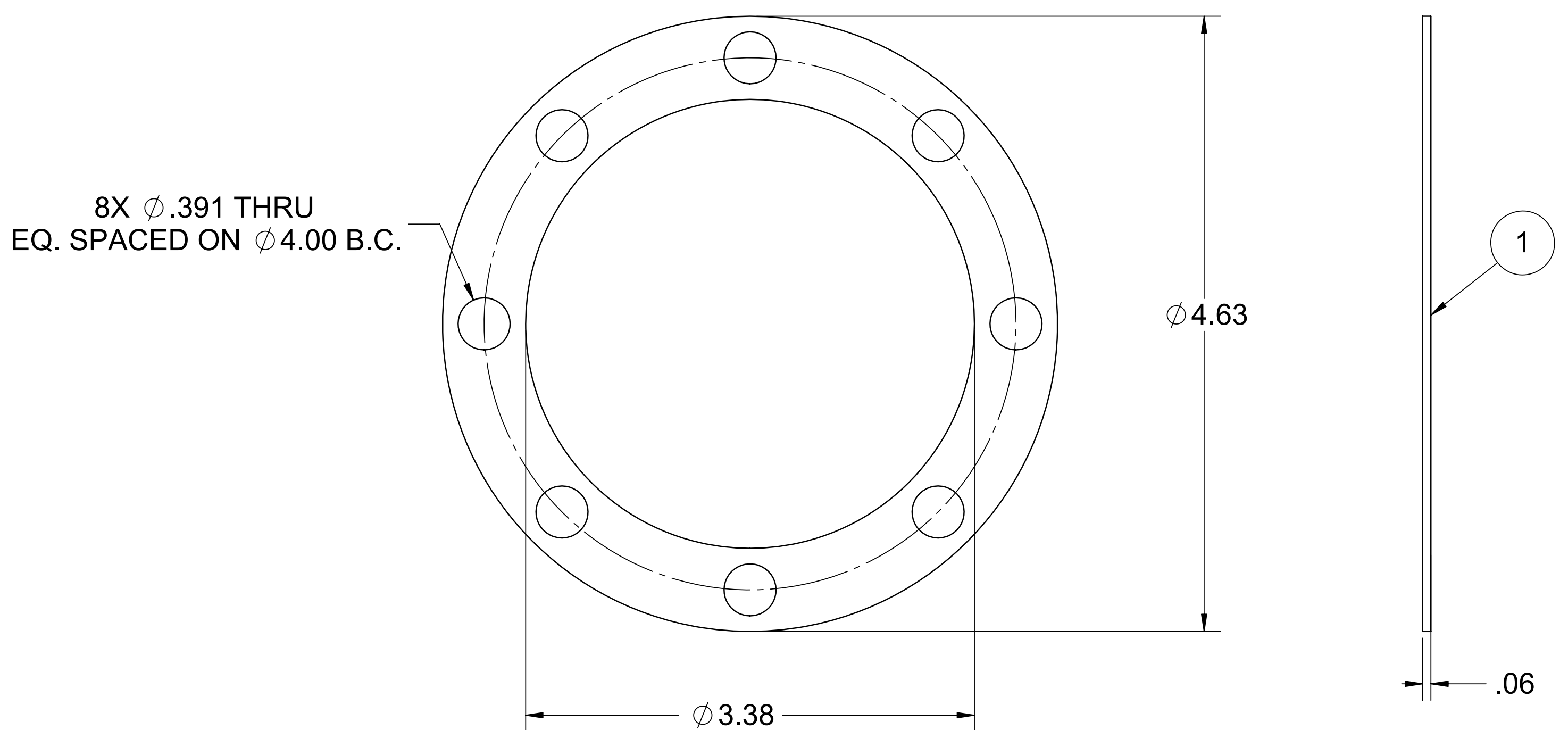

\begin{tabular}{|c|c|c|c|c|}
\hline 1 & SNG1004.10B & $\begin{array}{c}\text { THERMICULITE } \\
815\end{array}$ & GASKET, BOTTOM BELLOWS FLANGE & 1 \\
\hline ITEM & PART NUMBER & MATERIAL & DESCRIPTION & QTY. \\
NO. & PAT & S
\end{tabular}

\begin{tabular}{|c|c|c|c|c|c|c|}
\hline UNLESS OTHERWISE SPECIFIED: & & & & \multirow{3}{*}{\multicolumn{3}{|c|}{$\begin{array}{l}\text { ARIZONA PUBLIC SERVICE } \\
400 \text { N. 5th Street } \\
\text { Phoenix, Az. } 85003\end{array}$}} \\
\hline $\begin{array}{l}\text { DIMENSION ARE EN MIILLIMETERS } \\
\text { ANGULAR: OLERANACES: }\end{array}$ & DRAWN & D. WAIBEL & $12 / 24 / 07$ & & & \\
\hline 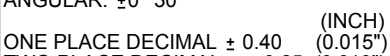 & CHECKED & & & & & \\
\hline 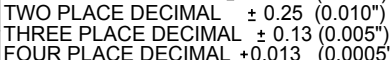 & ENG APPR. & & & \multirow{2}{*}{$\begin{array}{l}\text { PROJE } \\
\text { TITLE: }\end{array}$} & \multicolumn{2}{|c|}{ COAL TO SNG } \\
\hline $\begin{array}{l}\text { SURFACE FINISH } 63 \text {, UNLESS NOTED } \\
\text { SUTE }\end{array}$ & MFG APPR. & & & & \multicolumn{2}{|c|}{ KINETICS REACTOR } \\
\hline 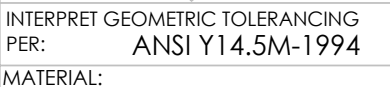 & \multirow{3}{*}{$\begin{array}{l}\text { SI } \\
\text { METRI } \\
\text { COMME }\end{array}$} & & \multirow{3}{*}{ 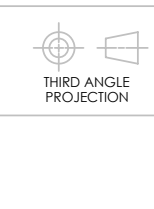 } & \multicolumn{3}{|c|}{ GASKET, BOTTOM BELLOWS FLANGE } \\
\hline $\begin{array}{l}\text { MAIERALE: THERMICULITE } 815 \\
\text { FNSH: }\end{array}$ & & $\begin{array}{lll}0 & \mathrm{MM} & 25 \\
= & 1 & 1\end{array}$ & & \multirow{2}{*}{${ }_{\text {SC }}^{\text {SIZE }}$} & $\begin{array}{l}\text { DWG. NO. } \\
\text { SNG } 1004.10\end{array}$ & REV \\
\hline SIMILAR & & & & & ALE: $1: 1 \quad$ WEIGHT: & $1 \mathrm{OF} 1$ \\
\hline
\end{tabular}


DIMENSION AND TOLERANCING PER ANSI Y14.5-1994.

2 GASKET TO BE SOFT CUT, CRITICAL SERVICE SERIES, FLEXITALLIC

THERMICULITE 815 WITH .004" 316SS TANGED REINFORCEMENT.

$8 X \varnothing .391 \mathrm{THRU}$

EQ. SPACED ON $\varnothing 4.00$ B.C.
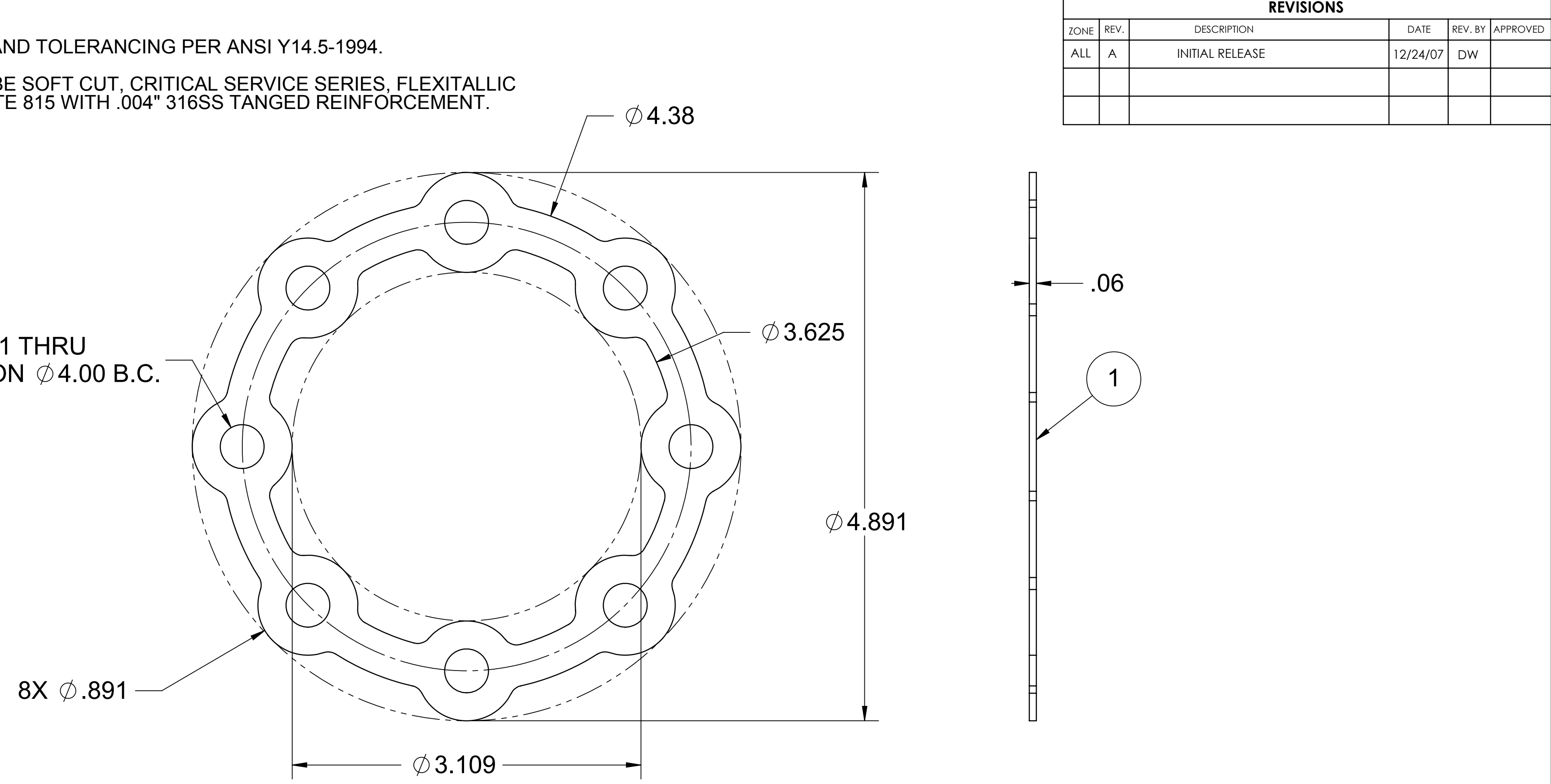

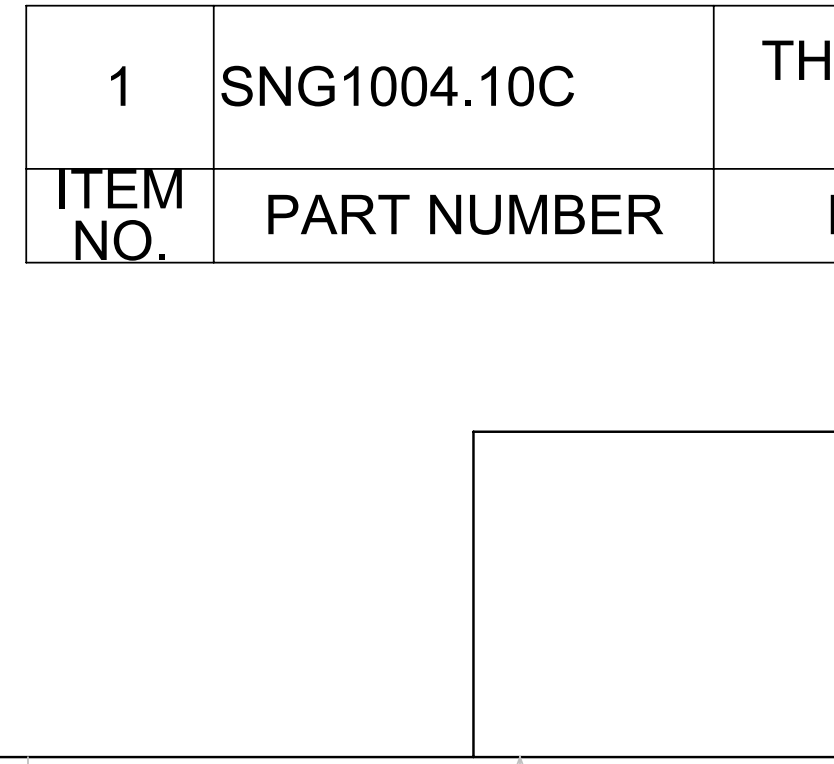

\begin{tabular}{|c|c|c|c|c|c|c|}
\hline 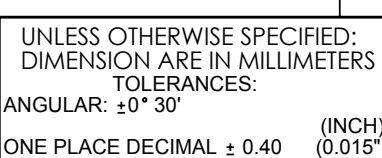 & $\begin{array}{ll}\text { DRAWN } \\
\text { CHECKED }\end{array}$ & \begin{tabular}{c|c|} 
DAFE \\
D. WABBEL \\
\end{tabular} & $\begin{array}{c}\text { NAME } \\
12 / 24 / 07 \\
\end{array}$ & \multicolumn{3}{|c|}{$\begin{array}{l}\text { ARIZONA PUBLIC SERVICE } \\
400 \mathrm{~N} \text {. } 5 \text { ht Street } \\
\text { Phoenix, AZ. } 85003\end{array}$} \\
\hline 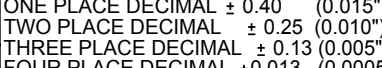 & ENG APPR. & & & \multicolumn{3}{|c|}{ COAL TO SNG } \\
\hline 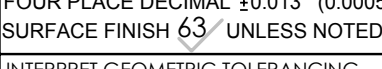 & MEG APR. & & & \multirow{2}{*}{\multicolumn{3}{|c|}{$\begin{array}{l}\text { TITLE: } \quad \text { KINETICS REACTOR } \\
\text { GASKET, BOTTOM BELLOWS FLANGE }\end{array}$}} \\
\hline 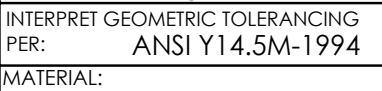 & $\mathrm{SI}$ & & & & & \\
\hline $\begin{array}{l}\text { THERMICULITE } 815 \\
\text { FNISH: } \\
\end{array}$ & \multirow{2}{*}{\multicolumn{2}{|c|}{$\begin{array}{l}\text { METRIC } \\
\text { COMMENS: }\end{array}$}} & \multirow[t]{2}{*}{ 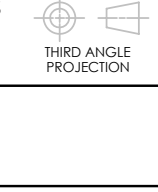 } & B & \multirow{2}{*}{$\begin{array}{l}\text { SWG. NO. } \\
\text { SNG } 1004.10\end{array}$} & C \\
\hline & & & & & & $1 O F 1$ \\
\hline
\end{tabular}


2 GASKET TO BE SOFT CUT, CRITICAL SERVICE SERIES, FLEXITALLIC

THERMICULITE 815, WITH .004" 316SS TANGED REINFORCEMENT.
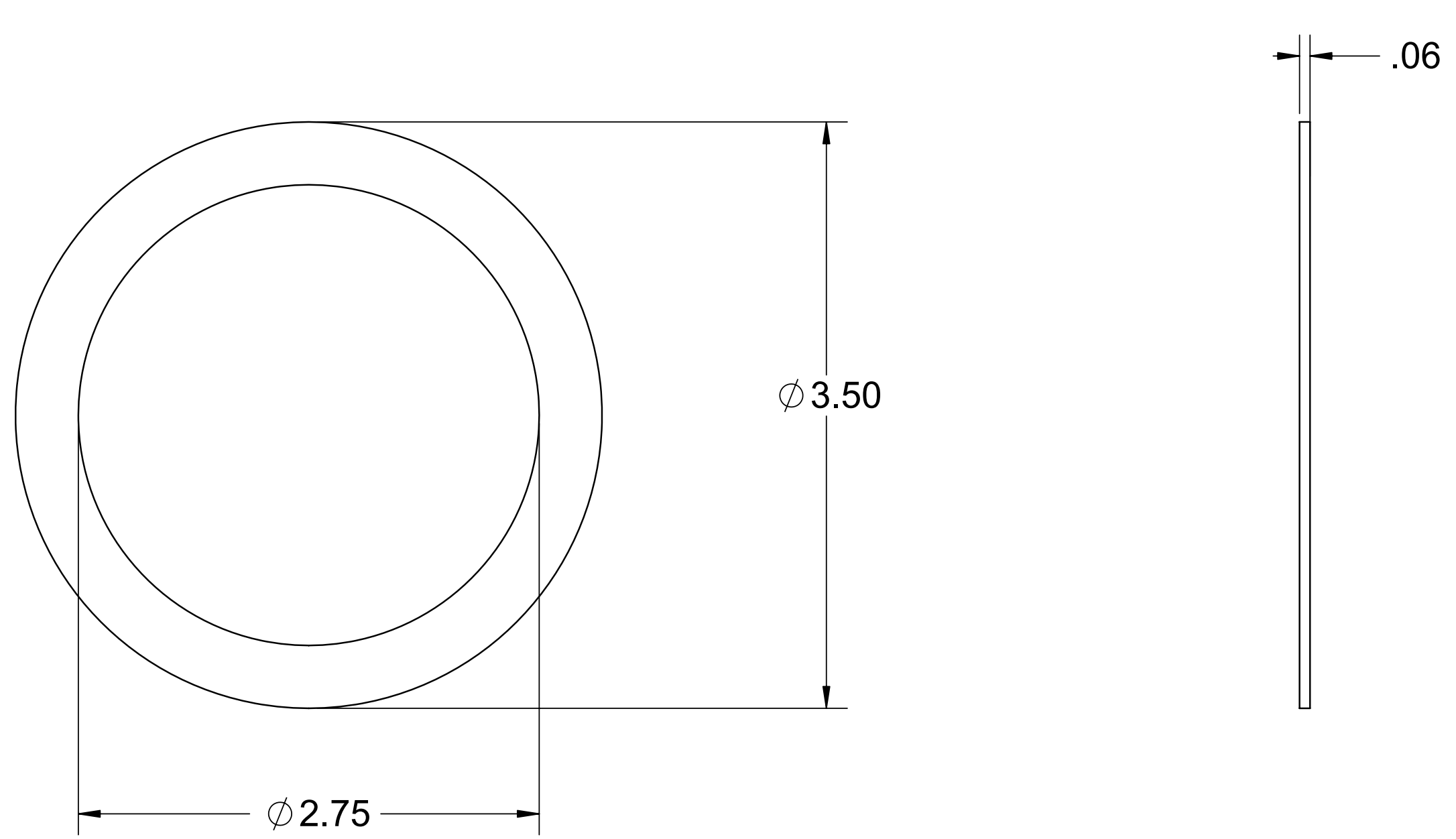

\begin{tabular}{|c|c|c|c|c|}
\hline 1 & SNG1004.11B & $\begin{array}{c}\text { THERMICULITE } \\
815\end{array}$ & GASKET, TOP BELLOWS FLANGE & 1 \\
\hline $\begin{array}{c}\text { ITEM } \\
\text { NO. }\end{array}$ & PART NUMBER & MATERIAL & DESCRIPTION & QTY. \\
\hline
\end{tabular}

\begin{tabular}{|c|c|c|c|}
\hline 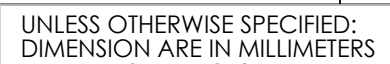 & & DATE & NAME \\
\hline $\begin{array}{l}\text { TNLERANCES: } \\
\text { ANGULAR: } 0^{\circ} 30^{\circ}\end{array}$ & DRAWN & D. WAIBEL & $12 / 24 / 07$ \\
\hline 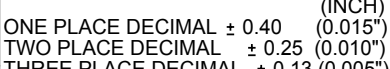 & CHECKED & & \\
\hline 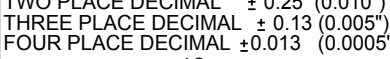 & ENG APPR. & & \\
\hline 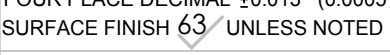 & MFG APPR. & & \\
\hline 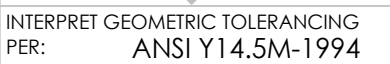 & Q.A. & & \\
\hline MAERRAL: THERMICULITE 815 & Sl & $\begin{cases}\mathrm{MM} & 25 \\
C & 25\end{cases}$ & 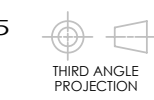 \\
\hline & сомм & & \\
\hline
\end{tabular}
ARIZONA PUBLIC SERVICE 400 N. 5th Street
Phoenix, Az. 85003
PROJECT: COAL TO SNG TIILE: KINETICS REACTOR GASKET, TOP BELLOWS FLANGE

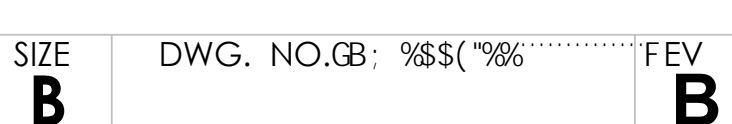
SCALE: 1:1 WEIGHT: SHEET I OF 1 
8 MCMASTER-CARR PIN:94768A110, 18-8 STAINLESS STEEL

\section{GENERAL NOTIES:}
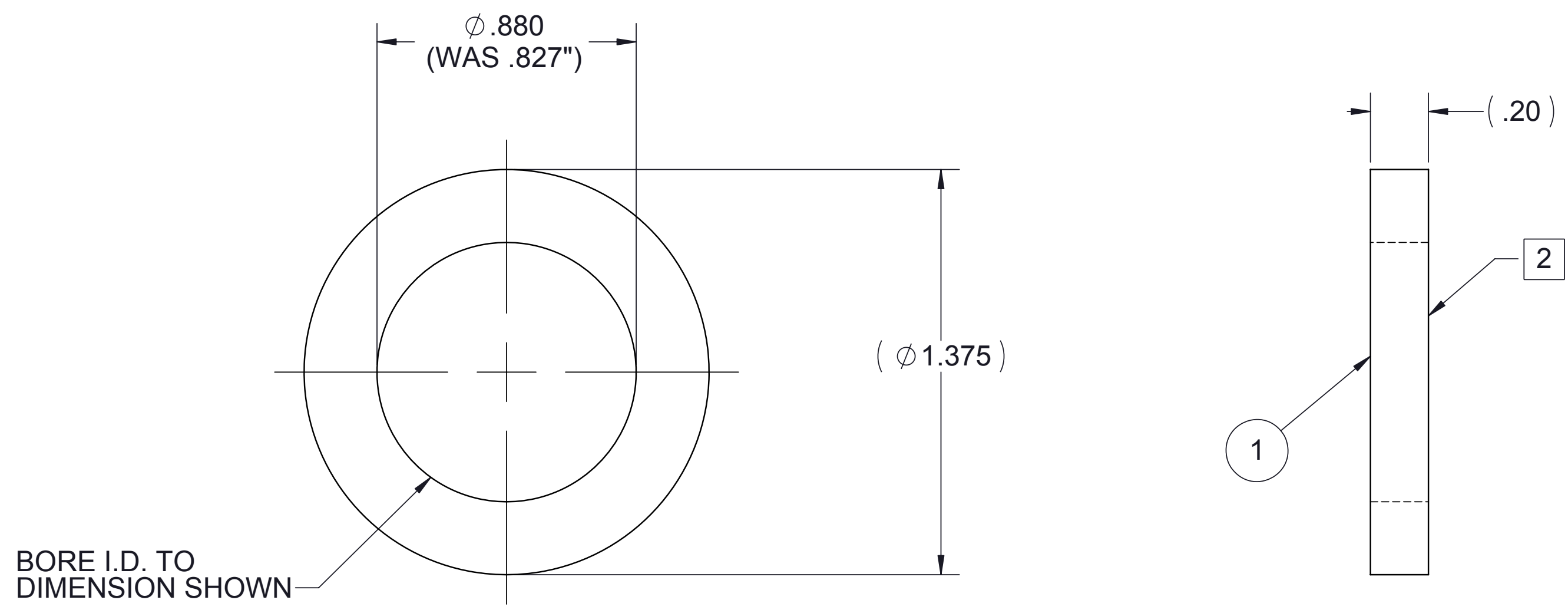

\begin{tabular}{|c|c|c|c|c|}
\hline 1 & SNG1004.12A & 18-8 STAINLESS & WASHER, MODIFIED, .88" ID & 1 \\
\hline $\begin{array}{l}\text { ITEM } \\
\text { NO. }\end{array}$ & PART NUMBER & MATERIAL & DESCRIPTION & QTY. \\
\hline
\end{tabular}

\begin{tabular}{|c|c|c|c|c|c|c|}
\hline 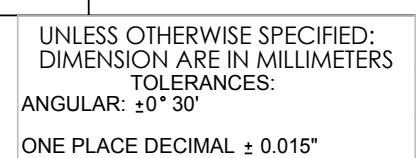 & $\begin{array}{l}\frac{D R A W N}{} \\
\text { CHECEED }\end{array}$ & \begin{tabular}{|c|} 
DATE \\
$01 / 09 / 08$
\end{tabular} & $\begin{array}{c}\text { NaME } \\
\text { D. WABELE }\end{array}$ & \multirow{2}{*}{\multicolumn{3}{|c|}{$\begin{array}{l}\text { ARIZONA PUBLIC SERVICE } \\
400 \mathrm{~N} \text {. 5th Street } \\
\text { Phoenix, Az. } 85003 \\
\text { COAL To SNG }\end{array}$}} \\
\hline 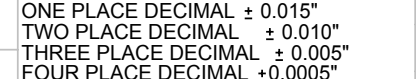 & ENG APRR. & & & & & \\
\hline 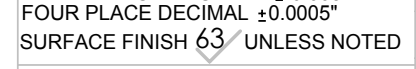 & MEG APPR. & & & \multicolumn{3}{|c|}{ KINETICS REACTOR } \\
\hline 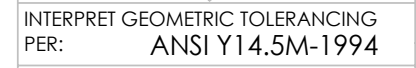 & & & & \multicolumn{3}{|c|}{ WASHER, MODIFIED, . .88" ID } \\
\hline $\begin{array}{l}\text { SEEE BOM } \\
\text { FNSS: BOM }\end{array}$ & \multirow[t]{2}{*}{$\begin{array}{l}\text { MER } \\
\text { COMM } \\
\text { COMn }\end{array}$} & 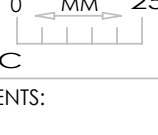 & 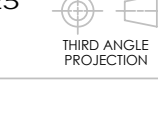 & & SNGIO & A \\
\hline & & & & & WEIGHT & \\
\hline
\end{tabular}


ALL D DIM. 9.562 WAS 9.750, ADDED ITEM 2 10/18/071 DW GENERAL NOTES: 1

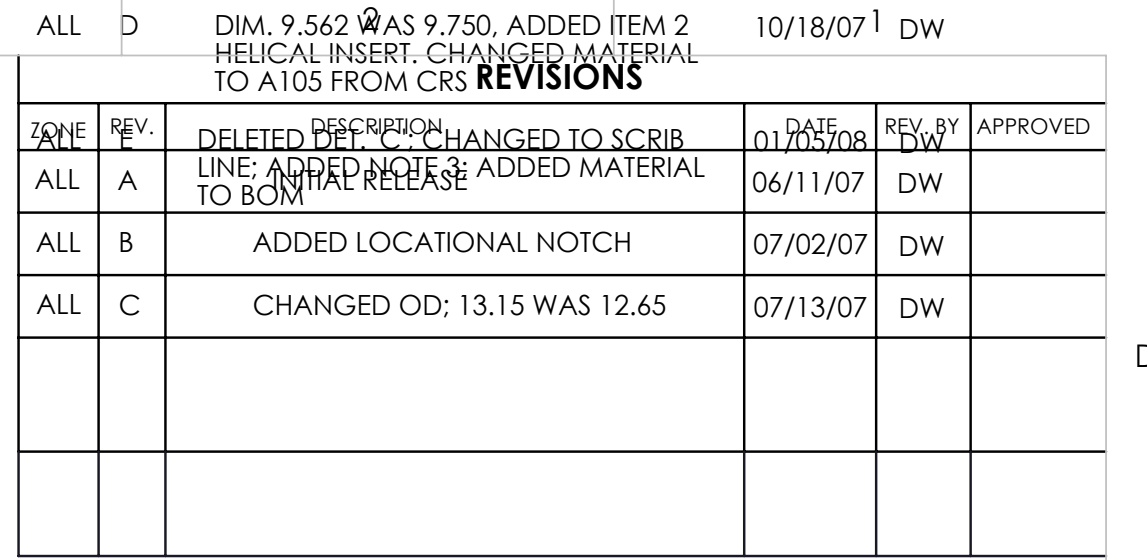

D $\square \square$

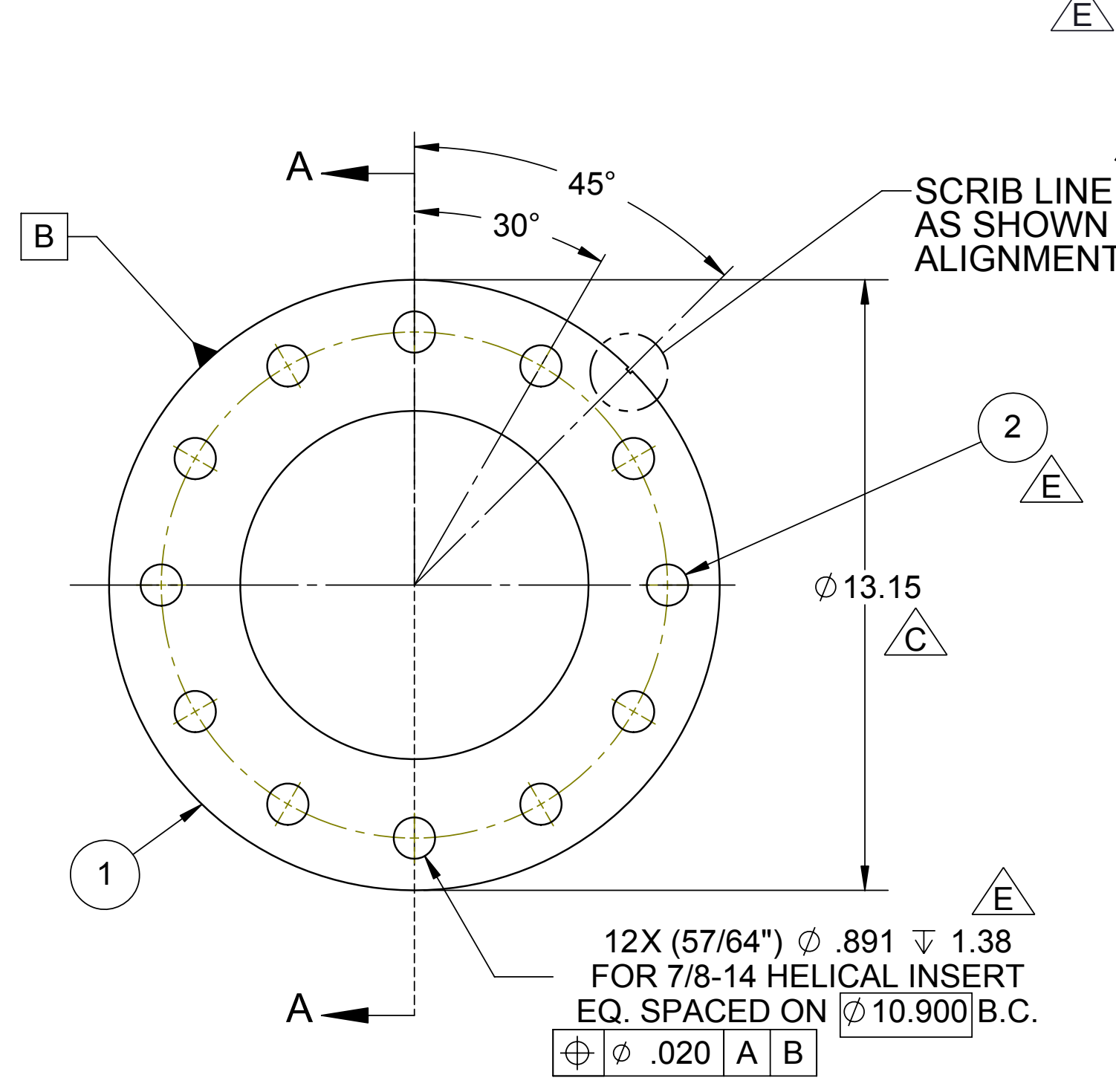

E SCRIB LINE IN LOCATION ASER

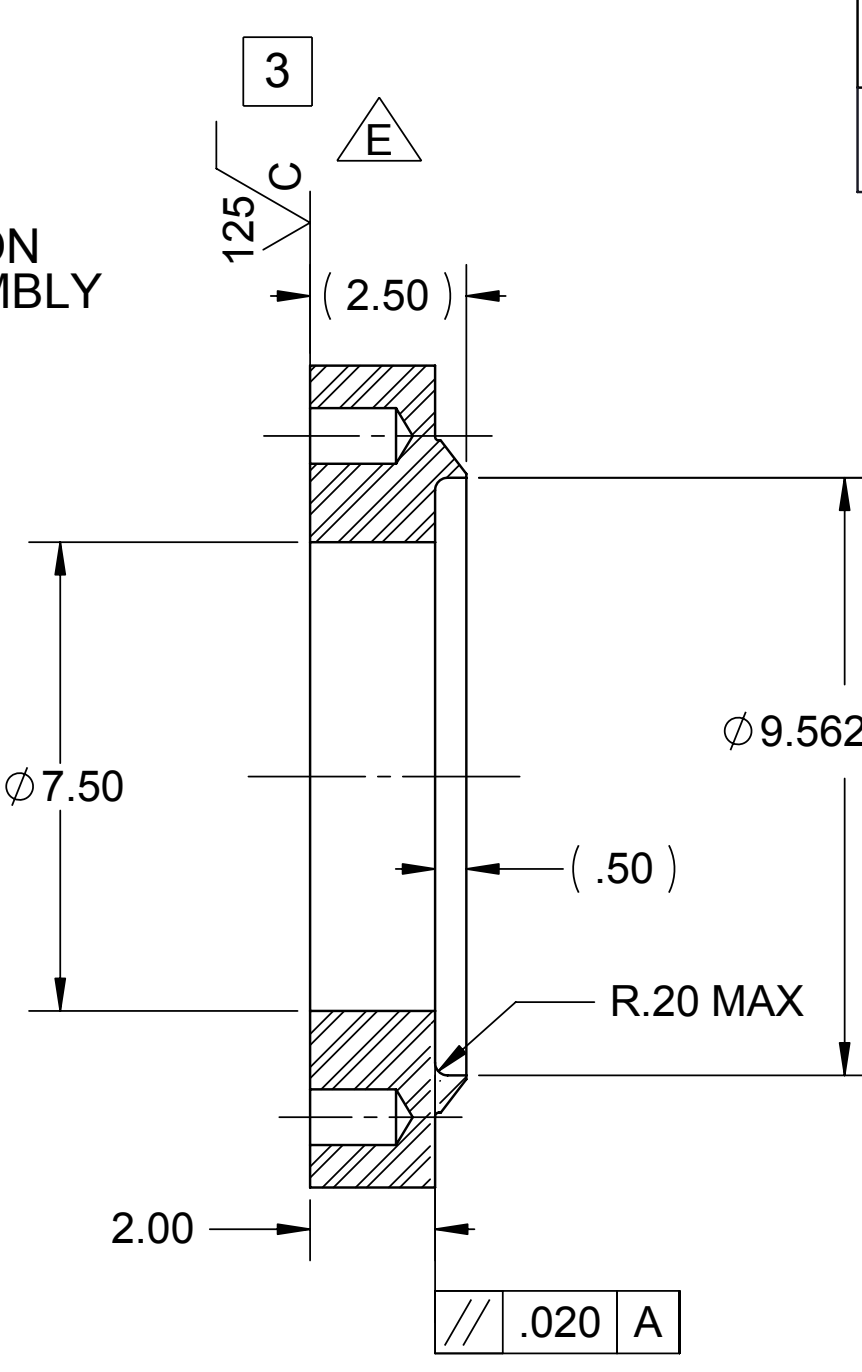

SECTION A-A

A

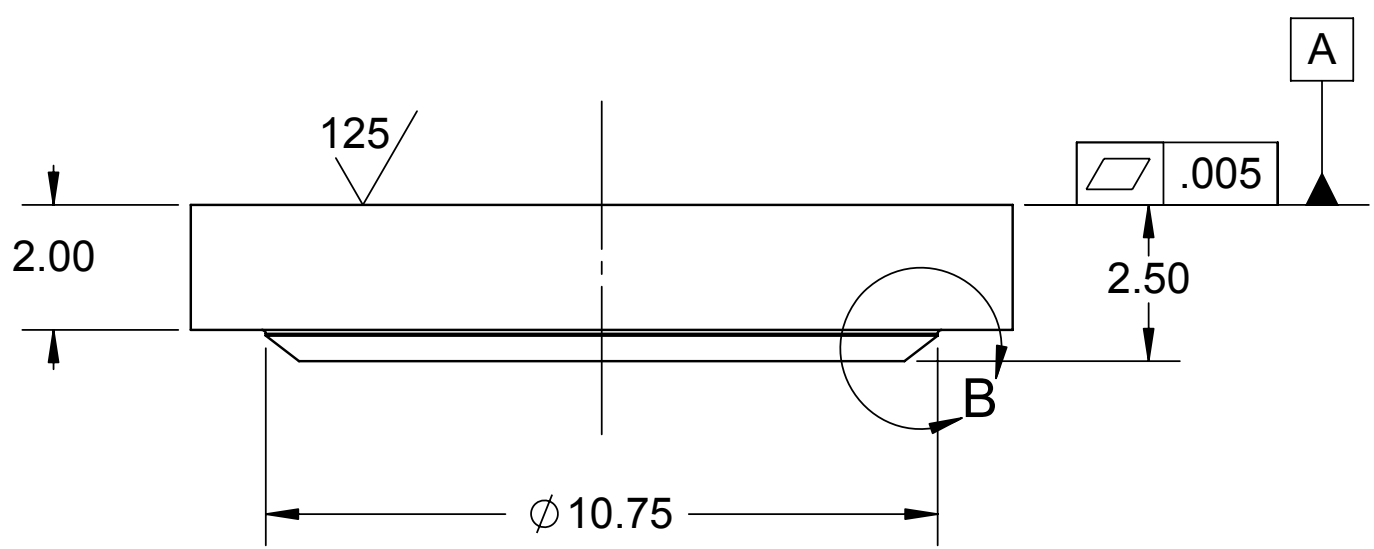

\begin{tabular}{|c|l|c|c|c|}
\hline 2 & $91732 A 240$ & $18-8$ STAINLESS STEEL & $\begin{array}{c}\text { HELICAL INSERT, 7/8 } \times 1.3 " \text { LG } \\
\text { (MCMASTER-CARR) }\end{array}$ & 12 \\
\hline 1 & SNG1004.2E & $\begin{array}{c}\text { CARBON STEEL, SA105 } \\
\text { GRADE 2, SA-266 }\end{array}$ & $\begin{array}{c}\text { FLANGE, REACTOR OUTER } \\
\text { SHELL, FOOT }\end{array}$ & 1 \\
\hline TTEM & PART NUMBER & MATERIAL & DESCRIPTION & QTY. \\
NO. & PRT & M
\end{tabular}

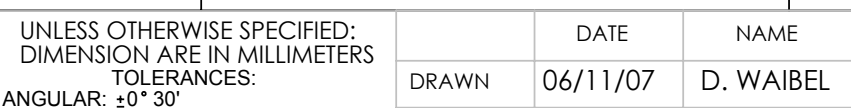

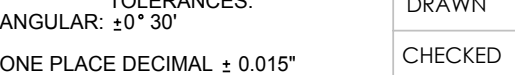

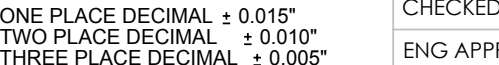
OUR PLACE DECLMAL :.0.0005"

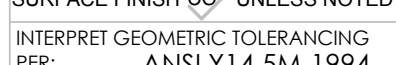
MAERAL:

SI (2) .020 B

$\Delta$ $+.005$ SEEBOM E SMMLARTO 


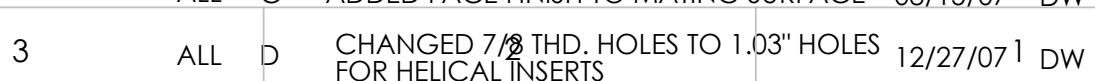

\section{GENERAL NOTES:}

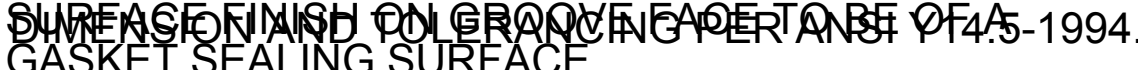

2 BREAK AND DEBURR ALL SHARP EDGES.

D $\square$ DRILL FOR 1"-14 × 1.5" LG. HELICAL INSERT, (MCMASTER-CARR) P/N: 917322027 OR OD THIS COMPONENT WITH HELICAL INSERTS INSTALLED.
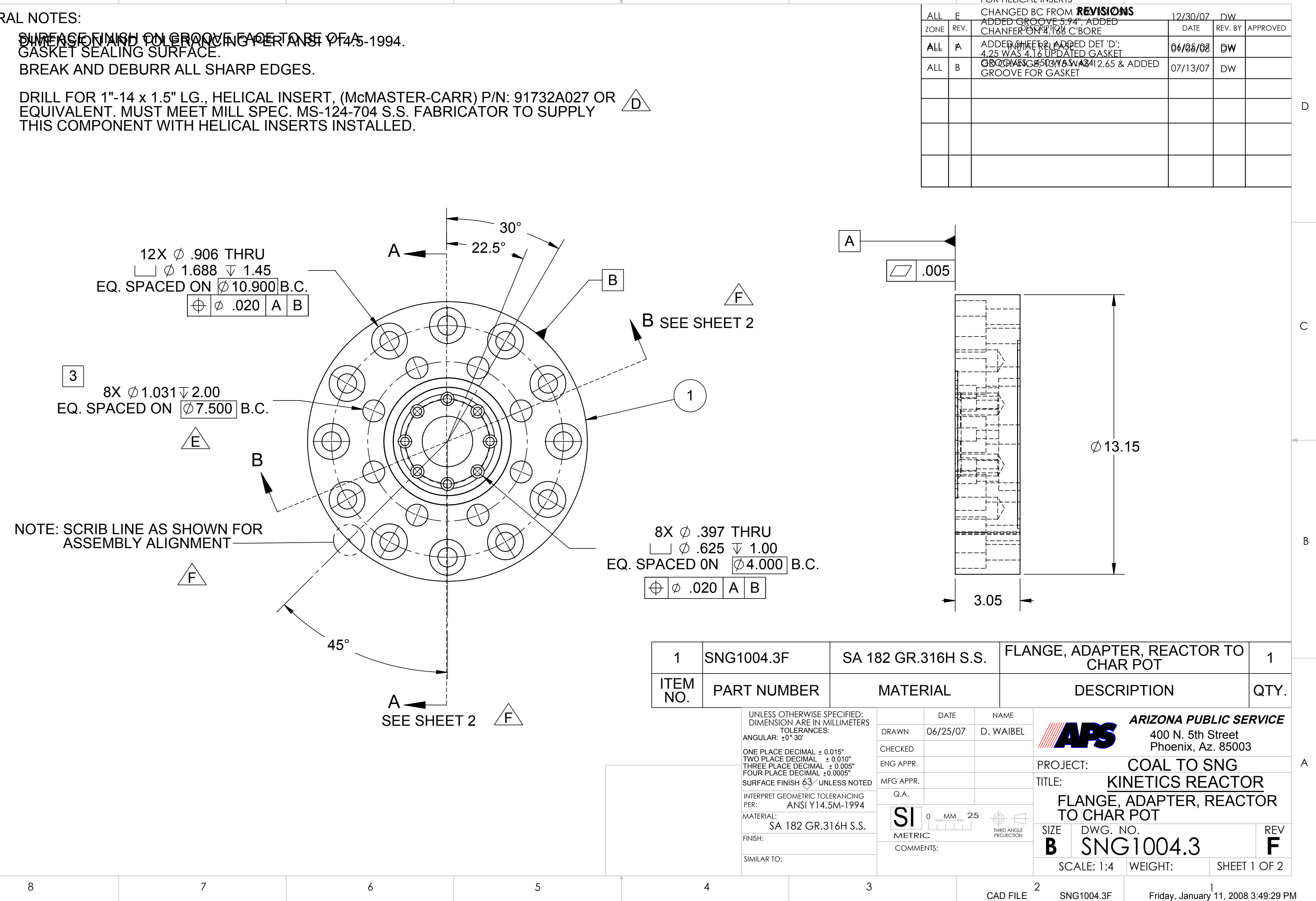


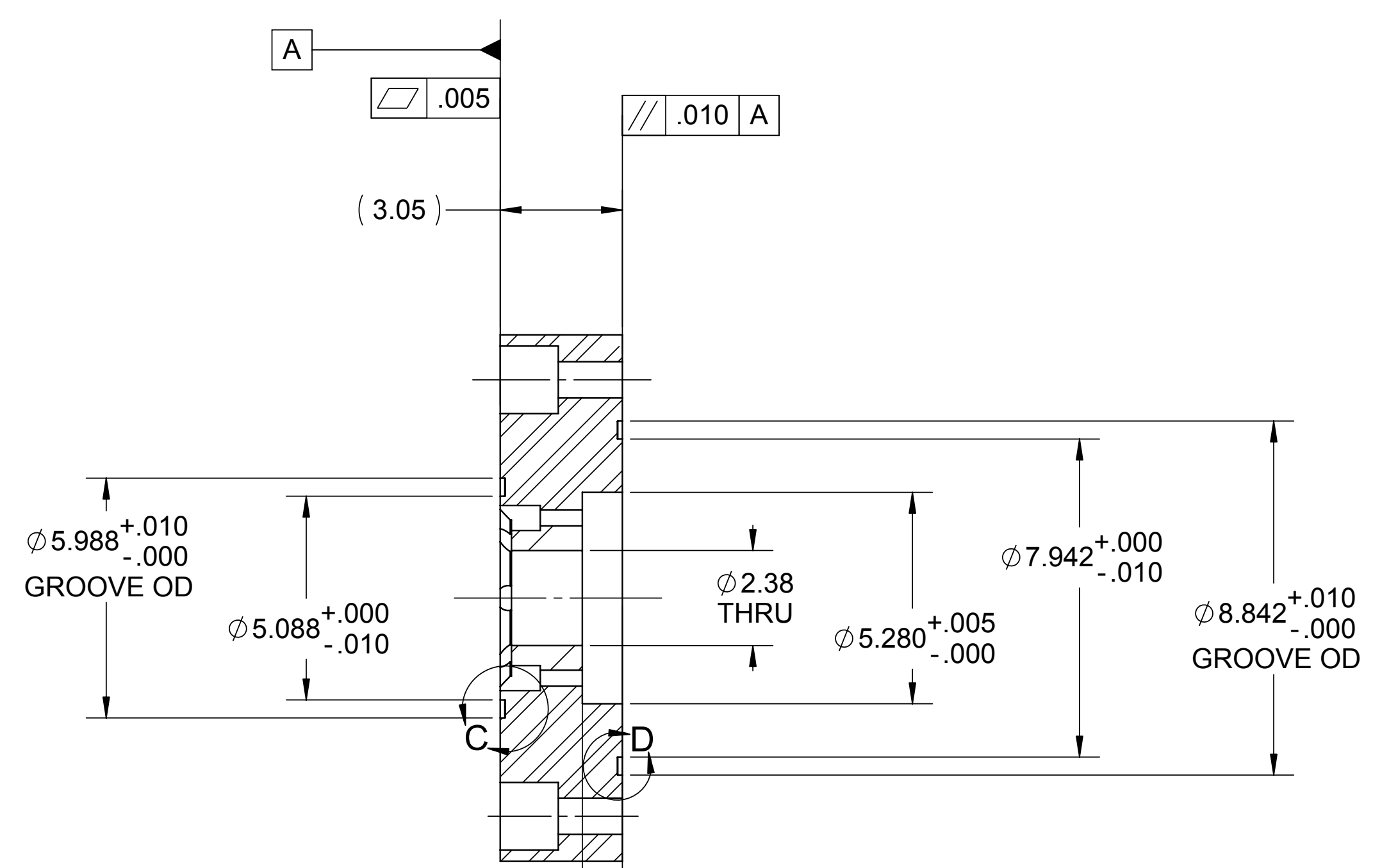

B

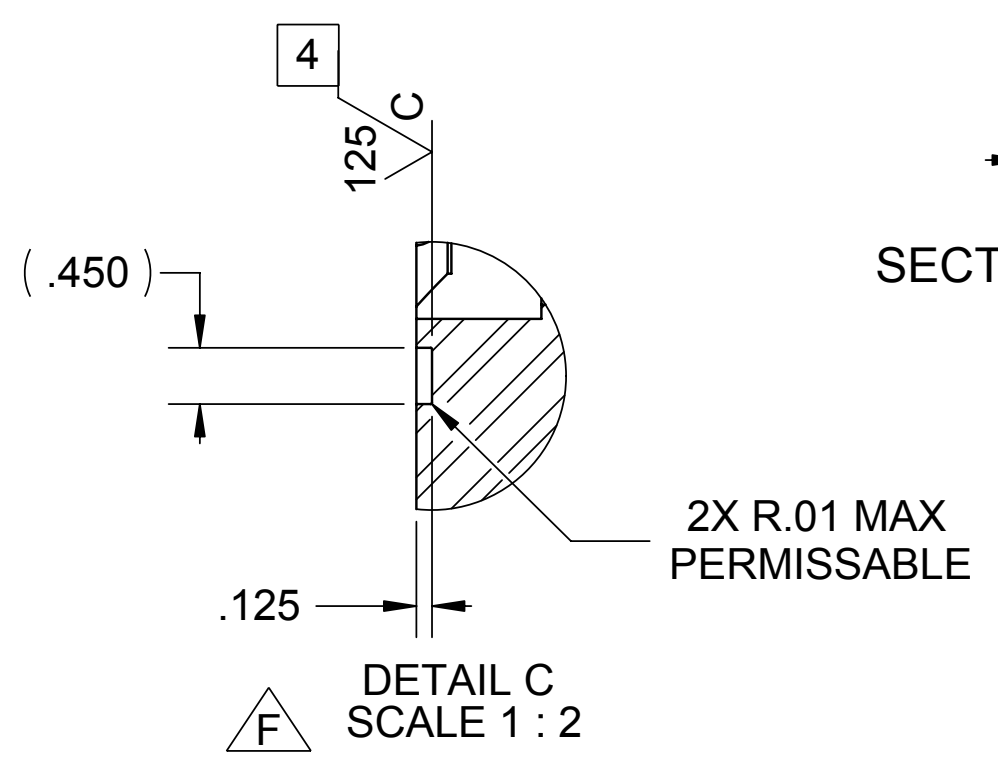

SECTION A-A

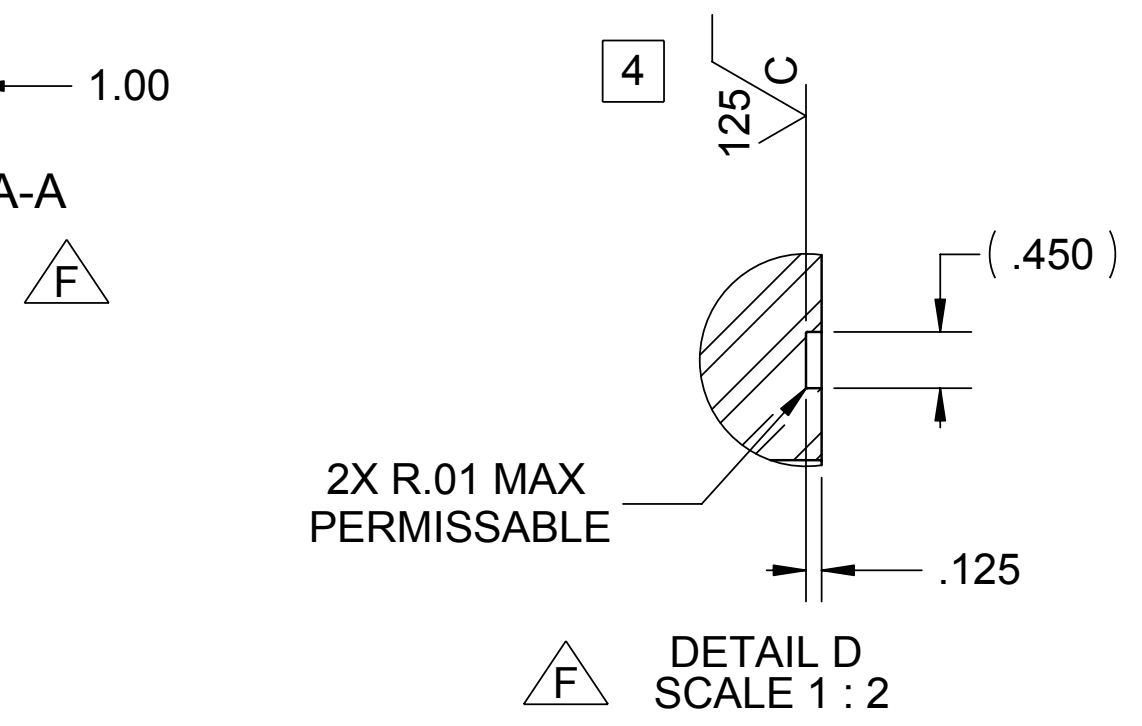

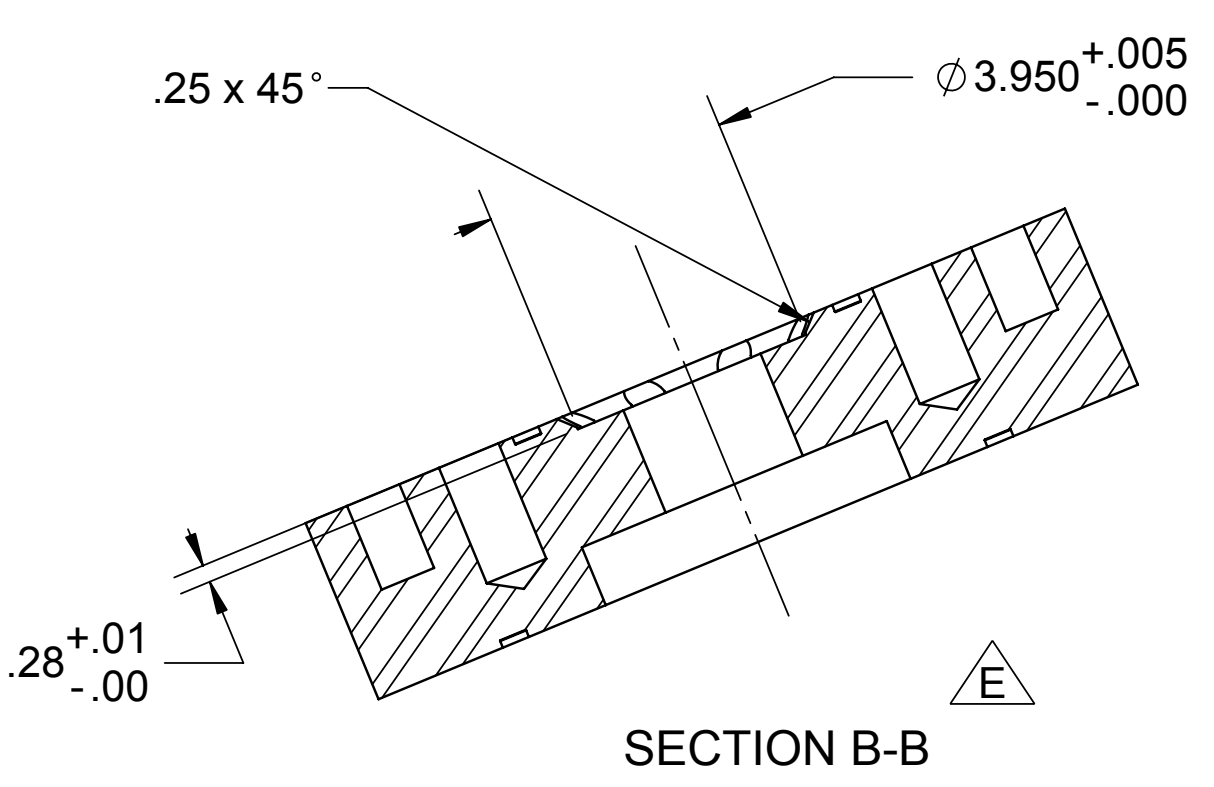

UIIIIIT ARIZONA PUBLIC SERVICE 400 N. 5th Street
Phoenix, Az. 85003 \begin{tabular}{lc} 
& COAL TO SNG \\
\hline PROJECT: & KINETICS REACTOR
\end{tabular} \begin{tabular}{|l}
\hline TITLE: \\
\hline FLANGE, ADAPTER, REACTOR
\end{tabular} TO CHAR POTS

\section{B SNG1004.3 F}

SCALE: 1:4 WEIGHT: SHEET 2 OF 2 
BREAK ALL EDGES 5 [20] MAX ALL INSIDE RADII

SURFACE FINISH AS INDICATED, SHOULD ALSO BE TO A QUALITY

DESIGN BASIS: ASME SECTION 8, DIVISION 1, 2004

STRESS BASIS: ASME SECTION 2 DIVISION D, 2005

D

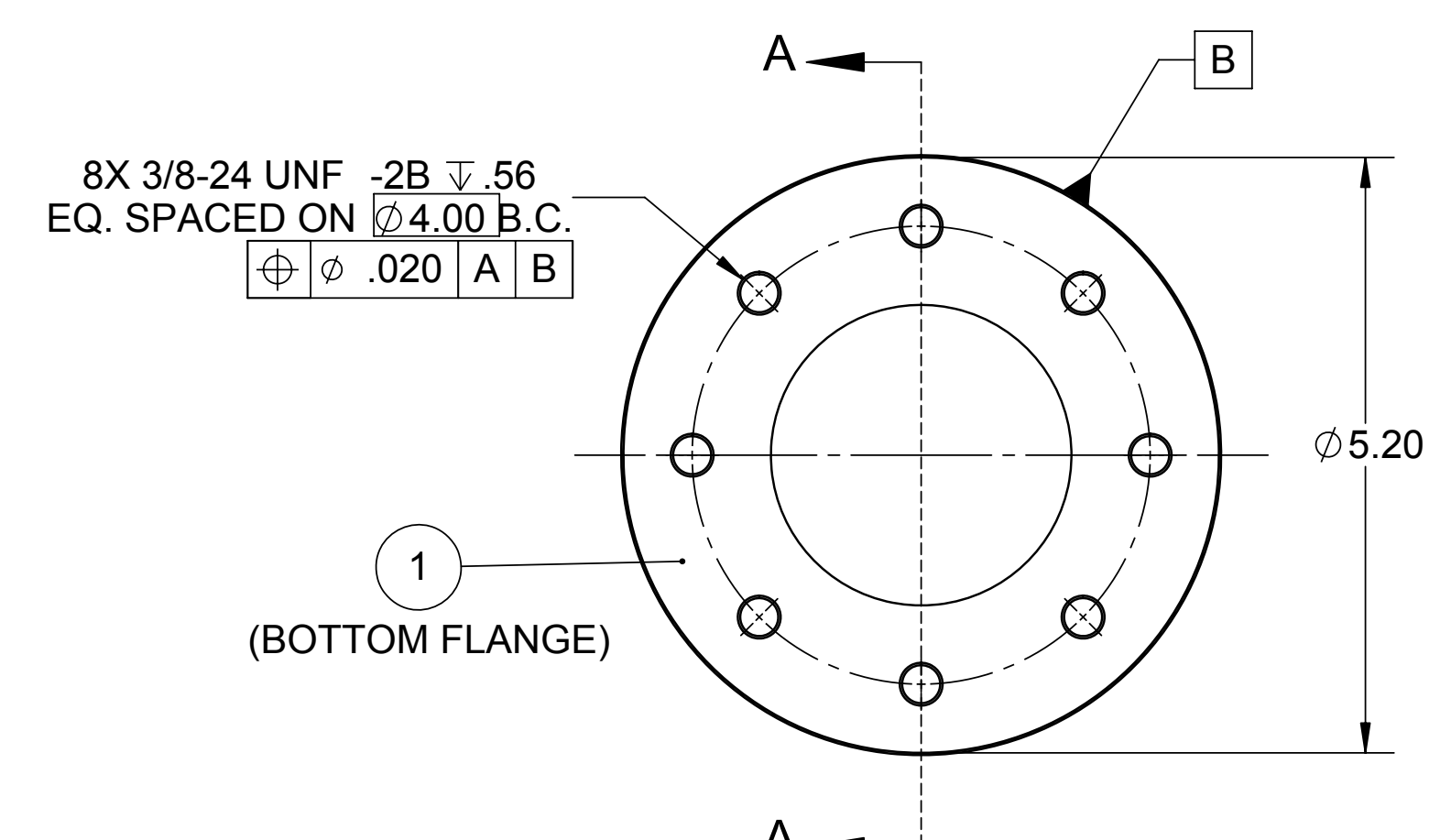

$8 \times \varnothing .391 \mathrm{THRU}$

EQ. SPACED ON $\varnothing 4.00$ B.C.

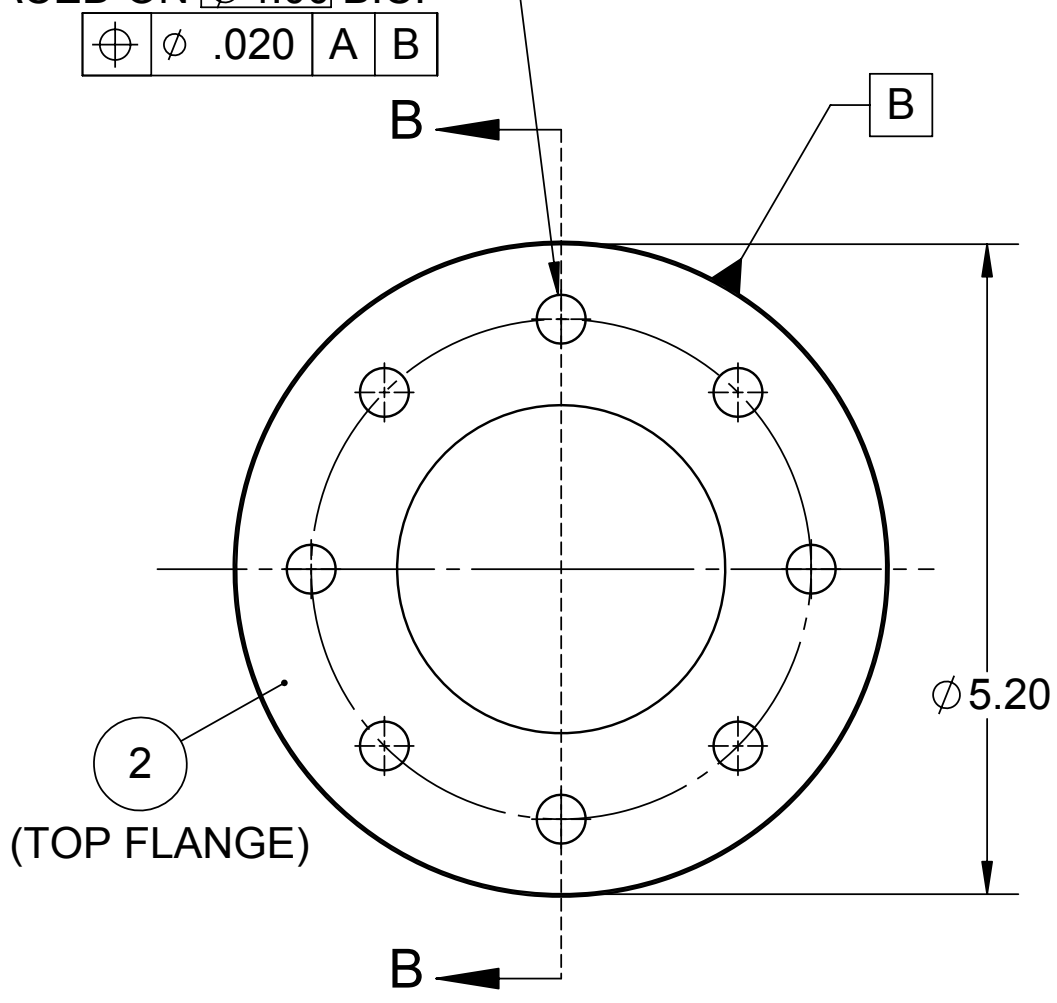

A

$3 \stackrel{\leftrightarrow}{\stackrel{0}{*}}$

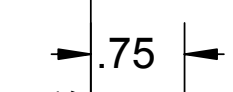

$-.75-$

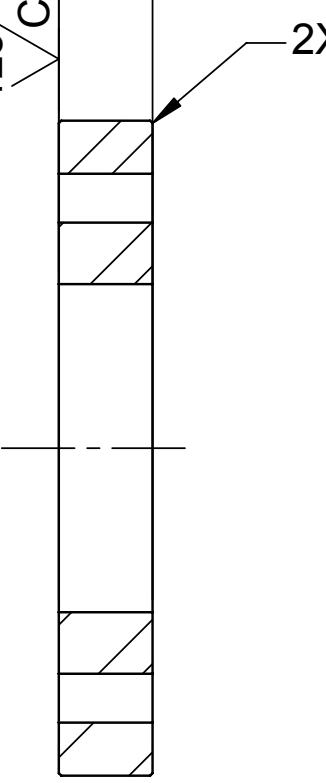

SECTION B-B

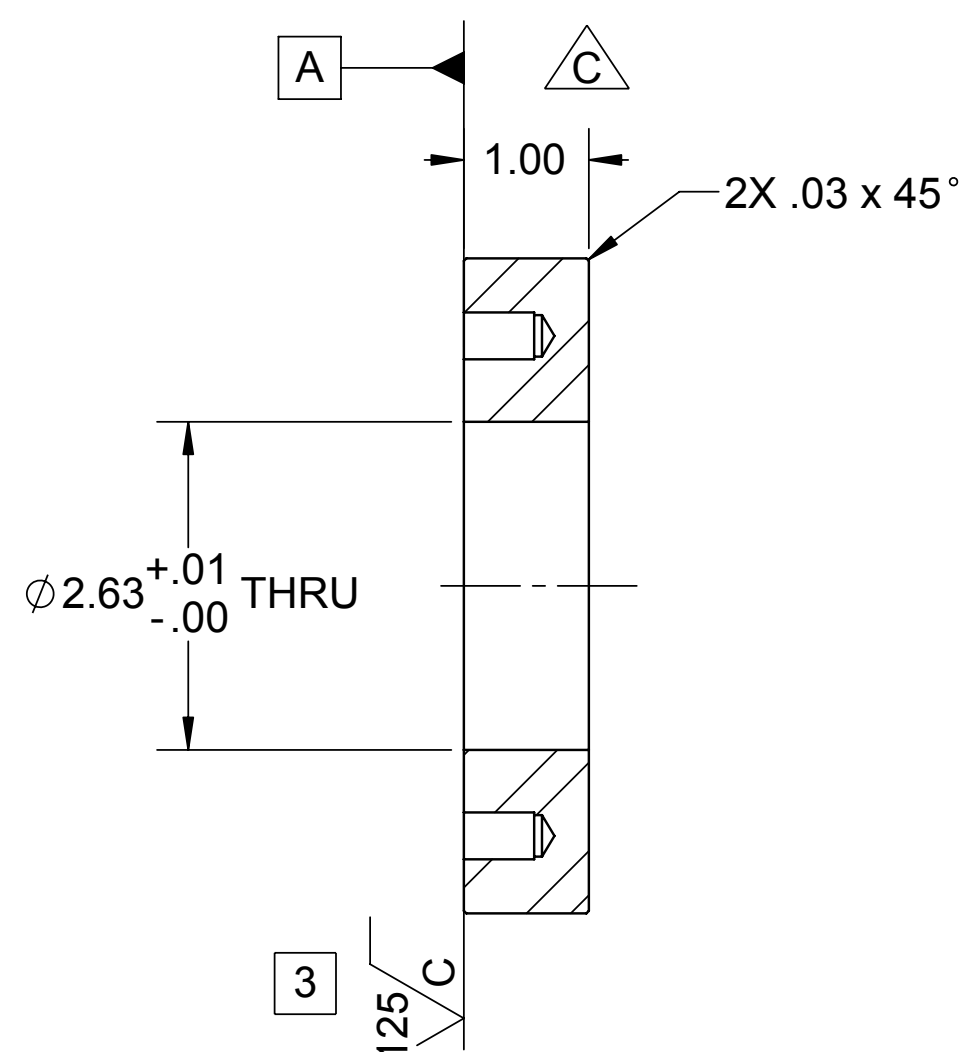

SECTION A-A

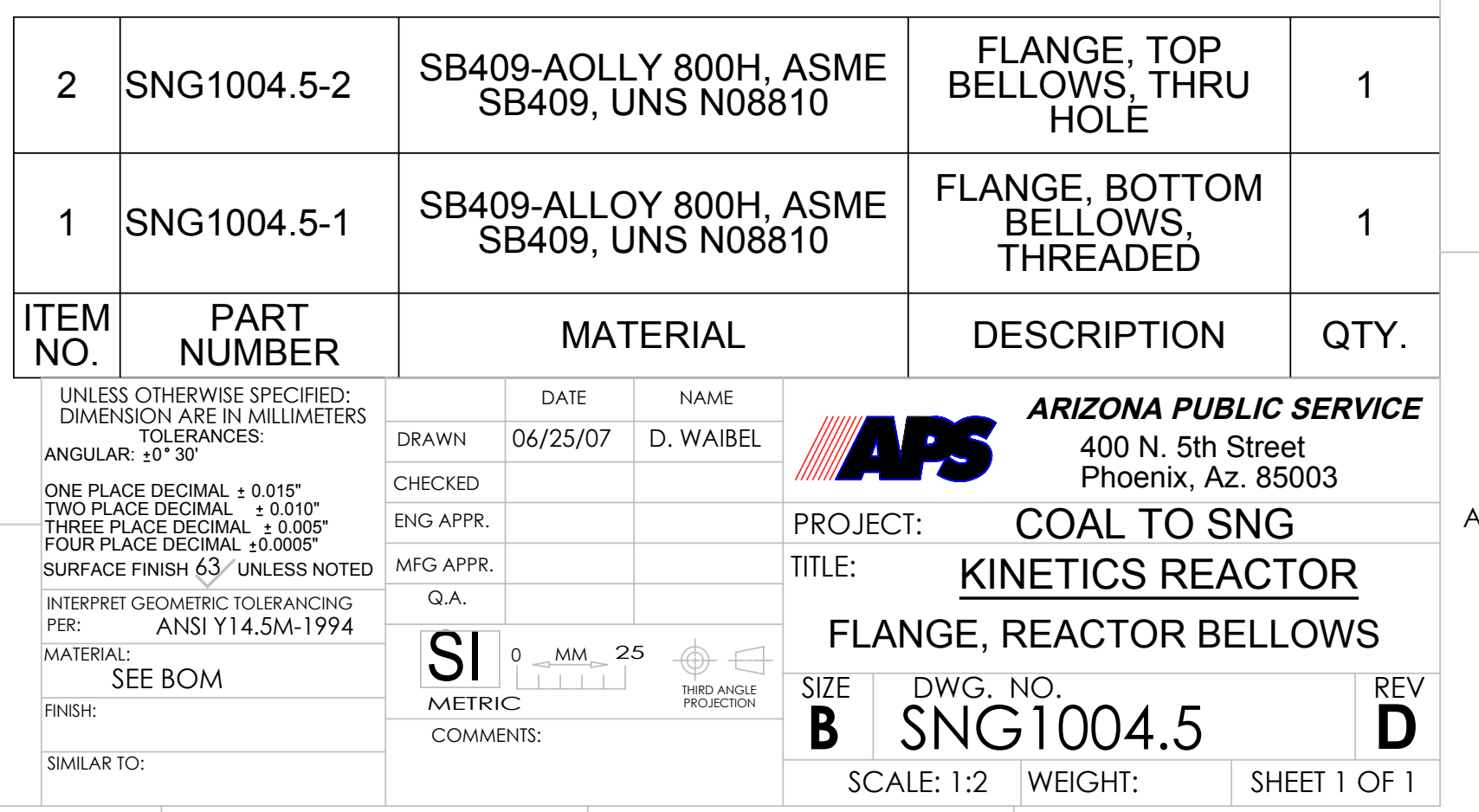


1 DIMENSION AND TOLERANCING PER ANSI Y14.5-1994.

2 GASKET TO BE STYLE 'R', (CRITICAL SERVICE SERIES) SEE BOM BELOW

REVISIONS

\begin{tabular}{|l|l|l|c|l|l|}
\hline \multicolumn{7}{|c|}{ REVISIONS } \\
\hline ZONE & REV. & DESCRIPTION & DATE & REV. BY & APPROVED \\
\hline ALL & A & INITIAL RELEASE & $05 / 16 / 07$ & I.T. & \\
\hline ALL & B & CHANGED GASKET FROM FLAT TO STYLE 'R' & $08 / 03 / 07$ & D.W. & \\
\hline & & & & & \\
\hline
\end{tabular}
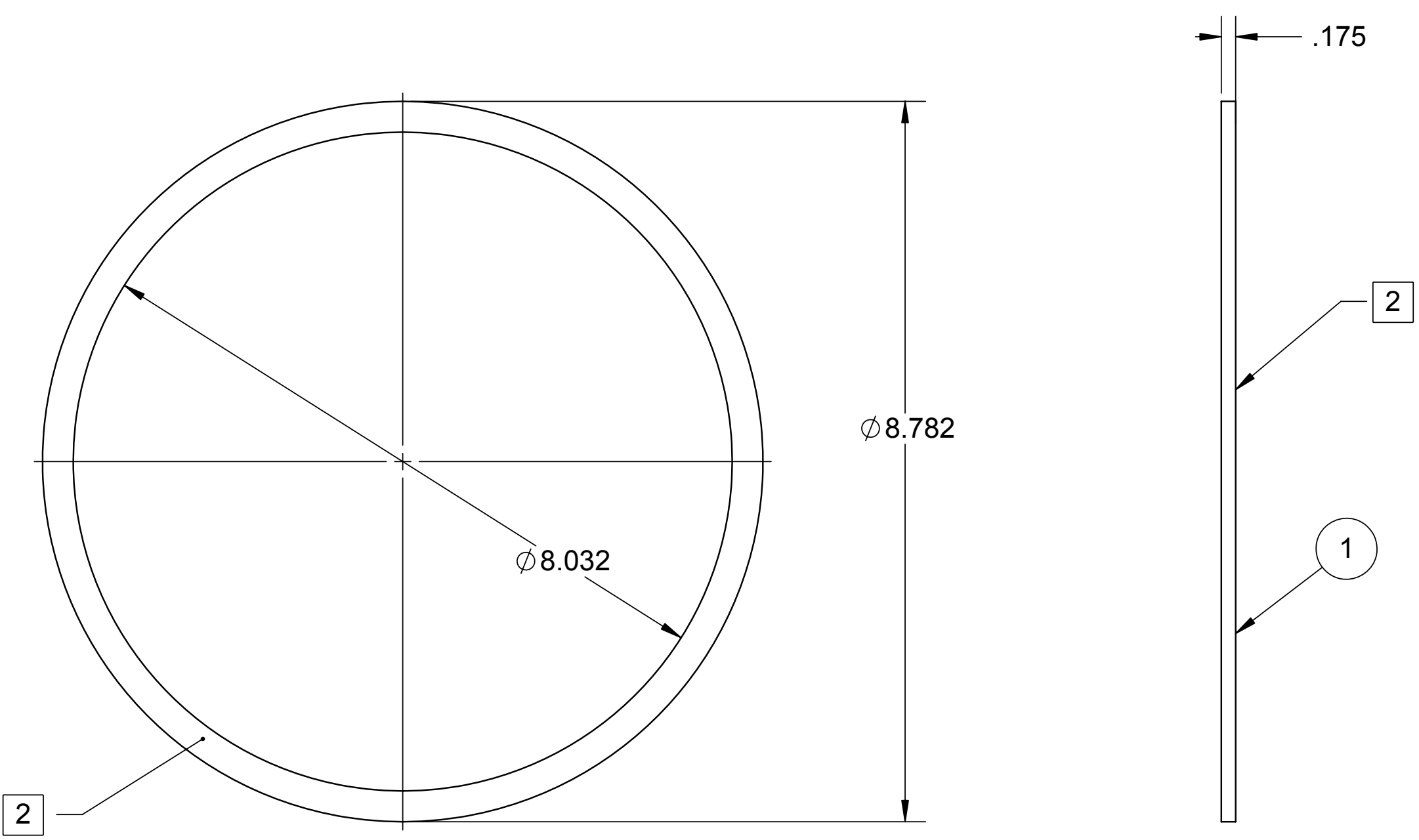

\begin{tabular}{|c|c|c|c|c|c|}
\hline 1 & SNG1004.7C & \multicolumn{3}{|c|}{$\begin{array}{c}\text { R STYLE, SWG INCONEL } 625 \text { WINDING } \\
\text { WITH THERMICULITE 835 FILLER } \\
\text { (CTRITICAL SERVICE) }\end{array}$} & \\
\hline $\begin{array}{l}\text { ITEM } \\
\text { NO. }\end{array}$ & PART NUMBER & \multicolumn{3}{|l|}{ MATERIAL } & \\
\hline & & \multirow{5}{*}{ 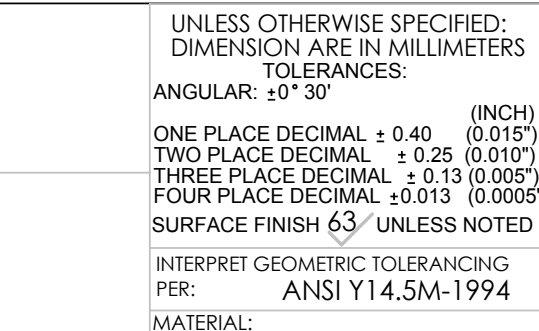 } & & DATE & NAME \\
\hline & & & DRAWN & I. TAYLOR & $05 / 16 / 07$ \\
\hline & & & $\begin{array}{l}\text { CHECKED } \\
\text { ENG APPR. }\end{array}$ & & \\
\hline & & & MFG APPR & & \\
\hline & & & Q.A. & & \\
\hline & & $\begin{array}{l}\text { MATERIAL: } \\
\text { SEE BOM }\end{array}$ & Sl & {$\left[\begin{array}{ll}M M_{-} 2 \\
C\end{array}\right.$} & 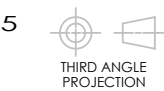 \\
\hline
\end{tabular}

GASKET, REACTOR FOOT

DESCRIPTION

ARIZONA PUBLIC SERVICE 400 N. 5th Street
Phoenix, Az. 85003 PROJECT: COAL TO SNG TITLE: KINETICS REACTOR GASKET, REACTOR FOOT FINISH: METRIC 


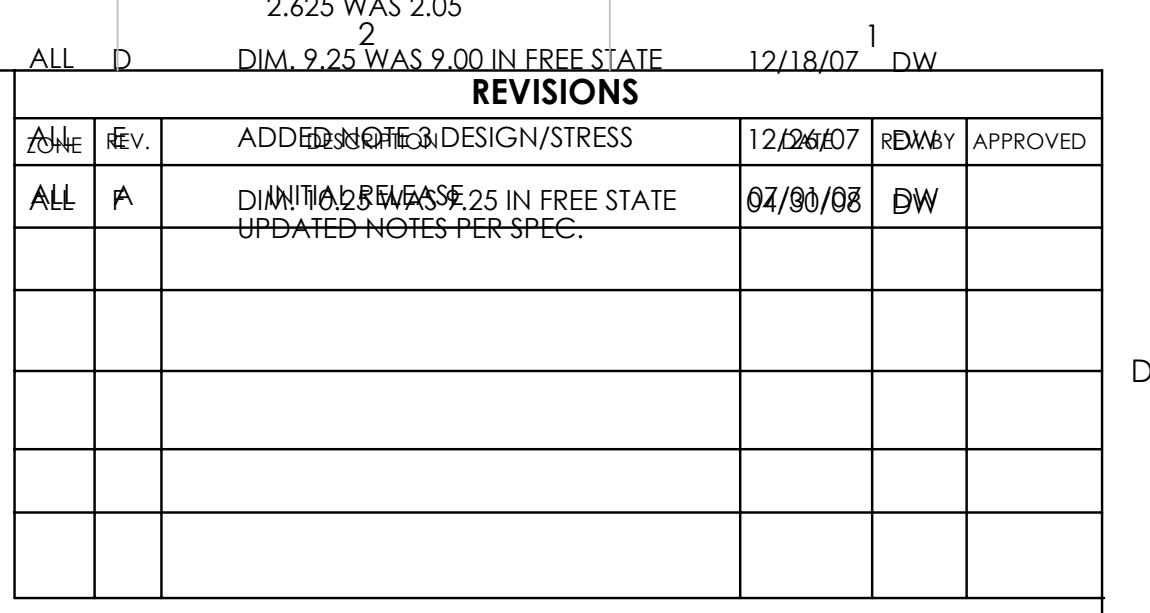

в GENERAL NOTES: AXIAL SPRING RATE 162 BSIIN OVERALL (10.25") IS AT ROOM TEMPERATURE WITH NO COMPRESSION OR TENSIONS.

INSTALL BELLOWS TO PRODUCE APPROX. 1.4" OF TENSION (REQUIRES 240LBS DRAW FORCE) DESIGN BASIS: ASME SECTION 8, DIVISION 1, 2004
STRESS BASIS: ASME SECTION 2, DIVISION D, 2005
E
3

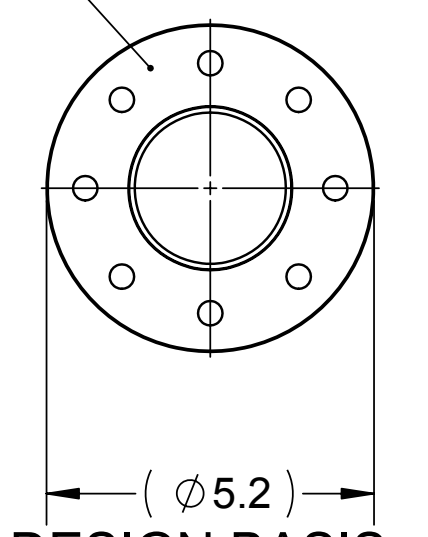

DESIGN BASIS:

ASME SECTION 8, DIVISION 1, 2004

1 BELLOWS; BELLOWS MATERIAL, SB409-ALLOY 800H

2 COLLAR MATERIAL, SB409-ALLOY $800 \mathrm{H}$ DESIGN PRESSURE (Psig) -50 AXIAL COMPRESSION (IN) 3.000 AXIAL EXTENSION (IN) 0.000

10.25

N FREE STATE
(3)

(1) 2
3

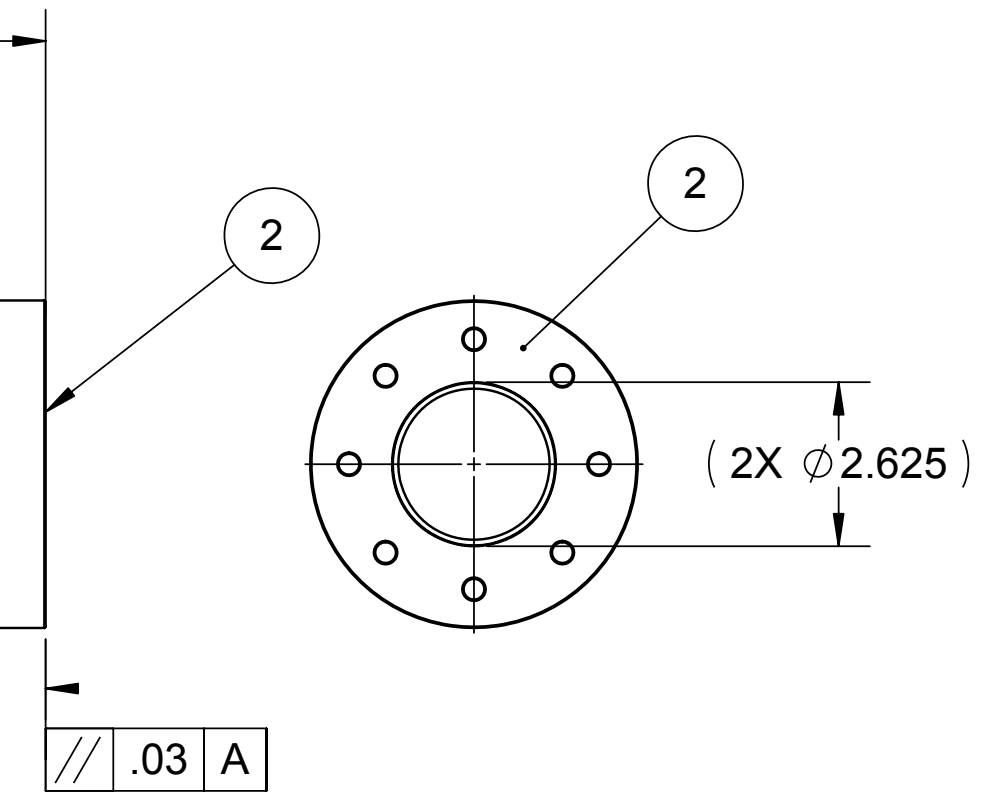

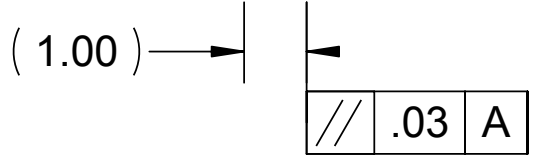

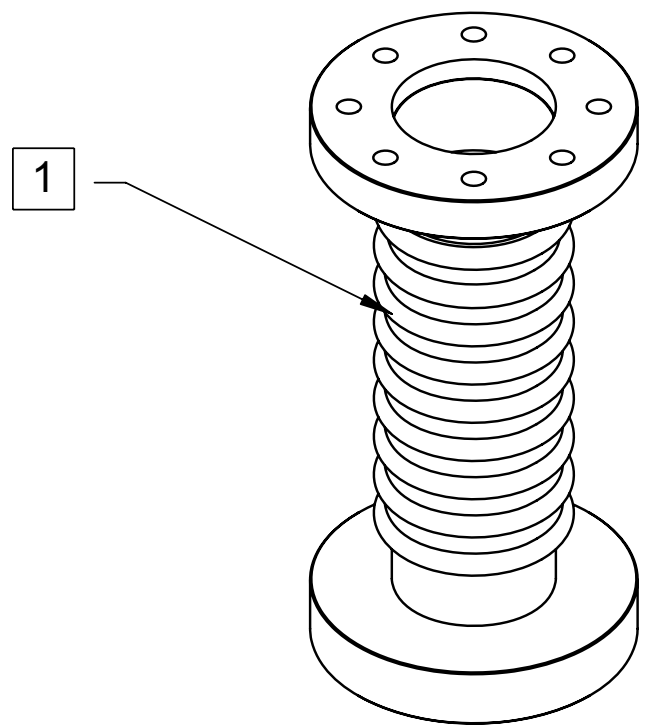

ISO VIEW

(NOT TO SCALE)

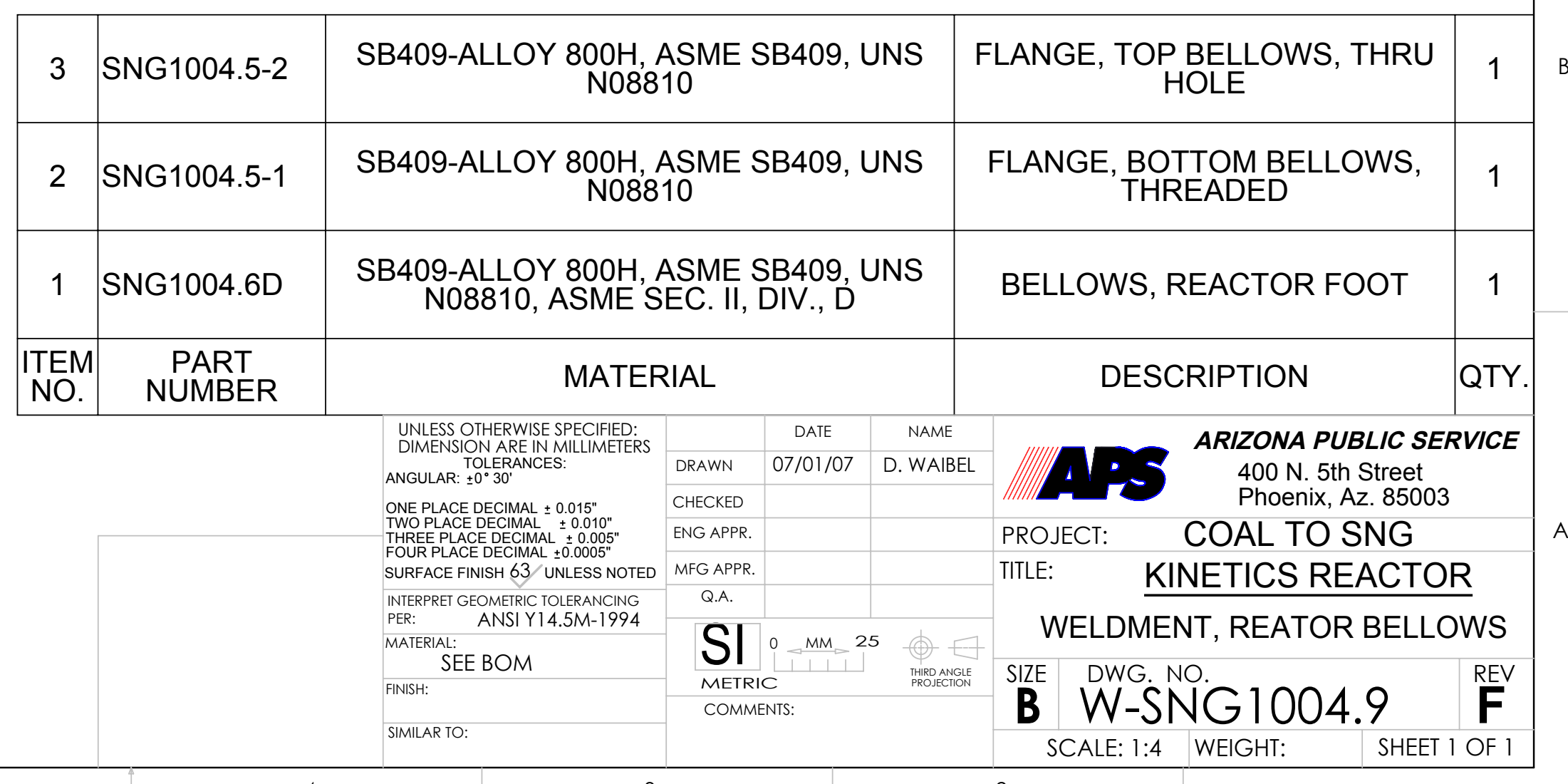


GENERAL NOTES:

ALL MATING SURFACES SHOULD BE CLEAN AND FREE OR ANY DEBURR BEFORE ASSEMBLY.
ALIGNE ALL COMPONENTS TO ORIENTATION AS SHOWN, OR DESCRIBED.

2 TOROUE BOLTS, FOR HYDROTEST TOROUE TO 107 FT, / LBS. (20,000 PSI BOLT STRESS) FOLLOW GASKET

GASKET, ITEM 5, TO BE USED BETWEEN VALVE FLANGES ONLY AS SHOWN.

GASKET, ITEM 6, TO BE USED AT BLIND FLANGE ON LOWER CHAR POT ONLY.

MATERIAL: 316, CARBON CONTENT MUST BE EQUAL TO, OR ABOVE .04\%

SEE SHEET 2 FOR DETAILS AND BILL OF MATERIAL.

REFERENCE OR WORK WITH RESPECTIVE DRAWINGS FOR WELDING PROCEDURES,
AND DETAILS OF FABRICATED COMPONENTS.

REFERENCE DRAWINGS

W-SNG1005.2 WELDMENT, CHAR POT UPPER

W-SNG1005.10 WELDMENT, CHAR POT LOWER

W-SNG1005.18 WELDMENT, CHAR POT PRESSURE BOUNDARY
2

()

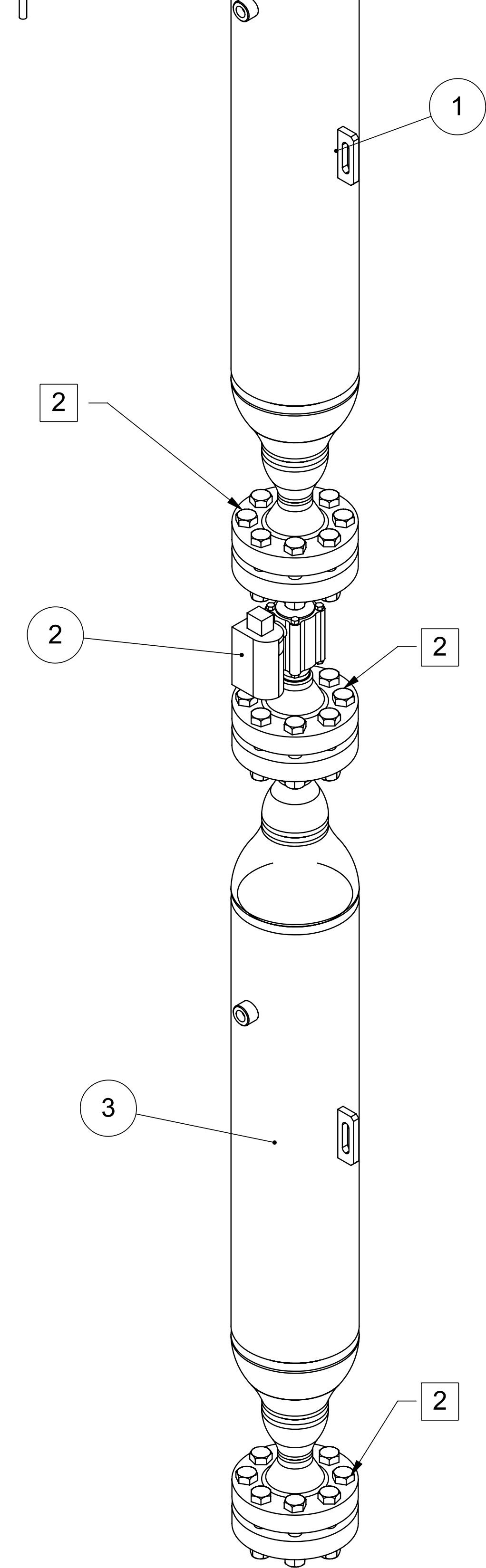

\begin{tabular}{|c|c|c|c|c|c|c|}
\hline & ZONE & REV. & DESCRIPTION & DATE & REV. BY & APPROVED \\
\hline & Aul & A & INITIAL RELEASE & 07/06/07 & D.W. & \\
\hline & ALL & B & 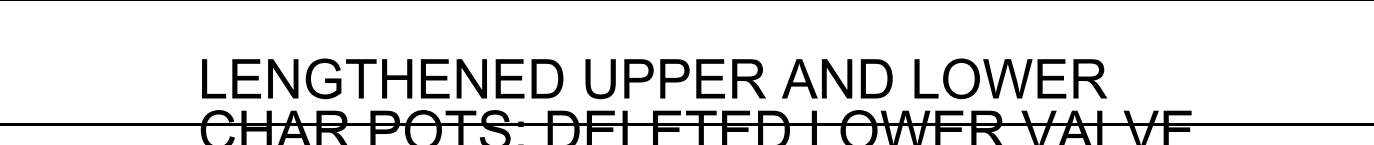 & $08 / 28 / 07$ & D.W. & \\
\hline & ALL & c & ADDED COUPLINGS TO WELDMENTS & 10/19/07 & D.W. & \\
\hline & ALL & $D$ & ADDED NOTES 3, 4, AND 5, ALSO ADDED SHEET 2 & $12 / 06 / 07$ & D.W. & \\
\hline & ALL & E & ITEM 1; W-SNG1005.18D WAS W-SNG1005.18C & 12/30/07 & D.W. & \\
\hline & ALL & $\mathrm{F}$ & ADDED LITFING LUGS TO ASSEMBLIES & $03 / 09 / 08$ & D.W. & \\
\hline
\end{tabular}

REVISIONS 


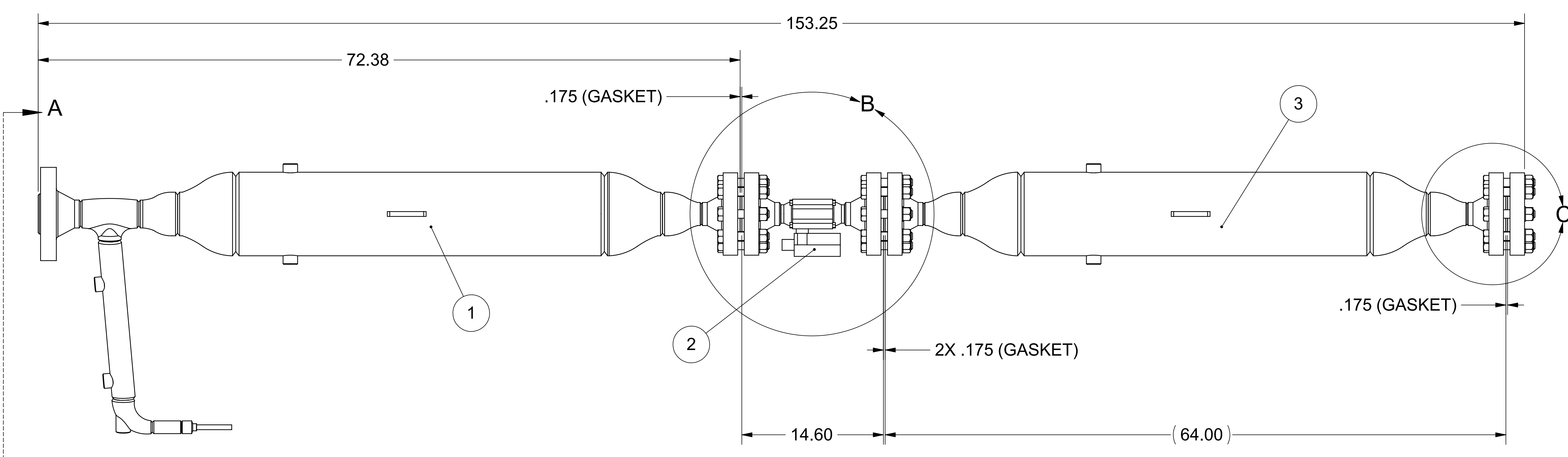

\begin{tabular}{|c|c|c|c|c|}
\hline 9 & SNG1009.14 & ALLOY ST & 7/8' FLAT WASHER & 24 \\
\hline 8 & SNG1009.10 & ALLOY ST & 7/8-9UNC-2B HEX HEAVY NUT SA194 GR B7 & 24 \\
\hline 7 & SNG1009.5 & ALLOY ST & 7/8-9UNC-2A x 5.75LG SA193 GR B7 & 24 \\
\hline 6.6 & SNG1005.22A & 316 ST STL 5 & PLATE, LIFTING LUG, CHAR POT & 2 \\
\hline 6.5 & SNG1005.13B & 316 ST STL 5 & $\begin{array}{c}\text { 1/2"-6000\#-FGD THD HALF CPLG SA182 GR F316 } \\
\text { ASME B16.11 }\end{array}$ & 2 \\
\hline 6.4 & SNG1005.11C & 316 ST STL 5 & \begin{tabular}{|l|} 
PIPE 8"-SCH.80.500" WALL ST STL SMLS SA312 TP \\
316 SS ASME B36.19
\end{tabular} & 1 \\
\hline 6.3 & SNG1005.5A & 316 ST STL 5 & $\begin{array}{l}\text { 8" } 4 \text { 4" CONC RED.WTR ST STL SCH.80 BW SA403 } \\
\text { WP-316SS ASMEB B16.9 }\end{array}$ & 2 \\
\hline 6.2 & SNG1005.7A & 316 ST STL 5 & 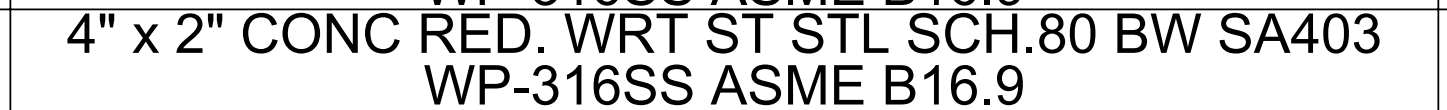 & 2 \\
\hline 6.1 & SNG1005.9A & 316 ST STL 5 & $\begin{array}{l}\text { TO } 250 \text { AARH FACE FINISH SA182 GRF316 ASME } \\
\text { B16.5 }\end{array}$ & 2 \\
\hline 6 & W-SNG1005.10F & 316 ST STL 5 & WELDMENT, CHAR POT LOWER & 1 \\
\hline 5 & SNG1005.20A & 316L / THERMICULITE & 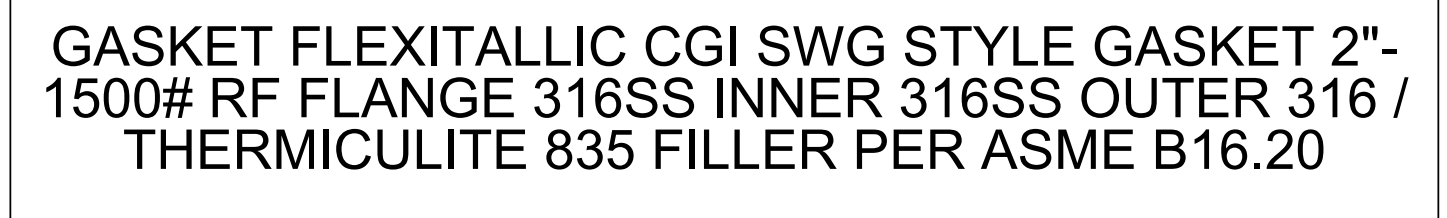 & 1 \\
\hline 4 & SNG1005.19A & \begin{tabular}{|l|} 
625/THEONEL \\
835
\end{tabular} & 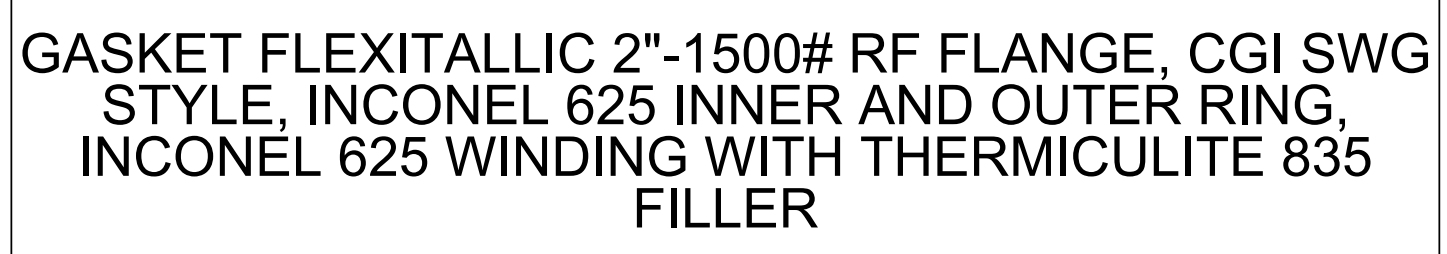 & 2 \\
\hline 3 & SNG1005.16A & $316 \mathrm{ST}$ STL & $\begin{array}{c}\text { 2"- BLIND FGD ST STL } 1500 \# \text { RF SA } 182 \text { GRF316 } \\
\text { ASME B16.5 }\end{array}$ & 1 \\
\hline 2.2 & SNG1005.9A & 316 ST STL & $\begin{array}{c}\text { 2"-WNF FGD ST STL 1500\# RF BORE FOR SCH.80 } 125 \\
\text { TO } 250 \text { AARH FACE FINSH BA182 GRF316 ASME } \\
\text { B16.5 }\end{array}$ & 2 \\
\hline 2.1 & TBD & ST STL & 1" BV SERIES, WITH 2" RF FLANGE ENDS & 1 \\
\hline 2 & W-SNG1005.11A & 316 S.S. & VALVE, (AOV) CHAR POTS & 1 \\
\hline 1.6 & SNG1008.7A & 316 ST STL & \begin{tabular}{|l|} 
TUBING, 1/2"-065" WALL, 316 ST STL, SA213 TP 316 \\
ASTM A229, PER ASME B3113
\end{tabular} & 1 \\
\hline 1.5 & $\begin{array}{l}\text { SS-16-MPW-A- } \\
\text { 8TSW }\end{array}$ & $316 S \mathrm{SS}$ & 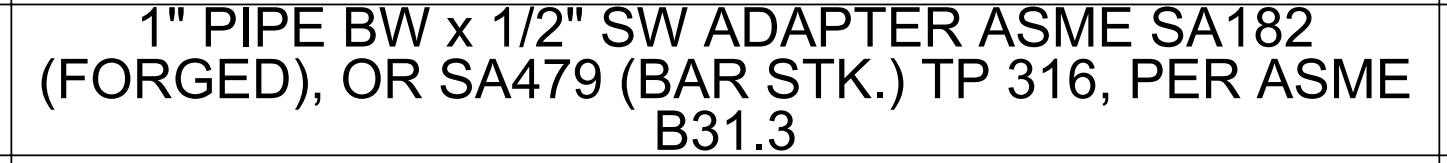 & 1 \\
\hline 1.4 & SNG0001-6A & 316 ST STL 5 & \begin{tabular}{|c|} 
PIPE 1"-SCH.80. $179 "$ WALL ST STL SMLS SA312 TP \\
316SS ASME B36.19
\end{tabular} & 1 \\
\hline 1.3 & SNG0001-4A & 316 ST STL 5 & $\begin{array}{l}\text { 2" x 1" ELL RED. WRT ST STL SCH.80 BW SA403 WP- } \\
316 S S \text { ASME B16.9 }\end{array}$ & 1 \\
\hline 1.2 & SNG0001-5A & 316 ST STL 5 & $\begin{array}{c}\text { PIPE 2"-SCH.80.218 WALL ST STL SMLS SA312 TP } \\
316 S \text { ASME B36.19 }\end{array}$ & 1 \\
\hline 1.1.9 & SNG1005.22A & 316 ST STL 5 & PLATE, LIFTING LUG, CHAR POT & 2 \\
\hline 1.1.8 & SNG1005.9A & 316 ST STL 5 & $\begin{array}{c}\text { 2"-WNF FGD ST STL 1500\# RF BORE FOR SCH.80 } 125 \\
\text { TO } 250 \text { AARH FACE FINSH SA182 GRF316 ASME } \\
\text { B16.5 }\end{array}$ & 1 \\
\hline 1.1.7 & SNG1005.13B & 316 ST STL 5 & $\begin{array}{l}\text { 1/2"-6000\#-FGD THD HALF CPLG SA182 GR F316 } \\
\text { ASME B16.11 }\end{array}$ & 2 \\
\hline 1.1.6 & SNG1005.17B & 316 ST STL 5 & \begin{tabular}{|l|}
$21 / 2 " \times 2 "$ TEE RED. WRT ST STL SCH.80 BW SA403 \\
WP-316SS ASME B16.9
\end{tabular} & 1 \\
\hline 1.1.5 & SNG1005.7A & 316 ST STL 5 & $\begin{array}{c}\text { 4" x 2" CONC RED. WRT ST STL SCH.80 BW SA403 } \\
\text { WP-316SS ASME B16.9 }\end{array}$ & 1 \\
\hline 1.1 .4 & SNG1005.6C & 316 ST STL 5 & $\begin{array}{l}\text { PIPE 8"-SCH.80.500" WALL ST TSL SMLS SA312 TP } \\
316 S S \text { ASME B36.19 }\end{array}$ & 1 \\
\hline 1.1 .3 & SNG1005.5A & 316 ST STL 5 & $\begin{array}{c}" \text { × 4" CONC RED. WRT ST STL SCH. } 80 \text { BW SA403 } \\
\text { WP-316SS ASME B16.9 }\end{array}$ & 2 \\
\hline 1.1.2 & SNG1005.4A & 316 ST STL 5 & 4" 22 1/2" CONC RED WRT ST STL SCH.80 BW SA403 & 1 \\
\hline 1.1.1 & SNG1005.3B & 316 ST STL 5 & $\begin{array}{c}2112^{2} \text { "WF FGD ST STL 1500\# RF BORE FOR SCH.80 } \\
125 \text { TO } 250 \text { AARH FACE FINSH SA182 GRF316 ASME } \\
\text { B16.5 }\end{array}$ & 1 \\
\hline 1.1 & W-SNG1005.2G & 316 ST STL 5 & WELDMENT, CHAR POT UPPER & 1 \\
\hline 1 & W-SNG1005.18E & 316 ST STL 5 & $\begin{array}{c}\text { WELDMENT, UPPER CHAR POT PRESSURE } \\
\text { BOUNDARY }\end{array}$ & 1 \\
\hline TEM & PART NUMBER & MATERIAL & DESCRIPTION & QTY. \\
\hline
\end{tabular}

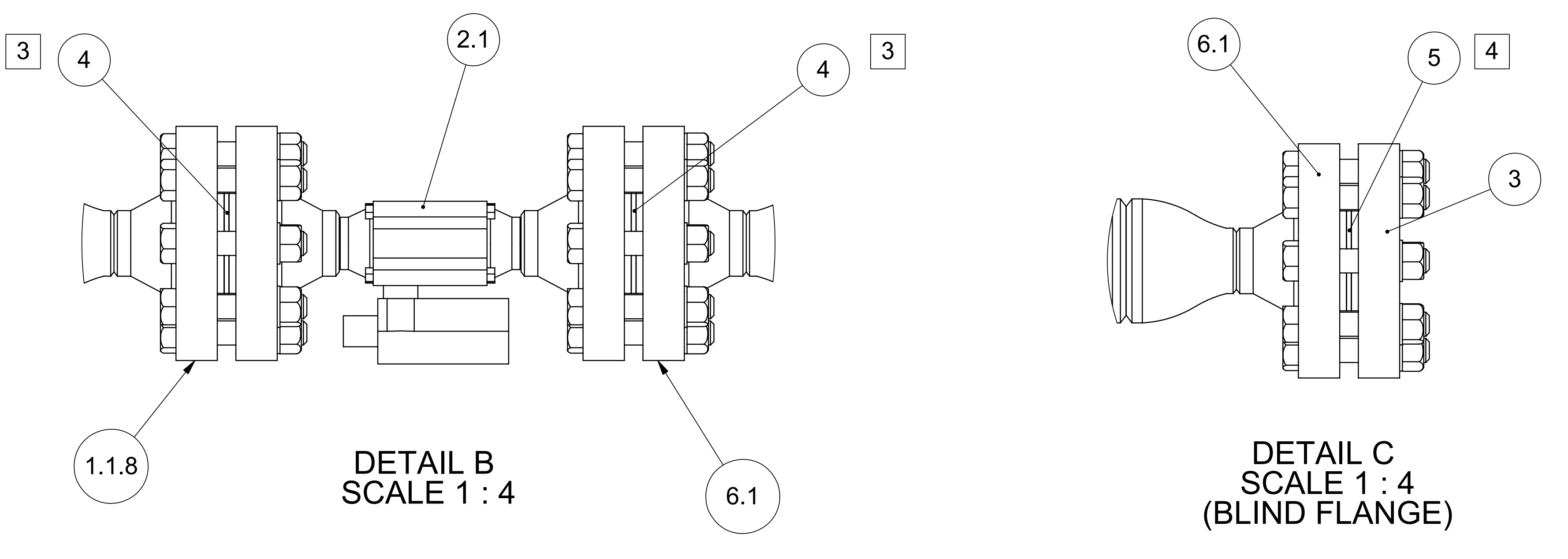

SECTION A-A 

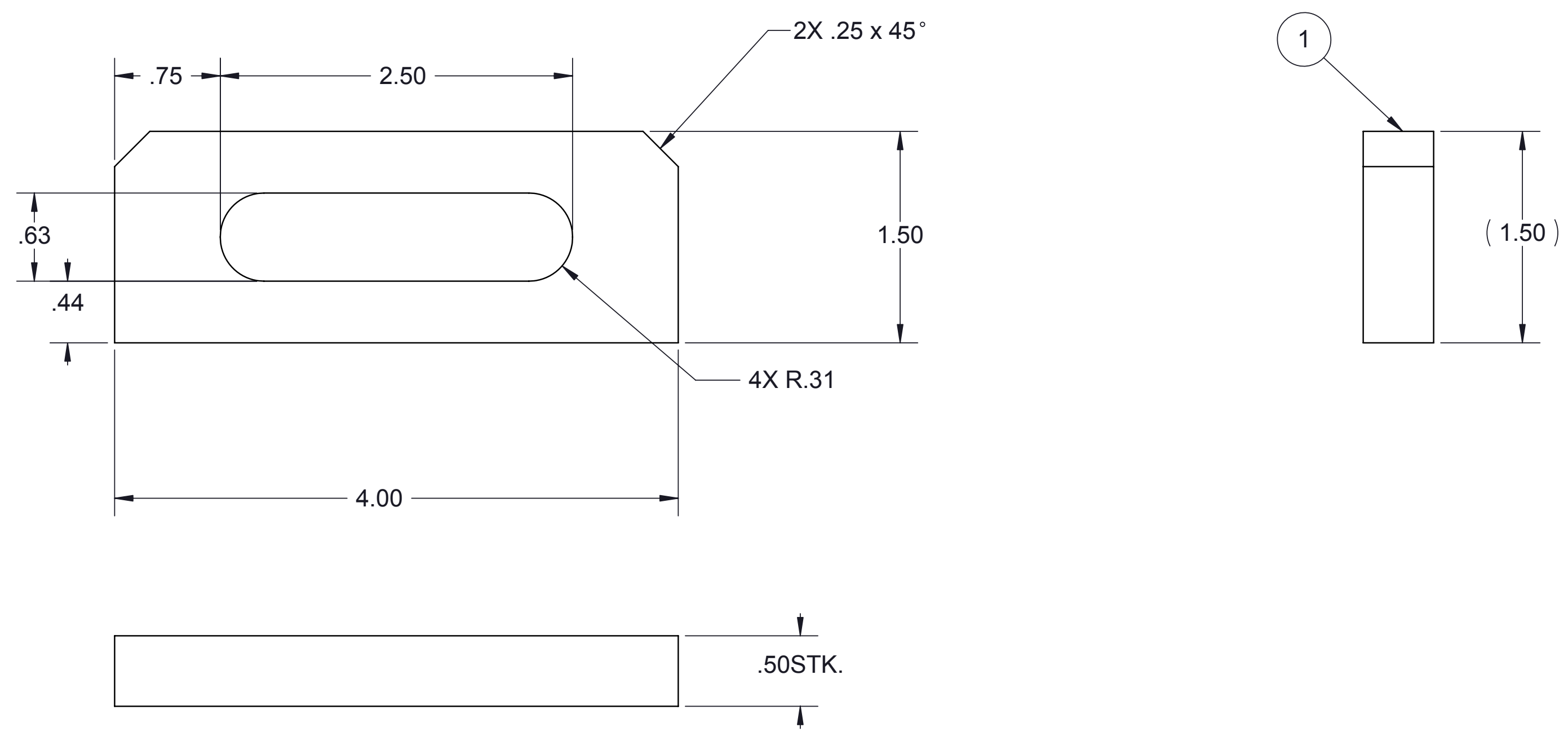

\begin{tabular}{|c|c|c|c|c|c|c|c|c|c|c|}
\hline 1 & SNG1005.22A & 2 & \multicolumn{2}{|c|}{316 ST STL } & & \multicolumn{4}{|c|}{ PLATE, LIFTING LUG, CHAR POT } & 1 \\
\hline $\begin{array}{l}\text { ITEM } \\
\text { NO. }\end{array}$ & PART NUMBER & & \multicolumn{2}{|c|}{ MATERIAL } & & \multicolumn{4}{|c|}{ DESCRIPTION } & QTY. \\
\hline & \multirow{4}{*}{\multicolumn{2}{|c|}{ 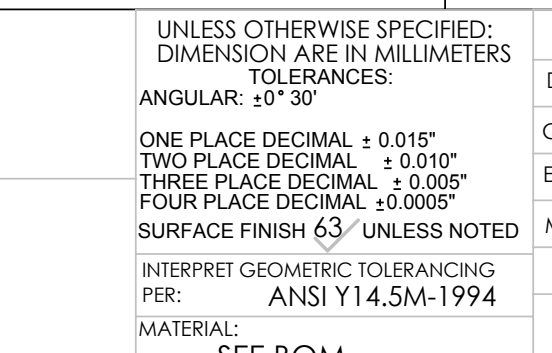 }} & DRAWN & \begin{tabular}{|c} 
DATE \\
$03 / 09 / 08$
\end{tabular} & $\begin{array}{l}\text { NAME } \\
\text { D. WAIBEL }\end{array}$ & \multicolumn{5}{|c|}{$\begin{array}{l}\text { ARIZONA PUBLIC SERVICE } \\
\text { 400 N. 5th Street } \\
\text { Phoenix, Az. } 85003\end{array}$} \\
\hline & & & $\begin{array}{l}\text { CHECKED } \\
\text { ENG APPR. }\end{array}$ & & & \multicolumn{5}{|c|}{ PROJECT: $\quad$ COAL TO SNG } \\
\hline & & & MFG APPR. & & & \multicolumn{5}{|c|}{ TITLE: $\quad$ KINETICS REACTOR } \\
\hline & & & Q.A. & & & \multicolumn{5}{|c|}{ PLATE, LIFTING LUG CHAR POT } \\
\hline & $\begin{array}{l}\text { MAEERAL: } \\
\text { FENSH: }\end{array}$ & & \multicolumn{2}{|c|}{$\mathrm{SI}_{M \mathrm{TRIC}}$} & 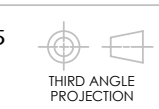 & SIZE & \multicolumn{2}{|c|}{ DWG. 1} & | & SEV \\
\hline & SIMILAR TO: & & CAD FILE & & NG1005.22A & SCA & E: 1:1 & WEIGHT: & SHEET & OF 1 \\
\hline
\end{tabular}


GENERAL NOTES:

ALL WELDS TO MEET PRESSURE VESSEL CODE, ASME SECTION 8, DIVISION 1 ,
UNLESS NOTED OTHERWISE.

INSPECTION OF WELDS TO BE 100\% X-RAY, UNLESS SPECIFIED OTHERWISE.

FITTINGS, AND PIPE, TO BE IN ACCORDANCE WITH ANSI B16.9. FLANGES IN

ACCORDANCE WITH ANSI B16.5. GASKETS IN ACCORDANCEWITH, ASME B16.20
THREADOLETS,AND ANY BUTTWELDE ITITINGS ARE TO BE END PREPPED
PER ASME B16.25.

4 THREADOLET'S TO BE AT 6:00 AND 12:00 POSITIONS AS SHOWN.

5. MATERIAL: 316 ST. STL., CARBON CONTENT MUST BE EQUAL TO, OR ABOVE. 04\% E

REVISIONS

\begin{tabular}{|c|c|c|c|c|c|}
\hline \multicolumn{6}{|c|}{ 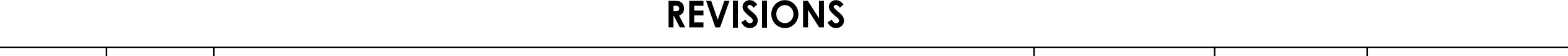 } \\
\hline ZONE & REV. & DESCRIPTION & DATE & REV. BY & APPROVED \\
\hline ALL & A & INITIAL RELEASE & $07 / 09 / 07$ & D.W. & \\
\hline ALL & B & 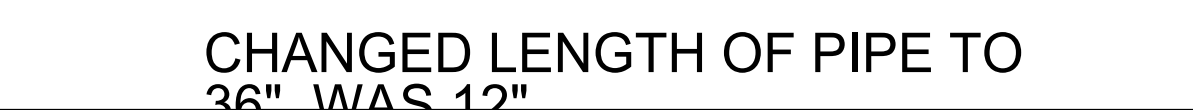 & 08/28/07 & D.W. & \\
\hline ALL & c & ADDED ITEM 5 WELDOLET & 10/19/07 & D.W. & \\
\hline ALL & D & $\begin{array}{l}\text { ADDED MATERIALCALL OUT TO BOM, } \\
\text { ADDED WELD NOTES, AND CORRECTED } \\
\text { WELDS TO BE FULL PEN. }\end{array}$ & 12/01/07 & D.W. & \\
\hline ALL & E & ADDED NOTE 5; MATERIAL SPEC, AND & 12/06/07 & D.W. & \\
\hline ALL & $\mathrm{F}$ & $\begin{array}{l}\text { ADDED ITEM; } 6 \text {, LIFTING LUG, CHANGED } \\
\text { ALLMATERAL TO } 316 \text { ST STL ST }\end{array}$ & 03/09/08 & D.W. & \\
\hline ALL & G & DIM. 44.00 WAS 30.25; ROTATED LUGS 70Deg & 04/30/08 & D.w. & \\
\hline
\end{tabular}

6

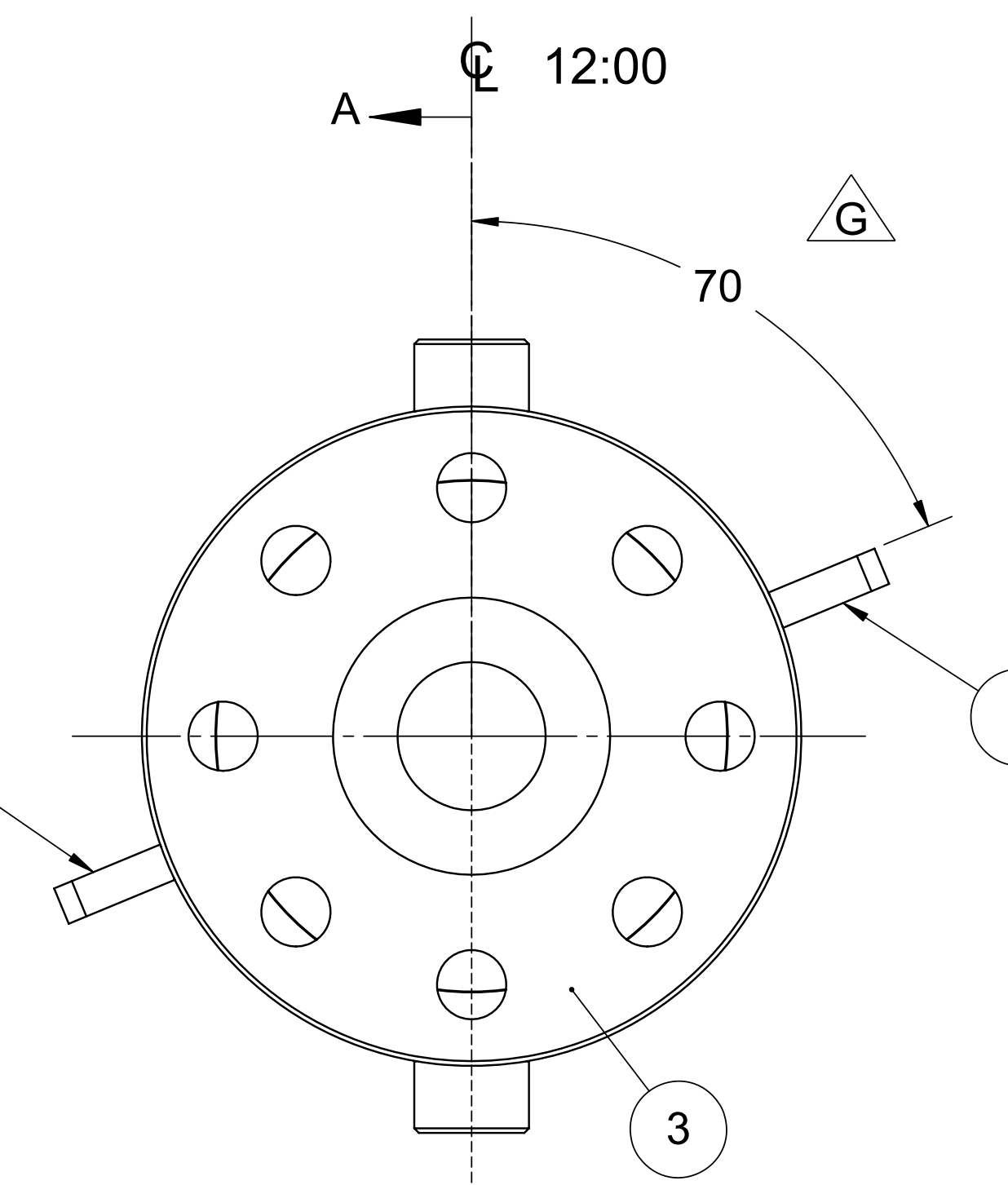

SEE SHEET 2
A

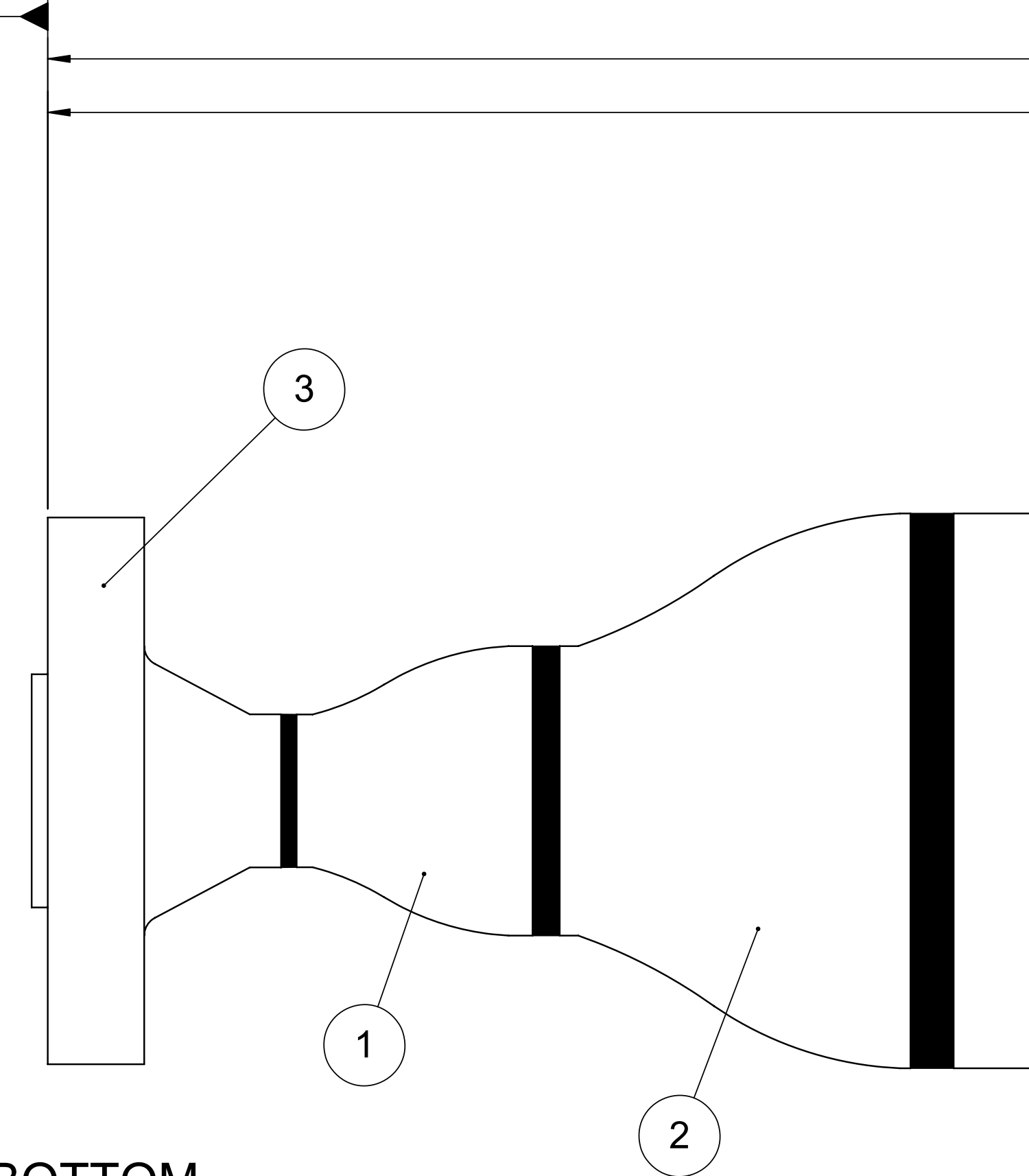

BOTTOM
$2 X @ 180^{\circ} 44.00$

$\widehat{G}$

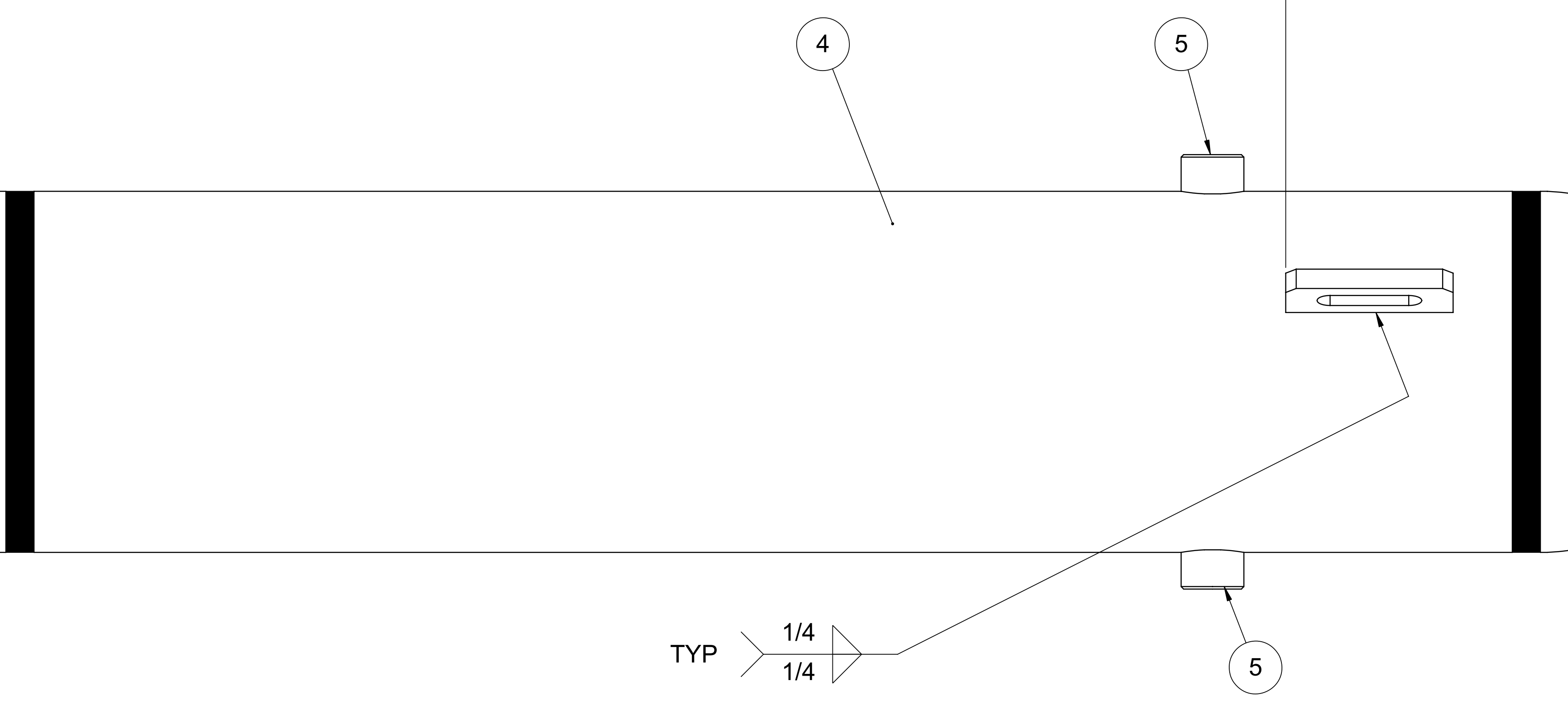

3
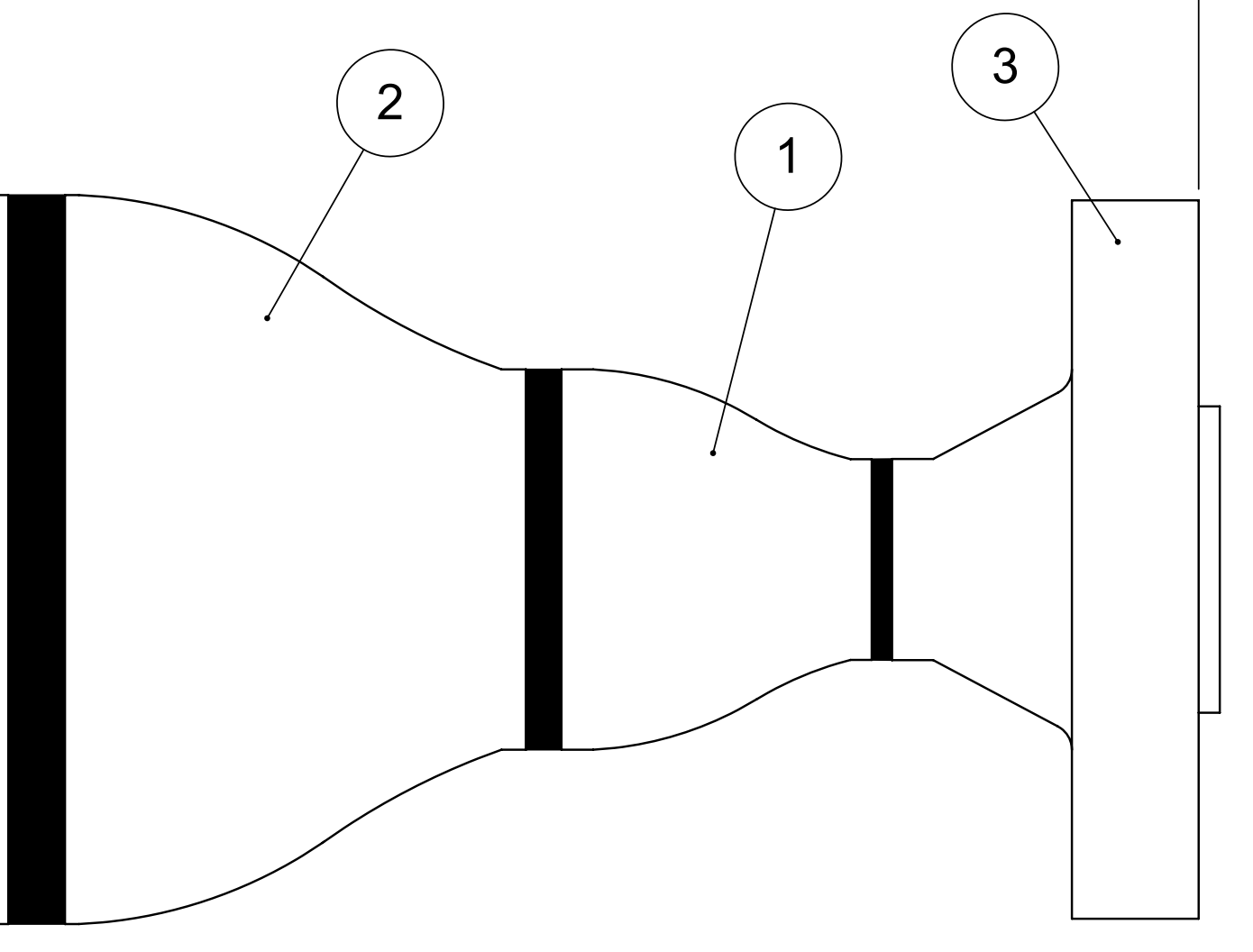

TOP

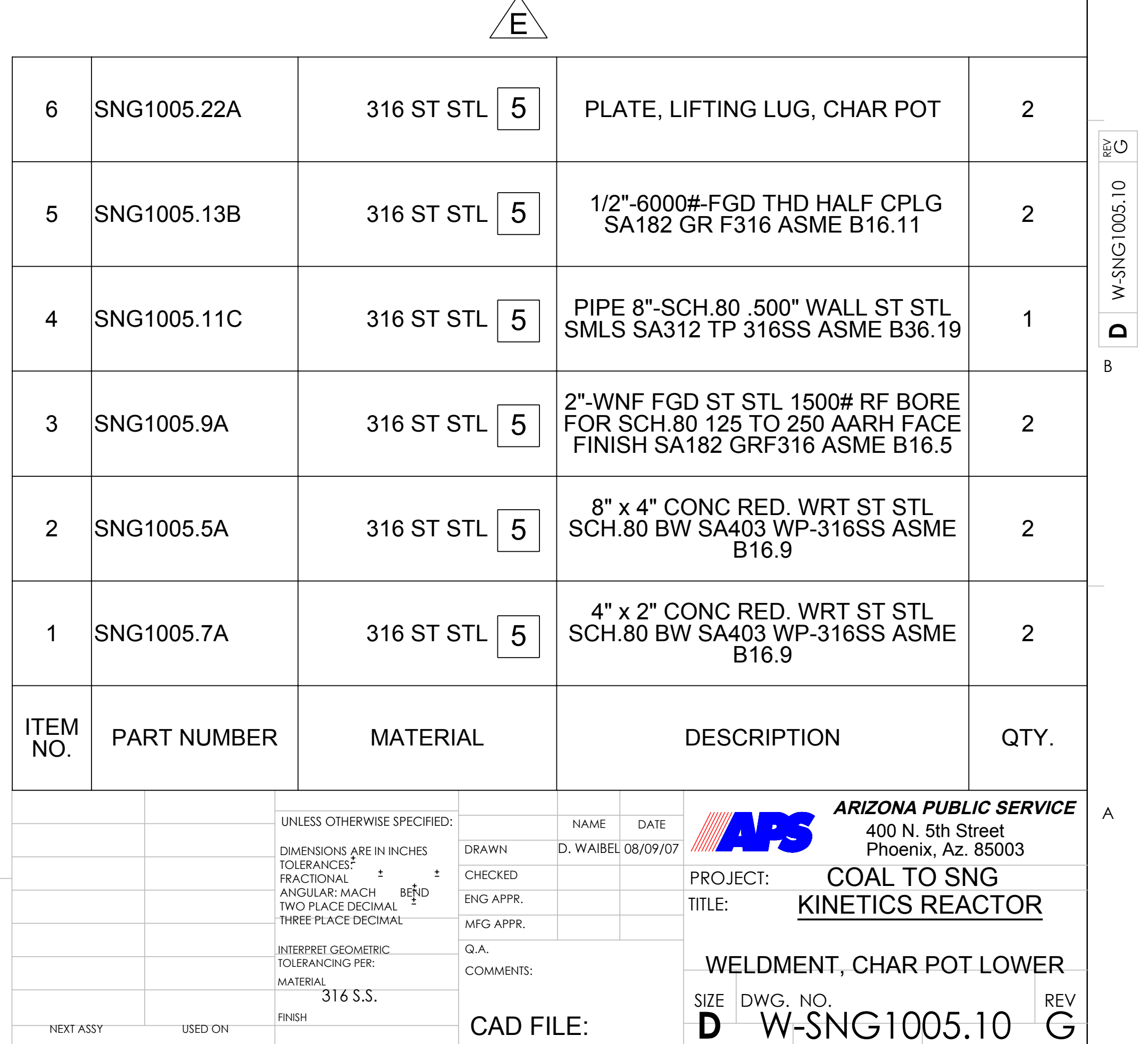




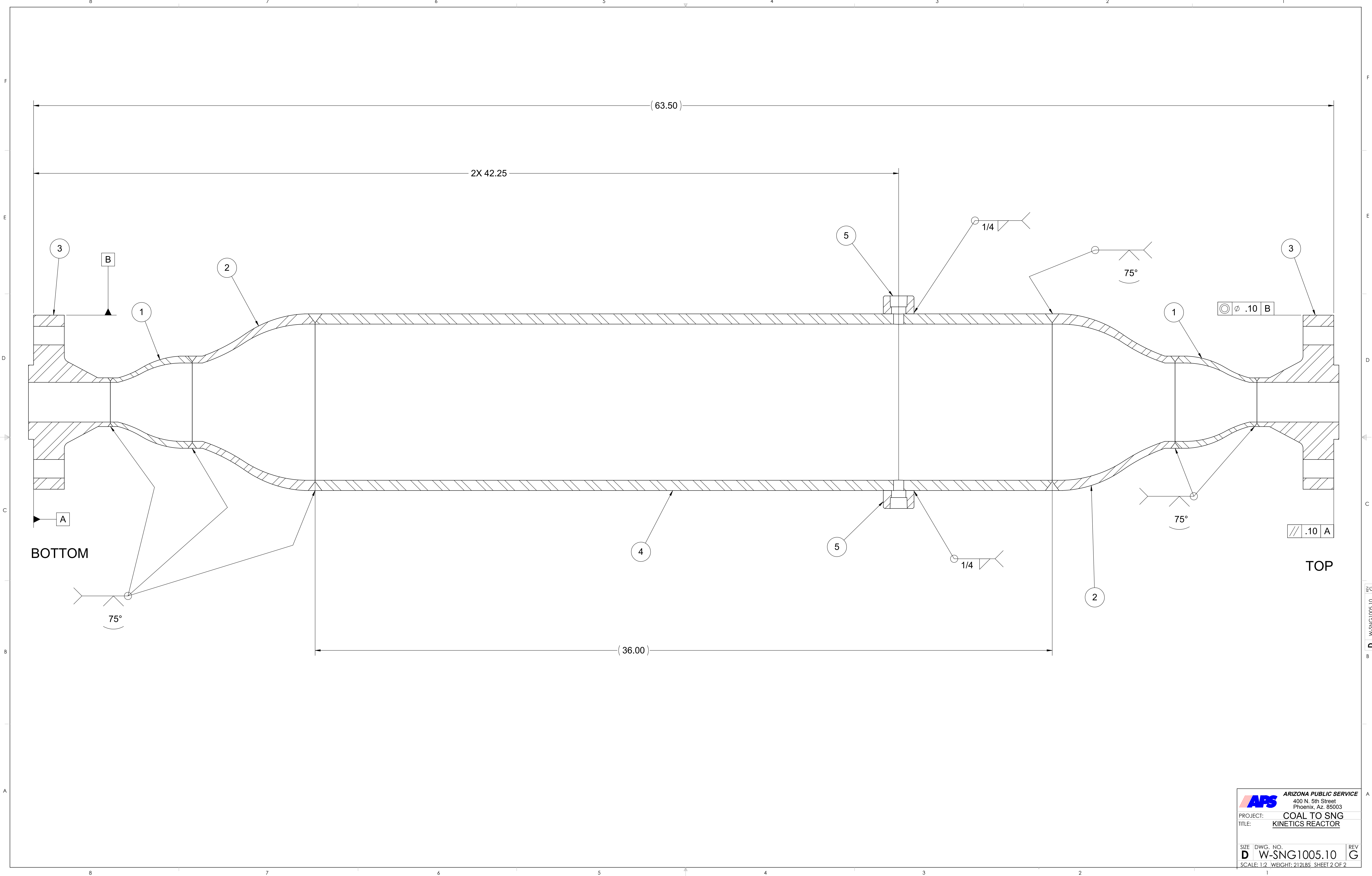



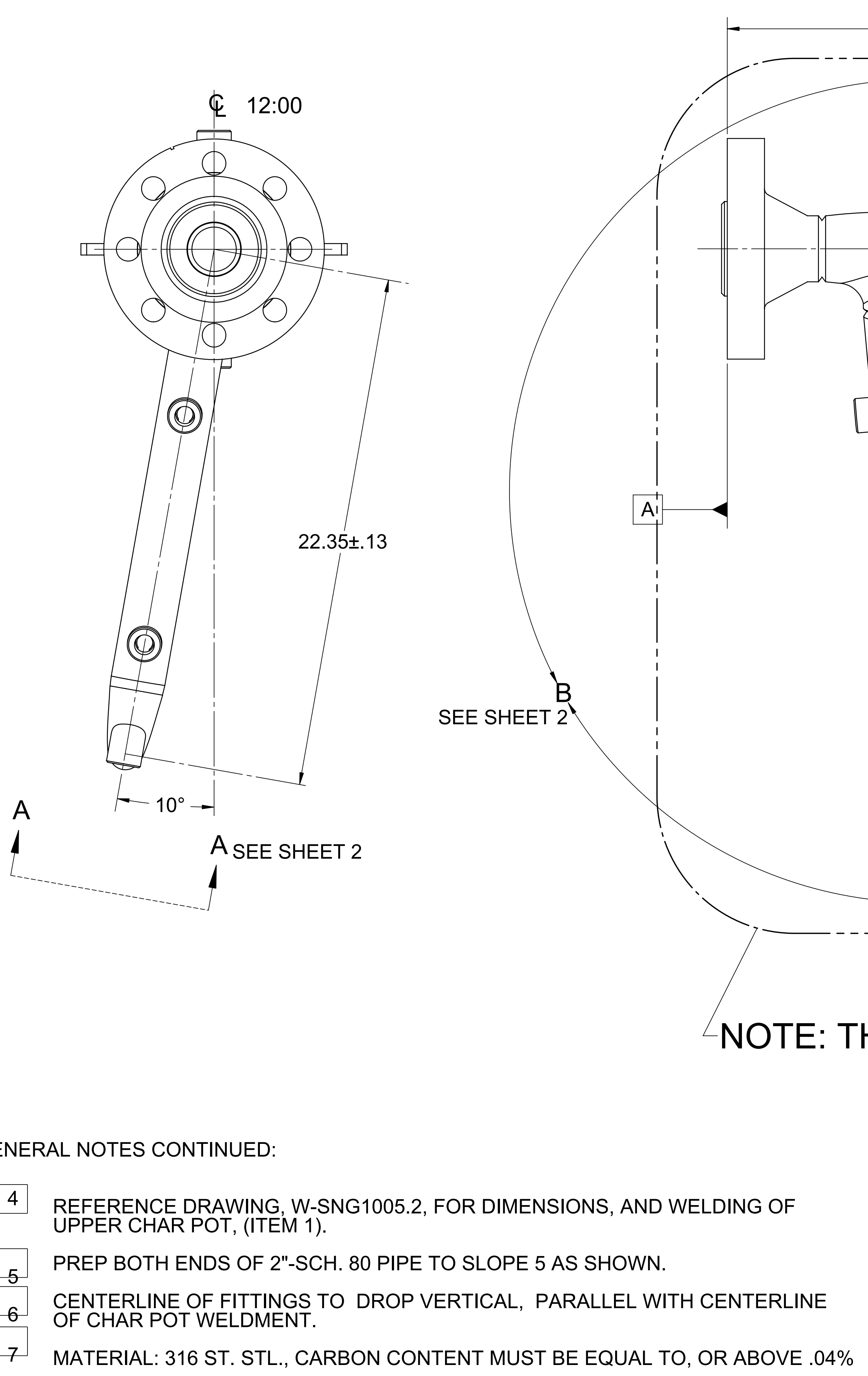

$(71.88)$

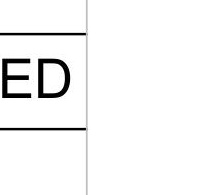
.

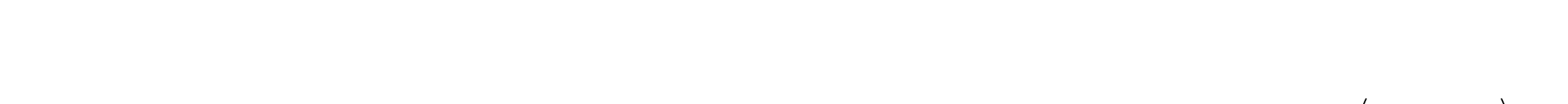

\section{1}

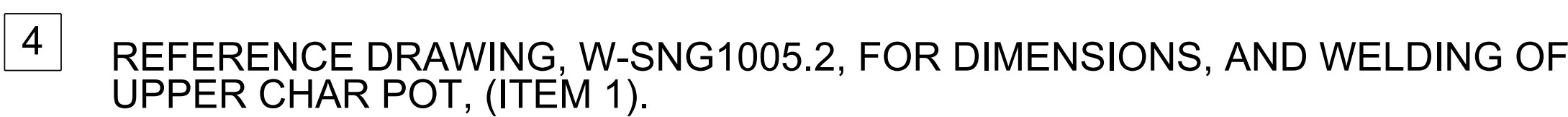
5 PREP BOTH ENDS OF 2"-SCH. 80 PIPE TO SLOPE 5 AS SHOWN.
6 CENTERLINE OF FITTINGS TO DROP VERTICAL, PARALLEL WITH CENTERLINE
7 MATERIAL: $316 \mathrm{ST}$. STL., CARBON CONTENT MUST BE EQUAL TO, OR ABOVE. 04\%

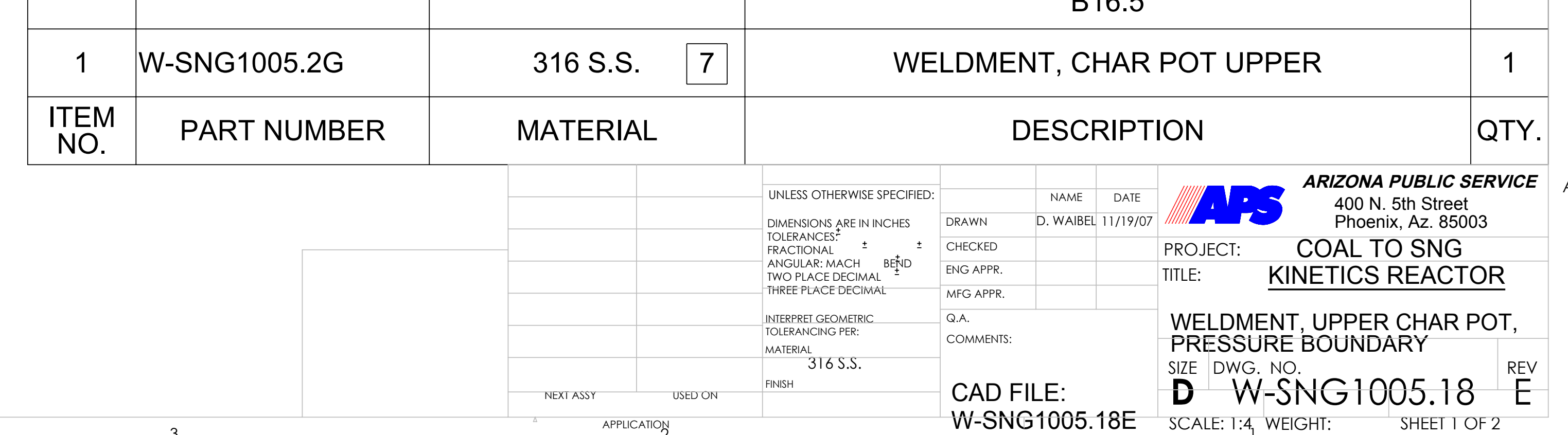



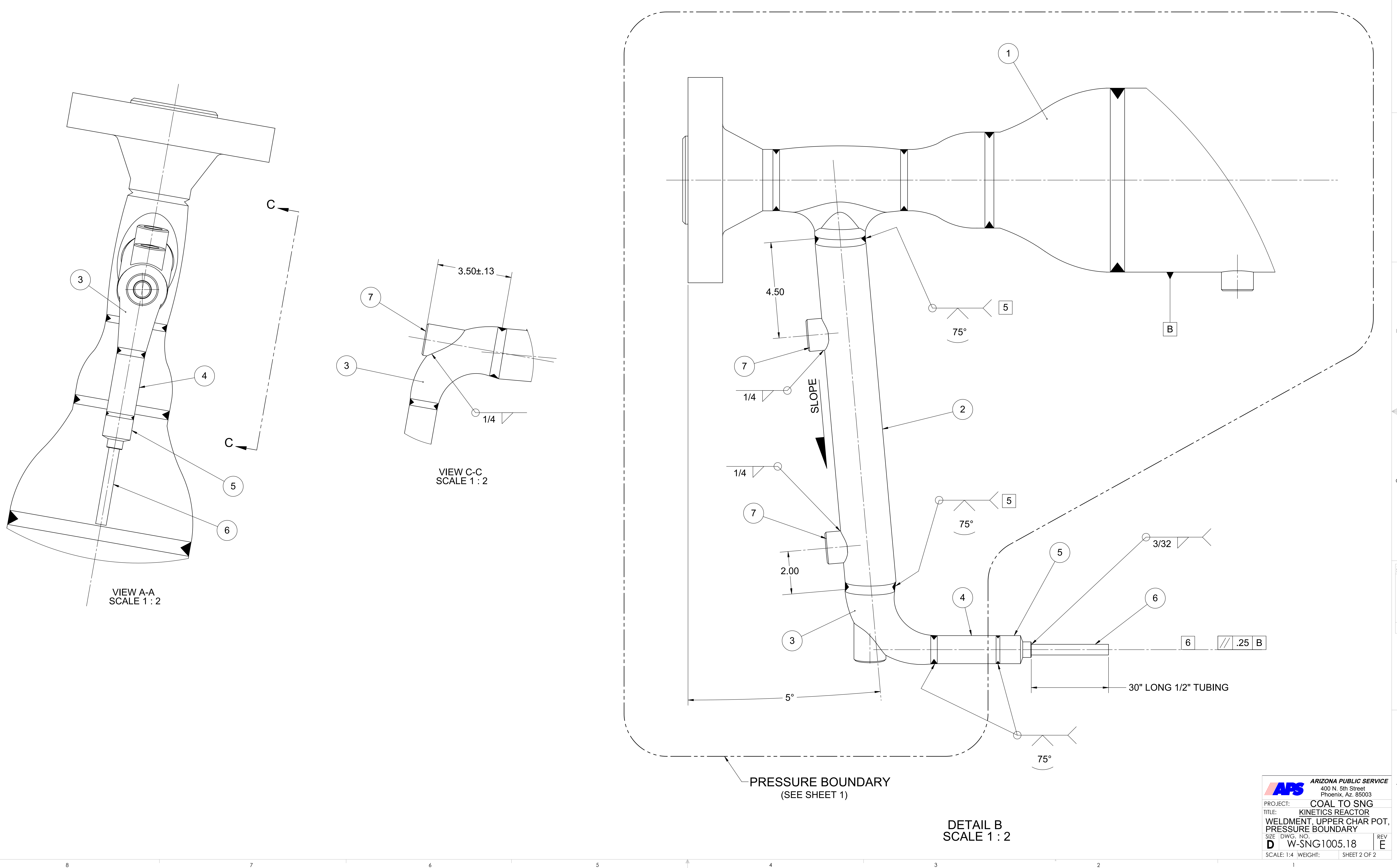


\begin{tabular}{|c|c|c|c|c|c|}
\hline ZONE & REV. & DESCRIPTION & DATE & REV. BY & APPROVE \\
\hline ALL & & INITIAL RELEASE & $07 / 05 / 07$ & D.W. & \\
\hline ALL & B & 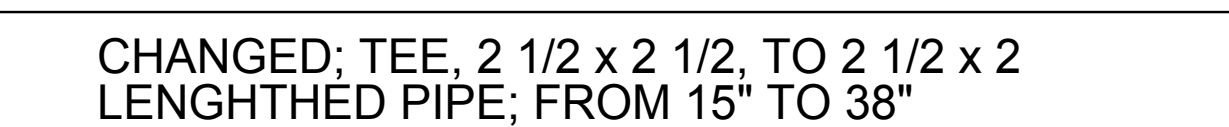 & $08 / 06 / 107$ & D.W. & \\
\hline ALt & $c$ & ADDEDITEM 7, WELDOLET COUPLING & 101490707 & Dw & \\
\hline ALL & D & ADDED MATERIAL TO BOM, ADDED WELD NOTES, & 12/01/07 & D.W. & \\
\hline ALL & E & ADDED NOTTE 5, MATERIAL SPEC. AND CORRECTED & 12/06/107 & D.W. & \\
\hline ALL & $\mathrm{F}$ & ADDED FLANGE FACE DETAIL ON SHEET 2 & 12/30/07 & D.W. & \\
\hline ALL & G & ADDED ITEM ; 9 , LIFTING LUG & $03 / 09 / 08$ & D.w. & \\
\hline
\end{tabular}

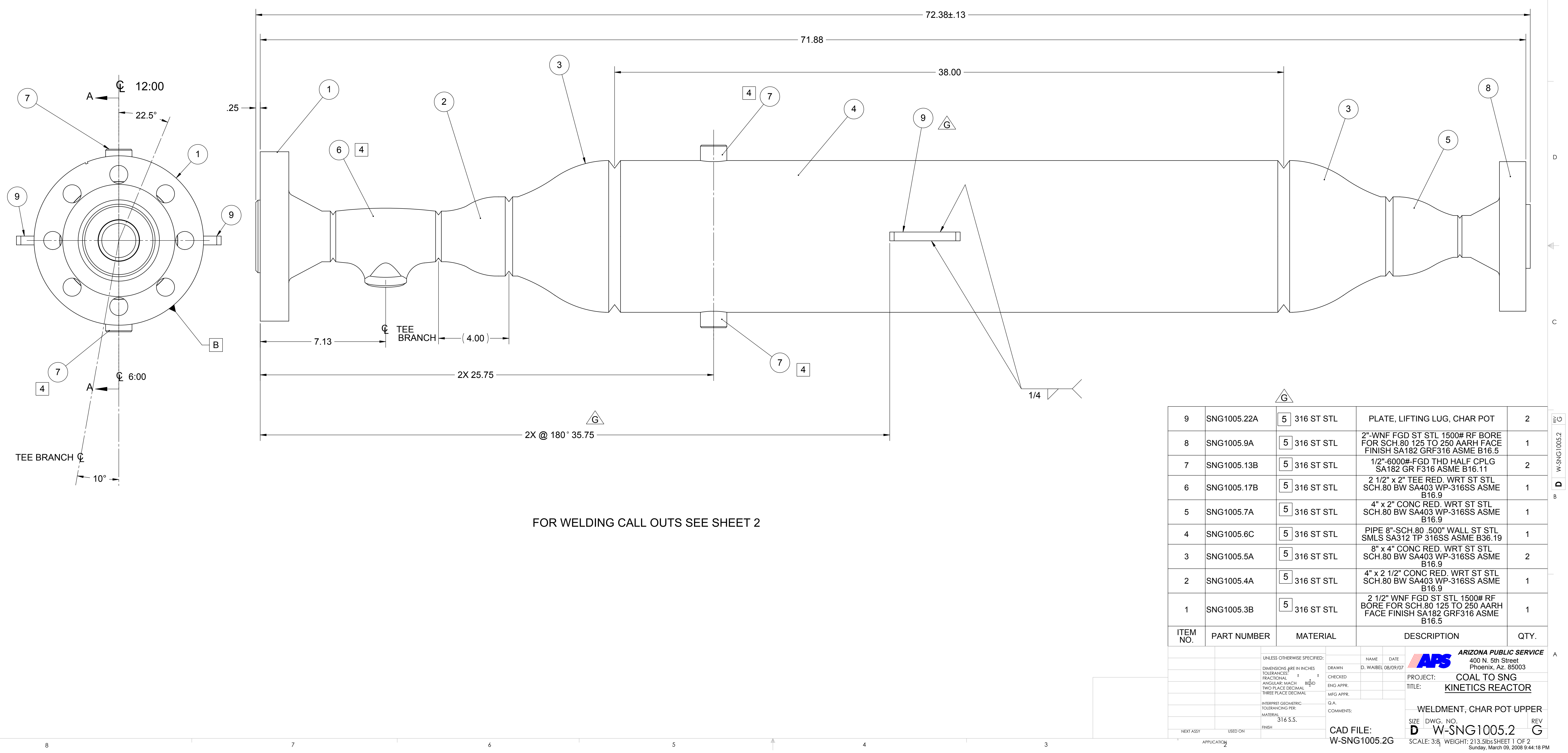



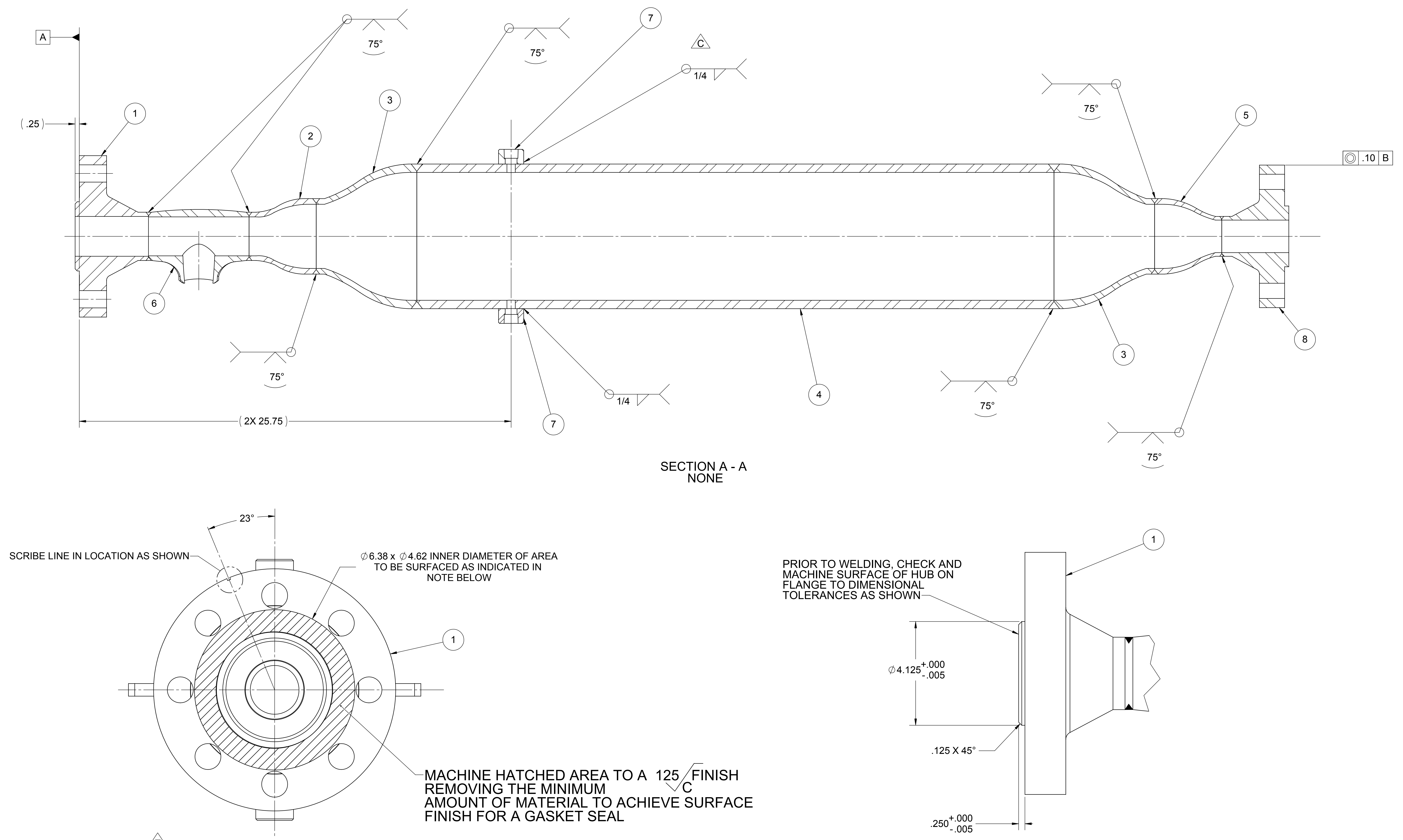

FLANGE, (ITEM 1) MACHINING PRIOR TO WELDING 


\section{GENERAL NOTES:}

1 SEE SHEET 2 FOR ORTHO VIEWS, SECTIONS, AND DETALLS.

2 MATERIAL: 316 ST STL, CARBON CONTENT MUST BE EQUAL TO, OR ABOVE .04\%. 3 TC PAD, ITEM 5, IS 1" 1 1" $\times 1 / 8$ " THICK, REFERENCE
4

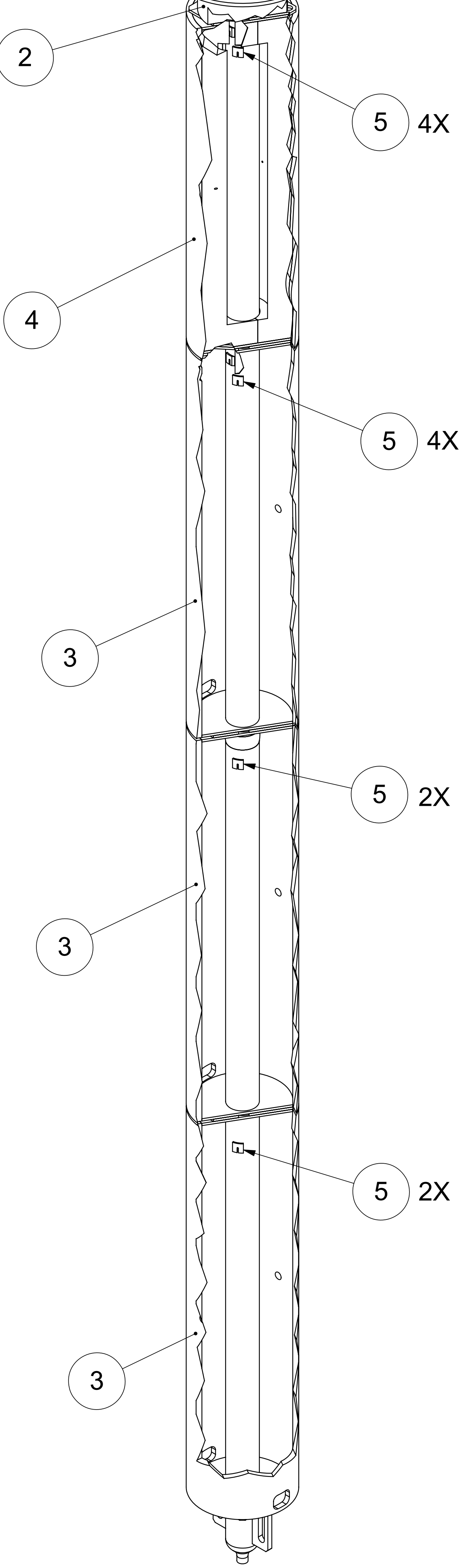

\begin{tabular}{|c|c|c|c|c|c|c|}
\hline 5 & SNG1006.12A & INCONEL 625 & \multicolumn{3}{|c|}{ PLATE, TC PAD } & 12 \\
\hline 4 & A-SNG1006.11A & CERAMIC & \multicolumn{3}{|c|}{$\begin{array}{l}\text { ASSEMBLY, HEATER W/CERAMIC INSULATION BLANKET, } \\
\text { 30"LG. CERAMIC }\end{array}$} & 1 \\
\hline 3 & A-SNG1006.7A & CERAMIC & \multicolumn{3}{|c|}{$\begin{array}{l}\text { ASSEMBLY, HEATER W/CERAMIC INSULATION BLANKET, } \\
\text { 36"LG, CERAMIC }\end{array}$} & 3 \\
\hline 2 & SNG1006.5A & CERAMIC & \multicolumn{3}{|c|}{ TOP INSULATION } & 1 \\
\hline 1 & W-SNG1006.14 & SEE WELDMENT & \multicolumn{3}{|c|}{ WELDMENT, PRE-HEATER TUBING, W/SUPPORTS } & 1 \\
\hline 1 & SNG1006.2C & INCONEL 625 & \multicolumn{3}{|c|}{ LOWER, 3"-DIA., ROUND x 90"LG. INCONEL 625} & 1 \\
\hline $\begin{array}{l}\text { ITEM } \\
\text { NO. }\end{array}$ & PART NUMBER & MATERIAL & \multicolumn{3}{|c|}{ DESCRIPTION } & /QTY. \\
\hline & & & \multicolumn{4}{|c|}{ 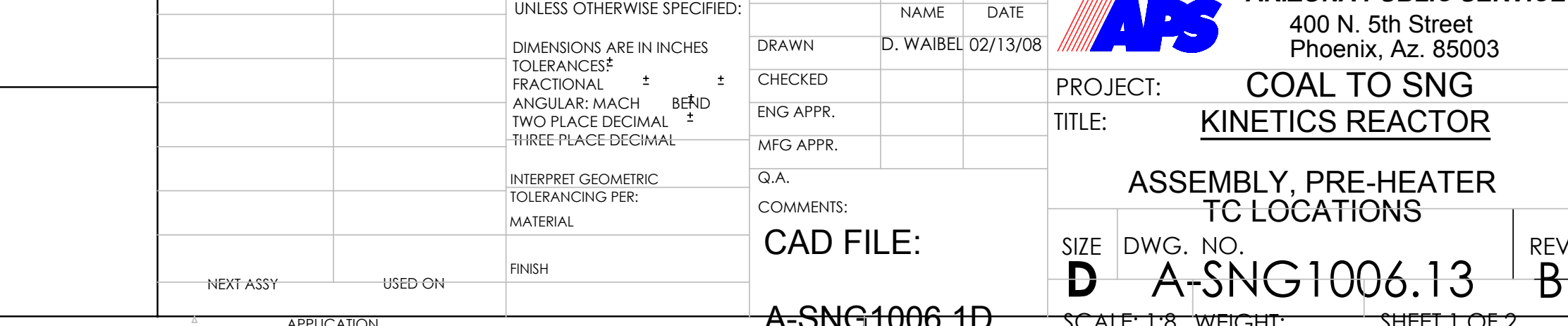 } \\
\hline
\end{tabular}




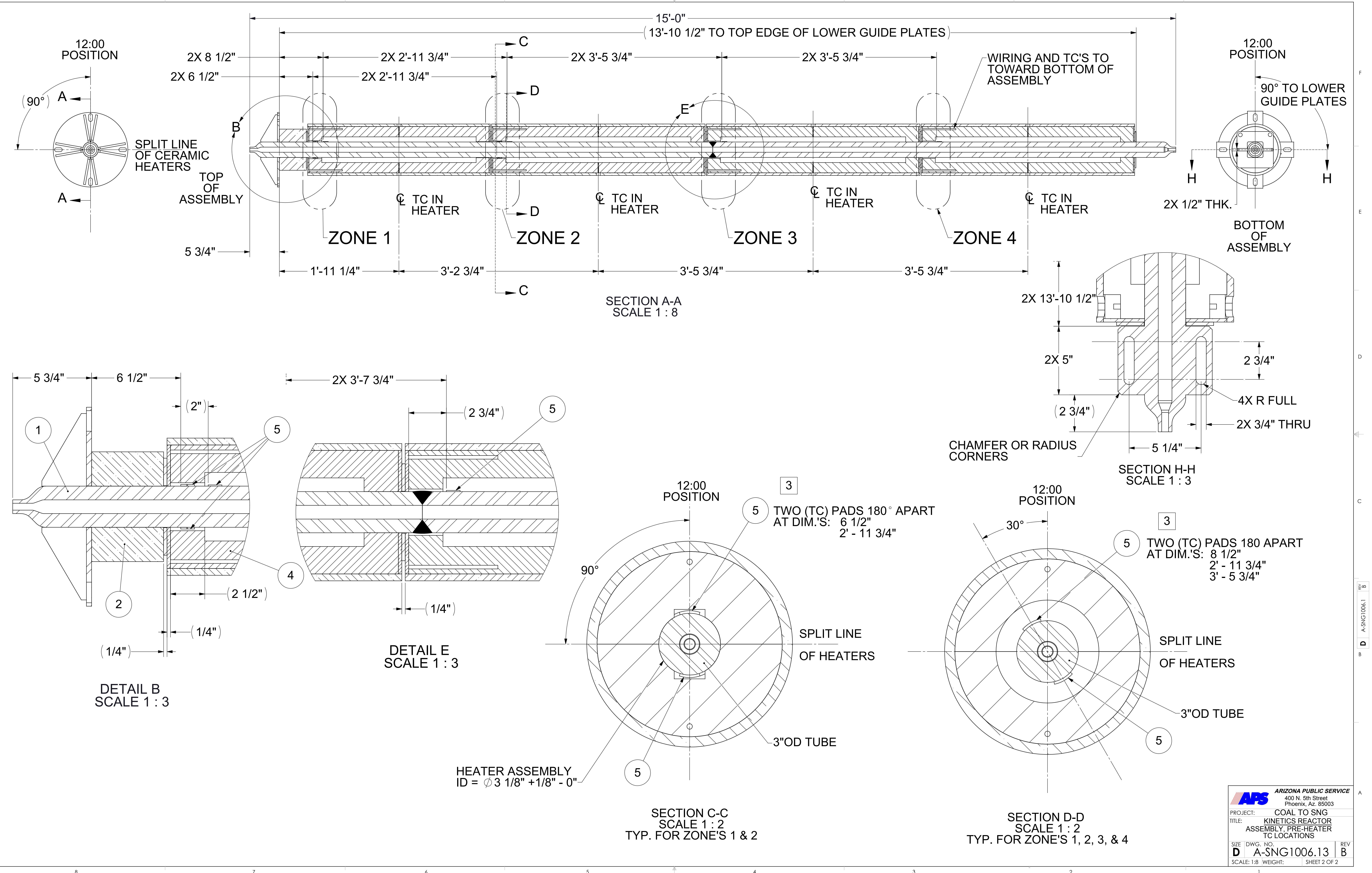




\section{GENERAL NOTES:}

1 SEE SHEET 2 FOR ORTHO VIEWS, SECTIONS, AND DETAILS.

2 MATERIAL: 316 ST STL, CARBON CONTENT

MUST BE EQUAL TO, OR ABOVE .04\%.

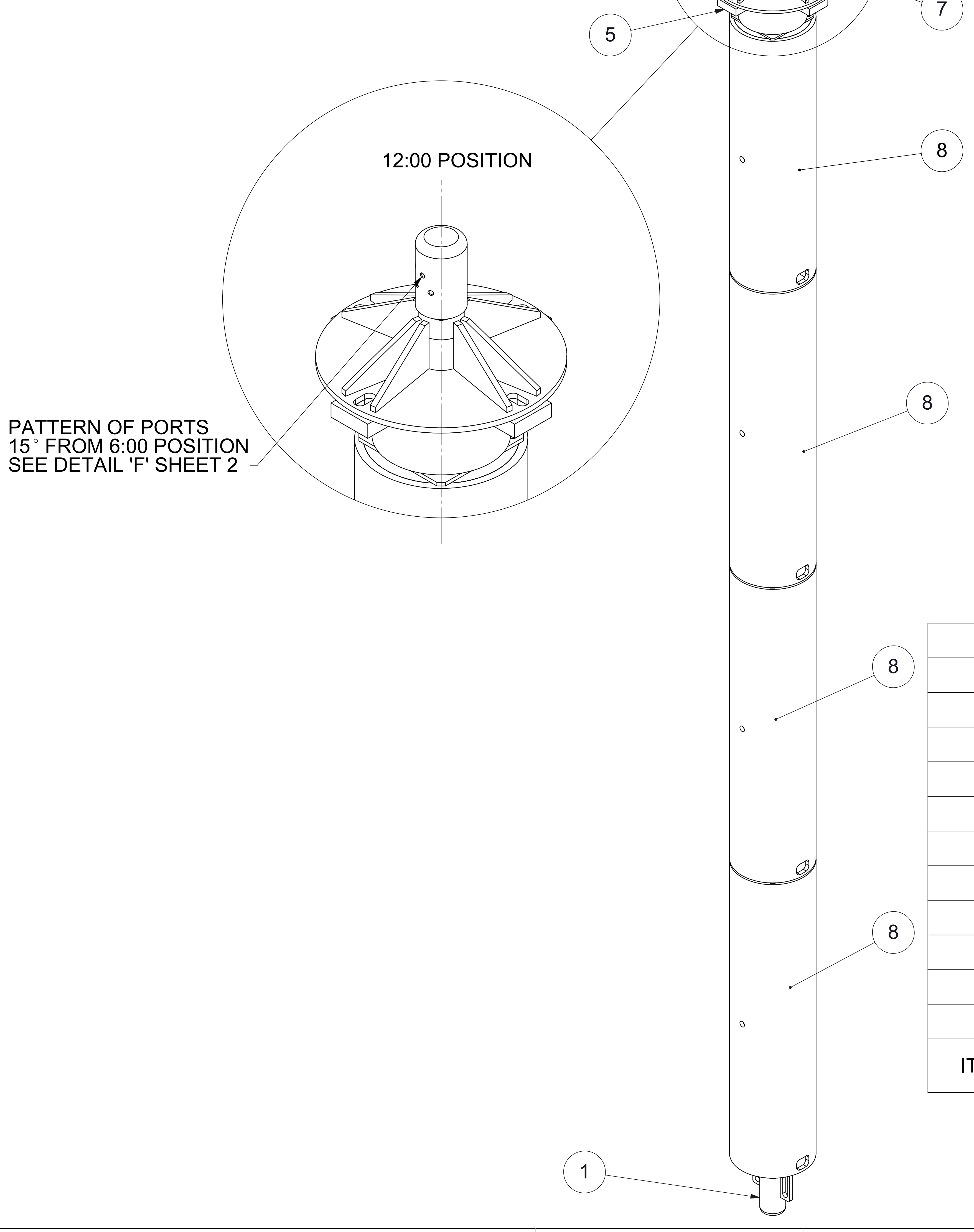

8

ALL A INITIAL DESIGN LAYOUT $02 / 13 / 08$ DW

\begin{tabular}{l|l|l} 
ALL & B & ITEM 7. TOP MOUTN CHANGED \\
TO SLOTS INSTEAD OF HOLES
\end{tabular}

\begin{tabular}{l|l|l} 
ALL & $\mathrm{C}$ & $\begin{array}{l}\text { OVERALL LENGTH CHANGE } \\
\text { FROM 12' TO 15', ADDED }\end{array}$
\end{tabular} FROM 12' TO 15', ADDED

03/16/08 DW HEATER

\begin{tabular}{l|l|l} 
ALL & D & REVISED LOWER GUIDE \\
PLATES FOR CLEARANCE IN
\end{tabular} PLATES FOR CLEARA

$07 / 15 / 08$ DW REACTOR FRAME

07/28/08 DW

\begin{tabular}{l|l|l} 
ALL & E & REVISED LOWER GUIDE \\
PLATES FOR THERMAL
\end{tabular} EXPANSION \begin{tabular}{l|l|l} 
ALL & F & $\begin{array}{l}\text { ADDED HEAD WITH PORTS } \\
\text { ITEM 12 }\end{array}$ \\
\hline
\end{tabular} 08/07/08 DW

11/19/08 DW

\begin{tabular}{|c|}
\hline 12 \\
\hline 11 \\
\hline 10 \\
\hline 9 \\
\hline 8 \\
\hline 7 \\
\hline 6 \\
\hline 5 \\
\hline 4 \\
\hline 3 \\
\hline 2 \\
\hline 1 \\
\hline ITEM NO. \\
\hline
\end{tabular}

\begin{tabular}{|l|r|r|}
\hline SNG1006.15 & INCONEL 625 \\
\hline SNG1006.12A & INCONEL 625 \\
\hline A-SNG1006.11A & CERAMIC \\
\hline SNG1006.10 & CERAMIC \\
\hline SNG1006.9 & CERAMIC \\
\hline W-SNG1006.8B & 2 & $\begin{array}{r}\text { 316 ST STL } \\
\hline \text { A-SNG1006.7A }\end{array}$ \\
\hline SNG1006.6B & CERAMIC \\
\hline SNG1006.5A & CERAMIC \\
\hline SNG1006.4A & 2 & CERAMIC \\
\hline SNG1006.3C & & 316 ST STL \\
\hline SNG1006.2E & INCONEL 62 \\
\hline PART NUMBER & INCONEL 62 \\
\hline
\end{tabular}

ROUND BAR, PRE-HEATER TOP PLATE, TC PAD ASSEMBLY, HEATER W/CERAMIC INSULATION BLANKET, SHEET, 1/4" THICK CERAMIC FIBER BLANKET, 1/2" THICK CERAMIC FIBER WRAP WELDMENT, PRE-HEATER TOP MOUNTING ASSEMBLY, HEATER W/CERAMIC INSULATION BLANKET, TOP, MOUNT INSULATION, 1" THICK TOP INSULATION

PLATE, SPIDER PRE-HEAT UPPER, 3" Dia., x 90"LG. INCONEL 625 LOWER, 3"-DIA., ROUND x 90"LG. INCONEL 625 DESCRIPTION 


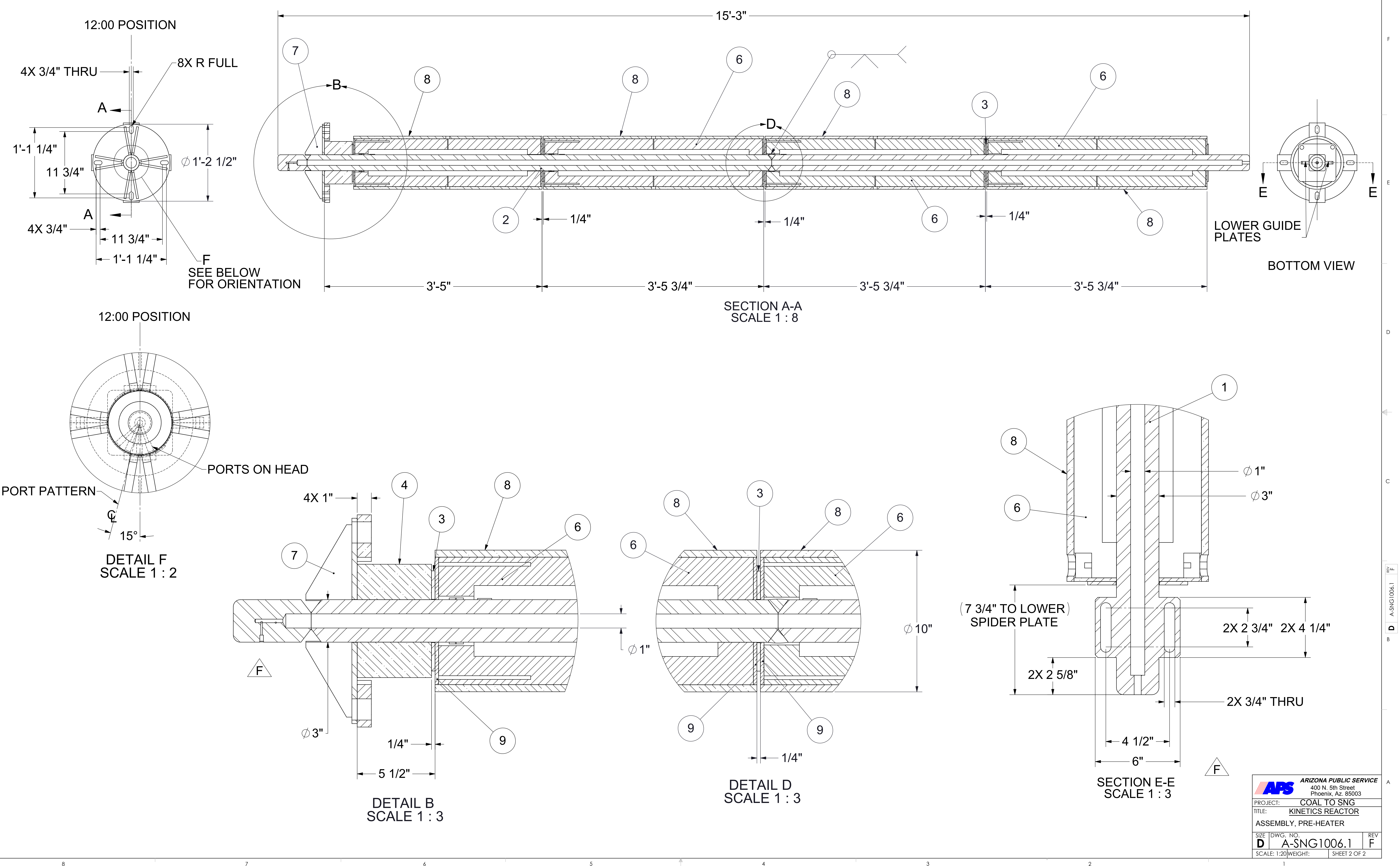


DIMENSION AND TOLERANCING PER ANSI Y14.5-1994.

2 BREAK ALL EDGES .5 [.20] MAX. ALL INSIDE RADII

2.4 [.09] MAX. UNLESS NOTED OTHERWISE.
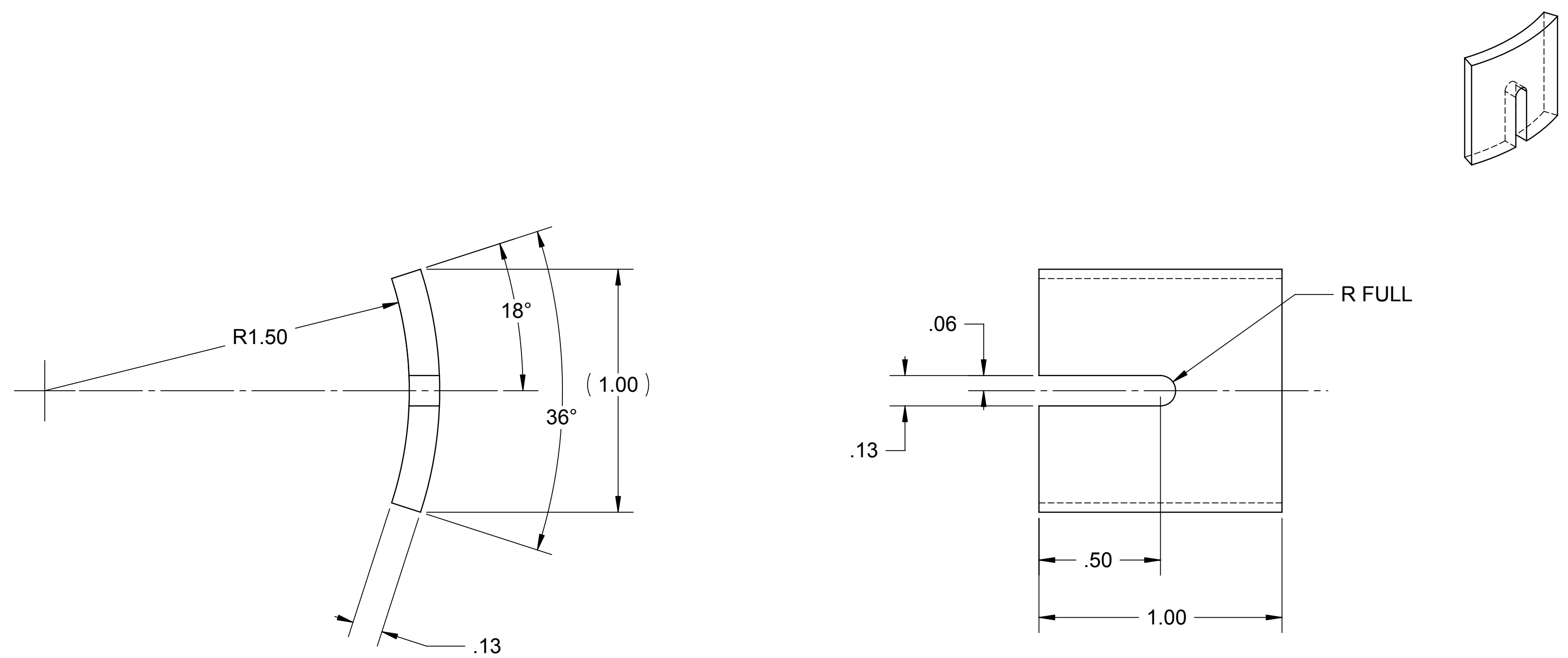

\begin{tabular}{|c|c|c|c|}
\hline $\begin{array}{l}\text { UNLESS OTHERWISE SPECIFIED: } \\
\text { DIMENIIN ARE IN MLIMTERS }\end{array}$ & & $\begin{array}{l}\text { DATE } \\
\end{array}$ & NAME \\
\hline 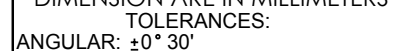 & DRAWN & 07/16/08 & D. WAIBEL \\
\hline ONE PLACE DECIMAL & CHECKED & & \\
\hline 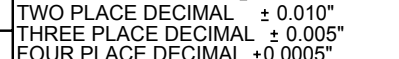 & ENG APPR. & & \\
\hline $\begin{array}{l}\text { FFURR P PACACDECCIMA I0.0000" } \\
\text { SURFACE FINISH } 63 \text { UNLSS NOTED }\end{array}$ & MFG APPR. & & \\
\hline $\begin{array}{l}\text { INTERRRET GEOMERTIC TOLRANCING } \\
\text { PER: }\end{array}$ & Q.A. & & \\
\hline $\begin{array}{l}\text { MATERIAL: } \\
\text { INCONEL } 625 \\
\text { FINSH: NONE }\end{array}$ & 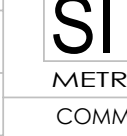 & $\begin{array}{l}\text { ENTS: } \\
\text { EN }\end{array}$ & 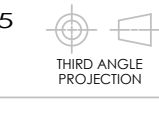 \\
\hline SIMLIAR TO: & CAD FILE & & NG1006.12A \\
\hline
\end{tabular}


GENERAL NOTES:

1. BREAK ALL EDGES, AND DEBURR, REMOVE ALL SHARP CORNERS.

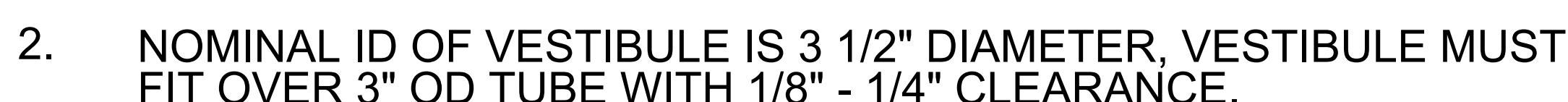
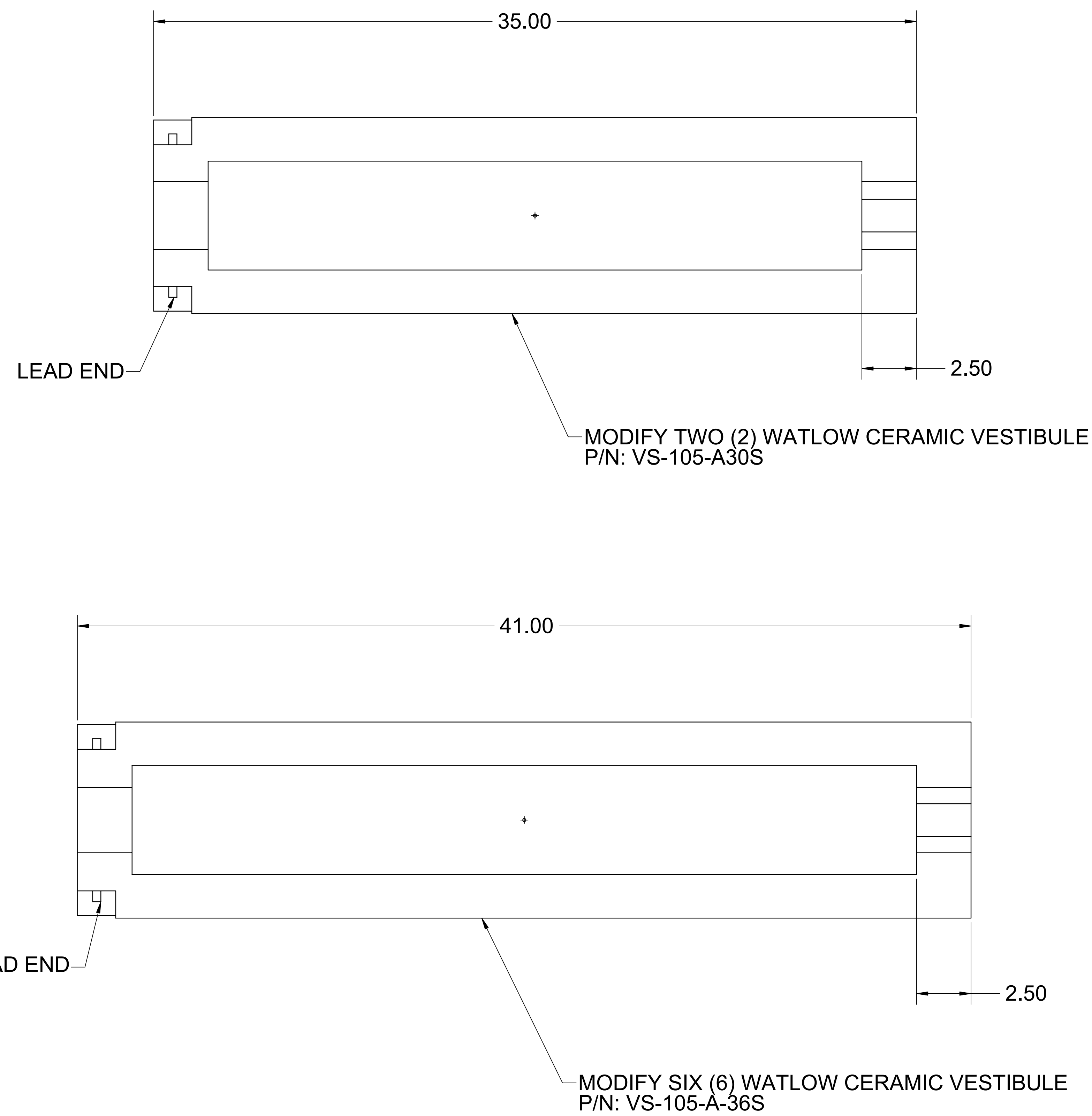

LEAD END

N: VS-105-A-36S
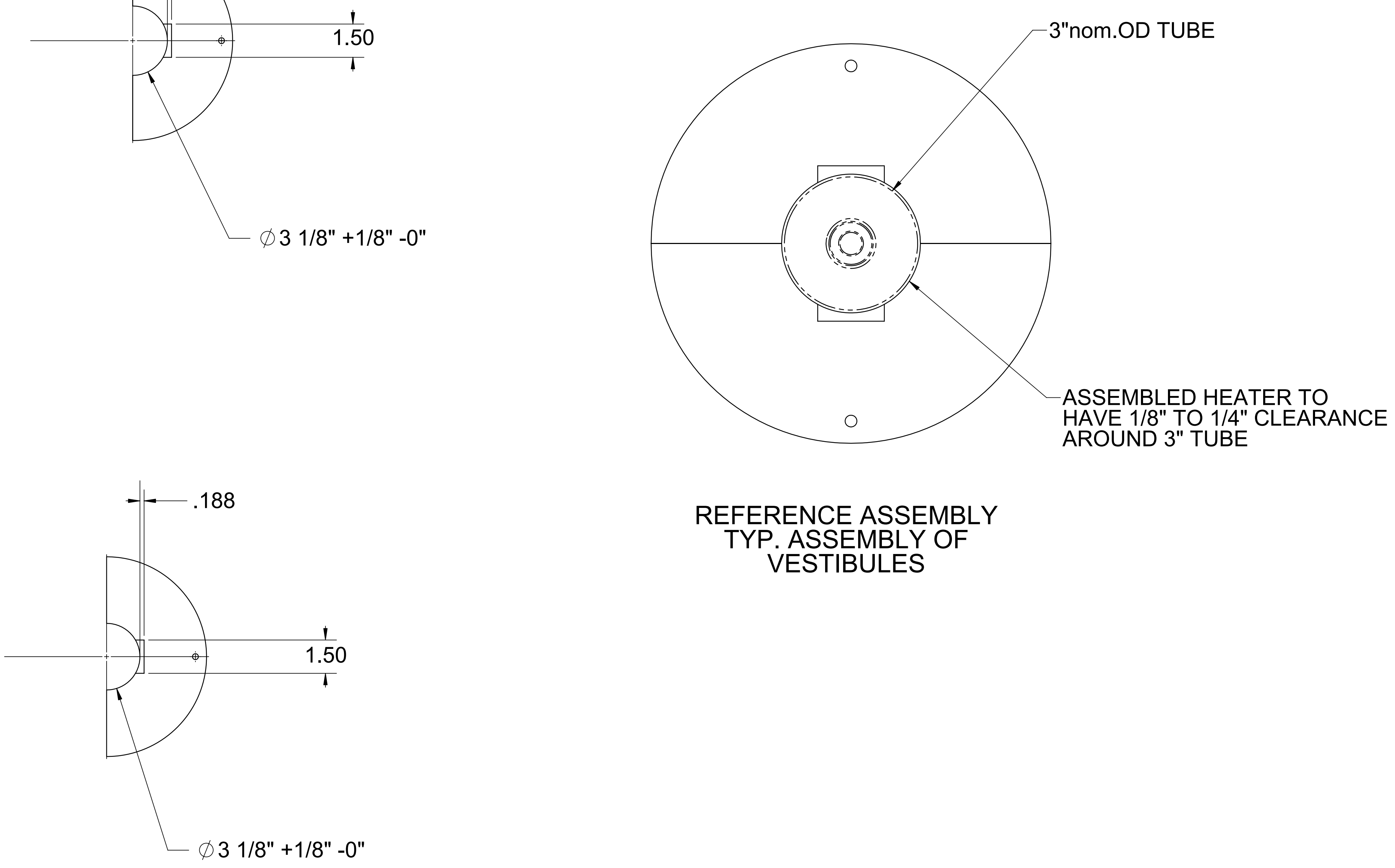

REFERENCE ASSEMBLY

TYP. ASSEMBLY
VESTIBULES

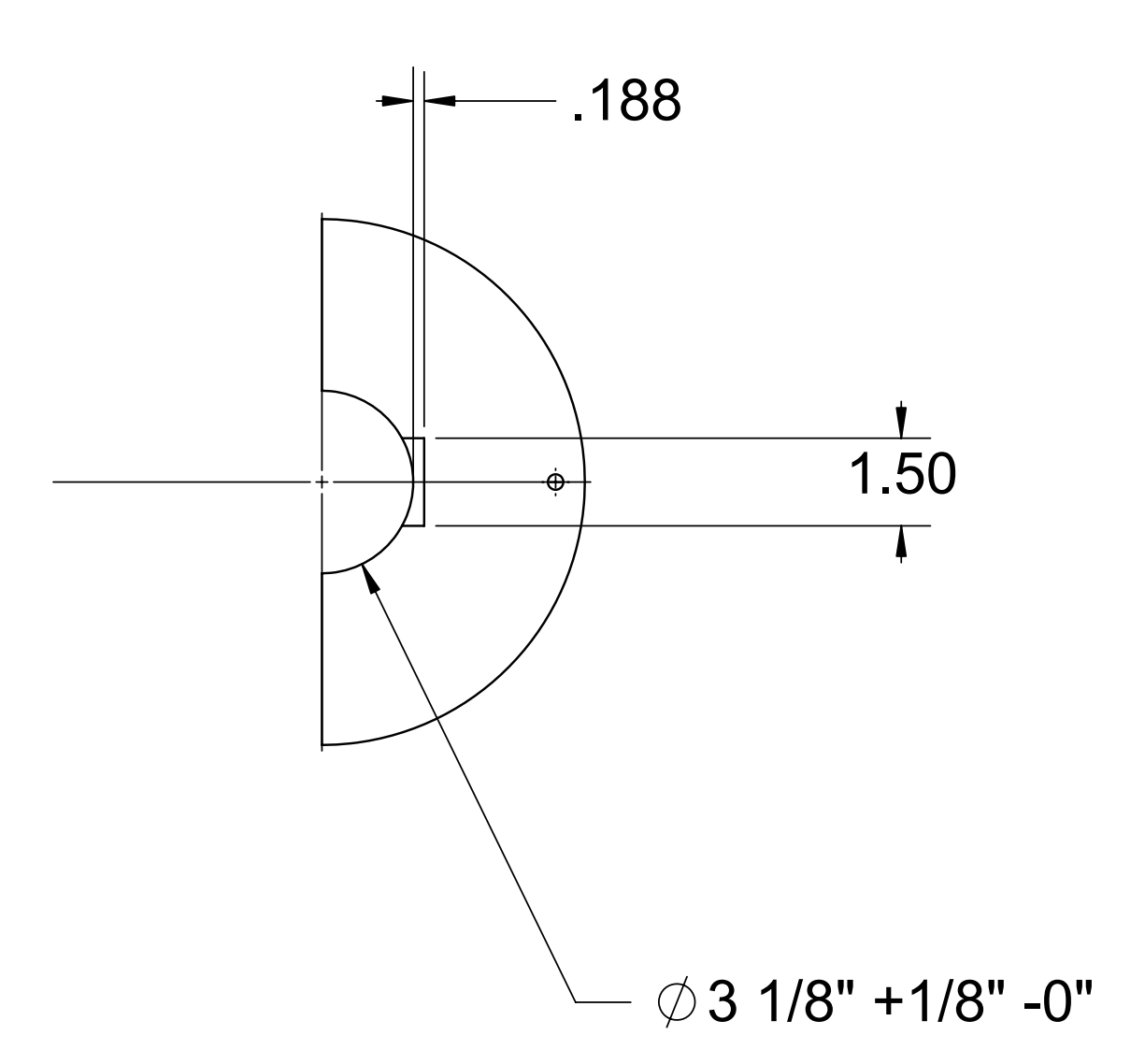

HAVE $1 / 8 "$ TO $1 / 4$
AROUND $3 "$ TUBE 


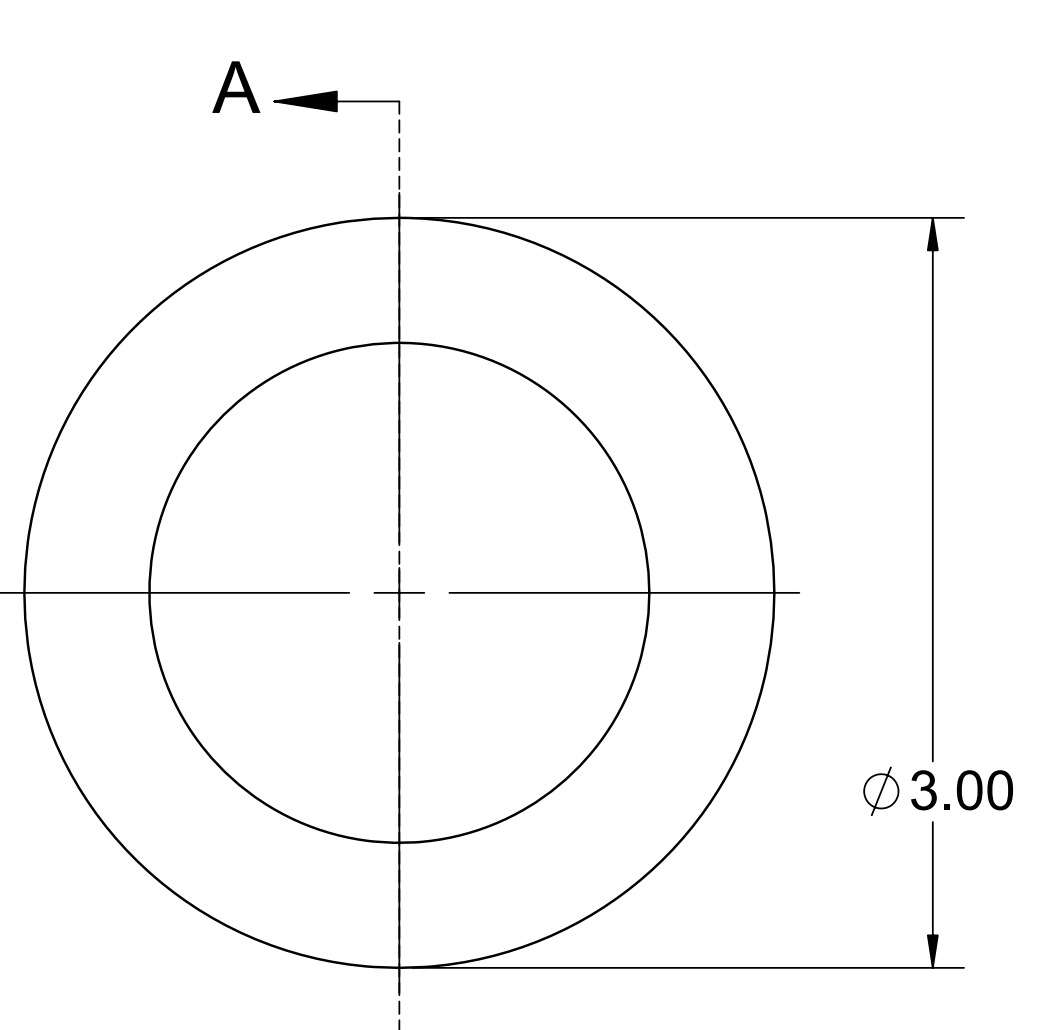

A-

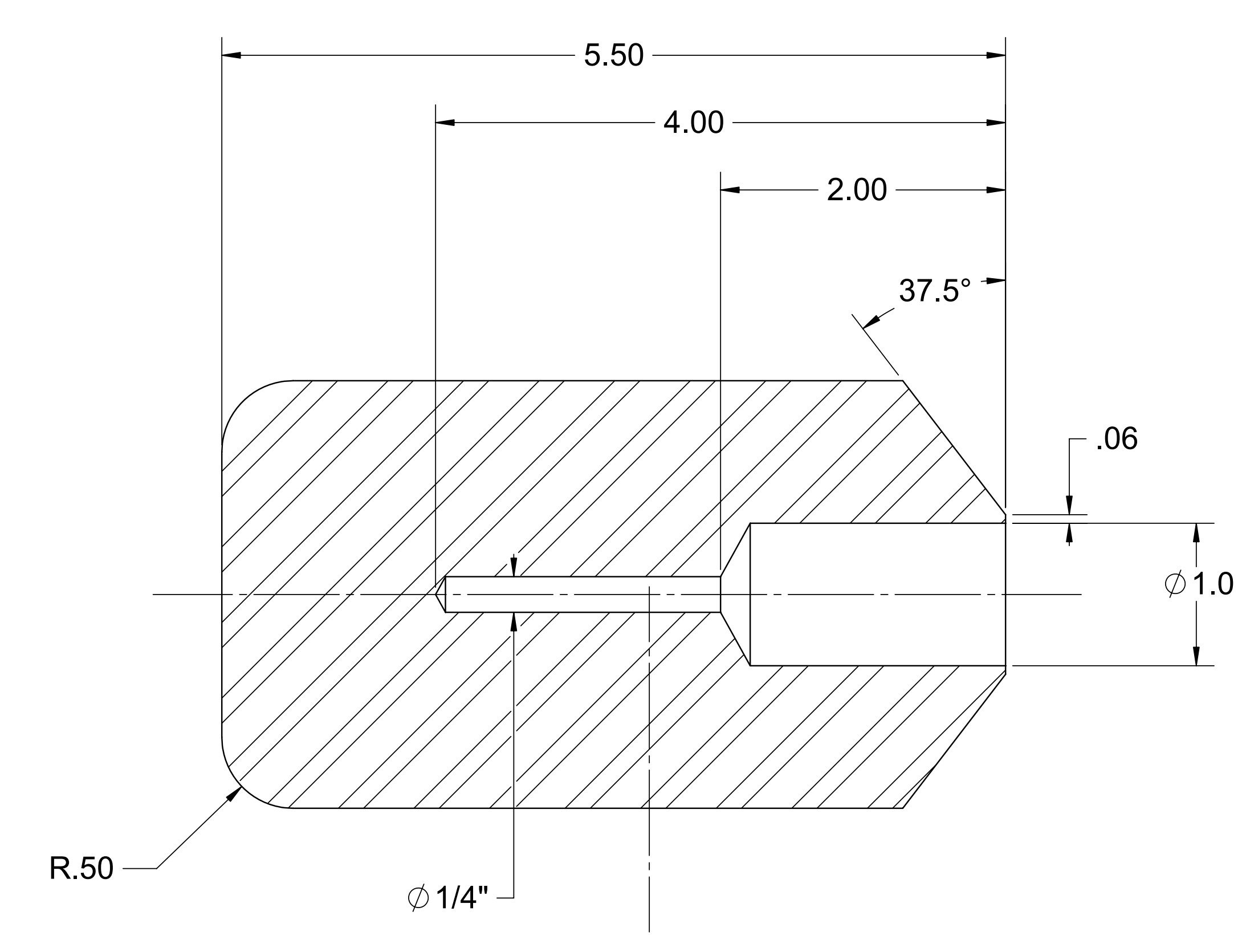

SECTION A-A
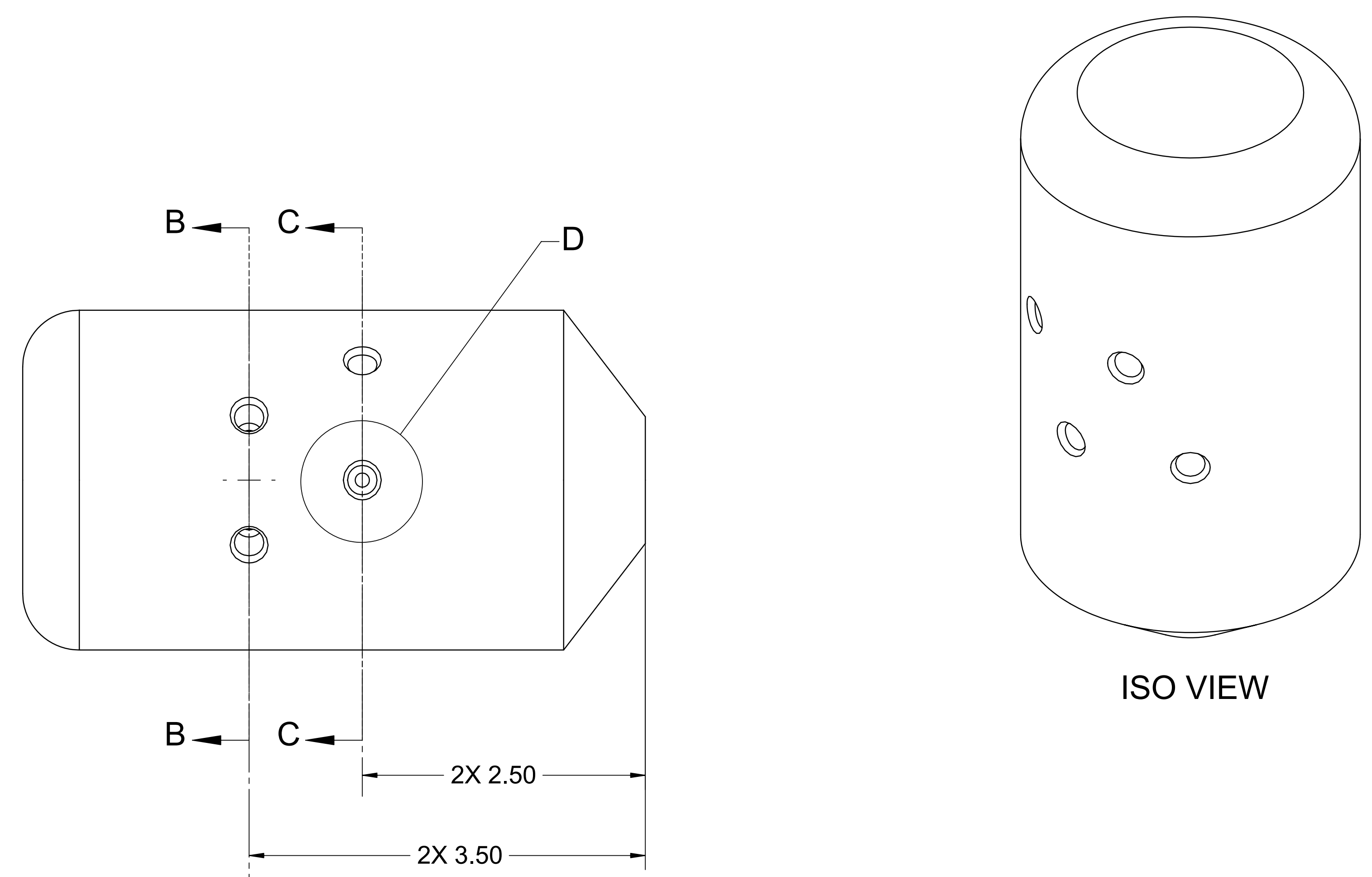

ISO VIEW

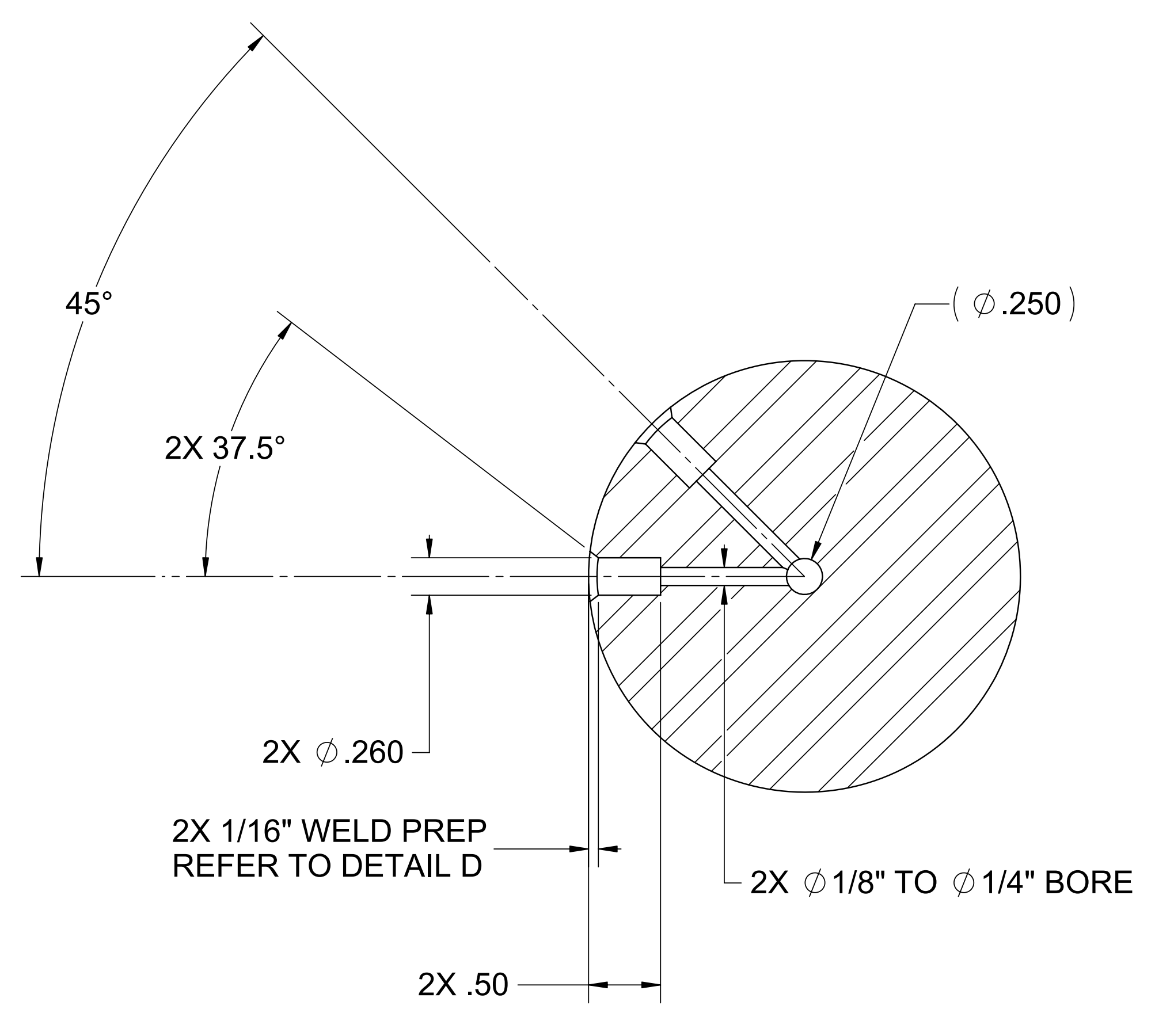

SECTION C-C

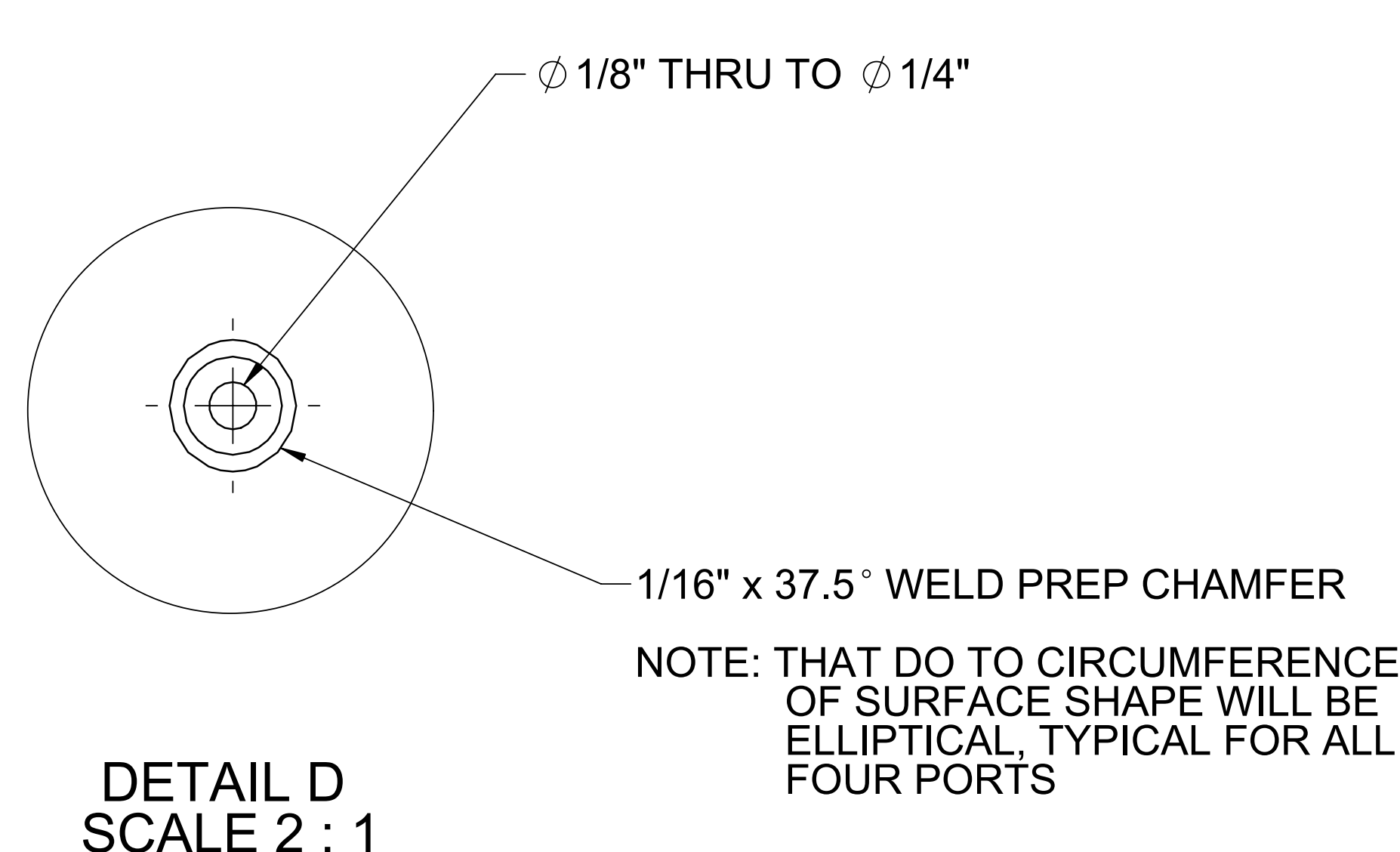

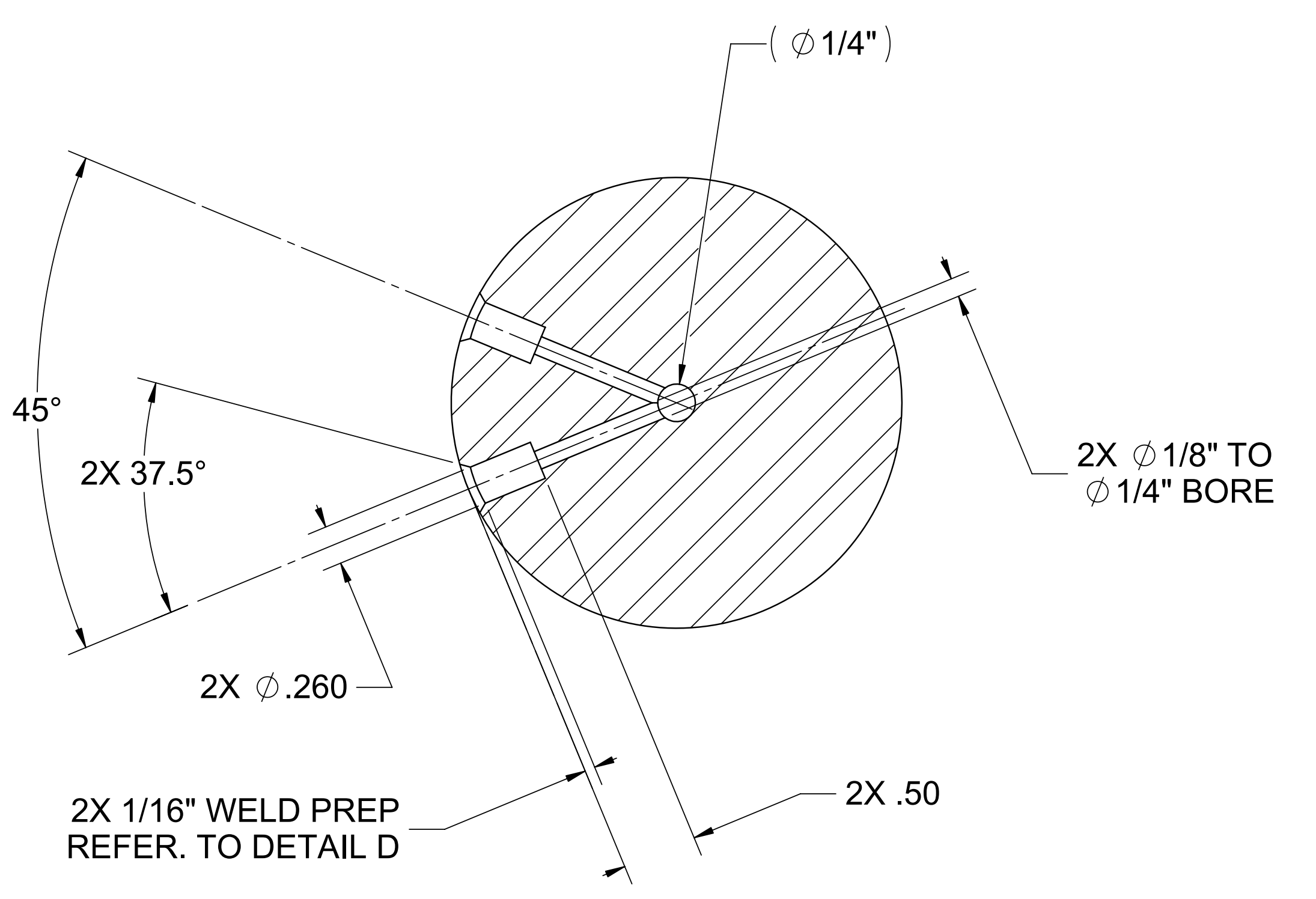

SECTION B-B

\begin{tabular}{|c|c|c|c|c|c|}
\hline 1 & SNG1006.15 & INCONEL 625 & \multicolumn{2}{|c|}{ ROUND BAR, PRE-HEATER TOP } & 1 \\
\hline ITEM NO. & PART NUMBER & MATERIAL & \multicolumn{2}{|r|}{ DESCRIPTION } & QTY. \\
\hline & & 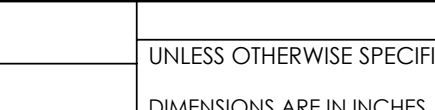 & & NTME & SERVICE \\
\hline & & 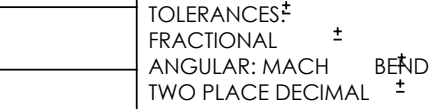 & 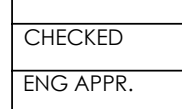 & \multicolumn{2}{|c|}{ 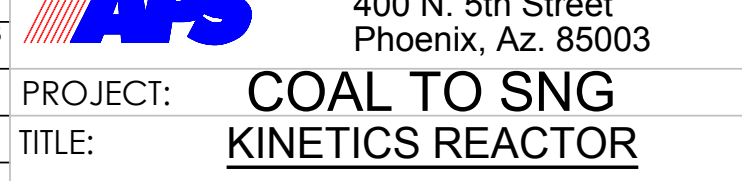 } \\
\hline & & 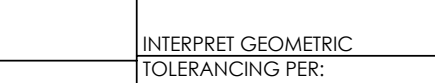 & 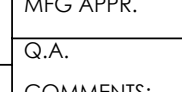 & \multicolumn{2}{|c|}{ ROUND BAR, PRE-HEATER TOP } \\
\hline & 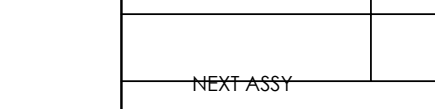 & masert & CAD FI & 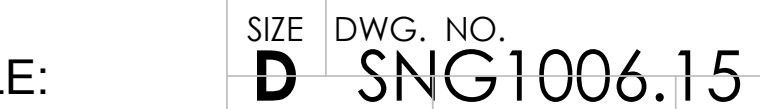 & $\stackrel{\mathrm{REV}}{\mathrm{A}}$ \\
\hline
\end{tabular}



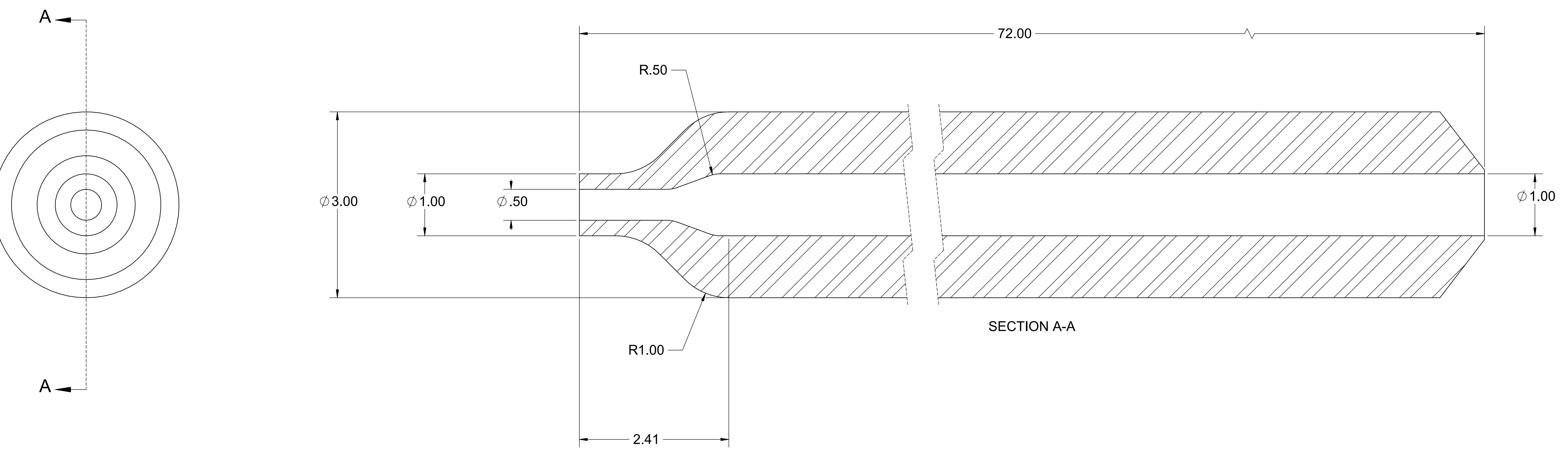

\begin{tabular}{l|l|l|l}
1 & SNG1006.3A & INCONEL 625 & UPPER, 3" Dia., x 72"LG. INCONEL 625
\end{tabular} 
1 ALL WELDS TO MEET PRESSURE VESSEL CODE, ASME SECTION 8, DIVISION 1 ,

2 INSPECTION OF WELDS TO BE 100\% X-RAY, UNLESS SPECIFIED OTHERWISE.

3 MATERIAL: 316, CARBON CONTENT MUST BE EQUAL TO, OR ABOVE .04\%
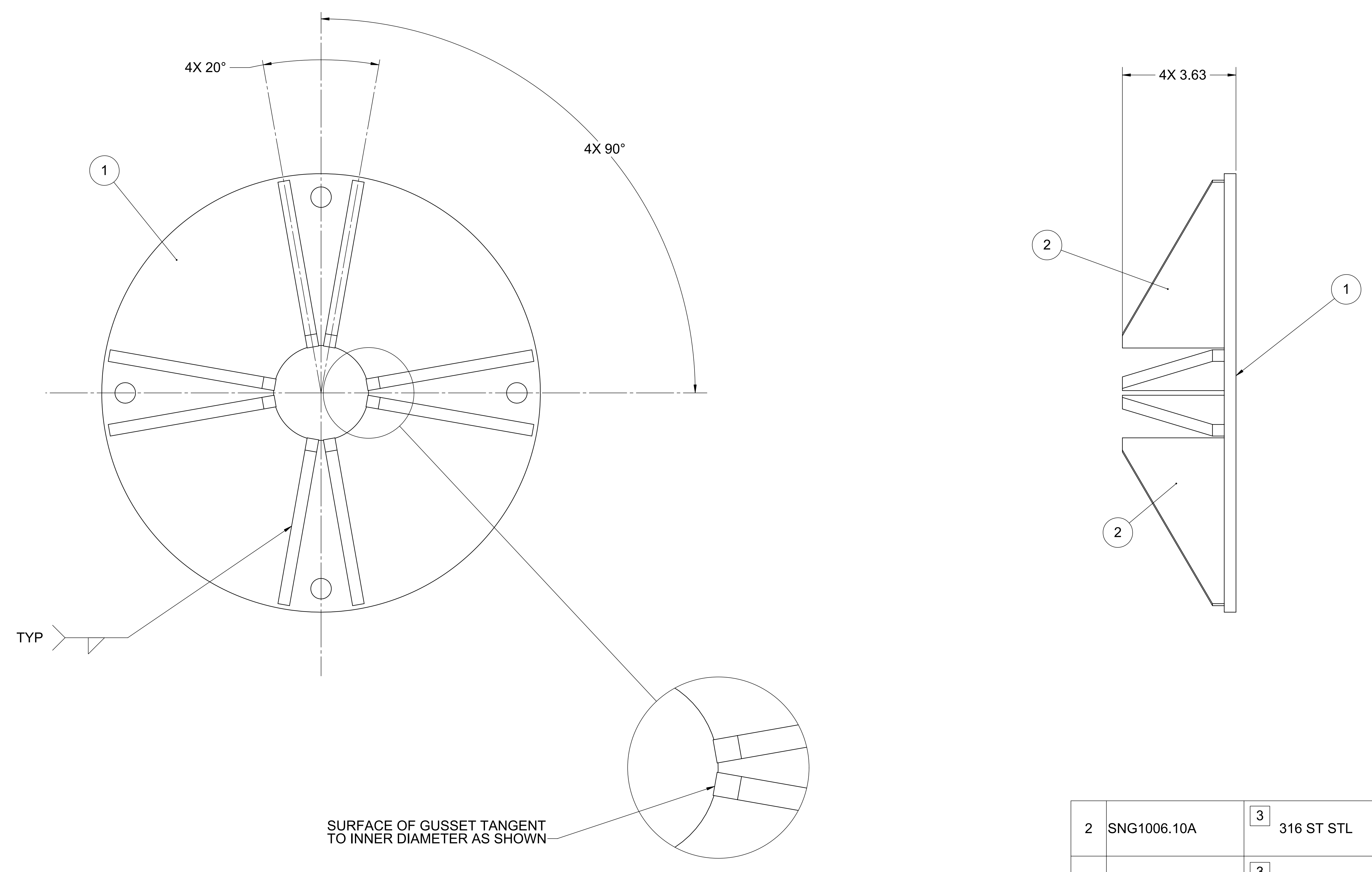

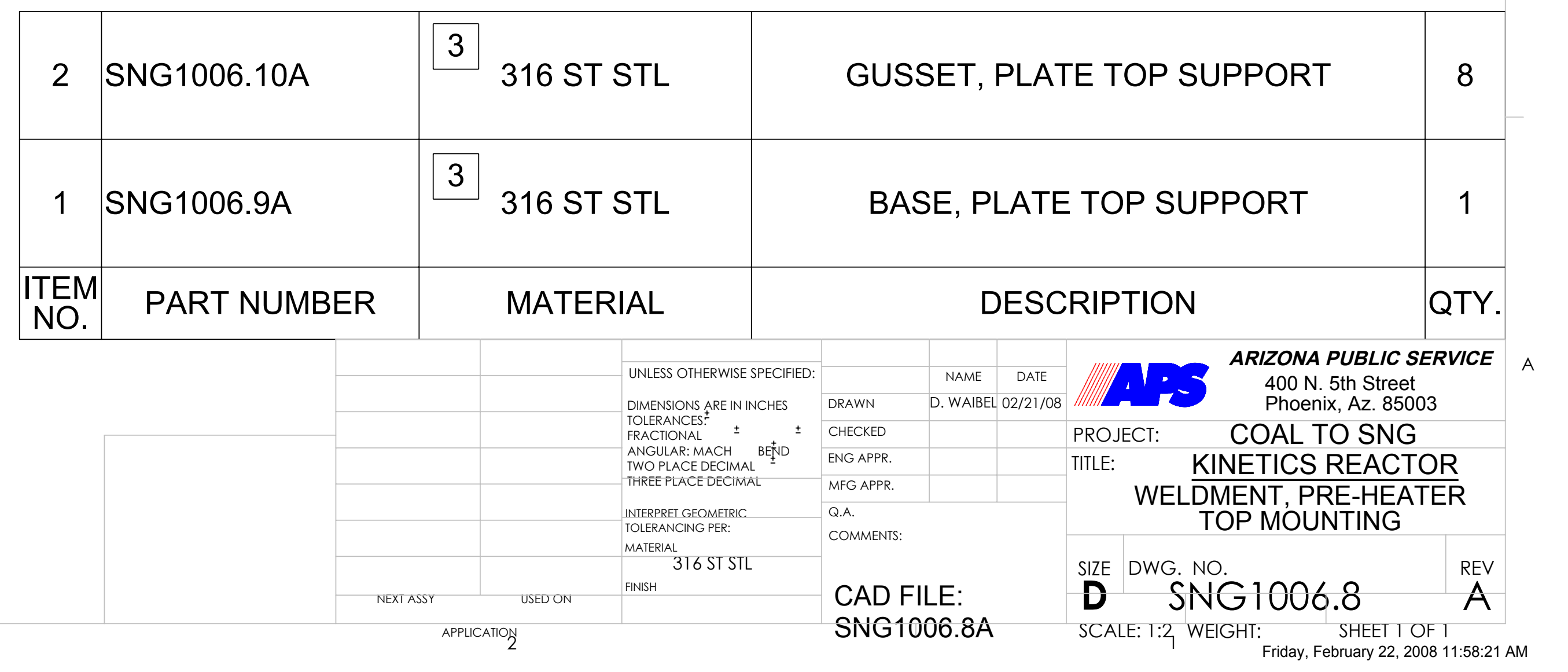




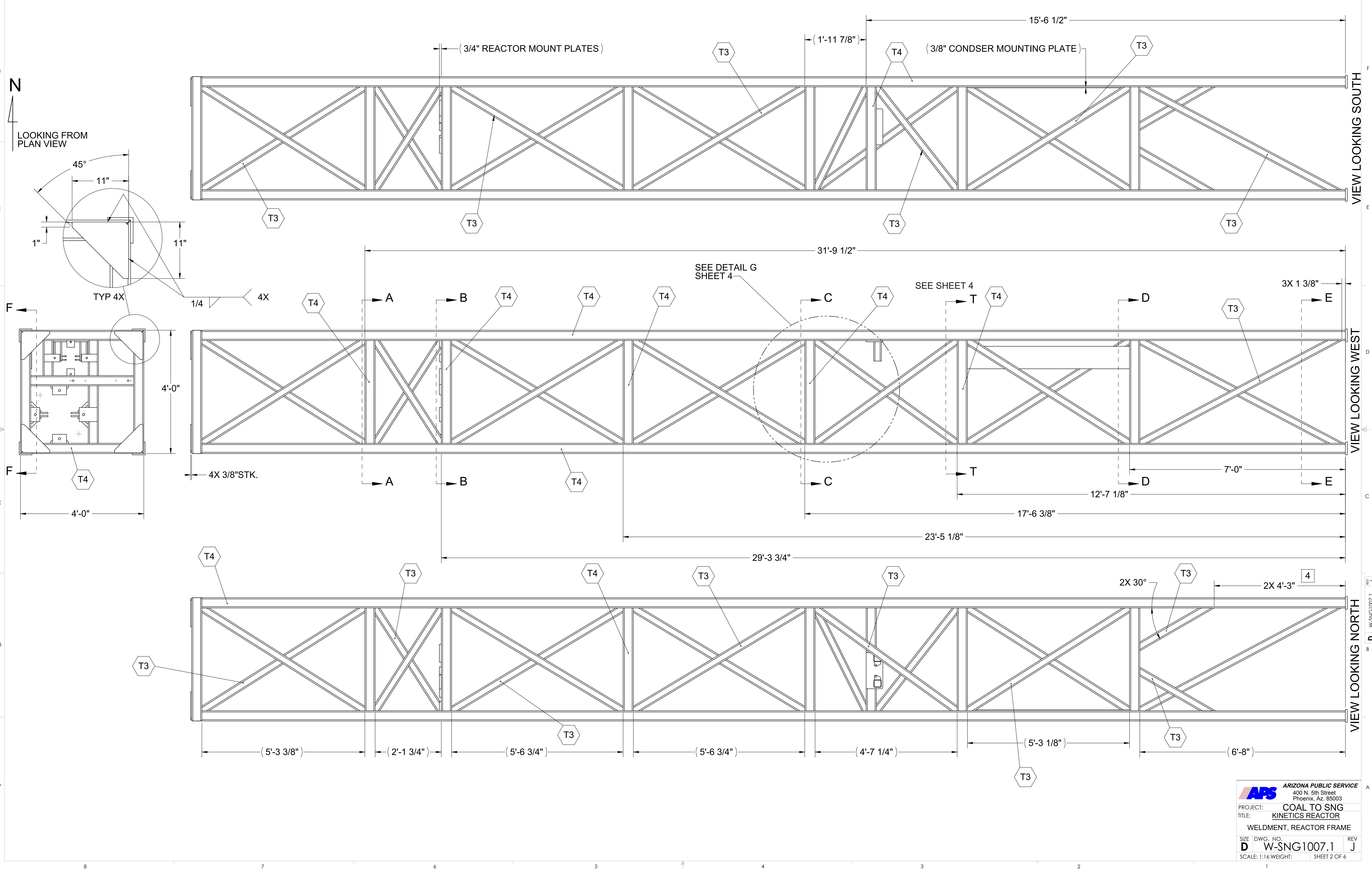



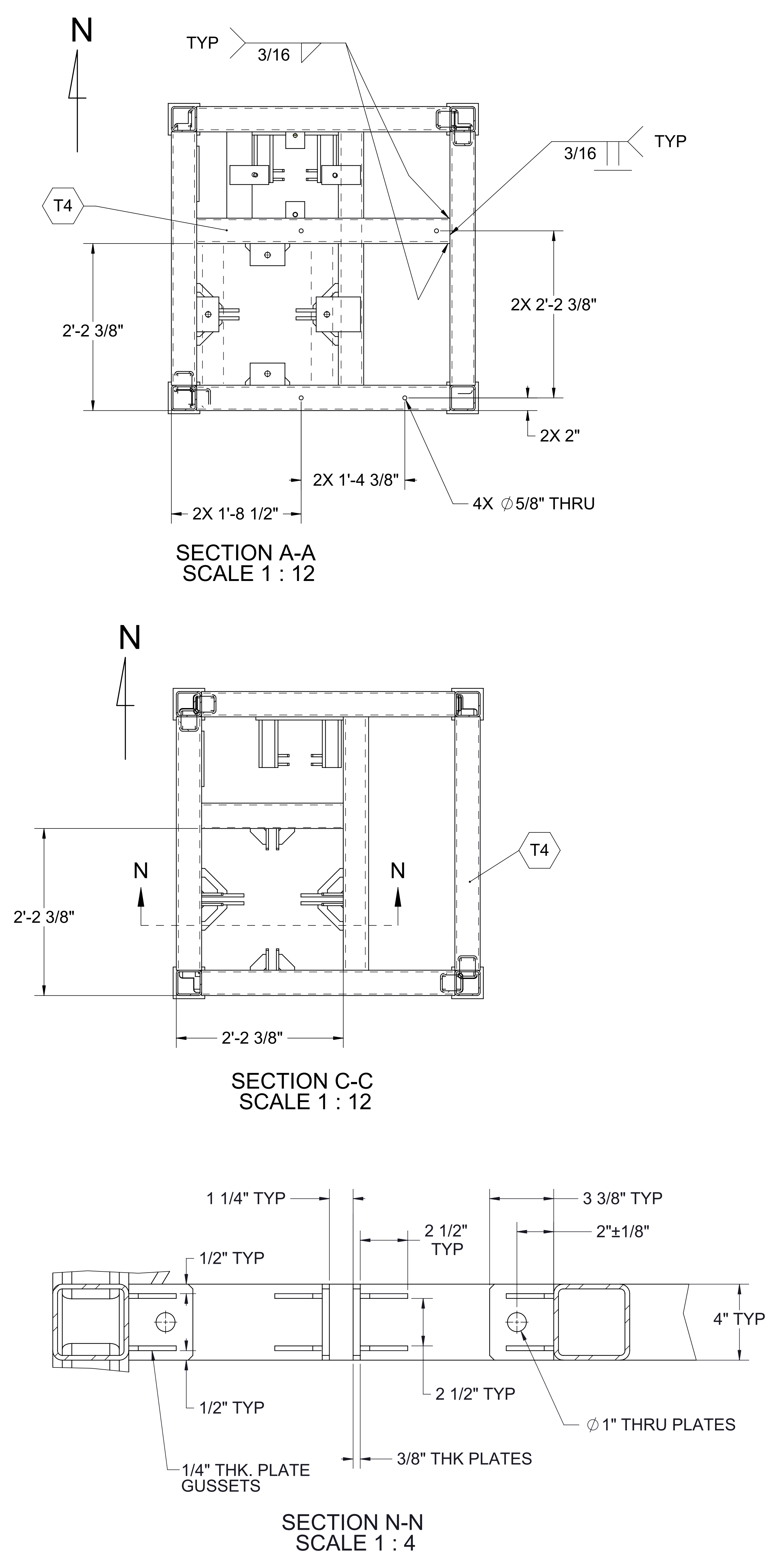
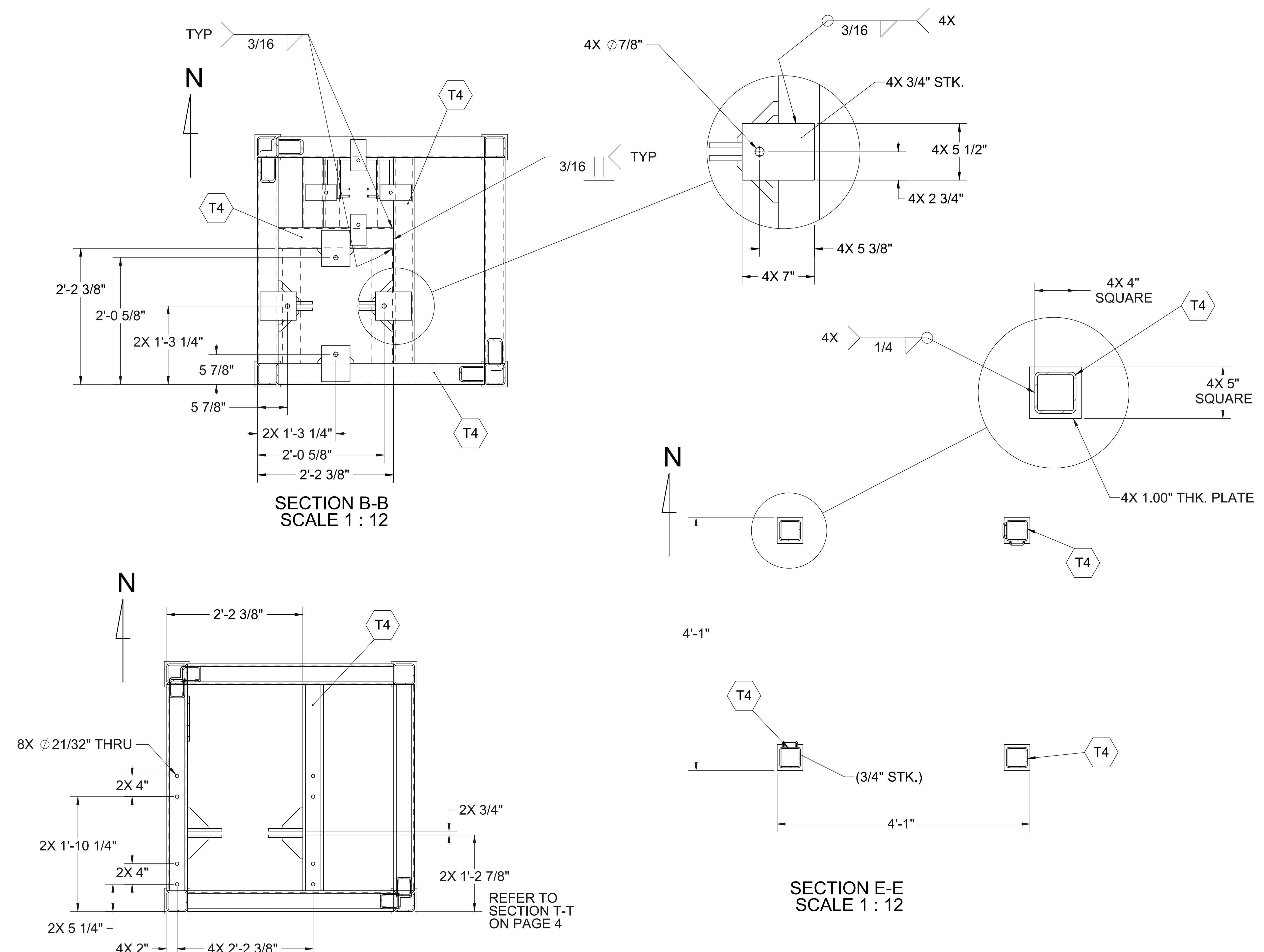

SECTION E-E
SCALE $1: 12$

SECTION D-D
SCALE $1: 12$

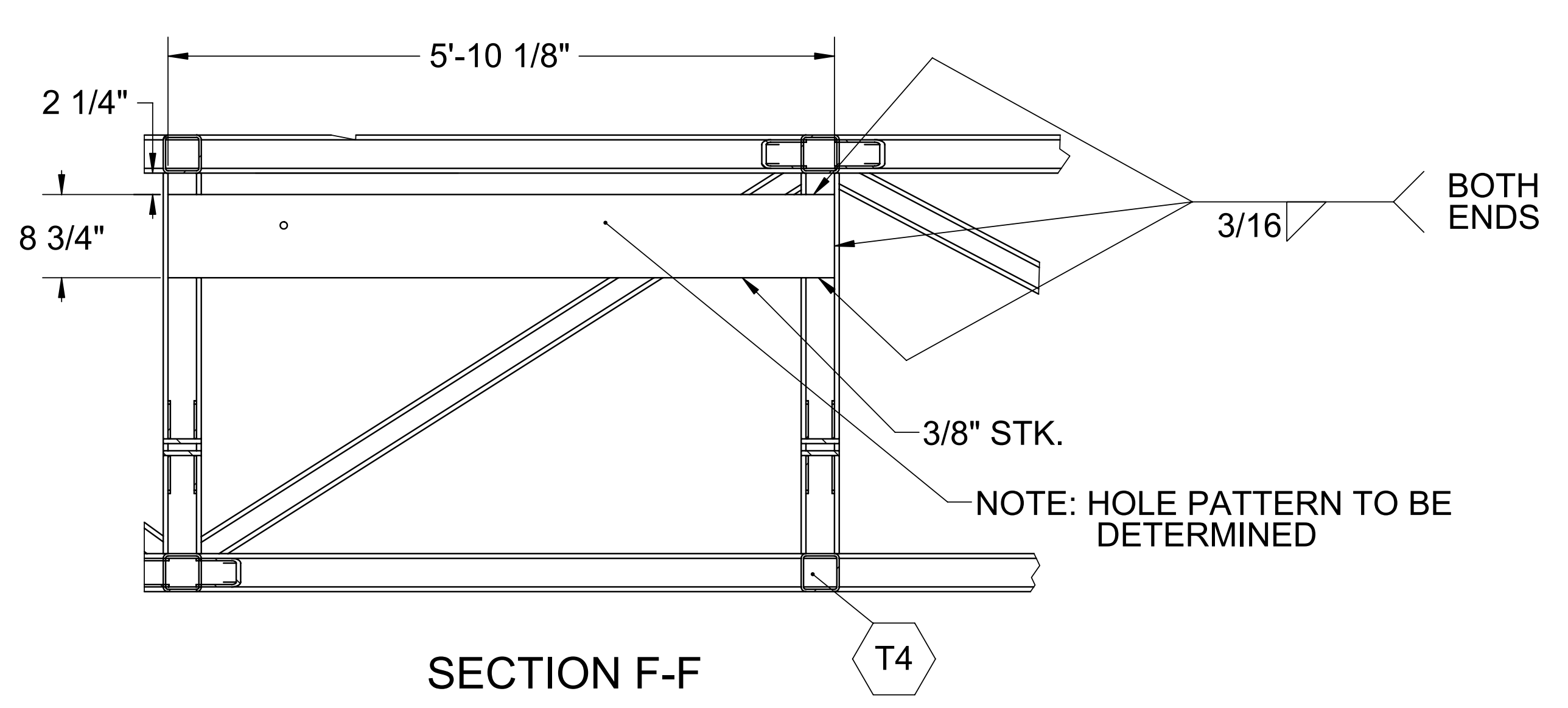

A.S.S 


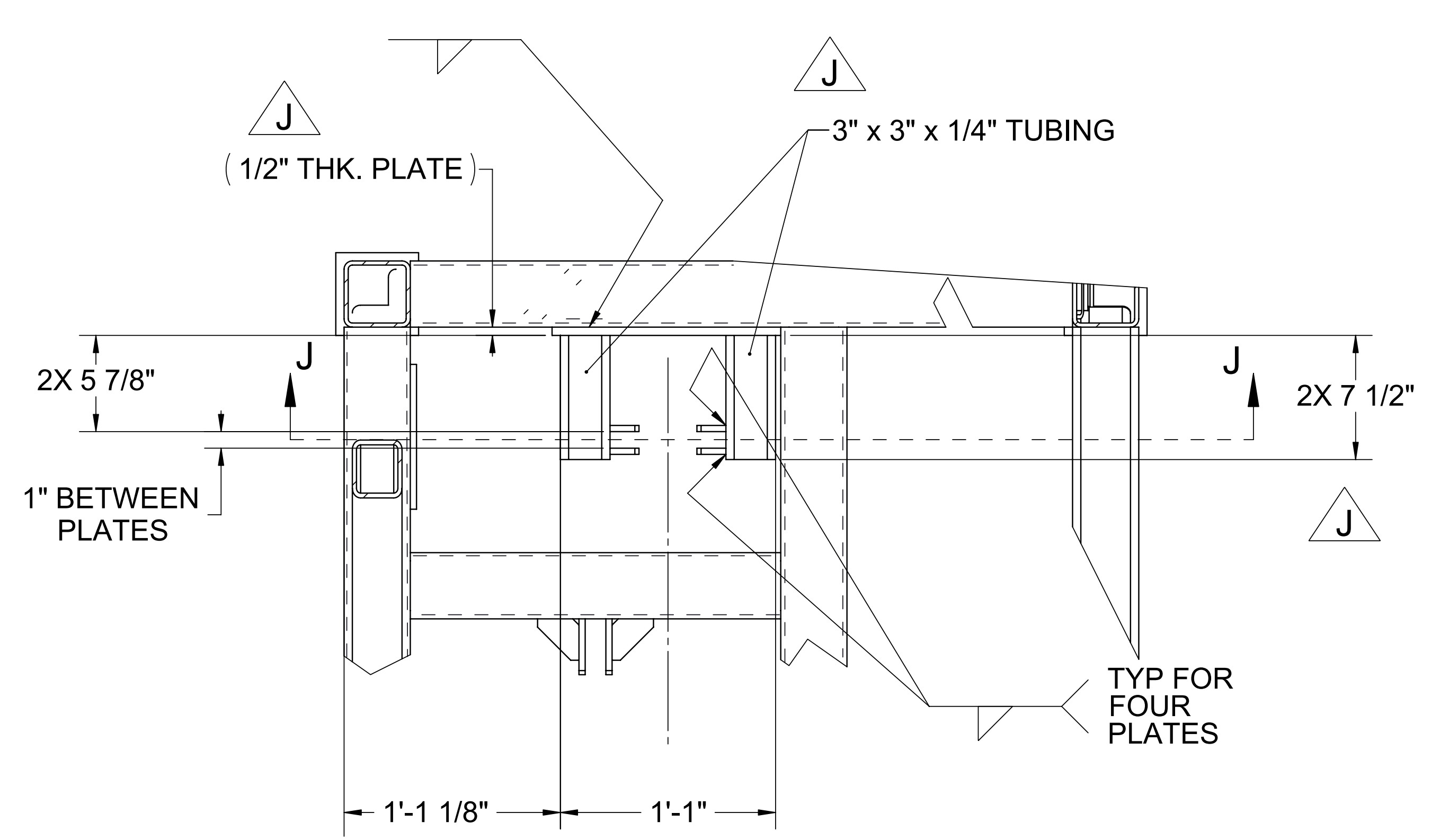

SECTION H-H
SCALE $1: 8$

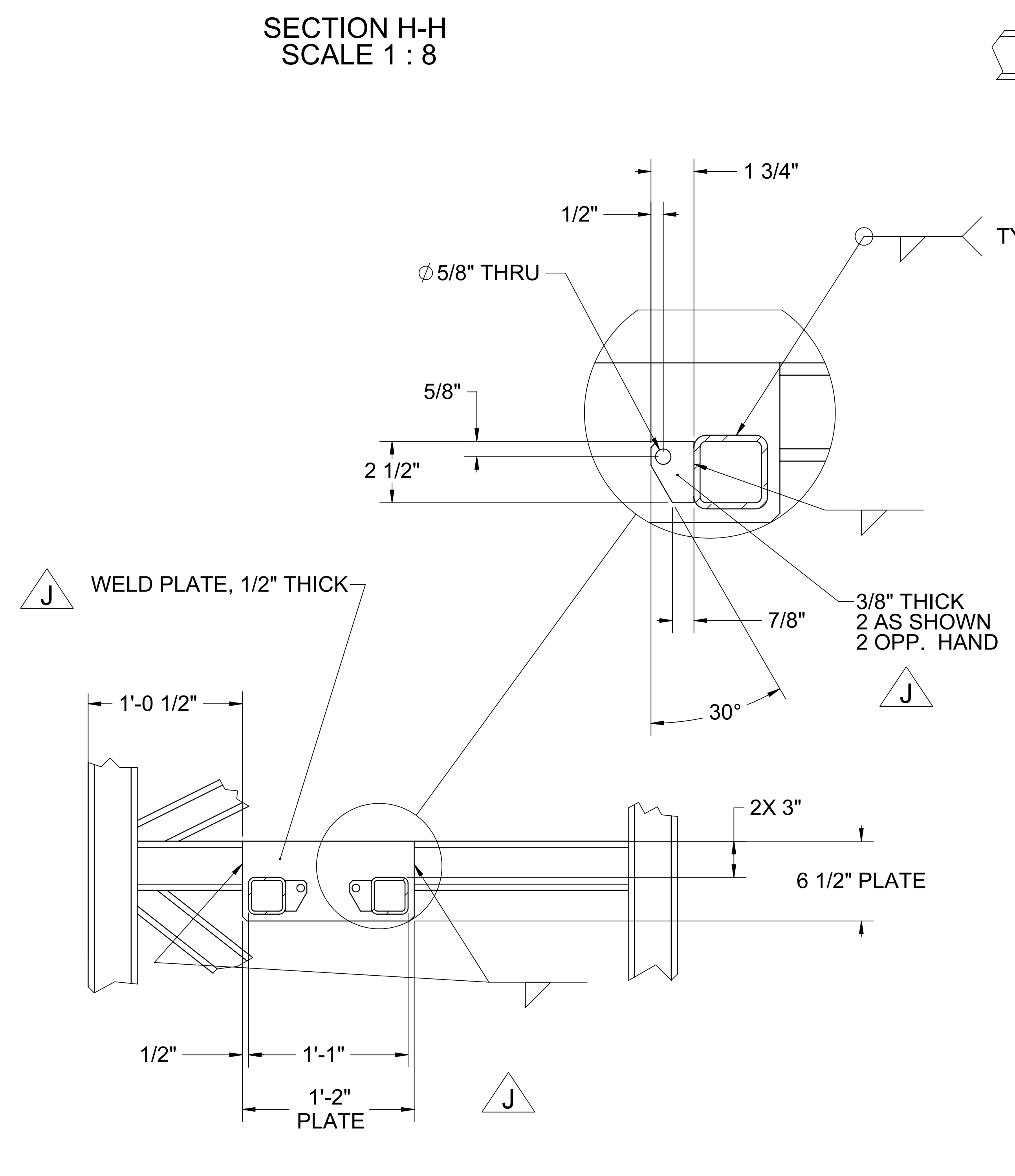

SECTION J-J
SCALE $1: 8$

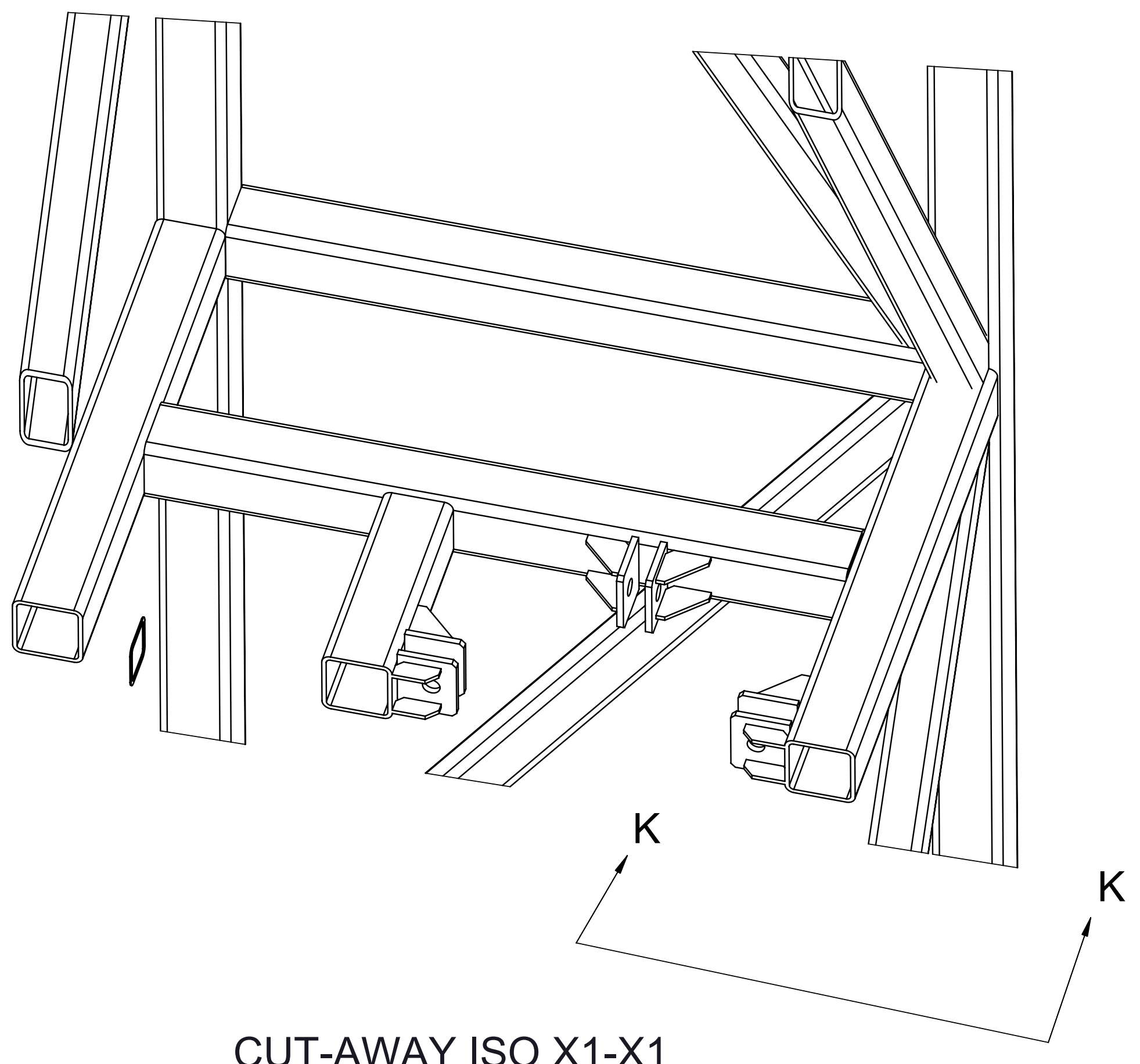

CUT-AWAY ISO X1-X1

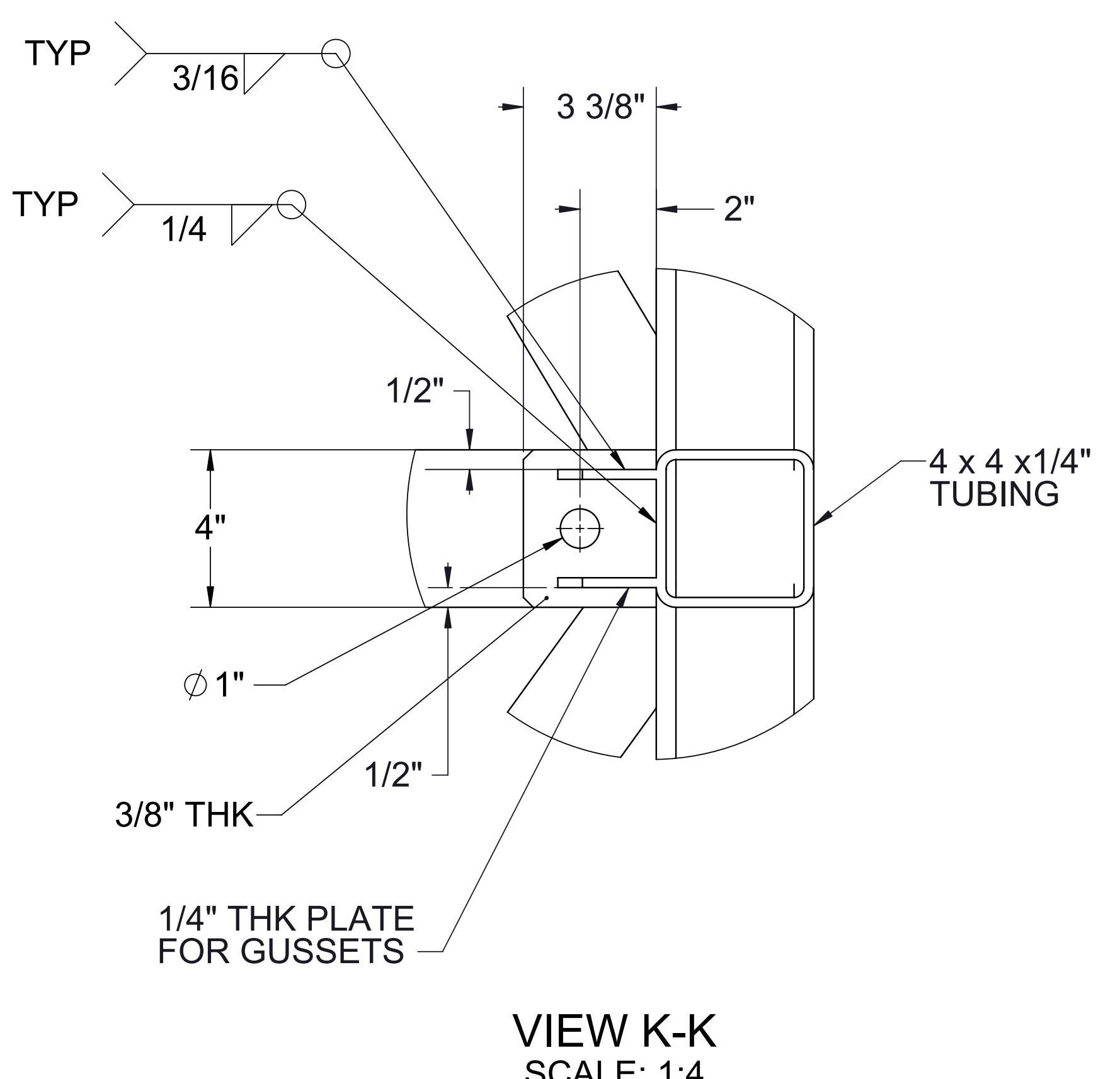
COALTOSNG
NEITISREACTOR

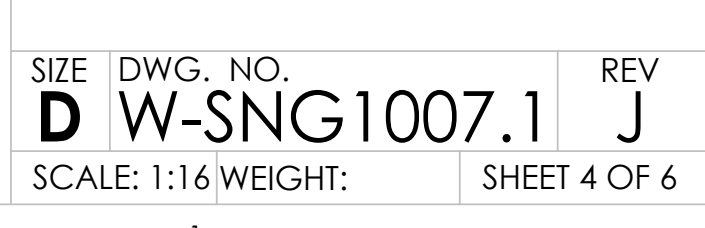




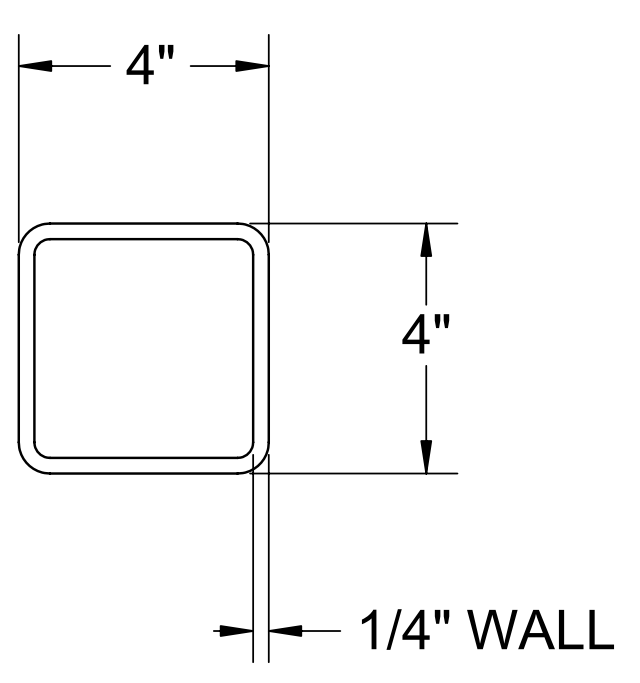

1) MAIN RISER
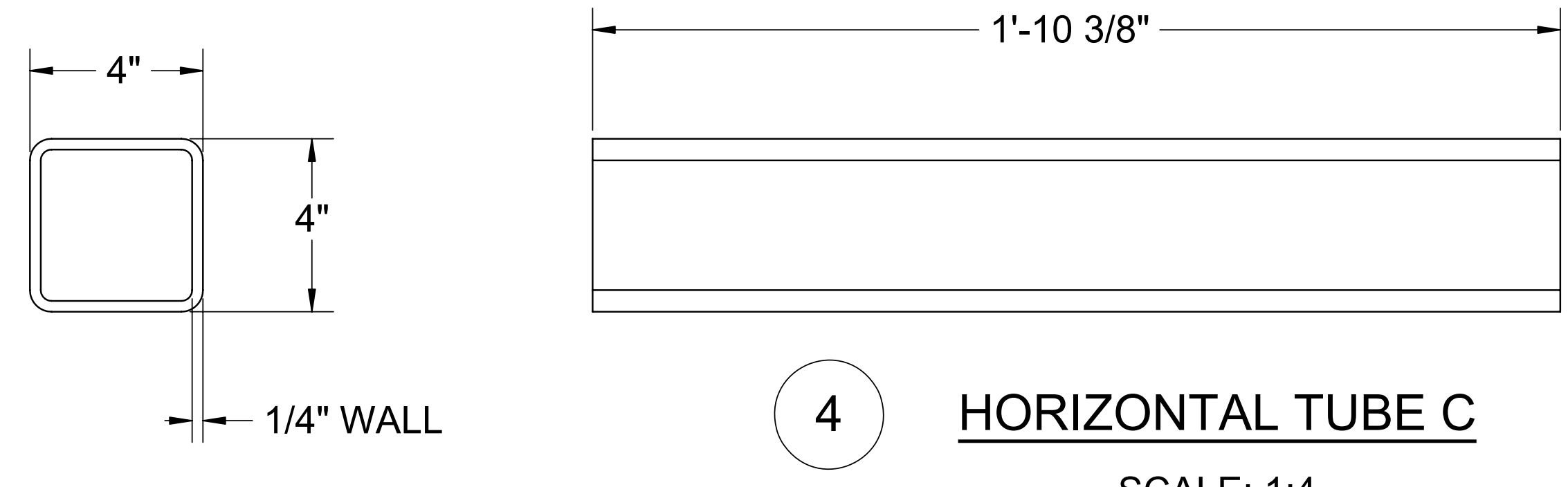

4) HORIZONTAL TUBE C

$$
\text { SCALE: 1:4 }
$$

$$
\underbrace{\sum_{3 " 1}^{1 "}}_{-1 / 4^{\prime \prime} \text { WALL }}
$$

(7) TUBE

SCALE: 1:4

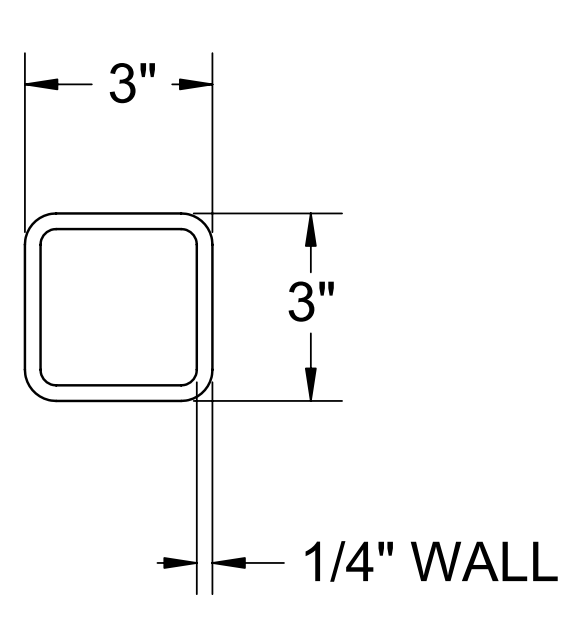

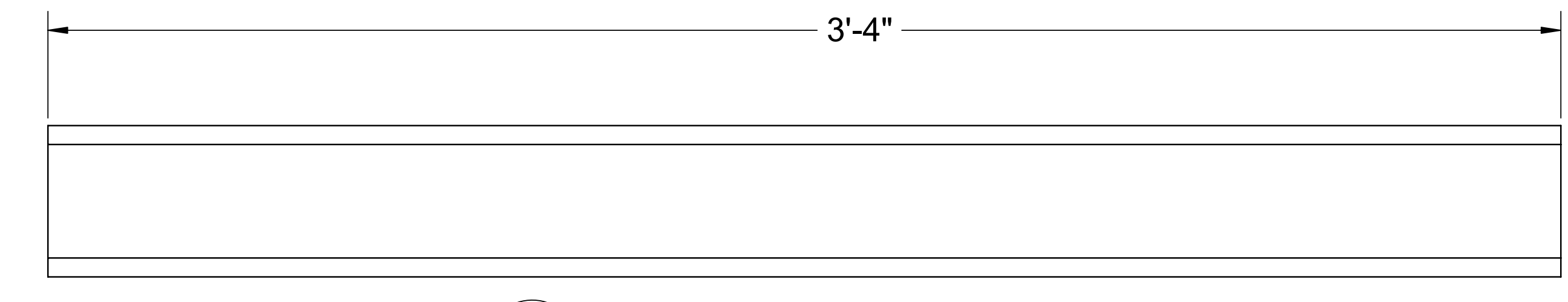

2) HORIZONTAL TUBE A$$
\text { SCALE: } 1: 4
$$
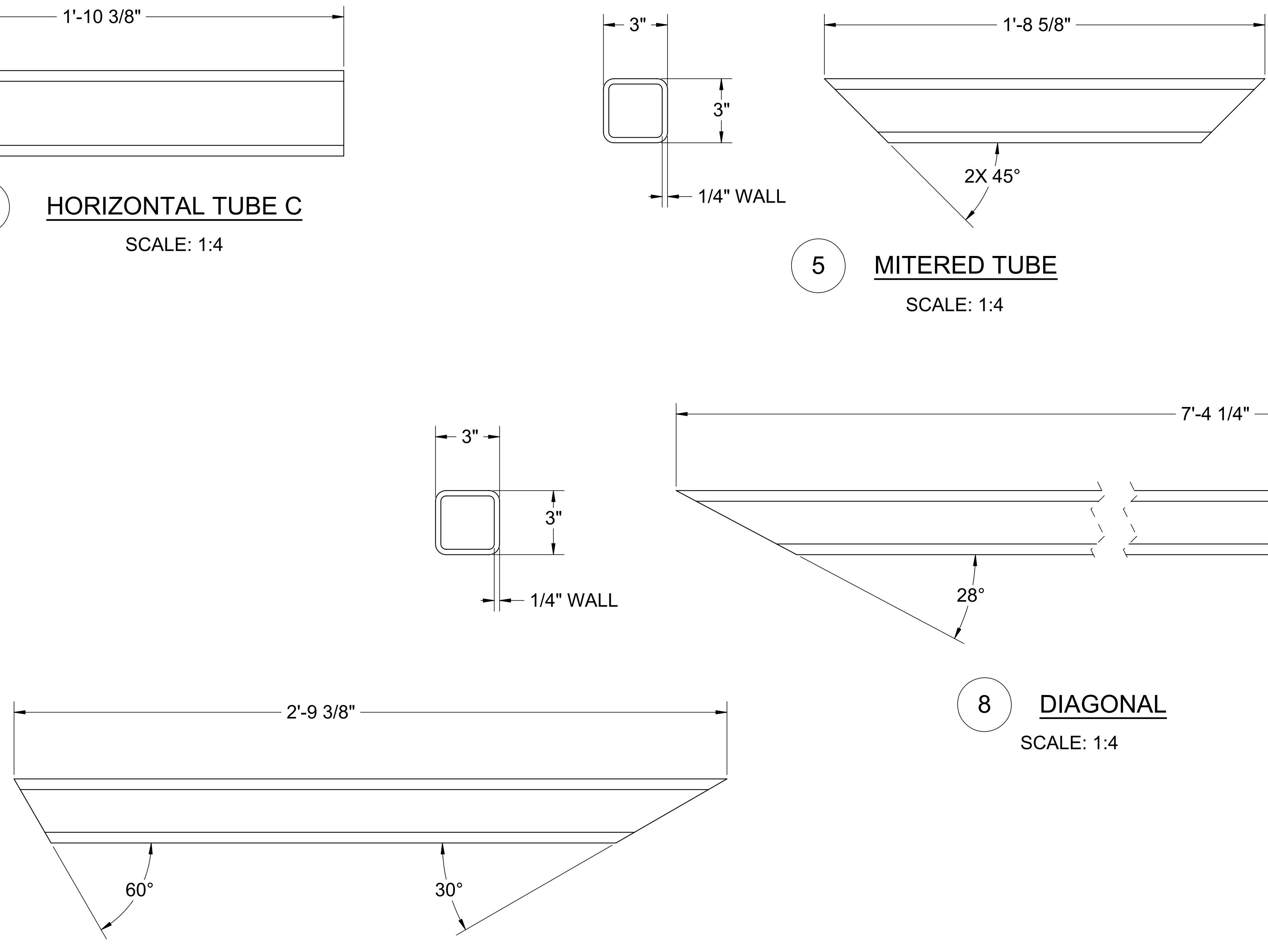

5) MITERED TUBE

SCALE: 1:4

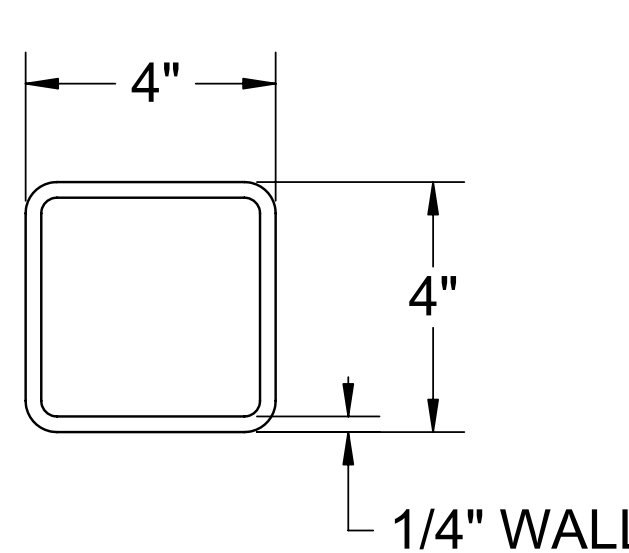

1/4" WALL

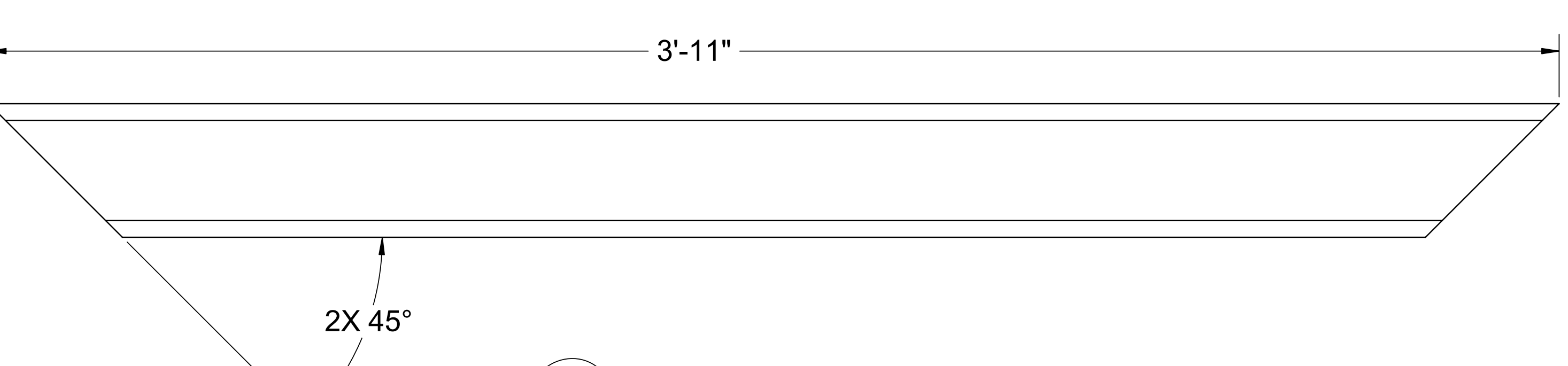

3 MITERED, HORIZONTAL TUBE B

SCALE: 1:4
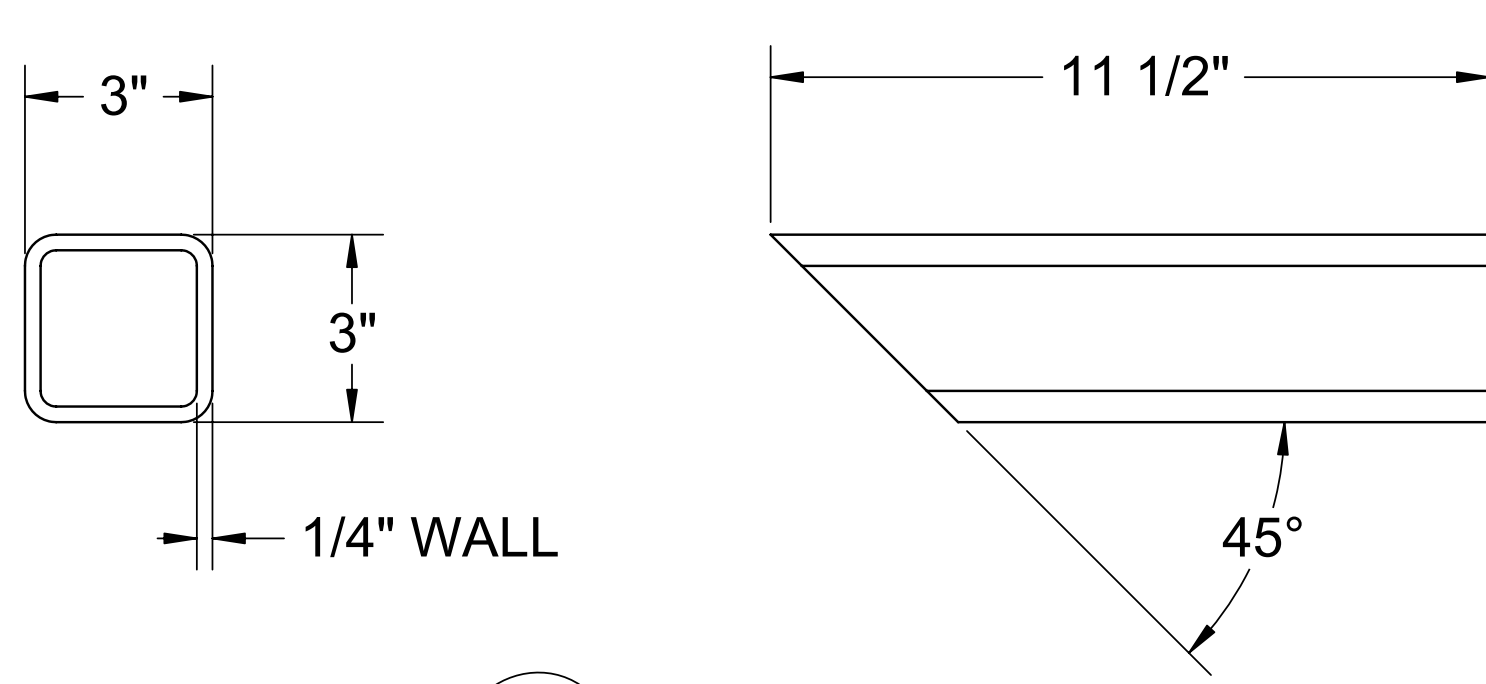

6 MITERED TUBE SCALE: $1 ; 4$

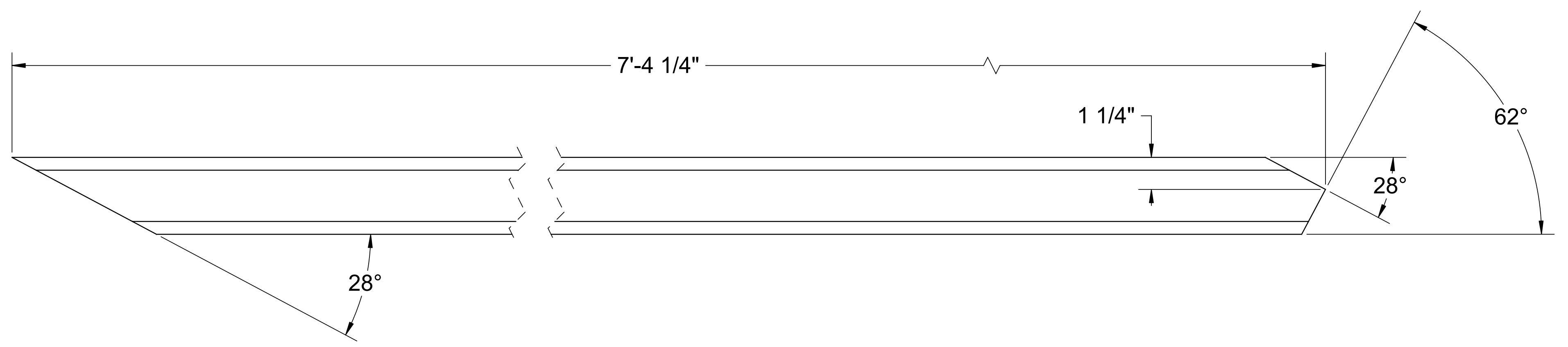

8 DIAGONAL SCALE: 1:4

9) DIAGONAL SCALE: 1:4 
at $\quad-\quad x$ 


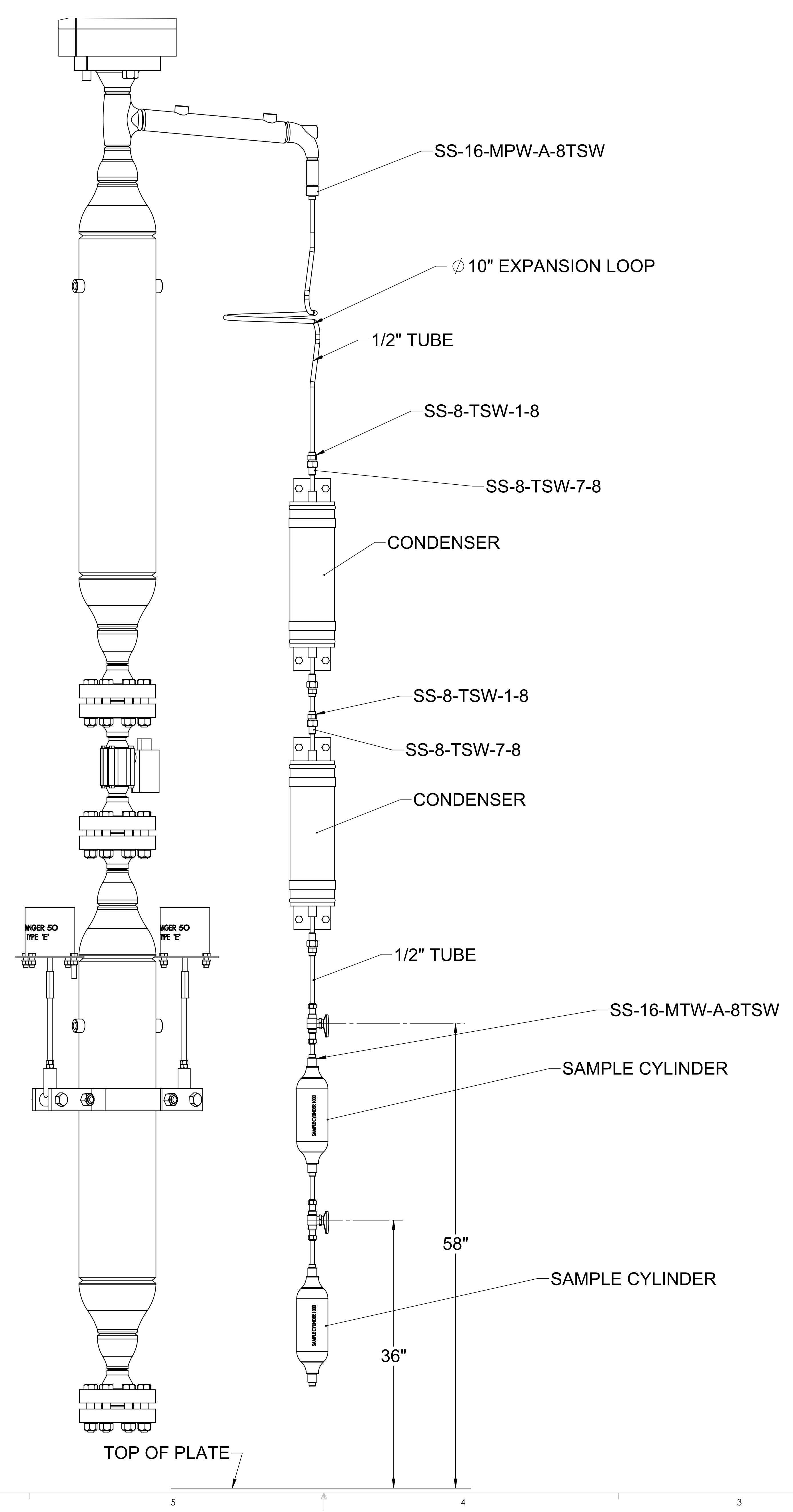




\section{GENERAL NOTES:}

1 DIMENSION AND TOLERANCING PER ANSI Y14.5-1994.

2 GASKET TO BE STYLE 'R', (CRITICAL SERVICE SERIES) SEE BOM BELOW
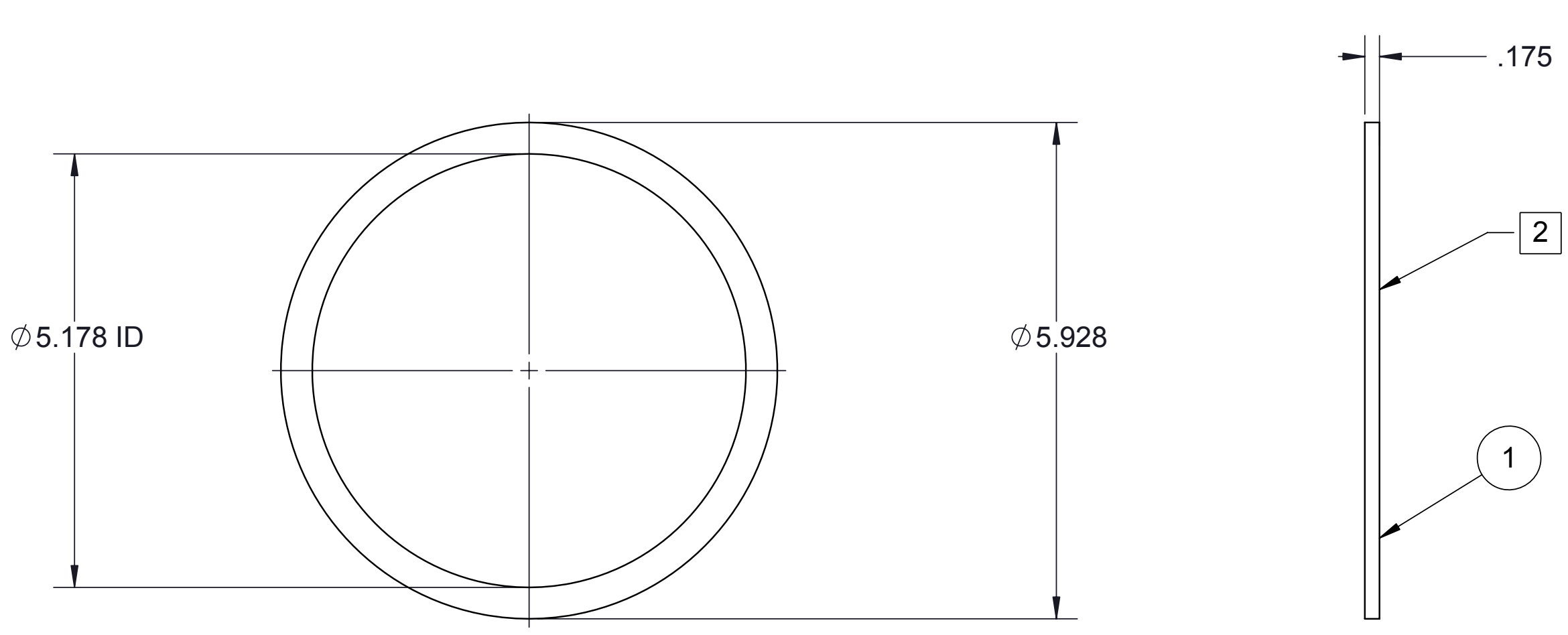

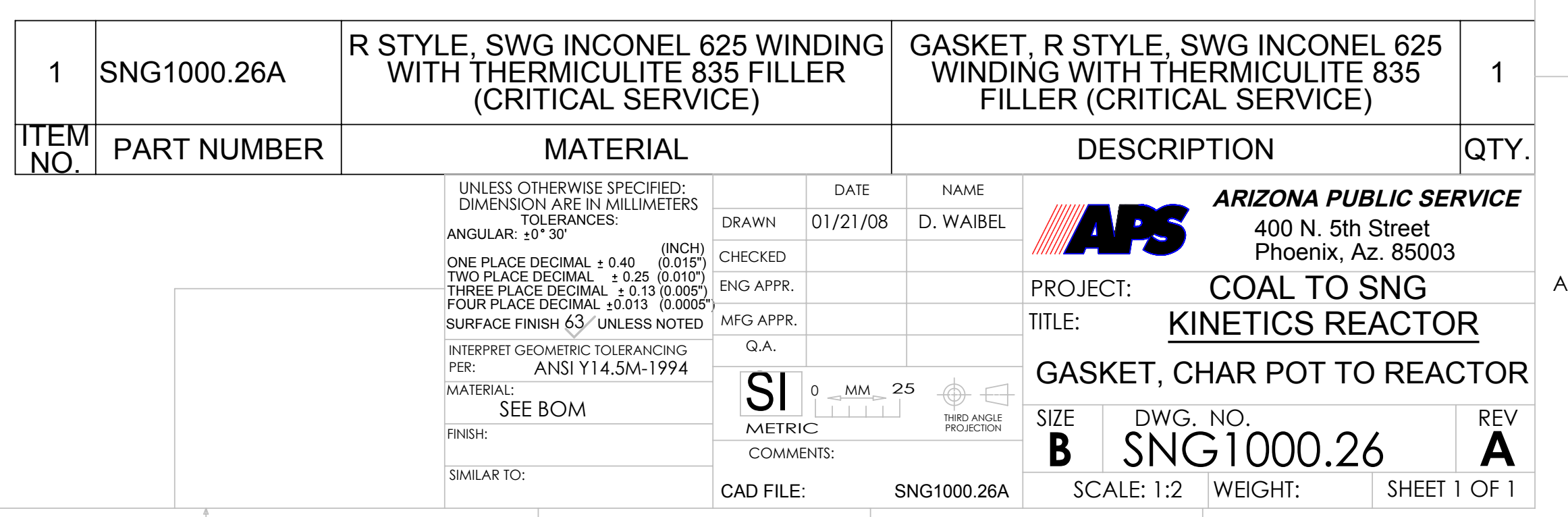


1 DIMENSION AND TOLERANCING PER ANSI Y14.5-1994.

2 GASKET TO BE STYLE 'R', (CRITICAL SERVICE SERIES) SEE BOM BELOW.
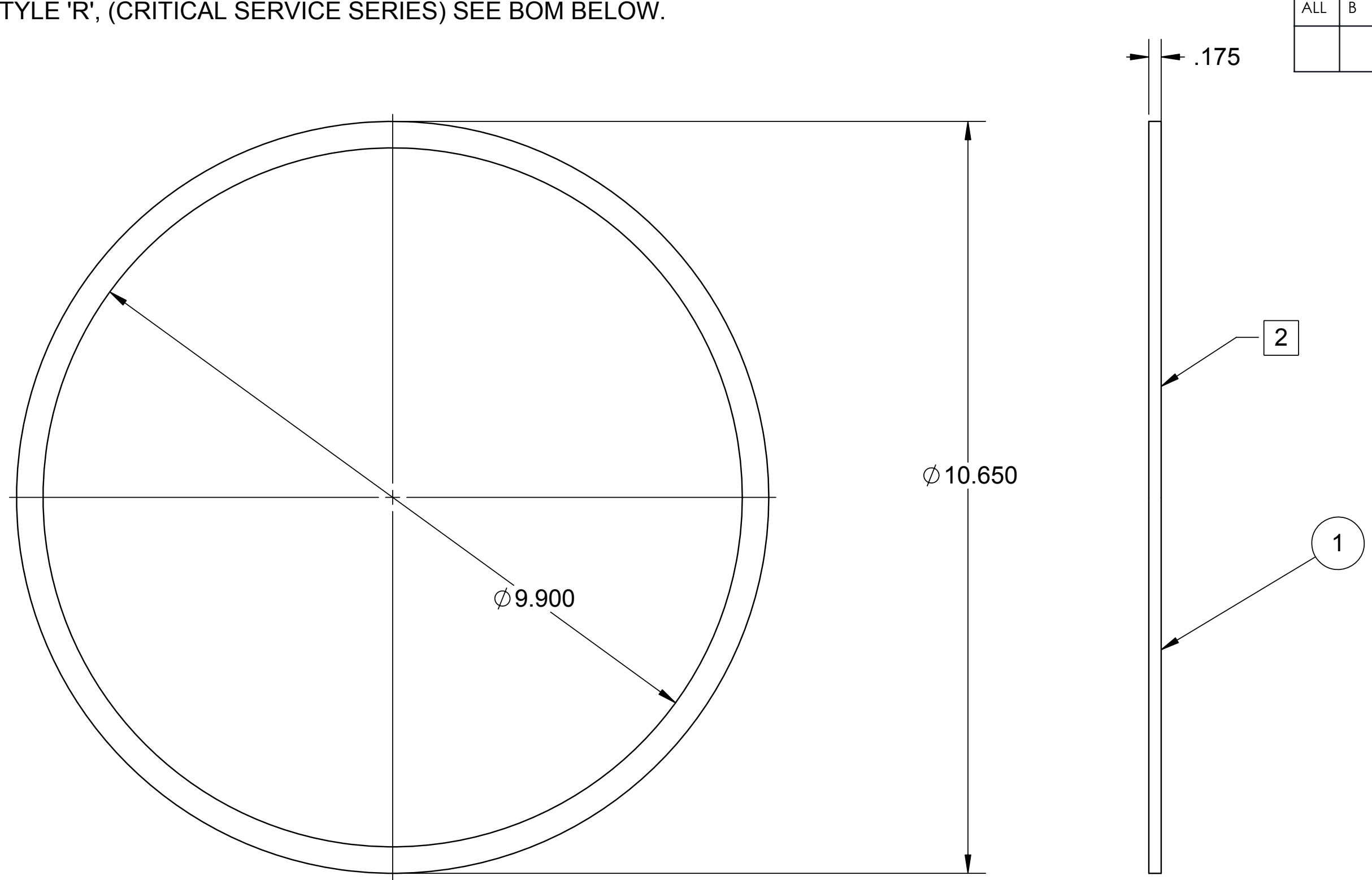

\begin{tabular}{|c|c|c|c|c|c|}
\hline 1 & SNG1003.9C & \multicolumn{4}{|c|}{$\begin{array}{l}\text { R STYLE, SWG INCONEL } 625 \\
\text { WINDING WITH THERMICULITE } 835 \\
\text { FILLER (CRITICAL SERVICE) }\end{array}$} \\
\hline $\begin{array}{l}\text { TTEM } \\
\text { NO. }\end{array}$ & PART NUMBER & \multicolumn{4}{|c|}{ MATERIAL } \\
\hline & & \multirow{7}{*}{ 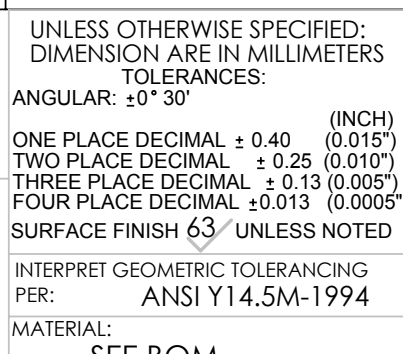 } & & DATE & NAME \\
\hline & & & DRAWN & 1. TAYLOR & 05/16/07 \\
\hline & & & CHECKED & & \\
\hline & & & ENG APPR. & & \\
\hline & & & MFG APPR & & \\
\hline & & & Q.A. & & \\
\hline & & & Sil & $c=\frac{M M}{2} 25$ & $\underbrace{}_{\substack{\text { thito } \\
\text { pron }}}$ \\
\hline & & & COMN & & \\
\hline
\end{tabular}

GASKET, REACTOR HEAD

DESCRIPTION

ARIZONA PUBLIC SERVICE IIIII $\quad \begin{aligned} & 400 \text { N. 5th Street } \\ & \text { Phoenix, Az. } 85003\end{aligned}$

PROJECT: $\quad$ COAL TO SNG IITLE: KINETICS REACTOR GASKET, REACTOR HEAD 
DIMENSION AND TOLERANCING PER ANSI Y14 5-1994.

2 GASKET TO BE SOFT CUT, CRITICAL SERVICE SERIES, FLEXITALLIC THERMICULITE 815 WITH .004" 316SS TANGED REINFORCEMENT.

\begin{tabular}{|l|c|c|c|c|}
\hline \multicolumn{4}{c}{ REVISIONS } \\
\hline ZONE & REV. & DESCRPTION & & \\
\hline ALL & A & INITIAL RELEASE & REV. BY APPROV \\
\hline & & $12 / 24 / 07$ & DW \\
\hline
\end{tabular}

D

$\varnothing .391 \mathrm{THRU}$ EQ. SPACED ON $\varnothing 4.00$ B.C.
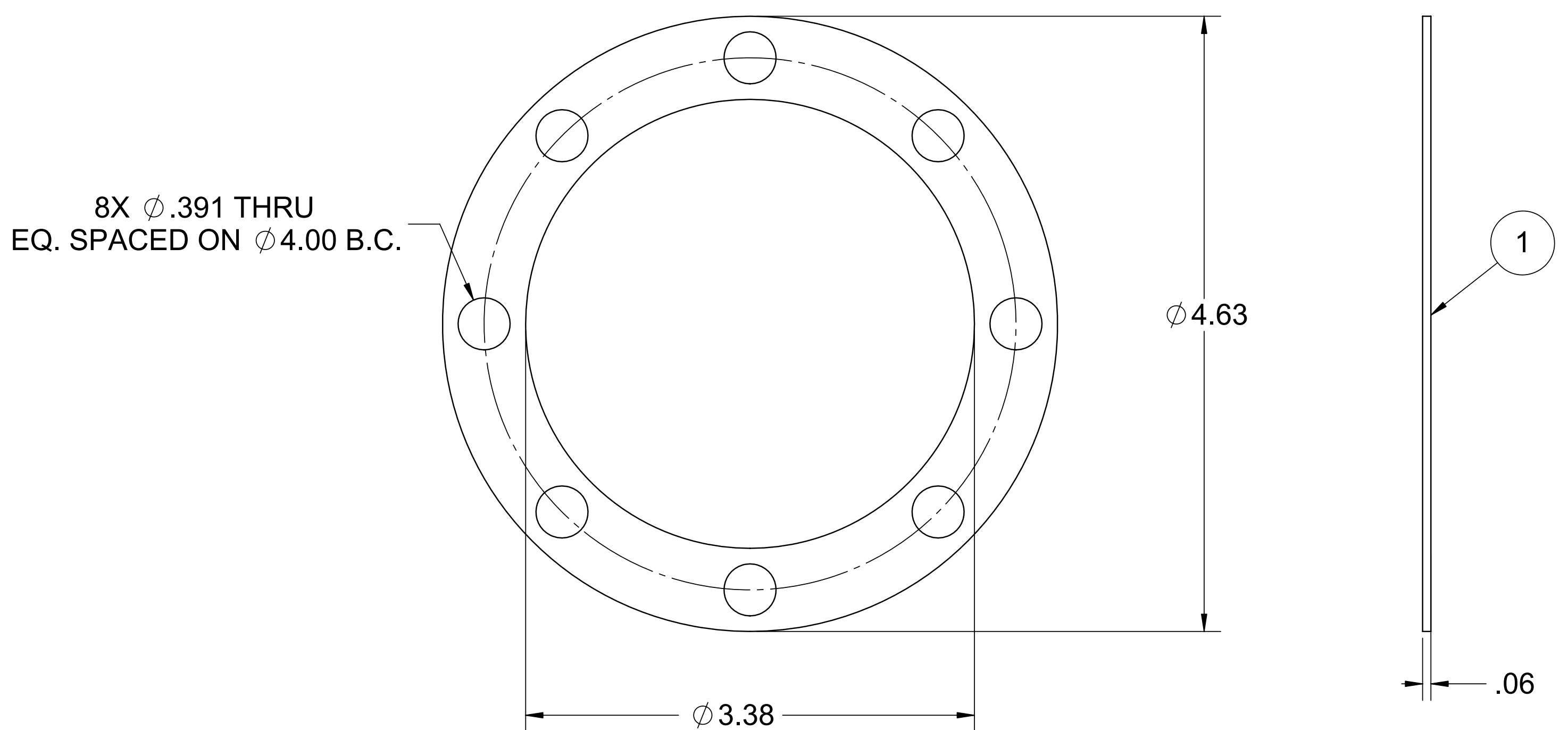

\begin{tabular}{|c|c|c|c|c|}
\hline 1 & SNG1004.10B & $\begin{array}{c}\text { THERMICULITE } \\
815\end{array}$ & GASKET, BOTTOM BELLOWS FLANGE & 1 \\
\hline ITEM & PART NUMBER & MATERIAL & DESCRIPTION & QTY. \\
NO. & PAT & S
\end{tabular}

\begin{tabular}{|c|c|c|c|c|c|c|}
\hline UNLESS OTHERWISE SPECIFIED: & & & & \multirow{3}{*}{\multicolumn{3}{|c|}{$\begin{array}{l}\text { ARIZONA PUBLIC SERVICE } \\
400 \text { N. 5th Street } \\
\text { Phoenix, Az. } 85003\end{array}$}} \\
\hline $\begin{array}{l}\text { DIMENSION ARE EN MIILLIMETERS } \\
\text { ANGULAR: OLERANACES: }\end{array}$ & DRAWN & D. WAIBEL & $12 / 24 / 07$ & & & \\
\hline 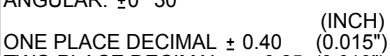 & CHECKED & & & & & \\
\hline 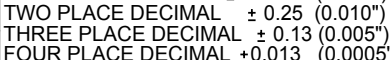 & ENG APPR. & & & \multirow{2}{*}{$\begin{array}{l}\text { PROJE } \\
\text { TITLE: }\end{array}$} & \multicolumn{2}{|c|}{ COAL TO SNG } \\
\hline $\begin{array}{l}\text { SURFACE FINISH } 63 \text {, UNLESS NOTED } \\
\text { SUTE }\end{array}$ & MFG APPR. & & & & \multicolumn{2}{|c|}{ KINETICS REACTOR } \\
\hline 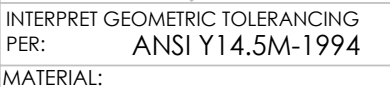 & \multirow{3}{*}{$\begin{array}{l}\text { SI } \\
\text { METRI } \\
\text { COMME }\end{array}$} & & \multirow{3}{*}{ 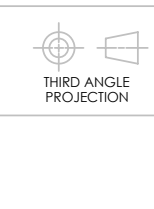 } & \multicolumn{3}{|c|}{ GASKET, BOTTOM BELLOWS FLANGE } \\
\hline $\begin{array}{l}\text { MAIERALE: THERMICULITE } 815 \\
\text { FNSH: }\end{array}$ & & $\begin{array}{lll}0 & \mathrm{MM} & 25 \\
= & 1 & 1\end{array}$ & & \multirow{2}{*}{${ }_{\text {SC }}^{\text {SIZE }}$} & $\begin{array}{l}\text { DWG. NO. } \\
\text { SNG } 1004.10\end{array}$ & REV \\
\hline SIMILAR & & & & & ALE: $1: 1 \quad$ WEIGHT: & $1 \mathrm{OF} 1$ \\
\hline
\end{tabular}


DIMENSION AND TOLERANCING PER ANSI Y14.5-1994.

2 GASKET TO BE SOFT CUT, CRITICAL SERVICE SERIES, FLEXITALLIC

THERMICULITE 815 WITH .004" 316SS TANGED REINFORCEMENT.

$8 X \varnothing .391 \mathrm{THRU}$

EQ. SPACED ON $\varnothing 4.00$ B.C.
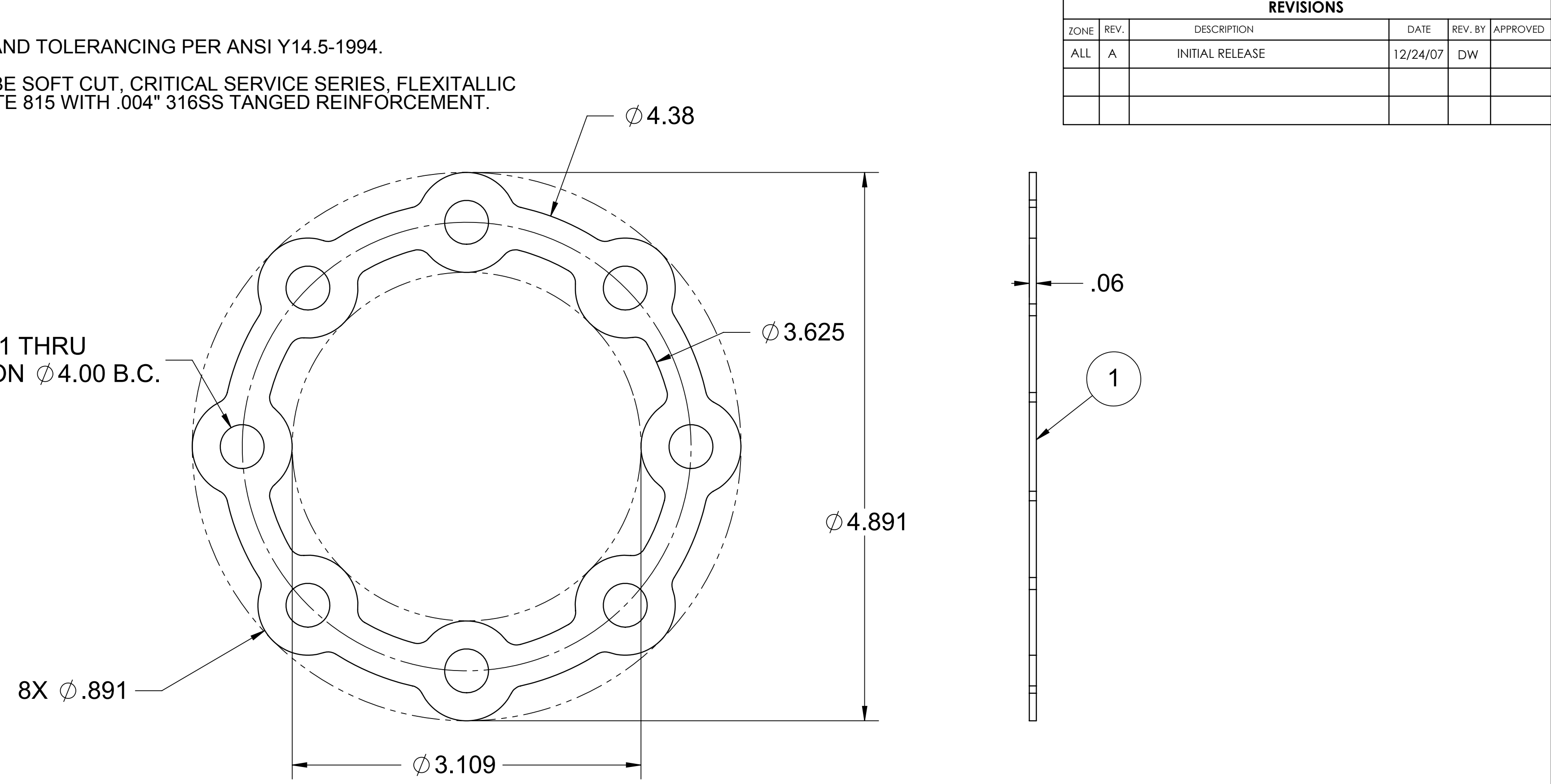

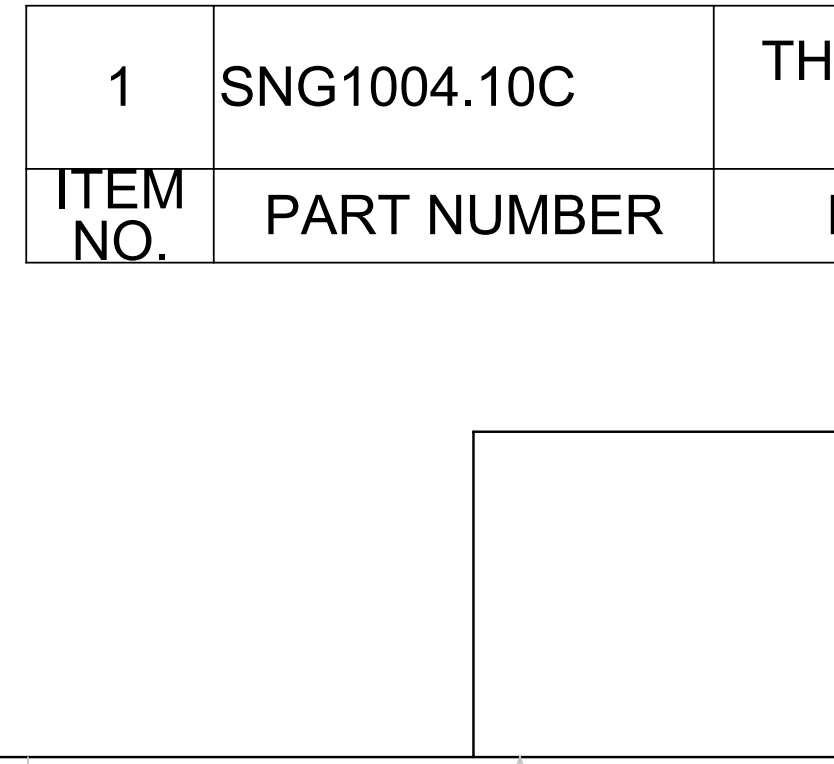

\begin{tabular}{|c|c|c|c|c|c|c|}
\hline 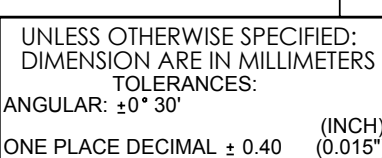 & $\begin{array}{ll}\text { DRAWN } \\
\text { CHECKED }\end{array}$ & \begin{tabular}{c|c|} 
DAFE \\
D. WABBEL \\
\end{tabular} & $\begin{array}{c}\text { NAME } \\
12 / 24 / 07 \\
\end{array}$ & \multicolumn{3}{|c|}{$\begin{array}{l}\text { ARIZONA PUBLIC SERVICE } \\
400 \mathrm{~N} \text {. } 5 \text { ht Street } \\
\text { Phoenix, AZ. } 85003\end{array}$} \\
\hline 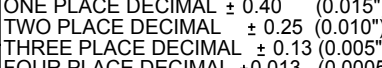 & ENG APPR. & & & \multicolumn{3}{|c|}{ COAL TO SNG } \\
\hline 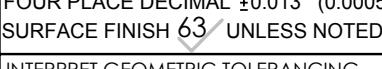 & MEG APR. & & & \multirow{2}{*}{\multicolumn{3}{|c|}{$\begin{array}{l}\text { TITLE: } \quad \text { KINETICS REACTOR } \\
\text { GASKET, BOTTOM BELLOWS FLANGE }\end{array}$}} \\
\hline 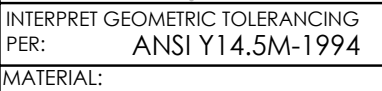 & $\mathrm{SI}$ & & & & & \\
\hline $\begin{array}{l}\text { THERMICULITE } 815 \\
\text { FNISH: } \\
\end{array}$ & \multirow{2}{*}{\multicolumn{2}{|c|}{$\begin{array}{l}\text { METRIC } \\
\text { COMMENS: }\end{array}$}} & \multirow[t]{2}{*}{ 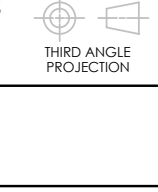 } & B & \multirow{2}{*}{$\begin{array}{l}\text { SWG. NO. } \\
\text { SNG } 1004.10\end{array}$} & C \\
\hline & & & & & & $1 O F 1$ \\
\hline
\end{tabular}


2 GASKET TO BE SOFT CUT, CRITICAL SERVICE SERIES, FLEXITALLIC

THERMICULITE 815, WITH .004" 316SS TANGED REINFORCEMENT.
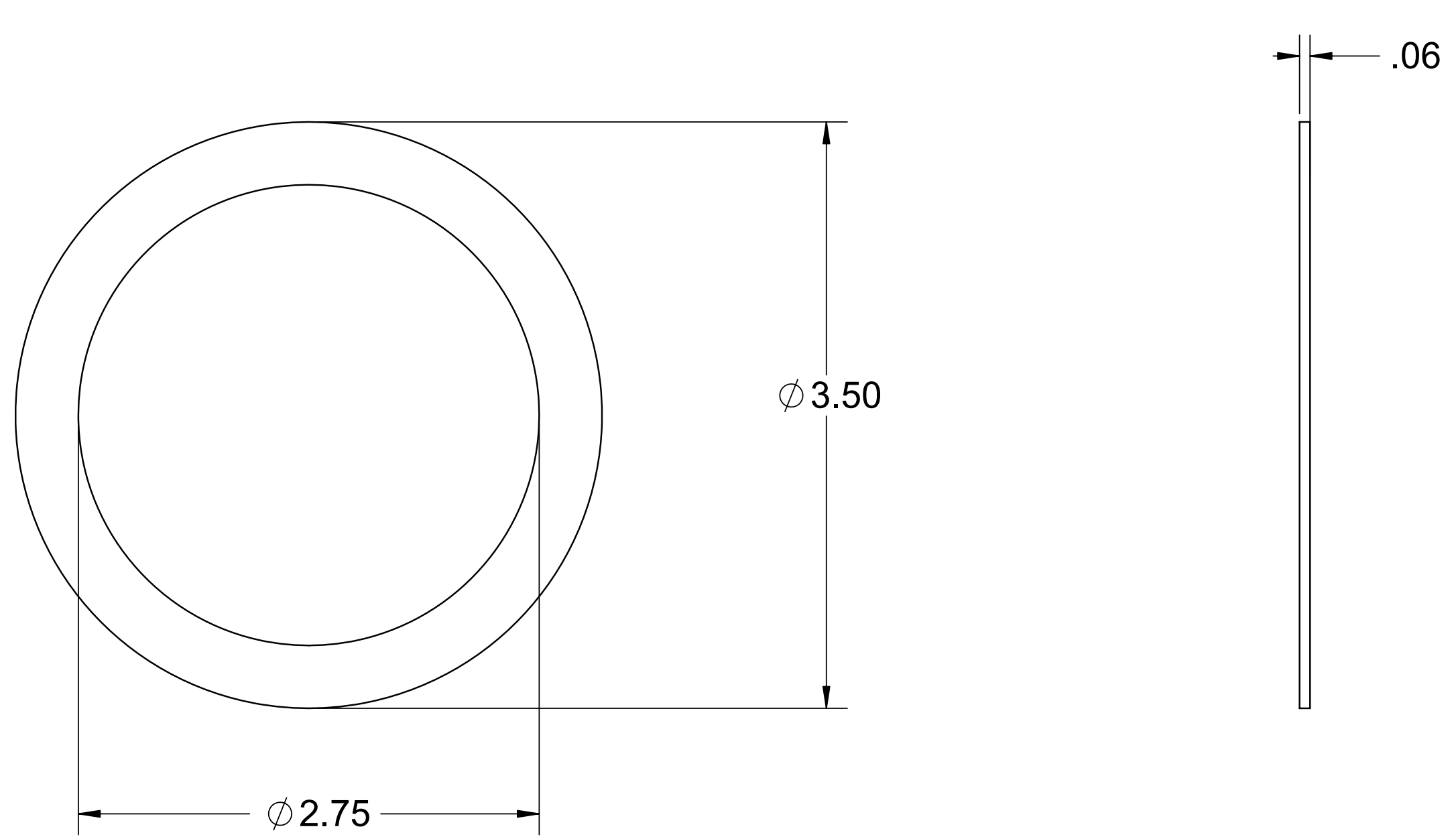

\begin{tabular}{|c|c|c|c|c|}
\hline 1 & SNG1004.11B & $\begin{array}{c}\text { THERMICULITE } \\
815\end{array}$ & GASKET, TOP BELLOWS FLANGE & 1 \\
\hline $\begin{array}{c}\text { ITEM } \\
\text { NO. }\end{array}$ & PART NUMBER & MATERIAL & DESCRIPTION & QTY. \\
\hline
\end{tabular}

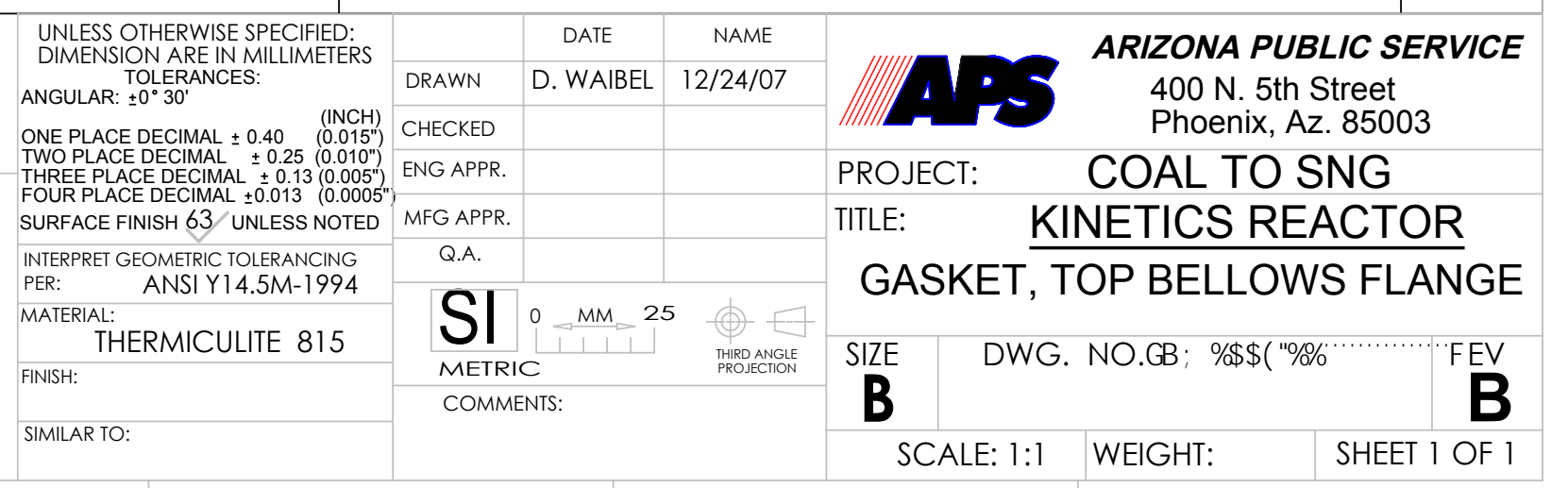


1 DIMENSION AND TOLERANCING PER ANSI Y14.5-1994.

2 GASKET TO BE STYLE 'R', (CRITICAL SERVICE SERIES) SEE BOM BELOW

REVISIONS

\begin{tabular}{|l|l|l|c|l|l|}
\hline \multicolumn{7}{|c|}{ REVISIONS } \\
\hline ZONE & REV. & DESCRIPTION & DATE & REV. BY & APPROVED \\
\hline ALL & A & INITIAL RELEASE & $05 / 16 / 07$ & I.T. & \\
\hline ALL & B & CHANGED GASKET FROM FLAT TO STYLE 'R' & $08 / 03 / 07$ & D.W. & \\
\hline & & & & & \\
\hline
\end{tabular}
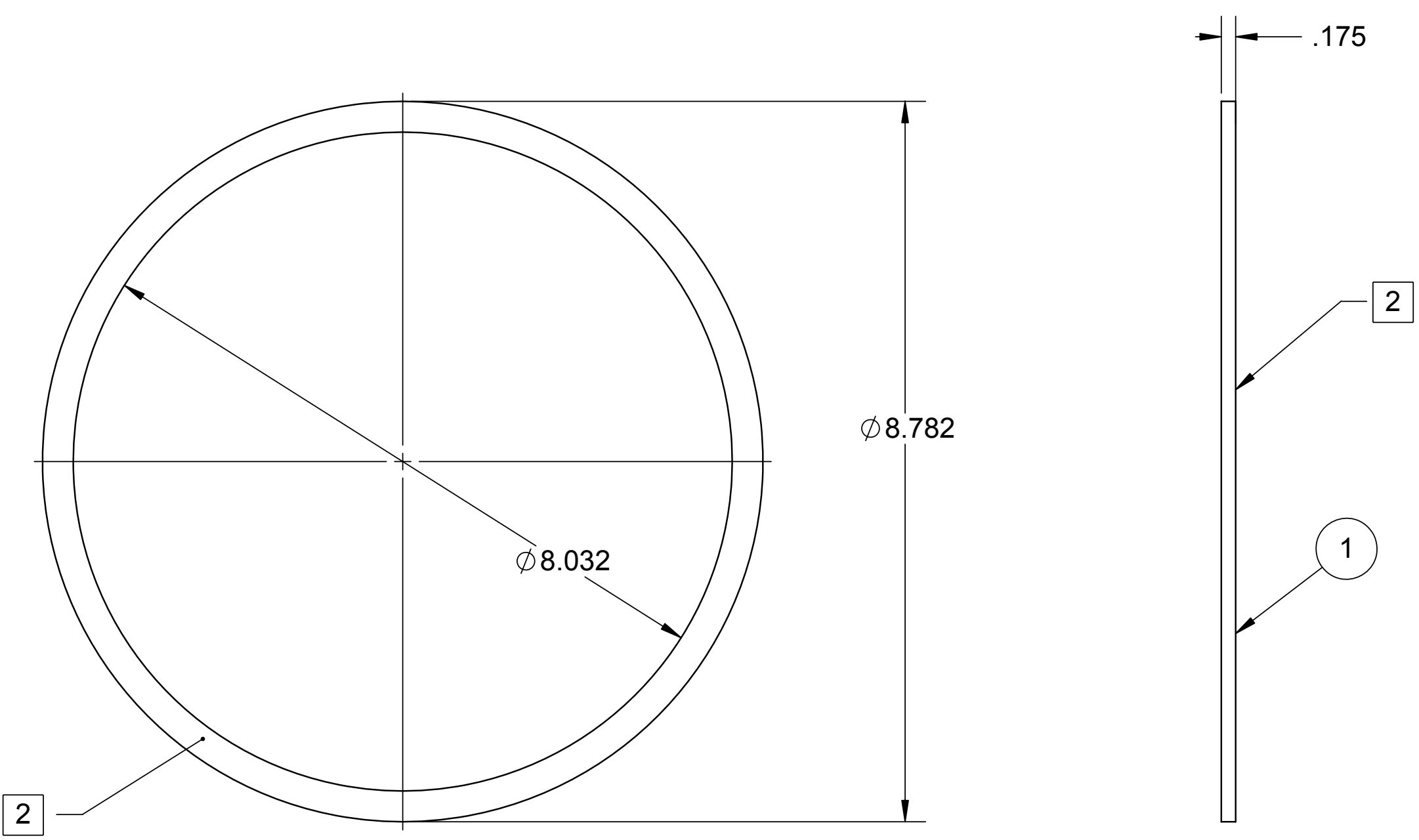

\begin{tabular}{|c|c|c|c|c|c|}
\hline 1 & SNG1004.7C & \multicolumn{3}{|c|}{$\begin{array}{c}\text { R STYLE, SWG INCONEL } 625 \text { WINDING } \\
\text { WITH THERMICULITE 835 FILLER } \\
\text { (CTRITICAL SERVICE) }\end{array}$} & \\
\hline $\begin{array}{l}\text { ITEM } \\
\text { NO. }\end{array}$ & PART NUMBER & \multicolumn{3}{|l|}{ MATERIAL } & \\
\hline & & \multirow{5}{*}{ 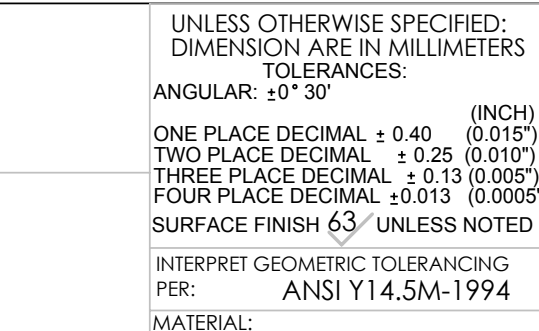 } & & DATE & NAME \\
\hline & & & DRAWN & I. TAYLOR & $05 / 16 / 07$ \\
\hline & & & $\begin{array}{l}\text { CHECKED } \\
\text { ENG APPR. }\end{array}$ & & \\
\hline & & & MFG APPR & & \\
\hline & & & Q.A. & & \\
\hline & & $\begin{array}{l}\text { MATERIAL: } \\
\text { SEE BOM }\end{array}$ & Sl & {$\left[\begin{array}{ll}M M_{-} 2 \\
C\end{array}\right.$} & 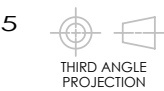 \\
\hline
\end{tabular}

GASKET, REACTOR FOOT

DESCRIPTION

ARIZONA PUBLIC SERVICE 400 N. 5th Street
Phoenix, Az. 85003 PROJECT: COAL TO SNG TITLE: KINETICS REACTOR GASKET, REACTOR FOOT FINISH: METRIC 


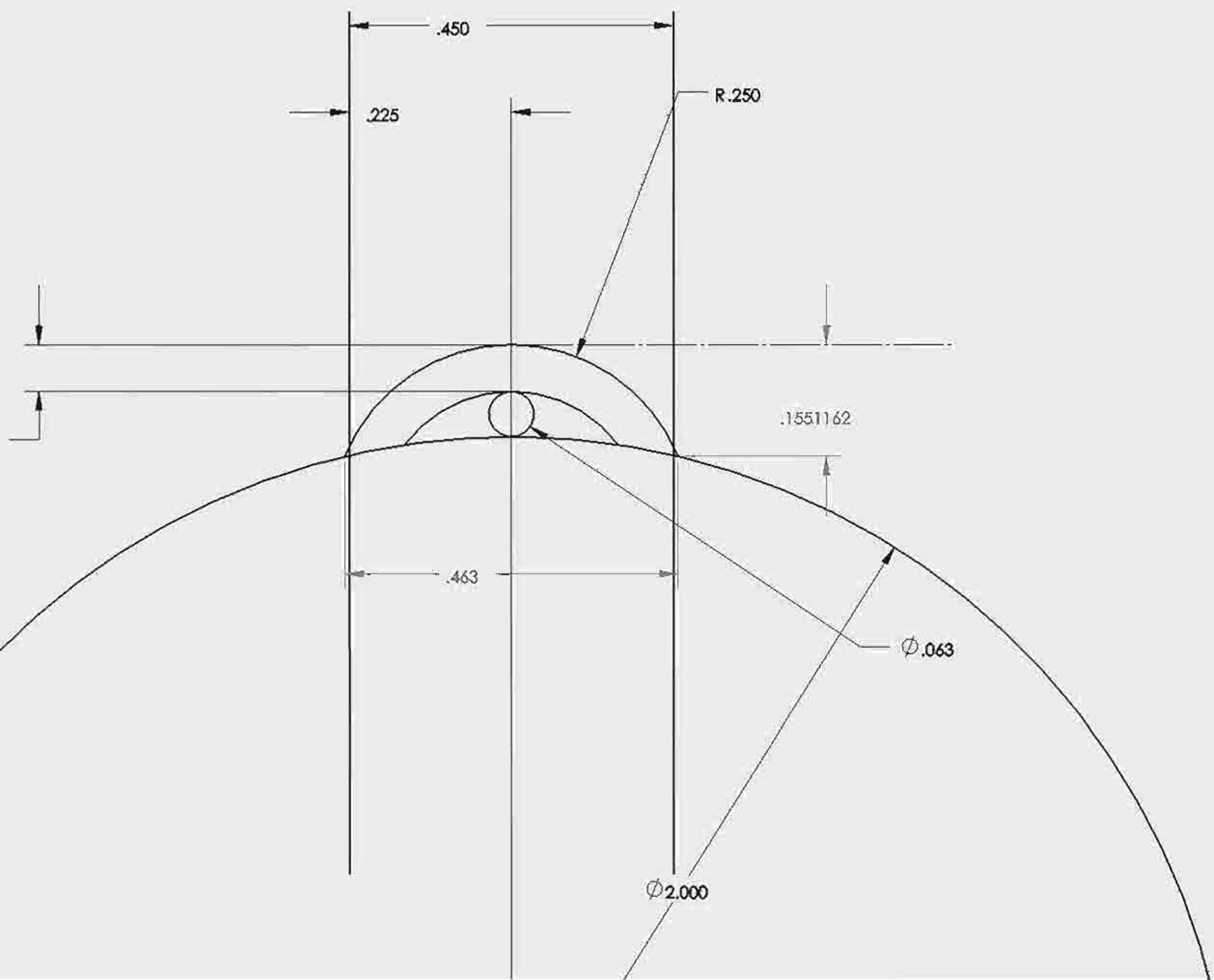


THIS PAGE INTENTIONALLY LEFT BLANK 


\title{
APPENDIX C
}

\section{Bench Scale Hydrogasifier Reactor}

\author{
Final Design from Gaspar Inc.
}


Development of a Hydrogasification Process for

Coproduction of SNG and Electrical Power from Western Coals

Final Report

THIS PAGE INTENTIONALLY LEFT BLANK 
1545 Whipple Avenue SW Canton, Ohio 44710

Phone: 330-477-2222

Fax: $330-477-2322$

www.gasparinc.com

\section{FINAL DATA PACKAGE}

\begin{tabular}{|r||l||}
\hline GASPAR JOB NUMBER(S): & 36094 \\
\hline CUSTOMIER: & ARIZONA PUBLIC SERVICE \\
\hline PURCHASE ORDER NUMBER: & 700521452 \\
\hline DESCRIPTION: & KINETICS REACTOR \\
\hline ITEM NUMBER(S): & N/A \\
\hline OTHER: & N/A \\
\hline
\end{tabular}

\section{DATA PACKAGE CONTENTS}

$\square$ DATAREPORT

$\square$ NAMEPLATE COPY

$\checkmark$ BILL OF MATERIAL

$\square$ MATERIAL TEST REPORTS

$\square$ NDE REPORTS

$\square$ HEAT TREAT CHARTS

$\square$ CALCLILATIONS

$\checkmark$ DRAWINGS

$\square$ OTHER: (LIST BELOW)

NOTE: 
FORM U-I MANUFACTLRER'S DATA REPORT FOR PRESSURE VESSELS

As Required by the Provisions of the ASME Boiler and Pressure Vessel Code Rules, Section VIII, Division 1

Manufactured and certified by: _Gaspar, Inc. 1545 Whipple Ave SW Canton, Ohio 44710

Manufactured for: $\quad$ Arizona Public Service Co. P. P. Box 53999 Phoenix, Az 85072

Location of installation

Unknown

(Name and adaress of Purchaser)

Type: Vertical

36094

(Horizontal, vertical, or sphere)
36094 Rev.5

(Drawing number) 2007 Edition

[Edition and Addenda (date)] Kinetics Reactor

(Name and address)

tor, jkt.. vessei, heat exch., etc.) 2550

National Board number

(Code Case number)
(Manufacturer's serial number)

2008

(Year built)

[Speciai Service per UG-120(d)]

'tems 6-11 incl. to be completer for single wall vessels, jackets of jacketed vessels, shell of heat exchangers, or chamber of multi-chamber vessels.

j. Shell (a) Number of course (s):

(b) Overall Length :

$13^{\prime}-11.50^{\prime \prime}$

\begin{tabular}{|c|c|c|c|c|c|c|c|c|c|c|c|c|}
\hline \multicolumn{2}{|c|}{ Course(s) } & Material & \multicolumn{2}{|c|}{ Thickness } & \multicolumn{3}{|c|}{ Long. Joint (Cat A) } & \multicolumn{3}{|c|}{ Circum Joint (Cat $A, B, \&$ ) } & \multicolumn{2}{|c|}{ Heat Treatment } \\
\hline Diameter & Length & Spec/Grade or Type & Nom. & Corr. & Type & Full, Spot, None & Eff. & Type & Full, Spot, None & Eff & Temp. & Time \\
\hline $10.75^{\prime \prime O D}$ & $13^{\prime}-11.50^{\prime \prime}$ & SA106-B & $.593^{\prime \prime}$ & $.06^{\prime \prime}$ & $S$ & None & $100 \%$ & 1 & Full & $100 \%$ & $\cdots$ & $\cdots$ \\
\hline & & & & & & & & & & & & \\
\hline & & & & & & & & & & & & \\
\hline
\end{tabular}

Heads: (a)

(a)

(b)

If removable, bolts used (describe other fastenings)

8.Type of jacket

(Material spec. number grade or type) (H.T. - time \& temp)

(b)

\begin{tabular}{|c|c|c|c|c|c}
\hline & (Material spec. number grade or type) (H.T. - time \& temp) \\
\hline $\begin{array}{c}\text { Hemispherical } \\
\text { Radius }\end{array}$ & $\begin{array}{c}\text { Flat } \\
\text { Diameter }\end{array}$ & Side to Pressure & \multicolumn{3}{c}{ Category A } \\
\cline { 2 - 6 } & & Convex Concave & Type & Full, Spot, None & Eff. \\
\hline & & & & & \\
\hline
\end{tabular}
Jacket closure

(Material spec. number grade, slze, number)

If bar, give dimensions

$--$

9. MAWP $\frac{1200}{\text { (Internal) }} \frac{\cdots}{\text { (External) }}$ psi at max temp.

o. Impact Test UCS-66(b) Exempt.

[Indicate yes or no and the component(s) impact tested]
If bolted, describe or sketch

Hydro., Pneumatic., or comb. test press. _____ Pneumatic 1320____ Proof Test

tems 12 and 13 to be completed for tube sections.

2. Tubesheet

\begin{tabular}{|c|c|}
\hline [Stationary (Material spec. number)] & [Diameter (subject to press.)] \\
\hline Floating (Material spec. number)] & (Diameter) \\
\hline
\end{tabular}

(Nominal thickness)
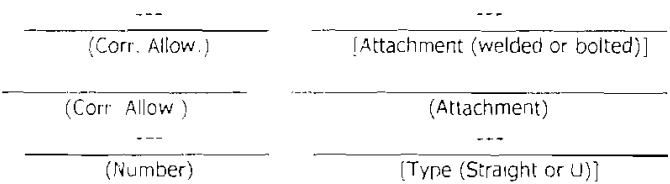

ens 14-18 incl. To be completed for inner chambers of jacketed vessels or channels of heat exchongers.

4. Shell (a) Number of course (s):

(b) Overall Length :

\begin{tabular}{|c|c|c|c|c|c|c|c|c|c|c|c|c|}
\hline \multicolumn{2}{|c|}{ Courses } & Materiai & \multicolumn{2}{|c|}{ Thickness } & \multicolumn{3}{|c|}{ Long. Joint (Cat $A)$} & \multicolumn{3}{|c|}{ Circum. Joint (Cat. $A, B, C$ ) } & \multicolumn{2}{|c|}{ Heat Treatment } \\
\hline Diameter & Length & Spec./Grade or Type & Nom. & Corr & Type & Full, Spot, None & Eff. & Type & Full, spot, None & Eff & Temp. & Time \\
\hline & & & & & & & & & & & & \\
\hline & & & & & & & & & & & & \\
\hline & & & & & & & & & & & & \\
\hline
\end{tabular}

5. Heads: (a)

(b)

If removable, bolts used (describe other Fastenings)

Bottom, Ends)
SA403-316H (Material spec. number grade or type) (H.T. - time \& temp)

\begin{tabular}{|l|c|c|c|}
\hline \multicolumn{2}{|c|}{ Thickness } & \multicolumn{2}{c|}{ Radius } \\
\hline Min. & Corr. & Crown & Knuckle \\
\hline $.500^{\prime \prime}$ & $.03^{\prime \prime}$ & $\ldots$ &..-- \\
\hline & & & \\
\hline
\end{tabular}

$$
\text { gs) }
$$

\begin{tabular}{|c|c|c|c|c|c|c|c|c|}
\hline \multicolumn{3}{|c|}{$\overline{\text { H.T. }}$ - time \& temp) } & \multicolumn{6}{|c|}{ (Material spec. number grade or type) (H.T. - ume \& temp) } \\
\hline \multirow{2}{*}{$\begin{array}{l}\text { Elliptical } \\
\text { Ratio }\end{array}$} & \multirow{2}{*}{$\begin{array}{c}\text { Conical } \\
\text { Apex Angle }\end{array}$} & \multirow{2}{*}{$\begin{array}{l}\text { Hemispherical } \\
\text { Radius }\end{array}$} & \multirow{2}{*}{$\begin{array}{c}\text { Flat } \\
\text { Diameter }\end{array}$} & \multicolumn{2}{|c|}{ Side to Pressure } & \multicolumn{3}{|c|}{ Category A } \\
\hline & & & & Convex & Concave & Type & Full, Spot, None & Eff. \\
\hline $2: 1$ & --. & -.. & $\cdots$ & ...- & Concave & $\ldots$ & $\ldots$ & - \\
\hline & & & & & & & & \\
\hline
\end{tabular}

(12) 1" Studs SA193-B7 (12) 1" Nuts SA194-2H

(Material spec. number grade, size, number) 
Form U-1 (Back)

MAWP

$\frac{1200}{\text { (Internal) }} \quad \frac{\cdots}{\text { (External) }} \quad$ psi at max temp.

$\frac{1000}{(\text { Internal) }}$

$\frac{-}{\text { (External) }}{ }^{\circ}$

Min. design metal temp.

5 of at

1200 psi

Impact Test UHA-51(d), UCS-66(b) Exempt.

Indicate yes or no and the component(s) impact tested

at test temperature of

Hydro., pneu., or comb. test press. ___ Pneumatic 1320____ Proof Test

Nozzles, inspection, and safety valve openings:

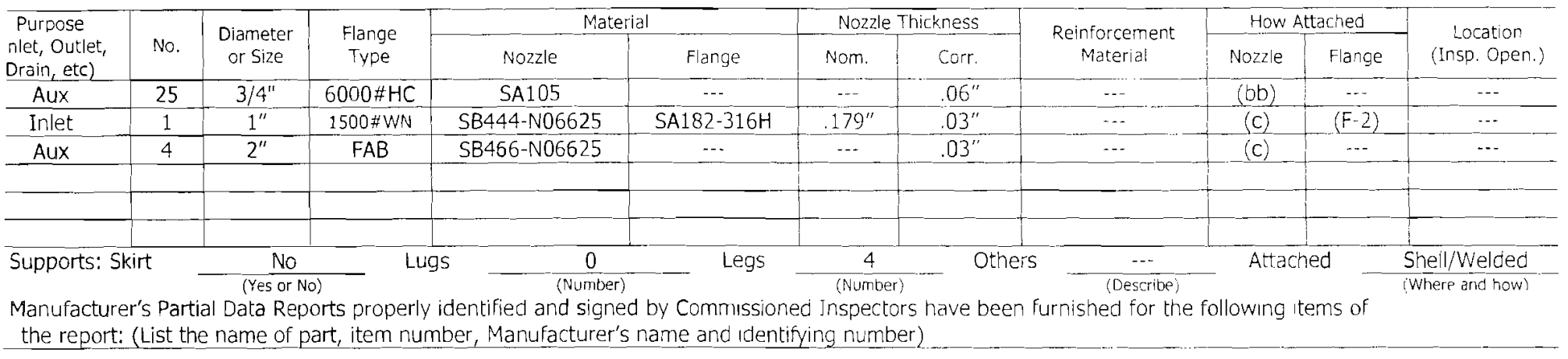

Remarks Bottom of reactor shell ends with a fab flange $2-7 / 8^{\prime \prime}$ thk $\times 13.15^{\prime \prime O D} \times 7-1 / 2 " \mathrm{ID}$ (SA516-70). Shell head flange is 3-1/16"thk $\times 14-5 / 8^{\prime \prime O D}$

9-9/16"ID (SA516-70). Top head flange is 3-3/4"thk $\times 14-5 / 8^{\prime \prime O D} \times 7-5 / 8^{\prime \prime I D ~(S A 182-316 H) . ~}$

\section{CERTIFICATE OF SHOP COMPLIANCE}

We certify that the statements in this report are correct and that all details of design. material, construction and workmanshn of this vessel conform to the ASME, BOILER AND PRESSURE VESSEL CODE. Section VIII. Division I.

Certificate of Authorization

Date $12-19-08$ Name

16.862

Expires luly 25.2011

$\frac{\text { Gaspar, lnc. }}{\text { (Manufacturer) }}$

CERTIFICATE OF SHOP INSPECTION

I, the undersigned, holding a valid commission issued by the National Board of Boiler and Pressure Vessel Inspectors and 0 the State or Province of Ohio___and employed by_OneBeacon America Insurance Company_ of _____ Lynn. Mass have inspected che pressure vessel described in this Manufacturer's Data Report on $12-19-2008$

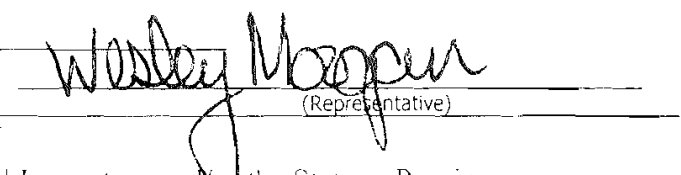
State that. to the best of my knowledge and belief, the Manufacturer has constructed this pressure vessel in accordance Wich ASME BOII ER AND PRFSSURE VESSEL CODE, Section VIIl, Division I. By signing this certiticate neither the Inspector nor hoher emplover makes any warmanty. expressed or implied. concerning the pressure vessel described in this Manufacturer's Data Report. Furthermore, ncither the Inspector nor his/her employer shall be liable in any manner for any personal injury or property damage or a loss of any kind arising from or connected with this inspection.

\section{Date 12-19-200E signed - X. (Authorized Inspector) _____ National Board (ind endorsements) State, Province and number. EERTIFICATE OF FIELD ASSEMBLY COMPLIANCE}

We certify that the statements on this report are correct and that the field assembiy construction of all parts of this vessel conlorms with the requirements of ASME BOILER AND PRESSURE VESSEL CODE Section VIIl. Division 1.U Certificale of Authorization No. Expires

Date Name Signed

\section{CERTIFICATE OF FIELD ASSEMBLY INSPECTION}

(Representative)

I. the undersigned, holding a valid commission issued by the National Board of Boiler and Pressure Vessel Inspectors and/or the State or Province and employed by have compared the statements in this Manufacturer's Data Report with the described pressure vessel not included in the certificate of shop inspection. have been and state that parts referred to as data items inspected by me and to the best of my knowledge and belief, the Manufacturer has construted and assembled this pressure vessel in accordance
with the ASME BOILER AND PRESSURE VESSEL. CODE. Section VIII. Division 1. The described vessel was inspected and subjected to a

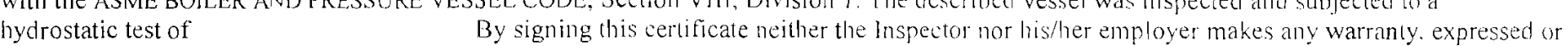
implied, concerning the pressure vessel described in this Manufacturer's Data Report. Furthermore. neither the Inspector nor his/her employer shall be liable in any manner for any personal injury or property damage or a loss of any kind arising from or connecled with this inspection.

Date

Signed

Commissions number) 


\section{1}

a

\section{CERTIFIED BY GASPAR, INC.} CANTON, OHIO

SHELL SIDE

$\begin{array}{ll}\text { MAWP } & \text { PSI AT } \\ \text { MDMT } & \text { OFAT } \\ \text { MAEWP } & \text { PSI AT }\end{array}$

T 1 Bf

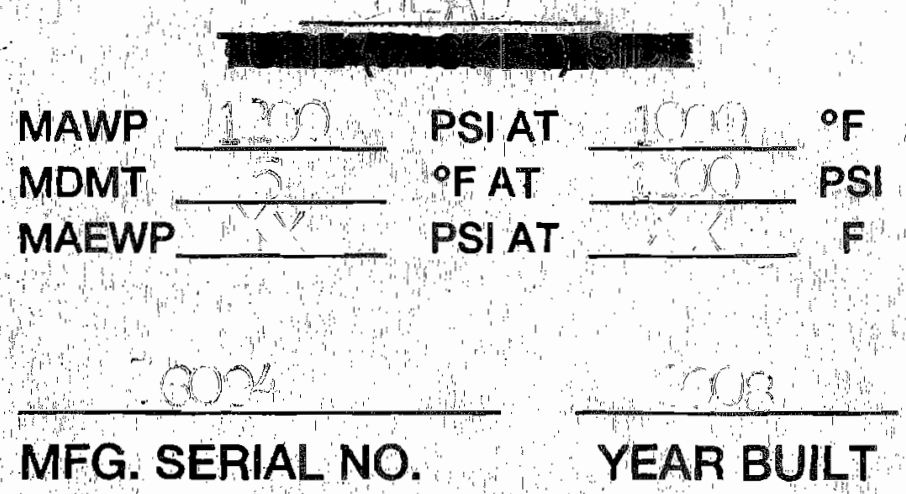




\begin{tabular}{|c|c|c|c|c|}
\hline Company Name & Project Name & \multicolumn{2}{|c|}{ Projegt Description } & \multirow{2}{*}{$\begin{array}{l}\text { Item Nilumber } \\
\text { PRTY\#3 }\end{array}$} \\
\hline ARIZONA PUBI_IC SERVICE & 36094 & $F A B(1) F$ & EACTOR UNIT & \\
\hline PONunnher & dwonumber & Enginger & Material Restri & \\
\hline 700521452 & 36094 & RCK & Dom & or West European Origin \\
\hline
\end{tabular}

\section{MRK REV IIY TYPE IIESCRIPTIDN}

LUCATIIN

MATERIAL $\quad L / \mathbb{L} \mathbb{P} / \mathbb{P}$

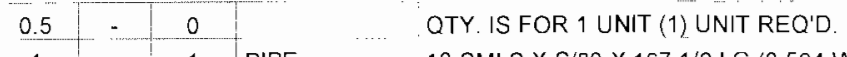

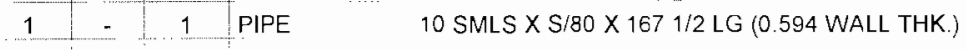

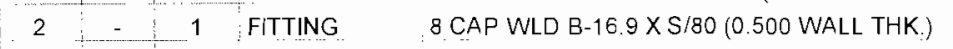

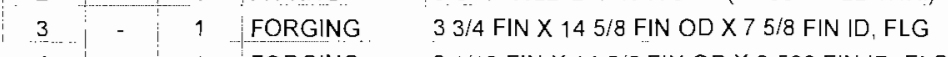

4. - 1 FORGING $31 / 16$ FIN X $145 / 8$ FIN OD X 9562 FIN ID FLG

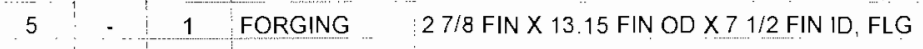

6 - 13 PLATE $37 / 8$ FIN $\times 13.15$ FIN OD X 2.38 FIN ID

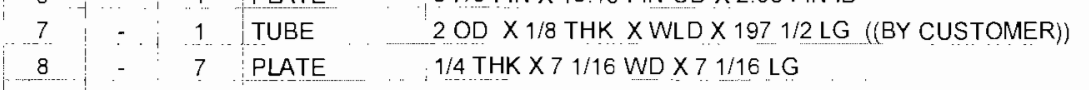

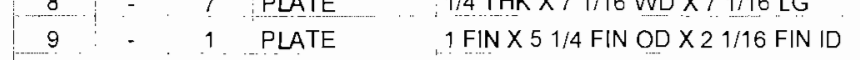

10. $1: 14$ TUBE $\quad 2$ WLD X 1/8 THKX 1 LG CUT IN 72 DEG. SEG.

\begin{tabular}{c|c:c:c|c}
10 & 1 & 0 & 1 PIPE MAKES 5 PARTS (MATERIAL BY CUSTOMER)) \\
11 & - & 1 & BAR/ROD & INJECTOR HUB (BY CUSTOMER) 3" OD X $31 / 2$ LG
\end{tabular}

\begin{tabular}{l|l|l|l|l|l|l}
112 & - & 4 & BAR/ROD INJECTOR HOUSING (BY CUSTOMER) $11 / 4$ OD $\times 2.20 \mathrm{LG}$
\end{tabular}

$13 . . . \quad 4 \quad$ EXP JNT. INJECTOR TUBE BELLOWS (BY CUSTOMER)

\begin{tabular}{l|l|l|l|l}
14 & 4 & 4 & TUBE & INJEC. HOUSEING TUBE (BY CUSTOMER) 2 OD $\times 3.69$ LG
\end{tabular}

$15:-1$ FCRGING 11500 B-16.5 RFWN (S/80 BORE)

\begin{tabular}{ll|l|ll}
16 & 1 & 0 & & DELETED \\
17 & - & 1 & GASKET & 175 THK X 10.65 OD X 9.910
\end{tabular}

$17-0.0$ R STYLE. SWG INCONEL 625 WINDING WITH

$17-0_{10 *}^{*}$ THERMICULITE 835 FILLER (CRITICAL SERVICE)

\begin{tabular}{|l:l:l|l}
18 & 2 & 1 & PIPE
\end{tabular}

\begin{tabular}{l:lll:l}
18 & 2 & 1 & 1 & PIPE \\
\hline 19 & - & $\vdots$ & 4 & PLATE \\
20 & - & 4 & PLATE
\end{tabular}

\begin{tabular}{|c|c:c|c|c}
20 & - & 4 & PLATE \\
\hline 21 & - & 7 & PLATE
\end{tabular}

\begin{tabular}{l:l|l|l|l|l|l}
21 & - & - & 4 & PLATE \\
22 & - & 4 & \\
\hline
\end{tabular}

$23 \ldots-4$ ! PLATE

\begin{tabular}{ll:l|l|l}
24 & - & 8 & PLATE
\end{tabular}

\begin{tabular}{l|l|l|l}
25 & - & 4 & PLATE
\end{tabular}

\begin{tabular}{l|l|l|l}
26 & - & 25 & FORGING
\end{tabular}

$27-1$

\begin{tabular}{l|l|l}
28 & 1 & 1
\end{tabular}

$28: 1: 0$

$\begin{array}{l:l:l}29 & 1 & 1 \\ & \text { GASKET }\end{array}$

\begin{tabular}{ll|l}
29 & 1 & 0
\end{tabular}

$30-1$ GASKET

\begin{tabular}{c:c}
30 & -1 \\
\hline 30 & -
\end{tabular}

$30-0$

\begin{tabular}{l|l|l|l}
31 & 2 & 8 & BOLTING
\end{tabular}

\begin{tabular}{l|l|l}
31 & 2 & 0
\end{tabular}

\begin{tabular}{l|l|l}
32 & 2 & 8
\end{tabular}

\begin{tabular}{|l|l|l}
32 & 2 & 8 \\
32 & 2 & 0 \\
\hline 33 & 2 & 8
\end{tabular}

\begin{tabular}{c:c|c}
33 & 2 & 8 \\
\hline 33 & 2 & 0 \\
\hline
\end{tabular}

\begin{tabular}{l|l:l}
34 & 2 & 8
\end{tabular}

$34: 2 \quad 0$

\begin{tabular}{l:l|l|l}
35 & 1 & 12 & BOLTING
\end{tabular}

$\begin{array}{ll:l:l}36 & 1 & 12 & \text { BAR/ROD }\end{array}$

$37:-8 \quad B O L T I N G$

\begin{tabular}{l|l|l|l|}
38 & - & 8 & BOLTING
\end{tabular}

\begin{tabular}{l|l|l|l|}
39 & 4 & 12 & BOLTING
\end{tabular}

\begin{tabular}{|l|l|l|l|}
\hline 40 & 4 & 12 & BOLTING \\
\hline
\end{tabular}

\begin{tabular}{l|l|l|l}
41 & -12 & BOLTING
\end{tabular}

\begin{tabular}{c|c:c:c}
42 & 2 & 0 & MISC \\
\hline 42 & 4 & 12 & MISC
\end{tabular}

\begin{tabular}{|l|l|l|l|}
43 & 2 & 12 & MISC
\end{tabular}

\begin{tabular}{|l|l|l|l|l}
\hline 43 & 2 & 0 & 1 \\
\hdashline 44 & 2 & 8 & MISC
\end{tabular}
1 SMLS X S/80 X $117 / 8$ LG

$1 / 2$ THK $\times 5$ WD $\times 5$ LG

$3 / 8$ THK X 3 WD X6 LG

$3 / 16$ THK $\times 1$ WD $\times 1$ LG

3/8 THK X $61 / 2$ WD X 7 LG

$1 / 2$ THKX 5 WD $\times 51 / 2 \mathrm{LG}$

$3 / 4$ THK X $411 / 16$ WD X 5 LG

$3 / 8$ THK X $23 / 16$ WD $\times 51 / 2$ LG

$3 / 4 \times 6000 \#$ NPT HALF COUPLING

BELLOWS ALLOY 800H W-SNG1004.9F (CUSTOMER SUPPLY)

$1 / 16$ THK X 4.63 OD X 3.38 ID (SEE DETAIL)

\# THERMICULITE 815

1/16 THK X $31 / 2$ OD X $25 / 8$ ID

\# THERMICULITE 815

175 THK X 8.782 OD X 8.032 ID

*** R STYLE, SWG INCONEL 625 WINDINGS

WITH THERMICULITE 835 FILLER

3/8 -24UNF SOC. HD CAP SCREW X 2 1/4 LG

(M) INCONEL 625

3/8 -24UNF STUD X $23 / 4$ LG

(M) INCONEL 625

3/8 -24UNF NUT X HEX HEAVY

(M) INCONEL 625

3/8 LOCKWASHER

(M) INCONEL 625

$7 / 8$-14UNF SOC. HD. CAP SCREW $\times 33 / 4$ LG

$11 / 2$ OD X 1 ID X $1 / 8$ THK.

1 -14UNF STUD $X 41 / 2$ LG

1 -14UNF NUT $X$ HEX HEAVY

1 -14UNF STUD $X 6$ LG

1-14UNF NUT X HEX HEAVY

1 WASHER $X$ TYPE A PLAIN SERIES $N$

MS-124 DASH\# 691 MCMASTER CARR NO $91732 A 030$

$1-14$ UNF X $11 / 2$ LG. THREADED INSERT W STI TAP

7/8 -14UNF X $15 / 16$ LG. THREADED INSERT W STI TAP

MS-124 DASH\# 664 MCMASTER CARR NO 91732 A549

1 -14UNF X 1 1/2 LG. THREADED INSERT W STI TAP
SHEIL

HEAD

HEAD FLG

SH. HD FLG

LOWER FLG

ADAP. FLG.

INNER TUBE

SPIDER SPTS

MOUNT FLG

INNER TC PAD

TOP HEAD

TOP HEAD

TOP HEAD

TOP HEAD

NOZ A

HEAD GASKET

NOZ A

GUIDE

GUIDE

TC PAD

SUPPORT

SUPPORT

SUPPORT

SUPPORT

NOZ CO1-C25

LOWER BEL

LOWER BEL

TOP BEL

ADAPTER FLG

BELLOWS

BELLOWS

BELLOWS

BEILOWS

ADAP PLATE

ADAP. PLATE

ADAP. PLATE

ADAP PLATE

HD FLG

HD FLG

HD FLG

HD FLG

LOWER FLG

HD FLG
(M)

(M)

(M)

(M)

SA-106B

SA-403-316H

SA-182-F316H

SA516-70

SA516-70

SA-182-316H

INCONEL 617 *

SA-240-304

SA-240-316H

INCONEL 617 *

INCONEL 625 * INCONEL $625^{*}$ INCONEL $625^{*}$

SB-446-N06625

SA-182-F316H

$x * *$

SB-444-N06625

SA-516-70

SA-516-70

SA-240-304

SA-516-70

SA-516-70

SA -516.70

SA-516-70

SA-105

W-SNG1004.9 F*

$\#$

\#

$\star * \star$

SA-193-B7

SA-479-304

SA-193-B8 2

SA-194-B8 2

SA-193-B7

SA-194-2H

STL

18-8 STN STL* 18-8 STN STL*

18-8 STN STL* 
$\begin{array}{l:l:l}44 & 2 & 0\end{array}$

\begin{tabular}{l|l|l}
45 & 4 \\
\hline 46 & 4
\end{tabular}

\begin{tabular}{l|l|l|l}
46 & 4 & 1 & FORGING
\end{tabular}

\begin{tabular}{ll|l|l|l}
47 & 4 & 1 & GASKET
\end{tabular}

\begin{tabular}{l|l|l|l}
48 & 4 & 4 & BOLTING
\end{tabular}

$49 \div 4 \quad 4 \quad$ BOLTING

$\begin{array}{l:l:l:l}50 & 4 & 1 & \text { :PLATE }\end{array}$

$\begin{array}{l:l:l:l}51 & 4 & 1 & \text { GASKET }\end{array}$

\begin{tabular}{l|l|l|l}
52 & 4 & 12 & BOLTING
\end{tabular}

$4: 12$, BOLTING

54 . 4 FORGING

\begin{tabular}{l|l|l|l}
55 & 4 & 4 & BAR/ROD
\end{tabular}

: MS-124 DASH\# 705 MCMASTER CARR NO 91732A027

DELETED

1 1500\# B-16.5 BLIND

$1 / 8$ THKXX11500\# STD RF

7/8-9UNC STUD X 4 3/4 LG

NOZA

NOZA

$7 / 8$-9UNC NUT $X$ HEX HEAVY

NOZ A

NOZA

2 FIN X $131 / 8$ OD

$1 / 8$ THK $\times 9$ OD $\times 8$ ID

$7 / 8-14$ UNC STUD $X 41 / 4 \mathrm{LG}$

SHELL

HYDRO COVER

7/8-14UNC NUT $X$ HEX HEAVY

COVER

COVER

$3 / 4$ NFT PIPE PLUG

80 FIN X 2 FIN OD (BY CUSTOMER)

C1-C25

$11,2,3,4$

* CUSTOMER WILL SUPPLY THIS ITEM

SA-105

GARLOCK 3200

SA-193-B7

SA-194-2H

SA-516-70

GARLOCK 3200

SA-193-B7

SA-194-2H

SA-105

SB-446-N06625"

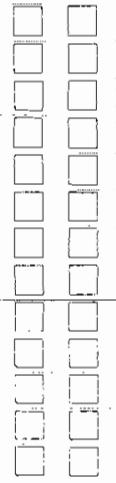




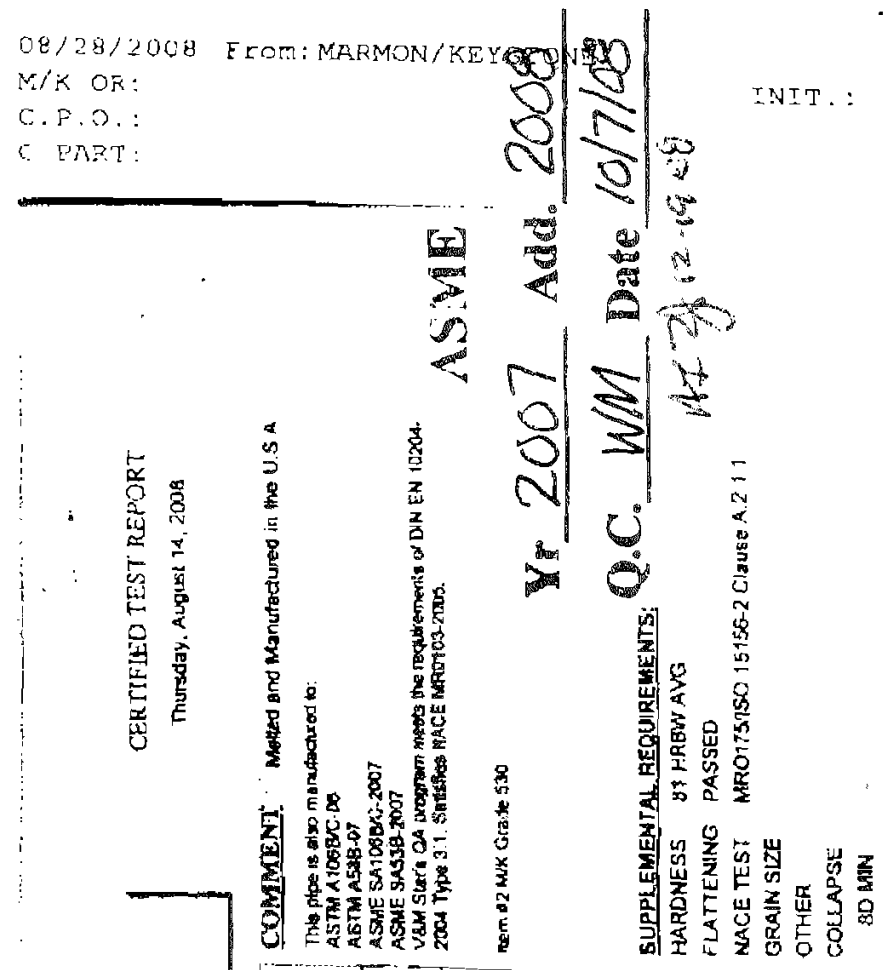

36094 MKI

To:

QR. REF

SLSLRS:

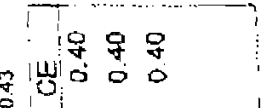

年

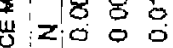

释 訚

15 8

$\infty$

웡

10 0 :

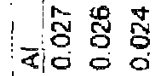

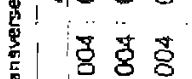

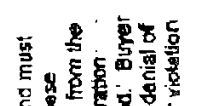

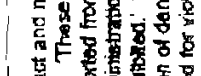

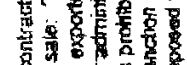

80

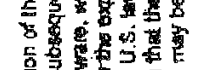

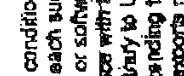

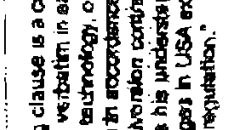

焉

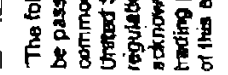

$>1000$

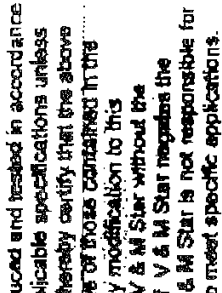

월: 용 :

|
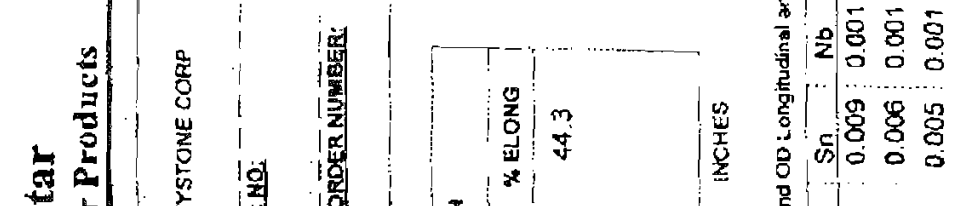

\begin{tabular}{l|l|l}
0 & 0 \\
0 & 0 & 0 \\
0
\end{tabular}

"ร.?

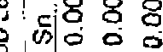

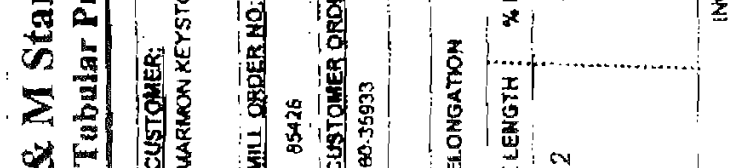

1........

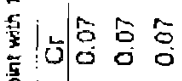

28

w

㲾

a

|....

|

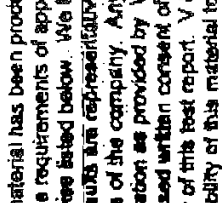

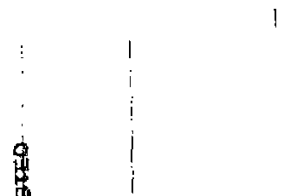

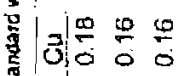

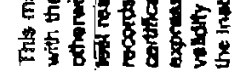

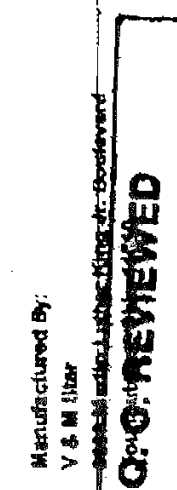

$\overrightarrow{8}$

品

कण

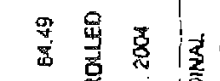

|

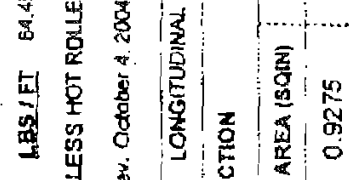

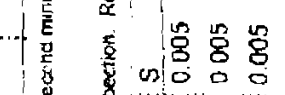

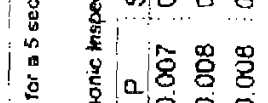

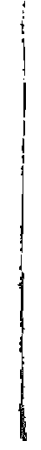

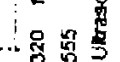

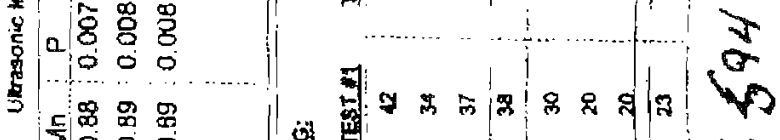

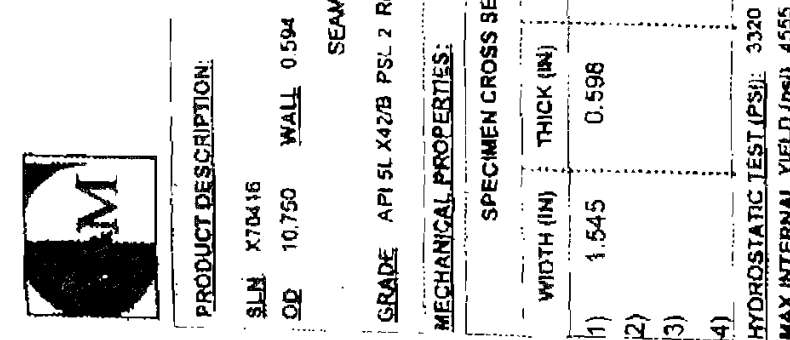

$\begin{array}{llll}2 & 0 & 0 & 0 \\ 0 & 0 & 0 & 0\end{array}$

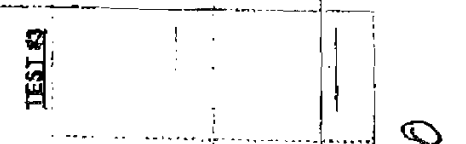

.

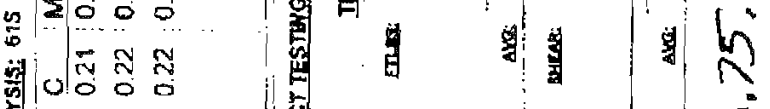

|

i $\quad i$

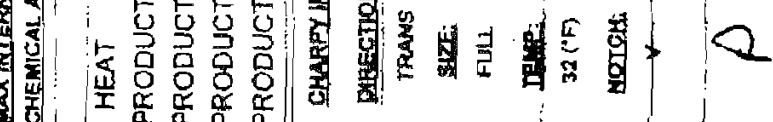

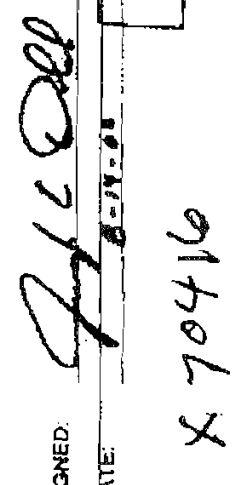

UY IS 
687 COUNTY ROAD 2201

CLEVELAND, TX 77327

PH (281) 727-7000 FAX (281) 727-7062

\section{CERTIFIED MATERIAL TEST REPORT}

\section{CERTIFIED IAW EN10204 3.1 (MADE IN USA)}

TO: GASPAR INC.

PO: 30884

SPECIFICATIONS:
WFF SO NO:
HEAT NO:

12241 45924

\section{DIM/TOL PER ASME B16.5}

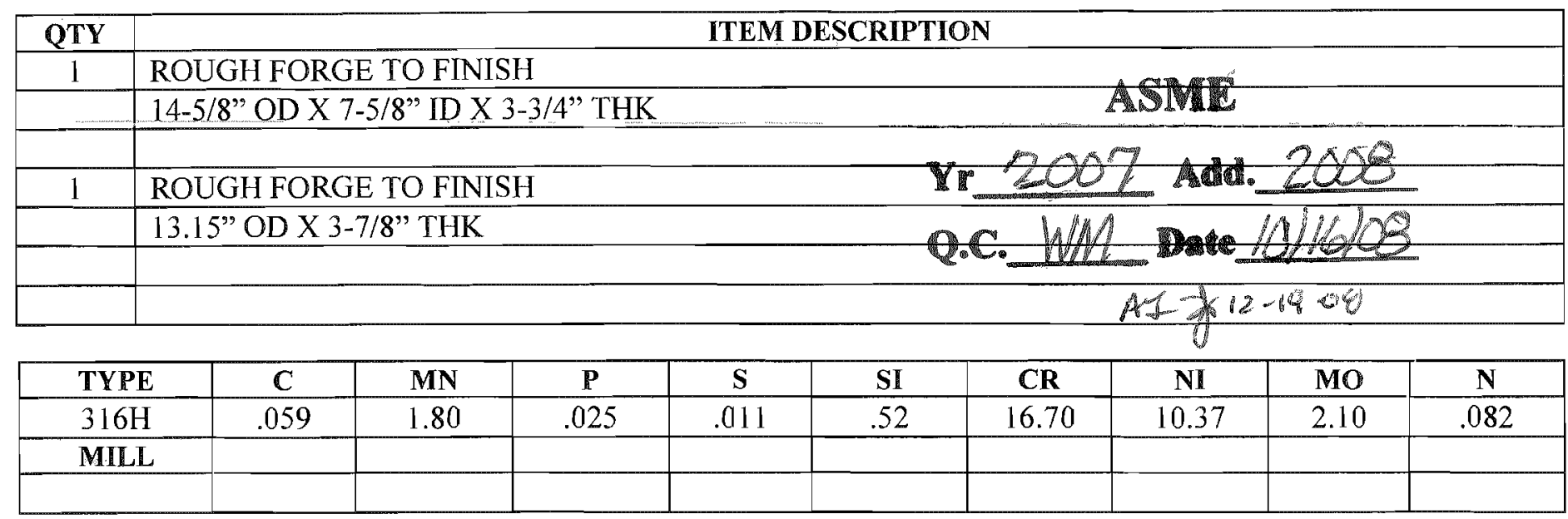

\begin{tabular}{|c|c|c|c|c|c|c|}
\hline HEAT NO. & YIELD . $\%$ OFF-PSI & TENSILE-PSI & \% ELONG & \% RED AREA & HARDNESS & GRAIN SIZE (ASTM) \\
\hline 45924 & 43,800 & 90,300 & 55.1 & 71.7 & & No. 5.0 \\
\hline & & & & & & \\
\hline & & & & & & \\
\hline
\end{tabular}

- NO WELDING PERFORMED ON THE FORGINGS OF THIS ORDER.

- MATERIAL HEREIN IS CERTIFIED FREE FROM ANY FORM OF MERCURY, RADIUM OR ALPHA PARTICLE CONTAMINATION

THROUGHOUT ALL PHASES OF MANUFACTURE AND SHIPMENT.

- THE FORGINGS ON THIS ORDER CONFORM TO THE SPECIFIED DIMENSIONAL REQUIREMENTS.

- MATERIAL MANUFACTURED IAW WFF QA PROGRAM QAM-WF07.0702.

- Solution annealed @ $1950^{\circ} \mathrm{F}$ and water quenched to below $300^{\circ} \mathrm{F}$ within less than three minutes.

WE CERTIFY THE MATERIAL DESCRIBED HEREIN HAS BEEN MANUFACTURED, TESTED AND EXAMINED IAW AND MEETS ALL THE REQUIREMENTS OF ABOVE PURCHASE ORDER AND REFERENCED SPECIFICATIONS.

RATE
ROSE KAY, QUALITY ASSURANCE ASSISTANT
WESTERN FORGE \& FLANGE CO.




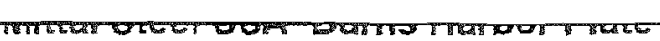
OUALITY ASSUZAFNEE
REPART OF TEST AND ANAYLES

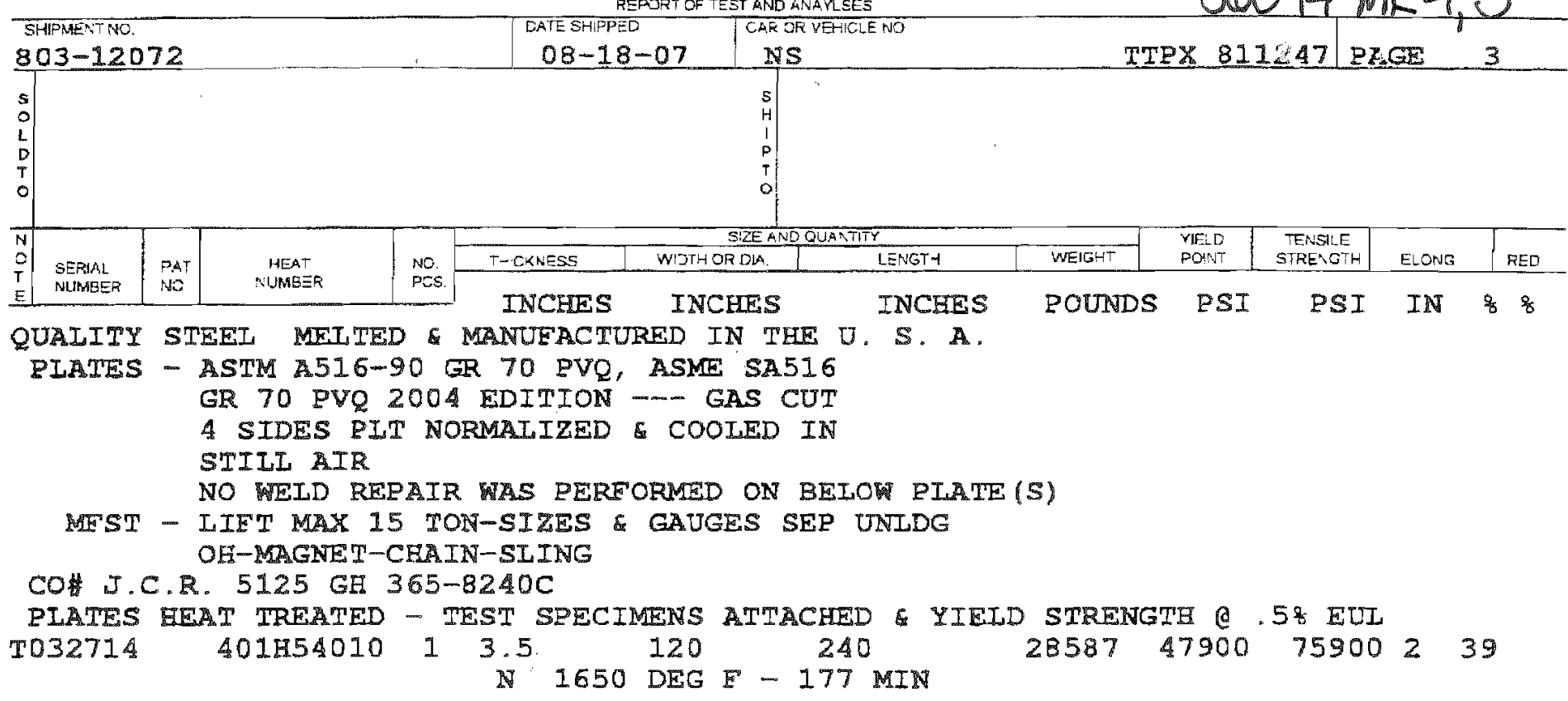

ASME
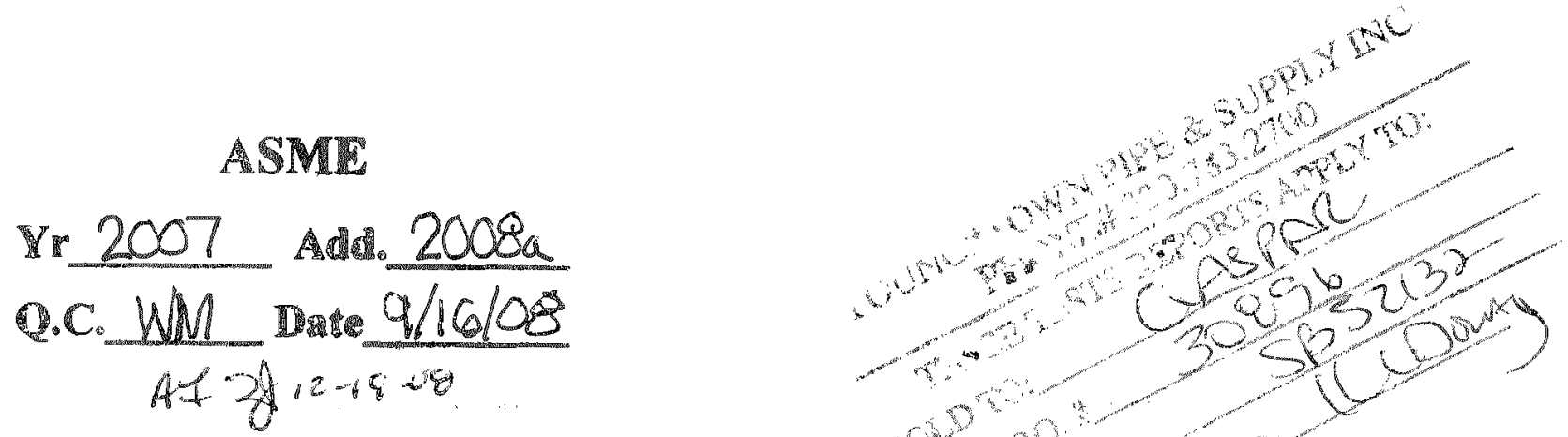

\begin{tabular}{|c|c|c|c|c|c|c|c|c|c|c|c|c|c|c|c|c|c|c|}
\hline \multirow{3}{*}{$\begin{array}{l}\text { SERIAL } \\
\text { FLIMIER }\end{array}$} & \multirow{3}{*}{$\begin{array}{l}\text { PAT } \\
\text { NO }\end{array}$} & \multirow{3}{*}{$\begin{array}{l}\text { HEAT } \\
\text { NUMBEF }\end{array}$} & \multirow{3}{*}{ HARE } & \multirow{3}{*}{ BENAD } & \multicolumn{14}{|c|}{ CHARPY IMPACT } \\
\hline & & & & & \multirow{2}{*}{$\begin{array}{r}\text {-HEKNESS } \\
\text { INCHES }\end{array}$} & \multirow[t]{2}{*}{ TYPE } & \multirow[t]{2}{*}{$32 E$} & \multirow{2}{*}{\multicolumn{2}{|c|}{ EIR \begin{tabular}{c|c} 
TEST \\
TEWD \\
\\
E
\end{tabular}}} & \multicolumn{3}{|c|}{ ENERGET LBS } & \multicolumn{3}{|c|}{ SHEARI\$? } & \multicolumn{3}{|c|}{ LAT EXP MIIUS } \\
\hline & & & & & & & & & & 1 & 2 & 2 & 7 & 2 & 3 & 1 & 2 & 3 \\
\hline
\end{tabular}

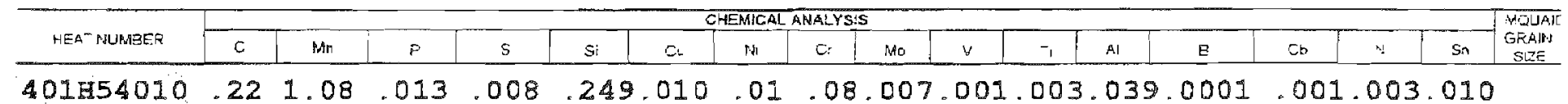

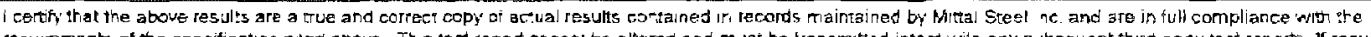

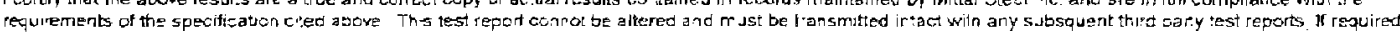

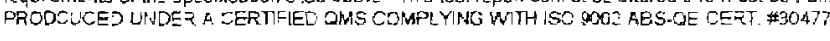

SUFV OUALTY ASSJRANCE

D. W. ELWOOD PER

WTRE
} 


\section{cals 1 CERTIFICATE OF CONFORMANCE

25 Gregg Street sway, CA 92064

PHONE 858-513-1300 FAX 858-513-6600

\begin{tabular}{|c|c|c|c|c|}
\hline $\begin{array}{l}\text { Customer } \\
\text { ARIZONA PUBLIC SERVICE CO }\end{array}$ & $\begin{array}{l}\text { P. No. } \\
700517805\end{array}$ & $\begin{array}{l}\text { VM Job No. } \\
33628 \text {. }\end{array}$ & $\begin{array}{l}\text { Pack slip No } \\
33628\end{array}$ & $\begin{array}{l}\text { Date } \\
2 / 20 / 2008\end{array}$ \\
\hline $\begin{array}{l}\text { Material } \\
\text { ALLOY } 617 / \text { AMS } 5889 \mathrm{~B}\end{array}$ & $\begin{array}{l}\text { Heat No. } \\
8617-4-8870\end{array}$ & $\begin{array}{l}\text { Specification } \\
\text { N/A }\end{array}$ & & \\
\hline
\end{tabular}

Quantity

$5 \mathrm{PCS}$

Description CUSTOMER FURNISHED MATERIAL

$2.0000 \times 125 \times 4$ R/LWELDED TUBNG $476^{\prime \prime} 45^{\prime}$

NELDED PER MPS 101 WITHOUT FLLLR WIRE

WE HEREBY CERTIFY THAT THE MANUFACTURED ITEMS STATED ABOVE ARE IN CONFORMANCE WITH THE APPLICABLE SPECIFICATIONS AS REQUIRED BY THE PURCHASE ORDER AND SUPPORTING DOCUMENTS. SPECIFIED PROCESSES

REQUIRING CERTIFICATION ARE LISTED BELOW OR ARE INCLUDED IN

ATTACHMENTS.

THIS MATERIAL HAS NOT COME INTO CONTACT WITH MERCURY OR LEAD

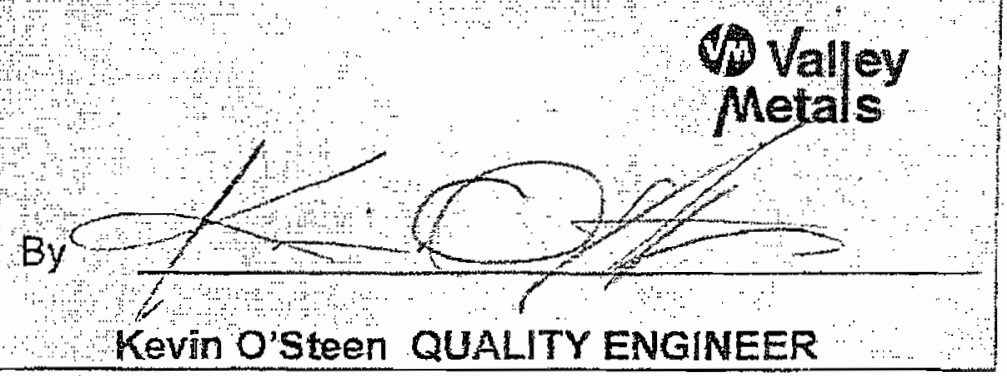

e informalton contalned in this test report represents only the material submitted to andtor tested by Valtey Melals. This document shail not be reproduced cept in full, without written approval of Valley Metals Quality Assurance. The recording of false, fictitious, or fraudulent statements or entries on the rtincate may be punished as a felony under tederal law. 


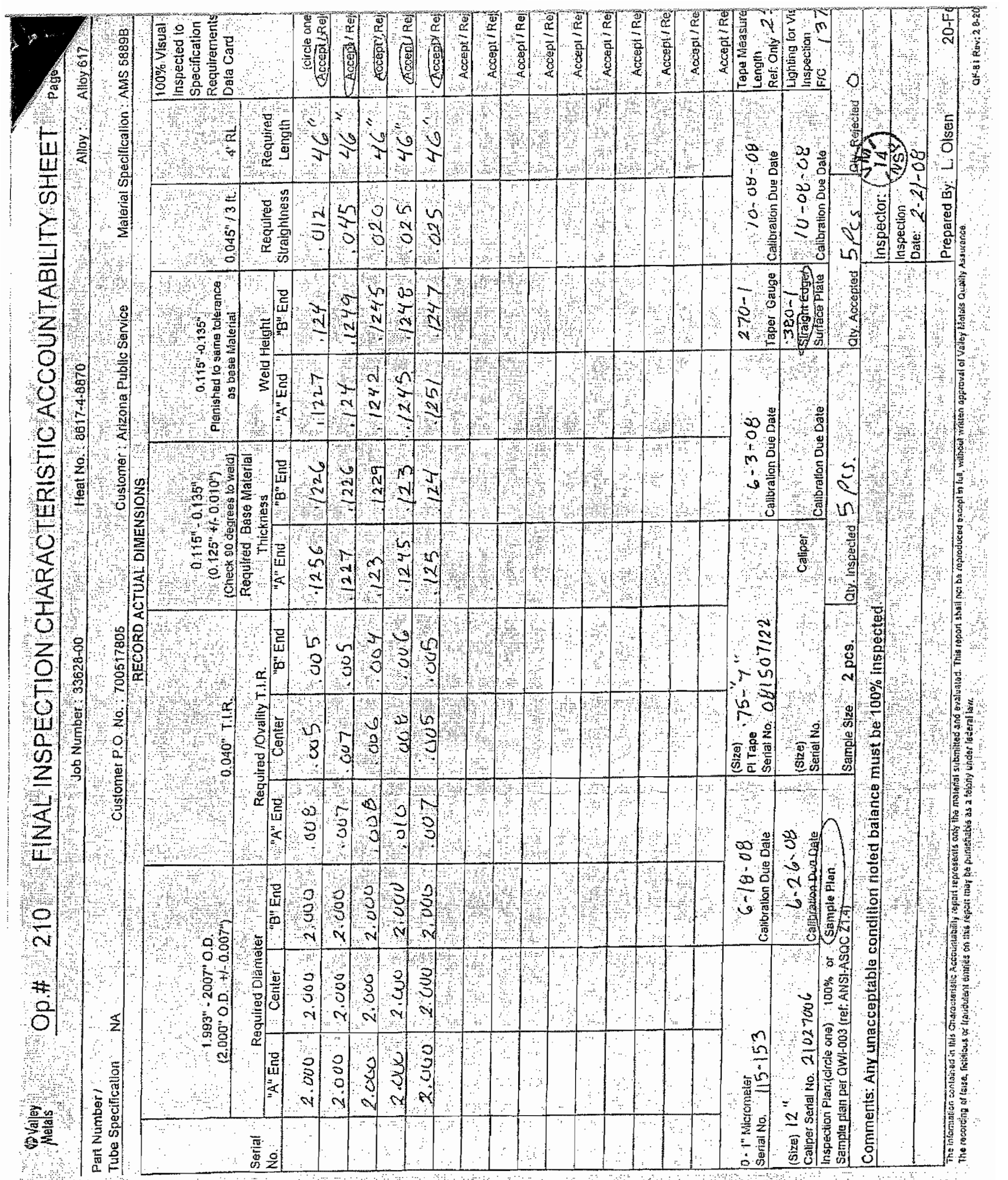



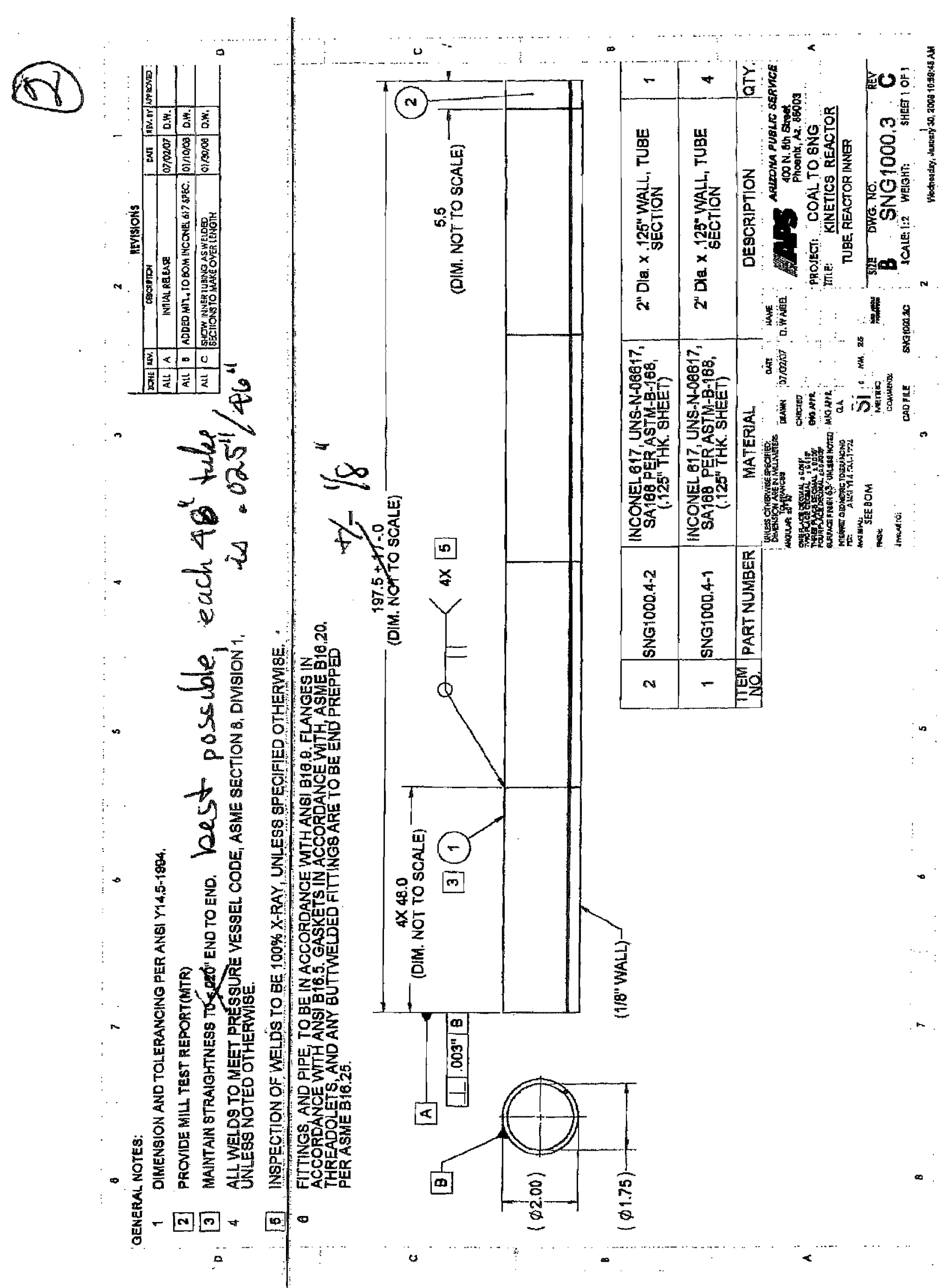


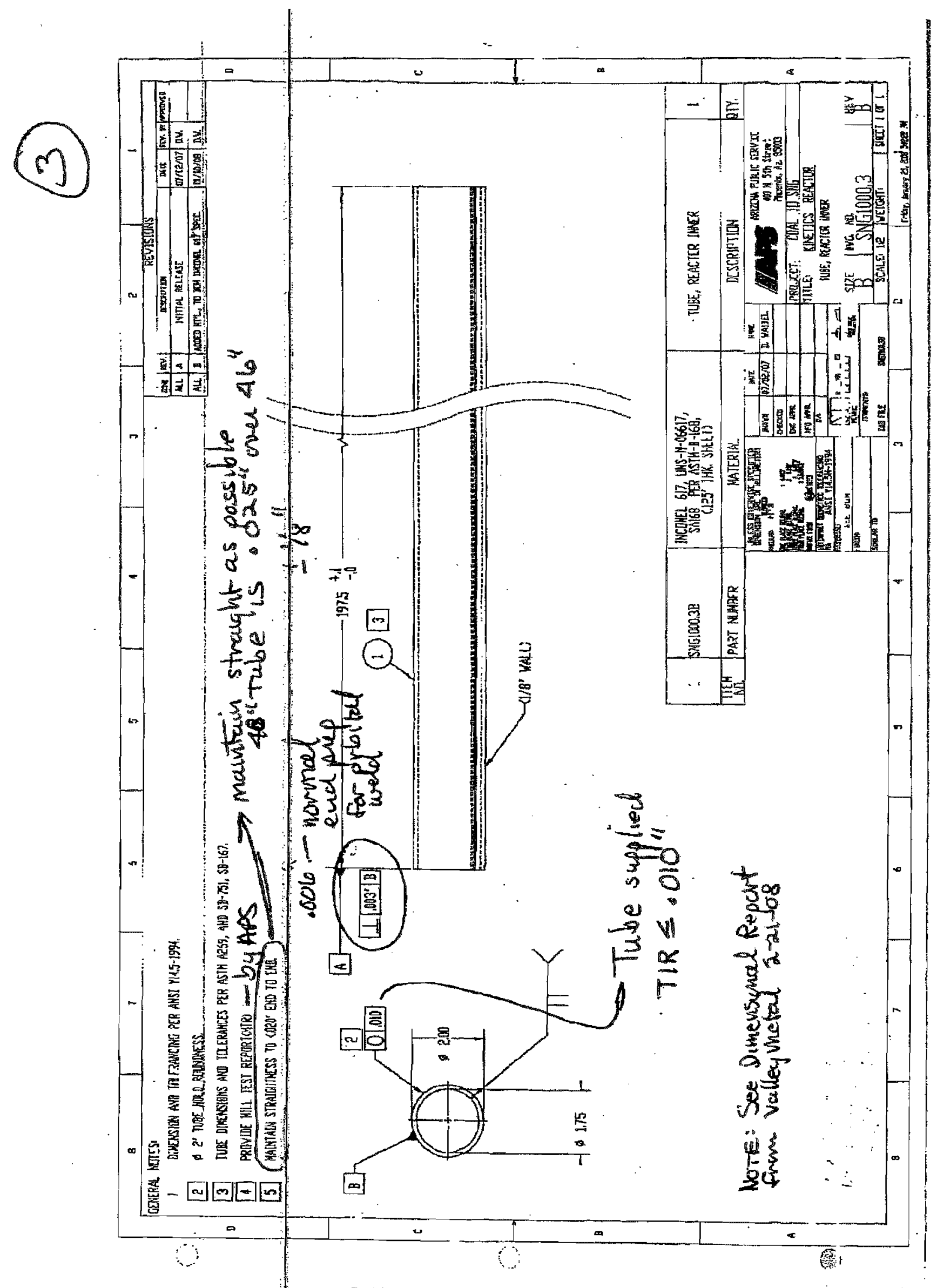




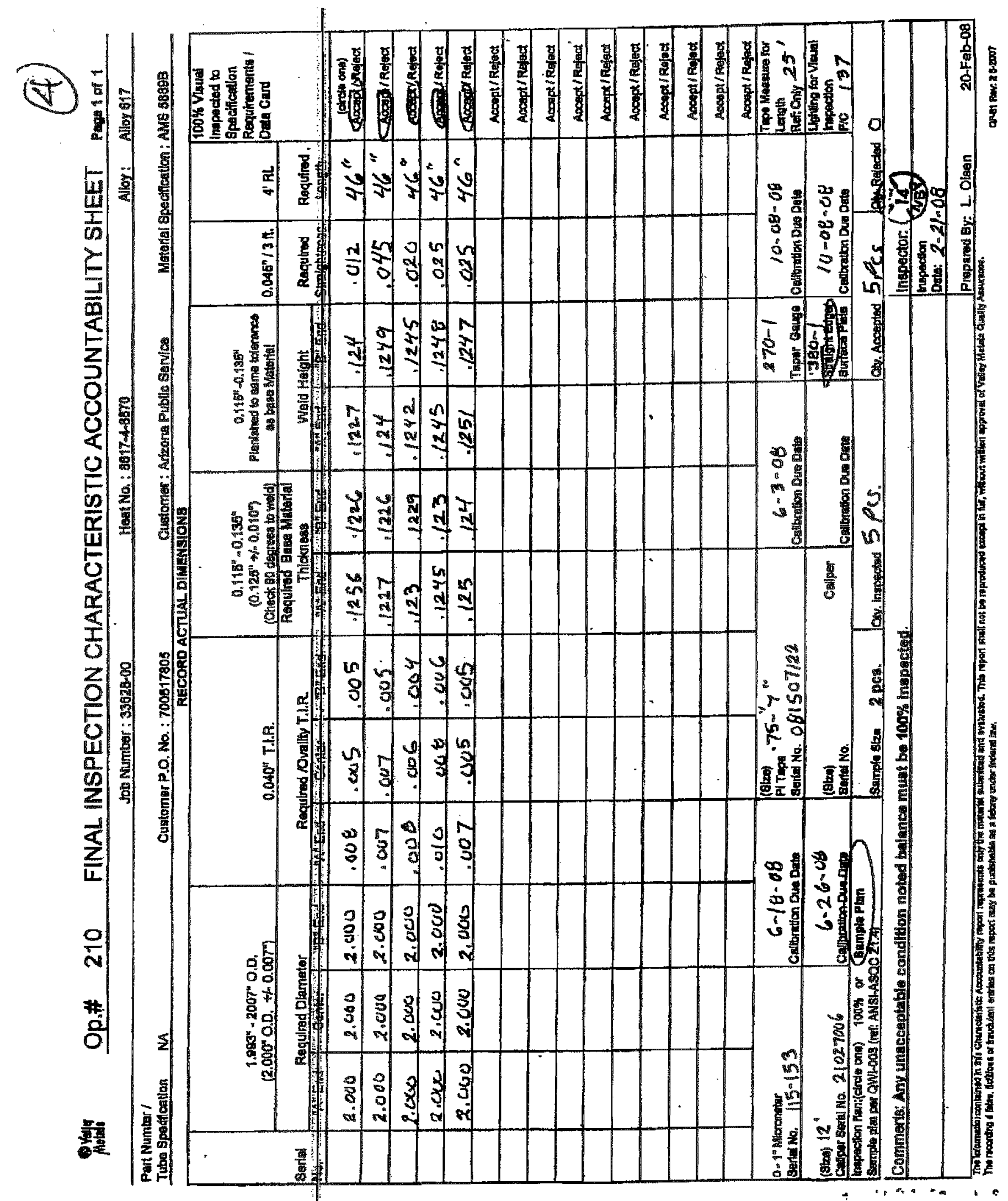




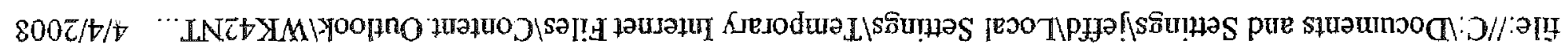

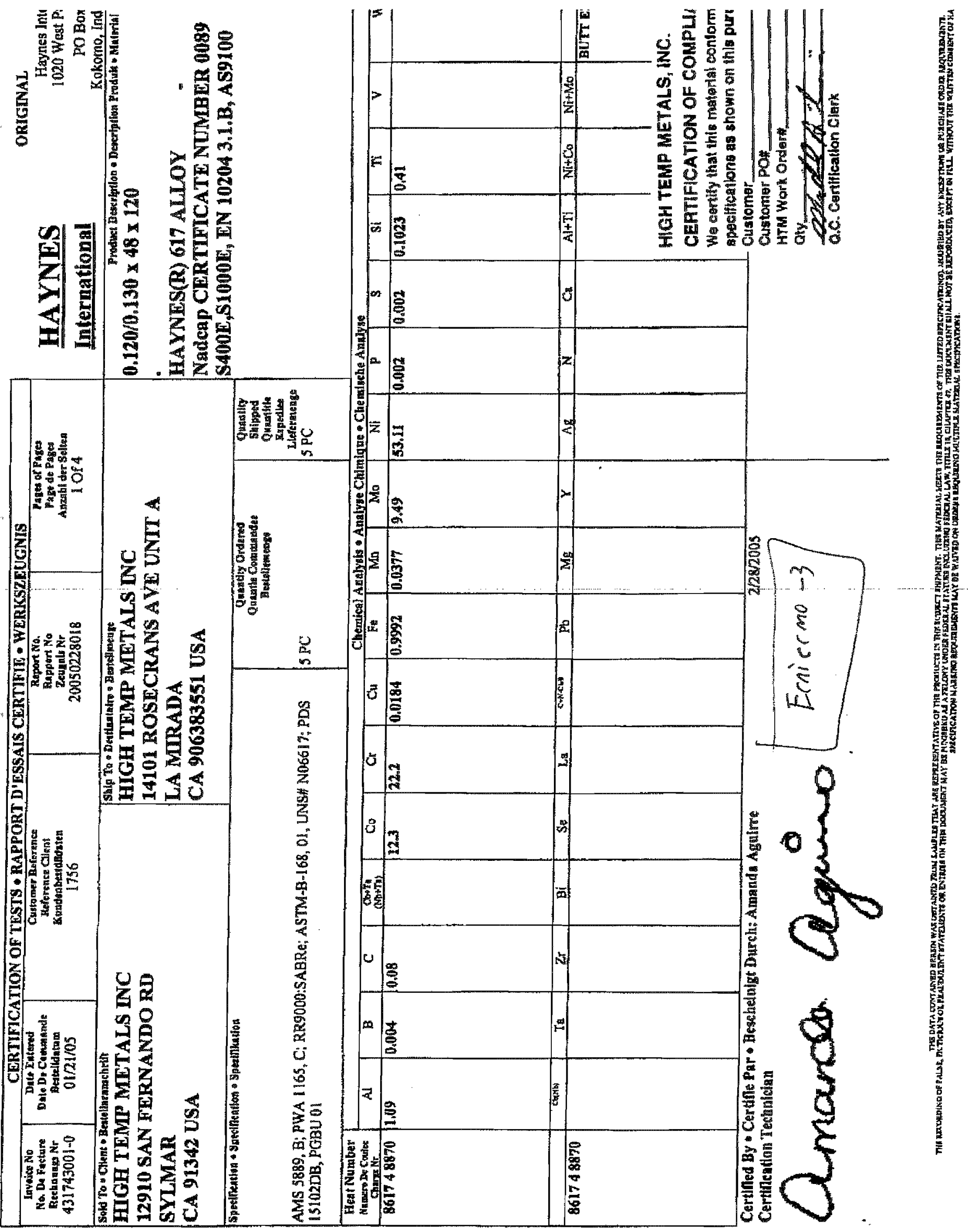




\section{VITI VALLEY INSPECTION SERVICE INC.}

759 N. Fenwick St., Allentown, PA 18109

Phone: $610-782-9310$

Fax: $610-782-9309$

RADIOGRAPHIC INSPECTION REPORT

Customer: $\mathrm{CV} / \mathrm{P}$

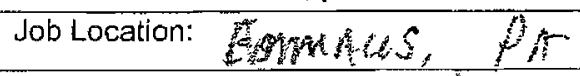

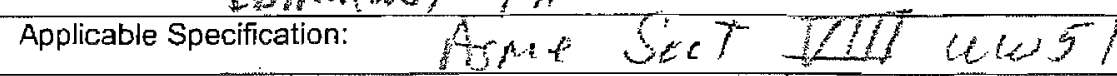

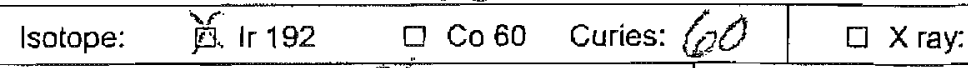

Mt. Type: $\quad 3 / s \quad$ Mtl. Thickness: 125

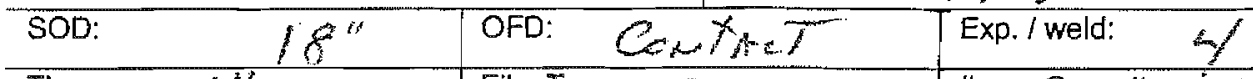

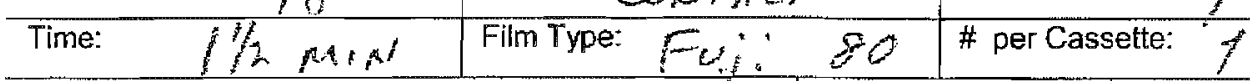

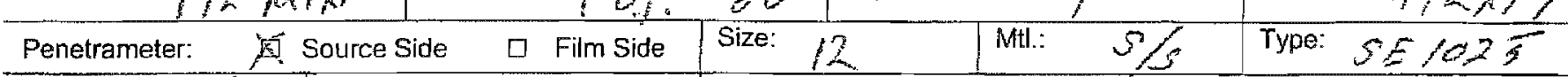

\begin{tabular}{l|l|l}
\hline Shim MLl: $5 / 5$ & Shim Thk. 250 & Technique Used $1 / 5 /-17 \%+100-6$ \\
\hline
\end{tabular}

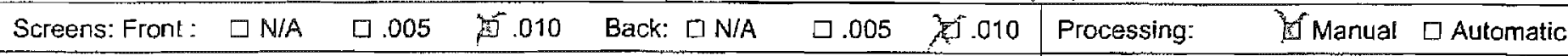

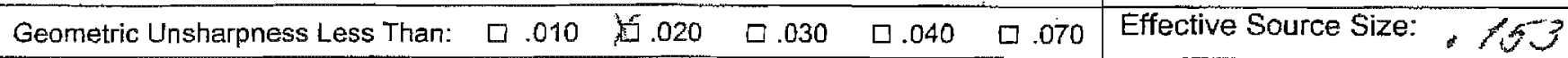

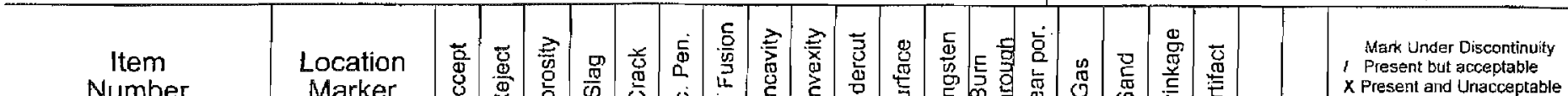

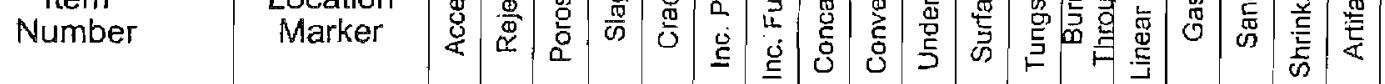

Remarks/Indications

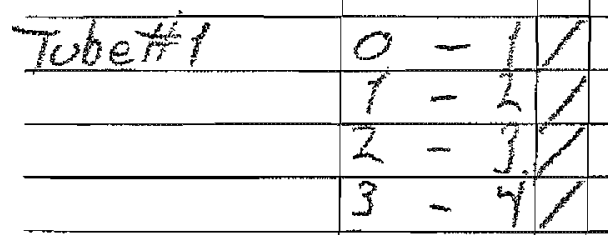

$\begin{aligned} 0 & -1 / 4 \\ 1 & -2 / 2 \\ 2 & =37 \\ 3 & -47\end{aligned}$

Tubet?

Tobet
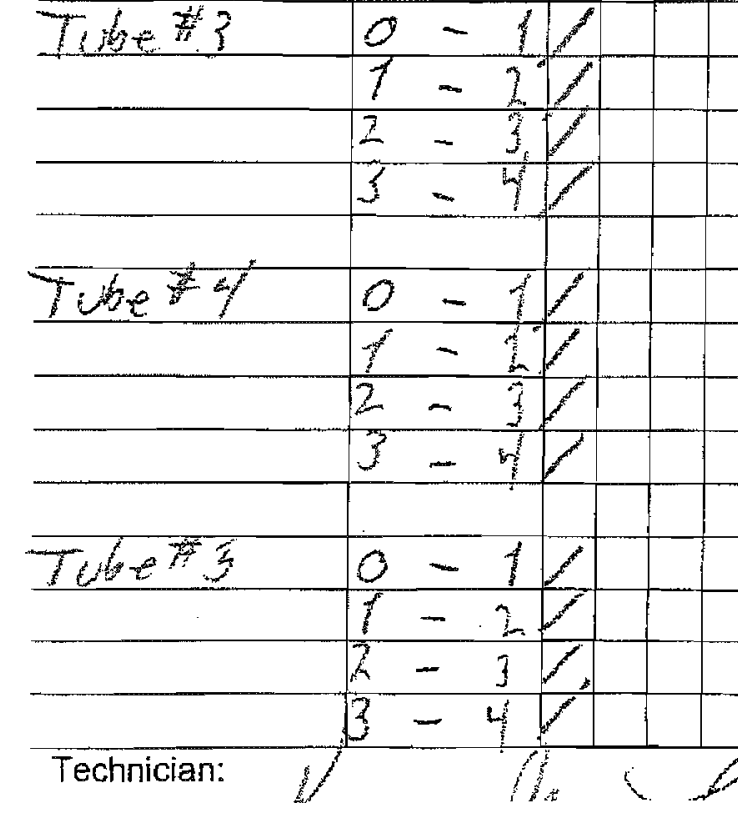


\section{$\sqrt{\text { ISI }}$ \\ VALLEY INSPECTION SERVICE INC.}

759 N. Fenwick St., Allentown, PA 18109

Phone: 610-782-9310

Fax: $610-782-9309$

RADIOGRAPHIC INSPECTION REPORT

Customer: $\mathrm{CHCP}$

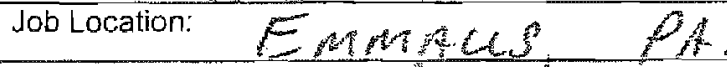

Applicable Specification: Amate Sec. $f, 3 / 3$

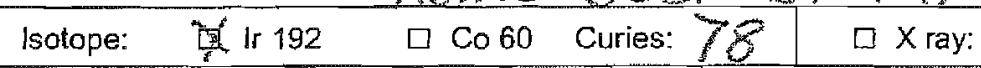

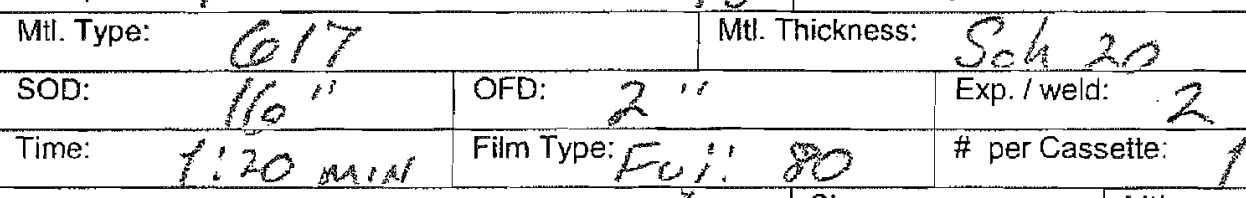

\begin{tabular}{ll|l|l|l|l|}
\hline Penetrameter: 図 Source Side $\square$ Film Side & Size: $/ 2$ & Mtl.:
\end{tabular}

Job\# 3408

Page $\quad l$ of $l$

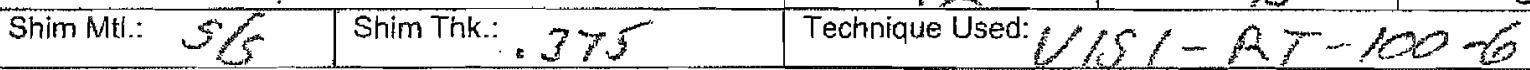

Screens: Front: $\square$ N/A $\square .005$ 商.010 Back: $\square$ N/A $\square .005 \quad$ 国.010 Processing: 酋Manual $\square$ Automatic

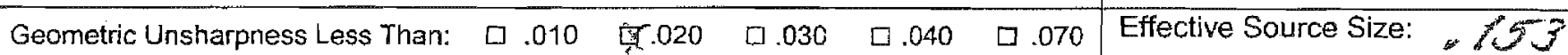

\begin{tabular}{|c|c|c|c|c|c|c|c|c|c|c|c|c|c|c|c|c|c|}
\hline $\begin{array}{l}\text { Item } \\
\text { Number }\end{array}$ & $\begin{array}{l}\text { Location } \\
\text { Marker }\end{array}$ & $\bar{q}$ & 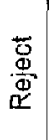 & $\begin{array}{l}\overrightarrow{\tilde{D}_{0}} \\
\overrightarrow{0}\end{array}$ & $\frac{0}{0}$ & 造 & 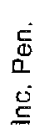 & 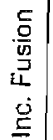 & 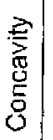 & 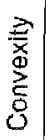 & 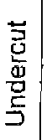 & 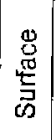 & $\begin{array}{l}\text { \$ } \\
\text { 兽 } \\
\text { 点 }\end{array}$ & 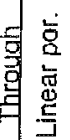 & 8 & 趸 & 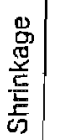 \\
\hline
\end{tabular}

Jo6\%3408

\begin{tabular}{|c|c|}
\hline$I 0 \# 1$ & $0^{\circ}$ \\
\hline$\omega-76$ & $90^{\circ}$ \\
\hline $70 \% 2$ & \\
\hline$\omega-76$ & $70^{\circ}$ \\
\hline
\end{tabular}

$k V$ :

$\mathrm{mA}:$ Size:

Diallength: $\frac{-12}{2}$

Film Size: $4 / 2 \times / 0$

Type: $5=1025$ 


\section{VISI \\ VALLEY INSPECTION SERVICE INC.}

759 N. Fenwick St., Allentown, PA 18109

Phone: $610-782-9310$

Fax: $610-782-9309$

RADIOGRAPHIC INSPECTION REPORT

Customer: $\quad C H I F$

Job Location: EMMA-CG,

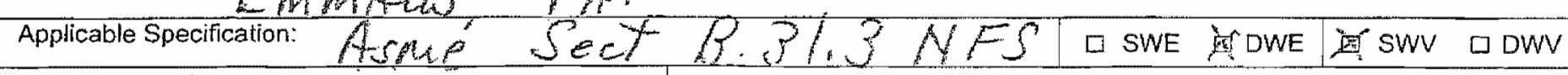

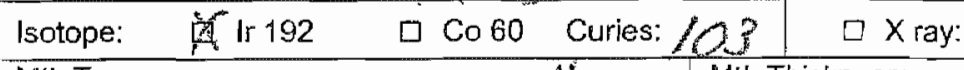

Mti. Type: 617 LNCQMell Mil. Thickness: 120

SOD: $16^{\text {if }}$ OFD: 2 OF"

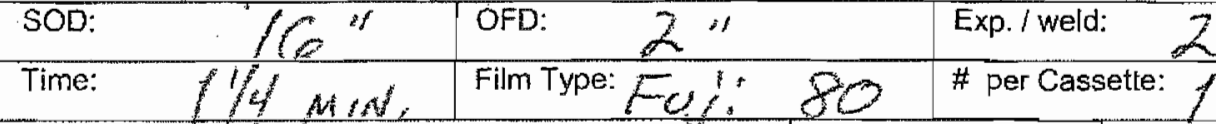

\begin{tabular}{l|l|l|l|l|}
\hline Penetrameter: & Mtl.:
\end{tabular} $k \mathrm{~V}$ :

mA: Size:

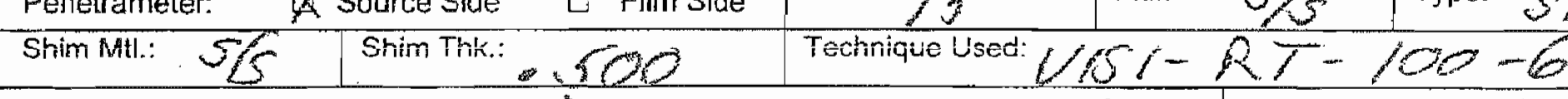

Screens: Front: $\square$ N/A $\square .005 \quad 0.010$ Back: $\square$ N/A $\square .005$ T.

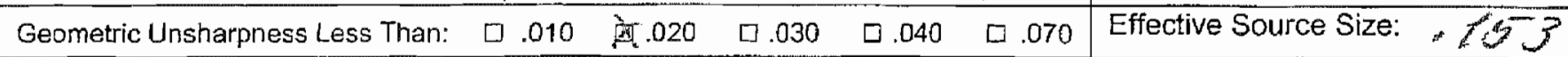

\begin{tabular}{|c|c|c|c|c|c|c|c|c|c|c|c|c|c|c|c|c|c|c|}
\hline $\begin{array}{l}\text { Item } \\
\text { Number }\end{array}$ & $\begin{array}{c}\text { Location } \\
\text { Marker }\end{array}$ & $\begin{array}{l}\text { 音 } \\
\text { 品 } \\
\end{array}$ & 薄 & 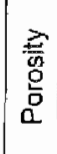 & $\frac{\text { 票 }}{\omega}$ & 惫 & 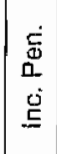 & & 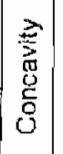 & 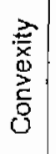 & $\begin{array}{l}\breve{\vec{u}} \\
\stackrel{\mathrm{g}}{\mathrm{g}} \\
\text { 宂 }\end{array}$ & 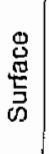 & 点 & 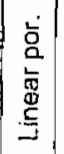 & 占 & $\begin{array}{l}\text { 鬲 } \\
\text { o }\end{array}$ & 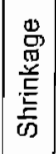 & 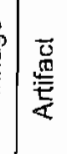 \\
\hline
\end{tabular}

3408

700511190

77

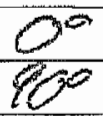

$\frac{1}{F}$

$0^{\circ}$

Technician:

$1 \times 9$

Level: 78

Date: $8-22-08$ 


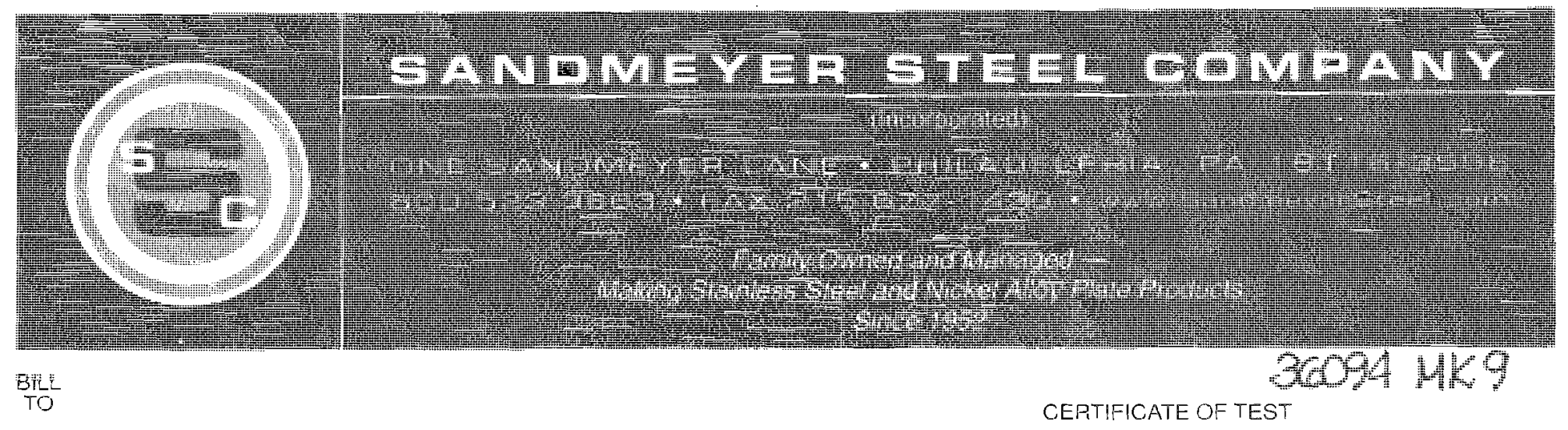

GASPAR INC.

1545 WHIPPLE AVE S.W. CANTON, OH 44710

CUSTOMER ORDERNO. 38885

DATE: $\varnothing 9 / 10 / 2008$
WE CERTIFY THAT THE CHEMICAL ANALYSIS ANO MECHANICAL TEST RESULTS APPEARING IN THIS CERTIFICATE ARE CORRECT AND TAUE AS REDORTED BY THE MANUFACTURER.

SANDMEYER STEEL COMPANY

T. BOHNSACK - MANAGER, QUALITY ASSURANCE QUALITY CONTROL DEPARTMENT

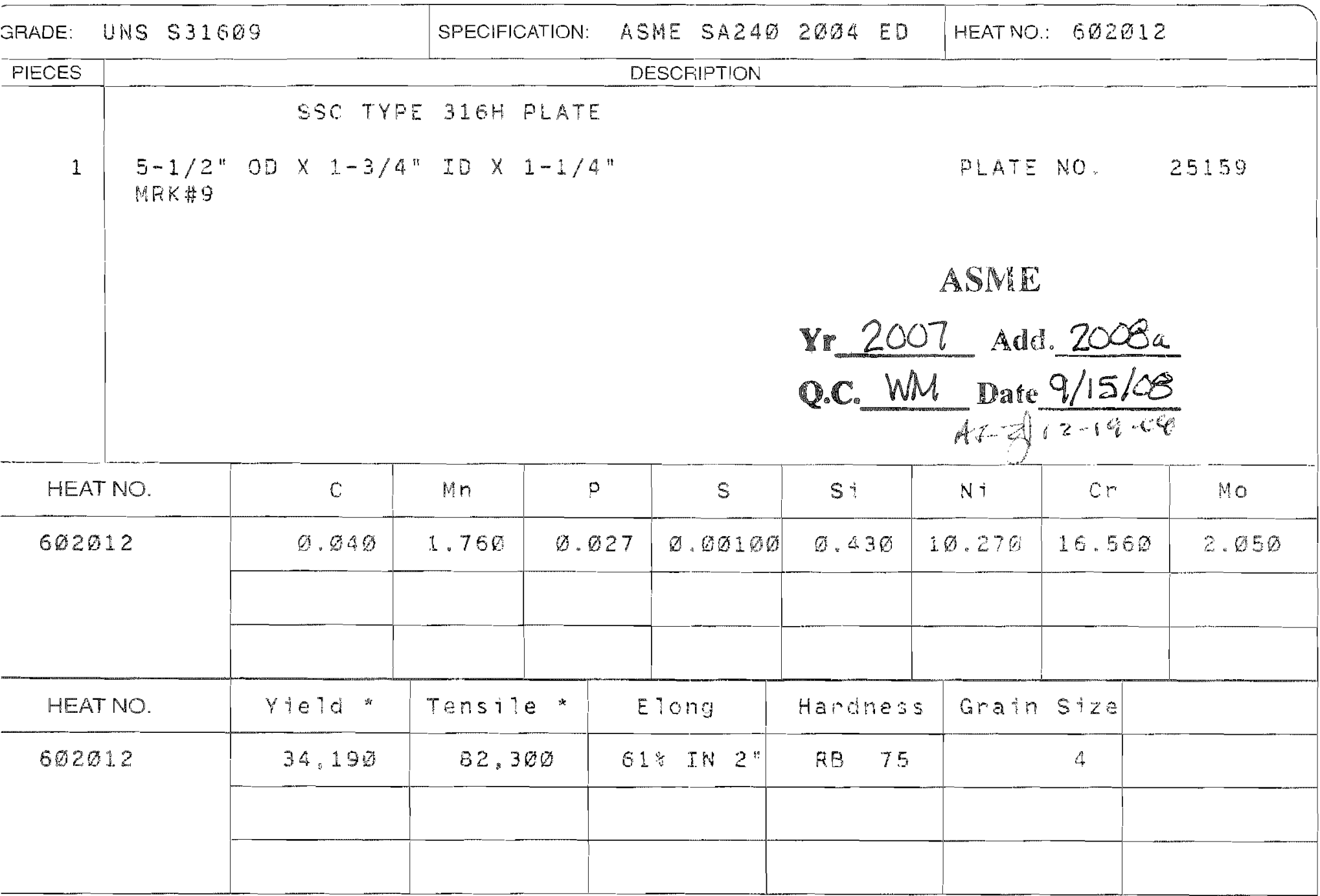

* LQS/IN2

MATERIAL SOLUTION ANNEALED AT 1950 DEGREES E MTNIMUM

AND WATER QUENCHED OR RAPIDLY COOLED BV AIR

MATERIAL MANUFACTURED EY AVESTAPOLARIT PLATE

THIS MATERIAL IS FREE FROM MERCURY CONTAMINATION 


\section{Avestapolarit Inc. \\ Plate Products}

Avestapolarit

An Oufokuvau Group coimpony

\section{Trrtificate of Antulusis and Trats}

OUR ORDER 232498\%O

HEAT \&PIECE $6020125 \mathrm{~A} / 3 / 31 / 03$

SOLD TO SANDMEYER STEEL COMPANY ONE SANDMEYER LANE

PHILADELPH.IA

PA 19116

SHIP TO SANDMEYER STEEL COMPANY ONE SANDMEYER LANE

YOUR OROER E DATE

PA 19116

60371

$0 / 00 / 00$

HEAT \& PIECE 602012

WEIGHT

FINISH

GRADE

DIMENSIONS
7292

1

$$
316 \mathrm{H}
$$

** MFG TN NEW CASTIE, IN, USA ASTMA240-01 ASMESA240-0102AD NO GRIPPER MARKS ASTM A262-01 PRAC A
ITEM DESCRIPTION

UNS - $\$ 31609$

254.000 EXAOT

25159

FROM SIABS IMPOETED FROM SWEDEM ASTMA480-01A ASMESA480-02AD NO WELD REPAIRS ASTM A262-01 PRAC E

PLATES \& TEST PCS SOLETION RNNEALED @ 1950 DTGRESP FAHRENIEIT MINIMUM.

THEN WATIR COOLED OR RIPIDLY COOLED BY AIFW

FREE OR KNOWN MERCURY CONTAMINATION

HOT ROLLED, ANNEALED \& PICKLED (HRAP)

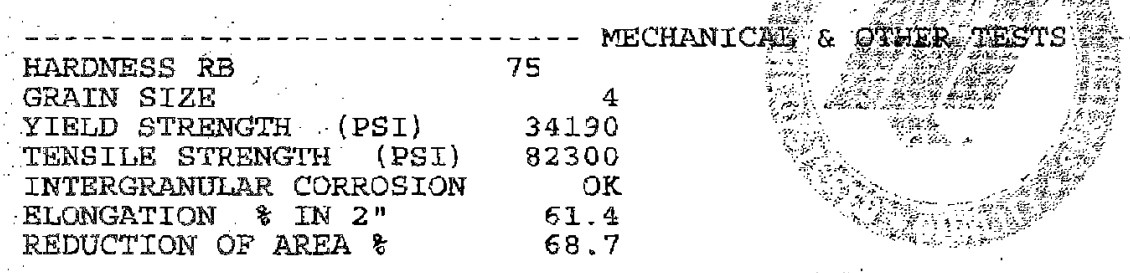

REDUCTION OF AREA :

CHEMICAT COMEOSTTION

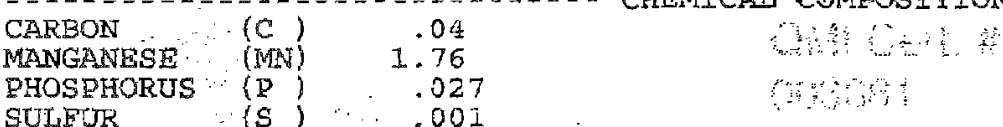

CHROMIUM (CR) 15.56

NICKEL $\quad \therefore$ (NI) 10.27

COBALT . (CO) . . . (CO

COPRER $\cdots($ CU) .27

MOLY (MO) 2.05

NTTROGEN : . (N) $\quad .04$

COLUMB IUM $\quad .006$ (CB)

IITANIUM $\cdots$ (TI)

ALINININUM $\ldots$ (AL) $\quad .003$

TIN $\quad$ (SN) .008

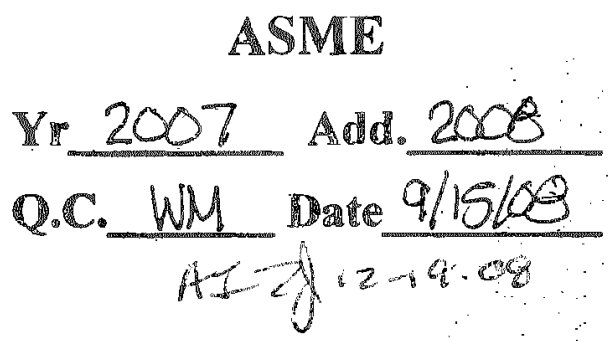

ASME

KNOWINGLY \& WILIFULLY FAISIFYING OR CONCEALING A MATERIAL FACT ON THIS FOFM, OR MARING FALSE, FICTITIOUS OR FRAUDULENT STATEMENTS OR REPRESENTATIONS HEREIN COULD CONSTITUTE I FELONY PUNISHABLE UNDER FEDRRAL STATUTES.

JAMES DOUBMAN, QUALITY ASSURANCE MANAGER

CERTIFICATE IN CONEORMANCE WITH DIN50049 3.1.3/EN10204 3.1.B

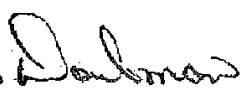

\section{Avestatholarit, Inc.}

Plate Prostucts

P.O. Box 370

New Castla, Indiana 47362 
CERTITICATE OF TESTS

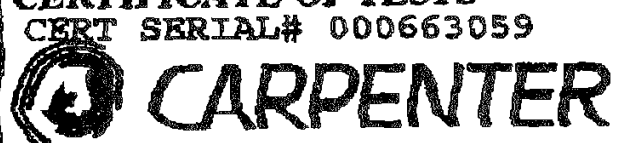

Carpenter Tochnology Compontion

101 West Bem Stret Reading, Pa. 19601

Tiel: (610) 2082000 (200) 33:-4522

\section{$09 / 17 / 08$}

CUSTOMER/AESTIUUV/CIIENT

FRY STEEL COMPANY

ATTW: IIARRY LONG

13325 MOLETTES ST

SANTA FE SPRING CA 90670

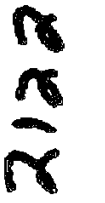

\section{ABNAHMEPRUETLEUGNIS}

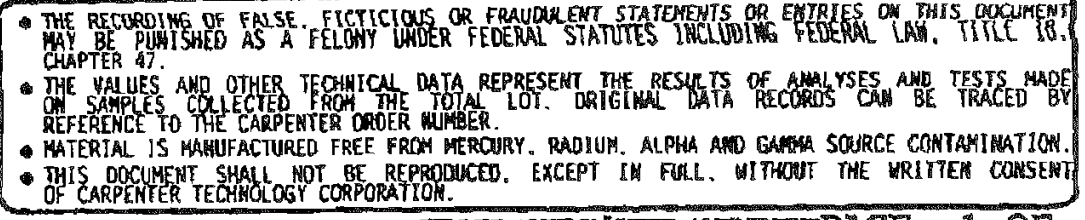
SEIIER/VER A UDER/VENDEURPAGE 1 OF 1

STOWER DRDER WO, /BESTELL-HR. /W DE COMWAMOE

$44999-7$ S\# 19870

$-\infty$
CARPEETER MO./HERKS-MR./T OE REFEREMCE INTERSE $\$ 86227-1$ 212279

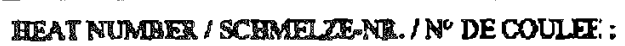

\section{9}

PRODUCT DESCRTPTION: PYROMET 625 HI TEMP AIIOY ANNEATED GROUND

SPECIFICATION: AMS 5666 REV T (05/,106)

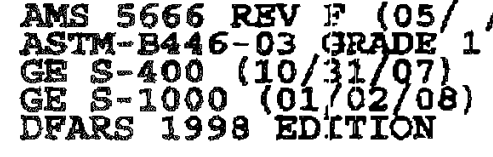

SIZE 3.000000 IN.(76.20 MV) RD BAR

TNGOT 2

PRIMARY HEAT CHEMISTRY (RT\%; : (TEST METHOD IS SHOWN IN PARENTHESIS)
0. 0 (COM)
0.02 (MRP)
0.02 (XR)
$0.002^{\text {(COM) }}$
LI .001
22.36
$60.79(X R F)$
$8.76($ XRE)
0.02 (XRP)
0.16 (OES)
$0 . \frac{1}{3} \frac{1}{1}$ (XRE)
3. ${ }_{5 B}$ (XRF)

RAN

FFY STEEL CO. CERTIFIES THAT THIS IS A TRUE COPY OF THE ORIGINAL MHL TEST PEPOAT NOW ON FILE
RECEIVED AND INSPECTED

GEAG SOPPLIER CODE 21100 / CARPENTER

\section{HARDNESS AS SHIPPBD $216 \mathrm{HB}$ ( $96 \mathrm{HRB}$ )}

YIRLP STREHYTH $(0.20$ \%) RSI (MPA)

BENSILESTREMGTH, KSI (MPA)

GRAIN SIZE PER ASTM B112: 8

5 MI K202 (30\%), $100 \mathrm{ML}$ HCL

\section{(MIDRADIUS) OCT 12008} $\left.\begin{array}{r}63.5 \\ 127.0 \\ 50.0\end{array} \quad \begin{array}{l}438 \\ 876\end{array}\right)$

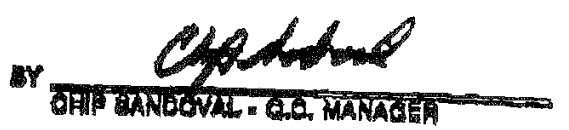

MATERIAI PRODUCED ON THIS OFDER MAS MELTED AND MANUFACTURED IN THE U.S.A.

MATERIAI HAS BEEN MELTED IN USA OR OUAI IFYING COUNIRY TO DFARS REQUIRE:-

MENTS $252,225-7014$ NITH ALTHANATE 1 FOR OUALIFYING COONIRY 225.872 . 1 .

CARPENTER 'S QUALITY MANAGEMIFNTT SYSTEM WAS REGTSTERED AS OF SBPTEMBER 2 2004 TO THE REOUIREMENTS OF ISO $9002: 2000$ APPROVAL CERTIFICATZ 07 -0869 BY PERFORMANCE REVIEW INSTITUTE CERTIFICATI OF TEST IS PREPARED IN ACCORDANCE WITH PARAGRAPH R 1 OF FN 10204 (DIN 50049 ). WE HBREBY CERTIFY THAT THE ABOUE TBST DATA ARE IN ACCORDANCE WITH THE PURCIUSE ORDER AND SPECIFICATION REQUIREMINTS.

MARGARET A TURNER MET RELEASE/REOUIREMENTS ANATYST CARPENIDER TECHAOLOGY CORPORATION 


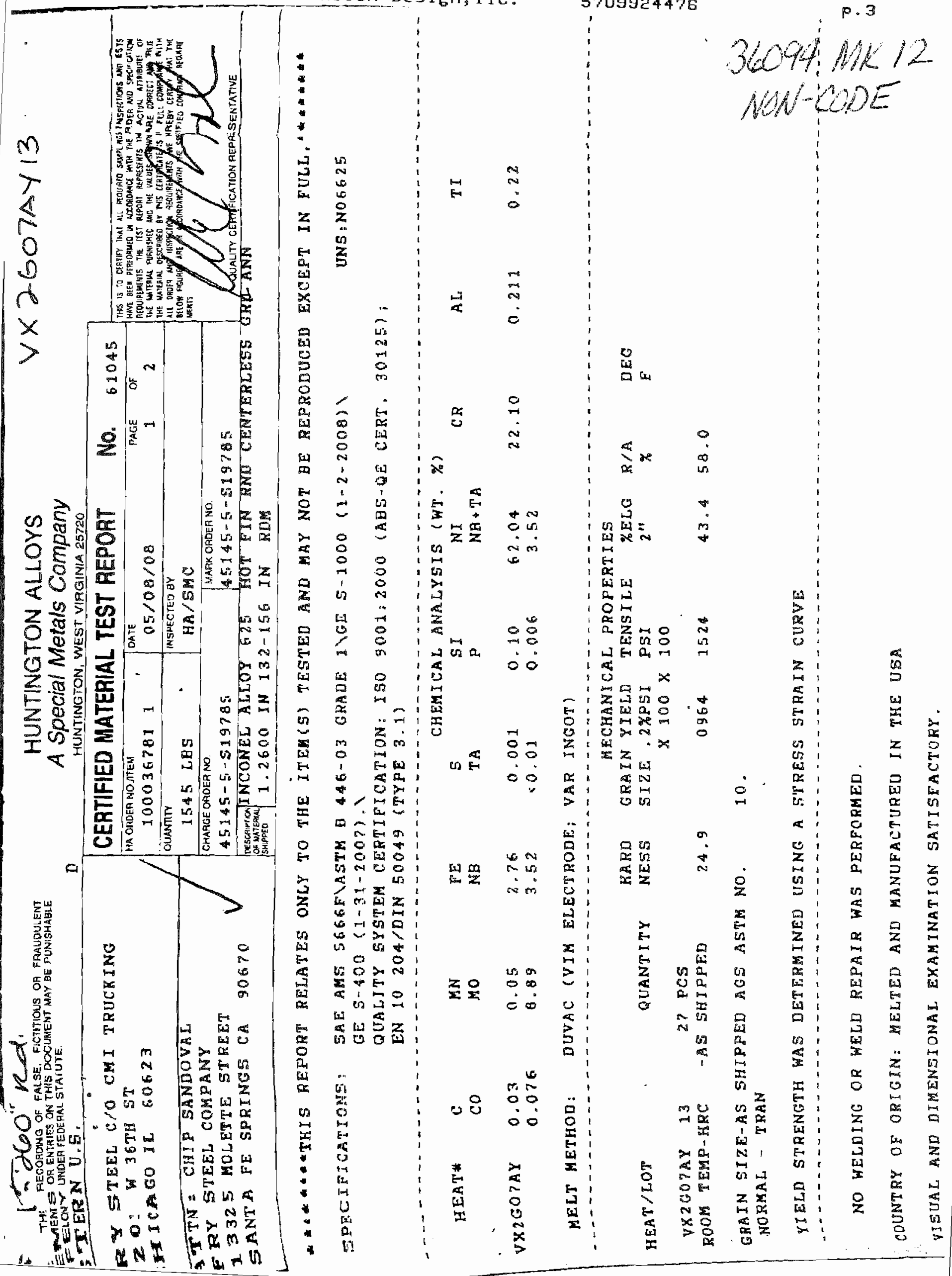




\section{CERTIFICATE OF TESTS \\ - CERT SERIAL\# 000653588 \\ (c. CARPENTER}

Capenter Technology Corporation 401 West Bern Stroet, Roading. Pa. 19801 Tel: (610) 2082000 (2000) 338-4592

\section{o6/30/08 CUSTOMER/MESTELLR/CITENT} FRY STEET COMPANY ATTN : ZARRY LONG 13325 MOLETTE ST SANTA PE SPRIMG CA 90670
ALIENAHMEPRUEFZEUGNIS CERTIFICAT DE CONTROLE

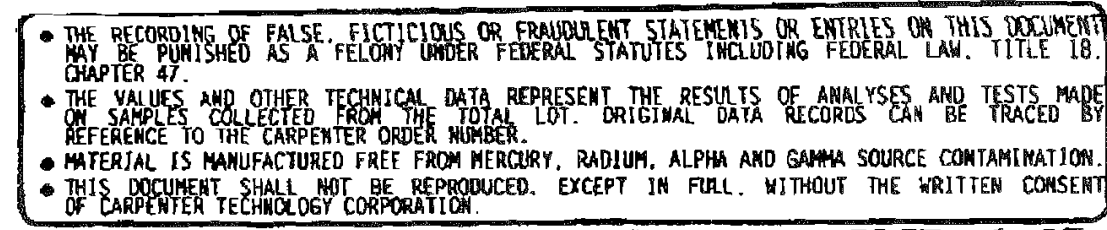
ASM

\section{SELLR/VRRKAUER/ VENDEURPAGE 1 OF}

\section{Y. 2007 Add. 2008 A3}

OC WM Date 12LULOS RAN

CUSTONER OROER MO./BESTELL - MR. INE OE COHAROE

45078-6 S\# 19845

พ89158

212194

HEAT NUMRER / SCHMER JE-RIR. / NO DE COULEE : CARPENTER NO./WERKS-NR. IN OE REFERERCE INTERME DATE/OATUM/OATE WEIGHT/GEWICHT/PDIDS

W89158

PRODUCT DESCRIPTION: PYROMET 625 HI TEMP ALLOY ANNEALED GROUND

PRODLCI DESCRIPTION: PYROMET 625 HI TEMP ALLOY ANNEALED GROUND

SPECIFICATION: AMS $5666 \mathrm{REV}$ F $(05 / / 06)$

$$
\begin{aligned}
& \text { ASTM-B446-03 GRADE } 1 \\
& \text { GE S-1000 (01 02/08) } \\
& \text { DFARS } 1998 \text { BDTTON } \\
& \text { GE S-400 (10)31707) }
\end{aligned}
$$

SIZE

2.375000 IN. ( $60.33 \mathrm{MA}) \mathrm{RD} \quad \mathrm{BAR}$

INGOT 2

PRIMARY HEAT CHEMISTRX (WTY): (TEST METHOD IS SHOWN IN PARENTHESIS)
$0 . \mathrm{C}_{4}$ (COM)
0.04 (KRF)
0.06 (XRF)
0. Do3 (COM)
LT. $.001^{\text {(COM) }}$
60.21 (XRF)
8. MO (XRF)
0.09 (XRF)
0.19 (OES)
$0 . \frac{T T}{2}$ (XRE)
0.01 (OES)
$C B+T A$
3.54
4.22 (XRF)

GEAG SUPPLIER CODE 21100 / CARPENTER

HARDNESS AS SHIPPED, HBN - 235

(MIDRADIUS)

YIELD STRENGTH, 10.20 , KSI (MPA)

TENSILE STREUGLH, KSI (MPA)

EIONGATION IN $2.00 \%$ "

$75 \cdot 0$
$136: 0$
938

GRAIN STZE PBR ASTM B112: 8 (10\%)

$55: 0$

$5 \mathrm{ML}$ H202(30\%), 100 H HCL.

10

(90)

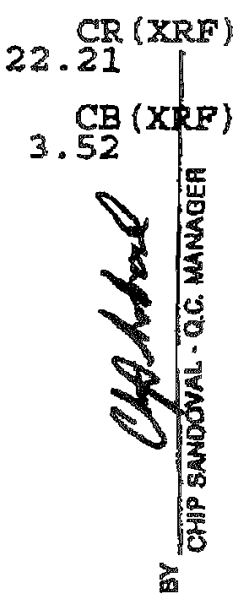

MATERTAI PRODOCED OA THIS OROMR MAS MELTED AND MANUFACTURED IN THE U.S.A.

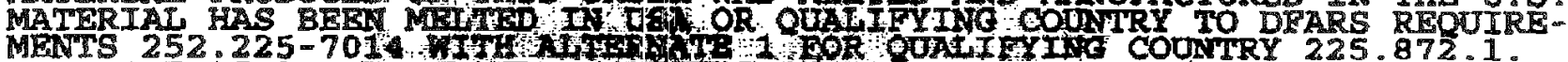

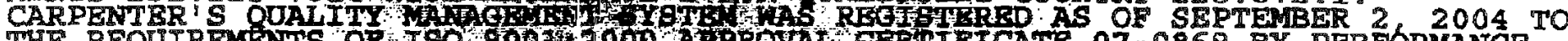

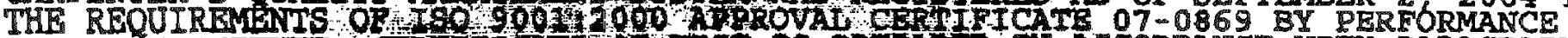

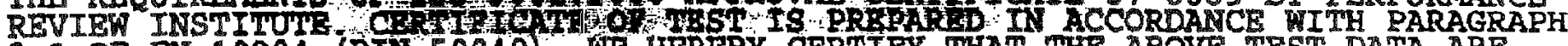
3.1 OP BN 10204 (DIN 50049 ) HE HEREBY CERTIFY THAT THE ABOVE TEST DATA ARE IN ACCORDANCE WIII THE PURCHISB ORDER AND SPECIFICATION REQUIREMENTS.

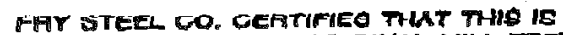

D A TAUE COPY OF THE ORIGINAL MILL TEST

REPOAT NOW ON FILE

RECEIVED AND INSPECTED

DAWN E BETESG 


\section{W. Maass

Sold to: ROBERT JAMES SALES, INC.

Cust. P.O.\#:

CG6153

2164 E. AURORA RD

Order\#:

167490

ISO 9001:2000

Certified TWINSBURG, OH 44087

Item

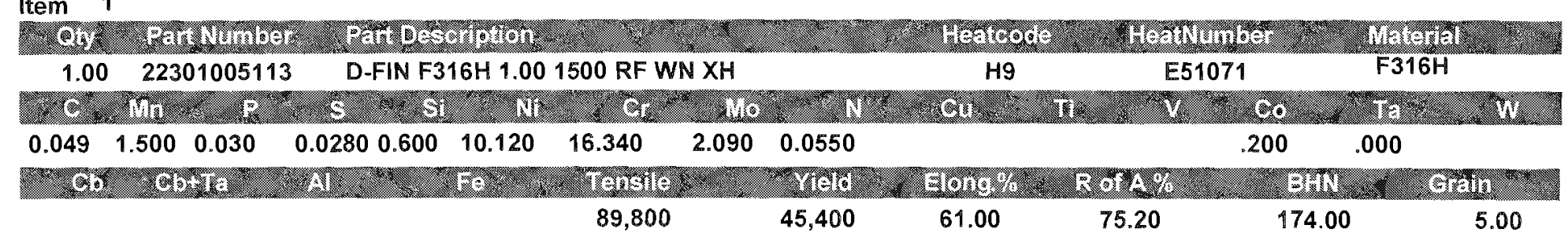

CVN: $-20 F, 171,154,163 \mathrm{ft-libs}$

Heat Treatment: Solution annealed at $1950 \mathrm{~F} / 1065 \mathrm{C}$ and water quenched Specification: AVA182-07a F316H; NACE MR-0175, MR-0103; B16.5-2003 as applicable Made in the USA

We certify that the material represented by this document has been tested and inspected and is in conformance with the purchase order, drawing('s) and specification requirements.

Approved by<smiles>CC(C)=CC=CC=CC(C)C</smiles>

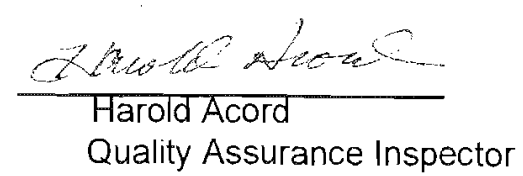

Page 1 of 1 
3004 MK 18

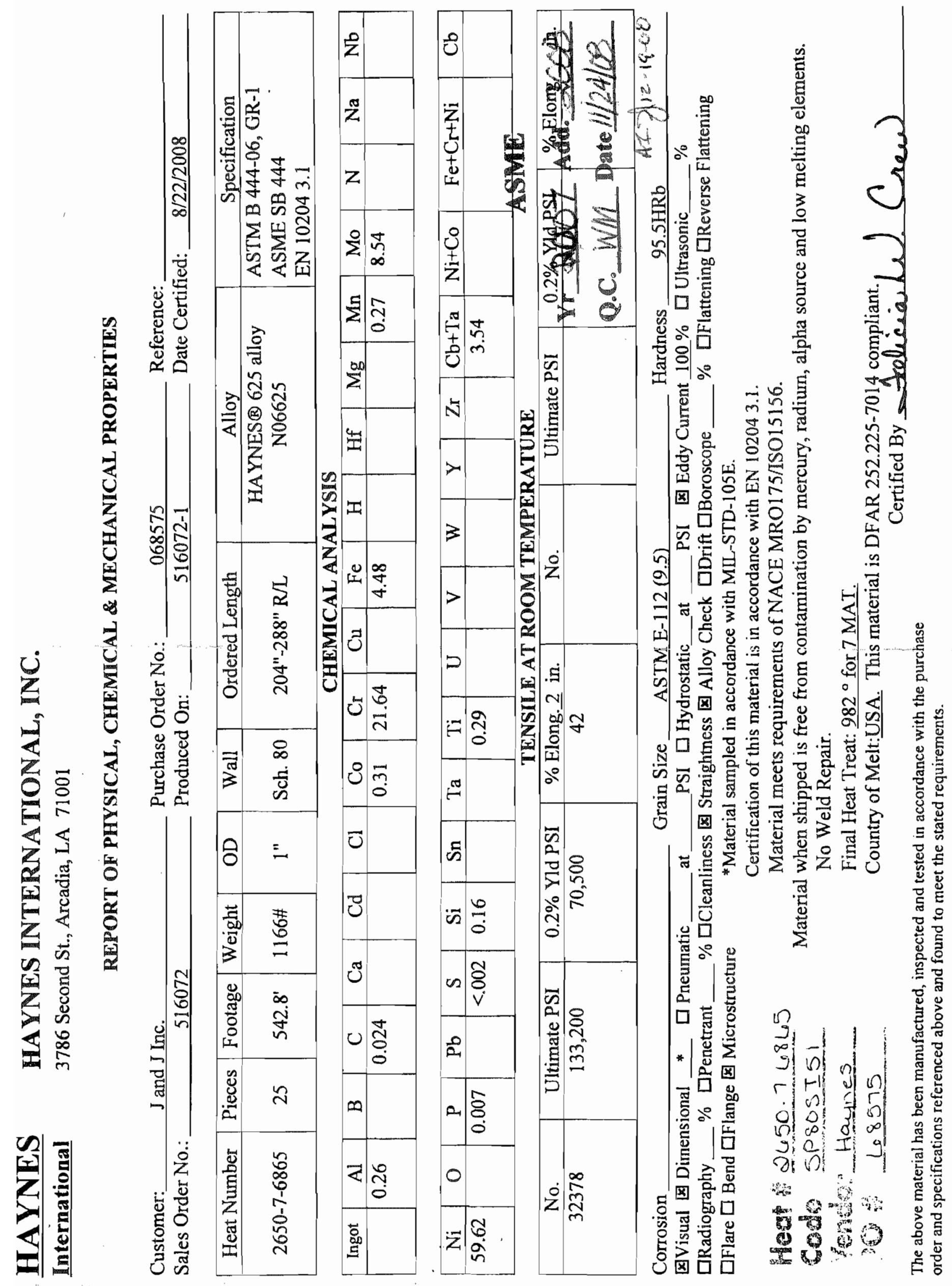


AMERICAN BOA

POST OFFICE BOX 1301

TELEPHONE (770)889-9400
INCORPORATED

CUMMING, GEORGIA 30040

TELECOPIER (770)889-0661

September 12, 2008

Form QA16,Rev.0,1-16-98

\section{MATERIAL TEST REPORTS}

\section{QA/QC DOCUMENT SUBMITTAL FORM 1A}

$X$ COMPLETE

PARTIAL

BOA Order No.: 1049967

Customer Contract No.: 700522797

Customer Name: PINNACLE WEST CAPITAL CORPORATION

Attention:

Product Type: EXPANSION JOINT

ABI Drawing Numbers: 1019759

Reference Documents:

Contract Type: COMMERIAL

Type Quality Control System Implemented: ISO 9001 \& ASME "U" STAMP

Special Contract Requirements:

Documents or Specifications Applicable to Material Test Reports:

Document Description

NATERIAL TEST REPORTS

NDE REPORTS

U2A DATA REPORT
Enclosed

Not
Enclosed

$\mathrm{X}$

$X$

$\mathrm{X}$ 
FORM U-2A MANUFACTURER'S PARTIAL DATA REPORT (ALTERNATIVE FORM)

A Part of a Pressure Vessel Fabricated by One Manufacturer for Another Manufacturer As Required by the Provisions of the ASME Code Rules, Section VIII, Division 1

1. Manufactured and certified by:

2. Manufactured for:

American Boa, lnc. 1420 Redi Road, Cumming, Georgia, United States, 30040

(Name and Address of manufacturer)

3. Location of Installation: Pinnacle West Capital Corporation P.O. Box 53940 Phoenix, AZ 85072-3940 Unknown (Name and address of purchaser)

4. Type Expansion Joint (Name and address of purchaser)

$118373 \& 118374$ (Description of vesscl part (shell, two-piece head, tube bundle)) none 1020044 Rev A (Nat't Bd No.)

5. ASME Code, Section Vlli, Div. 1: 2007 Edition

6. Shell (a) No. of course(s) 3

$$
\text { (Mig's Serial Number) }
$$

American Boa, Inc. (Drawing prepared by)

none none

(Drawing prepared by)
none

(b) Overall length (ft. \& in.) $0 \mathrm{ft} \& 10.25$ in

\begin{tabular}{|c|c|c|c|c|c|c|c|c|c|c|c|c|c|}
\hline \multicolumn{3}{|c|}{ Course(s) } & Material & \multicolumn{2}{|c|}{ Thickness } & \multicolumn{3}{|c|}{ Long.joini(Cat. A) } & \multicolumn{3}{|c|}{ Circum joint (Cat. $A, B \& C)$} & \multicolumn{2}{|c|}{ Heat treatment } \\
\hline No. & Diameter, in & Length $(f t$ \& in) & Spec/Grade or Type & Nom & Cort & Typ & Full,Spot, None & Eff & Type & Full,Spot,None & Eff & Temp. & Time \\
\hline 1 & 2 in & 7.5 in. & SB409Alloy $800 \mathrm{~h}$ & .012 & 0 & 1 & none & 1.0 & & & & & \\
\hline 1 & 2 in. & $5.2 \mathrm{in} . \mathrm{OD}$ & SB409Alloy $800 \mathrm{~h}$ & .75 & 0 & & None & .7 & & & & & \\
\hline 1 & 2 in. & 5.2 in. $O D$ & SB409Alloy $800 \mathrm{~h}$ & 1.0 & 0 & & None & .7 & & & & & \\
\hline
\end{tabular}

7. Heads (a) none (Mat'ISper No.,Grade or Type) H.T - Time \& Temp (b): none (Mat'I Spec No.,Grade or Type) H.T - Time \& Temp

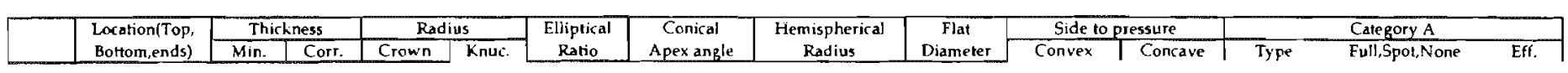

If removable, bolts used (describe other fastening) : none

8. MAWP: $35 \quad-50$ psi at max. temp. $1000 \quad 1000$ Mat', Sper. No., Grade, size, No.

8. MAWP: $\frac{35}{\text { (Internal) }} \frac{-50}{\text { (External) }}$ psi at max. temp.: $\frac{1000}{\text { (Internal) }} \frac{1000}{\text { (External) }}$ Min. Design metal temp: $70 \quad F$ at : $35 /-50$ psi 9. Impact test: Not required

10. Hydro,pneu.,or comb.test press: $\quad 46 \mathrm{psi}$

ponent(s) impact tested)

Proof test: none

11. Nozzles, inspection, and safety valve openings: none

\begin{tabular}{|c|c|c|c|c|c|c|c|c|c|c|c|}
\hline \multirow{2}{*}{$\begin{array}{l}\text { Purpose (inlet, } \\
\text { outlet,drain,etc) }\end{array}$} & \multirow[b]{2}{*}{ No. } & \multirow{2}{*}{$\begin{array}{c}\text { Diameler } \\
\text { or size }\end{array}$} & \multirow{2}{*}{$\begin{array}{c}\text { Flange } \\
\text { Type } \\
\end{array}$} & \multicolumn{2}{|c|}{ Material } & \multicolumn{2}{|c|}{ Nozzle Thickness } & \multirow{2}{*}{$\begin{array}{c}\begin{array}{c}\text { Reinforcement } \\
\text { Material }\end{array} \\
\end{array}$} & \multicolumn{2}{|c|}{ How Attached } & \multirow{2}{*}{$\begin{array}{c}\text { Lociation } \\
\text { (lnsp. Open. }\end{array}$} \\
\hline & & & & Nozzle & Flange & Nom. & Corr. & & Nozzle & Flange & \\
\hline & & & & & & & & & & & \\
\hline & & & & & & & & & & & \\
\hline & & & & & & & & & & & \\
\hline & & & & & & & & & & & \\
\hline
\end{tabular}

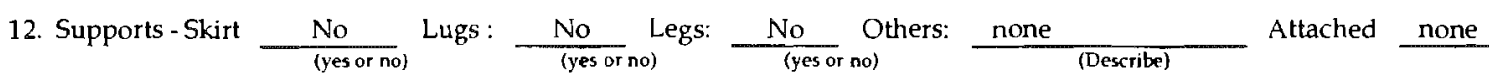

13. Remarks This part has been designed by American Boa, lnc. in accordance with EJMA and ASME Section VIII, Div. 1, 2007 ED. Axial Spring Rate: 201 Fatique Life: 166 Axial Movement: .0 in.,. Extension/Compression 3.00 in Loading Condition: Thermal Mvmt. Unit was helium leak check at $1 \times 10-6$.

\section{CERTIFICATE OF SHOP/FIELD COMPLIANCE}

We certify that the statements made in this report are correct and that all details of material, construction, and workmanship of this pressure vessel part conform to the ASME code for pressure vessels, Section Vlll, Division 1.
U certificate of Author
Date: $\quad 9 / 16 / 08$
No.: $\quad 24,594$
expires:
Name: American Boa, lnc. (Manufacturer)
Signed:

\section{CERTIFICATE OF SHOP/FIELD INSPECTION}

l, the undersigned,holding a valid commision issued by the National Board of Boiler and Pressure Vessel inspectors and/or the State or Province of Georgia and employed by: Arise lnc. of Brecksville, $\mathrm{OH}$ have inspected the pressure vessel part described in this Manufacturer's Data report on September16 , $20 \quad \overline{08}$ and state that, to the best of my krowledge and belief, the Manufacturer has constructed this pressure vessel part in accordance with ASME Code, Section VIll, Division 1. By signing this certificate neither the inspector nor his employer makes any warranty, expressed or implied, concerning the pressure vessel part described in the Manufacturer's Data

report. Furthermore, neither the inspector nor his exployf shal be flable in any manner for any personal injury or property damage or a loss of

any kind arising from or connected with this in
Date: $9 / 16 / 08 \quad$ Signed:

Signed:

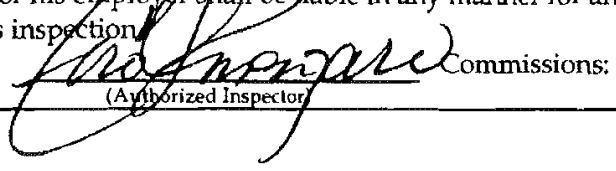




\section{AMERICAN BOA INC.}

Automotive Decoupling Joints - Flexible Hoses

\section{REPORT OF NONDESTRUCTIVE EXAMINATION}

Form QA 18 Rev.2, 1/04/08

Date 12 September, 2008

CUSTOMER NAME: PINNACLE WEST CAPITAL CORPORATION P.O. Number: 700522797

Reference Number as applicable:

ABI FACTORY / SALES ORDER NLTMBER: 1049967

SPECIFICATION FOR TEST : SEE BELOW

(criteria for test)

TEST PERFORMED: DYE PENETRANT

(Type of Test)

Note; (PT Testing is by Visible Penetrant components, which are, Penetrant Type SKL-SP, Cleaner Type SKC-S, Developer Type SKD-S2 or equivalents) Lighting utilized for examination was artificial, portable as needed to obtain necessary brightness.

PROCEDURE USED FOR TEST: 4.498 .002 REV.4

(AB1 Procedure number and Rev)

RESULTS OF TESTING:

\begin{tabular}{|l|l|l|c|c|c|}
\hline DATE & Part/Ser \# & DESCRIPTION & ACCEPT & REJECT & Mat' 1/Thickness \\
\hline $9 / 15 / 08$ & $118373 \&$ & Bellows seam welds \& & $x$ & $800 \mathrm{H}$ \\
& 118374 & attachment welds & .012 \\
\hline
\end{tabular}

\begin{tabular}{|c|c|c|c|c|c|}
\hline SIGNED By:: & TESTER & NDE LEVEL & Q.A. Mgr./ND & $\begin{array}{l}\text { rvisor } \\
\text { DE Level }\end{array}$ & $\begin{array}{l}\text { AUTH. INSPECTOR } \\
\text { Or Customer as } 3 p \text { licable }\end{array}$ \\
\hline Signatures $=$ & S.Walls & II & Eddie Hicks & II & 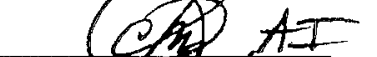 \\
\hline Date signed & $9-15-08$ & & $8-15-08$ & & 716108 \\
\hline
\end{tabular}




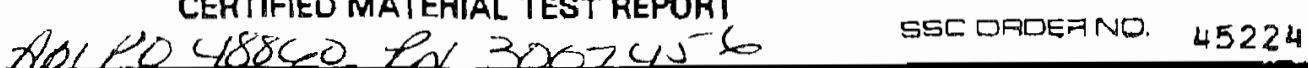

\section{GANDMEYER GTEEL COMPANY}

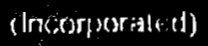

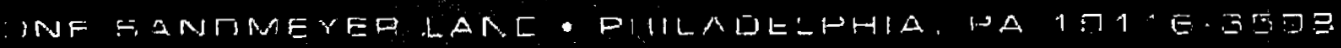

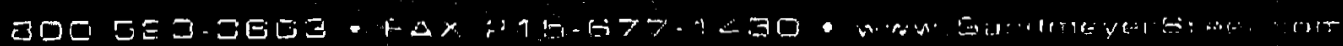

Family Owned and Maniacd -

Making Slainless Steel and Nickel Alloy Plate Produts

\section{Sirico: 1952}

BILL

TO

AMERICAN BOA INC.

P. O. BOX 1301

CUMMING. GA 30028
CERTIFICATE OF TEST

WE CERTIFY THAT THE CHEMICAL ANALYSIS AND MECHANICAL TESI RESULTS APPEARING IN THIS CERTIFICATE ARE CORRECT AND TRUE AS REPORTED BY THE MANUFACTURER.
CUSTOMER ORDER NO. 48860 REPLACE DATE: $09 / 08 / 08$

\begin{tabular}{|r|r|r|}
\hline GRADE, UNS NO8810 & SPECIFICAT \\
\hline PIECES & SSC ALLDY 800H A \\
2 & $\begin{array}{l}3 / 4 m \text { THK } \\
\text { PER DRAHING 3007456 REV.A } \\
\text { MACHINED }\end{array}$
\end{tabular}

I. BOHNSACK - MANAGER QUALITY ASSURANCE

\section{BUALITY CONTAOL OEPARTMENT}




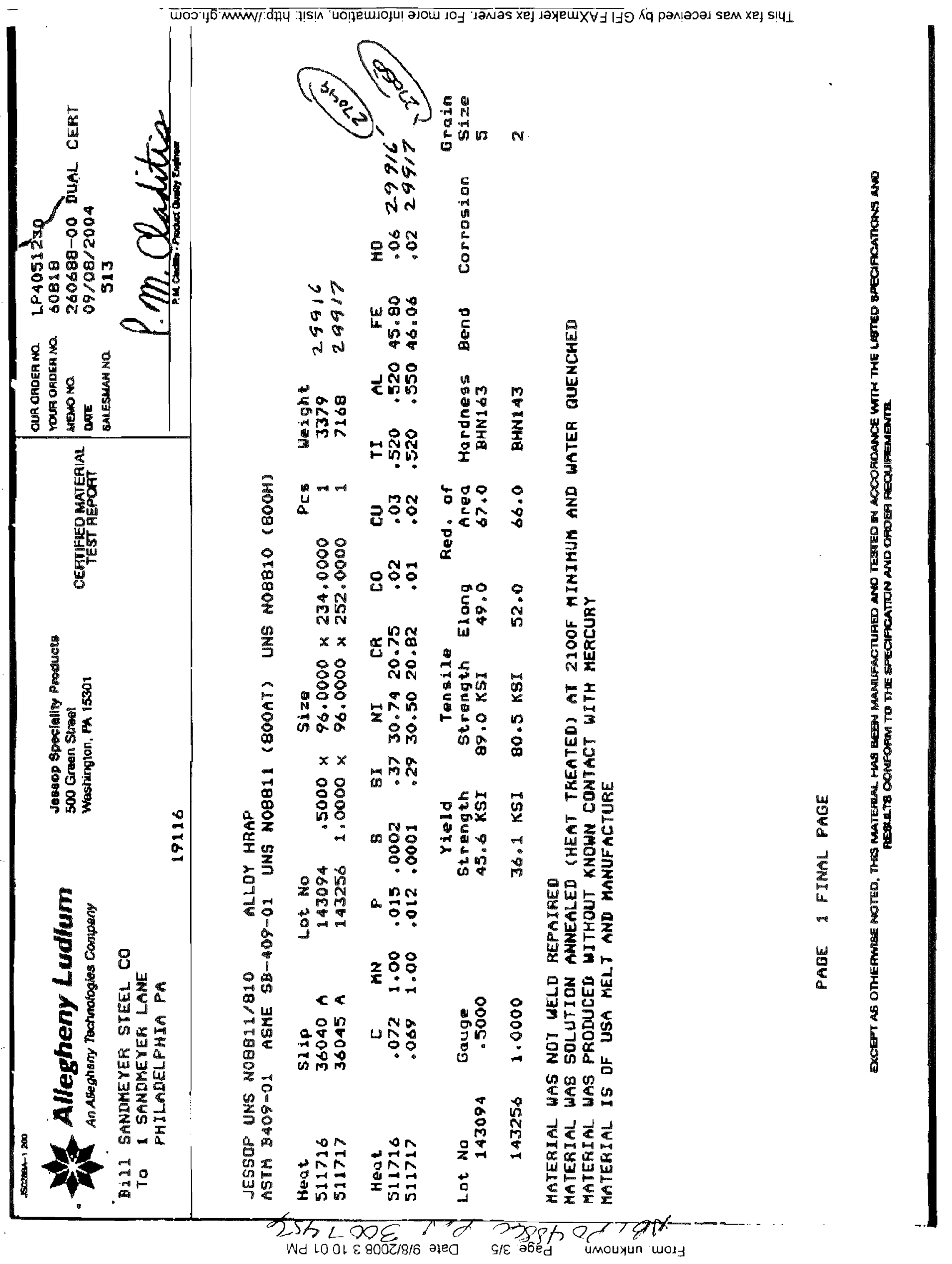




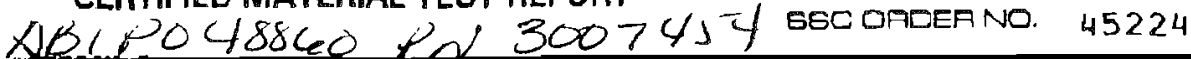

\section{SANDMEYEA STEEL COMPANY}

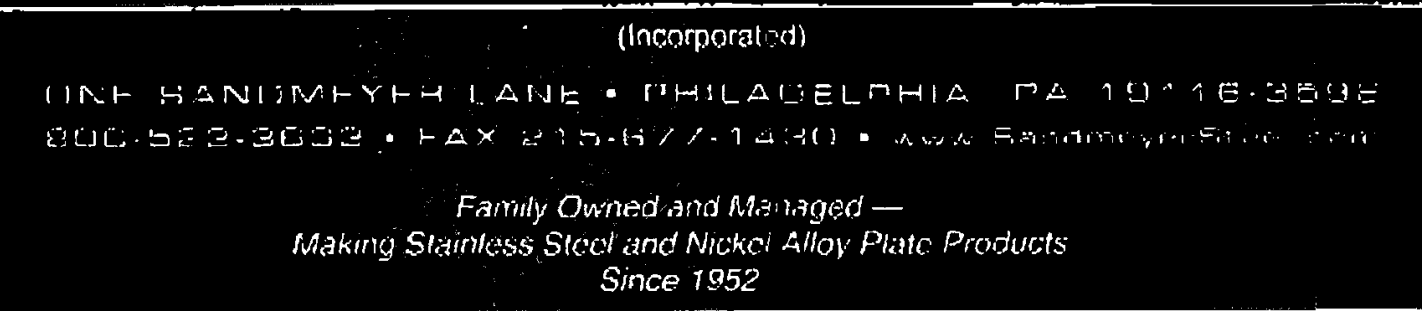

BIL

CERTIFICATE OF TEST

AMERICAN BOA INC.

P. O. BOX 1301

CUMMING. GA 30028
WE CERTIFY THAT THE CHEMICAL ANALYSIS ANO MECHANICAL TEST RESULTS APPEARING IN THIS CERTIFICATE ARE CORRECT AND IRUE AS REPORTED BY THE MANUFACTURER.

SANDMEYER STEEL COMPANY

CUSTOMER ORDER NO. DATE: $09 / 08 / 00$

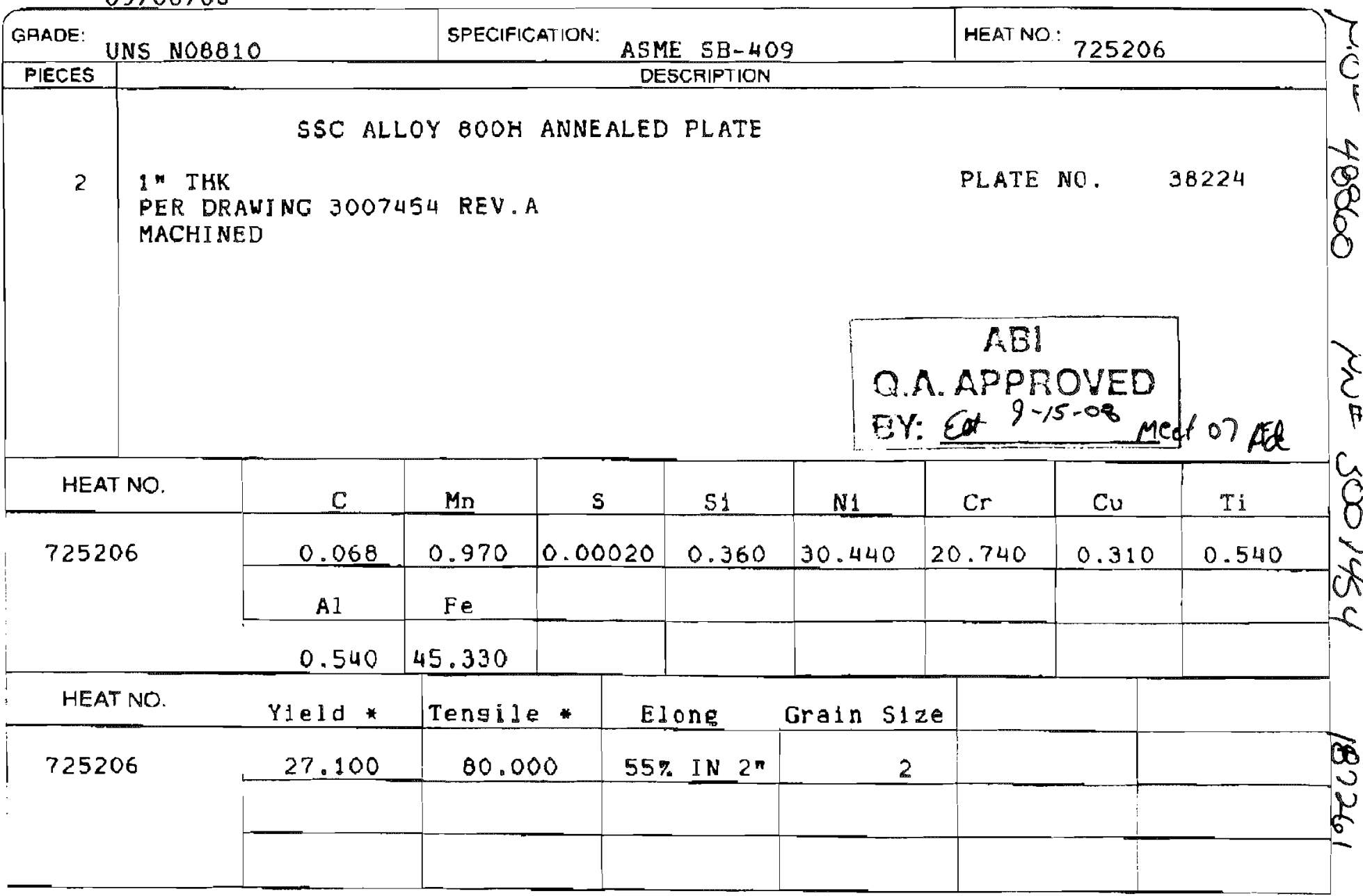




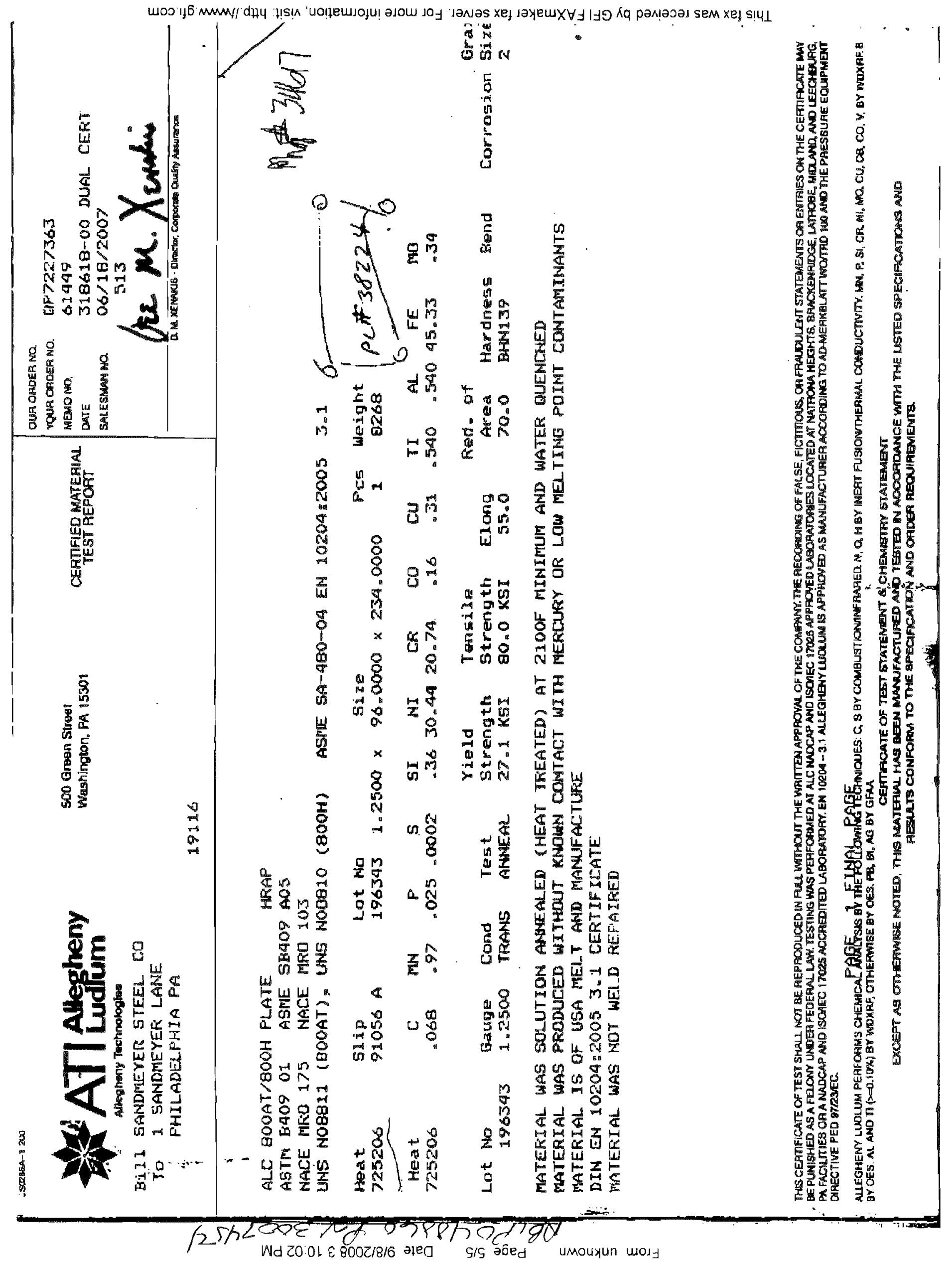




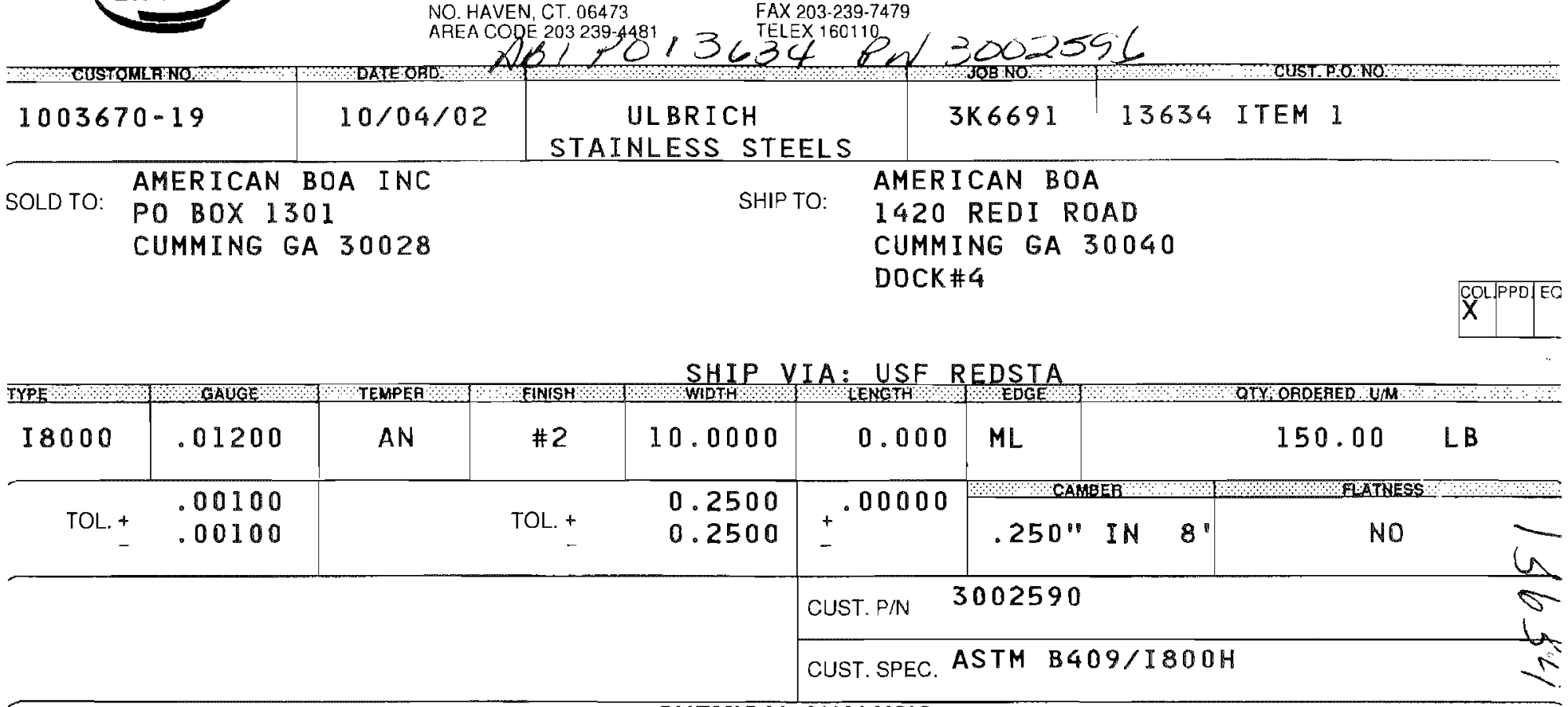

\begin{tabular}{lclc}
\hline HEAT NO. & \multicolumn{1}{c}{ CHI3BHH } & NICMICAL ANALYSIS \\
CARBON & $: .08$ & NICKEL & $: 30.48$ \\
MANGANESE & $: .96$ & ALUMINUM & $: .47$ \\
SILICON & $: .26$ & COPPER & $: .01$ \\
PHOSPHORUS & $: .016$ & TITANIUM & $: .54$ \\
SULPHUR & $: .001$ & IRON & $:$ BAL \\
CHROMIUM & $: 20.70$ & &
\end{tabular}

\begin{tabular}{|c|c|c|c|c|}
\hline \multicolumn{5}{|c|}{ MECHANICAL FROPERTIES AT ROOM TEMPERATURE } \\
\hline $\begin{array}{l}\text { TENSILE } \\
\text { YIELD } \\
\text { ELONG }\end{array}$ & $\begin{array}{l}(P S I): \\
(P S I): \\
(\%):\end{array}$ & $\begin{array}{l}92600 \\
47800 \\
41\end{array}$ & $\begin{array}{l}\text { HARDNESS : } 83 \text { HRB } \\
\text { GRAIN SIZE : } 9.5 \\
\text { HARDNBLTY : }\end{array}$ & $\begin{array}{l}\text { BEND TEST: } \\
\text { EMBR ITTLE: }\end{array}$ \\
\hline \multicolumn{5}{|c|}{ MECHANICAL PROPERTIES AFTER HEAT TREATMENT } \\
\hline $\begin{array}{l}\text { TENS I LE } \\
\text { YIELD }\end{array}$ & $\begin{array}{l}\text { (PSI): } \\
(P S I):\end{array}$ & & HARDNESS : & \\
\hline ELDNG & $(\%):$ & & HARDNBLTY : & \\
\hline
\end{tabular}

STRESS RUPTURE TEST:

MECHANICAL PROPERTIES AT
HARDNESS : DEG. F
HARDNBLTY :

OTHER PROPERTIES

TENSILE (PSI):

YIELD (PSI):

ELONG $(\%)$ :
HARDNESS : 83 HRB

GRAIN SIZE: 9.5

HARDNBLTY :
EMBRITTLE: 


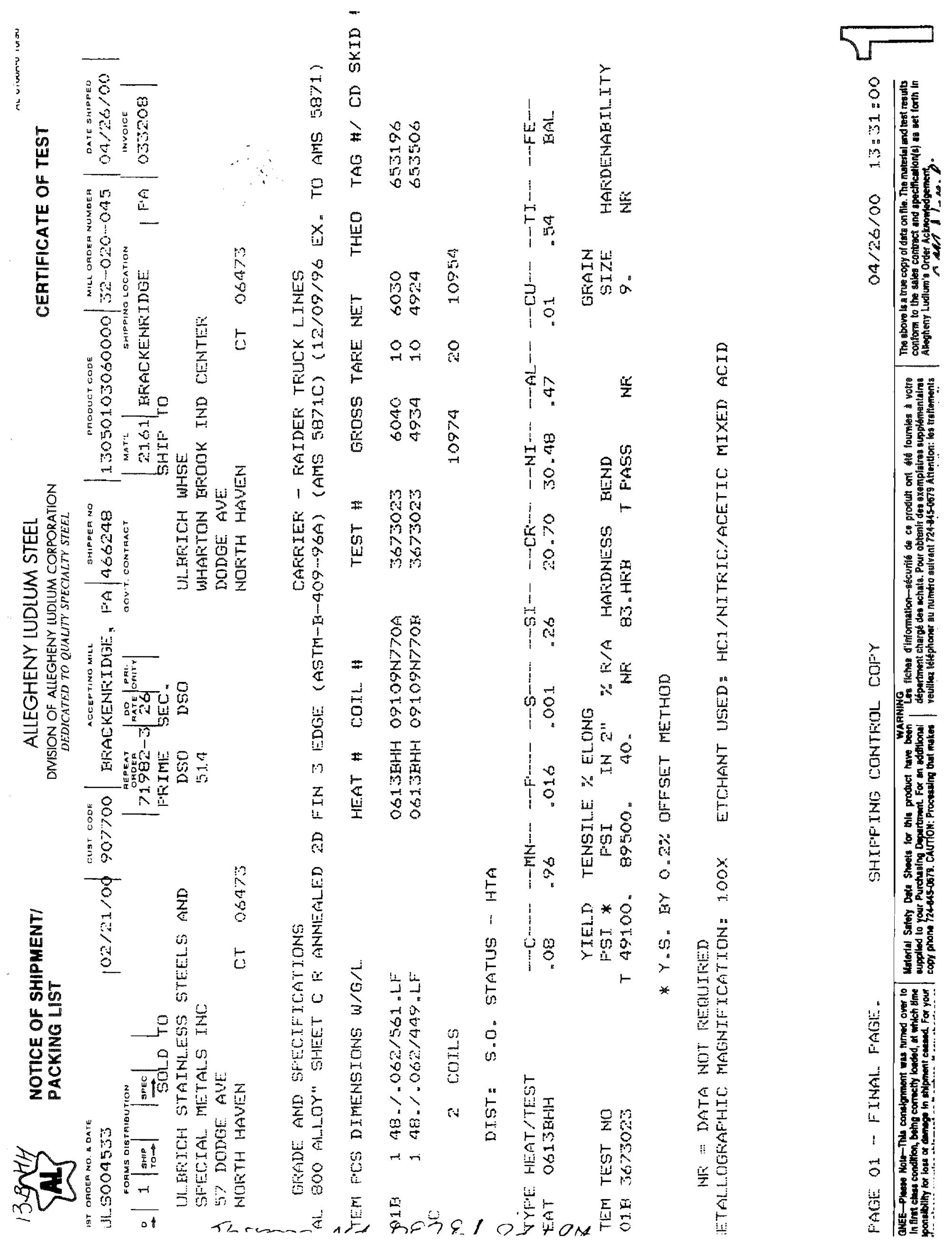




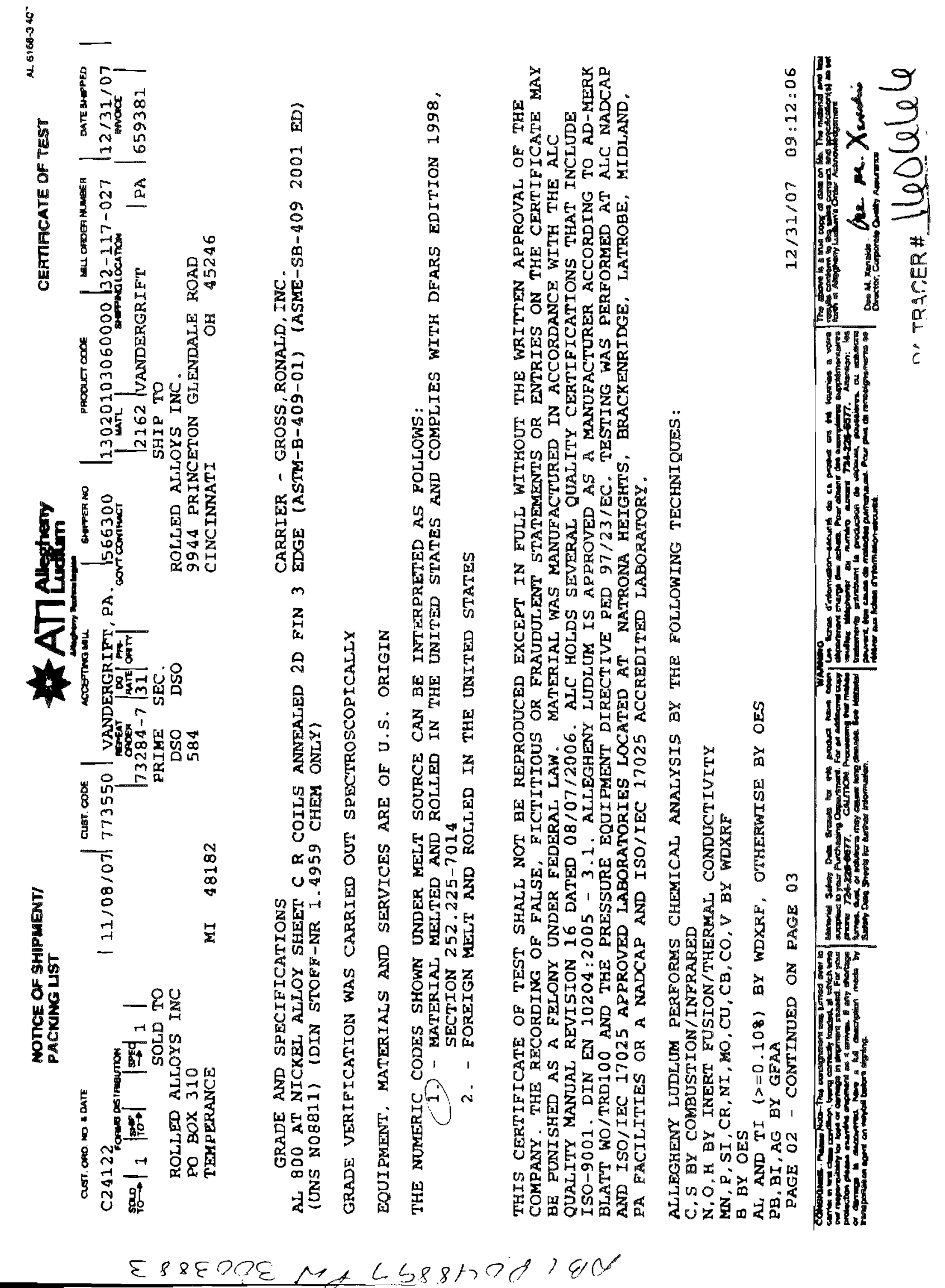




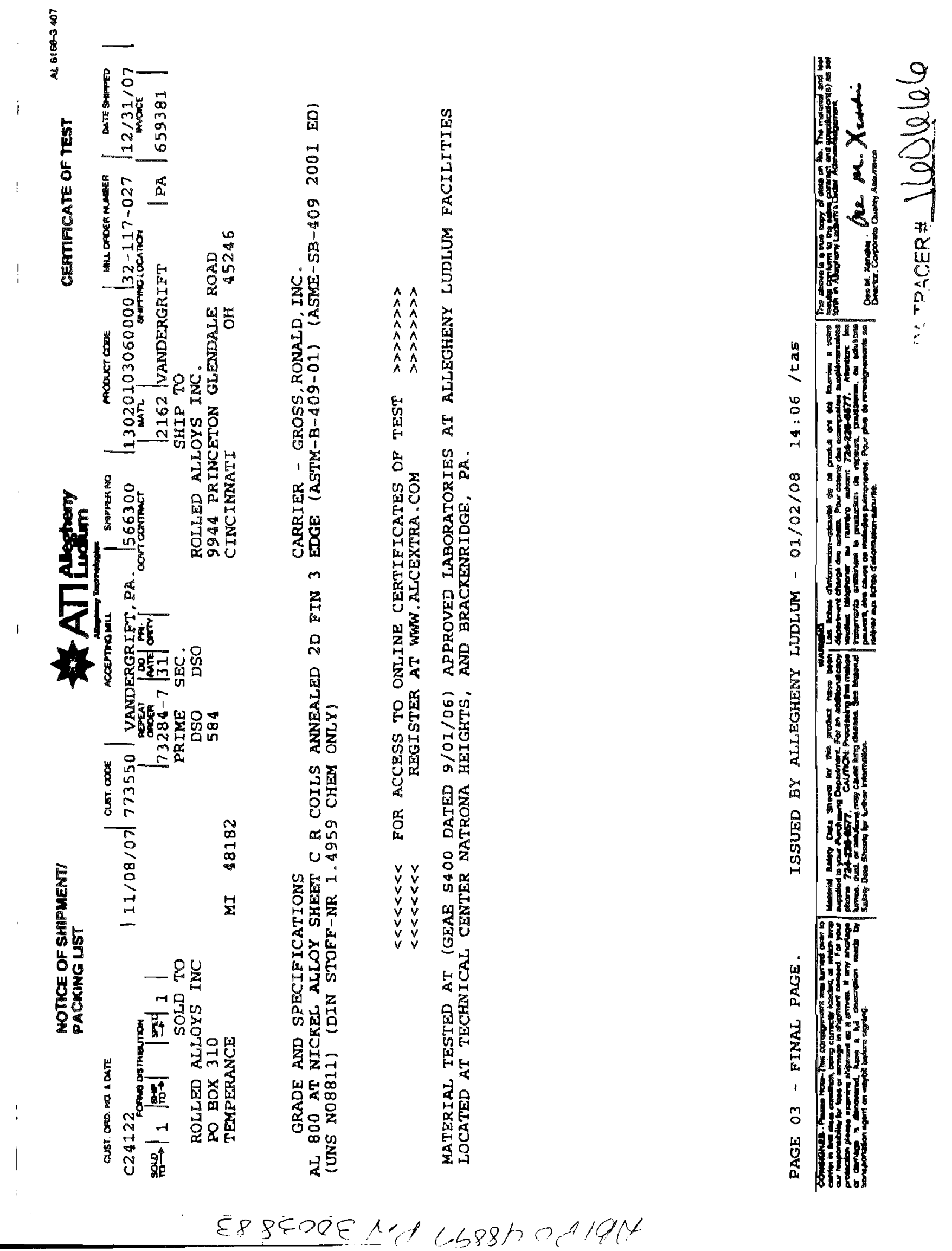


90.31307

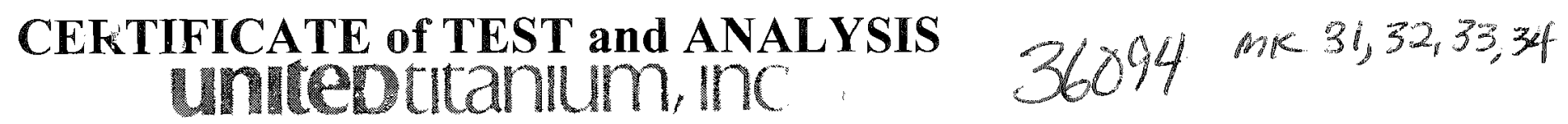

3450 OLD AIRPORT ROAD

WOOSTER, OHIO 44691

(330) 264-2111 or FAX (330) 263-1336

Quantity: 8.000 EA

Lot: M413410

Product Shipped: $\quad$ 1-08-519-12.25

3/8-24 X 2-1/4 SKT CAP A-625

Manufactured from:

Size: $\quad .625$ DIAMETER - DFARS

Alloy: ALLOY 625

Heat: D90Y

To: GASPAR INC.

1545 WHIPPLE AVE. S.W.

CANTON, OH 44710

USA

Customer P.O.: $\quad 31307$

Sales Order: $\quad 991359$

\section{SPFCIFICATIONS}

ASTM B446 N06625 Grade 1

\section{CHFMICAI COMPOSITION (INGOT ANATYYSIS)}

\begin{tabular}{|c|c|c|c|c|c|c|c|c|c|}
\hline AI & .25 & $\mathrm{C}$ & .040 & $\mathrm{Cb}$ & 3.79 & Co & .04 & Cr & 20.83 \\
\hline $\mathrm{Fe}$ & 4.42 & $\mathrm{Mn}$ & .03 & Mo & 8.37 & $\mathrm{Ni}$ & $\mathrm{BaI}$ & $\mathbf{P}$ & .003 \\
\hline $\mathbf{S}$ & .0004 & $S i$ & .03 & $\mathrm{~T} i$ & .29 & & & & \\
\hline
\end{tabular}

\section{MECHANICAI PROPFRTIES (RAW MATERTAL)}

\begin{tabular}{|c|c|c|c|c|c|c|}
\hline 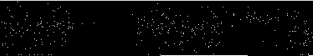 & MaCH & AI $P$ & ARIIFS & (RAW M & D) & \\
\hline HEAT TREATMENT & $\begin{array}{c}\text { TEMP } \\
{ }^{\circ} \mathrm{F} \\
\end{array}$ & $\begin{array}{l}\text { UTS } \\
\text { Ksi }\end{array}$ & $\begin{array}{c}28 \mathrm{Y} \text { YIELD } \\
\mathrm{Ksi}\end{array}$ & $\begin{array}{c}\text { EL } \\
8 \\
\end{array}$ & $\begin{array}{c}\mathrm{Ra} \\
8\end{array}$ & HARDNESS \\
\hline Annealed & ROOM & 136.1 & 82.6 & 57.0 & 63.9 & $24 \mathrm{RC}$ \\
\hline
\end{tabular}

NOTES :

- Material is free from Mercury Contamination

- Grain size meets specification requirements

\section{REMARKS :}

MELT SOURCE: ALLVAC - USA

This material is DFARS compliant.

This material is RoHs compliant.

United Titanium, Inc. certifies that these figures are true as taken directly from the mill test report supplied to us. The original is kept on file.
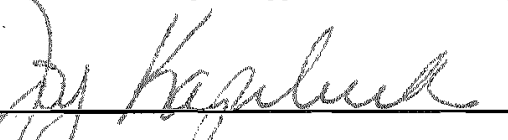


\section{CERTIFICATE of TEST and ANALYSIS undedulanIum, inc}

$$
\begin{aligned}
& 3450 \text { OLD AIRPORT ROAD } \\
& \text { WOOSTER, OHIO } 44691
\end{aligned}
$$

(330) 264-2111 or FAX (330) 263-1336

Quantity: 8.000 EA

Lot: M413420

Product Shipped: $\quad 1-08-569-12.75$

3/8-24 X 2-3/4 STUD A-625

Manufactured from:

Size: $\quad .375$ DIAMETER - DFARS

Alloy: ALLOY 625
Heat: D88P
To: GASPAR INC.

1545 WHIPPLE AVE. S.W.

CANTON, OH 44710

USA

Customer P.O.: $\quad 31307$

Sales Order:

\section{SPECIFICATIONS}

ASTM B446 N06625 Grade 1

\begin{tabular}{|c|c|c|c|c|c|c|c|c|c|}
\hline Al & .26 & $c$ & .036 & $\mathrm{Cb}+$ & 3.78 & Co & .13 & $\mathrm{Cr}$ & 20.95 \\
\hline Fe & 4.18 & $\mathrm{Mn}$ & .07 & Mo & 8.27 & $\mathrm{Ni}$ & $\mathrm{Bal}$ & $P$ & .007 \\
\hline$c$ & .0003 & Si & .11 & $T i$ & .29 & & & & \\
\hline
\end{tabular}

\section{CHFMICAT COMPOSITION (INEOT ANALYSIS)}

\begin{tabular}{|c|c|c|c|c|c|c|}
\hline HEAT TREATMENT & $\begin{array}{c}\text { TEMP } \\
{ }^{\circ} \mathrm{F} \\
\end{array}$ & $\begin{array}{l}\text { UTS } \\
\text { Ksi }\end{array}$ & $\begin{array}{c}.28 \mathrm{YIELD} \\
\mathrm{Ksi}\end{array}$ & $\begin{array}{c}\text { EL } \\
8 \\
\end{array}$ & $\begin{array}{c}\mathrm{Ra} \\
8\end{array}$ & HARDNE SS \\
\hline Annealed & ROOM & 130.7 & 76.4 & 57.3 & 59.7 & \\
\hline
\end{tabular}

\section{MFCHANICAL PROPFRTIES (RAK MATERIAT)}

NOTES :

- Material is free from Mercury Contamination

- Grain size meets specification requirements

\section{REMARKS :}

MELT SOURCE: ALLVAC, USA

This material is DFARS compliant.

This material is RoHS compliant. 


\section{Solar Atmospheres of Western PA \\ Certification}

Order No.: 39109

Date: $11 / 17 / 2008$

Entry Date: 10/10/2008

Page: 1 of 1

To:

GASPAR INC 1545 WIPPLE AVE SW

Purchase Order No.: 30966

Packing List No.:

CANTON

$\mathrm{OH} 44710$

Material: INCONEL 625

All work performed subject to Solar Atmospheres Terms Of Sale as presented on form SA-1 (01-00).

\begin{tabular}{r|l|c}
\hline Quantity & Part Number./ Part Name / Part Description & Pounds \\
\hline $4 \quad$ & MATERIAL INCONEL 625 & 10 \\
& ITEM\# 36095-0 \\
& 1/4" X 83 1/4" TUBE \\
2 & MATERIAL INCONEL 625 \\
& ITEM\# 36095-0 & \\
& 1/4" X 72 3/4" TUBE
\end{tabular}

Insp. Type Scale Minimum Maximum Number Other

Customer Requirements:

N/A

THIS IS TO CERTIFY THAT THE ABOVE NAMED PARTS WERE PROCESSED IN ACCORDANCE WITH YOUR PURCHASE ORDER REQUIREMENTS AND PROCESS HW8598 10/17/08 JNH.

FURNACE RUN \# 70-8598-2123

This certification is no guarantee of material performance, properties, or microstructure. Mechanical, physical, and/or metallurgical testing is not performed unless specifically itemized on your purchase order to Solar Atmosoheres.

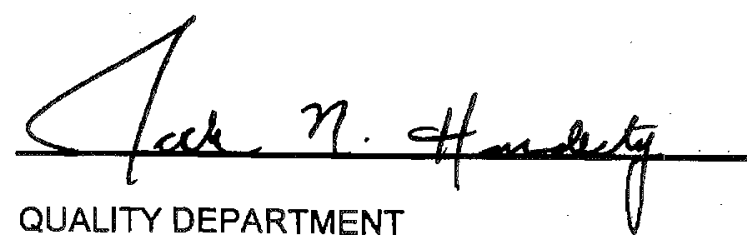

SOLAR ATMOSPHERES INC. 


\section{Solar Atmospheres of Western PA Certification}

Order No.: 39109

Date: $11 / 17 / 2008$

Entry Date: 10/10/2008

Page: 1 of 1

To:

GASPAR INC

1545 WIPPLE AVE SW

CANTON

$\mathrm{OH} 44710$

Purchase Order No:: 30966

Packing List No.:

87379

Material: INCONEL 625

All work performed subject to Solar Atmospheres Terms Of Sale as presented on form SA-1 (01-00).

\begin{tabular}{|c|c|c|}
\hline Quantity & Part Number / Part Name / Part Description & Pounds \\
\hline 4 & $\begin{array}{l}\text { MATERIAL INCONEL } 625 \\
\text { ITEM \# 36095-0 } \\
\text { 3" DIA X } 43 \text { 3/4" BAR }\end{array}$ & 420 \\
\hline 1 & $\begin{array}{l}\text { MATERIAL INCONEL } 625 \\
\text { ITEM\# 36095-0 } \\
\text { 3" DIA X 9" BAR }\end{array}$ & \\
\hline 1. & $\begin{array}{l}\text { MATERIAL INCONEL } 625 \\
\text { ITEM\# 36095-0 } \\
\text { 3" DIA X } 5 \text { 1/2" BAR }\end{array}$ & \\
\hline 1 & $\begin{array}{l}\text { MATERIAL INCONEL } 625 \\
\text { ITEM\# 36095-0 } \\
\text { 3" DIA X } 6 \text { 3/4" BAR }\end{array}$ & \\
\hline 1 & TEST PIECE & \\
\hline Insp. Typo & Scale Minimum Maximum & \\
\hline \multicolumn{3}{|c|}{ Customer Requirements: } \\
\hline N/A & . & \\
\hline
\end{tabular}

THIS IS TO CERTIFY THAT THE ABOVE NAMED PARTS WERE PROCESSED IN ACCORDANCE WITH YOUR PURCHASE ORDER REQUIREMENTS AND PROCESS HW8598 10/17/08 JNH.

FURNACE RUN \# 70-8598-2123

This certification is no guarantee of material performance, properties, or microstructure. Mechanical, physical, and/or metallurgical testing is not performed unless specifically itemized on your purchase order to Solar Atmosoheres.

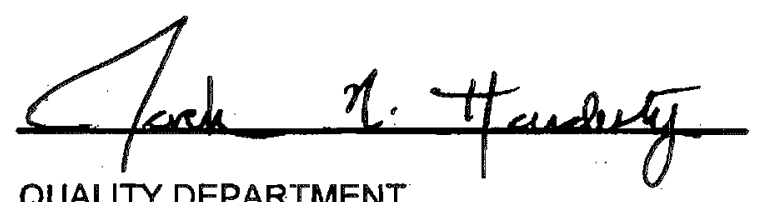

QUALITY DEPARTMENT

SOLAR ATMOSPHERES INC. 


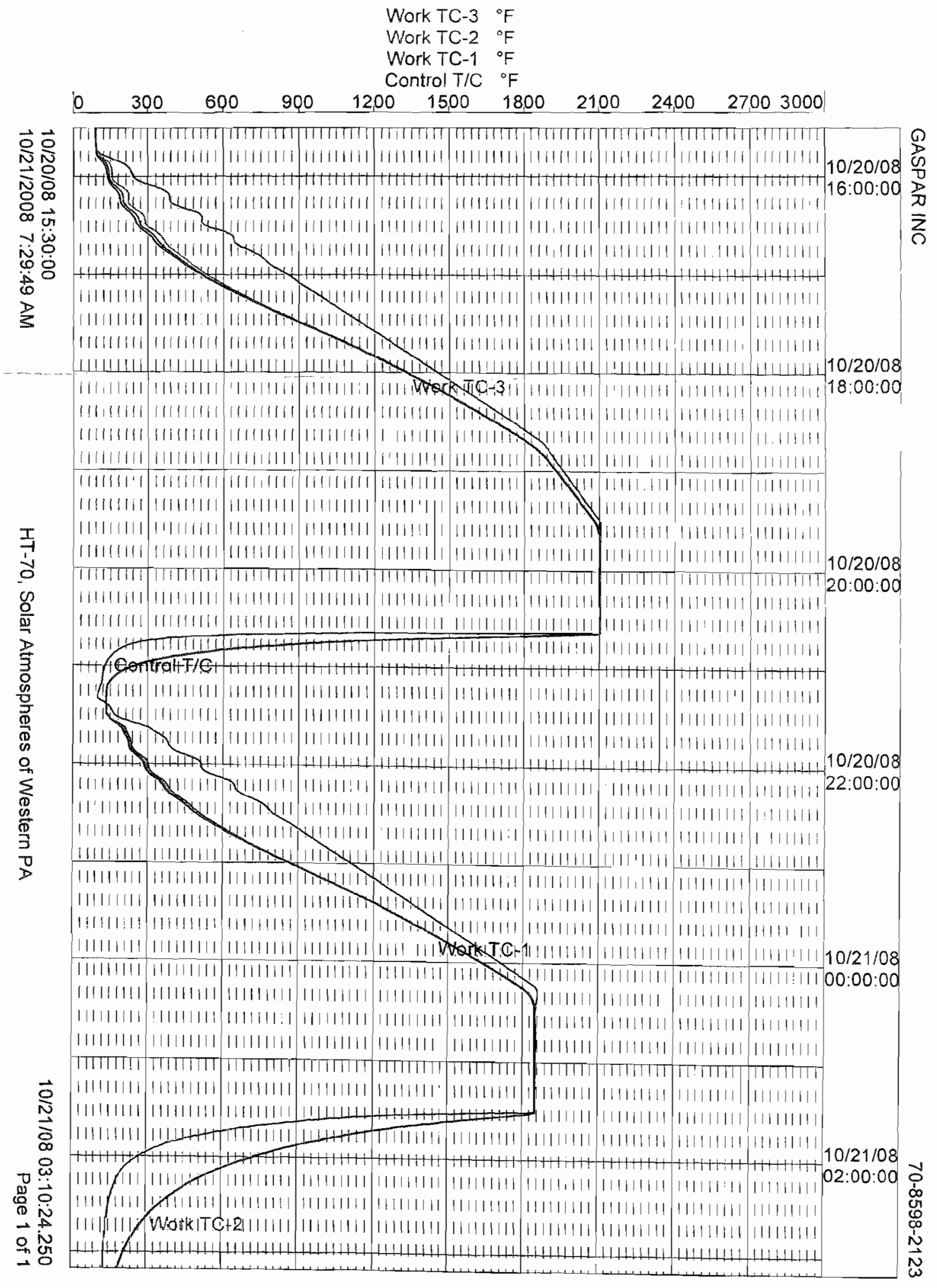


HW8598 10/17/08 JNH

SOLUTION ANNEAL / THERMALLY STABILIZE - INCONEL 625 - SOLAR SPEC -

GASPAR INC.

NOTE:

-RAMP RATE MAY BE ADJUSTED IF NECESSARY.

PROCESS:

1. PUMP DOWN TO A HARD VACUUM ( $1 \mathrm{X} 10$ - 3 TORR OR LOWER ).

2. RAMP AT $600^{\circ} \mathrm{F} / \mathrm{HOUR}$ TO $2100^{\circ} \mathrm{F} \pm 25^{\circ} \mathrm{F}$.

3. HOLD AT $2100^{\circ} \mathrm{F} \pm 25^{\circ} \mathrm{F}$ FOR $60-75$ MINUTES, WORK TC'S.

4. HELIUM 2 BAR QUENCH TO BELOW $175^{\circ} \mathrm{F}$, WORK TC'S.

5. PUMP DOWN TO A HARD VACUUM ( $1 \mathrm{X} 10$ - 3 TORR OR LOWER ).

6. RAMP AT $600^{\circ} \mathrm{F} / \mathrm{HOUR}$ TO $1850^{\circ} \mathrm{F} \pm 25^{\circ} \mathrm{F}$.

7. HOLD AT $1850^{\circ} \mathrm{F} \pm 25^{\circ} \mathrm{F}$ FOR $60-75$ MINUTES, WORK TC'S.

8. -5 ARGON QUENCH TO BELOW $200^{\circ} \mathrm{F}$, WORK TC'S 
Gaspar Inc.

1545 Whipple Ave. SW

Canton OH 44710
Job No.: A $\quad$ A-296-826

Date: $\quad 10-29-08$

Cust. PO\#: 31221

Description: $\quad 2$ samples $\quad 3^{\prime 2} \mathrm{Bia} \times 6^{\prime \prime} \mathrm{Bar} \cdot$ Project $36095=0$ $1 / 4$ " $\times 48$ " Tube

4 Spec: $\quad$ ASTM B446/ASME SB444 N06625 Gr. 2

Requirements (Mins):

Tube HTHVOO6 OI
Tensile, ksi

100

122
Yield. .2\% ksi 40

49.7
Elong. \% in 2"

30

61

Test Method: ASTM A370-08a

$\begin{array}{ccccc}\text { Requirements (Min.): } & \frac{\text { Tensile, ksi }}{100} & \frac{\text { Yield, .2\% ksi }}{40} & \frac{\text { Elong. \% in 4D }}{30} & \text { Red.of Area, \% } \\ \begin{array}{c}\text { Bar } \\ \text { HTHL01A }\end{array} & 110 & 47.3 & 62 & 56 \\ & & \text { Test Method: ASTM A370-08a } & \end{array}$

The above conforms to specifications listed.

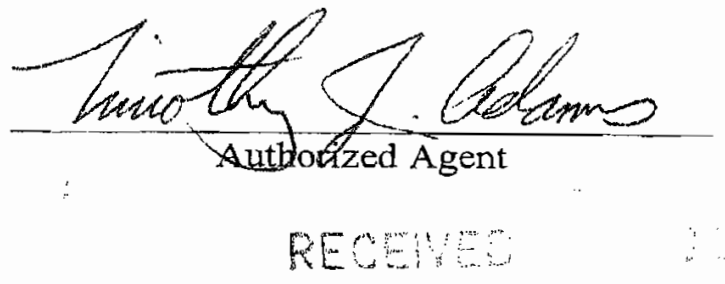




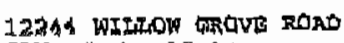

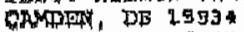

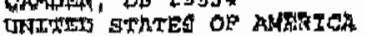

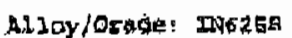

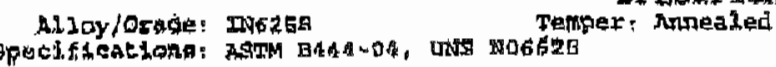

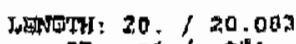

OD: $25,2.26 \mathrm{H}$

ID: $\dot{\sigma}^{25} 0$

Wa, 1: .0565/.0725

Q6y Bhyped; RT6

Date Brdphed: 3/9-2/0C

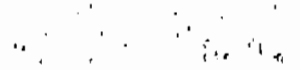

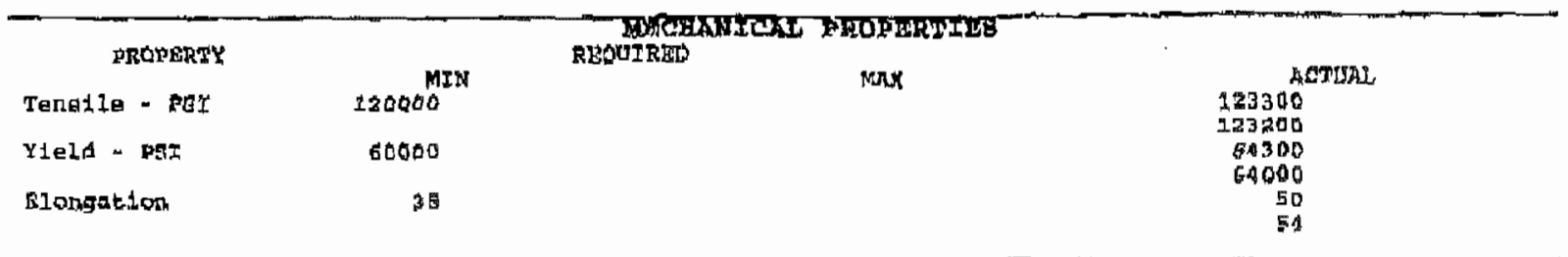

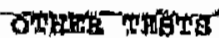

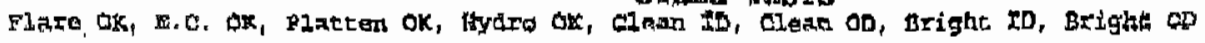

\begin{tabular}{|c|c|c|c|c|}
\hline $\begin{array}{l}\text { Hoge: } \\
\text { C: } 0.021 \\
\text { Cr: } 20.40 \\
T 1: 0.08\end{array}$ & $\begin{array}{l}\text { Mn: } 0,080 \\
\text { NL: } 03,02 \\
\text { ob: } 3,33\end{array}$ & $\begin{array}{l}\text { Meltex: Divy } \\
\text { P1 } 0.005 \\
\text { Ge: } 4.70\end{array}$ & $\begin{array}{l}\text { DSYBIS } \\
\text { g1 } 0.009 \\
\text { Mo: } \$, 27\end{array}$ & 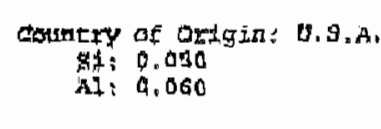 \\
\hline
\end{tabular}

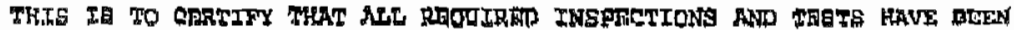

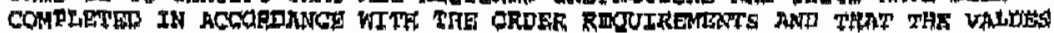

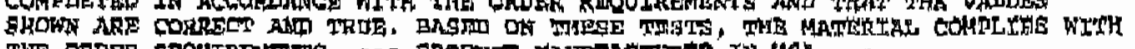

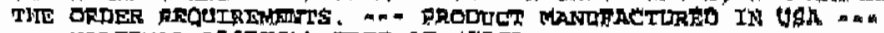

A.. MARFRIAL RRODUCG FHER OF RARCURY -

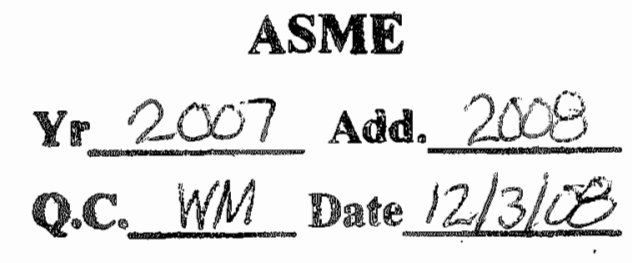

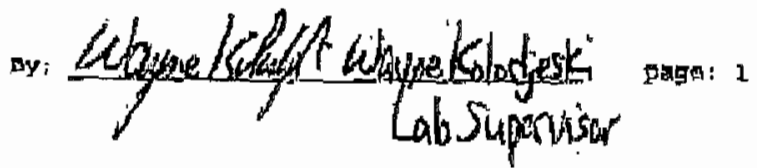

$D_{a}=0 \times 3-3-2066$ 


\section{BAKER INSPECTION GROUP}

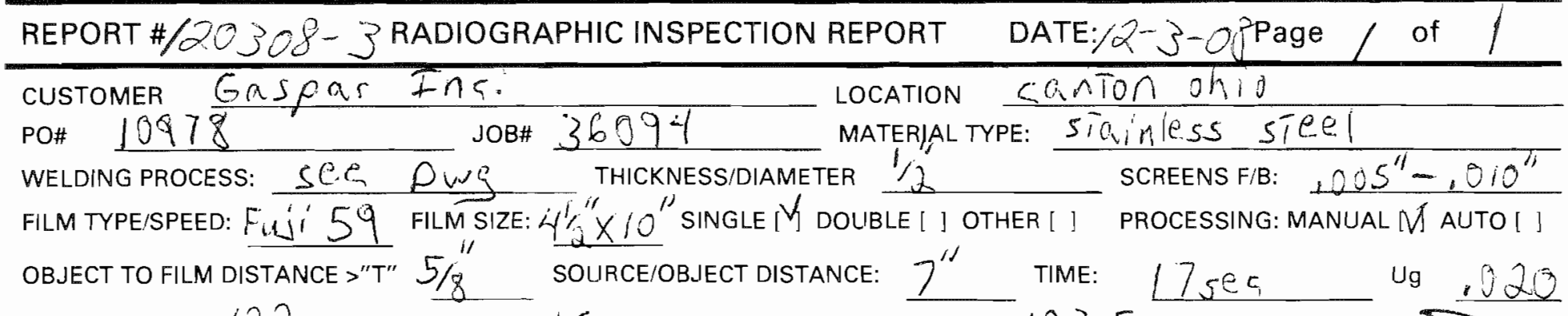

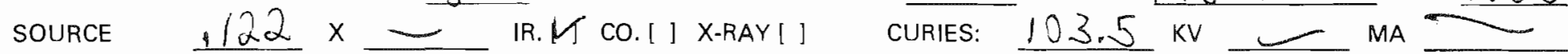
radiographic technique: Asme sec II Art 2 acceptance standard: Asme sec IIII uw 51

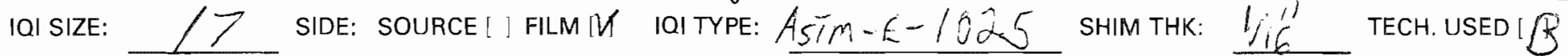
WE ASSUME NO RESPONSIBILITY FOR LOSSES OF ANY KIND DUE TO OUR INTERPRETATION OF THE QUALITY OF THE MATERIAL SUBMITTED. ALL DATA AND INFORMATION WILL BE HELD STRICTLY CONFIDENTIIAL.

\begin{tabular}{|c|c|c|c|c|c|c|c|c|c|c|c|}
\hline $\begin{array}{l}\text { SPECIMEN } \\
\text { IDENTIFICATION } \\
36094\end{array}$ & $\begin{array}{c}\text { FILM } \\
\text { INTERVAL } \\
\text { NUMBERS }\end{array}$ & 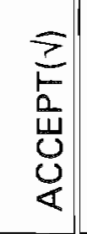 & 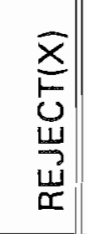 & 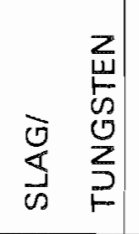 & $\begin{array}{l}\frac{Z}{c} \\
0 \\
0 \\
\frac{7}{0} \\
\Sigma\end{array}$ & 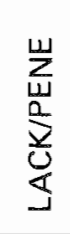 & $\begin{array}{l}z \\
\frac{0}{w n} \\
2 \\
\frac{1}{z} \\
0 \\
z\end{array}$ & 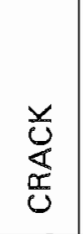 & $\begin{array}{l}\text { 岁 } \\
\frac{1}{4} \\
\frac{1}{\alpha^{2}} \\
\infty\end{array}$ & 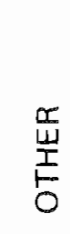 & $\begin{array}{l}\text { REMARKS AND/OR } \\
\text { DEFECT LOCATIONS } \\
\text { NOTE: N.A.D. = No Apparent } \\
\text { Defects }\end{array}$ \\
\hline \multirow[t]{14}{*}{$65+3$} & $0-1$ & $\sqrt{\prime}$ & & & & & & & $\zeta$ & & I.D \\
\hline & $1-2$ & $\sqrt{ }$ & & & & & & & $\zeta$ & & $I .0$ \\
\hline & $2-3$ & $\gamma$ & & & & & & & $\zeta$ & & I. O. \\
\hline & $3-0$ & $V$ & & & & & & & Y & & $I, D$ \\
\hline & & & & & & & & & & & \\
\hline & & & & & & & & & & & DUE TO WUELD \\
\hline & & & & & & & & & & & LPT FLANGE \\
\hline & & & & & & & & & & & MINIMUM FILM \\
\hline & & & & & & & & & & & COWERACE \\
\hline & & & & & & & & & & & \\
\hline & & & & & & & & & & & \\
\hline & & & & & & & & & & & AI REV EW E \\
\hline & & & & & & & & & & & $2 x-12-09-00$ \\
\hline & & & & & & & & & & & 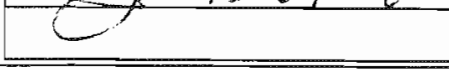 \\
\hline \multicolumn{2}{|c|}{ REVIEWER: $X / 2$} & \multicolumn{8}{|c|}{ RADIOGRAPHER: s. Lindsey } & \multicolumn{2}{|c|}{ CLIENT REVIEWER } \\
\hline \multicolumn{2}{|c|}{ SNT-TC1ALEVEL: } & \multicolumn{6}{|c|}{ SNT-TC1A LEVEL: II } & & & \multirow{2}{*}{\multicolumn{2}{|c|}{$\begin{array}{l}\text { Weldley Moroom } \\
\text { "C" = Diametervimited by }\end{array}$}} \\
\hline "A" = Pipe Diame & $\leq 31 / 2^{\prime \prime}$ & & $\mathrm{B}^{\prime \prime}=$ & ipe O.D & $\geq 31$ & & Unlin & nited & & & \\
\hline
\end{tabular}

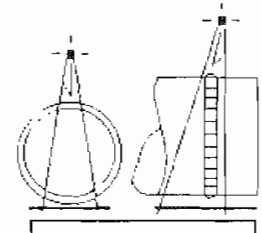

DWE/DWV

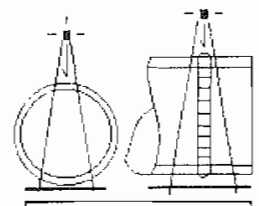

DWE/DWV

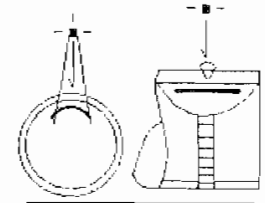

SWE/SWV

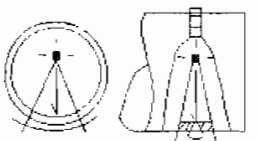

SWE/SWV

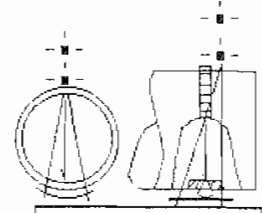

DWE/SWV

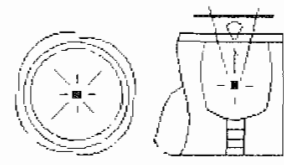

SWE/SWV 
36094 X-RAY MAP

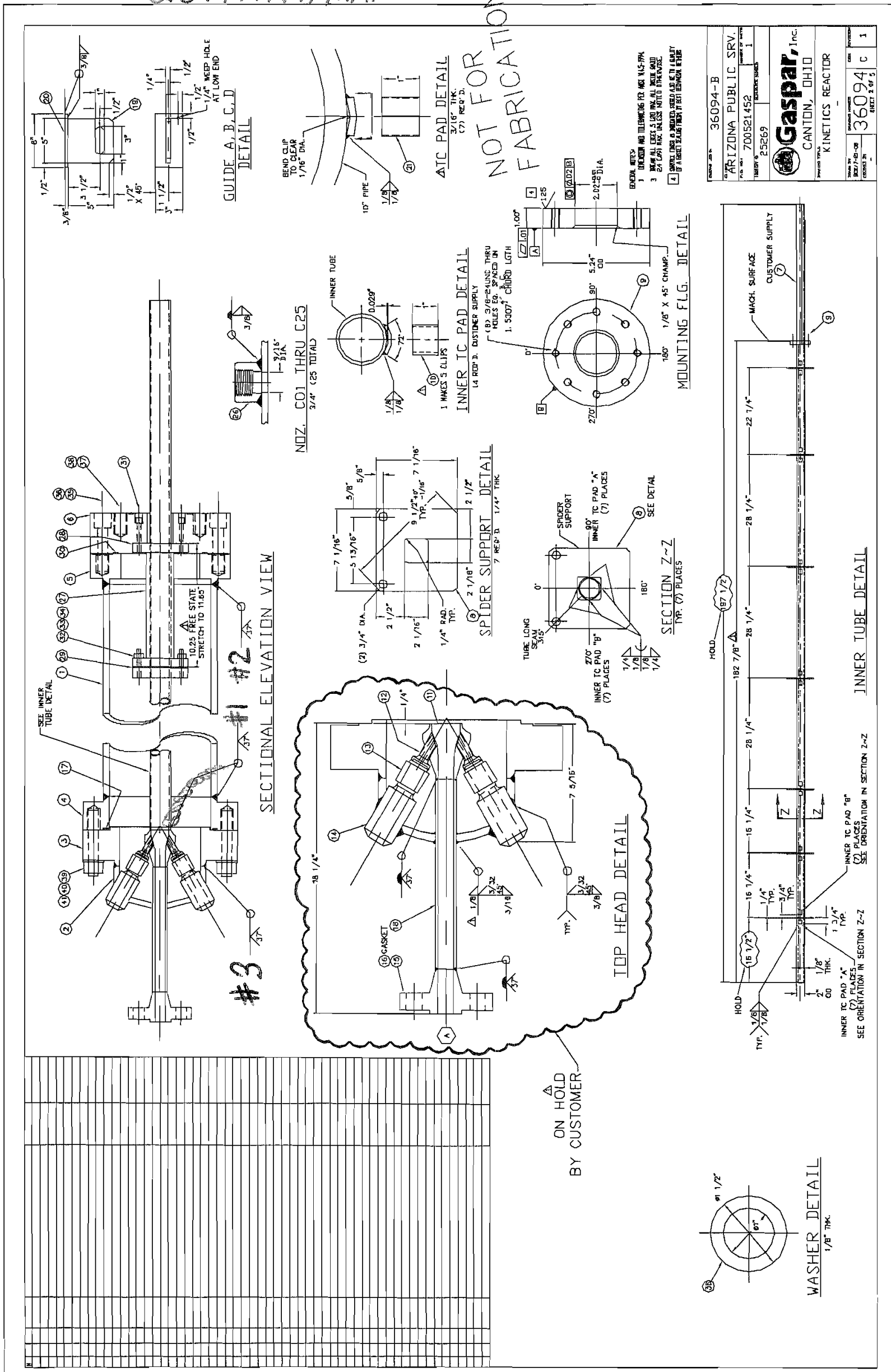




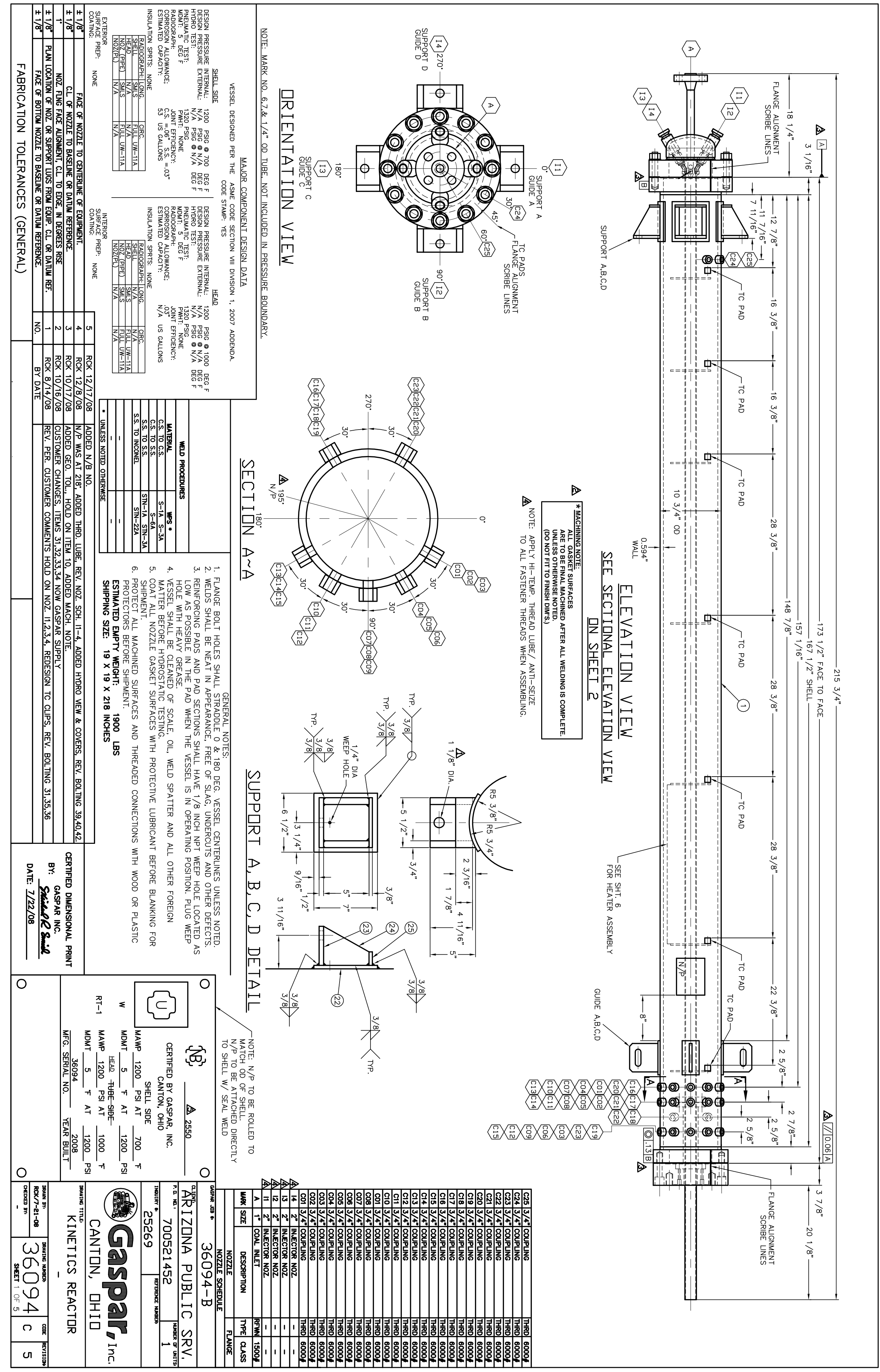




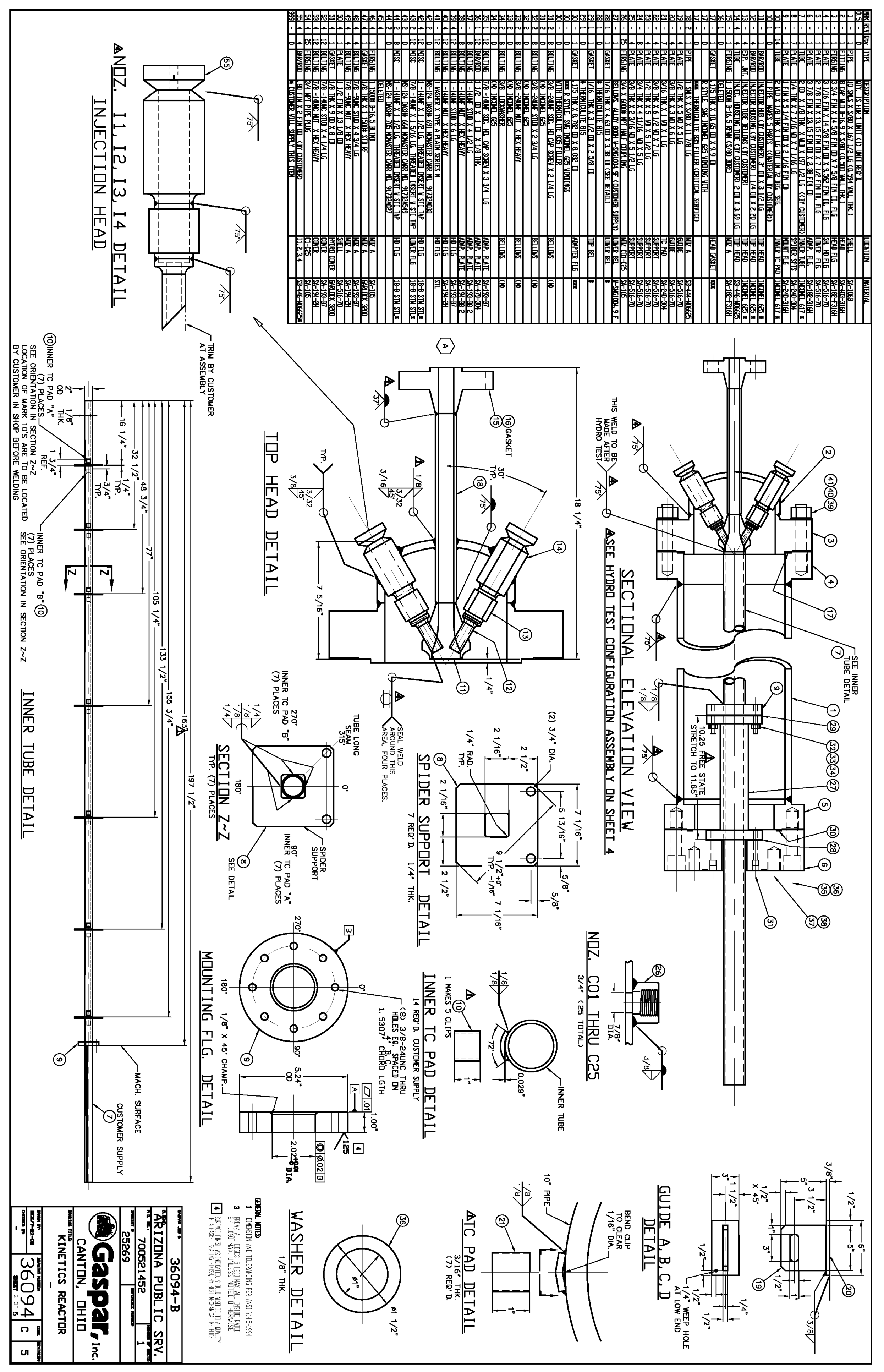




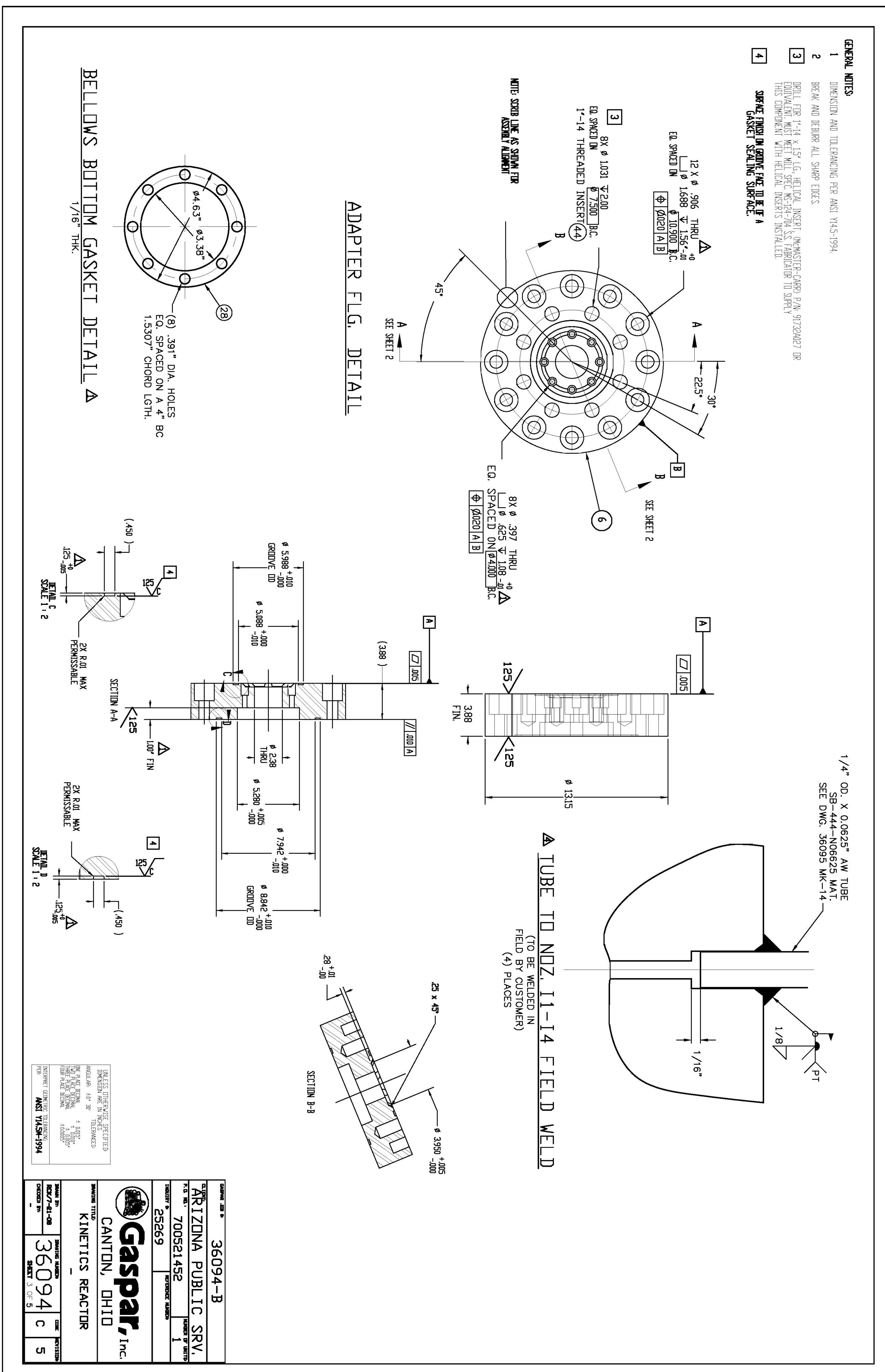



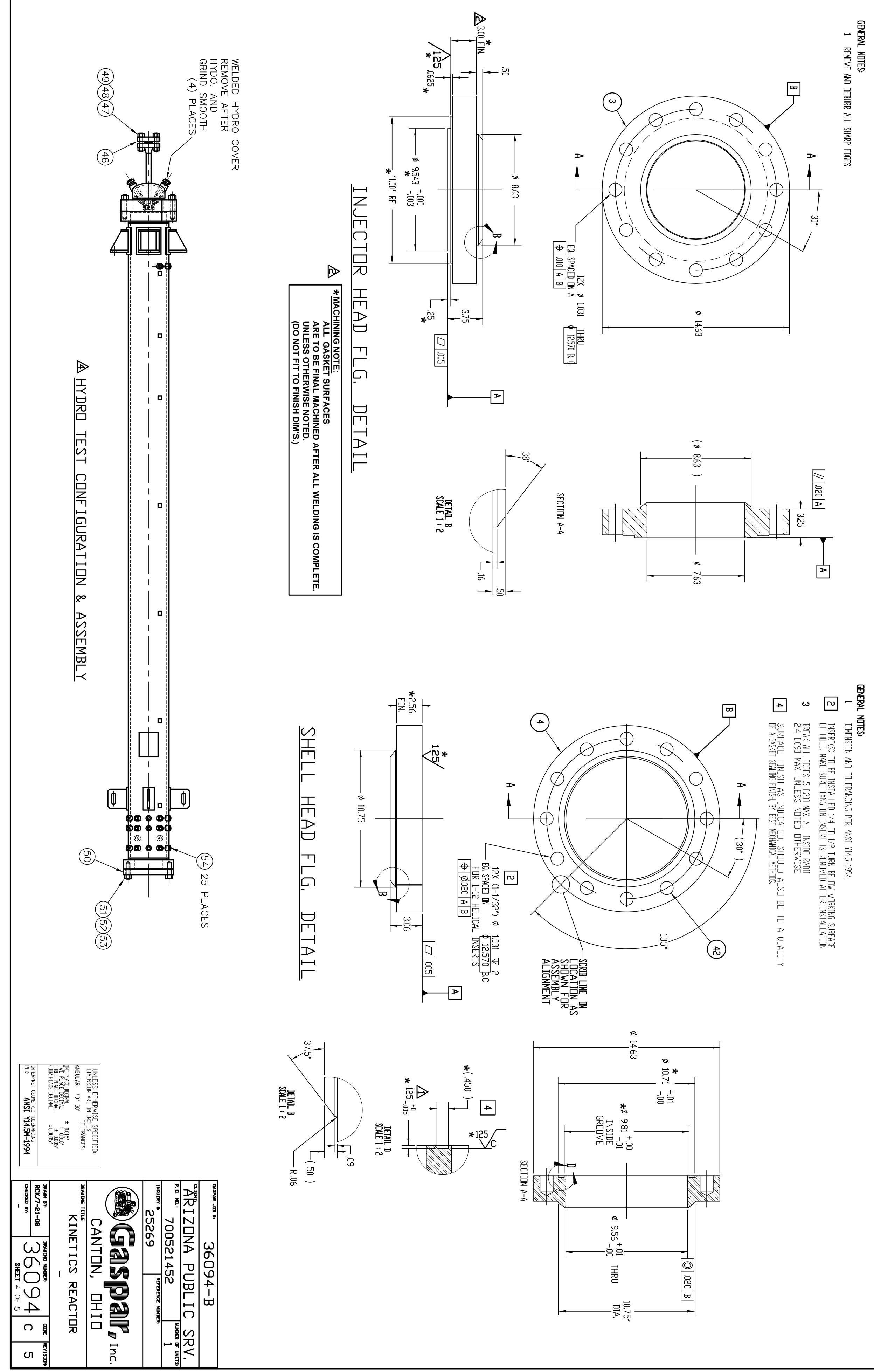

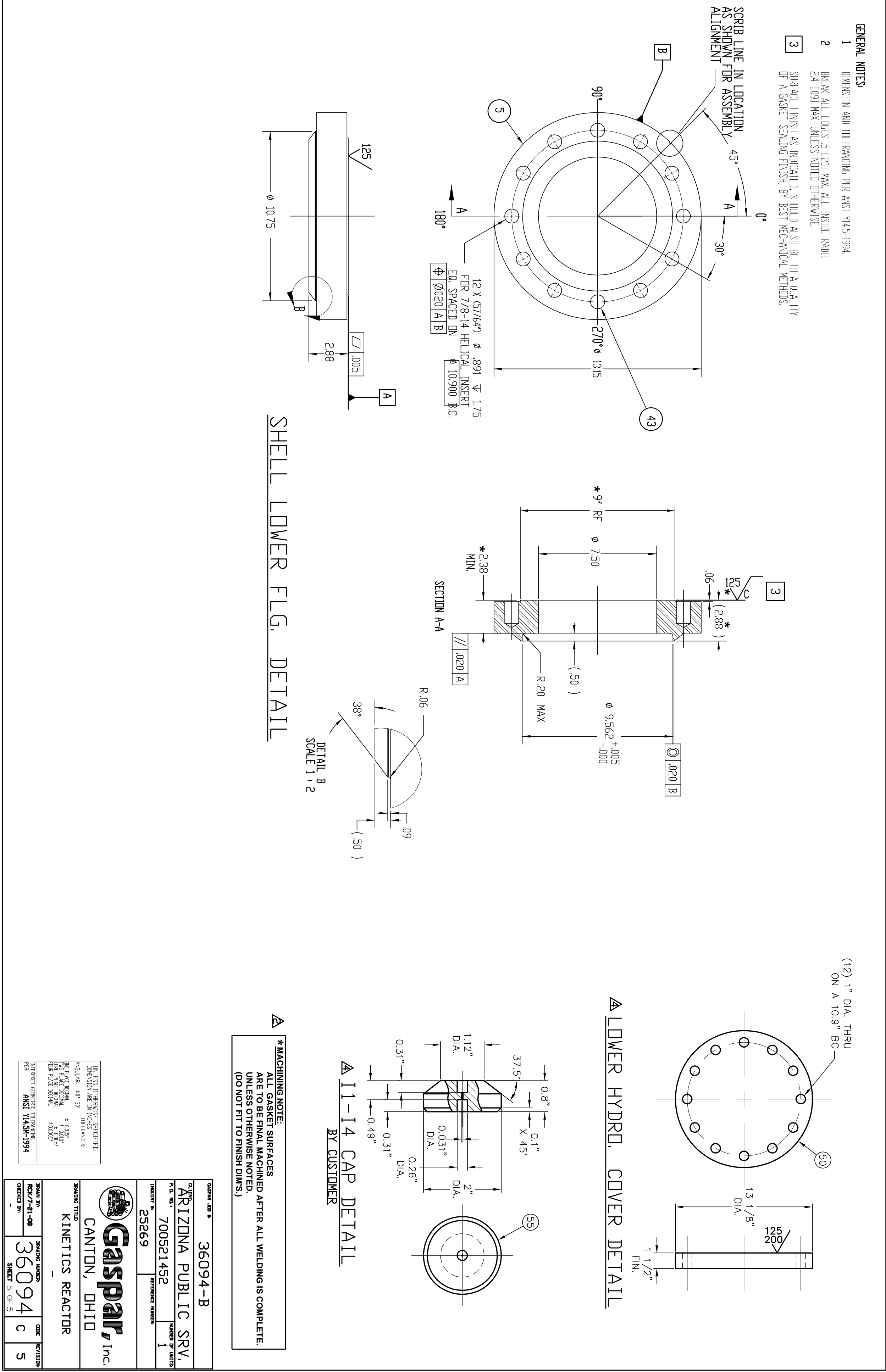


\section{CALCULATION PACKAGE}

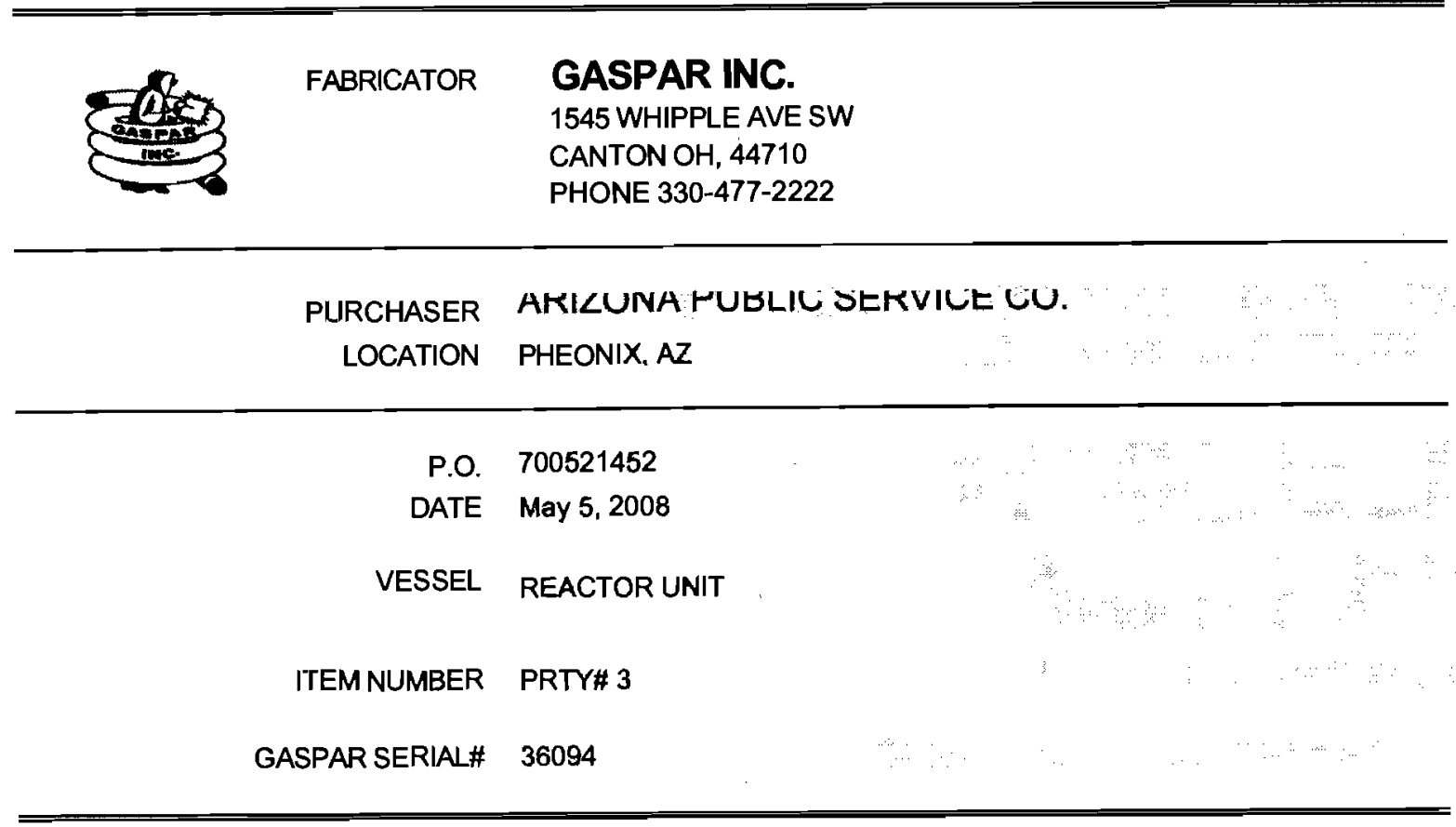

DEC 18, 2008: "FLANGE, SHELL HEAD" \& "FLANGE, REACTOR OUTER SHELL FOOT" WERE SA-105 MATERIAL.

DEC 10, 2008: ADDED INJECTOR CAP \& 1/4" OD TUBE CALCS

SEP 30, 2008: UPDATED NOZ "I1" THRU "I4" RADIAL ORIENTATION \& PROJECTION. PRESSURE TEST WAS PNEUMATIC.

\begin{tabular}{|c|c|c|}
\hline & 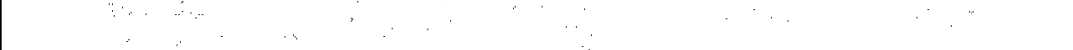 & \\
\hline & $\therefore$ & \\
\hline & $\therefore \therefore$ & 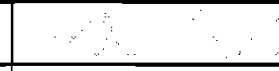 \\
\hline & - & $\cdots \cdots$ \\
\hline & क & \\
\hline & & 8 \\
\hline Dec 1B. 2008 & SEE NOTE ABOVE & Hateluan L Milk \\
\hline Dec 10, 2007 & SEE NOTE ABOVE & 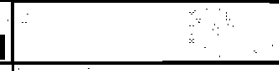 \\
\hline Sep 30, 2097 & SEE NOTE ABOVE & \\
\hline Jul 29; 2008 & FULL CALCULATION PACKAGE FOR APPROVAL & \\
\hline Jun 13,2008 & OUTER SHELL \& FLANGES ADDED TO CALCS & \\
\hline Jun 4, 2008 & FOR APPROVAL & \\
\hline DATE & DESCRIPTION & BY \\
\hline
\end{tabular}




\section{Table Of Contents}

1. Pressure Summary

2. Pneumatic Test

3. Weight Summary

4. INJECTOR WEIGHT (APPROX.)

5. INNER TUBE WEIGHT (APPROX)

6. HEATER WEIGHT, UPPER

7. HEATER WEIGHT, LOWER

8. CHAR POT WEIGHT

9. 8" WELD CAP SCH 80

10. Straight Flange on 8 " WELD CAP SCH 80

11. FLANGE, INJECTOR HEAD

12. FLANGE, SHELL HEAD

13. REACTOR OUTER SHELL

14. COAL INLET (A)

15. INJECTOR HOUSING (I1)

16. INJECTOR HOUSING (I2)

17. . INJECTOR HOUSING (I3)

18. INJECTOR HOUSING (I4)

19. COUPLING (CO1)

20. COUPLING $(\mathrm{CO2})$

21. COUPLING (C03)

22. COUPLING (C04)

23. COUPLING (CO5)

24. COUPLING (C06)

25. COUPLING (C07)

26. COUPLING (C08)

27. COUPLING (C09)

28. COUPLING (C10)

29. COUPLING (C11)

30. COUPLING (C12)

31. COUPLING (C13)

32. COUPLING (C14)

33. COUPLING (C15)

34. COUPLING (C16)

35. COUPLING (C17)

36. COUPLING (C18)

37. COUPLING (C19)

38. COUPLING (C20)

39. COUPLING (C21)

40. COUPLING (C22)

41. COUPLING (C23)

NOTE: EXTERNAL LOADS ON FLANGES NOT CONSIDERED IN DESIGN CALCULATIONS.

42. COUPLING (C24)

43. COUPLING (C25)

44. Wind Code

45. Seismic Code

46. SUPPORT LUGS

NOTE: "ADAPTER FLANGE, REACTOR TO CHAR POT" "KINETICS REACTOR INNER TUBE", AND 1/4" OD TUBE ARE NOT INCLUDED IN CODE PRESSURE BOUNDARY. 
47. FLANGE, REACTOR OUTER SHELL FOOT

48. ADAPTER FLANGE, REACTOR TO CHAR POT

49. INJECTOR CAP (I1 TO 14)

50. KINETICS REACTOR INNER TUBE

51. RUPTURE DESIGN FOR $1 / 4$ " TUBE

52. 1/4" TUBE TO VESSEL WELD 


\section{Pressure Summary}

\begin{tabular}{|c|c|c|c|c|c|c|}
\hline Identifier & $\begin{array}{c}\text { P } \\
\text { Design } \\
\text { ( psi) }\end{array}$ & $\begin{array}{c}\mathrm{T} \\
\text { Design } \\
\left({ }^{\circ} \mathrm{F}\right)\end{array}$ & $\underset{\text { ( psi) }}{\text { MAWP }}$ & $\begin{array}{c}\text { MDMT } \\
\left({ }^{\circ} \mathrm{F}\right)\end{array}$ & $\begin{array}{c}\text { MDMT } \\
\text { Exemption }\end{array}$ & $\begin{array}{l}\text { Impact } \\
\text { Tested }\end{array}$ \\
\hline$\underline{8}$ "WELD CAP SCH 80 & 1,200 & 1,000 & $1,596.11$ & -320 & Note 1 & No \\
\hline Straight Flange on $8^{\prime \prime}$ WELD CAP SCH 80 & 1,200 & 1,000 & $1,502.53$ & -320 & Note 2 & No \\
\hline REACTOR OUTER SHELL & 1,200 & 700 & $1,381.61$ & -21.5 & Note 3 & No \\
\hline FLANGE, INJECTOR HEAD & 1,200 & 700 & $1,258.75$ & -320 & Note 2 & No \\
\hline FLANGE, INJECTOR HEAD - Flange Hub & 1,200 & 700 & $1,378.83$ & -320 & Note 2 & No \\
\hline FLANGE, SHELL HEAD & 1,200 & 700 & $1,293.18$ & -49.49 & Note 4 & No \\
\hline FLANGE, SHELL HEAD - Flange Hub & 1,200 & 700 & $1,872.99$ & -55 & Note 5 & No \\
\hline SUPPORT LUGS & 1,200 & 700 & $1,258.75$ & N/A & N/A & N/A \\
\hline COAL INLET (A) & 1,200 & 1,000 & $1,263.39$ & -55 & Note 6 & No \\
\hline COUPLING (C01) & 1,200 & 700 & $1,381.47$ & -155 & Note 7 & No \\
\hline COUPLING (CO2) & 1,200 & 700 & $1,381.47$ & -155 & Note 7 & No \\
\hline COUPLING (C03) & 1,200 & 700 & $1,381.47$ & -155 & Note 7 & No \\
\hline COUPLING (C04) & 1,200 & 700 & $1,381.47$ & -155 & Note 7 & No \\
\hline COUPLING (C05) & 1,200 & 700 & $1,381.47$ & -155 & Note 7 & No \\
\hline COUPLING (C06) & 1,200 & 700 & $1,381.47$ & -155 & Note 7 & No \\
\hline COUPLING (C07) & 1,200 & 700 & $1,381.47$ & -155 & Note 7 & No \\
\hline COUPLING (C08) & 1,200 & 700 & $1,381.47$ & -155 & Note 7 & No \\
\hline COUPLING_(C09) & 1,200 & 700 & $1,381.47$ & -155 & Note 7 & No \\
\hline COUPLING (C10) & 1,200 & 700 & $1,381.47$ & -155 & Note 7 & No \\
\hline COUPLING (C11) & 1,200 & 700 & $1,381.47$ & -155 & Note 7 & No \\
\hline COUPLING (C12) & 1,200 & 700 & $1,381.47$ & -155 & Note 7 & No \\
\hline COUPLING (C13) & 1,200 & 700 & $1,381.47$ & -155 & Note 7 & No \\
\hline COUPLING $(\mathrm{Cl} 4)$ & 1,200 & 700 & $1,381.47$ & -155 & Note 7 & No \\
\hline COUPLING (C15) & 1,200 & 700 & $1,381.47$ & -155 & Note 7 & No \\
\hline COUPLING (C16) & 1,200 & 700 & $1,381.47$ & -155 & Note 7 & No \\
\hline COUPLING (C17) & 1,200 & 700 & $1,381.47$ & -155 & Note 7 & No \\
\hline COUPLING (C18) & 1,200 & 700 & $1,381.47$ & -155 & Note 7 & No \\
\hline COUPLING (C19) & 1,200 & 700 & $1,381.47$ & -155 & Note 7 & No \\
\hline COUPLING (C20) & 1,200 & 700 & $1,381.47$ & -155 & Note 7 & No \\
\hline COUPLING (C21) & 1,200 & 700 & $1,381,47$ & -155 & Note 7 & No \\
\hline COUPLING (C22) & 1,200 & 700 & $1,381.47$ & -155 & Note 7 & No \\
\hline COUPLING (C23) & 1,200 & 700 & $1,381.47$ & -155 & Note 7 & No \\
\hline COUPLING (C24) & 1,200 & 700 & $1,381.47$ & -155 & Note 7 & No \\
\hline COUPLING (C25) & 1,200 & 700 & $1,381.47$ & -155 & Note 7 & No \\
\hline INJECTOR HOUSING (I1) & 1,200 & 1,000 & $1,596.14$ & -325 & Note 8 & No \\
\hline
\end{tabular}




\begin{tabular}{|l|r|r|r|r|r|r|} 
INJECTOR HOUSING (I2) & 1,200 & 1,000 & $1,596.14$ & -325 & Note 8 & No \\
\hline INJECTOR HOUSING (I3) & 1,200 & 1,000 & $1,596.14$ & -325 & Note 8 & No \\
\hline INJECTOR HOUSING (I4) & 1,200 & 1,000 & $1,596.14$ & -325 & Note 8 & No \\
\hline
\end{tabular}

Chamber design MDMT is $5^{\circ} \mathrm{F}$

Chamber rated MDMT is $-21.5^{\circ} \mathrm{F} @ 1,258.75$ psi

Chamber MAWP hot \& corroded is $1,258.75$ psi @ $700^{\circ} \mathrm{F}$

This pressure chamber is not designed for external pressure.

\section{Notes for MDMT Rating:}

\begin{tabular}{|c|c|c|}
\hline $\begin{array}{c}\text { Note } \\
\#\end{array}$ & Exemption & Details \\
\hline 1. & Straight Flange governs MDMT & \\
\hline 2. & $\begin{array}{l}\text { Rated MDMT per UHA-51(d)(1)(b), (carbon content does not exceed } 0.1 \text { percent) } \\
=-320^{\circ} \mathrm{F}\end{array}$ & \\
\hline 3. & $\begin{array}{l}\text { Material impact test exemption temperature from Fig UCS- } 66 \text { Curve } \mathrm{B}=-5.1^{\circ} \mathrm{F} \\
\text { Fig UCS-66.1 MDMT reduction }=16.4^{\circ} \mathrm{F} \text {, (coincident ratio }=0.8359815 \text { ) }\end{array}$ & UCS- 66 governing thickness $=0.5198$ in \\
\hline 4. & $\begin{array}{l}\text { Flange impact test exemption temperature from Fig UCS-66 Curve } \mathrm{D}=-49.49^{\circ} \mathrm{F} \\
\text { UCS-66 governing thickness }=0.594 \text { in }\end{array}$ & $\begin{array}{l}\text { Bolts rated MDMT per Fig UCS- } 66 \text { note }(\mathrm{c})=- \\
55^{\circ} \mathrm{F}\end{array}$ \\
\hline 5. & $\begin{array}{l}\text { Material impact test exemption temperature from Fig UCS-66 Curve } \mathrm{D}=-53.74^{\circ} \\
\text { F } \\
\left.\text { Fig UCS-66.1 MDMT reduction }=38.2^{\circ} \mathrm{F} \text {, (coincident ratio }=0.6178278\right) \\
\text { Rated MDMT is governed by UCS- } 66(\mathrm{~b})(2)\end{array}$ & UCS-66 governing thickness $=0.5198$ in \\
\hline 6. & $\begin{array}{l}\text { Flange rating governs: } \\
\text { Flange rated MDMT }=-320^{\circ} \mathrm{F} \\
\text { Bolts rated MDMT per Fig UCS- } 66 \text { note }(e)=-55^{\circ} \mathrm{F}\end{array}$ & Per UHA-51(d)(1)(a) \\
\hline 7. & $\begin{array}{l}\text { Nozzle is impact test exempt to }-155^{\circ} \mathrm{F} \text { per } \mathrm{UCS}-66(\mathrm{~b})(3) \text { (coincident ratio }= \\
0.11344 \text { ). }\end{array}$ & \\
\hline 8. & Rated MDMT per UNF- $65=-325^{\circ} \mathrm{F}$ & \\
\hline
\end{tabular}




\section{Pneumatic Test}

\section{Shop test pressure determination} based on design $P$ per UG-100(b)

Shop pneumatic test gauge pressure is 1,320 psi at $70^{\circ} \mathrm{F}$ (the chamber design $\mathrm{P}=1,200 \mathrm{psi}$ )

The shop test is performed with the vessel in the horizontal position.

\begin{tabular}{|c|c|c|c|c|}
\hline Identifier & $\begin{array}{c}\text { Local test } \\
\text { pressure } \\
\text { psi }\end{array}$ & $\begin{array}{c}\text { Test liquid } \\
\text { static head } \\
\text { psi }\end{array}$ & $\begin{array}{c}\text { UG-100 } \\
\text { stress } \\
\text { ratio }\end{array}$ & $\begin{array}{c}\text { UG-100 } \\
\text { pressure } \\
\text { factor }\end{array}$ \\
\hline $8^{\prime \prime}$ WELD CAP SCH 80 & 1,320 & 0 & 1.3072 & 1.10 \\
\hline Straight Flange on 8 " WELD CAP SCH 80 & 1,320 & 0 & 1.3072 & 1.10 \\
\hline REACTOR OUTER SHELL & 1,320 & 0 & 1.0962 & 1.10 \\
\hline FLANGE, INJECTOR HEAD (1) & 1,320 & 0 & 1 & 1.10 \\
\hline FLANGE, SHELL HEAD & 1,320 & 0 & 1 & 1.10 \\
\hline COAL INLET (A) & 1,320 & 0 & 1.1472 & 1.10 \\
\hline COUPLING (C01) & 1,320 & 0 & 1.1628 & 1.10 \\
\hline COUPLING $(\mathrm{CO} 2)$ & 1,320 & 0 & 1.1628 & 1.10 \\
\hline COUPLING (C03) & 1,320 & 0 & 1.1628 & 1.10 \\
\hline COUPLING (C04) & 1,320 & 0 & 1.1628 & 1.10 \\
\hline COUPLING (C05) & 1,320 & 0 & 1.1628 & 1.10 \\
\hline COUPLING (C06) & 1,320 & 0 & 1.1628 & 1.10 \\
\hline COUPLING (C07) & 1,320 & 0 & 1.1628 & 1.10 \\
\hline COUPLING (C08) & 1,320 & 0 & 1.1628 & 1.10 \\
\hline COUPLING (C09) & 1,320 & 0 & 1.1628 & 1.10 \\
\hline COUPLING (C10) & 1,320 & 0 & 1.1628 & 1.10 \\
\hline COUPLING (C11) & 1,320 & 0 & 1.1628 & 1.10 \\
\hline COUPLING (C12) & 1,320 & 0 & 1.1628 & 1.10 \\
\hline COUPLING (C13) & 1,320 & 0 & 1.1628 & 1.10 \\
\hline COUPLING (C14) & 1,320 & 0 & 1.1628 & 1.10 \\
\hline COUPLING (C15) & 1,320 & 0 & 1.1628 & 1.10 \\
\hline COUPLING (C16) & 1,320 & 0 & 1.1628 & 1.10 \\
\hline COUPLING (C17) & 1,320 & 0 & 1.1628 & 1.10 \\
\hline COUPLING (C18) & 1,320 & 0 & 1.1628 & 1.10 \\
\hline COUPLING (C19) & 1,320 & 0 & 1.1628 & 1.10 \\
\hline COUPLING (C20) & 1,320 & 0 & 1.1628 & 1.10 \\
\hline COUPLING (C21) & 1,320 & 0 & 1.1628 & 1.10 \\
\hline COUPLING (C22) & 1,320 & 0 & 1.1628 & 1.10 \\
\hline COUPLING (C23) & 1,320 & 0 & 1.1628 & 1.10 \\
\hline COUPLING (C24) & 1,320 & 0 & 1.1628 & 1.10 \\
\hline COUPLING (C25) & 1,320 & 0 & 1.1628 & 1.10 \\
\hline
\end{tabular}




\begin{tabular}{|l|r|r|r|r|} 
INJECTOR HOUSING (I1) & 1,320 & 0 & 1.146 & 1.10 \\
\hline INJECTOR HOUSING (I2) & 1,320 & 0 & 1.146 & 1.10 \\
\hline INJECTOR HOUSING (I3) & 1,320 & 0 & 1.146 & 1.10 \\
\hline INJECTOR HOUSING (I4) & 1,320 & 0 & 1.146 & 1.10 \\
\hline
\end{tabular}

Notes:

(1) FLANGE, INJECTOR HEAD limits the UG-100 stress ratio.

(2) The zero degree angular position is assumed to be up, and the test liquid height is assumed to the top-most flange. 


\section{Weight Summary}

\begin{tabular}{|l|r|r|r|r|r|r|r|}
\hline \multirow{2}{*}{ Component } & \multicolumn{6}{|c|}{ Weight ( lb) Contributed by Vessel Elements } \\
\cline { 2 - 8 } & $\begin{array}{l}\text { Metal } \\
\text { New* }\end{array}$ & $\begin{array}{c}\text { Metal } \\
\text { Corroded* }\end{array}$ & $\begin{array}{c}\text { Insulation \& } \\
\text { Supports }\end{array}$ & Lining & $\begin{array}{c}\text { Piping } \\
\text { + Liquid }\end{array}$ & $\begin{array}{c}\text { Operating } \\
\text { Liquid }\end{array}$ & $\begin{array}{c}\text { Test } \\
\text { Liquid }\end{array}$ \\
\hline 8" WELD CAP SCH 80 & 13.8 & 12.9 & 0 & 0 & 0 & 0 & 0 \\
\hline REACTOR OUTER SHELL & 888.6 & 803.6 & 0 & 0 & 0 & 0 & 0 \\
\hline SUPPORT LUGS & 63.2 & 63.2 & 0 & 0 & 0 & 0 & 0 \\
\hline TOTAL: & $\mathbf{9 6 5 . 6}$ & $\mathbf{8 7 9 . 8}$ & $\mathbf{0}$ & $\mathbf{0}$ & $\mathbf{0}$ & $\mathbf{0}$ & $\mathbf{0}$ \\
\hline
\end{tabular}

* Shells with attached nozzles have weight reduced by material cut out for opening.

\begin{tabular}{|c|c|c|c|c|c|c|c|c|c|}
\hline \multirow{3}{*}{ Component } & \multicolumn{9}{|c|}{ Weight ( lb) Contributed by Attachments } \\
\hline & \multicolumn{2}{|c|}{ Body Flanges } & \multicolumn{2}{|c|}{$\begin{array}{c}\text { Nozzles \& } \\
\text { Flanges }\end{array}$} & \multirow{2}{*}{$\begin{array}{c}\text { Packed } \\
\text { Beds }\end{array}$} & \multirow{2}{*}{$\begin{array}{l}\text { Ladders \& } \\
\text { Platforms }\end{array}$} & \multirow{2}{*}{$\begin{array}{l}\text { Trays \& } \\
\text { Supports }\end{array}$} & \multirow{2}{*}{$\begin{array}{c}\text { Rings \& } \\
\text { Clips }\end{array}$} & \multirow{2}{*}{$\begin{array}{c}\text { Vertical } \\
\text { Loads }\end{array}$} \\
\hline & New & Corroded & New & Corroded & & & & & \\
\hline 8" WELD CAP SCH 80 & 109 & 108.3 & 16.6 & 15.8 & 0 & 0 & 0 & 0 & 0 \\
\hline REACTOR OUTER SHELL & 69.7 & 68.1 & 11.7 & 10.9 & 0 & 0 & 0 & 0 & $910^{*}$ \\
\hline TOTAL: & 178.8 & 176.4 & 28.3 & 26.7 & $\mathbf{0}$ & 0 & $\mathbf{0}$ & 0 & $910^{*}$ \\
\hline
\end{tabular}

* This number includes vertical loads which are not present in all conditions.

Vessel operating weight, Corroded: $1,993 \mathrm{lb}$

Vessel operating weight, New: $\quad 2,083 \mathrm{lb}$

Vessel empty weight, Corroded: $\quad 1,993 \mathrm{lb}$

Vessel empty weight, New: $\quad 2,083 \mathrm{lb}$

Vessel test weight, New: $\quad 1,323 \mathrm{lb}$

\section{Vessel center of gravity location - from datum - lift condition}

Vessel Lift Weight, New: 1,583 lb

Center of Gravity: $\quad$ 102.9366"

\section{Vessel Capacity}

Vessel Capacity** (New): 53 US gal

Vessel Capacity** (Corroded): 54 US gal

**The vessel capacity does not include volume of nozzle, piping or other attachments. 


\section{INJECTOR WEIGHT (APPROX.)}

$\begin{array}{ll}\text { Load Orientation } & \text { Vertical Load } \\ \text { Elevation above datum: } & 170^{\prime \prime} \\ \text { Direction angle: } & 0.00 \text { degrees }\end{array}$

Distance from center of vessel: $0^{\prime \prime}$

Magnitude of force: $\quad 30 \mathrm{lb}$

Present when operating: Yes

Included in vessel lift weight: Yes

Present when vessel is empty: Yes

Present during hydrotest: Yes 


\section{INNER TUBE WEIGHT (APPROX)}

$\begin{array}{ll}\text { Load Orientation } & \text { Vertical Load } \\ \text { Elevation above datum: } & 95^{\prime \prime} \\ \text { Direction angle: } & 0.00 \text { degrees }\end{array}$

Distance from center of vessel: 0 "

Magnitude of force:

$120 \mathrm{lb}$

Present when operating: Yes

Included in vessel lift weight: Yes

Present when vessel is empty: Yes

Present during hydrotest: Yes 


\section{HEATER WEIGHT, UPPER}
Load Orientation
Vertical Load
Elevation above datum:
$122.875^{\prime \prime}$
Direction angle:
0.00 degrees

Distance from center of vessel: $0^{\prime \prime}$

Magnitude of force:

$130 \mathrm{lb}$

Present when operating: Yes

Included in vessel lift weight: Yes

Present when vessel is empty: Yes

Present during hydrotest: No 


\section{HEATER WEIGHT, LOWER}

$\begin{array}{ll}\text { Load Orientation } & \text { Vertical Load } \\ \text { Elevation above datum: } & 42.875^{\prime \prime} \\ \text { Direction angle: } & 0.00 \text { degrees }\end{array}$

Distance from center of vessel: $0^{\prime \prime}$

Magnitude of force:

$130 \mathrm{lb}$

Present when operating: $\quad$ Yes

Included in vessel lift weight: Yes

Present when vessel is empty: Yes

Present during hydrotest: No 


\section{CHAR POT WEIGHT}

$\begin{array}{ll}\text { Load Orientation } & \text { Vertical Load } \\ \text { Elevation above datum: } & 0^{\prime \prime} \\ \text { Direction angle: } & 0.00 \text { degrees }\end{array}$

Distance from center of vessel: $0^{\prime \prime}$

Magnitude of force:

$500 \mathrm{lb}$

Present when operating: $\quad$ Yes

Included in vessel lift weight: No

Present when vessel is empty: Yes

Present during hydrotest: No 


\section{8" WELD CAP SCH 80}

\section{ASME Section VIII, Division 1, 2007 Edition}

Component:

Material Specification:

Straight Flange governs MDMT

Internal design pressure: $\mathrm{P}=1,200 \mathrm{psi} @ 1,000^{\circ} \mathrm{F}$

\section{Static liquid head:}

$\mathrm{P}_{\mathrm{s}}=0 \mathrm{psi}\left(\mathrm{SG}=1, \mathrm{H}_{\mathrm{s}}=0\right.$ " Operating head $)$

Corrosion allowance: $\quad$ Inner $\mathrm{C}=0.03 " \quad$ Outer $\mathrm{C}=0$ "

Design $\mathrm{MDMT}=5^{\circ} \mathrm{F}$

Rated MDMT $=-320^{\circ} \mathrm{F}$

No impact test performed

Material is not normalized

Material is not produced to fine grain practice

PWHT is not performed

Do not Optimize MDMT / Find MAWP
Seamless No RT

Full UW-11(a) Type 1

$$
\text { corr }=12.9 \mathrm{lb}
$$$$
\text { corr }=0.6 \mathrm{US} \mathrm{gal}
$$

Capacity*: new $=0.6 \mathrm{US}$ gal

* includes straight flange

Outer diameter $\quad=8.625^{\prime \prime}$

Minimum head thickness $\quad=0.4375^{\prime \prime}$

Head ratio D/2h $\quad=2$ (new)

Head ratio $\mathrm{D} / 2 \mathrm{~h}=1.9848$ (corroded)

Straight flange length $\mathrm{L}_{\text {sf }} \quad=1.68^{\prime \prime}$

Nominal straight flange thickness $t_{\text {sf }}=0.4375^{\prime \prime}$

\section{Results Summary}

The governing condition is internal pressure.

Minimum thickness per UG-16 $=0.0625^{\prime \prime}+0.03^{\prime \prime}=0.0925^{\prime \prime}$

Design thickness due to internal pressure $(t) \quad=0.343 "$

Maximum allowable working pressure (MAWP) $=1,596.11 \mathrm{psi}$

\section{K (Corroded)}

$$
\begin{aligned}
\mathrm{K} & =(1 / 6)^{*}\left[2+(\mathrm{D} /(2 * \mathrm{~h}))^{2}\right] \\
& =(1 / 6)^{*}\left[2+\left(7.81 /\left(2^{*} 1.9675\right)\right)^{2}\right] \\
& =0.989874
\end{aligned}
$$

$$
\begin{aligned}
& \mathbf{K}(\mathbf{N e w}) \\
& \begin{aligned}
\mathrm{K} & =(1 / 6)^{*}\left[2+\left(\mathrm{D} /\left(2^{*} \mathrm{~h}\right)\right)^{2}\right] \\
& =(1 / 6)^{*}\left[2+(7.75 /(2 * 1.9375))^{2}\right] \\
& =
\end{aligned}
\end{aligned}
$$

Design thickness for internal pressure, (Corroded at 1,000 ${ }^{\circ}$ F) Appendix 1-4(c) 
$\mathrm{t}=\mathrm{P} * \mathrm{D}_{\mathrm{o}}^{*} \mathrm{~K} /(2 * \mathrm{~S} * \mathrm{E}+2 * \mathrm{P} *(\mathrm{~K}-0.1))+$ Corrosion

$=1,200 * 8.625 * 0.989874 /(2 * 15,300 * 1+2 * 1,200 *(0.989874-0.1))+0.03$

$=0.343 "$

The head internal pressure design thickness is $\underline{0.343 "}$.

Maximum allowable working pressure, (Corroded at 1,000 $\left.{ }^{\circ} \mathrm{F}\right)$ Appendix 1-4(c)

$$
\begin{aligned}
\mathrm{P} & =2 * \mathrm{~S} * \mathrm{E}^{*} \mathrm{t} /\left(\mathrm{K}^{*} \mathrm{D}_{\mathrm{o}}-2 * \mathrm{t}^{*}(\mathrm{~K}-0.1)\right)-\mathrm{P}_{\mathrm{s}} \\
& =2 * 15,300^{*} 1 * 0.4075 /(0.989874 * 8.625-2 * 0.4075 *(0.989874-0.1))-0 \\
& =1,596.11 \mathrm{psi}
\end{aligned}
$$

The maximum allowable working pressure (MAWP) is $\underline{1,596.11} \mathrm{psi}$.

\% Forming strain - UHA-44(a)(2)(b)

$$
\begin{aligned}
\mathrm{EFE} & =\left(75^{*} \mathrm{t} / \mathrm{R}_{\mathrm{f}}\right) *\left(1-\mathrm{R}_{\mathrm{f}} / \mathrm{R}_{\mathrm{o}}\right) \\
& =\left(75^{*} 0.4375 / 1.5363\right)^{*}(1-1.5363 / \infty) \\
& =21.3588 \%
\end{aligned}
$$




\section{Straight Flange on 8" WELD CAP SCH 80}

ASME Section VIII Division 1, 2007 Edition

Component:

Material specification:

Rated MDMT per UHA-51(d)(1)(b), (carbon content does not exceed 0.1 percent) $=-320^{\circ} \mathrm{F}$

Straight Flange

SA-403 316H (II-D p. 78, ln. 8)
Internal design pressure: $\mathrm{P}=1,200 \mathrm{psi} @ 1,000^{\circ} \mathrm{F}$

Static liquid head:

$P_{\text {th }}=0 \mathrm{psi}\left(\mathrm{SG}=0, \mathrm{H}_{\mathrm{s}}=9.6584^{\prime \prime}\right.$, Horizontal test head $)$

Corrosion allowance $\quad$ Inner $\mathrm{C}=\mathbf{0 . 0 3 "} \quad$ Outer $\mathrm{C}=\mathbf{0}^{\prime \prime}$
Design MDMT $=5^{\circ} \mathrm{F}$
No impact test performed
Rated MDMT $=-320^{\circ} \mathrm{F}$
Material is not normalized
Material is not produced to Fine Grain Practice
PWHT is not performed

Radiography: Longitudinal joint - $\quad$ Seamless No RT

Circumferential joint - Full UW-11(a) Type 1

Estimated weight New $=5.5 \mathrm{lb} \quad$ corr $=5.1 \mathrm{lb}$

Capacity New $=0.34$ US gal corr $=0.35$ US gal

$\mathrm{OD} \quad=8.625^{\prime \prime}$

Length $\mathrm{L}_{\mathrm{c}}=1.68^{\prime \prime}$

$\mathrm{t} \quad=0.4375^{\prime \prime}$

Design thickness, (at $1,000{ }^{\circ}$ F) Appendix 1-1

$\mathrm{t}=\mathrm{P} * \mathrm{R}_{\mathrm{o}} /(\mathrm{S} * \mathrm{E}+0.40 * \mathrm{P})+$ Corrosion

$=1,200 * 4.3125 /(15,300 * 1.00+0.40 * 1,200)+0.03$

$=0.358^{\prime \prime}$

Maximum allowable working pressure, $\left(\right.$ at 1,000 ${ }^{\circ}$ F) Appendix 1-1

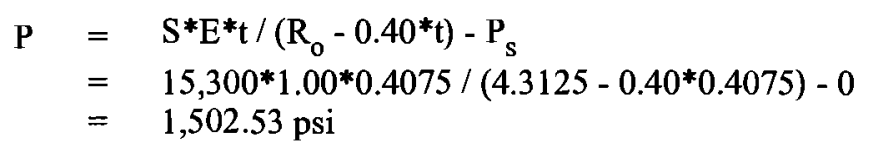

\% Forming strain - UHA-44(a)(2)(a)

$$
\begin{aligned}
\mathrm{EFE} & =\left(50 * \mathrm{t} / \mathrm{R}_{\mathrm{f}}\right) *\left(1-\mathrm{R}_{\mathrm{f}} / \mathrm{R}_{\mathrm{o}}\right) \\
& =(50 * 0.4375 / 4.0938)^{*}(1-4.0938 / \infty) \\
& =5.3435 \%
\end{aligned}
$$

\section{Design thickness $=0.358 "$}

The governing condition is due to internal pressure. 
The cylinder thickness of $0.4375^{\prime \prime}$ is adequate.

Thickness Required Due to Pressure + External Loads

\begin{tabular}{|c|c|c|c|c|c|c|c|c|}
\hline \multirow[t]{2}{*}{ Condition } & \multirow{2}{*}{$\begin{array}{l}\text { Pressure } \\
\text { P ( psi) }\end{array}$} & \multicolumn{2}{|c|}{$\begin{array}{l}\text { Allowable Stress } \\
\text { Before UG-23 Stress } \\
\text { Increase ( psi) }\end{array}$} & \multirow{2}{*}{$\begin{array}{c}\text { Temperature } \\
\left({ }^{\circ} \mathrm{F}\right)\end{array}$} & \multirow[t]{2}{*}{$\begin{array}{c}\text { Corrosion } \\
\text { C (in) }\end{array}$} & \multirow[t]{2}{*}{ Load } & \multirow{2}{*}{$\begin{array}{l}\text { Req'd Thk } \\
\text { Due to } \\
\text { Tension (in) }\end{array}$} & \multirow{2}{*}{$\begin{array}{l}\text { Req'd Thk Due to } \\
\text { Compression (in) }\end{array}$} \\
\hline & & $\mathbf{S}_{\mathrm{t}}$ & $\mathbf{S}_{\mathbf{c}}$ & & & & & \\
\hline \multirow{2}{*}{$\begin{array}{l}\text { Operating, Hot \& } \\
\text { Corroded }\end{array}$} & \multirow{2}{*}{1,200} & \multirow{2}{*}{15,300} & \multirow{2}{*}{9,945} & \multirow{2}{*}{1,000} & \multirow{2}{*}{0.03} & Wind & 0.1257 & 0.1257 \\
\hline & & & & & & Seismic & 0.1257 & 0.1256 \\
\hline \multirow{2}{*}{$\begin{array}{c}\text { Operating, Hot \& } \\
\text { New }\end{array}$} & \multirow{2}{*}{1,200} & \multirow{2}{*}{15,300} & \multirow{2}{*}{9,995} & \multirow{2}{*}{1,000} & \multirow{2}{*}{0} & Wind & 0.1247 & 0.1247 \\
\hline & & & & & & Seismic & 0.1247 & 0.1246 \\
\hline \multirow{2}{*}{ Empty, Corroded } & \multirow{2}{*}{0} & \multirow{2}{*}{20,000} & \multirow{2}{*}{14,142} & \multirow{2}{*}{0} & \multirow{2}{*}{0.03} & Wind & 0.0003 & 0.0003 \\
\hline & & & & & & Seismic & 0.0003 & 0.0004 \\
\hline \multirow{2}{*}{ Empty, New } & \multirow{2}{*}{0} & \multirow{2}{*}{20,000} & \multirow{2}{*}{14,203} & \multirow{2}{*}{0} & \multirow{2}{*}{0} & Wind & 0.0003 & 0.0003 \\
\hline & & & & & & Seismic & 0.0003 & 0.0004 \\
\hline $\begin{array}{l}\text { Hot Shut Down, } \\
\text { Corroded, Weight \& } \\
\text { Eccentric Moments } \\
\text { Only }\end{array}$ & 0 & 15,300 & 9,945 & 1,000 & 0.03 & Weight & 0.0005 & 0.0005 \\
\hline
\end{tabular}




\section{FLANGE, INJECTOR HEAD}

\section{ASME VIII-1, 2007 Edition, Appendix 2 Flange Calculations}

Flange is attached to:

Flange type:

Flange material specification:

Bolt material specification:

Bolt Description:

Internal design pressure, $\mathbf{P}$ :

Required flange thickness: $\mathrm{t}_{\mathrm{r}}=$
8" WELD CAP SCH 80

Weld neck integral

SA-182 F $316 \mathrm{H}<=5$ (low stress) (II-D p.

$74, \ln .32)$

SA-193 B7 Bolt $<=2$ 1/2 (II-D p. 348,

ln. 33)

1 in - 14 Thread

1,200 psi@ 900 F

2.9132 in

$1,258.75 \mathrm{psi} @ 700^{\circ} \mathrm{F}$

MAWP:

Corrosion allowance:

Bolt corrosion (root), $\mathrm{C}_{\text {bolt }}$ :

Design MDMT:

Rated MDMT:

Bore $=0.03$ in

0 in

$5^{\circ} \mathrm{F}$

$-320^{\circ} \mathrm{F}$

Estimated weight:

New $=109 \mathrm{lb}$
NOTE: EXTERNAL LOADS ON FLANGES NOT CONSIDERED IN DESIGN CALCULATIONS.

\section{Flange dimensions, new}

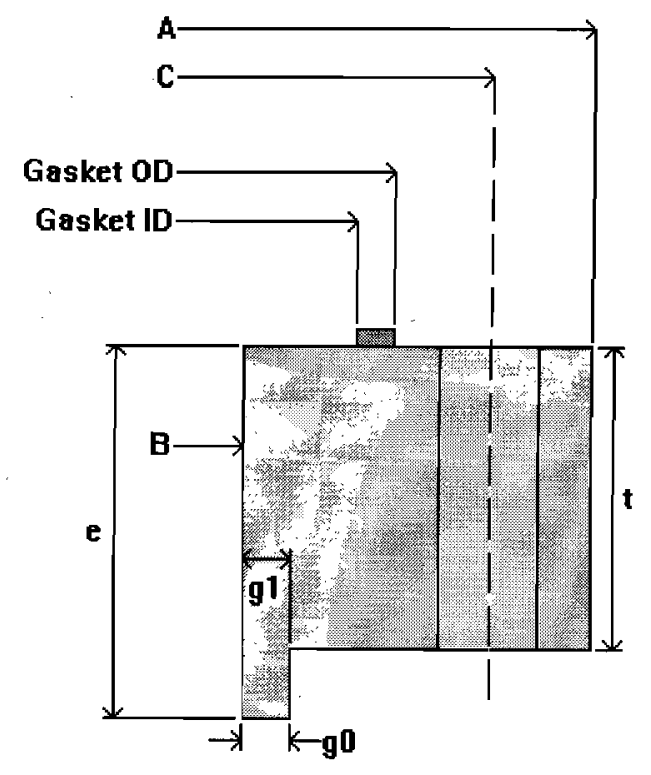

$$
\begin{aligned}
& \text { flange OD A }=14.625 \text { in } \\
& \text { bolt circle } \mathrm{C}=12.57 \mathrm{in} \\
& \text { gasket } \mathrm{OD}=10.65 \mathrm{in} \\
& \text { gasket ID }=9.9 \mathrm{in} \\
& \text { flange ID } \mathrm{B}=7.625 \mathrm{in} \\
& \text { thickness } \mathrm{t}=3 \mathrm{in} \\
& \text { bolting }=12-1 \text { in dia } \\
& \text { hub thickness } \mathrm{g}_{1}=0.5 \mathrm{in} \\
& \text { hub thickness } \mathrm{g}_{0}=0.5 \mathrm{in} \\
& \text { length } \mathrm{e}=3.705 \mathrm{in} \\
& \text { gasket factor } \mathrm{m}=3 \\
& \text { seating stress } \mathrm{y}=10,000 \mathrm{psi} \\
& \text { Gasket thickness } \mathrm{T}=0.175 \text { in } \\
& \\
& \text { NOTE: THICKNESS "t" INCLUDES } \\
& \text { 1/16" RAISED FACE. }
\end{aligned}
$$


Note: this flange is calculated as an integral type.

Flange calculations for Internal Pressure + Wind

\section{Longitudinal bending moment on flange}

$$
\begin{aligned}
& P_{\mathrm{m}}=16^{*} \mathrm{M}_{\mathrm{b}} /\left(\pi^{*} \mathrm{G}^{3}\right) \\
& =16^{*} 1,500 /\left(\pi^{*} 10.275^{3}\right) \\
& =7.0423 \mathrm{psi}
\end{aligned}
$$

\section{Axial load on flange}

$$
\begin{aligned}
& P_{\overline{\mathrm{I}}}=-4 * F /\left(-\pi^{*} \cdot G^{2}\right) \\
& =-4 * 500 /\left(\pi^{*} 10.275^{2}\right) \\
& =-6.03 \mathrm{psi}
\end{aligned}
$$

Total design load on flange (used for H - ref. Ill-1 NC-3658.1)

$$
\begin{aligned}
& =P+P_{s}+P_{m}+P_{r} \\
& =1,200+0+7.0423+-6.03 \\
& =1,201.0123 \mathrm{psi}
\end{aligned}
$$

\section{Gasket details from facing sketch 1(a) or (b), Column II}

Gasket width $N=0.375$ in

$\mathrm{b}_{0}=\mathrm{N} / 2=0.1875$ in

Effective gasket seating width, $b=b_{0}=0.1875$ in

$$
\begin{aligned}
& \mathrm{G}=(\text { gasket } \mathrm{OD}+\text { gasket ID }) / 2=(10.65+9.9) / 2=10.275 \text { in } \\
& \mathrm{h}_{\mathrm{G}}=(\mathrm{C}-\mathrm{G}) / 2=(12.57-10.275) / 2=1.1475 \text { in } \\
& \mathrm{h}_{\mathrm{D}}=\mathrm{R}+\mathrm{g}_{\mathrm{l}} / 2=1.9725+0.47 / 2=2.2075 \text { in } \\
& \mathrm{h}_{\mathrm{T}}=\left(\mathrm{R}+\mathrm{g}_{1}+\mathrm{h}_{\mathrm{G}}\right) / 2=(1.9725+0.47+1.1475) / 2=1.795 \text { in } \\
& \mathrm{H}_{\mathrm{p}}=2 * \mathrm{~b} * 3.14 * \mathrm{G} * \mathrm{~m} * \mathrm{P} \\
& =2 * 0.1875 * 3.14 * 10.275 * 3 * 1,200 \\
& =43,555.72 \mathrm{lb}_{\mathrm{f}} \\
& \mathrm{H}=0.785 * \mathrm{G}^{2 * \mathrm{P}} \\
& =0.785 * 10.275^{2 * 1,201.0123} \\
& =99,536.13 \mathrm{lb}_{\mathrm{f}} \\
& \mathrm{H}_{\mathrm{D}}=0.785^{*} \mathrm{~B}^{2 * \mathrm{P}} \\
& =0.785^{*} 7.685^{2 * 1,200} \\
& =55,633.79 \mathrm{lb}_{\mathrm{f}} \\
& \mathrm{H}_{\mathrm{T}}=\mathrm{H}-\mathrm{H}_{\mathrm{D}}
\end{aligned}
$$


$=99,536.13-55,633.79$

$=43,902.34 \mathrm{lb}_{\mathrm{f}}$

$\mathrm{W}_{\mathrm{m} 1}=\mathrm{H}+\mathrm{H}_{\mathrm{p}}$

$=99,536.13+43,555.72$

$=143,091.86 \mathrm{lb}_{\mathrm{f}}$

$\mathrm{W}_{\mathrm{m} 2}=3.14^{*} \mathrm{~b}^{*} \mathrm{G}^{*} \mathrm{y}$

$=3.14^{*} 0.1875^{*} 10.275^{*} 10,000$

$=60,494.06 \mathrm{lb}_{\mathrm{f}}$

Required bolt area, $A_{m}=$ greater of $A_{m 1}, A_{m 2}=5.723674 \mathrm{in}^{2}$

$A_{m l}=W_{m l} / S_{b}=143,091.9 / 25,000=5.7237 \mathrm{in}^{2}$

$\mathrm{A}_{\mathrm{m} 2}=\mathrm{W}_{\mathrm{m} 2} / \mathrm{S}_{\mathrm{a}}=60,494.06 / 25,000=2.4198 \mathrm{in}^{2}$

Total area for 12- 1 in dia bolts, corroded, $A_{b}=6.612$ in $^{2}$

$\mathrm{W}=\left(\mathrm{A}_{\mathrm{m}}+\mathrm{A}_{\mathrm{b}}\right) * \mathrm{~S}_{\mathrm{a}} / 2$

$=(5.7237+6.612)^{*} 25,000 / 2$

$=154,195.92 \mathrm{lb}_{\mathrm{f}}$

$\mathrm{M}_{\mathrm{D}}=\mathrm{H}_{\mathrm{D}} * \mathrm{~h}_{\mathrm{D}}=55,633.79 * 2.2075=122,811.6 \mathrm{lb}$-in

$\mathrm{M}_{\mathrm{T}}=\mathrm{H}_{\mathrm{T}} * \mathrm{~h}_{\mathrm{T}}=43,902.34 * 1.795=78,804.7 \mathrm{lb}$-in

$\mathrm{H}_{\mathrm{G}}=\mathrm{W}_{\mathrm{m} 1}-\mathrm{H}=143,091.9-99,536.13=43,555.73 \mathrm{lb}_{\mathrm{f}}$

$\mathrm{M}_{\mathrm{G}}=\mathrm{H}_{\mathrm{G}}{ }^{*} \mathrm{~h}_{\mathrm{G}}=43,555.73 * 1.1475=49,980.2 \mathrm{lb}$-in

$M_{o}=M_{D}+M_{T}+M_{G}=122,811.6+78,804.7+49,980.2=251,596.5 \mathrm{lb}$-in

$M_{g}=W^{*} h_{G}=154,195 \cdot 9 * 1 \cdot 1475=176,939.8 \mathrm{lb}$-in 
Hub and Flange Factors

$h_{0}=\left(B^{*} g_{0}\right)^{1 / 2}=\left(7.685^{*} 0.47\right)^{1 / 2}=1.9005$ in

From FIG. 2-7.1, where $K=A / B=14.625 / 7.685=1.9031$

$$
\begin{aligned}
& \mathrm{T}=1.5435 \quad \mathrm{Z}=1.7629 \quad \mathrm{Y}=3.1841 \quad \mathrm{U}=3.499 \\
& \mathrm{~h} / \mathrm{h}_{0}=0.371 \mathrm{~g}_{1} / \mathrm{g}_{0}=1 \\
& F=0.9089 \quad \mathrm{~V}=0.5501 \quad \mathrm{e}=\mathrm{F} / \mathrm{h}_{0}=0.4782 \\
& \mathrm{~d}=(\mathrm{U} / \mathrm{V}) \mathrm{h}_{0}{ }^{*} \mathrm{~g}_{0}{ }^{2}=(3.49902 / 0.5501) * 1.9005 * 0.47^{2} \\
& =2.6704 \mathrm{in}^{3}
\end{aligned}
$$

Stresses at operating conditions - VIII-1, Appendix 2-7

$\mathbf{f}=1$

$\mathrm{L}=\left(\mathrm{t}^{*} \mathrm{e}+1\right) / \mathrm{T}+\mathrm{t}^{3} / \mathrm{d}$

$=\left(3^{*} 0.4782+1\right) / 1.543522+3^{3} / 2.6704$

$=11.68838$

$\mathrm{S}_{\mathrm{H}}=\mathrm{f}^{*} \mathrm{M}_{\mathrm{o}} /\left(\mathrm{L}^{*} \mathrm{~g}_{1}{ }^{2 * \mathrm{~B})}\right.$

$=1 * 251,596.5 /\left(11.68838 * 0.47^{2 * 7.685}\right)$

$=12,680 \mathrm{psi}$

$\mathrm{S}_{\mathrm{R}}=(1.33 * \mathrm{t} * \mathrm{e}+1) * \mathrm{M}_{\mathrm{o}} /\left(\mathrm{L} * \mathrm{t}^{2} * \mathrm{~B}\right)$

$=(1.33 * 3 * 0.4782+1) * 251,596.5 /\left(11.68838 * 3^{2 * 7.685}\right)$

$=905 \mathrm{psi}$

$\mathrm{S}_{\mathrm{T}}=\mathrm{Y} * \mathrm{M}_{\mathrm{o}} /\left(\mathrm{t}^{2 * \mathrm{~B}}\right)-\mathrm{Z} * \mathrm{~S}_{\mathrm{R}}$

$=3.1841 * 251,596.5 /\left(3^{2 * 7.685}\right)-1.7629 * 905$

$=9,987 \mathrm{psi}$

Allowable stress $\mathrm{S}_{\mathrm{fo}}=12,100 \mathrm{psi}$

Allowable stress $S_{\text {no }}=15,300$ psi

$\mathrm{S}_{\mathrm{T}}$ does not exceed $\mathrm{S}_{\mathrm{fo}}$

$\mathrm{S}_{\mathrm{H}}$ does not exceed Min[ $\left.1.5 * \mathrm{~S}_{\mathrm{fo}}, 2.5 * \mathrm{~S}_{\mathrm{no}}\right]=18,150 \mathrm{psi}$

$\mathrm{S}_{\mathrm{R}}$ does not exceed $\mathrm{S}_{\mathrm{fo}}$

$0.5\left(\mathrm{~S}_{\mathrm{H}}+\mathrm{S}_{\mathrm{R}}\right)=6,792$ psi does not exceed $\mathrm{S}_{\mathrm{fo}}$

$0.5\left(\mathrm{~S}_{\mathrm{H}}+\mathrm{S}_{\mathrm{T}}\right)=11,333$ psi does not exceed $\mathrm{S}_{\mathrm{fo}}$

Stresses at gasket seating - VIII-1, Appendix 2-7

$\mathrm{S}_{\mathrm{H}}=\mathrm{f}^{*} \mathrm{M}_{\mathrm{g}}\left(\mathrm{L}^{*} \mathrm{~g}_{1}{ }^{2 * \mathrm{~B})}\right.$

$=1 * 176,939.8 /\left(11.68838 * 0.47^{2 * 7.685}\right)$

$=8,917 \mathrm{psi}$ 
$\mathrm{S}_{\mathrm{R}}=(1.33 * \mathrm{t} * \mathrm{e}+1) * \mathrm{M}_{\mathrm{g}} /\left(\mathrm{L} * \mathrm{t}^{2} * \mathrm{~B}\right)$

$=(1.33 * 3 * 0.4782+1)^{*} 176,939.8 /(11.68838 * 32 * 7.685)$

$=637 \mathrm{psi}$

$\mathrm{S}_{\mathrm{T}}=\mathrm{Y}^{*} \mathrm{M}_{\mathrm{g}} /\left(\mathrm{t}^{2 * \mathrm{~B})}-\mathrm{Z}^{*} \mathrm{~S}_{\mathrm{R}}\right.$

$=3.1841 * 176,939.8 /\left(3^{2 * 7.685}\right)-1.7629 * 637$

$=7,024 \mathrm{psi}$

Allowable stress $\mathrm{S}_{\mathrm{fa}}=20,000$ psi

Allowable stress $S_{\text {na }}=20,000$ psi

$\mathrm{S}_{\mathrm{T}}$ does not exceed $\mathrm{S}_{\mathrm{fa}}$

$\mathrm{S}_{\mathrm{H}}$ does not exceed $\mathrm{Min}\left[1.5 * \mathrm{~S}_{\mathrm{fa}}, 2.5 * \mathrm{~S}_{\mathrm{na}}\right]=30,000 \mathrm{psi}$

$S_{R}$ does not exceed $S_{f a}$

$0.5\left(\mathrm{~S}_{\mathrm{H}}+\mathrm{S}_{\mathrm{R}}\right)=4,777$ psi does not exceed $\mathrm{S}_{\mathrm{fa}}$

$0.5\left(\mathrm{~S}_{\mathrm{H}}+\mathrm{S}_{\mathrm{T}}\right)=7,970$ psi does not exceed $\mathrm{S}_{\text {fa }}$

Flange rigidity per VIII-1, Appendix 2-14

$\mathrm{J}=52.14 * \mathrm{~V} * \mathrm{M}_{\mathrm{o}} /\left(\mathrm{L}^{*} \mathrm{E}^{*} \mathrm{~g}_{0}{ }^{2 *} \mathrm{~K}_{\mathrm{I}} * \mathrm{~h}_{0}\right)$

$=52.14 * 0.5501 * 251,596.5 /\left(11.6884 * 24,800,000 * 0.47^{2 *} 0.3 * 1.9005\right)$

$=0.1976626$

The flange rigidity index $J$ does not exceed 1 ; satisfactory.

Flange calculations for Internal Pressure + Seismic

Longitudinal bending moment on flange

$\mathrm{P}_{\mathrm{m}}=16 * \mathrm{M}_{\mathrm{b}} /\left(\pi^{*} \mathrm{G}^{3}\right)$

$=16^{*} 1,500 /\left(\pi * 10.275^{3}\right)$

$=7.0423 \mathrm{psi}$

Axial load on flange

$P_{r}=-4 * F /\left(\pi * G^{2}\right)$

$=-4 * 500 /\left(\pi^{*} 10.275^{2}\right)$

$=-6.03 \mathrm{psi}$

Total design load on flange (used for $\mathrm{H}$ - ref. III-1 NC-3658.1)

$=\mathrm{P}+\mathrm{P}_{\mathrm{s}}+\mathrm{P}_{\mathrm{m}}+\mathrm{P}_{\mathrm{r}}$

$=1,200+0+7.0423+-6.03$

$=1,201.0123 \mathrm{psi}$

Gasket details from facing sketch 1(a) or (b), Column II

Gasket width $N=0.375$ in

$b_{0}=N / 2=0.1875$ in 
Effective gasket seating width, $b=b_{0}=0.1875$ in

$\mathrm{G}=($ gasket $\mathrm{OD}+$ gasket ID $) / 2=(10.65+9.9) / 2=10.275$ in

$h_{G}=(C-G) / 2=(12.57-10.275) / 2=1.1475$ in

$h_{D}=R+g_{1} / 2=1.9725+0.47 / 2=2.2075$ in

$\mathrm{h}_{\mathrm{T}}=\left(\mathrm{R}+\mathrm{g}_{1}+\mathrm{h}_{\mathrm{G}}\right) / 2=(1.9725+0.47+1.1475) / 2=1.795$ in

$\mathrm{H}_{\mathrm{p}}=2 * \mathrm{~b} * 3.14 * \mathrm{G} * \mathrm{~m} * \mathrm{P}$

$=2 * 0.1875 * 3.14 * 10.275 * 3 * 1,200$

$=43,555.72 \mathrm{lb}_{\mathrm{f}}$

$\mathrm{H}=0.785^{*} \mathrm{G}^{2 * \mathrm{P}}$

$=0.785 * 10.275^{2 * 1,201.0123}$

$=99,536.13 \mathrm{lb}_{\mathrm{f}}$

$\mathrm{H}_{\mathrm{D}}=0.785 * \mathrm{~B}^{2 * \mathrm{P}}$

$=0.785 * 7.685^{2 * 1,200}$

$=55,633.79 \mathrm{lb}_{\mathrm{f}}$

$\mathrm{H}_{\mathrm{T}}=\mathrm{H}-\mathrm{H}_{\mathrm{D}}$

$=99,536.13-55,633.79$

$=43,902.34 \mathrm{lb}_{\mathrm{f}}$

$\mathrm{W}_{\mathrm{m} 1}=\mathrm{H}+\mathrm{H}_{\mathrm{p}}$

$=99,536.13+43,555.72$

$=143,091.86 \mathrm{lb}_{\mathrm{f}}$

$\mathrm{W}_{\mathrm{m} 2}=3.14 * \mathrm{~b} * \mathrm{G} * \mathrm{y}$

$=3.14 * 0.1875 * 10.275 * 10,000$

$=60,494.061 \mathrm{~b}_{\mathrm{f}}$

Required bolt area, $A_{m}=$ greater of $A_{m 1}, A_{m 2}=5.723674 \mathrm{in}^{2}$

$A_{m 1}=W_{m 1} / S_{b}=143,091.9 / 25,000=5.7237 \mathrm{in}^{2}$

$\mathrm{A}_{\mathrm{m} 2}=\mathrm{W}_{\mathrm{m} 2} / \mathrm{S}_{\mathrm{a}}=60,494.06 / 25,000=2.4198 \mathrm{in}^{2}$

Total area for 12- 1 in dia bolts, corroded, $A_{b}=6.612$ in $^{2}$

$\mathrm{W}=\left(\mathrm{A}_{\mathrm{m}}+\mathrm{A}_{\mathrm{b}}\right) * \mathrm{~S}_{\mathrm{a}} / 2$

$=(5.7237+6.612) * 25,000 / 2$

$=154,195.92 \mathrm{lb}_{\mathrm{f}}$

$\mathrm{M}_{\mathrm{D}}=\mathrm{H}_{\mathrm{D}}{ }^{*} \mathrm{~h}_{\mathrm{D}}=55,633.79 * 2.2075=122,811.6 \mathrm{lb}-\mathrm{in}$

$\mathrm{M}_{\mathrm{T}}=\mathrm{H}_{\mathrm{T}}{ }^{*} \mathrm{~h}_{\mathrm{T}}=43,902.34 * 1.795=78,804.7 \mathrm{lb}$-in 


$$
\begin{aligned}
& \mathrm{H}_{\mathrm{G}}=\mathrm{W}_{\mathrm{m} 1}-\mathrm{H}=143,091.9-99,536 \cdot 13=43,555.73 \mathrm{lb}_{\mathrm{f}} \\
& \mathrm{M}_{\mathrm{G}}=\mathrm{H}_{\mathrm{G}}{ }^{*} \mathrm{~h}_{\mathrm{G}}=43,555.73 * 1.1475=49,980.2 \mathrm{lb}-\mathrm{in} \\
& \mathrm{M}_{\mathrm{o}}=\mathrm{M}_{\mathrm{D}}+\mathrm{M}_{\mathrm{T}}+\mathrm{M}_{\mathrm{G}}=122,811.6+78,804.7+49,980.2=251,596.5 \mathrm{lb}-\mathrm{in} \\
& \mathrm{M}_{\mathrm{g}}=\mathrm{W}^{*} \mathrm{~h}_{\mathrm{G}}=154,195.9 * 1.1475=176,939.8 \mathrm{lb} \text {-in }
\end{aligned}
$$




\section{Hub and Flange Factors}

$h_{0}=\left(B^{*} g_{0}\right)^{1 / 2}=\left(7.685^{*} 0.47\right)^{1 / 2}=1.9005$ in

From FIG. 2-7.1, where $\mathrm{K}=\mathrm{A} / \mathrm{B}=14.625 / 7.685=1.9031$

$$
\begin{aligned}
& \mathrm{T}=1.5435 \quad Z=1.7629 \quad \mathrm{Y}=3.1841 \quad \mathrm{U}=3.499 \\
& \mathrm{~h} / \mathrm{h}_{0}=0.371 \mathrm{~g} / \mathrm{g}_{0}=1 \\
& F=0.9089 \quad V=0.5501 \quad e=F / h_{0}=0.4782 \\
& \mathrm{~d}=(\mathrm{U} / \mathrm{V})^{*} \mathrm{~h}_{0}{ }^{*} \mathrm{~g}_{0}{ }^{2}=(3.49902 / 0.5501)^{*} 1.9005 * 0.47^{2} \\
& =2.6704 \mathrm{in}^{3}
\end{aligned}
$$

\section{Stresses at operating conditions - VIII-1, Appendix 2-7}

$f=1$

$\mathrm{L}=\left(\mathrm{t}^{*} \mathrm{e}+1\right) / \mathrm{T}+\mathrm{t}^{3} / \mathrm{d}$

$=\left(3^{*} 0.4782+1\right) / 1.543522+3^{3} / 2.6704$

$=11.68838$

$\mathrm{S}_{\mathrm{H}}=\mathrm{f}^{*} \mathrm{M}_{\mathrm{o}} /\left(\mathrm{L}^{*} \mathrm{~g}_{\mathrm{l}}{ }^{2 * \mathrm{~B})}\right.$

$=1 * 251,596.5 /\left(11.68838 * 0.47^{2 * 7.685}\right)$

$=12,680 \mathrm{psi}$

$\mathrm{S}_{\mathrm{R}}=\left(1.33^{*} \mathrm{t}^{*} \mathrm{e}+1\right) * \mathrm{M}_{\mathrm{o}} /\left(\mathrm{L}^{*} \mathrm{t}^{2 * \mathrm{~B}}\right)$

$=(1.33 * 3 * 0.4782+1) * 251,596.5 /(11.68838 * 32 * 7.685)$

$=905 \mathrm{psi}$

$$
\begin{aligned}
& \mathrm{S}_{\mathrm{T}}=\mathrm{Y}^{*} \mathrm{M}_{\mathrm{o}} /\left(\mathrm{t}^{2 * \mathrm{~B})}-\mathrm{Z}^{*} \mathrm{~S}_{\mathrm{R}}\right. \\
& =3.1841 * 251,596.5 /\left(3^{2 *} 7.685\right)-1.7629 * 905 \\
& =9,987 \mathrm{psi}
\end{aligned}
$$

Allowable stress $\mathrm{S}_{\mathrm{fo}}=12,100 \mathrm{psi}$

Allowable stress $S_{\text {no }}=15,300$ psi

$\mathrm{S}_{\mathrm{T}}$ does not exceed $\mathrm{S}_{\mathrm{fo}}$

$\mathrm{S}_{\mathrm{H}}$ does not exceed $\operatorname{Min}\left[1.5^{*} \mathrm{~S}_{\mathrm{fo}}, 2.5^{*} \mathrm{~S}_{\mathrm{no}}\right]=18,150 \mathrm{psi}$

$\mathrm{S}_{\mathrm{R}}$ does not exceed $\mathrm{S}_{\mathrm{fo}}$

$0.5\left(\mathrm{~S}_{\mathrm{H}}+\mathrm{S}_{\mathrm{R}}\right)=6,792$ psi does not exceed $\mathrm{S}_{\mathrm{fo}}$

$0.5\left(\mathrm{~S}_{\mathrm{H}}+\mathrm{S}_{\mathrm{T}}\right)=11,333$ psi does not exceed $\mathrm{S}_{\text {fo }}$

\section{Stresses at gasket seating - VIII-1, Appendix 2-7}

$\mathrm{S}_{\mathrm{H}}=\mathrm{f}^{*} \mathrm{M}_{\mathrm{g}} /\left(\mathrm{L}^{*} \mathrm{~g}_{\mathrm{j}}{ }^{2 * \mathrm{~B})}\right.$

$=1 * 176,939.8 /\left(11.68838 * 0.47^{2 * 7.685}\right)$

$=8,917 \mathrm{psi}$ 
$\mathrm{S}_{\mathrm{R}}=\left(1.33 * \mathrm{t}^{*} \mathrm{e}+1\right) * \mathrm{M}_{\mathrm{g}} /\left(\mathrm{L} * \mathrm{t}^{2 *} \mathrm{~B}\right)$

$=(1.33 * 3 * 0.4782+1)^{*} 176,939.8 /\left(11.68838 * 3^{2 *} 7.685\right)$

$=637 \mathrm{psi}$

$\mathrm{S}_{\mathrm{T}}=\mathrm{Y} * \mathrm{M}_{\mathrm{g}}\left(\mathrm{t}^{2 * \mathrm{~B})}-\mathrm{Z}^{*} \mathrm{~S}_{\mathrm{R}}\right.$

$=3.1841 * 176,939.8 /\left(3^{2 * 7.685}\right)-1.7629 * 637$

$=7,024 \mathrm{psi}$

Allowable stress $\mathrm{S}_{\mathrm{fa}}=20,000 \mathrm{psi}$

Allowable stress $S_{n a}=20,000$ psi

$\mathrm{S}_{\mathrm{T}}$ does not exceed $\mathrm{S}_{\mathrm{fa}}$

$\mathrm{S}_{\mathrm{H}}$ does not exceed $\operatorname{Min}\left[1.5^{*} \mathrm{~S}_{\mathrm{fa}}, 2.5 * \mathrm{~S}_{\mathrm{na}}\right]=30,000 \mathrm{psi}$

$\mathrm{S}_{\mathrm{R}}$ does not exceed $\mathrm{S}_{\mathrm{fa}}$

$0.5\left(\mathrm{~S}_{\mathrm{H}}+\mathrm{S}_{\mathrm{R}}\right)=4,777$ psi does not exceed $\mathrm{S}_{\mathrm{fa}}$

$0.5\left(\mathrm{~S}_{\mathrm{H}}+\mathrm{S}_{\mathrm{T}}\right)=7,970$ psi does not exceed $\mathrm{S}_{\mathrm{fa}}$

\section{Flange rigidity per VIII-1, Appendix 2-14}

$$
\begin{aligned}
& \mathrm{J}=52.14 * \mathrm{~V} * \mathrm{M}_{\mathrm{o}} /\left(\mathrm{L}^{*} \mathrm{E} * \mathrm{~g}_{0}{ }^{2 *} \mathrm{~K}_{\mathrm{I}} * \mathrm{~h}_{0}\right) \\
& =52.14 * 0.5501 * 251,596.5 /\left(11.6884 * 24,800,000 * 0.47^{2 * 0.3 * 1.9005)}\right. \\
& =0.1976626
\end{aligned}
$$

The flange rigidity index $\mathrm{J}$ does not exceed 1 ; satisfactory.

\section{FLANGE, INJECTOR HEAD - Flange hub}

\section{ASME Section VIII Division 1, 2007 Edition}

Component:

Material specification:

Flange hub

SA-182 F316H $<=5$ (low stress) (II-D p. 74 , ln. 32)

Internal design pressure: $\mathrm{P}=1,200 \mathrm{psi} @ 700^{\circ} \mathrm{F}$

Static liquid head:

$P_{\text {th }}=0 \mathrm{psi}\left(\mathrm{SG}=0, H_{\mathrm{s}}=9.5959 "\right.$, Horizontal test head $)$

Corrosion allowance $\quad$ Inner $\mathrm{C}=0.03^{\prime \prime} \quad$ Outer $\mathrm{C}=0^{\prime \prime}$

Design MDMT $=5^{\circ} \mathrm{F}$

Rated MDMT $=-320^{\circ} \mathrm{F}$

No impact test performed

Material is not normalized

Material is not produced to Fine Grain Practice

PWHT is not performed

Radiography: Longitudinal joint - $\quad$ Seamless No RT

Top circumferential joint - Full UW-1 1(a) Type 1

Bottom circumferential

joint - 


$$
\begin{aligned}
& \text { Estimated weight New }=2.4 \mathrm{lb} \quad \text { corr }=2.3 \mathrm{lb} \\
& \text { Capacity New }=0.14 \text { US gal corr }=0.14 \text { US gal } \\
& \mathrm{OD} \quad=8.625^{\prime \prime} \\
& \text { Length } \mathrm{L}_{\mathrm{c}}=0.705^{\prime \prime} \\
& \mathrm{t} \quad=0.5^{\prime \prime}
\end{aligned}
$$

\section{Design thickness, (at $\left.700^{\circ} \mathrm{F}\right)$ Appendix 1-1}

$$
\begin{aligned}
\mathrm{t} & =\mathrm{P}^{*} \mathrm{R}_{\mathrm{o}} /\left(\mathrm{S} * \mathrm{E}+0.40^{*} \mathrm{P}\right)+\text { Corrosion } \\
& =1,200^{*} 4.3125 /\left(12,100^{*} 1.00+0.40^{*} 1,200\right)+0.03 \\
& =0.4414^{\prime \prime}
\end{aligned}
$$

Maximum allowable working pressure, $\left(\right.$ at $^{700}{ }^{\circ} \mathrm{F}$ ) Appendix 1-1

\begin{tabular}{|c|c|c|c|c|c|c|c|c|}
\hline \multirow[t]{2}{*}{ Condition } & \multirow[t]{2}{*}{$\begin{array}{l}\text { Pressure } \\
\text { P ( psi) }\end{array}$} & \multicolumn{2}{|c|}{$\begin{array}{c}\text { Allowable Stress } \\
\text { Before UG-23 Stress } \\
\text { Increase ( psi) }\end{array}$} & \multirow{2}{*}{$\begin{array}{c}\text { Temperature } \\
\left({ }^{\circ} \mathbf{F}\right)\end{array}$} & \multirow{2}{*}{$\begin{array}{l}\text { Corrosion } \\
\text { C (in) }\end{array}$} & \multirow[t]{2}{*}{ Load } & \multirow{2}{*}{$\begin{array}{l}\text { Req'd Thk } \\
\text { Due to } \\
\text { Tension (in) }\end{array}$} & \multirow{2}{*}{$\begin{array}{l}\text { Req'd Thk Due to } \\
\text { Compression (in) }\end{array}$} \\
\hline & & $S_{t}$ & $\mathbf{S}_{\mathbf{c}}$ & & & & & \\
\hline \multirow{2}{*}{$\begin{array}{l}\text { Operating, Hot \& } \\
\text { Corroded }\end{array}$} & \multirow{2}{*}{1,200} & \multirow{2}{*}{12,100} & \multirow{2}{*}{11,639} & \multirow{2}{*}{700} & \multirow{2}{*}{0.03} & Wind & 0.1559 & 0.1558 \\
\hline & & & & & & Seismic & 0.1558 & 0.1557 \\
\hline \multirow{2}{*}{$\begin{array}{c}\text { Operating, Hot \& } \\
\text { New }\end{array}$} & \multirow{2}{*}{1,200} & \multirow{2}{*}{12,100} & \multirow{2}{*}{11,687} & \multirow{2}{*}{700} & \multirow{2}{*}{0} & Wind & 0.1546 & 0.1546 \\
\hline & & & & & & Seismic & 0.1546 & 0.1545 \\
\hline \multirow{2}{*}{ Empty, Corroded } & \multirow{2}{*}{0} & \multirow{2}{*}{20,000} & \multirow{2}{*}{14,265} & \multirow{2}{*}{0} & \multirow{2}{*}{0.03} & Wind & 0.0003 & 0.0003 \\
\hline & & & & & & Seismic & 0.0003 & 0.0004 \\
\hline \multirow[t]{2}{*}{ Empty, New } & \multirow[t]{2}{*}{0} & \multirow{2}{*}{20,000} & \multirow{2}{*}{14,318} & \multirow{2}{*}{0} & \multirow{2}{*}{0} & Wind & 0.0003 & 0.0004 \\
\hline & & & & & & Seismic & 0.0003 & 0.0004 \\
\hline $\begin{array}{c}\text { Hot Shut Down, } \\
\text { Corroded, Weight \& } \\
\text { Eccentric Moments } \\
\text { Only }\end{array}$ & 0 & 12,100 & 11,639 & 700 & 0.03 & Weight & 0.0005 & 0.0005 \\
\hline
\end{tabular}

$$
\begin{aligned}
\mathrm{P} & =\mathrm{S} * \mathrm{E}^{*} \mathrm{t} /\left(\mathrm{R}_{\mathrm{o}}-0.40 * \mathrm{t}\right)-\mathrm{P}_{\mathrm{S}} \\
& =12,100 * 1.00^{*} 0.47 /\left(4.3125-0.40^{*} 0.47\right)-0 \\
& =1,378.83 \mathrm{psi}
\end{aligned}
$$

\section{\% Forming strain - UHA-44(a)(2)(a)}

$$
\begin{aligned}
\mathrm{EFE} & =\left(50 * \mathrm{t} / \mathrm{R}_{\mathrm{f}}\right)^{*}\left(1-\mathrm{R}_{\mathrm{f}} / \mathrm{R}_{\mathrm{o}}\right) \\
& =(50 * 0.5 / 4.0625)^{*}(1-4.0625 / \infty) \\
& =6.1538 \%
\end{aligned}
$$

\section{Design thickness $=0.4414 "$}

The governing condition is due to internal pressure.

The cylinder thickness of $0.5^{\prime \prime}$ is adequate.

Thickness Required Due to Pressure + External Loads 
P.O.\#: 700521452 


\section{FLANGE, SHELL HEAD}

\section{ASME VIII-1, 2007 Edition, Appendix 2 Flange Calculations}

Flange is attached to:

Flange type:

Flange material specification:

Bolt material specification:

Bolt Description:

Internal design pressure, $P$ :

Required flange thickness: $t_{r}=$

Maximum allowable working pressure,

MAWP:

Corrosion allowance:

Bolt corrosion (root), $\mathrm{C}_{\text {bolt }}$ :

Design MDMT:

Rated MDMT:

Estimated weight:

$\mathrm{New}=69.7 \mathrm{lb}$

$348, \ln .33$ )

2.0507 in

0 in

$5^{\circ} \mathrm{F}$

$-49.49^{\circ} \mathrm{F}$
REACTOR OUTER SHELL (Top)

Weld neck integral

SA-516 70 (II-D p. 18, ln. 22)

SA-193 B7 Bolt $<=2$ 1/2 (II-D p.

1 in -14 Thread

$1,200 \mathrm{psi} @ 700^{\circ} \mathrm{F}$

NOTE: EXTERNAL LOADS ON FLANGES NOT

1,293.18 psi@700F

Bore $=0.06$ in

Flange $=0$ in

No impact test performed

Flange material is normalized

Material is not produced to fine grain practice

PWHT is not performed

corroded $=68.1 \mathrm{lb}$

Flange dimensions, new

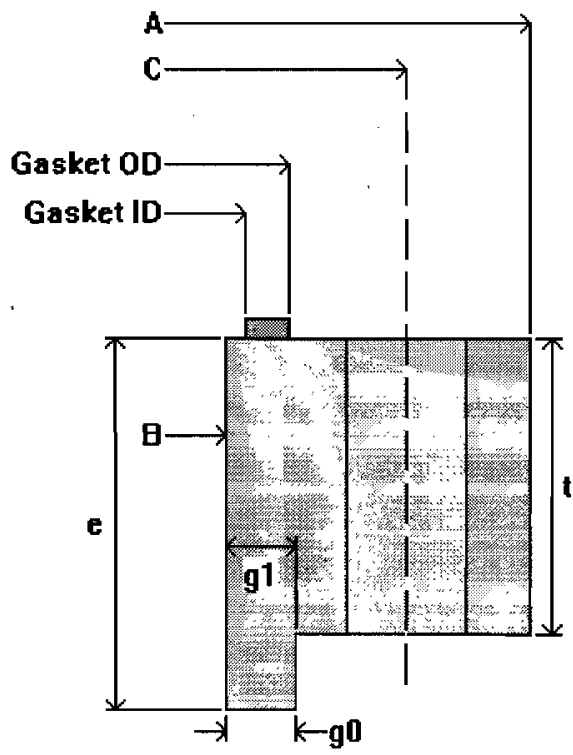

$$
\begin{aligned}
\text { flange OD A } & =14.625 \text { in } \\
\text { bolt circle } \mathrm{C} & =12.57 \mathrm{in} \\
\text { gasket } \mathrm{OD} & =10.65 \mathrm{in} \\
\text { gasket } \mathrm{ID} & =9.9 \mathrm{in} \\
\text { flange ID } \mathrm{B} & =9.56 \mathrm{in} \\
\text { thickness } \mathrm{t} & =2.4375 \mathrm{in} \\
\text { bolting } & =12-1 \mathrm{in} \mathrm{dia} \\
\text { hub thickness } \mathrm{g}_{1} & =0.594 \text { in } \\
\text { hub thickness } \mathrm{g}_{0} & =0.594 \text { in } \\
\text { length } \mathrm{e} & =3.0625 \mathrm{in} \\
\text { gasket factor } \mathrm{m} & =3 \\
\text { seating stress } \mathrm{y} & =10,000 \mathrm{psi} \\
\text { Gasket thickness } \mathrm{T} & =0.175 \mathrm{in}
\end{aligned}
$$

Note: this flange is calculated as an integral type. 


\section{Determination of Flange MDMT}

Flange impact test exemption temperature from Fig UCS-66 Curve $\mathrm{D}=-49.49^{\circ} \mathrm{F}$ UCS-66 governing thickness $=0.594$ in

Bolts rated MDMT per Fig UCS- 66 note $(\mathrm{c})=-55^{\circ} \mathrm{F}$

The rated flange MDMT is $-49.49^{\circ} \mathrm{F}$

\section{Flange calculations for Internal Pressure + Wind}

\section{Longitudinal bending moment on flange}

$\mathrm{P}_{\mathrm{m}}=16^{*} \mathrm{M}_{\mathrm{b}} /\left(\pi^{*} \mathrm{G}^{3}\right)$

$=16^{*} 1,500 /\left(\pi^{*} 10.275^{3}\right)$

$=7.0423 \mathrm{psi}$

\section{Axial load on flange}

$\mathrm{P}_{\mathrm{r}}=-4 * \mathrm{~F} /\left(\pi^{*} \mathrm{G}^{2}\right)$

$=-4 * 500 /\left(\pi^{*} 10.275^{2}\right)$

$=-6.03 \mathrm{psi}$

Total design load on flange (used for H - ref. III-1 NC-3658.1)

$=\mathrm{P}+\mathrm{P}_{\mathrm{s}}+\mathrm{P}_{\mathrm{m}}+\mathrm{P}_{\mathrm{r}}$

$=1,200+0+7.0423+-6.03$

$=1,201.0123 \mathrm{psi}$

\section{Gasket details from facing sketch 1(a) or (b), Column II}

Gasket width $\mathrm{N}=0.375$ in

$\mathrm{b}_{0}=\mathrm{N} / 2=0.1875$ in

Effective gasket seating width, $b=b_{0}=0.1875$ in

$\mathrm{G}=($ gasket $\mathrm{OD}+$ gasket ID $) / 2=(10.65+9.9) / 2=10.275$ in

$\mathrm{h}_{\mathrm{G}}=(\mathrm{C}-\mathrm{G}) / 2=(12.57-10.275) / 2=1.1475$ in

$\mathrm{h}_{\mathrm{D}}=\mathrm{R}+\mathrm{g}_{1} / 2=0.911+0.534 / 2=1.178$ in

$\mathrm{h}_{\mathrm{T}}=\left(\mathrm{R}+\mathrm{g}_{1}+\mathrm{h}_{\mathrm{G}}\right) / 2=(0.911+0.534+1.1475) / 2=1.2963$ in

$\mathrm{H}_{\mathrm{p}}=2 * \mathrm{~b} * 3 \cdot 14 * \mathrm{G} * \mathrm{~m} * \mathrm{P}$

$=2 * 0.1875 * 3.14 * 10.275 * 3 * 1,200$

$=43,555.72 \mathrm{lb}_{\mathrm{f}}$

$\mathrm{H}=0.785^{*} \mathrm{G}^{2} * \mathrm{P}$

$=0.785 * 10.275^{2 * 1,201.0123}$

$=99,536.13 \mathrm{lb}_{\mathrm{f}}$ 


$$
\begin{aligned}
& \mathrm{H}_{\mathrm{D}}=0.785^{*} \mathrm{~B}^{2 * \mathrm{P}} \\
& =0.785^{*} 9.68^{2 *} 1,200 \\
& =88,267.67 \mathrm{lb}_{\mathrm{f}} \\
& \mathrm{H}_{\mathrm{T}}=\mathrm{H}-\mathrm{H}_{\mathrm{D}} \\
& =99,536.13-88,267.67 \\
& =11,268.46 \mathrm{lb}_{\mathrm{f}} \\
& \mathrm{W}_{\mathrm{m} 1}=\mathrm{H}+\mathrm{H}_{\mathrm{p}} \\
& =99,536.13+43,555.72 \\
& =143,091.86 \mathrm{lb}_{\mathrm{f}} \\
& \mathrm{W}_{\mathrm{m} 2}=3.14 * \mathrm{~b}^{*} \mathrm{G}^{*} \mathrm{y} \\
& =3.14 * 0.1875 * 10.275 * 10,000 \\
& =60,494.06 \mathrm{lb}_{\mathrm{f}}
\end{aligned}
$$

Required bolt area, $A_{m}=$ greater of $A_{m 1}, A_{m 2}=5.723674 \mathrm{in}^{2}$

$A_{m 1}=W_{m l} / S_{b}=143,091.9 / 25,000=5.7237$ in $^{2}$

$A_{m 2}=W_{m 2} / S_{a}=60,494.06 / 25,000=2.4198 \mathrm{in}^{2}$

Total area for 12- 1 in dia bolts, corroded, $A_{b}=6.612 \mathrm{in}^{2}$

$\mathrm{W}=\left(\mathrm{A}_{\mathrm{m}}+\mathrm{A}_{\mathrm{b}}\right) * \mathrm{~S}_{\mathrm{a}} / 2$

$=(5.7237+6.612) * 25,000 / 2$

$=154,195.92 \mathrm{lb}_{\mathrm{f}}$

$\mathrm{M}_{\mathrm{D}}=\mathrm{H}_{\mathrm{D}}{ }^{*} \mathrm{~h}_{\mathrm{D}}=88,267.67 * 1.178=103,979.3 \mathrm{lb}$-in

$\mathrm{M}_{\mathrm{T}}=\mathrm{H}_{\mathrm{T}}{ }^{*} \mathrm{~h}_{\mathrm{T}}=11,268.46^{*} 1.2963=14,606.7 \mathrm{lb}$-in

$\mathrm{H}_{\mathrm{G}}=\mathrm{W}_{\mathrm{m} 1}-\mathrm{H}=143,091.9-99,536.13=43,555.73 \mathrm{lb}_{\mathrm{f}}$

$\mathrm{M}_{\mathrm{G}}=\mathrm{H}_{\mathrm{G}}{ }^{*} \mathrm{~h}_{\mathrm{G}}=43,555.73 * 1.1475=49,980.2 \mathrm{lb}$-in

$M_{o}=M_{D}+M_{T}+M_{G}=103,979.3+14,606.7+49,980.2=168,566.2 \mathrm{lb}-$ in

$\mathrm{M}_{\mathrm{g}}=\mathrm{W}^{*} \mathrm{~h}_{\mathrm{G}}=154,195.9 * 1.1475=176,939.8 \mathrm{lb}$-in 


\section{Hub and Flange Factors}

$\mathrm{h}_{0}=\left(\mathrm{B}^{*} \mathrm{~g}_{0}\right)^{1 / 2}=\left(9.68^{*} 0.534\right)^{1 / 2}=2.2736$ in

From FIG. 2-7.1, where $K=\mathrm{A} / \mathrm{B}=14.625 / 9.68=1.5108$

$$
\begin{aligned}
& \mathrm{T}=1.706 \quad \mathrm{Z}=2.5593 \quad \mathrm{Y}=4.8778 \quad \mathrm{U}=5.3603 \\
& \mathbf{h} / \mathrm{h}_{0}=0.2749 \quad \mathrm{~g}_{1} / \mathrm{g}_{0}=1 \\
& \mathrm{~F}=0.9089 \quad \mathrm{~V}=0.5501 \quad \mathrm{e}=\mathrm{F} / \mathrm{h}_{0}=0.3998 \\
& \mathrm{~d}=(\mathrm{U} / \mathrm{V}) * \mathrm{~h}_{0}{ }^{*} \mathrm{~g}_{0}{ }^{2}=(5.36026 / 0.5501) * 2.2736^{*} 0.534^{2} \\
& =6.3173 \mathrm{in}^{3}
\end{aligned}
$$

\section{Stresses at operating conditions - VIII-1, Appendix 2-7}

$$
\mathrm{f}=1
$$

$\mathrm{L}=\left(\mathrm{t}^{*} \mathrm{e}+1\right) / \mathrm{T}+\mathrm{t}^{3} / \mathrm{d}$

$=(2.4375 * 0.3998+1) / 1.705978+2.4375^{3} / 6.3173$

$=3.449832$

$\mathrm{S}_{\mathrm{H}}=\mathrm{f}^{*} \mathrm{M}_{\mathrm{o}} /\left(\mathrm{L}^{*} \mathrm{~g}_{1}^{2 * \mathrm{~B}}\right)$

$=1 * 168,566.2 /\left(3.449832 * 0.534^{2 * 9.68)}\right.$

$=17,702 \mathrm{psi}$

$\mathrm{S}_{\mathrm{R}}=\left(1.33 * \mathrm{t}^{*} \mathrm{e}+1\right) * \mathrm{M}_{\mathrm{o}} /\left(\mathrm{L} * \mathrm{t}^{2 * \mathrm{~B}}\right)$

$=(1.33 * 2.4375 * 0.3998+1) * 168,566.2 /\left(3.449832 * 2.4375^{2 * 9.68)}\right.$

$=1,951 \mathrm{psi}$

$\mathrm{S}_{\mathrm{T}}=\mathrm{Y}^{*} \mathrm{M}_{\mathrm{o}} /\left(\mathrm{t}^{2 *} \mathrm{~B}\right)-\mathrm{Z} * \mathrm{~S}_{\mathrm{R}}$

$=4.8778 * 168,566.2 /\left(2.4375^{2 * 9.68)}-2.5593 * 1,951\right.$

$=9,304 \mathrm{psi}$

Allowable stress $\mathrm{S}_{\mathrm{fo}}=18,100 \mathrm{psi}$

Allowable stress $S_{n o}=15,600$ psi

$S_{T}$ does not exceed $S_{\text {fo }}$

$\mathrm{S}_{\mathrm{H}}$ does not exceed $\operatorname{Min}\left[1.5 * \mathrm{~S}_{\mathrm{fo}}, 2.5 * \mathrm{~S}_{\mathrm{no}}\right]=27,150 \mathrm{psi}$

$\mathrm{S}_{\mathrm{R}}$ does not exceed $\mathrm{S}_{\mathrm{fo}}$

$0.5\left(S_{H}+S_{R}\right)=9,826$ psi does not exceed $S_{f o}$

$0.5\left(\mathrm{~S}_{\mathrm{H}}+\mathrm{S}_{\mathrm{T}}\right)=13,503$ psi does not exceed $\mathrm{S}_{\mathrm{fo}}$

Stresses at gasket seating - VIII-1, Appendix 2-7

$\mathrm{S}_{\mathrm{H}}=\mathrm{f}^{*} \mathrm{M}_{\mathrm{g}}\left(\mathrm{L} * \mathrm{~g}_{1}{ }^{2 *} \mathrm{~B}\right)$

$=1 * 176,939.8 /\left(3.449832 * 0.534^{2 * 9.68)}\right.$

$=18,581 \mathrm{psi}$ 
$\mathrm{S}_{\mathrm{R}}=\left(1.33 * \mathrm{t}^{*} \mathrm{e}+1\right) * \mathrm{M}_{\mathrm{g}} /\left(\mathrm{L} * \mathrm{t}^{2 *} \mathrm{~B}\right)$

$=(1.33 * 2.4375 * 0.3998+1) * 176,939.8 /\left(3.449832 * 2.4375^{2} * 9.68\right)$

$=2,048 \mathrm{psi}$

$\mathrm{S}_{\mathrm{T}}=\mathrm{Y} * \mathrm{M}_{\mathrm{g}} /\left(\mathrm{t}^{2 * \mathrm{~B})}-\mathrm{Z} * \mathrm{~S}_{\mathrm{R}}\right.$

$=4.8778 * 176,939.8 /\left(2.4375^{2 * 9.68)}-2.5593 * 2,048\right.$

$=9,767 \mathrm{psi}$

Allowable stress $\mathrm{S}_{\mathrm{fa}}=20,000$ psi

Allowable stress $\mathrm{S}_{\mathrm{na}}=17,100 \mathrm{psi}$

$\mathrm{S}_{\mathrm{T}}$ does not exceed $\mathrm{S}_{\mathrm{fa}}$

$\mathrm{S}_{\mathrm{H}}$ does not exceed $\operatorname{Min}\left[1.5 * \mathrm{~S}_{\mathrm{fa}}, 2.5 * \mathrm{~S}_{\mathrm{na}}\right]=30,000 \mathrm{psi}$

$S_{R}$ does not exceed $S_{\text {fa }}$

$0.5\left(\mathrm{~S}_{\mathrm{H}}+\mathrm{S}_{\mathrm{R}}\right)=10,314$ psi does not exceed $\mathrm{S}_{\mathrm{fa}}$

$0.5\left(\mathrm{~S}_{\mathrm{H}}+\mathrm{S}_{\mathrm{T}}\right)=14,174$ psi does not exceed $\mathrm{S}_{\mathrm{fa}}$

Flange rigidity per VIII-1, Appendix 2-14

$$
\begin{aligned}
& \mathrm{J}=52.14 * \mathrm{~V} * \mathrm{M}_{\mathrm{o}} /\left(\mathrm{L} * \mathrm{E}^{*} \mathrm{~g}_{0}{ }^{2 *} \mathrm{~K}_{\mathrm{I}}{ }^{*} \mathrm{~h}_{0}\right) \\
& =52.14 * 0.5501 * 168,566,2 /\left(3.4498 * 25,500,000 * 0.534^{2 *} 0.3 * 2.2736\right) \\
& =0.2825761
\end{aligned}
$$

The flange rigidity index $\mathrm{J}$ does not exceed 1 ; satisfactory.

\section{Flange calculations for Internal Pressure + Seismic}

\section{Longitudinal bending moment on flange}

$$
\begin{aligned}
& P_{m}=16^{*} M_{b} /\left(\pi^{*} G^{3}\right) \\
& =16^{*} 1,500 /\left(\pi * 10.275^{3}\right) \\
& =7.0423 \mathrm{psi}
\end{aligned}
$$

Axial load on flange

$$
\begin{aligned}
& P_{r}=-4 * F /\left(\pi * G^{2}\right) \\
& =-4 * 500 /\left(\pi * 10.275^{2}\right) \\
& =-6.03 \mathrm{psi}
\end{aligned}
$$

Total design load on flange (used for $\mathrm{H}$ - ref. III-1 NC-3658.1)

$$
\begin{aligned}
& =\mathbf{P}+\mathbf{P}_{\mathrm{s}}+\mathbf{P}_{\mathbf{m}}+\mathbf{P}_{\mathbf{r}} \\
& =1,200+0+7.0423+-6.03 \\
& =1,201.0123 \mathrm{psi}
\end{aligned}
$$

\section{Gasket details from facing sketch 1(a) or (b), Column II}

Gasket width $\mathrm{N}=0.375$ in

$\mathrm{b}_{0}=\mathrm{N} / 2=0.1875$ in 
Effective gasket seating width, $b=b_{0}=0.1875$ in

$\mathrm{G}=($ gasket $\mathrm{OD}+$ gasket $\mathrm{ID}) / 2=(10.65+9.9) / 2=10.275 \mathrm{in}$

$h_{G}=(C-G) / 2=(12.57-10.275) / 2=1.1475$ in

$h_{D}=R+g_{1} / 2=0.911+0.534 / 2=1.178$ in

$\mathrm{h}_{\mathrm{T}}=\left(\mathrm{R}+\mathrm{g}_{1}+\mathrm{h}_{\mathrm{G}}\right) / 2=(0.911+0.534+1.1475) / 2=1.2963$ in

$\mathrm{H}_{\mathrm{p}}=2 * \mathrm{~b} * 3.14 * \mathrm{G} * \mathrm{~m} * \mathrm{P}$

$=2 * 0.1875^{*} 3.14 * 10.275^{*} 3 * 1,200$

$=43,555.72 \mathrm{lb}_{\mathrm{f}}$

$\mathrm{H}=0.785^{*} \mathrm{G}^{2 *} \mathrm{P}$

$=0.785^{*} 10.275^{2 *} 1,201.0123$

$=99,536.13 \mathrm{lb}_{\mathrm{f}}$

$\mathrm{H}_{\mathrm{D}}=0.785^{*} \mathrm{~B}^{2 * \mathrm{P}}$

$=0.785^{*} 9.68^{2 * 1,200}$

$=88,267.67 \mathrm{lb}_{\mathrm{f}}$

$\mathrm{H}_{\mathrm{T}}=\mathrm{H}-\mathrm{H}_{\mathrm{D}}$

$=99,536.13-88,267.67$

$=11,268.46 \mathrm{lb}_{\mathrm{f}}$

$\mathrm{W}_{\mathrm{ml}}=\mathrm{H}+\mathrm{H}_{\mathrm{p}}$

$=99,536.13+43,555.72$

$=143,091.86 \mathrm{lb}_{\mathrm{f}}$

$\mathrm{W}_{\mathrm{m} 2}=3.14 * \mathrm{~b} * \mathrm{G} * \mathrm{y}$

$=3.14 * 0.1875 * 10.275 * 10,000$

$=60,494.06 \mathrm{lb}_{\mathrm{f}}$

Required bolt area, $A_{m}=$ greater of $A_{m 1}, A_{m 2}=5.723674 \mathrm{in}^{2}$

$A_{m 1}=W_{m 1} / S_{b}=143,091.9 / 25,000=5.7237 \mathrm{in}^{2}$

$A_{m 2}=W_{m 2} / S_{a}=60,494.06 / 25,000=2.4198$ in $^{2}$

Total area for $12-1$ in dia bolts, corroded, $A_{b}=6.612$ in $^{2}$

$\mathrm{W}=\left(\mathrm{A}_{\mathrm{m}}+\mathrm{A}_{\mathrm{b}}\right) * \mathrm{~S}_{\mathrm{a}} / 2$

$=(5.7237+6.612) * 25,000 / 2$

$=154,195.92 \mathrm{lb}_{\mathrm{f}}$

$\mathrm{M}_{\mathrm{D}}=\mathrm{H}_{\mathrm{D}}{ }^{*} \mathrm{~h}_{\mathrm{D}}=88,267.67 * 1.178=103,979.3 \mathrm{lb}$-in

$\mathrm{M}_{\mathrm{T}}=\mathrm{H}_{\mathrm{T}} \mathrm{h}_{\mathrm{T}}=11,268.46 * 1.2963=14,606.7 \mathrm{lb}$-in 


$$
\begin{aligned}
& \mathrm{H}_{\mathrm{G}}=\mathrm{W}_{\mathrm{m} 1}-\mathrm{H}=143,091.9-99,536.13=43,555.73 \mathrm{lb}_{\mathrm{f}} \\
& \mathrm{M}_{\mathrm{G}}=\mathrm{H}_{\mathrm{G}}{ }^{*} \mathrm{~h}_{\mathrm{G}}=43,555.73 * 1.1475=49,980.2 \mathrm{lb} \text {-in } \\
& \mathrm{M}_{\mathrm{o}}=\mathrm{M}_{\mathrm{D}}+\mathrm{M}_{\mathrm{T}}+\mathrm{M}_{\mathrm{G}}=103,979.3+14,606.7+49,980.2=168,566.2 \mathrm{lb} \text {-in } \\
& \mathrm{M}_{\mathrm{g}}=\mathrm{W}^{*} \mathrm{~h}_{\mathrm{G}}=154,195.9 * 1.1475=176,939.8 \mathrm{lb} \text {-in }
\end{aligned}
$$




\section{Hub and Flange Factors}

$\mathrm{h}_{0}=\left(\mathrm{B}^{*} \mathrm{~g}_{0}\right)^{1 / 2}=\left(9.68^{*} 0.534\right)^{1 / 2}=2.2736$ in

From FIG. 2-7.1, where $\mathrm{K}=\mathrm{A} / \mathrm{B}=14.625 / 9.68=1.5108$

$$
\begin{aligned}
& \mathrm{T}=1.706 \quad \mathrm{Z}=2.5593 \quad \mathrm{Y}=4.8778 \quad \mathrm{U}=5.3603 \\
& \mathrm{~h} / \mathrm{h}_{0}=0.2749 \quad \mathrm{~g}_{1} / \mathrm{g}_{0}=1 \\
& \mathrm{~F}=0.9089 \quad \mathrm{~V}=0.5501 \quad \mathrm{e}=\mathrm{F} / \mathrm{h}_{0}=0.3998 \\
& \mathrm{~d}=(\mathrm{U} / \mathrm{V})^{*} \mathrm{~h}_{0}{ }^{*} \mathrm{~g}_{0}{ }^{2}=(5.36026 / 0.5501)^{*} 2.2736^{*} 0.534^{2} \\
& =6.3173 \mathrm{in}^{3}
\end{aligned}
$$

\section{Stresses at operating conditions - VIII-1, Appendix 2-7}

$\mathbf{f}=1$

$\mathrm{L}=\left(\mathrm{t}^{*} \mathrm{e}+1\right) / \mathrm{T}+\mathrm{t}^{3} / \mathrm{d}$

$=\left(2.4375^{*} 0.3998+1\right) / 1.705978+2.4375^{3} / 6.3173$

$=3.449832$

$\mathrm{S}_{\mathrm{H}}=\mathrm{f}^{*} \mathrm{M}_{\mathrm{o}} /\left(\mathrm{L}^{*} \mathrm{~g}_{1}{ }^{2 * \mathrm{~B})}\right.$

$=1 * 168,566.2 /\left(3.449832 * 0.534^{2 * 9.68)}\right.$

$=17,702 \mathrm{psi}$

$S_{R}=(1.33 * t * e+1) * M_{o} /\left(L * t^{2 * B}\right)$

$=\left(1.33 * 2.4375^{*} 0.3998+1\right) * 168,566.2 /\left(3.449832 * 2.4375^{2 * 9.68)}\right.$

$=1,951 \mathrm{psi}$

$\mathrm{S}_{\mathrm{T}}=\mathrm{Y} * \mathrm{M}_{\mathrm{o}} /\left(\mathrm{t}^{2 * \mathrm{~B})}-\mathrm{Z} * \mathrm{~S}_{\mathrm{R}}\right.$

$=4.8778^{*} 168,566.2 /\left(2.4375^{2 * 9.68)}-2.5593 * 1,951\right.$

$=9,304 \mathrm{psi}$

Allowable stress $S_{\text {fo }}=18,100$ psi

Allowable stress $S_{\text {no }}=15,600 \mathrm{psi}$

$S_{\mathrm{T}}$ does not exceed $\mathrm{S}_{\mathrm{fo}}$

$\mathrm{S}_{\mathrm{H}}$ does not exceed Min[ $\left.1.5^{*} \mathrm{~S}_{\mathrm{fo}}, 2.5^{*} \mathrm{~S}_{\mathrm{no}}\right]=27,150 \mathrm{psi}$

$\mathrm{S}_{\mathrm{R}}$ does not exceed $\mathrm{S}_{\mathrm{fo}}$

$0.5\left(\mathrm{~S}_{\mathrm{H}}+\mathrm{S}_{\mathrm{R}}\right)=9,826$ psi does not exceed $\mathrm{S}_{\mathrm{fo}}$

$0.5\left(\mathrm{~S}_{\mathrm{H}}+\mathrm{S}_{\mathrm{T}}\right)=13,503$ psi does not exceed $\mathrm{S}_{\mathrm{fo}}$

\section{Stresses at gasket seating - VIII-1, Appendix 2-7}

$$
\begin{aligned}
& S_{H}=f^{*} M_{g}\left(L^{*} g_{1}{ }^{2 * B}\right) \\
& =1 * 176,939.8 /\left(3.449832 * 0.534^{2 * 9.68)}\right. \\
& =18,581 \mathrm{psi}
\end{aligned}
$$


$\mathrm{S}_{\mathrm{R}}=(1.33 * \mathrm{t} * \mathrm{e}+1) * \mathrm{M}_{\mathrm{g}} /\left(\mathrm{L}^{*} \mathrm{t}^{2} * \mathrm{~B}\right)$

$=(1.33 * 2.4375 * 0.3998+1) * 176,939.8 /\left(3.449832 * 2.4375^{2 * 9.68)}\right.$

$=2,048 \mathrm{psi}$

$\mathrm{S}_{\mathrm{T}}=\mathrm{Y}^{*} \mathrm{M}_{\mathrm{g}} /\left(\mathrm{t}^{2 * \mathrm{~B}}\right)-\mathrm{Z} * \mathrm{~S}_{\mathrm{R}}$

$=4.8778 * 176,939.8 /\left(2.4375^{2 * 9.68)}-2.5593 * 2,048\right.$

$=9,767 \mathrm{psi}$

Allowable stress $\mathrm{S}_{\mathrm{fa}}=20,000 \mathrm{psi}$

Allowable stress $\mathrm{S}_{\mathrm{na}}=17,100 \mathrm{psi}$

$S_{T}$ does not exceed $S_{f a}$

$\mathrm{S}_{\mathrm{H}}$ does not exceed $\operatorname{Min}\left[1.5 * \mathrm{~S}_{\mathrm{fa}}, 2.5 * \mathrm{~S}_{\mathrm{na}}\right]=30,000 \mathrm{psi}$

$\mathrm{S}_{\mathrm{R}}$ does not exceed $\mathrm{S}_{\mathrm{fa}}$

$0.5\left(\mathrm{~S}_{\mathrm{H}}+\mathrm{S}_{\mathrm{R}}\right)=10,314$ psi does not exceed $\mathrm{S}_{\mathrm{fa}}$

$0.5\left(\mathrm{~S}_{\mathrm{H}}+\mathrm{S}_{\mathrm{T}}\right)=14,174$ psi does not exceed $\mathrm{S}_{\mathrm{fa}}$

Flange rigidity per VIII-1, Appendix 2-14

$\mathrm{J}=52.14 * \mathrm{~V}^{*} \mathrm{M}_{\mathrm{o}} /\left(\mathrm{L}^{*} \mathrm{E}^{*} \mathrm{~g}_{0}{ }^{2 *} \mathrm{~K}_{\mathrm{I}} * \mathrm{~h}_{0}\right)$

$=52.14 * 0.5501 * 168,566.2 /\left(3.4498 * 25,500,000 * 0.5344^{2 * 0.3 * 2.2736)}\right.$

$=0.2825761$

The flange rigidity index $\mathrm{J}$ does not exceed 1 ; satisfactory.

\section{FLANGE, SHELL HEAD - Flange hub}

\section{ASME Section VIII Division 1, 2007 Edition}

Component:

Material specification:

SA-516 70 (II-D p. 18, ln. 22)

Fit

Fig UCS-66.1 MDMT reduction $=38.2^{\circ} \mathrm{F}$, (coincident ratio $=0.6178278$ )

Rated MDMT is governed by UCS-66(b)(2)

UCS-66 governing thickness $=0.5198$ in

Internal design pressure: $\mathrm{P}=1,200 \mathrm{psi} @ 700^{\circ} \mathrm{F}$

\section{Static liquid head:}

$P_{\text {th }}=0 \mathrm{psi}\left(\mathrm{SG}=0, \mathrm{H}_{\mathrm{s}}=10.5634\right.$ ", Horizontal test head $)$

Corrosion allowance $\quad$ Inner $\mathrm{C}=0.06 " \quad$ Outer $\mathrm{C}=0^{\prime \prime}$

Design MDMT $=5^{\circ} \mathrm{F}$

Rated MDMT $=-55^{\circ} \mathrm{F}$

No impact test performed

Material is normalized

Material is not produced to Fine Grain Practice

PWHT is not performed

Radiography: Longitudinal joint - Seamless No RT

Top circumferential joint - N/A

Bottom circumferential Full UW-11(a) Type 1 
joint -

Estimated weight New $=3.2 \mathrm{lb} \quad$ corr $=2.9 \mathrm{lb}$

Capacity New $=0.19$ US gal corr $=0.2$ US gal

OD $\quad=10.748^{\prime \prime}$

Length $\mathrm{L}_{\mathrm{c}}=0.625^{\prime \prime}$

$\mathrm{t} \quad=0.594^{\prime \prime}$

Design thickness, (at $700{ }^{\circ} \mathrm{F}$ ) Appendix 1-1

$\mathrm{t}=\mathrm{P} * \mathrm{R}_{\mathrm{o}} /(\mathrm{S} * \mathrm{E}+0.40 * \mathrm{P})+$ Corrosion

$=1,200 * 5.374 /(18,100 * 1.00+0.40 * 1,200)+0.06$

$=0.4071^{\prime \prime}$

Maximum allowable working pressure, (at $700^{\circ}$ F) Appendix 1-1

$$
\begin{aligned}
\mathrm{P} & =\mathrm{S} \mathrm{E}^{*} \mathrm{t} /\left(\mathrm{R}_{\mathrm{o}}-0.40^{*} \mathrm{t}\right)-\mathrm{P}_{\mathrm{S}} \\
& =18,100 * 1.00 * 0.534 /(5.374-0.40 * 0.534)-0 \\
& =1,872.99 \mathrm{psi}
\end{aligned}
$$

\% Extreme fiber elongation - UCS-79(d)

$$
\begin{aligned}
\mathrm{EFE} & =\left(50 * \mathrm{t} / \mathrm{R}_{\mathrm{f}}\right)^{*}\left(1-\mathrm{R}_{\mathrm{f}} / \mathrm{R}_{\mathrm{o}}\right) \\
& =(50 * 0.594 / 5.077)^{*}(1-5.077 / \infty) \\
& =5.8499 \%
\end{aligned}
$$

The extreme fiber elongation exceeds 5 percent. Heat treatment per UCS-56 may be required. See UCS-79(d)(4) or

\begin{tabular}{|c|c|c|c|c|c|c|c|c|}
\hline \multirow[t]{2}{*}{ Condition } & \multirow{2}{*}{$\begin{array}{c}\text { Pressure } \\
\text { P ( psi) }\end{array}$} & \multicolumn{2}{|c|}{$\begin{array}{l}\text { Allowable Stress } \\
\text { Before UG-23 Stress } \\
\text { Increase ( psi) }\end{array}$} & \multirow{2}{*}{$\begin{array}{c}\text { Temperature } \\
\left({ }^{\circ} \mathbf{F}\right)\end{array}$} & \multirow{2}{*}{$\begin{array}{l}\text { Corrosion } \\
\mathbf{C}(\mathbf{l n})\end{array}$} & \multirow[t]{2}{*}{ Load } & \multirow{2}{*}{$\begin{array}{c}\text { Req'd Thk } \\
\text { Due to } \\
\text { Tension (in) }\end{array}$} & \multirow{2}{*}{$\begin{array}{l}\text { Req'd Thk Due to } \\
\text { Compression (in) }\end{array}$} \\
\hline & & $S_{t}$ & $\mathbf{S}_{\mathbf{c}}$ & & & & & \\
\hline \multirow{2}{*}{$\begin{array}{l}\text { Operating, Hot \& } \\
\text { Corroded }\end{array}$} & \multirow{2}{*}{1,200} & \multirow{2}{*}{18,100} & \multirow{2}{*}{12,461} & \multirow{2}{*}{700} & \multirow{2}{*}{0.06} & Wind & 0.1321 & 0.132 \\
\hline & & & & & & Seismic & 0.1321 & 0.132 \\
\hline \multirow{2}{*}{$\begin{array}{c}\text { Operating, Hot \& } \\
\text { New }\end{array}$} & \multirow{2}{*}{1,200} & \multirow{2}{*}{18,100} & \multirow{2}{*}{12,641} & \multirow{2}{*}{700} & \multirow{2}{*}{0} & Wind & 0.1305 & 0.1303 \\
\hline & & & & & & Seismic & 0.1304 & 0.1303 \\
\hline \multirow{2}{*}{ Empty, Corroded } & \multirow{2}{*}{0} & \multirow{2}{*}{20,000} & \multirow{2}{*}{17,425} & \multirow{2}{*}{0} & \multirow{2}{*}{0.06} & Wind & 0.0001 & 0.0003 \\
\hline & & & & & & Seismic & 0.0002 & 0.0003 \\
\hline Empty, New & 0 & 20,000 & 17,452 & 0 & 0 & Wind & 0.0002 & 0.0003 \\
\hline
\end{tabular}
(5).

\section{Design thickness $=0.4071 "$}

The governing condition is due to internal pressure.

The cylinder thickness of $0.594 "$ is adequate.

Thickness Required Due to Pressure + External Loads 


\begin{tabular}{|c|c|c|c|c|c|c|c|c|}
\hline & & & & & & Seismic & 0.0002 & 0.0003 \\
\hline $\begin{array}{c}\text { Hot Shut Down, } \\
\text { Corroded, Weight \& } \\
\text { Eccentric Moments } \\
\text { Only }\end{array}$ & 0 & 18,100 & 12,461 & 700 & 0.06 & Weight & 0.0003 & 0.0003 \\
\hline
\end{tabular}




\section{TAPPED HOLES PER UG-43(d) $\&$ (g)}

FOR FLANGE SHELL HEAD

NOMINAL DIAMETER OF STUD/BOLT, ds = NUMBER OF THREADS PER INCH, tpi = ALLOW. STRESS OF STUD/BOLT MAT'L AT DESIGN TEMP, Sas = ALLOW. STRESS OF TAPPED MAT'L AT DESIGN TEMP, Sat =

MATERIAL THICKNESS TO BE TAPPED, $\mathrm{t}=$ CORROSION ALLOWANCE OF MATERIAL, ca =

REQUIRED THREAD ENGAGEMENT, te $=.75 \times \mathrm{ds} \times($ Sas $/$ Sat $)$

te $=1.307$

THREAD ENGAGEMENT NEED NOT EXCEED $11 / 2 \times \mathrm{ds}=1.7985$

MINIMUM. THREAD ENGAGEMENT, tem $=1.307$

1"-14 X 1 1/2" LONG HELICAL INSERT REQUIRED HOLE DRILL DEPTH: 2.00" (MIN.)

NOTE: INSERT MINIMUM TENSILE STRENGTH: 200,000 PSI > FLANGE MATERIAL MINIMUM TENSILE STRENGTH: 70,000 PSI 


\section{REACTOR OUTER SHELL}

\section{ASME Section VIII Division 1, 2007 Edition}

Component:

Material specification:

Pipe NPS and Schedule:

Material impact test exemption temperature from Fig UCS-66 Curve $\mathrm{B}=-5.1^{\circ} \mathrm{F}$

Fig UCS-66.1 MDMT reduction $=16.4^{\circ} \mathrm{F}$, (coincident ratio $=0.8359815$ )

UCS-66 governing thickness $=0.5198$ in

Internal design pressure: $\mathrm{P}=1,200 \mathrm{psi} @ 700^{\circ} \mathrm{F}$

Static liquid head:

$P_{\text {th }}=0 \mathrm{psi}\left(\mathrm{SG}=0, \mathrm{H}_{\mathrm{s}}=10.5644 "\right.$ ", Horizontal test head $)$

Corrosion allowance $\quad$ Inner $\mathrm{C}=0.06^{\prime \prime} \quad$ Outer $\mathrm{C}=0 "$

Design MDMT $=5^{\circ} \mathrm{F}$

Rated MDMT $=-21.5^{\circ} \mathrm{F}$

No impact test performed

Material is not normalized

Material is not produced to Fine Grain Practice

PWHT is not performed

Radiography: Longitudinal joint - Seamless No RT

Top circumferential joint - Full UW-11(a) Type 1

Bottom circumferential

joint -

Full UW-11(a) Type 1

Estimated weight New $=898.7 \mathrm{lb} \quad$ corr $=812.7 \mathrm{lb}$

Capacity $\quad$ New $=52.09$ US gal corr $=53.41$ US gal

OD $\quad=10.75^{\prime \prime}$

Length $\mathrm{L}_{\mathrm{c}}=167.5625^{\prime \prime}$

$\mathrm{t} \quad=0.594^{\prime \prime}$

Design thickness, (at $700{ }^{\circ}$ F) Appendix 1-1

$\mathrm{t}=\mathrm{P} * \mathrm{R}_{\mathrm{o}} /(\mathrm{S} * \mathrm{E}+0.40 * \mathrm{P})+$ Corrosion

$=1,200 * 5.375 /(15,600 * 1.00+0.40 * 1,200)+0.06$

$=0.4612^{\prime \prime}$

Maximum allowable working pressure, $\left(\right.$ at $700^{\circ} \mathrm{F}$ ) Appendix 1-1

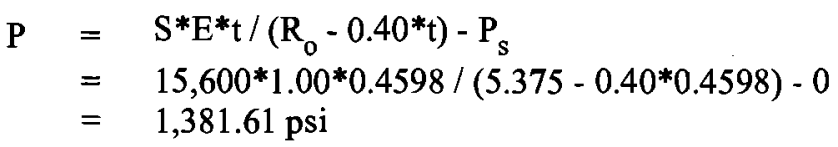

Design thickness $=0.4612 "$

The governing condition is due to internal pressure.

The cylinder thickness of $0.594 "$ is adequate. 


\section{Thickness Required Due to Pressure + External Loads}

\begin{tabular}{|c|c|c|c|c|c|c|c|c|c|}
\hline \multirow[t]{2}{*}{ Condition } & \multirow{2}{*}{$\begin{array}{l}\text { Pressure } \\
\text { P ( psi) }\end{array}$} & \multicolumn{2}{|c|}{$\begin{array}{l}\text { Allowable Stress } \\
\text { Before UG-23 Stress } \\
\text { Increase ( psi) }\end{array}$} & \multirow{2}{*}{$\begin{array}{c}\text { Temperature } \\
\left({ }^{\circ} \text { F) }\right.\end{array}$} & \multirow{2}{*}{$\begin{array}{l}\text { Corrosion } \\
\text { C (in) }\end{array}$} & \multirow[t]{2}{*}{ Location } & \multirow[t]{2}{*}{ Load } & \multirow{2}{*}{$\begin{array}{l}\text { Req'd Thk } \\
\text { Due to } \\
\text { Tension } \\
\text { (in) }\end{array}$} & \multirow{2}{*}{$\begin{array}{l}\text { Req'd Thk Due } \\
\text { to Compression } \\
\text { (in) }\end{array}$} \\
\hline & & $\mathbf{S}_{\mathbf{t}}$ & $\mathrm{S}_{\mathrm{c}}$ & & & & & & \\
\hline \multirow{4}{*}{$\begin{array}{l}\text { Operating, Hot \& } \\
\text { Corroded }\end{array}$} & \multirow{4}{*}{1,200} & \multirow{4}{*}{15,600} & \multirow{4}{*}{12,210} & \multirow{4}{*}{700} & \multirow{4}{*}{0.06} & \multirow{2}{*}{ Top } & Wind & 0.1529 & 0.1526 \\
\hline & & & & & & & Seismic & 0.1528 & 0.1526 \\
\hline & & & & & & \multirow{2}{*}{ Bottom } & Wind & 0.1653 & 0.1466 \\
\hline & & & & & & & Seismic & 0.1792 & 0.1333 \\
\hline \multirow{4}{*}{$\begin{array}{c}\text { Operating, Hot \& } \\
\text { New }\end{array}$} & \multirow{4}{*}{1,200} & \multirow{4}{*}{15,600} & \multirow{4}{*}{12,415} & \multirow{4}{*}{700} & \multirow{4}{*}{0} & \multirow{2}{*}{ Top } & Wind & 0.151 & 0.1507 \\
\hline & & & & & & & Seismic & 0.1509 & 0.1507 \\
\hline & & & & & & \multirow{2}{*}{ Bottom } & Wind & 0.1637 & 0.1448 \\
\hline & & & & & & & Seismic & 0.1786 & 0.1304 \\
\hline \multirow{4}{*}{ Empty, Corroded } & \multirow{4}{*}{0} & \multirow{4}{*}{17,100} & \multirow{4}{*}{17,100} & \multirow{4}{*}{0} & \multirow{4}{*}{0.06} & \multirow{2}{*}{ Top } & Wind & 0.0003 & 0.0005 \\
\hline & & & & & & & Seismic & 0.0004 & $0.000 \hat{5}$ \\
\hline & & & & & & \multirow{2}{*}{ Bottom } & Wind & 0.0111 & 0.006 \\
\hline & & & & & & & Seismic & 0.0237 & 0.0181 \\
\hline \multirow{4}{*}{ Empty, New } & & & & & & & Wind & 0.0003 & 0.0005 \\
\hline & 0 & 17100 & 17100 & I & 0 & Av & Seismic & 0.0004 & 0.0005 \\
\hline & & & & & & & Wind & 0.0113 & 0.006 \\
\hline & & & & & & Dovivint & Seismic & 0.0249 & 0.019 \\
\hline $\begin{array}{l}\text { Hot Shut Down, } \\
\text { Corroded, Weight }\end{array}$ & 0 & 15600 & 12710 & 700 & 006 & Top & Weight & 0.0007 & 0.0007 \\
\hline $\begin{array}{c}\text { \& Eccentric } \\
\text { Moments Only }\end{array}$ & & & & & 0.00 & Bottom & Weight & 0.0033 & 0.0033 \\
\hline
\end{tabular}




\section{COAL INLET (A)}

ASME Section VIII Division 1, 2007 Edition

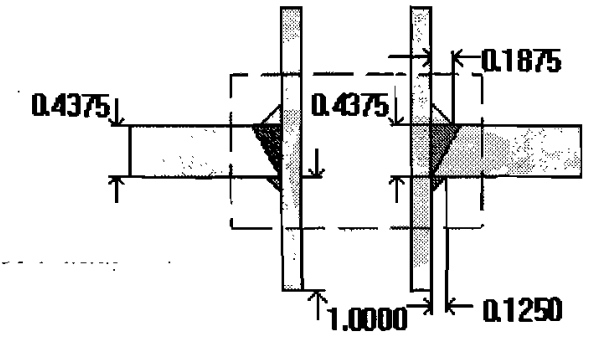

Note: round inside edges per UG-76(c)

Located on:

Liquid static head included:

Nozzle material specification:

Nozzle longitudinal joint efficiency:

Nozzle description:

Flange description:

Bolt Material:

Flange rated MDMT:

(Per UHA-51(d)(1)(a))

(Flange rated MDMT $=-320^{\circ} \mathrm{F}$

Bolts rated MDMT per Fig UCS-66 note $\left.(\mathrm{e})=-55^{\circ} \mathrm{F}\right)$

Liquid static head on flange:

ASME B16.5 flange rating MAWP:

ASME B16.5 flange rating MAP:

ASME B16.5 flange hydro test:

Nozzle orientation:

Calculated as hillside:

Local vessel minimum thickness:

End of nozzle to datum line:

Nozzle inside diameter, new:

Nozzle nominal wall thickness:

Nozzle corrosion allowance:

Projection available outside vessel, Lpr:

Internal projection, $h_{\text {new }}$ :

Projection available outside vessel to flange face, Lf: 13.9103 in

Distance to head center, $R$ :
0 in

$$
\begin{aligned}
& t_{w(l o w e r)}=0.4375 \text { in } \\
& \operatorname{Leg}_{41}=0.1875 \text { in } \\
& \operatorname{Leg}_{43}=0.125 \text { in } \\
& h_{\text {new }}=1 \mathrm{in}
\end{aligned}
$$

NOTE: EXTERNAL LOADS ON FLANGES NOT CONSIDERED IN DESIGN CALCULATIONS.

MAXIMUM ALLOWABLE LOADS ON NOZZLE NECKAXIAL: $500 \mathrm{lbf}$ IN DIRECTION OF VESSEL

SHEAR: $500 \mathrm{lbf}$

BENDING MOMENT: $1500 \mathrm{lb}$-in.

\section{8" WELD CAP SCH 80}

0 psi

SB-444 1 Annealed Smls pipe N06625 (II-D p. 226, ln. 7)

1

$1 "$ Sch 80 (XS)

1 inch Class 1500 WN A1 82 F316

SA-193 B7 Bolt $<=21 / 2$ (II-D p. 348, ln. 33)

$-55^{\circ} \mathrm{F}$

0 psi

$1820 \mathrm{psi} @ 1000^{\circ} \mathrm{F}$

3600 psi@70 $\mathrm{F}$

$5425 \mathrm{psi}$ @70 $\mathrm{F}$

$0^{\circ}$

no

0.4375 in

195.3175 in

0.957 in

0.179 in

0.03 in

12.5403 in

1 in

\section{Reinforcement Calculations for Internal Pressure}

Available reinforcement per UG-37 governs the MAWP of this nozzle. 


\begin{tabular}{|c|c|c|c|c|c|c|c|c|}
\multicolumn{8}{|c|}{$\begin{array}{c}\text { UG-37 Area Calculation Summary (in } \\
\text { 2) } \\
\text { For } \mathbf{P}=1263.39 \text { psi @ } 1000\end{array}$} & \multicolumn{2}{c|}{$\begin{array}{c}\text { UG-45 Nozzle Wall } \\
\text { Thickness Summary (in) } \\
\text { The nozzle passes UG-45 }\end{array}$} \\
\begin{tabular}{|c|c|c|c|c|c|c|c|} 
The opening is adequately reinforced \\
required
\end{tabular} & $\begin{array}{c}\mathbf{A} \\
\text { available }\end{array}$ & $\mathbf{A}_{1}$ & $\mathbf{A}_{2}$ & $\mathbf{A}_{3}$ & $\mathbf{A}_{5}$ & $\begin{array}{c}\mathbf{A} \\
\text { welds }\end{array}$ & $\mathbf{t}_{\text {req }}$ & $\mathbf{t}_{\text {min }}$ \\
\hline 0.3155 & 0.3156 & 0.1083 & 0.0946 & 0.0708 & -- & 0.0419 & 0.1464 & 0.1566 \\
\hline
\end{tabular}

\section{UG-41 Weld Failure Path Analysis Summary}

The nozzle is exempt from weld strength calculations per UW-15(b)(1)

\begin{tabular}{|c|r|r|c|}
\hline \multicolumn{4}{|c|}{ UW-16 Weld Sizing Summary } \\
\hline Weld description & $\begin{array}{r}\text { Required weld } \\
\text { throat size (in) }\end{array}$ & $\begin{array}{r}\text { Actual weld } \\
\text { throat size (in) }\end{array}$ & Status \\
\hline Nozzle to shell fillet $\left(\operatorname{Leg}_{41}\right)$ & 0.1043 & 0.1312 & weld size is adequate \\
\hline
\end{tabular}




\section{Applied Loads}

Radial load: $\quad \mathrm{P}_{\mathrm{r}}=\quad 500 \mathrm{lb}_{\mathrm{f}}$

Circumferential moment: $\mathrm{M}_{1}=\quad 0 \mathrm{lb}_{\mathrm{f}}$ in

Circumferential shear: $\quad \mathrm{V}_{2}=\quad 500 \mathrm{lb}_{\mathrm{f}}$

Longitudinal moment: $\quad \mathrm{M}_{2}=1,500 \mathrm{lb}_{\mathrm{f}}$ in

Longitudinal shear: $\quad \mathrm{v}_{1}=\quad 0 \mathrm{lb}_{\mathrm{f}}$

Torsion moment: $\quad \mathrm{M}_{\mathrm{t}}=\quad 0 \mathrm{lb}_{\mathrm{f}}$ in

Internal pressure: $\quad \mathbf{P}=1,263.387 \mathrm{psi}$

Head yield stress: $\quad \mathrm{S}_{\mathrm{y}}=17,000 \mathrm{psi}$

Maximum stresses due to the applied loads at the nozzle OD (includes pressure)

Mean dish radius $R_{m}=7.2436$ in

$\mathrm{U}=\mathrm{r}_{\mathrm{o}} / \operatorname{Sqr}\left(\mathrm{R}_{\mathrm{m}}{ }^{*} \mathrm{t}\right)=0.383$

Pressure stress intensity factor, $I=1$ (derived from PVP-Vol. 399, pages 77-82)

Local pressure stress $=I^{*} P * R_{i} / 2 * t=10,913.00 p s i$

Maximum combined stress $\left(\mathrm{P}_{\mathrm{L}}+\mathrm{P}_{\mathrm{b}}\right)=22,661 \mathrm{psi}$

Allowable combined stress $\left(\mathrm{P}_{\mathrm{L}}+\mathrm{P}_{\mathrm{b}}\right)=+-1.5 * \mathrm{~S}=+-22,950 \mathrm{psi}$

The maximum combined stress $\left(\mathrm{P}_{\mathrm{L}}+\mathrm{P}_{\mathrm{b}}\right)$ is within allowable limits.

Maximum local primary membrane stress $\left(\mathrm{P}_{\mathrm{L}}\right)=11,261 \mathrm{psi}$

Allowable local primary membrane stress $\left(\mathrm{P}_{\mathrm{L}}\right)=+-1.5 * \mathrm{~S}=+-22,950 \mathrm{psi}$

The local maximum primary membrane stress $\left(\mathrm{P}_{\mathrm{L}}\right)$ is within allowable limits. 


\begin{tabular}{|c|c|c|c|c|c|c|c|c|c|}
\hline \multicolumn{10}{|c|}{ Stresses at the nozzle OD per WRC Bulletin 107} \\
\hline Figure & value & $\mathbf{A}_{\mathbf{u}}$ & $\mathbf{A}_{\mathbf{l}}$ & $\mathbf{B}_{\mathbf{u}}$ & $\mathbf{B}_{1}$ & $\mathbf{C}_{\mathbf{u}}$ & $\mathbf{C}_{\mathbf{I}}$ & $\mathbf{D}_{\mathbf{u}}$ & $\mathbf{D}_{1}$ \\
\hline SR-2* & 0.1644 & -495 & -495 & -495 & -495 & -495 & -495 & -495 & -495 \\
\hline SR-2 & 0.1101 & $-1,989$ & 1,989 & $-1,989$ & 1,989 & $-1,989$ & 1,989 & $-1,989$ & 1,989 \\
\hline SR-3* & 0.1604 & 0 & 0 & 0 & 0 & 0 & 0 & 0 & 0 \\
\hline SR-3 & 0.3504 & 0 & 0 & 0 & 0 & 0 & 0 & 0 & 0 \\
\hline SR-3* & 0.1604 & -843 & -843 & 843 & 843 & 0 & 0 & 0 & 0 \\
\hline SR-3 & 0.3504 & $-11,054$ & 11,054 & 11,054 & $-11,054$ & 0 & 0 & 0 & 0 \\
\hline \multicolumn{2}{|c|}{ Pressure stress ${ }^{\star}$} & 10,913 & 10,913 & 10,913 & 10,913 & 10,913 & 10,913 & 10,913 & 10,913 \\
\hline \multicolumn{2}{|c|}{ Total $\mathbf{O}_{\mathbf{x}}$ stress } & $-3,468$ & 22,618 & 20,326 & 2,196 & 8,429 & 12,407 & 8,429 & 12,407 \\
\hline \multicolumn{2}{|c|}{$\begin{array}{c}\text { Membrane } O_{x} \\
\text { stress }^{\star}\end{array}$} & 9,575 & 9,575 & 11,261 & 11,261 & 10,418 & 10,418 & 10,418 & 10,418 \\
\hline SR-2* & 0.0494 & -149 & -149 & -149 & -149 & -149 & -149 & -149 & -149 \\
\hline SR-2 & 0.0337 & -609 & 609 & -609 & 609 & -609 & 609 & -609 & 609 \\
\hline SR-3* & 0.0493 & 0 & 0 & 0 & 0 & 0 & 0 & 0 & 0 \\
\hline SR-3 & 0.1048 & 0 & 0 & 0 & 0 & 0 & 0 & 0 & 0 \\
\hline SR-3* & 0.0493 & -259 & -259 & 259 & 259 & 0 & 0 & 0 & 0 \\
\hline SR-3 & 0.1048 & $-3,306$ & 3,306 & 3,306 & $-3,306$ & 0 & 0 & 0 & 0 \\
\hline \multicolumn{2}{|c|}{ Pressure stress ${ }^{\star}$} & 10,913 & 10,913 & 10,913 & 10,913 & 10,913 & 10,913 & 10,913 & 10,913 \\
\hline \multicolumn{2}{|c|}{ Total $O_{y}$ stress } & 6,590 & 14,420 & 13,720 & 8,326 & 10,155 & 11,373 & 10,155 & 11,373 \\
\hline \multicolumn{2}{|c|}{$\begin{array}{c}\text { Membrane } O_{y} \\
\text { stress }^{\star}\end{array}$} & 10,505 & 10,505 & 11,023 & 11,023 & 10,764 & 10,764 & 10,764 & 10,764 \\
\hline \multicolumn{2}{|c|}{ Shear from $\mathbf{M}_{\mathbf{t}}$} & 0 & 0 & 0 & 0 & 0 & 0 & 0 & 0 \\
\hline \multicolumn{2}{|c|}{ Shear from $V_{1}$} & 0 & 0 & 0 & 0 & 0 & 0 & 0 & 0 \\
\hline \multicolumn{2}{|c|}{ Shear from $V_{2}$} & 594 & 594 & -594 & -594 & 0 & 0 & 0 & 0 \\
\hline \multicolumn{2}{|c|}{$\begin{array}{c}\text { Total Shear } \\
\text { stress }\end{array}$} & 594 & 594 & -594 & -594 & 0 & 0 & 0 & 0 \\
\hline \multicolumn{2}{|c|}{$\begin{array}{c}\text { Combined } \\
\text { stress } \\
\left(\mathbf{P}_{L}+\mathbf{P}_{b}\right)\end{array}$} & 10,128 & 22,661 & 20,379 & 8,383 & 10,155 & 12,407 & 10,155 & 12,407 \\
\hline
\end{tabular}

Notes: (1) * denotes primary stress.

(2) The nozzle is assumed to be a rigid (solid) attachment.

\section{Longitudinal stress in the nozzle wall due to internal pressure + external loads}

$$
\begin{aligned}
& \sigma_{\mathrm{n}(\mathrm{Pm})}=\mathrm{P} * \mathrm{R}_{\mathrm{i}} /\left(2 * \mathrm{t}_{\mathrm{n}}\right)-\mathrm{P}_{\mathrm{r}} /\left(\pi *\left(\mathrm{R}_{\mathrm{o}}{ }^{2}-\mathrm{R}_{\mathrm{i}}{ }^{2}\right)\right)+\mathrm{M} * \mathrm{R}_{\mathrm{o}} / \mathrm{I} \\
& =1,263.387 * 0.5085 /(2 * 0.1266)-500 /\left(\pi *\left(0.6575^{2}-0.5085^{2}\right)\right)+1,500.00 * 0.6575 / 0.09427066 \\
& =12,082.57 \mathrm{psi}
\end{aligned}
$$

The average primary stress $\mathrm{P}_{m}$ (see Division 2 Appendix 4-138(b)) across the nozzle wall due to internal pressure + external loads is acceptable $(=\mathrm{S}=29,900.00 \mathrm{psi})$ 


\section{Shear stress in the nozzle wall due to external loads}

$$
\begin{aligned}
& \sigma_{\text {shear }}=\left(\mathrm{V}_{\mathrm{L}}^{2}+\mathrm{V}_{\mathrm{c}}^{2}\right)^{0.5} /\left(\pi^{*} \mathrm{R}_{\mathrm{i}}{ }^{*} \mathrm{t}_{\mathrm{n}}\right) \\
& =\left(0^{2}+500^{2}\right)^{0.5} /\left(\pi^{*} 0.5085^{*} 0.149\right) \\
& =2,101 \mathrm{psi} \\
& \sigma_{\text {torsion }}=\mathrm{M}_{\mathrm{t}} /\left(2^{*} \pi^{*} \mathrm{R}_{\mathrm{i}}^{2 *} \mathrm{t}_{\mathrm{n}}\right) \\
& =0 /\left(2^{*} \pi^{*} 0.5085^{2 *} 0.149\right) \\
& =0 \mathrm{psi} \\
& \sigma_{\text {total }}=\sigma_{\text {shear }}+\sigma_{\text {torsion }} \\
& =2,101+0 \\
& =2,101 \mathrm{psi}
\end{aligned}
$$

UG-45(c): The total combined shear stress $(2,101 \mathrm{psi})$ is below than the allowable $\left(0.7 * \mathrm{~S}_{\mathrm{n}}=0.7 * 29,900=20,930 \mathrm{psi}\right)$

\section{Reinforcement Calculations for MAP}

Available reinforcement per UG-37 governs the MAP of this nozzle.

\begin{tabular}{|c|c|c|c|c|c|c|c|c|}
\hline \multicolumn{7}{|c|}{$\begin{array}{c}\text { UG-37 Area Calculation Summary (in } \\
\text { For } \\
\text { P }=2166.6 \text { psi @ } \\
\text { The opening is adequately reinforced }\end{array}$} & \multicolumn{2}{c|}{$\begin{array}{c}\text { UG-45 Nozzle Wall } \\
\text { Thickness Summary (in) } \\
\text { The nozzle passes UG-45 }\end{array}$} \\
\hline $\begin{array}{c}\text { A } \\
\text { required }\end{array}$ & $\begin{array}{c}\text { A } \\
\text { available }\end{array}$ & $\mathbf{A}_{1}$ & $\mathbf{A}_{2}$ & $\mathbf{A}_{3}$ & $\mathbf{A}_{5}$ & $\begin{array}{c}\mathbf{A} \\
\text { welds }\end{array}$ & $\mathbf{t}_{\text {req }}$ & $\mathbf{t}_{\text {min }}$ \\
\hline 0.3857 & 0.3857 & 0.0426 & 0.1321 & 0.1602 & -- & 0.0508 & 0.1164 & 0.1566 \\
\hline
\end{tabular}

\section{UG-41 Weld Failure Path Analysis Summary}

The nozzle is exempt from weld strength calculations per UW-15(b)(1)

\begin{tabular}{|c|r|r|c|}
\hline \multicolumn{4}{|c|}{ UW-16 Weld Sizing Summary } \\
\hline Weld description & $\begin{array}{r}\text { Required weld } \\
\text { throat size (in) }\end{array}$ & $\begin{array}{r}\text { Actual weld } \\
\text { throat size (in) }\end{array}$ & Status \\
\hline Nozzle to shell fillet $\left(\operatorname{Leg}_{41}\right)$ & 0.1253 & 0.1312 & weld size is adequate \\
\hline
\end{tabular}




\section{INJECTOR HOUSING (I1)}

\section{ASME Section VIII Division 1, 2007 Edition}

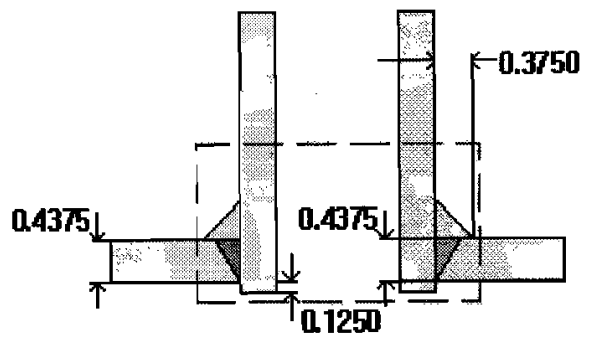

$$
\begin{aligned}
& t_{w(\text { lower })}=0.4375 \text { in } \\
& \operatorname{Leg}_{41}=0.375 \text { in } \\
& \operatorname{Leg}_{43}=0 \text { in } \\
& h_{\text {new }}=0.125 \text { in }
\end{aligned}
$$

Note: round inside edges per UG-76(c)

\section{Located on:}

Liquid static head included:

Nozzle material specification:

Nozzle longitudinal joint efficiency:

Nozzle orientation:

Calculated as hillside:

Local vessel minimum thickness:

End of nozzle to datum line:

Nozzle inside diameter, new:

Nozzle nominal wall thickness:

Nozzle corrosion allowance:

Projection available outside vessel, Lpr: 1.5294 in

Internal projection, $\mathrm{h}_{\text {new }}$ :

0.125 in

Distance to head center, $R$ :

\section{8" WELD CAP SCH 80}

$0 \mathrm{psi}$

SB-446 1 Annealed Bar <= 4 N06625 (II-D 226, In. 8)

1

$0^{\circ}$

no

0.4375 in

180.9425 in

1.24 in

0.38 in

0.03 in

\section{Reinforcement Calculations for Internal Pressure}

The vessel wall thickness governs the MAWP of this nozzle.

\begin{tabular}{|c|c|c|c|c|c|c|c|c|}
\hline \multicolumn{3}{|c|}{$\begin{array}{c}\text { UG-37 Area Calculation Summary } \\
\left.\text { (in }{ }^{2}\right) \\
\text { For } P=1596.14 \text { psi @ } 1000\end{array}$} & \multicolumn{2}{c|}{$\begin{array}{c}\text { UG-45 Nozzle Wall } \\
\text { Thickness Summary (in) } \\
\text { The nozzle passes UG-45 }\end{array}$} \\
\hline $\begin{array}{c}\text { The opening is adequately reinforced } \\
\text { required }\end{array}$ & $\begin{array}{c}\mathbf{A} \\
\text { available }\end{array}$ & $\mathbf{A}_{1}$ & $\mathbf{A}_{2}$ & $\mathbf{A}_{3}$ & $\mathbf{A}_{5}$ & $\begin{array}{c}\mathbf{A} \\
\text { welds }\end{array}$ & $\mathbf{t}_{\text {req }}$ & $\mathbf{t}_{\text {min }}$ \\
\hline 0.5298 & 0.6845 & -- & 0.5439 & -- & -- & 0.1406 & 0.1648 & 0.38 \\
\hline
\end{tabular}

\section{UG-41 Weld Failure Path Analysis Summary}

The nozzle is exempt from weld strength calculations per UW-15(b)(1)

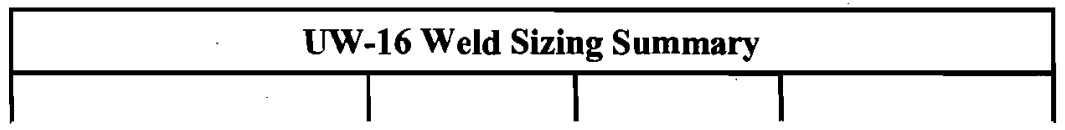




\begin{tabular}{|c|r|r|c|} 
Weld description & $\begin{array}{r}\text { Required weld } \\
\text { throat size (in) }\end{array}$ & $\begin{array}{r}\text { Actual weld } \\
\text { throat size (In) }\end{array}$ & Status \\
\hline Nozzle to shell fillet $\left(\operatorname{Leg}_{41}\right)$ & 0.245 & 0.2625 & weld size is adequate \\
\hline
\end{tabular}

\% Forming strain - UNF-79(a)(2)(a)

$$
\begin{aligned}
\text { EFE } & =\left(50^{*} t / R_{f}\right)^{*}\left(1-R_{f} / R_{o}\right) \\
& =\left(50^{*} 0.38 / 0.81\right)^{*}(1-0.81 / \infty) \\
& =23.4568 \%
\end{aligned}
$$

\begin{tabular}{|c|c|c|c|c|c|c|c|c|}
\hline \multicolumn{7}{|c|}{$\begin{array}{l}\text { UG-37 Area Calculation Summary }\left(\text { in }^{2}\right) \\
\text { For } P=2232.88 \text { psi @ } 70^{\circ} \mathrm{F} \\
\text { The opening is adequately reinforced }\end{array}$} & \multicolumn{2}{|c|}{$\begin{array}{l}\text { UG-45 Nozzle Wall } \\
\text { Thickness Summary (in) } \\
\text { The nozzle passes UG-45 }\end{array}$} \\
\hline$\underset{\text { required }}{\mathbf{A}}$ & $\underset{\text { available }}{\mathbf{A}}$ & $\mathbf{A}_{1}$ & $A_{2}$ & $\mathbf{A}_{3}$ & $A_{5}$ & $\begin{array}{c}\mathbf{A} \\
\text { welds }\end{array}$ & $t_{\text {req }}$ & $\mathbf{t}_{\min }$ \\
\hline 0.5425 & 0.775 & -- & 0.6344 & -- & -- & 0.1406 & 0.1348 & 0.38 \\
\hline
\end{tabular}

\section{Reinforcement Calculations for MAP}

The vessel wall thickness governs the MAP of this nozzle.

\section{UG-41 Weld Failure Path Analysis Summary}

The nozzle is exempt from weld strength calculations per UW-15(b)(1)

\begin{tabular}{|c|r|r|c|}
\hline \multicolumn{4}{|c|}{ UW-16 Weld Sizing Summary } \\
\hline Weld description & $\begin{array}{r}\text { Required weld } \\
\text { throat size (in) }\end{array}$ & $\begin{array}{r}\text { Actual weld } \\
\text { throat size (in) }\end{array}$ & Status \\
\hline Nozzle to shell fillet $\left(\operatorname{Leg}_{41}\right)$ & 0.25 & 0.2625 & weld size is adequate \\
\hline
\end{tabular}


DESC: REACTOR UNIT

P.O.\#: 700521452

\section{INJECTOR HOUSING (I2)}

\section{ASME Section VIII Division 1, 2007 Edition}

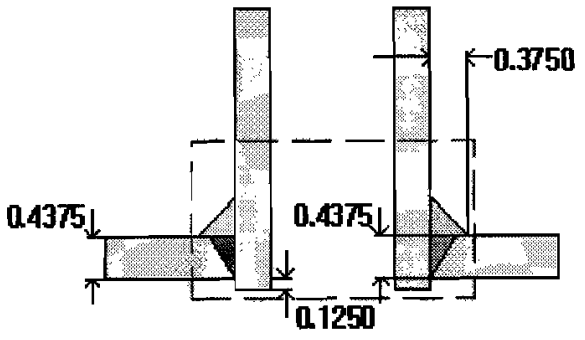

$$
\begin{aligned}
& t_{w(\text { lower) }}=0.4375 \text { in } \\
& \operatorname{Leg}_{41}=0.375 \text { in } \\
& \operatorname{Leg}_{43}=0 \text { in } \\
& h_{\text {new }}=0.125 \text { in }
\end{aligned}
$$

Note: round inside edges per UG-76(c)

Located on:

Liquid static head included:

Nozzle material specification:

Nozzle longitudinal joint efficiency:

Nozzle orientation:

Calculated as hillside:

Local vessel minimum thickness:

End of nozzle to datum line:

Nozzle inside diameter, new:

Nozzle nominal wall thickness:

Nozzle corrosion allowance:
8" WELD CAP SCH 80

$0 \mathrm{psi}$

SB-446 1 Annealed Bar <= 4 N06625 (II-D 226, In. 8)

1

$90^{\circ}$

no

0.4375 in

180.9425 in

1.24 in

0.38 in

0.03 in

Projection available outside vessel, Lpr: 1.5294 in

Internal projection, $h_{\text {new }}$ : $\quad 0.125$ in

\begin{tabular}{|c|c|c|c|c|c|c|c|c|}
\hline \multicolumn{7}{|c|}{$\begin{array}{c}\text { UG-37 Area Calculation Summary }\left(\mathrm{in}^{2}\right) \\
\text { For } P=1596.14 \text { psi @ } 1000^{\circ} \mathrm{F} \\
\text { The opening is adequately reinforced }\end{array}$} & \multicolumn{2}{|c|}{$\begin{array}{l}\text { UG-45 Nozzle Wall } \\
\text { Thickness Summary (in) } \\
\text { The nozzle passes UG-45 }\end{array}$} \\
\hline $\begin{array}{c}A \\
\text { required }\end{array}$ & $\underset{\text { available }}{A}$ & $A_{1}$ & $\mathbf{A}_{2}$ & $\mathbf{A}_{\mathbf{3}}$ & $A_{5}$ & $\begin{array}{c}\text { A } \\
\text { welds }\end{array}$ & $t_{\text {req }}$ & $t_{\min }$ \\
\hline 0.5298 & 0.6845 & -1 & 0.5439 & -- & - & 0.1406 & 0.1648 & 0.38 \\
\hline
\end{tabular}

Distance to head center, $\mathrm{R}$ : $\quad 3.875$ in

\section{Reinforcement Calculations for Internal Pressure}

The vessel wall thickness governs the MAWP of this nozzle.

\section{UG-41 Weld Failure Path Analysis Summary}

The nozzle is exempt from weld strength calculations per UW-15(b)(1)

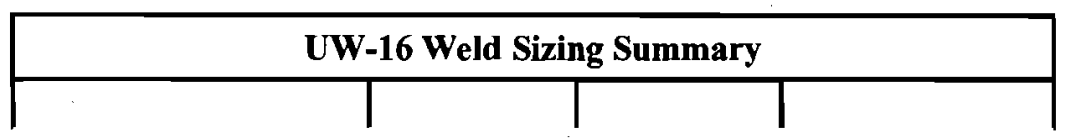




\begin{tabular}{|c|r|r|c|} 
Weld description & $\begin{array}{r}\text { Required weld } \\
\text { throat size (in) }\end{array}$ & $\begin{array}{r}\text { Actual weld } \\
\text { throat size (in) }\end{array}$ & Status \\
\hline Nozzle to shell fillet $\left(\operatorname{Leg}_{41}\right)$ & 0.245 & 0.2625 & weld size is adequate \\
\hline
\end{tabular}

\% Forming strain - UNF-79(a)(2)(a)

$$
\begin{aligned}
\mathrm{EFE} & =\left(50 *_{\mathrm{t}} / \mathrm{R}_{\mathrm{f}}\right) *\left(1-\mathrm{R}_{\mathrm{f}} / \mathrm{R}_{\mathrm{o}}\right) \\
& =(50 * 0.38 / 0.81)^{*}(1-0.81 / \infty) \\
& =23.4568 \%
\end{aligned}
$$

\begin{tabular}{|c|c|c|c|c|c|c|c|c|}
\hline \multicolumn{7}{|c|}{$\begin{array}{l}\text { UG-37 Area Calculation Summary }\left(\text { in }^{2}\right) \\
\text { For } P=2232.88 \text { psi @ } 70^{\circ} \mathrm{F} \\
\text { The opening is adequately reinforced }\end{array}$} & \multicolumn{2}{|c|}{$\begin{array}{l}\text { UG-45 Nozzle Wall } \\
\text { Thickness Summary (in) } \\
\text { The nozzle passes UG-45 }\end{array}$} \\
\hline $\begin{array}{c}A \\
\text { required }\end{array}$ & $\underset{\text { available }}{A}$ & $\mathbf{A}_{1}$ & $\mathbf{A}_{2}$ & $\mathbf{A}_{3}$ & $\mathbf{A}_{5}$ & $\begin{array}{c}\mathrm{A} \\
\text { welds }\end{array}$ & $t_{\text {req }}$ & $t_{\text {mln }}$ \\
\hline 0.5425 & 0.775 & -- & 0.6344 & -- & -- & 0.1406 & 0.1348 & 0.38 \\
\hline
\end{tabular}

\section{Reinforcement Calculations for MAP}

The vessel wall thickness governs the MAP of this nozzle.

\section{UG-41 Weld Failure Path Analysis Summary}

The nozzle is exempt from weld strength calculations per UW-15(b)(1)

\begin{tabular}{|c|r|r|c|}
\hline \multicolumn{4}{|c|}{ UW-16 Weld Sizing Summary } \\
\hline Weld description & $\begin{array}{r}\text { Required weld } \\
\text { throat size (in) })\end{array}$ & $\begin{array}{r}\text { Actual weld } \\
\text { throat size (in) }\end{array}$ & Status \\
\hline Nozzle to shell fillet $\left(\right.$ Leg $\left._{41}\right)$ & 0.25 & 0.2625 & weld size is adequate \\
\hline
\end{tabular}




\section{INJECTOR HOUSING (I3)}

\section{ASME Section VIII Division 1, 2007 Edition}

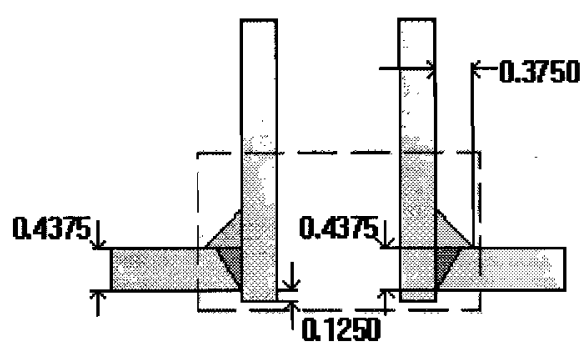

$$
\begin{aligned}
& \mathrm{t}_{\mathrm{w}(\text { lower) }}=0.4375 \mathrm{in} \\
& \operatorname{Leg}_{41}=0.375 \mathrm{in} \\
& \operatorname{Leg}_{43}=0 \mathrm{in} \\
& \mathrm{h}_{\text {new }}=0.125 \mathrm{in}
\end{aligned}
$$

Note: round inside edges per UG-76(c)

Located on:

Liquid static head included:

Nozzle material specification:

Nozzle longitudinal joint efficiency:

Nozzle orientation:

Calculated as hillside:

Local vessel minimum thickness:

End of nozzle to datum line:

Nozzle inside diameter, new:

Nozzle nominal wall thickness:

Nozzle corrosion allowance:

Projection available outside vessel, Lpr: 1.5294 in

Internal projection, $\mathrm{h}_{\text {new }}$ :

Distance to head center, $R$ :

0.125 in

3.875 in
8 " WELD CAP SCH 80

0 psi

SB-446 1 Annealed Bar <= 4 N06625 (II-D 226, In. 8)

1

$180^{\circ}$

no

0.4375 in

180.9425 in

1.24 in

0.38 in

0.03 in

\section{Reinforcement Calculations for Internal Pressure}

The vessel wall thickness governs the MAWP of this nozzle.

\begin{tabular}{|c|c|c|c|c|c|c|c|c|}
\hline \multicolumn{2}{|c|}{$\begin{array}{c}\text { UG-37 Area Calculation Summary } \\
\text { For } P=1596.14 \\
\text { Fsi } \\
\text { The opening is adequately reinforced }\end{array}$} & \multicolumn{2}{c|}{$\begin{array}{c}\text { UG-45 Nozzle Wall } \\
\text { Thickness Summary (in) } \\
\text { The nozzle passes UG-45 }\end{array}$} \\
\hline $\begin{array}{c}\text { A } \\
\text { required }\end{array}$ & $\begin{array}{c}\mathbf{A} \\
\text { available }\end{array}$ & $\mathbf{A}_{1}$ & $\mathbf{A}_{2}$ & $\mathbf{A}_{3}$ & $\mathbf{A}_{\mathbf{5}}$ & $\begin{array}{c}\mathbf{A} \\
\text { welds }\end{array}$ & $\mathbf{t}_{\text {req }}$ & $\mathbf{t}_{\text {min }}$ \\
\hline 0.5298 & 0.6845 & -- & 0.5439 & -- & -- & 0.1406 & 0.1648 & 0.38 \\
\hline
\end{tabular}

\section{UG-41 Weld Failure Path Analysis Summary}

The nozzle is exempt from weld strength calculations per UW-15(b)(1)

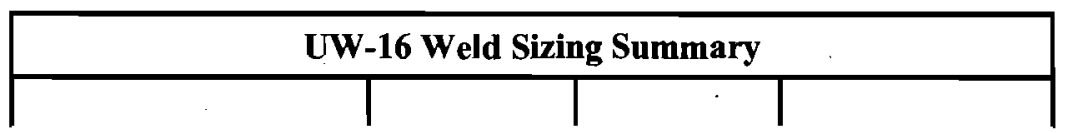




\begin{tabular}{|c|r|r|c|} 
Weld description & $\begin{array}{r}\text { Required weld } \\
\text { throat size (in) }\end{array}$ & $\begin{array}{r}\text { Actual weld } \\
\text { throat size (in) }\end{array}$ & Status \\
\hline Nozzle to shell fillet $\left(\operatorname{Leg}_{41}\right)$ & 0.245 & 0.2625 & weld size is adequate \\
\hline
\end{tabular}

\% Forming strain - UNF-79(a)(2)(a)

$$
\begin{aligned}
\mathrm{EFE} & =\left(50 * \mathrm{t} / \mathrm{R}_{\mathrm{f}}\right) *\left(1-\mathrm{R}_{\mathrm{f}} / \mathrm{R}_{\mathrm{o}}\right) \\
& =(50 * 0.38 / 0.81) *(1-0.81 / \infty) \\
& =23.4568 \%
\end{aligned}
$$

\begin{tabular}{|c|c|c|c|c|c|c|c|c|}
\hline \multicolumn{7}{|c|}{$\begin{array}{c}\text { UG-37 Area Calculation Summary }\left(\mathrm{in}^{2}\right) \\
\text { For } P=2232.88 \text { psi @ } 70^{\circ} \mathrm{F} \\
\text { The opening is adequately reinforced }\end{array}$} & \multicolumn{2}{|c|}{$\begin{array}{l}\text { UG-45 Nozzle Wall } \\
\text { Thickness Summary (in) } \\
\text { The nozzle passes UG-45 }\end{array}$} \\
\hline $\begin{array}{c}\mathrm{A} \\
\text { required }\end{array}$ & $\underset{\text { available }}{\mathbf{A}}$ & $A_{1}$ & $\mathbf{A}_{2}$ & $\mathbf{A}_{3}$ & $\mathbf{A}_{5}$ & $\begin{array}{c}\mathbf{A} \\
\text { welds }\end{array}$ & $\mathbf{t}_{\text {req }}$ & $\mathbf{t}_{\min }$ \\
\hline 0.5425 & 0.775 & -- & 0.6344 & -- & -- & 0.1406 & 0.1348 & 0.38 \\
\hline
\end{tabular}

\section{Reinforcement Calculations for MAP}

The vessel wall thickness governs the MAP of this nozzle.

\section{UG-41 Weld Failure Path Analysis Summary}

The nozzle is exempt from weld strength calculations per UW-15(b)(1)

\begin{tabular}{|c|r|r|c|}
\hline \multicolumn{4}{|c|}{ UW-16 Weld Sizing Summary } \\
\hline Weld description & $\begin{array}{r}\text { Required weld } \\
\text { throat size (in) }\end{array}$ & $\begin{array}{r}\text { Actual weld } \\
\text { throat size (in) }\end{array}$ & Status \\
\hline Nozzle to shell fillet $\left(\right.$ Leg $\left._{41}\right)$ & 0.25 & 0.2625 & weld size is adequate \\
\hline
\end{tabular}


INJECTOR HOUSING (I4)

\section{ASME Section VIII Division 1, 2007 Edition}

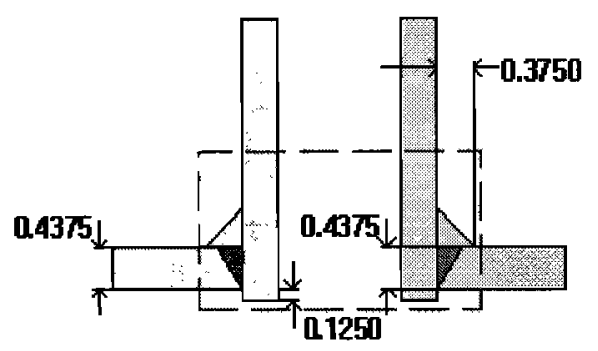

$$
\begin{aligned}
& t_{w(l o w e r)}=0.4375 \text { in } \\
& \operatorname{Leg}_{41}=0.375 \text { in } \\
& \operatorname{Leg}_{43}=0 \text { in } \\
& h_{\text {new }}=0.125 \text { in }
\end{aligned}
$$

Note: round inside edges per UG-76(c)

Located on:

Liquid static head included:

Nozzle material specification:

Nozzle longitudinal joint efficiency:

Nozzle orientation:

Calculated as hillside:

Local vessel minimum thickness:

End of nozzle to datum line:

Nozzle inside diameter, new:

Nozzle nominal wall thickness:

Nozzle corrosion allowance:

Projection available outside vessel, Lpr: 1.5294 in

Internal projection, $\mathrm{h}_{\text {new }}$ :

0.125 in

Distance to head center, $R$ :

\begin{tabular}{|c|c|c|c|c|c|c|c|c|}
\hline \multicolumn{7}{|c|}{$\begin{array}{l}\text { UG-37 Area Calculation Summary }\left(\mathrm{in}^{2}\right) \\
\text { For } P=1596.14 \text { psi @ } 1000{ }^{\circ} \mathrm{F} \\
\text { The opening is adequately reinforced }\end{array}$} & \multicolumn{2}{|c|}{$\begin{array}{c}\text { UG-45 Nozzle Wall } \\
\text { Thickness Summary (in) } \\
\text { The nozzle passes UG-45 }\end{array}$} \\
\hline $\begin{array}{c}A \\
\text { required }\end{array}$ & $\underset{\text { available }}{\mathbf{A}}$ & $A_{1}$ & $\mathbf{A}_{2}$ & $\mathbf{A}_{3}$ & $A_{5}$ & $\begin{array}{c}A \\
\text { welds }\end{array}$ & $\mathbf{t}_{\text {req }}$ & $t_{\min }$ \\
\hline 0.5298 & 0.6845 & -- & 0.5439 & -- & |-- & 0.1406 & 0.1648 & 0.38 \\
\hline
\end{tabular}

3.875 in

\section{Reinforcement Calculations for Internal Pressure}

The vessel wall thickness governs the MAWP of this nozzle.

\section{UG-41 Weld Failure Path Analysis Summary}

The nozzle is exempt from weld strength calculations per UW-15(b)(1)

\begin{tabular}{|l|l|l|l|}
\hline \multicolumn{3}{|c|}{ UW-16 Weld Sizing Summary } \\
\hline & & & \\
\hline
\end{tabular}




\begin{tabular}{|c|r|r|c|} 
Weld description & $\begin{array}{r}\text { Required weld } \\
\text { throat size (in) }\end{array}$ & $\begin{array}{r}\text { Actual weld } \\
\text { throat size (in) }\end{array}$ & Status \\
\hline Nozzle to shell fillet $\left(\operatorname{Leg}_{41}\right)$ & 0.245 & 0.2625 & weld size is adequate \\
\hline
\end{tabular}

\% Forming strain - UNF-79(a)(2)(a)

$$
\begin{aligned}
\mathrm{EFE} & =\left(50 *_{\mathrm{t}} / \mathrm{R}_{\mathrm{f}}\right)^{*}\left(1-\mathrm{R}_{\mathrm{f}} / \mathrm{R}_{\mathrm{o}}\right) \\
& =\left(50^{*} 0.38 / 0.81\right)^{*}(1-0.81 / \infty) \\
& =23.4568 \%
\end{aligned}
$$

\begin{tabular}{|c|c|c|c|c|c|c|c|c|}
\hline \multicolumn{7}{|c|}{$\begin{array}{l}\text { UG-37 Area Calculation Summary }\left(\text { in }^{2}\right) \\
\text { For } P=2232.88 \text { psi } @ 70^{\circ} \mathrm{F} \\
\text { The opening is adequately reinforced }\end{array}$} & \multicolumn{2}{|c|}{$\begin{array}{l}\text { UG-45 Nozzle Wall } \\
\text { Thickness Summary (in) } \\
\text { The nozzle passes UG-45 }\end{array}$} \\
\hline $\begin{array}{c}A \\
\text { required }\end{array}$ & \begin{tabular}{|c|}
$\mathbf{A}$ \\
available
\end{tabular} & $A_{1}$ & $A_{2}$ & $A_{3}$ & $A_{5}$ & $\begin{array}{c}\text { A } \\
\text { welds }\end{array}$ & $\mathbf{t}_{\text {req }}$ & $t_{\min }$ \\
\hline 0.5425 & 0.775 & -- & 0.6344 & -- & -- & 0.1406 & 0.1348 & 0.38 \\
\hline
\end{tabular}

\section{Reinforcement Calculations for MAP}

The vessel wall thickness governs the MAP of this nozzle.

\section{UG-41 Weld Failure Path Analysis Summary}

The nozzle is exempt from weld strength calculations per UW-15(b)(1)

\begin{tabular}{|c|r|r|c|}
\hline \multicolumn{4}{|c|}{ UW-16 Weld Sizing Summary } \\
\hline Weld description & $\begin{array}{l}\text { Required weld } \\
\text { throat size (in) }\end{array}$ & $\begin{array}{r}\text { Actual weld } \\
\text { throat size (in) }\end{array}$ & Status \\
\hline Nozzle to shell fillet $\left(\mathrm{Leg}_{41}\right)$ & 0.25 & 0.2625 & weld size is adequate \\
\hline
\end{tabular}




\section{COUPLING (C01)}

\section{ASME Section VIII Division 1, 2007 Edition}

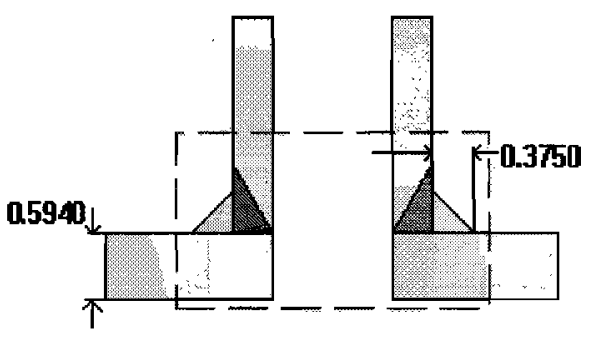

$$
\begin{aligned}
& t_{w(\text { lower })}=0 \text { in } \\
& \operatorname{Leg}_{41}=0.375 \text { in }
\end{aligned}
$$

Note: round inside edges per UG-76(c)

Located on:

Liquid static head included:

Nozzle material specification:

Nozzle longitudinal joint efficiency:

Nozzle description:

Nozzle orientation:

Local vessel minimum thickness:

Nozzle center line offset to datum line: 13.375 in

End of nozzle to shell center:

Nozzle inside diameter, new:

Nozzle nominal wall thickness:

Nozzle corrosion allowance:

Projection available outside vessel, Lpr: 1 in

\section{Reinforcement Calculations for Internal Pressure}

The vessel wall thickness governs the MAWP of this nozzle.

\begin{tabular}{|c|c|c|c|c|c|c|c|c|}
\hline \multicolumn{3}{|c|}{$\begin{array}{c}\text { UG-37 Area Calculation Summary } \\
\text { (in }{ }^{2} \text { ) }\end{array}$} \\
$\begin{array}{c}\text { For } \mathbf{P}=1381.47 \text { psi @ } \\
\text { The opening is adequately reinforced }\end{array}$ & \multicolumn{2}{c|}{$\begin{array}{c}\text { UG-45 Nozzle Wall } \\
\text { Thickness Summary (in) } \\
\text { The nozzle passes UG-45 }\end{array}$} \\
\hline $\begin{array}{c}\mathbf{A} \\
\text { required }\end{array}$ & $\begin{array}{c}\mathbf{A} \\
\text { available }\end{array}$ & $\mathbf{A}_{\mathbf{1}}$ & $\mathbf{A}_{\mathbf{2}}$ & $\mathbf{A}_{\mathbf{3}}$ & $\mathbf{A}_{\mathbf{5}}$ & $\begin{array}{c}\mathbf{A} \\
\text { welds }\end{array}$ & $\mathbf{t}_{\text {req }}$ & $\mathbf{t}_{\text {min }}$ \\
\hline 0.5103 & 0.5777 & -- & 0.4371 & -- & -- & 0.1406 & 0.0981 & 0.35 \\
\hline
\end{tabular}

UG-41 Weld Failure Path Analysis Summary

The nozzle is exempt from weld strength calculations per UW-15(b)(1)

\begin{tabular}{|l|l|l|l|}
\hline \multicolumn{4}{|c|}{ UW-16 Weld Sizing Summary } \\
\hline Weld description & $\begin{array}{l}\text { Required weld } \\
\text { throat size (in) }\end{array}$ & $\begin{array}{r}\text { Actual weld } \\
\text { throat size (in) }\end{array}$ & Status \\
\hline & & & \\
\hline
\end{tabular}




\begin{tabular}{|l|l|l|l|} 
Nozzle to shell fillet $\left(\operatorname{Leg}_{41}\right)$ & 0.224 & 0.2625 & weld size is adequate \\
\hline
\end{tabular}

Opening C02 is too close per UG-36(c)(3)(d) to allow an exemption per UG-36(c)(3)(a).

\section{Reinforcement Calculations for MAP}

The vessel wall thickness governs the MAP of this nozzle.

\begin{tabular}{|c|c|c|c|c|c|c|c|c|}
\hline \multicolumn{7}{|c|}{$\begin{array}{l}\text { UG-37 Area Calculation Summary }\left(\mathrm{in}^{2}\right) \\
\text { For } P=1719.91 \text { psi @ } 70^{\circ} \mathrm{F} \\
\text { The opening is adequately reinforced }\end{array}$} & \multicolumn{2}{|c|}{$\begin{array}{l}\text { UG-45 Nozzle Wall } \\
\text { Thickness Summary (in) } \\
\text { The nozzle passes UG-45 }\end{array}$} \\
\hline $\begin{array}{c}\mathbf{A} \\
\text { required }\end{array}$ & $\begin{array}{c}\text { A } \\
\text { available }\end{array}$ & $A_{1}$ & $\mathbf{A}_{2}$ & $\mathbf{A}_{3}$ & $\mathbf{A}_{5}$ & $\begin{array}{c}\text { A } \\
\text { welds }\end{array}$ & $\mathbf{t}_{\mathrm{req}}$ & $t_{\min }$ \\
\hline 0.5457 & 0.6698 & -- & 0.5292 & -- & -- & 0.1406 & 0.0727 & 0.35 \\
\hline
\end{tabular}

\section{UG-41 Weld Failure Path Analysis Summary}

The nozzle is exempt from weld strength calculations per UW-15(b)(1)

\begin{tabular}{|c|r|r|c|}
\hline \multicolumn{4}{|c|}{ UW-16 Weld Sizing Summary } \\
\hline Weld description & $\begin{array}{r}\text { Required weld } \\
\text { throat size (in) }\end{array}$ & $\begin{array}{r}\text { Actual weld } \\
\text { throat size (in) }\end{array}$ & Status \\
\hline Nozzle to shell fillet ( $\left.\operatorname{Leg}_{41}\right)$ & 0.245 & 0.2625 & weld size is adequate \\
\hline
\end{tabular}

Opening $\mathrm{C02}$ is too close per UG-36(c)(3)(d) to allow an exemption per UG-36(c)(3)(a). 
COUPLING (C02)

ASME Section VIII Division 1, 2007 Edition

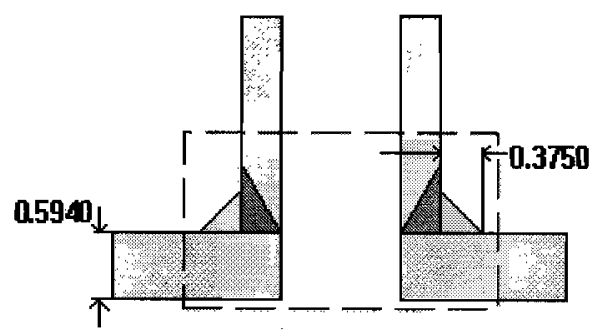

$$
\begin{aligned}
& t_{w(\text { lower })}=0 \text { in } \\
& \operatorname{Leg}_{41}=0.375 \text { in }
\end{aligned}
$$

Note: round inside edges per UG-76(c)

Located on:

Liquid static head included:

Nozzle material specification:

Nozzle longitudinal joint efficiency:

Nozzle description:

Nozzle orientation:

Local vessel minimum thickness:
REACTOR OUTER SHELL

0 psi

SA-105 (II-D p. 18, ln. 8)

1

$0.750^{\prime \prime}$ Class 6000 - threaded

$30^{\circ}$

0.5197 in

10.75 in

6.375 in

1.05 in

0.35 in

Nozzle corrosion allowance: $\quad 0.03$ in

Projection available outside vessel, Lpr: 1 in

\section{Reinforcement Calculations for Internal Pressure}

The vessel wall thickness governs the MAWP of this nozzle.

\begin{tabular}{|c|c|c|c|c|c|c|c|c|}
\hline \multicolumn{3}{|c|}{$\begin{array}{c}\text { UG-37 Area Calculation Summary }\left(\mathrm{in}^{2}\right) \\
\text { For P }=1381.47 \text { psi @ } \\
\text { The opening is adequately reinforced }\end{array}$} & \multicolumn{2}{c|}{$\begin{array}{c}\text { UG-45 Nozzle Wall } \\
\text { Thickness Summary (in) } \\
\text { The nozzle passes UG-45 }\end{array}$} \\
\hline $\begin{array}{c}\mathbf{A} \\
\text { required }\end{array}$ & $\begin{array}{c}\mathbf{A} \\
\text { available }\end{array}$ & $\mathbf{A}_{1}$ & $\mathbf{A}_{2}$ & $\mathbf{A}_{\mathbf{3}}$ & $\mathbf{A}_{\mathbf{5}}$ & $\begin{array}{c}\mathbf{A} \\
\text { welds }\end{array}$ & $\mathbf{t}_{\text {req }}$ & $\mathbf{t}_{\text {min }}$ \\
\hline 0.5103 & 0.5777 & -- & 0.4371 & -- & -- & 0.1406 & 0.0981 & 0.35 \\
\hline
\end{tabular}

UG-41 Weld Failure Path Analysis Summary

The nozzle is exempt from weld strength calculations per UW-15(b)(1)

\begin{tabular}{|l|l|l|l|}
\hline \multicolumn{4}{|c|}{ UW-16 Weld Sizing Summary } \\
\hline Weld description & $\begin{array}{l}\text { Required weld } \\
\text { throat size (in) }\end{array}$ & $\begin{array}{c}\text { Actual weld } \\
\text { throat size (in) }\end{array}$ & Status \\
\hline & & & \\
\hline
\end{tabular}




\begin{tabular}{|l|l|l|l|} 
Nozzle to shell fillet $\left(\operatorname{Leg}_{41}\right)$ & 0.224 & 0.2625 & weld size is adequate \\
\hline
\end{tabular}

Opening $\mathrm{C01}$ is too close per UG-36(c)(3)(d) to allow an exemption per UG-36(c)(3)(a).

\section{Reinforcement Calculations for MAP}

The vessel wall thickness governs the MAP of this nozzle.

\begin{tabular}{|c|c|c|c|c|c|c|c|c|}
\hline \multicolumn{3}{|c|}{$\begin{array}{c}\text { UG-37 Area Calculation Summary } \\
\text { (in }{ }^{2} \text { ) } \\
\text { For } \mathbf{P}=1719.91 \text { psi @ } \mathbf{7 0}^{\circ} \mathrm{F}\end{array}$} & \multicolumn{2}{c|}{$\begin{array}{c}\text { UG-45 Nozzle Wall } \\
\text { Thickness Summary (in) } \\
\text { The nozzen passes UG-45 }\end{array}$} \\
\hline $\begin{array}{c}\text { A } \\
\text { required }\end{array}$ & $\begin{array}{c}\mathbf{A} \\
\text { available }\end{array}$ & $\mathbf{A}_{1}$ & $\mathbf{A}_{\mathbf{2}}$ & $\mathbf{A}_{\mathbf{3}}$ & $\mathbf{A}_{\mathbf{5}}$ & $\begin{array}{c}\mathbf{A} \\
\text { welds }\end{array}$ & $\mathbf{t}_{\text {req }}$ & $\mathbf{t}_{\text {min }}$ \\
\hline 0.5457 & 0.6698 & -- & 0.5292 & -- & -- & 0.1406 & 0.0727 & 0.35 \\
\hline
\end{tabular}

\section{UG-41 Weld Failure Path Analysis Summary}

The nozzle is exempt from weld strength calculations per UW-15(b)(1)

\begin{tabular}{|c|r|r|c|}
\hline \multicolumn{4}{|c|}{ UW-16 Weld Sizing Summary } \\
\hline Weld description & $\begin{array}{r}\text { Required weld } \\
\text { throat size (in) }\end{array}$ & $\begin{array}{r}\text { Actual weld } \\
\text { throat size (in) }\end{array}$ & Status \\
\hline Nozzle to shell fillet $\left(\operatorname{Leg}_{41}\right)$ & 0.245 & 0.2625 & weld size is adequate \\
\hline
\end{tabular}

Opening C01 is too close per UG-36(c)(3)(d) to allow an exemption per UG-36(c)(3)(a). 
COUPLING (C03)

ASME Section VIII Division 1, 2007 Edition

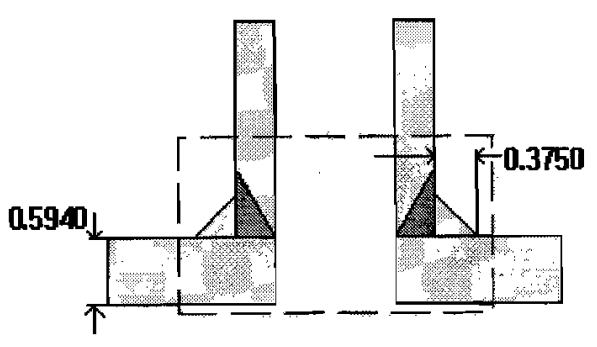

$$
\begin{aligned}
& t_{w(l o w e r)}=0 \text { in } \\
& \operatorname{Leg}_{41}=0.375 \text { in }
\end{aligned}
$$

Note: round inside edges per UG-76(c)

Located on:

Liquid static head included:

Nozzle material specification:

Nozzle longitudinal joint efficiency:

Nozzle description:

Nozzle orientation:

Local vessel minimum thickness:

End of nozzle to shell center: $\quad 6.375$ in

Nozzle inside diameter, new: $\quad 1.05$ in

Nozzle nominal wall thickness: $\quad 0.35$ in

Nozzle corrosion allowance: $\quad 0.03$ in

Projection available outside vessel, Lpr: 1 in

\section{Reinforcement Calculations for Internal Pressure}

The vessel wall thickness governs the MAWP of this nozzle.

\begin{tabular}{|c|c|c|c|c|c|c|c|c|}
\hline \multicolumn{3}{|c|}{$\begin{array}{c}\text { UG-37 Area Calculation Summary } \\
\text { (in } \\
\text { For } P=1381.47 \text { psi @ } \\
\text { The opening is adequately reinforced }\end{array}$} & \multicolumn{2}{c|}{$\begin{array}{c}\text { UG-45 Nozzle Wall } \\
\text { Thickness Summary (in) } \\
\text { The nozzle passes UG-45 }\end{array}$} \\
\hline $\begin{array}{c}\mathbf{A} \\
\text { required }\end{array}$ & $\begin{array}{c}\mathbf{A} \\
\text { available }\end{array}$ & $\mathbf{A}_{\mathbf{1}}$ & $\mathbf{A}_{\mathbf{2}}$ & $\mathbf{A}_{\mathbf{3}}$ & $\mathbf{A}_{\mathbf{5}}$ & $\begin{array}{c}\mathbf{A} \\
\text { welds }\end{array}$ & $\mathbf{t}_{\text {req }}$ & $\mathbf{t}_{\text {min }}$ \\
\hline 0.5103 & 0.5777 & -- & 0.4371 & -- & -- & 0.1406 & 0.0981 & 0.35 \\
\hline
\end{tabular}

\section{UG-41 Weld Failure Path Analysis Summary}

The nozzle is exempt from weld strength calculations per UW-15(b)(1)

\begin{tabular}{|c|c|c|c|}
\hline \multicolumn{4}{|c|}{ UW-16 Weld Sizing Summary } \\
\hline Weld description & $\begin{array}{r}\text { Required weld } \\
\text { throat size (in) }\end{array}$ & $\begin{array}{r}\text { Actual weld } \\
\text { throat size (in) }\end{array}$ & Status \\
\hline & & & \\
\hline
\end{tabular}




\begin{tabular}{|l|l|l|l|} 
Nozzle to shell fillet $\left(\operatorname{Leg}_{41}\right)$ & 0.224 & 0.2625 & weld size is adequate
\end{tabular}

\section{Reinforcement Calculations for MAP}

The vessel wall thickness governs the MAP of this nozzle.

\begin{tabular}{|c|c|c|c|c|c|c|c|c|}
\hline \multicolumn{7}{|c|}{$\begin{array}{l}\text { UG-37 Area Calculation Summary }\left(\mathrm{in}^{2}\right) \\
\text { For } P=1719.91 \text { psi } @ 70^{\circ} \mathrm{F} \\
\text { The opening is adequately reinforced }\end{array}$} & \multicolumn{2}{|c|}{$\begin{array}{l}\text { UG-45 Nozzle Wall } \\
\text { Thickness Summary (in) } \\
\text { The nozzle passes UG-45 }\end{array}$} \\
\hline $\begin{array}{c}\mathbf{A} \\
\text { required }\end{array}$ & $\underset{\text { available }}{\mathbf{A}}$ & $A_{1}$ & $\mathbf{A}_{2}$ & $A_{3}$ & $A_{5}$ & $\begin{array}{c}\text { A } \\
\text { welds }\end{array}$ & $\mathbf{t}_{\text {req }}$ & $t_{\min }$ \\
\hline 0.5457 & 0.6698 & -- & 0.5292 & -- & -- & 0.1406 & 0.0727 & 0.35 \\
\hline
\end{tabular}

\section{UG-41 Weld Failure Path Analysis Summary}

The nozzle is exempt from weld strength calculations per UW-15(b)(1)

\begin{tabular}{|c|r|r|c|}
\hline \multicolumn{4}{|c|}{ UW-16 Weld Sizing Summary } \\
\hline Weld description & $\begin{array}{r}\text { Required weld } \\
\text { throat size (in) }\end{array}$ & $\begin{array}{r}\text { Actual weld } \\
\text { throat size (in) }\end{array}$ & Status \\
\hline Nozzle to shell fillet $\left(\operatorname{Leg}_{41}\right)$ & 0.245 & 0.2625 & weld size is adequate \\
\hline
\end{tabular}




\section{ASME Section VIII Division 1, 2007 Edition}

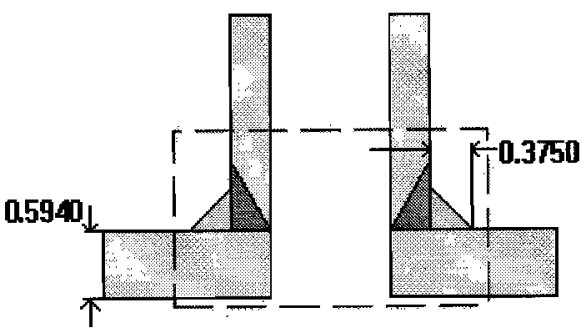

$$
\begin{aligned}
& \mathrm{t}_{\mathrm{w}(\text { lower })}=0 \text { in } \\
& \operatorname{Leg}_{41}=0.375 \text { in }
\end{aligned}
$$

Note: round inside edges per UG-76(c)

Located on:

Liquid static head included:

Nozzle material specification:

Nozzle longitudinal joint efficiency:

Nozzle description:

Nozzle orientation:

Local vessel minimum thickness:

Nozzle center line offset to datum line:

End of nozzle to shell center:

Nozzle inside diameter, new:

Nozzle nominal wall thickness:

Nozzle corrosion allowance:

Projection available outside vessel, Lpr: 1 in

\section{Reinforcement Calculations for Internal Pressure}

\begin{tabular}{|c|c|c|c|c|c|c|c|c|}
\hline \multicolumn{7}{|c|}{$\begin{array}{c}\text { UG-37 Area Calculation Summary }\left(\mathrm{in}^{2}\right) \\
\text { For } P=1381.47 \text { psi @ } 700^{\circ} \mathrm{F} \\
\text { The opening is adequately reinforced }\end{array}$} & \multicolumn{2}{|c|}{$\begin{array}{l}\text { UG-45 Nozzle Wall } \\
\text { Thickness Summary (in) } \\
\text { The nozzle passes UG-45 }\end{array}$} \\
\hline $\begin{array}{c}A \\
\text { required }\end{array}$ & $\underset{\text { available }}{A}$ & $\mathbf{A}_{1}$ & $A_{2}$ & $\mathbf{A}_{\mathbf{3}}$ & $\mathbf{A}_{5}$ & $\begin{array}{c}\text { A } \\
\text { welds }\end{array}$ & $t_{\text {req }}$ & $t_{\text {min }}$ \\
\hline 0.5103 & 0.5777 & -- & 0.4371 & -- & -- & 0.1406 & 0.0981 & 0.35 \\
\hline
\end{tabular}

The vessel wall thickness governs the MAWP of this nozzle.

\section{UG-41 Weld Failure Path Analysis Summary}

The nozzle is exempt from weld strength calculations per UW-15(b)(1)

\begin{tabular}{|c|c|c|c|}
\hline \multicolumn{4}{|c|}{ UW-16 Weld Sizing Summary } \\
\hline Weld description & $\begin{array}{r}\text { Required weld } \\
\text { throat size (in) }\end{array}$ & $\begin{array}{r}\text { Actual weld } \\
\text { throat size (in) }\end{array}$ & Status \\
\hline & & & \\
\hline
\end{tabular}




\begin{tabular}{|l|l|l|l|} 
Nozzle to shell fillet $\left(\operatorname{Leg}_{41}\right)$ & 0.224 & 0.2625 & weld size is adequate \\
\hline
\end{tabular}

\section{Reinforcement Calculations for MAP}

The vessel wall thickness governs the MAP of this nozzle.

\begin{tabular}{|c|c|c|c|c|c|c|c|c|}
\hline \multicolumn{7}{|c|}{$\begin{array}{c}\text { UG-37 Area Calculation Summary }\left(\mathrm{in}^{2}\right) \\
\text { For } \mathrm{P}=1719.91 \text { psi } @ 70^{\circ} \mathrm{F} \\
\text { The opening is adequately reinforced }\end{array}$} & \multicolumn{2}{|c|}{$\begin{array}{l}\text { UG-45 Nozzle Wall } \\
\text { Thickness Summary (in) } \\
\text { The nozzle passes UG-45 }\end{array}$} \\
\hline $\begin{array}{c} \\
\text { required }\end{array}$ & \begin{tabular}{|c|}
$\mathbf{A}$ \\
available
\end{tabular} & $\mathbf{A}_{1}$ & $\mathbf{A}_{2}$ & $\mathbf{A}_{\mathbf{3}}$ & $\mathbf{A}_{5}$ & $\begin{array}{c}A \\
\text { welds }\end{array}$ & $t_{\text {req }}$ & $t_{\min }$ \\
\hline 0.5457 & 0.6698 & -- & 0.5292 & -- & -- & 0.1406 & 0.0727 & 0.35 \\
\hline
\end{tabular}

UG-41 Weld Failure Path Analysis Summary

The nozzle is exempt from weld strength calculations per UW-15(b)(1)

\begin{tabular}{|c|r|r|c|}
\hline \multicolumn{4}{|c|}{ UW-16 Weld Sizing Summary } \\
\hline Weld description & $\begin{array}{l}\text { Required weld } \\
\text { throat size (in) }\end{array}$ & $\begin{array}{r}\text { Actual weld } \\
\text { throat size (in) }\end{array}$ & Status \\
\hline Nozzle to shell fillet $\left(\operatorname{Leg}_{41}\right)$ & 0.245 & 0.2625 & weld size is adequate \\
\hline
\end{tabular}




\section{COUPLING (C05)}

ASME Section VIII Division 1, 2007 Edition

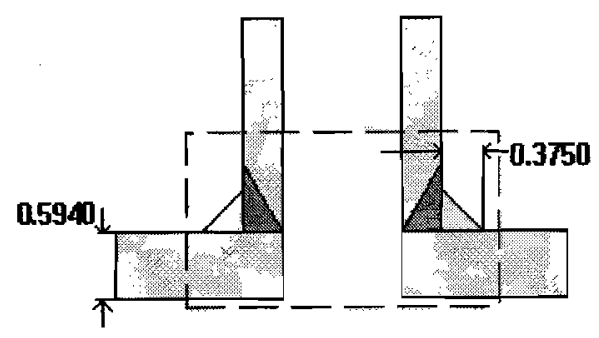

$$
\begin{aligned}
& t_{w(l o w e r)}=0 \text { in } \\
& \operatorname{Leg}_{41}=0.375 \text { in }
\end{aligned}
$$

Note: round inside edges per UG-76(c)

Located on:

Liquid static head included:

Nozzle material specification:

Nozzle longitudinal joint efficiency:

Nozzle description:

Nozzle orientation:

Local vessel minimum thickness:

Nozzle center line offset to datum line:

End of nozzle to shell center:

Nozzle inside diameter, new:

Nozzle nominal wall thickness:

Nozzle corrosion allowance:

Projection available outside vessel, Lpr: 1 in

\section{Reinforcement Calculations for Internal Pressure}

\begin{tabular}{|c|c|c|c|c|c|c|c|c|}
\hline \multicolumn{7}{|c|}{$\begin{array}{l}\text { UG-37 Area Calculation Summary }\left(\mathrm{in}^{2}\right) \\
\text { For } P=1381.47 \text { psi @ } 700^{\circ} \mathrm{F} \\
\text { The opening is adequately reinforced }\end{array}$} & \multicolumn{2}{|c|}{$\begin{array}{l}\text { UG-45 Nozzle Wall } \\
\text { Thickness Summary (in) } \\
\text { The nozzle passes UG-45 }\end{array}$} \\
\hline $\begin{array}{c}A \\
\text { required }\end{array}$ & \begin{tabular}{|c|} 
A \\
available \\
\end{tabular} & $\mathbf{A}_{1}$ & $\mathbf{A}_{2}$ & $A_{3}$ & $A_{5}$ & $\begin{array}{c}\text { A } \\
\text { welds }\end{array}$ & $\mathbf{t}_{\mathrm{req}}$ & $t_{\text {min }}$ \\
\hline 0.5103 & 0.5777 & -- & 0.4371 & -- & -- & 0.1406 & 0.0981 & 0.35 \\
\hline
\end{tabular}

The vessel wall thickness governs the MAWP of this nozzle.

\section{UG-41 Weld Failure Path Analysis Summary}

The nozzle is exempt from weld strength calculations per UW-15(b)(1)

\begin{tabular}{|c|c|c|c|}
\hline \multicolumn{4}{|c|}{ UW-16 Weld Sizing Summary } \\
\hline Weld description & $\begin{array}{l}\text { Required weld } \\
\text { throat size (in) }\end{array}$ & $\begin{array}{r}\text { Actual weld } \\
\text { throat size (in) }\end{array}$ & Status \\
\hline & & & \\
\hline
\end{tabular}




\begin{tabular}{|l|l|l|l|} 
Nozzle to shell fillet $\left(\operatorname{Leg}_{41}\right)$ & 0.224 & 0.2625 & weld size is adequate \\
\hline
\end{tabular}

Opening C01 is too close per UG-36(c)(3)(d) to allow an exemption per UG-36(c)(3)(a).

\section{Reinforcement Calculations for MAP}

The vessel wall thickness governs the MAP of this nozzle.

\begin{tabular}{|c|c|c|c|c|c|c|c|c|}
\hline \multicolumn{7}{|c|}{$\begin{array}{c}\text { UG-37 Area Calculation Summary }\left(\mathrm{in}^{2}\right) \\
\text { For } \mathrm{P}=1719.91 \text { psi } @ 70^{\circ} \mathrm{F} \\
\text { The opening is adequately reinforced } \\
\end{array}$} & \multicolumn{2}{|c|}{$\begin{array}{l}\text { UG-45 Nozzle Wall } \\
\text { Thickness Summary (in) } \\
\text { The nozzle passes UG-45 }\end{array}$} \\
\hline $\begin{array}{c}A \\
\text { required }\end{array}$ & $\begin{array}{c}\mathbf{A} \\
\text { available }\end{array}$ & $\mathbf{A}_{1}$ & $\mathbf{A}_{2}$ & $\mathbf{A}_{\mathbf{3}}$ & $\mathbf{A}_{5}$ & $\begin{array}{c}\text { A } \\
\text { welds }\end{array}$ & $t_{\text {req }}$ & $\mathbf{t}_{\min }$ \\
\hline 0.5457 & 0.6698 & -- & 0.5292 & -- & -- & 0.1406 & 0.0727 & 0.35 \\
\hline
\end{tabular}

\section{UG-41 Weld Failure Path Analysis Summary}

The nozzle is exempt from weld strength calculations per UW-15(b)(1)

\begin{tabular}{|c|r|r|c|}
\hline \multicolumn{4}{|c|}{ UW-16 Weld Sizing Summary } \\
\hline Weld description & $\begin{array}{r}\text { Required weld } \\
\text { throat size (in) }\end{array}$ & $\begin{array}{r}\text { Actual weld } \\
\text { throat size (in) }\end{array}$ & Status \\
\hline Nozzle to shell fillet $\left(\right.$ Leg $\left._{41}\right)$ & 0.245 & 0.2625 & weld size is adequate \\
\hline
\end{tabular}

Opening $\mathrm{C} 01$ is too close per UG-36(c)(3)(d) to allow an exemption per UG-36(c)(3)(a). 
COUPLING (C06)

ASME Section VIII Division 1, 2007 Edition

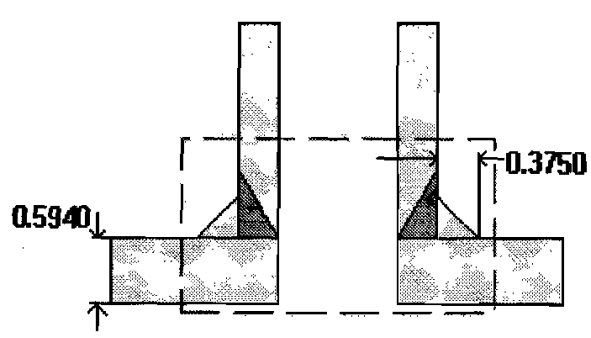

$$
\begin{aligned}
& t_{w(l o w e r)}=0 \text { in } \\
& \operatorname{Leg}_{41}=0.375 \text { in }
\end{aligned}
$$

Note: round inside edges per UG-76(c)

Located on:

Liquid static head included:

Nozzle material specification:

Nozzle longitudinal joint efficiency:

Nozzle description:

Nozzle orientation:

Local vessel minimum thickness:

Nozzle center line offset to datum line:

End of nozzle to shell center:

Nozzle inside diameter, new:

Nozzle nominal wall thickness:

Nozzle corrosion allowance:

Projection available outside vessel, Lpr: 1 in
REACTOR OUTER SHELL

$0 \mathrm{psi}$

SA-105 (II-D p. 18, ln. 8)

1

$0.750^{\prime \prime}$ Class 6000 - threaded $60^{\circ}$

0.5197 in

\section{Reinforcement Calculations for Internal Pressure}

The vessel wall thickness governs the MAWP of this nozzle.

\begin{tabular}{|c|c|c|c|c|c|c|c|c|}
\hline \multicolumn{3}{|c|}{$\begin{array}{c}\text { UG-37 Area Calculation Summary } \\
\text { (in }{ }^{2} \text { ) } \\
\text { For } \mathbf{P}=1381.47 \text { psi @ } \\
\text { The opening is adequately reinforced }\end{array}$} & \multicolumn{2}{c|}{$\begin{array}{c}\text { UG-45 Nozzle Wall } \\
\text { Thickness Summary (in) } \\
\text { The nozzle passes UG-45 }\end{array}$} \\
\hline $\begin{array}{c}\text { A } \\
\text { required }\end{array}$ & $\begin{array}{c}\mathbf{A} \\
\text { available }\end{array}$ & $\mathbf{A}_{1}$ & $\mathbf{A}_{2}$ & $\mathbf{A}_{3}$ & $\mathbf{A}_{\mathbf{5}}$ & $\begin{array}{c}\mathbf{A} \\
\text { welds }\end{array}$ & $\mathbf{t}_{\text {req }}$ & $\mathbf{t}_{\text {min }}$ \\
\hline 0.5103 & 0.5777 & -- & 0.4371 & -- & -- & 0.1406 & 0.0981 & 0.35 \\
\hline
\end{tabular}

\section{UG-41 Weld Failure Path Analysis Summary}

The nozzle is exempt from weld strength calculations per UW-15(b)(1)

\begin{tabular}{|c|c|c|c|}
\hline \multicolumn{4}{|c|}{ UW-16 Weld Sizing Summary } \\
\hline Weld description & $\begin{array}{l}\text { Required weld } \\
\text { throat size (in) }\end{array}$ & $\begin{array}{r}\text { Actual weld } \\
\text { throat size (in) }\end{array}$ & Status \\
\hline & & & \\
\hline
\end{tabular}




\begin{tabular}{|l|l|l|l|} 
Nozzle to shell fillet $\left(\operatorname{Leg}_{41}\right)$ & 0.224 & 0.2625 & weld size is adequate \\
\hline
\end{tabular}

\section{Reinforcement Calculations for MAP}

The vessel wall thickness governs the MAP of this nozzle.

\begin{tabular}{|c|c|c|c|c|c|c|c|c|}
\hline \multicolumn{7}{|c|}{$\begin{array}{c}\text { UG-37 Area Calculation Summary }\left(\mathrm{in}^{2}\right) \\
\text { For } \mathrm{P}=1719.91 \text { psi @ } 70^{\circ} \mathrm{F} \\
\text { The opening is adequately reinforced }\end{array}$} & \multicolumn{2}{|c|}{$\begin{array}{l}\text { UG-45 Nozzle Wall } \\
\text { Thickness Summary (in) } \\
\text { The nozzle passes UG-45 }\end{array}$} \\
\hline$\underset{\text { required }}{A}$ & $\underset{\text { available }}{A}$ & $A_{1}$ & $\mathbf{A}_{2}$ & $\mathbf{A}_{\mathbf{3}}$ & $A_{5}$ & $\begin{array}{c}A \\
\text { welds }\end{array}$ & $t_{\text {req }}$ & $t_{\min }$ \\
\hline 0.5457 & 0.6698 & -- & 0.5292 & -- & -- & 0.1406 & 0.0727 & 0.35 \\
\hline
\end{tabular}

\section{UG-41 Weld Failure Path Analysis Summary}

The nozzle is exempt from weld strength calculations per $U W-15(b)(1)$

\begin{tabular}{|c|r|r|c|}
\hline \multicolumn{4}{|c|}{ UW-16 Weld Sizing Summary } \\
\hline Weld description & $\begin{array}{r}\text { Required weld } \\
\text { throat size (in) }\end{array}$ & $\begin{array}{r}\text { Actual weld } \\
\text { throat size (in) }\end{array}$ & Status \\
\hline Nozzle to shell fillet $\left(\operatorname{Leg}_{41}\right)$ & 0.245 & 0.2625 & weld size is adequate \\
\hline
\end{tabular}


ASME Section VIII Division 1, 2007 Edition

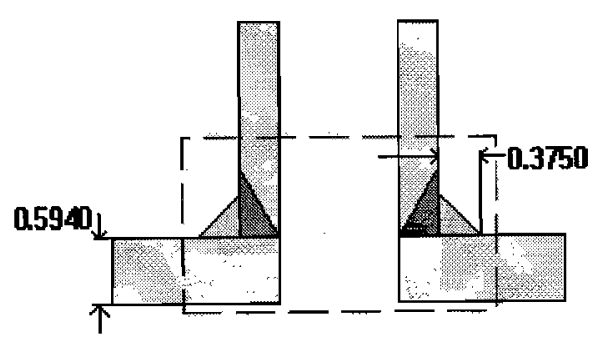

$$
\begin{aligned}
& t_{w(\text { lower })}=0 \text { in } \\
& \operatorname{Leg}_{41}=0.375 \text { in }
\end{aligned}
$$

Note: round inside edges per UG-76(c)

Located on:

Liquid static head included:

Nozzle material specification:

Nozzle longitudinal joint efficiency:

Nozzle description:

Nozzle orientation:

Local vessel minimum thickness:

Nozzle center line offset to datum line:

End of nozzle to shell center:

Nozzle inside diameter, new:

Nozzle nominal wall thickness:

Nozzle corrosion allowance:

Projection available outside vessel, Lpr: 1 in

\section{Reinforcement Calculations for Internal Pressure}

The vessel wall thickness governs the MAWP of this nozzle.

\begin{tabular}{|c|c|c|c|c|c|c|c|c|}
\hline $\begin{array}{c}\text { UG-37 Area Calculation Summary } \\
\left(\text { in }^{2} \text { ) }\right. \\
\text { For } \mathbf{P}=1381.47 \text { psi } @ 700^{\circ} \mathbf{F} \\
\text { The opening is adequately reinforced }\end{array}$ & \multicolumn{2}{c|}{$\begin{array}{c}\text { UG-45 Nozzle Wall } \\
\text { Thickness Summary (in) } \\
\text { The nozzle passes UG-45 }\end{array}$} \\
\hline $\begin{array}{c}\mathbf{A} \\
\text { required }\end{array}$ & $\begin{array}{c}\mathbf{A} \\
\text { available }\end{array}$ & $\mathbf{A}_{\mathbf{1}}$ & $\mathbf{A}_{\mathbf{2}}$ & $\mathbf{A}_{\mathbf{3}}$ & $\mathbf{A}_{\mathbf{5}}$ & $\begin{array}{c}\mathbf{A} \\
\text { welds }\end{array}$ & $\mathbf{t}_{\text {req }}$ & $\mathbf{t}_{\text {min }}$ \\
\hline 0.5103 & 0.5777 & -- & 0.4371 & -- & -- & 0.1406 & 0.0981 & 0.35 \\
\hline
\end{tabular}

UG-41 Weld Failure Path Analysis Summary

The nozzle is exempt from weld strength calculations per UW-15(b)(1)

\begin{tabular}{|c|c|c|c|}
\hline \multicolumn{4}{|c|}{ UW-16 Weld Sizing Summary } \\
\hline Weld description & $\begin{array}{l}\text { Required weld } \\
\text { throat size (in) }\end{array}$ & $\begin{array}{r}\text { Actual weld } \\
\text { throat size (in) }\end{array}$ & Status \\
\hline & & & \\
\hline
\end{tabular}




\begin{tabular}{|l|l|l|l|} 
Nozzle to shell fillet $\left(\operatorname{Leg}_{41}\right)$ & 0.224 & 0.2625 & weld size is adequate \\
\hline
\end{tabular}

\section{Reinforcement Calculations for MAP}

The vessel wall thickness governs the MAP of this nozzle.

\begin{tabular}{|c|c|c|c|c|c|c|c|c|}
\hline \multicolumn{7}{|c|}{$\begin{array}{l}\text { UG-37 Area Calculation Summary }\left(\mathrm{in}^{2}\right) \\
\text { For } P=1719.91 \text { psi @ } 70^{\circ} \mathrm{F} \\
\text { The opening is adequately reinforced }\end{array}$} & \multicolumn{2}{|c|}{$\begin{array}{c}\text { UG-45 Nozzle Wall } \\
\text { Thickness Summary (in) } \\
\text { The nozzle passes UG-45 } \\
\end{array}$} \\
\hline $\begin{array}{c}\mathbf{A} \\
\text { required }\end{array}$ & $\begin{array}{c}\mathbf{A} \\
\text { available }\end{array}$ & $A_{1}$ & $\mathbf{A}_{2}$ & $\mathbf{A}_{3}$ & $A_{5}$ & $\begin{array}{c}\text { A } \\
\text { welds }\end{array}$ & $\mathbf{t}_{\text {req }}$ & $t_{\min }$ \\
\hline 0.5457 & 0.6698 & -- & 0.5292 & -- & -- & 0.1406 & 0.0727 & 0.35 \\
\hline
\end{tabular}

\section{UG-41 Weld Failure Path Analysis Summary}

The nozzle is exempt from weld strength calculations per UW-15(b)(1)

\begin{tabular}{|c|r|r|c|}
\hline \multicolumn{4}{|c|}{ UW-16 Weld Sizing Summary } \\
\hline Weld description & $\begin{array}{r}\text { Required weld } \\
\text { throat size (in) }\end{array}$ & $\begin{array}{r}\text { Actual weld } \\
\text { throat size (ln) }\end{array}$ & Status \\
\hline Nozzle to shell fillet (Leg 4 ) & 0.245 & 0.2625 & weld size is adequate \\
\hline
\end{tabular}




\section{ASME Section VIII Division 1, 2007 Edition}

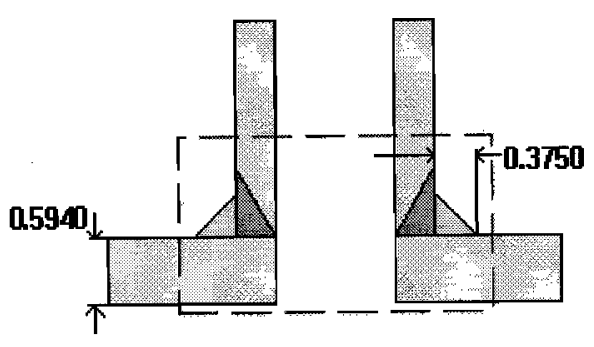

$$
\begin{aligned}
& t_{w(\text { lower })}=0 \text { in } \\
& \operatorname{Leg}_{41}=0.375 \text { in }
\end{aligned}
$$

Note: round inside edges per UG-76(c)

Located on:

Liquid static head included:

Nozzle material specification:

Nozzle longitudinal joint efficiency:

Nozzle description:

Nozzle orientation:

Local vessel minimum thickness:

Nozzle center line offset to datum line:

End of nozzle to shell center:

Nozzle inside diameter, new:

Nozzle nominal wall thickness:

Nozzle corrosion allowance:

Projection available outside vessel, Lpr: 1 in

\section{Reinforcement Calculations for Internal Pressure}

\begin{tabular}{|c|c|c|c|c|c|c|c|c|}
\hline \multicolumn{7}{|c|}{$\begin{array}{l}\text { UG-37 Area Calculation Summary }\left(\mathrm{in}^{2}\right) \\
\text { For } P=1381.47 \text { psi @ } 700^{\circ} \mathrm{F} \\
\text { The opening is adequately reinforced }\end{array}$} & \multicolumn{2}{|c|}{$\begin{array}{l}\text { UG-45 Nozzle Wall } \\
\text { Thickness Summary (in) } \\
\text { The nozzle passes UG-45 }\end{array}$} \\
\hline$\underset{\text { required }}{A}$ & $\underset{\text { available }}{\mathrm{A}}$ & $\mathbf{A}_{1}$ & $\mathbf{A}_{2}$ & $\mathbf{A}_{3}$ & $\mathbf{A}_{5}$ & $\begin{array}{c}A \\
\text { welds }\end{array}$ & $t_{\text {req }}$ & $t_{\min }$ \\
\hline 0.5103 & 0.5777 & -- & 0.4371 & -- & -- & 0.1406 & 0.0981 & 0.35 \\
\hline
\end{tabular}

The vessel wall thickness governs the MAWP of this nozzle.

\section{UG-41 Weld Failure Path Analysis Summary}

The nozzle is exempt from weld strength calculations per UW-15(b)(1)

\begin{tabular}{|c|c|c|c|}
\hline \multicolumn{4}{|c|}{ UW-16 Weld Sizing Summary } \\
\hline Weld description & $\begin{array}{l}\text { Required weld } \\
\text { throat size (in) }\end{array}$ & $\begin{array}{r}\text { Actual weld } \\
\text { throat size (in) }\end{array}$ & Status \\
\hline & & & \\
\hline
\end{tabular}




\begin{tabular}{|l|l|l|l|} 
Nozzle to shell fillet $\left(\mathrm{Leg}_{41}\right)$ & 0.224 & 0.2625 & weld size is adequate
\end{tabular}

Opening C04 is too close per UG-36(c)(3)(d) to allow an exemption per UG-36(c)(3)(a).

\section{Reinforcement Calculations for MAP}

The vessel wall thickness governs the MAP of this nozzle.

\begin{tabular}{|c|c|c|c|c|c|c|c|c|}
\hline \multicolumn{3}{|c|}{$\begin{array}{c}\text { UG-37 Area Calculation Summary } \\
\text { For } \text { in }^{2} \text { ) } \\
\text { F }=1719.91 \text { psi @ } \\
\text { The opening is adequately reinforced }\end{array}$} & \multicolumn{2}{c|}{$\begin{array}{c}\text { UG-45 Nozzle Wall } \\
\text { Thickness Summary (in) } \\
\text { The nozzle passes UG-45 }\end{array}$} \\
\hline $\begin{array}{c}\text { A } \\
\text { required }\end{array}$ & $\begin{array}{c}\mathbf{A} \\
\text { available }\end{array}$ & $\mathbf{A}_{1}$ & $\mathbf{A}_{2}$ & $\mathbf{A}_{3}$ & $\mathbf{A}_{3}$ & $\begin{array}{c}\mathbf{A} \\
\text { welds }\end{array}$ & $\mathbf{t}_{\text {req }}$ & $\mathbf{t}_{\text {min }}$ \\
\hline 0.5457 & 0.6698 & -- & 0.5292 & -- & -- & 0.1406 & 0.0727 & 0.35 \\
\hline
\end{tabular}

\section{UG-41 Weld Failure Path Analysis Summary}

The nozzle is exempt from weld strength calculations per UW-15(b)(1)

\begin{tabular}{|c|r|r|c|}
\hline \multicolumn{4}{|c|}{ UW-16 Weld Sizing Summary } \\
\hline Weld description & $\begin{array}{r}\text { Required weld } \\
\text { throat size (in) }\end{array}$ & $\begin{array}{r}\text { Actual weld } \\
\text { throat size (in) }\end{array}$ & Status \\
\hline Nozzle to shell fillet ( $\left.\mathrm{Leg}_{41}\right)$ & 0.245 & 0.2625 & weld size is adequate \\
\hline
\end{tabular}

Opening C04 is too close per UG-36(c)(3)(d) to allow an exemption per UG-36(c)(3)(a). 


\section{ASME Section VIII Division 1, 2007 Edition}

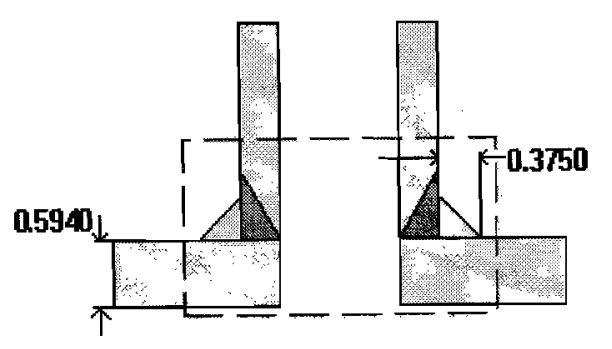

$$
\begin{aligned}
& t_{w(\text { lower })}=0 \text { in } \\
& \operatorname{Leg}_{41}=0.375 \text { in }
\end{aligned}
$$

Note: round inside edges per UG-76(c)

Located on:

Liquid static head included:

Nozzle material specification:

Nozzle longitudinal joint efficiency:

Nozzle description:

Nozzle orientation:

Local vessel minimum thickness:

End of nozzle to shell center:

Nozzle inside diameter, new:

Nozzle nominal wall thickness:

Nozzle corrosion allowance:

Projection available outside vessel, Lpr: 1 in

\section{Reinforcement Calculations for Internal Pressure}

\begin{tabular}{|c|c|c|c|c|c|c|c|c|}
\hline \multicolumn{7}{|c|}{$\begin{array}{c}\text { UG-37 Area Calculation Summary }\left(\mathrm{in}^{2}\right) \\
\text { For } P=1381.47 \text { psi } @ 700^{\circ} \mathrm{F} \\
\text { The opening is adequately reinforced }\end{array}$} & \multicolumn{2}{|c|}{$\begin{array}{l}\text { UG-45 Nozzle Wall } \\
\text { Thickness Summary (in) } \\
\text { The nozzle passes UG-45 }\end{array}$} \\
\hline$\underset{\text { required }}{\mathbf{A}}$ & $\underset{\text { available }}{\mathbf{A}}$ & $\mathbf{A}_{1}$ & $\mathbf{A}_{2}$ & $\mathbf{A}_{3}$ & $\mathbf{A}_{5}$ & $\begin{array}{c}\text { A } \\
\text { welds }\end{array}$ & $t_{\text {req }}$ & $\mathbf{t}_{\min }$ \\
\hline 0.5103 & 0.5777 & -- & 0.4371 & -- & -- & 0.1406 & 0.0981 & 0.35 \\
\hline
\end{tabular}

The vessel wall thickness governs the MAWP of this nozzle.

\section{UG-41 Weld Failure Path Analysis Summary}

The nozzle is exempt from weld strength calculations per $U W-15(b)(1)$

\begin{tabular}{|l|l|r|l|}
\hline \multicolumn{3}{|c|}{ UW-16 Weld Sizing Summary } \\
\hline Weld description & $\begin{array}{r}\text { Required weld } \\
\text { throat size (in) }\end{array}$ & $\begin{array}{r}\text { Actual weld } \\
\text { throat size (in) }\end{array}$ & Status \\
\hline & & & \\
\hline
\end{tabular}




$\mid$\begin{tabular}{l|l|l|l|} 
Nozzle to shell fillet $\left(\operatorname{Leg}_{41}\right)$ & 0.224 & 0.2625 & weld size is adequate
\end{tabular}

\section{Reinforcement Calculations for MAP}

The vessel wall thickness governs the MAP of this nozzle.

\begin{tabular}{|c|c|c|c|c|c|c|c|c|}
\hline \multicolumn{3}{|c|}{$\begin{array}{c}\text { UG-37 Area Calculation Summary } \\
\left.\text { (in }{ }^{2}\right) \\
\text { For } \mathbf{P}=1719.91 \text { psi @ } \\
\text { The opening is adequately reinforced }\end{array}$} & \multicolumn{2}{c|}{$\begin{array}{c}\text { UG-45 Nozzle Wall } \\
\text { Thickness Summary (in) } \\
\text { The nozzle passes UG-45 }\end{array}$} \\
\hline $\begin{array}{c}\text { A } \\
\text { required }\end{array}$ & $\begin{array}{c}\mathbf{A} \\
\text { available }\end{array}$ & $\mathbf{A}_{1}$ & $\mathbf{A}_{2}$ & $\mathbf{A}_{3}$ & $\mathbf{A}_{5}$ & $\begin{array}{c}\mathbf{A} \\
\text { welds }\end{array}$ & $\mathbf{t}_{\text {req }}$ & $\mathbf{t}_{\text {min }}$ \\
\hline 0.5457 & 0.6698 & -- & 0.5292 & -- & -- & 0.1406 & 0.0727 & 0.35 \\
\hline
\end{tabular}

UG-41 Weld Failure Path Analysis Summary

The nozzle is exempt from weld strength calculations per UW-15(b)(1)

\begin{tabular}{|c|r|r|c|}
\hline \multicolumn{4}{|c|}{ UW-16 Weld Sizing Summary } \\
\hline Weld description & $\begin{array}{r}\text { Required weld } \\
\text { throat size (in) }\end{array}$ & $\begin{array}{r}\text { Actual weld } \\
\text { throat size (in) }\end{array}$ & Status \\
\hline Nozzle to shell fillet $\left(\operatorname{Leg}_{41}\right)$ & 0.245 & 0.2625 & weld size is adequate \\
\hline
\end{tabular}


ASME Section VIII Division 1, 2007 Edition

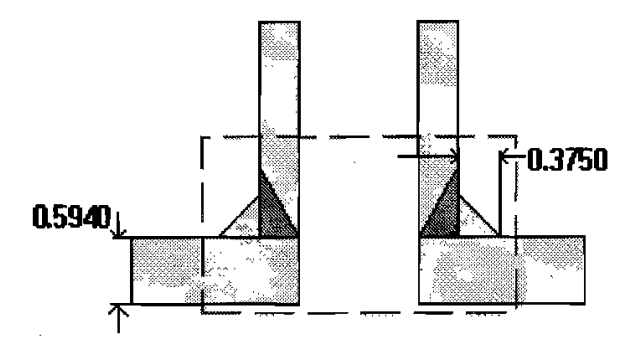

$$
\begin{aligned}
& t_{w(l o w e r)}=0 \text { in } \\
& \operatorname{Leg}_{41}=0.375 \text { in }
\end{aligned}
$$

Note: round inside edges per UG-76(c)

Located on:

Liquid static head included:

Nozzle material specification:

Nozzle longitudinal joint efficiency:

Nozzle description:

Nozzle orientation:

Local vessel minimum thickness:

Nozzle center line offset to datum line:

End of nozzle to shell center:

Nozzle inside diameter, new:

Nozzle nominal wall thickness:

Nozzle corrosion allowance:

Projection available outside vessel, Lpr: 1 in
REACTOR OUTER SHELL

$0 \mathrm{psi}$

SA-105 (II-D p. 18, ln. 8)

$0.750^{\prime \prime}$ Class 6000 - threaded

$120^{\circ}$

0.5197 in

13.375 in

6.375 in

1.05 in

0.35 in

0.03 in

\section{Reinforcement Calculations for Internal Pressure}

\begin{tabular}{|c|c|c|c|c|c|c|c|c|}
\hline \multicolumn{7}{|c|}{$\begin{array}{l}\text { UG-37 Area Calculation Summary }\left(\mathbf{i n}^{2}\right) \\
\text { For } P=1381.47 \mathrm{psi} @ 700^{\circ} \mathrm{F} \\
\text { The opening is adequately reinforced }\end{array}$} & \multicolumn{2}{|c|}{$\begin{array}{l}\text { UG-45 Nozzle Wall } \\
\text { Thickness Summary (in) } \\
\text { The nozzle passes UG-45 }\end{array}$} \\
\hline $\begin{array}{c}A \\
\text { required }\end{array}$ & $\begin{array}{c}\text { A } \\
\text { available }\end{array}$ & $\mathbf{A}_{1}$ & $\mathbf{A}_{2}$ & $\mathbf{A}_{\mathbf{3}}$ & $\mathbf{A}_{5}$ & $\begin{array}{c}\text { A } \\
\text { welds }\end{array}$ & $t_{\text {req }}$ & $\mathbf{t}_{\min }$ \\
\hline 0.5103 & 0.5777 & -- & 0.4371 & - & -- & 0.1406 & 0.0981 & 0.35 \\
\hline
\end{tabular}

The vessel wall thickness governs the MAWP of this nozzle.

\section{UG-41 Weld Failure Path Analysis Summary}

The nozzle is exempt from weld strength calculations per UW-15(b)(1)

\begin{tabular}{|c|c|c|c|}
\hline \multicolumn{3}{|c|}{ UW-16 Weld Sizing Summary } \\
\hline Weld description & $\begin{array}{l}\text { Required weld } \\
\text { throat size (in) }\end{array}$ & $\begin{array}{r}\text { Actual weld } \\
\text { throat size (in) }\end{array}$ & Status \\
\hline & & & \\
\hline
\end{tabular}




$\mid$ Nozzle to shell fillet $\left(\mathrm{Leg}_{41}\right)|\quad 0.224| 0.2625 \mid$ weld size is adequate

\section{Reinforcement Calculations for MAP}

The vessel wall thickness governs the MAP of this nozzle.

\begin{tabular}{|c|c|c|c|c|c|c|c|c|}
\hline \multicolumn{7}{|c|}{$\begin{array}{c}\text { UG-37 Area Calculation Summary }\left(\mathrm{in}^{2}\right) \\
\text { For } P=1719.91 \text { psi @ } 70^{\circ} \mathrm{F} \\
\text { The opening is adequately reinforced }\end{array}$} & \multicolumn{2}{|c|}{$\begin{array}{c}\text { UG-45 Nozzle Wall } \\
\text { Thickness Summary (in) } \\
\text { The nozzle passes UG-45 }\end{array}$} \\
\hline $\begin{array}{c}\mathrm{A} \\
\text { required }\end{array}$ & \begin{tabular}{|c|}
$A$ \\
available
\end{tabular} & $A_{1}$ & $\mathbf{A}_{2}$ & $\mathbf{A}_{3}$ & $A_{5}$ & $\begin{array}{c}\text { A } \\
\text { welds }\end{array}$ & $\mathrm{t}_{\text {req }}$ & $\mathbf{t}_{\min }$ \\
\hline 0.5457 & 0.6698 & - & 0.5292 & -- & -- & 0.1406 & 0.0727 & 0.35 \\
\hline
\end{tabular}

UG-41 Weld Failure Path Analysis Summary

The nozzle is exempt from weld strength calculations per UW-15(b)(1)

\begin{tabular}{|c|r|r|c|}
\hline \multicolumn{4}{|c|}{ UW-16 Weld Sizing Summary } \\
\hline Weld description & $\begin{array}{r}\text { Required weld } \\
\text { throat size (in) }\end{array}$ & $\begin{array}{r}\text { Actual weld } \\
\text { throat size (in) }\end{array}$ & Status \\
\hline Nozzle to shell fillet $\left(\operatorname{Leg}_{41}\right)$ & 0.245 & 0.2625 & weld size is adequate \\
\hline
\end{tabular}




\section{COUPLING (C11)}

\section{ASME Section VIII Division 1, 2007 Edition}

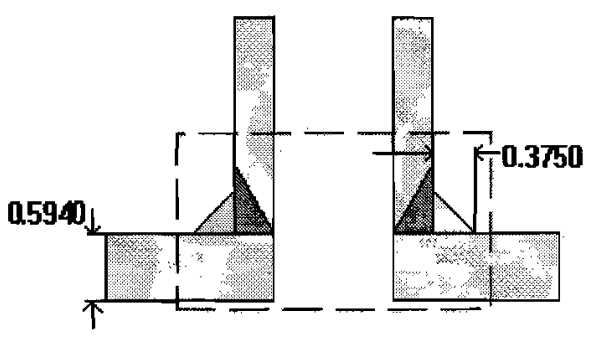

$$
\begin{aligned}
& t_{w(\text { lower })}=0 \text { in } \\
& \operatorname{Leg}_{41}=0.375 \text { in }
\end{aligned}
$$

Note: round inside edges per UG-76(e)

Located on:

Liquid static head included:

Nozzle material specification:

Nozzle longitudinal joint efficiency:

Nozzle description:

Nozzle orientation:

Local vessel minimum thickness:

Nozzle center line offset to datum line:

End of nozzle to shell center:

Nozzle inside diameter, new:

Nozzle nominal wall thickness:

Nozzle corrosion allowance:

\section{REACTOR OUTER SHELL}

$0 \mathrm{psi}$

SA-105 (II-D p. 18, ln. 8)

$0.750^{\prime \prime}$ Class 6000 - threaded

$120^{\circ}$

0.5197 in

10.75 in

6.375 in

1.05 in

0.35 in

0.03 in

Projection available outside vessel, Lpr: 1 in

\section{Reinforcement Calculations for Internal Pressure}

\begin{tabular}{|c|c|c|c|c|c|c|c|c|}
\hline \multicolumn{7}{|c|}{$\begin{array}{l}\text { UG-37 Area Calculation Summary }\left(\mathrm{in}^{2}\right) \\
\text { For } \mathrm{P}=1381.47 \text { psi } @ 700^{\circ} \mathrm{F} \\
\text { The opening is adequately reinforced }\end{array}$} & \multicolumn{2}{|c|}{$\begin{array}{l}\text { UG-45 Nozzle Wall } \\
\text { Thickness Summary (in) } \\
\text { The nozzle passes UG-45 }\end{array}$} \\
\hline$\underset{\text { required }}{A}$ & $\underset{\text { available }}{\mathbf{A}}$ & $A_{1}$ & $\mathbf{A}_{2}$ & $\mathbf{A}_{\mathbf{3}}$ & $A_{5}$ & $\begin{array}{c}\text { A } \\
\text { welds }\end{array}$ & $t_{\text {req }}$ & $\mathbf{t}_{\min }$ \\
\hline 0.5103 & 0.5777 & -- & 0.4371 & -- & -- & 0.1406 & 0.0981 & 0.35 \\
\hline
\end{tabular}

The vessel wall thickness governs the MAWP of this nozzle.

\section{UG-41 Weld Failure Path Analysis Summary}

The nozzle is exempt from weld strength calculations per UW-15(b)(1)

\begin{tabular}{|c|c|c|c|}
\hline \multicolumn{4}{|c|}{ UW-16 Weld Sizing Summary } \\
\hline Weld description & $\begin{array}{l}\text { Required weld } \\
\text { throat size (in) }\end{array}$ & $\begin{array}{r}\text { Actual weld } \\
\text { throat size (in) }\end{array}$ & Status \\
\hline & & & \\
\hline
\end{tabular}




$\mid$ Nozzle to shell fillet $\left(\operatorname{Leg}_{41}\right)|\quad 0.224| 0.2625 \mid$ weld size is adequate

Opening C07 is too close per UG-36(c)(3)(d) to allow an exemption per UG-36(c)(3)(a).

\section{Reinforcement Calculations for MAP}

The vessel wall thickness governs the MAP of this nozzle.

\begin{tabular}{|c|c|c|c|c|c|c|c|c|}
\hline \multicolumn{7}{|c|}{$\begin{array}{l}\text { UG-37 Area Calculation Summary }\left(\mathrm{in}^{2}\right) \\
\text { For } \mathrm{P}=1719.91 \text { psi } @ 70^{\circ} \mathrm{F} \\
\text { The opening is adequately reinforced }\end{array}$} & \multicolumn{2}{|c|}{$\begin{array}{l}\text { UG-45 Nozzle Wall } \\
\text { Thickness Summary (in) } \\
\text { The nozzle passes UG-45 }\end{array}$} \\
\hline$\underset{\text { required }}{A}$ & $\underset{\text { available }}{A}$ & $\mathbf{A}_{1}$ & $A_{2}$ & $\mathbf{A}_{\mathbf{3}}$ & $A_{5}$ & $\underset{\text { welds }}{A}$ & $t_{\text {req }}$ & $t_{\min }$ \\
\hline 0.5457 & 0.6698 & -- & 0.5292 & -- & -- & 0.1406 & 0.0727 & 0.35 \\
\hline
\end{tabular}

\section{UG-41 Weld Failure Path Analysis Summary}

The nozzle is exempt from weld strength calculations per UW-15(b)(1)

\begin{tabular}{|c|c|c|c|}
\hline \multicolumn{4}{|c|}{ UW-16 Weld Sizing Summary } \\
\hline Weld description & $\begin{array}{l}\text { Required weld } \\
\text { throat size (in) }\end{array}$ & $\begin{array}{r}\text { Actual weld } \\
\text { throat size (in) }\end{array}$ & Status \\
\hline Nozzle to shell fillet $\left(\operatorname{Leg}_{41}\right)$ & 0.245 & 0.2625 & weld size is adequate \\
\hline
\end{tabular}

Opening C07 is too close per UG-36(c)(3)(d) to allow an exemption per UG-36(c)(3)(a). 


\section{COUPLING (C12)}

\section{ASME Section VIII Division 1, 2007 Edition}

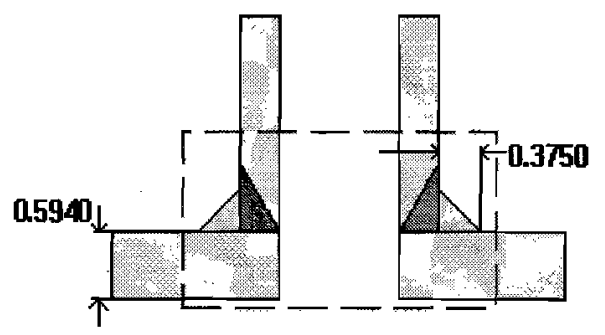

$$
\begin{aligned}
& t_{w(\text { lower })}=0 \text { in } \\
& \operatorname{Leg}_{41}=0.375 \text { in }
\end{aligned}
$$

Note: round inside edges per UG-76(c)

Located on:

Liquid static head included:

Nozzle material specification:

Nozzle longitudinal joint efficiency:

Nozzle description:

Nozzle orientation:

Local vessel minimum thickness:

Nozzle center line offset to datum line:

End of nozzle to shell center:

Nozzle inside diameter, new:

Nozzle nominal wall thickness:

Nozzle corrosion allowance:

Projection available outside vessel, Lpr: 1 in

\section{Reinforcement Calculations for Internal Pressure}

\begin{tabular}{|c|c|c|c|c|c|c|c|c|}
\hline \multicolumn{7}{|c|}{$\begin{array}{l}\text { UG-37 Area Calculation Summary }\left(\mathrm{in}^{2}\right) \\
\text { For } \mathrm{P}=1381.47 \text { psi @ } 700{ }^{\circ} \mathrm{F} \\
\text { The opening is adequately reinforced }\end{array}$} & \multicolumn{2}{|c|}{$\begin{array}{l}\text { UG-45 Nozzle Wall } \\
\text { Thickness Summary (in) } \\
\text { The nozzle passes UG-45 }\end{array}$} \\
\hline $\begin{array}{c}\mathbf{A} \\
\text { required }\end{array}$ & $\underset{\text { available }}{\mathbf{A}}$ & $\mathbf{A}_{\mathbf{1}}$ & $\mathbf{A}_{2}$ & $\mathbf{A}_{\mathbf{3}}$ & $\mathbf{A}_{5}$ & $\underset{\text { welds }}{\text { A }}$ & $\mathrm{t}_{\text {req }}$ & $\mathrm{t}_{\text {min }}$ \\
\hline 0.5103 & 0.5777 & - & 0.4371 & -. & -- & 0.1406 & 0.0981 & 0.35 \\
\hline
\end{tabular}

The vessel wall thickness governs the MAWP of this nozzle.

\section{UG-41 Weld Failure Path Analysis Summary}

The nozzle is exempt from weld strength calculations per UW-15(b)(1)

\begin{tabular}{|c|c|c|c|}
\hline \multicolumn{4}{|c|}{ UW-16 Weld Sizing Summary } \\
\hline Weld description & $\begin{array}{c}\text { Required weld } \\
\text { throat size (in) }\end{array}$ & $\begin{array}{r}\text { Actual weld } \\
\text { throat size (in) }\end{array}$ & Status \\
\hline & & & \\
\hline
\end{tabular}




\begin{tabular}{|l|l|l|l|} 
Nozzle to shell fillet $\left(\mathrm{Leg}_{41}\right)$ & 0.224 & 0.2625 & weld size is adequate \\
\hline
\end{tabular}

\section{Reinforcement Calculations for MAP}

The vessel wall thickness governs the MAP of this nozzle.

\begin{tabular}{|c|c|c|c|c|c|c|c|c|}
\hline \multicolumn{3}{|c|}{$\begin{array}{c}\text { UG-37 Area Calculation Summary }\left(\mathrm{in}^{2}\right) \\
\text { For P }=1719.91 \text { psi @ } \\
\text { The }\end{array}$} & \multicolumn{3}{c|}{$\begin{array}{c}\text { UG-45 Nozzle Wall } \\
\text { Thickness Summary (in) } \\
\text { The nozzle passes UG-45 }\end{array}$} \\
\hline $\begin{array}{c}\text { A } \\
\text { required }\end{array}$ & $\begin{array}{c}\mathrm{A} \\
\text { available }\end{array}$ & $\mathrm{A}_{1}$ & $\mathrm{~A}_{2}$ & $\mathrm{~A}_{3}$ & $\mathrm{~A}_{5}$ & $\begin{array}{c}\mathrm{A} \\
\text { welds }\end{array}$ & $\mathbf{t}_{\text {req }}$ & $\mathbf{t}_{\text {min }}$ \\
\hline 0.5457 & 0.6698 & -- & 0.5292 & -- & -- & 0.1406 & 0.0727 & 0.35 \\
\hline
\end{tabular}

\section{UG-41 Weld Failure Path Analysis Summary}

The nozzle is exempt from weld strength calculations per UW-15(b)(1)

\begin{tabular}{|c|r|r|c|}
\hline \multicolumn{4}{|c|}{ UW-16 Weld Sizing Summary } \\
\hline Weld description & $\begin{array}{r}\text { Required weld } \\
\text { throat size (in) }\end{array}$ & $\begin{array}{r}\text { Actual weld } \\
\text { throat size (in) }\end{array}$ & Status \\
\hline Nozzle to shell fillet $\left(\mathrm{Leg}_{41}\right)$ & 0.245 & 0.2625 & weld size is adequate \\
\hline
\end{tabular}


ASME Section VIII Division 1, 2007 Edition

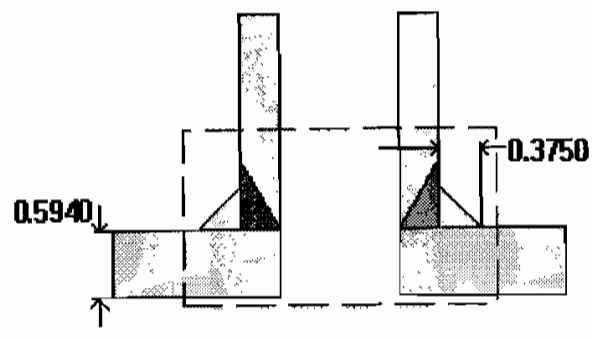

$$
\begin{aligned}
& t_{w(\text { lower })}=0 \text { in } \\
& \operatorname{Leg}_{41}=0.375 \text { in }
\end{aligned}
$$

Note: round inside edges per UG-76(c)

Located on:

Liquid static head included:

Nozzle material specification:

Nozzle longitudinal joint efficiency:

Nozzle description:

Nozzle orientation:

Local vessel minimum thickness:

Nozzle center line offset to datum line:

End of nozzle to shell center:

Nozzle inside diameter, new:

Nozzle nominal wall thickness:

Nozzle corrosion allowance:

Projection available outside vessel, Lpr: 1 in

\section{REACTOR OUTER SHELL}

$0 \mathrm{psi}$

SA-105 (II-D p. 18, ln. 8)

1

$0.750^{\prime \prime}$ Class 6000 - threaded

$150^{\circ}$

0.5197 in

13.375 in

6.375 in

1.05 in

0.35 in

0.03 in

\section{Reinforcement Calculations for Internal Pressure}

\begin{tabular}{|c|c|c|c|c|c|c|c|c|}
\hline \multicolumn{7}{|c|}{$\begin{array}{l}\text { UG-37 Area Calculation Summary }\left(\mathrm{in}^{2}\right) \\
\text { For } P=1381.47 \text { psi @ } 700^{\circ} \mathrm{F} \\
\text { The opening is adequately reinforced }\end{array}$} & \multicolumn{2}{|c|}{$\begin{array}{l}\text { UG-45 Nozzle Wall } \\
\text { Thickness Summary (in) } \\
\text { The nozzle passes UG-45 }\end{array}$} \\
\hline $\begin{array}{c}A \\
\text { required }\end{array}$ & $\underset{\text { available }}{A}$ & $A_{1}$ & $\mathbf{A}_{2}$ & $\mathbf{A}_{\mathbf{3}}$ & $A_{5}$ & $\begin{array}{c}\text { A } \\
\text { welds }\end{array}$ & $t_{\text {req }}$ & $t_{\min }$ \\
\hline 0.5103 & 0.5777 & -- & 0.4371 & -- & -- & 0.1406 & 0.0981 & 0.35 \\
\hline
\end{tabular}

The vessel wall thickness governs the MAWP of this nozzle.

\section{UG-41 Weld Failure Path Analysis Summary}

The nozzle is exempt from weld strength calculations per UW-15(b)(1)

\begin{tabular}{|l|l|l|l|}
\hline \multicolumn{4}{|c|}{ UW-16 Weld Sizing Summary } \\
\hline Weld description & $\begin{array}{l}\text { Required weld } \\
\text { throat size (in) }\end{array}$ & $\begin{array}{c}\text { Actual weld } \\
\text { throat size (in) }\end{array}$ & Status \\
\hline & & & \\
\hline
\end{tabular}




\begin{tabular}{|l|l|l|l|} 
Nozzle to shell fillet $\left(\operatorname{Leg}_{41}\right)$ & 0.224 & 0.2625 & weld size is adequate
\end{tabular}

\section{Reinforcement Calculations for MAP}

The vessel wall thickness governs the MAP of this nozzle.

\begin{tabular}{|c|c|c|c|c|c|c|c|c|}
\hline \multicolumn{7}{|c|}{$\begin{array}{l}\text { UG-37 Area Calculation Summary }\left(\text { in }^{2}\right) \\
\text { For } P=1719.91 \text { psi @ } 70^{\circ} \mathrm{F} \\
\text { The opening is adequately reinforced }\end{array}$} & \multicolumn{2}{|c|}{$\begin{array}{c}\text { UG-45 Nozzle Wall } \\
\text { Thickness Summary (in) } \\
\text { The nozzle passes UG-45 }\end{array}$} \\
\hline $\begin{array}{c}A \\
\text { required }\end{array}$ & $\underset{\text { available }}{A}$ & $\mathbf{A}_{1}$ & $\mathbf{A}_{2}$ & $\mathbf{A}_{3}$ & $A_{5}$ & $\begin{array}{c}\text { A } \\
\text { welds }\end{array}$ & $\mathbf{t}_{\text {req }}$ & $\mathbf{t}_{\min }$ \\
\hline 0.5457 & 0.6698 & -- & 0.5292 & -- & -- & 0.1406 & 0.0727 & 0.35 \\
\hline
\end{tabular}

\section{UG-41 Weld Failure Path Analysis Summary}

The nozzle is exempt from weld strength calculations per UW-15(b)(1)

\begin{tabular}{|c|r|r|c|}
\hline \multicolumn{4}{|c|}{ UW-16 Weld Sizing Summary } \\
\hline Weld description & $\begin{array}{r}\text { Required weld } \\
\text { throat size (in) }\end{array}$ & $\begin{array}{r}\text { Actual weld } \\
\text { throat size (in) }\end{array}$ & Status \\
\hline Nozzle to shell fillet $\left(\right.$ Leg $\left._{41}\right)$ & 0.245 & 0.2625 & weld size is adequate \\
\hline
\end{tabular}




\section{COUPLING (C14)}

ASME Section VIII Division 1, 2007 Edition

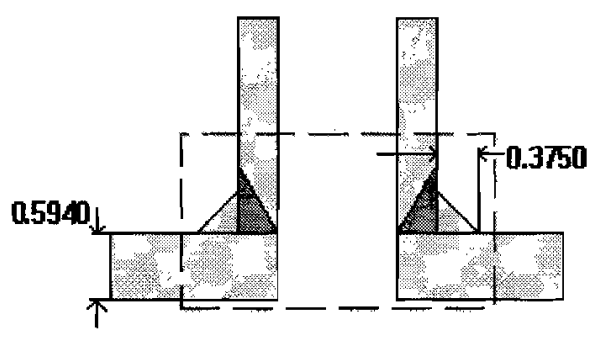

$$
\begin{aligned}
& t_{w(\text { lower })}=0 \text { in } \\
& \operatorname{Leg}_{41}=0.375 \text { in }
\end{aligned}
$$

Note: round inside edges per UG-76(c)

Located on:

Liquid static head included:

Nozzle material specification:

Nozzle longitudinal joint efficiency:

Nozzle description:

Nozzle orientation:

Local vessel minimum thickness:

Nozzle center line offset to datum line:

End of nozzle to shell center:

Nozzle inside diameter, new:

Nozzle nominal wall thickness:

Nozzle corrosion allowance:

Projection available outside vessel, Lpr: 1 in

\section{Reinforcement Calculations for Internal Pressure}

\begin{tabular}{|c|c|c|c|c|c|c|c|c|}
\hline \multicolumn{7}{|c|}{$\begin{array}{c}\text { UG-37 Area Calculation Summary }\left(\mathrm{in}^{2}\right) \\
\text { For } P=1381.47 \text { psi } @ 700^{\circ} \mathrm{F} \\
\text { The opening is adequately reinforced }\end{array}$} & \multicolumn{2}{|c|}{$\begin{array}{c}\text { UG-45 Nozzle Wall } \\
\begin{array}{c}\text { Thickness Summary (in) } \\
\text { The nozzle passes UG-45 }\end{array} \\
\end{array}$} \\
\hline $\begin{array}{c}\text { A } \\
\text { required }\end{array}$ & $\underset{\text { available }}{\text { A }}$ & $A_{1}$ & $\mathbf{A}_{2}$ & $\mathbf{A}_{\mathbf{3}}$ & $A_{5}$ & $\begin{array}{c}\mathrm{A} \\
\text { welds }\end{array}$ & $t_{\text {req }}$ & $\mathbf{t}_{\mathrm{min}}$ \\
\hline 0.5103 & 0.5777 & - & 0.4371 & -- & |-- & 0.1406 & 0.0981 & 0.35 \\
\hline
\end{tabular}

The vessel wall thickness governs the MAWP of this nozzle.

\section{UG-41 Weld Failure Path Analysis Summary}

The nozzle is exempt from weld strength calculations per UW-15(b)(1)

\begin{tabular}{|c|c|c|c|}
\hline \multicolumn{4}{|c|}{ UW-16 Weld Sizing Summary } \\
\hline Weld description & $\begin{array}{l}\text { Required weld } \\
\text { throat size (in) }\end{array}$ & $\begin{array}{r}\text { Actual weld } \\
\text { throat size (in) }\end{array}$ & Status \\
\hline & & & \\
\hline
\end{tabular}




\begin{tabular}{|l|l|l|l|} 
Nozzle to shell fillet $\left(\operatorname{Leg}_{41}\right)$ & 0.224 & 0.2625 & weld size is adequate \\
\hline
\end{tabular}

Opening $\mathrm{C10}$ is too close per UG-36(c)(3)(d) to allow an exemption per UG-36(c)(3)(a).

\section{Reinforcement Calculations for MAP}

The vessel wall thickness governs the MAP of this nozzle.

\begin{tabular}{|c|c|c|c|c|c|c|c|c|}
\hline $\begin{array}{c}\text { UG-37 Area Calculation Summary } \\
\text { For }{ }^{2} \text { ) } \\
\text { F }=1719.91 \text { psi @ 70 } \\
\text { The opening is adequately reinforced }\end{array}$ & \multicolumn{2}{c|}{$\begin{array}{c}\text { UG-45 Nozzle Wall } \\
\text { Thickness Summary (in) } \\
\text { The nozzle passes UG-45 }\end{array}$} \\
\hline $\begin{array}{c}\mathbf{A} \\
\text { required }\end{array}$ & $\begin{array}{c}\mathbf{A} \\
\text { available }\end{array}$ & $\mathbf{A}_{1}$ & $\mathbf{A}_{2}$ & $\mathbf{A}_{3}$ & $\mathbf{A}_{5}$ & $\begin{array}{c}\mathbf{A} \\
\text { welds }\end{array}$ & $\mathbf{t}_{\text {req }}$ & $\mathbf{t}_{\text {min }}$ \\
\hline 0.5457 & 0.6698 & -- & 0.5292 & -- & -- & 0.1406 & 0.0727 & 0.35 \\
\hline
\end{tabular}

\section{UG-41 Weld Failure Path Analysis Summary}

The nozzle is exempt from weld strength calculations per UW-15(b)(1)

\begin{tabular}{|c|r|r|c|}
\hline \multicolumn{4}{|c|}{ UW-16 Weld Sizing Summary } \\
\hline Weld description & $\begin{array}{r}\text { Required weld } \\
\text { throat size (in) }\end{array}$ & $\begin{array}{r}\text { Actual weld } \\
\text { throat size (in) }\end{array}$ & Status \\
\hline Nozzle to shell fillet $\left(\operatorname{Leg}_{41}\right)$ & 0.245 & 0.2625 & weld size is adequate \\
\hline
\end{tabular}

Opening $\mathrm{C10}$ is too close per UG-36(c)(3)(d) to allow an exemption per UG-36(c)(3)(a). 


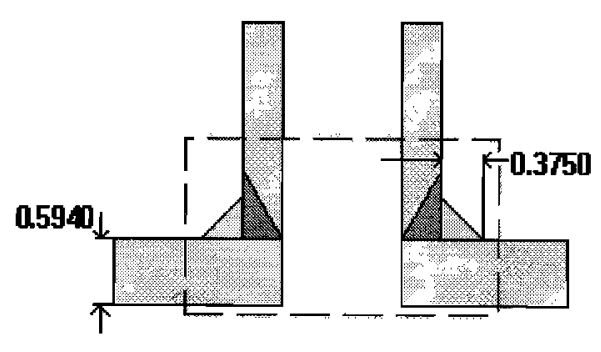

$$
\begin{aligned}
& t_{w(l o w e r)}=0 \text { in } \\
& \operatorname{Leg}_{41}=0.375 \text { in }
\end{aligned}
$$

Note: round inside edges per UG-76(c)

Located on:

Liquid static head included:

Nozzle material specification:

Nozzle longitudinal joint efficiency:

Nozzle description:

Nozzle orientation:

Local vessel minimum thickness:

Nozzle center line offset to datum line: 5

End of nozzle to shell center:

Nozzle inside diameter, new:

Nozzle nominal wall thickness:

Nozzle corrosion allowance:

Projection available outside vessel, Lpr: 1 in

\section{Reinforcement Calculations for Internal Pressure}

\begin{tabular}{|c|c|c|c|c|c|c|c|c|}
\hline \multicolumn{7}{|c|}{$\begin{array}{l}\text { UG-37 Area Calculation Summary }\left(\mathrm{in}^{2}\right) \\
\text { For } P=1381.47 \text { psi } @ 700^{\circ} \mathrm{F} \\
\text { The opening is adequately reinforced }\end{array}$} & \multicolumn{2}{|c|}{$\begin{array}{c}\text { UG-45 Nozzle Wall } \\
\text { Thickness Summary (in) } \\
\text { The nozzle passes UG-45 }\end{array}$} \\
\hline $\begin{array}{c}A \\
\text { required }\end{array}$ & $\underset{\text { available }}{\text { A }}$ & $\mathbf{A}_{1}$ & $\mathbf{A}_{2}$ & $\mathbf{A}_{3}$ & $\mathbf{A}_{5}$ & $\begin{array}{c}\text { A } \\
\text { welds }\end{array}$ & $\mathbf{t}_{\text {req }}$ & $t_{\min }$ \\
\hline 0.5103 & 0.5777 & -- & 0.4371 & -- & -- & 0.1406 & 0.0981 & 0.35 \\
\hline
\end{tabular}

The vessel wall thickness governs the MAWP of this nozzle.

\section{UG-41 Weld Failure Path Analysis Summary}

The nozzle is exempt from weld strength calculations per UW-15(b)(1)

\begin{tabular}{|c|c|c|c|}
\hline \multicolumn{4}{|c|}{ UW-16 Weld Sizing Summary } \\
\hline Weld description & $\begin{array}{l}\text { Required weld } \\
\text { throat size (in) }\end{array}$ & $\begin{array}{r}\text { Actual weld } \\
\text { throat size (in) }\end{array}$ & Status \\
\hline & & & \\
\hline
\end{tabular}




\begin{tabular}{|l|l|l|l|} 
Nozzle to shell fillet $\left(\operatorname{Leg}_{41}\right)$ & 0.224 & 0.2625 & weld size is adequate
\end{tabular}

\section{Reinforcement Calculations for MAP}

The vessel wall thickness governs the MAP of this nozzle.

\begin{tabular}{|c|c|c|c|c|c|c|c|c|}
\hline $\begin{array}{c}\text { UG-37 Area Calculation Summary } \\
\left(\mathbf{i n}^{2} \text { ) }\right. \\
\text { For } \mathbf{P}=1719.91 \text { psi @ } \\
\text { The opening is adequately reinforced }\end{array}$ & \multicolumn{2}{c|}{$\begin{array}{c}\text { UG-45 Nozzle Wall } \\
\text { Thickness Summary (in) } \\
\text { The nozzle passes UG-45 }\end{array}$} \\
\hline $\begin{array}{c}\mathbf{A} \\
\text { required }\end{array}$ & $\begin{array}{c}\mathbf{A} \\
\text { available }\end{array}$ & $\mathbf{A}_{\mathbf{1}}$ & $\mathbf{A}_{\mathbf{2}}$ & $\mathbf{A}_{\mathbf{3}}$ & $\mathbf{A}_{\mathbf{5}}$ & $\begin{array}{c}\mathbf{A} \\
\text { welds }\end{array}$ & $\mathbf{t}_{\text {req }}$ & $\mathbf{t}_{\text {min }}$ \\
\hline 0.5457 & 0.6698 & -- & 0.5292 & -- & -- & 0.1406 & 0.0727 & 0.35 \\
\hline
\end{tabular}

\section{UG-41 Weld Failure Path Analysis Summary}

The nozzle is exempt from weld strength calculations per UW-15(b)(1)

\begin{tabular}{|c|r|r|c|}
\hline \multicolumn{4}{|c|}{ UW-16 Weld Sizing Summary } \\
\hline Weld description & $\begin{array}{r}\text { Required weld } \\
\text { throat size (in) }\end{array}$ & $\begin{array}{r}\text { Actual weld } \\
\text { throat size (in) }\end{array}$ & Status \\
\hline Nozzle to shell fillet $\left(\right.$ Leg $\left._{41}\right)$ & 0.245 & 0.2625 & weld size is adequate \\
\hline
\end{tabular}




\section{COUPLING (C16)}

\section{ASME Section VIII Division 1, 2007 Edition}

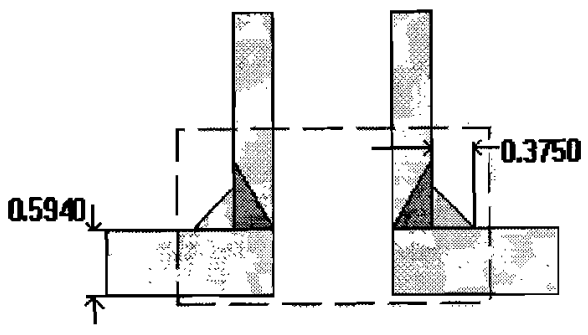

$$
\begin{aligned}
& t_{w(l o w e r)}=0 \text { in } \\
& \operatorname{Leg}_{41}=0.375 \text { in }
\end{aligned}
$$

Note: round inside edges per UG-76(c)

Located on:

Liquid static head included:

Nozzle material specification:

Nozzle longitudinal joint efficiency:

Nozzle description:

Nozzle orientation:

Local vessel minimum thickness:

Nozzle center line offset to datum line: 13.375 in

End of nozzle to shell center:

Nozzle inside diameter, new:

Nozzle nominal wall thickness:

Nozzle corrosion allowance:

Projection available outside vessel, Lpr: 1 in

\section{Reinforcement Calculations for Internal Pressure}

\begin{tabular}{|c|c|c|c|c|c|c|c|c|}
\hline \multicolumn{7}{|c|}{$\begin{array}{c}\text { UG-37 Area Calculation Summary }\left(\text { in }^{2}\right) \\
\text { For } P=1381.47 \text { psi @ } 700^{\circ} \mathrm{F} \\
\text { The opening is adequately reinforced }\end{array}$} & \multicolumn{2}{|c|}{$\begin{array}{l}\text { UG-45 Nozzle Wall } \\
\text { Thickness Summary (in) } \\
\text { The nozzle passes UG-45 }\end{array}$} \\
\hline \begin{tabular}{|c|} 
A \\
required
\end{tabular} & $\begin{array}{c}\text { A } \\
\text { available }\end{array}$ & $A_{1}$ & $A_{2}$ & $A_{3}$ & $A_{5}$ & $\begin{array}{c}\text { A } \\
\text { welds }\end{array}$ & $t_{\text {req }}$ & $t_{\text {min }}$ \\
\hline 0.5103 & 0.5777 & -- & 0.4371 & -- & -- & 0.1406 & 0.0981 & 0.35 \\
\hline
\end{tabular}

The vessel wall thickness governs the MAWP of this nozzle.

\section{UG-41 Weld Failure Path Analysis Summary}

The nozzle is exempt from weld strength calculations per UW-15(b)(1)

\begin{tabular}{|c|c|r|c|}
\hline \multicolumn{4}{|c|}{ UW-16 Weld Sizing Summary } \\
\hline Weld description & $\begin{array}{r}\text { Required weld } \\
\text { throat size (in) }\end{array}$ & $\begin{array}{r}\text { Actual weld } \\
\text { throat size (in) }\end{array}$ & Status \\
\hline & & & \\
\hline
\end{tabular}




\begin{tabular}{|l|l|l|l|} 
Nozzle to shell fillet $\left(\operatorname{Leg}_{41}\right)$ & 0.224 & 0.2625 & weld size is adequate \\
\hline
\end{tabular}

Opening $\mathrm{C17}$ is too close per UG-36(c)(3)(d) to allow an exemption per UG-36(c)(3)(a).

\section{Reinforcement Calculations for MAP}

The vessel wall thickness governs the MAP of this nozzle.

\begin{tabular}{|c|c|c|c|c|c|c|c|c|}
\hline \multicolumn{7}{|c|}{$\begin{array}{c}\text { UG-37 Area Calculation Summary }\left(\mathrm{in}^{2}\right) \\
\text { For } P=1719.91 \text { psi @ } 70^{\circ} \mathrm{F} \\
\text { The opening is adequately reinforced }\end{array}$} & \multicolumn{2}{|c|}{$\begin{array}{l}\text { UG-45 Nozzle Wall } \\
\text { Thickness Summary (in) } \\
\text { The nozzle passes UG-45 }\end{array}$} \\
\hline$\underset{\text { required }}{\mathbf{A}}$ & $\underset{\text { available }}{\mathbf{A}}$ & $\mathbf{A}_{1}$ & $A_{2}$ & $\mathbf{A}_{3}$ & $A_{3}$ & $\begin{array}{c}\text { A } \\
\text { welds }\end{array}$ & $t_{\text {req }}$ & $\mathbf{t}_{\min }$ \\
\hline 0.5457 & 0.6698 & -- & 0.5292 & -- & -- & 0.1406 & 0.0727 & 0.35 \\
\hline
\end{tabular}

\section{UG-41 Weld Failure Path Analysis Summary}

The nozzle is exempt from weld strength calculations per UW-15(b)(1)

\begin{tabular}{|c|r|r|c|}
\hline \multicolumn{4}{|c|}{ UW-16 Weld Sizing Summary } \\
\hline Weld description & $\begin{array}{r}\text { Required weld } \\
\text { throat size (in) }\end{array}$ & $\begin{array}{r}\text { Actual weld } \\
\text { throat size (in) }\end{array}$ & Status \\
\hline Nozzle to shell fillet (Leg $\left.{ }_{41}\right)$ & 0.245 & 0.2625 & weld size is adequate \\
\hline
\end{tabular}




\section{COUPLING (C17)}

\section{ASME Section VIII Division 1, 2007 Edition}

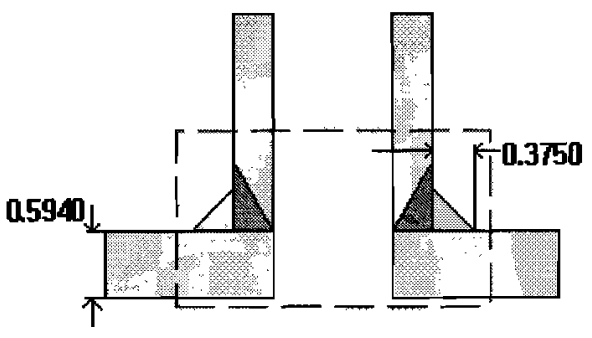

$$
\begin{aligned}
& t_{w(\text { lower })}=0 \text { in } \\
& \operatorname{Leg}_{41}=0.375 \text { in }
\end{aligned}
$$

Note: round inside edges per UG-76(c)

Located on:

Liquid static head included:

Nozzle material specification:

Nozzle longitudinal joint efficiency:

Nozzle description:

Nozzle orientation:

Local vessel minimum thickness:

Nozzle center line offset to datum line:

End of nozzle to shell center:

Nozzle inside diameter, new:

Nozzle nominal wall thickness:

Nozzle corrosion allowance:

Projection available outside vessel, Lpr: 1 in

\section{Reinforcement Calculations for Internal Pressure}

\begin{tabular}{|c|c|c|c|c|c|c|c|c|}
\hline \multicolumn{7}{|c|}{$\begin{array}{l}\text { UG-37 Area Calculation Summary }\left(\mathrm{in}^{2}\right) \\
\text { For } P=1381.47 \text { psi @ } 700^{\circ} \mathrm{F} \\
\text { The opening is adequately reinforced }\end{array}$} & \multicolumn{2}{|c|}{$\begin{array}{c}\text { UG-45 Nozzle Wall } \\
\text { Thickness Summary (in) } \\
\text { The nozzle passes UG-45 }\end{array}$} \\
\hline $\begin{array}{c}\mathbf{A} \\
\text { required }\end{array}$ & $\underset{\text { available }}{\mathbf{A}}$ & $A_{1}$ & $\mathbf{A}_{2}$ & $\mathbf{A}_{\mathbf{3}}$ & $\mathbf{A}_{5}$ & $\begin{array}{c}\text { A } \\
\text { welds }\end{array}$ & $t_{\text {req }}$ & $t_{\min }$ \\
\hline 0.5103 & 0.5777 & -- & 0.4371 & -- & -- & 0.1406 & 0.0981 & 0.35 \\
\hline
\end{tabular}

The vessel wall thickness governs the MAWP of this nozzle.

\section{UG-41 Weld Failure Path Analysis Summary}

The nozzle is exempt from weld strength calculations per UW-15(b)(1)

\begin{tabular}{|c|c|c|c|}
\hline \multicolumn{4}{|c|}{ UW-16 Weld Sizing Summary } \\
\hline Weld description & $\begin{array}{l}\text { Required weld } \\
\text { throat size (in) }\end{array}$ & $\begin{array}{r}\text { Actual weld } \\
\text { throat size (in) }\end{array}$ & Status \\
\hline & & & \\
\hline
\end{tabular}




\begin{tabular}{|l|l|l|l|} 
Nozzle to shell fillet $\left(\operatorname{Leg}_{4}\right)$ & 0.224 & 0.2625 & weld size is adequate \\
\hline
\end{tabular}

Opening C16 is too close per UG-36(c)(3)(d) to allow an exemption per UG-36(c)(3)(a).

\section{Reinforcement Calculations for MAP}

The vessel wall thickness governs the MAP of this nozzle.

\begin{tabular}{|c|c|c|c|c|c|c|c|c|}
\hline \multicolumn{7}{|c|}{$\begin{array}{l}\text { UG-37 Area Calculation Summary }\left(\mathrm{in}^{2}\right) \\
\text { For } \mathrm{P}=1719.91 \mathrm{psi} @ 70^{\circ} \mathrm{F} \\
\text { The opening is adequately reinforced }\end{array}$} & \multicolumn{2}{|c|}{$\begin{array}{l}\text { UG-45 Nozzle Wall } \\
\text { Thickness Summary (in) } \\
\text { The nozzle passes UG-45 }\end{array}$} \\
\hline $\begin{array}{c}\mathrm{A} \\
\text { required }\end{array}$ & $\underset{\text { available }}{A}$ & $A_{1}$ & $\mathbf{A}_{2}$ & $\mathbf{A}_{\mathbf{3}}$ & $A_{5}$ & $\begin{array}{c}\mathrm{A} \\
\text { welds }\end{array}$ & $\mathbf{t}_{\text {req }}$ & $\mathbf{t}_{\min }$ \\
\hline 0.5457 & 0.6698 & -- & 0.5292 & -- & -- & 0.1406 & 0.0727 & 0.35 \\
\hline
\end{tabular}

\section{UG-41 Weld Failure Path Analysis Summary}

The nozzle is exempt from weld strength calculations per UW-15(b)(1)

\begin{tabular}{|c|r|r|c|}
\hline \multicolumn{4}{|c|}{ UW-16 Weld Sizing Summary } \\
\hline Weld description & $\begin{array}{r}\text { Required weld } \\
\text { throat size (in) }\end{array}$ & $\begin{array}{r}\text { Actual weld } \\
\text { throat size (in) }\end{array}$ & Status \\
\hline Nozzle to shell fillet $\left(\right.$ Leg $\left._{41}\right)$ & 0.245 & 0.2625 & weld size is adequate \\
\hline
\end{tabular}

Opening C16 is too close per UG-36(c)(3)(d) to allow an exemption per UG-36(c)(3)(a). 


\section{COUPLING (C18)}

\section{ASME Section VIII Division 1, 2007 Edition}

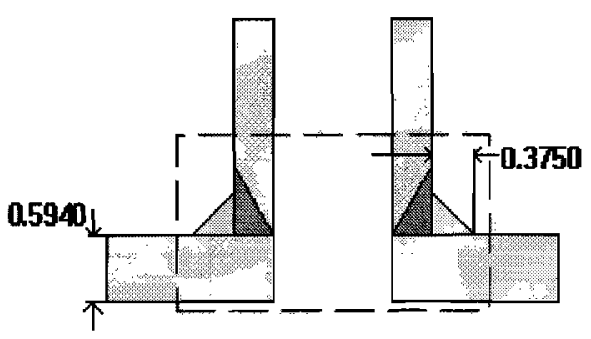

$$
\begin{aligned}
& t_{w(l o w e r)}=0 \text { in } \\
& \operatorname{Leg}_{41}=0.375 \text { in }
\end{aligned}
$$

Note: round inside edges per UG-76(c)

Located on:

Liquid static head included:

Nozzle material specification:

Nozzle longitudinal joint efficiency:

Nozzle description:

Nozzle orientation:

Local vessel minimum thickness:

Nozzle center line offset to datum line:

End of nozzle to shell center:

Nozzle inside diameter, new:

Nozzle nominal wall thickness:

Nozzle corrosion allowance:

Projection available outside vessel, Lpr: 1 in

\section{REACTOR OUTER SHELL}

0 psi

SA-105 (II-D p. 18, ln. 8)

1

$0.750^{\prime \prime}$ Class 6000 - threaded

$245^{\circ}$

0.5197 in

8.125 in

6.375 in

1.05 in

0.35 in

0.03 in

\section{Reinforcement Calculations for Internal Pressure}

The vessel wall thickness governs the MAWP of this nozzle.

\begin{tabular}{|c|c|c|c|c|c|c|c|c|}
\hline \multicolumn{3}{|c|}{$\begin{array}{c}\text { UG-37 Area Calculation Summary } \\
\text { For } \mathbf{P}=1381.47 \text { psi @ } \\
\text { The opening is adequately reinforced }\end{array}$} & \multicolumn{2}{c|}{$\begin{array}{c}\text { UG-45 Nozzle Wall } \\
\text { Thickness Summary (in) } \\
\text { The nozzle passes UG-45 }\end{array}$} \\
\hline $\begin{array}{c}\mathbf{A} \\
\text { required }\end{array}$ & $\begin{array}{c}\mathbf{A} \\
\text { available }\end{array}$ & $\mathbf{A}_{1}$ & $\mathbf{A}_{2}$ & $\mathbf{A}_{3}$ & $\mathbf{A}_{5}$ & $\begin{array}{c}\mathbf{A} \\
\text { welds }\end{array}$ & $\mathbf{t}_{\text {req }}$ & $\mathbf{t}_{\text {min }}$ \\
\hline 0.5103 & 0.5777 & -- & 0.4371 & -- & -- & 0.1406 & 0.0981 & 0.35 \\
\hline
\end{tabular}

UG-41 Weld Failure Path Analysis Summary

The nozzle is exempt from weld strength calculations per UW-15(b)(1)

\begin{tabular}{|c|c|c|c|}
\hline \multicolumn{3}{|c|}{ UW-16 Weld Sizing Summary } \\
\hline Weld description & $\begin{array}{c}\text { Required weld } \\
\text { throat size (in) }\end{array}$ & $\begin{array}{r}\text { Actual weld } \\
\text { throat size (in) }\end{array}$ & Status \\
\hline
\end{tabular}




\begin{tabular}{|l|l|l|l|} 
Nozzle to shell fillet $\left(\operatorname{Leg}_{41}\right)$ & 0.224 & 0.2625 & weld size is adequate \\
\hline
\end{tabular}

Opening C16 is too close per UG-36(c)(3)(d) to allow an exemption per UG-36(c)(3)(a).

\section{Reinforcement Calculations for MAP}

The vessel wall thickness governs the MAP of this nozzle.

\begin{tabular}{|c|c|c|c|c|c|c|c|c|}
\hline \multicolumn{7}{|c|}{$\begin{array}{l}\text { UG-37 Area Calculation Summary }\left(\text { in }^{2}\right) \\
\text { For } P=1719.91 \text { psi @ } 70^{\circ} \mathrm{F} \\
\text { The opening is adequately reinforced }\end{array}$} & \multicolumn{2}{|c|}{$\begin{array}{l}\text { UG-45 Nozzle Wall } \\
\text { Thickness Summary (in) } \\
\text { The nozzle passes UG-45 }\end{array}$} \\
\hline $\begin{array}{c}A \\
\text { required }\end{array}$ & $\underset{\text { available }}{\mathbf{A}}$ & $\mathbf{A}_{1}$ & $\mathbf{A}_{2}$ & $\mathbf{A}_{\mathbf{3}}$ & $\mathbf{A}_{5}$ & $\begin{array}{c}\text { A } \\
\text { welds }\end{array}$ & $\mathbf{t}_{\text {req }}$ & $t_{\min }$ \\
\hline 0.5457 & 0.6698 & -- & 0.5292 & -- & -- & 0.1406 & 0.0727 & 0.35 \\
\hline
\end{tabular}

\section{UG-41 Weld Failure Path Analysis Summary}

The nozzle is exempt from weld strength calculations per UW-15(b)(1)

\begin{tabular}{|c|r|r|c|}
\hline \multicolumn{4}{|c|}{ UW-16 Weld Sizing Summary } \\
\hline Weld description & $\begin{array}{r}\text { Required weld } \\
\text { throat size (in) }\end{array}$ & $\begin{array}{r}\text { Actual weld } \\
\text { throat size (in) }\end{array}$ & Status \\
\hline Nozzle to shell fillet $\left(\operatorname{Leg}_{41}\right)$ & 0.245 & 0.2625 & weld size is adequate \\
\hline
\end{tabular}

Opening $\mathrm{C17}$ is too close per UG-36(c)(3)(d) to allow an exemption per UG-36(c)(3)(a). 


\section{COUPLING (C19)}

\section{ASME Section VIII Division 1, 2007 Edition}

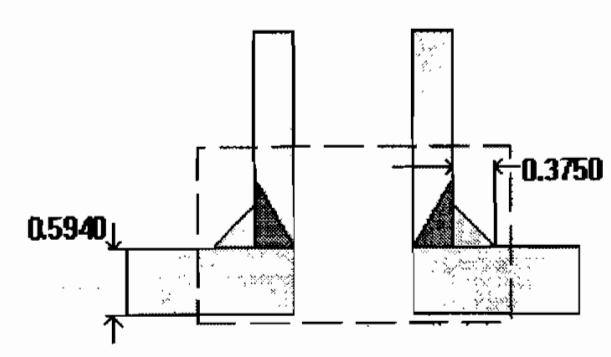

$$
\begin{aligned}
& t_{w(\text { lower })}=0 \text { in } \\
& \operatorname{Leg}_{41}=0.375 \text { in }
\end{aligned}
$$

Note: round inside edges per UG-76(c)

Located on:

Liquid static head included:

Nozzle material specification:

Nozzle longitudinal joint efficiency:

Nozzle description:

Nozzle orientation:

Local vessel minimum thickness:

Nozzle center line offset to datum line.

End of nozzle to shell center:

Nozzle inside diameter, new:

Nozzle nominal wall thickness:

Nozzle corrosion allowance:

Projection available outside vessel, Lpr: 1 in
REACTOR OUTER SHELL

0 psi

SA-105 (II-D p. 18, ln. 8)

1

$0.750^{\prime \prime}$ Class 6000 - threaded

$245^{\circ}$

0.5197 in

\section{Reinforcement Calculations for Internal Pressure}

\begin{tabular}{|c|c|c|c|c|c|c|c|c|}
\hline \multicolumn{7}{|c|}{$\begin{array}{l}\text { UG-37 Area Calculation Summary }\left(\mathrm{in}^{2}\right) \\
\text { For } P=1381.47 \text { psi } @ 700^{\circ} \mathrm{F} \\
\text { The opening is adequately reinforced }\end{array}$} & \multicolumn{2}{|c|}{$\begin{array}{l}\text { UG-45 Nozzle Wall } \\
\text { Thickness Summary (in) } \\
\text { The nozzle passes UG-45 }\end{array}$} \\
\hline $\begin{array}{c}A \\
\text { required }\end{array}$ & $\underset{\text { available }}{\text { A }}$ & $\mathbf{A}_{1}$ & $\mathbf{A}_{2}$ & $A_{3}$ & $A_{5}$ & $\begin{array}{c}\text { A } \\
\text { welds }\end{array}$ & $t_{\text {req }}$ & $t_{\min }$ \\
\hline 0.5103 & 0.5777 & -- & 0.4371 & -- & - & 0.1406 & 0.0981 & 0.35 \\
\hline
\end{tabular}

The vessel wall thickness governs the MAWP of this nozzle.

\section{UG-41 Weld Failure Path Analysis Summary}

The nozzle is exempt from weld strength calculations per UW-15(b)(1)

\begin{tabular}{|c|c|c|c|}
\hline \multicolumn{4}{|c|}{ UW-16 Weld Sizing Summary } \\
\hline Weld description & $\begin{array}{l}\text { Required weld } \\
\text { throat size (in) }\end{array}$ & $\begin{array}{r}\text { Actual weld } \\
\text { throat size (in) }\end{array}$ & Status \\
\hline & & & \\
\hline
\end{tabular}




\begin{tabular}{|l|l|l|l|} 
Nozzle to shell fillet $\left(\operatorname{Leg}_{41}\right)$ & 0.224 & 0.2625 & weld size is adequate \\
\hline
\end{tabular}

Opening C17 is too close per UG-36(c)(3)(d) to allow an exemption per UG-36(c)(3)(a).

\section{Reinforcement Calculations for MAP}

The vessel wall thickness governs the MAP of this nozzle.

\begin{tabular}{|c|c|c|c|c|c|c|c|c|}
\hline \multicolumn{7}{|c|}{$\begin{array}{l}\text { UG-37 Area Calculation Summary }\left(\mathrm{in}^{2}\right) \\
\text { For } P=1719.91 \mathrm{psi} @ 70^{\circ} \mathrm{F} \\
\text { The opening is adequately reinforced }\end{array}$} & \multicolumn{2}{|c|}{$\begin{array}{c}\text { UG-45 Nozzle Wall } \\
\text { Thickness Summary (in) } \\
\text { The nozzle passes UG-45 }\end{array}$} \\
\hline $\begin{array}{c}A \\
\text { required }\end{array}$ & $\underset{\text { available }}{A}$ & $\mathbf{A}_{1}$ & $\mathbf{A}_{2}$ & $\mathbf{A}_{\mathbf{3}}$ & $A_{5}$ & $\begin{array}{c}\text { A } \\
\text { welds }\end{array}$ & $\mathbf{t}_{\text {req }}$ & $t_{\min }$ \\
\hline 0.5457 & 0.6698 & - & 0.5292 & -- & -- & 0.1406 & 0.0727 & 0.35 \\
\hline
\end{tabular}

UG-41 Weld Failure Path Analysis Summary

The nozzle is exempt from weld strength calculations per UW-15(b)(1)

\begin{tabular}{|c|r|r|c|}
\hline \multicolumn{4}{|c|}{ UW-16 Weld Sizing Summary } \\
\hline Weld description & $\begin{array}{r}\text { Required weld } \\
\text { throat size (in) }\end{array}$ & $\begin{array}{r}\text { Actual weld } \\
\text { throat size (in) }\end{array}$ & Status \\
\hline Nozzle to shell fillet $\left(\mathrm{Leg}_{41}\right)$ & 0.245 & 0.2625 & weld size is adequate \\
\hline
\end{tabular}




\section{ASME Section VIII Division 1, 2007 Edition}

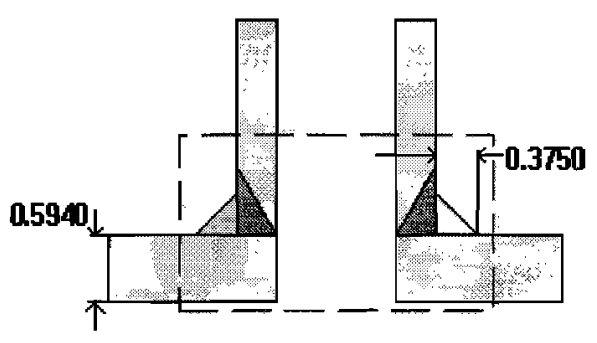

$$
\begin{aligned}
& t_{w(\text { lower })}=0 \text { in } \\
& \operatorname{Leg}_{41}=0.375 \text { in }
\end{aligned}
$$

Note: round inside edges per UG-76(c)

Located on:

Liquid static head included:

Nozzle material specification:

Nozzle longitudinal joint efficiency:

Nozzle description:

Nozzle orientation:

Local vessel minimum thickness:

Nozzle center line offset to datum line:

End of nozzle to shell center:

Nozzle inside diameter, new:

Nozzle nominal wall thickness:

Nozzle corrosion allowance:

Projection available outside vessel, Lpr: 1 in

\section{REACTOR OUTER SHELL}

$0 \mathrm{psi}$

SA-105 (II-D p. 18, ln. 8)

1

$0.750^{\prime \prime}$ Class 6000 - threaded

$295^{\circ}$

0.5197 in

13.375 in

6.375 in

1.05 in

0.35 in

0.03 in

\section{Reinforcement Calculations for Internal Pressure}

\begin{tabular}{|c|c|c|c|c|c|c|c|c|}
\hline \multicolumn{7}{|c|}{$\begin{array}{c}\text { UG-37 Area Calculation Summary }\left(\text { in }^{2}\right) \\
\text { For } P=1381.47 \text { psi @ } 700^{\circ} \mathrm{F} \\
\text { The opening is adequately reinforced }\end{array}$} & \multicolumn{2}{|c|}{\begin{tabular}{|c|} 
UG-45 Nozzle Wall \\
Thickness Summary (in \\
The nozzle passes UG-45
\end{tabular}} \\
\hline$\underset{\text { required }}{A}$ & $\underset{\text { available }}{A}$ & $A_{1}$ & $\mathbf{A}_{2}$ & $\mathbf{A}_{3}$ & $\mathbf{A}_{\mathbf{5}}$ & $\begin{array}{c}\text { A } \\
\text { welds }\end{array}$ & $\mathbf{t}_{\text {req }}$ & $\mathbf{t}_{\text {min }}$ \\
\hline 0.5103 & 0.5777 & -- & 0.4371 & -- & -- & 0.1406 & 0.0981 & 0.35 \\
\hline
\end{tabular}

The vessel wall thickness governs the MAWP of this nozzle.

\section{UG-41 Weld Failure Path Analysis Summary}

The nozzle is exempt from weld strength calculations per UW-15(b)(1)

\begin{tabular}{|c|c|c|c|}
\hline \multicolumn{4}{|c|}{ UW-16 Weld Sizing Summary } \\
\hline Weld description & $\begin{array}{l}\text { Required weld } \\
\text { throat size (in) }\end{array}$ & $\begin{array}{r}\text { Actual weld } \\
\text { throat size (in) }\end{array}$ & Status \\
\hline & & & \\
\hline
\end{tabular}




\begin{tabular}{|l|l|l|l|} 
Nozzle to shell fillet $\left(\operatorname{Leg}_{41}\right)$ & 0.224 & 0.2625 & weld size is adequate \\
\hline
\end{tabular}

Opening C21 is too close per UG-36(c)(3)(d) to allow an exemption per UG-36(c)(3)(a).

\section{Reinforcement Calculations for MAP}

The vessel wall thickness governs the MAP of this nozzle.

\begin{tabular}{|c|c|c|c|c|c|c|c|c|}
\hline \multicolumn{7}{|c|}{$\begin{array}{c}\text { UG-37 Area Calculation Summary }\left(\mathrm{in}^{2}\right) \\
\text { For } P=1719.91 \text { psi } @ 70^{\circ} \mathrm{F} \\
\text { The opening is adequately reinforced }\end{array}$} & \multicolumn{2}{|c|}{$\begin{array}{l}\text { UG-45 Nozzle Wall } \\
\text { Thickness Summary (in) } \\
\text { The nozzle passes UG-45 } \\
\end{array}$} \\
\hline $\begin{array}{c}\mathrm{A} \\
\text { required }\end{array}$ & $\begin{array}{c}\text { A } \\
\text { vailable }\end{array}$ & $A_{1}$ & $\mathbf{A}_{2}$ & $\mathbf{A}_{3}$ & $\mathbf{A}_{5}$ & $\begin{array}{c}\text { A } \\
\text { welds }\end{array}$ & $t_{\text {req }}$ & $t_{\min }$ \\
\hline 0.5457 & 0.6698 & -- & 0.5292 & -- & -- & 0.1406 & 0.0727 & 0.35 \\
\hline
\end{tabular}

\section{UG-41 Weld Failure Path Analysis Summary}

The nozzle is exempt from weld strength calculations per UW-15(b)(1)

\begin{tabular}{|c|r|r|c|}
\hline \multicolumn{4}{|c|}{ UW-16 Weld Sizing Summary } \\
\hline Weld description & $\begin{array}{l}\text { Required weld } \\
\text { throat size (in) }\end{array}$ & $\begin{array}{r}\text { Actual weld } \\
\text { throat size (in) }\end{array}$ & Status \\
\hline Nozzle to shell fillet $\left(\mathrm{Leg}_{41}\right)$ & 0.245 & 0.2625 & weld size is adequate \\
\hline
\end{tabular}




\section{ASME Section VIII Division 1, 2007 Edition}

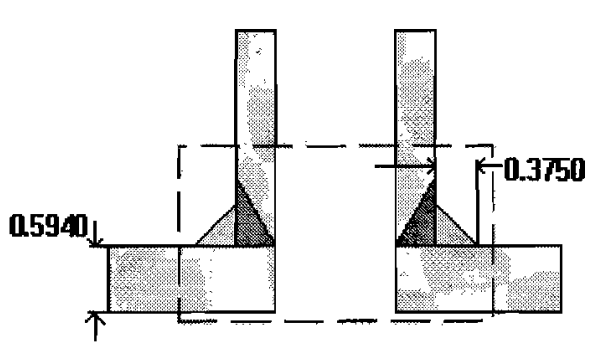

$$
\begin{aligned}
& t_{w(\text { lower })}=0 \text { in } \\
& \operatorname{Leg}_{41}=0.375 \text { in }
\end{aligned}
$$

Note: round inside edges per UG-76(c)

Located on:

Liquid static head included:

Nozzle material specification:

Nozzle longitudinal joint efficiency:

Nozzle description:

Nozzle orientation:

Local vessel minimum thickness:

Nozzle center line offset to datum line: 10.75 in

End of nozzle to shell center: $\quad 6.375$ in

Nozzle inside diameter, new: $\quad 1.05$ in

Nozzle nominal wall thickness: $\quad 0.35$ in

Nozzle corrosion allowance: $\quad 0.03$ in

Projection available outside vessel, Lpr: 1 in

\section{Reinforcement Calculations for Internal Pressure}

\begin{tabular}{|c|c|c|c|c|c|c|c|c|}
\hline \multicolumn{7}{|c|}{$\begin{array}{l}\text { UG-37 Area Calculation Summary }\left(\mathrm{in}^{2}\right) \\
\text { For } P=1381.47 \text { psi } @ 700^{\circ} \mathrm{F} \\
\text { The opening is adequately reinforced }\end{array}$} & \multicolumn{2}{|c|}{$\begin{array}{l}\text { UG-45 Nozzle Wall } \\
\text { Thickness Summary (in) } \\
\text { The nozzle passes UG-45 }\end{array}$} \\
\hline $\begin{array}{c}A \\
\text { required }\end{array}$ & \begin{tabular}{|c|}
$\mathbf{A}$ \\
available \\
\end{tabular} & $A_{1}$ & $A_{2}$ & $A_{3}$ & $A_{5}$ & $\begin{array}{c}\mathrm{A} \\
\text { welds }\end{array}$ & $t_{\text {req }}$ & $\mathbf{t}_{\min }$ \\
\hline 0.5103 & 0.5777 & -- & 0.4371 & -- & -- & 0.1406 & 0.0981 & 0.35 \\
\hline
\end{tabular}

The vessel wall thickness governs the MAWP of this nozzle.

\section{UG-41 Weld Failure Path Analysis Summary}

The nozzle is exempt from weld strength calculations per UW-15(b)(1)

\begin{tabular}{|c|c|c|c|}
\hline \multicolumn{4}{|c|}{ UW-16 Weld Sizing Summary } \\
\hline Weld description & $\begin{array}{l}\text { Required weld } \\
\text { throat size (in) }\end{array}$ & $\begin{array}{r}\text { Actual weld } \\
\text { throat size (in) }\end{array}$ & Status \\
\hline & & & \\
\hline
\end{tabular}




\begin{tabular}{|l|l|l|l|} 
Nozzle to shell fillet $\left(\operatorname{Leg}_{41}\right)$ & 0.224 & 0.2625 & weld size is adequate
\end{tabular}

Opening $\mathrm{C20}$ is too close per UG-36(c)(3)(d) to allow an exemption per UG-36(c)(3)(a).

\section{Reinforcement Calculations for MAP}

The vessel wall thickness governs the MAP of this nozzle.

\begin{tabular}{|c|c|c|c|c|c|c|c|c|}
\hline \multicolumn{7}{|c|}{$\begin{array}{l}\text { UG-37 Area Calculation Summary }\left(\mathrm{in}^{2}\right) \\
\text { For } \mathrm{P}=1719.91 \text { psi @ } 70^{\circ} \mathrm{F} \\
\text { The opening is adequately reinforced }\end{array}$} & \multicolumn{2}{|c|}{$\begin{array}{l}\text { UG-45 Nozzle Wall } \\
\text { Thickness Summary (in) } \\
\text { The nozzle passes UG-45 }\end{array}$} \\
\hline$\underset{\text { required }}{A}$ & $\underset{\text { available }}{\mathbf{A}}$ & $\mathbf{A}_{1}$ & $\mathbf{A}_{2}$ & $\mathbf{A}_{\mathbf{3}}$ & $\mathbf{A}_{5}$ & $\underset{\text { welds }}{\text { A }}$ & $t_{\text {req }}$ & $t_{\min }$ \\
\hline 0.5457 & 0.6698 & -- & 0.5292 & -- & -- & 0.1406 & 0.0727 & 0.35 \\
\hline
\end{tabular}

\section{UG-41 Weld Failure Path Analysis Summary}

The nozzle is exempt from weld strength calculations per UW-15(b)(1)

\begin{tabular}{|c|r|r|c|}
\hline \multicolumn{4}{|c|}{ UW-16 Weld Sizing Summary } \\
\hline Weld description & $\begin{array}{r}\text { Required weld } \\
\text { throat size (in) }\end{array}$ & $\begin{array}{r}\text { Actual weld } \\
\text { throat size (in) }\end{array}$ & Status \\
\hline Nozzle to shell fillet $\left(\operatorname{Leg}_{41}\right)$ & 0.245 & 0.2625 & weld size is adequate \\
\hline
\end{tabular}

Opening C20 is too close per UG-36(c)(3)(d) to allow an exemption per UG-36(c)(3)(a). 
COUPLING (C22)

ASME Section VIII Division 1, 2007 Edition

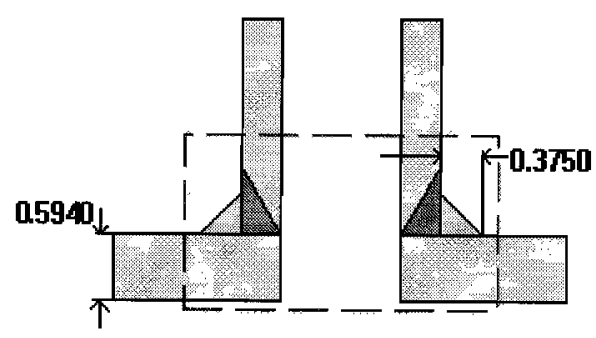

$\mathrm{t}_{\mathrm{w}(\text { lower })}=0$ in

$\operatorname{Leg}_{41}=0.375$ in

Note: round inside edges per UG-76(c)

Located on:

REACTOR OUTER SHELL

Liquid static head included:

0 psi

Nozzle material specification:

SA-105 (II-D p. 18, ln. 8)

Nozzle longitudinal joint efficiency:

Nozzle description:

Nozzle orientation:

$0.750^{\prime \prime}$ Class 6000 - threaded $295^{\circ}$

Local vessel minimum thickness: $\quad 0.5197$ in

Nozzle center line offset to datum line: 8.125 in

End of nozzle to shell center: $\quad 6.375$ in

Nozzle inside diameter, new: $\quad 1.05$ in

Nozzle nominal wall thickness: $\quad 0.35$ in

Nozzle corrosion allowance: $\quad 0.03$ in

Projection available outside vessel, Lpr: 1 in

\section{Reinforcement Calculations for Internal Pressure}

The vessel wall thickness governs the MAWP of this nozzle.

\begin{tabular}{|c|c|c|c|c|c|c|c|c|}
\hline \multicolumn{3}{|c|}{$\begin{array}{c}\text { UG-37 Area Calculation Summary }\left(\mathrm{in}^{2} \text { ) }\right. \\
\text { For } \mathbf{P}=1381.47 \text { psi @ } 700\end{array}$} & \multicolumn{2}{c|}{$\begin{array}{c}\text { UG-45 Nozzle Wall } \\
\text { Thickness Summary (in) } \\
\text { The nozzle passes UG-45 }\end{array}$} \\
\hline $\begin{array}{c}\text { The opening is adequately reinforced } \\
\text { required }\end{array}$ & $\begin{array}{c}\mathbf{A} \\
\text { available }\end{array}$ & $\mathbf{A}_{1}$ & $\mathbf{A}_{\mathbf{2}}$ & $\mathbf{A}_{\mathbf{3}}$ & $\mathbf{A}_{\mathbf{5}}$ & $\begin{array}{c}\mathbf{A} \\
\text { welds }\end{array}$ & $\mathbf{t}_{\text {req }}$ & $\mathbf{t}_{\text {min }}$ \\
\hline 0.5103 & 0.5777 & -- & 0.4371 & -- & -- & 0.1406 & 0.0981 & 0.35 \\
\hline
\end{tabular}

UG-41 Weld Failure Path Analysis Summary

The nozzle is exempt from weld strength calculations per UW-15(b)(1)

\begin{tabular}{|c|c|c|c|}
\hline \multicolumn{4}{|c|}{ UW-16 Weld Sizing Summary } \\
\hline Weld description & $\begin{array}{l}\text { Required weld } \\
\text { throat size (in) }\end{array}$ & $\begin{array}{r}\text { Actual weld } \\
\text { throat size (in) }\end{array}$ & Status \\
\hline & & & \\
\hline
\end{tabular}




\begin{tabular}{|l|l|l|l|} 
Nozzle to shell fillet $\left(\operatorname{Leg}_{41}\right)$ & 0.224 & 0.2625 & weld size is adequate \\
\hline
\end{tabular}

Opening $\mathrm{C20}$ is too close per UG-36(c)(3)(d) to allow an exemption per UG-36(c)(3)(a).

\section{Reinforcement Calculations for MAP}

The vessel wall thickness governs the MAP of this nozzle.

\begin{tabular}{|c|c|c|c|c|c|c|c|c|}
\hline \multicolumn{7}{|c|}{$\begin{array}{l}\text { UG-37 Area Calculation Summary }\left(\mathrm{in}^{2}\right) \\
\text { For } P=1719.91 \text { psi } @ 70^{\circ} \mathrm{F} \\
\text { The opening is adequately reinforced }\end{array}$} & \multicolumn{2}{|c|}{$\begin{array}{l}\text { UG-45 Nozzle Wall } \\
\text { Thickness Summary (in) } \\
\text { The nozzle passes UG-45 }\end{array}$} \\
\hline $\begin{array}{c}\text { A } \\
\text { required }\end{array}$ & \begin{tabular}{|c|} 
A \\
available \\
\end{tabular} & $A_{1}$ & $\mathbf{A}_{2}$ & $\mathbf{A}_{\mathbf{3}}$ & $\mathbf{A}_{\mathbf{5}}$ & $\begin{array}{c}\text { A } \\
\text { welds }\end{array}$ & $t_{\text {req }}$ & $t_{\text {min }}$ \\
\hline 0.5457 & 0.6698 & -- & 0.5292 & -- & -- & 0.1406 & 0.0727 & 0.35 \\
\hline
\end{tabular}

\section{UG-41 Weld Failure Path Analysis Summary}

The nozzle is exempt from weld strength calculations per UW-15(b)(1)

\begin{tabular}{|c|r|r|c|}
\hline \multicolumn{4}{|c|}{ UW-16 Weld Sizing Summary } \\
\hline Weld description & $\begin{array}{r}\text { Required weld } \\
\text { throat size (in) }\end{array}$ & $\begin{array}{r}\text { Actual weld } \\
\text { throat size (in) }\end{array}$ & Status \\
\hline Nozzle to shell fillet $\left(\right.$ Leg $\left._{41}\right)$ & 0.245 & 0.2625 & weld size is adequate \\
\hline
\end{tabular}

Opening C21 is too close per UG-36(c)(3)(d) to allow an exemption per UG-36(c)(3)(a). 
COUPLING (C23)

ASME Section VIII Division 1, 2007 Edition

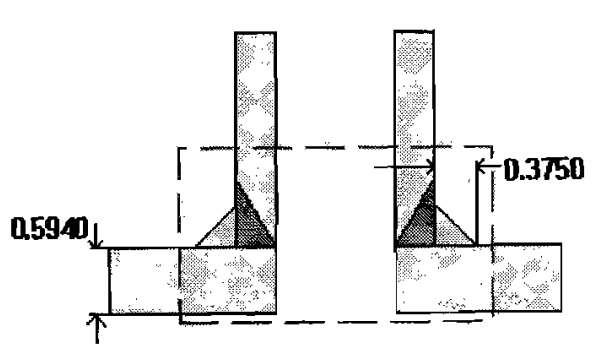

$$
\begin{aligned}
& t_{w(\text { lower })}=0 \text { in } \\
& \operatorname{Leg}_{41}=0.375 \text { in }
\end{aligned}
$$

Note: round inside edges per UG-7G(c)

Located on:

Liquid static head included:

Nozzle material specification:

Nozzle longitudinal joint efficiency:

Nozzle description:

Nozzle orientation:

Local vessel minimum thickness:

Nozzle center line offset to datum line:

End of nozzle to shell center:

Nozzle inside diameter, new:

Nozzle nominal wall thickness:

Nozzle corrosion allowance:

\section{REACTOR OUTER SHELL}

$0 \mathrm{psi}$

SA-105 (II-D p. 18, ln. 8)

1

$0.750^{\prime \prime}$ Class 6000 - threaded

$295^{\circ}$

0.5197 in

5.5 in

6.375 in

1.05 in

0.35 in

0.03 in

Projection available outside vessel, Lpr: 1 in

\section{Reinforcement Calculations for Internal Pressure}

\begin{tabular}{|c|c|c|c|c|c|c|c|c|}
\hline \multicolumn{7}{|c|}{$\begin{array}{l}\text { UG-37 Area Calculation Summary }\left(\mathrm{in}^{2}\right) \\
\text { For } \mathrm{P}=1381.47 \text { psi @ } 700^{\circ} \mathrm{F} \\
\text { The opening is adequately reinforced }\end{array}$} & \multicolumn{2}{|c|}{$\begin{array}{l}\text { UG-45 Nozzle Wall } \\
\text { Thickness Summary (in) } \\
\text { The nozzle passes UG-45 }\end{array}$} \\
\hline $\begin{array}{c}A \\
\text { required }\end{array}$ & $\begin{array}{c}\mathrm{A} \\
\text { available }\end{array}$ & $A_{1}$ & $\mathbf{A}_{2}$ & $A_{3}$ & $A_{5}$ & $\begin{array}{c}\text { A } \\
\text { welds }\end{array}$ & $t_{\text {req }}$ & $t_{\min }$ \\
\hline 0.5103 & 0.5777 & -- & 0.4371 & -- & - & 0.1406 & 0.0981 & 0.35 \\
\hline
\end{tabular}

The vessel wall thickness governs the MAWP of this nozzle.

\section{UG-41 Weld Failure Path Analysis Summary}

The nozzle is exempt from weld strength calculations per UW-15(b)(1)

\begin{tabular}{|c|c|c|c|}
\hline \multicolumn{4}{|c|}{ UW-16 Weld Sizing Summary } \\
\hline Weld description & $\begin{array}{l}\text { Required weld } \\
\text { throat size (in) }\end{array}$ & $\begin{array}{r}\text { Actual weld } \\
\text { throat size (in) }\end{array}$ & Status \\
\hline & & & \\
\hline
\end{tabular}




\begin{tabular}{|l|l|l|l|} 
Nozzle to shell fillet $\left(\mathrm{Leg}_{41}\right)$ & 0.224 & 0.2625 & weld size is adequate \\
\hline
\end{tabular}

Opening C21 is too close per UG-36(c)(3)(d) to allow an exemption per UG-36(c)(3)(a).

\section{Reinforcement Calculations for MAP}

The vessel wall thickness governs the MAP of this nozzle.

\begin{tabular}{|c|c|c|c|c|c|c|c|c|}
\hline \multicolumn{7}{|c|}{$\begin{array}{l}\text { UG-37 Area Calculation Summary }\left(\mathrm{in}^{2}\right) \\
\text { For } P=1719.91 \text { psi } @ 70^{\circ} \mathrm{F} \\
\text { The opening is adequately reinforced }\end{array}$} & \multicolumn{2}{|c|}{$\begin{array}{l}\text { UG-45 Nozzle Wall } \\
\text { Thickness Summary (in) } \\
\text { The nozzle passes UG-45 }\end{array}$} \\
\hline $\begin{array}{c}A \\
\text { required }\end{array}$ & \begin{tabular}{|c}
$\mathbf{A}$ \\
available
\end{tabular} & $\mathbf{A}_{1}$ & $\mathbf{A}_{2}$ & $\mathbf{A}_{3}$ & $\mathbf{A}_{5}$ & $\begin{array}{c}\text { A } \\
\text { welds }\end{array}$ & $\mathbf{t}_{\text {req }}$ & $\mathbf{t}_{\min }$ \\
\hline 0.5457 & 0.6698 & -- & 0.5292 & -- & -. & 0.1406 & 0.0727 & 0.35 \\
\hline
\end{tabular}

UG-41 Weld Failure Path Analysis Summary

The nozzle is exempt from weld strength calculations per UW-15(b)(1)

\begin{tabular}{|c|r|r|c|}
\hline \multicolumn{4}{|c|}{ UW-16 Weld Sizing Summary } \\
\hline Weld description & $\begin{array}{r}\text { Required weld } \\
\text { throat size (in) }\end{array}$ & $\begin{array}{r}\text { Actual weld } \\
\text { throat size (in) }\end{array}$ & Status \\
\hline Nozzle to shell fillet $\left(\operatorname{Leg}_{41}\right)$ & 0.245 & 0.2625 & weld size is adequate \\
\hline
\end{tabular}


COUPLING (C24)

ASME Section VIII Division 1, 2007 Edition

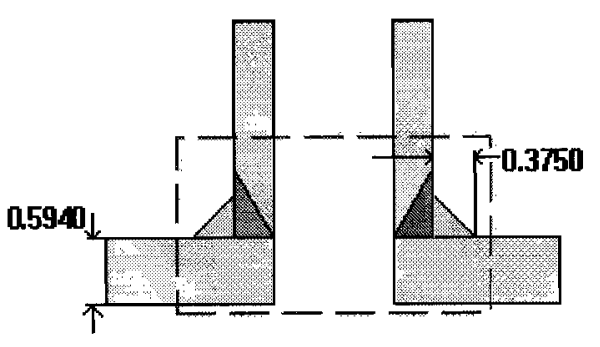

$$
\begin{aligned}
& t_{w(l o w e r)}=0 \text { in } \\
& \text { Leg }_{41}=0.375 \text { in }
\end{aligned}
$$

Note: round inside edges per UG-76(c)

Located on:

Liquid static head included:

Nozzle material specification:

Nozzle longitudinal joint efficiency:

Nozzle description:

Nozzle orientation:

Local vessel minimum thickness:

Nozzle center line offset to datum line:

End of nozzle to shell center:

Nozzle inside diameter, new:

Nozzle nominal wall thickness:

Nozzle corrosion allowance:

Projection available outside vessel, Lpr: 1 in

\section{Reinforcement Calculations for Internal Pressure}

\begin{tabular}{|c|c|c|c|c|c|c|c|c|}
\hline \multicolumn{7}{|c|}{$\begin{array}{l}\text { UG-37 Area Calculation Summary }\left(\mathrm{in}^{2}\right) \\
\text { For } \mathrm{P}=1381.47 \text { psi } @ 700^{\circ} \mathrm{F} \\
\text { The opening is adequately reinforced }\end{array}$} & \multicolumn{2}{|c|}{$\begin{array}{l}\text { UG-45 Nozzle Wall } \\
\text { Thickness Summary (in) } \\
\text { The nozzle passes UG-45 }\end{array}$} \\
\hline $\begin{array}{c}A \\
\text { required }\end{array}$ & $\underset{\text { available }}{\text { A }}$ & $\mathbf{A}_{1}$ & $\mathbf{A}_{2}$ & $\mathbf{A}_{\mathbf{3}}$ & $A_{5}$ & $\begin{array}{c}\text { A } \\
\text { welds }\end{array}$ & $\mathbf{t}_{\text {req }}$ & $\mathbf{t}_{\min }$ \\
\hline 0.5103 & 0.5777 & -- & 0.4371 & - & -- & 0.1406 & 0.0981 & 0.35 \\
\hline
\end{tabular}

The vessel wall thickness governs the MAWP of this nozzle.

\section{UG-41 Weld Failure Path Analysis Summary}

The nozzle is exempt from weld strength calculations per UW-15(b)(1)

\begin{tabular}{|c|c|c|c|}
\hline \multicolumn{4}{|c|}{ UW-16 Weld Sizing Summary } \\
\hline Weld description & $\begin{array}{l}\text { Required weld } \\
\text { throat size (in) }\end{array}$ & $\begin{array}{r}\text { Actual weld } \\
\text { throat size (in) }\end{array}$ & Status \\
\hline & & & \\
\hline
\end{tabular}




\begin{tabular}{|l|l|l|l|} 
Nozzle to shell fillet $\left(\operatorname{Leg}_{41}\right)$ & 0.224 & 0.2625 & weld size is adequate \\
\hline
\end{tabular}

\section{Reinforcement Calculations for MAP}

The vessel wall thickness governs the MAP of this nozzle.

\begin{tabular}{|c|c|c|c|c|c|c|c|c|}
\hline \multicolumn{7}{|c|}{$\begin{array}{c}\text { UG-37 Area Calculation Summary }\left(\mathrm{in}^{2}\right) \\
\text { For } P=1719.91 \mathrm{psi} @ 70^{\circ} \mathrm{F} \\
\text { The opening is adequately reinforced }\end{array}$} & \multicolumn{2}{|c|}{$\begin{array}{l}\text { UG-45 Nozzle Wall } \\
\text { Thickness Summary (in) } \\
\text { The nozzle passes UG-45 }\end{array}$} \\
\hline$\underset{\text { required }}{\text { A }}$ & $\underset{\text { available }}{\mathbf{A}}$ & $\mathbf{A}_{1}$ & $\mathbf{A}_{2}$ & $\mathbf{A}_{\mathbf{3}}$ & $\mathbf{A}_{5}$ & $\begin{array}{c}\text { A } \\
\text { welds }\end{array}$ & $t_{\text {req }}$ & $\mathbf{t}_{\min }$ \\
\hline 0.5457 & 0.6698 & -- & 0.5292 & -- & -- & 0.1406 & 0.0727 & 0.35 \\
\hline
\end{tabular}

\section{UG-41 Weld Failure Path Analysis Summary}

The nozzle is exempt from weld strength calculations per UW-15(b)(1)

\begin{tabular}{|c|r|r|c|}
\hline \multicolumn{4}{|c|}{ UW-16 Weld Sizing Summary } \\
\hline Weld description & $\begin{array}{r}\text { Required weld } \\
\text { throat size (in) }\end{array}$ & $\begin{array}{r}\text { Actual weld } \\
\text { throat size (in) }\end{array}$ & Status \\
\hline Nozzle to shell fillet $\left(\mathrm{Leg}_{41}\right)$ & 0.245 & 0.2625 & weld size is adequate \\
\hline
\end{tabular}


COUPLING (C25)

ASME Section VIII Division 1, 2007 Edition

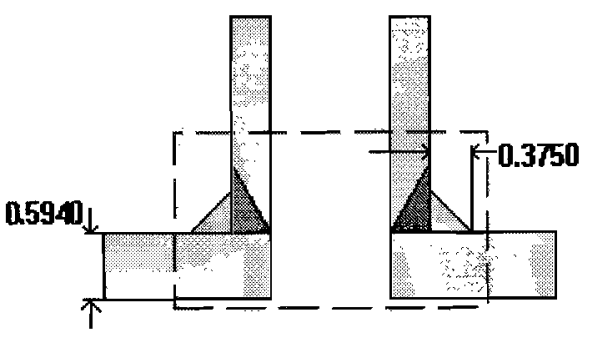

$$
\begin{aligned}
& t_{w(l o w e r)}=0 \text { in } \\
& \operatorname{Leg}_{41}=0.375 \text { in }
\end{aligned}
$$

Note: round inside edges per UG-76(c)

Located on:

Liquid static head included:

Nozzle material specification:

Nozzle longitudinal joint efficiency:

Nozzle description:

Nozzle orientation:

Local vessel minimum thickness:

Nozzle center line offset to datum line:

End of nozzle to shell center:

Nozzle inside diameter, new:

Nozzle nominal wall thickness:

Nozzle corrosion allowance:

Projection available outside vessel, Lpr: 1 in

\section{Reinforcement Calculations for Internal Pressure}

\begin{tabular}{|c|c|c|c|c|c|c|c|c|}
\hline \multicolumn{7}{|c|}{$\begin{array}{l}\text { UG-37 Area Calculation Summary }\left(\text { in }^{2}\right) \\
\text { For } P=1381.47 \text { psi @ } 700^{\circ} \mathrm{F} \\
\text { The opening is adequately reinforced }\end{array}$} & \multicolumn{2}{|c|}{$\begin{array}{l}\text { UG-45 Nozzle Wall } \\
\text { Thickness Summary (in) } \\
\text { The nozzle passes UG-45 }\end{array}$} \\
\hline $\begin{array}{c}A \\
\text { required }\end{array}$ & $\underset{\text { available }}{A}$ & $\mathbf{A}_{1}$ & $A_{2}$ & $\mathbf{A}_{3}$ & $A_{5}$ & $\begin{array}{c}\text { A } \\
\text { welds }\end{array}$ & $t_{\text {req }}$ & $t_{\min }$ \\
\hline 0.5103 & 0.5777 & -- & 0.4371 & -- & -- & 0.1406 & 0.0981 & 0.35 \\
\hline
\end{tabular}

The vessel wall thickness governs the MAWP of this nozzle.

\section{UG-41 Weld Failure Path Analysis Summary}

The nozzle is exempt from weld strength calculations per UW-15(b)(1)

\begin{tabular}{|c|c|c|c|}
\hline \multicolumn{4}{|c|}{ UW-16 Weld Sizing Summary } \\
\hline Weld description & $\begin{array}{l}\text { Required weld } \\
\text { throat size (in) }\end{array}$ & $\begin{array}{r}\text { Actual weld } \\
\text { throat size (in) }\end{array}$ & Status \\
\hline & & & \\
\hline
\end{tabular}




\begin{tabular}{|l|l|l|l|} 
Nozzle to shell fillet $\left(\operatorname{Leg}_{41}\right)$ & 0.224 & 0.2625 & weld size is adequate \\
\hline
\end{tabular}

\section{Reinforcement Calculations for MAP}

The vessel wall thickness governs the MAP of this nozzle.

\begin{tabular}{|c|c|c|c|c|c|c|c|c|}
\hline \multicolumn{7}{|c|}{$\begin{array}{c}\text { UG-37 Area Calculation Summary }\left(\mathrm{in}^{2}\right) \\
\text { For } \mathrm{P}=1719.91 \mathrm{psi}\left(\mathrm{70}^{\circ} \mathrm{F}\right. \\
\text { The opening is adequately reinforced }\end{array}$} & \multicolumn{2}{|c|}{$\begin{array}{l}\text { UG-45 Nozzle Wall } \\
\text { Thickness Summary (in) } \\
\text { The nozzle passes UG-45 }\end{array}$} \\
\hline$\underset{\text { required }}{A}$ & $\underset{\text { available }}{\mathbf{A}}$ & $\mathbf{A}_{1}$ & $\mathbf{A}_{2}$ & $\mathbf{A}_{\mathbf{3}}$ & $\mathbf{A}_{5}$ & $\begin{array}{c}\text { A } \\
\text { welds }\end{array}$ & $t_{\text {req }}$ & $\mathbf{t}_{\min }$ \\
\hline 0.5457 & 0.6698 & -- & 0.5292 & -- & -- & 0.1406 & 0.0727 & 0.35 \\
\hline
\end{tabular}

\section{UG-41 Weld Failure Path Analysis Summary}

The nozzle is exempt from weld strength calculations per UW-15(b)(1)

\begin{tabular}{|c|r|r|c|}
\hline \multicolumn{4}{|c|}{ UW-16 Weld Sizing Summary } \\
\hline Weld description & $\begin{array}{r}\text { Required weld } \\
\text { throat size (in) }\end{array}$ & $\begin{array}{r}\text { Actual weld } \\
\text { throat size (in) }\end{array}$ & Status \\
\hline Nozzle to shell fillet $\left(\mathrm{Leg}_{41}\right)$ & 0.245 & 0.2625 & weld size is adequate \\
\hline
\end{tabular}




\section{Wind Code}

\section{Building Code:}

Elevation of base above grade:

Increase effective outer diameter by: $0.0000 \mathrm{ft}$

Wind Force Coefficient Cf:

0.8500

Basic Wind Speed:, V:

Importance Factor:, I:

Exposure category:

Wind Directionality Factor, Kd:

Top Deflection Limit:

Topographic Factor, Kzt:

Enforce min. loading of $10 \mathrm{psf}$ :
$90.0000 \mathrm{mph}$

1.1500

$\mathrm{C}$

0.9500

6 in. per $100 \mathrm{ft}$.

1.0000

Yes

\section{Vessel Characteristics}

Vessel height, h:

Vessel Minimum Diameter, b

Operating, Corroded: $0.8958 \mathrm{ft}$

Empty, Corroded: $0.8958 \mathrm{ft}$

Fundamental Frequency, $\mathrm{n}_{1}$

Operating, Corroded: $7.5136 \mathrm{~Hz}$

Empty, Corroded: $8.0677 \mathrm{~Hz}$

Vacuum, Corroded: $7.5136 \mathrm{~Hz}$

Damping coefficient, $\beta$

Operating, Corroded: 0.0188

Empty, Corroded: 0.0200

Vacuum, Corroded: 0.0188

Table Lookup Values

\subsubsection{Basic Load Combinations for Allowable Stress Design}

The following load combinations are considered in accordance with ASCE section 2.4.1:

$$
\begin{array}{ll}
\text { 5. } & D+H+W \\
\text { Where } & \\
D \quad=\text { Dead load } \\
H \quad=\text { Pressure load } \\
W \quad=\text { Wind load }
\end{array}
$$

\section{Wind Deflection Reports:}

Operating, Corroded

Empty, Corroded

Wind Pressure Calculations

Wind Deflection Report: Operating, Corroded

\begin{tabular}{|l|l|l|l|l|l|l|l|} 
Elevation of & Effective & Elastic & Inertia & Platform & Total wind & bending & Deflection
\end{tabular}




\begin{tabular}{|c|c|c|c|c|c|c|c|c|}
\hline Component & $\begin{array}{c}\text { bottom above } \\
\text { base (in) }\end{array}$ & $\begin{array}{l}\text { OD } \\
\text { (ft) }\end{array}$ & $\begin{array}{c}\text { modulus } \\
\text { E }\left(10^{6} \mathrm{psi}\right)\end{array}$ & I $\left(\mathbf{f t}^{\mathbf{4}}\right)$ & $\begin{array}{l}\text { wind shear at } \\
\text { Bottom (lbf) }\end{array}$ & $\begin{array}{c}\text { shear at } \\
\text { Bottom (lbf) }\end{array}$ & $\begin{array}{c}\text { moment at } \\
\text { Bottom (lbf-ft) }\end{array}$ & at top (in) \\
\hline $\begin{array}{l}\text { 8" WELD CAP SCH } \\
80\end{array}$ & 10.7125 & 0.72 & 22.8 & * & 0 & 9 & 2 & 0 \\
\hline $\begin{array}{l}\text { REACTOR OUTER } \\
\text { SHELL (top) }\end{array}$ & 0 & 0.90 & 25.5 & 0.01081 & 0 & 21 & 16 & 0 \\
\hline $\begin{array}{l}\text { REACTOR OUTER } \\
\text { SHELL (bottom) }\end{array}$ & 0 & 0.90 & 25.5 & 0.01081 & 0 & 179 & 1,197 & 0.0161 \\
\hline
\end{tabular}

\section{Wind Deflection Report: Empty, Corroded}

\begin{tabular}{|c|c|c|c|c|c|c|c|c|}
\hline Component & $\begin{array}{c}\text { Elevation of } \\
\text { bottom above } \\
\text { base (in) }\end{array}$ & $\begin{array}{c}\text { Effective } \\
\text { OD } \\
\text { (ft) }\end{array}$ & $\begin{array}{c}\text { Elastic } \\
\text { modulus } \\
\text { E }\left(10^{6} \text { psi) }\right.\end{array}$ & $\begin{array}{c}\text { Inertia } \\
\text { I }\left(\mathbf{f t}^{4}\right)\end{array}$ & $\begin{array}{l}\text { Platform } \\
\text { wind shear at } \\
\text { Bottom (lbf) }\end{array}$ & $\begin{array}{l}\text { Total wind } \\
\text { shear at } \\
\text { Bottom (lbf) }\end{array}$ & $\begin{array}{c}\text { bending } \\
\text { moment at } \\
\text { Bottom (lbf-ft) }\end{array}$ & $\begin{array}{l}\text { Deflection } \\
\text { at top (in) }\end{array}$ \\
\hline $\begin{array}{l}\text { 8" WELD CAP SCH } \\
80\end{array}$ & 10.7125 & 0.72 & 28.3 & * & 0 & 9 & 2 & 0 \\
\hline $\begin{array}{l}\text { REACTOR OUTER } \\
\text { SHELL (top) }\end{array}$ & 0 & 0.90 & 29.4 & 0.01081 & 0 & 21 & 16 & 0 \\
\hline $\begin{array}{l}\text { REACTOR OUTER } \\
\text { SHELL (bottom) }\end{array}$ & 0 & 0.90 & 29.4 & 0.01081 & 0 & 179 & 1,197 & 0.014 \\
\hline
\end{tabular}

\section{Wind Pressure (WP) Calculations}

Gust Factor $\left(\mathrm{G}^{-}\right)$Calculations

$$
\begin{aligned}
\mathrm{Kz} & =2.01 *(\mathrm{Z} / \underline{\mathrm{Zg}})^{2 / \underline{\alpha}} \\
& =2.01 *(\mathrm{Z} / 900.0000))^{0.2105} \\
\mathrm{qz} & =0.00256 * \mathrm{Kz}^{*} \mathrm{Kzt} * \mathrm{Kd}^{*} \underline{\mathrm{V}}^{2} * \underline{\mathrm{I}} \mathrm{psf} \\
& =0.00256 * \mathrm{Kz} * 1.0000 * 0.9500 * 90.0000^{2} * 1.1500 \\
& =22.6541 * \mathrm{Kz} \\
\mathrm{WP} & =\mathrm{qz}^{*} \underline{\mathrm{G}}^{*} \underline{\mathrm{Cf}}\left(\text { Minimum } 10 \mathrm{lb} / \mathrm{ft}^{2}\right) \\
& =\mathrm{qz} * \underline{\mathrm{G}}^{-} * 0.8500\left(\text { Minimum } 10 \mathrm{lb} / \mathrm{ft}^{2}\right)
\end{aligned}
$$

\section{Design Wind Pressures}

\begin{tabular}{|c|c|c|c|c|c|c|}
\hline $\begin{array}{c}\text { Height Z } \\
(\text { ) }\end{array}$ & $\mathbf{K z}$ & $\begin{array}{c}\mathbf{q Z} \\
(\mathbf{p s f})\end{array}$ & $\begin{array}{c}\text { WP: Operating } \\
\text { (psf) }\end{array}$ & $\begin{array}{c}\text { WP: Empty } \\
\text { (psf) }\end{array}$ & $\begin{array}{c}\text { WP: hydrotest } \\
\text { (psf) }\end{array}$ & $\begin{array}{c}\text { WP: Vacuum } \\
\text { (psf) }\end{array}$ \\
\hline 15.0 & 0.8489 & 19.23 & 15.02 & 15.02 & N.A. & N.A. \\
\hline
\end{tabular}

Design Wind Force determined from: $F=$ Pressure * Af, where Af is the projected area.

\section{Gust Factor Calculations}

Operating, Corroded

Empty, Corroded

\section{Gust Factor Calculations: Operating, Corroded}




$$
\begin{aligned}
\mathrm{z}^{-} & =0.60 * \underline{\mathrm{h}} \\
& =0.60 * 1.5467 \\
& =15.0000 \\
\mathrm{I}_{\mathrm{z}^{-}} & =\underline{\mathrm{c}}^{*}(33 / \mathrm{z})^{1 / 6} \\
& =0.2000 *(33 / 15.0000)^{1 / 6} \\
& =0.2281 \\
\mathrm{~L}_{\mathrm{z}^{-}} & =\underline{1}^{*}\left(\mathrm{z}^{-} / 33\right)^{\mathrm{ep}} \\
& =500.0000 *(15.0000 / 33)^{0.2000} \\
& =427.0566 \\
\mathrm{Q} & =\operatorname{Sqr}\left(1 /\left(1+0.63 *\left((\underline{\mathrm{b}}+\underline{\mathrm{h}}) / \mathrm{L}_{\mathrm{z}}-\right)^{0.63}\right)\right) \\
& =\operatorname{Sqr}\left(1 /\left(1+0.63 *((0.8958+1.5467) / 427.0566)^{0.63}\right)\right) \\
& =0.9880 \\
\mathrm{G} & =0.925 *\left(1+1.7 * \mathrm{~g}_{\mathrm{Q}} * \mathrm{I}_{\mathrm{z}^{-}} * \mathrm{Q}\right) /\left(1+1.7 * \mathrm{~g}_{\underline{\mathrm{v}}}^{*} \mathrm{I}_{\mathrm{z}}-\right) \\
& =0.925 *\left(1+1.7 * 3.40^{*} 0.2281 * 0.9880\right) /(1+1.7 * 3.40 * 0.2281) \\
& =0.9187
\end{aligned}
$$

\section{Gust Factor Calculations: Empty, Corroded}

$$
\begin{aligned}
\mathrm{z}^{-} & =0.60 * \mathrm{~h} \\
& =0.60 * 1.5467 \\
& =15.0000 \\
\mathrm{I}_{\mathrm{z}^{-}} & =\underline{\mathrm{c}} *\left(33 / \mathrm{z}^{-}\right)^{1 / 6} \\
& =0.2000 *(33 / 15.0000)^{1 / 6} \\
& =0.2281 \\
\mathrm{~L}_{\mathrm{z}}- & =1^{*}\left(\mathrm{z}^{-} / 33\right)^{\mathrm{ep}} \\
& =500.0000 *(15.0000 / 33)^{0.2000} \\
& =427.0566 \\
\mathrm{Q} & =\mathrm{Sqr}\left(1 /\left(1+0.63 *\left((\underline{\mathrm{b}}+\underline{\mathrm{h}}) / \mathrm{L}_{\mathrm{z}}-\right)^{0.63}\right)\right) \\
& =\operatorname{Sqr}\left(1 /\left(1+0.63 *((0.8958+1.5467) / 427.0566)^{0.63}\right)\right) \\
& =0.9880 \\
\mathrm{G} & =0.925 *\left(1+1.7 * \mathrm{~g}_{\mathrm{Q}} * \mathrm{I}_{\mathrm{z}}{ }^{*} \mathrm{Q}\right) /\left(1+1.7 * \mathrm{~g}_{\underline{\mathrm{v}}} * \mathrm{I}_{\mathrm{z}}-\right) \\
& =0.925 *\left(1+1.7 * 3.40^{*} 0.2281 * 0.9880\right) /(1+1.7 * 3.40 * 0.2281) \\
& =0.9187
\end{aligned}
$$

\section{Table Lookup Values}

$$
\begin{array}{ll}
\alpha=9.5000, \mathrm{Zg}=900.0000^{\prime} & {[\text { Table 6-2, page 78] }} \\
\mathrm{c}^{\prime}=0.2000,1=500.0000, \mathrm{ep}=0.2000 & {[\text { Table 6-2, page 78] }} \\
\mathrm{a}^{-}=0.1538, \mathrm{~b}^{-}=0.6500 & {[\text { Table 6-2, page 78] }} \\
\mathrm{g}_{\mathrm{Q}}=3.40 & {[6.5 .8 .1 \text { page 26] }} \\
\mathrm{g}_{\mathrm{v}}=3.40 & {[6.5 .8 .1 \text { page 26] }}
\end{array}
$$




\section{Seismic Code}

Method of seismic analysis:

Site Class

Importance Factor:

Spectral Response Acceleration at short period (\% g)

Spectral Response Acceleration at period of $1 \mathrm{sec}(\% \mathrm{~g})$
Response Modification Coeficient from Table $15.4-2$

Acceleration based site co-efficient:

Velocity based site co-efficient:

Long-period transition period:

Redundancy factor:

User Defined Vertical Accelerations Considered:

Force Multiplier:

Minimum Weight Multiplier:
ASCE 7-05 ground supported

D

$$
\mathrm{I}=1.5000
$$

$\mathrm{S}_{\mathrm{s}}=40.00 \%$

g) $S_{1}=10.00 \%$

$\mathrm{R}=3.0000$

$\mathrm{F}_{\mathrm{a}}=1.4800$

$\mathrm{F}_{\mathrm{v}}=2.4000$

$\mathrm{T}_{\mathrm{L}}=12.0000$

$\rho=1.0000$

Yes

$=0.3333$

$=0.2000$

\subsubsection{Basic Load Combinations for Allowable Stress Design}

The following load combinations are considered in accordance with ASCE section 2.4.1:

5. $D+H+0.7 E=\left(1.0+V_{\text {Accel }}\right) D+H+0.7 \rho Q_{E}$

Where

$D \quad=$ Dead load

$H \quad=$ Pressure load

$E \quad=$ Seismic load $=\rho Q_{E}$

$V_{\text {Accel }}=$ User defined vertical acceleration

\section{Vessel Characteristics}

Vessel height: $1.5467 \mathrm{ft}$

Vessel Weight:

Operating, Corroded: 1,993 lb

Empty, Corroded: 1,993 lb

\section{Period of Vibration Calculation}

Fundamental Period, T:

Operating, Corroded: $0.133 \mathrm{sec}(\mathrm{f}=7.5 \mathrm{~Hz})$

Empty, Corroded: $0.124 \mathrm{sec}(\mathrm{f}=8.1 \mathrm{~Hz})$

The fundamental period of vibration $\mathrm{T}$ (above) is calculated using the Rayleigh method of approximation:

$\mathrm{T}=2 * \mathrm{PI} * \operatorname{Sqr}\left(\left\{\operatorname{Sum}\left(\mathrm{W}_{\mathrm{i}}^{*} \mathrm{y}_{\mathrm{i}}^{2}\right)\right\} /\left\{\mathrm{g} * \operatorname{Sum}\left(\mathrm{W}_{\mathrm{i}}^{*} \mathrm{y}_{\mathrm{i}}\right)\right\}\right)$, where

$\mathrm{W}_{\mathrm{i}}$ is the weight of the $\mathrm{i}^{\text {th }}$ lumped mass, and 
$\mathrm{y}_{\mathrm{i}}$ is its deflection when the system is treated as a cantilever beam.

\section{.Seismic Shear Reports:}

Operating, Corroded

Empty, Corroded

Base Shear Calculations

Seismic Shear Report: Operating, Corroded

\begin{tabular}{|l|r|r|r|r|r|}
\hline \multicolumn{1}{|c|}{ Component } & $\begin{array}{c}\text { Elevation of bottom } \\
\text { above base (in) }\end{array}$ & $\begin{array}{c}\text { Elastic modulus E } \\
\left(\mathbf{1 0}^{\mathbf{6}} \mathbf{p s i}\right)\end{array}$ & $\begin{array}{c}\text { Inertia I } \\
\left(\mathbf{f t}^{\mathbf{4}}\right)\end{array}$ & $\begin{array}{c}\text { Seismic shear at } \\
\text { Bottom (lbf) }\end{array}$ & $\begin{array}{c}\text { Bending Moment at } \\
\text { Bottom (lbf-ft) }\end{array}$ \\
\hline 8" WELD CAP SCH 80 & 10.7125 & $\mathbf{2 2 . 8}$ & & 3 & 1 \\
\hline REACTOR OUTER SHELL (top) & 0 & 25.5 & 0.0108 & 5 & $\mathbf{2 7 1}$ \\
\hline REACTOR OUTER SHELL (bottom) & 0 & 25.5 & 0.0108 & 2,894 \\
\hline *Moment of Inertia I varies over the length of the component \\
\hline
\end{tabular}

Seismic Shear Report: Empty, Corroded

\begin{tabular}{|c|c|c|c|c|c|}
\hline Component & $\begin{array}{l}\text { Elevation of bottom } \\
\text { above base (in) }\end{array}$ & $\begin{array}{c}\text { Elastic modulus } \mathrm{E} \\
\left(10^{6} \mathrm{psi}\right)\end{array}$ & $\begin{array}{c}\text { Inertia I } \\
\left(\text { (ft }^{4}\right)\end{array}$ & $\begin{array}{c}\text { Seismic shear at } \\
\text { Bottom (lbf) }\end{array}$ & $\begin{array}{l}\text { Bending Moment at } \\
\text { Bottom (lbf-ft) }\end{array}$ \\
\hline 8" WELD CAP SCH 80 & 10.7125 & 28.3 & * & 3 & 1 \\
\hline REACTOR OUTER SHELL (top) & 0 & 29.4 & 0.0108 & 5 & 5 \\
\hline REACTOR OUTER SHELL (bottom) & 0 & 29.4 & 0.0108 & 271 & 2,894 \\
\hline
\end{tabular}

\subsection{3: Maximum considered earthquake spectral response acceleration}

The maximum considered earthquake spectral response acceleration at short period, $\mathrm{S}_{M S}$

$\mathrm{S}_{M S}=\underline{\mathrm{F}}_{\underline{\mathrm{a}}} * \underline{\mathrm{S}}_{\underline{\mathrm{S}}}=1.4800 * 40.00 / 100=0.5920$

The maximum considered earthquake spectral response acceleration at $1 \mathrm{~s}$ period, $\mathrm{S}_{M I}$

$\mathrm{S}_{M I}=\mathrm{F}_{\underline{\mathrm{y}}} * \underline{\mathrm{S}}_{1}=2.4000 * 10.00 / 100=0.2400$

\subsection{4: Design spectral response acceleration parameters}

Design earthquake spectral response acceleration at short period, $\mathrm{S}_{D S}$

$\mathrm{S}_{D S}=2 / 3 * \mathrm{~S}_{M S}=2 / 3 * 0.5920=0.3947$

Design earthquake spectral response acceleration at $1 \mathrm{~s}$ period, $\mathrm{S}_{D I}$

$\mathrm{S}_{D I}=2 / 3 * \mathrm{~S}_{M I}=2 / 3 * 0.2400=0.1600$

\section{User Defined Vertical Acceleration Term, $\mathbf{V}_{\text {Accel }}$}

Factor is applied to dead load.

Compressive Side: $=1.0+\mathrm{V}_{\text {Accel }}$ 


\begin{tabular}{|l|r|r|r|r|}
\hline \multicolumn{5}{|c|}{$\mathbf{V}_{\text {Accel }}$ Term is: } \\
greater of (Force Mult * Base Shear / Weight) or (Min. Weight Mult.) \\
\hline \multicolumn{2}{|c|}{ Force multiplier = 0.3333} & \multicolumn{3}{|c|}{ Minimum Weight Multiplier = 0.2000} \\
\hline \multirow{2}{*}{ Condition } & Base Shear ( lbf) & Weight ( lb) & \multicolumn{2}{|c|}{ Force Mult * Shear } \\
& & & Weight & $\mathbf{V}_{\text {Accel }}$ \\
\hline Operating, Corroded & 275 & $1,992.9$ & 0.046 & 0.2 \\
\hline Operating, New & 288 & $2,082.6$ & 0.046 & 0.2 \\
\hline Empty, Corroded & 275 & $1,992.9$ & 0.046 & 0.2 \\
\hline Empty, New & 288 & $2,082.6$ & 0.046 & 0.2 \\
\hline
\end{tabular}

\section{Base Shear Calculations}

Operating, Corroded

Empty, Corroded

\section{Base Shear Calculations: Operating, Corroded}

\section{Paragraph 15.4.4: Period Determination}

Fundamental Period is taken from the Rayleigh method listed previously in this report. $\mathrm{T}=0.133 \mathrm{sec}$.

\subsection{1: Calculation of Seismic Response Coefficient}

$\mathrm{C}_{\mathrm{s}}$ is the value computed below, bounded by $\mathrm{C}_{\mathrm{s}} \mathrm{Min}$ and $\mathrm{C}_{\mathrm{s}} \mathrm{Max}$ : $\mathrm{C}_{\mathrm{s}}$ Min is 0.01 unless $\mathrm{S}_{1}>=0.6 \mathrm{~g}$, in which case eqn 12.8-6 is used. $\mathrm{C}_{\mathrm{s}} \mathrm{Max}$ calculated with $12.8-3$ because $(\mathrm{T}=0.133)<=\left(\mathrm{T}_{\mathrm{L}}=12.0000\right)$

$$
\begin{aligned}
& \mathrm{C}_{\mathrm{s}}=\underline{\mathrm{S}}_{\underline{D S}} /(\underline{\mathrm{R}} / \underline{\mathrm{I}})=0.3947 /(3.0000 / 1.5000)=0.1973 \\
& \mathrm{C}_{\mathrm{s}} \mathrm{Min}=0.01 \\
& \mathrm{C}_{\mathrm{s}} \mathrm{Max}=\underline{\mathrm{S}}_{\underline{D 1}} /(\mathrm{T} *(\underline{\mathrm{R}} / \underline{\mathrm{I}}))=0.1600 /(0.0444 *(3.0000 / 1.5000))=1.8026 \\
& \mathrm{C}_{\mathrm{s}}=0.1973
\end{aligned}
$$

\subsection{1: Calculation of Base Shear}

$$
\begin{aligned}
\mathrm{V} & =\mathrm{C}_{\mathrm{S}} * \underline{\mathrm{W}} \\
& =0.1973 * 1992.8973 \\
& =393.27 \mathrm{lb}
\end{aligned}
$$

\subsubsection{Seismic Load Combinations: Horizontal Seismic Load Effect, $E_{h}$}

$$
\begin{aligned}
\mathrm{Q}_{\mathrm{E}} & =\mathrm{V} \\
\mathrm{E}_{\mathrm{h}} & =0.7 * \rho * \mathrm{Q}_{\mathrm{E}}(\text { Only 70\% of seismic load considered as per Section 2.4.1) } \\
& =0.70 * 1.0000 * 393.27 \\
& =275.29 \mathrm{lb}
\end{aligned}
$$




\section{Base Shear Calculations: Empty, Corroded}

\section{Paragraph 15.4.4: Period Determination}

Fundamental Period is taken from the Rayleigh method listed previously in this report.

$\mathrm{T}=0.124 \mathrm{sec}$.

\subsection{1: Calculation of Seismic Response Coefficient}

$\mathrm{C}_{\mathrm{s}}$ is the value computed below, bounded by $\mathrm{C}_{\mathrm{s}}$ Min and $\mathrm{C}_{\mathrm{s}}$ Max:

$\mathrm{C}_{\mathrm{s}} \mathrm{Min}$ is 0.01 unless $\mathrm{S}_{1}>=0.6 \mathrm{~g}$, in which case eqn 12.8-6 is used.

$\mathrm{C}_{\mathrm{s}}$ Max calculated with $12.8-3$ because $(\mathrm{T}=0.124)<=\left(\mathrm{T}_{\mathrm{L}}=12.0000\right)$

$\mathrm{C}_{\mathrm{S}}=\underline{\mathrm{S}}_{\underline{D S}} /(\underline{\mathrm{R}} / \underline{\mathrm{I}})=0.3947 /(3.0000 / 1.5000)=0.1973$

$\mathrm{C}_{\mathrm{s}} \mathrm{Min}=0.01$

$\mathrm{C}_{\mathrm{s}} \mathrm{Max}=\underline{\mathrm{S}}_{\underline{D I}} /(\mathrm{T} *(\underline{\mathrm{R}} / \underline{\mathrm{I}}))=0.1600 /(0.0444 *(3.0000 / 1.5000))=1.8026$

$C_{s}=0.1973$

\subsection{1: Calculation of Base Shear}

$$
\begin{aligned}
\mathrm{V} & =\mathrm{C}_{\mathrm{s}} * \underline{\mathrm{W}} \\
& =0.1973 * 1992.8973 \\
& =393.27 \mathrm{lb}
\end{aligned}
$$

\subsubsection{Seismic Load Combinations: Horizontal Seismic Load Effect, $\mathbf{E}_{\mathbf{b}}$}

$\mathrm{Q}_{\mathrm{E}}=\mathrm{V}$

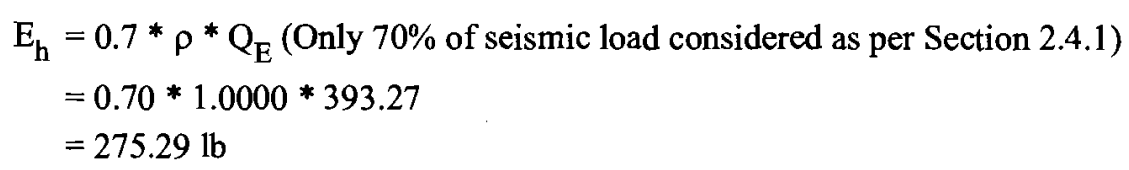




\section{SUPPORT LUGS}

Support material:

A36

This support is attached to:

Distance from baseplate to datum:

REACTOR OUTER SHELL

Local shell outer diameter, new:

162.875 in

Local shell thickness, new:

10.75 in

Local shell inner corrosion:

0.594 in

Local shell outer corrosion:

0.06 in

Lug allowable stress

Top plate width

Top plate thickness

Base plate width

Base plate thickness

Base plate load bearing width

Shell to center of load bearing area Gusset height

Gusset thickness

Gusset separation

Lug length, circumferential direction

0 in

$\mathrm{S}_{\mathrm{b}}=16,000 \mathrm{psi}$

$\mathrm{W}_{\mathrm{p}}=1.5$ in

$\mathrm{t}_{\mathrm{a}}=0.375_{\text {in }}$

$\mathrm{b}=4.625 \mathrm{in}$

$\mathrm{t}_{\mathrm{b}}=0.5$ in

$\mathrm{L}_{\mathrm{b}}=2$ in

$\mathrm{d}=3.6875 \mathrm{in}$

$\mathrm{h}=5$ in

$\mathrm{t}_{\mathrm{g}}=0.75$ in

$\mathrm{L}_{\mathrm{g}}=4$ in

$\mathrm{L}=5.5$ in

0.375 in

6.5 in

Pad width, circumferential direction:

7 in

Pad length, longitudinal direction:

Pad thickness

Pad attachment fillet weld size:

Radial/bending lug stiffness ratio:

$\mathrm{e}=0.375 \mathrm{in}$

0.25 in

Number of support lugs: 


\begin{tabular}{|c|c|c|c|c|c|c|c|c|c|c|c|c|c|}
\hline \multicolumn{14}{|c|}{ Stresses in Shell at Lug Supports } \\
\hline \multirow[b]{2}{*}{ Condition } & \multirow{2}{*}{$\begin{array}{c}\text { Total } \\
\text { Weight } \\
\text { (lb) }\end{array}$} & \multirow[b]{2}{*}{$\begin{array}{l}\text { Shear } \\
\text { V (lb) }\end{array}$} & \multirow{2}{*}{$\begin{array}{c}\text { Moment } \\
\text { M (ft- } \\
\text { lb) }\end{array}$} & \multirow[b]{2}{*}{$\begin{array}{c}\text { Lug } \\
\text { orient }\end{array}$} & \multicolumn{6}{|c|}{ Lug Loading } & \multicolumn{3}{|c|}{$\begin{array}{c}\text { Stress in Shell } \\
\text { (psi) }\end{array}$} \\
\hline & & & & & $\begin{array}{c}\mathbf{W} \\
(\mathbf{l b})\end{array} \mid$ & $\mid \begin{array}{c}\mathbf{P}_{\mathbf{r}} \\
\text { (lb) }\end{array}$ & $\begin{array}{l}V_{L} \\
\text { (lb) }\end{array}$ & $\begin{array}{c}\mathbf{M}_{\mathbf{L}} \\
\text { (ft-lb) }\end{array}$ & $\begin{array}{c}V_{c} \\
(l b)\end{array}$ & $\begin{array}{c}\mathbf{M}_{\mathrm{c}} \\
\text { (ft-Jb) }\end{array}$ & $\begin{array}{c}\text { Primary } \\
\text { Circ } \\
\left(P_{L}\right)\end{array}$ & $\begin{array}{c}\text { Primary } \\
\text { Long } \\
\left(P_{1}\right)\end{array}$ & $\begin{array}{c}\text { Combined } \\
P_{L}+P_{D}\end{array}$ \\
\hline \multirow{4}{*}{$\begin{array}{c}\text { Wind, operating, corroded } \\
\text { Attack angle }=0^{\circ}\end{array}$} & \multirow{4}{*}{1,993} & \multirow{4}{*}{200} & \multirow{4}{*}{1,181} & $0^{\circ}$ & 498 & -67 & -284 & -87.24 & 0 & 0.00 & 11,537 & 5,777 & 11,686 \\
\hline & & & & $90^{\circ}$ & 498 & 0 & 498 & 153.10 & 33 & 10.26 & 11,580 & 5,798 & 11,785 \\
\hline & & & & $180^{\circ}$ & 498 & 67 & 1,280 & 393.44 & 0 & 0.00 & 11,816 & 5,925 & 12,311 \\
\hline & & & & $270^{\circ}$ & 498 & 0 & 498 & 153.10 & -33 & 10.26 & 11,580 & 5,798 & 11,785 \\
\hline \multirow{4}{*}{$\begin{array}{c}\text { Wind, operating, corroded } \\
\text { Attack angle }=45^{\circ}\end{array}$} & \multirow{4}{*}{1,993} & \multirow{4}{*}{200} & \multirow{4}{*}{1,181} & $0^{\circ}$ & 498 & -35 & -55 & -16.85 & $|-35|$ & $10.88^{-}$ & 11,446 & 5,737 & 11,525 \\
\hline & & & & $90^{\circ}$ & 498 & -35 & -55 & -16.85 & 35 & 10.88 & 11,446 & 5,737 & 11,525 \\
\hline & & & & $180^{\circ}$ & 498 & 35 & 1,051 & 323.05 & 35 & 10.88 & 11,752 & 5,892 & 12,168 \\
\hline & & & & $270^{\circ}$ & 498 & 35 & 1,051 & 323.05 & $|-35|$ & $10.88^{-}$ & 11,752 & 5,892 & 12,168 \\
\hline \multirow{4}{*}{$\begin{array}{c}\text { Wind, operating, new } \\
\text { Attack angle }=0^{\circ}\end{array}$} & \multirow{4}{*}{2,083} & \multirow{4}{*}{200} & \multirow{4}{*}{1,181} & $0^{\circ}$ & 521 & -67 & -262 & -80.37 & 0 & 0.00 & 10,230 & 5,120 & 10,362 \\
\hline & & & & $90^{\circ}$ & 521 & 0 & 521 & 159.99 & 33 & 10.26 & 10,277 & 5,143 & 10,478 \\
\hline & & & & $180^{\circ}$ & 521 & 67 & 1,303 & 400.36 & 0 & 0.00 & 10,472 & 5,244 & 10,944 \\
\hline & & & & $270^{\circ}$ & 521 & 0 & 521 & 159.99 & -33 & 10.26 & 10,277 & 5,143 & 10,478 \\
\hline \multirow{4}{*}{$\begin{array}{l}\text { Wind, operating, new } \\
\text { Attack angle }=45^{\circ}\end{array}$} & \multirow{4}{*}{2,083} & \multirow{4}{*}{200} & \multirow{4}{*}{1,181} & $0^{\circ}$ & 521 & -35 & -32 & -9.97 & -35 & 10.88 & 10,153 & 5,091 & 10,226 \\
\hline & & & & $90^{\circ}$ & 521 & -35 & -32 & -9.97 & 35 & 10.88 & 10,153 & 5,091 & 10,226 \\
\hline & & & & $180^{\circ}$ & 521 & 35 & 1,074 & 329.96 & 35 & 10.88 & 10,420 & 5,217 & 10,818 \\
\hline & & & & $270^{\circ}$ & 521 & 35 & 1,074 & 329.96 & -35 & $10.88^{-}$ & 10,420 & 5,217 & 10,818 \\
\hline \multirow{4}{*}{$\begin{array}{l}\text { Wind, empty, corroded } \\
\text { Attack angle }=0^{\circ}\end{array}$} & & & & $0^{\circ}$ & 498 & -67 & -284 & -87.24 & 0 & 0.00 & 126 & 71 & 337 \\
\hline & & & & $90^{\circ}$ & 498 & 0 & 498 & \begin{tabular}{|l}
153.10 \\
\end{tabular} & 33 & 10.26 & -169 & -92 & 447 \\
\hline & 1,993 & 200 & 1,181 & $180^{\circ}$ & 498 & 67 & 1,280 & 393.44 & 0 & 0.00 & -465 & -255 & $-1,231$ \\
\hline & & & & $270^{\circ}$ & 498 & 0 & 498 & 153.10 & -33 & 10.26 & -169 & -92 & 447 \\
\hline & & & & $0^{\circ}$ & 498 & $|-35|$ & -55 & -16.85 & -35 & $10.88^{-}$ & 35 & 31 & 116 \\
\hline Wind, empty, corroded & 1003 & 200 & 181 & $90^{\circ}$ & 498 & -35 & -55 & -16.85 & 35 & 10.88 & 35 & 31 & 116 \\
\hline Attack angle $=45^{\circ}$ & & & 1,101 & $180^{\circ}$ & 498 & 35 & 1,051 & 323.05 & 35 & 10.88 & -373 & -204 & -987 \\
\hline & & & & $270^{\circ}$ & 498 & 35 & 1,051 & 323.05 & -35 & $10.88^{-}$ & -373 & -204 & -987 \\
\hline & & & & $0^{\circ}$ & 521 & -67 & -262 & -80.37 & 0 & 0.00 & 99 & 54 & 280 \\
\hline & & & & $90^{\circ}$ & 521 & 0 & 521 & 159.99 & 33 & 10.26 & -146 & -77 & 419 \\
\hline Attack angle $=0^{\circ}$ & 2,083 & 200 & 1,181 & $180^{\circ}$ & 521 & 67 & 1,303 & 400.36 & 0 & 0.00 & -391 & -208 & $-1,117$ \\
\hline & & & & $270^{\circ}$ & 521 & 0 & 521 & 159.99 & -33 & $10.26^{-}$ & -146 & -77 & 419 \\
\hline & & & & $0^{\circ}$ & 521 & -35 & -32 & -9.97 & -35 & $10.88^{-}$ & 22 & 25 & 96 \\
\hline $\begin{array}{l}\text { Wind, empty, new } \\
\text { Attack angle }=\mathbf{4 5 ^ { \circ }}\end{array}$ & 2,083 & 200 & 1,181 & $90^{\circ}$ & 521 & -35 & -32 & -9.97 & 35 & 10.88 & 22 & 25 & 96 \\
\hline & & & & $180^{\circ}$ & 521 & 35 & 1,074 & 329.96 & 35 & 10.88 & -315 & -167 & -901 \\
\hline & & & & & & & & & & & & & \\
\hline
\end{tabular}




\begin{tabular}{|c|c|c|c|c|c|c|c|c|c|c|c|c|c|}
\hline & & & & $270^{\circ}$ & 521 & 35 & 1,074 & 329.96 & -35 & 10.88 & -315 & -167 & -901 \\
\hline \multirow{4}{*}{$\begin{array}{c}\text { Seismic, operating, } \\
\text { corroded } \\
\text { Attack angle }=0^{\circ}\end{array}$} & \multirow{4}{*}{1,993} & \multirow{4}{*}{275} & \multirow{4}{*}{2,889} & $0^{\circ}$ & 498 & -92 & 1,415 & 434.74 & 0 & 0.00 & 11,933 & 5,992 & 12,560 \\
\hline & & & & $90^{\circ}$ & 598 & 0 & 598 & 183.72 & 46 & 14.10 & 11,614 & 5,817 & 11,860 \\
\hline & & & & $180^{\circ}$ & 598 & 92 & 2,511 & 771.56 & 0 & 0.00 & 12,221 & 6,147 & 13,210 \\
\hline & & & & $270^{\circ}$ & 598 & 0 & 598 & 183.72 & -46 & $14.10^{-}$ & 11,614 & 5,817 & 11,860 \\
\hline \multirow{4}{*}{$\begin{array}{c}\text { Seismic, operating, } \\
\quad \text { corroded } \\
\text { Attack angle }=45^{\circ}\end{array}$} & \multirow{4}{*}{1,993} & \multirow{4}{*}{275} & \multirow{4}{*}{2,889} & $0^{\circ}$ & 498 & -49 & -854 & 262.57 & -49 & 14.95 & 11,723 & 5,877 & 12,099 \\
\hline & & & & $90^{\circ}$ & 498 & -49 & -854 & 262.57 & 49 & 14.95 & 11,723 & 5,877 & 12,099 \\
\hline & & & & $180^{\circ}$ & 598 & 49 & 1,951 & 599.39 & 49 & 14.95 & 12,051 & 6,054 & 12,830 \\
\hline & & & & $270^{\circ}$ & 598 & 49 & 1,951 & 599.39 & -49 & 14.95 & 12,051 & 6,054 & 12,830 \\
\hline \multirow{4}{*}{$\begin{array}{l}\text { Seismic, operating, new } \\
\text { Attack angle }=0^{\circ}\end{array}$} & \multirow{4}{*}{2,083} & \multirow{4}{*}{288} & \multirow{4}{*}{3,001} & $0^{\circ}$ & 521 & -96 & 1,466 & $450.62^{-}$ & 0 & 0.00 & 10,579 & 5,305 & 11,189 \\
\hline & & & & $90^{\circ}$ & 625 & 0 & 625 & 191.99 & 48 & 14.73 & 10,307 & 5,159 & 10,548 \\
\hline & & & & $180^{\circ}$ & 625 & 96 & 2,612 & 802.60 & 0 & 0.00 & 10,829 & 5,431 & 11,792 \\
\hline & & & & $270^{\circ}$ & 625 & 0 & 625 & 191.99 & -48 & 14.73 & 10,307 & 5,159 & 10,548 \\
\hline \multirow{4}{*}{$\begin{array}{l}\text { Seismic, operating, new } \\
\text { Attack angle }=45^{\circ}\end{array}$} & \multirow{4}{*}{2,083} & \multirow{4}{*}{288} & \multirow{4}{*}{3,001} & $0^{\circ}$ & 521 & -51 & -884 & $271.77^{-}$ & -51 & $15.63^{-}$ & 10,399 & 5,209 & 10,763 \\
\hline & & & & $90^{\circ}$ & 521 & -51 & -884 & $271.77^{-}$ & 51 & 15.63 & 10,399 & 5,209 & 10,763 \\
\hline & & & & $180^{\circ}$ & 625 & 51 & 2,030 & 623.76 & 51 & 15.63 & 10,683 & 5,355 & 11,443 \\
\hline & & & & $270^{\circ}$ & 625 & 51 & 2,030 & 623.76 & -51 & 15.63 & 10,683 & 5,355 & 11,443 \\
\hline \multirow{4}{*}{$\begin{array}{c}\text { Seismic, empty, cor roded } \\
\text { Attack angle }=\infty^{\infty}\end{array}$} & \multirow{4}{*}{1,993} & \multirow{4}{*}{275} & \multirow{4}{*}{2,889} & $0^{\circ}$ & 498 & -92 & 1,415 & $434.74^{-}$ & 0 & 0.00 & 522 & 286 & 1,381 \\
\hline & & & & $90^{\circ}$ & 598 & 0 & 598 & 183.72 & 46 & 14.10 & -203 & -111 & -538 \\
\hline & & & & $180^{\circ}$ & 598 & 92 & 2,511 & 771.56 & 0 & 0.00 & -894 & -489 & $-2,366$ \\
\hline & & & & $270^{\circ}$ & 598 & 0 & 598 & 183.72 & -46 & $14.10^{-}$ & -203 & -111 & -538 \\
\hline \multirow{4}{*}{$\begin{array}{c}\text { Seismic, empty, corroded } \\
\text { Attack angle }=45^{\circ}\end{array}$} & \multirow{4}{*}{1,993} & \multirow{4}{*}{275} & \multirow{4}{*}{2,889} & $0^{\circ}$ & 498 & -49 & -854 & 262.57 & -49 & 14.95 & 312 & 171 & 826 \\
\hline & & & & $90^{\circ}$ & 498 & -49 & -854 & $262.57^{-}$ & 49 & 14.95 & 312 & 171 & 826 \\
\hline & & & & $180^{\circ}$ & 598 & 49 & 1,951 & 599.39 & 49. & 14.95 & -684 & -374 & $-1,811$ \\
\hline & & & & $270^{\circ}$ & 598 & 49 & 1,951 & 599.39 & -49 & 14.95 & -684 & -374 & $-1,811$ \\
\hline \multirow{4}{*}{$\begin{array}{l}\text { Seismic, empty, new } \\
\text { Attack angle }=0^{\circ}\end{array}$} & \multirow{4}{*}{2,083} & & & $0^{\circ}$ & 521 & -96 & 1,466 & 450.62 & 0 & 0.00 & 448 & 239 & 1,279 \\
\hline & & 288 & 3001 & $90^{\circ}$ & 625 & 0 & 625 & 191.99 & 48 & 14.73 & -176 & -93 & 503 \\
\hline & & 288 & 3,001 & $180^{\circ}$ & 625 & 96 & 2,612 & 802.60 & 0 & 0.00 & -770 & -409 & $-2,201$ \\
\hline & & & & $270^{\circ}$ & 625 & 0 & 625 & 191.99 & -48 & 14.73 & -176 & -93 & 503 \\
\hline & & & & $0^{\circ}$ & 521 & -51 & -884 & $271.77^{-}$ & -51 & 15.63 & 268 & 143 & 764 \\
\hline $\begin{array}{l}\text { Seismic, empty, new } \\
\text { Attack angle }=45^{\circ}\end{array}$ & 2,083 & 288 & 3,001 & $90^{\circ}$ & 521 & -51 & -884 & $271.77^{-}$ & 51 & 15.63 & 268 & 143 & 764 \\
\hline & & & & $180^{\circ}$ & 625 & 51 & 2,030 & 623.76 & 51 & 15.63 & -590 & -313 & $-1,686$ \\
\hline
\end{tabular}


P.O.\#: 700521452

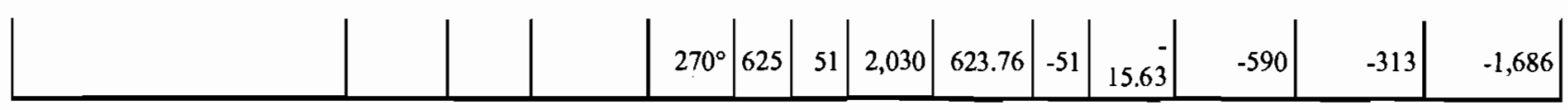


Applied Loads (Seismic, operating, corroded, Attack angle $=0^{\circ}$, lug orientation $=180^{\circ}$ )

$\begin{array}{lrc}\text { Radial load: } & \mathrm{P}_{\mathrm{r}}= & 91.76 \mathrm{lb}_{\mathrm{f}} \\ \text { Circumferential moment: } & \mathrm{M}_{\mathrm{c}}= & 0 \mathrm{lb}_{\mathrm{f}} \text { in } \\ \text { Circumferential shear: } & \mathrm{V}_{\mathrm{c}}= & 0 \mathrm{lb}_{\mathrm{f}} \\ \text { Longitudinal moment: } & \mathrm{M}_{\mathrm{L}}= & 9,258.76 \mathrm{lb}_{\mathrm{f}} \text { in } \\ \text { Longitudinal shear: } & \mathrm{V}_{\mathrm{L}}= & 2,510.85 \mathrm{lb}_{\mathrm{f}} \\ \text { Torsion moment: } & \mathrm{M}_{\mathrm{t}}= & 0 \mathrm{lb}_{\mathrm{f}} \text { in } \\ \text { Internal pressure: } & \mathrm{P}=1,258.749 \mathrm{psi} \\ \text { Mean shell radius: } & \mathrm{R}_{\mathrm{m}}= & 5.108 \mathrm{in} \\ \text { Local shell thickness: } & \mathrm{t}= & 0.534 \mathrm{in} \\ \text { Shell yield stress: } & \mathrm{S}_{\mathrm{y}}= & 25,100 \mathrm{psi}\end{array}$

Maximum stresses due to the applied loads at the pad edge (includes pressure)

$\mathrm{R}_{\mathrm{m}} / \mathrm{t}=9.5655$

$\mathrm{C}_{1}=3.25, \mathrm{C}_{2}=3.5$ in

Local circumferential pressure stress $=\mathrm{P}^{*} \mathrm{R}_{\mathrm{i}} / \mathrm{t}=11,411 \mathrm{psi}$

Local longitudinal pressure stress $=\mathrm{P}^{*} \mathrm{R}_{\mathrm{i}} / 2 \mathrm{t}=5,706 \mathrm{psi}$

Maximum combined stress $\left(\mathrm{P}_{\mathrm{L}}+\mathrm{P}_{\mathrm{b}}\right)=13,210 \mathrm{psi}$

Allowable combined stress $\left(\mathrm{P}_{\mathrm{L}}+\mathrm{P}_{\mathrm{b}}\right)=+-1.5^{*} \mathrm{~S}=+-23,400 \mathrm{psi}$

The maximum combined stress $\left(P_{L}+P_{b}\right)$ is within allowable limits.

Maximum local primary membrane stress $\left(\mathrm{P}_{\mathrm{L}}\right)=12,221 \mathrm{psi}$

Allowable local primary membrane $\left(P_{L}\right)=+-1.5 * S=+-23,400$ psi

The maximum local primary membrane stress $\left(\mathrm{P}_{\mathrm{L}}\right)$ is within allowable limits. 


\begin{tabular}{|c|c|c|c|c|c|c|c|c|c|c|}
\hline \multicolumn{11}{|c|}{ Stresses at the pad edge per WRC Bulletin 107} \\
\hline Figure & value & $\beta$ & $\mathbf{A}_{\mathbf{u}}$ & $\mathbf{A}_{\mathbf{l}}$ & $\mathbf{B}_{\mathbf{u}}$ & $\mathbf{B}_{1}$ & $\mathbf{C}_{\mathbf{u}}$ & $\mathbf{C}_{\mathbf{I}}$ & $\mathbf{D}_{\mathbf{u}}$ & $\mathbf{D}_{\mathbf{I}}$ \\
\hline $3 C^{*}$ & 0.7278 & 0.6905 & 0 & 0 & 0 & 0 & -24 & -24 & -24 & -24 \\
\hline $4 C^{*}$ & 1.2339 & 0.6729 & -42 & -42 & -42 & -42 & 0 & 0 & 0 & 0 \\
\hline $1 \mathrm{C}$ & 0.0566 & 0.6527 & 0 & 0 & 0 & 0 & -109 & 109 & -109 & 109 \\
\hline $2 \mathrm{C}-1$ & 0.0231 & 0.6527 & -45 & 45 & -45 & 45 & 0 & 0 & 0 & 0 \\
\hline $3 A^{*}$ & 0.4426 & 0.6522 & 0 & 0 & 0 & 0 & 0 & 0 & 0 & 0 \\
\hline $1 \mathrm{~A}$ & 0.0779 & 0.7444 & 0 & 0 & 0 & 0 & 0 & 0 & 0 & 0 \\
\hline $3 B^{*}$ & 0.9652 & 0.6685 & -852 & -852 & 852 & 852 & 0 & 0 & 0 & 0 \\
\hline 1B-1 & 0.0182 & 0.6716 & $-1,034$ & 1,034 & 1,034 & $-1,034$ & 0 & 0 & 0 & 0 \\
\hline \multicolumn{3}{|c|}{ Pressure stress } & 11,411 & 11,411 & 11,411 & 11,411 & 11,411 & 11,411 & 11,411 & 11,411 \\
\hline \multicolumn{3}{|c|}{$\begin{array}{c}\text { Total circumferential } \\
\text { stress }\end{array}$} & 9,438 & 11,596 & 13,210 & 11,232 & 11,278 & 11,496 & 11,278 & 11,496 \\
\hline \multicolumn{3}{|c|}{$\begin{array}{c}\text { Primary membrane } \\
\text { circumferential stress* }\end{array}$} & 10,517 & 10,517 & 12,221 & 12,221 & 11,387 & 11,387 & 11,387 & 11,387 \\
\hline $3 C^{*}$ & 0.7278 & 0.6729 & -24 & -24 & -24 & -24 & 0 & 0 & 0 & 0 \\
\hline $4 C^{*}$ & 1.2339 & 0.6905 & 0 & 0 & 0 & 0 & -42 & -42 & -42 & -42 \\
\hline $1 \mathrm{C}-1$ & 0.0448 & 0.676 & -86 & 86 & -86 & 86 & 0 & 0 & 0 & 0 \\
\hline $2 \mathrm{C}$ & 0.0354 & 0.676 & 0 & 0 & 0 & 0 & -68 & 68 & -68 & 68 \\
\hline $4 A^{*}$ & \begin{tabular}{|l|}
1.0387 \\
\end{tabular} & 0.6522 & 0 & 0 & 0 & 0 & 0 & 0 & 0 & 0 \\
\hline $2 \mathrm{~A}$ & 0.0418 & 0.7807 & 0 & 0 & 0 & 0 & 0 & 0 & 0 & 0 \\
\hline $4 B^{*}$ & 0.4574 & 0.6685 & -465 & -465 & 465 & 465 & 0 & 0 & 0 & 0 \\
\hline 2B-1 & 0.034 & 0.724 & $-1,791$ & 1,791 & 1,791 & $-1,791$ & 0 & 0 & 0 & 0 \\
\hline \multicolumn{3}{|c|}{ Pressure stress* } & 5,706 & 5,706 & 5,706 & 5,706 & 5,706 & 5,706 & 5,706 & 5,706 \\
\hline \multicolumn{3}{|c|}{ Total longitudinal stress } & 3,340 & 7,094 & 7,852 & 4,442 & 5,596 & 5,732 & 5,596 & 5,732 \\
\hline \multicolumn{3}{|c|}{$\begin{array}{l}\text { Primary membrane } \\
\text { longitudinal stress * }\end{array}$} & 5,217 & 5,217 & 6,147 & 6,147 & 5,664 & 5,664 & 5,664 & 5,664 \\
\hline \multicolumn{3}{|c|}{ Shear from $M_{t}$} & 0 & 0 & 0 & 0 & 0 & 0 & 0 & 0 \\
\hline \multicolumn{3}{|c|}{ Circ shear from $V_{c}$} & 0 & 0 & 0 & 0 & 0 & 0 & 0 & 0 \\
\hline \multicolumn{3}{|c|}{ Long shear from $V_{L}$} & 0 & 0 & 0 & 0 & -336 & -336 & 336 & 336 \\
\hline \multicolumn{3}{|c|}{ Total Shear stress } & 0 & 0 & 0 & 0 & -336 & -336 & 336 & 336 \\
\hline \multicolumn{3}{|c|}{$\begin{array}{c}\text { Combined stress } \\
\qquad\left(\mathbf{P}_{\mathrm{L}}+\mathbf{P}_{\mathrm{b}}\right)\end{array}$} & 9,438 & 11,596 & 13,210 & 11,232 & 11,298 & 11,516 & 11,298 & 11,516 \\
\hline
\end{tabular}

Note: * denotes primary stress.

Maximum stresses due to the applied loads at the lug edge (includes pressure)

$R_{m} / t=5.6194$

$\mathrm{C}_{1}=2.75, \mathrm{C}_{2}=2.9375$ in

Local circumferential pressure stress $=\mathrm{P}^{*} \mathrm{R}_{\mathrm{i}} / \mathrm{t}=11,411 \mathrm{psi}$ 
Local longitudinal pressure stress $=\mathrm{P}^{*} \mathrm{R}_{\mathrm{i}} / 2 \mathrm{t}=5,706 \mathrm{psi}$

Maximum combined stress $\left(\mathrm{P}_{\mathrm{L}}+\mathrm{P}_{\mathrm{b}}\right)=12,453 \mathrm{psi}$

Allowable combined stress $\left(\mathrm{P}_{\mathrm{L}}+\mathrm{P}_{\mathrm{b}}\right)=+-1.5 * \mathrm{~S}=+-23,400 \mathrm{psi}$

The maximum combined stress $\left(\mathrm{P}_{\mathrm{L}}+\mathrm{P}_{\mathrm{b}}\right)$ is within allowable limits.

Maximum local primary membrane stress $\left(\mathrm{P}_{\mathrm{L}}\right)=11,796 \mathrm{psi}$

Allowable local primary membrane $\left(\mathrm{P}_{\mathrm{L}}\right)=+-1.5 * \mathrm{~S}=+-23,400 \mathrm{psi}$

The maximum local primary membrane stress $\left(\mathrm{P}_{\mathrm{L}}\right)$ is within allowable limits. 


\begin{tabular}{|c|c|c|c|c|c|c|c|c|c|c|}
\hline \multicolumn{11}{|c|}{ Stresses at the lug edge per WRC Bulletin 107} \\
\hline Figure & value & $\beta$ & $\mathbf{A}_{\mathbf{u}}$ & $\mathbf{A}_{\mathrm{l}}$ & $\mathbf{B}_{\mathbf{u}}$ & $\mathbf{B}_{1}$ & $\mathbf{C}_{\mathbf{u}}$ & $\mathbf{C}_{1}$ & $\mathbf{D}_{\mathbf{u}}$ & $\mathbf{D}_{1}$ \\
\hline $3 C^{*}$ & 0.6173 & 0.5792 & 0 & 0 & 0 & 0 & -12 & -12 & -12 & -12 \\
\hline $4 C^{*}$ & 0.8157 & 0.5659 & -16 & -16 & -16 & -16 & 0 & 0 & 0 & 0 \\
\hline $1 \mathrm{C}$ & 0.0578 & 0.5507 & 0 & 0 & 0 & 0 & -39 & 39 & -39 & 39 \\
\hline $2 \mathrm{C}-1$ & 0.0377 & 0.5507 & -25 & 25 & -25 & 25 & 0 & 0 & 0 & 0 \\
\hline $3 A^{*}$ & 0.2848 & 0.5503 & 0 & 0 & 0 & 0 & 0 & 0 & 0 & 0 \\
\hline $1 \mathrm{~A}$ & 0.0858 & 0.6287 & 0 & 0 & 0 & 0 & 0 & 0 & 0 & 0 \\
\hline $3 \mathrm{~B}^{*}$ & 0.6496 & 0.5626 & -401 & -401 & 401 & 401 & 0 & 0 & 0 & 0 \\
\hline $1 \mathrm{~B}-1$ & 0.0293 & 0.5655 & -682 & 682 & 682 & -682 & 0 & 0 & 0 & 0 \\
\hline \multicolumn{3}{|c|}{ Pressure stress* } & 11,411 & 11,411 & 11,411 & 11,411 & 11,411 & 11,411 & 11,411 & 11,411 \\
\hline \multicolumn{3}{|c|}{$\begin{array}{c}\text { Total circumferential } \\
\text { stress }\end{array}$} & 10,287 & 11,701 & 12,453 & 11,139 & 11,360 & 11,438 & 11,360 & 11,438 \\
\hline \multicolumn{3}{|c|}{$\begin{array}{l}\text { Primary membrane } \\
\text { circumferential stress* }\end{array}$} & 10,994 & 10,994 & 11,796 & 11,796 & 11,399 & 11,399 & 11,399 & 11,399 \\
\hline $3 C^{*}$ & 0.6173 & 0.5659 & -12 & -12 & -12 & -12 & 0 & 0 & 0 & 0 \\
\hline $4 C^{*}$ & 0.8157 & 0.5792 & 0 & 0 & 0 & 0 & -16 & -16 & -16 & -16 \\
\hline 1C-1 & 0.0562 & 0.5683 & -37 & 37 & -37 & 37 & 0 & 0 & 0 & 0 \\
\hline $2 \mathrm{C}$ & 0.0394 & 0.5683 & 0 & 0 & 0 & 0 & -26 & 26 & -26 & 26 \\
\hline $4 A^{*}$ & 0.5731 & 0.5503 & 0 & 0 & 0 & 0 & 0 & 0 & 0 & 0 \\
\hline $2 \mathrm{~A}$ & 0.0489 & 0.6572 & 0 & 0 & 0 & 0 & 0 & 0 & 0 & 0 \\
\hline $4 B^{*}$ & 0.2522 & 0.5626 & -179 & -179 & 179 & 179 & 0 & 0 & 0 & 0 \\
\hline 2B-1 & 0.0522 & 0.6091 & $-1,128$ & 1,128 & 1,128 & \begin{tabular}{|l}
$-1,128$ \\
\end{tabular} & 0 & 0 & 0 & 0 \\
\hline \multicolumn{3}{|c|}{ Pressure stress* } & 5,706 & 5,706 & 5,706 & 5,706 & 5,706 & 5,706 & 5,706 & 5,706 \\
\hline \multicolumn{3}{|c|}{ Total longitudinal stress } & 4,350 & 6,680 & 6,964 & 4,782 & 5,664 & 5,716 & 5,664 & 5,716 \\
\hline \multicolumn{3}{|c|}{$\begin{array}{l}\text { Primary membrane } \\
\text { longitudinal stress* }\end{array}$} & 5,515 & 5,515 & 5,873 & 5,873 & 5,690 & 5,690 & 5,690 & 5,690 \\
\hline \multicolumn{3}{|c|}{ Shear from $M_{t}$} & 0 & 0 & 0 & 0 & 0 & 0 & 0 & 0 \\
\hline \multicolumn{3}{|c|}{ Circ shear from $v_{c}$} & 0 & 0 & 0 & 0 & 0 & 0 & 0 & 0 \\
\hline \multicolumn{3}{|c|}{ Long shear from $V_{L}$} & 0 & 0 & 0 & 0 & -235 & -235 & 235 & 235 \\
\hline \multicolumn{3}{|c|}{ Total Shear stress } & 0 & 0 & 0 & 0 & -235 & -235 & 235 & 235 \\
\hline \multicolumn{3}{|c|}{$\begin{array}{c}\text { Combined stress } \\
\qquad\left(P_{L}+P_{b}\right)\end{array}$} & 10,287 & 11,701 & 12,453 & 11,139 & 11,370 & 11,448 & 11,370 & 11,448 \\
\hline
\end{tabular}

Note: * denotes primary stress. 
Lug top plate required thickness, Bednar 5.2

$$
\begin{aligned}
& \mathrm{t}_{\mathrm{a}}=0.75 *\left(\mathrm{~V}_{\mathrm{L}}{ }^{*} \mathrm{~d} * \mathrm{~L}\right) /\left(\mathrm{S}_{\mathrm{b}} * \mathrm{~W}_{\mathrm{p}}{ }^{2 *} \mathrm{~h}\right) \\
& =0.75 *(2,611.86 * 3.6875 * 5.5) /\left(16,000 * 1.5^{2 * 5}\right) \\
& =0.2207 \mathrm{in}
\end{aligned}
$$

The top plate thickness of 0.375 in is adequate.

\section{Gusset plate required thickness, Bednar 5.2}

$$
\begin{aligned}
& \mathrm{S}_{\mathrm{c}}=12,000 /\left(1+(1 / 12,000)^{*}\left(\mathrm{~h} /\left(0.289 * \mathrm{t}_{\mathrm{g}}\right)\right)^{2}\right) \\
& =12,000 /\left(1+(1 / 12,000)^{*}(5 /(0.289 * 0.75))^{2}\right) \\
& =11,490 \mathrm{psi} \\
& \mathrm{t}_{\mathrm{g}}=\mathrm{V}_{\mathrm{L}}{ }^{*}(3 * \mathrm{~d}-\mathrm{b}) /\left(\mathrm{S}_{\mathrm{c}} * \mathrm{~b}^{2 *} \operatorname{Sin}(\alpha)^{2}\right) \\
& =2,611.86 *(3 * 3.6875-4.625) /\left(11,490 * 4.625^{2} * \operatorname{Sin}(57.995)^{2}\right) \\
& =0.0951 \mathrm{in}
\end{aligned}
$$

The gusset thickness of 0.75 in is adequate.

\section{Lug base plate required thickness}

From Escoe table 4-8 $(1 / \mathrm{b}=1.1563)$

$$
\begin{aligned}
& C_{x}=0.10526, C_{y}=-0.12056 \\
& f_{c}=V_{L} /\left(L_{b}{ }^{*} L\right) \\
& =2,611.86 /(2 * 5.5) \\
& =237 \mathrm{psi} \\
& M_{x}=C_{x}{ }^{*} f_{c}{ }^{*} L_{g}{ }^{2} \\
& =0.10526 * 237 * 4 \\
& =399.89 \text { in- }-1 b_{f} / \text { in } \\
& M_{y}=C_{y}{ }^{*} f_{c}{ }^{*} L_{b}{ }^{2} \\
& =-0.12056 * 237 * 2 \\
& =-114.5 \text { in- }-1 b_{f} / \text { in } \\
& M=\operatorname{Max}\left[A b s\left(M_{x}\right), A b s\left(M_{y}\right)\right] \\
& =\operatorname{Max}[\operatorname{Abs}(399.89), \operatorname{Abs}(-114.5)] \\
& =399.89 \text { lb-ft } \\
& t_{b}=\operatorname{Sqr}\left(6 * M / S_{b}\right) \\
& =\operatorname{Sqr}(6 * 399.9 / 16,000) \\
& =0.3872 \text { in }
\end{aligned}
$$

The base plate thickness of 0.5 in is adequate.

\section{Support Lug to Pad Fillet Weld Sizing - Bednar chapters 5.2 and 10.3}

Note: continuous welding is assumed for all support lug fillet welds. 
$d_{h}=t_{a}+h+t_{b}$

$\mathrm{L}_{\mathrm{w}}=2 *\left(\mathrm{~b}+\mathrm{d}_{\mathrm{h}}\right)=2 *(5.5+5.875)=22.75$ in

$\mathrm{Z}_{\mathrm{w}}=\mathrm{b} * \mathrm{~d}_{\mathrm{h}}+\mathrm{d}_{\mathrm{h}}{ }^{2} / 3=5.5^{*} 5.875+5.875^{2} / 3=43.82 \mathrm{in}^{2}$

$\mathrm{Z}_{\mathrm{z}}=\mathrm{d}_{\mathrm{h}}{ }^{*} \mathrm{~b}+\mathrm{b}^{2} / 3=5.875^{*} 5.5+5.5^{2} / 3=42.4 \mathrm{in}^{2}$

Shear $\mathrm{f}_{1}=\mathrm{V}_{\mathrm{L}} / \mathrm{L}_{\mathrm{w}}=2,611.86 / 22.75=115 \mathrm{lb} / \mathrm{in}$

Shear $f_{2}=V_{c} / L_{w}=0 / 22.75=0 \mathrm{lb} /$ in

Bending $f_{3}=$ larger absolute value of $M_{L} / Z_{w}$ or $M_{c} / Z_{z}$

$=\mathrm{M}_{\mathrm{L}} / \mathrm{Z}_{\mathrm{w}}$

$=9,631.2 / 43.82$

$=219.8 \mathrm{lb} / \mathrm{in}$

Resultant load $\mathrm{f}=\left(\mathrm{f}_{1}^{2}+\mathrm{f}_{2}^{2}+\mathrm{f}_{3}^{2}\right)^{1 / 2}$

$=\left(114.81^{2}+0^{2}+219.8^{2}\right)^{1 / 2}$

$=247.98 \mathrm{lb} / \mathrm{in}$

Required weld size $\mathrm{w}=\mathrm{F} /\left(0.707^{*} 0.55^{*} \mathrm{~S}_{\mathrm{a}}\right)$

$=247.98 /(0.707 * 0.55 * 15,600)$

$=0.0409$ in

The support lug fillet weld size of 0.375 in is adequate.

Support Lug Pad to Shell Fillet Weld Sizing - Bednar chapters 5.2 and 10.3

$\mathrm{L}_{\mathrm{w}}=2 *\left(\mathrm{~b}+\mathrm{d}_{\mathrm{h}}\right)=2 *(6.5+7)=27$ in

$\mathrm{Z}_{\mathrm{w}}=\mathrm{b}^{*} \mathrm{~d}_{\mathrm{h}}+\mathrm{d}_{\mathrm{h}}{ }^{2 / 3}=6.5^{*} 6.5+7^{2} / 3=61.83 \mathrm{in}^{2}$

$\mathrm{Z}_{\mathrm{z}}=\mathrm{d}_{\mathrm{h}}{ }^{*} \mathrm{~b}+\mathrm{b}^{2} / 3=7^{*} 6.5+6.5^{2} / 3=59.58 \mathrm{in}^{2}$

Shear $\mathrm{f}_{1}=\mathrm{V}_{\mathrm{L}} / \mathrm{L}_{\mathrm{w}}=2,611.86 / 27=97 \mathrm{lb} /$ in

Shear $\mathrm{f}_{2}=\mathrm{V}_{\mathrm{c}} / \mathrm{L}_{\mathrm{w}}=0 / 27=0 \mathrm{lb} / \mathrm{in}$

Bending $f_{3}=$ larger absolute value of $M_{L} / Z_{w}$ or $M_{c} / Z_{z}$

$=\mathrm{M}_{\mathrm{L}} / \mathrm{Z}_{\mathrm{w}}$

$=9,631.2 / 61.83$

$=155.76 \mathrm{lb} / \mathrm{in}$

Resultant load $\mathrm{f}=\left(\mathrm{f}_{1}^{2}+\mathrm{f}_{2}{ }^{2}+\mathrm{f}_{3}{ }^{2}\right)^{1 / 2}$

$=\left(96.74^{2}+0^{2}+155.76^{2}\right)^{1 / 2}$

$=183.36 \mathrm{lb} / \mathrm{in}$

Required weld size $w=F /\left(0.707 * 0.55^{*} \mathrm{~S}_{\mathrm{a}}\right)$

$=183.36 /(0.707 * 0.55 * 15,600)$

$=0.0302 \mathrm{in}$

The support lug pad fillet weld size of 0.25 in is adequate. 


\section{FLANGE DESIGN BASED ON ASME SECTION VIII DIV. 1. APP. 2}

\section{FLANGE DESCRIPTION: REVERSE FLANGE}

REACTOR OUTLET SHELL, FOOT

FIG. 2-13.2 LOOSE RING TYPE REVERSE FLANGE

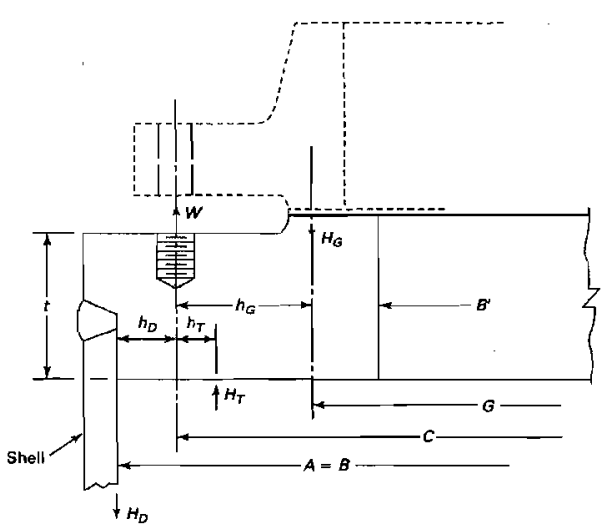

NOTE: EXTERNAL LOADS ON FLANGES NOT CONSIDERED IN DESIGN CALCLILATIONS.

NOTE: FLANGE THICKNESS (t) INCLLIDES 1/16" RAISED FACE.

(1a)

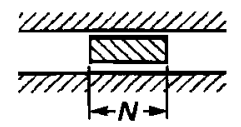

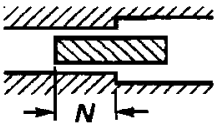

NOTE: FLANGE IS SA-516-70 MATERIAL DESIGN TEMP: 700 DEG. $F$

FLANGE THICKNESS (t): 2.375"

\section{DESIGN CASE}

PER APPENDIX 2, SKETCH 2-4 (DO NOT INPUT LETTERS) FLANGE IS 1=LOOSE, 2=INTEGRAL FACING SKETCH, TABLE 2-5.2 (1A-6) GASKET COLUMN 1 OR 2 GASKET COMPRESSION WIDTH, TABLE 2-5.2, w TEMADESIGN, $1=$ YES $0=$ NO DESIGN PRESSURE

I FOR INTERNAL, E FOR EXTERNAL (EXT P IS ENTERED AS A +VALUE)

LONGITUDINAL BENDING MOMENT ON FLANGE (IN-LB) AXIAL LOAD ON FLANGE (+ IS COMPRESSIVE, - IS TENSILE) (LBS) EQUIV. PRESSURE DUE TO MOMENT, Pm EQUIV. PRESSURE DUE TO AXIAL LOAD, Pr (NEGATIVE VALUES OF (Pm + Pr) ARE IGNORED (CONSERVATVE) TOTAL DESIGN LOAD ON FLANGE (FOR H CALCULATION PER III-1)

DESIGN TEMPERATURE CORROSION ALLOWANCE(FLG ID) CORROSION ALLOWANCE (HUB OD) APPENDIX S, MAX ALLOW FACTOR, $J$ NOM HUB THK, g1 NOM HUB THK, go W REVERSE FLANGE INSIDE DIA B SHELE TNSIDE DIA B FLANGE OUTSIDE DIA, A NOTE: IF SWNG BOLT DESIGN, ENTER OD AS INNER MOST SURFACE OF BOLT HOLE FLG THK, (CORRODED),

\begin{tabular}{r} 
BOLT QTY \\
NOM BOLT DIA \\
BOLT ROOT AREA \\
BOLT STRESS HOT, Sb \\
BOLT STRESS COLD, Sa \\
BOLT MATERIAL \\
\hline FLANGE MATERIAL STRESS COLD, Sfa \\
\hline FLANGE MATERIAL STRESS HOT, Sfo \\
\hline FLANGE MATERIAL MOD. E \\
FLANGE MATERIAL
\end{tabular}




\begin{tabular}{|c|c|c|c|}
\hline HUB LENGTH PER SKETCH, $\mathrm{h}$ & $\mathbf{0}$ & & \\
\hline MIN HUB LENGTH FOR SKETCH 6 FLANGES & $\mathrm{N} / \mathrm{A}$ & & \\
\hline MIN FILLET RADIUS FOR SKETCH $5 \& 6$ FLANGES & $\mathrm{N} / \mathrm{A}$ & & \\
\hline GASKET OD & 8.782 & & \\
\hline GASKET WIDTH, N & 0.375 & & \\
\hline GASKET THICKNESS, T & 0.175 & & \\
\hline BASIC GASKET SEATING WIDTH, bo & 0.1875 & & \\
\hline EFFECTIVE GASKET SEATING WIDTH, b & 0.1875 & & \\
\hline GASKET FACTOR, $m$ & 3 & & \\
\hline GASKET FACTOR, y & 10000 & & \\
\hline GASKET MATERIAL & FLEXITALLIC & & \\
\hline GASKET RIB SEATING WIDTH, $\mathrm{Nr}$ & 0 & & \\
\hline GASKET RIB TOTAL LENGTH, rl & 0 & & \\
\hline GASKET RIB FACTOR, $\mathbf{m}^{\prime}$ & 0 & & \\
\hline GASKET RIB FACTOR, $y^{\prime}$ & 0 & & \\
\hline GASKET RIB EFFECTIVE SEATING WIDTH, br & $\mathbf{0}$ & & \\
\hline (CORRODED), g1 & -0.06 & & \\
\hline (CORRODED), go & -0.06 & & \\
\hline BOLT CIRCLE DIA, C & 10,9 & & \\
\hline DIA AT GASKET LOAD REACTION, G & 8.407 & & \\
\hline NOTE: FOR LI FLGS INPUT G AS MIDPOINT OF FLGNS CONTACT & & & \\
\hline INSIDE DIA (CORRODED), B & 9.567 & & \\
\hline$W m 2$ & 49496.2 & & \\
\hline INPUT FOR FLANGED PAIRS (PER 2-5), Wm2 & 0 & & \\
\hline VALUE USED FOR FOLLOWING CALCS, Wm2 & 49496.2 & & \\
\hline $\mathrm{H}$ & 66578.3 & & \\
\hline $\mathrm{Hp}$ & 35637.3 & & \\
\hline$W_{\mathrm{m} 1}$ & 102215.6 & & \\
\hline INPUT FOR FLANGED PAIRS (PER 2-5), Wm1 & 0 & & \\
\hline VALUE USED FOR FOLLOWING CALCS, Wm1 & 102215.6 & & \\
\hline Am1 & 4.0886 & & \\
\hline Am2 & 1.9798 & & \\
\hline Am & 4.0886 & & \\
\hline$A b$ & 6.108 & & \\
\hline MAX. DEVELOPED BOLT STRESS & 16734.7 & & \\
\hline & AREA OK & & \\
\hline USE FULL BOLT LOAD FOR FLANGE DESIGN (Y/N) & $\mathrm{N}^{2} \mathrm{i}$ & & \\
\hline FULL BOLT LOAD, SEATING W & 152700 & & \\
\hline FULL BOLT LOAD, OPERATING W & 105057.6 & & \\
\hline GASKET SEATING LOAD USED IN CALCULATION, W & 127457.5 & & \\
\hline OPERATING LOAD USED IN CALCULATION, $W(\mathrm{Wm} 1)$ & 102215.6 & & \\
\hline BOLT TORQUE FRICTION FACTOR, $\mathrm{f}$ & 0.17 & & \\
\hline REQUIRED BOLT TORQUE FOR DESIGN, T (FT-LB) & 105.6 & & \\
\hline MIN GASKET WIDTH TO PREVENT CRUSH, Nmin & N/A & & \\
\hline $\begin{array}{l}\text { RECOMMENDED MIN. GASKET CONTACT WIDTHS FOR } \\
\text { SHEET AND COMPOSITE GASKETS, (TABLE 2-4) }\end{array}$ & $\begin{array}{c}\text { METAL TO METAL } \\
\text { N/A }\end{array}$ & & \\
\hline MOMENT CALCS---OPERATING & & & \\
\hline 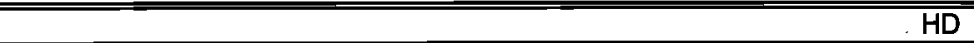 & 86218.9 & & \\
\hline HG & 35637.3 & & \\
\hline 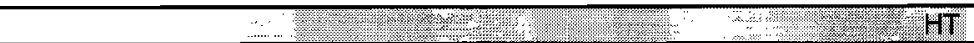 & -19640.6 & & \\
\hline $\mathrm{R}$ & 0.7265 & & \\
\hline 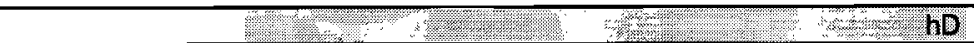 & 0.6665 & & \\
\hline hG & 1.2465 & & \\
\hline $\mathrm{s}+\mathrm{cos}-\mathrm{hT}$ & 0.9565 & & \\
\hline $\mathrm{MD}$ & 57464.9 & & \\
\hline MG & 44421.9 & & \\
\hline MT & -18786.2 & & \\
\hline Mo & 83100.6 & & \\
\hline
\end{tabular}




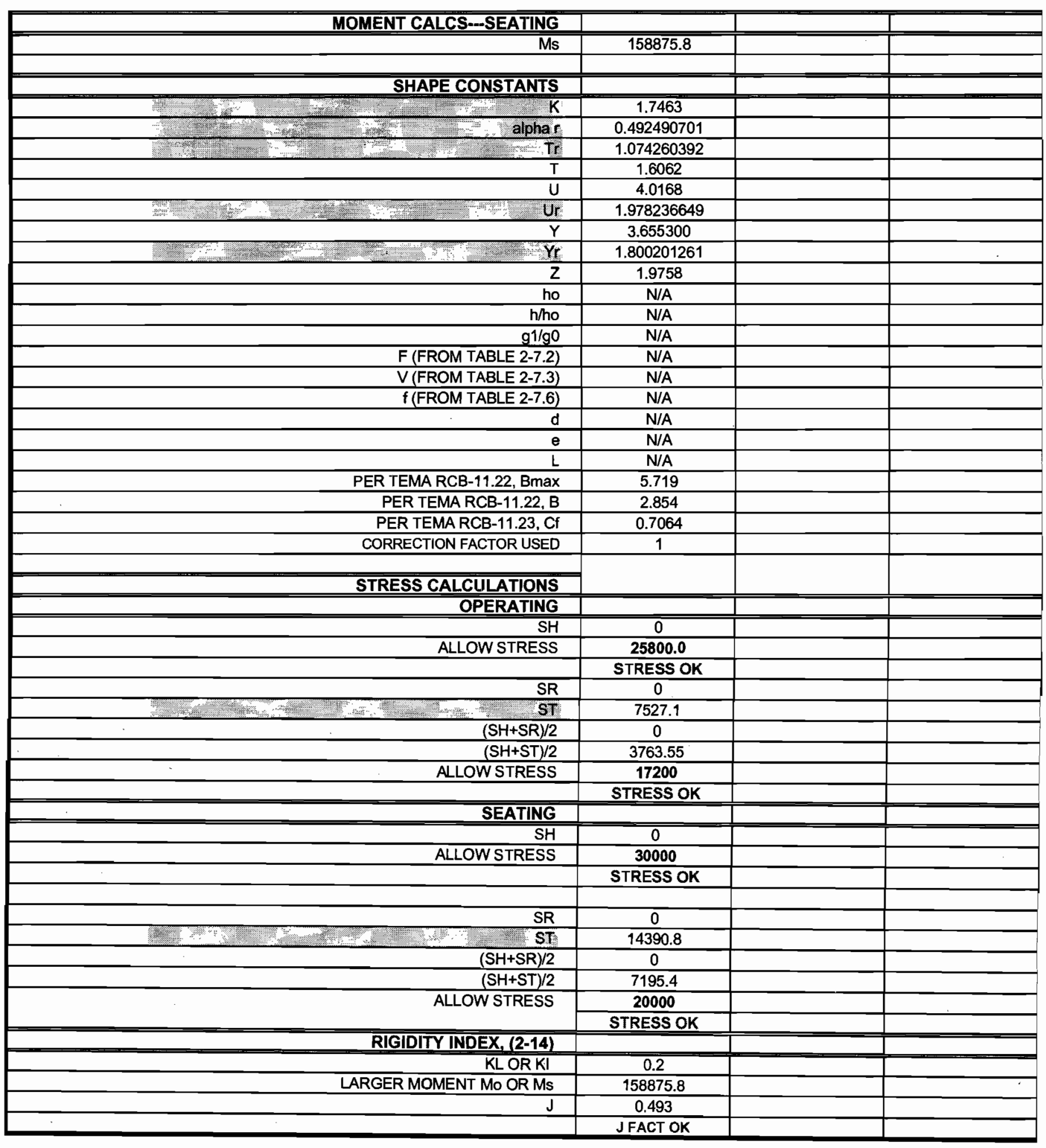




\section{TAPPED HOLES PER UG-43(d) \& (g)} FOR REACTOR OUTER SHELL, FOOT

NOMINAL DIAMETER OF STUD/BOLT, $d s=$ NUMBER OF THREADS PER INCH, tpi = ALLOW. STRESS OF STUD/BOLT MATL AT DESIGN TEMP, Sas = ALLOW. STRESS OF TAPPED MAT'L AT DESIGN TEMP, Sat =

MATERIAL THICKNESS TO BE TAPPED, $\mathrm{t}=$ CORROSION ALLOWANCE OF MATERIAL, ca =

REQUIRED THREAD ENGAGEMENT, te $=.75 \times \mathrm{ds} \times$ (Sas $/$ Sat)

te $=1.1457$

THREAD ENGAGEMENT NEED NOT EXCEED $11 / 2 \mathrm{x}$ ds $=1.5765$

MINIMUM THREAD ENGAGEMENT, tem $=\mathbf{1 . 1 4 5 7}$

7/8"-14 X 1.313" LONG INSERT REQUIRED HOLE DRILL DEPTH: 1.75"

NOTE: INSERT MINIMUM TENSILE STRENGTH: 200,000 PSI > FLANGE MATERIAL MINIMUM TENSILE STRENGTH: 70,000 PSI 


\section{ADAPTER FLANGE, REACTOR TO CHAR POT}

\begin{tabular}{l} 
NOTE: "ADAPTER FLANGE, REACTOR \\
TO CHAR POT" IS NOT INCLUDED IN \\
PRESSURE BOUNDARY. \\
\hline
\end{tabular}

NOTE: EXTERNAL LOADS ON FLANGES NOT CONSIDERED IN DESIGN CALCULATIONS.

\section{NOTE: ASSOCIATED VALUES ARE FROM REACTOR OUTLET SHELL, FOOT}

CHECK ADAPTER FLANGE CALCULATION AS BOLTED COVER WITH NO OPENINGS

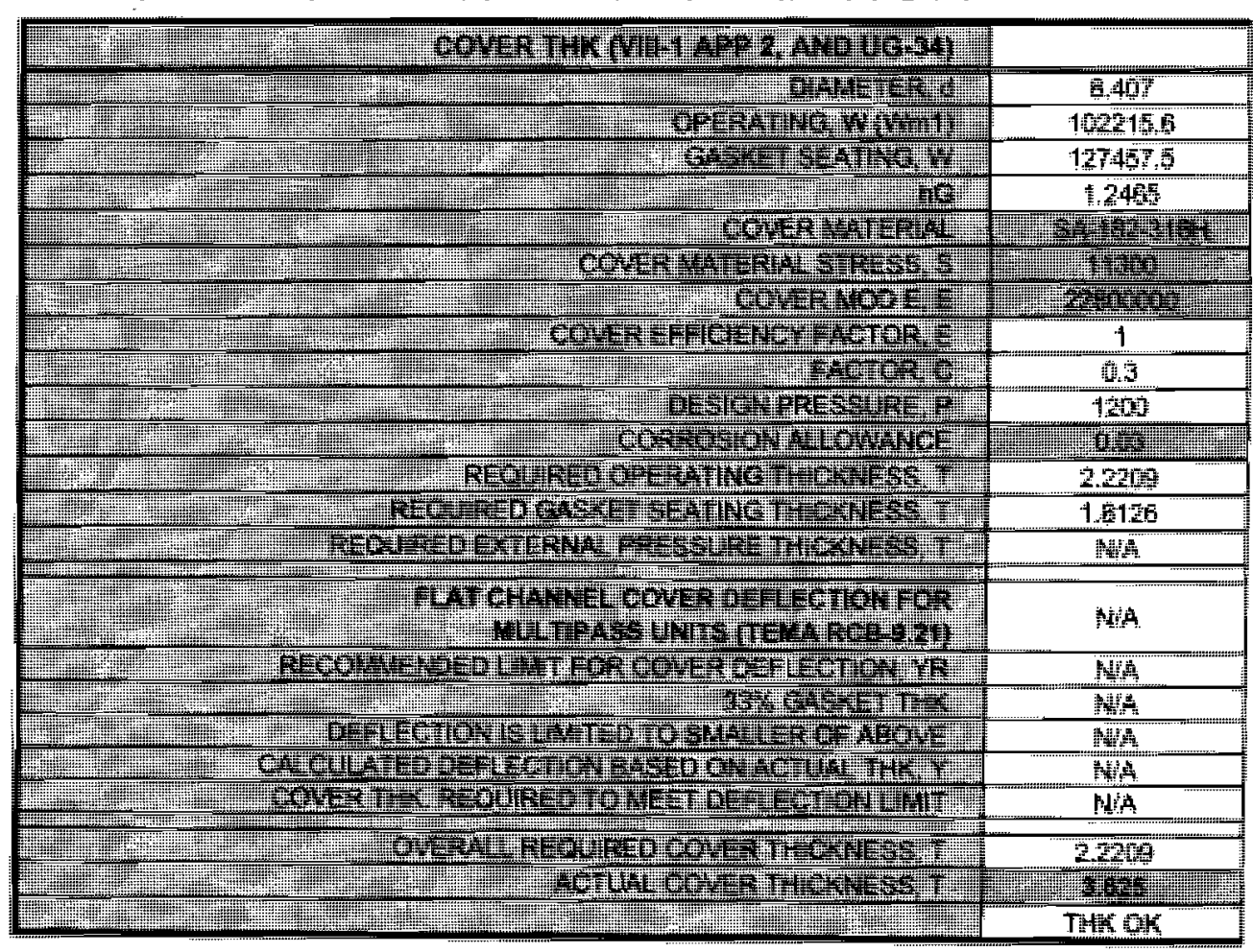

"CALCULATION CONTINUED NEXT PAGE UTILIZING "OVERALL REQUIRED COVER THICKNESS, T" = 2.23" 


\section{REACTOR TO CHAR POT ADAPTER FLANGE}

\section{ASME Section VIII Division 1, 2007 Edition}

$\begin{array}{ll}\text { Component: } & \text { Welded Cover } \\ \text { Material specification: } & \text { SA-182 F316H }<=5 \text { (low stress) (II-D p. 74, } \\ \text { ln. 32) }\end{array}$

Rated MDMT per UHA-51(d)(1)(b), (carbon content does not exceed 0.1 percent) $=-320^{\circ} \mathrm{F}$

Internal design pressure: $\mathrm{P}=1200.0000$ psi @ $1000.00^{\circ} \mathrm{F}$

Static liquid head:

$P_{t h}=0.0954 \mathrm{psi}\left(\mathrm{SG}=1.0000, \mathrm{H}_{\mathrm{s}}=2.6425^{\prime \prime}\right.$, Horizontal test head $)$

Corrosion allowance: $\quad$ Inner $\mathrm{C}=0.0300^{\prime \prime} \quad$ Outer $\mathrm{C}=0.0000^{\prime \prime}$

Design MDMT $=-20.00^{\circ} \mathrm{F}$

No impact test performed

Rated MDMT $=-320.00^{\circ} \mathrm{F}$ Material is not normalized Material is not produced to Fine Grain Practice PWHT is not performed

Radiography: $\quad$ Category A joints - $\quad$ Seamless No RT

Estimated weight: $\quad$ New $=84.1 \mathrm{lb} \quad$ corr $=83.4 \mathrm{lb}$

Head outside diameter $=11.7600^{\prime \prime}$

Cover thickness $=3.6250^{\prime \prime}$

Factor C from Fig. UG-34, sketch (b-2), (e through g)

$\mathrm{C}=0.33$ (worst case assumed)

Design thickness, (at $\left.1,000^{\circ} \mathrm{F}\right)$ UG-34 (c)(2)

$\mathrm{t}=\mathrm{d}^{*} \mathrm{Sqr}\left(\mathrm{C}^{*} \mathrm{P} /\left(\mathrm{S}^{*} \mathrm{E}\right)\right)+$ Corrosion

$=11.82 * \operatorname{Sqr}(0.33 * 1,200 /(11,300.00 * 1))+0.03$

$=2.2427$ in

Maximum allowable working pressure, $\left(\right.$ at $1,000^{\circ} \mathrm{F}$ )

$\mathrm{C}=\mathbf{0 . 3 3}$ (worst case assumed)

$\mathrm{P}=(\mathrm{S} * \mathrm{E} / \mathrm{C}) *(\mathrm{t} / \mathrm{d})^{2}-\mathrm{P}_{\mathrm{S}}$

$=(11,300.00 * 1 / 0.33) *(3.595 / 11.82)^{2}-0$

$=3,167.578 \mathrm{psi}$ 


\section{CENTER HOLE (C)}

\section{ASME Section VIII Division 1, 2007 Edition}

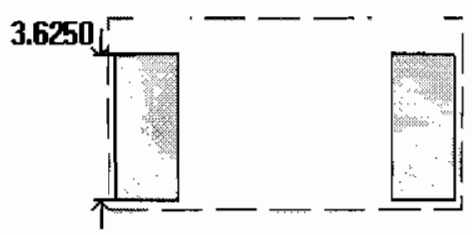

Note: round inside edges per UG-76(c)

Located on:

Local vessel minimum thickness: $\quad 3.625$ in

inside diameter, new:

5.285 in
REACTOR TO CHAR POT ADAPTER FLANGE 


\section{Reinforcement Calculations for Internal Pressure}

\begin{tabular}{|c|c|c|c|c|c|c|c|c|}
\hline \multicolumn{7}{|c|}{$\begin{array}{l}\text { UG-39 Area Calculation Summary }\left(\mathrm{in}^{2}\right) \\
\text { For } P=1213.16 \text { psi @ } 1000^{\circ} \mathrm{F} \\
\text { The opening is adequately reinforced }\end{array}$} & \multicolumn{2}{|c|}{$\begin{array}{l}\text { UG-45 Nozzle Wall } \\
\text { Thickness Summary (in) } \\
\text { The nozzle passes UG-45 }\end{array}$} \\
\hline$\underset{\text { required }}{A}$ & $\underset{\text { available }}{A}$ & $A_{1}$ & $A_{2}$ & $A_{3}$ & $A_{3}$ & $\begin{array}{c}\text { A } \\
\text { welds }\end{array}$ & $t_{\text {req }}$ & $t_{\min }$ \\
\hline 10.8791 & 10.8792 & 10.8792 & - & [- & - & - & 0.245 & 0.375 \\
\hline
\end{tabular}

Required thickness $t_{r}$ from UG-34

$$
\begin{aligned}
\mathrm{t}_{\mathrm{r}} & =\mathrm{d}^{*} \operatorname{Sqr}(\mathrm{C} * \mathrm{P} /(\mathrm{S} * \mathrm{E})) \\
& =11.82 * \operatorname{Sqr}(0.33 * 1,213.159 /(11,300 * 1)) \\
& =2.2248 \mathrm{in}
\end{aligned}
$$

Area required per UG-39

Allowable stresses: $S_{n}=15,300, S_{v}=11,300 p s i$

$\mathrm{f}_{\mathrm{rl}}=1$

$f_{r 2}=$ lesser of 1 or $S_{n} / S_{v}=1$ 


$$
\begin{aligned}
\mathrm{A} & =0.5 * \mathrm{~d}^{*} \mathrm{t}_{\mathrm{r}}+\mathrm{t}_{\mathrm{r}} * \mathrm{t}_{\mathrm{n}} *\left(1-\mathrm{f}_{\mathrm{r} 1}\right)+\text { Tapped hole area loss } \\
& =0.5 * 5.285 * 2.2248+2.2248 * 0.375 *(1-1)+5 \\
& =10.8791 \mathrm{in}^{2}
\end{aligned}
$$

Area available from FIG. UG-37.1

$$
\begin{aligned}
\mathrm{A}_{1}= & \text { larger of the following }=10.8792 \mathrm{in}^{2} \\
& =\mathrm{d}^{*}\left(\mathrm{E}_{1} * \mathrm{t}_{\mathrm{t}}-\mathrm{F}^{*} \mathrm{t}_{\mathrm{r}}\right)-2 * \mathrm{t}_{\mathrm{n}} *\left(\mathrm{E}_{1} *{ }_{\mathrm{t}}-\mathrm{F}^{*} \mathrm{t}_{\mathrm{T}}\right) *\left(1-\mathrm{f}_{\mathrm{r} 1}\right) \\
& =5.285^{*}(1 * 3.595-1 * 2.2248)-2 * 0.375 *(1 * 3.595-1 * 2.2248)^{*}(1-1) \\
& =7.2414 \text { in }^{2} \\
& =2 *\left(\mathrm{t}+\mathrm{t}_{\mathrm{n}}\right)^{*}\left(\mathrm{E}_{1}{ }^{*} \mathrm{t}-\mathrm{F}^{*} \mathrm{t}_{\mathrm{T}}\right)-2 * \mathrm{t}_{\mathrm{n}} *\left(\mathrm{E}_{1} * \mathrm{t}-\mathrm{F}^{*} \mathrm{t}_{\mathrm{r}}\right) *\left(1-\mathrm{f}_{\mathrm{r} 1}\right) \\
& =2 *(3.595+0.375)^{*}(1 * 3.595-1 * 2.2248)-2 * 0.375 *(1 * 3.595-1 * 2.2248)^{*}(1-1) \\
& =10.8792 \text { in }^{2}
\end{aligned}
$$

$$
\begin{aligned}
\text { Area } & =\mathrm{A}_{1}+\mathrm{A}_{2}+\mathrm{A}_{41} \\
& =10.8792+0+0 \\
& =\underline{10.8792} \mathrm{in}^{2}
\end{aligned}
$$

As Area $>=A$ the reinforcement is adequate. 


\section{TAPPED HOLES PER UG-43(d) \& (g)} ADAPTER FLANGE TO CHAR POT NOMINAL DIAMETER OF STUD/BOLT, ds = NUMBER OF THREADS PER INCH, tpi = ALLOW. STRESS OF STUID/BOLT MAT'L AT DESIGN TEMP, Sas = ALLOW. STRESS OF TAPPED MAT'L AT DESIGN TEMP, Sat =

MATERIAL THICKNESS TO BE TAPPED, $t=$ CORROSION ALLOWANCE OF MATERIAL, ca = REQUIRED THREAD ENGAGEMENT, te = te $=$ THREAD ENGAGEMENT NEED NOT EXCEED $11 / 2 \times \mathrm{ds}=$ MINIMUM THREAD ENGAGEMENT, tem =

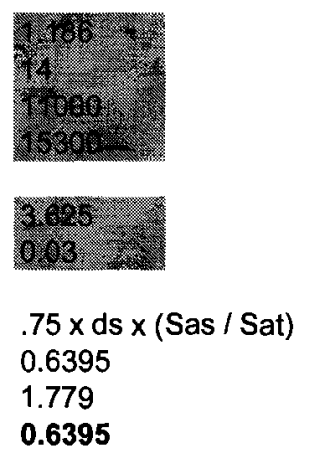

PER "MACHINERY'S HANDBOOK" SECTION "TAPS AND THREADING DIES" NUMBER OF THREADS TAPER TAPS ARE CHAMFERED NUMBER OF THREADS PLUG TAPS ARE CHAMFERED NUMBER OF THREADS BOTTOMING TAPS ARE CHAMFERED NUMBER OF THREADS FOR TAPER, $\mathrm{ntt}=$ LENGTH OF TAPER, It $=$

MIN. DEPTH OF HOLE IN MATERIAL TO PROVIDE FULL THREAD ENGAGEMENT, hd $=$ tem + It

(d) DRILLED HOLES TO BE TAPPED SHALL NOT PENETRATE WITHIN ONE-FOURTH OF THE WALL THICKNESS FROM THE INSIDE SURFACE OF THE VESSEL AFTER DEDUCTING CORROSION ALLOWANCE

MINIMUM THICKNESS OF MATERIAL TO BE TAPPED TO MEET UG-43(d)= $\begin{aligned} & \text { hd } \times(4 / 3)+c a \\ & \mathbf{1 . 2 6 3 6}\end{aligned}$

SINCE $t>1.2636$, DESIGN IS SATISFACTORY

NOTE- THE DATA BELOW TAKES THE EXTRA THREADING REQUIRED FOR THE HELICAL INSERT INTO ACCOUNT.

\section{TAPPED HOLES PER UG-43(d) \& (g)} ADAPTER FLANGE TO CHAR POT NOMINAL DIAMETER OF STUD/BOLT, ds = NUMBER OF THREADS PER INCH, tpi = ALLOW. STRESS OF STUD/BOLT MATL AT DESIGN TEMP, Sas = ALLOW. STRESS OF TAPPED MAT'L AT DESIGN TEMP, Sat =

MATERIAL THICKNESS TO BE TAPPED, $t=$ CORROSION ALLOWANCE OF MATERIAL, $\mathrm{ca}=$ REQUIRED THREAD ENGAGEMENT, te $=$ te $=$ THREAD ENGAGEMENT NEED NOT EXCEED $11 / 2 \times \mathrm{ds}=$ MINIMUM THREAD ENGAGEMENT, tem =

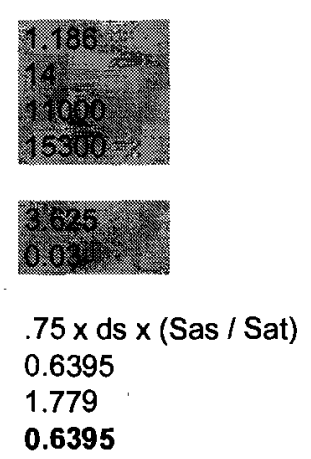

PER "MACHINERY'S HANDBOOK" SECTION "TAPS AND THREADING DIES" NUMBER OF THREADS TAPER TAPS ARE CHAMFERED NUMBER OF THREADS PLUG TAPS ARE CHAMFERED NUMBER OF THREADS BOTTOMING TAPS ARE CHAMFERED NUMBER OF THREADS FOR TAPER, $\mathrm{ntt}=$ LENGTH OF TAPER, It = 
(d) DRILLED HOLES TO BE TAPPED SHALL NOT PENETRATE WITHIN ONE-FOURTH OF THE WALL THICKNESS FROM THE INSIDE SURFACE OF THE VESSEL AFTER DEDUCTING CORROSION ALLOWANCE

MINIMUM THICKNESS OF MATERIAL TO BE TAPPED TO MEET UG-43(d) $=$ hd $x(4 / 3)+c a$ 2.6967

SINCE $t>2.6967$, DESIGN IS SATISFACTORY

1"-14 X 1 1/2" LONG HELICAL INSERT HOLE DRILL DEPTH: 2.00" (MIN.)

NOTE: INSERT MINIMUM TENSILE STRENGTH: 200,000 PSI > FLANGE MATERIAL

MINIMUM TENSILE STRENGTH: 75,000 PSI

NOTE: STUD/ BOLT MATERIAL IS SA-197-B16 


\section{INJECTOR CAP (I1 TO I4)}

\section{ASME Section VIII Division 1, 2007 Edition}

Component:

Material specification:

Rated MDMT per UNF- $65=-325^{\circ} \mathrm{F}$

Internal design pressure: $\mathrm{P}=1200 \mathrm{psi} @ 1000^{\circ} \mathrm{F}$

Static liquid head:

$P_{\text {th }}=0.04 \mathrm{psi}\left(\mathrm{SG}=1.0000, \mathrm{H}_{\mathrm{s}}=1.24\right.$ ", Horizontal test head $)$

Corrosion allowance: $\quad$ Inner $\mathrm{C}=0.03 "$

Design MDMT $=-20^{\circ} \mathrm{F}$

Rated MDMT $=-325^{\circ} \mathrm{F}$

Radiography: Category A joints -

Estimated weight: $\quad$ New $=0.2 \mathrm{lb}$

Head outside diameter $=2^{\prime \prime}$

Cover thickness $\quad=0.8^{\prime \prime}$

\section{Factor C from Fig. UG-34, sketch (h)}

Factor $\mathrm{C}=0.66$

Note: Factor C has been increased per UG-39(d)

Design thickness, (at 1,000 ${ }^{\circ}$ F) UG-34 (c)(2)

$\mathrm{t}=\mathrm{d}^{*} \operatorname{Sqr}\left(\mathrm{C}^{*} \mathrm{P} /(\mathrm{S} * \mathrm{E})\right)+$ Corrosion

$=1.3 * \operatorname{Sqr}\left(0.66^{*} 1,200 /\left(29,900^{*} 1\right)\right)+0.03$

$=0.2416$ in

Maximum allowable working pressure, (at $1,000^{\circ} \mathrm{F}$ )

$\mathrm{P}=(\mathrm{S} * \mathrm{E} / \mathrm{C}) *(\mathrm{t} / \mathrm{d})^{2}-\mathrm{P}_{\mathrm{S}}$

$=(29,900 * 1 / 0.66)^{*}(0.77 / 1.3)^{2}-0$

$=15,893.59 \mathrm{psi}$
Welded Cover

SB-446 1 Annealed Bar <= 4 N06625 (II-D

p. $226, \ln .8)$ 
KINETICS REACTOR INNER TUBE

MATERIAL: INCONEL 617

OD: 2"

WALL THICKNESS: $0.125 "$

CORROSION ALLOWANCE: $0.005^{\prime \prime}$

MAXIMUIM DIFFERENTIAL PRESSURE: 50 PSI

MAXIMUM TEMPERATURE: $1900^{\circ} \mathrm{F}$

DESIGN LIFE: 2000 HOURS

AXIAL LOAD: 50 LBS

MATERIAL PROPERTIES (@ 1900\%) - MATERIAL PROPERTIES FROM HAYNES INTERNATIONAL AND SPECIAL METALS PRODUCT SPECS.

MOD OF ELASTICITY (E): 18,800,000 PSI

POISSON'S RATIO: 0.32

TENSILE STG. (APPROX.):15,000 PSI

YIELD STG. (APPROX): 10,000 PSI

RUPTURE STG. AT 2,000 HRS (APPROX): 2,000 PSI

\section{DESIGN CONDITIONS/ ASSUMPTIONS}

-PER PRESSURE VESSEL DESIGN HANDBOOK 2ND ED. (BEDNAR)

SEC 3.1- THIN SHELL VESSEL IS R $t / \mathrm{t}>10$

$0.880 " / 0.120 "=7.3$, THEREFORE THIS IS NOT A THIN WALLED VESSEL

SEC 3.3 UNDER UNIFORM EXTERNAL PRESSURE - LENGTH OF CYLINDER IS A FACTOR FOR COLLAPSE IN THIN-WALLED CYLINDERS ONLY.

FOR COLUMN BUCKLING CALCULATIONS SPIDER SUPPORTS ARE NOT CONSIDERED (TUBE IS CONSIDERED AS A 163" LONG COLUMN WITH NO INTERMEDIATE SUPPORT) FOR A CONSERVATIVE ANALYSIS.

ALL CALCULATIONS CONSIDER THE TUBE IN THE CORRODED CONDITION (WALL THK = $0.120^{\prime \prime}$, ID = 1.760"). 


\section{CALCULATION}

AXIAL STRESS $(\sigma 1)=\mathrm{P} / \mathrm{A}=50 \mathrm{LBS} / 0.709 \mathrm{IN} \wedge 2=70.52 \mathrm{PSI}$

WHERE $A=$ CROSS SECTIONAL ARE OF TUBE $=0.709 \mathrm{IN}^{\wedge} 2$

CIRCUMFERENTIAL STRESS (FROM EXTERNAL PRESSURE)

PER ROARK'S $6^{\text {TH }}$ (YOUNG)- TABLE 32, CASE. 1C

$\sigma 2\left(\right.$ MAX) $=-q^{\star} 2^{\star} a^{\wedge} 2 /\left(a^{\wedge} 2-b^{\wedge} 2\right)=-50 \mathrm{PSI}^{\star} 2^{\star} 1^{\wedge} 2 /\left(1^{\wedge} 2-0.880^{\wedge} 2\right)=-443.13 \mathrm{PSI}$

$a=$ OUTER RADIUS OF TUBE
$b=$ INNER RADIUS OF TUBE
$q=$ PRESSURE

CIRCUMFERENTIAL STRESS (FROM INTERNAL PRESSURE)

PER ROARK'S $6^{\text {TH }}$ (YOUNG)- TABLE 32, CASE. 1 a

$\sigma 2(\mathrm{MAX})=\mathrm{q}^{\star}\left(\mathrm{a}^{\wedge} 2+\mathrm{b}^{\wedge} 2\right) /\left(\mathrm{a}^{\wedge} 2-\mathrm{b}^{\wedge} 2\right)=-50 \mathrm{PSI}^{\star}\left(1^{\wedge} 2+0.880^{\wedge} 2\right) /\left(1^{\wedge} 2-0.880^{\wedge} 2\right)=393.26 \mathrm{PSI}$

RADIAL STRESS (INTERNAL \& EXTERNAL PRESSURE

PER ROARK'S $6^{\text {TH }}$ (YOUNG)- TABLE 32, CASE. 1a \& 1c

$\sigma 3(\mathrm{MAX})=-\mathrm{q}=-50$ PSI

COMBINED STRESS $=\left(\sigma 1^{\wedge} 2+\sigma 2^{\wedge} 2+\sigma 3^{\wedge} 2\right)^{\wedge} 1 / 2=\left(70.52^{\wedge} 2+443.13^{\wedge} 2+50^{\wedge} 2\right)^{\wedge} 1 / 2$

$=\underline{451 \text { PSI }}$

COLUMN BUCKLING

PER ROARK'S $6^{\text {TH }}$ (YOUNG)- ART. 11.1 FORMULA (1)

$P / A=C^{*} P I^{\wedge} 2^{\star} E /(L / r)^{\wedge} 2=1^{\star}(3.14)^{\wedge} 2^{*} 18,800,000 /(163 / 0.660)^{\wedge} 2=3,039 \mathrm{PSI}$

NOTE: PIA = THE UNIT STRESS AT WHICH A LONG COLUMN FAILS BY ELASTIC INSTABILITY.

CHECK FOR TUBE FAILURE:

FROM STRESS: 451 PSI < 2,000 PSI (RUPTURE STG), TUBE STRESS IS ACCEPTABLE

FROM COLUMN BUCKLING: 451 PSI < 3,039 PSI, TUBE STRESS IS ACCEPTABLE 
P.O.\#: 700521452

API Standand 530/ / SO 137042001 (E)

RUPTURE DESIGN FOR $1 / 4^{n}$ OD TUBE

\begin{tabular}{|c|c|c|}
\hline \multicolumn{3}{|c|}{$\begin{array}{l}\text { BDO 13704 } \\
\text { CALCULATION 8HEET } \\
\text { (US customary units) }\end{array}$} \\
\hline Qutside diameter, inches & $D_{0}=0.25^{\prime \prime}$ & $D_{0}=0.25^{\prime \prime}$ \\
\hline Design pressure, psi (gauge) & $p_{e t}=1150 \mathrm{PSI}$ & $p_{\mathrm{r}}=1150 \mathrm{PSI}$ \\
\hline Maximum or equivalent metal temperature, ${ }^{\circ} F$ & $T_{\max }=1600 \mathrm{~F}$ & $T_{\max }=1600 \mathrm{~F}$ \\
\hline Temperature allowance, ${ }^{\circ} \mathrm{F}$ & $T_{A}=$ NONE & $T_{\mathrm{A}}=\mathrm{NONE}$ \\
\hline Design metal temperature, "F & $T_{\mathrm{d}}=1600 \mathrm{~F}$ & $T_{\mathrm{d}}=1600 \mathrm{~F}$ \\
\hline Design life, $\mathrm{h}$ & - & $L_{L L}=2000$ \\
\hline Allowable stress at $T_{d}$ & $\sigma_{\theta 1}=20,000 \mathrm{PSI}$ & $\sigma_{\mathrm{r}}=4000 \mathrm{PSI}$ \\
\hline Stress thickness, equation (2) or (4), inches & $\delta_{\alpha}=0.0070^{\prime \prime}$ & $\delta_{\sigma}=0.0314^{n}$ \\
\hline Corrosion allowance, inches & $\delta_{C A}=$ NONE & $\delta_{C_{A}}=$ NONE \\
\hline Conosion fraction, Figure $1, n=B=$ & - & $f_{\text {cor }}=\mathrm{N} / \mathrm{A}$ \\
\hline Minimum thickness, equation (3) or (5), inches & $\delta_{\min }=0.0070^{\prime \prime}$ & $\delta_{\min }=0.0314^{\prime \prime}$ \\
\hline
\end{tabular}

MATERIAL: SB-444 N06625 GR 2 (SOLUTION TREATED)

MINIMUM WALL THICKNESS OF TUBE: $0.0585^{"}$

ALLOWABLE STRESSES PER "SPECIAL METALS" ALLOY 625 CATALOG

NO CORROSION ALLOWANCE IS USED

NOTE: $1 / 4 " X 0.065$ NOM THK. TUBE NOT INCLUDED IN CODE

PRESSURE BOUNDARY. TUBE IS FIELD WELDED TO VESSEL.

RUPTURE DESIGN PER 4.4 EQN (4)

$$
\begin{aligned}
\delta_{\sigma}=\frac{p_{\mathrm{r}} D_{\mathrm{o}}}{2 \sigma_{\mathrm{r}}+p_{\mathrm{r}}} & =\left(1150^{*} 0.25\right) /\left(2^{*} 4000+1150\right)=0.0314 " \text { min. thickness required } \\
& 0.0585^{\prime \prime}>0.0314^{\prime \prime} \text { THEREFORE TUBE THICKNESS IS ADEQUATE }
\end{aligned}
$$

ELASTIC DESIGN CHECK PER 6.1 AND 4.3 EQN (2)

$$
\begin{aligned}
\delta_{\sigma}=\frac{p_{e l} D_{0}}{2 \sigma_{0+1}+P_{\theta}} & =\left(1150^{*} 0.25\right) /\left(2^{\star} 20,000+150\right)=0.0070^{n} \text { min. thickness required } \\
& 0.0585^{\prime \prime}>0.0070^{n} \text { THEREFORE TUBE THICKNESS IS ADEQUATE }
\end{aligned}
$$


TUBE TO VESSEL WELD

NOTE: TUBE AND TUBE TO VESSEL WELD NOT INCLUDED IN CODE PRESSLURE BOUNDARY

(5) WELDS REQUIRED

TUBES: 1/4" OD X 0.065" AVG WALL SB-444 N06625 GR.2

ASME VIII-1 2007 EDITION USED AS GUIDE

UW-16(E)(1) AND FIG. UW-16.1 (W-1)

WELDED FROM THE OUTSIDE ONLY

REQUIRED FILLET THROAT SIZE $=\operatorname{Tmin}{ }^{*} 1.25=0.065 * 1.25=0.08125$,

REQUIRED FILLET LEG SIZE $=0.08125 / 0.707=0.115 " \Rightarrow$ USE $1 / 8 ”$ LEG

NOTE: TUBE DOES NOT EXTEND TO INSIDE WALL OF VESSEL

ADDITIONAL 1/16" GROOVE WELD NOT REQUIRED BY FIG. UW-16.1 (W-1) IS USED; THEREFORE, THE WELD DESIGN IS CONSERVATIVE. 


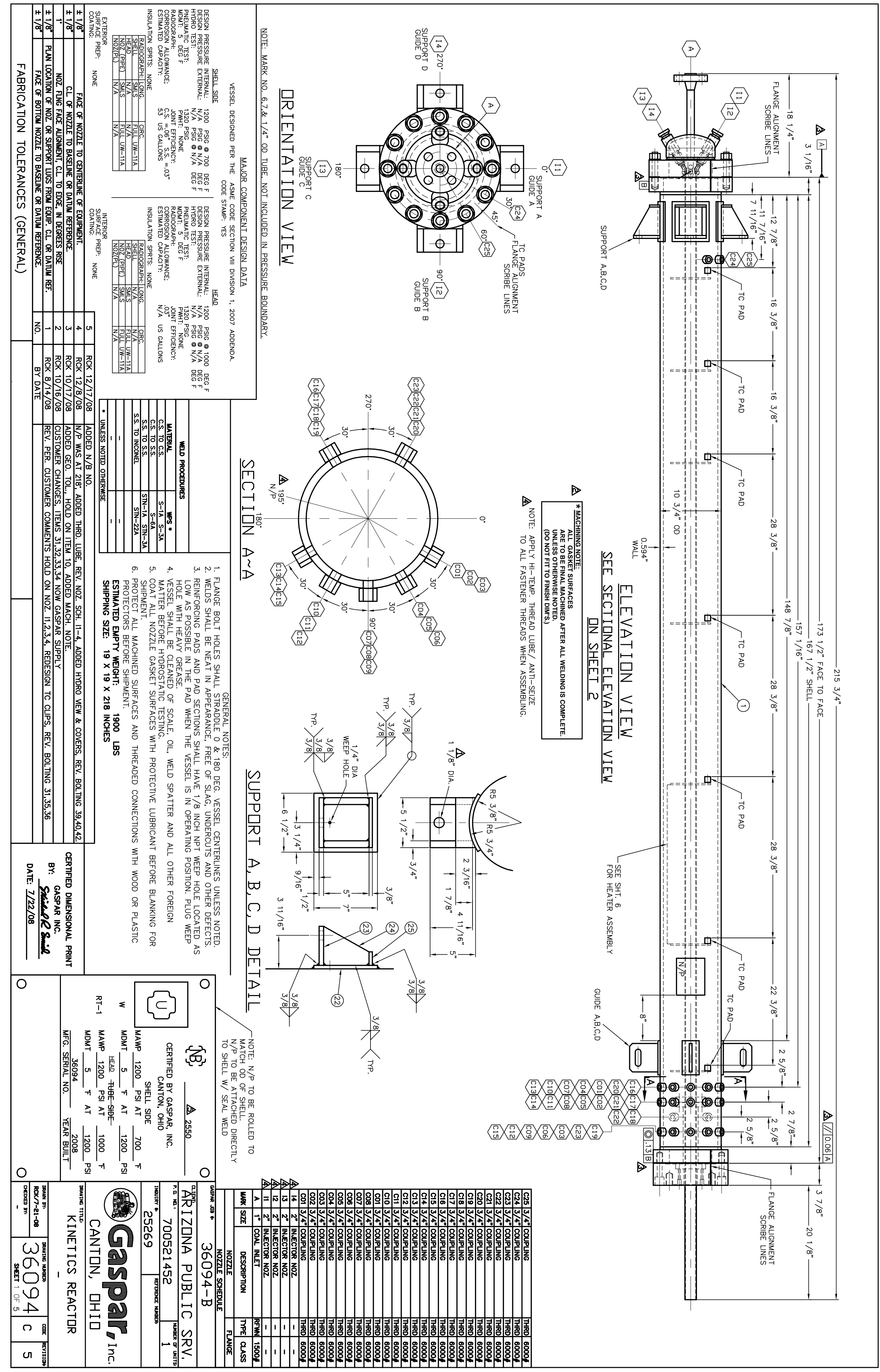




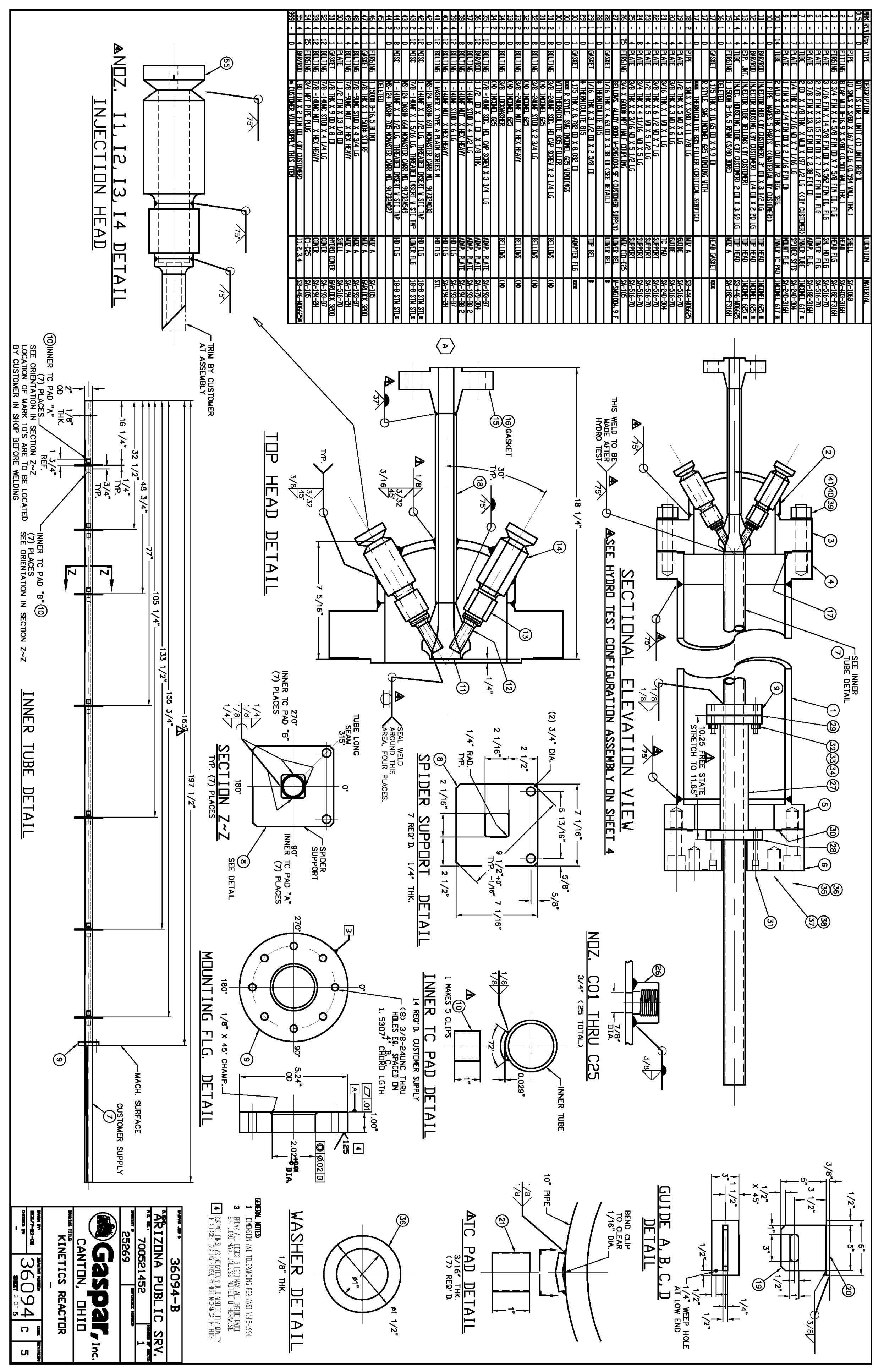




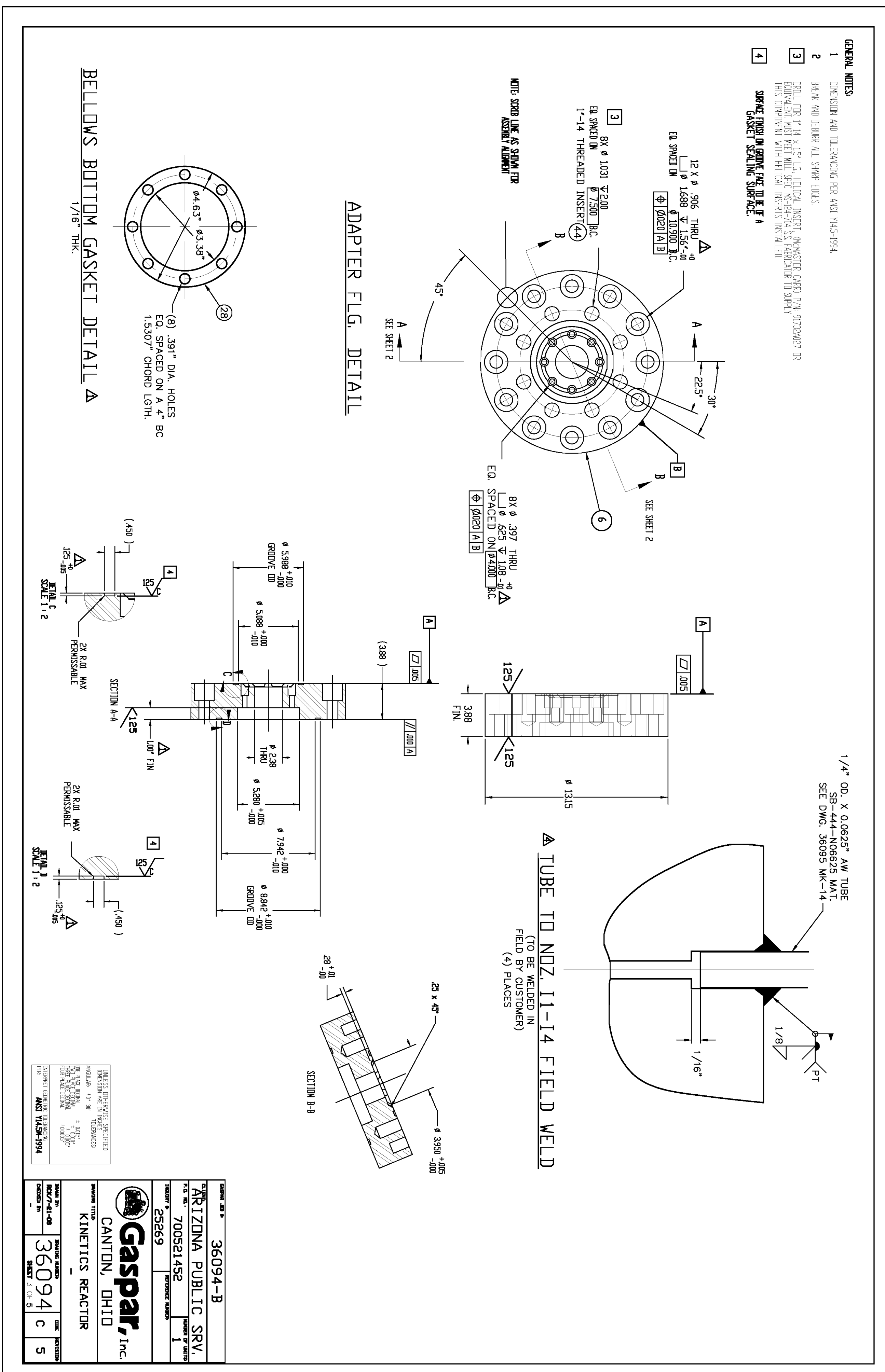



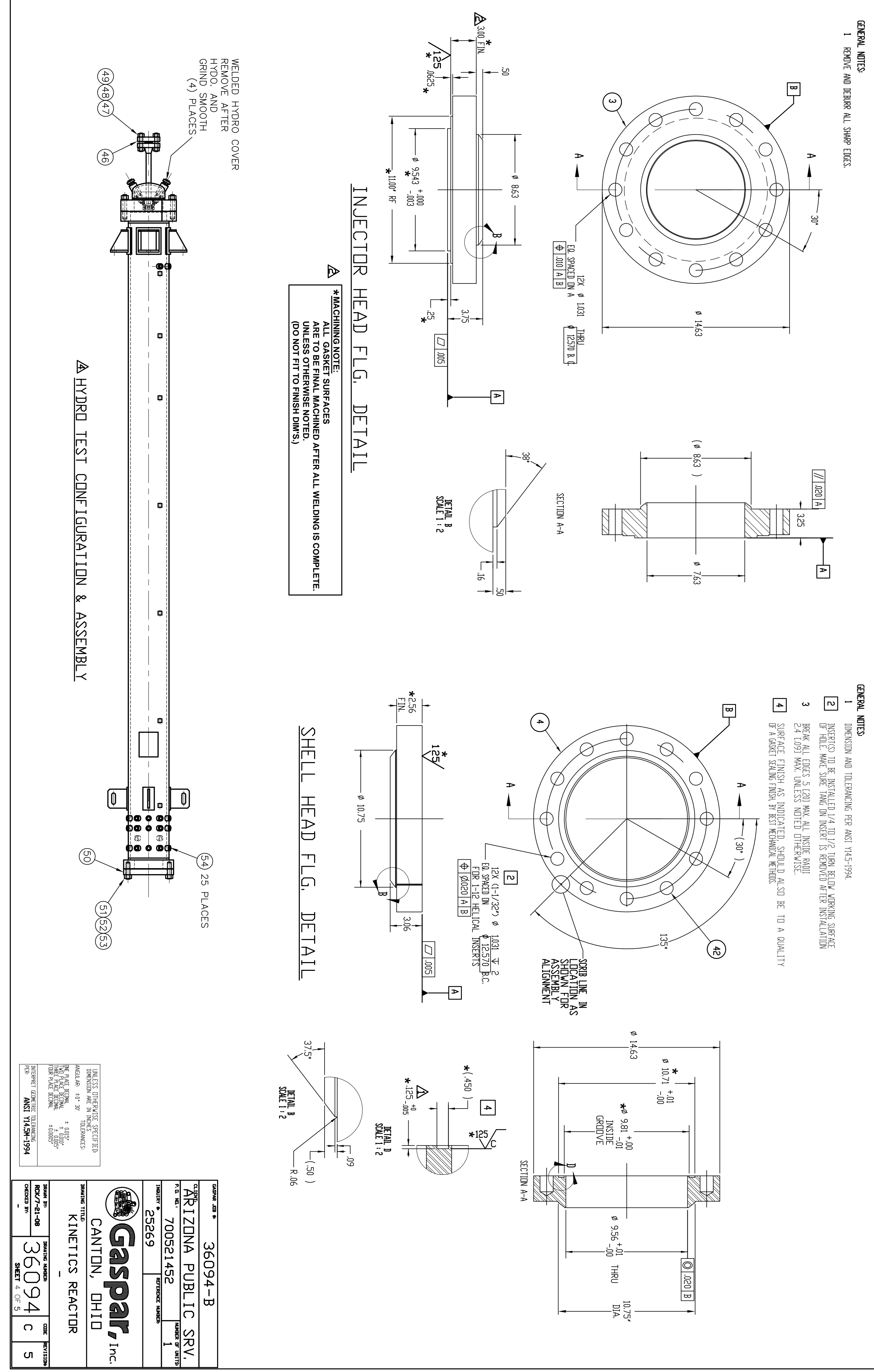

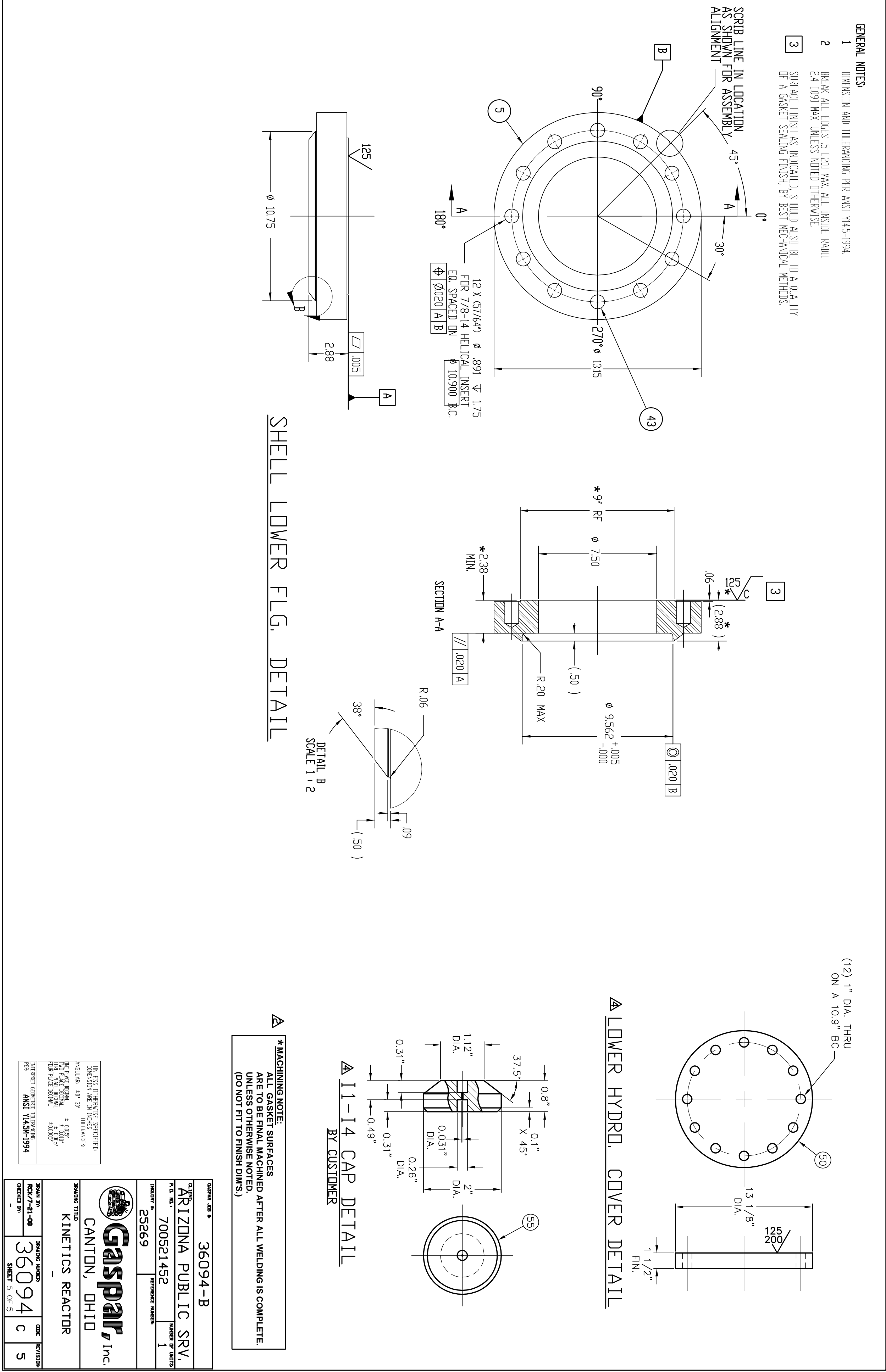


\title{
APPENDIX D
}

\section{Bench Scale Hydrogasifier Preheater}

\author{
Final Design from Gaspar Inc.
}


Development of a Hydrogasification Process for

Coproduction of SNG and Electrical Power from Western Coals

Final Report

THIS PAGE INTENTIONALLY LEFT BLANK 


\section{Gasparinc. $_{\text {. }}$ \\ WELDING \& FABRICATIONS}

1545 Whipple Avenue SW Canton, Ohio 44710

Phone: 330-477-2222

Fax: $330-477-2322$

www.gasparinc.com

\section{FINAL DATA PACKAGE}

\begin{tabular}{|r|l||}
\hline GASPAR JOB NUMBER(S): & 36095 \\
\hline CUSTOMER: & ARIZONA PUBLIC SERVICE CO. \\
\hline PUIRCHASE ORDER NUMBER: & 700521452 \\
\hline DESCRIPTION: & PREHEATER \\
\hline ITEM NUMBER(S): & N/A \\
\hline OTHER: & N/A \\
\hline
\end{tabular}

\section{DATA PACKAGE CONTENTS}

$\checkmark$ DATAREPORT

$\square$ NAMEPLATE COPY

[ BILL OF MATERIAL

$\square$ MATERIAL TEST REPORTS

$\square$ NDE REPORTS

$\square$ HEAT TREAT CHARTS

$\square$ CALCULATIONS

$\square$ DRAWINGS

$\square$ OTHER: (LIST BELOW)

NOTE: 


\section{FORM U-1A MANUFACTURER'S DATA REPORT FOR PRESSURE VESSELS}

(Alternative Form for Single Chamber, Completely Shop or Field Fabricated Vessels Only)

As Required by the Provisions of the ASME Boiler and Pressure Vessel Code Rules, Section VIII, Division I

1. Manufactured and certified by:

Gaspar, Inc. 1545 Whipple Ave SW Canton, Ohio 44710

(Name and address of manufacturer)

2. Manufactured for: $\quad$ Arizona Public Service Co. P.O. Box 53999 Phoenix, AZ 85072

3. Location of installation Unknown

4. Type: Vertical $36095 \quad-36095$ Rev.4

(Horizontal or vertical tank)

$-\frac{2551}{- \text { (National Board number) }} \frac{2008}{\text { (Year built) }}$

5. The chemical and physical properties of all parts meet the requirements of material specifications of the ASME BOILER AND PRESSURE VESSEL

CODE The design, construction, and workmanship conform to ASME Rules, Section VIII, Division 1

2007 Edition

to

. She

Addenda (Date)

$$
\text { --- }
$$

6. Shell $\frac{\text { SB466-N06625 }}{\text { Materiai spec. number. grade) }}$

7. Seams:

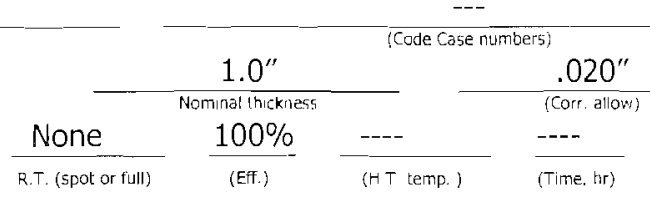

(b) Material SB466-N06625 Year

8. Heads: (a) Material (Spec, number, grade) [Special Servce per UG-120 $\{$ d $\}]$

\begin{tabular}{|c|c|c|c|c|c|c|c|c|c|c|}
\hline & $\begin{array}{l}\text { Location (Top, } \\
\text { Bottom, Ends) }\end{array}$ & $\begin{array}{l}\text { Minimum } \\
\text { Thickness }\end{array}$ & $\begin{array}{l}\text { Corrosion } \\
\text { Allowance }\end{array}$ & $\begin{array}{l}\text { Crown } \\
\text { Radius }\end{array}$ & $\begin{array}{l}\text { Knuckle } \\
\text { Radius }\end{array}$ & $\begin{array}{c}\text { Elliptical } \\
\text { Ratio }\end{array}$ & $\begin{array}{c}\text { Conical } \\
\text { Apex Angle }\end{array}$ & $\begin{array}{l}\text { Hemispherical } \\
\text { Radius }\end{array}$ & $\begin{array}{c}\text { Flat } \\
\text { Diameter }\end{array}$ & $\begin{array}{l}\text { Side to Pressure } \\
\text { (Convex or Concave) }\end{array}$ \\
\hline (a) & Top & $1 . \overline{0^{\prime \prime}}$ & $.020^{\prime \prime}$ & $-\cdots$ & $\cdots$ & --- & ---- & --- & $3^{\prime \prime}$ & ---- \\
\hline (b) & Bottom & $1.0^{\prime \prime}$ & $.020^{\prime \prime}$ & $-\cdots$ & --- & $-\ldots$ & $-\cdots$ & $-\cdots$ & $3^{\prime \prime}$ & --- \\
\hline
\end{tabular}

If removable, bolts used (describe other fastenings)

9. MAWP $\frac{1150}{\text { (Internal) }}$
Min. design metal temp. $\begin{array}{ccc}\frac{1150}{(\text { Internal) }} & & \text { (External) } \\ \text { Min. design metal temp. } & \frac{-20}{n n}\end{array}$ at max temp.

(Material spec. number, grade, size, number) 1600

$\begin{array}{clcc}\text { Min. design metal temp. } & \text { (External) } & & \text { (Internal) } \\ & -20 & \text { at } & 1150\end{array}$ Hydro. pneu., or comb. test press.

\section{Welded/Type 1}

$0^{\prime}-1.0^{\prime \prime}$
(Inner diameter)
ype 1

butt)] $14^{\prime}-5^{\prime \prime}$

$\frac{\text { Full }}{\text { [R.T.(spot or }}$

T. (spot or
fulli)]

Length (overall) $100 \%$ Four (Eff. \%) No. of courses

SB466-N06625 SB466-N06625

10. Nozzles, inspection, and safety valve openings:

\begin{tabular}{|c|c|c|c|c|c|c|c|c|}
\hline $\begin{array}{c}\text { Purpose } \\
\text { (Inlet, Outlet, Drain) }\end{array}$ & Number & $\begin{array}{l}\text { Diameter } \\
\text { or Size }\end{array}$ & Type & Material & \begin{tabular}{|l} 
Nominal \\
Thickness
\end{tabular} & $\begin{array}{l}\text { Reinforcement } \\
\text { Material }\end{array}$ & $\begin{array}{c}\text { How } \\
\text { Attached }\end{array}$ & Location \\
\hline Aux & 5 & $.260^{\prime \prime}$ & Bore & ----- & $\begin{array}{l}----- \\
\end{array}$ & $---\cdot$ & $\cdots$ & ---- \\
\hline & & & & & & & & \\
\hline & & & & & & & & \\
\hline & & & & & & & & \\
\hline 11. Supports: Skirt & No & Lugs & 0 & Legs & Oth & 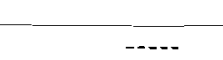 & Attached & hell/Welded \\
\hline
\end{tabular}

12. Remarks: Manufacturer's Partial Data Reports properly identified and signed by Commissioned Inspectors have been furnished for the following items of the report:

UNF-65 Exempt (impact test).

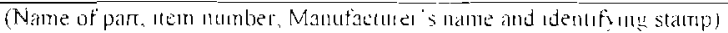

\section{CERTIFICATE OF SHOP/FIELD COMPLIANCE}

We certify that the statements made in this report are correct and that all details of design, material, construction and workmanship of this vessel conform to the ASME Boiler and pressure Vessel Code, Section VIII, Division 1. expires July 25,2011

Date $\quad 12-29 \cdot 06$ Co. Name

"U" Certificate of Authorization Number

16.862

Vesiel constructed by

Gaspar, Inc.

(Manufacturer)

Signed

\section{CERTIFICATE OF SHOP/FIELD INSPECTION}

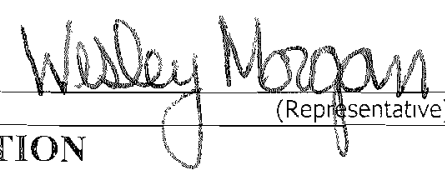

Gaspar, Inc.

at

J 545 Whipple $\wedge$ ve SW Canton, Ohio 44710

I. the undersigned, holding a valid commission issued by the National Board of Boiler and Pressure Vessel Inspectors and/or the State or Province of Ohio and employed by

OneBeacon Amcrica Insurance Company Lynn. Mass

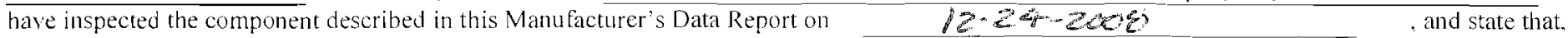
to the best of my knowledge and belief, the Manufacturer has constructed this pressure vessel in accordance with ASME Boiler and Pressure Vessel Code, Section VIII. Division 1. By signing this certificate neither the Inspector nor his/her employer makes any warranty. expressed or implied, coneerning the pressure vessel this described in this Manulacturer's Data Report. Furthemore, ncither the Inspector nor his/her enployer shall be liable in any manner for any personal injury or property damage or a loss of any kind arising from or connected with this inspection.

Date $01-06-200 \%$ Signed

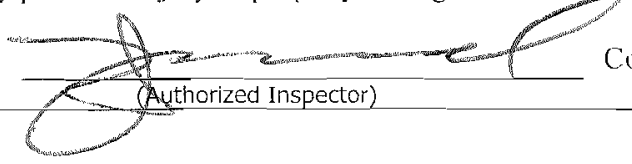

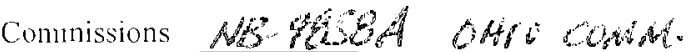
[National Board (incl endorsements) State, Prov, and number] 


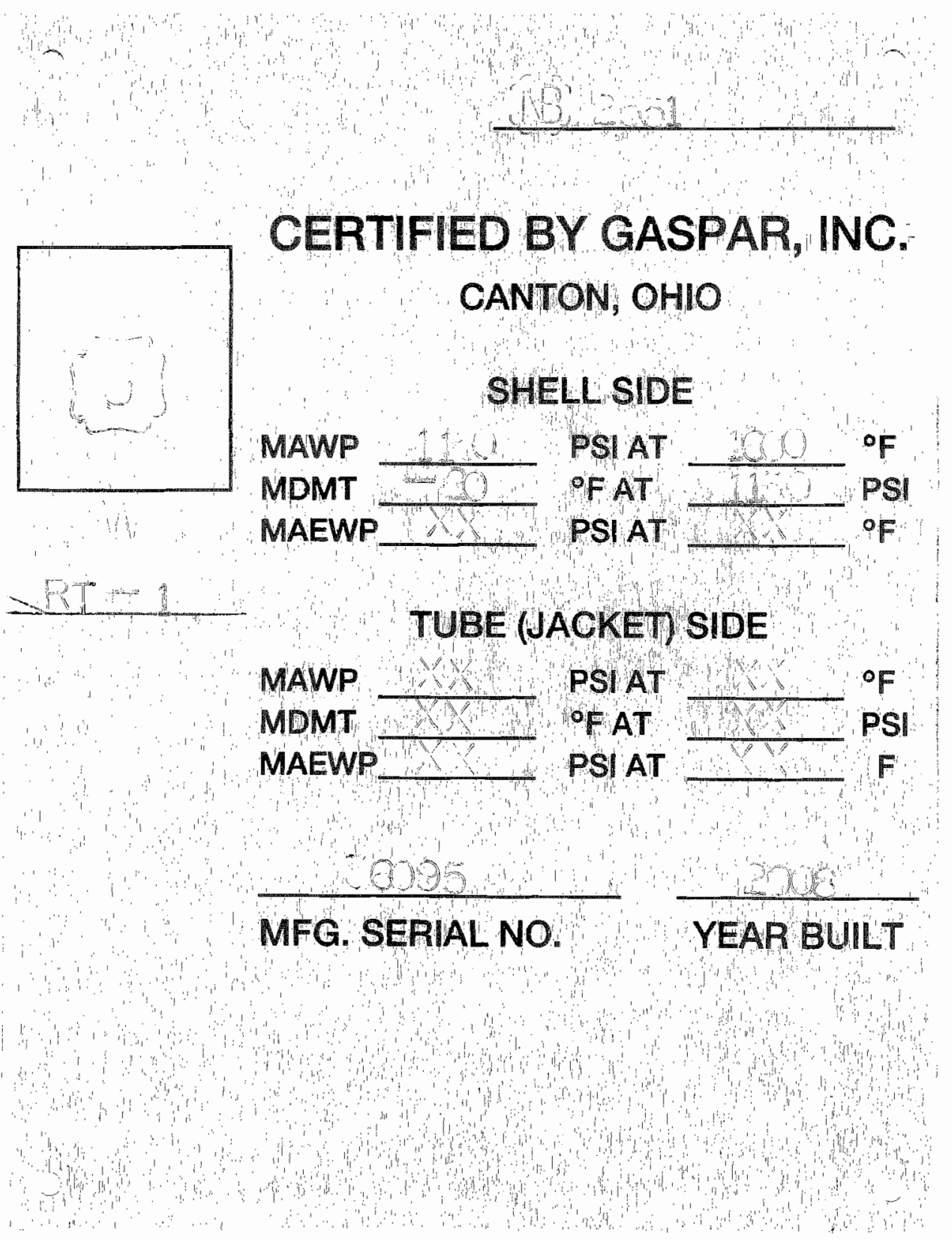




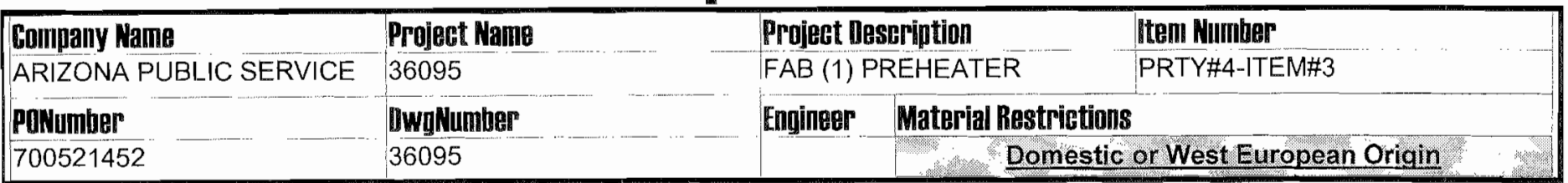

\section{MRK REV MIY TYPE IIESCRIPTION}

\begin{tabular}{|c|c|c|c|c|c|c|c|}
\hline Wh & LI & 10 & & 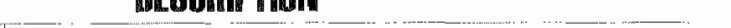 & & & \\
\hline 0.5 & - & & & (1) UNIT SHOWN (1) UNIT REQ'D & & & \\
\hline 1 & 2 & 1 & BAR/ROD & 3 DIA $\times 51 / 2$ LG & TOP HEAD & SB-446-N06625 & \\
\hline 1 & 2 & & & & & $\mathrm{GR} 2^{*}$ & \\
\hline 2 & 1 & 4 & BAR/ROD & $3 \mathrm{DIA} \times 431 / 4 \mathrm{LG}$ & SHELL & SB-446-No6625 & \\
\hline 2 & 1 & & & - - . - - & $\ldots$ & GR2* & \\
\hline 3. & 2 & 5 & PLATE & $1 / 4 \mathrm{FIN} \times 8 \mathrm{OD} \times 31 / 8 \mathrm{D}$ & SPIDER SPRT & $S A-240-316$ & \\
\hline 4 & 3. & 1 & PLATE & 7/16 THKX $141 / 2$ OD $\times 31 / 8 \mathrm{ID}$ & TOP MOUNT & $S A-240-316^{* *}$ & \\
\hline 5 & 2 & 4 & PLATE & $7 / 16$ THK $\times 23 / 4$ WD $\times 513 / 16 \mathrm{LG}$ & TOP MOUNT & SA-240-316 ** & \\
\hline 6 & 2 & 4 & PLATE & 7/16 THKX $\times 23 / 4$ WD $\times 513 / 16 \mathrm{LG}$ & TOP MOUNT & SA-240-316** & \\
\hline 7 & 2 & 1 & PLATE & $7 / 16 \mathrm{THK} \times 9$ OD $\times 31 / 8 \mathrm{lD}$ & TOP MOUNT & SA-240-316 $\ldots$ & \\
\hline 8 & 3 & 2 & PLATE & $7 / 16 \mathrm{THK} \times 2 \mathrm{WD} \times 41 / 4 \mathrm{LG}$ & LOWER SPRT CLIP & SA-240-316 & \\
\hline 9 & - & 1 & PLATE & $1 / 8 \mathrm{THK} \times 6 \mathrm{WD} \times 7 \mathrm{LG}$ & N/P & SA-240-304 & \\
\hline 10 & - & 1 & PLATE & $1 / 8$ THK $\times 2$ WD $\times 3$ LG & $\mathrm{N} / \mathrm{P}$ & SA-240-304 & \\
\hline 11 & 1 & 12 & TUBE & 2 WLD $\times 1 / 8$ THKX 1 LG CUT IN 72 DEG. SEG. & T/C PAD & INCONEL 617 & \\
\hline 11 & 1 & & & 1 PIPE MAKES 5 PARTS (MATERIAL BY CUSTOMER) & & & \\
\hline 12 & 1 & 1. & MISC & TURBULATOR- hITRAN MATRIX ELEMENT- $173^{\prime \prime}$ LG & SHELL & 316 SS & \\
\hline 12 & 1 & & & PART No. 25400-1A158-2FEA2-94D33-R-318A/4.00 & & & \\
\hline 13 & 2 & 1 & BAR/ROD & $3 \mathrm{DIA} \times 41 / 2 \mathrm{LG}$ & BTM HEAD & SB-446-N06625 & \\
\hline 13 & 2 & & & & & GR2* & \\
\hline 14 & 3 & & TUBE & $1 / 4$ OD X0.065 AW (SUPPLIED BY CUST) (SHIP LOOSE) & & SB-444-N06625 G & \\
\hline 999 & - & & NOTE & "HOT ROLLED OR FORGED & & & \\
\hline 999 & - & & & "A MINIMUM OF 2" EXTRA STOCK REQUIRED FOR CHEMICAL & & & \\
\hline 999 & - & & & ANALYSIS AFTER HEAT TREAT, THIS EXTRA LENGTH IS NOT & & & \\
\hline 999 & - & & & INCLUDED IN THE BILL OF MATERIAL. & & & \\
\hline 999 & - & & & *LONG DIMENSION OF OF BARIROD TOBE APPROX. & & & \\
\hline 999 & - & & & PARALLEL TO METAL FLOW LINES OF THE STOCK & & & \\
\hline 999 & - & & & **CARBON CONTENT EQUAL TO OR GREATER THAN .04\% & & & \\
\hline
\end{tabular}



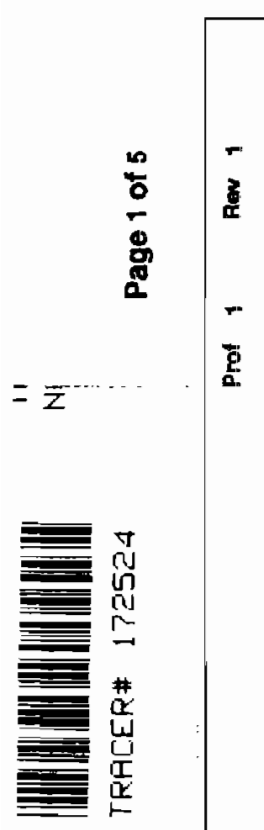

曷

홈

高弯

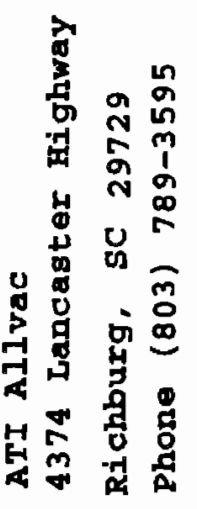

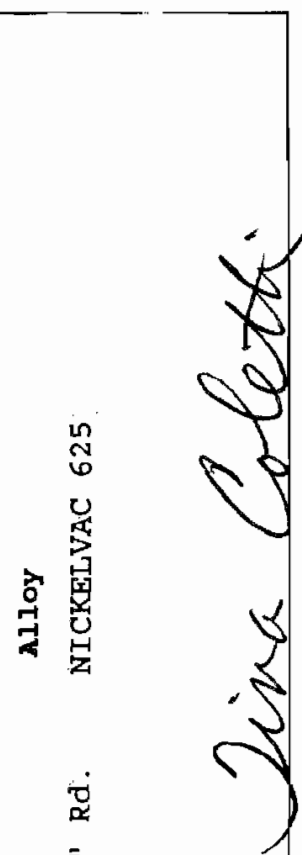

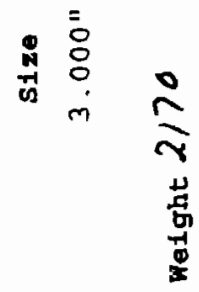

号

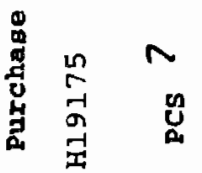
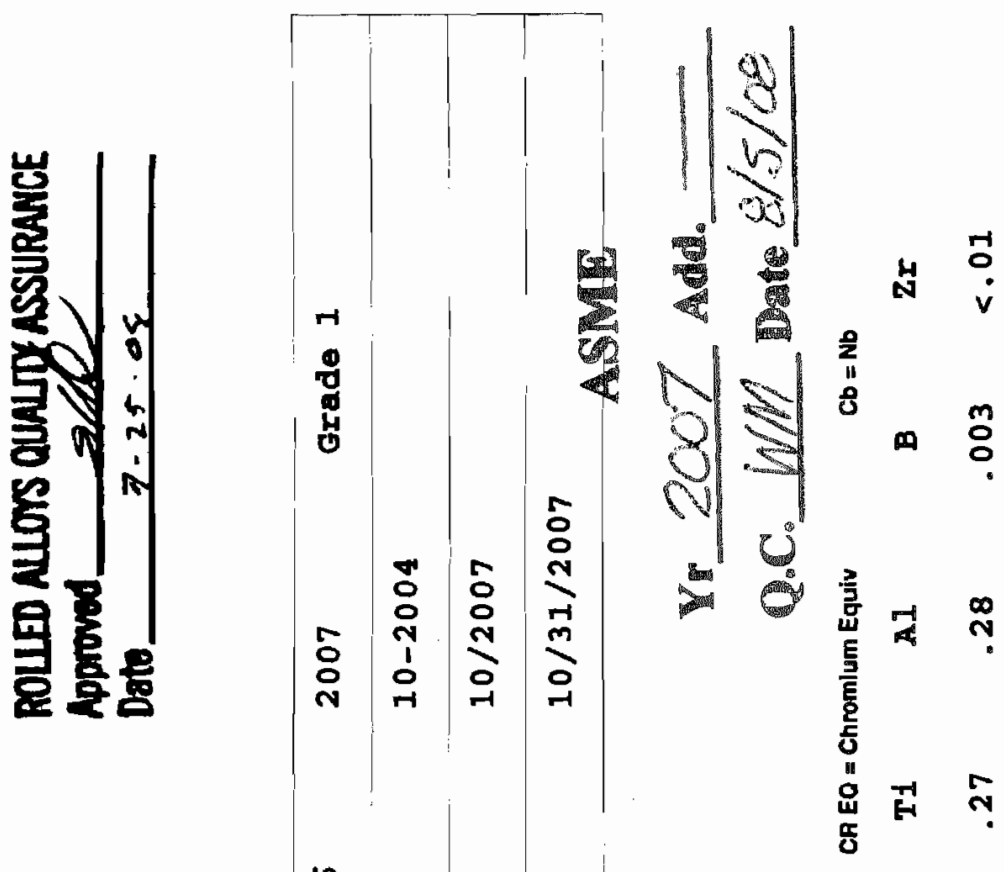

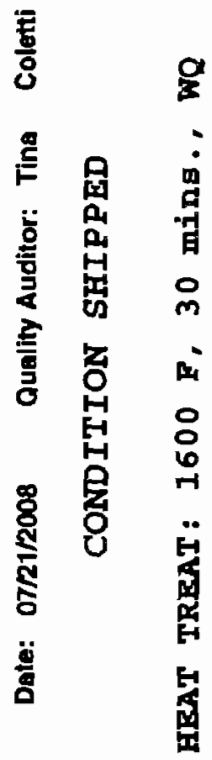

蒠

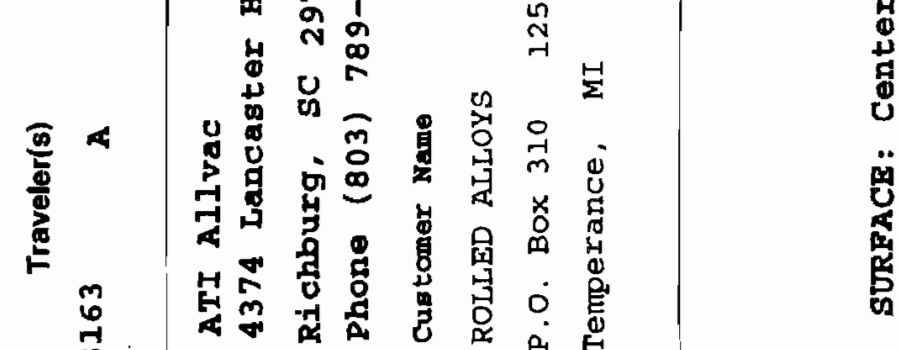

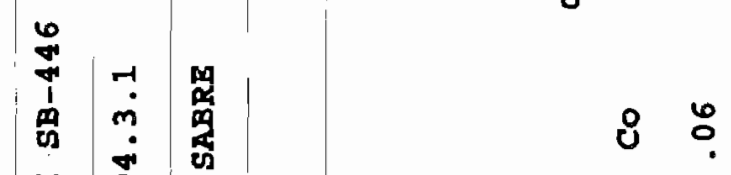

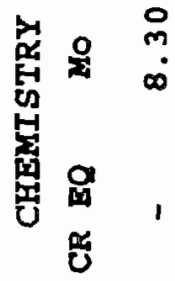

ثै $\begin{aligned} & \text { M } \\ & 0\end{aligned}$

ซึ :

皇

๓

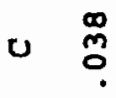

窗 
in

$\prod^{1}$

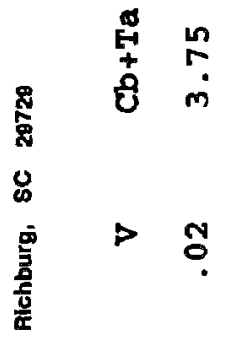

$=: \quad \frac{\mathrm{g}}{\mathrm{s}}$

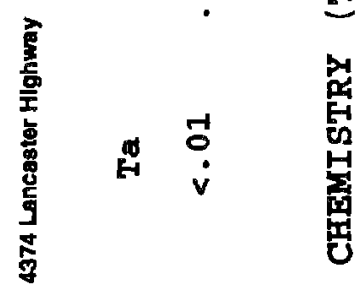

翣 $8 \stackrel{n}{m}$

ค $\stackrel{n}{\circ}$

홍

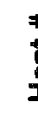

is<smiles>[CH]C=[Co]</smiles>

स $\stackrel{2}{2}$

(1)

8 :
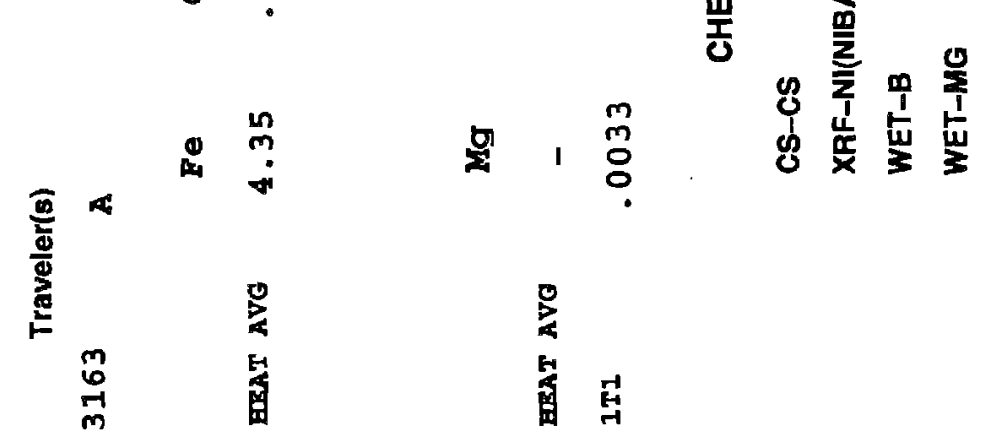
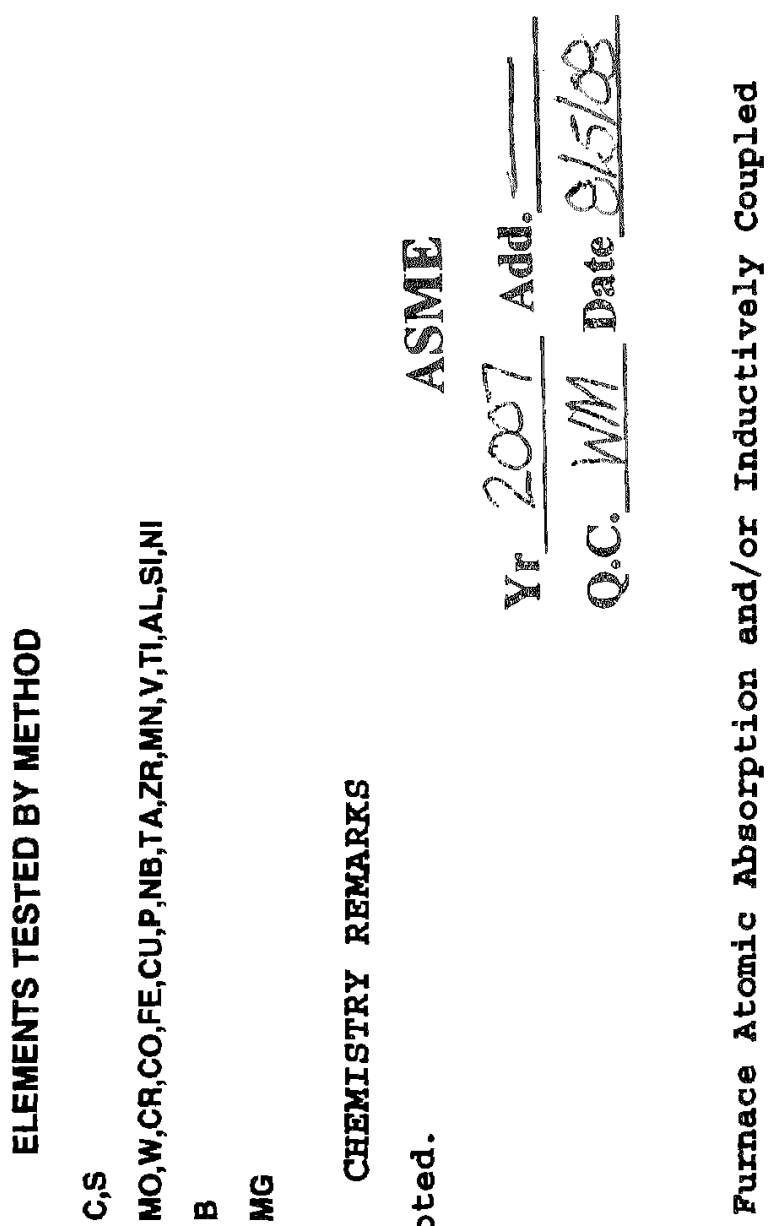

瓷
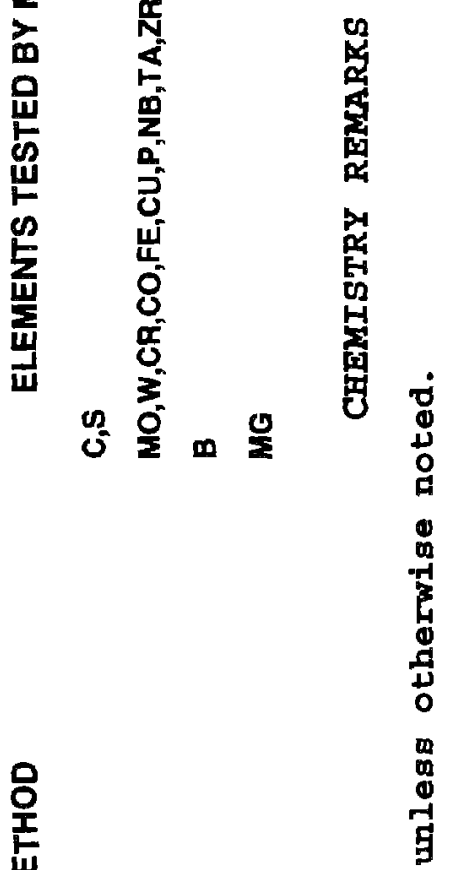

ย 5 व

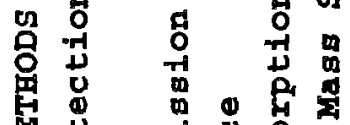

毁

경

点蛋

F 영 i 式

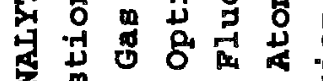

学

尊若

" I "

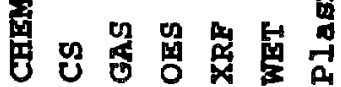


5
$\frac{0}{0}$
0
0
0
0
0

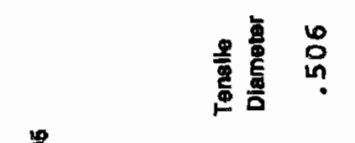

$\frac{p}{4}$

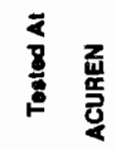

$$
\begin{aligned}
& \text { !I! }
\end{aligned}
$$

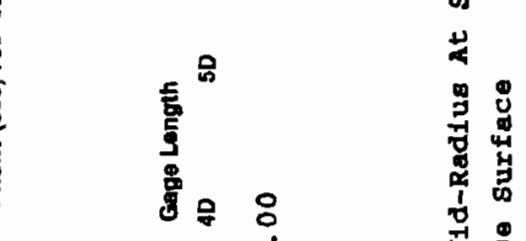

造

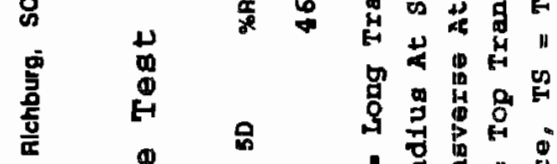

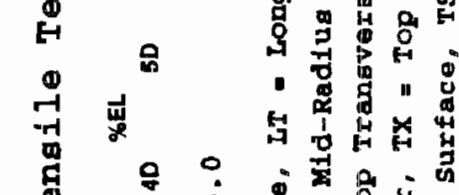

ब

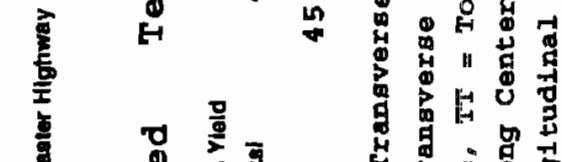

高

年

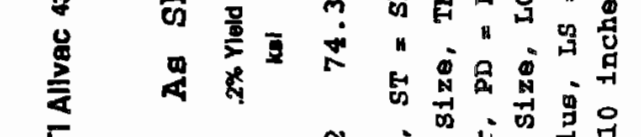

E

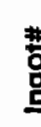

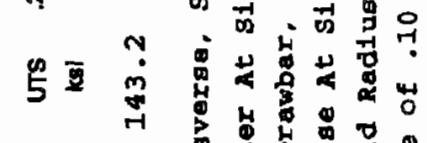

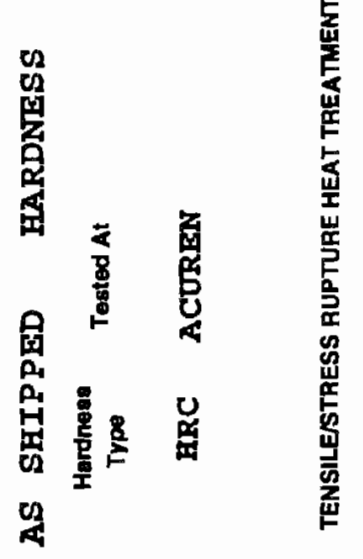

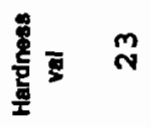

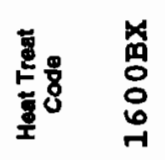

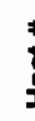

욷

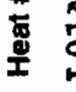

क्ञ

要

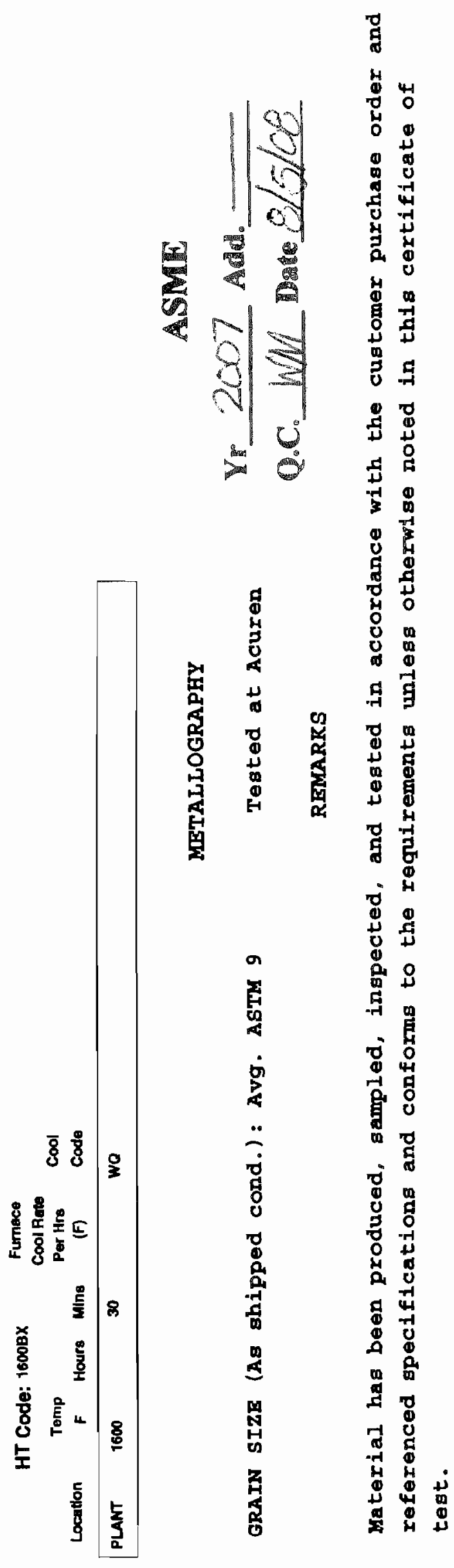




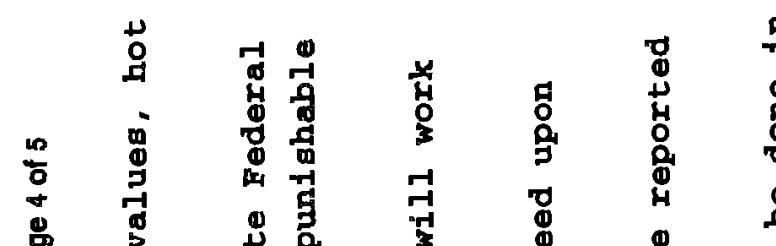

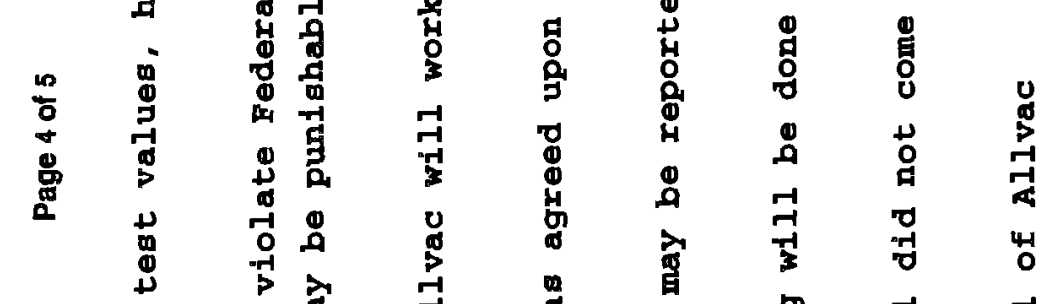

空

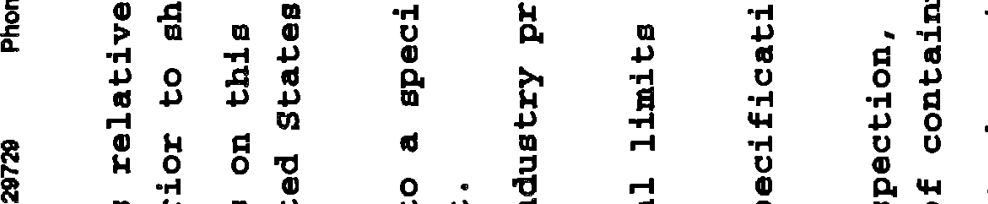

\&

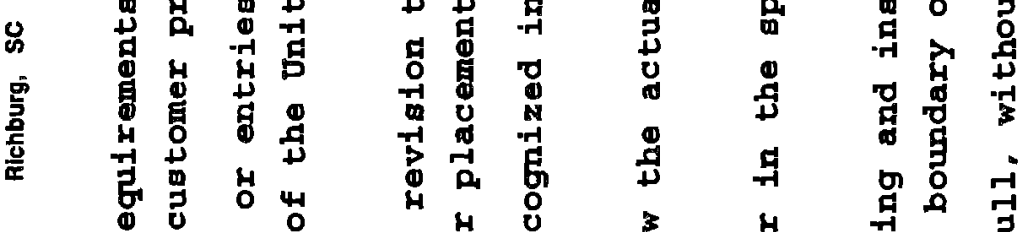

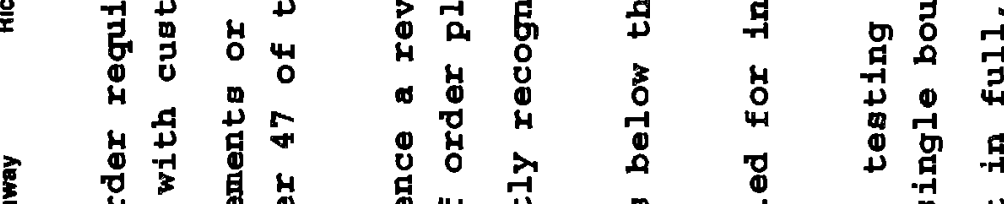

ก.

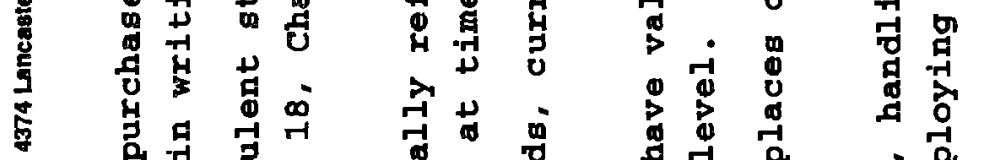

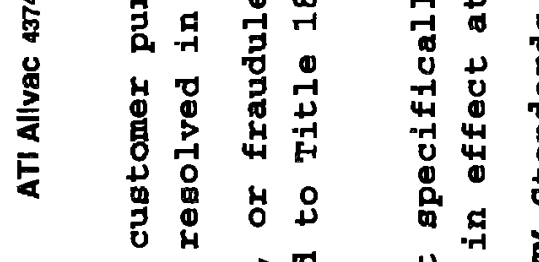

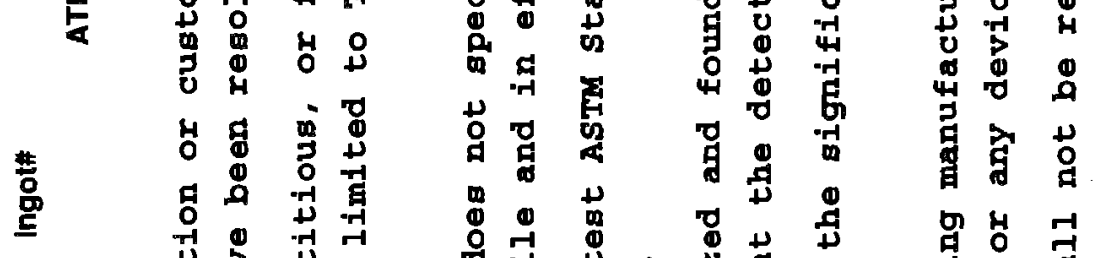

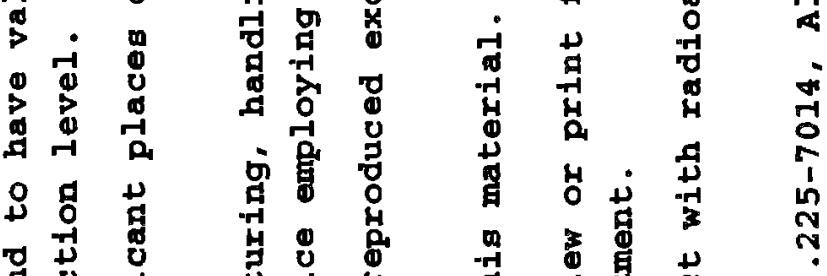

吾

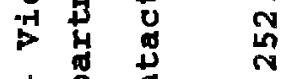

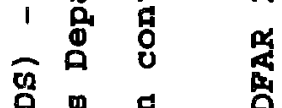

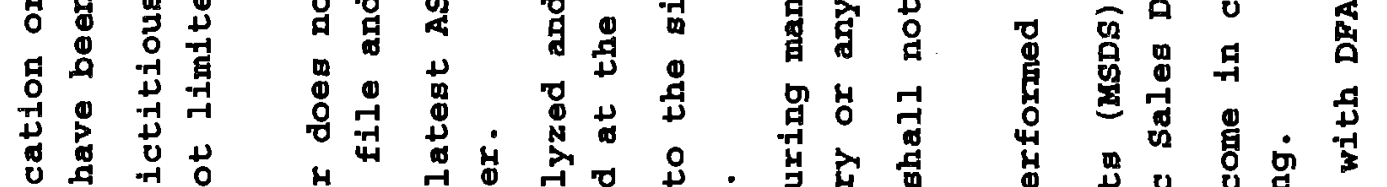

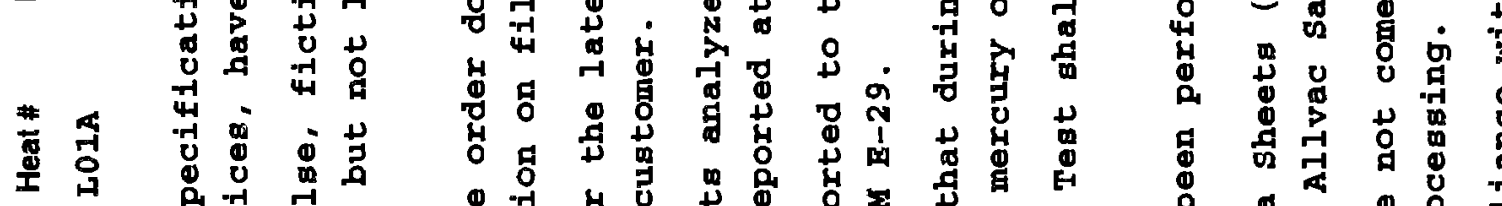

员

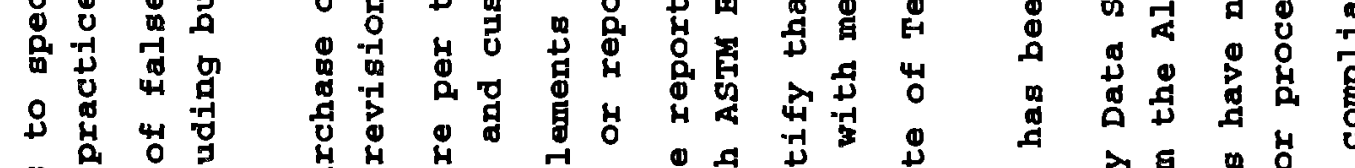

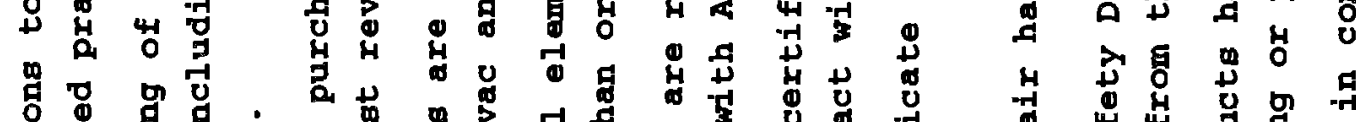

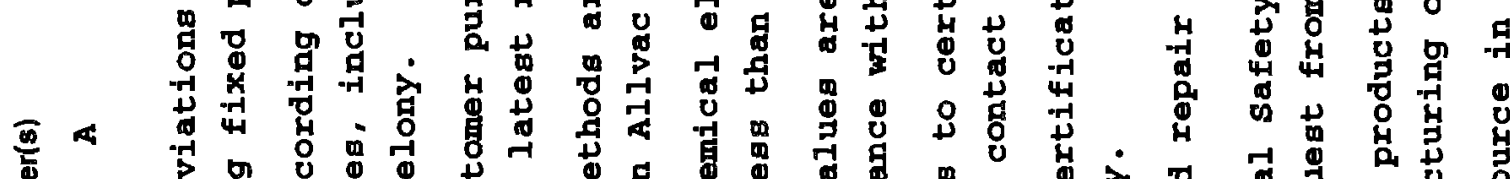

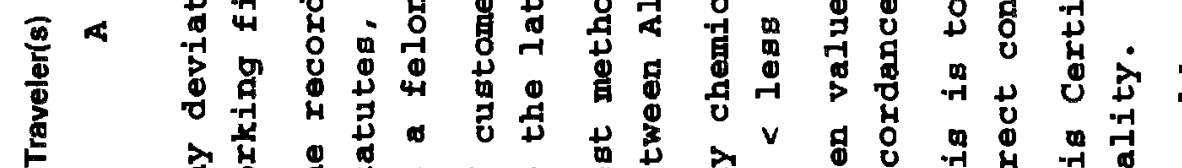

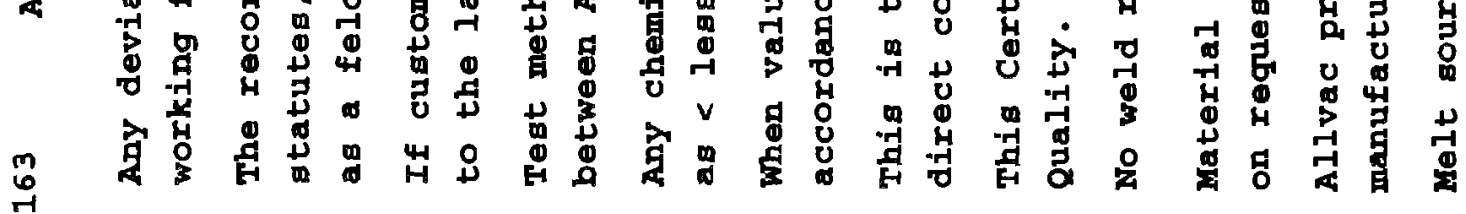




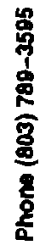

。

罢蛋思

象

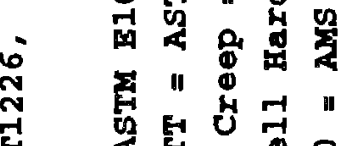

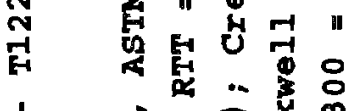

蛋

-

出 $\quad$ స

\&

竞

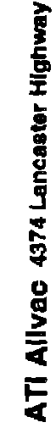

แก ก ...

花

రับ

E

में

ชั \%

点

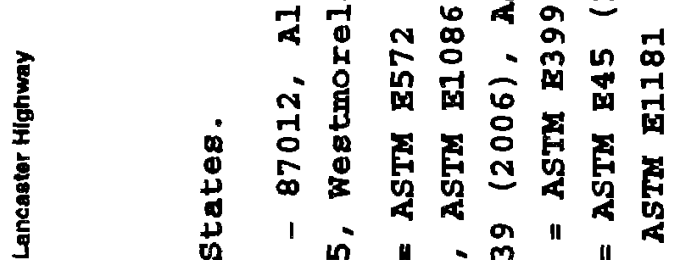

:

点

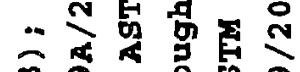

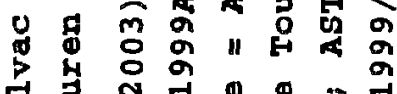

म

萻

o u

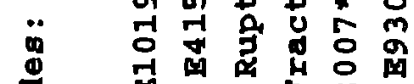

कृ

赑

불

重育

11 क

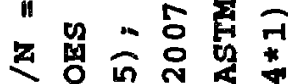

웅용

के

\%

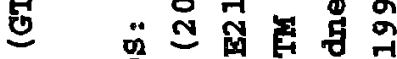

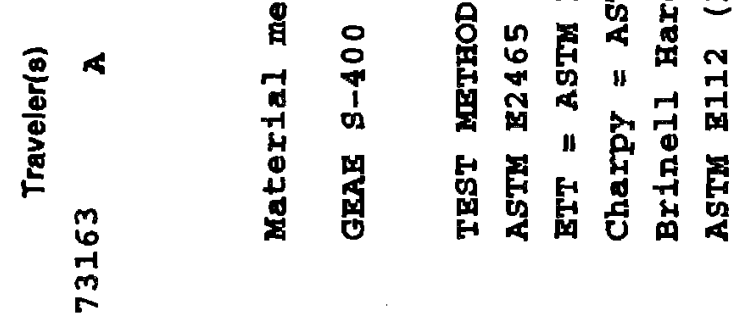

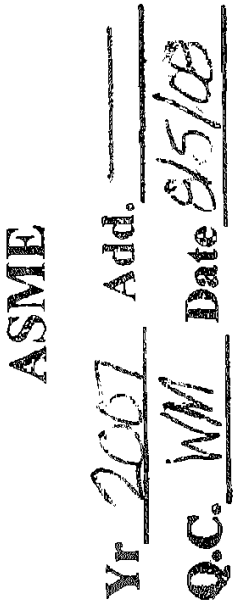




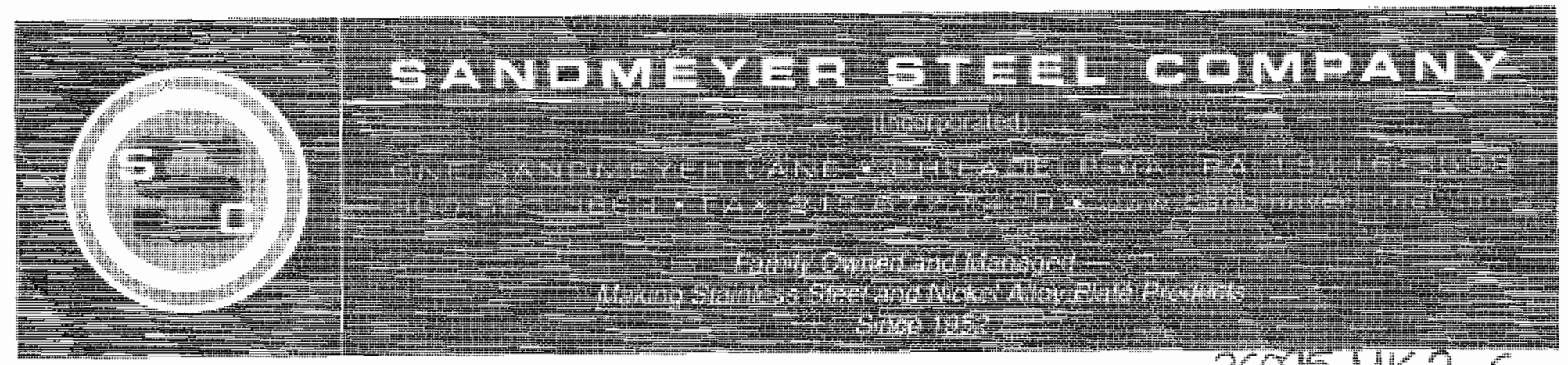

BIILL

TO

GASPAR INC.

1545 WHIPPLE AVE S.W.

CANTON, OH $4471 \%$

CUSTOMER ORDER NO. 31219

DATE: $10 / 28 / 2908$
CERTIFICATE OF TEST

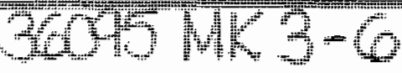

WE CERTIFY THAT THE CHEMTCAL ANALYSIS AND MECHANICAL TEST RESULTS APPEARING IN THIS CERTTFICATE ARE CORRECT AND TRUE AS REPORTED BY THE MANUFACTUREP.

SANDMEYER STEEL COMPANY

T. BOHNSACK - MANAGER, QUALITY ASSURANCE OUALITY CONTROL DEPARTMENT

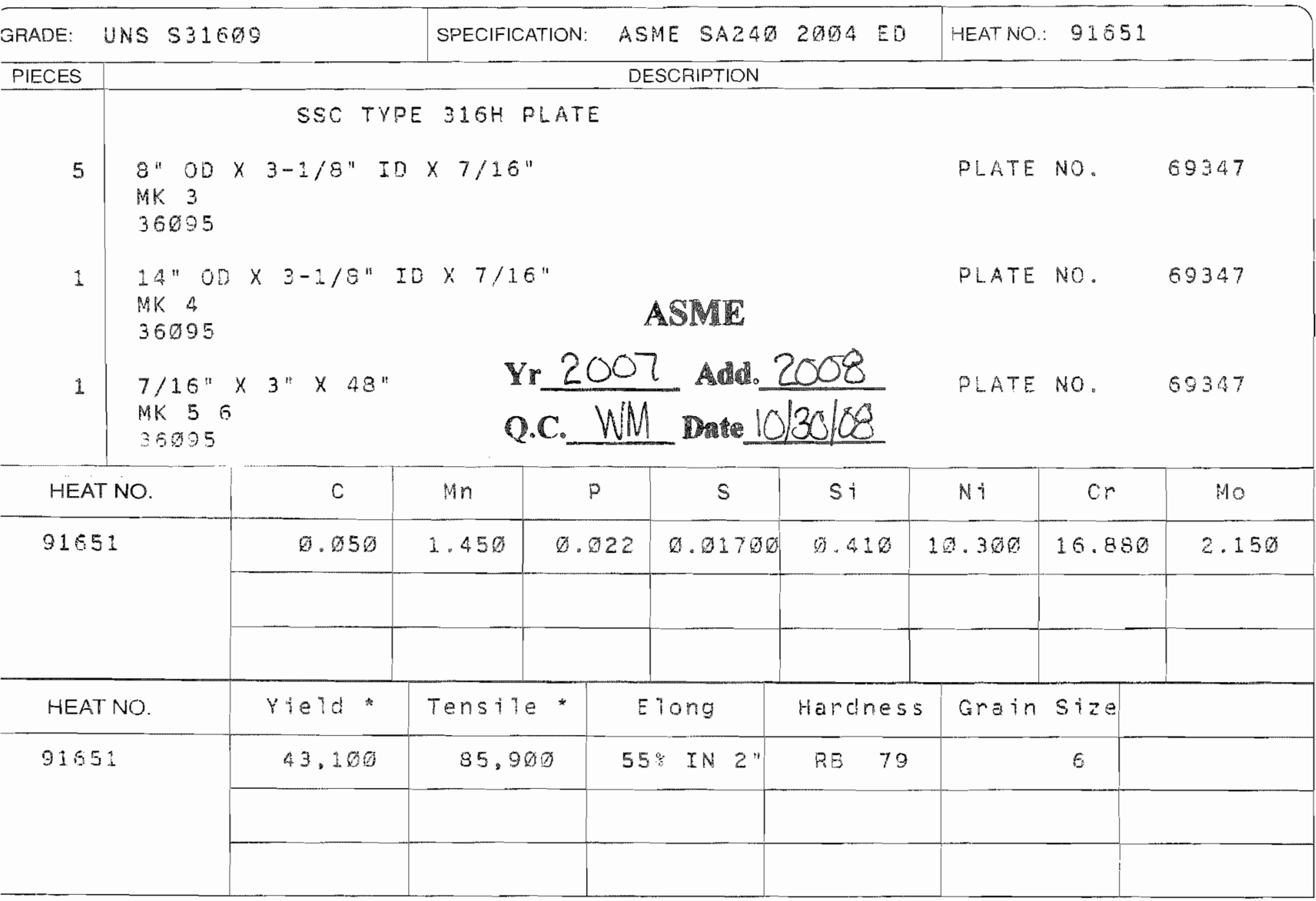

* LGS/INZ

MATERIAL SOLUTION ANNEALED AT 1950 DEGREES F MINIMUM

ANO WATER QUENCHED OR RAPIDLY COOLEO GY AIR

MATERIAL MANUFACTURED BY AVESTAPOLARIT PLATE

THIS MATERIAL IS FREE FROM MERCURY CONTAMINATION 


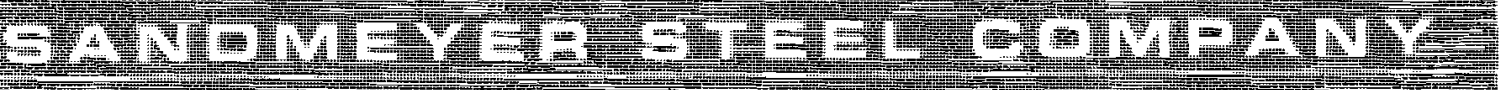

ETLi

GASPAR TNC.

1545 WHIPPLE AVE S.W.

CANTON, OH 44710

CUSTOMER ORDER NO. 31219

DATE: $\quad 10 / 28 / 2008$

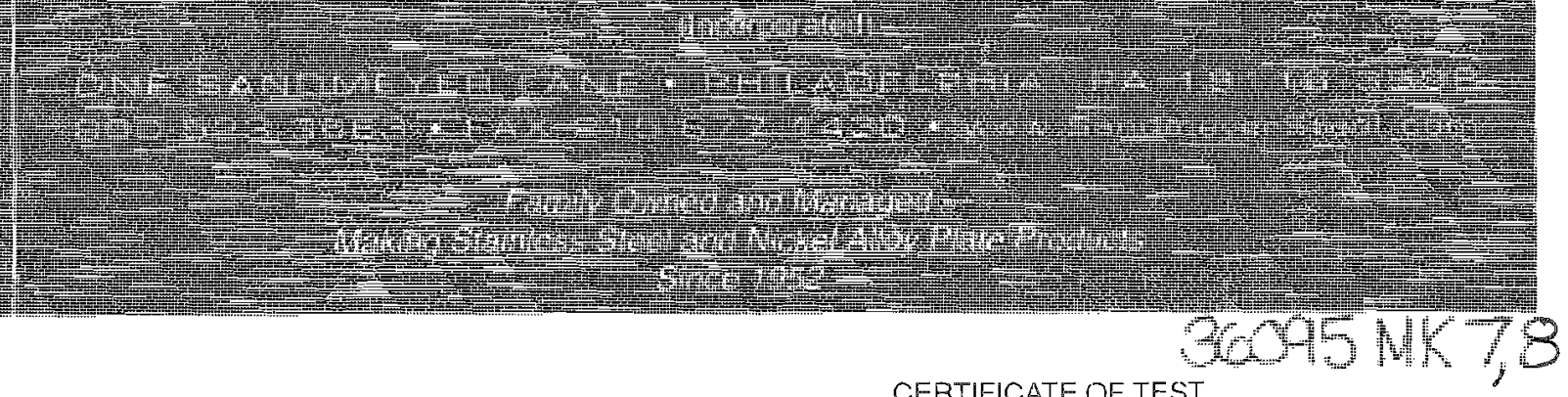

CERTIFICATE OF TEST

WE CERTIFY THAT THE CHEMICAL ANALYSIS AND MECHANICAL TEST RESULTS APPEARING IN THIS CERTIFICATE ARE CORRECT ANO TRUE AS REPORTED BY THE MANGFACTURER.

SANDMEYER STEEL COMPANY

T. BOHNSACK - MANAGER, QUALITY ASSURANCE QUALITY CONTROL DEPARTMENT

GRADE: UNS $\$ 31609$

SPECIFICATION: ASME $\$ A 24 D 2094$ ED

HEATNO: 9.1651

PIECES DESCRIPTION

SSC TYPE $316 H$ PLATE

$19000 \times 3-1 / 9: \operatorname{ID} \times 7 / 16 "$

PLATE NO.

69347

$M K \quad 7$

$\$ 6095$

$47 / 15^{\prime \prime} \times 2 \times 2-1 / 4 n$

348

ASMIL

PLATE NO.

69347

35093

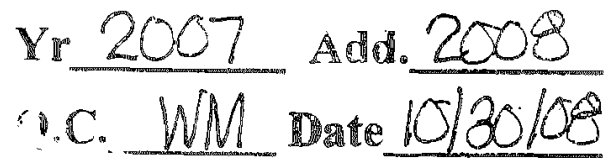

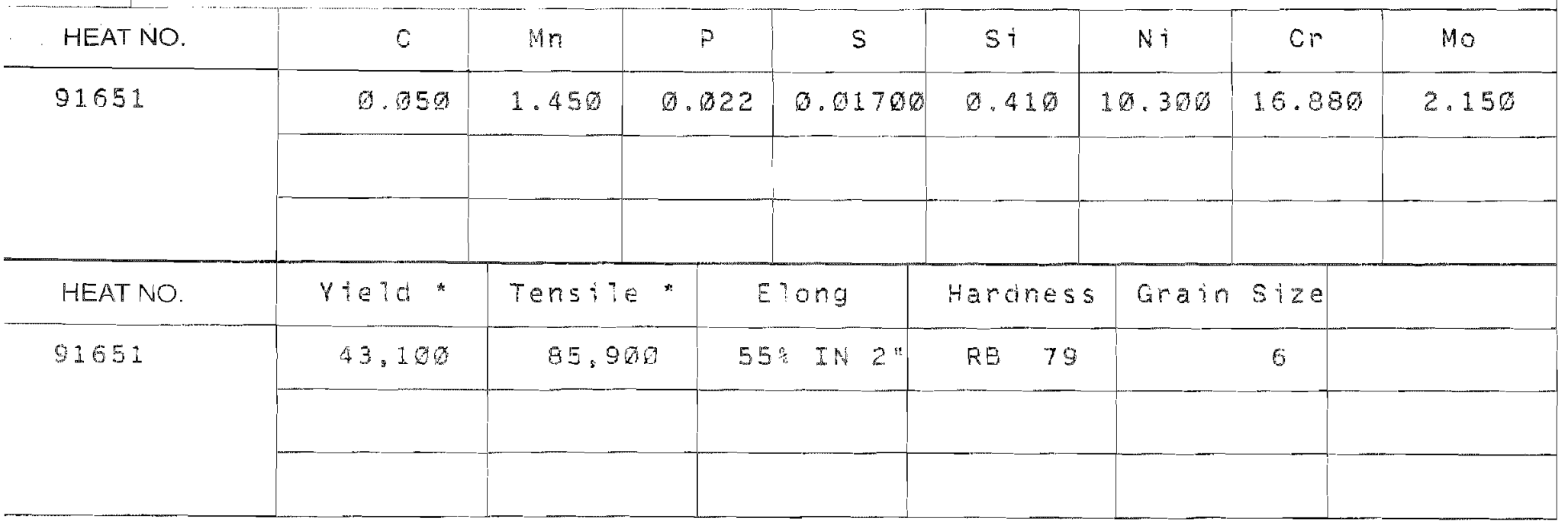

$*$ LBS/INZ

MATERIAL SOLUTION ANNEALED AT 1950 DEGREES F MINIMUM

ANS WATER QUENCHED OR RAPIDLY COOLED GY AIR

MATERIAL MANUFACTURED OY AVESTAPOLAFIT PLATE

THIS MATERIAL IS FREE FROM MERCURY CONTAMINATION 


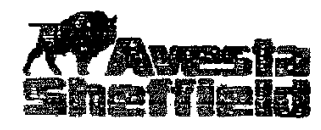

Avesta Sheffield East, Inc.

\section{Uertificate of Ânalyzis and Tegts}

OUR ORDER $\quad 95589 \quad-02$

SOLD TO: SANDMEYER STEEL COMPANY ONE SANDMEYER LANE

PHILADELPHIA
PA $\quad 19116$
HEAT \& PIECE 91651-1C

$5 / 13 / 97$

SHIP TO: SANDMEYER STEEL - CPU CONTACT STAN MATMERN $215-464-7100$

$782001-01$

YOUR ORDER \& DATE

29584

$5 / 09 / 97$

ITEM DESCRIPTION

HEAT \& PIECE $.91651-3167$

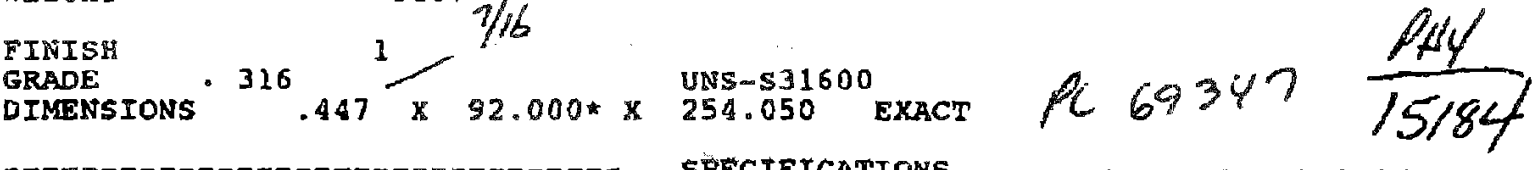

THE PRODUCTS LISTEO ON THIS MILI TEST REPORT SATISFY RREFERENCE CRITERION AS DEEINED IN ARTICLE AOI OF THE NORTH AMERICAN FREE TRADE AGREEMEN2. COUNTRY OF ORIGIN IS USA

ASTM A240-95B. MSME SA240-95

PLATES TEST FCS SOLUTION ANNEALED 1950 DEGREES FARENHEIT MINIMUM.

THEN WATER COOLED OR RAPIDLY COOLED BY AIR

FREE OF MERCURY CONTAMINATION

HOT ROLLED ANWEALED \& RICKLED \{HRAP)

$\begin{array}{lr}\text { HARDNESS RB } & 79 \\ \text { YIELD STRENGTH (PSI) } & 43100 \\ \text { TENSILE STRENGTH (PSI) } & 5900 \\ \text { BEND OKG } & \text { OK } \\ \text { INTERGRANULAR CORROSION } & \text { OR } \\ \text { ELONGATION IN 2\% } & 55.0 \\ \text { REDUCTION OF AREA } & 62.0\end{array}$

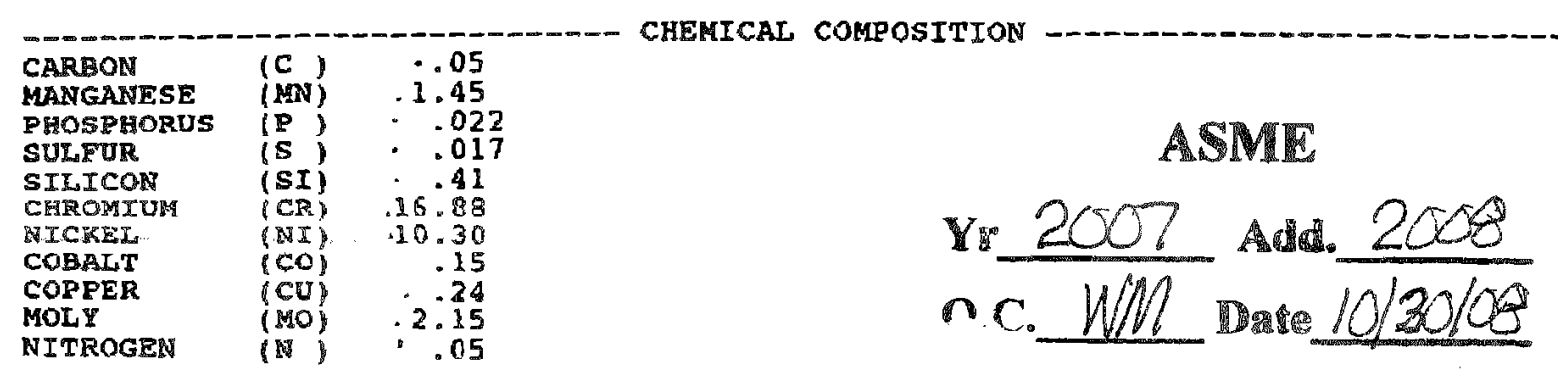

KNOWINGLY \& WILLULIY FALSIFYING OR CONCEALING A MATERIAL FACT ON THIS FORM, OR MAKIKG FALSE, EICTITIOUS OR FRAUDULENT STATEMENTS OR REPRESENTA2IONS HEREIN COULD CONSTITUTE A FELONY PUMISHABLE UNDER FEDERAL STATUTES.

J. BONGARDT" LAB MANAGER 
12244 WITROW GROUE ROAD

CANDORY, DE 19534

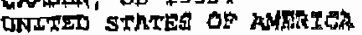

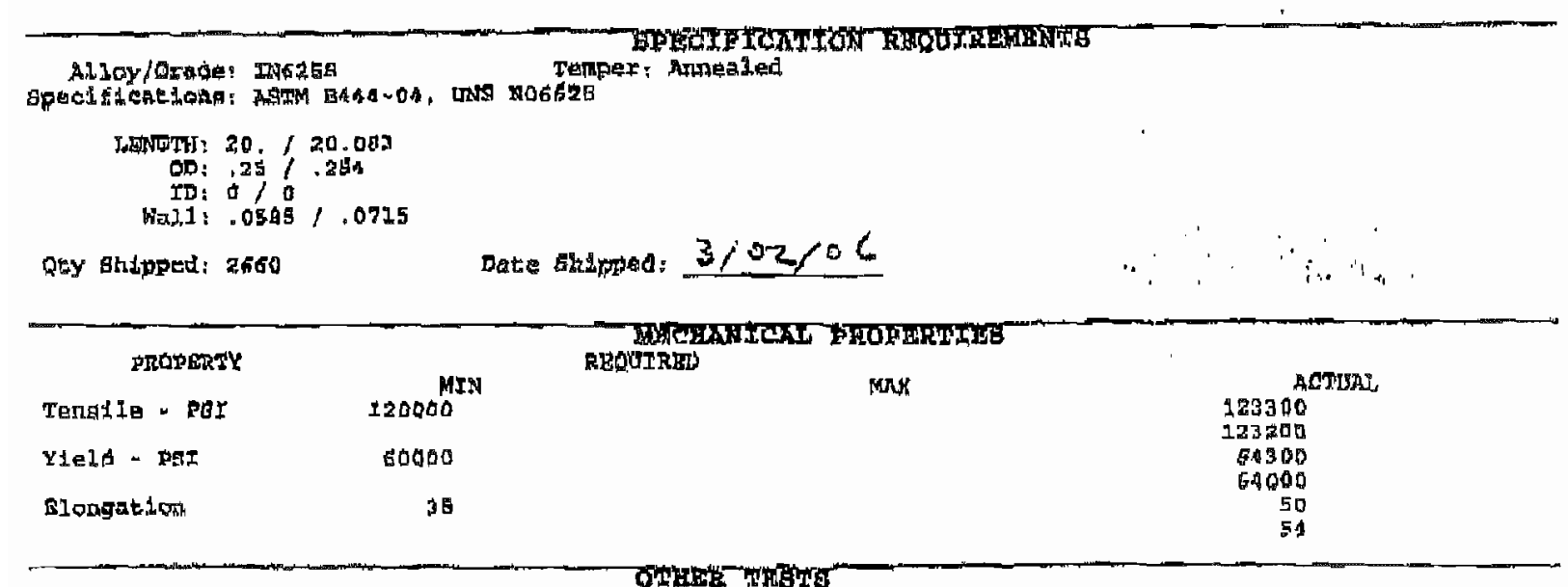

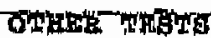

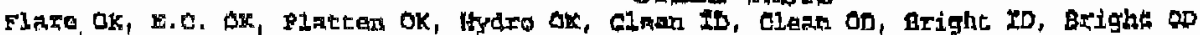

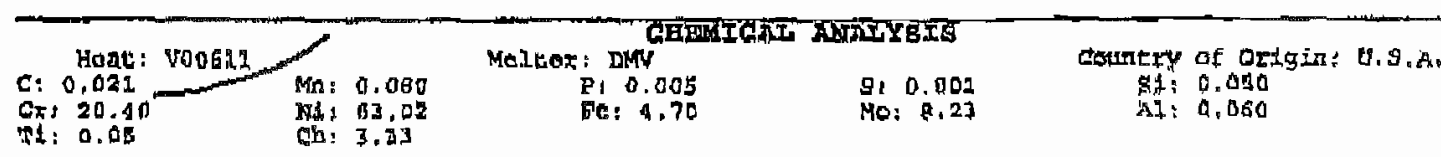

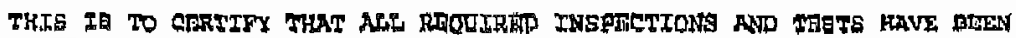

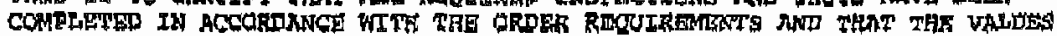

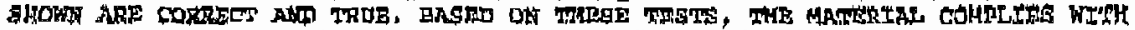

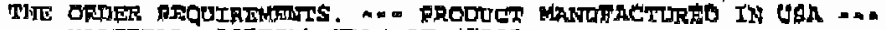

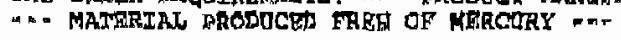

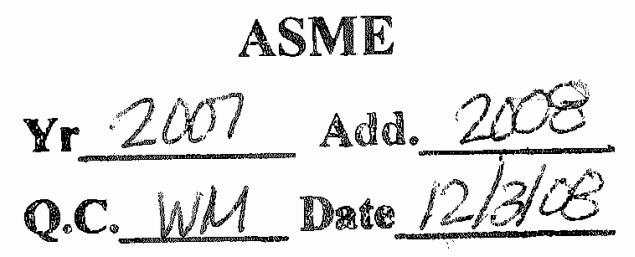

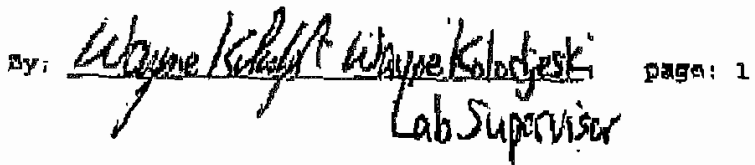

Datar, $3-2-206$ 


\section{Solar Atmospheres of Western PA \\ Certification}

To:

GASPAR INC

1545 WIPPLE AVE SW

CANTON

$\mathrm{OH} 44710$

All work performed subject to Solar Atmospheres Terms Of Sale as presented on form SA-1 (01-00).
Purchase Order No.: 30966

Packing List No.:

30095 MK $12,13,14$

Order No.: 39109

Date: $11 / 17 / 2008$

Entry Date: 10/10/2008

Page: 1 of 1

\begin{tabular}{r|r|r}
\hline Quantity & Part Number / Part Name / Part Description & Pounds \\
\hline 4 & IMATERIAL INCONEL 625 & 10 \\
& ITEM\# 36095-0 \\
& 1/4" X 83 1/4" TUBE \\
$2 \quad$ & MATERIAL INCONEL 625 \\
& ITEM\# 36095-0 \\
& 1/4" X 72 3/4" TUBE & \\
\hline
\end{tabular}

\begin{tabular}{ccc}
\hline Insp. Type Scale Minimum Maximum Number Other \\
\hline Customer Requirements: & \\
N/A & $\cdots$ & \\
\hline
\end{tabular}

THIS IS TO CERTIFY THAT THE ABOVE NAMED PARTS WERE PROCESSED IN ACCORDANCE WITH YOUR PURCHASE ORDER REQUIREMENTS AND PROCESS HW8598 10/17/08 JNH.

FURNACE RUN \# 70-8598-2123

This certification is no guarantee of material performance, properties, or microstructure. Mechanical, physical, and/or metallurgical testing is not performed unless specifically itemized on your purchase order to Solar Atmosoheres.

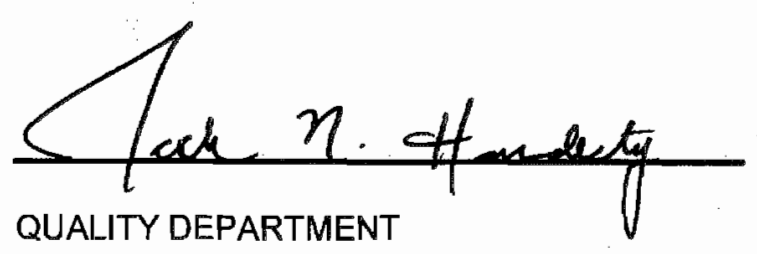

SOLAR ATMOSPHERES INC. 


\section{Solar Atmospheres of Western PA \\ Certification}

Order No.: 39109

Date: $11 / 17 / 2008$

Entry Date: $10 / 10 / 2008$

Page: 1 of 1

TO:

GASPAR INC

1545 WIPPLE AVE SW

CANTON

$\mathrm{OH} 44710$

Purchase Order No.: 30966

Packing List No.: 87379

Material: INCONEL 625

All work performed subject to Solar Atmospheres Terms Of Sale as presented on form SA-1 (01-00).

\begin{tabular}{|c|c|c|}
\hline Quantity & Part Number / Part Name / Part Description & Pounds \\
\hline 4 & $\begin{array}{l}\text { MATERIAL INCONEL } 625 \\
\text { ITEM \# 36095-0 } \\
\text { 3" DIA X 43 3/4" BAR }\end{array}$ & 420 \\
\hline 1 & $\begin{array}{l}\text { MATERIAL INCONEL } 625 \\
\text { ITEM\# } 36095-0 \\
\text { 3" DIA X 9" BAR }\end{array}$ & \\
\hline 1 & $\begin{array}{l}\text { MATERIAL INCONEL } 625 \\
\text { ITEM\# 36095-0 } \\
\text { 3" DIA X } 5 \text { 1/2" BAR }\end{array}$ & \\
\hline 1 & $\begin{array}{l}\text { MATERIAL INCONEL } 625 \\
\text { ITEM\# 36095-0 } \\
\text { 3" DIA X } 63 / 4 " \text { BAR }\end{array}$ & \\
\hline 1 & TEST PIECE & \\
\hline Insp. Type & Scale Minimum Maximum Number Other & \\
\hline \multicolumn{3}{|c|}{ Customer Requirements: } \\
\hline $\mathrm{N} / \mathrm{A}$ & . & \\
\hline
\end{tabular}

THIS IS TO CERTIFY THAT THE ABOVE NAMED PARTS WERE PROCESSED IN ACCORDANCE WITH YOUR PURCHASE ORDER REQUIREMENTS AND PROCESS HW8598 10/17/08 JNH.

FURNACE RUN \# 70-8598-2123

This certification is no guarantee of material performance, properties, or microstructure. Mechanical, physical, and/or metallurgical testing is not performed unless specifically itemized on your purchase order to Solar Atmosoheres.

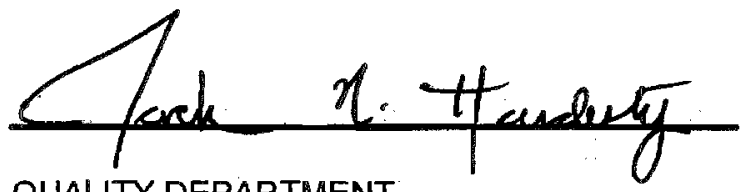

QUALITY DEPARTMENT SOLAR ATMOSPHERES INC. 


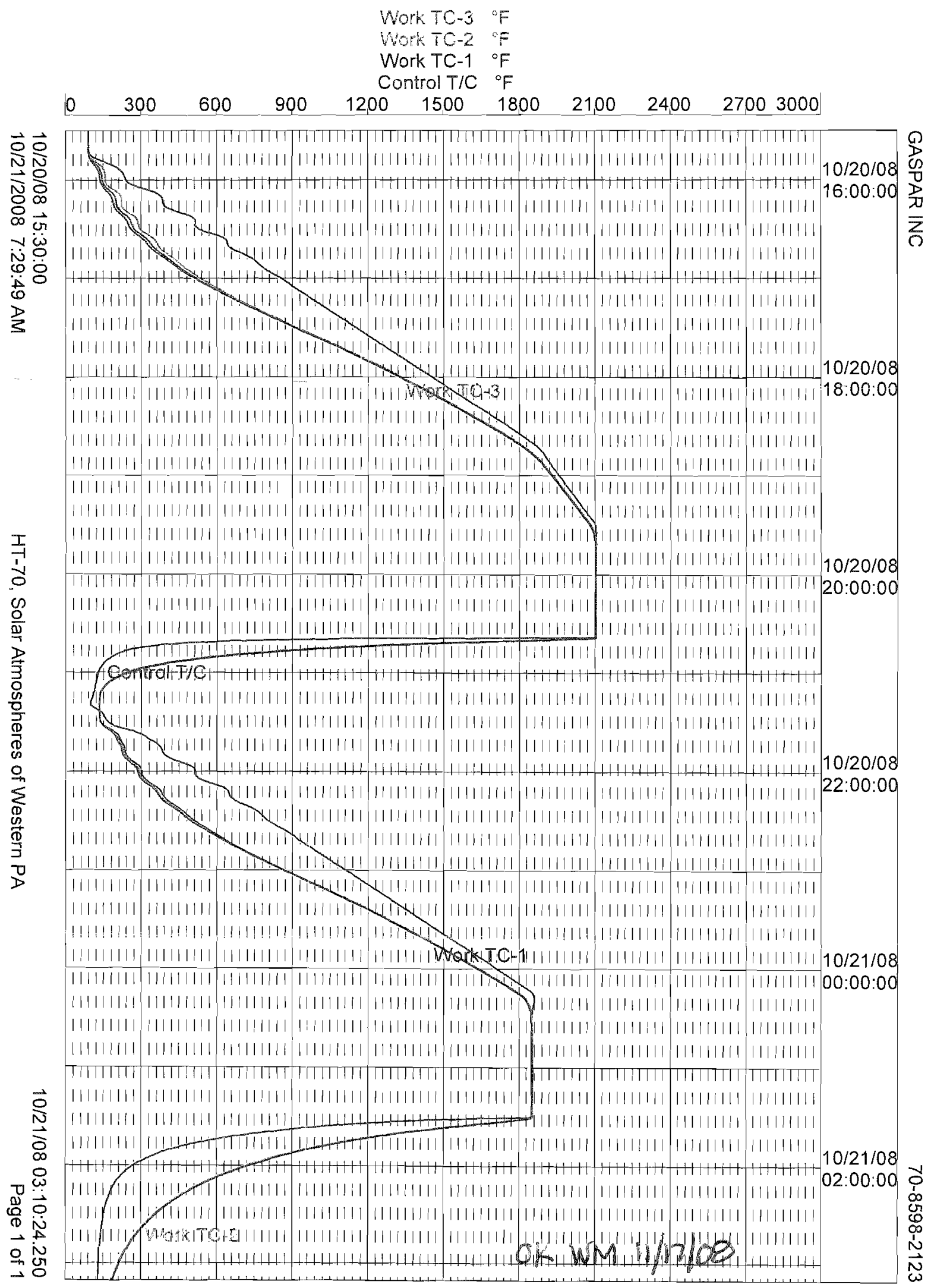


HW8598 10/17/08 NNH

SOLUTION ANNEAL / THERMALLY STABILIZE - INCONEL 625 - SOLAR SPEC GASPAR INC.

NOTE:

-RAMP RATE MAY BE ADJUSTED IF NECESSARY.

PROCESS:

1. PUMP DOWN TO A HARD VACUUM ( $1 \mathrm{X} 10$ - 3 TORR OR LOWER ).

2. RAMP AT $600^{\circ} \mathrm{F} / \mathrm{HOUR}$ TO $2100^{\circ} \mathrm{F} \pm 25^{\circ} \mathrm{F}$.

3. HOLD AT $2100^{\circ} \mathrm{F} \pm 25^{\circ} \mathrm{F}$ FOR $60-75$ MINUTES, WORK TC'S.

4. HELIUM 2 BAR QUENCH TO BELOW $175^{\circ} \mathrm{F}$, WORK TC'S.

5. PUMP DOWN TO A HARD VACUUM ( $1 \mathrm{X} 10$ - 3 TORR OR LOWER ).

6. RAMP AT $600^{\circ} \mathrm{F} / \mathrm{HOUR}$ TO $1850^{\circ} \mathrm{F} \pm 25^{\circ} \mathrm{F}$.

7. HOLD AT $1850^{\circ} \mathrm{F} \pm 25^{\circ} \mathrm{F}$ FOR $60-75$ MINUTES, WORK TC'S.

8. -5 ARGON QUENCH TO BELOW $200^{\circ} \mathrm{F}$, WORK TC'S 
$36095 M K 1,2,13,14$

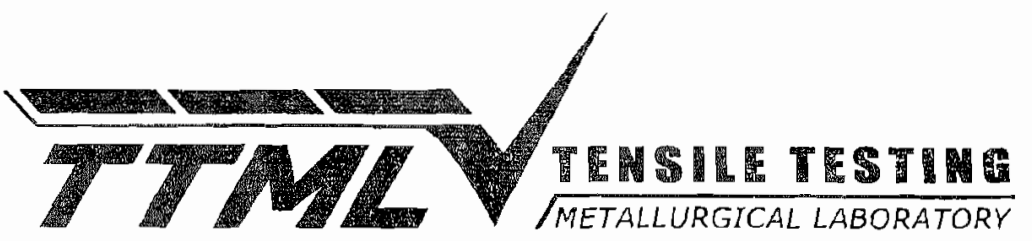

A DIVISION OF J.T. ADAMS CO., INC.

4520 WILLOW PARKWAY

CLEVELAND, OHIO 44125

PHONE (216) 641-3290

FAX (216) 641-1223

www.tensile.com

Gaspar Inc.

1545 Whipple Ave. SW

Canton $\mathrm{OH} \quad 44710$
Job No.: A8-296-826

Date: $\quad 10-29-08$

Cust. PO\#: $\quad 31221$

Description: $\quad 2$ samples “ 3" Dia.x 6" Bar $\quad$ Projectir 36095-e

$1 / 4 " x$ 48" Tube

4

Spec: $\quad$ ASTM B446/ASME SB44 N06625 Gr. 2

\begin{abstract}
Requirements (Min.):
Tube HTHVOCOI
\end{abstract}

$\underline{\text { Tensile. ksi }}$

100

122
Yield, .2\% ksi

40

49.7 $\frac{\text { Elong:\% in 2\% }}{30}$

61

Test Method: ASTM A370-08a

\section{Tensile, ksi \\ 100}

110
Yield. $.2 \% \mathrm{ksi}$

47.3

Test Method: ASTM A370-08a $\frac{\text { Elong. } \% \text { in } 4 \mathrm{D}}{30} \quad$ Redl. of Area, \%

62

HTHL01A

The above conforms to specifications listed.

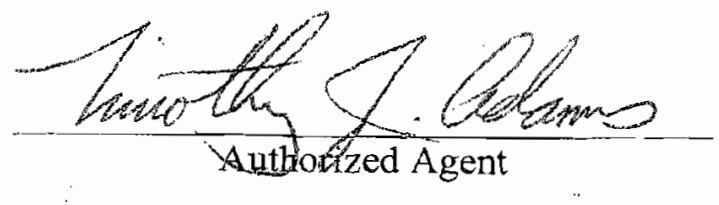

REOP: 


\section{BAKER INSPECTION GROUP}

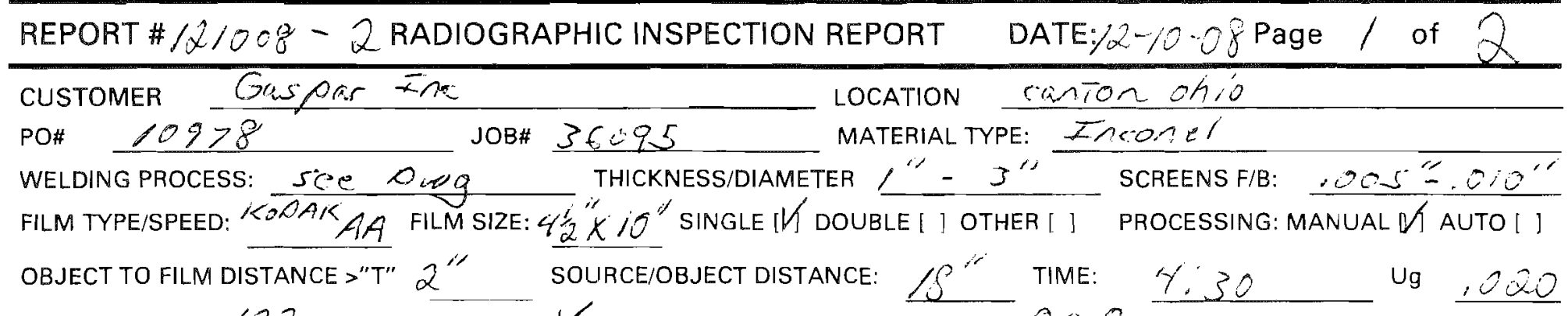

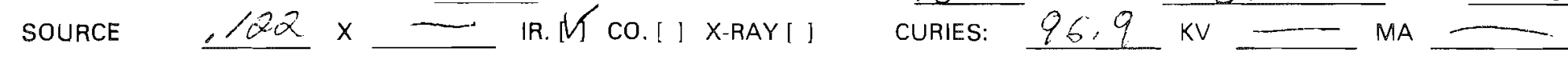

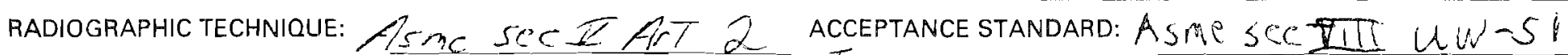

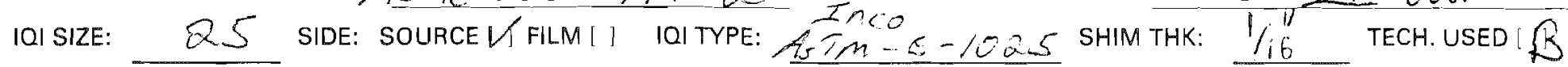
WE ASSUME NO RESPONSIBILITY FOR LOSSES OF ANY KIND DUE TO OUR INTERPRETATION OF THE QUALITY OF THE MATERIAL SUBMITTED. ALL DATA AND INFORMATION WILL BE HELD STRICTLY CONFIDENTIAL. ALL DATA ANDINEORMATION WILLBEHELDSTRICTLY CONFIDENTIAL.

\begin{tabular}{|c|c|c|c|c|c|c|c|c|c|c|c|}
\hline $\begin{array}{l}\text { SPECIMEN } \\
\text { IDENTIFICATION } \\
36095\end{array}$ & $\begin{array}{c}\text { FILM } \\
\text { INTERVAL } \\
\text { NUMBERS }\end{array}$ & $\begin{array}{l}\text { 㐔 } \\
\text { 岂 } \\
\text { 呆 }\end{array}$ & 髉 & 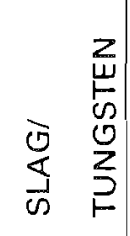 & $\begin{array}{l}\frac{\zeta}{w} \\
0 \\
\frac{1}{0} \\
0\end{array}$ & 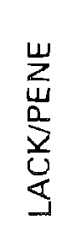 & \begin{tabular}{l|l}
2 & 0 \\
0 \\
0 \\
2 \\
$\frac{1}{2}$ \\
0 \\
2
\end{tabular} & 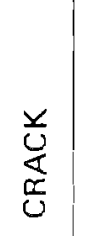 & 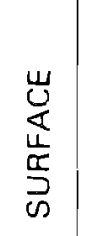 & 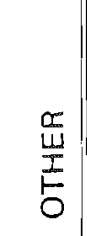 & $\begin{array}{l}\text { REMARKS AND/OR } \\
\text { DEFECT LOCATIONS } \\
\text { NOTE: N.A.D. = No Apparent } \\
\text { Defects }\end{array}$ \\
\hline \multirow[t]{6}{*}{$w_{s}+1$} & $v-0$ & 7 & & & & & & & & & $N, \triangle, D$, \\
\hline & $r-1$ & $V$ & & & & & & & & & -10 \\
\hline & $\not-2$ & 7 & & & & & & & & & \\
\hline & $V-3$ & 7 & & & & & & & & & \\
\hline & $V-4$ & 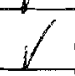 & & & & & & & & & 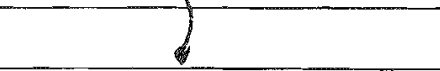 \\
\hline & $r-5$ & $\checkmark$ & & & & & & & & & $N, A, D$, \\
\hline & & & & & & & & & & & \\
\hline \multirow[t]{8}{*}{$\omega s \mathbb{2}$} & $v-0$ & $\sqrt{ }$ & & & & & & & & & $N A, D$. \\
\hline & $V-1$ & $\checkmark$ & & & & & & & & & $C$ \\
\hline & $r-2$ & $v$ & & & & & & & & & \\
\hline & $v-3$ & ir & & & & & & & & & \\
\hline & $V-4$ & $i$ & & & & & & & & & $\sqrt{ }$ \\
\hline & $r-5$ & 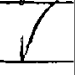 & & & & & & & & & $N, A, D$ \\
\hline & & & & & & & & & & & \\
\hline & & & & & & & & & & & \\
\hline \multirow{2}{*}{\multicolumn{2}{|c|}{ REVIEWER: }} & \multirow{2}{*}{\multicolumn{7}{|c|}{$\begin{array}{l}\text { RADIOGRAPHER: } S, L \text {, } \\
\text { SNT-TC1A LEVEL: } \mathbb{I}\end{array}$}} & & \multirow{2}{*}{\multicolumn{2}{|c|}{$\begin{array}{l}\text { CLENT REVIEWER } \\
\text { WOX RU MOOP }\end{array}$}} \\
\hline & & & & & & & & & & & \\
\hline
\end{tabular}

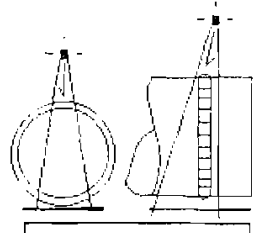

DWE/DWV

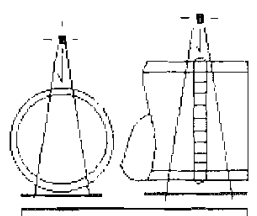

DWE/DWV
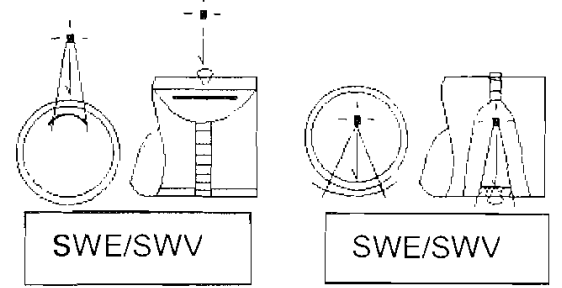

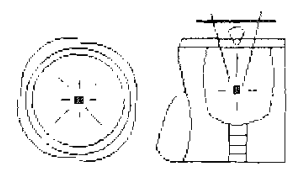

SWE/SWV 


\section{BAKER INSPECTION GROUP}

\section{REPORT \#/2/208-4 RADIOGRAPHIC INSPECTION REPORT DATE: $12 \% 12-98^{2}$ Page $/$ of 1}

CUSTOMER Gaspar InC

PO\#

WELDING PROCESS:

SCe LWO THICKN

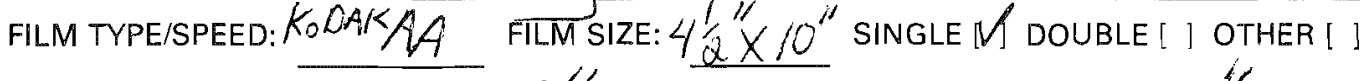

OBJeCT TO FILM DISTANCE >"T" 2 " SOURCE/OBJeCt Distance: 20 " TIME:

SOURCE I22 $\quad$ IR.K CO.I ] X-RAY[1 CURIES: 95.1

Radiographic technique: Asme sec If A.T 2 acceptance standard: Asme sec IIIII lic -51

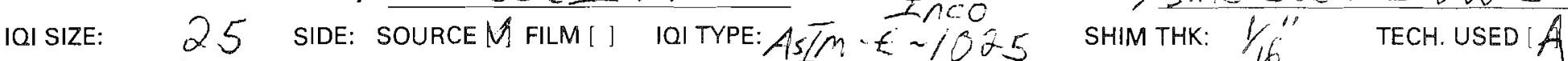
WE ASSUME NO RESPONSIBLITYY FOR LOSSES OF ANY KIND DUE TO OUR INTERPRETATION OF THE QUALITY OF THE MATERIAL SUBMITTED. ALL DATA AND INFORMATION WILL BE HELD STRICTLY CONFIDENTIAL.

\begin{tabular}{|c|c|c|c|c|c|c|c|c|c|c|c|}
\hline $\begin{array}{l}\text { SPECIMEN } \\
\text { IDENTIFICATION } \\
36095\end{array}$ & $\begin{array}{c}\text { FILM } \\
\text { INTERVAL } \\
\text { NUMBERS }\end{array}$ & 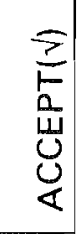 & 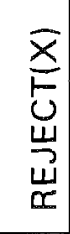 & 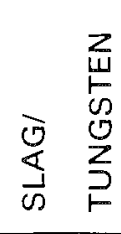 & 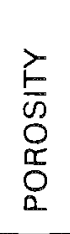 & 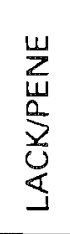 & $\begin{array}{l}z \\
\frac{0}{0} \\
\frac{1}{3} \\
\frac{1}{2} \\
0 \\
z\end{array}$ & 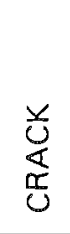 & 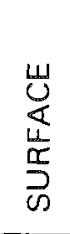 & 営 & $\begin{array}{l}\text { REMARKS AND/OR } \\
\text { DEFECT LOCATIONS } \\
\text { NOTE: N.A.D. = No Apparent } \\
\text { Defects }\end{array}$ \\
\hline \multirow[t]{14}{*}{$25^{*} 5$} & $M-0$ & $\checkmark$ & & & & & & & & & $N, A, D$ \\
\hline & $V-i$ & $\checkmark$ & & & & & & & & & $N, A, D$ \\
\hline & $11-2$ & $\checkmark$ & & & & & & & & & $N, A, D$ \\
\hline & $V-3$ & $\mathcal{L}$ & & & & & & & & & N.A,D. \\
\hline & $V-4$ & $\checkmark$ & & & & & & & & & $N, A, D$ \\
\hline & $V-5$ & $\checkmark$ & & & & & & & & & $N, A, D$ \\
\hline & & & & & & & & & & & \\
\hline & & & & & & & & & & & \\
\hline & & & & & & & & & & & \\
\hline & & & & & & & & & & & \\
\hline & & & & & & & & & & & \\
\hline & & & & & & & & & & & AI REUEWI \\
\hline & & & & & & & & & & & (X) $22-1900$ \\
\hline & & & & & & & & & & & \\
\hline
\end{tabular}

\section{REVIEWER: 7 Radographer: S, Lindsey SNT-TC1A LEVEL:}

"A" = Pipe Diameter $\leq 3^{1} / 2 "$
SINT-TC1A LEVEL: "B" = Pipe O.D. $\geq 3 \frac{1}{2} 2^{\prime \prime}$ to Unlimited
CLIENT REVIEWER

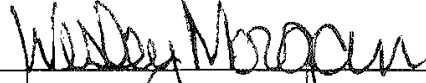

" $\mathrm{C}^{\prime}=$ Diamete limited by

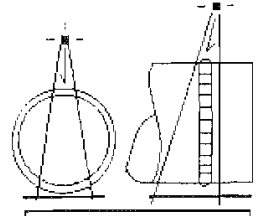

DWE/DWV

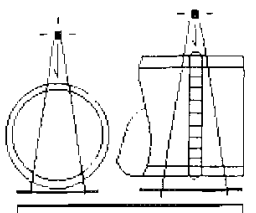

DWE/DWV

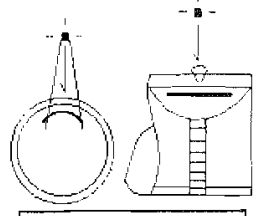

SWE/SWV

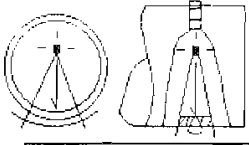

SWE/SWV

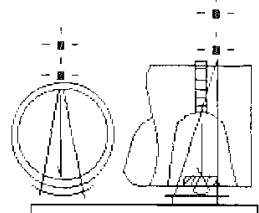

DWE/SWV

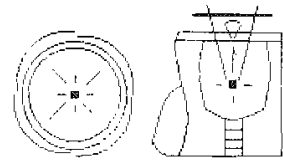

SWE/SWV

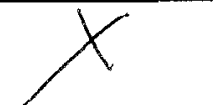




\section{GASPAR, INC. \\ LIQUID PENETRANT INSPECTION REPORT}

DATE: December 23, 2008

JOB \#: 36095

PT: PROCEDURE: PT-1 REV.7

PENETRANT TYPE : [X] Direct Visual

ACCEPTANCE STANDARD: [X] ASME Sect. VIII Div.I [ ] ASME B31.1 [ ] Other -

MATERIAL IDENTIFICATION

\begin{tabular}{ll}
\hline CLEANER: & SKC-S \\
\hline PENETRANT: & SKL-SP \\
\hline DEVELOPER: & SKD-S
\end{tabular}

LIGHTING EQUIPMENT: Portable AC Halogen single light of 100 watts.

COMPONENT DESCRIPTION / PC. \#: PREHEATER

DRAWING \#: 36095 REV.4

THICKNESS / DIMENSIONS: SEE DWG.

\begin{tabular}{|c|c|c|c|c|c|}
\hline $\begin{array}{c}\text { WELD ID OR } \\
\text { PART NUMBER }\end{array}$ & TEMP. & SURF. COND. & $\begin{array}{l}\text { ACCEPT/ } \\
\text { REJECT }\end{array}$ & $\begin{array}{l}\text { SIZE AND TYPE } \\
\text { OF INDICATION }\end{array}$ & REMARKS \\
\hline NON-PRESSURE WELDS & $60^{\circ} \mathrm{F}$ & AS WELDED & ACCEPT & NONE & \\
\hline & & & & & \\
\hline & & & & & \\
\hline & & & & & \\
\hline & & & & & \\
\hline & & & & & \\
\hline & & & & & \\
\hline & & & & & \\
\hline
\end{tabular}

ADDITIONAL REMARKS, SKETCHES, ETC.

PERFORMED BY:

JIM FREDERICK/LEVEL ॥ 


\section{CALCULATION PACKAGE}

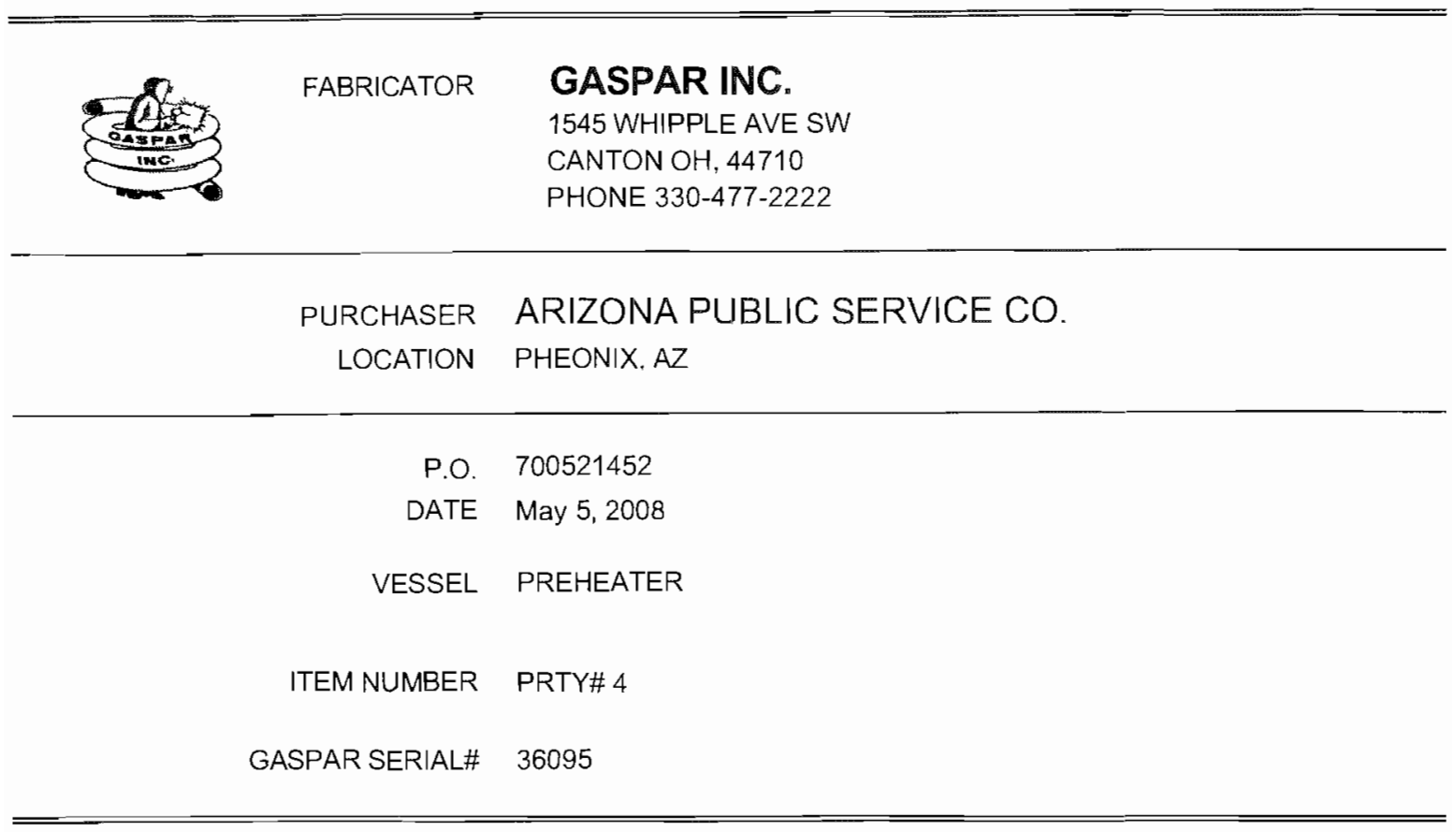

DEC 04, 2008: HEADS RE-DESIGNED, 1/4" TUBE ADDED TO CALCS, 1/4" TUBE: WELD DESIGN ADDED TO CALCS.

\begin{tabular}{|c|c|c|}
\hline & & \\
\hline & & \\
\hline & & \\
\hline & & \\
\hline & & \\
\hline & & \\
\hline & & \\
\hline & & \\
\hline Dec 4, 2008 & SEE NOTE ABOVE & 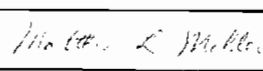 \\
\hline Sep 30,2008 & 3" VESSEL LENGTHS CHANGED & \\
\hline Jul 22, 2008 & FOR APPROVAL & \\
\hline DATE & DESCRIPTION & $\mathrm{BY}$ \\
\hline
\end{tabular}

AF ReVEU:

(2)- 


\section{Table Of Contents}

1. Pressure Summary

2. Weight Summary

3. Hydrostatic Test

4. HEATER \#1 WEIGHT

5. HEATER \#2 WEIGHT

6. HEATER \#3 WEIGHT

7. HEATER \#4 WEIGHT

8. HEAD TOP

9. 3" TUBE \#1

10. 3" TUBE $\# 2$

11. 3" TUBE \#3

12. 3" TUBE \#4

13. HEAD, BOTTOM

14. TOP MOUNT

15. SEISMIC CODE

16. WIND CODE

17. TOP HEAD OPENINGS

18. RUPTURE DESIGN FOR 1/4" TUBE

19. TUBE TO VESSEL WELD

NOTE: The Design Temperature of $1600.00^{\circ} \mathrm{F}$ is beyond the ASME Elasticity Data Range for SB-446-N06625 grade 2.

Elasticity (E) at $1600^{\circ} \mathrm{F}$ per "Special Metals" alloy 625 catalog is $21,500,000$ psi.

Elasticity $(\mathrm{E})$ at $1600^{\circ} \mathrm{F}$ used in calculations: $21,400,000 \mathrm{psi}$.

NOTE: PER ASIME D-II NOTE 1B:G23 FOR SB-446-N06625 GRADE 2:

THIS ALLOY IS SUBJECT TO SEVERE LOSS OF IMPACT STRENGTH AT ROOM TEMPERATURES AFTER EXPOSURE IN THE RANGE OF $1000^{\circ} \mathrm{F}$ TO $1400^{\circ} \mathrm{F}$ 


\section{Pressure Summary}

Pressure Summary for Chamber bounded by Top of vessell and Top of vessell

\begin{tabular}{|c|c|c|c|c|c|}
\hline Identifier & $\begin{array}{c}\mathbb{P} \\
\text { Design } \\
\text { ( psi) }\end{array}$ & $\begin{array}{c}\text { T } \\
\text { Design } \\
\left({ }^{\circ} \mathrm{F}\right)\end{array}$ & $\begin{array}{c}\text { MDMT } \\
\left({ }^{\circ} \mathrm{F}\right)\end{array}$ & $\begin{array}{c}\text { MDMT } \\
\text { Exemption }\end{array}$ & $\begin{array}{l}\text { Impact } \\
\text { Tested }\end{array}$ \\
\hline HEAD TOE & 1,150 & 1,600 & $\mathrm{~N} / \mathrm{A}$ & N/A & No \\
\hline 3" TUBE \#1 & 1,150 & 1,600 & $\mathrm{~N} / \mathrm{A}$ & N/A & No \\
\hline 3"TUBE +1 2 & 1,150 & 1,600 & $\mathrm{~N} / \mathrm{A}$ & $\mathrm{N} / \mathrm{A}$ & No \\
\hline 3" TUBE \#3 & 1,150 & 1,600 & $\mathrm{~N} / \mathrm{A}$ & $\mathrm{N} / \mathrm{A}$ & No \\
\hline 3"TUBE H4 & 1,150 & 1,600 & $\mathrm{~N} / \mathrm{A}$ & $\mathrm{N} / \mathrm{A}$ & No \\
\hline HEAD BOTTOM & 1,150 & 1,600 & $\mathrm{~N} / \mathrm{A}$ & N/A & No \\
\hline IOP MOUNT & 1,150 & 1,600 & $\mathrm{~N} / \mathrm{A}$ & $\mathrm{N} / \mathrm{A}$ & $\mathrm{N} / \mathrm{A}$ \\
\hline
\end{tabular}

Chamber Design MAWP hot \& corroded is 1,150 psi

This pressure chamber is not designed for external pressure. CHAMBER DESIGN MDMT IS $-20^{\circ} \mathrm{F}$

CHAMBER RATED MDMT IS $-325^{\circ} \mathrm{F} @ 1150$ ps

NOTE: The Design Temperature of $1600.00^{\circ} \mathrm{F}$ is beyond the ASME Elasticity Data Range for SB-446-N06625 grade 2.

Elasticity (E) at $1600^{\circ} \mathrm{F}$ per "Special Metals" alloy 625 catalog is $21,500,000$ psi.

Elasticity (E) at $1600^{\circ} \mathrm{F}$ used in calculations: $21,400,000 \mathrm{psi}$.

\section{NOTE:}

PER ASME D-II NOTE 1B:G23 FOR SB-446-N06625 GRADE 2:

THIS ALLOY IS SUBJECT TO SEVERE LOSS OF IMPACT STRENGTH AT ROOM TEMPERATURES AFTER EXPOSURE IN THE RANGE OF $1000^{\circ} \mathrm{F}$ TO $1400^{\circ} \mathrm{F}$ 


\section{Weight Summary}

\begin{tabular}{|c|c|c|c|c|c|c|c|}
\hline \multirow[b]{2}{*}{ Component } & \multicolumn{7}{|c|}{ Weight ( Ib) Contributed by Vessel Elements } \\
\hline & \begin{tabular}{|l|} 
Metal \\
New:
\end{tabular} & $\begin{array}{c}\text { Metal } \\
\text { Corroded }\end{array}$ & $\begin{array}{c}\text { Insulation \& } \\
\text { Supports }\end{array}$ & Lining & $\begin{array}{c}\text { Piping } \\
+ \text { Liquid } \\
\end{array}$ & $\begin{array}{c}\text { Operating } \\
\text { Liquid }\end{array}$ & $\begin{array}{c}\text { Test } \\
\text { Liquid } \\
\end{array}$ \\
\hline HEAD TOP & 10.2 & 10.1 & 0 & 0 & 0 & 0 & 0.2 \\
\hline 3" TUBE: \#1 & 80.3 & 79.4 & 0 & 0 & 0 & 0 & 1.4 \\
\hline 3"TUBE, +2 & 80.3 & 79.4 & 0 & 0 & 0 & 0 & 1.4 \\
\hline 3"TUBE:33 & 80.3 & 79.4 & 0 & 0 & 0 & 0 & 1.4 \\
\hline 3"TUBH:\#4 & 80.3 & 79.4 & 0 & 0 & 0 & 0 & 1.4 \\
\hline HEAD, BOTTOM & 8.4 & 8.3 & 0 & 0 & 0 & 0 & 0.1 \\
\hline IOPMOUNT & 38.5 & 38.5 & 0 & 0 & 0 & 0 & 0 \\
\hline TOTAL: & 378.1 & 374.4 & 0 & 0 & 0 & 0 & 5.8 \\
\hline
\end{tabular}

* Shells with attached nozzles have weight reduced by material cut out for opening.

\begin{tabular}{|c|c|c|c|c|c|c|c|c|c|}
\hline \multirow{3}{*}{ Component } & \multicolumn{9}{|c|}{ Weight ( lb) Contributed by Attachments } \\
\hline & \multicolumn{2}{|c|}{ Body Flanges } & \multicolumn{2}{|c|}{$\begin{array}{l}\text { Nozzles \& } \\
\text { Flanges }\end{array}$} & \multirow{2}{*}{$\begin{array}{c}\text { Packed } \\
\text { Beds }\end{array}$} & \multirow{2}{*}{$\begin{array}{c}\text { Ladders \& } \\
\text { Platforms }\end{array}$} & \multirow{2}{*}{$\begin{array}{c}\text { Trays \& } \\
\text { Supports }\end{array}$} & \multirow{2}{*}{$\begin{array}{l}\text { Rings \& } \\
\text { Clips }\end{array}$} & \multirow{2}{*}{$\begin{array}{c}\text { Vertical } \\
\text { Loads }\end{array}$} \\
\hline & New & Corroded & New & Corroded & & & & & \\
\hline HEAD, TOP & 0 & 0 & 0 & 0 & 0 & 0 & 0 & 0 & 0 \\
\hline 3" TUBE\#L & 0 & 0 & 0 & 0 & 0 & 0 & 0 & 0 & $50^{*}$ \\
\hline 3" TUBE $\# 2$ & 0 & 0 & 0 & 0 & 0 & 0 & 0 & 0 & $50^{*}$ \\
\hline 3" TUBE: & 0 & 0 & 0 & 0 & 0 & 0 & 0 & 0 & $50^{*}$ \\
\hline 3" TUBE 㙬 & 0 & 0 & 0 & 0 & 0 & 0 & 0 & 0 & $30^{*}$ \\
\hline$\underline{\mathrm{HE}} \mathrm{AD}, \mathrm{BO} \mathrm{TTOM}$ & 0 & 0 & 0 & 0 & 0 & 0 & 0 & 0 & 0 \\
\hline TOTAL: & 0 & 0 & 0 & 0 & 0 & 0 & 0 & 0 & $180^{*}$ \\
\hline
\end{tabular}

* This number includes vertical loads which are not present in all conditions.

Vessel operating weight, Corroded: $554 \mathrm{lb}$

Vessel operating weight, New: $558 \mathrm{lb}$

Vessel empty weight, Corroded: $554 \mathrm{lb}$

Vessel empty weight, New: $558 \mathrm{lb}$

Vessel test weight, New: $\quad 384 \mathrm{lb}$

\section{Vessel center of gravity location - from datum - lift condition}

Vessel Lift Weight, New: $558 \mathrm{lb}$

Center of Gravity: $\quad 95.3712^{\prime \prime}$

\section{Vessel Capacity}

Vessel Capacity** (New): 1 US gal

Vessel Capacity** (Corroded): 1 US gal

**The vessel capacity does not include volume of nozzle, piping or other attachments. 


\section{Hydrostatic Test}

Shop test pressure determination

P per UG-99(b)

Shop hydrostatic test gauge pressure is $15,736.819 \mathrm{psi}$ at $70^{\circ} \mathrm{F}$ (the chamber design $\mathrm{P}=1,150 \mathrm{psi}$ )

The shop test is performed with the vesse 1 in the horizontal position.

\begin{tabular}{|l|r|r|r|r|}
\hline \multicolumn{1}{|c|}{ Identifier } & $\begin{array}{c}\text { Local test } \\
\text { pressure } \\
\text { psi }\end{array}$ & $\begin{array}{c}\text { Test liquid } \\
\text { static head } \\
\text { psi }\end{array}$ & $\begin{array}{c}\text { UG-99 } \\
\text { stress } \\
\text { ratio }\end{array}$ & $\begin{array}{c}\text { UG-99 } \\
\text { pressure } \\
\text { factor }\end{array}$ \\
\hline HEAD, TOP (1) & $15,736.857$ & 0.038 & 10.5263 & 1.30 \\
\hline 3" TUBE\#1 & $15,736.857$ & 0.038 & 10.5263 & 1.30 \\
\hline 3" TUBE \#2 & $15,736.857$ & 0.038 & 10.5263 & 1.30 \\
\hline 3" TUBE \#3 & $15,736.857$ & 0.038 & 10.5263 & 1.30 \\
\hline 3" TUBE \#4 & $15,736.857$ & 0.038 & 10.5263 & 1.30 \\
\hline HEAD, BOTTOM & $15,736.857$ & 0.038 & 10.5263 & 1.30 \\
\hline
\end{tabular}

Notes:

(1) HEAD, TOP limits the UG-99 stress ratio.

(2) The zero degree angular position is assumed to be up, and the test liquid height is assumed to the top-most flange. 


\section{HEATER \#1 WEIGHT}

$\begin{array}{ll}\text { Load Orientation } & \text { Vertical Load } \\ \text { Elevation above datum: } & 151.375^{\prime \prime} \\ \text { Direction angle: } & 0.00 \text { degrees }\end{array}$

Distance from center of vessel: $0^{\prime \prime}$

Magnitude of force: $\quad 50 \mathrm{lb}$

Present when operating: Yes Included in vessel lift weight: Yes Present when vessel is empty: Yes Present during hydrotest: No 


\section{HEATER \#2 WEIGHT}

$\begin{array}{ll}\text { Load Orientation } & \text { Vertical Load } \\ \text { Elevation above datum: } & 109.375^{\prime \prime} \\ \text { Direction angle: } & 0.00 \text { degrees }\end{array}$

Distance from center of vessel: 0 "

Magnitude of force: $\quad 50 \mathrm{lb}$

Present when operating: Yes Included in vessel lift weight: Yes Present when vessel is empty: Yes Present during hydrotest: No 


\section{HEATER \#3 WEIGHT}

$\begin{array}{ll}\text { Load Orientation } & \text { Vertical Load } \\ \text { Elevation above datum: } & 67.375^{\prime \prime} \\ \text { Direction angle: } & 0.00 \text { degrees }\end{array}$

Distance from center of vessel: $0^{\prime \prime}$

Magnitude of force: $\quad 50 \mathrm{lb}$

Present when operating: Yes

Included in vessel lift weight: Yes

Present when vessel is empty: Yes

Present during hydrotest: No 


\section{HEATER \#4 WEIGHT}

$\begin{array}{ll}\text { Load Orientation } & \text { Vertical Load } \\ \text { Elevation above datum: } & 24.375^{\prime \prime} \\ \text { Direction angle: } & 0.00 \text { degrees }\end{array}$

Distance from center of vessel: $0^{\prime \prime}$

Magnitude of force: $\quad 30 \mathrm{lb}$

Present when operating: Yes Included in vessel lift weight: Yes Present when vessel is empty: Yes Present during hydrotest:

No 


\section{HEAD, TOP}

\section{ASME Section VIII Division 1, 2007 Edition}

\section{Component:}

Material specification:

Rated MDMT per UNF-65 $=-325^{\circ} \mathrm{F}$

Internal design pressure: $\mathrm{P}=1,150 \mathrm{psi} @ 1,600^{\circ} \mathrm{F}$

\section{Static liquid head:}

$P_{t h}=0.04 \mathrm{psi}\left(\mathrm{SG}=1, \mathrm{H}_{\mathrm{S}}=1.06^{\prime \prime}\right.$, Horizontal test head $)$

Corrosion allowance $\quad$ Inner $\mathrm{C}=0.02^{\prime \prime} \quad$ Outer $\mathrm{C}=0^{\prime \prime}$

Design MDMT $=-20^{\circ} \mathrm{F}$

Rated $\mathrm{MDMT}=-325^{\circ} \mathrm{F}$

No impact test performed

Material is not normalized

Material is not produced to Fine Grain Practice

PWHT is notperformed

Radiography: $\quad$ Longitudinal joint - Seamless No RT

Top circumferential joint - Fu1] UW-11(a) Type I

Bottom circumferential

joint -

Full UW-11(a) Type

$$
\begin{aligned}
& \text { Estimated weight New }=10.2 \mathrm{lb} \quad \text { corr }=10.1 \mathrm{lb} \\
& \text { Capacity New }=0.02 \mathrm{US} \text { gal corr }=0.02 \mathrm{US} \\
& \begin{array}{l}
\text { OD } \quad=3^{\prime \prime} \\
\text { Length } \mathrm{L}_{\mathrm{c}}=5.5^{\prime \prime} \\
\mathrm{t} \quad=0.97^{\prime \prime}
\end{array}
\end{aligned}
$$$$
\text { Capacity New }=0.02 \text { US gal corr }=0.02 \text { US gal }
$$

\section{Design thickness, (at $1,600^{\circ} \mathrm{F}$ ) Appendix 1-2}

$\mathrm{Z}=(\mathrm{S} * \mathrm{E}+\mathrm{P}) /(\mathrm{S} * \mathrm{E}-\mathrm{P})$

$=(1,900 * 1.00+1,150) /(1,900 * 1.00-1,150)$

$=4.0667$

$\mathrm{t}=\mathrm{R}_{0} *\left(\mathrm{Z}^{1 / 2}-1\right) / \mathrm{Z}^{1 / 2}+$ Corrosion

$=1.5^{*}\left(4.0667^{1 / 2}-1\right) / 4.0667^{1 / 2}+0.02$

$=0.7762^{\prime}$

\section{\% Forming Strain - UNF-79(a)(2)(a)}

$$
\begin{aligned}
\mathrm{EFE} & =\left(50 * t / \mathrm{R}_{\mathrm{f}}\right) *\left(1-\mathrm{R}_{\mathrm{f}} / \mathrm{R}_{\mathrm{o}}\right) \\
& =(50 * 0.97 / 1.015) *(1-1.015 / \infty) \\
& =47.7833 \%
\end{aligned}
$$




\section{Design thickness $=0.7762^{\prime \prime}$}

The governing condition is due to internal pressure.

The cylinder thickness of $0.97 "$ is adequate.

\section{Thickness Required Due to Pressure + External Loads}

\begin{tabular}{|c|c|c|c|c|c|c|c|c|}
\hline \multirow[t]{2}{*}{ Condition } & \multirow{2}{*}{$\begin{array}{l}\text { Pressure } \\
\text { P (psi) }\end{array}$} & \multicolumn{2}{|c|}{$\begin{array}{l}\text { Allowable Stress } \\
\text { Before UG-23 Stress } \\
\text { Increase ( psi) }\end{array}$} & \multirow{2}{*}{$\begin{array}{l}\text { Temperature } \\
\left({ }^{\circ} \mathrm{F}\right)\end{array}$} & \multirow{2}{*}{$\begin{array}{l}\text { Corrosion } \\
\text { C (in) }\end{array}$} & \multirow[t]{2}{*}{ Load } & \multirow{2}{*}{$\begin{array}{l}\text { Req'd Thk } \\
\text { Due to } \\
\text { Teusion (in) }\end{array}$} & \multirow{2}{*}{$\begin{array}{l}\text { Req'd Thk Due } \\
\text { to Compression } \\
\text { (in) }\end{array}$} \\
\hline & & $S_{t}$ & $S_{c}$ & & & & & \\
\hline \multirow{2}{*}{$\begin{array}{l}\text { Operating, Hot \& } \\
\text { Corroded }\end{array}$} & \multirow{2}{*}{1,150} & \multirow{2}{*}{1,900} & \multirow{2}{*}{1,900} & \multirow{2}{*}{1,600} & \multirow{2}{*}{0.02} & Wind & 0.126 & 0.1246 \\
\hline & & & & & & Seismic & 0.1253 & 0.1251 \\
\hline \multirow{2}{*}{$\begin{array}{c}\text { Operating, Hot \& } \\
\text { New }\end{array}$} & \multirow{2}{*}{1,150} & \multirow{2}{*}{1,900} & \multirow{2}{*}{1,900} & \multirow{2}{*}{1,600} & \multirow{2}{*}{0} & Wind & 0.1214 & 0.12 \\
\hline & & & & & & Seismic & 0.1207 & 0.1205 \\
\hline \multirow{2}{*}{ Empty, Corroded } & \multirow{2}{*}{0} & \multirow{2}{*}{26,700} & \multirow{2}{*}{20,000} & \multirow{2}{*}{0} & \multirow{2}{*}{0.02} & Wind & 0 & 0.0001 \\
\hline & & & & & & Seismic & 0.0001 & 0.0001 \\
\hline \multirow{2}{*}{ Empty, New } & \multirow{2}{*}{0} & \multirow{2}{*}{26,700} & \multirow{2}{*}{20,000} & \multirow{2}{*}{0} & \multirow{2}{*}{0} & Wind & 0 & 0.0001 \\
\hline & & & & & & Seismic & 0.0001 & 0.0001 \\
\hline $\begin{array}{l}\text { Hot Shut Down, } \\
\text { Conroded, Weight \& } \\
\text { Eccentric Moments } \\
\text { Only }\end{array}$ & 0 & 1,900 & 1,900 & 1,600 & 0.02 & Weight & 0.0008 & 0.0008 \\
\hline
\end{tabular}




\section{TOP HEAD, CHECK AS FLAT HEAD}

ASME Section VIII Division 1, 2007 Edition

Component:

Material specification:
Welded Cover

SB-446 N06625 Gr.2 SOL ANN BAR-

HIGH TEMP (user defined)

Internal design pressure: $\mathrm{P}=1150 \mathrm{psi} @ 1600^{\circ} \mathrm{F}$

Static liquid head:

$P_{t h}=0.04 \mathrm{psi}\left(\mathrm{SG}=1.0000, \mathrm{H}_{\mathrm{s}}=1.03 "\right.$, Horizontal test head $)$

Corrosion allowance: $\quad$ Inner $\mathrm{C}=0.02^{\prime \prime} \quad$ Outer $\mathrm{C}=0$ "

PWHT is notperformed

Radiography: $\quad$ Category A joints - $\quad$ Seamless No RT

Estimated weight: $\quad$ New $=0.2 \mathrm{lb} \quad$ corr $=0.2 \mathrm{lb}$

Head outside diameter $=2.94 "$

Cover thickness $\quad=1 "$

Inner corner radius $\quad=0.375^{\prime \prime}$

Factor $\mathbb{C}$ from $\mathbb{F i g}$. UG-34, sketch (b-2), (e through g)

$\mathrm{C}=0.33^{*} \operatorname{tr} / \mathrm{ts}=0.33^{*} 0.4942 / 0.95=0.1717$

As factor $\mathrm{C}<0.2$ let $\mathrm{C}=0.2$

Design thickness, (at $\left.1,600^{\circ} \mathrm{F}\right) \mathrm{UG}-34$ (c)(2)

$\mathrm{t}=\mathrm{d}^{*} \mathrm{Sqr}(\mathrm{C} * \mathrm{P} /(\mathrm{S} * \mathrm{E}))+$ Corrosion

$=1.04 * \operatorname{Sqr}(0.2 * 1,150 /(1,900 * 1))+0.02$

$=0.3818$ in

ACTUAL "COVER" THICKNESS $=1.75$ " $>0.3818$ ";

THEREFORE THE DESIGN IS ADEQUATE. 


\section{3" TUBE \#1}

\section{ASME Section VIII Division 1, 2007 Edition}

Component:

Material specification:

Rated MDMT per UNF-65 $=-325^{\circ} \mathrm{F}$

Internal design pressure: $\mathrm{P}=1,150$ psi @ $1,600^{\circ} \mathrm{F}$

\section{Static liquid head:}

$\mathrm{P}_{\mathrm{t}_{\mathrm{l}}}=0.04 \mathrm{psi}\left(\mathrm{SG}=1, \mathrm{H}_{\mathrm{S}}=1.06^{\prime \prime}\right.$, Horizontal test head $)$

Corrosion allowance $\quad$ Inner $\mathrm{C}=0.02^{\prime \prime} \quad$ Outer $\mathrm{C}=0 "$

Design MDMT $=-20^{\circ} \mathrm{F}$

Rated MDMT $=-325^{\circ} \mathrm{F}$

No impact test performed Material is not normalized Material is not produced to Fine Grain Practice PWHT is not performed

Radiography: Longitudinal joint - Seamless No RT Top circumferential joint - Full UW-11(a) Type 1 Bottom circumferential joint -

Full UW-11(a) Type 1

Estimated weight New $=80.3 \mathrm{lb} \quad$ corr $=79.4 \mathrm{lb}$

C.apacity New $=0.17$ US gal corr $=0.18$ US gal

$\mathrm{OD} \quad=3^{\prime \prime}$

Length $\mathrm{L}_{\mathrm{c}}=43.25^{\prime \prime}$

$\mathrm{t} \quad=0.97^{\prime \prime}$

Design thickness, (at $1,600^{\circ} \mathrm{F}$ ) Appendix 1-2

$$
\begin{aligned}
\mathrm{Z} & =\left(\mathrm{S}^{*} \mathrm{E}+\mathrm{P}\right) /\left(\mathrm{S}^{*} \mathrm{E}-\mathrm{P}\right) \\
& =\left(1,900^{*} 1.00+1,150\right) /\left(1,900^{*} 1.00-1,150\right) \\
& =4.0667 \\
\mathrm{t} & =\mathrm{R}_{\mathrm{o}}^{*}\left(\mathrm{Z}^{1 / 2}-1\right) / \mathrm{Z}^{1 / 2}+\text { Corrosion } \\
& =1.5 *\left(4.0667^{1 / 2}-1\right) / 4.0667^{1 / 2}+0.02 \\
& =0.7762^{\prime \prime}
\end{aligned}
$$

\% Forming Strain - UNF-79(a)(2)(a)

$$
\begin{aligned}
\operatorname{EFE} & =\left(50 * t / R_{f}\right) *\left(1-R_{f} / R_{0}\right) \\
& =(50 * 0.97 / 1.015) *(1-1.015 / \infty) \\
& =47.7833 \%
\end{aligned}
$$




\section{Required thickness, largest of the following + corrosion $=0.8826^{\prime \prime}$}

The governing condition is due to wind at the Bottom of the support in condition Operating, Hot \& Corroded The cylinder thickness of $0.97 "$ is adequate.

\section{Thickness Required Due to Pressure + External Loads}

\begin{tabular}{|c|c|c|c|c|c|c|c|c|c|}
\hline \multirow[t]{2}{*}{ Condition } & \multirow[t]{2}{*}{$\begin{array}{l}\text { Pressure } \\
P(\text { psi) }\end{array}$} & \multicolumn{2}{|c|}{$\begin{array}{l}\text { Allowable Stress } \\
\text { Before UG-23 } \\
\text { Stress Increase } \\
\text { (psi) }\end{array}$} & \multirow[t]{2}{*}{$\begin{array}{c}\text { Temperature } \\
\left({ }^{\circ} \mathrm{F}\right)\end{array}$} & \multirow[t]{2}{*}{$\begin{array}{l}\text { Corrosion } \\
\text { C (in) }\end{array}$} & \multirow[t]{2}{*}{ Location } & \multirow[t]{2}{*}{ Load } & \multirow{2}{*}{$\begin{array}{l}\text { Req'd Thk } \\
\text { Due to } \\
\text { Tension } \\
\text { (in) }\end{array}$} & \multirow[t]{2}{*}{$\begin{array}{l}\text { Req'd Thk Due } \\
\text { to Compression } \\
\text { (in) }\end{array}$} \\
\hline & & $S_{t}$ & $S_{c}$ & & & & & & \\
\hline \multirow{4}{*}{$\begin{array}{l}\text { Operating, Hot } \\
\text { \& Corroded }\end{array}$} & \multirow{4}{*}{1,150} & \multirow{4}{*}{1,900} & \multirow{4}{*}{1,900} & \multirow{4}{*}{1,600} & \multirow{4}{*}{0.02} & \multirow{2}{*}{ Top } & Wind & 0.1266 & 0.1233 \\
\hline & & & & & & & Seismic & 0.125 & 0.1247 \\
\hline & & & & & & \multirow{2}{*}{ Bottom } & Wind & 0.8626 & 0.5425 \\
\hline & & & & & & & Seismic & 0.7789 & 0.452 \\
\hline \multirow{4}{*}{$\begin{array}{l}\text { Operating, Hot } \\
\text { \& New }\end{array}$} & \multirow{4}{*}{1,150} & \multirow{4}{*}{1,900} & \multirow{4}{*}{1,900} & \multirow{4}{*}{1,600} & \multirow{4}{*}{0} & \multirow{2}{*}{ Тор } & Wind & 0.122 & 0.1186 \\
\hline & & & & & & & Seismic & 0.1204 & 0.1201 \\
\hline & & & & & & \multirow{2}{*}{ Bottom } & Wind & 0.8725 & 0.5604 \\
\hline & & & & & & & Seismic & 0.7915 & 0.4725 \\
\hline \multirow{4}{*}{$\begin{array}{l}\text { Empty, } \\
\text { Corroded }\end{array}$} & \multirow{4}{*}{0} & \multirow{4}{*}{26,700} & \multirow{4}{*}{20,000} & \multirow{4}{*}{0} & \multirow{4}{*}{0.02} & \multirow{2}{*}{ Top } & Wind & 0 & 0.0003 \\
\hline & & & & & & & Seismic & 0.0001 & 0.0001 \\
\hline & & & & & & $0 t$ & Wind & 0.0524 & 0.0635 \\
\hline & & & & & & 6utons & Seismic & 0.0465 & 0.0549 \\
\hline \multirow{4}{*}{ Empty, New } & \multirow{4}{*}{0} & & & & & Ton & Wind & 0 & 0.0003 \\
\hline & & 26700 & 20000 & 0 & 1 & $10 \mathrm{p}$ & Seismic & 0.0001 & 0.0001 \\
\hline & & & & & & $\mathrm{B}$ & Wind & 0.0535 & 0.0648 \\
\hline & & & & & & Dotion & Seismic & 0.0477 & 0.0564 \\
\hline $\begin{array}{l}\text { Hot Shut Down, } \\
\text { Corroded } \\
\text { Weight \& }\end{array}$ & 0 & 1,900 & 1.900 & 1600 & 002 & Top & Weight & 0.0013 & 0.0013 \\
\hline $\begin{array}{c}\text { Eccentric } \\
\text { Moments Only }\end{array}$ & & & & & & Botrom & Weight & 0.0409 & 0.0409 \\
\hline
\end{tabular}


Allowable Compressive Stress, Hot and Corroded- $\mathrm{S}_{\mathrm{cHC}}$, (table NFN-18)

From Jawad \& Farr GUIDEBOOK 1998 (Eq. 2.25)

$$
\begin{aligned}
\mathrm{C}_{\mathrm{f}} & =1-0.90 *\left(1-\mathrm{e}^{((-1 / 1 / 16) * \operatorname{Sqr}(\operatorname{Ro} / \mathrm{t}))}\right) \\
& =1-0.90 *\left(1-\mathrm{e}^{\left((-\mathrm{l} / 16)^{*} \operatorname{Sqr}(\mathrm{L} .5000 / 0.9500)\right)}\right) \\
& =0.932023 \\
\mathrm{~B} & =0.10 * 0.60 * \mathrm{C}_{\mathrm{f}} * \mathrm{E} /\left(\mathrm{R}_{\mathrm{o}} / \mathrm{t}\right) \\
& =0.10 * 0.60 * 0.9320 * 21400000.0000 /(1.5000 / 0.9500) \\
& =757920.875000 \\
\mathrm{~S} & =1,900 / 1.00=1,900 \mathrm{psi} \\
\mathrm{S}_{\mathrm{cHC}} & =\min (\mathrm{B}, \mathrm{S})=1,900 \mathrm{psi}
\end{aligned}
$$

\section{Operating, Hot \& Corroded, Wind, Above Support Point}

$$
\begin{aligned}
& \mathrm{t}_{\mathrm{p}}=\mathrm{P} * \mathrm{R} /\left(2 * \mathrm{~S}_{\mathrm{t}} * \mathrm{~K}_{\mathrm{s}}{ }^{*} \mathrm{E}_{\mathrm{c}}+0.40 *|\mathrm{P}|\right) \quad \text { (Pressure) } \\
& =1,150 * 0.55 /(2 * 1,900 * 1.20 * 1.00+0.40 *|1,150|) \\
& =0.126^{\prime \prime} \\
& \mathrm{t}_{\mathrm{m}}=\mathrm{M} /\left(\pi * \mathrm{R}_{\mathrm{m}}{ }^{2} * \mathrm{~S}_{\mathrm{t}} * \mathrm{~K}_{\mathrm{s}}{ }^{*} \mathrm{E}_{\mathrm{c}}\right) \\
& \text { (bending) } \\
& =13 /\left(\pi * 1.025^{2 *} 1,900 * 1.20 * 1.00\right) \\
& =0.0017^{\prime \prime} \\
& \mathrm{t}_{\mathrm{w}}=\mathrm{W} /\left(2 * \pi * \mathrm{R}_{\mathrm{m}}{ }^{*} \mathrm{~S}_{\mathrm{t}} * \mathrm{~K}_{\mathrm{s}} * \mathrm{E}_{\mathrm{c}}\right) \\
& =15.6 /(2 * \pi * 1.025 * 1,900 * 1.20 * 1.00) \\
& =0.0011 " \\
& t_{t}=t_{p}+t_{m}-t_{w} \\
& =0.126+0.0017-(0.0011) \\
& =\underline{0.126 \underline{6}^{\prime \prime}} \\
& \mathrm{t}_{\mathrm{c}}=\left|\mathrm{t}_{\mathrm{nc}}+\mathrm{t}_{\mathrm{wc}}-\mathrm{t}_{\mathrm{pc}}\right| \\
& \text { (total, net tensile) } \\
& =|0.0017+(0.0011)-(0.126)| \\
& =0.1233^{\prime \prime}
\end{aligned}
$$




\section{Operating, Hot \& Corroded, Wind, Below Support Point}

$$
\begin{aligned}
\mathrm{t}_{\mathrm{p}} & =\mathrm{P} * \mathrm{R} /\left(2 * \mathrm{~S}_{\mathrm{t}}^{*} \mathrm{~K}_{\mathrm{s}} * \mathrm{E}_{\mathrm{c}}+0.40 *|\mathrm{P}|\right) \quad \text { (Pressure) } \\
& =1,150 * 0.55 /(2 * 1,900 * 1.20 * 1.00+0.40 *|1,150|) \\
& =0.126^{\prime \prime} \\
\mathrm{t}_{\mathrm{m}} & =\mathrm{M} /\left(\pi^{*} \mathrm{R}_{\mathrm{m}}{ }^{2} \mathrm{~S}_{\mathrm{t}} * \mathrm{~K}_{\mathrm{s}}{ }^{*} \mathrm{E}_{\mathrm{c}}\right) \\
& =5,287 /\left(\pi^{*} 1.025^{2} * 1,900 * 1.20 * 1.00\right) \\
& =0.7026^{\prime \prime} \\
\mathrm{t}_{\mathrm{w}} & =\mathrm{W} /\left(2 * \pi * \mathrm{R}_{\mathrm{m}} * \mathrm{~S}_{\mathrm{t}} * \mathrm{~K}_{\mathrm{s}} * \mathrm{E}_{\mathrm{c}}\right) \\
& =-500.3 /\left(2 * \pi^{*} 1.025^{*} 1,900 * 1.20 * 1.00\right) \\
& =-0.0341 " \\
\mathrm{t}_{\mathrm{t}} & =\mathrm{t}_{\mathrm{p}}+\mathrm{t}_{\mathrm{m}}-\mathrm{t}_{\mathrm{w}} \\
& =0.126+0.7026-(-0.0341) \\
& =\underline{0.8626 "} \quad \text { (Weight) } \\
\mathrm{t}_{\mathrm{c}} & =\mathrm{t}_{\mathrm{mc}}+\mathrm{t}_{\mathrm{wc}}-\mathrm{t}_{\mathrm{pc}} \\
& =0.7026+(-0.0341)-(0.126) \\
& =\underline{0.5425 "} \quad \text { (total required, tensile) }
\end{aligned}
$$

\section{Operating, Hot \& Corroded, Seismic, Above Support Point}

$$
\begin{array}{rlr}
t_{\mathrm{p}} & =\mathrm{P} * \mathrm{R} /\left(2 * \mathrm{~S}_{\mathrm{t}} * \mathrm{~K}_{\mathrm{s}} * \mathrm{E}_{\mathrm{c}}+0.40 * \mathrm{P} \mid\right) \quad \text { (Pressure) } \\
& =1,150 * 0.55 /(2 * 1,900 * 1.20 * 1.00+0.40 *|1,150|) \\
& =0.126 " \\
\mathrm{t}_{\mathrm{m}} & =\mathrm{M} /\left(\pi * \mathrm{R}_{\mathrm{n}}{ }^{2} * \mathrm{~S}_{\mathrm{t}} * \mathrm{~K}_{\mathrm{s}} * \mathrm{E}_{\mathrm{c}}\right) & \text { (bending) } \\
& =0 /\left(\pi * 1.025^{2 *} 1,900 * 1.20 * 1.00\right) \\
& =0 " \\
\mathrm{t}_{\mathrm{w}} & =\mathrm{W} /\left(2 * \pi * \mathrm{R}_{\mathrm{m}} * \mathrm{~S}_{\mathrm{t}} * \mathrm{~K}_{\mathrm{s}} * \mathrm{E}_{\mathrm{c}}\right) \\
& =15.6 /\left(2 * \pi * 1.025^{*} 1,900^{*} 1.20 * 1.00\right) \\
& =0.0011 "
\end{array}
$$




$$
\begin{array}{rlr}
\mathrm{t}_{\mathrm{t}} & =\mathrm{t}_{\mathrm{p}}+\mathrm{t}_{\mathrm{m}}-\mathrm{t}_{\mathrm{w}} & \text { (total required, tensile) } \\
& =0.126+0-(0.0011) \\
& =\underline{0.125 "} \\
\mathrm{t}_{\mathrm{wc}} & =(1+\mathrm{VAccel}) * \mathrm{~W} /\left(2^{*} \pi^{*} \mathrm{R}_{\mathrm{m}} * \mathrm{~S}_{\mathrm{t}} * \mathrm{~K}_{\mathrm{s}} * \mathrm{E}_{\mathrm{c}}\right) & \text { (Weight) } \\
& =1.20 * 15.6 /\left(2 * \pi^{*} 1.025^{*} 1,900^{*} 1.20 * 1.00\right) \\
& =0.0013^{\prime \prime} \\
\mathrm{t}_{\mathrm{c}} & =\left|\mathrm{t}_{\mathrm{nc}}+\mathrm{t}_{\mathrm{wc}}-\mathrm{t}_{\mathrm{pc}}\right| \\
& =|0+(0.0013)-(0.126)| \\
& =0.1247^{\prime \prime}
\end{array}
$$

\section{Operating, Hot \& Corroded, Seismic, Below Support Point}

$$
\begin{aligned}
& \mathrm{t}_{\mathrm{p}}=\mathrm{P} * \mathrm{R} /\left(2 * \mathrm{~S}_{\mathrm{t}} * \mathrm{~K}_{\mathrm{s}} * \mathrm{E}_{\mathrm{c}}+0.40 *|\mathrm{P}|\right) \\
& \text { (Pressure) } \\
& =1,150 * 0.55 /(2 * 1,900 * 1.20 * 1.00+0.40 *|1,150|) \\
& =0.126^{\prime \prime} \\
& \mathrm{t}_{\mathrm{m}}=\mathrm{M} /\left(\pi * \mathrm{R}_{\mathrm{m}}{ }^{2} \mathrm{~S}_{\mathrm{t}} * \mathrm{~K}_{\mathrm{s}}{ }^{*} \mathrm{E}_{\mathrm{c}}\right) \\
& =4,606 /\left(\pi * 1.025^{2 * 1,900 * 1.20 * 1.00)}\right. \\
& =0.612^{\prime \prime} \\
& \mathrm{t}_{\mathrm{w}}=(1+\text { VAccel }) * \mathrm{~W} /\left(2 * \pi * \mathrm{R}_{\mathrm{m}} * \mathrm{~S}_{\mathrm{t}} * \mathrm{~K}_{\mathrm{s}} * \mathrm{E}_{\mathrm{c}}\right) \\
& \text { (Weight) } \\
& =1.20^{*}-500.3 /(2 * \pi * 1.025 * 1,900 * 1.20 * 1.00) \\
& =-0.0409^{\prime \prime} \\
& t_{\mathrm{t}}=\mathrm{t}_{\mathrm{p}}+\mathrm{t}_{\mathrm{m}}-\mathrm{t}_{\mathrm{w}} \\
& =0.126+0.612-(-0.0409) \\
& =\underline{0.7789^{\prime \prime}} \\
& \mathrm{t}_{\mathrm{wc}}=\mathrm{W} /\left(2 * \pi * \mathrm{R}_{\mathrm{m}} * \mathrm{~S}_{\mathrm{c}} * \mathrm{~K}_{\mathrm{s}}\right) \\
& =-500.3 /(2 * \pi * 1.025 * 1,900 * 1.20) \\
& =-0.0341^{\prime \prime}
\end{aligned}
$$




$$
\begin{aligned}
\mathbf{t}_{c} & =\mathbf{t}_{m c}+t_{w c}-t_{p c} \\
& =0.612+(-0.0341)-(0.126) \\
& =0.452^{\prime \prime}
\end{aligned}
$$

(total required, compressive) 
DESC: PREHEATER

P.O.\#: 700521452

\section{3" TUBE \#2}

\section{ASME Section VIII Division 1, 2007 Edition}

Component:

Material specification:

Rated MDMT per UNF-65 $=-325^{\circ} \mathrm{F}$

Internal design pressure: $\mathrm{P}=1,150 \mathrm{psi} @ 1,600^{\circ} \mathrm{F}$

\section{Static liquid head:}

$P_{\text {th }}=0.04 \mathrm{psi}\left(\mathrm{SG}=1, \mathrm{H}_{\mathrm{s}}=1.06^{\prime \prime}\right.$, Horizontal test head $)$

Corrosion allowance Inner $\mathrm{C}=0.02^{\prime \prime}$

Outer $\mathrm{C}=0^{\prime \prime}$
Cylinder

SB-4462 Solution ann. Bar N06625 (low stress) (II-D p. 222, $\ln , 40$ )

$$
\begin{aligned}
& \text { Design MDMT }=-20^{\circ} \mathrm{F} \quad \text { No impact test performed } \\
& \text { Rated MDMT }=-325^{\circ} \mathrm{F} \quad \text { Material is not normalized } \\
& \text { Material is not produced to Fine Grain Practice } \\
& \text { Radiograpliy: Longitudinal joint - Seamless No RT } \\
& \text { Top circumferential joint - Full UW-11(a) Type I } \\
& \text { Estimated weight New }=80.3 \mathrm{lb} \quad \text { corr }=79.4 \mathrm{lb} \\
& \text { Capacity New }=0.17 \text { US gal corr }=0.18 \text { US gal } \\
& \mathrm{OD} \quad=3^{\prime \prime} \\
& \text { Length } L_{c}=43.25^{\prime \prime} \\
& \mathrm{t} \quad=0.97^{\prime \prime}
\end{aligned}
$$$$
\text { PWHT is notperformed }
$$$$
\begin{aligned}
& \text { Bottom circumferential Full UW-11(a) Type I } \\
& \text { joint - }
\end{aligned}
$$

\section{Design thickness, (at $1,600^{\circ} \mathrm{F}$ ) Appendix 1-2}

$$
\begin{aligned}
\mathrm{Z} & =\left(\mathrm{S}^{*} \mathrm{E}+\mathrm{P}\right) /(\mathrm{S} * \mathrm{E}-\mathrm{P}) \\
& =\left(1,900^{*} 1.00+1,150\right) /\left(1,900^{*} 1.00-1,150\right) \\
& =4.0667 \\
\mathrm{t} & =\mathrm{R}_{0}^{*}\left(\mathrm{Z}^{1 / 2}-1\right) / \mathrm{Z}^{1 / 2}+\text { Corrosion } \\
& =1.5 *\left(4.0667^{1 / 2}-1\right) / 4.0667^{1 / 2}+0.02 \\
& =0.7762^{\prime \prime}
\end{aligned}
$$

\% Forming Strain - UNF-79(a)(2)(a)

$$
\begin{aligned}
\mathrm{EFE} & =\left(50 * \mathrm{t} / \mathrm{R}_{\mathrm{f}}\right) *\left(1-\mathrm{R}_{\mathrm{f}} / \mathrm{R}_{\mathrm{o}}\right) \\
& =(50 * 0.97 / 1.015) *(1-1.015 / \infty) \\
& =47.7833 \%
\end{aligned}
$$




\section{Design thickness $=0.7762^{\prime \prime}$}

The governing condition is due to internal pressure.

The cylinder thickness of $0.97^{\prime \prime}$ is adequate.

Thickness Required Due to Pressure + External Loads

\begin{tabular}{|c|c|c|c|c|c|c|c|c|}
\hline \multirow[t]{2}{*}{ Condition } & \multirow{2}{*}{$\begin{array}{l}\text { Pressure } \\
\text { P ( psi) }\end{array}$} & \multicolumn{2}{|c|}{$\begin{array}{c}\text { Allowable Stress } \\
\text { Before UG-23 Stress } \\
\text { Increase ( psi) }\end{array}$} & \multirow{2}{*}{$\begin{array}{l}\text { Temperature } \\
\left({ }^{\circ} \mathrm{F}\right)\end{array}$} & \multirow{2}{*}{$\begin{array}{l}\text { Corrosion } \\
\text { C (in) }\end{array}$} & \multirow[t]{2}{*}{ Load } & \multirow{2}{*}{$\begin{array}{l}\text { Req'd Thk } \\
\text { Due to } \\
\text { Tension (in) }\end{array}$} & \multirow{2}{*}{$\begin{array}{l}\text { Req'd Thk Due } \\
\text { to Compression } \\
\text { (in) }\end{array}$} \\
\hline & & $s_{1}$ & $S_{c}$ & & & & & \\
\hline \multirow{2}{*}{$\begin{array}{l}\text { Operating, Hot \& } \\
\text { Corroded }\end{array}$} & \multirow{2}{*}{1,150} & \multirow{2}{*}{1,900} & \multirow{2}{*}{1,900} & \multirow{2}{*}{1,600} & \multirow{2}{*}{0.02} & Wind & 0.5675 & 0.2642 \\
\hline & & & & & & Seismic & 0.5531 & 0.2447 \\
\hline \multirow{2}{*}{$\begin{array}{c}\text { Operating, Hot \& } \\
\text { New }\end{array}$} & \multirow{2}{*}{1,150} & \multirow{2}{*}{1,900} & \multirow{2}{*}{1,900} & \multirow{2}{*}{1,600} & \multirow{2}{*}{0} & Wind & 0.5716 & 0.2766 \\
\hline & & & & & & Seismic & 0.5598 & 0.2596 \\
\hline \multirow{2}{*}{ Empty, Corroded } & \multirow{2}{*}{0} & \multirow{2}{*}{26,700} & \multirow{2}{*}{20,000} & \multirow{2}{*}{0} & \multirow{2}{*}{0.02} & Wind & 0.0314 & 0.0371 \\
\hline & & & & & & Seismic & 0.0304 & 0.0352 \\
\hline \multirow{2}{*}{ Empty, New } & \multirow{2}{*}{0} & \multirow{2}{*}{26,700} & \multirow{2}{*}{20,000} & \multirow{2}{*}{0} & \multirow{2}{*}{0} & Wind & 0.032 & 0.0378 \\
\hline & & & & & & Seismic & 0.0312 & 0.0362 \\
\hline $\begin{array}{l}\text { Hot Shut Down, } \\
\text { Coroded, Weight \& } \\
\text { Eccentric Moments } \\
\text { Only }\end{array}$ & 0 & 1,900 & 1.900 & 1.600 & 0.02 & Weight & 0.0308 & 0.0308 \\
\hline
\end{tabular}




\section{3" TUBE \#3}

\section{ASME Section VIrI Division 1, 2007 Edition}

Component:

Material specification:

Rated MDMT per UNF-65 $=-325^{\circ} \mathrm{F}$

Internal design pressure: $\mathrm{P}=1,150 \mathrm{psi} @ 1,600{ }^{\circ} \mathrm{F}$

\section{Static liquid head:}

$\mathrm{P}_{\mathrm{th}}=0.04 \mathrm{psi}\left(\mathrm{SG}=1, \mathrm{H}_{\mathrm{S}}=1.06 "\right.$, Horizontal test head $)$

Corrosion allowance $\quad$ Inner $\mathrm{C}=0.02^{\prime \prime} \quad$ Outer $\mathrm{C}=0 "$

Design MDMT $=-20^{\circ} \mathrm{F}$

Rated MDMT $=-325^{\circ} \mathrm{F}$

No impact test performed

Material is not normalized

Material is not produced to Fine Grain Practice

PWHT is notperformed

Radiography: Longitudinal joint - Seamless No RT

Top circumferential joint - Full UW-11(a) Type 1

Bottom circumferential joint -

Full UW-11(a) Type 1

$$
\begin{aligned}
& \text { Estimated weight New }=80.3 \mathrm{lb} \quad \text { corr }=79.4 \mathrm{lb} \\
& \text { Capacity } \quad \text { New }=0.17 \text { US gal corr }=0.18 \text { US gal } \\
& \mathrm{OD} \quad=3^{\prime \prime} \\
& \text { Length } L_{c}=43.25^{\prime \prime} \\
& \mathrm{t} \quad=0.97^{\prime \prime}
\end{aligned}
$$

\section{Design thickness, (at $\mathbb{1}, 600^{\circ} \mathrm{F}$ ) Appendix 1-2}

$$
\begin{aligned}
\mathrm{Z} & =(\mathrm{S} * \mathrm{E}+\mathrm{P}) /\left(\mathrm{S}^{*} \mathrm{E}-\mathrm{P}\right) \\
& =\left(1,900^{*} 1.00+1,150\right) /\left(1,900^{*} 1.00-1,150\right) \\
& =4.0667 \\
\mathrm{t} & =\mathrm{R}_{\mathrm{o}}^{*}\left(\mathrm{Z}^{1 / 2}-1\right) / \mathrm{Z}^{1 / 2}+\text { Corrosion } \\
& =1.5^{*}\left(4.0667^{1 / 2}-1\right) / 4.0667^{1 / 2}+0.02 \\
& =0.7762^{\prime \prime}
\end{aligned}
$$

\section{\% Forming Strain - UNF-79(a)(2)(a)}

$$
\begin{aligned}
\mathrm{EFE} & =\left(50 * \mathrm{t} / \mathrm{R}_{\mathrm{f}}\right) *\left(1-\mathrm{R}_{\mathrm{f}} / \mathrm{R}_{\mathrm{o}}\right) \\
& =(50 * 0.97 / 1.015) *(1-1.015 / \infty) \\
& =47.7833 \%
\end{aligned}
$$




\section{Design thickness $=0.7762^{\prime \prime}$}

The governing condition is due to internal pressure.

The cylinder thickness of $0.97^{\prime \prime}$ is adequate.

Thickness Required Due to Pressure + External Loads

\begin{tabular}{|c|c|c|c|c|c|c|c|c|}
\hline \multirow[t]{2}{*}{ Condition } & \multirow{2}{*}{$\begin{array}{l}\text { Pressure } \\
\text { P ( psi) }\end{array}$} & \multicolumn{2}{|c|}{$\begin{array}{l}\text { Allowable Stress } \\
\text { Before UG-23 Stress } \\
\text { Increase ( psi) }\end{array}$} & \multirow{2}{*}{$\begin{array}{l}\text { Temperature } \\
\left({ }^{\circ} \mathrm{F}\right)\end{array}$} & \multirow{2}{*}{$\begin{array}{l}\text { Corrosioll } \\
\text { C (in) }\end{array}$} & \multirow[t]{2}{*}{ Load } & \multirow{2}{*}{$\begin{array}{l}\text { Req'd Thk } \\
\text { Due to } \\
\text { Tension (ill) }\end{array}$} & \multirow{2}{*}{$\begin{array}{l}\text { Req'd Thk Due } \\
\text { to Compression } \\
\text { (in) }\end{array}$} \\
\hline & & $S_{t}$ & $S_{c}$ & & & & & \\
\hline \multirow{2}{*}{$\begin{array}{l}\text { Operating, Hot \& } \\
\text { Corroded }\end{array}$} & \multirow{2}{*}{1,150} & \multirow{2}{*}{1,900} & \multirow{2}{*}{1,900} & \multirow{2}{*}{1,600} & \multirow{2}{*}{0.02} & Wind & 0.3339 & 0.0482 \\
\hline & & & & & & Seismic & 0.3405 & 0.0515 \\
\hline \multirow{2}{*}{$\begin{array}{c}\text { Operating, Hot \& } \\
\text { New }\end{array}$} & \multirow{2}{*}{1,150} & \multirow{2}{*}{1,900} & \multirow{2}{*}{1,900} & \multirow{2}{*}{$\mathrm{I}, 600$} & \multirow{2}{*}{0} & Wind & 0.3334 & 0.0563 \\
\hline & & & & & & Seismic & 0.3416 & 0.0611 \\
\hline \multirow{2}{*}{ Empry, Conoded } & \multirow{2}{*}{0} & \multirow{2}{*}{26,700} & \multirow{2}{*}{20,000} & \multirow{2}{*}{0} & \multirow{2}{*}{0.02} & Wind & 0.0148 & 0.0166 \\
\hline & & & & & & Seismic & 0.0153 & 0.0169 \\
\hline \multirow{2}{*}{ Empty, New } & \multirow{2}{*}{0} & \multirow{2}{*}{26,700} & \multirow{2}{*}{20,000} & \multirow{2}{*}{0} & \multirow{2}{*}{0} & Wind & 0.0151 & 0.0169 \\
\hline & & & & & & Seismic & 0.0157 & 0.0173 \\
\hline $\begin{array}{l}\text { Hor Shur Down, } \\
\text { Corroded. Weight \& } \\
\text { Eccentric Moments } \\
\text { Only }\end{array}$ & 0 & $\mathrm{I}, 900$ & 1,900 & 1,600 & 0.02 & Weight & 0.0202 & 0.0202 \\
\hline
\end{tabular}




\section{3" TUBE \#4}

ASME Section VIII Division 1, 2007 Edition

Component:

Material specification:

Rated MDMT per UNF- $65=-325^{\circ} \mathrm{F}$

Internal design pressure: $\mathrm{P}=1,150 \mathrm{psi} @ 1,600^{\circ} \mathrm{F}$
Cylinder

SB-446 2 Solution ann. Bar N06625 (low stress) (II-D p. 222, In. 40)

\section{Static liquid head:}

$P_{\text {th }}=0.04 \mathrm{psi}\left(\mathrm{SG}=1, \mathrm{H}_{\mathrm{S}}=1.06 "\right.$, Horizontal test head $)$

Corrosion allowance $\quad$ Inner $C=0.02^{\prime \prime} \quad$ Outer $C=0 "$

$\begin{array}{ll}\text { Design MDMT }=-20^{\circ} \mathrm{F} & \text { No impact test performed } \\ \text { Rated MDMT }=-325^{\circ} \mathrm{F} & \text { Material is not normalized } \\ & \text { Material is not produced to Fine Grain Practice } \\ & \text { PWHT is not performed }\end{array}$

Radiography: Longitudinal joint - Seamless No RT

Top circumferential joint - Full UW-11(a) Type 1

Bottom circumferential joint -

Full UW-11(a) Type 1

$$
\begin{aligned}
& \text { Estimated weight New }=80.3 \mathrm{lb} \quad \text { corr }=79.4 \mathrm{lb} \\
& \text { Capacity } \quad \text { New }=0.17 \text { US gal corr }=0.18 \text { US gal } \\
& \begin{array}{ll}
\text { OD } \quad=3^{\prime \prime} \\
\text { Length } \mathrm{L}_{\mathrm{c}}=43.25^{\prime \prime} \\
\mathrm{t} \quad=0.97^{\prime \prime}
\end{array}
\end{aligned}
$$

\section{Design thickness, (at 1,600 ${ }^{\circ}$ ) Appendix 1-2}

$$
\begin{aligned}
\mathrm{Z} & =(\mathrm{S} * \mathrm{E}+\mathrm{P}) /(\mathrm{S} * \mathrm{E}-\mathrm{P}) \\
& =\left(1,900^{*} 1.00+1,150\right) /(1,900 * 1.00-1,150) \\
& =4.0667 \\
\mathrm{t} & =\mathrm{R}_{\mathrm{O}}{ }^{*}\left(\mathrm{Z}^{1 / 2}-1\right) / \mathrm{Z}^{1 / 2}+\text { Corrosion } \\
& =1.5 *\left(4.0667^{1 / 2}-1\right) / 4.0667^{1 / 2}+0.02 \\
& =0.7762 "
\end{aligned}
$$

\% Forming Strain - UNF-79(a)(2)(a)

$$
\begin{aligned}
\mathrm{EFE} & =\left(50 * \mathrm{t} / \mathrm{R}_{\mathrm{f}}\right) *\left(1-\mathrm{R}_{\mathrm{f}} / \mathrm{R}_{\mathrm{o}}\right) \\
& =(50 * 0.97 / 1.015) *(1-1.015 / \infty) \\
& =47.7833 \%
\end{aligned}
$$




\section{Design thickness $=0.7762^{\prime \prime}$}

The governing condition is due to internal pressure.

The cylinder thickness of $0.97^{\prime \prime}$ is adequate.

Thickness Required Due to Pressure + External Loads

\begin{tabular}{|c|c|c|c|c|c|c|c|c|}
\hline \multirow[t]{2}{*}{ Condition } & \multirow{2}{*}{$\begin{array}{l}\text { Pressure } \\
\text { P (psi) }\end{array}$} & \multicolumn{2}{|c|}{$\begin{array}{c}\text { Allowable Stress } \\
\text { Before UG-23 Stress } \\
\text { Increase (psi) }\end{array}$} & \multirow{2}{*}{$\begin{array}{l}\text { Temperatıre } \\
\left({ }^{\circ} \mathrm{F}\right)\end{array}$} & \multirow{2}{*}{$\begin{array}{l}\text { Corrosion } \\
\text { C (in) }\end{array}$} & \multirow[t]{2}{*}{ Load } & \multirow{2}{*}{$\begin{array}{l}\text { Req'd Thk } \\
\text { Due to } \\
\text { Teusiou (in) }\end{array}$} & \multirow{2}{*}{$\begin{array}{l}\text { Req'd Thk Due } \\
\text { to Compression } \\
\text { (iil) }\end{array}$} \\
\hline & & $S_{t}$ & $S_{c}$ & & & & & \\
\hline \multirow{2}{*}{$\begin{array}{l}\text { Operating, Hot \& } \\
\text { Corroded }\end{array}$} & \multirow{2}{*}{1,150} & \multirow{2}{*}{1,900} & \multirow{2}{*}{1,900} & \multirow{2}{*}{1,600} & \multirow{2}{*}{0.02} & Wind & 0.1866 & 0.0814 \\
\hline & & & & & & Seismic & 0.1907 & 0.079 \\
\hline \multirow{2}{*}{$\begin{array}{l}\text { Operating, Hot \& } \\
\text { New }\end{array}$} & \multirow{2}{*}{1.150} & \multirow{2}{*}{1.900} & \multirow{2}{*}{1,900} & \multirow{2}{*}{1,600} & \multirow{2}{*}{0} & Wind & 0.1832 & 0.0759 \\
\hline & & & & & & Seismic & 0.1878 & 0.073 \\
\hline \multirow{2}{*}{ Empty, Corroded } & \multirow{2}{*}{0} & \multirow{2}{*}{26,700} & \multirow{2}{*}{20,000} & \multirow{2}{*}{0} & \multirow{2}{*}{0.02} & Wind & 0.0043 & 0.0042 \\
\hline & & & & & & Seismic & 0.0046 & 0.0045 \\
\hline \multirow{2}{*}{ Empty, New } & \multirow{2}{*}{0} & \multirow{2}{*}{26.700} & \multirow{2}{*}{20,000} & \multirow{2}{*}{0} & \multirow{2}{*}{0} & Wind & 0.0044 & 0.0043 \\
\hline & & & & & & Seismic & 0.0047 & 0.0046 \\
\hline $\begin{array}{l}\text { Hot Shut Down, } \\
\text { Corroded, Weight \& } \\
\text { Eccentric Moments } \\
\text { Only }\end{array}$ & 0 & 1,900 & 1,900 & 1.600 & 0.02 & Weight & 0.0096 & 0.0096 \\
\hline
\end{tabular}




\section{HEAD, BOTTOM}

\section{ASME Section VIII Division 1, 2007 Edition}

Component:

Material specification:

Rated MDMT per UNF- $65=-325^{\circ} \mathrm{F}$

Interna1 design pressure: $\mathrm{P}=1,150 \mathrm{psi} @ 1,600^{\circ} \mathrm{F}$
Cylinder

SB-4462 Solution ann. Bar N06625 (low stress) (II-D p. 222, ln. 40)

\section{Static liquid head:}

$\mathrm{P}_{\mathrm{th}}=0.04 \mathrm{psi}\left(\mathrm{SG}=1, \mathrm{H}_{\mathrm{S}}=1.06 "\right.$, Horizontal test head $)$

Corrosion allowance $\quad$ Inner $\mathrm{C}=0.02^{\prime \prime} \quad$ Outer $\mathrm{C}=0 "$

$$
\begin{aligned}
& \text { Design MDMT }=-20^{\circ} \mathrm{F} \\
& \text { No impact test performed } \\
& \text { Rated MDMT }=-325^{\circ} \mathrm{F} \\
& \text { Material is not produced to Fine Grain Practice } \\
& \mathrm{E}=1 \\
& \text { Estimated weight New }=8.4 \mathrm{lb} \quad \text { corr }=8.3 \mathrm{lb} \\
& \text { Capacity New }=0.02 \text { US gal corr }=0.02 \text { US gal } \\
& \mathrm{OD} \quad=3^{\prime \prime} \\
& \text { Length } \mathrm{L}_{\mathrm{c}}=4.5^{\text {" }} \\
& \mathrm{t} \quad=0.97^{\prime \prime}
\end{aligned}
$$$$
\text { Material is not normalized }
$$$$
\text { PWHT is notperformed }
$$

Top circumferential joint - Full UW-11(a) Type 1

\section{SEAMLESS}

\section{Design thickness, (at $1,600^{\circ} \mathrm{F}$ ) Appendix 1-2}

$$
\begin{aligned}
\mathrm{Z} & =(\mathrm{S} * \mathrm{E}+\mathrm{P}) /(\mathrm{S} * \mathrm{E}-\mathrm{P}) \\
& =\left(1,900^{*} 1.00+1,150\right) /\left(1,900^{*} 1.00-1,150\right) \\
& =4.0667 \\
\mathrm{t} & =\mathrm{R}_{0} *\left(\mathrm{Z}^{1 / 2}-1\right) / \mathrm{Z}^{1 / 2}+\text { Corrosion } \\
& =1.5 *\left(4.0667^{1 / 2}-1\right) / 4.0667^{1 / 2}+0.02 \\
& =0.7762^{\prime \prime}
\end{aligned}
$$

\% Forming Strain - UNF-79(a)(2)(a)

$$
\begin{aligned}
\mathrm{EFE} & =\left(50 * \mathrm{t} / \mathrm{R}_{\mathrm{f}}\right) *\left(1-\mathrm{R}_{\mathrm{f}} / \mathrm{R}_{\mathrm{o}}\right) \\
& =(50 * 0.97 / 1.015) *(1-1.015 / \infty) \\
& =47.7833 \%
\end{aligned}
$$


P.O.\#: 700521452

Design thickness $=0.7762^{\prime \prime}$

The governing condition is due to internal pressure.

The cylinder thickness of $0.97^{\prime \prime}$ is adequate.

Thickness Required Due to Pressure + External Loads

\begin{tabular}{|c|c|c|c|c|c|c|c|c|}
\hline \multirow[t]{2}{*}{ Condition } & \multirow{2}{*}{$\begin{array}{l}\text { Pressure } \\
\mathbf{P}(\text { psi) }\end{array}$} & \multicolumn{2}{|c|}{$\begin{array}{l}\text { Allowable Stress } \\
\text { Before UG-23 Stress } \\
\text { Increase ( psi) }\end{array}$} & \multirow{2}{*}{$\begin{array}{l}\text { Temperature } \\
\left({ }^{\circ} \mathrm{F}\right)\end{array}$} & \multirow{2}{*}{$\begin{array}{l}\text { Corrosion } \\
\quad C \text { (in) }\end{array}$} & \multirow[t]{2}{*}{ Load } & \multirow{2}{*}{$\begin{array}{l}\text { Req'd Thk } \\
\text { Due to } \\
\text { Tension (in) }\end{array}$} & \multirow{2}{*}{$\begin{array}{l}\text { Req'd Thk Due } \\
\text { to Compression } \\
\text { (in) }\end{array}$} \\
\hline & & $S_{t}$ & $\mathrm{~S}_{\mathrm{c}}$ & & & & & \\
\hline \multirow{2}{*}{$\begin{array}{l}\text { Operating. Hot \& } \\
\text { Corroded }\end{array}$} & \multirow{2}{*}{1,150} & \multirow{2}{*}{1,900} & \multirow{2}{*}{1,900} & \multirow{2}{*}{1,600} & \multirow{2}{*}{0.02} & Wind & 0.127 & 0.1261 \\
\hline & & & & & & Seismic & 0.1271 & 0.1261 \\
\hline \multirow{2}{*}{$\begin{array}{c}\text { Operating, Hot \& } \\
\text { New }\end{array}$} & \multirow{2}{*}{1,150} & \multirow{2}{*}{1,900} & \multirow{2}{*}{1,900} & \multirow{2}{*}{1,600} & \multirow{2}{*}{0} & Wind & 0.1225 & 0.1215 \\
\hline & & & & & & Seismic & 0.1225 & 0.1215 \\
\hline \multirow{2}{*}{ Empty, Corroded } & \multirow{2}{*}{0} & \multirow{2}{*}{26,700} & \multirow{2}{*}{20,000} & \multirow{2}{*}{0} & \multirow{2}{*}{0.02} & Wind & 0.0001 & 0 \\
\hline & & & & & & Seismic & 0.0001 & 0 \\
\hline \multirow{2}{*}{ Empty, New } & \multirow{2}{*}{0} & \multirow{2}{*}{26,700} & \multirow{2}{*}{20,000} & \multirow{2}{*}{0} & \multirow{2}{*}{0} & Wind & 0.0001 & 0 \\
\hline & & & & & & Seismic & 0.0001 & 0 \\
\hline $\begin{array}{l}\text { Hot Shut Down, } \\
\text { Corroded, Weight \& } \\
\text { Eccentric Monents } \\
\text { Only }\end{array}$ & 0 & 1,900 & 1,900 & 1,600 & 0.02 & Weight & 0.0007 & 0.0007 \\
\hline
\end{tabular}




\section{TOP MOUNT}

Support material:

This support is attached to:

Distance from baseplate to datum:

Local shell outer diameter, new:

Local shell thickness, new:

Local shell inner corrosion:

Local shell outer corrosion:

Lug allowable stress

Top plate width

Top plate thickness

Base plate width

Base plate thickness

Base plate load bearing width

Shell to center of load bearing area

Gusset height

Gusset thickness

Gusset separation

Number of ring girder support locations:
SA-240-316 (0.04\% CARBON OR HIGHER)

3" TUBE \#1

170 in

3 in

0.97 in

0.02 in

0 in

$\mathrm{S}_{\mathrm{b}}=1,800 \mathrm{psi}$

$\mathrm{W}_{\mathrm{p}}=3$ in

$\mathrm{t}_{\mathrm{a}}=0.4375$ in

$\mathrm{b}=5.75$ in

$\mathrm{t}_{\mathrm{b}}=0.4375 \mathrm{in}$

$\mathrm{L}_{\mathrm{b}}=2.25$ in

$\mathrm{d}=5.125$ in

$\mathrm{h}=2.75 \mathrm{in}$

$\mathrm{t}_{\mathrm{g}}=0.4375$ in

$\mathrm{L}_{\mathrm{g}}=1.75$ in

4 


\begin{tabular}{|c|c|c|c|c|c|c|c|c|c|c|c|}
\hline \multicolumn{12}{|c|}{ Stresses in Shell and Ring Girder Supports } \\
\hline \multirow{3}{*}{ Load } & \multirow{3}{*}{ Condition } & \multirow{3}{*}{$\begin{array}{l}\text { Weight } \\
\text { Supported } \\
\text { (lb) }\end{array}$} & \multirow{3}{*}{$\begin{array}{c}\text { Base } \\
\text { Moment } \\
\text { (ft-lb) }\end{array}$} & \multicolumn{4}{|c|}{ At Supports (psi) } & \multicolumn{4}{|c|}{ Between Supports (psi) } \\
\hline & & & & \multicolumn{2}{|c|}{ Top Ring } & \multicolumn{2}{|c|}{ Base Ring } & \multicolumn{2}{|c|}{ Top Ring } & \multicolumn{2}{|c|}{ Base Ring } \\
\hline & & & & In Shell & In Ring & In Shell & In Ring & In Shell & In Ring & In Shell & In Ring \\
\hline Wind & operating, corroded & 554 & 440 & 1,172 & -283 & 1,295 & 181 & 1,006 & -125 & 1.407 & 101 \\
\hline Wind & operating, new & 558 & 440 & 1,135 & -278 & 1,258 & 179 & 972 & -123 & 1,367 & 100 \\
\hline Wind & empty, corroded & 554 & 440 & -69 & -283 & 55 & 181 & -235 & -125 & 166 & 101 \\
\hline Wind & empty, new & 558 & 440 & -68 & -278 & 55 & 179 & -231 & -123 & 164 & 100 \\
\hline Seismic & operating, corroded & 554 & 384 & 1.175 & -271 & 1,293 & 173 & 1,016 & -119 & 1,400 & 97 \\
\hline Seismic & operating, new & 558 & 386 & 1,137 & -268 & 1,256 & 172 & 981 & -118 & 1,361 & 96 \\
\hline Seismic & empty, corroded & 554 & 384 & -66 & -271 & 52 & 173 & -225 & -119 & 159 & 97 \\
\hline Seismic & empty, new & 558 & 386 & -66 & -268 & 53 & 172 & -222 & -118 & 158 & 96 \\
\hline
\end{tabular}

Stress at the support, at the top ring due to wind (operating, corroded)

$\mathrm{S}_{\mathrm{p}}=\mathrm{P}^{*} \mathrm{R}_{\mathrm{m}} / \mathrm{t}$

$=1,150 * 1.025 / 0.95$

$=1,241 \mathrm{psi}$

Reaction at the support (Bednar 5.2)

$\mathrm{F}_{\mathrm{r}}=48 * \mathrm{M} /(\mathrm{N} * \mathrm{D})+\mathrm{W} / \mathrm{N}$

$=48 * 439.5 /(4 * 13.25)+554.38 / 4$

$=536.68 \mathrm{lb}_{\mathrm{f}}$

Reaction force on ring $\mathbb{P}_{\mathbf{f}}$ acting perpendicular to the vessel longitudinal axis

$\mathrm{P}_{\mathrm{f}}=\mathrm{F}_{\mathrm{r}} * \mathrm{~d} /\left(\mathrm{h}+0.5 *\left(\mathrm{t}_{\mathrm{a}}+\mathrm{t}_{\mathrm{b}}\right)\right)$

$=536.68 * 5.125 /(2.75+0.5 *(0.4375+0.4375))$

$=862.89 \mathrm{lb}_{\mathrm{f}}$

$\mathrm{M}_{1}=0.5 * \mathrm{P}_{\mathrm{f}}^{*} \mathrm{R}_{\mathrm{c}}^{*}((1 / \alpha)-\operatorname{Cot}(\alpha))$

$=0.5^{*} 862.89 * 1.8675^{*}((1 / 0.7854)-\operatorname{Cot}(45))$

$=220.2 \mathrm{lb}$-in

$\mathrm{T}_{\mathrm{l}}=\left(\mathrm{P}_{\mathrm{f}} / \operatorname{Tan}(\alpha)\right) / 2$

$=(862.89 / \operatorname{Tan}(45)) / 2$

$=431.45 \mathrm{lb} f$

\section{Stress in the ring}

$\mathrm{S}=-\left(\mathrm{T}_{1} / \mathrm{A}_{\mathrm{r}}\right)-\left(\mathrm{M}_{1} * \mathrm{y} / \mathrm{I}\right)$

$=-(431.45 / 3.08)-(220.2 * 2.6325 / 4.0526)$

$=-283 \mathrm{psi}$

Stress in the top ring is acceptable $(<=2,850 \mathrm{psi})$ 
Stress at the support, at the base ring due to wind (operating, corroded)

$\mathrm{S}_{\mathrm{p}}=\mathrm{P} * \mathrm{R}_{\mathrm{m}} / \mathrm{t}$

$=1,150 * 1.025 / 0.95$

$=1,241 \mathrm{psi}$

Reaction at the support (Bednar 5.2)

$\mathrm{F}_{\mathrm{r}}=48 * \mathrm{M} /(\mathrm{N} * \mathrm{D})+\mathrm{W} / \mathrm{N}$

$=48 * 439.5 /(4 * 13.25)+554.38 / 4$

$=536.68 \mathrm{lb}_{\mathrm{f}}$

Reaction force on ring $\mathbb{P}_{\mathbf{f}}$ acting perpendicular to the vessel longitudinal axis

$\mathrm{P}_{\mathrm{f}}=\mathrm{F}_{\mathrm{T}}{ }_{\mathrm{T}} \mathrm{d} /\left(\mathrm{h}+0.5 *\left(\mathrm{t}_{\mathrm{a}}+\mathrm{t}_{\mathrm{b}}\right)\right)$

$=536.68 * 5.125 /(2.75+0.5 *(0.4375+0.4375))$

$=862.89 \mathrm{lb}_{\mathrm{f}}$

$\mathrm{M}_{1}=0.5 * \mathrm{P}_{\mathrm{f}} * \mathrm{R}_{\mathrm{c}} *((1 / \alpha)-\operatorname{Cot}(\alpha))$

$=0.5 * 862.89 * 2.9941 *((1 / 0.7854)-\operatorname{Cot}(45))$

$=353 \mathrm{lb}-\mathrm{in}$

$\mathrm{T}_{1}=\left(\mathrm{P}_{\mathrm{f}} / \operatorname{Tan}(\alpha)\right) / 2$

$=(862.89 / \operatorname{Tan}(45)) / 2$

$=431.45 \mathrm{lb}_{\mathrm{f}}$

Stress in the ring

$\mathrm{S}=\left(\mathrm{T}_{1} / \mathrm{A}_{\mathrm{r}}\right)+\left(\mathrm{M}_{1} * \mathrm{y} / \mathrm{I}\right)$

$=(431.45 / 4.28)+(353 * 4.2559 / 18.701)$

$=\underline{181} \mathrm{psi}$

Stress in the base ring is acceptable $(<=2,850 \mathrm{psi})$

Stress between supports, at the base ring due to wind (operating, corroded)

$\mathrm{S}_{\mathrm{p}}=\mathrm{P} * \mathrm{R}_{\mathrm{m}} / \mathrm{t}$

$=1,150^{*} 1.025 / 0.95$

$=1,241 \mathrm{psi}$

Reaction at the support (Bednar 5.2)

$\mathrm{F}_{\mathrm{r}}=48 * \mathrm{M} /(\mathrm{N} * \mathrm{D})+\mathrm{W} / \mathrm{N}$

$=48 * 439.5 /(4 * 13.25)+554.38 / 4$

$=536.68 \mathrm{lb}_{\mathrm{f}}$

Reaction force on ring $\mathbb{P}_{\mathbf{f}}$ acting perpendicular to the vessel longitudinal axis

$\mathrm{P}_{\mathrm{f}}=\mathrm{F}_{\mathrm{r}}{ }^{*} \mathrm{~d} /\left(\mathrm{h}+0.5 *\left(\mathrm{t}_{\mathrm{a}}+\mathrm{t}_{\mathrm{b}}\right)\right)$

$=536.68 * 5.125 /(2.75+0.5 *(0.4375+0.4375))$ 
$=862.89 \mathrm{lb}_{\mathrm{f}}$

$\mathrm{M}_{2}=0.5 * \mathrm{P}_{\mathrm{f}}{ }^{*} \mathrm{R}_{\mathrm{c}}{ }^{*}((1 / \alpha)-1 / \operatorname{Sin}(\alpha))$

$=0.5 * 862.89 * 2.9941 *((1 / 0.7854)-1 / \operatorname{Sin}(45))$

$=182.1 \mathrm{lb}-$ in

$\mathrm{T}_{2}=\left(\mathrm{P}_{\mathrm{f}} / \operatorname{Sin}(\alpha)\right) / 2$

$=(862.89 / \operatorname{Sin}(45)) / 2$

$=610.16 \mathrm{lb}_{\mathrm{f}}$

Stress in the shell

$\mathrm{S}=\left(\mathrm{T}_{2} / \mathrm{A}_{\mathrm{r}}\right)+\left(\mathrm{M}_{2} * \mathrm{y} / \mathrm{I}\right)+\mathrm{S}_{\mathrm{p}}$

$=(610.16 / 4.28)+(182.1 * 2.4441 / 18.701)+1,241$

$=1.407 \mathrm{psi}$

Stress in the base ring is acceptable $(<=2,850 \mathrm{psi})$

Stress at the support, at the top ring due to seismic (operating, corroded)

$\mathrm{S}_{\mathrm{p}}=\mathrm{P} * \mathrm{R}_{\mathrm{m}} / \mathrm{t}$

$=1,150^{*} 1.025 / 0.95$

$=1,241 \mathrm{psi}$

Reaction at the support (Bednar 5.2)

$\mathrm{F}_{\mathrm{r}}=48 * \mathrm{M} /\left(\mathrm{N}^{*} \mathrm{D}\right)+(1+\mathrm{VAcce} 1)^{*} \mathrm{~W} / \mathrm{N}$

$=48 * 383.8 /(4 * 13.25)+(1+0.2) * 554.38 / 4$

$=513.89 \mathrm{lb}_{\mathrm{f}}$

Reaction force on ring $\mathbb{P}_{f}$ acting perpendicular to the vessel longitudinal axis

$\mathrm{P}_{\mathrm{f}}=\mathrm{F}_{\mathrm{t}}{ }^{*} \mathrm{~d} /\left(\mathrm{h}+0.5 *\left(\mathrm{t}_{\mathrm{a}}+\mathrm{t}_{\mathrm{b}}\right)\right)$

$=513.89 * 5.125 /(2.75+0.5 *(0.4375+0.4375))$

$=826.26 \mathrm{lb}_{\mathrm{f}}$

$\mathrm{M}_{1}=0.5 * \mathrm{P}_{\mathrm{f}} \mathrm{R}_{\mathrm{c}}^{*}((1 / \alpha)-\operatorname{Cot}(\alpha))$

$=0.5 * 826.26 * 1.8675 *((1 / 0.7854)-\operatorname{Cot}(45))$

$=210.8 \mathrm{lb}$-in

$\mathrm{T}_{1}=\left(\mathrm{P}_{\mathrm{f}} / \operatorname{Tan}(\alpha)\right) / 2$

$=(826.26 / \operatorname{Tan}(45)) / 2$

$=413.13 \mathrm{lb}_{\mathrm{f}}$

\section{Stress in the shell}

$\mathrm{S}=-\left(\mathrm{T}_{1} / \mathrm{A}_{\mathrm{r}}\right)+\left(\mathrm{M}_{1} * \mathrm{y} / \mathrm{I}\right)+\mathrm{S}_{\mathrm{p}}$

$=-(413.13 / 3.08)+(210.8 * 1.3175 / 4.0526)+1,241$

$=\underline{1.175} \mathrm{psi}$

Stress in the top ring is acceptable $(<=2,850 \mathrm{psi})$ 
CUST: ARIZONA PUBLIC SERVICE CO

DESC: PREHEATER

P.O.\#: 700521452 


\section{Gusset plate required thickness, Bednar 5.2}

$\mathrm{S}_{\mathrm{c}}=1,350 /\left(1+(1 / 1,350) *\left(\mathrm{~h} /\left(0.289 * \mathrm{t}_{\mathrm{g}}\right)\right)^{2}\right)$

$=1,350 /\left(1+(1 / 1,350) *(2.75 /(0.289 * 0.4375))^{2}\right)$

$=1,000 \mathrm{psi}$

$\mathrm{t}_{\mathrm{g}}=\mathrm{V}_{\mathrm{L}} *\left(3^{*} \mathrm{~d}-\mathrm{b}\right) /\left(\mathrm{S}_{\mathrm{c}} * \mathrm{~b}^{2 *} \operatorname{Sin}(\alpha)^{2}\right)$

$=537.61 *(3 * 5.125-5.75) /\left(1,000 * 5.75^{2 *} \operatorname{Sin}(45)^{2}\right)$

$=0.3131 \mathrm{in}$

The gusset thickness of 0.4375 in is adequate.

\section{Lug base plate required thickness}

From Escoe table 4-8 $(1 / \mathrm{b}=3.2857)$

$C_{x}=0.133, C_{y}=-0.125$

$\mathrm{f}_{\mathrm{c}}=\mathrm{V}_{\mathrm{L}} /\left(\mathrm{L}_{\mathrm{b}}{ }^{*} \mathrm{~L}\right)$

$=537.61 /(2.25 * 3.625)$

$=66 \mathrm{psi}$

$\mathrm{M}_{\mathrm{x}}=\mathrm{C}_{\mathrm{x}} * \mathrm{f}_{\mathrm{c}} * \mathrm{~L}_{\mathrm{g}}^{2}$

$=0.133 * 66^{*} 1.75^{2}=26.85 \mathrm{in}-1 \mathrm{~b}_{\mathrm{f}}$ in

$\mathrm{t}_{\mathrm{b}}=\operatorname{Sqr}\left(6 * \mathrm{M}_{\mathrm{x}} / \mathrm{S}_{\mathrm{b}}\right)$

$=\operatorname{Sqr}(6 * 26.9 / 1,800)$

$=0.2992 \mathrm{in}$

The base plate thickness of $0.4375 \mathrm{in}$ is adequate. 


\section{Seismic Code}

Method of seismic analysis:

Site Class

Importance Factor:

Spectral Response Acceleration at short period ( $\% g$ )

Spectral Response Acceleration at period of $1 \mathrm{sec}(\% \mathrm{~g})$

Response Modification Coeficient from Table 15.42

Acceleration based site co-efficient:

Velocity based site co-efficient:

Long-period transition period:

Redundancy factor:

User Defined Vertical Accelerations Considered:

Force Multiplier:

Minimum Weight Multiplier:
ASCE 7-05 ground supported

$\mathrm{D}$

$\mathrm{I}=1.1500$

$\mathrm{S}_{\mathrm{s}}=40.00 \%$

$\mathrm{S}_{1}=8.00 \%$

$\mathrm{R}=3.0000$

$F_{\mathrm{a}}=1.4800$

$\mathrm{F}_{\mathrm{v}}=2.4000$

$\mathrm{T}_{\mathrm{L}}=12.0000$

$p=1.0000$

Yes

$=0.3333$

$=0.2000$

12.4.2.3 Basic Load Combinations for Allowable Stress Design

The following load combinations are considered in accordance with ASCE section 2.4.1:

5. $D+H+0.7 E=\left(1.0+V_{\text {Accel }}\right) D+H+0.7 \rho Q_{E}$

Where

$D \quad=$ Dead load

$H \quad=$ Pressure load

$E \quad=$ Seismic load $=\rho Q_{E}$

$V_{\text {Accel }}=$ User defined vertical acceleration

\section{Vessel Characteristics}

Vessel height: $0.7083 \mathrm{ft}$

Vessel Weight:

Operating, Corroded: $554 \mathrm{lb}$

Empty, Corroded: $554 \mathrm{lb}$ 


\section{Period of Vibration Calculation}

Fundamental Period, T:

Operating, Corroded: $0.447 \mathrm{sec}(\mathrm{f}=2.2 \mathrm{~Hz})$

Empty, Corroded: $0.404 \mathrm{sec}(\mathrm{f}=2.5 \mathrm{~Hz})$

The fundamental period of vibration $\mathrm{T}$ (above) is calculated using the Rayleigh method of approximation:

$\mathrm{T}=2 * \mathrm{PI} * \operatorname{Sqr}\left(\left\{\operatorname{Sum}\left(\mathrm{W}_{\mathrm{i}} * \mathrm{y}_{\mathrm{i}}^{2}\right)\right\} /\left\{\mathrm{g} * \operatorname{Sum}\left(\mathrm{W}_{\mathrm{i}} * \mathrm{y}_{\mathrm{i}}\right)\right\}\right)$, where

$\mathrm{W}_{\mathrm{i}}$ is the weight of the $\mathrm{i}^{\text {th }}$ lumped mass, and

$y_{i}$ is its deflection when the system is treated as a cantilever beam.

\section{Seismic Shear Reports:}

Operating, Corroded

Base Shear Calculations

Seismic Shear Report: Operating, Corroded

\begin{tabular}{|l|l|l|l|}
\multicolumn{1}{|c|}{ Component } & $\begin{array}{c}\text { Elevation of bottom } \\
\text { above base (in) }\end{array}$ & $\begin{array}{c}\text { Inertia I I } \\
\left(\mathbf{f t}^{4}\right)\end{array}$ & $\begin{array}{c}\text { Seismic shear at } \\
\text { Bottom (lbf) }\end{array}$ \\
\hline HEAD, TOP & 3 & 0.0002 & 0 \\
\hline 3" TUBE \#1 (top) & 0 & 0.0002 & 0 \\
\hline 3" TUBE \#1 (bottom) & 0 & 0.0002 & 59 \\
\hline 3" TUBE \#2 & -40.25 & 0.0002 & 55 \\
\hline 3" TUBE \#3 & -83.5 & 0.0002 & 44 \\
\hline 3" TUBE \#4 & -126.75 & 0.0002 & 25 \\
\hline HEAD, BOTTOM & -170 & 0.0002 & 2 \\
\hline
\end{tabular}

\subsection{3: Maximum considered earthquake spectral response acceleration}

The maximum considered earthquake spectral response acceleration at short period, $\mathrm{S}_{M S}$

$\mathrm{S}_{M S}=\underline{\mathrm{F}}_{\underline{\mathrm{a}}} * \underline{\mathrm{S}}_{\mathrm{S}}=1.4800 * 40.00 / 100=0.5920$

The maximum considered earthquake spectral response acceleration at $1 \mathrm{~s}$ period, $\mathrm{S}_{M I}$

$\mathrm{S}_{M I}=\underline{\mathrm{F}}_{\mathrm{Y}} * \underline{\mathrm{S}}_{1}=2.4000 * 8.00 / 100=0.1920$ 


\subsection{4: Design spectral response acceleration parameters}

Design earthquake spectral response acceleration at short period, $\mathrm{S}_{D S}$

$\mathrm{S}_{D, S}=2 / 3 * \mathrm{~S}_{M S}=2 / 3 * 0.5920=0.3947$

Design earthquake spectral response acceleration at $1 \mathrm{~s}$ period, $S_{D 1}$

$S_{D I}=2 / 3 * S_{i l l}=2 / 3 * 0.1920=0.1280$

\section{User Defined Vertical Acceleration Term, $V_{\text {Accel }}$}

Factor is applied to dead load.

Compressive Side: $=1.0+\mathrm{V}_{\text {Accel }}$

\begin{tabular}{|c|c|c|c|c|}
\hline \multicolumn{5}{|c|}{$\begin{array}{l}\qquad V_{\text {Accel }} \text { Term is: } \\
\text { greater of (Force Mult * Base Shear/Weight) or (Min. Weight Mult.) }\end{array}$} \\
\hline \multicolumn{2}{|c|}{ Force multiplier $=0.3333$} & \multicolumn{3}{|c|}{ Minimum Weight Multiplier $=0.2000$} \\
\hline Condition & Base Shear ( lbf) & Weight ( Ib) & $\frac{\text { Force Mult * Shear }}{\text { Weight }}$ & $V_{\text {Accel }}$ \\
\hline Operating, Corroded & 59 & 554.4 & 0.0353 & 0.2 \\
\hline Operating, New & 59 & 558.1 & 0.0353 & 0.2 \\
\hline Empty, Corroded & 59 & 554.4 & 0.0353 & 0.2 \\
\hline Empty, New & 59 & 558.1 & 0.0353 & 0.2 \\
\hline
\end{tabular}

Base Shear Calculations

Operating, Corroded

Empty, Corroded

Base Shear Calculations: Operating, Corroded

\section{Paragraph 15.4.4: Period Determination}

Fundamental Period is taken from the Rayleigh method listed previously in this report.

$\mathrm{T}=0.447 \mathrm{sec}$.

\subsection{1: Calculation of Seismic Response Coefficient}

$\mathrm{C}_{\mathrm{s}}$ is the value computed below, bounded by $\mathrm{C}_{\mathrm{s}} \mathrm{Min}$ and $\mathrm{C}_{\mathrm{s}} \mathrm{Max}$ : 
$\mathrm{C}_{\mathrm{S}} \mathrm{Min}$ is 0.01 unless $\mathrm{S}_{\mathrm{l}}>0.6 \mathrm{~g}$, in which case eqn $12.8-6$ is used.

$\mathrm{C}_{\mathrm{S}} \mathrm{Max}$ calculated with $12.8-3$ because $(\mathrm{T}=0.447)<=\left(\mathrm{T}_{\mathrm{L}}=12.0000\right)$

$\mathrm{C}_{\mathrm{s}}=\underline{\mathrm{S}_{\underline{\underline{S}}}} /(\underline{\mathrm{R}} / \mathrm{I})=0.3947 /(3.0000 / 1.1500)=0.1513$

$\mathrm{C}_{\mathrm{S}} \mathrm{Min}=0.01$

$\mathrm{C}_{\mathrm{s}} \operatorname{Max}=\underline{\mathrm{S}_{D I}} /(\mathrm{T} *(\underline{\mathrm{R}} / \underline{\mathrm{I}}))=0.1280 /(0.0263 *(3.0000 / 1.1500))=1.8691$

$\mathrm{C}_{\mathrm{s}}=0.1513$

\subsection{1: Calculation of Base Shear}

$$
\begin{aligned}
\mathrm{V} & =\mathrm{C}_{\mathrm{s}} * \underline{\mathrm{W}} \\
& =0.1513 * 554.3804 \\
& =83.87 \mathrm{lb}
\end{aligned}
$$

\subsubsection{Seismic Load Combinations: Horizontal Seismic Load Effect, $\mathbb{E}_{\mathrm{h}}$}

$\mathrm{Q}_{\mathrm{E}}=\mathrm{V}$

$$
\begin{aligned}
\mathrm{E}_{\mathrm{h}} & =0.7 * \rho * \mathrm{Q}_{\mathrm{E}}(\text { Only } 70 \% \text { of seismic load considered as per Section 2.4.1) } \\
& =0.70 * 1.0000 * 83.87 \\
& =58.71 \mathrm{lb}
\end{aligned}
$$

\section{Base Shear Calculations: Empty, Corroded}

\section{Paragraph 15.4.4: Period Determination}

Fundamental Period is taken from the Rayleigh method listed previously in this report.

$\mathrm{T}=0.404 \mathrm{sec}$.

\subsection{1: Calculation of Seismic Response Coefficient}

$\mathrm{C}_{\mathrm{s}}$ is the value computed below, bounded by $\mathrm{C}_{\mathrm{s}} \mathrm{Min}$ and $\mathrm{C}_{\mathrm{s}} \mathrm{Max}$ :

$\mathrm{C}_{\mathrm{s}}$ Min is 0.01 unless $\mathrm{S}_{1}>=0.6 \mathrm{~g}$, in which case eqn 12.8-6 is used.

$\mathrm{C}_{\mathrm{s}}$ Max calculated with $12.8-3$ because $(\mathrm{T}=0.404)<=\left(\mathrm{T}_{\mathrm{L}}=12.0000\right)$

$\mathrm{C}_{\mathrm{s}}=\underline{\mathrm{S}_{D S}} /(\underline{\mathrm{R}} / \mathrm{I})=0.3947 /(3.0000 / 1.1500)=0.1513$

$\mathrm{C}_{\mathrm{s}} \mathrm{Min}=0.01$

$\mathrm{C}_{\mathrm{S}} \mathrm{Max}=\underline{\mathrm{S}}_{\underline{D I}} /(\mathrm{T} *(\underline{\mathrm{R}} / \underline{\mathrm{I}}))=0.1280 /(0.0263 *(3.0000 / 1.1500))=1.8691$

$\mathrm{C}_{\mathrm{s}}=0.1513$ 


\subsection{1: Calculation of Base Shear}

$$
\begin{aligned}
\mathrm{V} & =\mathrm{C}_{\mathrm{s}} * \underline{\mathrm{W}} \\
& =0.1513 * 554.3804 \\
& =83.87 \mathrm{lb}
\end{aligned}
$$

12.4.2.1 Seismic Load Combinations: Horizontal Seismic $\mathbb{L o a d}$ Effect, $\mathbb{E}_{\mathrm{h}}$ $\mathrm{Q}_{\mathrm{E}}=\mathrm{V}$

$\mathrm{E}_{\mathrm{h}}=0.7 * \rho * \mathrm{Q}_{\mathrm{E}}($ Only $70 \%$ of seismic load considered as per Section 2.4.1)

$=0.70 * 1.0000 * 83.87$

$=58.71 \mathrm{lb}$

MAXIMUM SEISMIC SHEAR: $59 \mathrm{lbf} ; 59 \mathrm{lbf} / 15 \mathrm{ft}=3.9 \mathrm{lbf} / \mathrm{ft}$ 


\section{Wind Code}

Building Code:

Elevation of base above grade:

\section{ASCE 7-05}

$19.0000 \mathrm{ft}$

Increase effective outer diameter by: $0.5833 \mathrm{ft}$

Wind Force Coefficient Cf:

0.8000

Basic Wind Speed:, V: $90.0000 \mathrm{mph}$

Importance Factor:, I: 1.1500

Exposure category. $\mathrm{C}$

Wind Directionality Factor, Kd: $\quad 0.9500$

Topographic Factor, Kzt: $\quad 1.0000$

Enforce min. loading of $10 \mathrm{psf}$ No

\section{Vessel Characteristics}

Vessel height, h:

Vessel Minimum Diameter, $\mathrm{b}$

Operating, Corroded: $0.2500 \mathrm{ft}$

Empty, Corroded: $0.2500 \mathrm{ft}$

Fundamental Frequency, $n_{t}$

Operating, Corroded: $2.2373 \mathrm{~Hz}$

Empty, Corroded: $2.4757 \mathrm{~Hz}$

Vacuum, Corroded: $2.2373 \mathrm{~Hz}$

Damping coefficient, $\beta$

Operating, Corroded: 0.0189

Empty, Corroded: 0.0205

Vacuum, Corroded: 0.0189

Table Lookup Values

2.4.1 Basic Load Combinations for Allowable Stress Design

The following load combinations are considered in accordance with ASCE section 2.4.1:

5. $D+H+W$

Where

$D \quad=$ Dead load

$H \quad=$ Pressure load 
$W \quad=$ Wind load

\section{Wind Deflection Reports:}

Operating, Corroded

Wind Pressure Calculations

\section{Wind Deflection Report: Operating, Corroded}

\begin{tabular}{|c|c|c|c|c|}
\hline Component & $\begin{array}{c}\text { Elevation of } \\
\text { botton above } \\
\text { base (in) }\end{array}$ & $\begin{array}{c}\text { Effective OD } \\
\text { (ft) }\end{array}$ & $\begin{array}{c}\text { Inertia } \\
\text { I }\left(\mathrm{ft}^{+}\right)\end{array}$ & $\begin{array}{l}\text { Total wind } \\
\text { shear at } \\
\text { Bottom (lb) }\end{array}$ \\
\hline HEAD, TOP & 3 & 0.83 & 0.0001883 & 6 \\
\hline 3" TUBE \#1 (top) & 0 & 0.83 & 0.0001883 & 9 \\
\hline 3" TUBE \#1 (bottom) & 0 & 0.83 & 0.0001883 & 175 \\
\hline 3" TUBE \#2 & -40.25 & 0.83 & 0.0001883 & 133 \\
\hline 3" TUBE \#3 & -83.5 & 0.83 & 0.0001883 & 90 \\
\hline 3" TUBE \#4 & -126.75 & 0.83 & 0.0001883 & 47 \\
\hline HEAD, BOTTOM & -170 & 0.83 & 0.0001883 & 4 \\
\hline
\end{tabular}

\section{Wind Pressure (WP) Calculations}

Gust Factor $\left(\mathrm{G}^{-}\right)$Calculations

$$
\begin{aligned}
\mathrm{Kz} & =2.01 *(\mathrm{Z} / \underline{\mathrm{Zg}})^{2 / \underline{\underline{\alpha}}} \\
& =2.01 *(\mathrm{Z} / 900.0000)^{0.2105} \\
\mathrm{qz} & =0.00256 * \mathrm{Kz} * \mathrm{Kzt} * \mathrm{Kd} * \mathrm{~V}^{2} * \underline{\mathrm{I} p f} \\
& =0.00256 * \mathrm{Kz} * 1.0000 * 0.9500 * 90.0000^{2} * 1.1500 \\
& =22.6541 * \mathrm{Kz} \\
\mathrm{WP} & =\mathrm{qz}^{*} \underline{\mathrm{G}^{-}} * \underline{\mathrm{Cf}} \\
& =\mathrm{qz}^{*} \underline{\mathrm{G}^{-}} * 0.8000
\end{aligned}
$$

\begin{tabular}{|c|c|c|c|c|c|c|}
\hline $\begin{array}{l}\text { Height } \mathbb{Z} \\
\text { () }\end{array}$ & $\mathbb{K} Z$ & $\begin{array}{c}q z \\
(p s f)\end{array}$ & $\begin{array}{l}\text { WP: Operating } \\
\text { (psf) }\end{array}$ & $\begin{array}{l}\text { WP: Empty } \\
\text { (psf) }\end{array}$ & $\begin{array}{l}\text { WP: hydrotest } \\
\text { (psf) }\end{array}$ & $\begin{array}{l}\text { WP: Vacuum } \\
\text { (psf) }\end{array}$ \\
\hline 15.0 & 0.8489 & 19.23 & 14.18 & 14.18 & N.A. & N.A. \\
\hline 20.0 & 0.9019 & 20.43 & 15.06 & 15.06 & N.A. & N.A. \\
\hline
\end{tabular}

\section{Design Wind Pressures}


Design Wind Force determined from: $\mathrm{F}=$ Pressure * Af, where Af is the projected area.

\section{Gust Factor Calculations}

Operating, Corroded

Empty, Corroded

\section{Gust Factor Calculations: Operating, Corroded}

$$
\begin{aligned}
\mathrm{z}^{-} & =0.60 * \underline{\mathrm{h}} \\
& =0.60 * 0.7083 \\
& =15.0000 \\
\mathrm{I}_{\mathrm{z}^{-}} & =\underline{\mathrm{c}} *\left(33 / \mathrm{z}^{-}\right)^{1 / 6} \\
& =0.2000 *(33 / 15.0000)^{1 / 6} \\
& =0.2281 \\
\mathrm{~L}_{\mathrm{z}^{-}} & =\underline{\underline{1}} *\left(\mathrm{z}^{-} / 33\right)^{\underline{\mathrm{p}}} \\
& =500.0000 *(15.0000 / 33)^{0.2000} \\
& =427.0566 \\
\mathrm{Q} & =\mathrm{Sqr}\left(1 /\left(1+0.63 *\left((\underline{\mathrm{b}}+\underline{\mathrm{h}}) / \mathrm{L}_{\mathrm{z}^{-}}\right)^{0.63}\right)\right) \\
& =\mathrm{Sqr}\left(1 /\left(1+0.63 *((0.2500+0.7083) / 427.0566)^{0.63}\right)\right) \\
& =0.9933 \\
\mathrm{G} & =0.925 *\left(1+1.7 * \mathrm{~g}_{\mathrm{Q}} * \mathrm{I}_{\mathrm{z}^{-}} * \mathrm{Q}\right) /\left(1+1.7 * \mathrm{~g}_{\underline{\underline{v}}} * \mathrm{I}_{\mathrm{z}^{-}}\right) \\
& =0.925 *\left(1+1.7 * 3.40^{*} 0.2281 * 0.9933\right) /(1+1.7 * 3.40 * 0.2281) \\
& =0.9215
\end{aligned}
$$

\section{Gust Factor Calculations: Empty, Corroded}

$$
\begin{aligned}
\mathrm{z}^{-} & =0.60 * \underline{\mathrm{h}} \\
& =0.60 * 0.7083 \\
& =15.0000 \\
\mathrm{I}_{\mathrm{z}^{-}} & =\underline{\mathrm{c}} *\left(33 / \mathrm{z}^{-}\right)^{1 / 6} \\
& =0.2000 *(33 / 15.0000)^{1 / 6} \\
& =0.2281 \\
\mathrm{~L}_{\mathrm{z}^{-}} & =\underline{\mathrm{J}} *\left(\mathrm{z}^{-} / 33\right)^{\underline{c} \mathrm{p}} \\
& =500.0000 *(15.0000 / 33)^{0.2000} \\
& =427.0566 \\
\mathrm{Q} & =\operatorname{Sqr}\left(1 /\left(1+0.63 *\left((\underline{\mathrm{b}}+\underline{\mathrm{h}}) / \mathrm{L}_{\mathbf{z}^{-}}\right)^{0.63}\right)\right) \\
& =\operatorname{Sqr}\left(1 /\left(1+0.63 *((0.2500+0.7083) / 427.0566)^{0.63}\right)\right)
\end{aligned}
$$




$$
\begin{aligned}
& =0.9933 \\
\mathrm{G} & =0.925 *\left(1+1.7 * \mathrm{~g}_{\mathrm{Q}} * \mathrm{I}_{\mathrm{z}^{-}} * \mathrm{Q}\right) /\left(1+1.7 * \mathrm{~g}_{\underline{\underline{v}}} * \mathrm{I}_{\mathrm{z}^{*}}\right) \\
& =0.925 *(1+1.7 * 3.40 * 0.2281 * 0.9933) /(1+1.7 * 3.40 * 0.2281) \\
& =0.9215
\end{aligned}
$$

Table Lookup Values

$\alpha=9.5000, \mathrm{Zg}=900.0000^{\circ} \quad$ [Table 6-2, page 78]

$c=0.2000,1=500.0000$, ep $=0.2000 \quad[$ Table $6-2$, page 78]

$\mathrm{a}^{-}=0.1538, \mathrm{~b}^{-}=0.6500 \quad$ [Table 6-2, page 78]

$\mathrm{g}_{\mathrm{Q}}=3.40 \quad$ [6.5.8.1 page 26]

$\mathrm{g}_{\mathrm{v}}=3.40 \quad[6.5 .8 .1$ page 26]

MAXIMUM WIND SHEAR: $175 \mathrm{lbf} / 15 \mathrm{ft}=11.7 \mathrm{lbf} / \mathrm{ft}$

WIND SHEAR IS GREATER THAIN SEISMIC SHEAR (175 lbf $>59$ lbf)

CHECK VESSEL AS A BEAM: 
DESC: PREHEATER

P.O.\#: 700521452

Archon Beam Design Program

INPUT DATA

Beam Type $=$ CUSTOM

Beam Classification $=\mathrm{P}$

Total Length $=14.15(\mathrm{ft})$

Unbraced Length $=14.15(\mathrm{ft})$

Young's Modulus $=2.14 \mathrm{E}+07(\mathrm{psi})$

Moment of Inertia $=3.927\left(\mathrm{in}^{\wedge} 4\right)$

Section Modlus $=2.618\left(i n^{\wedge} 3\right)$

Left end is fixed, Right end is Pinned.

LOADS

( 1) Level Unif. Load $=11.60(\mathrm{lbs} / \mathrm{ft})$ Starting $0(\mathrm{ft})$ from the left side.

Over a length of $14.15(\mathrm{ft})$.

OUTPUT

Max. Moinent $=-290.3(\mathrm{ft}-1 \mathrm{bs})$

Max. Shear $=102.6$ (libs)

Reaction R1 = 102.6 (lbs)

Reaction $R 2=61.55(\mathrm{lbs})$

MAX MOMENT (M) FROM SEISMIC/ WIND: $290.3 \mathrm{ft}$-lbS

MAX MOMENT (M) USED IN CODE CALCULATIONS: $433.17 \mathrm{ft}$-lbs $=5,198 \mathrm{in}-\mathrm{lbs}$ 


\section{TOP HEAD OPENINGS}

PER ASME VIII-1 2007 EDITION

4 RADIAL OPENINGS REQUIRED

OPENING SIZE: 0.26" DIA

PER UG-36 (C)(3)(D):

MINIMUIM SPACING BETWEEN OPENING CENTERS $=\left((1+1.5 \operatorname{COS}(\text { THETA }))^{*}(\mathrm{~d} 1+\mathrm{d} 2)\right.$

$=\left(1+1.5 \operatorname{COS}\left(45^{\circ}\right)\right)^{*}(0.26+0.26)=1.072^{\prime \prime}$

MAXIMUM ACTUAL SPACING BETWEEN OPENING CENTERS $=1.18$ "

1.18”> 1.072; THEREFORE, PER UG-36 (C)(3) NO ADDITIONAL REINFORCEMENT REQUIRED 
RUPTURE DESIGN FOR 1/4" OD TUBE

\begin{tabular}{|c|c|c|}
\hline \multicolumn{3}{|c|}{$\begin{array}{c}15013704 \\
\text { CALCULATION SHEET } \\
\text { (US customary units) }\end{array}$} \\
\hline Outside diameter, inches & $D_{0}=0.25^{\prime \prime}$ & $D_{0}=0.25^{\prime \prime}$ \\
\hline Design pressure, psi (gauge) & $p_{\mathrm{El}}=1150 \mathrm{PSI}$ & $p_{\mathrm{r}}=1150 \mathrm{PSI}$ \\
\hline Maximum or equivalent metal temperature, ${ }^{\circ} \mathrm{F}$ & $T_{\max }=1600 \mathrm{~F}$ & $\Gamma_{\max }=1600 \mathrm{~F}$ \\
\hline Temperature allowance, ${ }_{3}^{\circ} \mathrm{F}$ & $T_{A}=N O N E$ & $T_{A}=$ NONE \\
\hline Design metal temperature, ${ }^{\circ} \mathrm{F}$ & $\tau_{\mathrm{d}}=1600 \mathrm{~F}$ & $T_{\mathrm{d}}=1600 \mathrm{~F}$ \\
\hline Design life, h & - & ${ }^{t} \mathrm{DL}=2000$ \\
\hline Allowable stress at $T_{\mathrm{d}}$ & $v_{e l}=20,000 \mathrm{PSI}$ & $\sigma_{\mathrm{r}}=4000 \mathrm{PSI}$ \\
\hline Stress thickness, equation (2) or (4), inches & $s_{c r}=0.0070^{\prime \prime}$ & $\delta_{, t}=0.0314^{\prime \prime}$ \\
\hline Corrosion allowance, inches & $\phi_{\mathrm{CA}}=\mathrm{NONE}$ & $\phi_{\mathrm{CA}}=$ NONE \\
\hline Corrosion fraction, Figure $1, n=B=$ & $+\infty$ & $f_{\mathrm{CO}}=\mathrm{N} / \mathrm{A}$ \\
\hline Ainimum thickness, equation (3) or (5), inches & $a_{\text {min }}=0.0070^{\prime \prime}$ & $\phi_{\text {min }}=0.0314^{\prime \prime}$ \\
\hline
\end{tabular}

MATERIAL: SB-444 N06625 GR 2 (SOLUTION TREATED)

MINIMUM WALL THICKNESS OF TUBE: $0.0585^{\prime \prime}$

ALLOWABLE STRESSES PER "SPECIAL METALS" ALLOY 625 CATALOG

NO CORROSION ALLOWANCE IS USED

NOTE: $1 / 4 " X 0.065$ NOM THK. TUBE NOT INCLUDED IN CODE PRESSURE BOUNDARY. TUBE IS FIELD WELDED TO VESSEL.

RUPTURE DESIGN PER 4.4 EQN (4)

$$
\begin{aligned}
\delta_{\sigma}=\frac{p_{r} D_{o}}{2 \sigma_{r}+p_{r}} & =\left(1150^{*} 0.25\right) /\left(2^{\star} 4000+1150\right)=0.0314^{\prime \prime} \text { min. thickness required } \\
& 0.0585^{\prime \prime}>0.0314^{\prime \prime} \text { THEREFORE TUBB THICKNESS IS ADEQUATE }
\end{aligned}
$$

ELASTIC DESIGN CHECK PER 6.1 AND 4.3 EQN (2)

$$
\begin{aligned}
\delta_{\sigma}=\frac{p_{e l} D_{\mathrm{o}}}{2 \sigma_{\mathrm{el}}+p_{\mathrm{el}}} & =\left(1150^{*} 0.25\right) /\left(2^{\star} 20,000+150\right)=0.0070^{\prime \prime} \text { min. thickness required } \\
& 0.0585^{\prime \prime}>0.0070^{\prime \prime} \text { THEREFORE TUBE THICKNESS IS ADEQUATE }
\end{aligned}
$$


TUBE TO VESSEL WELD

NOTE: TUBE AND TUBE TO VESSEL WELD NOT INCLUDED IN CODE PRESSURE BOUNDARY

(5) WELDS REQUIRED

TUBES: 1/4" OD X 0.065" AVG WALL SB-444 N06625 GR.2

ASME VIII-1 2007 EDITION USED AS GUIDE

UW-16(E)(1) AND FIG. UW-16.1 (W-1)

WELDED FROM THE OUTSIDE ONLY

REQUIRED FILLET THROAT SIZE $=\operatorname{Tmin} * 1.25=0.065^{*} 1.25=0.08125 "$

REQUIRED FILLET LEG SIZE $=0.08125 / 0.707=0.115 " \Rightarrow$ USE $1 / 8 "$ LEG

NOTE: TUBE DOES NOT EXTEND TO INSIDE WALL OF VESSEL

ADDITIONAL 1/16" GROOVE WELD NOT REQUIRED BY FIG. UW-16.1 (W-1) IS USED; THEREFORE, THE WELD DESIGN IS CONSERVATIVE. 


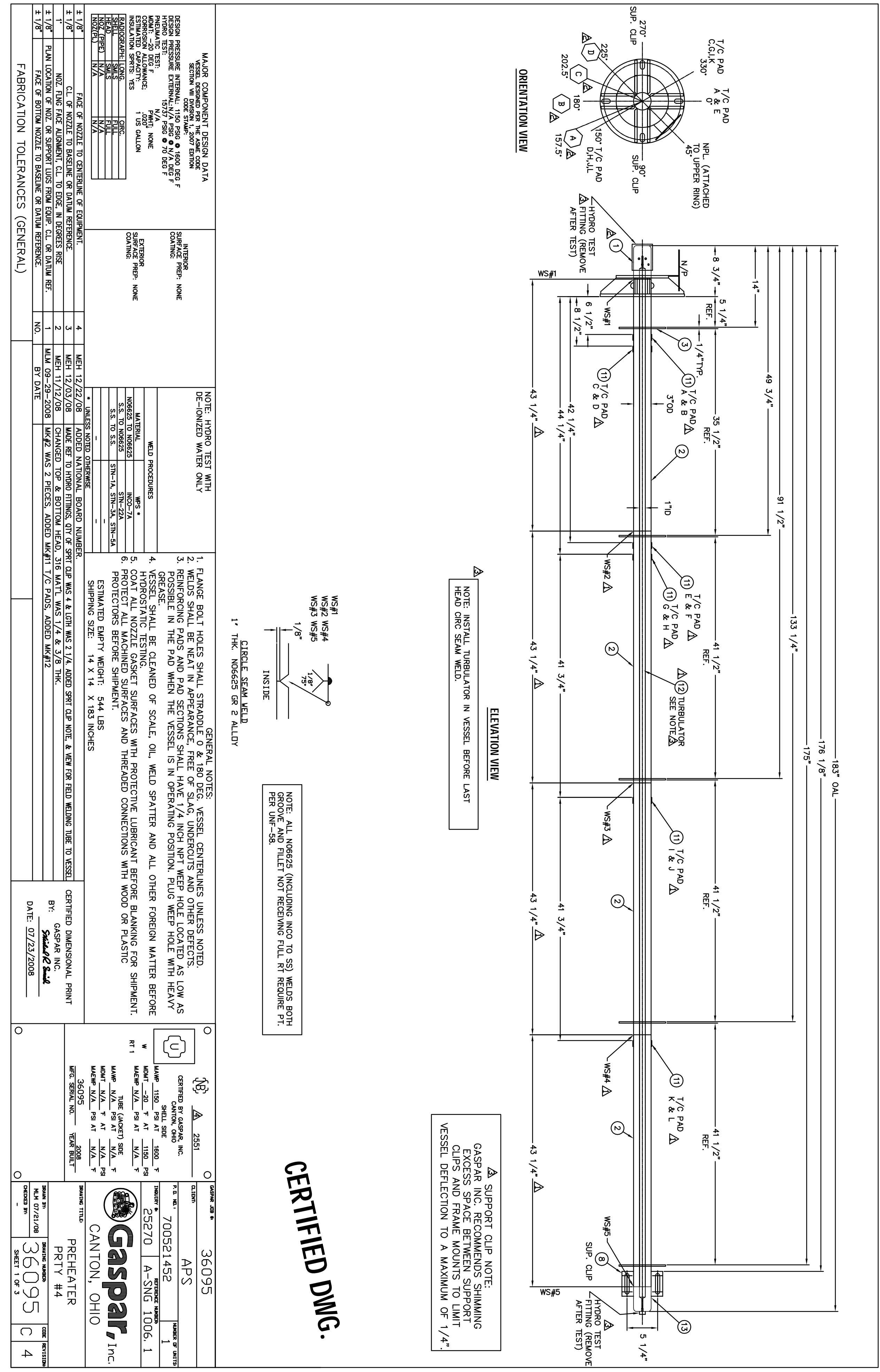




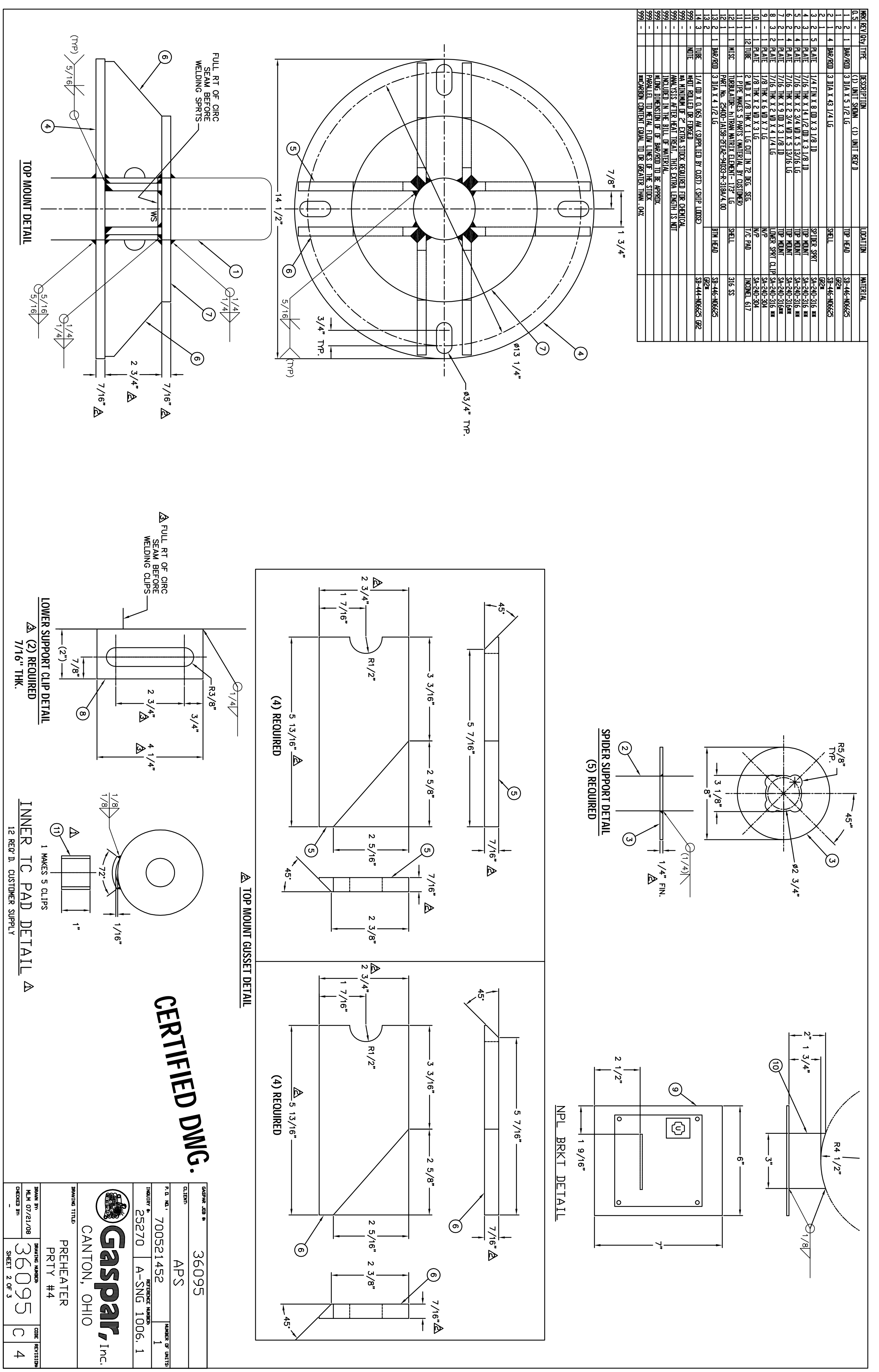




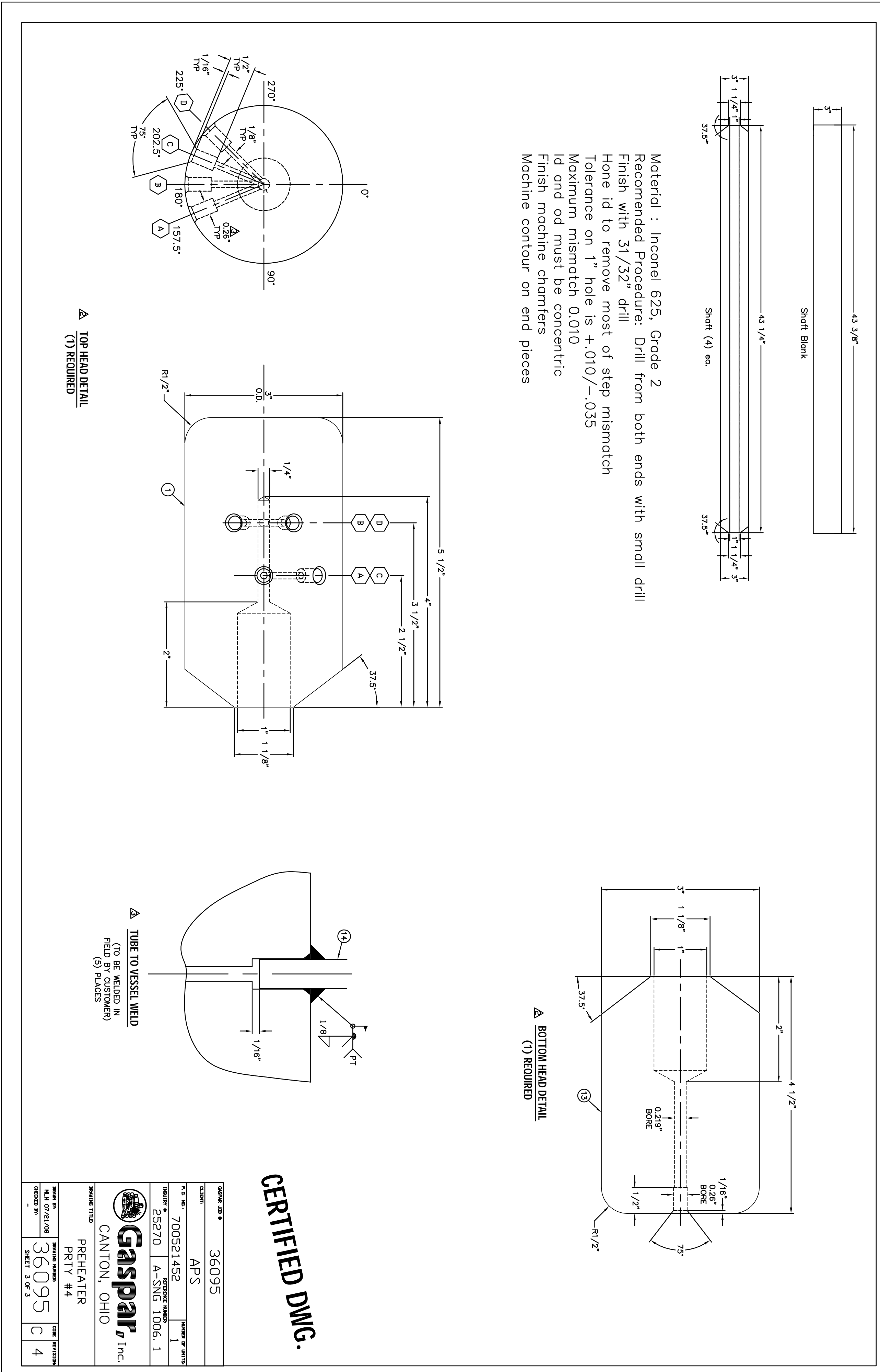




$$
\text { I }
$$




\section{APPENDIX E}

\section{Bench Scale Hydrogasifier Coal Feeder}

Final Design from Gaspar Inc. 
Development of a Hydrogasification Process for

Coproduction of SNG and Electrical Power from Western Coals

Final Report

THIS PAGE INTENTIONALLY LEFT BLANK 


\section{Gaspar $_{\text {inc. }}$}

WELDING \& FABRICATIONS

1545 Whlpple Avenue SW Canton. Ohio 44710

Phone: $330-477-2222$

Fax: $330-477-2322$

urum gasparinc.com

\section{FINAL DATA PACKAGE}

\begin{tabular}{|r|l|}
\hline \hline GASPAR JOB NUMBER(S): & 36056 \\
\hline CUSTOMER: & ARIZONA PUBLIC SERVICE \\
\hline PURCHASE OROER NUMBER: & 700521452 \\
\hline DESCRJPTION: & COAL FEEDER \\
\hline JTEM NUMEER(S): & N/A \\
\hline OTHER: & N/A \\
\hline
\end{tabular}

\section{DATA PACKAGE CONTENTS}

$\square$ DATA REPORT

$\square$ NAMEPLATE COPY

$\square$ EILL OF MATERIAL

๑ MATERIAL TEST REPORTS

$\square$ NDE REPORTS

$\square$ HEAT TREAT CHARTS

$\square$ CALCULATIONS

D DRAWINGS

$\square$ OTHER: (LIST BELOW)

\begin{tabular}{||l|l|}
\hline NOTE: \\
\hline
\end{tabular}




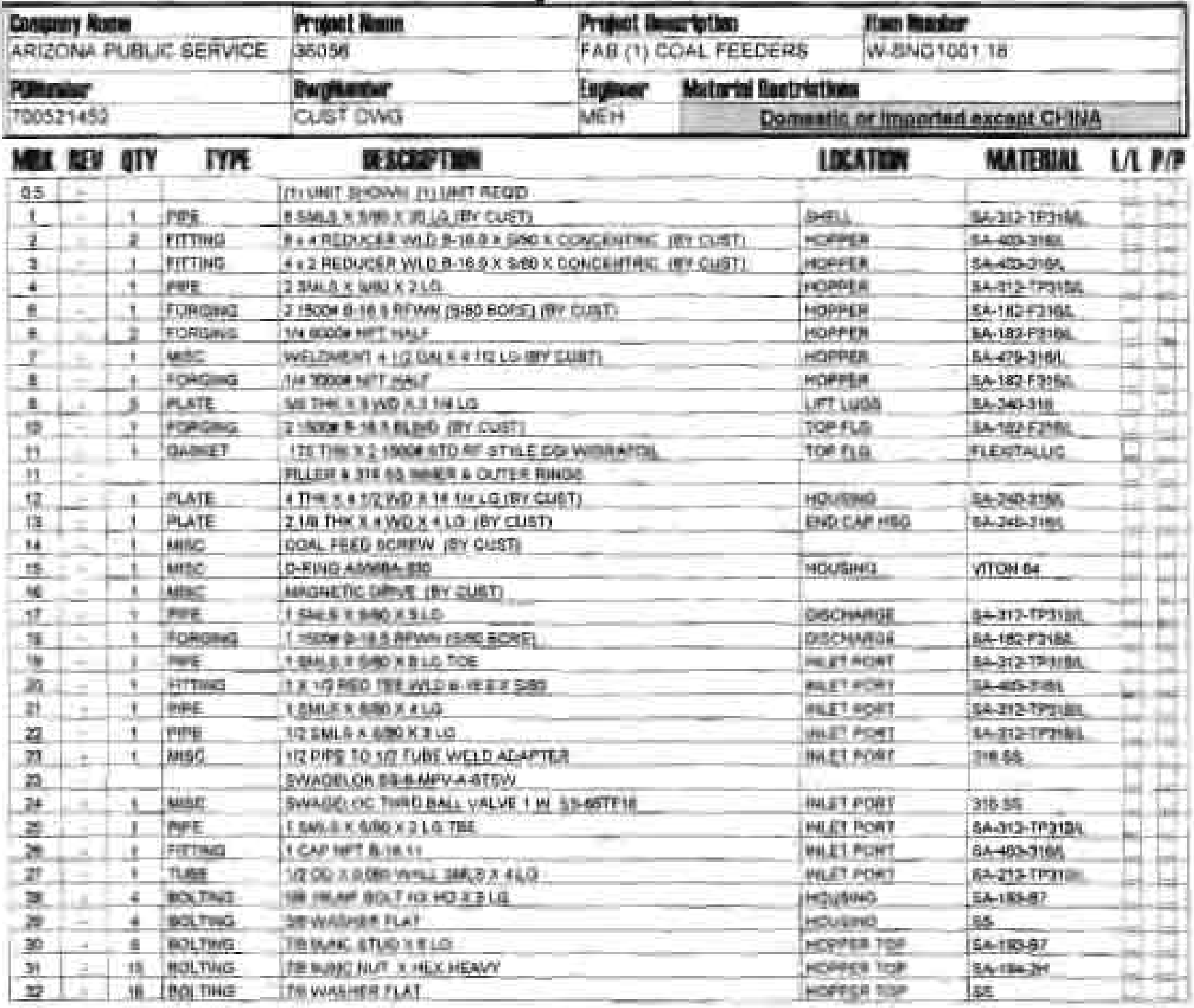




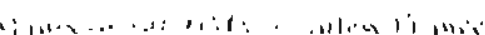

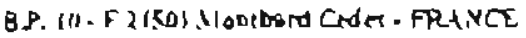

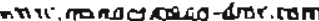

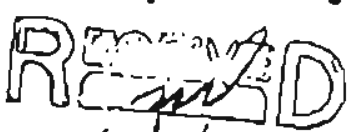

P. Q. \#

$12 / 7 / 07$
1

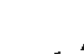

MANNESMANN

DMV STAINLESS

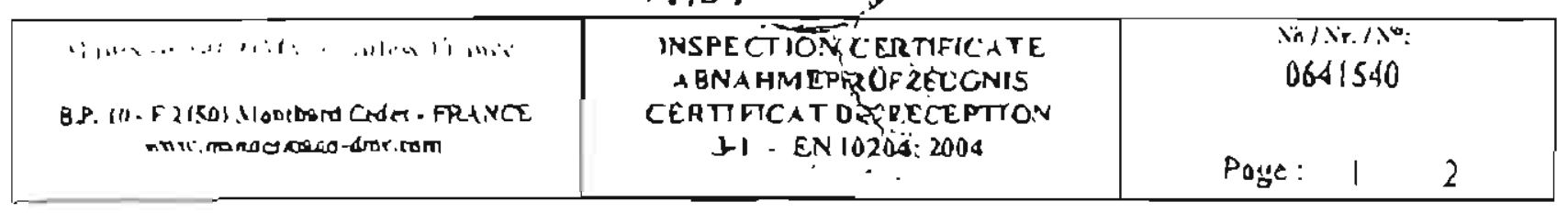

CUSTOMER ! Kunde ICITAI!: WARREN ALLOY VALVE \& FITT NGS

CUSTONERS PURCHASER ORDER No/KLndenauftragonr.I Hode dlent: 102753

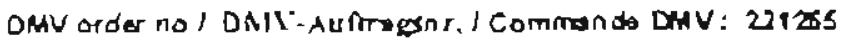

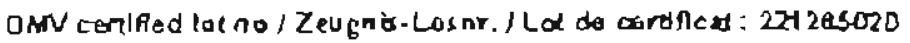

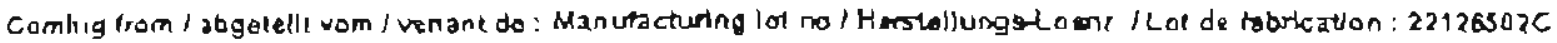

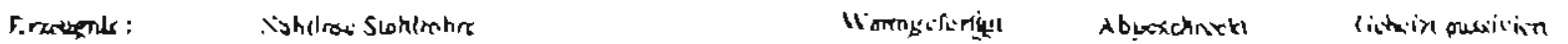

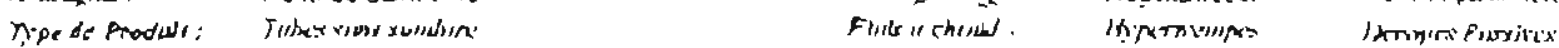

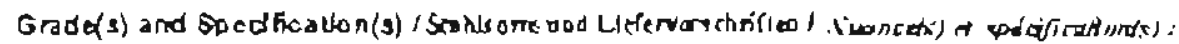

Trinki.

TS: 16

ISTM $\wedge$ III- $-13 \mathrm{~B}$

ASME $5 \lambda-3 \mathrm{Al}=\mathrm{ED}$. (NA.

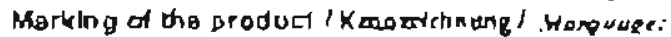

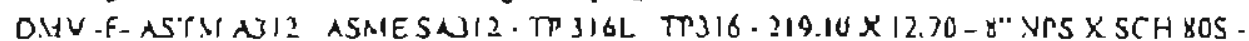

HFAT : 5NYJIOS - SNLL - I2IIASODO. FR ANCE

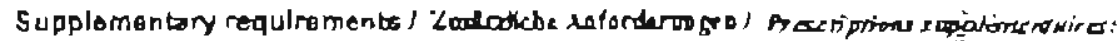

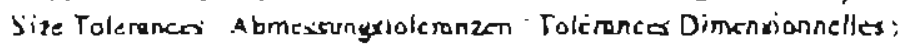
ULiolding io ASTM \& .112-NSA

\begin{tabular}{|c|c|c|c|c|c|c|}
\hline Po in & Vident & Tanl Leted & 00 & WV.T & M so Lengh & llor Lamenh \\
\hline 2 & $812 \mathrm{~kg}$ & $12501 \mathrm{~m}$ & $219.10 \mathrm{mrn}$ & (x) & alva man & d265 ताru \\
\hline & $1790.1 \%$ Lod & $11.01 \mathrm{FT}$ & 8" NPS & $S C$ W & $20,15 \mathrm{Fl}$ & $2 \pi .5 \overline{F r}$ \\
\hline
\end{tabular}

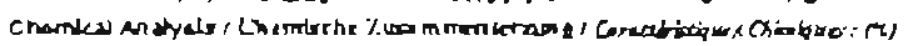

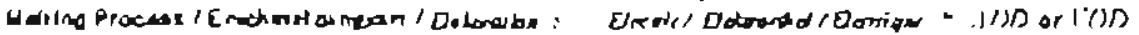

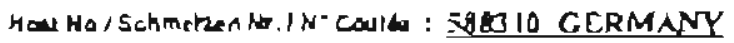

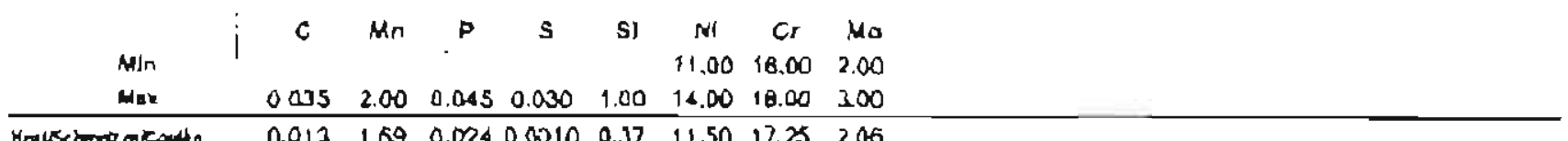

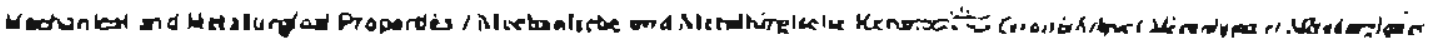

\begin{tabular}{|c|c|c|c|c|}
\hline TEHSILE TES & T AT & $Y S a 2 \%(M P A)$ & 7. 51 MPA & Eangadon ( $50 \perp \pi n)$ \\
\hline V.ugforghtil & & 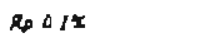 & en & 24 \\
\hline 7raten & & aer: & & \\
\hline Limat & He veremat: & $1 / n 300$ & $11 / \pi) / J$ & S. ע. \\
\hline $1 V_{107}$ & Rusulis: & jor & $\Leftrightarrow$ & 10:T \\
\hline
\end{tabular}

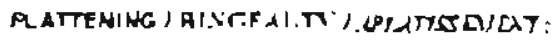

WrOT WIOS

HARD NE95 TEST IIALE'R', DLRTTE:

W107 Requiement: $c=$ ZUHRC

CORROGION/ hUHARSIUN/ CORAOSRON:

Wro7

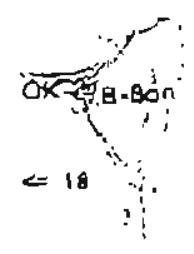

RaQuinmon: : ASTHA262/E

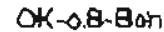




\section{MANNESMANN DMV STAINLESS}

\begin{tabular}{|c|c|c|c|c|}
\hline \multirow{2}{*}{ 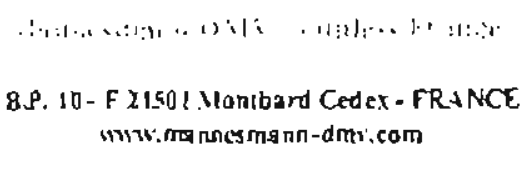 } & \multirow{2}{*}{ 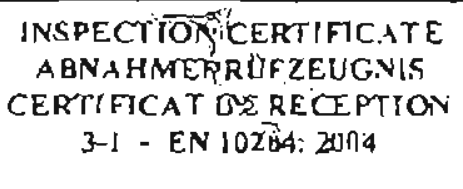 } & \multicolumn{3}{|c|}{0641540} \\
\hline & & Page: & 2 & 2 \\
\hline
\end{tabular}

DMV order no/ DMV -Aufrezsnt, / Commande CMV: 221265

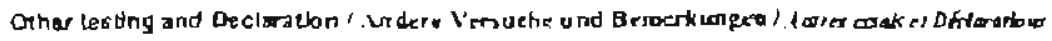

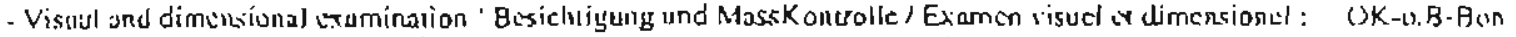

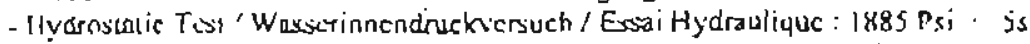

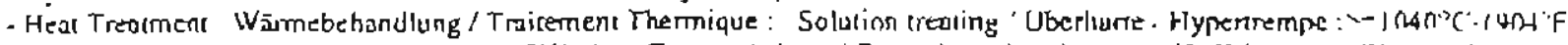

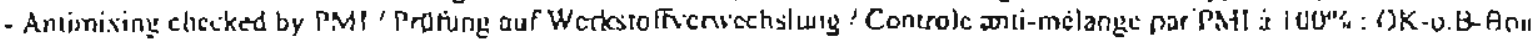

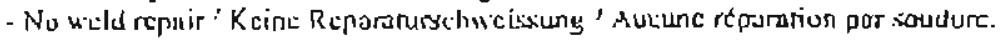

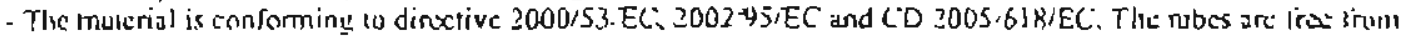

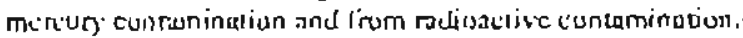

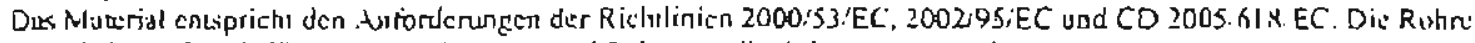

sind irei von Quecksitherserwireinigungen und frei von radiaskiter Veruntemigung.

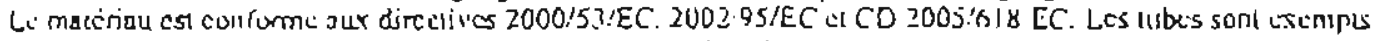

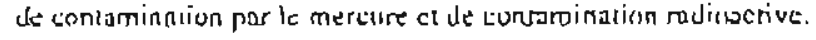

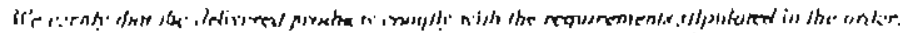

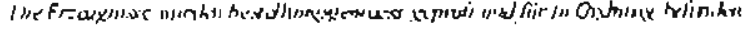

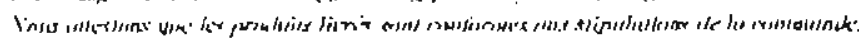

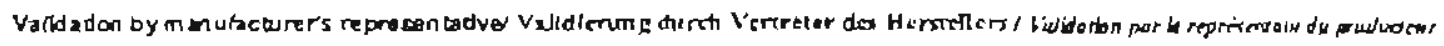

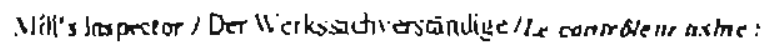

Dulc catidion : $31 / 1012006$

MTIRE LARCHER

$\div-$

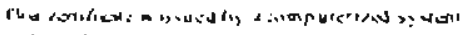
II. ل ‥

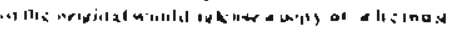

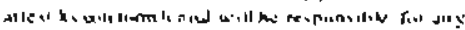

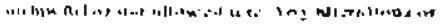

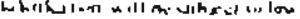

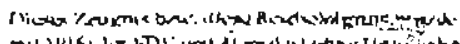

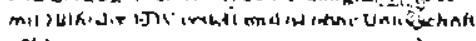
r.allite

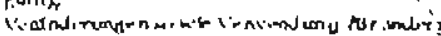

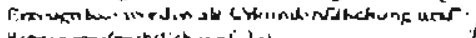

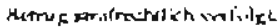

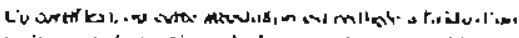

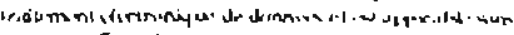

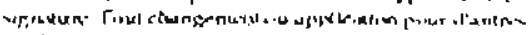

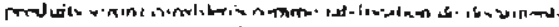
:

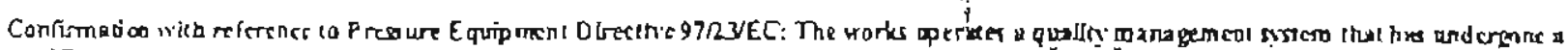

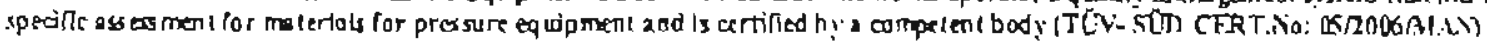




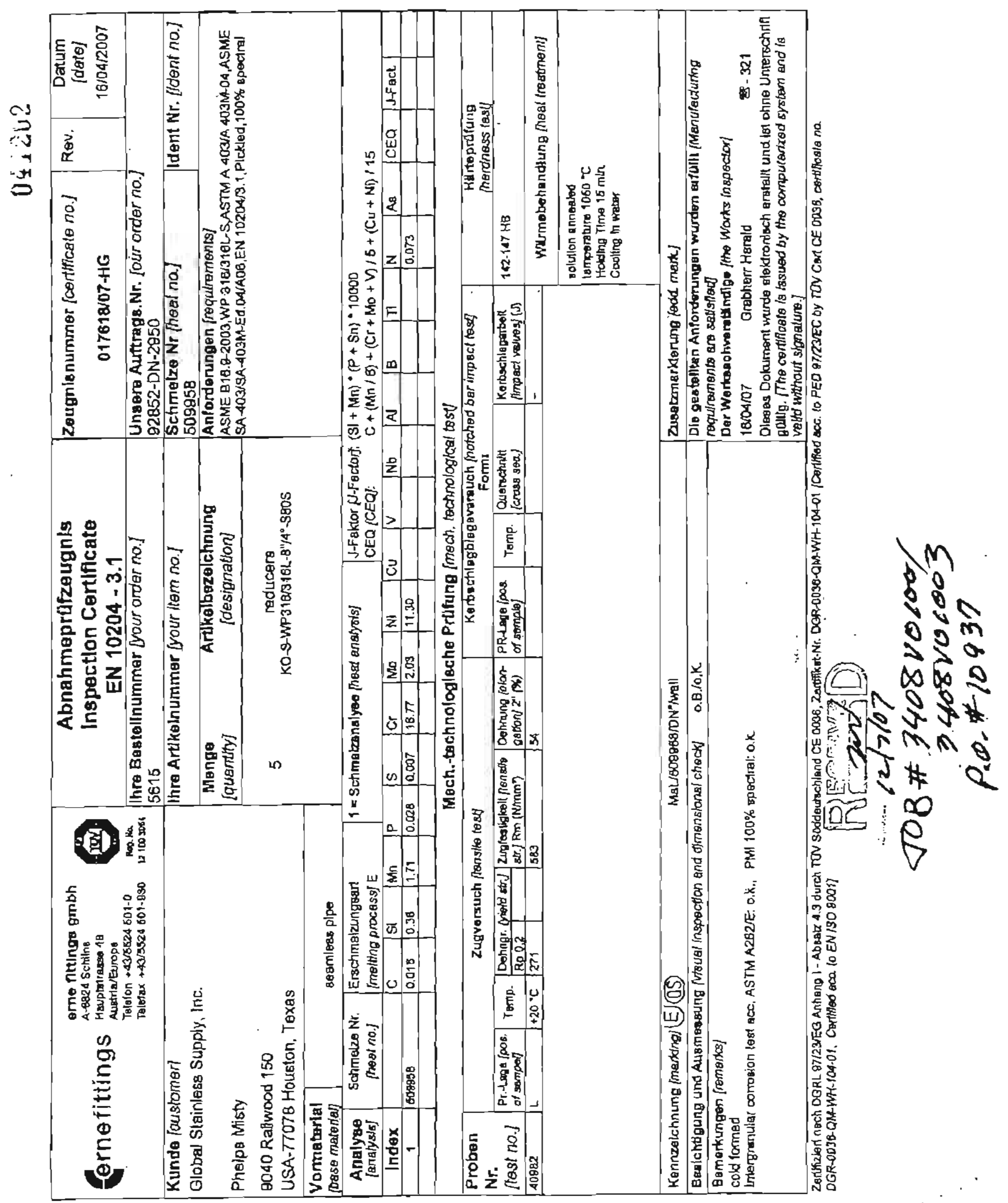


$M K 3$

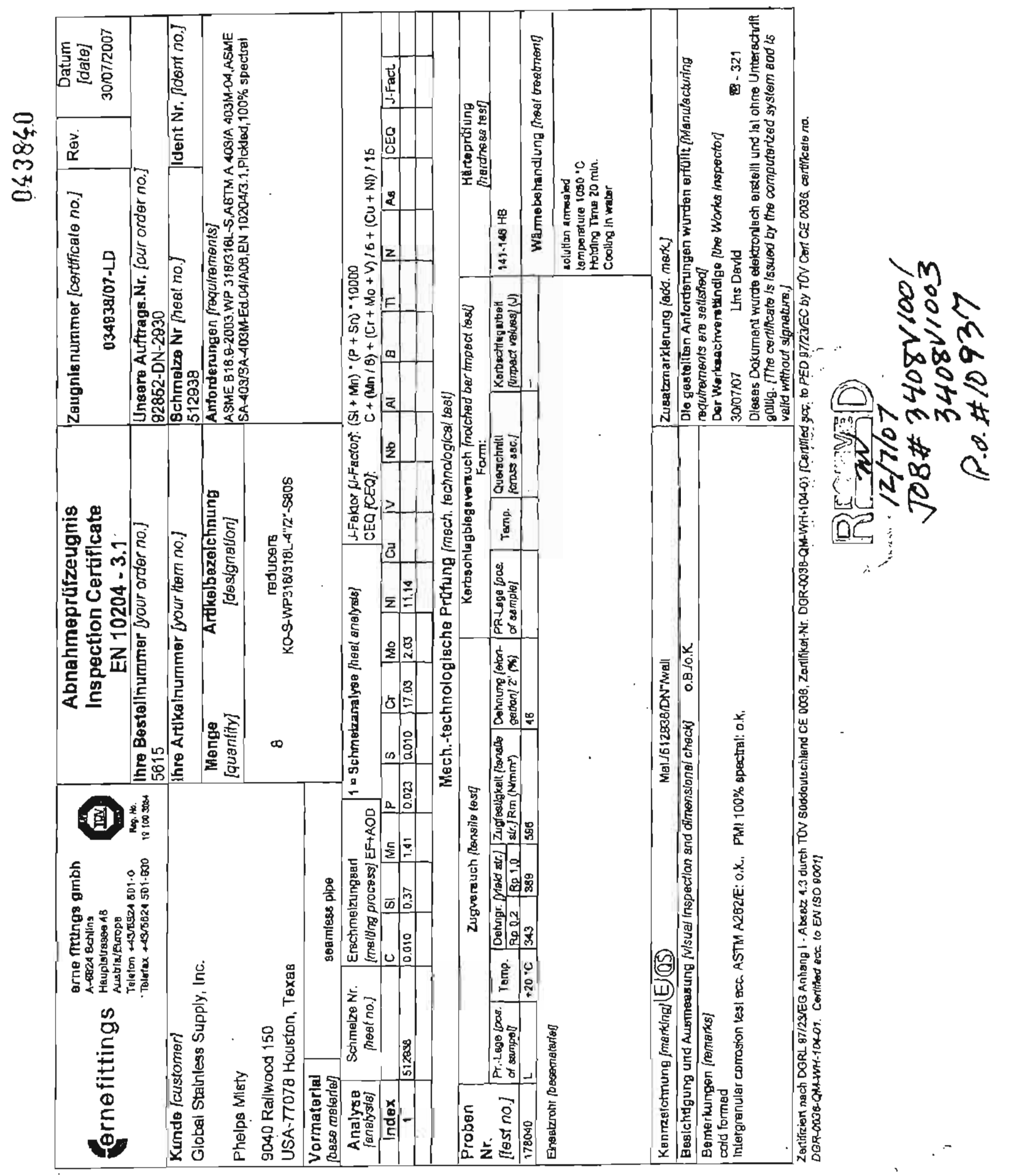


CERTIFIED MATERIAL TEST REPORT31056 MK 4

\begin{tabular}{|c|c|}
\hline \multicolumn{2}{|l|}{ Vetsis. } \\
\hline vote $6020 t$ & IP $316 / 316 t$ \\
\hline Coatition & Solbtion lazces \\
\hline Heat $\mathrm{ka}$. & 504188 \\
\hline $\sin$ & $2 \times 3 C H 80.8$ \\
\hline Lost" & 23.951281 \\
\hline plests & 17 \\
\hline Yes Ielant & 6.457 LBS \\
\hline
\end{tabular}

Date Ver. 23, 2007
Sheot to. 604238

Contrate MD. HA+PAZOZS

Patking LIII Ko. E158ss

Eusole No. $10 \quad-32$

COSANYO SPECIAL STEEL CO., LTD. Tecbnical Adoinitiration Debartenos Qualitis Assarance Crove.

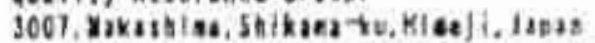
TEL (079) 235-6029

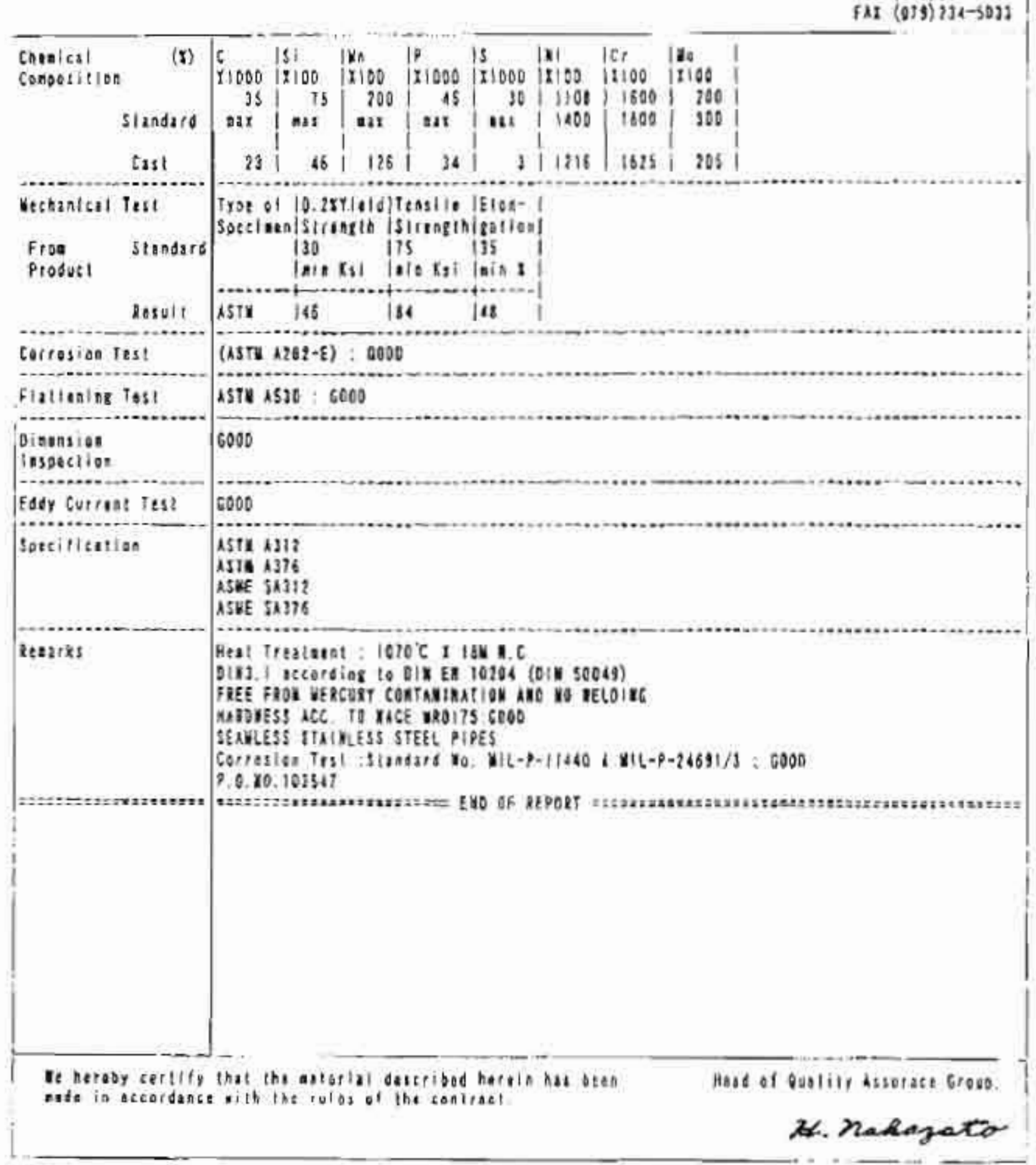


Material Test Certification Certificate Conforms to EN 10204/3 1

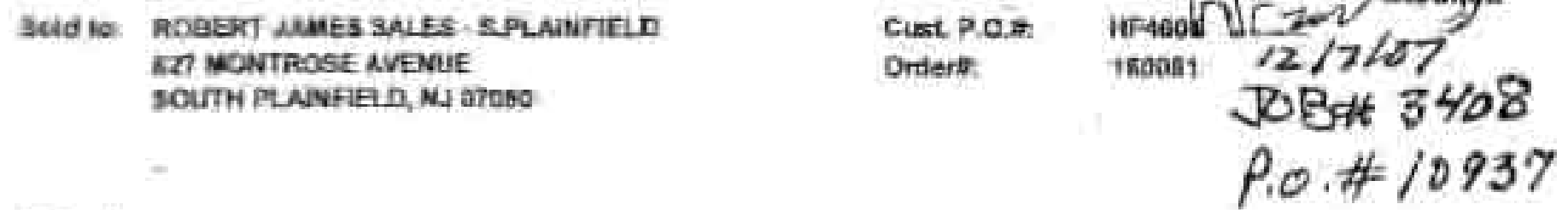

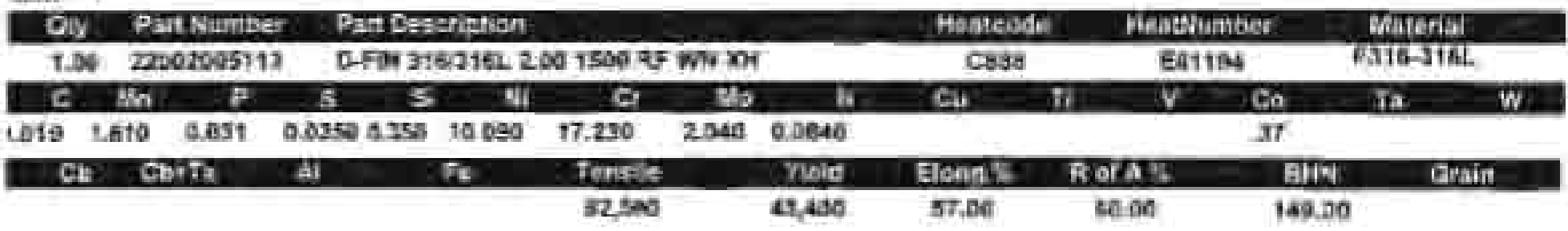

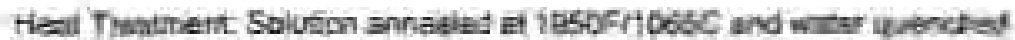

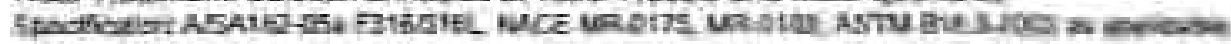

Maoe unt ins LA

Wer cerofy that the watenal reprosented by the documunt has boen tested and mseacted

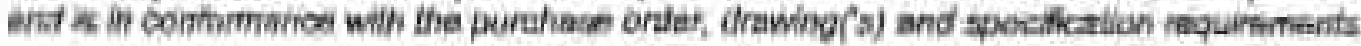




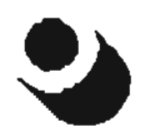

SCHOELLER

BLECKMANN

EDELSTAHLROHR

STAMLESS-STAINLCSS

HAHTLI IUM ERTOLG

ABNAHREPRŌFZEDGNIS B - TASPECTION CBRTIPICATE B CERTIFICAT DE RECEPTION PAR L'OSINE 3.1 .8 C.C.P.U. nacb/accoxding to OBHORM/DIN EN 10 204-3.1.B
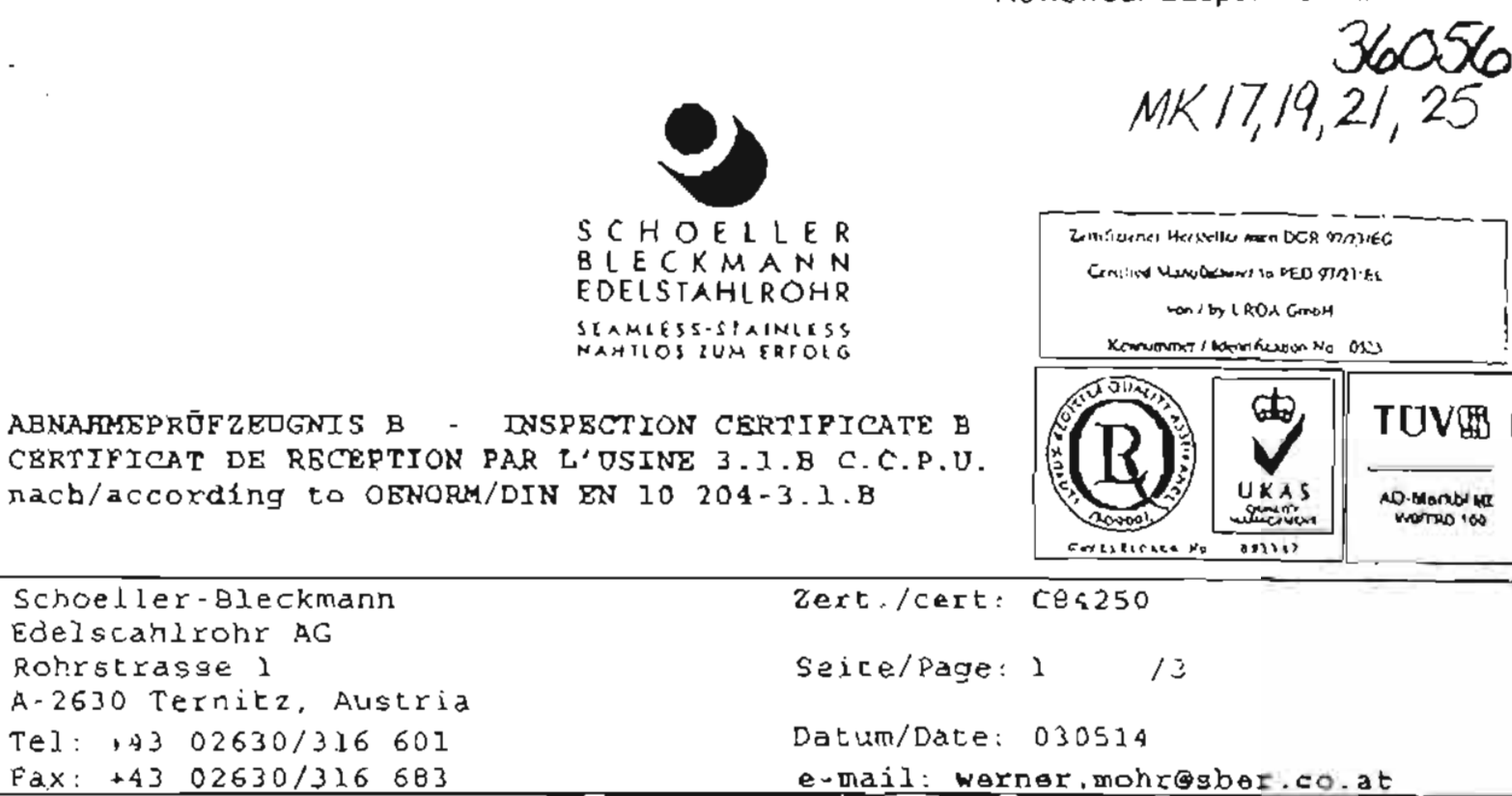

Beateller/Purcbaser/Commetant

SCHOELLER BLECMMANN PIPE \& TUBE INC.

S月30, BRYSTONE DRIVE

HOUSTON, TEXAS 77041

LSA

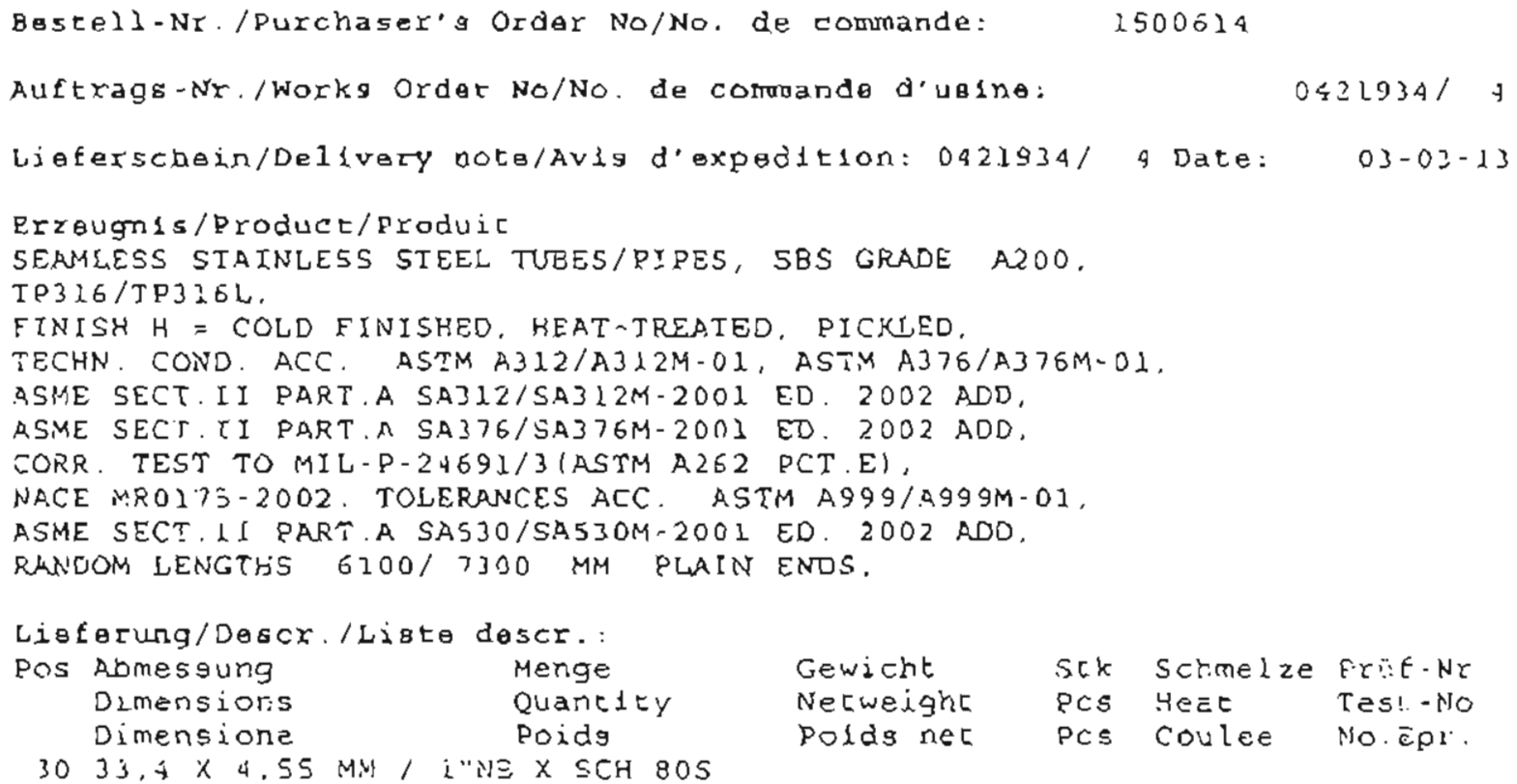

Gewicht Nerweight polds nec

Sck Scrmelie Errit.Nr PCs Heet Tes!-no PCs Coulee Mo.èpl.

$1939.00 \mathrm{KG}$

$$
\text { 67 } 7 \div 443 \quad 135 \div 5
$$

F.rgeunis der PrürongenrTest Resultroultal des exsals:

Die gestellect Antorderunger sinu erfulle.

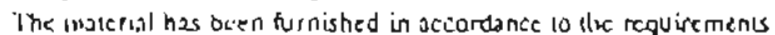

l.e uiaterial a ele erauvé conforme aux exigesces.

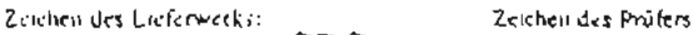

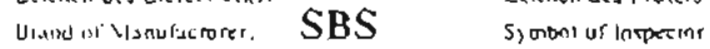
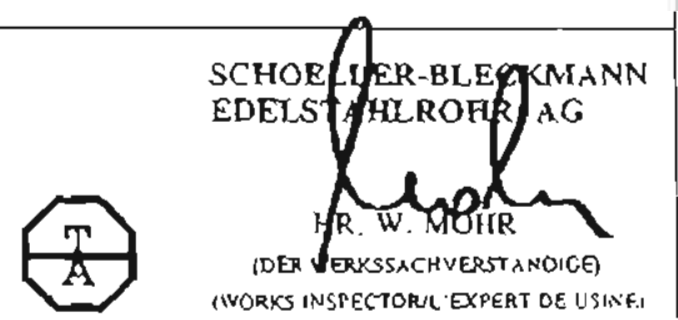

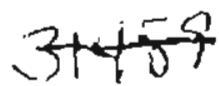

Aftrans: : 


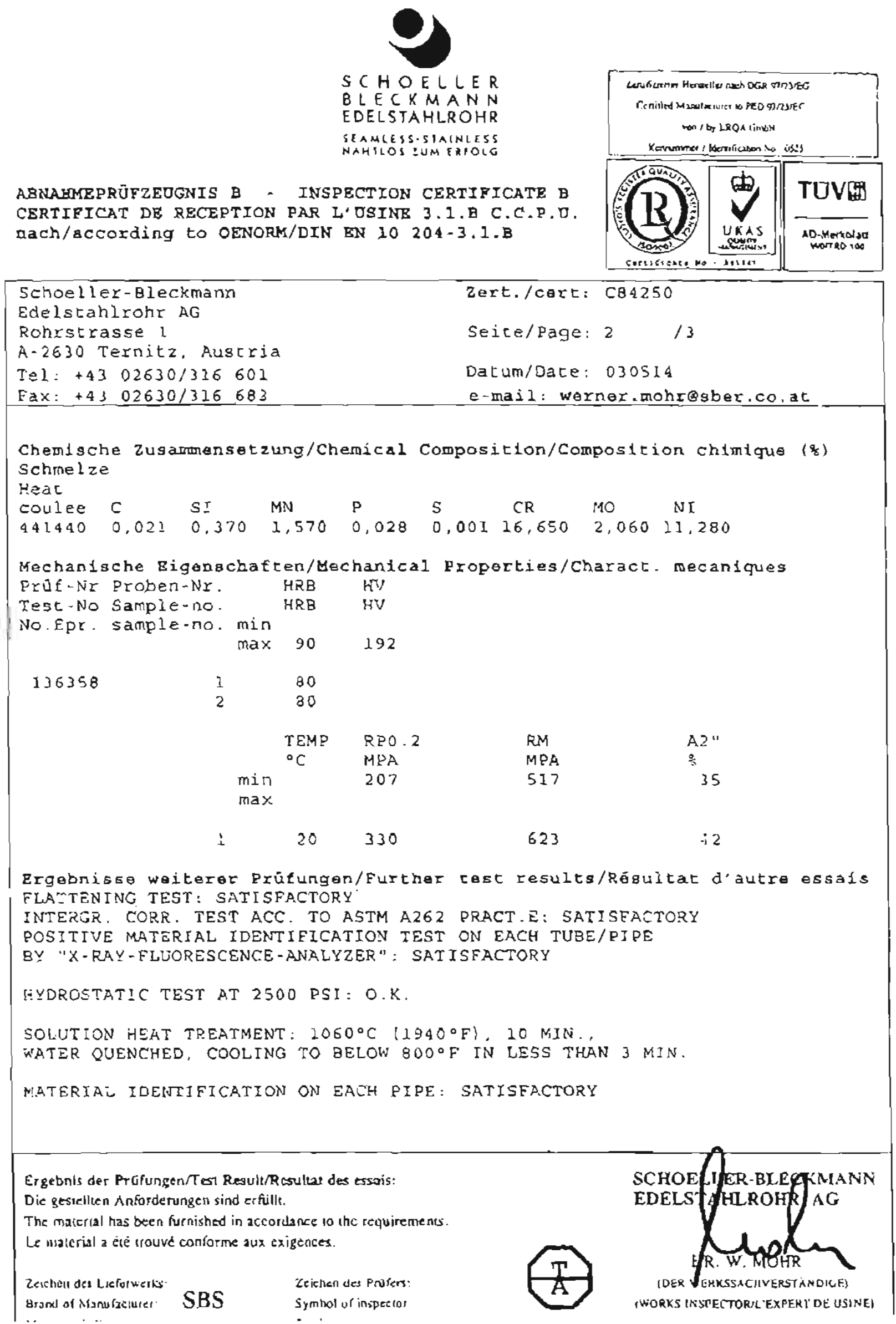




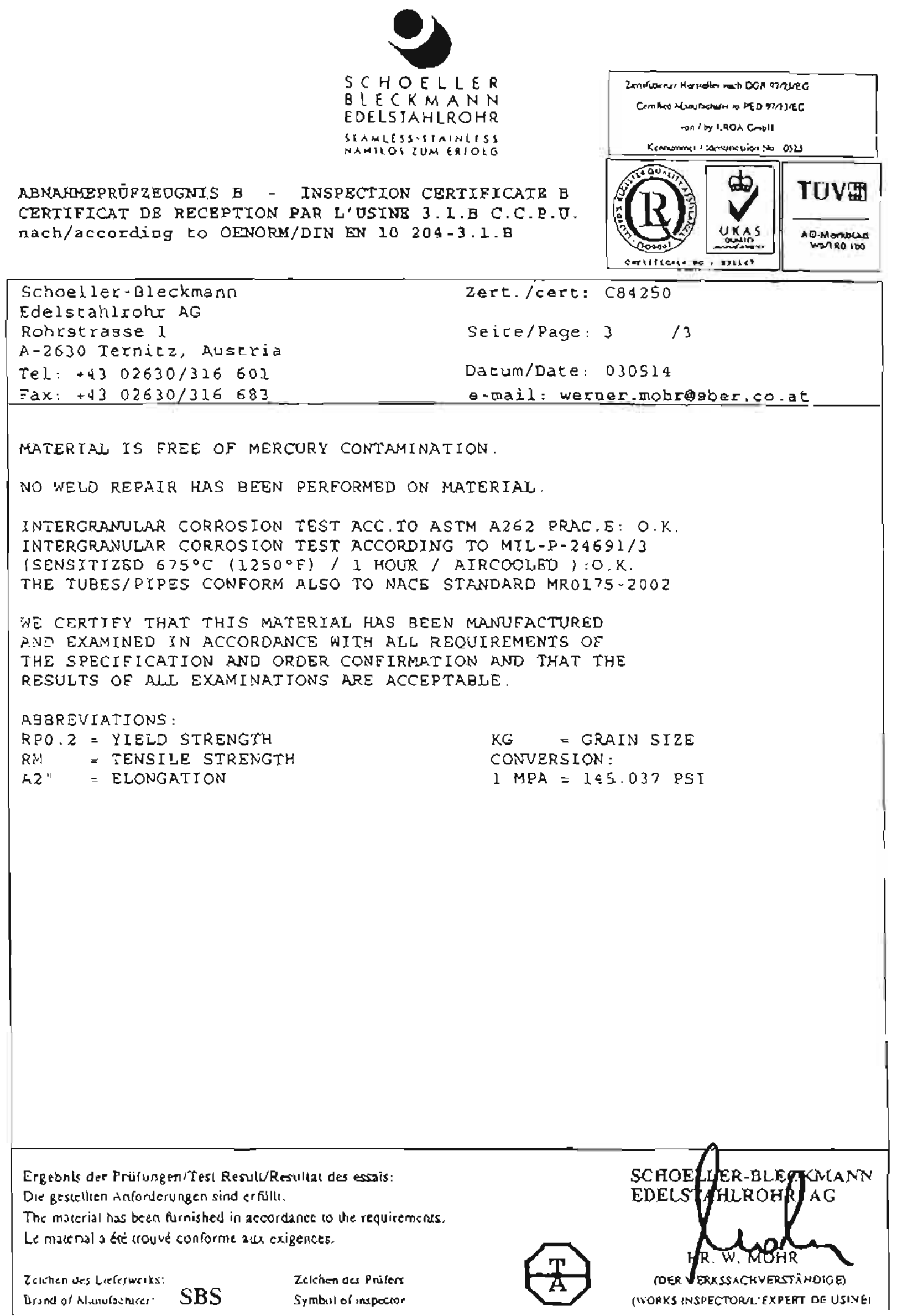




\section{MATERIAL TEST REPORT}

DATE: 7/15/05

SOLD TO: WARREN ALLOY VALVE \& FTTTING-HOUSTON SHIP TO:

213774

ORDER NO: 101328

TTEM DESCRIPTION

1 1500\# WN RF XH/S80 316/316L A182/SA182 ASTM 02/ASME A02

\begin{tabular}{lllllllll}
$\mathrm{C} \%$ & $\mathrm{Mn} \%$ & $\mathrm{Si} \%$ & $\mathrm{~S} \%$ & $\mathrm{P} \%$ & $\mathrm{Cr} \%$ & $\mathrm{Ni} \%$ & $\mathrm{Mo} \%$ & $\mathrm{~N} \%$ \\
\hline 0.026 & 1.75 & 0.39 & 0.024 & 0.043 & 16.68 & 10.00 & 2.08 & 0.0725
\end{tabular}

\begin{tabular}{cclll} 
TENSILE $(p s i)$ & YIELD $(p s i)$ & ELONG $\%$ & REDUCTION \% & BHN \\
\hline 79,670 & 41,992 & 56.40 & 71.50 & 156
\end{tabular}

SOLUTION ANNEALED AT 1050 DEGREES CELSIUS AND WATER QUENCHED.

IN COMPLLANCE WITH NACE MRO 175-02

3.1.b DIN 50049/EN 10204
HEAT NO.

67143

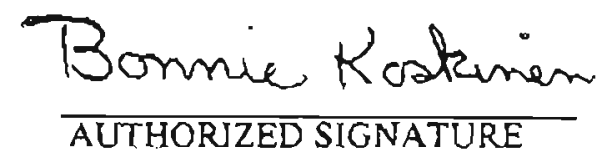

Voice: (989) 686-0350

Fax: (989) 686-0399

Toll Free: (800) 248-5060 


\section{EDTKCordoration}

\section{INSPECTION CERTIFICATE}

1syt-1 kbes angerag

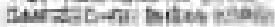

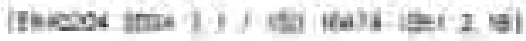

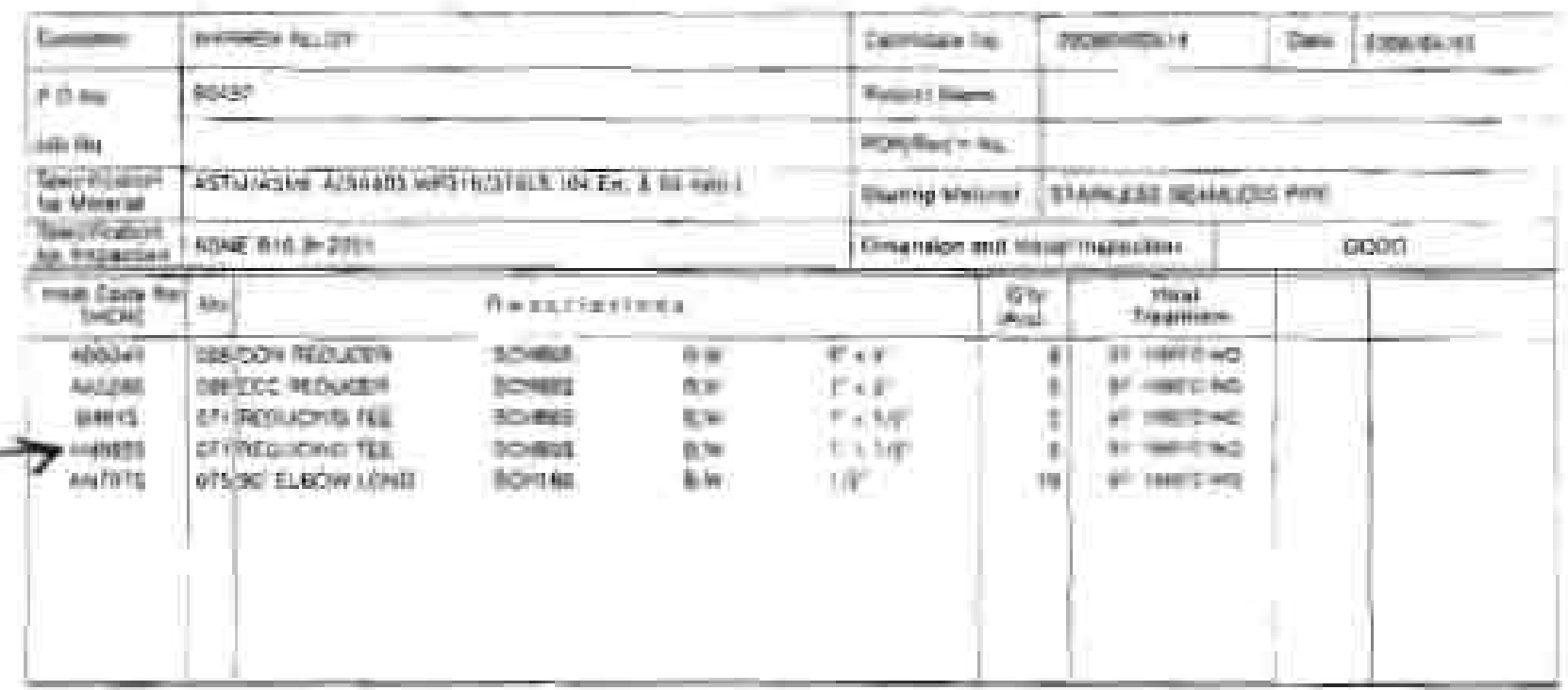

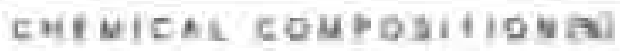

\begin{tabular}{|c|c|c|c|c|c|c|c|c|c|c|}
\hline \multirow{3}{*}{ my cay wit } & ased. & 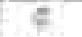 & t & $\omega$ & $\theta$ & $\mathbf{s}$ & E & C. & (a) & n-then \\
\hline & 1601 & ien & 76 & tia & 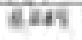 & 52 & $16=7$ & $10=$ & $\sqrt{2 x}$ & \\
\hline & dis: & & & & $=$ & $=$ & ve all & it te & 4int & \\
\hline comes & I & हाजा: & $8 x$ & h, & в & Doon & 10 in & 1818 & Bar & nutom \\
\hline Gables & k & teas & exti & 101 & Hate & in not & u we & 163 & 2.54 & CHangwry \\
\hline tans & $E$ & antes & , 4 it & ints & Hate & boz: & $11 \pm 2$ & $14: 14$ & 1,10 & SAtand \\
\hline hersen & + & A abd & 4.41 & 10 & Wast & it anz & 120 & in ia & pal & Jabrosacilit \\
\hline isters & 4 & 50 & ox & in & thes. & atul & ats & $x+20$ & $F *$ & Conalivican \\
\hline
\end{tabular}

WECHANICAL PUOPEATIES

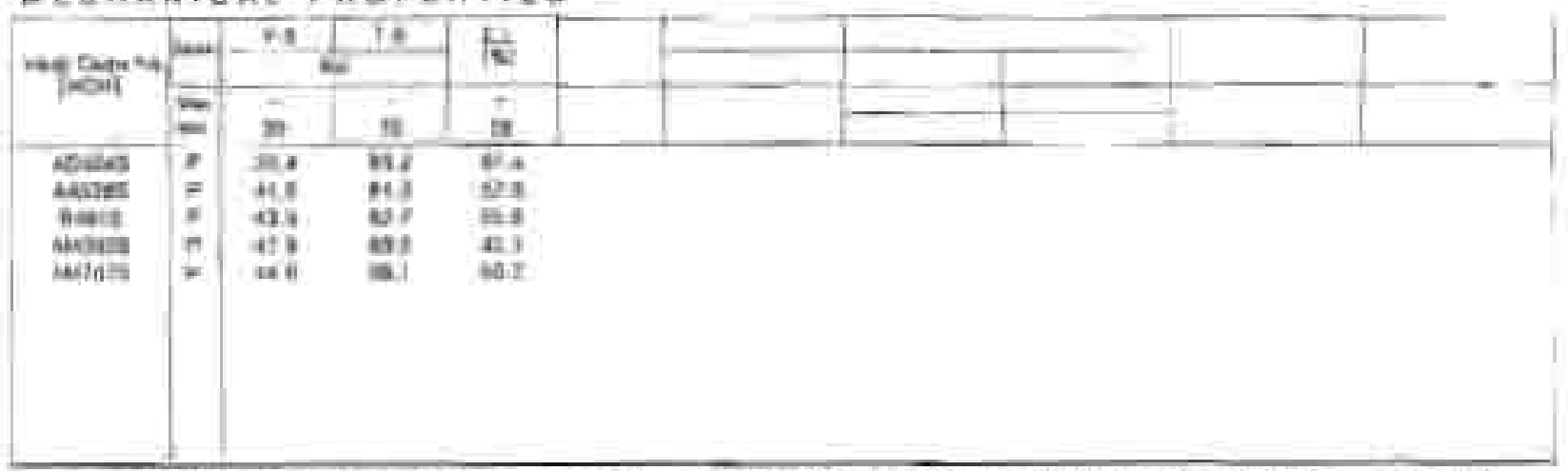

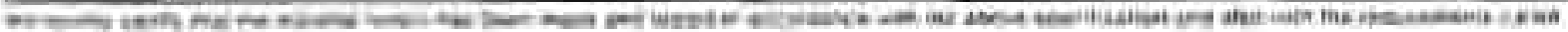

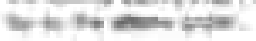

tonat

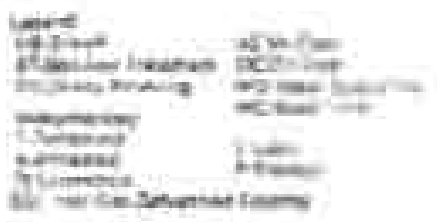




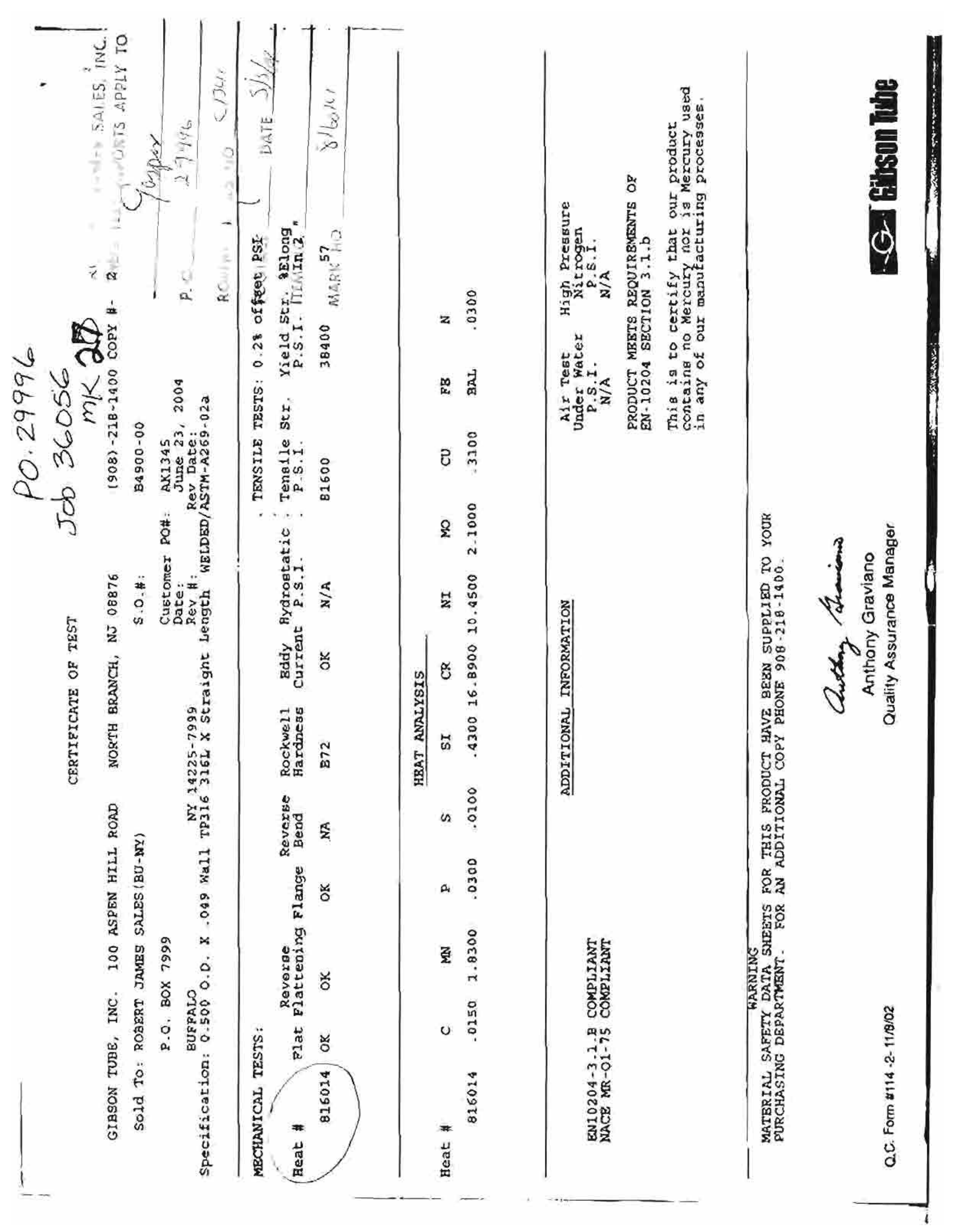


36056

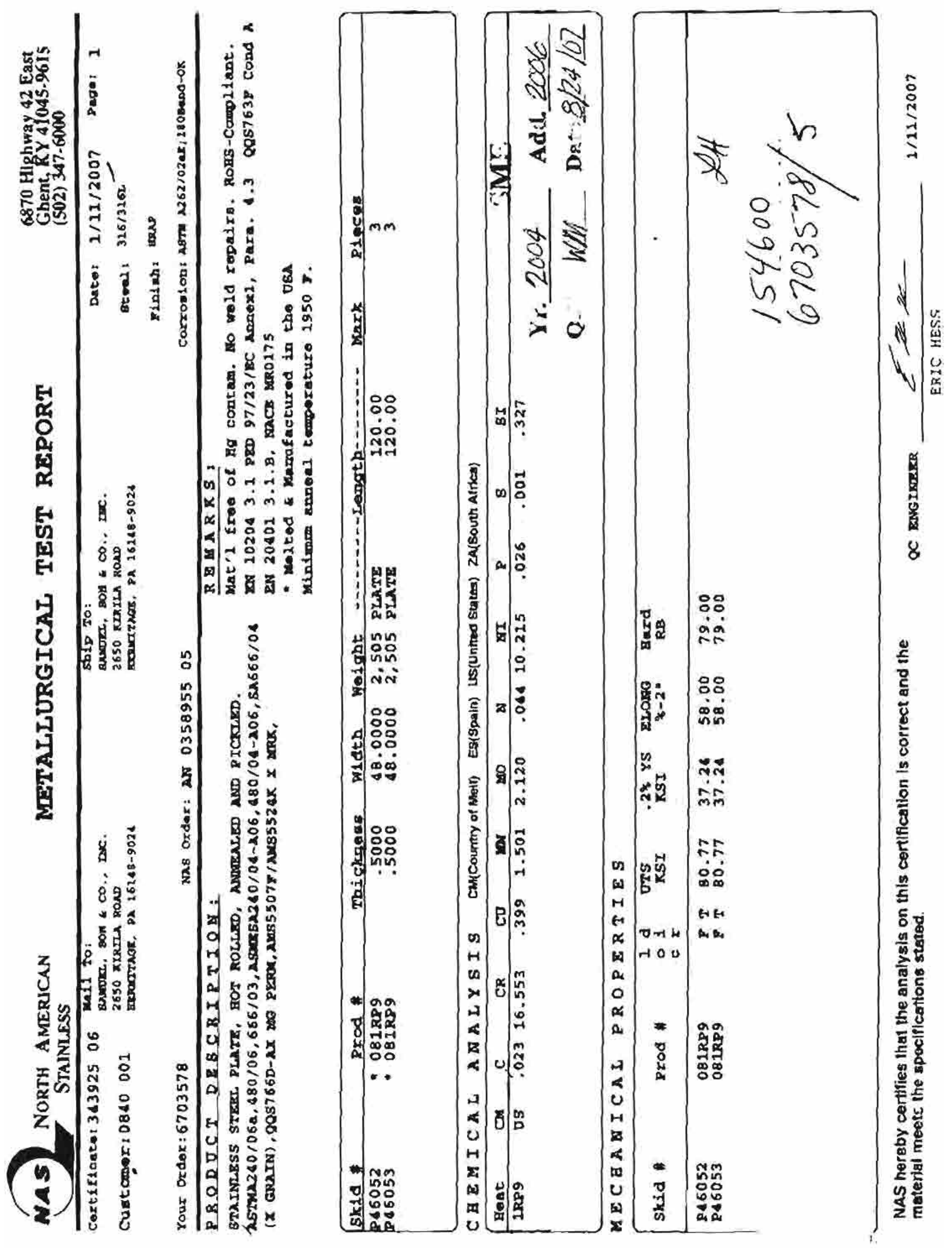




\section{BAKER INSPECTION GROUP}

REPORT \#05/508-G RADIOGRAPHIC INSPECTION REPORT DATE: 05/15/OE Page 1 of 1 CUSTOMER GASPAB LOCATION CULTENI,OMIO POA 10978 MOBH $3505 \mathrm{C}$ MATERIALTYPE: STANLESS STEEL

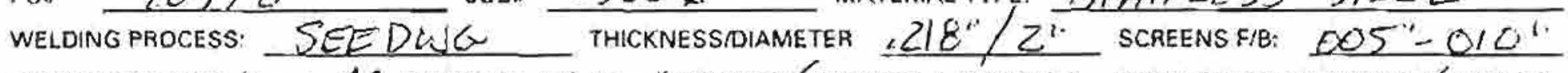

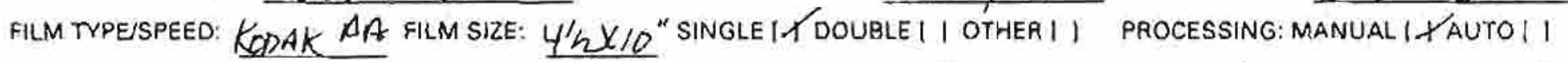

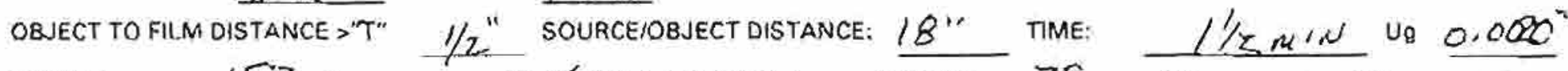
SOURCE $\quad 157 \times$ _ $18 . M$ CO.11 X-RAYI1 CURIES: 35 KV - MA -

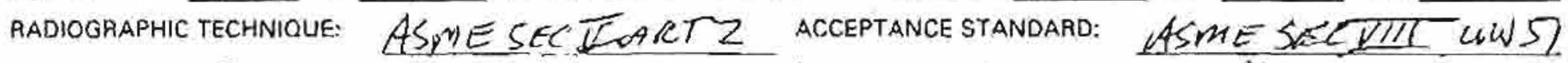
IQI SIZE: $/ 2$ SIDE: SOURCE U FILMII IOI TYPE: ASTME $10 Z 5$ SHIMTHK: $1 / 2$ "TECH. USEDIS WE ASSUME NO RESPONSIBILTY FOR LOSSES OF ANY KIND DUE TO OUR INTERPRETATION OF TME QUALITY OF THE MATERIAL SUBMITTED. ALL DATA AND INFORMATION WILL BE HELD STAICTLY CONFIDENTIAL.

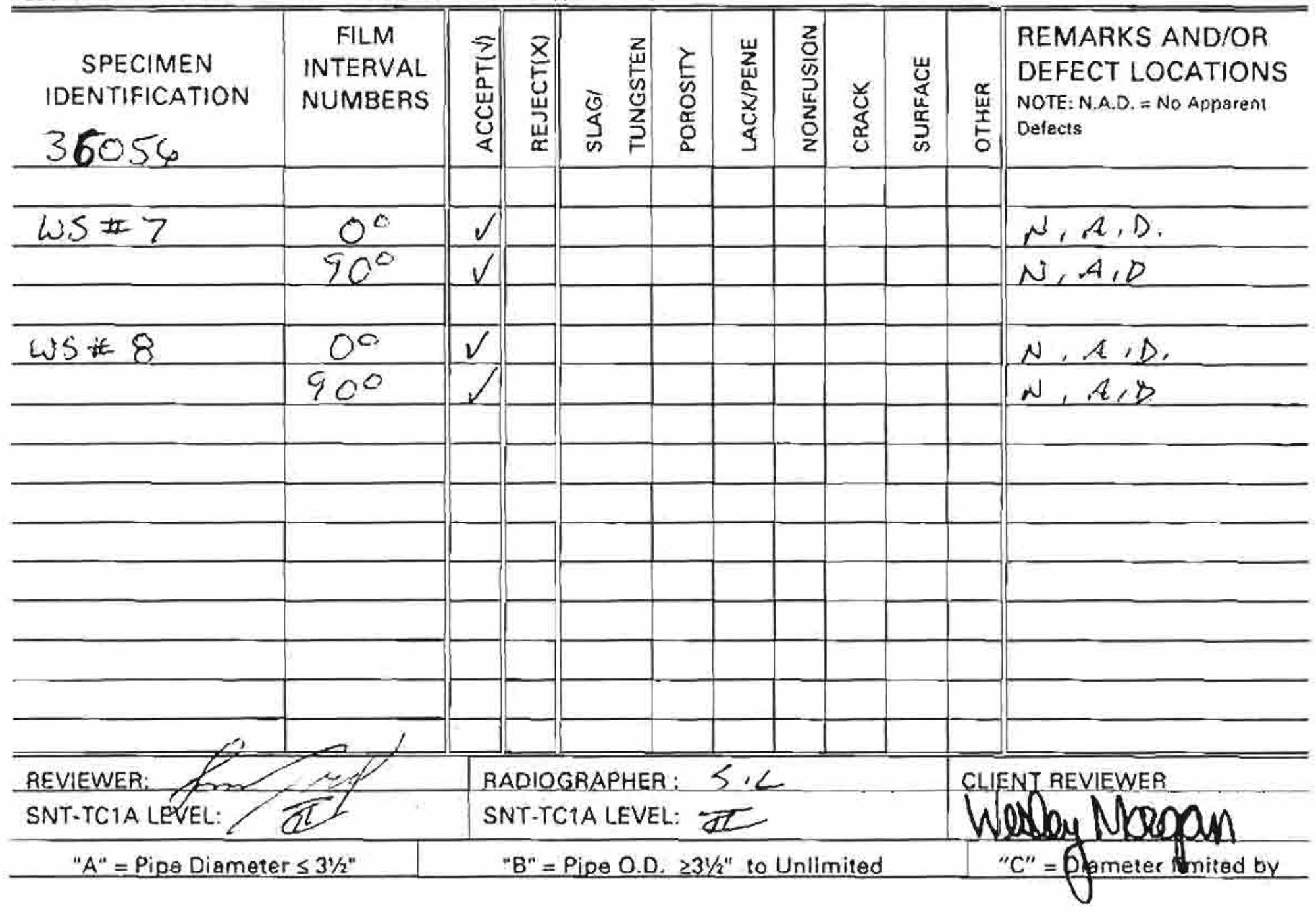

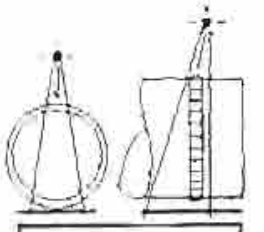

DWEIDWV

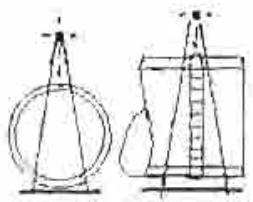

DWEIDWV

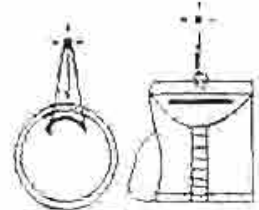

SWEISWN

K

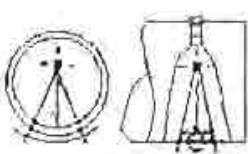

SWEISWN

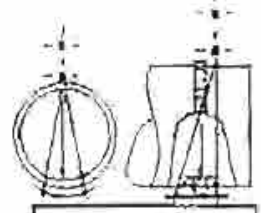

DWEISWV *

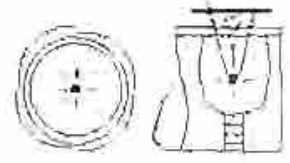

SWE/SWV 


\section{BAKER INSPECTION GROUP}

REPORT $=05 / 508-\&$ RADIOGRAPHIC INSPECTION REPORT DATEIOS/IS/OB Fage i of I

SUSTOMER GASPAR L LOCAMON CANTDNGDAlO

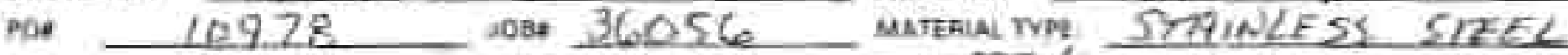

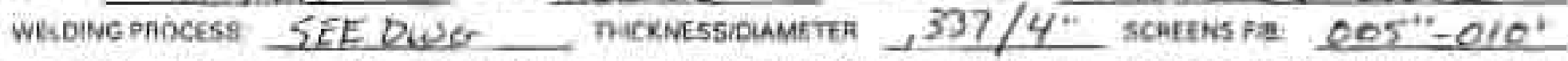

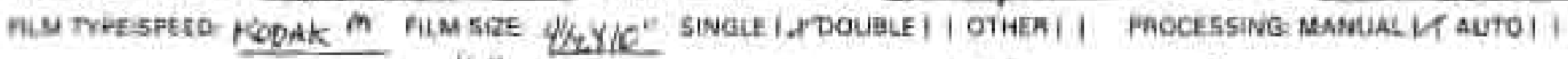

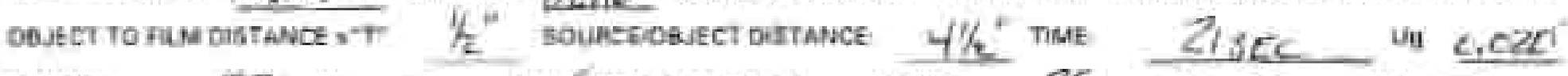

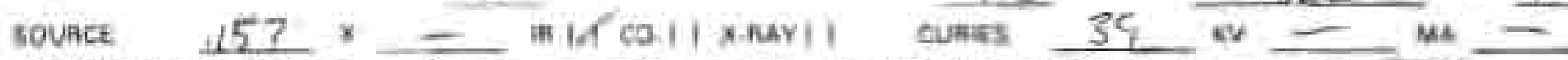

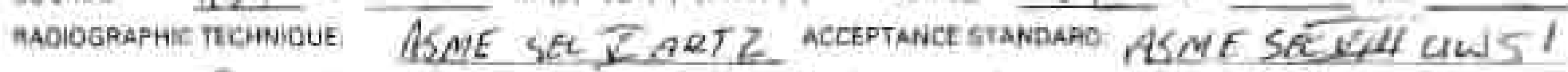

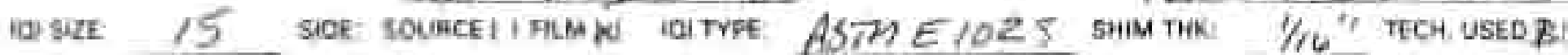

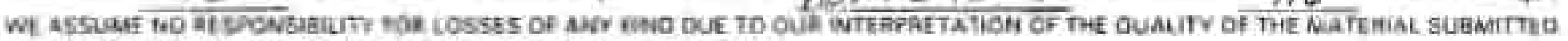

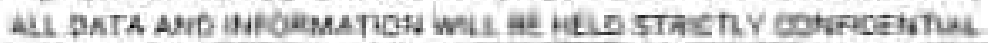

\begin{tabular}{|c|c|c|c|c|c|c|c|c|c|c|c|}
\hline $\begin{array}{l}\text { SPECIMEN } \\
\text { IDENTHFICATION } \\
3605 C\end{array}$ & $\begin{array}{l}\text { FILM } \\
\text { INTERVAL } \\
\text { NUMBERS }\end{array}$ & $\begin{array}{l}\frac{3}{5} \\
\frac{2}{4} \\
\frac{1}{2} \\
\end{array}$ & $\begin{array}{l}\frac{\mathrm{x}}{\mathrm{E}} \\
\frac{\mathrm{w}}{\mathrm{H}} \\
\mathrm{a}\end{array}$ & $\begin{array}{ll} & \frac{z}{6} \\
3 & \frac{6}{3} \\
\frac{4}{3} & \frac{2}{2} \\
\end{array}$ & $\frac{\frac{k}{5}}{\frac{5}{2}}$ & $\begin{array}{l}\frac{u}{2} \\
\frac{4}{5} \\
\frac{2}{4} \\
5\end{array}$ & $\begin{array}{l}z \\
\frac{2}{3} \\
\frac{3}{2} \\
\frac{9}{z}\end{array}$ & $\frac{d}{d}$ & $\begin{array}{l}\frac{y}{4} \\
\frac{2}{5} \\
\end{array}$ & $\frac{\pi}{4}$ & 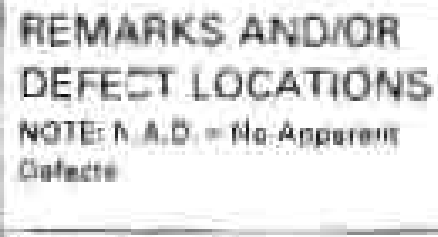 \\
\hline \multirow[t]{3}{*}{$\cos A 2$} & $0-1$ & $\mathcal{L}$ & & & & & & & & & $N=1 . D$ \\
\hline & $1-2$ & L & & & & & & & & & $N, A, D$, \\
\hline & $2-0$ & 1 & & & & & & & & & $A=A^{2}$ \\
\hline \multirow[t]{3}{*}{ wStk 6} & $c-1$ & 1 & & & & & & & & & $\Delta, B_{i} D_{i}$ \\
\hline & $1-2$ & 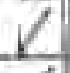 & & & & & & & & & $A, Q, D$ \\
\hline & $z-c$ & & & & & & & & & & $N-A, D$. \\
\hline
\end{tabular}
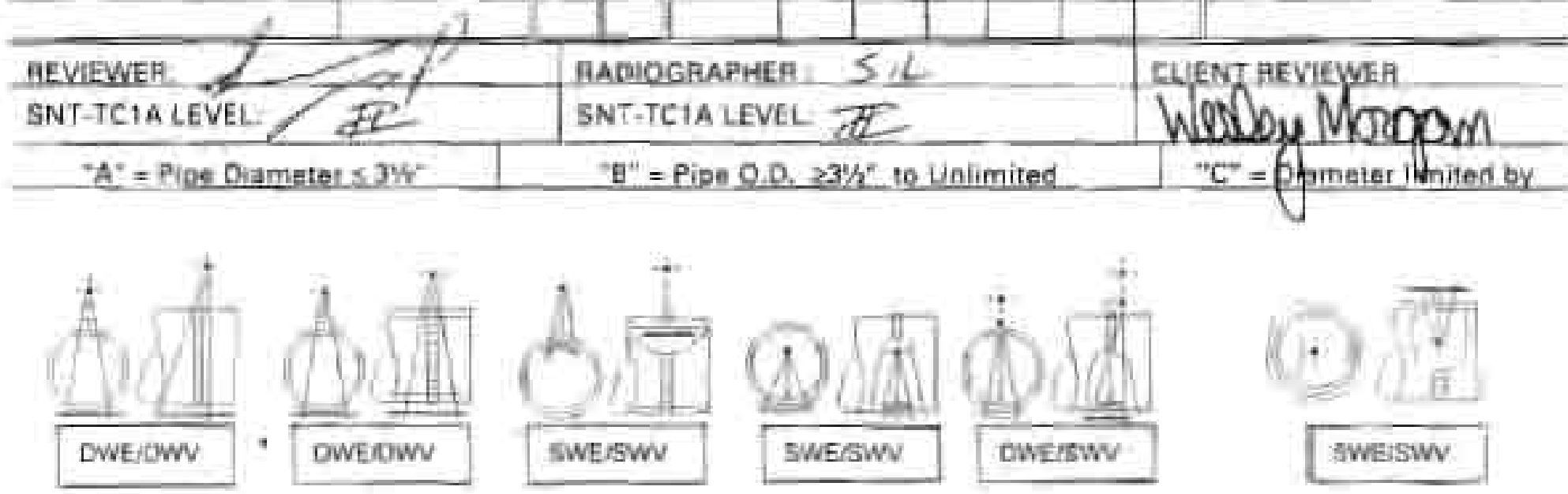


\section{BAKER INSPECTION GROUP}

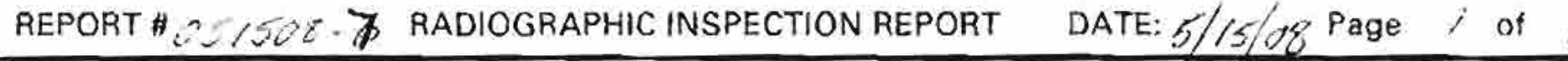

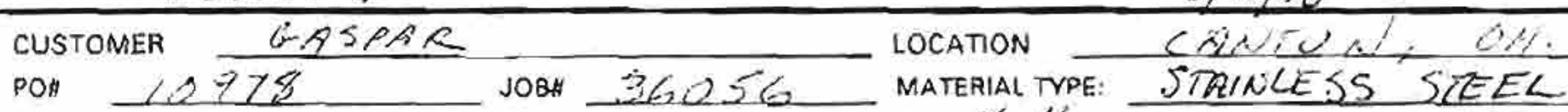

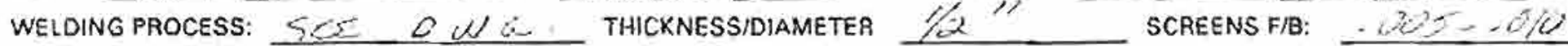

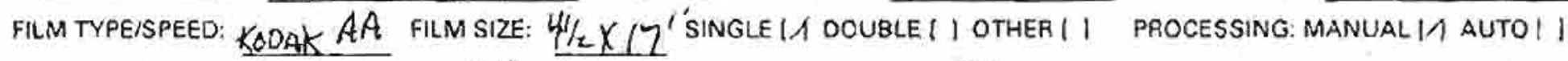

OBJECT TO FILM DISTANCE > "T" $1 / 2$ " SOURCE/OBJECT OISTANCE: $8^{\prime \prime}$ TIME: $1 \mathrm{~m}, \mathrm{~N}$. SOURCE $\quad 157 \times$ - IR.W CO.11 X-RAYI1 CUAIES: $39.1 \mathrm{KV}=\mathrm{MA}-$

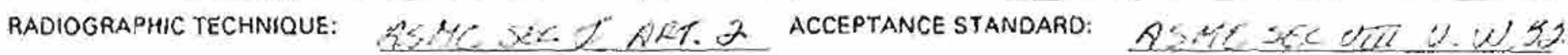
IOI SIZE: 18 SIOE: SOURCEIIFILMN IOITYPE: ASThE 747 SHIMTHK: N/VT TECH USEDIB WE ASSUME NO RESPONSIEILITY FOR LOSSES OF ANY KINO DUE TO OUR INTERPRETATION OF THE OUALITY OF THE MATERLAL SUBMITRED. ALL DATA AND INFORMATION WILL BE HELD STRICTLY CONFDENTIAL.

\begin{tabular}{|c|c|c|c|c|c|c|c|c|c|c|c|}
\hline $\begin{array}{l}\text { SPECIMEN } \\
\text { IDENTIFICATION } \\
3606\end{array}$ & $\begin{array}{l}\text { FILM } \\
\text { INTERVAL } \\
\text { NUMBERS }\end{array}$ & 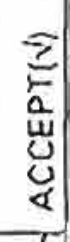 & 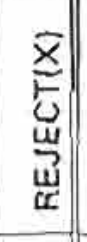 & $\begin{array}{ll} & z \\
0 & 5 \\
0 & 0 \\
5 & 2 \\
0 & 2 \\
\end{array}$ & $\begin{array}{l}\frac{\hbar}{5} \\
0 \\
\text { E } \\
0 \\
a \\
\end{array}$ & 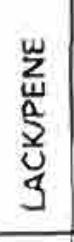 & $\begin{array}{l}z \\
\frac{2}{9} \\
\frac{3}{2} \\
\frac{1}{2} \\
\frac{0}{2} \\
\end{array}$ & 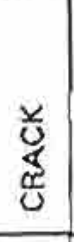 & 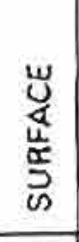 & 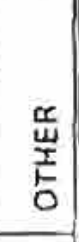 & $\begin{array}{l}\text { REMARKS AND/OR } \\
\text { DEFECT LOCATIONS } \\
\text { NOTE: N.A.D. = No APDarent } \\
\text { Detects }\end{array}$ \\
\hline \multirow[t]{3}{*}{$x 1,17 /$} & $0-1$ & $\sqrt{ }$ & & & & & & & & & $N, A, D$. \\
\hline & $1-2$ & $\sqrt{ }$ & & & & & & & & & $N, A \cdot D$ \\
\hline & $z-0$ & $\sqrt{ }$ & & & & & & & & & $N, A, D$, \\
\hline & & & & & & & & & & & \\
\hline \multirow{11}{*}{ Gist 5} & C. . 1 & $V$ & & & & & & & $\checkmark$ & & I.D. $\quad \mu, \lambda$.$\rangle .$ \\
\hline & $1-2$ & $\downarrow$ & & & & & & & & & $N, A, D$ \\
\hline & $2-0$ & 1 & & & & & & & & & $N, n, D$. \\
\hline & & & & & & & & & & & \\
\hline & & & & & & & & & & & \\
\hline & & & & & & & & & & & \\
\hline & & & & & & & & & & & \\
\hline & & & & & & & & & & & \\
\hline & & & & & & & & & & & \\
\hline & & & & & & & & & & & \\
\hline & & & & & & & & & & & \\
\hline \multirow{2}{*}{\multicolumn{2}{|c|}{$\begin{array}{l}\text { REVIEWER: } \\
\text { SNT-TC1A LEVEL: }\end{array}$}} & \multirow{2}{*}{\multicolumn{6}{|c|}{$\begin{array}{l}\text { RADIOGRAPHER: } \frac{S, L}{\pi} \\
\text { SNT-TCIA LEVEL: }\end{array}$}} & & & \multicolumn{2}{|c|}{ CLIENT REVIEWER } \\
\hline & & & & & & & & & & & Wloy Moran \\
\hline \multicolumn{2}{|c|}{ "A" $=$ Pipe Diameter $\leqslant 3 Y_{2}$ " } & \multicolumn{8}{|c|}{ "B" $=$ Pipe $0 . D .231 / 2$ " to Unlimited } & & $c^{\prime \prime}=$ piameter i mited by \\
\hline
\end{tabular}

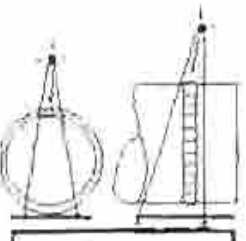

DWEIOWN

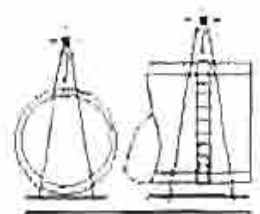

OWEIDWV

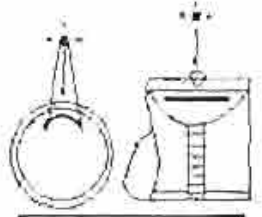

SWE/SWV

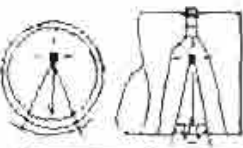

SWEISWV
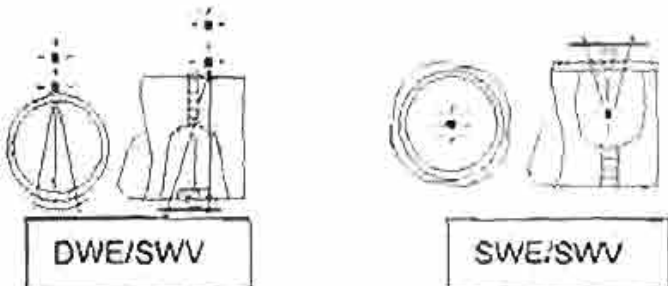

SWE'SWV 


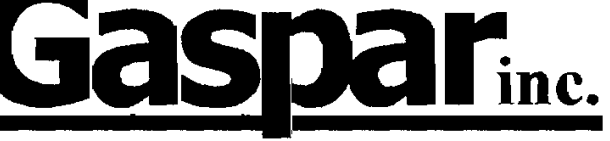

WELDING\& FABRICATIONS

1545 Whipple Avenue SW • Canton, Ohio 44710

Phone: 330-477-2222

Fax: $330-477-2322$

CERTIFICATE OF HYDROSTATIC/PNEUMATIC TEST

DATE: MAY 22, 2008

GASPAR JOB\#: 36056

CUSTOMER: ARIZONA PUBLIC SERVICE

CUSTOMER PO\#: 700521452

PRODUCT DESCRIPTION: COAL FEEDER ASSEMBLY

TEST PRESSURE: 1320 PSIG

TYPE OF TEST: PNEUMATIC

HOLD TIME OF TEST: 15 MINUTES

INSPECTED BY: WESLEY MORGAN

RESULTS: NO APPARENT LEAKS

NO PHYSICAL DEFORMATION

NOTES: 


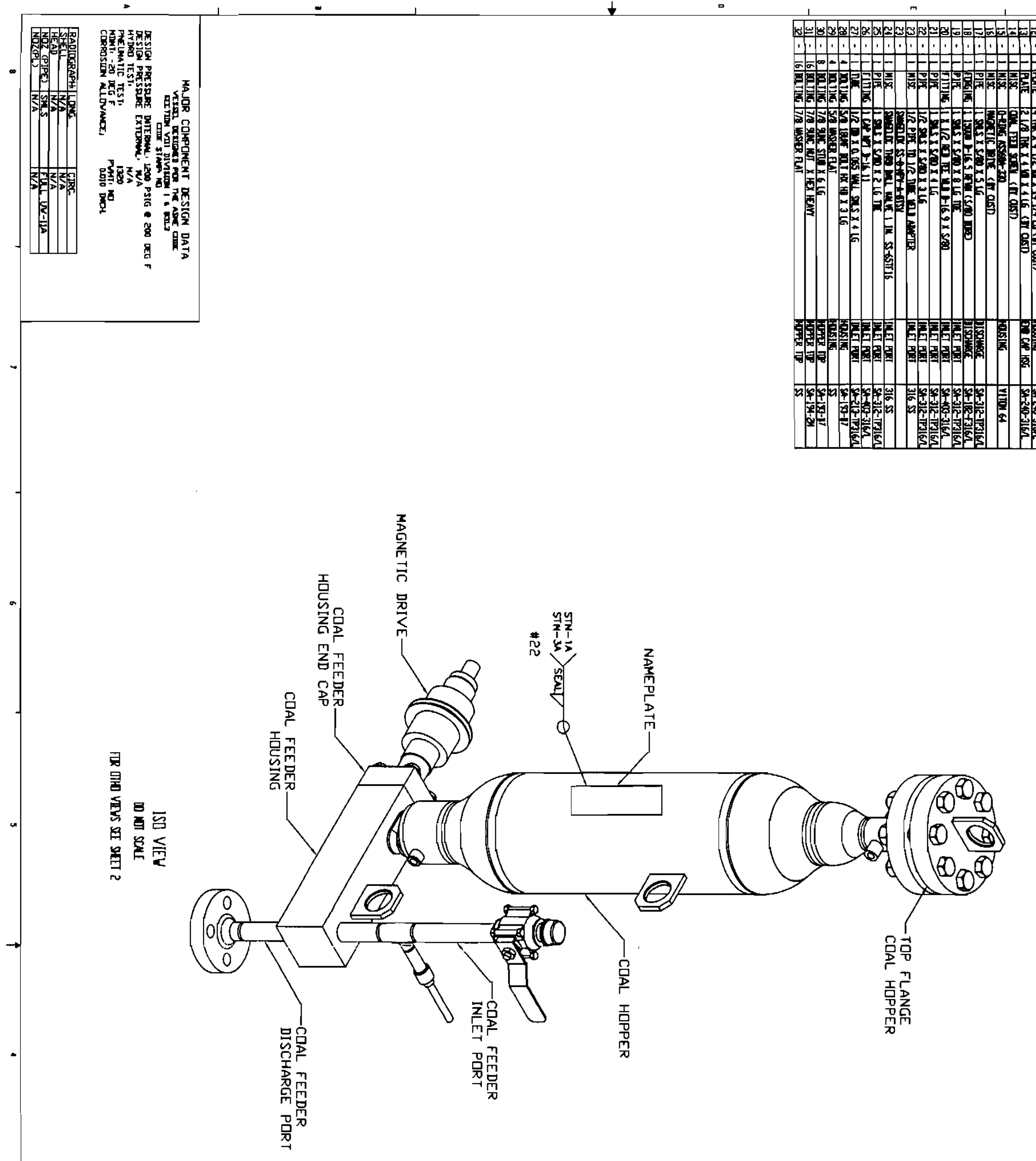



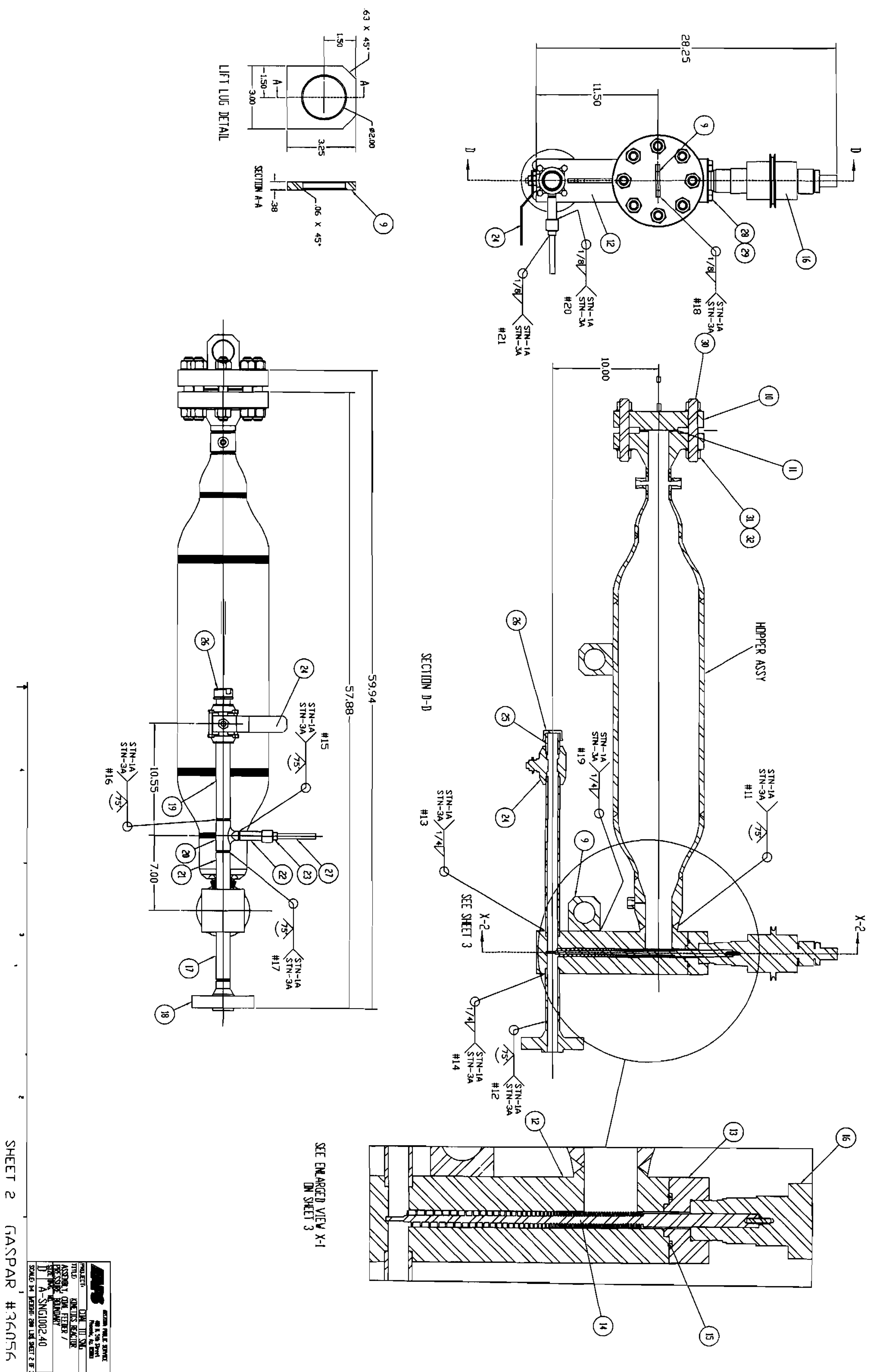

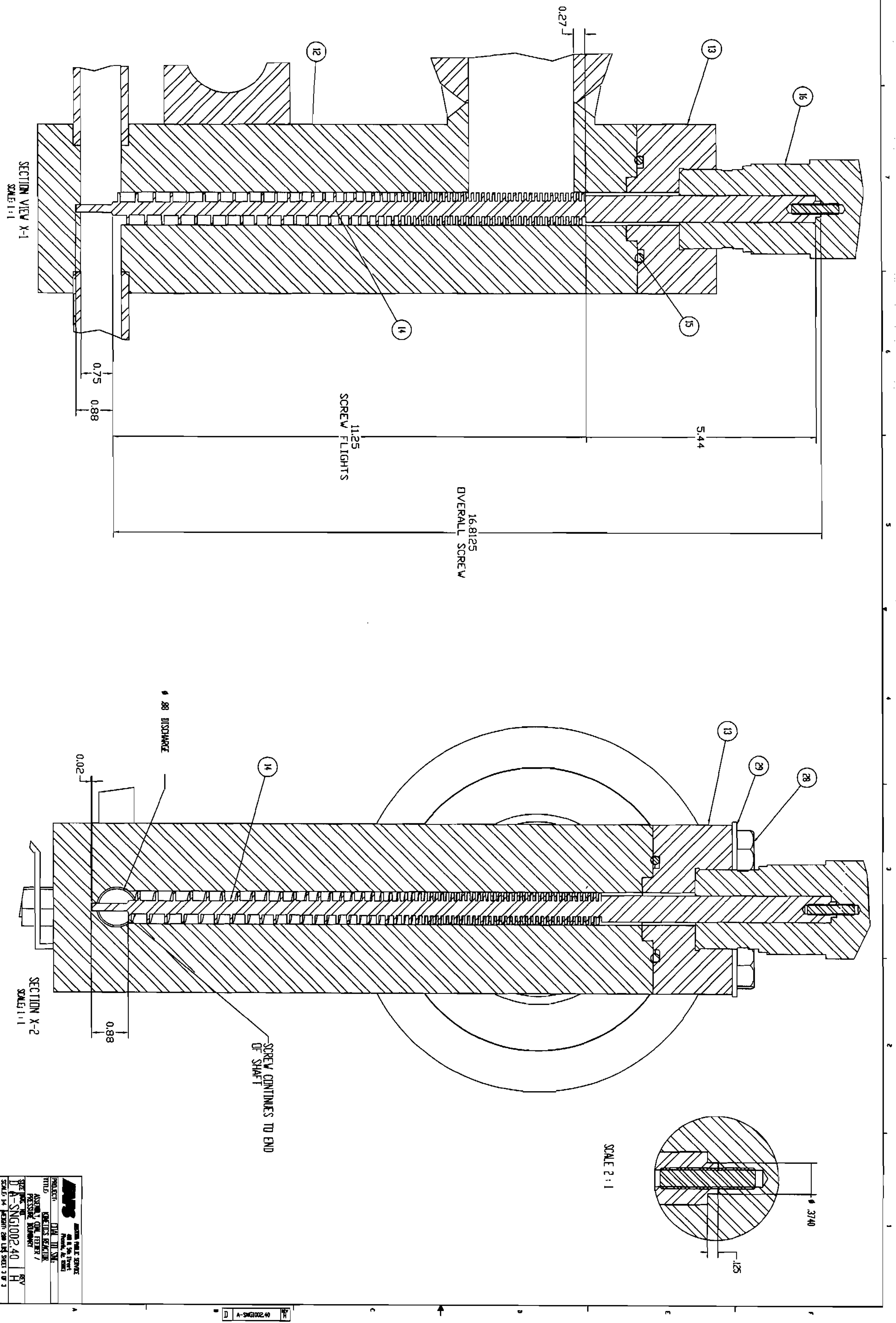


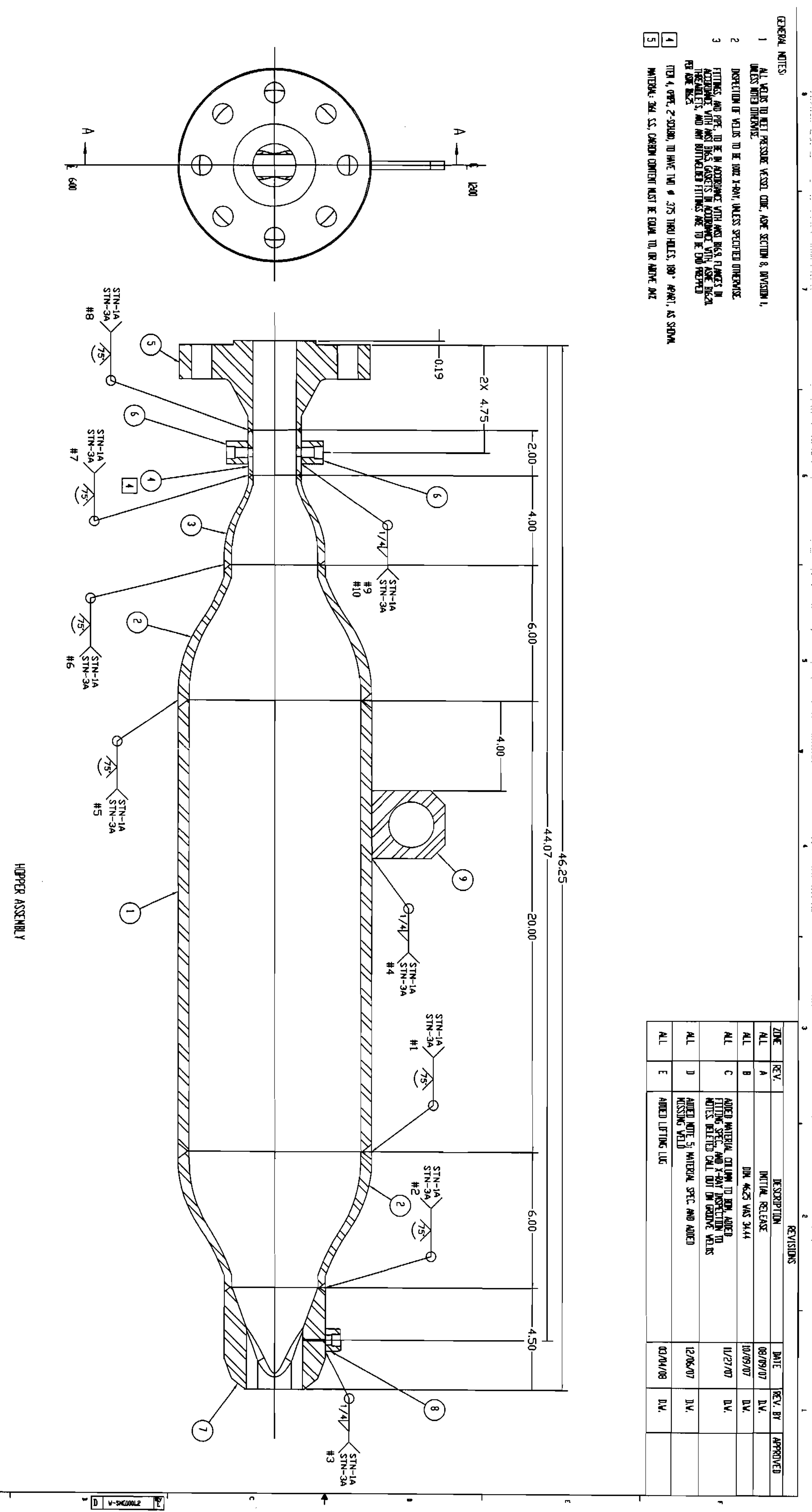




\section{Gasparinc.}

\section{WELDING \& FABRICATIONS}

1545 Whipple Ave S.W. - Canton. Ohio 44710

Phone: $330-477-2222$

Fax: $330-477-2322$

\section{CERTIFICATE OF COMPLIANCE}

\section{CUSTOMER:}

GASPAR JOB NUMBER:

DRAWING NUMBER:

CUSTOMER PO NUMBER:

ITEM(S):
ARIZONA PUBLIC SERVICE

\begin{tabular}{l}
\hline 36063 \\
\hline W.SNG1002.48 REV.B \\
\hline 700521452 \\
\hline COAL FEEDER FRAME \\
\hline
\end{tabular}

THIS CERTIFICATE OF COMPLIANCE AFFIRMS THAT, YO THE BEST OF OUR KNOWLEDGE. THE REFERENCED EOUIPMENT WAS FABRICATED AND INSPECTED IN ACCORDANCE W|TH THE PURCHASE ORDER, CONTRACT, AND APPLICABLE CODE REQUIREMENTS (IF ANY).

ADDITIONAL INFORMATION:

NAME: WESLEYMORGAN

TITLE: QUALITY CONTROL MANAGER

DATE: $\quad 5 / 21108$ 


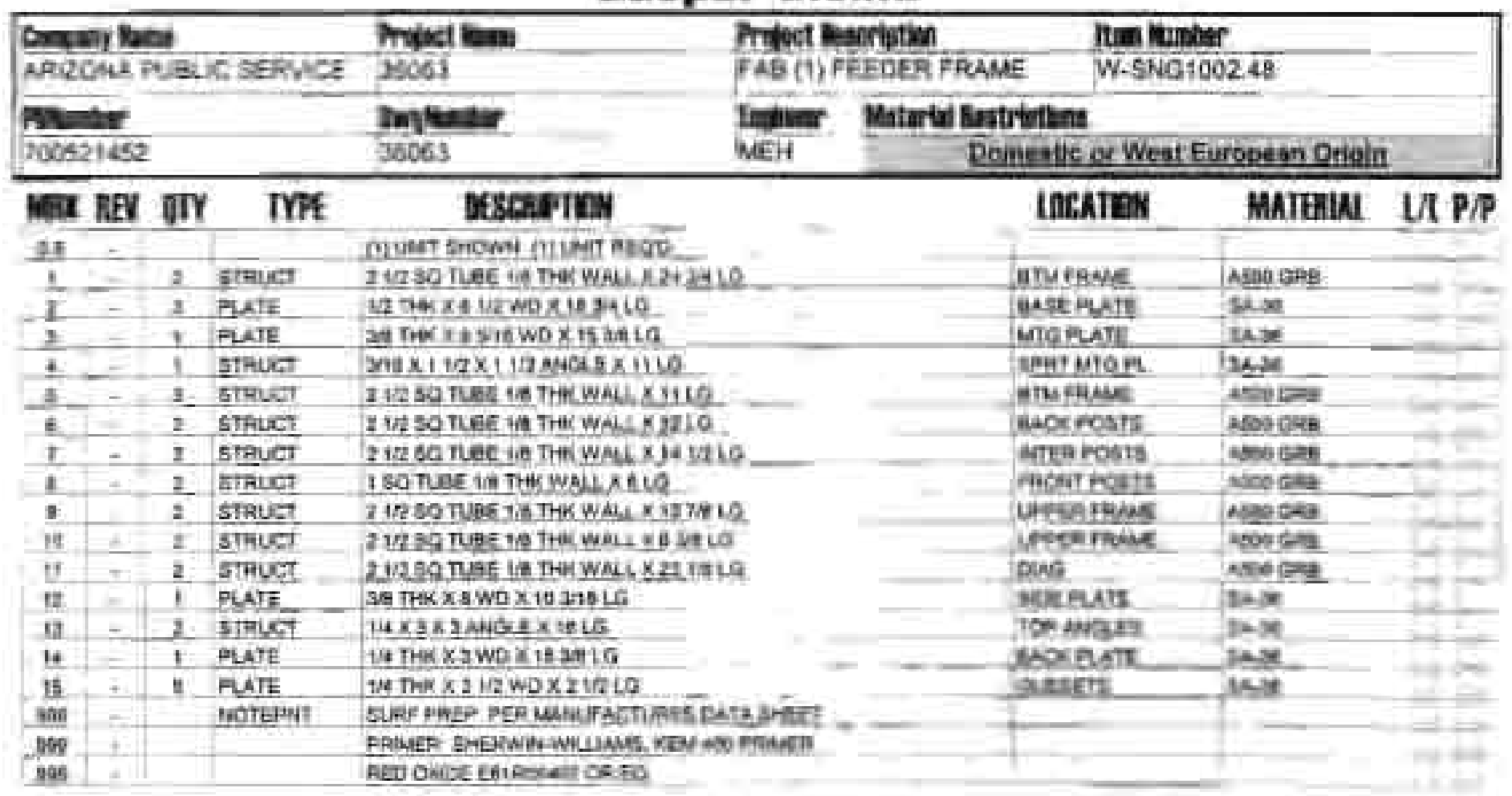




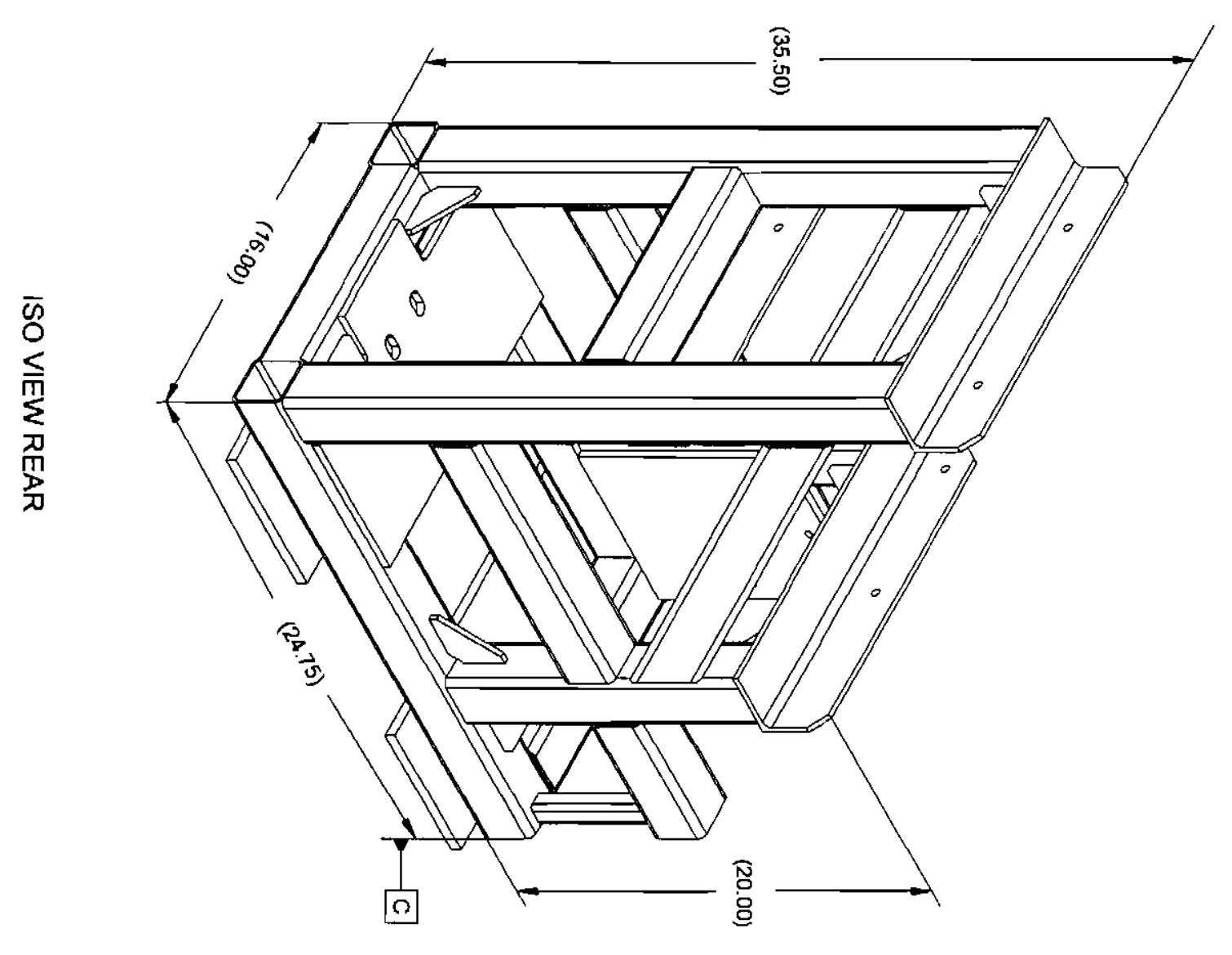

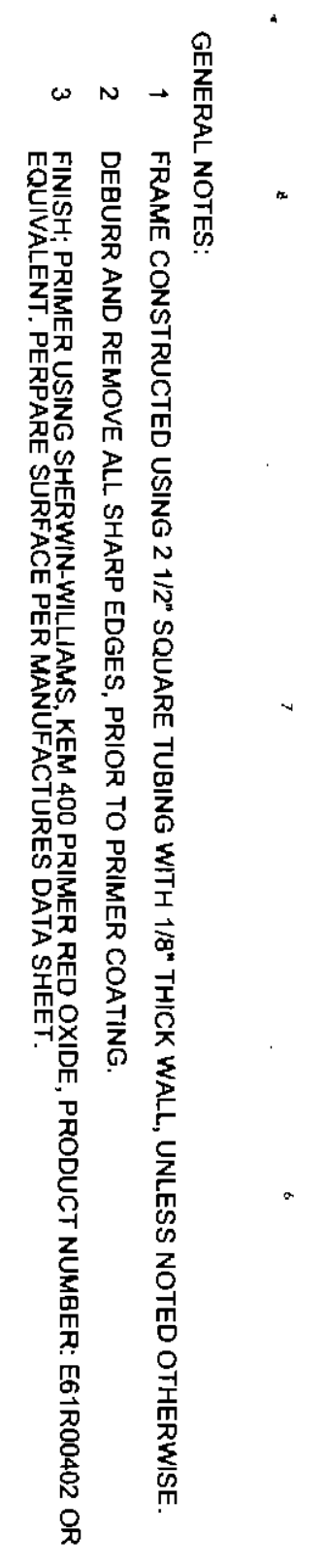
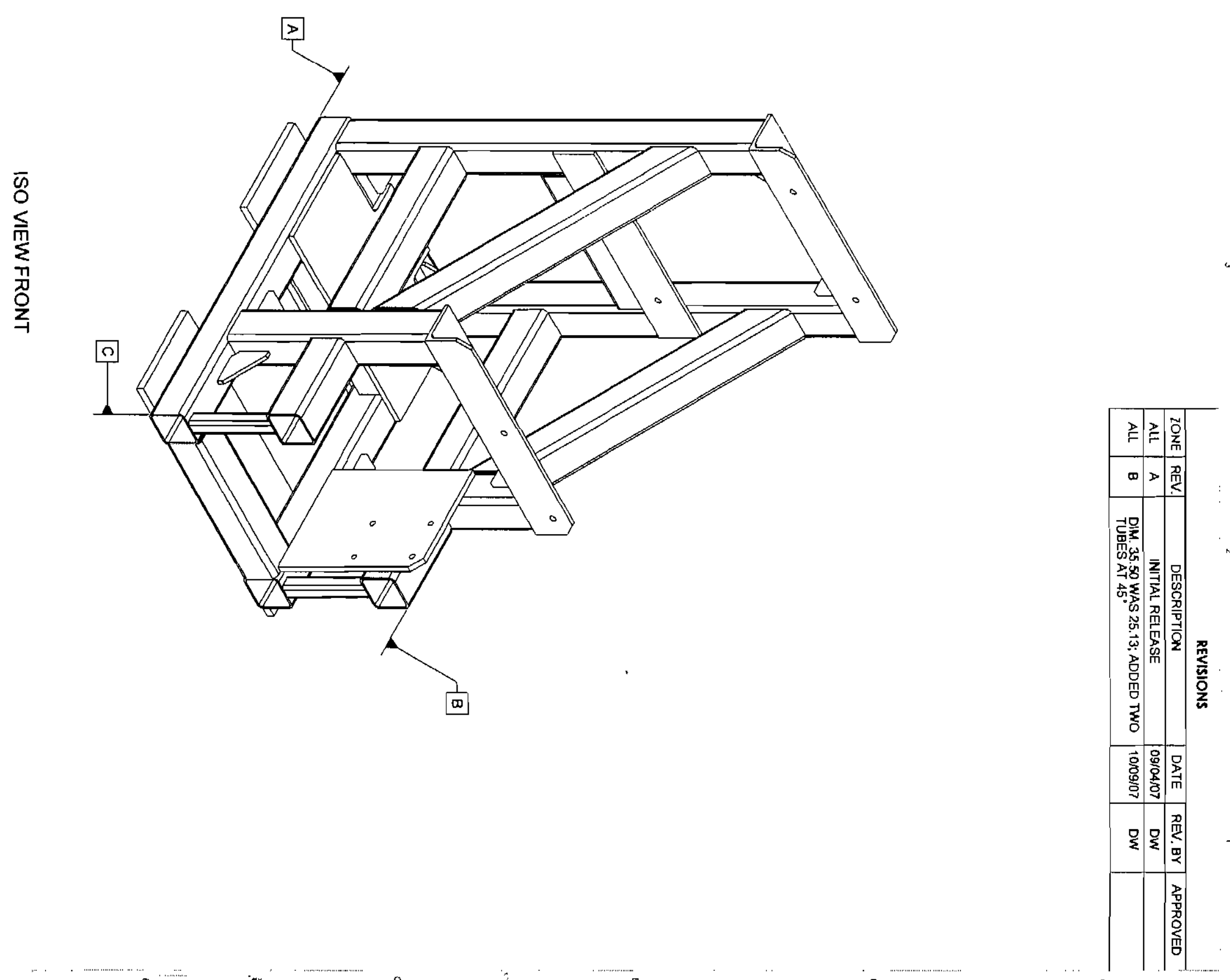

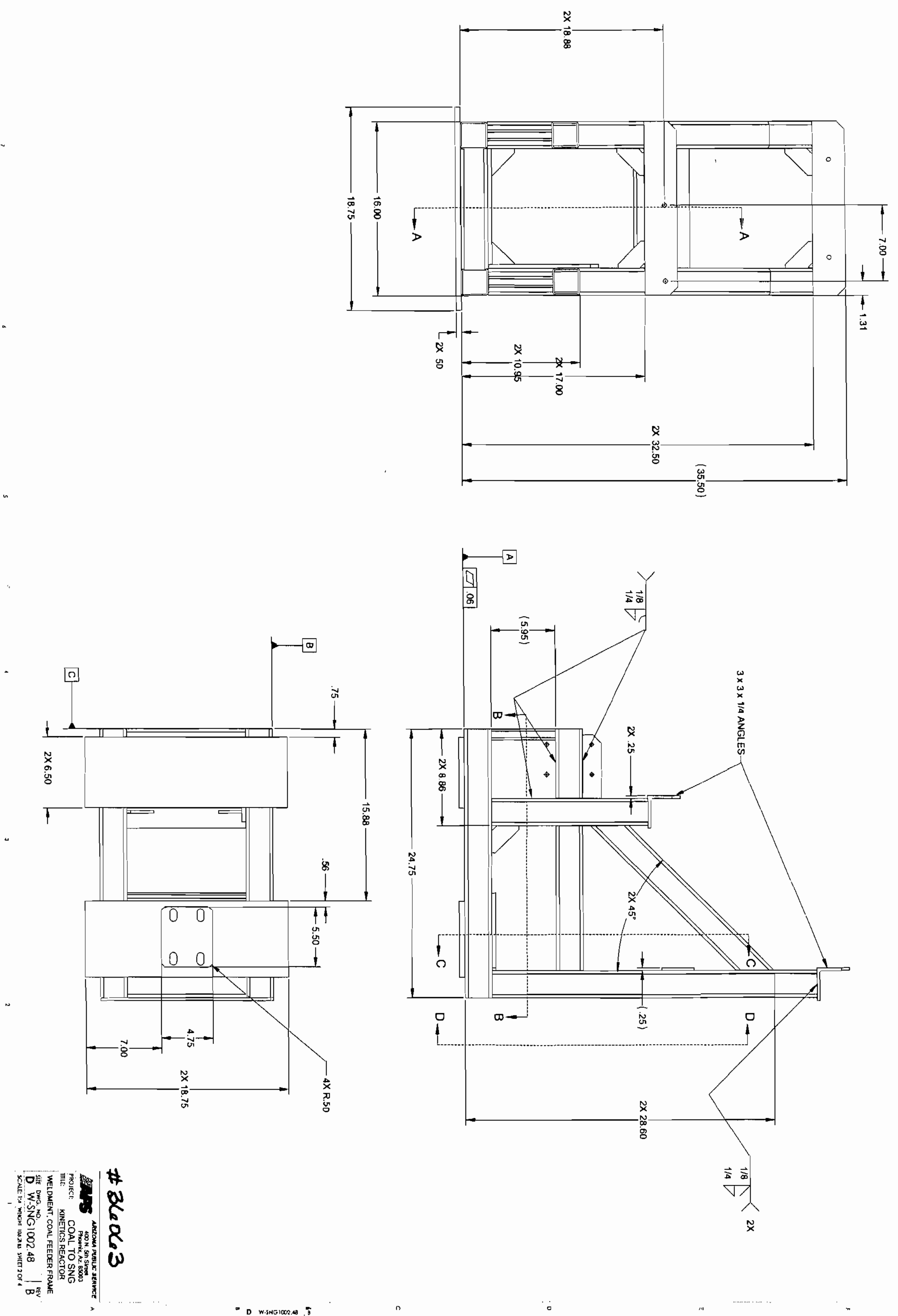


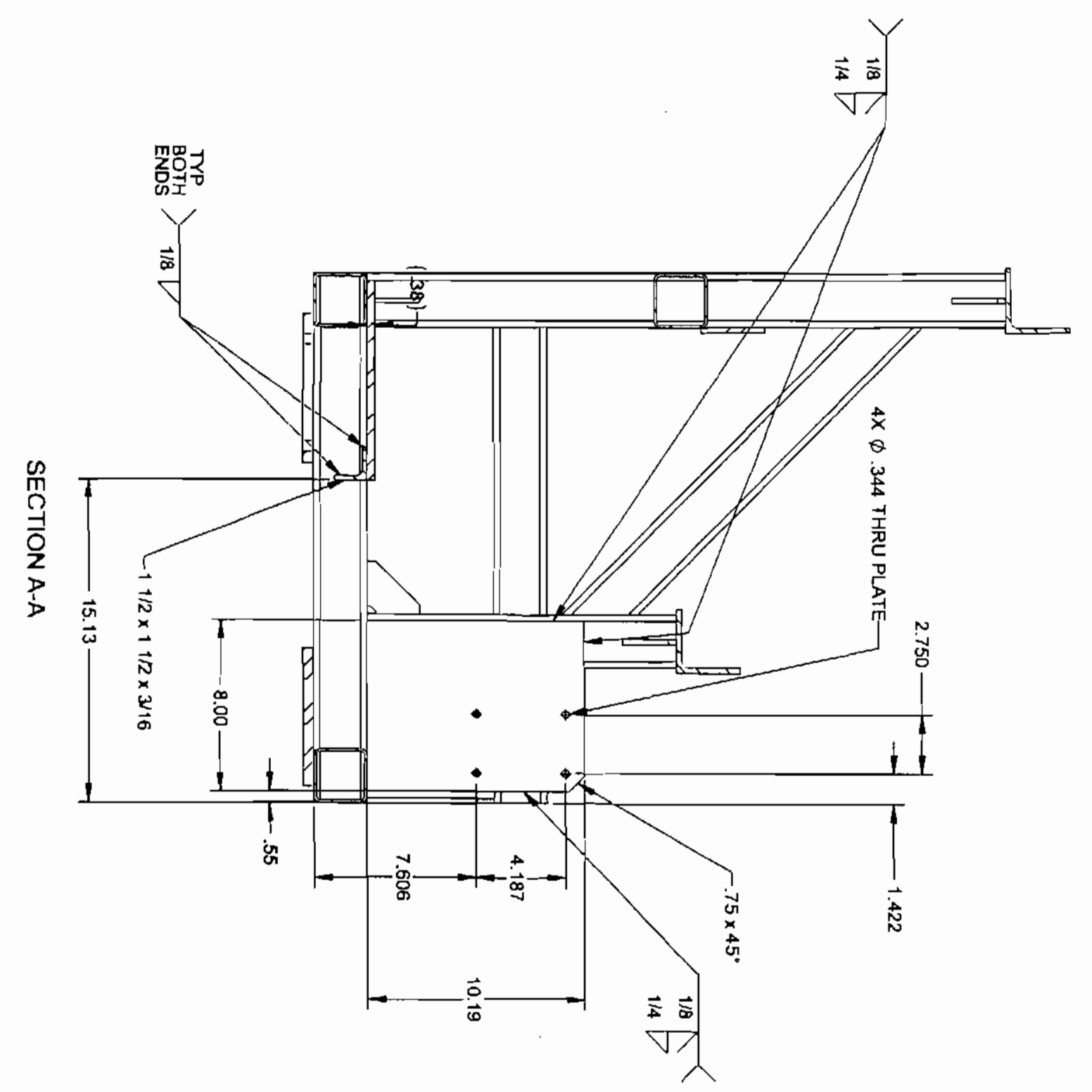

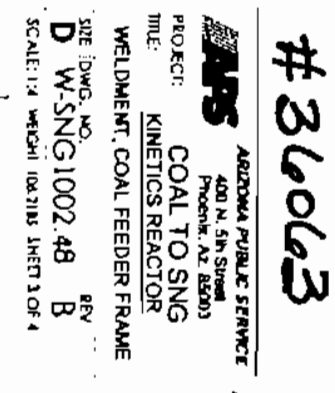

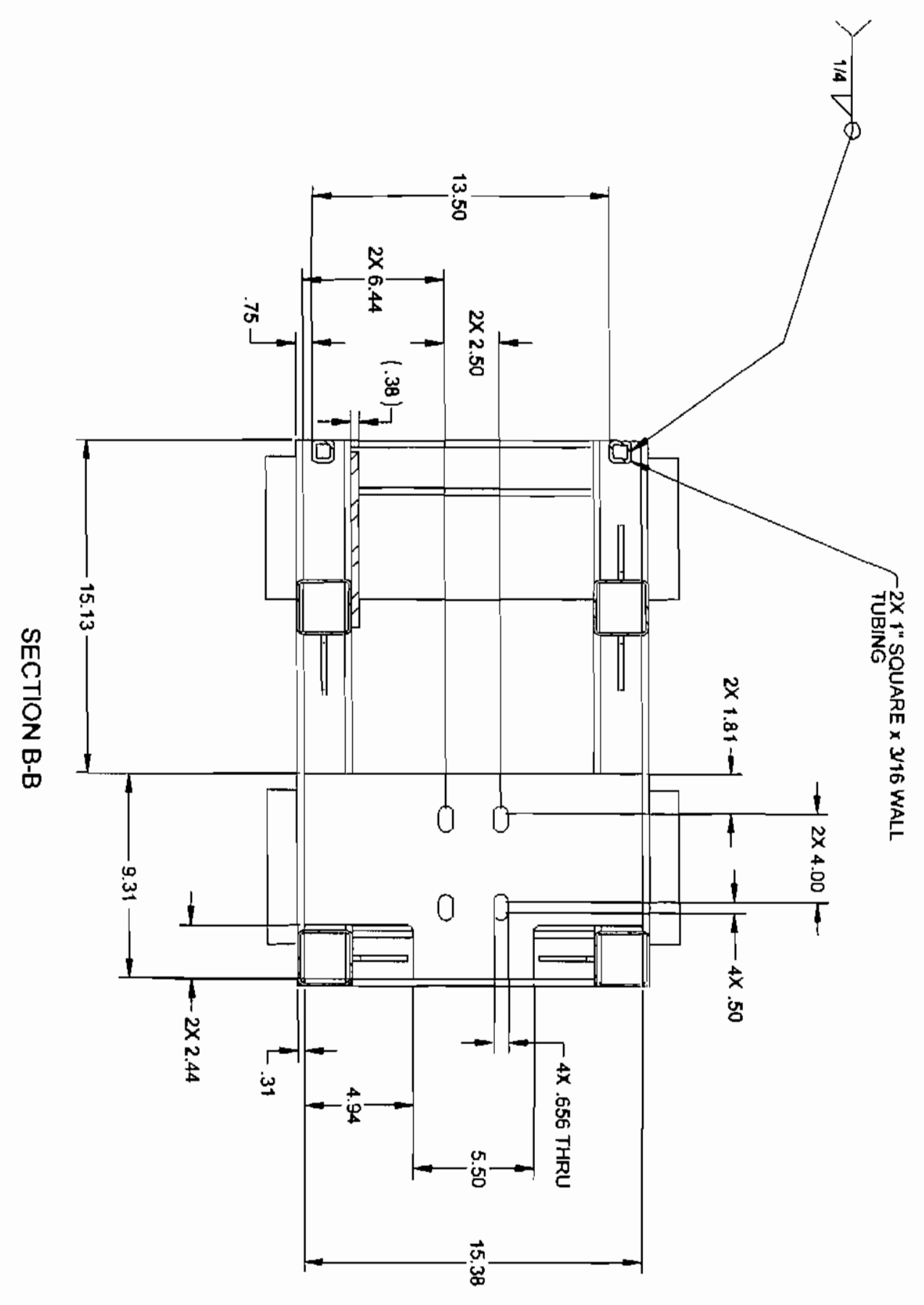



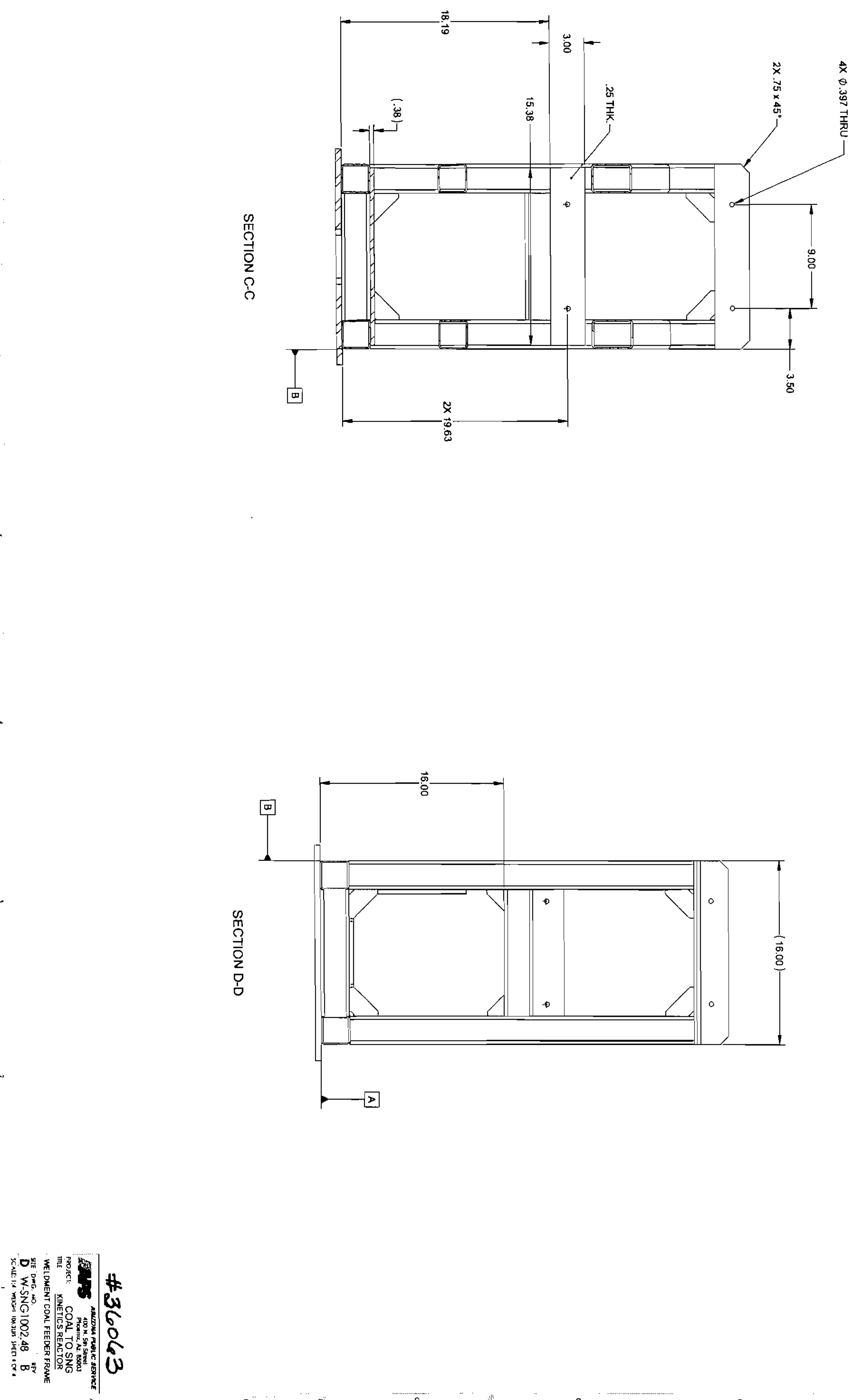


$$
\text { I }
$$




\title{
APPENDIX $F$
}

\section{Bench Scale Hydrogasifier Upper CharPot Assembly}

\author{
Final Design from Gaspar Inc.
}


Development of a Hydrogasification Process for

Coproduction of SNG and Electrical Power from Western Coals

Final Report

THIS PAGE INTENTIONALLY LEFT BLANK 
WELDING \& FABRICATIONS

1545 Whipple Avenue SW Canton, Ohio 44710

Phone: 330-477-2222

Fax: $330-477-2322$

www.gasparinc.com

\section{FINAL DATA PACKAGE}

\begin{tabular}{|r|l|}
\hline GASPAR JOB NUMIBER(S): & 36093 \\
\hline CUSTOMER: & ARIZONA PUIBLIC SERVICE \\
\hline PURCHASE ORDER NUMBER: & 700521452 \\
\hline DESCRIPTION: & UPPER CHAR POT \\
\hline ITEM NUMBER(S): & N/A \\
\hline OTHER: & N/A \\
\hline
\end{tabular}

DATA PACKAGE CONTENTS

$\square$ DATA REPORT

$\square$ NAMEPLATE COPY

$\square$ BILL OF MATERIAL

$\square$ MATERIAL TEST REPORTS

$\square$ NDE REPORTS

$\square$ HEAT TREAT CHARTS

$\square$ CALCULATIONS

$\square$ DRAWINGS

$\square$ OTHER: (LIST BELOW)

NOTE: 


\section{FORM U-1A MANUFACTURER'S DATA REPORT FOR PRESSURE VESSELS}

(Alternative Form for Single Chamber, Completely Shop or Field Fabricated Vessels Only)

As Required by the Provisions of the ASME Boiler and Pressure Vessel Code Rules, Section VIII, Division 1

1. Manufactured and certified by:

Gaspar, Inc. 1545 Whipple Ave SW Canton, Ohio 44710

(Name and address of manufacturer)

2. Manufactured for: $\quad$ Arizona Public Service Co. P.O. Box 53999 Phoenix, AZ 85072

3. Location of installation

4. Type: Vertical Unknown

(thorizontal or vertical tank)

(Name and address)
36093 Rev. 3

$\frac{2527}{\text { (National Board number) }}$

2008

(Year built)

5. The chemical and physical properties of all parts meet the requirements of material specifications of the ASME BOILER AND PRESSURE VESSEL

CODE The design, construction, and workmanship conform to ASME Rules, Section VIII, Division 1

2007 Edition

to

\begin{tabular}{|c|c|}
\hline 6. Shell & $\begin{array}{l}\text { Addenda (Date) } \\
\text { SA312-316H }\end{array}$ \\
\hline & $\begin{array}{l}\text { Material spec. number. grade) } \\
\text { Smls }\end{array}$ \\
\hline & , lap \\
\hline
\end{tabular}

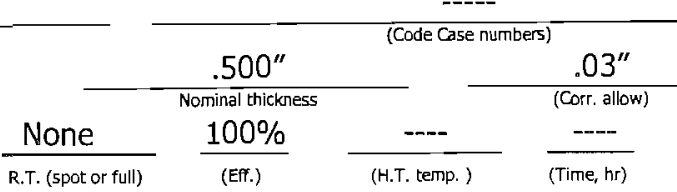

(b) Material Year

8. Heads: (a) Material

$\mathrm{SA} 403-316 \mathrm{H}$

(Spec, number, grade)

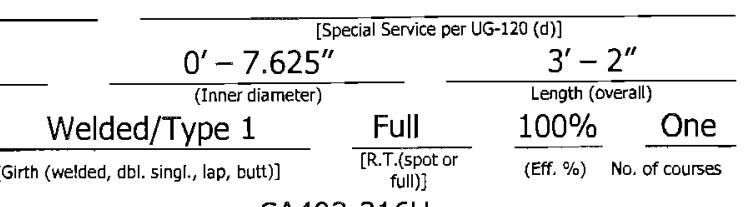

\begin{tabular}{|c|c|c|c|c|c|c|c|c|c|c|}
\hline & $\begin{array}{l}\text { Location (Top, } \\
\text { Bottom, Ends) }\end{array}$ & $\begin{array}{l}\text { Minimum } \\
\text { Thickness }\end{array}$ & $\begin{array}{l}\text { Corrosion } \\
\text { Allowance }\end{array}$ & $\begin{array}{l}\text { Crown } \\
\text { Radius } \\
\end{array}$ & $\begin{array}{l}\text { Knuckle } \\
\text { Radius }\end{array}$ & $\begin{array}{c}\text { Elliptical } \\
\text { Ratio }\end{array}$ & $\begin{array}{c}\text { Conical } \\
\text { Apex Angle } \\
\end{array}$ & $\begin{array}{l}\text { Hemispherical } \\
\text { Radius }\end{array}$ & $\begin{array}{c}\text { Flat } \\
\text { Diameter }\end{array}$ & $\begin{array}{c}\text { Side to Pressure } \\
\text { (Convex or Concave) }\end{array}$ \\
\hline (a) & Top & $.337^{\prime \prime}$ & $.03^{\prime \prime}$ & $-\cdots$ & $\ldots$ & $\ldots-$ & $*$ & $-\cdots-$ & $-\cdots$ & Concave \\
\hline (b) & Bottom & $.337^{\prime \prime}$ & $.03^{\prime \prime}$ & $\overline{-\cdots}$ & $-\cdots$ & --- & * & $\cdots$ & --- & Concave \\
\hline
\end{tabular}

If removable, bolts used (describe other fastenings)

9. MAWP

$$
\begin{aligned}
& \frac{1150}{\text { (Internal) }} \\
& \frac{---}{-20}
\end{aligned}
$$

at max temp.

(Material spec. number, grade, size, number)

Min. design metal temp. -20 at 1150 1000

(Internal)

SA403-316H

10. Nozzles, inspection, and safety valve openings:

\begin{tabular}{|c|c|c|c|c|c|c|c|c|}
\hline $\begin{array}{c}\text { Purpose } \\
\text { (Inlet, Outlet, Drain) }\end{array}$ & Number & $\begin{array}{l}\text { Diameter } \\
\text { or Size }\end{array}$ & Type & Material & \begin{tabular}{|l|} 
Nominal \\
Thickness \\
\end{tabular} & $\begin{array}{l}\text { Reinforcement } \\
\text { Material }\end{array}$ & $\begin{array}{c}\text { How } \\
\text { Attached }\end{array}$ & Location \\
\hline Aux & 1 & $2^{\prime \prime}$ & $1500 \# W N$ & $\mathrm{SA} 403-316 \mathrm{H}$ & $.218^{\prime \prime}$ & - & Butt Weld & - \\
\hline Aux & 1 & $2-1 / 2^{\prime \prime}$ & $1500 \# W N$ & SA403-316H & $.218^{\prime \prime}$ & $\cdots$ & Butt Weld & $-\cdots$ \\
\hline & & & & & & & & \\
\hline & & & & & & & & \\
\hline 11. Supports: Skirt & $\frac{\text { No }}{\text { Yes or No) }}$ & Lugs & $\frac{2}{\text { (No.) }}$ & Legs & Othe & $\frac{---}{\text { (Describe) }}$ & Attached & $\begin{array}{l}\text { hell/Welded } \\
\text { here and How) }\end{array}$ \\
\hline
\end{tabular}

\begin{tabular}{|l}
\hline \\
\hline \\
\hline
\end{tabular}

12. Remarks: Manufacturer's Partial Data Reports properly identified and signed by Commissioned Inspectors have been furnished for the following items of the report:

(Name of part, item number, Manufacturer's name and identifying stanp)

UHA-51(d) Exempt (impact test). Item 8: *Heads are standard 8" $\times 4^{\prime \prime}$ concentric reducers (ANSI B16.9).

\section{CERTIFICATE OF SHOP/FIELD COMPLIANCE}

We certify that the statements made in this report are correct and that all details of design, material, construction and workmanship of this vessel conform to the ASME Boiler and pressure Vessel Code, Section Vlll, Division 1.

expires July 25,2011

Date $9-12-08$ Co. Name

"U" Certificate of Authorization Number

16,862

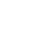

Vessel constructed by $\frac{\text { Gaspar, lnc. }}{\text { (Manufacturer) }}$

Signed

\section{CERTIFICATE OF SHOP/FIELD INSPECTION}

Vessel constructed by Gaspar, lnc.

at

1545 Whipple Ave SW Canton, Ohio 44710

I, the undersigned, holding a valid commission issued by the National Board of Boiler and Pressure Vessel Inspectors and/or the State or Province of Ohio and employed by OneBeacon America Insurance Company Lynn, Mass

have inspected the component described in this Manufacturer's Data Report on to the best of my knowledge and belief, the Manufacturer has constructed this pressure vessel in accordance with ASME Boiler and Pressure Vessel Code, Section VIII, Division l. By signing this certificate neither the Inspector nor his/her employer makes any warranty, expressed or implied, concerning the pressure vessel this described in this Manufacturer's Data Report. Furthermore, neither the Inspector nor his/her employer shall be liable in any manner for any personal injury or property damage or a loss of any kind arising from or connected with this inspection.

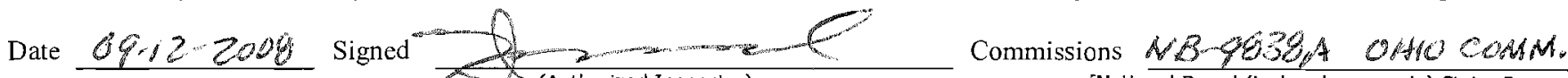




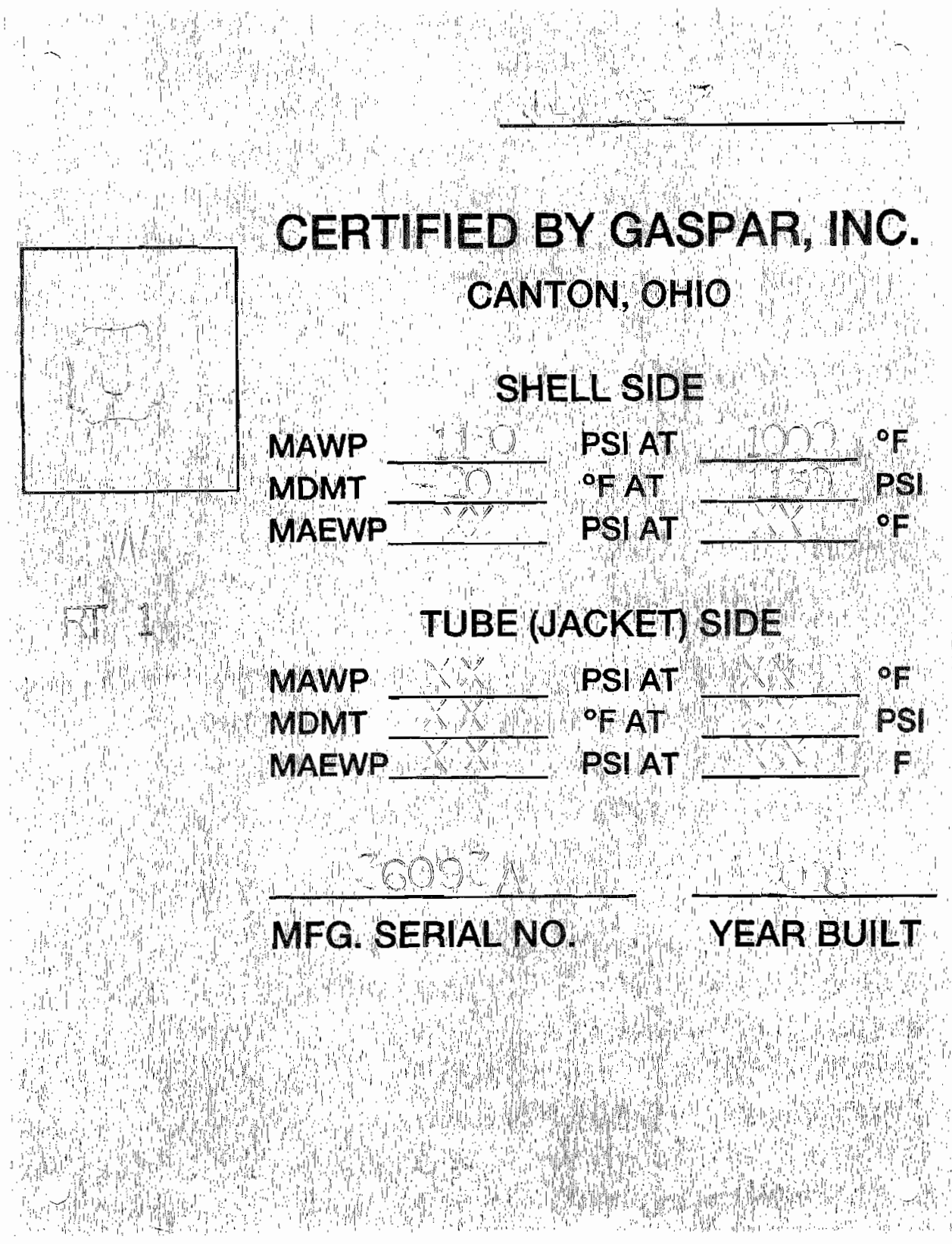




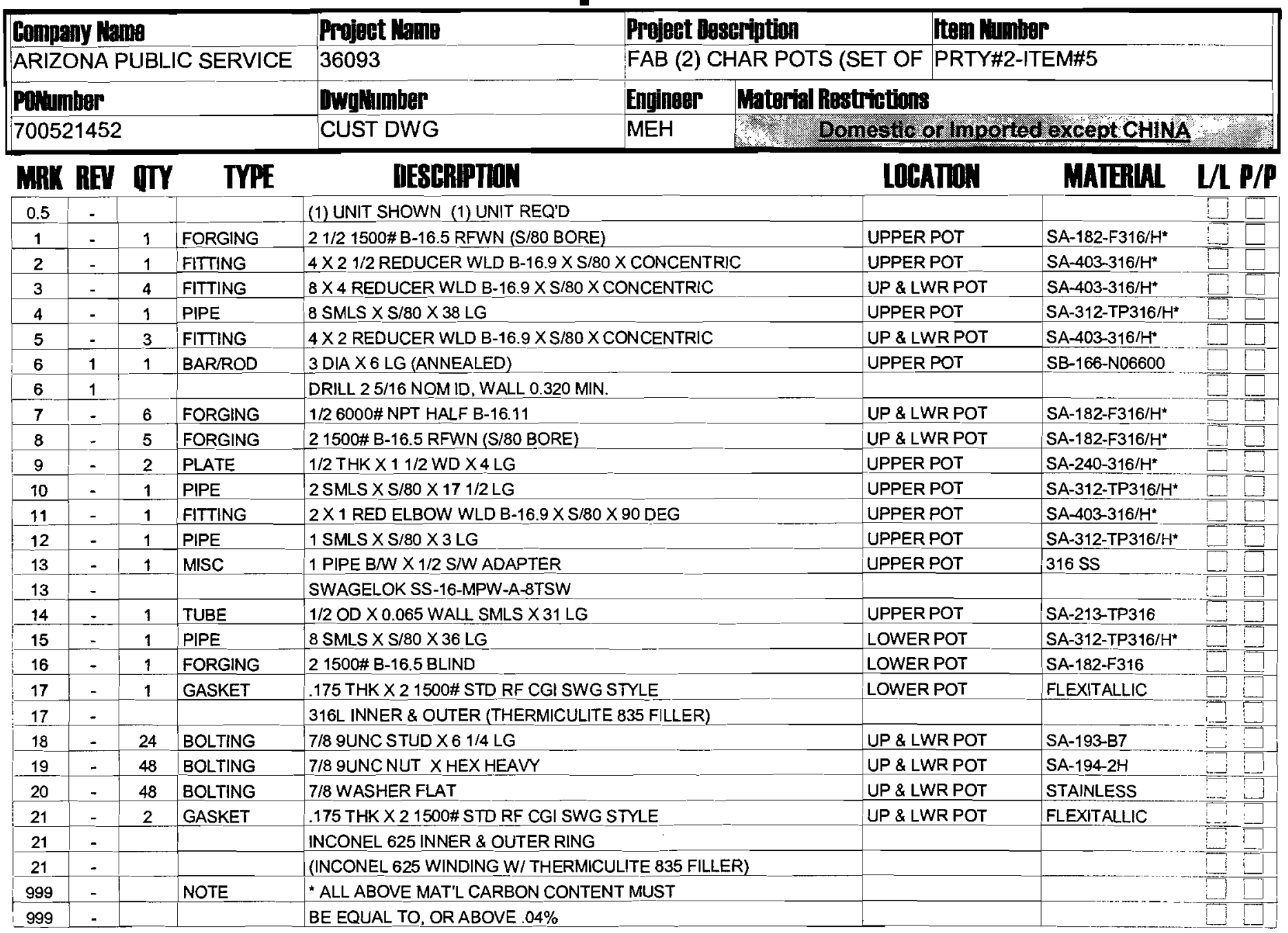




\section{WeSTERN FORGE \& FLANGe CO.}

687 COUNTY ROAD 2201

CLEVELAND, TX 77327

PH (281) 727-7000 FAX (281) 727-7062

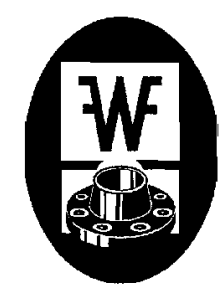

\section{CERTIFIED MATERIAL TEST REPORT}

\section{CERTIFIED IAW EN10204 3.1 (MADE IN USA)}

TO: GASPAR INC.

PO: 30214

SPECIFICATIONS:
ASME SA182 F316H

DIM/TOL PER ASME B16.5

\begin{tabular}{|c|c|}
\hline QTY & ITEM DESCRPTION \\
\hline 1 & $21 / 2 \% 1500 \#$ WN RF S80 \\
\hline & PROJECT\# 36093-1 \\
\hline & \\
\hline & \\
\hline & \\
\hline
\end{tabular}

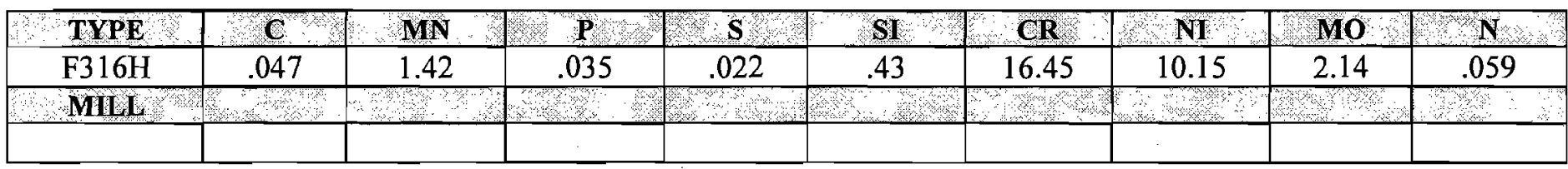

\begin{tabular}{|c|c|c|c|c|c|c|}
\hline HEAT NO & YIELD $2 \%$ OFF-PSI & TENSILE-PSI & \% EEONG & $\%$ RED AREA & HABDNESS & GRAIN SIZE (ASTM) \\
\hline 28122 & 59,690 & 85,300 & 67.0 & 82.8 & & No. 4.0 \\
\hline & & & & & & \\
\hline & & & & & & \\
\hline
\end{tabular}

- NO WELDING PERFORMED ON THE FORGINGS OF THIS ORDER.

- MATERLAL HEREIN IS CERTIFIED FREE FROM ANY FORM OF MERCURY, RADIUM OR ALPHA PARTICLE CONTAMINATION

THROUGHOUT ALL PHASES OF MANUFACTURE AND SHIPMENT:

- THE FORGINGS ON THIS ORDER CONFORM TO THE SPECIFIED DIMENSIONAL REQUIREMENTS.

- MATERLAL MANUFACTURED LAW WFF QA PROGRAM QAM-WF07.0702.

- Solution annealed @ $1950^{\circ} \mathrm{F}$ and water quenched to below $300^{\circ} \mathrm{F}$ within less than three minutes.

WE CERTIFY THE MATERIAL DESCRIBED HEREIN HAS BEEN MANUFACTURED, TESTED AND EXAMINED LAW AND MEETS ALL THE REQUIREMENTS OF ABOVE PURCHASE ORDER AND REFERENCED SPECIFICATIONS.

$07 / 24 / 08$
DATE

This document shall not be reproduced, except in full, without written approval of Western Forge \& Flange. The recording of false, fictitious or fraudulent statements or entries on this document may be punishable as felony under federal statutes. 


\section{TAYLOR FORGE STAINLESS}

P. O. BOX 610

SOMERVILLE, NJ 08876

Phone: (908)722-1313

FAX: (908)722-2943

\section{SOLD TO:}

GASPAR INC.

1545 WHIPPLE AVE. SW

CANTON, OH 44710

\section{Material Test Report}

Page : 1 of 1

SALES ORDER \# : 0000012238

\section{SHIP TO:}

GASPAR INC.

1545 WHIPPLE AVE. SW

CANTON, $\mathrm{OH} 44710$

CUST P.O.\#: 30236

TAG NUMBER: 30236, PPD-CHG

DATE SHIPPED: $/ 0 /$

\begin{tabular}{c|r|r}
\hline ITEM \# & QTY & ITEM DESCRIPTION \\
\hline 1 & 1 & $4 \times 21 / 2$ CR S/80 WP316HS SA403
\end{tabular}

STARTING MATERIAL

PIPE, A312-07, 316/316H

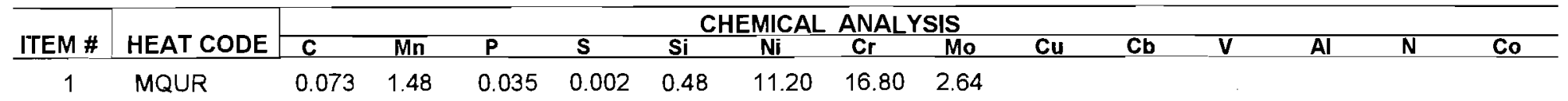

\begin{tabular}{c|lllll}
\hline & & & & PHYSICAL PROPERTIES \\
\cline { 2 - 5 } & $\begin{array}{l}\text { TENSILE } \\
\text { (PSI) }\end{array}$ & $\begin{array}{l}\text { YIELD } \\
\text { (PSI) }\end{array}$ & $\begin{array}{l}\text { ELONG } \\
(\mathbf{2} \text { IN) }\end{array}$ & \\
\hline 1 & 89600 & 58000 & 50.00 & 0.00
\end{tabular}

\begin{tabular}{c|cc}
\hline ITEM \# & SLIPPLEMENTAL INFORMATION \\
\hline 1 & GRAIN SIZE: ACTUAL NO. 6.5 &
\end{tabular}

SOLUTION ANNEALED, WATER QUENCHED

ASME SA403 2004 ED; ASTM A403-O6; EN 10204/3.1 ASME B16.9

THE FITTINGS WERE MANUFACTURED, SAMPLED, TESTED AND INSPECTED IN

ACCORDANCE WITH THE SPECIFICATIONS AND FOUND TO MEET THE REQUIREMENTS.

THE MATERIAL WAS MANUFACTURED, TESTED AND INSPECTED WITHOUT MERCURY

CONTAMINATION. NO WELD REPAIRS.

NACE MR0175

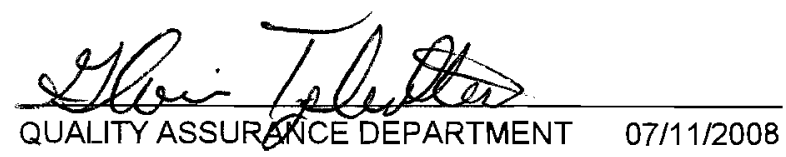




\section{TAYLOR FORGE STAINLESS}

P. O. BOX 610

SOMERVILLE, NJ 08876

Phone: (908)722-1313

FAX: (908)722-2943
Material Test Report

Page : 1 of 1

SALES ORDER \# : 0000012238

\section{SOLD TO:}

GASPAR INC

1545 WHIPPLE AVE. SW

CANTON, OH 44710
SHIP TO:

GASPAR INC.

1545 WHIPPLE AVE. SW

CANTON, OH 44710
CUST P.O.\#: 30236

TAG NUMBER: 30236, PPD-CHG

\begin{tabular}{c|r|l}
\hline ITEM \# & QTY & ITEM DESCRIPTION \\
\hline 2 & 2 & $8 \times 4$ CR S/80 WP316HS SA403
\end{tabular}

DATE SHIPPED: $10 /$

STARTING MATERIAL

PIPE, A312-07, 316/316H

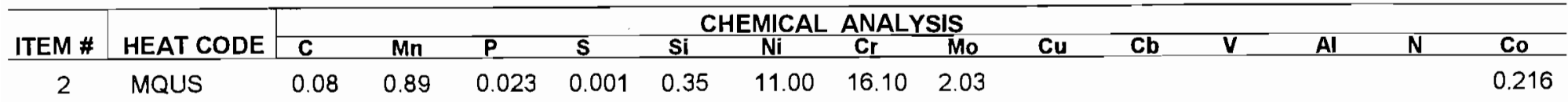

\begin{tabular}{|c|c|c|c|c|}
\hline & & & & PHYSICAL PROPERTIES \\
\hline ITEM \# & $\begin{array}{l}\text { TENSILE } \\
\text { (PSI) }\end{array}$ & $\begin{array}{l}\text { YIELD } \\
\text { (PSI) }\end{array}$ & $\begin{array}{l}\text { ELONG } \\
(2 \text { IN) }\end{array}$ & \\
\hline 2 & 76200 & 32500 & 77.00 & 0.00 \\
\hline
\end{tabular}

\begin{tabular}{c|cc}
\hline ITEM \# & & SUPPLEMENTAL INFORMATION \\
\hline 2 & GRAIN SIZE ACUTAL: ASTM NO 4.0
\end{tabular}

SOLUTION ANNEALED, WATER QUENCHED .

ASME SA403 2004 ED; ASTM A403-O6; EN 10204/3.1 ASME B16.9

THE FITTINGS WERE MANUFACTURED, SAMPLED, TESTED AND INSPECTED IN ACCORDANCE WITH THE SPECIFICATIONS AND FOUND TO MEET THE REQUIREMENTS. THE MATERIAL WAS MANUFACTURED, TESTED AND INSPECTED WITHOUT MERCURY CONTAMINATION. NO WELD REPAIRS. 
TAYLOR FORGE STAINLESS

P. O. BOX 610

SOMERVILLE, NJ 08876

Phone: (908)722-1313

FAX: (908)722-2943
Material Test Report

Page : 1 of 1

SALES ORDER \# : 0000012238

\section{SOLD TO:}

GASPAR INC.

1545 WHIPPLE AVE. SW

CANTON, OH 44710
SHUP TO:

GASPAR INC.

1545 WHIPPLE AVE. SW

CANTON, OH 44710
CUST P.O.\#: 30236

\begin{tabular}{l|l|l}
\hline ITEM \# & QTY & ITEM DESCRIPTION
\end{tabular}

$228 \times 4$ CR S/80 WP316HS SA403
TAG NUMBER: 30236, PPD-CHG

DATE SHIPPED: / $/$

STARTING MATERIAL

PIPE, A312-07, 316/316H

\begin{tabular}{|c|c|c|c|c|c|c|c|c|c|c|c|c|c|c|c|}
\hline \multirow[b]{2}{*}{ ITEM \# } & \multirow[b]{2}{*}{ HEAT CODE } & \multicolumn{12}{|c|}{ CHEMICAL ANALYSIS } & \multirow[b]{2}{*}{$\mathbf{N}$} & \\
\hline & & C & Mn & $\mathbf{P}$ & $\mathbf{S}$ & Si & $\mathrm{Ni}$ & $\mathrm{Cr}$ & $\mathrm{Mo}$ & $\mathbf{C u}$ & $\mathrm{Cb}$ & $\bar{V}$ & Al & & $\mathrm{Co}_{0}$ \\
\hline 2 & MQUS & 0.08 & 0.89 & 0.023 & 0.001 & 0.35 & 11.00 & 16.10 & 2.03 & & & & & & 0.216 \\
\hline
\end{tabular}

\begin{tabular}{|c|c|c|c|c|}
\hline \multirow[b]{2}{*}{ ITEM \# } & \multicolumn{4}{|r|}{ PHYSICAL PROPERTIES } \\
\hline & $\begin{array}{l}\text { TENSILE } \\
\text { (PSI) }\end{array}$ & $\begin{array}{l}\text { YIELD } \\
\text { (PSI) }\end{array}$ & $\begin{array}{l}\text { ELONG } \\
\text { (2 IN) }\end{array}$ & \\
\hline 2 & 76200 & 32500 & 77.00 & 0.00 \\
\hline
\end{tabular}

\begin{tabular}{c|cc}
\hline TTEM \# & & SUPPLEMENTAL INFORMATION \\
\hline 2 & GRAIN SIZE ACUTAL: ASTM NO 4.0
\end{tabular}

SOLUTION ANNEALED, WATER QUENCHED

ASME SA403 2004 ED; ASTM A403-O6; EN 10204/3.1 ASME B16.9

THE FITTINGS WERE MANUFACTURED, SAMPLED, TESTED AND INSPECTED IN

ACCORDANCE WITH THE SPECIFICATIONS AND FOUND TO MEET THE REQUIREMENTS.

THE MATERIAL WAS MANUFACTURED, TESTED AND INSPECTED WITHOUT MERCURY

CONTAMINATION. NO WELD REPAIRS.

NACE MR0175

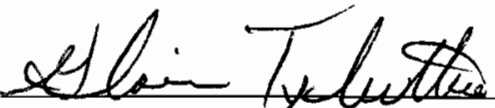

QUALITY ASSURAMCE DEPARTMENT 
INSPECTION CERTIFICATE

$\cdots$

SISHTOMO MLTAL INDISTRIES, LTD. STEEL TUBE HORK'S

IIIGASHJ-MILKOJ LRA. AMGGASAKI, JAPAN

CLRTIFICATL N0. : OYYUI947

PAGE: I

DATE: 2007-07-26

CUSTOHER TTJOFA PIIE SIPPLY INC.

ORDER RO. : $: 902033$ ITEH NO. 14

SHIPPER :SUNITOAO CORPORATION 057 KE:Q 06I3/5 7P!2S30650I

COMUIOUITY :SEAALESS IOT FIMISIIED STAINLESS STEEL PIPE HITH PLAIN SQUARE CUT ENDS

STMDARD :ASTH A312-05A / ASHE 2004 (A05) SA-312 TP316 ASTM A312-05A / ASME 2004 (AD5) SA-312 TPI16II SPECIJICATION :POA $¥ 2 S U H-1 A$ REV. 7

ASTW A376-04 / ASME 2004 (AD5) SA-376 TP316 ASTY A376-04 / ASHE 2004 (1D5) SA-376 TP316H

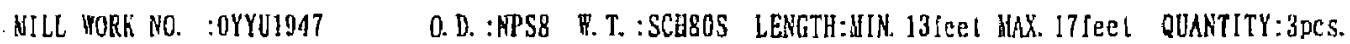
TOTAL LENGTH: 46. 48fecl MASS: $316 \mathrm{~kg}$

HEAT NA. PRODHCTS PCS.

1421613

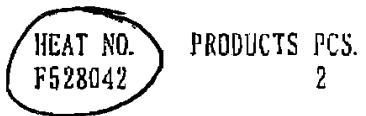

WEAT TREATNENT: SOLUTION TREATED (2102 FX2min. H.Q.)

CIEMIICAL COMPOSITION(⿻)

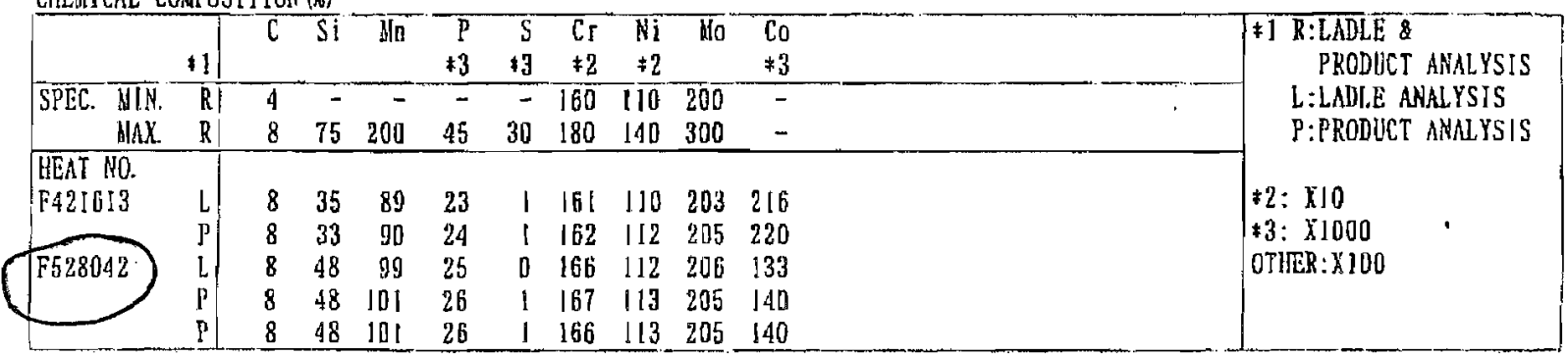

TENSILE TEST

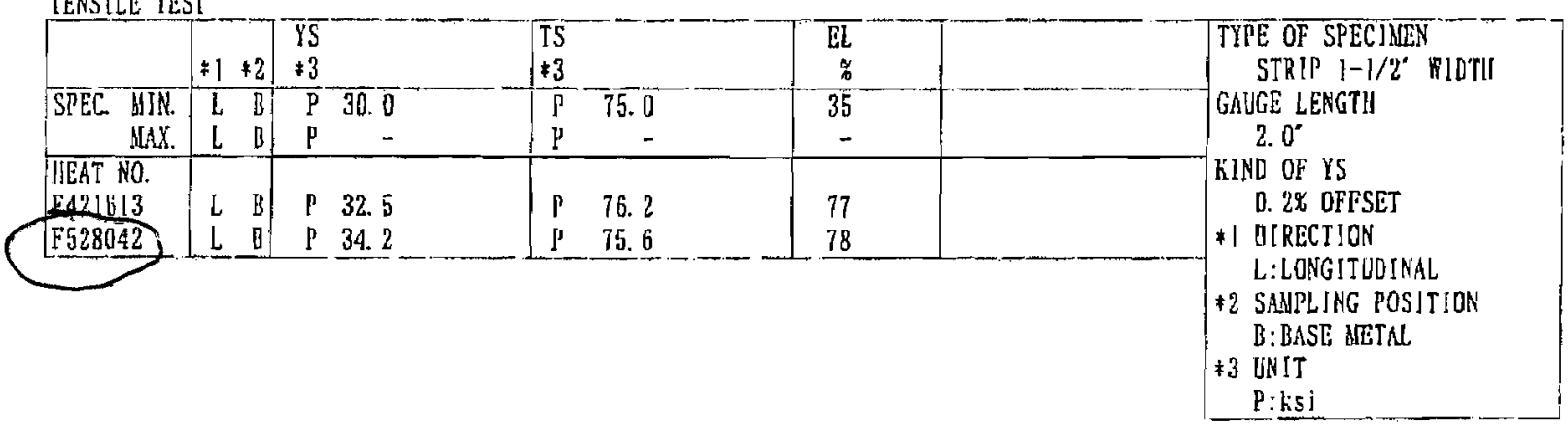

AUSTENITE GRA LN SIZE (ASTH E]J2)

\begin{tabular}{|r|r|}
\hline SPEC WIN. & -1 \\
NAA & 7.0 \\
\hline HEAT MO. & \\
F421613 & 3.8 \\
F528012 & 3.7 \\
& 3.5 \\
\hline
\end{tabular}

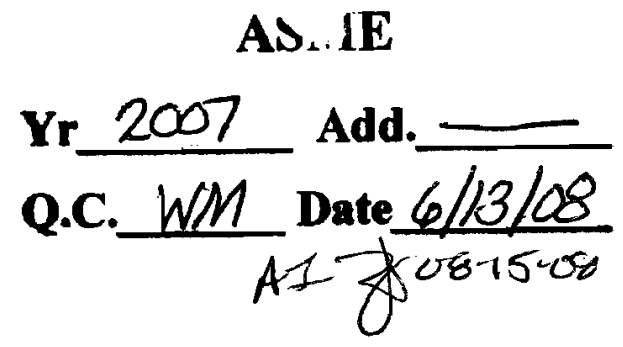

WE HEREDY CERTIFY THAT THE MATERIAL HEREIN DESCRIBED HAS BEEN BMNUIACTURED, SAMPLED, TESTED, AND INSPECTED IN ACCORDANCE MITH ABOVE STANDARD AND SPECSFJCATION ANO SATJSFIES PIIE REQUIREMENTS. 


\author{
INSPECTION CERTIFICATE
}
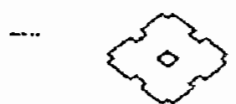

SLTITOHO
I. NISHINO-CHO. IS STEEL TUBE MORRS

CERTIPICATE NO. : OYYUIOA?
IGASIII-WLIRIJIEGA, ANAGASALT, JAFAM

PAGE: 2
BATE: $2007-07-26$

LORLOSION TEST OAIL-P-J14AD\} :ACCEPTABLE

CORRDSION TEST (MIL-P-24691/g) :GLARANTEE

FLATTENING TEST:ACCELTAALE

VISUIAL \& DILENSIONS:ACCEPTABLE

HYDROSTATIC TEST I70OPS I:ACCEPTABLE

EN10204 3.

NO MELD KEPANR

LERCURY FREE

NACE MROOI75 ILARDNESS (IIRC 22 UIAX. ) :GLARANTEED

THE MTERJAL PAS COOLED TO IIELOW BUO DEG F IN 3 MINUTES BR LESS

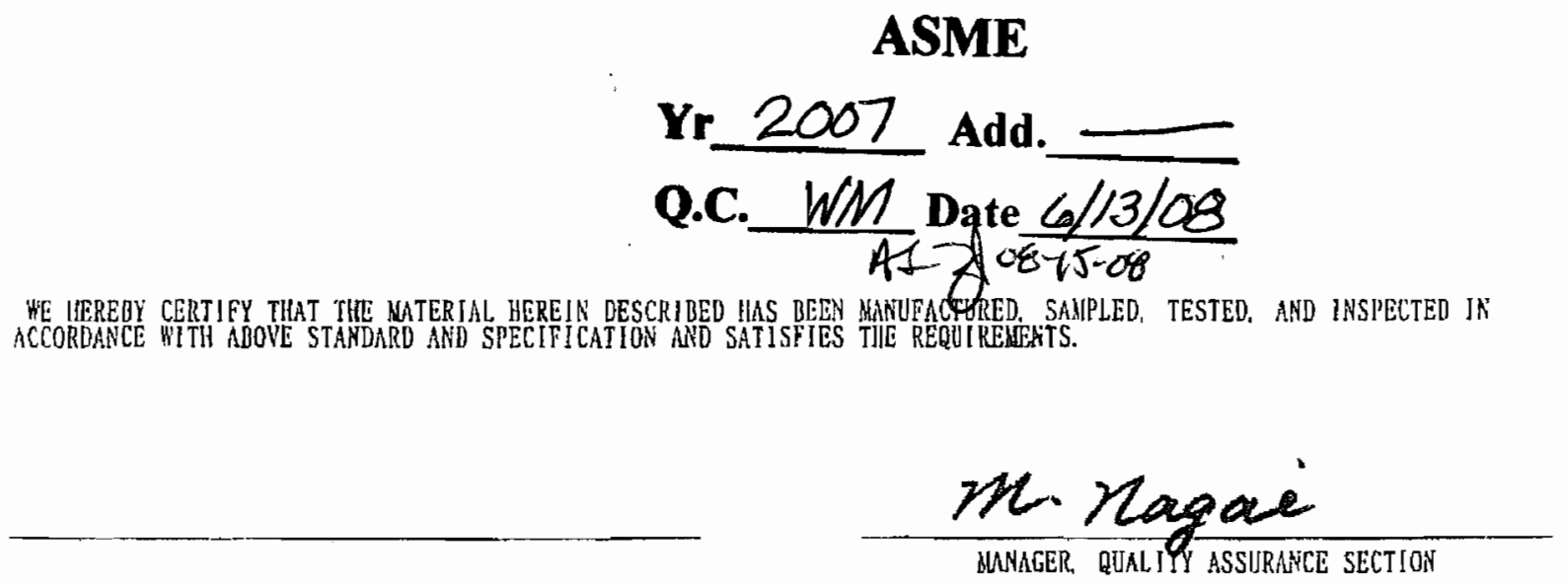


TAYLOR FORGE STAINLESS

P. O. BOX 610

SOMERVILLE, NJ 08876

Phone: (908)722-1313

FAX: (908)722-2943
Material Test Report

Page: 1 of 1

SALES ORDER \# : 0000012238

\section{SOLD TO:}

GASPAR INC.

1545 WHIPPLE AVE. SW

CANTON, OH 44710

\section{SHIP TO:}

GASPAR INC.

1545 WHIPPLE AVE. SW

CANTON, OH 44710
CUST P.O.\#: 30236

TAG NUMBER: 30236, PPD-CHG

\begin{tabular}{c|r|r|}
\hline ITEM \# & QTY & ITEM DESCRIPTION \\
\hline 3 & 3 & $4 \times 2$ CR S/80 WP316HS SA403
\end{tabular}

DATE SHIPPED: $/ 0 /$

STARTING MATERIAL

PIPE, A312-07, 316/316H

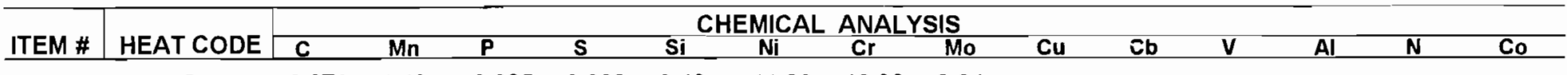

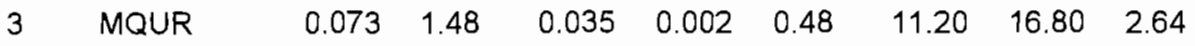

\begin{tabular}{c|llll}
\hline \multirow{2}{*}{ ITEM \# } & $\begin{array}{l}\text { TENSILE } \\
\text { (PSI) }\end{array}$ & $\begin{array}{l}\text { YIELD } \\
\text { (PSI) }\end{array}$ & $\begin{array}{l}\text { ELONG } \\
\text { (2 IN) }\end{array}$ & PHYSICAL PROPERTIES \\
\hline 3 & 89600 & 58000 & 50.00 & 0.00
\end{tabular}

\begin{tabular}{c|cc}
\hline ITEM \# & & SLIPPLEMENTAL INFORMATION \\
\hline 3 & GRAIN SIZE: ACTUAL NO. 6.5 &
\end{tabular}

SOLUTION ANNEALED, WATER QUENCHED .

ASME SA403 2004 ED; ASTM A403-O6; EN 10204/3.1 ASME B16.9

THE FITTINGS WERE MANUFACTURED, SAMPLED, TESTED AND INSPECTED IN

ACCORDANCE WITH THE SPECIFICATIONS AND FOUND TO MEET THE REQUIREMENTS.

THE MATERIAL WAS MANUFACTURED, TESTED AND INSPECTED WITHOUT MERCURY CONTAMINATION. NO WELD REPAIRS.

NACE MR0175

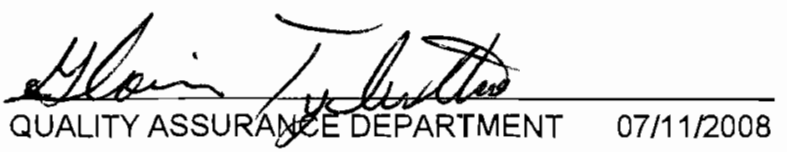




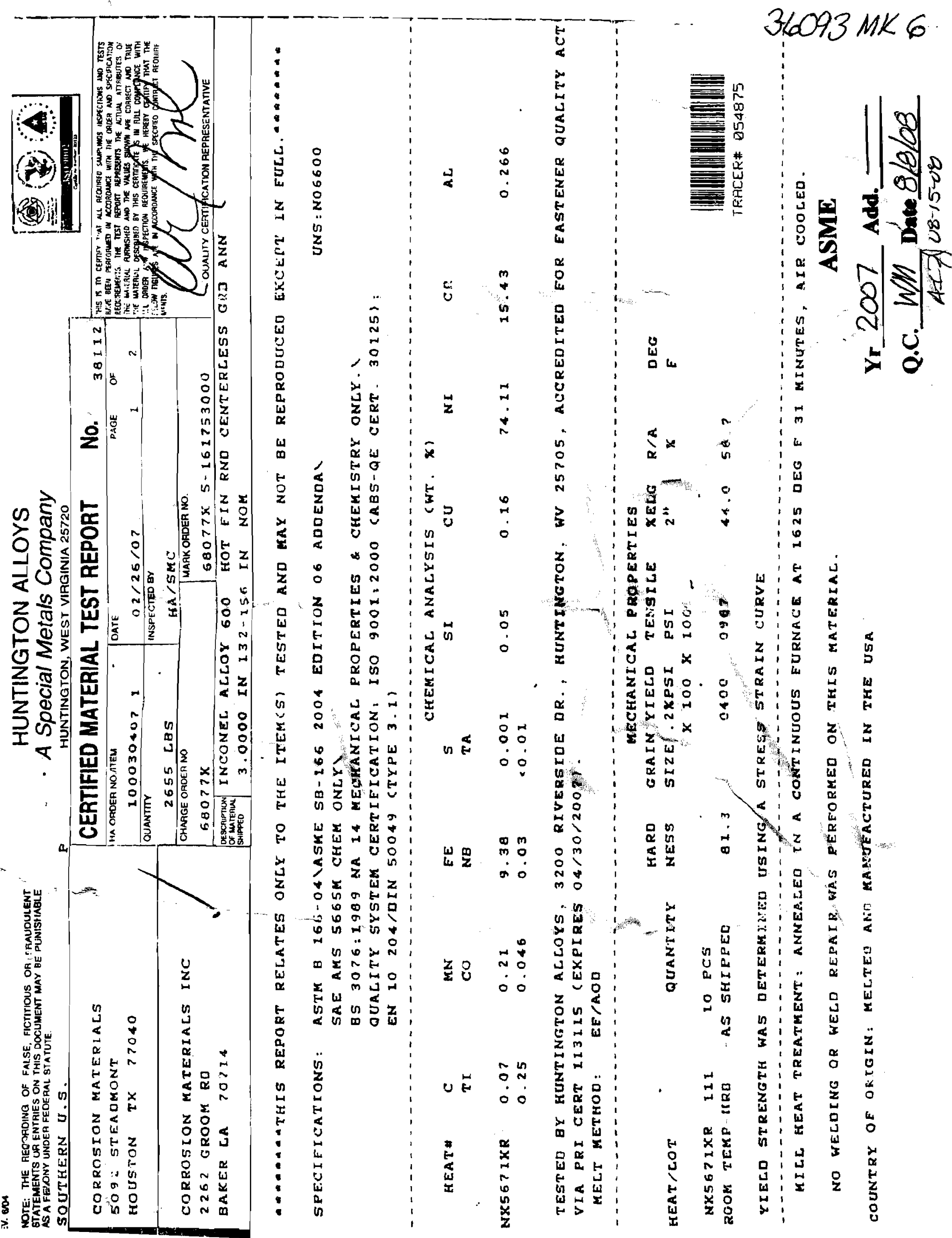




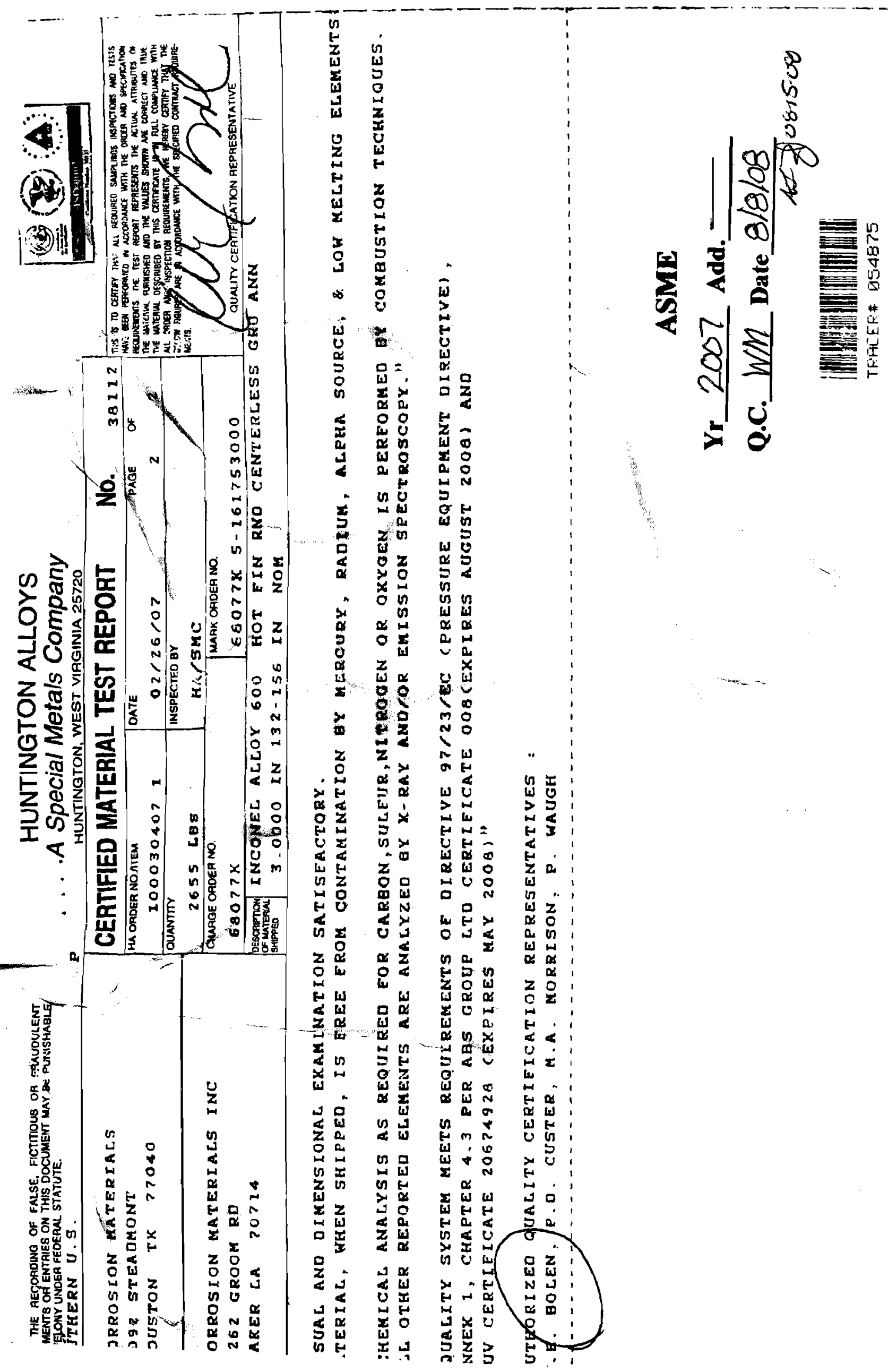


$P 0.3023 \%$

PENNSYLVANIA MACHINE WORKS, INC. 201 BETHEL AVENUE ASTON, PENNSYLVANIA 19014 (610) 497-3300 - FAX (610) 497-3325 www.pennusa.com

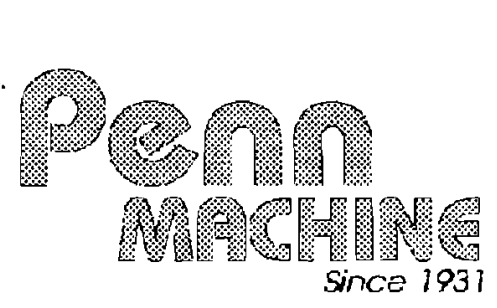

$93 \mathrm{mk} 7$

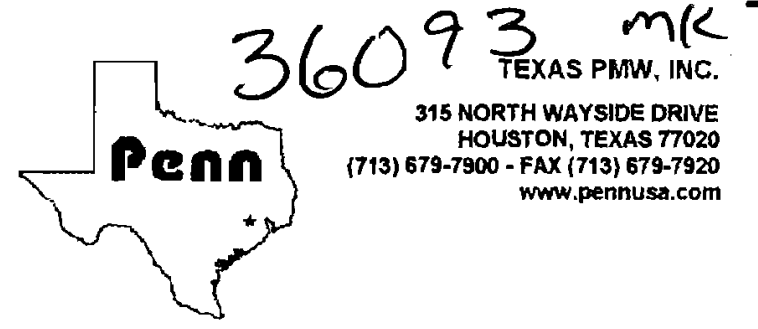

M I L L T E S T

$\begin{array}{llllll}\text { R } & \text { E } & \mathbf{O} & \mathrm{O} & \mathbf{R} & \mathbf{T}\end{array}$

ROBERT-JAMES - TWINSBURG

P.O. BOX 590

T'WINSBURG, OH 44087

P.O. cg4571

ORDER \# 01411275

$6 / 19 / 08$

$1 / 2$ 6MTD HFCP $316 \mathrm{H}$

QTY : $\quad 6$

HEAT CODE : $\mathbf{B 6 1 9 4 1}$

SPECIFICATION: A182

HEAT TREATMENT: SOLUTION ANNEALED

MATERIAL: F316H

UNS NUMBER: S31609

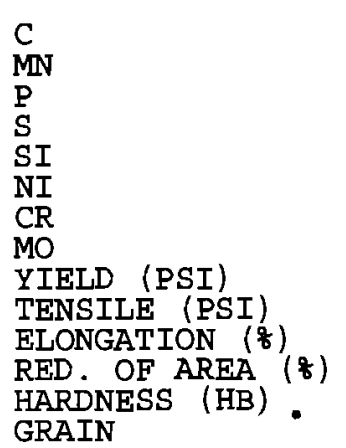

ACTUAL MEASUREMENT

.046

1.680

.029

.025

.280

10.560

17.450

2.040

37500

81500

58.000

77.000

137

4.50
SPEC. YEAR: 04

SPECIFICATION

$\begin{array}{rr}\text { MIN } 8 & \text { MAX } \% \\ .040 & .100\end{array}$

$.040 \quad 2.000$

.045

.030

1.000

$10.000 \quad 14.000$

$16.000 \quad 18.000$

2.000

30000

75000

30.000

50.000

6.00
SPEC. REV :

\section{SOLUTION ANNEALED}

ACTUAL MEASUREMENT $1900 \mathrm{~F}$

\section{SPECIFICATION \\ MINIMUM}

MATERIAL I/A/W ASME-SEC.II SA SPECIFICATION-CERTIFICATE I/A/W EN10204 TYPE 3.1

MATERIAL MEETS NACE-MRO175-2003

MATERIAL IS CAPABLE OF PASSING A CORROSION TEST TO ASTM-A262 PRACTICE "E"

MATERIAL MEETS NACE-MRO103-2007

MERCURY FREE MATERIAL - NO WELD REPAIRED MATERIAL - MADE IN THE U.S.A.

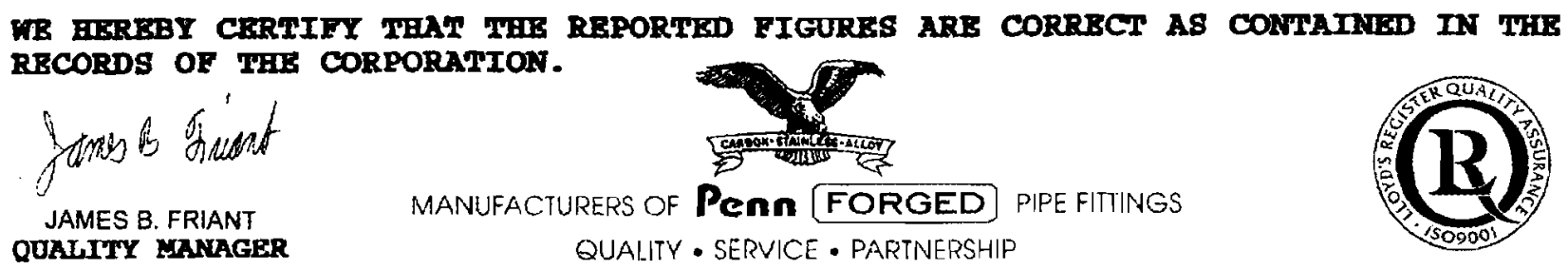


687 COUNTY ROAD 2201

CLEVELAND, TX 77327

PH (281) 727-7000 FAX (281) 727-7062

\section{CERTIFIED MATERIAL TEST REPORT}

CERTIFIED IAW EN10204 3.1 (MADE IN USA)

\begin{tabular}{|c|c|c|c|}
\hline TO: GASPAR INC. & & WFF SO NO: & 11752 \\
\hline PO: $\quad 30214$ & & HEAT NO: & 23988 \\
\hline SPECIFICATIONS: & $\begin{array}{l}\text { ASME SA182 F316H } \\
\text { DIM/TOL PER ASME B16.5 }\end{array}$ & & \\
\hline
\end{tabular}

\begin{tabular}{|c|l|}
\hline OTY & TEM DESCRIPTION \\
\hline 5 & 2" $1500 \#$ WN RF S80 \\
\hline & PROJECT\# 36093-8 \\
\hline & \\
\hline & \\
\hline & \\
\hline
\end{tabular}

\begin{tabular}{|c|c|c|c|c|c|c|c|c|c|}
\hline TYPE & $\mathbf{C}$ & $\mathbf{M N}$ & $\mathbf{P}$ & $\mathbf{S}$ & $\mathbf{S I}$ & $\mathbf{C R}$ & $\mathbf{N I}$ & $\mathbf{M O}$ & $\mathbf{N}$ \\
\hline F316H & .047 & 1.45 & .031 & .001 & .54 & 16.20 & 10.10 & 2.13 & .043 \\
\hline MILL & & & & & & & & & \\
\hline
\end{tabular}

\begin{tabular}{|c|c|c|c|c|c|c|}
\hline HEAT No, & VIELD . $\%$ OFF-PSI & TENSIGE-PSI & \% ELONG. & $\%$ RED AREA & HARDNESS & GRAN SIZE. (ASTM) \\
\hline 23988 & 41,950 & 82,050 & 63.7 & 81.1 & & No. 5.0 \\
\hline & & & & & & \\
\hline & & & & & & \\
\hline
\end{tabular}

- NO WELDING PERFORMED ON THE FORGINGS OF THIS ORDER.

- MATERIAL HEREIN IS CERTIFIED FREE FROM ANY FORM OF MERCURY, RADIUM OR ALPHA PARTICLE CONTAMINATION

THROUGHOUT ALL PHASES OF MANUFACTURE AND SHIPMENT.

- THE FORGINGS ON THIS ORDER CONFORM TO THE SPECIFIED DIMENSIONAL REQUIREMENTS.

- MATERIAL MANUFACTURED IAW WFF QA PROGRAM QAM-WF07.0702.

- Solution annealed @ $1950^{\circ} \mathrm{F}$ and water quenched to below $300^{\circ} \mathrm{F}$ within less than three minutes.

WE CERTIFY THE MATERIAL DESCRIBED HEREIN HAS BEEN MANUFACTURED, TESTED AND EXAMINED IAW AND MEETS ALL THE REQUIREMENTS OF ABOVE PURCHASE ORDER AND REFERENCED SPECIFICATIONS.

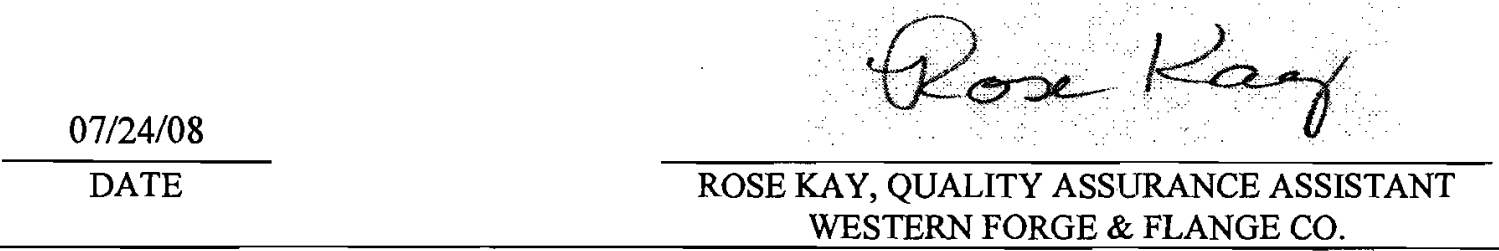

This document shall not be reproduced, except in full, without written approval of Western Forge \& Flange. The recording of false, fictitious or fraudulent statements or entries on this document may be punishable as felony under federal statutes. 

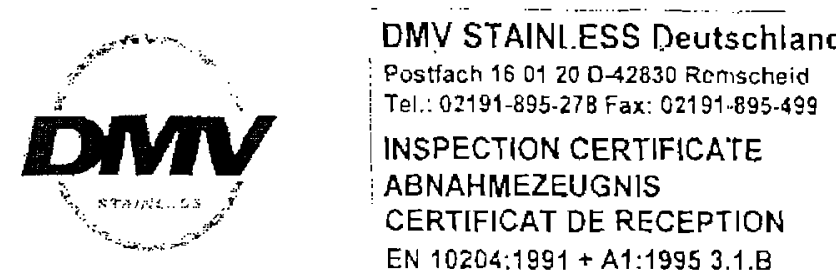

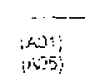

Nr.No/N". (403)
$01156-050613$
0080067815
Shatt / $909=\quad 1 / 2$

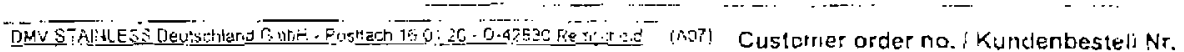
DMV STAINLESS USA INC. 12050 W. LITTLE YORK No de cornmande client:

USA - HOUSTON TX 77041
USA

\section{2}

(AOB) DNV order no / OMV Auftrags-Nr. I No. de cominande OMV

\section{5}

(8202124/005)

Part number:

\section{(Ais) Purchaser/Basteller/Client}

Grade and Specifications

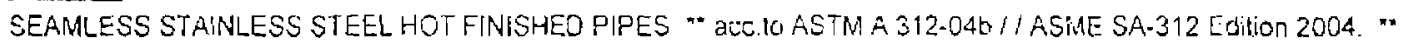
HEAT TREATED CONDITION ACC.1O STANDARD and SPECIFICATION * PICKLED * PLAII ENDS, SQUATE CUT, DEBURRED * Size tolerances acc. to ASTM A 312-04b ** RANDOM LENGTH 17,000 - 24,000 feet ** Ouantity Talerance $+10,0 / 10.0 \% *$ TP316H $:$ TP316 $\cdots$

Nahtiose warmgefertigte Edelstahlronre ** nach ASTM A 312-04b / I ASME SA-312 ED. 04. * wärmebehandelt ** gebeizt * glath, senkrecht geschnilten, emigralet "* Aomessungstoleranzrorm. ASTM A 312-04b ** Herstellïnge 17,000-24.000 fee: ** Mengentoleranz + 10,0i 10,0\% * TP316H/TP316 *

PIPES ACIER INOX SANS SOUDURE FINIS A CHAUD * SelON ASTM A 312-04D//ASME SA-312 ED. O4. * A LEIAT TRAITE SELON NORME ET SPECIFICATION. " DECAPE * ExLímItés LISSES, COLPEES D'EQUERRE. EBAVUREES " Tolérances dimensiommelles selon ASTM A 312-040" LOVGUEURS COURANTES 17.003 - 24,000 pieds ** Tolerance sur quantite $+10,0 /-10.0 \% *$ TP3 $16 \mathrm{H} / \mathrm{TP}^{2} 16^{* *}$

Mariking of the product / Kennzeichung/Marquaqes

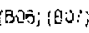

DMV-D - TP $316 H$ - TP 316 - HEAT - 2 X SCH BOS AW . ASTM A 312 - ASME SA 312 - LOT NO - SML - GERMANY - WA.

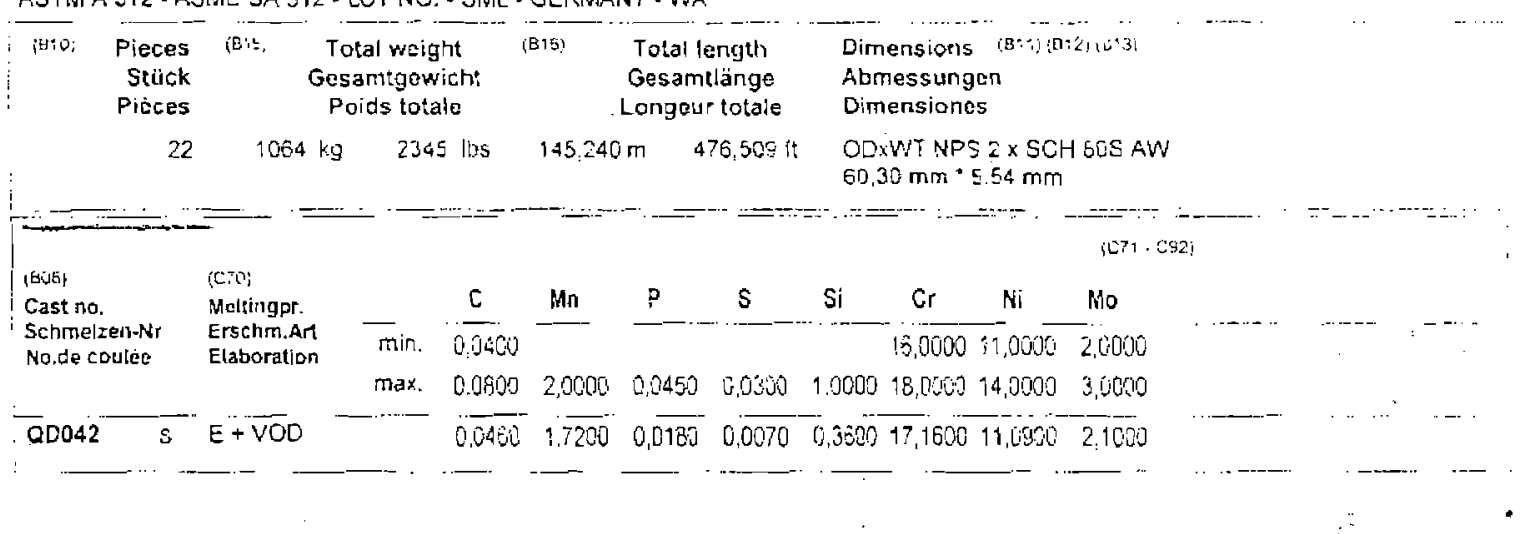

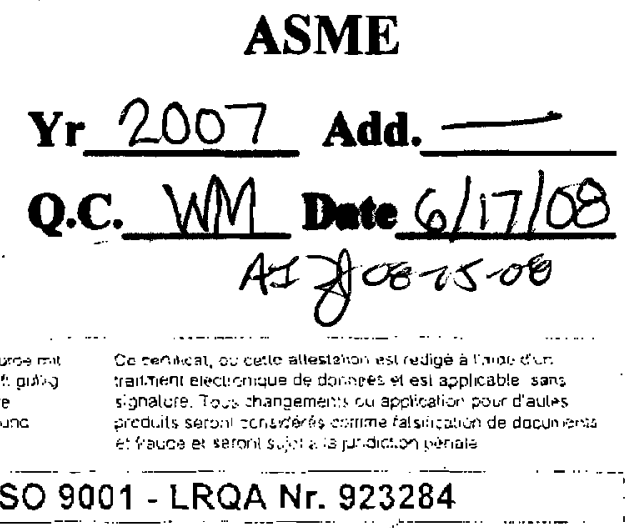

Trus terthicite is is suec by a no ilorized synlum and is

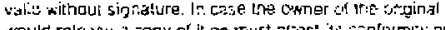

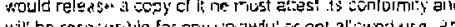

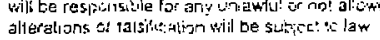

-.. ...... - -

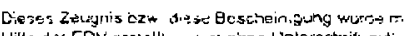

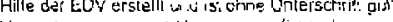

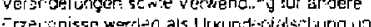
Serecing

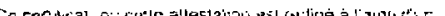

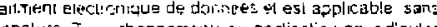

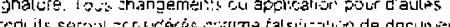

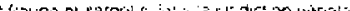




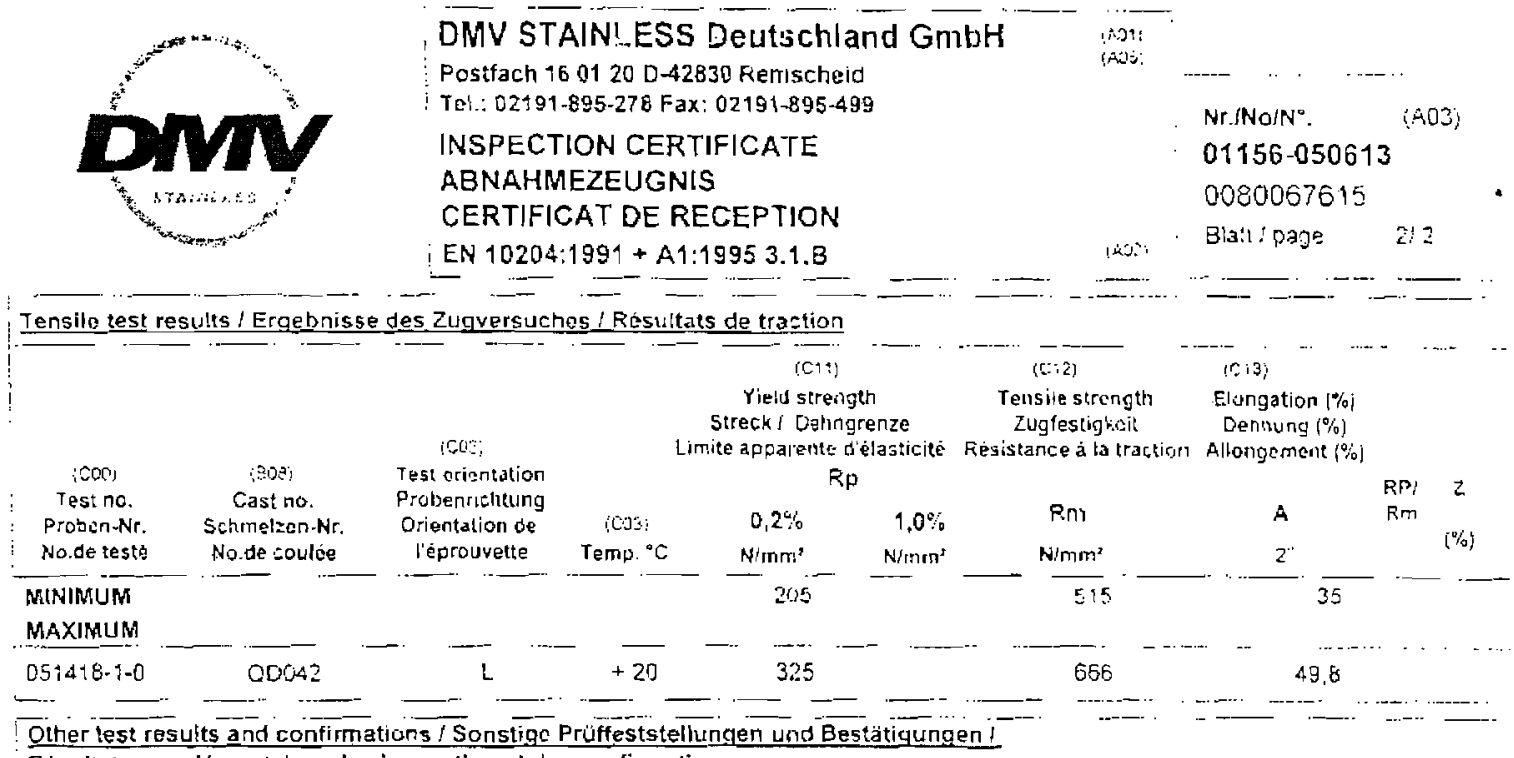

Tensilo test results / Ergebnisse des Zugversuches / Resultats de traction

Other test results and confirmations / Sonstion Pruffeststellungen und Bestätigungen I

Résultats supplementaires des inspection et des confimations

VISUAL AND DIMENSIONAL INSPECTION SATISFACTORY (DO1)

MATERIAL IDENTIFICATION TEST OF ALL TUBES BY SPECTROGRAPHIC ANALYSIS: SATISFACTOPY

FLATTENING TEST: SATISFACTORY :C5O)

GRAIN SIZE ? ACC. TO ASTME 112

HYDROSTATIC TEST, 170 BAR (1BAR=10DKPA): SATISFACTORY

HEAT TREATMENT: SOLUTION HERT TREATED

BESICHTIGUNG UND MASSKONTROLLE: EESTANDEN :O01)

VERWECHSLUNGSPRUEFUNG ALLER ROHRE DURCH SPECTRAL-ANALYSE: BESTANDEN

RINGFALTVERSUCH: BESTANDEN (C5O)

KORNGROESSE 7 GEMAESS ASTM E 112

INNENORUCKVERSUCH MIT WASSER 170 EAR (13AR=10OKPA). BESTANDEN

WAERMEBEHANDLUNG: LOESUNGSGEGLUEHT

INSPECTIONET CONTROLE DIMENSIONEL: SATISFNISANT (DO1)

ESSAI ANTI MELANGE SUR TOUS LES TUBES PAR ANAL YSE SPECTRALE. SATISFAISANT

ESSAI D'APLATISSEMENT: SATISFAISANT (C50)

DINENSION DU GREIN 7 CONFORME ASTME 112

ESSAJ HYDRAULIQUE: 170 BAR 1 16AR=100KPA): PASSE

TRAITEMENT THERMIOUE: RECUIT

The products have been tested in accordance with the purchase specification and are found to be satisfied.

Wir bestätigen, das die Erzeugnisse bestellungsgemäß geprüft und für in Ordnung belunden wurden.

Nous attestons que les produits livrés sont conformes aux stipulations de ia commande

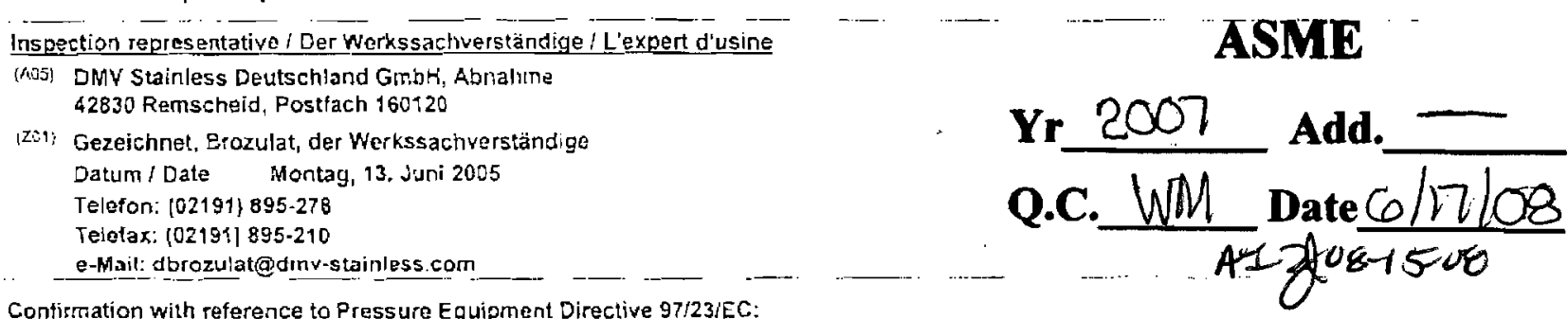

Confirmation with reference to Pressure Equipment Directive 97/23/EC:

The works operates a quality management system that has undergone a specific assessment for materials for pressure equipment and is certified by a competent body (TÜV-Cert No: 01202811/Q-020025)

Bestätigung in Bezug auf Druckgeräterichtlinie 97/23/EG:

Das Werk wendet ein Qualitätsmanagementsystom an, das in Bezug auf Werkstoffe für Oruckgeräte einer spezilischen Bewertung Lnterzogen wurde und yon einer zuständigen Stelle (TÜV-Cert.No: 01202811/Q-020025) zertifiziert ist.

Confirmation concermant la Dircctive Equipements sous Pression 97/23/EC

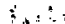

L'usine applique un systéme de management de la qualité qui a fait lobjet d'une évaiuation spécifique pour les matériauz pour équipements sous pression etc qui est certifié par un organisme compétent

(TUeV.Cert.No: 01202811/Q-020025)

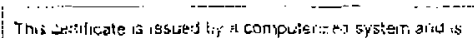

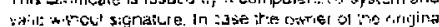

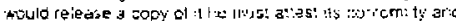

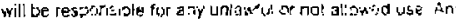

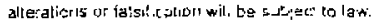

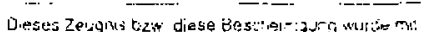

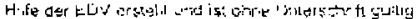

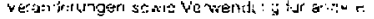

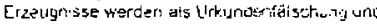

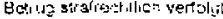

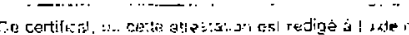

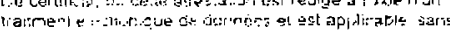

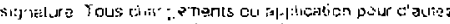

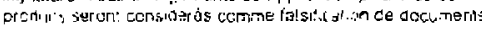

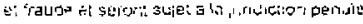


SOLD TO:

ROBERT JAMES SALES, INC.

P.O. BOX 590

TWINSBURG, OH 44087
SALES ORDER \# : 0000012240
CUST P.O.\#: CG4572

\begin{tabular}{l|l|l}
\hline ITEM \# & QTY & ITEM DESCRIPTION \\
\hline
\end{tabular}

212 LR 90 S/80 WP316HS AVSA403
TAG NUMBER: CG4572, PPD-CHG

SHIP TO:

GASPAR INC.

1545 WHIPPLE AVE S.W!

CANTON, OH 44710

\begin{tabular}{|c|c|c|c|c|c|c|c|c|c|c|c|c|c|c|c|}
\hline \multirow[b]{2}{*}{ ITEM \# } & \multirow[b]{2}{*}{ HEAT CODE } & \multicolumn{13}{|c|}{ CHEMICAL ANALYSIS } & \multirow[b]{2}{*}{ Co } \\
\hline & & C & $\mathrm{Mn}$ & $P$ & $S$ & Si & $\mathrm{Ni}$ & $\mathrm{Cr}$ & Mo & $\mathrm{Cu}$ & $\mathrm{Cb}$ & $\mathbf{V}$ & Al & $\mathbf{N}$ & \\
\hline 2 & MPMK & 0.06 & 1.14 & 0.037 & 0.011 & 0.49 & 12.36 & 16.80 & 2.12 & & & & & & 0.096 \\
\hline
\end{tabular}

\begin{tabular}{c|llll}
\hline & \multicolumn{3}{|c}{} & PHYSICAL PROPERTIES \\
\cline { 2 - 5 } & $\begin{array}{l}\text { TENSILE } \\
\text { (PSI) }\end{array}$ & $\begin{array}{l}\text { YIELD } \\
\text { (PSI) }\end{array}$ & $\begin{array}{l}\text { ELONG } \\
\text { (2 IN) }\end{array}$ \\
\hline ITEM \# & & & & \\
\hline 2 & 80400 & 41800 & 49.00 & 0.00
\end{tabular}

\begin{tabular}{c|c}
\hline ITEM \# & SUPPLEMENTAL INFORMATION \\
\hline 2 & GRAIN SIZE: MPMK-ACTUAL:DUPLEX ASTM NO. 6 \& ASTM NO. 4.0
\end{tabular}

SOLUTION ANNEALED, WATER QUENCHED .

ASME SA403 2004 ED; ASTM A403-O6; EN 10204/3.1 ASME B16.9

THE FITTINGS WERE MANUFACTURED, SAMPLED, TESTED AND INSPECTED IN

ACCORDANCE WITH THE SPECIFICATIONS AND FOUND TO MEET THE REQUIREMENTS

THE MATERIAL WAS MANUFACTURED, TESTED AND INSPECTED WITHOUT MERCURY

CONTAMINATION. NO WELD REFAIRS.

NACE MR0175

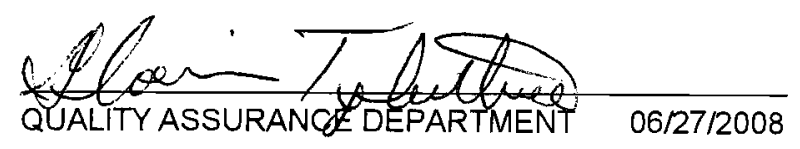




\section{MK 12}

DMV STAINLESS Italia s.r.I

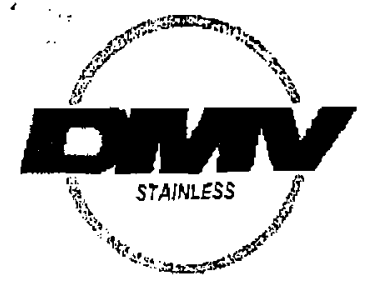

Via Pio, 3024062 Costa Volpino (BG)

Tel : 035.975.611 - Fax :035.971624

e-mail: dmvitaly@dmv-stainless.com

INSPECTION CERTIFICATE

3.1.B - EN 10204 : 1991+A1 : 1995

Purchaser

Address

Customers Order

DMV Ref. Order / Item

Part number
DMV STAINLESS USA INC. .

12050 W. LITTLE YORK HOUSTON.

0D00211080 date 09/03/2004.

$0,000211107 / 000009$.

N.A.

Product

Seamless Stainless Steel Hot Finished Pipes Solution Annealed Pickled

Plain Ends Square Cut Deburr

Specification

Grade

Tolerances

Marking

Heat

54446

Tot.

Heat 54446 Melting Process

Chemical analisys (\%)

\begin{tabular}{|l|rrrrrrrrr}
\hline \\
Min & \multicolumn{2}{c}{$\mathbf{C}$} & Si & Mn & $\mathbf{P}$ & $\mathbf{S}$ & $\mathbf{C r}$ & $\mathbf{M o}$ & $\mathbf{N i}$ \\
Max & 0.040 & & & & & 16.000 & 2.000 & 11.000 \\
Heat & 0.080 & 1.0000 & 2.00 & 0.045 & 0.0300 & 18.000 & 3.000 & 14.000 \\
\hline
\end{tabular}

Mechanical and Metallurgical Properties

Tensile test (at $20^{\circ} \mathrm{C}$ )

$\begin{array}{lrrrr} & \text { Direct. } & \text { Y.S. } 0,2 \% & \text { U.T.S. } & \text { El } 50 \mathrm{~mm} \\ \text { Long/Trans } & \mathrm{ksi} & \mathrm{ksi} & \% \\ \text { Min } & & 30,00 & 75,00 & 35.00 \\ \text { No } 175148 & \text { L } & 48.62 & 93.99 & 48.00\end{array}$

FLATTENING TEST

Required

ASTM A 999

CORROSION TEST

Required

ASTM A 262 PR E

MICROGRAPHIC EXAMINATION

No 175148 Grain size required max 7 , result 6,5. According to ASTM E 112

No 176020 Grain size required max 7 , result 6,5. According to ASTM E 112

Other tests and declarations

Heat treatment

$1958^{\circ} \mathrm{F}$

Antimixing checked by PMI

Visual and dimensional examination

Eddy current testing

ASTM A 999

\section{ASME}

\section{Yr 2007 Add.}

Q.C. WM Date 6 67108

$$
\text { AI 2008-15:08 }
$$

Result OK

Result OK 


\section{DMV STAINLESS Italia s.r.I}

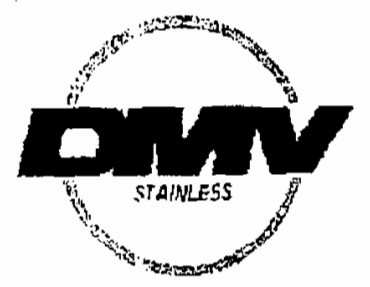

Via Pio, 3024062 Costa Volpino (BG)

Tel : 035.975.611 - Fax :035.971624

8-mail: dmvltaly@dmv-stainless.com

\section{INSPECTION CERTIFICATE}

3.1.B - EN $10204: 1991+A 1: 1995$

\section{No weld repair}

Tubes are free from mercury contamination

We certify that the delivered products comply with the request of the order

Corrosion test acc.to MIL-P-2469, 3: satisfactory

Date 09-Sep-2004

Mill inspector F. RAZZITT!

\section{ASME \\ Yr 2007 Add. \\ Q.C. WM Date $6 / 17 / 08$ \\ AI $80,15.00$}

Confirmation with reference to Pressure Equipment Directive 97123/EC:

The works operates a quality management system that has undergone a specific assessment for materials for pressure equipment and is certified by a competent body (TUV-CERT.No: 7012002/NUC)

This certificate is issued by a computerized system and is valid without signature. In case the oumer of the original conificato wouid release a copy of he must altest its conformity to the original one, taking upon himself the responsibility for any anlawful or not allowed use. Any alteration and/or falsification will be subject to the law 


\section{Certificate of Compliance/Typlcal Materlal Certfflcation (EN 10204-2.2)}

\begin{tabular}{|c|c|c|}
\hline Dlstrlbutor & Customer & Customer PO\# \\
\hline $\begin{array}{l}\text { Abbott Valve \& Gitting Co. } \\
8090 \text { Cochran Rosd } \\
\text { Solon, OH } 44139\end{array}$ & $\begin{array}{l}\text { Gaspar } \\
1545 \text { Whipple Ave SW } \\
\text { Canton, OH. } 44710\end{array}$ & 30188 \\
\hline No. Part Number & Qty & \\
\hline
\end{tabular}

Bwagelok products referenced above are manufactured from material purchased and certifted as being in aceordancs with the specification(s) listed bslow.

The material stipulations included in this certification do not inelude such components as shap plngs, Eprings, balls, a-rings, gaskets, Jam nuts, space collars, seals, locking dogs, lanyards, or sleeves.

Stainlèss steel materlal has passed the Intergranular Corrosion Test requirements of EN ISO 3851-2, Melhod A, and/or ASTM A-262 Practice A or E.

All parte were cleaned and peckaged in accordance with Swagelok Specilications.

Typical mechanical andior chemical analysis of the material used in the manufacture of the swagelok produets involved are ifisted below. These values are average values determined from a sample of certified material test reporta, Actual values for a materlal heat may vary from those indicated.

\begin{tabular}{lll} 
& \multicolumn{2}{c}{ MATERIAL \$TANDARDS } \\
Components & Materlal & Standards \\
\hline Shaped Fittings & F316 Stainless Sterl Forglings & A\&TM A1B2, ASME BA1B2 \\
Straight Fittings & 316 Stalnless Steel Bar & A8TM A276, ASTM A47\%, ASME SA479
\end{tabular}

\begin{tabular}{|c|c|c|c|c|c|c|c|c|c|c|}
\hline \multicolumn{11}{|c|}{ MECHANICAL PROPERTIES } \\
\hline Components / Materials & \multicolumn{2}{|c|}{$\begin{array}{l}\text { Yield Sirength } \\
(\mathrm{ks} / \mathrm{MP} a)\end{array}$} & \multicolumn{3}{|c|}{$\begin{array}{c}\text { Tensile Strength } \\
(\mathrm{ksi} / \mathrm{MPa})\end{array}$} & \multicolumn{2}{|c|}{$\begin{array}{c}\text { Elongation } \\
(\%)\end{array}$} & $\begin{array}{c}\% \text { Red. } \\
\text { Area }\end{array}$ & \multicolumn{2}{|c|}{ Hardness } \\
\hline $\begin{array}{l}\text { Shaped Fittings } \\
\text { Straight Fittings }\end{array}$ & & $48 / 331$ & & \multicolumn{2}{|c|}{$90 / 621$} & $\begin{array}{l}31 \\
38\end{array}$ & & $\begin{array}{l}76 \\
73\end{array}$ & \multicolumn{2}{|c|}{ HRC 22} \\
\hline \multicolumn{11}{|c|}{ CHEMICAL ANALYSIS } \\
\hline Components / Materlals & $\mathrm{c}$ & $\mathrm{Cr}_{\mathrm{r}}$ & $\mathrm{Mn}$ & Mo & $\mathbf{N}$ & Ni & $\mathbf{P}$ & 8 & $\mathrm{Si}$ & co \\
\hline $\begin{array}{l}\text { Shaped Fittings } \\
\text { Stralght Fittings }\end{array}$ & $\begin{array}{l}0.043 \\
0.040\end{array}$ & $\begin{array}{l}17.16 \\
17.51\end{array}$ & $\begin{array}{l}1.57 \\
1.71\end{array}$ & $\begin{array}{l}2.09 \\
2.27\end{array}$ & $\begin{array}{l}0.042 \\
0.037\end{array}$ & $\begin{array}{l}12.28 \\
12.99\end{array}$ & 0.028 & $\begin{array}{l}0,025 \\
0,025\end{array}$ & $\begin{array}{l}0.60 \\
0.60\end{array}$ & 0.23 \\
\hline
\end{tabular}

The Swagelok product specified above were manufactured in accordence with Swagelok Company's Ouality Assurance Manual (lalest revision, rev|slon $H_{1}$ dated December 10, 2007). Swagelok Compeny's Qually System is approved to ISO 9001 (BSt Certificate \# FMO1729). 
$\% \%[$ [TO:1-330-425-9395, GASPAR, INC, DAVE C]

Robert James Sales

Twinsburg, $\mathrm{OH} 44087$

330-425-9116

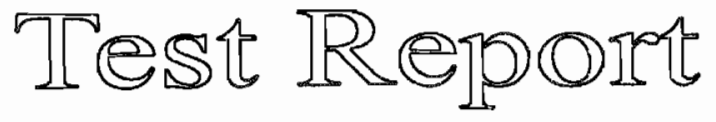

Date : 09/03/08

CG6154

509542-CCP

To: GASPAR, INC

1545 WHIPPLE AVE SW

CANTON, OH 44710

ATTN: BOB | 830879

\begin{tabular}{|c|}
\hline PRODUCT DESCRIPTION \\
\hline $1 / 2 \times 065(16 \mathrm{GA}) \mathrm{ST} 316$ \\
\hline
\end{tabular}

Heat \# :

J0210-154

Misc Note 1 :

Spec :

ASTM A213/269

Misc Note 2 :

Finish:

A\&P CF

C :

0.042

Eddy Hydro :

OK

$\mathrm{Si}$ :

0.430

Yield (psi) :

37000

Mn :

0.740

Tensile (psi) :

89000

$P$ :

0.035

Elong (\%/2") :

50.000

$S$ :

0.010

Hardness RB :

88

$\mathrm{Ni}$ :

11.150

Flattening :

OK

$\mathrm{Cr}$ :

16.350

Flaring :

OK

Mo:

2.150

Rev Flt/Bend:

$\mathrm{Ti}$ :

$\mathrm{Cb}+\mathrm{Ta}$ :

Flange:

OK

Int. Cor/Embr :

$\mathrm{Fe}$ :

Passiv. :

$\mathrm{Cu}$ :

Al :

Grain Size :

Misc Note:

A/SA213-99A, A26901

N:

Co:

Certified - The above information is an extract of data furnished to us by the producing mill.

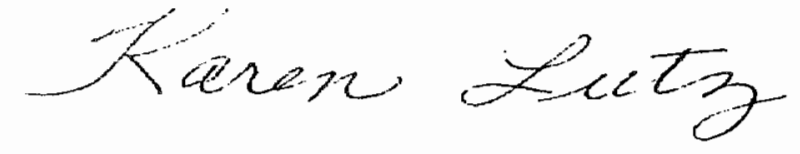

Authorized Signature

ASME

$\% \%[S E N D]$

Yr.2007 Add. $2008 \mathrm{a}$

Q.C.WII Date $9 / 8 / 08$ 
687 COUNTY ROAD 2201

CLEVELAND, TX 77327

PH (281) 727-7000 FAX (281) 727-7062

\section{CERTIFIED MATERIAL TEST REPORT}

\section{CERTIFIED IAW EN10204 3.1 (MADE IN USA)}

\begin{tabular}{|ll|l|l|}
\hline TO: $\quad$ GASPAR INC. & WFF SO NO: & \multicolumn{1}{c|}{ 11752 } \\
\hline PO: 30214 & ASME SA182 F316 & HEAT NO: & 8688074 \\
\hline SPECIFICATIONS: & DIM/TOL PER ASME B 16.5 & & \\
\hline
\end{tabular}

\begin{tabular}{|c|l|l|}
\hline OTY & \multicolumn{1}{|c|}{ TTEM DESCRIPTION } \\
\hline 1 & 2" 1500\# BL RF \\
\hline & PROJECT\# 36093-16 \\
\hline & \\
\hline & \\
\hline & \\
\hline
\end{tabular}

\begin{tabular}{|c|c|c|c|c|c|c|c|c|c|}
\hline TYPE & $\mathbf{C}$ & MN & P & S & SI & CR & NI & MO & N \\
\hline F316 & .047 & 1.70 & .026 & .02 & .43 & 16.86 & 10.21 & 2.10 & .049 \\
\hline MULL & & & & & & & & & \\
\hline
\end{tabular}

\begin{tabular}{|c|c|c|c|c|c|c|}
\hline HEATNO & YIEU D $2 \%$ OFEPSI & TENSII E PSI & \%LLONG & \%RED AREA & H ARDNESS & GRANSWE (ASTM) \\
\hline 8688074 & 40,700 & 81,360 & 57.8 & 55.7 & & \\
\hline & & & & & & \\
\hline & & & & & & \\
\hline
\end{tabular}

- NO WELDING PERFORMED ON THE FORGINGS OF THIS ORDER.

- MATERIAL HEREIN IS CERTIFIED FREE FROM ANY FORM OF MERCURY, RADIUM OR ALPHA PARTICLE CONTAMINATION

THROUGHOUT ALL PHASES OF MANUFACTURE AND SHIPMENT.

- THE FORGINGS ON THIS ORDER CONFORM TO THE SPECIFIED DIMENSIONAL REQUIREMENTS.

- MATERIAL MANUFACTURED IAW WFF QA PROGRAM QAM-WF07.0702.

- Solution annealed @1950 $\mathrm{F}$ and water quenched to below $300^{\circ} \mathrm{F}$ within less than three minutes.

WE CERTIFY THE MATERLAL DESCRIBED HEREIN HAS BEEN MANUFACTURED, TESTED AND EXAMINED IAW AND MEETS ALL THE REQUIREMENTS OF ABOVE PURCHASE ORDER AND REFERENCED SPECIFICATIONS.

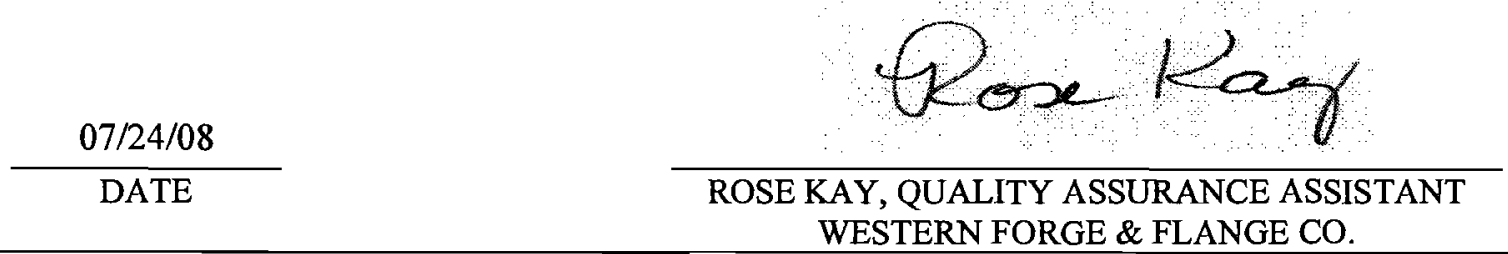

This document shall not be reproduced, except in full, without written approval of Western Forge \& Flange. The recording of false, fictitious or fraudulent statements or entries on this document may be punishable as felony under federal statutes. 


\section{REPORT \# 08/808-3 RADIOGRAPHIC INSPECTION REPORT DATE: $8 / 18 / 08$ Page 1 of $\mathrm{Z}$}

\section{CUSTOMER GASPAR}

PO\# 10978

POHELDTOCES:

WELDING PROCESS: SEE DIUG

JOB\# $36093 A$

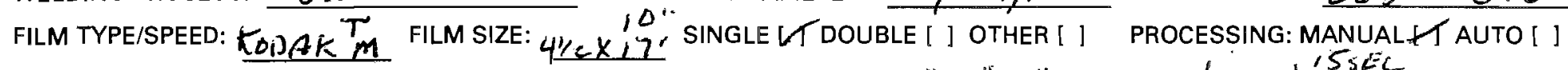

\section{CANTON,OH10}

MATERIAL TYPE: STTANLESS STEEL

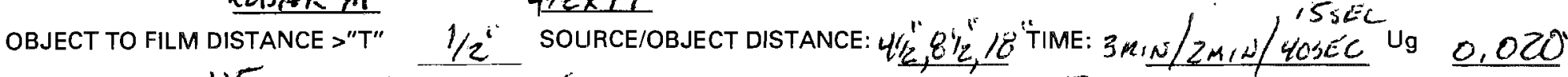

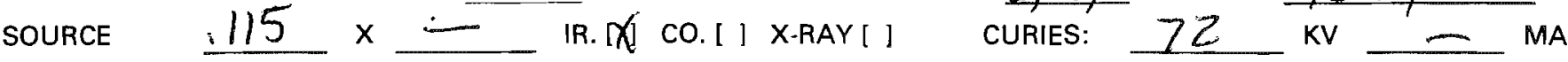
RADIOGRAPHIC TECHNIOUE: AESME SEC I ART 2 ACCEPTANCE STANDARD: $145 \mathrm{ME}$ SEC VIILC ON 51

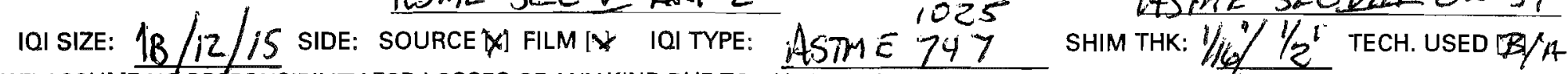
WE ASSUME NO RESPONSIBILITY FOR LOSSES OF ANY KIND DUE TO OUR INTERPRETATION OF THE QUALITY OF THE MATERIAL SUBMITTED. ALL DATA AND INFORMATION WILL BE HELD STRICTLY CONFIDENTIAL.

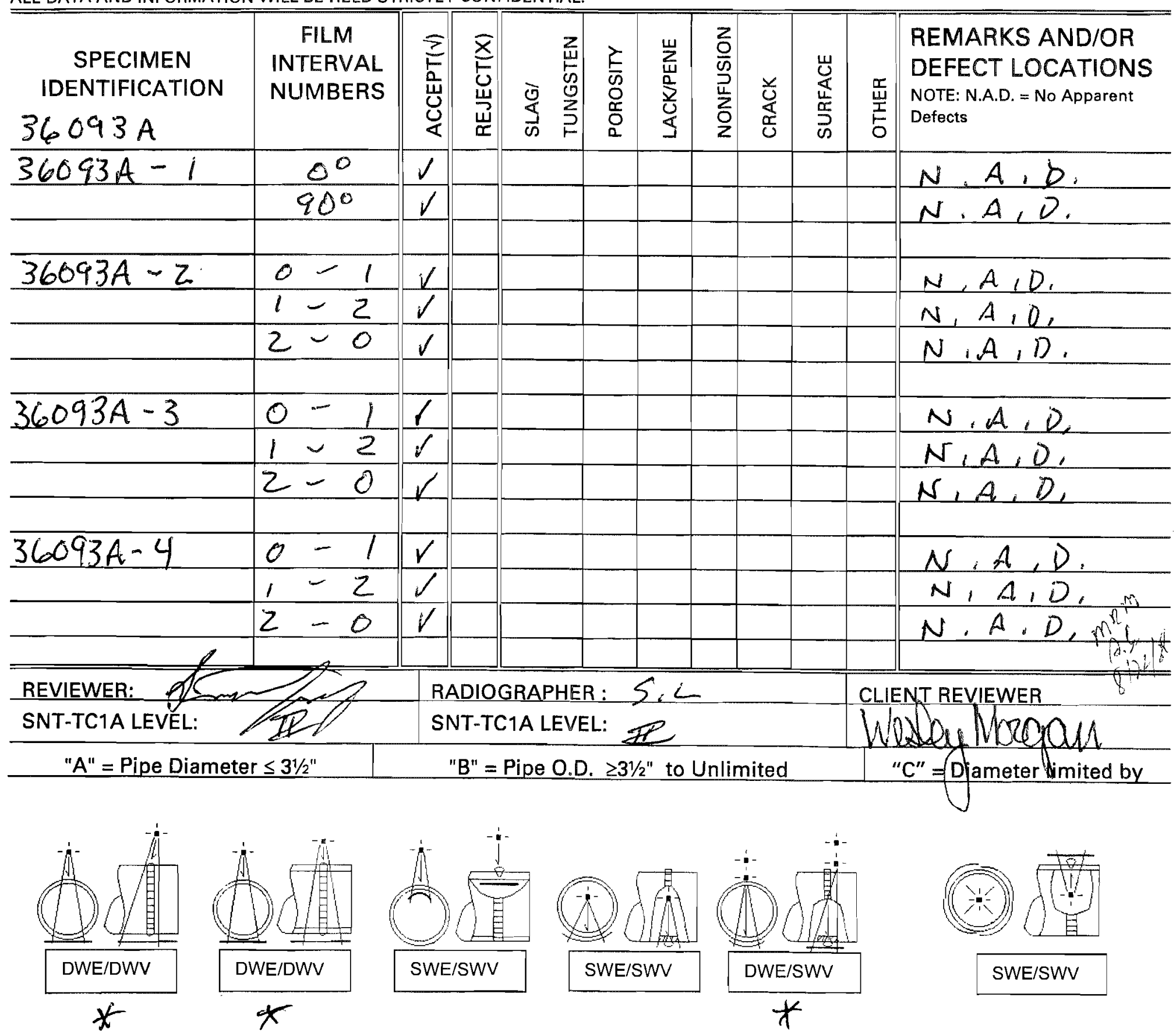


BAKER INSPECTION GROUP

\begin{tabular}{|c|c|c|c|}
\hline REPORT \#081808-4 & RADIOGRAPHIC INSPECTION REPORT & DATE: & Page $z$ of $z$ \\
\hline GASPA & & C & 01410 \\
\hline $109 \overline{78}$ & лов\# 360934 & & SOANLESS STEEL \\
\hline
\end{tabular}

WE ASSUME NO RESPONSIBILITY FOR LOSSES OF ANY KIND DUE TO OUR INTERPRETATION OF THE QUALITY OF THE MATERIAL SUBMITTED. ALL DATA AND INFORMATION WILL BE HELD STRICTLY CONFIDENTIAL.

\begin{tabular}{|c|c|c|c|c|c|c|c|c|c|c|c|}
\hline $\begin{array}{c}\text { SPECIMEN } \\
\text { IDENTIFICATION } \\
36093 \mathrm{~A}\end{array}$ & $\begin{array}{c}\text { FILM } \\
\text { INTERVAL } \\
\text { NUMBERS }\end{array}$ & 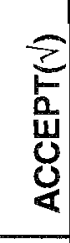 & 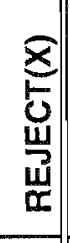 & 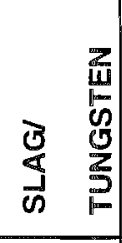 & 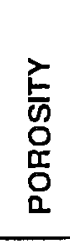 & 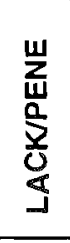 & $\begin{array}{l}\mathbf{z} \\
\frac{0}{9} \\
\frac{u}{z} \\
\mathbf{0} \\
\end{array}$ & 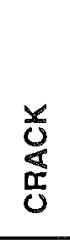 & 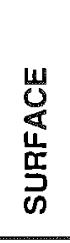 & $\begin{array}{l}\stackrel{\frac{n}{4}}{T} \\
\stackrel{5}{5} \\
\end{array}$ & $\begin{array}{l}\text { REMARKS AND/OR } \\
\text { DEFECT LOCATIONS } \\
\text { NOTE: N.A.D. = No Apparent } \\
\text { Defects }\end{array}$ \\
\hline \multirow[t]{3}{*}{$36093 A-5$} & $0-1$ & $V$ & & & & & & & $\checkmark$ & & $I, D_{r}$ \\
\hline & $1-2$ & $\checkmark$ & & & & & & & & & $N, A, D$. \\
\hline & $2-0$ & $V$ & & & & & & & & & $N, A, D$. \\
\hline \multirow{2}{*}{$36093 A-6$} & $0^{\circ}$ & $d$ & & & & & & & & & 16 \\
\hline & 900 & 1 & & & & & & & & & $N, A, D$ \\
\hline
\end{tabular}

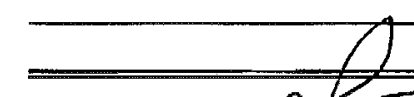

REVIEWER:

SNT-TC1A LEVEL: 


\section{GASPAR, INC. \\ LIQUID PENETRANT INSPECTION REPORT}

DATE: September 11, 2008

JOB \#: $36093 \mathrm{~A}$

PT: PROCEDURE: PT-1 REV.7

PENETRANT TYPE : $[\mathrm{X}]$ Direct Visual

ACCEPTANCE STANDARD: [X] ASNIE Sect. VIII Div.I [ ] ASME B31.1 [ ] Other -

MATERIAL IDENTIFICATION

\begin{tabular}{ll}
\hline CLEANER: & SKC-S \\
\hline PENETRANT: & SKL-SP \\
\hline DEVELOPER: & SKD-S
\end{tabular}

LIGHTING EQUIPMENT: Portable AC Halogen single light of 100 watts.

COMPONENT DESCRIPTION / PC. \#: LOWER CHAR POT

DRAWING \#: 36093 REV.2

THICKNESS / DIMIENSIONS: SEE DWG.

\begin{tabular}{|c|c|c|c|c|c|}
\hline $\begin{array}{c}\text { WELD ID OR } \\
\text { PART NUMBER }\end{array}$ & TEMP. & SURF. COND. & $\begin{array}{c}\text { ACCEPT/ } \\
\text { REJECT }\end{array}$ & $\begin{array}{c}\text { SIZE AND TYPE } \\
\text { OF INDICATION }\end{array}$ & REMARKS \\
\hline ALL PRESSURE WELDS & $70^{\circ} \mathrm{F}$ & AS WELDED & ACCEPT & NONE & \\
\hline & & & & & \\
\hline & & & & & \\
\hline & & & & & \\
\hline & & & & & \\
\hline & & & & & \\
\hline & & & & & \\
\hline
\end{tabular}

ADDITIONAL REMARKS, SKETCHES, ETC.

PERFORMED BY:

JIM FREDERICKJLEVEL II 


\section{CALCULATION PACKAGE}

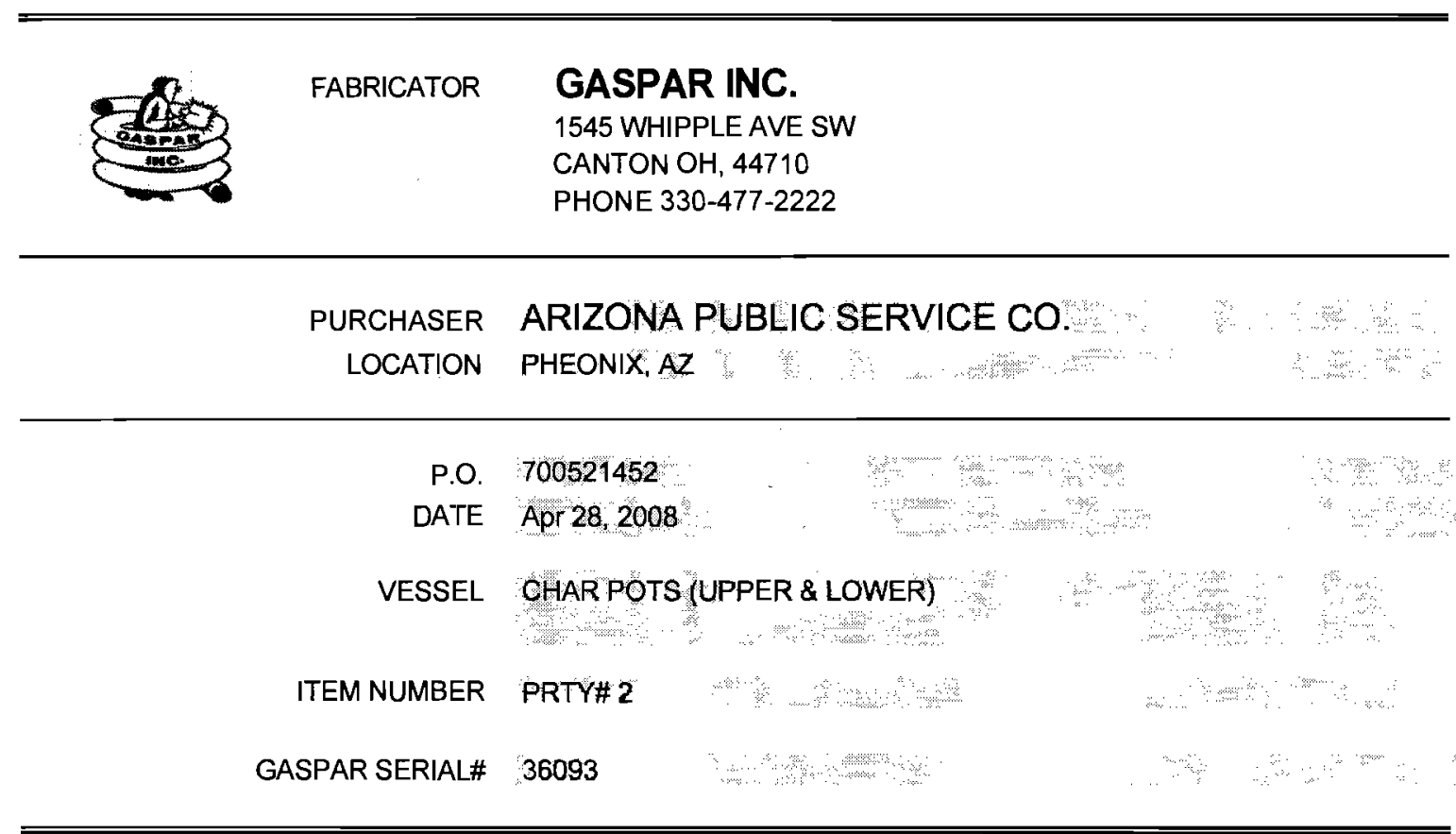

AUG 13, 2008: CHANGED LOWER CHAR POT PRESSURE TEST FROM HYDRO TO PNEUMATIC

AUG 06, 2008: 2 1/2" PIPE 316H MATERIAL CHANGED TO 3" OD INCONEL 600 MATERIAL.

\begin{tabular}{|c|c|c|}
\hline 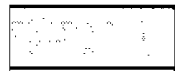 & 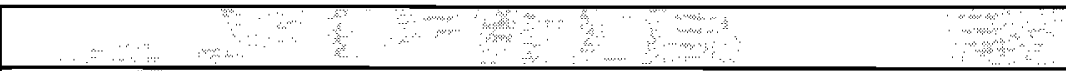 & $m$ \\
\hline$\therefore$ & s.t.n. & 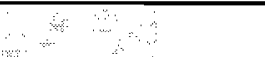 \\
\hline & 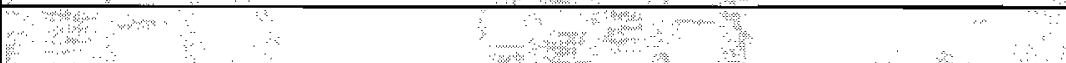 & $=3$ \\
\hline & 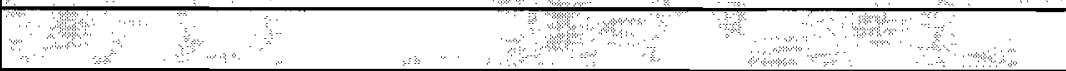 & (n) \\
\hline & 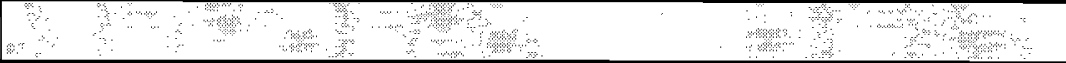 & 3. \\
\hline ste & 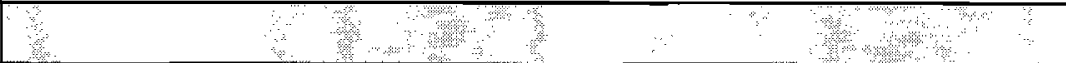 & 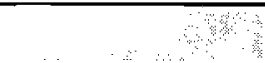 \\
\hline . & S & a \\
\hline 3 & (a) & ?as \\
\hline Aug 14,2008 & कर & Whather $<$ hiteles \\
\hline Aug 6, 2008 & SEE NOTE $\quad \vdots \quad$ (MLM) & s. \\
\hline Jul 31,2008 & CALCULATIONS PER ASME VIII-1 $\quad$ (MLM) & . \\
\hline May 8, 2008 & FOR APPROVAL & \\
\hline DATE & DESCRIPTION & BY \\
\hline
\end{tabular}


Table of Contents

\section{COMPONENTS INCLUDED IN PRESSURE BOUNDARY}

2. UPPER CHAR POT

3. LOWER CHAR POT 


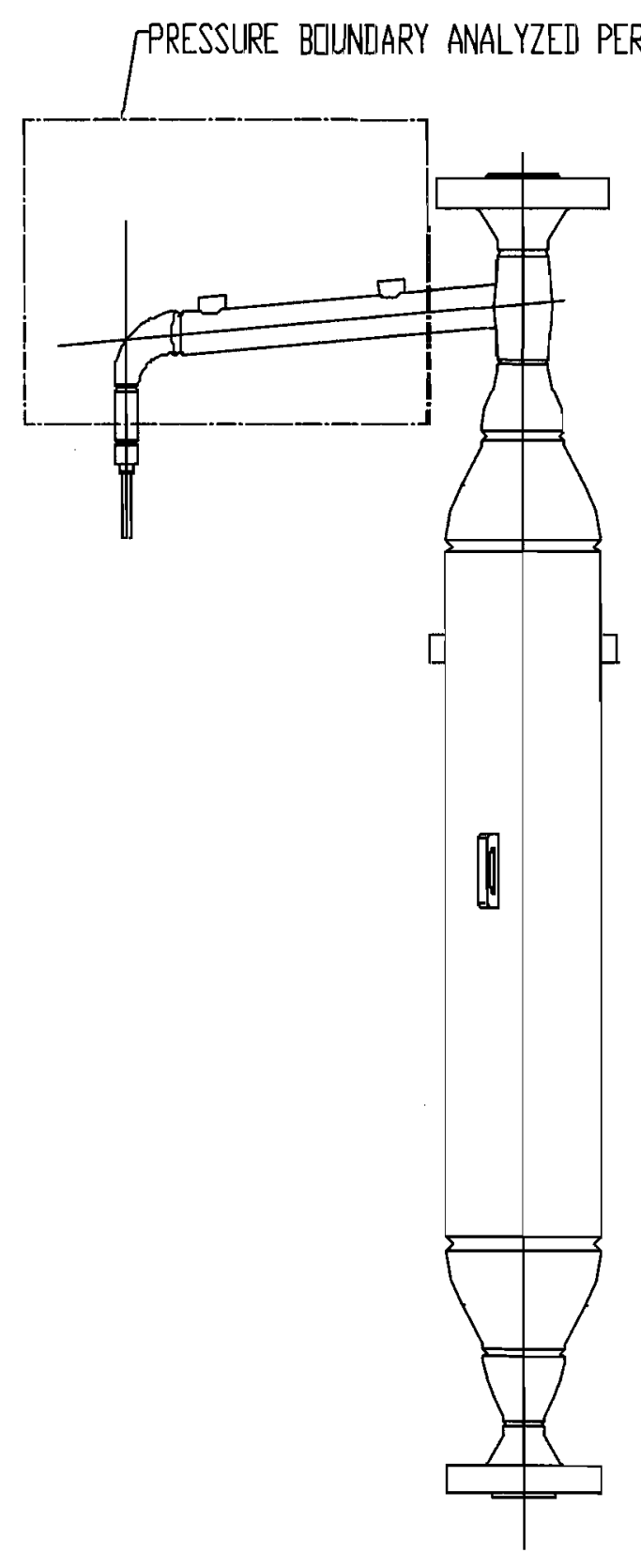

[PRESSURE BIUNDARY- ASME VIII-1

ASME B31,3
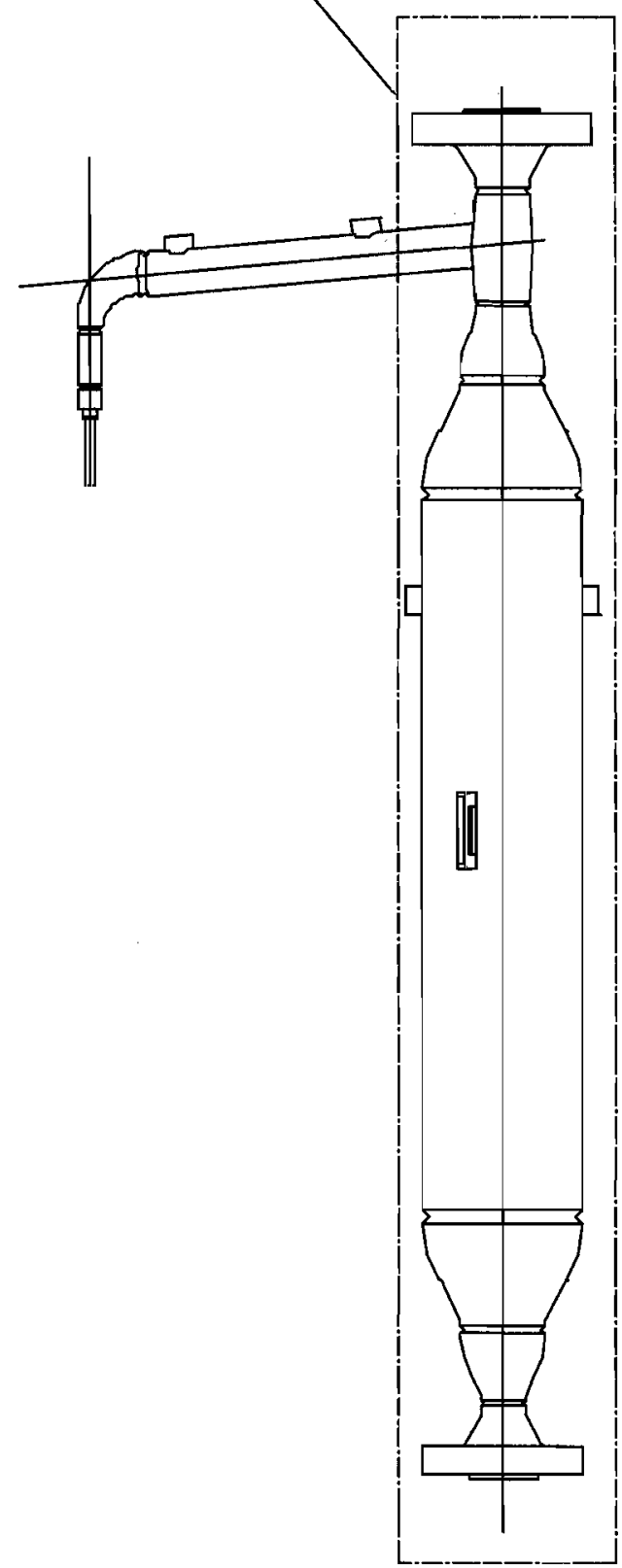


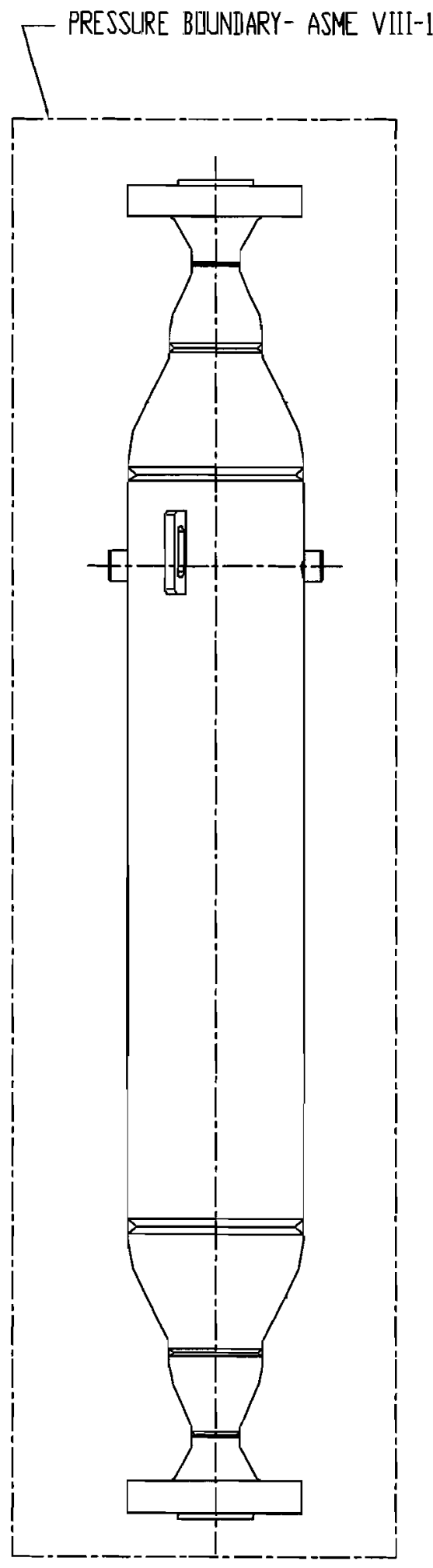




\section{Table Of Contents}

1. Pressure Summary

2. Hydrostatic Test

3. Weight Summary

4. $21 / 2^{\prime \prime}$ WN FLANGE \#1500 (MODIFIED)

5. 3 OD" PIPE

6. $4^{\prime \prime} \times 21 / 2^{\prime \prime}$ REDUCER B 16.9. UPPPER (SCH XS) 2 1/2" END

7. $4^{\prime \prime} \mathrm{X} 21 / 2^{\prime \prime}$ REDUCER B 16.9. UPPER (SCH XS) 4" END

8. 8" X 4" REDUCER B 16.9. UPPER (SCH XS) 4" END

9. 8" X 4" REDUCER B16.9. UPPER (SCH XS) 8" END

10. 8 " PIPE

11. $8^{\prime \prime} X 4^{\prime \prime}$ REDUCER B 16.9. LOWER (SCH XS) 8" END

12. 8" X 4" REDUCER B 16.9. LOWER (SCH XS) 4" END

13. 4" X 2" REDUCER B 16.9. LOWER (SCH XS) 4" END

14. 4" X 2" REDUCER B 16.9. LOWER (SCH XS) 2" END

15. 2" RFWN FLANGE 1500\#

16. 2" BRANCH (B1)

17. 1/2" HALF-COUPLING (7a)

18. 1/2" HALF-COUPLING (7b)

19. Wind Code

20. Seismic Code 


\section{Pressure Summary}

\begin{tabular}{|c|c|c|c|c|c|c|}
\hline Identifier & $\begin{array}{c}P \\
\text { Design } \\
(\mathrm{psi})\end{array}$ & $\begin{array}{c}\mathrm{T} \\
\text { Design } \\
\left({ }^{\circ} \mathrm{F}\right)\end{array}$ & $\begin{array}{c}\text { MAWP } \\
\text { ( psi) }\end{array}$ & $\begin{array}{c}\text { MDMT } \\
\left({ }^{\circ} \mathrm{F}\right)\end{array}$ & $\begin{array}{c}\text { MDMT } \\
\text { Exemption }\end{array}$ & $\begin{array}{l}\text { Impact } \\
\text { Tested }\end{array}$ \\
\hline 3 OD" PIPE & 1,150 & 1,000 & 1,250 & -325 & Note 1 & No \\
\hline 4" X 2 1/2" REDUCER B 16.9. UPPER (SCH XS) $21 / 2 "$ END & 1,150 & 1,000 & $2,397.87$ & -320 & Note 2 & No \\
\hline 4" X 2 1/2" REDUCER B16.2 UPPER (SCH XS) 4" END & 1,150 & 1,000 & $1,891.09$ & -320 & Note 2 & No \\
\hline 8" X 4" REDUCER B16.9. UPPER (SCH XS) 4" END & 1,150 & 1,000 & $1,891.09$ & -320 & Note 2 & No \\
\hline 8" X 4" REDUCER B16.9, UPPER (SCH XS) 8" END & 1,150 & 1,000 & $1,502.53$ & -320 & Note 2 & No \\
\hline 8" PIPE & 1,150 & 1,000 & $1,502.53$ & -320 & Note 2 & No \\
\hline 8" X 4" REDUCER B16.9. LOWER (SCH XS) 8" END & 1,150 & 1,000 & $1,502.53$ & -320 & Note 2 & No \\
\hline 8" X 4" REDUCER B 16.9. LOWER (SCH XS) 4" END & 1,150 & 1,000 & $1,891.09$ & -320 & Note 2 & No \\
\hline 4" X 2" REDUCER B16.9. LOWER (SCH XS) 4" END & 1,150 & 1,000 & $1,891.09$ & -320 & Note 2 & No \\
\hline 4" X 2" REDUCER B16.9. LOWER (SC1I XS) 2" END & 1,150 & 1,000 & $2,193.3$ & -320 & Note 2 & No \\
\hline ASME B16.5/16.47 Blind on 2" RFWN FLANGE 1500\# & 1,150 & 1,000 & 1,820 & -55 & Note 3,4 & No \\
\hline 2 I/2" WN FLANGE \#1500 (MODIFIED) & 1,150 & 1,000 & $1,169.51$ & -320 & Note 2 & No \\
\hline 21/2" WN FLANGE \#1500 (MOD1F1ED) - Flange Hub & 1,150 & 1,000 & $2,075.87$ & -320 & Note 5 & No \\
\hline 2" RFWN FLANGE 1500\# & 1,150 & 1,000 & 1,820 & -55 & Note 3,4 & No \\
\hline 1/2" HALF-COUPLING (7a) & 1,150 & 1,000 & $1,502.52$ & -320 & Note 6 & No \\
\hline 1/2" HALF-COUPLING (7b) & 1,150 & 1,000 & $1,502.52$ & -320 & Note 6 & No \\
\hline 2" BRANCH (B1) & 1,150 & 1,000 & 1,250 & -320 & Note 2 & No \\
\hline
\end{tabular}

Chamber design MDMT is $-20^{\circ} \mathrm{F}$

Chamber rated MDMT is $-55^{\circ} \mathrm{F} @ 1,169.51$ psi

Chamber MAWP was used in the MDMT determination

This pressure chamber is not designed for external pressure.

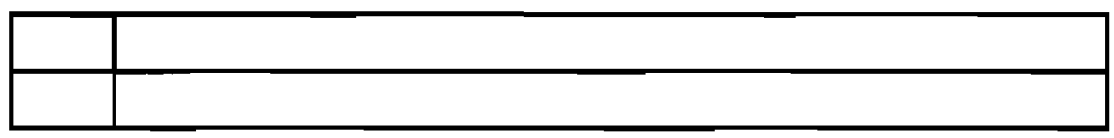

Notes for MDMT Rating:

\begin{tabular}{|r|l|l|}
\hline $\begin{array}{c}\text { Note } \\
\#\end{array}$ & \multicolumn{1}{|c|}{ Exemption } & \multicolumn{1}{|c|}{ Details } \\
\hline I. & Rated MDMT per UNF-65=-325 ${ }^{\circ} \mathrm{F}$ & \\
\hline 2. & $\begin{array}{l}\text { Rated MDMT per UHA }-51(\mathrm{~d})(1)(\mathrm{b}), \text { (carbon content does not exceed } 0.1 \\
\text { percent })=-320^{\circ} \mathrm{F}\end{array}$ & \\
\hline & &
\end{tabular}




\begin{tabular}{|r|l|l|}
\hline 3. & Per UHA -51 $(\mathrm{d})(1)(\mathrm{b})$ & \\
\hline 4. & Flange rated MDMT $=-320^{\circ} \mathrm{F}$ & $\begin{array}{l}\text { Bolts rated MDMT per Fig UCS-66 note }(\mathrm{e}) \\
=-55^{\circ} \mathrm{F}\end{array}$ \\
\hline 5. & Impact test exempt per UHA-51(g)(coincident ratio $=0.33389)$ & \\
\hline 6. & Impact test exempt per UHA-51 $(\mathrm{g})($ coincident ratio $=0.0909)$ & \\
\hline
\end{tabular}




\section{Hydrostatic Test}

Shop test pressure determination based on design P per UG-99(b)

Shop hydrostatic test gauge pressure is $1,495 \mathrm{psi}$ at $70^{\circ} \mathrm{F}$ (the chamber design $\mathrm{P}=1,150 \mathrm{psi}$ )

The shop test is performed with the vessel in the horizontal position.

\begin{tabular}{|l|r|r|r|r|}
\hline \multicolumn{1}{|c|}{ Identifier } & $\begin{array}{c}\text { Local test } \\
\text { pressure } \\
\text { psi }\end{array}$ & $\begin{array}{c}\text { Test liquid } \\
\text { static head } \\
\text { psi }\end{array}$ & $\begin{array}{c}\text { UG-99 } \\
\text { stress } \\
\text { ratio }\end{array}$ & $\begin{array}{c}\text { UG-99 } \\
\text { pressure } \\
\text { factor }\end{array}$ \\
\hline 3 OD" PIPE & $1,495.232$ & 0.232 & 3.2714 & 1.30 \\
\hline 4" X 2 1/2" REDUCER B16.9, UPPER (SCH XS) 2 1/2" END & $1,495.233$ & 0.233 & 1.3072 & 1.30 \\
\hline 4" X 2 1/2" REDUCER B16.9, UPPER (SCH XS) 4" END & $1,495.26$ & 0.26 & 1.3072 & 1.30 \\
\hline 8" X 4" REDUCER B16.9, UPPER (SCH XS) 4" END & $1,495.26$ & 0.26 & 1.3072 & 1.30 \\
\hline 8" X 4" REDUCER B16.9, UPPER (SCH XS) 8" END & $1,495.329$ & 0.329 & 1.3072 & 1.30 \\
\hline 8" PIPE & $1,495.327$ & 0.327 & 1.3072 & 1.30 \\
\hline 8" X 4" REDUCER B16.9, LOWER (SCH XS) 8" END & $1,495.329$ & 0.329 & 1.3072 & 1.30 \\
\hline 8" X 4" REDUCER B16.9, LOWER (SCH XS) 4" END & $1,495.26$ & 0.26 & 1.3072 & 1.30 \\
\hline 4" X 2" REDUCER B16.9, LOWER (SCH XS) 4"END & $1,495.26$ & 0.26 & 1.3072 & 1.30 \\
\hline 4" X 2" REDUCER B16.9, LOWER (SCH XS) 2" END & $1,495.225$ & 0.226 & 1.3072 & 1.30 \\
\hline 2" RFWN FLANGE 1500\# & $1,495.227$ & 0.227 & N/A & 1.30 \\
\hline 2 1/2" WN FLANGE \#1500 (MODIFIED) (1) & $1,495.231$ & 0.231 & 1 & 1.30 \\
\hline 1/2" HALF-COUPLING (7a) & $1,495.034$ & 0.034 & 1.7699 & 1.30 \\
\hline 1/2" HALF-COUPLING (7b) & $1,495.379$ & 0.379 & 1.7699 & 1.30 \\
\hline 2" BRANCH (B1) & $1,495.88$ & 0.88 & 1.3072 & 1.30 \\
\hline
\end{tabular}

Notes:

(1) 2 1/2" WN FLANGE \#1500 (MODIFIED) limits the UG-99 stress ratio.

(2) The zero degree angular position is assumed to be up, and the test liquid height is assumed to the top-most flange. 


\section{Weight Summary}

\begin{tabular}{|c|c|c|c|c|c|c|c|}
\hline \multirow[b]{2}{*}{ Component } & \multicolumn{7}{|c|}{ Weight ( Ib) Contributed by Vessel Elements } \\
\hline & $\begin{array}{l}\text { Metal } \\
\text { New* }\end{array}$ & $\begin{array}{c}\text { Metal } \\
\text { Corroded * }\end{array}$ & $\begin{array}{c}\text { Insulation } \\
\& \\
\text { Supports }\end{array}$ & Lining & $\begin{array}{c}\text { Piping } \\
+ \\
\text { Liquid }\end{array}$ & $\begin{array}{c}\text { Operating } \\
\text { Liquid }\end{array}$ & $\begin{array}{c}\text { Test } \\
\text { Liquid }\end{array}$ \\
\hline $30 D^{\prime \prime}$ PIPE & 4.4 & 4.1 & 0 & 0 & 0 & 0 & 3.5 \\
\hline $\begin{array}{l}4 " \times 2 \text { 1/2" REDUCER B16.9. UPPER (SCHXS) } 2 \text { 1/2" } \\
\text { END }\end{array}$ & 1.2 & 1 & 0 & 0 & 0 & 0 & 0.3 \\
\hline 4" X 2 1/2" REDUCER B16.9. UPPER (SCH XS) 4" END & 2.3 & 2 & 0 & 0 & 0 & 0 & 0.9 \\
\hline 8" X 4" REDUCER B 16.9. UPPER (SCH XS) 4" END & 3.4 & 3.1. & 0 & 0 & 0 & 0 & 1.3 \\
\hline 8" X 4" REDUCER B16.9. UPPER (SCH XS) 8" END & 9.8 & 9.2 & 0 & 0 & 0 & 0 & 5.1 \\
\hline$\underline{8 " \mathrm{PIPE}}$ & 140.1 & 132.2 & 0 & 0 & 0 & 0 & 62.7 \\
\hline 8" X 4" REDUCER B16.9, LOWER (SCH XS) 8" END & 9.8 & 9.2 & 0 & 0 & 0 & 0 & 5.1 \\
\hline 8" X 4" REDUCER B 16.9, LOWER (SCH XS) 4" END & 3.4 & 3.1 & 0 & 0 & 0 & 0 & 1.3 \\
\hline 4" X 2" REDUCER B 16.9. LOWER (SCH XS) 4" END & 2.3 & 2 & 0 & 0 & 0 & 0 & 0.9 \\
\hline 4" X 2" REDUCER B 16.9, LOWER (SCH XS) 2" END & 0.8 & 0.6 & 0 & 0 & 0 & 0 & 0.2 \\
\hline ASME B16.5/16.47 Blind on 2" RFWN FLANGE 1500\# & 32 & 32 & 0 & 0 & 0 & 0 & 0 \\
\hline Support Skirt \#1 & 3.8 & 3.8 & 0 & 0 & 0 & 0 & 0 \\
\hline TOTAL: & 213.2 & 202.3 & 0 & $\mathbf{0}$ & $\mathbf{0}$ & 0 & 81.2 \\
\hline
\end{tabular}

* Shells with attached nozzles have weight reduced by material cut out for opening.

\begin{tabular}{|c|c|c|c|c|c|c|c|c|c|}
\hline \multirow{3}{*}{ Component } & \multicolumn{9}{|c|}{ Weight ( lb) Contributed by Attachments } \\
\hline & \multicolumn{2}{|c|}{ Body Flanges } & \multicolumn{2}{|c|}{$\begin{array}{l}\text { Nozzles \& } \\
\text { Flanges }\end{array}$} & \multirow{2}{*}{$\begin{array}{l}\text { Packed } \\
\text { Beds }\end{array}$} & \multirow{2}{*}{$\begin{array}{c}\text { Ladders } \\
\& \\
\text { Platforms }\end{array}$} & \multirow{2}{*}{$\begin{array}{l}\text { Trays \& } \\
\text { Supports }\end{array}$} & \multirow{2}{*}{$\begin{array}{c}\text { Rings } \\
\& \\
\text { Clips }\end{array}$} & \multirow{2}{*}{$\begin{array}{c}\text { Vertical } \\
\text { Loads }\end{array}$} \\
\hline & New & Corroded & New & Corroded & & & & & \\
\hline 3 OD" PIPE & 35.9 & 35.6 & 7.9 & 6.9 & 0 & 0 & 0 & 0 & 0 \\
\hline $\begin{array}{l}4 " \mathrm{X} 2 \text { 1/2" REDUCER B 16.9, UPPER (SCH } \\
\mathrm{XS} 21 / 2 " \mathrm{END}\end{array}$ & 0 & 0 & 0 & 0 & 0 & 0 & 0 & 0 & 0 \\
\hline $\begin{array}{l}\text { 4"X } 2 \text { 1/2" REDUCER B 16.9. UPPER (SCH } \\
\text { XS) 4" END }\end{array}$ & 0 & 0 & 0 & 0 & 0 & 0 & 0 & 0 & 0 \\
\hline $\begin{array}{l}8^{\prime \prime} \times 4^{\prime \prime} \text { REDUCER B } 16.9 \text {. UPPER (SCH XS) } \\
4^{\prime \prime} \mathrm{END}\end{array}$ & 0 & 0 & 0 & 0 & 0 & 0 & 0 & 0 & 0 \\
\hline $\begin{array}{l}\text { 8"X 4" REDUCER B 16.9. UPPER (SCH XS) } \\
8 \text { " END }\end{array}$ & 0 & 0 & 0 & 0 & 0 & 0 & 0 & 0 & 0 \\
\hline 8" PIPE & 0 & 0 & 0.7 & 0.7 & 0 & 0 & 0 & 0 & 0 \\
\hline $\begin{array}{l}\text { 8" X 4" REDUCER B16.9. LOWER (SCH } \\
\text { XS) 8" END }\end{array}$ & 0 & 0 & 0 & 0 & 0 & 0 & 0 & 0 & 0 \\
\hline $\begin{array}{l}\text { 8" X 4" REDUCER B16.9, LOWER (SCH } \\
\text { XS) 4" END }\end{array}$ & 0 & 0 & 0 & 0 & 0 & 0 & 0 & 0 & 0 \\
\hline $\begin{array}{l}\text { 4" X 2" REDUCER B16.9, LOWER (SCH } \\
\text { XS) 4" END }\end{array}$ & 0 & 0 & 0 & 0 & 0 & 0 & 0 & 0 & 0 \\
\hline $\begin{array}{l}\text { 4" X 2" REDUCER B16.9. LOWER (SCH } \\
\text { XS) 2" END }\end{array}$ & 25 & 25 & 0 & 0 & 0 & 0 & 0 & 0 & 0 \\
\hline $\begin{array}{l}\text { ASME B } 16.5 / 16.47 \text { Blind on 2" RFWN } \\
\text { FLANGE } 1500 \#\end{array}$ & 0 & 0 & 0 & 0 & 0 & 0 & 0 & 0 & 0 \\
\hline Support Skirt \#1 & 0 & 0 & 0 & 0 & 0 & 0 & 0 & 0 & 0 \\
\hline TOTAL: & 60.9 & 60.6 & 8.6 & 7.6 & $\mathbf{0}$ & 0 & $\mathbf{0}$ & 0 & $\mathbf{0}$ \\
\hline
\end{tabular}


Vessel operating weight, Corroded: $270 \mathrm{lb}$

Vessel operating weight, New: $283 \mathrm{lb}$

Vessel empty weight, Corroded: $270 \mathrm{lb}$

Vessel empty weight, New: $\quad 283 \mathrm{lb}$

Vessel test weight, New: $\quad 364 \mathrm{lb}$

\section{Vessel center of gravity location - from datum - lift condition}

Vessel Lift Weight, New:283 lb

Center of Gravity: $\quad 36.285$ I" $^{\prime \prime}$

\section{Vessel Capacity}

Vessel Capacity** (New): 9 US gal

Vessel Capacity** (Corroded): 10 US gal

${ }^{* *}$ The vessel capacity does not include volume of nozzle, piping or other attachments. 


\section{1/2" WN FLANGE \#1500 (MODIFIED)}

\section{ASME VIII-1, 2007 Edition, Appendix 2 Flange Calculations}

Flange is attached to:

Flange type:

Flange material specification:

Bolt material specification:

Bolt Description:

Internal design pressure, $\mathrm{P}$ :

Required flange thickness: $t_{r}=$

Maximum allowable working pressure, MAWP:

Corrosion allowance:

Bolt corrosion (root), $\mathrm{C}_{\text {bolt }}$ :

Design MDMT:

Rated MDMT:

Estimated weight:

Flange dimensions, new
3 OD" PIPE (Top)

Weld neck integral

SA-182 F3 16H $<=5$ (low stress) (II-D

p. $74, \ln .32)$

SA-193 B8 2 Bolt $(3 / 4<t<=1$ ) (II-D

p. $356, \ln .27$ )

1 in Coarse Thread

1,150 psi@1,000F

1.5402 in

1,169.51 psi @ $1,000^{\circ} \mathrm{F}$

Bore $=0.03$ in

Flange $=0$ in

0 in

$-20^{\circ} \mathrm{F}$

$-320^{\circ} \mathrm{F}$

New $=35.9 \mathrm{lb}$
No impact test performed

Flange material is not normalized Material is not produced to fine grain practice

PWHT is not performed corroded $=35.6 \mathrm{lb}$ 


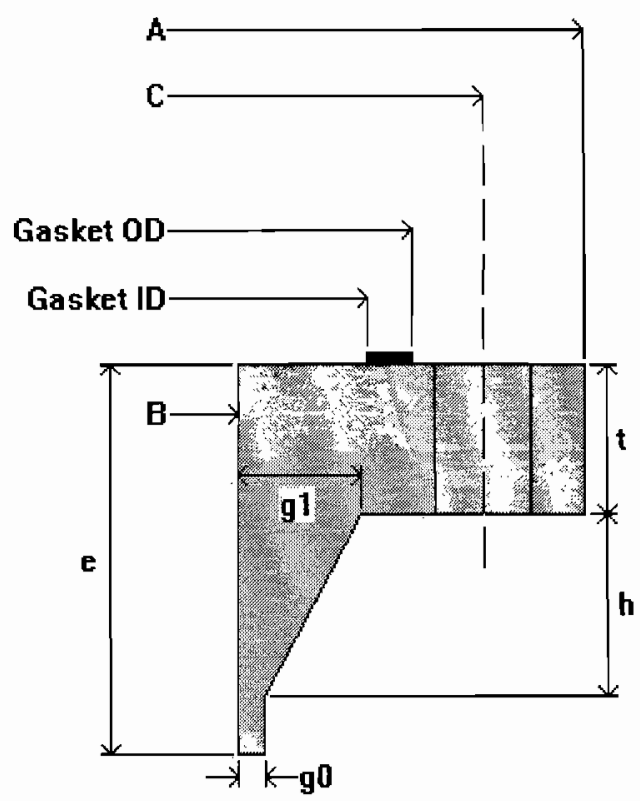

$$
\begin{aligned}
\text { flange OD A } & =9.625 \mathrm{in} \\
\text { bolt circle } \mathrm{C} & =7.5 \mathrm{in} \\
\text { gasket OD } & =5.988 \mathrm{in} \\
\text { gasket ID } & =5.088 \mathrm{in} \\
\text { flange ID B } & =2.323 \mathrm{in} \\
\text { thickness } \mathrm{t} & =1.55 \mathrm{in} \\
\text { bolting } & =8.1 \mathrm{in} \mathrm{dia}
\end{aligned}
$$

hub thickness $\mathrm{g}_{1}=1.3028$ in

hub thickness $\mathrm{g}_{0}=0.276$ in

hub length $\mathrm{h}=1.876 \mathrm{in}$

length $\mathrm{e}=4.0404$ in

gasket factor $\mathrm{m}=3$

seating stress y $=10,000.00 \mathrm{psi}$

Note: this flange is calculated as an integraltype.

\section{Flange calculations for Internal Pressure + Wind}

\section{Gasket details from facing sketch 1(a) or (b), Column II}

Gasket width $N=0.45$ in

$\mathrm{b}_{0}=\mathrm{N} / 2=0.225 \mathrm{in}$

Effective gasket seating width, $b=b_{0}=0.225$ in

$\mathrm{G}=(\mathrm{OD}$ of contact face + gasket ID $) / 2=5.538 \mathrm{in}$

$\mathrm{h}_{\mathrm{G}}=(\mathrm{C}-\mathrm{G}) / 2=(7.5-5.538) / 2=0.981$ in

$\mathrm{h}_{\mathrm{D}}=\mathrm{R}+\mathrm{g}_{1} / 2=1.2857+1.2728 / 2=1.9221 \mathrm{in}$

$\mathrm{h}_{\mathrm{T}}=\left(\mathrm{R}+\mathrm{g}_{1}+\mathrm{h}_{\mathrm{G}}\right) / 2=(1.2857+1.2728+0.981) / 2=1.7698$ in

$\mathrm{H}_{\mathrm{p}}=2 * \mathrm{~b} * 3.14 * \mathrm{G} * \mathrm{~m} * \mathrm{P}$

$=2 * 0.225 * 3.14 * 5.538 * 3 * 1,150$

$=26,996.92 \mathrm{lb}_{\mathrm{f}}$

$\mathrm{H}=0.785^{*} \mathrm{G}^{2 * \mathrm{P}}$

$=0.785^{*} 5.538^{2 *} 1,150$ 


$$
\begin{aligned}
& =27,686.84 \mathrm{lb}_{\mathrm{f}} \\
& \mathrm{H}_{\mathrm{D}}=0.785^{*} \mathrm{~B}^{2 * \mathrm{P}} \\
& =0.785 * 2.383^{2 * 1,150} \\
& =5,126.44 \mathrm{lb}_{\mathrm{f}} \\
& \mathrm{H}_{\mathrm{T}}=\mathrm{H}-\mathrm{H}_{\mathrm{D}} \\
& =27,686.84-5,126.44 \\
& =22,560.41 \mathrm{lb} \\
& \mathrm{W}_{\mathrm{m} 1}=\mathrm{H}+\mathrm{H}_{\mathrm{p}} \\
& =27,686.84+26,996.92 \\
& =54,683.77 \mathrm{lb}_{\mathrm{f}} \\
& \mathrm{W}_{\mathrm{m} 2}=3.14 * \mathrm{~b}^{*} \mathrm{G}^{*} \mathrm{y} \\
& =3.14 * 0.225 * 5.538 * 10,000 \\
& =39,125.98 \mathrm{lb}_{\mathrm{f}}
\end{aligned}
$$

Required bolt area, $A_{m}=$ greater of $A_{m 1}, A_{m 2}=2.734188 \mathrm{in}^{2}$

$$
\mathrm{A}_{\mathrm{ml}}=\mathrm{W}_{\mathrm{ml}} / \mathrm{S}_{\mathrm{b}}=54,683.77 / 20,000=2.7342 \mathrm{in}^{2}
$$

$$
\mathrm{A}_{\mathrm{m} 2}=\mathrm{W}_{\mathrm{m} 2} / \mathrm{S}_{\mathrm{a}}=39,125.98 / 20,000=1.9563 \mathrm{in}^{2}
$$

Total area for 8- 1 in dia bolts, corroded, $\mathrm{A}_{\mathrm{b}}=4.408 \mathrm{in}^{2}$

$$
\begin{aligned}
& \mathrm{W}=\left(\mathrm{A}_{\mathrm{m}}+\mathrm{A}_{\mathrm{b}}\right)^{*} \mathrm{~S}_{\mathrm{a}} / 2 \\
& =(2.7342+4.408)^{*} 20,000 / 2 \\
& =71,421.88 \mathrm{lb}_{\mathrm{f}} \\
& \mathrm{M}_{\mathrm{D}}=\mathrm{H}_{\mathrm{D}}{ }^{*} \mathrm{~h}_{\mathrm{D}}=5,126.44^{*} 1.9221=9,853.5 \mathrm{lb}-\mathrm{in} \\
& \mathrm{M}_{\mathrm{T}}=\mathrm{H}_{\mathrm{T}}{ }^{*} \mathrm{~h}_{\mathrm{T}}=22,560.41 * 1.7698=39,926.3 \mathrm{lb} \text {-in } \\
& \mathrm{H}_{\mathrm{G}}=\mathrm{W}_{\mathrm{m} 1}-\mathrm{H}=54,683.77-27,686.84=26,996.92 \mathrm{lb}_{\mathrm{f}} \\
& \mathrm{M}_{\mathrm{G}}=\mathrm{H}_{\mathrm{G}}{ }^{*} \mathrm{~h}_{\mathrm{G}}=26,996.92 * 0.981=26,484 \mathrm{lb}-\mathrm{in} \\
& \mathrm{M}_{\mathrm{o}}=\mathrm{M}_{\mathrm{D}}+\mathrm{M}_{\mathrm{T}}+\mathrm{M}_{\mathrm{G}}=9,853.5+39,926.3+26,484=76,263.8 \mathrm{lb}-\mathrm{in} \\
& \mathrm{M}_{\mathrm{g}}=\mathrm{W}^{*} \mathrm{~h}_{\mathrm{G}}=71,421.88 * 0.981=70,064.9 \mathrm{lb}-\mathrm{in}
\end{aligned}
$$




\section{Hub and Flange Factors}

$\mathrm{h}_{0}=\left(\mathrm{B}^{*} \mathrm{~g}_{0}\right)^{1 / 2}=(2.383 * 0.246)^{1 / 2}=0.7656$ in

From FIG. 2-7.1, where $\mathrm{K}=\mathrm{A} / \mathrm{B}=9.625 / 2.383=4.039$

$$
\begin{array}{lll}
\mathrm{T}=1.003 & \mathrm{Z}=1.1306 & \mathrm{Y}=1.4349 \quad \mathrm{U}=1.5768 \\
\mathrm{~h} / \mathrm{h}_{0}=2.4502 & \mathrm{~g}_{1} / \mathrm{g}_{0}=5.174 \\
\mathrm{~F}=0.4731 & \mathrm{~V}=0.0152 \quad \mathrm{e}=\mathrm{F} / \mathrm{h}_{0}=0.6179 \\
\mathrm{~d}=(\mathrm{U} / \mathrm{V})^{*} \mathrm{~h}_{0}{ }^{*} \mathrm{~g}_{0}{ }^{2}=(1.576849 / 0.0152)^{*} 0.7656^{*} 0.246^{2} \\
=4.8108 \mathrm{in}^{3}
\end{array}
$$

\section{Stresses at operating conditions - VIII-1, Appendix 2-7}

$\mathrm{f}=1$

$\mathrm{L}=\left(\mathrm{t}^{*} \mathrm{e}+1\right) / \mathrm{T}+\mathrm{t}^{3} / \mathrm{d}$

$=\left(1.55^{*} 0.6179+1\right) / 1.003027+1.55^{3} / 4.8108$

$=2.725902$

$\mathrm{S}_{\mathrm{H}}=\mathrm{f}^{*} \mathrm{M}_{\mathrm{o}} /\left(\mathrm{L} * \mathrm{~g}_{1}{ }^{2 *} \mathrm{~B}\right)$

$=1 * 76,263.8 /\left(2.725902 * 1.2728^{2 *} 2.383\right)$

$=7,247 \mathrm{psi}$

$\mathrm{S}_{\mathrm{R}}=(1.33 * \mathrm{t} * \mathrm{e}+1) * \mathrm{M}_{\mathrm{o}} /\left(\mathrm{L} * \mathrm{t}^{2} * \mathrm{~B}\right)$

$=\left(1.33^{*} 1.55^{*} 0.6179+1\right) * 76,263.8 /\left(2.725902 * 1.55^{2 *} 2.383\right)$

$=11,112 \mathrm{psi}$

$\mathrm{S}_{\mathrm{T}}=\mathrm{Y}^{*} \mathrm{M}_{\mathrm{o}} /\left(\mathrm{t}^{2 * \mathrm{~B}}\right)-\mathrm{Z} * \mathrm{~S}_{\mathrm{R}}$

$=1.4349 * 76,263.8 /\left(1.55^{2} * 2.383\right)-1.1306 * 11,112$

$=6,552 \mathrm{psi}$

Allowable stress $\mathrm{S}_{\mathrm{fo}}=11,300 \mathrm{psi}$

Allowable stress $\mathrm{S}_{\text {no }}=7,000 \mathrm{psi}$

$\mathrm{S}_{\mathrm{T}}$ does not exceed $\mathrm{S}_{\mathrm{fo}_{\mathrm{o}}}$

$\mathrm{S}_{\mathrm{H}}$ does not exceed $\operatorname{Min}\left[1.5 * \mathrm{~S}_{\mathrm{fo}}, 2.5 * \mathrm{~S}_{\mathrm{no}}\right]=16,950 \mathrm{psi}$

$\mathrm{S}_{\mathrm{R}}$ does not exceed $\mathrm{S}_{\mathrm{fo}}$

$0.5\left(\mathrm{~S}_{\mathrm{H}}+\mathrm{S}_{\mathrm{R}}\right)=9,179$ psi does not exceed $\mathrm{S}_{\mathrm{fo}}$

$0.5\left(\mathrm{~S}_{\mathrm{H}}+\mathrm{S}_{\mathrm{T}}\right)=6,899$ psi does not exceed $\mathrm{S}_{\text {fo }}$

Stresses at gasket seating - VIII -1, Appendix 2-7

$$
\begin{aligned}
& \mathrm{S}_{\mathrm{H}}=\mathrm{f}^{*} \mathrm{M}_{\mathrm{g}} /\left(\mathrm{L}^{*} \mathrm{~g}_{1}{ }^{2 * \mathrm{~B})}\right. \\
& =1 * 70,064.9 /\left(2.725902 * 1.2728^{2 *} 2.383\right)
\end{aligned}
$$




$$
\begin{aligned}
& =6,658 \mathrm{psi} \\
& \mathrm{S}_{\mathrm{R}}=\left(1.33^{*} \mathrm{t}^{*} \mathrm{e}+1\right)^{*} \mathrm{M}_{\mathrm{g}} /\left(\mathrm{L}^{*} \mathrm{t}^{2 * \mathrm{~B}}\right) \\
& =\left(1.33^{*} 1.55^{*} 0.6179+1\right)^{*} 70,064.9 /\left(2.725902 * 1.55^{2 * 2.383)}\right. \\
& =10,208 \mathrm{psi} \\
& \mathrm{S}_{\mathrm{T}}=\mathrm{Y}^{*} \mathrm{M}_{\mathrm{g}} /\left(\mathrm{t}^{2 *} \mathrm{~B}\right)-\mathrm{Z}^{*} \mathrm{~S}_{\mathrm{R}} \\
& =1.4349^{*} 70,064.9 /\left(1.55^{2 *} 2.383\right)-1.1306^{*} 10,208 \\
& =6,019 \mathrm{psi}
\end{aligned}
$$

Allowable stress $\mathrm{S}_{\mathrm{fa}}=20,000 \mathrm{psi}$

Allowable stress $\mathrm{S}_{\mathrm{na}}=22,900 \mathrm{psi}$

$\mathrm{S}_{\mathrm{T}}$ does not exceed $\mathrm{S}_{\mathrm{fa}}$

$\mathrm{S}_{\mathrm{H}}$ does not exceed $\operatorname{Min}\left[1.5^{*} \mathrm{~S}_{\mathrm{fa}}, 2.5^{*} \mathrm{~S}_{\mathrm{na}}\right]=30,000 \mathrm{psi}$

$\mathrm{S}_{\mathrm{R}}$ does not exceed $\mathrm{S}_{\mathrm{fa}}$

$0.5\left(\mathrm{~S}_{\mathrm{H}}+\mathrm{S}_{\mathrm{R}}\right)=8,433$ psi does not exceed $\mathrm{S}_{\mathrm{fa}}$

$0.5\left(\mathrm{~S}_{\mathrm{H}}+\mathrm{S}_{\mathrm{T}}\right)=6,339$ psi does not exceed $\mathrm{S}_{\text {fa }}$

\section{Flange rigidity per VIII-1, Appendix 2-14}

$$
\begin{aligned}
& \mathrm{J}=52.14 * \mathrm{~V}^{*} \mathrm{M}_{\mathrm{o}} /\left(\mathrm{L}^{*} \mathrm{E}^{*} \mathrm{~g}_{0}{ }^{2 * \mathrm{~K}_{\mathrm{I}}} \mathrm{h}_{0}\right) \\
& =52.14 * 0.0152 * 76,263.8 /\left(2.7259 * 22,800,000 * 0.246^{2 *} 0.3 * 0.7656\right) \\
& =0.06990274
\end{aligned}
$$

The flange rigidity index $\mathbf{J}$ does not exceed 1 ; satisfactory.

\section{1/2" WN FLANGE \#1500 (MODIFIED) - Flange hub}

\section{ASME Section VIII Division 1, 2007 Edition}

Component:

Material specification:

Impact test exempt per UHA $-51(\mathrm{~g})($ coincident ratio $=0.33389)$

Internal design pressure: $\mathrm{P}=1,150 \mathrm{psi} @ 1,000^{\circ} \mathrm{F}$

\section{Static liquid head:}

Not Considered

Corrosion allowance Inner $\mathrm{C}=0.03 "$

$$
\text { Outer } \mathrm{C}=0 "
$$

Design MDMT $=-20^{\circ} \mathrm{F}$

Rated MDMT $=-320^{\circ} \mathrm{F}$

No impact test performed Material is not normalized

Material is not produced to Fine Grain Practice PWHT is not performed

Radiography: Longitudinal joint - $\quad$ Seamless No RT 
Top circumferential joint - N/A

Bottom circumferential joint -

Full UW-11(a) Type 1

Estimated weight New $=1.1 \mathrm{lb} \quad$ corr $=1 \mathrm{lb}$

Capacity $\quad$ New $=0.03$ US gal corr $=0.04$ US gal

$\mathrm{OD} \quad=2.875^{\prime \prime}$

Length $\mathrm{L}_{\mathrm{c}}=1.876^{\prime \prime}$

$\mathrm{t} \quad=0.276^{\prime \prime}$

Design thickness, (at $\left.1,000{ }^{\circ} \mathrm{F}\right)$ Appendix 1-1

$\mathrm{t}=\mathrm{P}^{*} \mathrm{R}_{\mathrm{o}} /\left(\mathrm{S} * \mathrm{E}+0.40^{*} \mathrm{P}\right)+$ Corrosion

$=1,150 * 1.4375 /(11,300 * 1.00+0.40 * 1,150)+0.03$

$=0.1706^{\prime \prime}$

Maximum allowable working pressure, $\left(\right.$ at $\left.^{1,000}{ }^{\circ} \mathrm{F}\right)$ Appendix 1-1

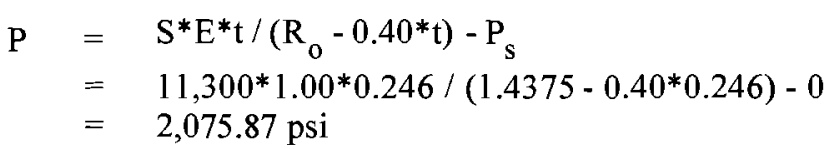

\% Forming Strain - UHA-44(a)(2)(a)

$$
\begin{aligned}
\mathrm{EFE} & =\left(50 * t / \mathrm{R}_{\mathrm{f}}\right) *\left(1-\mathrm{R}_{\mathrm{f}} / \mathrm{R}_{\mathrm{o}}\right) \\
& =(50 * 0.276 / 1.2995) *(1-1.2995 / \infty) \\
& =10.6195 \%
\end{aligned}
$$

Design thickness $=0.1706^{\prime \prime}$

The governing condition is due to internal pressure.

\begin{tabular}{|c|c|c|c|c|c|c|c|c|}
\hline \multirow[t]{2}{*}{ Condition } & \multirow{2}{*}{$\begin{array}{l}\text { Pressure } \\
\text { P ( psi) }\end{array}$} & \multicolumn{2}{|c|}{$\begin{array}{c}\text { Allowable Stress } \\
\text { Before UG-23 Stress } \\
\text { Increase ( psi) }\end{array}$} & \multirow{2}{*}{$\begin{array}{c}\text { Temperature } \\
\left({ }^{\circ} \mathrm{F}\right)\end{array}$} & \multirow{2}{*}{$\begin{array}{c}\text { Corrosion } \\
\mathrm{C} \text { (in) }\end{array}$} & \multirow[t]{2}{*}{ Load } & \multirow{2}{*}{$\begin{array}{l}\text { Req'd Thk } \\
\text { Due to } \\
\text { Tension (in) }\end{array}$} & \multirow{2}{*}{$\begin{array}{l}\text { Req'd Thk Due } \\
\text { to Compression } \\
\text { (in) }\end{array}$} \\
\hline & & $\mathbf{S}_{\mathbf{t}}$ & $\mathrm{S}_{\mathrm{c}}$ & & & & & \\
\hline \multirow{2}{*}{$\begin{array}{l}\text { Operating, Hot \& } \\
\text { Corroded }\end{array}$} & \multirow{2}{*}{1,150} & \multirow{2}{*}{11,300} & \multirow{2}{*}{10,369} & \multirow{2}{*}{1,000} & \multirow{2}{*}{0.03} & Wind & 0.0497 & 0.0496 \\
\hline & & & & & & Seismic & 0.05 & 0.0494 \\
\hline \multirow{2}{*}{$\begin{array}{c}\text { Operating, Hot \& } \\
\text { New }\end{array}$} & \multirow{2}{*}{1,150} & \multirow{2}{*}{11,300} & \multirow{2}{*}{10,454} & \multirow{2}{*}{1,000} & \multirow{2}{*}{0} & Wind & 0.0485 & 0.0484 \\
\hline & & & & & & Seismic & 0.0488 & 0.0481 \\
\hline \multirow{2}{*}{ Empty, Corroded } & \multirow{2}{*}{0} & \multirow{2}{*}{20,000} & \multirow{2}{*}{14,651} & \multirow{2}{*}{0} & \multirow{2}{*}{0.03} & Wind & 0 & 0 \\
\hline & & & & & & Seismic & 0.0002 & 0.0002 \\
\hline & & & & & & Wind & 0 & 0 \\
\hline
\end{tabular}

The cylinder thickness of $0.276^{\prime \prime}$ is adequate.

Thickness Required Due to Pressure + External Loads 


\begin{tabular}{|c|r|r|r|r|r|r|r|r|} 
Empty, New & 0 & 20,000 & 14,737 & 0 & 0 & Seismic & 0.0002 & 0.0002 \\
\hline $\begin{array}{c}\text { Hot Shut Down, } \\
\text { Corroded, Weight \& } \\
\begin{array}{c}\text { Eccentric Moments } \\
\text { Only }\end{array}\end{array}$ & 0 & 11,300 & 10,369 & 1,000 & 0.03 & Weight & 0 & 0 \\
\hline
\end{tabular}




\section{OD" PIPE}

\section{ASME Section VIII Division 1, 2007 Edition}

Component:

Material specification:

Rated MDMT per UNF- $65=-325^{\circ} \mathrm{F}$

Internal design pressure: $\mathrm{P}=1,150 \mathrm{psi} @ 1,000^{\circ} \mathrm{F}$

\section{Static liquid head:}

$P_{\text {th }}=0.23 \mathrm{psi}\left(\mathrm{SG}=1, \mathrm{H}_{\mathrm{S}}=6.43\right.$ ", Horizontal test head $)$

Corrosion allowance

$$
\text { Inner } \mathrm{C}=0.03 "
$$

Outer $\mathrm{C}=0$ "
Cylinder

SB-166 Annealed

N06600 (II-D p. 222, ln. 3)
Design MDMT $=-20^{\circ} \mathrm{F}$

Rated MDMT $=-325^{\circ} \mathrm{F}$
No impact test performed

Material is not normalized

Material is not produced to Fine Grain Practice

PWHT is not performed

Radiography: Longitudinal joint - Seamless No RT

Top circumferential joint - Full UW-11(a) Type 1

Bottom circumferential

joint -

Full UW-11(a) Type 1

Estimated weight $\mathrm{New}=4.8 \mathrm{lb} \quad$ corr $=4.4 \mathrm{lb}$

Capacity $\quad \mathrm{New}=0.11$ US gal corr $=0.12$ US gal

$$
\begin{aligned}
\mathrm{OD} & =3^{\prime \prime} \\
\text { Length } \mathrm{L}_{\mathrm{c}} & =6^{\prime \prime} \\
\mathrm{t} & =0.32^{\prime \prime}
\end{aligned}
$$

\section{Design thickness, (at $1,000^{\circ}$ F) Appendix 1-1}

$$
\begin{aligned}
\mathrm{t} & =\mathrm{P}^{*} \mathrm{R}_{\mathrm{o}} /\left(\mathrm{S}^{*} \mathrm{E}+0.40 * \mathrm{P}\right)+\text { Corrosion } \\
& =1,150^{*} 1.5 /\left(7,000^{*} 1.00+0.40^{*} 1,150\right)+0.03 \\
& =0.2613^{\prime \prime}
\end{aligned}
$$

Maxim um allowable working pressure, (at 1,000 ${ }^{\circ}$ F) Appendix 1-1

$$
\begin{aligned}
\mathrm{P} & =\mathrm{S}^{*} \mathrm{E}^{*} \mathrm{t} /\left(\mathrm{R}_{\mathrm{o}}-0.40 * \mathrm{t}\right)-\mathrm{P}_{\mathrm{S}} \\
& =7,000 * 1.00 * 0.25 /\left(1.5-0.40^{*} 0.25\right)-0 \\
& =1,250 \mathrm{psi}
\end{aligned}
$$

Design thickness $=0.2613^{\prime \prime}$

The governing condition is due to internal pressure.

The cylinder thickness of $0.32 "$ is adequate. 
DESC: CHAR POTS, UPPER \& LOWER

P.O.\#: 700521452

Thickness Required Due to Pressure + External Loads

\begin{tabular}{|c|c|c|c|c|c|c|c|c|}
\hline \multirow[t]{2}{*}{ Condition } & \multirow{2}{*}{$\begin{array}{l}\text { Pressure } \\
\text { P ( psi) }\end{array}$} & \multicolumn{2}{|c|}{$\begin{array}{l}\text { Allowable Stress } \\
\text { Before UG-23 Stress } \\
\text { Increase ( psi) }\end{array}$} & \multirow{2}{*}{$\begin{array}{c}\text { Temperature } \\
\left({ }^{\circ} \mathrm{F}\right)\end{array}$} & \multirow{2}{*}{$\begin{array}{l}\text { Corrosion } \\
\text { C (in) }\end{array}$} & \multirow[t]{2}{*}{ Load } & \multirow{2}{*}{$\begin{array}{l}\text { Req'd Thk } \\
\text { Due to } \\
\text { Tension (in) }\end{array}$} & \multirow{2}{*}{$\begin{array}{l}\text { Req'd Thk Due } \\
\text { to Compression } \\
\text { (in) }\end{array}$} \\
\hline & & $\mathbf{S}_{\mathbf{t}}$ & $\mathbf{S}_{\mathbf{c}}$ & & & & & \\
\hline \multirow{2}{*}{$\begin{array}{l}\text { Operating, Hot \& } \\
\text { Corroded }\end{array}$} & \multirow{2}{*}{1,150} & \multirow{2}{*}{7,000} & \multirow{2}{*}{7,000} & \multirow{2}{*}{1,000} & \multirow{2}{*}{0.03} & Wind & 0.083 & 0.0769 \\
\hline & & & & & & Seismic & 0.0846 & 0.0752 \\
\hline \multirow{2}{*}{$\begin{array}{l}\text { Operating, Hot \& } \\
\text { New }\end{array}$} & \multirow{2}{*}{1,150} & \multirow{2}{*}{7,000} & \multirow{2}{*}{7,000} & \multirow{2}{*}{1,000} & \multirow{2}{*}{0} & Wind & 0.0814 & 0.0744 \\
\hline & & & & & & Seismic & 0.0831 & 0.0727 \\
\hline \multirow{2}{*}{ Empty, Corroded } & \multirow{2}{*}{0} & \multirow{2}{*}{22,900} & \multirow{2}{*}{13,800} & \multirow{2}{*}{0} & \multirow{2}{*}{0.03} & Wind & 0.0007 & 0.0019 \\
\hline & & & & & & Seismic & 0.0012 & 0.0027 \\
\hline \multirow{2}{*}{ Empty, New } & \multirow{2}{*}{0} & \multirow{2}{*}{22,900} & \multirow{2}{*}{13,800} & \multirow{2}{*}{0} & \multirow{2}{*}{0} & Wind & 0.0009 & 0.0021 \\
\hline & & & & & & Seismic & 0.0014 & 0.003 \\
\hline $\begin{array}{l}\text { Hot Shut Down, } \\
\text { Corroded, Weight \& } \\
\text { Eccentric Moments } \\
\text { Only }\end{array}$ & 0 & 7,000 & 7,000 & 1,000 & 0.03 & Weight & 0.0025 & 0.0041 \\
\hline
\end{tabular}




\section{4" X 2 1/2" REDUCER B16.9, UPPER (SCH XS) 2 1/2" END}

\section{ASME Section VIII Division 1, 2007 Edition}

Component: Cylinder

Material specification: $\quad$ SA-403 316H (II-D p. 78, ln. 8)

Rated MDMT per UHA-51(d)(1)(b), (carbon content does not exceed 0.1 percent) $=-320^{\circ} \mathrm{F}$

Internal design pressure: $\mathrm{P}=1,150 \mathrm{psi} @ 1,000^{\circ} \mathrm{F}$

Static liquid head:

$\mathrm{P}_{\text {th }}=0.23 \mathrm{psi}\left(\mathrm{SG}=1, \mathrm{H}_{\mathrm{S}}=6.4455\right.$ ", Horizontal test head $)$

Corrosion allowance $\quad$ Inner $\mathrm{C}=0.03 " \quad$ Outer $\mathrm{C}=0 "$

Design MDMT $=-20^{\circ} \mathrm{F} \quad$ No impact test performed

Rated MDMT $=-320^{\circ} \mathrm{F} \quad$ Material is not normalized

Material is not produced to Fine Grain Practice

PWHT is not performed

\section{Radiography: Longitudinal joint - Seamless No RT}

Top circumferential joint - Full UW-11(a) Type 1

Bottom circumferential joint -

Full UW-11(a) Type 1

$$
\begin{aligned}
\text { Estimated weight New } & =1.2 \mathrm{lb} \quad \text { corr }=1 \mathrm{lb} \\
\text { Capacity } \quad \mathrm{New} & =0.04 \text { US gal corr }=0.04 \text { US gal }
\end{aligned}
$$

$$
\begin{aligned}
\text { OD } & =2.875^{\prime \prime} \\
\text { Length } L_{\mathbf{c}} & =2^{\prime \prime} \\
\mathrm{t} & =0.242 "
\end{aligned}
$$

\section{Design thickness, (at $1,000^{\circ}$ F) Appendix 1-1}

$$
\begin{aligned}
\mathrm{t} & =\mathrm{P}^{*} \mathrm{R}_{\mathrm{o}} /\left(\mathrm{S}^{*} \mathrm{E}+0.40^{*} \mathrm{P}\right)+\text { Corrosion } \\
& =1,150^{*} 1.4375 /\left(15,300^{*} 1.00+0.40^{*} 1,150\right)+0.03 \\
& =0.1349^{\prime \prime}
\end{aligned}
$$

Maximum allowable working pressure, (at 1,000 ${ }^{\circ}$ F) Appendix 1-1

$$
\begin{aligned}
\mathrm{P} & =\mathrm{S}^{*} \mathrm{E}^{*} \mathrm{t} /\left(\mathrm{R}_{\mathrm{o}}-0.40^{*} \mathrm{t}\right)-\mathrm{P}_{\mathrm{S}} \\
& =15,300^{*} 1.00^{*} 0.212 /\left(1.4375-0.40^{*} 0.212\right)-0 \\
& =2,397.87 \mathrm{psi}
\end{aligned}
$$

\% Forming Strain - UHA-44(a)(2)(a)

$$
\begin{aligned}
\mathrm{EFE} & =\left(50 * \mathrm{t} / \mathrm{R}_{\mathrm{f}}\right) *\left(1-\mathrm{R}_{\mathrm{f}} / \mathrm{R}_{\mathrm{o}}\right) \\
& =(50 * 0.242 / 1.3165) *(1-1.3165 / \infty) \\
& =9.1910 \%
\end{aligned}
$$




\section{Design thickness $=0.1349^{\prime \prime}$}

The governing condition is due to internal pressure.

The cylinder thickness of 0.242 " is adequate.

Thickness Required Due to Pressure + External Loads

\begin{tabular}{|c|c|c|c|c|c|c|c|c|}
\hline \multirow[t]{2}{*}{ Condition } & \multirow{2}{*}{$\begin{array}{c}\text { Pressure } \\
\text { P ( psi) }\end{array}$} & \multicolumn{2}{|c|}{$\begin{array}{l}\text { Allowable Stress } \\
\text { Before UG-23 Stress } \\
\text { Increase ( psi) }\end{array}$} & \multirow{2}{*}{$\begin{array}{c}\text { Temperature } \\
\left({ }^{\circ} \mathrm{F}\right)\end{array}$} & \multirow{2}{*}{$\begin{array}{l}\text { Corrosion } \\
\mathrm{C} \text { (in) }\end{array}$} & \multirow[t]{2}{*}{ Load } & \multirow{2}{*}{$\begin{array}{l}\text { Req'd Thk } \\
\text { Due to } \\
\text { Tension (in) }\end{array}$} & \multirow{2}{*}{$\begin{array}{l}\text { Req'd Thk Due } \\
\text { to Compression } \\
\text { (in) }\end{array}$} \\
\hline & & $\mathbf{S}_{\mathbf{t}}$ & $\mathbf{S}_{\mathbf{c}}$ & & & & & \\
\hline \multirow{2}{*}{$\begin{array}{l}\text { Operating, Hot \& } \\
\text { Corroded }\end{array}$} & \multirow{2}{*}{1,150} & \multirow{2}{*}{15,300} & \multirow{2}{*}{10,261} & \multirow{2}{*}{1,000} & \multirow{2}{*}{0.03} & Wind & 0.0391 & 0.0361 \\
\hline & & & & & & Seismic & 0.04 & 0.0351 \\
\hline \multirow{2}{*}{$\begin{array}{c}\text { Operating, Hot \& } \\
\text { New }\end{array}$} & \multirow{2}{*}{1,150} & \multirow{2}{*}{15,300} & \multirow{2}{*}{10,357} & \multirow{2}{*}{1,000} & \multirow{2}{*}{0} & Wind & 0.0384 & 0.0349 \\
\hline & & & & & & Seismic & 0.0394 & 0.0339 \\
\hline \multirow{2}{*}{ Empty, Corroded } & \multirow{2}{*}{0} & \multirow{2}{*}{20,000} & \multirow{2}{*}{14,528} & \multirow{2}{*}{0} & \multirow{2}{*}{0.03} & Wind & 0.0009 & 0.0019 \\
\hline & & & & & & Seismic & 0.0016 & 0.003 \\
\hline \multirow{2}{*}{ Empty, New } & \multirow{2}{*}{0} & \multirow{2}{*}{20,000} & \multirow{2}{*}{14,638} & \multirow{2}{*}{0} & \multirow{2}{*}{0} & Wind & 0.0011 & 0.0021 \\
\hline & & & & & & Seismic & 0.0018 & 0.0032 \\
\hline $\begin{array}{l}\text { Hot Shut Down, } \\
\text { Corroded, Weight \& } \\
\text { Eccentric Moments } \\
\text { Only }\end{array}$ & 0 & 15,300 & 10,261 & 1,000 & 0.03 & Weight & 0.0012 & 0.0029 \\
\hline
\end{tabular}




\section{4" X 2 1/2" REDUCER B16.9, UPPER (SCH XS) 4" END}

\section{ASME Section VIII Division 1, 2007 Edition}

$\begin{array}{ll}\text { Component: } & \text { Cylinder } \\ \text { Material specification: } & \text { SA-403 316H (II-D p. 78, In. 8) }\end{array}$

Rated MDMT per UHA-51(d)(1)(b), (carbon content does not exceed 0.1 percent $)=-320^{\circ} \mathrm{F}$

Internal design pressure: $\mathrm{P}=1,150 \mathrm{psi} @ 1,000^{\circ} \mathrm{F}$

Static liquid head:

$P_{\text {th }}=0.26 \mathrm{psi}\left(\mathrm{SG}=1, \mathrm{H}_{\mathrm{S}}=7.205 "\right.$, Horizontal test head $)$

Corrosion allowance $\quad$ Inner $\mathrm{C}=0.03^{\prime \prime} \quad$ Outer $\mathrm{C}=0 "$

Design MDMT $=-20^{\circ} \mathrm{F} \quad$ No impact test performed

Rated MDMT $=-320^{\circ} \mathrm{F} \quad$ Material is not normalized

Material is not produced to Fine Grain Practice

PWHT is not performed

Radiography: Longitudinal joint - Seamless No RT

Top circumferential joint - Full UW-11(a) Type 1

Bottom circumferential

joint -

Full UW-11(a) Type 1

Estimated weight New $=2.3 \mathrm{lb} \quad$ corr $=2 \mathrm{lb}$

Capacity $\quad$ New $=0.1$ US gal corr $=0.11$ US gal

$\mathrm{OD} \quad=4.5^{\prime \prime}$

Length $\mathrm{L}_{\mathrm{c}}=2^{\prime \prime}$

$\mathrm{t} \quad=0.295^{\prime \prime}$

\section{Design thickness, (at $1,000^{\circ}$ F) Appendix 1-1}

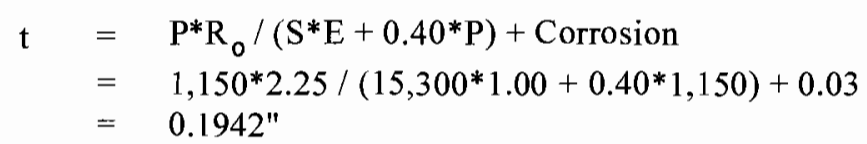

Maximum allowable working pressure, (at $1,000^{\circ} \mathrm{F}$ ) Appendix 1-1

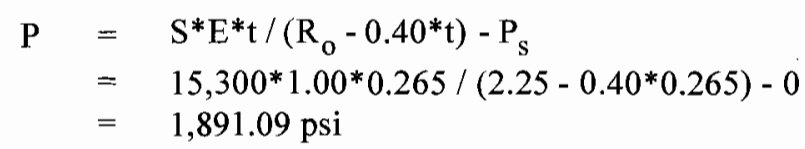

\% Forming Strain - UHA-44(a)(2)(a)

$$
\begin{aligned}
\mathrm{EFE} & =\left(50 * \mathrm{t} / \mathrm{R}_{\mathrm{f}}\right) *\left(1-\mathrm{R}_{\mathrm{f}} / \mathrm{R}_{\mathrm{o}}\right) \\
& =(50 * 0.295 / 2.1025) *(1-2.1025 / \infty) \\
& =7.0155 \%
\end{aligned}
$$




\section{Design thickness $=0.1942 "$}

The governing condition is due to internal pressure.

The cylinder thickness of $0.295^{\prime \prime}$ is adequate.

Thickness Required Due to Pressure + External Loads

\begin{tabular}{|c|c|c|c|c|c|c|c|c|}
\hline \multirow[t]{2}{*}{ Condition } & \multirow{2}{*}{$\begin{array}{l}\text { Pressure } \\
\text { P ( psi) }\end{array}$} & \multicolumn{2}{|c|}{$\begin{array}{l}\text { Allowable Stress } \\
\text { Before UG-23 Stress } \\
\text { Increase ( psi) }\end{array}$} & \multirow{2}{*}{$\begin{array}{c}\text { Temperature } \\
\left({ }^{\circ} \mathrm{F}\right)\end{array}$} & \multirow{2}{*}{$\begin{array}{l}\text { Corrosion } \\
\text { C (in) }\end{array}$} & \multirow[t]{2}{*}{ Load } & \multirow{2}{*}{$\begin{array}{l}\text { Req'd Thk } \\
\text { Due to } \\
\text { Tension (in) }\end{array}$} & \multirow{2}{*}{$\begin{array}{l}\text { Req'd Thk Due } \\
\text { to Compression } \\
\text { (in) }\end{array}$} \\
\hline & & $S_{t}$ & $S_{c}$ & & & & & \\
\hline \multirow{2}{*}{$\begin{array}{l}\text { Operating, Hot \& } \\
\text { Corroded }\end{array}$} & \multirow{2}{*}{1,150} & \multirow{2}{*}{15,300} & \multirow{2}{*}{10,100} & \multirow{2}{*}{1,000} & \multirow{2}{*}{0.03} & Wind & 0.0618 & 0.0606 \\
\hline & & & & & & Seismic & 0.0623 & 0.0601 \\
\hline \multirow{2}{*}{$\begin{array}{c}\text { Operating, Hot \& } \\
\text { New }\end{array}$} & \multirow{2}{*}{1,150} & \multirow{2}{*}{15,300} & \multirow{2}{*}{10,177} & \multirow{2}{*}{1,000} & \multirow{2}{*}{0} & Wind & 0.061 & 0.0596 \\
\hline & & & & & & Seismic & 0.0614 & 0.059 \\
\hline \multirow{2}{*}{ Empty, Corroded } & \multirow{2}{*}{0} & \multirow{2}{*}{20,000} & \multirow{2}{*}{14,332} & \multirow{2}{*}{0} & \multirow{2}{*}{0.03} & Wind & 0.0003 & 0.0009 \\
\hline & & & & & & Seismic & 0.0007 & 0.0014 \\
\hline \multirow{2}{*}{ Empty, New } & \multirow{2}{*}{0} & \multirow{2}{*}{20,000} & \multirow{2}{*}{14,425} & \multirow{2}{*}{0} & \multirow{2}{*}{0} & Wind & 0.0004 & 0.001 \\
\hline & & & & & & Seismic & 0.0007 & 0.0015 \\
\hline $\begin{array}{l}\text { Hot Shut Down, } \\
\text { Corroded, Weight \& } \\
\text { Eccentric Moments } \\
\text { Only }\end{array}$ & 0 & 15,300 & 10,100 & 1,000 & 0.03 & Weight & 0.0004 & 0.0013 \\
\hline
\end{tabular}




\section{8" X 4" REDUCER B16.9, UPPER (SCH XS) 4" END}

\section{ASME Section VIII Division 1, 2007 Edition}

\section{Component:}

Material specification:

Rated MDMT per UHA-51(d)(1)(b), (carbon content does not exceed 0.1 percent) $=-320^{\circ} \mathrm{F}$
Cylinder

SA-403 316H (II-D p. 78, ln. 8)

Internal design pressure: $\mathrm{P}=1,150 \mathrm{psi} @ 1,000^{\circ} \mathrm{F}$

Static liquid head:

$P_{t h}=0.26 \mathrm{psi}\left(\mathrm{SG}=1, \mathrm{H}_{\mathrm{s}}=7.205^{\prime \prime}\right.$, Horizontal test head $)$

Corrosion allowance $\quad$ Inner $\mathrm{C}=0.03^{\prime \prime} \quad$ Outer $\mathrm{C}=0 "$

Design MDMT $=-20^{\circ} \mathrm{F}$

Rated MDMT $=-320^{\circ} \mathrm{F}$

No impact test performed

Material is not normalized

Material is not produced to Fine Grain Practice

PWHT is not performed

Radiography: Longitudinal joint - Seamless No RT

Top circumferential joint - Full UW-11(a) Type 1

Bottom circumferential joint -

Full UW-11(a) Type 1

$$
\begin{aligned}
\text { Estimated weight New } & =3.4 \mathrm{lb} \quad \text { corr }=3.1 \mathrm{lb} \\
\text { Capacity } \quad \mathrm{New} & =0.16 \mathrm{US} \text { gal corr }=0.16 \mathrm{US} \text { gal }
\end{aligned}
$$

$$
\begin{aligned}
\mathrm{OD} & =4.5^{\prime \prime} \\
\text { Length } \mathrm{L}_{\mathrm{c}} & =3^{\prime \prime} \\
\mathrm{t} & =0.295^{\prime \prime}
\end{aligned}
$$

\section{Design thickness, $\left(\right.$ at $\left.^{1,000}{ }^{\circ} \mathrm{F}\right)$ Appendix 1-1}

$$
\begin{aligned}
\mathrm{t} & =\mathrm{P} * \mathrm{R}_{\mathrm{o}} /\left(\mathrm{S}^{*} \mathrm{E}+0.40^{*} \mathrm{P}\right)+\text { Corrosion } \\
& =1,150^{*} 2.25 /\left(15,300^{*} 1.00+0.40^{*} 1,150\right)+0.03 \\
& =0.1942^{*}
\end{aligned}
$$

Maximum allowable working pressure, (at 1,000 ${ }^{\circ}$ F) Appendix 1-1

$$
\begin{aligned}
\mathrm{P} & =\mathrm{S}^{*} \mathrm{E}^{*} \mathrm{t} /\left(\mathrm{R}_{\mathrm{o}}-0.40^{*} \mathrm{t}\right)-\mathrm{P}_{\mathrm{S}} \\
& =15,300^{*} 1.00^{*} 0.265 /\left(2.25-0.40^{*} 0.265\right)-0 \\
& =1,891.09 \mathrm{psi}
\end{aligned}
$$

\% Forming Strain - UHA-44(a)(2)(a)

$$
\begin{aligned}
\mathrm{EFE} & =\left(50 * \mathrm{t} / \mathrm{R}_{\mathrm{f}}\right) *\left(1-\mathrm{R}_{\mathrm{f}} / \mathrm{R}_{\mathrm{o}}\right) \\
& =(50 * 0.295 / 2.1025) *(1-2.1025 / \infty) \\
& =7.0155 \%
\end{aligned}
$$




\section{Design thickness $=0.1942 "$}

The governing condition is due to internal pressure.

The cylinder thickness of $0.295^{\prime \prime}$ is adequate.

Thickness Required Due to Pressure + External Loads

\begin{tabular}{|c|c|c|c|c|c|c|c|c|}
\hline \multirow[t]{2}{*}{ Condition } & \multirow{2}{*}{$\begin{array}{l}\text { Pressure } \\
\text { P ( psi) }\end{array}$} & \multicolumn{2}{|c|}{$\begin{array}{c}\text { Allowable Stress } \\
\text { Before UG-23 Stress } \\
\text { Increase ( psi) }\end{array}$} & \multirow{2}{*}{$\begin{array}{c}\text { Temperature } \\
\left({ }^{\circ} \mathbf{F}\right)\end{array}$} & \multirow{2}{*}{$\begin{array}{l}\text { Corrosion } \\
\text { C (in) }\end{array}$} & \multirow[t]{2}{*}{ Load } & \multirow{2}{*}{$\begin{array}{c}\text { Req'd Thk } \\
\text { Due to } \\
\text { Tension (in) }\end{array}$} & \multirow{2}{*}{$\begin{array}{l}\text { Req'd Thk Due } \\
\text { to Compression } \\
\text { (in) }\end{array}$} \\
\hline & & $\mathbf{S}_{\mathbf{t}}$ & $\mathbf{S}_{\mathbf{c}}$ & & & & & \\
\hline \multirow{2}{*}{$\begin{array}{l}\text { Operating, Hot \& } \\
\text { Corroded }\end{array}$} & \multirow{2}{*}{1,150} & \multirow{2}{*}{15,300} & \multirow{2}{*}{10,100} & \multirow{2}{*}{1,000} & \multirow{2}{*}{0.03} & Wind & 0.0619 & 0.0605 \\
\hline & & & & & & Seismic & 0.0624 & 0.0599 \\
\hline \multirow{2}{*}{$\begin{array}{c}\text { Operating, Hot \& } \\
\text { New }\end{array}$} & \multirow{2}{*}{1,150} & \multirow{2}{*}{15,300} & \multirow{2}{*}{10,177} & \multirow{2}{*}{1,000} & \multirow{2}{*}{0} & Wind & 0.061 & 0.0595 \\
\hline & & & & & & Seismic & 0.0616 & 0.0589 \\
\hline \multirow{2}{*}{ Empty, Corroded } & \multirow{2}{*}{0} & \multirow{2}{*}{20,000} & \multirow{2}{*}{14,332} & \multirow{2}{*}{0} & \multirow{2}{*}{0.03} & Wind & 0.0003 & 0.001 \\
\hline & & & & & & Seismic & 0.0008 & 0.0016 \\
\hline \multirow{2}{*}{ Empty, New } & \multirow{2}{*}{0} & \multirow{2}{*}{20,000} & \multirow{2}{*}{14,425} & \multirow{2}{*}{0} & \multirow{2}{*}{0} & Wind & 0.0004 & 0.001 \\
\hline & & & & & & Seismic & 0.0009 & 0.0017 \\
\hline $\begin{array}{l}\text { Hot Shut Down, } \\
\text { Corroded, Weight \& } \\
\text { Eccentric Moments } \\
\text { Only }\end{array}$ & 0 & 15,300 & 10,100 & 1,000 & 0.03 & Weight & 0.0004 & 0.0013 \\
\hline
\end{tabular}




\section{8" X 4" REDUCER B16.9, UPPER (SCH XS) 8" END}

\section{ASME Section VIII Division 1, 2007 Edition}

Component: $\quad$ Cylinder

Material specification: $\quad$ SA-403 $316 \mathrm{H}$ (II-D p. 78, $\ln .8$ )

Rated MDMT per UHA-51(d)(1)(b), (carbon content does not exceed 0.1 percent) $=-320^{\circ} \mathrm{F}$

Internal design pressure: $\mathrm{P}=1,150 \mathrm{psi} @ 1,000^{\circ} \mathrm{F}$

Static liquid head:

$\mathrm{P}_{\mathrm{th}}=0.33 \mathrm{psi}\left(\mathrm{SG}=1, \mathrm{H}_{\mathrm{s}}=9.125^{\prime \prime}\right.$, Horizontal test head $)$

Corrosion allowance $\quad$ Inner $\mathrm{C}=0.03^{\prime \prime} \quad$ Outer $\mathrm{C}=0$ "

Design MDMT $=-20^{\circ} \mathrm{F} \quad$ No impact test performed

Rated MDMT $=-320^{\circ} \mathrm{F} \quad$ Material is not normalized

Material is not produced to Fine Grain Practice

PWHT is notperformed

Radiography: Longitudinal joint - Seamless No RT

Top circumferential joint - Full UW-11(a) Type 1

Bottom circumferential $\quad$ Full UW-11(a) Type 1
joint -

Estimated weight New $=9.8 \mathrm{lb} \quad$ corr $=9.2 \mathrm{lb}$

Capacity New $=0.61$ US gal corr $=0.62$ US gal

$\mathrm{OD}=8.625^{\prime \prime}$

Length $\mathrm{L}_{\mathrm{c}}=3^{\prime \prime}$

$\mathrm{t} \quad=0.4375^{\prime \prime}$

Design thickness, $\left(\right.$ at $\left.1,000^{\circ} \mathrm{F}\right)$ Appendix 1-1

$\mathrm{t}=\mathrm{P}^{*} \mathrm{R}_{\mathrm{o}} /(\mathrm{S} * \mathrm{E}+0.40 * \mathrm{P})+$ Corrosion

$=1,150 * 4.3125 /(15,300 * 1.00+0.40 * 1,150)+0.03$

$=0.3447^{\prime \prime}$

Maximum allowable working pressure, (at 1,000 ${ }^{\circ} \mathrm{F}$ ) Appendix 1 -1

$\mathrm{P}=\mathrm{S} * \mathrm{E}^{*} \mathrm{t} /\left(\mathrm{R}_{\mathrm{o}}-0.40 * \mathrm{t}\right)-\mathrm{P}_{\mathrm{S}}$

$=15,300 * 1.00 * 0.4075 /(4.3125-0.40 * 0.4075)-0$

$=1,502.53 \mathrm{psi}$

\% Forming Strain - UHA-44(a)(2)(a)

$$
\begin{aligned}
\mathrm{EFE} & =\left(50 * \mathrm{t} / \mathrm{R}_{\mathrm{f}}\right) *\left(1-\mathrm{R}_{\mathrm{f}} / \mathrm{R}_{\mathrm{o}}\right) \\
& =(50 * 0.4375 / 4.0938) *(1-4.0938 / \infty) \\
& =5.3435 \%
\end{aligned}
$$




\section{Design thickness $=0.3447^{\prime \prime}$}

The governing condition is due to internal pressure.

The cylinder thickness of $0.4375^{\prime \prime}$ is adequate.

Thickness Required Due to Pressure + External Loads

\begin{tabular}{|c|c|c|c|c|c|c|c|c|}
\hline \multirow[t]{2}{*}{ Condition } & \multirow{2}{*}{$\begin{array}{c}\text { Pressure } \\
\text { P ( psi) }\end{array}$} & \multicolumn{2}{|c|}{$\begin{array}{c}\text { Allowable Stress } \\
\text { Before UG-23 Stress } \\
\text { Increase ( psi) }\end{array}$} & \multirow{2}{*}{$\begin{array}{c}\text { Temperature } \\
\left({ }^{\circ} \mathbf{F}\right)\end{array}$} & \multirow{2}{*}{$\begin{array}{l}\text { Corrosion } \\
\mathrm{C} \text { (in) }\end{array}$} & \multirow[t]{2}{*}{ Load } & \multirow{2}{*}{$\begin{array}{l}\text { Req'd Thk } \\
\text { Due to } \\
\text { Tension (in) }\end{array}$} & \multirow{2}{*}{$\begin{array}{l}\text { Req'd Thk Due } \\
\text { to Compression } \\
\text { (in) }\end{array}$} \\
\hline & & $\mathbf{S}_{\mathbf{t}}$ & $\mathbf{S}_{\mathbf{c}}$ & & & & & \\
\hline \multirow{2}{*}{$\begin{array}{l}\text { Operating, Hot \& } \\
\text { Corroded }\end{array}$} & \multirow{2}{*}{1,150} & \multirow{2}{*}{15,300} & \multirow{2}{*}{9,945} & \multirow{2}{*}{1,000} & \multirow{2}{*}{0.03} & Wind & 0.1209 & 0.1205 \\
\hline & & & & & & Seismic & 0.121 & 0.1203 \\
\hline \multirow{2}{*}{$\begin{array}{c}\text { Operating, Hot \& } \\
\text { New }\end{array}$} & \multirow{2}{*}{1,150} & \multirow{2}{*}{15,300} & \multirow{2}{*}{9,995} & \multirow{2}{*}{1,000} & \multirow{2}{*}{0} & Wind & 0.1199 & 0.1195 \\
\hline & & & & & & Seismic & 0.1201 & 0.1193 \\
\hline \multirow{2}{*}{ Empty, Corroded } & \multirow{2}{*}{0} & \multirow{2}{*}{20,000} & \multirow{2}{*}{14,142} & \multirow{2}{*}{0} & \multirow{2}{*}{0.03} & Wind & 0.0001 & 0.0004 \\
\hline & & & & & & Seismic & 0.0002 & 0.0006 \\
\hline \multirow{2}{*}{ Empty, New } & \multirow{2}{*}{0} & \multirow{2}{*}{20,000} & \multirow{2}{*}{14,203} & \multirow{2}{*}{0} & \multirow{2}{*}{0} & Wind & 0.0001 & 0.0004 \\
\hline & & & & & & Seismic & 0.0002 & 0.0006 \\
\hline $\begin{array}{l}\text { Hot Shut Down, } \\
\text { Corroded, Weight \& } \\
\text { Eccentric Moments } \\
\text { Only }\end{array}$ & 0 & 15,300 & 9,945 & 1,000 & 0.03 & Weight & 0 & 0.0005 \\
\hline
\end{tabular}




\section{8" PIPE}

ASME Section VIII Division 1, 2007 Edition

Component:

Material specification:

Pipe NPS and Schedule:

Rated MDMT per UHA-51(d)(1)(b), (carbon content does not exceed 0.1 percent) $=-320^{\circ} \mathrm{F}$
Cylinder

SA-312 TP316H Wld \& smls pipe (II-D p.

$78, \ln .1)$

8 " Sch 80 S (XS)

Internal design pressure: $\mathrm{P}=1,150 \mathrm{psi} @ 1,000^{\circ} \mathrm{F}$

\section{Static liquid head:}

$P_{\text {th }}=0.33$ psi $\left(\mathrm{SG}=1, H_{\mathrm{s}}=9.0625^{\prime \prime}\right.$, Horizontal test head $)$

Corrosion allowance $\quad$ Inner $\mathrm{C}=0.03^{\prime \prime} \quad$ Outer $\mathrm{C}=0$ "

$\begin{array}{ll}\text { Design MDMT }=-20^{\circ} \mathrm{F} & \text { No impact test performed } \\ \text { Rated MDMT }=-320^{\circ} \mathrm{F} & \text { Material is not normalized } \\ & \text { Material is not produced to Fine Grain Practice } \\ & \text { PWHT is not performed }\end{array}$

Radiography: Longitudinal joint - Seamless No RT

Top circumferential joint - Full UW-11(a) Type 1

Bottom circumferential joint -

Full UW-11(a) Type 1

Estimated weight New $=140.6 \mathrm{lb} \quad$ corr $=132.7 \mathrm{lb}$

Capacity New $=7.51$ US gal corr $=7.63$ US gal

$\mathrm{OD}=8.625^{\prime \prime}$

Length $\mathrm{L}_{\mathrm{c}}=38^{\prime \prime}$

$\mathrm{t}$

$=0.5^{\prime \prime}$

Design thickness, (at $1,000^{\circ} \mathrm{F}$ ) Appendix 1-1

$\mathrm{t}=\mathrm{P} * \mathrm{R}_{\mathrm{o}} /(\mathrm{S} * \mathrm{E}+0.40 * \mathrm{P})+$ Corrosion

$=1,150 * 4.3125 /(15,300 * 1.00+0.40 * 1,150)+0.03$

$=0.3447^{\prime \prime}$

Maximum allowable working pressure, $\left(\right.$ at $^{1,000}{ }^{\circ} \mathrm{F}$ ) Appendix 1-1

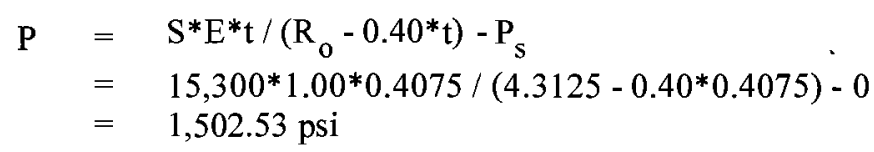

\section{Design thickness $=0.3447^{\prime \prime}$}

The governing condition is due to internal pressure.

The cylinder thickness of 0.5 " is adequate. 
Thickness Required Due to Pressure + External Loads

\begin{tabular}{|c|c|c|c|c|c|c|c|c|}
\hline \multirow[t]{2}{*}{ Condition } & \multirow{2}{*}{$\begin{array}{l}\text { Pressure } \\
\text { P ( psi) }\end{array}$} & \multicolumn{2}{|c|}{$\begin{array}{l}\text { Allowable Stress } \\
\text { Before UG-23 Stress } \\
\text { Increase ( psi) }\end{array}$} & \multirow{2}{*}{$\begin{array}{l}\text { Temperature } \\
\left({ }^{\circ} \mathrm{F}\right)\end{array}$} & \multirow{2}{*}{$\begin{array}{c}\text { Corrosion } \\
\text { C (in) }\end{array}$} & \multirow[t]{2}{*}{ Load } & \multirow{2}{*}{$\begin{array}{l}\text { Req'd Thk } \\
\text { Due to } \\
\text { Tension (in) }\end{array}$} & \multirow{2}{*}{$\begin{array}{l}\text { Req'd Thk Due } \\
\text { to Compression } \\
\text { (in) }\end{array}$} \\
\hline & & $S_{t}$ & $\mathbf{S}_{\mathbf{c}}$ & & & & & \\
\hline \multirow{2}{*}{$\begin{array}{l}\text { Operating, Hot \& } \\
\text { Corroded }\end{array}$} & \multirow{2}{*}{1,150} & \multirow{2}{*}{15,300} & \multirow{2}{*}{9,945} & \multirow{2}{*}{1,000} & \multirow{2}{*}{0.03} & Wind & 0.1195 & 0.1173 \\
\hline & & & & & & Seismic & 0.1199 & 0.1169 \\
\hline \multirow{2}{*}{$\begin{array}{c}\text { Operating, Hot \& } \\
\text { New }\end{array}$} & \multirow{2}{*}{1,150} & \multirow{2}{*}{15,300} & \multirow{2}{*}{9,995} & \multirow{2}{*}{1,000} & \multirow{2}{*}{0} & Wind & 0.1186 & 0.1164 \\
\hline & & & & & & Seismic & 0.119 & 0.1158 \\
\hline \multirow{2}{*}{ Empty, Corroded } & \multirow{2}{*}{0} & \multirow{2}{*}{20,000} & \multirow{2}{*}{14,142} & \multirow{2}{*}{0} & \multirow{2}{*}{0.03} & Wind & 0.0005 & 0.0016 \\
\hline & & & & & & Seismic & 0.0008 & 0.0021 \\
\hline \multirow{2}{*}{ Empty, New } & \multirow{2}{*}{0} & \multirow{2}{*}{20,000} & \multirow{2}{*}{14,203} & \multirow{2}{*}{0} & \multirow{2}{*}{0} & Wind & 0.0005 & 0.0017 \\
\hline & & & & & & Seismic & 0.0009 & 0.0022 \\
\hline $\begin{array}{l}\text { Hot Shut Down, } \\
\text { Corroded, Weight \& } \\
\text { Eccentric Moments } \\
\text { Only }\end{array}$ & 0 & 15,300 & 9,945 & 1,000 & 0.03 & Weight & 0.0005 & 0.001 \\
\hline
\end{tabular}




\section{8" X 4" REDUCER B16.9, LOWER (SCH XS) 8" END}

ASME Section VIII Division 1, 2007 Edition

Component: $\quad$ Cylinder

Material specification: $\quad$ SA-403 $316 \mathrm{H}$ (II-D p. 78, ln. 8)

Rated MDMT per UHA-51(d)(1)(b), (carbon content does not exceed 0.1 percent) $=-320{ }^{\circ} \mathrm{F}$

Internal design pressure: $\mathrm{P}=1,150 \mathrm{psi} @ 1,000^{\circ} \mathrm{F}$

Static liquid head:

$P_{\text {th }}=0.33$ psi $\left(S G=1, H_{s}=9.125^{\prime \prime}\right.$, Horizontal test head $)$

Corrosion allowance $\quad$ Inner $\mathrm{C}=0.03^{\prime \prime} \quad$ Outer $\mathrm{C}=0 "$

Design MDMT $=-20^{\circ} \mathrm{F} \quad$ No impact test performed

Rated MDMT $=-320^{\circ} \mathrm{F} \quad$ Material is not normalized

Material is not produced to Fine Grain Practice

PWHT is not performed

Radiography: $\quad$ Longitudinal joint - $\quad$ Seamless No RT

Top circumferential joint - Full UW-11(a) Type 1

Bottom circumferential

joint -

Full UW-11(a) Type 1

Estimated weight New $=9.8 \mathrm{lb} \quad$ corr $=9.2 \mathrm{lb}$

Capacity New $=0.61$ US gal corr $=0.62$ US gal

$\begin{aligned} \text { OD } & =8.625^{\prime \prime} \\ \text { Length } \mathrm{L}_{\mathrm{c}} & =3^{\prime \prime} \\ \mathrm{t} & =0.4375^{\prime \prime}\end{aligned}$

Design thickness, (at $1,000^{\circ} \mathrm{F}$ ) Appendix 1-1

$\mathrm{t}=\mathrm{P}^{*} \mathrm{R}_{\mathrm{o}} /\left(\mathrm{S} * \mathrm{E}+0.40^{*} \mathrm{P}\right)+$ Corrosion

$=1,150 * 4.3125 /(15,300 * 1.00+0.40 * 1,150)+0.03$

$=0.3447^{\prime \prime}$

Maximum allowable working pressure, (at 1,000 ${ }^{\circ} \mathrm{F}$ ) Appendix 1-1

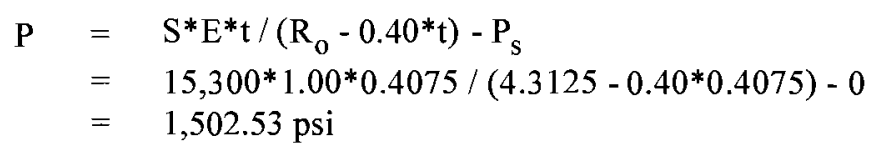

\% Forming Strain - UHA-44(a)(2)(a)

$$
\begin{aligned}
\mathrm{EFE} & =\left(50 * \mathrm{t} / \mathrm{R}_{\mathrm{f}}\right) *\left(1-\mathrm{R}_{\mathrm{f}} / \mathrm{R}_{\mathrm{o}}\right) \\
& =(50 * 0.4375 / 4.0938) *(1-4.0938 / \infty) \\
& =5.3435 \%
\end{aligned}
$$


Design thickness $=0.3447^{\prime \prime}$

The governing condition is due to internal pressure.

The cylinder thickness of $0.4375^{\prime \prime}$ is adequate.

Thickness Required Due to Pressure + External Loads

\begin{tabular}{|c|c|c|c|c|c|c|c|c|}
\hline \multirow[t]{2}{*}{ Condition } & \multirow{2}{*}{$\begin{array}{l}\text { Pressure } \\
\text { P (psi) }\end{array}$} & \multicolumn{2}{|c|}{$\begin{array}{l}\text { Allowable Stress } \\
\text { Before UG-23 Stress } \\
\text { Increase ( psi) }\end{array}$} & \multirow{2}{*}{$\begin{array}{c}\text { Temperature } \\
\left({ }^{\circ} \mathbf{F}\right)\end{array}$} & \multirow{2}{*}{$\begin{array}{c}\text { Corrosion } \\
\text { C (in) }\end{array}$} & \multirow[t]{2}{*}{ Load } & \multirow{2}{*}{$\begin{array}{l}\text { Req'd Thk } \\
\text { Due to } \\
\text { Tension (in) }\end{array}$} & \multirow{2}{*}{$\begin{array}{l}\text { Req'd Thk Due } \\
\text { to Compression } \\
\text { (in) }\end{array}$} \\
\hline & & $S_{\mathbf{t}}$ & $S_{c}$ & & & & & \\
\hline \multirow{2}{*}{$\begin{array}{l}\text { Operating, Hot \& } \\
\text { Corroded }\end{array}$} & \multirow{2}{*}{1,150} & \multirow{2}{*}{15,300} & \multirow{2}{*}{9,945} & \multirow{2}{*}{1,000} & \multirow{2}{*}{0.03} & Wind & 0.1216 & 0.1191 \\
\hline & & & & & & Seismic & 0.1219 & 0.1187 \\
\hline \multirow{2}{*}{$\begin{array}{c}\text { Operating, Hot \& } \\
\text { New }\end{array}$} & \multirow{2}{*}{1,150} & \multirow{2}{*}{15,300} & \multirow{2}{*}{9,995} & \multirow{2}{*}{1,000} & \multirow{2}{*}{0} & Wind & 0.1206 & 0.1182 \\
\hline & & & & & & Seismic & 0.121 & 0.1177 \\
\hline \multirow{2}{*}{ Empty, Corroded } & \multirow{2}{*}{0} & \multirow{2}{*}{20,000} & \multirow{2}{*}{14,142} & \multirow{2}{*}{0} & \multirow{2}{*}{0.03} & Wind & 0.0006 & 0.0018 \\
\hline & & & & & & Seismic & 0.0009 & 0.0022 \\
\hline \multirow{2}{*}{ Empty, New } & \multirow{2}{*}{0} & \multirow{2}{*}{20,000} & \multirow{2}{*}{14,203} & \multirow{2}{*}{0} & \multirow{2}{*}{0} & Wind & 0.0006 & 0.0018 \\
\hline & & & & & & Seismic & 0.0009 & 0.0024 \\
\hline $\begin{array}{l}\text { Hot Shut Down, } \\
\text { Corroded, Weight \& } \\
\text { Eccentric Moments } \\
\text { Only }\end{array}$ & 0 & 15,300 & 9,945 & 1,000 & 0.03 & Weight & 0.0005 & 0.001 \\
\hline
\end{tabular}




\section{8" X 4" REDUCER B16.9, LOWER (SCH XS) 4" END}

\section{ASME Section VIII Division 1, 2007 Edition}

Component:

Material specification:

Rated MDMT per UHA-51(d)(1)(b), (carbon content does not exceed 0.1 percent) $=-320^{\circ} \mathrm{F}$
Cylinder

SA-403 316H (II-D p. 78, ln. 8)

Internal design pressure: $\mathrm{P}=1,150$ psi @ $1,000^{\circ} \mathrm{F}$

Static liquid head:

$P_{\text {th }}=0.26 \mathrm{psi}\left(\mathrm{SG}=1, \mathrm{H}_{\mathrm{s}}=7.205^{\prime \prime}\right.$, Horizontal test head $)$

Corrosion allowance $\quad$ Inner $\mathrm{C}=0.03^{\prime \prime} \quad$ Outer $\mathrm{C}=0^{\prime \prime}$

$\begin{array}{ll}\text { Design MDMT }=-20^{\circ} \mathrm{F} & \text { No impact test performed } \\ \text { Rated MDMT }=-320^{\circ} \mathrm{F} & \text { Material is not normalized } \\ & \text { Material is not produced to Fine Grain Practice } \\ & \text { PWHT is not performed }\end{array}$

Radiography: $\quad$ Longitudinal joint - $\quad$ Seamless No RT

Top circumferential joint - Full UW-11(a) Type 1 Bottom circumferential joint -

Full UW-11(a) Type 1

$$
\begin{aligned}
& \text { Estimated weight } \mathrm{New}=3.4 \mathrm{lb} \quad \text { corr }=3.1 \mathrm{lb} \\
& \text { Capacity } \quad \mathrm{New}=0.16 \mathrm{US} \text { gal corr }=0.16 \mathrm{US} \text { gal } \\
& \text { OD }=4.5^{\prime \prime} \\
& \begin{aligned}
\text { Length } \mathrm{L}_{\mathrm{c}}=3^{\prime \prime} \\
\mathrm{t} \quad=0.295^{\prime \prime}
\end{aligned}
\end{aligned}
$$

\section{Design thickness, (at $1,000^{\circ} \mathrm{F}$ ) Appendix 1-1}

$$
\begin{aligned}
\mathrm{t} & =\mathrm{P} * \mathrm{R}_{\mathrm{o}} /(\mathrm{S} * \mathrm{E}+0.40 * \mathrm{P})+\text { Corrosion } \\
& =1,150^{*} 2.25 /\left(15,300^{*} 1.00+0.40^{*} 1,150\right)+0.03 \\
& =0.1942 "
\end{aligned}
$$

Maximum allowable working pressure, (at 1,000 ${ }^{\circ} \mathrm{F}$ ) Appendix 1-1

$$
\begin{aligned}
\mathrm{P} & =\mathrm{S} * \mathrm{E}^{*} \mathrm{t} /\left(\mathrm{R}_{\mathrm{o}}-0.40^{*} \mathrm{t}\right)-\mathrm{P}_{\mathrm{S}} \\
& =15,300^{*} 1.00^{*} 0.265 /\left(2.25-0.40^{*} 0.265\right)-0 \\
& =1,891.09 \mathrm{psi}
\end{aligned}
$$

\% Forming Strain - UHA-44(a)(2)(a)

$$
\begin{aligned}
\mathrm{EFE} & =\left(50 * t / \mathrm{R}_{\mathrm{f}}\right) *\left(1-\mathrm{R}_{\mathbf{f}} / \mathrm{R}_{\mathrm{o}}\right) \\
& =(50 * 0.295 / 2.1025) *(1-2.1025 / \infty) \\
& =7.0155 \%
\end{aligned}
$$




\section{Design thickness $=0.1942^{\prime \prime}$}

The governing condition is due to internal pressure.

The cylinder thickness of $0.295^{\prime \prime}$ is adequate.

Thickness Required Due to Pressure + External Loads

\begin{tabular}{|c|c|c|c|c|c|c|c|c|}
\hline \multirow[t]{2}{*}{ Condition } & \multirow{2}{*}{$\begin{array}{l}\text { Pressure } \\
\text { P ( psi) }\end{array}$} & \multicolumn{2}{|c|}{$\begin{array}{c}\text { Allowable Stress } \\
\text { Before UG-23 Stress } \\
\text { Increase ( psi) }\end{array}$} & \multirow{2}{*}{$\begin{array}{l}\text { Temperature } \\
\left({ }^{\circ} \mathrm{F}\right)\end{array}$} & \multirow{2}{*}{$\begin{array}{l}\text { Corrosion } \\
\text { C (in) }\end{array}$} & \multirow[t]{2}{*}{ Load } & \multirow{2}{*}{$\begin{array}{c}\text { Req'd Thk } \\
\text { Due to } \\
\text { Tension (in) }\end{array}$} & \multirow{2}{*}{$\begin{array}{l}\text { Req'd Thk Due } \\
\text { to Compression } \\
\text { (in) }\end{array}$} \\
\hline & & $\mathbf{S}_{\mathfrak{t}}$ & $S_{c}$ & & & & & \\
\hline \multirow{2}{*}{$\begin{array}{l}\text { Operating, Hot } \& \\
\text { Corroded }\end{array}$} & \multirow{2}{*}{1,150} & \multirow{2}{*}{15,300} & \multirow{2}{*}{10,100} & \multirow{2}{*}{1,000} & \multirow{2}{*}{0.03} & Wind & 0.0655 & 0.0555 \\
\hline & & & & & & Seismic & 0.0668 & 0.0541 \\
\hline \multirow{2}{*}{$\begin{array}{c}\text { Operating, Hot \& } \\
\text { New }\end{array}$} & \multirow{2}{*}{1,150} & \multirow{2}{*}{15,300} & \multirow{2}{*}{10,177} & \multirow{2}{*}{1,000} & \multirow{2}{*}{0} & Wind & 0.0647 & 0.0544 \\
\hline & & & & & & Seismic & 0.0663 & 0.0527 \\
\hline \multirow{2}{*}{ Empty, Corroded } & \multirow{2}{*}{0} & \multirow{2}{*}{20,000} & \multirow{2}{*}{14,332} & \multirow{2}{*}{0} & \multirow{2}{*}{0.03} & Wind & 0.0032 & 0.0062 \\
\hline & & & & & & Seismic & 0.0042 & 0.0078 \\
\hline \multirow{2}{*}{ Empty, New } & \multirow{2}{*}{0} & \multirow{2}{*}{20,000} & \multirow{2}{*}{14,425} & \multirow{2}{*}{0} & \multirow{2}{*}{0} & Wind & 0.0032 & 0.0064 \\
\hline & & & & & & Seismic & 0.0044 & 0.0082 \\
\hline $\begin{array}{l}\text { Hot Shut Down, } \\
\text { Corroded, Weight \& } \\
\text { Eccentric Moments } \\
\text { Only }\end{array}$ & 0 & 15,300 & 10,100 & 1,000 & 0.03 & Weight & 0.0006 & 0.0025 \\
\hline
\end{tabular}




\section{4" X 2" REDUCER B16.9, LOWER (SCH XS) 4" END}

ASME Section VIII Division 1, 2007 Edition

Component:

Material specification:

Rated MDMT per UHA-51(d)(1)(b), (carbon content does not exceed 0.1 percent) $=-320{ }^{\circ} \mathrm{F}$

Cylinder

SA-403 316 H (II-D p. $78, \ln .8$ )
Internal design pressure: $\mathrm{P}=1,150 \mathrm{psi} @ 1,000^{\circ} \mathrm{F}$

Static liquid head:

$P_{\text {th }}=0.26 \mathrm{psi}\left(\mathrm{SG}=1, \mathrm{H}_{\mathrm{s}}=7.205^{\prime \prime}\right.$, Horizontal test head $)$

Corrosion allowance $\quad$ Inner $\mathrm{C}=0.03^{\prime \prime} \quad$ Outer $\mathrm{C}=0 "$

Design MDMT $=-20^{\circ} \mathrm{F} \quad$ No impact test performed

Rated MDMT $=-320^{\circ} \mathrm{F} \quad$ Material is not normalized

Material is not produced to Fine Grain Practice

PWHT is notperformed

Radiography: $\quad$ Longitudinal joint - $\quad$ Seamless No RT

Top circumferential joint - Full UW-11(a) Type 1

Bottom circumferential joint -

Full UW-11(a) Type 1

Estimated weight New $=2.3 \mathrm{lb} \quad$ corr $=2 \mathrm{lb}$

Capacity $\quad$ New $=0.1$ US gal corr $=0.11$ US gal

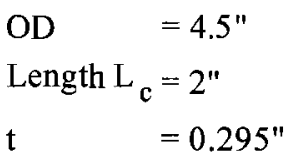

Design thickness, (at $1,000^{\circ} \mathrm{F}$ ) Appendix 1-1

$\mathrm{t}=\mathrm{P} * \mathrm{R}_{\mathrm{o}} /(\mathrm{S} * \mathrm{E}+0.40 * \mathrm{P})+$ Corrosion

$=1,150 * 2.25 /(15,300 * 1.00+0.40 * 1,150)+0.03$

$=0.1942^{\prime \prime}$

Maximum allowable working pressure, (at 1,000 ${ }^{\circ}$ F) Appendix 1-1

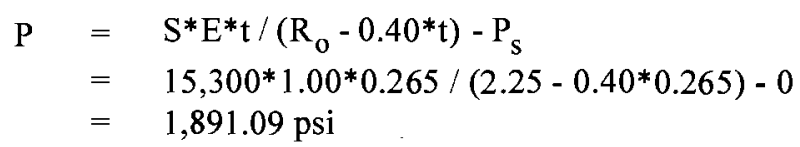

\% Forming Strain - UHA-44(a)(2)(a)

$$
\begin{aligned}
\mathrm{EFE} & =\left(50 * \mathrm{t} / \mathrm{R}_{\mathrm{f}}\right) *\left(1-\mathrm{R}_{\mathrm{f}} / \mathrm{R}_{\mathrm{o}}\right) \\
& =(50 * 0.295 / 2.1025) *(1-2.1025 / \infty) \\
& =7.0155 \%
\end{aligned}
$$




\section{Design thickness $=0.1942 "$}

The governing condition is due to internal pressure.

The cylinder thickness of $0.295^{\prime \prime}$ is adequate.

Thickness Required Due to Pressure + External Loads

\begin{tabular}{|c|c|c|c|c|c|c|c|c|}
\hline \multirow[t]{2}{*}{ Condition } & \multirow{2}{*}{$\begin{array}{l}\text { Pressure } \\
\text { P ( psi) }\end{array}$} & \multicolumn{2}{|c|}{$\begin{array}{l}\text { Allowable Stress } \\
\text { Before UG-23 Stress } \\
\text { Increase ( psi) }\end{array}$} & \multirow{2}{*}{$\begin{array}{l}\text { Temperature } \\
\left({ }^{\circ} \mathrm{F}\right)\end{array}$} & \multirow{2}{*}{$\begin{array}{l}\text { Corrosion } \\
\text { C (in) }\end{array}$} & \multirow[t]{2}{*}{ Load } & \multirow{2}{*}{$\begin{array}{l}\text { Req'd Thk } \\
\text { Due to } \\
\text { Tension (in) }\end{array}$} & \multirow{2}{*}{$\begin{array}{l}\text { Req'd Thk Due } \\
\text { to Compression } \\
\text { (in) }\end{array}$} \\
\hline & & $S_{t}$ & $\mathbf{S}_{\mathbf{c}}$ & & & & & \\
\hline \multirow{2}{*}{$\begin{array}{l}\text { Operating, Hot \& } \\
\text { Corroded }\end{array}$} & \multirow{2}{*}{1,150} & \multirow{2}{*}{15,300} & \multirow{2}{*}{10,100} & \multirow{2}{*}{1,000} & \multirow{2}{*}{0.03} & Wind & 0.0659 & 0.0552 \\
\hline & & & & & & Seismic & 0.0671 & 0.0538 \\
\hline \multirow{2}{*}{$\begin{array}{c}\text { Operating, Hot \& } \\
\text { New }\end{array}$} & \multirow{2}{*}{1,150} & \multirow{2}{*}{15,300} & \multirow{2}{*}{10,177} & \multirow{2}{*}{1,000} & \multirow{2}{*}{0} & Wind & 0.065 & 0.0541 \\
\hline & & & & & & Seismic & 0.0665 & 0.0524 \\
\hline \multirow{2}{*}{ Empty, Corroded } & \multirow{2}{*}{0} & \multirow{2}{*}{20,000} & \multirow{2}{*}{14,332} & \multirow{2}{*}{0} & \multirow{2}{*}{0.03} & Wind & 0.0034 & 0.0066 \\
\hline & & & & & & Seismic & 0.0044 & 0.0081 \\
\hline \multirow{2}{*}{ Empty, New } & \multirow{2}{*}{0} & \multirow{2}{*}{20,000} & \multirow{2}{*}{14,425} & \multirow{2}{*}{0} & \multirow{2}{*}{0} & Wind & 0.0035 & 0.0068 \\
\hline & & & & & & Seismic & 0.0046 & 0.0086 \\
\hline $\begin{array}{l}\text { Hot Shut Down, } \\
\text { Corroded, Weight \& } \\
\text { Eccentric Moments } \\
\text { Only }\end{array}$ & 0 & 15,300 & 10,100 & 1,000 & 0.03 & Weight & 0.0006 & 0.0025 \\
\hline
\end{tabular}




\section{4" X 2" REDUCER B16.9, LOWER (SCH XS) 2" END}

\section{ASME Section VIII Division 1, 2007 Edition}

Component:

Material specification:

Rated MDMT per UHA-51(d)(l)(b), (carbon content does not exceed 0.1 percent) $=-320^{\circ} \mathrm{F}$

Static liquid head:

$\mathrm{P}_{\text {th }}=0.23 \mathrm{psi}\left(\mathrm{SG}=1, \mathrm{H}_{\mathrm{s}}=6.2465^{\prime}\right.$, Horizontal test head $)$

Corrosion allowance $\quad$ Inner $\mathrm{C}=0.03^{\prime \prime} \quad$ Outer $\mathrm{C}=0^{\prime \prime}$

$\begin{array}{ll}\text { Design MDMT }=-20^{\circ} \mathrm{F} & \text { No impact test performed } \\ \text { Rated MDMT }=-320^{\circ} \mathrm{F} & \text { Material is not normalized } \\ & \text { Material is not produced to Fine Grain Practice } \\ & \text { PWHT is not performed }\end{array}$

Radiography: $\quad$ Longitudinal joint - $\quad$ Seamless No RT

Top circumferential joint - Full UW-11(a) Type 1

Bottom circumferential joint -

Full UW-11(a) Type 1

Estimated weight New $=0.8 \mathrm{lb} \quad$ corr $=0.6 \mathrm{lb}$

Capacity New $=0.03$ US gal corr $=0.03$ US gal

OD $=2.375^{\prime \prime}$
Length $\mathrm{L}_{\mathrm{c}}=2^{\prime \prime}$
$\quad=0.191^{\prime \prime}$

Design thickness, (at $1,000^{\circ}$ F) Appendix 1-1

$\mathrm{t}=\mathrm{P}^{*} \mathrm{R}_{\mathrm{o}} /(\mathrm{S} * \mathrm{E}+0.40 * \mathrm{P})+$ Corrosion

$=1,150 * 1.1875 /(15,300 * 1.00+0.40 * 1,150)+0.03$

$=0.1167^{\prime \prime}$

Maximum allowable working pressure, (at 1,000 $\left.{ }^{\circ} \mathrm{F}\right)$ Appendix 1-1

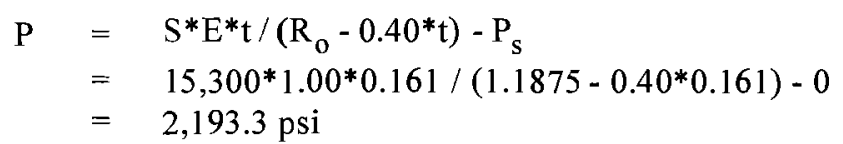

\% Forming Strain - UHA-44(a)(2)(a)

$$
\begin{aligned}
\mathrm{EFE} & =\left(50 * \mathrm{t} / \mathrm{R}_{\mathrm{f}}\right) *\left(1-\mathrm{R}_{\mathrm{f}} / \mathrm{R}_{\mathrm{o}}\right) \\
& =(50 * 0.191 / 1.092) *(1-1.092 / \infty) \\
& =8.7454 \%
\end{aligned}
$$




\section{Design thickness $=0.1167^{\prime \prime}$}

The governing condition is due to internal pressure.

The cylinder thickness of $0.191^{\prime \prime}$ is adequate.

Thickness Required Due to Pressure + External Loads

\begin{tabular}{|c|c|c|c|c|c|c|c|c|}
\hline \multirow[t]{2}{*}{ Condition } & \multirow{2}{*}{$\begin{array}{l}\text { Pressure } \\
\text { P ( psi) }\end{array}$} & \multicolumn{2}{|c|}{$\begin{array}{c}\text { Allowable Stress } \\
\text { Before UG-23 Stress } \\
\text { Increase ( psi) }\end{array}$} & \multirow{2}{*}{$\begin{array}{c}\text { Temperature } \\
\left({ }^{\circ} \mathbf{F}\right)\end{array}$} & \multirow{2}{*}{$\begin{array}{l}\text { Corrosion } \\
\text { C (in) }\end{array}$} & \multirow[t]{2}{*}{ Load } & \multirow{2}{*}{$\begin{array}{l}\text { Req'd Thk } \\
\text { Due to } \\
\text { Tension (in) }\end{array}$} & \multirow{2}{*}{$\begin{array}{l}\text { Req'd Thk Due } \\
\text { to Compression } \\
\text { (in) }\end{array}$} \\
\hline & & $S_{1}$ & $\mathbf{S}_{\mathbf{c}}$ & & & & & \\
\hline \multirow{2}{*}{$\begin{array}{l}\text { Operating, Hot \& } \\
\text { Corroded }\end{array}$} & \multirow{2}{*}{1,150} & \multirow{2}{*}{15,300} & \multirow{2}{*}{10,201} & \multirow{2}{*}{1,000} & \multirow{2}{*}{0.03} & Wind & 0.0532 & 0.0066 \\
\hline & & & & & & Seismic & 0.0571 & 0.0023 \\
\hline \multirow{2}{*}{$\begin{array}{c}\text { Operating, Hot \& } \\
\text { New }\end{array}$} & \multirow{2}{*}{1,150} & \multirow{2}{*}{15,300} & \multirow{2}{*}{10,324} & \multirow{2}{*}{1,000} & \multirow{2}{*}{0} & Wind & 0.053 & 0.0047 \\
\hline & & & & & & Seismic & 0.0582 & 0.0016 \\
\hline \multirow{2}{*}{ Empty, Corroded } & \multirow{2}{*}{0} & \multirow{2}{*}{20,000} & \multirow{2}{*}{14,454} & \multirow{2}{*}{0} & \multirow{2}{*}{0.03} & Wind & 0.0164 & 0.0266 \\
\hline & & & & & & Seismic & 0.0194 & 0.0312 \\
\hline \multirow{2}{*}{ Empty, New } & \multirow{2}{*}{0} & \multirow{2}{*}{20,000} & \multirow{2}{*}{14,604} & \multirow{2}{*}{0} & \multirow{2}{*}{0} & Wind & 0.017 & 0.0274 \\
\hline & & & & & & Seismic & 0.021 & 0.0332 \\
\hline $\begin{array}{l}\text { Hot Shut Down, } \\
\text { Corroded, Weight \& } \\
\text { Eccentric Moments } \\
\text { Only }\end{array}$ & 0 & 15,300 & 10,201 & 1,000 & 0.03 & Weight & 0 & 0.0067 \\
\hline
\end{tabular}




\section{2" RFWN FLANGE 1500\#}

Flange description:

Bolt Material:

Flange rated MDMT:

(Per UHA-51(d)(1)(b))

(Flange rated MDMT $=-320^{\circ} \mathrm{F}$

Bolts rated MDMT per Fig UCS -66 note $(\mathrm{e})=-55^{\circ} \mathrm{F}$ )

Liquid static head on flange:

ASME B16.5 flange rating MAWP:

ASME B 16.5 flange rating MAP:

ASME B16.5 flange hydro test:
2 inch Class 1500 WN A 182 F316H

SA-193 B7 Bolt $<=21 / 2$

$-55^{\circ} \mathrm{F}$

\section{$0 \mathrm{psi}$}

1820 psi@ $1000^{\circ} \mathrm{F}$

$3600 \mathrm{psi}$ @70 $\mathrm{F}$

5425 psi@ $90^{\circ} \mathrm{F}$ 


\section{2" BRANCH (B1)}

\section{ASME Section VIII Division 1, 2007 Edition}

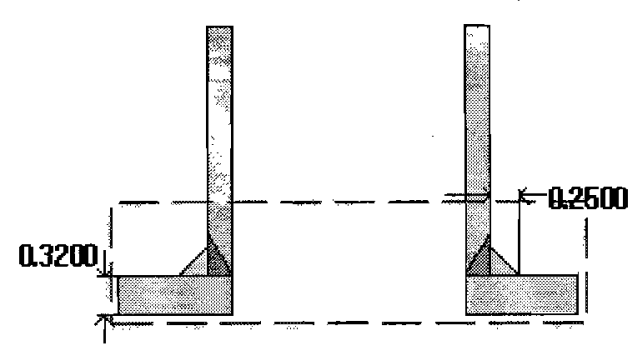

$$
\begin{aligned}
& \mathrm{t}_{\mathrm{w}(\text { lower })}=0 \mathrm{in} \\
& \operatorname{Leg}_{41}=0.25 \mathrm{in}
\end{aligned}
$$

Note: round inside edges per UG-76(c)

Located on:

3 OD" PIPE

Liquid static head included:

$0 \mathrm{psi}$

Nozzle material specification:

SA-312 TP316H Wld \& smls pipe (II-D p. $78, \ln .1$ )

Nozzle longitudinal joint efficiency:

1

$2 "$ Sch 80 S (XS)

Nozzle description:

$190^{\circ}$

Local vessel minimum thickness:

0.28 in

Nozzle center/shell outer surface intersection to datum: 69.25 in

End of nozzle to shell center:

19.25 in

1.939 in

Nozzle inside diameter, new:

0.218 in

Nozzle nominal wall thickness:

0.03 in

Nozzle corrosion allowance:

17.7139 in

Projection available outside vessel, Lpr:

$-5^{\circ}$ from radial

Nozzle is tilted:

\section{Reinforcement Calculations for Internal Pressure}

\begin{tabular}{|c|c|c|c|c|c|c|c|c|}
\hline \multicolumn{7}{|c|}{$\begin{array}{l}\text { Appendix 1-10 Maximum Local Primary Membrane Stress } \\
\text { For } \mathrm{P}=1250 \mathrm{psi} @ 1000{ }^{\circ} \mathrm{F}\end{array}$} & \multicolumn{2}{|c|}{$\begin{array}{l}\text { UG-45 Nozzle Wall } \\
\text { Thickness Summary (in) } \\
\text { The nozzle passes UG-45 }\end{array}$} \\
\hline$\underset{\text { (psi) }}{\mathbf{P}_{\mathbf{L}}}$ & $\begin{array}{c}\mathbf{s}_{\text {allow }} \\
\text { (psi) }\end{array}$ & $\begin{array}{c}A_{1} \\
\left(\text { in }^{2}\right)\end{array}$ & $\begin{array}{c}\mathbf{A}_{2} \\
\left(\mathbf{i n}^{2}\right)\end{array}$ & $\begin{array}{c}\mathbf{A}_{\mathbf{3}} \\
\left(\mathbf{i n}^{2}\right)\end{array}$ & $\begin{array}{c}\mathbf{A}_{5} \\
\left(\text { in }^{2}\right)\end{array}$ & $\begin{array}{c}\text { A } \\
\text { welds } \\
\left(\mathbf{i n}^{2}\right)\end{array}$ & $\begin{array}{l}t_{\text {req }} \\
\text { (in) }\end{array}$ & $\begin{array}{l}t_{\min } \\
\text { (in) }\end{array}$ \\
\hline \multicolumn{7}{|c|}{ This nozzle is exempt from area calculations per UG-36(c)(3)(a) } & 0.1648 & 0.1908 \\
\hline
\end{tabular}

The vessel wall thickness governs the MAWP of thisnozzle.

Division 2 Part 4.5 Strength of Nozzle Attachment Welds Summary Average Shear Stress in Weld

\begin{tabular}{|l|l|l|l|l|l|l|l|l|}
\hline & $\mathbf{L}_{\tau}$ & $\mathbf{L}_{41 \mathrm{~T}}$ & $\mathbf{L}_{42 \mathrm{~T}}$ & $\mathbf{L}_{43 \mathrm{~T}}$ & $\mathbf{f}_{\text {welds }}$ & $\tau$ & $\mathbf{S}$ & Over \\
\hline
\end{tabular}




\begin{tabular}{|c|c|c|c|c|c|c|c|c|}
$k_{y}$ & (in) & (in) & (in) & (in) & (lb $b_{\mathbf{f}}$ & (psi) & (psi) & stressed \\
\hline 1 & 1.8683 & 0.1768 & 0 & 0 & $1,517.52$ & 4,073 & 7,000 & No \\
\hline
\end{tabular}

\begin{tabular}{|c|r|r|c|}
\hline \multicolumn{4}{|c|}{ UW-16 Weld Sizing Sum mary } \\
\hline Weld description & $\begin{array}{l}\text { Required weld } \\
\text { throat size (in) }\end{array}$ & $\begin{array}{r}\text { Actual weld } \\
\text { throat size (in) }\end{array}$ & Status \\
\hline Nozzle to shell fillet $\left(\mathrm{Leg}_{41}\right)$ & 0.1316 & 0.175 & weld size is adequate \\
\hline
\end{tabular}

This opening does not require reinforcement per UG-36(c)(3)(a)

\section{Reinforcement Calculations for MAP}

The thickness requirements of UG-45 govern the MAP of this nozzle.

\begin{tabular}{|c|c|c|c|c|c|c|c|c|}
\hline \multicolumn{7}{|c|}{$\begin{array}{l}\text { Appendix 1-10 Maximum Local Primary Membrane Stress } \\
\text { For } \mathrm{P}=3519.48 \mathrm{psi} @ 7^{\circ} \mathrm{F}\end{array}$} & \multicolumn{2}{|c|}{$\begin{array}{l}\text { UG-45 Nozzle Wall } \\
\text { Thickness Sum mary (in) } \\
\text { The nozzle passes UG-45 }\end{array}$} \\
\hline $\begin{array}{c}\mathbf{P}_{\mathbf{L}} \\
\text { (psi) }\end{array}$ & $\begin{array}{c}\mathbf{S}_{\text {allow }} \\
\text { (psi) }\end{array}$ & $\begin{array}{c}A_{1} \\
\left(\text { in }^{2}\right)\end{array}$ & $\begin{array}{c}\mathbf{A}_{2} \\
\left(\mathrm{in}^{2}\right)\end{array}$ & $\begin{array}{c}\mathbf{A}_{3} \\
\left(\mathbf{i n}^{2}\right)\end{array}$ & $\begin{array}{r}\mathbf{A}_{5} \\
\left(\text { in }^{2}\right)\end{array}$ & $\begin{array}{c}\text { A } \\
\text { welds } \\
\left(\text { in }^{2}\right)\end{array}$ & $\begin{array}{l}\mathbf{t}_{\text {req }} \\
\text { (in) }\end{array}$ & $\begin{array}{l}t_{\min } \\
\text { (in) }\end{array}$ \\
\hline \multicolumn{7}{|c|}{ This nozzle is exempt from area calculations per UG-36(c)(3)(a) } & 0.1907 & 0.1908 \\
\hline
\end{tabular}

Division 2 Part 4.5 Strength of Nozzle Attachment Welds Summary Average Shear Stress in Weld

\begin{tabular}{|c|c|c|c|c|c|c|c|c|}
\hline $\mathbf{k}_{\mathbf{y}}$ & $\begin{array}{c}\mathbf{L}_{\tau} \\
(\mathbf{i n})\end{array}$ & $\begin{array}{c}\mathbf{L}_{41 T} \\
(\mathbf{i n})\end{array}$ & $\begin{array}{c}\mathbf{L}_{42 T} \\
(\text { in) }\end{array}$ & $\begin{array}{c}\mathbf{L}_{43 T} \\
(\mathbf{i n})\end{array}$ & $\begin{array}{c}\mathbf{f}_{\text {welds }} \\
\left(\mathbf{l b}_{\mathbf{f}}\right)\end{array}$ & $\begin{array}{c}\tau \\
(\mathbf{p s i})\end{array}$ & $\begin{array}{c}\mathbf{S} \\
(\mathbf{p s i})\end{array}$ & $\begin{array}{c}\text { Over } \\
\text { stressed }\end{array}$ \\
\hline 1 & 1.8682 & 0.1768 & 0 & 0 & $4,041.71$ & 9,950 & 22,900 & No \\
\hline
\end{tabular}

\begin{tabular}{|c|r|r|c|}
\hline \multicolumn{4}{|c|}{ UW-16 Weld Sizing Summary } \\
\hline Weld description & $\begin{array}{r}\text { Required weld } \\
\text { throat size (in) }\end{array}$ & $\begin{array}{r}\text { Actual weld } \\
\text { throat size (in) }\end{array}$ & Status \\
\hline Nozzle to shell fillet $\left(\right.$ Leg $\left._{41}\right)$ & 0.1526 & 0.175 & weld size is adequate \\
\hline
\end{tabular}

This opening does not require reinforcement per UG-36(c)(3)(a) 


\section{1/2" HALF-COUPLING (7a)}

\section{ASME Section VIII Division 1, 2007 Edition}

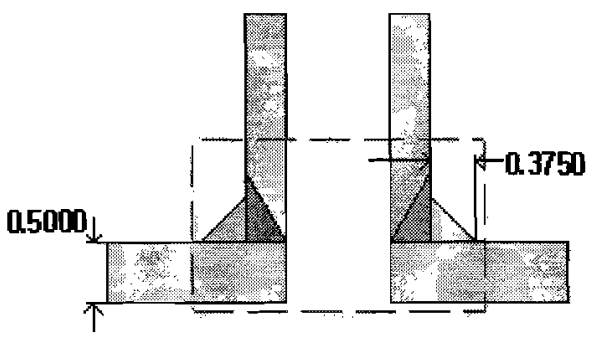

$$
\begin{aligned}
& \mathrm{t}_{\mathrm{w}(\text { lower })}=0 \mathrm{in} \\
& \operatorname{Leg}_{41}=0.375 \mathrm{in}
\end{aligned}
$$

Note. round inside edges per $\mathrm{UG}-706(\mathrm{c})$

Located on:

Liquid static head included:

Nozzle material specification:

Nozzle longitudinal joint efficiency:

Nozzle description:

Nozzle orientation:

Local vessel minimum thickness:

Nozzle center line offset to datum line:

End of nozzle to shell center:

Nozzle inside diameter, new:

Nozzle nominal wall thickness:

Nozzle corrosion allowance:

Projection available outside vessel, Lpr: 0.9375 in

0.4375 in
8" PIPE

0 psi

SA-182 F316H $<=5$ (low stress) (II-D p. 74, ln. 32)

1

$0.500 "$ Class 6000 - threaded

$0^{\circ}$

48.75 in

5.25 in

0.84 in

0.33 in

0.03 in

\section{Reinforcement Calculations for Internal Pressure}

\begin{tabular}{|c|c|c|c|c|c|c|c|c|}
\hline \multicolumn{7}{|c|}{$\begin{array}{l}\text { UG-37 Area Calculation Summary }\left(\mathrm{in}^{2}\right) \\
\text { For } \mathrm{P}=1502.52 \mathrm{psi} @ 1000^{\circ} \mathrm{F}\end{array}$} & \multicolumn{2}{|c|}{$\begin{array}{l}\text { UG-45 Nozzle Wall } \\
\text { Thickness Summary (in) } \\
\text { The nozzle passes UG-45 }\end{array}$} \\
\hline$\underset{\text { required }}{A}$ & $\underset{\text { available }}{\mathrm{A}}$ & $\mathbf{A}_{1}$ & $\mathbf{A}_{2}$ & $\mathbf{A}_{3}$ & $A_{5}$ & $\begin{array}{c}\text { A } \\
\text { welds }\end{array}$ & $\mathbf{t}_{\text {req }}$ & $\mathbf{t}_{\min }$ \\
\hline \multicolumn{7}{|c|}{ This nozzle is exempt from area calculations per UG-36(c)(3)(a) } & 0.1247 & 0.33 \\
\hline
\end{tabular}

The vessel wall thickness governs the MAWP of this nozzle.

UG-41 Weld Failure Path Analysis Summary

The nozzle is exempt from weld strength calculations per UW-15(b)(2)

\begin{tabular}{|l|l|l|}
\hline \multicolumn{3}{|c|}{ UW -16 Weld Sizing Summary } \\
\hline & &
\end{tabular}




\begin{tabular}{|c|r|r|c|} 
Weld description & $\begin{array}{r}\text { Required weld } \\
\text { throat size (in) }\end{array}$ & $\begin{array}{r}\text { Actual weld } \\
\text { throat size (in) }\end{array}$ & Status \\
\hline Nozzle to shell fillet $\left(\operatorname{Leg}_{41}\right)$ & 0.21 & 0.2625 & weld size is adequate \\
\hline
\end{tabular}

This opening does not require reinforcement per UG-36(c)(3)(a)

\section{Reinforcement Calculations for MAP}

The vessel wall thickness governs the MAP of this nozzle.

\begin{tabular}{|c|c|c|c|c|c|c|c|c|}
\hline \multicolumn{7}{|c|}{$\begin{array}{l}\text { UG-37 Area Calculation Summary }\left(\text { in }^{2}\right) \\
\text { For } P=2114.8 \mathrm{psi} @ 70^{\circ} \mathrm{F}\end{array}$} & \multicolumn{2}{|c|}{$\begin{array}{c}\text { UG-45 Nozzle Wall } \\
\text { Thickness Summary (in) } \\
\text { The nozzle passes UG-45 }\end{array}$} \\
\hline$\stackrel{A}{\text { required }}$ & $\underset{\text { available }}{\mathbf{A}}$ & $\mathbf{A}_{1}$ & $\mathbf{A}_{2}$ & $\mathbf{A}_{3}$ & $\mathbf{A}_{5}$ & $\begin{array}{c}\text { A } \\
\text { welds }\end{array}$ & $\mathbf{t}_{\text {req }}$ & $\mathbf{t}_{\mathrm{min}}$ \\
\hline \multicolumn{7}{|c|}{ This nozzle is exempt from area calculations per UG-36(c)(3)(a) } & 0.0761 & 0.33 \\
\hline
\end{tabular}

\section{UG-41 Weld Failure Path Analysis Summary}

The nozzle is exempt from weld strength calculations per UW-15(b)(2)

\begin{tabular}{|c|r|r|c|}
\hline \multicolumn{4}{|c|}{ UW-16 Weld Sizing Summary } \\
\hline Weld description & $\begin{array}{r}\text { Required weld } \\
\text { throat size (in) }\end{array}$ & $\begin{array}{r}\text { Actual weld } \\
\text { throat size (in) }\end{array}$ & Status \\
\hline Nozzle to shell fillet $\left(\right.$ Leg $\left._{41}\right)$ & 0.231 & 0.2625 & weld size is adequate \\
\hline
\end{tabular}

This opening does not require reinforcement per UG-36(c)(3)(a) 


\section{1/2" HALF-COUPLING (7b)}

\section{ASME Section VIII Division 1, 2007 Edition}

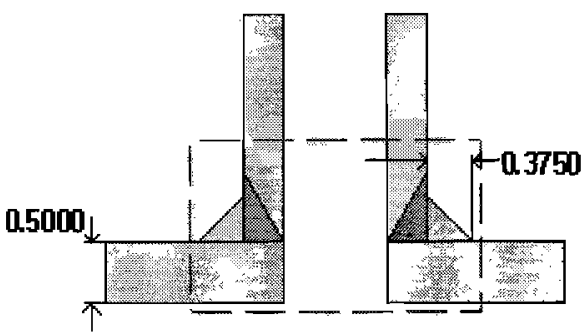

$$
\mathrm{t}_{\mathrm{w}(\text { lower })}=0 \text { in }
$$$$
\operatorname{Leg}_{41}=0.375 \text { in }
$$

Note: round inside edges per UG-76(c)

Located on:

Liquid static head included:

Nozzle material specification:

Nozzle longitudinal joint efficiency:

Nozzle description:

Nozzle orientation:

Local vessel minimum thickness:

Nozzle center line offset to datum line: 48.75 in

End of nozzle to shell center:

Nozzle inside diameter, new:

Nozzle nominal wall thickness:

Nozzle corrosion allowance:

Projection available outside vessel, Lpr: 0.9375 in
8" PIPE

0 psi

SA-182 F316H $<=5$ (low stress) (II-D p. 74, In. 32)

1

$0.500^{\prime \prime}$ Class 6000 - threaded

$180^{\circ}$

0.4375 in

5.25 in

0.84 in

0.33 in

0.03 in

\section{Reinforcement Calculations for Internal Pressure}

The vessel wall thickness governs the MAWP of this nozzle.

\begin{tabular}{|c|c|c|c|c|c|c|c|c|}
\hline \multicolumn{7}{|c|}{$\begin{array}{l}\text { UG-37 Area Calculation Summary }\left(\text { in }^{2}\right) \\
\text { For } P=1502.52 \text { psi } @ 1000^{\circ} \mathrm{F}\end{array}$} & \multicolumn{2}{|c|}{\begin{tabular}{|c|} 
UG-45 Nozzle Wall \\
Thickness Su m mary (in) \\
The nozzle passes UG-45 \\
\end{tabular}} \\
\hline$\underset{\text { required }}{\text { A }}$ & $\underset{\text { available }}{\text { A }}$ & $A_{1}$ & $\mathbf{A}_{2}$ & $\mathbf{A}_{3}$ & $\mathbf{A}_{5}$ & $\underset{\text { welds }}{\mathbf{A}}$ & $t_{\text {req }}$ & $t_{\min }$ \\
\hline \multicolumn{7}{|c|}{ This nozzle is exempt from area calculations perUG-36(c)(3)(a) } & 0.1247 & 0.33 \\
\hline
\end{tabular}

\section{UG-41 Weld Failure Path Analysis Summary}

The nozzle is exempt from weld strength calculations per UW-15(b)(2)

\begin{tabular}{|l|l|l|}
\hline \multicolumn{3}{|c|}{ UW-16 Weld Sizing Summary } \\
\hline $\mid$ & & \\
\hline
\end{tabular}




\begin{tabular}{|c|r|r|c|} 
Weld description & $\begin{array}{r}\text { Required weld } \\
\text { throat size (in) }\end{array}$ & $\begin{array}{r}\text { Actual weld } \\
\text { throat size (in) }\end{array}$ & Status \\
\hline Nozzle to shell fillet $\left(\mathrm{Leg}_{41}\right)$ & 0.21 & 0.2625 & weld size is adequate \\
\hline
\end{tabular}

This opening does not require reinforcement per UG-36(c)(3)(a)

\section{Reinforcement Calculations for MAP}

The vessel wall thickness governs the MAP of this nozzle.

\begin{tabular}{|c|c|c|c|c|c|c|c|c|}
\hline \multicolumn{7}{|c|}{ 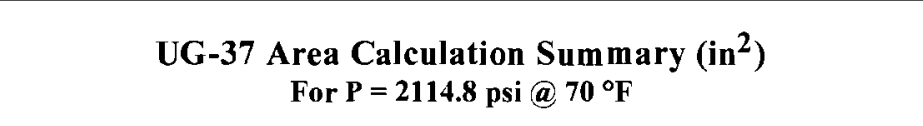 } & \multicolumn{2}{|c|}{$\begin{array}{l}\text { UG-45 Nozzle Wall } \\
\text { Thickness Summary (in) } \\
\text { The nozzle passes UG-45 }\end{array}$} \\
\hline $\begin{array}{c}\text { A } \\
\text { required }\end{array}$ & $\underset{\text { available }}{\text { A }}$ & $\mathbf{A}_{1}$ & $\mathbf{A}_{2}$ & $\mathbf{A}_{3}$ & $\mathbf{A}_{5}$ & $\begin{array}{c}\text { A } \\
\text { welds }\end{array}$ & $t_{\text {req }}$ & $\mathbf{t}_{\min }$ \\
\hline \multicolumn{7}{|c|}{ This nozzle is exempt from area calculations per UG-36(c)(3)(a) } & 0.0761 & 0.33 \\
\hline
\end{tabular}

\section{UG-41 Weld Failure Path Analysis Summary}

The nozzle is exempt from weld strength calculations per UW-15(b)(2)

\begin{tabular}{|c|r|r|c|}
\hline \multicolumn{4}{|c|}{ UW-16 Weld Sizing Sum mary } \\
\hline Weld description & $\begin{array}{r}\text { Required weld } \\
\text { throat size (in) }\end{array}$ & $\begin{array}{r}\text { Actual weld } \\
\text { throat size (in) }\end{array}$ & Status \\
\hline Nozzle to shell fillet $\left(\operatorname{Leg}_{41}\right)$ & 0.231 & 0.2625 & weld size is adequate \\
\hline
\end{tabular}

This opening does not require reinforcement per UG-36(c)(3)(a) 


\section{Wind Code}

Building Code:

Elevation of base above grade:

Increase effective outer diameter by: $0.0000 \mathrm{ft}$

Wind Force Coefficient Cf:

0.8400

Basic Wind Speed:, V:

$85.0000 \mathrm{mph}$

Importance Factor:, I:

1.1500

Exposure category:

$\mathrm{C}$

Wind Directionality Factor, Kd: $\quad 0.9500$

Topographic Factor, Kzt: $\quad 1.0000$

Enforce min. loading of $10 \mathrm{psf}$ : No

\section{Vessel Characteristics}

Vessel height, h:

$6.3584 \mathrm{ft}$

Vessel Minimum Diameter, $b$

Operating, Corroded: $0.1979 \mathrm{ft}$

Empty, Corroded: $0.1979 \mathrm{ft}$

Fundamental Frequency, $\mathrm{n}_{1}$

Operating, Corroded: $7.7056 \mathrm{~Hz}$

Empty, Corroded: $8.5724 \mathrm{~Hz}$

Vacuum, Corroded: $7.7056 \mathrm{~Hz}$

Damping coefficient, $\beta$

Operating, Corroded: 0.0179

Empty, Corroded: 0.0200

Vacuum, Corroded: 0.0179

Table Lookup Values

\subsubsection{Basic Load Combinations for Allowable Stress Design}

The following load combinations are considered in accordance with ASCE section 2.4.1:

5. $D+H+W$

Where

$D \quad=$ Dead load

$H \quad=$ Pressure load

$W \quad=$ Wind load

Wind Deflection Reports:

Operating, Corroded

Empty, Corroded

Wind Pressure Calculations

Wind Deflection Report: Operating, Corroded

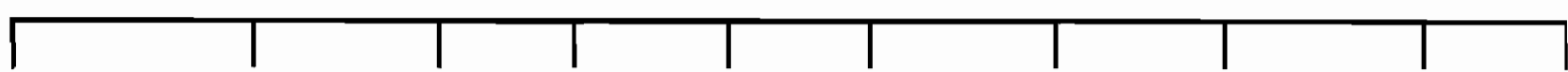




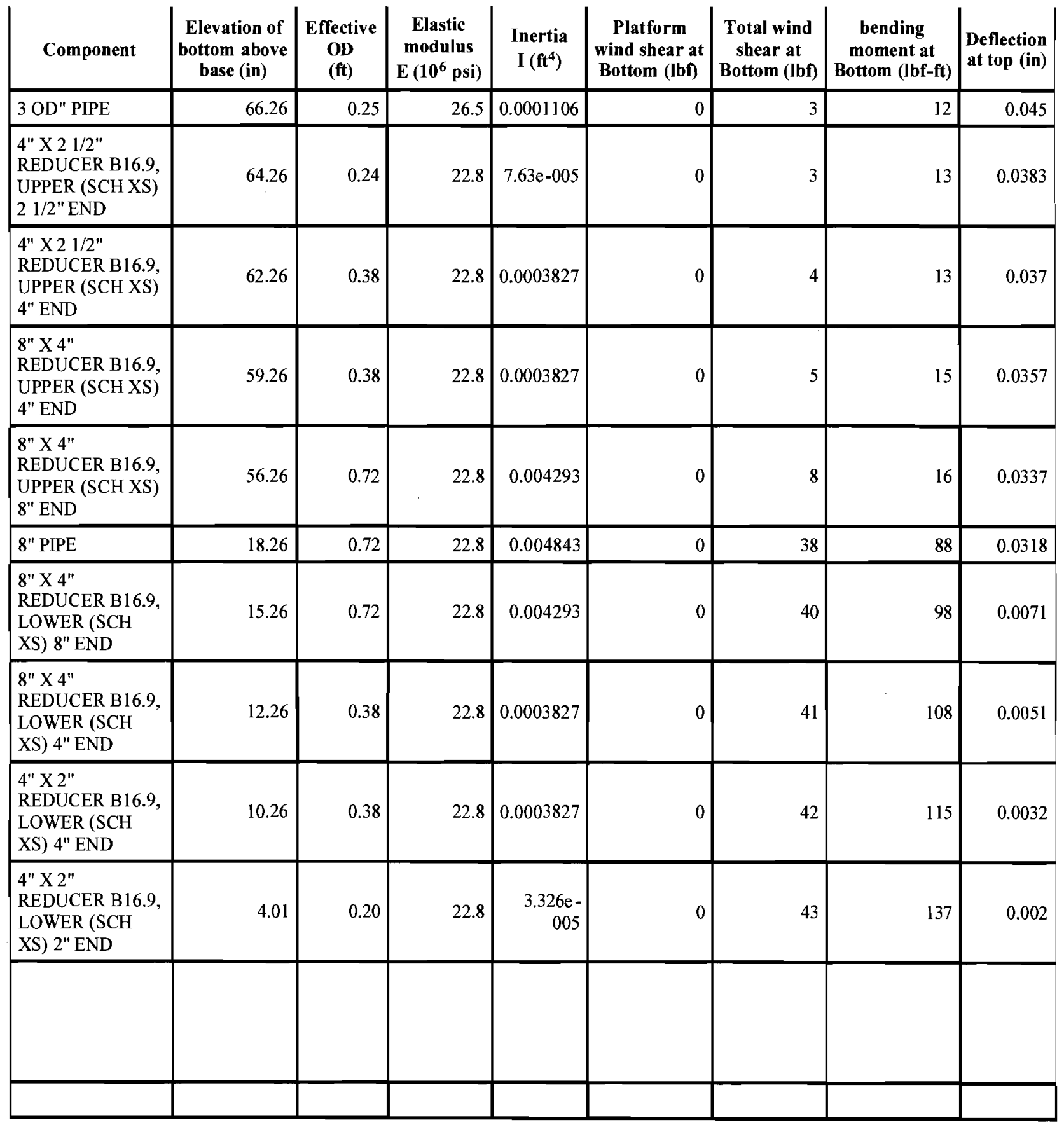

\section{Wind Deflection Report: Empty, Corroded}

\begin{tabular}{|c|c|c|c|c|c|c|c|c|}
\hline Component & $\begin{array}{c}\text { Elevation of } \\
\text { bottom above } \\
\text { base (in) }\end{array}$ & $\begin{array}{c}\text { Effective } \\
\text { OD } \\
\text { (ft) }\end{array}$ & $\begin{array}{c}\text { Elastic } \\
\text { modulus } \\
\text { E }\left(10^{6} \mathrm{psi}\right)\end{array}$ & $\begin{array}{c}\text { Inertia } \\
\text { I }\left(\mathrm{ft}^{4}\right)\end{array}$ & $\begin{array}{c}\text { Platform } \\
\text { wind shear at } \\
\text { Bottom (lbf) }\end{array}$ & $\begin{array}{c}\text { Total wind } \\
\text { shear at } \\
\text { Bottom (lbf) }\end{array}$ & $\begin{array}{c}\text { bending } \\
\text { moment at } \\
\text { Bottom (Ibf-ft) }\end{array}$ & $\begin{array}{l}\text { Deflection } \\
\text { at top (in) }\end{array}$ \\
\hline 3 OD" PIPE & 66.26 & 0.25 & 31.0 & 0.0001106 & 0 & 3 & 12 & 0.0364 \\
\hline $\begin{array}{l}4 " X 21 / 2 " \\
\text { REDUCER B } 16.9 \text {, } \\
\text { UPPER (SCH XS) } \\
21 / 2 " \text { END }\end{array}$ & 64.26 & 0.24 & 28.3 & $7.63 e-005$ & 0 & 3 & 13 & 0.031 \\
\hline & & & & & & & & \\
\hline
\end{tabular}




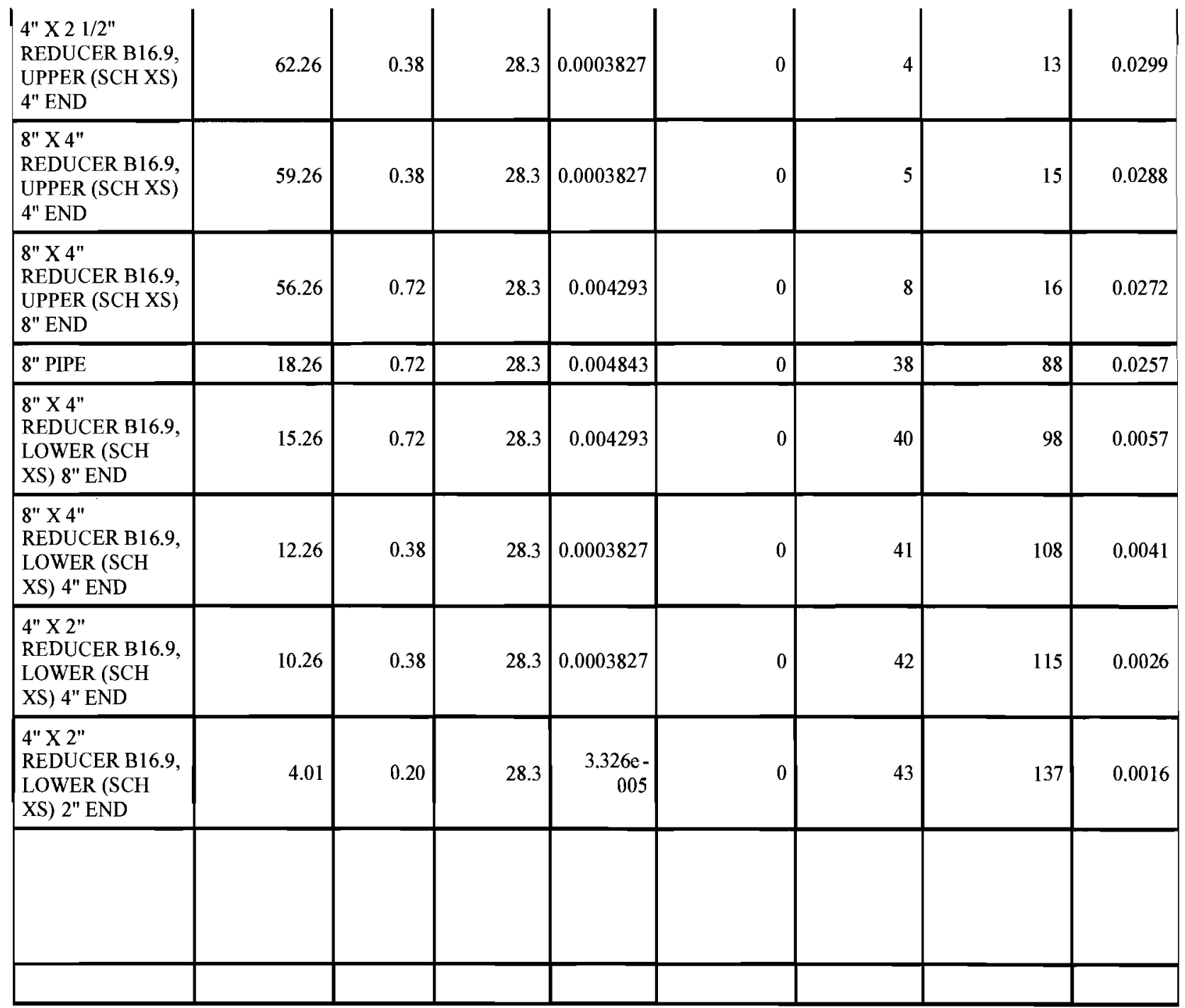

\section{Wind Pressure (WP) Calculations}

Gust Factor $\left(\mathrm{G}^{-}\right)$Calculations

$$
\begin{aligned}
\mathrm{Kz} & =2.01 *(\mathrm{Z} / \underline{\mathrm{Zg}})^{2 / \underline{\alpha}} \\
& =2.01 *(\mathrm{Z} / 900.0000)^{0.2105} \\
\mathrm{qz} & =0.00256 * \mathrm{Kz} * \mathrm{Kzt}^{*} \mathrm{Kd}^{*} \underline{\mathrm{V}}^{2} * \underline{\mathrm{I}} \mathrm{psf} \\
& =0.00256^{*} \mathrm{Kz} * 1.0000 * 0.9500 * 85.0000^{2} * 1.1500 \\
& =20.2069 * \mathrm{Kz} \\
\mathrm{WP} & =\mathrm{qz}^{*} \underline{\mathrm{G}}^{-} * \underline{\mathrm{Cf}} \\
& =\mathrm{qz}^{*} \underline{\mathrm{G}}^{-} * 0.8400
\end{aligned}
$$

\section{Design Wind Pressures}

\begin{tabular}{|c|c|c|c|c|c|c|}
\hline $\begin{array}{c}\text { Height } \mathbf{Z} \\
(')\end{array}$ & $\mathrm{Kz}$ & $\begin{array}{c}\mathrm{qz} \\
(\mathrm{psf})\end{array}$ & $\begin{array}{c}\text { WP: Operating } \\
\text { (psf) }\end{array}$ & $\begin{array}{c}\text { WP: Empty } \\
\text { (psf) }\end{array}$ & $\begin{array}{c}\text { WP: hydrotest } \\
\text { (psf) }\end{array}$ & $\begin{array}{c}\text { WP: Vacuum } \\
\text { (psf) }\end{array}$ \\
\hline
\end{tabular}




\begin{tabular}{|l|l|l|l|l|l|l|}
15.0 & 0.8489 & 17.15 & 13.16 & 13.16 & N.A. & N.A. \\
\hline
\end{tabular}

Design Wind Force determined from: $F=$ Pressure * Af, where Af is the projected area.

\section{Gust Factor Calculations}

Operating, Corroded

Empty, Corroded

\section{Gust Factor Calculations: Operating, Corroded}

$$
\begin{aligned}
\mathrm{z}^{-} & =0.60 * \underline{\mathrm{h}} \\
& =0.60 * 6.3584 \\
& =15.0000 \\
\mathrm{I}_{\mathrm{z}^{-}} & =\underline{\mathrm{c}}^{*}\left(33 / \mathrm{z}^{-}\right)^{1 / 6} \\
& =0.2000 *(33 / 15.0000)^{1 / 6} \\
& =0.2281 \\
\mathrm{~L}_{\mathrm{z}^{-}} & =\underline{1}^{*}\left(\mathrm{z}^{-} / 33\right)^{\mathrm{ep}} \\
& =500.0000 *(15.0000 / 33)^{0.2000} \\
& =427.0566 \\
\mathrm{Q} & =\mathrm{Sqr}\left(1 /\left(1+0.63 *\left((\mathrm{~b}+\underline{\mathrm{h}}) / \mathrm{L}_{\mathrm{z}}\right)^{0.63}\right)\right) \\
& =\mathrm{Sqr}\left(1 /\left(1+0.63 *((0.1979+6.3584) / 427.0566)^{0.63}\right)\right) \\
& =0.9781 \\
\mathrm{G} & =0.925 *\left(1+1.7 * \mathrm{~g}_{-\mathrm{Q}}^{*} \mathrm{I}_{\mathrm{z}}{ }^{*} \mathrm{Q}\right) /\left(1+1.7 * \mathrm{~g}_{\underline{\mathrm{v}}}^{*} \mathrm{I}_{\mathrm{z}^{-}}\right) \\
& =0.925 *\left(1+1.7 * 3.40^{*} 0.2281 * 0.9781\right) /(1+1.7 * 3.40 * 0.2281) \\
& =0.9135
\end{aligned}
$$

\section{Gust Factor Calculations: Empty, Corroded}

$$
\begin{aligned}
& \mathrm{z}^{-}=0.60 * \underline{\mathrm{L}} \\
& =0.60 * 6.3584 \\
& =15.0000 \\
& \mathrm{I}_{\mathrm{z}^{-}}=\mathrm{c}^{*}\left(33 / \mathrm{z}^{-}\right)^{1 / 6} \\
& =0.2000 *(33 / 15.0000)^{1 / 6} \\
& =0.2281 \\
& \mathrm{~L}_{\mathrm{z}}^{-}=\underline{1}^{*}\left(\mathrm{z}^{-} / 33\right)^{\mathrm{e} p} \\
& =500.0000 *(15.0000 / 33)^{0.2000} \\
& =427.0566 \\
& \mathrm{Q}=\operatorname{Sqr}\left(1 /\left(1+0.63^{*}\left((\underline{\mathrm{b}}+\mathrm{h}) / \mathrm{L}_{\mathrm{z}^{-}}\right)^{0.63}\right)\right) \\
& =\operatorname{Sqr}\left(1 /\left(1+0.63 *((0.1979+6.3584) / 427.0566)^{0.63}\right)\right) \\
& =0.9781 \\
& \mathrm{G}=0.925 *\left(1+1.7 * \mathrm{~g}_{\underline{Q}} * \mathrm{I}_{\mathrm{z}}{ }^{*} \mathrm{Q}\right) /\left(1+1.7 * \mathrm{~g}_{\underline{\mathrm{v}}} * \mathrm{I}_{\mathrm{z}}\right) \\
& =0.925 *(1+1.7 * 3.40 * 0.2281 * 0.9781) /(1+1.7 * 3.40 * 0.2281)
\end{aligned}
$$




$$
=0.9135
$$

\section{Table Lookup Values}

$$
\begin{array}{lr}
\alpha=9.5000, \mathrm{Zg}=900.0000^{\prime} & {[\text { Table 6-2, page 78] }} \\
\mathrm{c}=0.2000,1=500.0000, \text { ep }=0.2000 & {[\text { Table 6-2, page 78] }} \\
\mathrm{a}^{-}=0.1538, \mathrm{~b}^{-}=0.6500 & {[\text { Table 6-2, page 78] }} \\
\mathrm{g}_{\mathrm{Q}}=3.40 & {[6.5 .8 .1 \text { page 26] }} \\
\mathrm{g}_{\mathrm{v}}=3.40 & {[6.5 .8 .1 \text { page 26] }}
\end{array}
$$




\section{Seismic Code}

Method of seismic analysis:

Site Class

Importance Factor:

Spectral Response Acceleration at short period (\%g)

Spectral Response Acceleration at period of $1 \mathrm{sec}(\% \mathrm{~g}) \mathrm{S}_{1}=10.00 \%$

Response Modification Coeficient from Table 15.4-2

Acceleration based site co-efficient:

$\mathrm{R}=3.0000$

$\mathrm{F}_{\mathrm{a}}=1.4800$

Velocity based site co-efficient:

Long-period transition period:

Redundancy factor:

User Defined Vertical Accelerations Considered:

Force Multiplier:

Minimum Weight Multiplier:

$\mathrm{T}_{\mathrm{L}}=12.0000$

$\rho=1.0000$

Yes

$=0.3333$

$=0.2000$

\section{ASCE 7-05 ground supported}

$\mathrm{D}$

$\mathrm{I}=1.5000$

$\mathrm{S}_{\mathrm{S}}=40.00 \%$

\subsubsection{Basic Load Combinations for Allowable Stress Design}

The following load combinations are considered in accordance with ASCE section 2.4.1:

5. $D+H+0.7 E=\left(1.0+V_{\text {Acce }}\right) D+H+0.7 \rho Q_{E}$

Where

$D \quad=$ Dead load

$H \quad=$ Pressure load

$E \quad=$ Seismic load $=\rho Q_{E}$

$V_{\text {Accel }}=$ User defined vertical acceleration

\section{Vessel Characteristics}

Vessel height: $6.3584 \mathrm{ft}$

Vessel Weight:

Operating, Corroded: $270 \mathrm{lb}$

Empty, Corroded: $270 \mathrm{lb}$

\section{Period of Vibration Calculation}

Fundamental Period, T:

Operating, Corroded: $0.130 \sec (f=7.7 \mathrm{~Hz})$

Empty, Corroded: $0.117 \mathrm{sec}(\mathrm{f}=8.6 \mathrm{~Hz})$

The fundamental period of vibration $\mathrm{T}$ (above) is calculated using the Rayleigh method of approximation:

$\mathrm{T}=2 * \mathrm{PI} * \operatorname{Sqr}\left(\left\{\operatorname{Sum}\left(\mathrm{W}_{\mathrm{i}}{ }^{*} \mathrm{y}_{\mathrm{i}}{ }^{2}\right)\right\} /\left\{\mathrm{g} * \operatorname{Sum}\left(\mathrm{W}_{\mathrm{i}}^{*} \mathrm{y}_{\mathrm{i}}\right)\right\}\right)$, where 
$\mathrm{W}_{\mathrm{i}}$ is the weight of the $i^{\text {th }}$ lumped mass, and

$y_{i}$ is its deflection when the system is treated as a cantilever beam.

Seismic Shear Reports:

Operating, Corroded

Empty, Corroded

Base Shear Calculations

Seismic Shear Report: Operating, Corroded

\begin{tabular}{|c|c|c|c|c|c|}
\hline Component & $\begin{array}{l}\text { Elevation of bottom } \\
\text { above base (in) }\end{array}$ & $\begin{array}{c}\text { Elastic modulus } E \\
\left(10^{6} \mathrm{psi}\right)\end{array}$ & $\begin{array}{c}\text { Inertia I } \\
\left(\mathrm{ft}^{4}\right)\end{array}$ & $\begin{array}{c}\text { Seismic shear at } \\
\text { Bottom (lbf) }\end{array}$ & $\begin{array}{l}\text { Bending Moment at } \\
\text { Bottom (lbf- ft) }\end{array}$ \\
\hline 3 OD" PIPE & 66.26 & 26.5 & 0.0001 & 13 & 19 \\
\hline $\begin{array}{l}4 " \text { X } 2 \text { 1/2" REDUCER B16.9, } \\
\text { UPPER (SCH XS) } 2 \text { 1/2" END }\end{array}$ & 64.26 & 22.8 & 0.0001 & 13 & 21 \\
\hline $\begin{array}{l}\text { 4" X } 2 \text { 1/2" REDUCER B16.9, } \\
\text { UPPER (SCH XS) 4" END }\end{array}$ & 62.26 & 22.8 & 0.0004 & 14 & 23 \\
\hline $\begin{array}{l}\text { 8" X 4" REDUCER B16.9, UPPER } \\
\text { (SCH XS) 4" END }\end{array}$ & 59.26 & 22.8 & 0.0004 & 14 & 27 \\
\hline $\begin{array}{l}\text { 8" X 4" REDUCER B16.9, UPPER } \\
\text { (SCH XS) 8" END }\end{array}$ & 56.26 & 22.8 & 0.0043 & 16 & 30 \\
\hline 8" PIPE & 18.26 & 22.8 & 0.0048 & 35 & 118 \\
\hline $\begin{array}{l}\text { 8" X 4" REDUCER B16.9, LOWER } \\
\text { (SCH XS) 8" END }\end{array}$ & 15.26 & 22.8 & 0.0043 & 36 & 126 \\
\hline $\begin{array}{l}\text { 8" X 4" REDUCER B16.9, LOWER } \\
\text { (SCH XS) 4" END }\end{array}$ & 12.26 & 22.8 & 0.0004 & 36 & 135 \\
\hline $\begin{array}{l}\text { 4" X 2" REDUCER B16.9, LOWER } \\
\text { (SCH XS) 4" END }\end{array}$ & 10.26 & 22.8 & 0.0004 & 36 & 141 \\
\hline $\begin{array}{l}\text { 4" X 2" REDUCER B16.9, LOWER } \\
\text { (SCH XS) 2" END }\end{array}$ & 4.01 & 22.8 & 0.0000 & 37 & 160 \\
\hline $\begin{array}{l}\text { ASME B16.5/16.47 Blind on 2" } \\
\text { RFWN FLANGE 1500\# (top) }\end{array}$ & 4 & 20.4 & 0.0124 & 37 & 160 \\
\hline Support Skirt \#1 & 0 & 29.3 & 0.001519 & 37 & 173 \\
\hline
\end{tabular}

\section{Seismic Shear Report: Empty, Corroded}

\begin{tabular}{|l|r|r|r|r|r|}
\hline \multicolumn{1}{|c|}{ Component } & $\begin{array}{c}\text { Elevation of bottom } \\
\text { above base (in) }\end{array}$ & $\begin{array}{r}\text { Elastic modulus E } \\
\left(\mathbf{1 0 ^ { 6 }} \mathbf{~ p s i )}\right.\end{array}$ & $\begin{array}{c}\text { Inertia I } \\
\left(\mathbf{f t}^{4}\right)\end{array}$ & $\begin{array}{c}\text { Seismic shear at } \\
\text { Bottom (lbf) }\end{array}$ & $\begin{array}{c}\text { Bending Moment at } \\
\text { Bottom (lbf-ft) }\end{array}$ \\
\hline 3 OD" PIPE & 66.26 & 31.0 & 0.0001 & 13 & 13 \\
\hline $\begin{array}{l}\text { 4" X 2 1/2" REDUCER B16.9, } \\
\text { UPPER (SCH XS) 2 1/2" END }\end{array}$ & 64.26 & 28.3 & 0.0001 & 21 \\
\hline $\begin{array}{l}\text { 4" X 2 1/2" REDUCER B16.9, } \\
\text { UPPER (SCH XS) 4" END }\end{array}$ & 62.26 & 28.3 & 0.0004 & & 14 \\
\hline $\begin{array}{l}\text { 8" X 4" REDUCER B16.9, UPPER } \\
\text { (SCH XS) 4" END }\end{array}$ & 59.26 & 28.3 & 0.0004 & & 14 \\
\hline $\begin{array}{l}\text { 8" X 4" REDUCER B16.9, UPPER } \\
\text { (SCH XS) 8" END }\end{array}$ & 56.26 & 28.3 & 0.0043 & & 27 \\
\hline
\end{tabular}




\begin{tabular}{|c|c|c|c|c|c|}
\hline 8" PIPE & 18.26 & 28.3 & 0.0048 & 35 & 118 \\
\hline $\begin{array}{l}\text { 8" X 4" REDUCER B16.9, LOWER } \\
\text { (SCH XS) 8" END }\end{array}$ & 15.26 & 28.3 & 0.0043 & 36 & 126 \\
\hline $\begin{array}{l}\text { 8" X 4" REDUCER B16.9, LOWER } \\
\text { (SCH XS) 4" END }\end{array}$ & 12.26 & 28.3 & 0.0004 & 36 & 135 \\
\hline $\begin{array}{l}\text { 4" X 2" REDUCER B16.9, LOWER } \\
\text { (SCH XS) 4" END }\end{array}$ & 10.26 & 28.3 & 0.0004 & 36 & 141 \\
\hline $\begin{array}{l}\text { 4" X 2" REDUCER B16.9, LOWER } \\
\text { (SCH XS) 2" END }\end{array}$ & 4.01 & 28.3 & 0.0000 & 37 & 160 \\
\hline $\begin{array}{l}\text { ASME B16.5/16.47 Blind on 2" } \\
\text { RFWN FLANGE 1500\# (top) }\end{array}$ & 4 & 29.4 & 0.0124 & 37 & 160 \\
\hline Support Skirt \#1 & 0 & 29.4 & 0.001519 & 37 & 173 \\
\hline
\end{tabular}

\subsection{3: Maximum considered earthquake spectral response acceleration}

The maximum considered earthquake spectral response acceleration at short period, $\mathrm{S}_{M S}$

$\mathrm{S}_{M S}=\mathrm{E}_{\mathrm{a}} * \underline{\mathrm{S}}=1.4800 * 40.00 / 100=0.5920$

The maximum considered earthquake spectral response acceleration at $1 \mathrm{~s}$ period, $\mathrm{S}_{M 1}$

$\mathrm{S}_{M l}=\underline{\mathrm{E}}_{\underline{\mathrm{V}}} * \underline{\mathrm{S}}_{\underline{\mathrm{l}}}=2.4000 * 10.00 / 100=0.2400$

\subsection{4: Design spectral response acceleration parameters}

Design earthquake spectral response acceleration at short period, $\mathrm{S}_{D S}$

$\mathrm{S}_{D S}=2 / 3 * \mathrm{~S}_{M S}=2 / 3 * 0.5920=0.3947$

Design earthquake spectral response acceleration at $1 \mathrm{~s}$ period, $\mathrm{S}_{D I}$

$\mathrm{S}_{D 1}=2 / 3 * \mathrm{~S}_{M 1}=2 / 3 * 0.2400=0.1600$

User Defined Vertical Acceleration Term, $V_{\text {Accel }}$

Factor is applied to dead load.

Compressive Side: $=1.0+\mathrm{V}_{\text {Accel }}$

\section{$V_{\text {Accel }}$ Term is:}

greater of (Force Mult * Base Shear / Weight) or (Min. Weight Mult.)

\begin{tabular}{|l|r|r|r|r|}
\hline \multicolumn{2}{|c|}{ Force multiplier $=0.3333$} & \multicolumn{3}{|c|}{ Minimum Weight Multiplier $=0.2000$} \\
\hline Condition & Base Shear ( Ibf) & Weight ( Ib) & $\begin{array}{l}\text { Force Mult * Shear } \\
\text { Weight }\end{array}$ & V Accel \\
\hline Operating, Corroded & 37 & 270.5 & 0.046 & 0.2 \\
\hline Operating, New & 39 & 282.6 & 0.046 & 0.2 \\
\hline Empty, Corroded & 37 & 270.5 & 0.046 & 0.2 \\
\hline Empty, New & 39 & 282.6 & 0.046 & 0.2 \\
\hline
\end{tabular}




\section{Base Shear Calculations}

Operating, Corroded

Empty, Corroded

\section{Base Shear Calculations: Operating, Corroded}

\section{Paragraph 15.4.4: Period Determination}

Fundamental Period is taken from the Rayleigh method listed previously in this report.

$\mathrm{T}=0.130 \mathrm{sec}$.

\subsection{1: Calculation of Seismic Response Coefficient}

$\mathrm{C}_{\mathrm{s}}$ is the value computed below, bounded by $\mathrm{C}_{\mathrm{s}} \mathrm{Min}$ and $\mathrm{C}_{\mathrm{s}} \mathrm{Max}$ :

$\mathrm{C}_{\mathrm{s}} \mathrm{Min}$ is 0.01 unless $\mathrm{S}_{1}>=0.6 \mathrm{~g}$, in which case eqn $12.8-6$ is used.

$\mathrm{C}_{\mathrm{S}}$ Max calculated with $12.8-3$ because $(\mathrm{T}=0.130)<=\left(\mathrm{T}_{\mathrm{L}}=12.0000\right)$

$\mathrm{C}_{\mathrm{S}}=\underline{\mathrm{S}}_{\underline{D S}} /(\underline{\mathrm{R}} / \mathrm{D})=0.3947 /(3.0000 / 1.5000)=0.1973$

$\mathrm{C}_{\mathrm{s}} \mathrm{Min}=0.01$

$\mathrm{C}_{\mathrm{s}} \mathrm{Max}=\underline{\mathrm{S}}_{\underline{D I}} /(\mathrm{T} *(\mathrm{R} / \mathrm{I}))=0.1600 /(0.1281 *(3.0000 / 1.5000))=0.6244$

$\mathrm{C}_{\mathrm{s}}=0.1973$

\subsection{1: Calculation of Base Shear}

$$
\begin{aligned}
\mathrm{V} & =\mathrm{C}_{\mathrm{S}} * \underline{\mathrm{W}} \\
& =0.1973 * 270.4562 \\
& =53.37 \mathrm{lb}
\end{aligned}
$$

\subsubsection{Seismic Load Combinations: Horizontal Seismic Load Effect, $\mathbf{E}_{\mathbf{h}}$}

$\mathrm{Q}_{\mathrm{E}}=\mathrm{V}$

$E_{h}=0.7 * \rho * Q_{E}($ Only $70 \%$ of seismic load considered as per Section 2.4.1)

$=0.70 * 1.0000 * 53.37$

$=37.36 \mathrm{lb}$

Base Shear Calculations: Empty, Corroded

\section{Paragraph 15.4.4: Period Determination}

Fundamental Period is taken from the Rayleigh method listed previously in this report. $\mathrm{T}=0.117 \mathrm{sec}$.

\subsection{1: Calculation of Seismic Response Coefficient}

$\mathrm{C}_{\mathrm{S}}$ is the value computed below, bounded by $\mathrm{C}_{\mathrm{s}} \mathrm{Min}$ and $\mathrm{C}_{\mathrm{s}} \mathrm{Max}$ :

$\mathrm{C}_{\mathrm{s}} \mathrm{Min}$ is 0.01 unless $\mathrm{S}_{1}>=0.6 \mathrm{~g}$, in which case eqn 12.8-6 is used.

$\mathrm{C}_{\mathrm{s}}$ Max calculated with $12.8-3$ because $(\mathrm{T}=0.117)<=\left(\mathrm{T}_{\mathrm{L}}=12.0000\right)$ 


$$
\begin{aligned}
& \mathrm{C}_{\mathrm{s}}=\underline{\mathrm{S}}_{\underline{ } \mathrm{S}} /(\underline{\mathrm{R}} / \mathrm{I}=0.3947 /(3.0000 / 1.5000)=0.1973 \\
& \mathrm{C}_{\mathrm{s}} \operatorname{Min}=0.01 \\
& \mathrm{C}_{\mathrm{s}} \mathrm{Max}=\underline{\mathrm{S}}_{\underline{D I}} /(\mathrm{T} *(\underline{\mathrm{R}} / \mathrm{I}))=0.1600 /(0.1167 *(3.0000 / 1.5000))=0.6858 \\
& \mathrm{C}_{\mathrm{s}}=0.1973
\end{aligned}
$$

\subsection{1: Calculation of Base Shear}

$$
\begin{aligned}
\mathrm{V} & =\mathrm{C}_{\mathrm{s}} * \underline{\mathrm{W}} \\
& =0.1973 * 270.4562 \\
& =53.37 \mathrm{lb}
\end{aligned}
$$

12.4.2.1 Seismic Load Combinations: Horizontal Seismic Load Effect, $E_{h}$

$$
\begin{aligned}
\mathrm{Q}_{\mathrm{E}} & =\mathrm{V} \\
\mathrm{E}_{\mathrm{h}} & =0.7 * \rho * \mathrm{Q}_{\mathrm{E}}(\text { Only } 70 \% \text { of seismic load considered as per Section 2.4.1) } \\
& =0.70 * 1.0000 * 53.37 \\
& =37.36 \mathrm{lb}
\end{aligned}
$$


CALCULATIONS PER ASME B31.3 2006 EDITION

NOTE: THESE CALCULATIONS INCLUDE UPPER CHAR POT ONLY

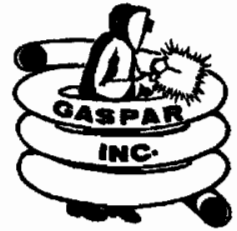

PER SECTION 304.1.1(A) EQUATION (2) AND SECTION 304.1.2 EQUATION \#3A $\operatorname{tm}=\left(P^{*} \mathrm{Do} /\left(2^{*}\left(S^{*} E^{*} W+P^{*} Y\right)\right)\right)+c$

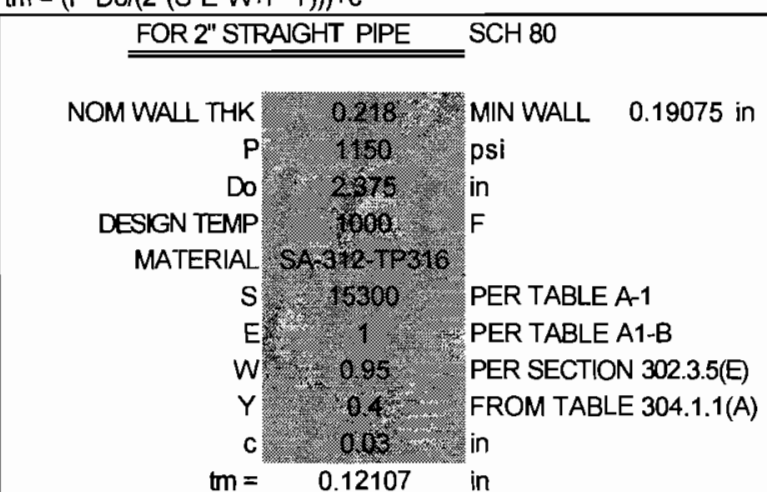

SINCE THE MIN WAU THK IS GREATER THAN tm, THE THICKNESS IS ADEQUATE

PER SECTION 304.1.1(A) EQUATION (2) AND SECTION 304.1.2 EQUATION \#3A $\mathrm{tm}=\left(\mathrm{P}^{*} \mathrm{Do} /\left(2^{*}\left(\mathrm{~S}^{*} \mathrm{E}^{*} \mathrm{~W}+\mathrm{P}^{*} \mathrm{Y}\right)\right)\right)+\mathrm{C}$

\begin{tabular}{|c|c|}
\hline FOR 1" STRAIGHT PIPE & $\mathrm{SCH} 80$ \\
\hline 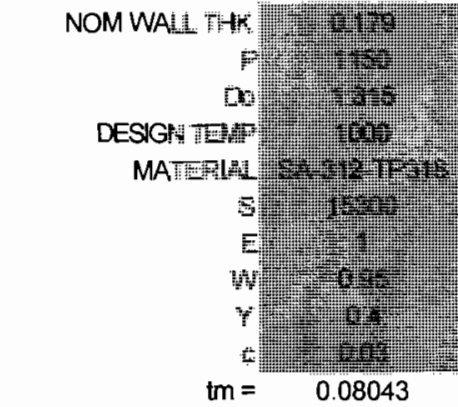 & 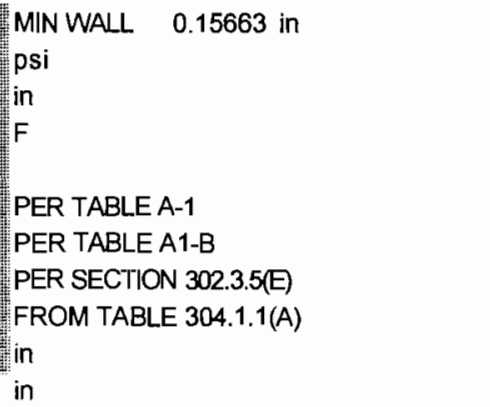 \\
\hline SINCE THE MIN WAL THK IS GREATER & R THAN tm, THE THICKNESS IS ADEQUATE \\
\hline
\end{tabular}

PER SECTION 304.1.1(A) EQUATION (2), SEC. 304.1.5 EQUATION \#3A, SEC. 326.1, 326.2, AND TABLE 326.1 STANDARDS SEC. 304.3.2(B), SEC. 328.5, 328.5.4; ASME B16.11 SEC 2.1 
ASME B16.11 TABLE 2, ASME B1.20.1 NPT

$\mathrm{tm}=\left(\mathrm{P}^{*} \mathrm{Do} /\left(2^{*}\left(\mathrm{~S}^{*} \mathrm{E}^{*} \mathrm{~W}+\mathrm{P}^{*} \mathrm{Y}\right)\right)\right)+\mathrm{c}$

FOR B16.11 1/2" NPT THREADED FITTING $\quad$ CLASS 6000

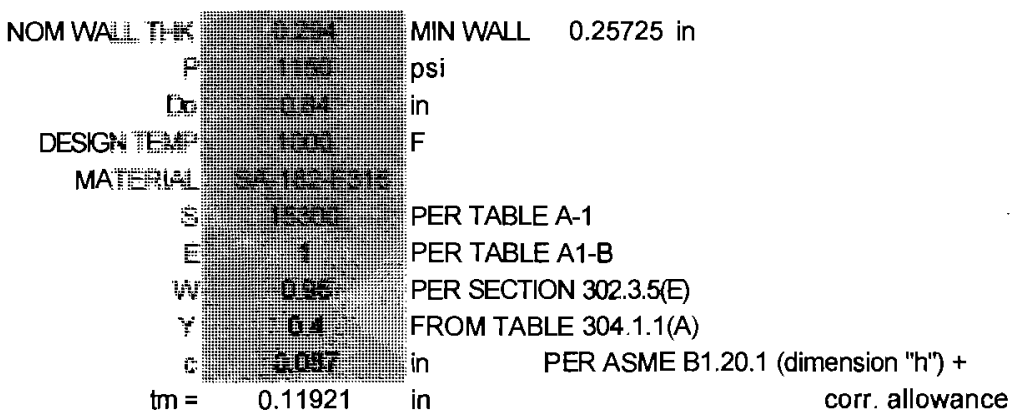

SINCE THE MIN WALL THK IS GREATER THAN tm, THE THICKNESS IS ADEQUATE

REQUIRED WELD SIZE ON 2" PIPE

PER SEC. 328.5.4(C,D) \& FIG. 328.5.4D

FULL PENETRATION GROOVE WELD FINISHED WTH COVER FILLET WELD

FILLET WELD SZE: tc = LESSER OF 0.7 Tb OR 1/4"

tc $=\quad 0.2058$ in. FILLET WELD ROOT DEPTH

2. 3 S.

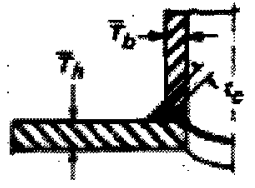

FIG. 328.5.4D (1)

PER SECTION 304.1.1(A) EQUATION (2), SEC. 304.1.5 EQUATION \#3A,

SEC. 326.1, 326.2, AND TABLE 326.1 STANDARDS; ASME B16.9 SEC. 2.1

$\mathrm{tm}=\left(P^{*} \mathrm{Do} /\left(2^{*}\left(\mathrm{~S}^{*} \mathrm{E}^{*} \mathrm{~W}+\mathrm{P}^{*} \mathrm{Y}\right)\right)\right)+\mathrm{C}$

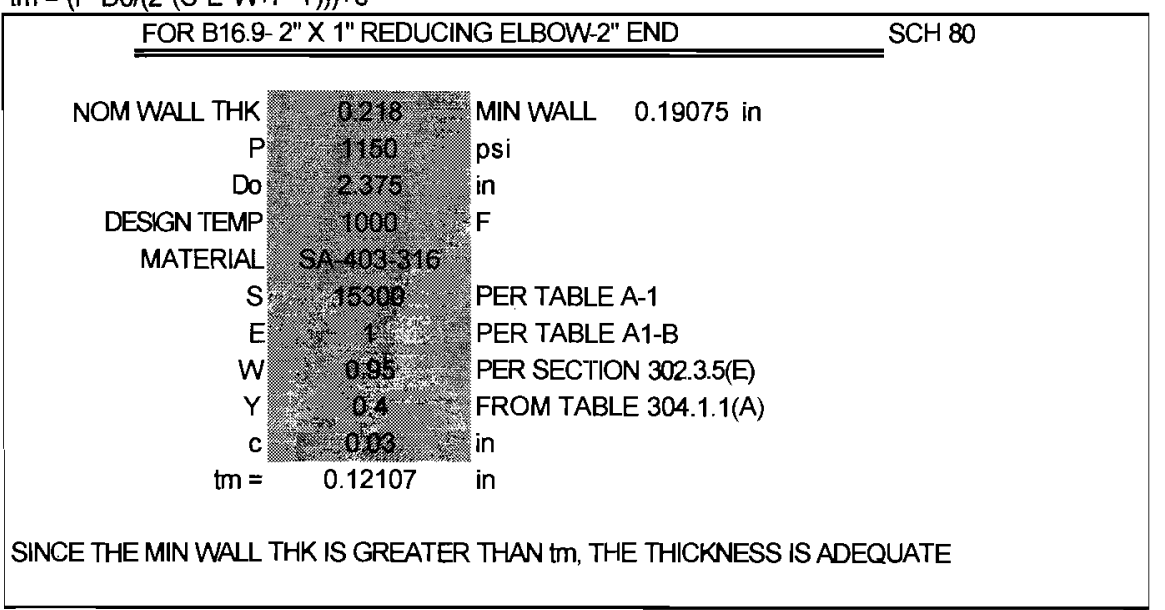

PER SECTION 304.1.1(A) EQUATION (2), SEC. 304.1.5 EQUATION \#3A, SEC. 326.1, 326.2, AND TABLE 326.1 STANDARDS; ASME B16.9 SEC. 2.1 $\mathrm{tm}=\left(\mathrm{P}^{*} \mathrm{Do} /\left(2^{*}\left(\mathrm{~S}^{*} \mathrm{E}^{*} \mathrm{~W}+\mathrm{P}^{*} \mathrm{Y}\right)\right)\right)+\mathrm{c}$

FOR B16.9-2"X 1" REDUCING ELBOW-1" END
NOM WALL,




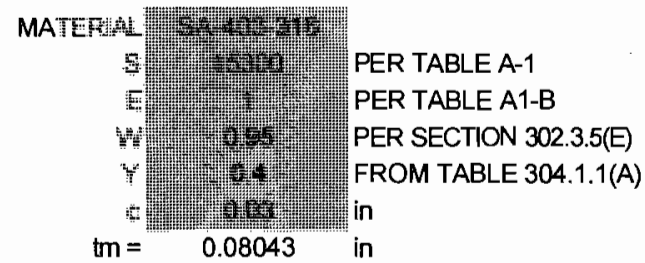

SINCE THE MIN WALL THK IS GREATER THAN tm, THE THICKNESS IS ADEQUATE

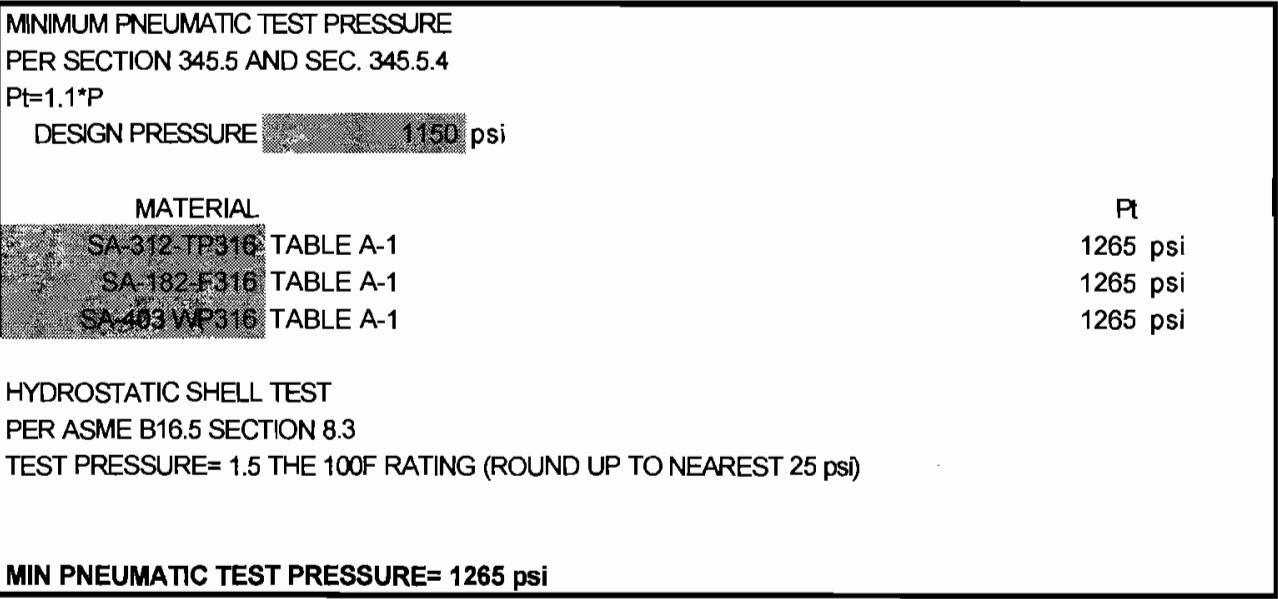




\section{Table Of Contents}

1. Pressure Summary

2. Pneumatic Test

3. Weight Summary

4. 2" RFWN FLANGE 1500\#, LPPER

5. 4" X 2" REDUCER B 16.9. UPPER (SCH XS) 2" END

6. 4" X 2" REDUCER B 16.9. UPPER (SCH XS) 4" END

7. 8"X 4" REDUCER B16.9. UPPER (SCH XS) 4" END

8. 8 "X 4" REDUCER B16.9, UPPER (SCH XS) 8" END

9. $8^{\prime \prime} \mathrm{PIPE}$

10. $8^{\prime \prime}$ X 4" REDUCER B 16.9, LOWER (SCH XS) 8" END

11. 8" X 4" REDUCER B16.9, LOWER (SCH XS) 4" END

12. $4 " \mathrm{X}$ 2" B16.9 REDUCER, LOWER (SCH XS) 4" END

13. 4" X 2" B 16.9 REDUCER, LOWER (SCH XS) 2" END

14. 2" RFWN FLANGE 1500\#, LOWER

15. ASME B 16.5/16.47 Blind on 2" RFWN FLANGE 1500\#. LOWER

16. 1/2" HALF-COUPLING (7a)

17. 1/2" HALF-COUPLING (7b)

18. Wind Code

19. Seismic Code 


\section{Pressure Summary}

\begin{tabular}{|c|c|c|c|c|c|c|}
\hline Identifier & $\begin{array}{c}\mathbf{P} \\
\text { Design } \\
(\mathbf{p s i})\end{array}$ & $\begin{array}{c}\mathbf{T} \\
\text { Design } \\
\left({ }^{\circ} \mathbf{F}\right)\end{array}$ & $\begin{array}{c}\text { MAWP } \\
(\text { psi) }\end{array}$ & $\begin{array}{c}\text { MDMT } \\
\left({ }^{\circ} \mathrm{F}\right)\end{array}$ & $\begin{array}{c}\text { MDMT } \\
\text { Exemption }\end{array}$ & $\begin{array}{l}\text { Impact } \\
\text { Tested }\end{array}$ \\
\hline 4" X 2" REDUCER B16.9. UPPER (SCH XS) 2" END & 1,150 & 1,000 & $2,193.3$ & -320 & Note 1 & No \\
\hline 4" X 2" REDUCER B16.9. UPPER (SCH XS) 4" END & 1,150 & 1,000 & $1,891.09$ & -320 & Note 1 & No \\
\hline 8"X 4" REDUCER B16.9. UPPER (SCH XS) 4" END & 1,150 & 1,000 & $1,891.09$ & -320 & Note 1 & No \\
\hline 8" X 4" REDUCER B 16.9. UPPER (SCH XS) 8" END & 1,150 & 1,000 & $1,502.53$ & -320 & Note 1 & No \\
\hline 8" PIPE & 1,150 & 1,000 & $1,502.53$ & -320 & Note 1 & No \\
\hline 8" X 4" REDUCER B16.9. IOWER (SCH XS) 8" END) & 1,150 & 1,000 & $1,502.53$ & -320 & Note 1 & No \\
\hline 8" X 4" REDUCER B16.9. LOWER (SCH XS) 4" END & 1,150 & 1,000 & $1,891.09$ & -320 & Note 1 & No \\
\hline 4" X 2" B16.9 REDUCER LOWER (SCH XS) 4" END & 1,150 & 1,000 & $1,891.09$ & -320 & Note 1 & No \\
\hline 4" X 2" B16.9 REDUCER LOWER (SCH XS) 2" END & 1,150 & $\mathrm{I}, 000$ & $2,193.3$ & -320 & Note 1 & No \\
\hline ASME B16.5/16.47 Blind on 2" RFWN FLANGE 1500\# IOWER & 1,150 & 1,000 & 1,820 & -55 & Note 2,3 & No \\
\hline 2" RFWN FLANGE 1500\#. UPPER & 1,150 & 1,000 & 1,820 & -320 & Note 2 & No \\
\hline 2" RFWN FLANGE 1500\#, LOWER & 1,150 & 1,000 & 1,820 & -55 & Note 2,3 & No \\
\hline 1/2" HALF-COUPLING (7a) & $1, I 50$ & 1,000 & $1,502.52$ & -320 & Note 4 & No \\
\hline 1/2" HALF-COUPLING (7b) & 1,150 & 1,000 & $1,502.52$ & -320 & Note 4 & No \\
\hline
\end{tabular}

Chamber design MDMT is $-20^{\circ} \mathrm{F}$

Chamber rated MDMT is $-55^{\circ} \mathrm{F} @ 1,502.52 \mathrm{psi}$

This pressure chamber is not designed for external pressure.

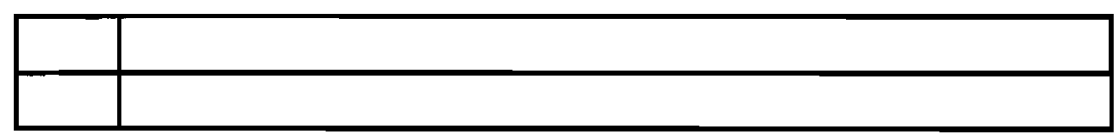

Notes for MDMT Rating:

\begin{tabular}{|r|l|l|}
\hline $\begin{array}{c}\text { Note } \\
\#\end{array}$ & \multicolumn{1}{|c|}{ Exemption } & \multicolumn{1}{c|}{ Details } \\
\hline 1. & $\begin{array}{l}\text { Rated MDMT per UHA }-51(\mathrm{~d})(1)(\mathrm{b}), \text { (carbon content does not exceed } 0.1 \\
\text { percent })=-320^{\circ} \mathrm{F}\end{array}$ & \\
\hline 2. & Per UHA $-51(\mathrm{~d})(1)(\mathrm{b})$ & \\
\hline 3. & Flange rated MDMT $=-320^{\circ} \mathrm{F}$ & $\begin{array}{l}\text { Bolts rated MDMT per Fig UCS-66 note (e) } \\
=-55^{\circ} \mathrm{F}\end{array}$ \\
\hline 4. & Impact test exempt per UHA-51(g)(coincident ratio $=0.11801)$ & \\
\hline
\end{tabular}




\section{Pneumatic Test}

\section{Shop test pressure determination} based on design $P$ per UG-100(b)

Shop pneumatic test gauge pressure is $1,653.608 \mathrm{psi}$ at $70^{\circ} \mathrm{F}$ (the chamber design $\mathrm{P}=1,150 \mathrm{psi}$ )

\begin{tabular}{|l|r|r|r|r|}
\hline \multicolumn{1}{|c|}{ Identifier } & $\begin{array}{c}\text { Local test } \\
\text { pressure } \\
\text { psi }\end{array}$ & $\begin{array}{c}\text { Test liquid } \\
\text { static head } \\
\text { psi }\end{array}$ & $\begin{array}{c}\text { UG-100 } \\
\text { stress } \\
\text { ratio }\end{array}$ & $\begin{array}{c}\text { UG-100 } \\
\text { press ure } \\
\text { factor }\end{array}$ \\
\hline 4" X 2" REDUCER B16.9, UPPER (SCH XS) 2" END (1) & $1,653.608$ & 0 & 1.3072 & 1.10 \\
\hline 2" RFWN FLANGE 1500\#, UPPER & $1,653.608$ & 0 & N/A & 1.10 \\
\hline 4" X 2" REDUCER B16.9, UPPER (SCH XS) 4" END & $1,653.608$ & 0 & 1.3072 & 1.10 \\
\hline 8" X 4" REDUCER B16.9, UPPER (SCH XS) 4" END & $1,653.608$ & 0 & 1.3072 & 1.10 \\
\hline 8" X 4" REDUCER B16.9, UPPER (SCH XS) 8" END & $1,653.608$ & 0 & 1.3072 & 1.10 \\
\hline 8" PIPE & $1,653.608$ & 0 & 1.3072 & 1.10 \\
\hline 8" X 4" REDUCER B16.9, LOWER (SCH XS) 8"END & $1,653.608$ & 0 & 1.3072 & 1.10 \\
\hline 8" X 4" REDUCER B16.9, LOWER (SCH XS) 4"END & $1,653.608$ & 0 & 1.3072 & 1.10 \\
\hline 4" X 2" B16.9 REDUCER, LOWER (SCH XS) 4"END & $1,653.608$ & 0 & 1.3072 & 1.10 \\
\hline 4" X 2" B16.9 REDUCER, LOWER (SCH XS) 2" END & $1,653.608$ & 0 & 1.3072 & 1.10 \\
\hline 2" RFWN FLANGE 1500\#, LOWER & $1,653.608$ & 0 & N/A & 1.10 \\
\hline 1/2" HALF-COUPLING (7a) & $1,653.608$ & 0 & 1.7699 & 1.10 \\
\hline 1/2" HALF-COUPLING (7b) & $1,653.608$ & 0 & 1.7699 & 1.10 \\
\hline
\end{tabular}

\section{Notes:}

(1) 4" X 2" REDUCER B16.9, UPPER (SCH XS) 2" END limits the UG-100 stress ratio.

(2) The zero degree angular position is assumed to be up, and the test liquid height is assumed to the top-most flange. 


\section{Weight Sum mary}

\begin{tabular}{|c|c|c|c|c|c|c|c|}
\hline \multirow[b]{2}{*}{ Component } & \multicolumn{7}{|c|}{ Weight ( Ib) Contributed by Vessel Elements } \\
\hline & $\begin{array}{l}\text { Metal } \\
\text { New* }\end{array}$ & $\begin{array}{c}\text { Metal } \\
\text { Corroded * }\end{array}$ & $\begin{array}{c}\text { Insulation } \\
\& \\
\text { Supports }\end{array}$ & Lining & $\begin{array}{c}\text { Piping } \\
+ \\
\text { Liquid }\end{array}$ & $\begin{array}{l}\text { Operating } \\
\text { Liquid }\end{array}$ & $\begin{array}{l}\text { Test } \\
\text { Liquid }\end{array}$ \\
\hline 4"X 2" REDUCER B16.9, UPPER (SCH XS) 2" END & 0.8 & 0.6 & 0 & 0 & 0 & 0 & 0.2 \\
\hline 4" X 2" REDUCER B 16.9. UPPER (SCH XS) 4" END & 2.3 & 2 & 0 & 0 & 0 & 0 & 0.9 \\
\hline 8" X 4" REDUCER B16.9. UPPER (SCH XS) 4" END & 3.4 & 3.1 & 0 & 0 & 0 & 0 & 1.3 \\
\hline 8" X 4" REDUCER B 16.9. UPPER (SCH XS) 8" END & 9.8 & 9.2 & 0 & 0 & 0 & 0 & 5.1 \\
\hline 8" PIPE & 132.7 & 125.2 & 10 & 0 & 0 & 0 & 59.4 \\
\hline 8" X 4" REDUCER B 16.9, LOWER (SCH XS) 8" END & 9.8 & 9.2 & 0 & 0 & 0 & 0 & 5.1 \\
\hline 8" X 4" REDUCER B16.9. LOWER (SCH XS) 4" END & 3.4 & 3.1 & 0 & 0 & 0 & 0 & 1.3 \\
\hline 4" X 2" B16.9 REDUCER. LOWER (SCH XS) 4" END & 2.3 & 2 & 0 & 0 & 0 & 0 & 0.9 \\
\hline 4" X 2" B16.9 REDUCER. LOWER (SCH XS) 2" END & 0.8 & 0.6 & 0 & 0 & 0 & 0 & 0.2 \\
\hline $\begin{array}{l}\text { ASME B16.5/16.47 Blind on 2" RFWN FLANGE } 1500 \# \text {. } \\
\text { LOWER }\end{array}$ & 32 & 32 & 0 & 0 & 0 & 0 & 0 \\
\hline Support Skitt \#1 & 1.9 & 1.9 & 0 & 0 & 0 & 0 & 0 \\
\hline TOTAL: & 199 & 189 & 0 & 0 & $\mathbf{0}$ & 0 & 74.4 \\
\hline
\end{tabular}

* Shells with attached nozzles have weight reduced by material cut out for opening.

\begin{tabular}{|c|c|c|c|c|c|c|c|c|c|}
\hline \multirow{3}{*}{ Component } & \multicolumn{9}{|c|}{ Weight ( lb) Contributed by Attachments } \\
\hline & \multicolumn{2}{|c|}{ Body Flanges } & \multicolumn{2}{|c|}{$\begin{array}{l}\text { Nozzles \& } \\
\text { Flanges }\end{array}$} & \multirow{2}{*}{$\begin{array}{c}\text { Packed } \\
\text { Beds }\end{array}$} & \multirow{2}{*}{$\begin{array}{l}\text { Ladders } \\
\& \\
\text { Platforms }\end{array}$} & \multirow{2}{*}{$\begin{array}{l}\text { Trays \& } \\
\text { Supports }\end{array}$} & \multirow{2}{*}{$\begin{array}{c}\text { Rings } \\
\& \\
\text { Clips }\end{array}$} & \multirow{2}{*}{$\begin{array}{c}\text { Vertical } \\
\text { Loads }\end{array}$} \\
\hline & New & Corroded & New & Corroded & & & & & \\
\hline $\begin{array}{l}4^{\prime \prime} \times 2 " \text { REDUCER B16.9 UPPER (SCH XS) } \\
2^{2 "} \mathrm{END}\end{array}$ & 25 & 25 & 0 & 0 & 0 & 0 & 0 & 0 & 0 \\
\hline $\begin{array}{l}\text { 4" X 2" REDUCER B16.9.UPPER (SCH XS) } \\
\text { 4" END }\end{array}$ & 0 & 0 & 0 & 0 & 0 & 0 & 0 & 0 & 0 \\
\hline $\begin{array}{l}\text { 8" X 4" REDUCER B 16.9. UPPER (SCH XS) } \\
\text { 4" END }\end{array}$ & 0 & 0 & 0 & 0 & 0 & 0 & 0 & 0 & 0 \\
\hline $\begin{array}{l}\text { 8" X 4" REDUCER B16.9, UPPER (SCH XS) } \\
\text { 8" END }\end{array}$ & 0 & 0 & 0 & 0 & 0 & 0 & 0 & 0 & 0 \\
\hline 8" PIPE & 0 & 0 & 0.7 & 0.7 & 0 & 0 & 0 & 0 & 0 \\
\hline $\begin{array}{l}\text { 8" X 4" REDUCER B16.9. LOWER (SCH } \\
\text { XS) 8" END }\end{array}$ & 0 & 0 & 0 & 0 & 0 & 0 & 0 & 0 & 0 \\
\hline $\begin{array}{l}\frac{8 " \mathrm{X} 4 " \mathrm{REDUCER} \text { B } 16.9, \text { LOWER (SCH }}{\mathrm{XS}) 4^{\prime \prime} \mathrm{END}} \\
\end{array}$ & 0 & 0 & 0 & 0 & 0 & 0 & 0 & 0 & 0 \\
\hline $\begin{array}{l}\text { 4" X 2" B16.9 REDUCER, LOWER (SCH } \\
\text { XS) 4" END }\end{array}$ & 0 & 0 & 0 & 0 & 0 & 0 & 0 & 0 & 0 \\
\hline $\begin{array}{l}\text { 4" X 2" B16.9 REDUCER. LOWER (SCH } \\
\text { XS) 2" END }\end{array}$ & 25 & 25 & 0 & 0 & 0 & 0 & 0 & 0 & 0 \\
\hline $\begin{array}{l}\text { ASME B } 16.5 / 16.47 \text { Blind on 2" RFWN } \\
\text { FLANGE 1500\#. LOWER }\end{array}$ & 0 & 0 & 0 & 0 & 0 & 0 & 0 & 0 & 0 \\
\hline Support Skirt \#I & 0 & 0 & 0 & 0 & 0 & 0 & 0 & 0 & 0 \\
\hline TOTAL: & 50 & 50 & 0.7 & 0.7 & $\mathbf{0}$ & $\mathbf{0}$ & $\overline{0}$ & 0 & 0 \\
\hline
\end{tabular}

Vessel operating weight, Corroded: $240 \mathrm{lb}$ 
Vessel operating weight, New: $250 \mathrm{lb}$

Vessel empty weight, Corroded: $240 \mathrm{lb}$

Vessel empty weight, New: $250 \mathrm{lb}$

Vessel test weight, New: $\quad 324 \mathrm{lb}$

\section{Vessel center of gravity location - from datum - lift condition}

Vessel Lift Weight, New:250 lb

Center of Gravity: $\quad 27.7778 "$

\section{Vessel Capacity}

Vessel Capacity** (New): 9 US gal

Vessel Capacity** (Corroded): 9 US gal

**The vessel capacity does not include volume of nozzle, piping or other attachments. 


\title{
2" RFWN FLANGE 1500\#, UPPER
}

\author{
Flange description: \\ 2 inch Class 1500 WN A 182 F316H \\ Bolt Material: \\ SB-166 Annealed Bolt N06600 \\ Flange rated MDMT: \\ $-320^{\circ} \mathrm{F}$ \\ (Per UHA-51(d)(1)(b)) \\ Liquid static head on flange: \\ $0 \mathrm{psi}$ \\ ASME B16.5 flange rating MAWP: $1820 \mathrm{psi} @ 1000^{\circ} \mathrm{F}$ \\ ASME B 16.5 flange rating MAP: $3600 \mathrm{psi} @ 70^{\circ} \mathrm{F}$ \\ ASME B16.5 flange hydro test: $\quad 5425$ psi @ $70^{\circ} \mathrm{F}$
}




\section{4" X 2" REDUCER B16.9, UPPER (SCH XS) 2" END}

\section{ASME Section VIII Division 1, 2007 Edition}

$\begin{array}{ll}\text { Component: } & \text { Cylinder } \\ \text { Material specification: } & \text { SA-403 } 316 \text { H (II-D p. 78, } \ln .8)\end{array}$

Rated MDMT per UHA-51(d)(1)(b), (carbon content does not exceed 0.1 percent) $=-320^{\circ} \mathrm{F}$

Internal design pressure: $\mathrm{P}=1,150 \mathrm{psi} @ 1,000^{\circ} \mathrm{F}$

Static liquid head:

$\mathrm{P}_{\text {th }}=0.23 \mathrm{psi}\left(\mathrm{SG}=1, \mathrm{H}_{\mathrm{s}}=6.2465^{\prime \prime}\right.$, Horizontal test head $)$

Corrosion allowance $\quad$ Inner $\mathrm{C}=0.03^{\prime \prime} \quad$ Outer $\mathrm{C}=0$ "

Design MDMT $=-20^{\circ} \mathrm{F} \quad$ No impact test performed

Rated MDMT $=-320^{\circ} \mathrm{F}$ Material is not normalized

Material is not produced to Fine Grain Practice

PWHT is not performed

Radiography: $\quad$ Longitudinal joint - $\quad$ Seamless No RT

Top circumferential joint - Full UW-11(a) Type 1

Bottom circumferential

joint -

Full UW-11(a) Type 1

Estimated weight New $=0.8 \mathrm{lb} \quad$ corr $=0.6 \mathrm{lb}$

Capacity New $=0.03$ US gal corr $=0.03$ US gal

$\mathrm{OD} \quad=2.375^{\prime \prime}$

Length $\mathrm{L}_{\mathrm{c}}=2^{\prime \prime}$

t $=0.191^{\prime \prime}$

Design thickness, (at $1,000^{\circ} \mathrm{F}$ ) Appendix 1-1

$\mathrm{t}=\mathrm{P}^{*} \mathrm{R}_{0} /\left(\mathrm{S} * \mathrm{E}+0.40^{*} \mathrm{P}\right)+$ Corrosion

$=1,150 * 1.1875 /(15,300 * 1.00+0.40 * 1,150)+0.03$

$=0.1167^{\prime \prime}$

Maximum allowable working pressure, (at 1,000 $\left.{ }^{\circ} \mathrm{F}\right)$ Appendix 1 -1

$$
\begin{aligned}
\mathrm{P} & =\mathrm{S}^{*} \mathrm{E}^{*} \mathrm{t} /\left(\mathrm{R}_{\mathrm{o}}-0.40^{*} \mathrm{t}\right)-\mathrm{P}_{\mathrm{S}} \\
& =15,300^{*} 1.00^{*} 0.161 /\left(1.1875-0.40^{*} 0.161\right)-0 \\
& =2,193.3 \mathrm{psi}
\end{aligned}
$$

\% Forming Strain - UHA-44(a)(2)(a)

$$
\begin{aligned}
\mathrm{EFE} & =\left(50 * \mathrm{t} / \mathrm{R}_{\mathrm{f}}\right) *\left(1-\mathrm{R}_{\mathrm{f}} / \mathrm{R}_{\mathrm{o}}\right) \\
& =(50 * 0.191 / 1.092) *(1-1.092 / \infty) \\
& =8.7454 \%
\end{aligned}
$$




\section{Design thickness $=0.1167^{\prime \prime}$}

The governing condition is due to internal pressure.

The cylinder thickness of $0.191 "$ is adequate.

Thickness Required Due to Pressure + External Loads

\begin{tabular}{|c|c|c|c|c|c|c|c|c|}
\hline \multirow[t]{2}{*}{ Condition } & \multirow{2}{*}{$\begin{array}{c}\text { Pressure } \\
\text { P ( psi) }\end{array}$} & \multicolumn{2}{|c|}{$\begin{array}{c}\text { Allowable Stress } \\
\text { Before UG-23 Stress } \\
\text { Increase ( psi) }\end{array}$} & \multirow{2}{*}{$\begin{array}{c}\text { Temperature } \\
\left({ }^{\circ} \mathbf{F}\right)\end{array}$} & \multirow{2}{*}{$\begin{array}{l}\text { Corrosion } \\
\text { C (in) }\end{array}$} & \multirow[t]{2}{*}{ Load } & \multirow{2}{*}{$\begin{array}{l}\text { Req'd Thk } \\
\text { Due to } \\
\text { Tension (in) }\end{array}$} & \multirow{2}{*}{$\begin{array}{l}\text { Req'd Thk Due } \\
\text { to Compression } \\
\text { (in) }\end{array}$} \\
\hline & & $S_{t}$ & $\mathbf{S}_{\mathrm{c}}$ & & & & & \\
\hline \multirow{2}{*}{$\begin{array}{l}\text { Operating, Hot \& } \\
\text { Corroded }\end{array}$} & \multirow{2}{*}{1,150} & \multirow{2}{*}{15,300} & \multirow{2}{*}{10,201} & \multirow{2}{*}{1,000} & \multirow{2}{*}{0.03} & Wind & 0.0316 & 0.0315 \\
\hline & & & & & & Seismic & 0.032 & 0.0311 \\
\hline \multirow{2}{*}{$\begin{array}{c}\text { Operating, Hot \& } \\
\text { New }\end{array}$} & \multirow{2}{*}{1,150} & \multirow{2}{*}{15,300} & \multirow{2}{*}{10,324} & \multirow{2}{*}{1,000} & \multirow{2}{*}{0} & Wind & 0.0307 & 0.0306 \\
\hline & & & & & & Seismic & 0.0311 & 0.0301 \\
\hline \multirow{2}{*}{ Empty, Corroded } & \multirow{2}{*}{0} & \multirow{2}{*}{20,000} & \multirow{2}{*}{14,454} & \multirow{2}{*}{0} & \multirow{2}{*}{0.03} & Wind & 0.0001 & 0.0003 \\
\hline & & & & & & Seismic & 0.0002 & 0.0007 \\
\hline \multirow{2}{*}{ Empty, New } & \multirow{2}{*}{0} & \multirow{2}{*}{20,000} & \multirow{2}{*}{14,604} & \multirow{2}{*}{0} & \multirow{2}{*}{0} & Wind & 0.0002 & 0.0003 \\
\hline & & & & & & Seismic & 0.0002 & 0.0007 \\
\hline $\begin{array}{l}\text { Hot Shut Down, } \\
\text { Corroded, Weight \& } \\
\text { Eccentric Moments } \\
\text { Only }\end{array}$ & 0 & 15,300 & 10,201 & 1,000 & 0.03 & Weight & 0.0004 & 0.0004 \\
\hline
\end{tabular}




\section{4" X 2" REDUCER B16.9, UPPER (SCH XS) 4" END}

\section{ASME Section VIII Division 1, 2007 Edition}

Component: $\quad$ Cylinder

Material specification: $\quad$ SA-403 $316 \mathrm{H}$ (II-D p. $78, \ln .8)$

Rated MDMT per UHA-51(d)(1)(b), (carbon content does not exceed 0.1 percent) $=-320^{\circ} \mathrm{F}$

Internal design pressure: $\mathrm{P}=1,150$ psi @ $1,000^{\circ} \mathrm{F}$

Static liquid head:

$\mathrm{P}_{\mathrm{th}}=0.26 \mathrm{psi}\left(\mathrm{SG}=1, \mathrm{H}_{\mathrm{s}}=7.205^{\prime}\right.$, Horizontal test head $)$

Corrosion allowance $\quad$ Inner $\mathrm{C}=0.03^{\prime \prime} \quad$ Outer $\mathrm{C}=0$ "

Design MDMT $=-20^{\circ} \mathrm{F} \quad$ No impact test performed

Rated MDMT $=-320^{\circ} \mathrm{F} \quad$ Material is not normalized

Material is not produced to Fine Grain Practice

PWHT is not performed

Radiography: $\quad$ Longitudinal joint - $\quad$ Seamless No RT

Top circumferential joint - Full UW-11(a) Type 1

Bottom circumferential joint -

Full UW-11(a) Type 1

Estimated weight New $=2.3 \mathrm{lb} \quad$ corr $=2 \mathrm{lb}$

Capacity New $=0.1 \mathrm{US}$ gal corr $=0.11 \mathrm{US}$ gal

$$
\begin{array}{ll}
\text { OD } & =4.5^{\prime \prime} \\
\text { Length } \mathrm{L}_{\mathrm{c}} & =2^{\prime \prime} \\
\mathrm{t} & =0.295^{\prime \prime}
\end{array}
$$

Design thickness, (at $1,000^{\circ} \mathrm{F}$ ) Appendix 1-1

$$
\begin{aligned}
\mathrm{t} & =\mathrm{P} * \mathrm{R}_{\mathrm{o}} /(\mathrm{S} * \mathrm{E}+0.40 * \mathrm{P})+\text { Corrosion } \\
& =1,150 * 2.25 /(15,300 * 1.00+0.40 * 1,150)+0.03 \\
& =0.1942
\end{aligned}
$$

Maximum allowable working pressure, (at 1,000 ${ }^{\circ} \mathrm{F}$ ) Appendix 1 -1

$$
\begin{aligned}
\mathrm{P} & =\mathrm{S} * \mathrm{E}^{*} \mathrm{t} /\left(\mathrm{R}_{\mathrm{o}}-0.40 * \mathrm{t}\right)-\mathrm{P}_{\mathrm{S}} \\
& =15,300 * 1.00 * 0.265 /(2.25-0.40 * 0.265)-0 \\
& =1,891.09 \mathrm{psi}
\end{aligned}
$$

\% Forming Strain - UHA-44(a)(2)(a)

$$
\begin{aligned}
\operatorname{EFE} & =\left(50 * t / R_{f}\right) *\left(1-R_{f} / R_{0}\right) \\
& =(50 * 0.295 / 2.1025) *(1-2.1025 / \infty) \\
& =7.0155 \%
\end{aligned}
$$




\section{Design thickness $=0.1942 "$}

The governing condition is due to internal pressure.

The cylinder thickness of $0.295^{\prime \prime}$ is adequate.

Thickness Required Due to Pressure + External Loads

\begin{tabular}{|c|c|c|c|c|c|c|c|c|}
\hline \multirow[t]{2}{*}{ Condition } & \multirow{2}{*}{$\begin{array}{l}\text { Pressure } \\
\text { P ( psi) }\end{array}$} & \multicolumn{2}{|c|}{$\begin{array}{c}\text { Allowable Stress } \\
\text { Before UG-23 Stress } \\
\text { Increase ( psi) }\end{array}$} & \multirow{2}{*}{$\begin{array}{l}\text { Temperature } \\
\left({ }^{\circ} \mathrm{F}\right)\end{array}$} & \multirow{2}{*}{$\begin{array}{l}\text { Corrosion } \\
\text { C (in) }\end{array}$} & \multirow[t]{2}{*}{ Load } & \multirow{2}{*}{$\begin{array}{l}\text { Req'd Thk } \\
\text { Due to } \\
\text { Tension (in) }\end{array}$} & \multirow{2}{*}{$\begin{array}{l}\text { Req'd Thk Due } \\
\text { to Compression } \\
\text { (in) }\end{array}$} \\
\hline & & $S_{t}$ & $\mathbf{S}_{\mathbf{c}}$ & & & & & \\
\hline \multirow{2}{*}{$\begin{array}{l}\text { Operating, Hot \& } \\
\text { Corroded }\end{array}$} & \multirow{2}{*}{1,150} & \multirow{2}{*}{15,300} & \multirow{2}{*}{10,100} & \multirow{2}{*}{1,000} & \multirow{2}{*}{0.03} & Wind & 0.0613 & 0.0613 \\
\hline & & & & & & Seismic & 0.0615 & 0.0611 \\
\hline \multirow{2}{*}{$\begin{array}{c}\text { Operating, Hot \& } \\
\text { New }\end{array}$} & \multirow{2}{*}{1,150} & \multirow{2}{*}{15,300} & \multirow{2}{*}{10,177} & \multirow{2}{*}{1,000} & \multirow{2}{*}{0} & Wind & 0.0604 & 0.0603 \\
\hline & & & & & & Seismic & 0.0605 & 0.0601 \\
\hline \multirow{2}{*}{ Empty, Corroded } & \multirow{2}{*}{0} & \multirow{2}{*}{20,000} & \multirow{2}{*}{14,332} & \multirow{2}{*}{0} & \multirow{2}{*}{0.03} & Wind & 0.0001 & 0.0002 \\
\hline & & & & & & Seismic & 0.0001 & 0.0003 \\
\hline \multirow{2}{*}{ Empty, New } & \multirow{2}{*}{0} & \multirow{2}{*}{20,000} & \multirow{2}{*}{14,425} & \multirow{2}{*}{0} & \multirow{2}{*}{0} & Wind & 0.0001 & 0.0002 \\
\hline & & & & & & Seismic & 0.0001 & 0.0003 \\
\hline $\begin{array}{l}\text { Hot Shut Down, } \\
\text { Corroded, Weight \& } \\
\text { Eccentric Moments } \\
\text { Only }\end{array}$ & 0 & 15,300 & 10,100 & 1,000 & 0.03 & Weight & 0.0002 & 0.0002 \\
\hline
\end{tabular}




\section{8" X 4" REDUCER B16.9, UPPER (SCH XS) 4" END}

ASME Section VIII Division 1, 2007 Edition

Component: Cylinder

Material specification: $\quad$ SA-403 316H (II-D p. 78, ln. 8)

Rated MDMT per UHA-51(d)(1)(b), (carbon content does not exceed 0.1 percent) $=-320^{\circ} \mathrm{F}$

Internal design pressure: $\mathrm{P}=1,150 \mathrm{psi} @ 1,000^{\circ} \mathrm{F}$

Static liquid head:

$P_{\text {th }}=0.26 \mathrm{psi}\left(\mathrm{SG}=1, \mathrm{H}_{\mathrm{S}}=7.205^{\prime \prime}\right.$, Horizontal test head $)$

Corrosion allowance $\quad$ Inner $\mathrm{C}=0.03 " \quad$ Outer $\mathrm{C}=0 "$

Design MDMT $=-20^{\circ} \mathrm{F} \quad$ No impact test performed

Rated MDMT $=-320^{\circ} \mathrm{F} \quad$ Material is not normalized

Material is not produced to Fine Grain Practice

PWHT is not performed

Radiography: $\quad$ Longitudinal joint - $\quad$ Seamless No RT

Top circumferential joint - Full UW-11(a) Type 1

Bottom circumferential joint -

Full UW-11(a) Type 1

Estimated weight New $=3.4 \mathrm{lb} \quad$ corr $=3.1 \mathrm{lb}$

Capacity New $=0.16$ US gal corr $=0.16$ US gal

$\mathrm{OD} \quad=4.5^{\prime \prime}$

Length $\mathrm{L}_{\mathrm{c}}=3^{\prime \prime}$

$\mathrm{t} \quad=0.295^{\prime \prime}$

Design thickness, (at $1,000{ }^{\circ}$ F) Appendix 1-1

$\mathrm{t}=\mathrm{P}^{*} \mathrm{R}_{\mathrm{o}} /(\mathrm{S} * \mathrm{E}+0.40 * \mathrm{P})+$ Corrosion

$=1,150 * 2.25 /(15,300 * 1.00+0.40 * 1,150)+0.03$

$=0.1942 "$

Maximum allowable working pressure, (at 1,000 ${ }^{\circ}$ F) Appendix 1-1

$$
\begin{aligned}
\mathrm{P} & =\mathrm{S} * \mathrm{E}^{*} \mathrm{t} /\left(\mathrm{R}_{\mathrm{o}}-0.40 * \mathrm{t}\right)-\mathrm{P}_{\mathrm{S}} \\
& =15,300^{*} 1.00 * 0.265 /(2.25-0.40 * 0.265)-0 \\
& =1,891.09 \mathrm{psi}
\end{aligned}
$$

$\%$ Forming Strain - UHA-44(a)(2)(a)

$$
\begin{aligned}
\mathrm{EFE} & =\left(50 * \mathrm{t} / \mathrm{R}_{\mathrm{f}}\right) *\left(1-\mathrm{R}_{\mathrm{f}} / \mathrm{R}_{\mathrm{o}}\right) \\
& =(50 * 0.295 / 2.1025) *(1-2.1025 / \infty) \\
& =7.0155 \%
\end{aligned}
$$




\section{Design thickness $=0.1942 "$}

The governing condition is due to internal pressure.

The cylinder thickness of $0.295^{\prime \prime}$ is adequate.

Thickness Required Due to Pressure + External Loads

\begin{tabular}{|c|c|c|c|c|c|c|c|c|}
\hline \multirow[t]{2}{*}{ Condition } & \multirow{2}{*}{$\begin{array}{l}\text { Pressure } \\
\text { P ( psi) }\end{array}$} & \multicolumn{2}{|c|}{$\begin{array}{l}\text { Allowable Stress } \\
\text { Before UG-23 Stress } \\
\text { Increase ( psi) }\end{array}$} & \multirow{2}{*}{$\begin{array}{l}\text { Temperature } \\
\left({ }^{\circ} \mathrm{F}\right)\end{array}$} & \multirow{2}{*}{$\begin{array}{l}\text { Corrosion } \\
\text { C (in) }\end{array}$} & \multirow[t]{2}{*}{ Load } & \multirow{2}{*}{$\begin{array}{l}\text { Req'd Thk } \\
\text { Due to } \\
\text { Tension (in) }\end{array}$} & \multirow{2}{*}{$\begin{array}{l}\text { Req'd Thk Due } \\
\text { to Compression } \\
\text { (in) }\end{array}$} \\
\hline & & $\mathbf{S}_{\mathbf{t}}$ & $\mathbf{S}_{\mathbf{c}}$ & & & & & \\
\hline \multirow{2}{*}{$\begin{array}{l}\text { Operating, Hot \& } \\
\text { Corroded }\end{array}$} & \multirow{2}{*}{1,150} & \multirow{2}{*}{15,300} & \multirow{2}{*}{10,100} & \multirow{2}{*}{1,000} & \multirow{2}{*}{0.03} & Wind & 0.0613 & 0.0612 \\
\hline & & & & & & Seismic & 0.0616 & 0.061 \\
\hline \multirow{2}{*}{$\begin{array}{c}\text { Operating, Hot } \& \\
\text { New }\end{array}$} & \multirow{2}{*}{1,150} & \multirow{2}{*}{15,300} & \multirow{2}{*}{10,177} & \multirow{2}{*}{1,000} & \multirow{2}{*}{0} & Wind & 0.0604 & 0.0603 \\
\hline & & & & & & Seismic & 0.0606 & 0.06 \\
\hline \multirow{2}{*}{ Empty, Corroded } & \multirow{2}{*}{0} & \multirow{2}{*}{20,000} & \multirow{2}{*}{14,332} & \multirow{2}{*}{0} & \multirow{2}{*}{0.03} & Wind & 0.0001 & 0.0002 \\
\hline & & & & & & Seismic & 0.0001 & 0.0005 \\
\hline \multirow{2}{*}{ Empty, New } & \multirow{2}{*}{0} & \multirow{2}{*}{20,000} & \multirow{2}{*}{14,425} & \multirow{2}{*}{0} & \multirow{2}{*}{0} & Wind & 0.0001 & 0.0002 \\
\hline & & & & & & Seismic & 0.0001 & 0.0005 \\
\hline $\begin{array}{l}\text { Hot Shut Down, } \\
\text { Corroded, Weight \& } \\
\text { Eccentric Moments } \\
\text { Only }\end{array}$ & 0 & 15,300 & 10,100 & 1,000 & 0.03 & Weight & 0.0002 & 0.0002 \\
\hline
\end{tabular}




\section{8" X 4" REDUCER B16.9, UPPER (SCH XS) 8" END}

ASME Section VIII Division 1, 2007 Edition

Component: $\quad$ Cylinder

Material specification: $\quad$ SA-403 $316 \mathrm{H}$ (II-D p. 78, ln. 8)

Rated MDMT per UHA-51(d)(1)(b), (carbon content does not exceed 0.1 percent) $=-320^{\circ} \mathrm{F}$

Internal design pressure: $\mathrm{P}=1,150$ psi @ $1,000^{\circ} \mathrm{F}$

Static liquid head:

$\mathrm{P}_{\mathrm{th}}=0.33 \mathrm{psi}\left(\mathrm{SG}=1, \mathrm{H}_{\mathrm{s}}=9.125^{\prime}\right.$, Horizontal test head $)$

Corrosion allowance $\quad$ Inner $\mathrm{C}=0.03^{\prime \prime} \quad$ Outer $\mathrm{C}=0^{\prime \prime}$

Design MDMT $=-20^{\circ} \mathrm{F} \quad$ No impact test performed

Rated MDMT $=-320^{\circ} \mathrm{F} \quad$ Material is not normalized

Material is not produced to Fine Grain Practice

PWHT is notperformed

Radiography: $\quad$ Longitudinal joint - $\quad$ Seamless No RT

Top circumferential joint - Full UW-11(a) Type 1

Bottom circumferential

joint -

Full UW-11(a) Type 1

Estimated weight New $=9.8 \mathrm{lb} \quad$ corr $=9.2 \mathrm{lb}$

Capacity New $=0.61$ US gal corr $=0.62$ US gal

OD $\quad=8.625^{\prime \prime}$

Length $\mathrm{L}_{\mathrm{c}}=3^{\prime \prime}$

$\mathrm{t} \quad=0.4375^{\prime \prime}$

Design thickness, (at $1,000^{\circ}$ F) Appendix 1-1

$\mathrm{t}=\mathrm{P}^{*} \mathrm{R}_{\mathrm{o}} /\left(\mathrm{S} * \mathrm{E}+0.40^{*} \mathrm{P}\right)+$ Corrosion

$=1,150 * 4.3125 /(15,300 * 1.00+0.40 * 1,150)+0.03$

$=0.3447^{\prime \prime}$

Maximum allowable working pressure, (at 1,000 $\left.{ }^{\circ} \mathrm{F}\right)$ Appendix 1 -1

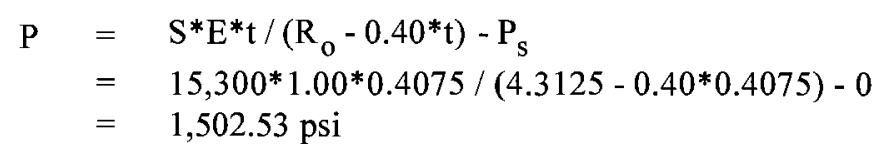

\% Forming Strain - UHA-44(a)(2)(a)

$$
\begin{aligned}
\mathrm{EFE} & =\left(50 * \mathrm{t} / \mathrm{R}_{\mathrm{f}}\right) *\left(1-\mathrm{R}_{\mathrm{f}} / \mathrm{R}_{\mathrm{o}}\right) \\
& =(50 * 0.4375 / 4.0938) *(1-4.0938 / \infty) \\
& =5.3435 \%
\end{aligned}
$$




\section{Design thickness $=0.3447^{\prime \prime}$}

The governing condition is due to internal pressure.

The cylinder thickness of $0.4375^{\prime \prime}$ is adequate.

Thickness Required Due to Pressure + External Loads

\begin{tabular}{|c|c|c|c|c|c|c|c|c|}
\hline \multirow[t]{2}{*}{ Condition } & \multirow{2}{*}{$\begin{array}{l}\text { Pressure } \\
\text { P ( psi) }\end{array}$} & \multicolumn{2}{|c|}{$\begin{array}{c}\text { Allowable Stress } \\
\text { Before UG-23 Stress } \\
\text { Increase ( psi) }\end{array}$} & \multirow{2}{*}{$\begin{array}{c}\text { Temperature } \\
\left({ }^{\circ} \mathrm{F}\right)\end{array}$} & \multirow{2}{*}{$\begin{array}{l}\text { Corrosion } \\
\text { C (in) }\end{array}$} & \multirow[t]{2}{*}{ Load } & \multirow{2}{*}{$\begin{array}{l}\text { Req'd Thk } \\
\text { Due to } \\
\text { Tension (in) }\end{array}$} & \multirow{2}{*}{$\begin{array}{l}\text { Req'd Thk Due } \\
\text { to Compression } \\
\text { (in) }\end{array}$} \\
\hline & & $\mathbf{S}_{\mathbf{t}}$ & $\mathbf{S}_{\mathbf{c}}$ & & & & & \\
\hline \multirow{2}{*}{$\begin{array}{l}\text { Operating, Hot \& } \\
\text { Corroded }\end{array}$} & \multirow{2}{*}{1,150} & \multirow{2}{*}{15,300} & \multirow{2}{*}{9,945} & \multirow{2}{*}{1,000} & \multirow{2}{*}{0.03} & Wind & 0.1207 & 0.1207 \\
\hline & & & & & & Seismic & 0.1208 & 0.1206 \\
\hline \multirow{2}{*}{$\begin{array}{c}\text { Operating, Hot \& } \\
\text { New }\end{array}$} & \multirow{2}{*}{1,150} & \multirow{2}{*}{15,300} & \multirow{2}{*}{9,995} & \multirow{2}{*}{1,000} & \multirow{2}{*}{0} & Wind & 0.1198 & 0.1197 \\
\hline & & & & & & Seismic & 0.1199 & 0.1196 \\
\hline \multirow{2}{*}{ Empty, Corroded } & \multirow{2}{*}{0} & \multirow{2}{*}{20,000} & \multirow{2}{*}{14,142} & \multirow{2}{*}{0} & \multirow{2}{*}{0.03} & Wind & 0.0001 & 0.0001 \\
\hline & & & & & & Seismic & 0 & 0.0002 \\
\hline \multirow{2}{*}{ Empty, New } & \multirow{2}{*}{0} & \multirow{2}{*}{20,000} & \multirow{2}{*}{14,203} & \multirow{2}{*}{0} & \multirow{2}{*}{0} & Wind & 0.0001 & 0.0001 \\
\hline & & & & & & Seismic & 0 & 0.0002 \\
\hline $\begin{array}{c}\text { Hot Shut Down, } \\
\text { Corroded, Weight \& } \\
\text { Eccentric Moments } \\
\text { Only }\end{array}$ & 0 & 15,300 & 9,945 & 1,000 & 0.03 & Weight & 0.0002 & 0.0002 \\
\hline
\end{tabular}




\section{8" PIPE}

ASME Section VIII Division 1, 2007 Edition

Component:

Material specification:

Pipe NPS and Schedule:
Cylinder

SA-312 TP316H Wld \& smls pipe (II-D p.

$78, \ln .1)$

$8 "$ Sch 80 S (XS)

Rated MDMT per UHA-51(d)(1)(b), (carbon content does not exceed 0.1 percent) $=-320^{\circ} \mathrm{F}$

Internal design pressure: $\mathrm{P}=1,150 \mathrm{psi} @ 1,000^{\circ} \mathrm{F}$

Static liquid head:

$P_{\text {th }}=0.33 \mathrm{psi}\left(\mathrm{SG}=1, \mathrm{H}_{\mathrm{s}}=9.0625^{\prime \prime}\right.$, Horizontal test head $)$

Corrosion allowance $\quad$ Inner $\mathrm{C}=0.03^{\prime \prime} \quad$ Outer $\mathrm{C}=0 "$

Design MDMT $=-20^{\circ} \mathrm{F}$

No impact test performed

Rated MDMT $=-320^{\circ} \mathrm{F}$

Material is not normalized

Material is not produced to Fine Grain Practice

PWHT is not performed

Radiography: Longitudinal joint - Seamless No RT

Top circumferential joint - Full UW-11(a) Type 1

Bottom circumferential Full UW-11(a) Type 1
joint -

Estimated weight $\mathrm{New}=133.2 \mathrm{lb} \quad$ corr $=125.7 \mathrm{lb}$

Capacity $\quad$ New $=7.12$ US gal corr $=7.23$ US gal

$\mathrm{OD}=8.625^{\prime \prime}$

Length $\mathrm{L}_{\mathrm{c}}=36^{\prime \prime}$

$\mathrm{t} \quad=0.5^{\prime \prime}$

Design thickness, $\left(\right.$ at $^{1,000}{ }^{\circ}$ F) Appendix 1-1

$\mathrm{t}=\mathrm{P}^{*} \mathrm{R}_{\mathrm{o}} /\left(\mathrm{S} * \mathrm{E}+0.40^{*} \mathrm{P}\right)+$ Corrosion

$=1,150 * 4.3125 /(15,300 * 1.00+0.40 * 1,150)+0.03$

$=0.3447^{\prime \prime}$

Maximum allowable working pressure, $\left(\right.$ at $\left.^{1,000^{\circ}}{ }^{\circ}\right)$ Appendix 1-1

$$
\begin{aligned}
\mathrm{P} & =\mathrm{S}^{*} \mathrm{E}^{*} \mathrm{t} /\left(\mathrm{R}_{\mathrm{o}}-0.40^{*} \mathrm{t}\right)-\mathrm{P}_{\mathrm{S}} \\
& =15,300^{*} 1.00^{*} 0.4075 /\left(4.3125-0.40^{*} 0.4075\right)-0 \\
& =1,502.53 \mathrm{psi}
\end{aligned}
$$

Design thickness $=0.3447^{\prime \prime}$

The governing condition is due to internal pressure.

The cylinder thickness of $0.5^{\prime \prime}$ is adequate. 
Thickness Required Due to Pressure + External Loads

\begin{tabular}{|c|c|c|c|c|c|c|c|c|}
\hline \multirow[t]{2}{*}{ Condition } & \multirow{2}{*}{$\begin{array}{l}\text { Pressure } \\
\text { P ( psi) }\end{array}$} & \multicolumn{2}{|c|}{$\begin{array}{l}\text { Allowable Stress } \\
\text { Before UG-23 Stress } \\
\text { Increase ( psi) }\end{array}$} & \multirow{2}{*}{$\begin{array}{l}\text { Temperature } \\
\left({ }^{\circ} \mathbf{F}\right)\end{array}$} & \multirow{2}{*}{$\begin{array}{c}\text { Corrosion } \\
\text { C (in) }\end{array}$} & \multirow[t]{2}{*}{ Load } & \multirow{2}{*}{$\begin{array}{l}\text { Req'd Thk } \\
\text { Due to } \\
\text { Tension (in) }\end{array}$} & \multirow{2}{*}{$\begin{array}{l}\text { Req'd Thk Due } \\
\text { to Compression } \\
\text { (in) }\end{array}$} \\
\hline & & $\mathbf{S}_{\mathbf{t}}$ & $\mathbf{S}_{\mathbf{c}}$ & & & & & \\
\hline \multirow{2}{*}{$\begin{array}{l}\text { Operating, Hot \& } \\
\text { Corroded }\end{array}$} & \multirow{2}{*}{1,150} & \multirow{2}{*}{15,300} & \multirow{2}{*}{9,945} & \multirow{2}{*}{1,000} & \multirow{2}{*}{0.03} & Wind & 0.1193 & 0.1177 \\
\hline & & & & & & Seismic & 0.1195 & 0.1175 \\
\hline \multirow{2}{*}{$\begin{array}{l}\text { Operating, Hot \& } \\
\text { New }\end{array}$} & \multirow{2}{*}{1,150} & \multirow{2}{*}{15,300} & \multirow{2}{*}{9,995} & \multirow{2}{*}{1,000} & \multirow{2}{*}{0} & Wind & 0.1183 & 0.1168 \\
\hline & & & & & & Seismic & 0.1186 & 0.1165 \\
\hline \multirow{2}{*}{ Empty, Corroded } & \multirow{2}{*}{0} & \multirow{2}{*}{20,000} & \multirow{2}{*}{14,142} & \multirow{2}{*}{0} & \multirow{2}{*}{0.03} & Wind & 0.0003 & 0.0012 \\
\hline & & & & & & Seismic & 0.0005 & 0.0015 \\
\hline \multirow{2}{*}{ Empty, New } & \multirow{2}{*}{0} & \multirow{2}{*}{20,000} & \multirow{2}{*}{14,203} & \multirow{2}{*}{0} & \multirow{2}{*}{0} & Wind & 0.0003 & 0.0012 \\
\hline & & & & & & Seismic & 0.0005 & 0.0016 \\
\hline $\begin{array}{l}\text { Hot Shut Down, } \\
\text { Corroded, Weight \& } \\
\text { Eccentric Moments } \\
\text { Only }\end{array}$ & 0 & 15,300 & 9,945 & 1,000 & 0.03 & Weight & 0.0007 & 0.0007 \\
\hline
\end{tabular}




\section{8" X 4" REDUCER B16.9, LOWER (SCH XS) 8" END}

ASME Section VIII Division 1, 2007 Edition

Component:

Material specification:

Rated MDMT per UHA-51(d)(1)(b), (carbon content does not exceed 0.1 percent) $=-320^{\circ} \mathrm{F}$
Cylinder

SA-403 $316 \mathrm{H}$ (II-D p. $78, \ln .8)$

Internal design pressure: $P=1,150 \mathrm{psi} @ 1,000^{\circ} \mathrm{F}$

Static liquid head:

$\mathrm{P}_{\mathrm{th}}=0.33 \mathrm{psi}\left(\mathrm{SG}=1, \mathrm{H}_{\mathrm{S}}=9.125 "\right.$ ", Horizontal test head $)$

Corrosion allowance $\quad$ Inner $\mathrm{C}=0.03 " \quad$ Outer $\mathrm{C}=0$ "

$\begin{array}{ll}\text { Design MDMT }=-20^{\circ} \mathrm{F} & \text { No impact test performed } \\ \text { Rated MDMT }=-320^{\circ} \mathrm{F} & \text { Material is not normalized } \\ & \text { Material is not produced to Fine Grain Practice } \\ & \text { PWHT is not performed }\end{array}$

Radiography: $\quad$ Longitudinal joint - $\quad$ Seamless No RT

Top circumferential joint - Full UW-11(a) Type 1

Bottom circumferential joint -

Estimated weight New $=9.8 \mathrm{lb} \quad$ corr $=9.2 \mathrm{lb}$

Capacity New $=0.61$ US gal corr $=0.62$ US gal

$\begin{aligned} \text { OD } & =8.625^{\prime \prime} \\ \text { Length } \mathrm{L}_{\mathrm{c}} & =3^{\prime \prime} \\ \mathrm{t} & =0.4375^{\prime \prime}\end{aligned}$

Design thickness, $\left(\right.$ at $\left.^{1,000}{ }^{\circ} \mathrm{F}\right)$ Appendix 1-1

$\mathrm{t}=\mathrm{P} * \mathrm{R}_{\mathrm{o}} /(\mathrm{S} * \mathrm{E}+0.40 * \mathrm{P})+$ Corrosion

$=1,150 * 4.3125 /(15,300 * 1.00+0.40 * 1,150)+0.03$

$=0.3447^{\prime \prime}$

Maximum allowable working pressure, $\left(\right.$ at $\left.1,000^{\circ} \mathrm{F}\right)$ Appendix 1-1

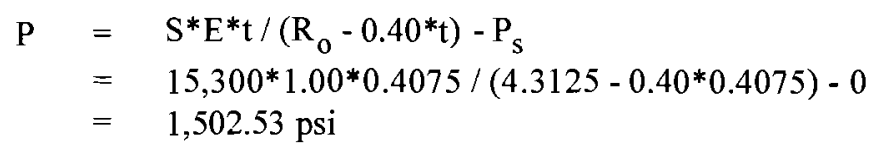

\% Forming Strain - UHA-44(a)(2)(a)

$$
\begin{aligned}
\mathrm{EFE} & =\left(50 * \mathrm{t} / \mathrm{R}_{\mathrm{f}}\right) *\left(1-\mathrm{R}_{\mathrm{f}} / \mathrm{R}_{\mathrm{o}}\right) \\
& =(50 * 0.4375 / 4.0938) *(1-4.0938 / \infty) \\
& =5.3435 \%
\end{aligned}
$$




\section{Design thickness $=0.3447^{\prime \prime}$}

The governing condition is due to internal pressure.

The cylinder thickness of $0.4375 "$ is adequate.

Thickness Required Due to Pressure + External Loads

\begin{tabular}{|c|c|c|c|c|c|c|c|c|}
\hline \multirow[t]{2}{*}{ Condition } & \multirow{2}{*}{$\begin{array}{l}\text { Pressure } \\
\text { P ( psi) }\end{array}$} & \multicolumn{2}{|c|}{$\begin{array}{c}\text { Allowable Stress } \\
\text { Before UG-23 Stress } \\
\text { Increase ( psi) }\end{array}$} & \multirow{2}{*}{$\begin{array}{c}\text { Temperature } \\
\left({ }^{\circ} \mathrm{F}\right)\end{array}$} & \multirow{2}{*}{$\begin{array}{c}\text { Corrosion } \\
\text { C (in) }\end{array}$} & \multirow[t]{2}{*}{ Load } & \multirow{2}{*}{$\begin{array}{l}\text { Req'd Thk } \\
\text { Due to } \\
\text { Tension (in) }\end{array}$} & \multirow{2}{*}{$\begin{array}{l}\text { Req'd Thk Due } \\
\text { to Compression } \\
\text { (in) }\end{array}$} \\
\hline & & $\mathbf{S}_{\mathrm{t}}$ & $\mathbf{S}_{\mathbf{c}}$ & & & & & \\
\hline \multirow{2}{*}{$\begin{array}{l}\text { Operating, Hot \& } \\
\text { Corroded }\end{array}$} & \multirow{2}{*}{1,150} & \multirow{2}{*}{15,300} & \multirow{2}{*}{9,945} & \multirow{2}{*}{1,000} & \multirow{2}{*}{0.03} & Wind & 0.1213 & 0.1195 \\
\hline & & & & & & Seismic & 0.1215 & 0.1193 \\
\hline \multirow{2}{*}{$\begin{array}{c}\text { Operating, Hot \& } \\
\text { New }\end{array}$} & \multirow{2}{*}{1,150} & \multirow{2}{*}{15,300} & \multirow{2}{*}{9,995} & \multirow{2}{*}{1,000} & \multirow{2}{*}{0} & Wind & 0.1203 & 0.1186 \\
\hline & & & & & & Seismic & 0.1206 & 0.1183 \\
\hline \multirow{2}{*}{ Empty, Corroded } & \multirow{2}{*}{0} & \multirow{2}{*}{20,000} & \multirow{2}{*}{14,142} & \multirow{2}{*}{0} & \multirow{2}{*}{0.03} & Wind & 0.0004 & 0.0013 \\
\hline & & & & & & Seismic & 0.0005 & 0.0016 \\
\hline \multirow{2}{*}{ Empty, New } & \multirow{2}{*}{0} & \multirow{2}{*}{20,000} & \multirow{2}{*}{14,203} & \multirow{2}{*}{0} & \multirow{2}{*}{0} & Wind & 0.0004 & 0.0014 \\
\hline & & & & & & Seismic & 0.0005 & 0.0017 \\
\hline $\begin{array}{l}\text { Hot Shut Down, } \\
\text { Corroded, Weight \& } \\
\text { Eccentric Moments } \\
\text { Only }\end{array}$ & 0 & 15,300 & 9,945 & 1,000 & 0.03 & Weight & 0.0007 & 0.0007 \\
\hline
\end{tabular}




\section{8" X 4" REDUCER B16.9, LOWER (SCH XS) 4" END}

\section{ASME Section VIII Division 1, 2007 Edition}

Component:

Material specification:

Rated MDMT per UHA-51(d)(1)(b), (carbon content does not exceed 0.1 percent) $=-320^{\circ} \mathrm{F}$
Cylinder

SA-403 $316 \mathrm{H}$ (II-D p. $78, \ln .8)$

Internal design pressure: $\mathrm{P}=1,150$ psi @ $1,000^{\circ} \mathrm{F}$

Static liquid head:

$P_{\text {th }}=0.26 \mathrm{psi}\left(\mathrm{SG}=1, \mathrm{H}_{\mathrm{s}}=7.205^{\prime}\right.$, Horizontal test head $)$

Corrosion allowance $\quad$ Inner $\mathrm{C}=0.03^{\prime \prime} \quad$ Outer $\mathrm{C}=0$ "

Design MDMT $=-20^{\circ} \mathrm{F}$

Rated $\mathrm{MDMT}=-320^{\circ} \mathrm{F}$
No impact test performed Material is not normalized Material is not produced to Fine Grain Practice PWHT is not performed

Radiography: Longitudinal joint Seamless No RT Top circumferential joint - Full UW-11(a) Type 1 Bottom circumferential joint -

Estimated weight New $=3.4 \mathrm{lb} \quad$ corr $=3.1 \mathrm{lb}$

Capacity New $=0.16$ US gal corr $=0.16$ US gal

$$
\begin{array}{ll}
\text { OD } & =4.5^{\prime \prime} \\
\text { Length } \mathrm{L}_{\mathrm{c}} & =3^{\prime \prime} \\
\mathrm{t} & =0.295^{\prime \prime}
\end{array}
$$

\section{Design thickness, (at $1,000^{\circ} \mathrm{F}$ ) Appendix 1-1}

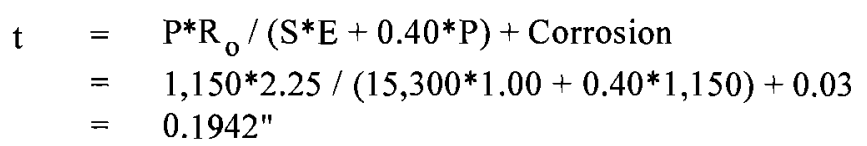

Maximum allowable working pressure, (at 1,000 $\left.{ }^{\circ} \mathrm{F}\right)$ Appendix 1-1

$$
\begin{aligned}
\mathrm{P} & =\mathrm{S} * \mathrm{E}^{*} \mathrm{t} /\left(\mathrm{R}_{\mathrm{o}}-0.40 * \mathrm{t}\right)-\mathrm{P}_{\mathrm{S}} \\
& =15,300 * 1.00 * 0.265 /(2.25-0.40 * 0.265)-0 \\
& =1,891.09 \mathrm{psi}
\end{aligned}
$$

\% Forming Strain - UHA-44(a)(2)(a)

$$
\begin{aligned}
\mathrm{EFE} & =\left(50 * \mathrm{t} / \mathrm{R}_{\mathrm{f}}\right) *\left(1-\mathrm{R}_{\mathrm{f}} / \mathrm{R}_{\mathrm{o}}\right) \\
& =(50 * 0.295 / 2.1025) *(1-2.1025 / \infty) \\
& =7.0155 \%
\end{aligned}
$$




\section{Design thickness $=0.1942 "$}

The governing condition is due to internal pressure.

The cylinder thickness of $0.295^{\prime \prime}$ is adequate.

Thickness Required Due to Pressure + External Loads

\begin{tabular}{|c|c|c|c|c|c|c|c|c|}
\hline \multirow[t]{2}{*}{ Condition } & \multirow{2}{*}{$\begin{array}{c}\text { Pressure } \\
\text { P ( psi) }\end{array}$} & \multicolumn{2}{|c|}{$\begin{array}{l}\text { Allowable Stress } \\
\text { Before UG-23 Stress } \\
\text { Increase ( psi) }\end{array}$} & \multirow{2}{*}{$\begin{array}{c}\text { Temperature } \\
\left({ }^{\circ} \mathrm{F}\right)\end{array}$} & \multirow{2}{*}{$\begin{array}{l}\text { Corrosion } \\
\text { C (in) }\end{array}$} & \multirow[t]{2}{*}{ Load } & \multirow{2}{*}{$\begin{array}{l}\text { Req'd Thk } \\
\text { Due to } \\
\text { Tension (in) }\end{array}$} & \multirow{2}{*}{$\begin{array}{l}\text { Req'd Thk Due } \\
\text { to Compression } \\
\text { (in) }\end{array}$} \\
\hline & & $\mathbf{S}_{\mathbf{t}}$ & $S_{c}$ & & & & & \\
\hline \multirow{2}{*}{$\begin{array}{l}\text { Operating, Hot \& } \\
\text { Corroded }\end{array}$} & \multirow{2}{*}{1,150} & \multirow{2}{*}{15,300} & \multirow{2}{*}{10,100} & \multirow{2}{*}{1,000} & \multirow{2}{*}{0.03} & Wind & 0.0644 & 0.057 \\
\hline & & & & & & Seismic & 0.065 & 0.0562 \\
\hline \multirow{2}{*}{$\begin{array}{c}\text { Operating, Hot \& } \\
\text { New }\end{array}$} & \multirow{2}{*}{1,150} & \multirow{2}{*}{15,300} & \multirow{2}{*}{10,177} & \multirow{2}{*}{1,000} & \multirow{2}{*}{0} & Wind & 0.0634 & 0.0559 \\
\hline & & & & & & Seismic & 0.0643 & 0.055 \\
\hline \multirow{2}{*}{ Empty, Corroded } & \multirow{2}{*}{0} & \multirow{2}{*}{20,000} & \multirow{2}{*}{14,332} & \multirow{2}{*}{0} & \multirow{2}{*}{0.03} & Wind & 0.0023 & 0.0047 \\
\hline & & & & & & Seismic & 0.0028 & 0.0056 \\
\hline \multirow{2}{*}{ Empty, New } & \multirow{2}{*}{0} & \multirow{2}{*}{20,000} & \multirow{2}{*}{14,425} & \multirow{2}{*}{0} & \multirow{2}{*}{0} & Wind & 0.0023 & 0.0048 \\
\hline & & & & & & Seismic & 0.0029 & 0.0058 \\
\hline $\begin{array}{l}\text { Hot Shut Down, } \\
\text { Corroded, Weight \& } \\
\text { Eccentric Moments } \\
\text { Only }\end{array}$ & 0 & 15,300 & 10,100 & 1,000 & 0.03 & Weight & 0.0013 & 0.0013 \\
\hline
\end{tabular}




\section{4" X 2" B16.9 REDUCER, LOWER (SCH XS) 4" END}

\section{ASME Section VIII Division 1, 2007 Edition}

\section{Component:}

Material specification:

Rated MDMT per UHA-51(d)(1)(b), (carbon content does not exceed 0.1 percent) $=-320^{\circ} \mathrm{F}$
Cylinder

SA-403 316H (II-D p. 78, ln. 8)

Internal design pressure: $\mathrm{P}=1,150 \mathrm{psi} @ 1,000^{\circ} \mathrm{F}$

Static liquid head:

$\mathrm{P}_{\text {th }}=0.26 \mathrm{psi}\left(\mathrm{SG}=1, \mathrm{H}_{\mathrm{s}}=7.205^{\prime \prime}\right.$, Horizontal test head $)$

Corrosion allowance $\quad$ Inner $\mathrm{C}=\mathbf{0 . 0 3 "} \quad$ Outer $\mathrm{C}=0 "$

Design MDMT $=-20^{\circ} \mathrm{F}$

Rated MDMT $=-320^{\circ} \mathrm{F}$
No impact test performed

Material is not normalized

Material is not produced to Fine Grain Practice

PWHT is not performed

Radiography: Longitudinal joint - $\quad$ Seamless No RT

Top circumferential joint - Full UW-1 1(a) Type 1

Bottom circumferential joint -

Full UW-11(a) Type 1

Estimated weight New $=2.3 \mathrm{lb} \quad$ corr $=2 \mathrm{lb}$

Capacity $\quad$ New $=0.1$ US gal corr $=0.11$ US gal

$$
\begin{aligned}
\mathrm{OD} & =4.5^{\prime \prime} \\
\text { Length } \mathrm{L}_{\mathrm{c}} & =2^{\prime \prime} \\
\mathrm{t} & =0.295^{\prime \prime}
\end{aligned}
$$

\section{Design thickness, (at $1,000^{\circ} \mathrm{F}$ ) Appendix 1-1}

$$
\begin{aligned}
\mathbf{t} & =\mathrm{P}^{*} \mathrm{R}_{\mathrm{o}} /\left(\mathrm{S}^{*} \mathrm{E}+0.40^{*} \mathrm{P}\right)+\text { Corrosion } \\
& =1,150^{*} 2.25 /\left(15,300^{*} 1.00+0.40^{*} 1,150\right)+0.03 \\
& =0.1942^{\prime \prime}
\end{aligned}
$$

Maximum allowable working pressure, $\left(\right.$ at $\left.1,000^{\circ} \mathrm{F}\right)$ Appendix 1-1

$$
\begin{aligned}
\mathrm{P} & =\mathrm{S}^{*} \mathrm{E}^{*} \mathrm{t} /\left(\mathrm{R}_{\mathrm{o}}-0.40^{*} \mathrm{t}\right)-\mathrm{P}_{\mathrm{s}} \\
& =15,300^{*} 1.00^{*} 0.265 /\left(2.25-0.40^{*} 0.265\right)-0 \\
& =1,891.09 \mathrm{psi}
\end{aligned}
$$

\% Forming Strain - UHA-44(a)(2)(a)

$$
\begin{aligned}
\mathrm{EFE} & =\left(50 * t / \mathrm{R}_{\mathrm{f}}\right) *\left(1-\mathrm{R}_{\mathrm{f}} / \mathrm{R}_{\mathrm{o}}\right) \\
& =(50 * 0.295 / 2.1025) *(1-2.1025 / \infty) \\
& =7.0155 \%
\end{aligned}
$$




\section{Design thickness $=0.1942 "$}

The governing condition is due to internal pressure.

The cylinder thickness of 0.295 " is adequate.

Thickness Required Due to Pressure + External Loads

\begin{tabular}{|c|c|c|c|c|c|c|c|c|}
\hline \multirow[t]{2}{*}{ Condition } & \multirow{2}{*}{$\begin{array}{l}\text { Pressure } \\
\text { P ( psi) }\end{array}$} & \multicolumn{2}{|c|}{$\begin{array}{c}\text { Allowable Stress } \\
\text { Before UG-23 Stress } \\
\text { Increase ( psi) }\end{array}$} & \multirow{2}{*}{$\begin{array}{c}\text { Temperature } \\
\left({ }^{\circ} \mathbf{F}\right)\end{array}$} & \multirow{2}{*}{$\begin{array}{c}\text { Corrosion } \\
\text { C (in) }\end{array}$} & \multirow[t]{2}{*}{ Load } & \multirow{2}{*}{$\begin{array}{l}\text { Req'd Thk } \\
\text { Due to } \\
\text { Tension (in) }\end{array}$} & \multirow{2}{*}{$\begin{array}{l}\text { Req'd Thk Due } \\
\text { to Compression } \\
\text { (in) }\end{array}$} \\
\hline & & $S_{t}$ & $S_{c}$ & & & & & \\
\hline \multirow{2}{*}{$\begin{array}{l}\text { Operating, Hot \& } \\
\text { Corroded }\end{array}$} & \multirow{2}{*}{1,150} & \multirow{2}{*}{15,300} & \multirow{2}{*}{10,100} & \multirow{2}{*}{1,000} & \multirow{2}{*}{0.03} & Wind & 0.0646 & 0.0567 \\
\hline & & & & & & Seismic & 0.0653 & 0.0559 \\
\hline \multirow{2}{*}{$\begin{array}{c}\text { Operating, Hot \& } \\
\text { New }\end{array}$} & \multirow{2}{*}{1,150} & \multirow{2}{*}{15,300} & \multirow{2}{*}{10,177} & \multirow{2}{*}{1,000} & \multirow{2}{*}{0} & Wind & 0.0637 & 0.0556 \\
\hline & & & & & & Seismic & 0.0645 & 0.0547 \\
\hline \multirow{2}{*}{ Empty, Corroded } & \multirow{2}{*}{0} & \multirow{2}{*}{20,000} & \multirow{2}{*}{14,332} & \multirow{2}{*}{0} & \multirow{2}{*}{0.03} & Wind & 0.0025 & 0.005 \\
\hline & & & & & & Seismic & 0.003 & 0.0059 \\
\hline \multirow{2}{*}{ Empty, New } & \multirow{2}{*}{0} & \multirow{2}{*}{20,000} & \multirow{2}{*}{14,425} & \multirow{2}{*}{0} & \multirow{2}{*}{0} & Wind & 0.0025 & 0.0051 \\
\hline & & & & & & Seismic & 0.0031 & 0.0061 \\
\hline $\begin{array}{c}\text { Hot Shut Down, } \\
\text { Corroded, Weight \& } \\
\text { Eccentric Moments } \\
\text { Only }\end{array}$ & 0 & 15,300 & 10,100 & 1,000 & 0.03 & Weight & 0.0013 & 0.0013 \\
\hline
\end{tabular}




\section{4" X 2" B16.9 REDUCER, LOWER (SCH XS) 2" END}

\section{ASME Section VIII Division 1, 2007 Edition}

\section{Component:}

Material specification:

Rated MDMT per UHA-51(d)(1)(b), (carbon content does not exceed 0.1 percent) $=-320^{\circ} \mathrm{F}$
Cylinder

SA-403 316H (II-D p. 78, In. 8)

Internal design pressure: $\mathrm{P}=1,150$ psi @ $1,000^{\circ} \mathrm{F}$

Static liquid head:

$P_{\text {th }}=0.23 \mathrm{psi}\left(\mathrm{SG}=1, \mathrm{H}_{\mathrm{s}}=6.2465^{\prime}\right.$, Horizontal test head $)$

Corrosion allowance $\quad$ Inner $\mathrm{C}=0.03^{\prime \prime} \quad$ Outer $\mathrm{C}=0^{\prime \prime}$

Design $\mathrm{MDMT}=-20^{\circ} \mathrm{F}$

Rated MDMT $=-320^{\circ} \mathrm{F}$
No impact test performed

Material is not normalized

Material is not produced to Fine Grain Practice

PWHT is not performed

Radiography: $\quad$ Longitudinal joint - $\quad$ Seamless No RT

Top circumferential joint - Full UW-11(a) Type 1

Bottom circumferential joint -

Full UW-11(a) Type 1

Estimated weight New $=0.8 \mathrm{lb} \quad$ corr $=0.6 \mathrm{lb}$

Capacity New $=0.03$ US gal corr $=0.03$ US gal

$\begin{array}{ll}\text { OD } & =2.375^{\prime \prime} \\ \text { Length } \mathrm{L}_{\mathrm{c}} & =2^{\prime \prime} \\ \mathrm{t} & =0.191^{\prime \prime}\end{array}$

Design thickness, (at $1,000^{\circ} \mathrm{F}$ ) Appendix 1-1

$\mathrm{t}=\mathrm{P}^{*} \mathrm{R}_{\mathrm{o}} /(\mathrm{S} * \mathrm{E}+0.40 * \mathrm{P})+$ Corrosion

$=1,150 * 1.1875 /(15,300 * 1.00+0.40 * 1,150)+0.03$

$=0.1167^{\prime \prime}$

Maximum allowable working pressure, (at 1,000 ${ }^{\circ} \mathrm{F}$ ) Appendix 1-1

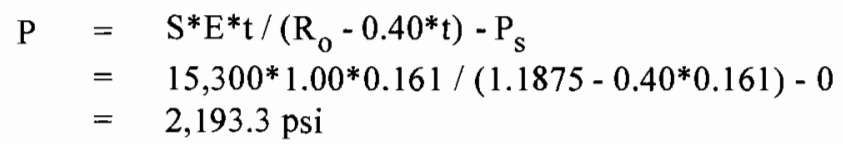

\% Forming Strain - UHA-44(a)(2)(a)

$$
\begin{aligned}
\mathrm{EFE} & =\left(50 * \mathrm{t} / \mathrm{R}_{\mathrm{f}}\right) *\left(1-\mathrm{R}_{\mathrm{f}} / \mathrm{R}_{\mathrm{o}}\right) \\
& =(50 * 0.191 / 1.092) *(1-1.092 / \infty) \\
& =8.7454 \%
\end{aligned}
$$




\section{Design thickness $=0.1167^{\prime \prime}$}

The governing condition is due to internal pressure.

The cylinder thickness of $0.191 "$ is adequate.

Thickness Required Due to Pressure + External Loads

\begin{tabular}{|c|c|c|c|c|c|c|c|c|}
\hline \multirow[t]{2}{*}{ Condition } & \multirow{2}{*}{$\begin{array}{l}\text { Pressure } \\
\text { P ( psi) }\end{array}$} & \multicolumn{2}{|c|}{$\begin{array}{c}\text { Allowable Stress } \\
\text { Before UG-23 Stress } \\
\text { Increase ( psi) }\end{array}$} & \multirow{2}{*}{$\begin{array}{l}\text { Temperature } \\
\left({ }^{\circ} \mathbf{F}\right)\end{array}$} & \multirow{2}{*}{$\begin{array}{c}\text { Corrosion } \\
\text { C (in) }\end{array}$} & \multirow[t]{2}{*}{ Load } & \multirow{2}{*}{$\begin{array}{l}\text { Req'd Thk } \\
\text { Due to } \\
\text { Tension (in) }\end{array}$} & \multirow{2}{*}{$\begin{array}{l}\text { Req'd Thk Due } \\
\text { to Compression } \\
\text { (in) }\end{array}$} \\
\hline & & $S_{t}$ & $\mathbf{S}_{\mathbf{c}}$ & & & & & \\
\hline \multirow{2}{*}{$\begin{array}{l}\text { Operating, Hot \& } \\
\text { Corroded }\end{array}$} & \multirow{2}{*}{1,150} & \multirow{2}{*}{15,300} & \multirow{2}{*}{10,201} & \multirow{2}{*}{1,000} & \multirow{2}{*}{0.03} & Wind & 0.0482 & 0.0121 \\
\hline & & & & & & Seismic & 0.0498 & 0.0101 \\
\hline \multirow{2}{*}{$\begin{array}{c}\text { Operating, Hot \& } \\
\text { New }\end{array}$} & \multirow{2}{*}{1,150} & \multirow{2}{*}{15,300} & \multirow{2}{*}{10,324} & \multirow{2}{*}{1,000} & \multirow{2}{*}{0} & Wind & 0.0476 & 0.0106 \\
\hline & & & & & & Seismic & 0.0501 & 0.0078 \\
\hline \multirow{2}{*}{ Empty, Corroded } & \multirow{2}{*}{0} & \multirow{2}{*}{20,000} & \multirow{2}{*}{14,454} & \multirow{2}{*}{0} & \multirow{2}{*}{0.03} & Wind & 0.0126 & 0.0208 \\
\hline & & & & & & Seismic & 0.0138 & 0.0229 \\
\hline \multirow{2}{*}{ Empty, New } & \multirow{2}{*}{0} & \multirow{2}{*}{20,000} & \multirow{2}{*}{14,604} & \multirow{2}{*}{0} & \multirow{2}{*}{0} & Wind & 0.0129 & 0.0212 \\
\hline & & & & & & Seismic & 0.0148 & 0.0242 \\
\hline $\begin{array}{c}\text { Hot Shut Down, } \\
\text { Corroded, Weight \& } \\
\text { Eccentric Moments } \\
\text { Only }\end{array}$ & 0 & 15,300 & 10,201 & 1,000 & 0.03 & Weight & 0.0029 & 0.0029 \\
\hline
\end{tabular}




\section{2" RFWN FLANGE 1500\#.LOWER}

Flange description:

Bolt Material:

Flange rated MDMT:

(Per UHA-51(d)(1)(b))

(Flange rated $\mathrm{MDMT}=-320^{\circ} \mathrm{F}$

Bolts rated MDMT per Fig UCS-66 note $\left.(\mathrm{e})=-55^{\circ} \mathrm{F}\right)$

Liquid static head on flange:

ASME B16.5 flange rating MAWP:

ASME B 16.5 flange rating MAP:

ASME B16.5 flange hydro test:
2 inch Class 1500 WN A 182 F316H

SA-193 B7 Bolt $<=21 / 2$

$-55^{\circ} \mathrm{F}$

\section{$0 \mathrm{psi}$}

1820 psi @ $1000^{\circ} \mathrm{F}$

3600 psi @ $70^{\circ} \mathrm{F}$

$5425 \mathrm{psi} @ 70^{\circ} \mathrm{F}$ 


\section{ASME B16.5/16.47 Blind on 2" RFWN FLANGE 1500\#, LOWER}

This is an ASME B16.5/16.47 rated blind flange.

Flange description:

Bolt Material:

Flange rated MDMT:

(Per UHA-51(d)(1)(b))

(Flange rated MDMT $=-320^{\circ} \mathrm{F}$

Bolts rated MDMT per Fig UCS- 66 note $\left.(e)=-55^{\circ} \mathrm{F}\right)$

Liquid static head on flange:

ASME B16.5 flange rating MAWP:

ASME B16.5 flange rating MAP:

ASME B16.5 flange hydro test:
2 inch Class $1500 \mathrm{WN}$ A182 F316H

SA-193 B7 Bolt $<=21 / 2$

$-55^{\circ} \mathrm{F}$

$0 \mathrm{psi}$

$1820 \mathrm{psi} @ 1000^{\circ} \mathrm{F}$

$3600 \mathrm{psi} @ 70^{\circ} \mathrm{F}$

$5425 \mathrm{psi} @ 70^{\circ} \mathrm{F}$ 


\section{1/2" HALF-COUPLING (7a)}

ASME Section VIII Division 1, 2007 Edition

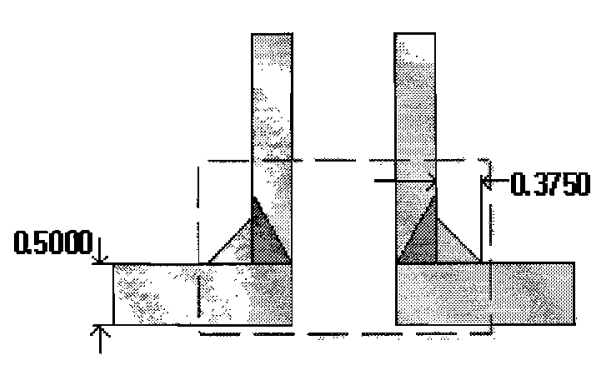

$$
\begin{aligned}
& \mathrm{t}_{\mathrm{w}(\text { lower })}=0 \mathrm{in} \\
& \operatorname{Leg}_{41}=0.375 \mathrm{in}
\end{aligned}
$$

Note: round inside edges per UG-76(c)

Located on:

Liquid static head included:

Nozzle material specification:

Nozzle longitudinal joint efficiency:

Nozzle description:

Nozzle orientation:

Local vessel minimum thickness:

Nozzle center line offset to datum line:

End of nozzle to shell center:

Nozzle inside diameter, new:

Nozzle nominal wall thickness:

Nozzle corrosion allowance:

Projection available outside vessel, Lpr: 0.9375 in

\section{Reinforcement CaIculations for Internal Pressure}

\begin{tabular}{|c|c|c|c|c|c|c|c|c|}
\hline \multicolumn{7}{|c|}{$\begin{array}{l}\text { UG-37 Area Calculation Summary }\left(\text { in }^{2}\right) \\
\text { For } P=1502.52 \text { psi @ } 1000^{\circ} \mathrm{F}\end{array}$} & \multicolumn{2}{|c|}{$\begin{array}{l}\text { UG-45 Nozzle Wall } \\
\text { Thickness Summary (in) } \\
\text { The nozzle passes UG-45 }\end{array}$} \\
\hline$\underset{\text { required }}{A}$ & $\underset{\text { available }}{\text { A }}$ & $\mathbf{A}_{1}$ & $\mathbf{A}_{2}$ & $\mathbf{A}_{3}$ & $\mathbf{A}_{5}$ & $\begin{array}{c}\text { A } \\
\text { welds }\end{array}$ & $t_{\text {req }}$ & $t_{\text {min }}$ \\
\hline \multicolumn{7}{|c|}{ This nozzle is exempt from area calculations perUG-36(c)(3)(a) } & 0.1247 & 0.33 \\
\hline
\end{tabular}

The vessel wall thickness governs the MAWP of this nozzle.

\section{UG-41 Weld Failure Path Analysis Summary}

The nozzle is exempt from weld strength calculations per UW-15(b)(2)

\section{UW-16 Weld Sizing Summary}




\begin{tabular}{|c|r|r|c|} 
Weld description & $\begin{array}{r}\text { Required weld } \\
\text { throat size (in) }\end{array}$ & $\begin{array}{r}\text { Actual weld } \\
\text { throat size (in) }\end{array}$ & Status \\
\hline Nozzle to shell fillet $\left(\operatorname{Leg}_{41}\right)$ & 0.21 & 0.2625 & weld size is adequate \\
\hline
\end{tabular}

This opening does not require reinforcement per UG-36(c)(3)(a)

\section{Reinforcement Calculations for MAP}

The vessel wall thickness governs the MAP of this nozzle.

\begin{tabular}{|c|c|c|c|c|c|c|c|c|}
\hline \multicolumn{7}{|c|}{$\begin{array}{l}\text { UG-37 Area Calculation Summary }\left(\text { in }^{2}\right) \\
\text { For } P=2114.8 \mathrm{psi} @ 7^{\circ} \mathrm{F}\end{array}$} & \multicolumn{2}{|c|}{$\begin{array}{c}\text { UG-45 Nozzle Wall } \\
\text { Thickness Summary (in) } \\
\text { The nozzle passes UG-45 }\end{array}$} \\
\hline$\underset{\text { required }}{\text { A }}$ & $\underset{\text { available }}{\mathbf{A}}$ & $\mathbf{A}_{1}$ & $\mathbf{A}_{2}$ & $\mathbf{A}_{3}$ & $\mathbf{A}_{\mathbf{s}}$ & $\begin{array}{c}\text { A } \\
\text { welds }\end{array}$ & $t_{\text {req }}$ & $\mathbf{t}_{\min }$ \\
\hline \multicolumn{7}{|c|}{ This nozzle is exempt from area calculations per UG-36(c)(3)(a) } & 0.0761 & 0.33 \\
\hline
\end{tabular}

\section{UG-41 Weld Failure Path Analysis Summary}

The nozzle is exempt from weld strength calculations per UW-15(b)(2)

\begin{tabular}{|c|r|r|c|}
\hline \multicolumn{4}{|c|}{ UW -16 Weld Sizing Summary } \\
\hline Weld description & $\begin{array}{r}\text { Required weld } \\
\text { throat size (in) }\end{array}$ & $\begin{array}{r}\text { Actual weld } \\
\text { throat size (in) }\end{array}$ & Status \\
\hline Nozzle to shell fillet ( $\left.\mathrm{Leg}_{41}\right)$ & 0.231 & 0.2625 & weld size is adequate \\
\hline
\end{tabular}

This opening does not require reinforcement per UG-36(c)(3)(a) 


\section{1/2" HALF-COUPLING (7b)}

ASME Section VIII Division 1, 2007 Edition

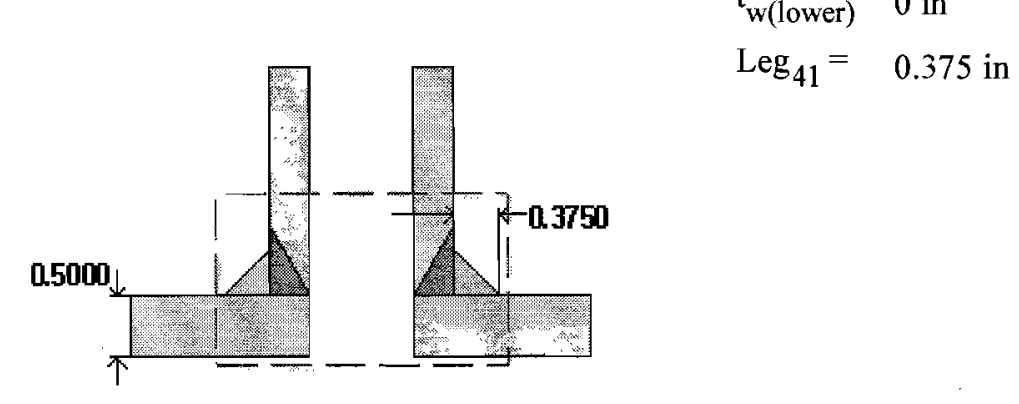

Note round inside edges per UG-76(c)

Located on:

Liquid static head included:

Nozzle material specification:

Nozzle longitudinal joint efficiency:

Nozzle description:

Nozzle orientation:

Local vessel minimum thickness:

Nozzle center line offset to datum line:

End of nozzle to shell center:

Nozzle inside diameter, new:

Nozzle nominal wall thickness:

Nozzle corrosion allowance:

Projection available outside vessel, Lpr: 0.9375 in

\section{Reinforcement Calculations for Internal Pressure}

The vessel wall thickness governs the MAWP of this nozzle.

\begin{tabular}{|c|c|c|c|c|c|c|c|c|}
\hline \multicolumn{8}{|c|}{$\begin{array}{c}\text { UG-37 Area Calculation Summary }\left(\text { in }^{2}\right) \\
\text { For P = 1502.52 psi @ } 1000{ }^{\circ} \mathbf{F}\end{array}$} & \multicolumn{2}{c|}{$\begin{array}{c}\text { UG-45 Nozzle Wall } \\
\text { Thickness Summary (in) } \\
\text { The nozzle passes UG-45 }\end{array}$} \\
\hline $\begin{array}{c}\text { A } \\
\text { required }\end{array}$ & $\begin{array}{c}\text { A } \\
\text { available }\end{array}$ & $\mathbf{A}_{1}$ & $\mathbf{A}_{2}$ & $\mathbf{A}_{3}$ & $\mathbf{A}_{5}$ & $\begin{array}{c}\text { A } \\
\text { welds }\end{array}$ & $\mathbf{t}_{\text {req }}$ & $\mathbf{t}_{\text {min }}$ \\
\hline This nozzle is exempt from area calculations per UG-36(c)(3)(a) & 0.1247 & 0.33 \\
\hline
\end{tabular}

\section{UG-41 Weld Failure Path Analysis Summary}

The nozzle is exempt from weld strength calculations per $U W-15(b)(2)$

UW-16 Weld Sizing Summary
8" PIPE

SA-182 F316H $<=5$ (low stress) (II-D p. 74, ln. 32)

$0.500^{\prime \prime}$ Class 6000 - threaded

5.25 in

.84 in

0.33 in

0.03 in 


\begin{tabular}{|c|r|r|c|}
\hline Weld description & $\begin{array}{r}\text { Required weld } \\
\text { throat size (in) }\end{array}$ & $\begin{array}{r}\text { Actual weld } \\
\text { throat size (in) }\end{array}$ & Status \\
\hline Nozzle to shell fillet $\left(\mathrm{Leg}_{41}\right)$ & 0.21 & 0.2625 & weld size is adequate \\
\hline
\end{tabular}

This opening does not require reinforcement per UG-36(c)(3)(a)

\section{Reinforcement Calculations for MAP}

The vessel wall thickness governs the MAP of this nozzle.

\begin{tabular}{|c|c|c|c|c|c|c|c|c|}
\hline \multicolumn{7}{|c|}{ 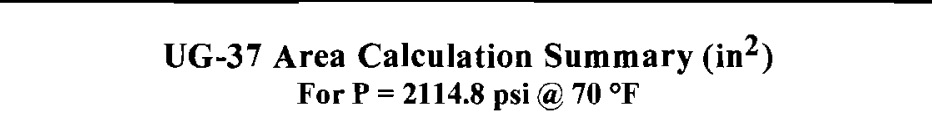 } & \multicolumn{2}{|c|}{$\begin{array}{l}\text { UG-45 Nozzle Wall } \\
\text { Thickness Summary (in) } \\
\text { The nozzle passes UG-45 }\end{array}$} \\
\hline$\underset{\text { required }}{A}$ & $\underset{\text { available }}{\text { A }}$ & $\mathbf{A}_{1}$ & $\mathbf{A}_{2}$ & $\mathbf{A}_{3}$ & $A_{5}$ & $\begin{array}{c}\text { A } \\
\text { welds }\end{array}$ & $t_{\text {req }}$ & $t_{\min }$ \\
\hline \multicolumn{7}{|c|}{ This nozzle is exempt from area calculations per UG-36(c)(3)(a) } & 0.0761 & 0.33 \\
\hline
\end{tabular}

\section{UG-41 Weld Failure Path Analysis Summary}

The nozzle is exempt from weld strength calculations per UW-15(b)(2)

\begin{tabular}{|c|r|r|c|}
\hline \multicolumn{4}{|c|}{ UW-16 Weld Sizing Summary } \\
\hline Weld description & $\begin{array}{r}\text { Required weld } \\
\text { throat size (in) }\end{array}$ & $\begin{array}{r}\text { Actual weld } \\
\text { throat size (in) }\end{array}$ & Status \\
\hline Nozzle to shell fillet $\left(\operatorname{Leg}_{41}\right)$ & 0.231 & 0.2625 & weld size is adequate \\
\hline
\end{tabular}

This opening does not require reinforcement per UG-36(c)(3)(a) 


\section{Wind Code}

Building Code:

ASCE 7-05

Elevation of base above grade: $\quad 0.0000 \mathrm{ft}$

Increase effective outer diameter by: $0.0000 \mathrm{ft}$

Wind Force Coefficient Cf: $\quad 0.8300$

Basic Wind Speed:, V: $\quad 85.0000 \mathrm{mph}$

Importance Factor:, I: $\quad 1.1500$

Exposure category: $\mathrm{C}$

Wind Directionality Factor, Kd: $\quad 0.9500$

Topographic Factor, Kzt: $\quad 1.0000$

Enforce min. loading of $10 \mathrm{psf}$ : No

\section{Vessel Characteristics}

Vessel height, h: $\quad 5.5425 \mathrm{ft}$

Vessel Minimum Diameter, $\mathbf{b}$

Operating, Corroded: $0.1979 \mathrm{ft}$

Empty, Corroded: $0.1979 \mathrm{ft}$

Fundamental Frequency, $\mathrm{n}_{1}$

Operating, Corroded: $9.6455 \mathrm{~Hz}$

Empty, Corroded: $10.7384 \mathrm{~Hz}$

Vacuum, Corroded: $9.5611 \mathrm{~Hz}$

Damping coefficient, $\beta$

Operating, Corroded: 0.0179

Empty, Corroded: 0.0200

Vacuum, Corroded: 0.0177

Table Lookup Values

\subsubsection{Basic Load Combinations for Allowable Stress Design}

The following load combinations are considered in accordance with ASCE section 2.4.1:

5. $D+H+W$

Where

$D \quad=$ Dead load

$H \quad=$ Pressure load

$W \quad=$ Wind load

\section{Wind Deflection Reports:}

Operating, Corroded

Empty, Corroded

Wind Pressure Calculations

Wind Deflection Report: Operating, Corroded

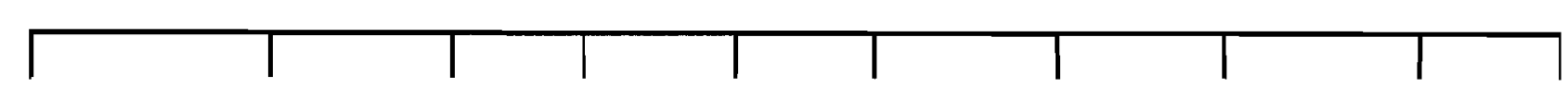




\begin{tabular}{|c|c|c|c|c|c|c|c|c|}
\hline Component & $\begin{array}{c}\text { Elevation of } \\
\text { bottom above } \\
\text { base (in) }\end{array}$ & $\begin{array}{c}\text { Effective } \\
\text { OD } \\
\text { (ft) }\end{array}$ & $\begin{array}{c}\text { Elastic } \\
\text { modulus } \\
\text { E }\left(10^{6} \mathrm{psi}\right)\end{array}$ & $\begin{array}{c}\text { Inertia } \\
\text { I }\left(\mathrm{ft}^{\mathbf{4}}\right)\end{array}$ & $\begin{array}{l}\text { Platform } \\
\text { wind shear at } \\
\text { Bottom (lbf) }\end{array}$ & $\begin{array}{c}\text { Total wind } \\
\text { shear at } \\
\text { Bottom (lbf) }\end{array}$ & \begin{tabular}{|c|} 
bending \\
moment at \\
Bottom (lbf- $\mathrm{ft}$ )
\end{tabular} & $\begin{array}{l}\text { Deflection } \\
\text { at top (in) }\end{array}$ \\
\hline $\begin{array}{l}4 " \text { X 2" } \\
\text { REDUCER B 16.9, } \\
\text { UPPER(SCH XS) } \\
\text { 2" END }\end{array}$ & 60.26 & 0.20 & 22.8 & $\begin{array}{r}3.326 \mathrm{e}- \\
005\end{array}$ & 0 & 1 & 0 & 0.03 \\
\hline $\begin{array}{l}\text { 4" X 2" } \\
\text { REDUCER B16.9, } \\
\text { UPPER (SCH XS) } \\
\text { 4" END }\end{array}$ & 58.26 & 0.38 & 22.8 & 0.0003827 & 0 & 2 & 1 & 0.0269 \\
\hline $\begin{array}{l}\text { 8" X 4" } \\
\text { REDUCER B16.9, } \\
\text { UPPER (SCH XS) } \\
\text { 4" END }\end{array}$ & 55.26 & 0.38 & 22.8 & 0.0003827 & 0 & 3 & 1 & 0.026 \\
\hline $\begin{array}{l}\text { 8" X 4" } \\
\text { REDUCER B16.9, } \\
\text { UPPER (SCH XS) } \\
\text { 8" END }\end{array}$ & 52.26 & 0.72 & 22.8 & 0.004293 & 0 & 6 & 2 & 0.0245 \\
\hline 8" PIPE & 16.26 & 0.72 & 22.8 & 0.004843 & 0 & 34 & 62 & 0.023 \\
\hline $\begin{array}{l}\text { 8" X 4" } \\
\text { REDUCER B16.9, } \\
\text { LOWER (SCH } \\
\text { XS) 8" END }\end{array}$ & 13.26 & 0.72 & 22.8 & 0.004293 & 0 & 36 & 70 & 0.0053 \\
\hline $\begin{array}{l}8^{\prime \prime} \text { X 4" } \\
\text { REDUCER B16.9, } \\
\text { LOWER (SCH } \\
\text { XS) 4" END }\end{array}$ & 10.26 & 0.38 & 22.8 & 0.0003827 & 0 & 37 & 80 & 0.0039 \\
\hline $\begin{array}{l}\text { 4" X 2" B16.9 } \\
\text { REDUCER, } \\
\text { LOWER (SCH } \\
\text { XS) 4" END }\end{array}$ & 8.26 & 0.38 & 22.8 & 0.0003827 & 0 & 38 & 86 & 0.0024 \\
\hline $\begin{array}{l}\text { 4" X 2" B16.9 } \\
\text { REDUCER, } \\
\text { LOWER (SCH } \\
\text { XS) 2" END }\end{array}$ & 2.01 & 0.20 & 22.8 & $\begin{array}{r}3.326 \mathrm{e}- \\
005\end{array}$ & 0 & 40 & 106 & 0.0015 \\
\hline $\begin{array}{l}\text { ASME } \\
\text { B16.5/16.47 Blind } \\
\text { on 2" RFWN } \\
\text { FLANGE 1500\#, } \\
\text { LOWER (top) }\end{array}$ & 2 & 0.71 & 20.4 & 0.01236 & 0 & 40 & 106 & 0 \\
\hline & & & & & & & & \\
\hline
\end{tabular}

\section{Wind Deflection Report: Empty, Corroded}

\begin{tabular}{|c|c|c|c|c|c|c|c|c|}
\hline Component & $\begin{array}{c}\text { Elevation of } \\
\text { bottom above } \\
\text { base (in) }\end{array}$ & $\begin{array}{c}\text { Effective } \\
\text { OD } \\
\text { (ft) }\end{array}$ & $\begin{array}{c}\text { Elastic } \\
\text { modulus } \\
\text { E }\left(\mathbf{1 0}^{6}{ }^{\mathrm{psi}}\right)\end{array}$ & $\begin{array}{c}\text { Inertia } \\
\mathbf{I}\left(\mathbf{f t}^{\mathbf{4}}\right)\end{array}$ & $\begin{array}{l}\text { Platform } \\
\text { wind shear at } \\
\text { Bottom (lbf) }\end{array}$ & $\begin{array}{c}\text { Total wind } \\
\text { shear at } \\
\text { Bottom (Ibf) }\end{array}$ & $\begin{array}{c}\text { bending } \\
\text { moment at } \\
\text { Bottom (lbf-ft) }\end{array}$ & $\begin{array}{l}\text { Deflection } \\
\text { at top (in) }\end{array}$ \\
\hline $\begin{array}{l}\text { 4" X 2" } \\
\text { REDUCER B16.9, } \\
\text { UPPER (SCH XS) } \\
\text { 2" END }\end{array}$ & 60.26 & 0.20 & 28.3 & $\begin{array}{r}3.326 \mathrm{e}- \\
005\end{array}$ & 0 & 1 & 0 & 0.0242 \\
\hline $\begin{array}{l}4^{\prime \prime} \times 2 " \\
\text { REDUCER B16.9, } \\
\text { UPPER (SCH XS) }\end{array}$ & 58.26 & 0.38 & 28.3 & 0.0003827 & 0 & 2 & 1 & 0.0217 \\
\hline
\end{tabular}




\begin{tabular}{|c|c|c|c|c|c|c|c|c|}
\hline 4" END & & & & & & & & \\
\hline $\begin{array}{l}\text { 8" X 4" } \\
\text { REDUCER B16.9, } \\
\text { UPPER (SCH XS) } \\
\text { 4" END }\end{array}$ & 55.26 & 0.38 & 28.3 & 0.0003827 & 0 & 3 & 1 & 0.0209 \\
\hline $\begin{array}{l}\text { 8" X 4" } \\
\text { REDUCER B16.9, } \\
\text { UPPER (SCH XS) } \\
\text { 8" END }\end{array}$ & 52.26 & 0.72 & 28.3 & 0.004293 & 0 & 6 & 2 & 0.0198 \\
\hline 8" PIPE & 16.26 & 0.72 & 28.3 & 0.004843 & 0 & 34 & 62 & 0.0186 \\
\hline $\begin{array}{l}\text { 8" X 4" }^{\prime \prime} \\
\text { REDUCER B16.9. } \\
\text { LOWER (SCH } \\
\text { XS) 8" END }\end{array}$ & 13.26 & 0.72 & 28.3 & 0.004293 & 0 & 36 & 70 & 0.0043 \\
\hline $\begin{array}{l}\text { 8" X 4" } \\
\text { REDUCER B16.9, } \\
\text { LOWER (SCH } \\
\text { XS) 4" END }\end{array}$ & 10.26 & 0.38 & 28.3 & 0.0003827 & 0 & 37 & 80 & 0.0031 \\
\hline $\begin{array}{l}\text { 4" X 2" B16.9 } \\
\text { REDUCER, } \\
\text { LOWER (SCH } \\
\text { XS) 4" END }\end{array}$ & 8.26 & 0.38 & 28.3 & 0.0003827 & 0 & 38 & 86 & 0.002 \\
\hline $\begin{array}{l}\text { 4" X 2" B16.9 } \\
\text { REDUCER, } \\
\text { LOWER (SCH } \\
\text { XS) 2" END }\end{array}$ & 2.01 & 0.20 & 28.3 & $\begin{array}{r}3.326 \mathrm{e}- \\
005\end{array}$ & 0 & 40 & 106 & 0.0012 \\
\hline $\begin{array}{l}\text { ASME } \\
\text { B16.5/16.47 Blind } \\
\text { on 2" RFWN } \\
\text { FLANGE 1500\#, } \\
\text { LOWER (top) }\end{array}$ & 2 & 0.71 & 29.4 & 0.01236 & 0 & 40 & 106 & 0 \\
\hline & & & & & & & & \\
\hline
\end{tabular}

\section{Wind Pressure (WP) Calculations}

Gust Factor $\left(\mathrm{G}^{-}\right)$Calculations

$$
\begin{aligned}
\mathrm{Kz} & =2.01 *(\mathrm{Z} / \underline{\mathrm{Zg}})^{2 / \underline{\alpha}} \\
& =2.01 *(\mathrm{Z} / 900.0000)^{0.2105} \\
\mathrm{qz} & =0.00256 * \mathrm{Kz}^{*} \mathrm{Kzt}^{*} \mathrm{Kd}^{*} \underline{\mathrm{V}}^{2 *} \underline{\mathrm{I} p \mathrm{ff}} \\
& =0.00256 * \mathrm{Kz}^{*} 1.0000 * 0.9500 * 85.0000^{2} * 1.1500 \\
& =20.2069 * \mathrm{Kz} \\
\mathrm{WP} & =\mathrm{qz}^{*} \underline{\mathrm{G}^{-}} * \underline{\mathrm{Cf}} \\
& =\mathrm{qz}^{*} \underline{\mathrm{G}^{-}} * 0.8300
\end{aligned}
$$

\section{Design Wind Pressures}

\begin{tabular}{|c|c|c|c|c|c|c|}
\hline $\begin{array}{c}\text { Height Z } \\
\text { (') }\end{array}$ & $\mathbf{K z}$ & $\begin{array}{c}\mathbf{q z} \\
\text { (psf) }\end{array}$ & $\begin{array}{c}\text { WP: Operating } \\
\text { (psf) }\end{array}$ & $\begin{array}{c}\text { WP: Empty } \\
\text { (psf) }\end{array}$ & $\begin{array}{c}\text { WP: hydrotest } \\
\text { (psf) }\end{array}$ & $\begin{array}{c}\text { WP: Vacuum } \\
\text { (psf) }\end{array}$ \\
\hline 15.0 & 0.8489 & 17.15 & 13.02 & 13.02 & N.A. & N.A. \\
\hline
\end{tabular}


Design Wind Force determined from: $F=$ Pressure $*$ Af, where Af is the projected area.

\section{Gust Factor Calculations}

Operating, Corroded

Empty, Corroded

\section{Gust Factor Calculations: Operating, Corroded}

$$
\begin{aligned}
\mathrm{z}^{-} & =0.60 * \underline{\mathrm{h}} \\
& =0.60 * 5.5425 \\
& =15.0000 \\
\mathrm{I}_{\mathrm{z}}^{-} & =\underline{\mathrm{c}}^{*}\left(33 / \mathrm{z}^{-}\right)^{1 / 6} \\
& =0.2000 *(33 / 15.0000)^{1 / 6} \\
& =0.2281 \\
\mathrm{~L}_{\mathrm{z}} & =\underline{\mathrm{I}}^{*}\left(\mathrm{z}^{-} / 33\right)^{\mathrm{ep}} \\
& =500.0000 *(15.0000 / 33)^{0.2000} \\
& =427.0566 \\
\mathrm{Q} & =\operatorname{Sqr}\left(1 /\left(1+0.63 *\left((\mathrm{~b}+\underline{\mathrm{h}}) / \mathrm{L}_{\mathrm{z}}\right)^{0.63}\right)\right) \\
& =\mathrm{Sqr}\left(1 /\left(1+0.63 *((0.1979+5.5425) / 427.0566)^{0.63}\right)\right) \\
& =0.9798 \\
\mathrm{G} & =0.925 *\left(1+1.7 * \mathrm{~g}_{\mathrm{Q}} * \mathrm{I}_{\mathrm{z}^{-}}{ }^{*} \mathrm{Q}\right) /\left(1+1.7 * \mathrm{~g}_{\mathrm{V}}{ }^{*} \mathrm{I}_{\mathrm{z}^{-}}\right) \\
& =0.925 *\left(1+1.7 * 3.40^{*} 0.2281 * 0.9798\right) /(1+1.7 * 3.40 * 0.2281) \\
& =0.9144
\end{aligned}
$$

\section{Gust Factor Calculations: Empty, Corroded}

$$
\begin{aligned}
\mathrm{z}^{-} & =0.60 * \underline{\mathrm{h}} \\
& =0.60 * 5.5425 \\
& =15.0000 \\
\mathrm{I}_{\mathrm{z}^{-}} & =\underline{\mathrm{c}}^{*}\left(33 / \mathrm{z}^{-}\right)^{1 / 6} \\
& =0.2000 *(33 / 15.0000)^{1 / 6} \\
& =0.2281 \\
\mathrm{~L}_{\mathrm{z}^{-}} & =\underline{\underline{I}}^{*}\left(\mathrm{z}^{-} / 33\right)^{\mathrm{ep}} \\
& =500.0000 *(15.0000 / 33)^{0.2000} \\
& =427.0566 \\
\mathrm{Q} & =\operatorname{Sqr}\left(1 /\left(1+0.63 *\left((\mathrm{~b}+\underline{\mathrm{h}}) / \mathrm{L}_{\mathrm{z}^{-}}\right)^{0.63}\right)\right) \\
& =\operatorname{Sqr}\left(1 /\left(1+0.63 *((0.1979+5.5425) / 427.0566)^{0.63}\right)\right) \\
& =0.9798 \\
\mathrm{G} & =0.925 *\left(1+1.7 * \mathrm{~g}_{\underline{Q}}^{*} \mathrm{I}_{\mathrm{z}^{-}} * \mathrm{Q}\right) /\left(1+1.7 * \underline{\mathrm{g}}^{*} \mathrm{I}_{\mathrm{z}^{-}}\right) \\
& =0.925 *\left(1+1.7 * 3.40^{*} 0.2281 * 0.9798\right) /(1+1.7 * 3.40 * 0.2281) \\
& =0.9144
\end{aligned}
$$

Table Lookup Values 


$$
\begin{array}{lr}
\alpha=9.5000, \mathrm{Zg}=900.0000^{\prime} & {[\text { Table 6-2, page 78] }} \\
\mathrm{c}=0.2000,1=500.0000, \mathrm{ep}=0.2000 & {[\text { Table 6-2, page 78] }} \\
\mathrm{a}^{-}=0.1538, \mathrm{~b}^{-}=0.6500 & {[\text { Table 6-2, page 78] }} \\
\mathrm{g}_{\mathrm{Q}}=3.40 & {[6.5 .8 .1 \text { page 26] }} \\
\mathrm{g}_{\mathrm{v}}=3.40 & {[6.5 .8 .1 \text { page 26] }}
\end{array}
$$




\section{Seismic Code}

Method of seismic analysis:

Site Class

Importance Factor:

Spectral Response Acceleration at short period (\%g)

Spectral Response Acceleration at period of $1 \mathrm{sec}(\% \mathrm{~g})$

Response Modification Coeficient from Table 15.4-2

Acceleration based site co-efficient:

Velocity based site co-efficient:

Long-period transition period:

Redundancy factor:

User Defined Vertical Accelerations Considered:

Force Multiplier:

Minimum Weight Multiplier:
ASCE 7-05 ground supported

D

$\mathrm{I}=1.5000$

$\mathrm{S}_{\mathrm{S}}=40.00 \%$

$S_{1}=10.00 \%$

$\mathrm{R}=3.0000$

$\mathrm{F}_{\mathrm{a}}=1.4800$

$F_{v}=2.4000$

$\mathrm{T}_{\mathrm{L}}=12.0000$

$\rho=1.0000$

Yes

$=0.3333$

$=0.2000$

\subsubsection{Basic Load Combinations for Allowable Stress Design}

The following load combinations are considered in accordance with ASCE section 2.4.1:

5. $D+H+0.7 E=\left(1.0+V_{\text {Accel }}\right) D+H+0.7 \rho Q_{E}$

Where

$D \quad=$ Dead load

$H \quad=$ Pressure load

$E \quad=$ Seismic load $=\rho Q_{E}$

$V_{\text {Accel }}=$ User defined vertical acceleration

\section{Vessel Characteristics}

Vessel height: $5.5425 \mathrm{ft}$ Vessel Weight:

Operating, Corroded: $240 \mathrm{lb}$

Empty, Corroded: $240 \mathrm{lb}$

\section{Period of Vibration Calculation}

Fundamental Period, T:

Operating, Corroded: $0.104 \mathrm{sec}(f=9.6 \mathrm{~Hz})$

Empty, Corroded: $0.093 \mathrm{sec}(\mathrm{f}=10.7 \mathrm{~Hz})$

The fundamental period of vibration $\mathrm{T}$ (above) is calculated using the Rayleigh method of approximation:

$\mathrm{T}=2 * \mathrm{PI} * \operatorname{Sqr}\left(\left\{\operatorname{Sum}\left(\mathrm{W}_{\mathrm{i}} * \mathrm{y}_{\mathrm{i}}^{2}\right)\right\} /\left\{\mathrm{g} * \operatorname{Sum}\left(\mathrm{W}_{\mathrm{i}}^{*} \mathrm{y}_{\mathrm{i}}\right)\right\}\right)$, where 
$\mathrm{W}_{\mathrm{i}}$ is the weight of the $\mathrm{i}^{\text {th }}$ lumped mass, and

$y_{i}$ is its deflection when the system is treated as a cantilever beam.

\section{Seismic Shear Reports:}

Operating. Corroded

Empty, Corroded

Base Shear Calculations

Seismic Shear Report: Operating, Corroded

\begin{tabular}{|c|c|c|c|c|c|}
\hline Component & $\begin{array}{l}\text { Elevation of bottom } \\
\text { above base (in) }\end{array}$ & $\begin{array}{l}\text { Elastic modulus } E \\
\left(10^{6} \mathrm{psi}\right)\end{array}$ & $\begin{array}{c}\text { Inertia I } \\
\left(\mathrm{ft}^{4}\right)\end{array}$ & $\begin{array}{l}\text { Seismic shear at } \\
\text { Bottom (lbf) }\end{array}$ & $\begin{array}{l}\text { Bending Moment at } \\
\text { Bottom (Ibf-ft) }\end{array}$ \\
\hline $\begin{array}{l}\text { 4" X 2" REDUCER B } 16.9, \text { UPPER } \\
\text { (SCH XS) 2" END }\end{array}$ & 60.26 & 22.8 & 0.0000 & 8 & 3 \\
\hline $\begin{array}{l}\text { 4" X 2" REDUCER B16.9, UPPER } \\
\text { (SCH XS) 4" END }\end{array}$ & 58.26 & 22.8 & 0.0004 & 8 & 4 \\
\hline $\begin{array}{l}\text { 8" X 4" REDUCER B } 16.9 \text {, UPPER } \\
\text { (SCH XS) 4" END }\end{array}$ & 55.26 & 22.8 & 0.0004 & 9 & 6 \\
\hline $\begin{array}{l}\text { 8" X 4" REDUCER B16.9, UPPER } \\
\text { (SCH XS) 8" END }\end{array}$ & 52.26 & 22.8 & 0.0043 & 11 & 9 \\
\hline 8" PIPE & 16.26 & 22.8 & 0.0048 & 31 & 78 \\
\hline $\begin{array}{l}\text { 8" X 4" REDUCER B } 16.9, \text { LOWER } \\
\text { (SCH XS) 8" END }\end{array}$ & 13.26 & 22.8 & 0.0043 & 32 & 86 \\
\hline $\begin{array}{l}\text { 8" X 4" REDUCER B16.9, LOWER } \\
(\mathrm{SCH} \text { XS) 4" END }\end{array}$ & 10.26 & 22.8 & 0.0004 & 32 & 94 \\
\hline $\begin{array}{l}\text { 4" X 2" B16.9 REDUCER, LOWER } \\
\text { (SCH XS) 4" END }\end{array}$ & 8.26 & 22.8 & 0.0004 & 32 & 99 \\
\hline $\begin{array}{l}\text { 4" X 2" B16.9 REDUCER, LOWER } \\
\text { (SCH XS) 2" END }\end{array}$ & 2.01 & 22.8 & 0.0000 & 33 & 116 \\
\hline $\begin{array}{l}\text { ASME B16.5/16.47 Blind on } 2 " \\
\text { RFWN FLANGE 1500\#, LOWER } \\
\text { (top) }\end{array}$ & 2 & 20.4 & 0.0124 & 33 & 116 \\
\hline & & & & & \\
\hline
\end{tabular}

\section{Seismic Shear Report: Empty, Corroded}

\begin{tabular}{|l|r|r|r|r|r|}
\hline \multicolumn{1}{|c|}{ Component } & $\begin{array}{c}\text { Elevation of bottom } \\
\text { above base (in) }\end{array}$ & $\begin{array}{r}\text { Elastic modulus E } \\
(\mathbf{1 0} \mathbf{~ p s i )}\end{array}$ & $\begin{array}{c}\text { Inertia I } \\
\left(\mathbf{f t}^{\mathbf{4}}\right)\end{array}$ & $\begin{array}{c}\text { Seismic shear at } \\
\text { Bottom (lbf) }\end{array}$ & $\begin{array}{c}\text { Bending Moment at } \\
\text { Bottom (lbf-ft) }\end{array}$ \\
\hline $\begin{array}{l}\text { 4" X 2" REDUCER B16.9, UPPER } \\
\text { (SCH XS) 2" END }\end{array}$ & 60.26 & 28.3 & 0.0000 & 8 \\
\hline $\begin{array}{l}\text { 4" X 2" REDUCER B16.9, UPPER } \\
\text { (SCH XS) 4" END }\end{array}$ & 58.26 & 28.3 & 0.0004 & 8 & 4 \\
\hline $\begin{array}{l}\text { 8" X 4" REDUCER B16.9, UPPER } \\
\text { (SCH XS) 4" END }\end{array}$ & 55.26 & 28.3 & 0.0004 & 9 & \\
\hline $\begin{array}{l}\text { 8" X 4" REDUCER B16.9, UPPER } \\
\text { (SCH XS) 8" END }\end{array}$ & 52.26 & 28.3 & 0.0043 & & 11 \\
\hline 8" PIPE & 16.26 & 28.3 & 0.0048 & & 31 \\
\hline
\end{tabular}




\begin{tabular}{|l|r|r|r|r|r|}
$\begin{array}{l}\text { 8" X 4" REDUCER B16.9, LOWER } \\
\text { (SCH XS) 8" END }\end{array}$ & 13.26 & 28.3 & 0.0043 & 32 & 86 \\
\hline $\begin{array}{l}\text { 8" X 4" REDUCER B16.9, LOWER } \\
\text { (SCH XS) 4" END }\end{array}$ & 10.26 & 28.3 & 0.0004 & 32 & 94 \\
\hline $\begin{array}{l}\text { 4" X 2" B 16.9 REDUCER, LOWER } \\
\text { (SCH XS) 4" END }\end{array}$ & 8.26 & 28.3 & 0.0004 & 32 & 99 \\
\hline $\begin{array}{l}\text { 4" X 2" B16.9 REDUCER, LOWER } \\
\text { (SCH XS) 2" END }\end{array}$ & 2.01 & 28.3 & 0.0000 & 33 & 116 \\
\hline $\begin{array}{l}\text { ASME B16.5/16.47 Blind on 2" } \\
\text { RFWN FLANGE 1500\#, LOWER } \\
\text { (top) }\end{array}$ & 2 & 29.4 & 0.0124 & 33 & 116 \\
\hline & & & & & \\
\hline
\end{tabular}

\subsection{3: Maximum considered earthquake spectral response acceleration}

The maximum considered earthquake spectral response acceleration at short period, $\mathrm{S}_{M S}$

$\mathrm{S}_{M S}=\underline{\mathrm{F}}_{\underline{\mathrm{a}}} * \underline{\mathrm{S}}_{\underline{\mathrm{S}}}=1.4800 * 40.00 / 100=0.5920$

The maximum considered earthquake spectral response acceleration at $1 \mathrm{~s}$ period, $\mathrm{S}_{M 1}$

$\mathrm{S}_{M 1}=\underline{\mathrm{F}}_{\underline{\mathrm{v}}} * \underline{\mathrm{S}}_{1}=2.4000 * 10.00 / 100=0.2400$

\subsection{4: Design spectral response acceleration parameters}

Design earthquake spectral response acceleration at short period, $\mathrm{S}_{D S}$

$\mathrm{S}_{D S}=2 / 3 * \mathrm{~S}_{M S}=2 / 3 * 0.5920=0.3947$

Design earthquake spectral response acceleration at $1 \mathrm{~s}$ period, $\mathrm{S}_{D 1}$

$\mathrm{S}_{D I}=2 / 3 * \mathrm{~S}_{M 1}=2 / 3 * 0.2400=0.1600$

\section{User Defined Vertical Acceleration Term, $\mathbf{V}_{\text {Accel }}$}

Factor is applied to dead load.

Compressive Side: $=1.0+\mathrm{V}_{\text {Accel }}$

\section{$V_{\text {Accel }}$ Term is:}

greater of (Force Mult * Base Shear / Weight) or (Min. Weight Mult.)

\begin{tabular}{|l|r|r|r|r|}
\hline \multicolumn{2}{|c|}{ Force multiplier $=0.3333$} & \multicolumn{3}{|c|}{ Minimum Weight Multiplier $=0.2000$} \\
\hline Condition & Base Shear ( Ibf) & Weight ( Ib) & $\begin{array}{r}\text { Force Mult * Shear } \\
\text { Weight }\end{array}$ & V $_{\text {Accel }}$ \\
\hline Operating, Corroded & 33 & 239.6 & 0.046 & 0.2 \\
\hline Operating, New & 34 & 249.8 & 0.046 & 0.2 \\
\hline Empty, Corroded & 33 & 239.6 & 0.046 & 0.2 \\
\hline Empty, New & 34 & 249.8 & 0.046 & 0.2 \\
\hline
\end{tabular}




\section{Base Shear Calculations}

\section{Operating, Corroded}

Empty, Corroded

\section{Base Shear Calculations: Operating, Corroded}

\section{Paragraph 15.4.4: Period Determination}

Fundamental Period is taken from the Rayleigh method listed previously in this report.

$\mathrm{T}=0.104 \mathrm{sec}$.

\subsection{1: Calculation of Seismic Response Coefficient}

$\mathrm{C}_{\mathrm{s}}$ is the value computed below, bounded by $\mathrm{C}_{\mathrm{s}}$ Min and $\mathrm{C}_{\mathrm{s}}$ Max:

$\mathrm{C}_{\mathrm{s}}$ Min is 0.01 unless $\mathrm{S}_{1}>=0.6 \mathrm{~g}$, in which case eqn $12.8-6$ is used.

$\mathrm{C}_{\mathrm{S}}$ Max calculated with $12.8-3$ because $(\mathrm{T}=0.104)<=\left(\mathrm{T}_{\mathrm{L}}=12.0000\right)$

$$
\begin{aligned}
& \mathrm{C}_{\mathrm{s}}=\underline{\mathrm{S}}_{\underline{D S}} /(\underline{\mathrm{R}} / \underline{\mathrm{I}})=0.3947 /(3.0000 / 1.5000)=0.1973 \\
& \mathrm{C}_{\mathrm{s}} \operatorname{Min}=0.01 \\
& \mathrm{C}_{\mathrm{s}} \mathrm{Max}=\underline{\mathrm{S}}_{\underline{D I}} /(\mathrm{T} *(\underline{\mathrm{R}} / \mathrm{I}))=0.1600 /(0.1037 *(3.0000 / 1.5000))=0.7716 \\
& \mathrm{C}_{\mathrm{s}}=0.1973
\end{aligned}
$$

\subsection{1: Calculation of Base Shear}

$$
\begin{aligned}
\mathrm{V} & =\mathrm{C}_{\mathrm{S}} * \underline{\mathrm{W}} \\
& =0.1973 * 239.6326 \\
& =47.29 \mathrm{lb}
\end{aligned}
$$

\subsubsection{Seismic Load Combinations: Horizontal Seismic Load Effect, $\mathbf{E}_{h}$}

$$
\begin{aligned}
\mathrm{Q}_{\mathrm{E}} & =\mathrm{V} \\
\mathrm{E}_{\mathrm{h}} & =0.7 * \rho * \mathrm{Q}_{\mathrm{E}}(\text { Only } 70 \% \text { of seismic load considered as per Section 2.4.1) } \\
& =0.70 * 1.0000 * 47.29 \\
& =33.10 \mathrm{lb}
\end{aligned}
$$

\section{Base Shear Calculations: Empty, Corroded}

\section{Paragraph 15.4.4: Period Determination}

Fundamental Period is taken from the Rayleigh method listed previously in this report. $\mathrm{T}=0.093 \mathrm{sec}$.

\subsection{1: Calculation of Seismic Response Coefficient}

$\mathrm{C}_{\mathrm{s}}$ is the value computed below, bounded by $\mathrm{C}_{\mathrm{s}}$ Min and $\mathrm{C}_{\mathrm{s}}$ Max:

$\mathrm{C}_{\mathrm{S}} \mathrm{Min}$ is 0.01 unless $\mathrm{S}_{1}>=0.6 \mathrm{~g}$, in which case eqn $12.8-6$ is used.

$\mathrm{C}_{\mathrm{s}}$ Max calculated with $12.8-3$ because $(\mathrm{T}=0.093)<=\left(\mathrm{T}_{\mathrm{L}}=12.0000\right)$ 


$$
\begin{aligned}
& \mathrm{C}_{\mathrm{s}}=\underline{\mathrm{S}}_{\underline{D S}} /(\underline{\mathrm{R}} / \underline{\mathrm{I}})=0.3947 /(3.0000 / 1.5000)=0.1973 \\
& \mathrm{C}_{\mathrm{s}} \operatorname{Min}=0.01 \\
& \mathrm{C}_{\mathrm{s}} \operatorname{Max}=\underline{\mathrm{S}}_{\underline{D I}} /(\mathrm{T} *(\underline{\mathrm{R}} / \underline{\mathrm{I}}))=0.1600 /(0.0931 *(3.0000 / 1.5000))=0.8591 \\
& \mathrm{C}_{\mathrm{s}}=0.1973
\end{aligned}
$$

\subsection{1: Calculation of Base Shear}

$$
\begin{aligned}
\mathrm{V} & =\mathrm{C}_{\mathrm{S}} * \underline{\mathrm{W}} \\
& =0.1973 * 239.6326 \\
& =47.29 \mathrm{lb}
\end{aligned}
$$

\subsubsection{Seismic Load Combinations: Horizontal Seismic Load Effect, $E_{h}$}

$$
\begin{aligned}
\mathrm{Q}_{\mathrm{E}} & =\mathrm{V} \\
\mathrm{E}_{\mathrm{h}} & =0.7 * \rho * \mathrm{Q}_{\mathrm{E}}(\text { Only } 70 \% \text { of seismic load considered as per Section 2.4.1) } \\
& =0.70 * 1.0000 * 47.29 \\
& =33.10 \mathrm{lb}
\end{aligned}
$$




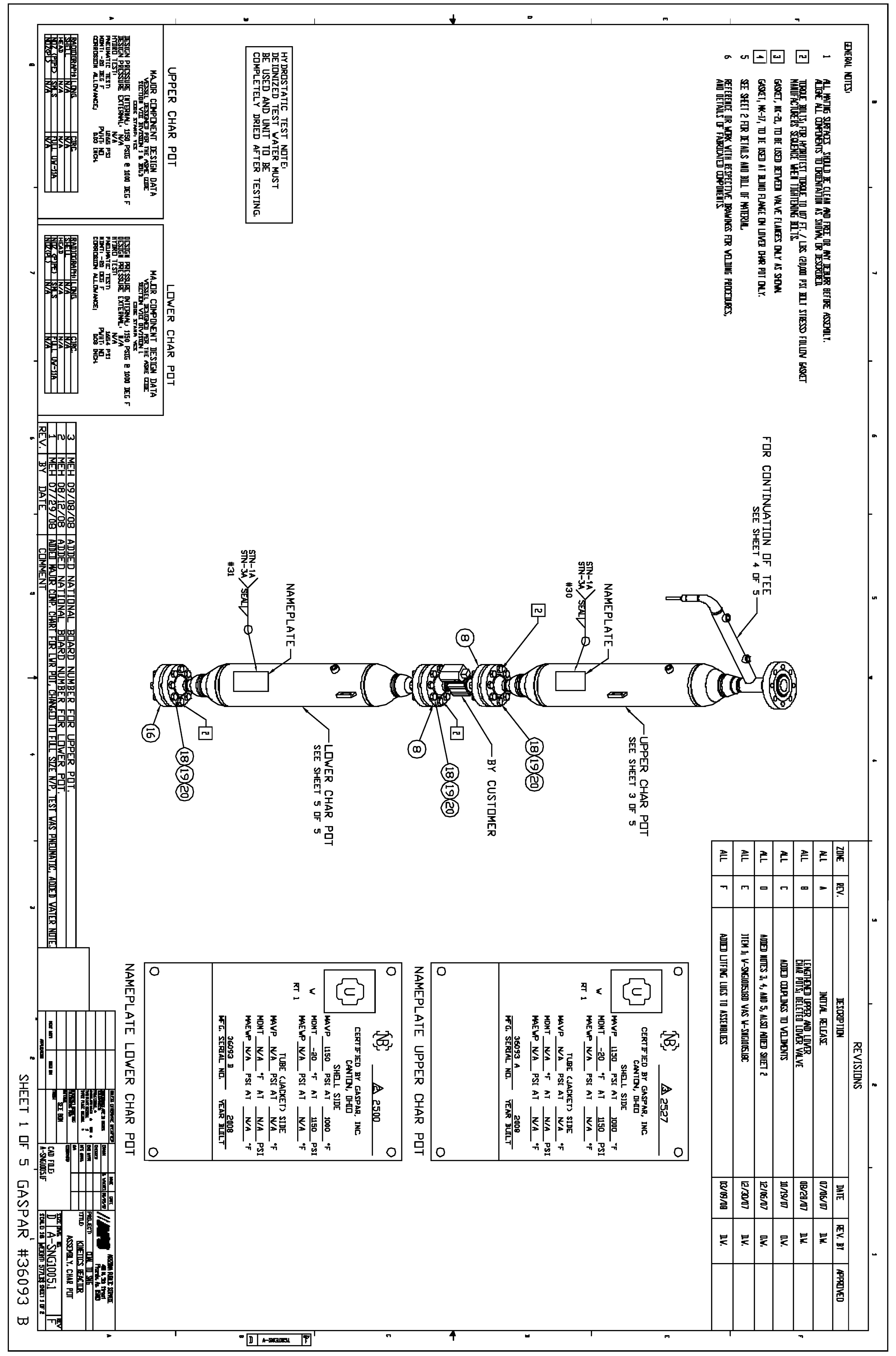




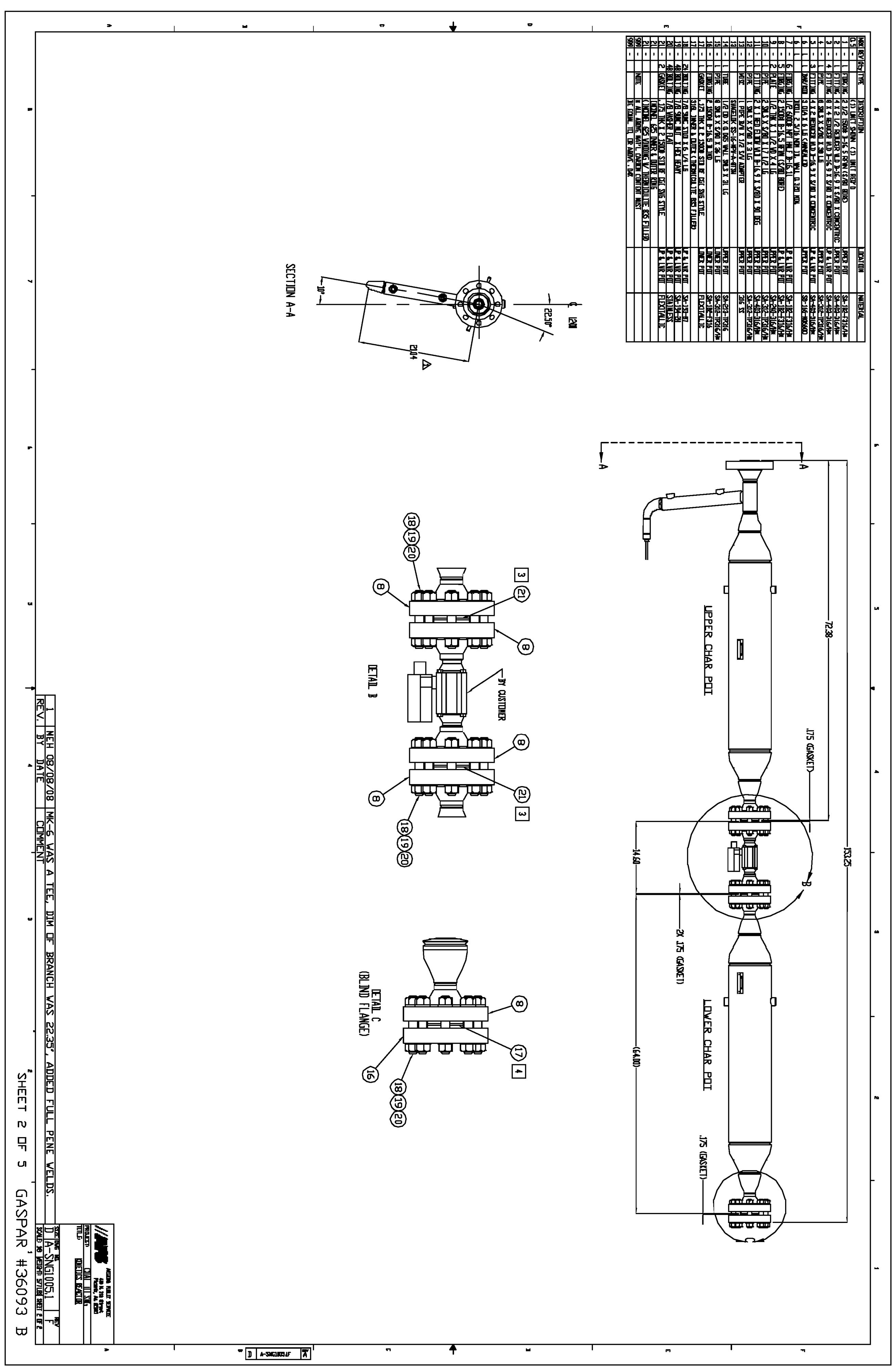




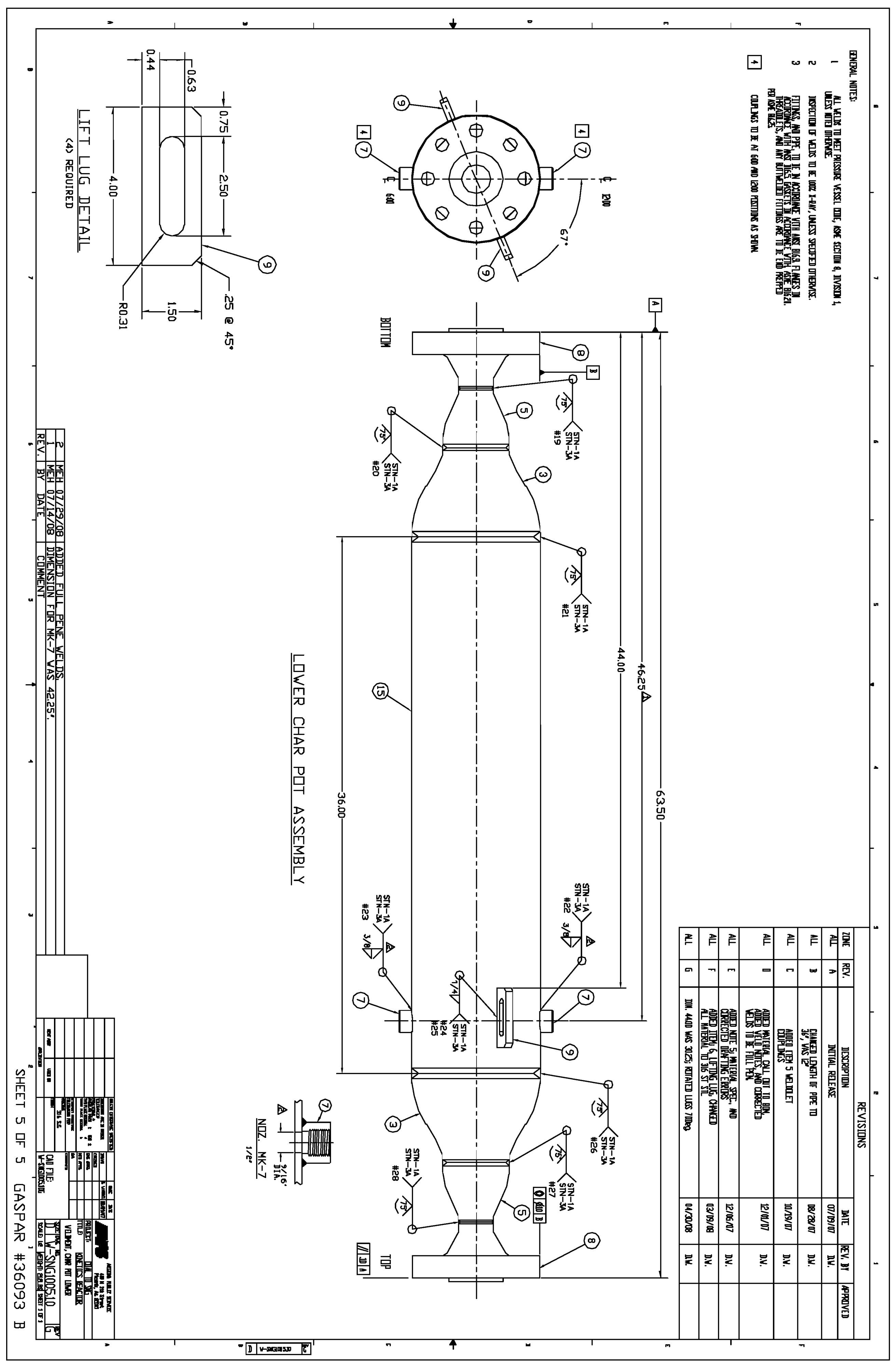




$$
\text { I }
$$




\title{
APPENDIX G
}

\section{Bench Scale Hydrogasifier Lower CharPot Assembly}

\author{
Final Design from Gaspar Inc.
}


Development of a Hydrogasification Process for

Coproduction of SNG and Electrical Power from Western Coals

Final Report

THIS PAGE INTENTIONALLY LEFT BLANK 


\section{Gaspar ine. \\ WELDING \& FABRICATIONS}

1545 Whipple Avenue SW Canton, Ohio 44710

Phone: 330-477-2222

Fax: $330-477-2322$

www.gasparinc.com

\section{FINAL DATA PACKAGE}

\begin{tabular}{|r|l||}
\hline GASPAR JOB NUMBER(S): & 36093 \\
\hline CUSTOMER: & ARIZONA PUBLIC SERVICE \\
\hline PURCHASE ORDER NUMBER: & 700521452 \\
\hline DESCRIPTION: & LOWER CHAR POT \\
\hline ITEM NUMBER(S): & N/A \\
\hline OTHER: & N/A \\
\hline
\end{tabular}

DATA PACKAGE CONTENTS

$\square$ DATA REPORT

$\square$ NAMEPLATE COPY

$\square$ BILL OF MATERIAL

$\square$ MATERIAL TEST REPORTS

$\square$ NDE REPORTS

$\square$ HEAT TREAT CHARTS

$\square$ CALCULATIONS

$\square$ DRAWINGS

$\square$ OTHER: (LIST BELOW)

NOTE: 
FORM U-1A MANUFACTURER'S DATA REPORT FOR PRESSURE VESSELS

(Alternative Form for Single Chamber, Completely Shop or Field Fabricated Vessels Only)

As Required by the Provisions of the ASME Boiler and Pressure Vessel Code Rules, Section VIII, Division 1

1. Manufactured and certified by:

Gaspar, Inc. 1545 Whipple Ave SW Canton, Ohio 44710

2. Manufactured for:

Arizona Public Service Co. P.O. Box 53999 Phoenix, AZ 85072

3. Location of installation

Unknown

4. Type:

Vertical

36093B

(Name and address of purchaser)

5. The chemical and physical properties of all parts meet the requirements of material specifications of the ASME BOILER AND PRESSURE VESSEL

CODE The design, construction, and workmanship conform to ASME Rules, Section VIII, Division 1

(Name and address)

36093 Rev. 2

(Drawing number)

(National Board number)

2008

(rear built)

to

6. Shell $\frac{\text { Adsenda (Date) }}{\frac{S A 312-316 \mathrm{H}}{\text { 7. Seams: }}}$

8. Heads: (a) Material

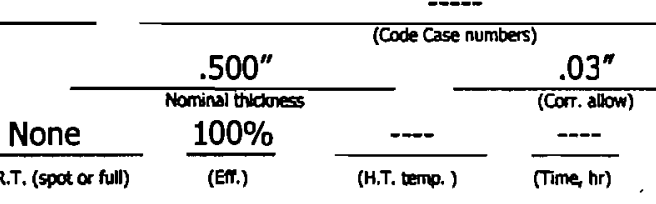

SA403-316H

(Spec. number, grade)

(b) Material
2007 Edition

Year

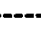

\begin{tabular}{|c|c|c|c|c|c|c|c|c|c|c|}
\hline & \multicolumn{6}{|c|}{ (Spec. number, grade) } & \multicolumn{4}{|c|}{ (spec. number, grade) } \\
\hline & $\begin{array}{l}\text { Location (Top, } \\
\text { Bottom, Ends) }\end{array}$ & $\begin{array}{l}\text { Minimum } \\
\text { Thlckness }\end{array}$ & $\begin{array}{l}\text { Corrosion } \\
\text { Allowance }\end{array}$ & $\begin{array}{l}\text { Crown } \\
\text { Radlus }\end{array}$ & $\begin{array}{l}\text { Knuckle } \\
\text { Radius }\end{array}$ & $\begin{array}{l}\text { Elliptical } \\
\text { Ratio }\end{array}$ & $\begin{array}{c}\text { Conical } \\
\text { Apex Angle }\end{array}$ & $\begin{array}{c}\text { Hemispherical } \\
\text { Radius }\end{array}$ & $\begin{array}{c}\text { Flat } \\
\text { Diameter }\end{array}$ & $\begin{array}{c}\text { Side to Pressure } \\
\text { (Convex or Concave) }\end{array}$ \\
\hline (a) & Top & $.337^{\prime \prime}$ & $.03^{n}$ & --- & $-\cdots$ & ---- & * & -- & ---- & Concave \\
\hline (b) & Bottom & $.337^{\prime \prime}$ & $.03^{\prime \prime}$ & $\overline{---}$ & $\cdots$ & $\overline{-\ldots}$ & * & $\overline{----}$ & --- & Concave \\
\hline
\end{tabular}

If removable, bolts used (describe other fastenings)

9. MAWP

1150

(Internal)

Min. design metal temp. $\quad \begin{array}{ll}-20 \\ \end{array}$ $-20$

10. Nozzles, inspection, and safety valve openings:

\begin{tabular}{|c|c|c|c|c|c|c|c|c|}
\hline $\begin{array}{c}\text { Purpose } \\
\text { (Inlet, Outlet, Drain) }\end{array}$ & Number & $\begin{array}{l}\text { Diameter } \\
\text { or Size }\end{array}$ & Type & Material & \begin{tabular}{|l|} 
Nominal \\
Thickness \\
\end{tabular} & $\begin{array}{c}\text { Reinforcement } \\
\text { Material }\end{array}$ & $\begin{array}{c}\text { How } \\
\text { Attached }\end{array}$ & Location \\
\hline Aux & 2 & $2^{n}$ & 1500\#WN & SA403-316H & $.218^{\prime \prime}$ & ---.- & Butt Weld & $\ldots$ \\
\hline & & & & & & & & \\
\hline & & & & & & & & \\
\hline & & & & & & & & \\
\hline & & & & & & & & \\
\hline 11. Supports: Skirt & $\frac{\text { No }}{\text { Yes or No) }}$ & Lugs & $\begin{array}{c}2 \\
\text { (No.) }\end{array}$ & Legs & Oth & $\begin{array}{c}---- \\
\text { (Describe) }\end{array}$ & Attached & $\begin{array}{l}\text { ell/Welded } \\
\text { ere and How) }\end{array}$ \\
\hline
\end{tabular}

12. Remarks: Manufacturer's Partial Data Reports properly identified and signed by Commissioned Inspectors have been furnished for the following items of the report:

(Name of part, item number, Manufacturer's name and identifying stamp)

UHA-51(d) Exempt (impact test). Item 8: *Heads are standard 8" $\times$ 4" concentric reducers (ANSI B16.9). Item 10: The two 2" 1500\#WN Flanges are welded to standard 4" $\times 2$ " concentric reducers (ANSI B16.9).

\section{CERTIFICATE OF SHOP/FIELD COMPLIANCE}

We certify that the statements made in this report are correct and that all details of design, material, construction and workmanship of this vessel conform to the ASME Boiler and pressure Vessel Code, Section VIII, Division I. expires July 25, 2011

Date $8+15-08$ Co. Name

Gaspar, Inc
(Manufacturer)

"U" Certificate of Authorization Number

16,862

\section{CERTIFICATE OF SHOP/FIELD INSPECTION \\ Gaspar, Inc \\ at \\ 1545 Whipple Ave SW Canton, Ohio 44710}

Vessel constructed by

I, the undersigned, holding a valid commission issued by the National Board of Boiler and Pressure Vessel Inspectors and/or the State or Province of

$$
\text { Ohio and employed by }
$$
OneBeacon America Insurance Company Lynn, Mass

have inspected the component described in this Manufacturer's Data Report on , O8 15,2008 , and state that, to the best of my knowledge and belief, the Manufacturer has constructed this pressure vessel in accordance with ASME Boiler and Pressure Vessel Code, Section VIII, Division 1. By signing this certificate neither the Inspector nor his/her employer makes any warranty, expressed or implied, concerning the pressure vessel this described in this Manufacturer's Data Report. Furthermore, neither the Inspector nor his/her employer shall be liable in any manner for any personal injury or property damage or a loss of any kind arising from or connected with this inspection.

Date $0815 \cdot 2008$ signed

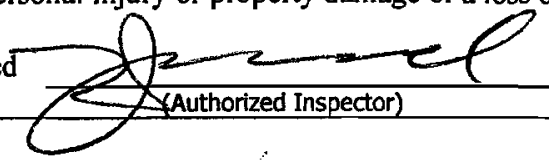




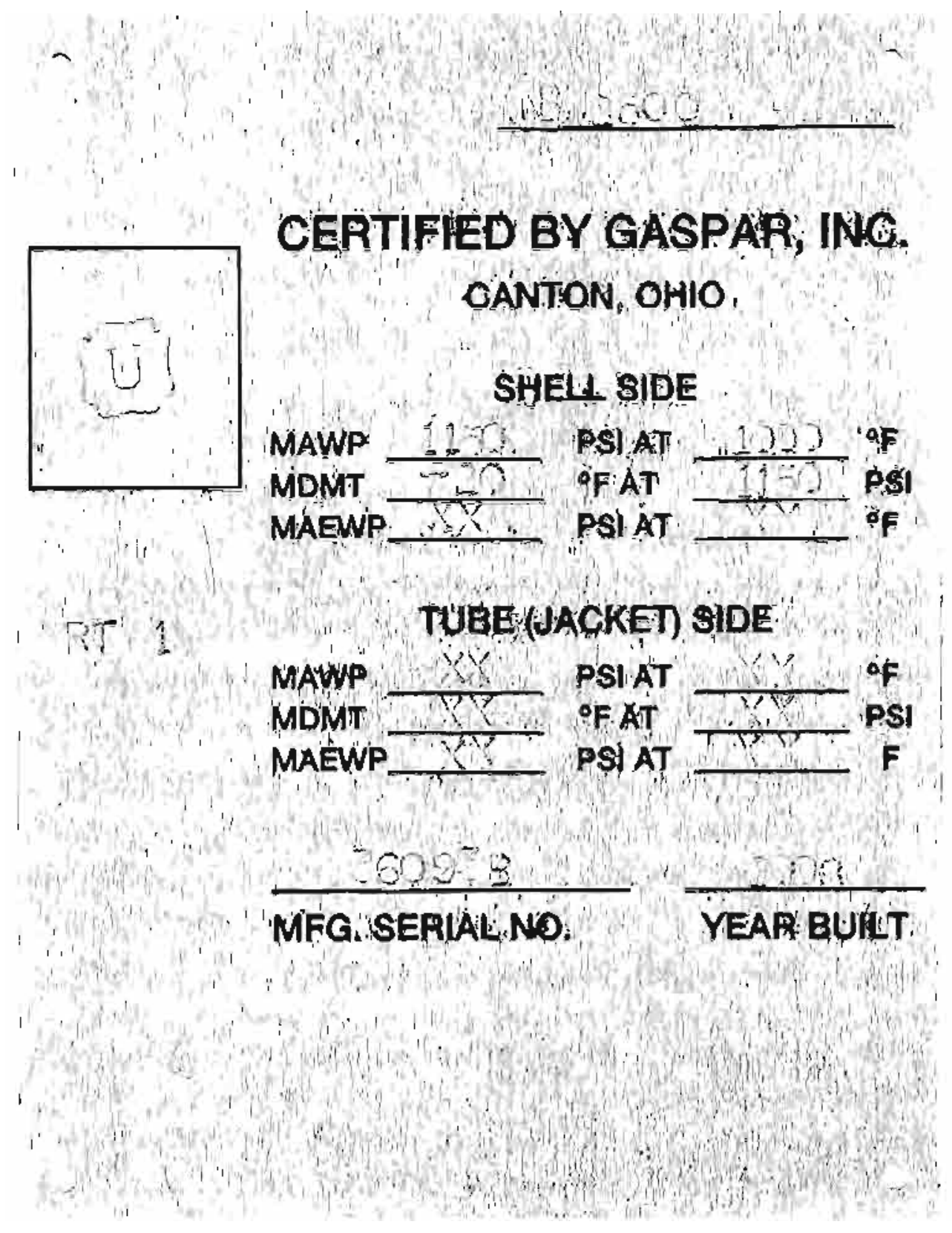




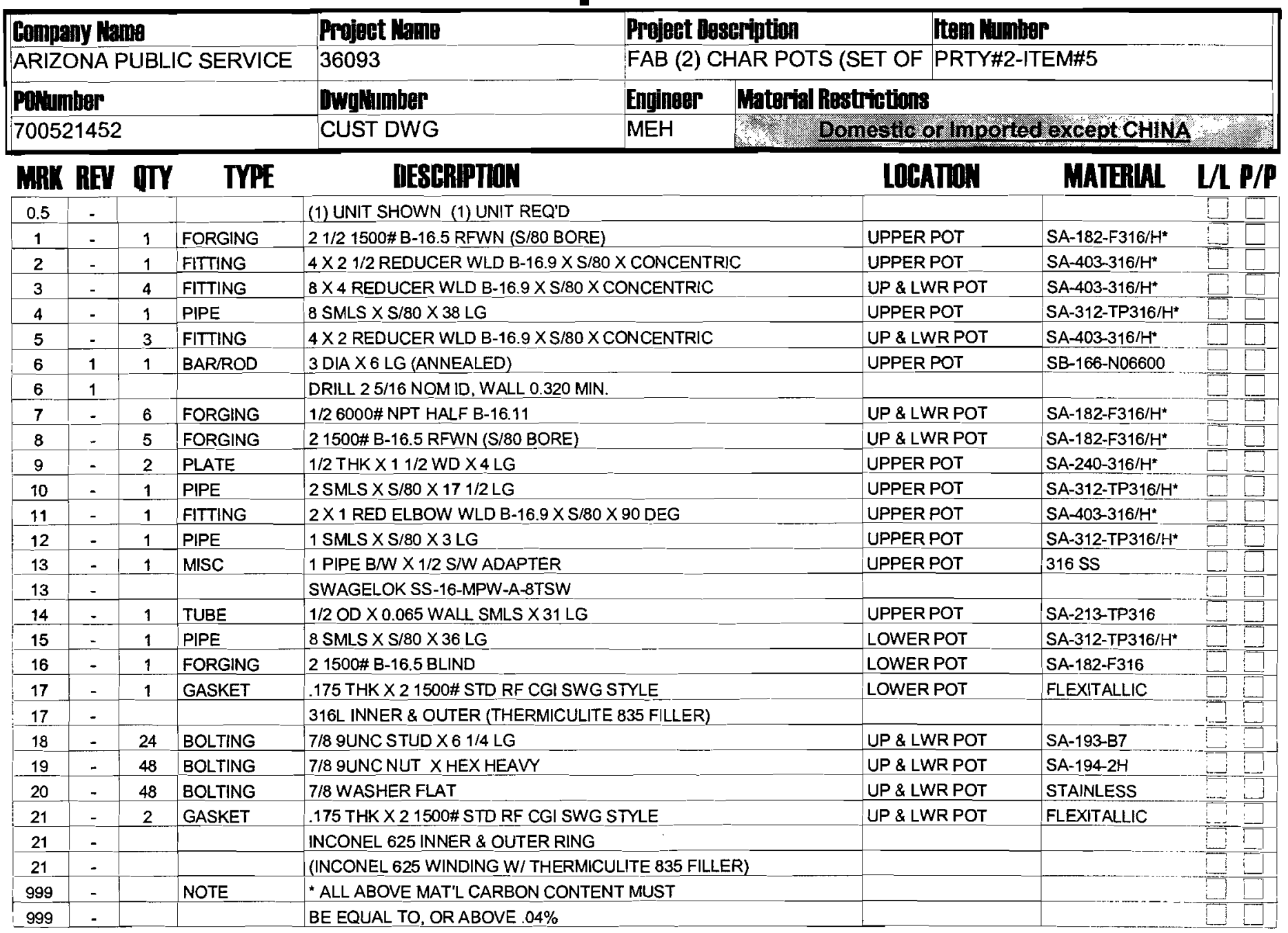




\section{WeSTERN FORGE \& FLANGe CO.}

687 COUNTY ROAD 2201

CLEVELAND, TX 77327

PH (281) 727-7000 FAX (281) 727-7062

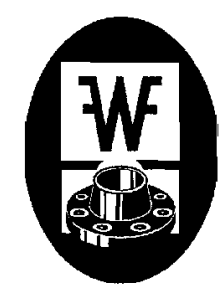

\section{CERTIFIED MATERIAL TEST REPORT}

\section{CERTIFIED IAW EN10204 3.1 (MADE IN USA)}

TO: GASPAR INC.

PO: 30214

SPECIFICATIONS:
ASME SA182 F316H

DIM/TOL PER ASME B16.5

\begin{tabular}{|c|c|}
\hline QTY & ITEM DESCRPTION \\
\hline 1 & $21 / 2 \% 1500 \#$ WN RF S80 \\
\hline & PROJECT\# 36093-1 \\
\hline & \\
\hline & \\
\hline & \\
\hline
\end{tabular}

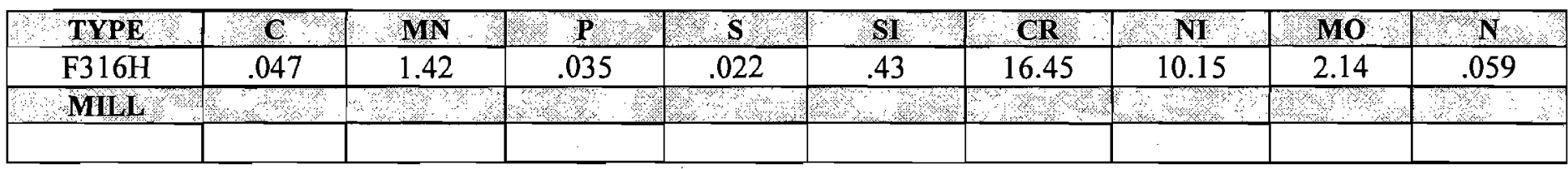

\begin{tabular}{|c|c|c|c|c|c|c|}
\hline HEAT NO & YIELD $2 \%$ OFF-PSI & TENSILE-PSI & \% EEONG & $\%$ RED AREA & HABDNESS & GRAIN SIZE (ASTM) \\
\hline 28122 & 59,690 & 85,300 & 67.0 & 82.8 & & No. 4.0 \\
\hline & & & & & & \\
\hline & & & & & & \\
\hline
\end{tabular}

- NO WELDING PERFORMED ON THE FORGINGS OF THIS ORDER.

- MATERLAL HEREIN IS CERTIFIED FREE FROM ANY FORM OF MERCURY, RADIUM OR ALPHA PARTICLE CONTAMINATION

THROUGHOUT ALL PHASES OF MANUFACTURE AND SHIPMENT:

- THE FORGINGS ON THIS ORDER CONFORM TO THE SPECIFIED DIMENSIONAL REQUIREMENTS.

- MATERLAL MANUFACTURED LAW WFF QA PROGRAM QAM-WF07.0702.

- Solution annealed @ $1950^{\circ} \mathrm{F}$ and water quenched to below $300^{\circ} \mathrm{F}$ within less than three minutes.

WE CERTIFY THE MATERIAL DESCRIBED HEREIN HAS BEEN MANUFACTURED, TESTED AND EXAMINED LAW AND MEETS ALL THE REQUIREMENTS OF ABOVE PURCHASE ORDER AND REFERENCED SPECIFICATIONS.

$07 / 24 / 08$
DATE

This document shall not be reproduced, except in full, without written approval of Western Forge \& Flange. The recording of false, fictitious or fraudulent statements or entries on this document may be punishable as felony under federal statutes. 


\section{TAYLOR FORGE STAINLESS}

P. O. BOX 610

SOMERVILLE, NJ 08876

Phone: (908)722-1313

FAX: (908)722-2943

\section{SOLD TO:}

GASPAR INC.

1545 WHIPPLE AVE. SW

CANTON, OH 44710

\section{Material Test Report}

Page : 1 of 1

SALES ORDER \# : 0000012238

\section{SHIP TO:}

GASPAR INC.

1545 WHIPPLE AVE. SW

CANTON, $\mathrm{OH} 44710$

CUST P.O.\#: 30236

TAG NUMBER: 30236, PPD-CHG

DATE SHIPPED: $/ 0 /$

\begin{tabular}{c|r|r}
\hline ITEM \# & QTY & ITEM DESCRIPTION \\
\hline 1 & 1 & $4 \times 21 / 2$ CR S/80 WP316HS SA403
\end{tabular}

STARTING MATERIAL

PIPE, A312-07, 316/316H

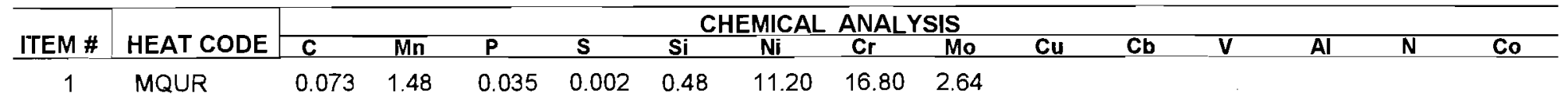

\begin{tabular}{c|lllll}
\hline & & & & PHYSICAL PROPERTIES \\
\cline { 2 - 5 } & $\begin{array}{l}\text { TENSILE } \\
\text { (PSI) }\end{array}$ & $\begin{array}{l}\text { YIELD } \\
\text { (PSI) }\end{array}$ & $\begin{array}{l}\text { ELONG } \\
(\mathbf{2} \text { IN) }\end{array}$ & \\
\hline 1 & 89600 & 58000 & 50.00 & 0.00
\end{tabular}

\begin{tabular}{c|cc}
\hline ITEM \# & SLIPPLEMENTAL INFORMATION \\
\hline 1 & GRAIN SIZE: ACTUAL NO. 6.5 &
\end{tabular}

SOLUTION ANNEALED, WATER QUENCHED

ASME SA403 2004 ED; ASTM A403-O6; EN 10204/3.1 ASME B16.9

THE FITTINGS WERE MANUFACTURED, SAMPLED, TESTED AND INSPECTED IN

ACCORDANCE WITH THE SPECIFICATIONS AND FOUND TO MEET THE REQUIREMENTS.

THE MATERIAL WAS MANUFACTURED, TESTED AND INSPECTED WITHOUT MERCURY

CONTAMINATION. NO WELD REPAIRS.

NACE MR0175

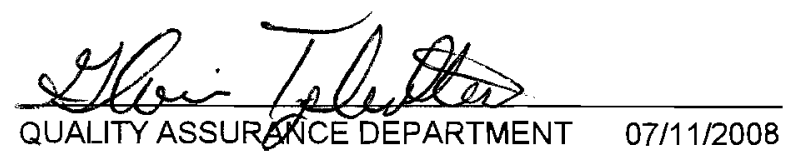




\section{TAYLOR FORGE STAINLESS}

P. O. BOX 610

SOMERVILLE, NJ 08876

Phone: (908)722-1313

FAX: (908)722-2943
Material Test Report

Page : 1 of 1

SALES ORDER \# : 0000012238

\section{SOLD TO:}

GASPAR INC

1545 WHIPPLE AVE. SW

CANTON, OH 44710
SHIP TO:

GASPAR INC.

1545 WHIPPLE AVE. SW

CANTON, OH 44710
CUST P.O.\#: 30236

TAG NUMBER: 30236, PPD-CHG

\begin{tabular}{c|r|l}
\hline ITEM \# & QTY & ITEM DESCRIPTION \\
\hline 2 & 2 & $8 \times 4$ CR S/80 WP316HS SA403
\end{tabular}

DATE SHIPPED: $10 /$

STARTING MATERIAL

PIPE, A312-07, 316/316H

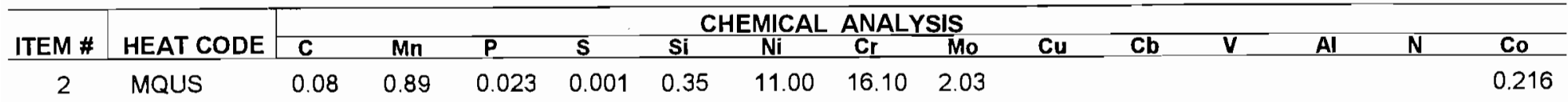

\begin{tabular}{|c|c|c|c|c|}
\hline & & & & PHYSICAL PROPERTIES \\
\hline ITEM \# & $\begin{array}{l}\text { TENSILE } \\
\text { (PSI) }\end{array}$ & $\begin{array}{l}\text { YIELD } \\
\text { (PSI) }\end{array}$ & $\begin{array}{l}\text { ELONG } \\
(2 \text { IN) }\end{array}$ & \\
\hline 2 & 76200 & 32500 & 77.00 & 0.00 \\
\hline
\end{tabular}

\begin{tabular}{c|cc}
\hline ITEM \# & & SUPPLEMENTAL INFORMATION \\
\hline 2 & GRAIN SIZE ACUTAL: ASTM NO 4.0
\end{tabular}

SOLUTION ANNEALED, WATER QUENCHED .

ASME SA403 2004 ED; ASTM A403-O6; EN 10204/3.1 ASME B16.9

THE FITTINGS WERE MANUFACTURED, SAMPLED, TESTED AND INSPECTED IN ACCORDANCE WITH THE SPECIFICATIONS AND FOUND TO MEET THE REQUIREMENTS. THE MATERIAL WAS MANUFACTURED, TESTED AND INSPECTED WITHOUT MERCURY CONTAMINATION. NO WELD REPAIRS. 
TAYLOR FORGE STAINLESS

P. O. BOX 610

SOMERVILLE, NJ 08876

Phone: (908)722-1313

FAX: (908)722-2943
Material Test Report

Page : 1 of 1

SALES ORDER \# : 0000012238

\section{SOLD TO:}

GASPAR INC.

1545 WHIPPLE AVE. SW

CANTON, OH 44710
SHUP TO:

GASPAR INC.

1545 WHIPPLE AVE. SW

CANTON, OH 44710
CUST P.O.\#: 30236

\begin{tabular}{l|l|l}
\hline ITEM \# & QTY & ITEM DESCRIPTION
\end{tabular}

$228 \times 4$ CR S/80 WP316HS SA403
TAG NUMBER: 30236, PPD-CHG

DATE SHIPPED: / $/$

STARTING MATERIAL

PIPE, A312-07, 316/316H

\begin{tabular}{|c|c|c|c|c|c|c|c|c|c|c|c|c|c|c|c|}
\hline \multirow[b]{2}{*}{ ITEM \# } & \multirow[b]{2}{*}{ HEAT CODE } & \multicolumn{12}{|c|}{ CHEMICAL ANALYSIS } & \multirow[b]{2}{*}{$\mathbf{N}$} & \\
\hline & & C & Mn & $\mathbf{P}$ & $\mathbf{S}$ & Si & $\mathrm{Ni}$ & $\mathrm{Cr}$ & $\mathrm{Mo}$ & $\mathbf{C u}$ & $\mathrm{Cb}$ & $\bar{V}$ & Al & & $\mathrm{Co}_{0}$ \\
\hline 2 & MQUS & 0.08 & 0.89 & 0.023 & 0.001 & 0.35 & 11.00 & 16.10 & 2.03 & & & & & & 0.216 \\
\hline
\end{tabular}

\begin{tabular}{|c|c|c|c|c|}
\hline \multirow[b]{2}{*}{ ITEM \# } & \multicolumn{4}{|r|}{ PHYSICAL PROPERTIES } \\
\hline & $\begin{array}{l}\text { TENSILE } \\
\text { (PSI) }\end{array}$ & $\begin{array}{l}\text { YIELD } \\
\text { (PSI) }\end{array}$ & $\begin{array}{l}\text { ELONG } \\
\text { (2 IN) }\end{array}$ & \\
\hline 2 & 76200 & 32500 & 77.00 & 0.00 \\
\hline
\end{tabular}

\begin{tabular}{c|cc}
\hline TTEM \# & & SUPPLEMENTAL INFORMATION \\
\hline 2 & GRAIN SIZE ACUTAL: ASTM NO 4.0
\end{tabular}

SOLUTION ANNEALED, WATER QUENCHED

ASME SA403 2004 ED; ASTM A403-O6; EN 10204/3.1 ASME B16.9

THE FITTINGS WERE MANUFACTURED, SAMPLED, TESTED AND INSPECTED IN

ACCORDANCE WITH THE SPECIFICATIONS AND FOUND TO MEET THE REQUIREMENTS.

THE MATERIAL WAS MANUFACTURED, TESTED AND INSPECTED WITHOUT MERCURY

CONTAMINATION. NO WELD REPAIRS.

NACE MR0175

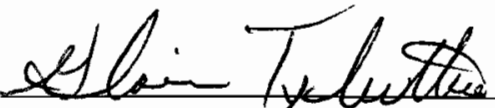

QUALITY ASSURAMCE DEPARTMENT 
INSPECTION CERTIFICATE

$\cdots$

SISHTOMO MLTAL INDISTRIES, LTD. STEEL TUBE HORK'S

IIIGASHJ-MILKOJ LRA. AMGGASAKI, JAPAN

CLRTIFICATL N0. : OYYUI947

PAGE: I

DATE: 2007-07-26

CUSTOHER TTJOFA PIIE SIPPLY INC.

ORDER RO. : $: 902033$ ITEH NO. 14

SHIPPER :SUNITOAO CORPORATION 057 KE:Q 06I3/5 7P!2S30650I

COMUIOUITY :SEAALESS IOT FIMISIIED STAINLESS STEEL PIPE HITH PLAIN SQUARE CUT ENDS

STMDARD :ASTH A312-05A / ASHE 2004 (A05) SA-312 TP316 ASTM A312-05A / ASME 2004 (AD5) SA-312 TPI16II SPECIJICATION :POA $¥ 2 S U H-1 A$ REV. 7

ASTW A376-04 / ASME 2004 (AD5) SA-376 TP316 ASTY A376-04 / ASHE 2004 (1D5) SA-376 TP316H

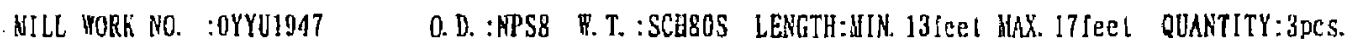
TOTAL LENGTH: 46. 48fecl MASS: $316 \mathrm{~kg}$

HEAT NA. PRODHCTS PCS.

1421613

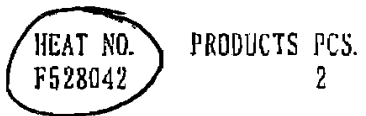

WEAT TREATNENT: SOLUTION TREATED (2102 FX2min. H.Q.)

CIEMIICAL COMPOSITION(⿻)

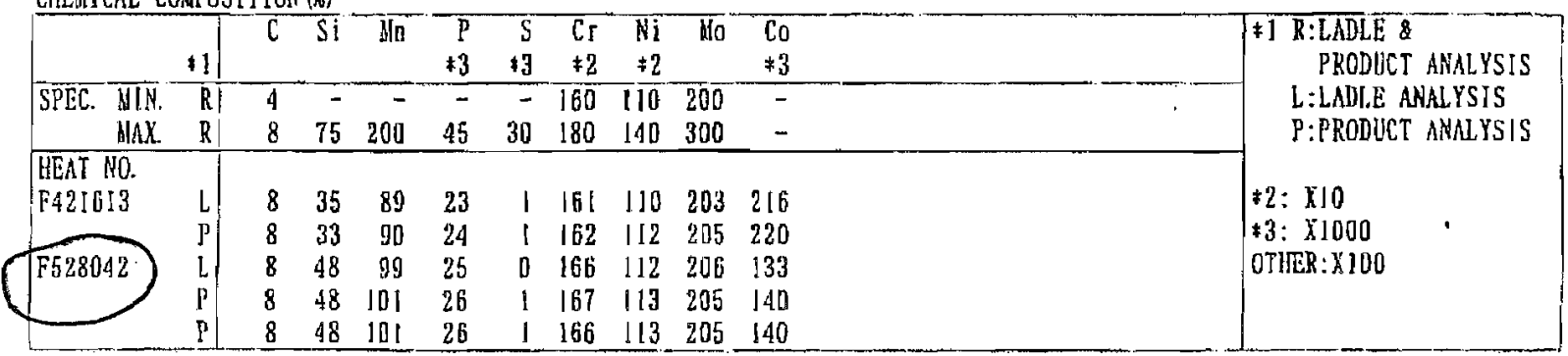

TENSILE TEST

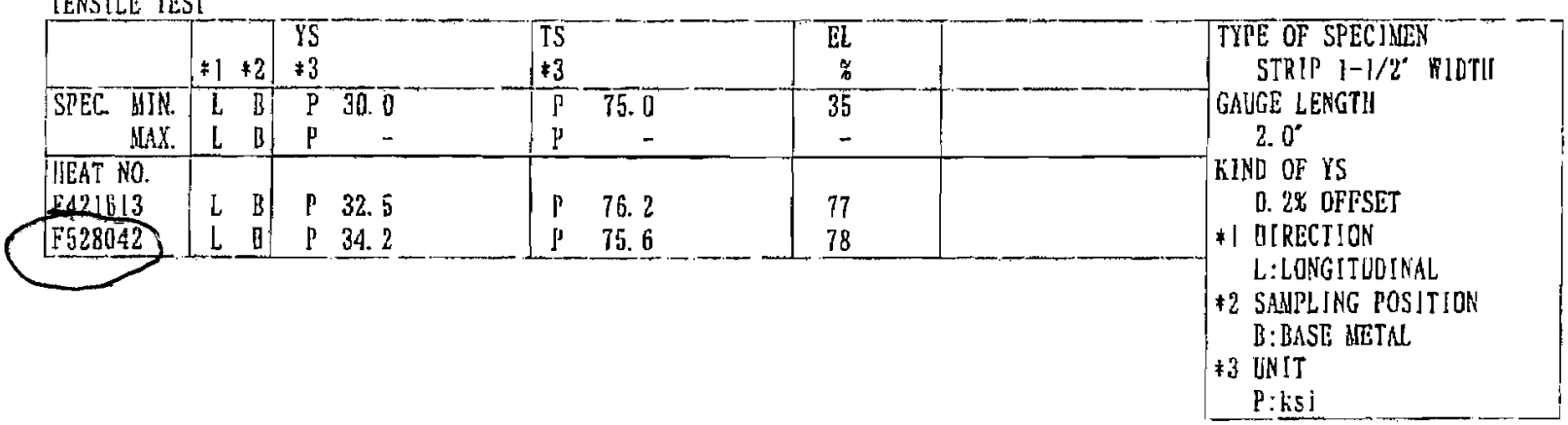

AUSTENITE GRA LN SIZE (ASTH E]J2)

\begin{tabular}{|r|r|}
\hline SPEC WIN. & -1 \\
NAA & 7.0 \\
\hline HEAT MO. & \\
F421613 & 3.8 \\
F528012 & 3.7 \\
& 3.5 \\
\hline
\end{tabular}

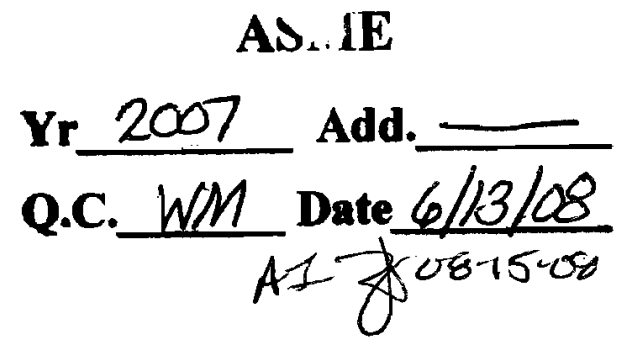

WE HEREDY CERTIFY THAT THE MATERIAL HEREIN DESCRIBED HAS BEEN BMNUIACTURED, SAMPLED, TESTED, AND INSPECTED IN ACCORDANCE MITH ABOVE STANDARD AND SPECSFJCATION ANO SATJSFIES PIIE REQUIREMENTS. 


\author{
INSPECTION CERTIFICATE
}
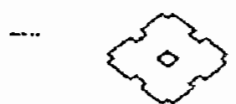

SLTITOHO
I. NISHINO-CHO. IS STEEL TUBE MORRS

CERTIPICATE NO. : OYYUIOA?
IGASIII-WLIRIJIEGA, ANAGASALT, JAFAM

PAGE: 2
BATE: $2007-07-26$

LORLOSION TEST OAIL-P-J14AD\} :ACCEPTABLE

CORRDSION TEST (MIL-P-24691/g) :GLARANTEE

FLATTENING TEST:ACCELTAALE

VISUIAL \& DILENSIONS:ACCEPTABLE

HYDROSTATIC TEST I70OPS I:ACCEPTABLE

EN10204 3.

NO MELD KEPANR

LERCURY FREE

NACE MROOI75 ILARDNESS (IIRC 22 UIAX. ) :GLARANTEED

THE MTERJAL PAS COOLED TO IIELOW BUO DEG F IN 3 MINUTES BR LESS

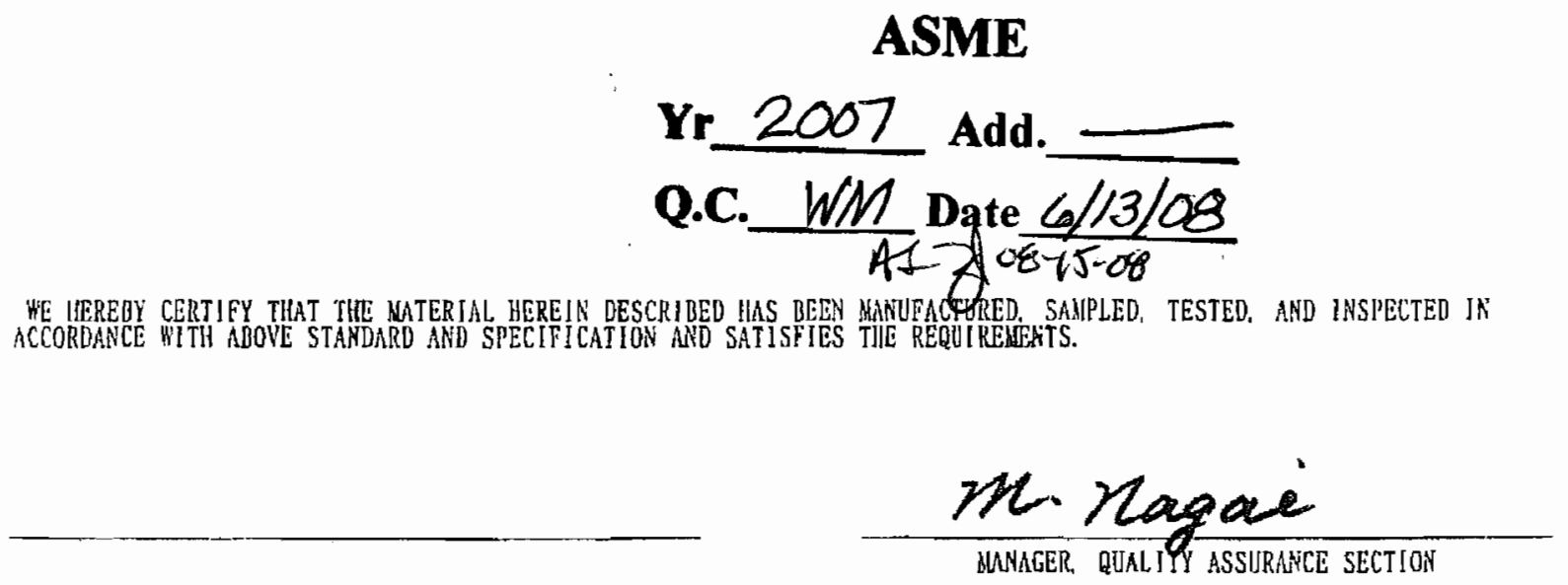


TAYLOR FORGE STAINLESS

P. O. BOX 610

SOMERVILLE, NJ 08876

Phone: (908)722-1313

FAX: (908)722-2943
Material Test Report

Page: 1 of 1

SALES ORDER \# : 0000012238

\section{SOLD TO:}

GASPAR INC.

1545 WHIPPLE AVE. SW

CANTON, OH 44710

\section{SHIP TO:}

GASPAR INC.

1545 WHIPPLE AVE. SW

CANTON, OH 44710
CUST P.O.\#: 30236

TAG NUMBER: 30236, PPD-CHG

\begin{tabular}{c|r|r|}
\hline ITEM \# & QTY & ITEM DESCRIPTION \\
\hline 3 & 3 & $4 \times 2$ CR S/80 WP316HS SA403
\end{tabular}

DATE SHIPPED: $/ 0 /$

STARTING MATERIAL

PIPE, A312-07, 316/316H

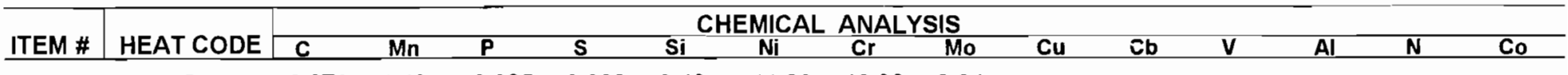

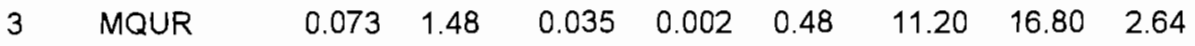

\begin{tabular}{c|llll}
\hline \multirow{2}{*}{ ITEM \# } & $\begin{array}{l}\text { TENSILE } \\
\text { (PSI) }\end{array}$ & $\begin{array}{l}\text { YIELD } \\
\text { (PSI) }\end{array}$ & $\begin{array}{l}\text { ELONG } \\
\text { (2 IN) }\end{array}$ & PHYSICAL PROPERTIES \\
\hline 3 & 89600 & 58000 & 50.00 & 0.00
\end{tabular}

\begin{tabular}{c|cc}
\hline ITEM \# & & SLIPPLEMENTAL INFORMATION \\
\hline 3 & GRAIN SIZE: ACTUAL NO. 6.5 &
\end{tabular}

SOLUTION ANNEALED, WATER QUENCHED .

ASME SA403 2004 ED; ASTM A403-O6; EN 10204/3.1 ASME B16.9

THE FITTINGS WERE MANUFACTURED, SAMPLED, TESTED AND INSPECTED IN

ACCORDANCE WITH THE SPECIFICATIONS AND FOUND TO MEET THE REQUIREMENTS.

THE MATERIAL WAS MANUFACTURED, TESTED AND INSPECTED WITHOUT MERCURY CONTAMINATION. NO WELD REPAIRS.

NACE MR0175

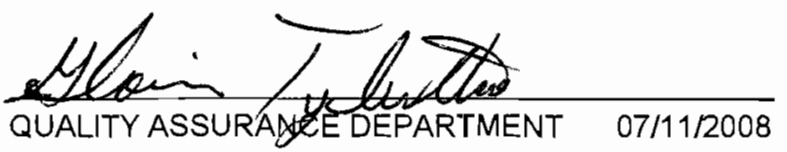




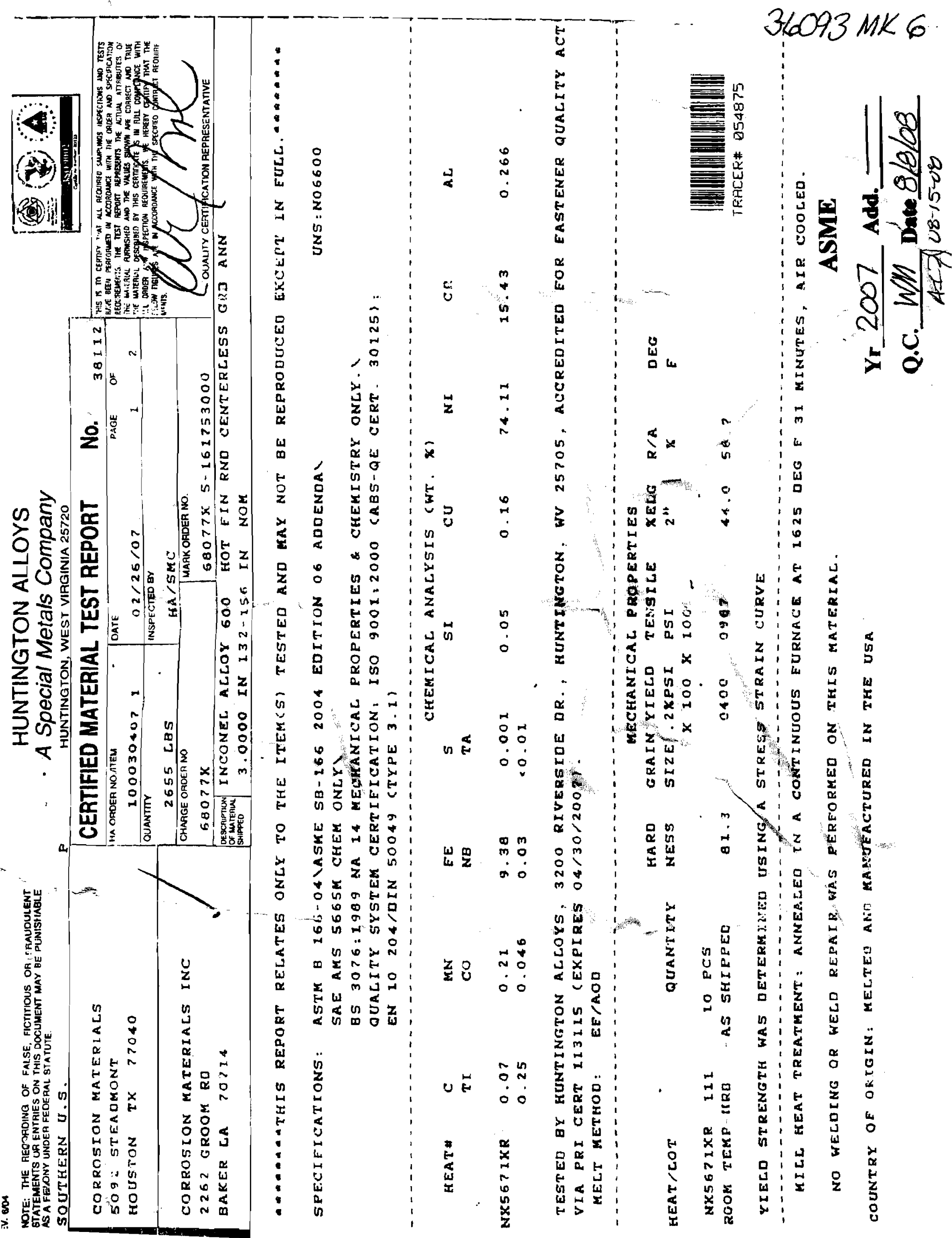




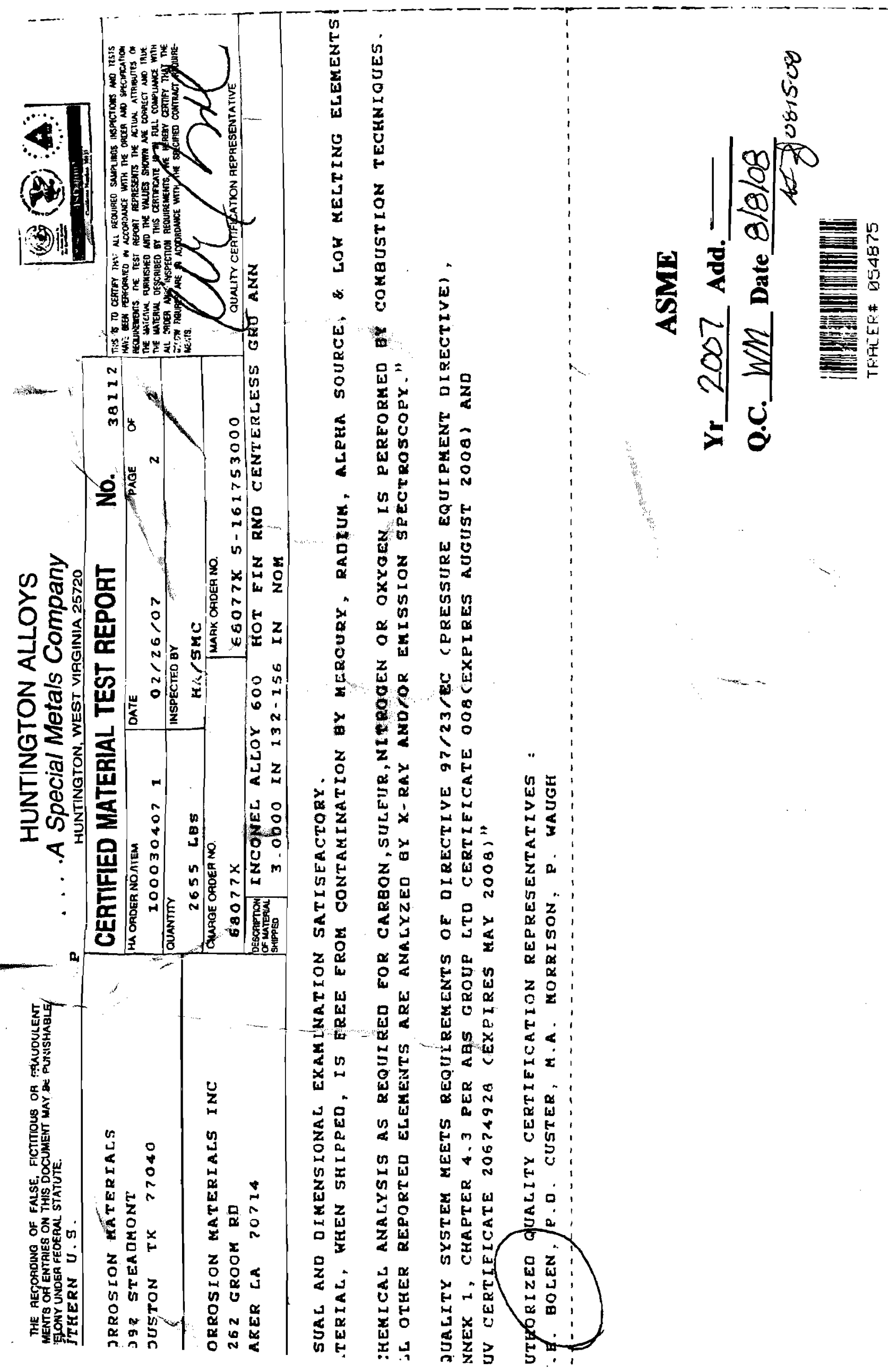


$P 0.3023 \%$

PENNSYLVANIA MACHINE WORKS, INC. 201 BETHEL AVENUE ASTON, PENNSYLVANIA 19014 (610) 497-3300 - FAX (610) 497-3325 www.pennusa.com

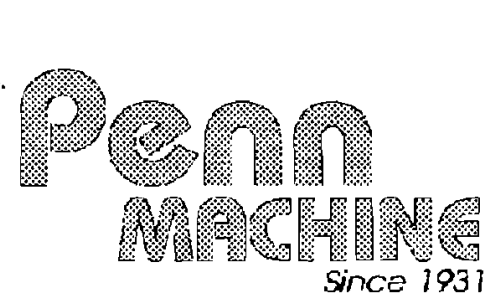

$93 \mathrm{mk} 7$

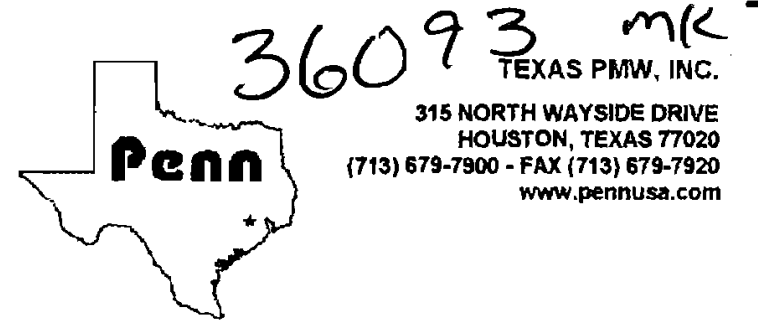

M I L L T E S T

$\begin{array}{llllll}\text { R } & \text { E } & \mathbf{O} & \mathrm{O} & \mathbf{R} & \mathbf{T}\end{array}$

ROBERT-JAMES - TWINSBURG

P.O. BOX 590

T'WINSBURG, OH 44087

P.O. cg4571

ORDER \# 01411275

$6 / 19 / 08$

$1 / 2$ 6MTD HFCP $316 \mathrm{H}$

QTY : $\quad 6$

HEAT CODE : $\mathbf{B 6 1 9 4 1}$

SPECIFICATION: A182

HEAT TREATMENT: SOLUTION ANNEALED

MATERIAL: F316H

UNS NUMBER: S31609

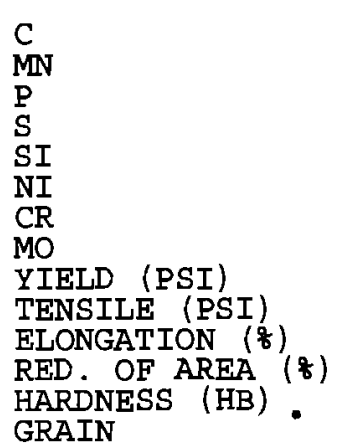

ACTUAL MEASUREMENT

.046

1.680

.029

.025

.280

10.560

17.450

2.040

37500

81500

58.000

77.000

137

4.50
SPEC. YEAR: 04

SPECIFICATION

$\begin{array}{rr}\text { MIN } 8 & \text { MAX } \% \\ .040 & .100\end{array}$

$.040 \quad 2.000$

.045

.030

1.000

$10.000 \quad 14.000$

$16.000 \quad 18.000$

2.000

30000

75000

30.000

50.000

6.00
SPEC. REV :

\section{SOLUTION ANNEALED}

ACTUAL MEASUREMENT $1900 \mathrm{~F}$

\section{SPECIFICATION \\ MINIMUM}

MATERIAL I/A/W ASME-SEC.II SA SPECIFICATION-CERTIFICATE I/A/W EN10204 TYPE 3.1

MATERIAL MEETS NACE-MRO175-2003

MATERIAL IS CAPABLE OF PASSING A CORROSION TEST TO ASTM-A262 PRACTICE "E"

MATERIAL MEETS NACE-MRO103-2007

MERCURY FREE MATERIAL - NO WELD REPAIRED MATERIAL - MADE IN THE U.S.A.

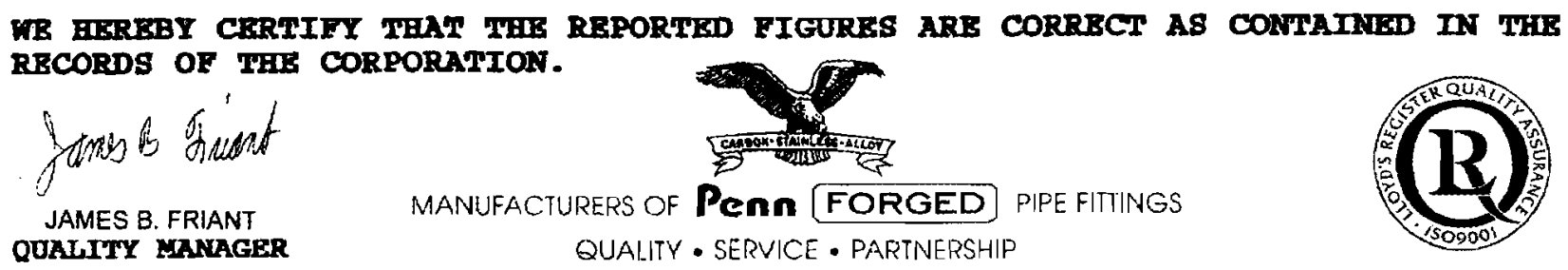


687 COUNTY ROAD 2201

CLEVELAND, TX 77327

PH (281) 727-7000 FAX (281) 727-7062

\section{CERTIFIED MATERIAL TEST REPORT}

CERTIFIED IAW EN10204 3.1 (MADE IN USA)

\begin{tabular}{|c|c|c|c|}
\hline TO: GASPAR INC. & & WFF SO NO: & 11752 \\
\hline PO: $\quad 30214$ & & HEAT NO: & 23988 \\
\hline SPECIFICATIONS: & $\begin{array}{l}\text { ASME SA182 F316H } \\
\text { DIM/TOL PER ASME B16.5 }\end{array}$ & & \\
\hline
\end{tabular}

\begin{tabular}{|c|l|}
\hline OTY & TEM DESCRIPTION \\
\hline 5 & 2" $1500 \#$ WN RF S80 \\
\hline & PROJECT\# 36093-8 \\
\hline & \\
\hline & \\
\hline & \\
\hline
\end{tabular}

\begin{tabular}{|c|c|c|c|c|c|c|c|c|c|}
\hline TYPE & $\mathbf{C}$ & $\mathbf{M N}$ & $\mathbf{P}$ & $\mathbf{S}$ & $\mathbf{S I}$ & $\mathbf{C R}$ & $\mathbf{N I}$ & $\mathbf{M O}$ & $\mathbf{N}$ \\
\hline F316H & .047 & 1.45 & .031 & .001 & .54 & 16.20 & 10.10 & 2.13 & .043 \\
\hline MILL & & & & & & & & & \\
\hline
\end{tabular}

\begin{tabular}{|c|c|c|c|c|c|c|}
\hline HEAT No, & VIELD . $\%$ OFF-PSI & TENSIGE-PSI & \% ELONG. & $\%$ RED AREA & HARDNESS & GRAN SIZE. (ASTM) \\
\hline 23988 & 41,950 & 82,050 & 63.7 & 81.1 & & No. 5.0 \\
\hline & & & & & & \\
\hline & & & & & & \\
\hline
\end{tabular}

- NO WELDING PERFORMED ON THE FORGINGS OF THIS ORDER.

- MATERIAL HEREIN IS CERTIFIED FREE FROM ANY FORM OF MERCURY, RADIUM OR ALPHA PARTICLE CONTAMINATION

THROUGHOUT ALL PHASES OF MANUFACTURE AND SHIPMENT.

- THE FORGINGS ON THIS ORDER CONFORM TO THE SPECIFIED DIMENSIONAL REQUIREMENTS.

- MATERIAL MANUFACTURED IAW WFF QA PROGRAM QAM-WF07.0702.

- Solution annealed @ $1950^{\circ} \mathrm{F}$ and water quenched to below $300^{\circ} \mathrm{F}$ within less than three minutes.

WE CERTIFY THE MATERIAL DESCRIBED HEREIN HAS BEEN MANUFACTURED, TESTED AND EXAMINED IAW AND MEETS ALL THE REQUIREMENTS OF ABOVE PURCHASE ORDER AND REFERENCED SPECIFICATIONS.

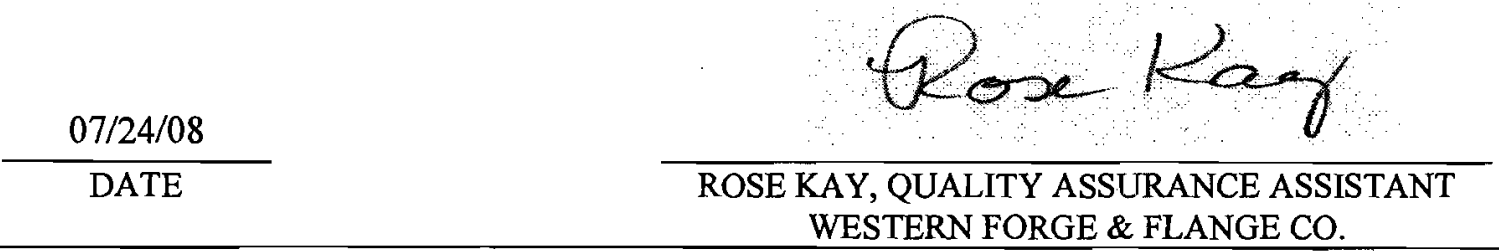

This document shall not be reproduced, except in full, without written approval of Western Forge \& Flange. The recording of false, fictitious or fraudulent statements or entries on this document may be punishable as felony under federal statutes. 

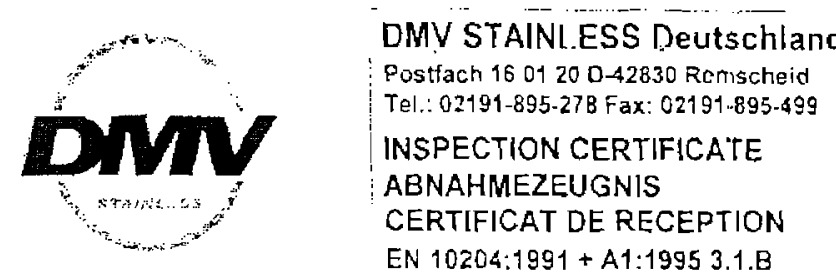

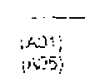

Nr.No/N". (403)
$01156-050613$
0080067815
Shatt / $909=\quad 1 / 2$

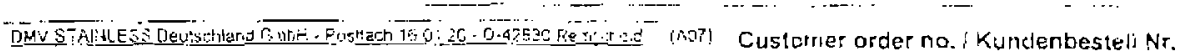
DMV STAINLESS USA INC. 12050 W. LITTLE YORK No de cornmande client:

USA - HOUSTON TX 77041
USA

\section{2}

(AOB) DNV order no / OMV Auftrags-Nr. I No. de cominande OMV

\section{5}

(8202124/005)

Part number:

\section{(Ais) Purchaser/Basteller/Client}

Grade and Specifications

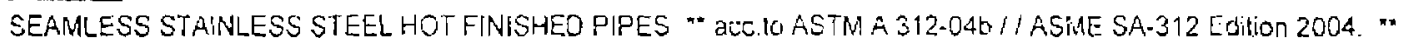
HEAT TREATED CONDITION ACC.1O STANDARD and SPECIFICATION * PICKLED * PLAII ENDS, SQUATE CUT, DEBURRED * Size tolerances acc. to ASTM A 312-04b ** RANDOM LENGTH 17,000 - 24,000 feet ** Ouantity Talerance $+10,0 / 10.0 \% *$ TP316H $:$ TP316 $\cdots$

Nahtiose warmgefertigte Edelstahlronre ** nach ASTM A 312-04b / I ASME SA-312 ED. 04. * wärmebehandelt ** gebeizt * glath, senkrecht geschnilten, emigralet "* Aomessungstoleranzrorm. ASTM A 312-04b ** Herstellïnge 17,000-24.000 fee: ** Mengentoleranz + 10,0i 10,0\% * TP316H/TP316 *

PIPES ACIER INOX SANS SOUDURE FINIS A CHAUD * SelON ASTM A 312-04D//ASME SA-312 ED. O4. * A LEIAT TRAITE SELON NORME ET SPECIFICATION. " DECAPE * ExLímItés LISSES, COLPEES D'EQUERRE. EBAVUREES " Tolérances dimensiommelles selon ASTM A 312-040" LOVGUEURS COURANTES 17.003 - 24,000 pieds ** Tolerance sur quantite $+10,0 /-10.0 \% *$ TP3 $16 \mathrm{H} / \mathrm{TP}^{2} 16^{* *}$

Mariking of the product / Kennzeichung/Marquaqes

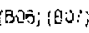

DMV-D - TP $316 H$ - TP 316 - HEAT - 2 X SCH BOS AW . ASTM A 312 - ASME SA 312 - LOT NO - SML - GERMANY - WA.

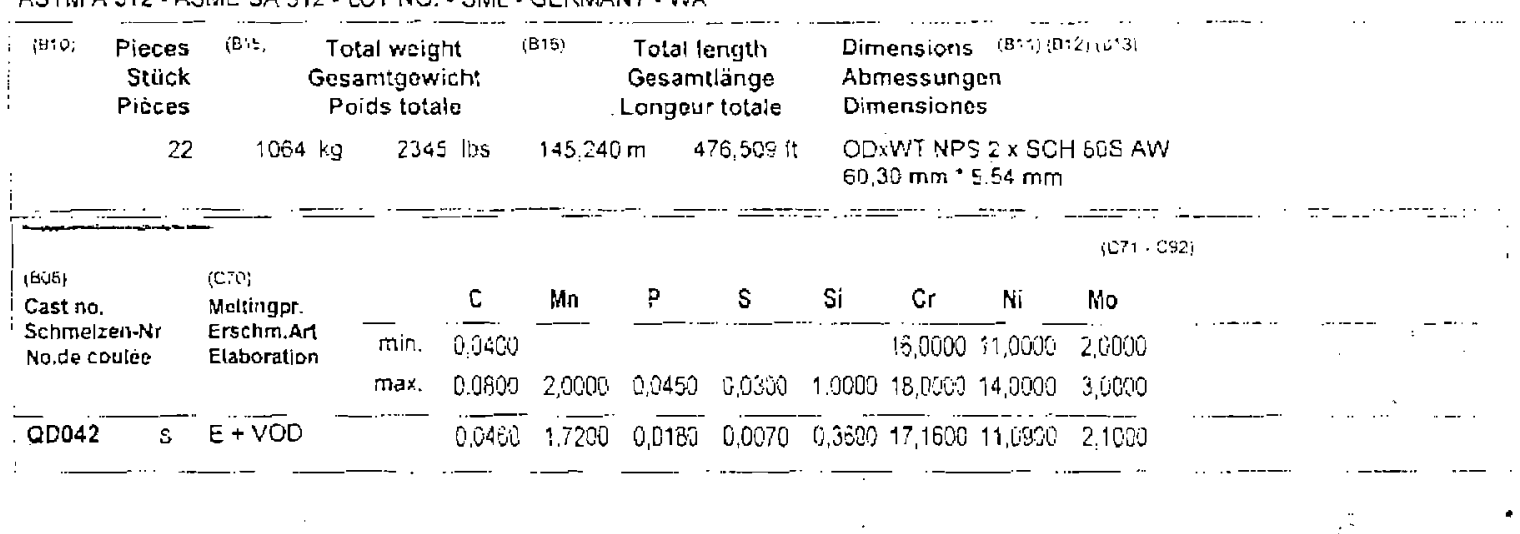

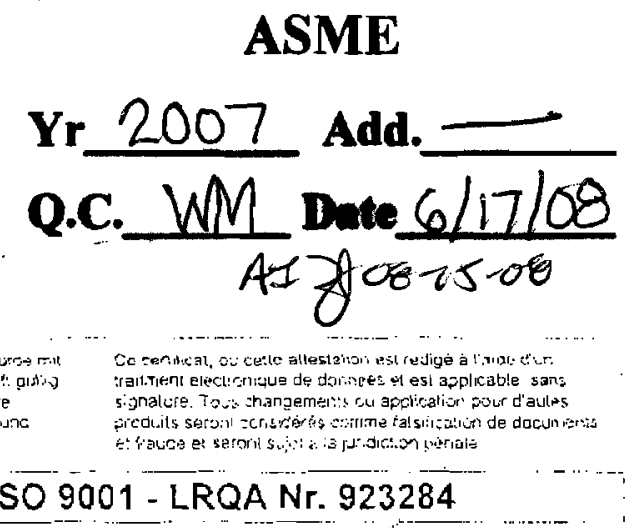

Trus terthicite is is suec by a no ilorized synlum and is

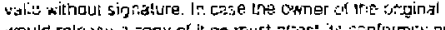

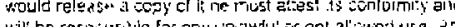

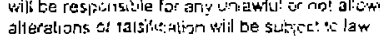

-.. ...... - -

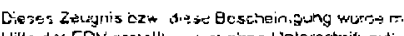

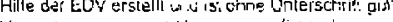

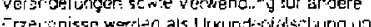
Serecing

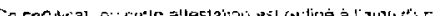

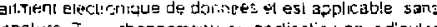

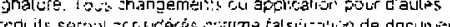

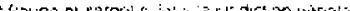




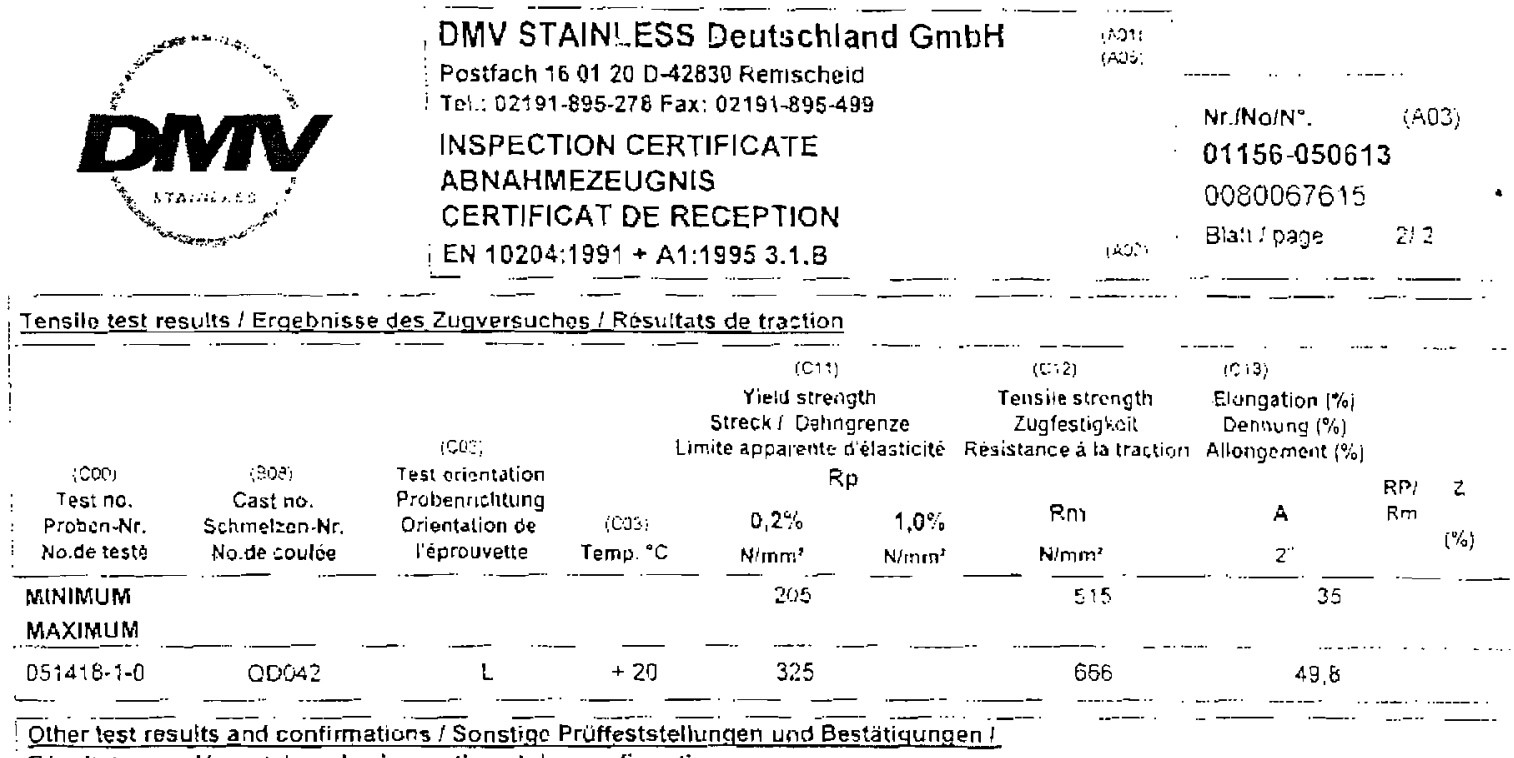

Tensilo test results / Ergebnisse des Zugversuches / Resultats de traction

Other test results and confirmations / Sonstion Pruffeststellungen und Bestätigungen I

Résultats supplementaires des inspection et des confimations

VISUAL AND DIMENSIONAL INSPECTION SATISFACTORY (DO1)

MATERIAL IDENTIFICATION TEST OF ALL TUBES BY SPECTROGRAPHIC ANALYSIS: SATISFACTOPY

FLATTENING TEST: SATISFACTORY :C5O)

GRAIN SIZE ? ACC. TO ASTME 112

HYDROSTATIC TEST, 170 BAR (1BAR=10DKPA): SATISFACTORY

HEAT TREATMENT: SOLUTION HERT TREATED

BESICHTIGUNG UND MASSKONTROLLE: EESTANDEN :O01)

VERWECHSLUNGSPRUEFUNG ALLER ROHRE DURCH SPECTRAL-ANALYSE: BESTANDEN

RINGFALTVERSUCH: BESTANDEN (C5O)

KORNGROESSE 7 GEMAESS ASTM E 112

INNENORUCKVERSUCH MIT WASSER 170 EAR (13AR=10OKPA). BESTANDEN

WAERMEBEHANDLUNG: LOESUNGSGEGLUEHT

INSPECTIONET CONTROLE DIMENSIONEL: SATISFNISANT (DO1)

ESSAI ANTI MELANGE SUR TOUS LES TUBES PAR ANAL YSE SPECTRALE. SATISFAISANT

ESSAI D'APLATISSEMENT: SATISFAISANT (C50)

DINENSION DU GREIN 7 CONFORME ASTME 112

ESSAJ HYDRAULIQUE: 170 BAR 1 16AR=100KPA): PASSE

TRAITEMENT THERMIOUE: RECUIT

The products have been tested in accordance with the purchase specification and are found to be satisfied.

Wir bestätigen, das die Erzeugnisse bestellungsgemäß geprüft und für in Ordnung belunden wurden.

Nous attestons que les produits livrés sont conformes aux stipulations de ia commande

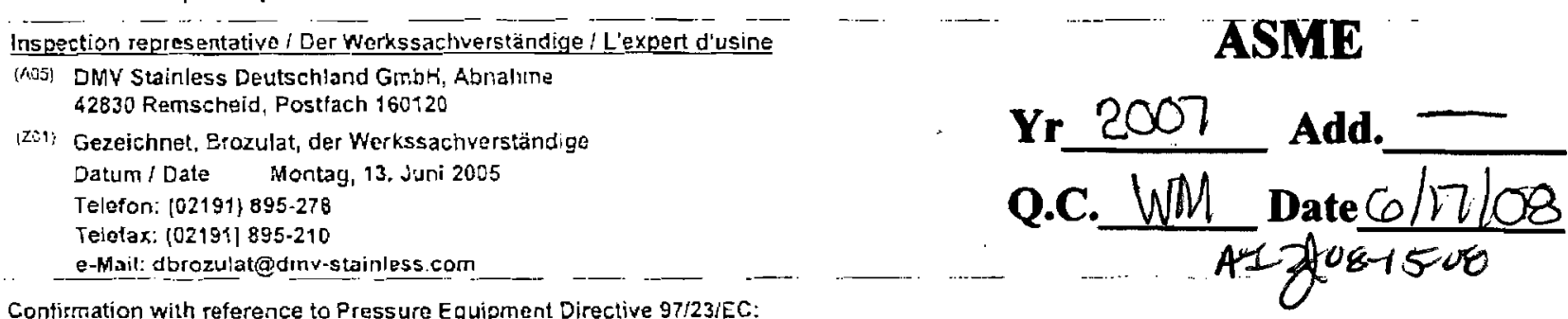

Confirmation with reference to Pressure Equipment Directive 97/23/EC:

The works operates a quality management system that has undergone a specific assessment for materials for pressure equipment and is certified by a competent body (TÜV-Cert No: 01202811/Q-020025)

Bestätigung in Bezug auf Druckgeräterichtlinie 97/23/EG:

Das Werk wendet ein Qualitätsmanagementsystom an, das in Bezug auf Werkstoffe für Oruckgeräte einer spezilischen Bewertung Lnterzogen wurde und yon einer zuständigen Stelle (TÜV-Cert.No: 01202811/Q-020025) zertifiziert ist.

Confirmation concermant la Dircctive Equipements sous Pression 97/23/EC

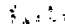

L'usine applique un systéme de management de la qualité qui a fait lobjet d'une évaiuation spécifique pour les matériauz pour équipements sous pression etc qui est certifié par un organisme compétent

(TUeV.Cert.No: 01202811/Q-020025)

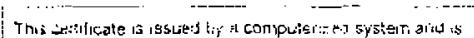

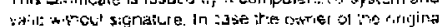

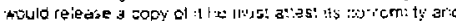

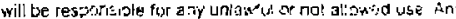

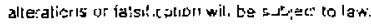

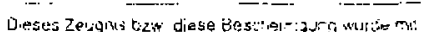

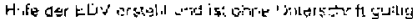

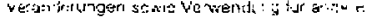

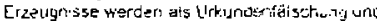

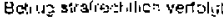

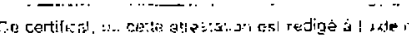

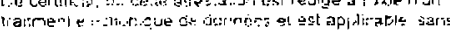

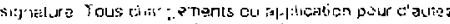

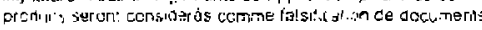

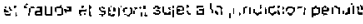


SOLD TO:

ROBERT JAMES SALES, INC.

P.O. BOX 590

TWINSBURG, OH 44087
SALES ORDER \# : 0000012240
CUST P.O.\#: CG4572

\begin{tabular}{l|l|l}
\hline ITEM \# & QTY & ITEM DESCRIPTION \\
\hline
\end{tabular}

212 LR 90 S/80 WP316HS AVSA403
TAG NUMBER: CG4572, PPD-CHG

SHIP TO:

GASPAR INC.

1545 WHIPPLE AVE S.W!

CANTON, OH 44710

\begin{tabular}{|c|c|c|c|c|c|c|c|c|c|c|c|c|c|c|c|}
\hline \multirow[b]{2}{*}{ ITEM \# } & \multirow[b]{2}{*}{ HEAT CODE } & \multicolumn{13}{|c|}{ CHEMICAL ANALYSIS } & \multirow[b]{2}{*}{ Co } \\
\hline & & C & $\mathrm{Mn}$ & $P$ & $S$ & Si & $\mathrm{Ni}$ & $\mathrm{Cr}$ & Mo & $\mathrm{Cu}$ & $\mathrm{Cb}$ & $\mathbf{V}$ & Al & $\mathbf{N}$ & \\
\hline 2 & MPMK & 0.06 & 1.14 & 0.037 & 0.011 & 0.49 & 12.36 & 16.80 & 2.12 & & & & & & 0.096 \\
\hline
\end{tabular}

\begin{tabular}{c|llll}
\hline & \multicolumn{3}{|c}{} & PHYSICAL PROPERTIES \\
\cline { 2 - 5 } & $\begin{array}{l}\text { TENSILE } \\
\text { (PSI) }\end{array}$ & $\begin{array}{l}\text { YIELD } \\
\text { (PSI) }\end{array}$ & $\begin{array}{l}\text { ELONG } \\
\text { (2 IN) }\end{array}$ \\
\hline ITEM \# & & & & \\
\hline 2 & 80400 & 41800 & 49.00 & 0.00
\end{tabular}

\begin{tabular}{c|c}
\hline ITEM \# & SUPPLEMENTAL INFORMATION \\
\hline 2 & GRAIN SIZE: MPMK-ACTUAL:DUPLEX ASTM NO. 6 \& ASTM NO. 4.0
\end{tabular}

SOLUTION ANNEALED, WATER QUENCHED .

ASME SA403 2004 ED; ASTM A403-O6; EN 10204/3.1 ASME B16.9

THE FITTINGS WERE MANUFACTURED, SAMPLED, TESTED AND INSPECTED IN

ACCORDANCE WITH THE SPECIFICATIONS AND FOUND TO MEET THE REQUIREMENTS

THE MATERIAL WAS MANUFACTURED, TESTED AND INSPECTED WITHOUT MERCURY

CONTAMINATION. NO WELD REFAIRS.

NACE MR0175

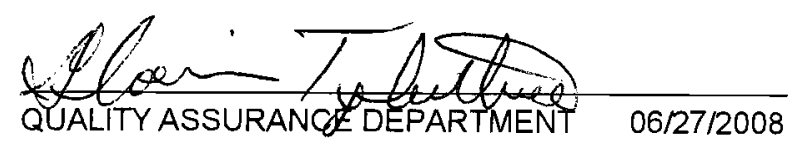




\section{MK 12}

DMV STAINLESS Italia s.r.I

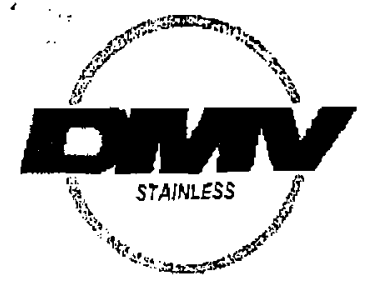

Via Pio, 3024062 Costa Volpino (BG)

Tel : 035.975.611 - Fax :035.971624

e-mail: dmvitaly@dmv-stainless.com

INSPECTION CERTIFICATE

3.1.B - EN 10204 : 1991+A1 : 1995

Purchaser

Address

Customers Order

DMV Ref. Order / Item

Part number
DMV STAINLESS USA INC. .

12050 W. LITTLE YORK HOUSTON.

0D00211080 date 09/03/2004.

$0,000211107 / 000009$.

N.A.

Product

Seamless Stainless Steel Hot Finished Pipes Solution Annealed Pickled

Plain Ends Square Cut Deburr

Specification

Grade

Tolerances

Marking

Heat

54446

Tot.

Heat 54446 Melting Process

Chemical analisys (\%)

\begin{tabular}{|l|rrrrrrrrr}
\hline \\
Min & \multicolumn{2}{c}{$\mathbf{C}$} & Si & Mn & $\mathbf{P}$ & $\mathbf{S}$ & $\mathbf{C r}$ & $\mathbf{M o}$ & $\mathbf{N i}$ \\
Max & 0.040 & & & & & 16.000 & 2.000 & 11.000 \\
Heat & 0.080 & 1.0000 & 2.00 & 0.045 & 0.0300 & 18.000 & 3.000 & 14.000 \\
\hline
\end{tabular}

Mechanical and Metallurgical Properties

Tensile test (at $20^{\circ} \mathrm{C}$ )

$\begin{array}{lrrrr} & \text { Direct. } & \text { Y.S. } 0,2 \% & \text { U.T.S. } & \text { El } 50 \mathrm{~mm} \\ \text { Long/Trans } & \mathrm{ksi} & \mathrm{ksi} & \% \\ \text { Min } & & 30,00 & 75,00 & 35.00 \\ \text { No } 175148 & \text { L } & 48.62 & 93.99 & 48.00\end{array}$

FLATTENING TEST

Required

ASTM A 999

CORROSION TEST

Required

ASTM A 262 PR E

MICROGRAPHIC EXAMINATION

No 175148 Grain size required max 7 , result 6,5. According to ASTM E 112

No 176020 Grain size required max 7 , result 6,5. According to ASTM E 112

Other tests and declarations

Heat treatment

$1958^{\circ} \mathrm{F}$

Antimixing checked by PMI

Visual and dimensional examination

Eddy current testing

ASTM A 999

\section{ASME}

\section{Yr 2007 Add.}

Q.C. WM Date 6 67108

$$
\text { AI 2008-15:08 }
$$

Result OK

Result OK 


\section{DMV STAINLESS Italia s.r.I}

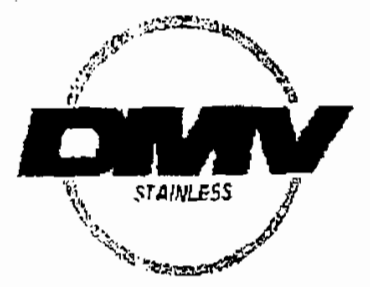

Via Pio, 3024062 Costa Volpino (BG)

Tel : 035.975.611 - Fax :035.971624

8-mail: dmvltaly@dmv-stainless.com

\section{INSPECTION CERTIFICATE}

3.1.B - EN $10204: 1991+A 1: 1995$

\section{No weld repair}

Tubes are free from mercury contamination

We certify that the delivered products comply with the request of the order

Corrosion test acc.to MIL-P-2469, 3: satisfactory

Date 09-Sep-2004

Mill inspector F. RAZZITT!

\section{ASME \\ Yr 2007 Add. \\ Q.C. WM Date $6 / 17 / 08$ \\ AI $80,15.00$}

Confirmation with reference to Pressure Equipment Directive 97123/EC:

The works operates a quality management system that has undergone a specific assessment for materials for pressure equipment and is certified by a competent body (TUV-CERT.No: 7012002/NUC)

This certificate is issued by a computerized system and is valid without signature. In case the oumer of the original conificato wouid release a copy of he must altest its conformity to the original one, taking upon himself the responsibility for any anlawful or not allowed use. Any alteration and/or falsification will be subject to the law 


\section{CERTIFICATE OF TEST}

\begin{tabular}{|c|c|c|c|c|}
\hline CERTIFICATION \# & CERTIFICATION DATE & MILL ORDER \# & ITEM\# & CUSTOMER PO\# \\
\hline 135430 & $07 / 20 / 2007$ & 0551805 & & \\
\hline
\end{tabular}

Sold To:

CORROSION MATERIALS

P.O. BOX 630

BAKER, LA 70714

USA

Ship To:

CORROSION MATERIALS

2262 GROOM ROAD

BAKER, LA 70714

USA

\begin{tabular}{|l|l|}
\hline PRODUCT DESCRIPTION & PRODUCT SPECIFICA \\
\hline BRIGHT ANNEALED $0.500^{\prime O D} \times 0.065^{\prime \prime}$ AW UNS N06600 (SMLS) & $\begin{array}{l}\text { ASTM-B-163-04/B-167-05a, } \\
\text { ASME-SB-163-04/SB-167-04 }\end{array}$
\end{tabular}

Chamicul Analygte

\begin{tabular}{|c|c|c|c|c|c|c|c|c|c|c|c|c|c|c|c|c|c|c|}
\hline at" & $\begin{array}{l}\text { Selt } \\
\text { source }\end{array}$ & $c$ & $\mathrm{ma}$ & $\mathbf{P}$ & $\mathbf{s}$ & $\mathbf{s i}$ & $\mathrm{CI}$ & $\mathbf{N I}$ & Mo & $\mathrm{cu}$ & co & $\mathbf{N}$ & TI & $\mathrm{Cb}+\mathrm{Ta}$ & Al & Fe & พ & $\mathbf{v}$ \\
\hline & USA & 0.030 & .210 & 0.005 & .003 & 0.060 & 15.680 & 73.790 & 0.000 & 0.190 & 0.067 & 0.000 & 0.210 & 0.000 & 0.312 & 9.390 & 0.000 & 0.000 \\
\hline $5605 X R-C$ & USA & 0.030 & 0.220 & 0.005 & 0.003 & 0.070 & 15.660 & 73.830 & 0.000 & 0.190 & 0.068 & 0.000 & 0.210 & 0.000 & 0.308 & 9.350 & 0.000 & 0.000 \\
\hline
\end{tabular}

Whanical Teets

\begin{tabular}{|c|c|c|c|c|c|c|c|}
\hline Beat & Lot & Piecos & Eootrige & $\begin{array}{c}\text { Tensilo } \\
\text { Strength (PaI) }\end{array}$ & $\begin{array}{c}\text { Yield } \\
\text { strangth (PeI) }\end{array}$ & $\begin{array}{l}\text { 8riongation } \\
\text { in 2" }\end{array}$ & Hardness (RB ) \\
\hline NX5605XR & 1 & 51 & 1020 & 94800 & 51100 & 43 & 83 \\
\hline
\end{tabular}

Flare Test - Pass

Hydrostatic Test - Pass

Heat treated at $1800 \mathrm{~F} \min$ for sufficient time to achieve uniform temperature throughout the wall thickness and rapidly atmoshperically quenched.

The raw material used to produce this tubing is DFARS compliant.

Melt Method - EF/AOD.

- Cold drawn - Made in the USA

- Produced in accordance with EN 102043.1

- Produced without contact with Mercury or other metals that are liquid at ambient temperature.

- No class I ozone depleting chemicals were used in the manufacture of this product.

- Produced without weld repair.
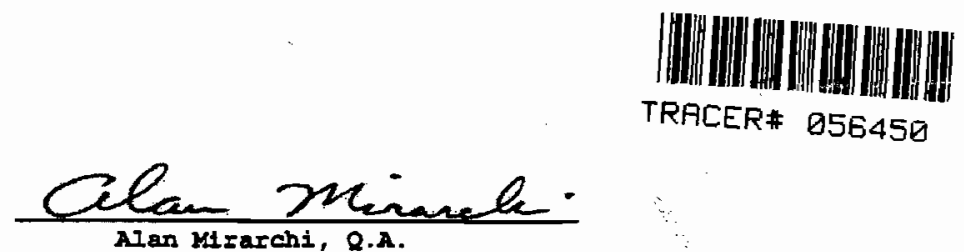

The Results shown above are certified to be a correct statement of records that were derived from testing of samples of the material.

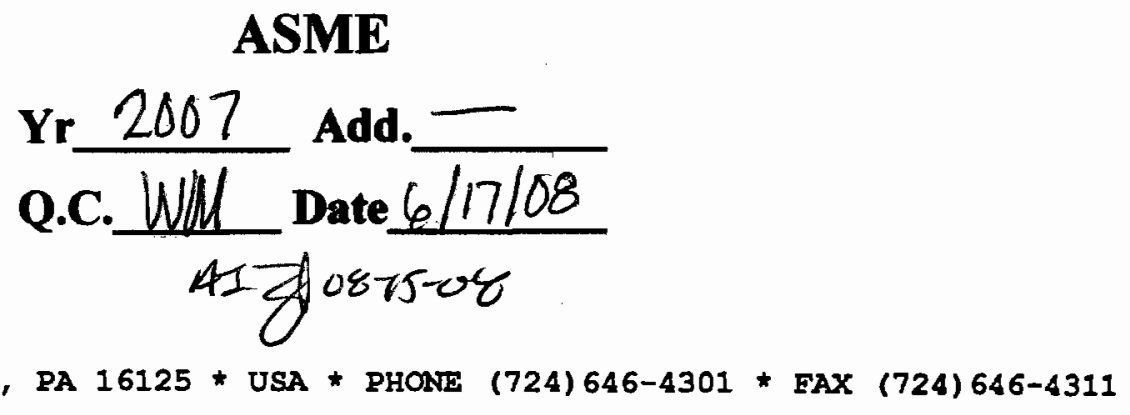


687 COUNTY ROAD 2201

CLEVELAND, TX 77327

PH (281) 727-7000 FAX (281) 727-7062

\section{CERTIFIED MATERIAL TEST REPORT}

\section{CERTIFIED IAW EN10204 3.1 (MADE IN USA)}

\begin{tabular}{|ll|l|l|}
\hline TO: $\quad$ GASPAR INC. & WFF SO NO: & \multicolumn{1}{c|}{ 11752 } \\
\hline PO: 30214 & ASME SA182 F316 & HEAT NO: & 8688074 \\
\hline SPECIFICATIONS: & DIM/TOL PER ASME B 16.5 & & \\
\hline
\end{tabular}

\begin{tabular}{|c|l|l|}
\hline OTY & \multicolumn{1}{|c|}{ TTEM DESCRIPTION } \\
\hline 1 & 2" 1500\# BL RF \\
\hline & PROJECT\# 36093-16 \\
\hline & \\
\hline & \\
\hline & \\
\hline
\end{tabular}

\begin{tabular}{|c|c|c|c|c|c|c|c|c|c|}
\hline TYPE & $\mathbf{C}$ & MN & P & S & SI & CR & NI & MO & N \\
\hline F316 & .047 & 1.70 & .026 & .02 & .43 & 16.86 & 10.21 & 2.10 & .049 \\
\hline MULL & & & & & & & & & \\
\hline
\end{tabular}

\begin{tabular}{|c|c|c|c|c|c|c|}
\hline HEATNO & YIEU D $2 \%$ OFEPSI & TENSII E PSI & \%LLONG & \%RED AREA & H ARDNESS & GRANSWE (ASTM) \\
\hline 8688074 & 40,700 & 81,360 & 57.8 & 55.7 & & \\
\hline & & & & & & \\
\hline & & & & & & \\
\hline
\end{tabular}

- NO WELDING PERFORMED ON THE FORGINGS OF THIS ORDER.

- MATERIAL HEREIN IS CERTIFIED FREE FROM ANY FORM OF MERCURY, RADIUM OR ALPHA PARTICLE CONTAMINATION

THROUGHOUT ALL PHASES OF MANUFACTURE AND SHIPMENT.

- THE FORGINGS ON THIS ORDER CONFORM TO THE SPECIFIED DIMENSIONAL REQUIREMENTS.

- MATERIAL MANUFACTURED IAW WFF QA PROGRAM QAM-WF07.0702.

- Solution annealed @1950 $\mathrm{F}$ and water quenched to below $300^{\circ} \mathrm{F}$ within less than three minutes.

WE CERTIFY THE MATERLAL DESCRIBED HEREIN HAS BEEN MANUFACTURED, TESTED AND EXAMINED IAW AND MEETS ALL THE REQUIREMENTS OF ABOVE PURCHASE ORDER AND REFERENCED SPECIFICATIONS.

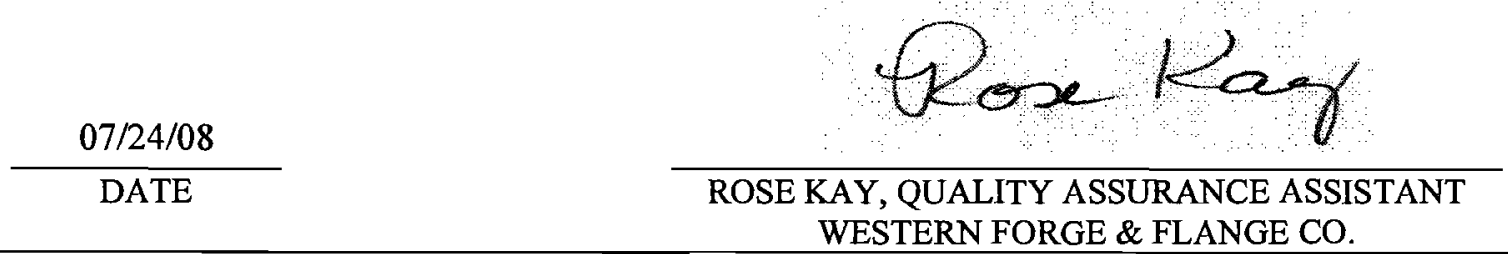

This document shall not be reproduced, except in full, without written approval of Western Forge \& Flange. The recording of false, fictitious or fraudulent statements or entries on this document may be punishable as felony under federal statutes. 
REPORT \# 080208- 2 RADIOGRAPHIC INSPECTION REPORT DATE: $8 / 02 / 08$ Page 1 of 2

CUSTOMER GATSPAR

PO\# 10978

WELDING PROCESS: SEE DUG- THICKNESS/DIAMETER, 218/,337/.500 SCREENS FIB: $005-010^{\prime}$

LOCATION CANTON.OHIO

MATERIAL TYPE: STAINLESS STEEL

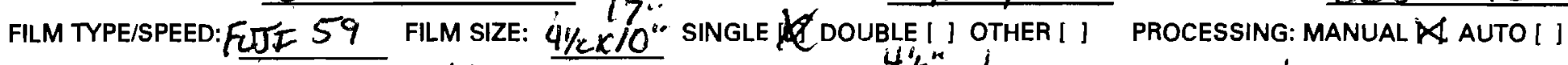

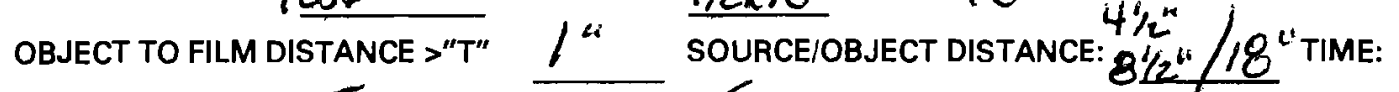
SOURCE $\quad 115 \times[$ IR. [T CO.II X-RAYI] CURIES: 29 LM,N/ZM,N Ug $0.020^{\prime}$

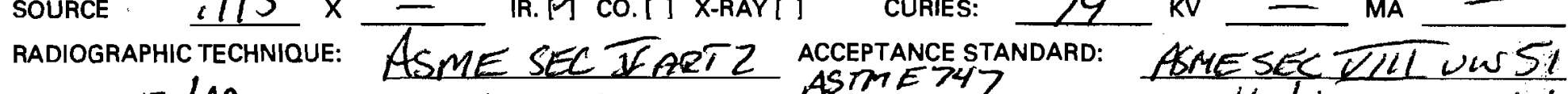

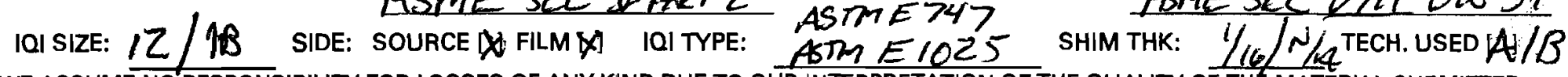
WE ASSUME NO RESPONSIBILITY FOR LOSSES OF ANY KIND DUE TO OUR INTERPRETATION OF THE QUALITY OF THE MATERIAL SUBMITTED. ALL DATA AND INFORMATION WILL BE HELD STRICTLY CONFIDENTIAL.

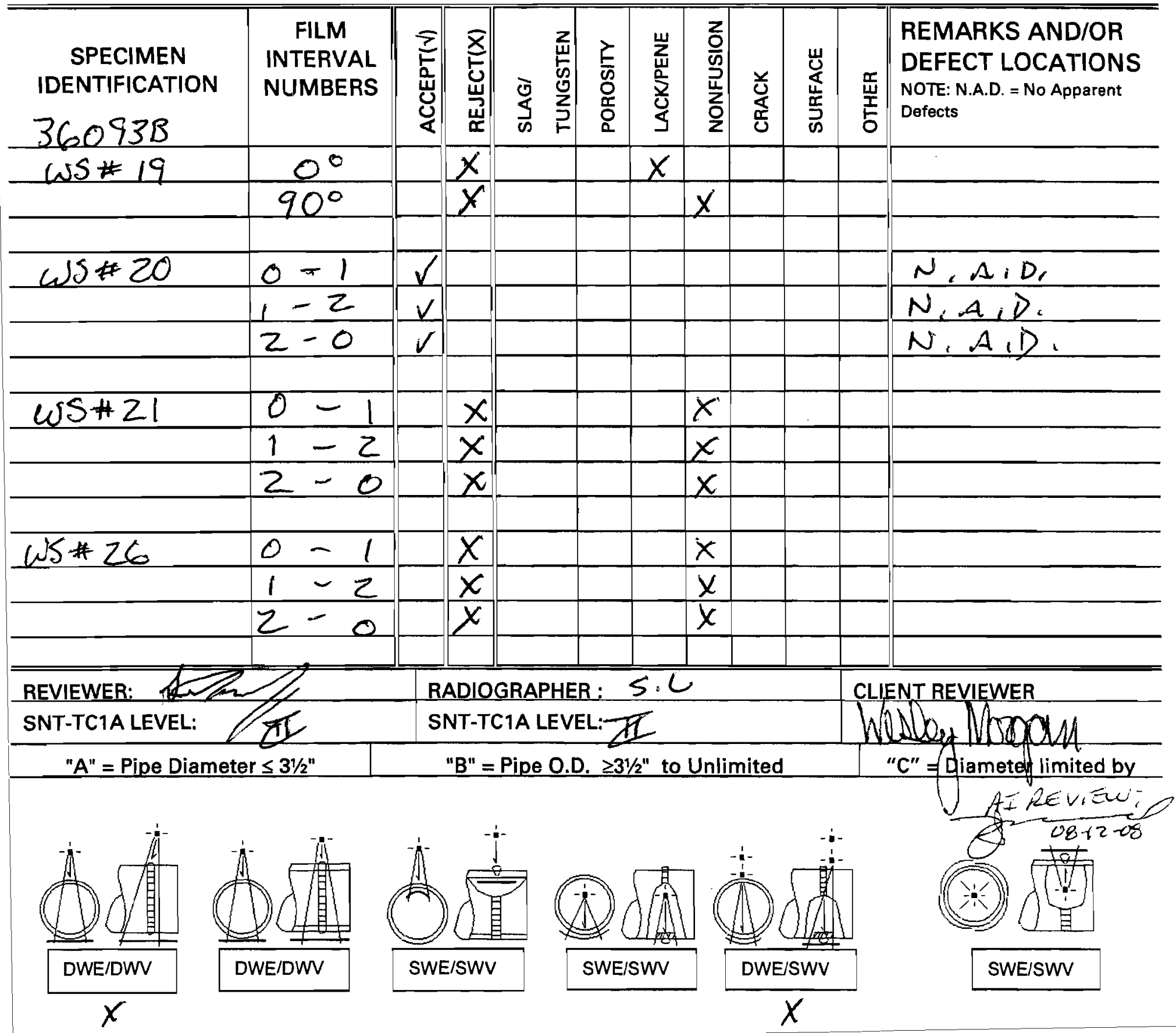




\section{REPORT \# 081208 - 2 RADIOGRAPHIC INSPECTION REPORT DATE:08/12/08Page 1 of 1} \begin{tabular}{lll}
\hline CUSTOMER GASPAR & & LOCATION CANTON, OHIO \\
PO\# 10978 & JOB\# $36093 B$ & MATERIAL TYPE: STAINLESS STEEL
\end{tabular} WELDING PROCESS: SEE DUJG THICKNESSIDIAMETER $218 / 1 / 2 \% 337$ SCREENS F/B: $205^{\circ}-010^{\circ}$

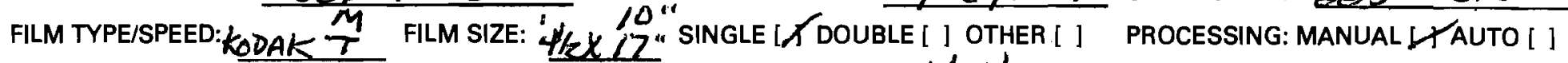

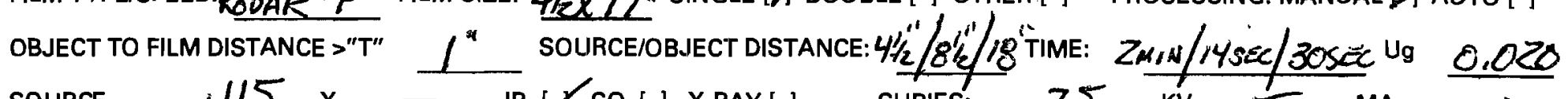

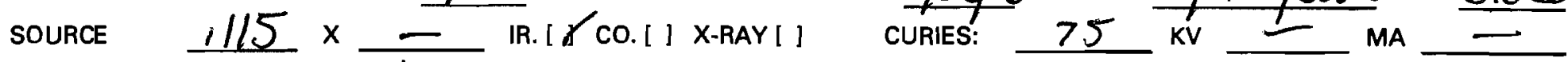

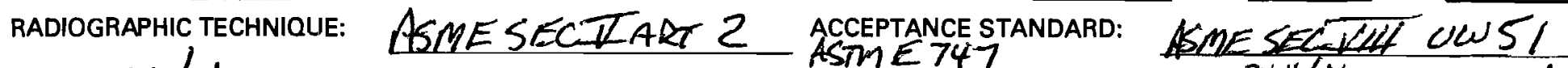
IOI SIZE: $12 / 1 B$ SIDE: SOURCE $X$ FILM IOI TYPE: ASTME 1025 SHIM THK: $3 / 4 / N / A$ TECH. USED IA/B WE ASSUME NO RESPONSIBILITY FOR LOSSES OF ANY KIND DUE TO OUR INTERPRETATION OF THE QUALITY OF THE MATERIAL SUBMITTED. ALL DATA AND INFORMATION WILL BE HELD STRICTLY CONFIDENTIAL.

\begin{tabular}{|c|c|c|c|c|c|c|c|c|c|c|c|}
\hline $\begin{array}{l}\text { SPECIMEN } \\
\text { IDENTIFICATION } \\
360938\end{array}$ & $\begin{array}{c}\text { FILM } \\
\text { INTERVAL } \\
\text { NUMBERS }\end{array}$ & 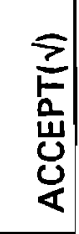 & 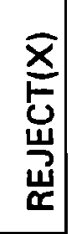 & 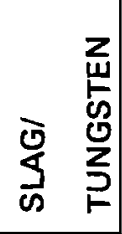 & $\begin{array}{l}\frac{z}{0} \\
0 \\
0 \\
0 \\
0\end{array}$ & 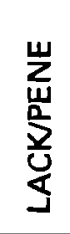 & $\begin{array}{l}z \\
\frac{2}{c o} \\
\frac{1}{u} \\
z \\
\frac{2}{z}\end{array}$ & 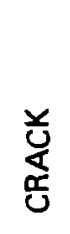 & 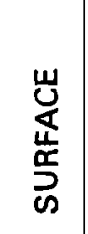 & $\begin{array}{l}\frac{\mathbb{4}}{\mathrm{T}} \\
\frac{\mathrm{J}}{\sigma}\end{array}$ & $\begin{array}{l}\text { REMARKS AND/OR } \\
\text { DEFECT LOCATIONS } \\
\text { NOTE: N.A.D. = No Apparent } \\
\text { Defects }\end{array}$ \\
\hline$\omega 5 \# 19$ & $0^{\circ}$ & $\sqrt{ }$ & & & & & & & & & $N, A, D$. \\
\hline NEW WELD & $90^{\circ}$ & $\sqrt{ }$ & & & & & & & & & $N, A, D$, \\
\hline$\omega S \# 21$ & $0-1$ & $\checkmark$ & & & & & & & & & $N A, D$ \\
\hline NEW WELD & $1-2$ & $\sqrt{ }$ & & & & & & & & & $N A, D$, \\
\hline & $z-0$ & $\sqrt{ }$ & & & & & & & & & $N, A, D$. \\
\hline & & & & & & & & & & & \\
\hline WS\#26 & $0-1$ & $\checkmark$ & & & & & & & & & $N, A, D$. \\
\hline NEW WECD & $1-2$ & $\sqrt{ }$ & & & & & & & & & $N, A, D$. \\
\hline & $2-0$ & $\sqrt{ }$ & & & & & & & & & $N, A, D$ \\
\hline WS\#27 RI & $2-0$ & $\checkmark$ & & & & & & & $\checkmark$ & & Fii) \\
\hline & & & & & & & & & & & \\
\hline WS\# $28 R I$ & $0^{\circ}$ & $\checkmark$ & & & & & & & & & $N, A, D$ \\
\hline
\end{tabular}

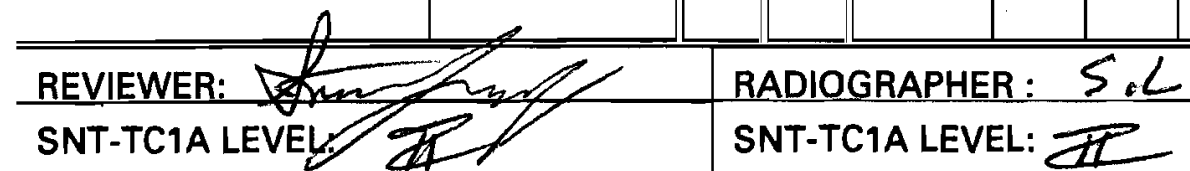

"A" = Pipe Diameter $\leq 31 / 2$ "

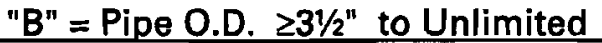

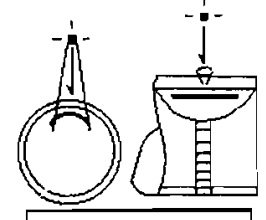

SWE/SWV

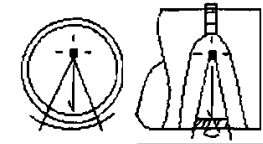

SWE/SWV

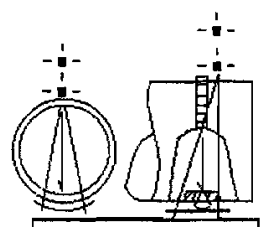

DWE/SWV
CLIENT REVIEWER

Weslog Mogan "C" $\mathrm{C}$ Dameter l mited by AI $A E V I E W:-1$ $\mathbb{Z}^{2} 0815-08$

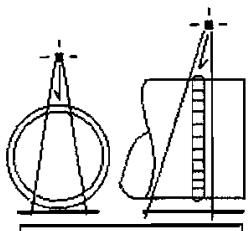

DWE/DWV
DWE/DWV
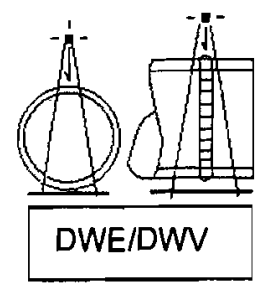


\section{GASPAR, INC. \\ LIQUID PENETRANT INSPECTION REPORT}

DATE: August 15, 2008

JOB \#: 36093B

PT: PROCEDURE: PT-1 REV.7

PENETRANT TYPE : [X] Direct Visual

ACCEPTANCE STANDARD: [X] ASME Sect. VIII Div.I [ ] ASME B31.1 [ ] Other -

MATERIAL IDENTIFICATION

\begin{tabular}{ll}
\hline CLEANER: & SKC-S \\
\hline PENETRANT: & SKL-SP \\
\hline DEVELOPER: & SKD-S
\end{tabular}

LIGHTING EQUIPMENT: Portable AC Halogen single light of 100 watts.

COMPONENT DESCRIPTION / PC. \#: LOWER CHAR POT

DRAWING \#: 36093 REV.2

THICKNESS / DIMENSIONS: SEE DWG.

\begin{tabular}{|c|c|c|c|c|c|}
\hline $\begin{array}{c}\text { WELD ID OR } \\
\text { PART NUMBER }\end{array}$ & TEMP. & SURF. COND. & $\begin{array}{c}\text { ACCEPT/ } \\
\text { REJECT }\end{array}$ & $\begin{array}{c}\text { SIZE AND TYYE } \\
\text { OF INDICATION }\end{array}$ & REMARKS \\
\hline ALL PRESSURE WELDS & $70^{\circ} \mathrm{F}$ & AS WELDED & ACCEPT & NONE & \\
\hline & & & & & \\
\hline & & & & & \\
\hline & & & & & \\
\hline & & & & & \\
\hline & & & & & \\
\hline & & & & & \\
\hline & & & & & \\
\hline
\end{tabular}

ADDITIONAL REMARKS, SKETCHES, ETC.

PERFORMED BY:

JIM FREDERICK/LEVEL II 


\section{CALCULATION PACKAGE}

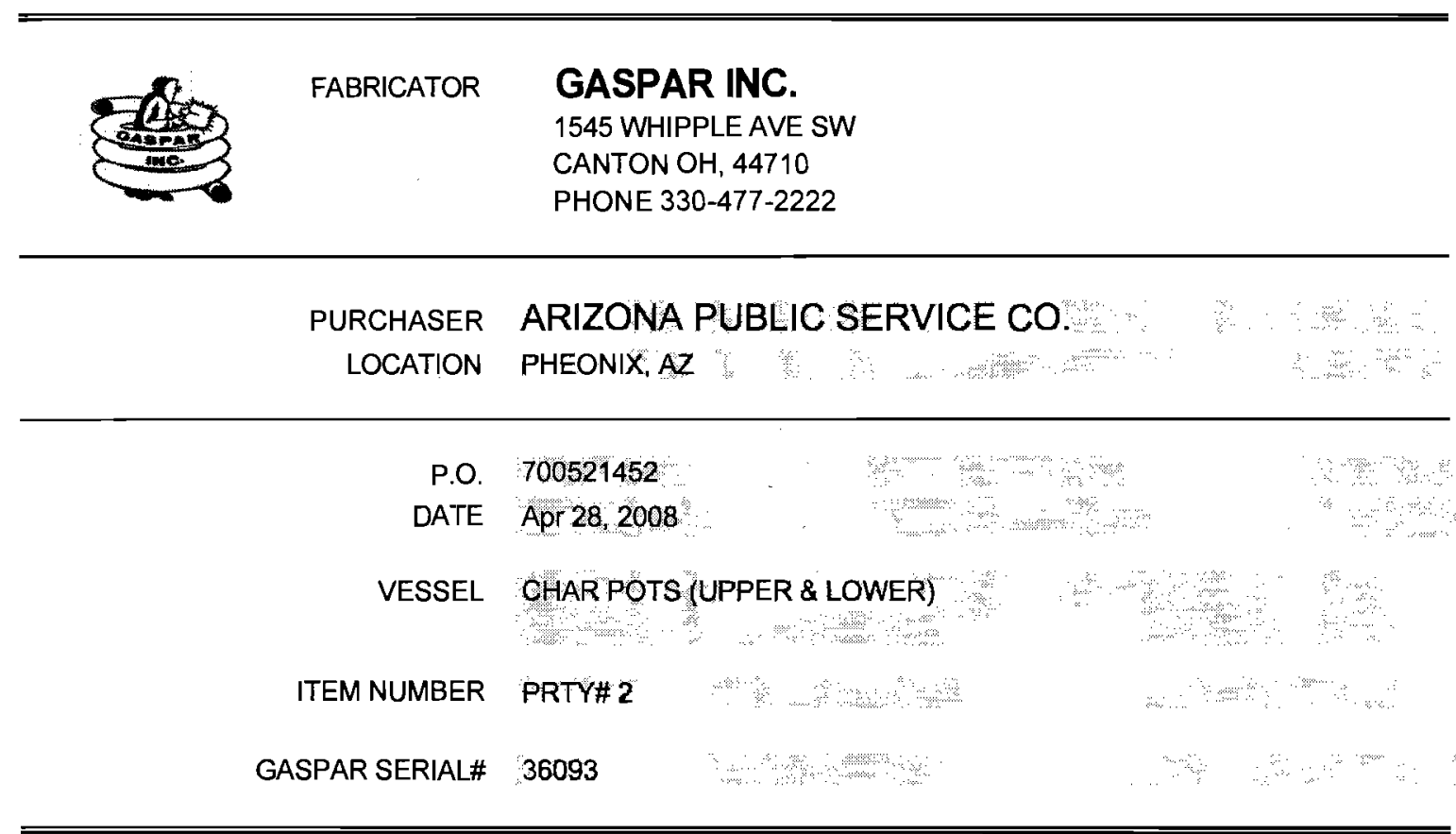

AUG 13, 2008: CHANGED LOWER CHAR POT PRESSURE TEST FROM HYDRO TO PNEUMATIC

AUG 06, 2008: 2 1/2" PIPE 316H MATERIAL CHANGED TO 3" OD INCONEL 600 MATERIAL.

\begin{tabular}{|c|c|c|}
\hline 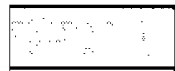 & 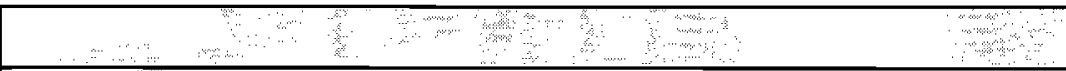 & $m$ \\
\hline$\therefore$ & s.t.n. & 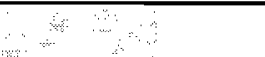 \\
\hline & 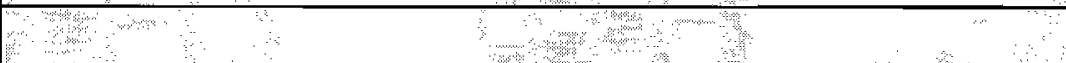 & $=3$ \\
\hline & 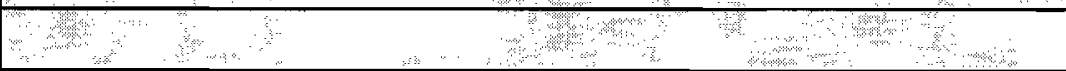 & (n) \\
\hline & 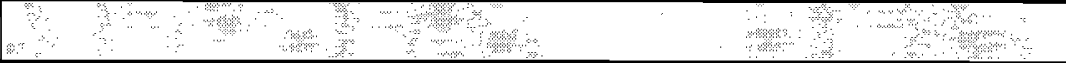 & 3. \\
\hline ste & 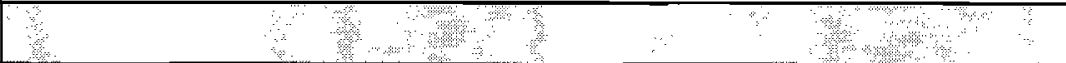 & 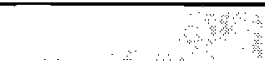 \\
\hline . & S & a \\
\hline 3 & (a) & ?as \\
\hline Aug 14,2008 & कर & Whather $<$ hiteles \\
\hline Aug 6, 2008 & SEE NOTE $\quad \vdots \quad$ (MLM) & s. \\
\hline Jul 31,2008 & CALCULATIONS PER ASME VIII-1 $\quad$ (MLM) & . \\
\hline May 8, 2008 & FOR APPROVAL & \\
\hline DATE & DESCRIPTION & BY \\
\hline
\end{tabular}


Table of Contents

\section{COMPONENTS INCLUDED IN PRESSURE BOUNDARY}

2. UPPER CHAR POT

3. LOWER CHAR POT 


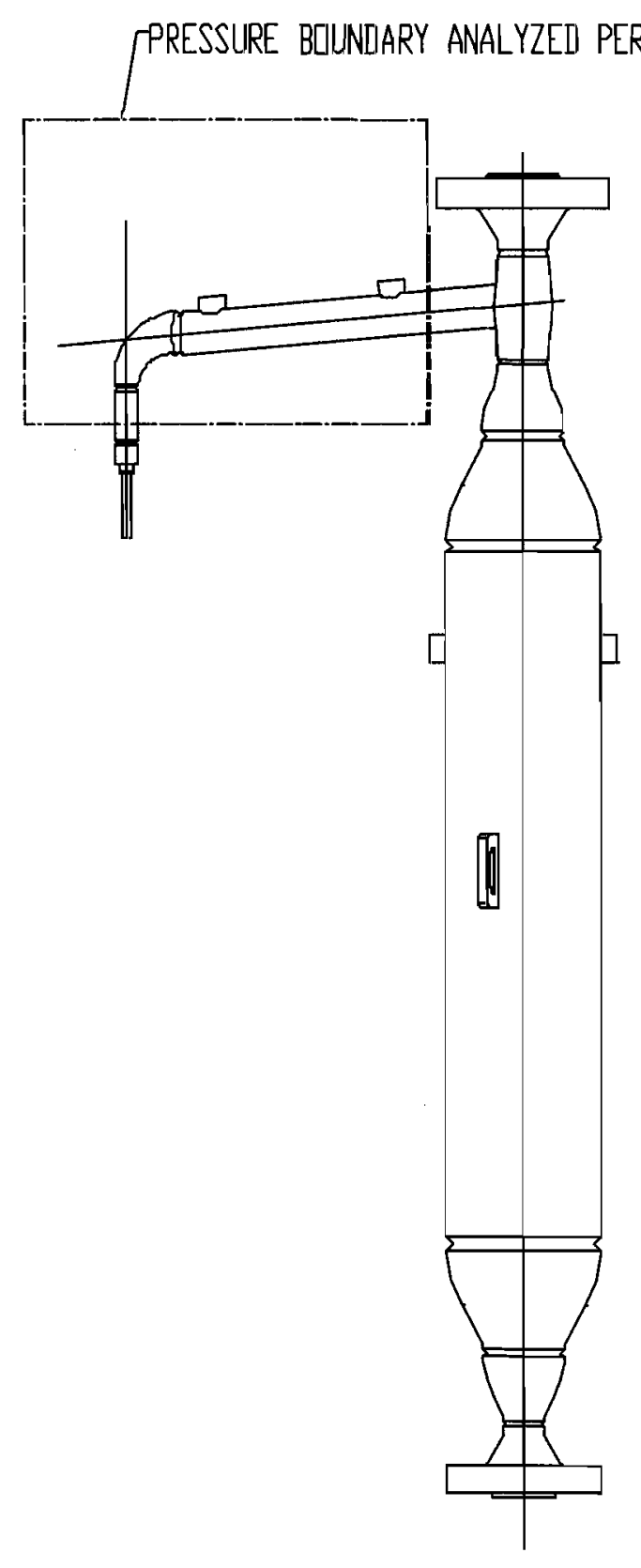

[PRESSURE BIUNDARY- ASME VIII-1

ASME B31,3
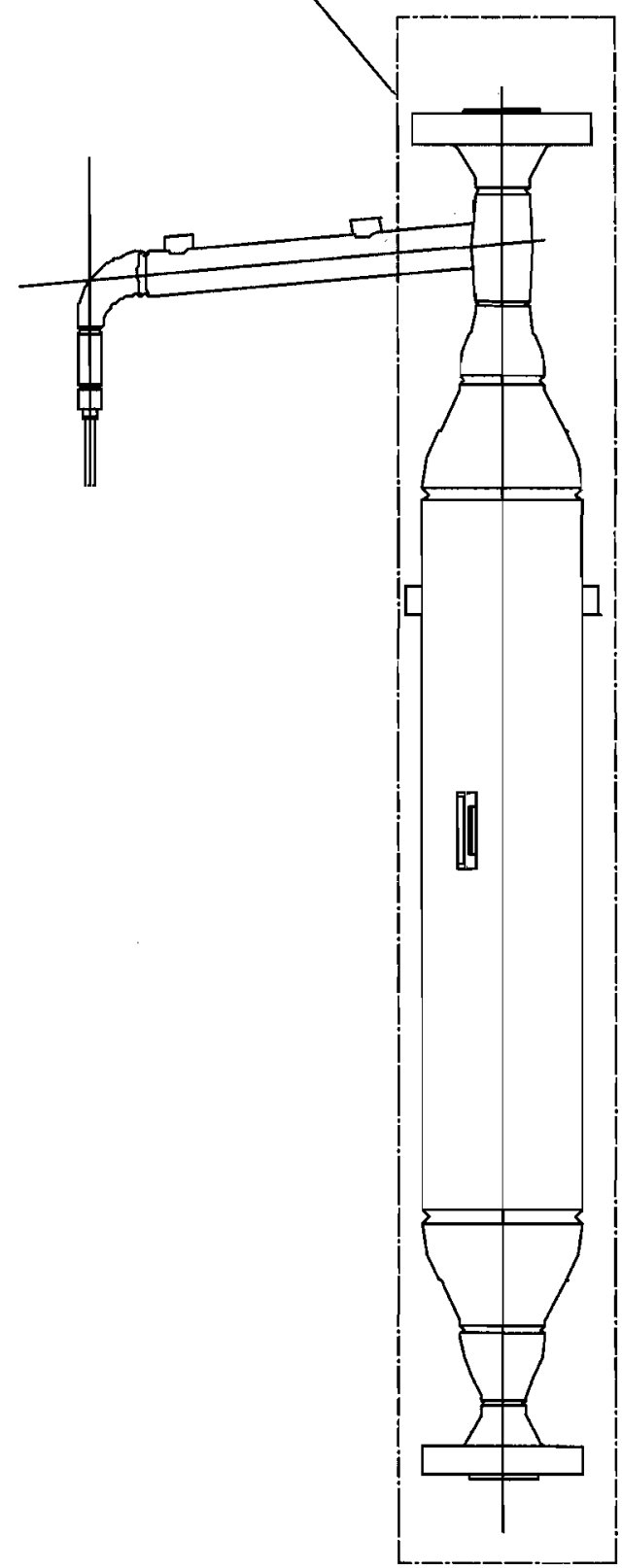


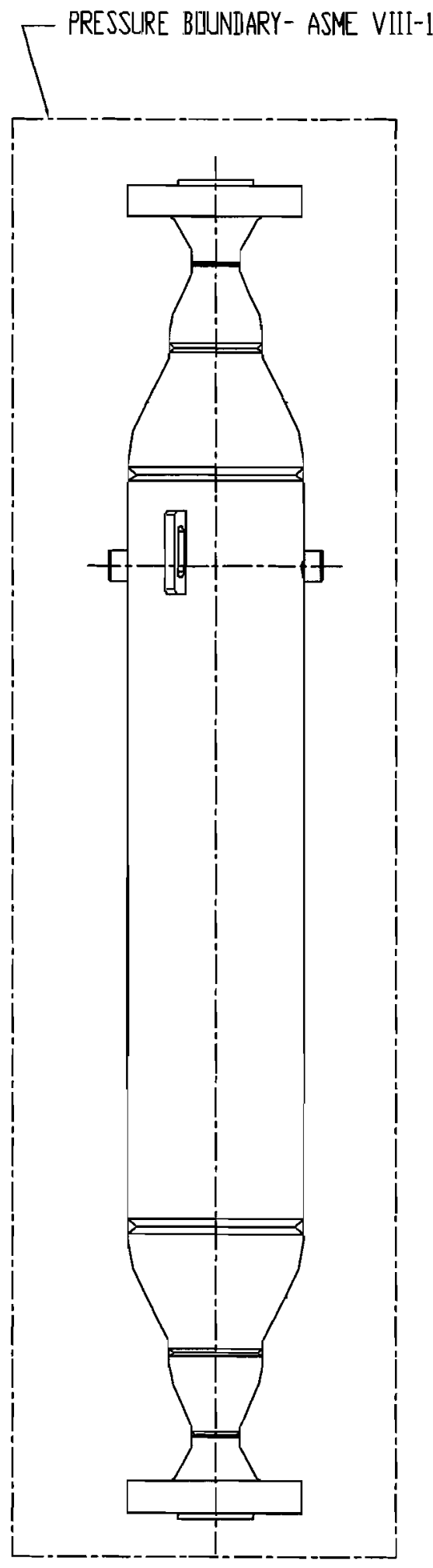




\section{Table Of Contents}

1. Pressure Summary

2. Hydrostatic Test

3. Weight Summary

4. $21 / 2^{\prime \prime}$ WN FLANGE \#1500 (MODIFIED)

5. 3 OD" PIPE

6. $4^{\prime \prime} \times 21 / 2^{\prime \prime}$ REDUCER B 16.9. UPPPER (SCH XS) 2 1/2" END

7. $4^{\prime \prime} \mathrm{X} 21 / 2^{\prime \prime}$ REDUCER B 16.9. UPPER (SCH XS) 4" END

8. 8" X 4" REDUCER B 16.9. UPPER (SCH XS) 4" END

9. 8" X 4" REDUCER B16.9. UPPER (SCH XS) 8" END

10. 8 " PIPE

11. $8^{\prime \prime} X 4^{\prime \prime}$ REDUCER B 16.9. LOWER (SCH XS) 8" END

12. 8" X 4" REDUCER B 16.9. LOWER (SCH XS) 4" END

13. 4" X 2" REDUCER B 16.9. LOWER (SCH XS) 4" END

14. 4" X 2" REDUCER B 16.9. LOWER (SCH XS) 2" END

15. 2" RFWN FLANGE 1500\#

16. 2" BRANCH (B1)

17. 1/2" HALF-COUPLING (7a)

18. 1/2" HALF-COUPLING (7b)

19. Wind Code

20. Seismic Code 


\section{Pressure Summary}

\begin{tabular}{|c|c|c|c|c|c|c|}
\hline Identifier & $\begin{array}{c}P \\
\text { Design } \\
(\mathrm{psi})\end{array}$ & $\begin{array}{c}\mathrm{T} \\
\text { Design } \\
\left({ }^{\circ} \mathrm{F}\right)\end{array}$ & $\begin{array}{c}\text { MAWP } \\
\text { ( psi) }\end{array}$ & $\begin{array}{c}\text { MDMT } \\
\left({ }^{\circ} \mathrm{F}\right)\end{array}$ & $\begin{array}{c}\text { MDMT } \\
\text { Exemption }\end{array}$ & $\begin{array}{l}\text { Impact } \\
\text { Tested }\end{array}$ \\
\hline 3 OD" PIPE & 1,150 & 1,000 & 1,250 & -325 & Note 1 & No \\
\hline 4" X 2 1/2" REDUCER B 16.9. UPPER (SCH XS) $21 / 2 "$ END & 1,150 & 1,000 & $2,397.87$ & -320 & Note 2 & No \\
\hline 4" X 2 1/2" REDUCER B16.2 UPPER (SCH XS) 4" END & 1,150 & 1,000 & $1,891.09$ & -320 & Note 2 & No \\
\hline 8" X 4" REDUCER B16.9. UPPER (SCH XS) 4" END & 1,150 & 1,000 & $1,891.09$ & -320 & Note 2 & No \\
\hline 8" X 4" REDUCER B16.9, UPPER (SCH XS) 8" END & 1,150 & 1,000 & $1,502.53$ & -320 & Note 2 & No \\
\hline 8" PIPE & 1,150 & 1,000 & $1,502.53$ & -320 & Note 2 & No \\
\hline 8" X 4" REDUCER B16.9. LOWER (SCH XS) 8" END & 1,150 & 1,000 & $1,502.53$ & -320 & Note 2 & No \\
\hline 8" X 4" REDUCER B 16.9. LOWER (SCH XS) 4" END & 1,150 & 1,000 & $1,891.09$ & -320 & Note 2 & No \\
\hline 4" X 2" REDUCER B16.9. LOWER (SCH XS) 4" END & 1,150 & 1,000 & $1,891.09$ & -320 & Note 2 & No \\
\hline 4" X 2" REDUCER B16.9. LOWER (SC1I XS) 2" END & 1,150 & 1,000 & $2,193.3$ & -320 & Note 2 & No \\
\hline ASME B16.5/16.47 Blind on 2" RFWN FLANGE 1500\# & 1,150 & 1,000 & 1,820 & -55 & Note 3,4 & No \\
\hline 2 I/2" WN FLANGE \#1500 (MODIFIED) & 1,150 & 1,000 & $1,169.51$ & -320 & Note 2 & No \\
\hline 21/2" WN FLANGE \#1500 (MOD1F1ED) - Flange Hub & 1,150 & 1,000 & $2,075.87$ & -320 & Note 5 & No \\
\hline 2" RFWN FLANGE 1500\# & 1,150 & 1,000 & 1,820 & -55 & Note 3,4 & No \\
\hline 1/2" HALF-COUPLING (7a) & 1,150 & 1,000 & $1,502.52$ & -320 & Note 6 & No \\
\hline 1/2" HALF-COUPLING (7b) & 1,150 & 1,000 & $1,502.52$ & -320 & Note 6 & No \\
\hline 2" BRANCH (B1) & 1,150 & 1,000 & 1,250 & -320 & Note 2 & No \\
\hline
\end{tabular}

Chamber design MDMT is $-20^{\circ} \mathrm{F}$

Chamber rated MDMT is $-55^{\circ} \mathrm{F} @ 1,169.51$ psi

Chamber MAWP was used in the MDMT determination

This pressure chamber is not designed for external pressure.

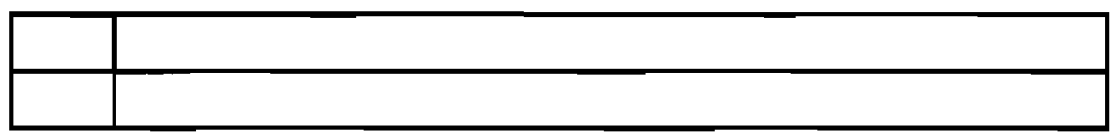

Notes for MDMT Rating:

\begin{tabular}{|r|l|l|}
\hline $\begin{array}{c}\text { Note } \\
\#\end{array}$ & \multicolumn{1}{|c|}{ Exemption } & \multicolumn{1}{|c|}{ Details } \\
\hline I. & Rated MDMT per UNF-65=-325 ${ }^{\circ} \mathrm{F}$ & \\
\hline 2. & $\begin{array}{l}\text { Rated MDMT per UHA }-51(\mathrm{~d})(1)(\mathrm{b}), \text { (carbon content does not exceed } 0.1 \\
\text { percent })=-320^{\circ} \mathrm{F}\end{array}$ & \\
\hline & &
\end{tabular}




\begin{tabular}{|r|l|l|}
\hline 3. & Per UHA -51 $(\mathrm{d})(1)(\mathrm{b})$ & \\
\hline 4. & Flange rated MDMT $=-320^{\circ} \mathrm{F}$ & $\begin{array}{l}\text { Bolts rated MDMT per Fig UCS-66 note }(\mathrm{e}) \\
=-55^{\circ} \mathrm{F}\end{array}$ \\
\hline 5. & Impact test exempt per UHA-51(g)(coincident ratio $=0.33389)$ & \\
\hline 6. & Impact test exempt per UHA-51 $(\mathrm{g})($ coincident ratio $=0.0909)$ & \\
\hline
\end{tabular}




\section{Hydrostatic Test}

Shop test pressure determination based on design P per UG-99(b)

Shop hydrostatic test gauge pressure is $1,495 \mathrm{psi}$ at $70^{\circ} \mathrm{F}$ (the chamber design $\mathrm{P}=1,150 \mathrm{psi}$ )

The shop test is performed with the vessel in the horizontal position.

\begin{tabular}{|l|r|r|r|r|}
\hline \multicolumn{1}{|c|}{ Identifier } & $\begin{array}{c}\text { Local test } \\
\text { pressure } \\
\text { psi }\end{array}$ & $\begin{array}{c}\text { Test liquid } \\
\text { static head } \\
\text { psi }\end{array}$ & $\begin{array}{c}\text { UG-99 } \\
\text { stress } \\
\text { ratio }\end{array}$ & $\begin{array}{c}\text { UG-99 } \\
\text { pressure } \\
\text { factor }\end{array}$ \\
\hline 3 OD" PIPE & $1,495.232$ & 0.232 & 3.2714 & 1.30 \\
\hline 4" X 2 1/2" REDUCER B16.9, UPPER (SCH XS) 2 1/2" END & $1,495.233$ & 0.233 & 1.3072 & 1.30 \\
\hline 4" X 2 1/2" REDUCER B16.9, UPPER (SCH XS) 4" END & $1,495.26$ & 0.26 & 1.3072 & 1.30 \\
\hline 8" X 4" REDUCER B16.9, UPPER (SCH XS) 4" END & $1,495.26$ & 0.26 & 1.3072 & 1.30 \\
\hline 8" X 4" REDUCER B16.9, UPPER (SCH XS) 8" END & $1,495.329$ & 0.329 & 1.3072 & 1.30 \\
\hline 8" PIPE & $1,495.327$ & 0.327 & 1.3072 & 1.30 \\
\hline 8" X 4" REDUCER B16.9, LOWER (SCH XS) 8" END & $1,495.329$ & 0.329 & 1.3072 & 1.30 \\
\hline 8" X 4" REDUCER B16.9, LOWER (SCH XS) 4" END & $1,495.26$ & 0.26 & 1.3072 & 1.30 \\
\hline 4" X 2" REDUCER B16.9, LOWER (SCH XS) 4"END & $1,495.26$ & 0.26 & 1.3072 & 1.30 \\
\hline 4" X 2" REDUCER B16.9, LOWER (SCH XS) 2" END & $1,495.225$ & 0.226 & 1.3072 & 1.30 \\
\hline 2" RFWN FLANGE 1500\# & $1,495.227$ & 0.227 & N/A & 1.30 \\
\hline 2 1/2" WN FLANGE \#1500 (MODIFIED) (1) & $1,495.231$ & 0.231 & 1 & 1.30 \\
\hline 1/2" HALF-COUPLING (7a) & $1,495.034$ & 0.034 & 1.7699 & 1.30 \\
\hline 1/2" HALF-COUPLING (7b) & $1,495.379$ & 0.379 & 1.7699 & 1.30 \\
\hline 2" BRANCH (B1) & $1,495.88$ & 0.88 & 1.3072 & 1.30 \\
\hline
\end{tabular}

Notes:

(1) 2 1/2" WN FLANGE \#1500 (MODIFIED) limits the UG-99 stress ratio.

(2) The zero degree angular position is assumed to be up, and the test liquid height is assumed to the top-most flange. 


\section{Weight Summary}

\begin{tabular}{|c|c|c|c|c|c|c|c|}
\hline \multirow[b]{2}{*}{ Component } & \multicolumn{7}{|c|}{ Weight ( Ib) Contributed by Vessel Elements } \\
\hline & $\begin{array}{l}\text { Metal } \\
\text { New* }\end{array}$ & $\begin{array}{c}\text { Metal } \\
\text { Corroded * }\end{array}$ & $\begin{array}{c}\text { Insulation } \\
\& \\
\text { Supports }\end{array}$ & Lining & $\begin{array}{c}\text { Piping } \\
+ \\
\text { Liquid }\end{array}$ & $\begin{array}{c}\text { Operating } \\
\text { Liquid }\end{array}$ & $\begin{array}{c}\text { Test } \\
\text { Liquid }\end{array}$ \\
\hline $30 D^{\prime \prime}$ PIPE & 4.4 & 4.1 & 0 & 0 & 0 & 0 & 3.5 \\
\hline $\begin{array}{l}4 " \times 2 \text { 1/2" REDUCER B16.9. UPPER (SCHXS) } 2 \text { 1/2" } \\
\text { END }\end{array}$ & 1.2 & 1 & 0 & 0 & 0 & 0 & 0.3 \\
\hline 4" X 2 1/2" REDUCER B16.9. UPPER (SCH XS) 4" END & 2.3 & 2 & 0 & 0 & 0 & 0 & 0.9 \\
\hline 8" X 4" REDUCER B 16.9. UPPER (SCH XS) 4" END & 3.4 & 3.1. & 0 & 0 & 0 & 0 & 1.3 \\
\hline 8" X 4" REDUCER B16.9. UPPER (SCH XS) 8" END & 9.8 & 9.2 & 0 & 0 & 0 & 0 & 5.1 \\
\hline$\underline{8 " \mathrm{PIPE}}$ & 140.1 & 132.2 & 0 & 0 & 0 & 0 & 62.7 \\
\hline 8" X 4" REDUCER B16.9, LOWER (SCH XS) 8" END & 9.8 & 9.2 & 0 & 0 & 0 & 0 & 5.1 \\
\hline 8" X 4" REDUCER B 16.9, LOWER (SCH XS) 4" END & 3.4 & 3.1 & 0 & 0 & 0 & 0 & 1.3 \\
\hline 4" X 2" REDUCER B 16.9. LOWER (SCH XS) 4" END & 2.3 & 2 & 0 & 0 & 0 & 0 & 0.9 \\
\hline 4" X 2" REDUCER B 16.9, LOWER (SCH XS) 2" END & 0.8 & 0.6 & 0 & 0 & 0 & 0 & 0.2 \\
\hline ASME B16.5/16.47 Blind on 2" RFWN FLANGE 1500\# & 32 & 32 & 0 & 0 & 0 & 0 & 0 \\
\hline Support Skirt \#1 & 3.8 & 3.8 & 0 & 0 & 0 & 0 & 0 \\
\hline TOTAL: & 213.2 & 202.3 & 0 & $\mathbf{0}$ & $\mathbf{0}$ & 0 & 81.2 \\
\hline
\end{tabular}

* Shells with attached nozzles have weight reduced by material cut out for opening.

\begin{tabular}{|c|c|c|c|c|c|c|c|c|c|}
\hline \multirow{3}{*}{ Component } & \multicolumn{9}{|c|}{ Weight ( lb) Contributed by Attachments } \\
\hline & \multicolumn{2}{|c|}{ Body Flanges } & \multicolumn{2}{|c|}{$\begin{array}{l}\text { Nozzles \& } \\
\text { Flanges }\end{array}$} & \multirow{2}{*}{$\begin{array}{l}\text { Packed } \\
\text { Beds }\end{array}$} & \multirow{2}{*}{$\begin{array}{c}\text { Ladders } \\
\& \\
\text { Platforms }\end{array}$} & \multirow{2}{*}{$\begin{array}{l}\text { Trays \& } \\
\text { Supports }\end{array}$} & \multirow{2}{*}{$\begin{array}{c}\text { Rings } \\
\& \\
\text { Clips }\end{array}$} & \multirow{2}{*}{$\begin{array}{c}\text { Vertical } \\
\text { Loads }\end{array}$} \\
\hline & New & Corroded & New & Corroded & & & & & \\
\hline 3 OD" PIPE & 35.9 & 35.6 & 7.9 & 6.9 & 0 & 0 & 0 & 0 & 0 \\
\hline $\begin{array}{l}4 " \mathrm{X} 2 \text { 1/2" REDUCER B 16.9, UPPER (SCH } \\
\mathrm{XS} 21 / 2 " \mathrm{END}\end{array}$ & 0 & 0 & 0 & 0 & 0 & 0 & 0 & 0 & 0 \\
\hline $\begin{array}{l}\text { 4"X } 2 \text { 1/2" REDUCER B 16.9. UPPER (SCH } \\
\text { XS) 4" END }\end{array}$ & 0 & 0 & 0 & 0 & 0 & 0 & 0 & 0 & 0 \\
\hline $\begin{array}{l}8^{\prime \prime} \times 4^{\prime \prime} \text { REDUCER B } 16.9 \text {. UPPER (SCH XS) } \\
4^{\prime \prime} \mathrm{END}\end{array}$ & 0 & 0 & 0 & 0 & 0 & 0 & 0 & 0 & 0 \\
\hline $\begin{array}{l}\text { 8"X 4" REDUCER B 16.9. UPPER (SCH XS) } \\
8 \text { " END }\end{array}$ & 0 & 0 & 0 & 0 & 0 & 0 & 0 & 0 & 0 \\
\hline 8" PIPE & 0 & 0 & 0.7 & 0.7 & 0 & 0 & 0 & 0 & 0 \\
\hline $\begin{array}{l}\text { 8" X 4" REDUCER B16.9. LOWER (SCH } \\
\text { XS) 8" END }\end{array}$ & 0 & 0 & 0 & 0 & 0 & 0 & 0 & 0 & 0 \\
\hline $\begin{array}{l}\text { 8" X 4" REDUCER B16.9, LOWER (SCH } \\
\text { XS) 4" END }\end{array}$ & 0 & 0 & 0 & 0 & 0 & 0 & 0 & 0 & 0 \\
\hline $\begin{array}{l}\text { 4" X 2" REDUCER B16.9, LOWER (SCH } \\
\text { XS) 4" END }\end{array}$ & 0 & 0 & 0 & 0 & 0 & 0 & 0 & 0 & 0 \\
\hline $\begin{array}{l}\text { 4" X 2" REDUCER B16.9. LOWER (SCH } \\
\text { XS) 2" END }\end{array}$ & 25 & 25 & 0 & 0 & 0 & 0 & 0 & 0 & 0 \\
\hline $\begin{array}{l}\text { ASME B } 16.5 / 16.47 \text { Blind on 2" RFWN } \\
\text { FLANGE } 1500 \#\end{array}$ & 0 & 0 & 0 & 0 & 0 & 0 & 0 & 0 & 0 \\
\hline Support Skirt \#1 & 0 & 0 & 0 & 0 & 0 & 0 & 0 & 0 & 0 \\
\hline TOTAL: & 60.9 & 60.6 & 8.6 & 7.6 & $\mathbf{0}$ & 0 & $\mathbf{0}$ & 0 & $\mathbf{0}$ \\
\hline
\end{tabular}


Vessel operating weight, Corroded: $270 \mathrm{lb}$

Vessel operating weight, New: $283 \mathrm{lb}$

Vessel empty weight, Corroded: $270 \mathrm{lb}$

Vessel empty weight, New: $\quad 283 \mathrm{lb}$

Vessel test weight, New: $\quad 364 \mathrm{lb}$

\section{Vessel center of gravity location - from datum - lift condition}

Vessel Lift Weight, New:283 lb

Center of Gravity: $\quad 36.285$ I" $^{\prime \prime}$

\section{Vessel Capacity}

Vessel Capacity** (New): 9 US gal

Vessel Capacity** (Corroded): 10 US gal

${ }^{* *}$ The vessel capacity does not include volume of nozzle, piping or other attachments. 


\section{1/2" WN FLANGE \#1500 (MODIFIED)}

\section{ASME VIII-1, 2007 Edition, Appendix 2 Flange Calculations}

Flange is attached to:

Flange type:

Flange material specification:

Bolt material specification:

Bolt Description:

Internal design pressure, $\mathrm{P}$ :

Required flange thickness: $t_{r}=$

Maximum allowable working pressure, MAWP:

Corrosion allowance:

Bolt corrosion (root), $\mathrm{C}_{\text {bolt }}$ :

Design MDMT:

Rated MDMT:

Estimated weight:

Flange dimensions, new
3 OD" PIPE (Top)

Weld neck integral

SA-182 F3 16H $<=5$ (low stress) (II-D

p. $74, \ln .32)$

SA-193 B8 2 Bolt $(3 / 4<t<=1$ ) (II-D

p. $356, \ln .27$ )

1 in Coarse Thread

1,150 psi@1,000F

1.5402 in

1,169.51 psi @ $1,000^{\circ} \mathrm{F}$

Bore $=0.03$ in

Flange $=0$ in

0 in

$-20^{\circ} \mathrm{F}$

$-320^{\circ} \mathrm{F}$

New $=35.9 \mathrm{lb}$
No impact test performed

Flange material is not normalized Material is not produced to fine grain practice

PWHT is not performed corroded $=35.6 \mathrm{lb}$ 


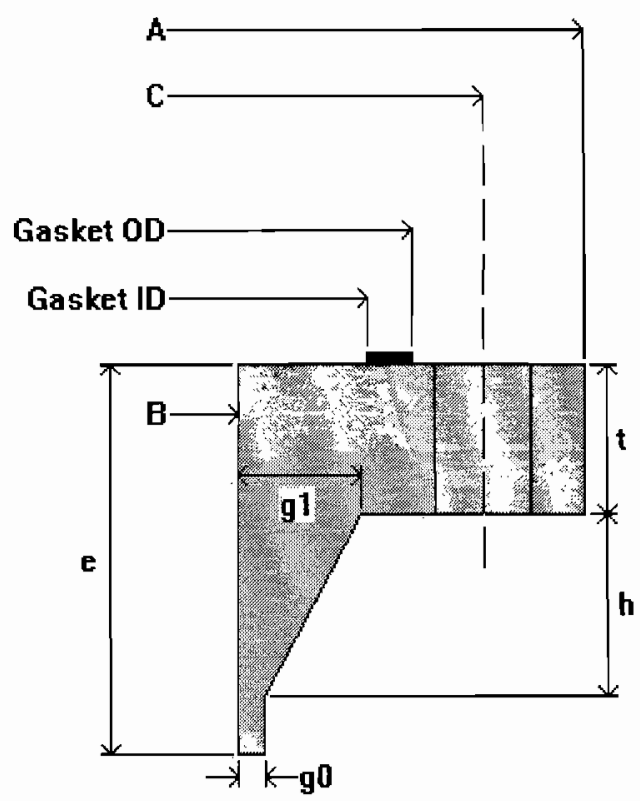

$$
\begin{aligned}
\text { flange OD A } & =9.625 \mathrm{in} \\
\text { bolt circle } \mathrm{C} & =7.5 \mathrm{in} \\
\text { gasket OD } & =5.988 \mathrm{in} \\
\text { gasket ID } & =5.088 \mathrm{in} \\
\text { flange ID B } & =2.323 \mathrm{in} \\
\text { thickness } \mathrm{t} & =1.55 \mathrm{in} \\
\text { bolting } & =8.1 \mathrm{in} \mathrm{dia}
\end{aligned}
$$

hub thickness $\mathrm{g}_{1}=1.3028$ in

hub thickness $\mathrm{g}_{0}=0.276$ in

hub length $\mathrm{h}=1.876 \mathrm{in}$

length $\mathrm{e}=4.0404$ in

gasket factor $\mathrm{m}=3$

seating stress y $=10,000.00 \mathrm{psi}$

Note: this flange is calculated as an integraltype.

\section{Flange calculations for Internal Pressure + Wind}

\section{Gasket details from facing sketch 1(a) or (b), Column II}

Gasket width $N=0.45$ in

$\mathrm{b}_{0}=\mathrm{N} / 2=0.225 \mathrm{in}$

Effective gasket seating width, $b=b_{0}=0.225$ in

$\mathrm{G}=(\mathrm{OD}$ of contact face + gasket ID $) / 2=5.538 \mathrm{in}$

$\mathrm{h}_{\mathrm{G}}=(\mathrm{C}-\mathrm{G}) / 2=(7.5-5.538) / 2=0.981$ in

$\mathrm{h}_{\mathrm{D}}=\mathrm{R}+\mathrm{g}_{1} / 2=1.2857+1.2728 / 2=1.9221 \mathrm{in}$

$\mathrm{h}_{\mathrm{T}}=\left(\mathrm{R}+\mathrm{g}_{1}+\mathrm{h}_{\mathrm{G}}\right) / 2=(1.2857+1.2728+0.981) / 2=1.7698$ in

$\mathrm{H}_{\mathrm{p}}=2 * \mathrm{~b} * 3.14 * \mathrm{G} * \mathrm{~m} * \mathrm{P}$

$=2 * 0.225 * 3.14 * 5.538 * 3 * 1,150$

$=26,996.92 \mathrm{lb}_{\mathrm{f}}$

$\mathrm{H}=0.785^{*} \mathrm{G}^{2 * \mathrm{P}}$

$=0.785^{*} 5.538^{2 *} 1,150$ 


$$
\begin{aligned}
& =27,686.84 \mathrm{lb}_{\mathrm{f}} \\
& \mathrm{H}_{\mathrm{D}}=0.785^{*} \mathrm{~B}^{2 * \mathrm{P}} \\
& =0.785 * 2.383^{2 * 1,150} \\
& =5,126.44 \mathrm{lb}_{\mathrm{f}} \\
& \mathrm{H}_{\mathrm{T}}=\mathrm{H}-\mathrm{H}_{\mathrm{D}} \\
& =27,686.84-5,126.44 \\
& =22,560.41 \mathrm{lb} \\
& \mathrm{W}_{\mathrm{m} 1}=\mathrm{H}+\mathrm{H}_{\mathrm{p}} \\
& =27,686.84+26,996.92 \\
& =54,683.77 \mathrm{lb}_{\mathrm{f}} \\
& \mathrm{W}_{\mathrm{m} 2}=3.14 * \mathrm{~b}^{*} \mathrm{G}^{*} \mathrm{y} \\
& =3.14 * 0.225 * 5.538 * 10,000 \\
& =39,125.98 \mathrm{lb}_{\mathrm{f}}
\end{aligned}
$$

Required bolt area, $A_{m}=$ greater of $A_{m 1}, A_{m 2}=2.734188 \mathrm{in}^{2}$

$$
\mathrm{A}_{\mathrm{ml}}=\mathrm{W}_{\mathrm{ml}} / \mathrm{S}_{\mathrm{b}}=54,683.77 / 20,000=2.7342 \mathrm{in}^{2}
$$

$$
\mathrm{A}_{\mathrm{m} 2}=\mathrm{W}_{\mathrm{m} 2} / \mathrm{S}_{\mathrm{a}}=39,125.98 / 20,000=1.9563 \mathrm{in}^{2}
$$

Total area for 8- 1 in dia bolts, corroded, $\mathrm{A}_{\mathrm{b}}=4.408 \mathrm{in}^{2}$

$$
\begin{aligned}
& \mathrm{W}=\left(\mathrm{A}_{\mathrm{m}}+\mathrm{A}_{\mathrm{b}}\right)^{*} \mathrm{~S}_{\mathrm{a}} / 2 \\
& =(2.7342+4.408)^{*} 20,000 / 2 \\
& =71,421.88 \mathrm{lb}_{\mathrm{f}} \\
& \mathrm{M}_{\mathrm{D}}=\mathrm{H}_{\mathrm{D}}{ }^{*} \mathrm{~h}_{\mathrm{D}}=5,126.44^{*} 1.9221=9,853.5 \mathrm{lb}-\mathrm{in} \\
& \mathrm{M}_{\mathrm{T}}=\mathrm{H}_{\mathrm{T}}{ }^{*} \mathrm{~h}_{\mathrm{T}}=22,560.41 * 1.7698=39,926.3 \mathrm{lb} \text {-in } \\
& \mathrm{H}_{\mathrm{G}}=\mathrm{W}_{\mathrm{m} 1}-\mathrm{H}=54,683.77-27,686.84=26,996.92 \mathrm{lb}_{\mathrm{f}} \\
& \mathrm{M}_{\mathrm{G}}=\mathrm{H}_{\mathrm{G}}{ }^{*} \mathrm{~h}_{\mathrm{G}}=26,996.92 * 0.981=26,484 \mathrm{lb}-\mathrm{in} \\
& \mathrm{M}_{\mathrm{o}}=\mathrm{M}_{\mathrm{D}}+\mathrm{M}_{\mathrm{T}}+\mathrm{M}_{\mathrm{G}}=9,853.5+39,926.3+26,484=76,263.8 \mathrm{lb}-\mathrm{in} \\
& \mathrm{M}_{\mathrm{g}}=\mathrm{W}^{*} \mathrm{~h}_{\mathrm{G}}=71,421.88 * 0.981=70,064.9 \mathrm{lb}-\mathrm{in}
\end{aligned}
$$




\section{Hub and Flange Factors}

$\mathrm{h}_{0}=\left(\mathrm{B}^{*} \mathrm{~g}_{0}\right)^{1 / 2}=(2.383 * 0.246)^{1 / 2}=0.7656$ in

From FIG. 2-7.1, where $\mathrm{K}=\mathrm{A} / \mathrm{B}=9.625 / 2.383=4.039$

$$
\begin{array}{lll}
\mathrm{T}=1.003 & \mathrm{Z}=1.1306 & \mathrm{Y}=1.4349 \quad \mathrm{U}=1.5768 \\
\mathrm{~h} / \mathrm{h}_{0}=2.4502 & \mathrm{~g}_{1} / \mathrm{g}_{0}=5.174 \\
\mathrm{~F}=0.4731 & \mathrm{~V}=0.0152 \quad \mathrm{e}=\mathrm{F} / \mathrm{h}_{0}=0.6179 \\
\mathrm{~d}=(\mathrm{U} / \mathrm{V})^{*} \mathrm{~h}_{0}{ }^{*} \mathrm{~g}_{0}{ }^{2}=(1.576849 / 0.0152)^{*} 0.7656^{*} 0.246^{2} \\
=4.8108 \mathrm{in}^{3}
\end{array}
$$

\section{Stresses at operating conditions - VIII-1, Appendix 2-7}

$\mathrm{f}=1$

$\mathrm{L}=\left(\mathrm{t}^{*} \mathrm{e}+1\right) / \mathrm{T}+\mathrm{t}^{3} / \mathrm{d}$

$=\left(1.55^{*} 0.6179+1\right) / 1.003027+1.55^{3} / 4.8108$

$=2.725902$

$\mathrm{S}_{\mathrm{H}}=\mathrm{f}^{*} \mathrm{M}_{\mathrm{o}} /\left(\mathrm{L} * \mathrm{~g}_{1}{ }^{2 *} \mathrm{~B}\right)$

$=1 * 76,263.8 /\left(2.725902 * 1.2728^{2 *} 2.383\right)$

$=7,247 \mathrm{psi}$

$\mathrm{S}_{\mathrm{R}}=(1.33 * \mathrm{t} * \mathrm{e}+1) * \mathrm{M}_{\mathrm{o}} /\left(\mathrm{L} * \mathrm{t}^{2} * \mathrm{~B}\right)$

$=\left(1.33^{*} 1.55^{*} 0.6179+1\right) * 76,263.8 /\left(2.725902 * 1.55^{2 *} 2.383\right)$

$=11,112 \mathrm{psi}$

$\mathrm{S}_{\mathrm{T}}=\mathrm{Y}^{*} \mathrm{M}_{\mathrm{o}} /\left(\mathrm{t}^{2 * \mathrm{~B}}\right)-\mathrm{Z} * \mathrm{~S}_{\mathrm{R}}$

$=1.4349 * 76,263.8 /\left(1.55^{2} * 2.383\right)-1.1306 * 11,112$

$=6,552 \mathrm{psi}$

Allowable stress $\mathrm{S}_{\mathrm{fo}}=11,300 \mathrm{psi}$

Allowable stress $\mathrm{S}_{\text {no }}=7,000 \mathrm{psi}$

$\mathrm{S}_{\mathrm{T}}$ does not exceed $\mathrm{S}_{\mathrm{fo}_{\mathrm{o}}}$

$\mathrm{S}_{\mathrm{H}}$ does not exceed $\operatorname{Min}\left[1.5 * \mathrm{~S}_{\mathrm{fo}}, 2.5 * \mathrm{~S}_{\mathrm{no}}\right]=16,950 \mathrm{psi}$

$\mathrm{S}_{\mathrm{R}}$ does not exceed $\mathrm{S}_{\mathrm{fo}}$

$0.5\left(\mathrm{~S}_{\mathrm{H}}+\mathrm{S}_{\mathrm{R}}\right)=9,179$ psi does not exceed $\mathrm{S}_{\mathrm{fo}}$

$0.5\left(\mathrm{~S}_{\mathrm{H}}+\mathrm{S}_{\mathrm{T}}\right)=6,899$ psi does not exceed $\mathrm{S}_{\text {fo }}$

Stresses at gasket seating - VIII -1, Appendix 2-7

$$
\begin{aligned}
& \mathrm{S}_{\mathrm{H}}=\mathrm{f}^{*} \mathrm{M}_{\mathrm{g}} /\left(\mathrm{L}^{*} \mathrm{~g}_{1}{ }^{2 * \mathrm{~B})}\right. \\
& =1 * 70,064.9 /\left(2.725902 * 1.2728^{2 *} 2.383\right)
\end{aligned}
$$




$$
\begin{aligned}
& =6,658 \mathrm{psi} \\
& \mathrm{S}_{\mathrm{R}}=\left(1.33^{*} \mathrm{t}^{*} \mathrm{e}+1\right)^{*} \mathrm{M}_{\mathrm{g}} /\left(\mathrm{L}^{*} \mathrm{t}^{2 * \mathrm{~B}}\right) \\
& =\left(1.33^{*} 1.55^{*} 0.6179+1\right)^{*} 70,064.9 /\left(2.725902 * 1.55^{2 * 2.383)}\right. \\
& =10,208 \mathrm{psi} \\
& \mathrm{S}_{\mathrm{T}}=\mathrm{Y}^{*} \mathrm{M}_{\mathrm{g}} /\left(\mathrm{t}^{2 *} \mathrm{~B}\right)-\mathrm{Z}^{*} \mathrm{~S}_{\mathrm{R}} \\
& =1.4349^{*} 70,064.9 /\left(1.55^{2 *} 2.383\right)-1.1306^{*} 10,208 \\
& =6,019 \mathrm{psi}
\end{aligned}
$$

Allowable stress $\mathrm{S}_{\mathrm{fa}}=20,000 \mathrm{psi}$

Allowable stress $\mathrm{S}_{\mathrm{na}}=22,900 \mathrm{psi}$

$\mathrm{S}_{\mathrm{T}}$ does not exceed $\mathrm{S}_{\mathrm{fa}}$

$\mathrm{S}_{\mathrm{H}}$ does not exceed $\operatorname{Min}\left[1.5^{*} \mathrm{~S}_{\mathrm{fa}}, 2.5^{*} \mathrm{~S}_{\mathrm{na}}\right]=30,000 \mathrm{psi}$

$\mathrm{S}_{\mathrm{R}}$ does not exceed $\mathrm{S}_{\mathrm{fa}}$

$0.5\left(\mathrm{~S}_{\mathrm{H}}+\mathrm{S}_{\mathrm{R}}\right)=8,433$ psi does not exceed $\mathrm{S}_{\mathrm{fa}}$

$0.5\left(\mathrm{~S}_{\mathrm{H}}+\mathrm{S}_{\mathrm{T}}\right)=6,339$ psi does not exceed $\mathrm{S}_{\text {fa }}$

\section{Flange rigidity per VIII-1, Appendix 2-14}

$$
\begin{aligned}
& \mathrm{J}=52.14 * \mathrm{~V}^{*} \mathrm{M}_{\mathrm{o}} /\left(\mathrm{L}^{*} \mathrm{E}^{*} \mathrm{~g}_{0}{ }^{2 * \mathrm{~K}_{\mathrm{I}}} \mathrm{h}_{0}\right) \\
& =52.14 * 0.0152 * 76,263.8 /\left(2.7259 * 22,800,000 * 0.246^{2 *} 0.3 * 0.7656\right) \\
& =0.06990274
\end{aligned}
$$

The flange rigidity index $\mathbf{J}$ does not exceed 1 ; satisfactory.

\section{1/2" WN FLANGE \#1500 (MODIFIED) - Flange hub}

\section{ASME Section VIII Division 1, 2007 Edition}

Component:

Material specification:

Impact test exempt per UHA $-51(\mathrm{~g})($ coincident ratio $=0.33389)$

Internal design pressure: $\mathrm{P}=1,150 \mathrm{psi} @ 1,000^{\circ} \mathrm{F}$

\section{Static liquid head:}

Not Considered

Corrosion allowance Inner $\mathrm{C}=0.03 "$

$$
\text { Outer } \mathrm{C}=0 "
$$

Design MDMT $=-20^{\circ} \mathrm{F}$

Rated MDMT $=-320^{\circ} \mathrm{F}$

No impact test performed Material is not normalized

Material is not produced to Fine Grain Practice PWHT is not performed

Radiography: Longitudinal joint - $\quad$ Seamless No RT 
Top circumferential joint - N/A

Bottom circumferential joint -

Full UW-11(a) Type 1

Estimated weight New $=1.1 \mathrm{lb} \quad$ corr $=1 \mathrm{lb}$

Capacity $\quad$ New $=0.03$ US gal corr $=0.04$ US gal

$\mathrm{OD} \quad=2.875^{\prime \prime}$

Length $\mathrm{L}_{\mathrm{c}}=1.876^{\prime \prime}$

$\mathrm{t} \quad=0.276^{\prime \prime}$

Design thickness, (at $\left.1,000{ }^{\circ} \mathrm{F}\right)$ Appendix 1-1

$\mathrm{t}=\mathrm{P}^{*} \mathrm{R}_{\mathrm{o}} /\left(\mathrm{S} * \mathrm{E}+0.40^{*} \mathrm{P}\right)+$ Corrosion

$=1,150 * 1.4375 /(11,300 * 1.00+0.40 * 1,150)+0.03$

$=0.1706^{\prime \prime}$

Maximum allowable working pressure, $\left(\right.$ at $\left.^{1,000}{ }^{\circ} \mathrm{F}\right)$ Appendix 1-1

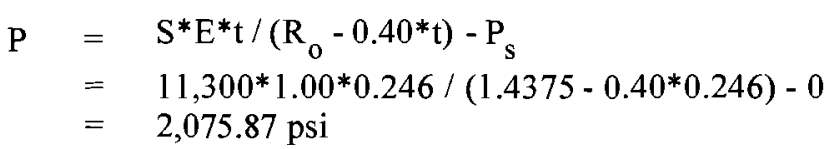

\% Forming Strain - UHA-44(a)(2)(a)

$$
\begin{aligned}
\mathrm{EFE} & =\left(50 * t / \mathrm{R}_{\mathrm{f}}\right) *\left(1-\mathrm{R}_{\mathrm{f}} / \mathrm{R}_{\mathrm{o}}\right) \\
& =(50 * 0.276 / 1.2995) *(1-1.2995 / \infty) \\
& =10.6195 \%
\end{aligned}
$$

Design thickness $=0.1706^{\prime \prime}$

The governing condition is due to internal pressure.

\begin{tabular}{|c|c|c|c|c|c|c|c|c|}
\hline \multirow[t]{2}{*}{ Condition } & \multirow{2}{*}{$\begin{array}{l}\text { Pressure } \\
\text { P ( psi) }\end{array}$} & \multicolumn{2}{|c|}{$\begin{array}{c}\text { Allowable Stress } \\
\text { Before UG-23 Stress } \\
\text { Increase ( psi) }\end{array}$} & \multirow{2}{*}{$\begin{array}{c}\text { Temperature } \\
\left({ }^{\circ} \mathrm{F}\right)\end{array}$} & \multirow{2}{*}{$\begin{array}{c}\text { Corrosion } \\
\mathrm{C} \text { (in) }\end{array}$} & \multirow[t]{2}{*}{ Load } & \multirow{2}{*}{$\begin{array}{l}\text { Req'd Thk } \\
\text { Due to } \\
\text { Tension (in) }\end{array}$} & \multirow{2}{*}{$\begin{array}{l}\text { Req'd Thk Due } \\
\text { to Compression } \\
\text { (in) }\end{array}$} \\
\hline & & $\mathbf{S}_{\mathbf{t}}$ & $\mathrm{S}_{\mathrm{c}}$ & & & & & \\
\hline \multirow{2}{*}{$\begin{array}{l}\text { Operating, Hot \& } \\
\text { Corroded }\end{array}$} & \multirow{2}{*}{1,150} & \multirow{2}{*}{11,300} & \multirow{2}{*}{10,369} & \multirow{2}{*}{1,000} & \multirow{2}{*}{0.03} & Wind & 0.0497 & 0.0496 \\
\hline & & & & & & Seismic & 0.05 & 0.0494 \\
\hline \multirow{2}{*}{$\begin{array}{c}\text { Operating, Hot \& } \\
\text { New }\end{array}$} & \multirow{2}{*}{1,150} & \multirow{2}{*}{11,300} & \multirow{2}{*}{10,454} & \multirow{2}{*}{1,000} & \multirow{2}{*}{0} & Wind & 0.0485 & 0.0484 \\
\hline & & & & & & Seismic & 0.0488 & 0.0481 \\
\hline \multirow{2}{*}{ Empty, Corroded } & \multirow{2}{*}{0} & \multirow{2}{*}{20,000} & \multirow{2}{*}{14,651} & \multirow{2}{*}{0} & \multirow{2}{*}{0.03} & Wind & 0 & 0 \\
\hline & & & & & & Seismic & 0.0002 & 0.0002 \\
\hline & & & & & & Wind & 0 & 0 \\
\hline
\end{tabular}

The cylinder thickness of $0.276^{\prime \prime}$ is adequate.

Thickness Required Due to Pressure + External Loads 


\begin{tabular}{|c|r|r|r|r|r|r|r|r|} 
Empty, New & 0 & 20,000 & 14,737 & 0 & 0 & Seismic & 0.0002 & 0.0002 \\
\hline $\begin{array}{c}\text { Hot Shut Down, } \\
\text { Corroded, Weight \& } \\
\begin{array}{c}\text { Eccentric Moments } \\
\text { Only }\end{array}\end{array}$ & 0 & 11,300 & 10,369 & 1,000 & 0.03 & Weight & 0 & 0 \\
\hline
\end{tabular}




\section{OD" PIPE}

\section{ASME Section VIII Division 1, 2007 Edition}

Component:

Material specification:

Rated MDMT per UNF- $65=-325^{\circ} \mathrm{F}$

Internal design pressure: $\mathrm{P}=1,150 \mathrm{psi} @ 1,000^{\circ} \mathrm{F}$

\section{Static liquid head:}

$P_{\text {th }}=0.23 \mathrm{psi}\left(\mathrm{SG}=1, \mathrm{H}_{\mathrm{S}}=6.43\right.$ ", Horizontal test head $)$

Corrosion allowance

$$
\text { Inner } \mathrm{C}=0.03 "
$$

Outer $\mathrm{C}=0$ "
Cylinder

SB-166 Annealed

N06600 (II-D p. 222, ln. 3)
Design MDMT $=-20^{\circ} \mathrm{F}$

Rated MDMT $=-325^{\circ} \mathrm{F}$
No impact test performed

Material is not normalized

Material is not produced to Fine Grain Practice

PWHT is not performed

Radiography: Longitudinal joint - Seamless No RT

Top circumferential joint - Full UW-11(a) Type 1

Bottom circumferential

joint -

Full UW-11(a) Type 1

Estimated weight $\mathrm{New}=4.8 \mathrm{lb} \quad$ corr $=4.4 \mathrm{lb}$

Capacity $\quad \mathrm{New}=0.11$ US gal corr $=0.12$ US gal

$$
\begin{aligned}
\mathrm{OD} & =3^{\prime \prime} \\
\text { Length } \mathrm{L}_{\mathrm{c}} & =6^{\prime \prime} \\
\mathrm{t} & =0.32^{\prime \prime}
\end{aligned}
$$

\section{Design thickness, (at $1,000^{\circ}$ F) Appendix 1-1}

$$
\begin{aligned}
\mathrm{t} & =\mathrm{P}^{*} \mathrm{R}_{\mathrm{o}} /\left(\mathrm{S}^{*} \mathrm{E}+0.40 * \mathrm{P}\right)+\text { Corrosion } \\
& =1,150^{*} 1.5 /\left(7,000^{*} 1.00+0.40^{*} 1,150\right)+0.03 \\
& =0.2613^{\prime \prime}
\end{aligned}
$$

Maxim um allowable working pressure, (at 1,000 ${ }^{\circ}$ F) Appendix 1-1

$$
\begin{aligned}
\mathrm{P} & =\mathrm{S}^{*} \mathrm{E}^{*} \mathrm{t} /\left(\mathrm{R}_{\mathrm{o}}-0.40 * \mathrm{t}\right)-\mathrm{P}_{\mathrm{S}} \\
& =7,000 * 1.00 * 0.25 /\left(1.5-0.40^{*} 0.25\right)-0 \\
& =1,250 \mathrm{psi}
\end{aligned}
$$

Design thickness $=0.2613^{\prime \prime}$

The governing condition is due to internal pressure.

The cylinder thickness of $0.32 "$ is adequate. 
DESC: CHAR POTS, UPPER \& LOWER

P.O.\#: 700521452

Thickness Required Due to Pressure + External Loads

\begin{tabular}{|c|c|c|c|c|c|c|c|c|}
\hline \multirow[t]{2}{*}{ Condition } & \multirow{2}{*}{$\begin{array}{l}\text { Pressure } \\
\text { P ( psi) }\end{array}$} & \multicolumn{2}{|c|}{$\begin{array}{l}\text { Allowable Stress } \\
\text { Before UG-23 Stress } \\
\text { Increase ( psi) }\end{array}$} & \multirow{2}{*}{$\begin{array}{c}\text { Temperature } \\
\left({ }^{\circ} \mathrm{F}\right)\end{array}$} & \multirow{2}{*}{$\begin{array}{l}\text { Corrosion } \\
\text { C (in) }\end{array}$} & \multirow[t]{2}{*}{ Load } & \multirow{2}{*}{$\begin{array}{l}\text { Req'd Thk } \\
\text { Due to } \\
\text { Tension (in) }\end{array}$} & \multirow{2}{*}{$\begin{array}{l}\text { Req'd Thk Due } \\
\text { to Compression } \\
\text { (in) }\end{array}$} \\
\hline & & $\mathbf{S}_{\mathbf{t}}$ & $\mathbf{S}_{\mathbf{c}}$ & & & & & \\
\hline \multirow{2}{*}{$\begin{array}{l}\text { Operating, Hot \& } \\
\text { Corroded }\end{array}$} & \multirow{2}{*}{1,150} & \multirow{2}{*}{7,000} & \multirow{2}{*}{7,000} & \multirow{2}{*}{1,000} & \multirow{2}{*}{0.03} & Wind & 0.083 & 0.0769 \\
\hline & & & & & & Seismic & 0.0846 & 0.0752 \\
\hline \multirow{2}{*}{$\begin{array}{l}\text { Operating, Hot \& } \\
\text { New }\end{array}$} & \multirow{2}{*}{1,150} & \multirow{2}{*}{7,000} & \multirow{2}{*}{7,000} & \multirow{2}{*}{1,000} & \multirow{2}{*}{0} & Wind & 0.0814 & 0.0744 \\
\hline & & & & & & Seismic & 0.0831 & 0.0727 \\
\hline \multirow{2}{*}{ Empty, Corroded } & \multirow{2}{*}{0} & \multirow{2}{*}{22,900} & \multirow{2}{*}{13,800} & \multirow{2}{*}{0} & \multirow{2}{*}{0.03} & Wind & 0.0007 & 0.0019 \\
\hline & & & & & & Seismic & 0.0012 & 0.0027 \\
\hline \multirow{2}{*}{ Empty, New } & \multirow{2}{*}{0} & \multirow{2}{*}{22,900} & \multirow{2}{*}{13,800} & \multirow{2}{*}{0} & \multirow{2}{*}{0} & Wind & 0.0009 & 0.0021 \\
\hline & & & & & & Seismic & 0.0014 & 0.003 \\
\hline $\begin{array}{l}\text { Hot Shut Down, } \\
\text { Corroded, Weight \& } \\
\text { Eccentric Moments } \\
\text { Only }\end{array}$ & 0 & 7,000 & 7,000 & 1,000 & 0.03 & Weight & 0.0025 & 0.0041 \\
\hline
\end{tabular}




\section{4" X 2 1/2" REDUCER B16.9, UPPER (SCH XS) 2 1/2" END}

\section{ASME Section VIII Division 1, 2007 Edition}

Component: Cylinder

Material specification: $\quad$ SA-403 316H (II-D p. 78, ln. 8)

Rated MDMT per UHA-51(d)(1)(b), (carbon content does not exceed 0.1 percent) $=-320^{\circ} \mathrm{F}$

Internal design pressure: $\mathrm{P}=1,150 \mathrm{psi} @ 1,000^{\circ} \mathrm{F}$

Static liquid head:

$\mathrm{P}_{\text {th }}=0.23 \mathrm{psi}\left(\mathrm{SG}=1, \mathrm{H}_{\mathrm{S}}=6.4455\right.$ ", Horizontal test head $)$

Corrosion allowance $\quad$ Inner $\mathrm{C}=0.03 " \quad$ Outer $\mathrm{C}=0 "$

Design MDMT $=-20^{\circ} \mathrm{F} \quad$ No impact test performed

Rated MDMT $=-320^{\circ} \mathrm{F} \quad$ Material is not normalized

Material is not produced to Fine Grain Practice

PWHT is not performed

\section{Radiography: Longitudinal joint - Seamless No RT}

Top circumferential joint - Full UW-11(a) Type 1

Bottom circumferential joint -

Full UW-11(a) Type 1

$$
\begin{aligned}
\text { Estimated weight New } & =1.2 \mathrm{lb} \quad \text { corr }=1 \mathrm{lb} \\
\text { Capacity } \quad \mathrm{New} & =0.04 \text { US gal corr }=0.04 \text { US gal }
\end{aligned}
$$

$$
\begin{aligned}
\text { OD } & =2.875^{\prime \prime} \\
\text { Length } L_{\mathbf{c}} & =2^{\prime \prime} \\
\mathrm{t} & =0.242 "
\end{aligned}
$$

\section{Design thickness, (at $1,000^{\circ}$ F) Appendix 1-1}

$$
\begin{aligned}
\mathrm{t} & =\mathrm{P}^{*} \mathrm{R}_{\mathrm{o}} /\left(\mathrm{S}^{*} \mathrm{E}+0.40^{*} \mathrm{P}\right)+\text { Corrosion } \\
& =1,150^{*} 1.4375 /\left(15,300^{*} 1.00+0.40^{*} 1,150\right)+0.03 \\
& =0.1349^{\prime \prime}
\end{aligned}
$$

Maximum allowable working pressure, (at 1,000 ${ }^{\circ}$ F) Appendix 1-1

$$
\begin{aligned}
\mathrm{P} & =\mathrm{S}^{*} \mathrm{E}^{*} \mathrm{t} /\left(\mathrm{R}_{\mathrm{o}}-0.40^{*} \mathrm{t}\right)-\mathrm{P}_{\mathrm{S}} \\
& =15,300^{*} 1.00^{*} 0.212 /\left(1.4375-0.40^{*} 0.212\right)-0 \\
& =2,397.87 \mathrm{psi}
\end{aligned}
$$

\% Forming Strain - UHA-44(a)(2)(a)

$$
\begin{aligned}
\mathrm{EFE} & =\left(50 * \mathrm{t} / \mathrm{R}_{\mathrm{f}}\right) *\left(1-\mathrm{R}_{\mathrm{f}} / \mathrm{R}_{\mathrm{o}}\right) \\
& =(50 * 0.242 / 1.3165) *(1-1.3165 / \infty) \\
& =9.1910 \%
\end{aligned}
$$




\section{Design thickness $=0.1349^{\prime \prime}$}

The governing condition is due to internal pressure.

The cylinder thickness of 0.242 " is adequate.

Thickness Required Due to Pressure + External Loads

\begin{tabular}{|c|c|c|c|c|c|c|c|c|}
\hline \multirow[t]{2}{*}{ Condition } & \multirow{2}{*}{$\begin{array}{c}\text { Pressure } \\
\text { P ( psi) }\end{array}$} & \multicolumn{2}{|c|}{$\begin{array}{l}\text { Allowable Stress } \\
\text { Before UG-23 Stress } \\
\text { Increase ( psi) }\end{array}$} & \multirow{2}{*}{$\begin{array}{c}\text { Temperature } \\
\left({ }^{\circ} \mathrm{F}\right)\end{array}$} & \multirow{2}{*}{$\begin{array}{l}\text { Corrosion } \\
\mathrm{C} \text { (in) }\end{array}$} & \multirow[t]{2}{*}{ Load } & \multirow{2}{*}{$\begin{array}{l}\text { Req'd Thk } \\
\text { Due to } \\
\text { Tension (in) }\end{array}$} & \multirow{2}{*}{$\begin{array}{l}\text { Req'd Thk Due } \\
\text { to Compression } \\
\text { (in) }\end{array}$} \\
\hline & & $\mathbf{S}_{\mathbf{t}}$ & $\mathbf{S}_{\mathbf{c}}$ & & & & & \\
\hline \multirow{2}{*}{$\begin{array}{l}\text { Operating, Hot \& } \\
\text { Corroded }\end{array}$} & \multirow{2}{*}{1,150} & \multirow{2}{*}{15,300} & \multirow{2}{*}{10,261} & \multirow{2}{*}{1,000} & \multirow{2}{*}{0.03} & Wind & 0.0391 & 0.0361 \\
\hline & & & & & & Seismic & 0.04 & 0.0351 \\
\hline \multirow{2}{*}{$\begin{array}{c}\text { Operating, Hot \& } \\
\text { New }\end{array}$} & \multirow{2}{*}{1,150} & \multirow{2}{*}{15,300} & \multirow{2}{*}{10,357} & \multirow{2}{*}{1,000} & \multirow{2}{*}{0} & Wind & 0.0384 & 0.0349 \\
\hline & & & & & & Seismic & 0.0394 & 0.0339 \\
\hline \multirow{2}{*}{ Empty, Corroded } & \multirow{2}{*}{0} & \multirow{2}{*}{20,000} & \multirow{2}{*}{14,528} & \multirow{2}{*}{0} & \multirow{2}{*}{0.03} & Wind & 0.0009 & 0.0019 \\
\hline & & & & & & Seismic & 0.0016 & 0.003 \\
\hline \multirow{2}{*}{ Empty, New } & \multirow{2}{*}{0} & \multirow{2}{*}{20,000} & \multirow{2}{*}{14,638} & \multirow{2}{*}{0} & \multirow{2}{*}{0} & Wind & 0.0011 & 0.0021 \\
\hline & & & & & & Seismic & 0.0018 & 0.0032 \\
\hline $\begin{array}{l}\text { Hot Shut Down, } \\
\text { Corroded, Weight \& } \\
\text { Eccentric Moments } \\
\text { Only }\end{array}$ & 0 & 15,300 & 10,261 & 1,000 & 0.03 & Weight & 0.0012 & 0.0029 \\
\hline
\end{tabular}




\section{4" X 2 1/2" REDUCER B16.9, UPPER (SCH XS) 4" END}

\section{ASME Section VIII Division 1, 2007 Edition}

$\begin{array}{ll}\text { Component: } & \text { Cylinder } \\ \text { Material specification: } & \text { SA-403 316H (II-D p. 78, In. 8) }\end{array}$

Rated MDMT per UHA-51(d)(1)(b), (carbon content does not exceed 0.1 percent $)=-320^{\circ} \mathrm{F}$

Internal design pressure: $\mathrm{P}=1,150 \mathrm{psi} @ 1,000^{\circ} \mathrm{F}$

Static liquid head:

$P_{\text {th }}=0.26 \mathrm{psi}\left(\mathrm{SG}=1, \mathrm{H}_{\mathrm{S}}=7.205 "\right.$, Horizontal test head $)$

Corrosion allowance $\quad$ Inner $\mathrm{C}=0.03^{\prime \prime} \quad$ Outer $\mathrm{C}=0 "$

Design MDMT $=-20^{\circ} \mathrm{F} \quad$ No impact test performed

Rated MDMT $=-320^{\circ} \mathrm{F} \quad$ Material is not normalized

Material is not produced to Fine Grain Practice

PWHT is not performed

Radiography: Longitudinal joint - Seamless No RT

Top circumferential joint - Full UW-11(a) Type 1

Bottom circumferential

joint -

Full UW-11(a) Type 1

Estimated weight New $=2.3 \mathrm{lb} \quad$ corr $=2 \mathrm{lb}$

Capacity $\quad$ New $=0.1$ US gal corr $=0.11$ US gal

$\mathrm{OD} \quad=4.5^{\prime \prime}$

Length $\mathrm{L}_{\mathrm{c}}=2^{\prime \prime}$

$\mathrm{t} \quad=0.295^{\prime \prime}$

\section{Design thickness, (at $1,000^{\circ}$ F) Appendix 1-1}

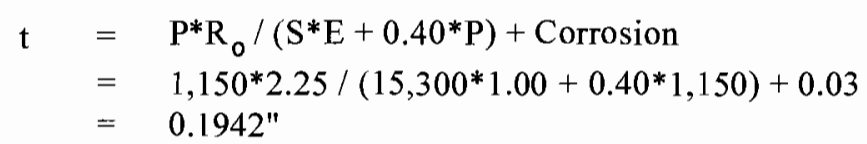

Maximum allowable working pressure, (at $1,000^{\circ} \mathrm{F}$ ) Appendix 1-1

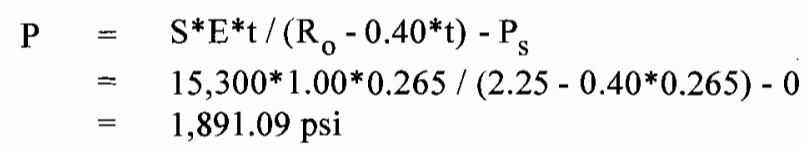

\% Forming Strain - UHA-44(a)(2)(a)

$$
\begin{aligned}
\mathrm{EFE} & =\left(50 * \mathrm{t} / \mathrm{R}_{\mathrm{f}}\right) *\left(1-\mathrm{R}_{\mathrm{f}} / \mathrm{R}_{\mathrm{o}}\right) \\
& =(50 * 0.295 / 2.1025) *(1-2.1025 / \infty) \\
& =7.0155 \%
\end{aligned}
$$




\section{Design thickness $=0.1942 "$}

The governing condition is due to internal pressure.

The cylinder thickness of $0.295^{\prime \prime}$ is adequate.

Thickness Required Due to Pressure + External Loads

\begin{tabular}{|c|c|c|c|c|c|c|c|c|}
\hline \multirow[t]{2}{*}{ Condition } & \multirow{2}{*}{$\begin{array}{l}\text { Pressure } \\
\text { P ( psi) }\end{array}$} & \multicolumn{2}{|c|}{$\begin{array}{l}\text { Allowable Stress } \\
\text { Before UG-23 Stress } \\
\text { Increase ( psi) }\end{array}$} & \multirow{2}{*}{$\begin{array}{c}\text { Temperature } \\
\left({ }^{\circ} \mathrm{F}\right)\end{array}$} & \multirow{2}{*}{$\begin{array}{l}\text { Corrosion } \\
\text { C (in) }\end{array}$} & \multirow[t]{2}{*}{ Load } & \multirow{2}{*}{$\begin{array}{l}\text { Req'd Thk } \\
\text { Due to } \\
\text { Tension (in) }\end{array}$} & \multirow{2}{*}{$\begin{array}{l}\text { Req'd Thk Due } \\
\text { to Compression } \\
\text { (in) }\end{array}$} \\
\hline & & $S_{t}$ & $S_{c}$ & & & & & \\
\hline \multirow{2}{*}{$\begin{array}{l}\text { Operating, Hot \& } \\
\text { Corroded }\end{array}$} & \multirow{2}{*}{1,150} & \multirow{2}{*}{15,300} & \multirow{2}{*}{10,100} & \multirow{2}{*}{1,000} & \multirow{2}{*}{0.03} & Wind & 0.0618 & 0.0606 \\
\hline & & & & & & Seismic & 0.0623 & 0.0601 \\
\hline \multirow{2}{*}{$\begin{array}{c}\text { Operating, Hot \& } \\
\text { New }\end{array}$} & \multirow{2}{*}{1,150} & \multirow{2}{*}{15,300} & \multirow{2}{*}{10,177} & \multirow{2}{*}{1,000} & \multirow{2}{*}{0} & Wind & 0.061 & 0.0596 \\
\hline & & & & & & Seismic & 0.0614 & 0.059 \\
\hline \multirow{2}{*}{ Empty, Corroded } & \multirow{2}{*}{0} & \multirow{2}{*}{20,000} & \multirow{2}{*}{14,332} & \multirow{2}{*}{0} & \multirow{2}{*}{0.03} & Wind & 0.0003 & 0.0009 \\
\hline & & & & & & Seismic & 0.0007 & 0.0014 \\
\hline \multirow{2}{*}{ Empty, New } & \multirow{2}{*}{0} & \multirow{2}{*}{20,000} & \multirow{2}{*}{14,425} & \multirow{2}{*}{0} & \multirow{2}{*}{0} & Wind & 0.0004 & 0.001 \\
\hline & & & & & & Seismic & 0.0007 & 0.0015 \\
\hline $\begin{array}{l}\text { Hot Shut Down, } \\
\text { Corroded, Weight \& } \\
\text { Eccentric Moments } \\
\text { Only }\end{array}$ & 0 & 15,300 & 10,100 & 1,000 & 0.03 & Weight & 0.0004 & 0.0013 \\
\hline
\end{tabular}




\section{8" X 4" REDUCER B16.9, UPPER (SCH XS) 4" END}

\section{ASME Section VIII Division 1, 2007 Edition}

\section{Component:}

Material specification:

Rated MDMT per UHA-51(d)(1)(b), (carbon content does not exceed 0.1 percent) $=-320^{\circ} \mathrm{F}$
Cylinder

SA-403 316H (II-D p. 78, ln. 8)

Internal design pressure: $\mathrm{P}=1,150 \mathrm{psi} @ 1,000^{\circ} \mathrm{F}$

Static liquid head:

$P_{t h}=0.26 \mathrm{psi}\left(\mathrm{SG}=1, \mathrm{H}_{\mathrm{s}}=7.205^{\prime \prime}\right.$, Horizontal test head $)$

Corrosion allowance $\quad$ Inner $\mathrm{C}=0.03^{\prime \prime} \quad$ Outer $\mathrm{C}=0 "$

Design MDMT $=-20^{\circ} \mathrm{F}$

Rated MDMT $=-320^{\circ} \mathrm{F}$

No impact test performed

Material is not normalized

Material is not produced to Fine Grain Practice

PWHT is not performed

Radiography: Longitudinal joint - Seamless No RT

Top circumferential joint - Full UW-11(a) Type 1

Bottom circumferential joint -

Full UW-11(a) Type 1

$$
\begin{aligned}
\text { Estimated weight New } & =3.4 \mathrm{lb} \quad \text { corr }=3.1 \mathrm{lb} \\
\text { Capacity } \quad \mathrm{New} & =0.16 \mathrm{US} \text { gal corr }=0.16 \mathrm{US} \text { gal }
\end{aligned}
$$

$$
\begin{aligned}
\mathrm{OD} & =4.5^{\prime \prime} \\
\text { Length } \mathrm{L}_{\mathrm{c}} & =3^{\prime \prime} \\
\mathrm{t} & =0.295^{\prime \prime}
\end{aligned}
$$

\section{Design thickness, $\left(\right.$ at $\left.^{1,000}{ }^{\circ} \mathrm{F}\right)$ Appendix 1-1}

$$
\begin{aligned}
\mathrm{t} & =\mathrm{P} * \mathrm{R}_{\mathrm{o}} /\left(\mathrm{S}^{*} \mathrm{E}+0.40^{*} \mathrm{P}\right)+\text { Corrosion } \\
& =1,150^{*} 2.25 /\left(15,300^{*} 1.00+0.40^{*} 1,150\right)+0.03 \\
& =0.1942^{*}
\end{aligned}
$$

Maximum allowable working pressure, (at 1,000 ${ }^{\circ}$ F) Appendix 1-1

$$
\begin{aligned}
\mathrm{P} & =\mathrm{S}^{*} \mathrm{E}^{*} \mathrm{t} /\left(\mathrm{R}_{\mathrm{o}}-0.40^{*} \mathrm{t}\right)-\mathrm{P}_{\mathrm{S}} \\
& =15,300^{*} 1.00^{*} 0.265 /\left(2.25-0.40^{*} 0.265\right)-0 \\
& =1,891.09 \mathrm{psi}
\end{aligned}
$$

\% Forming Strain - UHA-44(a)(2)(a)

$$
\begin{aligned}
\mathrm{EFE} & =\left(50 * \mathrm{t} / \mathrm{R}_{\mathrm{f}}\right) *\left(1-\mathrm{R}_{\mathrm{f}} / \mathrm{R}_{\mathrm{o}}\right) \\
& =(50 * 0.295 / 2.1025) *(1-2.1025 / \infty) \\
& =7.0155 \%
\end{aligned}
$$




\section{Design thickness $=0.1942 "$}

The governing condition is due to internal pressure.

The cylinder thickness of $0.295^{\prime \prime}$ is adequate.

Thickness Required Due to Pressure + External Loads

\begin{tabular}{|c|c|c|c|c|c|c|c|c|}
\hline \multirow[t]{2}{*}{ Condition } & \multirow{2}{*}{$\begin{array}{l}\text { Pressure } \\
\text { P ( psi) }\end{array}$} & \multicolumn{2}{|c|}{$\begin{array}{c}\text { Allowable Stress } \\
\text { Before UG-23 Stress } \\
\text { Increase ( psi) }\end{array}$} & \multirow{2}{*}{$\begin{array}{c}\text { Temperature } \\
\left({ }^{\circ} \mathbf{F}\right)\end{array}$} & \multirow{2}{*}{$\begin{array}{l}\text { Corrosion } \\
\text { C (in) }\end{array}$} & \multirow[t]{2}{*}{ Load } & \multirow{2}{*}{$\begin{array}{c}\text { Req'd Thk } \\
\text { Due to } \\
\text { Tension (in) }\end{array}$} & \multirow{2}{*}{$\begin{array}{l}\text { Req'd Thk Due } \\
\text { to Compression } \\
\text { (in) }\end{array}$} \\
\hline & & $\mathbf{S}_{\mathbf{t}}$ & $\mathbf{S}_{\mathbf{c}}$ & & & & & \\
\hline \multirow{2}{*}{$\begin{array}{l}\text { Operating, Hot \& } \\
\text { Corroded }\end{array}$} & \multirow{2}{*}{1,150} & \multirow{2}{*}{15,300} & \multirow{2}{*}{10,100} & \multirow{2}{*}{1,000} & \multirow{2}{*}{0.03} & Wind & 0.0619 & 0.0605 \\
\hline & & & & & & Seismic & 0.0624 & 0.0599 \\
\hline \multirow{2}{*}{$\begin{array}{c}\text { Operating, Hot \& } \\
\text { New }\end{array}$} & \multirow{2}{*}{1,150} & \multirow{2}{*}{15,300} & \multirow{2}{*}{10,177} & \multirow{2}{*}{1,000} & \multirow{2}{*}{0} & Wind & 0.061 & 0.0595 \\
\hline & & & & & & Seismic & 0.0616 & 0.0589 \\
\hline \multirow{2}{*}{ Empty, Corroded } & \multirow{2}{*}{0} & \multirow{2}{*}{20,000} & \multirow{2}{*}{14,332} & \multirow{2}{*}{0} & \multirow{2}{*}{0.03} & Wind & 0.0003 & 0.001 \\
\hline & & & & & & Seismic & 0.0008 & 0.0016 \\
\hline \multirow{2}{*}{ Empty, New } & \multirow{2}{*}{0} & \multirow{2}{*}{20,000} & \multirow{2}{*}{14,425} & \multirow{2}{*}{0} & \multirow{2}{*}{0} & Wind & 0.0004 & 0.001 \\
\hline & & & & & & Seismic & 0.0009 & 0.0017 \\
\hline $\begin{array}{l}\text { Hot Shut Down, } \\
\text { Corroded, Weight \& } \\
\text { Eccentric Moments } \\
\text { Only }\end{array}$ & 0 & 15,300 & 10,100 & 1,000 & 0.03 & Weight & 0.0004 & 0.0013 \\
\hline
\end{tabular}




\section{8" X 4" REDUCER B16.9, UPPER (SCH XS) 8" END}

\section{ASME Section VIII Division 1, 2007 Edition}

Component: $\quad$ Cylinder

Material specification: $\quad$ SA-403 $316 \mathrm{H}$ (II-D p. 78, $\ln .8$ )

Rated MDMT per UHA-51(d)(1)(b), (carbon content does not exceed 0.1 percent) $=-320^{\circ} \mathrm{F}$

Internal design pressure: $\mathrm{P}=1,150 \mathrm{psi} @ 1,000^{\circ} \mathrm{F}$

Static liquid head:

$\mathrm{P}_{\mathrm{th}}=0.33 \mathrm{psi}\left(\mathrm{SG}=1, \mathrm{H}_{\mathrm{s}}=9.125^{\prime \prime}\right.$, Horizontal test head $)$

Corrosion allowance $\quad$ Inner $\mathrm{C}=0.03^{\prime \prime} \quad$ Outer $\mathrm{C}=0$ "

Design MDMT $=-20^{\circ} \mathrm{F} \quad$ No impact test performed

Rated MDMT $=-320^{\circ} \mathrm{F} \quad$ Material is not normalized

Material is not produced to Fine Grain Practice

PWHT is notperformed

Radiography: Longitudinal joint - Seamless No RT

Top circumferential joint - Full UW-11(a) Type 1

Bottom circumferential $\quad$ Full UW-11(a) Type 1
joint -

Estimated weight New $=9.8 \mathrm{lb} \quad$ corr $=9.2 \mathrm{lb}$

Capacity New $=0.61$ US gal corr $=0.62$ US gal

$\mathrm{OD}=8.625^{\prime \prime}$

Length $\mathrm{L}_{\mathrm{c}}=3^{\prime \prime}$

$\mathrm{t} \quad=0.4375^{\prime \prime}$

Design thickness, $\left(\right.$ at $\left.1,000^{\circ} \mathrm{F}\right)$ Appendix 1-1

$\mathrm{t}=\mathrm{P}^{*} \mathrm{R}_{\mathrm{o}} /(\mathrm{S} * \mathrm{E}+0.40 * \mathrm{P})+$ Corrosion

$=1,150 * 4.3125 /(15,300 * 1.00+0.40 * 1,150)+0.03$

$=0.3447^{\prime \prime}$

Maximum allowable working pressure, (at 1,000 ${ }^{\circ} \mathrm{F}$ ) Appendix 1 -1

$\mathrm{P}=\mathrm{S} * \mathrm{E}^{*} \mathrm{t} /\left(\mathrm{R}_{\mathrm{o}}-0.40 * \mathrm{t}\right)-\mathrm{P}_{\mathrm{S}}$

$=15,300 * 1.00 * 0.4075 /(4.3125-0.40 * 0.4075)-0$

$=1,502.53 \mathrm{psi}$

\% Forming Strain - UHA-44(a)(2)(a)

$$
\begin{aligned}
\mathrm{EFE} & =\left(50 * \mathrm{t} / \mathrm{R}_{\mathrm{f}}\right) *\left(1-\mathrm{R}_{\mathrm{f}} / \mathrm{R}_{\mathrm{o}}\right) \\
& =(50 * 0.4375 / 4.0938) *(1-4.0938 / \infty) \\
& =5.3435 \%
\end{aligned}
$$




\section{Design thickness $=0.3447^{\prime \prime}$}

The governing condition is due to internal pressure.

The cylinder thickness of $0.4375^{\prime \prime}$ is adequate.

Thickness Required Due to Pressure + External Loads

\begin{tabular}{|c|c|c|c|c|c|c|c|c|}
\hline \multirow[t]{2}{*}{ Condition } & \multirow{2}{*}{$\begin{array}{c}\text { Pressure } \\
\text { P ( psi) }\end{array}$} & \multicolumn{2}{|c|}{$\begin{array}{c}\text { Allowable Stress } \\
\text { Before UG-23 Stress } \\
\text { Increase ( psi) }\end{array}$} & \multirow{2}{*}{$\begin{array}{c}\text { Temperature } \\
\left({ }^{\circ} \mathbf{F}\right)\end{array}$} & \multirow{2}{*}{$\begin{array}{l}\text { Corrosion } \\
\mathrm{C} \text { (in) }\end{array}$} & \multirow[t]{2}{*}{ Load } & \multirow{2}{*}{$\begin{array}{l}\text { Req'd Thk } \\
\text { Due to } \\
\text { Tension (in) }\end{array}$} & \multirow{2}{*}{$\begin{array}{l}\text { Req'd Thk Due } \\
\text { to Compression } \\
\text { (in) }\end{array}$} \\
\hline & & $\mathbf{S}_{\mathbf{t}}$ & $\mathbf{S}_{\mathbf{c}}$ & & & & & \\
\hline \multirow{2}{*}{$\begin{array}{l}\text { Operating, Hot \& } \\
\text { Corroded }\end{array}$} & \multirow{2}{*}{1,150} & \multirow{2}{*}{15,300} & \multirow{2}{*}{9,945} & \multirow{2}{*}{1,000} & \multirow{2}{*}{0.03} & Wind & 0.1209 & 0.1205 \\
\hline & & & & & & Seismic & 0.121 & 0.1203 \\
\hline \multirow{2}{*}{$\begin{array}{c}\text { Operating, Hot \& } \\
\text { New }\end{array}$} & \multirow{2}{*}{1,150} & \multirow{2}{*}{15,300} & \multirow{2}{*}{9,995} & \multirow{2}{*}{1,000} & \multirow{2}{*}{0} & Wind & 0.1199 & 0.1195 \\
\hline & & & & & & Seismic & 0.1201 & 0.1193 \\
\hline \multirow{2}{*}{ Empty, Corroded } & \multirow{2}{*}{0} & \multirow{2}{*}{20,000} & \multirow{2}{*}{14,142} & \multirow{2}{*}{0} & \multirow{2}{*}{0.03} & Wind & 0.0001 & 0.0004 \\
\hline & & & & & & Seismic & 0.0002 & 0.0006 \\
\hline \multirow{2}{*}{ Empty, New } & \multirow{2}{*}{0} & \multirow{2}{*}{20,000} & \multirow{2}{*}{14,203} & \multirow{2}{*}{0} & \multirow{2}{*}{0} & Wind & 0.0001 & 0.0004 \\
\hline & & & & & & Seismic & 0.0002 & 0.0006 \\
\hline $\begin{array}{l}\text { Hot Shut Down, } \\
\text { Corroded, Weight \& } \\
\text { Eccentric Moments } \\
\text { Only }\end{array}$ & 0 & 15,300 & 9,945 & 1,000 & 0.03 & Weight & 0 & 0.0005 \\
\hline
\end{tabular}




\section{8" PIPE}

ASME Section VIII Division 1, 2007 Edition

Component:

Material specification:

Pipe NPS and Schedule:

Rated MDMT per UHA-51(d)(1)(b), (carbon content does not exceed 0.1 percent) $=-320^{\circ} \mathrm{F}$
Cylinder

SA-312 TP316H Wld \& smls pipe (II-D p.

$78, \ln .1)$

8 " Sch 80 S (XS)

Internal design pressure: $\mathrm{P}=1,150 \mathrm{psi} @ 1,000^{\circ} \mathrm{F}$

\section{Static liquid head:}

$P_{\text {th }}=0.33$ psi $\left(\mathrm{SG}=1, H_{\mathrm{s}}=9.0625^{\prime \prime}\right.$, Horizontal test head $)$

Corrosion allowance $\quad$ Inner $\mathrm{C}=0.03^{\prime \prime} \quad$ Outer $\mathrm{C}=0$ "

$\begin{array}{ll}\text { Design MDMT }=-20^{\circ} \mathrm{F} & \text { No impact test performed } \\ \text { Rated MDMT }=-320^{\circ} \mathrm{F} & \text { Material is not normalized } \\ & \text { Material is not produced to Fine Grain Practice } \\ & \text { PWHT is not performed }\end{array}$

Radiography: Longitudinal joint - Seamless No RT

Top circumferential joint - Full UW-11(a) Type 1

Bottom circumferential joint -

Full UW-11(a) Type 1

Estimated weight New $=140.6 \mathrm{lb} \quad$ corr $=132.7 \mathrm{lb}$

Capacity New $=7.51$ US gal corr $=7.63$ US gal

$\mathrm{OD}=8.625^{\prime \prime}$

Length $\mathrm{L}_{\mathrm{c}}=38^{\prime \prime}$

$\mathrm{t}$

$=0.5^{\prime \prime}$

Design thickness, (at $1,000^{\circ} \mathrm{F}$ ) Appendix 1-1

$\mathrm{t}=\mathrm{P} * \mathrm{R}_{\mathrm{o}} /(\mathrm{S} * \mathrm{E}+0.40 * \mathrm{P})+$ Corrosion

$=1,150 * 4.3125 /(15,300 * 1.00+0.40 * 1,150)+0.03$

$=0.3447^{\prime \prime}$

Maximum allowable working pressure, $\left(\right.$ at $^{1,000}{ }^{\circ} \mathrm{F}$ ) Appendix 1-1

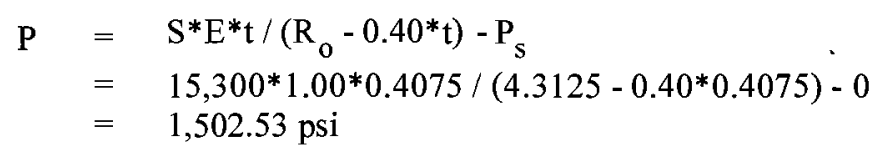

\section{Design thickness $=0.3447^{\prime \prime}$}

The governing condition is due to internal pressure.

The cylinder thickness of 0.5 " is adequate. 
Thickness Required Due to Pressure + External Loads

\begin{tabular}{|c|c|c|c|c|c|c|c|c|}
\hline \multirow[t]{2}{*}{ Condition } & \multirow{2}{*}{$\begin{array}{l}\text { Pressure } \\
\text { P ( psi) }\end{array}$} & \multicolumn{2}{|c|}{$\begin{array}{l}\text { Allowable Stress } \\
\text { Before UG-23 Stress } \\
\text { Increase ( psi) }\end{array}$} & \multirow{2}{*}{$\begin{array}{l}\text { Temperature } \\
\left({ }^{\circ} \mathrm{F}\right)\end{array}$} & \multirow{2}{*}{$\begin{array}{c}\text { Corrosion } \\
\text { C (in) }\end{array}$} & \multirow[t]{2}{*}{ Load } & \multirow{2}{*}{$\begin{array}{l}\text { Req'd Thk } \\
\text { Due to } \\
\text { Tension (in) }\end{array}$} & \multirow{2}{*}{$\begin{array}{l}\text { Req'd Thk Due } \\
\text { to Compression } \\
\text { (in) }\end{array}$} \\
\hline & & $S_{t}$ & $\mathbf{S}_{\mathbf{c}}$ & & & & & \\
\hline \multirow{2}{*}{$\begin{array}{l}\text { Operating, Hot \& } \\
\text { Corroded }\end{array}$} & \multirow{2}{*}{1,150} & \multirow{2}{*}{15,300} & \multirow{2}{*}{9,945} & \multirow{2}{*}{1,000} & \multirow{2}{*}{0.03} & Wind & 0.1195 & 0.1173 \\
\hline & & & & & & Seismic & 0.1199 & 0.1169 \\
\hline \multirow{2}{*}{$\begin{array}{c}\text { Operating, Hot \& } \\
\text { New }\end{array}$} & \multirow{2}{*}{1,150} & \multirow{2}{*}{15,300} & \multirow{2}{*}{9,995} & \multirow{2}{*}{1,000} & \multirow{2}{*}{0} & Wind & 0.1186 & 0.1164 \\
\hline & & & & & & Seismic & 0.119 & 0.1158 \\
\hline \multirow{2}{*}{ Empty, Corroded } & \multirow{2}{*}{0} & \multirow{2}{*}{20,000} & \multirow{2}{*}{14,142} & \multirow{2}{*}{0} & \multirow{2}{*}{0.03} & Wind & 0.0005 & 0.0016 \\
\hline & & & & & & Seismic & 0.0008 & 0.0021 \\
\hline \multirow{2}{*}{ Empty, New } & \multirow{2}{*}{0} & \multirow{2}{*}{20,000} & \multirow{2}{*}{14,203} & \multirow{2}{*}{0} & \multirow{2}{*}{0} & Wind & 0.0005 & 0.0017 \\
\hline & & & & & & Seismic & 0.0009 & 0.0022 \\
\hline $\begin{array}{l}\text { Hot Shut Down, } \\
\text { Corroded, Weight \& } \\
\text { Eccentric Moments } \\
\text { Only }\end{array}$ & 0 & 15,300 & 9,945 & 1,000 & 0.03 & Weight & 0.0005 & 0.001 \\
\hline
\end{tabular}




\section{8" X 4" REDUCER B16.9, LOWER (SCH XS) 8" END}

ASME Section VIII Division 1, 2007 Edition

Component: $\quad$ Cylinder

Material specification: $\quad$ SA-403 $316 \mathrm{H}$ (II-D p. 78, ln. 8)

Rated MDMT per UHA-51(d)(1)(b), (carbon content does not exceed 0.1 percent) $=-320{ }^{\circ} \mathrm{F}$

Internal design pressure: $\mathrm{P}=1,150 \mathrm{psi} @ 1,000^{\circ} \mathrm{F}$

Static liquid head:

$P_{\text {th }}=0.33$ psi $\left(S G=1, H_{s}=9.125^{\prime \prime}\right.$, Horizontal test head $)$

Corrosion allowance $\quad$ Inner $\mathrm{C}=0.03^{\prime \prime} \quad$ Outer $\mathrm{C}=0 "$

Design MDMT $=-20^{\circ} \mathrm{F} \quad$ No impact test performed

Rated MDMT $=-320^{\circ} \mathrm{F} \quad$ Material is not normalized

Material is not produced to Fine Grain Practice

PWHT is not performed

Radiography: $\quad$ Longitudinal joint - $\quad$ Seamless No RT

Top circumferential joint - Full UW-11(a) Type 1

Bottom circumferential

joint -

Full UW-11(a) Type 1

Estimated weight New $=9.8 \mathrm{lb} \quad$ corr $=9.2 \mathrm{lb}$

Capacity New $=0.61$ US gal corr $=0.62$ US gal

$\begin{aligned} \text { OD } & =8.625^{\prime \prime} \\ \text { Length } \mathrm{L}_{\mathrm{c}} & =3^{\prime \prime} \\ \mathrm{t} & =0.4375^{\prime \prime}\end{aligned}$

Design thickness, (at $1,000^{\circ} \mathrm{F}$ ) Appendix 1-1

$\mathrm{t}=\mathrm{P}^{*} \mathrm{R}_{\mathrm{o}} /\left(\mathrm{S} * \mathrm{E}+0.40^{*} \mathrm{P}\right)+$ Corrosion

$=1,150 * 4.3125 /(15,300 * 1.00+0.40 * 1,150)+0.03$

$=0.3447^{\prime \prime}$

Maximum allowable working pressure, (at 1,000 ${ }^{\circ} \mathrm{F}$ ) Appendix 1-1

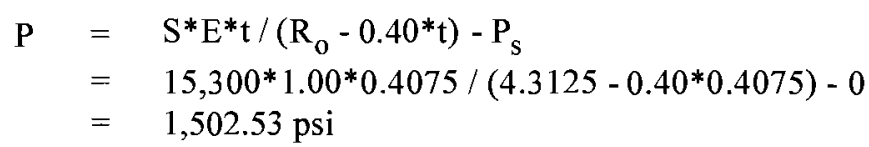

\% Forming Strain - UHA-44(a)(2)(a)

$$
\begin{aligned}
\mathrm{EFE} & =\left(50 * \mathrm{t} / \mathrm{R}_{\mathrm{f}}\right) *\left(1-\mathrm{R}_{\mathrm{f}} / \mathrm{R}_{\mathrm{o}}\right) \\
& =(50 * 0.4375 / 4.0938) *(1-4.0938 / \infty) \\
& =5.3435 \%
\end{aligned}
$$


Design thickness $=0.3447^{\prime \prime}$

The governing condition is due to internal pressure.

The cylinder thickness of $0.4375^{\prime \prime}$ is adequate.

Thickness Required Due to Pressure + External Loads

\begin{tabular}{|c|c|c|c|c|c|c|c|c|}
\hline \multirow[t]{2}{*}{ Condition } & \multirow{2}{*}{$\begin{array}{l}\text { Pressure } \\
\text { P (psi) }\end{array}$} & \multicolumn{2}{|c|}{$\begin{array}{l}\text { Allowable Stress } \\
\text { Before UG-23 Stress } \\
\text { Increase ( psi) }\end{array}$} & \multirow{2}{*}{$\begin{array}{c}\text { Temperature } \\
\left({ }^{\circ} \mathbf{F}\right)\end{array}$} & \multirow{2}{*}{$\begin{array}{c}\text { Corrosion } \\
\text { C (in) }\end{array}$} & \multirow[t]{2}{*}{ Load } & \multirow{2}{*}{$\begin{array}{l}\text { Req'd Thk } \\
\text { Due to } \\
\text { Tension (in) }\end{array}$} & \multirow{2}{*}{$\begin{array}{l}\text { Req'd Thk Due } \\
\text { to Compression } \\
\text { (in) }\end{array}$} \\
\hline & & $S_{\mathbf{t}}$ & $S_{c}$ & & & & & \\
\hline \multirow{2}{*}{$\begin{array}{l}\text { Operating, Hot \& } \\
\text { Corroded }\end{array}$} & \multirow{2}{*}{1,150} & \multirow{2}{*}{15,300} & \multirow{2}{*}{9,945} & \multirow{2}{*}{1,000} & \multirow{2}{*}{0.03} & Wind & 0.1216 & 0.1191 \\
\hline & & & & & & Seismic & 0.1219 & 0.1187 \\
\hline \multirow{2}{*}{$\begin{array}{c}\text { Operating, Hot \& } \\
\text { New }\end{array}$} & \multirow{2}{*}{1,150} & \multirow{2}{*}{15,300} & \multirow{2}{*}{9,995} & \multirow{2}{*}{1,000} & \multirow{2}{*}{0} & Wind & 0.1206 & 0.1182 \\
\hline & & & & & & Seismic & 0.121 & 0.1177 \\
\hline \multirow{2}{*}{ Empty, Corroded } & \multirow{2}{*}{0} & \multirow{2}{*}{20,000} & \multirow{2}{*}{14,142} & \multirow{2}{*}{0} & \multirow{2}{*}{0.03} & Wind & 0.0006 & 0.0018 \\
\hline & & & & & & Seismic & 0.0009 & 0.0022 \\
\hline \multirow{2}{*}{ Empty, New } & \multirow{2}{*}{0} & \multirow{2}{*}{20,000} & \multirow{2}{*}{14,203} & \multirow{2}{*}{0} & \multirow{2}{*}{0} & Wind & 0.0006 & 0.0018 \\
\hline & & & & & & Seismic & 0.0009 & 0.0024 \\
\hline $\begin{array}{l}\text { Hot Shut Down, } \\
\text { Corroded, Weight \& } \\
\text { Eccentric Moments } \\
\text { Only }\end{array}$ & 0 & 15,300 & 9,945 & 1,000 & 0.03 & Weight & 0.0005 & 0.001 \\
\hline
\end{tabular}




\section{8" X 4" REDUCER B16.9, LOWER (SCH XS) 4" END}

\section{ASME Section VIII Division 1, 2007 Edition}

Component:

Material specification:

Rated MDMT per UHA-51(d)(1)(b), (carbon content does not exceed 0.1 percent) $=-320^{\circ} \mathrm{F}$
Cylinder

SA-403 316H (II-D p. 78, ln. 8)

Internal design pressure: $\mathrm{P}=1,150$ psi @ $1,000^{\circ} \mathrm{F}$

Static liquid head:

$P_{\text {th }}=0.26 \mathrm{psi}\left(\mathrm{SG}=1, \mathrm{H}_{\mathrm{s}}=7.205^{\prime \prime}\right.$, Horizontal test head $)$

Corrosion allowance $\quad$ Inner $\mathrm{C}=0.03^{\prime \prime} \quad$ Outer $\mathrm{C}=0^{\prime \prime}$

$\begin{array}{ll}\text { Design MDMT }=-20^{\circ} \mathrm{F} & \text { No impact test performed } \\ \text { Rated MDMT }=-320^{\circ} \mathrm{F} & \text { Material is not normalized } \\ & \text { Material is not produced to Fine Grain Practice } \\ & \text { PWHT is not performed }\end{array}$

Radiography: $\quad$ Longitudinal joint - $\quad$ Seamless No RT

Top circumferential joint - Full UW-11(a) Type 1 Bottom circumferential joint -

Full UW-11(a) Type 1

$$
\begin{aligned}
& \text { Estimated weight } \mathrm{New}=3.4 \mathrm{lb} \quad \text { corr }=3.1 \mathrm{lb} \\
& \text { Capacity } \quad \mathrm{New}=0.16 \mathrm{US} \text { gal corr }=0.16 \mathrm{US} \text { gal } \\
& \text { OD }=4.5^{\prime \prime} \\
& \begin{aligned}
\text { Length } \mathrm{L}_{\mathrm{c}}=3^{\prime \prime} \\
\mathrm{t} \quad=0.295^{\prime \prime}
\end{aligned}
\end{aligned}
$$

\section{Design thickness, (at $1,000^{\circ} \mathrm{F}$ ) Appendix 1-1}

$$
\begin{aligned}
\mathrm{t} & =\mathrm{P} * \mathrm{R}_{\mathrm{o}} /(\mathrm{S} * \mathrm{E}+0.40 * \mathrm{P})+\text { Corrosion } \\
& =1,150^{*} 2.25 /\left(15,300^{*} 1.00+0.40^{*} 1,150\right)+0.03 \\
& =0.1942 "
\end{aligned}
$$

Maximum allowable working pressure, (at 1,000 ${ }^{\circ} \mathrm{F}$ ) Appendix 1-1

$$
\begin{aligned}
\mathrm{P} & =\mathrm{S} * \mathrm{E}^{*} \mathrm{t} /\left(\mathrm{R}_{\mathrm{o}}-0.40^{*} \mathrm{t}\right)-\mathrm{P}_{\mathrm{S}} \\
& =15,300^{*} 1.00^{*} 0.265 /\left(2.25-0.40^{*} 0.265\right)-0 \\
& =1,891.09 \mathrm{psi}
\end{aligned}
$$

\% Forming Strain - UHA-44(a)(2)(a)

$$
\begin{aligned}
\mathrm{EFE} & =\left(50 * t / \mathrm{R}_{\mathrm{f}}\right) *\left(1-\mathrm{R}_{\mathbf{f}} / \mathrm{R}_{\mathrm{o}}\right) \\
& =(50 * 0.295 / 2.1025) *(1-2.1025 / \infty) \\
& =7.0155 \%
\end{aligned}
$$




\section{Design thickness $=0.1942^{\prime \prime}$}

The governing condition is due to internal pressure.

The cylinder thickness of $0.295^{\prime \prime}$ is adequate.

Thickness Required Due to Pressure + External Loads

\begin{tabular}{|c|c|c|c|c|c|c|c|c|}
\hline \multirow[t]{2}{*}{ Condition } & \multirow{2}{*}{$\begin{array}{l}\text { Pressure } \\
\text { P ( psi) }\end{array}$} & \multicolumn{2}{|c|}{$\begin{array}{c}\text { Allowable Stress } \\
\text { Before UG-23 Stress } \\
\text { Increase ( psi) }\end{array}$} & \multirow{2}{*}{$\begin{array}{l}\text { Temperature } \\
\left({ }^{\circ} \mathrm{F}\right)\end{array}$} & \multirow{2}{*}{$\begin{array}{l}\text { Corrosion } \\
\text { C (in) }\end{array}$} & \multirow[t]{2}{*}{ Load } & \multirow{2}{*}{$\begin{array}{c}\text { Req'd Thk } \\
\text { Due to } \\
\text { Tension (in) }\end{array}$} & \multirow{2}{*}{$\begin{array}{l}\text { Req'd Thk Due } \\
\text { to Compression } \\
\text { (in) }\end{array}$} \\
\hline & & $\mathbf{S}_{\mathfrak{t}}$ & $S_{c}$ & & & & & \\
\hline \multirow{2}{*}{$\begin{array}{l}\text { Operating, Hot } \& \\
\text { Corroded }\end{array}$} & \multirow{2}{*}{1,150} & \multirow{2}{*}{15,300} & \multirow{2}{*}{10,100} & \multirow{2}{*}{1,000} & \multirow{2}{*}{0.03} & Wind & 0.0655 & 0.0555 \\
\hline & & & & & & Seismic & 0.0668 & 0.0541 \\
\hline \multirow{2}{*}{$\begin{array}{c}\text { Operating, Hot \& } \\
\text { New }\end{array}$} & \multirow{2}{*}{1,150} & \multirow{2}{*}{15,300} & \multirow{2}{*}{10,177} & \multirow{2}{*}{1,000} & \multirow{2}{*}{0} & Wind & 0.0647 & 0.0544 \\
\hline & & & & & & Seismic & 0.0663 & 0.0527 \\
\hline \multirow{2}{*}{ Empty, Corroded } & \multirow{2}{*}{0} & \multirow{2}{*}{20,000} & \multirow{2}{*}{14,332} & \multirow{2}{*}{0} & \multirow{2}{*}{0.03} & Wind & 0.0032 & 0.0062 \\
\hline & & & & & & Seismic & 0.0042 & 0.0078 \\
\hline \multirow{2}{*}{ Empty, New } & \multirow{2}{*}{0} & \multirow{2}{*}{20,000} & \multirow{2}{*}{14,425} & \multirow{2}{*}{0} & \multirow{2}{*}{0} & Wind & 0.0032 & 0.0064 \\
\hline & & & & & & Seismic & 0.0044 & 0.0082 \\
\hline $\begin{array}{l}\text { Hot Shut Down, } \\
\text { Corroded, Weight \& } \\
\text { Eccentric Moments } \\
\text { Only }\end{array}$ & 0 & 15,300 & 10,100 & 1,000 & 0.03 & Weight & 0.0006 & 0.0025 \\
\hline
\end{tabular}




\section{4" X 2" REDUCER B16.9, LOWER (SCH XS) 4" END}

ASME Section VIII Division 1, 2007 Edition

Component:

Material specification:

Rated MDMT per UHA-51(d)(1)(b), (carbon content does not exceed 0.1 percent) $=-320{ }^{\circ} \mathrm{F}$

Cylinder

SA-403 316 H (II-D p. $78, \ln .8$ )
Internal design pressure: $\mathrm{P}=1,150 \mathrm{psi} @ 1,000^{\circ} \mathrm{F}$

Static liquid head:

$P_{\text {th }}=0.26 \mathrm{psi}\left(\mathrm{SG}=1, \mathrm{H}_{\mathrm{s}}=7.205^{\prime \prime}\right.$, Horizontal test head $)$

Corrosion allowance $\quad$ Inner $\mathrm{C}=0.03^{\prime \prime} \quad$ Outer $\mathrm{C}=0 "$

Design MDMT $=-20^{\circ} \mathrm{F} \quad$ No impact test performed

Rated MDMT $=-320^{\circ} \mathrm{F} \quad$ Material is not normalized

Material is not produced to Fine Grain Practice

PWHT is notperformed

Radiography: $\quad$ Longitudinal joint - $\quad$ Seamless No RT

Top circumferential joint - Full UW-11(a) Type 1

Bottom circumferential joint -

Full UW-11(a) Type 1

Estimated weight New $=2.3 \mathrm{lb} \quad$ corr $=2 \mathrm{lb}$

Capacity $\quad$ New $=0.1$ US gal corr $=0.11$ US gal

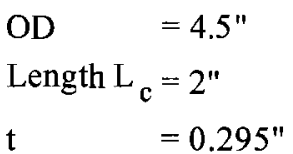

Design thickness, (at $1,000^{\circ} \mathrm{F}$ ) Appendix 1-1

$\mathrm{t}=\mathrm{P} * \mathrm{R}_{\mathrm{o}} /(\mathrm{S} * \mathrm{E}+0.40 * \mathrm{P})+$ Corrosion

$=1,150 * 2.25 /(15,300 * 1.00+0.40 * 1,150)+0.03$

$=0.1942^{\prime \prime}$

Maximum allowable working pressure, (at 1,000 ${ }^{\circ}$ F) Appendix 1-1

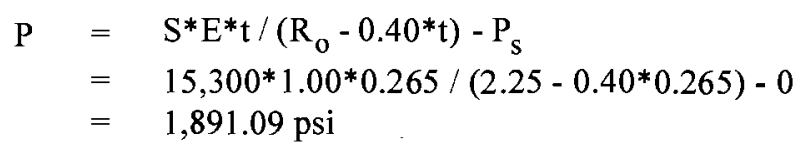

\% Forming Strain - UHA-44(a)(2)(a)

$$
\begin{aligned}
\mathrm{EFE} & =\left(50 * \mathrm{t} / \mathrm{R}_{\mathrm{f}}\right) *\left(1-\mathrm{R}_{\mathrm{f}} / \mathrm{R}_{\mathrm{o}}\right) \\
& =(50 * 0.295 / 2.1025) *(1-2.1025 / \infty) \\
& =7.0155 \%
\end{aligned}
$$




\section{Design thickness $=0.1942 "$}

The governing condition is due to internal pressure.

The cylinder thickness of $0.295^{\prime \prime}$ is adequate.

Thickness Required Due to Pressure + External Loads

\begin{tabular}{|c|c|c|c|c|c|c|c|c|}
\hline \multirow[t]{2}{*}{ Condition } & \multirow{2}{*}{$\begin{array}{l}\text { Pressure } \\
\text { P ( psi) }\end{array}$} & \multicolumn{2}{|c|}{$\begin{array}{l}\text { Allowable Stress } \\
\text { Before UG-23 Stress } \\
\text { Increase ( psi) }\end{array}$} & \multirow{2}{*}{$\begin{array}{l}\text { Temperature } \\
\left({ }^{\circ} \mathrm{F}\right)\end{array}$} & \multirow{2}{*}{$\begin{array}{l}\text { Corrosion } \\
\text { C (in) }\end{array}$} & \multirow[t]{2}{*}{ Load } & \multirow{2}{*}{$\begin{array}{l}\text { Req'd Thk } \\
\text { Due to } \\
\text { Tension (in) }\end{array}$} & \multirow{2}{*}{$\begin{array}{l}\text { Req'd Thk Due } \\
\text { to Compression } \\
\text { (in) }\end{array}$} \\
\hline & & $S_{t}$ & $\mathbf{S}_{\mathbf{c}}$ & & & & & \\
\hline \multirow{2}{*}{$\begin{array}{l}\text { Operating, Hot \& } \\
\text { Corroded }\end{array}$} & \multirow{2}{*}{1,150} & \multirow{2}{*}{15,300} & \multirow{2}{*}{10,100} & \multirow{2}{*}{1,000} & \multirow{2}{*}{0.03} & Wind & 0.0659 & 0.0552 \\
\hline & & & & & & Seismic & 0.0671 & 0.0538 \\
\hline \multirow{2}{*}{$\begin{array}{c}\text { Operating, Hot \& } \\
\text { New }\end{array}$} & \multirow{2}{*}{1,150} & \multirow{2}{*}{15,300} & \multirow{2}{*}{10,177} & \multirow{2}{*}{1,000} & \multirow{2}{*}{0} & Wind & 0.065 & 0.0541 \\
\hline & & & & & & Seismic & 0.0665 & 0.0524 \\
\hline \multirow{2}{*}{ Empty, Corroded } & \multirow{2}{*}{0} & \multirow{2}{*}{20,000} & \multirow{2}{*}{14,332} & \multirow{2}{*}{0} & \multirow{2}{*}{0.03} & Wind & 0.0034 & 0.0066 \\
\hline & & & & & & Seismic & 0.0044 & 0.0081 \\
\hline \multirow{2}{*}{ Empty, New } & \multirow{2}{*}{0} & \multirow{2}{*}{20,000} & \multirow{2}{*}{14,425} & \multirow{2}{*}{0} & \multirow{2}{*}{0} & Wind & 0.0035 & 0.0068 \\
\hline & & & & & & Seismic & 0.0046 & 0.0086 \\
\hline $\begin{array}{l}\text { Hot Shut Down, } \\
\text { Corroded, Weight \& } \\
\text { Eccentric Moments } \\
\text { Only }\end{array}$ & 0 & 15,300 & 10,100 & 1,000 & 0.03 & Weight & 0.0006 & 0.0025 \\
\hline
\end{tabular}




\section{4" X 2" REDUCER B16.9, LOWER (SCH XS) 2" END}

\section{ASME Section VIII Division 1, 2007 Edition}

Component:

Material specification:

Rated MDMT per UHA-51(d)(l)(b), (carbon content does not exceed 0.1 percent) $=-320^{\circ} \mathrm{F}$

Static liquid head:

$\mathrm{P}_{\text {th }}=0.23 \mathrm{psi}\left(\mathrm{SG}=1, \mathrm{H}_{\mathrm{s}}=6.2465^{\prime}\right.$, Horizontal test head $)$

Corrosion allowance $\quad$ Inner $\mathrm{C}=0.03^{\prime \prime} \quad$ Outer $\mathrm{C}=0^{\prime \prime}$

$\begin{array}{ll}\text { Design MDMT }=-20^{\circ} \mathrm{F} & \text { No impact test performed } \\ \text { Rated MDMT }=-320^{\circ} \mathrm{F} & \text { Material is not normalized } \\ & \text { Material is not produced to Fine Grain Practice } \\ & \text { PWHT is not performed }\end{array}$

Radiography: $\quad$ Longitudinal joint - $\quad$ Seamless No RT

Top circumferential joint - Full UW-11(a) Type 1

Bottom circumferential joint -

Full UW-11(a) Type 1

Estimated weight New $=0.8 \mathrm{lb} \quad$ corr $=0.6 \mathrm{lb}$

Capacity New $=0.03$ US gal corr $=0.03$ US gal

OD $=2.375^{\prime \prime}$
Length $\mathrm{L}_{\mathrm{c}}=2^{\prime \prime}$
$\quad=0.191^{\prime \prime}$

Design thickness, (at $1,000^{\circ}$ F) Appendix 1-1

$\mathrm{t}=\mathrm{P}^{*} \mathrm{R}_{\mathrm{o}} /(\mathrm{S} * \mathrm{E}+0.40 * \mathrm{P})+$ Corrosion

$=1,150 * 1.1875 /(15,300 * 1.00+0.40 * 1,150)+0.03$

$=0.1167^{\prime \prime}$

Maximum allowable working pressure, (at 1,000 $\left.{ }^{\circ} \mathrm{F}\right)$ Appendix 1-1

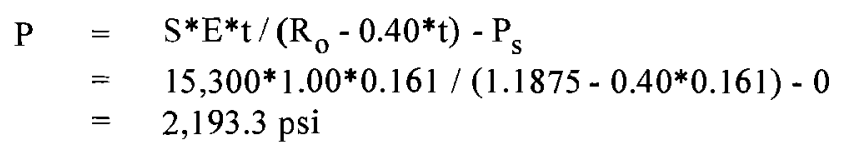

\% Forming Strain - UHA-44(a)(2)(a)

$$
\begin{aligned}
\mathrm{EFE} & =\left(50 * \mathrm{t} / \mathrm{R}_{\mathrm{f}}\right) *\left(1-\mathrm{R}_{\mathrm{f}} / \mathrm{R}_{\mathrm{o}}\right) \\
& =(50 * 0.191 / 1.092) *(1-1.092 / \infty) \\
& =8.7454 \%
\end{aligned}
$$




\section{Design thickness $=0.1167^{\prime \prime}$}

The governing condition is due to internal pressure.

The cylinder thickness of $0.191^{\prime \prime}$ is adequate.

Thickness Required Due to Pressure + External Loads

\begin{tabular}{|c|c|c|c|c|c|c|c|c|}
\hline \multirow[t]{2}{*}{ Condition } & \multirow{2}{*}{$\begin{array}{l}\text { Pressure } \\
\text { P ( psi) }\end{array}$} & \multicolumn{2}{|c|}{$\begin{array}{c}\text { Allowable Stress } \\
\text { Before UG-23 Stress } \\
\text { Increase ( psi) }\end{array}$} & \multirow{2}{*}{$\begin{array}{c}\text { Temperature } \\
\left({ }^{\circ} \mathbf{F}\right)\end{array}$} & \multirow{2}{*}{$\begin{array}{l}\text { Corrosion } \\
\text { C (in) }\end{array}$} & \multirow[t]{2}{*}{ Load } & \multirow{2}{*}{$\begin{array}{l}\text { Req'd Thk } \\
\text { Due to } \\
\text { Tension (in) }\end{array}$} & \multirow{2}{*}{$\begin{array}{l}\text { Req'd Thk Due } \\
\text { to Compression } \\
\text { (in) }\end{array}$} \\
\hline & & $S_{1}$ & $\mathbf{S}_{\mathbf{c}}$ & & & & & \\
\hline \multirow{2}{*}{$\begin{array}{l}\text { Operating, Hot \& } \\
\text { Corroded }\end{array}$} & \multirow{2}{*}{1,150} & \multirow{2}{*}{15,300} & \multirow{2}{*}{10,201} & \multirow{2}{*}{1,000} & \multirow{2}{*}{0.03} & Wind & 0.0532 & 0.0066 \\
\hline & & & & & & Seismic & 0.0571 & 0.0023 \\
\hline \multirow{2}{*}{$\begin{array}{c}\text { Operating, Hot \& } \\
\text { New }\end{array}$} & \multirow{2}{*}{1,150} & \multirow{2}{*}{15,300} & \multirow{2}{*}{10,324} & \multirow{2}{*}{1,000} & \multirow{2}{*}{0} & Wind & 0.053 & 0.0047 \\
\hline & & & & & & Seismic & 0.0582 & 0.0016 \\
\hline \multirow{2}{*}{ Empty, Corroded } & \multirow{2}{*}{0} & \multirow{2}{*}{20,000} & \multirow{2}{*}{14,454} & \multirow{2}{*}{0} & \multirow{2}{*}{0.03} & Wind & 0.0164 & 0.0266 \\
\hline & & & & & & Seismic & 0.0194 & 0.0312 \\
\hline \multirow{2}{*}{ Empty, New } & \multirow{2}{*}{0} & \multirow{2}{*}{20,000} & \multirow{2}{*}{14,604} & \multirow{2}{*}{0} & \multirow{2}{*}{0} & Wind & 0.017 & 0.0274 \\
\hline & & & & & & Seismic & 0.021 & 0.0332 \\
\hline $\begin{array}{l}\text { Hot Shut Down, } \\
\text { Corroded, Weight \& } \\
\text { Eccentric Moments } \\
\text { Only }\end{array}$ & 0 & 15,300 & 10,201 & 1,000 & 0.03 & Weight & 0 & 0.0067 \\
\hline
\end{tabular}




\section{2" RFWN FLANGE 1500\#}

Flange description:

Bolt Material:

Flange rated MDMT:

(Per UHA-51(d)(1)(b))

(Flange rated MDMT $=-320^{\circ} \mathrm{F}$

Bolts rated MDMT per Fig UCS -66 note $(\mathrm{e})=-55^{\circ} \mathrm{F}$ )

Liquid static head on flange:

ASME B16.5 flange rating MAWP:

ASME B 16.5 flange rating MAP:

ASME B16.5 flange hydro test:
2 inch Class 1500 WN A 182 F316H

SA-193 B7 Bolt $<=21 / 2$

$-55^{\circ} \mathrm{F}$

\section{$0 \mathrm{psi}$}

1820 psi@ $1000^{\circ} \mathrm{F}$

$3600 \mathrm{psi}$ @70 $\mathrm{F}$

5425 psi@ $90^{\circ} \mathrm{F}$ 


\section{2" BRANCH (B1)}

\section{ASME Section VIII Division 1, 2007 Edition}

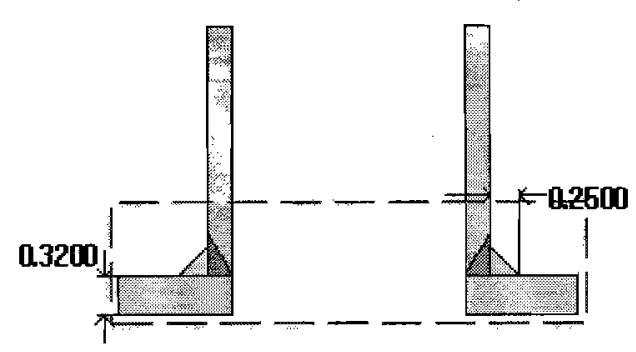

$$
\begin{aligned}
& \mathrm{t}_{\mathrm{w}(\text { lower })}=0 \mathrm{in} \\
& \operatorname{Leg}_{41}=0.25 \mathrm{in}
\end{aligned}
$$

Note: round inside edges per UG-76(c)

Located on:

3 OD" PIPE

Liquid static head included:

$0 \mathrm{psi}$

Nozzle material specification:

SA-312 TP316H Wld \& smls pipe (II-D p. $78, \ln .1$ )

Nozzle longitudinal joint efficiency:

1

$2 "$ Sch 80 S (XS)

Nozzle description:

$190^{\circ}$

Local vessel minimum thickness:

0.28 in

Nozzle center/shell outer surface intersection to datum: 69.25 in

End of nozzle to shell center:

19.25 in

1.939 in

Nozzle inside diameter, new:

0.218 in

Nozzle nominal wall thickness:

0.03 in

Nozzle corrosion allowance:

17.7139 in

Projection available outside vessel, Lpr:

$-5^{\circ}$ from radial

Nozzle is tilted:

\section{Reinforcement Calculations for Internal Pressure}

\begin{tabular}{|c|c|c|c|c|c|c|c|c|}
\hline \multicolumn{7}{|c|}{$\begin{array}{l}\text { Appendix 1-10 Maximum Local Primary Membrane Stress } \\
\text { For } \mathrm{P}=1250 \mathrm{psi} @ 1000{ }^{\circ} \mathrm{F}\end{array}$} & \multicolumn{2}{|c|}{$\begin{array}{l}\text { UG-45 Nozzle Wall } \\
\text { Thickness Summary (in) } \\
\text { The nozzle passes UG-45 }\end{array}$} \\
\hline$\underset{\text { (psi) }}{\mathbf{P}_{\mathbf{L}}}$ & $\begin{array}{c}\mathbf{s}_{\text {allow }} \\
\text { (psi) }\end{array}$ & $\begin{array}{c}A_{1} \\
\left(\text { in }^{2}\right)\end{array}$ & $\begin{array}{c}\mathbf{A}_{2} \\
\left(\mathbf{i n}^{2}\right)\end{array}$ & $\begin{array}{c}\mathbf{A}_{\mathbf{3}} \\
\left(\mathbf{i n}^{2}\right)\end{array}$ & $\begin{array}{c}\mathbf{A}_{5} \\
\left(\text { in }^{2}\right)\end{array}$ & $\begin{array}{c}\text { A } \\
\text { welds } \\
\left(\mathbf{i n}^{2}\right)\end{array}$ & $\begin{array}{l}t_{\text {req }} \\
\text { (in) }\end{array}$ & $\begin{array}{l}t_{\min } \\
\text { (in) }\end{array}$ \\
\hline \multicolumn{7}{|c|}{ This nozzle is exempt from area calculations per UG-36(c)(3)(a) } & 0.1648 & 0.1908 \\
\hline
\end{tabular}

The vessel wall thickness governs the MAWP of thisnozzle.

Division 2 Part 4.5 Strength of Nozzle Attachment Welds Summary Average Shear Stress in Weld

\begin{tabular}{|l|l|l|l|l|l|l|l|l|}
\hline & $\mathbf{L}_{\tau}$ & $\mathbf{L}_{41 \mathrm{~T}}$ & $\mathbf{L}_{42 \mathrm{~T}}$ & $\mathbf{L}_{43 \mathrm{~T}}$ & $\mathbf{f}_{\text {welds }}$ & $\tau$ & $\mathbf{S}$ & Over \\
\hline
\end{tabular}




\begin{tabular}{|c|c|c|c|c|c|c|c|c|}
$k_{y}$ & (in) & (in) & (in) & (in) & (lb $b_{\mathbf{f}}$ & (psi) & (psi) & stressed \\
\hline 1 & 1.8683 & 0.1768 & 0 & 0 & $1,517.52$ & 4,073 & 7,000 & No \\
\hline
\end{tabular}

\begin{tabular}{|c|r|r|c|}
\hline \multicolumn{4}{|c|}{ UW-16 Weld Sizing Sum mary } \\
\hline Weld description & $\begin{array}{l}\text { Required weld } \\
\text { throat size (in) }\end{array}$ & $\begin{array}{r}\text { Actual weld } \\
\text { throat size (in) }\end{array}$ & Status \\
\hline Nozzle to shell fillet $\left(\mathrm{Leg}_{41}\right)$ & 0.1316 & 0.175 & weld size is adequate \\
\hline
\end{tabular}

This opening does not require reinforcement per UG-36(c)(3)(a)

\section{Reinforcement Calculations for MAP}

The thickness requirements of UG-45 govern the MAP of this nozzle.

\begin{tabular}{|c|c|c|c|c|c|c|c|c|}
\hline \multicolumn{7}{|c|}{$\begin{array}{l}\text { Appendix 1-10 Maximum Local Primary Membrane Stress } \\
\text { For } \mathrm{P}=3519.48 \mathrm{psi} @ 7^{\circ} \mathrm{F}\end{array}$} & \multicolumn{2}{|c|}{$\begin{array}{l}\text { UG-45 Nozzle Wall } \\
\text { Thickness Sum mary (in) } \\
\text { The nozzle passes UG-45 }\end{array}$} \\
\hline $\begin{array}{c}\mathbf{P}_{\mathbf{L}} \\
\text { (psi) }\end{array}$ & $\begin{array}{c}\mathbf{S}_{\text {allow }} \\
\text { (psi) }\end{array}$ & $\begin{array}{c}A_{1} \\
\left(\text { in }^{2}\right)\end{array}$ & $\begin{array}{c}\mathbf{A}_{2} \\
\left(\mathrm{in}^{2}\right)\end{array}$ & $\begin{array}{c}\mathbf{A}_{3} \\
\left(\mathbf{i n}^{2}\right)\end{array}$ & $\begin{array}{r}\mathbf{A}_{5} \\
\left(\text { in }^{2}\right)\end{array}$ & $\begin{array}{c}\text { A } \\
\text { welds } \\
\left(\text { in }^{2}\right)\end{array}$ & $\begin{array}{l}\mathbf{t}_{\text {req }} \\
\text { (in) }\end{array}$ & $\begin{array}{l}t_{\min } \\
\text { (in) }\end{array}$ \\
\hline \multicolumn{7}{|c|}{ This nozzle is exempt from area calculations per UG-36(c)(3)(a) } & 0.1907 & 0.1908 \\
\hline
\end{tabular}

Division 2 Part 4.5 Strength of Nozzle Attachment Welds Summary Average Shear Stress in Weld

\begin{tabular}{|c|c|c|c|c|c|c|c|c|}
\hline $\mathbf{k}_{\mathbf{y}}$ & $\begin{array}{c}\mathbf{L}_{\tau} \\
(\mathbf{i n})\end{array}$ & $\begin{array}{c}\mathbf{L}_{41 T} \\
(\mathbf{i n})\end{array}$ & $\begin{array}{c}\mathbf{L}_{42 T} \\
(\text { in) }\end{array}$ & $\begin{array}{c}\mathbf{L}_{43 T} \\
(\mathbf{i n})\end{array}$ & $\begin{array}{c}\mathbf{f}_{\text {welds }} \\
\left(\mathbf{l b}_{\mathbf{f}}\right)\end{array}$ & $\begin{array}{c}\tau \\
(\mathbf{p s i})\end{array}$ & $\begin{array}{c}\mathbf{S} \\
(\mathbf{p s i})\end{array}$ & $\begin{array}{c}\text { Over } \\
\text { stressed }\end{array}$ \\
\hline 1 & 1.8682 & 0.1768 & 0 & 0 & $4,041.71$ & 9,950 & 22,900 & No \\
\hline
\end{tabular}

\begin{tabular}{|c|r|r|c|}
\hline \multicolumn{4}{|c|}{ UW-16 Weld Sizing Summary } \\
\hline Weld description & $\begin{array}{r}\text { Required weld } \\
\text { throat size (in) }\end{array}$ & $\begin{array}{r}\text { Actual weld } \\
\text { throat size (in) }\end{array}$ & Status \\
\hline Nozzle to shell fillet $\left(\right.$ Leg $\left._{41}\right)$ & 0.1526 & 0.175 & weld size is adequate \\
\hline
\end{tabular}

This opening does not require reinforcement per UG-36(c)(3)(a) 


\section{1/2" HALF-COUPLING (7a)}

\section{ASME Section VIII Division 1, 2007 Edition}

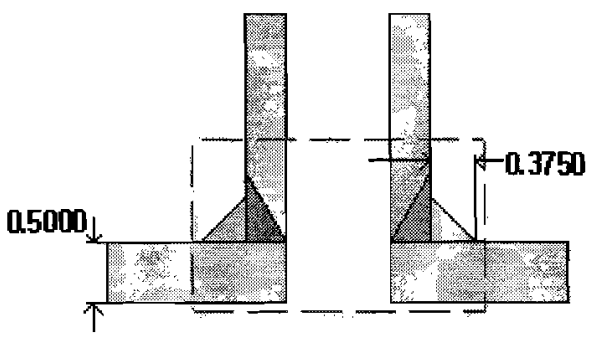

$$
\begin{aligned}
& \mathrm{t}_{\mathrm{w}(\text { lower })}=0 \mathrm{in} \\
& \operatorname{Leg}_{41}=0.375 \mathrm{in}
\end{aligned}
$$

Note. round inside edges per $\mathrm{UG}-706(\mathrm{c})$

Located on:

Liquid static head included:

Nozzle material specification:

Nozzle longitudinal joint efficiency:

Nozzle description:

Nozzle orientation:

Local vessel minimum thickness:

Nozzle center line offset to datum line:

End of nozzle to shell center:

Nozzle inside diameter, new:

Nozzle nominal wall thickness:

Nozzle corrosion allowance:

Projection available outside vessel, Lpr: 0.9375 in

0.4375 in
8" PIPE

0 psi

SA-182 F316H $<=5$ (low stress) (II-D p. 74, ln. 32)

1

$0.500 "$ Class 6000 - threaded

$0^{\circ}$

48.75 in

5.25 in

0.84 in

0.33 in

0.03 in

\section{Reinforcement Calculations for Internal Pressure}

\begin{tabular}{|c|c|c|c|c|c|c|c|c|}
\hline \multicolumn{7}{|c|}{$\begin{array}{l}\text { UG-37 Area Calculation Summary }\left(\mathrm{in}^{2}\right) \\
\text { For } \mathrm{P}=1502.52 \mathrm{psi} @ 1000^{\circ} \mathrm{F}\end{array}$} & \multicolumn{2}{|c|}{$\begin{array}{l}\text { UG-45 Nozzle Wall } \\
\text { Thickness Summary (in) } \\
\text { The nozzle passes UG-45 }\end{array}$} \\
\hline$\underset{\text { required }}{A}$ & $\underset{\text { available }}{\mathrm{A}}$ & $\mathbf{A}_{1}$ & $\mathbf{A}_{2}$ & $\mathbf{A}_{3}$ & $A_{5}$ & $\begin{array}{c}\text { A } \\
\text { welds }\end{array}$ & $\mathbf{t}_{\text {req }}$ & $\mathbf{t}_{\min }$ \\
\hline \multicolumn{7}{|c|}{ This nozzle is exempt from area calculations per UG-36(c)(3)(a) } & 0.1247 & 0.33 \\
\hline
\end{tabular}

The vessel wall thickness governs the MAWP of this nozzle.

UG-41 Weld Failure Path Analysis Summary

The nozzle is exempt from weld strength calculations per UW-15(b)(2)

\begin{tabular}{|l|l|l|}
\hline \multicolumn{3}{|c|}{ UW -16 Weld Sizing Summary } \\
\hline & &
\end{tabular}




\begin{tabular}{|c|r|r|c|} 
Weld description & $\begin{array}{r}\text { Required weld } \\
\text { throat size (in) }\end{array}$ & $\begin{array}{r}\text { Actual weld } \\
\text { throat size (in) }\end{array}$ & Status \\
\hline Nozzle to shell fillet $\left(\operatorname{Leg}_{41}\right)$ & 0.21 & 0.2625 & weld size is adequate \\
\hline
\end{tabular}

This opening does not require reinforcement per UG-36(c)(3)(a)

\section{Reinforcement Calculations for MAP}

The vessel wall thickness governs the MAP of this nozzle.

\begin{tabular}{|c|c|c|c|c|c|c|c|c|}
\hline \multicolumn{7}{|c|}{$\begin{array}{l}\text { UG-37 Area Calculation Summary }\left(\text { in }^{2}\right) \\
\text { For } P=2114.8 \mathrm{psi} @ 70^{\circ} \mathrm{F}\end{array}$} & \multicolumn{2}{|c|}{$\begin{array}{c}\text { UG-45 Nozzle Wall } \\
\text { Thickness Summary (in) } \\
\text { The nozzle passes UG-45 }\end{array}$} \\
\hline$\stackrel{A}{\text { required }}$ & $\underset{\text { available }}{\mathbf{A}}$ & $\mathbf{A}_{1}$ & $\mathbf{A}_{2}$ & $\mathbf{A}_{3}$ & $\mathbf{A}_{5}$ & $\begin{array}{c}\text { A } \\
\text { welds }\end{array}$ & $\mathbf{t}_{\text {req }}$ & $\mathbf{t}_{\mathrm{min}}$ \\
\hline \multicolumn{7}{|c|}{ This nozzle is exempt from area calculations per UG-36(c)(3)(a) } & 0.0761 & 0.33 \\
\hline
\end{tabular}

\section{UG-41 Weld Failure Path Analysis Summary}

The nozzle is exempt from weld strength calculations per UW-15(b)(2)

\begin{tabular}{|c|r|r|c|}
\hline \multicolumn{4}{|c|}{ UW-16 Weld Sizing Summary } \\
\hline Weld description & $\begin{array}{r}\text { Required weld } \\
\text { throat size (in) }\end{array}$ & $\begin{array}{r}\text { Actual weld } \\
\text { throat size (in) }\end{array}$ & Status \\
\hline Nozzle to shell fillet $\left(\right.$ Leg $\left._{41}\right)$ & 0.231 & 0.2625 & weld size is adequate \\
\hline
\end{tabular}

This opening does not require reinforcement per UG-36(c)(3)(a) 


\section{1/2" HALF-COUPLING (7b)}

\section{ASME Section VIII Division 1, 2007 Edition}

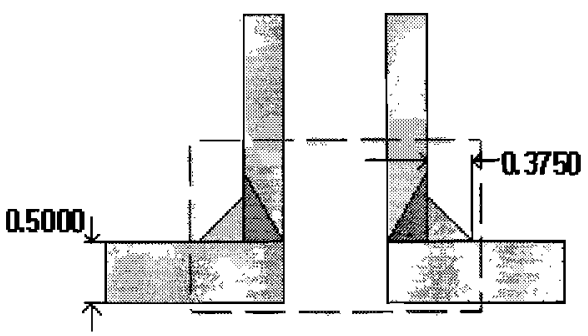

$$
\mathrm{t}_{\mathrm{w}(\text { lower })}=0 \text { in }
$$$$
\operatorname{Leg}_{41}=0.375 \text { in }
$$

Note: round inside edges per UG-76(c)

Located on:

Liquid static head included:

Nozzle material specification:

Nozzle longitudinal joint efficiency:

Nozzle description:

Nozzle orientation:

Local vessel minimum thickness:

Nozzle center line offset to datum line: 48.75 in

End of nozzle to shell center:

Nozzle inside diameter, new:

Nozzle nominal wall thickness:

Nozzle corrosion allowance:

Projection available outside vessel, Lpr: 0.9375 in
8" PIPE

0 psi

SA-182 F316H $<=5$ (low stress) (II-D p. 74, In. 32)

1

$0.500^{\prime \prime}$ Class 6000 - threaded

$180^{\circ}$

0.4375 in

5.25 in

0.84 in

0.33 in

0.03 in

\section{Reinforcement Calculations for Internal Pressure}

The vessel wall thickness governs the MAWP of this nozzle.

\begin{tabular}{|c|c|c|c|c|c|c|c|c|}
\hline \multicolumn{7}{|c|}{$\begin{array}{l}\text { UG-37 Area Calculation Summary }\left(\text { in }^{2}\right) \\
\text { For } P=1502.52 \text { psi } @ 1000^{\circ} \mathrm{F}\end{array}$} & \multicolumn{2}{|c|}{\begin{tabular}{|c|} 
UG-45 Nozzle Wall \\
Thickness Su m mary (in) \\
The nozzle passes UG-45 \\
\end{tabular}} \\
\hline$\underset{\text { required }}{\text { A }}$ & $\underset{\text { available }}{\text { A }}$ & $A_{1}$ & $\mathbf{A}_{2}$ & $\mathbf{A}_{3}$ & $\mathbf{A}_{5}$ & $\underset{\text { welds }}{\mathbf{A}}$ & $t_{\text {req }}$ & $t_{\min }$ \\
\hline \multicolumn{7}{|c|}{ This nozzle is exempt from area calculations perUG-36(c)(3)(a) } & 0.1247 & 0.33 \\
\hline
\end{tabular}

\section{UG-41 Weld Failure Path Analysis Summary}

The nozzle is exempt from weld strength calculations per UW-15(b)(2)

\begin{tabular}{|l|l|l|}
\hline \multicolumn{3}{|c|}{ UW-16 Weld Sizing Summary } \\
\hline $\mid$ & & \\
\hline
\end{tabular}




\begin{tabular}{|c|r|r|c|} 
Weld description & $\begin{array}{r}\text { Required weld } \\
\text { throat size (in) }\end{array}$ & $\begin{array}{r}\text { Actual weld } \\
\text { throat size (in) }\end{array}$ & Status \\
\hline Nozzle to shell fillet $\left(\mathrm{Leg}_{41}\right)$ & 0.21 & 0.2625 & weld size is adequate \\
\hline
\end{tabular}

This opening does not require reinforcement per UG-36(c)(3)(a)

\section{Reinforcement Calculations for MAP}

The vessel wall thickness governs the MAP of this nozzle.

\begin{tabular}{|c|c|c|c|c|c|c|c|c|}
\hline \multicolumn{7}{|c|}{ 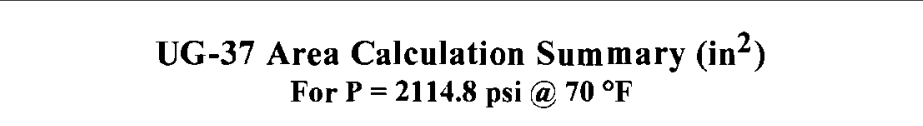 } & \multicolumn{2}{|c|}{$\begin{array}{l}\text { UG-45 Nozzle Wall } \\
\text { Thickness Summary (in) } \\
\text { The nozzle passes UG-45 }\end{array}$} \\
\hline $\begin{array}{c}\text { A } \\
\text { required }\end{array}$ & $\underset{\text { available }}{\text { A }}$ & $\mathbf{A}_{1}$ & $\mathbf{A}_{2}$ & $\mathbf{A}_{3}$ & $\mathbf{A}_{5}$ & $\begin{array}{c}\text { A } \\
\text { welds }\end{array}$ & $t_{\text {req }}$ & $\mathbf{t}_{\min }$ \\
\hline \multicolumn{7}{|c|}{ This nozzle is exempt from area calculations per UG-36(c)(3)(a) } & 0.0761 & 0.33 \\
\hline
\end{tabular}

\section{UG-41 Weld Failure Path Analysis Summary}

The nozzle is exempt from weld strength calculations per UW-15(b)(2)

\begin{tabular}{|c|r|r|c|}
\hline \multicolumn{4}{|c|}{ UW-16 Weld Sizing Sum mary } \\
\hline Weld description & $\begin{array}{r}\text { Required weld } \\
\text { throat size (in) }\end{array}$ & $\begin{array}{r}\text { Actual weld } \\
\text { throat size (in) }\end{array}$ & Status \\
\hline Nozzle to shell fillet $\left(\operatorname{Leg}_{41}\right)$ & 0.231 & 0.2625 & weld size is adequate \\
\hline
\end{tabular}

This opening does not require reinforcement per UG-36(c)(3)(a) 


\section{Wind Code}

Building Code:

Elevation of base above grade:

Increase effective outer diameter by: $0.0000 \mathrm{ft}$

Wind Force Coefficient Cf:

0.8400

Basic Wind Speed:, V:

$85.0000 \mathrm{mph}$

Importance Factor:, I:

1.1500

Exposure category:

$\mathrm{C}$

Wind Directionality Factor, Kd: $\quad 0.9500$

Topographic Factor, Kzt: $\quad 1.0000$

Enforce min. loading of $10 \mathrm{psf}$ : No

\section{Vessel Characteristics}

Vessel height, h:

$6.3584 \mathrm{ft}$

Vessel Minimum Diameter, $b$

Operating, Corroded: $0.1979 \mathrm{ft}$

Empty, Corroded: $0.1979 \mathrm{ft}$

Fundamental Frequency, $\mathrm{n}_{1}$

Operating, Corroded: $7.7056 \mathrm{~Hz}$

Empty, Corroded: $8.5724 \mathrm{~Hz}$

Vacuum, Corroded: $7.7056 \mathrm{~Hz}$

Damping coefficient, $\beta$

Operating, Corroded: 0.0179

Empty, Corroded: 0.0200

Vacuum, Corroded: 0.0179

Table Lookup Values

\subsubsection{Basic Load Combinations for Allowable Stress Design}

The following load combinations are considered in accordance with ASCE section 2.4.1:

5. $D+H+W$

Where

$D \quad=$ Dead load

$H \quad=$ Pressure load

$W \quad=$ Wind load

Wind Deflection Reports:

Operating, Corroded

Empty, Corroded

Wind Pressure Calculations

Wind Deflection Report: Operating, Corroded

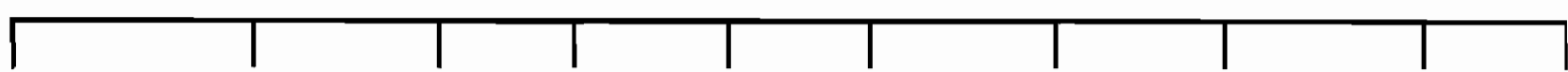




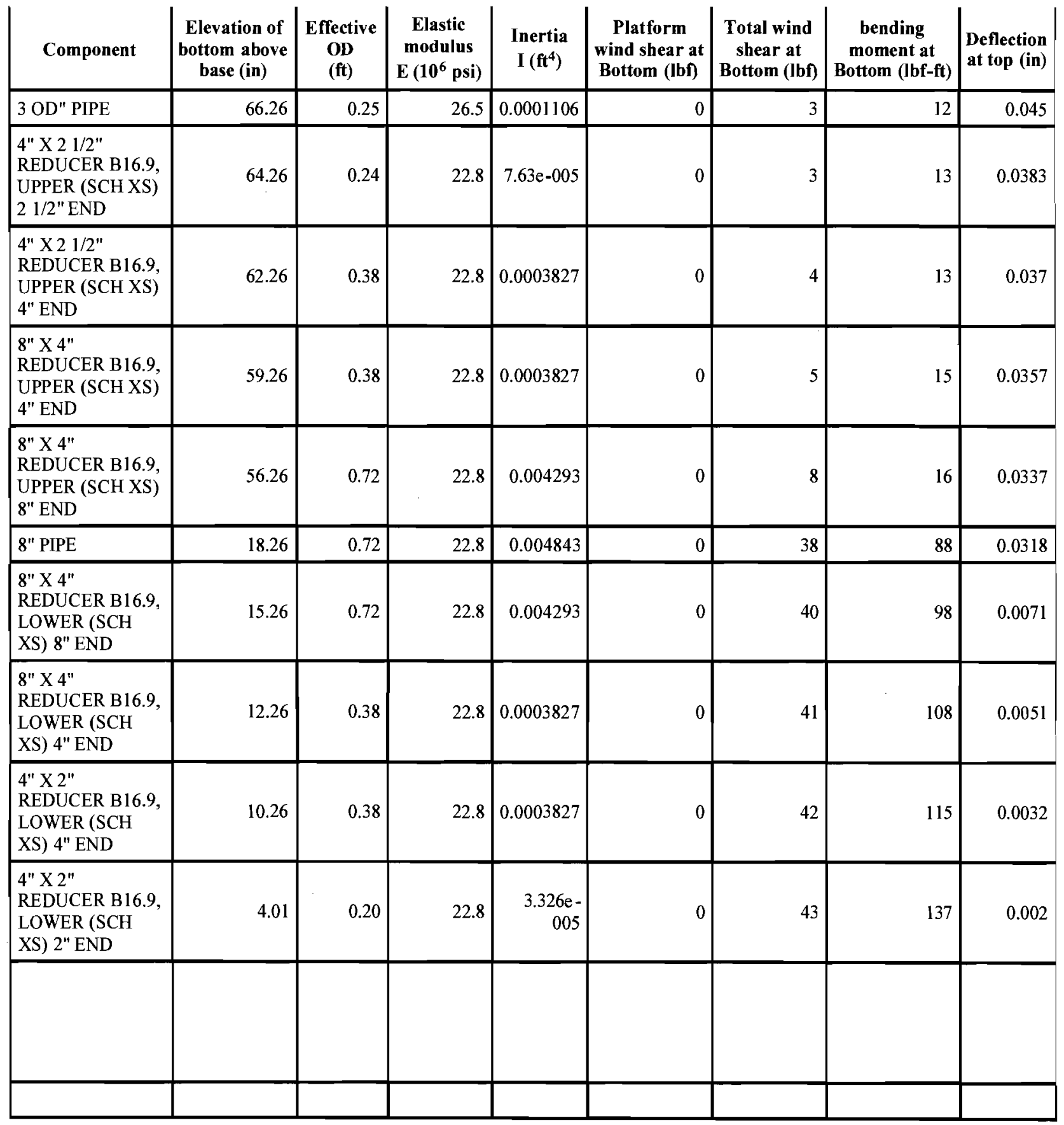

\section{Wind Deflection Report: Empty, Corroded}

\begin{tabular}{|c|c|c|c|c|c|c|c|c|}
\hline Component & $\begin{array}{c}\text { Elevation of } \\
\text { bottom above } \\
\text { base (in) }\end{array}$ & $\begin{array}{c}\text { Effective } \\
\text { OD } \\
\text { (ft) }\end{array}$ & $\begin{array}{c}\text { Elastic } \\
\text { modulus } \\
\text { E }\left(10^{6} \mathrm{psi}\right)\end{array}$ & $\begin{array}{c}\text { Inertia } \\
\text { I }\left(\mathrm{ft}^{4}\right)\end{array}$ & $\begin{array}{c}\text { Platform } \\
\text { wind shear at } \\
\text { Bottom (lbf) }\end{array}$ & $\begin{array}{c}\text { Total wind } \\
\text { shear at } \\
\text { Bottom (lbf) }\end{array}$ & $\begin{array}{c}\text { bending } \\
\text { moment at } \\
\text { Bottom (Ibf-ft) }\end{array}$ & $\begin{array}{l}\text { Deflection } \\
\text { at top (in) }\end{array}$ \\
\hline 3 OD" PIPE & 66.26 & 0.25 & 31.0 & 0.0001106 & 0 & 3 & 12 & 0.0364 \\
\hline $\begin{array}{l}4 " X 21 / 2 " \\
\text { REDUCER B } 16.9 \text {, } \\
\text { UPPER (SCH XS) } \\
21 / 2 " \text { END }\end{array}$ & 64.26 & 0.24 & 28.3 & $7.63 e-005$ & 0 & 3 & 13 & 0.031 \\
\hline & & & & & & & & \\
\hline
\end{tabular}




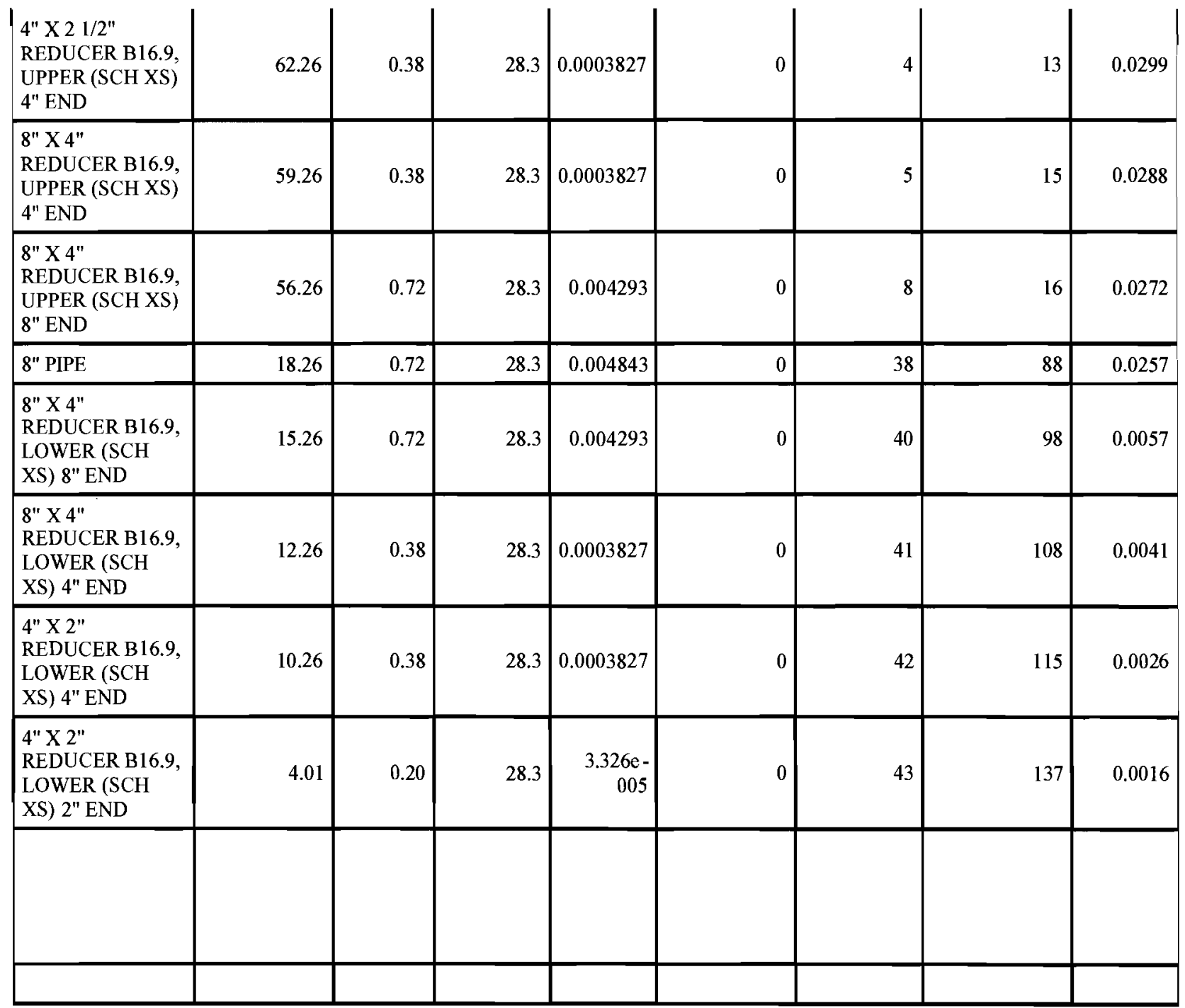

\section{Wind Pressure (WP) Calculations}

Gust Factor $\left(\mathrm{G}^{-}\right)$Calculations

$$
\begin{aligned}
\mathrm{Kz} & =2.01 *(\mathrm{Z} / \underline{\mathrm{Zg}})^{2 / \underline{\alpha}} \\
& =2.01 *(\mathrm{Z} / 900.0000)^{0.2105} \\
\mathrm{qz} & =0.00256 * \mathrm{Kz} * \mathrm{Kzt}^{*} \mathrm{Kd}^{*} \underline{\mathrm{V}}^{2} * \underline{\mathrm{I}} \mathrm{psf} \\
& =0.00256^{*} \mathrm{Kz} * 1.0000 * 0.9500 * 85.0000^{2} * 1.1500 \\
& =20.2069 * \mathrm{Kz} \\
\mathrm{WP} & =\mathrm{qz}^{*} \underline{\mathrm{G}}^{-} * \underline{\mathrm{Cf}} \\
& =\mathrm{qz}^{*} \underline{\mathrm{G}}^{-} * 0.8400
\end{aligned}
$$

\section{Design Wind Pressures}

\begin{tabular}{|c|c|c|c|c|c|c|}
\hline $\begin{array}{c}\text { Height } \mathbf{Z} \\
(')\end{array}$ & $\mathrm{Kz}$ & $\begin{array}{c}\mathrm{qz} \\
(\mathrm{psf})\end{array}$ & $\begin{array}{c}\text { WP: Operating } \\
\text { (psf) }\end{array}$ & $\begin{array}{c}\text { WP: Empty } \\
\text { (psf) }\end{array}$ & $\begin{array}{c}\text { WP: hydrotest } \\
\text { (psf) }\end{array}$ & $\begin{array}{c}\text { WP: Vacuum } \\
\text { (psf) }\end{array}$ \\
\hline
\end{tabular}




\begin{tabular}{|l|l|l|l|l|l|l|}
15.0 & 0.8489 & 17.15 & 13.16 & 13.16 & N.A. & N.A. \\
\hline
\end{tabular}

Design Wind Force determined from: $F=$ Pressure * Af, where Af is the projected area.

\section{Gust Factor Calculations}

Operating, Corroded

Empty, Corroded

\section{Gust Factor Calculations: Operating, Corroded}

$$
\begin{aligned}
\mathrm{z}^{-} & =0.60 * \underline{\mathrm{h}} \\
& =0.60 * 6.3584 \\
& =15.0000 \\
\mathrm{I}_{\mathrm{z}^{-}} & =\underline{\mathrm{c}}^{*}\left(33 / \mathrm{z}^{-}\right)^{1 / 6} \\
& =0.2000 *(33 / 15.0000)^{1 / 6} \\
& =0.2281 \\
\mathrm{~L}_{\mathrm{z}^{-}} & =\underline{1}^{*}\left(\mathrm{z}^{-} / 33\right)^{\mathrm{ep}} \\
& =500.0000 *(15.0000 / 33)^{0.2000} \\
& =427.0566 \\
\mathrm{Q} & =\mathrm{Sqr}\left(1 /\left(1+0.63 *\left((\mathrm{~b}+\underline{\mathrm{h}}) / \mathrm{L}_{\mathrm{z}}\right)^{0.63}\right)\right) \\
& =\mathrm{Sqr}\left(1 /\left(1+0.63 *((0.1979+6.3584) / 427.0566)^{0.63}\right)\right) \\
& =0.9781 \\
\mathrm{G} & =0.925 *\left(1+1.7 * \mathrm{~g}_{-\mathrm{Q}}^{*} \mathrm{I}_{\mathrm{z}}{ }^{*} \mathrm{Q}\right) /\left(1+1.7 * \mathrm{~g}_{\underline{\mathrm{v}}}^{*} \mathrm{I}_{\mathrm{z}^{-}}\right) \\
& =0.925 *\left(1+1.7 * 3.40^{*} 0.2281 * 0.9781\right) /(1+1.7 * 3.40 * 0.2281) \\
& =0.9135
\end{aligned}
$$

\section{Gust Factor Calculations: Empty, Corroded}

$$
\begin{aligned}
& \mathrm{z}^{-}=0.60 * \underline{\mathrm{L}} \\
& =0.60 * 6.3584 \\
& =15.0000 \\
& \mathrm{I}_{\mathrm{z}^{-}}=\mathrm{c}^{*}\left(33 / \mathrm{z}^{-}\right)^{1 / 6} \\
& =0.2000 *(33 / 15.0000)^{1 / 6} \\
& =0.2281 \\
& \mathrm{~L}_{\mathrm{z}}^{-}=\underline{1}^{*}\left(\mathrm{z}^{-} / 33\right)^{\mathrm{e} p} \\
& =500.0000 *(15.0000 / 33)^{0.2000} \\
& =427.0566 \\
& \mathrm{Q}=\operatorname{Sqr}\left(1 /\left(1+0.63^{*}\left((\underline{\mathrm{b}}+\mathrm{h}) / \mathrm{L}_{\mathrm{z}^{-}}\right)^{0.63}\right)\right) \\
& =\operatorname{Sqr}\left(1 /\left(1+0.63 *((0.1979+6.3584) / 427.0566)^{0.63}\right)\right) \\
& =0.9781 \\
& \mathrm{G}=0.925 *\left(1+1.7 * \mathrm{~g}_{\underline{Q}} * \mathrm{I}_{\mathrm{z}}{ }^{*} \mathrm{Q}\right) /\left(1+1.7 * \mathrm{~g}_{\underline{\mathrm{v}}} * \mathrm{I}_{\mathrm{z}}\right) \\
& =0.925 *(1+1.7 * 3.40 * 0.2281 * 0.9781) /(1+1.7 * 3.40 * 0.2281)
\end{aligned}
$$




$$
=0.9135
$$

\section{Table Lookup Values}

$$
\begin{array}{lr}
\alpha=9.5000, \mathrm{Zg}=900.0000^{\prime} & {[\text { Table 6-2, page 78] }} \\
\mathrm{c}=0.2000,1=500.0000, \text { ep }=0.2000 & {[\text { Table 6-2, page 78] }} \\
\mathrm{a}^{-}=0.1538, \mathrm{~b}^{-}=0.6500 & {[\text { Table 6-2, page 78] }} \\
\mathrm{g}_{\mathrm{Q}}=3.40 & {[6.5 .8 .1 \text { page 26] }} \\
\mathrm{g}_{\mathrm{v}}=3.40 & {[6.5 .8 .1 \text { page 26] }}
\end{array}
$$




\section{Seismic Code}

Method of seismic analysis:

Site Class

Importance Factor:

Spectral Response Acceleration at short period (\%g)

Spectral Response Acceleration at period of $1 \mathrm{sec}(\% \mathrm{~g}) \mathrm{S}_{1}=10.00 \%$

Response Modification Coeficient from Table 15.4-2

Acceleration based site co-efficient:

$\mathrm{R}=3.0000$

$\mathrm{F}_{\mathrm{a}}=1.4800$

Velocity based site co-efficient:

Long-period transition period:

Redundancy factor:

User Defined Vertical Accelerations Considered:

Force Multiplier:

Minimum Weight Multiplier:

$\mathrm{T}_{\mathrm{L}}=12.0000$

$\rho=1.0000$

Yes

$=0.3333$

$=0.2000$

\section{ASCE 7-05 ground supported}

$\mathrm{D}$

$\mathrm{I}=1.5000$

$\mathrm{S}_{\mathrm{S}}=40.00 \%$

\subsubsection{Basic Load Combinations for Allowable Stress Design}

The following load combinations are considered in accordance with ASCE section 2.4.1:

5. $D+H+0.7 E=\left(1.0+V_{\text {Acce }}\right) D+H+0.7 \rho Q_{E}$

Where

$D \quad=$ Dead load

$H \quad=$ Pressure load

$E \quad=$ Seismic load $=\rho Q_{E}$

$V_{\text {Accel }}=$ User defined vertical acceleration

\section{Vessel Characteristics}

Vessel height: $6.3584 \mathrm{ft}$

Vessel Weight:

Operating, Corroded: $270 \mathrm{lb}$

Empty, Corroded: $270 \mathrm{lb}$

\section{Period of Vibration Calculation}

Fundamental Period, T:

Operating, Corroded: $0.130 \sec (f=7.7 \mathrm{~Hz})$

Empty, Corroded: $0.117 \mathrm{sec}(\mathrm{f}=8.6 \mathrm{~Hz})$

The fundamental period of vibration $\mathrm{T}$ (above) is calculated using the Rayleigh method of approximation:

$\mathrm{T}=2 * \mathrm{PI} * \operatorname{Sqr}\left(\left\{\operatorname{Sum}\left(\mathrm{W}_{\mathrm{i}}{ }^{*} \mathrm{y}_{\mathrm{i}}{ }^{2}\right)\right\} /\left\{\mathrm{g} * \operatorname{Sum}\left(\mathrm{W}_{\mathrm{i}}^{*} \mathrm{y}_{\mathrm{i}}\right)\right\}\right)$, where 
$\mathrm{W}_{\mathrm{i}}$ is the weight of the $i^{\text {th }}$ lumped mass, and

$y_{i}$ is its deflection when the system is treated as a cantilever beam.

Seismic Shear Reports:

Operating, Corroded

Empty, Corroded

Base Shear Calculations

Seismic Shear Report: Operating, Corroded

\begin{tabular}{|c|c|c|c|c|c|}
\hline Component & $\begin{array}{l}\text { Elevation of bottom } \\
\text { above base (in) }\end{array}$ & $\begin{array}{c}\text { Elastic modulus } E \\
\left(10^{6} \mathrm{psi}\right)\end{array}$ & $\begin{array}{c}\text { Inertia I } \\
\left(\mathrm{ft}^{4}\right)\end{array}$ & $\begin{array}{c}\text { Seismic shear at } \\
\text { Bottom (lbf) }\end{array}$ & $\begin{array}{l}\text { Bending Moment at } \\
\text { Bottom (lbf- ft) }\end{array}$ \\
\hline 3 OD" PIPE & 66.26 & 26.5 & 0.0001 & 13 & 19 \\
\hline $\begin{array}{l}4 " \text { X } 2 \text { 1/2" REDUCER B16.9, } \\
\text { UPPER (SCH XS) } 2 \text { 1/2" END }\end{array}$ & 64.26 & 22.8 & 0.0001 & 13 & 21 \\
\hline $\begin{array}{l}\text { 4" X } 2 \text { 1/2" REDUCER B16.9, } \\
\text { UPPER (SCH XS) 4" END }\end{array}$ & 62.26 & 22.8 & 0.0004 & 14 & 23 \\
\hline $\begin{array}{l}\text { 8" X 4" REDUCER B16.9, UPPER } \\
\text { (SCH XS) 4" END }\end{array}$ & 59.26 & 22.8 & 0.0004 & 14 & 27 \\
\hline $\begin{array}{l}\text { 8" X 4" REDUCER B16.9, UPPER } \\
\text { (SCH XS) 8" END }\end{array}$ & 56.26 & 22.8 & 0.0043 & 16 & 30 \\
\hline 8" PIPE & 18.26 & 22.8 & 0.0048 & 35 & 118 \\
\hline $\begin{array}{l}\text { 8" X 4" REDUCER B16.9, LOWER } \\
\text { (SCH XS) 8" END }\end{array}$ & 15.26 & 22.8 & 0.0043 & 36 & 126 \\
\hline $\begin{array}{l}\text { 8" X 4" REDUCER B16.9, LOWER } \\
\text { (SCH XS) 4" END }\end{array}$ & 12.26 & 22.8 & 0.0004 & 36 & 135 \\
\hline $\begin{array}{l}\text { 4" X 2" REDUCER B16.9, LOWER } \\
\text { (SCH XS) 4" END }\end{array}$ & 10.26 & 22.8 & 0.0004 & 36 & 141 \\
\hline $\begin{array}{l}\text { 4" X 2" REDUCER B16.9, LOWER } \\
\text { (SCH XS) 2" END }\end{array}$ & 4.01 & 22.8 & 0.0000 & 37 & 160 \\
\hline $\begin{array}{l}\text { ASME B16.5/16.47 Blind on 2" } \\
\text { RFWN FLANGE 1500\# (top) }\end{array}$ & 4 & 20.4 & 0.0124 & 37 & 160 \\
\hline Support Skirt \#1 & 0 & 29.3 & 0.001519 & 37 & 173 \\
\hline
\end{tabular}

\section{Seismic Shear Report: Empty, Corroded}

\begin{tabular}{|l|r|r|r|r|r|}
\hline \multicolumn{1}{|c|}{ Component } & $\begin{array}{c}\text { Elevation of bottom } \\
\text { above base (in) }\end{array}$ & $\begin{array}{r}\text { Elastic modulus E } \\
\left(\mathbf{1 0 ^ { 6 }} \mathbf{~ p s i )}\right.\end{array}$ & $\begin{array}{c}\text { Inertia I } \\
\left(\mathbf{f t}^{4}\right)\end{array}$ & $\begin{array}{c}\text { Seismic shear at } \\
\text { Bottom (lbf) }\end{array}$ & $\begin{array}{c}\text { Bending Moment at } \\
\text { Bottom (lbf-ft) }\end{array}$ \\
\hline 3 OD" PIPE & 66.26 & 31.0 & 0.0001 & 13 & 13 \\
\hline $\begin{array}{l}\text { 4" X 2 1/2" REDUCER B16.9, } \\
\text { UPPER (SCH XS) 2 1/2" END }\end{array}$ & 64.26 & 28.3 & 0.0001 & 21 \\
\hline $\begin{array}{l}\text { 4" X 2 1/2" REDUCER B16.9, } \\
\text { UPPER (SCH XS) 4" END }\end{array}$ & 62.26 & 28.3 & 0.0004 & & 14 \\
\hline $\begin{array}{l}\text { 8" X 4" REDUCER B16.9, UPPER } \\
\text { (SCH XS) 4" END }\end{array}$ & 59.26 & 28.3 & 0.0004 & & 14 \\
\hline $\begin{array}{l}\text { 8" X 4" REDUCER B16.9, UPPER } \\
\text { (SCH XS) 8" END }\end{array}$ & 56.26 & 28.3 & 0.0043 & & 27 \\
\hline
\end{tabular}




\begin{tabular}{|c|c|c|c|c|c|}
\hline 8" PIPE & 18.26 & 28.3 & 0.0048 & 35 & 118 \\
\hline $\begin{array}{l}\text { 8" X 4" REDUCER B16.9, LOWER } \\
\text { (SCH XS) 8" END }\end{array}$ & 15.26 & 28.3 & 0.0043 & 36 & 126 \\
\hline $\begin{array}{l}\text { 8" X 4" REDUCER B16.9, LOWER } \\
\text { (SCH XS) 4" END }\end{array}$ & 12.26 & 28.3 & 0.0004 & 36 & 135 \\
\hline $\begin{array}{l}\text { 4" X 2" REDUCER B16.9, LOWER } \\
\text { (SCH XS) 4" END }\end{array}$ & 10.26 & 28.3 & 0.0004 & 36 & 141 \\
\hline $\begin{array}{l}\text { 4" X 2" REDUCER B16.9, LOWER } \\
\text { (SCH XS) 2" END }\end{array}$ & 4.01 & 28.3 & 0.0000 & 37 & 160 \\
\hline $\begin{array}{l}\text { ASME B16.5/16.47 Blind on 2" } \\
\text { RFWN FLANGE 1500\# (top) }\end{array}$ & 4 & 29.4 & 0.0124 & 37 & 160 \\
\hline Support Skirt \#1 & 0 & 29.4 & 0.001519 & 37 & 173 \\
\hline
\end{tabular}

\subsection{3: Maximum considered earthquake spectral response acceleration}

The maximum considered earthquake spectral response acceleration at short period, $\mathrm{S}_{M S}$

$\mathrm{S}_{M S}=\mathrm{E}_{\mathrm{a}} * \underline{\mathrm{S}}=1.4800 * 40.00 / 100=0.5920$

The maximum considered earthquake spectral response acceleration at $1 \mathrm{~s}$ period, $\mathrm{S}_{M 1}$

$\mathrm{S}_{M l}=\underline{\mathrm{E}}_{\underline{\mathrm{V}}} * \underline{\mathrm{S}}_{\underline{\mathrm{l}}}=2.4000 * 10.00 / 100=0.2400$

\subsection{4: Design spectral response acceleration parameters}

Design earthquake spectral response acceleration at short period, $\mathrm{S}_{D S}$

$\mathrm{S}_{D S}=2 / 3 * \mathrm{~S}_{M S}=2 / 3 * 0.5920=0.3947$

Design earthquake spectral response acceleration at $1 \mathrm{~s}$ period, $\mathrm{S}_{D I}$

$\mathrm{S}_{D 1}=2 / 3 * \mathrm{~S}_{M 1}=2 / 3 * 0.2400=0.1600$

User Defined Vertical Acceleration Term, $V_{\text {Accel }}$

Factor is applied to dead load.

Compressive Side: $=1.0+\mathrm{V}_{\text {Accel }}$

\section{$V_{\text {Accel }}$ Term is:}

greater of (Force Mult * Base Shear / Weight) or (Min. Weight Mult.)

\begin{tabular}{|l|r|r|r|r|}
\hline \multicolumn{2}{|c|}{ Force multiplier $=0.3333$} & \multicolumn{3}{|c|}{ Minimum Weight Multiplier $=0.2000$} \\
\hline Condition & Base Shear ( Ibf) & Weight ( Ib) & $\begin{array}{l}\text { Force Mult * Shear } \\
\text { Weight }\end{array}$ & V Accel \\
\hline Operating, Corroded & 37 & 270.5 & 0.046 & 0.2 \\
\hline Operating, New & 39 & 282.6 & 0.046 & 0.2 \\
\hline Empty, Corroded & 37 & 270.5 & 0.046 & 0.2 \\
\hline Empty, New & 39 & 282.6 & 0.046 & 0.2 \\
\hline
\end{tabular}




\section{Base Shear Calculations}

Operating, Corroded

Empty, Corroded

\section{Base Shear Calculations: Operating, Corroded}

\section{Paragraph 15.4.4: Period Determination}

Fundamental Period is taken from the Rayleigh method listed previously in this report.

$\mathrm{T}=0.130 \mathrm{sec}$.

\subsection{1: Calculation of Seismic Response Coefficient}

$\mathrm{C}_{\mathrm{s}}$ is the value computed below, bounded by $\mathrm{C}_{\mathrm{s}} \mathrm{Min}$ and $\mathrm{C}_{\mathrm{s}} \mathrm{Max}$ :

$\mathrm{C}_{\mathrm{s}} \mathrm{Min}$ is 0.01 unless $\mathrm{S}_{1}>=0.6 \mathrm{~g}$, in which case eqn $12.8-6$ is used.

$\mathrm{C}_{\mathrm{S}}$ Max calculated with $12.8-3$ because $(\mathrm{T}=0.130)<=\left(\mathrm{T}_{\mathrm{L}}=12.0000\right)$

$\mathrm{C}_{\mathrm{S}}=\underline{\mathrm{S}}_{\underline{D S}} /(\underline{\mathrm{R}} / \mathrm{D})=0.3947 /(3.0000 / 1.5000)=0.1973$

$\mathrm{C}_{\mathrm{s}} \mathrm{Min}=0.01$

$\mathrm{C}_{\mathrm{s}} \mathrm{Max}=\underline{\mathrm{S}}_{\underline{D I}} /(\mathrm{T} *(\mathrm{R} / \mathrm{I}))=0.1600 /(0.1281 *(3.0000 / 1.5000))=0.6244$

$\mathrm{C}_{\mathrm{s}}=0.1973$

\subsection{1: Calculation of Base Shear}

$$
\begin{aligned}
\mathrm{V} & =\mathrm{C}_{\mathrm{S}} * \underline{\mathrm{W}} \\
& =0.1973 * 270.4562 \\
& =53.37 \mathrm{lb}
\end{aligned}
$$

\subsubsection{Seismic Load Combinations: Horizontal Seismic Load Effect, $\mathbf{E}_{\mathbf{h}}$}

$\mathrm{Q}_{\mathrm{E}}=\mathrm{V}$

$E_{h}=0.7 * \rho * Q_{E}($ Only $70 \%$ of seismic load considered as per Section 2.4.1)

$=0.70 * 1.0000 * 53.37$

$=37.36 \mathrm{lb}$

Base Shear Calculations: Empty, Corroded

\section{Paragraph 15.4.4: Period Determination}

Fundamental Period is taken from the Rayleigh method listed previously in this report. $\mathrm{T}=0.117 \mathrm{sec}$.

\subsection{1: Calculation of Seismic Response Coefficient}

$\mathrm{C}_{\mathrm{S}}$ is the value computed below, bounded by $\mathrm{C}_{\mathrm{s}} \mathrm{Min}$ and $\mathrm{C}_{\mathrm{s}} \mathrm{Max}$ :

$\mathrm{C}_{\mathrm{s}} \mathrm{Min}$ is 0.01 unless $\mathrm{S}_{1}>=0.6 \mathrm{~g}$, in which case eqn 12.8-6 is used.

$\mathrm{C}_{\mathrm{s}}$ Max calculated with $12.8-3$ because $(\mathrm{T}=0.117)<=\left(\mathrm{T}_{\mathrm{L}}=12.0000\right)$ 


$$
\begin{aligned}
& \mathrm{C}_{\mathrm{s}}=\underline{\mathrm{S}}_{\underline{ } \mathrm{S}} /(\underline{\mathrm{R}} / \mathrm{I}=0.3947 /(3.0000 / 1.5000)=0.1973 \\
& \mathrm{C}_{\mathrm{s}} \operatorname{Min}=0.01 \\
& \mathrm{C}_{\mathrm{s}} \mathrm{Max}=\underline{\mathrm{S}}_{\underline{D I}} /(\mathrm{T} *(\underline{\mathrm{R}} / \mathrm{I}))=0.1600 /(0.1167 *(3.0000 / 1.5000))=0.6858 \\
& \mathrm{C}_{\mathrm{s}}=0.1973
\end{aligned}
$$

\subsection{1: Calculation of Base Shear}

$$
\begin{aligned}
\mathrm{V} & =\mathrm{C}_{\mathrm{s}} * \underline{\mathrm{W}} \\
& =0.1973 * 270.4562 \\
& =53.37 \mathrm{lb}
\end{aligned}
$$

12.4.2.1 Seismic Load Combinations: Horizontal Seismic Load Effect, $E_{h}$

$$
\begin{aligned}
\mathrm{Q}_{\mathrm{E}} & =\mathrm{V} \\
\mathrm{E}_{\mathrm{h}} & =0.7 * \rho * \mathrm{Q}_{\mathrm{E}}(\text { Only } 70 \% \text { of seismic load considered as per Section 2.4.1) } \\
& =0.70 * 1.0000 * 53.37 \\
& =37.36 \mathrm{lb}
\end{aligned}
$$


CALCULATIONS PER ASME B31.3 2006 EDITION

NOTE: THESE CALCULATIONS INCLUDE UPPER CHAR POT ONLY

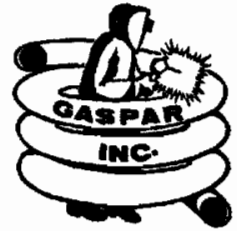

PER SECTION 304.1.1(A) EQUATION (2) AND SECTION 304.1.2 EQUATION \#3A $\operatorname{tm}=\left(P^{*} \mathrm{Do} /\left(2^{*}\left(S^{*} E^{*} W+P^{*} Y\right)\right)\right)+c$

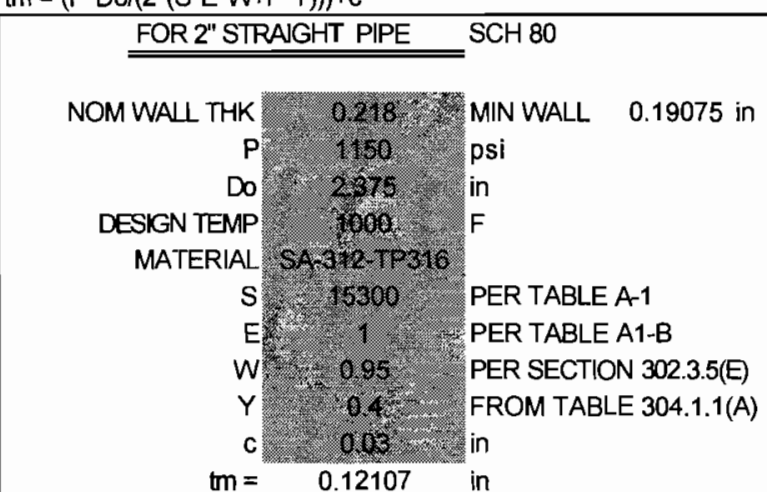

SINCE THE MIN WAU THK IS GREATER THAN tm, THE THICKNESS IS ADEQUATE

PER SECTION 304.1.1(A) EQUATION (2) AND SECTION 304.1.2 EQUATION \#3A $\mathrm{tm}=\left(\mathrm{P}^{*} \mathrm{Do} /\left(2^{*}\left(\mathrm{~S}^{*} \mathrm{E}^{*} \mathrm{~W}+\mathrm{P}^{*} \mathrm{Y}\right)\right)\right)+\mathrm{C}$

\begin{tabular}{|c|c|}
\hline FOR 1" STRAIGHT PIPE & $\mathrm{SCH} 80$ \\
\hline 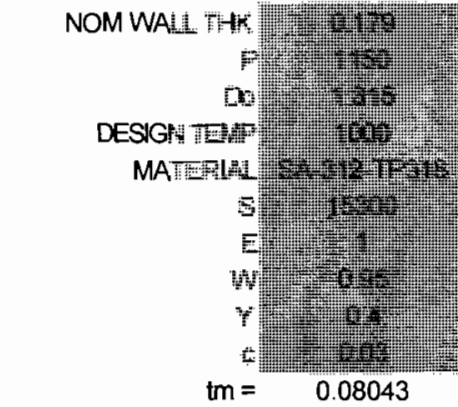 & 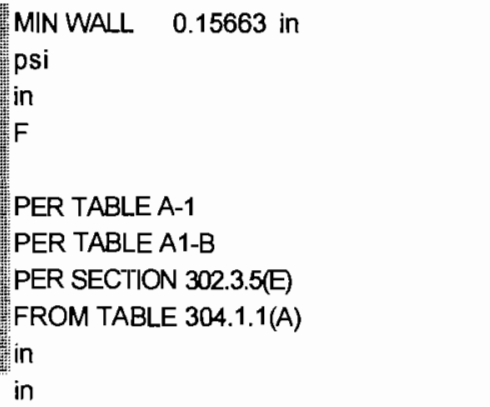 \\
\hline SINCE THE MIN WAL THK IS GREATER & R THAN tm, THE THICKNESS IS ADEQUATE \\
\hline
\end{tabular}

PER SECTION 304.1.1(A) EQUATION (2), SEC. 304.1.5 EQUATION \#3A, SEC. 326.1, 326.2, AND TABLE 326.1 STANDARDS SEC. 304.3.2(B), SEC. 328.5, 328.5.4; ASME B16.11 SEC 2.1 
ASME B16.11 TABLE 2, ASME B1.20.1 NPT

$\mathrm{tm}=\left(\mathrm{P}^{*} \mathrm{Do} /\left(2^{*}\left(\mathrm{~S}^{*} \mathrm{E}^{*} \mathrm{~W}+\mathrm{P}^{*} \mathrm{Y}\right)\right)\right)+\mathrm{c}$

FOR B16.11 1/2" NPT THREADED FITTING $\quad$ CLASS 6000

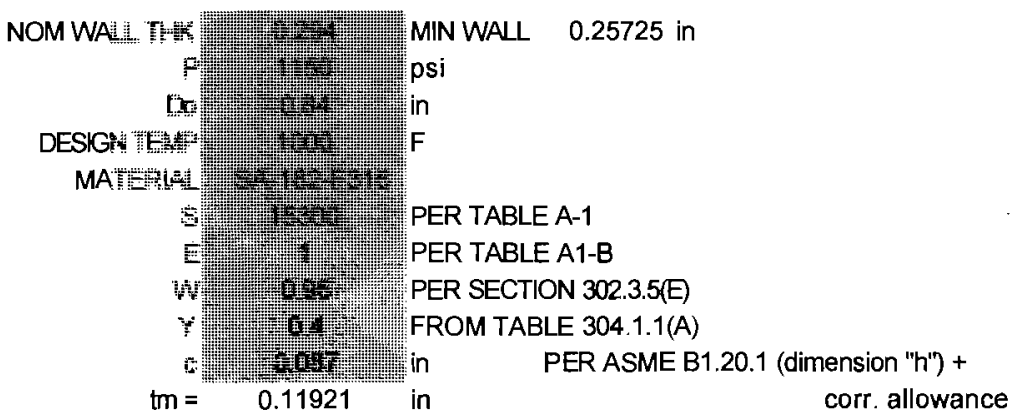

SINCE THE MIN WALL THK IS GREATER THAN tm, THE THICKNESS IS ADEQUATE

REQUIRED WELD SIZE ON 2" PIPE

PER SEC. 328.5.4(C,D) \& FIG. 328.5.4D

FULL PENETRATION GROOVE WELD FINISHED WTH COVER FILLET WELD

FILLET WELD SZE: tc = LESSER OF 0.7 Tb OR 1/4"

tc $=\quad 0.2058$ in. FILLET WELD ROOT DEPTH

2. 3 S.

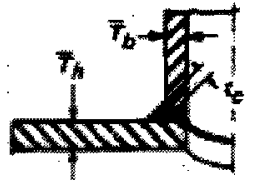

FIG. 328.5.4D (1)

PER SECTION 304.1.1(A) EQUATION (2), SEC. 304.1.5 EQUATION \#3A,

SEC. 326.1, 326.2, AND TABLE 326.1 STANDARDS; ASME B16.9 SEC. 2.1

$\mathrm{tm}=\left(P^{*} \mathrm{Do} /\left(2^{*}\left(\mathrm{~S}^{*} \mathrm{E}^{*} \mathrm{~W}+\mathrm{P}^{*} \mathrm{Y}\right)\right)\right)+\mathrm{C}$

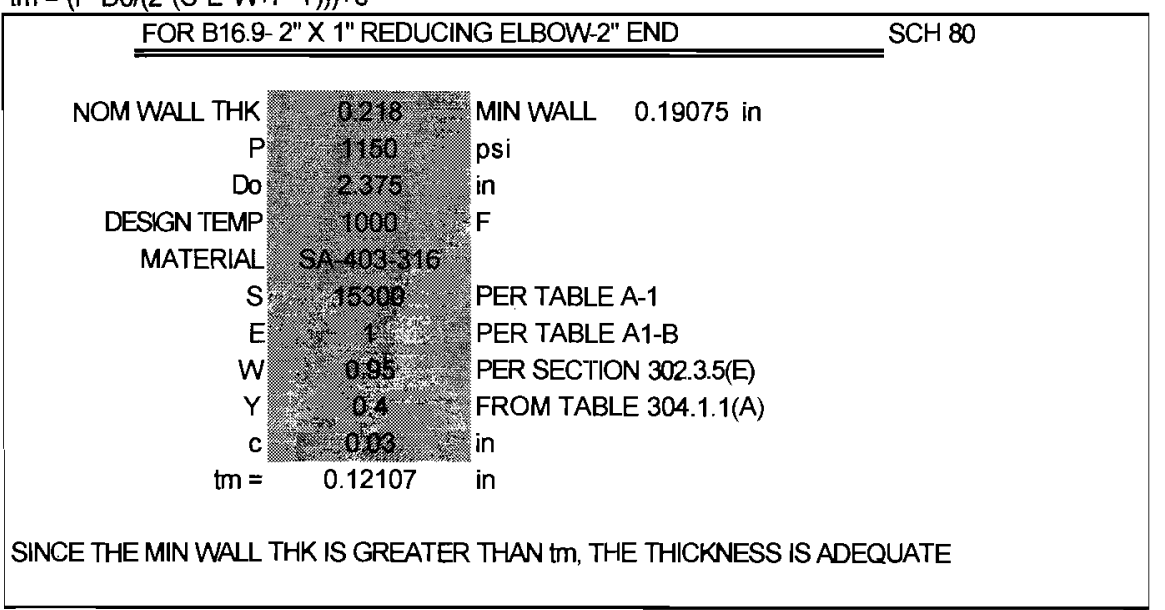

PER SECTION 304.1.1(A) EQUATION (2), SEC. 304.1.5 EQUATION \#3A, SEC. 326.1, 326.2, AND TABLE 326.1 STANDARDS; ASME B16.9 SEC. 2.1 $\mathrm{tm}=\left(\mathrm{P}^{*} \mathrm{Do} /\left(2^{*}\left(\mathrm{~S}^{*} \mathrm{E}^{*} \mathrm{~W}+\mathrm{P}^{*} \mathrm{Y}\right)\right)\right)+\mathrm{c}$

FOR B16.9-2"X 1" REDUCING ELBOW-1" END
NOM WALL,




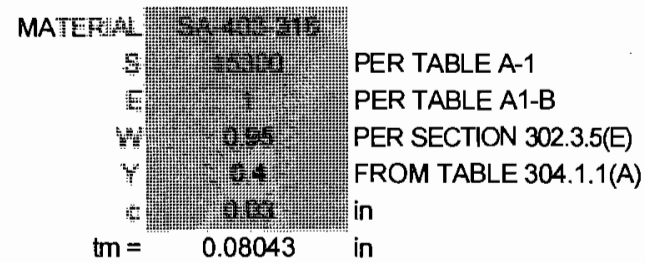

SINCE THE MIN WALL THK IS GREATER THAN tm, THE THICKNESS IS ADEQUATE

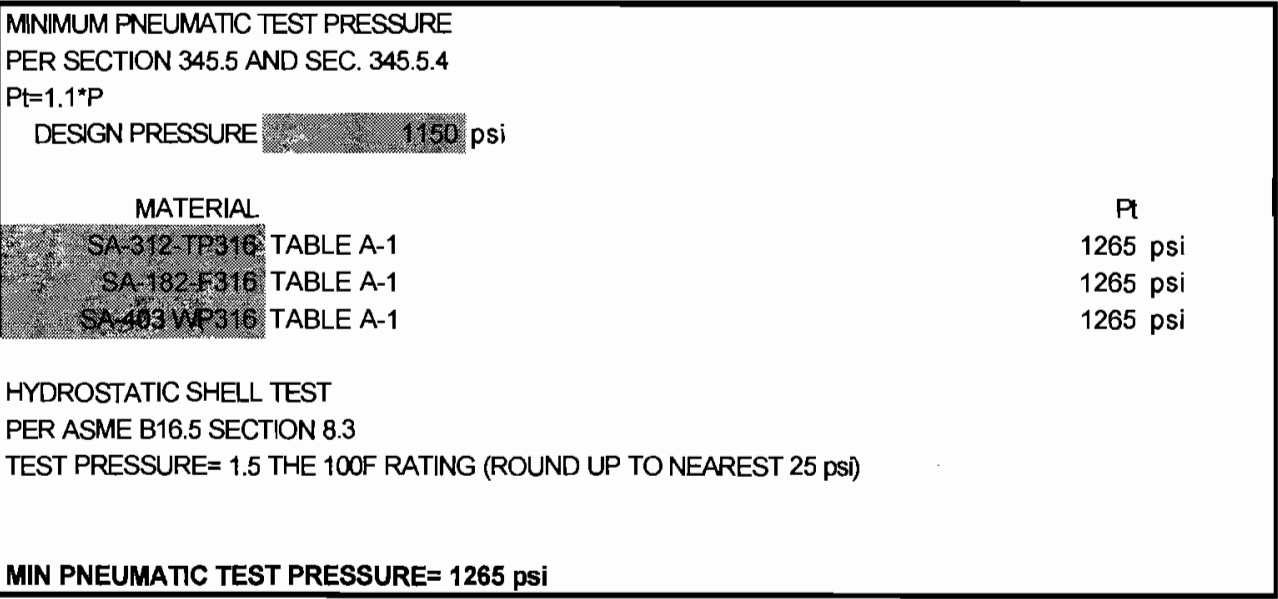




\section{Table Of Contents}

1. Pressure Summary

2. Pneumatic Test

3. Weight Summary

4. 2" RFWN FLANGE 1500\#, LPPER

5. 4" X 2" REDUCER B 16.9. UPPER (SCH XS) 2" END

6. 4" X 2" REDUCER B 16.9. UPPER (SCH XS) 4" END

7. 8"X 4" REDUCER B16.9. UPPER (SCH XS) 4" END

8. 8 "X 4" REDUCER B16.9, UPPER (SCH XS) 8" END

9. $8^{\prime \prime} \mathrm{PIPE}$

10. $8^{\prime \prime}$ X 4" REDUCER B 16.9, LOWER (SCH XS) 8" END

11. 8" X 4" REDUCER B16.9, LOWER (SCH XS) 4" END

12. $4 " \mathrm{X}$ 2" B16.9 REDUCER, LOWER (SCH XS) 4" END

13. 4" X 2" B 16.9 REDUCER, LOWER (SCH XS) 2" END

14. 2" RFWN FLANGE 1500\#, LOWER

15. ASME B 16.5/16.47 Blind on 2" RFWN FLANGE 1500\#. LOWER

16. 1/2" HALF-COUPLING (7a)

17. 1/2" HALF-COUPLING (7b)

18. Wind Code

19. Seismic Code 


\section{Pressure Summary}

\begin{tabular}{|c|c|c|c|c|c|c|}
\hline Identifier & $\begin{array}{c}\mathbf{P} \\
\text { Design } \\
(\mathbf{p s i})\end{array}$ & $\begin{array}{c}\mathbf{T} \\
\text { Design } \\
\left({ }^{\circ} \mathbf{F}\right)\end{array}$ & $\begin{array}{c}\text { MAWP } \\
(\text { psi) }\end{array}$ & $\begin{array}{c}\text { MDMT } \\
\left({ }^{\circ} \mathrm{F}\right)\end{array}$ & $\begin{array}{c}\text { MDMT } \\
\text { Exemption }\end{array}$ & $\begin{array}{l}\text { Impact } \\
\text { Tested }\end{array}$ \\
\hline 4" X 2" REDUCER B16.9. UPPER (SCH XS) 2" END & 1,150 & 1,000 & $2,193.3$ & -320 & Note 1 & No \\
\hline 4" X 2" REDUCER B16.9. UPPER (SCH XS) 4" END & 1,150 & 1,000 & $1,891.09$ & -320 & Note 1 & No \\
\hline 8"X 4" REDUCER B16.9. UPPER (SCH XS) 4" END & 1,150 & 1,000 & $1,891.09$ & -320 & Note 1 & No \\
\hline 8" X 4" REDUCER B 16.9. UPPER (SCH XS) 8" END & 1,150 & 1,000 & $1,502.53$ & -320 & Note 1 & No \\
\hline 8" PIPE & 1,150 & 1,000 & $1,502.53$ & -320 & Note 1 & No \\
\hline 8" X 4" REDUCER B16.9. IOWER (SCH XS) 8" END) & 1,150 & 1,000 & $1,502.53$ & -320 & Note 1 & No \\
\hline 8" X 4" REDUCER B16.9. LOWER (SCH XS) 4" END & 1,150 & 1,000 & $1,891.09$ & -320 & Note 1 & No \\
\hline 4" X 2" B16.9 REDUCER LOWER (SCH XS) 4" END & 1,150 & 1,000 & $1,891.09$ & -320 & Note 1 & No \\
\hline 4" X 2" B16.9 REDUCER LOWER (SCH XS) 2" END & 1,150 & $\mathrm{I}, 000$ & $2,193.3$ & -320 & Note 1 & No \\
\hline ASME B16.5/16.47 Blind on 2" RFWN FLANGE 1500\# IOWER & 1,150 & 1,000 & 1,820 & -55 & Note 2,3 & No \\
\hline 2" RFWN FLANGE 1500\#. UPPER & 1,150 & 1,000 & 1,820 & -320 & Note 2 & No \\
\hline 2" RFWN FLANGE 1500\#, LOWER & 1,150 & 1,000 & 1,820 & -55 & Note 2,3 & No \\
\hline 1/2" HALF-COUPLING (7a) & $1, I 50$ & 1,000 & $1,502.52$ & -320 & Note 4 & No \\
\hline 1/2" HALF-COUPLING (7b) & 1,150 & 1,000 & $1,502.52$ & -320 & Note 4 & No \\
\hline
\end{tabular}

Chamber design MDMT is $-20^{\circ} \mathrm{F}$

Chamber rated MDMT is $-55^{\circ} \mathrm{F} @ 1,502.52 \mathrm{psi}$

This pressure chamber is not designed for external pressure.

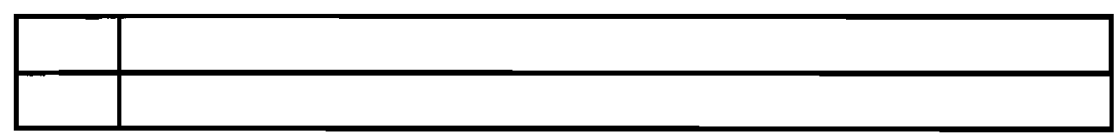

Notes for MDMT Rating:

\begin{tabular}{|r|l|l|}
\hline $\begin{array}{c}\text { Note } \\
\#\end{array}$ & \multicolumn{1}{|c|}{ Exemption } & \multicolumn{1}{c|}{ Details } \\
\hline 1. & $\begin{array}{l}\text { Rated MDMT per UHA }-51(\mathrm{~d})(1)(\mathrm{b}), \text { (carbon content does not exceed } 0.1 \\
\text { percent })=-320^{\circ} \mathrm{F}\end{array}$ & \\
\hline 2. & Per UHA $-51(\mathrm{~d})(1)(\mathrm{b})$ & \\
\hline 3. & Flange rated MDMT $=-320^{\circ} \mathrm{F}$ & $\begin{array}{l}\text { Bolts rated MDMT per Fig UCS-66 note (e) } \\
=-55^{\circ} \mathrm{F}\end{array}$ \\
\hline 4. & Impact test exempt per UHA-51(g)(coincident ratio $=0.11801)$ & \\
\hline
\end{tabular}




\section{Pneumatic Test}

\section{Shop test pressure determination} based on design $P$ per UG-100(b)

Shop pneumatic test gauge pressure is $1,653.608 \mathrm{psi}$ at $70^{\circ} \mathrm{F}$ (the chamber design $\mathrm{P}=1,150 \mathrm{psi}$ )

\begin{tabular}{|l|r|r|r|r|}
\hline \multicolumn{1}{|c|}{ Identifier } & $\begin{array}{c}\text { Local test } \\
\text { pressure } \\
\text { psi }\end{array}$ & $\begin{array}{c}\text { Test liquid } \\
\text { static head } \\
\text { psi }\end{array}$ & $\begin{array}{c}\text { UG-100 } \\
\text { stress } \\
\text { ratio }\end{array}$ & $\begin{array}{c}\text { UG-100 } \\
\text { press ure } \\
\text { factor }\end{array}$ \\
\hline 4" X 2" REDUCER B16.9, UPPER (SCH XS) 2" END (1) & $1,653.608$ & 0 & 1.3072 & 1.10 \\
\hline 2" RFWN FLANGE 1500\#, UPPER & $1,653.608$ & 0 & N/A & 1.10 \\
\hline 4" X 2" REDUCER B16.9, UPPER (SCH XS) 4" END & $1,653.608$ & 0 & 1.3072 & 1.10 \\
\hline 8" X 4" REDUCER B16.9, UPPER (SCH XS) 4" END & $1,653.608$ & 0 & 1.3072 & 1.10 \\
\hline 8" X 4" REDUCER B16.9, UPPER (SCH XS) 8" END & $1,653.608$ & 0 & 1.3072 & 1.10 \\
\hline 8" PIPE & $1,653.608$ & 0 & 1.3072 & 1.10 \\
\hline 8" X 4" REDUCER B16.9, LOWER (SCH XS) 8"END & $1,653.608$ & 0 & 1.3072 & 1.10 \\
\hline 8" X 4" REDUCER B16.9, LOWER (SCH XS) 4"END & $1,653.608$ & 0 & 1.3072 & 1.10 \\
\hline 4" X 2" B16.9 REDUCER, LOWER (SCH XS) 4"END & $1,653.608$ & 0 & 1.3072 & 1.10 \\
\hline 4" X 2" B16.9 REDUCER, LOWER (SCH XS) 2" END & $1,653.608$ & 0 & 1.3072 & 1.10 \\
\hline 2" RFWN FLANGE 1500\#, LOWER & $1,653.608$ & 0 & N/A & 1.10 \\
\hline 1/2" HALF-COUPLING (7a) & $1,653.608$ & 0 & 1.7699 & 1.10 \\
\hline 1/2" HALF-COUPLING (7b) & $1,653.608$ & 0 & 1.7699 & 1.10 \\
\hline
\end{tabular}

\section{Notes:}

(1) 4" X 2" REDUCER B16.9, UPPER (SCH XS) 2" END limits the UG-100 stress ratio.

(2) The zero degree angular position is assumed to be up, and the test liquid height is assumed to the top-most flange. 


\section{Weight Sum mary}

\begin{tabular}{|c|c|c|c|c|c|c|c|}
\hline \multirow[b]{2}{*}{ Component } & \multicolumn{7}{|c|}{ Weight ( Ib) Contributed by Vessel Elements } \\
\hline & $\begin{array}{l}\text { Metal } \\
\text { New* }\end{array}$ & $\begin{array}{c}\text { Metal } \\
\text { Corroded * }\end{array}$ & $\begin{array}{c}\text { Insulation } \\
\& \\
\text { Supports }\end{array}$ & Lining & $\begin{array}{c}\text { Piping } \\
+ \\
\text { Liquid }\end{array}$ & $\begin{array}{l}\text { Operating } \\
\text { Liquid }\end{array}$ & $\begin{array}{l}\text { Test } \\
\text { Liquid }\end{array}$ \\
\hline 4"X 2" REDUCER B16.9, UPPER (SCH XS) 2" END & 0.8 & 0.6 & 0 & 0 & 0 & 0 & 0.2 \\
\hline 4" X 2" REDUCER B 16.9. UPPER (SCH XS) 4" END & 2.3 & 2 & 0 & 0 & 0 & 0 & 0.9 \\
\hline 8" X 4" REDUCER B16.9. UPPER (SCH XS) 4" END & 3.4 & 3.1 & 0 & 0 & 0 & 0 & 1.3 \\
\hline 8" X 4" REDUCER B 16.9. UPPER (SCH XS) 8" END & 9.8 & 9.2 & 0 & 0 & 0 & 0 & 5.1 \\
\hline 8" PIPE & 132.7 & 125.2 & 10 & 0 & 0 & 0 & 59.4 \\
\hline 8" X 4" REDUCER B 16.9, LOWER (SCH XS) 8" END & 9.8 & 9.2 & 0 & 0 & 0 & 0 & 5.1 \\
\hline 8" X 4" REDUCER B16.9. LOWER (SCH XS) 4" END & 3.4 & 3.1 & 0 & 0 & 0 & 0 & 1.3 \\
\hline 4" X 2" B16.9 REDUCER. LOWER (SCH XS) 4" END & 2.3 & 2 & 0 & 0 & 0 & 0 & 0.9 \\
\hline 4" X 2" B16.9 REDUCER. LOWER (SCH XS) 2" END & 0.8 & 0.6 & 0 & 0 & 0 & 0 & 0.2 \\
\hline $\begin{array}{l}\text { ASME B16.5/16.47 Blind on 2" RFWN FLANGE } 1500 \# \text {. } \\
\text { LOWER }\end{array}$ & 32 & 32 & 0 & 0 & 0 & 0 & 0 \\
\hline Support Skitt \#1 & 1.9 & 1.9 & 0 & 0 & 0 & 0 & 0 \\
\hline TOTAL: & 199 & 189 & 0 & 0 & $\mathbf{0}$ & 0 & 74.4 \\
\hline
\end{tabular}

* Shells with attached nozzles have weight reduced by material cut out for opening.

\begin{tabular}{|c|c|c|c|c|c|c|c|c|c|}
\hline \multirow{3}{*}{ Component } & \multicolumn{9}{|c|}{ Weight ( lb) Contributed by Attachments } \\
\hline & \multicolumn{2}{|c|}{ Body Flanges } & \multicolumn{2}{|c|}{$\begin{array}{l}\text { Nozzles \& } \\
\text { Flanges }\end{array}$} & \multirow{2}{*}{$\begin{array}{c}\text { Packed } \\
\text { Beds }\end{array}$} & \multirow{2}{*}{$\begin{array}{l}\text { Ladders } \\
\& \\
\text { Platforms }\end{array}$} & \multirow{2}{*}{$\begin{array}{l}\text { Trays \& } \\
\text { Supports }\end{array}$} & \multirow{2}{*}{$\begin{array}{c}\text { Rings } \\
\& \\
\text { Clips }\end{array}$} & \multirow{2}{*}{$\begin{array}{c}\text { Vertical } \\
\text { Loads }\end{array}$} \\
\hline & New & Corroded & New & Corroded & & & & & \\
\hline $\begin{array}{l}4^{\prime \prime} \times 2 " \text { REDUCER B16.9 UPPER (SCH XS) } \\
2^{2 "} \mathrm{END}\end{array}$ & 25 & 25 & 0 & 0 & 0 & 0 & 0 & 0 & 0 \\
\hline $\begin{array}{l}\text { 4" X 2" REDUCER B16.9.UPPER (SCH XS) } \\
\text { 4" END }\end{array}$ & 0 & 0 & 0 & 0 & 0 & 0 & 0 & 0 & 0 \\
\hline $\begin{array}{l}\text { 8" X 4" REDUCER B 16.9. UPPER (SCH XS) } \\
\text { 4" END }\end{array}$ & 0 & 0 & 0 & 0 & 0 & 0 & 0 & 0 & 0 \\
\hline $\begin{array}{l}\text { 8" X 4" REDUCER B16.9, UPPER (SCH XS) } \\
\text { 8" END }\end{array}$ & 0 & 0 & 0 & 0 & 0 & 0 & 0 & 0 & 0 \\
\hline 8" PIPE & 0 & 0 & 0.7 & 0.7 & 0 & 0 & 0 & 0 & 0 \\
\hline $\begin{array}{l}\text { 8" X 4" REDUCER B16.9. LOWER (SCH } \\
\text { XS) 8" END }\end{array}$ & 0 & 0 & 0 & 0 & 0 & 0 & 0 & 0 & 0 \\
\hline $\begin{array}{l}\frac{8 " \mathrm{X} 4 " \mathrm{REDUCER} \text { B } 16.9, \text { LOWER (SCH }}{\mathrm{XS}) 4^{\prime \prime} \mathrm{END}} \\
\end{array}$ & 0 & 0 & 0 & 0 & 0 & 0 & 0 & 0 & 0 \\
\hline $\begin{array}{l}\text { 4" X 2" B16.9 REDUCER, LOWER (SCH } \\
\text { XS) 4" END }\end{array}$ & 0 & 0 & 0 & 0 & 0 & 0 & 0 & 0 & 0 \\
\hline $\begin{array}{l}\text { 4" X 2" B16.9 REDUCER. LOWER (SCH } \\
\text { XS) 2" END }\end{array}$ & 25 & 25 & 0 & 0 & 0 & 0 & 0 & 0 & 0 \\
\hline $\begin{array}{l}\text { ASME B } 16.5 / 16.47 \text { Blind on 2" RFWN } \\
\text { FLANGE 1500\#. LOWER }\end{array}$ & 0 & 0 & 0 & 0 & 0 & 0 & 0 & 0 & 0 \\
\hline Support Skirt \#I & 0 & 0 & 0 & 0 & 0 & 0 & 0 & 0 & 0 \\
\hline TOTAL: & 50 & 50 & 0.7 & 0.7 & $\mathbf{0}$ & $\mathbf{0}$ & $\overline{0}$ & 0 & 0 \\
\hline
\end{tabular}

Vessel operating weight, Corroded: $240 \mathrm{lb}$ 
Vessel operating weight, New: $250 \mathrm{lb}$

Vessel empty weight, Corroded: $240 \mathrm{lb}$

Vessel empty weight, New: $250 \mathrm{lb}$

Vessel test weight, New: $\quad 324 \mathrm{lb}$

\section{Vessel center of gravity location - from datum - lift condition}

Vessel Lift Weight, New:250 lb

Center of Gravity: $\quad 27.7778 "$

\section{Vessel Capacity}

Vessel Capacity** (New): 9 US gal

Vessel Capacity** (Corroded): 9 US gal

**The vessel capacity does not include volume of nozzle, piping or other attachments. 


\title{
2" RFWN FLANGE 1500\#, UPPER
}

\author{
Flange description: \\ 2 inch Class 1500 WN A 182 F316H \\ Bolt Material: \\ SB-166 Annealed Bolt N06600 \\ Flange rated MDMT: \\ $-320^{\circ} \mathrm{F}$ \\ (Per UHA-51(d)(1)(b)) \\ Liquid static head on flange: \\ $0 \mathrm{psi}$ \\ ASME B16.5 flange rating MAWP: $1820 \mathrm{psi} @ 1000^{\circ} \mathrm{F}$ \\ ASME B 16.5 flange rating MAP: $3600 \mathrm{psi} @ 70^{\circ} \mathrm{F}$ \\ ASME B16.5 flange hydro test: $\quad 5425$ psi @ $70^{\circ} \mathrm{F}$
}




\section{4" X 2" REDUCER B16.9, UPPER (SCH XS) 2" END}

\section{ASME Section VIII Division 1, 2007 Edition}

$\begin{array}{ll}\text { Component: } & \text { Cylinder } \\ \text { Material specification: } & \text { SA-403 } 316 \text { H (II-D p. 78, } \ln .8)\end{array}$

Rated MDMT per UHA-51(d)(1)(b), (carbon content does not exceed 0.1 percent) $=-320^{\circ} \mathrm{F}$

Internal design pressure: $\mathrm{P}=1,150 \mathrm{psi} @ 1,000^{\circ} \mathrm{F}$

Static liquid head:

$\mathrm{P}_{\text {th }}=0.23 \mathrm{psi}\left(\mathrm{SG}=1, \mathrm{H}_{\mathrm{s}}=6.2465^{\prime \prime}\right.$, Horizontal test head $)$

Corrosion allowance $\quad$ Inner $\mathrm{C}=0.03^{\prime \prime} \quad$ Outer $\mathrm{C}=0$ "

Design MDMT $=-20^{\circ} \mathrm{F} \quad$ No impact test performed

Rated MDMT $=-320^{\circ} \mathrm{F}$ Material is not normalized

Material is not produced to Fine Grain Practice

PWHT is not performed

Radiography: $\quad$ Longitudinal joint - $\quad$ Seamless No RT

Top circumferential joint - Full UW-11(a) Type 1

Bottom circumferential

joint -

Full UW-11(a) Type 1

Estimated weight New $=0.8 \mathrm{lb} \quad$ corr $=0.6 \mathrm{lb}$

Capacity New $=0.03$ US gal corr $=0.03$ US gal

$\mathrm{OD} \quad=2.375^{\prime \prime}$

Length $\mathrm{L}_{\mathrm{c}}=2^{\prime \prime}$

t $=0.191^{\prime \prime}$

Design thickness, (at $1,000^{\circ} \mathrm{F}$ ) Appendix 1-1

$\mathrm{t}=\mathrm{P}^{*} \mathrm{R}_{0} /\left(\mathrm{S} * \mathrm{E}+0.40^{*} \mathrm{P}\right)+$ Corrosion

$=1,150 * 1.1875 /(15,300 * 1.00+0.40 * 1,150)+0.03$

$=0.1167^{\prime \prime}$

Maximum allowable working pressure, (at 1,000 $\left.{ }^{\circ} \mathrm{F}\right)$ Appendix 1 -1

$$
\begin{aligned}
\mathrm{P} & =\mathrm{S}^{*} \mathrm{E}^{*} \mathrm{t} /\left(\mathrm{R}_{\mathrm{o}}-0.40^{*} \mathrm{t}\right)-\mathrm{P}_{\mathrm{S}} \\
& =15,300^{*} 1.00^{*} 0.161 /\left(1.1875-0.40^{*} 0.161\right)-0 \\
& =2,193.3 \mathrm{psi}
\end{aligned}
$$

\% Forming Strain - UHA-44(a)(2)(a)

$$
\begin{aligned}
\mathrm{EFE} & =\left(50 * \mathrm{t} / \mathrm{R}_{\mathrm{f}}\right) *\left(1-\mathrm{R}_{\mathrm{f}} / \mathrm{R}_{\mathrm{o}}\right) \\
& =(50 * 0.191 / 1.092) *(1-1.092 / \infty) \\
& =8.7454 \%
\end{aligned}
$$




\section{Design thickness $=0.1167^{\prime \prime}$}

The governing condition is due to internal pressure.

The cylinder thickness of $0.191 "$ is adequate.

Thickness Required Due to Pressure + External Loads

\begin{tabular}{|c|c|c|c|c|c|c|c|c|}
\hline \multirow[t]{2}{*}{ Condition } & \multirow{2}{*}{$\begin{array}{c}\text { Pressure } \\
\text { P ( psi) }\end{array}$} & \multicolumn{2}{|c|}{$\begin{array}{c}\text { Allowable Stress } \\
\text { Before UG-23 Stress } \\
\text { Increase ( psi) }\end{array}$} & \multirow{2}{*}{$\begin{array}{c}\text { Temperature } \\
\left({ }^{\circ} \mathbf{F}\right)\end{array}$} & \multirow{2}{*}{$\begin{array}{l}\text { Corrosion } \\
\text { C (in) }\end{array}$} & \multirow[t]{2}{*}{ Load } & \multirow{2}{*}{$\begin{array}{l}\text { Req'd Thk } \\
\text { Due to } \\
\text { Tension (in) }\end{array}$} & \multirow{2}{*}{$\begin{array}{l}\text { Req'd Thk Due } \\
\text { to Compression } \\
\text { (in) }\end{array}$} \\
\hline & & $S_{t}$ & $\mathbf{S}_{\mathrm{c}}$ & & & & & \\
\hline \multirow{2}{*}{$\begin{array}{l}\text { Operating, Hot \& } \\
\text { Corroded }\end{array}$} & \multirow{2}{*}{1,150} & \multirow{2}{*}{15,300} & \multirow{2}{*}{10,201} & \multirow{2}{*}{1,000} & \multirow{2}{*}{0.03} & Wind & 0.0316 & 0.0315 \\
\hline & & & & & & Seismic & 0.032 & 0.0311 \\
\hline \multirow{2}{*}{$\begin{array}{c}\text { Operating, Hot \& } \\
\text { New }\end{array}$} & \multirow{2}{*}{1,150} & \multirow{2}{*}{15,300} & \multirow{2}{*}{10,324} & \multirow{2}{*}{1,000} & \multirow{2}{*}{0} & Wind & 0.0307 & 0.0306 \\
\hline & & & & & & Seismic & 0.0311 & 0.0301 \\
\hline \multirow{2}{*}{ Empty, Corroded } & \multirow{2}{*}{0} & \multirow{2}{*}{20,000} & \multirow{2}{*}{14,454} & \multirow{2}{*}{0} & \multirow{2}{*}{0.03} & Wind & 0.0001 & 0.0003 \\
\hline & & & & & & Seismic & 0.0002 & 0.0007 \\
\hline \multirow{2}{*}{ Empty, New } & \multirow{2}{*}{0} & \multirow{2}{*}{20,000} & \multirow{2}{*}{14,604} & \multirow{2}{*}{0} & \multirow{2}{*}{0} & Wind & 0.0002 & 0.0003 \\
\hline & & & & & & Seismic & 0.0002 & 0.0007 \\
\hline $\begin{array}{l}\text { Hot Shut Down, } \\
\text { Corroded, Weight \& } \\
\text { Eccentric Moments } \\
\text { Only }\end{array}$ & 0 & 15,300 & 10,201 & 1,000 & 0.03 & Weight & 0.0004 & 0.0004 \\
\hline
\end{tabular}




\section{4" X 2" REDUCER B16.9, UPPER (SCH XS) 4" END}

\section{ASME Section VIII Division 1, 2007 Edition}

Component: $\quad$ Cylinder

Material specification: $\quad$ SA-403 $316 \mathrm{H}$ (II-D p. $78, \ln .8)$

Rated MDMT per UHA-51(d)(1)(b), (carbon content does not exceed 0.1 percent) $=-320^{\circ} \mathrm{F}$

Internal design pressure: $\mathrm{P}=1,150$ psi @ $1,000^{\circ} \mathrm{F}$

Static liquid head:

$\mathrm{P}_{\mathrm{th}}=0.26 \mathrm{psi}\left(\mathrm{SG}=1, \mathrm{H}_{\mathrm{s}}=7.205^{\prime}\right.$, Horizontal test head $)$

Corrosion allowance $\quad$ Inner $\mathrm{C}=0.03^{\prime \prime} \quad$ Outer $\mathrm{C}=0$ "

Design MDMT $=-20^{\circ} \mathrm{F} \quad$ No impact test performed

Rated MDMT $=-320^{\circ} \mathrm{F} \quad$ Material is not normalized

Material is not produced to Fine Grain Practice

PWHT is not performed

Radiography: $\quad$ Longitudinal joint - $\quad$ Seamless No RT

Top circumferential joint - Full UW-11(a) Type 1

Bottom circumferential joint -

Full UW-11(a) Type 1

Estimated weight New $=2.3 \mathrm{lb} \quad$ corr $=2 \mathrm{lb}$

Capacity New $=0.1 \mathrm{US}$ gal corr $=0.11 \mathrm{US}$ gal

$$
\begin{array}{ll}
\text { OD } & =4.5^{\prime \prime} \\
\text { Length } \mathrm{L}_{\mathrm{c}} & =2^{\prime \prime} \\
\mathrm{t} & =0.295^{\prime \prime}
\end{array}
$$

Design thickness, (at $1,000^{\circ} \mathrm{F}$ ) Appendix 1-1

$$
\begin{aligned}
\mathrm{t} & =\mathrm{P} * \mathrm{R}_{\mathrm{o}} /(\mathrm{S} * \mathrm{E}+0.40 * \mathrm{P})+\text { Corrosion } \\
& =1,150 * 2.25 /(15,300 * 1.00+0.40 * 1,150)+0.03 \\
& =0.1942
\end{aligned}
$$

Maximum allowable working pressure, (at 1,000 ${ }^{\circ} \mathrm{F}$ ) Appendix 1 -1

$$
\begin{aligned}
\mathrm{P} & =\mathrm{S} * \mathrm{E}^{*} \mathrm{t} /\left(\mathrm{R}_{\mathrm{o}}-0.40 * \mathrm{t}\right)-\mathrm{P}_{\mathrm{S}} \\
& =15,300 * 1.00 * 0.265 /(2.25-0.40 * 0.265)-0 \\
& =1,891.09 \mathrm{psi}
\end{aligned}
$$

\% Forming Strain - UHA-44(a)(2)(a)

$$
\begin{aligned}
\operatorname{EFE} & =\left(50 * t / R_{f}\right) *\left(1-R_{f} / R_{0}\right) \\
& =(50 * 0.295 / 2.1025) *(1-2.1025 / \infty) \\
& =7.0155 \%
\end{aligned}
$$




\section{Design thickness $=0.1942 "$}

The governing condition is due to internal pressure.

The cylinder thickness of $0.295^{\prime \prime}$ is adequate.

Thickness Required Due to Pressure + External Loads

\begin{tabular}{|c|c|c|c|c|c|c|c|c|}
\hline \multirow[t]{2}{*}{ Condition } & \multirow{2}{*}{$\begin{array}{l}\text { Pressure } \\
\text { P ( psi) }\end{array}$} & \multicolumn{2}{|c|}{$\begin{array}{c}\text { Allowable Stress } \\
\text { Before UG-23 Stress } \\
\text { Increase ( psi) }\end{array}$} & \multirow{2}{*}{$\begin{array}{l}\text { Temperature } \\
\left({ }^{\circ} \mathrm{F}\right)\end{array}$} & \multirow{2}{*}{$\begin{array}{l}\text { Corrosion } \\
\text { C (in) }\end{array}$} & \multirow[t]{2}{*}{ Load } & \multirow{2}{*}{$\begin{array}{l}\text { Req'd Thk } \\
\text { Due to } \\
\text { Tension (in) }\end{array}$} & \multirow{2}{*}{$\begin{array}{l}\text { Req'd Thk Due } \\
\text { to Compression } \\
\text { (in) }\end{array}$} \\
\hline & & $S_{t}$ & $\mathbf{S}_{\mathbf{c}}$ & & & & & \\
\hline \multirow{2}{*}{$\begin{array}{l}\text { Operating, Hot \& } \\
\text { Corroded }\end{array}$} & \multirow{2}{*}{1,150} & \multirow{2}{*}{15,300} & \multirow{2}{*}{10,100} & \multirow{2}{*}{1,000} & \multirow{2}{*}{0.03} & Wind & 0.0613 & 0.0613 \\
\hline & & & & & & Seismic & 0.0615 & 0.0611 \\
\hline \multirow{2}{*}{$\begin{array}{c}\text { Operating, Hot \& } \\
\text { New }\end{array}$} & \multirow{2}{*}{1,150} & \multirow{2}{*}{15,300} & \multirow{2}{*}{10,177} & \multirow{2}{*}{1,000} & \multirow{2}{*}{0} & Wind & 0.0604 & 0.0603 \\
\hline & & & & & & Seismic & 0.0605 & 0.0601 \\
\hline \multirow{2}{*}{ Empty, Corroded } & \multirow{2}{*}{0} & \multirow{2}{*}{20,000} & \multirow{2}{*}{14,332} & \multirow{2}{*}{0} & \multirow{2}{*}{0.03} & Wind & 0.0001 & 0.0002 \\
\hline & & & & & & Seismic & 0.0001 & 0.0003 \\
\hline \multirow{2}{*}{ Empty, New } & \multirow{2}{*}{0} & \multirow{2}{*}{20,000} & \multirow{2}{*}{14,425} & \multirow{2}{*}{0} & \multirow{2}{*}{0} & Wind & 0.0001 & 0.0002 \\
\hline & & & & & & Seismic & 0.0001 & 0.0003 \\
\hline $\begin{array}{l}\text { Hot Shut Down, } \\
\text { Corroded, Weight \& } \\
\text { Eccentric Moments } \\
\text { Only }\end{array}$ & 0 & 15,300 & 10,100 & 1,000 & 0.03 & Weight & 0.0002 & 0.0002 \\
\hline
\end{tabular}




\section{8" X 4" REDUCER B16.9, UPPER (SCH XS) 4" END}

ASME Section VIII Division 1, 2007 Edition

Component: Cylinder

Material specification: $\quad$ SA-403 316H (II-D p. 78, ln. 8)

Rated MDMT per UHA-51(d)(1)(b), (carbon content does not exceed 0.1 percent) $=-320^{\circ} \mathrm{F}$

Internal design pressure: $\mathrm{P}=1,150 \mathrm{psi} @ 1,000^{\circ} \mathrm{F}$

Static liquid head:

$P_{\text {th }}=0.26 \mathrm{psi}\left(\mathrm{SG}=1, \mathrm{H}_{\mathrm{S}}=7.205^{\prime \prime}\right.$, Horizontal test head $)$

Corrosion allowance $\quad$ Inner $\mathrm{C}=0.03 " \quad$ Outer $\mathrm{C}=0 "$

Design MDMT $=-20^{\circ} \mathrm{F} \quad$ No impact test performed

Rated MDMT $=-320^{\circ} \mathrm{F} \quad$ Material is not normalized

Material is not produced to Fine Grain Practice

PWHT is not performed

Radiography: $\quad$ Longitudinal joint - $\quad$ Seamless No RT

Top circumferential joint - Full UW-11(a) Type 1

Bottom circumferential joint -

Full UW-11(a) Type 1

Estimated weight New $=3.4 \mathrm{lb} \quad$ corr $=3.1 \mathrm{lb}$

Capacity New $=0.16$ US gal corr $=0.16$ US gal

$\mathrm{OD} \quad=4.5^{\prime \prime}$

Length $\mathrm{L}_{\mathrm{c}}=3^{\prime \prime}$

$\mathrm{t} \quad=0.295^{\prime \prime}$

Design thickness, (at $1,000{ }^{\circ}$ F) Appendix 1-1

$\mathrm{t}=\mathrm{P}^{*} \mathrm{R}_{\mathrm{o}} /(\mathrm{S} * \mathrm{E}+0.40 * \mathrm{P})+$ Corrosion

$=1,150 * 2.25 /(15,300 * 1.00+0.40 * 1,150)+0.03$

$=0.1942 "$

Maximum allowable working pressure, (at 1,000 ${ }^{\circ}$ F) Appendix 1-1

$$
\begin{aligned}
\mathrm{P} & =\mathrm{S} * \mathrm{E}^{*} \mathrm{t} /\left(\mathrm{R}_{\mathrm{o}}-0.40 * \mathrm{t}\right)-\mathrm{P}_{\mathrm{S}} \\
& =15,300^{*} 1.00 * 0.265 /(2.25-0.40 * 0.265)-0 \\
& =1,891.09 \mathrm{psi}
\end{aligned}
$$

$\%$ Forming Strain - UHA-44(a)(2)(a)

$$
\begin{aligned}
\mathrm{EFE} & =\left(50 * \mathrm{t} / \mathrm{R}_{\mathrm{f}}\right) *\left(1-\mathrm{R}_{\mathrm{f}} / \mathrm{R}_{\mathrm{o}}\right) \\
& =(50 * 0.295 / 2.1025) *(1-2.1025 / \infty) \\
& =7.0155 \%
\end{aligned}
$$




\section{Design thickness $=0.1942 "$}

The governing condition is due to internal pressure.

The cylinder thickness of $0.295^{\prime \prime}$ is adequate.

Thickness Required Due to Pressure + External Loads

\begin{tabular}{|c|c|c|c|c|c|c|c|c|}
\hline \multirow[t]{2}{*}{ Condition } & \multirow{2}{*}{$\begin{array}{l}\text { Pressure } \\
\text { P ( psi) }\end{array}$} & \multicolumn{2}{|c|}{$\begin{array}{l}\text { Allowable Stress } \\
\text { Before UG-23 Stress } \\
\text { Increase ( psi) }\end{array}$} & \multirow{2}{*}{$\begin{array}{l}\text { Temperature } \\
\left({ }^{\circ} \mathrm{F}\right)\end{array}$} & \multirow{2}{*}{$\begin{array}{l}\text { Corrosion } \\
\text { C (in) }\end{array}$} & \multirow[t]{2}{*}{ Load } & \multirow{2}{*}{$\begin{array}{l}\text { Req'd Thk } \\
\text { Due to } \\
\text { Tension (in) }\end{array}$} & \multirow{2}{*}{$\begin{array}{l}\text { Req'd Thk Due } \\
\text { to Compression } \\
\text { (in) }\end{array}$} \\
\hline & & $\mathbf{S}_{\mathbf{t}}$ & $\mathbf{S}_{\mathbf{c}}$ & & & & & \\
\hline \multirow{2}{*}{$\begin{array}{l}\text { Operating, Hot \& } \\
\text { Corroded }\end{array}$} & \multirow{2}{*}{1,150} & \multirow{2}{*}{15,300} & \multirow{2}{*}{10,100} & \multirow{2}{*}{1,000} & \multirow{2}{*}{0.03} & Wind & 0.0613 & 0.0612 \\
\hline & & & & & & Seismic & 0.0616 & 0.061 \\
\hline \multirow{2}{*}{$\begin{array}{c}\text { Operating, Hot } \& \\
\text { New }\end{array}$} & \multirow{2}{*}{1,150} & \multirow{2}{*}{15,300} & \multirow{2}{*}{10,177} & \multirow{2}{*}{1,000} & \multirow{2}{*}{0} & Wind & 0.0604 & 0.0603 \\
\hline & & & & & & Seismic & 0.0606 & 0.06 \\
\hline \multirow{2}{*}{ Empty, Corroded } & \multirow{2}{*}{0} & \multirow{2}{*}{20,000} & \multirow{2}{*}{14,332} & \multirow{2}{*}{0} & \multirow{2}{*}{0.03} & Wind & 0.0001 & 0.0002 \\
\hline & & & & & & Seismic & 0.0001 & 0.0005 \\
\hline \multirow{2}{*}{ Empty, New } & \multirow{2}{*}{0} & \multirow{2}{*}{20,000} & \multirow{2}{*}{14,425} & \multirow{2}{*}{0} & \multirow{2}{*}{0} & Wind & 0.0001 & 0.0002 \\
\hline & & & & & & Seismic & 0.0001 & 0.0005 \\
\hline $\begin{array}{l}\text { Hot Shut Down, } \\
\text { Corroded, Weight \& } \\
\text { Eccentric Moments } \\
\text { Only }\end{array}$ & 0 & 15,300 & 10,100 & 1,000 & 0.03 & Weight & 0.0002 & 0.0002 \\
\hline
\end{tabular}




\section{8" X 4" REDUCER B16.9, UPPER (SCH XS) 8" END}

ASME Section VIII Division 1, 2007 Edition

Component: $\quad$ Cylinder

Material specification: $\quad$ SA-403 $316 \mathrm{H}$ (II-D p. 78, ln. 8)

Rated MDMT per UHA-51(d)(1)(b), (carbon content does not exceed 0.1 percent) $=-320^{\circ} \mathrm{F}$

Internal design pressure: $\mathrm{P}=1,150$ psi @ $1,000^{\circ} \mathrm{F}$

Static liquid head:

$\mathrm{P}_{\mathrm{th}}=0.33 \mathrm{psi}\left(\mathrm{SG}=1, \mathrm{H}_{\mathrm{s}}=9.125^{\prime}\right.$, Horizontal test head $)$

Corrosion allowance $\quad$ Inner $\mathrm{C}=0.03^{\prime \prime} \quad$ Outer $\mathrm{C}=0^{\prime \prime}$

Design MDMT $=-20^{\circ} \mathrm{F} \quad$ No impact test performed

Rated MDMT $=-320^{\circ} \mathrm{F} \quad$ Material is not normalized

Material is not produced to Fine Grain Practice

PWHT is notperformed

Radiography: $\quad$ Longitudinal joint - $\quad$ Seamless No RT

Top circumferential joint - Full UW-11(a) Type 1

Bottom circumferential

joint -

Full UW-11(a) Type 1

Estimated weight New $=9.8 \mathrm{lb} \quad$ corr $=9.2 \mathrm{lb}$

Capacity New $=0.61$ US gal corr $=0.62$ US gal

OD $\quad=8.625^{\prime \prime}$

Length $\mathrm{L}_{\mathrm{c}}=3^{\prime \prime}$

$\mathrm{t} \quad=0.4375^{\prime \prime}$

Design thickness, (at $1,000^{\circ}$ F) Appendix 1-1

$\mathrm{t}=\mathrm{P}^{*} \mathrm{R}_{\mathrm{o}} /\left(\mathrm{S} * \mathrm{E}+0.40^{*} \mathrm{P}\right)+$ Corrosion

$=1,150 * 4.3125 /(15,300 * 1.00+0.40 * 1,150)+0.03$

$=0.3447^{\prime \prime}$

Maximum allowable working pressure, (at 1,000 $\left.{ }^{\circ} \mathrm{F}\right)$ Appendix 1 -1

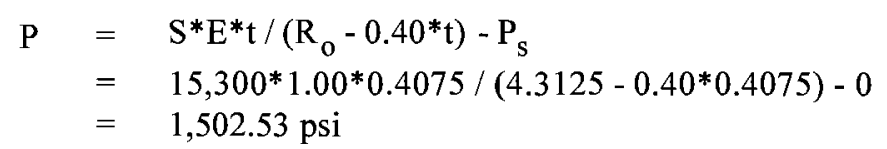

\% Forming Strain - UHA-44(a)(2)(a)

$$
\begin{aligned}
\mathrm{EFE} & =\left(50 * \mathrm{t} / \mathrm{R}_{\mathrm{f}}\right) *\left(1-\mathrm{R}_{\mathrm{f}} / \mathrm{R}_{\mathrm{o}}\right) \\
& =(50 * 0.4375 / 4.0938) *(1-4.0938 / \infty) \\
& =5.3435 \%
\end{aligned}
$$




\section{Design thickness $=0.3447^{\prime \prime}$}

The governing condition is due to internal pressure.

The cylinder thickness of $0.4375^{\prime \prime}$ is adequate.

Thickness Required Due to Pressure + External Loads

\begin{tabular}{|c|c|c|c|c|c|c|c|c|}
\hline \multirow[t]{2}{*}{ Condition } & \multirow{2}{*}{$\begin{array}{l}\text { Pressure } \\
\text { P ( psi) }\end{array}$} & \multicolumn{2}{|c|}{$\begin{array}{c}\text { Allowable Stress } \\
\text { Before UG-23 Stress } \\
\text { Increase ( psi) }\end{array}$} & \multirow{2}{*}{$\begin{array}{c}\text { Temperature } \\
\left({ }^{\circ} \mathrm{F}\right)\end{array}$} & \multirow{2}{*}{$\begin{array}{l}\text { Corrosion } \\
\text { C (in) }\end{array}$} & \multirow[t]{2}{*}{ Load } & \multirow{2}{*}{$\begin{array}{l}\text { Req'd Thk } \\
\text { Due to } \\
\text { Tension (in) }\end{array}$} & \multirow{2}{*}{$\begin{array}{l}\text { Req'd Thk Due } \\
\text { to Compression } \\
\text { (in) }\end{array}$} \\
\hline & & $\mathbf{S}_{\mathbf{t}}$ & $\mathbf{S}_{\mathbf{c}}$ & & & & & \\
\hline \multirow{2}{*}{$\begin{array}{l}\text { Operating, Hot \& } \\
\text { Corroded }\end{array}$} & \multirow{2}{*}{1,150} & \multirow{2}{*}{15,300} & \multirow{2}{*}{9,945} & \multirow{2}{*}{1,000} & \multirow{2}{*}{0.03} & Wind & 0.1207 & 0.1207 \\
\hline & & & & & & Seismic & 0.1208 & 0.1206 \\
\hline \multirow{2}{*}{$\begin{array}{c}\text { Operating, Hot \& } \\
\text { New }\end{array}$} & \multirow{2}{*}{1,150} & \multirow{2}{*}{15,300} & \multirow{2}{*}{9,995} & \multirow{2}{*}{1,000} & \multirow{2}{*}{0} & Wind & 0.1198 & 0.1197 \\
\hline & & & & & & Seismic & 0.1199 & 0.1196 \\
\hline \multirow{2}{*}{ Empty, Corroded } & \multirow{2}{*}{0} & \multirow{2}{*}{20,000} & \multirow{2}{*}{14,142} & \multirow{2}{*}{0} & \multirow{2}{*}{0.03} & Wind & 0.0001 & 0.0001 \\
\hline & & & & & & Seismic & 0 & 0.0002 \\
\hline \multirow{2}{*}{ Empty, New } & \multirow{2}{*}{0} & \multirow{2}{*}{20,000} & \multirow{2}{*}{14,203} & \multirow{2}{*}{0} & \multirow{2}{*}{0} & Wind & 0.0001 & 0.0001 \\
\hline & & & & & & Seismic & 0 & 0.0002 \\
\hline $\begin{array}{c}\text { Hot Shut Down, } \\
\text { Corroded, Weight \& } \\
\text { Eccentric Moments } \\
\text { Only }\end{array}$ & 0 & 15,300 & 9,945 & 1,000 & 0.03 & Weight & 0.0002 & 0.0002 \\
\hline
\end{tabular}




\section{8" PIPE}

ASME Section VIII Division 1, 2007 Edition

Component:

Material specification:

Pipe NPS and Schedule:
Cylinder

SA-312 TP316H Wld \& smls pipe (II-D p.

$78, \ln .1)$

$8 "$ Sch 80 S (XS)

Rated MDMT per UHA-51(d)(1)(b), (carbon content does not exceed 0.1 percent) $=-320^{\circ} \mathrm{F}$

Internal design pressure: $\mathrm{P}=1,150 \mathrm{psi} @ 1,000^{\circ} \mathrm{F}$

Static liquid head:

$P_{\text {th }}=0.33 \mathrm{psi}\left(\mathrm{SG}=1, \mathrm{H}_{\mathrm{s}}=9.0625^{\prime \prime}\right.$, Horizontal test head $)$

Corrosion allowance $\quad$ Inner $\mathrm{C}=0.03^{\prime \prime} \quad$ Outer $\mathrm{C}=0 "$

Design MDMT $=-20^{\circ} \mathrm{F}$

No impact test performed

Rated MDMT $=-320^{\circ} \mathrm{F}$

Material is not normalized

Material is not produced to Fine Grain Practice

PWHT is not performed

Radiography: Longitudinal joint - Seamless No RT

Top circumferential joint - Full UW-11(a) Type 1

Bottom circumferential Full UW-11(a) Type 1
joint -

Estimated weight $\mathrm{New}=133.2 \mathrm{lb} \quad$ corr $=125.7 \mathrm{lb}$

Capacity $\quad$ New $=7.12$ US gal corr $=7.23$ US gal

$\mathrm{OD}=8.625^{\prime \prime}$

Length $\mathrm{L}_{\mathrm{c}}=36^{\prime \prime}$

$\mathrm{t} \quad=0.5^{\prime \prime}$

Design thickness, $\left(\right.$ at $^{1,000}{ }^{\circ}$ F) Appendix 1-1

$\mathrm{t}=\mathrm{P}^{*} \mathrm{R}_{\mathrm{o}} /\left(\mathrm{S} * \mathrm{E}+0.40^{*} \mathrm{P}\right)+$ Corrosion

$=1,150 * 4.3125 /(15,300 * 1.00+0.40 * 1,150)+0.03$

$=0.3447^{\prime \prime}$

Maximum allowable working pressure, $\left(\right.$ at $\left.^{1,000^{\circ}}{ }^{\circ}\right)$ Appendix 1-1

$$
\begin{aligned}
\mathrm{P} & =\mathrm{S}^{*} \mathrm{E}^{*} \mathrm{t} /\left(\mathrm{R}_{\mathrm{o}}-0.40^{*} \mathrm{t}\right)-\mathrm{P}_{\mathrm{S}} \\
& =15,300^{*} 1.00^{*} 0.4075 /\left(4.3125-0.40^{*} 0.4075\right)-0 \\
& =1,502.53 \mathrm{psi}
\end{aligned}
$$

Design thickness $=0.3447^{\prime \prime}$

The governing condition is due to internal pressure.

The cylinder thickness of $0.5^{\prime \prime}$ is adequate. 
Thickness Required Due to Pressure + External Loads

\begin{tabular}{|c|c|c|c|c|c|c|c|c|}
\hline \multirow[t]{2}{*}{ Condition } & \multirow{2}{*}{$\begin{array}{l}\text { Pressure } \\
\text { P ( psi) }\end{array}$} & \multicolumn{2}{|c|}{$\begin{array}{l}\text { Allowable Stress } \\
\text { Before UG-23 Stress } \\
\text { Increase ( psi) }\end{array}$} & \multirow{2}{*}{$\begin{array}{l}\text { Temperature } \\
\left({ }^{\circ} \mathbf{F}\right)\end{array}$} & \multirow{2}{*}{$\begin{array}{c}\text { Corrosion } \\
\text { C (in) }\end{array}$} & \multirow[t]{2}{*}{ Load } & \multirow{2}{*}{$\begin{array}{l}\text { Req'd Thk } \\
\text { Due to } \\
\text { Tension (in) }\end{array}$} & \multirow{2}{*}{$\begin{array}{l}\text { Req'd Thk Due } \\
\text { to Compression } \\
\text { (in) }\end{array}$} \\
\hline & & $\mathbf{S}_{\mathbf{t}}$ & $\mathbf{S}_{\mathbf{c}}$ & & & & & \\
\hline \multirow{2}{*}{$\begin{array}{l}\text { Operating, Hot \& } \\
\text { Corroded }\end{array}$} & \multirow{2}{*}{1,150} & \multirow{2}{*}{15,300} & \multirow{2}{*}{9,945} & \multirow{2}{*}{1,000} & \multirow{2}{*}{0.03} & Wind & 0.1193 & 0.1177 \\
\hline & & & & & & Seismic & 0.1195 & 0.1175 \\
\hline \multirow{2}{*}{$\begin{array}{l}\text { Operating, Hot \& } \\
\text { New }\end{array}$} & \multirow{2}{*}{1,150} & \multirow{2}{*}{15,300} & \multirow{2}{*}{9,995} & \multirow{2}{*}{1,000} & \multirow{2}{*}{0} & Wind & 0.1183 & 0.1168 \\
\hline & & & & & & Seismic & 0.1186 & 0.1165 \\
\hline \multirow{2}{*}{ Empty, Corroded } & \multirow{2}{*}{0} & \multirow{2}{*}{20,000} & \multirow{2}{*}{14,142} & \multirow{2}{*}{0} & \multirow{2}{*}{0.03} & Wind & 0.0003 & 0.0012 \\
\hline & & & & & & Seismic & 0.0005 & 0.0015 \\
\hline \multirow{2}{*}{ Empty, New } & \multirow{2}{*}{0} & \multirow{2}{*}{20,000} & \multirow{2}{*}{14,203} & \multirow{2}{*}{0} & \multirow{2}{*}{0} & Wind & 0.0003 & 0.0012 \\
\hline & & & & & & Seismic & 0.0005 & 0.0016 \\
\hline $\begin{array}{l}\text { Hot Shut Down, } \\
\text { Corroded, Weight \& } \\
\text { Eccentric Moments } \\
\text { Only }\end{array}$ & 0 & 15,300 & 9,945 & 1,000 & 0.03 & Weight & 0.0007 & 0.0007 \\
\hline
\end{tabular}




\section{8" X 4" REDUCER B16.9, LOWER (SCH XS) 8" END}

ASME Section VIII Division 1, 2007 Edition

Component:

Material specification:

Rated MDMT per UHA-51(d)(1)(b), (carbon content does not exceed 0.1 percent) $=-320^{\circ} \mathrm{F}$
Cylinder

SA-403 $316 \mathrm{H}$ (II-D p. $78, \ln .8)$

Internal design pressure: $P=1,150 \mathrm{psi} @ 1,000^{\circ} \mathrm{F}$

Static liquid head:

$\mathrm{P}_{\mathrm{th}}=0.33 \mathrm{psi}\left(\mathrm{SG}=1, \mathrm{H}_{\mathrm{S}}=9.125 "\right.$ ", Horizontal test head $)$

Corrosion allowance $\quad$ Inner $\mathrm{C}=0.03 " \quad$ Outer $\mathrm{C}=0$ "

$\begin{array}{ll}\text { Design MDMT }=-20^{\circ} \mathrm{F} & \text { No impact test performed } \\ \text { Rated MDMT }=-320^{\circ} \mathrm{F} & \text { Material is not normalized } \\ & \text { Material is not produced to Fine Grain Practice } \\ & \text { PWHT is not performed }\end{array}$

Radiography: $\quad$ Longitudinal joint - $\quad$ Seamless No RT

Top circumferential joint - Full UW-11(a) Type 1

Bottom circumferential joint -

Estimated weight New $=9.8 \mathrm{lb} \quad$ corr $=9.2 \mathrm{lb}$

Capacity New $=0.61$ US gal corr $=0.62$ US gal

$\begin{aligned} \text { OD } & =8.625^{\prime \prime} \\ \text { Length } \mathrm{L}_{\mathrm{c}} & =3^{\prime \prime} \\ \mathrm{t} & =0.4375^{\prime \prime}\end{aligned}$

Design thickness, $\left(\right.$ at $\left.^{1,000}{ }^{\circ} \mathrm{F}\right)$ Appendix 1-1

$\mathrm{t}=\mathrm{P} * \mathrm{R}_{\mathrm{o}} /(\mathrm{S} * \mathrm{E}+0.40 * \mathrm{P})+$ Corrosion

$=1,150 * 4.3125 /(15,300 * 1.00+0.40 * 1,150)+0.03$

$=0.3447^{\prime \prime}$

Maximum allowable working pressure, $\left(\right.$ at $\left.1,000^{\circ} \mathrm{F}\right)$ Appendix 1-1

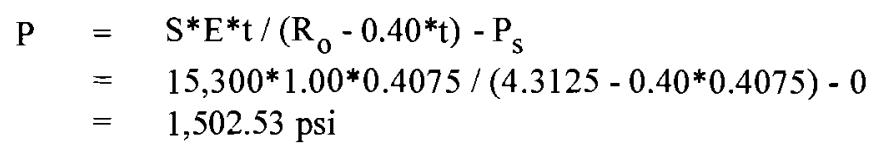

\% Forming Strain - UHA-44(a)(2)(a)

$$
\begin{aligned}
\mathrm{EFE} & =\left(50 * \mathrm{t} / \mathrm{R}_{\mathrm{f}}\right) *\left(1-\mathrm{R}_{\mathrm{f}} / \mathrm{R}_{\mathrm{o}}\right) \\
& =(50 * 0.4375 / 4.0938) *(1-4.0938 / \infty) \\
& =5.3435 \%
\end{aligned}
$$




\section{Design thickness $=0.3447^{\prime \prime}$}

The governing condition is due to internal pressure.

The cylinder thickness of $0.4375 "$ is adequate.

Thickness Required Due to Pressure + External Loads

\begin{tabular}{|c|c|c|c|c|c|c|c|c|}
\hline \multirow[t]{2}{*}{ Condition } & \multirow{2}{*}{$\begin{array}{l}\text { Pressure } \\
\text { P ( psi) }\end{array}$} & \multicolumn{2}{|c|}{$\begin{array}{c}\text { Allowable Stress } \\
\text { Before UG-23 Stress } \\
\text { Increase ( psi) }\end{array}$} & \multirow{2}{*}{$\begin{array}{c}\text { Temperature } \\
\left({ }^{\circ} \mathrm{F}\right)\end{array}$} & \multirow{2}{*}{$\begin{array}{c}\text { Corrosion } \\
\text { C (in) }\end{array}$} & \multirow[t]{2}{*}{ Load } & \multirow{2}{*}{$\begin{array}{l}\text { Req'd Thk } \\
\text { Due to } \\
\text { Tension (in) }\end{array}$} & \multirow{2}{*}{$\begin{array}{l}\text { Req'd Thk Due } \\
\text { to Compression } \\
\text { (in) }\end{array}$} \\
\hline & & $\mathbf{S}_{\mathrm{t}}$ & $\mathbf{S}_{\mathbf{c}}$ & & & & & \\
\hline \multirow{2}{*}{$\begin{array}{l}\text { Operating, Hot \& } \\
\text { Corroded }\end{array}$} & \multirow{2}{*}{1,150} & \multirow{2}{*}{15,300} & \multirow{2}{*}{9,945} & \multirow{2}{*}{1,000} & \multirow{2}{*}{0.03} & Wind & 0.1213 & 0.1195 \\
\hline & & & & & & Seismic & 0.1215 & 0.1193 \\
\hline \multirow{2}{*}{$\begin{array}{c}\text { Operating, Hot \& } \\
\text { New }\end{array}$} & \multirow{2}{*}{1,150} & \multirow{2}{*}{15,300} & \multirow{2}{*}{9,995} & \multirow{2}{*}{1,000} & \multirow{2}{*}{0} & Wind & 0.1203 & 0.1186 \\
\hline & & & & & & Seismic & 0.1206 & 0.1183 \\
\hline \multirow{2}{*}{ Empty, Corroded } & \multirow{2}{*}{0} & \multirow{2}{*}{20,000} & \multirow{2}{*}{14,142} & \multirow{2}{*}{0} & \multirow{2}{*}{0.03} & Wind & 0.0004 & 0.0013 \\
\hline & & & & & & Seismic & 0.0005 & 0.0016 \\
\hline \multirow{2}{*}{ Empty, New } & \multirow{2}{*}{0} & \multirow{2}{*}{20,000} & \multirow{2}{*}{14,203} & \multirow{2}{*}{0} & \multirow{2}{*}{0} & Wind & 0.0004 & 0.0014 \\
\hline & & & & & & Seismic & 0.0005 & 0.0017 \\
\hline $\begin{array}{l}\text { Hot Shut Down, } \\
\text { Corroded, Weight \& } \\
\text { Eccentric Moments } \\
\text { Only }\end{array}$ & 0 & 15,300 & 9,945 & 1,000 & 0.03 & Weight & 0.0007 & 0.0007 \\
\hline
\end{tabular}




\section{8" X 4" REDUCER B16.9, LOWER (SCH XS) 4" END}

\section{ASME Section VIII Division 1, 2007 Edition}

Component:

Material specification:

Rated MDMT per UHA-51(d)(1)(b), (carbon content does not exceed 0.1 percent) $=-320^{\circ} \mathrm{F}$
Cylinder

SA-403 $316 \mathrm{H}$ (II-D p. $78, \ln .8)$

Internal design pressure: $\mathrm{P}=1,150$ psi @ $1,000^{\circ} \mathrm{F}$

Static liquid head:

$P_{\text {th }}=0.26 \mathrm{psi}\left(\mathrm{SG}=1, \mathrm{H}_{\mathrm{s}}=7.205^{\prime}\right.$, Horizontal test head $)$

Corrosion allowance $\quad$ Inner $\mathrm{C}=0.03^{\prime \prime} \quad$ Outer $\mathrm{C}=0$ "

Design MDMT $=-20^{\circ} \mathrm{F}$

Rated $\mathrm{MDMT}=-320^{\circ} \mathrm{F}$
No impact test performed Material is not normalized Material is not produced to Fine Grain Practice PWHT is not performed

Radiography: Longitudinal joint Seamless No RT Top circumferential joint - Full UW-11(a) Type 1 Bottom circumferential joint -

Estimated weight New $=3.4 \mathrm{lb} \quad$ corr $=3.1 \mathrm{lb}$

Capacity New $=0.16$ US gal corr $=0.16$ US gal

$$
\begin{array}{ll}
\text { OD } & =4.5^{\prime \prime} \\
\text { Length } \mathrm{L}_{\mathrm{c}} & =3^{\prime \prime} \\
\mathrm{t} & =0.295^{\prime \prime}
\end{array}
$$

\section{Design thickness, (at $1,000^{\circ} \mathrm{F}$ ) Appendix 1-1}

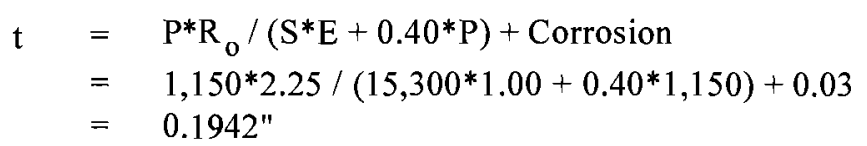

Maximum allowable working pressure, (at 1,000 $\left.{ }^{\circ} \mathrm{F}\right)$ Appendix 1-1

$$
\begin{aligned}
\mathrm{P} & =\mathrm{S} * \mathrm{E}^{*} \mathrm{t} /\left(\mathrm{R}_{\mathrm{o}}-0.40 * \mathrm{t}\right)-\mathrm{P}_{\mathrm{S}} \\
& =15,300 * 1.00 * 0.265 /(2.25-0.40 * 0.265)-0 \\
& =1,891.09 \mathrm{psi}
\end{aligned}
$$

\% Forming Strain - UHA-44(a)(2)(a)

$$
\begin{aligned}
\mathrm{EFE} & =\left(50 * \mathrm{t} / \mathrm{R}_{\mathrm{f}}\right) *\left(1-\mathrm{R}_{\mathrm{f}} / \mathrm{R}_{\mathrm{o}}\right) \\
& =(50 * 0.295 / 2.1025) *(1-2.1025 / \infty) \\
& =7.0155 \%
\end{aligned}
$$




\section{Design thickness $=0.1942 "$}

The governing condition is due to internal pressure.

The cylinder thickness of $0.295^{\prime \prime}$ is adequate.

Thickness Required Due to Pressure + External Loads

\begin{tabular}{|c|c|c|c|c|c|c|c|c|}
\hline \multirow[t]{2}{*}{ Condition } & \multirow{2}{*}{$\begin{array}{c}\text { Pressure } \\
\text { P ( psi) }\end{array}$} & \multicolumn{2}{|c|}{$\begin{array}{l}\text { Allowable Stress } \\
\text { Before UG-23 Stress } \\
\text { Increase ( psi) }\end{array}$} & \multirow{2}{*}{$\begin{array}{c}\text { Temperature } \\
\left({ }^{\circ} \mathrm{F}\right)\end{array}$} & \multirow{2}{*}{$\begin{array}{l}\text { Corrosion } \\
\text { C (in) }\end{array}$} & \multirow[t]{2}{*}{ Load } & \multirow{2}{*}{$\begin{array}{l}\text { Req'd Thk } \\
\text { Due to } \\
\text { Tension (in) }\end{array}$} & \multirow{2}{*}{$\begin{array}{l}\text { Req'd Thk Due } \\
\text { to Compression } \\
\text { (in) }\end{array}$} \\
\hline & & $\mathbf{S}_{\mathbf{t}}$ & $S_{c}$ & & & & & \\
\hline \multirow{2}{*}{$\begin{array}{l}\text { Operating, Hot \& } \\
\text { Corroded }\end{array}$} & \multirow{2}{*}{1,150} & \multirow{2}{*}{15,300} & \multirow{2}{*}{10,100} & \multirow{2}{*}{1,000} & \multirow{2}{*}{0.03} & Wind & 0.0644 & 0.057 \\
\hline & & & & & & Seismic & 0.065 & 0.0562 \\
\hline \multirow{2}{*}{$\begin{array}{c}\text { Operating, Hot \& } \\
\text { New }\end{array}$} & \multirow{2}{*}{1,150} & \multirow{2}{*}{15,300} & \multirow{2}{*}{10,177} & \multirow{2}{*}{1,000} & \multirow{2}{*}{0} & Wind & 0.0634 & 0.0559 \\
\hline & & & & & & Seismic & 0.0643 & 0.055 \\
\hline \multirow{2}{*}{ Empty, Corroded } & \multirow{2}{*}{0} & \multirow{2}{*}{20,000} & \multirow{2}{*}{14,332} & \multirow{2}{*}{0} & \multirow{2}{*}{0.03} & Wind & 0.0023 & 0.0047 \\
\hline & & & & & & Seismic & 0.0028 & 0.0056 \\
\hline \multirow{2}{*}{ Empty, New } & \multirow{2}{*}{0} & \multirow{2}{*}{20,000} & \multirow{2}{*}{14,425} & \multirow{2}{*}{0} & \multirow{2}{*}{0} & Wind & 0.0023 & 0.0048 \\
\hline & & & & & & Seismic & 0.0029 & 0.0058 \\
\hline $\begin{array}{l}\text { Hot Shut Down, } \\
\text { Corroded, Weight \& } \\
\text { Eccentric Moments } \\
\text { Only }\end{array}$ & 0 & 15,300 & 10,100 & 1,000 & 0.03 & Weight & 0.0013 & 0.0013 \\
\hline
\end{tabular}




\section{4" X 2" B16.9 REDUCER, LOWER (SCH XS) 4" END}

\section{ASME Section VIII Division 1, 2007 Edition}

\section{Component:}

Material specification:

Rated MDMT per UHA-51(d)(1)(b), (carbon content does not exceed 0.1 percent) $=-320^{\circ} \mathrm{F}$
Cylinder

SA-403 316H (II-D p. 78, ln. 8)

Internal design pressure: $\mathrm{P}=1,150 \mathrm{psi} @ 1,000^{\circ} \mathrm{F}$

Static liquid head:

$\mathrm{P}_{\text {th }}=0.26 \mathrm{psi}\left(\mathrm{SG}=1, \mathrm{H}_{\mathrm{s}}=7.205^{\prime \prime}\right.$, Horizontal test head $)$

Corrosion allowance $\quad$ Inner $\mathrm{C}=\mathbf{0 . 0 3 "} \quad$ Outer $\mathrm{C}=0 "$

Design MDMT $=-20^{\circ} \mathrm{F}$

Rated MDMT $=-320^{\circ} \mathrm{F}$
No impact test performed

Material is not normalized

Material is not produced to Fine Grain Practice

PWHT is not performed

Radiography: Longitudinal joint - $\quad$ Seamless No RT

Top circumferential joint - Full UW-1 1(a) Type 1

Bottom circumferential joint -

Full UW-11(a) Type 1

Estimated weight New $=2.3 \mathrm{lb} \quad$ corr $=2 \mathrm{lb}$

Capacity $\quad$ New $=0.1$ US gal corr $=0.11$ US gal

$$
\begin{aligned}
\mathrm{OD} & =4.5^{\prime \prime} \\
\text { Length } \mathrm{L}_{\mathrm{c}} & =2^{\prime \prime} \\
\mathrm{t} & =0.295^{\prime \prime}
\end{aligned}
$$

\section{Design thickness, (at $1,000^{\circ} \mathrm{F}$ ) Appendix 1-1}

$$
\begin{aligned}
\mathbf{t} & =\mathrm{P}^{*} \mathrm{R}_{\mathrm{o}} /\left(\mathrm{S}^{*} \mathrm{E}+0.40^{*} \mathrm{P}\right)+\text { Corrosion } \\
& =1,150^{*} 2.25 /\left(15,300^{*} 1.00+0.40^{*} 1,150\right)+0.03 \\
& =0.1942^{\prime \prime}
\end{aligned}
$$

Maximum allowable working pressure, $\left(\right.$ at $\left.1,000^{\circ} \mathrm{F}\right)$ Appendix 1-1

$$
\begin{aligned}
\mathrm{P} & =\mathrm{S}^{*} \mathrm{E}^{*} \mathrm{t} /\left(\mathrm{R}_{\mathrm{o}}-0.40^{*} \mathrm{t}\right)-\mathrm{P}_{\mathrm{s}} \\
& =15,300^{*} 1.00^{*} 0.265 /\left(2.25-0.40^{*} 0.265\right)-0 \\
& =1,891.09 \mathrm{psi}
\end{aligned}
$$

\% Forming Strain - UHA-44(a)(2)(a)

$$
\begin{aligned}
\mathrm{EFE} & =\left(50 * t / \mathrm{R}_{\mathrm{f}}\right) *\left(1-\mathrm{R}_{\mathrm{f}} / \mathrm{R}_{\mathrm{o}}\right) \\
& =(50 * 0.295 / 2.1025) *(1-2.1025 / \infty) \\
& =7.0155 \%
\end{aligned}
$$




\section{Design thickness $=0.1942 "$}

The governing condition is due to internal pressure.

The cylinder thickness of 0.295 " is adequate.

Thickness Required Due to Pressure + External Loads

\begin{tabular}{|c|c|c|c|c|c|c|c|c|}
\hline \multirow[t]{2}{*}{ Condition } & \multirow{2}{*}{$\begin{array}{l}\text { Pressure } \\
\text { P ( psi) }\end{array}$} & \multicolumn{2}{|c|}{$\begin{array}{c}\text { Allowable Stress } \\
\text { Before UG-23 Stress } \\
\text { Increase ( psi) }\end{array}$} & \multirow{2}{*}{$\begin{array}{c}\text { Temperature } \\
\left({ }^{\circ} \mathbf{F}\right)\end{array}$} & \multirow{2}{*}{$\begin{array}{c}\text { Corrosion } \\
\text { C (in) }\end{array}$} & \multirow[t]{2}{*}{ Load } & \multirow{2}{*}{$\begin{array}{l}\text { Req'd Thk } \\
\text { Due to } \\
\text { Tension (in) }\end{array}$} & \multirow{2}{*}{$\begin{array}{l}\text { Req'd Thk Due } \\
\text { to Compression } \\
\text { (in) }\end{array}$} \\
\hline & & $S_{t}$ & $S_{c}$ & & & & & \\
\hline \multirow{2}{*}{$\begin{array}{l}\text { Operating, Hot \& } \\
\text { Corroded }\end{array}$} & \multirow{2}{*}{1,150} & \multirow{2}{*}{15,300} & \multirow{2}{*}{10,100} & \multirow{2}{*}{1,000} & \multirow{2}{*}{0.03} & Wind & 0.0646 & 0.0567 \\
\hline & & & & & & Seismic & 0.0653 & 0.0559 \\
\hline \multirow{2}{*}{$\begin{array}{c}\text { Operating, Hot \& } \\
\text { New }\end{array}$} & \multirow{2}{*}{1,150} & \multirow{2}{*}{15,300} & \multirow{2}{*}{10,177} & \multirow{2}{*}{1,000} & \multirow{2}{*}{0} & Wind & 0.0637 & 0.0556 \\
\hline & & & & & & Seismic & 0.0645 & 0.0547 \\
\hline \multirow{2}{*}{ Empty, Corroded } & \multirow{2}{*}{0} & \multirow{2}{*}{20,000} & \multirow{2}{*}{14,332} & \multirow{2}{*}{0} & \multirow{2}{*}{0.03} & Wind & 0.0025 & 0.005 \\
\hline & & & & & & Seismic & 0.003 & 0.0059 \\
\hline \multirow{2}{*}{ Empty, New } & \multirow{2}{*}{0} & \multirow{2}{*}{20,000} & \multirow{2}{*}{14,425} & \multirow{2}{*}{0} & \multirow{2}{*}{0} & Wind & 0.0025 & 0.0051 \\
\hline & & & & & & Seismic & 0.0031 & 0.0061 \\
\hline $\begin{array}{c}\text { Hot Shut Down, } \\
\text { Corroded, Weight \& } \\
\text { Eccentric Moments } \\
\text { Only }\end{array}$ & 0 & 15,300 & 10,100 & 1,000 & 0.03 & Weight & 0.0013 & 0.0013 \\
\hline
\end{tabular}




\section{4" X 2" B16.9 REDUCER, LOWER (SCH XS) 2" END}

\section{ASME Section VIII Division 1, 2007 Edition}

\section{Component:}

Material specification:

Rated MDMT per UHA-51(d)(1)(b), (carbon content does not exceed 0.1 percent) $=-320^{\circ} \mathrm{F}$
Cylinder

SA-403 316H (II-D p. 78, In. 8)

Internal design pressure: $\mathrm{P}=1,150$ psi @ $1,000^{\circ} \mathrm{F}$

Static liquid head:

$P_{\text {th }}=0.23 \mathrm{psi}\left(\mathrm{SG}=1, \mathrm{H}_{\mathrm{s}}=6.2465^{\prime}\right.$, Horizontal test head $)$

Corrosion allowance $\quad$ Inner $\mathrm{C}=0.03^{\prime \prime} \quad$ Outer $\mathrm{C}=0^{\prime \prime}$

Design $\mathrm{MDMT}=-20^{\circ} \mathrm{F}$

Rated MDMT $=-320^{\circ} \mathrm{F}$
No impact test performed

Material is not normalized

Material is not produced to Fine Grain Practice

PWHT is not performed

Radiography: $\quad$ Longitudinal joint - $\quad$ Seamless No RT

Top circumferential joint - Full UW-11(a) Type 1

Bottom circumferential joint -

Full UW-11(a) Type 1

Estimated weight New $=0.8 \mathrm{lb} \quad$ corr $=0.6 \mathrm{lb}$

Capacity New $=0.03$ US gal corr $=0.03$ US gal

$\begin{array}{ll}\text { OD } & =2.375^{\prime \prime} \\ \text { Length } \mathrm{L}_{\mathrm{c}} & =2^{\prime \prime} \\ \mathrm{t} & =0.191^{\prime \prime}\end{array}$

Design thickness, (at $1,000^{\circ} \mathrm{F}$ ) Appendix 1-1

$\mathrm{t}=\mathrm{P}^{*} \mathrm{R}_{\mathrm{o}} /(\mathrm{S} * \mathrm{E}+0.40 * \mathrm{P})+$ Corrosion

$=1,150 * 1.1875 /(15,300 * 1.00+0.40 * 1,150)+0.03$

$=0.1167^{\prime \prime}$

Maximum allowable working pressure, (at 1,000 ${ }^{\circ} \mathrm{F}$ ) Appendix 1-1

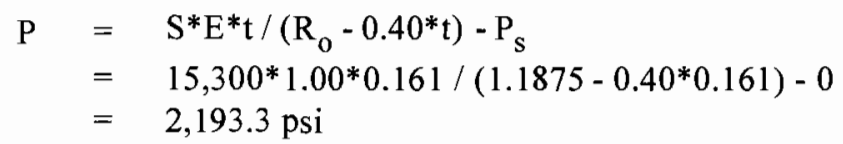

\% Forming Strain - UHA-44(a)(2)(a)

$$
\begin{aligned}
\mathrm{EFE} & =\left(50 * \mathrm{t} / \mathrm{R}_{\mathrm{f}}\right) *\left(1-\mathrm{R}_{\mathrm{f}} / \mathrm{R}_{\mathrm{o}}\right) \\
& =(50 * 0.191 / 1.092) *(1-1.092 / \infty) \\
& =8.7454 \%
\end{aligned}
$$




\section{Design thickness $=0.1167^{\prime \prime}$}

The governing condition is due to internal pressure.

The cylinder thickness of $0.191 "$ is adequate.

Thickness Required Due to Pressure + External Loads

\begin{tabular}{|c|c|c|c|c|c|c|c|c|}
\hline \multirow[t]{2}{*}{ Condition } & \multirow{2}{*}{$\begin{array}{l}\text { Pressure } \\
\text { P ( psi) }\end{array}$} & \multicolumn{2}{|c|}{$\begin{array}{c}\text { Allowable Stress } \\
\text { Before UG-23 Stress } \\
\text { Increase ( psi) }\end{array}$} & \multirow{2}{*}{$\begin{array}{l}\text { Temperature } \\
\left({ }^{\circ} \mathbf{F}\right)\end{array}$} & \multirow{2}{*}{$\begin{array}{c}\text { Corrosion } \\
\text { C (in) }\end{array}$} & \multirow[t]{2}{*}{ Load } & \multirow{2}{*}{$\begin{array}{l}\text { Req'd Thk } \\
\text { Due to } \\
\text { Tension (in) }\end{array}$} & \multirow{2}{*}{$\begin{array}{l}\text { Req'd Thk Due } \\
\text { to Compression } \\
\text { (in) }\end{array}$} \\
\hline & & $S_{t}$ & $\mathbf{S}_{\mathbf{c}}$ & & & & & \\
\hline \multirow{2}{*}{$\begin{array}{l}\text { Operating, Hot \& } \\
\text { Corroded }\end{array}$} & \multirow{2}{*}{1,150} & \multirow{2}{*}{15,300} & \multirow{2}{*}{10,201} & \multirow{2}{*}{1,000} & \multirow{2}{*}{0.03} & Wind & 0.0482 & 0.0121 \\
\hline & & & & & & Seismic & 0.0498 & 0.0101 \\
\hline \multirow{2}{*}{$\begin{array}{c}\text { Operating, Hot \& } \\
\text { New }\end{array}$} & \multirow{2}{*}{1,150} & \multirow{2}{*}{15,300} & \multirow{2}{*}{10,324} & \multirow{2}{*}{1,000} & \multirow{2}{*}{0} & Wind & 0.0476 & 0.0106 \\
\hline & & & & & & Seismic & 0.0501 & 0.0078 \\
\hline \multirow{2}{*}{ Empty, Corroded } & \multirow{2}{*}{0} & \multirow{2}{*}{20,000} & \multirow{2}{*}{14,454} & \multirow{2}{*}{0} & \multirow{2}{*}{0.03} & Wind & 0.0126 & 0.0208 \\
\hline & & & & & & Seismic & 0.0138 & 0.0229 \\
\hline \multirow{2}{*}{ Empty, New } & \multirow{2}{*}{0} & \multirow{2}{*}{20,000} & \multirow{2}{*}{14,604} & \multirow{2}{*}{0} & \multirow{2}{*}{0} & Wind & 0.0129 & 0.0212 \\
\hline & & & & & & Seismic & 0.0148 & 0.0242 \\
\hline $\begin{array}{c}\text { Hot Shut Down, } \\
\text { Corroded, Weight \& } \\
\text { Eccentric Moments } \\
\text { Only }\end{array}$ & 0 & 15,300 & 10,201 & 1,000 & 0.03 & Weight & 0.0029 & 0.0029 \\
\hline
\end{tabular}




\section{2" RFWN FLANGE 1500\#.LOWER}

Flange description:

Bolt Material:

Flange rated MDMT:

(Per UHA-51(d)(1)(b))

(Flange rated $\mathrm{MDMT}=-320^{\circ} \mathrm{F}$

Bolts rated MDMT per Fig UCS-66 note $\left.(\mathrm{e})=-55^{\circ} \mathrm{F}\right)$

Liquid static head on flange:

ASME B16.5 flange rating MAWP:

ASME B 16.5 flange rating MAP:

ASME B16.5 flange hydro test:
2 inch Class 1500 WN A 182 F316H

SA-193 B7 Bolt $<=21 / 2$

$-55^{\circ} \mathrm{F}$

\section{$0 \mathrm{psi}$}

1820 psi @ $1000^{\circ} \mathrm{F}$

3600 psi @ $70^{\circ} \mathrm{F}$

$5425 \mathrm{psi} @ 70^{\circ} \mathrm{F}$ 


\section{ASME B16.5/16.47 Blind on 2" RFWN FLANGE 1500\#, LOWER}

This is an ASME B16.5/16.47 rated blind flange.

Flange description:

Bolt Material:

Flange rated MDMT:

(Per UHA-51(d)(1)(b))

(Flange rated MDMT $=-320^{\circ} \mathrm{F}$

Bolts rated MDMT per Fig UCS- 66 note $\left.(e)=-55^{\circ} \mathrm{F}\right)$

Liquid static head on flange:

ASME B16.5 flange rating MAWP:

ASME B16.5 flange rating MAP:

ASME B16.5 flange hydro test:
2 inch Class $1500 \mathrm{WN}$ A182 F316H

SA-193 B7 Bolt $<=21 / 2$

$-55^{\circ} \mathrm{F}$

$0 \mathrm{psi}$

$1820 \mathrm{psi} @ 1000^{\circ} \mathrm{F}$

$3600 \mathrm{psi} @ 70^{\circ} \mathrm{F}$

$5425 \mathrm{psi} @ 70^{\circ} \mathrm{F}$ 


\section{1/2" HALF-COUPLING (7a)}

ASME Section VIII Division 1, 2007 Edition

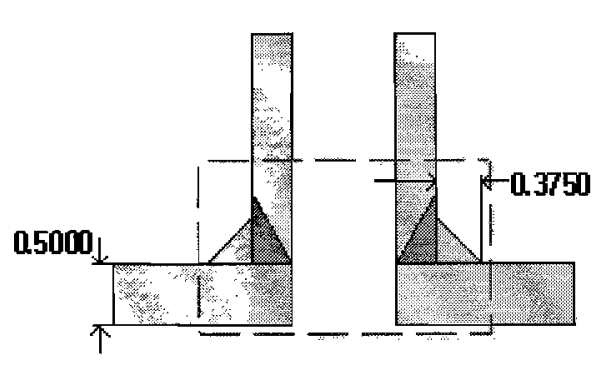

$$
\begin{aligned}
& \mathrm{t}_{\mathrm{w}(\text { lower })}=0 \mathrm{in} \\
& \operatorname{Leg}_{41}=0.375 \mathrm{in}
\end{aligned}
$$

Note: round inside edges per UG-76(c)

Located on:

Liquid static head included:

Nozzle material specification:

Nozzle longitudinal joint efficiency:

Nozzle description:

Nozzle orientation:

Local vessel minimum thickness:

Nozzle center line offset to datum line:

End of nozzle to shell center:

Nozzle inside diameter, new:

Nozzle nominal wall thickness:

Nozzle corrosion allowance:

Projection available outside vessel, Lpr: 0.9375 in

\section{Reinforcement CaIculations for Internal Pressure}

\begin{tabular}{|c|c|c|c|c|c|c|c|c|}
\hline \multicolumn{7}{|c|}{$\begin{array}{l}\text { UG-37 Area Calculation Summary }\left(\text { in }^{2}\right) \\
\text { For } P=1502.52 \text { psi @ } 1000^{\circ} \mathrm{F}\end{array}$} & \multicolumn{2}{|c|}{$\begin{array}{l}\text { UG-45 Nozzle Wall } \\
\text { Thickness Summary (in) } \\
\text { The nozzle passes UG-45 }\end{array}$} \\
\hline$\underset{\text { required }}{A}$ & $\underset{\text { available }}{\text { A }}$ & $\mathbf{A}_{1}$ & $\mathbf{A}_{2}$ & $\mathbf{A}_{3}$ & $\mathbf{A}_{5}$ & $\begin{array}{c}\text { A } \\
\text { welds }\end{array}$ & $t_{\text {req }}$ & $t_{\text {min }}$ \\
\hline \multicolumn{7}{|c|}{ This nozzle is exempt from area calculations perUG-36(c)(3)(a) } & 0.1247 & 0.33 \\
\hline
\end{tabular}

The vessel wall thickness governs the MAWP of this nozzle.

\section{UG-41 Weld Failure Path Analysis Summary}

The nozzle is exempt from weld strength calculations per UW-15(b)(2)

\section{UW-16 Weld Sizing Summary}




\begin{tabular}{|c|r|r|c|} 
Weld description & $\begin{array}{r}\text { Required weld } \\
\text { throat size (in) }\end{array}$ & $\begin{array}{r}\text { Actual weld } \\
\text { throat size (in) }\end{array}$ & Status \\
\hline Nozzle to shell fillet $\left(\operatorname{Leg}_{41}\right)$ & 0.21 & 0.2625 & weld size is adequate \\
\hline
\end{tabular}

This opening does not require reinforcement per UG-36(c)(3)(a)

\section{Reinforcement Calculations for MAP}

The vessel wall thickness governs the MAP of this nozzle.

\begin{tabular}{|c|c|c|c|c|c|c|c|c|}
\hline \multicolumn{7}{|c|}{$\begin{array}{l}\text { UG-37 Area Calculation Summary }\left(\text { in }^{2}\right) \\
\text { For } P=2114.8 \mathrm{psi} @ 7^{\circ} \mathrm{F}\end{array}$} & \multicolumn{2}{|c|}{$\begin{array}{c}\text { UG-45 Nozzle Wall } \\
\text { Thickness Summary (in) } \\
\text { The nozzle passes UG-45 }\end{array}$} \\
\hline$\underset{\text { required }}{\text { A }}$ & $\underset{\text { available }}{\mathbf{A}}$ & $\mathbf{A}_{1}$ & $\mathbf{A}_{2}$ & $\mathbf{A}_{3}$ & $\mathbf{A}_{\mathbf{s}}$ & $\begin{array}{c}\text { A } \\
\text { welds }\end{array}$ & $t_{\text {req }}$ & $\mathbf{t}_{\min }$ \\
\hline \multicolumn{7}{|c|}{ This nozzle is exempt from area calculations per UG-36(c)(3)(a) } & 0.0761 & 0.33 \\
\hline
\end{tabular}

\section{UG-41 Weld Failure Path Analysis Summary}

The nozzle is exempt from weld strength calculations per UW-15(b)(2)

\begin{tabular}{|c|r|r|c|}
\hline \multicolumn{4}{|c|}{ UW -16 Weld Sizing Summary } \\
\hline Weld description & $\begin{array}{r}\text { Required weld } \\
\text { throat size (in) }\end{array}$ & $\begin{array}{r}\text { Actual weld } \\
\text { throat size (in) }\end{array}$ & Status \\
\hline Nozzle to shell fillet ( $\left.\mathrm{Leg}_{41}\right)$ & 0.231 & 0.2625 & weld size is adequate \\
\hline
\end{tabular}

This opening does not require reinforcement per UG-36(c)(3)(a) 


\section{1/2" HALF-COUPLING (7b)}

ASME Section VIII Division 1, 2007 Edition

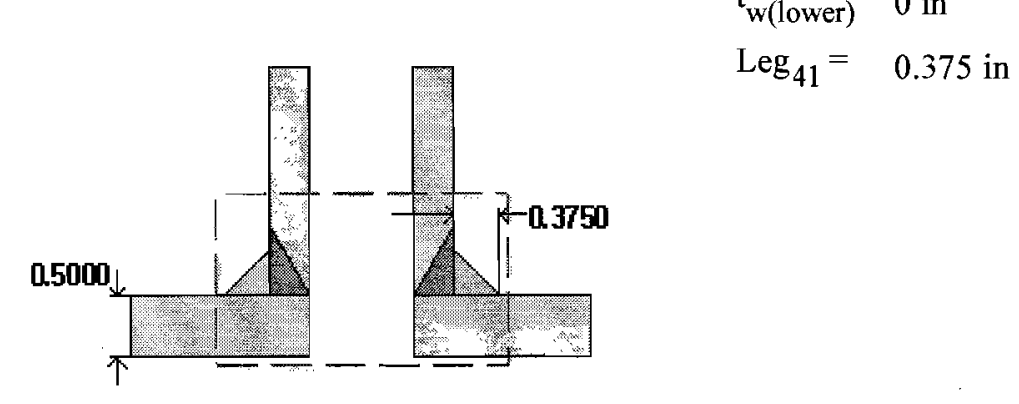

Note round inside edges per UG-76(c)

Located on:

Liquid static head included:

Nozzle material specification:

Nozzle longitudinal joint efficiency:

Nozzle description:

Nozzle orientation:

Local vessel minimum thickness:

Nozzle center line offset to datum line:

End of nozzle to shell center:

Nozzle inside diameter, new:

Nozzle nominal wall thickness:

Nozzle corrosion allowance:

Projection available outside vessel, Lpr: 0.9375 in

\section{Reinforcement Calculations for Internal Pressure}

The vessel wall thickness governs the MAWP of this nozzle.

\begin{tabular}{|c|c|c|c|c|c|c|c|c|}
\hline \multicolumn{8}{|c|}{$\begin{array}{c}\text { UG-37 Area Calculation Summary }\left(\text { in }^{2}\right) \\
\text { For P = 1502.52 psi @ } 1000{ }^{\circ} \mathbf{F}\end{array}$} & \multicolumn{2}{c|}{$\begin{array}{c}\text { UG-45 Nozzle Wall } \\
\text { Thickness Summary (in) } \\
\text { The nozzle passes UG-45 }\end{array}$} \\
\hline $\begin{array}{c}\text { A } \\
\text { required }\end{array}$ & $\begin{array}{c}\text { A } \\
\text { available }\end{array}$ & $\mathbf{A}_{1}$ & $\mathbf{A}_{2}$ & $\mathbf{A}_{3}$ & $\mathbf{A}_{5}$ & $\begin{array}{c}\text { A } \\
\text { welds }\end{array}$ & $\mathbf{t}_{\text {req }}$ & $\mathbf{t}_{\text {min }}$ \\
\hline This nozzle is exempt from area calculations per UG-36(c)(3)(a) & 0.1247 & 0.33 \\
\hline
\end{tabular}

\section{UG-41 Weld Failure Path Analysis Summary}

The nozzle is exempt from weld strength calculations per $U W-15(b)(2)$

UW-16 Weld Sizing Summary
8" PIPE

SA-182 F316H $<=5$ (low stress) (II-D p. 74, ln. 32)

$0.500^{\prime \prime}$ Class 6000 - threaded

5.25 in

.84 in

0.33 in

0.03 in 


\begin{tabular}{|c|r|r|c|}
\hline Weld description & $\begin{array}{r}\text { Required weld } \\
\text { throat size (in) }\end{array}$ & $\begin{array}{r}\text { Actual weld } \\
\text { throat size (in) }\end{array}$ & Status \\
\hline Nozzle to shell fillet $\left(\mathrm{Leg}_{41}\right)$ & 0.21 & 0.2625 & weld size is adequate \\
\hline
\end{tabular}

This opening does not require reinforcement per UG-36(c)(3)(a)

\section{Reinforcement Calculations for MAP}

The vessel wall thickness governs the MAP of this nozzle.

\begin{tabular}{|c|c|c|c|c|c|c|c|c|}
\hline \multicolumn{7}{|c|}{ 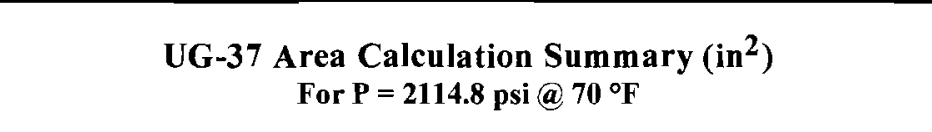 } & \multicolumn{2}{|c|}{$\begin{array}{l}\text { UG-45 Nozzle Wall } \\
\text { Thickness Summary (in) } \\
\text { The nozzle passes UG-45 }\end{array}$} \\
\hline$\underset{\text { required }}{A}$ & $\underset{\text { available }}{\text { A }}$ & $\mathbf{A}_{1}$ & $\mathbf{A}_{2}$ & $\mathbf{A}_{3}$ & $A_{5}$ & $\begin{array}{c}\text { A } \\
\text { welds }\end{array}$ & $t_{\text {req }}$ & $t_{\min }$ \\
\hline \multicolumn{7}{|c|}{ This nozzle is exempt from area calculations per UG-36(c)(3)(a) } & 0.0761 & 0.33 \\
\hline
\end{tabular}

\section{UG-41 Weld Failure Path Analysis Summary}

The nozzle is exempt from weld strength calculations per UW-15(b)(2)

\begin{tabular}{|c|r|r|c|}
\hline \multicolumn{4}{|c|}{ UW-16 Weld Sizing Summary } \\
\hline Weld description & $\begin{array}{r}\text { Required weld } \\
\text { throat size (in) }\end{array}$ & $\begin{array}{r}\text { Actual weld } \\
\text { throat size (in) }\end{array}$ & Status \\
\hline Nozzle to shell fillet $\left(\operatorname{Leg}_{41}\right)$ & 0.231 & 0.2625 & weld size is adequate \\
\hline
\end{tabular}

This opening does not require reinforcement per UG-36(c)(3)(a) 


\section{Wind Code}

Building Code:

ASCE 7-05

Elevation of base above grade: $\quad 0.0000 \mathrm{ft}$

Increase effective outer diameter by: $0.0000 \mathrm{ft}$

Wind Force Coefficient Cf: $\quad 0.8300$

Basic Wind Speed:, V: $\quad 85.0000 \mathrm{mph}$

Importance Factor:, I: $\quad 1.1500$

Exposure category: $\mathrm{C}$

Wind Directionality Factor, Kd: $\quad 0.9500$

Topographic Factor, Kzt: $\quad 1.0000$

Enforce min. loading of $10 \mathrm{psf}$ : No

\section{Vessel Characteristics}

Vessel height, h: $\quad 5.5425 \mathrm{ft}$

Vessel Minimum Diameter, $\mathbf{b}$

Operating, Corroded: $0.1979 \mathrm{ft}$

Empty, Corroded: $0.1979 \mathrm{ft}$

Fundamental Frequency, $\mathrm{n}_{1}$

Operating, Corroded: $9.6455 \mathrm{~Hz}$

Empty, Corroded: $10.7384 \mathrm{~Hz}$

Vacuum, Corroded: $9.5611 \mathrm{~Hz}$

Damping coefficient, $\beta$

Operating, Corroded: 0.0179

Empty, Corroded: 0.0200

Vacuum, Corroded: 0.0177

Table Lookup Values

\subsubsection{Basic Load Combinations for Allowable Stress Design}

The following load combinations are considered in accordance with ASCE section 2.4.1:

5. $D+H+W$

Where

$D \quad=$ Dead load

$H \quad=$ Pressure load

$W \quad=$ Wind load

\section{Wind Deflection Reports:}

Operating, Corroded

Empty, Corroded

Wind Pressure Calculations

Wind Deflection Report: Operating, Corroded

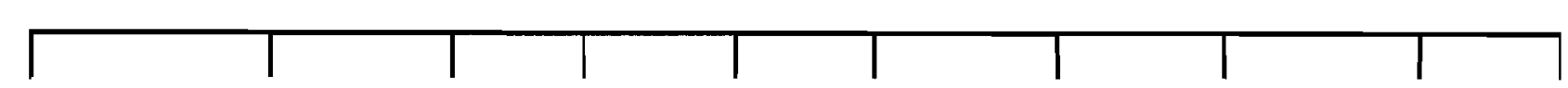




\begin{tabular}{|c|c|c|c|c|c|c|c|c|}
\hline Component & $\begin{array}{c}\text { Elevation of } \\
\text { bottom above } \\
\text { base (in) }\end{array}$ & $\begin{array}{c}\text { Effective } \\
\text { OD } \\
\text { (ft) }\end{array}$ & $\begin{array}{c}\text { Elastic } \\
\text { modulus } \\
\text { E }\left(10^{6} \mathrm{psi}\right)\end{array}$ & $\begin{array}{c}\text { Inertia } \\
\text { I }\left(\mathrm{ft}^{\mathbf{4}}\right)\end{array}$ & $\begin{array}{l}\text { Platform } \\
\text { wind shear at } \\
\text { Bottom (lbf) }\end{array}$ & $\begin{array}{c}\text { Total wind } \\
\text { shear at } \\
\text { Bottom (lbf) }\end{array}$ & \begin{tabular}{|c|} 
bending \\
moment at \\
Bottom (lbf- $\mathrm{ft}$ )
\end{tabular} & $\begin{array}{l}\text { Deflection } \\
\text { at top (in) }\end{array}$ \\
\hline $\begin{array}{l}4 " \text { X 2" } \\
\text { REDUCER B 16.9, } \\
\text { UPPER(SCH XS) } \\
\text { 2" END }\end{array}$ & 60.26 & 0.20 & 22.8 & $\begin{array}{r}3.326 \mathrm{e}- \\
005\end{array}$ & 0 & 1 & 0 & 0.03 \\
\hline $\begin{array}{l}\text { 4" X 2" } \\
\text { REDUCER B16.9, } \\
\text { UPPER (SCH XS) } \\
\text { 4" END }\end{array}$ & 58.26 & 0.38 & 22.8 & 0.0003827 & 0 & 2 & 1 & 0.0269 \\
\hline $\begin{array}{l}\text { 8" X 4" } \\
\text { REDUCER B16.9, } \\
\text { UPPER (SCH XS) } \\
\text { 4" END }\end{array}$ & 55.26 & 0.38 & 22.8 & 0.0003827 & 0 & 3 & 1 & 0.026 \\
\hline $\begin{array}{l}\text { 8" X 4" } \\
\text { REDUCER B16.9, } \\
\text { UPPER (SCH XS) } \\
\text { 8" END }\end{array}$ & 52.26 & 0.72 & 22.8 & 0.004293 & 0 & 6 & 2 & 0.0245 \\
\hline 8" PIPE & 16.26 & 0.72 & 22.8 & 0.004843 & 0 & 34 & 62 & 0.023 \\
\hline $\begin{array}{l}\text { 8" X 4" } \\
\text { REDUCER B16.9, } \\
\text { LOWER (SCH } \\
\text { XS) 8" END }\end{array}$ & 13.26 & 0.72 & 22.8 & 0.004293 & 0 & 36 & 70 & 0.0053 \\
\hline $\begin{array}{l}8^{\prime \prime} \text { X 4" } \\
\text { REDUCER B16.9, } \\
\text { LOWER (SCH } \\
\text { XS) 4" END }\end{array}$ & 10.26 & 0.38 & 22.8 & 0.0003827 & 0 & 37 & 80 & 0.0039 \\
\hline $\begin{array}{l}\text { 4" X 2" B16.9 } \\
\text { REDUCER, } \\
\text { LOWER (SCH } \\
\text { XS) 4" END }\end{array}$ & 8.26 & 0.38 & 22.8 & 0.0003827 & 0 & 38 & 86 & 0.0024 \\
\hline $\begin{array}{l}\text { 4" X 2" B16.9 } \\
\text { REDUCER, } \\
\text { LOWER (SCH } \\
\text { XS) 2" END }\end{array}$ & 2.01 & 0.20 & 22.8 & $\begin{array}{r}3.326 \mathrm{e}- \\
005\end{array}$ & 0 & 40 & 106 & 0.0015 \\
\hline $\begin{array}{l}\text { ASME } \\
\text { B16.5/16.47 Blind } \\
\text { on 2" RFWN } \\
\text { FLANGE 1500\#, } \\
\text { LOWER (top) }\end{array}$ & 2 & 0.71 & 20.4 & 0.01236 & 0 & 40 & 106 & 0 \\
\hline & & & & & & & & \\
\hline
\end{tabular}

\section{Wind Deflection Report: Empty, Corroded}

\begin{tabular}{|c|c|c|c|c|c|c|c|c|}
\hline Component & $\begin{array}{c}\text { Elevation of } \\
\text { bottom above } \\
\text { base (in) }\end{array}$ & $\begin{array}{c}\text { Effective } \\
\text { OD } \\
\text { (ft) }\end{array}$ & $\begin{array}{c}\text { Elastic } \\
\text { modulus } \\
\text { E }\left(\mathbf{1 0}^{6}{ }^{\mathrm{psi}}\right)\end{array}$ & $\begin{array}{c}\text { Inertia } \\
\mathbf{I}\left(\mathbf{f t}^{\mathbf{4}}\right)\end{array}$ & $\begin{array}{l}\text { Platform } \\
\text { wind shear at } \\
\text { Bottom (lbf) }\end{array}$ & $\begin{array}{c}\text { Total wind } \\
\text { shear at } \\
\text { Bottom (Ibf) }\end{array}$ & $\begin{array}{c}\text { bending } \\
\text { moment at } \\
\text { Bottom (lbf-ft) }\end{array}$ & $\begin{array}{l}\text { Deflection } \\
\text { at top (in) }\end{array}$ \\
\hline $\begin{array}{l}\text { 4" X 2" } \\
\text { REDUCER B16.9, } \\
\text { UPPER (SCH XS) } \\
\text { 2" END }\end{array}$ & 60.26 & 0.20 & 28.3 & $\begin{array}{r}3.326 \mathrm{e}- \\
005\end{array}$ & 0 & 1 & 0 & 0.0242 \\
\hline $\begin{array}{l}4^{\prime \prime} \times 2 " \\
\text { REDUCER B16.9, } \\
\text { UPPER (SCH XS) }\end{array}$ & 58.26 & 0.38 & 28.3 & 0.0003827 & 0 & 2 & 1 & 0.0217 \\
\hline
\end{tabular}




\begin{tabular}{|c|c|c|c|c|c|c|c|c|}
\hline 4" END & & & & & & & & \\
\hline $\begin{array}{l}\text { 8" X 4" } \\
\text { REDUCER B16.9, } \\
\text { UPPER (SCH XS) } \\
\text { 4" END }\end{array}$ & 55.26 & 0.38 & 28.3 & 0.0003827 & 0 & 3 & 1 & 0.0209 \\
\hline $\begin{array}{l}\text { 8" X 4" } \\
\text { REDUCER B16.9, } \\
\text { UPPER (SCH XS) } \\
\text { 8" END }\end{array}$ & 52.26 & 0.72 & 28.3 & 0.004293 & 0 & 6 & 2 & 0.0198 \\
\hline 8" PIPE & 16.26 & 0.72 & 28.3 & 0.004843 & 0 & 34 & 62 & 0.0186 \\
\hline $\begin{array}{l}\text { 8" X 4" }^{\prime \prime} \\
\text { REDUCER B16.9. } \\
\text { LOWER (SCH } \\
\text { XS) 8" END }\end{array}$ & 13.26 & 0.72 & 28.3 & 0.004293 & 0 & 36 & 70 & 0.0043 \\
\hline $\begin{array}{l}\text { 8" X 4" } \\
\text { REDUCER B16.9, } \\
\text { LOWER (SCH } \\
\text { XS) 4" END }\end{array}$ & 10.26 & 0.38 & 28.3 & 0.0003827 & 0 & 37 & 80 & 0.0031 \\
\hline $\begin{array}{l}\text { 4" X 2" B16.9 } \\
\text { REDUCER, } \\
\text { LOWER (SCH } \\
\text { XS) 4" END }\end{array}$ & 8.26 & 0.38 & 28.3 & 0.0003827 & 0 & 38 & 86 & 0.002 \\
\hline $\begin{array}{l}\text { 4" X 2" B16.9 } \\
\text { REDUCER, } \\
\text { LOWER (SCH } \\
\text { XS) 2" END }\end{array}$ & 2.01 & 0.20 & 28.3 & $\begin{array}{r}3.326 \mathrm{e}- \\
005\end{array}$ & 0 & 40 & 106 & 0.0012 \\
\hline $\begin{array}{l}\text { ASME } \\
\text { B16.5/16.47 Blind } \\
\text { on 2" RFWN } \\
\text { FLANGE 1500\#, } \\
\text { LOWER (top) }\end{array}$ & 2 & 0.71 & 29.4 & 0.01236 & 0 & 40 & 106 & 0 \\
\hline & & & & & & & & \\
\hline
\end{tabular}

\section{Wind Pressure (WP) Calculations}

Gust Factor $\left(\mathrm{G}^{-}\right)$Calculations

$$
\begin{aligned}
\mathrm{Kz} & =2.01 *(\mathrm{Z} / \underline{\mathrm{Zg}})^{2 / \underline{\alpha}} \\
& =2.01 *(\mathrm{Z} / 900.0000)^{0.2105} \\
\mathrm{qz} & =0.00256 * \mathrm{Kz}^{*} \mathrm{Kzt}^{*} \mathrm{Kd}^{*} \underline{\mathrm{V}}^{2 *} \underline{\mathrm{I} p \mathrm{ff}} \\
& =0.00256 * \mathrm{Kz}^{*} 1.0000 * 0.9500 * 85.0000^{2} * 1.1500 \\
& =20.2069 * \mathrm{Kz} \\
\mathrm{WP} & =\mathrm{qz}^{*} \underline{\mathrm{G}^{-}} * \underline{\mathrm{Cf}} \\
& =\mathrm{qz}^{*} \underline{\mathrm{G}^{-}} * 0.8300
\end{aligned}
$$

\section{Design Wind Pressures}

\begin{tabular}{|c|c|c|c|c|c|c|}
\hline $\begin{array}{c}\text { Height Z } \\
\text { (') }\end{array}$ & $\mathbf{K z}$ & $\begin{array}{c}\mathbf{q z} \\
\text { (psf) }\end{array}$ & $\begin{array}{c}\text { WP: Operating } \\
\text { (psf) }\end{array}$ & $\begin{array}{c}\text { WP: Empty } \\
\text { (psf) }\end{array}$ & $\begin{array}{c}\text { WP: hydrotest } \\
\text { (psf) }\end{array}$ & $\begin{array}{c}\text { WP: Vacuum } \\
\text { (psf) }\end{array}$ \\
\hline 15.0 & 0.8489 & 17.15 & 13.02 & 13.02 & N.A. & N.A. \\
\hline
\end{tabular}


Design Wind Force determined from: $F=$ Pressure $*$ Af, where Af is the projected area.

\section{Gust Factor Calculations}

Operating, Corroded

Empty, Corroded

\section{Gust Factor Calculations: Operating, Corroded}

$$
\begin{aligned}
\mathrm{z}^{-} & =0.60 * \underline{\mathrm{h}} \\
& =0.60 * 5.5425 \\
& =15.0000 \\
\mathrm{I}_{\mathrm{z}}^{-} & =\underline{\mathrm{c}}^{*}\left(33 / \mathrm{z}^{-}\right)^{1 / 6} \\
& =0.2000 *(33 / 15.0000)^{1 / 6} \\
& =0.2281 \\
\mathrm{~L}_{\mathrm{z}} & =\underline{\mathrm{I}}^{*}\left(\mathrm{z}^{-} / 33\right)^{\mathrm{ep}} \\
& =500.0000 *(15.0000 / 33)^{0.2000} \\
& =427.0566 \\
\mathrm{Q} & =\operatorname{Sqr}\left(1 /\left(1+0.63 *\left((\mathrm{~b}+\underline{\mathrm{h}}) / \mathrm{L}_{\mathrm{z}}\right)^{0.63}\right)\right) \\
& =\mathrm{Sqr}\left(1 /\left(1+0.63 *((0.1979+5.5425) / 427.0566)^{0.63}\right)\right) \\
& =0.9798 \\
\mathrm{G} & =0.925 *\left(1+1.7 * \mathrm{~g}_{\mathrm{Q}} * \mathrm{I}_{\mathrm{z}^{-}}{ }^{*} \mathrm{Q}\right) /\left(1+1.7 * \mathrm{~g}_{\mathrm{V}}{ }^{*} \mathrm{I}_{\mathrm{z}^{-}}\right) \\
& =0.925 *\left(1+1.7 * 3.40^{*} 0.2281 * 0.9798\right) /(1+1.7 * 3.40 * 0.2281) \\
& =0.9144
\end{aligned}
$$

\section{Gust Factor Calculations: Empty, Corroded}

$$
\begin{aligned}
\mathrm{z}^{-} & =0.60 * \underline{\mathrm{h}} \\
& =0.60 * 5.5425 \\
& =15.0000 \\
\mathrm{I}_{\mathrm{z}^{-}} & =\underline{\mathrm{c}}^{*}\left(33 / \mathrm{z}^{-}\right)^{1 / 6} \\
& =0.2000 *(33 / 15.0000)^{1 / 6} \\
& =0.2281 \\
\mathrm{~L}_{\mathrm{z}^{-}} & =\underline{\underline{I}}^{*}\left(\mathrm{z}^{-} / 33\right)^{\mathrm{ep}} \\
& =500.0000 *(15.0000 / 33)^{0.2000} \\
& =427.0566 \\
\mathrm{Q} & =\operatorname{Sqr}\left(1 /\left(1+0.63 *\left((\mathrm{~b}+\underline{\mathrm{h}}) / \mathrm{L}_{\mathrm{z}^{-}}\right)^{0.63}\right)\right) \\
& =\operatorname{Sqr}\left(1 /\left(1+0.63 *((0.1979+5.5425) / 427.0566)^{0.63}\right)\right) \\
& =0.9798 \\
\mathrm{G} & =0.925 *\left(1+1.7 * \mathrm{~g}_{\underline{Q}}^{*} \mathrm{I}_{\mathrm{z}^{-}} * \mathrm{Q}\right) /\left(1+1.7 * \underline{\mathrm{g}}^{*} \mathrm{I}_{\mathrm{z}^{-}}\right) \\
& =0.925 *\left(1+1.7 * 3.40^{*} 0.2281 * 0.9798\right) /(1+1.7 * 3.40 * 0.2281) \\
& =0.9144
\end{aligned}
$$

Table Lookup Values 


$$
\begin{array}{lr}
\alpha=9.5000, \mathrm{Zg}=900.0000^{\prime} & {[\text { Table 6-2, page 78] }} \\
\mathrm{c}=0.2000,1=500.0000, \mathrm{ep}=0.2000 & {[\text { Table 6-2, page 78] }} \\
\mathrm{a}^{-}=0.1538, \mathrm{~b}^{-}=0.6500 & {[\text { Table 6-2, page 78] }} \\
\mathrm{g}_{\mathrm{Q}}=3.40 & {[6.5 .8 .1 \text { page 26] }} \\
\mathrm{g}_{\mathrm{v}}=3.40 & {[6.5 .8 .1 \text { page 26] }}
\end{array}
$$




\section{Seismic Code}

Method of seismic analysis:

Site Class

Importance Factor:

Spectral Response Acceleration at short period (\%g)

Spectral Response Acceleration at period of $1 \mathrm{sec}(\% \mathrm{~g})$

Response Modification Coeficient from Table 15.4-2

Acceleration based site co-efficient:

Velocity based site co-efficient:

Long-period transition period:

Redundancy factor:

User Defined Vertical Accelerations Considered:

Force Multiplier:

Minimum Weight Multiplier:
ASCE 7-05 ground supported

D

$\mathrm{I}=1.5000$

$\mathrm{S}_{\mathrm{S}}=40.00 \%$

$S_{1}=10.00 \%$

$\mathrm{R}=3.0000$

$\mathrm{F}_{\mathrm{a}}=1.4800$

$F_{v}=2.4000$

$\mathrm{T}_{\mathrm{L}}=12.0000$

$\rho=1.0000$

Yes

$=0.3333$

$=0.2000$

\subsubsection{Basic Load Combinations for Allowable Stress Design}

The following load combinations are considered in accordance with ASCE section 2.4.1:

5. $D+H+0.7 E=\left(1.0+V_{\text {Accel }}\right) D+H+0.7 \rho Q_{E}$

Where

$D \quad=$ Dead load

$H \quad=$ Pressure load

$E \quad=$ Seismic load $=\rho Q_{E}$

$V_{\text {Accel }}=$ User defined vertical acceleration

\section{Vessel Characteristics}

Vessel height: $5.5425 \mathrm{ft}$ Vessel Weight:

Operating, Corroded: $240 \mathrm{lb}$

Empty, Corroded: $240 \mathrm{lb}$

\section{Period of Vibration Calculation}

Fundamental Period, T:

Operating, Corroded: $0.104 \mathrm{sec}(f=9.6 \mathrm{~Hz})$

Empty, Corroded: $0.093 \mathrm{sec}(\mathrm{f}=10.7 \mathrm{~Hz})$

The fundamental period of vibration $\mathrm{T}$ (above) is calculated using the Rayleigh method of approximation:

$\mathrm{T}=2 * \mathrm{PI} * \operatorname{Sqr}\left(\left\{\operatorname{Sum}\left(\mathrm{W}_{\mathrm{i}} * \mathrm{y}_{\mathrm{i}}^{2}\right)\right\} /\left\{\mathrm{g} * \operatorname{Sum}\left(\mathrm{W}_{\mathrm{i}}^{*} \mathrm{y}_{\mathrm{i}}\right)\right\}\right)$, where 
$\mathrm{W}_{\mathrm{i}}$ is the weight of the $\mathrm{i}^{\text {th }}$ lumped mass, and

$y_{i}$ is its deflection when the system is treated as a cantilever beam.

\section{Seismic Shear Reports:}

Operating. Corroded

Empty, Corroded

Base Shear Calculations

Seismic Shear Report: Operating, Corroded

\begin{tabular}{|c|c|c|c|c|c|}
\hline Component & $\begin{array}{l}\text { Elevation of bottom } \\
\text { above base (in) }\end{array}$ & $\begin{array}{l}\text { Elastic modulus } E \\
\left(10^{6} \mathrm{psi}\right)\end{array}$ & $\begin{array}{c}\text { Inertia I } \\
\left(\mathrm{ft}^{4}\right)\end{array}$ & $\begin{array}{l}\text { Seismic shear at } \\
\text { Bottom (lbf) }\end{array}$ & $\begin{array}{l}\text { Bending Moment at } \\
\text { Bottom (Ibf-ft) }\end{array}$ \\
\hline $\begin{array}{l}\text { 4" X 2" REDUCER B } 16.9, \text { UPPER } \\
\text { (SCH XS) 2" END }\end{array}$ & 60.26 & 22.8 & 0.0000 & 8 & 3 \\
\hline $\begin{array}{l}\text { 4" X 2" REDUCER B16.9, UPPER } \\
\text { (SCH XS) 4" END }\end{array}$ & 58.26 & 22.8 & 0.0004 & 8 & 4 \\
\hline $\begin{array}{l}\text { 8" X 4" REDUCER B } 16.9 \text {, UPPER } \\
\text { (SCH XS) 4" END }\end{array}$ & 55.26 & 22.8 & 0.0004 & 9 & 6 \\
\hline $\begin{array}{l}\text { 8" X 4" REDUCER B16.9, UPPER } \\
\text { (SCH XS) 8" END }\end{array}$ & 52.26 & 22.8 & 0.0043 & 11 & 9 \\
\hline 8" PIPE & 16.26 & 22.8 & 0.0048 & 31 & 78 \\
\hline $\begin{array}{l}\text { 8" X 4" REDUCER B } 16.9, \text { LOWER } \\
\text { (SCH XS) 8" END }\end{array}$ & 13.26 & 22.8 & 0.0043 & 32 & 86 \\
\hline $\begin{array}{l}\text { 8" X 4" REDUCER B16.9, LOWER } \\
(\mathrm{SCH} \text { XS) 4" END }\end{array}$ & 10.26 & 22.8 & 0.0004 & 32 & 94 \\
\hline $\begin{array}{l}\text { 4" X 2" B16.9 REDUCER, LOWER } \\
\text { (SCH XS) 4" END }\end{array}$ & 8.26 & 22.8 & 0.0004 & 32 & 99 \\
\hline $\begin{array}{l}\text { 4" X 2" B16.9 REDUCER, LOWER } \\
\text { (SCH XS) 2" END }\end{array}$ & 2.01 & 22.8 & 0.0000 & 33 & 116 \\
\hline $\begin{array}{l}\text { ASME B16.5/16.47 Blind on } 2 " \\
\text { RFWN FLANGE 1500\#, LOWER } \\
\text { (top) }\end{array}$ & 2 & 20.4 & 0.0124 & 33 & 116 \\
\hline & & & & & \\
\hline
\end{tabular}

\section{Seismic Shear Report: Empty, Corroded}

\begin{tabular}{|l|r|r|r|r|r|}
\hline \multicolumn{1}{|c|}{ Component } & $\begin{array}{c}\text { Elevation of bottom } \\
\text { above base (in) }\end{array}$ & $\begin{array}{r}\text { Elastic modulus E } \\
(\mathbf{1 0} \mathbf{~ p s i )}\end{array}$ & $\begin{array}{c}\text { Inertia I } \\
\left(\mathbf{f t}^{\mathbf{4}}\right)\end{array}$ & $\begin{array}{c}\text { Seismic shear at } \\
\text { Bottom (lbf) }\end{array}$ & $\begin{array}{c}\text { Bending Moment at } \\
\text { Bottom (lbf-ft) }\end{array}$ \\
\hline $\begin{array}{l}\text { 4" X 2" REDUCER B16.9, UPPER } \\
\text { (SCH XS) 2" END }\end{array}$ & 60.26 & 28.3 & 0.0000 & 8 \\
\hline $\begin{array}{l}\text { 4" X 2" REDUCER B16.9, UPPER } \\
\text { (SCH XS) 4" END }\end{array}$ & 58.26 & 28.3 & 0.0004 & 8 & 4 \\
\hline $\begin{array}{l}\text { 8" X 4" REDUCER B16.9, UPPER } \\
\text { (SCH XS) 4" END }\end{array}$ & 55.26 & 28.3 & 0.0004 & 9 & \\
\hline $\begin{array}{l}\text { 8" X 4" REDUCER B16.9, UPPER } \\
\text { (SCH XS) 8" END }\end{array}$ & 52.26 & 28.3 & 0.0043 & & 11 \\
\hline 8" PIPE & 16.26 & 28.3 & 0.0048 & & 31 \\
\hline
\end{tabular}




\begin{tabular}{|l|r|r|r|r|r|}
$\begin{array}{l}\text { 8" X 4" REDUCER B16.9, LOWER } \\
\text { (SCH XS) 8" END }\end{array}$ & 13.26 & 28.3 & 0.0043 & 32 & 86 \\
\hline $\begin{array}{l}\text { 8" X 4" REDUCER B16.9, LOWER } \\
\text { (SCH XS) 4" END }\end{array}$ & 10.26 & 28.3 & 0.0004 & 32 & 94 \\
\hline $\begin{array}{l}\text { 4" X 2" B 16.9 REDUCER, LOWER } \\
\text { (SCH XS) 4" END }\end{array}$ & 8.26 & 28.3 & 0.0004 & 32 & 99 \\
\hline $\begin{array}{l}\text { 4" X 2" B16.9 REDUCER, LOWER } \\
\text { (SCH XS) 2" END }\end{array}$ & 2.01 & 28.3 & 0.0000 & 33 & 116 \\
\hline $\begin{array}{l}\text { ASME B16.5/16.47 Blind on 2" } \\
\text { RFWN FLANGE 1500\#, LOWER } \\
\text { (top) }\end{array}$ & 2 & 29.4 & 0.0124 & 33 & 116 \\
\hline & & & & & \\
\hline
\end{tabular}

\subsection{3: Maximum considered earthquake spectral response acceleration}

The maximum considered earthquake spectral response acceleration at short period, $\mathrm{S}_{M S}$

$\mathrm{S}_{M S}=\underline{\mathrm{F}}_{\underline{\mathrm{a}}} * \underline{\mathrm{S}}_{\underline{\mathrm{S}}}=1.4800 * 40.00 / 100=0.5920$

The maximum considered earthquake spectral response acceleration at $1 \mathrm{~s}$ period, $\mathrm{S}_{M 1}$

$\mathrm{S}_{M 1}=\underline{\mathrm{F}}_{\underline{\mathrm{v}}} * \underline{\mathrm{S}}_{1}=2.4000 * 10.00 / 100=0.2400$

\subsection{4: Design spectral response acceleration parameters}

Design earthquake spectral response acceleration at short period, $\mathrm{S}_{D S}$

$\mathrm{S}_{D S}=2 / 3 * \mathrm{~S}_{M S}=2 / 3 * 0.5920=0.3947$

Design earthquake spectral response acceleration at $1 \mathrm{~s}$ period, $\mathrm{S}_{D 1}$

$\mathrm{S}_{D I}=2 / 3 * \mathrm{~S}_{M 1}=2 / 3 * 0.2400=0.1600$

\section{User Defined Vertical Acceleration Term, $\mathbf{V}_{\text {Accel }}$}

Factor is applied to dead load.

Compressive Side: $=1.0+\mathrm{V}_{\text {Accel }}$

\section{$V_{\text {Accel }}$ Term is:}

greater of (Force Mult * Base Shear / Weight) or (Min. Weight Mult.)

\begin{tabular}{|l|r|r|r|r|}
\hline \multicolumn{2}{|c|}{ Force multiplier $=0.3333$} & \multicolumn{3}{|c|}{ Minimum Weight Multiplier $=0.2000$} \\
\hline Condition & Base Shear ( Ibf) & Weight ( Ib) & $\begin{array}{r}\text { Force Mult * Shear } \\
\text { Weight }\end{array}$ & V $_{\text {Accel }}$ \\
\hline Operating, Corroded & 33 & 239.6 & 0.046 & 0.2 \\
\hline Operating, New & 34 & 249.8 & 0.046 & 0.2 \\
\hline Empty, Corroded & 33 & 239.6 & 0.046 & 0.2 \\
\hline Empty, New & 34 & 249.8 & 0.046 & 0.2 \\
\hline
\end{tabular}




\section{Base Shear Calculations}

\section{Operating, Corroded}

Empty, Corroded

\section{Base Shear Calculations: Operating, Corroded}

\section{Paragraph 15.4.4: Period Determination}

Fundamental Period is taken from the Rayleigh method listed previously in this report.

$\mathrm{T}=0.104 \mathrm{sec}$.

\subsection{1: Calculation of Seismic Response Coefficient}

$\mathrm{C}_{\mathrm{s}}$ is the value computed below, bounded by $\mathrm{C}_{\mathrm{s}}$ Min and $\mathrm{C}_{\mathrm{s}}$ Max:

$\mathrm{C}_{\mathrm{s}}$ Min is 0.01 unless $\mathrm{S}_{1}>=0.6 \mathrm{~g}$, in which case eqn $12.8-6$ is used.

$\mathrm{C}_{\mathrm{S}}$ Max calculated with $12.8-3$ because $(\mathrm{T}=0.104)<=\left(\mathrm{T}_{\mathrm{L}}=12.0000\right)$

$$
\begin{aligned}
& \mathrm{C}_{\mathrm{s}}=\underline{\mathrm{S}}_{\underline{D S}} /(\underline{\mathrm{R}} / \underline{\mathrm{I}})=0.3947 /(3.0000 / 1.5000)=0.1973 \\
& \mathrm{C}_{\mathrm{s}} \operatorname{Min}=0.01 \\
& \mathrm{C}_{\mathrm{s}} \mathrm{Max}=\underline{\mathrm{S}}_{\underline{D I}} /(\mathrm{T} *(\underline{\mathrm{R}} / \mathrm{I}))=0.1600 /(0.1037 *(3.0000 / 1.5000))=0.7716 \\
& \mathrm{C}_{\mathrm{s}}=0.1973
\end{aligned}
$$

\subsection{1: Calculation of Base Shear}

$$
\begin{aligned}
\mathrm{V} & =\mathrm{C}_{\mathrm{S}} * \underline{\mathrm{W}} \\
& =0.1973 * 239.6326 \\
& =47.29 \mathrm{lb}
\end{aligned}
$$

\subsubsection{Seismic Load Combinations: Horizontal Seismic Load Effect, $\mathbf{E}_{h}$}

$$
\begin{aligned}
\mathrm{Q}_{\mathrm{E}} & =\mathrm{V} \\
\mathrm{E}_{\mathrm{h}} & =0.7 * \rho * \mathrm{Q}_{\mathrm{E}}(\text { Only } 70 \% \text { of seismic load considered as per Section 2.4.1) } \\
& =0.70 * 1.0000 * 47.29 \\
& =33.10 \mathrm{lb}
\end{aligned}
$$

\section{Base Shear Calculations: Empty, Corroded}

\section{Paragraph 15.4.4: Period Determination}

Fundamental Period is taken from the Rayleigh method listed previously in this report. $\mathrm{T}=0.093 \mathrm{sec}$.

\subsection{1: Calculation of Seismic Response Coefficient}

$\mathrm{C}_{\mathrm{s}}$ is the value computed below, bounded by $\mathrm{C}_{\mathrm{s}}$ Min and $\mathrm{C}_{\mathrm{s}}$ Max:

$\mathrm{C}_{\mathrm{S}} \mathrm{Min}$ is 0.01 unless $\mathrm{S}_{1}>=0.6 \mathrm{~g}$, in which case eqn $12.8-6$ is used.

$\mathrm{C}_{\mathrm{s}}$ Max calculated with $12.8-3$ because $(\mathrm{T}=0.093)<=\left(\mathrm{T}_{\mathrm{L}}=12.0000\right)$ 


$$
\begin{aligned}
& \mathrm{C}_{\mathrm{s}}=\underline{\mathrm{S}}_{\underline{D S}} /(\underline{\mathrm{R}} / \underline{\mathrm{I}})=0.3947 /(3.0000 / 1.5000)=0.1973 \\
& \mathrm{C}_{\mathrm{s}} \operatorname{Min}=0.01 \\
& \mathrm{C}_{\mathrm{s}} \operatorname{Max}=\underline{\mathrm{S}}_{\underline{D I}} /(\mathrm{T} *(\underline{\mathrm{R}} / \underline{\mathrm{I}}))=0.1600 /(0.0931 *(3.0000 / 1.5000))=0.8591 \\
& \mathrm{C}_{\mathrm{s}}=0.1973
\end{aligned}
$$

\subsection{1: Calculation of Base Shear}

$$
\begin{aligned}
\mathrm{V} & =\mathrm{C}_{\mathrm{S}} * \underline{\mathrm{W}} \\
& =0.1973 * 239.6326 \\
& =47.29 \mathrm{lb}
\end{aligned}
$$

\subsubsection{Seismic Load Combinations: Horizontal Seismic Load Effect, $E_{h}$}

$$
\begin{aligned}
\mathrm{Q}_{\mathrm{E}} & =\mathrm{V} \\
\mathrm{E}_{\mathrm{h}} & =0.7 * \rho * \mathrm{Q}_{\mathrm{E}}(\text { Only } 70 \% \text { of seismic load considered as per Section 2.4.1) } \\
& =0.70 * 1.0000 * 47.29 \\
& =33.10 \mathrm{lb}
\end{aligned}
$$




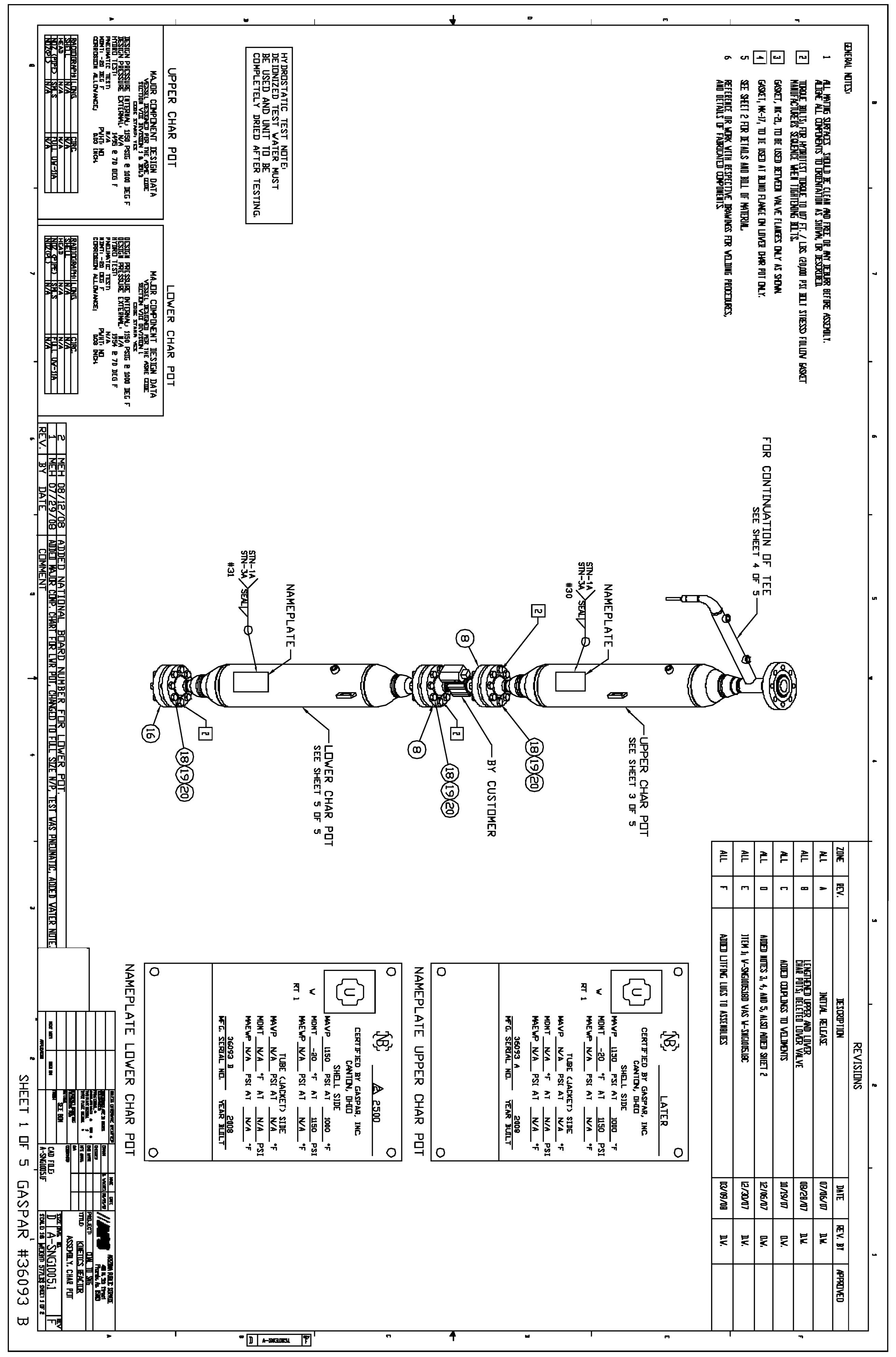




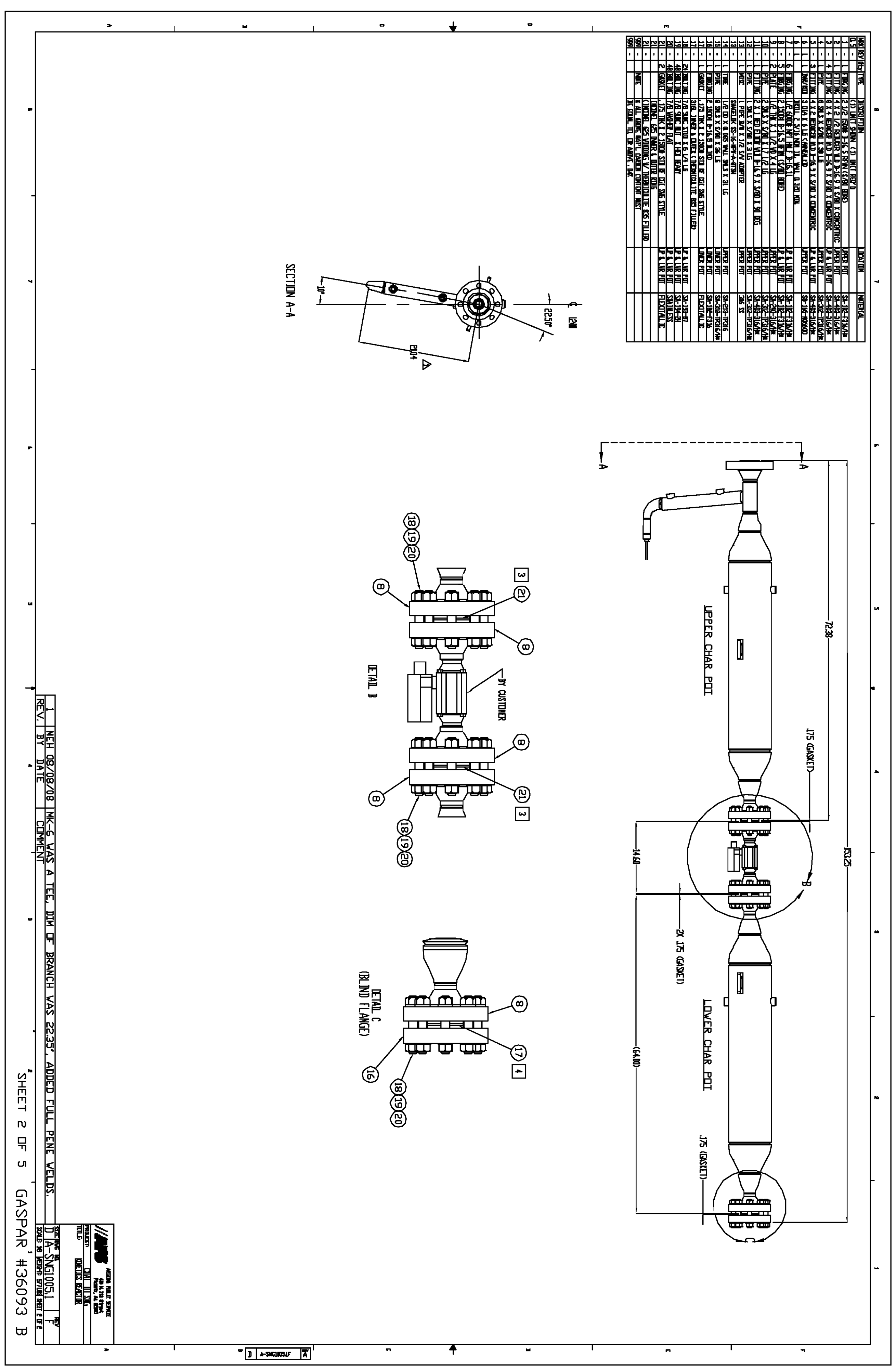




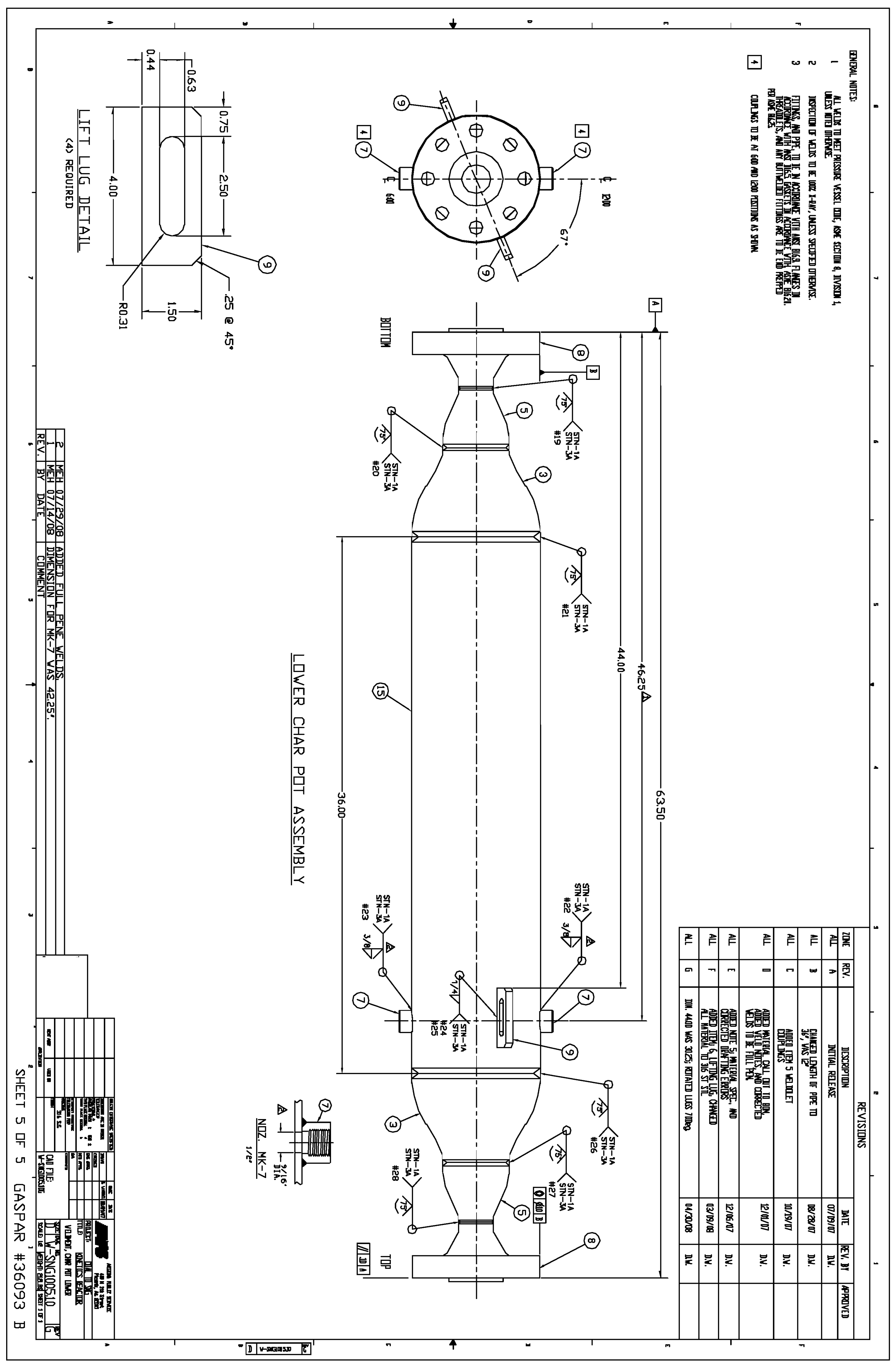




\title{
APPENDIX H
}

\section{Bench Scale Hydrogasification Testing Process}

\author{
PI\&D
}


Development of a Hydrogasification Process for

Coproduction of SNG and Electrical Power from Western Coals

Final Report

THIS PAGE INTENTIONALLY LEFT BLANK 


\title{
COAL TO SNG \\ BENCH SCALE TEST REACTOR \\ Process PI \& D
}

\author{
FINAL \\ MARCH 2010
}

\section{Arizona Public Service Company 400 NORTH 5TH STREET \\ Phoenix, AZ 85003}

\author{
Principal Investigator \\ Dr. Xiaolei Sun \\ 602.250.1510 \\ 602.250.1505 \\ xiaolei.sun@aps.com
}



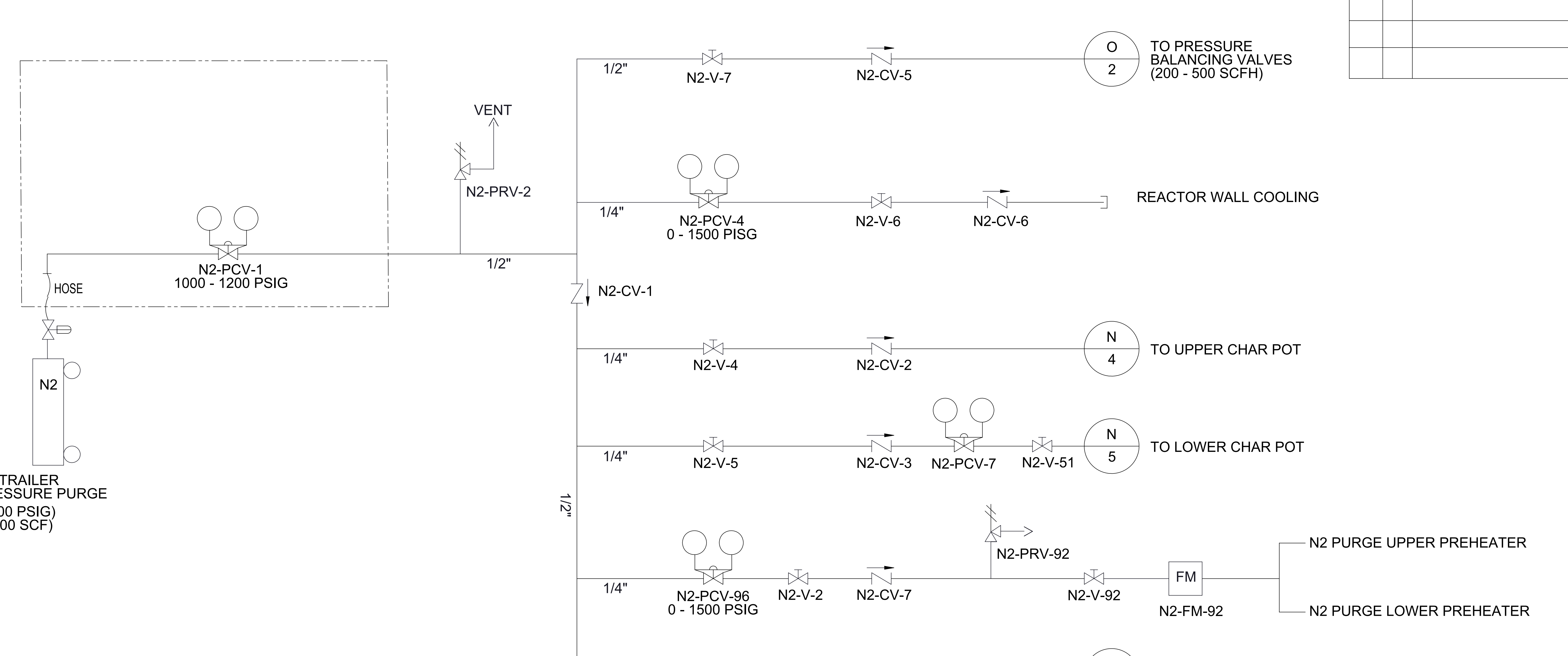

N2 TUBE TRALER
HIGH PRESSURE PURGE

$(2400 \mathrm{PSIC})$
$(3000 \mathrm{SCF})$
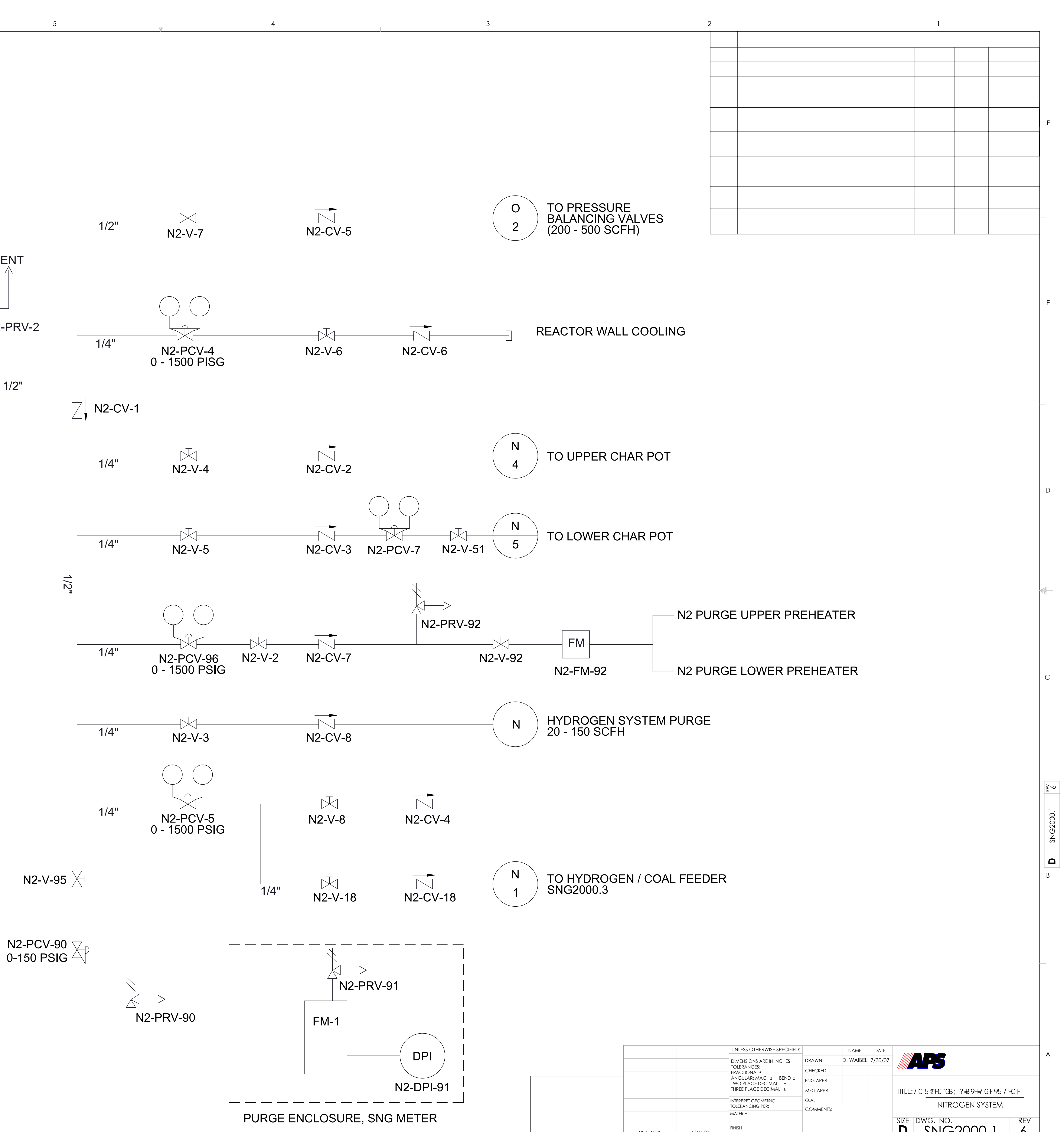

N HYDROGEN SYSTEM PURGE

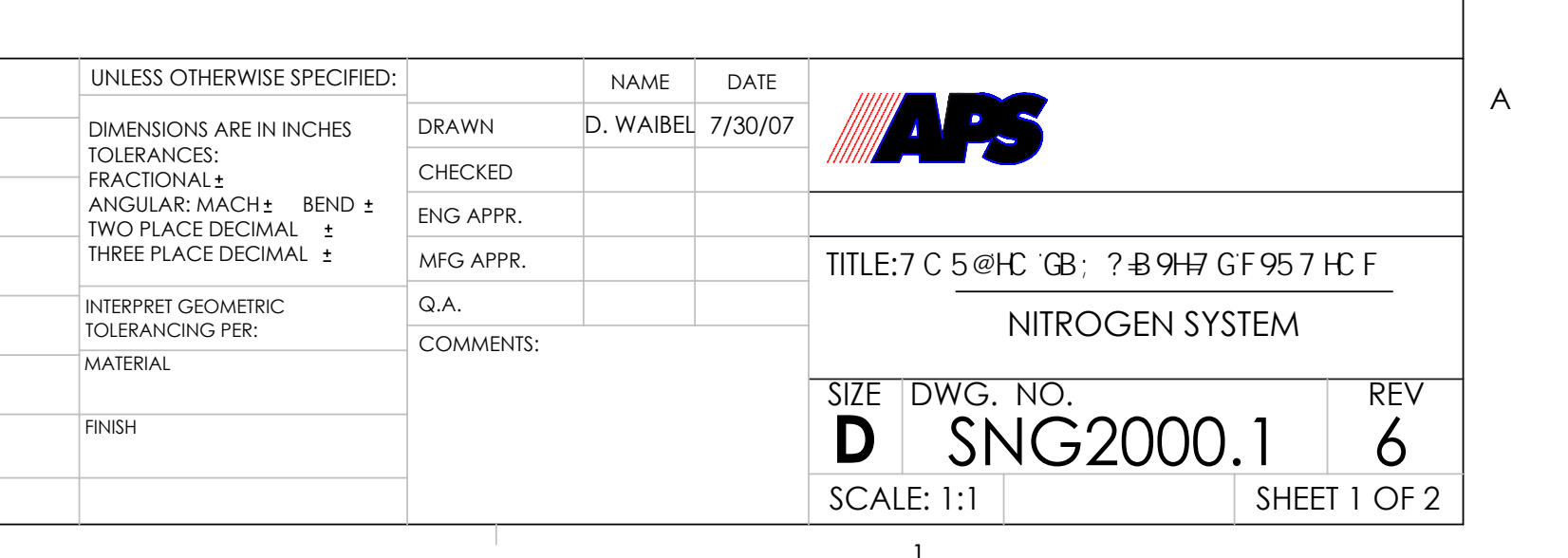




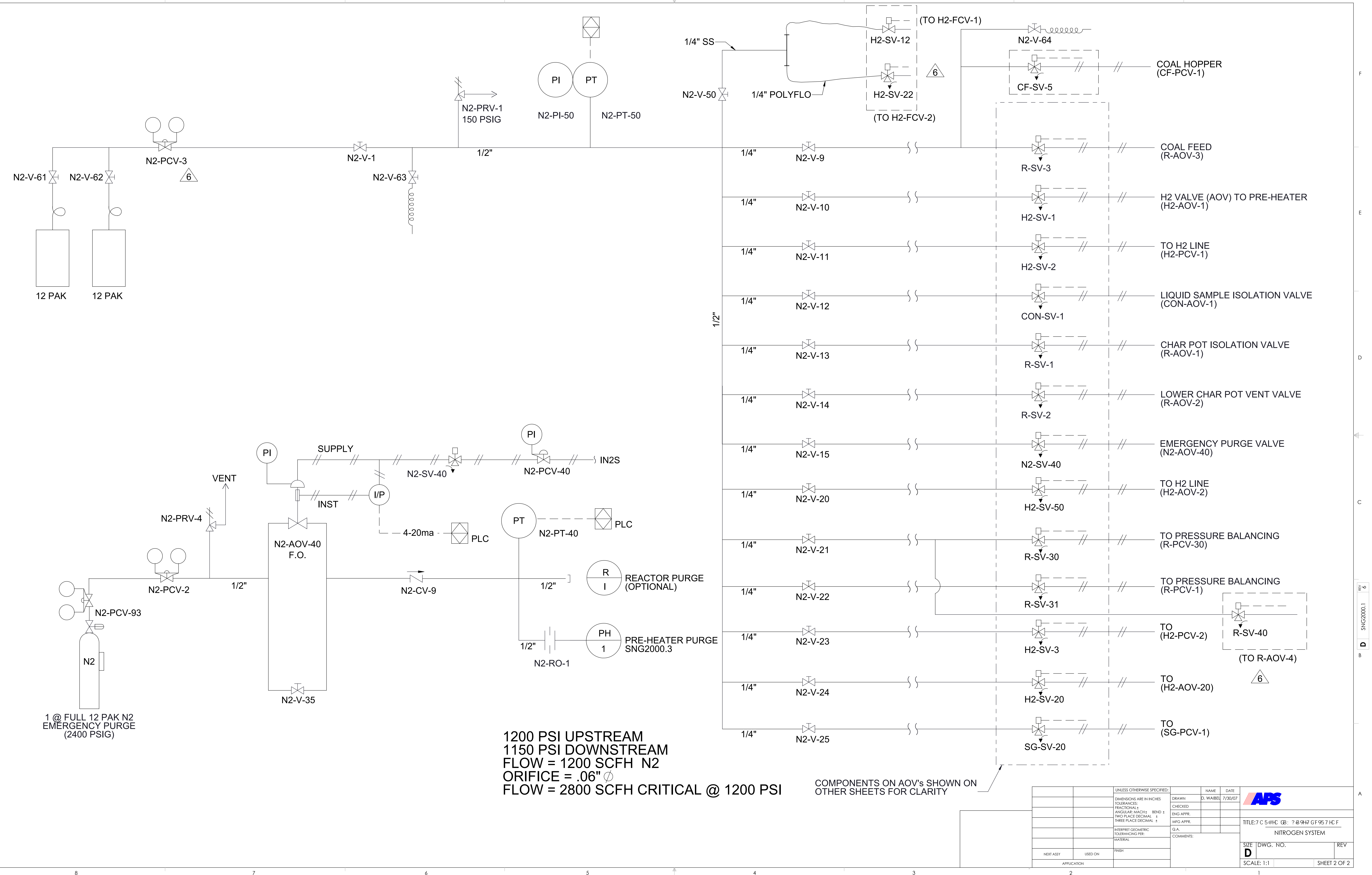



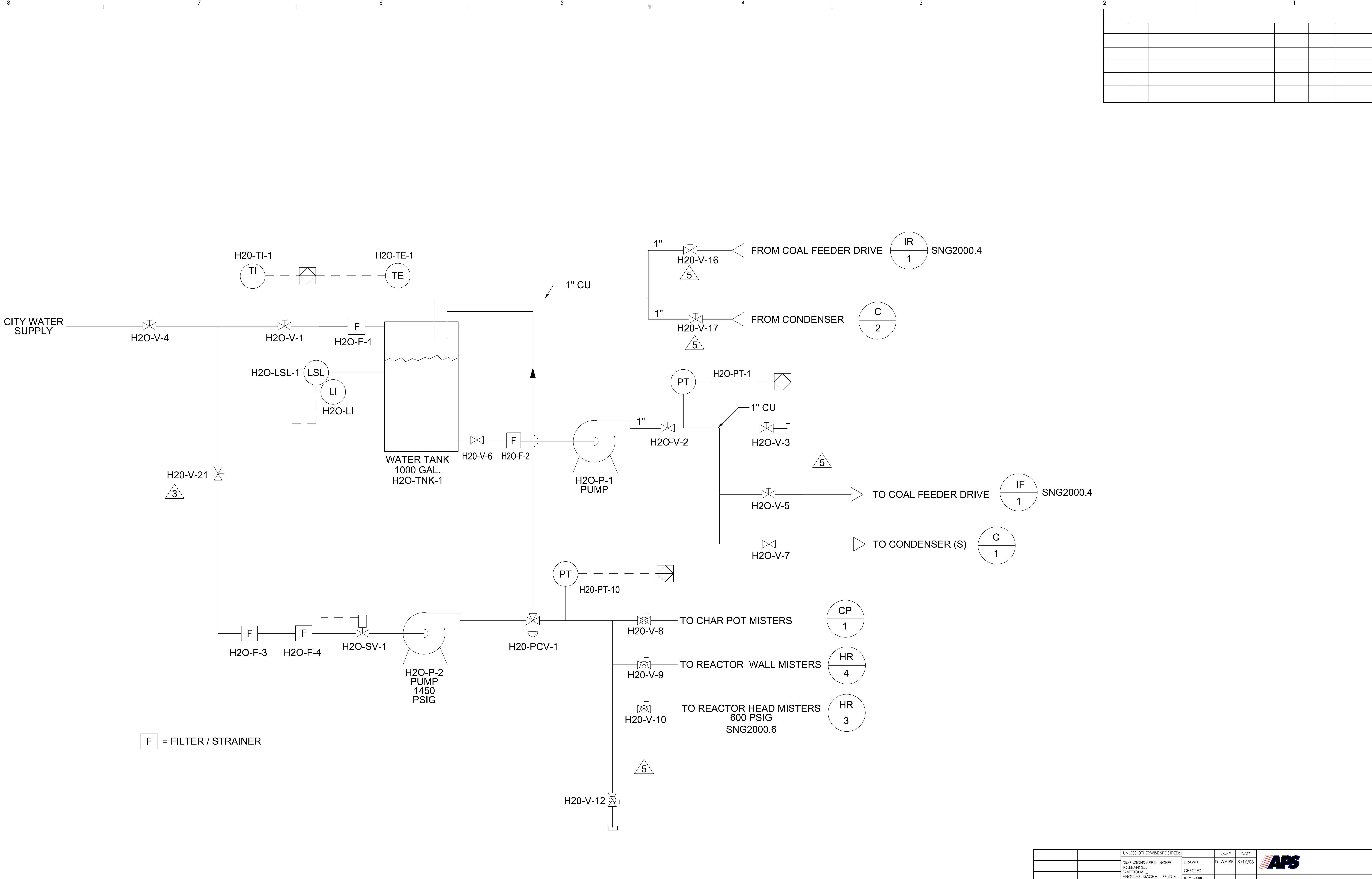


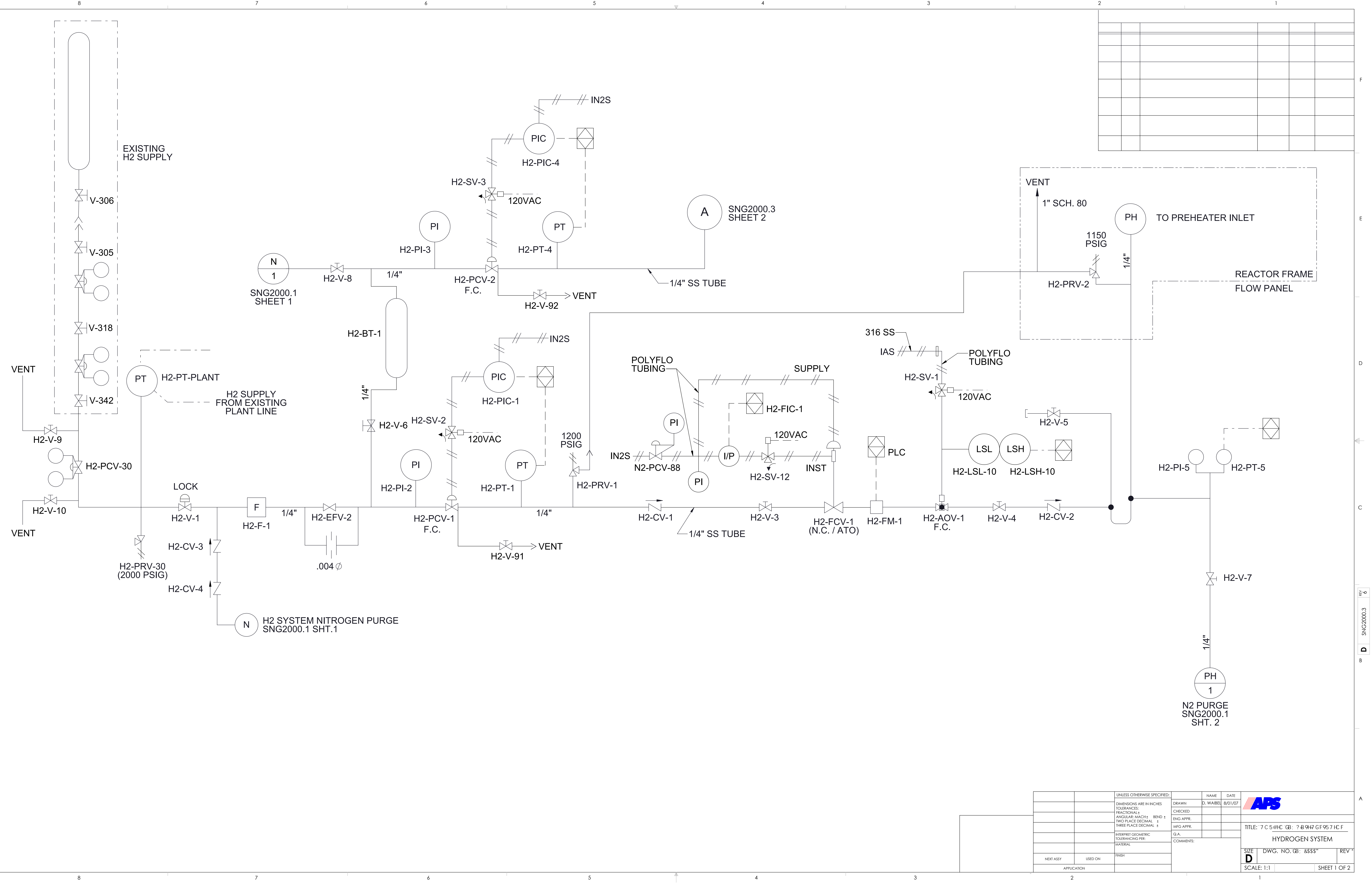




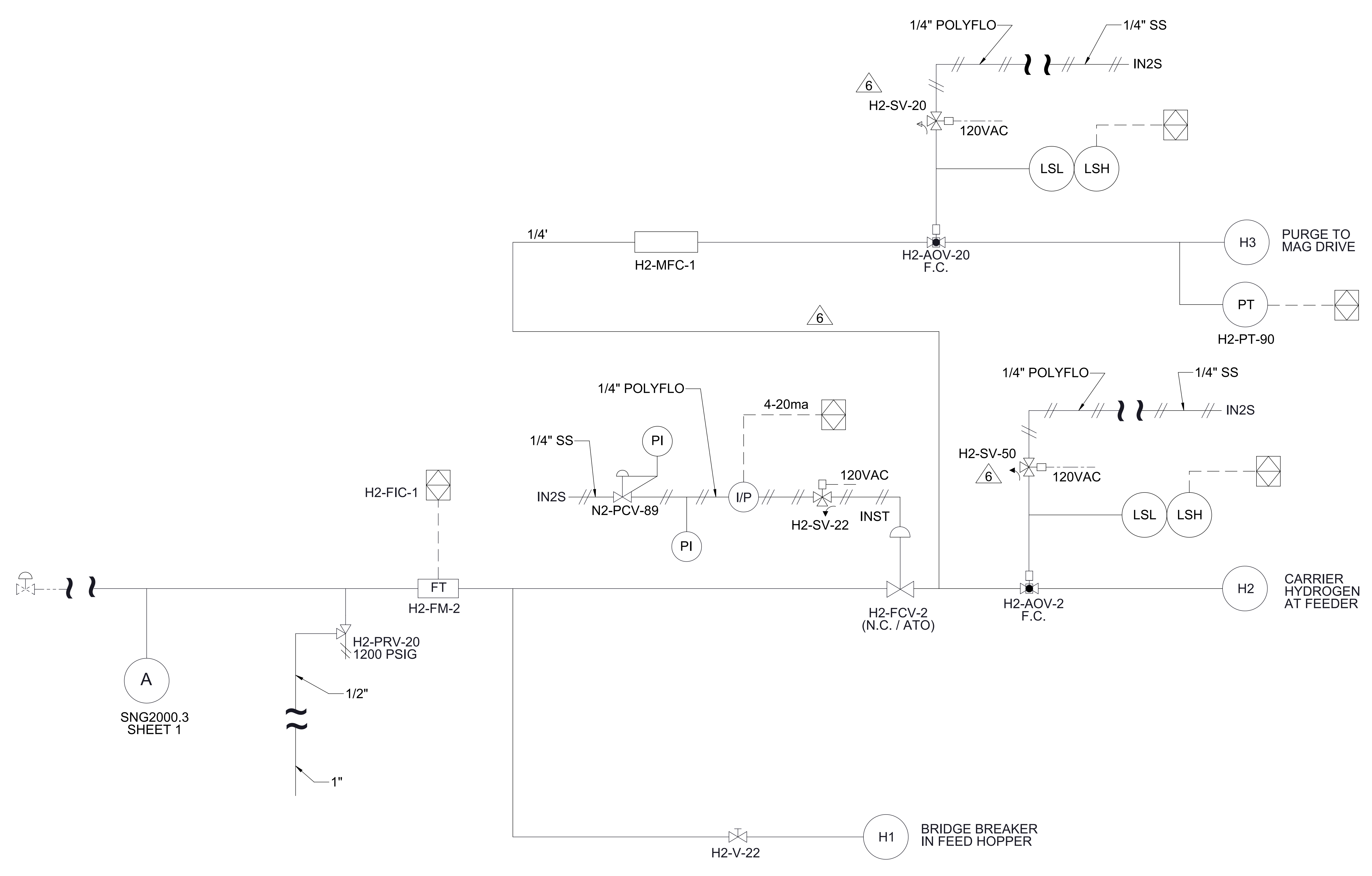



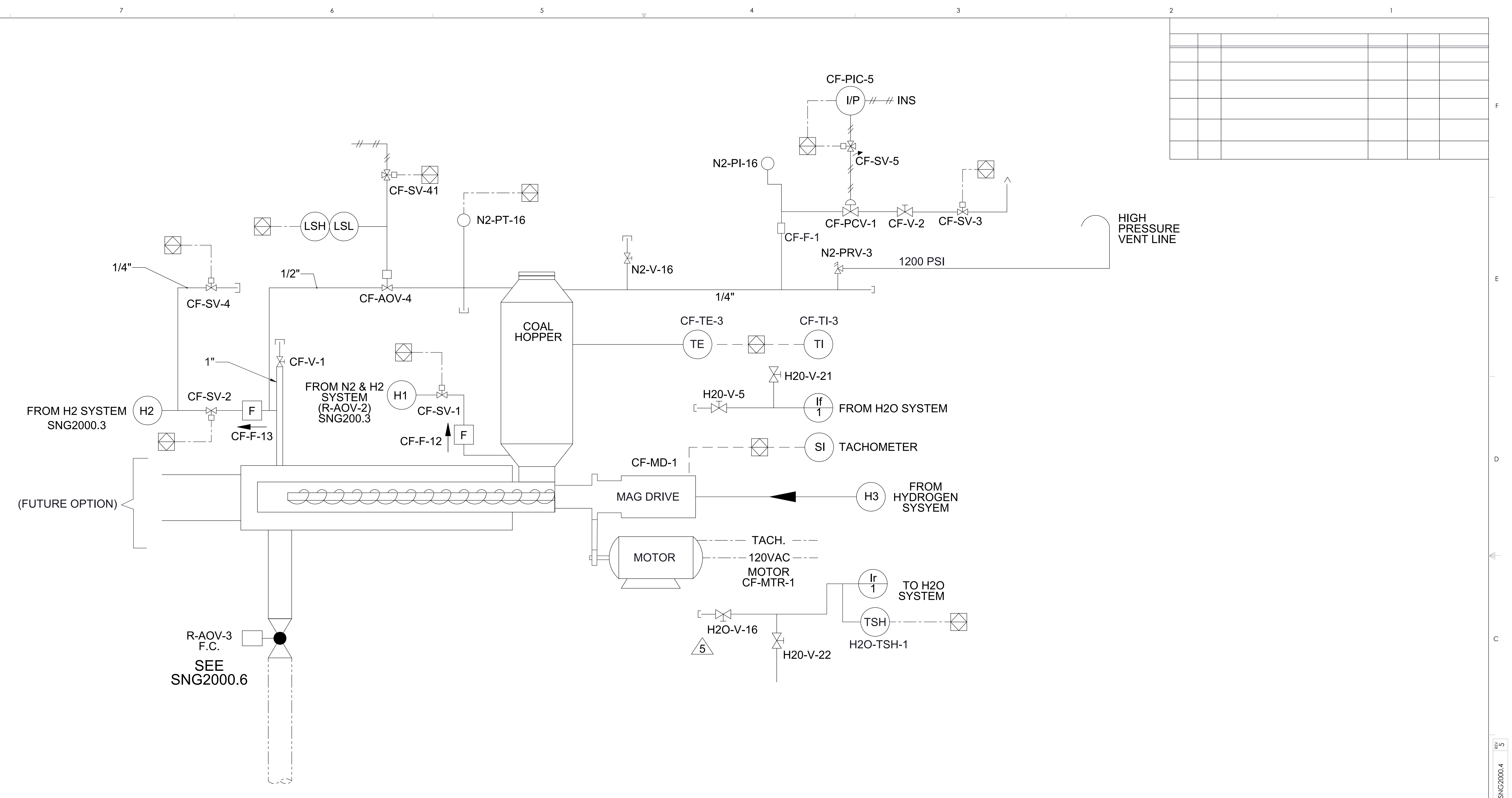

HIGH
RESSURE
ENT LINE 

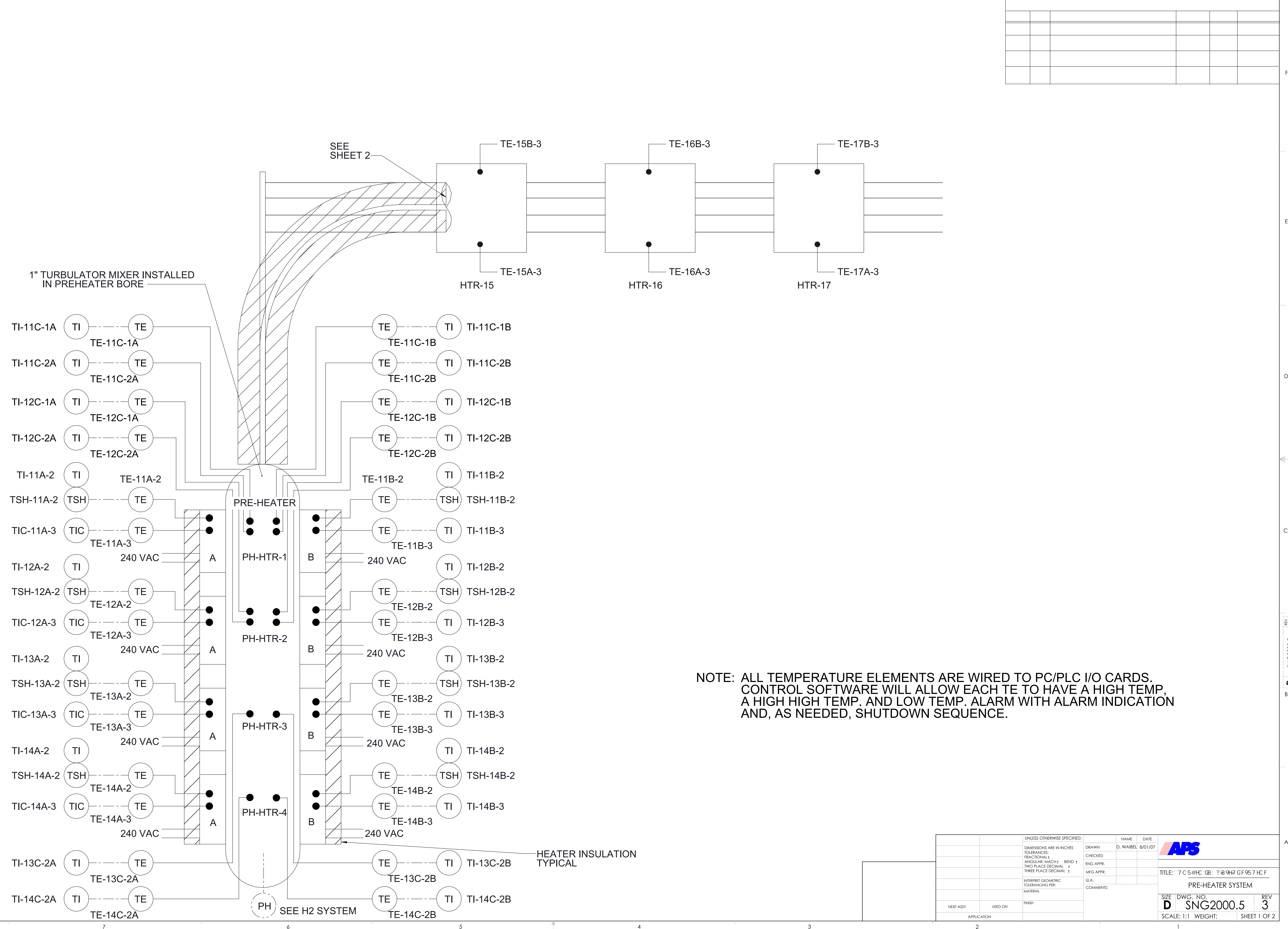


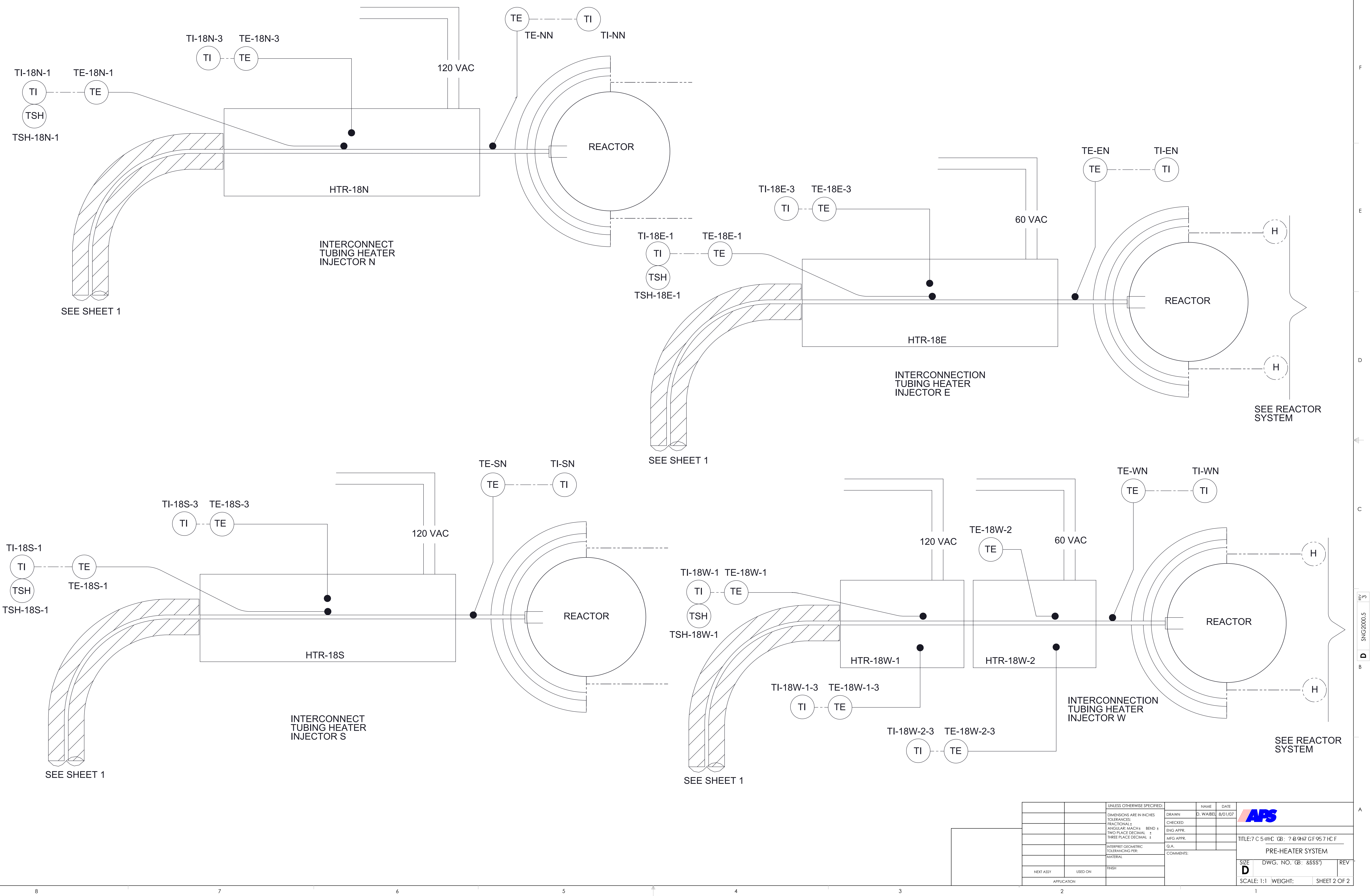




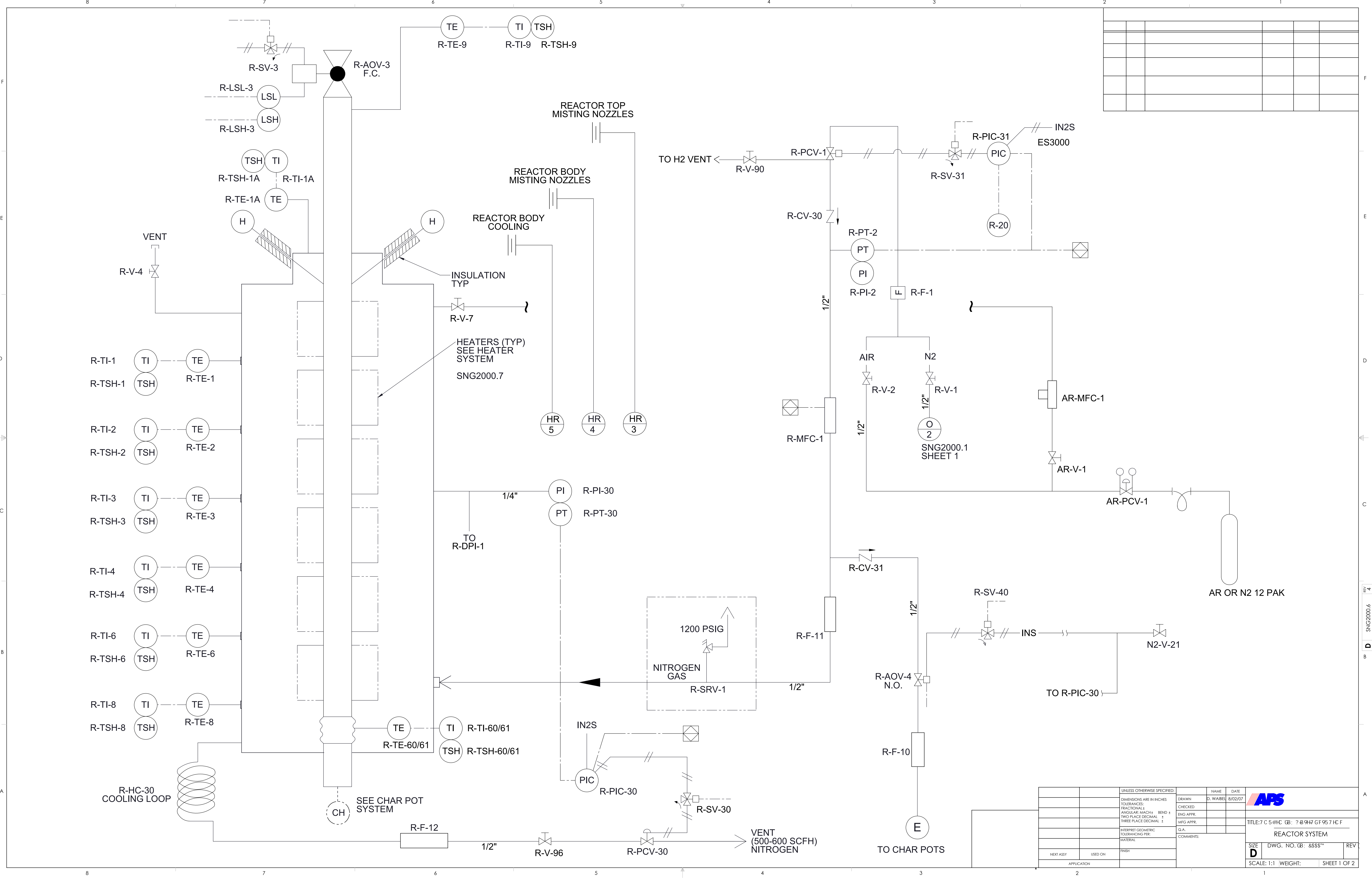




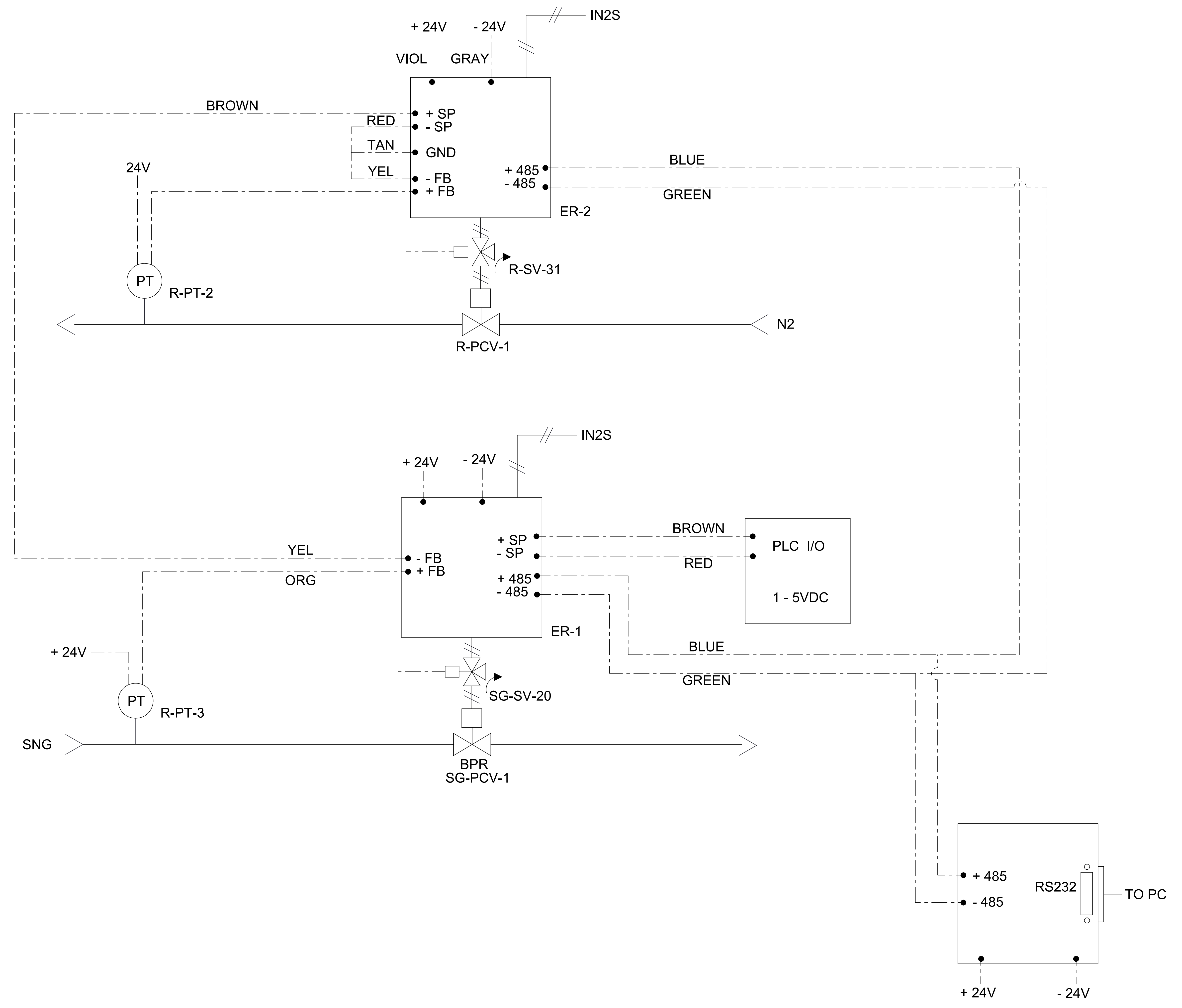




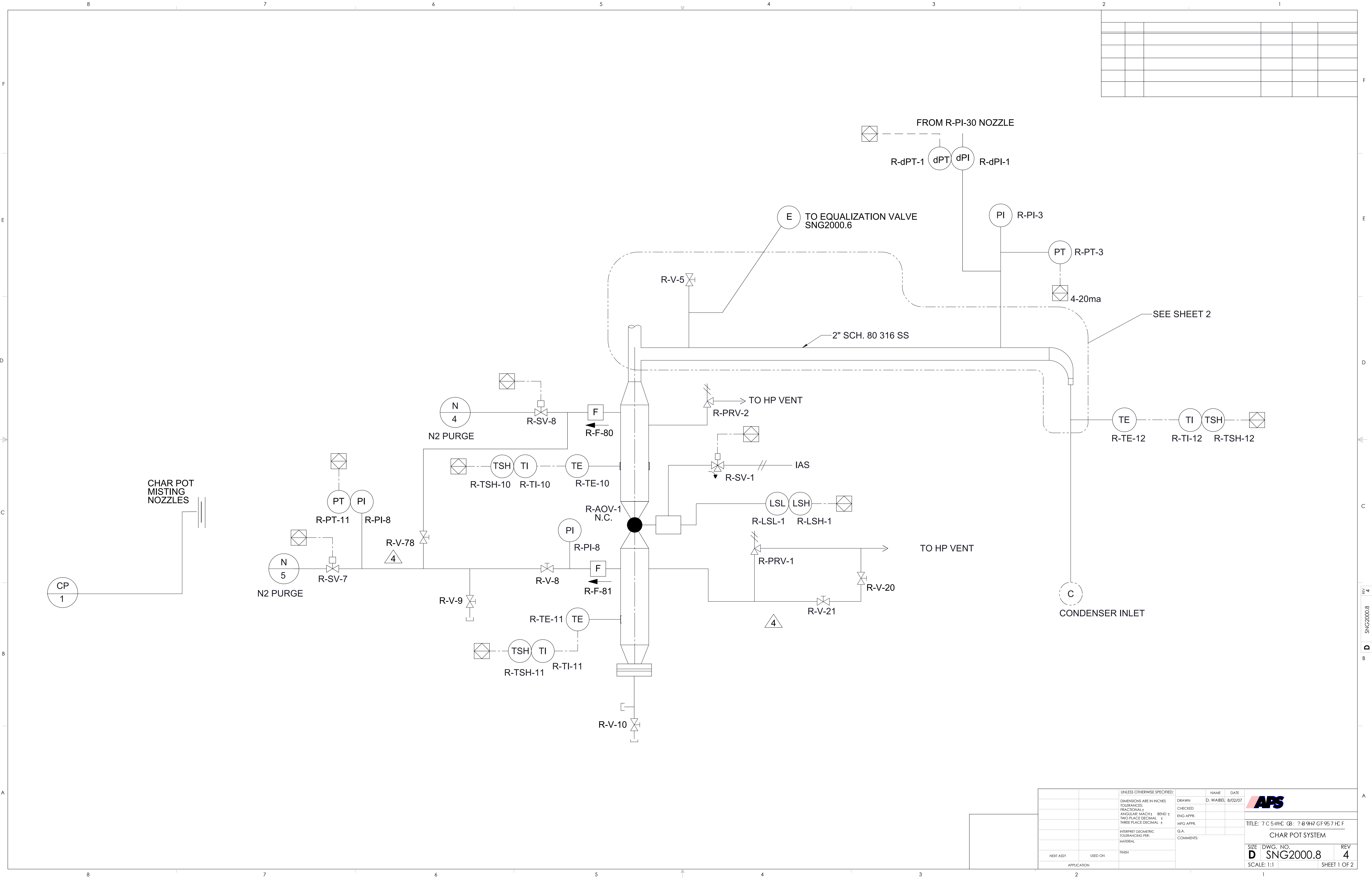




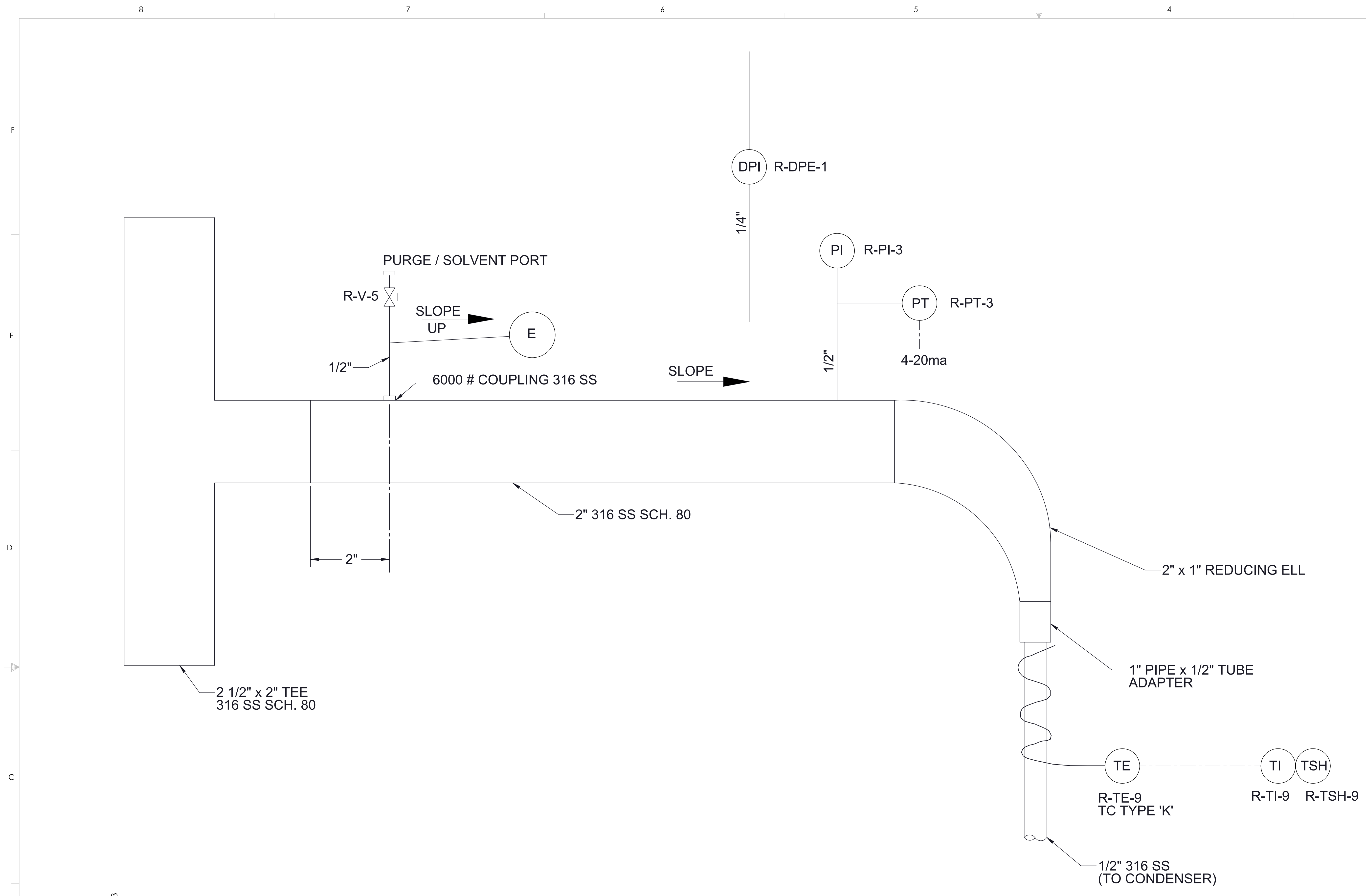




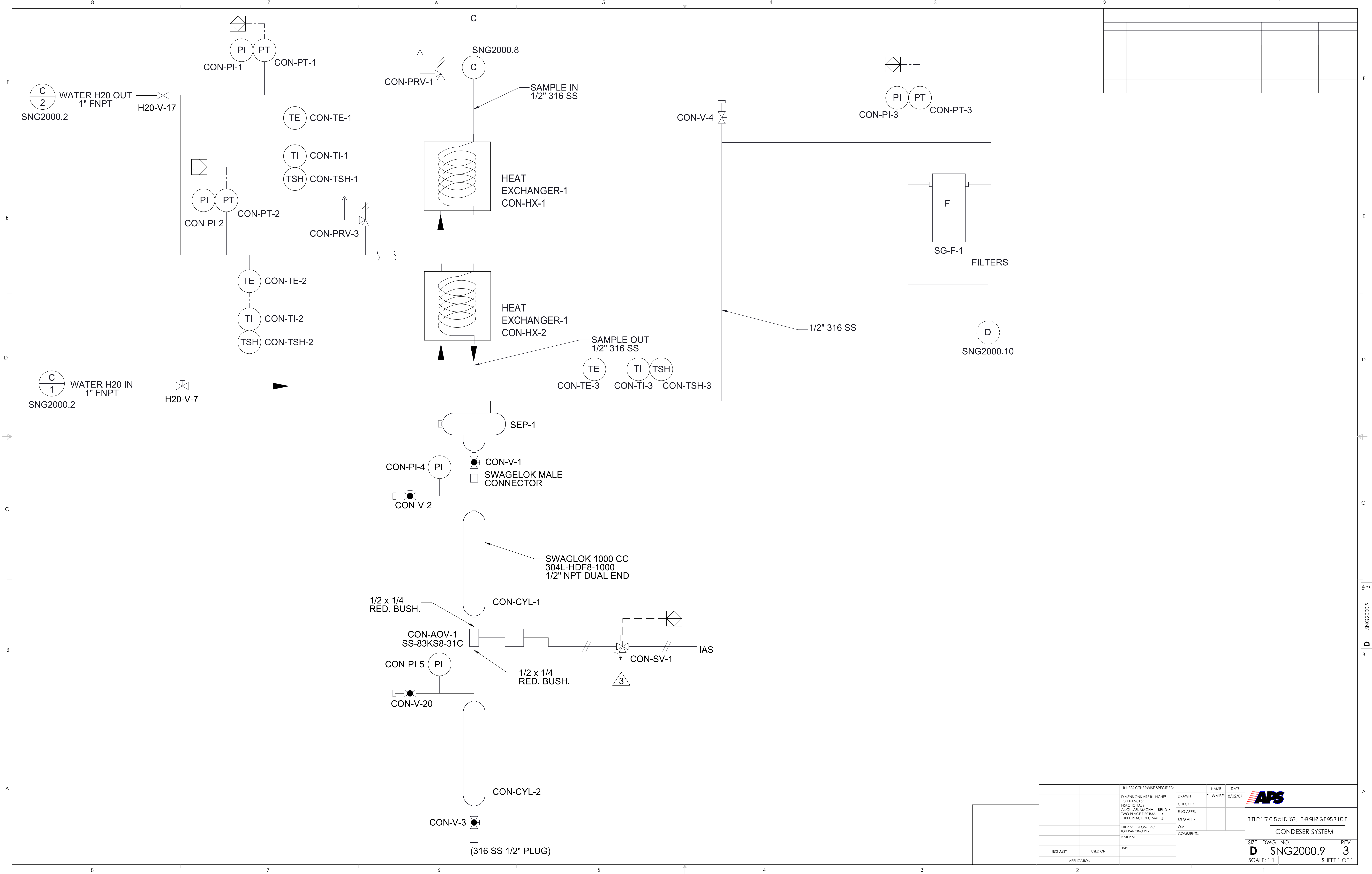



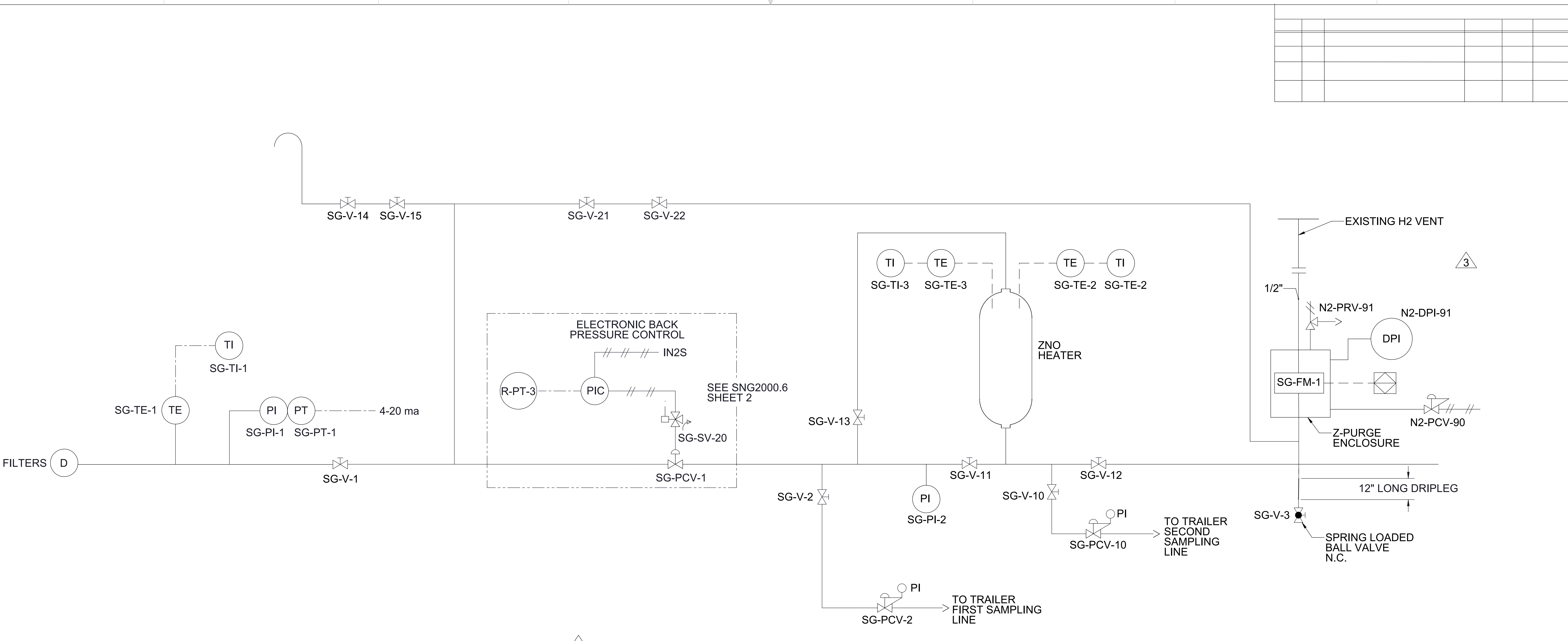

今

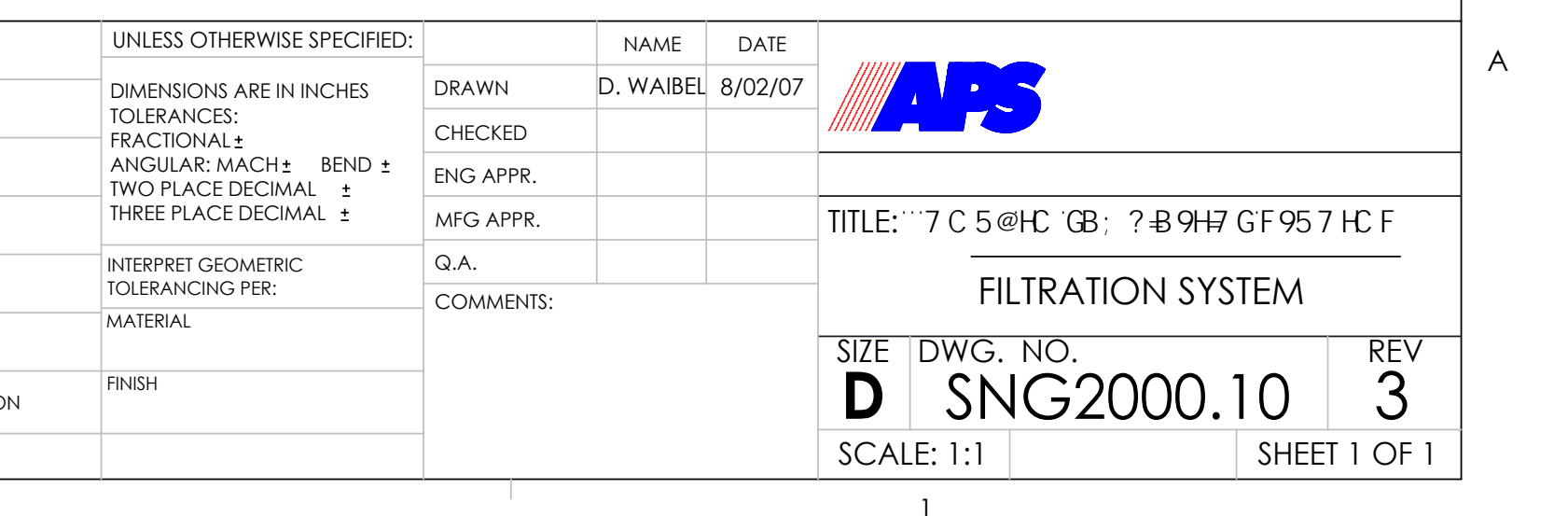




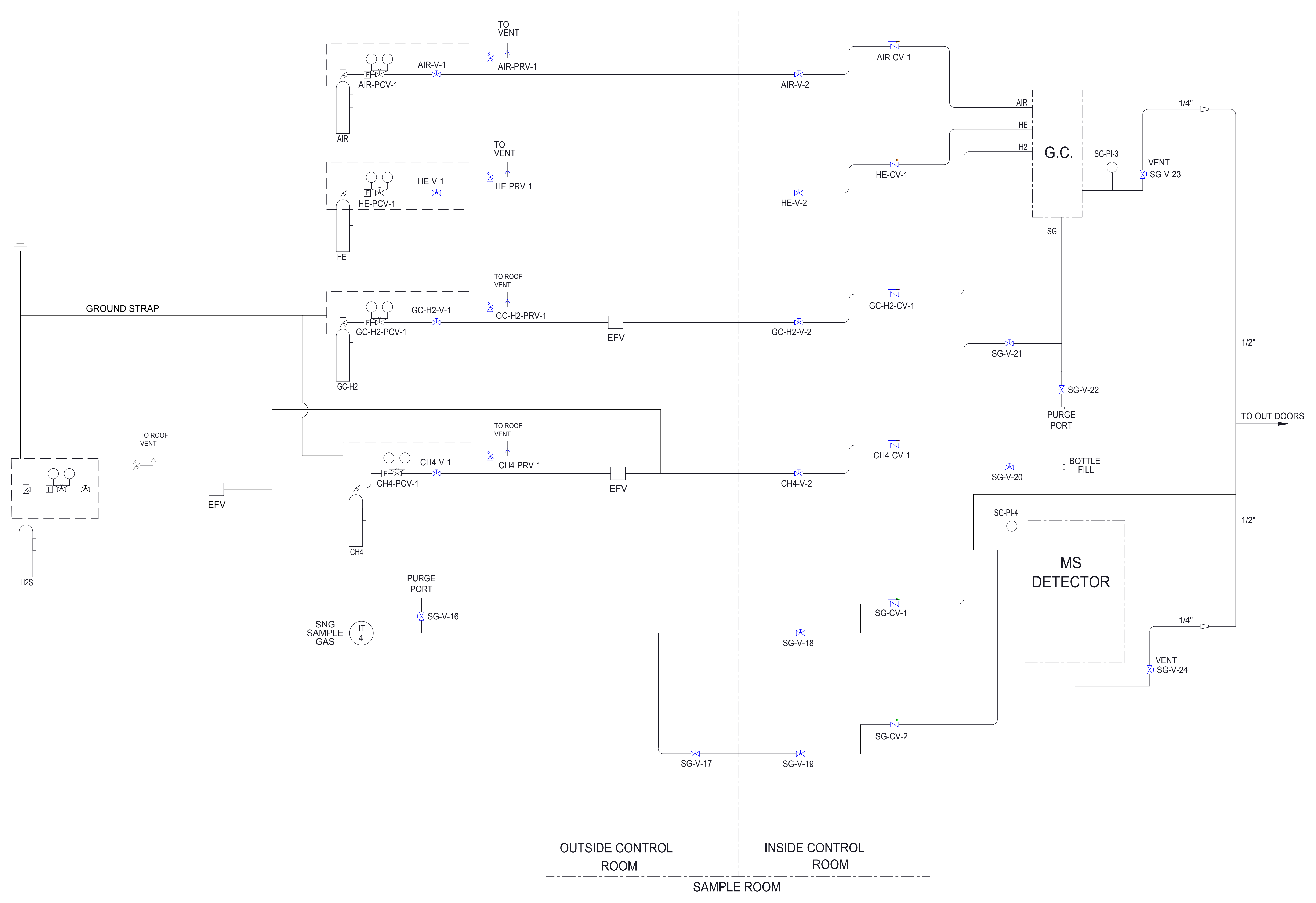




\title{
APPENDIX I
}

\section{Bench Scale Hydrogasification Testing Process}

\author{
Bill of Material
}


Development of a Hydrogasification Process for

Coproduction of SNG and Electrical Power from Western Coals

Final Report

THIS PAGE INTENTIONALLY LEFT BLANK 


\title{
COAL TO SNG \\ BENCH SCALE TEST REACTOR \\ BiLling OF MATERIAL (BOM)
}

\author{
FINAL \\ MARCH 2010
}

\author{
Arizona Public Service Company \\ 400 NORTH 5TH STREET \\ Phoenix, AZ 85003
}
Principal Investigator
Dr. Xiaolei Sun
602.250.1510
602.250.1505
xiaolei.sun@aps.com




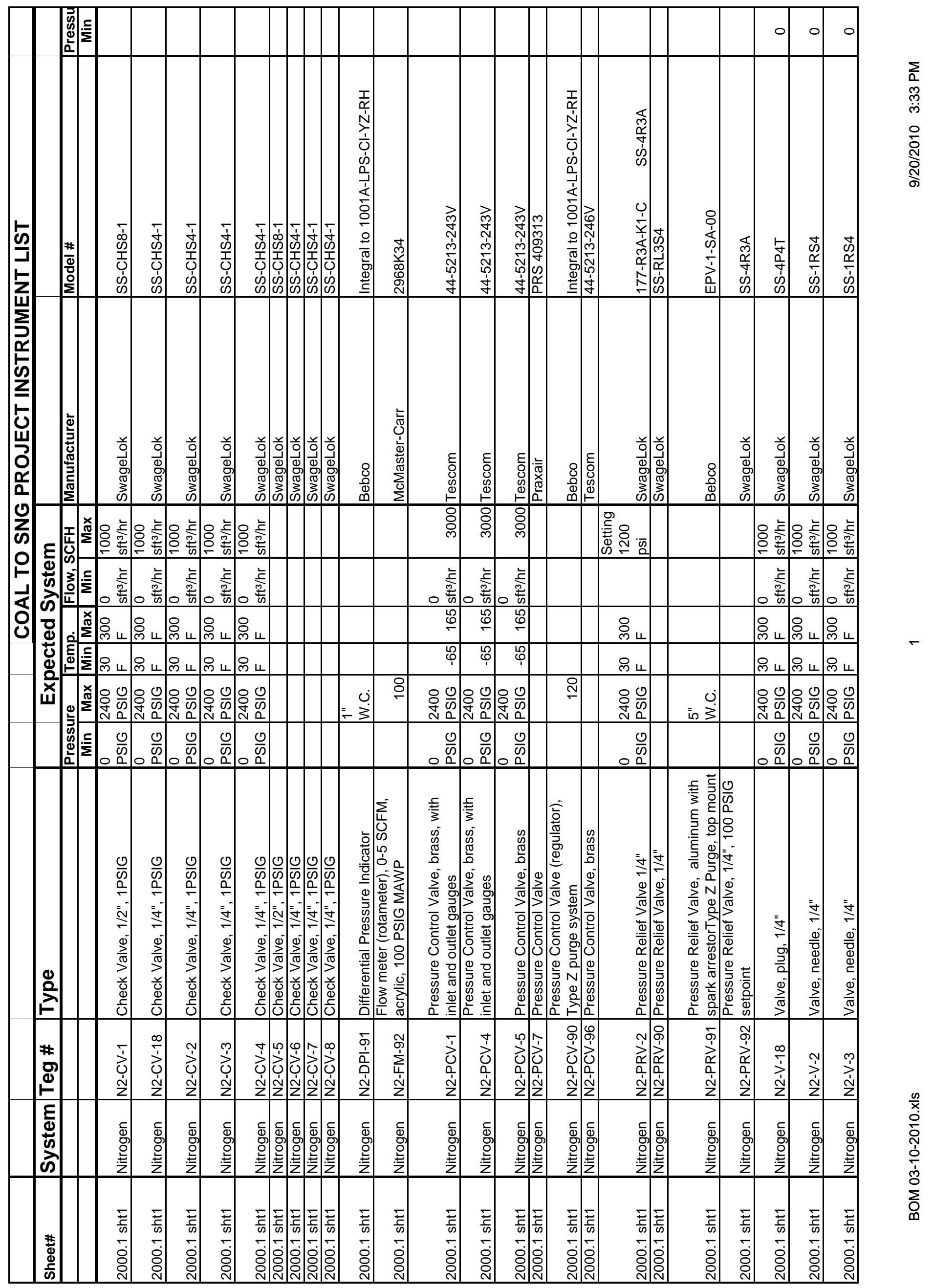




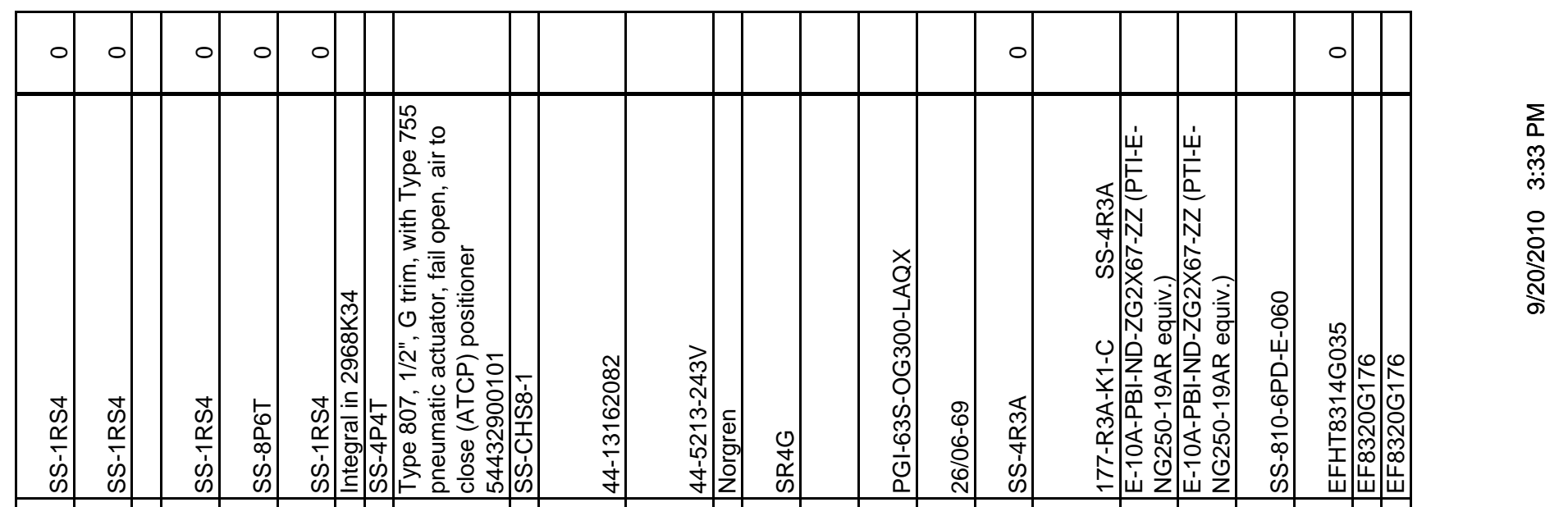

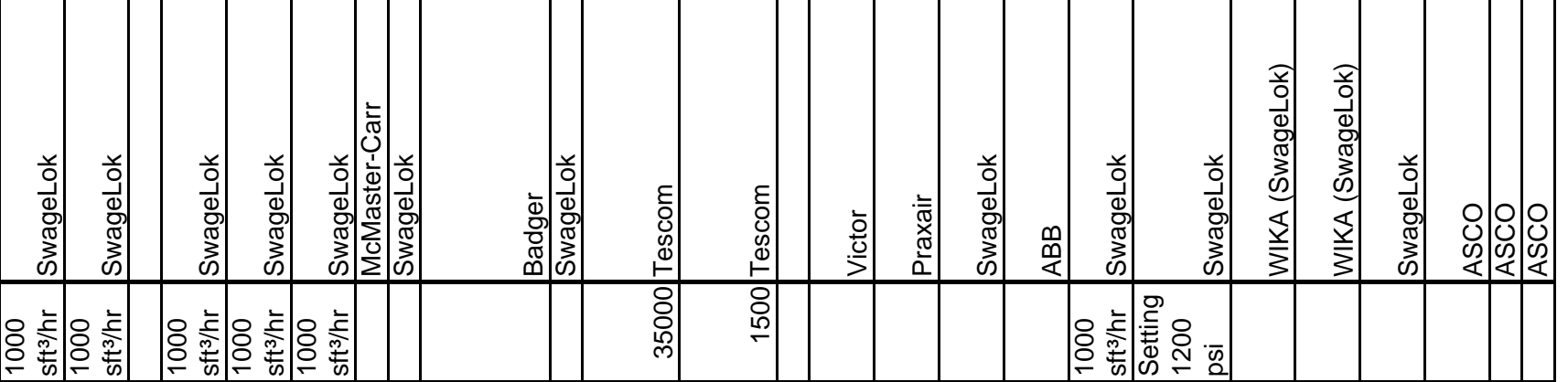

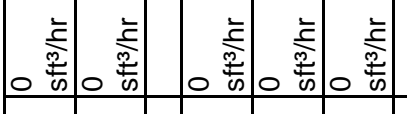

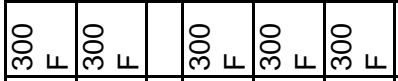

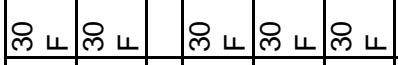

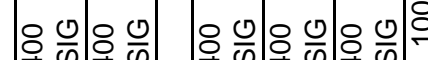

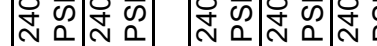

\begin{tabular}{|c|c|c|c|c|}
\hline$\frac{0}{\omega}$ & $\frac{0}{\omega}$ & $\frac{0}{\omega}$ & $\frac{0}{\omega}$ & $\frac{0}{\omega}$ \\
0 & 0 & 0 & 0 \\
0 & 0 & 0 \\
\hline
\end{tabular}

$+$

$+$

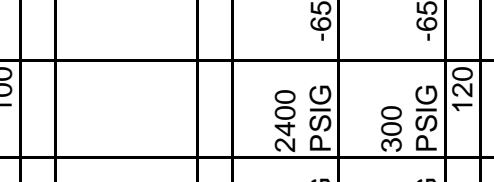

|ror
+

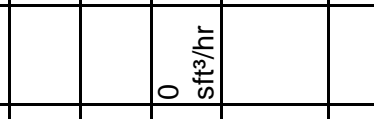

\begin{tabular}{|c|c|c|c|}
\hline ঠ্লি & ৪্ল & 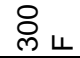 & ৪্ল \\
\hline
\end{tabular}

\begin{tabular}{|c|c|c|c|c|c|c|c|c|c|c|c|c|c|c|c|c|c|c|c|c|c|c|c|c|}
\hline $\begin{array}{l}\bar{n} \\
\circ \mathrm{a} \\
\end{array}$ & $\frac{0}{c}$ & & $\begin{array}{l}\bar{n} \\
0 \mathrm{n} \\
\end{array}$ & $\frac{0}{n}$ & $\begin{array}{l}\bar{n} \\
0 \stackrel{0}{n} \\
\end{array}$ & & & & & $\begin{array}{l}\frac{c}{n} \\
\circ \stackrel{0}{0}\end{array}$ & $\begin{array}{ll} & \frac{1}{N} \\
\circ & 0 \\
\end{array}$ & & & $\begin{array}{l}\vdots \\
\vdots \\
\end{array}$ & \begin{tabular}{|ll} 
& 5 \\
0 & 0 \\
\end{tabular} & $\begin{array}{l}\sum \\
\vdots \\
\end{array}$ & $\begin{array}{ll} & \frac{1}{n} \\
0 & 0 \\
\end{array}$ & $\begin{array}{ll} & \bar{n} \\
\circ & 0 \\
\end{array}$ & & \begin{tabular}{ll} 
& \multicolumn{1}{c}{} \\
0 & 0 \\
\end{tabular} & & & & \\
\hline 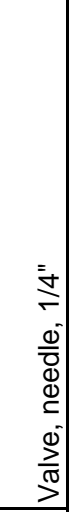 & 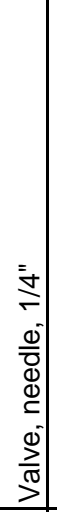 & & 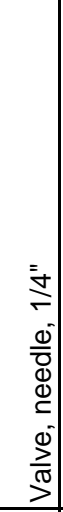 & 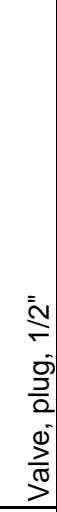 & 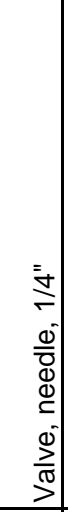 & 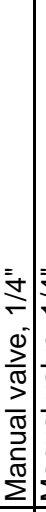 & 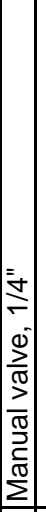 & $\begin{array}{l}0 \\
4 \\
0 \\
2 \\
0 \\
2 \\
0 \\
0 \\
0 \\
0 \\
0 \\
0 \\
0 \\
0 \\
0 \\
0\end{array}$ & 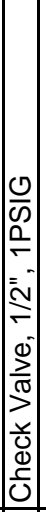 & 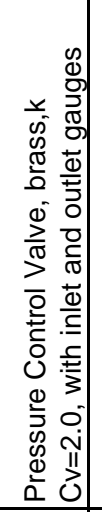 & 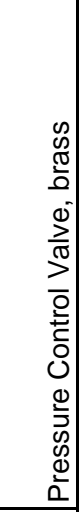 & 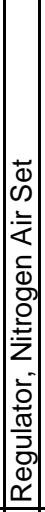 & 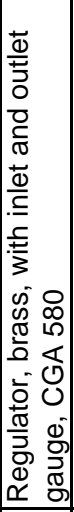 & 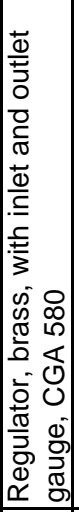 & 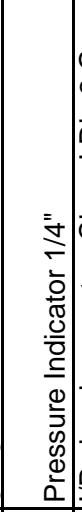 & 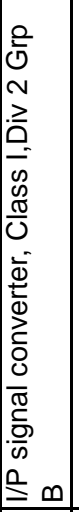 & 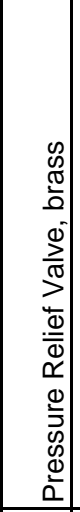 & 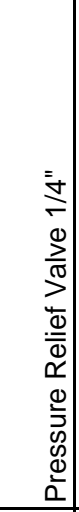 & 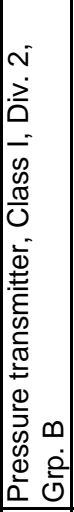 & 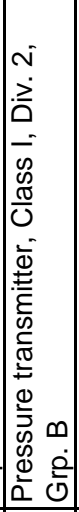 & 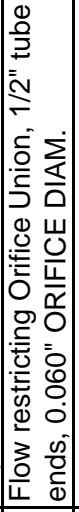 & 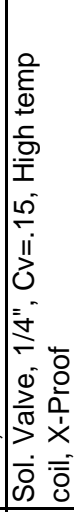 & 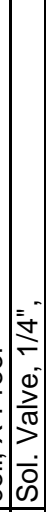 & 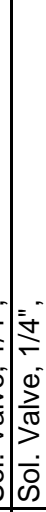 \\
\hline 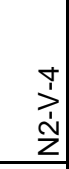 & 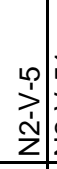 & 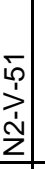 & 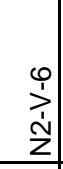 & $\begin{array}{l}\mathbf{1} \\
\dot{1} \\
\tilde{z} \\
z\end{array}$ & $\begin{array}{l}\infty \\
\stackrel{1}{1} \\
\stackrel{1}{1} \\
\stackrel{1}{Z} \\
\end{array}$ & & 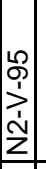 & 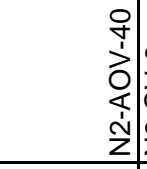 & 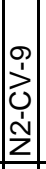 & $\begin{array}{l}\overrightarrow{1} \\
0 \\
\dot{1} \\
\sim \\
\end{array}$ & 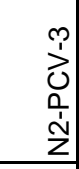 & 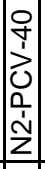 & 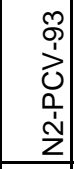 & 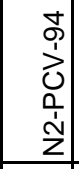 & $\begin{array}{l}\text { 워 } \\
\frac{1}{\alpha} \\
\stackrel{1}{1} \\
\stackrel{2}{z}\end{array}$ & $\begin{array}{l}\text { Oे } \\
\dot{u} \\
\frac{0}{0} \\
\grave{1} \\
z\end{array}$ & 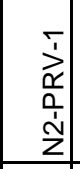 & 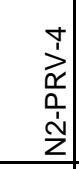 & 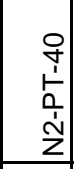 & 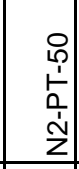 & 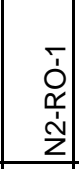 & $\begin{array}{l}\text { Pे } \\
\text { गे } \\
\text { के } \\
\text { Ṅ }\end{array}$ & 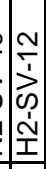 & 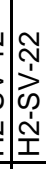 \\
\hline 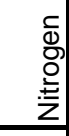 & $\begin{array}{l}\bar{c} \\
\frac{D}{D} \\
\stackrel{\underline{D}}{Z} \\
\end{array}$ & & 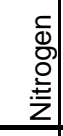 & 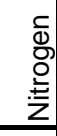 & 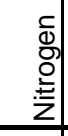 & & 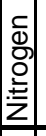 & 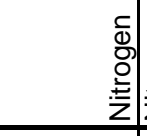 & 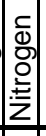 & 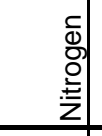 & 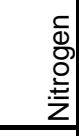 & 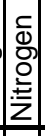 & 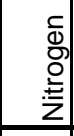 & 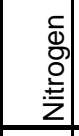 & 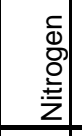 & 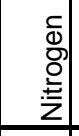 & 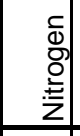 & 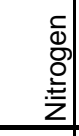 & 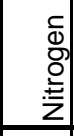 & 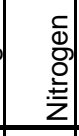 & $\begin{array}{l}\frac{c}{0} \\
\frac{0}{D} \\
\stackrel{ \pm}{Z} \\
\end{array}$ & 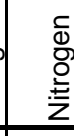 & 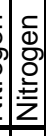 & 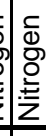 \\
\hline 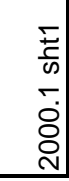 & 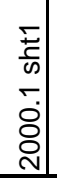 & & 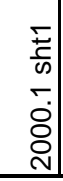 & 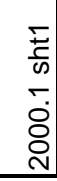 & 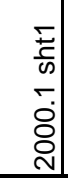 & 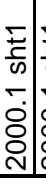 & 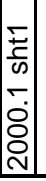 & 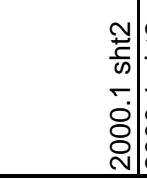 & 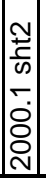 & 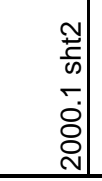 & 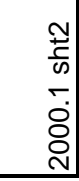 & 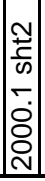 & 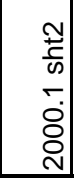 & 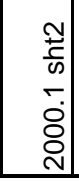 & 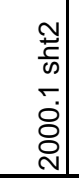 & 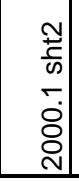 & 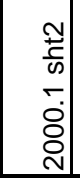 & 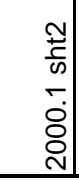 & 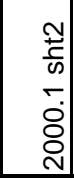 & 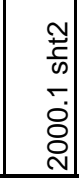 & 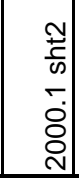 & 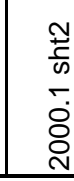 & 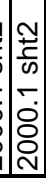 & 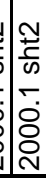 \\
\hline
\end{tabular}




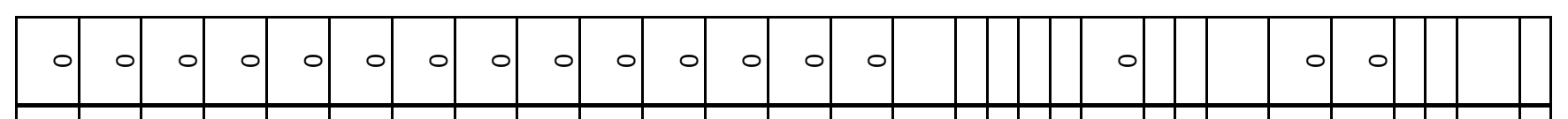

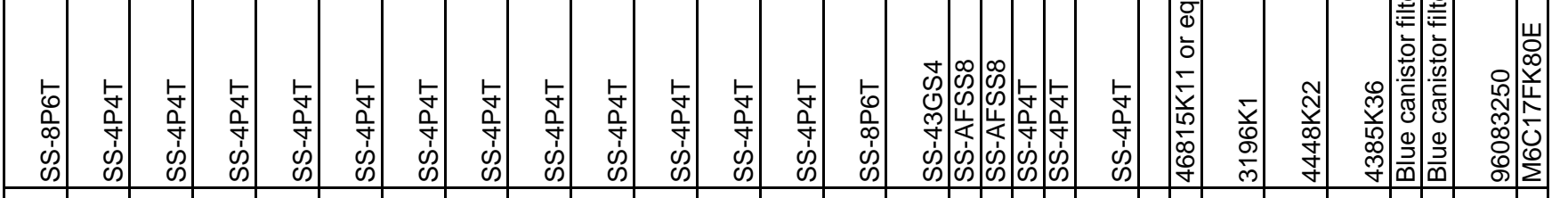

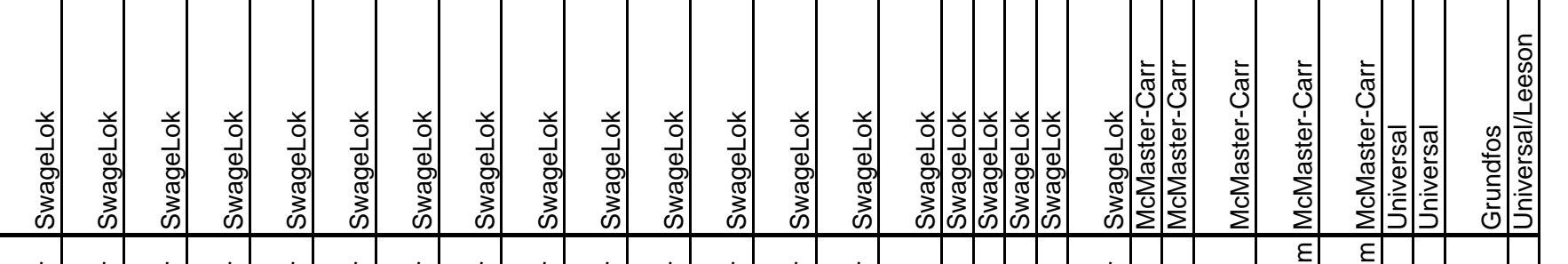

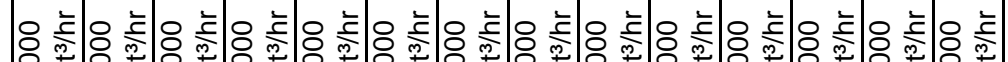

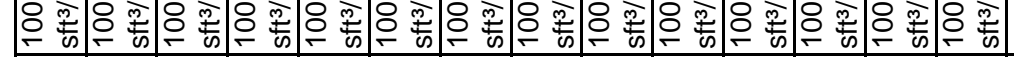

率

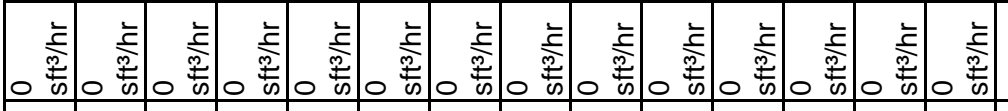

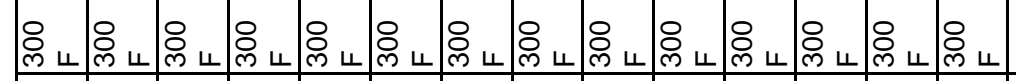

.

8484848484.848 .84 .84848484 .8484 .84

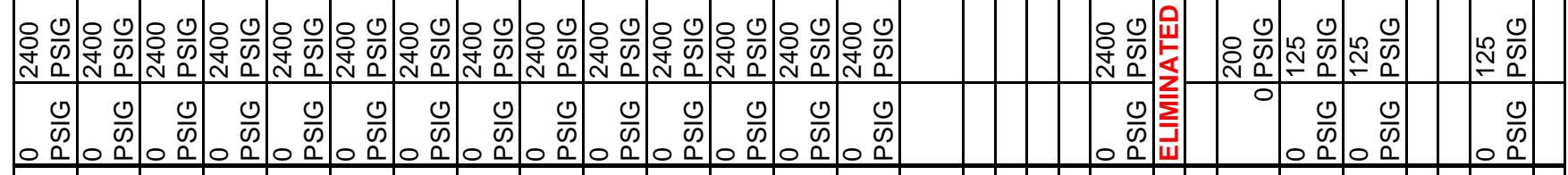

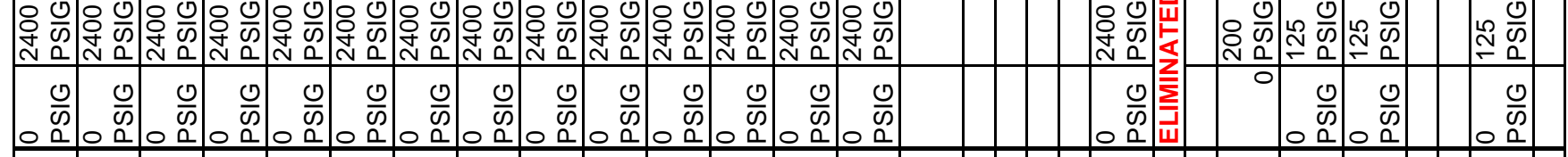

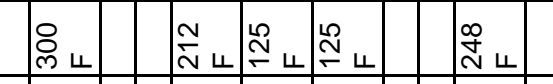




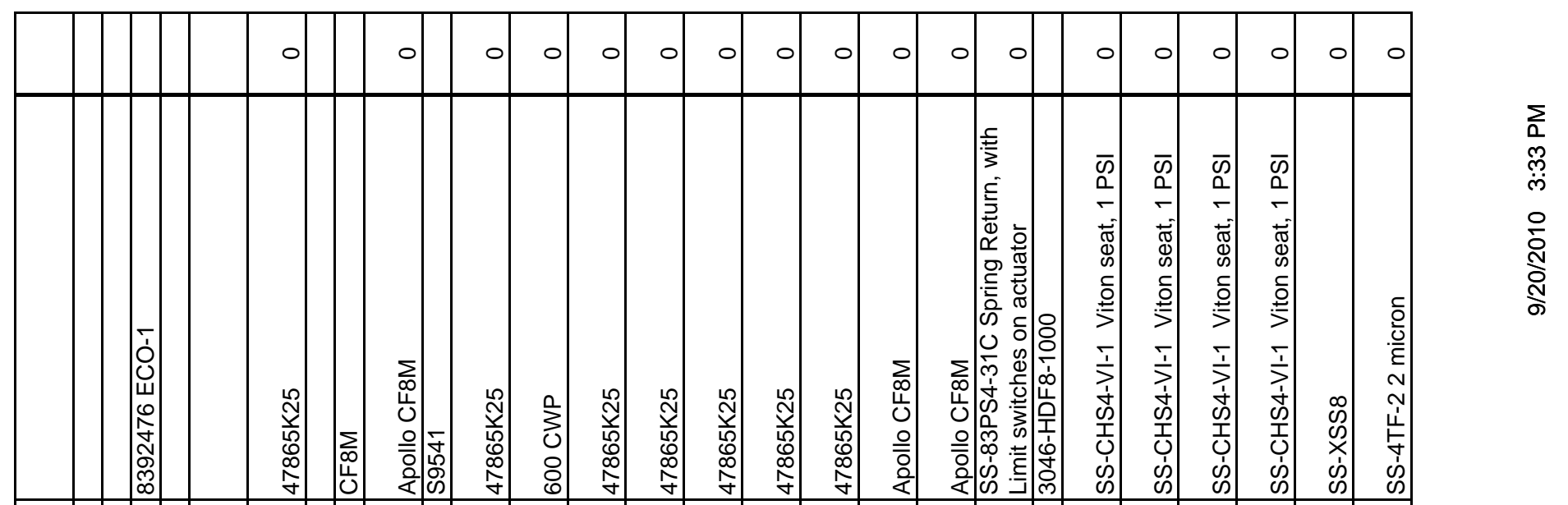

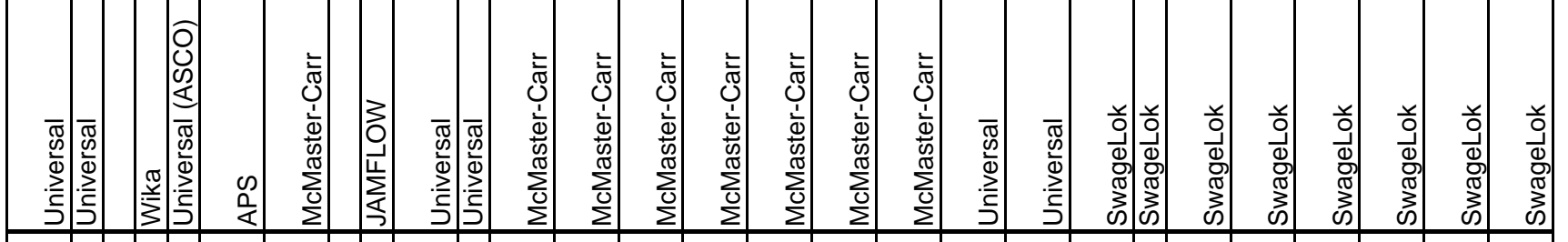
ठ

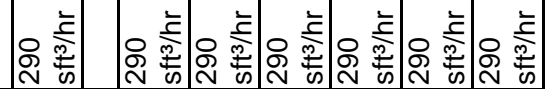

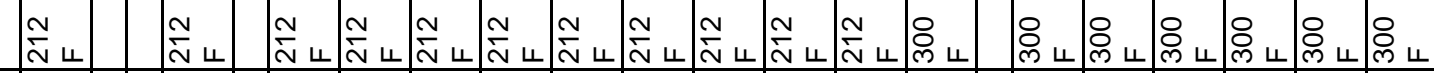

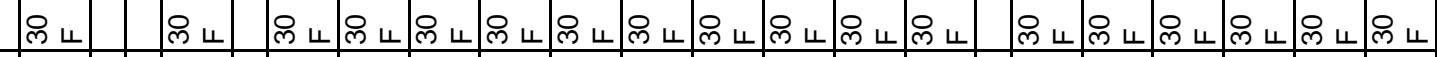

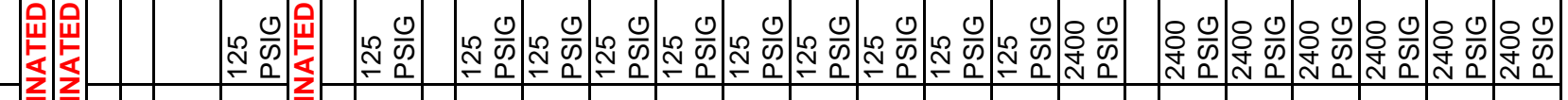

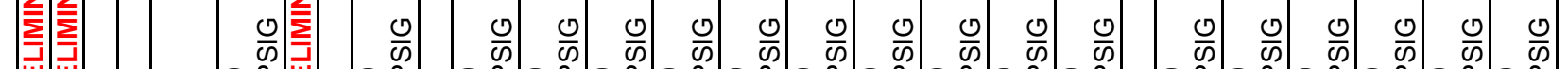

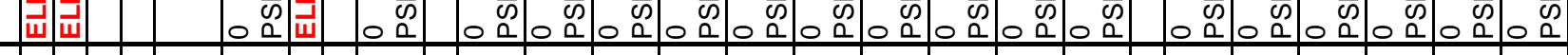

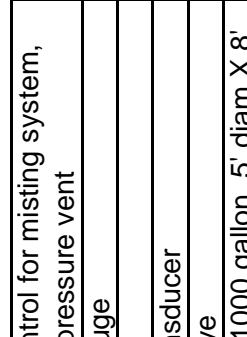

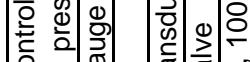

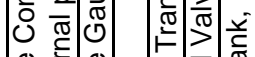

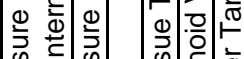

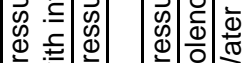

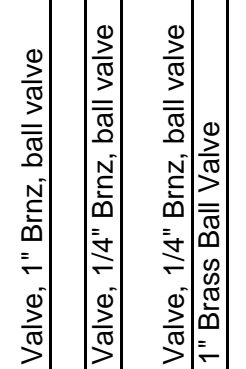

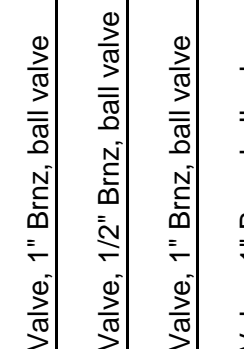

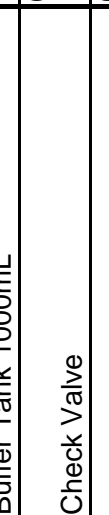

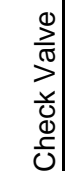

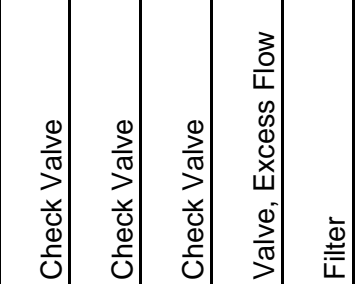

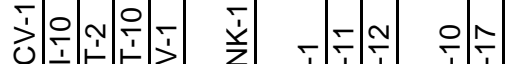

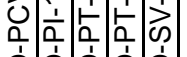

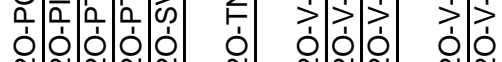

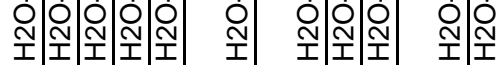

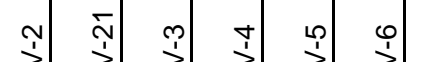

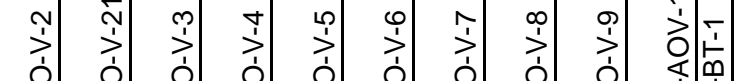

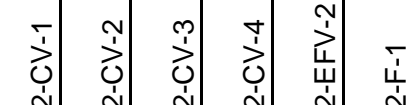

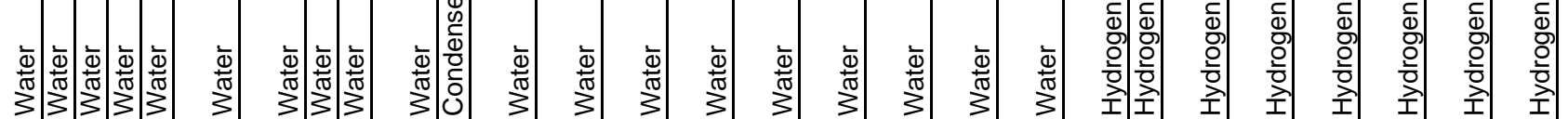

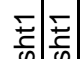

กง กางกาง

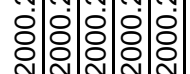

กาง

พ

$+$

-




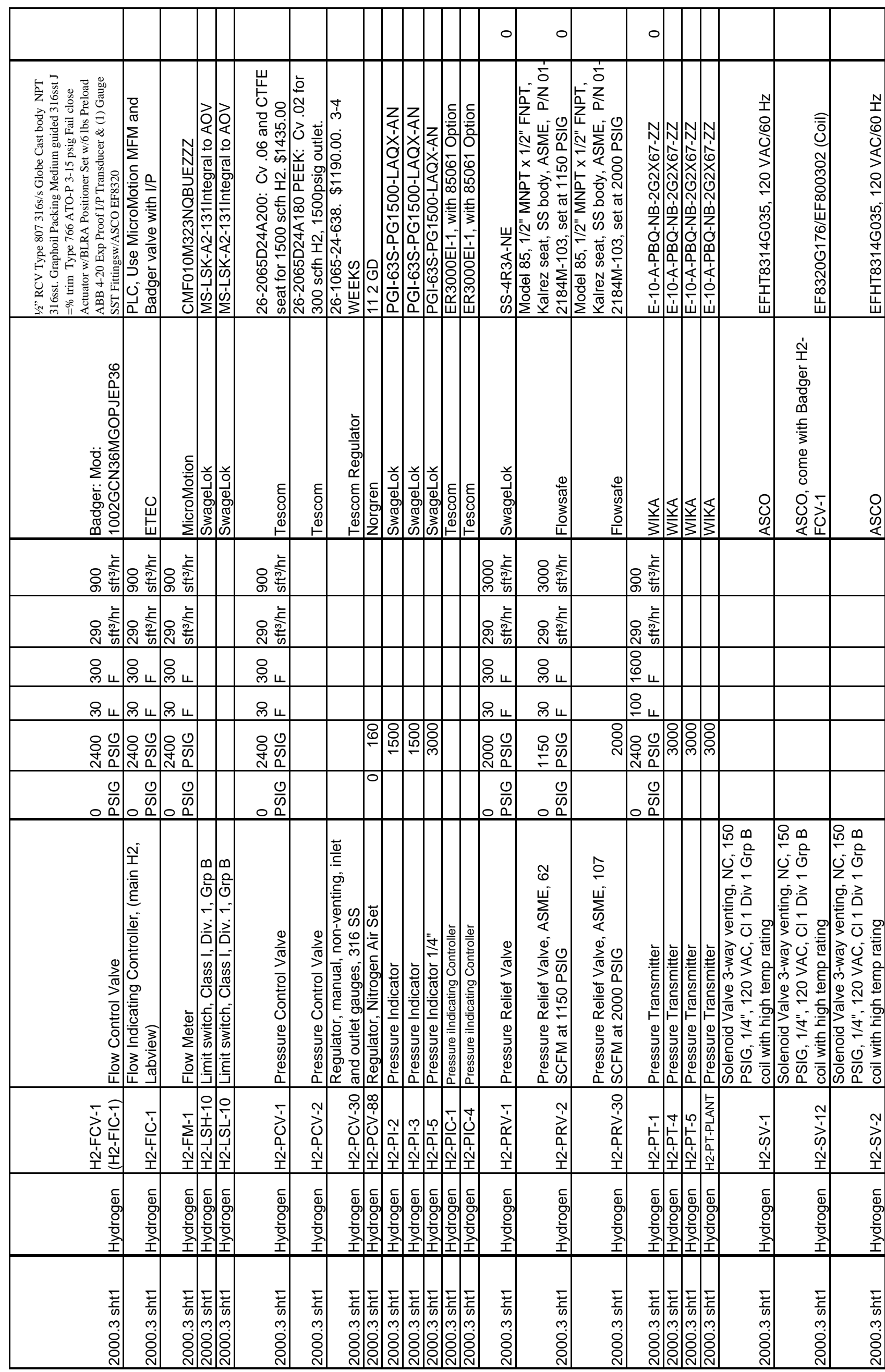




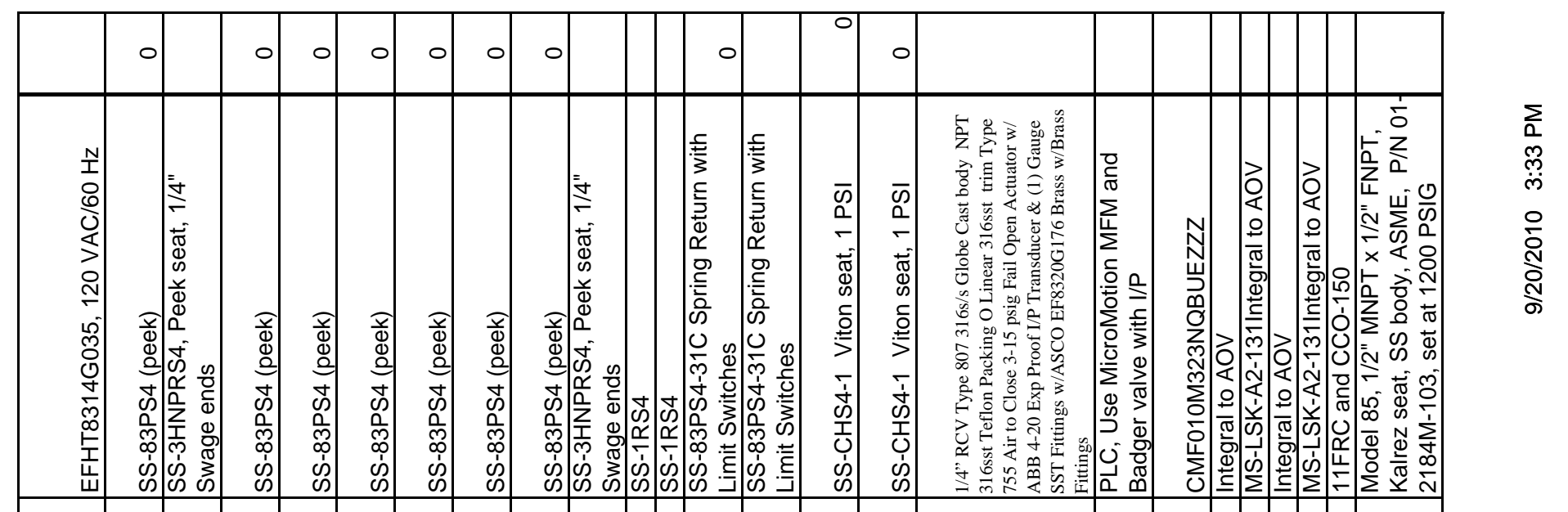

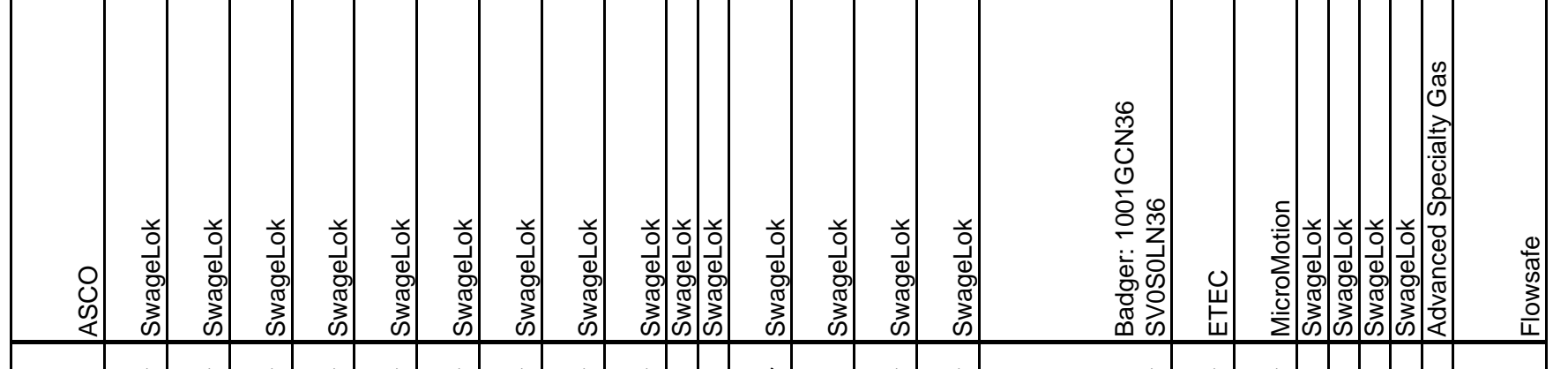

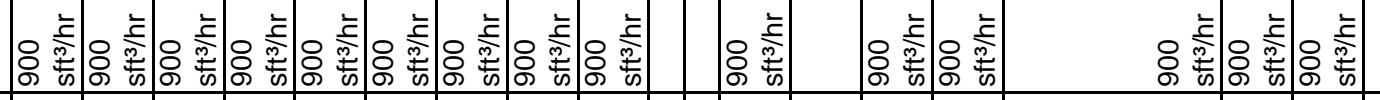

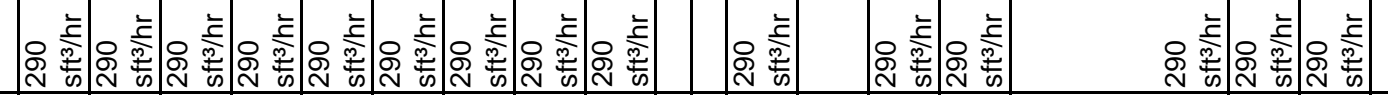

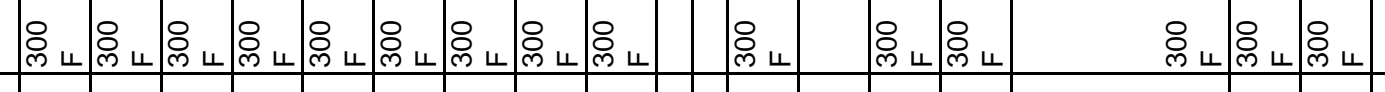

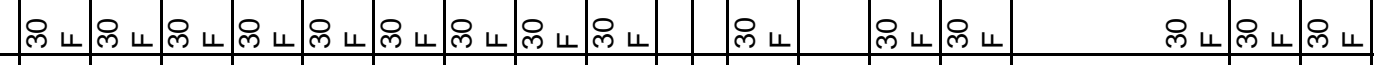

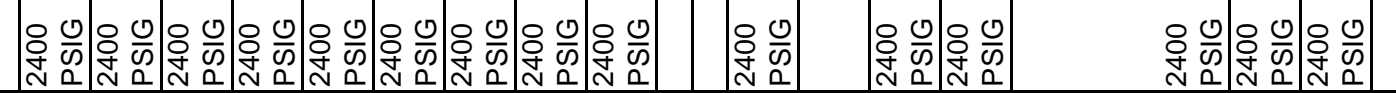

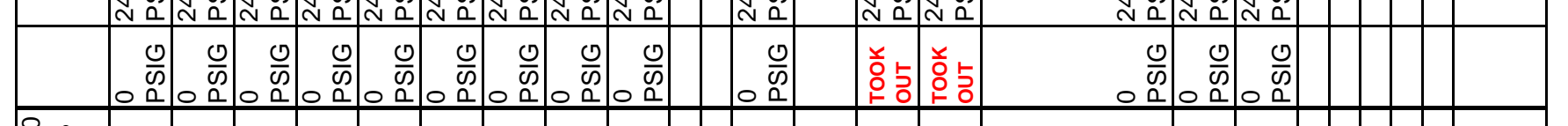

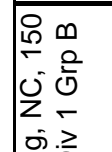

(1)

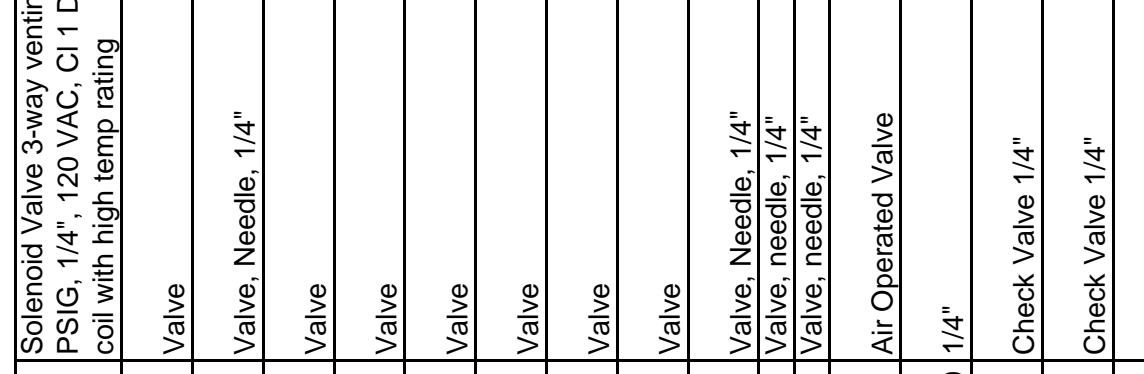

作




\begin{tabular}{|c|c|c|c|c|c|c|c|c|c|c|c|c|c|c|c|c|c|c|c|c|}
\hline & 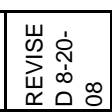 & 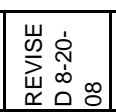 & 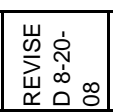 & 0 & & & & & & & & & & & & & & & & \\
\hline 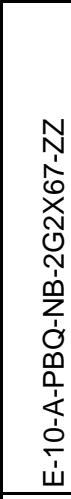 & 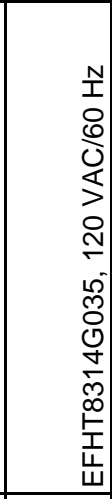 & 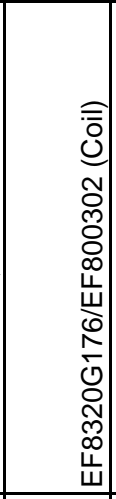 & 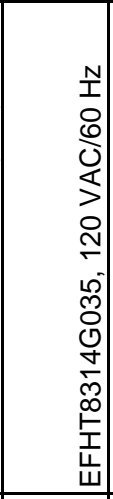 & 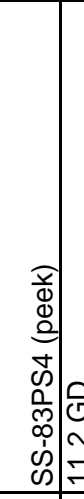 & $\begin{array}{l} \\
\\
0 \\
\vdots \\
- \\
- \\
\exists\end{array}$ & & 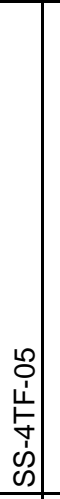 & 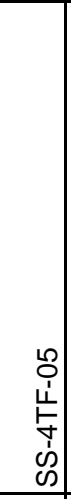 & 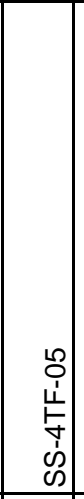 & $\begin{array}{l}\frac{0}{1} \\
\stackrel{1}{s} \\
\stackrel{2}{i} \\
\end{array}$ & & 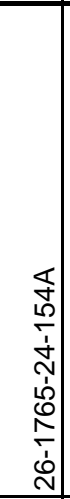 & & 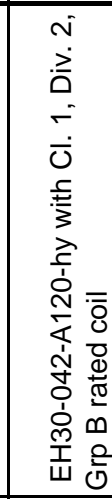 & 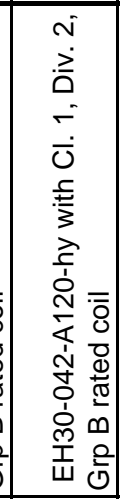 & 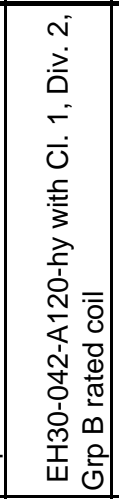 & 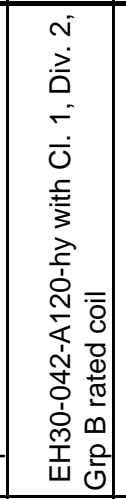 & 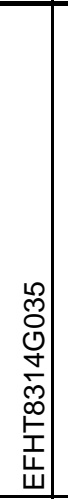 & 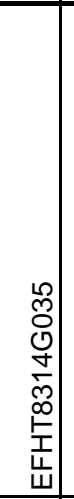 & 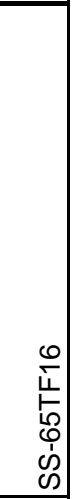 \\
\hline$\frac{5}{3}$ & $\begin{array}{l}8 \\
0 \\
\text { Q }\end{array}$ & 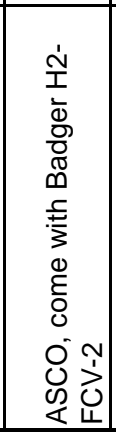 & $\begin{array}{l}0 \\
0 \\
0 \\
0\end{array}$ & 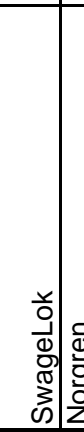 & 힌 & & 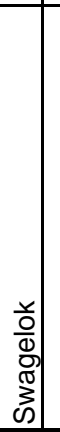 & $\begin{array}{l}\text { 음 } \\
\frac{0}{0} \\
\frac{\pi}{3} \\
0\end{array}$ & \begin{tabular}{|c|} 
\\
$\frac{1}{0}$ \\
$\stackrel{3}{3}$ \\
3 \\
0
\end{tabular} & $\begin{array}{l}0 \\
0 \\
0\end{array}$ & 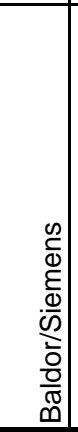 & $\begin{array}{c}\varepsilon \\
0 \\
0 \\
0 \\
-1\end{array}$ & & 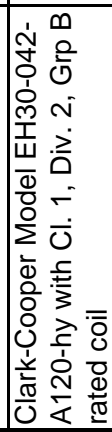 & 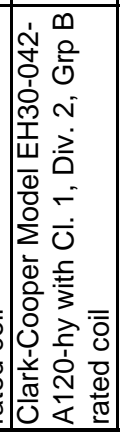 & 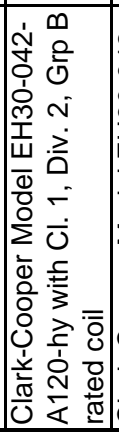 & 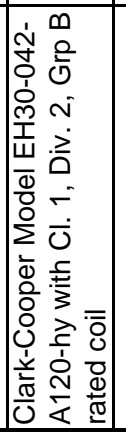 & $\begin{array}{l}0 \\
0 \\
0 \\
\end{array}$ & $\begin{array}{l}0 \\
0 \\
0\end{array}$ & 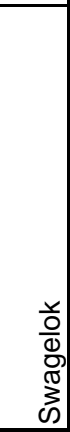 \\
\hline & & & & $8 \frac{\bar{z}}{\frac{m}{2}}$ & & & & & & & 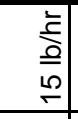 & & & & & & & & & \\
\hline & & & & 竞 & & & & & & & $\begin{array}{l}\bar{\equiv} \\
\underline{0} \\
\end{array}$ & & & ○旁 & ○旁 & $0 \stackrel{\frac{z}{c}}{\frac{m}{n}}$ & $0 \frac{\frac{z}{p}}{\frac{m}{\omega}}$ & & & \\
\hline & & & & $\dot{o}_{4}$ & & & & & & & 总 & & & 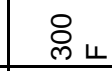 & $\stackrel{\circ}{u}_{4}$ & $\stackrel{p}{~}_{4}$ & $\stackrel{一}{u}_{4}$ & & & \\
\hline & & & & $\dot{m} \sim$ & & & & & & & ஜे u & & & ஜे & 户े & ஓ & ஜे & & & \\
\hline ষ্ল & & & & 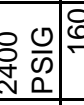 & 8 & & & & & & $\begin{array}{r}\frac{0}{n} \\
0 \\
0\end{array}$ & & & $\stackrel{O}{\stackrel{O}{N}}$ & 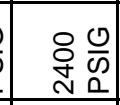 & 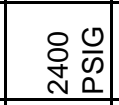 & 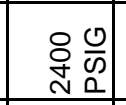 & & & \\
\hline & & & & \begin{tabular}{r|r} 
& $\frac{0}{w}$ \\
0 & 0 \\
0
\end{tabular} & 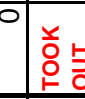 & & & & & & 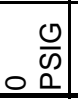 & & & $\frac{0}{\omega}$ & \begin{tabular}{rl|} 
& $\frac{0}{\omega}$ \\
$\circ$ & 0 \\
\end{tabular} & \begin{tabular}{|l|l|} 
& $\frac{0}{w}$ \\
0 & 0 \\
\end{tabular} & $\begin{array}{c}\frac{v}{\omega} \\
0 \\
0\end{array}$ & & & \\
\hline 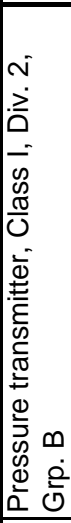 & 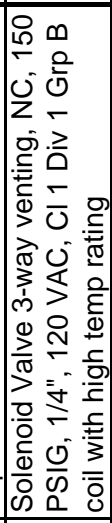 & 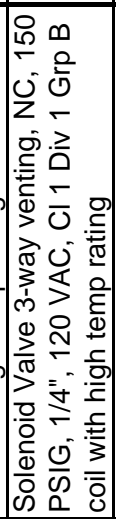 & 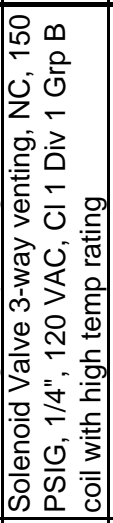 & 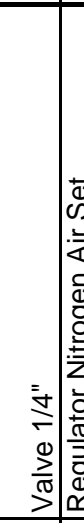 & 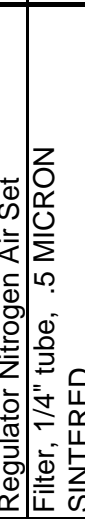 & 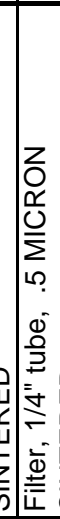 & 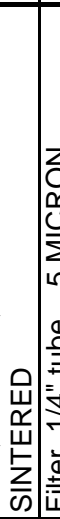 & 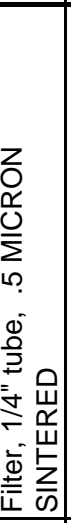 & 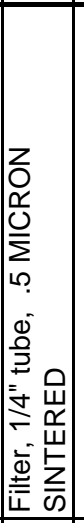 & 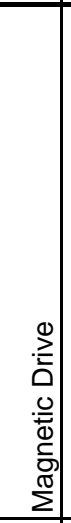 & $\begin{array}{c}0 \\
\times \\
0 \\
0 \\
1 \\
-1 \\
\overline{0} \\
\stackrel{0}{2} \\
\Sigma\end{array}$ & 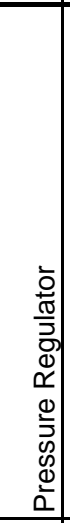 & 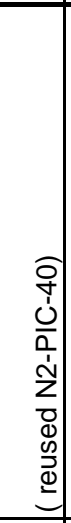 & 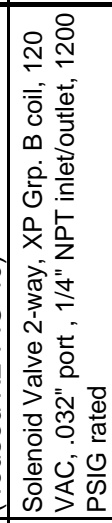 & 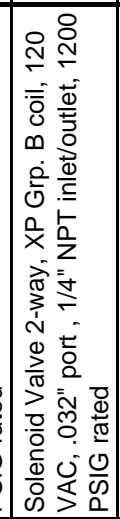 & 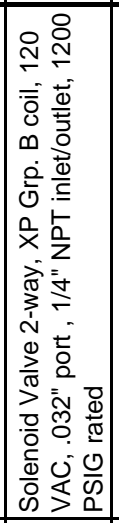 & 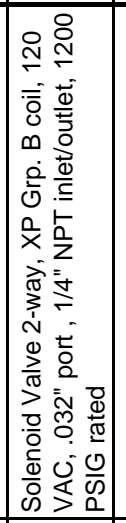 & $\begin{array}{l}\frac{0}{20} \\
\frac{10}{5} \\
0 \\
0 \\
\frac{0}{0} \\
\frac{0}{0} \\
0\end{array}$ & $\begin{array}{l}\frac{0}{\pi} \\
2 \\
\frac{0}{0} \\
\frac{0}{0} \\
\frac{0}{0} \\
0\end{array}$ & 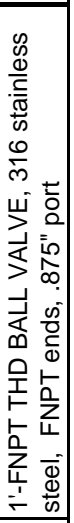 \\
\hline
\end{tabular}

\begin{tabular}{|c|c|c|c|c|c|c|c|c|c|c|c|c|c|c|c|c|c|c|c|}
\hline $\begin{array}{l}0 \\
0 \\
1 \\
\mathbf{1} \\
\mathbf{N} \\
\mathbf{I}\end{array}$ & 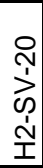 & 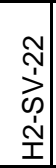 & 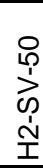 & 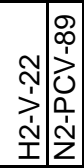 & $\begin{array}{l}\overrightarrow{1} \\
\dot{u} \\
\dot{u} \\
0\end{array}$ & $\begin{array}{l}\overrightarrow{1} \\
\vec{u} \\
\dot{U}\end{array}$ & $\begin{array}{l}\text { I } \\
\stackrel{4}{4} \\
\dot{u} \\
\end{array}$ & $\begin{array}{c}m \\
\overrightarrow{1} \\
\dot{\dot{1}} \\
\dot{\vec{u}}\end{array}$ & $\begin{array}{l}\overrightarrow{1} \\
\sum_{i} \\
\dot{u}\end{array}$ & 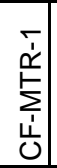 & $\begin{array}{l}-1 \\
\grave{d} \\
0 \\
\dot{1} \\
\end{array}$ & 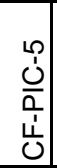 & $\begin{array}{l}\overbrace{0}^{-1} \\
\text { un } \\
\text { U. }\end{array}$ & $\begin{array}{l}\tilde{N} \\
\dot{1} \\
0 \\
\dot{1} \\
ن\end{array}$ & 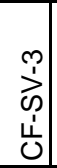 & 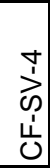 & 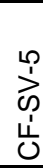 & $\begin{array}{l}\vec{y} \\
\dot{y} \\
0 \\
\dot{u} \\
0\end{array}$ & \\
\hline
\end{tabular}

\begin{tabular}{|c|c|c|c|c|c|c|c|c|c|c|c|c|c|c|c|c|c|c|c|}
\hline & 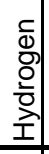 & $\begin{array}{l}\frac{1}{0} \\
\overline{0} \\
\frac{0}{0} \\
\stackrel{2}{1} \\
\end{array}$ & 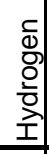 & 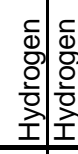 & 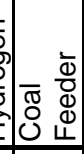 & 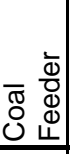 & 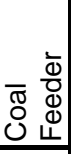 & 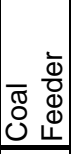 & 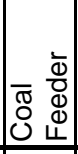 & 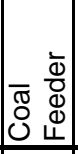 & 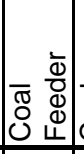 & 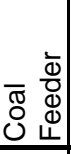 & 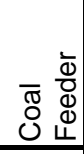 & 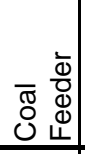 & 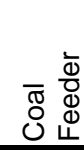 & 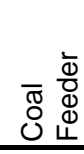 & 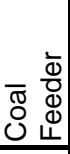 & 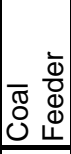 & 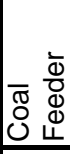 \\
\hline & 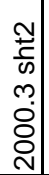 & 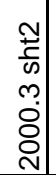 & $\begin{array}{l}\bar{c} \\
0 \\
\dot{0} \\
\dot{0} \\
\text { d. }\end{array}$ & 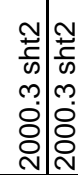 & : & 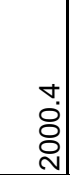 & రి & ठ্ঠ & $\begin{array}{l}\nabla \\
\vdots \\
\vdots \\
\vdots\end{array}$ & రి & $\left|\begin{array}{r} \pm \\
8 \\
8 \\
\end{array}\right|$ & 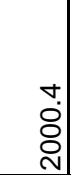 & 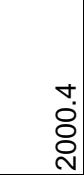 & $\begin{array}{l}\dot{⿱} \\
\dot{\delta} \\
\dot{D}\end{array}$ & $\begin{array}{l}\text { ণ } \\
\text { ঠे } \\
\text { ठ }\end{array}$ & ¿ें & 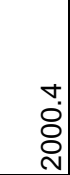 & $\begin{array}{l}+ \\
\dot{0} \\
0 \\
\text { N }\end{array}$ & \\
\hline
\end{tabular}



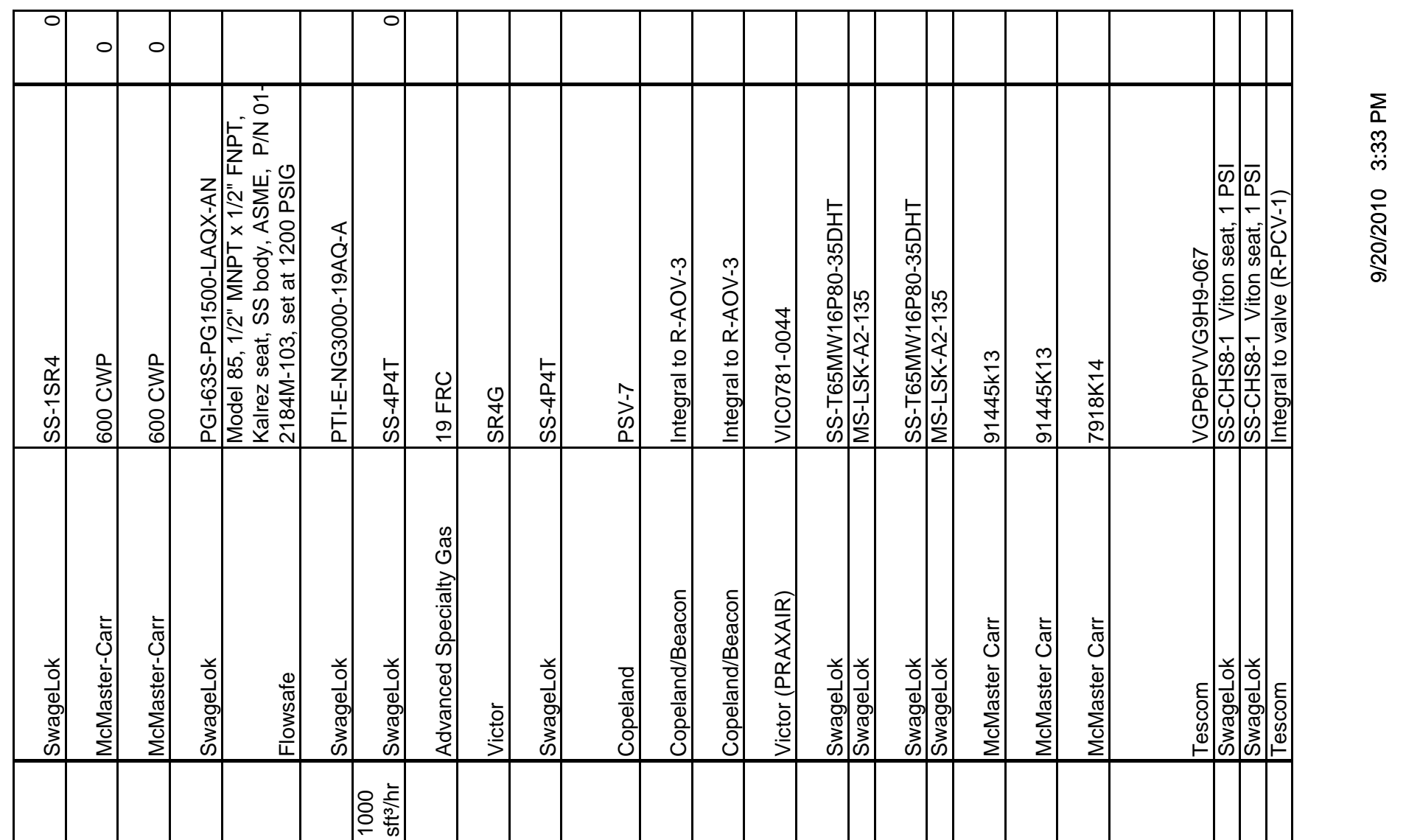

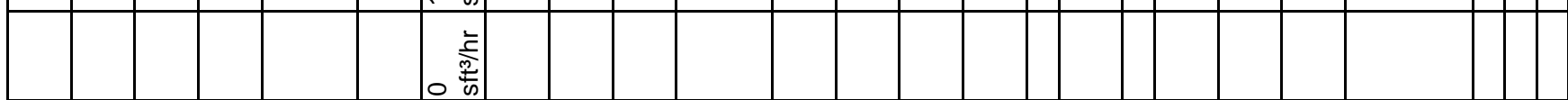
สี 4 สี

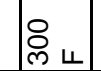

穴 色

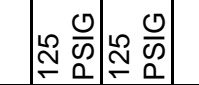

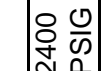

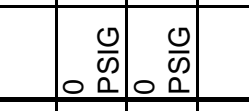

$\left|\begin{array}{r}0 \\ \frac{0}{n} \\ 0\end{array}\right|$

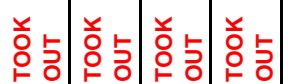

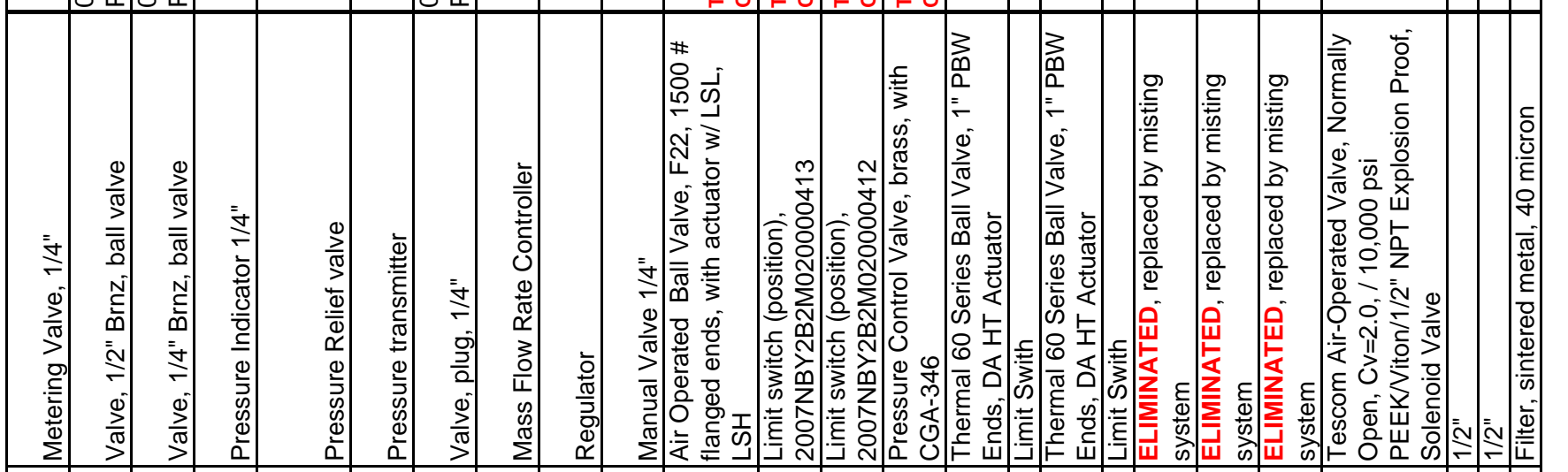

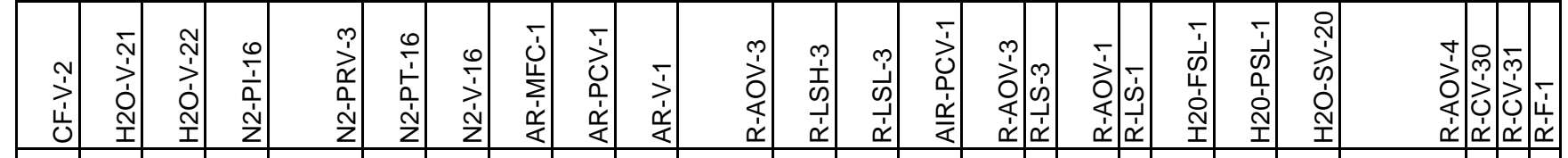

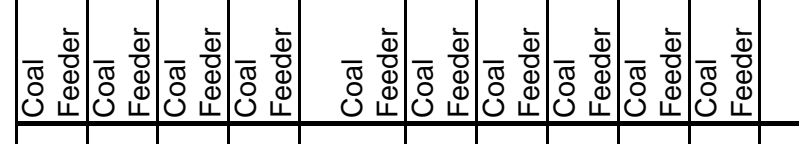

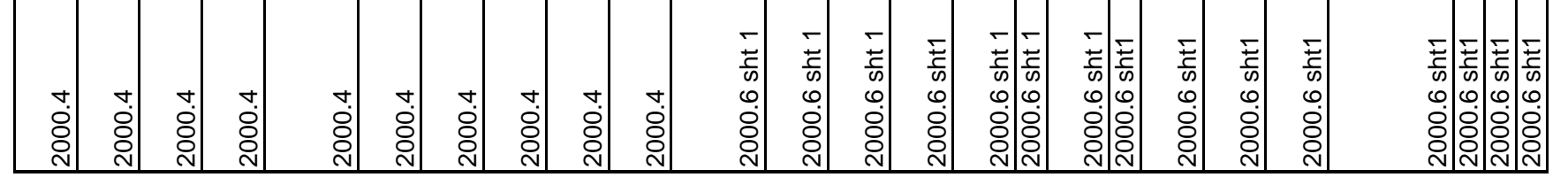




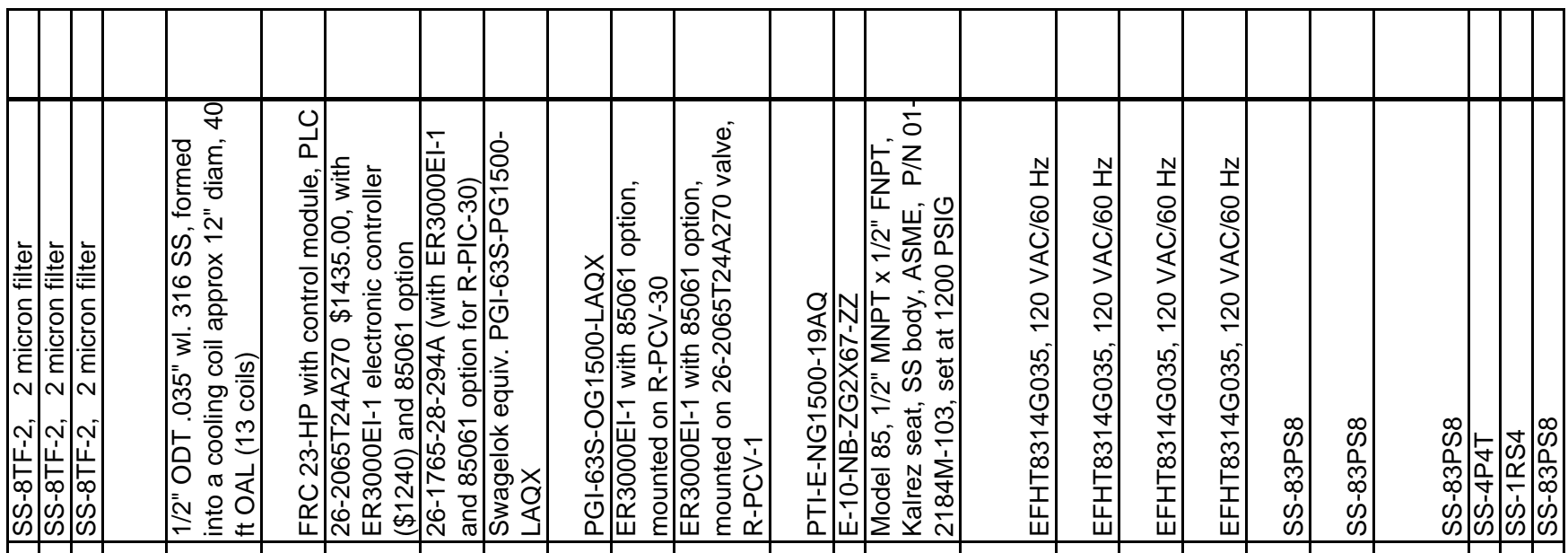

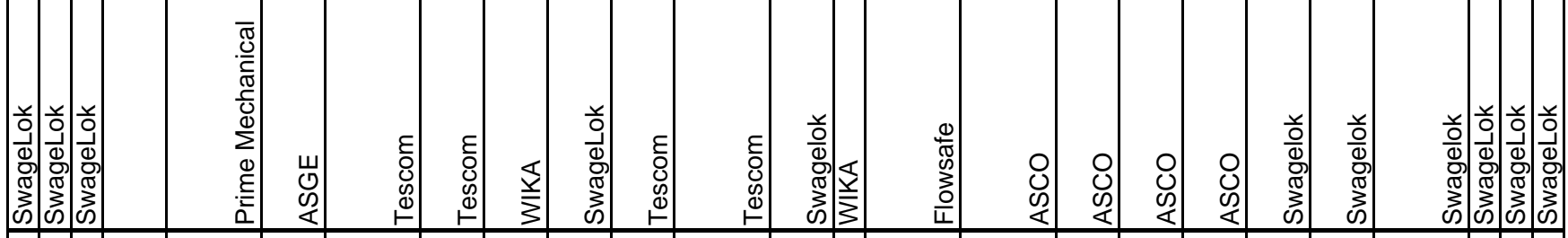

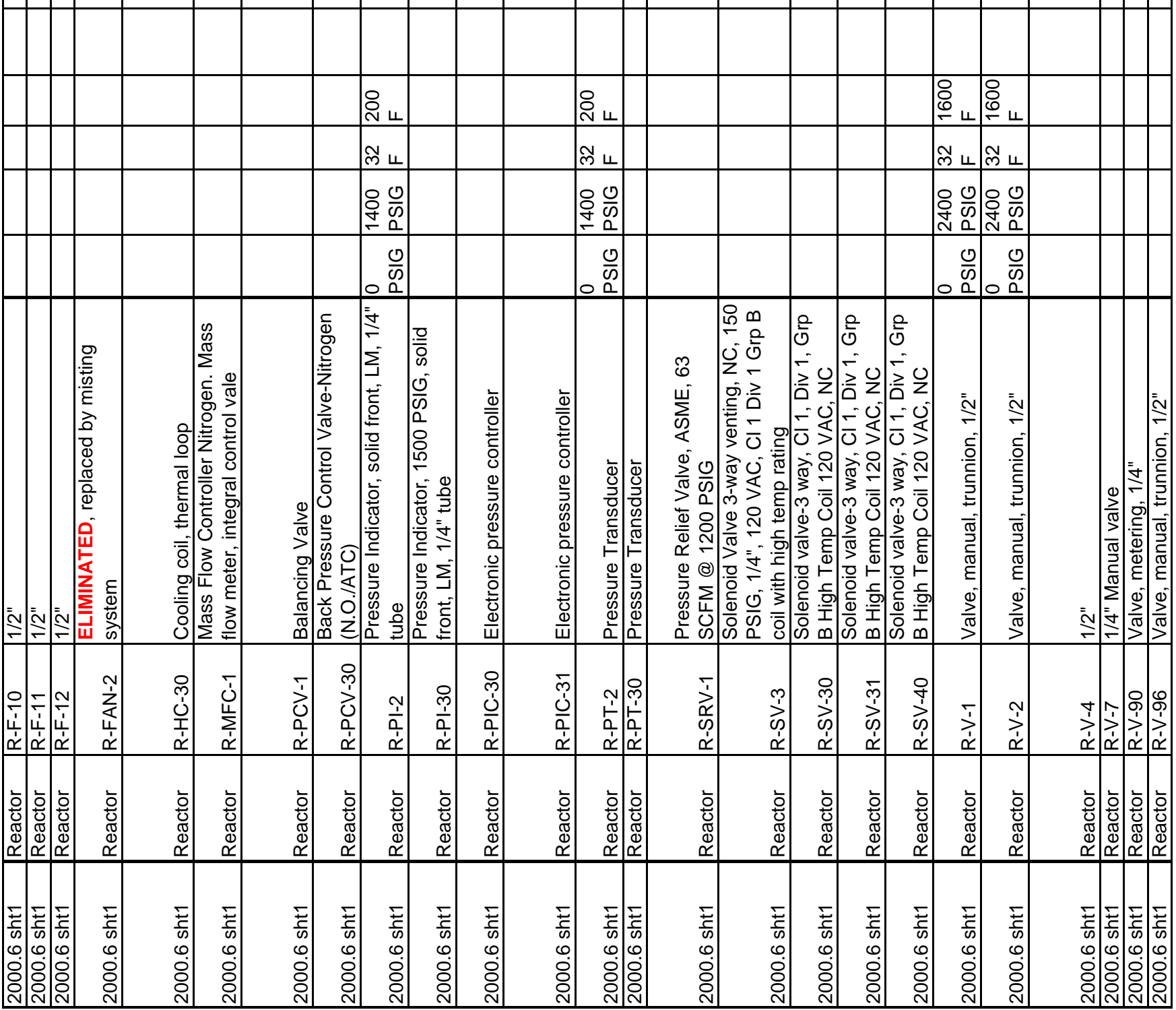




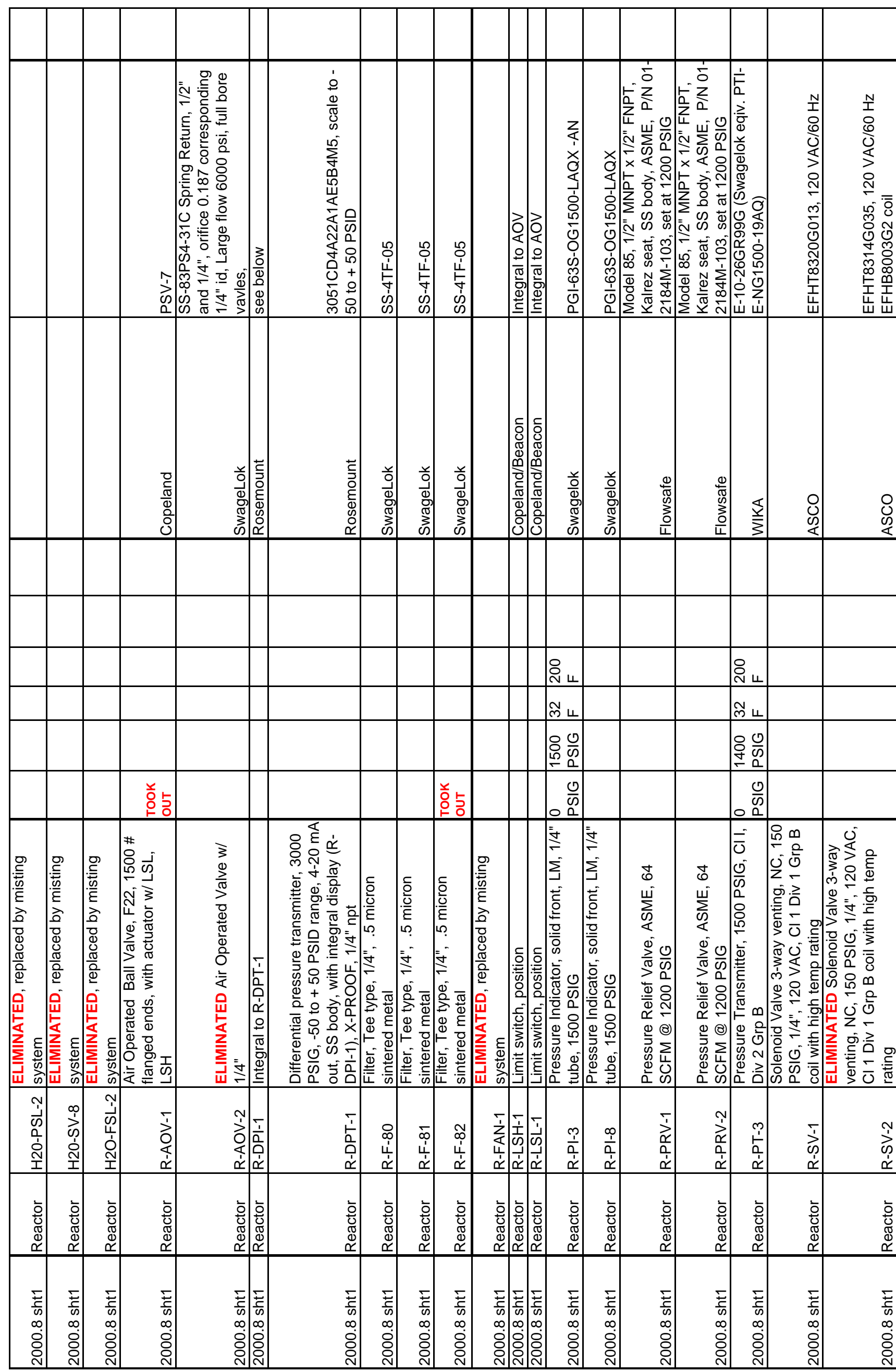




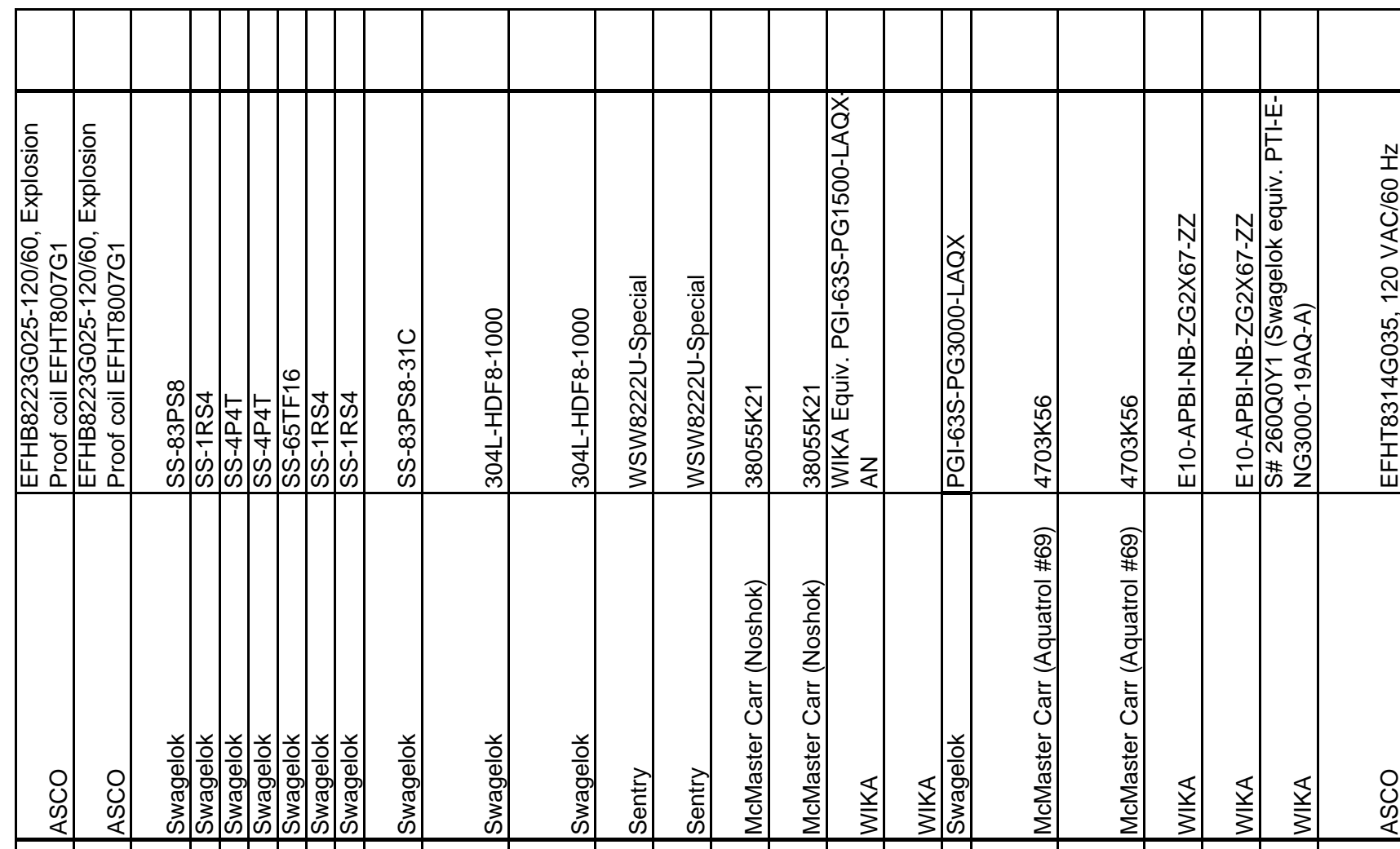

\begin{tabular}{|c|c|c|c|c|c|c|c|c|c|c|c|c|c|c|c|c|c|c|c|c|c|c|}
\hline & & $\begin{array}{l} \\
0 \\
0 \\
-1\end{array}$ & & & & & $\begin{array}{l}8 \\
0 \\
0 \\
-1\end{array}$ & & & 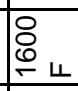 & 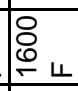 & 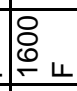 & 욱 & & ${ }_{7}^{8}$ & & $\begin{array}{l}\mathbb{Q}_{4} \\
\mathfrak{G}_{4}\end{array}$ & & \begin{tabular}{|l|} 
\\
0 \\
\end{tabular} & 욱 & & \\
\hline & & $\tilde{m}=$ & & & & & $\tilde{m}_{4}$ & & & $\tilde{m}_{L}$ & $\approx \sim$ & $\tilde{m}_{L}$ & $\tilde{m}=$ & & $\tilde{m}_{4}$ & & $\tilde{m} \leftarrow$ & & $\approx \sim$ & $\tilde{m}_{L}$ & & \\
\hline & & 沜 & & & & & 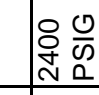 & & & 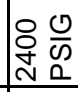 & & & & & & & & & ঠ্ল & ৪্ল $\frac{0}{\infty}$ & & \\
\hline & & $\frac{0}{N}$ & & & & & $\frac{0}{\omega}$ & & & $\frac{0}{\omega}$ & $\frac{0}{\omega}$ & $\frac{0}{n}$ & $\int_{0}^{\frac{0}{\omega}}$ & & $\begin{array}{r}0 \\
0 \\
0\end{array}$ & & $\begin{array}{r}\frac{0}{\omega} \\
00\end{array}$ & & $\begin{array}{ll}0 & 0 \\
0 & 0 \\
0\end{array}$ & $\frac{0}{\omega}$ & & \\
\hline 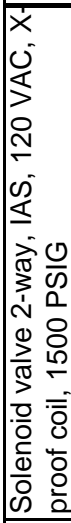 & 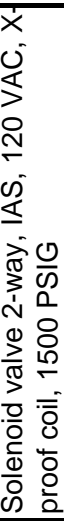 & 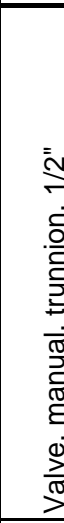 & 更 & 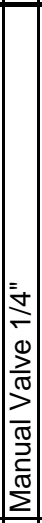 & 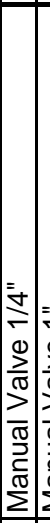 & 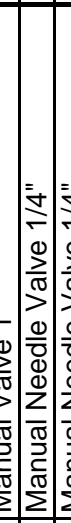 & 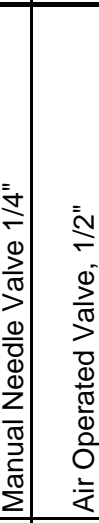 & 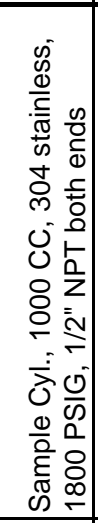 & 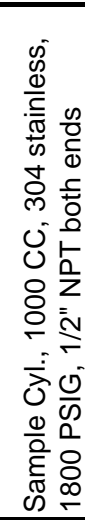 & 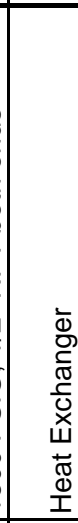 & 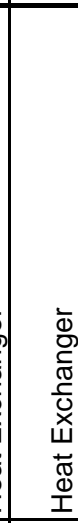 & 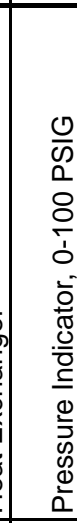 & $\begin{array}{l}0 \\
\bar{\omega} \\
0 \\
0 \\
0 \\
0 \\
0 \\
\overline{0} \\
\frac{0}{0} \\
\frac{1}{0} \\
0 \\
\overline{0} \\
0 \\
0 \\
0 \\
\end{array}$ & 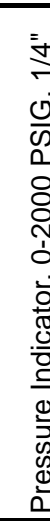 & & $\frac{0}{0}$ & 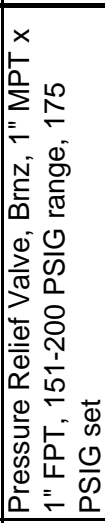 & 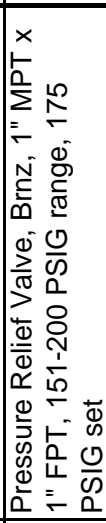 & 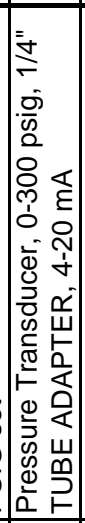 & 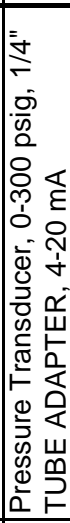 & 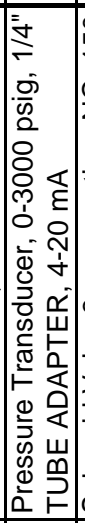 & 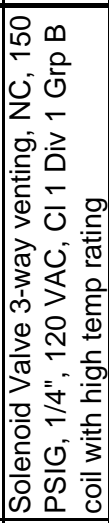 \\
\hline $\begin{array}{l}\hat{1} \\
\text { o } \\
\text { ¿ } \\
\end{array}$ & $\begin{array}{l}\infty \\
\dot{1} \\
0 \\
\dot{1} \\
\dot{\alpha}\end{array}$ & 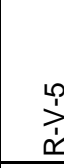 & & 虫 & $\frac{1}{\tilde{\alpha}}$ & 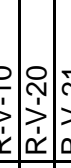 & 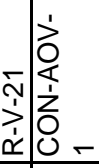 & $\begin{array}{l}\overrightarrow{1} \\
\dot{\lambda} \\
0 \\
\dot{2} \\
0 \\
0\end{array}$ & 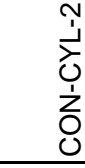 & $\begin{array}{l}\overrightarrow{1} \\
\ddot{x} \\
\mathbf{1} \\
\dot{3} \\
0 \\
0 \\
\end{array}$ & 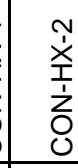 & $\begin{array}{l}\frac{1}{1} \\
\frac{1}{2} \\
\frac{1}{2} \\
0\end{array}$ & $\begin{array}{l}N \\
\frac{1}{0} \\
\dot{\lambda} \\
0 \\
0 \\
\end{array}$ & $\frac{9}{1}$ & & $\begin{array}{l}\vdots \\
\vdots \\
\vdots \\
\vdots \\
\vdots\end{array}$ & $\begin{array}{l}\lambda_{0}^{1} \\
\frac{x}{0} \\
\sum_{2} \\
0_{-1} \\
U_{-1}\end{array}$ & 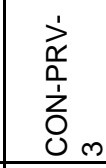 & $\begin{array}{l}-1 \\
\stackrel{1}{0} \\
0 \\
\grave{z} \\
0 \\
0\end{array}$ & $\begin{array}{l}N \\
\stackrel{1}{1} \\
\vdots \\
0 \\
0 \\
\end{array}$ & $\begin{array}{l}1 \\
\dot{1} \\
\grave{\partial} \\
0\end{array}$ & \\
\hline 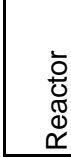 & $\begin{array}{l}\overline{0} \\
\stackrel{0}{0} \\
0 \\
\underline{\underline{u}} \\
\end{array}$ & 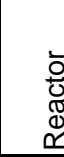 & & 10 & & 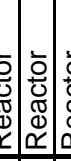 & $\begin{array}{l}\bar{\Phi} \\
0 \\
\bar{d} \\
\overline{0} \\
\overline{0} \\
0\end{array}$ & 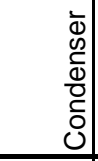 & $\bar{\varpi}$ & $\begin{array}{l}\bar{D} \\
0 \\
\bar{d} \\
0 \\
\overline{0} \\
\mathcal{u}\end{array}$ & $\begin{array}{l}\frac{1}{0} \\
0 \\
\bar{d} \\
\overline{0} \\
\overline{0} \\
0\end{array}$ & $\begin{array}{l}\bar{\Phi} \\
0 \\
\overline{0} \\
\overline{0} \\
\overline{0} \\
0\end{array}$ & 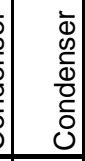 & d & & & $\begin{array}{l}\bar{\Phi} \\
\hat{\omega} \\
\frac{1}{0}\end{array}$ & $\begin{array}{l}\bar{\Phi} \\
\stackrel{\Phi}{c}\end{array}$ & $\begin{array}{l}\overline{\bar{q}} \\
\overline{0} \\
\bar{q} \\
\overline{0} \\
\bar{c}\end{array}$ & \begin{tabular}{|l}
$\bar{d}$ \\
0 \\
$\bar{d}$ \\
$\overline{0}$ \\
$\overline{0}$ \\
0
\end{tabular} & 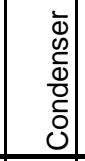 & $\bar{c}$ \\
\hline
\end{tabular}

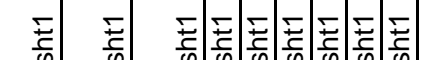

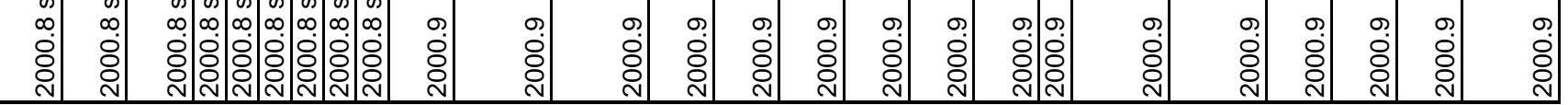




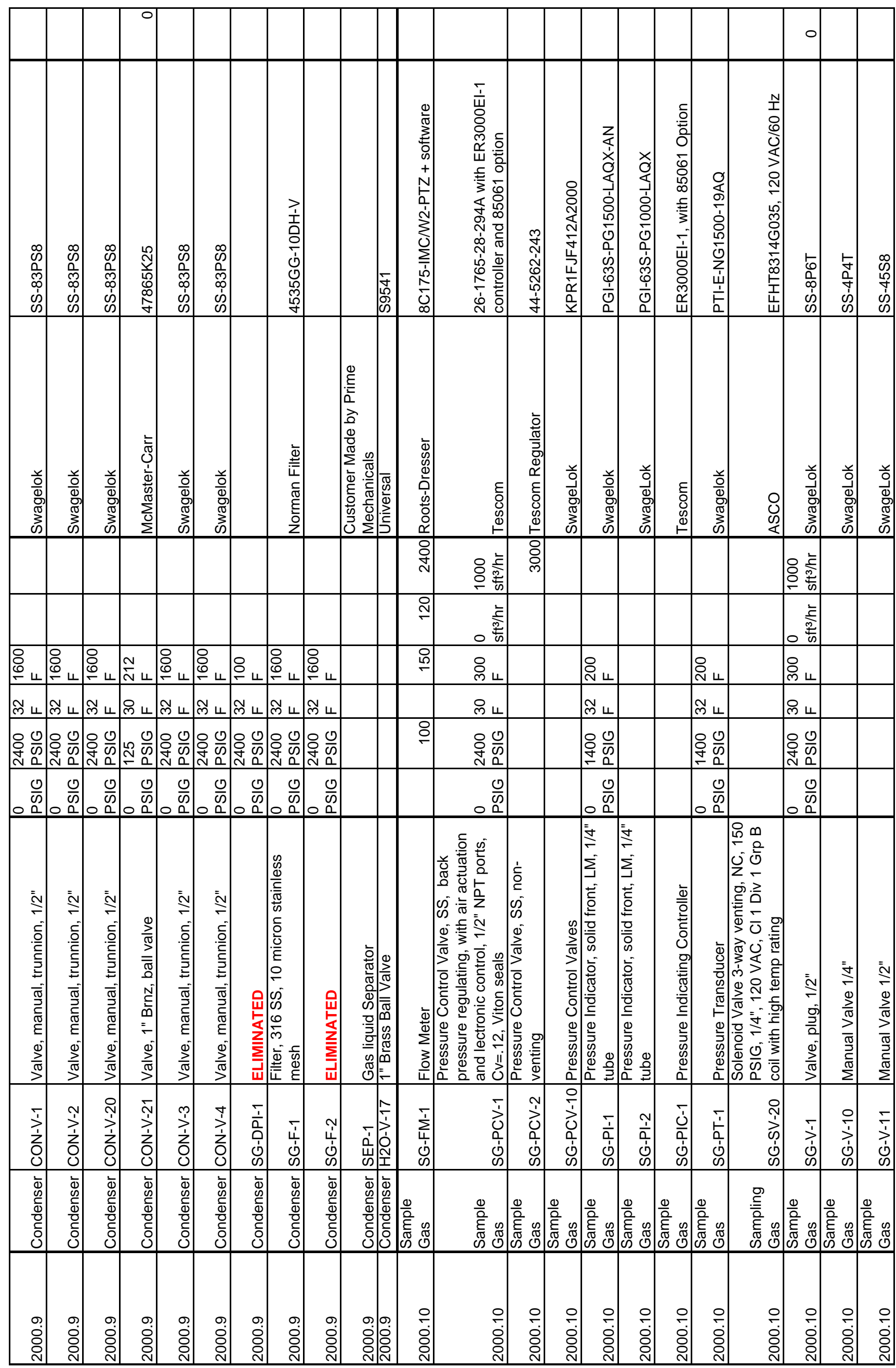




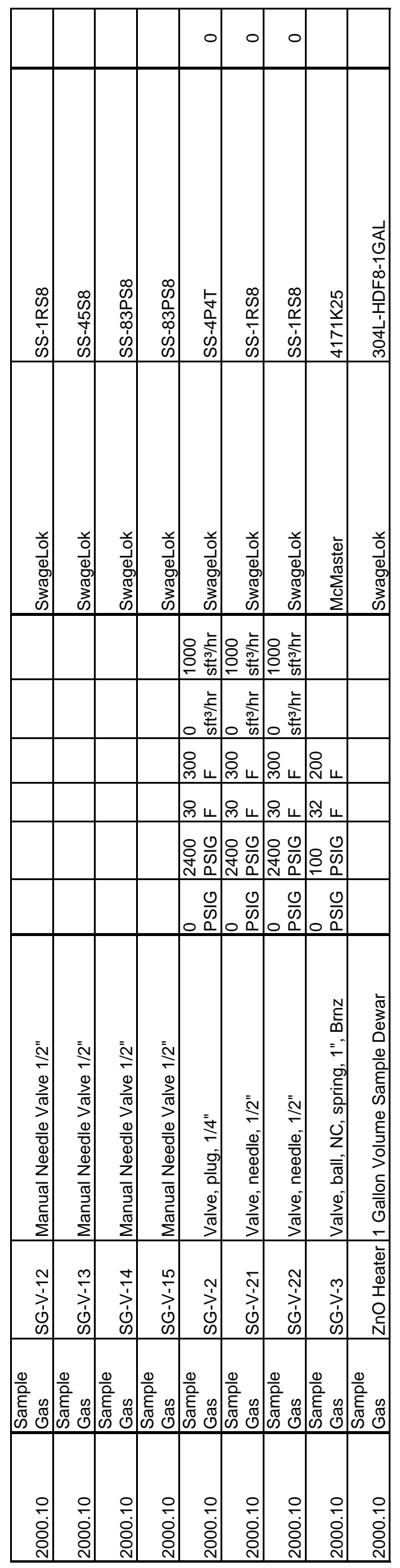




\title{
APPENDIX J
}

\section{Bench Scale Hydrogasification Testing}

\author{
Commissioning Procedure
}


Development of a Hydrogasification Process for

Coproduction of SNG and Electrical Power from Western Coals

Final Report

THIS PAGE INTENTIONALLY LEFT BLANK 


\title{
COAL TO SNG \\ Bench Scale Test Reactor COMMISSIONING PROCEDURE
}

FINAL

JULY 2010

\author{
Arizona Public Service Company \\ 400 NORTH 5TH STREET \\ Phoenix, AZ 85003
}

\author{
Principal Investigator \\ Dr. Xiaolei Sun \\ 602.250.1510 \\ 602.250.1505 \\ xiaolei.sun@aps.com
}




\section{COAL TO SNG \\ BENCH SCALE TEST REACTOR \\ COMMISSIONING PROCEDURE}

\section{TABLE OF CONTENTS}

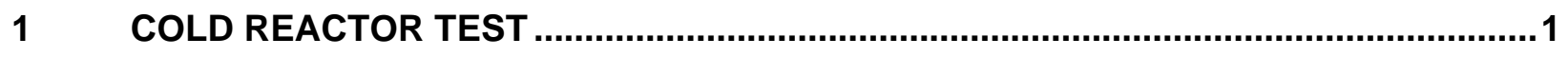

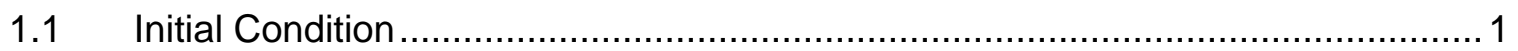

$1.2 \quad$ Main Line Process Functional Check Using Nitrogen ...................................... 1

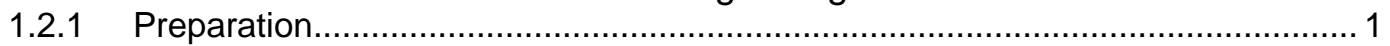

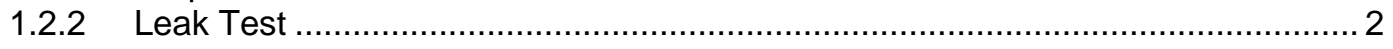

1.2.3 Flow Control Functional Check ....................................................................... 3

1.2.4 Temporal Plant Shutdown................................................................... 5

1.2.5 Flow Meter Calibration for R-MFC-1, H2-FCV-1 $\left(\mathrm{N}_{2}\right)$, and N2-RO-1 ..................... 6

1.2.6 Plant Depressurizing ............................................................................. 7

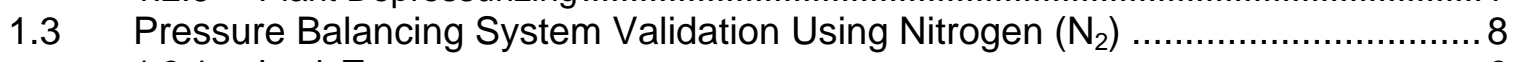

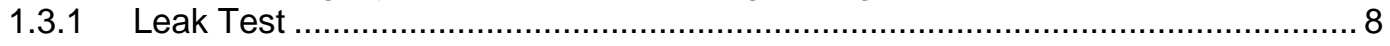

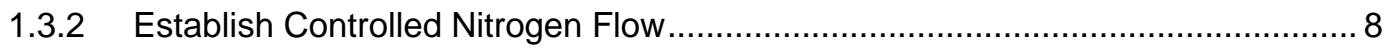

1.3.3 Balancing System Control Functional Check for Emergency Shutdown (ESD) .... 9

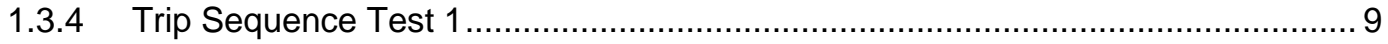

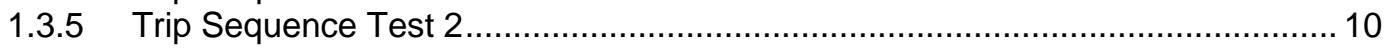

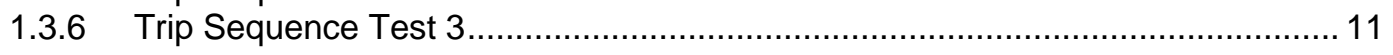

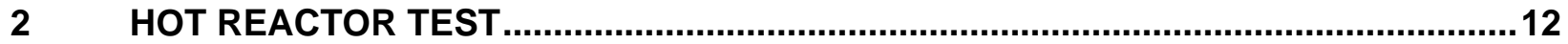

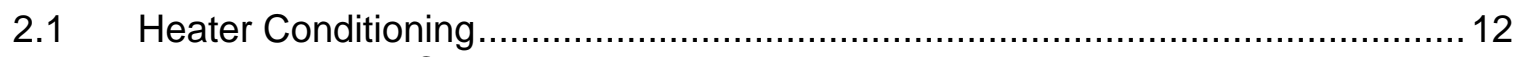

2.1.1 Establish Controlled Nitrogen and Air Flow .................................................. 12

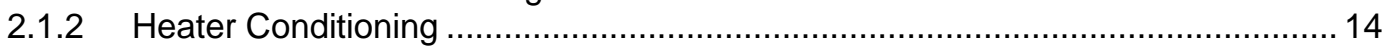

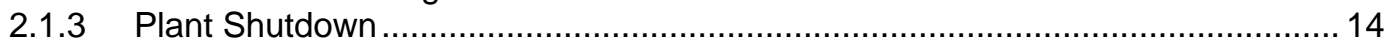

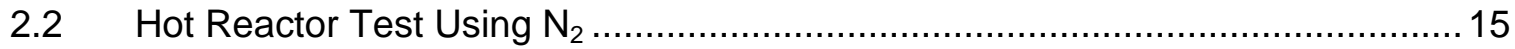

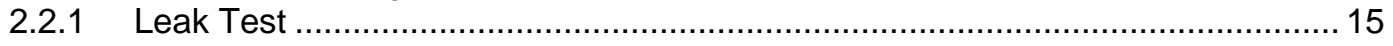

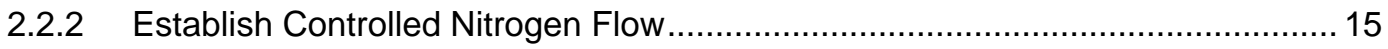

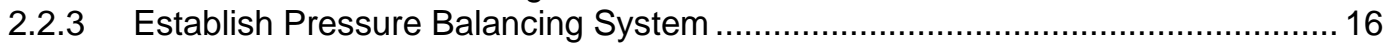

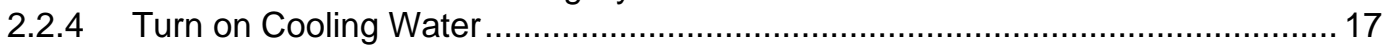

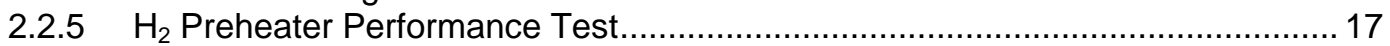

2.2.6 Reactor Heater Performance Test ............................................................... 20

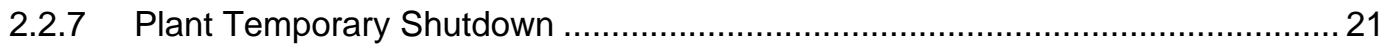

$2.3 \quad$ Hot Reactor Test using $\mathrm{H}_{2}$ (Low Flow) ............................................. 21

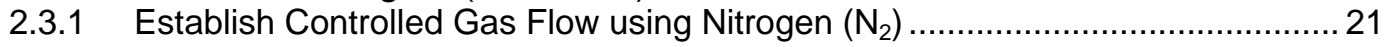

2.3.2 Establish Pressure Balancing System ........................................................ 22

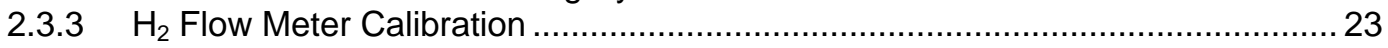

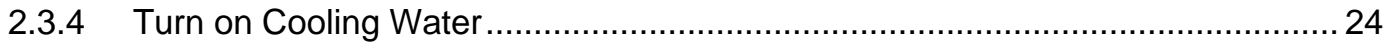

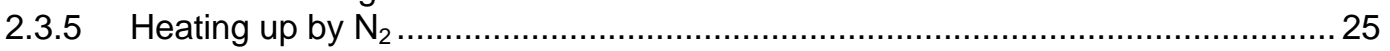

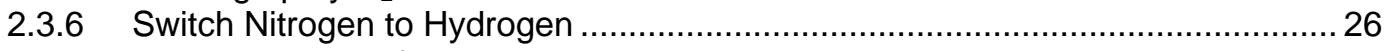

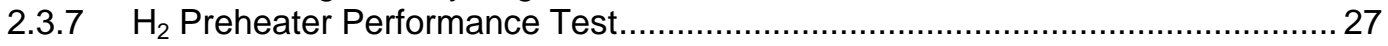

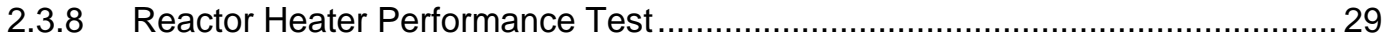

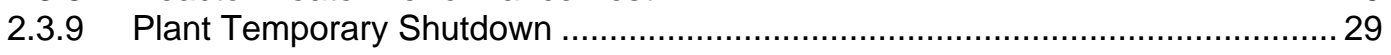

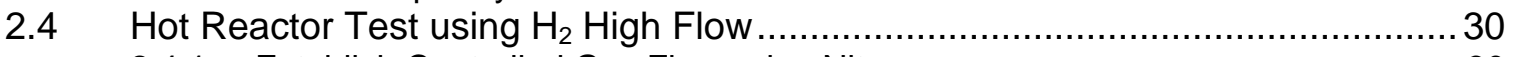

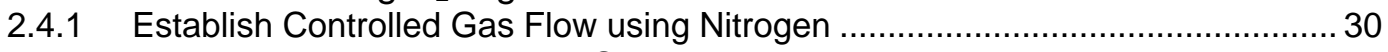

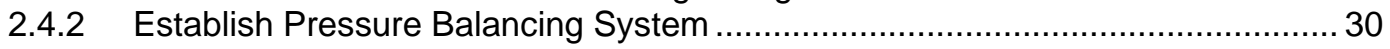

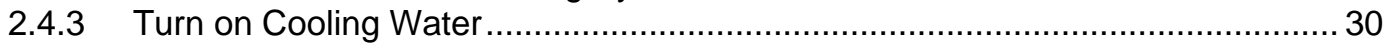




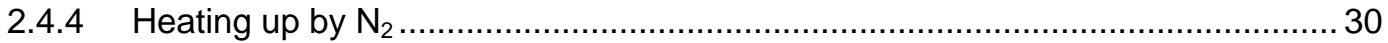

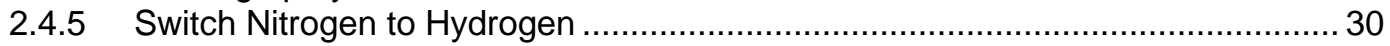

2.4.6 $\quad \mathrm{H}_{2}$ Preheater Performance Test............................................................... 31

2.4.7 Reactor Heater Performance Test ............................................................... 34

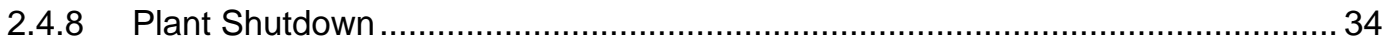

\section{List of Figures}

Figure 1 -Preheater 1 through 4 Temperature (degrees F) to Tube Length (inches)................ 17

Figure 2 - Preheaters 1 through 4 Temperature Profile and Preheaters Gas Temperatures Comparison to Outer and Inner Wall Temperatures.

Figure 3 - Relation between the Preheater Outputs, Temperatures, and Tube Temperatures

Depending on the Gas Flow.....

\section{List of Tables}

Table 1 - Leak Testing Tube Trailer Pressures, Capacities, and Available Volume ................. 2

Table $2-\mathrm{H} 2-\mathrm{PIC}-1 \mathrm{psig}$ and $\mathrm{sft}^{3} / \mathrm{h}$ and H2-FCV-1 Percent Opening ...................................2 23

Table 3 - H2-PIC-2 at psig and sft3/h with H2-FCV-2 percent opening ............................... 24

\section{Appendix}

Appendix 1 - Coal to SNG Bench Scale Test Reactor Normal Shutdown Lineup Checklist ........ 1 


\section{Cold Reactor Test}

\subsection{Initial Condition}

1. The system is in a normal shutdown lineup per Coal to SNG Bench Scale Test Reactor Normal Shutdown Lineup Checklist, Appendix 1.

2. All systems are secured and high-pressure lines are locked.

3. Confirm that all tagouts and locking devices are installed on the high-pressure gas lines per Appendix 1.

4. Verify that the reactor is cold and all heaters are secure.

5. Confirm that locking devices are installed on H2-HTR-1, -2, -3, and -4 and R-HTR-1, -2, $-3,-4,-5,-6$, and -7 .

6. Confirm that the coal hopper and feed system are empty and depressurized to 5 pounds per square inch gauge (psig). Also, confirm and check the following items:

a. C-MTR-1 is locked.

b. N2-PI-16 and N2-PT-16 are checked.

\subsection{Main Line Process Functional Check Using Nitrogen}

\subsubsection{Preparation}

Verify that power meter is operational and that the power usage is being recorded by the monitoring system during all modes of testing.

\subsubsection{Power}

Verify that power meter is operational and that the power usage is being recorded by the monitoring system during all modes of testing.

\subsubsection{Instrument Nitrogen Supply}

1. Verify that Nitrogen $\left(\mathrm{N}_{2}\right)$ air supply and that the pressure is more than 100 psig at N2-PT-50.

2. Check and open N2-V-1.

3. Check and open N2-V-9, -10, -11, -12, -13, -14, -15, -20, -21, -22, $-23,-24,-25$, and -50 .

1.2.1.3 $\quad \mathrm{N}_{2}$ Tube Trailer for Main Use

A $\mathrm{N}_{2}$ tube trailer, which is rated to 2400 psig with 55,000 standard cubic feet $\left(\mathrm{sft}^{3}\right)$ capacity, will be used for plant purge before operations, leak testing, and process use. This will have a usable capacity of about $27,000 \mathrm{sft}^{3}$; there will be 4 or 5 tests on the one trailer. When the pressure of tube trailer approaches 1500 psig, Test Manager must schedule the trailer's refilling.

1.2.1.4 $\quad \mathrm{N}_{2}$ PAK for Emergency

Verify that the $\mathrm{N}_{2}$ PAK is full through N2-PCV-2. 


\subsubsection{Temporary Tubing}

In order to simplify the pressure balancing system operation during initial startup operation and coal feeder calibration, take out R-CV-31 and temporarily insert short tube.

\subsubsection{Leak Test}

The leak test will be performed at three pressure levels, which are (1) 400, (2) 800 , and (3) 1000 psig. The required gas volume for pressurizing the plant to 1000 psig is about $730 \mathrm{sft}^{3}$. Before the leak testing, the pressure must be at least at 1500 to 1550 psig. Refer to Table 1 below:

Table 1 - Leak Testing Tube Trailer Pressures, Capacities, and Available Volume

\begin{tabular}{|c|c|c|}
\hline $\begin{array}{c}\text { Pressure of } \\
\text { Tube Trailer (psig) }\end{array}$ & $\begin{array}{c}\text { Capacity } \\
\mathbf{( s f t}^{3} \text { ) }\end{array}$ & $\begin{array}{c}\text { Available Volume to } \\
\mathbf{1 2 0 0} \text { psig } \mathbf{( s f t}^{3} \text { ) }\end{array}$ \\
\hline 2200 & 55,000 & 22,917 \\
\hline 2100 & 52,708 & 20,625 \\
\hline 2000 & 50,417 & 18,333 \\
\hline 1900 & 48,125 & 16,042 \\
\hline 1800 & 45,833 & 13,750 \\
\hline 1700 & 43,542 & 11,458 \\
\hline 1600 & 41,250 & 9,167 \\
\hline 1500 & 38,958 & 6,875 \\
\hline 1400 & 36,667 & 4,583 \\
\hline 1300 & 35,521 & 2,292 \\
\hline 1200 & 34,375 & 0 \\
\hline
\end{tabular}

1.2.2.1 Check and shut N2-V-7, N2-V-6, N2-V-4, N2-V-5, N2-V-2, N2-V-3, N2-V-8, N2-V-18/H2-V-5, H2-V-8, H2-V-9, H2-V-10/N2-V-16, CF-V-1, CF-SV-1, CF-SV-3, CF-SV-4/R-V-2, R-V-4/R-V-5, R-SV-7, R-SV8/CON-V-3, CON-V-4, CON-V-20/SG-V-21, SG-V-22, and SG-V-3.

1.2.2.2 Check and shut SG-PCV-1, R-AOV-2, and R-PCV-30.

1.2.2.3 Check and open H2-V-1, H2-V-6, H2-V-7, H2-V-3, H2-V-4/CF-SV2/R-V-1/CON-V-1, CON-V-2/SG-V-1, and SG-V-2.

1.2.2.4 Check and open H2-PCV-1, H2-FCV-1, H2-AOV-1, H2-PCV-2/H2FCV-2, H2-AOV-2, H2-MFC-1, H2-AOV-20/R-AOV-3, R-PCV-1, RMFC-1, and R-AOV-4/R-AOV-1/CON-AOV-1.

1.2.2.5 Set N2-PCV-1 secondary pressure at 1150 psig.

1.2.2.6 Set SG-PCV-1 setpoint at 400 (800, and 1000) psig and switch from manual control mode to auto control mode.

1.2.2.7 In half turn increments, open N2-V-3 and N2-V-7 so that they are fully open. Watch the pressure at H2-PT-1 and R-PT-2 and pressurize the system to 400 psig (800 and 1000) at SG-PT-1 and R-PT-30. 
1.2.2.8 In order to keep same pressurizing speed between R-PT-3 and R-PT30 , watch the time-history graph for both pressures, and adjust the opening of H2-PCV-1. (As the annulus volume is about three times larger than coal hopper plus the reactor tube plus char pot, the speed of pressurizing annulus will be slower.) The pressure difference must be kept within 50 psig.

1.2.2.9 When SG-PT-1 and R-PT-30 reach about 400 psig (800 and 1000), shut N2-V-3 and N2-V-7.

1.2.2.10 Perform leak test using liquid leak detector on flanges, joints, and the parts where was dismantled after the previous run for sampling and maintenance.

1. If a leakage is found out, try to tighten in accordance with the designed torque.

2. If the leakage is not stopped, open SG-PCV-1 to depressurize the system to ambient pressure and shut SG-PCV-1. (If the depressurizing speed is too slow, use SG-V-21 and SG-V-22 to bleed the pressure.)

3. Exchange the gasket and perform the following necessary actions:

a. Perform the $\mathrm{N}_{2}$ purge using subsection 2.2 steps for the $\mathrm{N}_{2}$ valve and vent valve, which are near the dismantled flange or joint.

b. Pressurize the plant in accordance with the subsection 1.2.2.7.

c. Make sure no leakage.

1.2.2.11 Repeat steps 1.2.2.6 through 1.2.2.10 at 800 psig for the second leak test, then repeat steps 1.2.2.6 through 1.2.2.10 at 1000 psig for the final leak test to make sure that there are no leaks in the system.

1.2.2.12 Make sure that there is no leaking. Check the liquid leak detector at 1000 psig, shut H2-V-1 and SG-V-1, and keep the plant at 1000 psig for 15 minutes.

1.2.2.13 Verify that the pressure did not change at SG-PT-1 and R-PT-30.

1.2.2.14 After the leak test was completed, check and shut H2-V-1, H2-PCV-1, H2-FCV-1, H2-PCV-2, H2-FCV-2, H2-MFC-1, R-PCV-1, R-MFC-1, (SG-V-21, SG-V-22), and open SG-V-1.

\subsubsection{Flow Control Functional Check}

1.2.3.1 Initial Condition: The plant pressure is kept at about 1000 psig after the leak test.

This functional check uses $\mathrm{N}_{2}$ to simulate $\mathrm{H}_{2}$ flow for tuning and better control of the pressure control valves (PCVs) and function control valves (FCVs). 
Follow these guidelines below to perform the flow control functional check:

\begin{tabular}{|c|c|c|c|c|c|c|c|c|}
\hline \multicolumn{4}{|c|}{ Simulating } & \multicolumn{3}{c|}{$\mathbf{N}_{2}$ flow $\left(\mathbf{s f t}^{3} / \mathbf{h}\right)$} & $\begin{array}{c}\text { Operation } \\
(\mathbf{h r})\end{array}$ & $\begin{array}{c}\text { Consumption } \\
\left.\mathbf{( s f t}^{3}\right)\end{array}$ \\
\hline $\mathrm{H}_{2} / \mathbf{C}$ & GRT & $\mathbf{H}_{2}$ main & $\mathbf{H}_{2}$ Carrier & Main & Carrier & Annulus & 4220 \\
\hline 0.4 & $10 \mathrm{sec}$ & $822 \mathrm{sft} 3 / \mathrm{h}$ & $91 \mathrm{stt}^{3} / \mathrm{h}$ & $220^{\mathrm{a}}$ & $24^{\mathrm{b}}$ & 600 & as 5.0 & 4 \\
\hline
\end{tabular}

1.2.3.2 Confirm $\mathrm{N}_{2}$ tube trailer has more than 1500 psig pressure.

1.2.3.3 Set N2-PCV-1 at 1150 psig.

1.2.3.4 Make trip bypass OFF on IN2-PI-1 $1_{\mathrm{LL}}$.

1.2.3.5 Check and open H2-V-3, H2-V-4, H2-V-6, H2-V-7, R-V-1, CON-V-1, and CON-V-2/SG-V-2.

1.2.3.6 Check and open H2-AOV-1, R-AOV-4, R-AOV-1, and CON-AOV-1.

1.2.3.7 Check and shut N2-V-7 and N2-V-3.

1.2.3.8 Check and shut H2-PCV-1, H2-FCV-1, H2-PCV-2, H2-FCV-2, H2AOV-2, H2-MFC-1, H2-AOV-20, R-PCV-1, R-MFC-1, and R-PCV-30.

1.2.3.9 Set SG-PIC-1 at $(\langle\mathrm{R}-\mathrm{PT}-3\rangle+10 \mathrm{psig})$, and switch from manual control mode to auto control mode.

1.2.3.10 In half turn increments, open N2-V-7 so that it is fully open.

1.2.3.11 Open R-PCV-1 in 1\% opening increments every 10 seconds until RPIC-31 indicates the same pressure as N2-PCV-1 secondary pressure.

1.2.3.12 Set R-MFC-1 setpoint at $10 \mathrm{sft}^{3} / \mathrm{h}$ and increase the setpoint $20 \mathrm{stt}^{3} / \mathrm{h}$ increments every 10 seconds to achieve $100 \mathrm{stt}^{3} / \mathrm{h}$.

1.2.3.13 Select $\mathrm{N}_{2}$ as fluid on $\mathrm{H} 2-\mathrm{FM}-1$ (Micro Motion flow meter).

1.2.3.14 In half turn increments, open N2-V-3 so that it is fully open.

1.2.3.15 Open $\mathrm{H} 2-\mathrm{PCV}-1$ in $1 \%$ opening increments every 10 seconds until $\mathrm{H} 2-\mathrm{PIC}-1$ indicates the same pressure as N2-PCV-1 secondary pressure.

1.2.3.16 Select flow indicator for $\mathrm{N}_{2}$ on $\mathrm{H} 2$-FIC-1.

1.2.3.17 Open $\mathrm{H} 2-\mathrm{FCV}-1$ in $1 \%$ opening increments every 10 seconds until you see the flow on the flow indicator ( $\mathrm{H} 2-\mathrm{FIC}-1)$ and verify that $\mathrm{N}_{2}$ starts to flow.

1.2.3.18 Set H2-FIC-1 setpoint at the flow rate on the indicator and switch from manual control mode to auto control mode.

1.2.3.19 Set SG-PCV-1 setpoint at 1000 psig.

1.2.3.20 Increase R-MFC-1 flow rate to $600 \mathrm{sft}^{3} / \mathrm{h}$ by changing the flow setpoint.

1.2.3.21 Increase H2-FIC-1 flow rate to $220 \mathrm{sft}^{3} / \mathrm{h}$ by changing the H2-FIC-1 setpoint. 
1.2.3.22 Adjust the opening of H2-PCV-1 and R-PCV-1 so as to achieve an appropriate opening on $\mathrm{H} 2-\mathrm{FCV}-1$ (about 65\%) and R-MFC-1. Record the openings of H2-PCV-1 and R-PCV-1 and use the instructions contained in the Coal to SNG Bench Scale Test Reactor Startup Procedure.

1.2.3.23 Check and open H2-V-6 and CF-SV-2.

1.2.3.24 Select $\mathrm{N}_{2}$ as fluid on $\mathrm{H} 2-\mathrm{FM}-2$ (Micro Motion flow meter).

1.2.3.25 Select flow Indicator for $\mathrm{N}_{2}$ on $\mathrm{H} 2-\mathrm{FIC}-2$.

1.2.3.26 Open H2-AOV-2 and H2-AOV-2O.

1.2.3.27 Open $\mathrm{H} 2-\mathrm{PCV}-2$ in $1 \%$ opening increments every 10 seconds until $\mathrm{H} 2-\mathrm{PIC}-4$ indicates the same pressure as N2-PCV-1's secondary pressure.

1.2.3.28 Check and open R-AOV-3.

1.2.3.29 Open $\mathrm{H} 2-\mathrm{FCV}-2$ in $1 \%$ opening increments until you see the flow on the flow indicator and verify that $\mathrm{N}_{2}$ starts to flow. If $\mathrm{H} 2-\mathrm{PIC}-4$ reduces rapidly, open $\mathrm{H} 2-\mathrm{PCV}-2$ to achieve stable flow rate.

1.2.3.30 Set H2-FCV-2 setpoint at the flow rate on the indicator and switch from manual control mode to auto control mode.

1.2.3.31 Increase $\mathrm{H} 2-\mathrm{FIC}-2$ to $24 \mathrm{stt}^{3} / \mathrm{h}$ by changing the setpoint. If the opening of $\mathrm{H} 2-\mathrm{FCV}-2$ fully opens, open $\mathrm{H} 2-\mathrm{PCV}-2$ to achieve appropriate opening on $\mathrm{H} 2-\mathrm{FCV}-2$.

1.2.3.32 Open H2-AOV-20.

1.2.3.33 Open $\mathrm{H} 2-\mathrm{MFC}-1$ by manual in 1\% opening increments until you see the flow on the flow indicator. If the coal feeder MAG drive seal is perfect, gas does not flow. In this case, keep H2-MFC-1 open at 50\%.

1.2.3.34 If a flow is indicated, try to set H2-MFC-1 setpoint at the flow rate on the indicator and switch from manual control mode to auto control mode.

1.2.3.35 If the flow increases with the opening, set H2-MFC-1 setpoint to achieve minimum and stable flow rate on $\mathrm{H} 2-\mathrm{MFC}-1$.

1.2.3.36 Check the stability of all auto control loop by observing the time history trend graph on the control screen.

1.2.3.37 If there is unstable control loop, turn on PID on the control loop in accordance with the Control Engineer's instruction.

\subsubsection{Temporal Plant Shutdown}

In order to make the flow meter calibration test continuously in next day, the plant will be kept at high-pressure $\mathrm{N}_{2}$.

1.2.4.1 Using manual control mode, manually shut H2-PCV-1, H2-PCV-2, and R-PCV-1.

1.2.4.2 Using manual control mode, manually shut N2-V-3 and N2-V-7.

1.2.4.3 Using manual control mode, manually shut SG-PCV-1.

${ }^{\circ} 2010$ Arizona Public Service All Rights Reserved 
1.2.4.4 Using manual control mode, manually shut SG-V-1 and keep the plant pressure at 1000 psig.

1.2.5 Flow Meter Calibration for R-MFC-1, H2-FCV-1 $\left(\mathrm{N}_{2}\right)$, and N2-RO-1

Initial Condition: The plant pressure is kept at about 1000 psig after the previous test.

This test is to check flow meters and make calibration using SG-FM-1.

1.2.5.1 For R-MFC-1 flow meter calibration, follow the following steps:

1. Confirm $\mathrm{N}_{2}$ tube trailer has more than 1500 psig pressure.

2. Confirm N2-PCV-1 setpoint at 1150 psig.

3. Check and open H2-AOV-1, R-AOV-4, R-AOV-1, and CON-AOV-1.

4. Check and shut N2-V-7 and N2-V-3.

5. Check and shut H2-PCV-1, H2-FCV-1, H2-PCV-2, H2-FCV-2, H2AOV-2, H2-MFC-1, H2-AOV-20, R-PCV-1, R-MFC-1, and R-PCV30.

6. Open SG-V-1.

7. Set SG-PIC-1 at R-PT-3 +10 psig and set R-MFC-1 to auto control mode for calibration.

8. In half turn increments, open N2-V-7 so that it is fully open.

9. Open R-PCV-1 in $1 \%$ opening increments every 10 seconds until R-PIC-31 indicates the same pressure as N2-PCV-1's secondary pressure.

10. Set R-MFC-1 setpoint at $10 \mathrm{stt}^{3} / \mathrm{h}$ and increase the setpoint 20 $\mathrm{stt}^{3} / \mathrm{h}$ incrementally every 10 seconds to achieve $100 \mathrm{stt}^{3} / \mathrm{h}$.

11. Set SG-PCV-1 setpoint at 1015 psig.

12. Increase R-MFC-1 flow rate to $600 \mathrm{sft}^{3} / \mathrm{h}$ by changing the flow setpoint.

13. Adjust R-PCV-1 opening (R-PT-2 pressure) to obtain stable control on R-MFC-1.

14. Measure and record the flow rate on SG-FM-1 at 600, 400, 200 $\mathrm{stt}^{3} / \mathrm{h}$ on R-MFC-1

1.2.5.2 For $\mathrm{H} 2-\mathrm{FCV}-1\left(\mathrm{~N}_{2}\right)$ flow meter calibration, follow these steps:

1. Select $\mathrm{N}_{2}$ as fluid on $\mathrm{H} 2-\mathrm{FM}-1$ (Micro Motion flow meter).

2. In half turn increments, open N2-V-3 so that it is fully open.

3. Open $\mathrm{H} 2-\mathrm{PCV}-1$ in $1 \%$ opening increments every 10 seconds until $\mathrm{H} 2-\mathrm{PIC}-1$ indicates the same pressure as N2-PCV-1 secondary pressure.

4. Select flow Indicator for $\mathrm{N}_{2}$ on $\mathrm{H} 2-\mathrm{FIC}-1$.

${ }^{\circ} 2010$ Arizona Public Service All Rights Reserved 
5. Open $\mathrm{H} 2-\mathrm{FCV}-1$ in $1 \%$ opening increments every 10 seconds until you see the flow on the flow indicator $\mathrm{H} 2-\mathrm{FIC}-1$ and verify that $\mathrm{N}_{2}$ starts to flow.

6. Set H2-FIC-1 setpoint at the flow rate on the indicator and switch from manual control mode to auto control mode.

7. Set SG-PCV-1 setpoint at 1000 psig.

8. Using manual control mode, manually shut R-PCV-1 and R-MFC-1.

9. Using manual control mode, manually shut N2-V-7.

10. Increase $\mathrm{H} 2-\mathrm{FIC}-1$ flow rate to $220 \mathrm{sft}^{3} / \mathrm{h}$ by changing the $\mathrm{H} 2-\mathrm{FIC}-1$ setpoint.

11. Adjust the opening of $\mathrm{H} 2-\mathrm{PCV}-1$ to achieve a stable control on $\mathrm{H} 2$ FCV-1.

12. Measure and record the flow rate on SG-FM-1 at 220, 150, and $100 \mathrm{stt}^{3} / \mathrm{h}$ on $\mathrm{H} 2-\mathrm{FM}-1$.

1.2.5.3 For N2-RO-1, flow meter calibration, follow these steps:

1. Using manual control mode, manually shut H2-PCV-1 and H2FCV-1.

2. Using manual control mode, manually shut N2-V-3.

3. Connect the $\mathrm{N}_{2}$ tube trailer to the inlet of emergency $\mathrm{N}_{2}$ line.

4. Set N2-PCV-2 at 1150 psig.

5. Set N2-PIC-40 at R-PT-5 - 10 psig and switch from manual control mode to auto control mode.

6. Input the trip signal intentionally as directed by Control Engineer so that N2-AOV-40 works as pressure control valve.

7. Measure the flow rates at N2-PIC-40 three setpoints (R-PT- $5+30$, +50 , and +70 psig) and find out the pressure difference through N2-RO-1 to achieve about $1000 \mathrm{sft}^{3} / \mathrm{h}$.

8. Switch from auto control mode to manual control mode and manually shut N2-AOV-40.

9. Manually shut N2-PCV-2 regulator and disconnect $\mathrm{N}_{2}$ tube trailer from the inlet of emergency $\mathrm{N}_{2}$ line.

\subsubsection{Plant Depressurizing}

When the flow meter calibration is completed, start plant depressurization. Follow these steps:

1.2.6.1 Set SG-PCV-1 program controller at 5 psig as the target pressure and 15 psig per minute as the ramp down speed.

1.2.6.2 Start the ramp down of plant pressure.

1.2.6.3 Shut N2-V-3 and N2-V-7.

1.2.6.4 Open by manual control mode H2-PCV-1, H2-PCV-2, and R-PCV-1. 
1.2.6.5 Watch the time history trend graph on H2-PIC-1, H2-PIC-4, N2-PT-16, R-PT-30, R-PT-3, and SG-PT-1.

1.2.6.6 If R-dPT-1 is going to go over 10 psig, slow down the ramp down speed so as to get R-dPT-1 within 10 psig. (Note: Remember to record the ramp down speed.)

1.2.6.7 When SG-PT-1 reaches 5 psig, shut by manual control mode SGPCV-1.

1.2.6.8 Keep the plant pressure at 5 psig.

\subsection{Pressure Balancing System Validation Using Nitrogen $\left(\mathrm{N}_{2}\right)$}

Install R-CV-31 in accordance with piping and instrumentation diagram (P\&ID).

\subsubsection{Leak Test}

Conduct the leak test following the subsection 1.2.2.

\subsubsection{Establish Controlled Nitrogen Flow}

Use these guidelines to establish the controlled nitrogen flow:

\begin{tabular}{|c|c|c|c|c|c|c|c|c|}
\hline \multicolumn{4}{|c|}{ Simulating } & \multicolumn{3}{|c|}{$\mathrm{N}_{2}$ flow $\left(\mathrm{sft}^{3} / \mathrm{h}\right)^{\mathrm{a}}$} & \multirow{2}{*}{$\begin{array}{l}\text { Operation } \\
\text { (hr) }\end{array}$} & \multirow{2}{*}{$\begin{array}{c}\text { Consumption } \\
\left(\mathrm{sft}^{3}\right)\end{array}$} \\
\hline $\mathrm{H}_{2} / \mathrm{C}$ & GRT & $\mathrm{H}_{2}$ main & $\mathrm{H}_{2}$ carrier & Main & Carrier & Annulus & & \\
\hline 0.4 & $10 \mathrm{sec}$ & $822 \mathrm{stt} 3 / \mathrm{h}$ & $91 \mathrm{stt}^{3} / \mathrm{h}$ & $220^{b}$ & $-c$ & 600 & as 5.0 & 4100 \\
\hline
\end{tabular}

1.3.2.1 Confirm that $\mathrm{N}_{2}$ trailer has 1600 psig pressure.

1.3.2.2 Set N2-PCV-1 at 1150 psig.

1.3.2.3 Check and open H2-V-3, H2-V-4, H2-V-6, H2-V-7, R-V-1, CON-V-1, CON-V-2, and SG-V-2.

1.3.2.4 Check and open H2-AOV-1, R-AOV-4, R-AOV-1, and CON-AOV-1.

1.3.2.5 Check and shut N2-V-7 and N2-V-3.

1.3.2.6 Check and shut H2-PCV-1, H2-FCV-1, H2-PCV-2, H2-FCV-2, H2AOV-2, H2-MFC-1, H2-AOV-20, R-PCV-1, R-MFC-1, and R-PCV-30.

1.3.2.7 Set SG-PCV-1 at $\langle\mathrm{R}-\mathrm{PI}-3\rangle+10 \mathrm{psig}$, and switch from manual control mode to auto control mode.

1.3.2.8 In half turn increments, open N2-V-7 so that it is fully open.

1.3.2.9 Open R-PCV-1 in 1\% opening increments every 10 seconds until RPIC-31 indicates the same pressure as N2-PCV-1's secondary pressure.

1.3.2.10 Set R-MFC-1 setpoint at $10 \mathrm{stt}^{3} / \mathrm{h}$ and increase the setpoint $20 \mathrm{st}^{3} / \mathrm{h}$ incremently every 10 seconds to achieve $100 \mathrm{stt}^{3} / \mathrm{h}$.

1.3.2.11 Select $\mathrm{N}_{2}$ as fluid on $\mathrm{H} 2-\mathrm{FM}-1$ (Micro Motion flow meter). 
1.3.2.12 In half turn increments, open N2-V-3 so that it is fully open.

1.3.2.13 Open H2-PCV-1 in 1\% opening increments every 10 seconds until $\mathrm{H} 2-\mathrm{PIC}-1$ indicates the same pressure as N2-PCV-1 secondary pressure.

1.3.2.14 Select flow Indicator for $\mathrm{N}_{2}$ on $\mathrm{H} 2-\mathrm{FIC}-1$.

1.3.2.15 Open $\mathrm{H} 2-\mathrm{FCV}-1$ in $1 \%$ opening increments every 10 seconds until you see the flow on the flow indicator $\mathrm{H} 2-\mathrm{FIC}-1$ and verify that $\mathrm{N}_{2}$ starts to flow.

1.3.2.16 Set H2-FIC-1 setpoint at the flow rate on the indicator and switch from manual control mode to auto control mode.

1.3.2.17 Set SG-PCV-1 setpoint at 1000 psig.

1.3.2.18 Increase R-MFC-1 flow rate to $350 \mathrm{stt}^{3} / \mathrm{h}$ by changing the flow setpoint.

1.3.2.19 Increase $\mathrm{H} 2-\mathrm{FIC}-1$ flow rate to $220 \mathrm{sft}^{3} / \mathrm{h}$ by changing the setpoint.

1.3.2.20 Adjust the opening of H2-PCV-1 and R-PCV-1 to achieve about $65 \%$ (an appropriate) opening on H2-FCV-1 and R-MFC-1.

1.3.2.21 Confirm proper responses for all pressure and flow instruments.

1.3.2.22 If there is unstable control loop, turn on PID on the control loop as directed by Control Engineer.

\subsubsection{Balancing System Control Functional Check for Emergency Shutdown (ESD)}

This test is to select a best sequence from three options to balance and perform ESD. Follow these steps:

1.3.3.1 Establish pressure balancing system.

1.3.3.2 Confirm that the auto control R-MFC-1 flow rate is stable.

1.3.3.3 Set R-PCV-30 setpoint at 1000 psig and switch from manual control mode to auto control mode.

1.3.3.4 Confirm proper response for R-PCV-30.

1.3.3.5 Shut R-AOV-4.

1.3.3.6 Confirm proper response for R-PCV-30.

1.3.3.7 Increase R-MFC-1 flow rate to $600 \mathrm{stt}^{3} / \mathrm{h}$ by changing the flow setpoint.

1.3.3.8 Set differential pressure $(\mathrm{dP})$ setpoint of program controller R-PT-30 = P-PT-3 + dP 0 psig and turn on the program controller.

1.3.3.9 Increase the dP setpoint in 3 psig increments to 15 psig to achieve RPT-30 at 1015 psig.

1.3.3.10 Confirm proper response for all pressure and flow instruments.

\subsubsection{Trip Sequence Test 1}

For the Trip Sequence Test 1 make sure of the following: (1) R-AOV-4 is open; and (2) change R-PCV-30 program controller dP setpoint to 0 psig. Other steps are as follows: 
1.3.4.1 Verify the emergency purge N2 Pak is full (at 2400 psig).

1.3.4.2 Set N2-PIC 40 setpoint at 1040 psig.

1.3.4.3 Confirm the Trip Sequence Test 1 is programmed in the Lab View Program.

1.3.4.4 Make the grouping of N2-PIC-40, H2-PT-5, R-PIC-31, R-PIC-30, RdPT-1, R-PT-3, and SG-PIC-1 on the time history trend graph on the control screen.

1.3.4.5 Activate the trip system by hitting manual trip button.

1.3.4.6 Confirm that H2-AOV-1 is shut, and R-AOV-4 and N2-AOV-40 are open.

1.3.4.7 Verify the proper responses for SG-PCV-1 and R-PCV-30.

1.3.4.8 Manually shut H2-FCV-1.

1.3.4.9 When R-PT-3 and R-PT-30 become stable, measure the flow rate at SG-FM-1 and estimate how the annulus purge $\mathrm{N}_{2}$ is divided and flowing to R-PCV-30 and SG-PCV-1.

1.3.4.10 When the flow measurement is finished, the test is finished.

1.3.4.11 Verify that all pressure and flow to R-MFC-1 is normal.

1.3.4.12 Push trip reset button on the Trip Bypass Screen.

1.3.4.13 Verify that H2-PT-1 is about same as the secondary pressure of H2-PCV-30.

1.3.4.14 Open H2-AOV-1 and shut N2-AOV-40.

1.3.4.15 Open $\mathrm{H} 2-\mathrm{FCV}-1$ in $1 \%$ opening increments every 10 seconds until you see the flow on the flow indicator and verify that $\mathrm{N}_{2}$ starts to flow.

1.3.4.16 Increase H2-FIC-1 flow rate to $220 \mathrm{stt}^{3} / \mathrm{h}$ by changing the flow setpoint.

1.3.4.17 Determine the proper responses for all pressure and flow instruments.

\subsubsection{Trip Sequence Test 2}

For Trip Sequence Test 2, perform the following steps: (1) change R-PCV-30 program controller dP setpoint to 0 psig; and (2) keep the R-AOV-4 shut.

1.3.5.1 Control Engineer changes the trip sequence to Test 2 action.

1.3.5.2 Change the emergency purge $\mathrm{N}_{2}$ Pak to a new full Pak.

1.3.5.3 Verify that N2-PIC 40 setpoint is at 1040 psig.

1.3.5.4 Using auto control mode, verify that R-MFC-1 flow rate is stable.

1.3.5.5 Shut R-AOV-4.

1.3.5.6 Confirm proper responses for R-PCV-30 and SG-PCV-1.

1.3.5.7 Set dP setpoint of program controller R-PT-30 = R-PT-3+dP 0 psig and turn on the R-PT-30 program controller.

1.3.5.8 Increase the $\mathrm{dP}$ setpoint in 3 psig increments to 15 psig to achieve 1015 psig at R-PI-30. 
1.3.5.9 Determine the proper responses for all pressure and flow instruments.

1.3.5.10 Confirm the grouping of N2-PIC-40, H2-PT-5, R-PIC-31, R-PIC-30, RdPT-1, R-PT-3, and SG-PIC-1 on the time history trend graph on the screen.

1.3.5.11 Activate the trip system by hitting manual trip button.

1.3.5.12 Confirm that H2-AOV-1 is shut and N2-AOV-40 is open.

1.3.5.13 Carefully observe the pressure changes on R-PT-30 and R-PT-3.

1.3.5.14 Verify the proper responses for SG-PCV-1 and R-PCV-30.

1.3.5.15 Using manual control mode, shut $\mathrm{H} 2-\mathrm{FCV}-1$.

1.3.5.16 When R-PT-3 and R-PT-30 become stable, the test is finished.

1.3.5.17 Verify that all pressures and R-MFC-1 flow are normal.

1.3.5.18 Push trip reset button on Trip Bypass Screen.

1.3.5.19 Open H2-AOV-1 and shut N2-AOV-40.

1.3.5.20 Open $\mathrm{H} 2-\mathrm{FCV}-1$ in $1 \%$ opening increments every 10 seconds until you see the flow on the flow indicator and verify the flow rate.

1.3.5.21 Increase H2-FCV-1 (H2-FM-1) flow rate to $220 \mathrm{stt}^{3} / \mathrm{h}$ by changing the flow setpoint.

1.3.5.22 Verify the proper responses for all pressure and flow instruments.

\subsubsection{Trip Sequence Test 3}

For Trip Sequence Test 3, the following steps must be performed: (1) open RAOV-4; (2) change R-PCV-30 program controller dP setpoint to 0 psig; and (3) shut R-AOV-4.

1.3.6.1 As directed by Control Engineer, change the trip sequence to Test 3 action.

1.3.6.2 Change the emergency purge $\mathrm{N}_{2}$ Pak to a new full Pak.

1.3.6.3 Verify that the N2-PIC 40 setpoint is set at 1040 psig.

1.3.6.4 Verify that the R-MFC-1 flow rate is stable.

1.3.6.5 Verify that R-PCV-30 setpoint at 1000 psig and switch from manual control mode to auto control mode.

1.3.6.6 Shut R-AOV-4.

1.3.6.7 Verify the proper responses for R-PCV-30 and SG-PCV-1.

1.3.6.8 Set dP setpoint of program controller R-PT-30 so that it equals R-PT$3+d P 0$ psig, and turn on the R-PT-30 program controller.

1.3.6.9 Increase the $\mathrm{dP}$ setpoint in 3 psig increments to 15 psig to achieve 1015 psig for R-PT-30.

1.3.6.10 Confirm the proper responses for all pressure and flow instruments. 
1.3.6.11 Confirm the grouping of N2-PIC-40, H2-PT-5, R-PIC-31, R-PIC-30, RdPT-1, R-PT-3, and SG-PIC-1 on the time history trend graph on the screen.

1.3.6.12 Activate the trip system by turning on the manual trip button.

1.3.6.13 Confirm that H2-AOV-1 is open, R-AOV-4 is shut, and N2-AOV-40 is open.

1.3.6.14 Carefully observe the pressure changes of R-PT-30 and R-PT-3

1.3.6.15 Verify proper response for SG-PCV-1 and R-PCV-30.

1.3.6.16 Using manual control mode, shut H2-FCV-1.

1.3.6.17 When R-PT-3 and R-PT-30 become stable, the test is finished.

1.3.6.18 Verify that all pressures and R-MFC-1 flow are normal.

1.3.6.19 Push trip reset button on Trip Bypass Screen.

1.3.6.20 Open H2-AOV-1 and shut N2-AOV-40.

1.3.6.21 Open R-AOV-4.

1.3.6.22 Using manual control mode, shut R-PCV-30; watch SG-PI-1 to make sure that there is no more than 5 psig pressure change.

1.3.6.23 Open $\mathrm{H} 2-\mathrm{FCV}-1$ in $1 \%$ opening increments every 10 seconds until you see the flow on the flow indicator and verify the flow rate.

1.3.6.24 Set SG-PCV-1 ramp down program controller at 5 psig as the target pressure and 15 psig per minute as the ramp down speed.

1.3.6.25 Start the ramp down of the plant's pressure.

1.3.6.26 Shut N2-V-3 and N2-V-7.

1.3.6.27 When SG-PT-1 reaches 5 psig, use manual control mode to shut SGPCV-1.

1.3.6.28 Set all valves in accordance with Appendix 1 in the normal shutdown line up.

\section{HOT REACTOR TEST}

\subsection{Heater Conditioning}

\subsubsection{Establish Controlled Nitrogen and Air Flow}

2.1.1.1 Connect Air 12 Pak to R-V-2.

2.1.1.2 Set Air 12 Pak regulator secondary pressure at 1200 psig.

2.1.1.3 Install needle valve temporally at the position of R-F-11.

2.1.1.4 Check and shut N2-V-7, N2-V-6, N2-V-4, N2-V-5, N2-V-2, N2-V-3, N2-V-8, N2-V-18, H2-V-1, H2-V-5, H2-V-6, H2-V-7, H2-V-8, H2-V-9, H2-V-10, N2-V-16, CF-V-1, CF-SV-1, CF-SV-2, CF-SV-3, CF-SV-4, R-AOV-3/R-V-2, R-V-4, R-V-5, R-SV-7, R-SV-8/CON-V-3, CON-V-4, CON-V-20, SG-V-21, SG-V-22, and SG-V-3. 
2.1.1.5 Check valves R-AOV-2, R-AOV-4, and R-PCV-30, and using manual control mode shut R-AOV-2, R-AOV-4, and R-PCV-30.

2.1.1.6 Check and open H2-V-3, H2-V-4/R-V-1, R-V-4/CON-V-1, CON-V2/SG-V-1, and SG-V-2.

2.1.1.7 Take out the plug on the end of R-V-4 tube.

2.1.1.8 Using manual control mode, open valves H2-AOV-1 and SG-PCV-1 all the way (100\%).

2.1.1.9 In half turn increments, open N2-V-7 so that it is fully opened.

2.1.1.10 Open R-PCV-1 in 1\% opening increments every 10 seconds until RPIC-31 indicates the same pressure as the secondary pressure Air 12 Pak Regulator.

2.1.1.11 Set R-MFC-1 setpoint at $10 \mathrm{stt}^{3} / \mathrm{h}$ and increase the setpoint $20 \mathrm{stt}^{3} / \mathrm{h}$ incremently every 10 seconds to achieve $100 \mathrm{stt}^{3} / \mathrm{h}$.

2.1.1.12 Select $\mathrm{N}_{2}$ as fluid on $\mathrm{H} 2-\mathrm{FM}-1$ (Micro Motion flow meter).

2.1.1.13 In half turn increments, open N2-V-3 so that it is fully open.

2.1.1.14 Open $\mathrm{H} 2-\mathrm{PCV}-1$ in $1 \%$ opening increments every 10 seconds until $\mathrm{H} 2-\mathrm{PIC}-1$ indicates the same pressure as N2-PCV-1 secondary pressure.

2.1.1.15 Select flow indicator $\mathrm{H} 2-\mathrm{FIC}-1$ for $\mathrm{N}_{2}$.

2.1.1.16 Open $\mathrm{H} 2-\mathrm{FCV}-1$ in $1 \%$ opening increments every 10 seconds until you see the flow on the flow indicator $\mathrm{H} 2-\mathrm{FIC}-1$, and verify that $\mathrm{N}_{2}$ starts to flow.

2.1.1.17 Set H2-FIC-1 setpoint at the flow rate on the indicator and set it on auto control mode.

2.1.1.18 Increase R-MFC-1 flow rate to $600 \mathrm{stt}^{3} / \mathrm{h}$ by changing the flow setpoint.

2.1.1.19 Increase H2-FIC-1 flow rate to $220 \mathrm{sft}^{3} / \mathrm{h}$ by changing the setpoint.

2.1.1.20 The reactor tube pressure R-PT-3 and annular space pressure $\mathrm{R}-\mathrm{PT}-30$ would be resigned to the situation (close to ambient pressure), but the R-dPT-1 has to be kept lower than 50 pounds per square inch (psi) under high temperature. If the R-dPT-1 cannot go over 50 psi (increasing both flow rates), the test manager has to decide to whether he needs to change either pressures or flow rate. 


\subsubsection{Heater Conditioning}

2.1.2.1 Verify that the power supply system is ready.

2.1.2.2 Confirm that the alarm setpoint on reactor heater's temperature.

2.1.2.3 Verify that all of heaters' switches are off and setpoint of power output is 0 .

2.1.2.4 Verify that all of trip triggers on heaters' temperature monitors have the Trip Bypass ON.

2.1.2.5 Set the Trip Bypass on R-TI-10 ${ }^{\mathrm{HH}}$ to OFF.

2.1.2.6 Check TIC-21,TE-21B-3,TE-21A-2,TE-21B-2,TE-21A-1,TE-21B-41 TE-21B-1, and TE-27A-1 (21 group) indicators on the time history trend graph on the screen.

2.1.2.7 Turn on the Reactor Heater Zone 1 and set TIC-21 to auto control mode.

2.1.2.8 Ramp the heater temperature up to $425^{\circ} \mathrm{F}$ by changing setpoint at $25^{\circ} \mathrm{F}$ increments per minute. Note: This is the actual heating up speed so be careful not to setpoint and change the speed.

2.1.2.9 Observe the air purge exhaust (smoke of binder) and analyze the $\mathrm{N}_{2}$ purge through the reactor tube.

2.1.2.10 Once the binder has out of gas (no visible smoke is coming out), increase $\mathrm{TIC}-21$ to $1600^{\circ} \mathrm{F}$ at $25^{\circ} \mathrm{F}$ increments per minute.

2.1.2.11 Monitor the reactor tube's temperature profile and observe (watch and listen) the reactor see if there is an indication of motion, hot spots, interferences with frame, etc.

2.1.2.12 Keep $1600^{\circ} \mathrm{F}$ for 30 minutes and de-energize the zone and proceed to next zone.

2.1.2.13 Repeat Steps 2.1.2.6 through Step 2.1.2.12 for Zone 2, then each subsequent zone. The Operator may decide to accelerate the conditioning processes by energizing more zones up to $425^{\circ} \mathrm{F}$ based on observing how the reactor responds during the conditioning of the first two zones.

\subsubsection{Plant Shutdown}

2.1.3.1 Continue to keep gas flow through reactor tube and annulus until all temperatures go below $200^{\circ} \mathrm{F}$.

2.1.3.2 Shut Air 12 Pak regulator valve and confirm that the annulus pressure has become ambient pressure.

2.1.3.3 Shut R-V-2 and R-V-4, and install the plug back at the end of R-V-4.

2.1.3.4 Shut N2-V-3 and confirm that the reactor's pressure on R-PT-3 and SG-PT-1 have become ambient pressure.

2.1.3.5 Open R-AOV-4.

2.1.3.6 Using manual control mode, open H2-PCV-1 and H2-FCV-1. 
2.1.3.7 Confirm that N2-PCV-4's secondary pressure setpoint is 200 psig.

2.1.3.8 Shut SG-PCV-1.

2.1.3.9 Open N2-V-6 and pressurize the reactor tube and annulus space to 150 psig.

2.1.3.10 Shut N2-V-6.

2.1.3.11 Open SG-PCV-1 and depressurize the reactor's tube and annulus space to 5 psig.

2.1.3.12 Shut SG-PCV-1.

2.1.3.13 Repeat Steps 2.1.3.9 through 2.1.3.8 two times.

2.1.3.14 Set all valves in accordance with Appendix $D$ the normal shutdown line up.

2.1.3.15 Keep the plant pressure at 5 psig.

\subsection{Hot Reactor Test Using $\mathrm{N}_{2}$}

\subsubsection{Leak Test}

Conduct the leak test following the subsection 1.2.2.

\subsubsection{Establish Controlled Nitrogen Flow}

Initial Condition: The plant pressure is kept at about 1000 psig after the leak test.

2.2.2.1 Confirm that the $\mathrm{N}_{2}$ tube trailer has more than 1500 psig pressure. See items below as reference:

Follow the steps below to perform the flow control functional check:

\begin{tabular}{|c|c|c|c|}
\hline \multicolumn{2}{|c|}{$\mathbf{N}_{2}$ Flow $\left(\mathbf{s f t}^{3} / \mathbf{h}\right)$} & \multirow{2}{*}{$\begin{array}{c}\text { Operation } \\
\text { (hr) }\end{array}$} & $\begin{array}{c}\text { Consumption } \\
\left(\mathbf{s f t}^{3}\right)\end{array}$ \\
\hline Process & Annulus & as 6.0 & 4920 \\
\hline $220^{a}$ & 600 & & 4 \\
\cline { 1 - 2 }
\end{tabular}

a $\mathrm{N}_{2}$ flow is at same FCV opening and upstream pressure as $\mathrm{H}_{2}$; the equivalent flow with high $\mathrm{H}_{2}$ flow is used for this test.

2.2.2.2 Set N2-PCV-1 at 1150 psig.

2.2.2.3 Check and open H2-V-3, H2-V-4, H2-V-6, H2-V-7, R-V-1, CON-V-1, CON-V-2, and SG-V-2.

2.2.2.4 Check and open H2-AOV-1, R-AOV-4, R-AOV-1, and CON-AOV-1.

2.2.2.5 Check and shut N2-V-7 and N2-V-3.

2.2.2.6 Check and shut H2-PCV-1, H2-FCV-1, H2-PCV-2, H2-FCV-2, H2AOV-2, H2-MFC-1, H2-AOV-20, R-AOV-3, R-PCV-1, R-MFC-1, and R-PCV-30.

2.2.2.7 Set SG-PCV-1 at R-PT-3 + 10 psig, and switch from manual control mode to auto control mode.

2.2.2.8 In half turn increments, open N2-V-7 so that it is fully open. 
2.2.2.9 Open R-PCV-1 in 1\% opening increments every 10 seconds until R-PIC-31 indicates the same pressure as N2-PCV-1's secondary pressure.

2.2.2.10 Set R-MFC-1 setpoint at $10 \mathrm{sft}^{3} / \mathrm{h}$ and increase the setpoint in 20 $\mathrm{sft}^{3} / \mathrm{h}$ incremently every 10 seconds to achieve $100 \mathrm{sft}^{3} / \mathrm{h}$.

2.2.2.11 Select $\mathrm{N}_{2}$ as fluid on $\mathrm{H}$ 2-FM-1 (Micro Motion flow meter).

2.2.2.12 In half turn increments, open N2-V-3 so that it is fully open.

2.2.2.13 Open H2-PCV-1 in 1\% opening increments every 10 seconds until $\mathrm{H} 2-\mathrm{PIC}-1$ indicates the same pressure as N2-PCV-1's secondary pressure.

2.2.2.14 Select flow indicator for $\mathrm{N}_{2}$ on $\mathrm{H} 2-\mathrm{FIC}-1$.

2.2.2.15 Open $\mathrm{H} 2-\mathrm{FCV}-1$ in $1 \%$ opening increments every 10 seconds until you see the flow on the flow indicator and verify that $\mathrm{N}_{2}$ starts to flow.

2.2.2.16 Set H2-FIC-1 setpoint at the flow rate on the indicator and switch from manual control mode to auto control mode.

2.2.2.17 Set SG-PCV-1 setpoint at 1000 psig.

2.2.2.18 Increase R-MFC-1 flow rate to $350 \mathrm{stt}^{3} / \mathrm{h}$ by changing the flow setpoint.

2.2.2.19 Increase H2-FCV-1 (H2-FM-1) flow rate to $220 \mathrm{stt}^{3} / \mathrm{h}$ by changing the flow setpoint (the opening will be about $65 \%$ with 90 psi of pressure drop).

2.2.2.20 Adjust the opening of H2-PCV-1 and R-PCV-1 to achieve an appropriate opening on $\mathrm{H} 2-\mathrm{FCV}-1$ and R-MFC-1.

2.2.2.21 Verify the proper responses for all pressure and flow instruments.

2.2.2.22 If there is unstable control loop, make a turning on PID on the control loop in accordance with Control Engineer's advice.

\subsubsection{Establish Pressure Balancing System}

2.2.3.1 Verify that the R-MFC-1 flow rate is stable and switch from manual control mode to auto control mode/

2.2.3.2 Set R-PCV-30 setpoint at 1000 psig and set it on auto control mode.

2.2.3.3 Verify the proper response for R-PCV-30.

2.2.3.4 Shut R-AOV-4.

2.2.3.5 Verify the proper response for R-PCV-30.

2.2.3.6 Increase R-MFC-1 flow rate to $600 \mathrm{stt}^{3} / \mathrm{h}$ by changing the flow setpoint.

2.2.3.7 Set dP setpoint of program controller R-PT-30 equals R-PT-3 + dP 0 psig and turn on the R-PT-30 program controller.

2.2.3.8 Increase the $\mathrm{dP}$ setpoint in 3 psig increments to 15 psig to achieve 1015 psig on R-PT-30.

2.2.3.9 Verify proper responses for all pressure and flow instrument. 


\subsubsection{Turn on Cooling Water}

2.2.4.1 Open $\mathrm{H} 2 \mathrm{O}-\mathrm{V}-1$ and verify that the tank is full.

2.2.4.2 Check and open $\mathrm{H} 2 \mathrm{O}-\mathrm{V}-2,-4,-5,-6,-7$, and -8; check and open $\mathrm{H} 2 \mathrm{O}-$ SV-8 and H2O-SV-2O.

\subsubsection{Turn on H2O-P-1.}

2.2.4.4 Open CON-V-21 and vent the air in the water line, verify that the water is circulating, and shut CON-V-21.

2.2.4.5 Verify that mist to the reactor head and char pot.

2.2.4.6 Verify CON-PI-1 and CON-PI-2 indicate pressure.

2.2.4.7 Set the Trip Bypass on H2O-FSL-2 ON to OFF and H2O-P-1 to OFF.

\subsection{5 $\mathrm{H}_{2}$ Preheater Performance Test}

Confirm the relation between the heater output, heater temperature, and tube temperature depending on gas flow. Refer to Figure 1.

Figure 1 -Preheater 1 through 4 Temperature (degrees F) to Tube Length (inches)

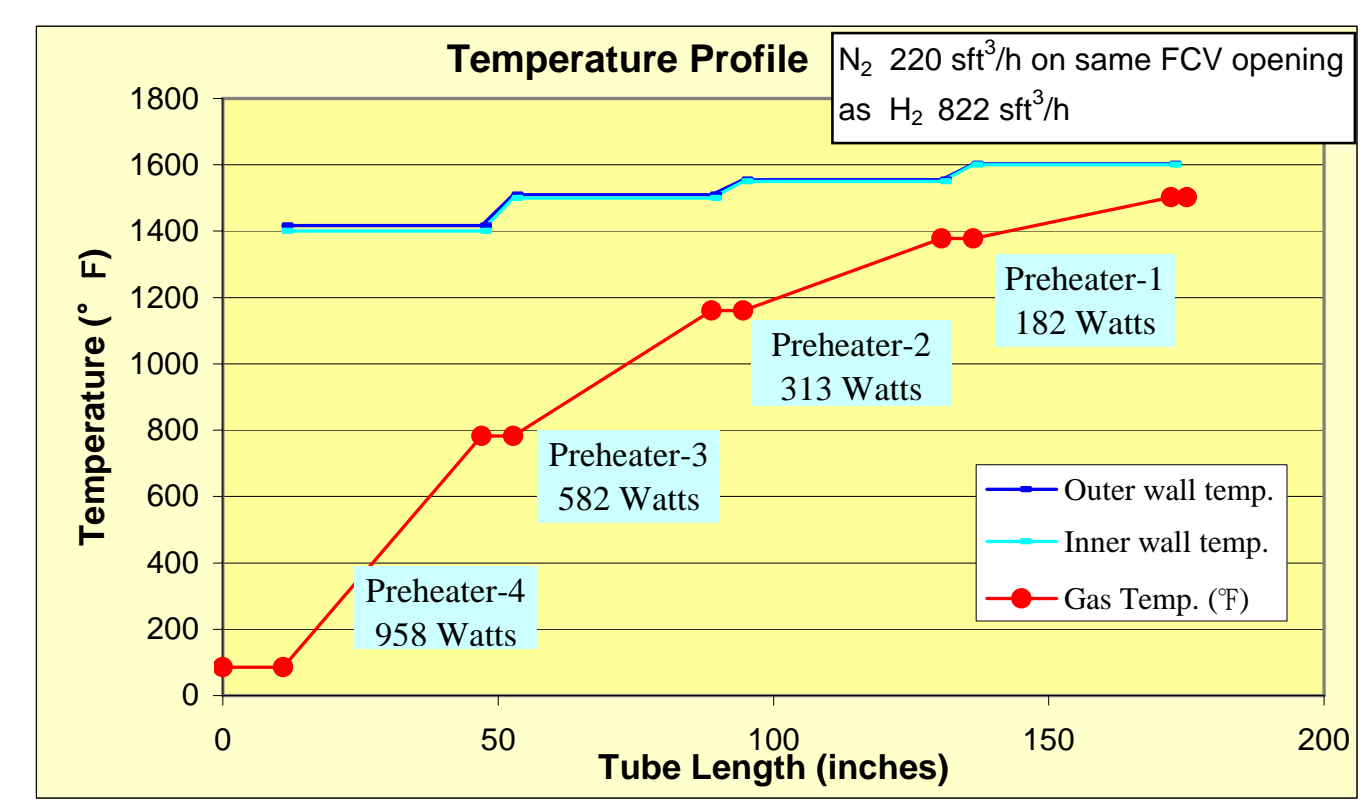

As the tube wall temperature in operation will be very close to the design temperature $\left(1650^{\circ} \mathrm{F}\right)$ especially on Preheater 1 , careful attention should be paid on the wall's temperature.

2.2.5.1 Verify that the power supply system is ready.

2.2.5.2 Confirm the alarm setpoint on all of heaters' related temperatures.

2.2.5.3 Verify that all of heaters are switched off, and setpoint of power output is at 0 .

2.2.5.4 Verify that all of trip triggers on heaters' temperature monitors have the Trip Bypass ON. 
2.2.5.5 Set the Trip Bypass on R-TI-10 ${ }^{\mathrm{HH}}$ to OFF.

2.2.5.6 Make the grouping of all preheaters' tube temperatures on the time history trend graph on the screen.

2.2.5.7 Turn on the $\mathrm{H}_{2}$ Preheaters PH-HTR-1, $-2,-3$, and -4 as well as Heater 5.

2.2.5.8 Set TIC-14A-3 program controller setpoint at $1000^{\circ} \mathrm{F}$ for the target temperature and at $25^{\circ} \mathrm{F}$ every minute as the ramp up speed and turn on the controller.

1. When TIC-14A-3 reaches to the target temperature, turn off the ramp up control and return to auto control mode.

2. Increase the TIC-14A-3 setpoint in $25^{\circ} \mathrm{F}$ increments every 1 to 2 minutes to achieve $1400{ }^{\circ} \mathrm{F}$ on $\mathrm{TI}-14 \mathrm{C}-2 \mathrm{~A} / 2 \mathrm{~B}$. Carefully watch for an overshoot on TIC-14A-3 and record the relation between power output and temperatures.

3. If the overshoot is over $25^{\circ} \mathrm{F}$ after PID tuning, switch TIC-14A3from auto control mode to auto control mode, and manually increase the TIC-14A-3's power output in $1 \%$ increments to achieve $1400^{\circ} \mathrm{F}$.

4. After TIC-14A-3 reaches $1400^{\circ} \mathrm{F}$, increase the heater output by manual control mode to achieve $1400^{\circ} \mathrm{F}$ for $\mathrm{TI}-14 \mathrm{C}-2 \mathrm{~A} / 2 \mathrm{~B}$.

5. Set TIC-14A-3 setpoint at temperature on the indicator and switch it from manual control mode to auto control mode.

6. Manually increase and adjust the heater output of PH-HTR-1, -2, 3 , and Heater 5 heater to achieve same temperature as TI-14A2A/2B on TI-13C-2A/2B, TI-12C-2A/2B and TI-11C-2A/2B, and TI$15 \mathrm{~A} / \mathrm{B} / \mathrm{C} / \mathrm{D}-2$.

7. When the PH-HTR-4 temperature is stable, record the temperature and heater output power.

8. Unless $\mathrm{TI}-14 \mathrm{C}-2 \mathrm{~A} / 2 \mathrm{~B}$ indicates abnormal temperature, turn the $\mathrm{TI}-$ $14 \mathrm{C}-2 \mathrm{~A} / 2 \mathrm{~B}^{\mathrm{HH}}$ trip bypass to OFF.

9. Surface temperature measurements use contact meter or infrared meter on the following:

a. Reactor top flange bolts and nuts.

b. Coal feed pipe to the reactor.

C. Reactor bottom flange bolts and nuts.

2.2.5.9 Set TIC-13A-3 setpoint at the temperature on the indicator and switch it to auto control mode. Follow these steps below:

1. Increase the TIC-13A-3 setpoint in $10^{\circ} \mathrm{F}$ increments every 1 to 2 minutes to achieve $1500^{\circ} \mathrm{F}$ on $\mathrm{TI}-13 \mathrm{C}-2 \mathrm{~A} / 2 \mathrm{~B}$. Carefully watch for an overshoot on TIC-13A-3 and record the relation between power output and temperatures. 
2. If the overshoot over $10^{\circ} \mathrm{F}$ after PID tuning, switch TIC-13A-3's auto control mode to manual control mode, and increase the power output carefully by manual control mode to achieve $1500{ }^{\circ} \mathrm{F}$ TIC-13A-3.

3. In manual control mode, set the TIC-13A-3 setpoint at the temperature on the indicator and switch it back to auto control mode.

4. Increase and adjust the heater output of PH-HTR-1, 2 and Heater 5 by manual control mode to achieve the same temperature as TI13A-2A/2B on TI-12C-2A/2B, TI-11C-2A/2B, and TI-15A/B/C/D-2.

5. When the temperature related with $\mathrm{PH}-\mathrm{HTR}-3$ are stable, record the temperature, and heater output power.

6. Unless $\mathrm{TI}-13 \mathrm{C}-2 \mathrm{~A} / 2 \mathrm{~B}$ indicates abnormal temperature, turn the trip bypass on $\mathrm{TI}-13 \mathrm{C}-2 \mathrm{~A} / 2 \mathrm{~B}^{\mathrm{HH}}$ to OFF.

7. Surface temperature measurements use contact meter or infrared meter on the following:

a. Reactor top flange bolts and nuts.

b. Coal feed pipe to the reactor.

C. Reactor bottom flange bolts and nuts.

2.2.5.10 On manual control mode, set TIC-12A-3 setpoint at the temperature on the indicator and switch to auto control mode. Follow these steps:

1. Increase the $\mathrm{TIC}-12 \mathrm{~A}-3$ setpoint in $5^{\circ} \mathrm{F}$ increments every 1 to 2 minutes to achieve $1550{ }^{\circ} \mathrm{F}$ on $\mathrm{TI}-12 \mathrm{C}-2 \mathrm{~A} / 2 \mathrm{~B}$. Carefully watch and do not overshoot on TIC-12A-3. Record the relation between power output and temperatures.

2. If the overshoot is over $5{ }^{\circ} \mathrm{F}$ after PID tuning, turn off auto control mode on TIC-12A-3, use TIC-12A-3's manual control mode to increase the power output carefully to achieve $1550{ }^{\circ} \mathrm{F}$.

3. If the control is manual control mode, set the TIC-12A-3 setpoint at the temperature on the indicator and turn on the auto control mode.

4. Increase and adjust the heater output of PH-HTR-1 and Heater 5 by manual control mode to achieve same temperature as TI-12A2A/2B on TI-11C-2A/2B and TI-15A/B/C/D-2.

5. When the temperatures related with PH-HTR-3 are stable, record the temperatures, and heater output power.

6. Unless $\mathrm{TI}-12 \mathrm{C}-2 \mathrm{~A} / 2 \mathrm{~B}$ and $\mathrm{TI}-12 \mathrm{C}-1 \mathrm{~A} / 1 \mathrm{~B}$ indicate abnormal temperatures, set the $\mathrm{TI}-12 \mathrm{C}-2 \mathrm{~A} / 2 \mathrm{BHH}$ and $\mathrm{TI}-12 \mathrm{C}-1 \mathrm{~A} / 1 \mathrm{BHH}$ trip bypass to OFF.

7. Surface temperature measurements use contact meter or infrared meter on the following:

a. Reactor top flange bolts and nuts. 
a. Coal feed pipe to the reactor.

a. Reactor bottom flange bolts and nuts.

2.2.5.11 Keep TIC-11A-3 manual control mode, and follow these instructions:

1. Increase the power output carefully by manual control mode and wait until the $\mathrm{TI}-11 \mathrm{C}-2 \mathrm{~A} / 2 \mathrm{~B}$ become stable, and check to make sure the temperature is under $1600^{\circ} \mathrm{F}$.

2. Repeat Step 1 (above) until the $\mathrm{TI}-11 \mathrm{C}-2 \mathrm{~A} / 2 \mathrm{~B}$ reaches to $1600^{\circ} \mathrm{F}$.

3. Set the TIC-11A-3 setpoint at the temperature on the indicator and switch it from auto control mode to manual control mode.

4. Using manual control mode, increase and adjust the Heater 5 to achieve the same temperature as TI-11A-2A/2B on TI-15-1.

5. When the temperature related to Heater PH-HTR-1 is stable, record the temperature.

6. Unless $\mathrm{TI}-11 \mathrm{C}-2 \mathrm{~A} / 2 \mathrm{~B}$ and $\mathrm{TI}-11 \mathrm{C}-1 \mathrm{~A} / 1 \mathrm{~B}$ indicate abnormal temperatures, set $\mathrm{TI}-11 \mathrm{C}-2 \mathrm{~A} / 2 \mathrm{~B}^{\mathrm{HH}}$ and $\mathrm{TI}-11 \mathrm{C}-1 \mathrm{~A} / 1 \mathrm{~B}^{\mathrm{HH}}$ trip bypass to OFF.

7. Surface temperature measurements use contact meter or infrared meter on the following:

a. Reactor top flange bolts and nuts.

b. Coal feed pipe to the reactor.

c. Reactor bottom flange bolts and nuts.

\subsubsection{Reactor Heater Performance Test}

The heater operation should be performed using temperature profile screen. Follow these steps for the reactor heaters' performance test:

2.2.6.1 Turn on the Reactor Heaters 1, 2, 3, 4, 5, 6, and 7 .

2.2.6.2 Set TIC-21 setpoint at the temperature on the indicator and switch from manual control mode to auto control mode.

2.2.6.3 Increase the TIC-21 setpoint $25^{\circ} \mathrm{F}$ increments to achieve the same temperature as the average of TI-15A/B/C/D-2 on the TE-22A-1. Observe carefully the behavior of TE-21A-1, TE-21B-4, TE-21B-1and TE-27A-1 due to the increase of the TIC-21 setpoint.

2.2.6.4 If the overshoot of TIC-21 is over $25^{\circ} \mathrm{F}$ after PID tuning, switch TIC21 from auto control mode to manual control mode and carefully increase the power.

2.2.6.5 Conduct the same operation on TIC-22, TIC-23, TIC-24, TIC-25, TIC26, and TIC-27.

2.2.6.6 Monitor R-TI-60/61 carefully. If the R-TI-60/61 goes over $900{ }^{\circ} \mathrm{F}$, first turn off Heater 7, and if necessary turn off Heater 6. 
2.2.6.7 When the temperature profile on reactor tube between Heater 2 and Heaters 5, 6, and 7 becomes roughly flat and stable, record the output of each heater and all of temperatures in the plant.

\subsubsection{Plant Temporary Shutdown}

2.2.7.1 Manually turn off all heaters.

2.2.7.2 Change the setpoints of R-PIC-30 program controller dP from 15 to 0 psig in 3 psig inclements every 5 minutes.

2.2.7.3 Turn off R-PIC-30's program controller.

2.2.7.4 When all heaters' TIs go below than $200{ }^{\circ} \mathrm{F}$, shut N2-V-3 and N2-V-7

2.2.7.5 Open R-AOV-4.

2.2.7.6 Using manual control mode, shut SG-PCV-1, R-PCV-30, and H2PCV-1.

2.2.7.7 Shut SG-V-1.

2.2.7.8 Keep the plant at about 1000 psig.

2.2.7.9 Set the tip bypass on H2O-FSL-2 to ON and H2O-P-1 to OFF.

\subsubsection{Turn off $\mathrm{H} 2 \mathrm{O}-\mathrm{P}-1$.}

\subsection{Hot Reactor Test using $\mathrm{H}_{2}$ (Low Flow)}

Initial Condition: The plant pressure is kept at about 1000 psig after the previous run.

\subsubsection{Establish Controlled Gas Flow using Nitrogen $\left(\mathrm{N}_{2}\right)$}

2.3.1.1 Confirm that $\mathrm{N}_{2}$ tube trailer has more than 1600 psig pressure and that it has sufficient $\mathrm{N}_{2}$ to operate the system. Follow this information to establish controlled gas flow using $\mathrm{N}_{2}$ :

\begin{tabular}{|c|c|c|c|c|c|}
\hline $\mathrm{H}_{2} / \mathrm{C}$ & $\begin{array}{c}\mathrm{N}_{2} \text { flow } \\
\left(\mathrm{sft}^{3} / \mathrm{h}\right) \\
\text { Process }\end{array}$ & $\begin{array}{c}\mathrm{H}_{2} \text { flow } \\
\left.\text { (sft }{ }^{3} / \mathrm{h}\right) \\
\text { Process }\end{array}$ & $\begin{array}{c}\mathrm{N}_{2} \text { flow } \\
\text { (sft/h) } \\
\text { Annulus }\end{array}$ & $\begin{array}{l}\text { Operation } \\
\text { (hr) }\end{array}$ & $\begin{array}{c}\mathrm{N}_{2} \text { Consumption } \\
\left(\mathrm{sft}^{3}\right)\end{array}$ \\
\hline 0.2 & $220^{a}$ & $267^{b}$ & 600 & as 6.0 & 4400 \\
\hline
\end{tabular}

2.3.1.2 Set N2-PCV-1 at 1150 psig.

2.3.1.3 Check and open H2-V-3, H2-V-4, H2-V-6, H2-V-7/R-V-1/CON-V-1, CON-V-2/SG-V-1, and SG-V-2.

2.3.1.4 Check and open H2-AOV-1/R-AOV-4/R-AOV-1/CON-AOV-1

2.3.1.5 Check and shut H2-PCV-1, H2-FCV-1, H2-PCV-2, H2-FCV-2, H2AOV-2, H2-MFC-1, H2-AOV-20, R-AOV-3, R-PCV-1, R-MFC-1, and R-PCV-30.

2.3.1.6 Set SG-PCV-1 at R-PT-3 +10 psig, and switch SG-PCV-1 to auto control mode. 
2.3.1.7 In half turn increments, open N2-V-7 so that it is fully open.

2.3.1.8 Open R-PCV-1 in $1 \%$ opening increments every 10 seconds until RPIC-31 indicates the same pressure as N2-PCV-1's secondary pressure.

2.3.1.9 Set R-MFC-1 setpoint at $10 \mathrm{stt}^{3} / \mathrm{h}$ and increase the setpoint $20 \mathrm{stt}^{3} / \mathrm{h}$ increments every 10 seconds to achieve $100 \mathrm{sft}^{3} / \mathrm{h}$.

2.3.1.10 In half turn increments, open N2-V-3 so that it is fully open.

2.3.1.11 Select $\mathrm{N}_{2}$ as fluid on $\mathrm{H} 2-\mathrm{FM}-1$ (Micro Motion flow meter).

2.3.1.12 Open $\mathrm{H} 2-\mathrm{PCV}-1$ in $1 \%$ opening increments every 10 seconds until $\mathrm{H} 2-\mathrm{PIC}-1$ indicates the same pressure as N2-PCV-1's secondary pressure.

2.3.1.13 Select $\mathrm{H} 2-\mathrm{FIC}-1$ flow indicator for $\mathrm{N}_{2}$.

2.3.1.14 Open $\mathrm{H} 2-\mathrm{FCV}-1$ in $1 \%$ opening increments every 10 seconds until you see the flow on the H2-FCV-1 flow indicator and verify that $\mathrm{N}_{2}$ starts to flow.

2.3.1.15 Set H2-FIC-1 setpoint at the flow rate on the indicator and switch from manual control mode to auto control mode.

2.3.1.16 Set SG-PCV-1 setpoint at 1000 psig.

2.3.1.17 Increase R-MFC-1 flow rate to $350 \mathrm{stt}^{3} / \mathrm{h}$ by changing the flow setpoint.

2.3.1.18 Increase H2-FIC-1 (H2-FM-1) flow rate to $220 \mathrm{sft}^{3} / \mathrm{h}$ on $\mathrm{N}_{2}$ flow scale by changing the flow setpoint.

2.3.1.19 Adjust the H2-PCV-1 opening to achieve an appropriate opening (about 65\%) on H2-FCV-1. Note: There is a 65\% opening at H2-PT-1 and it should be 1090 psig in theory.

2.3.1.20 If there is unstable control loop, make a turning on PID on the control loop in accordance with control engineer's advice.

\subsubsection{Establish Pressure Balancing System}

2.3.2.1 Using auto control mode, verify that R-MFC-1 flow rate is stable.

2.3.2.2 Set R-PCV-30 setpoint at 1000 psig and switch from manual control mode to auto control mode.

2.3.2.3 Verify the proper response for R-PCV-30.

2.3.2.4 Shut R-AOV-4.

2.3.2.5 Verify proper response for R-PCV-30.

2.3.2.6 Increase R-MFC-1 flow rate to $600 \mathrm{stt}^{3} / \mathrm{h}$ by changing the flow setpoint.

2.3.2.7 Set dP setpoint of program controller R-PT-30 at R-PT-3 + dP 0 psig and turn on R-PT-30 program controller.

2.3.2.8 Increase the $\mathrm{dP}$ setpoint in 3 psig increments to 15 psig to achieve 1015 psig for R-PT-30.

2.3.2.9 Verify the proper responses for all pressure and flow instruments. 


\subsection{3 $\mathrm{H}_{2}$ Flow Meter Calibration}

2.3.3.1 Operation of facility in $\mathrm{H}_{2}$ Park

1. Open V-306 in $\mathrm{H} 2$ Park.

2. Check and shut V-303, V-314, and V325 on Test Bed Panel.

3. Check and open V-305, V-318, V319, and V-342 on Test Bed Panel.

4. Set PCV-303 secondary pressure at 2400 psig.

\subsubsection{Operation on SNG Panel and Lab View}

1. Set H2-PCV-30 at 1200 psig.

2. Confirm H2-PT-PLANT shows 1200 psig.

3. Using manual control mode, fix $\mathrm{H} 2-\mathrm{FIC}-1 \%$ opening to present value.

4. Select $\mathrm{H}_{2}$ as fluid on $\mathrm{H} 2-\mathrm{FM}-1$ (Micro Motion flow meter).

5. Select flow indicator for $\mathrm{H}_{2}$ on $\mathrm{H} 2-\mathrm{FIC}-1$.

6. In half turn increments, open $\mathrm{H} 2-\mathrm{V}-1$ so that it is fully open.

7. In half turn increments, shut N2-V-3 so that it is fully shut.

8. Set H2-FIC-1 setpoint at the flow rate on the indicator and switch from manual control to auto control mode.

9. Calibrate $\mathrm{H} 2-\mathrm{FM}-1$ at 5 flow rates using SG-FM-1.

a. When the operator changes the setpoint, find out an appropriate interval of input to not a cause of pressure fluctuation of more than 5 psig on R-dPT-1.

b. Refer Table 2 as a guideline for the relation between flow rate and pressure and valve opening when H2-PT-5 is at 1000 psig.

Table 2 - H2-PIC-1 psig and $\mathrm{sft}^{3} / \mathrm{h}$ and H2-FCV-1 Percent Opening

\begin{tabular}{|c|c|c|}
\hline $\begin{array}{c}\text { H2-PIC-1 } \\
\text { (psig) }\end{array}$ & $\begin{array}{c}\text { H2-FIC-1 } \\
\text { (H2-FM-1) } \\
\text { (sft/h) }\end{array}$ & $\begin{array}{c}\text { H2-FCV-1 } \\
\text { \% opening }\end{array}$ \\
\hline 1090 & 822 & $65 \%$ \\
\hline- & 700 & - \\
\hline- & 500 & - \\
\hline- & 300 & - \\
\hline & 200 & $45 \%$ \\
\hline
\end{tabular}

2.3.3.3 Keep the flow rate H2-FCV-1 at $200 \mathrm{stt}^{3} / \mathrm{h}$.

2.3.3.4 Check and open H2-V-6 and CF-SV-2.

2.3.3.5 Select $\mathrm{H}_{2}$ as fluid on $\mathrm{H} 2-\mathrm{FM}-2$ (Micro Motion flow meter).

2.3.3.6 Select flow indicator for $\mathrm{H}_{2}$ on $\mathrm{H} 2-\mathrm{FIC}-2$. 
2.3.3.7 Open H2-AOV-2 and H2-AOV-2O.

2.3.3.8 Open H2-PCV-2 in 1\% opening increments every 10 seconds until $\mathrm{H} 2-\mathrm{PIC}-4$ indicates the same pressure as N2-PCV-1's secondary pressure.

2.3.3.9 Check and open R-AOV-3.

2.3.3.10 Open H2-FCV-2 in 1\% opening increments until you see the flow on the flow indicator and verify that $\mathrm{H}_{2}$ starts to flow. If $\mathrm{H} 2-\mathrm{PIC}-4$ reduces rapidly, open $\mathrm{H} 2-\mathrm{PCV}-2$ to achieve a stable flow rate

2.3.3.11 Set H2-FCV-2 setpoint at the flow rate on the indicator and switch to auto control mode.

2.3.3.12 Calibrate H2-FM-2 at 5 flow rates using SG-FM-1. Refer Table 3 as a guideline for the relation between flow rate and pressure and valve opening when N2-PT-16 is at 1000 psig.

Table 3 - H2-PIC-2 at psig and sft3/h with H2-FCV-2 percent opening

\begin{tabular}{|c|c|c|}
\hline $\begin{array}{c}\text { H2-PIC-2 } \\
\text { (psig) }\end{array}$ & $\begin{array}{c}\text { H2-FIC-2 } \\
\text { (H2-FM-2) } \\
\left.\text { (sft }{ }^{3} / \mathbf{h}\right)\end{array}$ & $\begin{array}{c}\text { H2-FCV-2 } \\
\text { \% opening }\end{array}$ \\
\hline 1045 & 100 & 63 \\
\hline- & 70 & - \\
\hline- & 50 & - \\
\hline 1010 & 30 & 43 \\
\hline
\end{tabular}

2.3.3.13 Using manual control mode, fix $\mathrm{H} 2-\mathrm{FIC}-1 \%$ opening to present value.

2.3.3.14 Using manual control mode, fix $\mathrm{H} 2-\mathrm{FIC}-2 \%$ opening to present value.

2.3.3.15 Select $\mathrm{N}_{2}$ as fluid for H2-FM-1 (Micro Motion flow meter).

2.3.3.16 Select $\mathrm{N}_{2}$ as fluid for H2-FM-2 (Micro Motion flow meter).

2.3.3.17 In half turn increments, open N2-V-3 so that it is fully open.

2.3.3.18 In half turn increments, shut $\mathrm{H} 2-\mathrm{V}-1$ so that it is fully shut.

2.3.3.19 Using manual control mode, shut H2-PCV-2 and H2-FCV-2.

2.3.3.20 Shut H2-AOV-2 and R-AOV-3.

2.3.3.21 Set H2-FIC-1 setpoint at the flow rate on the indicator and switch from manual control mode to auto control mode.

2.3.3.22 Increase $\mathrm{H} 2-\mathrm{FIC}-1$ (H2-FM-1) flow rate to $220 \mathrm{stt}^{3} / \mathrm{h}$ on $\mathrm{N}_{2}$ flow scale.

\subsubsection{Turn on Cooling Water}

2.3.4.1 Open $\mathrm{H} 2 \mathrm{O}-\mathrm{V}-1$ and verify that the tank is full.

2.3.4.2 Check and open H2O-V-2, 4, 5, 6, 7, and 8; H2O-SV-8; and H2O-SV20.

2.3.4.3 Turn on $\mathrm{H} 2 \mathrm{O}-\mathrm{P}-1$. 
2.3.4.4 Open CON-V-21 and vent the air in the water line; verify that the water is circulating and shut CON-V-21.

2.3.4.5 Verify the mists to the reactor head and char pot.

2.3.4.6 Verify CON-PI-1 and CON-PI-2 indicate the pressures.

2.3.4.7 Set the H2O-FSL-2 and H2O-P-1 trip bypasses on OFF.

2.3.5 Heating up by $\mathrm{N}_{2}$

2.3.5.1 Verify that the power supply system is ready.

2.3.5.2 Verify that the all of heaters are switched off and that the setpoint of power output is at 0 .

2.3.5.3 Verify that all of the trip triggers on heater's temperature trip bypasses are ON.

2.3.5.4 Set the trip bypass on $\mathrm{R}-\mathrm{TI}-10^{\mathrm{HH}}$ to OFF, $\mathrm{H} 2 \mathrm{O}-\mathrm{FSL}-2$ to $\mathrm{ON}$, and $\mathrm{H} 2 \mathrm{O}-$ P-1 to OFF.

2.3.5.5 The time history trend graph on the screen shows the preheater tube temperature.

2.3.5.6 Turn on the $\mathrm{H}_{2}$ Preheaters PH-HTR-1, $-2,-3$, and -4 .

2.3.5.7 Set TIC-14A-3 setpoint at $1400^{\circ} \mathrm{F}$ and set the TIC-14A-3 program controller at $25^{\circ} \mathrm{F}$ per minute and activate the ramp up controller .

Carefully watch for an overshoot on TIC-14A-3. If the overshoot is over $50^{\circ} \mathrm{F}$, turn off the auto control mode and switch to manual control mode on TIC-14A-3, and manually increase the power output to achieve $1400^{\circ} \mathrm{F}$.

2.3.5.8 Set TIC-13A-3 setpoint at $1500^{\circ} \mathrm{F}$ and set the TIC-13A-3 program controller at $25^{\circ} \mathrm{F}$ per minute and activate the ramp up controller.

Carefully watch for an overshoot on TIC-13A-3. If the overshoot is over $30^{\circ} \mathrm{F}$, turn off the auto control mode and switch to manual control mode on TIC-13A-3, and manually increase the power output to the achieve $1500^{\circ} \mathrm{F}$.

2.3.5.9 Set TIC-12A-3 setpoint at $1550^{\circ} \mathrm{F}$ and set TIC-12A-3 program controller at $25^{\circ} \mathrm{F}$ per minute and activate the ramp up controller.

Carefully watch for an overshoot on TIC-12A-3. If the overshoot is over $15^{\circ} \mathrm{F}$, turn off the auto control mode and switch to manual control mode on TIC-12A-3, and manually increase the power output to the achieve $1550^{\circ} \mathrm{F}$.

2.3.5.10 Set TIC-11A-3 setpoint at $1600^{\circ} \mathrm{F}$ and set TIC-11A-3 program controller at $25^{\circ} \mathrm{F}$ every minute and activate the ramp up controller.

Carefully watch for an overshoot on TIC-11A-3. If the overshoot is over $5^{\circ} \mathrm{F}$, turn off the auto control mode and switch to manual control mode on TIC-11A-3, and manually increase the power to the achieve $1600^{\circ} \mathrm{F}$. 
2.3.5.11 If the TI-11C-2A/B, TI-11C-1A/B, TI-12C-1A/B, and TI-12C-2A/B temperatures are normal, turn the $\mathrm{TI}-11 \mathrm{C}-1 \mathrm{~A} / \mathrm{B}^{\mathrm{HH}}, \mathrm{TI}-11 \mathrm{C}-2 \mathrm{~A} / \mathrm{B}^{\mathrm{HH}}$, TI$12 \mathrm{C}-1 \mathrm{~A} / \mathrm{B}^{\mathrm{HH}}, \mathrm{TI}-12 \mathrm{C}-2 \mathrm{~A} / \mathrm{B}^{\mathrm{HH}}, \mathrm{TI}-13 \mathrm{C}-1 \mathrm{~A} / \mathrm{B}^{\mathrm{HH}}$, and $\mathrm{TI}-14 \mathrm{C}-1 \mathrm{a} / \mathrm{B}^{\mathrm{HH}}$ trip bypasses to OFF.

2.3.5.12 Set and adjust the output of Preheater Zone 5 to achieve the same temperature as TI-11C-1A/1B on TIC-15A/B/C/D-2.

2.3.5.13 The time history trend graph on the screen should show reactor's tube temperature.

2.3.5.14 Turn on all of reactor Heaters1 to 5

2.3.5.15 Set TIC-21, $-22,-23,24$, and -25 setpoints at the temperature obtained in 2.2.6.7; set the TIC-21, -22, -23, 24, and -25 program controllers at $25^{\circ} \mathrm{F}$ per minute; and activate the controller.

2.3.5.16 After each heater reaches at the setpoint temperature, using manual control mode adjust the setpoint to achieve the average temperature of reactor tube between Heaters1 and 5 same as the reactor inlet temperature (the average temperature of $\mathrm{TI}-15 \mathrm{~A} / \mathrm{B} / \mathrm{C} / \mathrm{D}-2$ ).

\subsubsection{Switch Nitrogen to Hydrogen}

\subsubsection{Operation of Facility in $\mathrm{H}_{2}$ Park}

1. Open $\mathrm{V}-306$ in $\mathrm{H}_{2}$ Park.

2. Check and shut V-303, V-321, and V-325 on Test Bed Panel.

3. Check and open V-305, V-318, V-319, and V-342 on Test Bed Panel.

4. Set PCV-303 secondary pressure at 2400 psig.

\subsubsection{Operation on SNG Panel and Lab View}

1. Set H2-PCV-30 at 1200 psig.

2. Confirm H2-PT-PLANT shows 1200 psig.

3. Using manual control mode, fix the H2-FIC-1's opening to present value.

4. Using manual control mode, fix all of $\mathrm{H}_{2}$ Preheater and Reactor Heater outputs to present value.

5. Select $\mathrm{H}_{2}$ as fluid on $\mathrm{H} 2-\mathrm{FM}-1$ (Micro Motion flow meter).

6. Select flow indicator for $\mathrm{H}_{2}$ on $\mathrm{H} 2-\mathrm{FIC}-1$.

7. In half turn increments, open $\mathrm{H} 2-\mathrm{V}-1$ so that it is fully open.

8. In half turn increments, shut N2-V-3 so that it is fully shut.

9. Set $\mathrm{H} 2 \mathrm{FIC}-1$ setpoint at the flow rate on the indicator and switch from manual control mode to auto control mode.

10. Change H2-FCV-1 setpoint in about $50 \mathrm{sft}^{3} / \mathrm{h}$ increments from present value to $267 \mathrm{stt}^{3} / \mathrm{h}$.

11. In parallel with operation in Step 10 (above), adjust H2-PCV-1 opening to achieve a stable control point on H2-FIC-1. 
12. Confirm that H2-FCV-1 has proper response.

\subsection{7 $\mathrm{H}_{2}$ Preheater Performance Test}

Confirm the relation between the heater's outputs, heater's temperature, and tube's temperature depending on the gas flow. Figure 2 shows Preheaters 1 through 4 temperature profile and preheaters' comparison gas temperatures to outer and inner wall temperatures.

Figure 2 - Preheaters 1 through 4 Temperature Profile and Preheaters Gas Temperatures Comparison to Outer and Inner Wall Temperatures

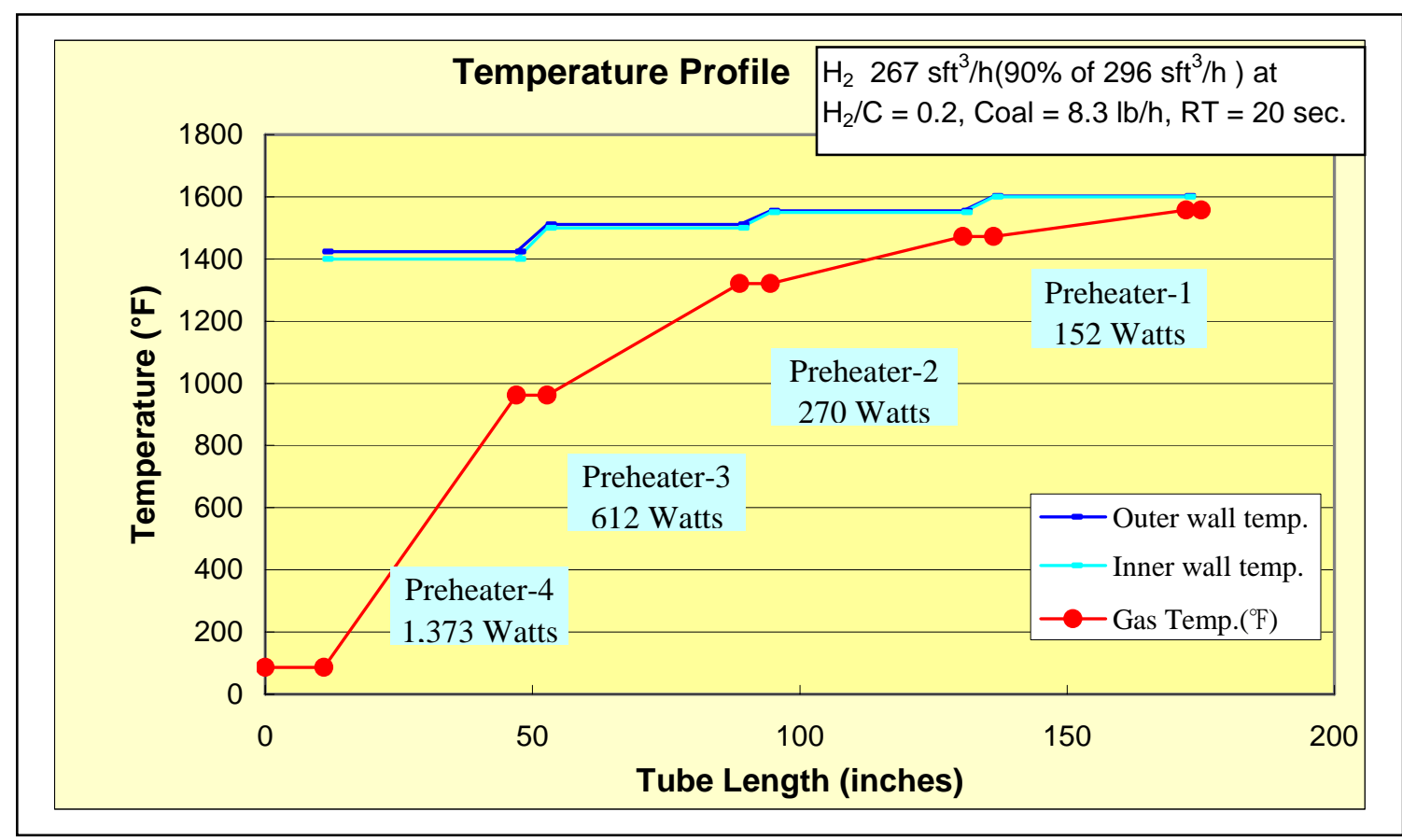

As the tube wall temperature in operation will be very close to the design temperature $\left(1650^{\circ} \mathrm{F}\right)$ especially on Preheater 1 , make sure to take precaution on the wall temperatures.

2.3.7.1 Set TIC-14A-3 setpoint at the temperature on the indicator. Follow these steps:

1. Increase the setpoint in $25^{\circ} \mathrm{F}$ increments per minute to the fastest rate to achieve $1400{ }^{\circ} \mathrm{F}$ at the $\mathrm{TI}-14 \mathrm{C}-2 \mathrm{~A} / \mathrm{B}$ (average). Carefully watch for an overshoot on $\mathrm{TIC}-14 \mathrm{~A}-3$ and record the relation between power output and temperatures.

2. If the overshoot is over $25^{\circ} \mathrm{F}$ after PID tuning, switch from auto control mode to manual control mode on TIC-14A-3, and manually increase the power output.

3. When $\mathrm{TI}-14 \mathrm{C}-2 \mathrm{~A} / 2 \mathrm{~B}$ reaches $1400^{\circ} \mathrm{F}$, set $\mathrm{TIC}-14 \mathrm{~A}-3$ setpoint at temperature on the indicator and switch from manual control mode to auto control mode.

4. When the PH-HTR-4 temperatures are stable, record the temperatures. 
5. Unless $\mathrm{TI}-14 \mathrm{C}-2 \mathrm{~A} / 2 \mathrm{~B}$ indicates abnormal temperature, switch $\mathrm{TI}-$ $14 \mathrm{C}-2 \mathrm{~A} / 2 \mathrm{~B}^{\mathrm{HH}}$ trip bypass to OFF.

2.3.7.2 Set TIC-13A-3 setpoint at the temperature on the indicator. Follow these steps:

1. Increase the setpoint to $10{ }^{\circ} \mathrm{F}$ in increments per minute to achieve $1500{ }^{\circ} \mathrm{F}$ on the $\mathrm{TI}-13 \mathrm{C}-2 \mathrm{~A} / \mathrm{B}$ (average). Carefully watch for an overshoot on TIC-13A-3 and record the relation between power output and temperatures.

2. If the overshoot is over $10^{\circ} \mathrm{F}$ after PID tuning, switch TIC-13A-3 from auto control mode to manual control mode and manually increase the power.

3. When $\mathrm{TI}-13 \mathrm{C}-2 \mathrm{~A} / 2 \mathrm{~B}$ reaches $1500{ }^{\circ} \mathrm{F}$, and the temperature related to PH-HTR-3 is stable, record the temperatures.

4. Unless $\mathrm{TI}-13 \mathrm{C}-2 \mathrm{~A} / 2 \mathrm{~B}$ indicates abnormal temperature, switch $\mathrm{TI}-$ $13 \mathrm{C}-2 \mathrm{~A} / 2 \mathrm{~B}^{\mathrm{HH}}$ trip bypass to OFF.

2.3.7.3 Set TIC-12A-3 setpoint at the temperature on the indicator. Follow these steps:

1. Increase the setpoint in $5^{\circ} \mathrm{F}$ increments per minute to achieve $1550{ }^{\circ} \mathrm{F}$ at the $\mathrm{TI}-12 \mathrm{C}-2 \mathrm{~A} / \mathrm{B}$ (average). Carefully watch not to overshoot TIC-12A-3 and record the relation between power output and temperatures.

2. If the overshoot is over $5^{\circ} \mathrm{F}$ after PID tuning, switch TIC-12A-3 from auto control mode to manual control mode and manually increase the power.

3. When $\mathrm{TI}-12 \mathrm{C}-2 \mathrm{~A} / 2 \mathrm{~B}$ reaches to $1550{ }^{\circ} \mathrm{F}$ and the temperature related to $\mathrm{PH}-\mathrm{HTR}-3$ is stable, record the temperatures.

4. Unless $\mathrm{TI}-12 \mathrm{C}-2 \mathrm{~A} / 2 \mathrm{~B}$ and $\mathrm{TI}-12 \mathrm{C}-1 \mathrm{~A} / 1 \mathrm{~B}$ indicate abnormal temperature, set the $\mathrm{TI}-12 \mathrm{C}-2 \mathrm{~A} / 2 \mathrm{~B}^{\mathrm{HH}}$ and $\mathrm{TI}-12 \mathrm{C}-1 \mathrm{~A} / 1 \mathrm{~B}^{\mathrm{HH}}$ trip bypasses to OFF.

2.3.7.4 Keep TIC-11A-3 control on the manual control mode. Follow these steps:

1. Using manual control mode, increase the power output; wait until the $\mathrm{TI}-11 \mathrm{C}-2 \mathrm{~A} / 2 \mathrm{~B}$ becomes stable and check to see if the temperature is under $1600^{\circ} \mathrm{F}$.

2. Repeat Step 1 (above) until the $\mathrm{TI}-11 \mathrm{C}-2 \mathrm{~A} / 2 \mathrm{~B}$ reaches $1600^{\circ} \mathrm{F}$.

3. Using the manual control mode, increase and adjust the heater output of Heater 5 to achieve same temperature as TI-11A-2A/2B on TI-15A/B/C/D-2.1.

4. When the temperature related to PH-HTR-1 is stable, record the temperature. 
5. Unless $\mathrm{TI}-11 \mathrm{C}-2 \mathrm{~A} / 2 \mathrm{~B}$ and $\mathrm{TI}-11 \mathrm{C}-1 \mathrm{~A} / 1 \mathrm{~B}$ indicates abnormal temperature, switch $\mathrm{TI}-11 \mathrm{C}-2 \mathrm{~A} / 2 \mathrm{~B}^{\mathrm{HH}}$ and $\mathrm{TI}-11 \mathrm{C}-1 \mathrm{~A} / 1 \mathrm{~B}^{\mathrm{HH}}$ trip bypasses to OFF.

6. Surface temperature measurements use contact meter or infrared meter on the following:

a. Reactor top flange bolts and nuts.

b. Coal feed pipe to the reactor.

c. Reactor bottom flange bolts and nuts.

\subsubsection{Reactor Heater Performance Test}

The heater operation should be performed using temperature profile screen. Follow these steps:

2.3.8.1 Turn on the Reactor Heaters 1, 2, 3, 4, 5, 6, and 7.

2.3.8.2 Set TIC-21 setpoint at the temperature on the indicator and switch from manual control mode to auto control mode.

2.3.8.3 Increase the TIC-21 setpoint $25^{\circ} \mathrm{F}$ increments to achieve the same temperature as the average of TI-15A/B/C/D-2 on the TE-22A-1. Due to the increase of the TIC-21 setpoint, carefully observe the behavior of TE-21A-1, TE-21B-4, TE-21B-1, and TE-27A-1.

2.3.8.4 If the overshoot of TIC-21 is over $25^{\circ} \mathrm{F}$ after PID tuning, switch TIC21 from auto control mode to manual control mode, and manually increase the power output.

2.3.8.5 Conduct the same operation on TIC-22, TIC-23, TIC-24, TIC-25, TIC26, and TIC-27 as shown in steps 2.3.8.2 through 2.3.8.4.

2.3.8.6 Carefully monitor R-TI-60/61. If the R-TI-60/61 goes over $900{ }^{\circ} \mathrm{F}$, first turn off Heater 7, then if necessary turn off Heater 6.

2.3.8.7 When the temperature profile on reactor tube between Heater 2 and Heaters 5, 6, and 7 becomes roughly flat and stable, record the output of each heater and all of temperatures on the plant.

\subsubsection{Plant Temporary Shutdown}

2.3.9.1 Manually turn off all heaters.

2.3.9.2 Keep $\mathrm{H}_{2}$ flow until all of tube temperatures for TIC-11A/B-3, TIC12A/B-3, TIC-13A/B-3 and TIC-14A/B-3 and heater temperatures for TIC-11A/B-3, TIC-12A/B-3, TIC-13A/B-3, and TIC-14A/B-3 are lower than $1000^{\circ} \mathrm{F}$.

2.3.9.3 Using manual control mode, set the H2-FIC-1 and fix the opening of H2-FCV-1.

2.3.9.4 Select flow indicator for $\mathrm{N}_{2}$ on $\mathrm{H} 2-\mathrm{FIC}-1$.

2.3.9.5 In half turn increments, open N2-V-3 so that it is fully open.

2.3.9.6 Select $\mathrm{N}_{2}$ as fluid on $\mathrm{H} 2-\mathrm{FM}-1$ (Micro Motion flow meter).

2.3.9.7 In half turn increments, shut $\mathrm{H} 2-\mathrm{V}-1$ so that it is fully shut.

${ }^{\circ} 2010$ Arizona Public Service All Rights Reserved 
2.3.9.8 Set H2-FIC-1 setpoint at the flow rate on the indicator and switch from manual control mode to auto control mode.

2.3.9.9 Increase H2-FIC-1 setpoint $20 \mathrm{stt}^{3} / \mathrm{h}$ increments to achieve $220 \mathrm{st}^{3} / \mathrm{h}$.

2.3.9.10 In parallel with $\mathrm{H} 2-\mathrm{FIC}-1$ operation, increase $\mathrm{H} 2-\mathrm{PI}-1$ by increasing $\mathrm{H} 2-\mathrm{PCV}-1$ opening to achieve about $65 \%$ on H2-FCV-1 (up to 1100 psig on $\mathrm{H} 2-\mathrm{PI}-1$ in theory).

2.3.9.11 When all heaters' $\mathrm{TI}$ are below $200^{\circ} \mathrm{F}$, change the R-PIC-30 program controller dP setpoint from 15 to 0 psig in 3 psig inclements every 5 minutes.

2.3.9.12 Turn off the R-PIC-30program controller.

2.3.9.13 Shut N2-V-3 and N2-V-7.

2.3.9.14 Open R-AOV-4.

2.3.9.15 Manually shut SG-PCV-1, R-PCV-30, and H2-PCV-1.

2.3.9.16 Shut SG-V-1 and keep the plant at about 1000 psig.

2.3.9.17 Switch $\mathrm{H} 2 \mathrm{O}-\mathrm{FSL}-2$ tip bypass to ON and H2O-P-1 tip bypass to OFF.

\subsubsection{Turn off H2O-P-1.}

\subsection{Hot Reactor Test using $\mathrm{H}_{2}$ High Flow}

Initial Condition: The plant pressure is kept at about 1000 psig after the previous run.

\subsubsection{Establish Controlled Gas Flow using Nitrogen}

Establish the $\mathrm{N}_{2}$ flow following Section 2.3.1.

\subsubsection{Establish Pressure Balancing System}

Establish the pressure balancing system following the Section 2.3.2.

\subsubsection{Turn on Cooling Water}

To turn on cooling water, follow Section 2.3.4.

2.4.4 Heating up by $\mathrm{N}_{2}$

For heating up the plant, follow Section 2.3.5.

\subsubsection{Switch Nitrogen to Hydrogen}

2.4.5.1 Facility in $\mathrm{H}_{2}$ Park Operation

1. Open V-306 in $\mathrm{H}_{2}$ Park.

2. Check and shut V-303, V-314, V-321, and V-325 on Test Bed Panel.

3. Check and open V-305, V-318, V319, and V-342 on Test Bed Panel.

4. Set PCV-303's secondary pressure at 2400 psig.

2.4.5.2 SNG Panel and Lab View Operation

${ }^{\circ} 2010$ Arizona Public Service All Rights Reserved 
1. Set H2-PCV-30 at 1200 psig.

2. Confirm H2-PT-PLANT shows 1200 psig.

3. Using manual control mode, set $\mathrm{H} 2-\mathrm{FIC}-1$ 1\% opening to present value.

4. Using the manual control mode, set all of $\mathrm{H}_{2}$ Preheaters' and Reactor Heaters' output at present value.

5. Select $\mathrm{H}_{2}$ as fluid on H2-FM-1 (Micro Motion flow meter).

6. Select $\mathrm{H} 2-\mathrm{FIC}-1$ flow indicator for $\mathrm{H}_{2}$.

7. In half turn increments, open $\mathrm{H} 2-\mathrm{V}-1$ so that it is fully open.

8. In half turn increments, shut N2-V-3 so that it is fully shut.

9. Set H2-FIC-1 setpoint at the flow rate on the indicator and switch from manual control mode to auto control mode.

10. Adjust H2-FIC-1 flow rate to $822 \mathrm{stt}^{3} / \mathrm{h}$ (Note: The H2-FCV-1 opening will be same as the N2-220's $\mathrm{stt}^{3} / \mathrm{h}$.).

11. Adjust $\mathrm{H} 2-\mathrm{PCV}-1$ opening to achieve a stable control point on $\mathrm{H} 2$ FIC-1.

12. Verify H2-FCV-1's proper response.

\subsection{6 $\mathrm{H}_{2}$ Preheater Performance Test}

Confirm the relation between the heater outputs, heater temperature, and tube temperature depending on gas flow. (Refer to Figure 3.) 
Figure 3 - Relation between the Preheater Outputs, Temperatures, and Tube Temperatures Depending on the Gas Flow

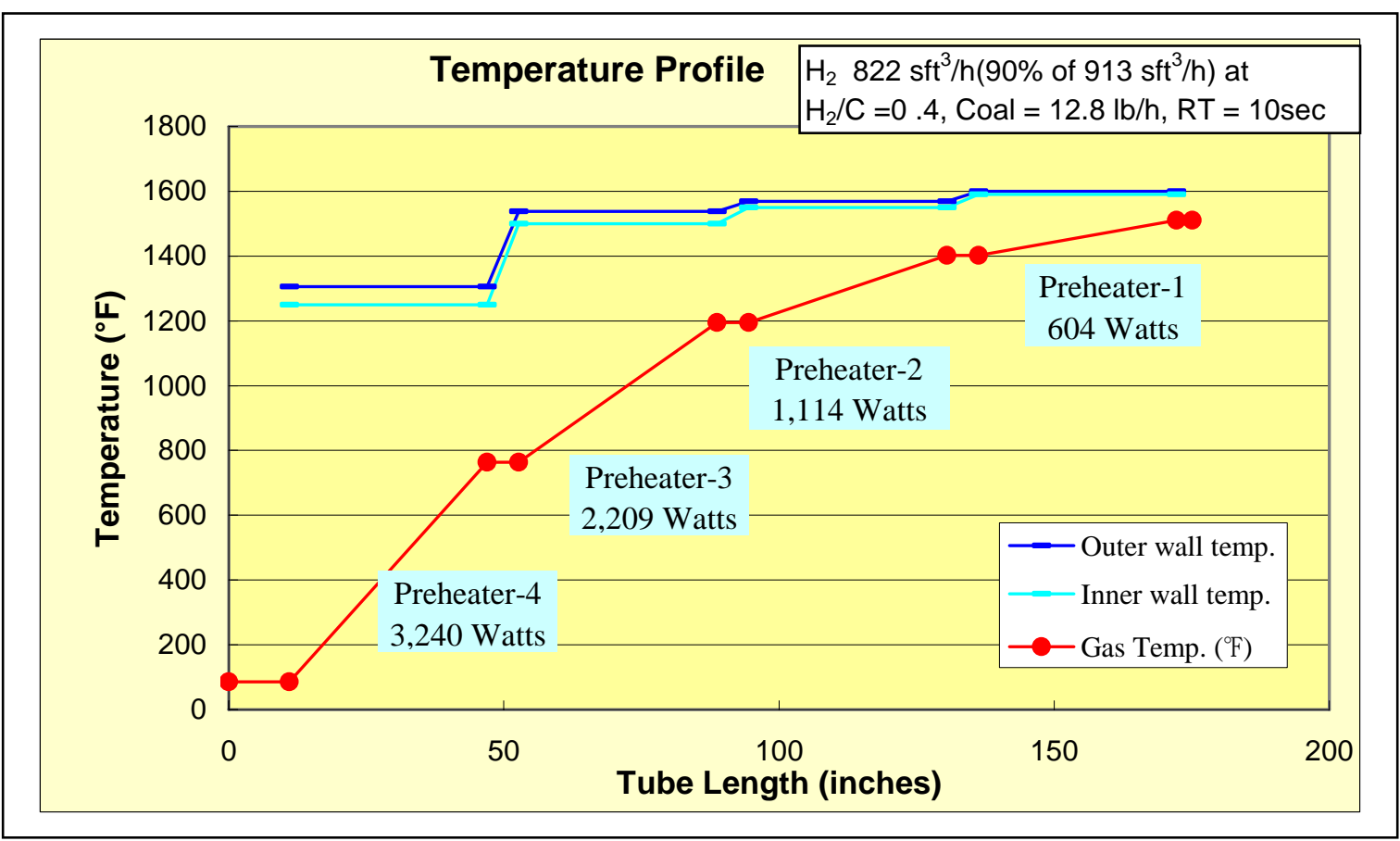

Because the tube wall temperature in operation will be very close to the design temperature $\left(1650^{\circ} \mathrm{F}\right)$ especially on Preheater 1 , pay careful attention to the preheaters' wall temperatures.

2.4.6.1 Set TIC-14A-3 setpoint at the temperature on the indicator. Follow these steps:

1. Increase the setpoint in $25^{\circ} \mathrm{F}$ increments per minute to achieve $1300{ }^{\circ} \mathrm{F}$ at the $\mathrm{TI}-14 \mathrm{C}-2 \mathrm{~A} / \mathrm{B}$ (average). Carefully watch for an overshoot on TIC-14A-3 and record the relation between power output and temperatures.

2. If the overshoot is over $25^{\circ} \mathrm{F}$ after PID tuning, switch TIC-14A-3 from auto control mode to manual control mode, and carefully manually increase the power output.

3. When $\mathrm{TI}-14 \mathrm{C}-2 \mathrm{~A} / 2 \mathrm{~B}$ reaches $1300{ }^{\circ} \mathrm{F}$, set $\mathrm{TIC}-14 \mathrm{~A}-3$ setpoint at temperature on the indicator and switch from manual control mode to auto control mode.

4. When the temperatures related to Heater PH-HTR-4 are stable, record the temperatures.

5. Unless $\mathrm{TI}-14 \mathrm{C}-2 \mathrm{~A} / 2 \mathrm{~B}$ indicates abnormal temperature, switch $\mathrm{TI}-$ $14 \mathrm{C}-2 \mathrm{~A} / 2 \mathrm{~B}^{\mathrm{HH}}$ trip bypass to OFF.

2.4.6.2 Set TIC-13A-3 setpoint at the temperature on the indicator. Follow these steps:

1. Increase the setpoint in $10^{\circ} \mathrm{F}$ increments per minute in the fastest case to achieve $1530^{\circ} \mathrm{F}$ at the $\mathrm{TI}-13 \mathrm{C}-2 \mathrm{~A} / \mathrm{B}$ (average). Carefully 
watch for an overshoot on $\mathrm{TIC}-13 \mathrm{~A}-3$ and record the relation between power output and temperatures.

2. If the overshoot is over $10^{\circ} \mathrm{F}$ after PID tuning, switch TIC-13A-3 from auto control mode to manual control mode, and carefully manually increase the power output.

3. When $\mathrm{TI}-13 \mathrm{C}-2 \mathrm{~A} / 2 \mathrm{~B}$ reaches to $1530{ }^{\circ} \mathrm{F}$, the Heater $\mathrm{PH}-\mathrm{HTR}-3$ temperature is stable, record the temperature.

4. Unless $\mathrm{TI}-13 \mathrm{C}-2 \mathrm{~A} / 2 \mathrm{~B}$ indicates abnormal temperature, make the $\mathrm{TI}-13 \mathrm{C}-2 \mathrm{~A} / 2 \mathrm{~B}^{\mathrm{HH}}$ trip bypass is OFF.

2.4.6.3 Set TIC-12A-3 setpoint at the temperature on the indicator. Follow these steps:

1. Increase the setpoint in $5^{\circ} \mathrm{F}$ increments per minute to achieve $1560{ }^{\circ} \mathrm{F}$ at the $\mathrm{TI}-12 \mathrm{C}-2 \mathrm{~A} / \mathrm{B}$ (average). Carefully watch for an overshoot on TIC-12A-3 and record the relation between power output and temperatures.

2. If the overshoot is over $5{ }^{\circ} \mathrm{F}$ after PID tuning, switch TIC-12A-3 from auto control mode to manual control mode, and manually increase the power output in $1 \%$ increments.

3. When TI-12C-2A/2B reaches to $1560{ }^{\circ} \mathrm{F}$ and the $\mathrm{PH}-\mathrm{HTR}-3$ temperature is stable, record the temperature.

4. Unless $\mathrm{TI}-12 \mathrm{C}-2 \mathrm{~A} / 2 \mathrm{~B}$ and $\mathrm{TI}-12 \mathrm{C}-1 \mathrm{~A} / 1 \mathrm{~B}$ indicate abnormal temperature, set the $\mathrm{TI}-12 \mathrm{C}-2 \mathrm{~A} / 2 \mathrm{~B}^{\mathrm{HH}}$, and $\mathrm{TI}-12 \mathrm{C}-1 \mathrm{~A} / 1 \mathrm{~B}^{\mathrm{HH}}$ trip bypasses to OFF.

2.4.6.4 Keep TIC-11A-3 control on the manual control mode. Follow these steps:

1. Manually increase the power output carefully, wait until the TI$11 \mathrm{C}-2 \mathrm{~A} / 2 \mathrm{~B}$ becomes stable, and check to make sure the temperature is under $1600^{\circ} \mathrm{F}$.

2. Repeat Step 1 (above) until the $\mathrm{TI}-11 \mathrm{C}-2 \mathrm{~A} / 2 \mathrm{~B}$ reaches $1600^{\circ} \mathrm{F}$.

3. Manually increase and adjust the Heater 5 to achieve same temperature as TI-11A-2A/2B on TI-15A/B/C/D-2.1.

4. When the temperatures related to Heater PH-HTR-1 are stable, record the temperatures.

5. Unless $\mathrm{TI}-11 \mathrm{C}-2 \mathrm{~A} / 2 \mathrm{~B}$ and $\mathrm{TI}-11 \mathrm{C}-1 \mathrm{~A} / 1 \mathrm{~B}$ indicate abnormal temperature, switch the $\mathrm{TI}-11 \mathrm{C}-2 \mathrm{~A} / 2 \mathrm{~B}^{\mathrm{HH}}$ trip bypass to OFF, and $\mathrm{TI}-11 \mathrm{C}-1 \mathrm{~A} / 1 \mathrm{~B}^{\mathrm{HH}}$ trip bypass to OFF.

6. Surface temperature measurements use contact meter or infrared meter on the following:

a. Reactor top flange bolts and nuts.

b. Coal feed pipe to the reactor.

c. Reactor bottom flange bolts and nuts. 


\subsubsection{Reactor Heater Performance Test}

The heater operation should be performed using temperature profile screen. Follow these steps:

2.4.7.1 Turn on the Reactor Heaters 1, 2, 3, 4, 5, 6, and 7.

2.4.7.2 Set TIC-21 setpoint at the temperature on the indicator and switch from manual control mode to auto control mode.

2.4.7.3 Increase the TIC-21 setpoint by $25^{\circ} \mathrm{F}$ increments to achieve the same temperature as the average temperature of TI-15A/B/C/D-2 on the TE-22A-1. Due to the increase of the TIC-21 setpoint, carefully observe the behavior of TE-21A-1, TE-21B-4, TE-21B-1and TE-27A-1.

2.4.7.4 If the overshoot of TIC-21 is over $25^{\circ} \mathrm{F}$ after PID tuning, switch to from auto control mode to manual control mode on TIC-21, and carefully manually increase the power output.

2.4.7.5 Follow the same steps as 2.4.7.2 through 2.4.7.4 for TIC-22, TIC-23, TIC-24, TIC-25, TIC-26, and TIC-27.

2.4.7.6 Carefully monitor R-TI-60/61. If the R-TI-60/61 goes over $900{ }^{\circ} \mathrm{F}$, first turn off Heater 7 and if necessary turn off Heater 6.

2.4.7.7 When the temperature profile on reactor tube between Heater 2, 5, 6, and 7 may become roughly flat and stable, record the output of each heater and all of temperatures on the plant.

\subsubsection{Plant Shutdown}

2.4.8.1 Manually turn off all heaters.

2.4.8.2 Keep $\mathrm{H}_{2}$ flow until all of tube temperatures for TIC-11A/B-3, TIC12A/B-3, TIC-13A/B-3, and TIC-14A/B-3 and heater temperatures for TIC-11A/B-3, TIC-12A/B-3, TIC-13A/B-3, and TIC-14A/B-3 are lower than $1000^{\circ} \mathrm{F}$.

2.4.8.3 Manually set the H2-FIC-1 and fix H2-FCV-1 opening.

2.4.8.4 Select $\mathrm{H} 2-\mathrm{FIC}-1$ flow indicator for $\mathrm{N}_{2}$.

2.4.8.5 In half turn increments, open N2-V-3 so that it is fully open.

2.4.8.6 Select $\mathrm{N}_{2}$ as fluid on $\mathrm{H} 2-\mathrm{FM}-1$ (Micro Motion flow meter).

2.4.8.7 In half turn increments, shut H2-V-1 so that it is fully shut.

2.4.8.8 Set H2-FIC-1 setpoint at the flow rate on the indicator and switch from manual control mode to auto control mode.

2.4.8.9 Adjust H2-FIC-1 flow rate at $220 \mathrm{stt}^{3} / \mathrm{h}$. Note: The H2-FCV-1 opening will be same as $\mathrm{H} 2-822$.

2.4.8.10 Adjust $\mathrm{H} 2-\mathrm{PCV}-1$ opening to achieve a stable control point on $\mathrm{H}$ 2-FIC1.

2.4.8.11 When all of the Heaters' $\mathrm{TI}$ reach below $200^{\circ} \mathrm{F}$, change the setpoint of R-PIC-30 program controller dP from 15 to 0 psig in 3 psig inclements every 5 minutes. 
2.4.8.12 Turn off the R-PIC-30 program controller.

2.4.8.13 Open R-AOV-4.

2.4.8.14 Manually shut R-PCV-30 so as to give a pressure change of more than 5 psig on SG-PT-1.

2.4.8.15 Check and open H2-PCV-2, H2-FCV-2, H2-AOV-20, CF-SV-2, and RAOV-3.

2.4.8.16 Set SG-PCV-1 ramp down program controller at 5 psig as the target pressure and $15 \mathrm{psig} / \mathrm{minute}$ as the ramp down speed.

2.4.8.17 Start the ramp down of plant pressure.

2.4.8.18 Shut N2-V-3 and N2-V-7.

2.4.8.19 When SG-PT-1 reaches 5 psig, manually shut SG-PCV-1.

2.4.8.20 Confirm that N2-PCV-4 and N2-PCV-5 secondary pressure setpoints are at 200 psig.

2.4.8.21 Open N2-V-6 and N2-V-8 to pressurize the plant.

2.4.8.22 When SG-PT-1 and R-PT-30 reach 150 psig, shut N2-V-6 and N2-V8.

2.4.8.23 Open SG-PCV-1 to de-pressurize the plant to 5 psig and close SGPCV-1.

2.4.8.24 Repeat Steps 2.4.8.21 through 2.4.8.23 three times.

2.4.8.25 Keep the plant pressure at 5 psig.

2.4.8.26 Set H2O-FSL-2 tip bypass to ON and H2O-P-1 tip bypass to OFF .

2.4.8.27 Turn off H2O-P-1.

2.4.8.28 Set all valves in accordance with Appendix D Normal Shutdown line up. 


\section{APPENDIX 1 - COAL TO SNG BENCH SCALE TEST REACTOR NORMAL SHUTDOWN LINEUP CHECKLIST}

\section{Coal to SNG Bench Scale Test Reactor Normal Shutdown Lineup Checklist}

Date Inspected:

Operator:

\begin{tabular}{|c|c|c|c|c|}
\hline $\begin{array}{c}\text { Component } \\
\text { Number }\end{array}$ & $\begin{array}{l}\text { Component } \\
\text { Description }\end{array}$ & $\begin{array}{c}\text { Required Position } \\
\text { (On, Off, Tagout, } \\
\text { Lockout, etc.) }\end{array}$ & Check & P\&ID Drawing \\
\hline $\mathrm{N} 2-\mathrm{V}-7$ & Valve & Shut & $\square$ & \multirow{9}{*}{$\begin{array}{c}\mathrm{N}_{2} \text { High Pressure } \\
\text { Purge Panel }\end{array}$} \\
\hline N2-V-6 & Valve & Shut & $\square$ & \\
\hline N2-V-4 & Valve & Shut & $\square$ & \\
\hline N2-V-5 & Valve & Shut & $\square$ & \\
\hline N2-V-2 & Valve & Shut & $\square$ & \\
\hline N2-V-3 & Valve & Shut & $\square$ & \\
\hline N2-V-8 & Valve & Shut & $\square$ & \\
\hline N2-V-18 & Valve & Shut & $\square$ & \\
\hline N2-V-95 & Valve & Shut & $\square$ & \\
\hline N2-V-1 & Valve & Open & $\square$ & \multirow{15}{*}{$\begin{array}{l}\text { Instrument } \\
\mathrm{N}_{2} \text { Panel }\end{array}$} \\
\hline $\mathrm{N} 2-\mathrm{V}-\mathrm{9}$ & Valve & Open & $\square$ & \\
\hline N2-V-10 & Valve & Open & $\square$ & \\
\hline N2-V-11 & Valve & Open & $\square$ & \\
\hline N2-V-12 & Valve & Open & $\square$ & \\
\hline N2-V-13 & Valve & Open & $\square$ & \\
\hline N2-V-14 & Valve & Open & $\square$ & \\
\hline N2-V-15 & Valve & Open & $\square$ & \\
\hline N2-V-20 & Valve & Open & $\square$ & \\
\hline N2-V-21 & Valve & Open & $\square$ & \\
\hline N2-V-22 & Valve & Open & $\square$ & \\
\hline N2-V-23 & Valve & Open & $\square$ & \\
\hline N2-V-24 & Valve & Open & $\square$ & \\
\hline N2-V-25 & Valve & Open & $\square$ & \\
\hline N2-V-50 & Valve & Open & $\square$ & \\
\hline N2-V-35 & Valve & Shut & $\square$ & Emergency Purge $\mathrm{N}_{2}$ \\
\hline 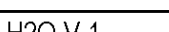 & Volno & Chut & $\square$ & \multirow{7}{*}{ Cooling Water Tank } \\
\hline $\mathrm{H} 2 \mathrm{O}-\mathrm{V}-1$ & Valve & Shut & $\square$ & \\
\hline $\mathrm{H} 2 \mathrm{O}-\mathrm{V}-2$ & Valve & Open & $\square$ & \\
\hline $\mathrm{H} 2 \mathrm{O}-\mathrm{V}-3$ & Valve & Open & $\square$ & \\
\hline $\mathrm{H} 2 \mathrm{O}-\mathrm{V}-4$ & Valve & Open & $\square$ & \\
\hline $\mathrm{H} 2 \mathrm{O}-\mathrm{P}-1$ & Pump & Off & $\square$ & \\
\hline H2O-P-2 & Pump & Off & $\square$ & \\
\hline $\mathrm{H} 2 \mathrm{O}-\mathrm{V}-7$ & Valve & Shut & $\square$ & In Frame \\
\hline
\end{tabular}

Page 1 


\section{APPENDIX 1 - COAL TO SNG BENCH SCALE TEST REACTOR NORMAL SHUTDOWN LINEUP CHECKLIST (CONTINUED)}

\section{Coal to SNG Bench Scale Test Reactor Normal Shutdown Lineup Checklist (Continued)}

\begin{tabular}{|c|c|c|c|c|}
\hline $\begin{array}{c}\text { Component } \\
\text { Number }\end{array}$ & $\begin{array}{l}\text { Component } \\
\text { Description }\end{array}$ & $\begin{array}{c}\text { Required Position } \\
\text { (On, Off, Tagout } \\
\text { Lockout, etc.) }\end{array}$ & Check & P\&ID Drawing \\
\hline $\mathrm{H} 2 \mathrm{O}-\mathrm{V}-8$ & Valve & Shut & $\square$ & \multirow[t]{5}{*}{ In Frame } \\
\hline $\mathrm{H} 2 \mathrm{O}-\mathrm{V}-9$ & Valve & Shut & $\square$ & \\
\hline $\mathrm{H} 2 \mathrm{O}-\mathrm{V}-10$ & Valve & Shut & $\square$ & \\
\hline $\mathrm{H} 2 \mathrm{O}-\mathrm{V}-16$ & Valve & Shut & $\square$ & \\
\hline $\mathrm{H} 2 \mathrm{O}-\mathrm{V}-17$ & Valve & Open & $\square$ & \\
\hline $\mathrm{H} 2-\mathrm{V}-1$ & Lock Valve & Shut - Lockout & $\square$ & \multirow{12}{*}{$\begin{array}{c}\text { Hydrogen } \\
\text { Panel }\end{array}$} \\
\hline $\mathrm{H} 2-\mathrm{V}-3$ & Valve & Open & $\square$ & \\
\hline $\mathrm{H} 2-\mathrm{V}-4$ & Valve & Open & $\square$ & \\
\hline $\mathrm{H} 2-\mathrm{V}-5$ & Valve & Shut & $\square$ & \\
\hline $\mathrm{H} 2-\mathrm{V}-6$ & Valve & Open & $\square$ & \\
\hline $\mathrm{H} 2-\mathrm{V}-7$ & Valve & Open & $\square$ & \\
\hline $\mathrm{H} 2-\mathrm{V}-8$ & Valve & Shut & $\square$ & \\
\hline $\mathrm{H} 2-\mathrm{V}-9$ & Valve & Shut & $\square$ & \\
\hline $\mathrm{H} 2-\mathrm{V}-10$ & Valve & Shut & $\square$ & \\
\hline $\mathrm{H} 2-\mathrm{V}-91$ & Valve & Shut & $\square$ & \\
\hline $\mathrm{H} 2-\mathrm{V}-92$ & Valve & Shut & $\square$ & \\
\hline $\mathrm{H} 2-\mathrm{V}-22$ & Valve & Shut & $\square$ & \\
\hline CF-V-1 & Valve & Shut & $\square$ & \multirow{3}{*}{ Coal Feeder Panel } \\
\hline CF-V-2 & Valve & Open & $\square$ & \\
\hline N2-V-16 & Valve & Shut & $\square$ & \\
\hline $\mathrm{H} 2 \mathrm{O}-\mathrm{V}-5$ & Valve & Open & $\square$ & \multirow{5}{*}{ In Frame } \\
\hline $\mathrm{H} 2 \mathrm{O}-\mathrm{V}-6$ & Valve & Open & $\square$ & \\
\hline $\mathrm{H} 2 \mathrm{O}-\mathrm{V}-21$ & Valve & Shut & $\square$ & \\
\hline $\mathrm{H} 2 \mathrm{O}-\mathrm{V}-22$ & Valve & Shut & $\square$ & \\
\hline C-MTR-1 & Motor & Off & $\square$ & \\
\hline$R-V-1$ & Valve & Open & & \multirow{5}{*}{ Balancing Panel } \\
\hline$R-V-2$ & Valve & Shut & $\square$ & \\
\hline R-V-6 & Valve & Open & $\square$ & \\
\hline R-V-96 & Valve & Shut & $\square$ & \\
\hline AR-V-1 & Valve & Shut & $\square$ & \\
\hline R-V-4 & Valve & Shut & $\square$ & \multirow{5}{*}{ In Frame } \\
\hline R-V-5 & Valve & Open & $\square$ & \\
\hline R-V-7 & Valve & Shut & $\square$ & \\
\hline R-V-8 & Valve & Shut & $\square$ & \\
\hline R-V-9 & Valve & Shut & $\square$ & \\
\hline
\end{tabular}

Page 2 


\section{APPENDIX 1 - COAL TO SNG BENCH SCALE TEST REACTOR NORMAL SHUTDOWN LINEUP CHECKLIST (CONTINUED)}

\section{Coal to SNG Bench Scale Test Reactor Normal Shutdown Lineup Checklist (Continued)}

\begin{tabular}{|c|c|c|c|c|}
\hline $\begin{array}{c}\text { Component } \\
\text { Number }\end{array}$ & $\begin{array}{l}\text { Component } \\
\text { Description }\end{array}$ & $\begin{array}{c}\text { Required Position } \\
\text { (On, Off, Tagout, } \\
\text { Lockout, etc.) }\end{array}$ & Check & P\&ID Drawing \\
\hline R-V-10 & Valve & Shut & $\square$ & \\
\hline R-V-78 & Valve & Shut & $\square$ & \\
\hline CON-V-1 & Valve & Open & $\square$ & \multirow{4}{*}{ In Frame } \\
\hline CON-V-2 & Valve & Shut & $\square$ & \\
\hline CON-V-3 & Valve & Shut & $\square$ & \\
\hline CON-V-20 & Valve & Shut & $\square$ & \\
\hline CON-V-4 & Valve & Shut & $\square$ & \multirow{3}{*}{ SNG Product Panel } \\
\hline SG-V-1 & Valve & Open & $\square$ & \\
\hline SG-V-2 & Valve & Shut (Commissioning) & $\square$ & \\
\hline SG-V-3 & Valve & Shut & $\square$ & \multirow{9}{*}{ SNG Product Panel } \\
\hline SG-V-10 & Valve & Open & $\square$ & \\
\hline SG-V-11 & Valve & Shut & $\square$ & \\
\hline SG-V-12 & Valve & Open & $\square$ & \\
\hline SG-V-13 & Valve & Open & $\square$ & \\
\hline SG-V-14 & Valve & Shut & $\square$ & \\
\hline SG-V-15 & Valve & Shut & $\square$ & \\
\hline SG-V-22 & Valve & Shut & $\square$ & \\
\hline SG-V-22 & Valve & Shut & $\square$ & \\
\hline N2-AOV-40 & Air Operated Valve & Shut & $\square$ & SNG2000.1 \\
\hline H2-PCV-1 & $\begin{array}{c}\text { Pressure Control } \\
\text { Valve }\end{array}$ & Shut & - & \multirow{4}{*}{$\begin{array}{c}\text { SNG2000.3 } \\
1 / 2\end{array}$} \\
\hline H2-FCV-1 & Flow Control Valve & Shut & $\square$ & \\
\hline $\mathrm{H} 2-\mathrm{AOV}-1$ & Air Operated Valve & Shut & $\square$ & \\
\hline H2-PCV-2 & $\begin{array}{c}\text { Pressure Control } \\
\text { Valve }\end{array}$ & Shut & - & \\
\hline $\mathrm{H} 2-\mathrm{FCV}-2$ & Flow Control Valve & Shut & $\square$ & \multirow{4}{*}{$\begin{array}{c}\text { SNG } 2000.3 \\
2 / 2\end{array}$} \\
\hline H2-MFC-1 & Flow Control Valve & Shut & $\square$ & \\
\hline H2-AOV-2 & Air Operated Valve & Shut & $\square$ & \\
\hline $\mathrm{H} 2-\mathrm{AOV}-20$ & Air Operated Valve & Shut & $\square$ & \\
\hline CF-SV-1 & Solenoid Valve & Shut & $\square$ & \multirow{6}{*}{ SNG2000.4 } \\
\hline CF-SV-2 & Solenoid Valve & Open & $\square$ & \\
\hline CF-SV-3 & Solenoid Valve & Shut & $\square$ & \\
\hline CF-SV-4 & Solenoid Valve & Shut & $\square$ & \\
\hline CF-PCV-1 & $\begin{array}{c}\text { Pressure Control } \\
\text { Valve }\end{array}$ & Shut & $\square$ & \\
\hline CF-AOV-4 & Air Operated Valve & Shut & $\square$ & \\
\hline
\end{tabular}

Page 3 


\section{APPENDIX 1 - COAL TO SNG BENCH SCALE TEST REACTOR NORMAL SHUTDOWN LINEUP CHECKLIST (CONTINUED)}

Coal to SNG Bench Scale Test Reactor Normal Shutdown Lineup Checklist (Continued)

\begin{tabular}{|c|c|c|c|c|}
\hline $\begin{array}{c}\text { Component } \\
\text { Number }\end{array}$ & $\begin{array}{l}\text { Component } \\
\text { Description }\end{array}$ & $\begin{array}{c}\text { Required Position } \\
\text { (On, Off, Tagout, } \\
\text { Lockout, etc.) }\end{array}$ & Check & P\&ID Drawing \\
\hline R-PCV-1 & $\begin{array}{c}\text { Pressure Control } \\
\text { Valve }\end{array}$ & Shut & $\square$ & \multirow{6}{*}{ SNG2000.6 } \\
\hline R-MFC-1 & Flow Control Valve & Shut & $\square$ & \\
\hline R-AOV-4 & Air Operated Valve & Open & $\square$ & \\
\hline R-PCV-30 & $\begin{array}{c}\text { Pressure Control } \\
\text { Valve }\end{array}$ & Shut & $\square$ & \\
\hline R-AOV-3 & Air Operated valve & Shut & $\square$ & \\
\hline AR-MFC-1 & Flow Control Valve & Shut & $\square$ & \\
\hline R-AOV-1 & Air Operated Valve & Open & $\square$ & \multirow{4}{*}{ SNG2000.8 } \\
\hline R-AOV-2 & Air Operated Valve & Shut & $\square$ & \\
\hline R-SV-7 & Solenoid Valve & Shut & $\square$ & \\
\hline R-SV-8 & Solenoid valve & Shut & $\square$ & \\
\hline CON-AOV-1 & Air Operated Valve & Open & $\square$ & SNG2000.9 \\
\hline SG-PCV-1 & $\begin{array}{c}\text { Pressure Control } \\
\text { Valve }\end{array}$ & Shut & $\square$ & SNG2000.10 \\
\hline
\end{tabular}




\title{
APPENDIX K
}

\section{Bench Scale Hydrogasification Testing}

\author{
Startup Procedure
}


Development of a Hydrogasification Process for

Coproduction of SNG and Electrical Power from Western Coals

Final Report

THIS PAGE INTENTIONALLY LEFT BLANK 


\title{
COAL TO SNG \\ BENCH SCALE TEST REACTOR STARTUP PROCEDURES
}

\author{
FINAL \\ JULY 2010 \\ Arizona Public Service Company \\ 400 NORTH 5TH STREET \\ Phoenix, AZ 85003
}

\author{
Principal Investigator \\ Dr. Xiaolei Sun \\ 602.250.1510 \\ 602.250.1505 \\ xiaolei.sun@aps.com
}




\section{COAL TO SNG \\ BENCH SCALE TEST REACTOR STARTUP PROCEDURES}

\section{TABLE OF CONTENTS}

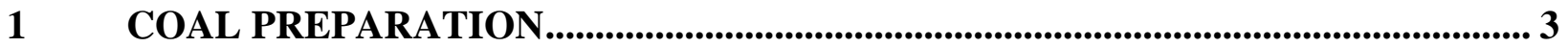

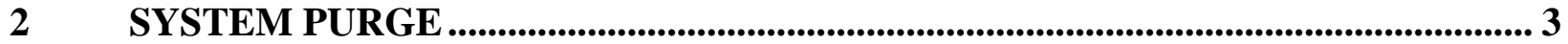

3 LEAK TEST AND PRESSURING THE PLANT .......................................................... 5

4 TEST OPERATION PREPARATION .......................................................................6 6

\section{APPENDiXES}

Appendix A - Torque Table A-1

Appendix B - Order of Bolt Tightening .......................................................................... A-1

Appendix C - Test Plan Data Sheet.. 


\section{CoAl Preparation}

\subsection{Coal/Silica Mixing}

One day before operation, follow these steps:

1.1.1. Weigh $19 \mathrm{lb}$. of coal and $1 \mathrm{lb}$. of fumed silica ion case of $5 \%$ mixture and pour into a large container. Mix the coal and silica by using power-drive hand-mixer to get uniform color for about one hour.

1.1.2. Make sampling of $0.5 \mathrm{lb}$ coal into the bottle from the bulk for coal analysis, and weigh $19 \mathrm{lb}$ of mixture and transfer it the big funnel for loading.

\subsection{Coal Loading to Coal Hopper}

One day before operation, ensure that the appropriate personal protective equipment (PPE) is used while filling the coal hopper. As a minimum, the operator shall wear a dust mask, gloves, apron, and safety glasses. Follow these steps:

\subsubsection{Shut and check shut R-AOV-3, CF-SV-1, CF-AOV-4, CF-PCV-1, and CF-SV-3.}

1.2.2 Open N2-V-16 and vent the coal hopper and check that the hopper is 0 psig at N2PT-16

1.2.3 Loosen the feed hopper flange bolts and remove the flange.

1.2.4 Pour the coal minimizing the amount of coal that goes airborne.

1.2.5 Clean the mating surfaces between the flange and the coal hopper by brushing away loose particulate matter or by using an approved solvent.

1.2.6 Use a new gasket, replace the hopper flange, and install and tighten all flange bolts in accordance with design torques in the Appendix A and bolt tightening order in the Appendix B.

\subsubsection{Shut N2-V-16}

\section{System PuRge}

One day before operation, follow these steps:

2.1 Ensure the system is in a normal shutdown lineup in Appendix D

2.2 Confirm that the N2 tube trailer has the pressure over 1600 psig.

2.3 Shut, Check Shut SG-PCV-1, R-PCV-30

2.4 Shut, check shut H2-FCV-1, H2-FCV-2, R-MFC-1(set point ; 0scf3/h)

2.5 Confirm regulator N2-PCV-1 set point at $1150 \mathrm{psig}$

2.6 Open N2-V-3 and N2-V-7 
2.7 Open, Check Open H2-V-1, H2-V-3, H2-V-4, H2-V-6/ R-V-1, R-V-6/ SG-V-1, SG-V-2

2.8 Open, Check Open H2-AOV-1, H2-AOV-2, H2-MFC-1(input the set point $2000 \mathrm{cc} / \mathrm{min}$ ), H2-AOV-20/ CF-SV-2/ R-AOV-1/ CON-AOV-1

2.9 Make the historical trend graph on the screen for R-PT-30, R-PT-3, N2-PT-16, CF-PT-1, SG-PT-1

2.10 Open, check open R-AOV-3

2.11 Set R-PCV-30 at 10 psig and auto control mode

2.12 Open R-PCV-1 to 5\% opening.

2.13 Open H2-PCV-1 to \% opening.

2.14 Set R-MFC-1 flow rate at $100 \mathrm{scf}^{3} / \mathrm{hr}$ and set auto control to start to pressurize the annulus space at first.

2.15 When R-PCV-30 reached at about 5 psig, and set H2-FCV-1 flow rate at $50 \mathrm{scf}^{3} / \mathrm{hr}$ on N2 and set auto control to start to pressurize reactor tube.

2.16 Increase R-MFC-1 flow rate to $300 \mathrm{scf} 3 / \mathrm{h}$ (with opening R-PCV-1) and increase/adjust also H2-FCV-1 flow rate (between $150-200 \mathrm{scf}^{3} / \mathrm{hr} h$ with opening H2-PCV-1) to keep R-dPT-1 within +10 psig.

2.17 Increase H2-PCV-1 and R-PCV-30 in 1\% increment along with the downstream pressure.

2.18 When R-PT-30 reached at about 160 psig, shut R-PCV-1 and H2-PCV-1.

2.19 Shut R-AOV-3.

2.20 Start to slowly depressurize the coal hopper by opening N2-V-16 manually.

2.21 (Note: The speed of depressurizing is same or less than as the speed of pressurizing. Make sure that there are no particles in the vent gas from N2-V-16)

2.22 Start to depressurize the reactor pressure by opening SG-V-21 and 22 to chase N2-PT-16 depressurizing.

2.23 Set R-PCV-30 set point at 0 psig and adjust SG-V-21/22 to keep the R-PT-3 less than RPT-30 and keep R-dPT-1 within 10 psig.

2.24 When N2-PT-16 reaches to 5 psig, make the N2-V-16 full open and close after the vent gas is stopped.

2.25 When R-PT-3 reaches to 5 psig, make the SG-V-21/22 full open and close SG-V-21/22 after the SG-PT-1 reaches to less than 0 psig.

2.26 Open R-AOV-3.

2.27 Set R-PCV-30 at 10 psig

2.28 Repeat 2.8 through 2.22 two times.

${ }^{\circledR} 2010$ Arizona Public Service All Rights Reserved 


\section{Leak Test And Pressuring the Plant}

These process steps listed below are performed with continuous operation from Section 2 System Purge:

3.1 Shut, Check Shut R-AOV-4, CF-AOV-4

3.2 Open, Check Open R-AOV-1, R-AOV-3

3.3 Set SG-PCV-1 set point at 400 psig and turn on auto control mode.

3.4 Open R-PCV-1 to $5 \%$ opening

3.5 Open $\mathrm{H} 2-\mathrm{PCV}-1$ to $5 \%$ opening

3.6 Set R-MFC-1 flow rate at $100 \mathrm{scf} 3 / \mathrm{h}$ and set auto control to start to pressurize the annulus space at first

3.7 When R-PCV-30 reached at about 5 psig, and set H2-FCV-1 flow rate at $50 \mathrm{scf}^{3} / \mathrm{hr}$ on N2 and set auto control to start to pressurize reactor tube.

3.8 Increase R-MFC-1 flow rate to $300 \mathrm{scf} 3 / \mathrm{h}$ (with opening R-PCV-1) and increase/adjust also H2-FCV-1 flow rate (between $150-200 \mathrm{scf}^{3} / \mathrm{hr}$ with opening H2-PCV-1) to keep RdPT-1 within +10 psig.

3.9 Increase H2-PCV-1 and R-PCV-30 in $1 \%$ increment along with the downstream pressure.

3.10 When the plant pressure get higher and higher, the H2-FM-1 is increased gradually in 5 $\mathrm{scf} 3 / \mathrm{h}$ increments and R-MFC- 1 is decreased gradually in $5 \mathrm{scf} 3 / \mathrm{h}$ increments to keep RdPT-1 between 4 and 10 psi.

3.11 When SG-PCV-1 start to control the reactor pressure, perform leak test using liquid leak detector on flanges, joints and the parts dismantled after the previous run for sampling and maintenance

3.11.1 If a leakage is found out, try to tighten in accordance with the designed torque.

3.11.2 If the leakage is not stopped, shut R-PCV-1, H2-PCV-1, H2-PCV-2 to stop gas flow in.

3.11.3 Open CF-SV-3 and set CF-PCV-1 ramp down conditions (start pressure and ramp down time) to depressurize the coal hopper at first.

3.11.4 Open SG-V-1-21 \& 22 to depressurize the reactor system to ambient pressure keeping the coal hopper pressure lower than the reactor tube (R-PI-3) by 30 psig.

3.11.5 Exchange the gasket and/or perform necessary action.

3.11.6 Perform the N2 purge referring section 2 procedure using N2 valve and vent valve where is nearest to the dismantled flange or joint.

3.11.7 Pressurize the plant in accordance with the procedure from 4.4

${ }^{\circledR} 2010$ Arizona Public Service All Rights Reserved 


\subsubsection{Make sure no leakage}

3.12 Change SG-PCV-1 set point at 800 and continue to pressurize the plant by increasing the inlet pressures (R-PCV-1 and H2-PCV-1) to 800 psig and perform the second leak test and then 800 PSIG as the final leak test to make sure no leakage in the system.

3.13 Record the H2-PCV-1 and R-PCV-1 valves opening.

3.14 After make sure that there is no leakage by liquid leak detector at 800 psig, shut H2PCV-1 \& R-PCV-1 and H2-FCV-1 \& R-MFC-1. The plant is kept automatically by SGPCV-1 setting.

3.15 Adjust the annulus pressure to get R-dPT-1 at 5 to 10 psig by flowing gas through RMFC-1.

3.16 Make the plant block by closing N2-V-3, N2-V-7, SG-V-1 and R-V-96.

3.17 Shut instrument N2 valve to the R-PCV-30 and small regulator valve on the H2-FCV-1 to save the instrument $\mathrm{N} 2$.

\section{TESt Operation Preparation}

\subsection{Prior Meeting}

Test Manager shall prepare the Test Condition Data Sheet prior to the test and call a prior meeting to verify the test condition according to the Test Plan.

\subsection{Miscellaneous Preparation}

\subsubsection{Labview Program and Power}

4.2.1.1 If the Labview Program shows any error, stop the program and shut down the computer and start again.

\subsubsection{Heater Control Panel}

1. Turn on the reactor heater power breaker

2. Confirm the system switch-on, which is on the left panel door.

4.2.1.3 Turn on the breaker switches for two pumps and coal feeder in the box.

4.2.2 Plant pressure: Verify that the decrease of plant pressure through, which means over night the pressure should be approximately 50 psi or below.

4.2.3 Instrument N2 supply: Verify the Instrument N2 supply from 16 N2 pack is higher than 500 psig. (Keep the spare 16 pack always).

4.2.4 Trip System: Make the trip "Bypass ON" on all of heater temperature triggers, R$\mathrm{TI}-10^{\mathrm{HH}}, \mathrm{H} 2 \mathrm{O}-\mathrm{PT}-2_{\mathrm{LL}}$.

4.2.5 N2 Tube Trailer: Verify the N2 tube trailer pressure is higher than 1600 psig. If the pressure is close to 1600 psig, make the refill order for next test operation. 
4.2.6 N2 PAK for Emergency: Verify that the N2 PAK is full (2400 psig) through N2PCV-2. However this system will not be used for a while.

4.2.7 Gas Chromatograph and Mass Spectrometer: Verify the GC/MS is ready to operate.

\section{TEST RuN}

\subsection{Plant block release}

5.1.1 Open instrument N2 valve to the R-PCV-30 and small regulator valve on the H2FCV-1 to get 30 psig.

\subsubsection{Open N2-V-3, N2-V-7, R-V-96 and SG-V-1}

5.2 Pressurizing and pressure balancing build up

5.2.1 Make trip bypass OFF on N2-PT-50 $0_{\mathrm{LL}}$

5.2.2 Confirm SG-PCV-1 set point at 1010 psig and set auto control mode.

5.2.3 Make the time history trend graph for flow rates.

5.2.4 Shut, check shut H2-PCV-1, R-PCV-1

5.2.5 Shut, Check shut H2-FCV-1 (0\% manual mode) and R-MFC-1 (0 $50 \mathrm{scf}^{3} / \mathrm{hr}$ set point)

5.2.6 Set R-PCV-30 dP set point at 10 psig and set auto control mode

5.2.7 Open H2-PCV-1 and R-PCV-1 to the valve opening which were recorded at the end of pressurizing in 3.13

5.2.8 IF the reading of R-dPT-1 is less than 5psig, set R-MFC-1 set point at $50 \mathrm{scf}^{3} / \mathrm{hr}$ and start to pressurize the annulus space. When the R-dPT- 1 approach to 5 psig, set H2-FCV-1 at $50 \mathrm{scf}^{3} / \mathrm{hr}$ on N2 scale and auto control mode.

5.2.9 IF the reading of R-dPT- 1 is higher than 5 psig, set H2-FCV-1 at $50 \mathrm{scf}^{3} / \mathrm{hr}$ on N2 scale and auto control mode to start pressurize the reactor tube side When the RdPT-1 approach to 5 psig, set R-MFC-1 set point at $50 \mathrm{scf}^{3} / \mathrm{hr}$ and start to pressurize the annulus space.

5.2.10 Increase H2-FCV-1 flow set point in $50 \mathrm{scf}^{3} / \mathrm{hr}$ increments gradually to 300 $\mathrm{scf}^{3} / \mathrm{hr}$ together with opening H2-PCV-1 and also adjusting the R-MFC-1 flow set point to keep R-dPT-1 between 5 and 10 psig. The final set point on H2-PCV-1 and R-PCV-1 will be 63-65\% to reach to 1010 psig on SG-PCV-1.

5.2.11 When the SG-PCV-1 start to control the plant pressure, stop to open H2-PCV-1 and R-PCV-1 and wait for the plant pressure is settle down.

5.2.12 Change the R-PCV-30 set point to 5 psig.

5.2.13 Increase R-MFC-1 flow rate according to the Test Plan Data Sheet

${ }^{\odot} 2010$ Arizona Public Service All Rights Reserved 


\subsubsection{Shut R-AOV-3}

5.2.15 Verify proper response of all pressure and flow instruments.

5.3 H2 in Operation of Facility in H2 Park

5.3.1 Check Open V-306 in H2 Park

5.3.2 Shut, Check shut V-330 on Test Bed Panel

5.3.3 Open, Check open V-305, V-318, and V342 on Test Bed Panel

5.3.4 Confirm PCV-300 secondary pressure at 4000 psig

5.3.5 Confirm PCV-303 secondary pressure at 1800 psig

Note: For operation on SNG panel and Labview, do the following steps

5.3.6 Verify H2-PCV-30 set point at 1200 psig

5.3.7 Be fixed H2-FCV-1 \% opening at present value on manual mode.

5.3.8 Open H2-V-1 at first

5.3.9 Shut N2-V-3 secondly

5.3.10 Select H2 as the fluid on H2-FIC-1 (Micro Motion flow meter).

5.3.11 When the flow indication on H2-FIC-1 become stable, set H2-FCV-1 set point at the flow rate on the indicator and set auto control mode.

5.3.12 Confirm proper response of H2-FCV-1

5.3.13 Change H2-FCV-1 set point in about $-50 \mathrm{sft} 3 / \mathrm{h}$ increments from the present value to get the $\mathrm{H} 2$ flow rate in accordance with the Test Plan Data Sheet.

5.3.14 In parallel with operation in step 5.3.13, adjust H2-PCV-1 opening to get a stable control point on $\mathrm{H} 2-\mathrm{FVC}-1$ (about $50 \%$ opening).

5.3.15 Confirm H2-FCV-1 proper response

5.3.16 Start the periodical Gas Chromatograph Analysis

5.4 Turn on Cooling Water

5.4.1 Open, check open H2O-V-4 and verify the tank water.

5.4.2 Open, check open H2O-V-7

5.4.3 Turn on the breaker lock lever on the connection boxes.

5.4.4 Switch on H2O-P-1

5.4.5 Confirm that the H2O-PT-1 indicate above 20 psig. 


\subsection{Heating Up}

5.5.1 Verify the all of heater switch off, and set point of power out put is 0 .

5.5.2 Verify all of trip triggers on heater temperature are 'Trip Bypass ON'.

\subsubsection{Shut R-AOV-3.}

5.5.4 Make the time history trend graphs on H2 Preheater, Connecting tube and reactor tube.

5.5.5 Double click the Heater-on switch on the screen

5.5.6 Set all of heater temperature (TIC-14A-3, TIC-13A-3, TIC-12A-3, TIC-11A-3) set point at $2000{ }^{\circ} \mathrm{F}$ and turn on all heaters to start the heating up.

5.5.7 Verify the all $\mathrm{H} 2$ pre-heater TC temperatures start heating up.

5.5.8 When either temperature of TC (TE-xxx-A3/B3) controlling the H2 pre-heater output hit the first high alarm point, change the heater set point according to the Test Plan Data Sheet.

5.5.9 Set PH5A (HTR-15/16/17) output at $10 \%$ and turn on the heaters.

5.5.10 Set PH5B (HTR-18N/18S) \& PH5C (HTR-18E/18W) output at $10 \%$ and turn on the heaters.

5.5.11 Verify all TC belonging to the connecting tube start heating up.

5.5.12 Increase PH5A, PH5B, and PH5C output in 10\% increments in about every 10 15 minutes to $70 \%$.

5.5.13 When either heater temperatures (TE-xxx-3) in PH5A, PH5B, and PH5C hit the first high alarm point, reduce/adjust the heater output to keep the heater temperature below than $1650 \mathrm{~F}$.

5.5.14 Set the heater output at $10 \%$ on the operating reactor heaters according to the Data Sheet and turn on heaters.

5.5.15 Increase the reactor tube heater output in 10\% increments looking at the tube temperature profile.

5.5.16 Adjust the reactor heater output to make the reaction temperature profile to be flat according to the Test Plan Data Sheet.

\subsection{Coal In}

5.6.1 Check shut H2-FCV-2.

5.6.2 Check Open H2-V-6, CF-SV-2, CF-SV-4.

5.6.3 Check Open H2-AOV-2, H2-AOV-20.

5.6.4 Verify H2-MFC-1 set point is $2000 \mathrm{cc} / \mathrm{min}$. 


\subsubsection{Open R-AOV-3.}

5.6.6 Open H2-PCV-2 to get the opening which is $1 \%$ lower than H2 PCV-1 opening.

5.6.7 Set H2-FCV-2 set point at the flow rate in the Test Plan Data Sheet and set auto control mode.

5.6.8 If the opening of H2-FCV-2 goes to full open, shut the H2-FCV-2 by manual mode.

5.6.9 Increase H2-PCV-2 opening in $0.1 \%$ increments to get the flow rate with a moderate valve opening on $\mathrm{H} 2-\mathrm{FCV}-2$ step by step.

5.6.10 If the opening of $\mathrm{H} 2-\mathrm{FCV}-2$ goes to $0 \%$ open, shut the H2-FCV-2 by manual mode.

5.6.11 Decrease H2-PCV-2 opening in 0.1\% increments to get the flow rate with a moderate valve opening on $\mathrm{H} 2-\mathrm{FCV}-2$ step by step.

5.6.12 Verify the H2-FCV-1 and H2-FCV-2 indicate the flow rates in the Test Plan Data Sheet, and stable flow.

5.6.13 Make the trip bypass OFF on DT-21A- $1^{\mathrm{HH}}$, DT-21B- $4^{\mathrm{HH}}$, DT-21B- $1^{\mathrm{HH}}$, DT-22A$1^{\mathrm{HH}}, \mathrm{DT}-22 \mathrm{~B}-4^{\mathrm{HH}}, \mathrm{DT}-22 \mathrm{~B}-1^{\mathrm{HH}}$

5.6.14 Set CF-MD-1 set point at 100 rpm on the motor speed and start the coal feeder.

5.6.15 Increase the CF-MD-1 set point in 100 rpm increments in every one minute to the final set point in the Test Plan Data Sheet, and confirm the coal-in by observing a temperature drop on the TE-21A-1/21B-4/21B-1/27A-1.

5.6.16 Adjust the No. 1 and No. 2 reactor heater output to reach the reactor temperature in accordance with the Test Plan Data Sheet, watching a temperature rise due to an exothermic reaction at the top part of reactor tube, and also adjusting the reactor heater power output to get a flat temperature profile (on the screen) at the target temperature.

5.6.17 Verify proper response of all pressure and temperature instruments.

5.6.18 Carefully keep watching the temperature on the TE-21A-1/21B-4/21B-1/27A-1. If those temperature start to increase simultaneously/simply suddenly, it indicate that the coal would be clogged between $\mathrm{H} 2$ nozzle and coal feeder.

1. Turn off the coal feeder

2. X Decrease No. 1 heater output to the value before the coal in.

3. Shut R-AOV-3 and wait for a while until N2-PT-16 reach to the same pressure as H2-PCV-2.

4. Open R-AOV-3 looking at the pressure trend graph and verify N2PT-16 return to the previous value 
5. If the N2-PT-16 does not return to the previous pressure, it means that the clogging is not cleared. Try to repeat items 3 and 4) several times, and if the clogging is not cleared still, report to the Test Manager.

6. If the N2-PT-16 returned to the previous pressure, verify the $\mathrm{H} 2$ FCV-2 return to the normal flow rate.

7. Turn on the Coal feeder and verify the reactor top zone temperature (the TE-21A-1/21B-4/21B-1/27A-1) start to drop to the previous temperature.

8. Adjust No. 1 heater output to get the temperature profile before the clogging.

\subsection{Product Gas Data Acquisition Start(steady state operation)}

5.7.1 Confirm that the gas component by GC Analysis is in a steady state (stable H2 and/or $\mathrm{CH} 4$ concentration in product gas).

5.7.2 Estimate the remaining coal weight in the hopper and run time which we can feed the coal considering the time spent since coal feed start in 5.6 operation

5.7.3 Decide the time (xx:xx) of data acquisition start.

5.7.4 Shut R-AOV-1 and CON-AOV-1 at xx:xx (starting time)

5.7.5 Verify that the GC and MS are operating properly.

5.8 Steady-State Operation

5.8.1 Keep adjusting the reactor heater output to bet flat temperature profile.

5.8.2 Continuously monitor system temperatures, pressures and flow rates.

$>$ If the reactor top zone temperatures (TE-21A-1/21B-4/21B-1/27A-1) start to increase simultaneously/simply suddenly, it indicates that the coal would be clogged between $\mathrm{H} 2$ nozzle and coal feeder. Execute the procedure in 5.6.18

If an alarm on pressure and flow rate in local auto control mode is on, perform the following steps

1. Check the time history trend and find out whether it is swinging or simple increase/decrease.

2. If it is a swinging, change the auto control mode to manual control. Adjust the valve opening to get correct number in accordance with the Test Plan Data Sheet. Make a tuning by changing P, I, D and return to auto control mode.

3. If it is a simple increase/decrease, change the auto control mode to manual control, and report to Test Manager and control engineer to find out the problem. 
$>$ If an alarm on pressure balancing system in program control mode is on

1. Turn off the program control.

2. Watch the control for a while by checking the time history trend whether the local auto control is stable.

3. If the problem is a swinging on the local control mode, take a required action mentioned above.

4. If it is a simple increase/decrease, change the auto control mode to manual control, and report to Test Manager and control engineer to find out the problem.

$>$ If high alarm on pre-heater tube temperature(TE-xxC-yy) is on,

1. Check the time history trend of the temperatures.

2. Decrease the temperature set point to clear the alarm set point.

3. If the temperature hit the heater off set point, turn on the heater after the temperature clear the alarm set point, and set lower set point than before.

4. Keep watching the trend and report the Test Manager

If high alarm on reactor tube temperature is on,

1. Check the time history trend of the temperatures.

2. Decrease the power output if needed.

3. If several TC which is close each other are going up, turn off the auto control mode on the heater related to the TC, and reduce the output of heater power.

4. Keep watching the trend and report to the Test Manager. 
APPENDIX A - TORQUE TABLE

\begin{tabular}{|c|c|c|c|c|c|c|c|c|}
\hline & \multirow{2}{*}{$\begin{array}{l}\text { Flange } \\
\text { size }\end{array}$} & \multirow[t]{2}{*}{ Gasket size } & \multicolumn{2}{|c|}{ Bolt } & \multirow{2}{*}{$\begin{array}{c}\text { 1st } \\
\text { Round } \\
\text { Torque } \\
\text { (Ft-Lbs) }\end{array}$} & \multirow{2}{*}{$\begin{array}{c}\text { 2nd } \\
\text { Round } \\
\text { Torque } \\
\text { (Ft-Lbs) }\end{array}$} & \multirow{2}{*}{$\begin{array}{c}\text { 3rd } \\
\text { Round } \\
\text { Torque } \\
\text { (Ft-Lbs) }\end{array}$} & \multirow{2}{*}{$\begin{array}{c}\text { 4th } \\
\text { Round } \\
\text { Torque } \\
\text { (Ft-Lbs) }\end{array}$} \\
\hline & & & Dia. & No. & & & & \\
\hline Coal Hopper Top & 2” & $\begin{array}{l}21 / 16 \times 2 \% / 16 \times 3 / 8 \times 5 \% / 8 \times \\
0.175\end{array}$ & 0.875 & 8 & 50 & 100 & 160 & 160 \\
\hline R-AOV-3 Upper & $1 ”$ & $\begin{array}{l}1 \frac{1}{16} \times 1 \frac{1}{4} \times 17 / 8 \times 3 \frac{1}{8} \times \\
0.175\end{array}$ & 0.875 & 4 & 35 & 70 & 115 & 115 \\
\hline R-AOV-3 Upper & $1 ”$ & $\downarrow$ & 0.875 & 4 & 35 & 70 & 115 & 115 \\
\hline Reactor Top & $10 ”$ & $9^{29 / 32} \times 10^{21 / 32} \times 0.175$ & $1 ”$ & 12 & 75 & 150 & 245 & 245 \\
\hline Reactor Bottom & $8 ”$ & $8^{1 / 32} \times 8^{25 / 32} \times 0.175$ & $0.875^{\prime}$ & 12 & 50 & 100 & 160 & 160 \\
\hline U. Char Pot Top & $2-1 / 2$ & $5^{11 / 64} \times 5^{59} / 64 \times 0.175$ & $1 ”$ & 8 & 75 & 150 & 245 & 245 \\
\hline U. Char Pot Bottom & 2” & $\begin{array}{l}2 \frac{1}{16} \times 2 \frac{5}{16} \times 3 \frac{3}{8} \times 5 \frac{5}{8} \times \\
0.175\end{array}$ & $0.875^{\prime}$ & 8 & 50 & 100 & 160 & 160 \\
\hline L. Char Pot Top & 2” & $\downarrow$ & 0.875 & 8 & 50 & 100 & 160 & 160 \\
\hline L. Char Pot Bottom & $2 ”$ & $\downarrow$ & 0.875 & 8 & 50 & 100 & 160 & 160 \\
\hline
\end{tabular}

\section{APPENDIX B - ORDER OF BOLT TIGHTENING}
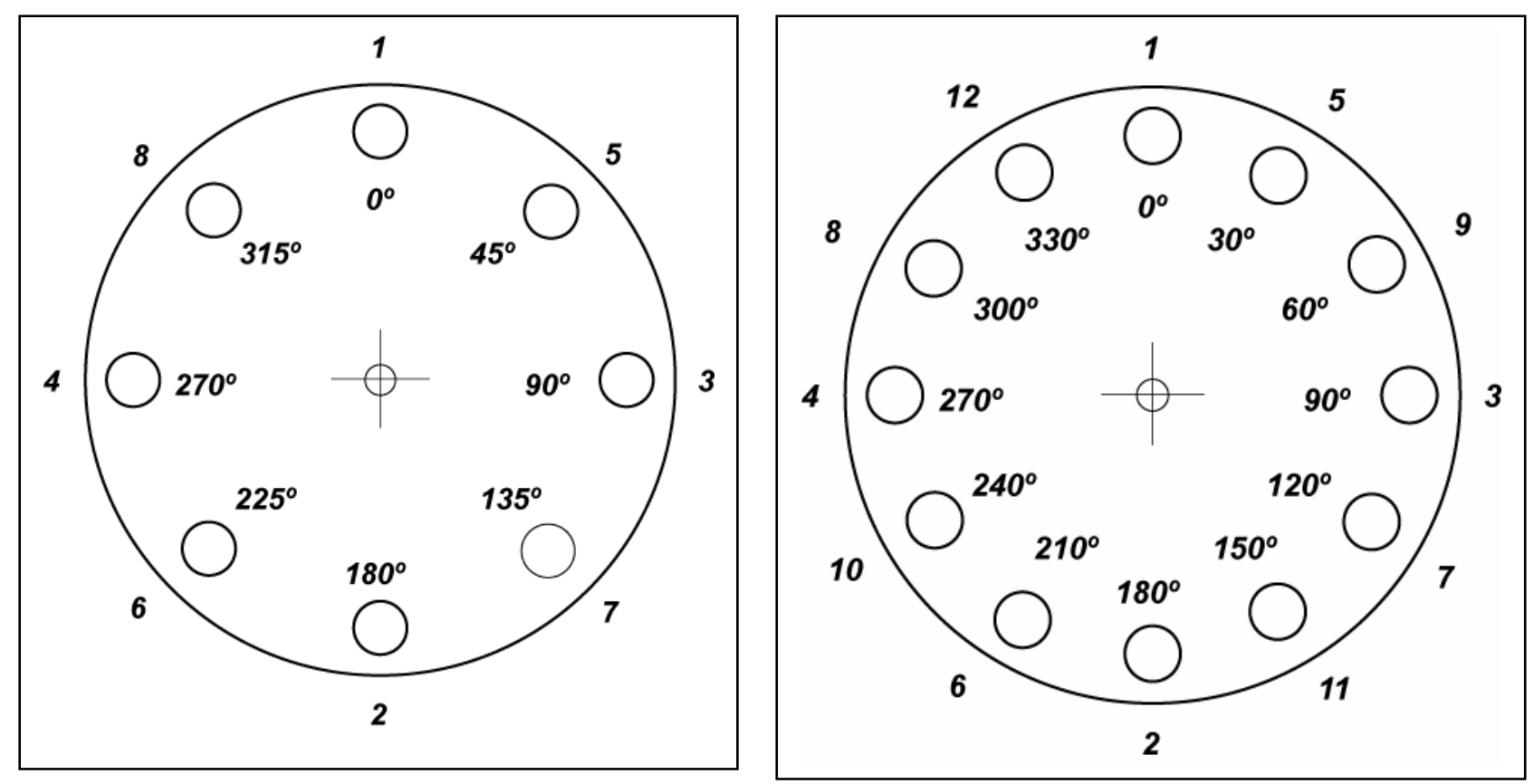


\section{APPENDIX C - TEST PLAN DATA SHEET}

\begin{tabular}{|c|c|c|c|c|c|}
\hline \multicolumn{6}{|c|}{ Test Plan Data Sheet } \\
\hline \multirow{2}{*}{\multicolumn{3}{|c|}{$\begin{array}{l}\text { Run Number } \\
\text { Date }\end{array}$}} & \multicolumn{2}{|c|}{ RUN $x x x x x x$} & \\
\hline & & & \multicolumn{2}{|l|}{ MM/DD/YY } & \\
\hline \multirow{4}{*}{\multicolumn{3}{|c|}{$\begin{array}{l}\text { Reaction } \\
\text { Condition }\end{array}$}} & Gas Residence Time & sec & \\
\hline & & & $\mathrm{H} 2 / \mathrm{C}$ ratio & wt/wt & \\
\hline & & & Reaction Pressure & psig & \\
\hline & & & Reaction Temperature & ${ }^{\circ} \mathrm{F}$ & \\
\hline \multicolumn{3}{|l|}{ Coal Feeder } & $\begin{array}{l}\text { Moter rpm setting } \\
\text { Coal Flow Rate }\end{array}$ & $\begin{array}{l}\mathrm{rpm} \\
\mathrm{lb} / \mathrm{h}\end{array}$ & \\
\hline \multicolumn{3}{|l|}{ N2 PCV Setting } & $\begin{array}{l}\text { N2-PCV-1 } \\
\text { N2-PCV-2 } \\
\text { N2-PCV-3 }\end{array}$ & $\begin{array}{r}1150 \text { psig } \\
1150 \text { psig } \\
110 \text { psig } \\
\end{array}$ & \\
\hline \multicolumn{3}{|l|}{ H2 PCV Setting } & $\begin{array}{l}\mathrm{H} 2-\mathrm{PCV}-30 \\
\mathrm{H} 2-\mathrm{PCV}-1 \\
\mathrm{H} 2-\mathrm{PCV}-2\end{array}$ & $\begin{array}{l}1200 \text { psig } \\
- \\
-\end{array}$ & \\
\hline \multicolumn{3}{|l|}{ Rx PCV-Setting } & $\begin{array}{l}\text { R-PCV-1 } \\
\text { R-dPT-30 } \\
\text { SG-PCV-1 }\end{array}$ & $\begin{array}{r} \\
\\
1010\end{array}$ & \\
\hline \multirow{3}{*}{\multicolumn{3}{|c|}{ H2 Flow Setting }} & $\mathrm{H} 2-\mathrm{FIC}-1$ & $\begin{array}{l}\text { scfh for } \mathrm{H} 2 \\
\text { scfh for } \mathrm{H} 2\end{array}$ & \begin{tabular}{|l|} 
during heating up \\
before coal in
\end{tabular} \\
\hline & & & H2-FIC-2 & scfh & \\
\hline & & & H2-FCV-20 & $\mathrm{cc} / \mathrm{min}$ & \\
\hline \multicolumn{3}{|l|}{ Rx Flow Setting } & R-MFC-1 & scfh & \\
\hline \multirow{17}{*}{$\begin{array}{l}\text { Temperature } \\
\text { Setting }\end{array}$} & \multirow{8}{*}{$\begin{array}{l}\mathrm{H} 2 \\
\text { pre- } \\
\text { heater }\end{array}$} & \multirow{4}{*}{$900 \frac{\mathrm{H} 2}{\mathrm{sft} 3 / \mathrm{h}}$} & TIC-11A-3 & $2000^{\circ} \mathrm{F}$ & \multirow{4}{*}{$\begin{array}{l}\text { heater set point } \\
\text { for startup }\end{array}$} \\
\hline & & & TIC-12A-3 & off $\quad{ }^{\circ} \mathrm{F}$ & \\
\hline & & & TIC-13A-3 & $2000^{\circ} \mathrm{F}$ & \\
\hline & & & TIC-14A-3 & OFF $\quad{ }^{\circ} \mathrm{F}$ & \\
\hline & & After & TIC-11A-3 & ${ }^{\circ} \mathrm{F}$ & at TE-11B-3=1560 F \\
\hline & & Tube & TIC-12A-3 & ${ }^{\circ} \mathrm{F}$ & at TE-12A-3=1560 F \\
\hline & & Temp get & TIC-13A-3 & ${ }^{\circ} \mathrm{F}$ & at TE-13A-3=1560 F \\
\hline & & to & TIC-14A-3 & ${ }^{\circ} \mathrm{F}$ & at TE-14A-3=1500 F \\
\hline & & & PH5A & $\%$ & \\
\hline & 1/4" col & nnecting & $\mathrm{PH} 5 \mathrm{~B}$ & $\%$ & start from , \& adjust \\
\hline & & & PH5C & $\%$ & \\
\hline & & & Heater \#1 & $\%$ & \\
\hline & & & Heater \#2 & $\%$ & \\
\hline & Reacto & Tube & Heater \#3 & $\%$ & \\
\hline & Heater & & Heater \#4 & $\%$ & start from , \& adjust \\
\hline & & & Heater \#5 & $\%$ & \\
\hline & & & Heater \#6 & $\%$ & \\
\hline & H2 pre- & heater & TI-11C-A/B & ${ }^{\circ} \mathrm{F}$ & \\
\hline & 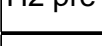 & & PH-TI-15 & $\mathrm{F}$ & \\
\hline Temperature & $\begin{array}{l}\text { Reactor t } \\
\text { Temp. an } \\
\text { flat part }\end{array}$ & $\begin{array}{l}\text { ube average } \\
\text { nong TE in }\end{array}$ & $\begin{array}{l}\text { TE-22A-1,TE-22B-1,TE-22B-4 } \\
\text { TE-23A-1,TE-23B-1,TE-23B-4 } \\
\text { TE-24A-1, TE-24B-1, TE-25A-1 }\end{array}$ & ${ }^{\circ} \mathrm{F}$ & \\
\hline Results ; & Runnin & $g$ time & & Runing time & \\
\hline & Upper & Char & $\mathrm{lb}$ & Lower Char & \\
\hline & Produc & tgas & $\operatorname{scf}^{3}$ & Product gas & \\
\hline & Oil sam & iple & $\mathrm{g}(7 \mathrm{ml})$ & Oil sample & \\
\hline & $\mathrm{H}_{2} \mathrm{O} \mathrm{sa}$ & mple & g & $\mathrm{H}_{2} \mathrm{O}$ sample & \\
\hline Comments & & & & & \\
\hline
\end{tabular}




\title{
APPENDIX L
}

\section{Bench Scale Hydrogasification Testing}

\author{
Shutdown Procedure
}


Development of a Hydrogasification Process for

Coproduction of SNG and Electrical Power from Western Coals

Final Report

THIS PAGE INTENTIONALLY LEFT BLANK 


\title{
COAL TO SNG \\ Bench Scale Test Reactor SHUTDOWN PROCEDURE
}

\author{
FINAL \\ JULY 2010
Arizona Public Service Company
400 NORTH 5TH STREET
PhoENIX, AZ 85003

\author{
Principal Investigator \\ Dr. Xiaolei Sun \\ 602.250.1510 \\ 602.250.1505 \\ xiaolei.sun@aps.com
}




\section{COAL TO SNG \\ BENCH SCALE TEST REACTOR \\ SHUTDOWN PROCEDURES}

\section{TABLE OF CONTENTS}

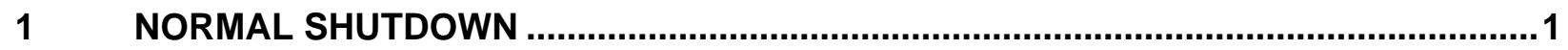

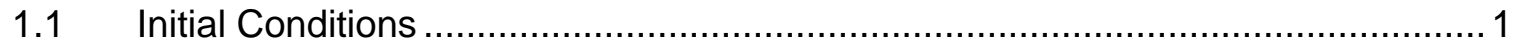

1.2 Coal Feeding Stop and Cool Down ..................................................... 1

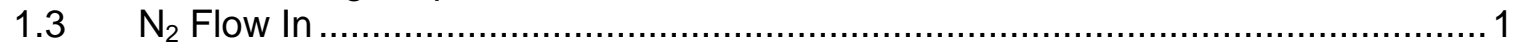

1.4 Depressurizing by Auto Control Mode for Ramp Down ................................... 2

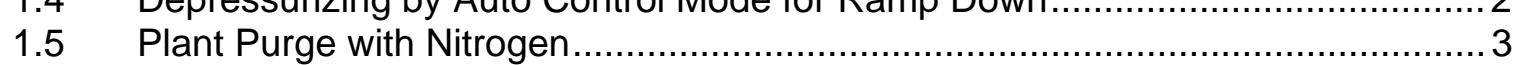

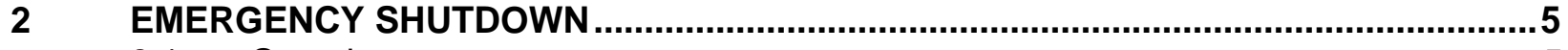

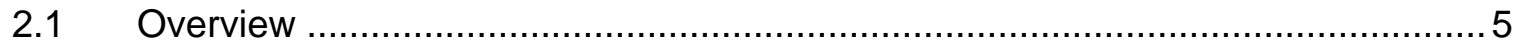

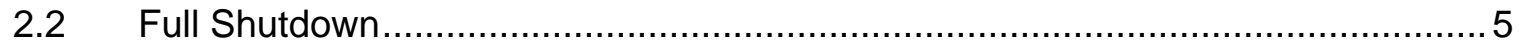

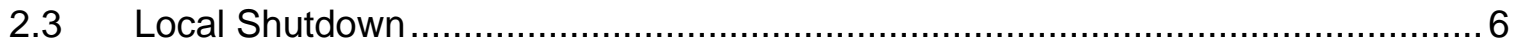

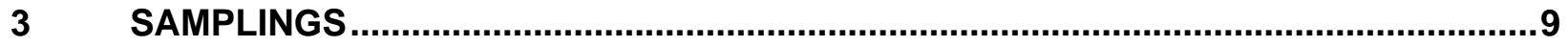

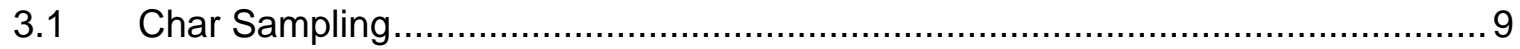

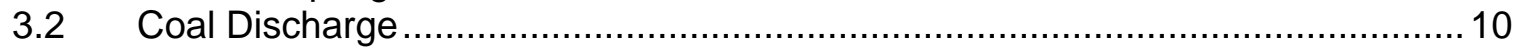

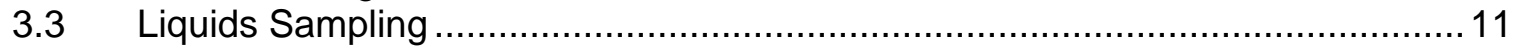

\section{APPENDIXES}

Appendix 1 - Test Plan Data Sheet ........................................................................... 1-1

Appendix 2 - Coal to SNG Bench Scale test Reactor Normal shutdown lineup checklist.........2-1 


\section{Normal ShUtdown}

\subsection{Initial Conditions}

Listed below are the initial conditions to be checked before starting the normal shutdown process:

1.1.1 The reactor is operating per the SNG Bench Scale Test Reactor Startup Procedure, subsections 5.7, Product Gas Data Acquisition Start Before SteadyState Operation, and 5.8 Steady-State Operation.

1.1.2 The required samples have been obtained on the Test Plan Data Sheet (Appendix A) and the end of data acquisition period is approaching, or Test Manager decides to do a shutdown.

1.1.3 Reactor temperatures and pressures have stabilized as expected and the system is ready for shutdown.

\subsection{Coal Feeding Stop and Cool Down}

Follow these steps for stopping coal feeding and cool down of the Bench-Scale Test Reactor:

1.2.1 Set the Trip Bypasses for TE-21A-1 ${ }^{\mathrm{HH}}$, TE-21B-4 ${ }^{\mathrm{HH}}$, TE-21B-1 ${ }^{\mathrm{HH}}$, TE-27A-1 ${ }^{\mathrm{HH}}$, $\mathrm{TE}-22 \mathrm{~A}-1^{\mathrm{HH}}$, and TE-22B-4 ${ }^{\mathrm{HH}}$ in the ON position.

1.2.2 Turn off the coal feeder and shut R-AOV-3 at the time which was decided in the Startup Procedure, subsection 5.7, Product Gas Data Acquisition Start Before Steady-State Operation.

1.2.3 Turn off the No. 1 Reactor Heater.

1.2.4 After 5 minutes from the coal feeder turn off, shut CON-V-1.

1.2.5 Shut H2-PCV-2.

1.2.6 Shut CF-AOV-4.

1.2.7 Turn off the $\mathrm{H}_{2}$ Preheater and Reactor Heater.

1.2.8 Keep $\mathrm{H}_{2}$ flow until all of preheater tube temperatures in TI-14C-1A/1B, TI-14C$2 \mathrm{~A} / 2 \mathrm{~B}, \mathrm{TI}-13 \mathrm{C}-1 \mathrm{~A} / 1 \mathrm{~B}, \mathrm{TI}-13 \mathrm{C}-2 \mathrm{~A} / 2 \mathrm{~B}, \mathrm{TI}-12 \mathrm{C}-2 \mathrm{~A} / 2 \mathrm{~B}$, and TI-11C-2A/2B and all of reactor tube temperatures in TIC-11A/B-3, TIC-12A/B-3, TIC-13A/B-3, and TIC14A/B-3 are lower than $1300^{\circ} \mathrm{F}$.

\section{$1.3 \quad \mathrm{~N}_{2}$ Flow In}

Follow these steps for $\mathrm{N}_{2}$ flow in:

1.3.1 Set $\mathrm{H} 2-\mathrm{FCV}-1$ to manual control mode and make the valve opening fix at the opening shown on the auto control mode. Open N2-V-3 in half turn increments to full open.

\subsubsection{Shut H2-V-1.}

1.3.3 Select flow Indicator for $\mathrm{N}_{2}$ on $\mathrm{H} 2-\mathrm{FCV}-1$. 
1.3.4 Set H2-FIC-1 setpoint at the flow rate on the indicator and switch from auto control mode to manual control mode.

1.3.5 Increase and adjust H2-FCV-1 setpoint to $220 \mathrm{stt}^{3} / \mathrm{h}$ and adjust H2-PCV-1 opening to get 50 to $60 \%$ opening on $\mathrm{H} 2-\mathrm{FCV}-1$.

1.3.6 On the Test Bed Panel, shut valves V-342 and V-306.

1.3.7 Keep the $\mathrm{N}_{2}$ flow for 15 minutes and then move to Depressurizing by Auto Control for Ramp Down, subsection 1.4, below.

\subsection{Depressurizing by Auto Control Mode for Ramp Down}

Follow these steps for depressurizing by auto control mode for ramp down:

1.4.1 Shut H2-PCV-1 and R-PCV-1 to stop $\mathrm{N}_{2}$ flow.

\subsubsection{Open CF-SV-3.}

1.4.3 For R-PCV-30, set dP setpoint at 15 psig.

1.4.4 For CF-PCV-1, set the dP setpoint at 40 psig.

1.4.5 Open R-V-78 char pot equalizing valve.

1.4.6 On SG-PCV-1, set the ramp down time at 90 minutes.

1.4.7 Turn on the ramp down and start the ramp down.

1.4.8 Verify that N2-PT-16 starts to decrease the pressure.

1.4.9 In order to keep R-PCV-30 higher than R-PT-3, pressurize the annulus space by setting R-MFC- 1 and by opening R-PCV-1 several times.

1.4.10 Verify that SG-PT-1 is switched on and start to decrease the pressure on R-PT30.

1.4.11 When the SG-PT-1 reaches about 50 psig or the auto ramp down does not work well, turn off SG-PCV-1 ramp down, and stop the ramp down. Switch from auto control mode to manual control mode to continue depressurization.

1.4.12 Manually shut CF-PCV-1 and CF-SV-3.

1.4.13 Remove the end cap on N2-V-16 and slightly open N2-V-16 to get about 3 psig per minute of depressurizing speed.

\subsubsection{Open R-AOV-4.}

1.4.15 Open SG-V-14 and SG-V-15 slightly to keep the same depressurizing speed on the coal hopper (N2-PT-16), but adjust the both valve openings to keep R-dPT-1 within 10 psig.

1.4.16 If the R-PT-11 does not pressurize R-PT-3 and SG-PT-1, open R-V-20 and R-V21 slightly to vent the lower char pot.

1.4.17 When N2-PT-16 reaches 10 psig, set N2-V-16 to full open then shut after the N2PT-16 shows ambient pressure or lower than 0 psig. 
1.4.18 When SG-PT-1 reaches 10 psig, set SG-V-14 and SG-V-15 to full open and shut after the SG-PT-1 shows ambient pressure or lower than 0 psig.

1.4.19 When R-PT-11 reaches 10 psig, turn R-V-20 and R-V-21 to full open and shut after the R-PT-11 shows ambient pressure or lower than 0 psig.

\subsection{Plant Purge with Nitrogen}

Follow these steps for plant purging with nitrogen:

1.5.1 Check and shut SG-PCV-1.

1.5.2 Check and shut CF-PCV-1.

1.5.3 Shut R-AOV-4.

1.5.4 Verify that R-dPT-1 is higher than 10 psig.

1.5.5 If R-dPT-1 is lower than 10 psig, pressurize the annulus space by opening RPCV-1 to $5 \%$ and set R-MFC-1 at $100 \mathrm{sft}^{3} / \mathrm{h}$ to $10 \mathrm{psig}$ for R-dPT-1. When the RPT-30 reaches 10 psig, shut R-PCV-1.

1.5.6 Open H2-PCV-1 to 5\% opening.

1.5.7 Set $\mathrm{H} 2-\mathrm{FCV}-1$ at $50 \mathrm{sft}^{3} / \mathrm{h}$ and switch from manual control mode to auto control mode to start to pressurizing the reactor tube.

1.5.8 When the R-dPT-1 approaches 5 psig, open R-PCV-1 to 5\% and set R-MFC-1 at $100 \mathrm{stt}^{3} / \mathrm{h}$ and switch from auto control mode to manual control mode to pressurize the annulus space.

1.5.9 Manually open $\mathrm{H} 2-\mathrm{FCV}-2$ to $100 \%$ and select $\mathrm{N}_{2}$ for flow indicator.

1.5.10 In order to pressurize the coal hopper, open H2-PCV-2 to get to $20 \mathrm{stt}^{3} / \mathrm{h}$ on $\mathrm{H} 2$ FM-2.

1.5.11 Increase H2-FCV-1 setpoint in $50 \mathrm{stt}^{3} / \mathrm{h}$ increments every 3 minutes to reach 150 $\mathrm{st}^{3} / \mathrm{h}$ by increasing $\mathrm{H} 2-\mathrm{PCV}-1$ in $1 \%$ increments.

1.5.12 Increase R-MFC-1 setpoint in $100 \mathrm{stt}^{3} / \mathrm{h}$ increments every 3 minutes to reach 300 $\mathrm{sft}^{3} / \mathrm{h}$ with increasing R-PCV-1 in 1\% increments.

1.5.13 Keep adjusting H2-FCV-1 flow rate and R-MFC-1 flow rate to keep R-dPT-1 within 10 psig.

1.5.14 Keep increasing H2-PCV-2 opening to keep about $20 \mathrm{stt}^{3} / \mathrm{h}$ on H2-FM-2 to pressurize to 150 psig on the N2-PT-16.

1.5.15 Keep increasing $\mathrm{H} 2-\mathrm{PCV}-1$ in $1 \%$ increments to pressurize to 150 psig for R-PT3 and SG-PT-1.

1.5.16 Keep increasing R-PCV-1 in 1\% increments to pressurize to 160 psig for R-PT30.

1.5.17 When R-PT-3/SG-PT-1 reaches to 150 psig, shut H2-PCV-1.

1.5.18 When R-PT-30 reaches to 160 psig, shut R-PCV-1.

${ }^{\circ} 2010$ Arizona Public Service All Rights Reserved 
1.5.19 When N2-PT-16 reaches to 150 psig, shut H2-PCV-2. Open N2-V-16 slightly to get about 3 psig per minute of depressurizing speed and start to depressurize the hopper.

1.5.20 Open N2-V-16 slightly to get about 3 psig per minute of depressurizing speed and start to depressurize the hopper.

\subsubsection{Open R-AOV-4.}

1.5.22 Open SG-V-14 and SG-V-15 slightly to keep the same depressurizing speed as coal hopper (N2-PT-16), but adjust the both valve openings to keep R-dPT-1 within 10 psig.

1.5.23 If the R-PT-11 does not follow to the R-PT-3 and SG-PT-1, open R-V-20 and R$\mathrm{V}$-21 slightly to vent the lower char pot.

1.5.24 When N2-PT-16 reaches 10 psig, set N2-V-16 full to open then shut after the N2PT-16 shows ambient pressure or lower than 0 psig.

1.5.25 When SG-PT-1 reached to 10 psig, fully open SG-V-14 and SG-V-15, and shut them after SG-PT-1 shows ambient pressure or lower than 0 psig.

1.5.26 When R-PT-11 reaches 10 psig, fully open R-V-20 and R-V-21 and shut them after R-PT-11 shows ambient pressure or lower than 0 psig.

1.5.27 Repeat steps 1.5.3 through 1.5.23.

1.5.28 At the second cycle, when N2-PT-16 and R-PT-3/SG-PT-1 reach 15 psig, shut N2-V-16, SG-V-14, SG-V-15, R-V-20, and R-V-21 to keep the plant pressure at about 15 psig.

1.5.29 When R-PT-30 reaches about 25 psig, shut R-PCV-30 and R-AOV-4 to keep the annulus pressure higher than reactor tube pressure.

1.5.30 Shut N2-V-3, N2 V-7, SG-V-1, and R-V 96 to block the plant.

1.5.31 Turn off the cooling water pump.

1.5.32 Turn off all heater breakers.

1.5.33 Verify that all valves are in the normal shutdown lineup as shown in Appendix B. 


\section{EMERGENCY SHUTDOWN}

\subsection{Overview}

An operator controls normal shutdown activities. The Operator activates and controls the alarms during normal shutdown. Emergency shutdown is not controlled by the Operator.

An Emergency Shutdown also occurs when there is a power failure and some instruments act as a battery backup so that the plant is safely shutdown.

During high-risk situations such as a thunderstorm, the Bench Scale Test Reactor (BSTR) should remain shutdown. This minimizes the risk of personnel injury due to lightning strikes as well as interruptions due to power outages.

If an emergency condition occurs, shutdown the reactor by pressing one of the emergency shutdown switches located near the test area. Evacuate all nonessential personnel from the area and perform actions as required by this procedure.

\subsection{Full Shutdown}

\subsubsection{Trigger Full Shutdown Conditions}

The following conditions will trigger a full shutdown:

1. Hitting the manual trip switch.

2. Power failure.

3. Instrument air pressure transmitter reaches the low-low setpoint.

4. R-dPT-1 reaches the high-high setpoint.

5. Char pot cooling system failure.

6. R-TI-10 high-high setpoint and H2O-FSL-2 is activated or H2O-P-1 is shut off.

\subsubsection{Automatic Actions Triggered}

The following things will happen automatically after an emergency shutdown is triggered:

1. Coal feeder stops.

2. H2-AOV-1, H2-AOV-2, and H2-AOV-20 shut.

3. N2-AOV-40 and R-AOV-4 open.

4. All heaters are off.

5. R-AOV-3 is shut.

2.2.3 The operator must confirm that the coal feeder stopped and ensures the following steps are performed: 


\subsubsection{H2-AOV-1, H2-AOV-2, and H2-AOV-40 are CLOSED.}

2.2.3.2 N2-AOV-40 and R-AOV-4 are OPEN.

2.2.3.3 All the heaters are OFF.

2.2.3.4 R-AOV-3 is CLOSED.

2.2.4 Shut H2-PCV-1, H2-PCV-2, and H2-FCV-2.

2.2.5 Verify that the proper response of all pressures and temperatures (especially on N2-PT-40, R-PT-3, R-PT-30 and SG-PT-1) (to confirm there is no big fluctuation or overshooting of all pressures and temperatures).

2.2.6 Confirm the condition that triggered the emergency shutdown has been identified and the condition is cleared before progressing with the other steps.

2.2.7 When the pressure on $\mathrm{N}_{2}$ Pack for emergency purge approaches 1200 psig, open N2-V-3.

2.2.8 Push the trip reset button and open $\mathrm{H} 2-\mathrm{AOV}-1$.

2.2.9 Select flow indicator $\mathrm{H} 2-\mathrm{FIC}-1$ to stop $\mathrm{N}_{2}$ flow.

2.2.10 Using manual control mode, adjust H2-FCV-1 opening to $70 \%$.

2.2.11 Open H2-PCV-1 (65\% opening) to get to 1085 psig on H2-PT-1.

2.2.12 Confirm that the $\mathrm{N}_{2}$ flow is about $220 \mathrm{sft}^{3} / \mathrm{hr}$ on $\mathrm{H} 2-\mathrm{FIC}-1$.

\subsubsection{Shut N2-AOV-40.}

2.2.14 Continue to cool the reactor.

2.2.15 Once all of temperature indicators on the $\mathrm{H}_{2}$ Preheater and the Reactor read below $1300^{\circ} \mathrm{F}$, move to the Subsection 1.4 followed by Subsection 1.5 in the normal shutdown.

\subsection{Local Shutdown}

Local Shutdown affects the Heaters and/or the Coal Feeder.

\subsubsection{Local Shutdown 1}

Local Shutdown 1 happens if the rate of change in one of the temperature sensors is at the top part of the reactor tube is too fast (over $100^{\circ} \mathrm{F}$ per second). It typically depends on the temperature increase in the reactor's top zone. If this does occur, follow these steps:

2.3.1.1 Confirm that the Coal Feeder stopped and R-AOV-3 is closed.

2.3.1.2 Depending on the temperature increase in the Reactor's top zone, turn off the No. 1 and No. 2 Reactor Heaters.

2.3.1.3 Verify proper response of all temperatures, flow rates, and pressures to make sure there is no big fluctuation of all these parameters.

2.3.1.4 Push the trip reset button.

${ }^{\circ} 2010$ Arizona Public Service All Rights Reserved 
2.3.1.5 Verify the pressure of H2-PCV-2 is higher than N2-PT-16.

2.3.1.6 Crack OPEN R-AOV-3 and wait for a several minutes.

2.3.1.7 If the blockage is not clear, shut R-AOV-3, and repeat steps 2.3.1.4 and 2.3.1.5 (above) two to three times to make sure that there are no blockages.

2.3.1.8 In the case the blockage is not cleared, turn off the entire heater and shut H2-V-6 and move to the Normal Shutdown, Subsection 1.2, of this procedure.

2.3.1.9 If the blockage is cleared, the Test Manager can decide if he wishes to continue the test or not.

Note: If the blockage has occurred during temperature stabilizing before R-AOV-1 shuts, the testing can be continued but the decision may depend on how much coal is remaining in the hopper.

2.3.1.10 If the Test Manager does not wish to continue the test, turn off the entire heater and shut $\mathrm{H} 2-\mathrm{V}-6$ and mover to the Normal Shutdown steps in subsection 1.2.

2.3.1.11 If the Test Manager decides to continue the test, there should be enough time to operate and coal is still in the hopper. After getting the process in stable condition, if R-AOV-1 and CON-AOV-1 were closed then open them.

2.3.1.12 Stabilize the Reactor's temperature following the operating data sheet, shown in Appendix A.

2.3.1.13 To re-start the process, go to Startup Procedure, subsection 1.2.

\subsubsection{Local Shutdown 2}

In case of the $\mathrm{H}_{2}$ Preheater Tube temperatures reach a high-high setpoint, the entire Preheater will be turned off. If the coal was being fed to the Reactor, the Coal Feeder must be powered off and R-AOV-3 is closed. Follow these steps:

2.3.2.1 Confirm which temperature controller (TC) hits high-high setpoint and turn off the Preheater.

2.3.2.2 If the temperature was jumped up to maximum value of the range abruptly, turn the temperature indicator ( $\mathrm{TI}$ ) trip bypass $\mathrm{ON}$.

2.3.2.3 When the temperature of trip trigger is cleared, push the trip reset button.

2.3.2.4 The Test Manager has to decide whether the shutdown operation can be continued or stopped.

2.3.2.5 If the setpoint of $\mathrm{H}_{2}$ Preheater can be reduced 10 or $20^{\circ} \mathrm{F}$, the operation will be continued.

2.3.2.6 If there is enough coal remaining in the coal hopper, the operation will be continued.

2.3.2.7 If the Test Manager decides to continue with the test, turn on the $\mathrm{H}_{2}$ Preheater and open R-AOV-3, R-AOV-1, and CON-AOV-1, then return 
to Startup Procedure, subsection 5.5, Heating Up.

2.3.2.8 If the Test Manager decides not to continue the test, turn off all of the Reactor Heaters, and go to the steps shown in Shutdown Procedure, subsection 1.2.

\subsubsection{Local Shutdown 3}

In case the reactor's tube temperature reaches a high-high setpoint, the entire reactor heater must be turned off. If the coal was being fed to the reactor, the coal feeder is powered off and R-AOV-3 is closed. Follow these steps:

2.3.3.1 Confirm which temperature controller (TC) hits the high-high setpoint and turn off its reactor heaters.

2.3.3.2 If the temperature was jumped up to maximum value of the range abruptly, turn the temperature indicator bypass ON.

2.3.3.3 When the temperature of trip trigger is cleared, push the trip reset button.

2.3.3.4 The Test Manager has to decide whether the operation can be continued or stopped. The Test Manager will consider the following items:

1. If the setpoint of $\mathrm{H}_{2}$ Preheater can be reduced, the operation will be continued.

2. If there is enough coal remaining in the coal hopper, the operation will be continued.

2.3.3.5 If the Testing Manager decides to continue the testing, turn on the $\mathrm{H}_{2}$ Preheater and open R-AOV-3, R-AOV-1, and CON-AOV-1, then return to Startup Procedure, subsection 5.5, Heating Up.

2.3.3.6 If the decision is not to continue, turn off the $\mathrm{H}_{2}$ Preheater, then go to the Shutdown Procedure, subsection 1.2

\subsubsection{Heater Shutdown}

Each heater on the $\mathrm{H}_{2}$ Preheater will be switched off when the temperature measuring Heater's element (xx-yy-3) and/or the temperature measuring insulation surface (xx-yy2) hits the high-high setpoint. Follow these steps:

2.3.4.1 If the Heater-Off is shown in the alarm annunciator, confirm which Heater was turned off and report it to the Test Manager.

2.3.4.2 If the temperature was jumped up to outside of the range abruptly, change the wiring to $\mathrm{B}$-side Heater. (As the initial wiring of temperature controller, A-side Heater TC (xx-yyA-3) is used to control the heater. If the A-side temperature controller failed, change the wiring to B-Side Heater's temperature controller (xx-yyB-3).)

2.3.4.3 When the temperature of shutdown heater is cleared, the Test Manager has to decide whether the operation can be continued or not. Follow these steps:

1. If the effect of heater off on the $\mathrm{H}_{2}$ temperature and/or the temperature profile on reactor tube is small, the test can be 
continued.

2. If there is a time for stabilizing the temperature and there is enough coal remaining in the coal hopper, the test can be continued.

2.3.4.4 If the Testing Manager decides to continue the testing, turn on the Heater. If the heater shutdown is performed during data acquisition period, open R-AOV-3, R-AOV-1, and CON-AOV-1, then return to Startup Procedure, subsection 5.5, Heating Up.

2.3.4.5 If the decision is not to continue the testing, move to the Section 1, Normal Shutdown, of this procedure.

\section{SAMPLINGS}

\subsection{Char Sampling}

Persons who perform the char samplings must wear a dust mask and safety glasses and follow these steps outlined and shown below:

3.1.1 Prepare metal can, with two plastic bags. The plastic bags must be large enough to fit over the metal can, the tubes, and the bottom of the char pot's pipe.

3.1.2 Put the can and plastic bag on the scale and set the scale reading to zero.

3.1.3 Set the can and plastic bag under the lower char pot and the plastic bag's open end must cover the char pot bottom pipe and R-V-10.

3.1.4 Shut R-V-78.

3.1.5 Verify that R-PT-11 shows 10 to 15 psig. If it is lower than $10 \mathrm{psig}$, pressurize the lower char pot with $\mathrm{N}_{2}$ by using N2-V-5, set N2-PCV-7 to 100 psig, use N2-V51 , open R-SV-7 and R-V-8. If it is higher than 15 psig, depressurize by opening $\mathrm{R}-\mathrm{V}-20$ and $\mathrm{R}-\mathrm{V}-21$.

3.1.6 Remove the end cap and open R-V-10 gradually and using the gas flow due to pressure release discharge the char in lower char pot so that the char flows through the tube into the plastic bag.

3.1.7 Insert the three 1-ft long $1 / 4$ tubes $\mathrm{N}_{2}$ blow tube from R-V-10 into the lower char pot, and use $\mathrm{N}_{2}$ to blow out the char on the char pot wall, and collect all char into the plastic bag.

3.1.8 Take the $\mathrm{N}_{2}$ blow tubes out.

3.1.9 Weigh the can plus the plastic bag with char and measure and record the net char weight, and put a char sampling into a plastic bottle.

3.1.10 Put the can and a new plastic bag on the scale, and set the scale reading zero.

3.1.11 Insert the three 1-ft long 1/4-inch $\mathrm{N}_{2}$ blow tubes back into the lower char pot.

3.1.12 Set the can and plastic bag under the lower char pot and join the plastic bag's open end completely covering the char pot bottom pipe and valve R-V-10. 
3.1.13 Verify that R-PT-3 shows 10 to 15 psig. If it is lower than 10 psig, pressurize the reactor tube with $\mathrm{N}_{2}$ by using $\mathrm{H} 2-\mathrm{PCV}-1$ and $\mathrm{H} 2-\mathrm{FCV}-1$. If it is higher than 15 psig, depressurize by opening SG-V-1, SG-V-14, and SG-V-15.

3.1.14 Open R-AOV-1 and discharge the char in the upper char pot with the gas flow due to pressure release.

3.1.15 Add other $\mathrm{N}_{2}$ blow tube (three 1-ft long 1/4-inch tubes) and blow $\mathrm{N}_{2}$ to blow out the char on the char pot wall, and collect all char into the same plastic bag.

3.1.16 Take all six of $\mathrm{N}_{2}$ blow tubes out of the char pots.

3.1.17 Weigh the can plus the plastic bag with char. Measure and record the net char weight.

3.1.18 Mix the char collected from upper char pot using drive stirrer for about 5 minutes and take the char sampling for the char analysis.

\subsection{Coal Discharge}

After char sampling, the remaining coal in the coal feeder should be discharged from the char pot and feeder to keep uniformity of coal for the next run. Follow these steps:

3.2.1 Verify N2-PT-16 shows 10 to 15 psig. If it is lower than 10 psig, pressurize the coal hopper with $\mathrm{N}_{2}$ by using $\mathrm{H} 2-\mathrm{PCV}-2$ and $\mathrm{H} 2-\mathrm{FCV}-2$. If it is higher than 15 psig, depressurize by opening N2-V-16.

3.2.2 Put the can and a new plastic bag on the scale and set the scale reading zero.

3.2.3 Set the can and plastic bag under the lower char pot and joint the plastic bag's open end completely covering the char pot bottom pipe and R-V-10.

3.2.4 Check and open H2-AOV-20, H2-MFC-1(set $2000 \mathrm{cc} / \mathrm{min}), \mathrm{H} 2-\mathrm{AOV}-2$, and CFSV-2.

3.2.5 Open R-AOV-3 and discharge the coal in the hopper with the gas flow due to pressure releasing.

3.2.6 Remove the top flange on the coal hopper and check to make sure that the coal hopper is empty.

3.2.7 If coal remains in the hopper, turn on the coal feeder and discharge all of the coal in the hopper.

3.2.8 Verify that all coal runs out and turn off the coal feeder.

3.2.9 Weigh the can plus plastic bag with coal, and measure and record the net coal weight.

\subsubsection{Open N2-V-3.}

3.2.11 Open H2-PCV-1 to 15\% opening.

3.2.12 Set H2-FCV-1 setpoint at $300 \mathrm{stt}^{3} / \mathrm{h}$ and switch from manual control mode to auto control mode in order to make sure that $\mathrm{N}_{2}$ flows into reactor tube. 
3.2.13 Shut H2-PCV-1 and H2-FCV-1 so that there are no more coal particles coming out of the char unit and going into the plastic bag.

3.2.14 Shut R-V-10 and set the end plugs back onto the char pot's bottom.

\subsection{Liquids Sampling}

Persons who work with liquid sampling must wear a dust mask, plastic gloves, and safety glasses. They must follow these steps in sampling liquids:

3.3.1 Prepare several sample grass bottles (500 cc), solvent (acetone), and an empty can.

3.3.2 Fill liquid sprayer with acetone.

3.3.3 Remove two caps at the end of vent line on two liquid pots, and one cap on the liquid pot's bottom end.

3.3.4 Open CON-V-2 and CON-V-20 slightly and depressurize the two liquid pots to the ambient pressure keeping upper pot pressure slightly higher than lower char pot's pressure.

3.3.5 Open CON-V-2 and CON-V-20 fully.

3.3.6 Set the sampling bottle at the outlet of CON-CYL-2.

3.3.7 Open CON-V-3 and discharge the lower pot liquids into the sampling bottle.

3.3.8 Blow $\mathrm{N}_{2}$ from $\mathrm{CON}-\mathrm{V}-203$ to make sure all liquids are discharged.

3.3.9 Set other glass bottle at the outlet of CON-CYL-2.

3.3.10 Pressurize the liquid sprayer with $\mathrm{N}_{2}$ and spray about $100 \mathrm{cc}$ Acetone from CON$\mathrm{V}-20$ to wash the pot wall.

3.3.11 Blow $\mathrm{N}_{2}$ from CON-V-20 for a while and dry out the Acetone.

3.3.12 Shut off CON-V-20.

3.3.13 Set the sampling bottle at the outlet of CON-CYL-2.

3.3.14 Open CON-AOV-1 and discharge the upper pot liquids into the sampling bottle.

3.3.15 Blow $\mathrm{N}_{2}$ from CON-V-2 3 to make sure all liquids discharged.

3.3.16 Set other grass bottle at the outlet of CON-CYL-2.

3.3.17 Shut off CON-V-2 and remove the plug on the nozzle on the SEP-1.

3.3.18 Pressurize the liquid sprayer with $\mathrm{N}_{2}$ and spray about $300 \mathrm{cc}$ Acetone from the nozzle on the SEP-1 to wash the SEP-1 and upper pots wall.

3.3.19 Blow $\mathrm{N}_{2}$ from the same nozzle to dry out the Acetone.

3.3.20 Check shut of CON-V-2, CON-V-20, and CON-V-3, and reset three caps at the tube ends and one plug on SEP-1. 
APPENDIX 1 - TEST PLAN DATA SHEET

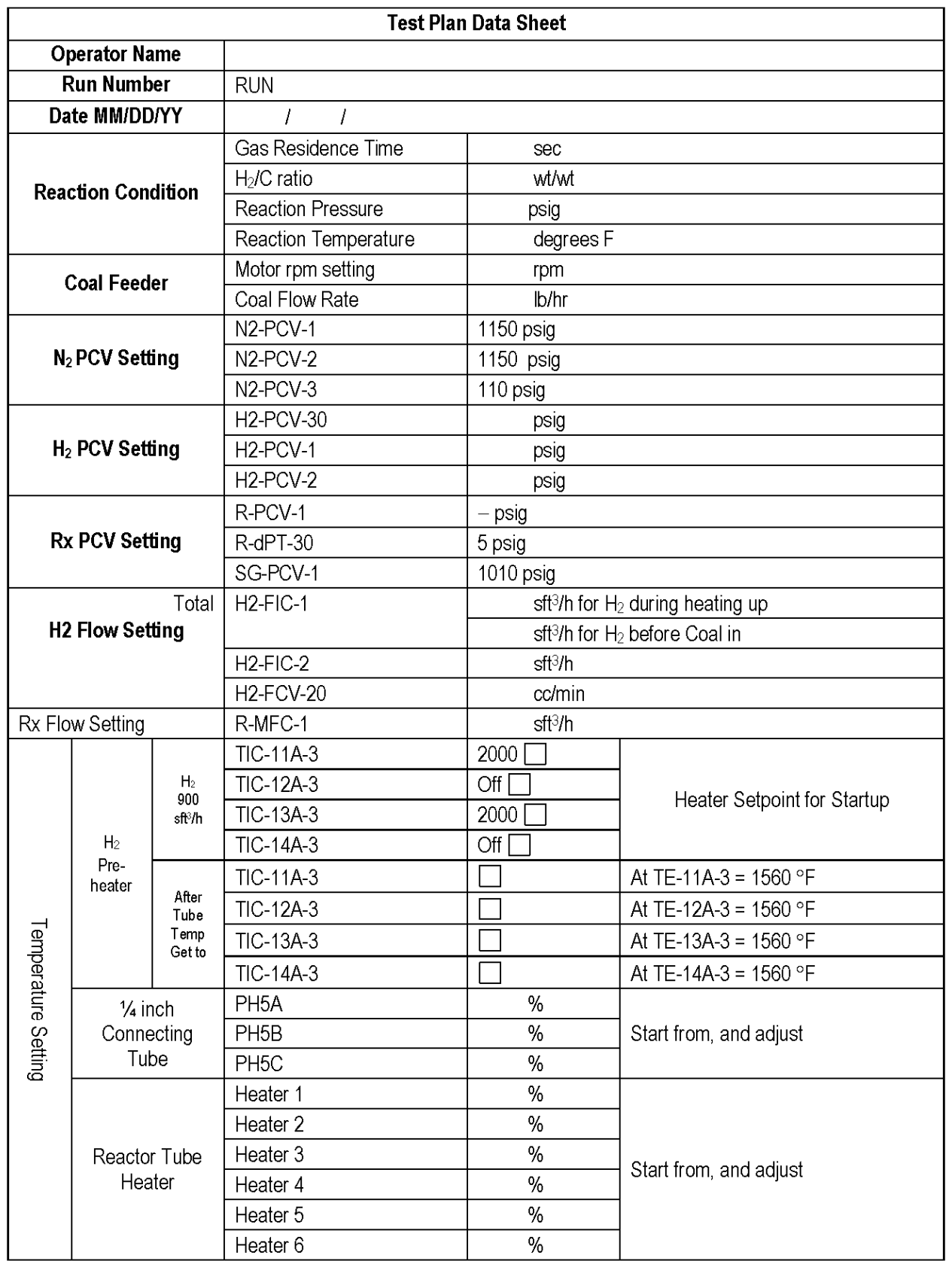

Page 1 


\section{APPENDIX 1 - TEST PLAN DATA SHEET (CONTD)}

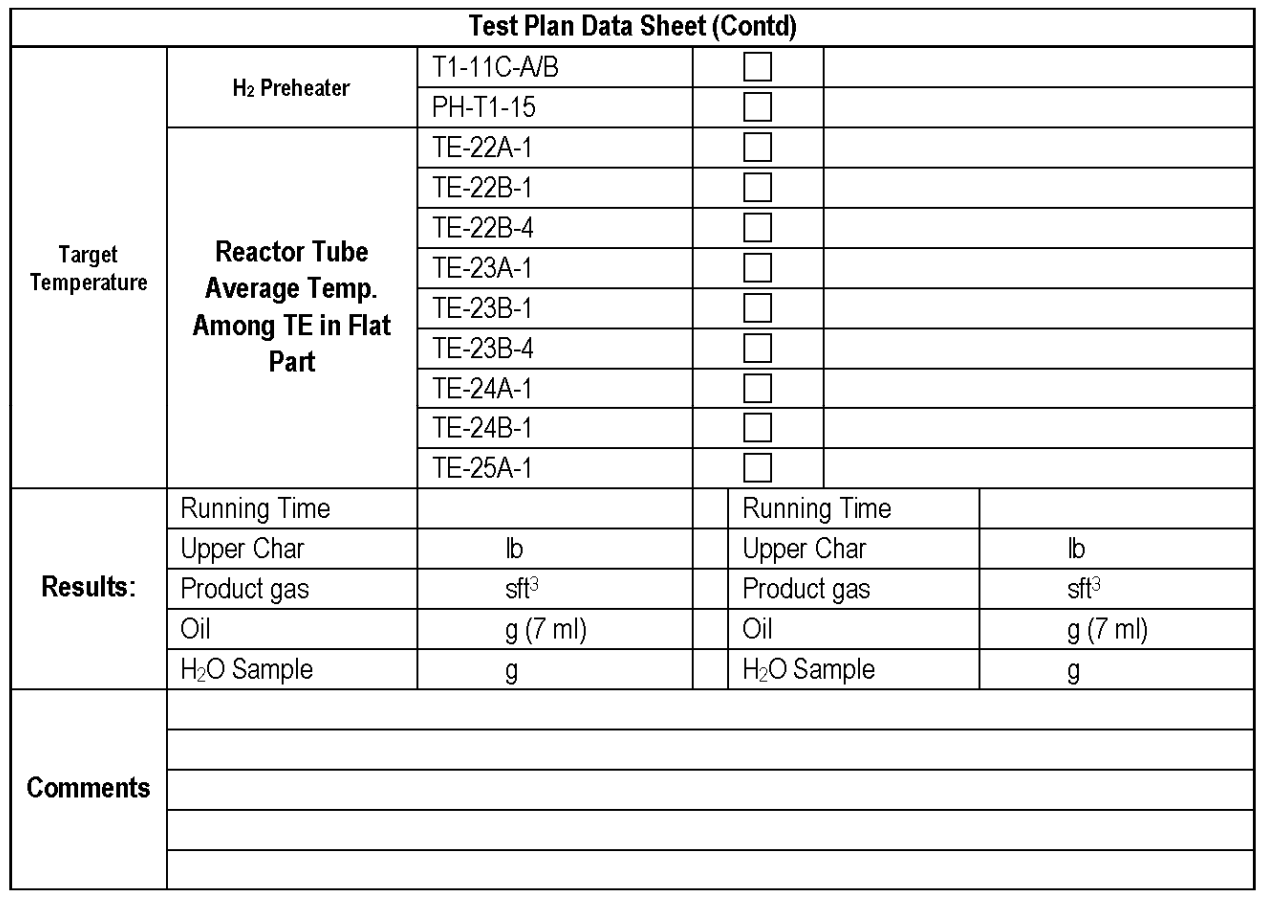


APPENDIX 2 - COAL TO SNG BENCH SCALE TEST REACTOR NORMAL SHUTDOWN LINEUP CHECKLIST

\section{Coal to SNG Bench Scale Test Reactor Normal Shutdown Lineup Checklist}

Date Inspected:

Operator:

\begin{tabular}{|c|c|c|c|c|}
\hline $\begin{array}{c}\text { Component } \\
\text { Number }\end{array}$ & $\begin{array}{l}\text { Component } \\
\text { Description }\end{array}$ & $\begin{array}{c}\text { Required Position } \\
\text { (On, Off, Tagout, } \\
\text { Lockout, etc.) }\end{array}$ & Check & P\&ID Drawing \\
\hline $\mathrm{N} 2-\mathrm{V}-7$ & Valve & Shut & $\square$ & \multirow{9}{*}{$\begin{array}{l}\mathrm{N}_{2} \text { High Pressure } \\
\text { Purge Panel }\end{array}$} \\
\hline N2-V-6 & Valve & Shut & $\square$ & \\
\hline N2-V-4 & Valve & Shut & $\square$ & \\
\hline N2-V-5 & Valve & Shut & $\square$ & \\
\hline N2-V-2 & Valve & Shut & $\square$ & \\
\hline N2-V-3 & Valve & Shut & $\square$ & \\
\hline N2-V-8 & Valve & Shut & $\square$ & \\
\hline N2-V-18 & Valve & Shut & $\square$ & \\
\hline N2-V-95 & Valve & Shut & $\square$ & \\
\hline N2-V-1 & Valve & Open & $\square$ & \multirow{15}{*}{$\begin{array}{c}\text { Instrument } \\
\mathrm{N}_{2} \text { Panel }\end{array}$} \\
\hline N2-V-9 & Valve & Open & $\square$ & \\
\hline N2-V-10 & Valve & Open & $\square$ & \\
\hline N2-V-11 & Valve & Open & $\square$ & \\
\hline N2-V-12 & Valve & Open & $\square$ & \\
\hline N2-V-13 & Valve & Open & $\square$ & \\
\hline N2-V-14 & Valve & Open & $\square$ & \\
\hline N2-V-15 & Valve & Open & $\square$ & \\
\hline N2-V-20 & Valve & Open & $\square$ & \\
\hline N2-V-21 & Valve & Open & $\square$ & \\
\hline N2-V-22 & Valve & Open & $\square$ & \\
\hline N2-V-23 & Valve & Open & $\square$ & \\
\hline N2-V-24 & Valve & Open & $\square$ & \\
\hline N2-V-25 & Valve & Open & $\square$ & \\
\hline N2-V-50 & Valve & Open & $\square$ & \\
\hline N2-V-35 & Valve & Shut & $\square$ & Emergency Purge $\mathrm{N}_{2}$ \\
\hline $\mathrm{H} 2 \mathrm{O}-\mathrm{V}-1$ & Valve & Shut & $\square$ & \multirow{6}{*}{ Cooling Water Tank } \\
\hline $\mathrm{H} 2 \mathrm{O}-\mathrm{V}-2$ & Valve & Open & $\square$ & \\
\hline $\mathrm{H} 2 \mathrm{O}-\mathrm{V}-3$ & Valve & Open & $\square$ & \\
\hline $\mathrm{H} 2 \mathrm{O}-\mathrm{V}-4$ & Valve & Open & $\square$ & \\
\hline $\mathrm{H} 2 \mathrm{O}-\mathrm{P}-1$ & Pump & Off & $\square$ & \\
\hline $\mathrm{H} 2 \mathrm{O}-\mathrm{P}-2$ & Pump & Off & $\square$ & \\
\hline $\mathrm{H} 2 \mathrm{O}-\mathrm{V}-7$ & Valve & Shut & $\square$ & In Frame \\
\hline
\end{tabular}

Page 1 
APPENDIX 2 - COAL TO SNG BENCH SCALE TEST REACTOR NORMAL SHUTDOWN LINEUP CHECKLIST (CONTD)

Coal to SNG Bench Scale Test Reactor Normal Shutdown Lineup Checklist (Continued)

\begin{tabular}{|c|c|c|c|c|}
\hline $\begin{array}{c}\text { Component } \\
\text { Number }\end{array}$ & $\begin{array}{l}\text { Component } \\
\text { Description }\end{array}$ & $\begin{array}{c}\text { Required Position } \\
\text { (On, Off, Tagout, } \\
\text { Lockout, etc.) }\end{array}$ & Check & P\&ID Drawing \\
\hline $\mathrm{H} 2 \mathrm{O}-\mathrm{V}-8$ & Valve & Shut & $\square$ & \multirow[t]{5}{*}{ In Frame } \\
\hline $\mathrm{H} 2 \mathrm{O}-\mathrm{V}-9$ & Valve & Shut & $\square$ & \\
\hline $\mathrm{H} 2 \mathrm{O}-\mathrm{V}-10$ & Valve & Shut & $\square$ & \\
\hline $\mathrm{H} 2 \mathrm{O}-\mathrm{V}-16$ & Valve & Shut & $\square$ & \\
\hline $\mathrm{H} 2 \mathrm{O}-\mathrm{V}-17$ & Valve & Open & $\square$ & \\
\hline $\mathrm{H} 2 \mathrm{~V} 1$ & Lock Valve & Shut Lockout & $\square$ & \multirow{12}{*}{$\begin{array}{c}\text { Hydrogen } \\
\text { Panel }\end{array}$} \\
\hline $\mathrm{H} 2-\mathrm{V}-3$ & Valve & Open & $\square$ & \\
\hline $\mathrm{H} 2-\mathrm{V}-4$ & Valve & Open & $\square$ & \\
\hline $\mathrm{H} 2-\mathrm{V}-5$ & Valve & Shut & $\square$ & \\
\hline H2-V-6 & Valve & Open & $\square$ & \\
\hline $\mathrm{H} 2-\mathrm{V}-7$ & Valve & Open & $\square$ & \\
\hline $\mathrm{H} 2-\mathrm{V}-8$ & Valve & Shut & $\square$ & \\
\hline $\mathrm{H} 2-\mathrm{V}-\mathrm{g}$ & Valve & Shut & $\square$ & \\
\hline $\mathrm{H} 2-\mathrm{V}-10$ & Valve & Shut & $\square$ & \\
\hline $\mathrm{H} 2-\mathrm{V}-91$ & Valve & Shut & $\square$ & \\
\hline $\mathrm{H} 2-\mathrm{V}-92$ & Valve & Shut & $\square$ & \\
\hline $\mathrm{H} 2-\mathrm{V}-22$ & Valve & Shut & $\square$ & \\
\hline CF-V-1 & Valve & Shut & $\square$ & \multirow{3}{*}{ Coal Feeder Panel } \\
\hline CF-V-2 & Valve & Open & $\square$ & \\
\hline N2-V-16 & Valve & Shut & $\square$ & \\
\hline $\mathrm{H} 2 \mathrm{O}-\mathrm{V}-5$ & Valve & Open & & \multirow{5}{*}{ In Frame } \\
\hline H2O-V-6 & Valve & Open & $\square$ & \\
\hline $\mathrm{H} 2 \mathrm{O}-\mathrm{V}-21$ & Valve & Shut & $\square$ & \\
\hline $\mathrm{H} 2 \mathrm{O}-\mathrm{V}-22$ & Valve & Shut & $\square$ & \\
\hline C-MTR-1 & Motor & Off & $\square$ & \\
\hline$R-V-1$ & Valve & Open & & \multirow{5}{*}{ Balancing Panel } \\
\hline R-V -2 & Valve & Shut & $\square$ & \\
\hline R-V-6 & Valve & Open & $\square$ & \\
\hline R-V-96 & Valve & Shut & $\square$ & \\
\hline AR-V-1 & Valve & Shut & 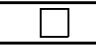 & \\
\hline R-V-4 & Valve & Shut & $\square$ & \multirow{5}{*}{ In Frame } \\
\hline R-V-5 & Valve & Open & $\square$ & \\
\hline R-V-7 & Valve & Shut & $\square$ & \\
\hline R-V-8 & Valve & Shut & $\square$ & \\
\hline R-V -9 & Valve & Shut & $\square$ & \\
\hline
\end{tabular}

Page 2 
APPENDIX 2 - COAL TO SNG BENCH SCALE TEST REACTOR NORMAL SHUTDOWN LINEUP CHECKLIST (CONTD)

\section{Coal to SNG Bench Scale Test Reactor Normal Shutdown Lineup Checklist (Continued)}

\begin{tabular}{|c|c|c|c|c|}
\hline $\begin{array}{c}\text { Component } \\
\text { Number }\end{array}$ & $\begin{array}{l}\text { Component } \\
\text { Description }\end{array}$ & $\begin{array}{c}\text { Required Position } \\
\text { (On, Off, Tagout, } \\
\text { Lockout, etc.) }\end{array}$ & Check & P\&ID Drawing \\
\hline R-V-10 & Valve & Shut & $\square$ & \\
\hline R-V-78 & Valve & Shut & $\square$ & \\
\hline$C O N-Y-1$ & Valve & Onen & $\Pi$ & \multirow{4}{*}{ In Frame } \\
\hline CON-V-2 & Valve & Shut & $\square$ & \\
\hline CON-V-3 & Valve & Shut & $\square$ & \\
\hline CON-V-20 & Valve & Shut & $\square$ & \\
\hline CON-V-4 & Valve & Shut & $\square$ & \multirow{3}{*}{ SNG Product Panel } \\
\hline SG-V-1 & Valve & Open & $\square$ & \\
\hline SG-V-2 & Valve & Shut (Commissioning) & $\square$ & \\
\hline SG-V-3 & Valve & Shut & $\square$ & \multirow{9}{*}{ SNG Product Panel } \\
\hline SG-V-10 & Valve & Open & $\square$ & \\
\hline SG-V-11 & Valve & Shut & $\square$ & \\
\hline SG-V-12 & Valve & Open & $\square$ & \\
\hline SG-V-13 & Valve & Open & $\square$ & \\
\hline SG-V-14 & Valve & Shut & $\square$ & \\
\hline SG-V-15 & Valve & Shut & $\square$ & \\
\hline SG-V-22 & Valve & Shut & $\square$ & \\
\hline SG-V-22 & Valve & Shut & $\square$ & \\
\hline N2-AOV-40 & Air Operated Valve & Shut & $\square$ & SNG2000.1 \\
\hline $\mathrm{H} 2-\mathrm{PCV}-1$ & $\begin{array}{c}\text { Pressure Control } \\
\text { Valve }\end{array}$ & Shut & $\square$ & \multirow{4}{*}{$\begin{array}{c}\text { SNG2000.3 } \\
1 / 2\end{array}$} \\
\hline H2-FCV-1 & Flow Control Valve & Shut & $\square$ & \\
\hline H2-AOV-1 & Air Operated Valve & Shut & $\square$ & \\
\hline H2-PCV-2 & $\begin{array}{c}\text { Pressure Control } \\
\text { Valve }\end{array}$ & Shut & $\square$ & \\
\hline $\mathrm{H} 2-\mathrm{FCV}-2$ & Flow Control Valve & Shut & $\square$ & \multirow{4}{*}{$\begin{array}{c}\text { SNG } 2000.3 \\
2 / 2\end{array}$} \\
\hline H2-MFC-1 & Flow Control Valve & Shut & $\square$ & \\
\hline $\mathrm{H} 2-\mathrm{AOV}-2$ & Air Operated Valve & Shut & $\square$ & \\
\hline H2-AOV-20 & Air Operated Valve & Shut & $\square$ & \\
\hline CF-SV-1 & Solenoid Valve & Shut & $\square$ & \multirow{6}{*}{ SNG2000.4 } \\
\hline CF-SV-2 & Solenoid Valve & Open & $\square$ & \\
\hline CF-SV-3 & Solenoid Valve & Shut & $\square$ & \\
\hline CF-SV-4 & Solenoid Valve & Shut & $\square$ & \\
\hline CF-PCV-1 & $\begin{array}{c}\text { Pressure Control } \\
\text { Valve }\end{array}$ & Shut & $\square$ & \\
\hline CF-AOV-4 & Air Operated Valve & Shut & $\square$ & \\
\hline
\end{tabular}

Page 3 


\section{APPENDIX 2 - COAL TO SNG BENCH SCALE TEST REACTOR NORMAL SHUTDOWN LINEUP CHECKLIST (CONTD)}

Coal to SNG Bench Scale Test Reactor

Normal Shutdown Lineup Checklist (Continued)

\begin{tabular}{|c|c|c|c|c|}
\hline $\begin{array}{c}\text { Component } \\
\text { Number }\end{array}$ & $\begin{array}{l}\text { Component } \\
\text { Description }\end{array}$ & $\begin{array}{c}\text { Required Position } \\
\text { (On, Off, Tagout, } \\
\text { Lockout, etc.) }\end{array}$ & Check & P\&ID Drawing \\
\hline R-PCV-1 & $\begin{array}{c}\text { Pressure Control } \\
\text { Valve }\end{array}$ & Shut & $\square$ & \multirow{6}{*}{ SNG2000.6 } \\
\hline R-MFC-1 & Flow Control Valve & Shut & $\square$ & \\
\hline R-AOV-4 & Air Operated Valve & Open & $\square$ & \\
\hline R-PCV-30 & $\begin{array}{c}\text { Pressure Control } \\
\text { Valve }\end{array}$ & Shut & $\square$ & \\
\hline R-AOV-3 & Air Operated valve & Shut & $\square$ & \\
\hline AR-MFC-1 & Flow Control Valve & Shut & $\square$ & \\
\hline R-AOV-1 & Air Operated Valve & Open & $\square$ & \multirow{4}{*}{ SNG2000.8 } \\
\hline R-AOV-2 & Air Operated Valve & Shut & $\square$ & \\
\hline R-SV-7 & Solenoid Valve & Shut & $\square$ & \\
\hline R-SV-8 & Solenoid valve & Shut & $\square$ & \\
\hline CON-AOV-1 & Air Operated Valve & Open & $\square$ & SNG2000.9 \\
\hline SG-PCV-1 & $\begin{array}{c}\text { Pressure Control } \\
\text { Valve }\end{array}$ & Shut & $\square$ & SNG2000.10 \\
\hline
\end{tabular}


THIS PAGE INTENTIONALLY LEFT BLANK 


\section{APPENDIX M}

\section{Process Design, Plant Design,}

\section{and Systems Analysis and Cost Engineering}


Integrated Energy System with Beneficial Carbon Dioxide $\left(\mathrm{CO}_{2}\right)$ Use

Final Report

THIS PAGE INTENTIONALLY LEFT BLANK. 


\section{Preliminary Engineering Package for Hydrogasification / Substitute Natural Gas Commercial Scale Facility Conceptual Design}

Prepared for:

Arizona Public Service Company

Prepared by:

W WorleyParsons resources \& energy

WorleyParsons Group Inc.

April 19, 2011

Final Report 


\section{NOTICE}

This Preliminary Engineering Package was prepared by WorleyParsons Group, Inc. (WorleyParsons) as an account of work contracted by and for the benefit of the Arizona Public Service Company. This package is a conceptual design and costing effort for a novel yet unproven hydrogasification / substitute natural gas (SNG) process. The overall process has been developed by joint discussions, analyses, and the best judgment of the APS Project Team. By necessity, many design basis inputs, including the gasifier performance, have been assumed based on the team's knowledge and best understanding to date. As possible, the team researched existing literature to make informed decisions based on the lessons from past gasifier / process development activities, and information available to the team members in the public domain, and/or past project experiences. APS provided gasifier performance for the bench scale test reactor.

WorleyParsons has relied upon this information and information from other team members in the preparation of this design basis document and has not independently verified that the information is accurate, complete or applicable. Key assumptions need to be confirmed during subsequent work. As such, WorleyParsons does not assume any liability for the use or misuse of the information in this report.

Decisions and actions based upon the information in this design basis and the subsequent conceptual design effort should acknowledge the preliminary nature of the current analysis. The design and cost data are only suited for planning and budget estimation purposes only, and are not of sufficient depth of detail to justify major capital investment.

Should the project continue to move forward, an experienced reactor/ gasifier designer will need to join the team to further the development of the hydrogasification reactor concepts and design elements. WorleyParsons is an architect/engineering firm and is not a reactor designer. In addition, a patent review for possible patent infringement should be performed prior to continued development of the process.

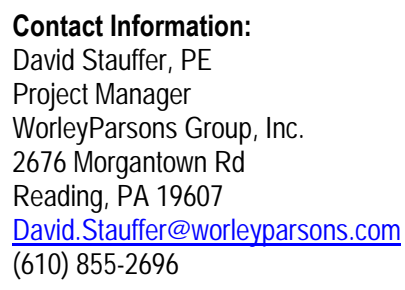

WorleyParsons Report No. APS-0-LI-011-0001 


\section{TABLE OF CONTENTS}

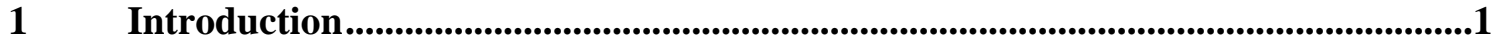

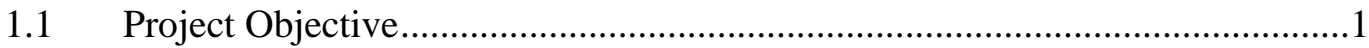

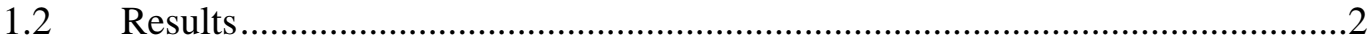

1.3 Report Approach/ Structure/ Content ..........................................................

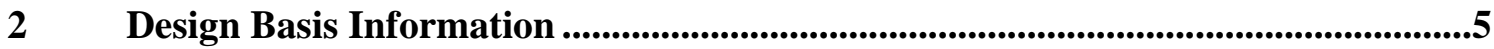

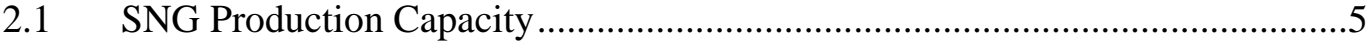

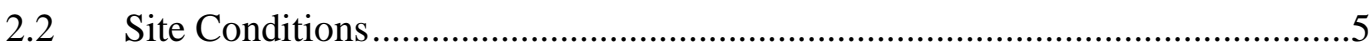

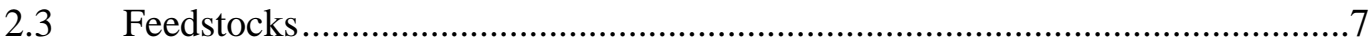

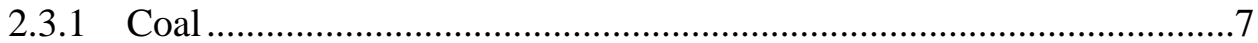

2.3.2 Externally Supplied Hydrogen and Oxygen ........................................9

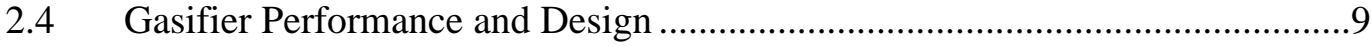

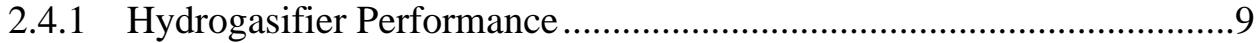

2.4.2 Hydrogasifier Model ....................................................................11

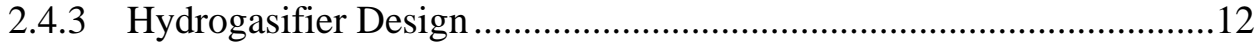

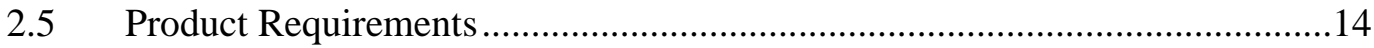

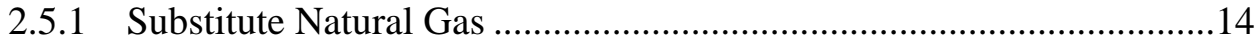

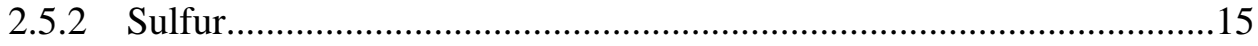

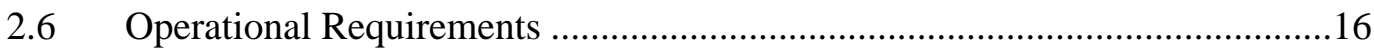

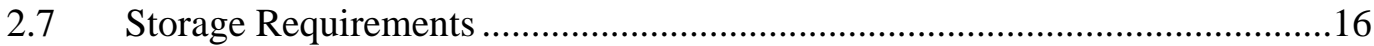

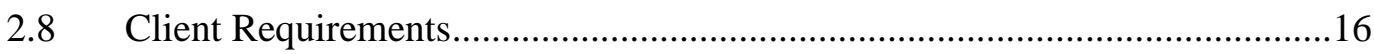

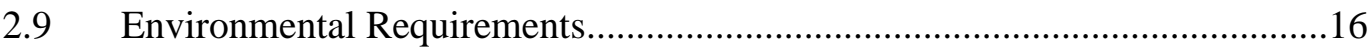

3 Hydrogasification Configuration Selection ............................................................19

$4 \quad$ Engineering Package ............................................................................................23

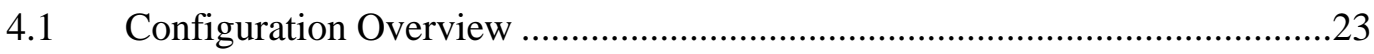

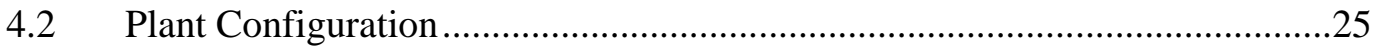

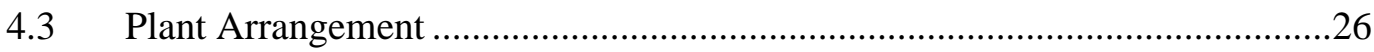

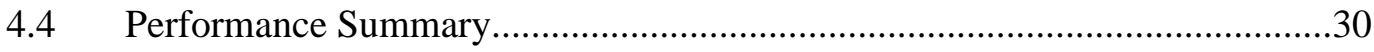

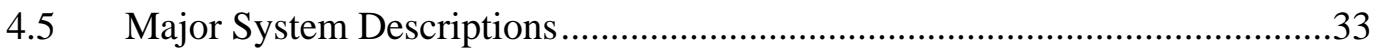

4.5.1 Coal Handling System .....................................................................33

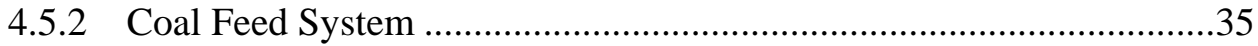

4.5.3 Hydrogasification Reactor .............................................................36

4.5.4 $\mathrm{H}_{2}$ Production and Handling ...........................................................39

4.5.5 Raw Gas Cooling and Entrained Particulate Removal .......................39

4.5.6 Mercury Removal .................................................................................40

4.5.7 Acid Gas Removal and Sulfur Recovery ..........................................40

4.5.8 Char Combustor ..........................................................................44

4.5.9 Ash / Waste Handling System .........................................................43 
4.5.10 Deoxidation, and $\mathrm{CO}_{2}$ Compression and Handling ..........................44

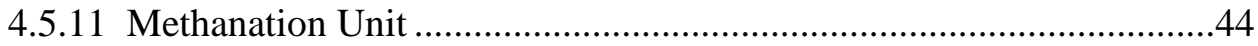

4.5.12 Cogeneration System ...............................................................45

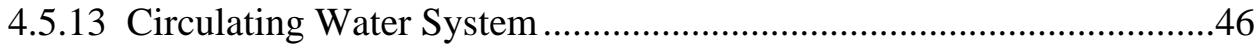

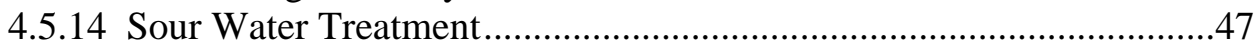

4.5.15 Water and Waste Water Treatment....................................................47

4.5.16 Flare System..........................................................................48

4.5.17 Accessory Electric Plant .............................................................49

4.5.18 Instrumentation and Control .......................................................49

5 Construction, Commissioning and Schedule ...................................................50

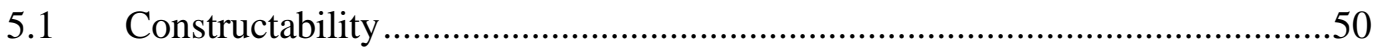

5.2 Initial Start-Up and Commissioning .......................................................52

5.2.1 Initial Start-Up and Commissioning Process ....................................53

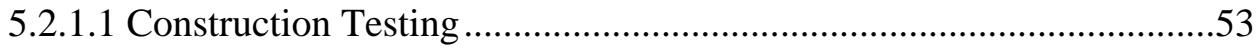

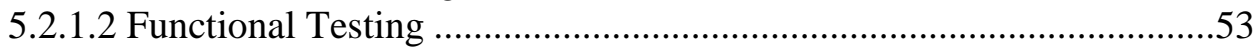

5.2.1.3 Operational / Performance Testing ....................................................54

5.2.2 Initial Start-Up and Commissioning Personnel .................................54

5.2.3 Start-Up Manual........................................................................54

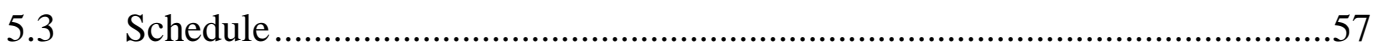

$6 \quad$ Start-up Sequence and Operating Procedures..............................................................62

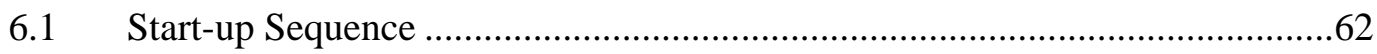

6.1.1 System Interrelationships........................................................62

6.1.2 Start-up Sequence .....................................................................63

6.2 Operating Procedure Manuals.................................................................66

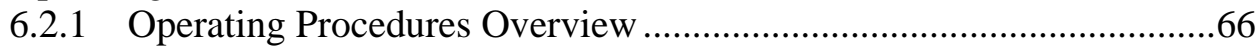

6.2.2 Example Table of Contents.............................................................67

6.2.3 Introduction to the Operating Manual................................................68

6.2.3.1 Integrated Plant Procedures ............................................................68

6.2.3.2 Localized Island Operating Procedures .............................................68

6.2.3.3 System Operating Procedures .........................................................69

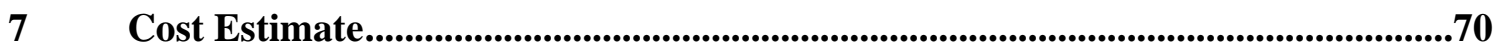

7.1 Cost Estimating Approach .................................................................... 70

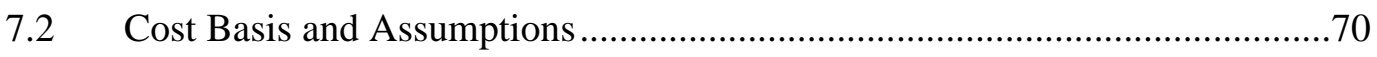

7.2.1 Capital Costs Basis and Assumptions...............................................71

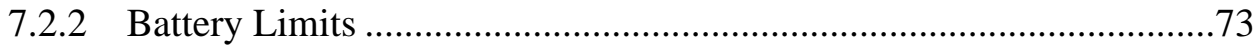

7.2.3 Operations and Maintenance Cost Basis and Assumptions ..................73

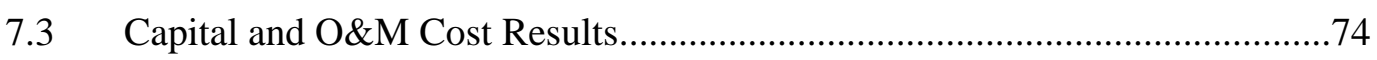

7.3.1 Total Indicative Price ................................................................... 74

7.3.2 Operating and Maintenance (O\&M) Costs .........................................75

7.4 Cost of Product Estimate Basis ................................................................... 
7.4.1 Hydrogen Cost / Electrolysis Basis.

7.4.2 Oxygen Cost / Electrolysis Basis

7.5 Cost of Product (Cost of Hydrogen, Cost of SNG)

References..

\section{Appendices}
A Design Basis
B Process Flow Diagrams
C Material Balance Tables
D Water Balance Diagram
E Major Equipment List
F Capital Cost Details
G Operating Cost Details
$\mathrm{H} \quad$ Hydrogen Source Review for Electrolysis 


\section{LIST OF EXHIBITS}

Exhibit 1-1 Commercial Hydrogasification Facility Key Features ........................................

Exhibit 2-1 Site Ambient Conditions.............................................................................6

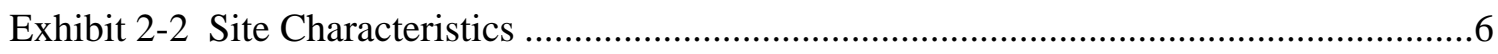

Exhibit 2-3 Fruitland Coal Analysis and Properties ...........................................................8

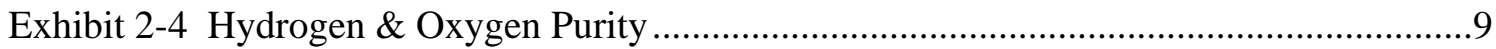

Exhibit 2-5 Hydrogasifier Performance .......................................................................... 10

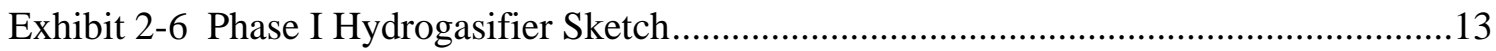

Exhibit 2-7 Natural Gas Pipeline Quality Specifications ...................................................14

Exhibit 2-8 Natural Gas Pipeline Quality Specification Summary .......................................15

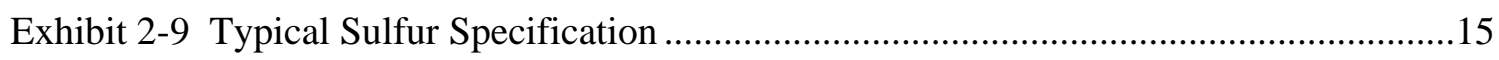

Exhibit 3-1 Phase I Block Flow Diagram of the Hydrogasification SNG Process .................19

Exhibit 4-1 Block Flow Diagram of the Base Hydrogasification SNG Process.....................24

Exhibit 4-2 Hydrogasification / SNG Process Configuration and Design Redundancy.........26

Exhibit 4-3 Hydrogasification Facility General Arrangement.............................................29

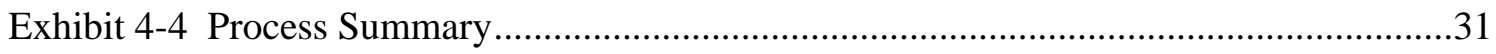

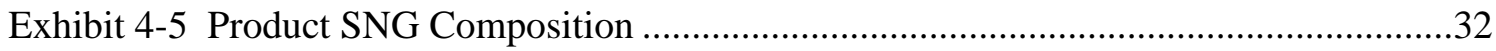

Exhibit 4-6 Electric Power Co-Generation Performance.......................................................33

Exhibit 4-7 Coal Handling System Technical Requirements ..................................................35

Exhibit 4-8 Commercial Scale Hydrogasifier Concept .....................................................38

Exhibit 4-9 Hydrogasifier Design Conditions ..................................................................38

Exhibit 4-10 Typical CrystaSulf Process .........................................................................41

Exhibit 4-11 Ash Handling System Technical Requirements and Design Basis....................43

Exhibit 4-12 Hydrogasification Plant Cooling Tower Duty ...............................................47

Exhibit 5-1 Recent Procurement Lead Times (as of Dec 2010) ..........................................58

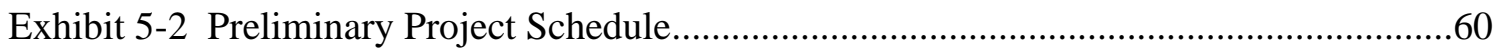

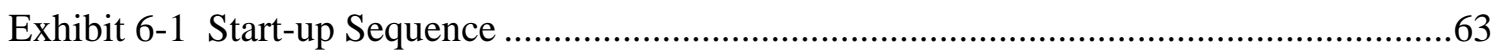

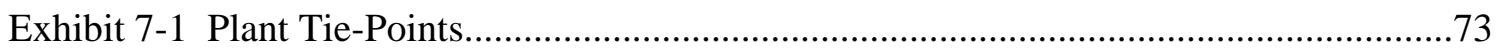

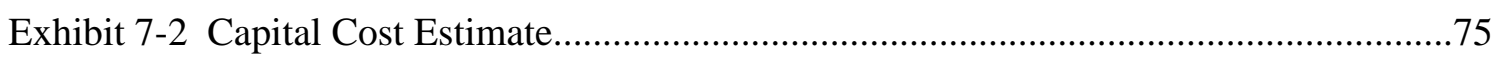

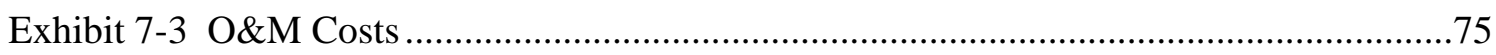

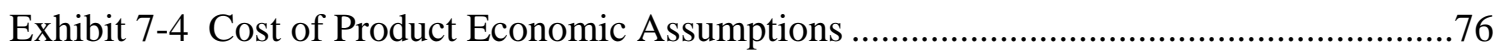

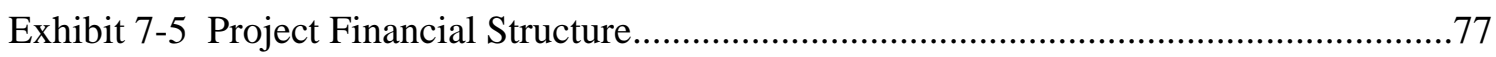

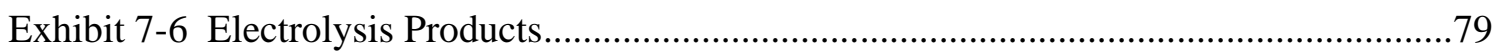

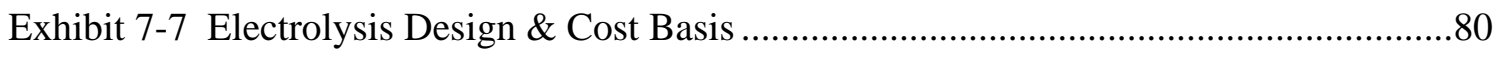

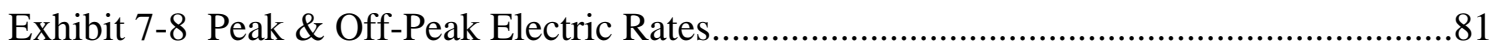

Exhibit 7-9 Peak \& Off-Peak Electric Rate Chart ................................................................81

Exhibit 7-10 Breakdown of Costs for Levelized Cost of Hydrogen Production .....................83 
Exhibit 7-11 Sensitivity of First Year Cost of Hydrogen Production for Variation in Electricity Costs.

Exhibit 7-12 Breakdown of Costs and Revenue of Hydrogasification Process with

Resulting SNG Production Cost.

Exhibit 7-13 Sensitivity of First Year Cost of SNG Production for Variation in Listed Cost Categories.

Exhibit 7-14: NYMEX Henry-Hub Natural Gas Price - Past 12 Months 
Revision Record: Appendices

\begin{tabular}{|c|c|c|c|}
\hline Appendix & Document Title & Document Number & Revision \\
\hline A & $\begin{array}{l}\text { Design Basis, Arizona Public Service } \\
\text { Hydrogasification / Substitute Natural Gas, } \\
\text { Conceptual Design Study Commercial Scale Facility }\end{array}$ & NA & I \\
\hline B & $\begin{array}{l}\text { Process Flow Diagrams } \\
\text { Block Flow Diagram } \\
\text { Gasification \& Radiant Cooler } \\
\text { Syngas Cooling \& Sulfur Removal } \\
\text { Methanation } \\
\text { Methanation Product Cooling and Compression } \\
\text { Char Combustor } \\
\text { Deoxidation and Recycle } \\
\text { Sour Water Stripper } \\
\text { Steam and Feedwater System }\end{array}$ & $\begin{array}{l}\text { APSS-1-SK-021-305-105 } \\
\text { APSS-1-SK-021-305-301 } \\
\text { APSS-1-SK-021-305-302 } \\
\text { APSS-1-SK-021-305-303 } \\
\text { APSS-1-SK-021-305-304 } \\
\text { APSS-1-SK-021-305-305 } \\
\text { APSS-1-SK-021-305-306 } \\
\text { APSS-1-SK-021-305-307 } \\
\text { APSS-1-SK-021-305-308 }\end{array}$ & $\begin{array}{l}B \\
E \\
C \\
E \\
F \\
E \\
D \\
D \\
D\end{array}$ \\
\hline C & $\begin{array}{l}\text { Material Balances } \\
\text { Gasification Section \& Radiant Cooler } \\
\text { Syngas Cooling \& Sulfur Removal } \\
\text { Methanation } \\
\text { SNG Cooling \& Compression } \\
\text { Char Combustion } \\
\text { Deoxidation \& Recycle } \\
\text { Sour Water Stripping } \\
\text { Steam Cycle } \\
\end{array}$ & $\begin{array}{l}\text { Streams } 101-113 \\
\text { Streams } 202-206 \\
\text { Streams } 301-309 \\
\text { Streams } 401-406 \\
\text { Streams } 501-510 \\
\text { Streams } 601-604 \\
\text { Streams } 701-703 \\
\text { Streams } 801-826\end{array}$ & $\begin{array}{l}01 / 20 / 11 \\
01 / 20 / 11 \\
01 / 20 / 11 \\
01 / 20 / 11 \\
01 / 20 / 11 \\
01 / 20 / 11 \\
01 / 20 / 11 \\
01 / 20 / 11 \\
\end{array}$ \\
\hline D & Water Balance Diagram & APSS-1-DW-021-305-500 & B \\
\hline E & Major Equipment List & NA & $01 / 20 / 11$ \\
\hline $\mathbf{F}$ & Capital Costs Details & NA & $01 / 28 / 11$ \\
\hline G & Operating Costs Details & NA & $01 / 28 / 11$ \\
\hline $\mathbf{H}$ & Hydrogen Source Review for Electrolysis & NA & $A$ \\
\hline
\end{tabular}





\section{Introduction}

This report represents a preliminary engineering package for the commercial-scale facility utilizing the hydrogasification process to produce Substitute Natural Gas (SNG). Section 1.1 presents the project objectives, Section 1.2 presents the key results of the project, and Section 1.3 presents the overall approach and structure of the report.

\subsection{Project Objective}

The objective of this Phase II 2010 update of the Phase I 2007 coal hydrogasification/ substitute natural gas (SNG) conceptual design effort is two fold:

1. To update the gasifier performance and its affect on the envisioned configuration, based on the Bench Scale Test Reactors (BSTR) initial results. Key gasifier performance parameters to be updated include:

a. Carbon conversion from $70 \%$ to approximately 52\%,

b. Methane syngas levels,

c. Disposition of coal sulfur between char and syngas, and

d. Quantity / analysis of gasifier tars and oils (no tars and oils are expected at $\left.1750^{\circ} \mathrm{F}\right)$,

e. Hydrogen to coal ratio (as influenced by both the gasifier and methanator),

f. Oxygen to coal ratio (as required for the thermal balance).

2. To update the configuration and cost estimate for changes that result from the intervening years including:

a. Cost escalation, and

b. Technological advances, if any.

The ultimate objective of the overall three (3) Phase project is to develop and demonstrate an engineering-scale, coal hydrogasification, based process for co-production of substitute natural gas (SNG) and electricity, with near-zero emissions meeting the following performance targets:

1. Overall process efficiency greater than $50 \%$,

2. SNG cost less than $\$ 5 / M M B T U$,

3. Capture and sequestration of $\mathrm{CO}_{2}$ equivalent to $90 \%$ of emissions from power production $^{\mathrm{a}}$,

4. Reduce water usage as compared to partial oxidation gasification/syngas methanation process.

5. Capability of accepting hydrogen (preferably from a renewable source) as a supplemental source of energy

The proposed cycle converts rather than captures $\mathrm{CO}_{2}$ as the process recycles the produced $\mathrm{CO}_{2}$ and utilizes it for the production of SNG. In this way, the produced $\mathrm{CO}_{2}$ is sequestered as fuel. 
6. Ability to use Western sub-bituminous coals. (In Phase I \& II, only a single design coal is considered), and

7. The capacity target for a single commercial-scale gasifier module is to process 1,000 short tons (dry) per day of coal.

The objective of this Phase II Task is to develop a conceptual design for the novel hydrogasification process and to examine the feasibility of a commercial-scale hydrogasification project. This preliminary package includes engineering and cost estimates for the Phase II conceptual effort of the Commercial-Scale facility.

\subsection{Results}

The key design features of a commercial scale hydrogasification facility are presented in Exhibit $1-1$. 
Exhibit 1-1 Commercial Hydrogasification Facility Key Features

\begin{tabular}{|c|c|}
\hline Parameter & Value \\
\hline $\begin{array}{l}\text { Output } \\
\text { SNG } \\
\text { Gross Electric Power } \\
\text { Net Electric Power } \\
\text { Elemental Sulfur } \\
\end{array}$ & $\begin{array}{l}\text { 119.7 MMSCFD (Note A) } \\
\text { 241.4 MWe (Note B) } \\
\text { 201.0 MWe (Note C) } \\
\text { 20.6 TPD }\end{array}$ \\
\hline $\begin{array}{l}\text { Plant Configuration } \\
\text { Gasifiers } \\
\text { Steam Turbines } \\
\text { Syngas Cooling and Conditioning } \\
\text { Oxygen Blown Char Combustor } \\
\text { SNG Conditioning }\end{array}$ & $\begin{array}{l}\text { Three gasifiers, 1,000 tpd each (Note D) } \\
\text { One steam turbine-generator, } 241 \mathrm{MWe} \\
\text { Three trains serving three gasifiers, } 384 \text { MMSCFD } \\
\text { Single system serving three gasifiers, } 1,584 \text { tpd of char } \\
\text { Single train serving three gasifiers, } 120 \text { MMSCFD }\end{array}$ \\
\hline $\begin{array}{l}\text { Consumables } \\
\text { Coal } \\
\text { Hydrogen } \\
\text { Oxygen } \\
\text { Water }\end{array}$ & $\begin{array}{l}3,235 \text { TPD } \\
354 \text { MMSCFD } \\
83 \mathrm{MMSCFD} \\
2,456 \mathrm{gpm}\end{array}$ \\
\hline$\frac{\text { Capital Costs (in Jun. } 2010 \text { USD) }}{\text { Bare Erected Capital Costs }}$ & $\$ 886,902,000$ \\
\hline $\begin{array}{l}\text { O \& M Costs (in Jun. 2010 USD) (Note } \\
\text { E) } \\
\text { Fixed Operating Cost } \\
\text { Routine Maintenance Material } \\
\text { Water } \\
\text { Chemicals } \\
\text { Fuel (@ \$29.5/ton) } \\
\text { Hydrogen (@ \$3.50/kg, or } \$ 1.59 / \mathrm{lb}) \\
\text { Oxygen } \\
\text { Ash Disposal (@ } \$ 19.37 / \text { ton ash, and } \\
\quad \$ 500 / \text { ton Hg sorbent) } \\
\text { Sulfur ByProduct Credit (@ \$30/ton) }\end{array}$ & $\begin{array}{c}\$ 12,535,000 . / \text { year } \\
\$ 7,347,000 . / \text { year } \\
\$ 1,358,000 . / \text { year } \\
\$ 9,930,000 . / \text { year } \\
\$ 26,919,000 . / \text { year } \\
\$ 873,185,000 . / \text { year } \\
\$ 0 . / \text { year (included in } \mathrm{H}_{2} \text { electrolysis cost) } \\
\$ 5,285,000 . / \text { year } \\
\\
(\$ 180,000 .) \text { / year }\end{array}$ \\
\hline Land Area Requirement & 70 acres \\
\hline
\end{tabular}

Notes:
A. At $60^{\circ} \mathrm{F}, 14.73$ psia
B. On generator terminals at $20 \mathrm{kV}$
C. On $230 \mathrm{kV}$ main step up transformer terminals.
D. Fruitland coal, Dry basis, $\mathrm{HHV}=10,710 \mathrm{Btu} / \mathrm{lb}$
E. O\&M costs reflect an $80 \%$ capacity factor.

\subsection{Report Approach/ Structure/ Content}

This report represents a broad engineering assessment for the project that should supply sufficiently accurate information to support system planning studies, preliminary cost and economic assessments, and plant siting evaluations. The conceptual design engineering 
presented in this report lays the technical foundation for selecting design concepts and equipment, and defines the key design features, functional systems and structure, system and equipment design constraints, plant performance, and plant costs.

This report focuses on developing the preliminary technical and cost information to support project planning and analysis. As the project evolves, a follow-on detailed design process will be required to facilitate continued feasibility assessment, permitting/licensing, equipment procurement, construction and operation of the new facility.

The performance of the hydrogasification facility is evaluated using ASPEN based gasification system models developed by WorleyParsons. Typically these models are based on commercial gasification processes which have been calibrated to actual plant characteristics over several decades of consulting for the US Department of Energy, Electric Power research Institute, and private sector clients. However, the work described herein is not based on an existing commercial gasification process, but a developmental concept. The models developed for the APS hydrogasification reactor are based on the BSTR results along with WorleyParsons' understanding of how to extend the BSTR results to the anticipated commercial scale reactor conditions. This performance should be confirmed with an engineering scale test should APS be interested in continuing the hydrogasification project. The cost estimates are based on a factored approach and are not a product of a detailed "bottoms-up" design or cost estimating. The developed cost represents the use of the aforementioned models coupled with engineering and cost estimating judgment.

The evaluation scope included performing steady-state simulations of the various technological islands, developing heat and mass balances and estimating plant performance to support equipment sizing and capital and operating costs estimates. Equipment lists were developed for the hydrogasification facility in support of the plant capital cost estimates.

The key performance and economic parameters of the hydrogasification facility are summarized in Section 1.2.

The evaluation basis details, including site ambient conditions, fuel composition and environmental targets, are provided in Section 2.

In Section 3, the base hydrogasification process selected by APS for development is presented.

Over the course of the evaluation, the configuration evolved. The final hydrogasification plant configuration, along with the performance summary, plant arrangement and major systems descriptions are presented in Section 4.

Constructability and construction schedule of the new facility are discussed in Section 5.

The preliminary information regarding new plant start-up and operation is provided in Section 06.

The new hydrogasification facility capital and operating costs are evaluated in Section 7. 


\section{Design Basis Information}

This section contains a summary of essential technical and functional requirements that were used as a basis in establishing conceptual design for the commercial-scale hydrogasification / SNG process evaluated in this report. Further design criteria details are provided in the Design Basis for the Conceptual Design of the Commercial Scale Facility (Appendix A).

\subsection{SNG Production Capacity}

A fundamental design criterion for the commercial-scale process is the SNG production level listed below.

1. The target SNG production for the commercial scale process has been selected as approximately 120 MMSCFD [1], and will be based on three (3) gasifier modules.

a. This SNG production level has been selected considering the capacity of the Dakota Gasification facility and publicly available information regarding SNG facilities proposed by others. The Dakota Gasification facility produces 160 MMSCFD of SNG and is the only SNG facility in the United States. A presentation by ConocoPhillips on SNG at the 2006 Gasification Technology Conference presented three SNG production schemes ranging from 90 to 115 MMSCFD [2]. Since the 1000 TPD hydrogasifier produces approximately 40 MMSCFD, three gasifier modules will produce approximately 120 MMSCFD.

b. In subsequent phases of this project, APS will want to consider additional factors such as the spare NG pipeline capacity, the availability of externally generated $\mathrm{H}_{2}$, the impact of economies of scale, market conditions, and the desires and financial considerations of the project development team.

\subsection{Site Conditions}

The design will be based on site conditions as presented in Exhibit 2-1. 
Exhibit 2-1 Site Ambient Conditions

\begin{tabular}{|l|c|}
\hline \multicolumn{1}{|c|}{ Parameter } & Value \\
\hline Location & South Western United States (Note A) \\
\hline Elevation, ft (above MSL) & 5,500 (Note B) \\
\hline Barometric Pressure, psia & 12.0 (Note B) \\
\hline Design Ambient Temperature, Dry Bulb, ${ }^{\circ} \mathrm{F}$ & 95 \\
Maximum & -3 \\
Minimum & 60 \\
Average & \\
Design Ambient Wet Bulb Temperature, ${ }^{\circ} \mathrm{F}$ & 65 \\
Summer Design & 50 \\
\hline Design Relative Humidity, \%RH & 77.27 \\
\hline Ambient Air Composition, Vol\%: & 0.93 \\
Nitrogen & 20.73 \\
Argon & 1.04 \\
Oxygen & 0.03 \\
Water & 100.00 \\
\hline Carbon Dioxide & Total \\
\hline .
\end{tabular}

Notes:

A. Assumed site is based on ambient conditions and other characteristics of the Four Corners Station, near Fruitland, San Juan County, New Mexico

B. Elevation and pressure are largely irrelevant if pressurized $\mathrm{H}_{2}$ and $\mathrm{O}_{2}$ are supplied over the fence.

Site characteristics are presented in Exhibit 2-2.

\section{Exhibit 2-2 Site Characteristics}

\begin{tabular}{|l|l|}
\hline \multicolumn{1}{|c|}{ Parameter } & \multicolumn{1}{c|}{ Value } \\
\hline Cost Basis & Farmington, New Mexico \\
\hline Topography & Level \\
\hline Size, acres & 200, (i.e., the site is not constrained) \\
\hline Access & $\begin{array}{l}\text { Land-locked, having access by major state highway, 2 miles north of the } \\
\text { plant. Nearest railroad hub is located in Gallup, New Mexico, 60 miles } \\
\text { from the site. }\end{array}$ \\
\hline Ash Disposal & Off Site \\
\hline Coal Delivery & $\begin{array}{l}\text { In 200 tons/load trucks from BHP Navajo Coal Company mine, located } \\
\text { about 1 } 1 / 2 \text { miles distant }\end{array}$ \\
\hline Water & Artificial cooling lake with water impounded from the Sun Juan River \\
\hline Waste Water & Zero Liquid Discharge (i.e., No evaporation pond) \\
\hline
\end{tabular}

The following design considerations are site-specific, and will not be quantified for this preliminary report of the commercial scale facility. Allowances for normal conditions and 
construction will be included in the cost estimates. Typically the consideration of these factors does not have a significant impact on the cost unless the site specific situation is unusual or extreme.

Flood plain considerations.

$>$ Existing soil/site conditions (except that the use of piles has been assumed).

$>$ Rainfall/snowfall criteria.

$>$ Seismic design.

$>$ Buildings/enclosures.

$>$ Fire protection.

$>$ Local code height requirements.

$>$ Noise regulations - Impact on site and surrounding area.

\section{$2.3 \quad$ Feedstocks}

This section documents the coal analysis and composition of the externally supplied hydrogen and oxygen.

\subsubsection{Coal}

Gas Technology Institute (GTI) performed the coal analysis on the Fruitland coal samples that were supplied by APS. Following are the coal composition and properties as reported by GTI. These values will be the design basis for the process modeling work. 
Exhibit 2-3 Fruitland Coal Analysis and Properties ${ }^{b}$

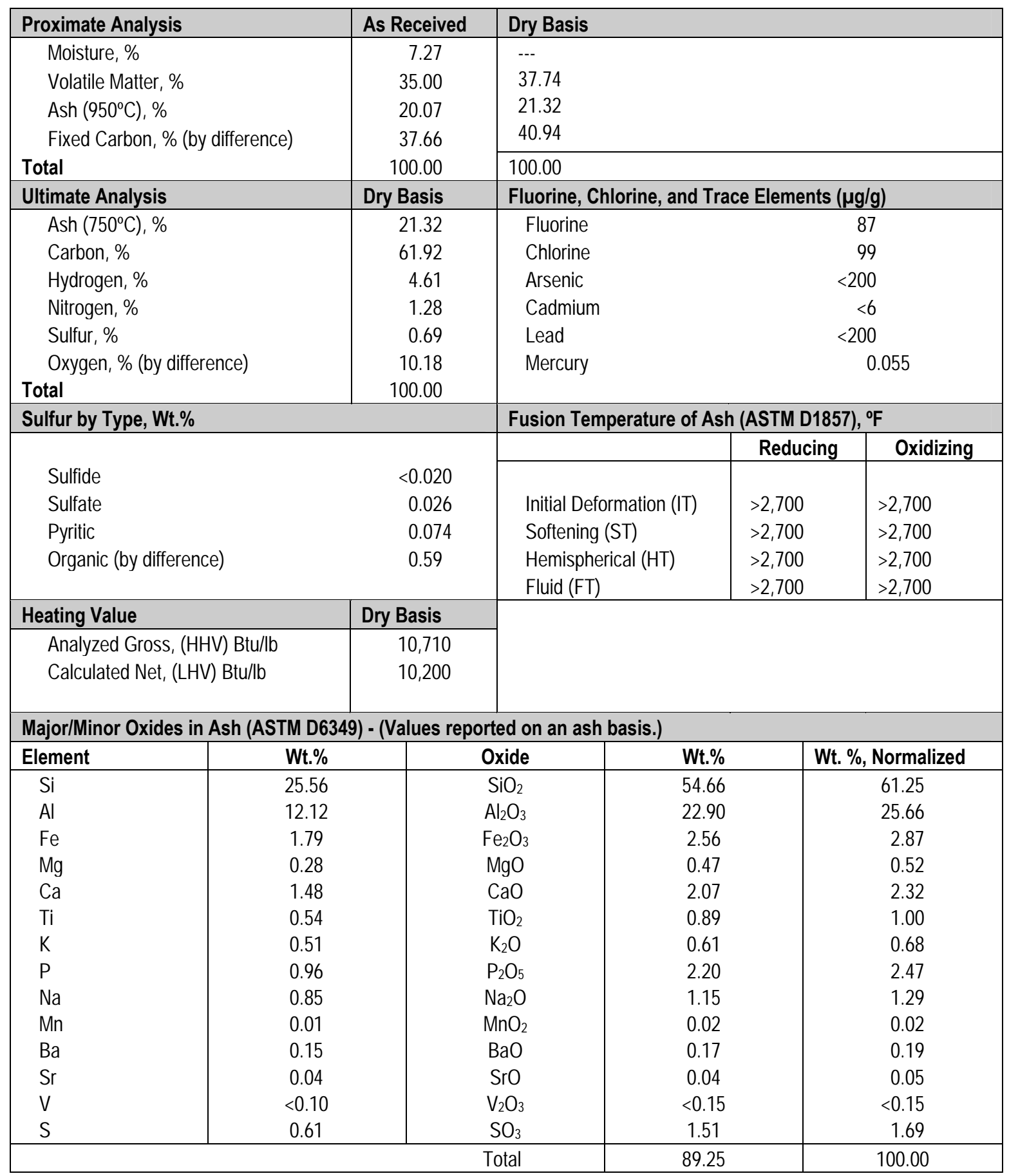

${ }^{\text {b }}$ CRS Sample Login No: 061131-001 (received 3/10/06) 
The coal feed into the gasifier will be pulverized such that approximately $70 \%$ will pass a 200 mesh screen. [3].

\subsubsection{Externally Supplied Hydrogen and Oxygen}

Hydrogen is assumed to be delivered as a compressed gas at $104^{\circ} \mathrm{F}$ and 1150 psig to the plant boundary via a dedicated pipeline. Hydrogen will be free of sulfur, chlorine, potassium, and particulate matter.

High pressure oxygen is assumed to be delivered as a compressed gas at $104^{\circ} \mathrm{F}$ and 1120 psig to the plant boundary via a dedicated pipeline. [4]

Low pressure oxygen is assumed to be delivered as a compressed gas at ambient temperature and 50 psig to the plant boundary via a dedicated pipeline.

Hydrogen and oxygen characteristics are based on an electrolysis process and are presented in Exhibit 2-4.

\section{Exhibit 2-4 Hydrogen \& Oxygen Purity}

\begin{tabular}{|c|c|}
\hline Electrolysis Product & Purity \\
\hline Hydrogen purity & $>99.99 \%$ \\
\hline Oxygen purity & $>99.5 \%$ \\
\hline
\end{tabular}

No on-site bulk storage will be assumed within the gasification facility battery limits, as the electrolysis unit will be compressed of many parallel units providing high reliability and will have the ability to quickly respond to the instantaneous demand.

\subsection{Gasifier Performance and Design}

APS has designed, built and tested a bench scale test reactor (BSTR) in order to determine the performance of such a hydrogasification reactor based on the Fruitland coal. The performance and design of a commercial scale hydrogasifier as developed by APS is presented below.

\subsubsection{Hydrogasifier Performance}

The operating conditions and performance of the hydrogasifier assuming the Fruitland coal feedstock are presented in Exhibit 2-5. 
Exhibit 2-5 Hydrogasifier Performance

\begin{tabular}{|c|c|c|c|}
\hline Specification Parameter & Value & Reference & Notes \\
\hline Gasifier Operating Temperature & $1750^{\circ} \mathrm{F}$ & [5] & \\
\hline Gasifier Effluent Pressure & 1000 psig & [5] & \\
\hline $\mathrm{H}_{2} /$ Coal (mass ratio) & 0.2 & [5] & $\begin{array}{l}\text { May be too low with recycled } \mathrm{CO}_{2} \text {. Allow to } \\
\text { float according to Methanation requirements }\end{array}$ \\
\hline $\mathrm{H}_{2}$ Injection Temperature & ca $1350^{\circ} \mathrm{F}$ & [5] & Lower if required by available materials \\
\hline $\mathrm{H}_{2} / \mathrm{O} 2$ burner & Possibly & [5] & Depends on $\mathrm{HB}$ requirement. \\
\hline Carbon Conversion (wt \%) & $51.84 \%$ & [5] & Conversion of coal constituents to syngas \\
\hline $\mathrm{CC}$ into $\mathrm{CH}_{4}$ & $46.30 \%$ & [5] & Result of BSTR \& subject to change. \\
\hline $\mathrm{CC}$ into $\mathrm{CO}$ & $5.24 \%$ & [5] & Result of BSTR \& subject to change. \\
\hline $\mathrm{CC}$ into $\mathrm{CO}_{2}$ & $0.00 \%$ & [5] & Result of BSTR \& subject to change. \\
\hline $\mathrm{CC}$ into $\mathrm{C}_{2} \mathrm{H}_{6}$ & $0.30 \%$ & [5] & Result of BSTR \& subject to change. \\
\hline CC into BTX & $0.00 \%$ & [5] & \\
\hline CC into Oil & $0.00 \%$ & [5] & \\
\hline Conversion to Syngas (wt \%) & & & Conversion of coal constituents to syngas \\
\hline S Conversion & $81.7 \%$ & [5] & \\
\hline H Conversion & $82.4 \%$ & [5] & \\
\hline N Conversion & $68.9 \%$ & [5] & \\
\hline O Conversion & $99.7 \%$ & [5] & \\
\hline Raw Syngas Composition (mol\%) & & & Result of BSTR \& subject to change. \\
\hline $\mathrm{H}_{2}$ & 0.6628 & [5] & \\
\hline $\mathrm{CH}_{4}$ & 0.2362 & [5] & \\
\hline $\mathrm{H}_{2} \mathrm{O}$ & 0.0631 & [5] & \\
\hline $\mathrm{N}_{2}$ & & [5] & \\
\hline $\mathrm{CO}$ & 0.0267 & [5] & \\
\hline $\mathrm{CO}_{2}$ & & [5] & \\
\hline $\mathrm{NH}_{3}$ & 0.0076 & [5] & \\
\hline $\mathrm{H}_{2} \mathrm{~S}$ & 0.0028 & [5] & \\
\hline $\mathrm{C}_{6} \mathrm{H}_{6}$ & & [5] & \\
\hline $\mathrm{C}_{2} \mathrm{H}_{6}$ & 0.0008 & [5] & \\
\hline Total Syngas & 1.0000 & [5] & \\
\hline
\end{tabular}

The information in Exhibit 2-5 is based on APS assumption of the commercial scale gasifier operating conditions. It is possible that many factors may cause the assumed performance for the 
commercial scale gasifier to drift away from those above (in addition to factors typically considered as a part of scale-up process, such as surface area to volume ratio). The factors that may cause the performance to change include the following:

1. Compared to the BSTR results, the commercial scale gasifier will be operated at 1000 psig, instead of the 800 psig utilized in the BSTR performance test.

2. Compared to the BSTR results which utilized a $\mathrm{H}_{2}$ carrier gas, a $\mathrm{CO}_{2}$ carrier gas will be used in the commercial scale reactor.

3. Compared to the BSTR arrangement, which utilized electric heaters, the commercial scale reactor utilizes the exothermic heat of reaction from injected $\mathrm{O}_{2}$ and an increased $\mathrm{H}_{2}$ flow to preheat the remaining $\mathrm{H}_{2}$ and to allow the hydrogasifier to achieve the desired operating condition of $1750^{\circ} \mathrm{F}$. Approximately $10 \%$ of the incoming $\mathrm{H}_{2}$ is consumed by the $\mathrm{O}_{2}$ feed.

4. With the relatively low carbon conversion ratio of approximately $50 \%$, the $\mathrm{CO}_{2}$ produced from the char combustion that ultimately ends up in the methanator, may require an increased hydrogen to coal ratio fed into the hydrogasifier.

To address these factors, WorleyParsons has developed a simplified gasifier model based on the BSTR performance in order to extend the gasifier performance to other conditions. This model is discussed briefly below.

\subsubsection{Hydrogasifier Model}

In order to capitalize on the actual BSTR testing while also allowing deviations from the BSTR test results as necessitated by changes required by the commercial scale reactor design conditions, a simple gasifier model was constructed by WorleyParsons. Inputs to the simplified model included the as-tested coal and char analyses, and the February 182010 test data results provided by APS [6]. Based on these inputs, an empirically-based "approach to equilibrium" gasifier model was set up with seven (7) independent gasification reactions. The temperature approaches to each reaction were adjusted to match as closely as possible the measured BSTR results. The simplified model matched the BSTR test results of $\mathrm{H}_{2}$ and $\mathrm{CH}_{4}$ to within $1.0 \%$ and $0.2 \%$ respectively. The predicted carbon partitioning between $\mathrm{CO}$ and $\mathrm{CO}_{2}$ as reflected by the ratio of $\mathrm{CO} /\left(\mathrm{CO}+\mathrm{CO}_{2}\right)$ was $97 \%$ for the model and $100 \%$ based on the measured data. Unfortunately the lack of a water balance for the test data prevented assessment of the model's accuracy for the predicted water value. The amount of water would affect the disposition of CO, $\mathrm{CO}_{2}, \mathrm{H}_{2} \mathrm{O}$ and $\mathrm{H}_{2}$ through the water gas shift reaction, and possibly the amount of methane. In

\footnotetext{
c The reader should understand that the "approach to equilibrium” modeling is not the same as using an equilibrium model. An equilibrium model assumes that the reactor provides sufficient time for the reactions to achieve equilibrium. The reality of limited residence-time reactors implies that many reactions will not achieve equilibrium as a result of "slow" reaction kinetics. The "approach to equilibrium" method allows for incorporation of this kinetic affect based on test data by determining an empirically-based artificial temperature where the equilibrium composition matches the composition of the actual kinetically limited reaction. The difference between the artificial temperature and the actual temperature is known as the approach to equilibrium $(\Delta \mathrm{T}$ equilibrium). This method allows for deviations from the test conditions to be estimated, and as such is more accurate than using an equilibrium model or in using the test data while ignoring the affects of these deviations.
} 
light of the above values, the empirical model was judged to be a good as possible based on test data.

The overall modeling approach utilized for the commercial scale application is summarized below.

1. The gasification reactions are modeled with the "approach to equilibrium" methodology, developed from applicable BSTR results.

2. The overall carbon conversion level is set to approximately $52 \%$ per the BSTR results

3. The reactor temperature is set to $1750^{\circ} \mathrm{F}$, per direction from APS.

4. The amount of supplied oxygen is varied so that the ASPEN gasifier model achieves an energy balance. (Equivalently the reader can consider ASPEN as varying the $\mathrm{O}_{2}$ flow to achieve the $1750^{\circ} \mathrm{F}$ target temperature)

5. The amount of $\mathrm{H}_{2}$ fed into the gasifier is varied to achieve the proper stoichiometric ratio for the Methanator. (i.e., 3:1 $\mathrm{H}_{2} / \mathrm{CO}$ and 4:1 $\mathrm{H}_{2} / \mathrm{CO}_{2}$ )

The above modeling approach allows the gasifier performance and effluent composition to reflect the changes in $\mathrm{H}_{2}, \mathrm{O}_{2}, \mathrm{~N}_{2}$ and $\mathrm{CO}_{2}$ feed rates and pressure from the test conditions.

\subsubsection{Hydrogasifier Design}

The design of the hydrogasifier for this second phase of the project is under the control of APS, who had been considering both the ARCH and Rockwell Type designs. However, since APS is now terminating the hydrogasification project and had many unanswered design decisions, APS has requested that the gasifier design be based on the Phase I design presented in Reference [7]. A sketch of the Phase I hydrogasifier from that reference is presented in Exhibit 2-6. The project team has allowed the design to evolve from this original vision as the project evolved. For example, although the fundamental design of the gasifier is unchanged from Phase I, the hydrogen heating has been modified and incorporated into the raw gas cooler and gasifier as described in Section 4. It is important that the reactor design, performance and cost are all consistent. Since the bench scale test reactor (BSTR) is essentially a drop tube reactor, the original hydrogasification design is consistent with that simple vision. 
Exhibit 2-6 Phase I Hydrogasifier Sketch

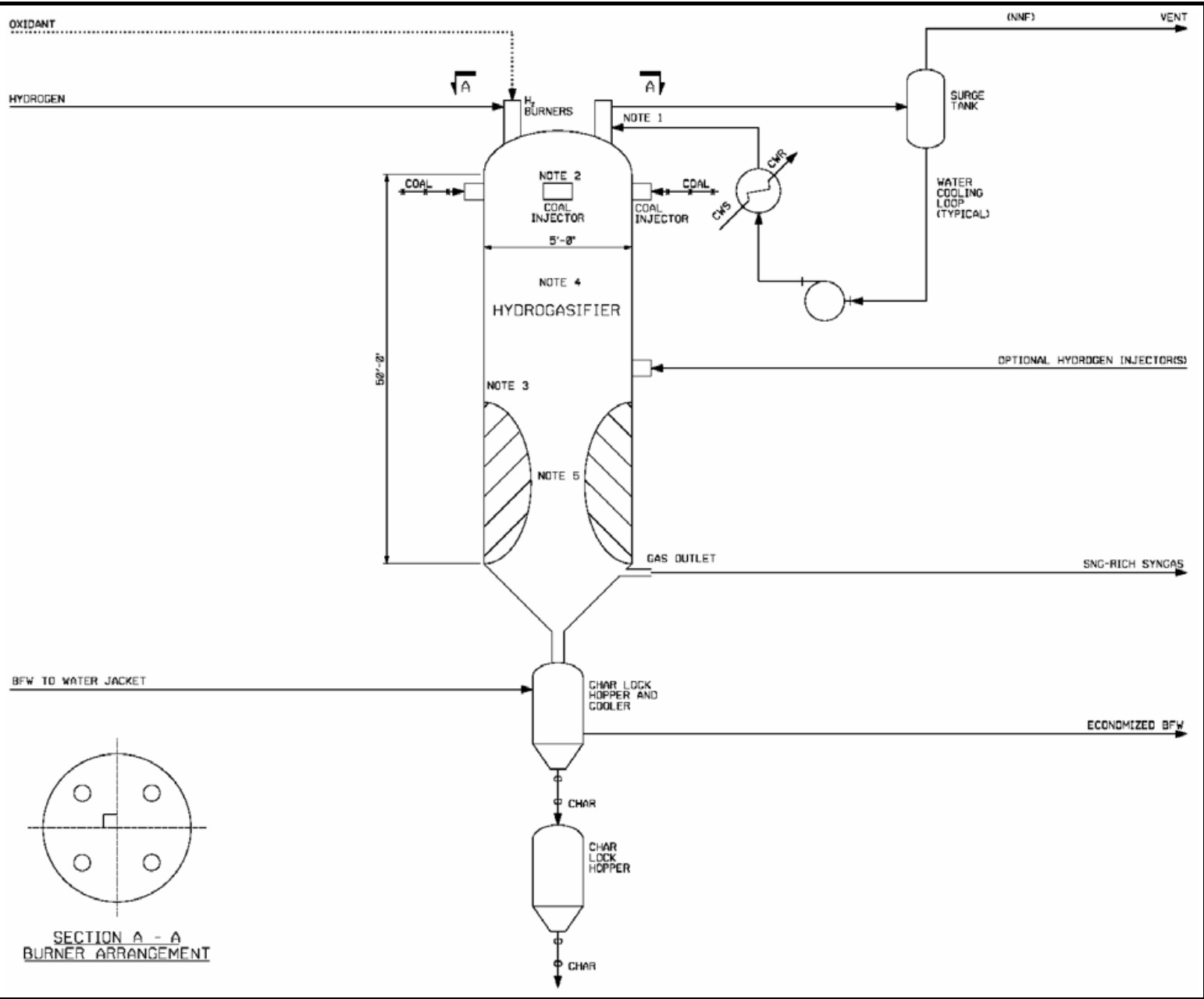

Reference:[7], p 38 Drawing APSS-1-SK-021-305-001-RA

Notes (Phase I):

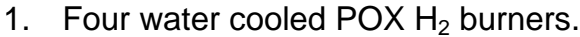

2. Four micronized coal injectors spaced 90 degrees apart. Coal injectors to be cooled.

3. Gasifier refractory lined inside edge of refractory liner shown.

4. Gasifier volume $900 \mathrm{ft}^{3}$.

5. Internal converging / diverging zone. 


\subsection{Product Requirements}

\subsubsection{Substitute Natural Gas}

El Paso Gas provided the following natural gas pipeline specification [8] for gas to be received by them in the Four Corners region. These requirements are shown below in Exhibit 2-7. These requirements are summarized in Exhibit 2-8.

\section{Exhibit 2-7 Natural Gas Pipeline Quality Specifications}

General Specifications. Shipper warrants that all natural gas received by El Paso at any mainline receipt point(s) shall conform to the following specifications and must be, in El Paso's reasonable judgment, otherwise merchantable:

(a) Liquids - The gas shall be free of water and hydrocarbons in liquid form at the temperature and pressure at which the gas is received. The gas shall in no event contain water vapor in excess of seven (7) pounds per million standard cubic feet.

(b) Hydrocarbon Dew Point - The hydrocarbon dew point of the gas received shall not exceed twenty degrees Fahrenheit $\left(20^{\circ} \mathrm{F}\right)$ at normal pipeline operating pressures.

(c) Total Sulfur - The gas shall not contain more than five (5) grains of total sulfur, which includes hydrogen sulfide, carbonyl sulfide, carbon disulfide, mercaptans, and mono-, di- and poly-sulfides, per one hundred (100) standard cubic feet. The gas shall also meet the following individual specifications for hydrogen sulfide, mercaptan sulfur or organic sulfur:

(i) Hydrogen Sulfide - The gas shall not contain more than one-quarter (0.25) grain of hydrogen sulfide per one hundred (100) standard cubic feet.

(ii) Mercaptan Sulfur - The mercaptan sulfur content shall not exceed more than three-quarters (0.75) grain per one hundred (100) standard cubic feet.

(iii) Organic Sulfur - The organic sulfur content shall not exceed one and one-quarter (1.25) grains per one hundred standard cubic feet, which includes mercaptans, mono-, di- and poly-sulfides, but it does not include hydrogen sulfide, carbonyl sulfide or carbon disulfide.

(d) Oxygen - The oxygen content shall not exceed two-tenths of one percent (0.2\%) by volume and every reasonable effort shall be made to keep the gas delivered free of oxygen.

(e) Carbon Dioxide - The gas shall not have a carbon dioxide content in excess of two percent (2\%) by volume, except for gas acceptable under Sections 5.2 and 5.3.

(f) Diluents -The gas shall not at any time contain in excess of three percent (3\%) total diluents (the total combined carbon dioxide, nitrogen, helium, oxygen, and any other diluent compound) by volume

(g) Dust, Gums and Solid Matter - The gas shall be commercially free of dust, gums and other solid matter.

(h) Heating Value - The gas shall have a heating value of not less than 967 Btu per cubic foot.

(i) Temperature - The gas received by El Paso shall be at temperatures not in excess of one hundred twenty degrees Fahrenheit $\left(120^{\circ} \mathrm{F}\right)$ nor less than fifty degrees Fahrenheit $\left(50^{\circ} \mathrm{F}\right)$. Any party tendering gas at a temperature standard less than fifty degrees Fahrenheit $\left(50^{\circ} \mathrm{F}\right)$ shall receive a waiver of such standard only if a test has been conducted in accordance with procedures set forth in Section 5.12(b) hereof, and the results from such test demonstrate that the particular segment of the pipeline tested can be safely operated below the fifty degrees Fahrenheit $\left(50^{\circ} \mathrm{F}\right)$ temperature standard.

(j) Deleterious Substances - The gas shall not contain deleterious substances in concentrations that are hazardous to health, injurious to pipeline facilities or adversely affect merchantability.

The pipelines in northern Arizona are running at 845 - 895 psig maximum. 
Exhibit 2-8 Natural Gas Pipeline Quality Specification Summary

\begin{tabular}{|c|c|}
\hline Specification Parameter & Value \\
\hline Pipeline Pressure & 895 psig (maximum) \\
\hline Pipeline Temperature Requirement & $\begin{array}{c}50-120^{\circ} \mathrm{F} \\
<50^{\circ} \mathrm{F} \text { by waiver }\end{array}$ \\
\hline HHV, Btu/scf ( with scf @ 60 F, 14.73 psi) & $>967$ \\
\hline Diluents $\left(\mathrm{CO}_{2}, \mathrm{~N}_{2}, \mathrm{He}, \mathrm{O}_{2},+\right.$ other $) \%$ vol. & $<3 \%$ \\
\hline $\mathrm{CO}_{2}, \%$ volume & $<2 \%$ \\
\hline $\mathrm{O}_{2}, \%$ volume & $<0.2 \%$ \\
\hline $\mathrm{CO}$ & Not specified, (see diluent) \\
\hline $\mathrm{H}_{2}$ & $<3 \mathrm{~mol} \%[9]$ \\
\hline Liquids & None, at receiving $\mathrm{T}$ \\
\hline Water & $<7 \mathrm{lbs} / \mathrm{MMSCF}$ \\
\hline Hydrocarbon Dew Point, ${ }^{\circ} \mathrm{F}$ & $<20^{\circ} \mathrm{F}$ at pipeline op pressure \\
\hline Total Sulfur, & 5 grains/100 scf \\
\hline $\mathrm{H}_{2} \mathrm{~S}$ & 0.25 grains/ $100 \mathrm{scf}$ \\
\hline Mercaptan S & 0.75 grains/ $100 \mathrm{scf}$ \\
\hline $\begin{array}{l}\text { Organic } \mathrm{S} \text { (includes mercaptans, mono- di- and } \\
\text { poly-sulfides, but excluding } \mathrm{H}_{2} \mathrm{~S}, \mathrm{COS}, \mathrm{CS}_{2}\end{array}$ & 1.25 grains/ $100 \mathrm{scf}$ \\
\hline
\end{tabular}

Since the maximum natural gas pipeline pressure can reach 895 psig, the SNG product delivery pressure at the compressor outlet will be assumed to be 960 psia to allow a 50 psi driving force for injection into the NG pipeline.

The existing natural gas pipeline is assumed to be located within 5 miles from the site boundary and is reported to have a spare capacity of 2 billion cubic feet per day.

\subsubsection{Sulfur}

Sulfur is a commodity, which can be used in a wide range of applications. Most sulfur is converted to sulfuric acid, which can be utilized in industrial processes. However, its primary use is in manufacturing phosphate fertilizers.

A typical set of sulfur product characteristics from the Montana Sulphur and Chemical Company is shown in Exhibit 2-9. [10]

\section{Exhibit 2-9 Typical Sulfur Specification}

\begin{tabular}{|l|c|}
\hline \multicolumn{1}{|c|}{ Parameter } & Value \\
\hline Sulfur Content, wt \% min & 99.9 \\
\hline Moisture, wt \% max & 0.5 \\
\hline Reduced Carbon, wt \% max & 0.05 \\
\hline Ash, wt \% max & 0.01 \\
\hline
\end{tabular}




\begin{tabular}{|l|c|}
\hline \multicolumn{1}{|c|}{ Parameter } & Value \\
\hline Acidity, wt \% max & 0.02 \\
\hline Color / Appearance & Light Yellow \\
\hline Odor & Sweet to a Mercaptan-Like Odor \\
\hline
\end{tabular}

\subsection{Operational Requirements}

The SNG process is expected to be a base-loaded unit. The following operational requirements are specified:

- Turndown to a minimum of $80 \%$ will be achievable for an indefinite time without flaring.

- On-stream time: The design on-stream factor shall be 292 days per year, which is consistent with a Target Availability $~ 80 \%$.

- In order to minimize the use of electricity for electrolysis during the summer peak, the plant will be scheduled for annual maintenance during the month of July.

\subsection{Storage Requirements}

The coal yard shall be sized for 7 days storage. The coal yard will not be covered.

No bulk oxygen or hydrogen storage will be associated with the SNG plant. The oxygen and hydrogen will be supplied on demand. The electrolysis plant will be comprised of hundreds of units, each with a turn down capability to $20 \%$ or less. In addition, electrolysis units are able to ramp nearly instantaneously. A small storage volume will be provided for operational considerations.

\subsection{Client Requirements}

The following guidance has been provided by APS.

1. No routine flaring will be allowed. Flaring for start-up, shutdown and upset conditions will be allowed. An elevated flare is planned. During normal operation, small flare streams can be combusted in the oxygen blown CFB combustor.

2. As much as possible, only commercial technology should be utilized. Where developmental technology is required, it will be identified in the system descriptions.

3. The site need only be planned for a single SNG facility. That is, accommodations will not be made for a future unit.

\subsection{Environmental Requirements}

Considering that the hydrogasification project does not include a combustion turbine or directly release any combustion products, traditional air emission limits are not relevant. The one source of combustion in the process is the fluidized bed oxygen-blown combustor that burns the gasification char. However, these combustion products, nearly pure $\mathrm{CO}_{2}$, are recycled back into the process and ultimately are converted into additional SNG. Therefore emission requirements will be placed on the end user of the SNG, such as a GTCC, and not on the process itself. 
Nevertheless, the project will need to obtain the appropriate permits that will cover the following:

- Flares, for controlling air emissions during start-up and upset conditions (as applicable),

- Cooling towers (as applicable),

- Water makeup

- Material handling and storage (unloading/loading, conveyor belts, transfer points, silos, bin vents, etc.) for

$$
\begin{array}{ll}
\circ & \text { Coal } \\
\circ & \text { Ash, and } \\
\circ & \text { Sulfur }
\end{array}
$$

- Wastewater treatment plant plan approval

- Waste disposal site, if ash and/or sulfur are not beneficially reused

These permits/approvals are not unique to the process, but would be typical of any industrial project.

In summary, it is anticipated that there will not be any process air emission values that will influence the plant design. Instead, the natural gas quality specification will form a part of the process design requirements.

Furthermore, the process will convert the produced $\mathrm{CO}_{2}$ to SNG, such that at least $90 \%$ of the carbon in the gasified coal will be contained in the resulting fuel. In addition, as described in the next section, the plant design will utilize a Zero Liquid Discharge (ZLD) system to eliminate the discharge of wastewater. 


\section{Hydrogasification Configuration Selection}

Several hydrogasification process configurations were considered and evaluated between 2007 and 2010. [7, 11, 12] The hydrogasification process selected by APS as the basis for the current Phase II engineering/cost estimating tasks is the Phase I configuration presented in Exhibit 3-1.

[13]

\section{Exhibit 3-1 Phase I Block Flow Diagram of the Hydrogasification SNG Process}

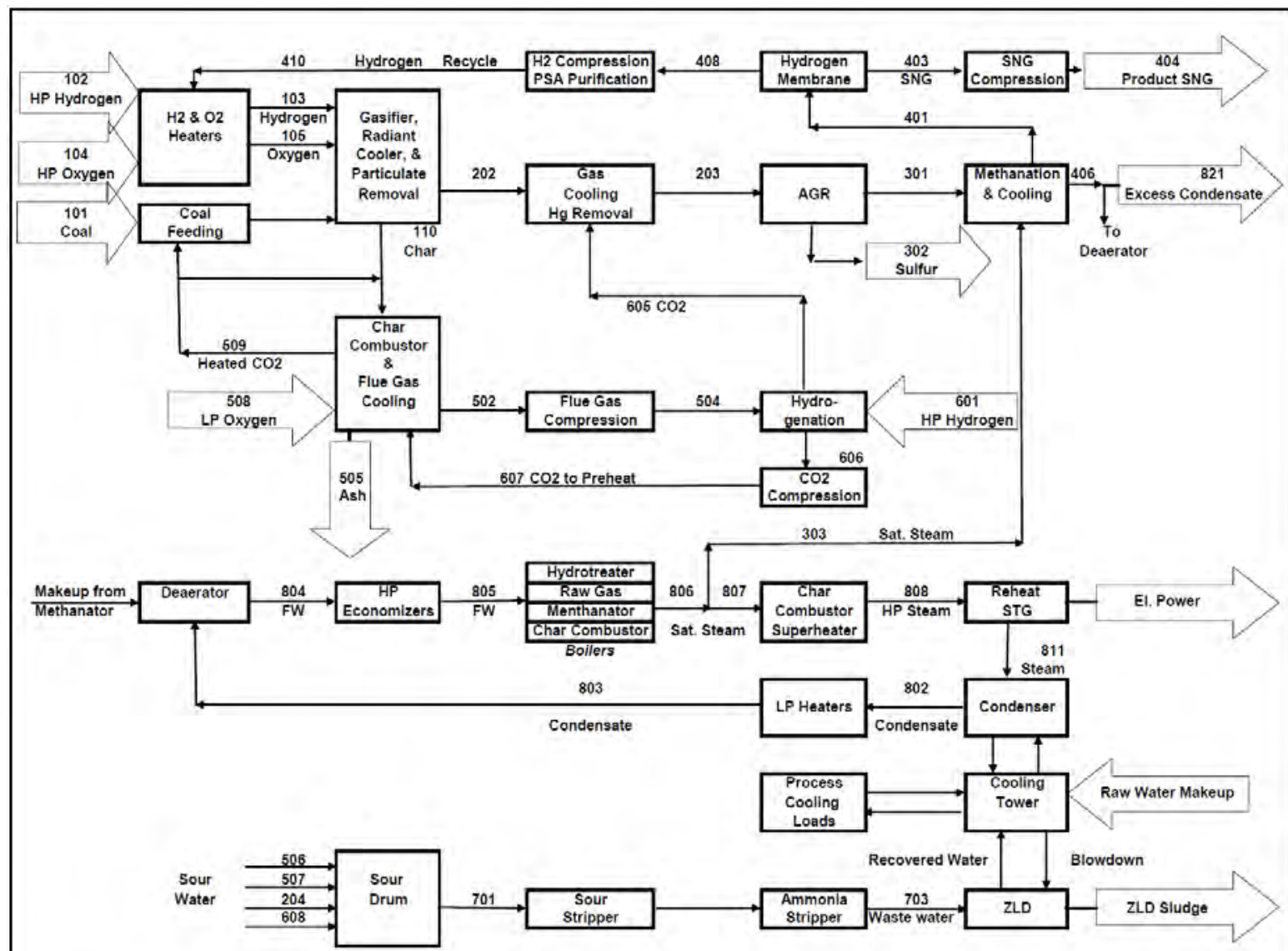

Note: This Phase I BFD was selected by APS as the base configuration for the present Phase II evaluation. Changes to this base configuration were implemented during the Phase II conceptual design effort and are documented below.

The configuration presented in Exhibit 3-1 formed the starting point for the present Phase II effort. The ground rules of this effort allowed for the configuration to evolve over the course of the study. The result of the Phase II configuration evolution is presented in Exhibit 4-1. The rest of this section will briefly highlight the changes that were made between the Phase I BFD and the Phase II BFD. A full description of the Phase II process is presented in Section 4.

The major changes incorporated into the Phase II process configuration compared to the Phase I process are listed below. 
1. Methanator Optimization. In Phase II, the methanation process was optimized by the utilization of two bulk methanation reactors, and a single trim reactor. The first bulk reactor is designed for high temperature catalyst and a recycle loop to limit the thermal rise resulting from the exothermic methanation reactions. Interstage heat exchange was incorporated to maximize methane production while simultaneously producing high quality heat sufficient for the production of superheated high pressure steam. In contrast, the Phase I methanator was an isothermal reactor with integral heat removal that produced only saturated steam.

2. Removal of the Hydrogen Membrane. With the optimized methanation process, the excess hydrogen remaining in the SNG product fell below the $3 \% \mathrm{H}_{2}$ specification without requiring the $\mathrm{H}_{2}$ membrane. Thus the $\mathrm{H}_{2}$ membrane originally utilized in Phase I was eliminated in Phase II.

3. Removal of the Pressure Swing Absorber (PSA). In Phase I, the PSA was utilized to remove methane from the $\mathrm{H}_{2}$ rich gas, separated by the Hydrogen membrane, prior to being recycled back to the gasifier. Minimization of methane recycled to the gasifier was introduced to maximize production of methane within the gasifier, thus allowing for smaller methanation reactors. In Phase II, the need for the PSA was eliminated along with the elimination of the hydrogen membrane.

4. Elimination of the Hydrogen Recycle Stream. With the elimination of the $\mathrm{H}_{2}$ membrane and PSA, the $\mathrm{H}_{2}$ recycle and $\mathrm{H}_{2}$ recycle compressor were eliminated in Phase II.

5. Elimination of the Methanator Steam Feed. As a result of the optimization of the methanation process, a high recycle rate was utilized for thermal moderation and humidification, thus eliminating the need for steam injection used in Phase I. This allowed for the steam turbine generator to produce additional power.

6. Elimination of the Process Condensate Going to the Deaerator. In Phase I, significant process condensate was collected from the cooled methanation product gas. In Phase I, the condensate was supplied to the steam cycle since large makeup quantities were required to supply the methanation steam feed. In Phase II, the process condensate was divorced from the steam cycle for simplicity and since the steam cycle makeup requirements were greatly reduced upon the elimination of the process steam injection prior to the methanator.

7. High Temperature $\mathrm{H}_{2}$ Heater. In order to minimize the amount of required oxygen and hydrogen, a high temperature $\left(1250^{\circ} \mathrm{F}\right) \mathrm{H}_{2}$ heater was introduced in Phase II within the raw syngas cooler. In Phase $\mathrm{I}$, the $\mathrm{H}_{2}$ was heated to $550^{\circ} \mathrm{F}$ via steam condensation.

8. Relocated Deoxidation Process. The deoxidation process is utilized to eliminate free oxygen in the $\mathrm{CO}_{2}$ rich stream before forwarding to other processes that can't tolerate oxygen. In Phase I, the deoxidation stage (formerly identified as hydrogenation) was located before the oxy-combustor's $\mathrm{CO}_{2}$ product stream was split into two streams for the methanator feed and material transport. In Phase II, the deoxidation process was located after the split, such that only the $\mathrm{CO}_{2}$ stream going to the methanator is deoxidized. In Phase II, the oxygen bearing $\mathrm{CO}_{2}$ stream used for pulverized coal feed was not deoxidized as the oxygen level of $5 \%$ by volume is judged to be sufficiently low 
enough to preclude explosion hazards. This judgment should be confirmed or refined as the project moves forward.

\section{Key Process Changes.}

In addition to the major configuration changes mentioned above, various process parameters where also changed in Phase II compared to Phase I. Key process parameter changes are highlighted below.

1. The Phase I, the gasification temperature had been $1600^{\circ} \mathrm{F}$. In Phase II, the gasification temperature was increased to $1750^{\circ} \mathrm{F}$ to eliminate the production of oil byproducts.

2. The Phase I, the carbon conversion to syngas was taken as $70 \%$ with $30 \%$ remaining in the solid stream leaving the gasifier as char. The Phase II carbon conversion is taken as $52 \%$, based upon the BSTR results.

3. In Phase I, approximately half of the generated $\mathrm{CO}_{2}$ stream was used to transport the coal and char. In Phase II, only $20 \%$ of the $\mathrm{CO}_{2}$ was utilized in the coal transport, while air is utilized to feed the char. This will minimize the $\mathrm{CO}_{2}$ release, increase safety, and maximize the methane production.

Although other process changes have been incorporated into the Phase II analysis, they are discussed in the other report sections where appropriate. 


\section{Engineering Package}

\subsection{Configuration Overview}

Over the course of this hydrogasification project, several hydrogasification process configurations were considered and evaluated. Section 3 presents the base configuration selected by APS for WorleyParsons to evaluate and improve if possible. The hydrogasification process developed during this Phase II engineering/cost estimating tasks is presented in Exhibit 4-1. 
Exhibit 4-1 Block Flow Diagram of the Base Hydrogasification SNG Process

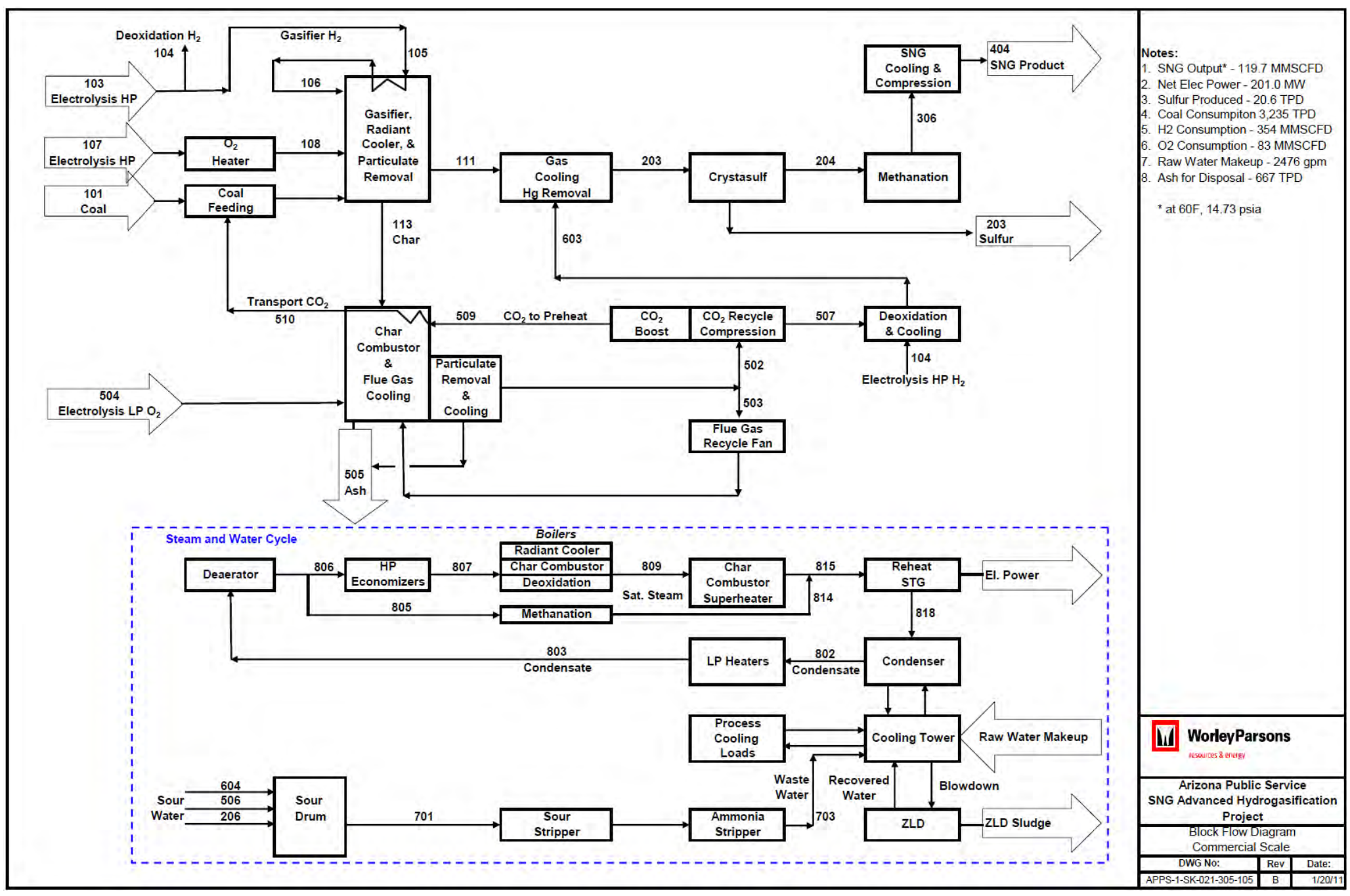


Coal, hydrogen, oxygen and a $\mathrm{CO}_{2}$ carrier gas stream are all fed into the hydrogasification reactor. A minimal amount of oxygen is introduced to help in providing the required gasification temperature of approximately $1750^{\circ} \mathrm{F}$. Both the oxygen and hydrogen are preheated to minimize the required oxygen. The oxygen is preheated in an external heater, while the hydrogen is heated within the radiant syngas cooler. The oxygen will be introduced with the hydrogen to partially oxidize the hydrogen, producing a hot hydrogen/steam stream which will come in contact with the coal transported into the gasifier by $\mathrm{CO}_{2}$. The hot hydrogen will begin the hydrogasification reaction with the coal. Approximately 52\% of the coal carbon will gasify with approximately $48 \%$ of the coal carbon remaining in the solid stream leaving the gasifier.

The solids leaving the gasifier will be combusted with an $\mathrm{O}_{2}$ stream in order to generate a nearly pure $\mathrm{CO}_{2}$ stream. The generated $\mathrm{CO}_{2}$ stream will be split into 2 streams, with approximately $20 \%$ being used to transport the coal and $80 \%$ being combined with syngas going to the methanator. The $\mathrm{CO}_{2}$ stream going to the gasifier will be cooled and compressed and reheated prior to being re-introduced into the gasifier with the coal. The $\mathrm{CO}_{2}$ stream going to the methanator will be cooled and compressed and mixed with the gas stream leaving the gasifier. Since there will be some excess $\mathrm{O}_{2}$ left after combustion, hydrogen will be introduced to catalytically combine with the remaining $\mathrm{O}_{2}$ and $\mathrm{SO}_{2}$. The mixed stream will be cooled and sent to a CrystaSulf desulfurizer process and then onto a methanation block consisting of a series of heat exchanger and catalytic reactors. The methanator catalyst requires a very low sulfur level to prevent poisoning.

The raw SNG leaving the methanation block will be dried and compressed.

Hot raw fuel gas exits the gasifier at approximately $1750^{\circ} \mathrm{F} / 1000$ psia. The fuel gas and residual char leaving the Radiant Raw Gas Cooler are cooled to $655^{\circ} \mathrm{F}$ raising high-pressure steam and preheating hydrogen gas. High pressure steam is also generated in the evaporative surfaces of the Char Combustor and the deoxidizer and Methanator gas coolers. High pressure steam is superheated in the Char Combustor and utilized in the steam turbine generator (STG) to produce electric power.

The lower portion of the BFD focuses on the collection of process water, waste heat, the generation of steam, and the integration of a steam turbine generator for the production of electricity.

\subsection{Plant Configuration}

Hydrogasification plant configuration and sparing philosophy of the major hydrogasification / SNG process components are presented in Exhibit 4-2. 
Exhibit 4-2

Hydrogasification I SNG Process Configuration and Design Redundancy

\begin{tabular}{|l|l|c|}
\hline \multicolumn{1}{|c|}{ System } & \multicolumn{1}{|c|}{ Description } & Quantity/Capacity \\
\hline Gasifier & APS Hydrogasification Reactor & $3 \times 33 \%$ \\
\hline Raw Syngas Cooler $/ \mathrm{H}_{2}$ Heater & RSC includes $\mathrm{H}_{2}$ heater & $3 \times 33 \%$ \\
\hline Fuel Feed (per Gasifier) & Dry feed (Stamet Posimetric pump) & $\begin{array}{c}9 \times 16.7 \% \\
{[\text { i.e. } 3 \times 50 \%, \text { per Gasifier] }}\end{array}$ \\
\hline Coal Preparation (per Plant) & $\begin{array}{l}\text { Pulverized Coal Mills } \\
\text { (70\% through 200 mesh.) }\end{array}$ & $3 \times 50 \%$ \\
\hline Hydrogen / Oxygen Generator & Electrolysis Unit (not in cost basis) & $\mathrm{NA}$ \\
\hline Char Combustor & Oxygen blown CFB & $1 \times 100 \%$ \\
\hline $\mathrm{CO}_{2}$ Recycle System & $\mathrm{CO}_{2}$ cooling/reheat/compression & $1 \times 100 \%$ \\
\hline Mercury Removal & Sulfur impregnated carbon bed & $1 \times 100 \%$ \\
\hline Acid Gas Removal & CrystaSulf, physical solvent & $1 \times 100 \%$ \\
\hline Sulfur Recovery & Via CrystaSulf Process & $1 \times 100 \%$ \\
\hline Methanation Unit & Catalytic & $1 \times 100 \%$ \\
\hline Flare System & Free standing elevated flare & $1 \times 100 \%$ \\
\hline
\end{tabular}

Since the hydrogasification plant will be co-producing electric power, the sparing philosophy of the Power Island will follow the established Good Engineering Practice (GEP) in the power plant design to achieve high availability / reliability. Except for the prime movers, large electrical equipment, and a few select equipment items, adequate sparing will be provided.

General guidelines on sparing are presented below:

1. Prime Movers (Steam Turbine Generators) : 1 x100\%

2. Heat Recovery Steam Generators: $1 \times 100 \%$

3. Step Up and Auxiliary Transformers: 1x100\%

4. Cooling Tower: 1 x 100\%,

5. Boiler Feed Pumps: $2 \times 100 \%$

6. Condensate Pumps: 2x100\%

7. Closed Cooling Water Pumps: 2x100\%

8. Circulating Water Pumps: $2 \times 50 \%$

9. Miscellaneous Other Pumps: 2x100\%

\subsection{Plant Arrangement}

The estimated space requirement for the new hydrogasification facility is approximately 70 acres, excluding a buffer zone. The site is designed to be accessible by automobiles and mine trucks. The hydrogasification plant components are arranged in several technological islands 
separated by access roads and with adequate space for construction, operations, and maintenance. Major technological islands include:

Coal handling Island - Coal receiving, storage and reclaim systems

Gasification Island - $\quad$ Hydrogasifiers and Char Combustor

Process Island - $\quad$ Syngas conditioning, Methanation, $\mathrm{CH}_{4}$ and $\mathrm{CO}_{2}$ processing systems

Power Island - $\quad$ Steam turbine systems, and water storage and treatment systems

Balance of plant Island - Cooling tower

Switch yard - $\quad$ High and medium voltage electrical equipment

\section{Buildings and Structures}

The following buildings are included in the preliminary design scope.

Steam turbine building

Warehouse

Administration and service building
Water Treatment building

Coal crusher building

Circulation Water Pump house
Machine shop

Runoff water pump house

Waste treatment building (Zero Liquid Discharge)

Pile foundations are provided for the following support structures, and other plant components.

Coal Storage Bins

Pipe Rack

Crusher Building

Conveyors

Mill House

$>$ Oxygen Custody Transfer and Preheaters

$>$ Hydrogen Custody Transfer

$>$ Coal Pneumatic Transfer System

$>$ Hydrogasification Section

$>$ Gasifier Cooling

$>$ CrystaSulf Sulfur Removal

$>$ Sulfur Storage

$>$ Methanation Unit
$>$ Flare

$>$ Hydrogen Separation

> Char Combustor

$>\mathrm{CO}_{2}$ Compression

$>$ Hydrogenation Unit

Sour Water Stripper

> Steam Turbine Generator

Transformers

SNG Compression and Custody Transfer

$>$ Emergency Diesel Generator

$>$ Switchyard

Cooling Tower

Ash Silo 
Hydrogasification facility general; arrangement is presented in Exhibit 4-3. 


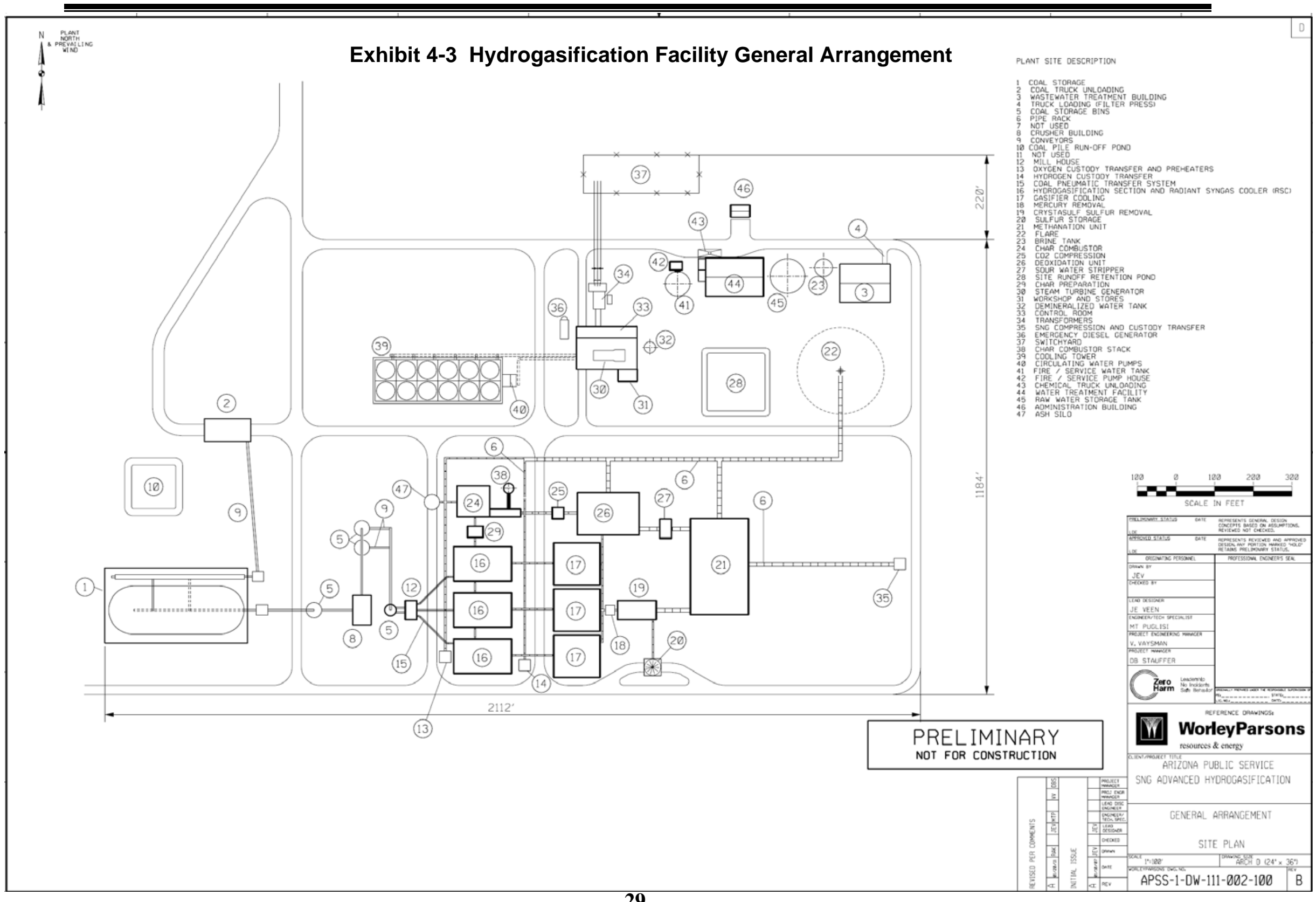




\subsection{Performance Summary}

The Aspen Plus, Version 20.0, software by Aspen Technology, Inc has been utilized to model the operation of the hydrogasification plant.

The summary of computer simulation results for process systems is presented in Exhibit 4-4, and electric power co-generation performance is presented in Exhibit 4-6.

The following engineering diagrams are presented in the appendices of this report:

- Process Flow Diagrams Appendix B

- Heat and Mass Balances Appendix C

- Water Balance diagram Appendix D 


\section{Exhibit 4-4}

\section{Process Summary}

\begin{tabular}{|l|c|}
\hline Gasifier & \\
\hline Coal Flow (AR), TPD & 3,235 \\
Coal Flow (Dry), TPD & 3,000 \\
Oxygen Flow, TPD & 783 \\
Hydrogen Flow, TPD & 929 \\
Carbon Conversion, \% & 52 \\
\hline Char Combustor & \\
\hline Char flow, TPD & 1,584 \\
Oxygen flow, TPD & 2,718 \\
Carbon Conversion, \% & 97 \\
Ash flow, TPD & 667 \\
\hline SNG & \\
\hline SNG flow, MMSCFD (Note A) & 119.7 \\
SNG Pressure, psia & 910 \\
SNG HHV, Btu/scf (Note A) & 967.5 \\
\hline CrystaSulf & \\
\hline Sulfur Recovery, \% & $99 \%$ \\
Sulfur Produced, TPD & 20.6 \\
Process Condensate ${ }^{\text {d }}$ & \\
\hline Flow, gpm & 726 \\
Methanation & 99.99 \\
CO Conversion, \% & 95.66 \\
CO ${ }_{2}$ Conversion, \% & \\
Deoxidization & 1080 \\
\hline Hydrogen Flow, TPD & \\
Temperature, ${ }^{\circ} \mathrm{F}$ & \\
\hline
\end{tabular}

Notes:

A. At $60^{\circ} \mathrm{F}, 14.73 \mathrm{psia}$

The Product SNG composition is presented Exhibit 4-5

d The process condensate is largely a result of the water produced during the methanation process, which is ultimately condensed out from the product SNG stream. 
Exhibit 4-5

Product SNG Composition

\begin{tabular}{|l|l|r|}
\hline \multicolumn{2}{|c|}{ Constituent } & \multicolumn{1}{c|}{ Value } \\
\hline Hydrogen & $\mathrm{H}_{2}$ & $2.63 \%$ \\
Methane & $\mathrm{CH}_{4}$ & $94.76 \%$ \\
Nitrogen & $\mathrm{N}_{2}$ & $0.87 \%$ \\
Carbon Monoxide & $\mathrm{CO}$ & $0.00 \%$ \\
Carbon Dioxide & $\mathrm{CO}_{2}$ & $1.75 \%$ \\
\hline \multicolumn{2}{|c|}{ Total } & $100.00 \%$ \\
\hline
\end{tabular}

It is noted that the carbon dioxide level is approximately $1.75 \%$ which is below the El Paso gas pipeline specification of $<2.0 \%$ as listed in Exhibit 2-8, and that the diluent level $\left(\mathrm{N}_{2}+\mathrm{CO}_{2}\right)$ totals $2.62 \%$, which is also below the El Paso gas specification of $<3.0 \%$. Originally, both of these specifications were violated in the March 2007 report [4]. The implementation of a multi-stage adiabatic methanation process with interstage cooling, in lieu of the isothermal methanation process that had been employed in Phase I, brings all of the SNG parameters into compliance with the SNG specifications. The SNG HHV of 967.5 Btu/SCF is also in compliance with the specification of $>967 \mathrm{Btu} / \mathrm{SCF}$. 
Exhibit 4-6 Electric Power Co-Generation Performance

\begin{tabular}{|c|c|}
\hline STG Gross Power at 20 kV Generator Terminals, kWe & 241,390 \\
\hline \multicolumn{2}{|l|}{ AUXILIARY LOAD SUMMARY } \\
\hline \multicolumn{2}{|l|}{13.8 kV Auxiliary Loads, kWe } \\
\hline $\mathrm{CO}_{2}$ Recycle Compressor & 12,390 \\
\hline HP FW Pump & 3,880 \\
\hline Subtotal Electrically-Driven Auxiliaries@13.8 kV, kWe & $-16,270$ \\
\hline Auxiliary Step-down Transformer 20 kV / 13.8 kV, kWe & -200 \\
\hline \multicolumn{2}{|l|}{4.16 kV Auxiliary Loads, kWe } \\
\hline Pulverizers & 1,220 \\
\hline Stamet Pumps & 2,240 \\
\hline Ash Handling & 3,740 \\
\hline Air Locking Gas Compressor & 1,140 \\
\hline Flue Gas Recycle Fan & 220 \\
\hline CrystaSulf & 1,960 \\
\hline Methanation Recycle Compressor & 2,120 \\
\hline SNG Compressor & 540 \\
\hline CW Pump & 2,460 \\
\hline Condensate Pump & 200 \\
\hline Plant Air Compressor & 400 \\
\hline Subtotal Electrically-Driven Auxiliaries@4.16 kV, kWe & $-16,240$ \\
\hline Auxiliary Step-down Transformer 13.8 kV / 4.16 kV, kWe & -120 \\
\hline \multicolumn{2}{|l|}{480 V Auxiliary Loads, kWe } \\
\hline Coal Handling & 2,390 \\
\hline Waste Water Treatment (ZLD) & 1,200 \\
\hline Cooling Tower Fans & 1,830 \\
\hline Miscellaneous Pumps/Blowers & 1,490 \\
\hline Subtotal 480 V loads, kWe (Note A) & $-6,910$ \\
\hline \multicolumn{2}{|l|}{ Auxiliary Step-down transformer 4.16 kV / 480 V losses, kWe } \\
\hline Subtotal Auxiliary Loads@20 kV, kWe & $-39,770$ \\
\hline Net Power Output at $20 \mathrm{kV}, \mathrm{kWe}$ & 201,620 \\
\hline Main Step-Up Transformer 20 kV / 230 kV losses, kWe & -600 \\
\hline Net Power Output at 230 kV, kWe & 201,020 \\
\hline
\end{tabular}

Notes:

A. All motors smaller than $250 \mathrm{hp}$ are connected at $480 \mathrm{~V}$. These include cooling tower fan motors, coal and ash handling systems and other miscellaneous loads.

\subsection{Major System Descriptions}

The descriptions of Hydrogasification, Power Islands and Balance of Plant (BOP) systems are provided in this section. Major equipment lists are contained in the Appendix E.

\subsubsection{Coal Handling System}

The function of the coal handling system is to unload, convey, store, and reclaim the coal delivered to the plant. The scope of the system is from the truck unloading station and coal receiving hoppers up to and including the slide gate valves on the outlet of the coal storage silos. 
New coal receiving, storage and reclaim systems will be sized for nominal 3300 tpd of Fruitland subbituminous coal.

\section{Coal Receiving and Storage}

The coal will be delivered to the site by 200 tons/load trucks from BHP Navajo Coal Company mine, located about $1 \frac{1}{2}$ miles distant. The unloading will be done by trucks dumping the coal into two receiving hoppers, equipped with grizzlies. Coal is withdrawn from each hopper by a single belt feeder. The 3" x 0 coal is discharged from the belt feeder onto a belt conveyor that includes a belt scale and "as-received" sample system. The coal is then conveyed to the storage pile. The storage pile is formed by a linear traveling stacker that builds a 7 day storage pile.

\section{Coal Preparation System}

Coal is delivered to the coal preparation system silos from the coal storage system via a reclaim system. The coal is reclaimed from the pile by rotary plows in a concrete tunnel under the pile. Reclaimed coal from the storage pile is conveyed by a reclaim conveyor. The reclaim conveyor includes a belt scale, magnetic separator and an "as-fired" sample system. The reclaim conveyor deposits the coal into a surge bin in the crusher building. A belt feeder withdraws coal from the surge bin and conveys it to the crusher where it is reduced to $1 \frac{1 / 4}{4} \times \mathrm{x} 0$. From the crusher, the coal is deposited onto a conveyor that lifts the coal to the top of the two (2) coal storage bins. A series of diverter gates and conveyors distribute the coal to the desired storage bin.

From the crushed coal silos, coal is fed into coal pulverizers with a heated air stream which dries the coal from the $7.27 \%$ as received moisture to $4.0 \%$ moisture. The air is heated via a low pressure extraction stream taken from the LP steam turbine. The coal is pulverized in three mills ( 3 x 50\%) to the fineness of $70 \%$ passing through a 200 mesh sieve ${ }^{e}$. Pulverized coal is carried pneumatically to the pulverized coal silos (two silos per each gasifier, total of six silos per plant). Coal particles are separated from the carrier gas in sleeve filters. Milled coal is stored in silos at atmospheric pressure with inert $\left(\mathrm{CO}_{2}\right)$ gas blanket.

\section{Technical Requirements and Design Basis}

The technical requirements and design basis for the coal handling system are presented in Exhibit 4-7. 


\section{Exhibit 4-7}

Coal Handling System Technical Requirements

\begin{tabular}{|c|c|c|}
\hline Parameter & Value & Comment \\
\hline \multicolumn{3}{|l|}{ Coal burn rate } \\
\hline Coal burn rate at MCR & $3,235 \mathrm{tpd}=135 \mathrm{tph}$ & Based on the $100 \%$ MCR rating for the plant \\
\hline Nominal design coal burn rate & $3,300 \mathrm{tpd}=137.5 \mathrm{tph}$ & \\
\hline Coal delivered to the plant & & $\begin{array}{l}\text { Mine trucks; Two } 8 \text {-hour shifts per day, no trucks } \\
\text { received at night. ( } 7 \text { days per week) }\end{array}$ \\
\hline Trucks per day at MCR & 17 & Truck capacity 200 tons \\
\hline Receiving System Load at MCR & 202 tph & \\
\hline Receiving System Design Capacity & 240 tph & Includes $15 \%$ capacity margin \\
\hline Coal Reclaim/Crushing & & Two 8-hour shifts, 7 days per week \\
\hline Reclaim/Crushing rate & 202 tph & \\
\hline Reclaim/Crushing Design Capacity & $240 \mathrm{tph}$ & Includes $15 \%$ capacity margin \\
\hline Crusher Design Capacity & 120 tph & $2 \times 50 \%$ \\
\hline Crushed Coal Storage Capacity & 1,620 tons & $8 \mathrm{hr}$ night shift plus $4 \mathrm{hrs}$ margin \\
\hline $\begin{array}{l}\text { Time required to refill Crushed coal } \\
\text { storage at MCR operation }\end{array}$ & $15 \mathrm{hrs}$ & Task should be completed during two 8-hours shifts \\
\hline $\begin{array}{l}\text { Duration to deplete Crushed coal } \\
\text { storage at MCR operation }\end{array}$ & 5 days & With one $50 \%$ crusher out of service \\
\hline Coal Milling & & Three 8-hour shifts per day, 7 days per week \\
\hline Milling rate & 135 tph & Based on the $100 \%$ MCR rating for the plant \\
\hline Design Milling Rate & $150 \mathrm{tph}$ & Includes $15 \%$ capacity margin \\
\hline Design Mill Capacity & $75 \mathrm{tph}$ & $3 \times 50 \%$ \\
\hline Milled Coal Storage & 600 tons & Four hours \\
\hline$\underline{\text { Storage pile }}$ & & Liners, run-off collection, and treatment systems \\
\hline Storage size & 23,000 tons & 7 days at nominal burn rate \\
\hline
\end{tabular}

\subsubsection{Coal Feed System}

The coal feed system for each hydrogasifier is comprised of 3 trains, each sized for $50 \%$ of coal feed requirement per gasifier unit, or $16.7 \%$ of total hydrogasification plant coal feed rate of 3,300 tpd. The coal is drawn from the pulverized coal silo and fed by Stamet posimetric solids pumps to a pneumatic conveyor. Coal is pressurized and fluidized with carbon dioxide gas, and transported to horizontally-opposed feed injectors on the hydrogasification reactor.

There is a potential that the utilization of $\mathrm{CO}_{2}$ as an inert/transport agent for the milled coal may result in $\mathrm{CO}_{2}$ reacting with surface moisture in the coal to form a carbonic acid. Presence of carbonic acid may cause relatively high corrosion rates in the milled coal handling and storage equipment. However, the extent of this potential problem is not known at this time and, thus, no 
special provisions related to acid corrosion are included in the current equipment design, other than the nominal drying associated with heated air contacting the coal in the pulverization process. The potential for carbonic acid induced corrosion will need to be better defined and addressed during the detailed design phase of the project.

\subsubsection{Hydrogasification Reactor}

Process flow diagram of the gasification section system is shown on Drawing APSS-1-SK-021305-301 (Appendix B).

The design of the hydrogasification reactor is based on the collaborative ideas of the APS organized team endeavoring to produce SNG from western coal. The root concept of this design is the exploitation of the hydrogen-coal reaction to produce methane $\left(\mathrm{CH}_{4}\right)$ induced by the moderately elevated temperatures $\left(>1,500^{\circ} \mathrm{F}\right)$. This reaction is typically referred to as hydrogasification. A preliminary concept for a commercial-scale hydrogasification reactor is described below. The proposed operating conditions for this reactor are 1,000 psia and $1,750^{\circ} \mathrm{F}$. The operating temperature of $1,750^{\circ} \mathrm{F}$ was chosen to preclude the production of oils as learned during via the BSTR testing program $[\mathbf{1 4}]^{\mathrm{f}}$.

An illustrative sketch of the hydrogasification concept is shown in Exhibit 4-8. The gasifier is envisioned as a refractory-lined vessel with internal dimensions of about 5 foot diameter and 50 foot in height. The bottom third of the gasifier will be shaped with steel and refractory blocks such that a converging-diverging section is affected to aid in the phase separation of the particulate and syngas. The overall internal dimensions of the gasifier would yield an approximate volume of 900 cubic feet.

Four partial oxidation burners will be located on the extreme top of the vessel. These burners will fire hydrogen in the presence of an oxidant consisting of 99.95 percent oxygen. Approximately $10 \%$ of the incoming hydrogen will be burned to provide a high temperature hydrogen-rich gas stream, along with a small amount of water vapor. The burners will be arranged to provide a tangential swirl and with 90 degrees of separation from one another. The burners will be cooled by a circulating water system with external indirect heat exchange to cooling water or other suitable heat sink.

Due to the high reaction rates and laminar flame speeds, it is recommended to start-up the gasifier on $\mathrm{CH}_{4}$ versus considering $\mathrm{H}_{2}$. Even though there may not be any manufacturers available to supply an existing burner which is capable of starting up on $\mathrm{CH}_{4}$ and loading up to a pressure of 1000 psi conditions on $\mathrm{H}_{2}$ fuel, it is possible to design one. The burner can be designed to accommodate multiple passages for both $\mathrm{CH}_{4}$ and $\mathrm{H}_{2}$ fuels to be burnt simultaneously during the transfers. Also, it is possible to design multiple plenums and orifices in the same burner to operate $\mathrm{H}_{2}$ at low pressures and high pressure conditions. One common burner for start-up, shutdown and full load operating condition is possibly available from several manufacturers today. However, it may not be up to the pressure of 1000 psi. Gas Turbine manufacturers can operate their $\mathrm{H}_{2}$ burners at pressures up to 300 psi.

f During the BSTR testing program, APS discovered that operation at $1,625^{\circ} \mathrm{F}$ produced oils, while operation at $1,750^{\circ} \mathrm{F}$ did not. APS therefore decided to analyze the gasifier performance at 1,750 . 
The hydrogen-rich gas stream will be directed downward into the gasifier. Pulverized coal will be injected perpendicularly into the hydrogen-rich gas stream. Four coal injectors will be used. The injectors will be arranged 90 degrees from one another. The injectors may or may not be cooled with an external cooling water loop similar to that of the hydrogen partial oxidation burners.

Hot hydrogen will contact the coal resulting in the production of $\mathrm{CH}_{4}$. The reaction is exothermic and will result in a bulk gas temperature increase. Downward motion of the gas and solid particles through the reactor will approximate plug-flow in character with some interphase slip between the suspend coal / char particles and the gas. It is anticipated that 52 percent of the coal carbon can be hydrogasified in this manner. The remaining 48 percent will exit the gasifier as char and primarily consist of carbon and ash with small amounts of hydrogen, nitrogen, and sulfur present.

Gas and particles will travel the length of the vessel, converging and accelerating, and then diverging and de-accelerating in the bottom third of the reactor. This motion is intended to focus the path of the pulverized particles towards the bottom of the gasifier and into the char hopper system. Gaseous components, such as $\mathrm{CH}_{4}, \mathrm{H}_{2}, \mathrm{~N}_{2}, \mathrm{H}_{2} \mathrm{~S}$, and $\mathrm{HCl}$, will exit from an outlet nozzle located at the terminus of the diverging zone.

The converging-diverging zone is shown because there is some concern as to the ease with which the pulverized particles will be separated from the gas phase. The concern is that the individual solid and gas phases would be indistinguishable. This will make phase separation very difficult. Other possible separation strategies include internal baffling, and/or the use of ceramic or metallic candle filters. A combination of cyclone and candle filters has been assumed in this design. Entrained particulate removal system is described in Section 4.5.5.

A char hopper system is illustrated at the bottom of the hydrogasifier. The first char lock hopper is designed to cool the char to perhaps $212^{\circ} \mathrm{F}$. The second char hopper is for depressurization to a secondary process. 


\section{Exhibit 4-8 Commercial Scale Hydrogasifier Concept}

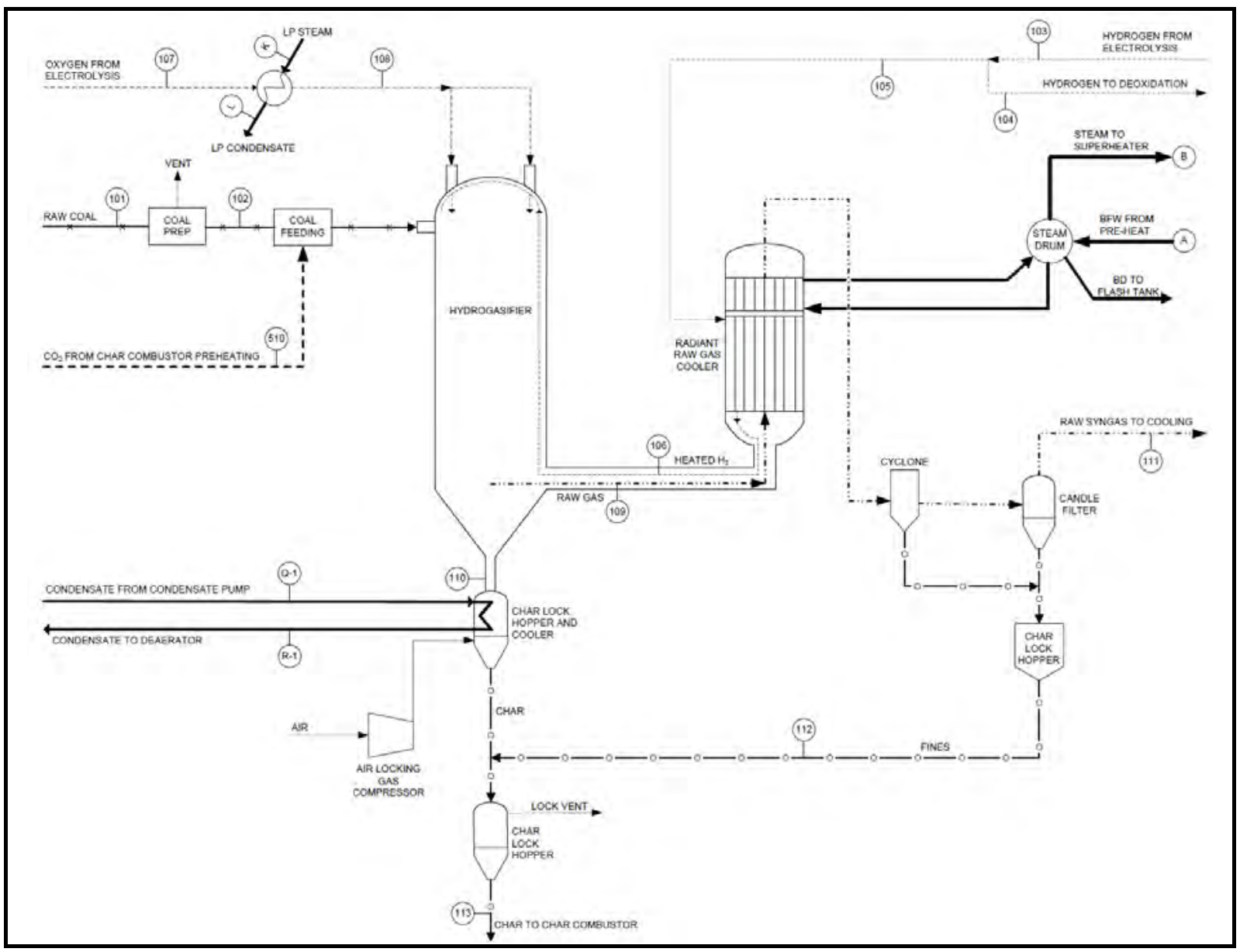

Key design features are presented in Exhibit 4-9.

\section{Exhibit 4-9 Hydrogasifier Design Conditions}

\begin{tabular}{|l|c|l|}
\hline Design Parameter & Design Value & Notes \\
\hline Gasifier type & Rockwell type & Single Stage, Entrained Flow, Hydrogasification \\
\hline Gas flow direction & Down flow & Single pass, no recycle. \\
\hline $\begin{array}{l}\text { Gasifier dimensions } \\
\mathrm{D} \times \mathrm{h}\end{array}$ & $\begin{array}{c}\text { 5' dia, } \times 50^{\prime} \text { high } \\
\left(900 \mathrm{ft}^{3}\right)\end{array}$ & Dimensions are for 1000 tpd dry coal throughput. \\
\hline Thermal Protection & Refractory & \\
\hline Coal Feeding & Coal lock hoppers & Fed by GE/Stamet Posimetric Solids pump \\
\hline Char Removal & Char lock hoppers & \\
\hline Location of coal injectors & Top Side & Four injectors, oriented tangentially for swirl. \\
\hline Location of $\mathrm{H}_{2}$ Injectors & Top & Number of injectors -4 \\
\hline
\end{tabular}




\begin{tabular}{|c|c|c|}
\hline Design Parameter & Design Value & Notes \\
\hline Location of $\mathrm{O}_{2}$ injectors & Top & Number of injectors -4 \\
\hline Gas Phase separation & $\begin{array}{c}\text { Acceleration } \\
\text { De-acceleration }\end{array}$ & $\begin{array}{l}\text { Other design features may be added, such as a } \\
\text { cyclone }\end{array}$ \\
\hline $\begin{array}{c}\mathrm{H}_{2} \text { Conditions } \\
\mathrm{T}, \\
\mathrm{P}, \\
\text { flow rate }\end{array}$ & $\begin{array}{c}1250^{\circ} \mathrm{F} \\
1150 \mathrm{psig} \\
77,433 \mathrm{lb} / \mathrm{hr}\end{array}$ & $\begin{array}{l}1250^{\circ} \mathrm{F} \text { based on suitability of materials to service. } \\
P=1000+\text { injector } \& \text { heater dPs of } 100+50 \\
\text { Flow rate as required for methanation }\end{array}$ \\
\hline $\begin{array}{c}\mathrm{O}_{2} \text { Conditions } \\
\mathrm{T}, \\
\mathrm{P}, \\
\text { flow rate }\end{array}$ & $\begin{array}{c}200^{\circ} \mathrm{F} \\
1120 \mathrm{psig} \\
65,246 \mathrm{lb} / \mathrm{hr}\end{array}$ & $\begin{array}{l}\mathrm{P}=1000+\text { injector } \mathrm{dP} \text { of } 80+\text { heater } \mathrm{dP} \text { of } 40= \\
\text { Flow rate, as required to achieve } 1750^{\circ} \mathrm{F} \text { gasifier } \mathrm{T}\end{array}$ \\
\hline Design Coal Flow & 3000 TPD & Assumed scaled up size of 1000 TPD per gasifier. \\
\hline $\begin{array}{c}\text { Coal Transport Gas } \\
\text { Gas } \\
\mathrm{T}, \\
\mathrm{P}, \\
\text { Flow rate }\end{array}$ & $\begin{array}{c}\mathrm{CO}_{2} \\
750^{\circ} \mathrm{F} \\
1105 \mathrm{psia} \\
40,644 \mathrm{lb} / \mathrm{hr}\end{array}$ & $\begin{array}{l}\mathrm{P}=1000+\text { heater, transport \& line } \mathrm{dP} \text { of } 65,20 \& 20 \\
\text { The } \mathrm{CO}_{2} \text { flow will be based on } 350 \mathrm{~kg} \text { coal } / \mathrm{m}^{3} \\
\text { carrier gas. }\end{array}$ \\
\hline
\end{tabular}

\subsection{4 $\quad \mathrm{H}_{2}$ Production and Handling}

The electrolysis unit is the sole source of $\mathrm{H}_{2}$ for the hydrogasifier. However, per the boundary limit set by APS and NETL, the electrolysis unit is outside of the engineering scope of this study.

\subsubsection{Raw Gas Cooling and Entrained Particulate Removal}

Process flow diagram of the gasifier cooling system is shown on Drawing APSS-1-SK-021-305301 as well as APSS-1-SK-021-305-302 (Appendix B).

The crude raw gas leaves the hydrogasifier at approximately $1750^{\circ} \mathrm{F}$ and contains a small quantity of char. Heat is recovered from this syngas in the Radiant Raw Syngas Cooler (RSC) which is comprised of a hydrogen heater bank and a high pressure saturated steam generator.

Near ambient temperature hydrogen enters the RSC and flows through the hydrogen heater banks in a downward direction, countercurrent to the syngas flow. The countercurrent flow will facilitate the heat transfer with minimal heat transfer area, while the upward syngas flow will aid in the disengagement of entrained char. Approximately $80 \%$ of the heat exchanged from the raw syngas is absorbed in the hydrogen heater. The remaining $20 \%$ is absorbed via the steam generator.

Heating the hydrogen to $1250^{\circ} \mathrm{F}$ at more than 1000 psia is challenging service. With hot hydrogen at $1250^{\circ} \mathrm{F}$ and the hot raw syngas at approximately $1750^{\circ} \mathrm{F}$, the metal temperatures will be on the order of $1500^{\circ} \mathrm{F}$. Finding metals to contain hydrogen with a 1000 psid pressure differential at $1500^{\circ} \mathrm{F}$ is not feasible. Therefore the heat exchange surface was envisioned as being completely within the pressure containment of the RSC. With this design approach, the differential pressure across the $1500^{\circ} \mathrm{F}$ heat exchange tubes is constrained to less than 150 psid. 
This design requires that the heated hydrogen not leave the gasifier/ RSC pressure boundary. As such the hot hydrogen is transported from the RSC to the gasifier via the internals of the gasifier and the pipe connecting the gasifier and RSC.

The partially cooled raw syngas exiting the RSC passes through a cyclone and a candle filter with the collected solids drained to the collecting hopper. The cleaned gas is piped to the Acid Gas Removal system via several heat exchangers that further recover heat from the gas for the feedwater heating.

Ash is removed from the cyclone and candle filter drains to a collecting hopper from which it passes into the lock hopper pressure letdown system. The char is then fed to the pelletizing system for the oxygen blown char-burning CFB.

\subsubsection{Mercury Removal}

Mercury removal process for the hydrogasification plant is based on Eastman Chemical's experience which uses carbon beds for mercury removal at its syngas facility in Kingsport, Tennessee [15]. Mercury removal is accomplished by a packed bed of sulfur-impregnated activated carbon. A bed of sulfur-impregnated activated carbon with approximately a 20-second superficial gas residence time would achieve more than 90 percent reduction of mercury in addition to removal some portions of other volatile heavy metals such as arsenic.

The packed carbon bed vessel is located upstream of the AGR unit and at a temperature near $100^{\circ} \mathrm{F}$. Allowable gas velocities are limited by considerations of particle entrainment, bed agitation, and pressure drop. The bed density of $30 \mathrm{lb} / \mathrm{ft}^{3}$ is based on the Calgon Carbon Corporation HGR-P sulfur-impregnated pelletized activated carbon [16]. These parameters determined the amount of carbon needed and the size of the vessels.

Eastman Chemicals replaces its bed every 18 to 24 months. For this study a 24 month carbon replacement cycle was assumed. Under these assumptions, the mercury loading in the bed would build up to almost $3.6 \mathrm{wt} \%$. Mercury capacity of sulfur-impregnated carbon can be as high as $20 \mathrm{wt} \%$. [17]. The mercury laden carbon is considered to be a hazardous waste, and the disposal cost estimate reflects this categorization.

\subsubsection{Acid Gas Removal and Sulfur Recovery}

CrystaSulf process is selected for the Acid Gas Removal (AGR) unit and sulfur recovery. The low sulfur Fruitland coal and the hydrogasifier reactions produce a syngas containing $\sim 1250$ ppmv of sulfur compounds, which corresponds to a relatively small amount of sulfur ( 20 TPD) to be recovered in the process. CrystaSulf is reported to have an economic advantage over the physical or chemical solvent AGR combined with a Claus Sulfur Recovery Unit (SRU) for sulfur recovery rates up to 20 to 30 TPD [18].

CrystaSulf is a sulfur recovery process that removes hydrogen sulfide and $\mathrm{SO}_{2}$ from gas streams and coverts it into sulfur. The CrystaSulf process utilizes a proprietary non-aqueous solution and operating conditions that promote liquid-phase conversion of $\mathrm{H}_{2} \mathrm{~S}$ and $\mathrm{SO}_{2}$ to elemental sulfur. The CrystaSulf is a solution that dissolves elemental sulfur and rejects product water because it is hydrophobic. 
$\mathrm{H}_{2} \mathrm{~S}$ is removed from the sour syngas in a tray countercurrent absorber, where $\mathrm{H}_{2} \mathrm{~S}$ reacts with dissolved sulfur dioxide in the circulating CrystaSulf scrubbing solution according to the Claus liquid process reaction to produce dissolved elemental sulfur. The CrystaSulf solution has a high solubility for sulfur, which remains totally dissolved at the process operating temperature. The sweet syngas from the absorber, with sulfur compounds volumetric concentration in low single digits of parts per million exits the system. Typical CrystaSulf process is illustrated in Exhibit 4-10.

\section{Exhibit 4-10 \\ Typical CrystaSulf Process}

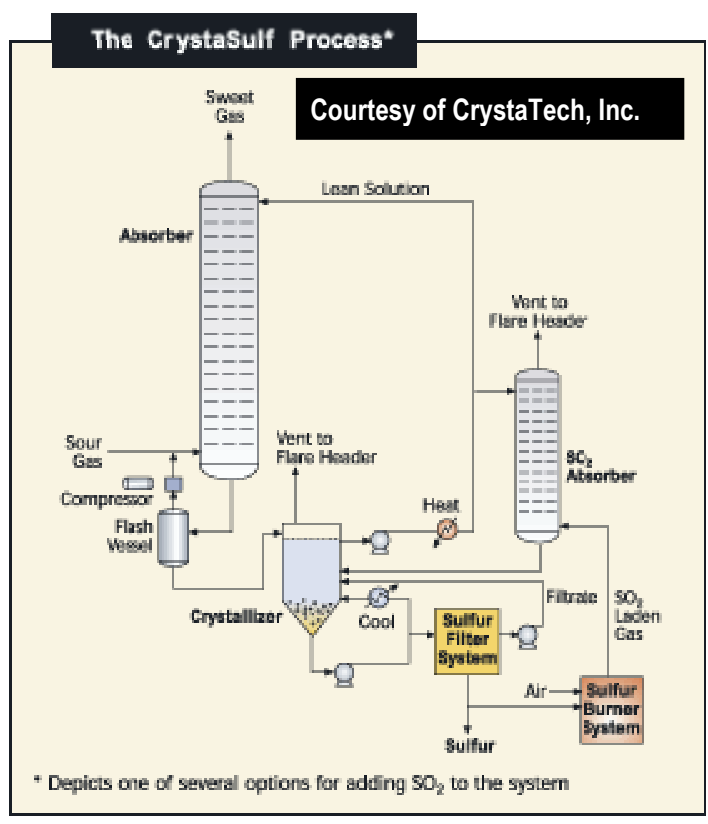

Rich solution from the absorber passes to a flash tank, where the CrystaSulf solution is flashed down to near atmospheric pressure, producing flash gas stream that is recycled upstream from the absorber.

The solution stream from the flash tank is fed to crystallizer, where a cooling loop causes the formation of crystalline sulfur. The CrystaSulf solution is cooled to a temperature sufficiently below the absorber temperature so that the solid sulfur forms in crystallizer. The higher operating temperature elsewhere in the system prevents sulfur crystallization and assures plugging free operation.

The regenerated CrystaSulf solution is re-heated by exchanging heat with the crystallizer cooling system and returned to the top of the absorber.

The slurry of crystalline sulfur from the crystallizer is fed to a filter or centrifuge that produces filter cake of elemental sulfur. A low-boiling wash solvent is used to wash a filter cake and remove the residual CrystaSulf solution from the sulfur. The CrystaSulf solution/wash solvent stream from the filter is fed to a solvent recovery for separation, where it rinsed with water. The 
recovered CrystaSulf solution, the wash solvent and the water are recycled to their respective processes.

Since the inlet sour syngas does not have the necessary $\mathrm{H}_{2} \mathrm{~S}: \mathrm{SO}_{2}$ ratio of $2: 1$, additional $\mathrm{SO}_{2}$ is introduced to the process, and carried into the absorber with lean CrystaSulf solution. The $\mathrm{SO}_{2}$ is produced by burning some elemental sulfur. The $\mathrm{SO}_{2}$ laden gas from the sulfur burner system is added to the CrystaSulf solution through a scrubber column on the sulfur burner exhaust.

\subsubsection{Char Combustor}

The process flow diagram of the Char Combustor system is shown on Drawing APSS-1-SK-021305-305 (Appendix B). The oxygen blown CFB steam generator system described herein is based on results obtained by the Alstom Power Inc. during their Phase 2 pilot scale testing of an oxygen blown CFB [19].

The solids leaving the gasifier are combusted with an $\mathrm{O}_{2}$ stream in order to generate a nearly pure $\mathrm{CO}_{2}$ stream. This is accomplished in an oxygen blown circulating fluidized bed boiler (CFB). The thermal and chemical energy in the char supplies the energy needed to raise $1800 \mathrm{psig} / 1000^{\circ} \mathrm{F} / 1000^{\circ} \mathrm{F}$ steam for the steam turbine. Approximately 52 percent of carbon in the coal is converted in the hydrogasification reactor. The remaining 48 percent exits the gasifier vessel as a char, and primarily consist of ash and carbon with small amounts of hydrogen, nitrogen, and sulfur. The residual carbon-rich char from the gasifier is cooled in a stripper/ cooler, depressurized, pelletized to particle size of approximately 0.25-inch and stored in a containment vessel before being injected into the CFB boiler for final combustion.

The average size of char particles coming out of the gasifier is expected to be $~ 50$ microns or less. The CFB process requires feedstock size of approximately 0.25-inch. Agglomeration of the char to a nominal size of 0.125 to 0.25 of an inch will be accomplished in a pelletizer. Pelletizer binding agent type is not known at this time, and can only be determined by testing. Binding agent properties are desired to be very similar to coal ash to minimize any contaminants that would dilute the $\mathrm{CO}_{2}$ combustion product.

In the boiler, residual carbon-rich char is reacted with a preheated mixture of oxygen and recirculated flue gas in the Combustor section of the Circulating Fluidized Bed (CFB) system. The oxygen supply is provided over the fence from an Electrolysis Unit. The products of combustion (comprised of primarily $\mathrm{CO}_{2}$ and $\mathrm{H}_{2} \mathrm{O}$ vapor and unreacted hot solids) leave the combustor, and flow through a cyclone, where most of the hot solids are removed. The hot solids are recirculated to the combustor. Draining hot solids through a water-cooled fluidized bed ash cooler controls solids inventory in the system, while recovering heat from the hot ash. The flue gas leaving the cyclone is cooled in an economizer located in the convection pass of the system. The flue gas leaving the convection pass heat exchanger sections is further cooled in an oxidant heater. The oxygen stream from the Electrolysis Unit is mixed with a small stream of recirculated flue gas and the mixture is preheated in the Preheater. The flue gas leaving the preheater heater is cleaned of fine particulate matter in the Particulate Removal system. Flue gas stream leaving the Char Combustor consists primarily of $\mathrm{CO}_{2}$ with some water vapor and excess $\mathrm{O}_{2}$. Water is subsequently condensed out of the $\mathrm{CO}_{2}$ stream in cooling, knockout and 
compression steps, and prior to $\mathrm{CO}_{2}$ stream being recycled back to the gasifier or sent to the deoxidation system for $\mathrm{O}_{2}$ removal.

\subsubsection{Ash / Waste Handling System}

The oxygen-blown CFB Char Combustor operates at non-slagging conditions. Therefore both the solids produced from the bottom of the char combustor as well as those entrained with the flue gas are classified as "ash" products.

The function of the ash handling system is to provide for conveying, preparing, storing, and disposing of the fly ash and bottom ash produced on a daily basis by the boiler.

The bottom ash and flyash are cooled and depressurized via separate pathways. Both ash streams are combined and transferred to the ash silo. The ash silo is sized for a nominal holdup capacity of 72 hours of full-load operation. At periodic intervals, ash-hauling trucks will transit the unloading station underneath the silo and remove a quantity of ash for disposal.

The scope of the system is from the baghouse hoppers and hydrobins to the truck filling stations. The fly ash collected in the baghouse is conveyed to the fly ash storage silo. A pneumatic transport system using low-pressure air from a blower provides the transport mechanism for the fly ash. Fly ash is discharged through a wet unloader, which conditions the fly ash and conveys it through a telescopic unloading chute into a truck for disposal.

The bottom ash from the boiler is fed into a clinker grinder. The clinker grinder is provided to break up any clinkers that may form. From the clinker grinders, the bottom ash is sluiced to hydrobins for dewatering and offsite removal by truck.

Ash from the economizer hoppers and pyrites (rejected from the coal pulverizers) is conveyed by hydraulic means (water) to the economizer/pyrites transfer tank. This material is then sluiced on a periodic basis to the hydrobins. Exhibit 4-11 presents the technical requirements and design basis for the ash handling system.

Exhibit 4-11

Ash Handling System Technical Requirements and Design Basis

\begin{tabular}{|l|l|}
\hline $\begin{array}{l}\text { Bottom Ash and Fly Ash Rates } \\
\text { Bottom ash generation rate } \\
\text { Fly ash generation rate }\end{array}$ & $\begin{array}{l}16,700 \mathrm{lb} / \mathrm{h}(8.3 \mathrm{tph}) \\
38,900 \mathrm{lb} / \mathrm{h}(19.5 \mathrm{tph})\end{array}$ \\
\hline Bottom Ash & $10 \mathrm{tph}$ \\
$\quad$ Clinker grinder capacity & $15 \mathrm{tph}$ \\
Conveying rate to hydrobins & \\
\hline Fly Ash & $20 \mathrm{tph}$ \\
Collection rate & $50 \mathrm{tph}$ \\
Conveying rate from precipitator and & \\
air heaters & $2,000 \mathrm{tons}$ \\
Silo capacity (72-hour storage) & $40 \mathrm{tph}$ \\
Wet unloader capacity & \\
\hline
\end{tabular}




\subsubsection{Deoxidation, and $\mathrm{CO}_{2}$ Compression and Handling}

Process flow diagram of the Deoxidation and $\mathrm{CO}_{2}$ Compression and Handling system is shown on Drawing APSS-1-SK-021-305-305 and-306 (Appendix B)

$\mathrm{CO}_{2}$ from the Char Combustor System's cooler and knock out drum is compressed in a multistage intercooled $\mathrm{CO}_{2}$ Recycle Compressor to around 1100 psig before being split into two streams. Approximately $15 \%$ of the compressed $\mathrm{CO}_{2}$ is compressed further prior to being heated in the oxycombustor and ultimately utilized as transport gas in the gasifier coal feed system. The remaining $\sim 85 \%$ is sent to the Deoxidation system.

In the Deoxidation system, excess oxygen is removed from the $\mathrm{CO}_{2}$ stream by injecting hydrogen into the stream and using two stages of catalytic beds. The deoxidation feed is first preheated to approximately $670^{\circ} \mathrm{F}$ using reactor product or a start-up heater before it is sent to the first reactor stage. Hydrogen is then injected into the stream and the $\mathrm{CO}_{2}+\mathrm{H}_{2}$ stream enters the first reactor stage where hydrogen and about $50 \%$ of the oxygen react. Only about $50 \%$ of the oxygen is allowed to react to limit the exotherm across the bed. The product stream exits at about $1090^{\circ} \mathrm{F}$ and is cooled by a feed/product heat exchanger. This cooled stream is further reacted with hydrogen in a second reactor stage where the remaining oxygen is reacted to water.

The second stage operates at similar inlet/outlet temperatures and exotherm as the first stage. Products from the second stage are cooled using a cooling train including high pressure steam generation, HP Boiler feedwater economization, and condensate preheat. Following the cooling train at approximately $110^{\circ} \mathrm{F}$, condensed water is removed in the $\mathrm{CO}_{2}$ knock out drum before it is sent to the sour water stripper. $\mathrm{CO}_{2}$ from the Knock Out Drum is mixed with the gasifier raw syngas prior to the mercury removal process. Ultimately this $\mathrm{CO}_{2}$ is further cleaned of sulfur in the CrystaSulf unit and sent to the methanator where it is converted to methane.

\subsubsection{Methanation Unit}

Process flow diagram of the methanation system is shown on Drawing APSS-1-SK-021-305-303 and 304 (Appendix B).

Desulfurized syngas from the CrystaSulf system is preheated in two Feed/ Product Exchangers to about $500^{\circ} \mathrm{F}$ before it enters the Zinc Oxide $(\mathrm{ZnO})$ guard bed to remove any remaining sulfur which would poison the Methanation reactor catalyst. The Methanation system is comprised of two bulk methanation reactors with recycle, followed by a trim reactor. The Methanation process reactions are as follows:

$$
\begin{aligned}
& 3 \mathrm{H}_{2}+\mathrm{CO} \rightarrow \mathrm{H}_{2} \mathrm{O}+\mathrm{CH}_{4} \\
& 4 \mathrm{H}_{2}+\mathrm{CO}_{2} \rightarrow 2 \mathrm{H}_{2} \mathrm{O}+\mathrm{CH}_{4}
\end{aligned}
$$

The preheated syngas stream leaving the $\mathrm{ZnO}$ bed mixes with a recycle stream that acts to limit the temperature rise across the first reactor by dilution prior to entering the first bulk reactor. The mixed reactor feed enters the first bulk reactor where the hydrogen reacts with the majority of carbon monoxide and carbon dioxide to produce methane and water. The first bulk reactor uses a high temperature catalyst that can withstand the exhaust temperature of approximately $1020^{\circ} \mathrm{F}$. Heat is removed from the exhaust stream by cooling to approximately $650^{\circ} \mathrm{F}$ and generating superheated high pressure steam. Approximately $78 \%$ of the exhaust is recycled back 
to the reactor feed. The recycled stream is cooled in a recycle feed/product heat exchanger followed by water cooling where water is knocked out as a result. The cooled recycle stream is then compressed and reheated to approximately $550^{\circ} \mathrm{F}$ prior to mixing in with the feed stream as diluent.

The first bulk reactor effluent that is not recycled is sent to the second bulk methanation reactor at approximately $650^{\circ} \mathrm{F}$ where additional $\mathrm{CO}$ and $\mathrm{CO}_{2}$ are converted to methane. The effluent temperature is around $800^{\circ} \mathrm{F}$. Heat is recovered from this stream for additional steam generation and economization, cooling the stream to approximately $500^{\circ} \mathrm{F}$. The cooled methanation effluent enters the trim reactor which brings the $\mathrm{CO}, \mathrm{CO}_{2}$ and $\mathrm{H}_{2}$ to low mole percentage levels of $0.003 \%, 1.75 \%$ and $2.63 \%$ (dry basis) respectively. The trim reactor effluent of approximately $580^{\circ} \mathrm{F}$ is utilized for sweet feed preheating and economization, cooling the stream to approximately $350^{\circ} \mathrm{F}$. Additional cooling/condensation is provided by cooling water before the SNG product stream is sent for drying in the Triethylene Glycol (TEG) unit.

Following the methanation cooling system, the process condensate is flashed to about atmospheric pressure. The overheads from the low pressure flash are sent to the Char Combustor while the condensate is sent to waste water treatment.

Water vapor from the TEG Unit is vented. The dried SNG stream is compressed to 910 psia before it is exported as SNG product.

\subsubsection{Cogeneration System}

Process flow diagram of the electric power cogeneration system is shown on Drawing APSS-1SK-021-305-308 (Appendix B)

\section{Steam Turbine}

The steam turbine is designed for a long-term operation at maximum continuous rating (MCR) with throttle control valves 95\% open. It is also capable of a short-term 5 percent over pressure/valves wide open (OP/VWO) condition (16 hours).

The steam turbine is tandem compound type, consisting of an HP-IP and two LP (double flow) sections enclosed in three casings, designed for condensing single reheat operation, and equipped with unregulated extractions and four-flow exhaust.

\section{Steam System}

The function of the main steam system is to convey main steam from the Char Combustor superheater outlet to the high-pressure turbine stop valve. The function of the reheat system is to convey steam from the HP turbine exhaust to the Char Combustor reheater, and to the turbine reheat stop valves. Main steam system will be operating at $1800 \mathrm{psig} / 1000^{\circ} \mathrm{F}$, and reheat system will be operating at $1000^{\circ} \mathrm{F}$.

\section{Feedwater System}

The function of the feedwater system is to pump the feedwater stream from the deaerator storage tank to the CFB (char burner) steam drum. Two 100 percent capacity boiler feed pumps are provided. Each pump is provided with inlet and outlet isolation valves, and an outlet check 
valve. Minimum flow recirculation to prevent overheating and cavitation of the pumps during start-up and low loads is provided by an automatic recirculation valve and associated piping that discharges back to the deaerator storage tank. Pneumatic flow control valves control the recirculation flow.

The feedwater pumps are supplied with instrumentation to monitor and alarm on low oil pressure, or high bearing temperature. Feedwater pump suction pressure and temperature are also monitored. In addition, the suction of each boiler feed pump is equipped with a start-up strainer.

\subsubsection{Circulating Water System}

The circulating water system supplies cooling water to the condenser to condense the main turbine exhaust steam. The system also supplies cooling water to the auxiliary cooling system. The heat transferred from the steam to the circulating water in the condenser is removed by a mechanical draft cooling tower.

The system consists of two 50 percent capacity vertical circulating water pumps. The circulating water pumps are vertical wet pit pumps, with a single stage impellor. The piping system is equipped with butterfly isolation valves and all required expansion joints.

The condenser is a single-pass, horizontal type with divided water boxes. There are two separate circulating water circuits in each box. One-half of the condenser can be removed from service for cleaning or plugging tubes. This can be done during normal operation at reduced load.

The condenser is equipped with an air extraction system to evacuate the condenser steam space for removal of noncondensable gases during steam turbine operation and to rapidly reduce the condenser pressure from atmospheric pressure before unit start-up and admission of steam to the condenser.

The auxiliary cooling water system is a closed-loop system. Plate and frame heat exchangers with circulating water as the cooling medium are provided. This system provides cooling water to the methanation, AGR, syngas cooler, air and carbon dioxide compressors, lube oil coolers, turbine generator, feedwater pumps, etc. All pumps, vacuum breakers, air release valves, instruments, controls, etc. are included for a complete operable system.

\section{Cooling Tower}

A mechanical draft, fiberglass structure, counter-flow cooling tower is provided for the circulating water heat sink.

The cooling tower is comprised of two linear array of 6 cells, each containing a 200 hp fan that induces airflow through the tower fill. Hot circulating water from the condenser is sprayed on to the spray deck above the fill, and falls down in films over the fill with a counterflow of air rising and cooling the water. Approximately $80 \%$ of the heat transfer occurs by evaporation of the water, with the remaining $20 \%$ occurring by sensible heat transfer to the air.

The cooled water falls into the cooling tower basin underneath the fill, and is directed to a wet pit pump suction chamber. The circulating water pumps take suction from this pump chamber, 
circulating the water to and through the steam condenser and back to the tower spray deck, completing the circuit.

Exhibit 4-12 shows the contribution of different plant systems to the cooling tower duty, which directly drives the cooling tower evaporation rate and blowdown rate.

Exhibit 4-12

Hydrogasification Plant Cooling Tower Duty

\begin{tabular}{|c|c|c|}
\hline System & Duty, $10^{6} \mathrm{Btu} / \mathrm{h}$ & Contribution \\
\hline Condenser duty & 1239.07 & $73.6 \%$ \\
\hline Sour Syngas cooler & 21.8 & $1.3 \%$ \\
\hline AGR Crystasulf & 16.4 & $1.0 \%$ \\
\hline Methanator cooling & 278.0 & $16.5 \%$ \\
\hline Flue gas from CFB cooling & 43.9 & $2.6 \%$ \\
\hline $\mathrm{CO}_{2}$ Compression duty & 48.0 & $2.8 \%$ \\
\hline Sour Water/Ammonia Stripper, & 37.1 & $2.2 \%$ \\
\hline Total & 1684.4 & $100.0 \%$ \\
\hline
\end{tabular}

\subsubsection{Sour Water Treatment}

Process flow diagram of sour water treatment system is shown on Drawing APSS-1-SK-021305-307 (Appendix B). The sour gas stripper removes $\mathrm{NH}_{3}, \mathrm{H}_{2} \mathrm{~S}$, and other impurities from the liquid waste streams of the syngas and flue gas coolers throughout the plant. The sour gas stripper consists of the sour drum that accumulates sour water from the coolers. Sour water from the drum flows to the sour stripper, which consists of a packed column with a steam-heated reboiler. Sour gas is stripped from the liquid and sent to the char-burning CFB unit. The liquid from the Sour Water Stripper is sent to the Ammonia Stripper where the ammonia is removed and the resulting liquid stream is sent to the Waste Water Treatment system.

\subsubsection{Water and Waste Water Treatment}

Water and waste water treatment is accomplished by the Zero Liquid Discharge (ZLD) system. The preliminary Water Balance is shown on Drawing APSS-1-DW-021-305-500 (Appendix D).

Plant water supply will provide service water, fire protection system water, potable water, steam cycle demineralized makeup water, and makeup water to the circulating water system (wet cooling tower system basis). Additional makeup water to the circulating water system will come from reuse of treated wastewaters and storm water runoff. Blowdown from the circulating water system will be treated via a zero liquid discharge (ZLD) system to concentrate and remove solids, and recycle treated wastewater back to the circulating water system. The steam cycle makeup will be provided from process condensate of the Methanation system.

A raw water tank and a filtered water/fire water tank will be provided to store water supplied from the water supply source. Depending on the hydrogasification plant location and reliability 
of the plant water supply, the two tanks may be combined as one in final design. Filtered water will be provided to the service water system.

Plant water supply will be directed to the cooling tower basin as required to makeup for evaporation losses, dust suppression water use, gasifier/stripper process consumption, and moisture losses with waste solids to landfill. The water balance is based on five cycles of concentration in the circulating water system. Cooling tower blowdown (CTBD) will be used for dust suppression and gasifier/sour stripper process needs. Excess CTBD will be directed to the ZLD system clarifier for chemical conditioning for solids removal and reuse. Clarifier sludge solids will be dewatered via a filter press and be directed to a landfill for disposal. Clarifier effluent will be concentrated in a wastewater RO system. RO concentrate will be sent to a brine concentrator system consisting of an evaporator and a crystallizer. A portion of the salt solids slurry from the crystallizer will be dewatered in a pneumatic pressure filter with the filtrate being returned to the crystallizer. The dewatered salt solids will be directed to a landfill for disposal.

Wastewater RO permeate, evaporator distillate, crystallizer condensate and boiler blowdown will be recycled as makeup to the circulating water system. Plant drains, storm water runoff, coal pile runoff, and gasifier/sour stripper process blowdown will be individually treated and reused as makeup to the circulating water system.

A brine holding tank, concentrate holding tank and sludge holding tank will be provided to allow for start-up and shutdown of the treatment systems. Chemical feed systems will be provided as required for treatment processes. Bulk liquid chemical storage tanks or totes will be provided depending on specific chemical demands. Chemical silo/slurry makeup systems will be provided for bulk solid chemicals.

Spare process and chemical feed pumps will be provided throughout the water and wastewater treatment system. Connections will be provided to allow use of contracted, trailer-mounted demineralizers during the plant start-up.

The wastewater treatment clarification system, RO package, and ZLD brine concentration package are all single $100 \%$ capacity units. Emergency 24-hour storage of cooling tower blowdown will be provided either via increased volume built into the cooling tower basin or via an emergency blowdown storage pond to allow for short term maintenance. The brine holding tank and concentrate tank will provide additional maintenance flexibility.

\subsubsection{Flare System}

Hydrogasification plant will be equipped with a single $100 \%$ capacity elevated and fully automated flare system. The purpose of the flare system is venting and disposal of waste gases from the gasifier and syngas cleaning systems any time during operation.

The flare system is comprised of multiple relief valves discharging into a common header, knockout drum for liquids separation, and self-supporting, refractory-lined, carbon steel flare stack. The integrated ignition system complete with multiple propane-fueled pilot burners, and monitoring instrumentation is capable of instantaneous initiation and maintaining of stable burning throughout the period of waste gas flow. 
The flare system will be designed to support planned and emergency flaring events. The examples of planned flaring events would be gasifier start-up, shutdown, and ramping. The emergency flaring events could occur as a result of upset operating condition. Waste gas during the planned flaring events would be treated to reduce environmental impact. Flaring of untreated waste gases will be limited to emergency events.

The flare stack location, height and exclusion zone around the stack will be selected based on the allowable radiation exposure limits guidance provided in API RP 521 [20].

\subsubsection{Accessory Electric Plant}

The accessory electric plant consists of switchgear and control equipment, generator equipment, station service equipment, conduit and cable trays, and wire and cable. It also includes the main power transformer, required foundations, and standby equipment.

\subsubsection{Instrumentation and Control}

An integrated plant-wide control and monitoring system (DCS) is provided. The DCS is a redundant microprocessor-based, functionally distributed system. The control room houses an array of multiple video monitor and keyboard units. The Monitor/keyboard units are the primary interface between the generating process and operations personnel. The DCS incorporates plant monitoring and control functions for all the major plant equipment. The DCS is designed to provide 99.5 percent availability. The plant equipment and the DCS are designed for automatic response to load changes from minimum load to 100 percent. Start-up and shutdown routines are implemented as supervised manual, with operator selection of modular automation routines available. 


\section{Construction, Commissioning and Schedule}

A preliminary constructability analysis, startup/commissioning process description and a construction/ start-up schedule are presented in the following sections.

\subsection{Constructability}

This section presents a preliminary constructability analysis of the commercial-scale facility including determination of novel construction processes, definition of construction methods, and identification of construction packages.

The constructability analysis is, by definition, very preliminary, since the project has not identified a specified site and detailed design documentation is not available to provide a more in depth analysis. As such, the following assumptions form the basis of this constructability analysis:

1. All engineering and procurement will be substantially complete prior to the start of major construction activities.

2. The entire site will require pilings and major construction activities cannot be started until all the piling work has been completed.

3. Due to unknown site conditions, we have not taken into account the relocation of existing utilities.

4. The site will have sufficient space to accommodate the following:

a. Pre-fabrication areas

b. Contractor office and tool trailers

c. Material/Equipment storage (Inside and outside storage)

d. Worker parking

e. Wash and toilet facilities

f. Break/Lunch areas

g. Access through two (2) separate points to allow for construction activities at two different areas.

The layout of the site is very clean and it can accommodate "novel" construction processes/pre fabrication. The following are areas that should be considered for pre - fabrication during FEED and final design:

1. Pre-fabrication of electrical duct banks

2. Pre-fabrication of integrated pipes/pipe rack

3. Pre-fabricated MCC room (with pre - installed and pre-wired equipment) 
4. Consideration of any filtration/process areas that can be shop assembled and skid mounted: Specifically, the following components should be considered for "shopfabrication":

a. Hydrogasifier

b. Cyclones

c. HRSG and heat exchangers (Sectioning and/or modularization should be considered), and

d. Conveyors

At this stage of the constructability analysis, it is estimated that the pre-fabrication of the above listed items could save 1- 3 months of construction time.

Without taking into account any specific pre-fabrication activities, construction will be conventional. It is expected that concrete pilings with pile caps that will bear the loads for the equipment will be utilized as the civil design. Equipment slabs with spread footings may be utilized to support major equipment. A pedestal type foundation will be utilized to support the STG. Buildings will be constructed on slab on finish grade.

Special precautions will be necessary during concrete pouring operations, for major structural foundations (i.e. - steam turbine generator, steam generators, and the char combustor), during high ambient temperatures. Concrete pours above $80^{\circ} \mathrm{F}$ will require special temperature control measures.

It is envisioned at this time that one large crane (up to 1,000 tons) may be needed for the major portions of the project and that 2-3 cranes (up to 250 tons) may be needed for selected portions of the project.

The bottom of the pipe rack should be kept at least $25 \mathrm{ft}$ above ground level to allow for the access of small cranes and other maintenance equipment. The general arrangement presented in Exhibit 4-3 is based on road access from both the south and north side of the plant for crane and other maintenance equipment. If the specifics of the selected site do not facilitate this level of access, then the location of the pipe racks should be reconsidered for the possible relocation to the southern side of the present arrangement or what ever is dictated by the specifics of that site.

Safety management will be critical for this project, due to worker/site exposure to heavy lifts, gases, and possible high ambient temperatures. These factors need to be taken into account on the specific Construction Management plan for the site. The possibility of siltation and run-off and spills need to be addressed during the project Construction Management plan. Noise pollution and required mitigating measures, especially during construction and start - up, need to be addressed during the project Construction Management plan.

The following are the construction packages that are envisioned at this time (all packages are "lump sum, firm price contract packages"):

1. Site clearing and grubbing (Includes site preparation and excavation for the retaining ponds, and installation of silt fences and other construction environmental protection devices.) 
2. Pile installation and major foundations/slabs (Includes "civil" construction of retaining pond - less liners)

3. Mechanical (Includes equipment, piping, pipe racks, etc. Includes structural steel for CFB and process equipment)

4. Electrical (Includes high voltage and low voltage - below grade and above grade. Includes structural supports for cable tray and aerial conduit runs)

5. Switch yard (T\&D) (Includes towers/poles (if any), and structural components for distribution lines and main transformer)

6. Buildings and roads (Could be grouped together with other civil activities. Includes structural steel for buildings and architectural finishes)

7. Landscaping

8. Painting and insulation (Could be grouped together with other activities)

9. Cooling tower

10. Liners for retaining ponds

An EPCM contract should be strongly considered for this project to maximize the savings on major procurements, to improve on the expected construction schedule time frame, and to have a highly qualified staff on site to ensure that the project goals are met. Key components that will provide benefits to the client via an EPCM contract are as follows:

1. Engineering: On going constructability review of the project as the design progresses. This will maximize opportunities for pre-fabrication and mitigate coordination problems between the disciplines when the construction phase of the project starts.

2. Procurement: On time coordination of engineering - procurement - construction, to ensure that the required equipment/materials are on site when needed.

3. Construction Management: Will have a highly skilled staff on site to manage the project and to ensure that all project goals (safety, health, environment, cost and schedule) are met.

Above all, having complete engineering packages for the procurement and for the construction bidding process will reduce change orders and provide more accurate construction pricing. Construction management available early on this process will assist the prospective contractors in preparing a more responsive bid for the project.

\subsection{Initial Start-Up and Commissioning}

The Initial Start-up and Commissioning of an involved process takes careful planning to ensure the execution of the project has a minimal number of unforeseen challenges. The creation of policies and procedures that control and direct the initial start-up and commissioning process are paramount to this effort. The overall philosophy of the control of start-up and commissioning should have two facets: Administrative Control and Technical Control. These two facets are defined in the Start-up Administrative Manual. This document controls all aspects of start-up 
from organization to technical testing documentation. Each section will be described below in greater detail. A discussion will also be made of a probable start-up sequence including possible start-up team members, site mobilization, commissioning and start-up, requirements of owner's operators, training, and performance testing.

\subsubsection{Initial Start-Up and Commissioning Process}

The process used for the initial start-up and commissioning of a project can be divided into three phases: 1) Construction Testing, 2) Functional Testing, and 3) Operational/ Performance Testing. The requirements of these phases are decided upon between the Owner and the Contractor during the initial negotiations of the contract. These requirements can be tailored to meet the needs of the Owner. Even though the details may change certain milestones for the project are met during these phases.

\subsubsection{Construction Testing}

Construction Testing involves the testing of equipment prior to, during, and/or after installation. Many tests are conducted on equipment to determine if it is the condition to perform the task for which it was procured. Electric motors are checked for proper insulation resistance and proper direction of rotation, pumps and motors are properly aligned to prevent damage and ensure efficient operation. These tests would primarily be performed by the construction trades and the results documented. The documentation from these tests would then be used to support the turn over of systems from the Construction Contractor to the Initial Start-Up and Commissioning Contractor. Once construction is complete and all turnover documentation has been reviewed the Construction Contractor and the Initial Start-Up and Commissioning Contractor would conduct a joint walk down of the system to ensure the system is complete and there are no outstanding issues such as missing insulation, wrong type of valve, etc. Any discrepancies would be recorded on a punch list to ensure the items are eventually completed. If there are no major problems then the care, custody and control of the system would pass from the Construction Contractor to the Initial Start-Up and Commissioning Contractor. Once the care, custody, and control of the system changes, then the initial start-up and commissioning for that system would progress to the next phase, Functional Testing.

\subsubsection{Functional Testing}

Functional Testing, as the name implies, deals with testing the different functions of the system to ensure they operate properly. This type of testing may require other systems to be operational in order for the testing to be conducted. The first thing to be performed upon systems during this phase of testing is a flush of the piping to remove any contaminants from the system left over from construction. Then if a hydrostatic test is required of the system that test would be performed. The functional items tested are operation of alarms, trips and interlocks. These tests would include any local controls as well as remote locations such as the control room. The Distributed Control System (DCS) logic would be included in this testing for the purpose of finding any errors in the control logic. Other tests to ensure pumps, fans, and control valves are working properly would be conducted also. The purpose of this testing is to ensure the system is ready and able to be operated in the automatic mode. Once a system has been verified to be functional by the completion of the functional test procedure all data from the tests would be 
recorded and compiled within the system turnover book along with all of the data from the construction testing. The Owner and the Initial Start-Up and Commissioning Contractor would then perform a joint walk down of the system to ensure there are no outstanding issues and the punch list for that system is completed. If the Owner agrees then care, custody, and control would pass from the Contractor to the Owner for that system.

\subsubsection{Operational / Performance Testing}

Once all functional testing is complete operational and performance testing can take place. These tests vary widely and are decided upon by the Owner depending on what requirements the Owner desires. For instance, if the project is an entire power plant the Owner might require testing to ensure plant performance falls into specifications for heat rate and electric power generation. The testing required for this project would entail ensuring the gasifier, char combustor, syngas cleanup, methanator and steam turbine are all operating within parameters determined during the detailed design phase of the project.

\subsubsection{Initial Start-Up and Commissioning Personnel}

The start-up staff would most likely consist of a start-up manager, assistant start-up manager, and a turnover coordinator for the overall management of the initial start-up and commissioning of the project. The number of required personnel for the Initial Start-up and Commissioning of the project would depend on the schedule for completion of the systems. The construction of major systems should be structured around staggered completion dates allowing for the minimum number of additional personnel. This will require forethought and planning on the part of the Engineer, Construction Contractor, and the Start-up group. A goal of three (3) Mechanical Commissioning Leads, three (3) Electrical Leads, and three (3) Instrumentation \& Control Leads for a total of nine (9) additional personnel supported by trades utilized from construction should be utilized. It is premature to speculate about personnel levels beyond this at this stage of development. The start-up schedule for a facility on this scale would require heavy planning and close coordination of all entities involved. The start-up schedule would dovetail into the construction schedule and the Start-up Manager and the construction manager would work together closely throughout the project. They would tackle any challenges to the schedule together. The construction craft labor would perform many of the initial checks required of the equipment such as motor checks, and pump inspections. These items will be documented and the documentation will be collected into a system turnover book. As the name implies, each system will have its own book and the organization of the book is governed by the system turnover procedure listed above. This documentation along with completed functional test procedures will be turned over to the Owner to prove the system is complete and functioning in accordance with contractual requirements. The Turnover Coordinator would be responsible for ensuring the turnover books were being completed in accordance with the procedure as well as coordinating walk downs of systems.

\subsubsection{Start-Up Manual}

A start-up manual can take different forms from one project to another. It is important to note however, regardless of its form, the manual will contain certain things to ensure that the plant is commissioned to the industry standard and to minimize warranty claims and rework. The proper 
administration of the initial start-up and commissioning process can determine the success or failure of the entire project. The administration encompasses the management and control of everything from the schedule, to the punch list, to the interface between start-up and other organizations. The basis of the control of these different items is the Start-up Administrative Manual. This manual contains the policies and procedures necessary to control how the start-up is conducted. Below is an example table of contents from a Start-up Administrative Manual with a brief description of each section.

\section{WP-01 Introduction to the Start-up Administrative Manual}

This procedure introduces and outlines the purposes of the manual. It also defines abbreviations and terms and contains a brief description of each procedure.

\section{WP-02 Start-up and Commissioning Organization}

This procedure describes the Start-up and Commissioning organization and its various components; it contains an organizational chart and describes the relationships between Start-up and interfacing organizations.

\section{WP-03 Initial Start-up Program}

This procedure describes the activities to be performed as part of the Initial Start-up Program.

\section{WP-04 Resolution of Engineering Problems}

This procedure describes methods to be used by Start-up to report engineering problems and request or document engineering changes.

\section{WP-05 Construction/Start-up and Commissioning Interface}

This procedure defines activities to be performed by Start-up and Construction during the Startup phases.

\section{WP-07 Start-up Turnback to Construction Procedure}

This procedure describes the method to be used to return a system or portion of a system to Construction for correction of a significant deficiency or change to a system.

\section{WP-08 System Turnover, Release Procedure}

This procedure outlines the preparation and utilization of turnover packages and the steps to be followed in the transfer of control of systems from Construction to Start-up and then to the Owner. It also describes the establishment and maintenance of System Turnover Punch Lists.

\section{WP-09 Borrowed Material Report}

This procedure outlines the steps to be taken when it becomes necessary to replace defective material with material borrowed from the on-site supplies of Operations or Construction. It also describes how replacement or repaired material is to be used to replace the borrowed material.

\section{WP-10 Vendor Representatives}


This procedure describes the methods to be used in monitoring and documenting vendor service requested by Start-up and outlines the preparation required to make the most efficient use of vendor time.

\section{WP-11 Start-up Purchasing}

This procedure describes the manner in which Start-up is to go about purchasing necessary test equipment, vendor service, repair service spare parts or replacement parts required during the start-up phase.

\section{WP-12 Initial Start-up Schedule}

This procedure consists of a graphical representation of Start-up activities versus time. Activities will be broken down by Start-up System and Key items such as system turnover dates and duration of activities will be included.

\section{WP-13 Checkout and Commissioning - Electrical Equipment and Systems}

This procedure defines how electrical equipment and systems are to be tested and placed in service during the operational phase of the project.

\section{WP-14 Checkout and Commissioning - Instrumentation and Controls}

This procedure defines how instrumentation and control systems and components are to be calibrated, tested and made operational during the start-up phase of the project.

\section{WP-15 Checkout and Commissioning - Mechanical Equipment and Systems}

This procedure defines how mechanical equipment and systems are to be tested and placed in service during the operational phase of the project.

\section{WP-16 Construction/Start-up-Commissioning Activity Interface}

This procedure presents the activity interface responsibility diagram for system completion and testing and includes a description of its use and purpose.

\section{WP-17 Start-up-Commissioning Progress Reporting}

This procedure establishes methods used for monitoring and reporting Start-up-Commissioning progress and establishes the frequency for updating and issuing reports.

\section{WP-18 Project Punch List}

This procedure defines the mechanism used for identifying and monitoring open work items on systems/components turned over to Start-up-Commissioning.

\section{WP-19 Test Equipment Control}

This procedure establishes the requirements for control and maintenance of Start-upCommissioning test equipment that is to be used during the Start-up-Commissioning Program.

\section{WP-20 Temporary Modification Control}

This procedure establishes the requirements for authorizing temporary modifications, for identifying temporary modifications, and for restoring temporary modifications after system/component turnover. 


\section{WP-21 Equipment Maintenance Program}

This procedure establishes the methods for interfacing with the Owner's Operation \& Maintenance group to ensure that equipment is properly maintained following turnover to Startup-Commissioning.

\section{WP-22 Turnover Tracking System}

This procedure establishes the process for tracking system turnover packages from the scheduled turnover date to Start-up-Commissioning through completion of the testing and commissioning.

These procedures provide for the control and management of initial start-up and commissioning activities from mobilization of the site to the end of performance testing and complete operational turn-over to the Owner. The technical procedures for mechanical, electrical, and instrumentation \& control check and commissioning are generic in nature and the specific procedure for each system would be developed separately. For example, the mechanical section contains the generic procedure for aligning a pump and its prime mover. As part of the commissioning procedure for each system a list of pumps would be generated for that system that needed to be aligned.

\subsection{Schedule}

This section presents a preliminary level 1 schedule for the commercial-scale facility design, construction and start up in Exhibit 5-2. This schedule is based upon the duration of major engineering, procurement, construction and start-up activities.

The total project is expected to take approximately $5 \frac{1 / 4}{4}$ years from the engineering notice to proceed (NTP) to commercial operation. Early permitting activities are expected to precede the NTP by approximately 6 months. Preliminary engineering and the development of bid packages for major equipment is forecast to take approximately 1 year. The critical path bid to award process is forecast to take approximately four months.

The overall procurement phase of the project includes equipment design, fabrication, and delivery and is estimated to span $23 / 4$ years, with the gasification reactor being on the critical path. A chart of procurement lead times for common power plant equipment is presented in Exhibit 5-1, and provides the procurement time utilized in the overall project schedule. The schedule presented herein is consistent with the lead times experienced in 2010. Of course, market conditions and these lead times are subject to change and must be evaluated as the project implementation draws near. 


\section{Exhibit 5-1 Recent Procurement Lead Times (as of Dec 2010)}

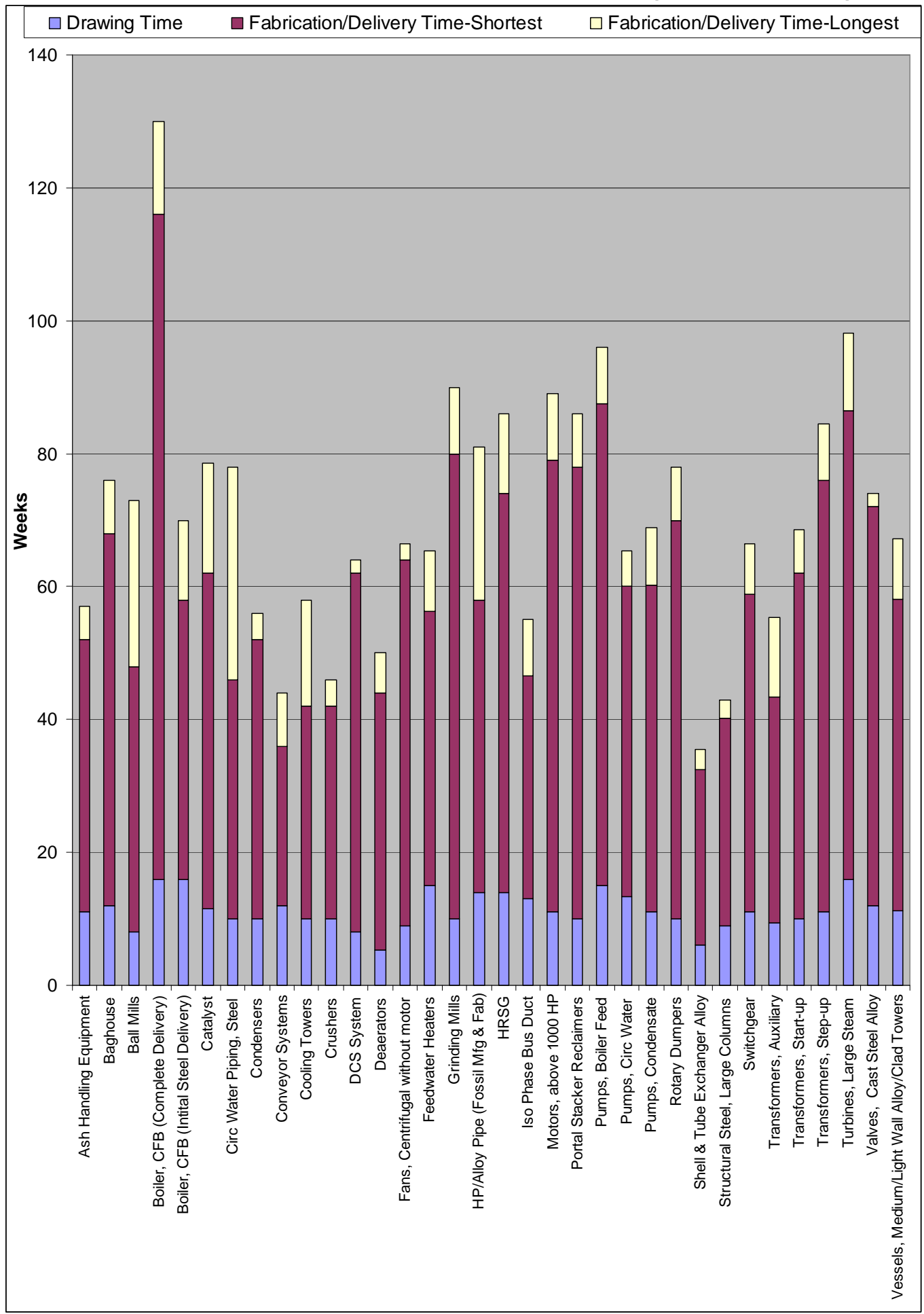


Project construction is forecast to span approximately 26 months. This construction schedule is based on a single shift 50-hour construction week, with night shifts envisioned for major welding activities. The schedule accounts for some minor pre-fabrication of components. Due to the generic nature of the site, it does not account for relocation of existing utilities. An assumption has been made that the entire site will require piling and that major construction cannot start until all piling work has been completed in that area. The initiation of the pile driving could be delayed somewhat from what is indicated in the developed schedule, thus shortening the total construction duration, without extending the completion schedule. Nevertheless, the piling is assumed to be started reasonable early to provide a buffer for surprises. Since the piles are not on the critical path, this assumption has no impact on the overall project duration. The construction phase concludes with demobilization, site clean up, and paving. This activity will be initiated after the major work features are installed to prevent damage to the new roads.

Start-up activities are forecast to last about 10 months, and are scheduled begin about 6 months before the completion of the gasifier itself. This will allow functional testing to be performed on systems completed prior to the availability of the syngas.

Again, these assumptions join together to forecast a total project duration of approximately $5 \frac{1}{4}$ years from engineering notice to proceed to commercial operation. A major driver in the overall project schedule is the estimated 33 month procurement cycle for award to delivery of the gasification reactor.

Historically, a 7" thick clad wall vessel, like the APS gasifier ${ }^{\mathrm{g}}$, would be ring forged as plate rolling has only been economic for 6" thick walls or less. Several years ago, with the heat up of the heavy wall equipment market, several vendors in the far east (e.g., Japan) have extended their capability of rolling plate up to a maximum of 8”. The turnkey procurement ${ }^{\mathrm{h}}$ cycle for forged ring manufacturing is approximately 40 to 42 months ExW ${ }^{\mathrm{i}}$. The turnkey procurement cycle for rolled plate manufacturing is forecast to be approximately 30 to 32 months ExW. With transportation from the far east, we have forecast 33 months for the gasification reactor for award to delivery based on a rolled plate manufacturing process. Should the reactor design be changed to more than 8" thickness, this would add an additional 10 months onto the total project duration.

g The precise gasifier wall thickness will not be determined until it is designed and certified according to the appropriate ASME pressure vessel code. The wall thickness is presumed to be approximately 5 to 7”. Until the final design, it will be presumed to be 7” for the scheduling considerations.

h In this context, “turnkey procurement” includes engineering and review.

i "ExW" is an Incoterms abbreviation for Ex works. Delivery is excluded. 


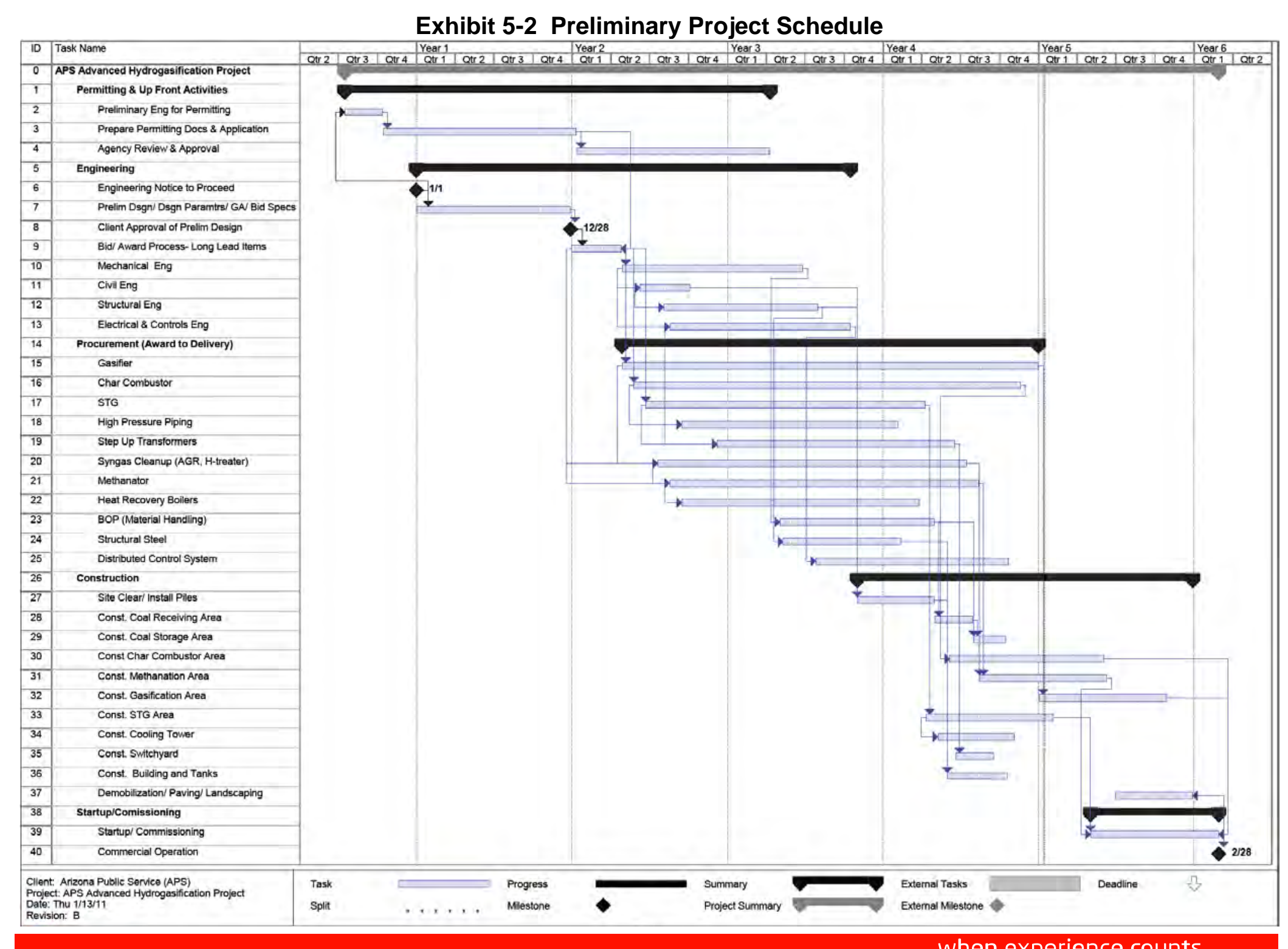

60

when experience counts 


\section{Start-up Sequence and Operating Procedures}

Section 6 provides an introduction to two operational issues: The development of a Plant Startup Sequence and a preliminary outline for the Plant Operating Procedures. Section 6.1 presents information on a plant start-up sequence and discusses the cold start-up of the entire SNG plant including the Hydrogasifier Island, Char Combustor Island, Power Island, and the Syngas Processing Island. The start-up sequence will provide insight to the interrelationships between the diverse systems comprising the plant.

Section 6.2 discusses a proposed organization for the Plant Operating Procedures manual. It provides a basic table of contents for the procedures for the safe and efficient operation of the plant. This section also presents the envisioned structure of these procedures and the reasoning underlying the structure.

\subsection{Start-up Sequence}

The start-up procedure that follows represents an initial birds-eye view procedure that is consistent with the preliminary conceptual design status of the process itself. The start-up sequence will need to be reviewed and revised as the conceptual design proceeds towards its final design. The many complex systems that form the overall process have many inter dependences that will require detailed design engineering before all the relationships can be finalized and properly understood. Nevertheless, a general start-up sequence is presented here that highlights the interrelationships and sequencing of the major systems.

\subsubsection{System Interrelationships}

It is important to understand the basic interrelationships between the major systems prior to discussing the overall start-up sequence. As such, the reader should reference the preceding system descriptions and the simplified block diagram found in Exhibit 4-1.

The generation of the SNG product is an involved process that is the result of the coordinated effort of many individual systems. All of these systems are interdependent upon one another. If one is not operating, then the others cannot operate or operate at a reduced efficiency. It is important to grasp the significance of this fact when discussing operations, especially discussing a start-up sequence. Knowing which system needs to be online prior to another system is the foundation upon which the start-up procedure is based.

Based upon the understanding of the system interrelationships and requirements, an overall cold start start-up sequence presented in Exhibit 6-1 was developed. 
Exhibit 6-1 Start-up Sequence

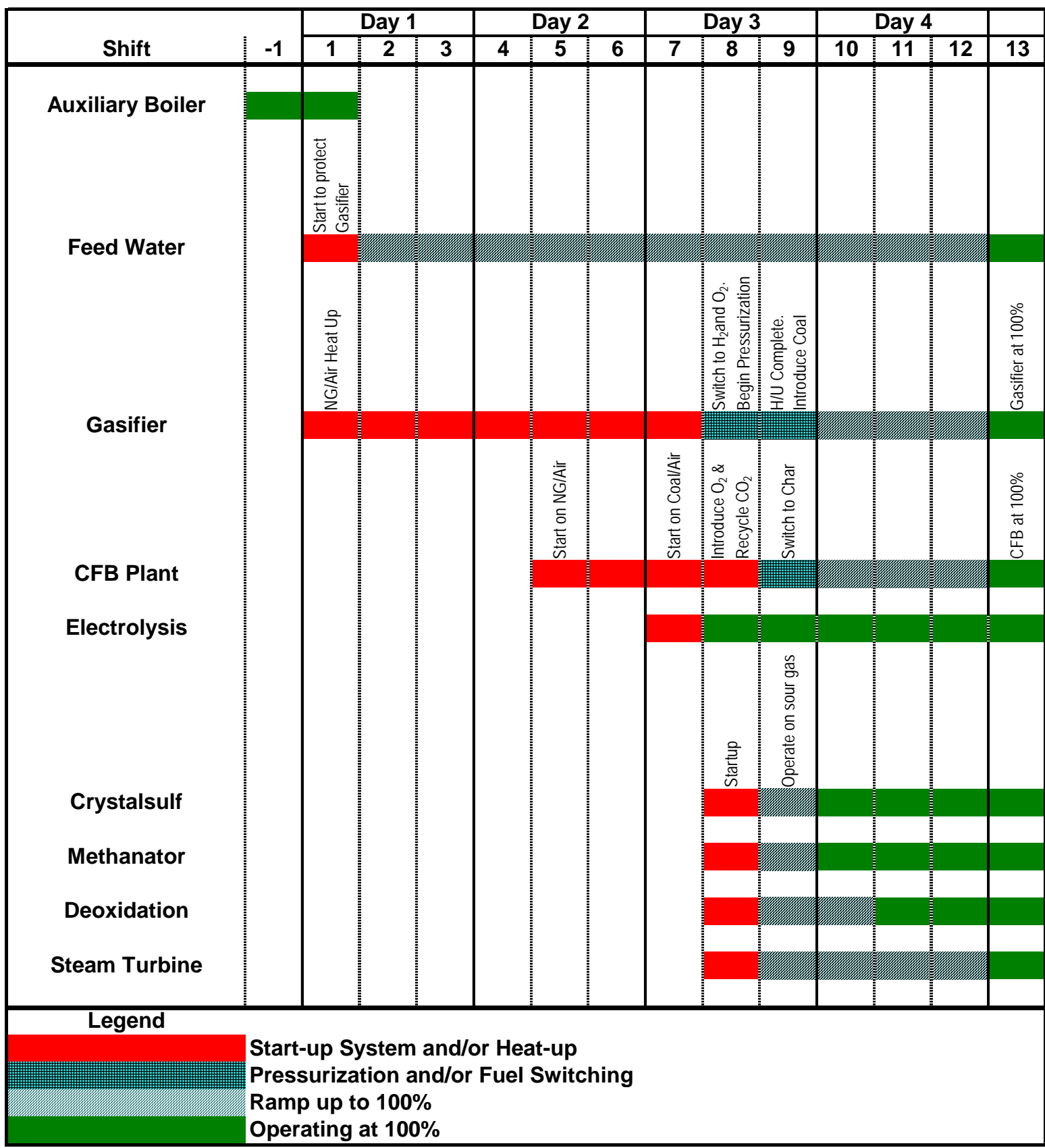

\subsubsection{Start-up Sequence}

The following start-up sequence is based upon a cold start and assumes that all essential services will be available. These services include water, steam, electric power, natural gas, hydrogen and oxygen gases, instrument air and plant service air. 


\section{Gasification Island - Preheat Gasifier}

The sequence begins with a purge of the gasifier and the start-up of the feed water system. The gasifier is purged to ensure that there is not a buildup of combustible or explosive gases in the vessel prior to lighting off the start-up burners. The feed water system is started to ensure heat removal from the radiant raw gas cooler during the hydrogasifier vessel heat-up and to keep the start-up burners cool. The gasifier heat-up will be accomplished using natural gas as a start-up fuel at atmospheric pressure. Combustion gases will leave the gasifier via the raw gas outlet and will be vented via the flare or a start-up stack. The heat-up will require approximately 8 eighthour shifts, or 64 hours ( 2.5days). In lieu of natural gas, the heat-up could also be accomplished via the hydrogen and oxygen gases which are available on site. However, natural gas is more economical and is also readily available on site. In addition, there are many technical challenges to overcome in order to use hydrogen as the start-up fuel. For reference, these challenges are because hydrogen has the following characteristics:

- High reaction rates,

- Highest laminar flame speed of any fuel, due to its reactivity,

- Risk of overheating due to its high laminar flame speed,

- High flame pressure fluctuations, and

- High stoichiometric combustor flame temperature.

Additional considerations for the hydrogen combustion are presented in Section 4.5.3

Heating up of the gasifier with natural gas will require a burner designed for natural gas and an accompanying air handling system ${ }^{\mathrm{j}}$. It is envisioned that the gasifier hydrogen/oxygen burner can be designed to handle natural gas/air combustion for start-up. This may also serve to simplify the design of the gasifier by minimizing the penetrations into the gasifier and number of burners requiring water cooling.

After approximately 7 shifts, or 56 hours of heating, the gasifier will be nearing its desired operating condition and will be nearing its ability to accept and gasify coal. Before the gasifier can accept coal, the syngas cleanup system should be ready to receive the coal syngas, and the gasifier should be brought to pressure. So during the $8^{\text {th }}$ shift, the gasifier start-up burners will be transitioned from natural gas and air, to hydrogen and oxygen, through the gradual introduction of hydrogen and oxygen and via the gradual reduction of natural gas and air. The pressurization of the hydrogasifier vessel will begin by throttling start-up flow control valves in the exhaust gas lines.

At this same time, during the $8^{\text {th }}$ shift, the syngas processing system will be started up so that the gasifier will be able to accept coal and have the syngas cleaned up in the $9^{\text {th }}$ shift. The start-up of these systems is described separately. The introduction of coal will require $\mathrm{CO}_{2}$ for coal transport. The Char combustor will be capable of producing $\mathrm{CO}_{2}$ at the beginning of the $9^{\text {th }}$ shift.

j Although $\mathrm{O}_{2}$ is available for combustion, it is being supplied by the electrolysis unit, so since natural gas is being used instead of $\mathrm{H}_{2}$ gas, no $\mathrm{O}_{2}$ will be available from the electrolysis unit. Thus natural gas / air blown startup heating is envisioned. This startup decision can be re-evaluated in the next phase. 
Approximately 8 hours later, or during the $9^{\text {th }}$ shift since the beginning of the start-up, the hydrogasifier will begin to operate on coal as well as hydrogen and oxygen and start ramping up to $100 \%$ operation. Once the hydrogasifier is operating on coal, the Char Combustor will begin switching from a fuel source of coal and char to $100 \%$ char. The ratio of char to coal will change in direct proportion to the operational level of the hydrogasifier.

\section{CFB Char Combustor Island}

Once the gasifier heat-up has reached the $4^{\text {th }}$ shift, or $32^{\text {nd }}$ hour, the Char Combustor should commence start-up to ensure that sufficient steam and carbon dioxide are available when they are required. The Char Combustor requires approximately 4 shifts or 32 hours to complete its heatup. Prior to the start up of the CFB, the start-up of the auxiliary boiler is required in order to provide steam for deaeration and start-up. This auxiliary boiler can be started up in several hours. Steam of the auxiliary boiler will be sparged in the deaerator and steam turbine condenser to decrease oxygen level in the condensate and feed water.

The CFB is purged to ensure that there is not a buildup of combustible or explosive gasses in the vessel prior to lighting off the start-up burners. The feedwater system is started to ensure heat removal from the CFB heat exchange surfaces during the furnace heat-up. The CFB heat-up will be accomplished using natural gas as a start-up fuel. Combustion gases will be evacuated from the CFB by the Flue Gas Recycle fan, which during the start up will be diverted to discharge in the start-up stack. The purpose of the CFB heat up is to dry out and heat up refractory in the boiler, and increase temperature of inert material in the furnace bed to an ignition temperature of a start-up coal. Once the desired temperature of the inert material is reached, the bed will be fluidized using a start-up fan, and a start-up coal will be introduced. The firing rate of the natural gas start-up burners will be gradually reduced as coal feed and air flow rates are increased. When normal operating temperature of bed inert material is reached, natural gas burners will be turned off, air velocity will be gradually increased, and a circulating movement of solids in the boiler will be established. At this point the CFB is expected to operate at approximately $30 \%$ of its rated capacity. Steam produced by the CFB will be dumped into the steam turbine condenser via steam bypass system.

The CFB transition from air blowing to oxygen blowing will be accomplished by gradually increasing flue gas recirculation into the furnace while injecting oxygen into the flue gas stream, and proportionally reducing start up air fan flow rate. Once complete transition to the oxygen blowing is accomplished, and the CFB is shifted to recycle operation, the system will start providing carbon dioxide for the coal feeding system of the hydrogasifier. During this time char will be gradually introduced in the coal feed stream of the CFB. Once CFB operation is transferred to $100 \%$ char combustion, the CFB will be ramped up to its full rated capacity.

\section{Syngas Cleanup and Processing Island}

After approximately 7 shifts, or 56 hours, many actions will be required beginning with the startup of major supporting equipment including the CrystaSulf, the methanator, and the deoxidizer. These systems are needed to treat the syngas produced by the hydrogasifier. As such, these system need to be operational prior to the addition of coal to the gasifier, unless the air permit would allow operation of the flare during this stage of the start-up. Here we are assuming that 
the plant will be designed to minimize flaring. At the beginning of the $9^{\text {th }}$ shift, the CrystaSulf, methanator, and deoxidizer systems will all be ready to receive the sour gas from the gasifier. The flare may be used during the $9^{\text {th }}$ shift should the gas cleanup system not be fully able to handle any of the gaseous streams that do not meet process or pipeline specifications.

These syngas cleanup systems are relatively straightforward and easy to start-up. The CrystaSulf is a low temperature system. Start-up heaters will be incorporated on the methanator and deoxidizer. All systems will be purged and heated as appropriate prior to start-up.

Upon generation of SNG that is capable of introduction into the NG pipeline, the SNG boost compressor may also need to be started depending upon the pressure requirement of the natural gas pipeline which can vary during the year.

\section{Steam Turbine Generator Island - Star-tup}

The steam turbine is used to produce electricity from steam that is produced from heat generated in other parts of the process. As such, the turbine will be brought up to speed based on the availability of steam. The steam system receives steam input from the Char Combustor, the radiant raw gas cooler, the methanator, and the hydrogenator. In order for the steam turbine to operate at $100 \%$ power all of the systems supplying steam must be operating at that level also. Therefore, the steam turbine ramp up will be directly proportional to the ramp up of the hydrogasifier. The ramp up to full operational capacity will require the monitoring of many different indications, understanding how all of the systems will react to changes in other systems.

\subsection{Operating Procedure Manuals}

This section presents an overview of the Operating Procedures and an example table of contents for the same.

\subsubsection{Operating Procedures Overview}

The operating procedures listed in the following section are generic in nature and are based upon the engineering package developed for this report. The actual number of procedures needed and their specific content will need to be revisited after final design engineering is complete for the commercial-scale facility. The number of procedures would most likely increase once all of the engineering is completed.

There are several important factors that must be considered in the development of effective operating procedures.

1. First, the procedure author(s) must completely understand the process, and all the inputs and outputs of that process. The many different complex systems that make up the facility will be interrelated in many different ways. In order to create effective procedures, the affect of these systems upon each other must be understood. This will allow operation actions to keep the process operating at optimum efficiency.

2. Second, the procedures must reflect the control philosophy developed for the control system and alert the operator to the major actions that the control system will automatically undertake. This will ensure that the control system is operating properly and that the operators understand what is happening in the process at all times. 
3. Last, the procedures must be concise and easy for a trained operator to understand.

In short, the development of final procedures for the process should wait until the engineering is complete and the entire control scheme is envisioned.

\subsubsection{Example Table of Contents}

During the final design phase, the plant operating procedures manual will need to be developed. A possible outline of this document is presented below. First, is the procedures manual for the operation of the integrated plant. Next are procedures for the major plant systems.

\section{Integrated Plant Procedures}

1. Integrated Start-up Procedure

2. Integrated Shutdown Procedure

\section{Hydrogasifier Island Operating Procedures}

1. Start-up Procedure

2. Burner Fuel Switching and Gasifier Pressurization Procedure

3. Gasifier Ramp to Normal Operation

4. Normal Shutdown Procedure

5. Emergency Shutdown Procedure

\section{III.Hydrogasifier Island and CFB Island Auxiliaries Operating Procedures}
1. Start-up Fuel Gas System
2. Air Handling System
3. Coal Handling System
4. Hydrogen and Oxygen Systems
5. Char Handling System
6. Radiant Raw Gas Cooler System
7. Sour Water System
8. Sour Gas System
9. Flare System
10. Carbon Dioxide System

\section{IV.SynGas Island Operating Procedures}
1. SynGas System
2. CrystaSulf System
3. Methanator System
4. Deoxidation System
5. SynGas Compression System

\section{CFB Island Operating Procedures}

1. Start-up Procedure

2. Recycle Operating Procedure

3. Normal Shutdown Procedure 
4. Emergency Shutdown Procedure

\section{Power Island Operating Procedures}

1. Main Steam System

2. Steam Turbine System

3. Auxiliary Steam System

4. Steam Drains System

5. Steam Drum Blowdown System

6. Circulating Water System

7. Auxiliary Cooling Water

8. Feedwater System

9. Condensate System

10. Cycle Makeup System

11. Condenser Air Extraction System

12. Cycle Chemical Feed System

13. Sampling System

14. Circulating Water Chemical Feed System

\section{Plant Auxiliary Systems}

1. Compressed Air System

2. Fire Water System

3. Service Water System

4. Waste Water System

5. Zero Liquid Discharge System

Again, as the design advances, the content and organization of these manuals will likely be revised as the design evolves.

\subsubsection{Introduction to the Operating Manual}

There are three types of procedures presented in the preceding table of contents, which may not be obvious from the list of titles above. The types of procedures include an overall integrated plant procedure, localized island operating procedures, and system operating procedures. These three types are described below.

\subsubsection{Integrated Plant Procedures}

The Integrated Start-up and Shutdown Procedures are the key tools to ensure the smooth operation of the plant through these involved processes. Most operating mistakes occur during these time periods due to the many complex procedures that must be followed at the same time. These procedures combine steps from the individual system procedures to allow the operator to understand the interconnections between the different systems and major pieces of equipment.

\subsubsection{Localized Island Operating Procedures}

The various equipment islands are technically complex pieces of equipment with equally challenging operations. These procedures integrate operations from the many systems and major 
pieces of equipment that comprise these islands. While these procedures do not cover all of the operations of the supporting systems they walk the operator through the overall operation of the islands to prevent operational mistakes by referencing multiple procedures.

\subsubsection{System Operating Procedures}

The equipment islands are comprised of many supporting systems that allow them to operate properly. Each system has its own operating procedure consisting of: a description of the purpose of the system, pre-start requirements, system start-up, normal operation, system shutdown, system valve line-up, and system electrical line-up. These procedures cover all operational situations for the system and are the key operational reference. Portions of these procedures are incorporated into the Integrated Plant Procedures as well as the Localized Island Operating Procedures. 


\section{Cost Estimate}

This section presents information on the cost estimating approach, the cost basis, and the estimates themselves.

\subsection{Cost Estimating Approach}

The approach to capital cost development is a combination of WorleyParsons estimates of selected specific major systems to supplement the costs from an in-house cost model that develops capital costs for the entire hydrogasification plant. The format includes separate evaluation of major systems and sub-systems for the entire plant. These costs are determined with several levels of complexity depending on the specific system being estimated. The capital cost at the level of Total Field Cost (TFC) includes equipment, materials, and installation labor.

The resulting capital cost is provided on an estimate form that recognizes each cost account for the plant. Each account in the estimate contains separate costs for equipment, materials, and installation labor. These costs comprise the TFC, also referred to as the Bare Erected Cost (BEC). The Total Capital Cost consists of equipment and material costs, direct and indirect labor costs, engineering costs, other costs and contingencies for the total hydrogasification plant. The estimate does not include financing costs or any additional costs that would be the responsibility of the plant owner. The WorleyParsons model helps assure that plant estimates are consistently evaluated and that all relevant process scope is included. When more than one case is evaluated, this approach produces costs that indicate normalized and unbiased results that reflect generic differences.

The cost of plant operating and maintenance is included for the hydrogasification plant. These costs were developed on an average annual cost basis. This operating and maintenance cost estimate includes the following:

- Fuel cost

- Fixed Operating Cost

○ Operating labor

- Maintenance labor and material

○ Administrative and support labor

- Variable, non-fuel operating costs

○ Consumables

○ Waste disposal

○ Other operating costs (By-Products)

\subsection{Cost Basis and Assumptions}

The section summarizes the cost basis and assumptions for the capital cost estimate, the plant battery limits for the cost estimate, and basis and assumptions for the operating cost estimate. 


\subsubsection{Capital Costs Basis and Assumptions}

- The capital cost estimate accuracy is $+/-30 \%$ and is based on a combination of recent historical cost data, in-house cost evaluations of similar plant system configurations, and vendor input.

- The estimate includes all anticipated costs for equipment and materials, installation labor, professional services (Engineering, CM, and Start-up), and contingency.

- Escalation to a future period of performance and Owner's costs are excluded.

- The estimates are presented in June 2010 dollars.

- Labor costs are based on a prevailing wages for a union labor force for the Northwest New Mexico region.

- Labor is based on a 50-hour work-week (5-10s). No additional incentives such as per-diems or bonuses offered to attract craft labor are included.

- Construction Overtime: Spot overtime (approximately 3\%)

- The estimate is based upon an EPCM contracting approach.

- While not included at this time, labor incentives may ultimately be required to attract and retain skilled labor depending on the amount of competing work and the availability of skilled craft in the area at the time the projects proceed to construction. The types and amounts of incentives will vary based on project location and timing relative to other work. The impact can be significant; in some cases adding as much as $25 \%$ to the cost of labor.

- Contingency is included in the estimate to account for the uncertainty in equipment design, performance, and cost. The overall contingency in approximately $18.9 \%$. It is derived based on a weighted analysis in which contingency is evaluated individually for each major cost element.

- The estimates exclude all taxes with the exception of payroll taxes.

- Owner's costs, including interest during construction, are excluded.

- The estimate is for a first hydrogasification unit installation. The estimate does not represent near-term mature technology plant, or "nth plant." It does include a contingency allowance for costs associated with a first-of-a-kind plant.

- The site is assumed to be free from above ground or below ground obstructions. No allowance has been made for removal/relocation of interferences.

- The site is assumed to be free from hazardous materials. No allowance has been made for removal/remediation of hazardous materials or soils.

- The site is assumed to be free of archeological artifacts. No allowances have been made for the removal of archeological finds. 
- The site conditions are assumed to be a "brownfield" quality site, with a relatively level site elevation and with all utility connections available at the site fence boundary.

- The estimate boundary limit is defined to include all new or modified systems or structures within the plant fence line. Offsite pipelines, rail facilities, transmission lines, etc. are excluded from the estimate.

- The WorleyParsons historical cost data applied in this evaluation consists of recent project cost data including individual equipment components, bulk material unit pricing, and labor costs. The proportionate breakdown of these costs is derived from actual completion cost data.

- The distribution of quotation data from suppliers supporting advanced technology projects is extremely limited due to market conditions and the preliminary development status of the subject technologies.

- The cost development of the gasification equipment, process unit equipment, and major power block equipment is based on the engineered equipment parameters, capacity data, flow information and process data related to the mass and energy balances.

- A significant amount of process equipment in this cost estimate has been estimated using ASPEN ICARUS. ASPEN ICARUS is used by companies to analyze the potential cost of capital projects, including new plants and revamps for existing facilities. The software is based on the ICARUS evaluation engine that utilizes industry standards, design codes and detailed real-world engineering and construction information. ASPEN ICARUS technology does not rely only on capacity factored curves for equipment pricing or factors to estimate total installed costs from equipment capacities. It follows a unique approach where equipment and the associated bulk material and labor costs are developed from a comprehensive design based installation model. This model is calibrated so that quantity based cost information is based upon actual historical installed project data.

\section{Owners Costs}

Owner's costs are excluded from the estimate. Typical Owner's costs include, but are not limited to, the following:

- Permits \& Licensing ( other than construction permits )

- Land Acquisition / Rights of Way Costs

- Economic Development

- Project Development Costs

- Environmental Impact Costs

- Excessive Noise Abatement

- Local Facilitation Costs

- Improvements to Existing Roads or Infrastructure

- Legal Fees 
- Wetland Mitigation

- Interconnection Agreements

- Fuel Purchase Agreements

- Owner's Engineering / Project \& Construction Management Staff

- Plant Operators during Start-up

- Electricity consumed during Start-up

- Fuel and Reagent consumed during Start-up

- Initial Fuel \& Reagent Inventory ${ }^{\mathrm{k}}$

- Operating Spare Parts

- Mobile Equipment for use during Plant Operations

- Furnishings for new Office, Warehouse and Laboratory

- Financing Costs

- Owner's Contingency

\subsubsection{Battery Limits}

The summary of plant tie-points is presented in Exhibit 7-1.

Exhibit 7-1 Plant Tie-Points

\begin{tabular}{|l|l|l|}
\hline \multicolumn{1}{|c|}{ Tie-Point } & \multicolumn{1}{c|}{ Location } & \multicolumn{1}{c|}{ Properties } \\
\hline Coal & At mine truck coal unloading & Exhibit 2-3 \\
\hline Hydrogen & At site boundary & $1150 \mathrm{psia} / 104^{\circ} \mathrm{F}$, Exhibit 2-4 \\
\hline HP Oxygen & At site boundary & $1120 \mathrm{psia} / 104^{\circ} \mathrm{F}$ \\
\hline LP Oxygen & At site boundary & $50 \mathrm{psia} / 77^{\circ} \mathrm{F}$ \\
\hline $\begin{array}{l}\text { NG (for start- } \\
\text { up) }\end{array}$ & At site boundary & $860 \mathrm{psia} / 50$-120 $\mathrm{F}$, Exhibit 2-7, Exhibit 2-8 \\
\hline SNG (product) & At site boundary & $910 \mathrm{psia} / 104^{\circ} \mathrm{F}$, Exhibit 2-7, Exhibit 2-8 \\
\hline Sulfur & At sulfur unloading station & Elemental, Exhibit 2-9 \\
\hline Ash & Outlet of the ash storage silo & Dry \\
\hline Water & Existing Cooling Lake & Appendix A (DBD), Exhibit 2-13 \\
\hline Waste Water & $\begin{array}{l}\text { At waste water ZLD treatment } \\
\text { facility }\end{array}$ & ZLD sludge \\
\hline Electric Power & $\begin{array}{l}\text { At site connections to utility } \\
\text { switchyard }\end{array}$ & Dead End 230 kV Tower on Site \\
\hline
\end{tabular}

\subsubsection{Operations and Maintenance Cost Basis and Assumptions}

Annual Operating and Maintenance (O\&M) costs are based on and include the following:

- $\mathrm{O} \& \mathrm{M}$ estimate is the average annual cost.

- Evaluation Plant Capacity factor is $80 \%$.

k Although the initial reagent fill is excluded for the TPC, the initial fill can be found in Appendix G. 
- Cost of fuel is included. (\$29.50/ton) [21]

- Average operator labor rate is $\$ 34.65$.

- Operator labor burden is $30 \%$ of base labor, and Overhead charge is $25 \%$ of all plant labor.

- Average annual maintenance material and labor

- Consumables costs are based on the expected full load consumption (resulting costs are adjusted for the stated plant capacity factor) and corresponding unit costs for the following:

○ Water Makeup

○ Water Treatment Chemicals

- Mercury Removal Bed

○ Deoxidation Reactor

- Waste Water Carbon Bed

○ ZnO Guard Beds

- Methanation Reactor

○ CrystaSulf (Solution/Chemicals)

○ TEG(Make-Up)

o Oxygen

○ Hydrogen

○ Waste Disposal

○ Sulfur By-Product Credit

\subsection{Capital and O\&M Cost Results}

The capital cost estimate and operating and maintenance costs are presented below.

\subsubsection{Total Indicative Price}

A summary of the capital cost estimate is shown in Exhibit 7-2. Additional capital cost details are presented in Appendix F. 
Exhibit 7-2 Capital Cost Estimate

\begin{tabular}{|c|c|c|c|c|c|c|c|c|}
\hline \begin{tabular}{|l|} 
Acct \\
No.
\end{tabular} & Item/Description & \begin{tabular}{|c|}
$\begin{array}{c}\text { Equipment } \\
\text { Cost }\end{array}$ \\
\end{tabular} & $\begin{array}{c}\text { Material } \\
\text { Cost }\end{array}$ & $\begin{array}{c}\text { Labor } \\
\text { Cost }\end{array}$ & \begin{tabular}{|c|} 
Bare Erected \\
Cost \$ \\
\end{tabular} & $\begin{array}{l}\text { Eng'g CM } \\
\text { H.O.\& Fee }\end{array}$ & \begin{tabular}{|c|} 
Contingency \\
Project \\
\end{tabular} & $\begin{array}{c}\text { Total Plant } \\
\text { Cost \$ }\end{array}$ \\
\hline 1 & Coal Handling \& Preparation & $\$ 27,273$ & $\$ 6,787$ & $\$ 13,071$ & $\$ 47,131$ & $\$ 4,165$ & $\$ 11,264$ & $\$ 62,559$ \\
\hline 2 & Gasifier \& Accessories & $\$ 42,659$ & $\$ 7,308$ & $\$ 23,555$ & $\$ 73,521$ & $\$ 7,131$ & $\$ 30,321$ & $\$ 110,973$ \\
\hline 3 & ASU \& Hydrogen Plant Plant & $\$ 0$ & $\$ 0$ & $\$ 0$ & $\$ 0$ & $\$ 0$ & $\$ 0$ & $\$ 0$ \\
\hline 4 & Raw Syngas Particulate Removal \& Cooling & $\$ 47,071$ & $\$ 562$ & $\$ 9,372$ & $\$ 57,004$ & $\$ 5,493$ & $\$ 12,499$ & $\$ 74,996$ \\
\hline 5 & Acid Gas Removal / Sulfur Recovery & $\$ 9,347$ & $\$ 5,341$ & $\$ 8,007$ & $\$ 22,695$ & $\$ 1,788$ & $\$ 6,121$ & $\$ 30,603$ \\
\hline 7 & Other Gas Processes & $\$ 69,919$ & $\$ 19,444$ & $\$ 42,103$ & $\$ 131,467$ & $\$ 12,858$ & $\$ 28,865$ & $\$ 173,190$ \\
\hline 10 & Char Combustor, Ducting \& Stack & $\$ 53,246$ & $\$ 2,557$ & $\$ 5,122$ & $\$ 60,926$ & $\$ 4,201$ & $\$ 22,565$ & $\$ 87,692$ \\
\hline 11 & Steam Turbone Generator & $\$ 73,538$ & $\$ 1,103$ & $\$ 15,035$ & $\$ 89,676$ & $\$ 7,701$ & $\$ 12,275$ & $\$ 109,652$ \\
\hline 12 & Feedwater \& Misc BOP Systems & $\$ 28,309$ & $\$ 8,179$ & $\$ 11,956$ & $\$ 48,444$ & $\$ 3,932$ & $\$ 13,572$ & $\$ 65,948$ \\
\hline 13 & Cooling Water System & $\$ 12,719$ & $\$ 8,097$ & $\$ 10,167$ & $\$ 30,982$ & $\$ 2,797$ & $\$ 6,147$ & $\$ 39,926$ \\
\hline 14 & Ash Handling System & $\$ 4,028$ & $\$ 2,301$ & $\$ 1,780$ & $\$ 8,110$ & $\$ 737$ & $\$ 1,354$ & $\$ 10,201$ \\
\hline 15 & Accessory Electric Plant & $\$ 11,414$ & $\$ 6,827$ & $\$ 11,989$ & $\$ 30,229$ & $\$ 2,620$ & $\$ 5,608$ & $\$ 38,457$ \\
\hline 16 & Insturmentation \& Control & $\$ 5,540$ & $\$ 1,427$ & $\$ 4,146$ & $\$ 11,113$ & $\$ 996$ & $\$ 1,737$ & $\$ 13,845$ \\
\hline 17 & Improvement to Site & $\$ 2,483$ & $\$ 17,448$ & $\$ 10,249$ & $\$ 30,180$ & $\$ 2,888$ & $\$ 9,920$ & $\$ 42,988$ \\
\hline 18 & Buildings \& Structures & $\$ 0$ & $\$ 9,816$ & $\$ 9,195$ & $\$ 19,011$ & $\$ 1,686$ & $\$ 5,174$ & $\$ 25,871$ \\
\hline & TOTAL COST & $\$ 387,544$ & $\$ 97,196$ & $\$ 175,747$ & $\$ 660,487$ & $\$ 58,992$ & $\$ 167,423$ & $\$ 886,902$ \\
\hline
\end{tabular}

\subsubsection{Operating and Maintenance (O\&M) Costs}

The estimated annual operating and maintenance costs are summarized in Exhibit 7-3. Additional O\&M cost details are presented in Appendix G.

Exhibit 7-3 O\&M Costs

\begin{tabular}{|l|c|}
\hline \multicolumn{1}{|c|}{ Parameter } & Value \\
\hline O \& M Costs (in Jun 2011 USD) (Note & \\
\hline A) & \\
Fixed Operating Cost & $\$ 12,535,000 . /$ year \\
Routine Maintenance Material & $\$ 7,347,000 . /$ year \\
Water & $\$ 1,358,000 . /$ year \\
\hline Chemicals & $\$ 9,930,000 . /$ year \\
Fuel (@ \$29.5/ton) & $\$ 26,919,000 . /$ year \\
Hydrogen (@ \$3.50/kg, or $\$ 1.59 / / b)$ & $\$ 873,184,000 . /$ year \\
Oxygen (note B) & $\$ 0 . /$ year \\
Ash Disposal (@ $\$ 19.37 /$ ton) & $\$ 5,279,000 . /$ year \\
Hg Sorbent Disposal (@\$500/ton) & $\$ 5,000 . /$ year \\
Sulfur ByProduct Credit (@ $\$ 30 /$ ton) & $(\$ 180,000$.$) / year$ \\
\hline
\end{tabular}

Notes:

A. O\&M costs reflect an $80 \%$ capacity factor.

B. The $\mathrm{O}_{2}$ cost is $\$ 0 /$ ton as it is included in the $\mathrm{H}_{2}$ electrolysis cost. 


\subsection{Cost of Product Estimate Basis}

The economic performance has been assessed using the NETL Power Systems Financial Model. The Power Systems Financial Model is a discounted cash flow based financial model developed by NETL that is used to evaluate long-term costs and investment criteria for advanced energy systems. The model incorporates detailed accounting of the financing structure, interest during construction, depreciation, senior and subordinated debt, debt payments, and escalation of feedstock, O\&M, and product prices, among many other financial and engineering parameters. The model can also be utilized to compute a cost of product (COP). For the current SNG project, the COP of SNG is a key measure of the economic feasibility of the project. The calculated COP is the price at which the product SNG must be sold in order to offset:

1. Project capital costs

2. Project operating costs,

3. Debt service, and to

4. Provide the expected rate of return to its equity investors.

The COP for the SNG will be calculated by first calculating the COP for hydrogen generated from an electrolysis project. Both COP analyses will be based on the economic assumptions recommended by NETL in 2008 [22] and utilized by a commonly cited NETL reference report from November 2010 [23]. These economic assumptions are presented in Exhibit 7-4.

Exhibit 7-4 Cost of Product Economic Assumptions

\begin{tabular}{|c|c|c|}
\hline Parameter & Value & Ref. \\
\hline \multicolumn{3}{|l|}{ TAXES } \\
\hline Income Tax Rate, Effective & $38 \%$ (34\% Federal, 6\% State) & [23] \\
\hline Capital Depreciation & 20 years, declining balance & [23] \\
\hline Investment Tax Credit & $0 \%$ & {$[22,23]$} \\
\hline Tax Holiday & 0 years & {$[22,23]$} \\
\hline \multicolumn{3}{|l|}{ CONTRACTING AND FINANCING TERMS } \\
\hline Contracting Strategy & $\begin{array}{l}\text { Engineering Procurement Construction } \\
\text { Management (owner assumes project risks } \\
\text { for performance, schedule and cost) }\end{array}$ & [23] \\
\hline Type of Debt Financing & $\begin{array}{l}\text { Non-Recourse (collateral that secures debt } \\
\text { is limited to the real assets of the project) }\end{array}$ & {$[22,23]$} \\
\hline Repayment Term of Debt & 15 years & {$[22,23]$} \\
\hline Grace Period on Debt Repayment & 0 years & {$[22,23]$} \\
\hline Debt Reserve Fund & None & [23] \\
\hline \multicolumn{3}{|l|}{ ANALYSIS TIME PERIODS } \\
\hline Capital Expenditure Period & 5 Years & [23] \\
\hline Operational Period & 30 years & {$[22,23]$} \\
\hline Economic Analysis Period (used for IRROE) & $\begin{array}{l}35 \text { Years (capital expenditure period plus } \\
\text { operational period) }\end{array}$ & [23] \\
\hline \multicolumn{3}{|l|}{ TREATMENT OF CAPITAL COSTS } \\
\hline $\begin{array}{l}\text { Capital Cost Escalation During Capital } \\
\text { Expenditure Period (nominal annual rate) }\end{array}$ & $2.0 \%$ & $\begin{array}{l}\text { page } \\
87\end{array}$ \\
\hline
\end{tabular}




\begin{tabular}{|l|l|l|}
\hline \multicolumn{1}{|c|}{ Parameter } & \multicolumn{1}{|c|}{ Value } & Ref. \\
\hline $\begin{array}{l}\text { Distribution of Total Overnight Capital over the } \\
\text { Capital Expenditure Period (before escalation) }\end{array}$ & 5-Year Period: 10\%, 30\%, 25\%, 20\%, 15\% & {$[23]$} \\
\hline Working Capital & zero for all parameters & {$[23]$} \\
\hline $\begin{array}{l}\text { \% of Total Overnight Capital that is } \\
\text { Depreciated }\end{array}$ & $\begin{array}{l}100 \% \text { (this assumption introduces a very } \\
\text { small error even if a substantial amount of } \\
\text { TOC is actually non-depreciable) }\end{array}$ & {$[23]$} \\
\hline ESCALATION OF OPERATING REVENUES AND COSTS & \\
\hline $\begin{array}{l}\text { Escalation of COE (revenue), O\&M Costs, and } \\
\text { Fuel Costs (nominal annual rate) }\end{array}$ & $2.0 \%$ & page \\
\hline
\end{tabular}

Project financing ${ }^{1}$ will be the financing structure utilized for the SNG project.

The COP will be calculated using financial parameters as shown inExhibit 7-5, which are based on assumed high technology and commodity risk for the project. (Source: Table 7-5 "Financial Structure for High-Risk Fuels Projects” [22]). The NETL reference defines advanced technology projects and or fuels projects as high risk. Thus the recommended cost of debt and equity are higher than electric generation projects cited within the reference document.

The cost of debt for the high-risk fuel project has been set as the LIBOR rate plus 6\% [22]. Since the 1 year LIBOR rate has been between $1.2 \%$ and $0.8 \%$ since November 2009 and February 2011, (the present) respectively, this analysis will utilize a nominal 1 year LIBOR of $1.0 \%$ [24]. Thus the cost of debt will be set as $7.0 \%$.

\section{Exhibit 7-5 \\ Project Financial Structure}

\begin{tabular}{|c|c|c|c|}
\hline Type of Security & \% of Total & $\begin{array}{c}\text { Current (Nominal) } \\
\text { Dollar Cost }\end{array}$ & $\begin{array}{c}\text { Weighted Current } \\
\text { Cost of Capital }\end{array}$ \\
\hline Debt & $50 \%$ & $\begin{array}{c}7.0 \% \\
\text { (LIBOR }+6 \%)\end{array}$ & 3.5 \\
\hline Equity & $50 \%$ & $20 \%$ & 10 \\
\hline Weighted Average & $100 \%$ & & $13.5 \%$ \\
\hline
\end{tabular}

In addition, the following financial parameters will be applied:

- Credits or debits for $\mathrm{CO}_{2}$ emissions are not accounted

\footnotetext{
l “Project financing," also known as "non-recourse financing” is in contrast to corporate financing. Nonrecourse debt is secured by a pledge of collateral, which typically will be the real assets of the project. The liability is limited to the collateral of these assets. Project financing is technically defined as the financing of long-term infrastructure or industrial projects based on non-recourse financing, and where debt and equity are paid back from the cashflow generated by the project. Project financing is typically accounted for off the balance sheet, while corporate finance will be on balance sheet, and the corporation would hold a general liability for the amount of the loan. [22]
} 
- All costs (e.g., fuel costs, O\&M costs) and product price are assumed to escalate at an annual nominal rate of $2 \%$. Capital costs are assumed to escalate at the same rate during the construction period. The escalation rate is based on the GDP Chain-type Price Index reported in Table 1 of the EIA's Annual Energy Outlook 2011 (Early Release Overview), which was projected to grow at an annual nominal rate of 1.8\% between 2009 and 2035. [25] 


\subsubsection{Hydrogen Cost / Electrolysis Basis}

One of the most significant cost contributors to the cost of the SNG (cost of product) is the cost of hydrogen. As such, defining the basis of the hydrogen cost is an important input to the cost of SNG product analysis.

The electrolysis unit will be excluded from the SNG plant engineering, performance and cost. The electrolysis unit cost will only be considered in this analysis to the extent that it is an input to the Hydrogen cost.

\subsubsection{Electrolysis}

APS has engaged several electrolysis vendors over the life of the project. Communication with Hydrogen Technologies, (formerly Norsk Hydro Electrolysers AS) was particular useful as they helped APS understand how the electrolysis technology might develop for such a high volume application. The electrolysis product requirements provided to Hydrogen Tehcnologies is presented in Exhibit 7-6.

\section{Exhibit 7-6 Electrolysis Products}

\begin{tabular}{|l|l|l|}
\hline Product & Parameter & Notes \\
\hline High Pressure Hydrogen & $\begin{array}{l}\text { Purity }=99.99 \%, \\
\mathrm{P}=1150 \mathrm{psig} \\
\mathrm{T}=104^{\circ} \mathrm{F}\left(40^{\circ} \mathrm{C}\right)\end{array}$ & $\begin{array}{l}\text { Requires De-oxygenation to reach specified purity } \\
\text { The product pressure is based on a gasifier operating pressure of } 1000 \\
\text { psig, } 100 \text { psid for flow control/ injection, and } 50 \text { psid for heating. }\end{array}$ \\
\hline \multirow{3}{*}{ High Pressure Oxygen } & $\begin{array}{l}\text { Purity }=99.5 \%, \\
\mathrm{P}=1120 \mathrm{psig} \\
\mathrm{T}=104^{\circ} \mathrm{F}\left(40^{\circ} \mathrm{C}\right)\end{array}$ & $\begin{array}{l}\text { The product pressure is based on a gasifier operating pressure of } 1000 \\
\text { psig, } 100 \text { psid for flow control/ injection, and } 20 \text { psid for heating. }\end{array}$ \\
\hline \multirow{2}{*}{ Low Pressure Oxygen } & $\begin{array}{l}\mathrm{Purity}=99.5 \%, \\
\mathrm{P}=50 \mathrm{psig}\end{array}$ & For oxy-combustion. \\
\hline
\end{tabular}

Reference: [26] 
The cost of Hydrogen will be developed from the parameters based in Exhibit 7-7.

\section{Exhibit 7-7 Electrolysis Design \& Cost Basis}

\begin{tabular}{|c|c|c|}
\hline Product & Parameter & Notes \\
\hline Energy Consumption of Plant & $\begin{array}{l}5.5 \mathrm{kWh} / \mathrm{Nm} 3 \text { of } \mathrm{H}_{2} \text {, [equivalent to: } \\
66 \mathrm{MWh} / \text { day @ 100\% capacity factor } \\
\text { for a single } 500 \mathrm{Nm} 3 / \mathrm{h} \text { unit.] }\end{array}$ & $\begin{array}{l}\text { Equivalent to } 62 \mathrm{kWh} / \mathrm{kg} \mathrm{H} 2 \text {, or } 63.7 \% \mathrm{HHV} \text {. } \\
\text { This consumption accounts for the product delivery } \\
\text { pressure and purity). Ref [27] }\end{array}$ \\
\hline Electrolysis Technology & 500 Nm3/h Atmospheric Alkaline Unit & \\
\hline $\begin{array}{l}\text { Cost of Electrolysis Plant } \\
\text { (for } 500 \mathrm{Nm} 3 / \mathrm{h} \text { capacity) }\end{array}$ & $\begin{array}{l}\text { 1.35M USD (2010) at } 70 \% \text { reduction } \\
\text { Basis [27]: } \\
4.5 \mathrm{M} \text { USD ( } 2010 \text { base }- \text { single unit) } \\
\text { High volume cost reduction of } 60-70 \%\end{array}$ & $\begin{array}{l}\text { This cost is for the plant, not just the electrolysis unit. } \\
\text { The cost covers the deoxo unit for oxygen removal } \\
\text { from the } \mathrm{H} 2 \text { and product pressurization. } \\
\text { High volume refers to several hundred units. }\end{array}$ \\
\hline $\begin{array}{l}\text { Cost of Electrolysis Plant } \\
\text { (for } 500 \mathrm{Nm} 3 / \mathrm{h} \text { capacity), } \\
\text { Installed }\end{array}$ & 2.565 USD (2010), Installed & $\begin{array}{l}\text { The installed cost will be developed from the above } \\
\text { equipment only cost through the utilization of a } 1.9 \\
\text { factor, and the equipment cost of } \$ 1.35 \mathrm{M} \text {. }\end{array}$ \\
\hline Basis of the Electrolysis Plant & $\begin{array}{l}\text { Electrolysis Unit } \\
\text { Water Purification (filtration, RO) } \\
\text { KOH storage and Mixing Tank } \\
\text { Stepdown Transformers \& Rectifiers } \\
\text { Product Purification, drying, and } \\
\text { compression }\end{array}$ & $\begin{array}{l}\text { Excluded from the cost scope from STATOIL are: } \\
\text { Installation } \\
\text { Utilities (cooling water, air) }\end{array}$ \\
\hline Operational Cost & $3 \%$ of the investment cost per year & $\begin{array}{l}\text { According to Norsk Hydro, now Hydrogen } \\
\text { Technologies, this would cover the major overhauls } \\
\text { that typically take place every } 7^{\text {th }} \text { year. [28] }\end{array}$ \\
\hline Electrolyzer cell replacement & $\begin{array}{l}\text { Every } 7 \text { years. } \\
\text { Cost }=35 \% \text { of original cost }\end{array}$ & $\begin{array}{l}\text { per NREL Reference [29]. This is redundant of the } \\
\text { operational cost above. The above will be used. }\end{array}$ \\
\hline Full Time Employee & 1 FTE per 8 units (@ 500 Nm3/h) & $\begin{array}{l}\text { Per Ref } 29 \text {, vendors quoted } 5-10 \text { for } 50,000 \mathrm{~kg} / \mathrm{d} \text {, } \\
\text { which is equivalent to } 45(500 \mathrm{Nm} 3 / \mathrm{h}) \text { units. This is } 1 \\
\text { FTE per } 4.5 \text { to } 9 \text { units. }\end{array}$ \\
\hline
\end{tabular}

References: [26, 27, 28, 29]

\subsubsection{Electric Power Supply}

Hydrogen will be produced on an as needed basis utilizing the electric rate at the time of production. APS has indicated that the projected electric prices for the Four Corners plant are as presented in Exhibit 7-8 and Exhibit 7-9. Since the electric demand and rates are the highest in July, the scheduled outage will be scheduled for the month of July. Thus, the annual average electric rate, excluding July, of \$33.26/MWh will be utilized in the development of the Hydrogen cost. The electric rate of $\$ 33.26 / \mathrm{MWh}$ reflects the composite rate of the on- and off-peak rates for 11 months of the year. 
Exhibit 7-8 Peak \& Off-Peak Electric Rates

\begin{tabular}{|c|r|r|r|}
\hline & \multicolumn{3}{|c|}{ Four Corners Power Cost } \\
\hline Future Month & $\begin{array}{r}\text { On-Peak } \\
\text { (\$/MWh) }\end{array}$ & $\begin{array}{c}\text { Off-Peak } \\
\text { (\$/MWh) }\end{array}$ & $\begin{array}{c}\text { Diff } \\
\text { (\$/MWh) }\end{array}$ \\
\hline $9 / 1 / 2010$ & $\$ 35.09$ & $\$ 24.34$ & $\$ 10.75$ \\
\hline $10 / 1 / 2010$ & $\$ 32.65$ & $\$ 23.00$ & $\$ 9.65$ \\
\hline $11 / 1 / 2010$ & $\$ 33.00$ & $\$ 25.75$ & $\$ 7.25$ \\
\hline $12 / 1 / 2010$ & $\$ 36.65$ & $\$ 27.25$ & $\$ 9.40$ \\
\hline $1 / 1 / 2011$ & $\$ 39.34$ & $\$ 28.76$ & $\$ 10.58$ \\
\hline $2 / 1 / 2011$ & $\$ 38.15$ & $\$ 27.86$ & $\$ 10.30$ \\
\hline $3 / 1 / 2011$ & $\$ 36.51$ & $\$ 26.64$ & $\$ 9.87$ \\
\hline $4 / 1 / 2011$ & $\$ 34.12$ & $\$ 20.40$ & $\$ 13.72$ \\
\hline $5 / 1 / 2011$ & $\$ 36.89$ & $\$ 22.09$ & $\$ 14.80$ \\
\hline $6 / 1 / 2011$ & $\$ 46.49$ & $\$ 28.76$ & $\$ 17.72$ \\
\hline $7 / 1 / 2011$ & $\$ 54.38$ & $\$ 35.34$ & $\$ 19.04$ \\
\hline $8 / 1 / 2011$ & $\$ 53.67$ & $\$ 35.30$ & $\$ 18.37$ \\
\hline & & & \\
\hline & $\$ 39.74$ & $\$ 27.12$ & \\
\hline Average (12 Months) & $\$ 38.41$ & $\$ 26.38$ & \\
\hline Average (minus July) & & & \\
\hline & 96.0 & 72.0 & \\
\hline Hours Per Week & $\$ 33.26$ & & \\
\hline Ave (minus Jul) Composite & & & \\
\hline Note: & & & \\
\hline & & & \\
\hline
\end{tabular}
1. Future prices as of $8 / 19 / 10$
2. Off-peak hours are 8 hours (10pm-6 am) M-Sat, \& all day Sunday.
Reference: [30]

\section{Exhibit 7-9 Peak \& Off-Peak Electric Rate Chart}

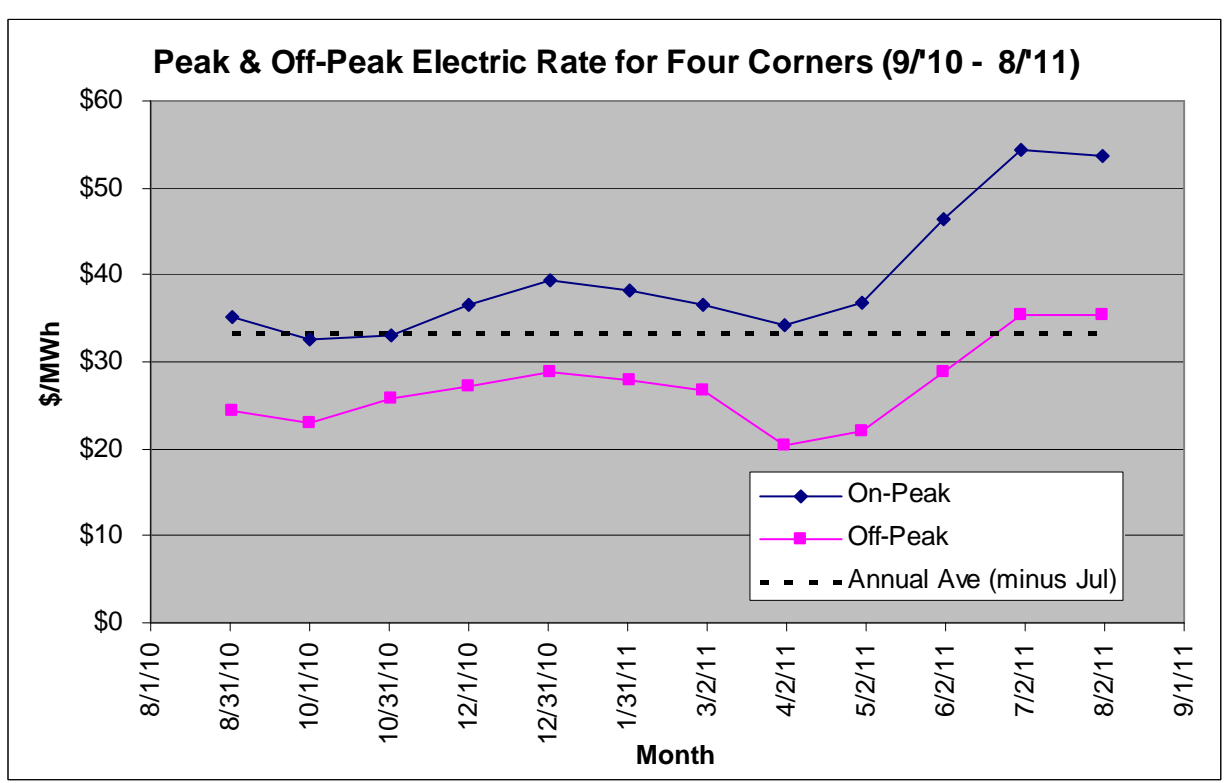




\subsubsection{Product Storage}

Since the electrolysis electric power supply will not be limited to off-peak power, bulk storage of the product gases will not be required. Electrolysis units are able to start up quickly, turn down quickly and follow the instantaneous demand requirement of the gasification plant. As such, no product storage will be considered as part of the electrolysis plant.

\subsubsection{Oxygen Cost I Electrolysis Basis}

Typically oxygen for gasification is supplied from a dedicated ASU and is quite expensive. However, since the oxygen will be co-generated by the electrolysis process and will be generated in excess compared to the hydrogen, and since the underlying cost of electrolysis will be covered by the hydrogen cost, the cost of oxygen will be free at the plant boundary.

\subsection{Cost of Product (Cost of Hydrogen, Cost of SNG)}

An economic assessment was performed to determine the first year production cost of SNG using the NETL Power Systems Financial Model Version 6.1. Using the first year production cost allows for the simple comparison of the assessment results to the current natural gas market.

The resulting first year cost of product for hydrogen is $\$ 3.49 / \mathrm{kg}$ (Jun 2010 USD). For reference, a September 2009 NETL study, based on different assumptions predicted a cost of hydrogen of $\$ 3.00 / \mathrm{kg}^{\mathrm{m}}$. A breakdown of the levelized ${ }^{\mathrm{n}}$ cost of product is shown in Exhibit 7-10. This Exhibit clearly illustrates that electricity costs dominate the cost of $\mathrm{H}_{2}$ production. To understand the influence of the variation of the electricity cost on the cost of $\mathrm{H}_{2}$ production, a sensitivity study was performed with a $\pm 20 \%$ variation in electricity costs. The results in Exhibit 7-11 illustrate that this variation in electricity cost results in a $\pm 12 \%$ variation in the cost of $\mathrm{H}_{2}$ production.

\footnotetext{
m Excerpts of the 2009 NETL study, along with other public domain information regarding the generation of hydrogen via electrolysis is presented in Appendix $H$ of this report.

n The levelized cost of product (COP), will by definition, be different than the first year COP. The levelized COP is calculated from the discounted SNG revenue stream per MMBtu of SNG required to cover the discounted expenditures assuming that the COP is escalated at a nominal annual rate of $0 \%$. That is, this COP is level or contstant over the 30 year operating life. In contrast, the analysis utilized to develop the first year COP accounts for an non-zero escalation of the SNG COP. In both cases the NPV of the revenue from the SNG will cover the NPV of the expenditures. The first year COP allow for a comparison to today's cost of natural gas. The levelized COP allows for the presentation of the cost components.
} 


\section{Exhibit 7-10 Breakdown of Costs for Levelized Cost of Hydrogen Production}

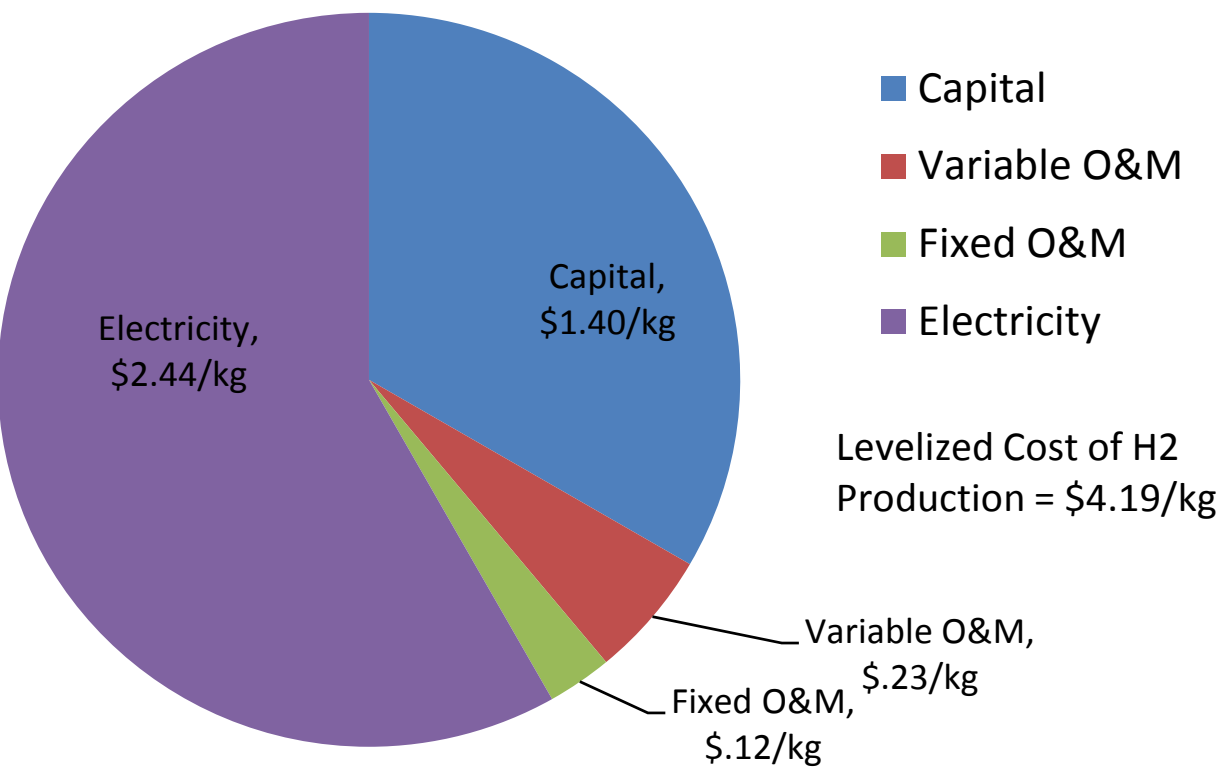

Exhibit 7-11 Sensitivity of First Year Cost of Hydrogen Production for Variation in Electricity Costs.

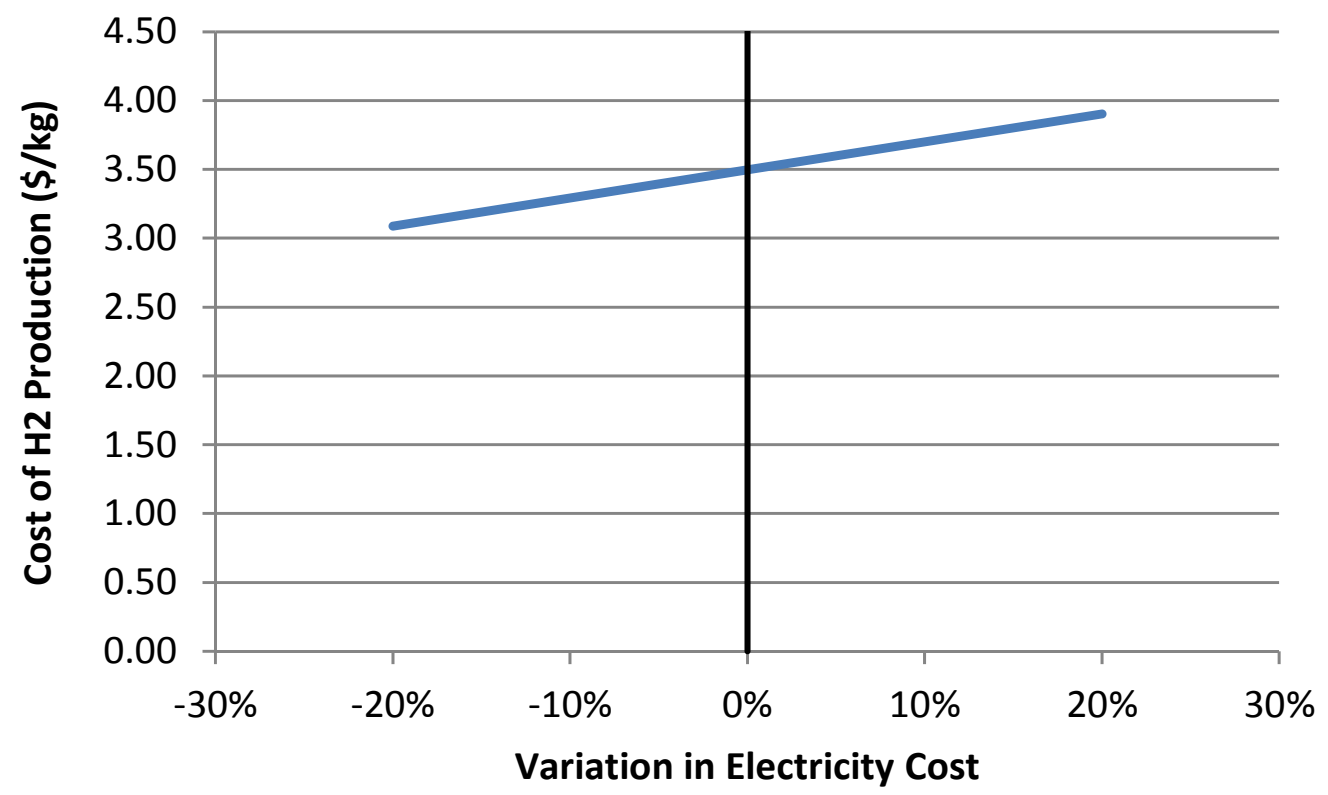

Based on the parameters defined in the above Exhibits and the hydrogen production cost of $\$ 3.49 / \mathrm{kg}$, the resulting first year production cost for SNG is $\$ 30.15 / \mathrm{MMBtu}$. This is significantly above the current price of natural gas of about $\$ 4$ to 5/MMBtu [31] as shown in 
Exhibit 7-14. To further understand the high SNG cost associated with this process, a breakdown of the process costs and revenues is provided in Exhibit 7-12. This exhibit clearly illustrates that the hydrogen cost to the process leads to the high SNG production cost. Therefore, to reduce the SNG production cost to a more competitive level, the hydrogen costs to the process need to be reduced through either greatly decreasing the amount of hydrogen required by the process and/or finding a lower costs method for producing hydrogen.

\section{Exhibit 7-12 Breakdown of Costs and Revenue of Hydrogasification Process with Resulting SNG Production Cost.}

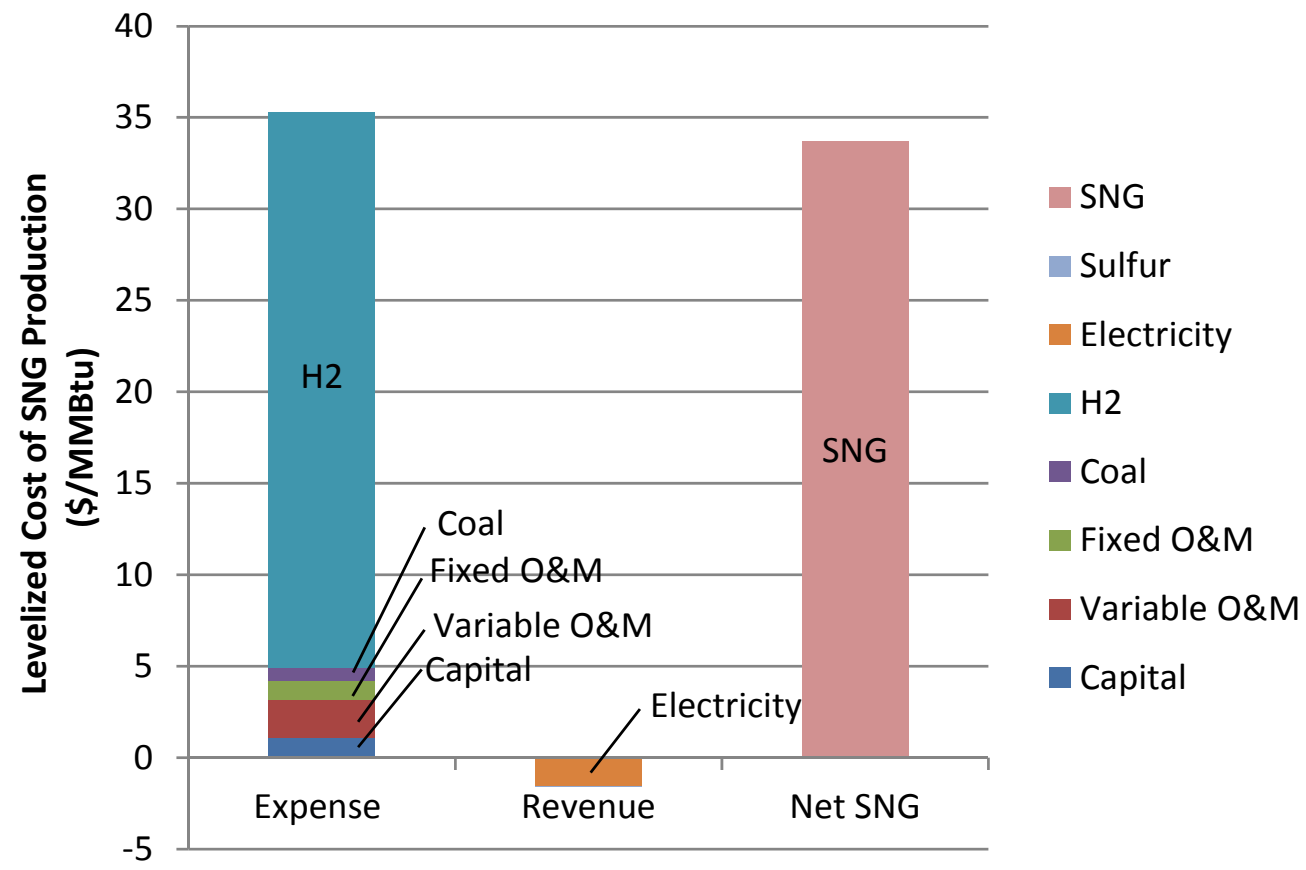

The sensitivity of the cost of SNG production to $\mathrm{H}_{2}$ cost, electricity costs, coal costs, and capital costs is illustrated in Exhibit 7-13. For the $\mathrm{H}_{2}$ cost sensitivity study, the cost of $\mathrm{H}_{2}$ was assumed to vary independently of the other costs. For the electricity sensitivity study, the impact of the electricity cost on the revenue from electricity sales and the production cost of $\mathrm{H}_{2}$ was taken into account. The results of this sensitivity study show that the variation in $\mathrm{H}_{2}$ and electricity costs have the greatest impact on the SNG production costs, while variations in the coal and capital costs have little impact. In Exhibit 7-13, it is important to note that the 12\% SNG variation resulting for the $20 \%$ variation in electricity costs is driven by large change in hydrogen cost, and not the electric revenue. The cost of SNG variation that results from a $20 \%$ variation in $\mathrm{H}_{2}$, electricity, coal and capital costs are $17.4 \%, 11.8 \%, 0.4 \%$ and $2.4 \%$ respectively. 


\section{Exhibit 7-13 Sensitivity of First Year Cost of SNG Production} for Variation in Listed Cost Categories.

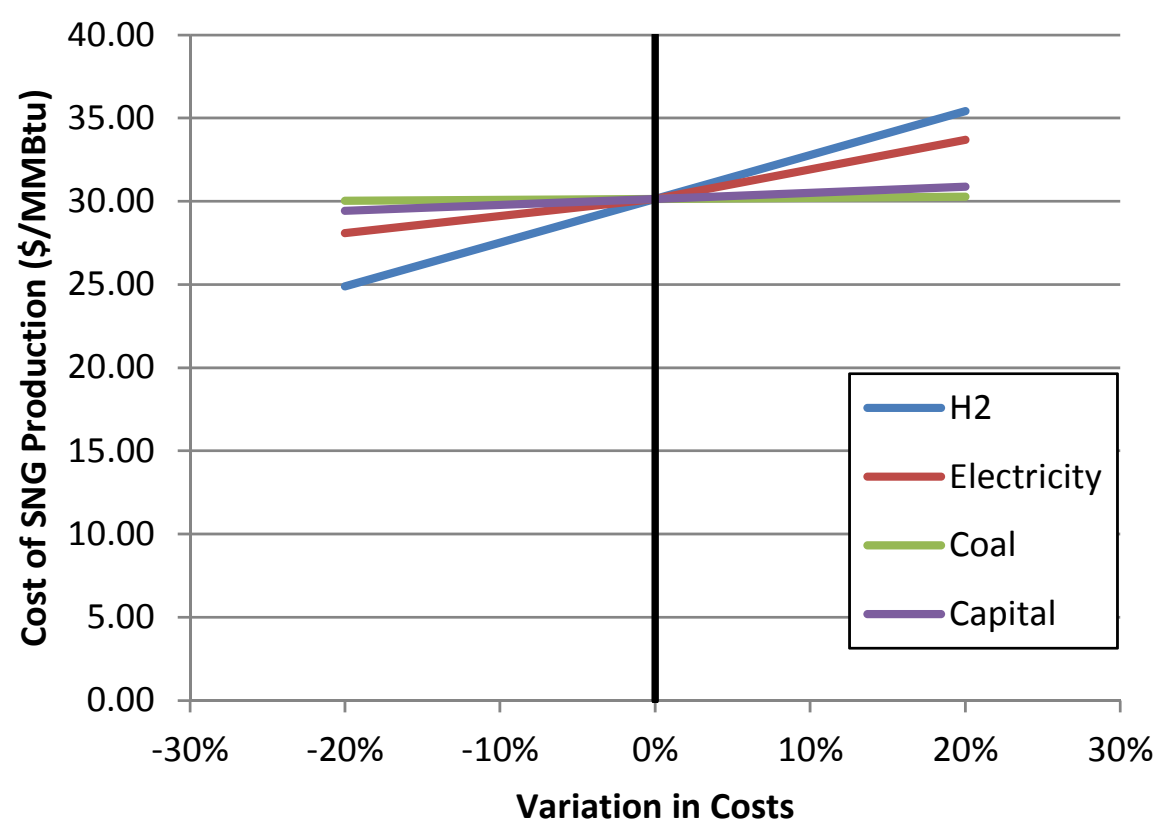

For reference, the NYMEX Henry Hub natural gas price for the past year is presented in Exhibit 7-14.

Exhibit 7-14: NYMEX Henry-Hub Natural Gas Price - Past 12 Months

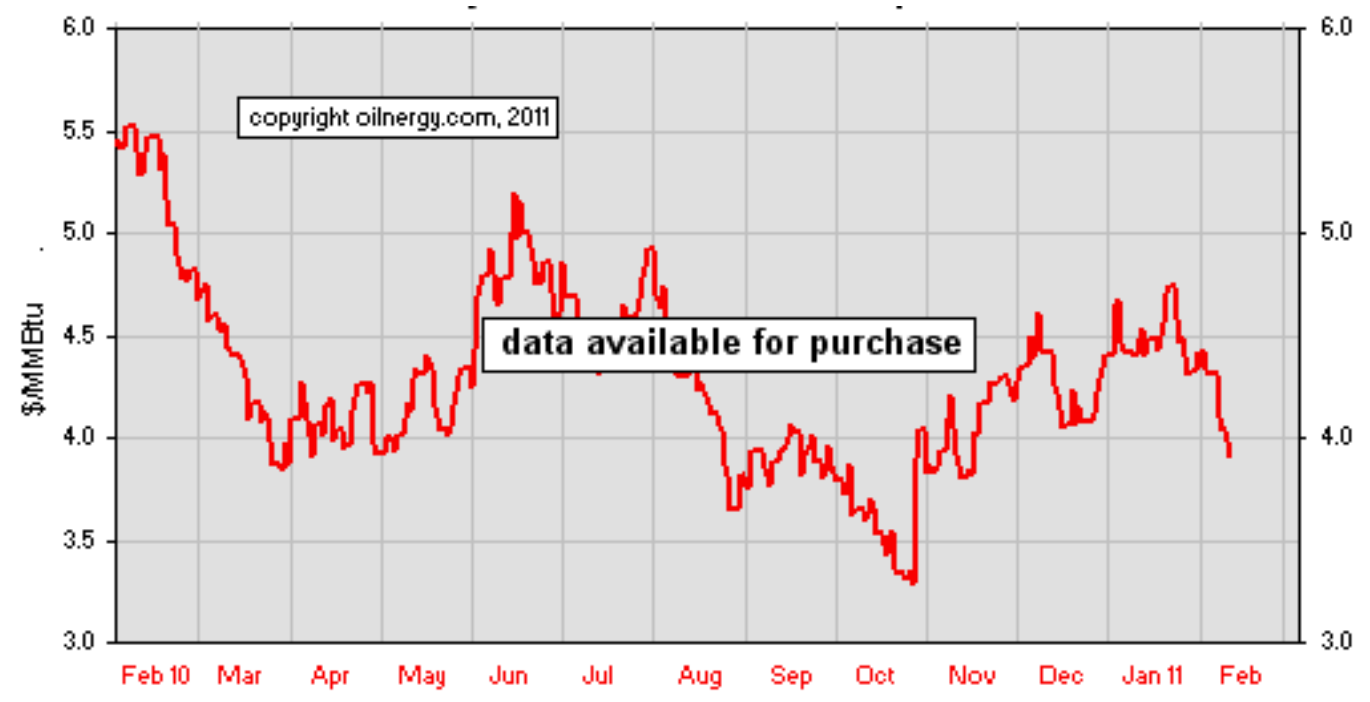

Reference: [31], courtesy of www.oilnergy.com. 


\section{References}

1 Design Criteria Telecon with APS, 11/06/06

2 Cliff Keeler, ConocoPhillips, "Substitute Natural Gas (SNG), Scrubbing the Carbon in Coal and Petcoke,” October 2, 2006, Gasification Technology conference, Washington DC, as found on http://www.gasification.org/Docs/2006_Papers/21CKEEL.pdf, as of November 1, 2006.

3 Email from Xiaolei “Sally” Sun (APS) to David Stauffer (WorleyParsons), RE: Conference call notes regarding the DBD, August 13, 2010.

4 “APS Advanced Hydrogasification / SNG Preliminary Engineering Package (PEP)” Scope of Work document transmitted by email from Juanita Rodriguez (APS) to David Stauffer (WorleyParsons) dated June 23, 2010.

5 Gasifier Performance Input as attached to email from Juanita Rodriguez (APS) to David Stauffer (WorleyParsons) dated June 23, 2010.

6 Email from Xiaolei "Sally" Sun (APS) to David Stauffer (WorleyParsons), RE: APS data input for WP phase II modeling, March 9, 2010.

7 "Preliminary Engineering Package for the Hydrogasification/ Substitute Natural Gas Commercial Scale Facility, Conceptual Design,” Prepared by WorleyParsons, for APS, dated March 22, 2007, Rev D.

8 Email from Anders Johnson, El Paso Gas, to Sally Sun, APS, dated September 8, 2006.

9 E-mail from Anders T. Johnson, Director, Gas Control and Facility Planning, El Paso Tennessee Gas Pipeline, to Sally Sun, APS, dated March 8, 2007

10 Montana Sulphur and Chemical Company Specification Sheet. Yellowstone Brand High Purity Sulfur Prills. 05 October 2006.

11 "Heat and Mass Balance for the Algae Integration \& Hydrogasification/ Substitute Natural Gas Commercial Scale Facility, Conceptual Design,” Prepared by WorleyParsons, for APS, dated March 8, 2007, Rev A.

12 Email from David Stauffer (WorleyParsons) to Raymond Hobbs (APS), RE: Algae Integration Process Model Preliminary Results, June 26, 2009.

13 “APS Advanced Hydrogasification / SNG Preliminary Engineering Package (PEP)” Scope of Work document transmitted by email from Juanita Rodriguez (APS) to David Stauffer (WorleyParsons) dated June 23, 2010.

14 Email from Xiaolei "Sally” Sun (APS) to David Stauffer (WorleyParsons), RE: APS Bench Data, February 1, 2010.

15 M.D. Rutkowski, M.G. Klett, R.C. Maxwell, Parsons, The Cost of Mercury Removal in an IGCC Plant, Gasification Technologies Public Policy Workshop, October 1, 2002, Washington, DC, http://www.gasification.org

16 http://www.calgoncarbon.com/bulletins/HGR-P.htm

17 http://www.calgoncarbon.com/bulletins/TYPE_HGR.htm 
18 Curtis O. Rueter, Kenneth E. DeBerry, Kenneth E. McIntosh, and Dennis A. Dalrymple, (CrystaTech, Inc.), CrystaSulf Process for Recovering Sulfur from Gas Streams, presented at the North Texas Gas Processors Association Chapter meeting, April 4, 2000.

19 Nsakala ya Nsakala, Gregory N. Liljedahl, David G. Turek, Greenhouse Gas Emissions Control by Oxygen Firing in Circulating Fluidized Bed Boilers, Phase 2 - Pilot Scale Testing and Updated Performance and Economic s for Oxygen Fired CFB with CO2 Capture, Alstom Power Inc. Power Plant Laboratories, prepared for the US DOE NETL, October 2004.

20 Guidance for Pressure-Relieving and Depressuring Systems, API Recommended Practice 521, American Petroleum Institute.

21 Email from Xiaolei “Sally” Sun (APS) to David Stauffer (WorleyParsons), RE: Coal Price, January 25, 2011.

22 DOE/NETL-401/090808 - "Recommended Project Finance Structures for the Economic Analysis of Fossil-Based Energy Projects,” DOE/NETL-401/090808, September 2008.

23 DOE/NETL-2010/1397 - "Cost and Performance Baseline for Fossil Energy Plants, Volume 1: Bituminous Coal and Natural Gas to Electricity,” Revision 2, November 2010.

24 “1 Year LIBOR - Rate, Definition \& Historical Graph,” www.moneycafe.com/library/libor.htm, February 15, 2011.

25 “Annual Energy Outlook 2011 Early Release Overview,” U.S. Energy Information Administration, as found on www.eia.gov/forecasts/aeo/pdf January, 12, 2011.

26 Email from Donna Rennemo (Hydrogen Technologies) to Xiaolei “Sally” Sun (APS) and David Stauffer (WorleyParsons), RE: Electrolysis Performance and Cost Confirmation, October 7, 2010.

27 Email from Donna Rennemo (Hydrogen Technologies) to Xiaolei “Sally” Sun (APS), RE: Electrolysis Performance, September 28, 2010.

28 Norsk Hydro Electrolysers AS Memo from Roy Grelland, provided to Ray Hobbs, dated 2006-07-12.

29 Current (2009) State-of-the-Art Hydrogen Production Cost Estimate Using Water Electolysis, Independent Review, published for the U.S. DOE Hydrogen Program, NREL/BK-6A1-46676, September 2009.

30 Email from Xiaolei “Sally” Sun (APS) to David Stauffer (WorleyParsons), RE: APS Peak / Off Peak Electric Rates, August 23, 2010.

31 “NYMEX Henry-Hub Natural Gas Price,” as presented on http://www.oilnergy.com/1gnymex.htm, February 16, 2011. 


\section{Appendices}

Appendix A: Design Basis Document

Appendix B: Process Flow Diagrams

Appendix C: Material Balances

Appendix D: Water Balance Diagrams

Appendix E: Major Equipment List

Appendix F: Capital Cost Details

Appendix G: Operating Cost Details

Appendix H: Hydrogen Source Review for Electrolysis 


\section{WW WorleyParsons

\section{Appendix A: \\ Design Basis Document}




\title{
WorleyParsons
}

resources \& energy

\author{
Design Basis \\ Arizona Public Service \\ Hydrogasification / Substitute Natural Gas \\ Conceptual Design Study \\ Commercial Scale Facility \\ (Gasifier Performance Update)
}

January 12, 2011

Revision I 


\section{NOTICE}

This Design Basis Document was prepared by WorleyParsons Resources and Energy Inc. as an account of work contracted by and for the benefit of the Arizona Public Service Company. This document forms the basis of a conceptual design and costing effort of a novel yet unproven hydrogasification / substitute natural gas (SNG) process. The overall process has been developed by joint discussions, analyses, and the best judgment of the APS Project Team. By necessity, many design basis inputs, including the gasifier performance, have been assumed based on the team's knowledge and best understanding to date. As possible, the team researched existing literature to make informed decisions based on the lessons from past gasifier / process development activities, and information available to the team members in the public domain, and/or past project experiences. APS provided gasifier performance for the bench scale test reactor.

WorleyParsons Resources and Energy Inc. has relied upon this information and information from other team members in the preparation of this design basis document and has not independently verified that the information is accurate, complete or applicable. As such, WorleyParsons does not assume any liability for the use or misuse of the information in this report.

Decisions and actions based upon the information in this design basis and the subsequent conceptual design effort should acknowledge the preliminary nature of the current analysis. The design and cost data to be developed are only suited for planning and budget estimation purposes, and are not of sufficient depth of detail to justify major capital investment.

Should the project continue to move forward, an experienced reactor/ gasifier designer will need to further develop the hydrogasification reactor concepts and design elements. WorleyParsons is an architect/engineering firm and is not a reactor designer. In addition, a patent review for possible patent infringement should be performed prior to continued development of the process. 


\section{TABLE OF CONTENTS}

$1 \quad$ Objective / Introduction .....................................................................................................1

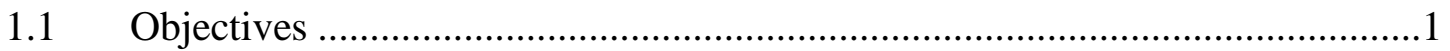

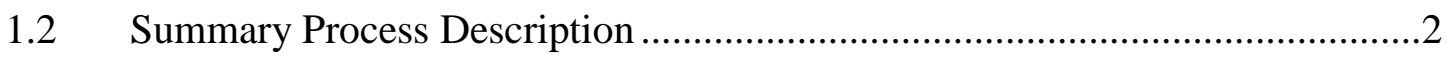

1.3 Document Organization ..................................................................................

2 Design Basis Information - Commercial Scale ...........................................................5

$2.1 \quad$ SNG Production Capacity.....................................................................

$2.2 \quad$ Site Conditions....................................................................................

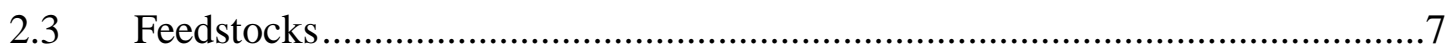

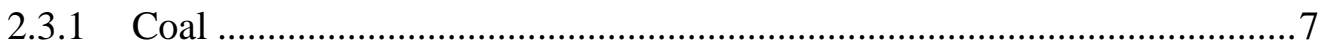

2.3.2 Externally Supplied Hydrogen and Oxygen ..............................................

$2.4 \quad$ Gasifier Performance and Design ....................................................................

2.4.1 Hydro-gasifier Performance .....................................................................

2.4.2 Hydro-gasifier Design ...........................................................................

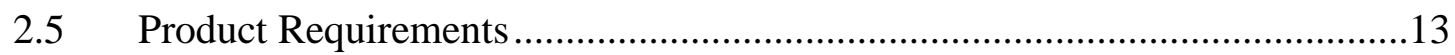

2.5.1 Substitute Natural Gas .........................................................................13

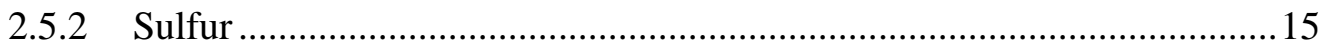

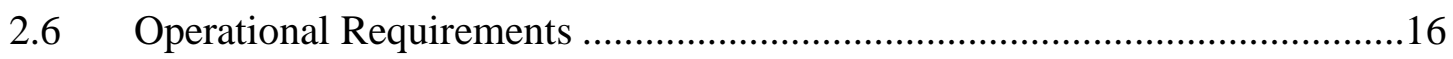

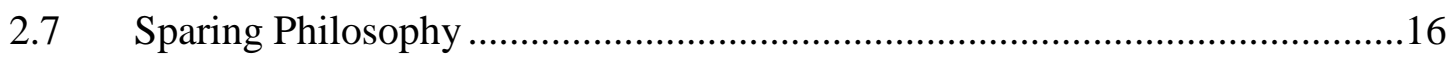

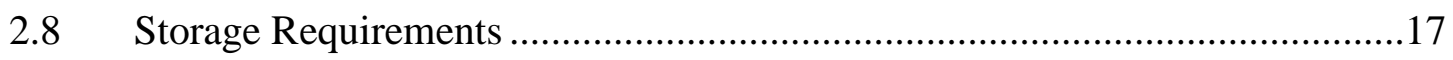

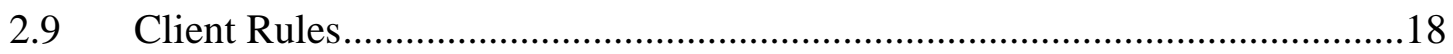

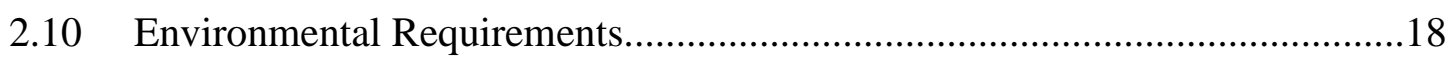

2.11 Balance of Plant Inputs ........................................................................18

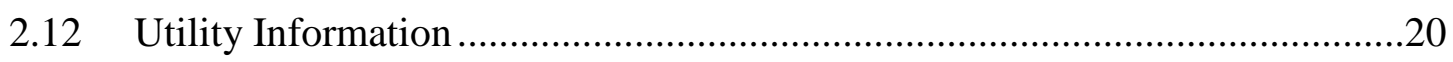

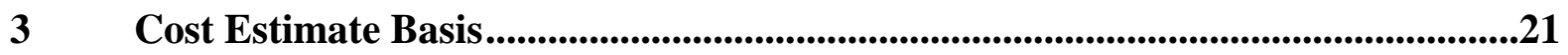

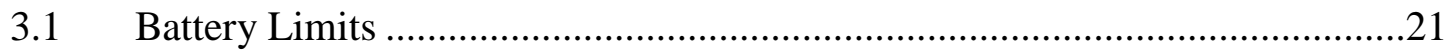

3.2 Capital Cost Estimate Basis .........................................................................21

3.3 Cost of Product Estimate Basis......................................................................22

3.3.1 Hydrogen Cost / Electrolysis Basis ..........................................................22

3.3.2 Oxygen Cost / Electrolysis Basis ............................................................26

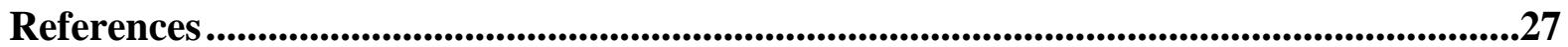




\section{LIST OF EXHIBITS}

Exhibit 1-1 Block Flow Diagram of the Base Hydrogasification SNG Process.......................2

Exhibit 2-1 Site Ambient Conditions........................................................................6

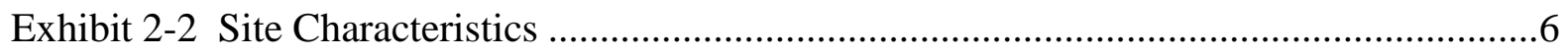

Exhibit 2-3 Fruitland Coal Analysis and Properties ..........................................................8

Exhibit 2-4 Hydrogen \& Oxygen Purity ........................................................................

Exhibit 2-5 Hydrogasifier Performance.........................................................................10

Exhibit 2-6 Hydrogasifier Sketch ....................................................................................12

Exhibit 2-7 Hydrogasifier Design Conditions - Preliminary Values ...................................13

Exhibit 2-8 Natural Gas Pipeline Quality Specifications ...............................................14

Exhibit 2-9 Natural Gas Pipeline Quality Specification Summary .....................................15

Exhibit 2-10 Typical Sulfur Specification .........................................................................16

Exhibit 2-11 Hydrogasification / SNG Process Configuration and Design Redundancy.......17

Exhibit 2-12 Balance of Plant Assumptions ...................................................................19

Exhibit 2-13 Site Water Characteristics.........................................................................20

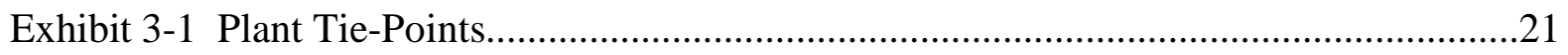

Exhibit 3-2 Cost Estimate Basis ...........................................................................21

Exhibit 3-3 Project Financial Structure........................................................................22

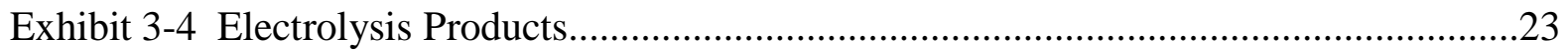

Exhibit 3-5 Electrolysis Design \& Cost Basis ................................................................24

Exhibit 3-6 Peak \& Off-Peak Electric Rates...................................................................25

Exhibit 3-7 Peak \& Off-Peak Electric Rate Chart .............................................................26 


\section{Revision Record}

\begin{tabular}{|c|c|l|}
\hline Revision & Date & \multicolumn{1}{c|}{ Content } \\
\hline A & $10 / 19 / 2006$ & Draft - pre-release \\
\hline B & $11 / 01 / 2006$ & Draft in progress - For APS Feedback. \\
\hline C & $11 / 22 / 2006$ & Incorporates APS inputs to date. \\
\hline D & $12 / 08 / 2006$ & Incorporates latest APS comments \\
\hline E & $02 / 08 / 2007$ & $\begin{array}{l}\text { Minor edits for consistency with Engineering Package. E.g. Cost basis date, } \\
\text { number of pulverizer trains. }\end{array}$ \\
\hline F & $06 / 03 / 2009$ & $\begin{array}{l}\text { Update analysis with newest APS prediction of gasifier carbon conversion. } \\
\text { Streamlined document: Deleted Sec 3 (Configuration justification), deleted } \\
\text { Sec 4 (Old Nexant H\&MB), and deleted Sec 5 (system descriptions). }\end{array}$ \\
\hline G & $8 / 2 / 2010$ & Update for Bench Scale Test Reactor results and revised BFD. \\
\hline H & $10 / 25 / 2010$ & Update for electrolysis, electric cost, gas storage, gasifier, among other. \\
\hline I & $1 / 12 / 2011$ & Update coal fineness remark and miscellaneous editorial improvements \\
\hline & & \\
\hline
\end{tabular}




\section{Objective / Introduction}

This section presents the objective of the conceptual design effort, an introduction to the process itself, and an overview of the organization of this document.

\section{$1.1 \quad$ Objectives}

The objective of this 2010 update of the 2007 coal hydrogasification/ substitute natural gas (SNG) conceptual design effort is two fold:

1. To update the gasifier performance and its affect on the envisioned configuration, based on the Bench Scale Test Reactors (BSTR) initial results. Key gasifier performance parameters to be updated include:

a. Carbon Conversion from $70 \%$ to approximately 52\%,

b. Methane syngas levels,

c. Disposition of coal sulfur between char and syngas, and

d. Quantity / analysis of gasifier tars and oils (no tars and oils are expected at $1750^{\circ} \mathrm{F}$ ),

e. Hydrogen to Coal ratio (as influenced by both the gasifier and methanator),

f. Oxygen to coal ratio (as required for the thermal balance).

2. To update the Configuration and cost estimate for changes that result from the intervening years including:

a. Cost escalation, and

b. Technological advances, if any.

The objective of the overall three (3) Phase project is to develop and demonstrate an engineering-scale, coal hydrogasification-based process for the co-production of substitute natural gas (SNG) and electricity -with near-zero emissions meeting the following performance targets:

1. Overall process efficiency greater than $50 \%$,

2. SNG cost less than $\$ 5 / M M B T U$,

3. Capture and sequestration of $\mathrm{CO}_{2}$ equivalent to $90 \%$ of emissions from power production ${ }^{\mathrm{a}}$,

4. Reduce water usage as compared to partial oxidation gasification/syngas methanation process,

5. Capability of accepting hydrogen (preferably from a renewable source) as a supplemental source of energy,

6. Ability to use low-rank Western coals. (In Phase I, only a single design coal is considered), and

7. The capacity target for a single commercial-scale gasifier module is to process 1,000 short tons (dry) per day of coal. 
The objective of the Phase I Tasks, is to develop a conceptual design for the novel hydrogasification process and to examine the feasibility of a commercial scale hydrogasification project. This Design Basis document identifies the criteria that will be used in developing the engineering and cost estimate for this Phase I conceptual effort of the Commercial Scale facility.

\subsection{Summary Process Description}

Several hydrogasification process configurations were considered and evaluated between 2007 and 2010. The hydrogasification process selected by APS as the basis for the updated Phase I engineering/cost estimating tasks is presented in Exhibit 1-1. [1]

\section{Exhibit 1-1 Block Flow Diagram of the Base Hydrogasification SNG Process}

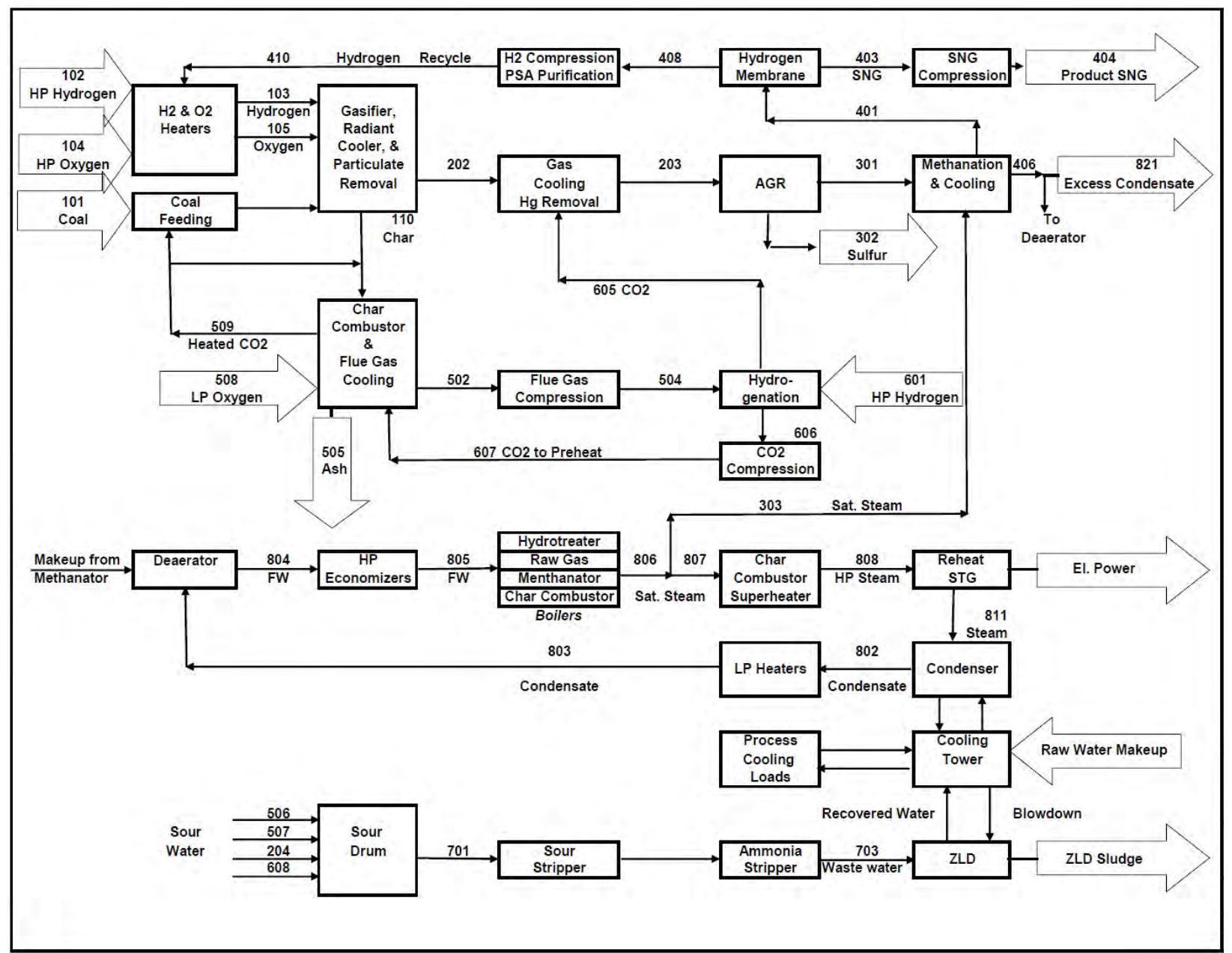

Note: This BFD was selected by APS as the base configuration, although changes during the conceptual design are permitted.

Coal, hydrogen, oxygen and a $\mathrm{CO}_{2}$ recycle stream are all fed into the hydrogasification reactor. A minimal amount of oxygen is introduced to help in providing the required gasification temperature of approximately $1750^{\circ} \mathrm{F}$. The oxygen required will be minimized by maximizing the preheating for the incoming feedstocks. If needed, the oxygen will be introduced with the hydrogen to partially oxidize the hydrogen, producing a hot hydrogen/steam stream which will come in contact with the coal fed into the gasifier. The hot hydrogen will begin the hydrogasification reaction with the coal. 
Approximately $52 \%$ of the coal carbon will gasify with approximately $48 \%$ of the coal carbon remaining in the solid stream leaving the gasifier.

The solids leaving the gasifier will be combusted with an $\mathrm{O}_{2}$ stream in order to generate a nearly pure $\mathrm{CO}_{2}$ stream. The generated $\mathrm{CO}_{2}$ stream will be split into 2 streams, with approximately one half being used to transport the coal and char, and the other half feeding the methanator. The $\mathrm{CO}_{2}$ stream going to the gasifier will be cooled and compressed and reheated prior to being re-introduced into the gasifier with the coal. Additionally, a $\mathrm{CO}_{2}$ stream will be utilized to transport the ash from the gasifier ash hoppers. The $\mathrm{CO}_{2}$ stream going to the syngas conditioning and methanation processes will be cooled and compressed and mixed with the gas stream leaving the gasifier. Since there will be excess $\mathrm{O}_{2}$ left after combustion, hydrogen will be introduced to catalytically combine with the remaining $\mathrm{O}_{2}$ and $\mathrm{SO}_{2}$. The mixed stream will be cooled and sent to a CrystaSulf desulfurizer process and then onto a methanation block consisting of a series of heat exchanger and catalytic reactors. The methanator catalyst requires a very low sulfur level to prevent poisoning.

The raw SNG leaving the methanation block will be dried and sent to a hydrogen membrane separation unit if required. This will remove most of the hydrogen while maintaining the SNG at high pressure. Methane will be removed from the hydrogen rich stream by a Pressure swing Absorber (PSA) unit in order to maximize the direct conversion of the coal carbon to methane. The separated hydrogen will be recycled back to the gasifier to minimize the required hydrogen. An additional hydrogen stream will be used in the hydrogenation reaction and used to remove the excess $\mathrm{O}_{2}$ from the oxyburner. Preliminary analysis indicates that it may be possible to eliminate the hydrogen membrane, PSA and hydrogen recycle compressor by careful design of the methanation process. This will be investigated and implemented as possible.

The lower portion of the BFD focuses on the collection of process water, waste heat, the generation of steam, and the integration of a steam turbine generator for the production of electricity.

\subsection{Document Organization}

This design basis document is organized with the following structure.

Section 1: Objective / Introduction

Section 2: Design Basis Information Commercial Scale

Section 3: Cost Basis Information
This Section

The technical design basis information for the Commercial Scale Process

The cost basis for the technical process

This version of the design basis document was streamlined from previous editions by the elimination of non-essential information ${ }^{\mathrm{b}}$.

b Specifically, the following non-essential sections were eliminated. Section 3: Hydrogasification Configuration Selection - eliminated because there is no longer a question of which configuration will be utilized; Section 4: Heat \& Mass Balance Basis for Selected Gasifier - eliminated because the section documented input from Nexant which is now irrelevant; and Section 5: Major System Descriptions - eliminated since it is more appropriate for inclusion in the Preliminary Engineering Package. 


\section{Design Basis Information - Commercial Scale}

The following sections form the design basis for the commercial scale hydrogasification/ SNG process.

\subsection{SNG Production Capacity}

A fundamental design criteria for the commercial scale process is the SNG production level listed below.

1. The target SNG production for the commercial scale process has been selected as approximately 120 MMSCFD [2], and will be based on three (3) gasifiers modules.

This SNG production level has been selected considering the capacity of the Dakota Gasification facility and publicly available information regarding SNG facilities proposed by others. The Dakota Gasification facility produces 160 MMSCFD of SNG and is the only SNG facility in the world. A Presentation by ConocoPhillips on SNG at the 2006 Gasification Technology Conference presented three SNG production schemes ranging from 90 to 115 MMSCFD [3]. Since the 1000 TPD hydrogasifier produces approximately 40 MMSCFD, three gasifier modules will produce approximately 120 MMSCFD.

In subsequent phases of this project, APS will want to consider additional factors such as the spare NG pipeline capacity, the availability of externally generated $\mathrm{H}_{2}$, the impact of economies of scale, market conditions, and the desires and financial considerations of the project development team.

\subsection{Site Conditions}

The design will be based on site conditions as presented in Exhibit 2-1. 
Exhibit 2-1

Site Ambient Conditions

\begin{tabular}{|c|c|}
\hline Parameter & Value \\
\hline Location & South Western United States ${ }^{c}$ \\
\hline Elevation, ft (above MSL) & $5,500 *$ \\
\hline Barometric Pressure, psia & $12.0^{*}$ \\
\hline $\begin{array}{l}\text { Design Ambient Temperature, Dry Bulb, }{ }^{\circ} \mathrm{F} \\
\text { Maximum } \\
\text { Minimum } \\
\text { Average }\end{array}$ & $\begin{array}{l}95 \\
-3 \\
60\end{array}$ \\
\hline $\begin{array}{l}\text { Design Ambient Wet Bulb Temperature, }{ }^{\circ} \mathrm{F} \\
\text { Summer Design }\end{array}$ & 65 \\
\hline Design relative Humidity, \%RH & 50 \\
\hline $\begin{array}{l}\text { Ambient Air Composition, Vol\%: } \\
\text { Nitrogen } \\
\text { Argon } \\
\text { Oxygen } \\
\text { Water } \\
\text { Carbon Dioxide } \\
\text { Total }\end{array}$ & $\begin{array}{r}77.27 \\
0.93 \\
20.73 \\
1.04 \\
0.03 \\
100.00\end{array}$ \\
\hline
\end{tabular}

Site characteristics ${ }^{\mathrm{c}}$ are presented in Exhibit 2-2.

Exhibit 2-2

Site Characteristics

\begin{tabular}{|l|l|}
\hline Cost Basis & Farmington, New Mexico \\
\hline Topography & Level \\
\hline Size, acres & 200, (the site is not constrained) \\
\hline Access & $\begin{array}{l}\text { Land-locked, having access by major state highway, 2 miles north of the } \\
\text { plant. Nearest railroad hub is located in Gallup, New Mexico, 60 miles } \\
\text { from the site. }\end{array}$ \\
\hline Ash Disposal & Off Site \\
\hline Coal Delivery & $\begin{array}{l}\text { In } 200 \text { tons/load trucks from BHP Navajo Coal Company mine, located } \\
\text { about } 1 \frac{1}{2} \text { miles distant }\end{array}$ \\
\hline Water & Artificial cooling lake with water impounded from the Sun Juan River \\
\hline Waste water & Zero Liquid Discharge (i.e., No evaporation pond) \\
\hline
\end{tabular}

The following design considerations are site-specific, and will not be quantified for this preliminary study of the commercial scale facility. Allowances for normal conditions and construction will be

c Assumed site is based on ambient conditions and other characteristics of the Four Corner Station, near

Fruitland, San Juan County, New Mexico. 
included in the cost estimates. Typically the consideration of these factors do not have a significant impact on the cost unless the site specific situation is unusual or extreme.

Flood plain considerations.

Existing soil/site conditions.

$>$ Water discharges and reuse.

$>$ Rainfall/snowfall criteria.

$>$ Seismic design.

$>$ Buildings/enclosures.

$>$ Fire protection.

Local code height requirements.

Noise regulations - Impact on site and surrounding area.

\section{$2.3 \quad$ Feedstocks}

This section documents the Coal analysis and composition of the externally supplied hydrogen and oxygen.

\subsubsection{Coal}

Gas Technology Institute (GTI) performed the coal analysis on the Fruitland coal samples that were supplied by APS. Following are the coal composition and properties as reported by GTI. These values will be the design basis for the process modeling work. 


\section{Exhibit 2-3 Fruitland Coal Analysis and Properties ${ }^{d}$}

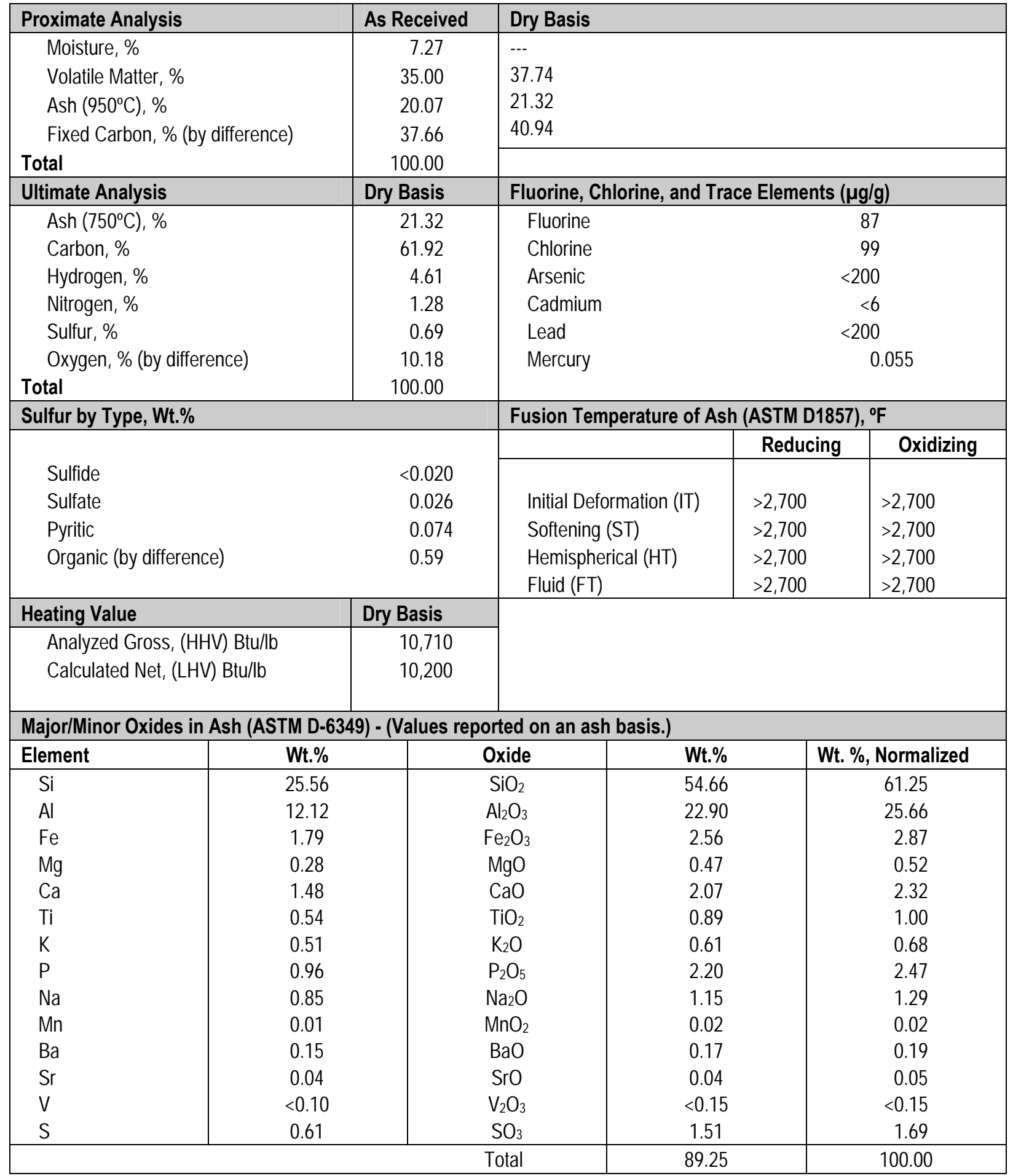

The coal feed into the gasifier will be pulverized such that approximately $70 \%$ will pass a 200 mesh screen. [4].

${ }^{\mathrm{d}}$ CRS Sample Login No: 061131-001 (received 3/10/06) and Login No. 061131-002 (received 3/24/06) 


\subsubsection{Externally Supplied Hydrogen and Oxygen}

Hydrogen is assumed to be delivered as a compressed gas at $104^{\circ} \mathrm{F}$ and 1150 psig to the plant boundary via a dedicated pipeline. Hydrogen will be free of sulfur, chlorine, potassium, and particulate matter. Hydrogen characteristics are based on an electrolysis process and are presented in Exhibit 2-4.

\section{Exhibit 2-4 Hydrogen \& Oxygen Purity}

\begin{tabular}{|c|c|}
\hline Electrolysis Product & Purity \\
\hline Hydrogen purity & $>99.99 \%$ \\
\hline Oxygen purity & $>99.5 \%$ \\
\hline
\end{tabular}

High pressure oxygen is assumed to be delivered as a compressed gas at $104^{\circ} \mathrm{F}$ and 1120 psig to the plant boundary via a dedicated pipeline. [1]

Low pressure oxygen is assumed to be delivered as a compressed gas at ambient temperature at 50 psig to the plant boundary via a dedicated pipeline.

No on-site bulk storage will be assumed within the gasification facility battery limits, as the electrolysis unit will be compressed of many parallel units providing high reliability and will have the ability to quickly respond to the instantaneous demand.

\subsection{Gasifier Performance and Design}

APS has designed, built and tested a bench scale test reactor (BSTR) in order to determine the performance of such a hydrogasification reactor based on the fruitland coal. The performance and design of a commercial scale hydrogasifier as developed by APS is presented below.

\subsubsection{Hydro-gasifier Performance}

The operating conditions and performance of the hydrogasifier assuming the Fruitland coal are presented in Exhibit 2-5. 


\section{Exhibit 2-5 Hydrogasifier Performance}

\begin{tabular}{|c|c|c|c|}
\hline Specification Parameter & Value & Reference & Notes \\
\hline Gasifier Operating Temperature & $1750^{\circ} \mathrm{F}$ & [5] & \\
\hline Gasifier Effluent Pressure & 1000 psig & [5] & \\
\hline $\mathrm{H} 2 /$ Coal (mass ratio) & 0.2 & [5] & $\begin{array}{l}\text { May be too low with recycled } \mathrm{CO} 2 \text {. Allow to } \\
\text { float according to Methanation requirements. }\end{array}$ \\
\hline H2 Injection Temperature & ca 1350 & [5] & Lower if required by available materials \\
\hline $\mathrm{H} 2 / \mathrm{O} 2$ burner & Possibly & [5] & Depends on HB requirement. \\
\hline Carbon Conversion (wt \%) & $51.84 \%$ & [5] & Conversion of coal constituents to syngas \\
\hline $\mathrm{CC}$ into $\mathrm{CH} 4$ & $46.30 \%$ & [5] & Result of BSTR \& subject to change. \\
\hline $\mathrm{CC}$ into $\mathrm{CO}$ & $5.24 \%$ & [5] & Result of BSTR \& subject to change. \\
\hline $\mathrm{CC}$ into $\mathrm{CO} 2$ & $0.00 \%$ & [5] & Result of BSTR \& subject to change. \\
\hline $\mathrm{CC}$ into $\mathrm{C} 2 \mathrm{H} 6$ & $0.30 \%$ & [5] & Result of BSTR \& subject to change. \\
\hline CC into BTX & $0.00 \%$ & [5] & \\
\hline CC into Oil & $0.00 \%$ & [5] & \\
\hline Conversion to Syngas (wt \%) & & & Conversion of coal constituents to syngas \\
\hline S Conversion & $81.7 \%$ & [5] & \\
\hline H Conversion & $82.4 \%$ & [5] & \\
\hline N Conversion & $68.9 \%$ & [5] & \\
\hline O Conversion & $99.7 \%$ & [5] & \\
\hline Raw Syngas Compostion (mol\%) & & & Result of BSTR \& subject to change. \\
\hline $\mathrm{H} 2$ & 0.6628 & [5] & \\
\hline $\mathrm{CH} 4$ & 0.2362 & [5] & \\
\hline $\mathrm{H} 2 \mathrm{O}$ & 0.0631 & [5] & \\
\hline N2 & & [5] & \\
\hline $\mathrm{CO}$ & 0.0267 & [5] & \\
\hline $\mathrm{CO} 2$ & & [5] & \\
\hline $\mathrm{NH} 3$ & 0.0076 & [5] & \\
\hline $\mathrm{H} 2 \mathrm{~S}$ & 0.0028 & [5] & \\
\hline $\mathrm{C} 6 \mathrm{H} 6$ & & [5] & \\
\hline $\mathrm{C} 2 \mathrm{H} 6$ & 0.0008 & [5] & \\
\hline Total Syngas & 1.0000 & [5] & \\
\hline
\end{tabular}

The information in Exhibit 2-5 is based on APS assumption of the commercial scale gasifier operating conditions. This performance should be revisited as the analysis moves forward. It is 
possible that many factors may cause the assumed performance to drift away from those above. The factors that may cause the performance to change may include the following:

1. Compared to the BSTR results, the commercial scale gasifier will be operated at 1000 psig, instead of the 800 psig utilized in the BSTR performance test.

2. Compared to the BSTR results which utilized a N2 carrier gas, a $\mathrm{CO}_{2}$ carrier gas will be used.

3. Compared to the BSTR arrangement, which utilized electric heaters, oxygen may be required to achieve the desired operating condition of $1750^{\circ} \mathrm{F}$ within the hydrogasifier.

4. With the relatively low carbon conversion ratio of approximately $50 \%$, the $\mathrm{CO}_{2}$ produced from the Char combustion that ultimately ends up in the methanator, may require an increased $\mathrm{H}$ to coal ratio fed into the hydrogasifier.

To address these factors, WorleyParsons has developed a simplified gasifier model based on the BSTR performance in order to extend the gasifier performance to other conditions.

\subsubsection{Hydro-gasifier Design}

The design of the hydrogasifier for this phase of the project is under the control of APS, who had been considering both the ARCH and Rockwell Type designs. However, since APS is now terminating the hydrogasification project and had may unanswered design decisions, APS has requested that the gasifier design be based on that presented in Reference [6]. A sketch of the hydrogasifier from that reference is presented in Exhibit 2-6. The project team will allow the design to evolve from this original vision as the project evolves. It is important that the reactor design, performance and cost are all consistent. Since the Bench scale test reactor (BSTR) is essentially a drop tube reactor, the original hydrogasification design is consistent with that simple vision.

Key design features are presented in Exhibit 2-7. 


\section{Exhibit 2-6 Hydrogasifier Sketch}

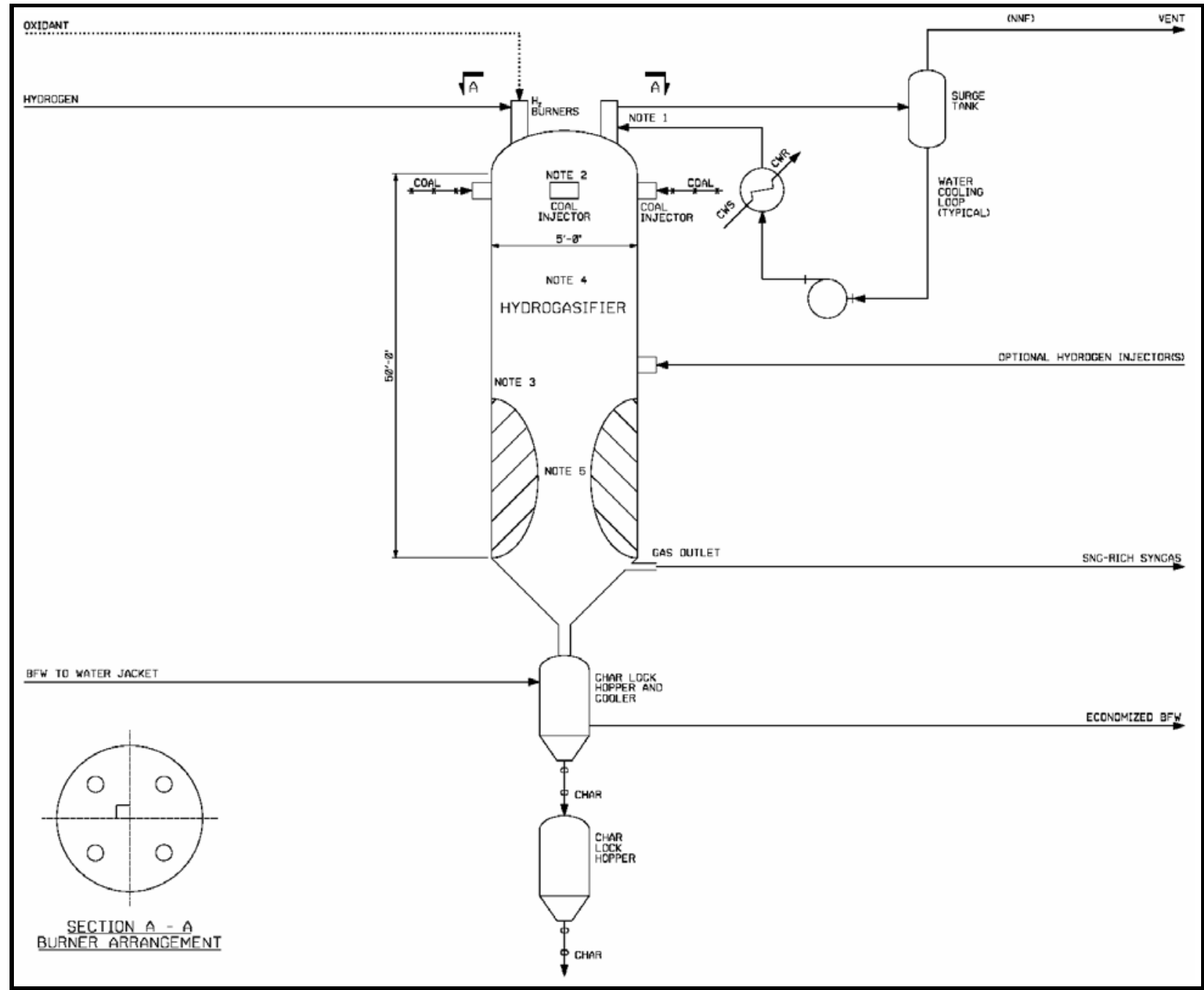

Reference:[6], p 38 Drawing APSS-1-SK-021-305-001-RA 


\section{Exhibit 2-7 Hydrogasifier Design Conditions - Preliminary Values}

\begin{tabular}{|c|c|c|}
\hline Design Parameter & Design Value & Notes \\
\hline Gasifier type & Rockwell type & Single Stage, Entrained Flow, Hydrogasification \\
\hline Gas flow direction & Down flow & Single pass, no recycle. \\
\hline $\begin{array}{l}\text { Gasifier dimensions } \\
\mathrm{D} \times \mathrm{h} \times \text { thickness }\end{array}$ & $\begin{array}{l}\text { 5'dia, x 50'high } \\
\quad(900 \mathrm{ft} 3)\end{array}$ & Dimensions are for 1000 tpd dry coal throughput. \\
\hline Thermal Protection & Refractory & \\
\hline Coal Feeding & Coal lock hoppers & Fed by GE/Stamet Posimetric Solids pump \\
\hline Char Removal & Char lock hoppers & \\
\hline Location of coal injectors & Top Side & Four injectors, oriented for swirl. \\
\hline Location of $\mathrm{H} 2$ Injectors & Top & Number of injectors -4 \\
\hline Location of $\mathrm{O} 2$ injectors & Top & Number of injectors -4 \\
\hline Gas Phase separation & $\begin{array}{c}\text { Acceleration } \\
\text { De-acceleration }\end{array}$ & $\begin{array}{l}\text { Other design features may be added, such as a } \\
\text { cyclone }\end{array}$ \\
\hline $\begin{array}{c}\text { H2 Conditions } \\
\text { T, } \\
\text { P, } \\
\text { flow rate }\end{array}$ & $\begin{array}{l}\text { Up to } 1350 \mathrm{~F} \\
1150 \text { psig } \\
\text { as required }\end{array}$ & $\begin{array}{l}\text { Temperature up to } 1350 \mathrm{~F} \text { per H\&MB } \\
\mathrm{P}=1000+\text { injector } \& \text { heater } \mathrm{dPs} \text { of } 100+50 \\
\text { Flow rate as required for methanation }\end{array}$ \\
\hline $\begin{array}{l}\text { O2 Conditions } \\
\text { T, } \\
\text { P, } \\
\text { flow rate }\end{array}$ & $\begin{array}{l}200 \mathrm{~F} \\
1120 \text { psig } \\
\text { as required }\end{array}$ & $\begin{array}{l}\text { (If needed) } \\
P=1000+\text { injector } \mathrm{dP} \text { of } 100+\text { heater dp of } 20= \\
\text { Flow rate, if required to achieve } 1750 \mathrm{~F}\end{array}$ \\
\hline Design Coal Flow & 1000 TPD & Assumed scaled up size \\
\hline $\begin{array}{c}\text { Coal Transport Gas } \\
\text { Gas } \\
\mathrm{T}, \\
\mathrm{P}, \\
\text { Flow rate }\end{array}$ & $\begin{array}{c}\mathrm{CO} 2 \\
750 \mathrm{~F} \\
\mathrm{TBD} \\
350 \mathrm{~kg} / \mathrm{m}^{3}\end{array}$ & $\begin{array}{l}\mathrm{P}=1000+\mathrm{dP} \text { for transport } \\
\text { The } \mathrm{CO}_{2} \text { flow will be based on } 350 \mathrm{~kg} \mathrm{coal} / \mathrm{m}^{3} \text {. }\end{array}$ \\
\hline $\begin{array}{l}\text { Cooling water } \\
\text { requirement }\end{array}$ & TBD & For injectors, (duty, $\mathrm{T}$, flow) \\
\hline
\end{tabular}

\subsection{Product Requirements}

\subsubsection{Substitute Natural Gas}

El Paso Gas provided the following natural gas pipeline specification [7] for gas to be received by them in the Four Corners region. These requirements are shown below in Exhibit 2-8. These requirements are summarized in Exhibit 2-9. 


\section{Exhibit 2-8 Natural Gas Pipeline Quality Specifications}

General Specifications. Shipper warrants that all natural gas received by El Paso at any mainline receipt point(s) shall conform to the following specifications and must be, in El Paso's reasonable judgment, otherwise merchantable:

(a) Liquids - The gas shall be free of water and hydrocarbons in liquid form at the temperature and pressure at which the gas is received. The gas shall in no event contain water vapor in excess of seven (7) pounds per million standard cubic feet.

(b) Hydrocarbon Dew Point - The hydrocarbon dew point of the gas received shall not exceed twenty degrees Fahrenheit $\left(20^{\circ} \mathrm{F}\right)$ at normal pipeline operating pressures.

(c) Total Sulfur - The gas shall not contain more than five (5) grains of total sulfur, which includes hydrogen sulfide, carbonyl sulfide, carbon disulfide, mercaptans, and mono-, di- and poly-sulfides, per one hundred (100) standard cubic feet. The gas shall also meet the following individual specifications for hydrogen sulfide, mercaptan sulfur or organic sulfur:

(i) Hydrogen Sulfide - The gas shall not contain more than one-quarter $(0.25)$ grain of hydrogen sulfide per one hundred (100) standard cubic feet.

(ii) Mercaptan Sulfur - The mercaptan sulfur content shall not exceed more than three-quarters $(0.75)$ grain per one hundred (100) standard cubic feet.

(iii) Organic Sulfur - The organic sulfur content shall not exceed one and one-quarter (1.25) grains per one hundred standard cubic feet, which includes mercaptans, mono-, di- and poly-sulfides, but it does not include hydrogen sulfide, carbonyl sulfide or carbon disulfide.

(d) Oxygen - The oxygen content shall not exceed two-tenths of one percent ( $0.2 \%)$ by volume and every reasonable effort shall be made to keep the gas delivered free of oxygen.

(e) Carbon Dioxide - The gas shall not have a carbon dioxide content in excess of two percent (2\%) by volume, except for gas acceptable under Sections 5.2 and 5.3 .

(f) Diluents -The gas shall not at any time contain in excess of three percent (3\%) total diluents (the total combined carbon dioxide, nitrogen, helium, oxygen, and any other diluent compound) by volume

(g) Dust, Gums and Solid Matter - The gas shall be commercially free of dust, gums and other solid matter.

(h) Heating Value - The gas shall have a heating value of not less than 967 Btu per cubic foot.

(i) Temperature - The gas received by El Paso shall be at temperatures not in excess of one hundred twenty degrees Fahrenheit $\left(120^{\circ} \mathrm{F}\right)$ nor less than fifty degrees Fahrenheit $\left(50^{\circ} \mathrm{F}\right)$. Any party tendering gas at a temperature standard less than fifty degrees Fahrenheit $\left(50^{\circ} \mathrm{F}\right)$ shall receive a waiver of such standard only if a test has been conducted in accordance with procedures set forth in Section 5.12(b) hereof, and the results from such test demonstrate that the particular segment of the pipeline tested can be safely operated below the fifty degrees Fahrenheit $\left(50^{\circ} \mathrm{F}\right)$ temperature standard.

(j) Deleterious Substances - The gas shall not contain deleterious substances in concentrations that are hazardous to health, injurious to pipeline facilities or adversely affect merchantability.

The pipelines in northern Arizona are running at 845 - 895 psig maximum. 
Exhibit 2-9 Natural Gas Pipeline Quality Specification Summary

\begin{tabular}{|c|c|}
\hline Specification Parameter & Value \\
\hline Pipeline Pressure & 895 psig (maximum) \\
\hline Pipeline Temperature Requirement & $\begin{array}{l}50-120^{\circ} \mathrm{F} \\
<50^{\circ} \mathrm{F} \text { by waiver }\end{array}$ \\
\hline HHV, Btu/scf ( with scf @ 60 F, 14.73 psi) & $>967$ \\
\hline Total Diluents $\left(\mathrm{CO}_{2}, \mathrm{~N}_{2}, \mathrm{He}, \mathrm{O}_{2},+\right.$ other $) \%$ vol. & $<3 \%$ \\
\hline $\mathrm{CO}_{2}, \%$ volume & $<2 \%$ \\
\hline $\mathrm{O}_{2}, \%$ volume & $<0.2 \%$ \\
\hline $\mathrm{CO}$ & Not specified, (see diluent) \\
\hline $\mathrm{H}_{2}$ & $<3 \mathrm{~mol} \%[8]$ \\
\hline Liquids & None, at receiving $\mathrm{T}$ \\
\hline Water & $<7 \mathrm{lbs} / \mathrm{MMSCF}$ \\
\hline Hydrocarbon Dew Point, ${ }^{\circ} \mathrm{F}$ & $<20^{\circ} \mathrm{F}$ at pipeline op pressure \\
\hline Total Sulfur, & 5 grains/100 scf \\
\hline $\mathrm{H}_{2} \mathrm{~S}$ & 0.25 grains/ $100 \mathrm{scf}$ \\
\hline Mercaptan S & 0.75 grains/ $100 \mathrm{scf}$ \\
\hline $\begin{array}{l}\text { Organic S (includes mercaptans, mono- di- and } \\
\text { poly-sulfides, but excluding } \mathrm{H}_{2} \mathrm{~S}, \mathrm{COS}, \mathrm{CS}_{2}\end{array}$ & 1.25 grains/ $100 \mathrm{scf}$ \\
\hline
\end{tabular}

Since the maximum natural gas pipeline pressure can reach 895 psig, the SNG product delivery pressure at the compressor outlet will be assumed to be 960 psia to allow a 50 psi driving force for injection into the NG pipeline.

The existing natural gas pipeline is assumed to be located within 5 miles from the site boundary and has a spare capacity of 2 billion cubic feet per day.

\subsubsection{Sulfur}

Sulfur is a commodity, which can be used in a wide range of applications. Most sulfur is converted to sulfuric acid, which can be utilized in industrial processes. However, its primary use is in manufacturing phosphate fertilizers.

A typical set of sulfur product characteristics from the Montana Sulphur and Chemical Company is shown in Exhibit 2-10. [10] 


\section{Exhibit 2-10 Typical Sulfur Specification}

\begin{tabular}{|l|c|}
\hline \multicolumn{1}{|c|}{ Parameter } & Value \\
\hline Sulfur Content, wt \% min & 99.9 \\
\hline Moisture, wt \% max & 0.5 \\
\hline Reduced Carbon, wt \% max & 0.05 \\
\hline Ash, wt \% max & 0.01 \\
\hline Acidity, wt \% max & 0.02 \\
\hline Color / Appearance & Light Yellow \\
\hline Odor & Sweet to a Mercaptan-Like Odor \\
\hline
\end{tabular}

\subsection{Operational Requirements}

The SNG process is expected to be a base-loaded unit. The following operational requirements are specified:

- Turndown to a minimum of $80 \%$ will be achievable for an indefinite time without flaring.

- On-stream time: The design on-stream factor shall be 292 days per year, which is consistent with a Target Availability $\sim 80 \%$.

- In order to minimize the use of electricity for electrolysis during the summer peak, the plant will be scheduled for annual maintenance during the month of July.

\subsection{Sparing Philosophy}

The sparing philosophy of the major hydrogasification/SNG process components are presented in Exhibit 2-11. 
Exhibit 2-11

Hydrogasification / SNG Process Configuration and Design Redundancy

\begin{tabular}{|l|l|c|}
\hline \multicolumn{1}{|c|}{ System } & \multicolumn{1}{|c|}{ Description } & Quantity/Capacity \\
\hline Gasifier & APS Hydrogasification Reactor & $3 \times 33 \%$ \\
\hline Fuel Feed (per Gasifier) & Dry feed (Stamet Posimetric pump) & $\begin{array}{c}9 \times 16.7 \% \\
{\left[\begin{array}{c}\text { i.e., } 3 \times 50 \%, \\
\text { per Gasifier }\end{array}\right.}\end{array}$ \\
\hline Syngas Scrubbing & $\begin{array}{l}\text { Pulverized Coal Mills } \\
\text { (70\% through 200 mesh.) }\end{array}$ & $3 \times 50 \%$ \\
\hline Hydrogen / Oxygen Generator & Tray counterflow & $1 \times 100 \%$ \\
\hline Hydrogen / Oxygen Storage & Electrolysis Unit (not in cost basis) & $\mathrm{NA}$ \\
\hline Char Combustor & $\begin{array}{l}\text { Type to be determined (in cost basis) } \\
\text { Not bulk storage, but small storage } \\
\text { volume for operational ease. }\end{array}$ & $1 \times 100 \%$ \\
\hline CO ${ }_{2}$ Recycle System & Oxygen blown CFB & $1 \times 100 \%$ \\
\hline Mercury Removal & CO ${ }_{2}$ cooling/reheat/compression & $1 \times 100 \%$ \\
\hline Acid Gas Removal & Sulfur impregnated carbon bed & $1 \times 100 \%$ \\
\hline Sulfur Recovery & CrystaSulf, physical solvent & $1 \times 100 \%$ \\
\hline Methanation unit & Via CrystaSulf Process & $1 \times 100 \%$ \\
\hline SNG Cleanup System & Catalytic & $1 \times 100 \%$ \\
\hline Flare System & Membrane/PSA (as appropriate) & $1 \times 100 \%$ \\
\hline
\end{tabular}

Since the Hydrogasification plant will be co-producing electric power, the sparing philosophy of the Power Island will follow the established Good Engineering Practice (GEP) in the power plant design to achieve high availability /reliability. Except for the prime movers, large electrical equipment, and a few select equipment, adequate sparing will be provided.

General guidelines on sparing are presented below:

1. Prime Movers (Steam Turbine Generators) : 1 x100\%

2. Heat Recovery Steam Generators: 1x100\%

3. Step Up and Auxiliary Transformers: 1x100\%

4. Cooling Tower: 1 x 100\%, if applicable

5. Boiler Feed Pumps: 2x100\%

6. Condensate Pumps: 2x100\%

7. Closed Cooling Water Pumps: 2x100\%

8. Circulating Water Pumps: 2x50\%

9. Miscellaneous Other Pumps: 2x100\%

\section{$2.8 \quad$ Storage Requirements}

The coal yard shall be sized for 7 days storage. The coal yard will not be covered. 
No bulk oxygen or hydrogen storage will be associated with the SNG plant. The oxygen and hydrogen will be supplied on demand. The electrolysis plant will be comprised of hundreds of units, each which can turn down to $20 \%$ or less. In addition, electrolysis units are able to ramp nearly instantaneously. A small storage volume will be provided for operational considerations.

\section{$2.9 \quad$ Client Rules}

The following guidance has been provided by APS.

1. No routine flaring will be allowed. Flaring for startup, shutdown and upset conditions will be allowed. An elevated flare is planned. During normal operation, small flare streams can be combusted in the oxygen blown CFB combustor.

2. As much as possible, only commercial technology should be utilized. Where developmental technology is required, it will be identified in the system descriptions.

3. The site need only be planned for a single SNG facility. That is, accommodations will not be made for a future unit.

\subsection{Environmental Requirements}

Considering that the hydrogasification project does not include a combustion turbine or directly release any combustion products, traditional air emission limits are not relevant. The one source of combustion in the process is the fluidized bed oxygen-blown combustor that burns the gasification char. However, these combustion products, nearly pure $\mathrm{CO}_{2}$, are recycled back into the process and ultimately are converted into additional SNG. Therefore emission requirements will be placed on the end user of the SNG, such as a GTCC, and not on the process itself.

Nevertheless, the project will need to obtain the appropriate permits that will cover the following:

- Flares, for controlling air emissions during startup and upset conditions (as applicable),

- Cooling towers (as applicable),

- Material handling and storage (unloading/loading, conveyor belts, transfer points, silos, bin vents, etc.) for

○ Coal

○ Ash, and

- Sulfur

- Wastewater treatment plant plan approval, if the zero liquid discharge system is not used.

- Waste disposal site, if ash and/or sulfur are not beneficially reused

These permits/approvals are not unique to the process, but would be typical of any industrial project.

In summary, it is anticipated that there will not be any process air emission values that will influence the plant design. Instead, the natural gas quality specification will form a part of the process design requirements.

Furthermore, the process will convert the produced $\mathrm{CO}_{2}$ to SNG, such that at least $90 \%$ of the carbon in the gasified coal will be contained in the resulting fuel. In addition, as described in the next section, the plant design will utilize a Zero Liquid Discharge (ZLD) system to eliminate the discharge of wastewater.

\subsection{Balance of Plant Inputs}

The process will be based on the Balance of plant assumptions presented in Exhibit 2-12. 
Exhibit 2-12

Balance of Plant Assumptions

\begin{tabular}{|c|c|}
\hline Plant Characteristic & Basis \\
\hline \multicolumn{2}{|l|}{ Fuel and Other Storage } \\
\hline Coal & $\begin{array}{l}\text { Coal pile sized for } 7 \text { days of operation at MCR while firing } \\
\text { the design Fruitland coal. }\end{array}$ \\
\hline Product Sulfur & 72 hours \\
\hline Ash & 72 hours \\
\hline Solids Handling Crew Operation & $(2$ shifts $\times 8$ hr $) \times 7$ days/week \\
\hline Hydrogen Storage & No bulk storage. Small operational storage. \\
\hline Oxygen Storage & No bulk storage. Small operational storage. \\
\hline \multicolumn{2}{|l|}{ System Inputs } \\
\hline Steam Turbine generator & Vendor Standard Designs \\
\hline Cooling system (if required) & Evaporative mechanical draft cooling tower \\
\hline Grid Interconnection voltage & $\begin{array}{l}\text { The new Hydro Gasification plant will utilize new dedicated } \\
\text { HV, MV and LV systems independent from the existing plant } \\
\text { systems. The new facility will be connected to the existing } \\
\text { APS switchyard at } 230 \mathrm{kV} \text {. The existing APS switchyard } \\
\text { capacity is assumed to be sufficient and no modifications } \\
\text { should be included in the Hydro Gasification plant scope. }\end{array}$ \\
\hline \multicolumn{2}{|l|}{ Water and Waste Water } \\
\hline Makeup Water & Plant makeup will be provided from the cooling lake \\
\hline Process Wastewater & $\begin{array}{l}\text { Process waste water (including gasification and BOP } \\
\text { processes) and storm water that contacts equipment } \\
\text { surfaces will be collected and treated in ZLD facility. }\end{array}$ \\
\hline Sanitary Waste Disposal & $\begin{array}{l}\text { Design will include a packaged domestic sewage treatment } \\
\text { plant with effluent discharged to the Zero Liquid Discharge } \\
\text { (ZLD) system. Sludge will be hauled off site. Packaged } \\
\text { sanitary waste plant will be sized for } 1,500 \text { gallons per day. }\end{array}$ \\
\hline Water Discharge & Zero liquid discharge \\
\hline
\end{tabular}

The characteristics of makeup/cooling water assumed in the study are presented in Exhibit 2-13. [11] 


\section{Exhibit 2-13 \\ Site Water Characteristics}

\begin{tabular}{|c|c|}
\hline Parameter & Value \\
\hline \multicolumn{2}{|l|}{ Temperature, [12] } \\
\hline Coldest, ${ }^{\circ} \mathrm{F}$ & 68 \\
\hline Hottest, ${ }^{\circ} \mathrm{F}$ & 94 \\
\hline $\mathrm{pH}$ & 8.2 \\
\hline Specific Conductance, at $25^{\circ} \mathrm{C}, \mu \mathrm{mhos}$ & 1240 \\
\hline Alkalinity, "P" as $\mathrm{CaCO}_{3}, \mathrm{ppm}$ & 0 \\
\hline Alkalinity, "M" as $\mathrm{CaCO}_{3}, \mathrm{ppm}$ & 105 \\
\hline Acidity, Free Mineral, as $\mathrm{CaCO}_{3}, \mathrm{ppm}$ & \\
\hline Sulfur, Total, as $\mathrm{SO}_{4}, \mathrm{ppm}$ & 455 \\
\hline Chloride, as $\mathrm{Cl}$, ppm & 49 \\
\hline Hardness, Total, as $\mathrm{CaCO}_{3}$, ppm & 378 \\
\hline Calcium Hardness, Total, as $\mathrm{CaCO}_{3}$, ppm & 229 \\
\hline Magnesium Hardness, Total, as $\mathrm{CaCO}_{3}$, ppm & 147 \\
\hline Copper, Total, as Cu, ppm & $<0.05$ \\
\hline Iron, Total, as Fe, ppm & 0.13 \\
\hline Sodium, as $\mathrm{Na}$, ppm & 126 \\
\hline Phosphate, Ortho-, as $\mathrm{PO}_{4}, \mathrm{ppm}$ & $<0.2$ \\
\hline Silica, Total, as $\mathrm{SIO}_{2}, \mathrm{ppm}$ & 7.5 \\
\hline Solids, Total Suspended mg/l & $<10$ \\
\hline Solids, Total Dissolved $\mathrm{mg} / \mathrm{l}$, at $105^{\circ} \mathrm{tab}$ & 914 \\
\hline Carbon, Total Organic, as C, ppm & 5.6 \\
\hline
\end{tabular}

\subsection{Utility Information}

Since the project will not be constructed at a facility with a predefined set of utility conditions (like would be found at a petrochemical facility), the required utility steam and condensate/feedwater conditions will be chosen as appropriate for the project as the design requirements solidify. 


\section{Cost Estimate Basis}

\subsection{Battery Limits}

The summary of plant tie-points is presented in Exhibit 3-1.

Exhibit 3-1

Plant Tie-Points

\begin{tabular}{|l|l|l|}
\hline \multicolumn{1}{|c|}{ Tie-Point } & \multicolumn{1}{c|}{ Location } & \multicolumn{1}{c|}{ Properties } \\
\hline Coal & At mine truck coal unloading & Exhibit 2-3 \\
\hline Hydrogen & At site boundary & $1150 \mathrm{psia} / 104^{\circ} \mathrm{F}$, Exhibit 2-4 \\
\hline HP Oxygen & At site boundary & $1120 \mathrm{psia} / 104^{\circ} \mathrm{F}$ \\
\hline LP Oxygen & At site boundary & $50 \mathrm{psia} / 77^{\circ} \mathrm{F}$ \\
\hline NG (for startup) & At site boundary & $860 \mathrm{psia} / 50-120^{\circ} \mathrm{F}$, Exhibit 2-8, Exhibit 2-9 \\
\hline SNG (product) & At site boundary & $910 \mathrm{psia} / 104^{\circ} \mathrm{F}$, Exhibit 2-8, Exhibit 2-9 \\
\hline Sulfur & At sulfur unloading station & Elemental, Exhibit 2-10 \\
\hline Ash & Outlet of the ash storage silo & Dry \\
\hline Water & Existing cooling lake & Exhibit 2-13 \\
\hline Waste Water & At waste water ZLD treatment facility & ZLD sludge \\
\hline Electric Power & $\begin{array}{l}\text { At site connections to utility } \\
\text { switchyard }\end{array}$ & Dead End 230 kV Tower on Site \\
\hline
\end{tabular}

\subsection{Capital Cost Estimate Basis}

Total Plant Costs (TPC) will be estimated based on the major assumptions presented in Exhibit 3-2.

\section{Exhibit 3-2 Cost Estimate Basis}

Estimate Basis

First Year of Operation

Contract Type

Labor

Construction Week

Construction Overtime

Site Size

Site Condition

Foundations

Coal Storage

Ash Disposal
2010 (Jun)

2016 [Unless adjusted by project schedule.]

EPCM

Union

50 hours

Spot overtime (approximately 3\%)

200 acres (i.e., unrestrained)

Level

Piles

7 Days of pile storage

Off site 


$\begin{array}{ll}\text { Switchyard } & \text { Not Included } \\ \text { Water Supply } & \text { At the site boundary, per Exhibit 2-13 } \\ \text { Start Up Fuel } & \text { Natural Gas } \\ \text { Gas Tie-In } & \text { At Site Boundary } \\ \text { Off Site Road Access } & \text { Assumed in Place } \\ \text { Cooling Method } & \text { Evaporative mechanical draft cooling tower } \\ \text { Power Tie-In } & \text { Dead End Tower on Site Included } \\ \text { Typical Owner's Cost } & \text { Not Included }\end{array}$

\subsection{Cost of Product Estimate Basis}

The economic performance will be assessed using the NETL Power Systems Financial Model. The Power Systems Financial Model is a discounted cash flow based financial model developed by NETL that is used to evaluate long-term costs and investment criteria for advanced energy systems. The model incorporates detailed accounting of the financing structure, interest during construction, depreciation, senior and subordinated debt, debt payments, and escalation of feedstock, O\&M, and product prices, among many other financial and engineering parameters. The model also computes a cost of product (COP). COP is a key measure of the economic feasibility of a project. This is the price at which the product SNG must be sold in order to offset:

1. project capital costs

2. project operating costs,

3. debt service, and

4. Provide the expected rate of return to its equity investors.

COP will be calculated using financial parameters as shown in Exhibit 3-3, which are based on assumed high technology and commodity risk for the project. (Source: Table 7-5 "Financial Structure for High-Risk Fuels Projects” [13]).

Exhibit 3-3
Project Financial Structure

\begin{tabular}{|c|c|c|c|}
\hline Type of Security & \% of Total & $\begin{array}{c}\text { Current (Nominal) } \\
\text { Dollar Cost }\end{array}$ & $\begin{array}{c}\text { Weighted Current } \\
\text { Cost of Capital }\end{array}$ \\
\hline Debt & $50 \%$ & $9.5 \%$ & 4.75 \\
\hline Equity & $50 \%$ & $20 \%$ & 10 \\
\hline
\end{tabular}

In addition, the following financial parameters will be applied:

- The internal rate of return for the equity portion of the investment (IRROE) is assumed to be a market standard of $20 \%$ per reference [13] recommendations.

- Debt term is assumed to be 15 years, which is the industry standard. The IRROE is calculated over the project life, which will typically be 30 years.

- A 30-year levelization and 5-year construction/capital expenditure period will be utilized 
- Credits or debits for $\mathrm{CO}_{2}$ emissions are not accounted

- All costs (e.g., fuel costs, O\&M costs) and product price are assumed to escalate at an annual nominal rate of $2 \%$. Capital costs are assumed to escalate at the same rate during the construction period. The escalation rate is based on the GDP Chain-type Price Index reported in Table 1 of the EIA's Annual Energy Outlook 2011 (Early Release Overview), which was projected to grow at an annual nominal rate of $1.8 \%$ between 2009 and 2035 . [14]

\subsubsection{Hydrogen Cost I Electrolysis Basis}

One of the most significant cost contributors to the cost of the SNG (cost of product) is the cost of hydrogen. As such, definition the basis of the hydrogen cost is an important input to the cost of SNG product analysis.

The electrolysis unit will be excluded from the SNG plant engineering, performance and cost. The electrolysis unit cost will only be considered in this analysis to the extent that it is an input to the Hydrogen cost.

\subsubsection{Electrolysis}

APS has engaged several electrolysis vendors over the life of the project. Communication with Hydrogen Technologies, (formerly Norsk Hydro Electrolysers AS) was particular useful as they helped APS understand how the electrolysis technology might develop for such a high volume application. The electrolysis product requirements provided to Hydrogen Tehcnologies is presented in Exhibit 3-4.

\section{Exhibit 3-4 Electrolysis Products}

\begin{tabular}{|l|l|l|}
\hline Product & Parameter & Notes \\
\hline High Pressure Hydrogen & $\begin{array}{l}\text { Purity }=99.99 \%, \\
\mathrm{P}=1150 \mathrm{psig} \\
\mathrm{T}=104^{\circ} \mathrm{F}\left(40^{\circ} \mathrm{C}\right)\end{array}$ & $\begin{array}{l}\text { Requires De-oxygenation to reach specified purity } \\
\text { The product pressure is based on a gasifier operating pressure of } 1000 \\
\text { psig, } 100 \text { psid for flow control/ injection, and } 50 \text { psid for heating. }\end{array}$ \\
\hline High Pressure Oxygen & $\begin{array}{l}\text { Purity }=99.5 \%, \\
\mathrm{P}=1120 \mathrm{psig} \\
\mathrm{T}=104^{\circ} \mathrm{F}\left(40^{\circ} \mathrm{C}\right)\end{array}$ & $\begin{array}{l}\text { The product pressure is based on a gasifier operating pressure of } 1000 \\
\text { psig, } 100 \text { psid for flow control/ injection, and } 20 \text { psid for heating. }\end{array}$ \\
\hline Low Pressure Oxygen & $\begin{array}{l}\mathrm{Purity}=99.5 \%, \\
\mathrm{P}=50 \mathrm{psig} \\
\mathrm{T}=77^{\circ} \mathrm{F}\end{array}$ & For oxy-combustion. \\
\hline
\end{tabular}

Reference: [15] 
The cost of Hydrogen will be developed from the parameters based in Exhibit 3-5.

\section{Exhibit 3-5 Electrolysis Design \& Cost Basis}

\begin{tabular}{|c|c|c|}
\hline Product & Parameter & Notes \\
\hline Energy Consumption of Plant & $5.5 \mathrm{kWh} / \mathrm{Nm} 3$ of $\mathrm{H} 2$ & $\begin{array}{l}\text { Equivalent to } 62 \mathrm{kWh} / \mathrm{kg} \mathrm{H} 2 \text {, or } 63.7 \% \mathrm{HHV} \text {. } \\
\text { This consumption accounts for the product delivery } \\
\text { pressure and purity). Ref [16] }\end{array}$ \\
\hline Electrolysis Technology & 500 Nm3/h Atmospheric Alkaline Unit & \\
\hline $\begin{array}{l}\text { Cost of Electrolysis Plant } \\
\text { (for } 500 \mathrm{Nm} 3 / \mathrm{h} \text { capacity) }\end{array}$ & $\begin{array}{l}1.35 \text { USD }(2010) \text { at } 70 \% \text { reduction } \\
\text { Basis [16]: } \\
4.5 M \text { USD (2010 base }- \text { single unit) } \\
\text { High volume cost reduction of } 60-70 \% \\
\end{array}$ & $\begin{array}{l}\text { This cost is for the plant, not just the electrolysis unit. } \\
\text { The cost covers the deoxo unit for oxygen removal } \\
\text { from the } \mathrm{H} 2 \text { and product pressurization. } \\
\text { High volume refers to several hundred units. }\end{array}$ \\
\hline Basis of the Electrolysis Plant & $\begin{array}{l}\text { Electrolysis Unit } \\
\text { Water Purification (filtration, RO) } \\
\text { KOH storage and Mixing Tank } \\
\text { Stepdown Transformers \& Rectifiers } \\
\text { Product Purification, drying, and } \\
\quad \text { compression }\end{array}$ & $\begin{array}{l}\text { Excluded from the cost scope from STATOIL are: } \\
\text { Installation } \\
\text { Utilities (cooling water, air) }\end{array}$ \\
\hline Operational Cost & $3 \%$ of the investment cost per year & $\begin{array}{l}\text { According to Norsk Hydro, now Hydrogen } \\
\text { Technologies, this would cover the major overhauls } \\
\text { that typically take place every } 7^{\text {th }} \text { year. [17] }\end{array}$ \\
\hline Electrolyzer cell replacement & $\begin{array}{l}\text { Every } 7 \text { years. } \\
\text { Cost }=35 \% \text { of original cost }\end{array}$ & $\begin{array}{l}\text { per NREL Reference [18]. This is redundant of the } \\
\text { operational cost above. The above will be used. }\end{array}$ \\
\hline Full Time Employee & 1 FTE per 8 units (@ 500 Nm3/h) & $\begin{array}{l}\text { Per Ref } 18 \text {, vendors quoted } 5-10 \text { for } 50,000 \mathrm{~kg} / \mathrm{d} \text {, } \\
\text { which is equivalent to } 45(500 \mathrm{Nm} 3 / \mathrm{h}) \text { units. This is } 1 \\
\text { FTE per } 4.5 \text { to } 9 \text { units. }\end{array}$ \\
\hline
\end{tabular}

References: $[15,16,17,18]$

\subsubsection{Electric Power Supply}

Hydrogen will be produced on an as needed basis utilizing the electric rate at the time of production. APS has indicated that the projected electric prices for the Four Corners plant are as presented in Exhibit 3-6 and Exhibit 3-7. Since the electric demand and rates are the highest in July, the scheduled outage will be scheduled for the month of July. Thus, the annual average electric rate, excluding July, of \$33.26/MWh will be utilized in the development of the Hydrogen cost. The electric rate of $\$ 33.26 / \mathrm{MWh}$ reflects the composite rate of the on- and off-peak rates for 11 months of the year. 
Exhibit 3-6 Peak \& Off-Peak Electric Rates

\begin{tabular}{|c|r|r|r|}
\hline & \multicolumn{3}{|c|}{ Four Corners Power Cost } \\
\hline Future Month & $\begin{array}{r}\text { On-Peak } \\
\text { (\$/MWh) }\end{array}$ & $\begin{array}{r}\text { Off-Peak } \\
\text { (\$/MWh) }\end{array}$ & $\begin{array}{c}\text { Diff } \\
\text { (\$/MWh) }\end{array}$ \\
\hline $9 / 1 / 2010$ & $\$ 35.09$ & $\$ 24.34$ & $\$ 10.75$ \\
\hline $10 / 1 / 2010$ & $\$ 32.65$ & $\$ 23.00$ & $\$ 9.65$ \\
\hline $11 / 1 / 2010$ & $\$ 33.00$ & $\$ 25.75$ & $\$ 7.25$ \\
\hline $12 / 1 / 2010$ & $\$ 36.65$ & $\$ 27.25$ & $\$ 9.40$ \\
\hline $1 / 1 / 2011$ & $\$ 39.34$ & $\$ 28.76$ & $\$ 10.58$ \\
\hline $2 / 1 / 2011$ & $\$ 38.15$ & $\$ 27.86$ & $\$ 10.30$ \\
\hline $3 / 1 / 2011$ & $\$ 36.51$ & $\$ 26.64$ & $\$ 9.87$ \\
\hline $4 / 1 / 2011$ & $\$ 34.12$ & $\$ 20.40$ & $\$ 13.72$ \\
\hline $5 / 1 / 2011$ & $\$ 36.89$ & $\$ 22.09$ & $\$ 14.80$ \\
\hline $6 / 1 / 2011$ & $\$ 46.49$ & $\$ 28.76$ & $\$ 17.72$ \\
\hline $7 / 1 / 2011$ & $\$ 54.38$ & $\$ 35.34$ & $\$ 19.04$ \\
\hline $8 / 1 / 2011$ & $\$ 53.67$ & $\$ 35.30$ & $\$ 18.37$ \\
\hline & & & \\
\hline Average (12 Month) & $\$ 39.74$ & $\$ 27.12$ & \\
\hline Average (minus July) & $\$ 38.41$ & $\$ 26.38$ & \\
\hline & & & \\
\hline Hours Per Week & 96.0 & 72.0 & \\
\hline Ave (minus Jul) Composite & $\$ 33.26$ & & \\
\hline Note: & & & \\
\hline
\end{tabular}

1. Future prices as of $8 / 19 / 10$

2. Off-peak hours are 8 hours (10pm-6 am) M-Sat, \& all day Sunday.

Reference: [19] 


\section{Exhibit 3-7 Peak \& Off-Peak Electric Rate Chart}

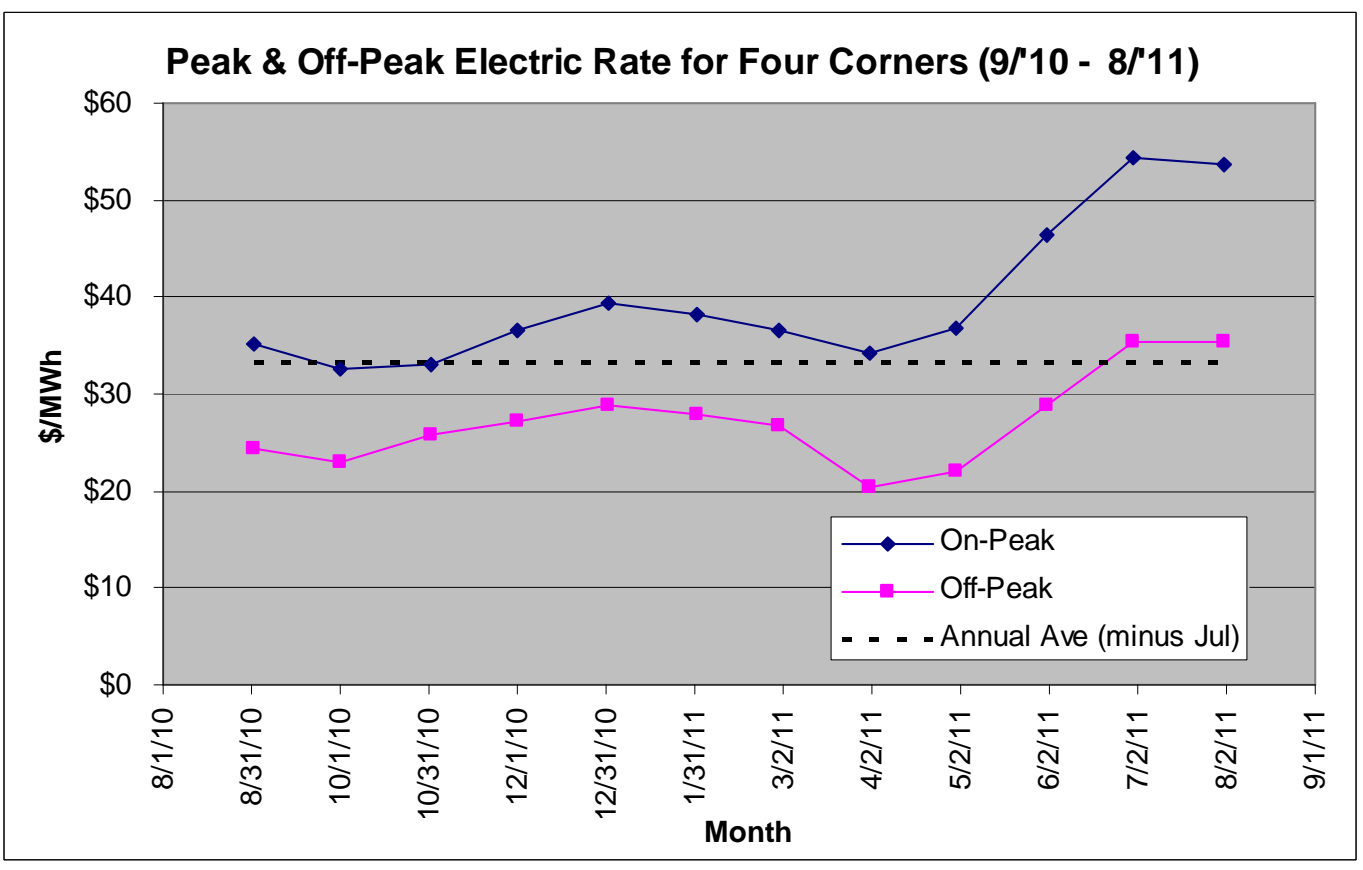

\subsubsection{Product Storage}

Since the electrolysis electric power supply will not be limited to off-peak power, bulk storage of the product gases will not be required. Electrolysis units are able to start up quickly, turn down quickly and follow the instantaneous demand requirement of the gasification plant. As such, no product storage will be considered as part of the electrolysis plant.

\subsubsection{Oxygen Cost / Electrolysis Basis}

Typically oxygen for gasification is supplied from a dedicated ASU and is quite expensive. However, since the oxygen will be co-generated by the electrolysis process and will be generated in excess compared to the hydrogen, and since the underlying cost of electrolysis will be covered by the hydrogen cost, the cost of oxygen will be free at the plant boundary. 


\section{References}

1 “APS Advanced Hydrogasification / SNG Preliminary Engineering Package (PEP)" Scope of Work document transmitted by email from Juanita Rodriguez (APS) to David Stauffer (WorleyParsons) dated June 23, 2010.

2 Design Criteria Telecon with APS, 11/06/06.

3 Cliff Keeler, ConocoPhillips, "Substitute Natural Gas (SNG), Scrubbing the Carbon in Coal and Petcoke," October 2, 2006, Gasification Technology conference, Washington DC, as found on http://www.gasification.org/Docs/2006_Papers/21CKEEL.pdf, as of November 1, 2006.

4 Email from Xiaolei "Sally" Sun (APS) to David Stauffer (WorleyParsons), RE: Conference call notes regarding the DBD, August 13, 2010.

5 Gasifier Performance Input as attached to email from Juanita Rodriguez (APS) to David Stauffer (WorleyParsons) dated June 23, 2010.

6 "Preliminary Engineering Package for the Hydrogasification/ Substitute Natural Gas Commercial Scale Facility, Conceptual Design,” Prepared by WorleyParsons, for APS, dated march 22, 2007, Rev D.

7 Email from Anders Johnson, El Paso Gas, to Sally Sun, APS, dated September 8, 2006.

8 E-mail from Anders T. Johnson, Director, Gas Control and Facility Planning, El Paso Tennessee Gas Pipeline, to Sally Sun, APS, dated March 8, 2007

10 Montana Sulphur and Chemical Company Specification Sheet. Yellowstone Brand High Purity Sulfur Prills. 05 October 2006.

11 Water analysis Report, GE Infrastructure Water \& Process Technologies, sampled 08/07/2005, reported 10/03/2005, transmitted via APS e-mail message dated 11/13/2006.

12 Email from Bruce Salisbury to Raymond Hobbs, dated November 20, 2006.

13 DOE/NETL-401/090808 - Recommended Project Finance Structures for the Economic Analysis of FossilBased Energy Projects, DOE/NETL-401/090808, September 2008.

14 “Annual Energy Outlook 2011 Early Release Overview,” U.S. Energy Information Administration, as found on www.eia.gov/forecasts/aeo/pdf January, 12, 2011.

15 Email from Donna Rennemo (Hydrogen Technologies) to Xiaolei "Sally" Sun (APS) and David Stauffer (WorleyParsons), RE: Electrolysis Performance and Cost Confirmation, October 7, 2010.

16 Email from Donna Rennemo (Hydrogen Technologies) to Xiaolei "Sally" Sun (APS), RE: Electrolysis Performance, September 28, 2010.

17 Norsk Hydro Electrolysers AS Memo from Roy Grelland, provided to Ray Hobbs, dated 2006-07-12.

18 Current (2009) State-of-the-Art Hydrogen Production Cost Estimate Using Water Electolysis, Independent Review, published for the U.S. DOE Hydrogen Program, NREL/BK-6A1-46676, September 2009.

19 Email from Xiaolei "Sally" Sun (APS) to David Stauffer (WorleyParsons), RE: APS Peak / Off Peak Electric Rates, August 23, 2010. 


\section{WW WorleyParsons \\ resources \& energy}

APS Hydrogasification SNG

Preliminary Engineering Package

Appendices

\section{Appendix B: \\ Process Flow Diagrams}




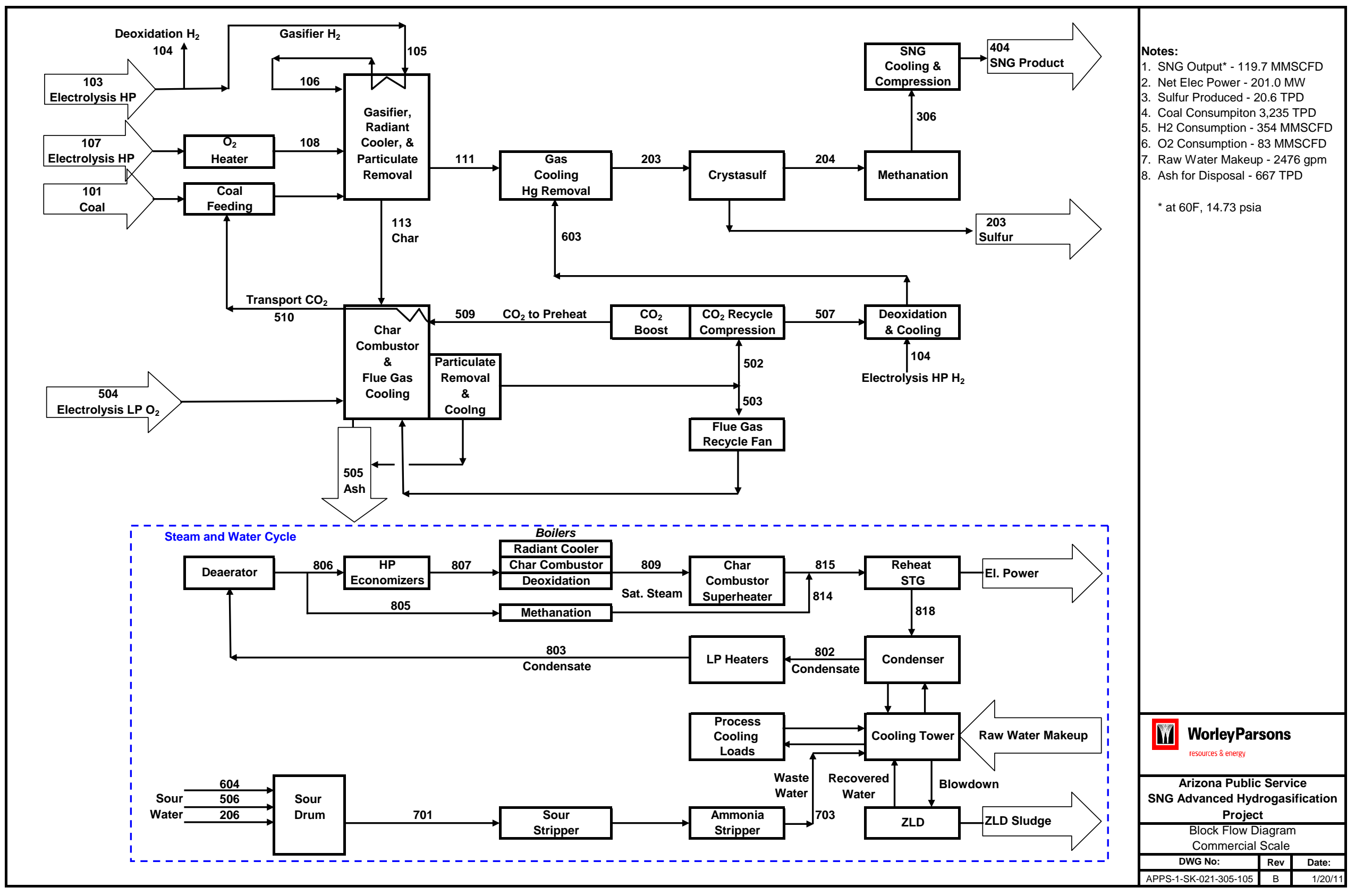




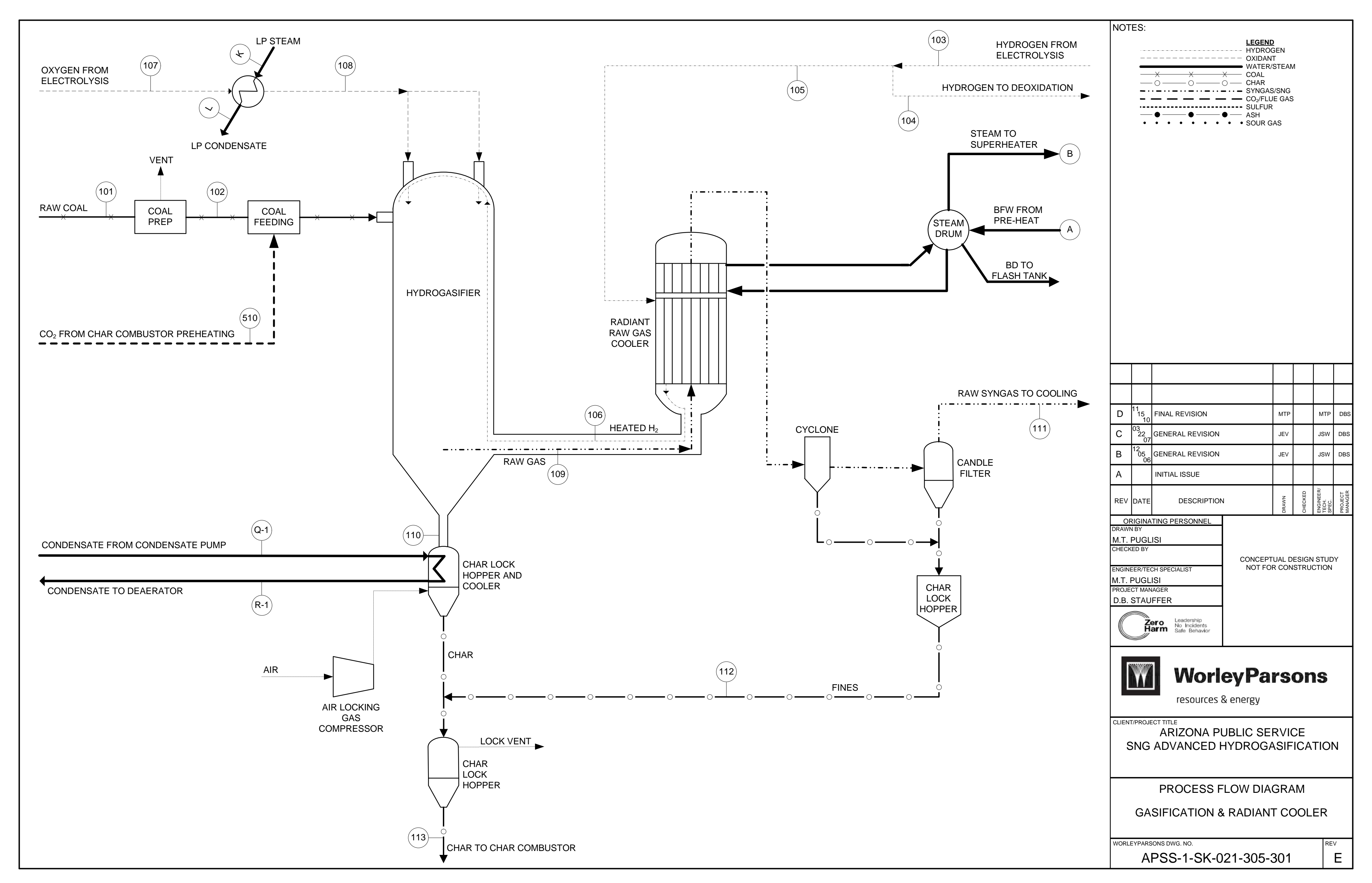



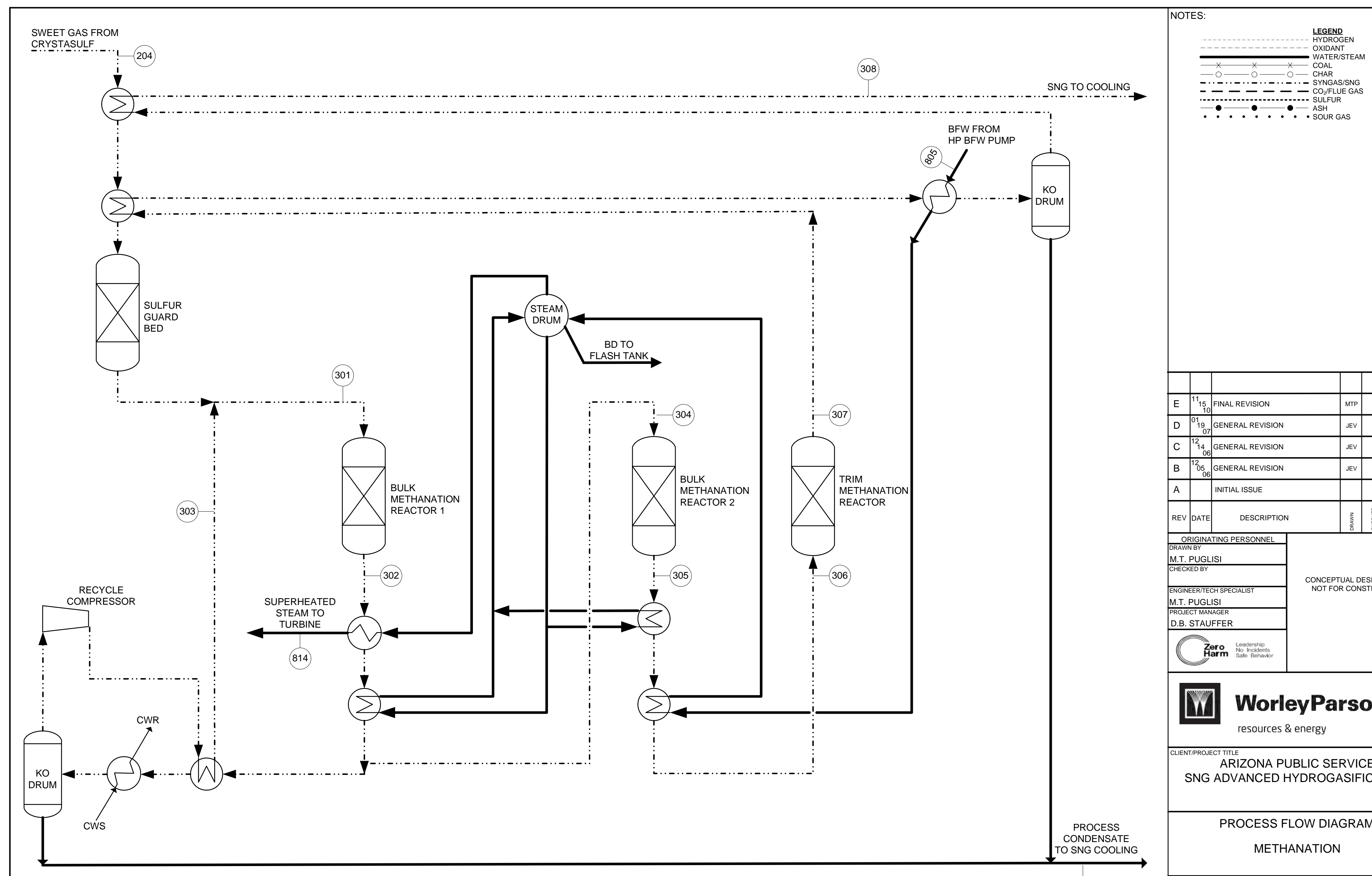

309

APSS-1-SK-021-305-303 


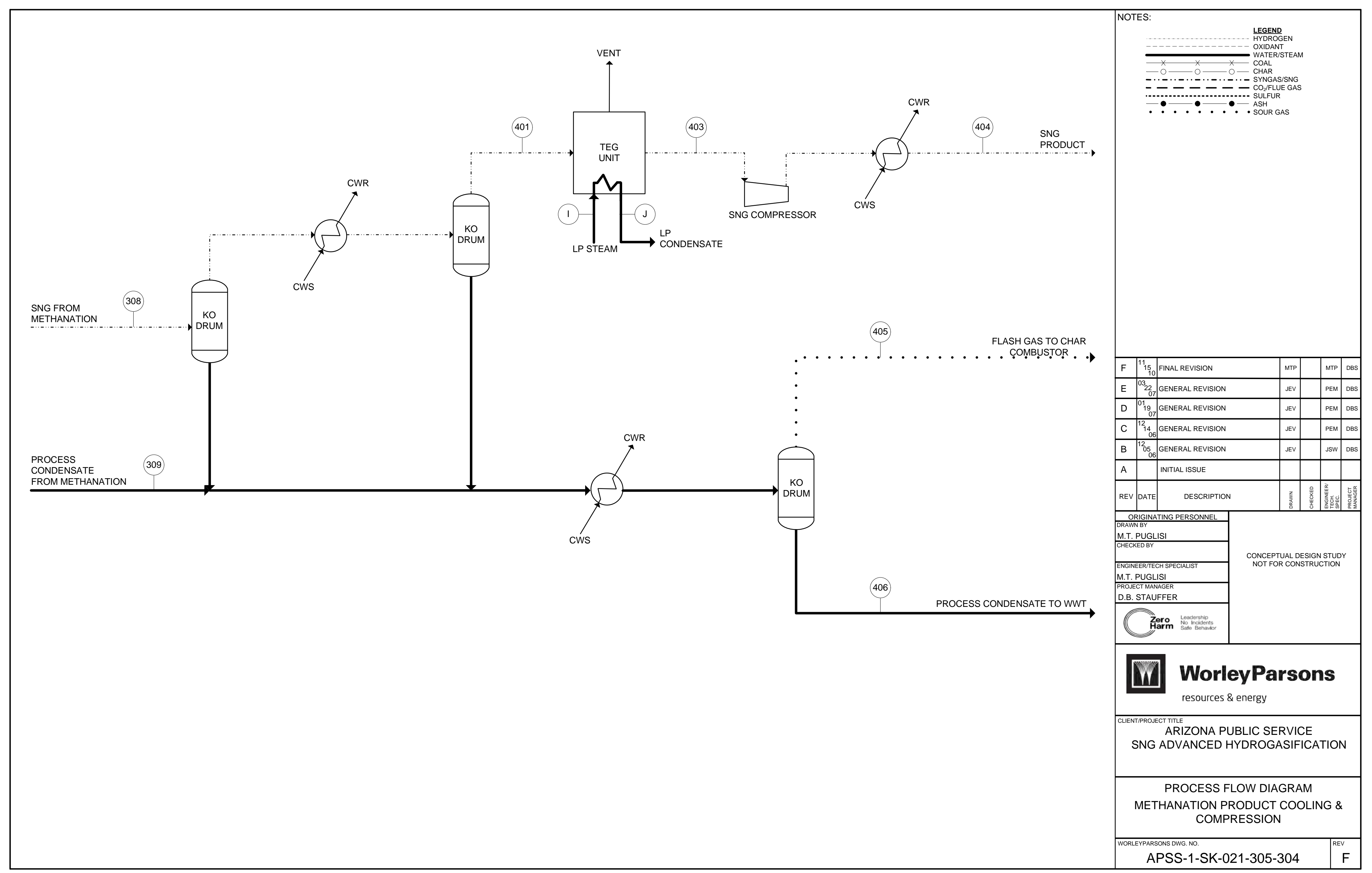




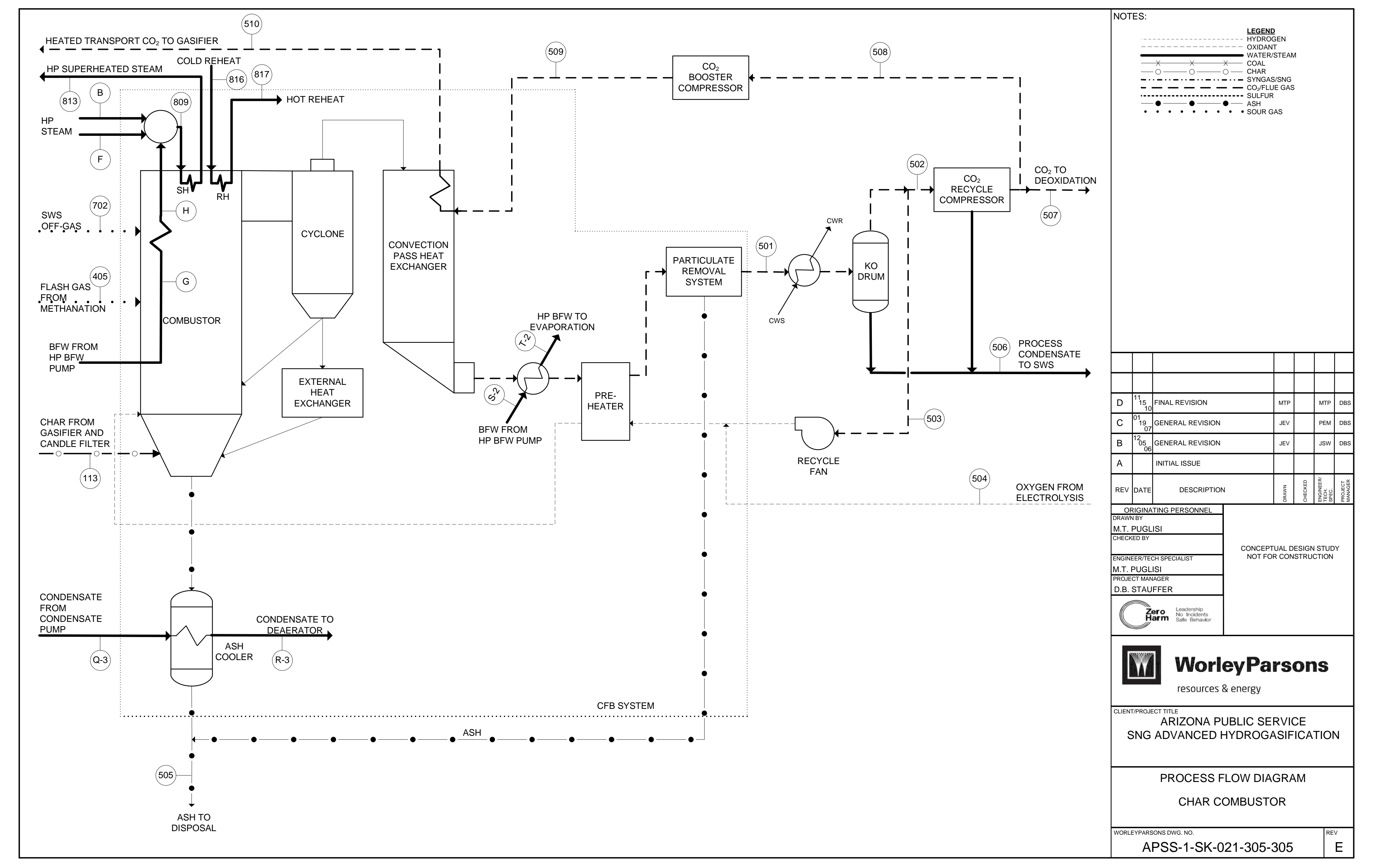




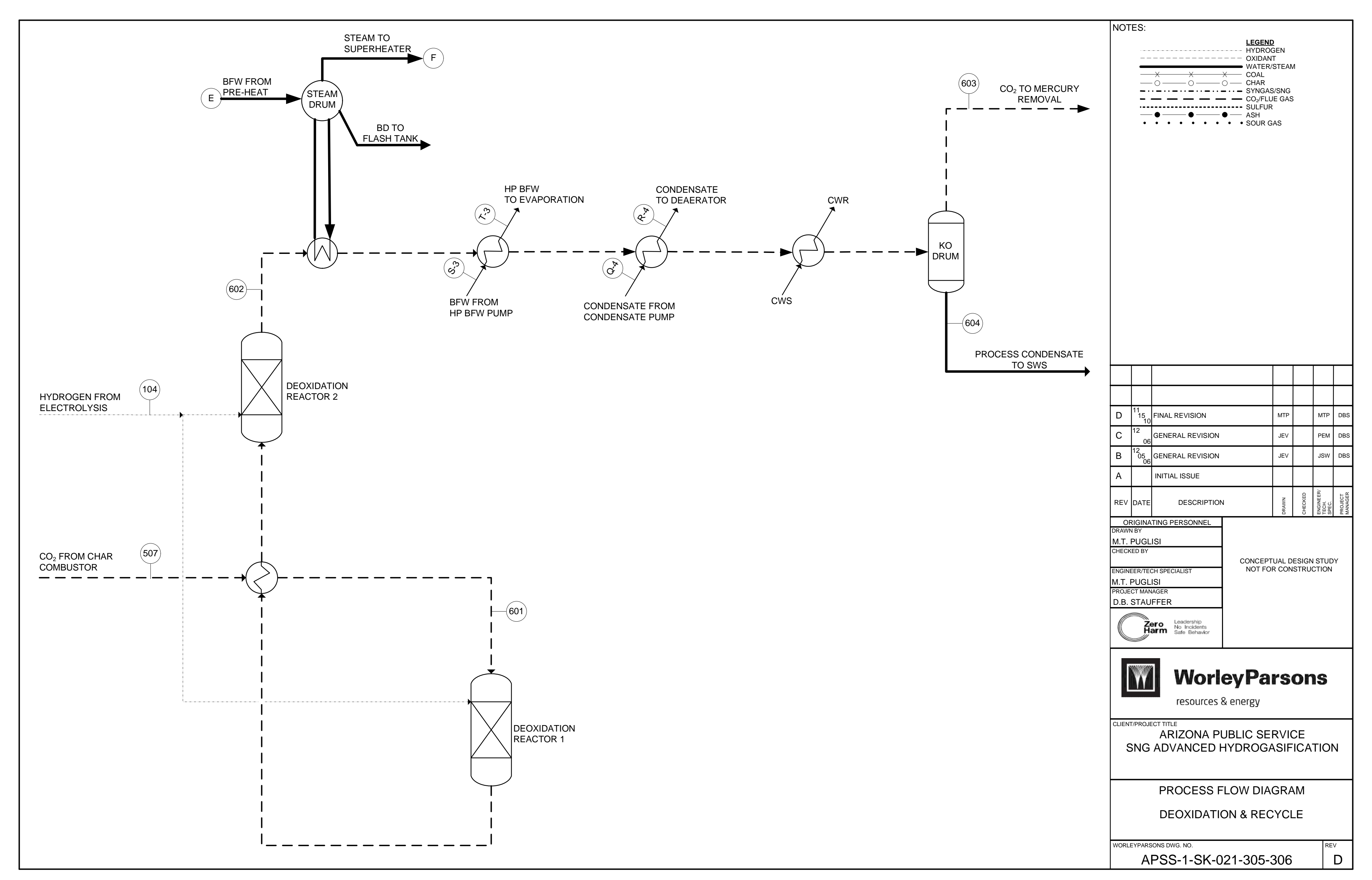




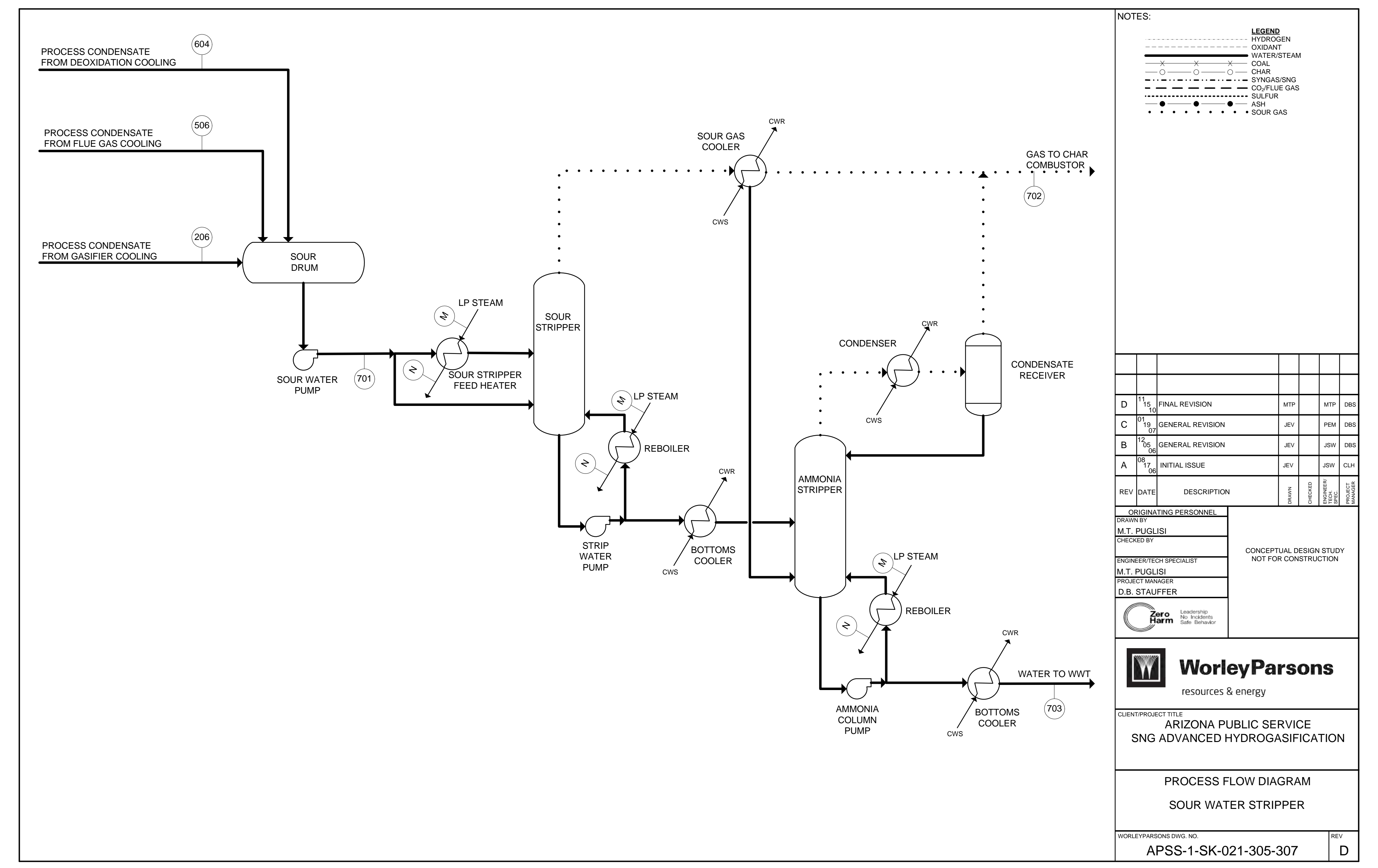




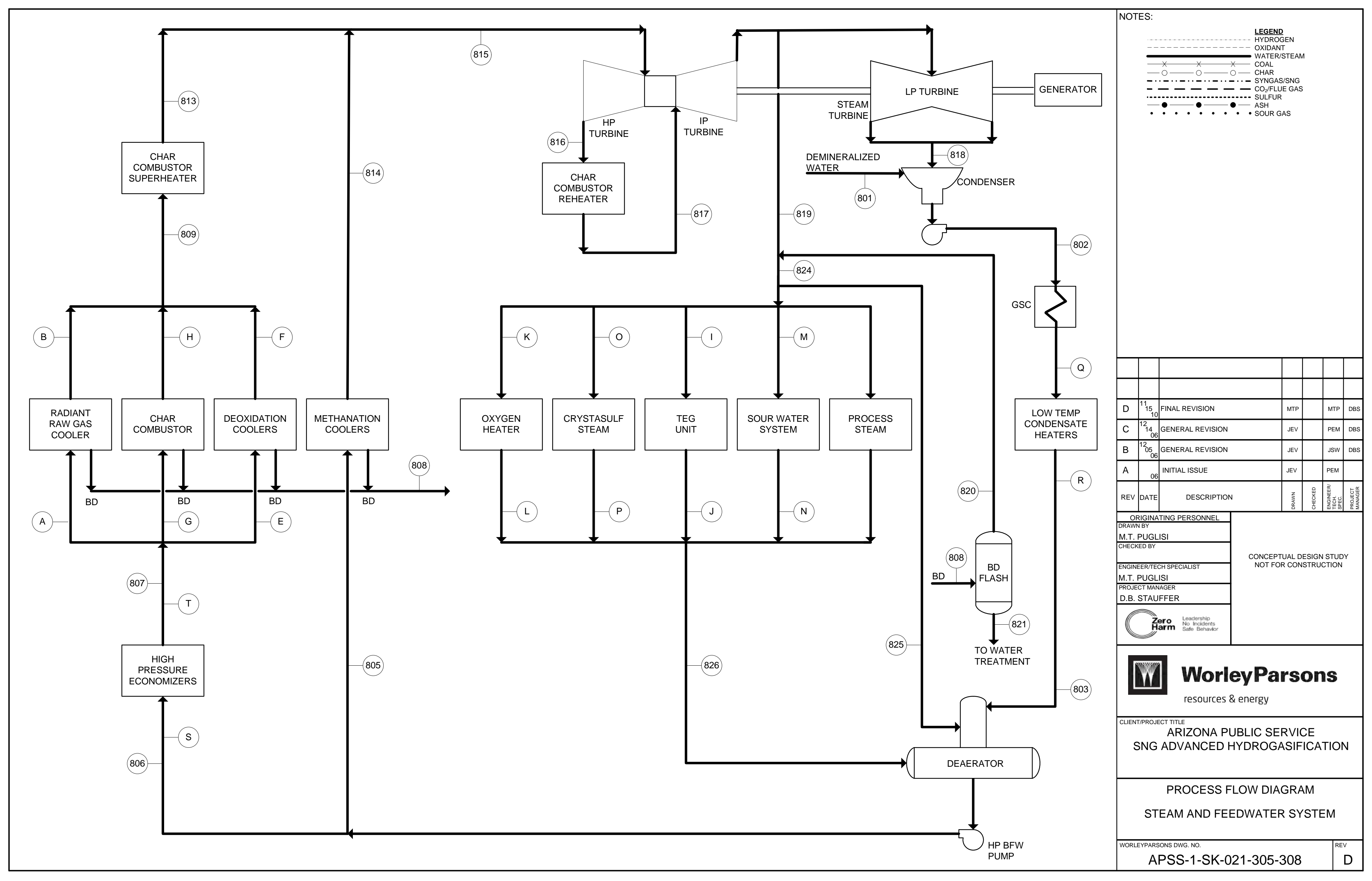




\section{Appendix C: \\ Material Balances}


WVI WorleyParsons resources \& energy

\begin{tabular}{|c|c|c|c|c|c|c|c|c|c|c|c|c|c|c|c|c|}
\hline$\longdiv { \text { PFD Stream No. } }$ & 10 & & \multicolumn{2}{|c|}{102} & \multicolumn{2}{|c|}{103} & \multicolumn{2}{|c|}{104} & \multicolumn{2}{|c|}{105} & \multicolumn{2}{|c|}{106} & \multicolumn{2}{|c|}{107} & \multicolumn{2}{|c|}{108} \\
\hline DESCRIPTION & \multicolumn{2}{|c|}{ Raw Coal Feed } & \multicolumn{2}{|c|}{ Dried Coal Feed } & \multicolumn{2}{|c|}{$\begin{array}{l}\text { Hydrogen from } \\
\text { Electrolysis }\end{array}$} & \multicolumn{2}{|c|}{$\begin{array}{l}\text { Hydrogen to } \\
\text { Deoxidation }\end{array}$} & \multicolumn{2}{|c|}{$\begin{array}{l}\text { Hydrogen to } \\
\text { Radiant Cooler }\end{array}$} & \multicolumn{2}{|c|}{$\begin{array}{c}\text { Heated Hydrogen to } \\
\text { Gasifier }\end{array}$} & \multicolumn{2}{|c|}{$\begin{array}{l}\text { Oxygen from } \\
\text { Electrolysis }\end{array}$} & \multicolumn{2}{|c|}{ Oxygen to Gasifier } \\
\hline \multicolumn{17}{|c|}{ Vapor \& Liquid } \\
\hline V\&L Mixture Components & Ibmol/hr & Mol \% & Ibmol/hr & Mol \% & Ibmol/hr & Mol \% & Ibmol/hr & $\mathrm{Mol} \%$ & Ibmol/hr & Mol \% & $\mathrm{Ibmol} / \mathrm{hr}$ & Mol \% & Ibmol/hr & Mol \% & Ibmol/hr & Mol \% \\
\hline $\mathrm{CH}_{4}$ & 0.0 & 0.000 & 0.0 & 0.000 & 0.0 & 0.000 & 0.0 & 0.000 & 0.0 & 0.000 & 0.0 & 0.000 & 0.0 & 0.000 & 0.0 & 0.000 \\
\hline $\mathrm{C}_{2} \mathrm{H}_{6}$ & 0.0 & 0.000 & 0.0 & 0.000 & 0.0 & 0.000 & 0.0 & 0.000 & 0.0 & 0.000 & 0.0 & 0.000 & 0.0 & 0.000 & 0.0 & 0.000 \\
\hline $\mathrm{CO}$ & 0.0 & 0.000 & 0.0 & 0.000 & 0.0 & 0.000 & 0.0 & 0.000 & 0.0 & 0.000 & 0.0 & 0.000 & 0.0 & 0.000 & 0.0 & 0.000 \\
\hline $\mathrm{CO}_{2}$ & 0.0 & 0.000 & 0.0 & 0.000 & 0.0 & 0.000 & 0.0 & 0.000 & 0.0 & 0.000 & 0.0 & 0.000 & 0.0 & 0.000 & 0.0 & 0.000 \\
\hline $\mathrm{H}_{2}$ & 0.0 & 0.000 & 0.0 & 0.000 & $38,919.7$ & 99.990 & 569.1 & 99.990 & $38,350.6$ & 99.990 & $38,350.6$ & 99.990 & 10.2 & 0.500 & 10.2 & 0.500 \\
\hline $\mathrm{H}_{2} \mathrm{O}$ (and ionic species) & $1,088.9$ & 100.000 & 578.7 & 100.000 & 0.0 & 0.000 & 0.0 & 0.000 & 0.0 & 0.000 & 0.0 & 0.000 & 0.0 & 0.000 & 0.0 & 0.000 \\
\hline $\mathrm{H}_{2} \mathrm{~S}$ & 0.0 & 0.000 & 0.0 & 0.000 & 0.0 & 0.000 & 0.0 & 0.000 & 0.0 & 0.000 & 0.0 & 0.000 & 0.0 & 0.000 & 0.0 & 0.000 \\
\hline $\mathrm{N}_{2}$ & 0.0 & 0.000 & 0.0 & 0.000 & 0.0 & 0.000 & 0.0 & 0.000 & 0.0 & 0.000 & 0.0 & 0.000 & 0.0 & 0.000 & 0.0 & 0.000 \\
\hline $\mathrm{NH}_{3}$ & 0.0 & 0.000 & 0.0 & 0.000 & 0.0 & 0.000 & 0.0 & 0.000 & 0.0 & 0.000 & 0.0 & 0.000 & 0.0 & 0.000 & 0.0 & 0.000 \\
\hline $\mathrm{O}_{2}$ & 0.0 & 0.000 & 0.0 & 0.000 & 3.9 & 0.010 & 0.1 & 0.010 & 3.8 & 0.010 & 3.8 & 0.010 & $2,038.4$ & 99.500 & $2,038.4$ & 99.500 \\
\hline $\mathrm{SO}_{2}$ & 0.0 & 0.000 & 0.0 & 0.000 & 0.0 & 0.000 & 0.0 & 0.000 & 0.0 & 0.000 & 0.0 & 0.000 & 0.0 & 0.000 & 0.0 & 0.000 \\
\hline TOTAL & $1,088.9$ & 100.000 & 578.7 & 100.000 & $38,923.6$ & 100.000 & 569.1 & 100.000 & $38,354.4$ & 100.000 & $38,354.4$ & 100.000 & $2,048.6$ & 100.000 & $2,048.6$ & 100.000 \\
\hline V\&L Mixture Components & $\mathrm{tb} / \mathrm{hr}$ & Mass \% & $\mathrm{Ib} / \mathrm{hr}$ & Mass \% & $\mathrm{Ib} / \mathrm{hr}$ & Mass \% & $\mathrm{lb} / \mathrm{hr}$ & Mass $\%$ & $\mathrm{lb} / \mathrm{hr}$ & Mass \% & $\mathrm{lb} / \mathrm{hr}$ & Mass \% & $\mathrm{lb} / \mathrm{hr}$ & Mass \% & $\mathrm{Ib} / \mathrm{hr}$ & Mass \% \\
\hline $\mathrm{CH}_{4}$ & 0 & 0.000 & 0 & 0.000 & $\overline{0}$ & 0.000 & 0 & 0.000 & 0 & 0.000 & 0 & 0.000 & 0 & 0.000 & 0 & 0.000 \\
\hline $\mathrm{C}_{2} \mathrm{H}_{6}$ & 0 & 0.000 & 0 & 0.000 & 0 & 0.000 & 0 & 0.000 & 0 & 0.000 & 0 & 0.000 & 0 & 0.000 & 0 & 0.000 \\
\hline $\mathrm{CO}$ & 0 & 0.000 & 0 & 0.000 & 0 & 0.000 & 0 & 0.000 & 0 & 0.000 & 0 & 0.000 & 0 & 0.000 & 0 & 0.000 \\
\hline $\mathrm{CO}_{2}$ & 0 & 0.000 & 0 & 0.000 & 0 & 0.000 & 0 & 0.000 & 0 & 0.000 & 0 & 0.000 & 0 & 0.000 & 0 & 0.000 \\
\hline $\mathrm{H}_{2}$ & 0 & 0.000 & 0 & 0.000 & 78,457 & 99.842 & 1,147 & 99.842 & 77,310 & 99.842 & 77,310 & 99.842 & 21 & 0.032 & 21 & 0.032 \\
\hline $\mathrm{H}_{2} \mathrm{O}$ (and ionic species) & 19,600 & 100.000 & 10,417 & 100.000 & 0 & 0.000 & 0 & 0.000 & 0 & 0.000 & 0 & 0.000 & 0 & 0.000 & 0 & 0.000 \\
\hline $\mathrm{H}_{2} \mathrm{~S}$ & 0 & 0.000 & 0 & 0.000 & 0 & 0.000 & 0 & 0.000 & 0 & 0.000 & 0 & 0.000 & 0 & 0.000 & 0 & 0.000 \\
\hline $\mathrm{N}_{2}$ & 0 & 0.000 & 0 & 0.000 & 0 & 0.000 & 0 & 0.000 & 0 & 0.000 & 0 & 0.000 & 0 & 0.000 & 0 & 0.000 \\
\hline $\mathrm{NH}_{3}$ & 0 & 0.000 & 0 & 0.000 & 0 & 0.000 & 0 & 0.000 & 0 & 0.000 & 0 & 0.000 & 0 & 0.000 & 0 & 0.000 \\
\hline $\mathrm{O}_{2}$ & 0 & 0.000 & 0 & 0.000 & 125 & 0.158 & 2 & 0.158 & 123 & 0.158 & 123 & 0.158 & 65,226 & 99.968 & 65,226 & 99.968 \\
\hline $\mathrm{SO}_{2}$ & 0 & 0.000 & 0 & 0.000 & 0 & 0.000 & 0 & 0.000 & 0 & 0.000 & 0 & 0.000 & 0 & 0.000 & 0 & 0.000 \\
\hline TOTAL & 19,600 & 100.000 & 10,417 & 100.000 & 78,582 & 100.000 & 1,149 & 100.000 & 77,433 & 100.000 & 77,433 & 100.000 & 65,246 & 100.000 & 65,246 & 100.000 \\
\hline \multicolumn{17}{|l|}{ V\&L Mixture Components } \\
\hline Total Flow, acfm & \multirow{2}{*}{\multicolumn{2}{|c|}{$\begin{array}{ll}-- \\
---\end{array}$}} & & - & 3,5 & & & 51 & $\overline{3,4}$ & & 10,6 & & $\overline{1}$ & & & 17 \\
\hline Vapor Frac & & & & - & 1.0 & & $\overline{1.0}$ & & 1.0 & & 1.0 & & 1.0 & & 1.0 & \\
\hline Temperature, $\mathrm{F}$ & 60 & & 60 & & 10 & & 10 & & 102 & & 1,250 & & 104 & & 200 & \\
\hline Pressure, psia & 12 & & 12 & & 1,16 & & 1,16 & & 1,162 & & 1,112 & & 1,135 & & 1,095 & \\
\hline Enthalpy, Btu/lb & & $\cdots$ & & -- & 104 & & 104. & & 104. & & 4,104 & & -2 & & 21. & \\
\hline Heat Capacity, Btu/lb R & & -- & & -- & 3.4 & & 3.4 & & 3.4 & & 3.5 & & 0.2 & & 0.2 & \\
\hline Density, Ib/ft ${ }^{3}$ & & -- & & -- & 0.3 & & 0.3 & & 0.3 & & 0.1 & & 6.2 & & 5.0 & \\
\hline Molecular Weight & & -- & & - & 2.0 & & 2.0 & & 2.0 & & 2.0 & & 31.8 & & 31.8 & \\
\hline & & & & & & & Solid & & & & & & & & & \\
\hline Solid Components & $\mathrm{Tb} / \mathrm{h}$ & & $\mathrm{Tb} / \mathrm{t}$ & & $\overline{\mathrm{Ib} /}$ & & $\overline{l b} / \mathrm{l}$ & & $\mathrm{Tb} / \mathrm{t}$ & & $\mathrm{Tb} / \mathrm{h}$ & & $\mathrm{Tb} / \mathrm{h}$ & & Tb/l & \\
\hline Coal (dry) & 250,0 & & 250,0 & & & $\overline{0}$ & & $\overline{0}$ & & $\overline{0}$ & & $\overline{0}$ & & $\overline{0}$ & & $\overline{0}$ \\
\hline Char & & 0 & & 0 & & 0 & & 0 & & 0 & & 0 & & 0 & & 0 \\
\hline Ash & & 0 & & 0 & & 0 & & 0 & & 0 & & 0 & & 0 & & 0 \\
\hline Sulfur & & 0 & & 0 & & 0 & & 0 & & 0 & & 0 & & 0 & & 0 \\
\hline TOTAL & 250,0 & & 250,0 & & & 0 & & 0 & & 0 & & 0 & & 0 & & 0 \\
\hline
\end{tabular}

1. Results based on bench scale reactor test results communicated by APS. 
WW WorleyParsons resources \& energy

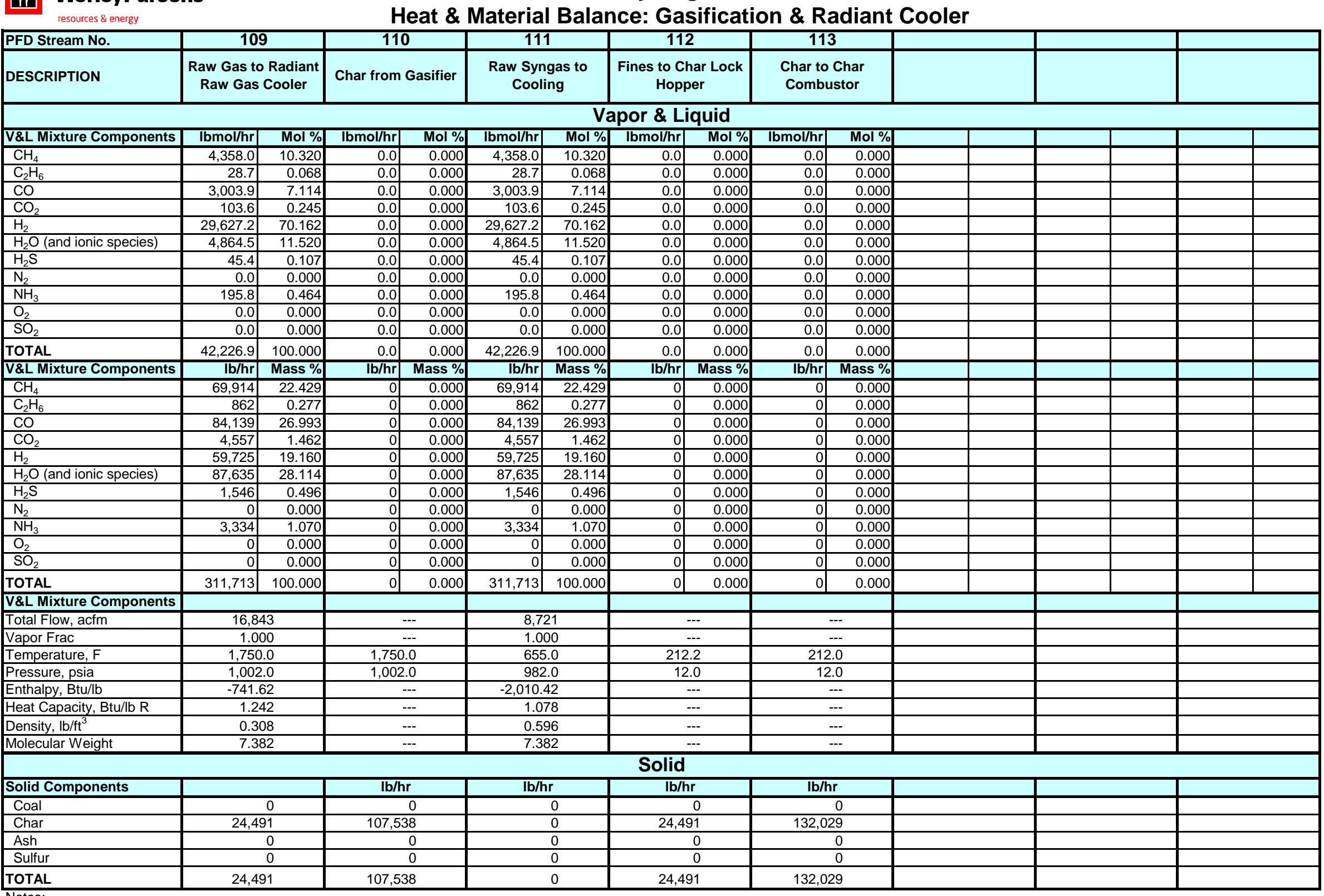

1. Results based on bench scale reactor test results communicated by APS. Heat \& Material Balance: Gasification \& Radiant Cooler

\begin{tabular}{ll|l|l|l|l}
\hline & 0.000 & 0.0 & 0.000 \\
\hline & 0.0 & 0.000 \\
\hline
\end{tabular}

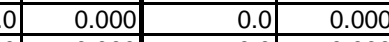

\begin{tabular}{l|l|}
0.0 & 0.000 \\
\hline
\end{tabular}

0.000

Arizona Public Service Advanced Hydrogasification I SNG -- Commercial Scale 
WiI WorleyParsons esources \& energy

\begin{tabular}{|l|l}
\hline PFD Stream No. & \\
\hline DESCRIPTION & \\
\hline
\end{tabular}

\begin{tabular}{|c|c|}
\hline 201 & \\
\hline $\begin{array}{c}\text { Cooled Syngas to } \\
\text { KO Drum }\end{array}$ & $\begin{array}{c}\text { Co } \\
\text { M }\end{array}$ \\
\hline
\end{tabular}
202 \begin{tabular}{c|c|c}
203 \\
\hline
\end{tabular} \begin{tabular}{c|c}
$\begin{array}{c}\text { Cooled Syngas to } \\
\text { Mercury Removal }\end{array}$ & $\begin{array}{c}\text { Reheated Syngas } \\
\text { Crystasulf }\end{array}$ \\
\hline
\end{tabular}

Arizona Public Service Advanced Hydrogasification I SNG -- Commercial Scale

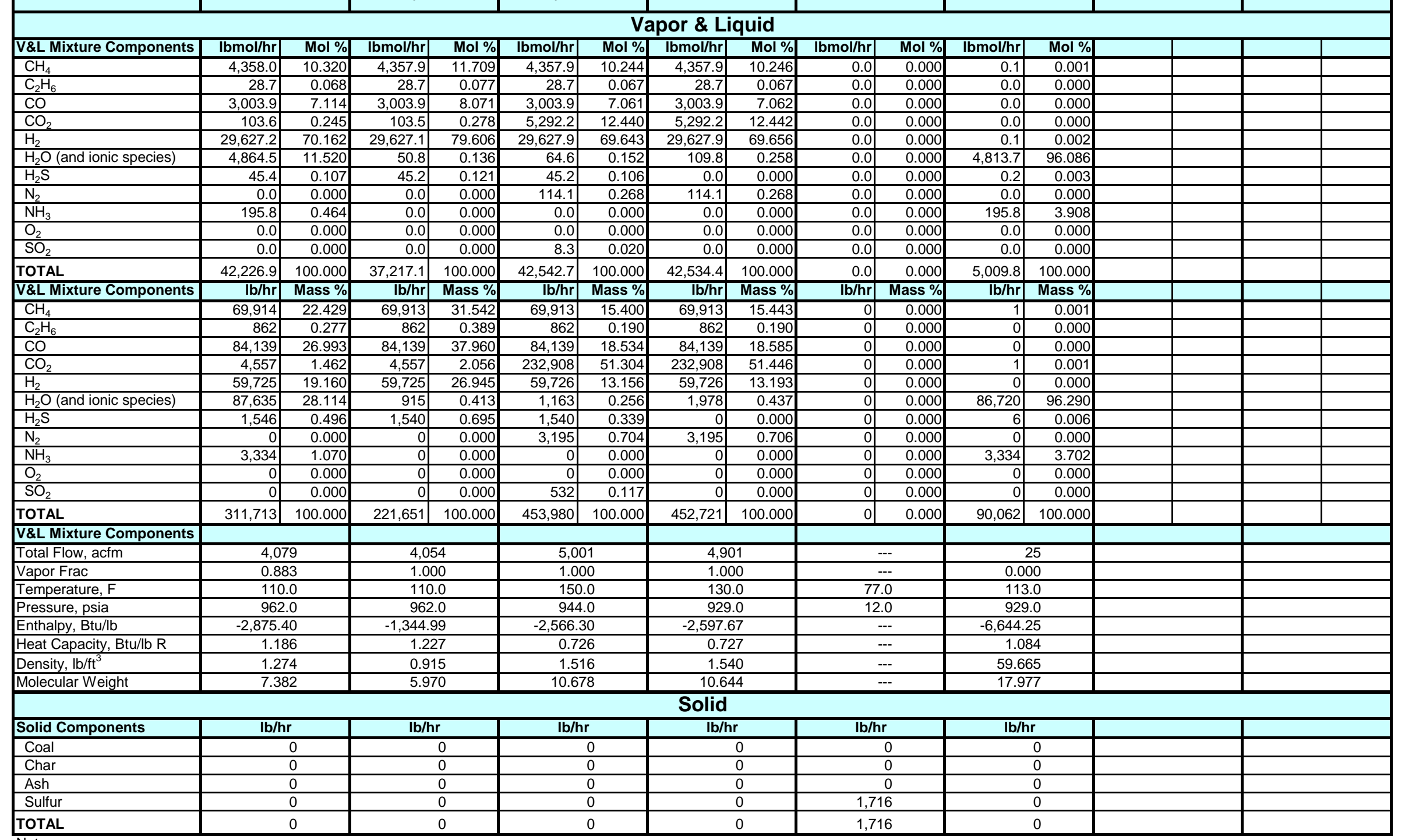

Notes:

1. Results based on bench scale reactor test results communicated by APS.

\section{: Syngas Cooling \& Sulfur Removal}

204

Sweet Gas to

weet Gas to

1.

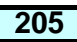

205

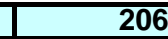

Process Condensat

to SWS

te

$(2$

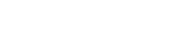

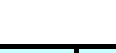

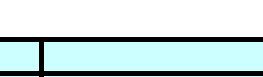


WiI WorleyParsons

resources \& energy

\begin{tabular}{|c|c|c|c|c|c|c|c|c|c|c|c|c|c|c|c|c|}
\hline PFD Stream No. & \multicolumn{2}{|c|}{301} & \multicolumn{2}{|c|}{302} & \multicolumn{2}{|c|}{303} & \multicolumn{2}{|c|}{304} & \multicolumn{2}{|c|}{305} & \multicolumn{2}{|c|}{306} & \multicolumn{2}{|c|}{307} & \multicolumn{2}{|c|}{308} \\
\hline DESCRIPTION & \multicolumn{2}{|c|}{ Bulk Reactor 1 Inlet } & \multicolumn{2}{|c|}{$\begin{array}{c}\text { Bulk Reactor } 1 \\
\text { Outlet }\end{array}$} & \multicolumn{2}{|c|}{ Recycled Syngas } & \multicolumn{2}{|c|}{ Bulk Reactor 2 Inlet } & \multicolumn{2}{|c|}{$\begin{array}{c}\text { Bulk Reactor } 2 \\
\text { Outlet }\end{array}$} & \multicolumn{2}{|c|}{ Trim Reactor Inlet } & \multicolumn{2}{|c|}{ Trim Reactor Outlet } & \multicolumn{2}{|c|}{ SNG to Cooling } \\
\hline \multicolumn{17}{|c|}{ Vapor \& Liquid } \\
\hline V\&L Mixture Components & $\mid \mathrm{bmol} / \mathrm{hr}$ & Mol \% & Ibmol/hr & Mol \% & Ibmol/hr & Mol \% & $\mid \mathrm{bmol} / \mathrm{hr}$ & Mol \% & Ibmol/hr & Mol \% & Ibmol/hr & Mol \% & $\mid \mathrm{bmol} / \mathrm{hr}$ & Mol \% & Ibmol/hr & Mol \% \\
\hline $\mathrm{CH}_{4}$ & $46,027.1$ & 36.299 & $53,428.1$ & 47.656 & $41,669.1$ & 49.450 & $11,754.2$ & 47.656 & $12,248.0$ & 51.729 & $12,248.0$ & 51.729 & $12,474.5$ & 53.714 & $12,472.1$ & 70.881 \\
\hline $\mathrm{C}_{2} \mathrm{H}_{6}$ & 28.7 & 0.023 & 0.0 & 0.000 & 0.0 & 0.000 & 0.0 & 0.000 & 0.0 & 0.000 & 0.0 & 0.000 & 0.0 & 0.000 & 0.0 & 0.000 \\
\hline $\mathrm{CO}$ & $3,372.8$ & 2.660 & 472.9 & 0.422 & 368.9 & 0.438 & 104.0 & 0.422 & 8.6 & 0.036 & 8.6 & 0.036 & 0.4 & 0.002 & 0.4 & 0.002 \\
\hline $\mathrm{CO}_{2}$ & $8,296.3$ & 6.543 & $3,852.4$ & 3.436 & $3,004.1$ & 3.565 & 847.5 & 3.436 & 449.1 & 1.897 & 449.1 & 1.897 & 230.8 & 0.994 & 230.6 & 1.310 \\
\hline $\mathrm{H}_{2}$ & $40,703.0$ & 32.100 & $14,199.1$ & 12.665 & $11,075.1$ & 13.143 & $3,123.8$ & 12.665 & $1,244.0$ & 5.254 & $1,244.0$ & 5.254 & 346.1 & 1.490 & 346.0 & 1.967 \\
\hline $\mathrm{H}_{2} \mathrm{O}$ (and ionic species) & $27,853.7$ & 21.967 & $39,641.4$ & 35.359 & $27,743.9$ & 32.924 & $8,721.1$ & 35.359 & $9,613.3$ & 40.602 & $9,613.3$ & 40.602 & $10,058.1$ & 43.309 & $4,432.7$ & 25.192 \\
\hline $\mathrm{H}_{2} \mathrm{~S}$ & 0.0 & 0.000 & 0.0 & 0.000 & 0.0 & 0.000 & 0.0 & 0.000 & 0.0 & 0.000 & 0.0 & 0.000 & 0.0 & 0.000 & 0.0 & 0.000 \\
\hline $\mathrm{N}_{2}$ & 518.4 & 0.409 & 518.4 & 0.462 & 404.3 & 0.480 & 114.0 & 0.462 & 114.0 & 0.482 & 114.0 & 0.482 & 114.0 & 0.491 & 114.0 & 0.648 \\
\hline $\mathrm{NH}_{3}$ & 0.0 & 0.000 & 0.0 & 0.000 & 0.0 & 0.000 & 0.0 & 0.000 & 0.0 & 0.000 & 0.0 & 0.000 & 0.0 & 0.000 & 0.0 & 0.000 \\
\hline $\mathrm{O}_{2}$ & 0.0 & 0.000 & 0.0 & 0.000 & 0.0 & 0.000 & 0.0 & 0.000 & 0.0 & 0.000 & 0.0 & 0.000 & 0.0 & 0.000 & 0.0 & 0.000 \\
\hline $\mathrm{SO}_{2}$ & 0.0 & 0.000 & 0.0 & 0.000 & 0.0 & 0.000 & 0.0 & 0.000 & 0.0 & 0.000 & 0.0 & 0.000 & 0.0 & 0.000 & 0.0 & 0.000 \\
\hline TOTAL & $126,799.9$ & 100.000 & $112,112.3$ & 100.000 & $84,265.5$ & 100.000 & $24,664.7$ & 100.000 & $23,677.1$ & 100.000 & $23,677.1$ & 100.000 & $23,224.1$ & 100.000 & $17,595.8$ & 100.000 \\
\hline V\&L Mixture Components & $\mathrm{Tb} / \mathrm{hr}$ & Mass \% & $\mathrm{Ib} / \mathrm{hr}$ & Mass \% & $\mathrm{Ib} / \mathrm{hr}$ & Mass \% & $\mathrm{Tb} / \mathrm{hr}$ & Mass $\%$ & $\mathrm{~Tb} / \mathrm{hr}$ & Mass \% & Tb/hr & Mass $\%$ & $\mid \mathrm{lb} / \mathrm{hr}$ & Mass \% & Tb/hr & Mass \% \\
\hline $\mathrm{CH}_{4}$ & 738,402 & 41.086 & 857,136 & 47.692 & 668,487 & 49.720 & 188,570 & 47.692 & 196,492 & 49.696 & 196,492 & 49.696 & 200,126 & 50.615 & 200,087 & 68.059 \\
\hline $\mathrm{C}_{2} \mathrm{H}_{6}$ & 862 & 0.048 & 0 & 0.000 & 0 & 0.000 & 0 & 0.000 & 0 & 0.000 & 0 & 0.000 & 0 & 0.000 & 0 & 0.000 \\
\hline $\mathrm{CO}$ & 94,472 & 5.257 & 13,247 & 0.737 & 10,333 & 0.769 & 2,914 & 0.737 & 242 & 0.061 & 242 & 0.061 & 11 & 0.003 & 11 & 0.004 \\
\hline $\mathrm{CO}_{2}$ & 365,118 & 20.316 & 169,541 & 9.434 & 132,211 & 9.833 & 37,299 & 9.434 & 19,765 & 4.999 & 19,765 & 4.999 & 10,159 & 2.569 & 10,147 & 3.451 \\
\hline $\mathrm{H}_{2}$ & 82,052 & 4.566 & 28,624 & 1.593 & 22,326 & 1.661 & 6,297 & 1.593 & 2,508 & 0.634 & 2,508 & 0.634 & 698 & 0.176 & 698 & 0.237 \\
\hline $\mathrm{H}_{2} \mathrm{O}$ (and ionic species) & 501,792 & 27.920 & 714,150 & 39.736 & 499,815 & 37.175 & 157,113 & 39.736 & 173,187 & 43.802 & 173,187 & 43.802 & 181,200 & 45.828 & 79,856 & 27.163 \\
\hline $\mathrm{H}_{2} \mathrm{~S}$ & 0 & 0.000 & 0 & 0.000 & 0 & 0.000 & 0 & 0.000 & 0 & 0.000 & 0 & 0.000 & 0 & 0.000 & 0 & 0.000 \\
\hline $\mathrm{N}_{2}$ & 14,522 & 0.808 & 14,522 & 0.808 & 11,326 & 0.842 & 3,195 & 0.808 & 3,195 & 0.808 & 3,195 & 0.808 & 3,195 & 0.808 & 3,194 & 1.087 \\
\hline $\mathrm{NH}_{3}$ & 0 & 0.000 & 0 & 0.000 & 0 & 0.000 & 0 & 0.000 & 0 & 0.000 & 0 & 0.000 & 0 & 0.000 & 0 & 0.000 \\
\hline $\mathrm{O}_{2}$ & 0 & 0.000 & 0 & 0.000 & 0 & 0.000 & 0 & 0.000 & 0 & 0.000 & 0 & 0.000 & 0 & 0.000 & 0 & 0.000 \\
\hline $\mathrm{SO}_{2}$ & 0 & 0.000 & 0 & 0.000 & 0 & 0.000 & 0 & 0.000 & 0 & 0.000 & 0 & 0.000 & 0 & 0.000 & 0 & 0.000 \\
\hline TOTAL & $1,797,221$ & 100.000 & $1,797,220$ & 100.000 & $1,344,498$ & 100.000 & 395,388 & 100.000 & 395,388 & 100.000 & 395,388 & 100.000 & 395,388 & 100.000 & 293,993 & 100.000 \\
\hline \multicolumn{17}{|l|}{ V\&L Mixture Components } \\
\hline Total Flow, acfm & 24,5 & & 32,9 & & 16,2 & & $\overline{5,4}$ & & $\overline{5,9}$ & & $\overline{4,4}$ & & 4,7 & & 2,5 & \\
\hline Vapor Frac & 1.0 & & 1.0 & & 1.00 & & 1.0 & & 1.0 & & 1.0 & & 1.0 & & 0.8 & \\
\hline Temperature, $\mathrm{F}$ & 534 & & 1,020 & & 553 & & 653 & & 801 & & 500 & & 569 & & 332 & \\
\hline Pressure, psia & 912 & & 902 & & 912 & & 890 & & 880 & & 870 & & 860 & & 845 & \\
\hline Enthalpy, Btu/lb & $-3,036$ & & $-3,036$ & & $-3,276$. & & $-3,303$ & & $-3,303$. & & $-3,508$ & & $-3,508$ & & $-3,052$ & \\
\hline Heat Capacity, Btu/lb R & 0.6 & & 0.7 & & 0.6 & & 0.6 & & 0.7 & & 0.6 & & 0.6 & & 0.7 & \\
\hline Density, $\mathrm{lb} / \mathrm{ft}^{3}$ & 1.2 & & 0.9 & & 1.3 & & 1.2 & & 1.1 & & 1.4 & & 1.3 & & 1.9 & \\
\hline Molecular Weight & 14.1 & & 16.0 & & 15.9 & & 16.0 & & 16.6 & & 16.6 & & 17.0 & & 16.7 & \\
\hline & & & & & & & Solid & & & & & & & & & \\
\hline Solid Components & $\overline{\mathrm{Ib} / \mathrm{h}}$ & & $\overline{\mathrm{Ib} / \mathrm{h}}$ & & $\mathrm{Ib} / \mathrm{h}$ & & $\mathrm{Ib} / \mathrm{h}$ & & Ib/l & & $\mathrm{Ib} / \mathrm{t}$ & & $\mathrm{Ib} / \mathrm{h}$ & & $\mathrm{Ib} / \mathrm{t}$ & \\
\hline Coal & & 0 & & 0 & & 0 & & 0 & & 0 & & 0 & & 0 & & $\overline{0}$ \\
\hline Char & & 0 & & 0 & & 0 & & 0 & & 0 & & 0 & & 0 & & 0 \\
\hline Ash & & 0 & & 0 & & 0 & & 0 & & 0 & & 0 & & 0 & & 0 \\
\hline \begin{tabular}{|l|} 
Sulfur \\
\end{tabular} & & 0 & & 0 & & 0 & & 0 & & 0 & & 0 & & 0 & & 0 \\
\hline TOTAL & & 0 & & 0 & & 0 & & 0 & & 0 & & 0 & & 0 & & 0 \\
\hline
\end{tabular}

1. Results based on bench scale reactor test results communicated by APS.

Heat \& Material Balance: Methanation 
WII WorleyParsons resources \& energy

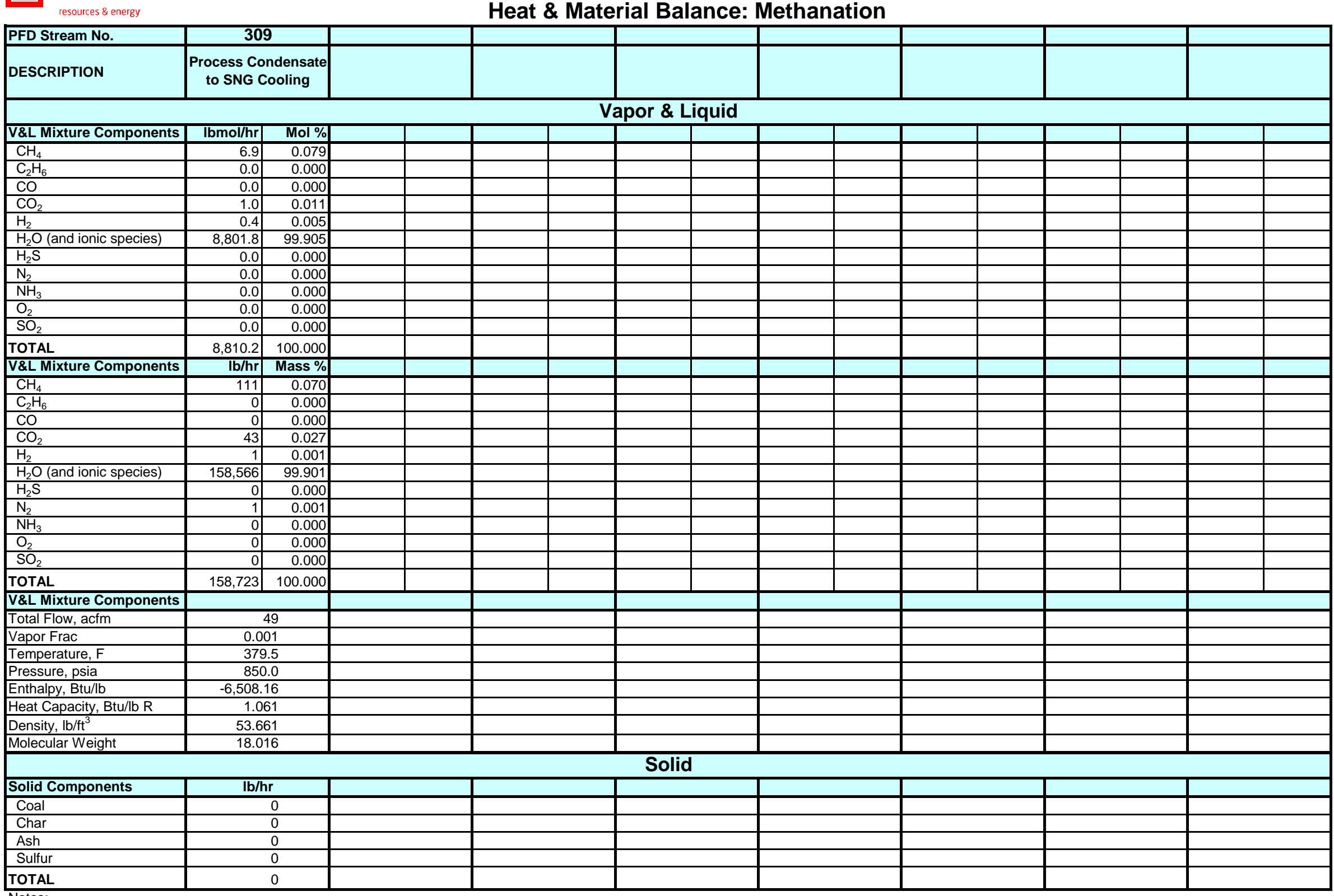

1. Results based on bench scale reactor test results communicated by APS 
WiI WorleyParsons esources \& energy

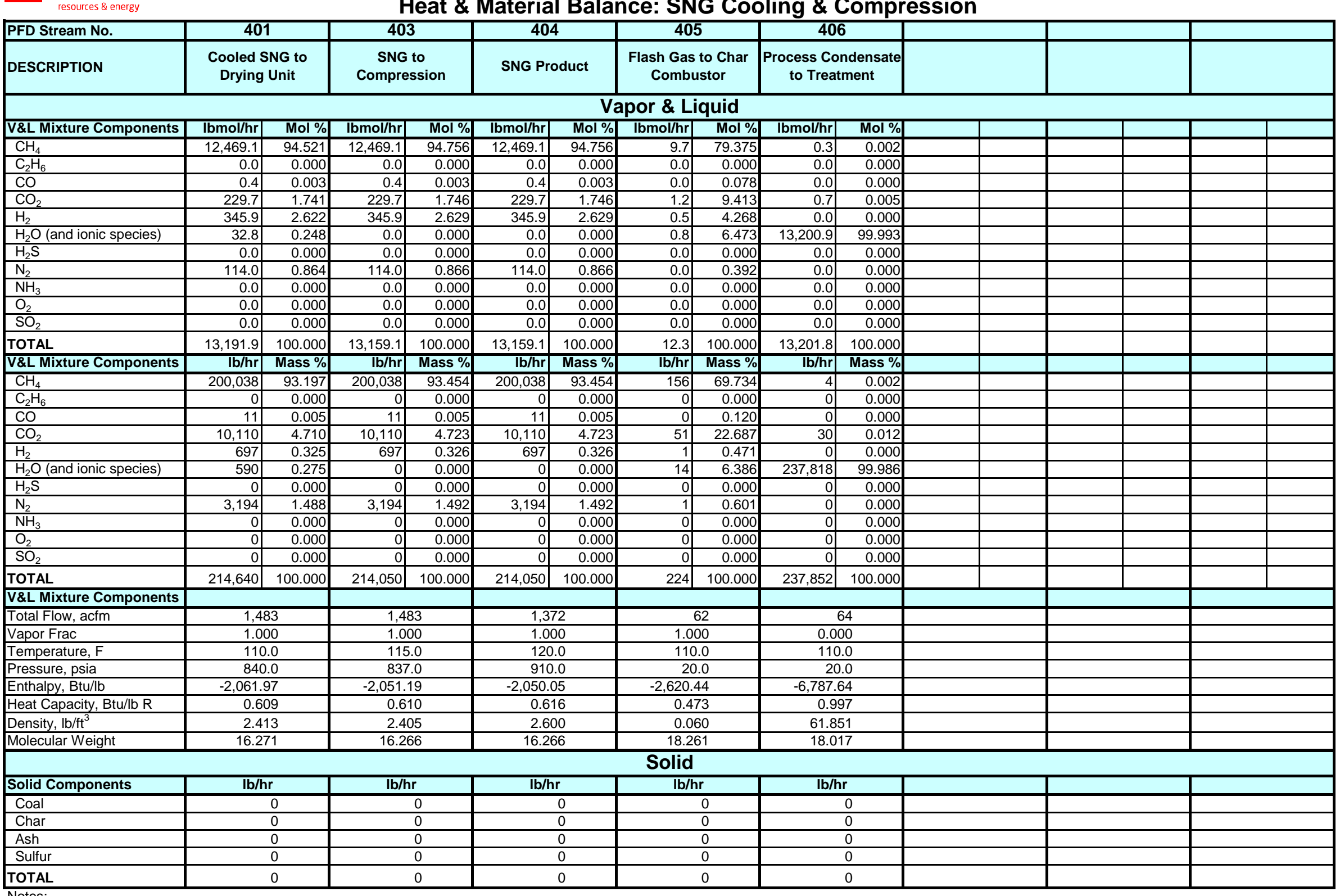

1. Results based on bench scale reactor test results communicated by APS. Heat \& Material Balance: SNG Cooling \& Compression 
IVI] WorleyParsons

esources \& energy

\begin{tabular}{|c|c|c|c|c|c|c|c|c|c|c|c|c|c|c|c|c|}
\hline PFD Stream No. & \multicolumn{2}{|c|}{501} & \multicolumn{2}{|c|}{502} & \multicolumn{2}{|c|}{503} & \multicolumn{2}{|c|}{504} & \multicolumn{2}{|c|}{505} & \multicolumn{2}{|c|}{506} & \multicolumn{2}{|c|}{507} & \multicolumn{2}{|c|}{508} \\
\hline DESCRIPTION & \multicolumn{2}{|c|}{$\begin{array}{c}\text { Flue Gas from } \\
\text { Particulate Removal } \\
\text { System }\end{array}$} & \multicolumn{2}{|c|}{$\mathrm{CO}_{2}$ to Compression } & \multicolumn{2}{|c|}{$\begin{array}{l}\text { Flue Gas to Recycle } \\
\text { Fan }\end{array}$} & \multicolumn{2}{|c|}{$\begin{array}{l}\text { Oxygen from } \\
\text { Electrolysis }\end{array}$} & \multicolumn{2}{|c|}{$\begin{array}{l}\text { Combustor Ash to } \\
\text { Disposal }\end{array}$} & \multicolumn{2}{|c|}{$\begin{array}{c}\text { Process Condensate } \\
\text { to SWS }\end{array}$} & \multicolumn{2}{|c|}{$\mathrm{CO}_{2}$ to Deoxidation } & \multicolumn{2}{|c|}{$\begin{array}{l}\text { Transport } \mathrm{CO}_{2} \text { to } \\
\text { Fan }\end{array}$} \\
\hline \multicolumn{17}{|c|}{ Vapor \& Liquid } \\
\hline V\&L Mixture Components & Ibmol/hr & Mol \% & Ibmol/hr & Mol\% & Ibmol/hr & Mol \% & Ibmol/hr & Mol \% & Ibmol/hr & Mol \% & Ibmol/hr & Mol \% & Ibmol/hr & Mol \% & Ibmol/hr & Mol \% \\
\hline $\mathrm{CH}_{4}$ & 0.0 & 0.000 & 0.0 & 0.000 & 0.0 & 0.000 & 0.0 & 0.000 & 0.0 & 0.000 & 0.0 & 0.000 & 0.0 & 0.000 & 0.0 & 0.000 \\
\hline $\mathrm{C}_{2} \mathrm{H}_{6}$ & 0.0 & 0.000 & 0.0 & 0.000 & 0.0 & 0.000 & 0.0 & 0.000 & 0.0 & 0.000 & 0.0 & 0.000 & 0.0 & 0.000 & 0.0 & 0.000 \\
\hline $\mathrm{CO}$ & 0.0 & 0.000 & 0.0 & 0.000 & 0.0 & 0.000 & 0.0 & 0.000 & 0.0 & 0.000 & 0.0 & 0.000 & 0.0 & 0.000 & 0.0 & 0.000 \\
\hline $\mathrm{CO}_{2}$ & $8,833.0$ & 78.701 & $6,062.4$ & 86.199 & $2,770.6$ & 86.199 & 0.0 & 0.000 & 0.0 & 0.000 & 0.0 & 0.003 & $5,189.0$ & 92.446 & 873.3 & 92.446 \\
\hline $\mathrm{H}_{2}$ & 0.0 & 0.000 & 0.0 & 0.000 & 0.0 & 0.000 & 35.6 & 0.500 & \begin{tabular}{l|l}
0.0 \\
\end{tabular} & 0.000 & 0.0 & 0.000 & 0.0 & 0.000 & 0.0 & 0.000 \\
\hline $\mathrm{H}_{2} \mathrm{O}$ (and ionic species) & $1,698.6$ & 15.134 & 495.7 & 7.049 & 226.6 & 7.049 & 0.0 & 0.000 & 0.0 & 0.000 & $1,451.5$ & 99.996 & 17.6 & 0.313 & 3.0 & 0.313 \\
\hline $\mathrm{H}_{2} \mathrm{~S}$ & 0.0 & 0.000 & 0.0 & 0.000 & 0.0 & 0.000 & 0.0 & 0.000 & 0.0 & 0.000 & 0.0 & 0.000 & 0.0 & 0.000 & 0.0 & 0.000 \\
\hline $\mathrm{N}_{2}$ & 194.2 & 1.730 & 133.3 & 1.895 & 60.9 & 1.895 & 0.0 & 0.000 & 0.0 & 0.000 & 0.0 & 0.000 & 114.1 & 2.032 & 19.2 & 2.032 \\
\hline $\mathrm{NH}_{3}$ & 0.0 & 0.000 & 0.0 & 0.000 & 0.0 & 0.000 & 0.0 & 0.000 & \begin{tabular}{l|l}
0.0 \\
\end{tabular} & 0.000 & 0.0 & 0.000 & 0.0 & 0.000 & 0.0 & 0.000 \\
\hline $\mathrm{O}_{2}$ & 483.5 & 4.308 & 331.8 & 4.718 & 151.7 & 4.718 & \begin{tabular}{l|l}
$7,077.2$ \\
\end{tabular} & 99.500 & 0.0 & 0.000 & 0.0 & 0.000 & 284.0 & 5.060 & 47.8 & 5.060 \\
\hline $\mathrm{SO}_{2}$ & 14.2 & 0.127 & 9.8 & 0.139 & 4.5 & 0.139 & \begin{tabular}{l|l}
0.0 \\
\end{tabular} & 0.000 & \begin{tabular}{l|l}
0.0 \\
\end{tabular} & 0.000 & 0.0 & 0.000 & 8.3 & 0.149 & 1.4 & 0.149 \\
\hline TOTAL & $11,223.4$ & 100.000 & $7,033.0$ & 100.000 & $3,214.2$ & 100.000 & $7,112.8$ & 100.000 & 0.0 & 0.000 & $1,451.5$ & 100.000 & $5,613.1$ & 100.000 & 944.7 & 100.000 \\
\hline V\&L Mixture Components & $\mathrm{Tb} / \mathrm{hr}$ & Mass \% & $\mathrm{Tb} / \mathrm{hr}$ & Mass \% & $\mathrm{Tb} / \mathrm{hr}$ & Mass \% & $\mid \mathrm{Tb} / \mathrm{hr}$ & Mass \% & $\mathrm{Ib} / \mathrm{hr}$ & Mass \% & Tb/hr & Mass \% & $\mathrm{Tb} / \mathrm{hr}$ & Mass \% & $\overline{\mathrm{Ib} / \mathrm{hr}}$ & Mass \% \\
\hline $\mathrm{CH}_{4}$ & 0 & 0.000 & 0 & 0.000 & 0 & 0.000 & 0 & 0.000 & 0 & 0.000 & 0 & 0.000 & 0 & 0.000 & 0 & 0.000 \\
\hline $\mathrm{C}_{2} \mathrm{H}_{6}$ & 0 & 0.000 & 0 & 0.000 & 0 & 0.000 & 0 & 0.000 & 0 & 0.000 & 0 & 0.000 & 0 & 0.000 & 0 & 0.000 \\
\hline $\mathrm{CO}$ & 0 & 0.000 & 0 & 0.000 & 0 & 0.000 & 0 & 0.000 & 0 & 0.000 & 0 & 0.000 & 0 & 0.000 & 0 & 0.000 \\
\hline $\mathrm{CO}_{2}$ & 388,737 & 88.117 & 266,804 & 91.776 & 121,934 & 91.776 & 0 & 0.000 & 0 & 0.000 & 2 & 0.008 & 228,369 & 94.561 & \begin{tabular}{l|l|}
38,433 \\
\end{tabular} & 94.561 \\
\hline $\mathrm{H}_{2}$ & 0 & 0.000 & 0 & 0.000 & 0 & 0.000 & 72 & 0.032 & 0 & 0.000 & 0 & 0.000 & 0 & 0.000 & 0 & 0.000 \\
\hline $\mathrm{H}_{2} \mathrm{O}$ (and ionic species) & 30,600 & 6.936 & 8,931 & 3.072 & 4,082 & 3.072 & 0 & 0.000 & 0 & 0.000 & 26,149 & 99.990 & 316 & 0.131 & 53 & 0.131 \\
\hline $\mathrm{H}_{2} \mathrm{~S}$ & 0 & 0.000 & 0 & 0.000 & 0 & 0.000 & 0 & 0.000 & 0 & 0.000 & 0 & 0.000 & 0 & 0.000 & 0 & 0.000 \\
\hline $\mathrm{N}_{2}$ & 5,439 & 1.233 & 3,733 & 1.284 & 1,706 & 1.284 & 0 & 0.000 & 0 & 0.000 & 0 & 0.000 & 3,195 & 1.323 & 538 & 1.323 \\
\hline $\mathrm{NH}_{3}$ & 0 & 0.000 & 0 & 0.000 & 0 & 0.000 & 0 & 0.000 & 0 & 0.000 & 0 & 0.000 & 0 & 0.000 & 0 & 0.000 \\
\hline $\mathrm{O}_{2}$ & 15,472 & 3.507 & 10,619 & 3.653 & 4,853 & 3.653 & 226,463 & 99.968 & 0 & 0.000 & 0 & 0.000 & 9,089 & 3.763 & 1,530 & 3.763 \\
\hline $\mathrm{SO}_{2}$ & 911 & 0.206 & 625 & 0.215 & 286 & 0.215 & 0 & 0.000 & 0 & 0.000 & 0 & 0.002 & 535 & 0.221 & 90 & 0.221 \\
\hline TOTAL & 441,158 & 100.000 & 290,711 & 100.000 & 132,860 & 100.000 & 226,534 & 100.000 & 0 & 0.000 & 26,152 & 100.000 & 241,504 & 100.000 & 40,644 & 100.000 \\
\hline \multicolumn{17}{|l|}{ V\&L Mixture Components } \\
\hline Total Flow, acfm & $\overline{96,9}$ & & 45,7 & & 20,8 & & $\overline{11,53}$ & & & -- & & 7 & & $\overline{31}$ & & 98 \\
\hline Vapor Frac & 1.0 & & 1.0 & & 1.0 & & 1.00 & & & -- & 0.0 & & 1.0 & & 1.00 & \\
\hline Temperature, $\mathrm{F}$ & 300 & & 110 & & 110 & & 104 & & 212. & & 110 & & 231 & & 231. & \\
\hline Pressure, psia & 15 & & 15 & & 15 & & 62 & & 12. & .0 & & & 1,017 & & 1,017 & \\
\hline Enthalpy, Btu/lb & $-3,739$. & & $-3,702$ & & $-3,702$ & & 5.4 & & & -- & $-6,830$. & & $-3,632$ & & $-3,632.7$ & \\
\hline Heat Capacity, Btu/lb R & 0.2 & & 0.2 & & 0.2 & & 0.22 & & & - & 1.0 & & 0.2 & & 0.28 & \\
\hline Density, Ib/ft ${ }^{3}$ & 0.0 & & 0.1 & & 0.1 & & 0.32 & & & - & 59.3 & & 6.9 & & 6.92 & \\
\hline Molecular Weight & 39.3 & & 41.3 & & 41.3 & & 31.84 & & & -- & 18.0 & & 43.0 & & 43.02 & \\
\hline & & & & & & & Solid & & & & & & & & & \\
\hline Solid Components & $\mathrm{Ib} / \mathrm{h}$ & & $\mathrm{Ib} / \mathrm{h}$ & & $\mathrm{Ib} / \mathrm{h}$ & & $\mathrm{Ib} / \mathrm{h}$ & & $\mathrm{Ib} / \mathrm{h}$ & & $\mathrm{Ib} / \mathrm{h}$ & & $\mathrm{Ib} / \mathrm{h}$ & & $\mathrm{Ib} / \mathrm{h}$ & \\
\hline Coal & & 0 & & 0 & & $\overline{0}$ & & $\overline{0}$ & & 0 & & $\overline{0}$ & & $\overline{0}$ & & 0 \\
\hline Char & & 0 & & 0 & & 0 & & 0 & & 0 & & 0 & & 0 & & 0 \\
\hline Ash & & 0 & & 0 & & 0 & & 0 & 55,55 & & & 0 & & 0 & & 0 \\
\hline Sulfur & & 0 & & 0 & & 0 & & 0 & & 0 & & 0 & & 0 & & 0 \\
\hline TOTAL & & 0 & & 0 & & 0 & & 0 & 55,55 & & & 0 & & 0 & & 0 \\
\hline
\end{tabular}

Notes:

1. Results based on bench scale reactor test results communicated by APS.

Arizona Public Service Advanced Hydrogasification / SNG -- Commercial Scale Heat \& Material Balance: Char Combustor

Electrolys

Disposal
Dish

Condensat

\begin{tabular}{|r|r|r|r|r|r|}
\hline Mol \% & lbmol/hr & Mol \% & lbmol/hr & Mol \% \\
\hline
\end{tabular}

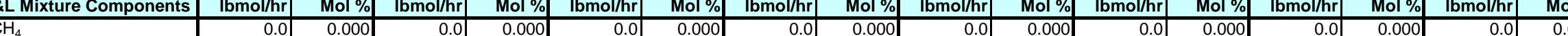


WiVI WorleyParsons esources \& energy

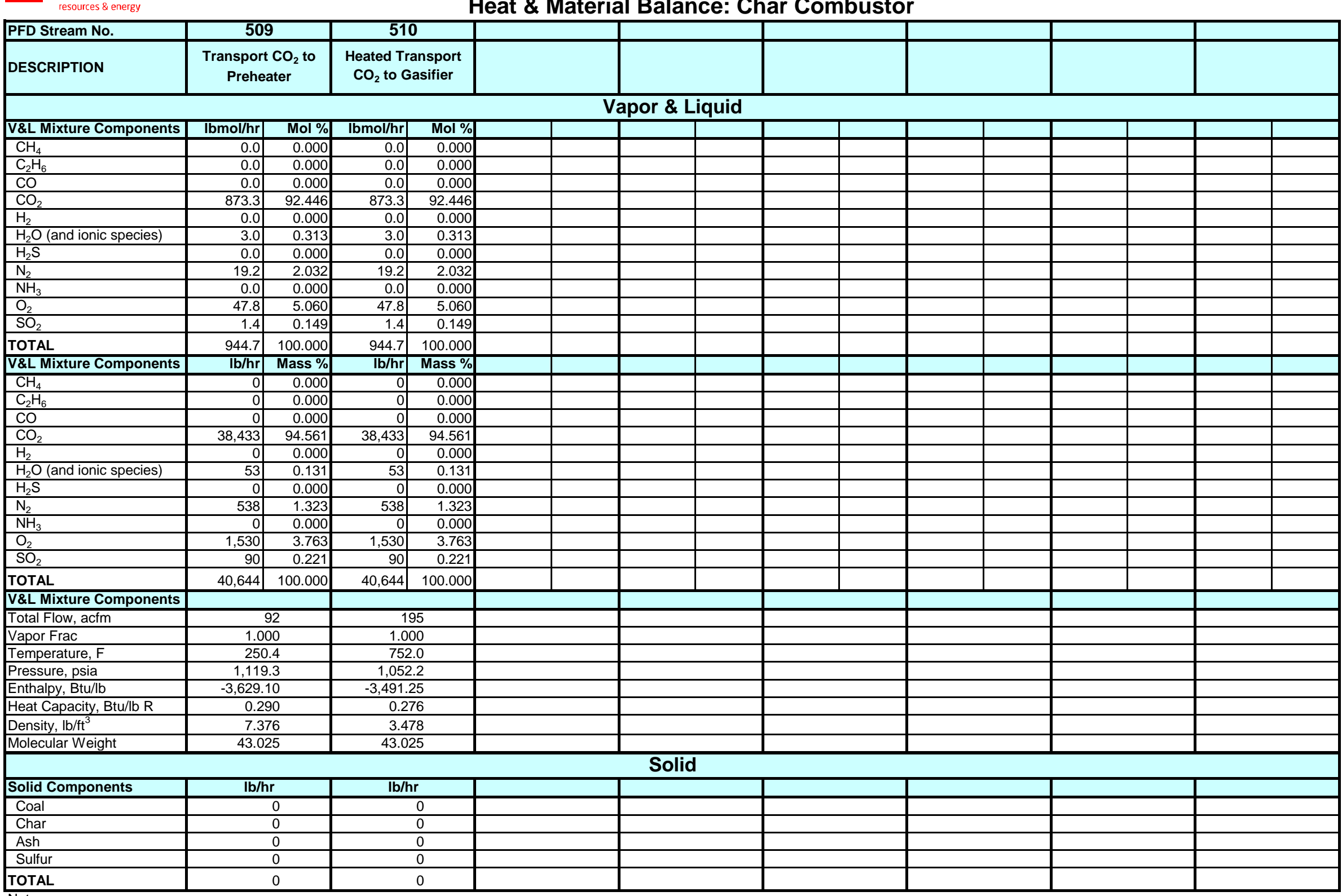

1. Results based on bench scale reactor test results communicated by APS. 
WiI WorleyParsons esources \& energy

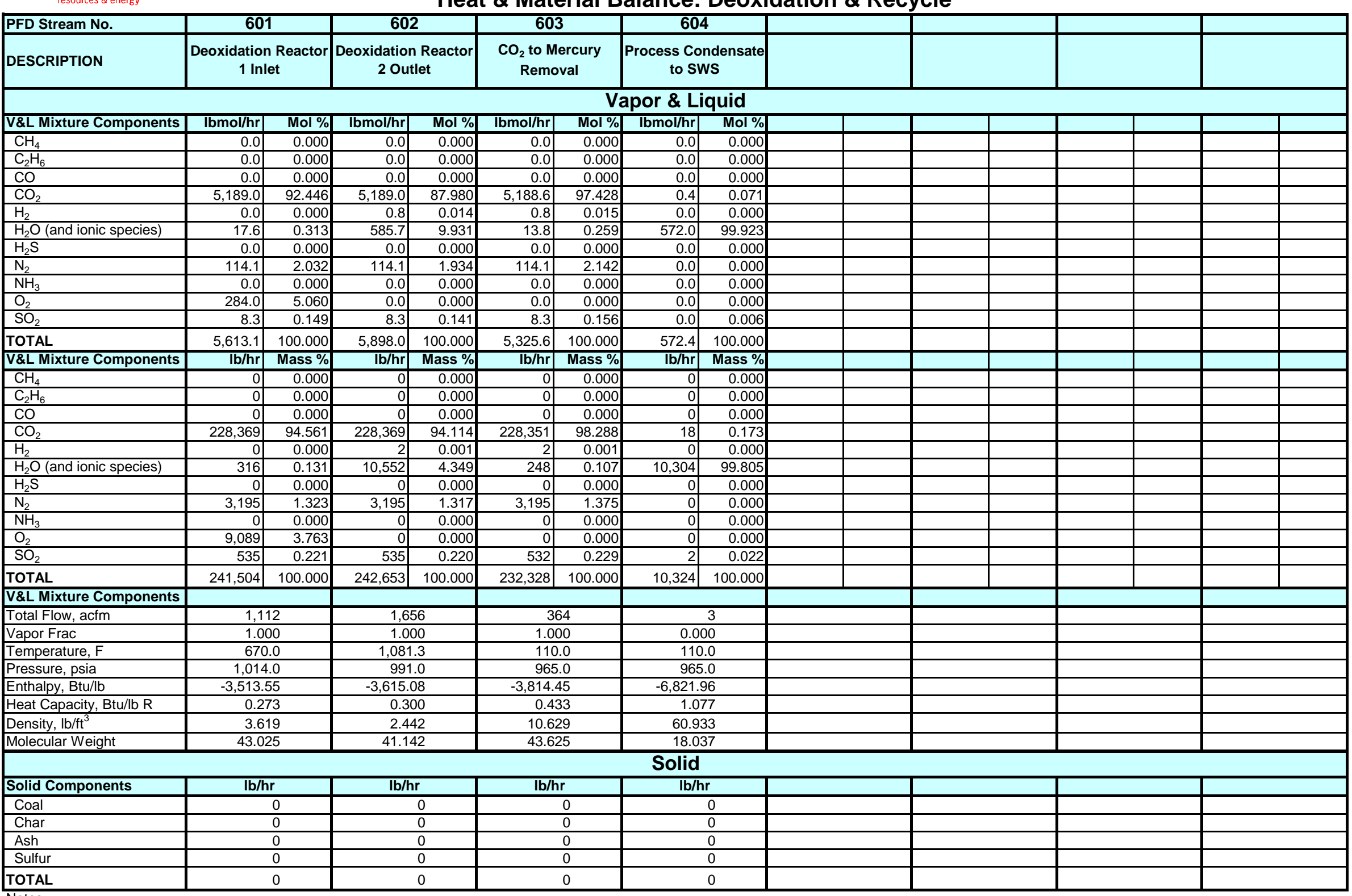

Notes:

1. Results based on bench scale reactor test results communicated by APS. Heat \& Material Balance: Deoxidation \& Recycle

Condensate

Arizona Public Service Advanced Hydrogasification / SNG -- Commercial Scale 
WiI WorleyParsons resources \& energy

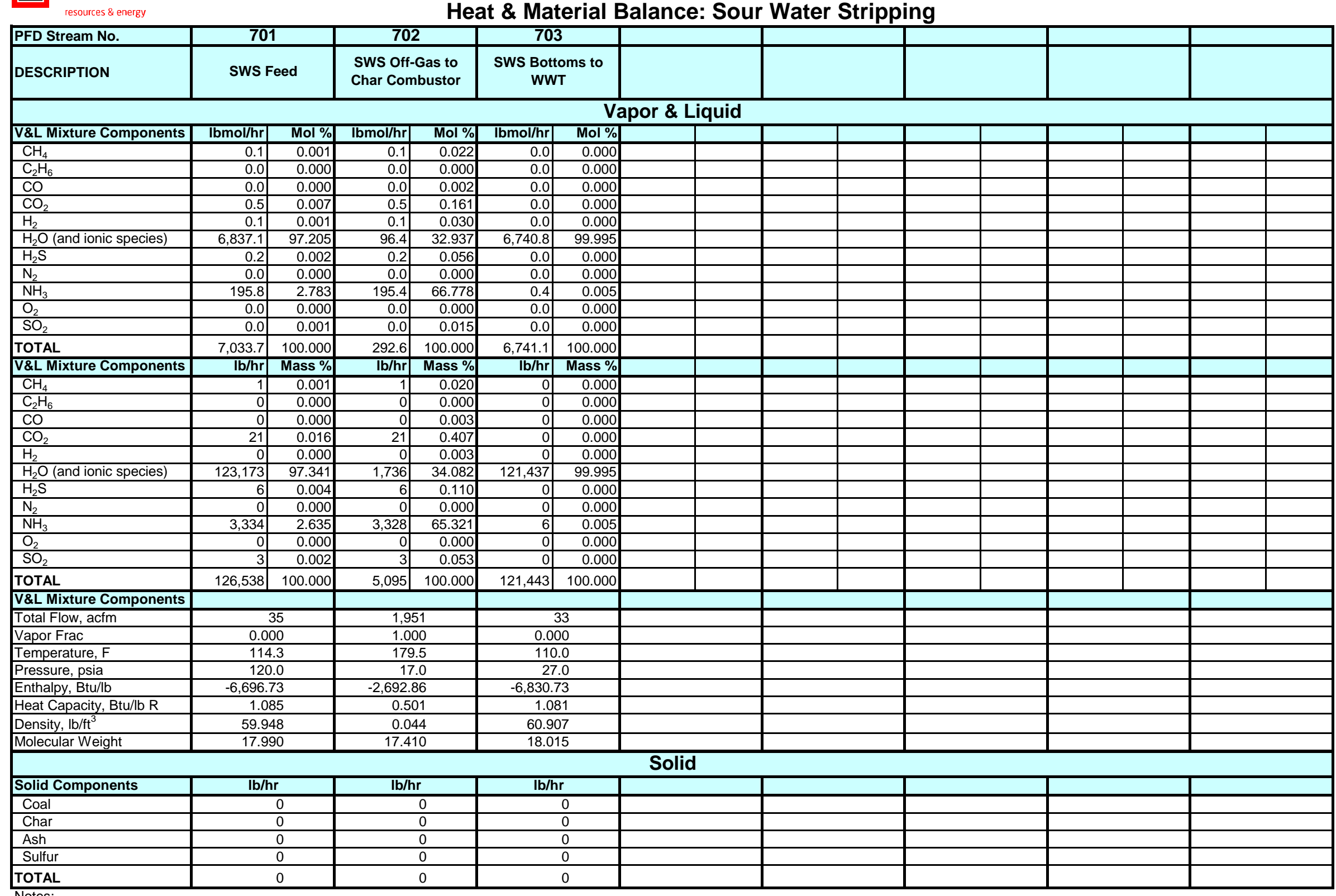

Notes:

1. Results based on bench scale reactor test results communicated by APS.

Arizona Public Service Advanced Hydrogasification / SNG -- Commercial Scale Heat \& Material Balance: Sour Water Stripping 


\begin{tabular}{|c|c|c|c|c|c|c|c|c|c|c|c|c|c|c|c|c|}
\hline DESCRIPTION & Demineraliz & ed Water & $\begin{array}{r}\text { ST Conde } \\
\text { LT He }\end{array}$ & $\begin{array}{l}\text { nsate to } \\
\text { ters }\end{array}$ & \multicolumn{2}{|c|}{$\begin{array}{l}\text { ST Condensate to } \\
\text { Deaerator }\end{array}$} & \multicolumn{2}{|c|}{$\begin{array}{l}\text { HP BFW to } \\
\text { Methanation }\end{array}$} & \multicolumn{2}{|c|}{$\begin{array}{l}\text { HP BFW to HP } \\
\text { Economizer }\end{array}$} & \multicolumn{2}{|c|}{$\begin{array}{c}\text { Economized HP } \\
\text { BFW to HP } \\
\text { Evaporators } \\
\end{array}$} & \multicolumn{2}{|c|}{$\begin{array}{c}\text { HP Steam } \\
\text { Blowdown to Flash } \\
\text { Drum } \\
\end{array}$} & \multicolumn{2}{|c|}{$\begin{array}{l}\text { HP Steam to Char } \\
\text { Combustor } \\
\text { Superheater }\end{array}$} \\
\hline \multicolumn{17}{|c|}{ Vapor \& Liquid } \\
\hline V\&L Mixture Components & $\mathrm{Ibmol} / \mathrm{hr}$ & Mol \% & $\mathrm{Ibmol} / \mathrm{hr}$ & Mol \% & Ibmol/hr & Mol \% & Ibmol/hr & $\mathrm{Mol} \%$ & $\mathrm{lbmol} / \mathrm{hr}$ & Mol \% & $\mathrm{lbmol} / \mathrm{hr}$ & Mol \% & Ibmol/hr & $\mathrm{Mol} \%$ & $\mathrm{Ibmol} / \mathrm{hr}$ & $\mathrm{Mol} \%$ \\
\hline $\mathrm{CH}_{4}$ & 0.0 & 0.000 & 0.0 & 0.000 & 0.0 & 0.000 & 0.0 & 0.000 & 0.0 & 0.000 & 0.0 & 0.000 & 0.0 & 0.000 & 0.0 & 0.000 \\
\hline $\mathrm{C}_{2} \mathrm{H}_{6}$ & 0.0 & 0.000 & 0.0 & 0.000 & 0.0 & 0.000 & 0.0 & 0.000 & 0.0 & 0.000 & 0.0 & 0.000 & 0.0 & 0.000 & 0.0 & 0.000 \\
\hline $\mathrm{CO}$ & 0.0 & 0.000 & 0.0 & 0.000 & 0.0 & 0.000 & 0.0 & 0.000 & 0.0 & 0.000 & 0.0 & 0.000 & 0.0 & 0.000 & 0.0 & 0.000 \\
\hline $\mathrm{CO}_{2}$ & 0.0 & 0.000 & 0.0 & 0.000 & 0.0 & 0.000 & 0.0 & 0.000 & 0.0 & 0.000 & 0.0 & 0.000 & 0.0 & 0.000 & 0.0 & 0.000 \\
\hline $\mathrm{H}_{2}$ & 0.0 & 0.000 & 0.0 & 0.000 & 0.0 & 0.000 & 0.0 & 0.000 & 0.0 & 0.000 & 0.0 & 0.000 & 0.0 & 0.000 & 0.0 & 0.000 \\
\hline $\mathrm{H}_{2} \mathrm{O}$ (and ionic species) & 988.1 & 100.000 & $72,769.5$ & 100.000 & $72,769.5$ & 100.000 & $26,614.1$ & 100.000 & $51,344.5$ & 100.000 & $51,344.5$ & 100.000 & $1,045.7$ & 100.000 & $50,831.1$ & 100.000 \\
\hline $\mathrm{H}_{2} \mathrm{~S}$ & 0.0 & 0.000 & 0.0 & 0.000 & 0.0 & 0.000 & 0.0 & 0.000 & 0.0 & 0.000 & 0.0 & 0.000 & 0.0 & 0.000 & 0.0 & 0.000 \\
\hline $\mathrm{N}_{2}$ & 0.0 & 0.000 & 0.0 & 0.000 & 0.0 & 0.000 & 0.0 & 0.000 & 0.0 & 0.000 & 0.0 & 0.000 & 0.0 & 0.000 & 0.0 & 0.000 \\
\hline $\mathrm{NH}_{3}$ & 0.0 & 0.000 & 0.0 & 0.000 & 0.0 & 0.000 & 0.0 & 0.000 & 0.0 & 0.000 & 0.0 & 0.000 & 0.0 & 0.000 & 0.0 & 0.000 \\
\hline $\mathrm{O}_{2}$ & 0.0 & 0.000 & 0.0 & 0.000 & 0.0 & 0.000 & 0.0 & 0.000 & 0.0 & 0.000 & 0.0 & 0.000 & 0.0 & 0.000 & 0.0 & 0.000 \\
\hline $\mathrm{SO}_{2}$ & 0.0 & 0.000 & 0.0 & 0.000 & 0.0 & 0.000 & 0.0 & 0.000 & 0.0 & 0.000 & 0.0 & 0.000 & 0.0 & 0.000 & 0.0 & 0.000 \\
\hline TOTAL & 988.1 & 100.000 & $72,769.5$ & 100.000 & $72,769.5$ & 100.000 & $26,614.1$ & 100.000 & $51,344.5$ & 100.000 & $51,344.5$ & 100.000 & $1,045.7$ & 100.000 & $50,831.1$ & 100.000 \\
\hline V\&L Mixture Components & $\mathrm{Ib} / \mathrm{hr}$ & Mass \% & $\mathrm{Ib} / \mathrm{hr}$ & Mass \% & $\mathrm{Ib} / \mathrm{hr}$ & Mass $\%$ & Ib/hr & Mass \% & $\mathrm{Ib} / \mathrm{hr}$ & Mass \% & $\mathrm{Ib} / \mathrm{hr}$ & Mass \% & $\mathrm{Ib} / \mathrm{hr}$ & Mass \% & $\mathrm{Ib} / \mathrm{hr}$ & Mass \% \\
\hline $\mathrm{CH}_{4}$ & 0 & 0.000 & 0 & 0.000 & 0 & 0.000 & 0 & 0.000 & 0 & 0.000 & 0 & 0.000 & 0 & 0.000 & 0 & 0.000 \\
\hline $\mathrm{C}_{2} \mathrm{H}_{6}$ & 0 & 0.000 & 0 & 0.000 & 0 & 0.000 & 0 & 0.000 & 0 & 0.000 & 0 & 0.000 & 0 & 0.000 & 0 & 0.000 \\
\hline $\mathrm{CO}$ & 0 & 0.000 & 0 & 0.000 & 0 & 0.000 & 0 & 0.000 & 0 & 0.000 & 0 & 0.000 & 0 & 0.000 & 0 & 0.000 \\
\hline $\mathrm{CO}_{2}$ & 0 & 0.000 & 0 & 0.000 & 0 & 0.000 & 0 & 0.000 & 0 & 0.000 & 0 & 0.000 & 0 & 0.000 & 0 & 0.000 \\
\hline $\mathrm{H}_{2}$ & 0 & 0.000 & 0 & 0.000 & 0 & 0.000 & 0 & 0.000 & 0 & 0.000 & 0 & 0.000 & 0 & 0.000 & 0 & 0.000 \\
\hline $\mathrm{H}_{2} \mathrm{O}$ (and ionic species) & 17,800 & 100.000 & $1,310,964$ & 100.000 & $1,310,964$ & 100.000 & 479,460 & 100.000 & 924,987 & 100.000 & 924,987 & 100.000 & 18,839 & 100.000 & 915,735 & 100.000 \\
\hline $\mathrm{H}_{2} \mathrm{~S}$ & 0 & 0.000 & 0 & 0.000 & 0 & 0.000 & 0 & 0.000 & 0 & 0.000 & 0 & 0.000 & 0 & 0.000 & 0 & 0.000 \\
\hline $\mathrm{N}_{2}$ & 0 & 0.000 & 0 & 0.000 & 0 & 0.000 & 0 & 0.000 & 0 & 0.000 & 0 & 0.000 & 0 & 0.000 & 0 & 0.000 \\
\hline $\mathrm{NH}_{3}$ & 0 & 0.000 & 0 & 0.000 & 0 & 0.000 & 0 & 0.000 & 0 & 0.000 & 0 & 0.000 & 0 & 0.000 & 0 & 0.000 \\
\hline $\mathrm{O}_{2}$ & 0 & 0.000 & 0 & 0.000 & 0 & 0.000 & 0 & 0.000 & 0 & 0.000 & 0 & 0.000 & 0 & 0.000 & 0 & 0.000 \\
\hline $\mathrm{SO}_{2}$ & 0 & 0.000 & 0 & 0.000 & 0 & 0.000 & 0 & 0.000 & 0 & 0.000 & 0 & 0.000 & 0 & 0.000 & 0 & 0.000 \\
\hline TOTAL & 17,800 & 100.000 & $1,310,964$ & 100.000 & $1,310,964$ & 100.000 & 479,460 & 100.000 & 924,987 & 100.000 & 924,987 & 100.000 & 18,839 & 100.000 & 915,735 & 100.000 \\
\hline \multicolumn{17}{|l|}{ V\&L Mixture Components } \\
\hline Total Flow, acfm & \multicolumn{2}{|r|}{2} & \multicolumn{2}{|c|}{117} & \multicolumn{2}{|c|}{125} & & $\overline{6}$ & & 88 & & $\overline{02}$ & & 3 & & $\overline{94}$ \\
\hline Vapor Frac & 0.0 & & 0.0 & & 0.0 & & 0.00 & & 0.0 & & 0.0 & & 0.0 & & 1.0 & \\
\hline Temperature, $\mathrm{F}$ & 59 & & 101 & & 27 & & 291 & & 29 & & 48 & & 632 & & 632 & \\
\hline Pressure, psia & 20 & & 150 & & 14 & & 2,250 & & 2,25 & & 1,95 & & 1,950 & & 1,950 & \\
\hline Enthalpy, Btu/lb & $-6,838$. & & $-6,796$. & & $-6,624$ & & $-6,601$ & & $-6,601$ & & $-6,400$ & & $-6,200$ & & $-5,724$ & \\
\hline Density, lb/ftt ${ }^{3}$ & 62.3 & & 62.0 & & 58.1 & & 58.1 & & 58.1 & & 50.5 & & 39.3 & & 5.1 & \\
\hline \begin{tabular}{|l} 
Molecular Weight \\
\end{tabular} & 18.0 & & 18.0 & & 18.0 & & 18.0 & & 18.0 & & 18.0 & & 18.0 & & 18.0 & \\
\hline & & & & & & & Solid & & & & & & & & & \\
\hline Solid Components & $\mathrm{Ib} / \mathrm{h}$ & & $\mathrm{Ib} / \mathrm{l}$ & & $\mid \mathrm{lb} / \mathrm{t}$ & & $\mathrm{Ib} / \mathrm{h}$ & & $\overline{\mid l b /}$ & & $\mid \mathrm{lb} / \mathrm{t}$ & & $\mid \mathrm{lb} /$ & & $\mathrm{Ib} / \mathrm{h}$ & \\
\hline Coal & & $\overline{0}$ & & $\overline{0}$ & & $\overline{0}$ & & $\overline{0}$ & & $\overline{0}$ & & $\overline{0}$ & & $\overline{0}$ & & $\overline{0}$ \\
\hline Char & & 0 & & 0 & & 0 & & 0 & & 0 & & 0 & & 0 & & 0 \\
\hline Ash & & 0 & & 0 & & 0 & & 0 & & 0 & & 0 & & 0 & & 0 \\
\hline Sulfur & & 0 & & 0 & & 0 & & 0 & & 0 & & 0 & & 0 & & 0 \\
\hline TOTAL & & 0 & & 0 & & 0 & & 0 & & 0 & & 0 & & 0 & & 0 \\
\hline
\end{tabular}

Notes:

1. Results based on bench scale reactor test results communicated by APS. 
WiI WorleyParsons

Arizona Public Service Advanced Hydrogasification I SNG -- Commercial Scale

esources \& energ

Heat \& Material Balance: Steam Cycle

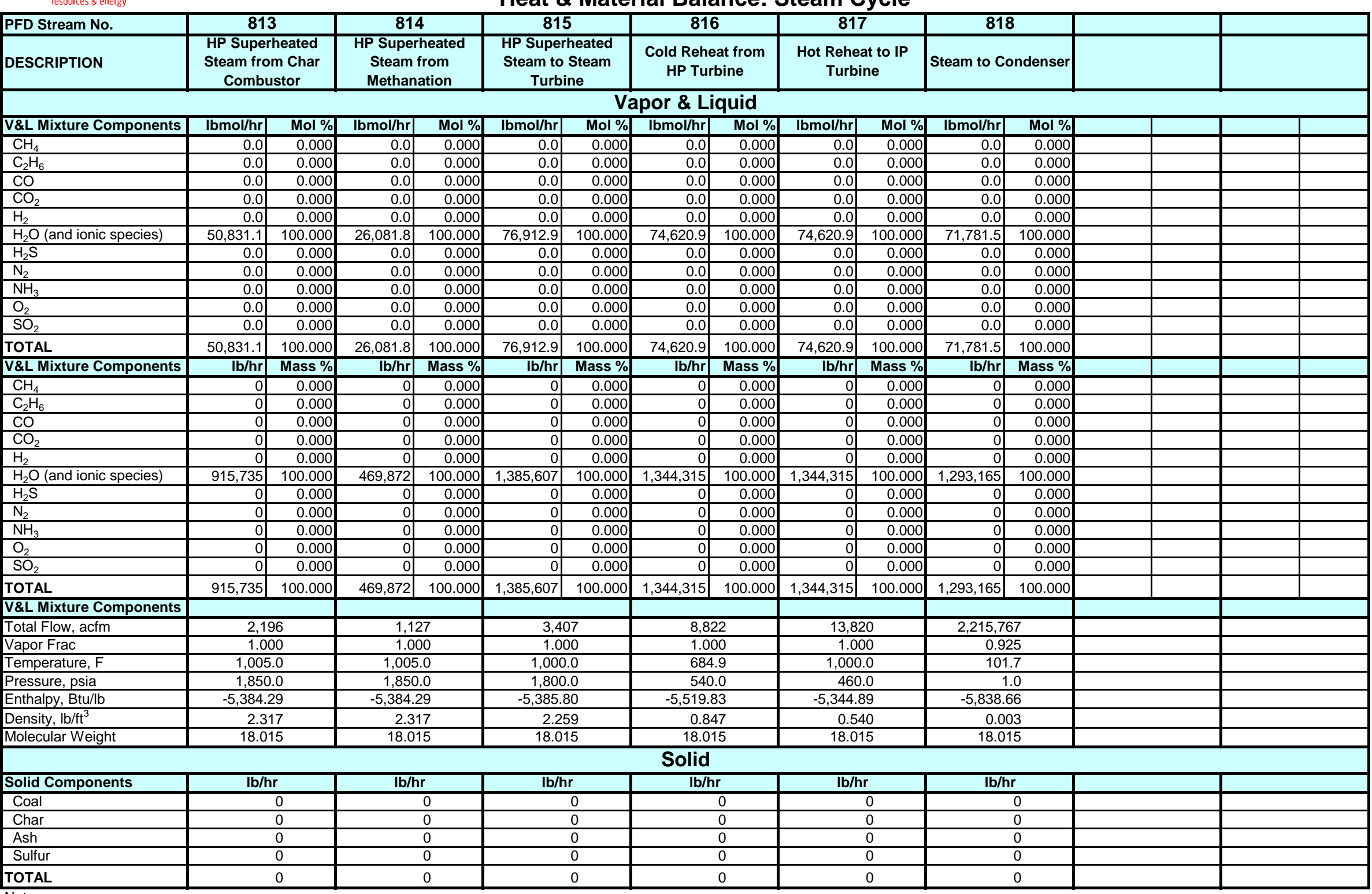

1. Results based on bench scale reactor test results communicated by APS. 


\begin{tabular}{|c|c|c|c|c|c|c|c|c|c|c|c|c|c|c|c|}
\hline PFD Stream No. & \multicolumn{2}{|c|}{819} & \multicolumn{2}{|c|}{820} & \multicolumn{2}{|c|}{821} & \multicolumn{2}{|c|}{824} & \multicolumn{2}{|c|}{825} & \multicolumn{2}{|c|}{826} & & & \\
\hline DESCRIPTION & \multicolumn{2}{|c|}{ LP Extraction } & \multicolumn{2}{|c|}{$\begin{array}{l}\text { LP Steam from } \\
\text { Blowdown Flash }\end{array}$} & \multicolumn{2}{|c|}{ Blowdown to WWT } & \multicolumn{2}{|c|}{$\begin{array}{l}\text { LP Steam to } \\
\text { Processes \& } \\
\text { Deaerator }\end{array}$} & \multicolumn{2}{|c|}{$\begin{array}{l}\text { LP Steam to } \\
\text { Deaerator }\end{array}$} & \multicolumn{2}{|c|}{$\begin{array}{l}\text { Process Steam } \\
\text { Condensate to } \\
\text { Deaerator }\end{array}$} & & & \\
\hline \multicolumn{13}{|c|}{ Vapor \& Liquid } & & & \\
\hline V\&L Mixture Components & Ibmol/hr & Mol \% & Ibmol/hr & Mol \% & Ibmol/hr & Mol \% & Ibmol/hr & $\mathrm{Mol} \%$ & lbmol/hr & Mol \% & Ibmol/hr & Mol \% & & & \\
\hline $\mathrm{CH}_{4}$ & 0.0 & 0.000 & 0.0 & 0.000 & 0.0 & 0.000 & 0.0 & 0.000 & 0.0 & 0.000 & 0.0 & 0.000 & & & \\
\hline $\mathrm{C}_{2} \mathrm{H}_{6}$ & 0.0 & 0.000 & 0.0 & 0.000 & 0.0 & 0.000 & 0.0 & 0.000 & 0.0 & 0.000 & 0.0 & 0.000 & & & \\
\hline $\mathrm{CO}$ & 0.0 & 0.000 & 0.0 & 0.000 & 0.0 & 0.000 & 0.0 & 0.000 & 0.0 & 0.000 & 0.0 & 0.000 & & & \\
\hline $\mathrm{CO}_{2}$ & 0.0 & 0.000 & 0.0 & 0.000 & 0.0 & 0.000 & 0.0 & 0.000 & 0.0 & 0.000 & 0.0 & 0.000 & & & \\
\hline $\mathrm{H}_{2}$ & 0.0 & 0.000 & 0.0 & 0.000 & 0.0 & 0.000 & 0.0 & 0.000 & 0.0 & 0.000 & 0.0 & 0.000 & & & \\
\hline $\mathrm{H}_{2} \mathrm{O}$ (and ionic species) & $5,131.4$ & 100.000 & 449.4 & 100.000 & 596.3 & 100.000 & $5,580.8$ & 100.000 & \begin{tabular}{c|}
972.2 \\
\end{tabular} & 100.000 & $4,608.7$ & 100.000 & & & \\
\hline $\mathrm{H}_{2} \mathrm{~S}$ & 0.0 & 0.000 & 0.0 & 0.000 & 0.0 & 0.000 & 0.0 & 0.000 & 0.0 & 0.000 & 0.0 & 0.000 & & & \\
\hline $\mathrm{N}_{2}$ & 0.0 & 0.000 & 0.0 & 0.000 & 0.0 & 0.000 & 0.0 & 0.000 & 0.0 & 0.000 & 0.0 & 0.000 & & & \\
\hline $\mathrm{NH}_{3}$ & 0.0 & 0.000 & 0.0 & 0.000 & 0.0 & 0.000 & 0.0 & 0.000 & 0.0 & 0.000 & 0.0 & 0.000 & & & \\
\hline $\mathrm{O}_{2}$ & 0.0 & 0.000 & 0.0 & 0.000 & 0.0 & 0.000 & 0.0 & 0.000 & 0.0 & 0.000 & 0.0 & 0.000 & & & \\
\hline $\mathrm{SO}_{2}$ & 0.0 & 0.000 & 0.0 & 0.000 & 0.0 & 0.000 & 0.0 & 0.000 & 0.0 & 0.000 & 0.0 & 0.000 & & & \\
\hline TOTAL & $5,131.4$ & 100.000 & 449.4 & 100.000 & 596.3 & 100.000 & $5,580.8$ & 100.000 & 972.2 & 100.000 & $4,608.7$ & 100.000 & & & \\
\hline V\&L Mixture Components & $\mathrm{Ib} / \mathrm{hr}$ & Mass \% & $\mathrm{Ib} / \mathrm{hr}$ & Mass \% & $\mathrm{Ib} / \mathrm{hr}$ & Mass \% & $\mathrm{Ib} / \mathrm{hr}$ & Mass \% & Ib/hr & Mass \% & $\mathrm{Ib} / \mathrm{hr}$ & Mass \% & & & \\
\hline $\mathrm{CH}_{4}$ & 0 & 0.000 & 0 & 0.000 & 0 & 0.000 & 0 & 0.000 & 0 & 0.000 & 0 & 0.000 & & & \\
\hline $\mathrm{C}_{2} \mathrm{H}_{6}$ & 0 & 0.000 & 0 & 0.000 & 0 & 0.000 & 0 & 0.000 & 0 & 0.000 & 0 & 0.000 & & & \\
\hline $\mathrm{CO}$ & 0 & 0.000 & 0 & 0.000 & 0 & 0.000 & 0 & 0.000 & 0 & 0.000 & 0 & 0.000 & & & \\
\hline $\mathrm{CO}_{2}$ & 0 & 0.000 & 0 & 0.000 & 0 & 0.000 & 0 & 0.000 & 0 & 0.000 & 0 & 0.000 & & & \\
\hline $\mathrm{H}_{2}$ & 0 & 0.000 & 0 & 0.000 & 0 & 0.000 & 0 & 0.000 & 0 & 0.000 & 0 & 0.000 & & & \\
\hline $\mathrm{H}_{2} \mathrm{O}$ (and ionic species) & 92,443 & 100.000 & 8,097 & 100.000 & 10,742 & 100.000 & 100,540 & 100.000 & 17,514 & 100.000 & 83,026 & 100.000 & & & \\
\hline $\mathrm{H}_{2} \mathrm{~S}$ & 0 & 0.000 & 0 & 0.000 & 0 & 0.000 & 0 & 0.000 & 0 & 0.000 & 0 & 0.000 & & & \\
\hline $\mathrm{N}_{2}$ & 0 & 0.000 & 0 & 0.000 & 0 & 0.000 & 0 & 0.000 & 0 & 0.000 & 0 & 0.000 & & & \\
\hline $\mathrm{NH}_{3}$ & 0 & 0.000 & 0 & 0.000 & 0 & 0.000 & 0 & 0.000 & 0 & 0.000 & 0 & 0.000 & & & \\
\hline $\mathrm{O}_{2}$ & 0 & 0.000 & 0 & 0.000 & 0 & 0.000 & 0 & 0.000 & 0 & 0.000 & 0 & 0.000 & & & \\
\hline $\mathrm{SO}_{2}$ & 0 & 0.000 & 0 & 0.000 & 0 & 0.000 & 0 & 0.000 & 0 & 0.000 & 0 & 0.000 & & & \\
\hline TOTAL & 92,443 & 100.000 & 8,097 & 100.000 & 10,742 & 100.000 & 100,540 & 100.000 & 17,514 & 100.000 & 83,026 & 100.000 & & & \\
\hline \multicolumn{16}{|l|}{ V\&L Mixture Components } \\
\hline Total Flow, acfm & \multicolumn{2}{|c|}{4,150} & \multicolumn{2}{|c|}{262} & \multicolumn{2}{|r|}{1} & \multicolumn{2}{|c|}{4,413} & 76 & & & $\overline{8}$ & & & \\
\hline Vapor Frac & 1.0 & & 1.0 & & 0.0 & & 1.0 & & 1.00 & & 0.0 & & & & \\
\hline Temperature, $\mathrm{F}$ & 570 & & 30 & & 307 & & $54 \varepsilon$ & & 548 & & 29 & & & & \\
\hline Pressure, psia & 75 & & & & 75 & & 75 & & 75 & & & .0 & & & \\
\hline Enthalpy, Btu/lb & $-5,549$. & & $-5,684$ & & $-6,588$. & & $-5,560$. & & $-5,560.6$ & & $-6,598$ & & & & \\
\hline Density, Ib/ft ${ }^{3}$ & 0.1 & & 0.1 & & 57.0 & & 0.1 & & 0.12 & & 57.3 & & & & \\
\hline Molecular Weight & 18.0 & & 18.0 & & 18.0 & & 18.0 & & 18.01 & & 18.0 & & & & \\
\hline & & & & & & & Solid & & & & & & & & \\
\hline Solid Components & $\mathrm{Ib} / \mathrm{t}$ & & $\overline{|l b /|}$ & & $\mathrm{Tb} / \mathrm{h}$ & & $\mathrm{Tb} / \mathrm{t}$ & & $\mathrm{Ib} / \mathrm{h}$ & & $\mathrm{Ib} /$ & & & & \\
\hline Coal & & 0 & & $\overline{0}$ & & $\overline{0}$ & & $\overline{0}$ & & $\overline{0}$ & & $\overline{0}$ & & & \\
\hline Char & & 0 & & 0 & & 0 & & 0 & & 0 & & 0 & & & \\
\hline Ash & & 0 & & 0 & & 0 & & 0 & & 0 & & 0 & & & \\
\hline Sulfur & & 0 & & 0 & & 0 & & 0 & & 0 & & 0 & & & \\
\hline TOTAL & & 0 & & 0 & & 0 & & 0 & & 0 & & 0 & & & \\
\hline
\end{tabular}

Notes:

1. Results based on bench scale reactor test results communicated by APS. 


\section{WW WorleyParsons \\ resources \& energy}

\section{Appendix D: \\ Water Balance Diagram}




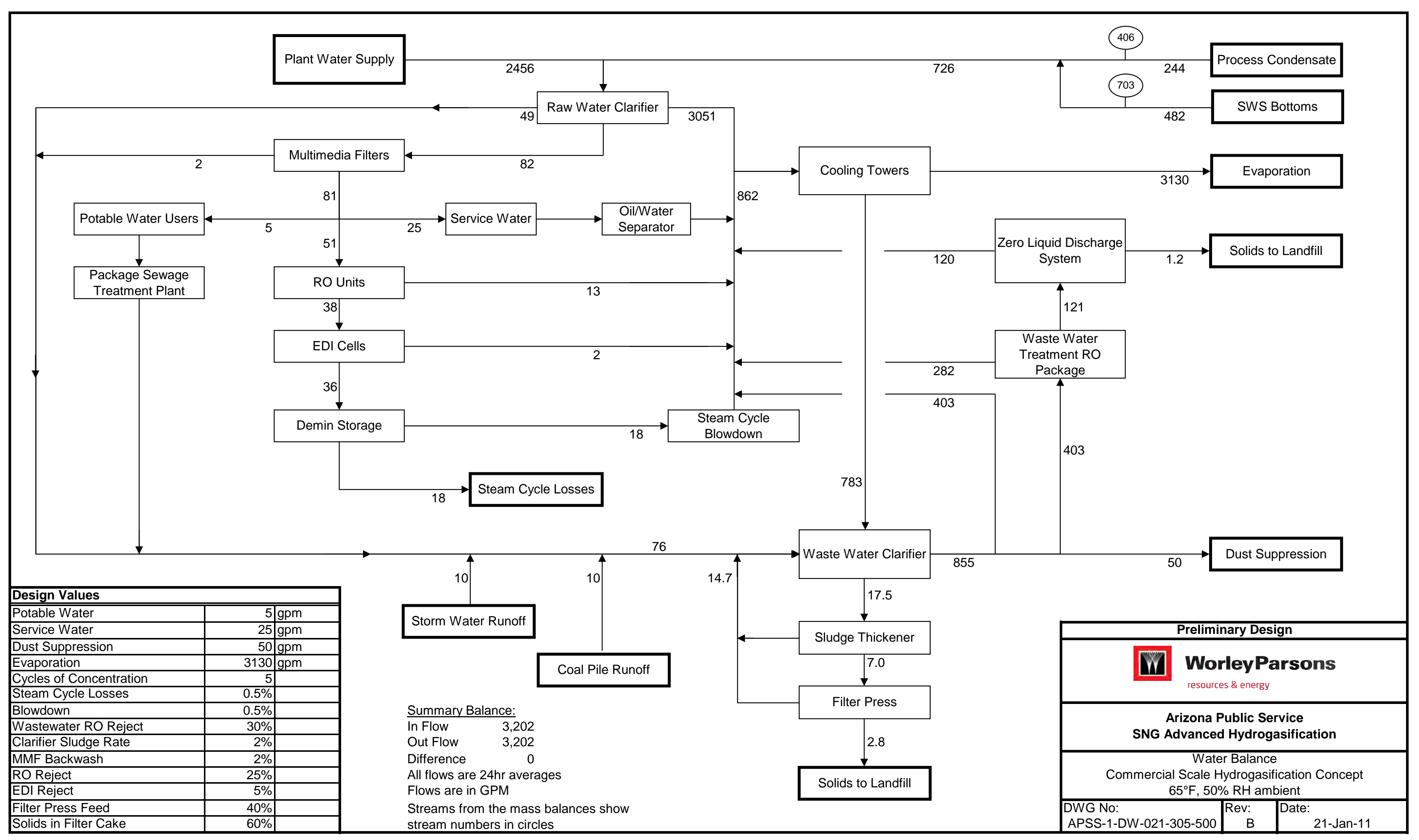




\section{Appendix E: \\ Major Equipment List}




\section{ACCOUNT 1 COAL HANDLING AND PREPARATION}

\section{ACCOUNT 1.1 COAL RECEIVING AND STORAGE}

\begin{tabular}{|c|c|c|c|c|}
\hline Equipment No. & Description & Type & Design Condition & Qty \\
\hline 1 & Truck Dump station & $\begin{array}{l}\text { Receiving hopper with } \\
\text { grizzly and dust collector }\end{array}$ & 240 tons & 1 \\
\hline 2 & Feeder & Belt & 240 tph & 1 \\
\hline 3 & Conveyor No. 1 & Belt w/scale & 240 tph & 1 \\
\hline 4 & Transfer House \#1 & & & 1 \\
\hline 5 & Conveyor No. 2 & Belt & 240 tph & 1 \\
\hline 6 & $\begin{array}{l}\text { As-Received Coal Sampling } \\
\text { System }\end{array}$ & Two-stage & $\mathrm{N} / \mathrm{A}$ & 1 \\
\hline 7 & Stacker Conveyor & Belt, linear & 240 tph & 1 \\
\hline 8 & Telescoping chute & & & 1 \\
\hline 9 & Coal Reclaim conveyor \#1 & Belt & 240 tph & 1 \\
\hline 10 & Transfer House \#2 & $\begin{array}{l}\text { w/dust collector and } \\
\text { w/magnetic separator }\end{array}$ & & 1 \\
\hline 11 & Coal Reclaim Conveyor No. 2 & Belt & 240 tph & 1 \\
\hline 12 & Crusher feed conveyor & Belt & 240 tph & 1 \\
\hline 13 & Coal Surge Bin w/ Vent Filter & Compartment & 120 ton & 1 \\
\hline 14 & Crusher Feeder & Belt & 240 tph & 1 \\
\hline 15 & Crusher & $\begin{array}{l}\text { Impact reduction swing } \\
\text { hammer }\end{array}$ & $\begin{array}{l}3 " x 0-1-1 / 4 " x 0 \\
120 \mathrm{tph}\end{array}$ & 2 \\
\hline 16 & As-Fired Coal Sampling System & & & 1 \\
\hline 17 & Coal storage Conveyor No. 1 & Belt & 240 tph & 1 \\
\hline 18 & Coal storage Conveyor \#2 & Belt & 240 tph & 1 \\
\hline 19 & Coal storage Conveyor \#3 & Belt & 240 tph & 1 \\
\hline 20 & Crushed Coal Silo & $\begin{array}{l}\text { w/ Vent Filter and Slide } \\
\text { Gates, Forced Ventilation, } \\
\text { Inert gas Blanket system }\end{array}$ & 810 ton & 2 \\
\hline
\end{tabular}




\section{ACCOUNT 1.2 COAL RECLAIM AND PREPARATION}

\begin{tabular}{|c|l|l|l|c|}
\hline Equipment No. & \multicolumn{1}{|c|}{ Description } & \multicolumn{1}{c|}{ Type } & \multicolumn{1}{c|}{ Design Condition } & Qty \\
\hline 1 & Feeder & Belt & 150 tph & 2 \\
\hline 2 & Conveyor No. 1 & Belt & 150 tph & 1 \\
\hline 3 & Transfer House \#1 & w/dust collector & & 1 \\
\hline 4 & Mill Feed Hopper & Vertical, double hopper & 100 tons & 1 \\
\hline 5 & Feeder & Belt & 150 tph & 2 \\
\hline 6 & Gravimetric Feeder & $\begin{array}{l}\text { W/ variable frequency drive } \\
\text { and magnetic separator }\end{array}$ & 150 tph & 2 \\
\hline 7 & Mill & Vendor Design & $\begin{array}{l}75 \text { tph } \\
\text { Inlet: 1-1/4" x 0", } \\
\text { Final 70\% through } \\
\text { mesh 200 }\end{array}$ & 3 \\
\hline
\end{tabular}

\section{ACCOUNT 2 GASIFIER AND GASIFIER FEED SYSTEM}

\section{ACCOUNT 2.1 GASIFIER}

\begin{tabular}{|c|l|l|l|c|}
\hline Equipment No. & \multicolumn{1}{|c|}{ Description } & \multicolumn{1}{|c|}{ Type } & Design Condition & Qty \\
\hline 1 & Pulverized Coal Silo & $\begin{array}{l}\text { Bolted CS, Forced } \\
\text { Ventilation, Inert Gas } \\
\text { Blanket system }\end{array}$ & 100 tons & 6 \\
\hline 2 & Milled Coal Pneumatic system & $\begin{array}{l}\text { Pressurized, } \mathrm{CO}_{2} \text { Gas, } \\
\text { including cyclone } \\
\text { separators, dust baghouse, } \\
\text { exhaust \& GR fans }\end{array}$ & 150 tph & 9 \\
\hline 3 & Solids Feed System & $\begin{array}{l}\text { Stamet Posimetric solids } \\
\text { pumps }\end{array}$ & 25 tph, 1,000 psig & 9 \\
\hline
\end{tabular}




\begin{tabular}{|c|c|c|c|c|}
\hline Equipment No. & Description & Type & Design Condition & Qty \\
\hline 4 & APS Hydrogasification System & $\begin{array}{l}\text { Pressurized, dry-feed, } \\
\text { Refractory-lined, } \\
\text { Down-flow, entrained bed } \\
\text { Non-slagging }\end{array}$ & $\begin{array}{l}8 \mathrm{ft} \text { ID, } 55 \mathrm{ft} \text { T/T } \\
316 \mathrm{SS} \text { clad - 4-inch CS } \\
5 \text {-inch surface refractory } \\
\text { 12-inch refractory brick } \\
1,000 \mathrm{psig} / 1,750^{\circ} \mathrm{F} \\
4 \text { coal feed injectors } \\
4 \text { water-cooled } \\
\text { hydrogen POX burners } \\
\text { POX burner cooling loop } \\
\text { exchanger }\end{array}$ & 3 \\
\hline 5 & Char Lock Hopper and Cooler & $\begin{array}{l}\text { Tube bundle } \\
\text { Refractory-lined }\end{array}$ & $\begin{array}{l}\text { Vessel: } \\
3 \mathrm{ft} \mathrm{ID,} 6 \mathrm{ft} \mathrm{T/T} \\
316 \mathrm{SS} \text { clad - 2-inch CS } \\
\text { 5-inch surface refractory } \\
\text { 12-inch refractory brick } \\
\text { 1,000 psig / 1,750 } \mathrm{F} \\
\text { Tube Bundle: } \\
33 \mathrm{MMBtu} / \mathrm{hr} \\
\text { CrMo }\end{array}$ & 3 \\
\hline 6 & Char Lock Hopper & $\begin{array}{l}\text { Vertical with conical } \\
\text { discharge }\end{array}$ & $\begin{array}{l}3 \mathrm{ft} \mathrm{ID,} 6 \mathrm{ft} \mathrm{T/T} \\
316 \mathrm{SS} \text { clad - 2-inch CS } \\
5 \text {-inch surface refractory } \\
12 \text {-inch refractory brick } \\
1,000 \mathrm{psig} / 250^{\circ} \mathrm{F}\end{array}$ & 3 \\
\hline 7 & Oxygen Heater & Shell and Tube & $\begin{array}{l}1 \mathrm{MMBtu} / \mathrm{hr} \\
25 \mathrm{ft}^{2} \\
\text { Shell: } 65 \mathrm{psig} / 490^{\circ} \mathrm{F} \\
\text { Tubes:1,125 psig } / 200^{\circ} \mathrm{F} \\
\text { B165 N04400 Annealed }\end{array}$ & 3 \\
\hline 8 & Air Locking Gas Compressor & Centrifugal & $\begin{array}{l}1,450 \mathrm{acfm} \\
0 / 1,000 \text { psig } \\
650 \mathrm{hp} \\
\text { CS/CS Impeller }\end{array}$ & 3 \\
\hline
\end{tabular}

Note: Pressures and temperatures are normal operating conditions. Ratings, capacities, and duties are nominal design values. 


\section{ACCOUNT 3}

AIR SEPARATION UNIT (ASU)

N/A - Oxygen supplied by electrolysis unit.

\section{ACCOUNT 4 RAW SYNGAS PARTICULATE REMOVAL AND COOLING}

\section{ACCOUNT 4.1 PARICULATE REMOVAL}

\begin{tabular}{|c|c|c|c|c|}
\hline Equipment No. & Description & Type & Design Condition & Qty \\
\hline 1 & Cyclone System & $\begin{array}{l}\text { Refractory-lined with } \\
\text { Vortex finder enclosed } \\
\text { in pressure shell }\end{array}$ & $\begin{array}{l}9,700 \mathrm{acfm} \\
970 \mathrm{psig} / 660^{\circ} \mathrm{F} \\
27,000 \mathrm{lb} / \mathrm{hr} \text { solids loading } \\
\text { CS shell }\end{array}$ & 1 \\
\hline 2 & Candle Filter System & $\begin{array}{l}\text { Pall's PSS iron } \\
\text { aluminide w/CPP inert } \\
\text { gas blow back, or } \\
\text { equal }\end{array}$ & $\begin{array}{l}9,700 \mathrm{acfm} \\
970 \mathrm{psig} / 660^{\circ} \mathrm{F} \\
1 \text { micron removal } \\
\text { efficiency }\end{array}$ & 1 \\
\hline 3 & Cyclone Char Lock Hopper & $\begin{array}{l}\text { Vertical with conical } \\
\text { discharge }\end{array}$ & $\begin{array}{l}3 \mathrm{ft} \mathrm{ID,} 6 \mathrm{ft} \mathrm{T/T} \\
\text { 2-inch CS } \\
\text { 5-inch surface refractory } \\
\text { 7-inch refractory brick } \\
970 \mathrm{psig} / 660^{\circ} \mathrm{F}\end{array}$ & 1 \\
\hline
\end{tabular}

\section{ACCOUNT 4.2 SYNGAS COOLING}

\begin{tabular}{|c|l|l|l|c|}
\hline Equipment No. & \multicolumn{1}{|c|}{ Description } & \multicolumn{1}{|c|}{ Type } & \multicolumn{1}{|c|}{ Design Condition } & Qty \\
\hline 1 & Hydrogen Heater & Pressurized, & $115 \mathrm{MMBtu} / \mathrm{hr}$ & 3 \\
& & Refractory-lined, & $18,100 \mathrm{ft}{ }^{2}$ & \\
& & Upflow & $1,000 \mathrm{psig} / 1,750^{\circ} \mathrm{F}$ \\
& & & $316 \mathrm{SS}$ clad - 4-inch CS \\
& & & 5 -inch surface refractory \\
& & & 12 -inch refractory brick \\
& & & Hydrogen Side: & \\
& & & 1,150 psig/1,250 $\mathrm{F}$ & \\
\end{tabular}




\begin{tabular}{|c|c|c|c|c|}
\hline Equipment No. & Description & Type & Design Condition & Qty \\
\hline 2 & Radiant Raw Gas Cooler (WHB) & $\begin{array}{l}\text { Gas-tight membrane } \\
\text { water wall evaporation } \\
\text { surfaces enclosed in } \\
\text { pressure shell, and } \\
\text { connected to the } \\
\text { steam drum (natural or } \\
\text { assisted circulation per } \\
\text { Vendor design) }\end{array}$ & $\begin{array}{l}30 \mathrm{MMBtu} / \mathrm{hr} \\
3,000 \mathrm{ft}^{2} \\
\text { Gas Side: } \\
990 \mathrm{psig} / 900^{\circ} \mathrm{F} \\
\text { Steam Side: } \\
1,940 \mathrm{psig} / 630^{\circ} \mathrm{F} \\
\text { CrMo/CrMo }\end{array}$ & 3 \\
\hline 3 & Syngas Cooler \#1 & Shell and Tube & $\begin{array}{l}150 \mathrm{MMBtu} / \mathrm{hr} \\
43,300 \mathrm{ft}^{2} \\
\text { Shell: } 970 \mathrm{psig} / 660^{\circ} \mathrm{F} \\
\text { Tubes: } 2,240 \mathrm{psig} / 475^{\circ} \mathrm{F} \\
316 \mathrm{clad} \mathrm{CrMo} / \mathrm{CS}\end{array}$ & 1 \\
\hline 4 & Syngas Cooler \#2 & Shell and Tube & $\begin{array}{l}110 \mathrm{MMBtu} / \mathrm{hr} \\
46,700 \mathrm{ft}^{2} \\
\text { Shell: } 140 \mathrm{psig} / 270^{\circ} \mathrm{F} \\
\text { Tubes: } 960 \mathrm{psig} / 310^{\circ} \mathrm{F} \\
\text { CS/316 clad CrMo }\end{array}$ & 1 \\
\hline 5 & Syngas Cooler \#3 & Shell and Tube & $\begin{array}{l}25 \mathrm{MMBtu} / \mathrm{hr} \\
9,900 \mathrm{ft}^{2} \\
\text { Shell: } 50 \mathrm{psig} / 100^{\circ} \mathrm{F} \\
\text { Tubes: } 950 \mathrm{psig} / 160^{\circ} \mathrm{F} \\
\text { CS/316 clad CrMo }\end{array}$ & 1 \\
\hline 6 & Raw Gas KO Drum & $\begin{array}{l}\text { Vertical with mist } \\
\text { eliminator }\end{array}$ & $\begin{array}{l}5 \mathrm{ft} \mathrm{dia}, 20 \mathrm{ft} \mathrm{T} / \mathrm{T} \\
950 \mathrm{psig} / 160^{\circ} \mathrm{F} \\
316 \mathrm{clad} \mathrm{CrMo}\end{array}$ & 1 \\
\hline 7 & Steam Drum & Horizontal & $\begin{array}{l}7 \mathrm{ft} \mathrm{dia}, 20 \mathrm{ft} \mathrm{T} / \mathrm{T} \\
1,940 \mathrm{psig} / 630^{\circ} \mathrm{F} \\
\mathrm{CS}\end{array}$ & 1 \\
\hline
\end{tabular}

ACCOUNT 4.3 MERCURY REMOVAL

\begin{tabular}{|c|l|l|l|c|}
\hline Equipment No. & \multicolumn{1}{|c|}{ Description } & \multicolumn{1}{c|}{ Type } & \multicolumn{1}{c|}{ Design Condition } & Qty \\
\hline 1 & Mercury Removal Unit & $\begin{array}{l}\text { Sulfur-impregnated } \\
\text { carbon bed }\end{array}$ & $\begin{array}{l}5,300 \mathrm{acfm} \\
\text { Operating: } 950 \mathrm{psia} / 115^{\circ} \mathrm{F} \\
\text { Design: } 1,050 \mathrm{psig} / 200^{\circ} \mathrm{F}\end{array}$ & 1 \\
& & & & \\
\hline
\end{tabular}




\section{ACCOUNT 5 ACID GAS REMOVAL AND SULFUR RECOVERY}

\begin{tabular}{|c|l|l|l|c|}
\hline Equipment No. & \multicolumn{1}{|c|}{ Description } & \multicolumn{1}{|c|}{ Type } & \multicolumn{1}{c|}{ Design Condition } & Qty \\
\hline \multirow{2}{*}{1} & Acid Gas Removal /Sulfur & CrystaSulf non-aqueous & Sour gas: & 1 \\
& Recovery & sulfur recovery process. & $930 \mathrm{psia} / 110^{\circ} \mathrm{F} / 500,000$ & \\
& & Vendor design & Ib/h \\
& & Includes syngas preheater & $430 \mathrm{MMSCFD}$ \\
& & & $1,250 \mathrm{ppm}$ total sulfur \\
& & & Sweet gas: \\
& & & 10 ppm total sulfur \\
& & & Elemental sulfur: 20 tpd & \\
\hline
\end{tabular}

\section{ACCOUNT $6 \quad$ SULFUR RECOVERY \& TGTU}

N/A - Sulfur recovery done through AGR. 


\section{ACCOUNT 7 OTHER GAS PROCESSES}

\section{ACCOUNT 7.1 METHANATION SYSTEM}

\begin{tabular}{|c|c|c|c|c|}
\hline Equipment No. & Description & Type & Design Condition & Qty \\
\hline 1 & Sulfur Guard Bed & Fixed-Bed & $\begin{array}{l}14 \mathrm{ft} \mathrm{dia}, 14 \mathrm{ft} \mathrm{T/T} \\
900 \mathrm{psig} / 500^{\circ} \mathrm{F} \\
\text { Top: Distribution Plate } \\
\text { Bottom: Johnson screen } \\
\text { with ceramic balls to } \\
\text { tangent line. } \\
\text { CS }\end{array}$ & 1 \\
\hline 2 & Bulk Methanation Reactor 1 & Fixed-Bed & $\begin{array}{l}13.5 \mathrm{ft} \text { dia, } 17.5 \mathrm{ft} \mathrm{T/T} \\
900 \mathrm{psig} / 1,060^{\circ} \mathrm{F} \\
\text { Top: Distribution Plate } \\
\text { Bottom: Johnson screen } \\
\text { with ceramic balls to } \\
\text { tangent line. } \\
\text { CrMo }\end{array}$ & 3 \\
\hline 3 & Bulk Methanation Reactor 2 & Fixed-Bed & $\begin{array}{l}14 \mathrm{ft} \mathrm{dia}, 14 \mathrm{ft} \mathrm{T/T} \\
880 \mathrm{psig} / 830^{\circ} \mathrm{F} \\
\text { Top: Distribution Plate } \\
\text { Bottom: Johnson screen } \\
\text { with ceramic balls to } \\
\text { tangent line. } \\
\text { CrMo }\end{array}$ & 1 \\
\hline 4 & Trim Methanation Reactor & Fixed-Bed & $\begin{array}{l}14 \mathrm{ft} \mathrm{dia}, 14 \mathrm{ft} \mathrm{T/T} \\
860 \mathrm{psig} / 580^{\circ} \mathrm{F} \\
\text { Top: Distribution Plate } \\
\text { Bottom: Johnson screen } \\
\text { with ceramic balls to } \\
\text { tangent line. } \\
\text { CrMo }\end{array}$ & 1 \\
\hline 5 & Feed/Product Exchanger 1 & Shell and Tube & $\begin{array}{l}45 \mathrm{MMBtu} / \mathrm{hr} \\
26,000 \mathrm{ft}^{2} \\
\text { Shell: } 840 \mathrm{psig} / 380^{\circ} \mathrm{F} \\
\text { Tubes: } 920 \mathrm{psig} / 250^{\circ} \mathrm{F} \\
\text { CrMo/CrMo }\end{array}$ & 1 \\
\hline
\end{tabular}




\begin{tabular}{|c|c|c|c|c|}
\hline Equipment No. & Description & Type & Design Condition & Qty \\
\hline 6 & Feed/Product Exchanger 2 & Shell and Tube & $\begin{array}{l}95 \mathrm{MMBtu} / \mathrm{hr} \\
80,300 \mathrm{ft}^{2} \\
\text { Shell: } 850 \mathrm{psig} / 580^{\circ} \mathrm{F} \\
\text { Tubes: } 915 \mathrm{psig} / 500^{\circ} \mathrm{F} \\
\text { CrMo/CrMo }\end{array}$ & 1 \\
\hline 7 & HP Steam Superheater & Shell and Tube & $\begin{array}{l}175 \mathrm{MMBtu} / \mathrm{hr} \\
57,500 \mathrm{ft}^{2} \\
\text { Shell: } 1,950 \mathrm{psig} / 1,000^{\circ} \mathrm{F} \\
\text { Tubes: } 890 \mathrm{psig} / 1,060^{\circ} \mathrm{F} \\
\text { CrMo/CrMo }\end{array}$ & 1 \\
\hline 8 & HP Steam Generator 1 & Shell and Tube & $\begin{array}{l}340 \mathrm{MMBtu} / \mathrm{hr} \\
32,400 \mathrm{ft}^{2} \\
\text { Shell: } 1,940 \mathrm{psig} / 630^{\circ} \mathrm{F} \\
\text { Tubes: } 890 \mathrm{psig} / 930^{\circ} \mathrm{F} \\
\text { CrMo/CrMo }\end{array}$ & 1 \\
\hline 9 & HP Steam Generator 2 & Shell and Tube & $\begin{array}{l}50 \mathrm{MMBtu} / \mathrm{hr} \\
6,600 \mathrm{ft}^{2} \\
\text { Shell: } 1,940 \mathrm{psig} / 630 \mathrm{~F} \\
\text { Tubes: } 870 \mathrm{psig} / 830 \mathrm{~F} \\
\text { CS/CrMo }\end{array}$ & 1 \\
\hline 10 & BFW Heater 1 & Shell and Tube & $\begin{array}{l}55 \mathrm{MMBtu} / \mathrm{hr} \\
19,600 \mathrm{ft}^{2} \\
\text { Shell: } 2,240 \mathrm{psig} / 350^{\circ} \mathrm{F} \\
\text { Tubes: } 850 \mathrm{psig} / 410^{\circ} \mathrm{F} \\
\text { CS/CrMo }\end{array}$ & 1 \\
\hline 11 & BFW Heater 2 & Shell and Tube & $\begin{array}{l}45 \mathrm{MMBtu} / \mathrm{hr} \\
8,200 \mathrm{ft}^{2} \\
\text { Shell: } 2,240 \mathrm{psig} / 430^{\circ} \mathrm{F} \\
\text { Tubes: } 870 \mathrm{psig} / 650^{\circ} \mathrm{F} \\
\text { CS/CrMo }\end{array}$ & 1 \\
\hline 12 & Recycle Inlet/Outlet Exchanger & Shell and Tube & $\begin{array}{l}125 \mathrm{MMBtu} / \mathrm{hr} \\
60,300 \mathrm{ft}^{2} \\
\text { Shell: } 870 \mathrm{psig} / 650^{\circ} \mathrm{F} \\
\text { Tubes: } 900 \mathrm{psig} / 550^{\circ} \mathrm{F} \\
\text { CrMo/CrMo }\end{array}$ & 1 \\
\hline
\end{tabular}




\begin{tabular}{|c|c|c|c|c|}
\hline Equipment No. & Description & Type & Design Condition & Qty \\
\hline 13 & Recycle Trim Cooler & Shell and Tube & $\begin{array}{l}145 \mathrm{MMBtu} / \mathrm{hr} \\
7,900 \mathrm{ft}^{2} \\
\text { Shell: } 50 \mathrm{psig} / 100^{\circ} \mathrm{F} \\
\text { Tubes: } 880 \mathrm{psig} / 520^{\circ} \mathrm{F} \\
\text { CS/SS }\end{array}$ & 1 \\
\hline 14 & SNG Trim Cooler & Shell and Tube & $\begin{array}{l}100 \mathrm{MMBtu} / \mathrm{hr} \\
18,000 \mathrm{ft}^{2} \\
\text { Shell: } 50 \mathrm{psig} / 100^{\circ} \mathrm{F} \\
\text { Tubes: } 840 \mathrm{psig} / 350^{\circ} \mathrm{F} \\
\text { CS/SS }\end{array}$ & 1 \\
\hline 15 & Process Condensate Cooler & Shell and Tube & $\begin{array}{l}55 \mathrm{MMBtu} / \mathrm{hr} \\
\text { 10,000 ft } \\
\text { Shell: } 50 \mathrm{psig} / 100^{\circ} \mathrm{F} \\
\text { Tubes: } 940 \mathrm{psig} / 380^{\circ} \mathrm{F} \\
\text { CS/CS }\end{array}$ & 1 \\
\hline 16 & Steam Drum & Horizontal & $\begin{array}{l}11 \mathrm{ft} \mathrm{dia}, 30 \mathrm{ft} \mathrm{T/T} \\
1,940 \mathrm{psig} / 630^{\circ} \mathrm{F} \\
\mathrm{CS}\end{array}$ & 1 \\
\hline 17 & Recycle KO Drum & Vertical & $\begin{array}{l}12 \mathrm{ft} \mathrm{dia}, 20 \mathrm{ft} \mathrm{T} / \mathrm{T} \\
880 \mathrm{psig} / 400^{\circ} \mathrm{F} \\
\text { SS }\end{array}$ & 2 \\
\hline 18 & Methanation KO Drum 1 & Vertical & $\begin{array}{l}8.5 \mathrm{ft} \mathrm{dia}, 15.5 \mathrm{ft} \mathrm{T} / \mathrm{T} \\
840 \mathrm{psig} / 380^{\circ} \mathrm{F} \\
\text { SS }\end{array}$ & 1 \\
\hline 19 & Methanation KO Drum 2 & Vertical & $\begin{array}{l}8 \mathrm{ft} \mathrm{dia}, 15 \mathrm{ft} \mathrm{T} / \mathrm{T} \\
830 \mathrm{psig} / 350^{\circ} \mathrm{F} \\
\mathrm{SS}\end{array}$ & 1 \\
\hline 20 & SNG Trim Cooler KO Drum & Vertical & $\begin{array}{l}6.5 \mathrm{ft} \mathrm{dia}, 13 \mathrm{ft} \mathrm{T/T} \\
830 \mathrm{psig} / 110^{\circ} \mathrm{F} \\
\text { SS }\end{array}$ & 1 \\
\hline 21 & Recycle Compressor & Centrifugal & $\begin{array}{l}13,500 \mathrm{acfm} \\
870 \mathrm{psig} / 900 \mathrm{psig} \\
3,350 \mathrm{hp} \\
\text { CS/SS Impeller }\end{array}$ & 1 \\
\hline
\end{tabular}




\section{ACCOUNT 7.2 DEOXIDATION SYSTEM}

\begin{tabular}{|c|c|c|c|c|}
\hline $\begin{array}{c}\text { Equipment } \\
\text { No. }\end{array}$ & Description & Type & Design Condition & Qty \\
\hline 1 & Deoxidation Reactor 1 & Fixed-Bed & $\begin{array}{l}6 \mathrm{ft} \text { dia, } 13 \mathrm{ft} \mathrm{T/T} \\
1,000 \text { psig } / 1,090^{\circ} \mathrm{F} \\
\text { Top: Distribution Plate } \\
\text { Bottom: Johnson screen } \\
\text { with ceramic balls to } \\
\text { tangent line. } \\
\text { CrMo }\end{array}$ & 1 \\
\hline 2 & Deoxidation Reactor 2 & Fixed-Bed & $\begin{array}{l}6 \mathrm{ft} \text { dia, } 13 \mathrm{ft} \mathrm{T/T} \\
990 \mathrm{psig} / 1,080^{\circ} \mathrm{F} \\
\text { Top: Distribution Plate } \\
\text { Bottom: Johnson screen } \\
\text { with ceramic balls to } \\
\text { tangent line. } \\
\text { CrMo }\end{array}$ & 1 \\
\hline 3 & Product/Feed Exchanger & Shell and Tube & $\begin{array}{l}32 \mathrm{MMBtu} / \mathrm{hr} \\
7,400 \mathrm{ft}^{2} \\
\text { Shell: } 990 \mathrm{psig} / 1,090^{\circ} \mathrm{F} \\
\text { Tubes: } 1,000 \mathrm{psig} / 670^{\circ} \mathrm{F} \\
\text { CrMo/CrMo }\end{array}$ & 1 \\
\hline 4 & HP Steam Generator & Shell and Tube & $\begin{array}{l}32 \mathrm{MMBtu} / \mathrm{hr} \\
2,200 \mathrm{ft}^{2} \\
\text { Shell: } 980 \mathrm{psig} / 1,080^{\circ} \mathrm{F} \\
\text { Tubes: } 1,940 \mathrm{psig} / 630^{\circ} \mathrm{F} \\
\text { CS/CrMo }\end{array}$ & 1 \\
\hline 5 & BFW Heater & Shell and Tube & $\begin{array}{l}26 \mathrm{MMBtu} / \mathrm{hr} \\
7,500 \mathrm{ft}^{2} \\
\text { Shell: } 970 \mathrm{psig} / 660^{\circ} \mathrm{F} \\
\text { Tubes: } 2,240 \mathrm{psig} / 480^{\circ} \mathrm{F} \\
\text { CS/CrMo }\end{array}$ & 1 \\
\hline 6 & Condensate Heater & Shell and Tube & $\begin{array}{l}22 \mathrm{MMBtu} / \mathrm{hr} \\
7,800 \mathrm{ft}^{2} \\
\text { Shell: } 140 \mathrm{psig} / 270^{\circ} \mathrm{F} \\
\text { Tubes: } 970 \mathrm{psig} / 310^{\circ} \mathrm{F} \\
\text { CS/SS }\end{array}$ & 1 \\
\hline
\end{tabular}




\begin{tabular}{|c|c|c|c|c|}
\hline $\begin{array}{c}\text { Equipment } \\
\text { No. }\end{array}$ & Description & Type & Design Condition & Qty \\
\hline 7 & Trim Cooler & Shell and Tube & $\begin{array}{l}6 \mathrm{MMBtu} / \mathrm{hr} \\
2,000 \mathrm{ft}^{2} \\
\text { Shell: } 50 \mathrm{psig} / 100^{\circ} \mathrm{F} \\
\text { Tubes: } 960 \mathrm{psig} / 160^{\circ} \mathrm{F} \\
\text { CS/SS }\end{array}$ & 1 \\
\hline 8 & Steam Drum & Horizontal & $\begin{array}{l}5 \mathrm{ft} \mathrm{dia}, 15 \mathrm{ft} \mathrm{T/T} \\
1,940 \mathrm{psig} / 630^{\circ} \mathrm{F} \\
\mathrm{CS}\end{array}$ & 1 \\
\hline 9 & Condensate KO Drum & Vertical & $\begin{array}{l}5 \mathrm{ft} \mathrm{dia,} 11.5 \mathrm{ft} \mathrm{T} / \mathrm{T} \\
950 \mathrm{psig} / 110^{\circ} \mathrm{F} \\
\mathrm{SS}\end{array}$ & 1 \\
\hline
\end{tabular}

\section{ACCOUNT 7.3 SNG PURIFICATION AND COMPRESSION}

\begin{tabular}{|c|c|c|c|c|}
\hline Equipment No. & Description & Type & Design Condition & Qty \\
\hline 1 & SNG Product Cooler & Shell and Tube & $\begin{array}{l}2 \mathrm{MMBtu} / \mathrm{hr} \\
2,000 \mathrm{ft}^{2} \\
\text { Shell: } 50 \mathrm{psig} / 100^{\circ} \mathrm{F} \\
\text { Tubes: } 900 \mathrm{psig} / 130^{\circ} \mathrm{F} \\
\text { CS/CS }\end{array}$ & 1 \\
\hline 2 & Gas Dryer & $\begin{array}{l}\text { Triethelyne glycol } \\
\text { (TEG) Desiccant } \\
\text { System }\end{array}$ & $\begin{array}{l}235,000 \mathrm{lb} / \mathrm{hr} \\
1,600 \mathrm{acfm} \\
0.25 \% \mathrm{H}_{2} \mathrm{O} \text { in } \\
-40^{\circ} \mathrm{F} \text { dew point out }\end{array}$ & 1 \\
\hline 3 & SNG Product Compression & Centrifugal & $\begin{array}{l}1,500 \mathrm{acfm} \\
830 \mathrm{psig} / 900 \mathrm{psig} \\
965 \mathrm{hp} \\
\text { CS/CS Impeller }\end{array}$ & 1 \\
\hline
\end{tabular}




\section{ACCOUNT $7.4 \quad$ SOUR WATER SYSTEM}

\begin{tabular}{|c|c|c|c|c|}
\hline Equipment No. & Description & Type & Design Condition & Qty \\
\hline 1 & Sour Water Stripper & $\begin{array}{l}\text { Vertical } \\
\text { Packed Column }\end{array}$ & $\begin{array}{l}5 \mathrm{ft} \text { dia, } 55 \mathrm{ft} \mathrm{T/T} \\
100 \mathrm{psig} / 250^{\circ} \mathrm{F} \\
\text { Carbon steel shell, } 316 \mathrm{SS} \\
\text { internals } \\
\text { 1-inch ceramic Raschig } \\
\text { rings }\end{array}$ & 1 \\
\hline 2 & Ammonia Stripper & $\begin{array}{l}\text { Vertical } \\
\text { Packed Column }\end{array}$ & $\begin{array}{l}4 \mathrm{ft} \mathrm{dia,} 60 \mathrm{ft} \mathrm{T/T} \\
20 \mathrm{psig} / 250^{\circ} \mathrm{F} \\
\text { Carbon steel shell, } 316 \mathrm{SS} \\
\text { internals } \\
\text { 1-inch ceramic Raschig } \\
\text { rings }\end{array}$ & 1 \\
\hline 3 & Sour Water Pump & $\begin{array}{l}\text { Horizontal, } \\
\text { Centrifugal }\end{array}$ & 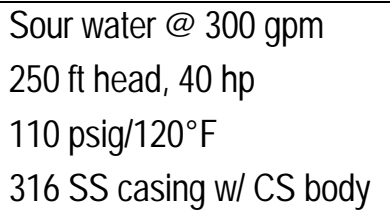 & 2 \\
\hline 4 & Stripper Column Pump & $\begin{array}{l}\text { Horizontal, } \\
\text { Centrifugal }\end{array}$ & $\begin{array}{l}\text { Sour water @ } 325 \text { gpm } \\
100 \text { ft head, } 20 \text { hp } \\
20 \text { psig/ } 180^{\circ} \mathrm{F} \\
316 \text { SS casing w/ CS body }\end{array}$ & 2 \\
\hline 5 & Ammonia Column Pump & $\begin{array}{l}\text { Horizontal, } \\
\text { Centrifugal }\end{array}$ & $\begin{array}{l}\text { Sour water @ } 300 \text { gpm } \\
100 \text { ft head, } 20 \text { hp } \\
10 \text { psig/ } 180^{\circ} \mathrm{F} \\
316 \text { SS casing w/ CS body }\end{array}$ & 2 \\
\hline 6 & Sour Stripper Feed Heater & Shell and Tube & $\begin{array}{l}1165 \mathrm{MMBtu} / \mathrm{hr} \\
1,000 \mathrm{ft}^{2} \\
\text { Shell: } \quad 60 \mathrm{psig} / 550^{\circ} \mathrm{F} \\
\text { Tubes: } 110 \mathrm{psig} / 260^{\circ} \mathrm{F} \\
\text { CS/SS }\end{array}$ & 1 \\
\hline 7 & Sour Stripper Reboiler & Shell and Tube & $\begin{array}{l}7 \mathrm{MMBtu} / \mathrm{hr} \\
800 \mathrm{ft}^{2} \\
\text { Shell: } \quad 60 \mathrm{psig} / 550^{\circ} \mathrm{F} \\
\text { Tubes: } 20 \mathrm{psig} / 250^{\circ} \mathrm{F} \\
\text { CS/SS }\end{array}$ & 1 \\
\hline
\end{tabular}




\begin{tabular}{|c|c|c|c|c|}
\hline Equipment No. & Description & Type & Design Condition & Qty \\
\hline 8 & Stripper Bottoms Cooler & Shell and Tube & $\begin{array}{l}3 \mathrm{MMBtu} / \mathrm{hr} \\
370 \mathrm{ft}^{2} \\
\text { Shell: } \quad 50 \mathrm{psig} / 100^{\circ} \mathrm{F} \\
\text { Tubes: } 20 \mathrm{psig} / 250^{\circ} \mathrm{F} \\
\text { CS/SS }\end{array}$ & 1 \\
\hline 9 & Sour Gas Cooler & $\begin{array}{l}\text { Shell and Tube w/ } \\
\text { condensate drain }\end{array}$ & $\begin{array}{l}1 \mathrm{MMBtu} / \mathrm{hr} \\
250 \mathrm{ft}{ }^{2} \\
\text { Shell: } \quad 50 \mathrm{psig} / 100^{\circ} \mathrm{F} \\
\text { Tubes: } 20 \mathrm{psig} / 210^{\circ} \mathrm{F} \\
\text { CS/SS }\end{array}$ & 1 \\
\hline 10 & Ammonia Stripper Reboiler & Shell and Tube & $\begin{array}{l}23 \mathrm{MMBtu} / \mathrm{hr} \\
2,550 \mathrm{ft}{ }^{2} \\
\text { Shell: } \quad 60 \mathrm{psig} / 550^{\circ} \mathrm{F} \\
\text { Tubes: } 20 \mathrm{psig} / 250^{\circ} \mathrm{F} \\
\text { CS/SS }\end{array}$ & 1 \\
\hline 11 & Ammonia Stripper Condenser & Shell and Tube & $\begin{array}{l}17 \mathrm{MMBtu} / \mathrm{hr} \\
4,750 \mathrm{ft}^{2} \\
\text { Shell: } 50 \mathrm{psig} / 100^{\circ} \mathrm{F} \\
\text { Tubes: } 20 \mathrm{psig} / 220^{\circ} \mathrm{F} \\
\text { CS/SS }\end{array}$ & 1 \\
\hline 12 & $\begin{array}{l}\text { Ammonia Stripper Bottoms } \\
\text { Cooler }\end{array}$ & Shell and Tube & $\begin{array}{l}21 \mathrm{MMBtu} / \mathrm{hr} \\
5,100 \mathrm{ft}^{2} \\
\text { Shell: } 50 \mathrm{psig} / 100^{\circ} \mathrm{F} \\
\text { Tubes: } 20 \mathrm{psig} / 250^{\circ} \mathrm{F} \\
\text { CS/SS }\end{array}$ & 1 \\
\hline 13 & Sour Water Drum & Horizontal Tank & $\begin{array}{l}14 \mathrm{ft} \mathrm{dia}, 115 \mathrm{ft} \mathrm{T} / \mathrm{T} \\
100 \mathrm{psig} / 200^{\circ} \mathrm{F} \\
316 \mathrm{clad} \text { CS }\end{array}$ & 1 \\
\hline 14 & $\begin{array}{l}\text { Ammonia Stripper Condensate } \\
\text { Receiver }\end{array}$ & Horizontal Tank & $\begin{array}{l}4 \mathrm{ft} \mathrm{dia,} 9 \mathrm{ft} \mathrm{T} / \mathrm{T} \\
50 \mathrm{psig} / 200^{\circ} \mathrm{F} \\
316 \mathrm{clad} \text { CS }\end{array}$ & 1 \\
\hline
\end{tabular}


ACCOUNT $7.5 \quad$ FLARE STACK

\begin{tabular}{|c|l|l|l|c|}
\hline Equipment No. & \multicolumn{1}{|c|}{ Description } & \multicolumn{1}{c|}{ Type } & Design Condition & Qty \\
\hline 1 & Flare Stack & $\begin{array}{l}\text { Self-supporting, carbon } \\
\text { steel, stainless steel } \\
\text { top, pilot ignition }\end{array}$ & $18 \mathrm{MMSCFH}$ at $110^{\circ} \mathrm{F}$ & 1 \\
\hline
\end{tabular}

ACCOUNT $8 \quad$ POLYGEN OPTIONS

NA

ACCOUNT 9 COMBUSTION TURBINE AND AUXILIARIES

NA

ACCOUNT 10 CHAR COMBUSTOR

\begin{tabular}{|c|c|c|c|c|}
\hline Equipment No. & Description & Type & Design Condition & Qty \\
\hline 1 & Char Combustor & $\begin{array}{l}\text { Oxygen-blown, pelletized char } \\
\text { fired, circulating fluidized bed } \\
\text { boiler, } \\
\text { Includes: } \\
\text { Char feeding system, } \\
\text { Oxygen feed and flue gas } \\
\text { recirculation systems, } \\
\text { Economizer, steam superheater, } \\
\text { reheater, furnace loop } \\
\text { evaporative equipment and drum, } \\
\text { Backpass heat recovery } \\
\text { equipment, } \\
\text { Tubular } \mathrm{CO}_{2} \text { and oxidant } \\
\text { preheaters, } \\
\text { External bubbling bed heat } \\
\text { exchanger, } \\
\text { Ash removal system, } \\
\text { Supporting structural steel } \\
\text { Materials per vendor }\end{array}$ & $\begin{array}{l}\text { Char Feed - } \\
75 \mathrm{tph} \\
\text { Steam Superheater - } \\
360 \mathrm{MMBtu} / \mathrm{hr} \text {, } \\
1,040,000 \mathrm{lb} / \mathrm{hr}, \\
1,840 \mathrm{psig} / 1,000^{\circ} \mathrm{F} \\
\text { Steam Reheater - } \\
220 \mathrm{MMBtu} / \mathrm{hr} \text {, } \\
1,510,000 \mathrm{lb} / \mathrm{hr}, \\
610 \mathrm{psig} / 1,000^{\circ} \mathrm{F} \\
\text { Steam Evaporator - } \\
620 \mathrm{MMBtu} / \mathrm{hr}, \\
875,000 \mathrm{lb} / \mathrm{hr}, \\
1,940 \mathrm{psig} / 630^{\circ} \mathrm{F} \\
\text { Economizer - } \\
35 \mathrm{MMBtu} / \mathrm{hr}, \\
165,000 \mathrm{lb} / \mathrm{hr}, \\
2,240 \mathrm{psig} / 475^{\circ} \mathrm{F} \\
\mathrm{CO}{ }_{2} \text { Preheater - } \\
8 \mathrm{MMBtu} / \mathrm{hr}, \\
1,100 \mathrm{psig} / 750^{\circ} \mathrm{F} \\
\text { Oxidant Preheater - } \\
12 \mathrm{MMBtu} / \mathrm{hr}, \\
5 \mathrm{psig} / 250^{\circ} \mathrm{F} \\
\text { Ash Removal - } \\
60,000 \mathrm{lb} / \mathrm{hr}\end{array}$ & 1 \\
\hline
\end{tabular}




\begin{tabular}{|c|c|c|c|c|}
\hline Equipment No. & Description & Type & Design Condition & Qty \\
\hline 2 & Char Pelletizer & $\begin{array}{l}\text { Mars Mineral Model P160 Disc } \\
\text { Pelletizer, with binder spray } \\
\text { system, or equal }\end{array}$ & $\begin{array}{l}25 \text { tph, } 75 \text { hp drive motor; } \\
67 \text { gpm binder supply flow } \\
\text { rate; } \\
\text { In: } 50 \mu \text { (average) } \\
\text { Out: } 0.25 \text { by } 0.125 \text { inch }\end{array}$ & 3 \\
\hline 3 & Flue Gas Trim Cooler & Shell and Tube & $\begin{array}{l}45 \mathrm{MMBtu} / \mathrm{hr} \\
8,900 \mathrm{ft}^{2} \\
\text { Shell: } 50 \mathrm{psig} / 100^{\circ} \mathrm{F} \\
\text { Tubes: } 5 \mathrm{psig} / 300^{\circ} \mathrm{F} \\
\text { CS/SS clad }\end{array}$ & 1 \\
\hline 4 & Flue Gas KO Drum & Vertical & $\begin{array}{l}11 \mathrm{ft} \mathrm{dia}, 18.5 \mathrm{ft} \mathrm{T} / \mathrm{T} \\
5 \mathrm{psig} / 110^{\circ} \mathrm{F} \\
\mathrm{SS} \text { clad }\end{array}$ & 1 \\
\hline 5 & $\begin{array}{l}\text { Flue Gas Recirculation } \\
\text { Fan }\end{array}$ & Centrifugal & $\begin{array}{l}12,000 \mathrm{acfm} \\
4 \mathrm{psig} / 6 \mathrm{psig} \\
190 \mathrm{hp} \\
\mathrm{CS}\end{array}$ & 2 \\
\hline 6 & $\mathrm{CO}_{2}$ Compressor & $\begin{array}{l}\text { Centrifugal, } \\
\text { Multistage, } \\
\text { Intercooled with KO drums }\end{array}$ & $\begin{array}{l}50,000 \text { acfm } \\
4 \text { psig/1,010 psig } \\
22,000 \mathrm{hp} \\
55 \text { MMBtu/hr Cooling Duty } \\
\text { SS/SS Impellers }\end{array}$ & 2 \\
\hline 7 & $\begin{array}{l}\mathrm{CO}_{2} \text { Booster } \\
\text { Compressor }\end{array}$ & Centrifugal & $\begin{array}{l}110 \mathrm{acfm} \\
1,000 \mathrm{psig} / 1,110 \mathrm{psig} \\
80 \mathrm{hp} \\
\mathrm{CS}\end{array}$ & 1 \\
\hline 8 & $\begin{array}{l}\text { Char Pneumatic } \\
\text { System }\end{array}$ & $\begin{array}{l}\text { Pressurized, } \mathrm{CO}_{2} \mathrm{Gas} \text {, including } \\
\text { cyclone separators, dust } \\
\text { baghouse, exhaust \& FGR fans }\end{array}$ & 75 tph & 1 \\
\hline 9 & Char Silo & $\begin{array}{l}\text { w/ Vent Filter and Rotary Valves, } \\
\text { Forced Ventilation }\end{array}$ & 700 ton & 1 \\
\hline 10 & Char Feeder & Belt & 75 tph & 1 \\
\hline 11 & Char Conveyor & Belt & 75 tph & 1 \\
\hline 12 & Char Conveyor & Belt w/ scale & 75 tph & 1 \\
\hline
\end{tabular}




\begin{tabular}{|c|l|l|l|c|}
\hline Equipment No. & \multicolumn{1}{|c|}{ Description } & \multicolumn{1}{c|}{ Type } & \multicolumn{1}{c|}{ Design Condition } & Qty \\
\hline 13 & Startup Stack & $\begin{array}{l}\text { Reinforced concrete with a single } \\
\text { fiber glass reinforced plastic liner }\end{array}$ & $\begin{array}{l}50 \% \text { MCR - max. } \\
\text { Liner ID 7 ft } \\
\text { Height } 170 \mathrm{ft}\end{array}$ & 1 \\
\hline
\end{tabular}

\section{ACCOUNT 11 STEAM TURBINE GENERATOR AND AUXILIARIES}

\begin{tabular}{|c|c|c|c|c|}
\hline Equipment No. & Description & Type & Design Condition & Qty \\
\hline 1 & $\begin{array}{l}\text { Steam Turbine Generator and } \\
\text { Ancillaries Package }\end{array}$ & $\begin{array}{l}\text { Reheat, Tandem } \\
\text { compound HP, IP, and } \\
\text { two-flow LP turbines }\end{array}$ & $\begin{array}{l}240 \mathrm{MWe} \\
1,800 \mathrm{psia} / 1000^{\circ} \mathrm{F} / 1000^{\circ} \mathrm{F}\end{array}$ & 1 \\
\hline 2 & Steam Turbine Generator & $\begin{array}{l}\text { Hydrogen cooled, } \\
\text { static excitation }\end{array}$ & $\begin{array}{l}270 \text { MVA, } 0.9 \text { p.f., } 60 \mathrm{HZ}, 3- \\
\text { ph }\end{array}$ & 1 \\
\hline 3 & Steam Bypass & & $\begin{array}{l}\text { 30\% steam flow @ design } \\
\text { steam conditions }\end{array}$ & 1 \\
\hline 4 & Bearing Lube Oil Coolers & Plate and frame & & 2 \\
\hline 5 & Bearing Lube Oil Conditioner & $\begin{array}{l}\text { Pressure filter closed } \\
\text { loop }\end{array}$ & & 1 \\
\hline 6 & Control System & $\begin{array}{l}\text { Digital electro- } \\
\text { hydraulic }\end{array}$ & & 1 \\
\hline 7 & Generator Coolers & Plate and frame & & 2 \\
\hline 8 & Hydrogen Seal Oil System & Closed loop & & 1 \\
\hline 9 & Surface Condenser & $\begin{array}{l}\text { Single pass, divided } \\
\text { waterbox }\end{array}$ & $\begin{array}{l}1,300,000 \mathrm{lb} / \mathrm{h} \\
2.0 \mathrm{in} \mathrm{HgA} \\
75^{\circ} \mathrm{F} \mathrm{CW}, 20^{\circ} \mathrm{F} \text { rise } \\
\text { Hotwell storage }-5 \mathrm{~min}\end{array}$ & 1 \\
\hline 10 & Condenser Vacuum Pumps & Rotary, water sealed & $\begin{array}{l}\text { 2,500/25 scfm } \\
\text { (hogging/holding) }\end{array}$ & 2 \\
\hline
\end{tabular}

ACCOUNT 12 BOP, STEAM \& WATER SYSTEMS

\section{ACCOUNT 12.1 FEEDWATER AND CONDENSATE}

\begin{tabular}{|c|l|l|l|c|}
\hline Equipment No. & \multicolumn{1}{|c|}{ Description } & \multicolumn{1}{|c|}{ Type } & \multicolumn{1}{c|}{ Design Condition } & Qty \\
\hline 1 & Condensate Storage Tank & $\begin{array}{l}\text { Vertical, cylindrical, } \\
\text { outdoor, 304 SS }\end{array}$ & $100,000 \mathrm{gal}$ & 1 \\
\hline 2 & Condensate Pumps & Vertical canned & $\begin{array}{l}2,900 \mathrm{gpm}, 420 \mathrm{ft}, \\
\text { El. motor } 380 \mathrm{hp}\end{array}$ & 2 \\
\hline
\end{tabular}




\begin{tabular}{|c|l|l|l|c|}
\hline Equipment No. & \multicolumn{1}{|c|}{ Description } & \multicolumn{1}{c|}{ Type } & \multicolumn{1}{c|}{ Design Condition } & Qty \\
\hline 3 & Deaerator & Horizontal spray type & $\begin{array}{l}3,100 \mathrm{gpm} \\
40 \mathrm{psig}, 5 \mathrm{ppb} \mathrm{O}_{2}, \\
5 \text { min storage tank }\end{array}$ & 1 \\
\hline 4 & $\begin{array}{l}\text { High Pressure } \\
\text { Feedwater Pump }\end{array}$ & $\begin{array}{l}\text { Horizontal, multi- } \\
\text { staged, centrifugal }\end{array}$ & $\begin{array}{l}3,100 \mathrm{gpm}, 6,300 \mathrm{ft} \\
\text { El. motor } 7,000 \mathrm{hp}\end{array}$ & 2 \\
\hline
\end{tabular}

\section{ACCOUT 12.2 BOP EQUIPMENT}

\begin{tabular}{|c|c|c|c|c|}
\hline Equipment No. & Description & Type & Design Condition & Qty \\
\hline 1 & Service Air Compressors & $\begin{array}{l}\text { Reciprocating, single } \\
\text { stage, double acting, } \\
\text { horizontal }\end{array}$ & 100 psig, $500 \mathrm{cfm}$ & 2 \\
\hline 2 & Instrument Air Dryers & Duplex, regenerative & $500 \mathrm{cfm}$ & 2 \\
\hline 3 & Raw Water Booster Pumps & SS, single suction & $\begin{array}{l}125 \mathrm{ft}, 2 \times 100 \% @ 2,500 \\
\text { gpm each }\end{array}$ & 2 \\
\hline 4 & Raw Water tank & Vertical, cylindrical & $\begin{array}{l}1,800,000 \mathrm{gal}-12 \mathrm{hr} \\
\text { storage }\end{array}$ & 1 \\
\hline 5 & $\begin{array}{l}\text { Cooling Tower Water Makeup } \\
\text { Pumps }\end{array}$ & $\begin{array}{l}\text { Horizontal centrifugal, } \\
\text { double suction }\end{array}$ & $\begin{array}{l}100 \mathrm{ft}, 2 \times 100 \% @ 4,000 \\
\text { gpm each }\end{array}$ & 2 \\
\hline 6 & $\begin{array}{l}\text { Wastewater Treatment System } \\
\text { Clarification }\end{array}$ & $\begin{array}{l}\text { Clarifier, including } \\
\text { sludge pumps (4) }\end{array}$ & $900 \mathrm{gpm}$ & 1 \\
\hline 7 & Sludge Dewatering & $\begin{array}{l}\text { Filter Press, including } \\
\text { filter press feed pumps } \\
\text { (2) }\end{array}$ & $70 \mathrm{ft}^{3}$ & 1 \\
\hline 8 & Service Water Pumps & SS, single suction & $160 \mathrm{ft}, 50 \mathrm{gpm}$ & 2 \\
\hline 9 & Fire Water Tank & Vertical, cylindrical & $600,000 \mathrm{gal}$ & 1 \\
\hline 10 & Fire Service Booster Pump & $\begin{array}{l}\text { Two-stage horizontal } \\
\text { centrifugal }\end{array}$ & $250 \mathrm{ft}, 1,000 \mathrm{gpm}$ & 1 \\
\hline 11 & Engine-Driven Fire Pump & $\begin{array}{l}\text { Vertical turbine, diesel } \\
\text { engine }\end{array}$ & $350 \mathrm{ft}, 1,000 \mathrm{gpm}$ & 1 \\
\hline 12 & Closed Cooling Water Pumps & Horizontal, centrifugal & $\begin{array}{l}70 \mathrm{ft}, 2 \times 100 \% @ 27,000 \\
\text { gpm each }\end{array}$ & 2 \\
\hline 13 & Closed Cooling Heat Exchangers & Plate & $250 \mathrm{MMBtu} / \mathrm{h}$ & 2 \\
\hline
\end{tabular}




\begin{tabular}{|c|c|c|c|c|}
\hline Equipment No. & Description & Type & Design Condition & Qty \\
\hline 14 & Chemical Feed Systems & $\begin{array}{l}\text { Sodium hypochlorite, } \\
\text { Sodium bisulfite, } \\
\text { antiscalant, } \\
\text { polymer, } \\
\text { caustic, } \\
\text { acid, } \\
\text { incl. diaphragm-type } \\
\text { chemical metering } \\
\text { pumps }\end{array}$ & $\begin{array}{l}\text { 1-50 gph each nominal, } 2 \\
\text { pumps per system }\end{array}$ & 6 \\
\hline 15 & Demineralized Water Treatment & $\begin{array}{l}\text { Multimedia filter, } \\
\text { cartridge filter, RO } \\
\text { pump, and reverse } \\
\text { osmosis (RO) } \\
\text { membrane assembly, } \\
\text { air scour blower, EDI }\end{array}$ & $100 \mathrm{gpm}$ & 1 \\
\hline 16 & $\begin{array}{l}\text { Demineralized water transfer } \\
\text { pumps }\end{array}$ & Horizontal, centrifugal & 100 gpm @100 ft & 2 \\
\hline 17 & Conductivity Sensors & & & 15 \\
\hline 18 & $\begin{array}{l}\text { Wastewater Treatment Reverse } \\
\text { Osmosis Package }\end{array}$ & $\begin{array}{l}\text { Multimedia filter, } \\
\text { cartridge filter, RO } \\
\text { pump, and reverse } \\
\text { osmosis (RO) } \\
\text { membrane assembly, } \\
\text { air scour blower }\end{array}$ & $410 \mathrm{gpm}$ & 1 \\
\hline 19 & $\begin{array}{l}\text { Zero Liquid Discharge } \\
\text { Equalization Tanks }\end{array}$ & $\begin{array}{l}\text { Brine holding tank, } \\
\text { Concentrate tank }\end{array}$ & $\begin{array}{l}500,000 \mathrm{gal} \\
100,000 \mathrm{gal}\end{array}$ & $\begin{array}{c}1 \\
\text { ea. }\end{array}$ \\
\hline 20 & Zero Liquid Discharge System & $\begin{array}{l}\text { Evaporator, } \\
\text { Crystallizer, distillate } \\
\text { and condensate tanks, } \\
\text { pumps, vapor } \\
\text { compressors, chemical } \\
\text { feed pumps, misc } \\
\text { tanks, etc. }\end{array}$ & $125 \mathrm{gpm}$ nominal & 1 \\
\hline 21 & $\begin{array}{l}\text { Zero Liquid Discharge } \\
\text { Dewatering }\end{array}$ & $\begin{array}{l}\text { Pneumatic Pressure } \\
\text { Filter, filtrate tank, } \\
\text { pump }\end{array}$ & 30 gpm max. feed & 1 \\
\hline 22 & Auxiliary Boiler & $\begin{array}{l}\text { Shop fab., water tube, } \\
\text { NG fired }\end{array}$ & $\begin{array}{l}40,000 \mathrm{lb} / \mathrm{h} \\
400 \mathrm{psig}, 650^{\circ} \mathrm{F}\end{array}$ & 1 \\
\hline 23 & Chemical Storage Tanks & $\begin{array}{l}\text { Caustic, Acid, Sodium } \\
\text { Hypochlorite, } \\
\text { Coagulant }\end{array}$ & 6,000 gal. each nominal & 4 \\
\hline
\end{tabular}




\begin{tabular}{|c|l|l|l|c|}
\hline Equipment No. & \multicolumn{1}{|c|}{ Description } & \multicolumn{1}{c|}{ Type } & \multicolumn{1}{c|}{ Design Condition } & Qty \\
\hline 24 & Bulk Silo, Slurry Feed Systems & $\begin{array}{l}\text { Lime, Soda Ash or } \\
\text { Other, including slurry } \\
\text { tank, mixer, pumps. etc }\end{array}$ & $2,000 \mathrm{ft}^{3}$ nominal & 2 \\
\hline 25 & Sludge Holding Tank & FRP with Mixer & $6,000 \mathrm{gal} \mathrm{nominal}$ & 1 \\
\hline 26 & Oil/Water Separator & & $25 \mathrm{gpm}$ & 1 \\
\hline
\end{tabular}

\section{ACCOUNT 13 COOLING WATER SYSTEM}

\begin{tabular}{|c|l|l|l|c|}
\hline Equipment No. & \multicolumn{1}{|c|}{ Description } & \multicolumn{1}{|c|}{ Type } & \multicolumn{1}{c|}{ Design Condition } & Qty \\
\hline 1 & Circ. Water Pumps & Vertical wet pit & $\begin{array}{l}93,000 \mathrm{gpm}, \text { @ } 100 \mathrm{ft} \\
\text { El. motor 3,300 hp }\end{array}$ & 2 \\
& & & $\begin{array}{l}65^{\circ} \mathrm{F} \mathrm{WB} \\
75^{\circ} \mathrm{F} \text { CWT } \\
95^{\circ} \mathrm{F} \mathrm{HWT} \\
1,850 \mathrm{MMBtu} / \mathrm{h}\end{array}$ & 1 \\
& Cooling Tower & Evaporative, \\
& & mechanical draft, multi- & & \\
& & & & \\
\hline
\end{tabular}

\section{ACCOUNT 14 ASH HANDLING}

\begin{tabular}{|c|l|l|l|c|}
\hline Equipment No. & \multicolumn{1}{|c|}{ Description } & \multicolumn{1}{|c|}{ Type } & \multicolumn{1}{c|}{ Design Condition } & Qty \\
\hline 1 & Bottom Ash Cooler & Screw & 9 tph & 1 \\
\hline 2 & Fly Ash Cooler & Screw & 20 tph & 1 \\
\hline 3 & Ash pneumatic system & Vacuum & 30 tph & 1 \\
\hline 4 & Ash Silo w/ Slide Gate Valves & Reinforced concrete & 2,000 tons & 1 \\
\hline 5 & Truck Loading Station & w/dust collector & 45 tph & 1 \\
\hline 6 & Telescoping Loading Chute & -- & 45 tph & 1 \\
\hline
\end{tabular}




\section{ACCOUNT 15 ACCESORIES ELECTRICAL PLANT}

\begin{tabular}{|c|l|l|l|c|}
\hline Equipment No. & \multicolumn{1}{|c|}{ Description } & \multicolumn{1}{|c|}{ Type } & \multicolumn{1}{c|}{ Design Condition } & Qty \\
\hline 1 & STG Step-up Transformer & Oil-filled & $\begin{array}{l}230 \mathrm{kV} / 20 \mathrm{kV}, 220 \mathrm{MVA}, \\
3-\mathrm{ph}, 60 \mathrm{~Hz}\end{array}$ & 1 \\
\hline 2 & Start-up Transformer & Oil-filled & $\begin{array}{l}20 \mathrm{kV} / 13.8 \mathrm{kV}, 20 \mathrm{MVA}, \\
3-\mathrm{ph}, 60 \mathrm{~Hz}\end{array}$ & 1 \\
\hline 3 & Auxiliary Transformer & Oil-filled & $\begin{array}{l}20 \mathrm{kV} / 13.8 \mathrm{kV}, 49 \mathrm{MVA}, \\
3-\mathrm{ph}, 60 \mathrm{~Hz}\end{array}$ & 1 \\
\hline 4 & Auxiliary Transformer & Oil-filled & $\begin{array}{l}13.8 \mathrm{kV} / 4.16 \mathrm{kV}, 29 \mathrm{MVA}, \\
3-\mathrm{ph}, 60 \mathrm{~Hz}\end{array}$ & 1 \\
\hline 5 & LV Auxiliary Transformer & Dry ventilated & $\begin{array}{l}4.16 \mathrm{kV} / 0.48 \mathrm{kV}, 9 \mathrm{MVA}, \\
3-\mathrm{ph}, 60 \mathrm{~Hz}\end{array}$ & 1 \\
\hline 6 & Emergency Diesel Generator & $\begin{array}{l}\text { Sized for emergency } \\
\text { shutdown }\end{array}$ & $\begin{array}{l}3,600 \mathrm{~kW}, 480 \mathrm{~V}, 3-\mathrm{ph}, \\
60 \mathrm{~Hz}\end{array}$ & 1 \\
\hline
\end{tabular}

\section{ACCOUNT 16 INSTRUMENTATION AND CONTROL}

\begin{tabular}{|c|l|l|l|c|}
\hline Equipment No. & \multicolumn{1}{|c|}{ Description } & \multicolumn{1}{|c|}{ Type } & \multicolumn{1}{c|}{ Design Condition } & Qty \\
\hline 1 & DCS - Main Control & $\begin{array}{l}\text { Monitor/keyboard; } \\
\text { Operator printer - laser } \\
\text { color; } \\
\text { Eng. Printer - laser } \\
\text { color }\end{array}$ & $\begin{array}{l}\text { Operator Stations/Printers } \\
\text { Engineering. } \\
\text { Stations/Printers }\end{array}$ & 4 \\
\hline 2 & DCS - Processor & $\begin{array}{l}\text { Microprocessor with } \\
\text { Redundant } \\
\text { Input/Output }\end{array}$ & 1 \\
\hline 3 & DCS - Data System & $\begin{array}{l}\text { Optical Disk/Tape } \\
\text { Backup }\end{array}$ & $\begin{array}{l}\text { Historical Archive, Trends } \\
\text { Logger, Report, } \\
\text { Performance Monitoring }\end{array}$ & 1 \\
\hline 4 & DCS - Data Highway & Fiber optic & $\begin{array}{l}\text { Fully redundant, } \\
\text { 25\% spare }\end{array}$ & \\
\hline
\end{tabular}


WW WorleyParsons

resources \& energy
APS Hydrogasification SNG

Preliminary Engineering Package Appendices

Appendix F: Capital Cost Details

when experience counts

1/21/2011 3:58 PM U:IPubliclAPS_DOELAPS Phase II SNG PEP Update 2010-07 onlReportl2011 Jan RpttAppendicesIAPS Appendix Cover Pages.doc 


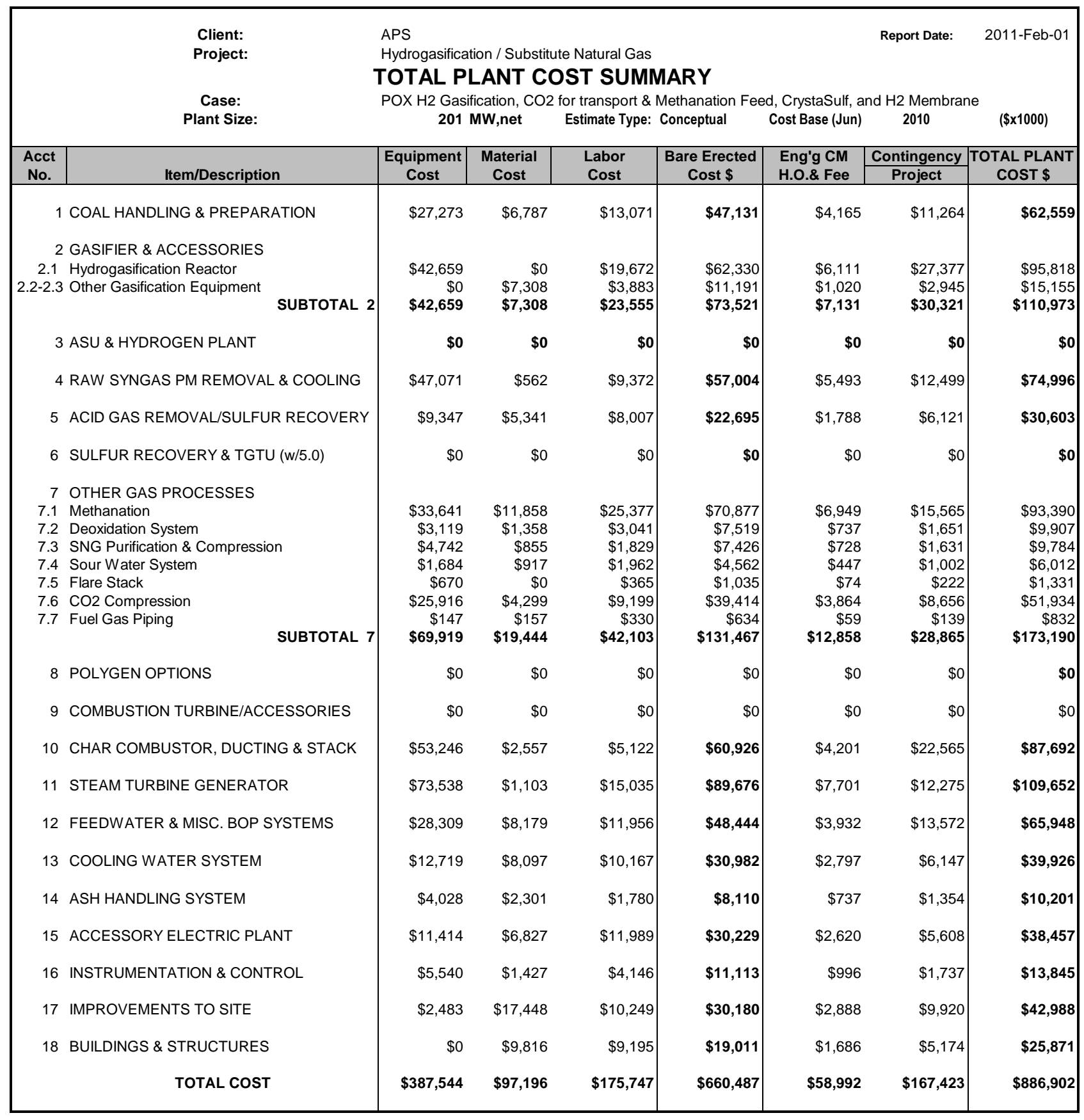




\section{Appendix G: Operating Cost Details}




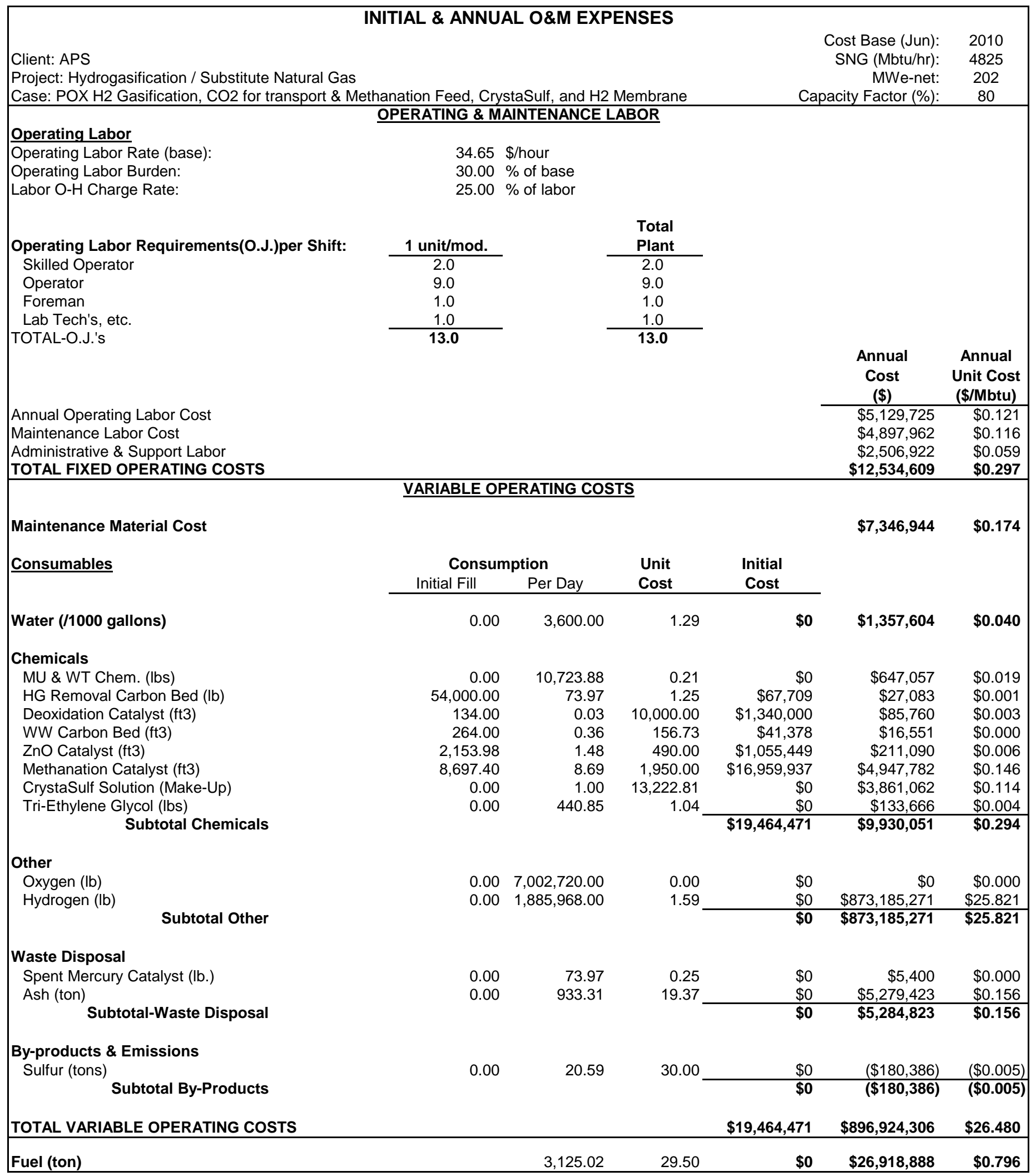




\section{WW WorleyParsons \\ resources \& energy}

\section{Appendix H:}

\section{Hydrogen Source Review for Electrolysis}




\section{Hydrogen Source Review for Electrolysis}

Prepared for:

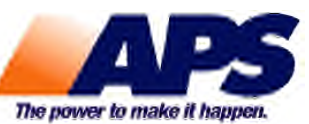

Arizona Public Service Company

Prepared by:

W WorleyParsons

resources \& energy

WorleyParsons Group Inc.

February 16, 2011

Revision A 


\section{NOTICE}

This document was prepared by WorleyParsons with the objective of performing a high level review of hydrogen production through electrolysis. The document examines both public available and project specific information regarding electrolysis.

This effort was not meant to be an exhaustive review of electrolysis processes. 


\section{TABLE OF CONTENTS}

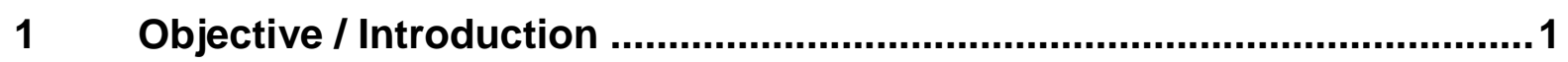

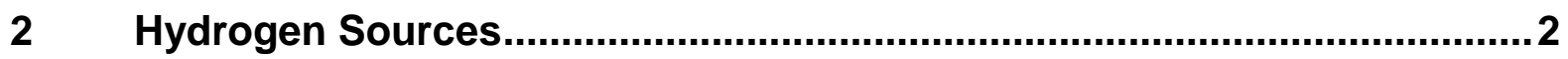

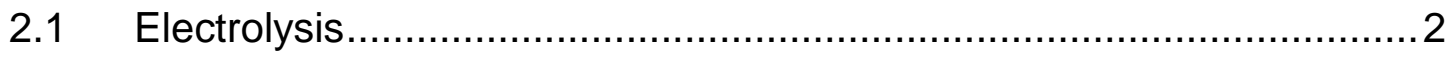

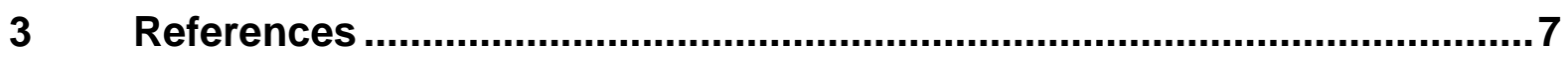

\section{LIST OF EXHIBITS}

Exhibit 2-1 Commercial or Near Commercial Hydrogen Production PEM and Alkaline Electrolysis Technology .................................................. 2

Exhibit 2-2 Estimate Performance and Cost of Electrolyzers ................................ 3

Exhibit 2-3 Centralized Plant (50,000 kg/day $\mathrm{H}_{2}$ Production) Base Case Input

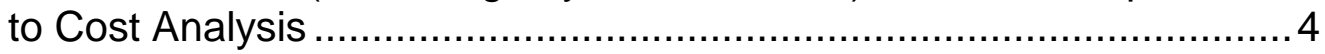

Exhibit 2-4 Breakdown of Centralized Plant Base Case Hydrogen Production

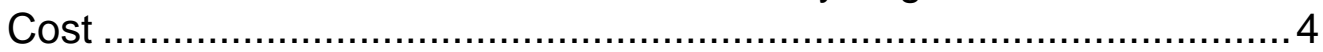

Exhibit 2-5 Sensitivity Analysis Tornado Chart for Centralized Plant Base

Case..... 5 


\section{Revision Record}

\begin{tabular}{|c|c|l|}
\hline Revision & Date & \multicolumn{1}{|c|}{ Content } \\
\hline A & $2 / 16 / 2011$ & Issued with report for reference \\
\hline & & \\
\hline & & \\
\hline & & \\
\hline & & \\
\hline & & \\
\hline & & \\
\hline & & \\
\hline & & \\
\hline
\end{tabular}




\section{Objective / Introduction}

Arizona Public Service (APS) has been developing a process/project known as the APS Hydrogasification / SNG project. The primary objective of the project has been the development of synthetic natural gas (SNG) from indigenous coal. The project hydrogasification process requires a supply of approximately 79,000 lb/hr (930,000 kg/day) of hydrogen $\left(\mathrm{H}_{2}\right)$. APS has decided that hydrogen is best supplied by electrolysis.

The primary objective of this document is to provide a high level review of electrolysis performance and cost values to allow the reader to interpret the values utilized within the SNG analysis.

This paper provides a high level review of $\mathrm{H}_{2}$ generation from electrolysis, including technical and cost information documented with the public domain by electrolysis vendors, information provided by one electrolysis vendor specific to the APS SNG process, as well as information taken from a 2009 independent review sponsored by USDOE. 


\section{Hydrogen Sources}

The base case hydrogen sourcing for the APS hydrogasification process has always been electrolysis. Other hydrogen sources such as steam methane reforming (SMR) and gasification are also possible, but were not selected by APS for implementation in the process analysis. This paper documents publicly available information regarding electrolysis.

\section{$2.1 \quad$ Electrolysis}

The base hydrogen source proposed by APS from the very beginning has always been electrolysis. The use of electrolysis allowed for renewable wind energy to be utilized as well as excess nuclear power which are GHG neutral and have been indicated as being low cost energy sources, particularly at certain times of day and year. Both of these electric energy sources offer advantages to APS.

Hydrogen Technologies, formerly known as Norsk Hydro, and IHT are suppliers of large capacity commercial electrolyzers, up to $760 \mathrm{Nm}^{3} / \mathrm{hr}(1,635 \mathrm{~kg} /$ day $)$. Other suppliers of commercial or near commercial alkaline and polymer electrolyte membrane (PEM) electrolyzers, but at smaller unit capacities, include those identified in Exhibit 2-1.

\section{Exhibit 2-1}

\section{Commercial or Near Commercial Hydrogen Production PEM and Alkaline Electrolysis} Technology

\begin{tabular}{|l|c|c|c|c|}
\hline Supplier & Location & Technology & $\begin{array}{c}\text { Production } \\
\text { Capacity (kg/day) }\end{array}$ & $\begin{array}{c}\mathbf{H}_{2} \text { Product } \\
\text { Pressure (psi) }\end{array}$ \\
\hline Avalance & United States & Unipolar Alkaline & Up to 10 & Up to 6,500 \\
\hline Giner & United States & Bipolar PEM & Up to 8 & Up to 1,250 \\
\hline H2 Technologies & Norway & Bipolar Alkaline & Up to 1,000 & Atmospheric \\
\hline Hydrogenics & United States & Bipolar PEM & Up to 127 & Up to 363 \\
\hline IHT & Switzerland & Bipolar Alkaline & Up to 1,500 & Up to 464 \\
\hline Proton & United States & Bipolar PEM & Up to 13 & Up to 435 \\
\hline
\end{tabular}

Reference [1, Table 1]

According to Hydrogen Technologies' website [2], they currently provide atmospheric electrolyzers with capacities up to about $485 \mathrm{Nm}^{3} / \mathrm{hr}$ of $\mathrm{H}_{2}$ (about $1,040 \mathrm{~kg} / \mathrm{day}$ ). Hydrogen Technologies indicate that they are developing a pressurized electrolyzer operating at up to 30 barg (450 psia). In two memos provided to WorleyParsons by APS, Hydrogen Technologies provided an estimate of the electric power requirement and costs for a $500 \mathrm{Nm}^{3} / \mathrm{hr} \mathrm{H}_{2}$ electrolyzer unit if a single unit was built, along with an estimate for future units assuming the development of more efficient manufacturing techniques and design optimization for high-volume applications [4,5]. The estimates provided in the memo and performance estimates obtained from the Hydrogen technology website are shown in Exhibit 2-2. 
Exhibit 2-2

Estimate Performance and Cost of Electrolyzers

\begin{tabular}{|c|c|c|c|c|c|}
\hline Supplier & $\begin{array}{l}\text { Electrolyzer } \\
\text { Type }\end{array}$ & Capacity & $\begin{array}{l}\text { Specific Electric } \\
\text { Power Required }\end{array}$ & Cost Today & Future Cost \\
\hline \multirow{3}{*}{$\begin{array}{l}\text { Hydrogen } \\
\text { Technologies, } \\
\text { Statoil } \\
\text { (formerly Norsk } \\
\text { Hydro) }\end{array}$} & $\begin{array}{l}\text { Pressurized } \\
\text { alkaline } \\
\text { (30 barg) } \\
\text { (future) [2] }\end{array}$ & \multirow{3}{*}{$\begin{array}{l}500 \mathrm{Nm}^{3} / \mathrm{hr} \text { [2] } \\
(1,075 \mathrm{~kg} / \text { day })\end{array}$} & $\begin{array}{l}4.7 \mathrm{kWh} / \mathrm{Nm}^{3} \mathrm{H}_{2}[4] \\
(53 \mathrm{kWh} / \mathrm{kg}) \\
(74.5 \% \mathrm{HHV})\end{array}$ & $\begin{array}{l}3.1 \mathrm{M} €[4] \\
(\$ 3.9 \mathrm{M})-2006 \$ \\
{\left[\$ 3,600 /\left(\mathrm{kg} / \text { day } \mathrm{H}_{2}\right)\right]} \\
{[\$ 1,660 / \mathrm{kWe} \text { in }]}\end{array}$ & $\begin{array}{l}750-900 \mathrm{~K} €(\$ 950 \mathrm{~K})- \\
2006 \$[4] \\
{\left[\$ 880 /\left(\mathrm{kg} / \mathrm{day} \mathrm{H}_{2}\right)\right]} \\
{[\$ 400 / \mathrm{kWe} \text { in }]}\end{array}$ \\
\hline & $\begin{array}{l}\text { Atmospheric } \\
\text { alkaline [2] }\end{array}$ & & $\begin{array}{l}4.3 \mathrm{kWh} / \mathrm{Nm}^{3} \mathrm{H}_{2}[2] \\
(48 \mathrm{kWh} / \mathrm{kg}) \\
(81.5 \% \mathrm{HHV})\end{array}$ & Not available & Not available \\
\hline & $\begin{array}{l}\begin{array}{l}\text { Atmospheric } \\
\text { alkaline [5] } \\
\text { (delivered to }\end{array} \\
\underline{\underline{1150 \text { psig, \& }}} \\
\underline{99.99 \% \mathrm{H}_{2}}\end{array}$ & & $\begin{array}{l}5.5 \mathrm{kWh} / \mathrm{Nm}^{3} \mathrm{H}_{2}[5] \\
(62 \mathrm{kWh} / \mathrm{kg}) \\
(63.7 \% \mathrm{HHV})\end{array}$ & $\begin{array}{l}\$ 4.5 \mathrm{M}(\mathrm{Y} 2010 \$)[5] \\
{\left[\$ 4,200 /\left(\mathrm{kg} / \text { day } \mathrm{H}_{2}\right)\right]} \\
{[\$ 1,640 / \mathrm{kWe} \text { in }]}\end{array}$ & $\begin{array}{l}60 \% \text { to } 70 \% \text { for high } \\
\text { volume cost reduction, [5] } \\
\text { i.e., } \$ 1.35-1.8 \mathrm{M} \\
{\left[\$ 1260-1670 /\left(\mathrm{kg} / \mathrm{day} \mathrm{H}_{2}\right)\right]} \\
{[\$ 490-650 / \mathrm{kWe} \mathrm{in]}}\end{array}$ \\
\hline \multirow{2}{*}{ IHT } & $\begin{array}{l}\text { Pressurized } \\
\text { alkaline } \\
\text { (Lurgi Tech.) } \\
\text { (30 bar) [3] }\end{array}$ & $\begin{array}{l}\text { to } 760 \mathrm{Nm}^{3} / \mathrm{hr} \\
(1,630 \mathrm{~kg} / \text { day }) \\
{[3]}\end{array}$ & $\begin{array}{l}4.3-4.6 \mathrm{kWh} / \mathrm{Nm}^{3} \\
\mathrm{H}_{2} \text { dry [3] } \\
(48-51 \mathrm{kWh} / \mathrm{kg}) \\
(81.5-76.2 \% \mathrm{HHV})\end{array}$ & Not available & Not available \\
\hline & $\begin{array}{l}\text { Atmospheric } \\
\text { alkaline } \\
\text { (Bamag Tech) } \\
\text { [3] }\end{array}$ & $\begin{array}{l}\text { to } 330 \mathrm{Nm}^{3} / \mathrm{hr} \\
(710 \mathrm{~kg} / \mathrm{day}) \\
{[3]}\end{array}$ & $\begin{array}{l}4.2-4.54 \\
\mathrm{kWh} / \mathrm{Nm}^{3} \mathrm{H}_{2} \text { dry [3] } \\
(47-51 \mathrm{kWh} / \mathrm{kg}) \\
(83.4-77.2 \% \mathrm{HHV})\end{array}$ & Not available & Not available \\
\hline
\end{tabular}

References: $[2,3,4,5]$

IHT's website indicates that they supply both atmospheric electrolyzers with unit capacities up to $330 \mathrm{Nm}^{3} / \mathrm{hr}(710 \mathrm{~kg} /$ day) and pressurized electrolyzers (based on the Lurgi pressurized electrolyzer) operating at 30 barg (450 psia) with capacities up to $760 \mathrm{Nm}^{3} / \mathrm{hr}$ $(1,630 \mathrm{~kg} / \mathrm{day})$ [3]. The estimated performance of the IHT atmospheric and pressurized electrolyzers, as provided by their website, is also shown in Exhibit 2-2. Cost information on the IHT electrolyzers was not available from the website. The information indicate that the specific power consumption is similar for the Hydrogen technologies and IHT technologies.

A major source of current (2009) state-of-the-art cost estimates for hydrogen from electrolysis is a report prepared for the U.S. Department of Energy (DOE) National Renewable Energy Laboratory. This comprehensive report contains cost information for both "forecourt" refueling stations, based on a design capacity of 1,500 kg/day of $\mathrm{H}_{2}$, and centralized electrolysis plants with total capacity of about $50,000 \mathrm{~kg} /$ day. The later would be most applicable to the APS project. The centralized plant was based on multiple electrolyzers with unit capacities no greater than 1,000 kg/day, consistent with the largest unit available from Hydrogen Technologies but slightly smaller than the largest pressurized unit available from IHT.

The DOE report provides a centralized plant Base Case estimate of about $\$ 3.00 / \mathrm{kg} \mathrm{H}_{2}$ (\$2005) based on the input shown in Exhibit 2-3. The total depreciable cost for the Base Case was based on an electrolyzer cost of $\$ 1000$ per kilogram per day of $\mathrm{H}_{2}$ production. A breakdown of the cost in terms of capital, fixed O\&M, electricity cost, and other variable 
costs reproduced in Exhibit 2-4 indicates that electricity cost represents about $76 \%$ of the total cost of hydrogen production via electrolysis.

\section{Exhibit 2-3}

Centralized Plant $\left(50,000 \mathrm{~kg} /\right.$ day $\mathrm{H}_{2}$ Production) Base Case Input to Cost Analysis

\begin{tabular}{|l|c|c|}
\hline \multicolumn{1}{|c|}{ Central Production } & Vendor Range & Panel Base Case \\
\hline & & \\
\hline Total Depreciable Capital Cost (M\$) & $\$ 17.9$ to $\$ 56.3$ & $\$ 50$ \\
\hline Energy use (kWh/kg) & 48 to 59 & 50 \\
\hline Electricity Price & NA & $0.045 \$ / \mathbf{k W h}$ \\
\hline Electrolyzer Cells Capital Replacement (M\$) & $\$ 1.2$ to $\$ 19.7$ & $\$ 12.5$ \\
(\% of Total Depreciable Capital) & $6 \%$ to $35 \%$ & $25 \%$ \\
\hline Eletrolyzer Cell Replacement Interval (yrs) & 7 to 10 & 7 \\
\hline Working Capital (\% of Change in Operating Costs) & NA & $5 \%$ \\
\hline FTE's & 5 to 10 & 10 \\
\hline Production Maintenance (\% of Total Depr. Capital) & $1 \%$ to $3 \%$ & $2 \%$ \\
\hline Process Water (gal/kg-H2) & 2.6 to 2.9 & 2.5 \\
\hline Cooling Water (gal/kg-H2) & 0.1 to 290 & 290 \\
\hline All other Variable Costs (k\$/yr) & NG & $\$ 0$ \\
\hline Op. Capacity Factor (\%) & $98-99.5$ & $98 \%$ \\
\hline Start-up time (months) & 6 & 6 \\
\hline Land Cost (\$acre) & NA & $\$ 50,000$ \\
\hline
\end{tabular}

Reference [1, Table 4]

\section{Exhibit 2-4}

\section{Breakdown of Centralized Plant Base Case Hydrogen Production Cost}

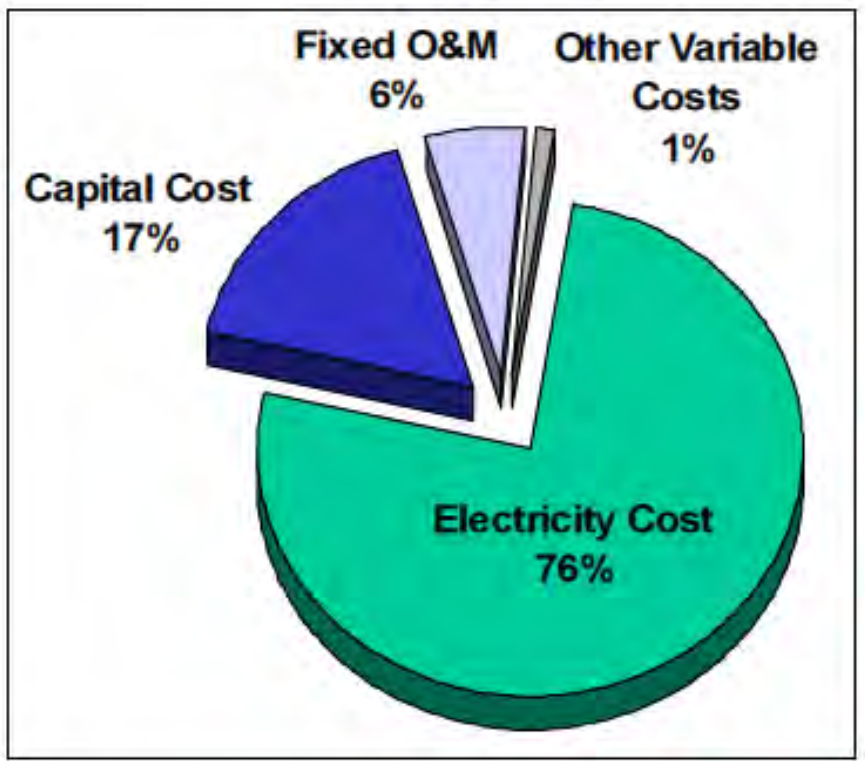

Reference [1, Figure 9]

The DOE report also provides a most useful "tornado" chart, reproduced in Exhibit 2-5, that shows the estimated variation in hydrogen production cost from the Base Case cost resulting from variations in the component costs. The range of values for the parameters 
was selected based on the reviewers' best estimate for the tenth-percentile and ninetiethpercentile likely values for the parameters of importance.

\section{Exhibit 2-5}

\section{Sensitivity Analysis Tornado Chart for Centralized Plant Base Case}

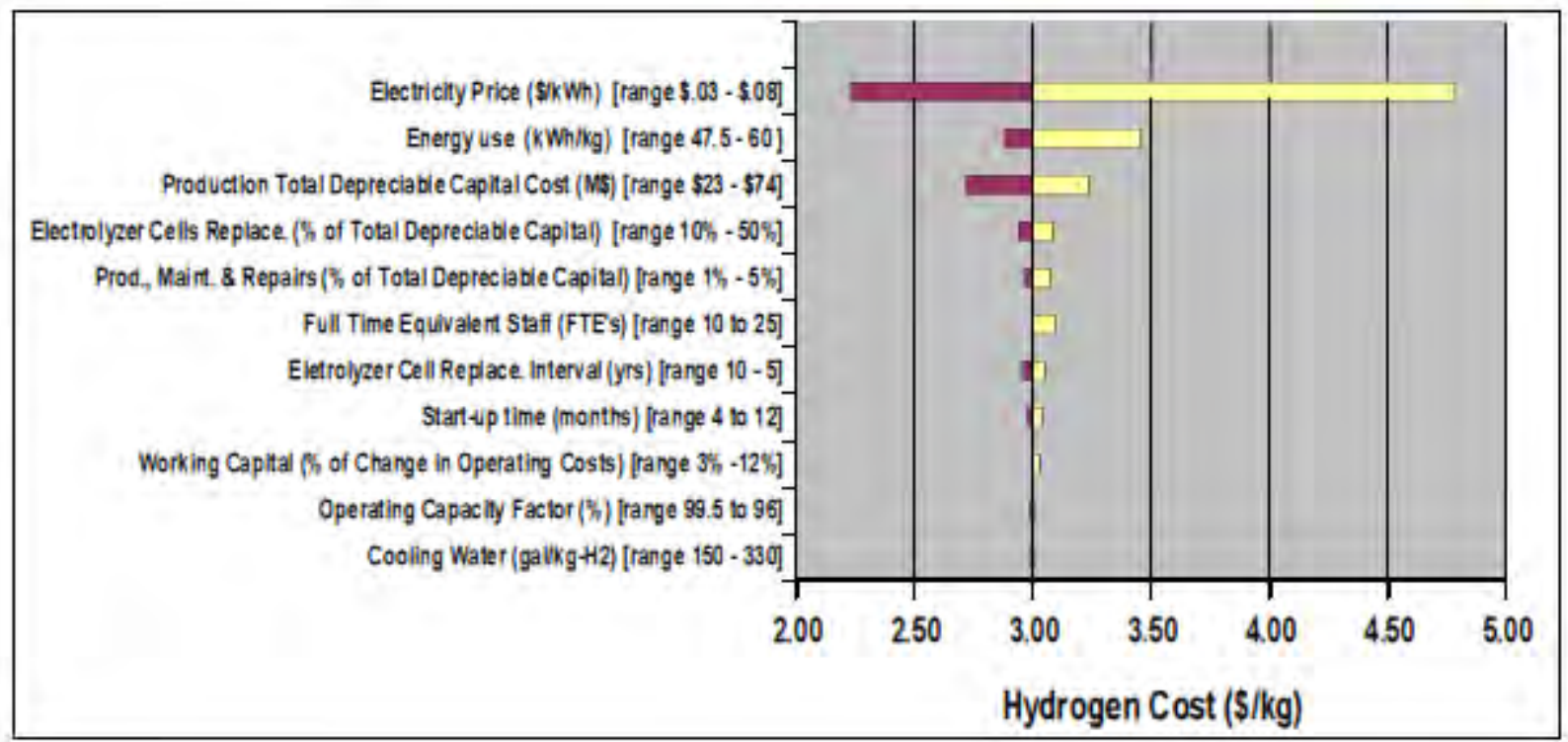

Reference [1, Figure 6].

The $\$ 1000$ per kilogram per day electrolyzer cost assumed in the DOE study is close to the $\$ 880$ per kilogram per day future price of pressurized electrolyzers estimated by Hydrogen Technologies assuming high volume, more efficient manufacturing techniques and design optimization for high volume applications. It is notably lower than the \$1260-1670 per kilogram per day future price of the atmospheric electrolyzer estimated by Hydrogen Technologies for the specific purity and pressure requirement of the SNG project. For perspective, it is significantly lower than the $\$ 3600$ to $\$ 4200$ per kilogram per day current day price estimate of Hydrogen Technologies taking into account today's designs and manufacturing capabilities. The capital cost range utilized in the "tornado" chart in Exhibit 25 corresponds to $\$ 460$ and 1480 per kilogram per day ${ }^{1}$.

The conclusion from the independent review panel summary report of 2009, found that:

"The current (2009) state-of-the art plant gate cost for hydrogen from a central electrolysis operation ranges from $\$ 2.70 / \mathrm{kg}-\mathrm{H}_{2}$ to $\$ 3.50 / \mathrm{kg}-\mathrm{H}_{2}$ with a base-case estimate of $\$ 3.00 / \mathrm{kg}-\mathrm{H}_{2}$. These costs are evaluated at an assumed renewablebased electricity cost of $\$ 0.045 / \mathrm{kWh}$, which was supplied to the Panel by DOE and based on wind-generated electricity." [1] These costs are Y2005 USD.

1 The base case capital cost of $\$ 50$ million for the $50,000 \mathrm{~kg} \mathrm{H}_{2} / \mathrm{d}$ corresponds to a specific capital cost of $\$ 1000 / \mathrm{kg} \mathrm{H}_{2} / \mathrm{d}$. Thus the tornado plot capital cost range of $\$ 23$ to 74 million corresponds to a specific capital cost of $\$ 460$ to $\$ 1480 / \mathrm{kg} \mathrm{H}_{2} / \mathrm{d}$. 
In Section 7.5 of the accompanying SNG report [6], the APS SNG project specific $\mathrm{H}_{2}$ production price was estimated to be $\$ 3.50 / \mathrm{kg}-\mathrm{H}_{2}$ (Y2010), which at the upper end of the review panels cost range. The SNG report utilized electric rates provided by APS, electrolyzer costs provided by Hydrogen Technologies, and capacity factor of $80 \%$ specific to the project as opposed to the $98 \%$ utilized in the review panel report. Thus the review panel report provides collaboration towards the credibility of the $\$ 3.50 / \mathrm{kg}-\mathrm{H}_{2}$ price developed within the body of the report. The $\$ 3.50 / \mathrm{kg}-\mathrm{H}_{2}$ value developed for the SNG project analysis is considered superior to the generic values of the review document since this value is based on APS SNG project specific information. 


\section{References}

1 "Current (2009) State-of-the-Art Hydrogen Production Cost Estimate Using Water Electrolysis," Independent Review Published for the U.S. Department of Energy Hydrogen Program, NERL/BK-6A1-46676, September 2009.

2 http://www3.statoil.com/hydrogentechnologies, as of 2010-03-10 and 2011-02-16.

3 http://iht.ch/technologie/electrolysis/industry/home.html, as of 2011-02-16.

4 Memo from Roy Grelland (Norsk Hydro Electrolysers, AS) to J.D. Serber \& Roy Hobbs (APS), Re: Solar to hydrogen plant in Arizona, US, 2006-07-12.

5 Memo from Atle Taalesen, Regional Sales Director (Statoil, Hydrogen Technologies) to Sally Sun (APS), Re: Solar to hydrogen plant in Arizona, US, 2010-10-07.

6 "Preliminary Engineering Package for Hydrogasification/ Substitute Natural Gas Commercial Scale Facility- Conceptual Design," prepared for Arizona Public Service, prepared by WorleyParsons, 2011-02-16. 
THIS PAGE INTENTIONALLY LEFT BLANK 